\title{
Proceedings of RIKEN BNL Research Center Workshop
}

BNL-100766-2013

\section{in Light of Recent dAu vs pPb Controls April 15-17, 2013 \\ RIKEN/RBRC Workshop Jet Quenching at RHIC vs LHC}

Volume 115

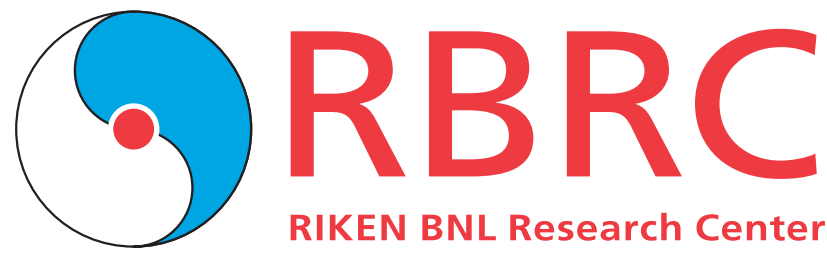




\section{Disclaimer}

This work was prepared as an account of work sponsored by an agency of the United States Government. Neither the United States Government nor any agency thereof, nor any of their employees, nor any of their contractors, subcontractors or their employees, makes any warranty, express or implied, or assumes any legal liability or responsibility for the accuracy, completeness, or any third party's use or the results of such use of any information, apparatus, product, or process disclosed, or represents that its use would not infringe privately owned rights. Reference herein to any specific commercial product, process, or service by trade name, trademark, manufacturer, or otherwise, does not necessarily constitute or imply its endorsement, recommendation, or favoring by the United States Government or any agency thereof or its contractors or subcontractors. The views and opinions of authors expressed herein do not necessarily state or reflect those of the United States Government or any agency thereof.

Notice: This manuscript has been authored by employees of Brookhaven Science Associates, LLC under Contract No. DE-AC02-98CH10886 with the U.S. Department of Energy. The publisher by accepting the manuscript for publication acknowledges that the United States Government retains a non-exclusive, paid-up, irrevocable, world-wide license to publish or reproduce the published form of this manuscript, or allow others to do so, for United States Government purposes. 


\section{Preface to the Series}

The RIKEN BNL Research Center (RBRC) was established in April 1997 at Brookhaven National Laboratory. It is funded by the "Rikagaku Kenkyusho" (RIKEN, The Institute of Physical and Chemical Research) of Japan. The Memorandum of Understanding between RIKEN and BNL, initiated in 1997 , has been renewed in 2002, 2007 and again in 2012. The Center is dedicated to the study of strong interactions, including spin physics, lattice QCD, and RHIC physics through the nurturing of a new generation of young physicists.

The RBRC has both a theory and experimental component. The RBRC Theory Group and the RBRC Experimental Group consists of a total of 25-30 researchers. Positions include the following: full time RBRC Fellow, half-time RHIC Physics Fellow, and full-time post-doctoral Research Associate. The RHIC Physics Fellows hold joint appointments with RBRC and other institutions and have tenure track positions at their respective universities or BNL. To date, RBRC has over 95 graduates (Fellows and Post-docs) of which approximately 40 theorists and 20 experimenters have already attained tenure positions at major institutions worldwide.

Beginning in 2001 a new RIKEN Spin Program (RSP) category was implemented at RBRC. These appointments are joint positions of RBRC and RIKEN and include the following positions in theory and experiment: RSP Researchers, RSP Research Associates and Young Researchers, who are mentored by senior RBRC Scientists. A number of RIKEN Jr. Research Associates and Visiting Scientists also contribute to the physics program at the Center.

RBRC has an active workshop program on strong interaction physics with each workshop focused on a specific physics problem. In most cases all the talks are made available on the RBRC website. In addition, highlights to each speaker's presentation are collected to form proceedings which can therefore be made available within a short time after the workshop. To date there are over one hundred proceeding volumes available.

A 10 teraflops RBRC QCDOC computer funded by RIKEN, Japan, was unveiled at a dedication ceremony at BNL on May 26, 2005. This supercomputer was designed and built by individuals from Columbia University, IBM, BNL, RBRC, and the University of Edinburgh, with the U.S. D.O.E. Office of Science providing infrastructure support at BNL. Physics results were reported at the RBRC QCDOC Symposium following the dedication. QCDSP, a 0.6 teraflops parallel processor, dedicated to lattice QCD, was begun at the Center on February 19, 1998, was completed on August 28, 1998, and was decommissioned in 2006. It was awarded the Gordon Bell Prize for price performance in 1998. QCDOC was decommissioned in May 2012. The next generation computer in this sequence, QCDCQ (600 Teraflops), is currently operational and is expected to produce many more interesting discoveries in the future.

\section{N. P. Samios, Director \\ March 2013}

*Work performed under the auspices of U.S.D.O.E. Contract No. DE-AC02-98CH10886. 


\section{Introduction}

In the last decade of the RHIC physics program, studies of jet quenching has played an important role in the discovery and detailed characterization of the strongly interacting Quark-Gluon Plasma. However, these studies also generated several critical puzzles, such as the azimuthal anisotropy of jet quenching, heavy flavor energy loss and initial state suppression, hinting at missing ingredients in our current understanding of jet quenching. Now only two years into the data taking, LHC has produced new measurements on a variety of existing and new jet quenching observables, over a much broader kinematic range.

In addition, in April 2013 new initial state control data via $\mathrm{p}+\mathrm{A}$ collisions have become available from both RHIC and LHC. With these fresh RHIC and LHC inputs combined, we will be poised to achieve a much better understanding of the hard probes for the quark-gluon matter (such as initial state saturation effects, effective jet medium couplings and tomographic structures) and the implication of these observables about the evolving properties of the Quark-Gluon Plasma from RHIC to LHC.

The purpose of this Workshop was to critically review the recent progress on the theory and phenomenology of jet quenching including both RHIC and LHC data, to develop a consistent unifed picture of jet quenching from RHIC to LHC, and to formulate new research efforts for the future.

Specific topics addressed included:

- To what extent do we achieve control over initial state effects in light of the new dAu and $\mathrm{pPb}$ data?

- How do the medium properties change from RHIC to LHC?

- Can we achieve precision by combining the vast amount of data from RHIC and LHC?

- How does the jet quenching response to fluctuating initial geometry (jet tomography) evolve from RHIC to LHC?

\section{Workshop Organizers:}

Miklos Gyulassy (Columbia University)

Jiangyong Jia (BNL)

Jinfeng Liao (IU/RBRC) 


\section{Contents}

\section{Presentations:}

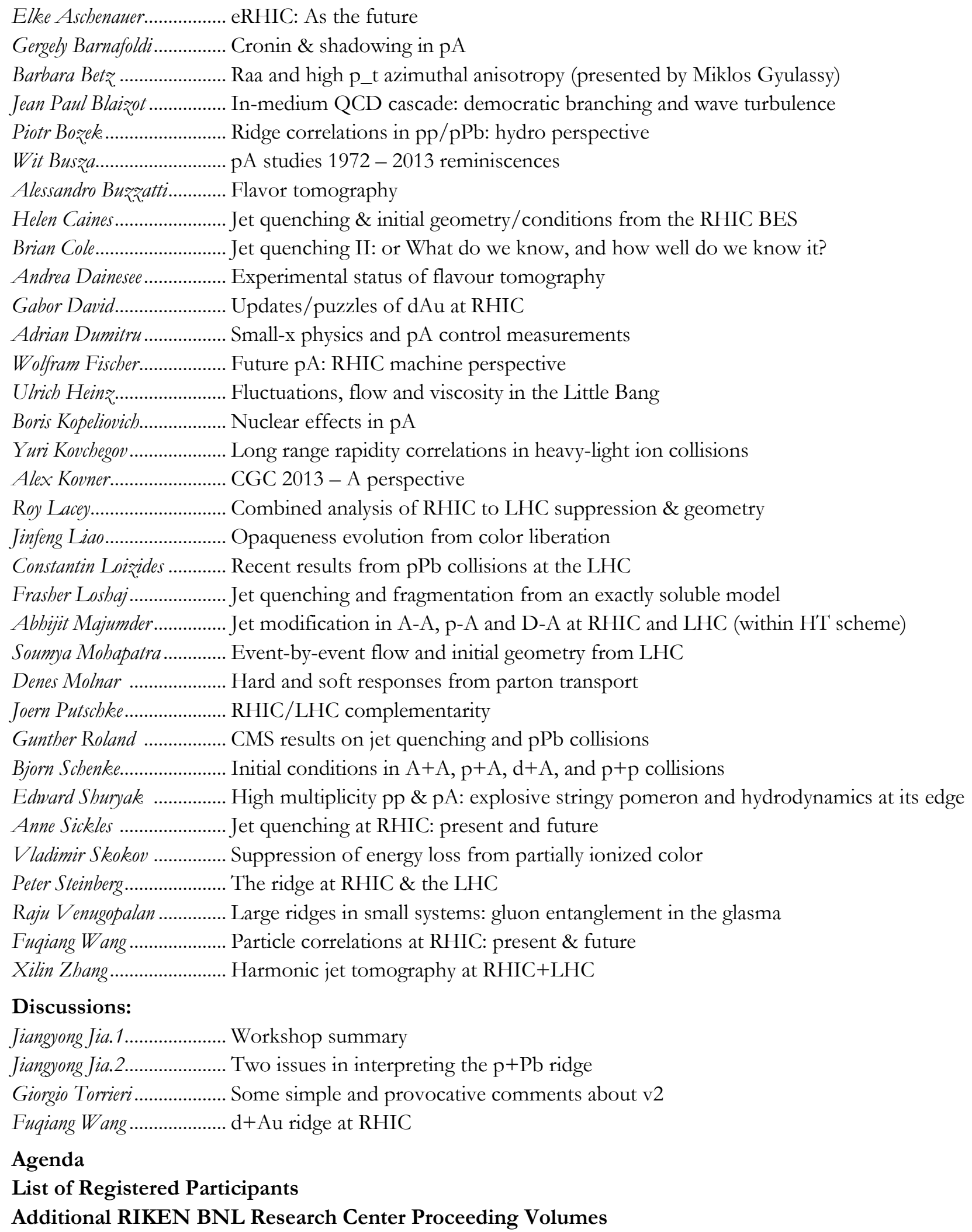

\section{Agenda}




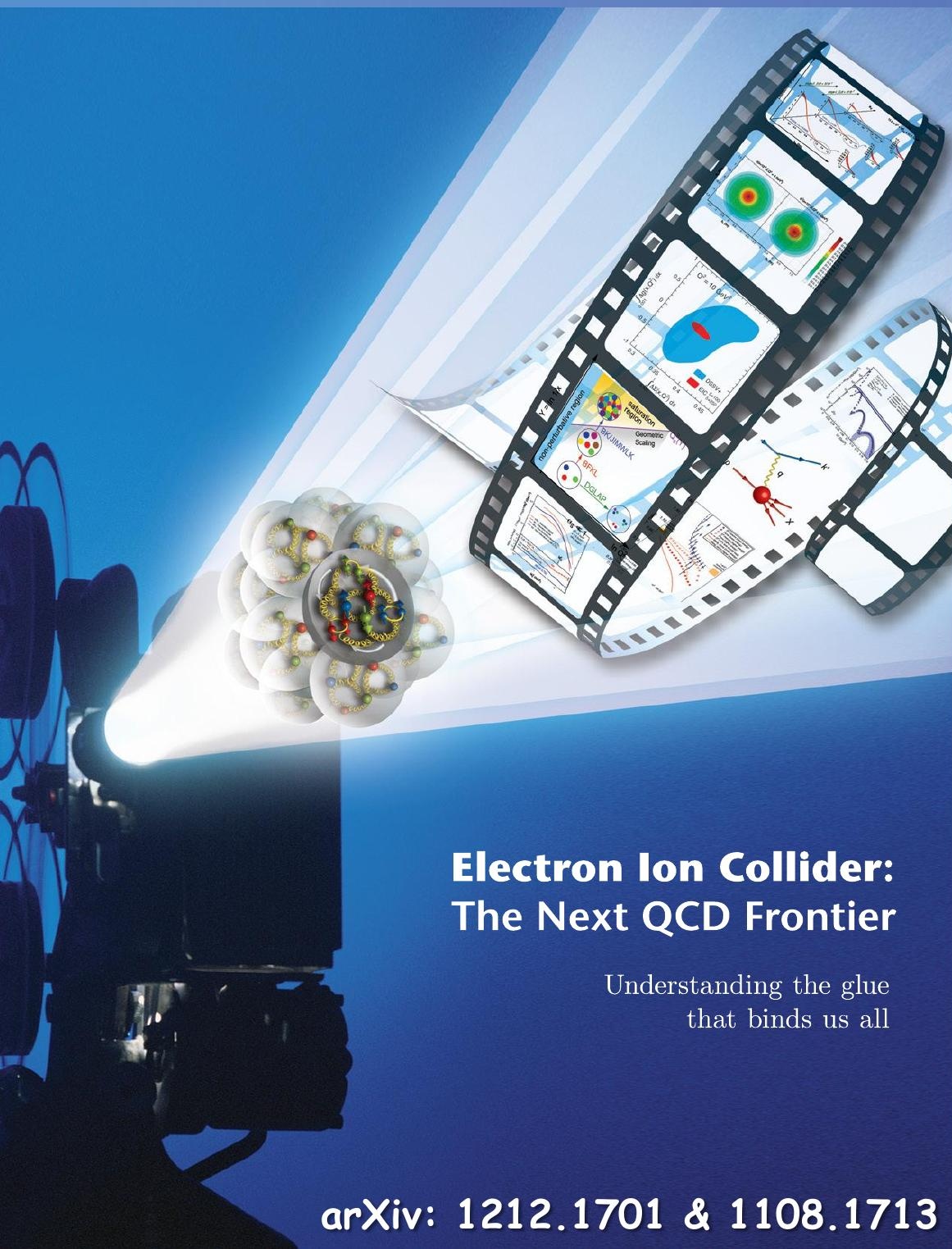

AS THE FUTURE

THANKS TO

BNL EIC-TASKFORCE and ERHIC-CAD-TEAM

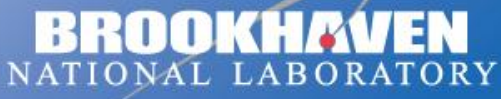

a passion for discovery

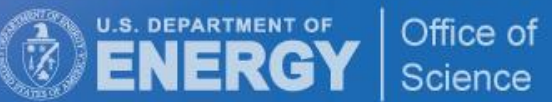




\section{Electron accelerator}

to be build

Unpolarized and polarized leptons 5-20 (30) GeV

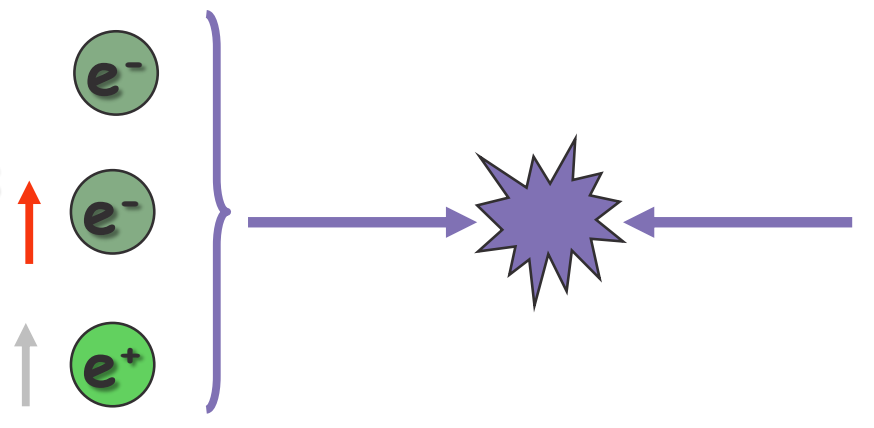

$70 \% e^{-}$beam polarization goal polarized positrons?

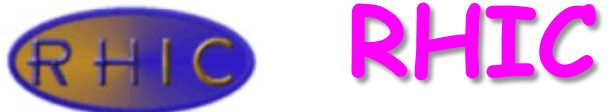

Existing $=\$ 2 B$

p $\uparrow$ Polarized protons 50-250 GeV

Light ions (d, Si, Cu) Heavy ions $(A u, U)$ $50-100 \mathrm{GeV} / \mathrm{u}$

Polarized light ions $\mathrm{He}^{3} 166 \mathrm{GeV} / \mathrm{u}$

Center mass energy range: $\sqrt{s}=30-200 \mathrm{GeV}$ : L 100-1000xHera longitudinal and transverse polarization for $\mathrm{p} / \mathrm{He}^{3}$ possible

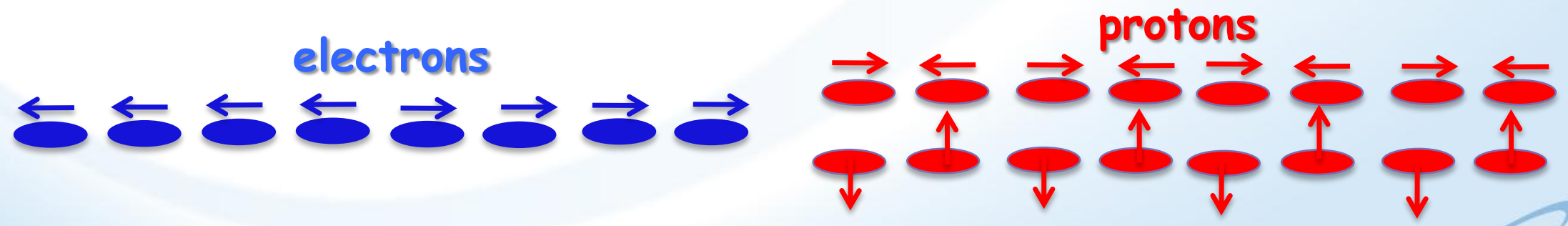




\section{ELECTRON BEAM EVOLUTION IN ERHIC'S ERL}

$0.60 \mathrm{GeV}$

All energies scale proportionally by adding SRF cavities to the injector

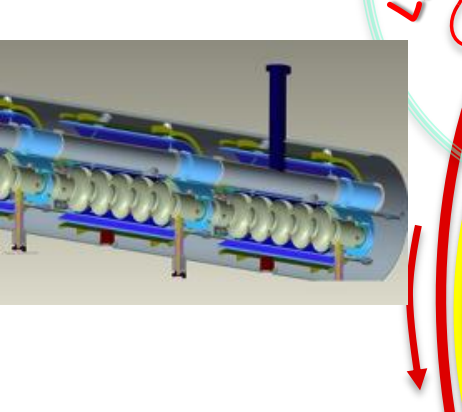

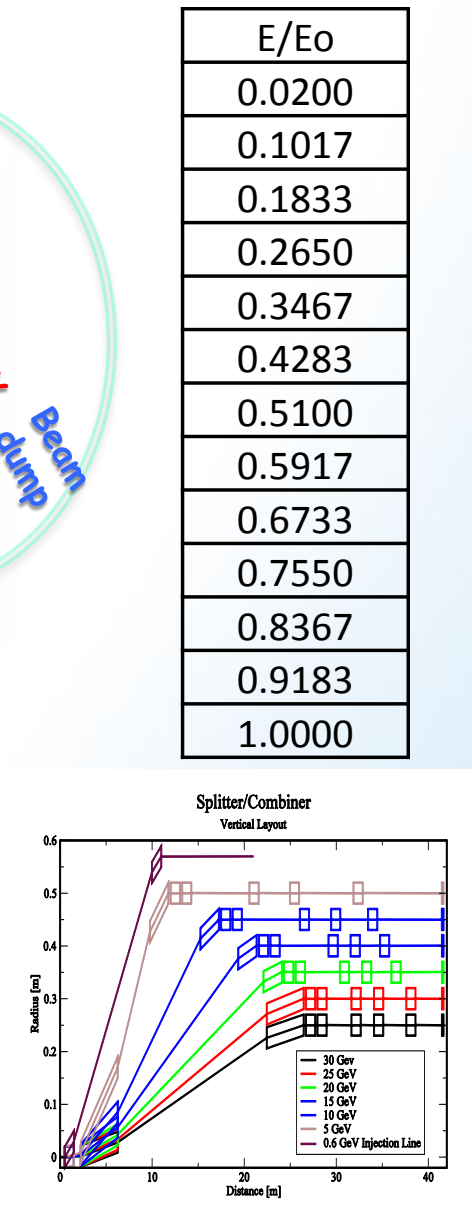

ERL:

energy recovery linac

BRDOKHEYEN

E.C. Aschenauer
$100 \mathrm{~m}$ up as energy is increasing

ESTAR $30 \mathrm{GeV}$ Animationdits byy Nail Tacupas 


\section{ERHIC R\&D HIGHLIGHTS AND LUMINOSITY}

\section{Challenge}

\section{Increase/reduction beyond}

the state of the art

\section{Polarized electron gun}

Coherent Electron Cooling

Multi-pass SRF ERL

\section{Crab crossing}

Understanding beam-beam effects

$\beta^{\star}=5 \mathrm{~cm}$

Multi-pass SRF ERL

Feedback for kink instability suppression

Space charge effect compensation

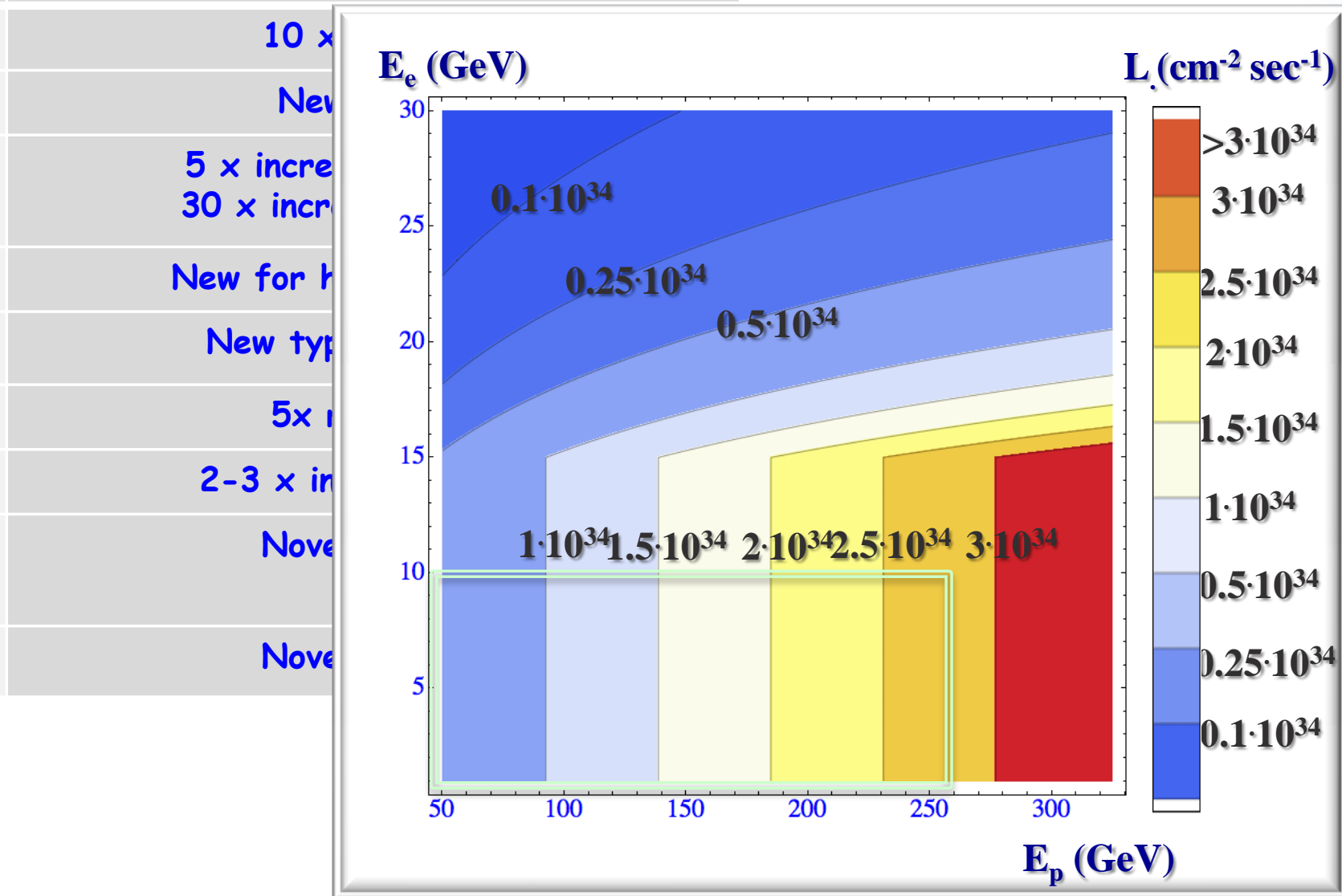

Hourglass the pinch effects are included. Space charge effects are compensated.

- Energy of electrons can be selected at any desirable value at or below $30 \mathrm{GeV}$

a The luminosity does not depend on the electron beam energy below or at $20 \mathrm{GeV}$

- The luminosity falls as $E_{e}^{-4}$ at energies above $20 \mathrm{GeV}$

The luminosity is proportional to the hadron beam energy: $L \sim E_{h} / E_{\text {top }}$ 


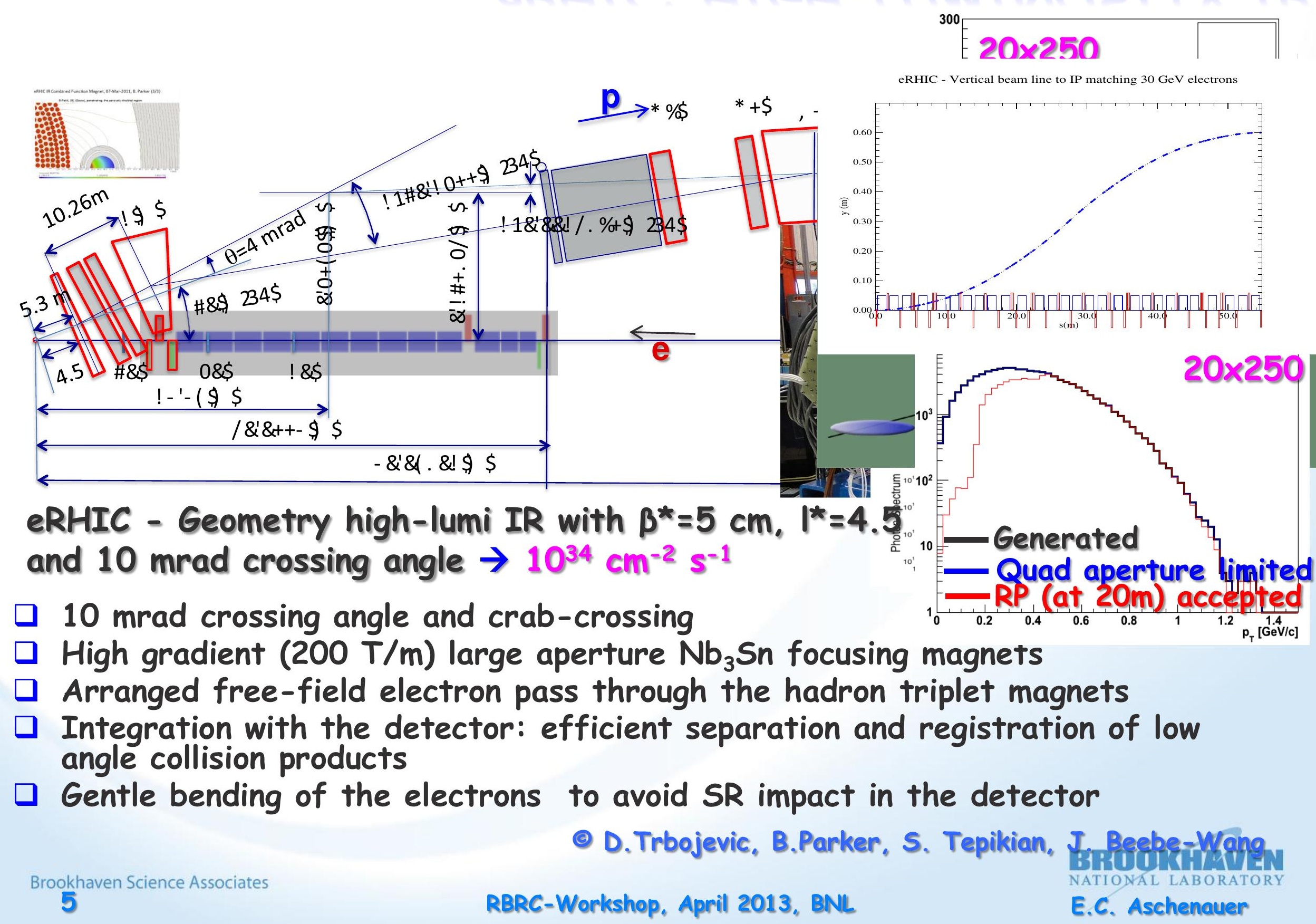




\section{BNL: $1^{\text {ST }}$ DETECTOR DESIGN CONCEPT}

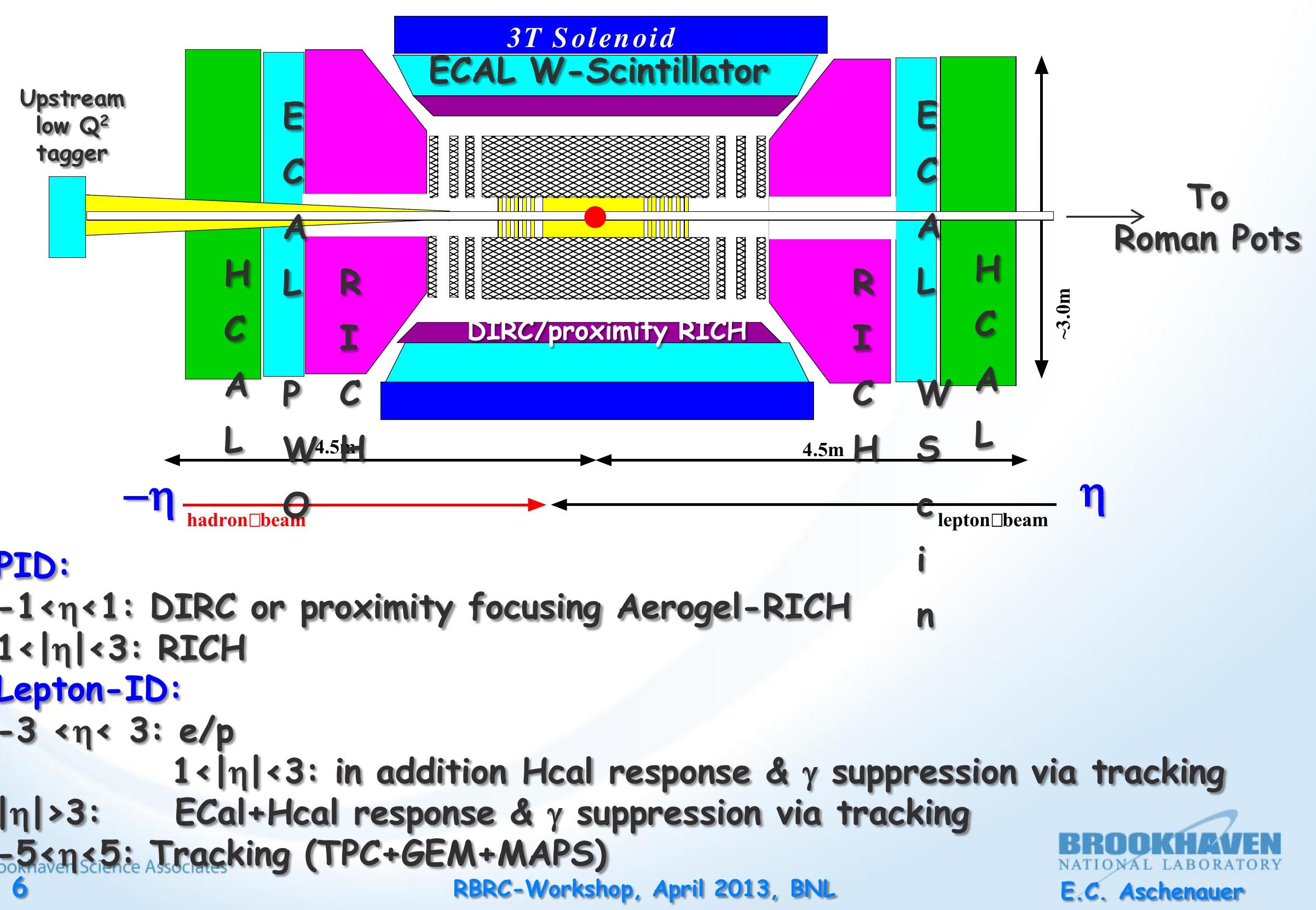




\section{VIBRANT DETECTOR R\&D PROGRAM}

Calorimetry

D-Scintillator \& W-Si

$>$ compact and high resolution

$>$ Crystal calorimeters PbW \& BGO

BNL, Indiana University, Penn State Univ,., UCLA, USTC, TAN

a Pre-Shower

W-Si

LYSO pixel array with readout via $X-Y$ WLS

Univ. Tecnica Valparaiso

$\square$ PID via Cerent

More info nttps:llwiki.b on EIC-Detector
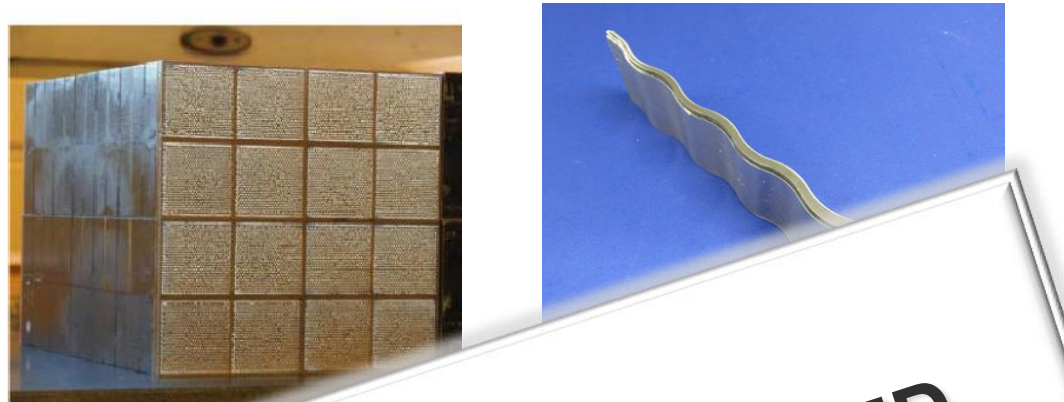

wiana Univ., USTC, VECC, ANL
U. ART

nl. $90^{\circ}$

\section{Trakking}

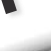

BNL, Florida Ins

$>\mu$-Vertex: central and forward based on MAPS

Central: TPC/HBD provides low mass.

good momentum, $d E / d x$, eID

Fast Layer: $\mu$-Megas or PImMS

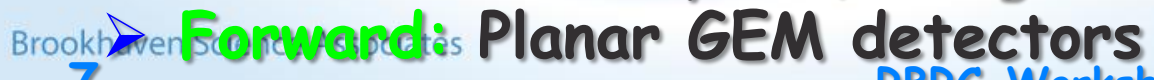

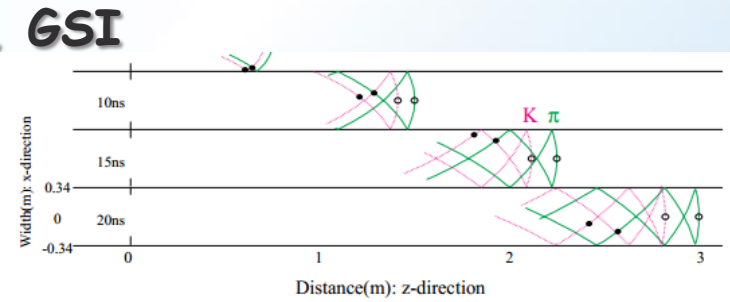




\section{MOST COMPELLING EIC SCIENCE QUESTIONS}

How are sea quarks and gluons and their spin distributed
in space and momentum inside the nucleon?
How are these quark and gluon distributions correlated with the
over all nucleon properties, such as spin direction?
What is the role of the motion of sea quarks and gluons
in building the nucleon spin?

How does the nuclear environment affect the distribution of quarks and gluons and their interaction in nuclei?

How does the transverse spatial distribution of gluons compare to that in the nucleon?

How does matter respond to fast moving color charge passing through yt?

Is this response different for light and heavy quarks?

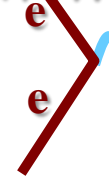

Where does the saturation of gluon densities set in?

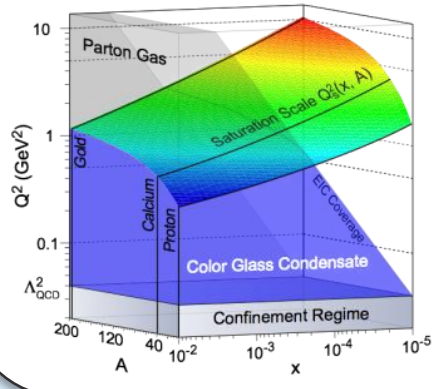

Is there a simple boundary that separates the region from the more dilute quark gluon matter? If so how do the distributions of quarks and gluons change as one crosses the boundary?

Does this saturation produce matter of universal properties in the nucleon and all nuclei viewed at nearly the speed of light? 


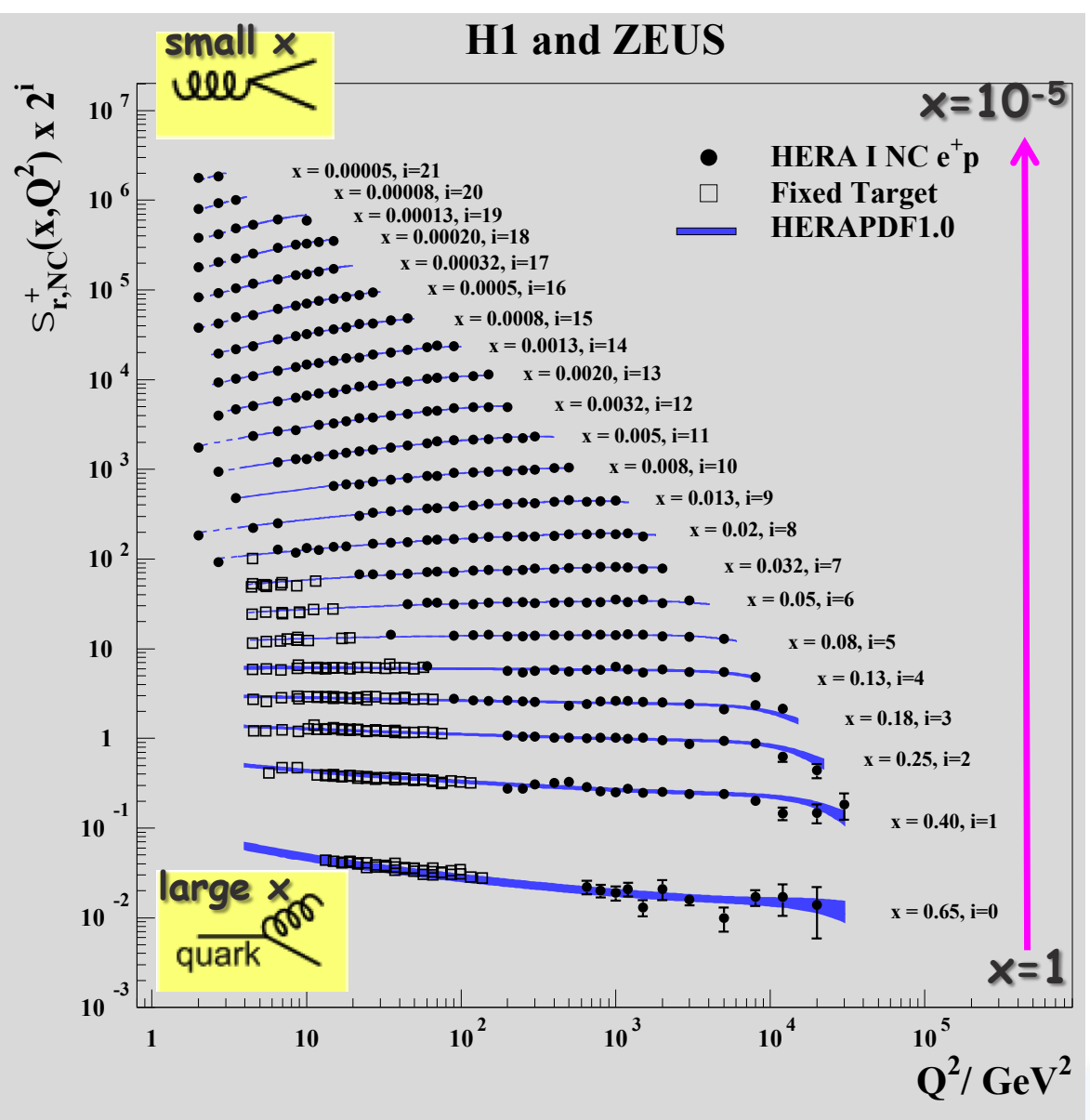

$$
\frac{d^{2} \sigma_{e^{\mp} p}^{N C}}{d x d Q^{2}}=\frac{2 \pi \alpha_{e m}^{2} Y_{+}}{x Q^{4}}(F_{2}-\frac{y^{2}}{Y_{+}} F_{L} \pm \frac{Y_{-}}{Y_{+}} \underbrace{}_{3} F_{3})
$$

\section{Obse Gluon density dominates rions}

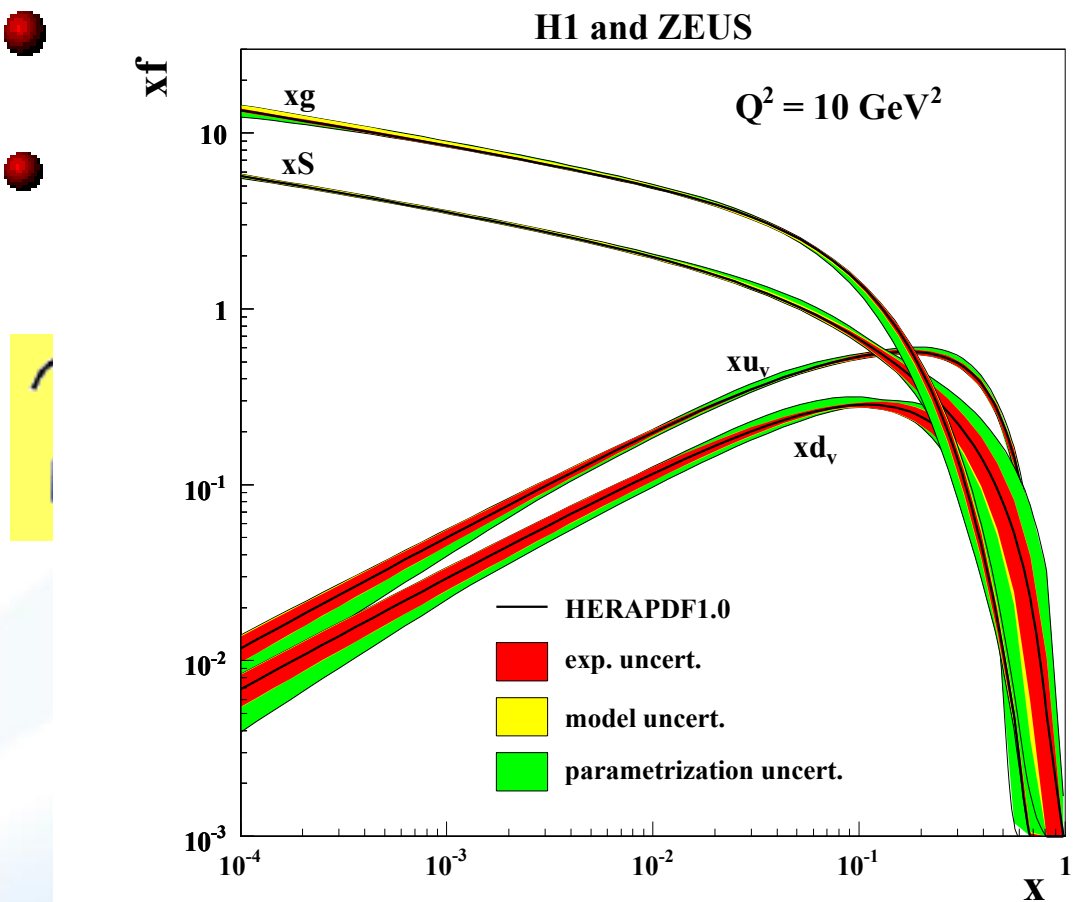




\section{DEEP INELASTIC SCATTERING}

\section{Kinematics:}

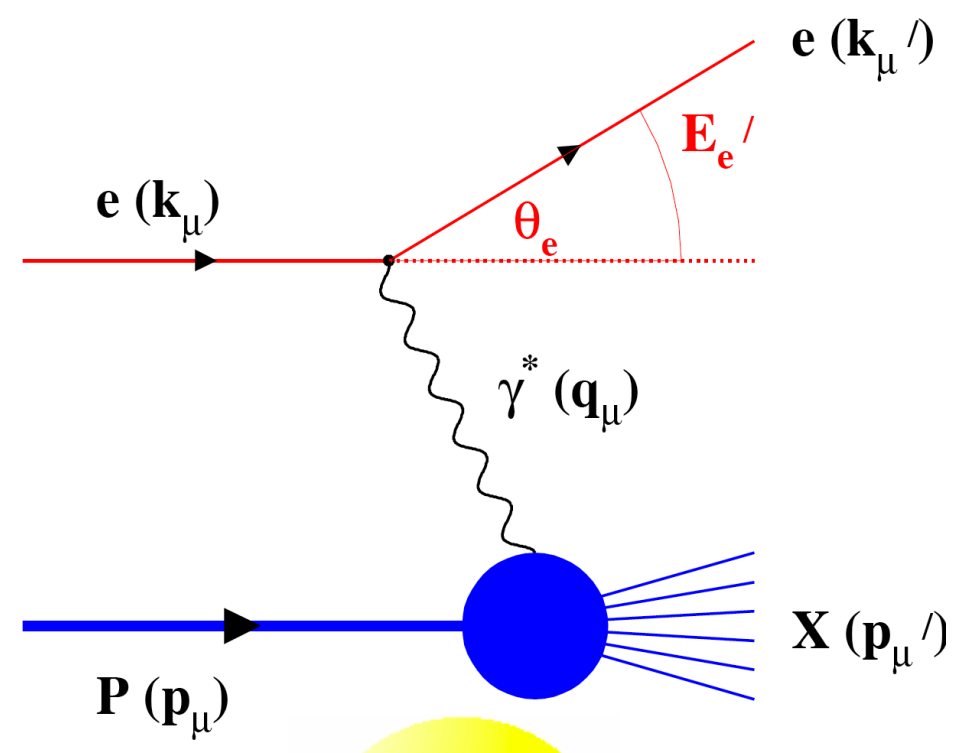

$$
\begin{aligned}
& Q^{2}=-q^{2}=-\left(k_{\mu}-k_{\mu}^{\prime}\right)^{2} \quad \begin{array}{l}
\text { Measure of } \\
\text { resolution }
\end{array} \\
& Q^{2}=2 E_{e} E_{e}^{\prime}\left(1-\cos \Theta_{e^{\prime}}\right) \text { power } \\
& y=\frac{p q}{p k}=1-\frac{E_{e}^{\prime}}{E_{e}} \cos ^{2}\left(\frac{\theta_{e}^{\prime}}{2}\right) \begin{array}{l}
\text { Measure of } \\
\text { inelasticity }
\end{array} \\
& x=\frac{Q^{2}}{2 p q}=\frac{Q^{2}}{s y} \begin{array}{l}
\text { Measure of } \\
\begin{array}{l}
\text { momentum } \\
\text { fraction of } \\
\text { struck quark }
\end{array}
\end{array}
\end{aligned}
$$

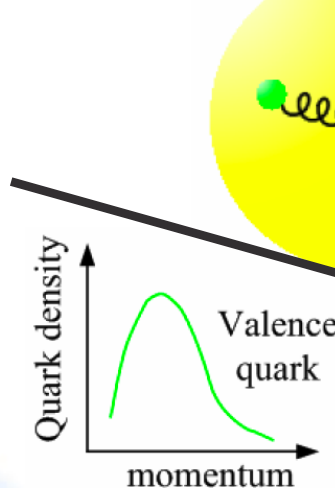

fraction $x$
Quark splits into gluon splits into quarks ... momentum
Gluon splits into quarks 


\section{THE PATH TO IMAGING QUARKS AND GLUONS}

a PDFs do not resolve transverse momenta or positions in the nucleon

a fast moving nucleon turns into a 'pizza' but transverse size remains about $1 \mathrm{fm}$

\section{compelling questions}

how are quarks and gluons spatially distributed

how do they move in the transverse plane

do they orbit and do we have access to spin-orbit correlations

$\rightarrow$ required set of measurements \& theoretical concepts

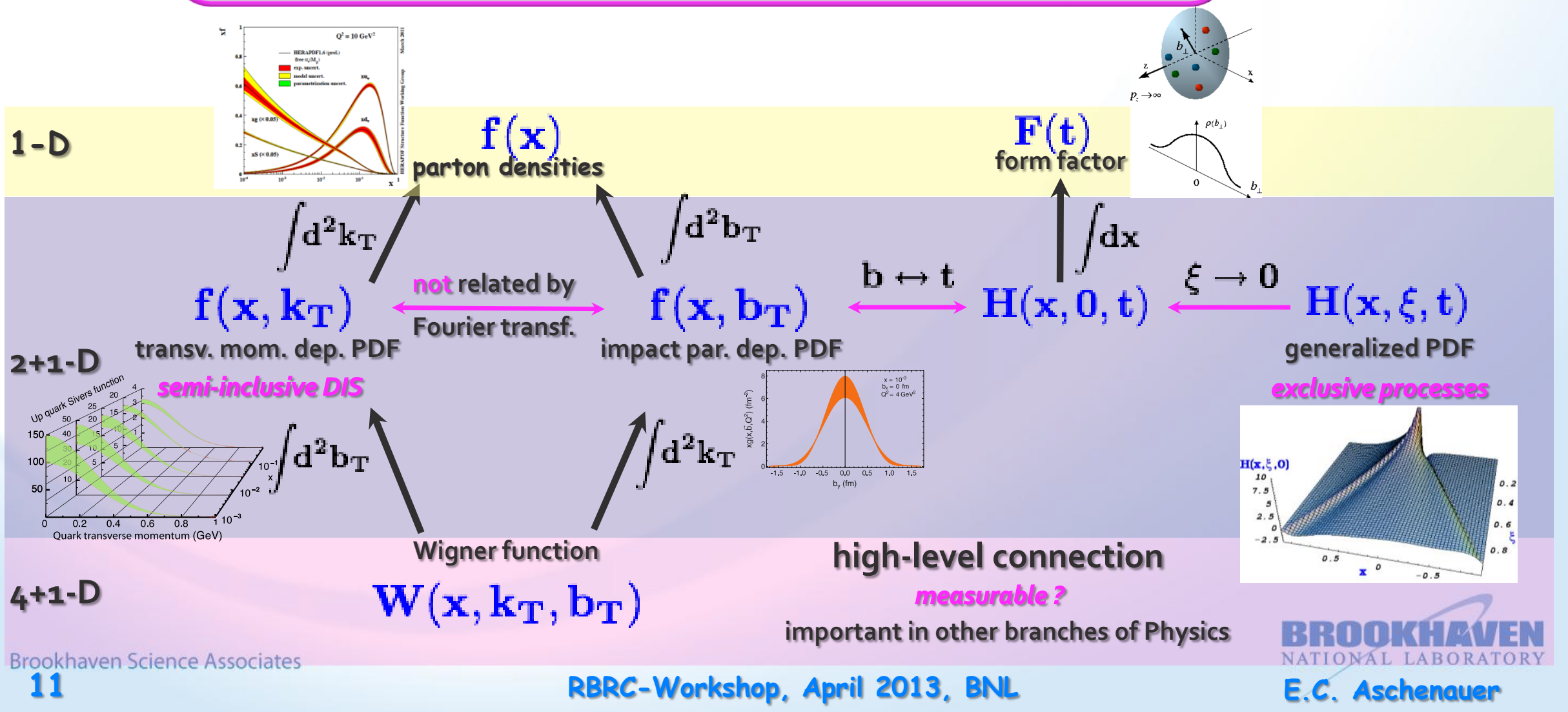




\section{BEYOND FORM FACTORS AND QUARK DISTRIBUTIONS}

\section{Generalized Parton Distributions}

X. Ji, D. Mueller, A. Radyushkin (1994-1997)

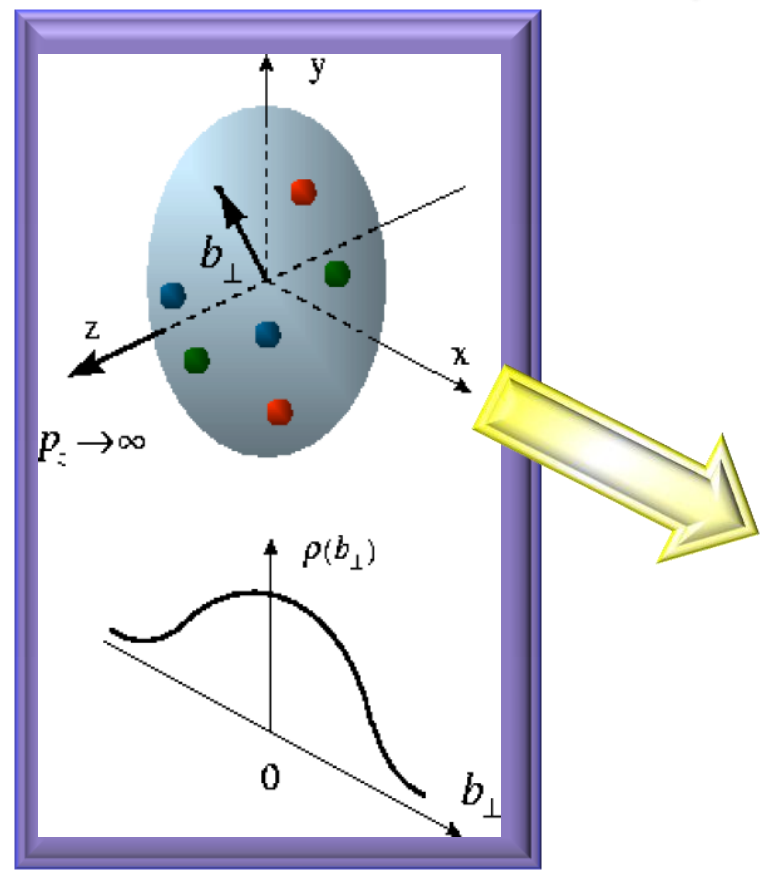

Proton form factors, transverse charge \& current densities

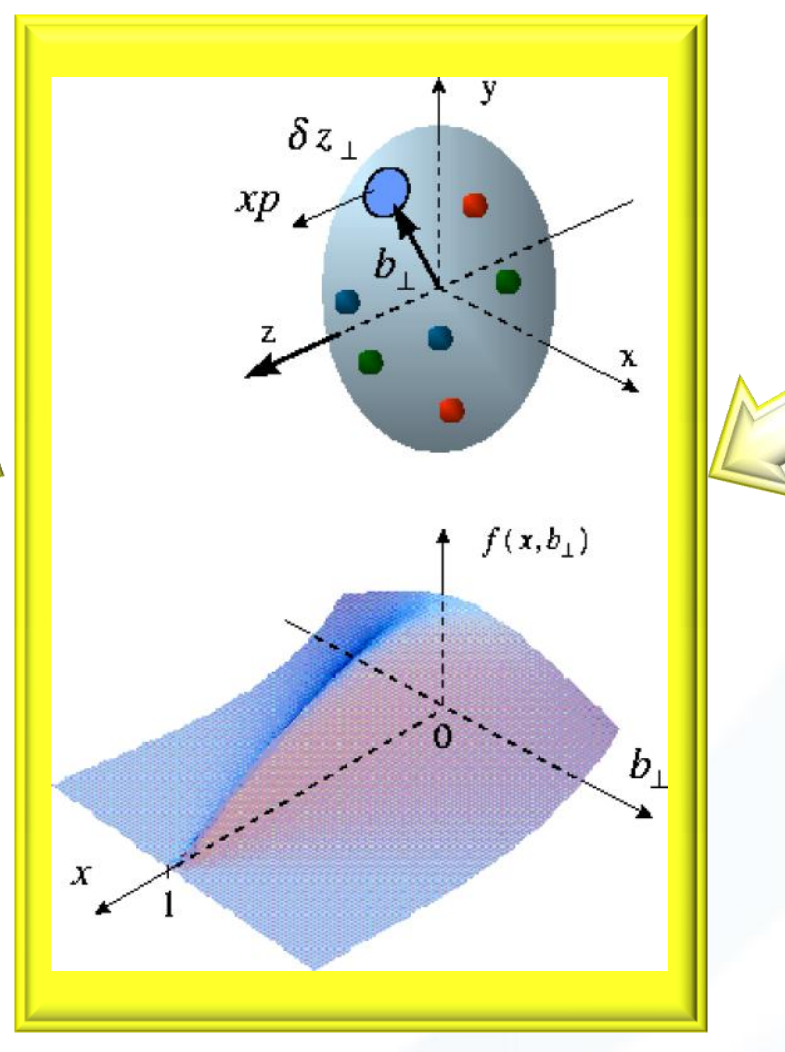

Correlated quark momentum and helicity distributions in transverse space - GPDs

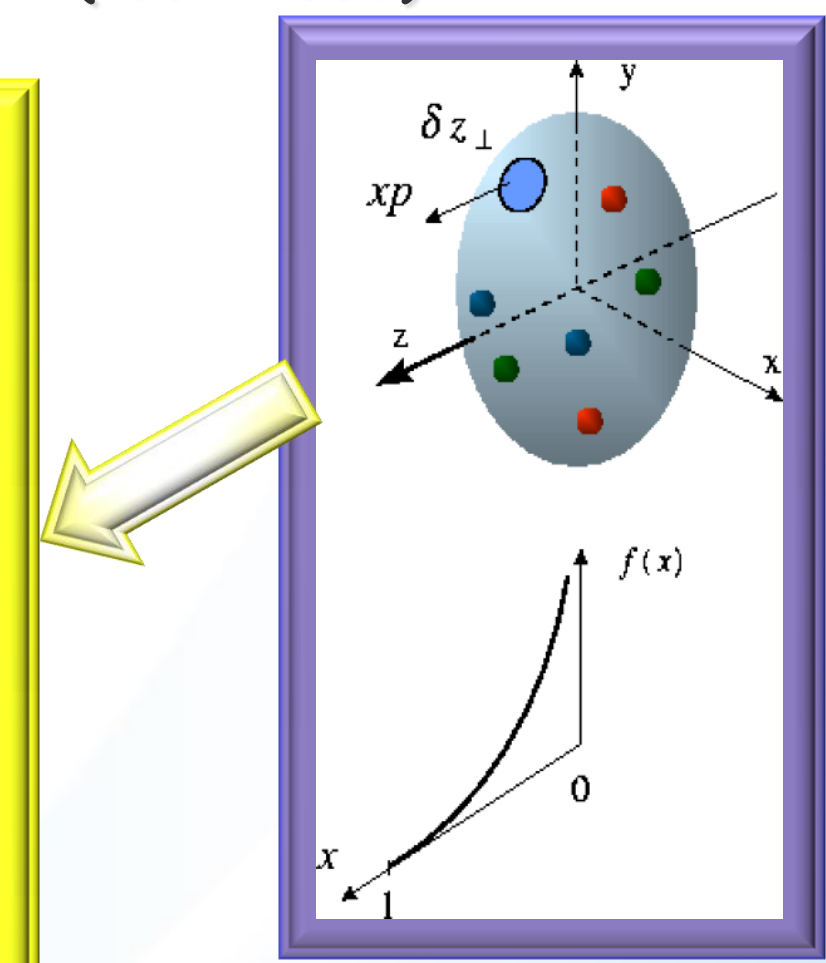

Structure functions, quark longitudinal momentum \& helicity distributions 
How are GPDs characterized?

unpolarized

$$
\begin{array}{ll}
H^{q}(x, \xi, t) & \tilde{\boldsymbol{H}}^{q}(\boldsymbol{x}, \xi, t) \begin{array}{c}
\text { conserve nucleon helicity } \\
\boldsymbol{H}^{q}(\boldsymbol{x}, \mathbf{0}, \mathbf{0})=\boldsymbol{q}, \tilde{\boldsymbol{H}}^{q}(\boldsymbol{x}, \mathbf{0}, \mathbf{0})=\Delta \boldsymbol{q}
\end{array} \\
E^{q}(x, \xi, t) & \tilde{\boldsymbol{E}}^{q}(\boldsymbol{x}, \xi, t) \quad \begin{array}{c}
\text { flip nucleon helicity } \\
\text { not accessible in DIS }
\end{array}
\end{array}
$$

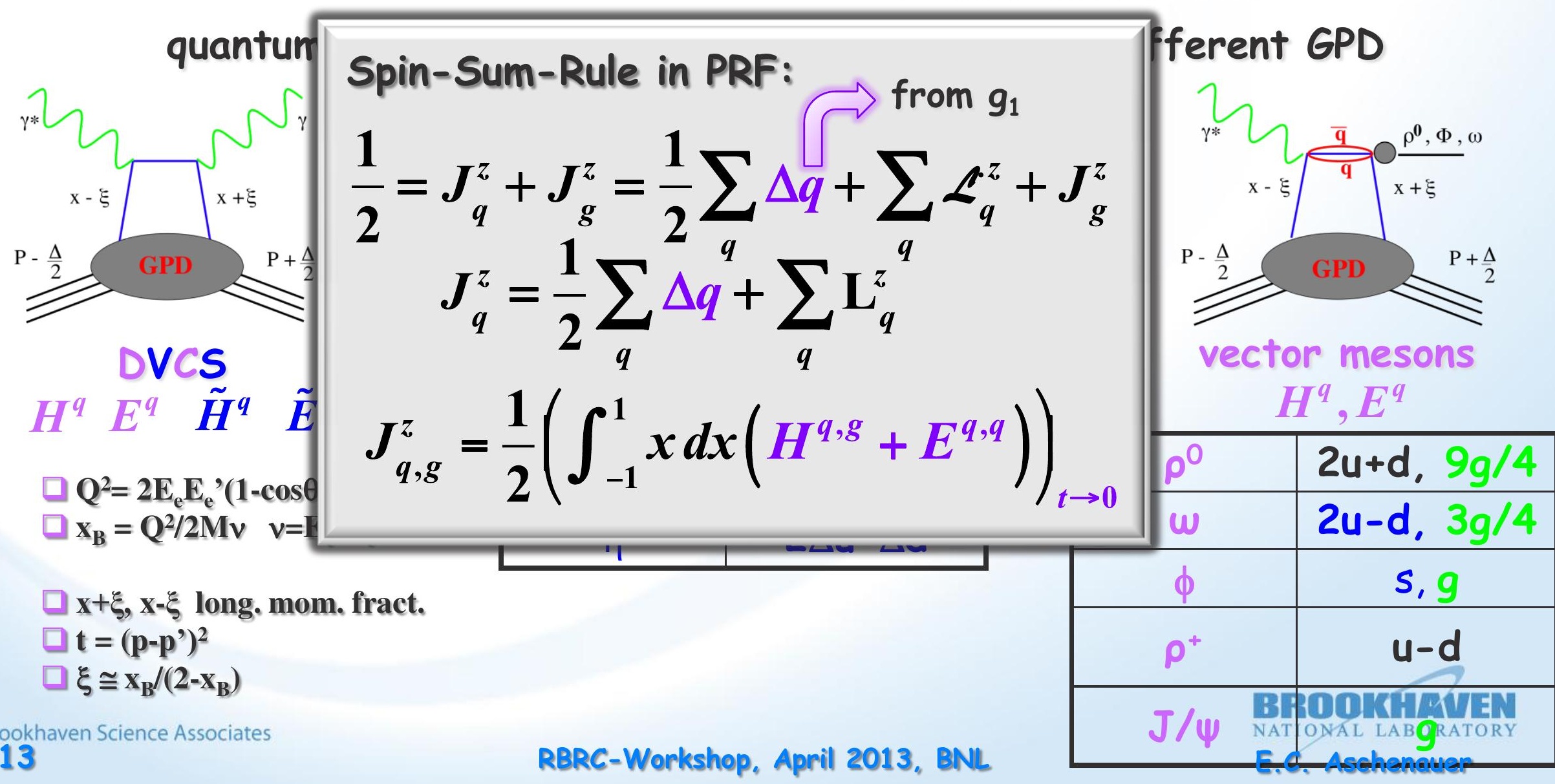




\section{THE DVCS PHASE SPACE}

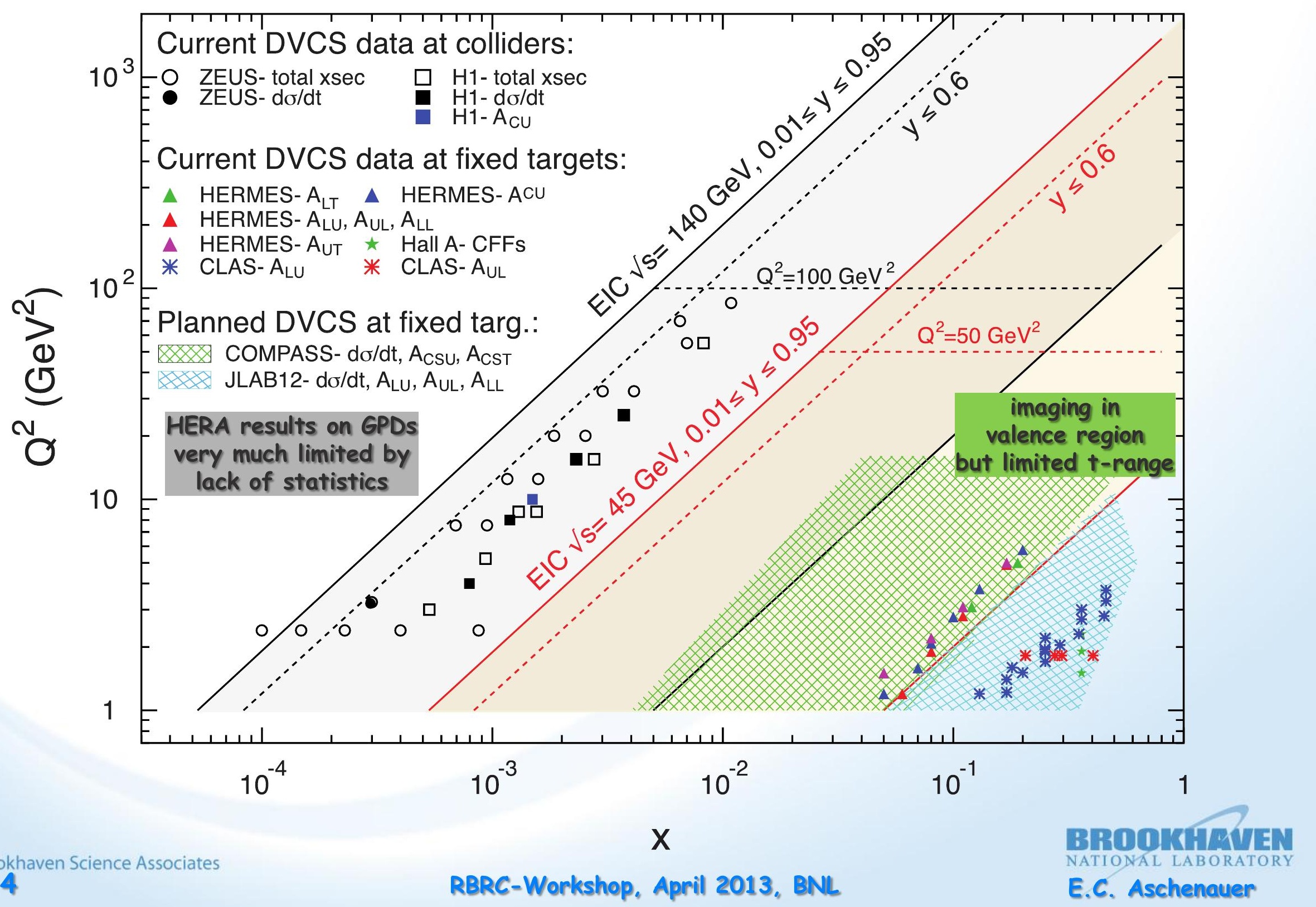


WHAT WILL WE LEARN ABOUT $20+1$ STRUCTURE OF THE PROTON GPD $H$ and $E$ dedflunction of $t, x$ and $Q^{2}$

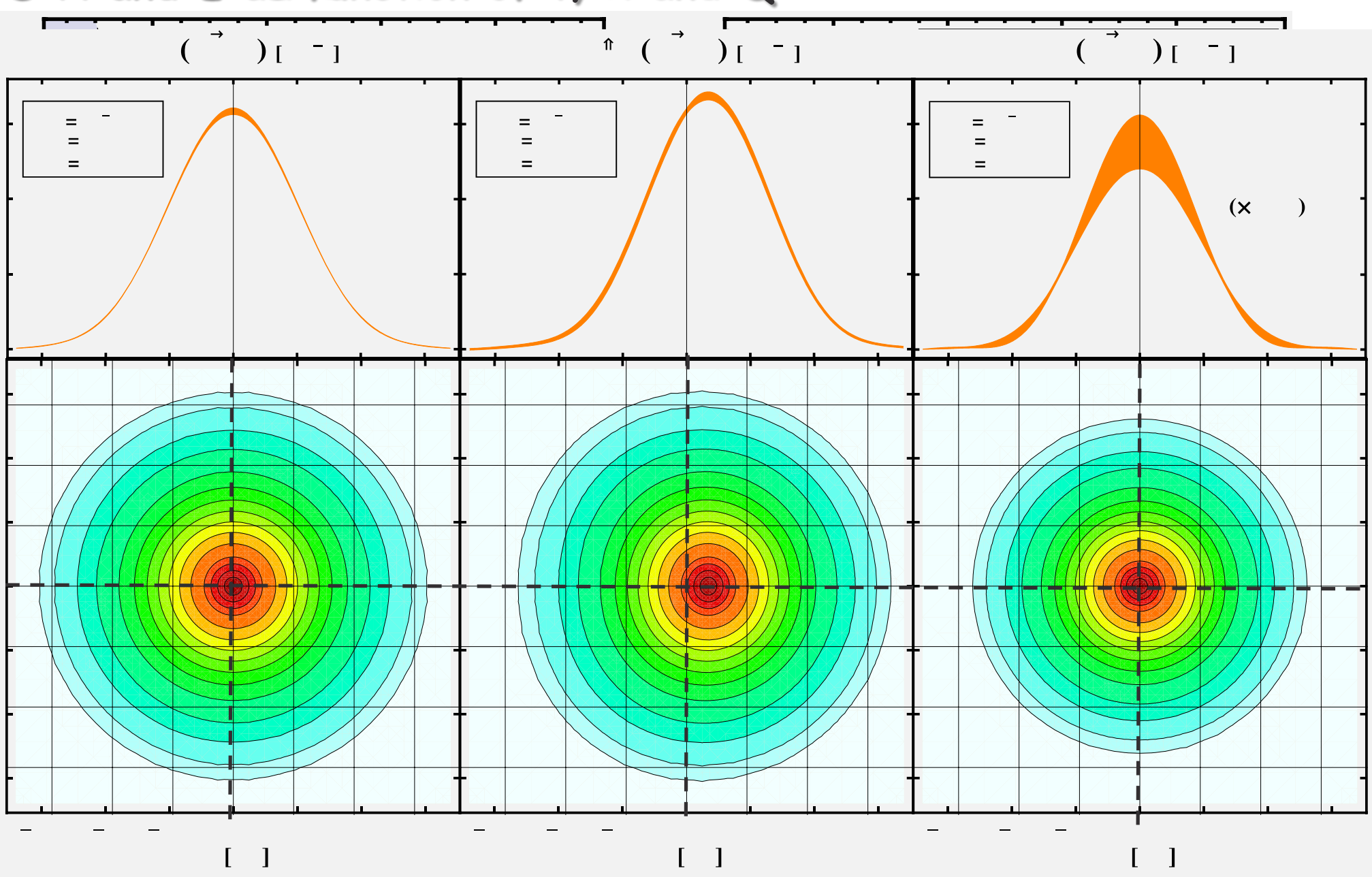

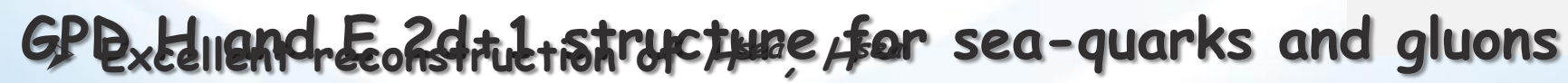

$-\left[\begin{array}{ll}- & 1\end{array}\right.$ and good reconstruction of the (from $d \sigma / d t)$ 


\section{THE EA PHYSICS PROGRAM}

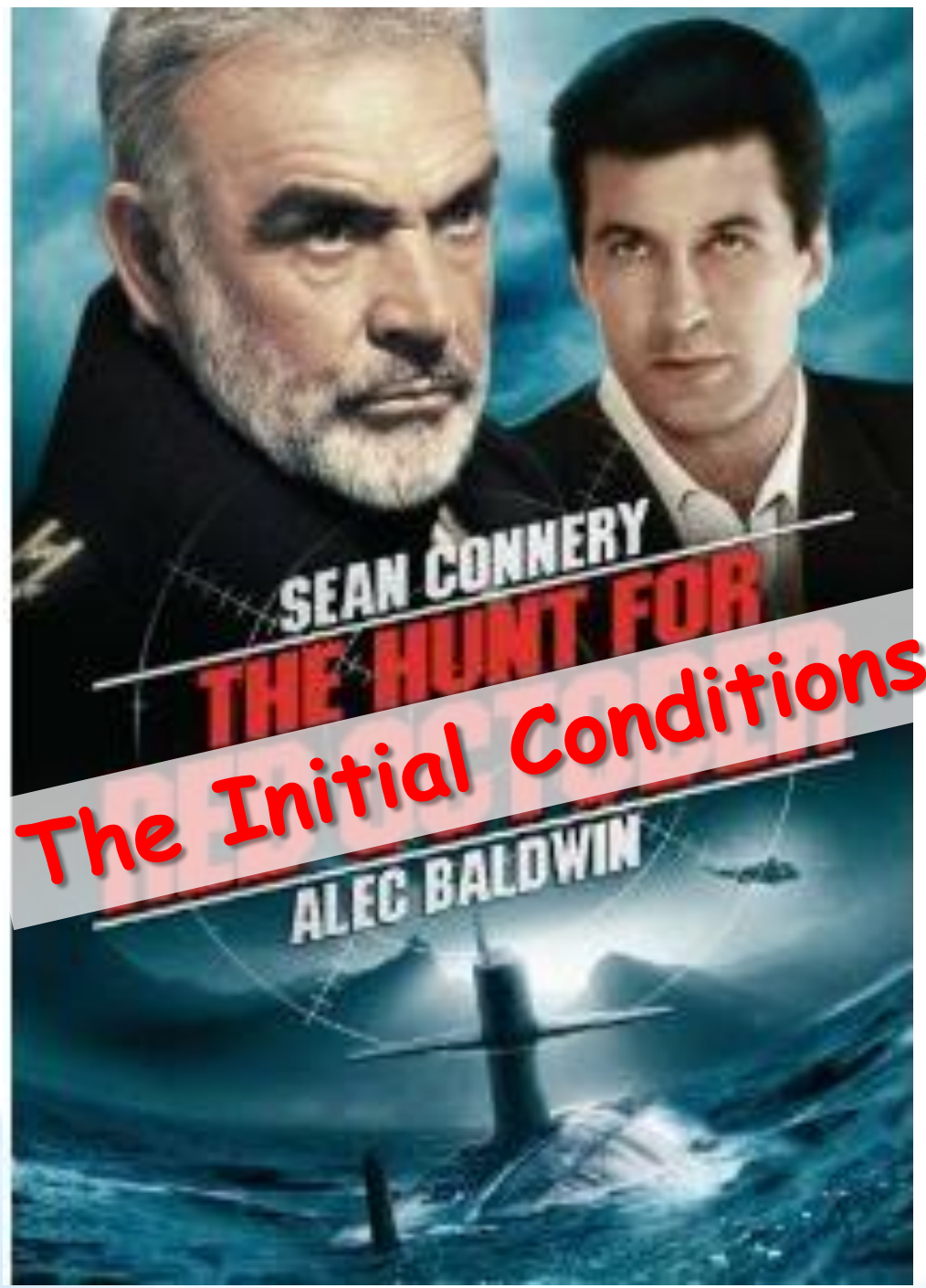

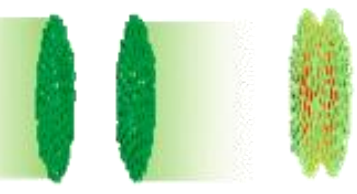

Color Glass

Condensates

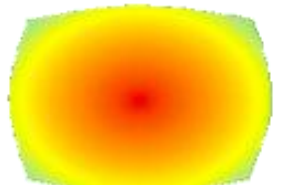

Glasma

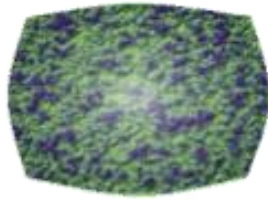

sQGP perfect fluid

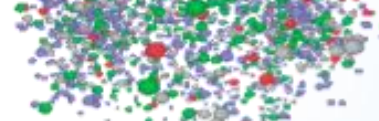

Hadron

Gas

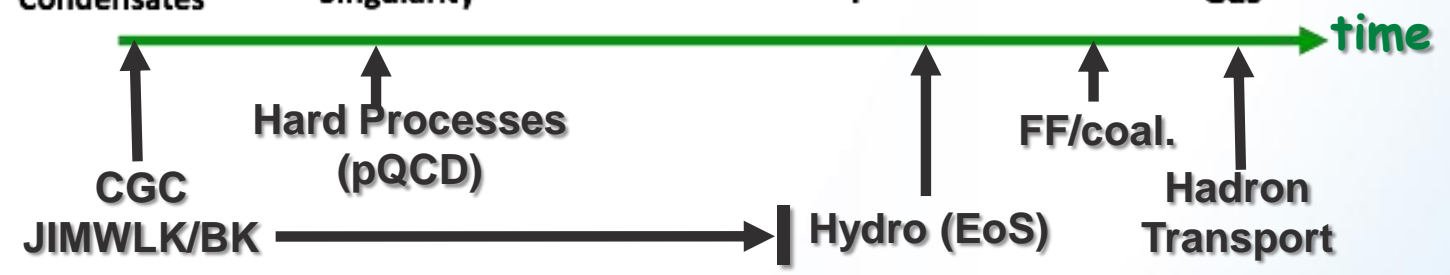

Our understanding of some fundamental properties of the Glasma, SQGP and Hadron Gas depend strongly on our knowledge of the initial state!

$\Longrightarrow 3$ conundrums of the initial state:

1. What is the spatial transverse distributions of nucleons and gluons?

2. How much does the spatial distribution fluctuate? Lumpiness, hot-spots etc.

3. How saturated is the initial state of the nucleus? 


\section{HOW MANY GLUONS HAVE SPACE IN A PROTON?}

at small $x$ linear evolution gives strongly rising $g(x)$

$>$ cannot go on forever

small $x$ / higher energy

$\square B K / J I M W L K$ non-linear evolution includes recombination effects $\rightarrow$ saturation

$>$ Dynamically generated scale Saturation Scale: $Q^{2}(x)$

- Increases with energy or decreasing $x$

$>$ Scale with $Q^{2} / Q^{2}{ }_{s}(x)$ instead of $x$ and $Q^{2}$

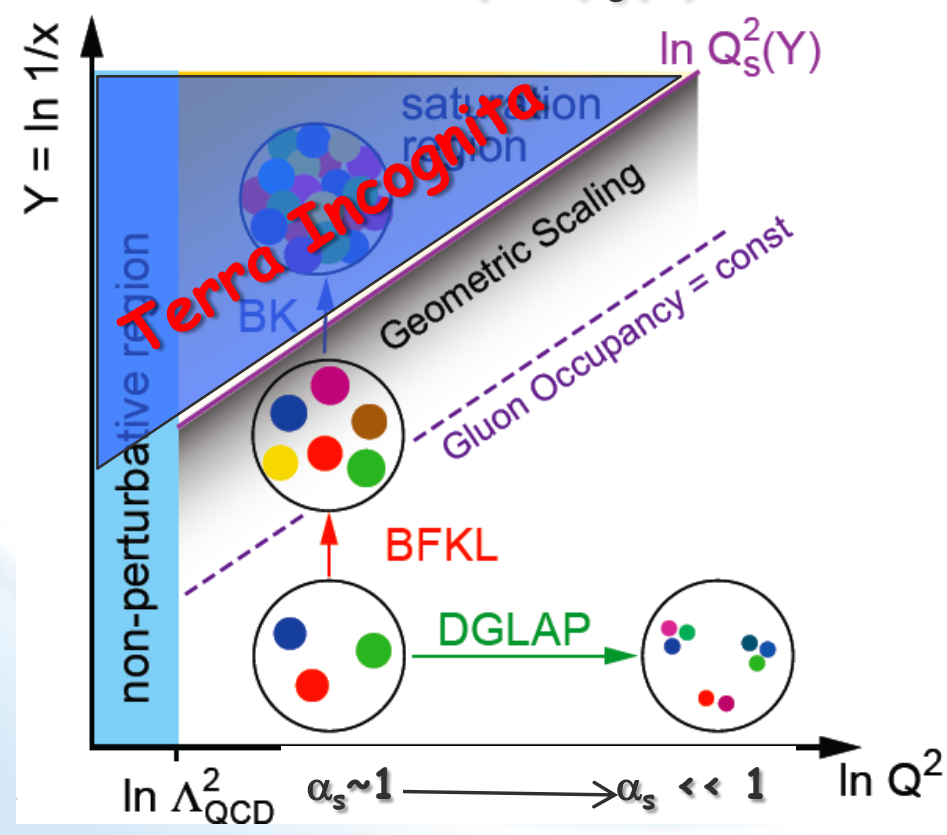

$x=P_{\text {parton }} / P_{\text {nucleon }}$ 


\section{INCLUSIVE DIS IN EA: NUCLEAR PDFS}

$$
\frac{d^{2} \quad \boldsymbol{e A \rightarrow e X}}{\boldsymbol{d x d Q ^ { 2 }}}=\frac{4}{\boldsymbol{x} \boldsymbol{Q}^{4}}\left[\left(\begin{array}{lll}
1 & y & \frac{y^{2}}{2}
\end{array}\right) F_{2}\left(x, Q^{2}\right) \quad \frac{y^{2}}{2} F_{L}\left(x, Q^{2}\right)\right]
$$

$>$ Expect strong non-linear effects in $F_{L}$
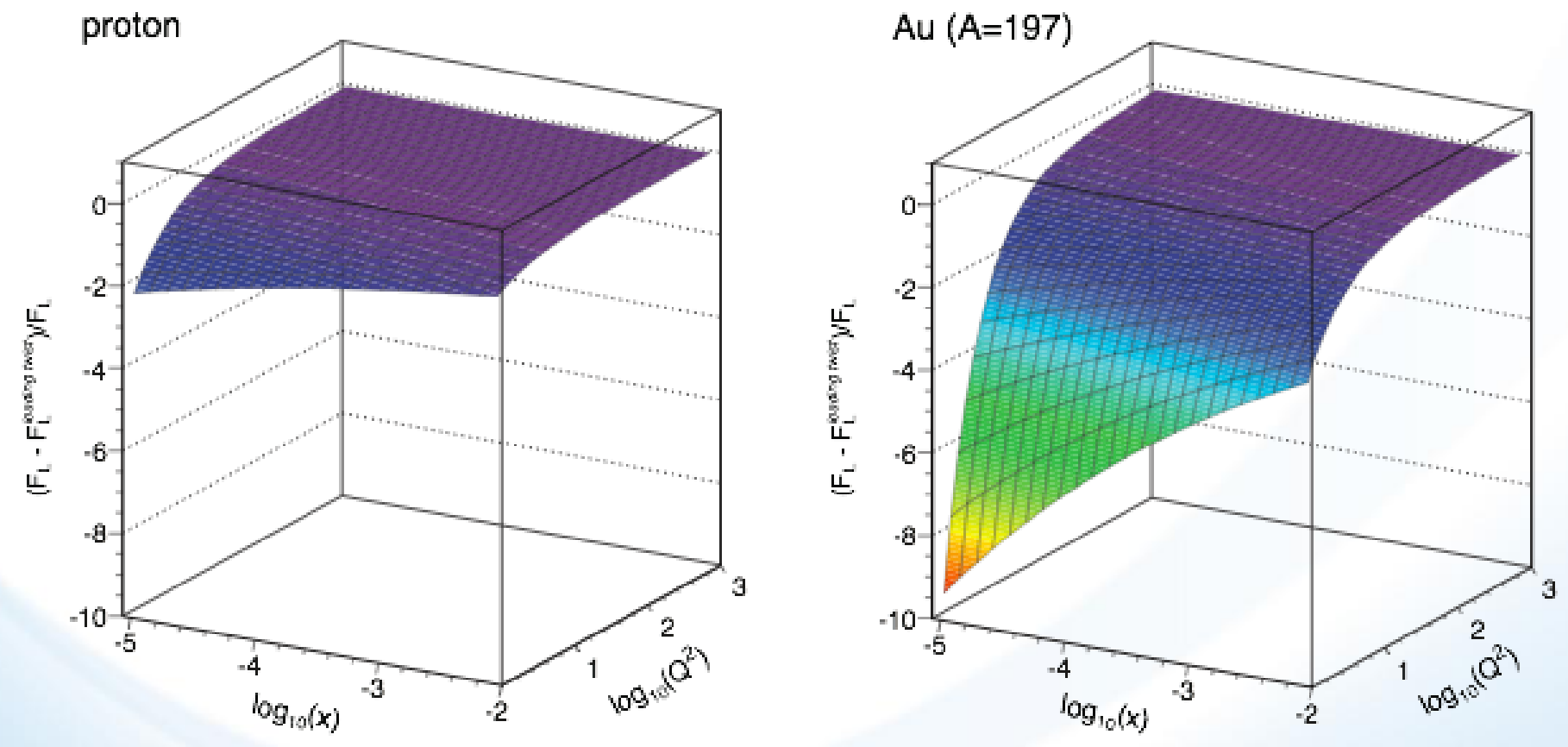

Relative contributions of higher twist effects to $F_{L}$ amplified in $\mathrm{eA}$

Dipole model ( $J$. Bartels et al.) 


\section{INCLUSIVE DIS IN EA: NUCLEAR PDFS}
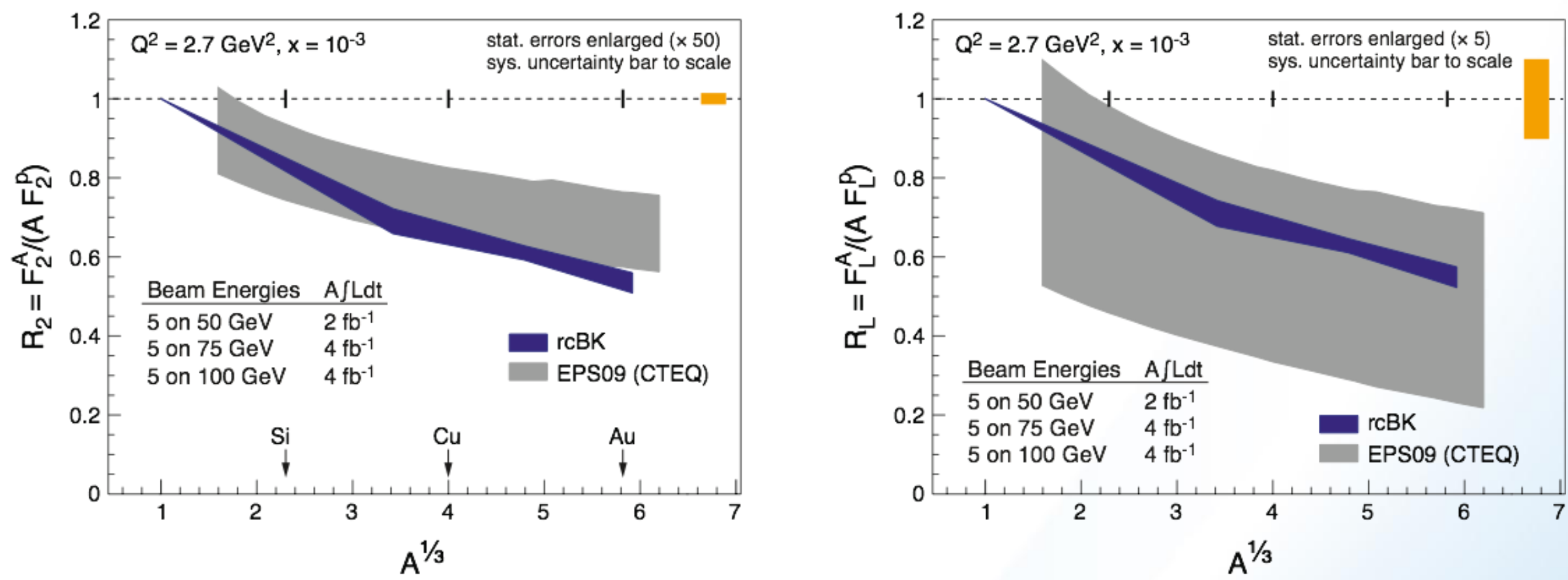

$>$ measurement of $F_{L}$ requires running at different $\square s$

$>F_{2}, F_{L}$ : negligible stat. error, systematics dominated

$>$ A dependence helps to discriminate between linear and non-linear (saturation) models

$>$ Precision nPDF: Huge impact on pA, AA programs 


\section{$h-h$ FORWARD CORRELATION IN $p(d) A$ AT RHIC}

side-view

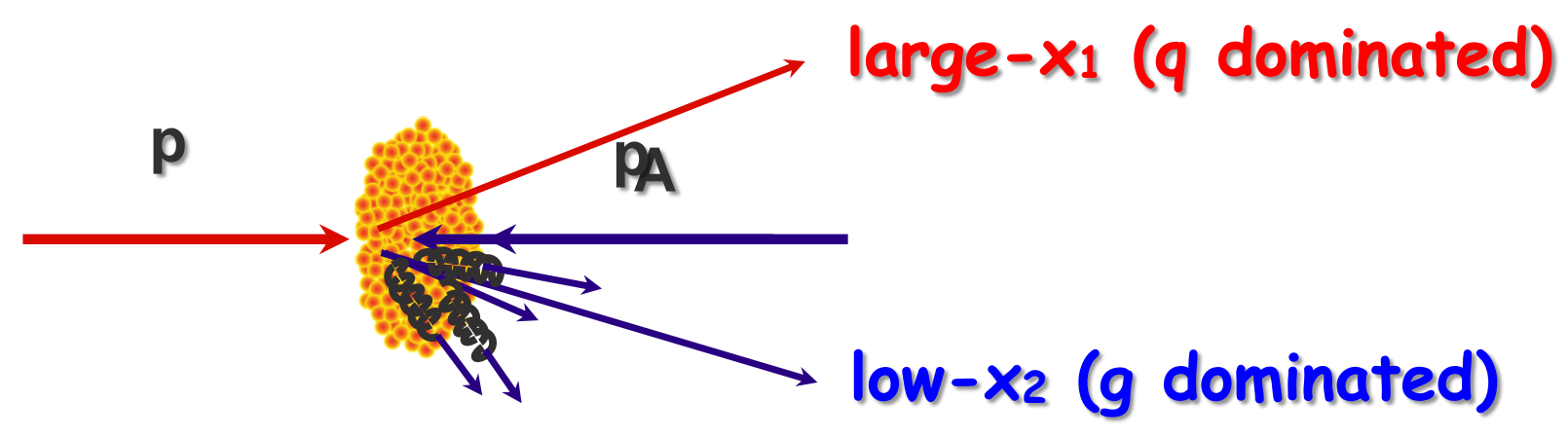

beam-view

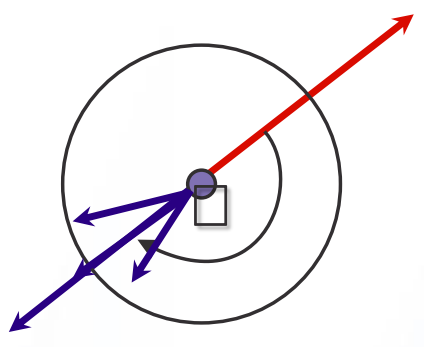

Low gluon density (pp): PQCD predicts $2 \rightarrow 2$ process

$\Rightarrow$ back-to-back di-jet
High gluon density (pA):

$2 \rightarrow$ many process

$\Rightarrow$ expect broadening of away-side

$>$ Small $-x$ evolution $\leftrightarrow$ multiple emissions

$>$ Multiple emissions $\rightarrow$ broadening

$>$ Back-to-back jets (here leading hadrons) may get broadening in PT with a spread of the order of Qs

First prediction by: $C$. Marquet ('07)

Latest review: Stasto, Xiao, Yuan arXiv:1109.1817 (Sep. '11) 


\section{FORW}

\section{1 question, 2 answers}

Initial state saturation model

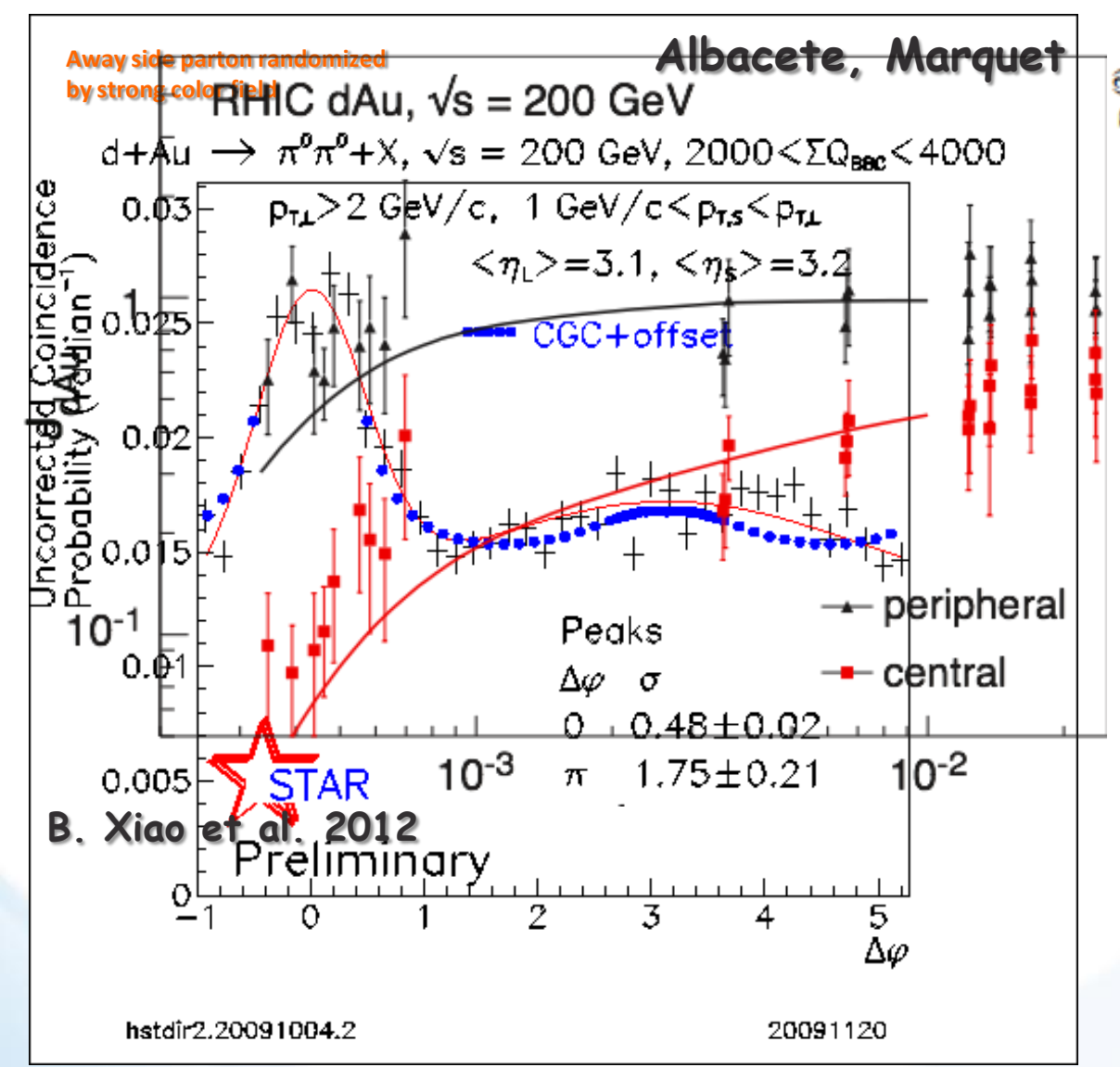

"Non-initial state" shadowing model

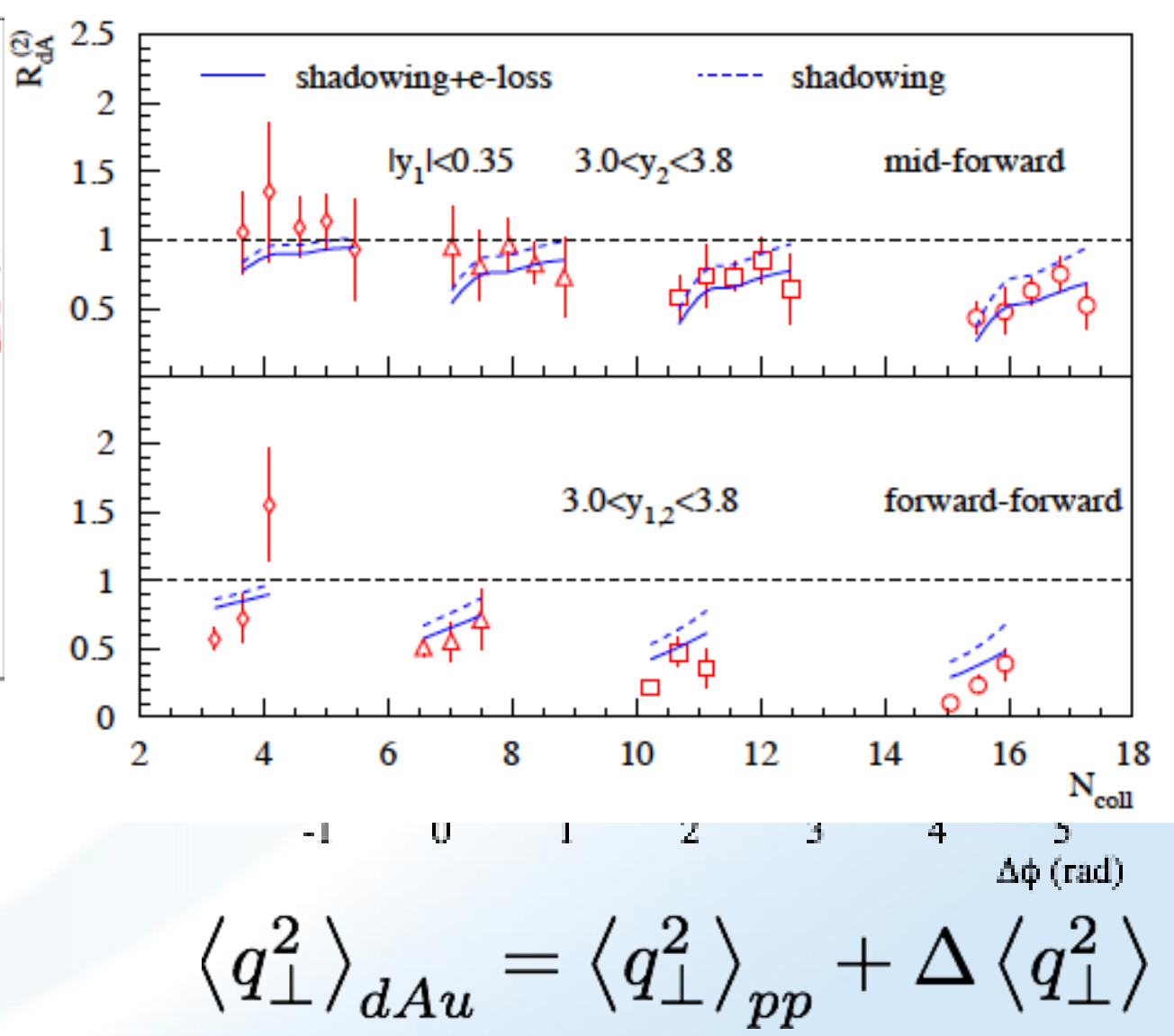




\section{DIHADRON CORRELATIONS IN EA AT EIC}

EIC:

D Extract the spatial multi-gluon correlations and study their nonlinear evolution

o essential for understanding the transition from a deconfined into a confined state.

Advantage over $p(d) A$ :

$>$ eA experimentally much cleaner

o no "spectator" background to subtract

- Access to the exact kinematics of the DIS process $\left(x, Q^{2}\right)$
Perfect saturation signature:

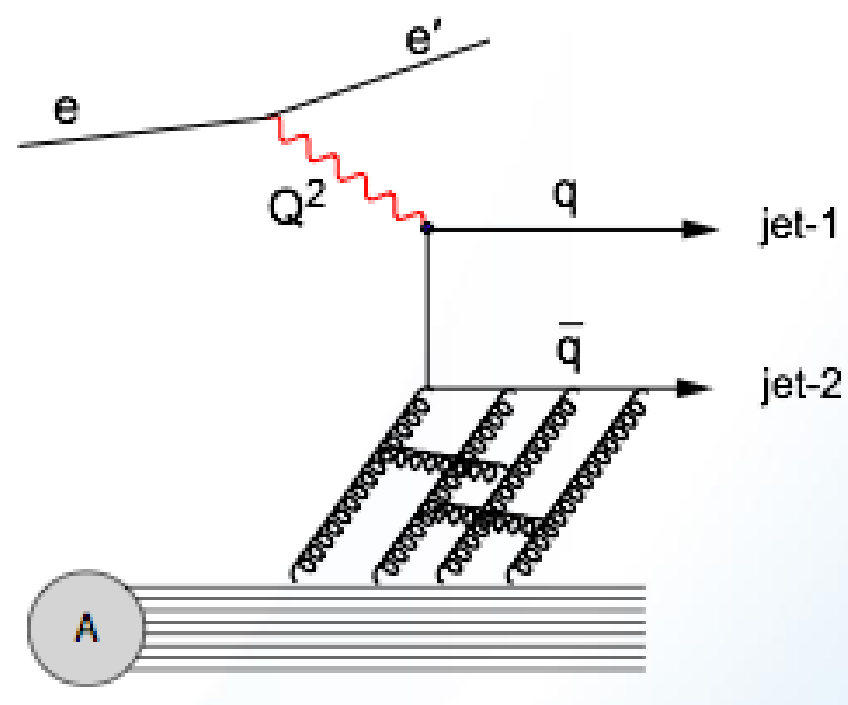

Either jets or use leading hadrons from jets (dihadrons) 


\section{EIC: EA DIHADRON CORRELATIONS STUDIES}

Theory: Saturation

Dominguez, Xiao, Yuan, Lee, Zheng '11/12

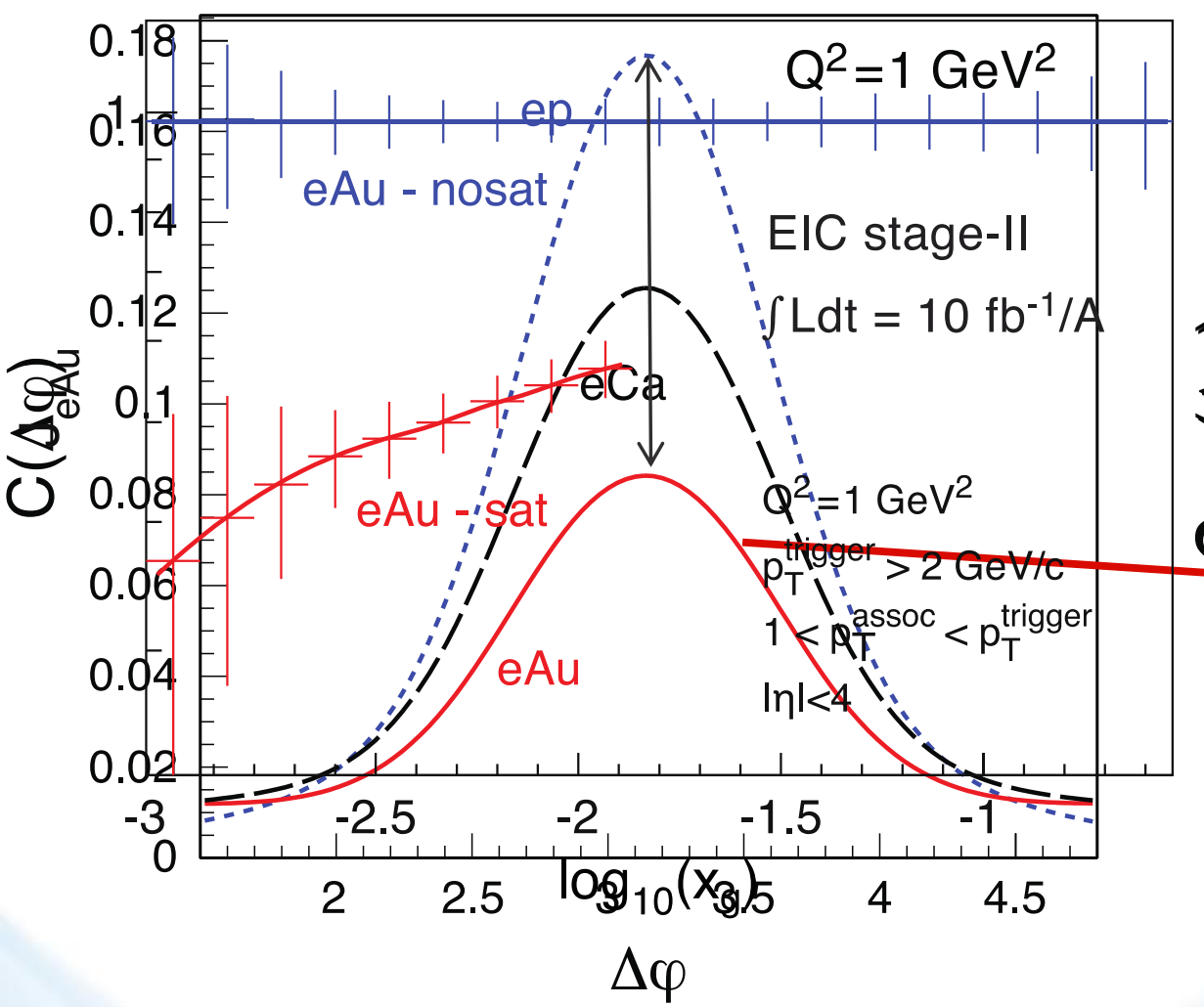

Exp: Saturation versus "conventional" scenario J.H. Lee, L. Zheng (CCNU) '11/12

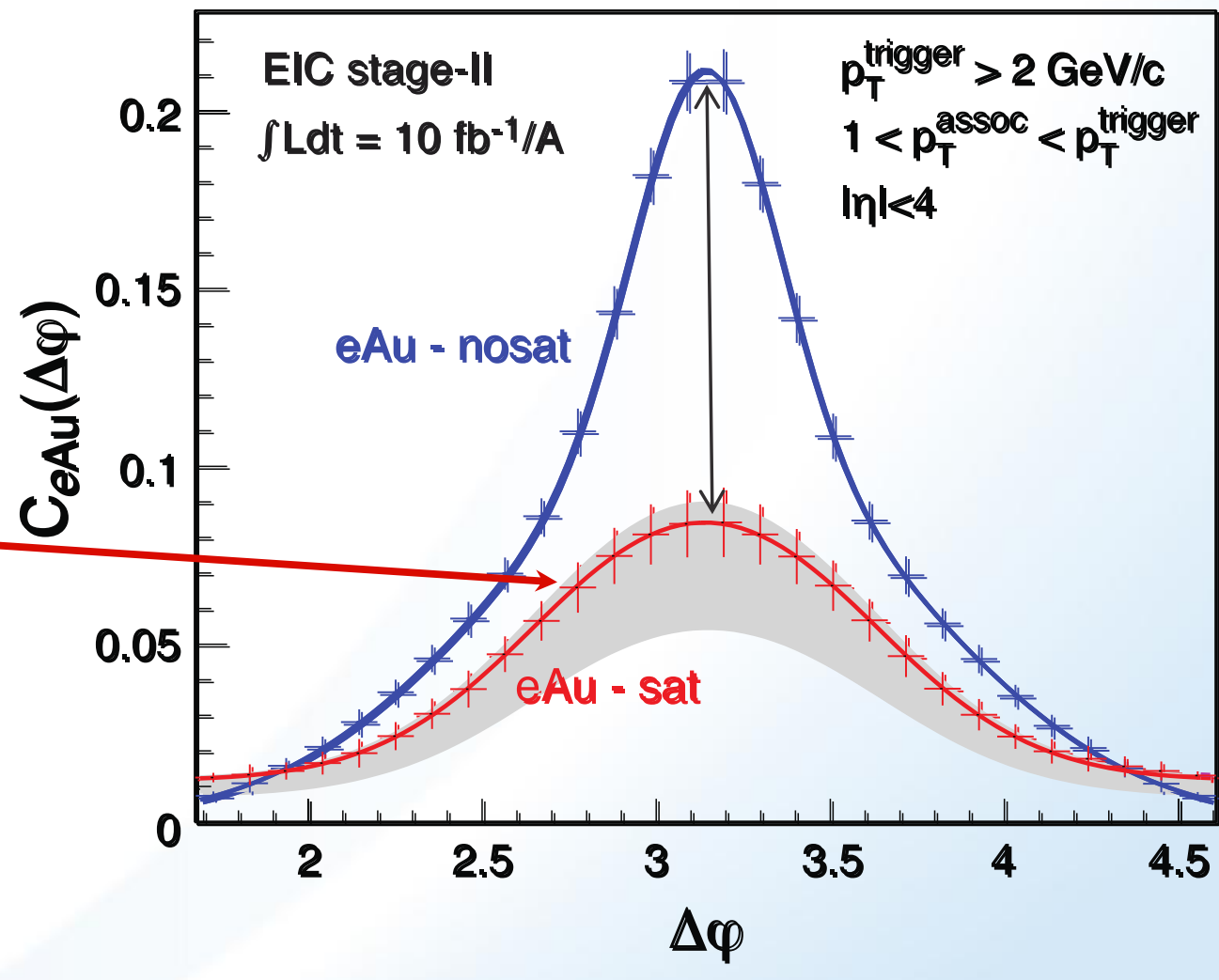

- eA-MC: Pythia6.4 + nPDF (EPS09) + nuclear geometry from DPMJetIII without PS

- Here for $10 \mathrm{fb}^{-1} / \mathrm{A}$ ( 20 weeks), std. experimental cuts

- Clear signal, pronounced differences between sat and no-sat 


\section{HARD DIFFRACTION IN DIS AT SMALLX}

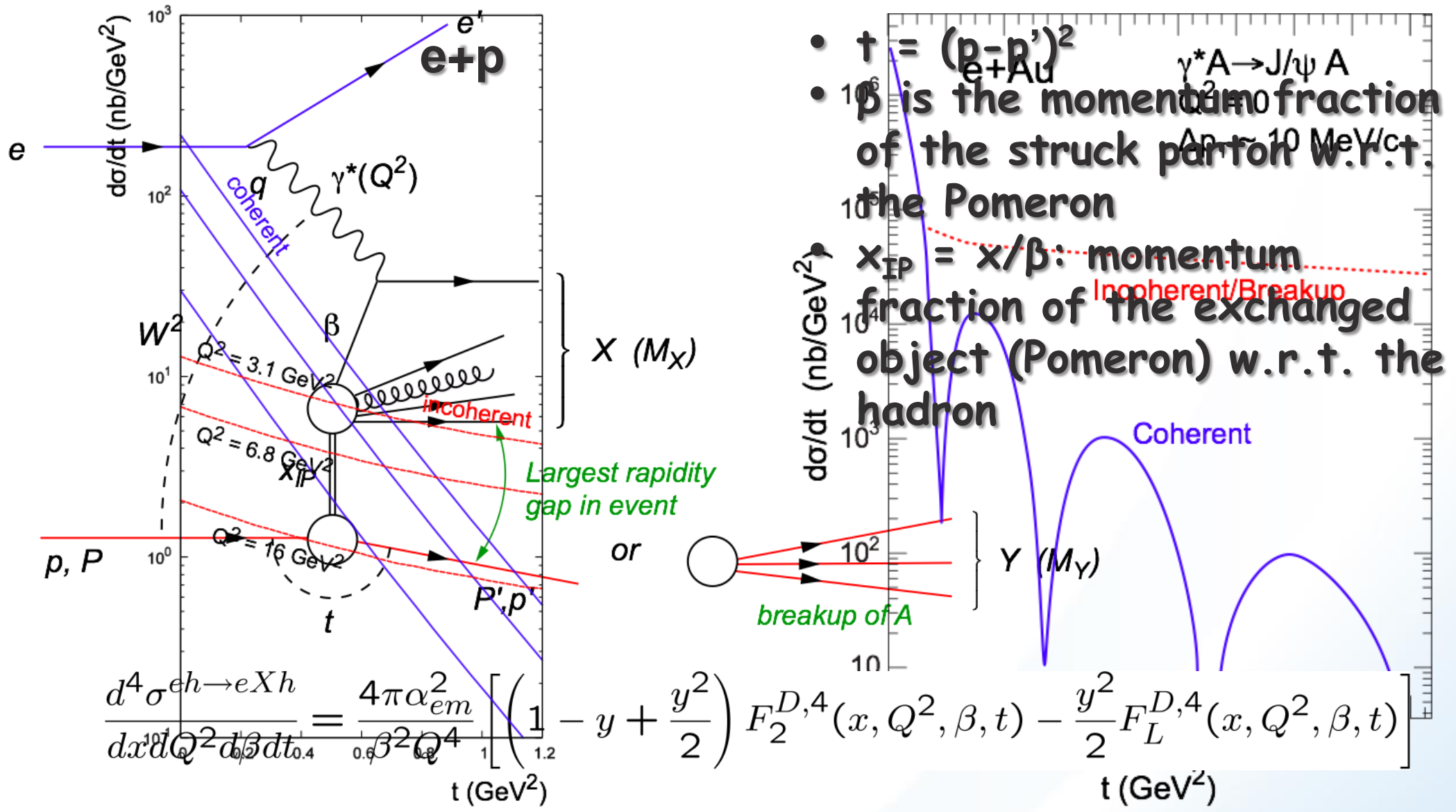

- Diffraction in e+p:

- coherent $\Leftrightarrow p$ intact

- incoherent $\Leftrightarrow$ breakup of $p$

- HERA: $15 \%$ of all events are hard diffractive
- Diffraction in e+A:

- coherent diffraction (nuclei intact)

- breakup into nucleons (nucleons intact)

- incoherent diffraction

- Predictions: $\sigma_{\text {diff }} / \sigma_{\text {tot }}$ in etAur25-40\% 


\section{WHY IS DIFFRACTION SO IMPORTANT}

a Sensitive to spatial gluon distribution

$\frac{d}{d t} \frac{\text { Fourier Transformation }}{\text { of Source Density }{ }_{g}(b)}$

Hot topic:

$>$ Lumpiness?

$>$ Just Wood-Saxon+nucleon g(b)

Incoherent case: measure fluctuations/lumpiness in $g_{A}(b)$

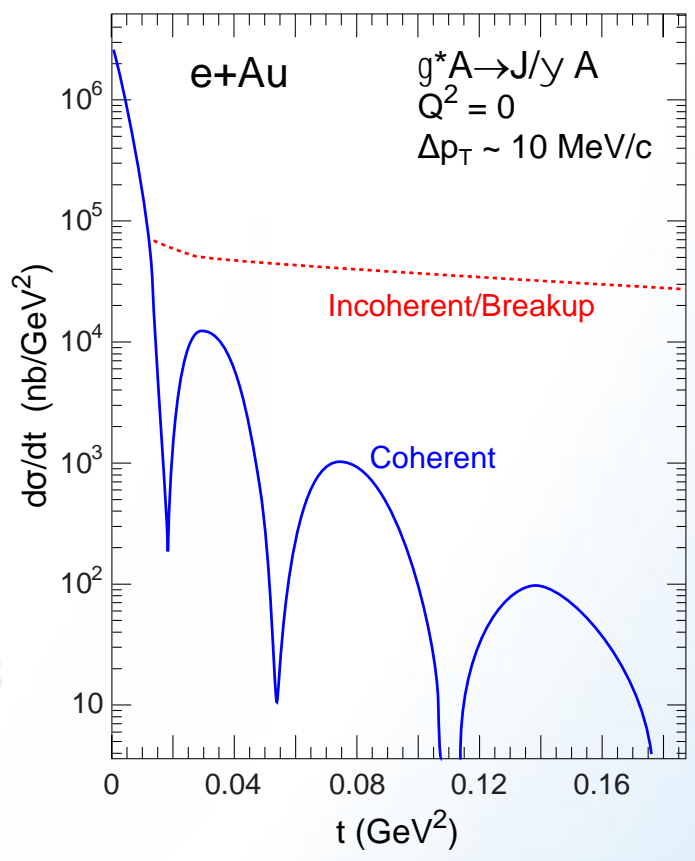

- Sensitive to gluon momentum distributions

$$
\begin{aligned}
& >\sigma \sim g\left(x, Q^{2}\right)^{2} \\
& \frac{d \sigma^{\gamma^{*} p \rightarrow p V}}{d t} \sim \mid \int \Psi_{V}^{*} \frac{\left.d \sigma_{q \bar{q}} \Psi e^{-i b \Delta}\right|^{2} b}{d^{2} b} \\
& \frac{d \sigma_{q \bar{q}} \sim r^{2} \alpha_{s} x g\left(x, \mu^{2}\right) T(b)}{d^{2} b}
\end{aligned}
$$

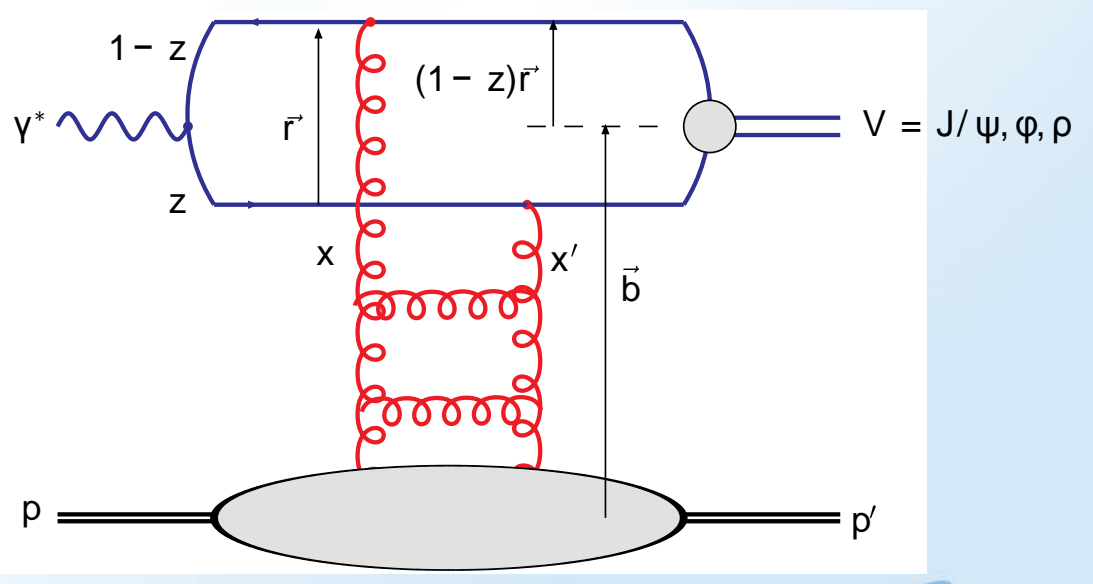




\section{RATIO OF DIFFRACTIVE TO TOTAL CROSS-SECTION}

- Black disc limit characterized by $\sigma_{\text {diff }} / \sigma_{\text {tot }}=1 / 2$ (Hera sees $1 / 7$ ) - Large fraction of diffractive event is unambiguous signature for reaching the saturated limit

Fraction of low-mass coherent diffraction in ep and eA at EIC:

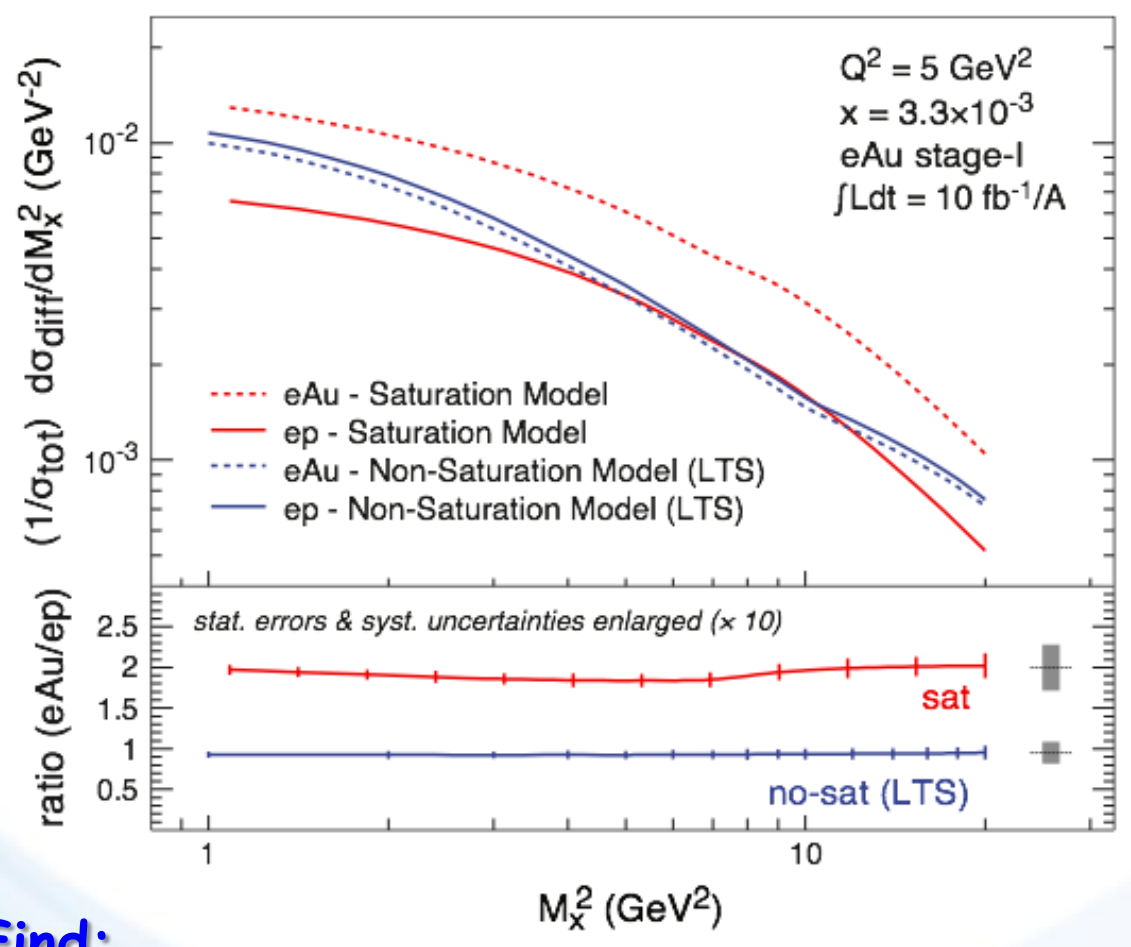

Find:

- w/o non-linear effects eA/ep ratio stays roughly one

- non-linear effects enhance odiff in eA scattering RBRC-Workshop, April 2013, BNL

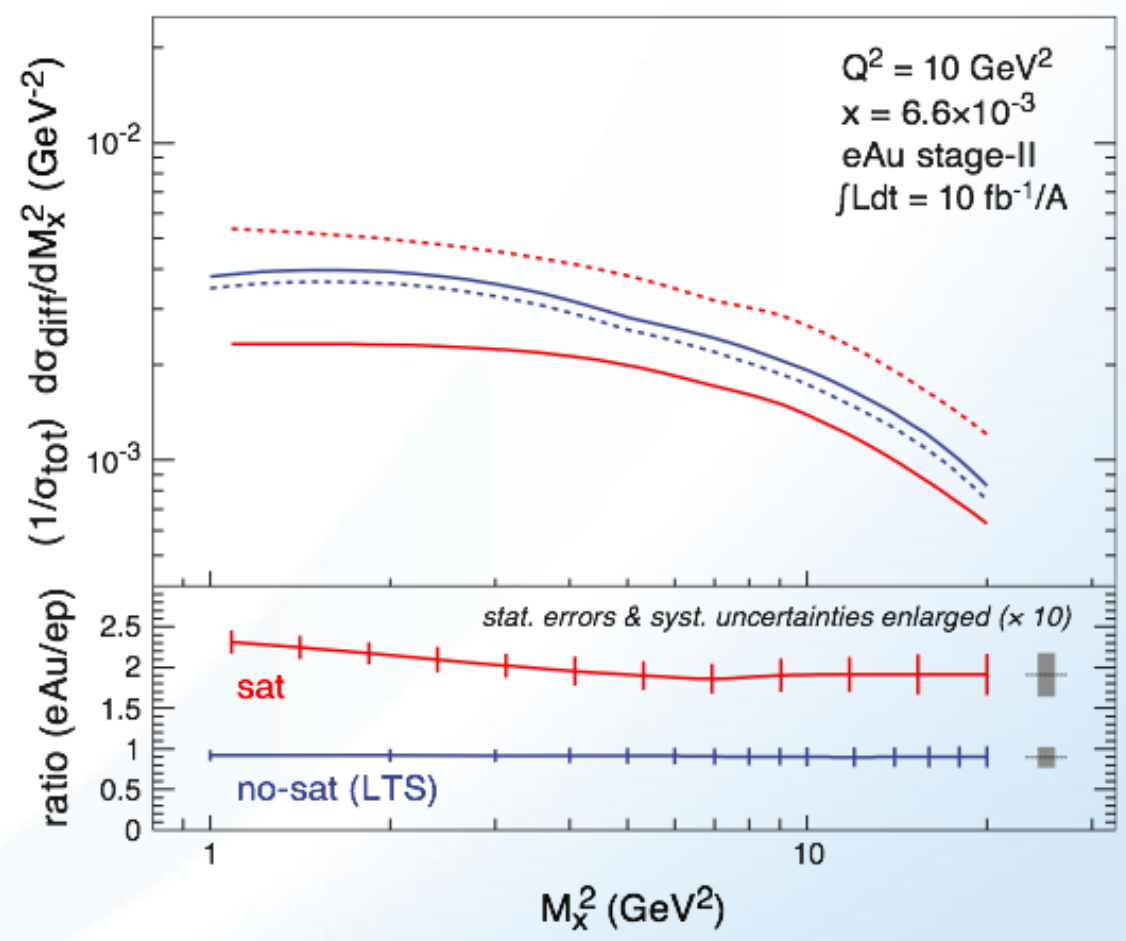

Day-1 signature for Saturation

NATIONAL LABORATORY

BOC. Aschenauer 


\section{EXCLUSIVE VECTOR MESON PRODUCTION}

$>$ Unique probe - allows to measure momentum transfer $t$ in eA diffraction

$\rightarrow$ in general, one cannot detect the outgoing nucleus and its momentum

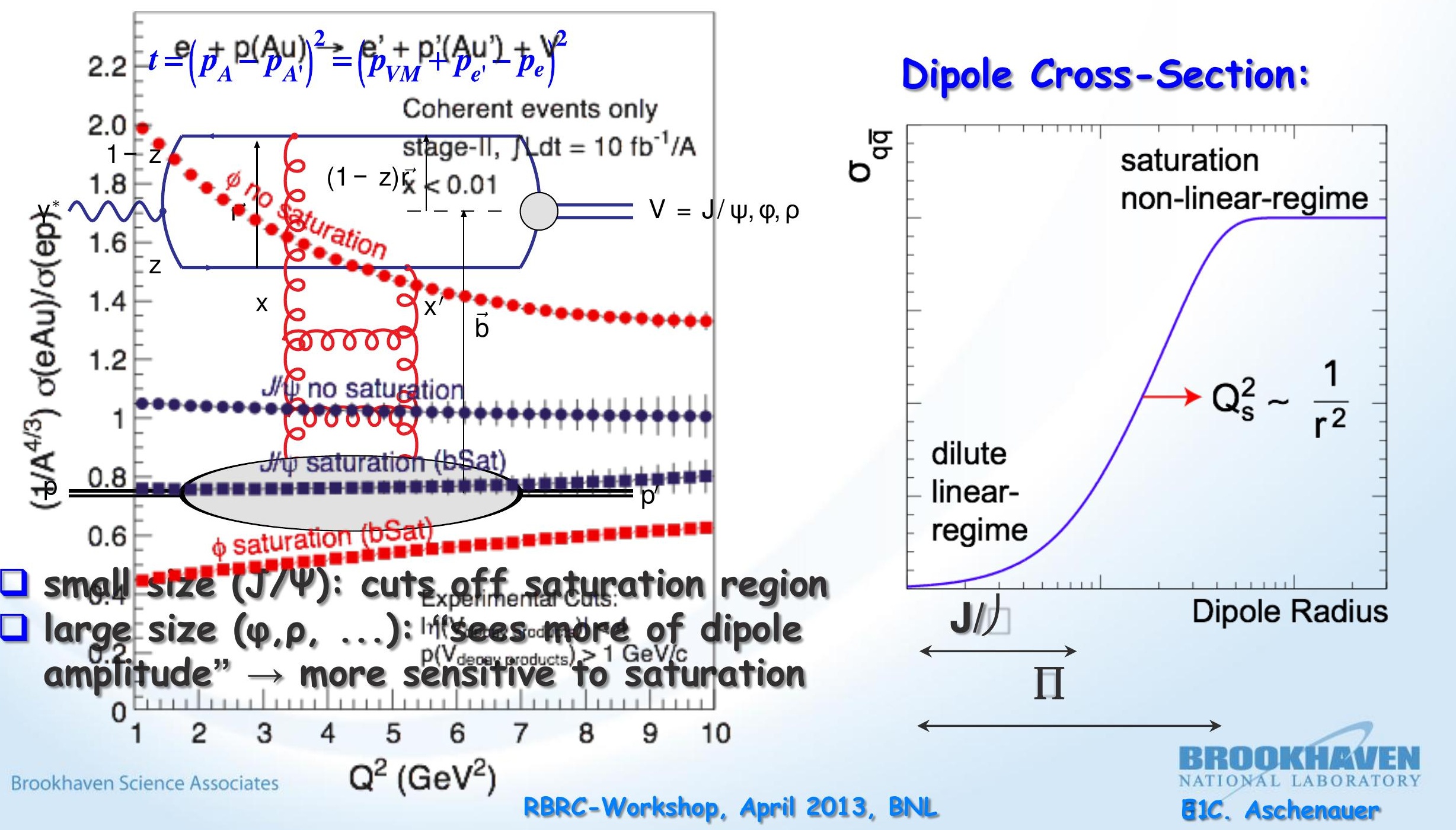



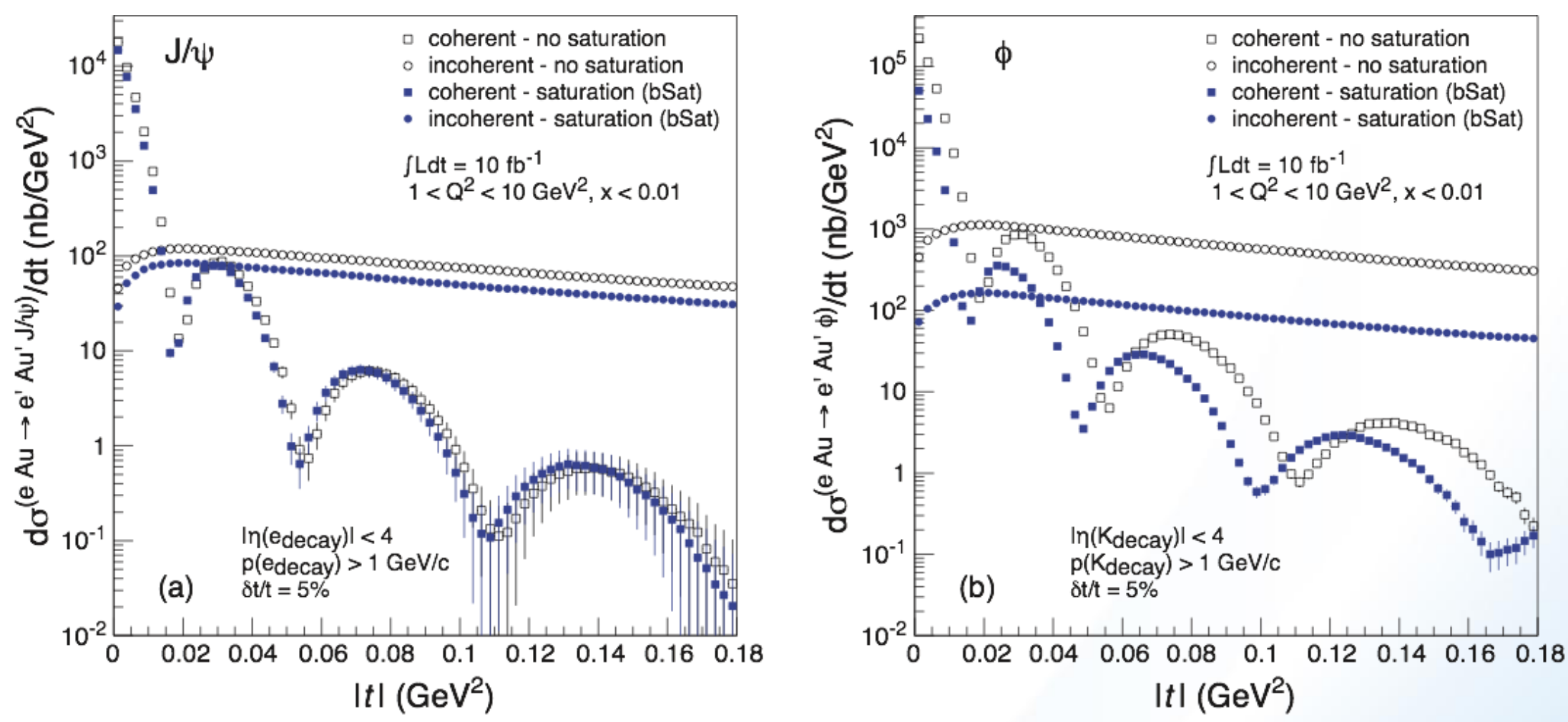

$>$ Goal: going after the source distribution of gluons through Fourier transform of $d \sigma / d t$

$>$ Find: Typical diffractive pattern for coherent (non-breakup) part

$>$ As expected: $J / \Psi$ less sensitive to saturation effects than larger $\Phi$-meson 
$>$ Idea: momentum transfer $t$ conjugate to transverse position $\left(b_{T}\right)$ o coherent part probes "shape of black disc"

o incoherent part (dominant at large $t$ ) sensitive to "lumpiness" of the source (fluctuations, hot spots, ...)

Spatial source distribution: $F(b) \sim \frac{1}{2 \pi} \int_{0}^{\infty} d \Delta \Delta J_{o}(\Delta b) \sqrt{\frac{d \sigma}{d t}}$

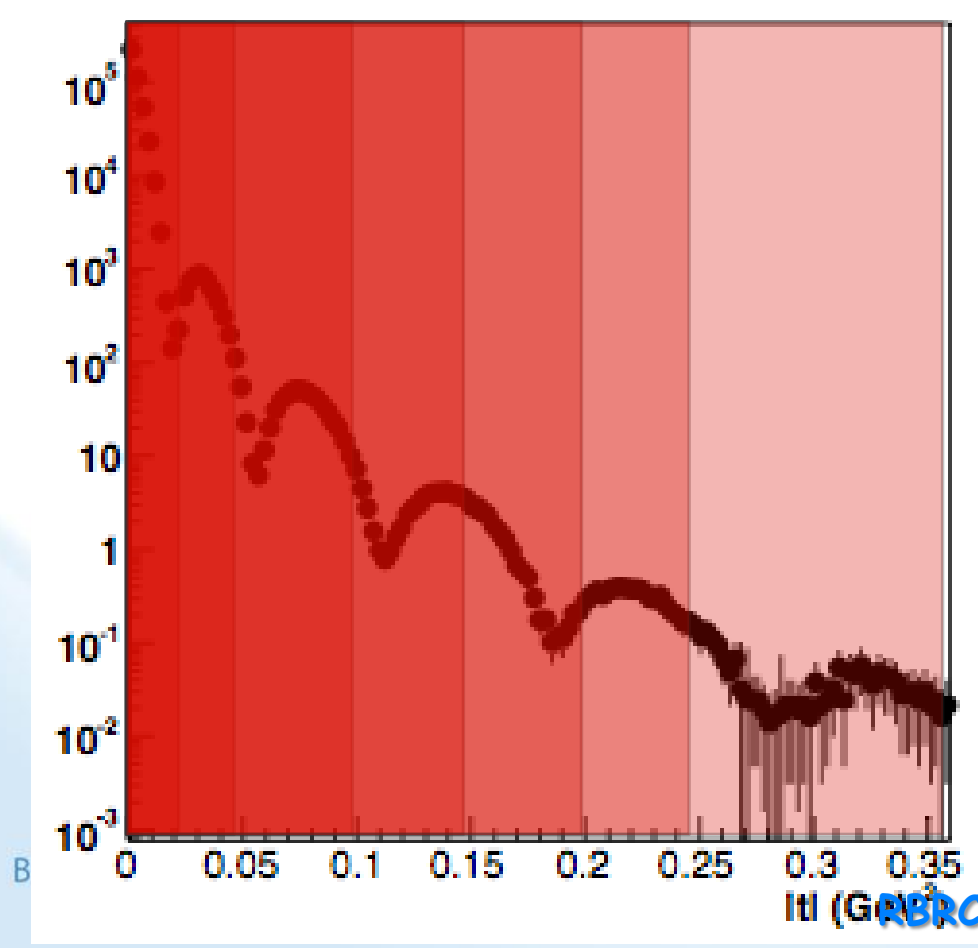

$t=\Delta^{2} /(1-x) \approx \Delta^{2} \quad($ for small $x)$

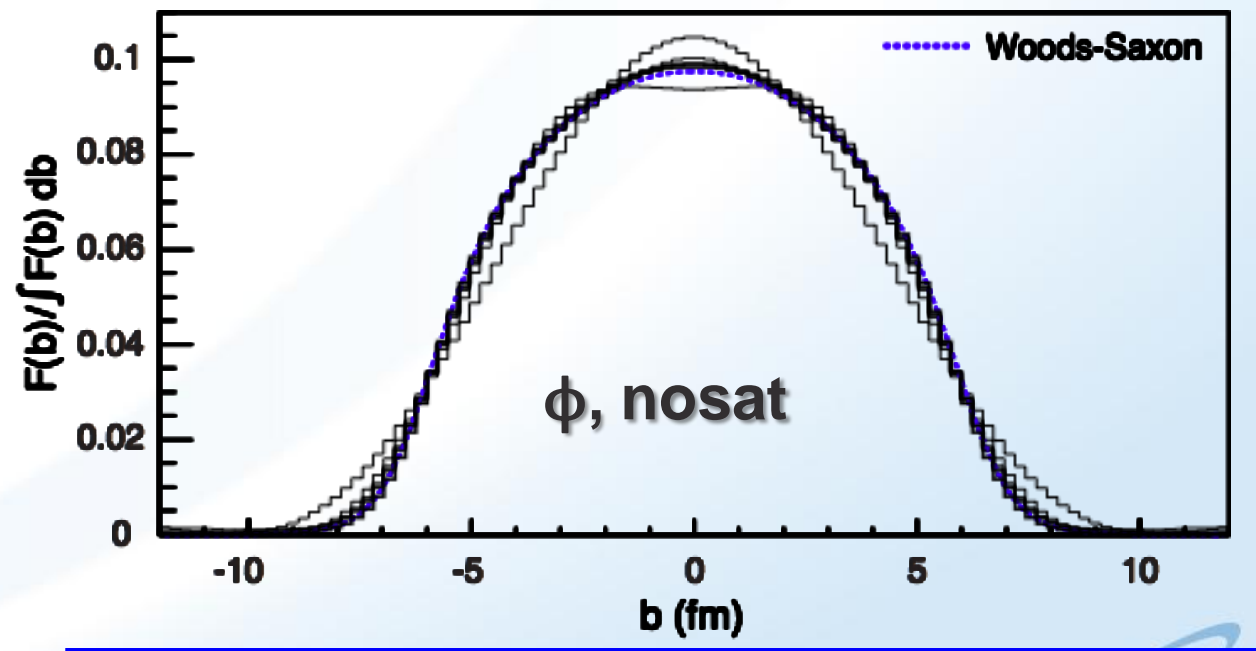

Golden eA measurement for EIC 
DEEP INELASTIC SCATTERING - VACUUM

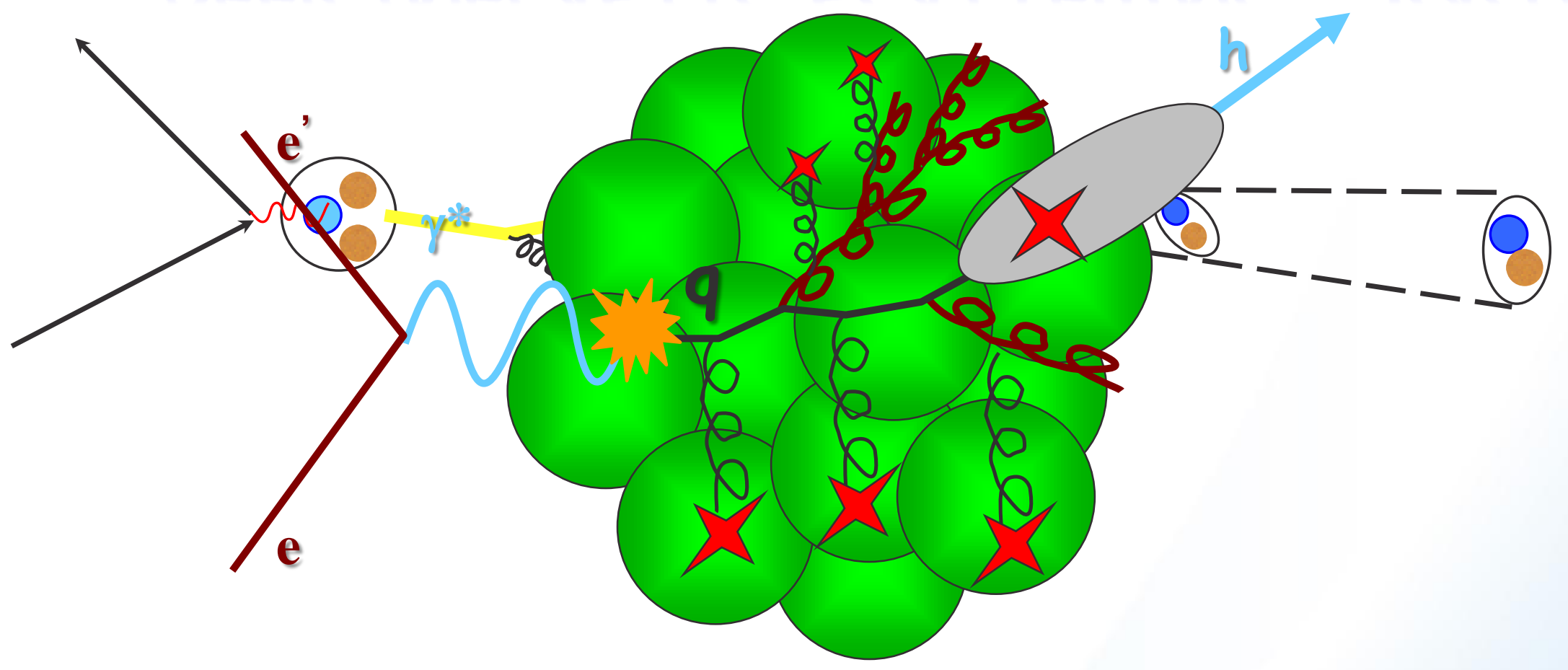

\section{What happens if we add a nuclear medium}

\section{Observables:}

Broadening:

Attenuation:
$\Delta p_{t}{ }^{2}$ linked directly with saturation scale ratio of hadron production in $A$ to $d$ modifications of nPDF cancel out

$$
\begin{gathered}
p_{t}^{2}=\left\langle p_{t}^{2}\right\rangle_{A}\left\langle p_{t}^{2}\right\rangle_{p} \\
R_{A}^{h}\left(Q^{2}, x, z, p_{t}, \quad\right)
\end{gathered}
$$




\section{WHAT DO WE KNOW AND WHAT CAN EIC DO}

\section{Hermes:}

$E_{e}=27 \mathrm{GeV} \rightarrow \sqrt{s}=7.2 \mathrm{G}$

$E_{h}=2-15 \mathrm{GeV}$
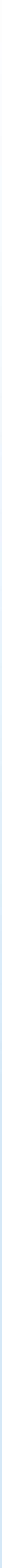


\section{TAKE AWAY MESSAGE}

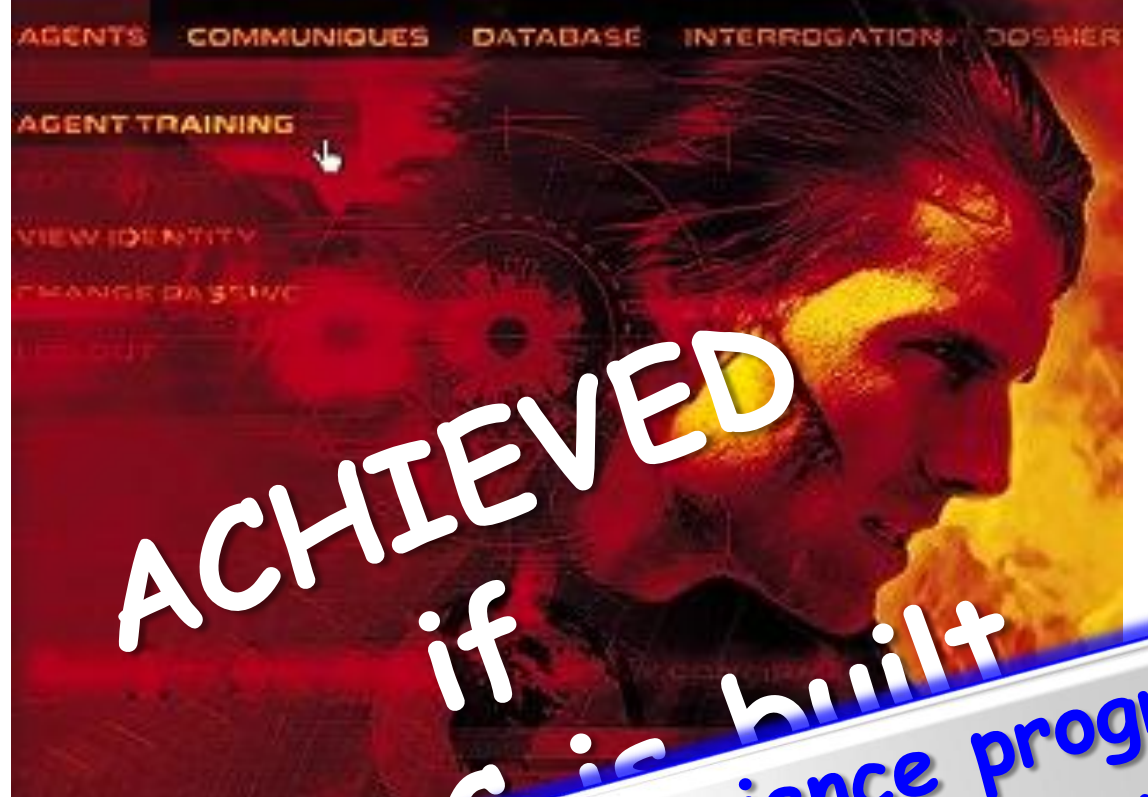

D entire science progy

The EIC will profoundly impact our understanding of QCD

with its high energy, high luminosity

eA and polarized ep collisions

$\square$ ep: precision studies a TMDs, and GPD s min tied to a com uniquely tied collider ucleon ever electron ion collider without ycture future high

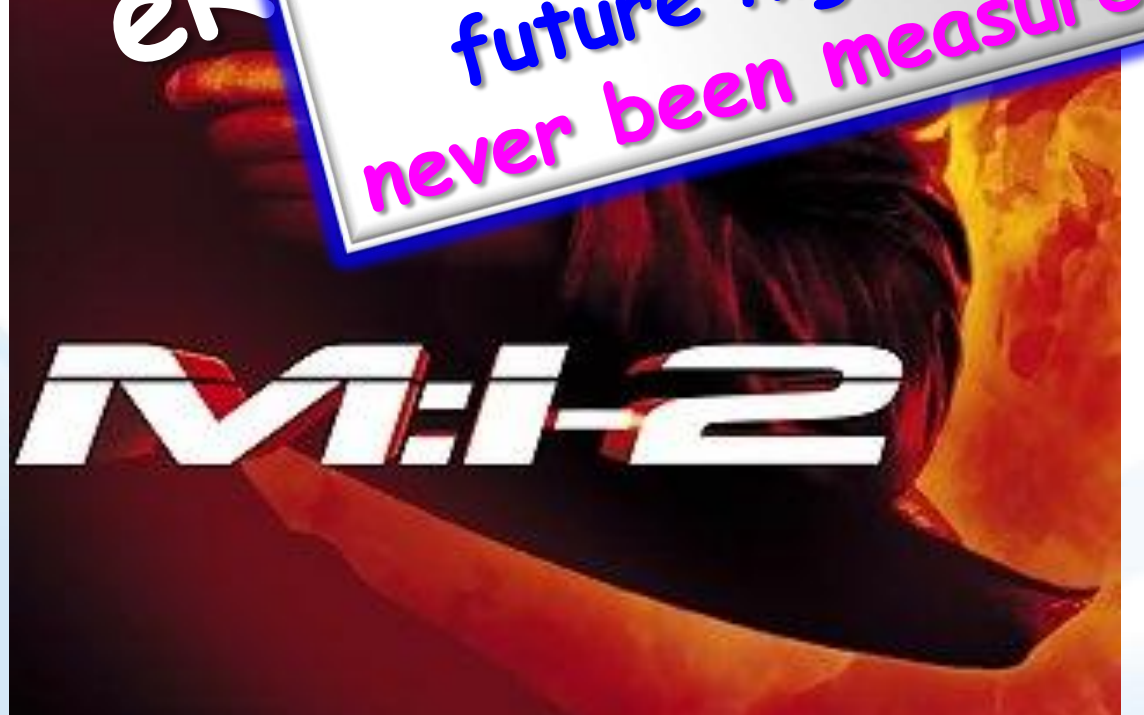

non-linear QCD/saturation/higher twist effects, properties of glue (momentum \& space-time). understand how fast partons interact as they traverse nuclear matter. new insight into fragmentation processes, clarification of the nature of pomerons. 
BACKUP 


\section{POSSIBLE SCHEDULE TO REALIZE ERHIC}

Fiscal year

RHIC I physics

RHIC II upgrades

RHIC II physics

sPHENIX/STAR upgrades

RHIC physics with upgrades

eRHIC

R\&D (CD0-CD1)

PED (CD1-CD2)

Baselined (CD2-CD3)

Construction (CD3-CD4)

eRHIC detector

eRHIC physics

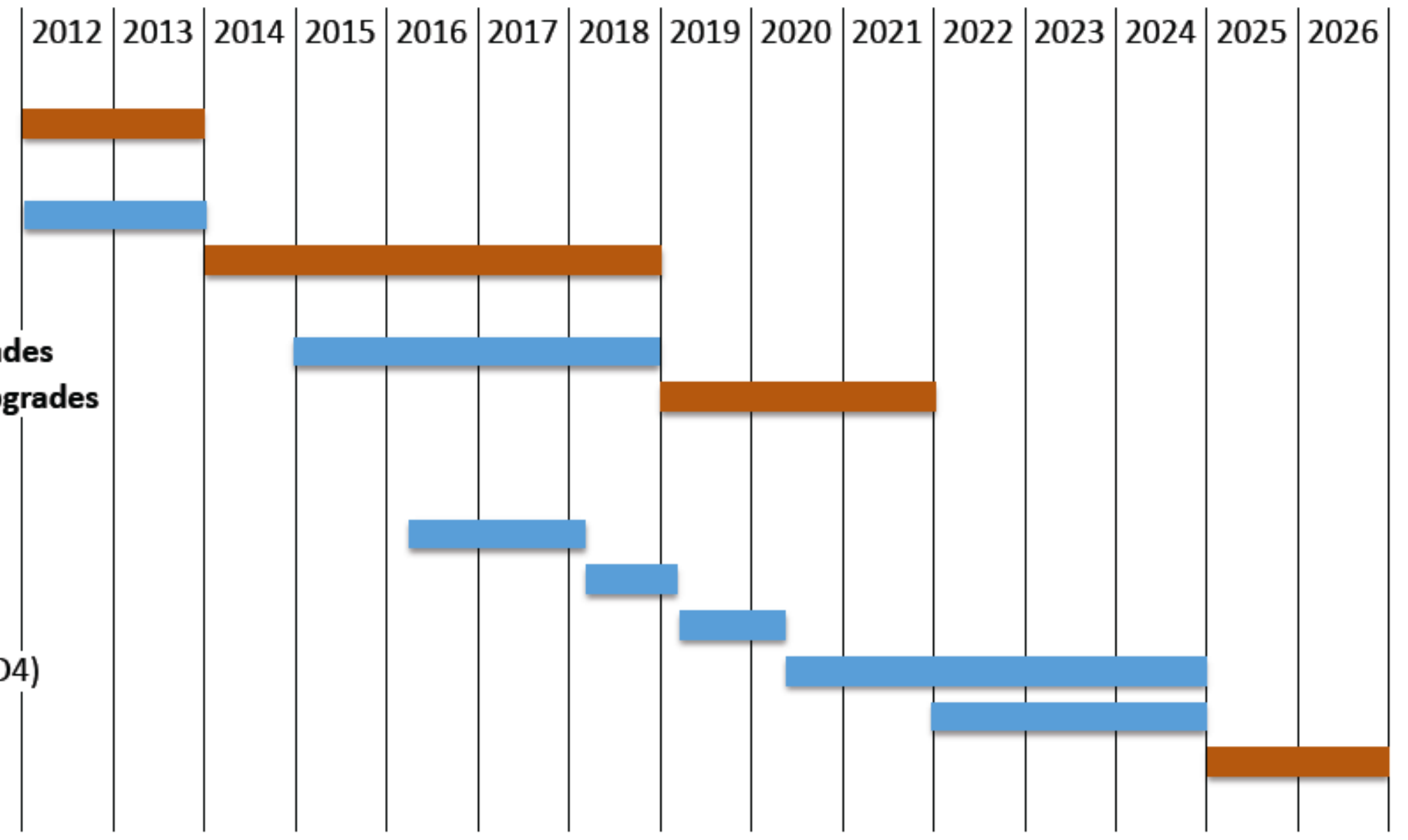

Projects/Construction

Operations 


\section{INTERNATIONAL CONTEXT}

Electron-"Ion" colliders in the past and future:

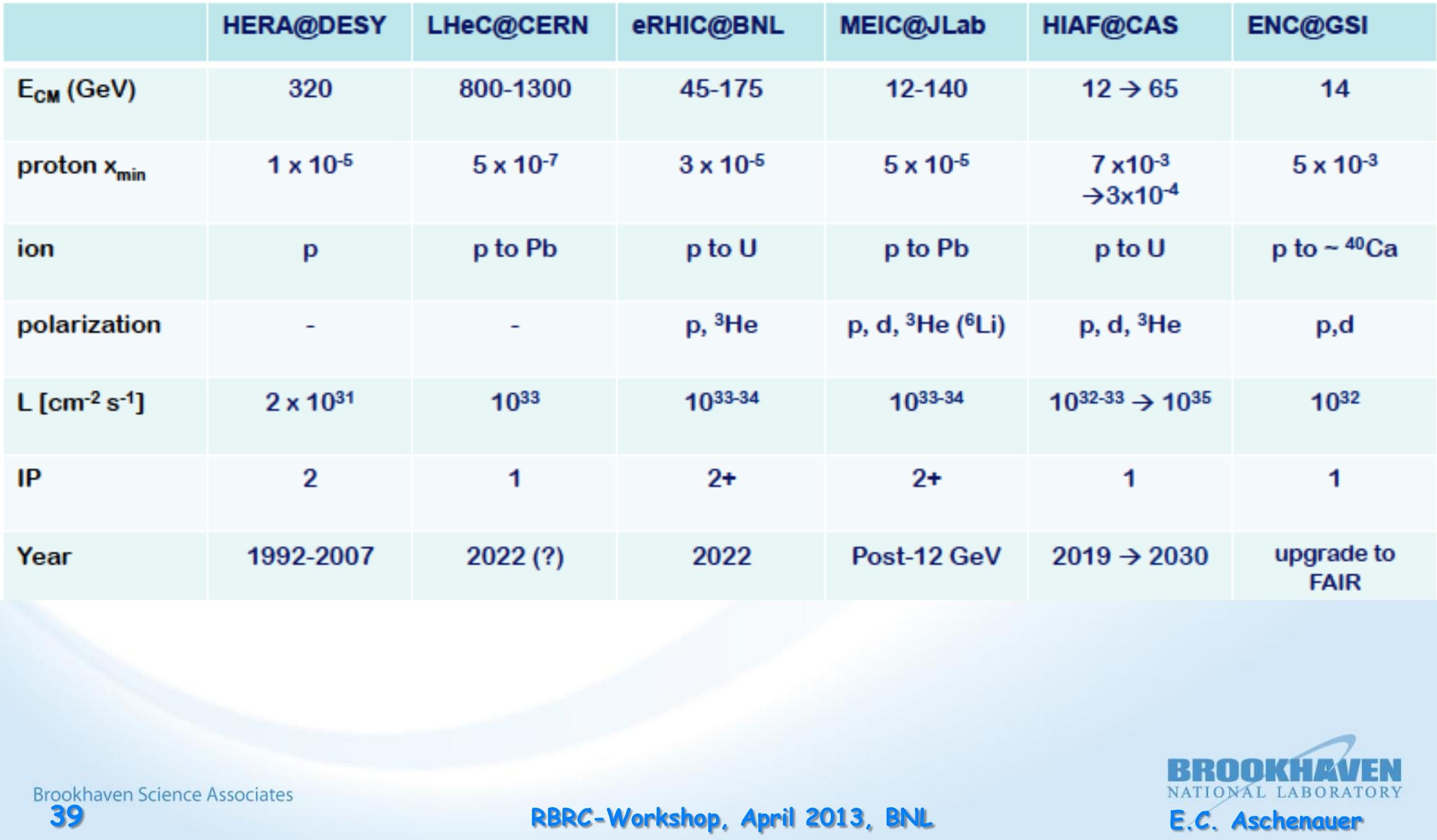




\section{AN ELECTRON ION COLLIDER IN THE US}

\section{Requirements:}

- High Luminosity > $10^{33} \mathrm{~cm}^{-2} \mathrm{~s}^{-1}$

$\square$ Flexible center of mass energies

$\square$ Electrons and protons/light nuclei polarised

$\square$ Wide range of nuclear beams

$\square$ a wide acceptance detector with good PID (e/h and $\pi, K, p)$

$\square$ wide acceptance for protons from elastic reactions and neutrons from nuclear breakup 


\section{eRHIC: design luminosity}

\begin{tabular}{|c|c|c|c|c|c|}
\hline & e & $\mathbf{p}$ & ${ }^{2} \mathrm{He}^{3}$ & ${ }^{79} A u^{197}$ & ${ }^{92} U^{238}$ \\
\hline Energy, GeV & 20 & 250 & 167 & 100 & 100 \\
\hline CM energy, GeV & & 100 & 82 & 63 & 63 \\
\hline Number of bunches/distance between bunches & 107 nsec & 111 & 111 & 111 & 111 \\
\hline Bunch intensity (nucleons), $10^{11}$ & 0.36 & 4 & 6 & 6 & 6 \\
\hline Bunch charge, $n C$ & 5.8 & 64 & 60 & 39 & 40 \\
\hline Beam current, $m A$ & 50 & 556 & 556 & 335 & 338 \\
\hline Normalized emittance of hadrons, $95 \%, \mathrm{~mm} \mathrm{mrad}$ & & 1.2 & 1.2 & 1.2 & 1.2 \\
\hline Normalized emittance of electrons, $\mathrm{rms}, \mathrm{mm} \mathrm{mrad}$ & & 16 & 24 & 40 & 40 \\
\hline Polarization, \% & 80 & 70 & 70 & none & none \\
\hline rms bunch length, $\mathrm{cm}$ & 0.2 & 5 & 5 & 5 & 5 \\
\hline$\beta^{*}, \mathrm{~cm}$ & 5 & 5 & 5 & 5 & 5 \\
\hline Luminosity per nucleon, $\times 10^{34} \mathrm{~cm}^{-2} \mathrm{~s}^{-1}$ & & 2.7 & 2.7 & 1.6 & 1.7 \\
\hline
\end{tabular}

Hourglass the pinch effects are included. Space charge effects are compensated. Energy of electrons can be selected at any desirable value at or below $30 \mathrm{GeV}$ The luminosity does not depend on the electron beam energy below or at $20 \mathrm{GeV}$ The luminosity falls as $E_{e}^{-4}$ at energies above $20 \mathrm{GeV}$ The luminosity is proportional to the hadron beam energy: $L \sim E_{h} / E_{\text {top }}$ 


\section{MODELING THE DETECTOR IN GEANT}

$\mu$-vertex detector:

- 6 layers with [30..160] $\mathrm{mm}$ radius

$0.37 \% X_{0}$ in acceptance per layer simulated precisely;

a digitization c cinale disarete nixels one-to-one from MC noints

\section{Forward/b}

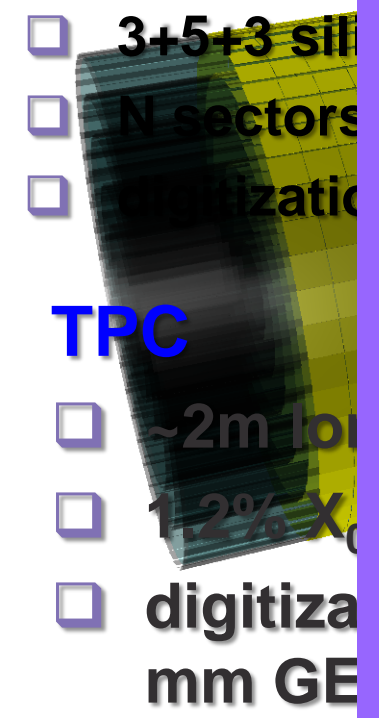

Forward $t$

3 disks behind th

$\square$ rather precise ST

$\square$ digitization: 100

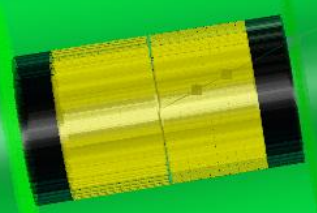

nd $1 \times 5$

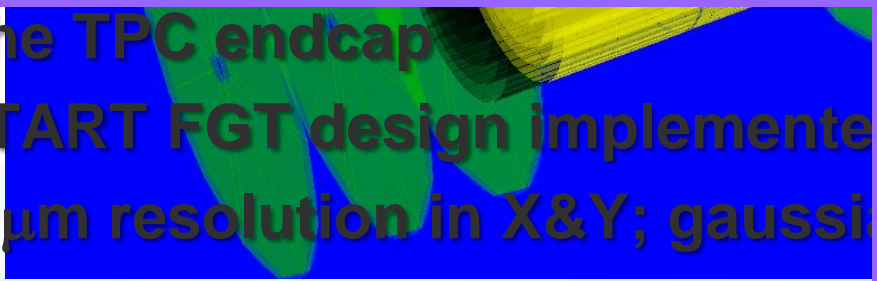

RBRC-Workshop, April 2013, BNL 


\section{THE PILLARS OF THE ERHIC PHYSICS PROGRAM}

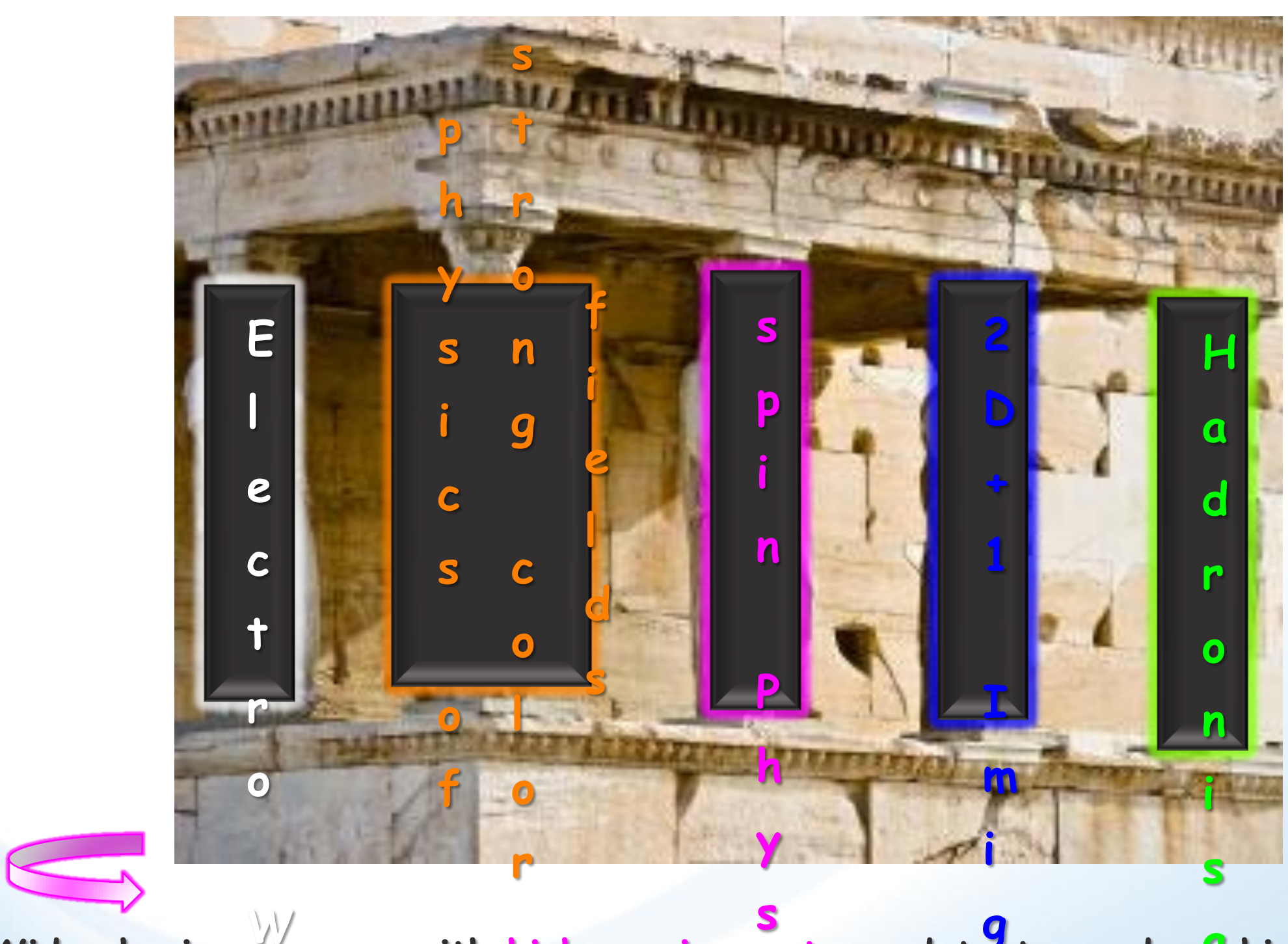

Wide physics program with high requirements on detector and machine performance ¿ 


\section{THE HADRONIC MASS PUZZLE}

quark

C.D. Roberts, Prog. Part. Nudl. Phys. 61 (2008) 50

nucleon

M. Bhagwat \& P.C. Tandy, AIP Conf. Prec. 842 (2006) 225-227

In QCD, all "constants" of quantum mechanics are actually strongly momentum dependent: couplings, number density, mass, etc.

- So, a quark's mass depends on its momentum.

$\square$ Mass function can calculated and is depicted here.

in agreement: the vast bulk of the light-quark mass comes from a cloud of gluons, dragged along by the quark as it propagates.

- Continuum- and Lattice-QCD

$\square$ Running gluon mass

Gluon is massless in UV, in agreement with PQCD

- Massive in infrared

a $m_{G}(0)=0.67-0.81 \mathrm{GeV}$

a $m_{G}\left(m_{G}{ }^{2}\right)=0.53-0.64 \mathrm{GeV}$

$\square$ DSE prediction confirmed by numerical simulations of lattice-regularised QCD 44
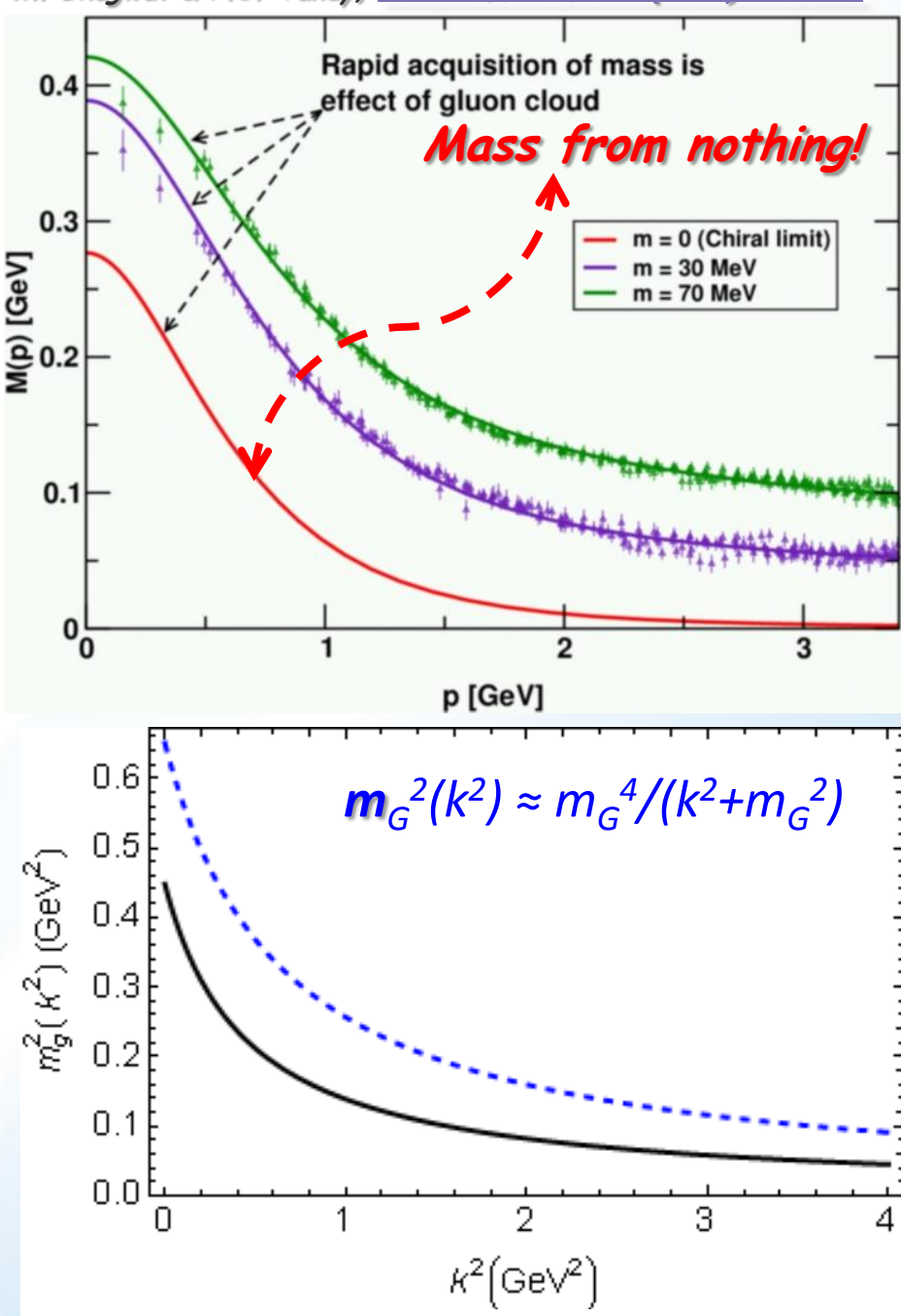

Qin et al., Phys. Rev. C 84042202 (Rapid Comm.) 


\section{CONSTRAIN J VIA GPDE}

observables sensitive to $E$ :

( $J_{q}$ input parameter in ansatz for $E$ ) $\square$ DVCS $A_{U T}:$ HERMES

$\square$ nDVCS $A_{L U}:$ Hall $A$

Hermes DVCS-TTSA [arXiv: 0802.2499]: $\quad A_{U T}^{\sin \left(\phi-\phi_{T}\right) \cos \phi} \sim \operatorname{Im}\left(F_{2} H-F_{1} E\right)$

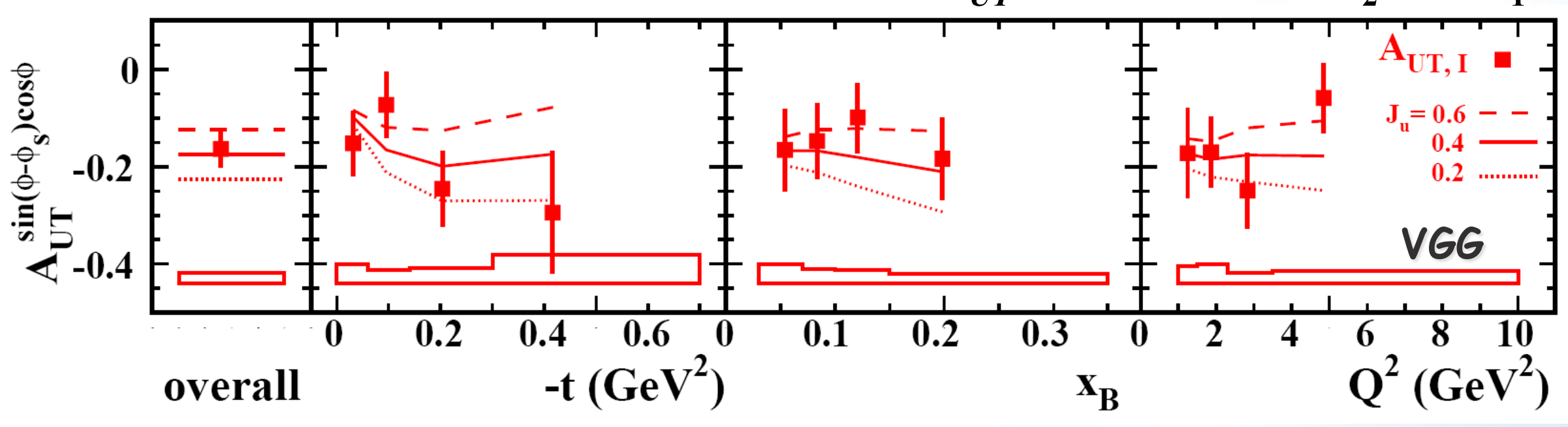

eRHIC:

HERMES like $A_{U T}$ $20 \mathrm{GeV} \times 250 \mathrm{GeV}$ Lumi: $2 \times 50 \mathrm{fb}^{-1}$

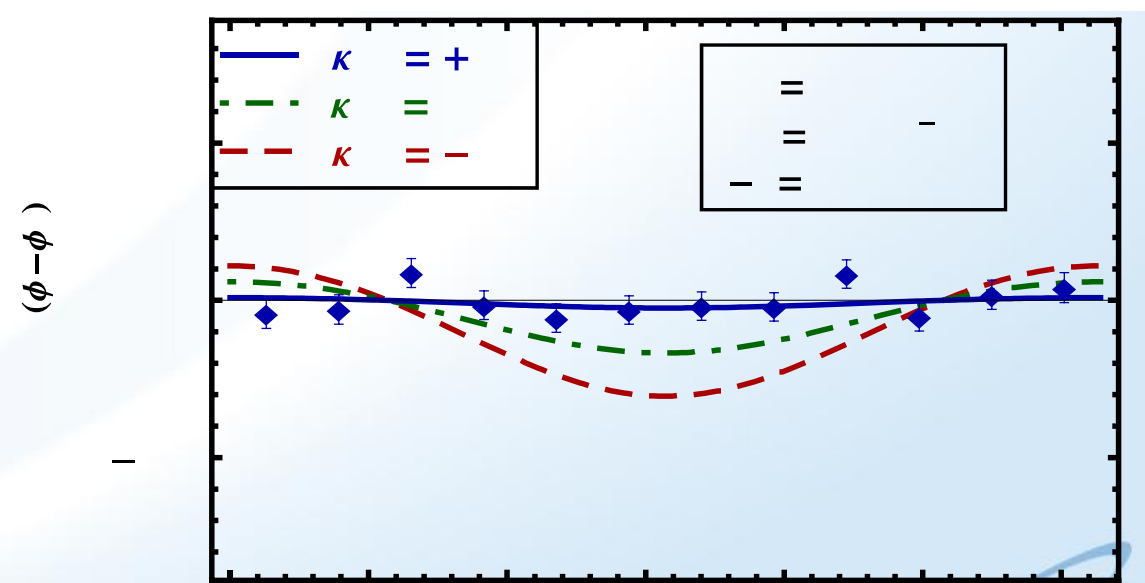

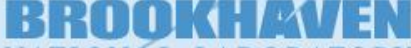
NATIONAL LABORATORY RBRC-Workshop, April 2013, BNL 


\section{DIFFERENT DVCS ASYMMETRIES}

\section{arXivi1304.0077}

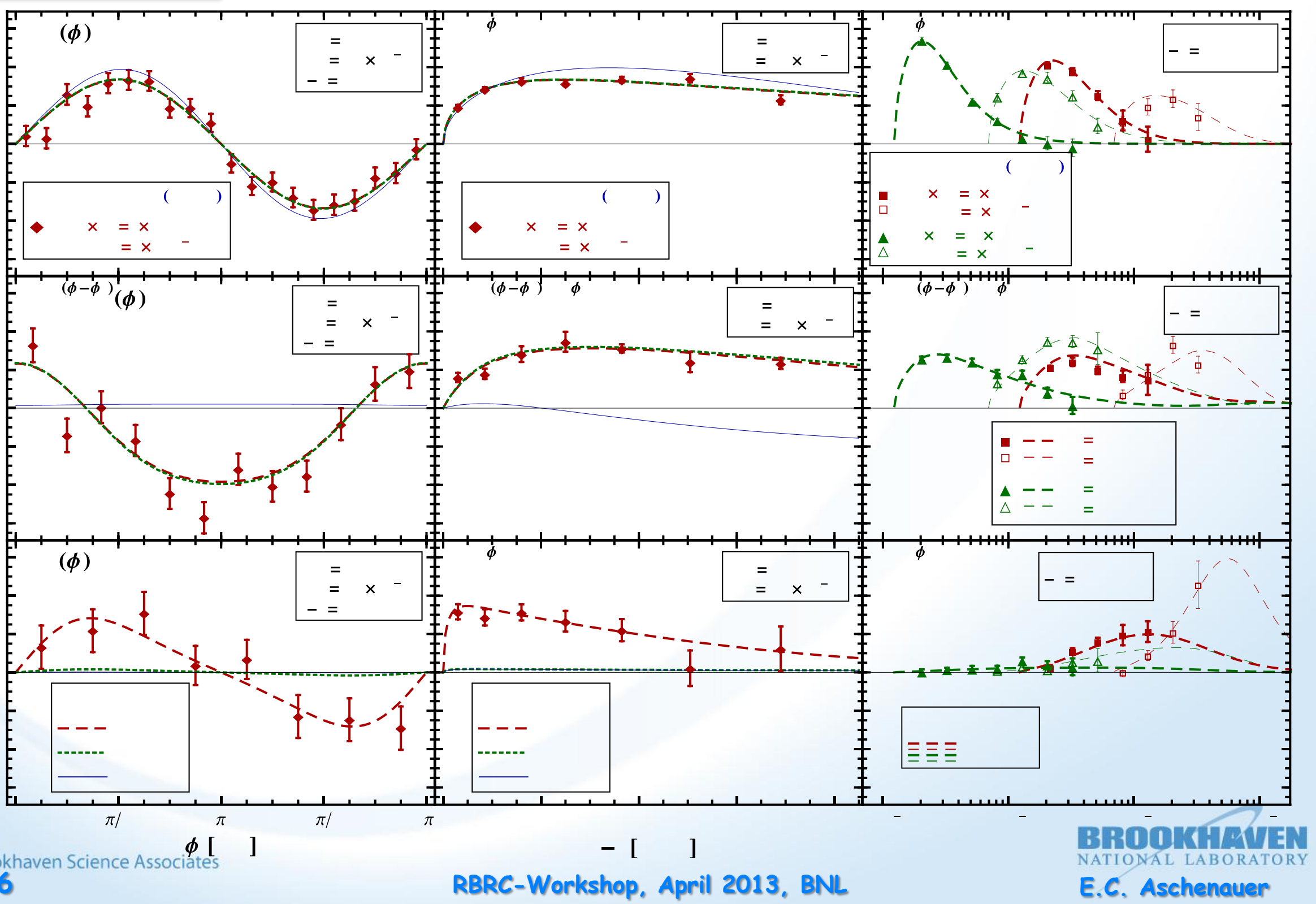




\section{HARD DIFFRACTION IN DIS AT SMALLX}

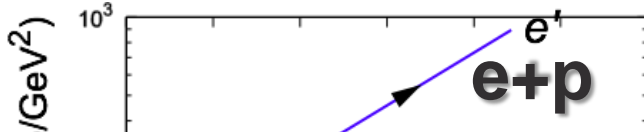

$$
{ }_{10^{6}} e_{0}^{ \pm} A \overline{\bar{u}}\left(p-p^{\prime}\right)^{2} A \rightarrow J / \psi A
$$

Diffraction Analogy: plane monochromatic wave incident on a circular screen of radius $\mathbf{R}$
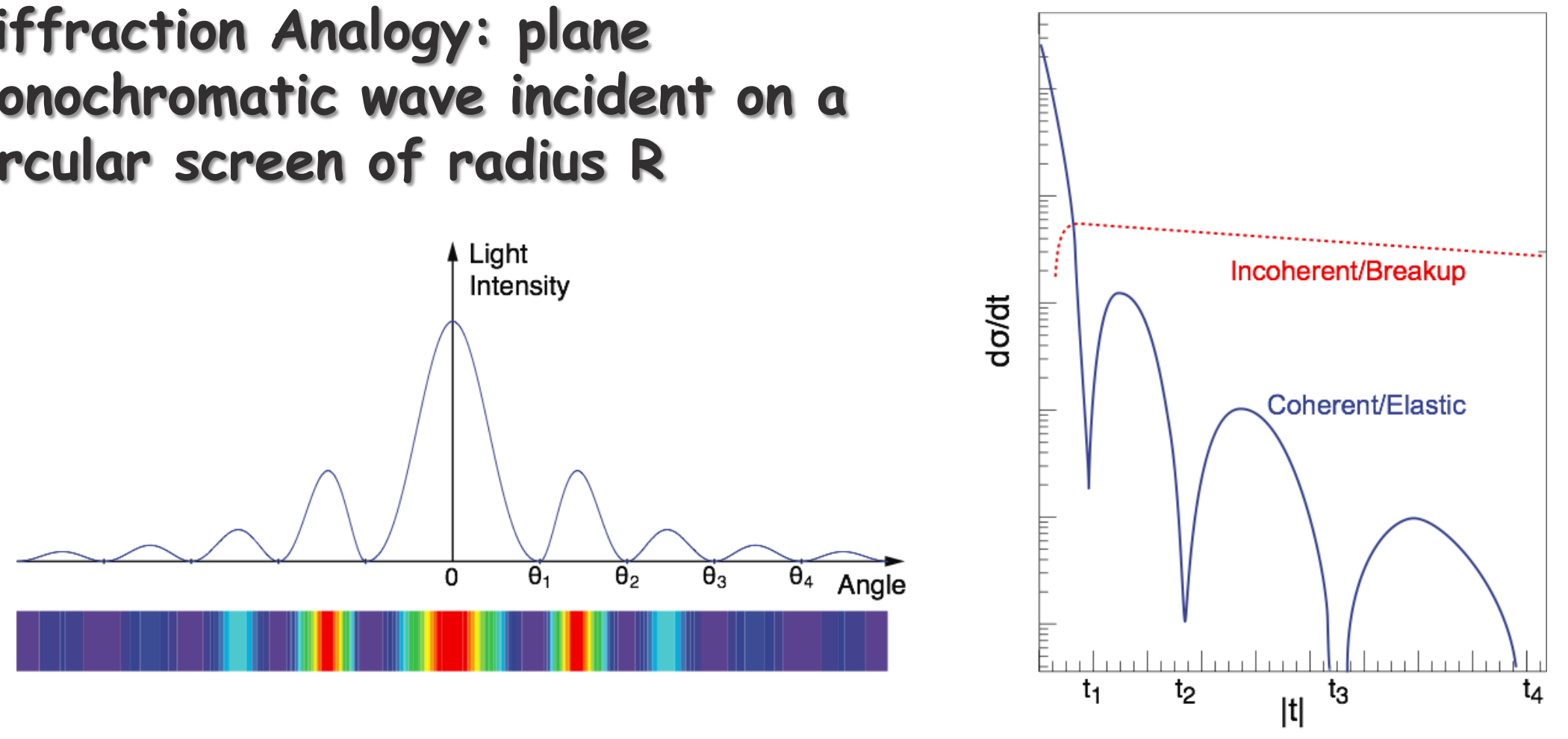

- incoherent $\Leftrightarrow$ breakup of $p$

- HERA: $15 \%$ of all events are hard diffractive

- breakup into nucleons (nucleons intact)

- incoherent diffraction

- Predictions: $\sigma_{\text {diff }} / \sigma_{\text {tot }}$ in etAur $25-40 \%$ 


\section{LARGE RAPIDITY GAP METHOD (LRG)}

$\square$ Identify Most Forward Going

Particle (MFP)

$>$ Works at HERA but at higher $\sqrt{s}$

$>$ EIC smaller beam rapidities

Diffractive $\rho^{0}$ production at EIC: $n$ of MFP

M. Lamont' '10

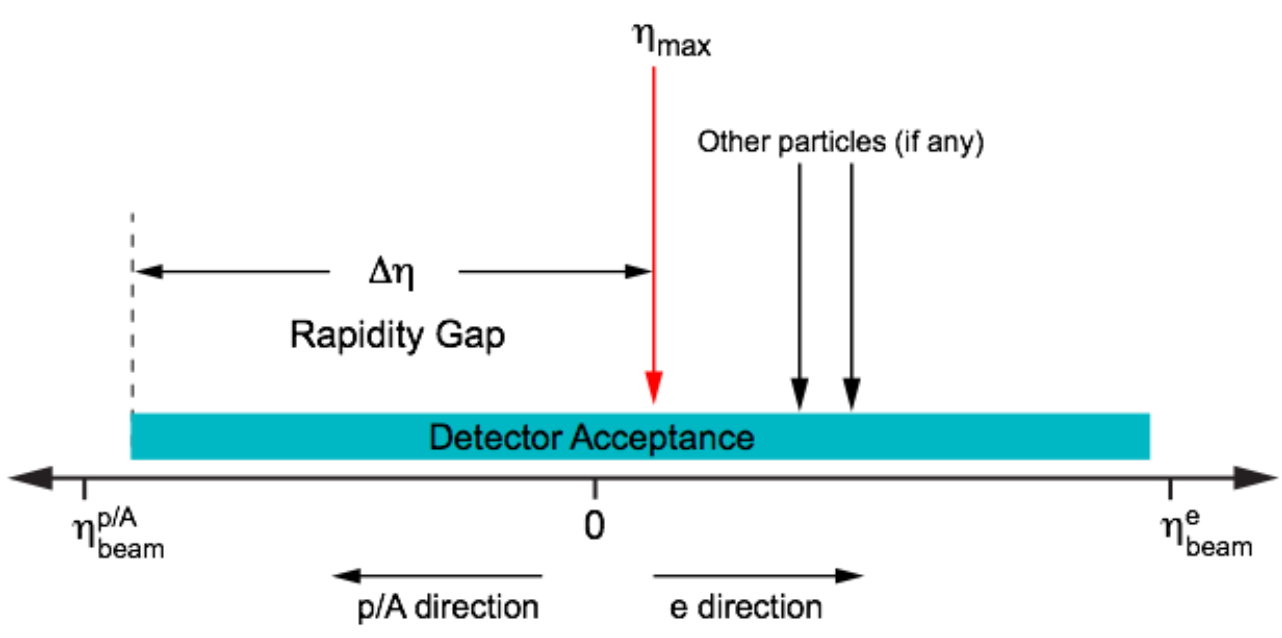

Hermeticity requirement:

- needs just to detector presence

- does not need momentum or PID

- simulations: $\sqrt{s}$ not a show stopper for EIC (can achieve 1\% contamination, $80 \%$ efficiency)

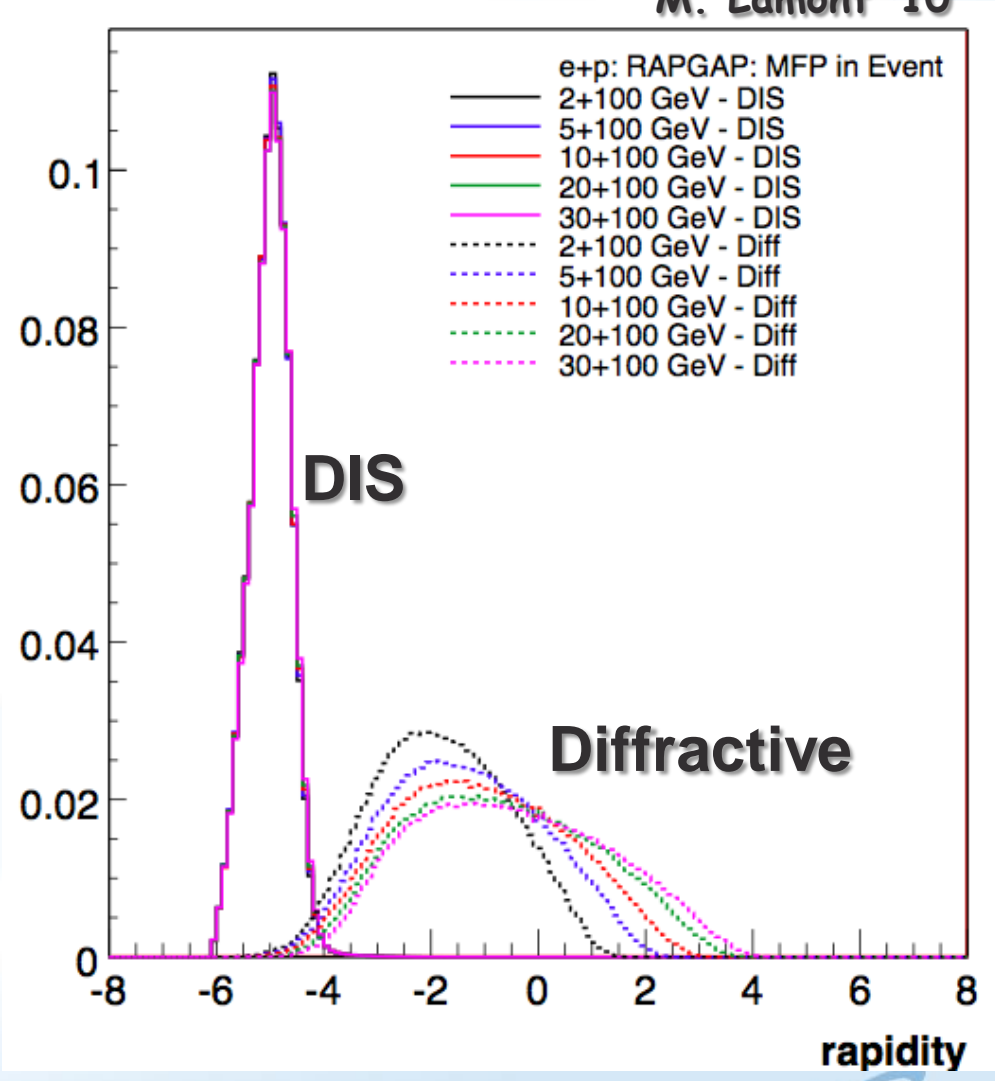




\section{BEST KNOWLEDGE OF QS IN EA}
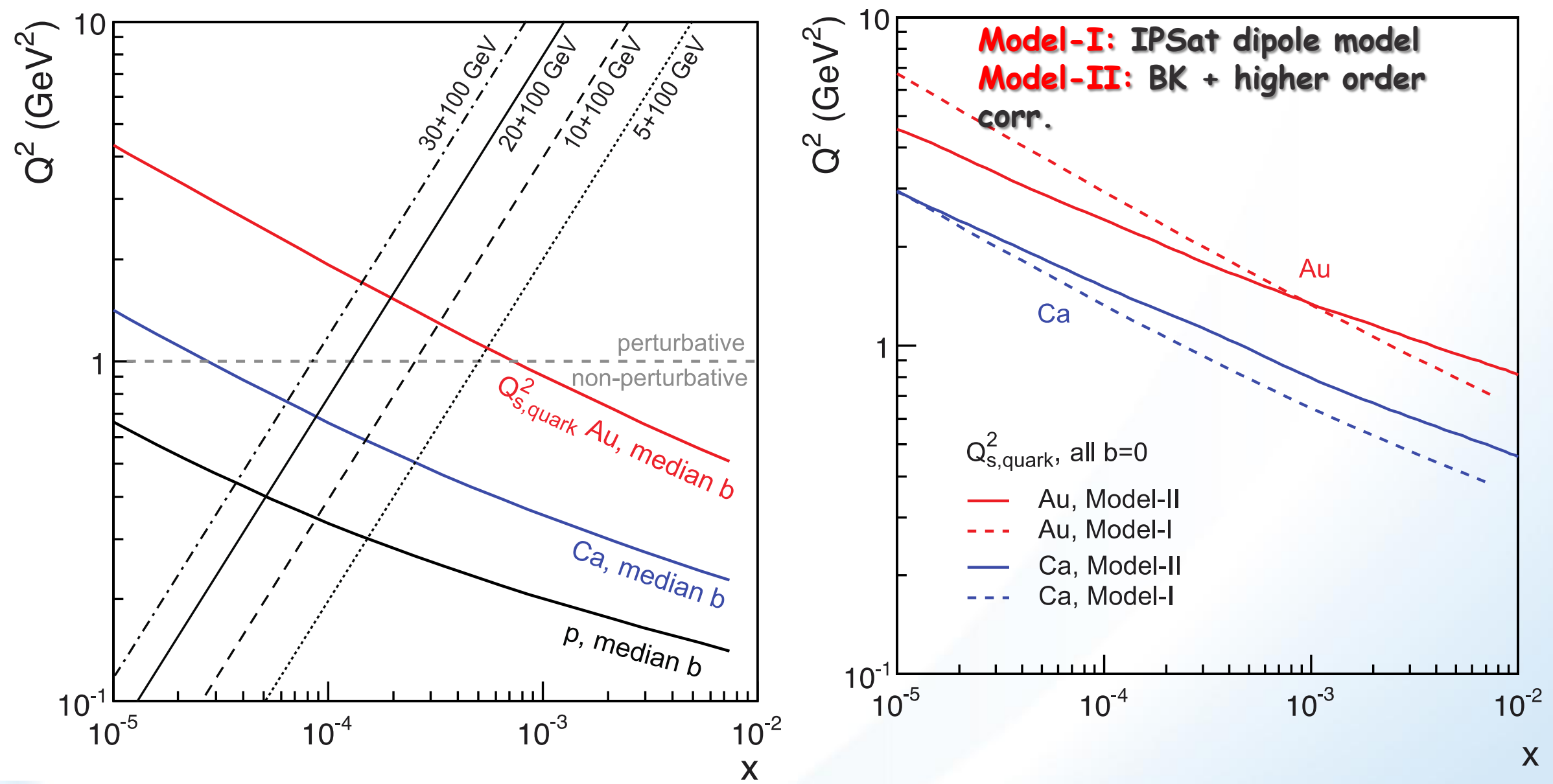

Enhancement of $Q_{s}$ with $A$

$\Rightarrow$ saturation regime reached at significantly lower energy in nuclei 


$$
\frac{d^{2} \sigma^{e p \rightarrow e X}}{d x d Q^{2}}=\frac{4 \pi \alpha_{e . m .}^{2}}{x Q^{4}}\left[\left(1-y+\frac{y^{2}}{2}\right) F_{2}\left(x, Q^{2}\right)-\frac{y^{2}}{2} F_{L}\left(x, Q^{2}\right)\right]
$$

In practice use reduced cross-section:

$$
\begin{aligned}
\sigma_{r}=\left(\frac{d^{2} \sigma}{d x d Q^{2}}\right) \frac{x Q^{4}}{2 \pi \alpha^{2}\left[1+(1-y)^{2}\right]} & =F_{2}\left(x, Q^{2}\right)-\frac{y^{2}}{1+(1-y)^{2}} F_{L} \\
& =F_{2}\left(x, Q^{2}\right)-\frac{y^{2}}{Y^{+}} F_{L}\left(x, Q^{2}\right)
\end{aligned}
$$

$\square$ How to extract $F_{L}$

$>$ Need different values of $y^{2} / y^{+}$

$>F_{L}$ slope of $\sigma_{r}$ vs $y^{2} / y^{+}$

$>F_{2}$ intercept of $\sigma_{r}$ vs $y^{2} / y^{+}$with $y-$ axis

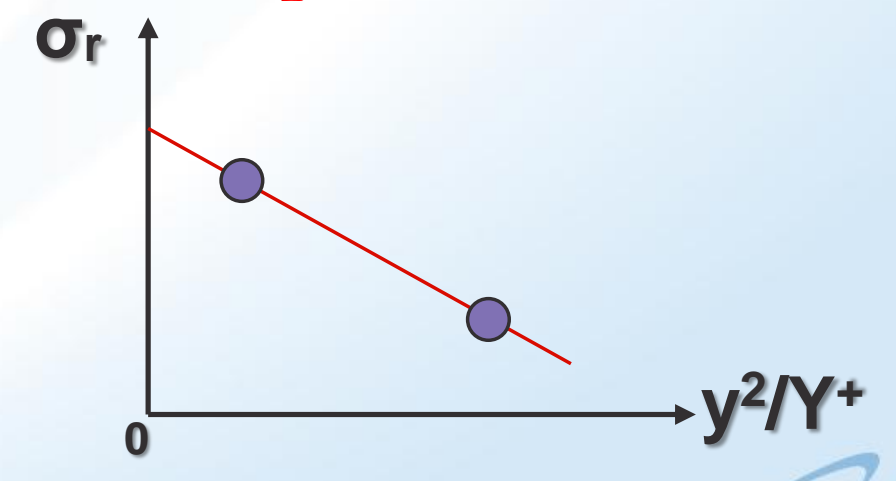




\section{MEASURING FL WITH THE EIC (II)}

In order to extract $F_{L}$ one needs at least two measurements of the inclusive cross section with "wide" span in inelasticity parameter y $\left(Q^{2}=5 x y\right)$

$F_{L}$ requires runs at various $\sqrt{ } s \Rightarrow$ longer program

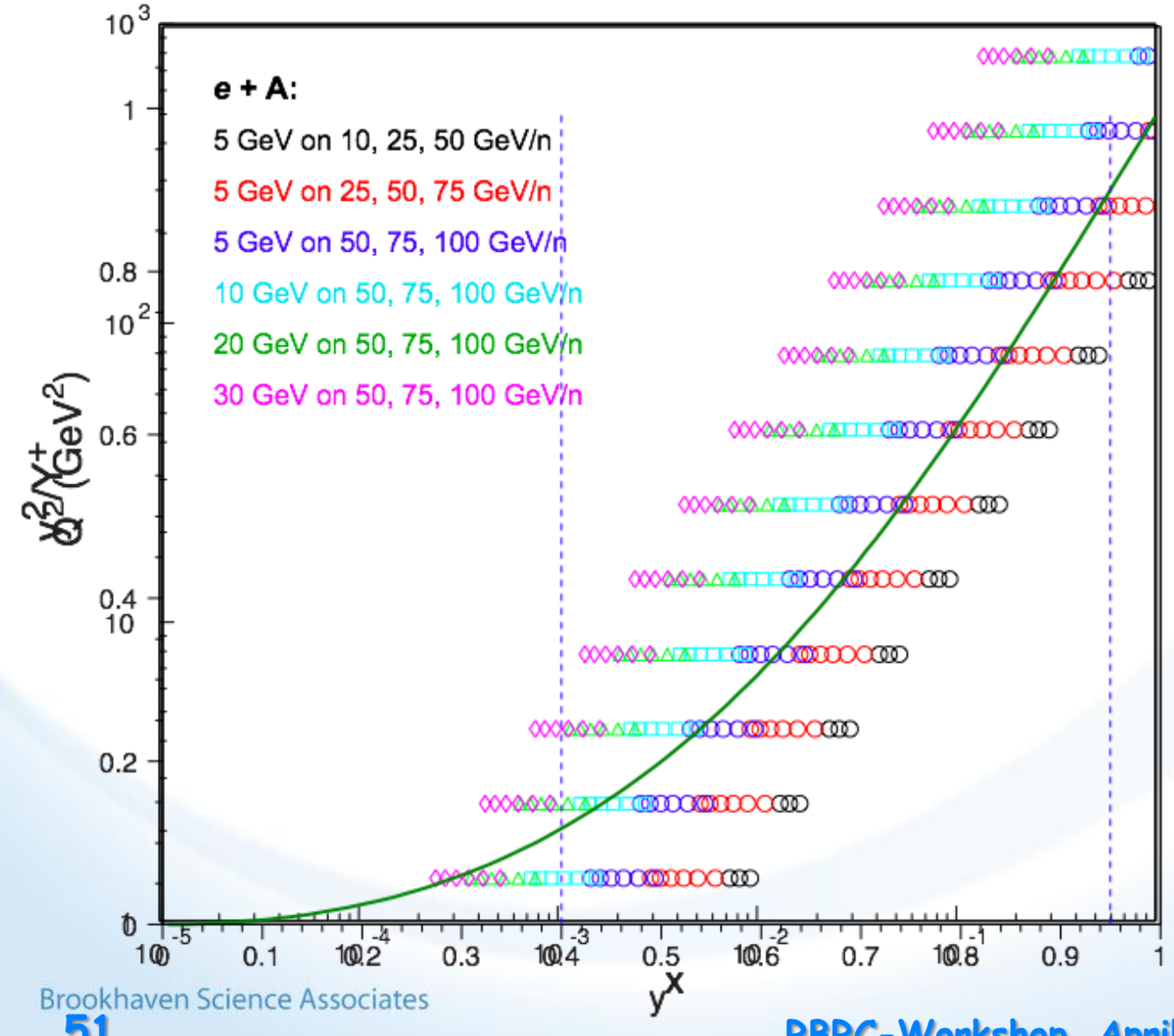

51

\section{EIC studies:}

- Statistical error is negligible in essentially whole range

- Systematical Error

Calibration

Normalization

$>$ Experiment

Radiative Corrections 


\section{PREPARATION OF DIS AND SIDIS MOCK DATA}

- PEPSI MC to generate $\sigma^{+*}$ and $\sigma^{+-}$with LO GRSV PDFs

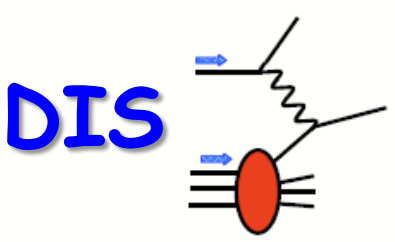

inclusive final-state

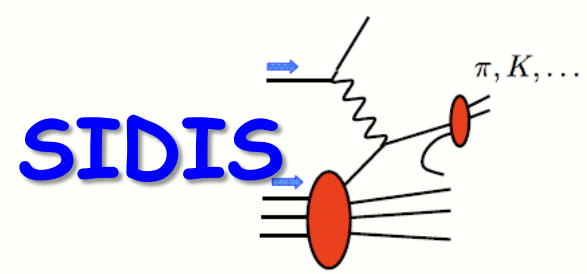

identified charged pions and kaons

assume modest $10 \mathrm{fb}^{-1}$ for each energy, $70 \%$ beam polarizations $Q^{2}>1 \mathrm{GeV}^{2}, 0.01<y<0.95$, invariant mass $W^{2}>10 \mathrm{GeV}^{2}$ depolarization factor of virtual photon $D\left(y, Q^{2}\right)>0.1$ (cuts on small $y$ ) scattered lepton: $1^{\circ}<\theta_{\text {elec }}<179^{\circ}$ and $p_{\text {elec }}>0.5 \mathrm{GeV}$ hadron: Phadr $>1 \mathrm{GeV}, 0.2<z<0.9$. $10<\theta_{\text {hadr }}<179^{\circ}$

- use rel. uncertainties of data to generate mock data by randomizing around DSSV+ by $1-\sigma$

- SIDIS: incl typical 5\% (10\%) uncertainty for pion (kaon) frag. fcts (frombss analysis) 52 


\section{SCALING VIOLATIONS AT SMALL $X$}

rough small-x approximation to $\mathbf{Q}^{2}$-evolution:

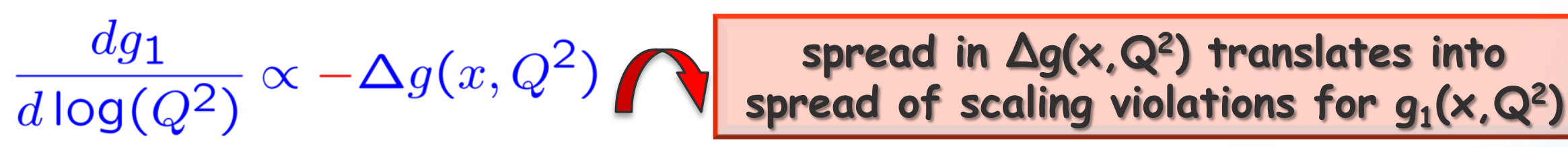

- need $x$-bins with a least two $Q^{2}$ values to compute derivative (limits $x$ reach somewhat)

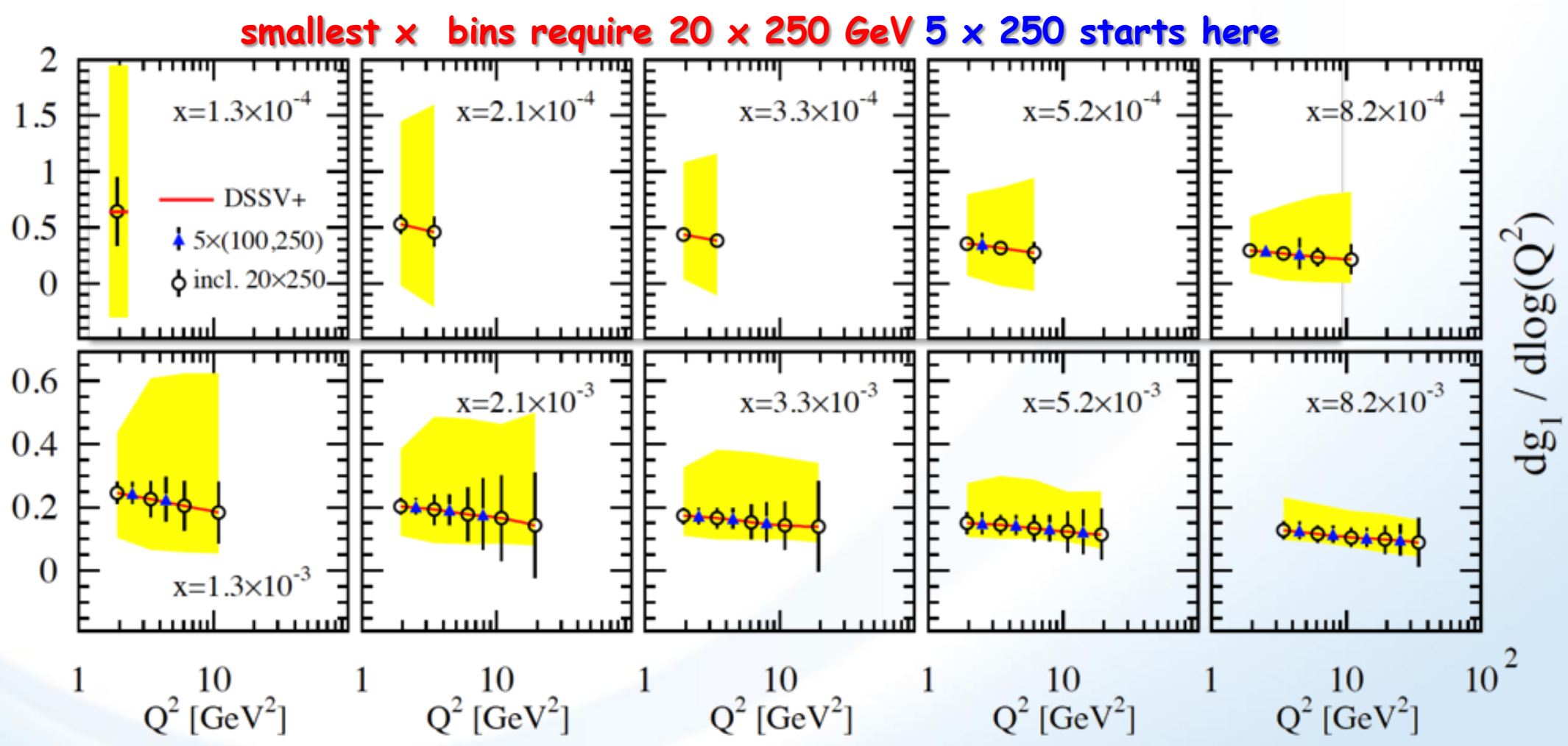

- error bars for moderate $10 \mathrm{fb}^{-1}$ per c.m.s. energy: bands parameterize current DSSV+ uncertainties 


\section{DVCS ASYMMETRIES}

$d \sigma \sim\left(\tau_{B H}^{*} \tau_{D V C S}+\tau_{D V C S}^{*} \tau_{B H}\right)+\left|\tau_{B H}\right|^{2}+\left|\tau_{D V C S}\right|^{2}$

$\Rightarrow$ different charges: $e^{+} e^{-:}$

$\Delta \sigma_{\mathrm{c}} \sim \cos \phi \cdot \operatorname{Re}\{\mathrm{H}+\xi \tilde{H}+\ldots\}$

$\stackrel{\mathrm{H}}{\| \longrightarrow}$

$\rightarrow$ polarization observables:

$\Delta \sigma_{\mathrm{LU}} \sim \sin \phi \cdot \operatorname{Im}\{H+\xi / H+K=\}$

$\Delta \sigma_{\mathrm{UL}} \sim \sin \phi \cdot \operatorname{Im}\{\tilde{H}+\xi K+\ldots\}$

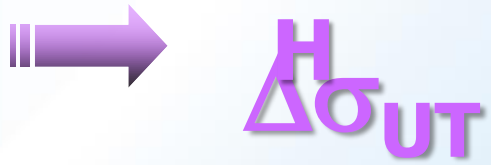

$\Delta \sigma_{U T} \sim \sin \phi \cdot \operatorname{Im}\{k(H-E) \neq \ldots\}$

$\xi=x_{B} /\left(2-x_{B}\right) \quad k=t / 4 M^{2} \quad$ kinematically suppressed 


\section{KINEMATICS OF BREAKUP NEUTRONS}

Results from GEMINI++ for $50 \mathrm{GeV} \mathrm{Au}$

theta distribution of neutrons at $\mathrm{E}^{*}=10 \mathrm{MeV}$

\begin{tabular}{|l|}
\hline \multicolumn{2}{|c|}{ histoTheta10 } \\
\hline Entries 9143
\end{tabular}

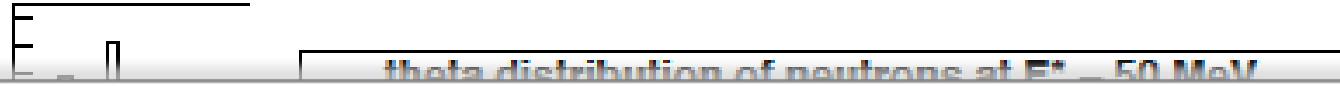

Results:

With an aperture of \pm 3 mrad we are in relative good shape

- enough "detection" power for $t>0.025 \mathrm{GeV}^{2}$

- below $t \sim 0.02 \mathrm{GeV}^{2}$ we have to look into photon detection

- Is it needed?

Question:

- For some physics rejection power for incoherent is needed $\sim 10^{4}$

$\rightarrow$ How efficient can the ZDCs be made?
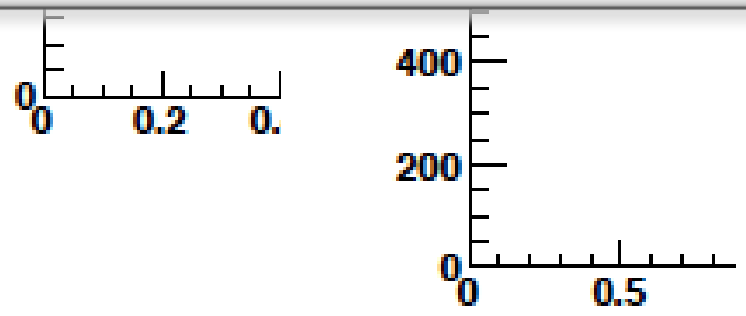

by Thomas Ullrich

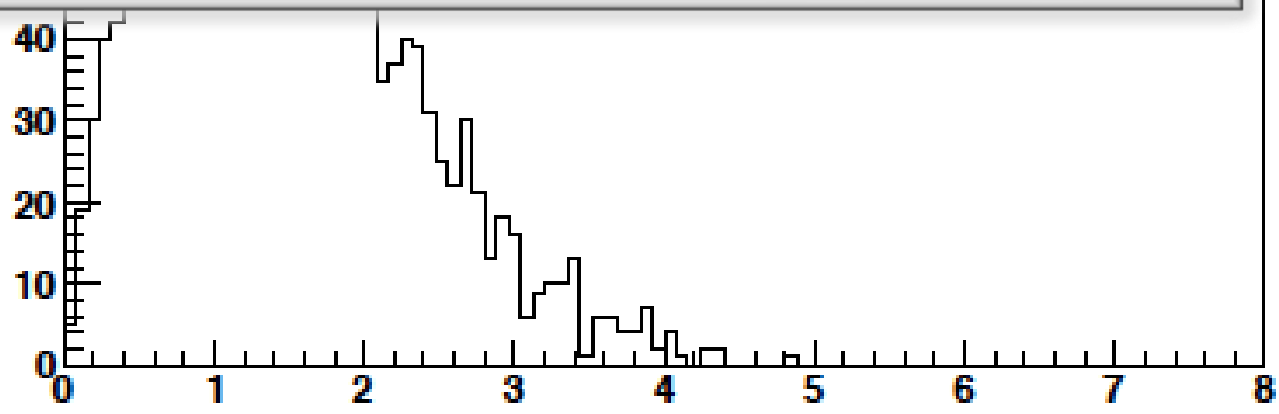

mrad 


\section{DIFFRACTIVE PHYSICS: P' KINEMATICS}

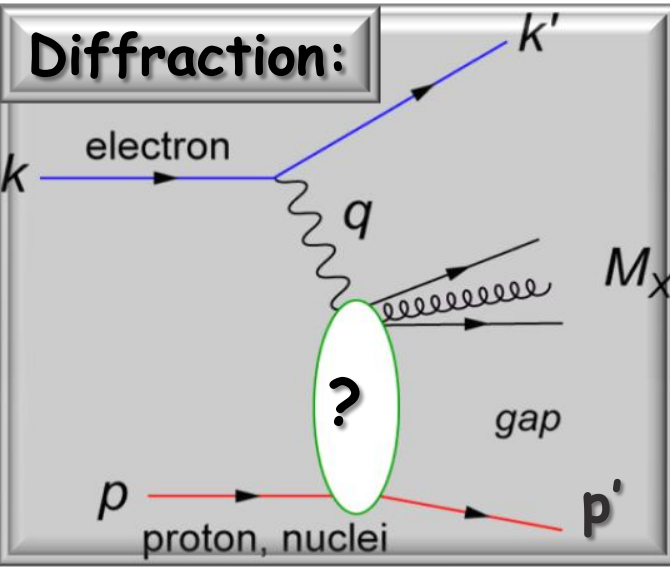

Simulations by J.H Lee

$$
t=\left(p_{4}-p_{2}\right)^{2}=2\left[\left(m_{p}^{\text {in }} \cdot m_{p}^{\text {out }}\right)-\left(E^{\text {in }} E^{\text {out }}-p_{z}^{\text {in }} p_{z}^{\text {out }}\right)\right]
$$

$\rightarrow$ "Roman Pots" acceptance studies see later

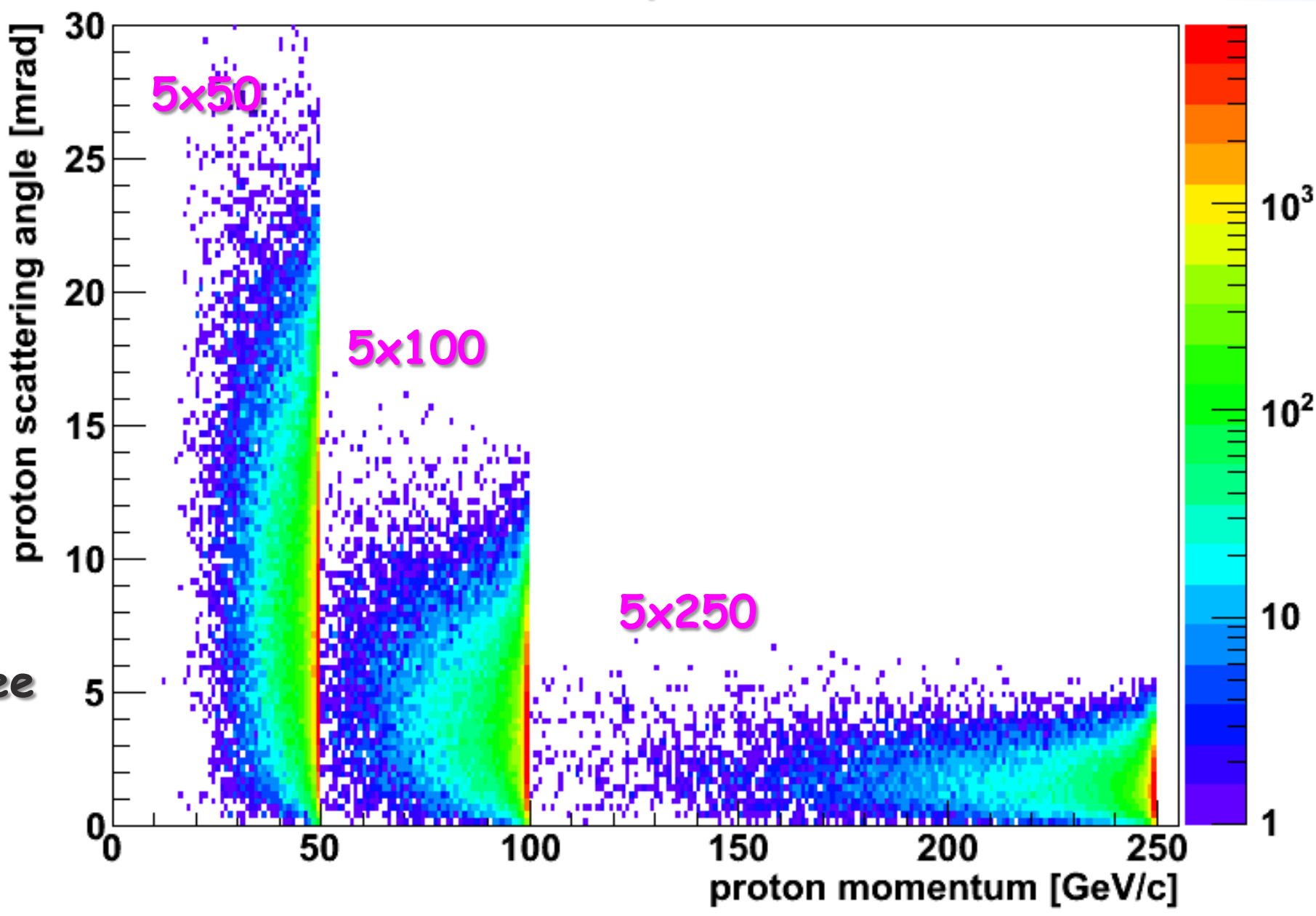




\section{PROTON DISTRIBUTION IN Y VS X AT S=20 M}

without quadrupole aperture limit
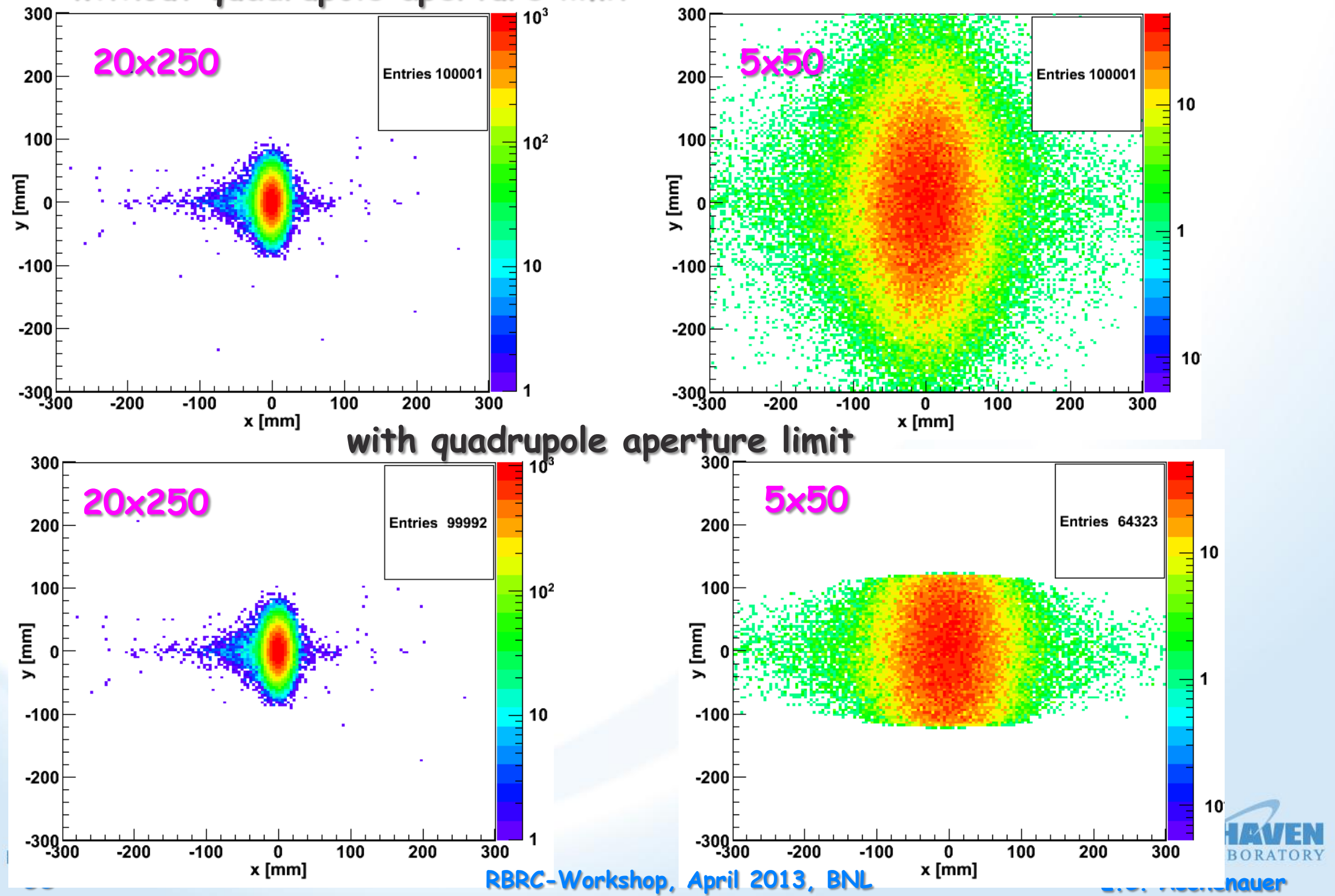
ACCEPIED IN ROMAN POI (EXAMPLE) A!

$S=20 \mathrm{M}$
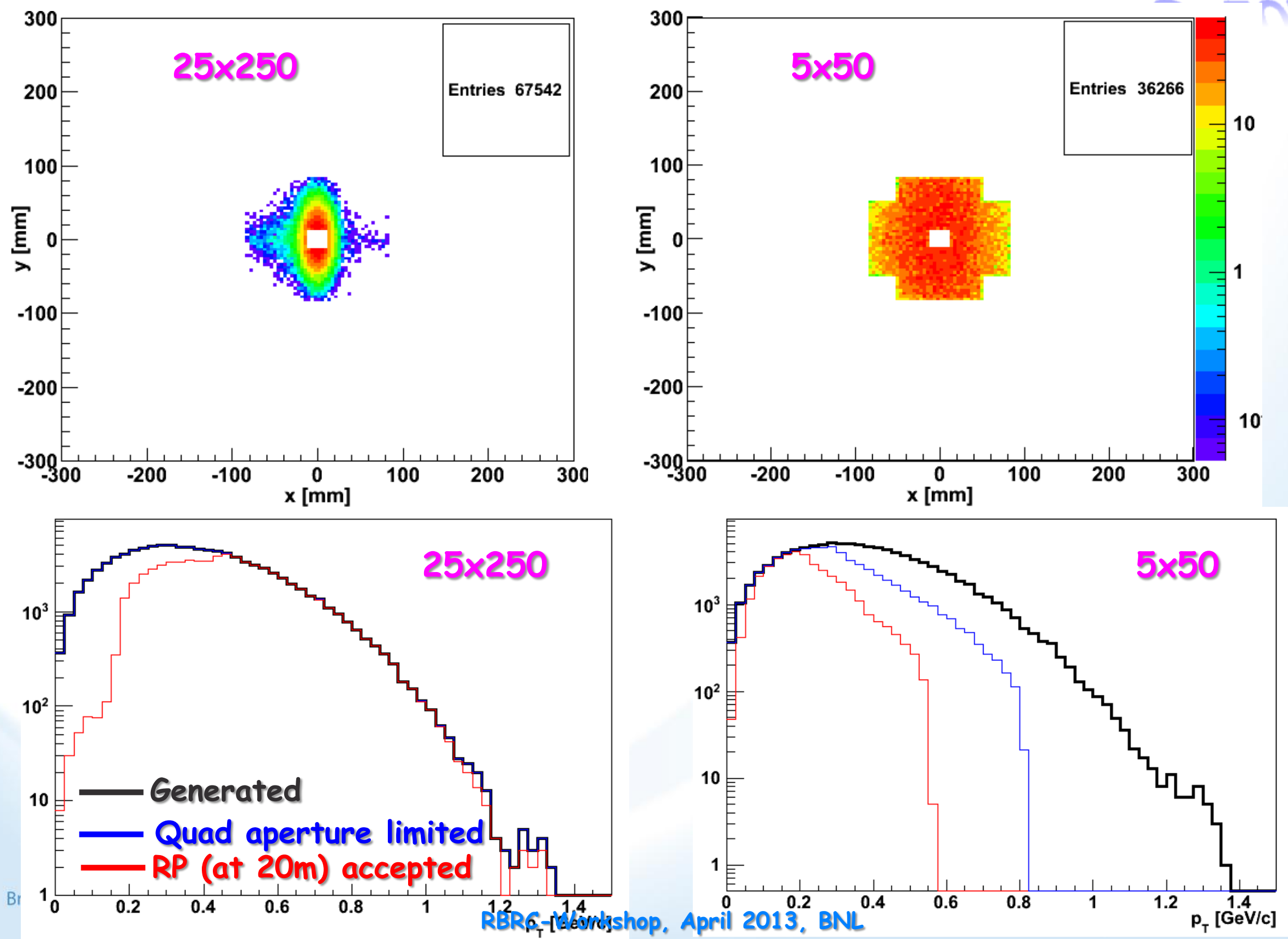


\section{Cronin and Shadowing in $p A$}

(PRC80 014903 2009; PRC85, 024903, 2012)

Gergely Gábor Barnaföldi, J. Barrette, M. Gyulassy,

Sz. Harangozó, P. Lévai, G. Papp, V. Topor Pop

Wigner RCP RMI of the HAS, Eötvös Loránd University,

Columbia University, Mc Gill University 


\section{OUTLINE}

- Motivation for $p A / d A u$ collisions

- pA/dAu collisions from $30 \mathrm{AGeV}$ to $5 \mathrm{ATeV}$

- How latest results fit into the picture.

- What phenomenology have we leart by the 'Cronin effect' so far?

- Nuclear shadowing

- Suppression at low-x

- High- $\mathrm{p}_{\mathrm{T}}$ nuclear effects @ midrapidity \& large y

- Cronin effect

- Enhancement in pA

- Saturated Glauber picture and the Cronin effect 


\section{MOTIVATION}

- Cronin effect at SPS energies

Brown et al: PRD11 (1975) 3105, Ric.Sci.Edu.Perm Suppl. 122 (2003) 541
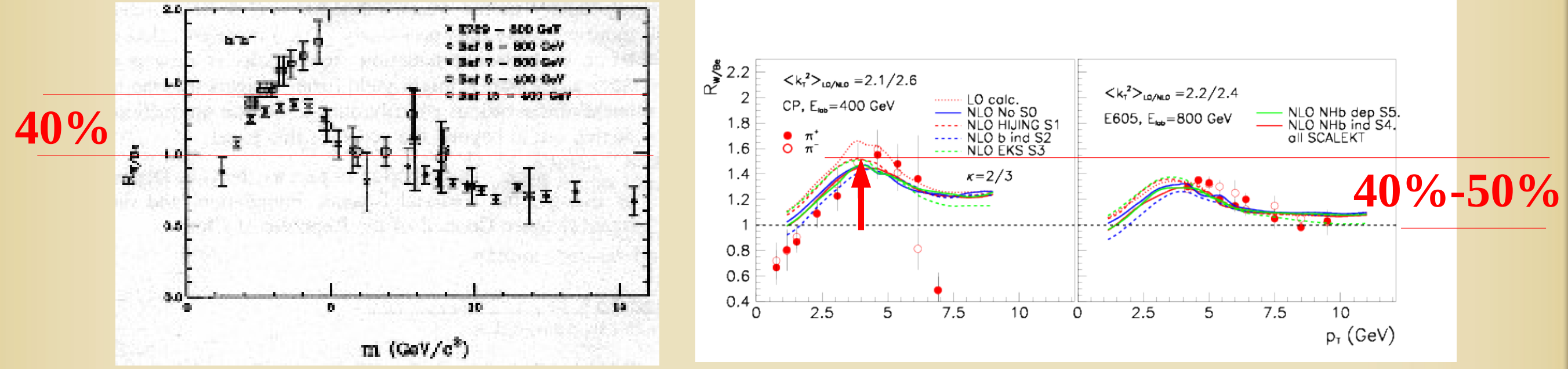

- RHIC analysis on dAu and AuAu
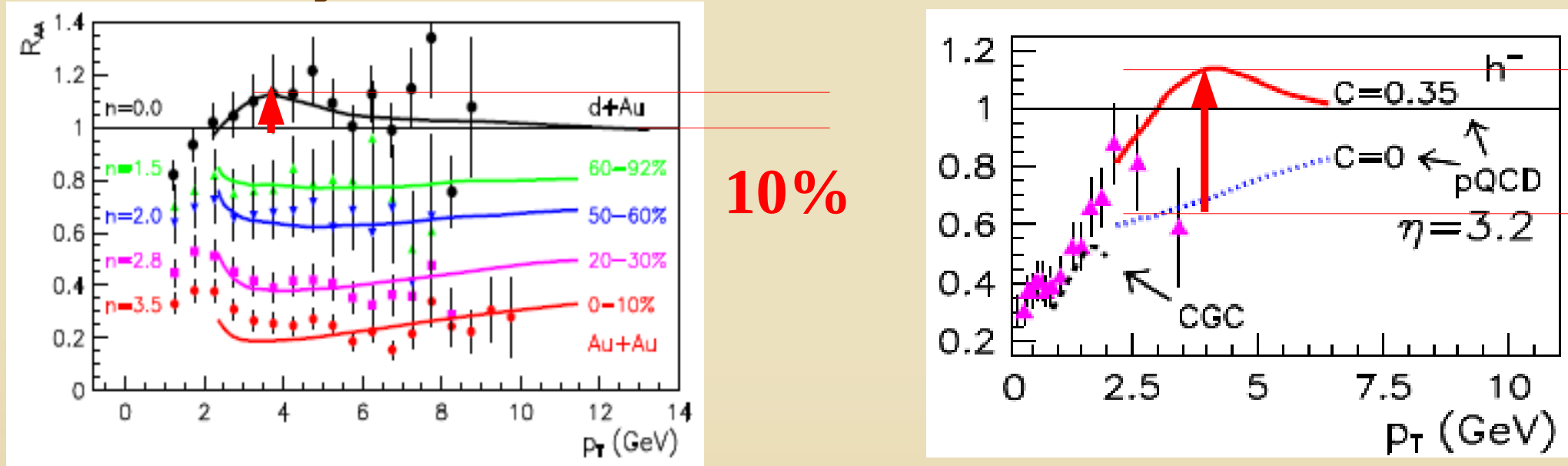

???

G.G. Barnaföldi: Cronin \& Shadowing in pA 


\section{MOTIVATION}

- Motivation for $p P b / d A u$ collisions

Preliminary dAu data from PHENIX@QM12 (B. Sahlmueller)
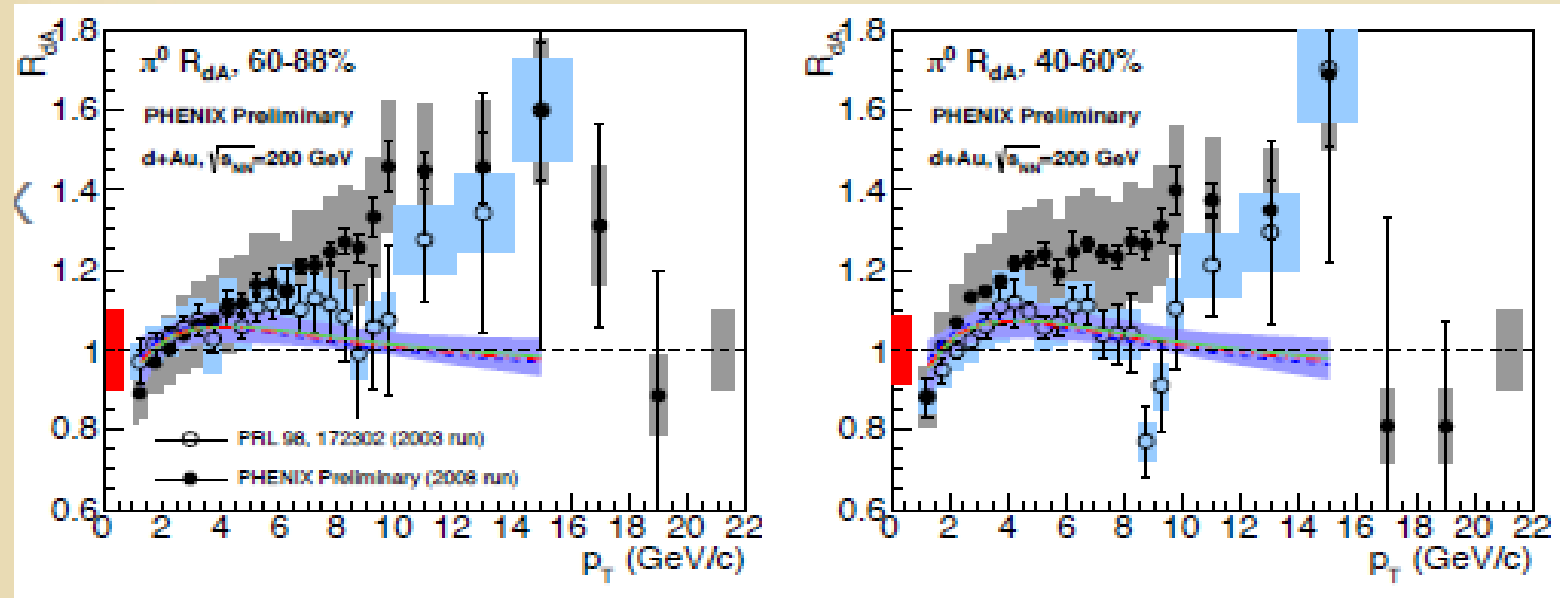

2008 data is not final,
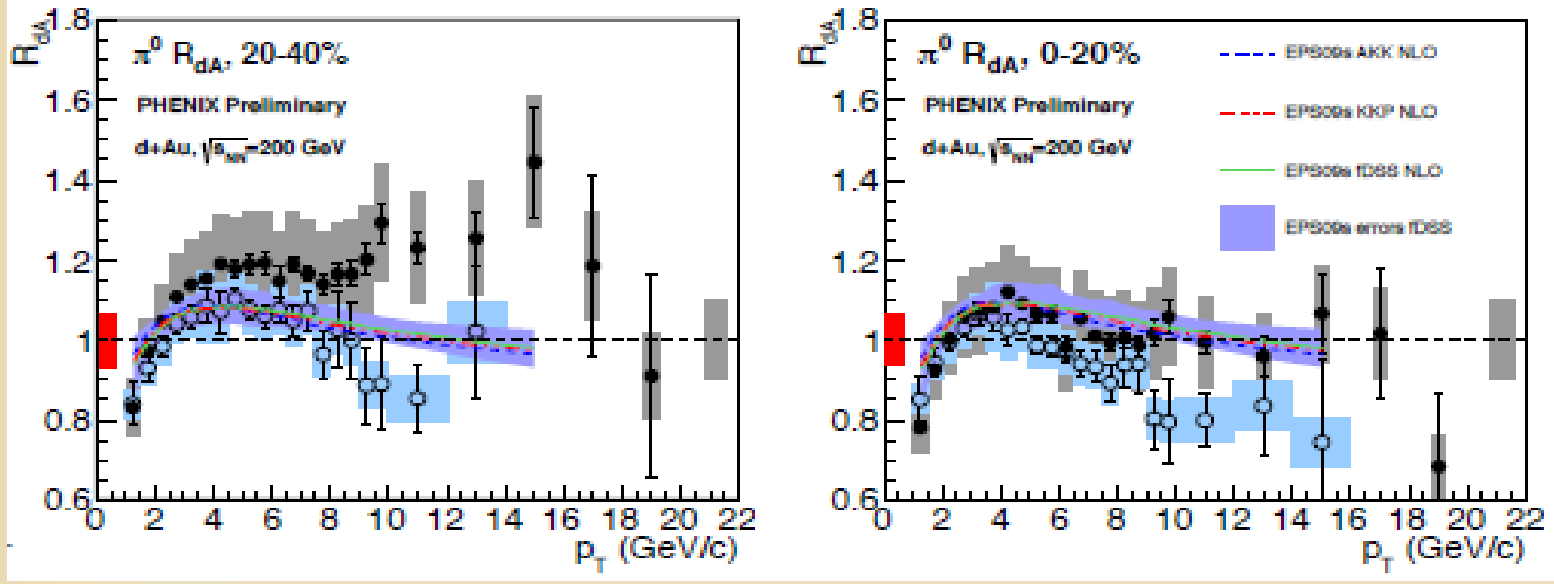
but differ at $p_{T}>6 \mathrm{GeV} / \mathrm{c}$ in all centralities from data taken in 2003

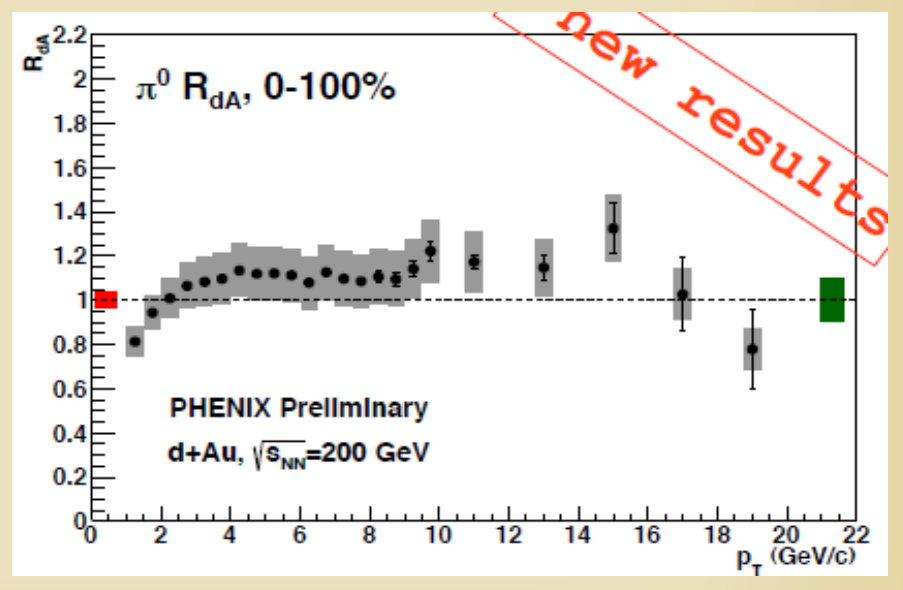




\section{MOTIVATION}

- Motivation for $p P b / d A u$ collisions

The RHIC MB is similar to the ALICE LHC MB data
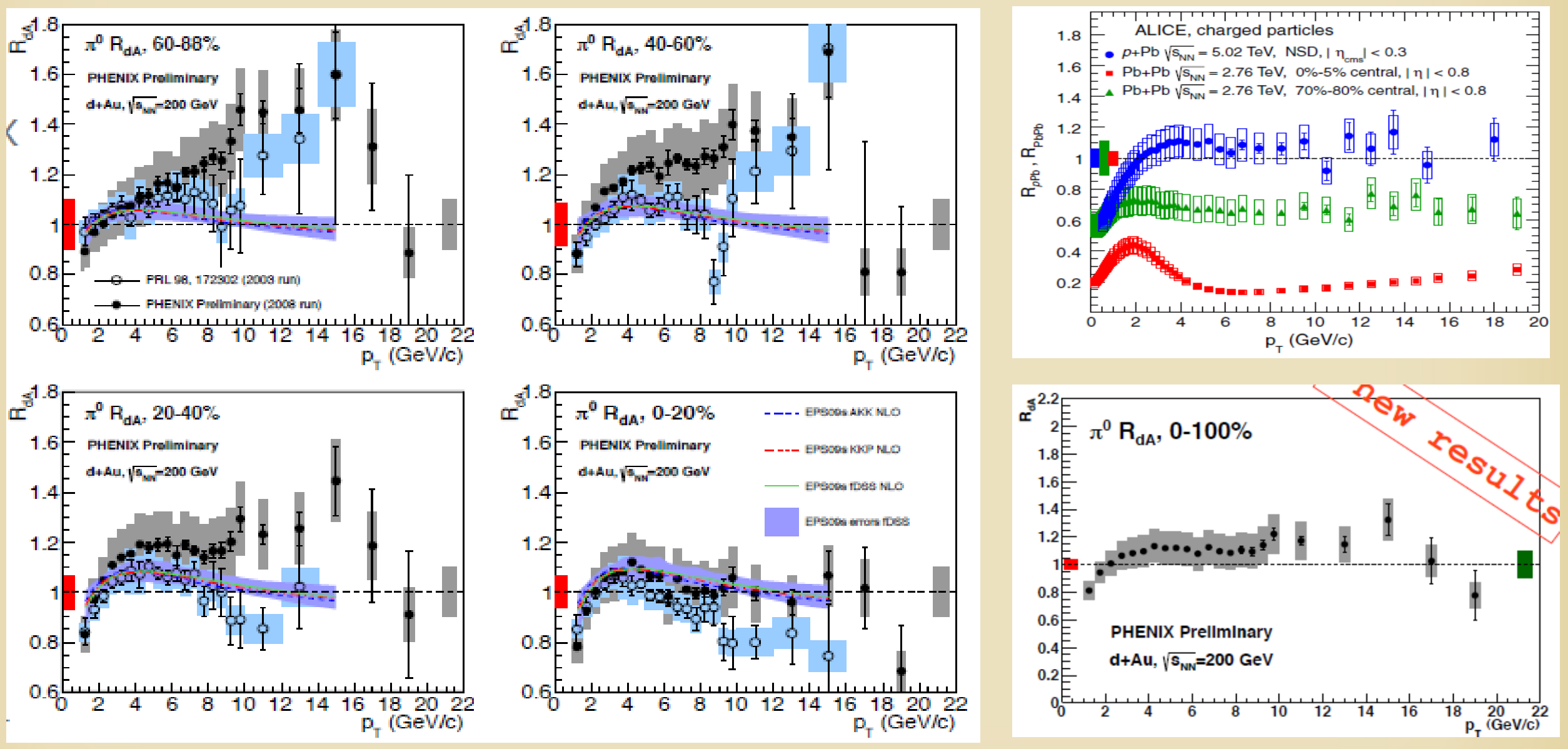

G.G. Barnaföldi: Cronin \& Shadowing in pA 


\section{What have we learnt about Cronin so far?}

- Overall properties of MB case:

CM energy dependence: Higher the sqrt(s) lower the peak

No relevant change in the place of the peak

Shape is similar, but hard to say anything on width

$\begin{array}{cccc}\begin{array}{c}\text { C.M. Energy } \\ \text { [GeV] }\end{array} & \begin{array}{c}\text { Peak' s place } \\ p_{\mathrm{T}}[\mathrm{GeV} / \mathrm{c}]\end{array} & \begin{array}{c}\text { Max effect. } \\ {[\%]}\end{array} & \text { Type } \\ 27.4 & 4 & 50 \% & \mathrm{pW} / \mathrm{pBe} \\ 38.8 & 4 & 40 \% & \mathrm{pW} / \mathrm{pBe} \\ 200 & 4 & 10-15 \% & \mathrm{dAu} / \mathrm{pp} \\ 5020 & 4 & 10 \% & \mathrm{pPb} / \mathrm{pp}\end{array}$

- Centrality \& Rapidity dependence

Anomalous increase in pheripheral collisions

Suppression of the peak in forward $(y>0)$ : less or no peak

No measurement in the backward $(y<0)$ : ???? 


\section{What have we learnt about Cronin so far?}

- Overall properties of MB case:

CM energy dependence: Higher the sqrt(s) lower the peak

No relevant change in the place of the peak

Shape is similar, but hard to say anything on width

\begin{tabular}{|c|c|c|c|c|}
\hline $\begin{array}{l}\text { C.M. Energy } \\
\text { [GeV] }\end{array}$ & $\begin{array}{c}\text { Peak's place } \\
p_{\mathrm{T}}[\mathrm{GeV} / \mathrm{c}]\end{array}$ & $\begin{array}{c}\text { Max effect. } \\
\text { [\%] }\end{array}$ & Type & Peak in $x$ \\
\hline 27.4 & 4 & $50 \%$ & pW/pBe & 0.3 \\
\hline 38.8 & 4 & $40 \%$ & pW/pBe & 0.2 \\
\hline 200 & 4 & $10-15 \%$ & dAu/pp & 0.04 \\
\hline 5020 & 4 & $10 \%$ & $\mathrm{pPb} / \mathrm{pp}$ & 0.002 \\
\hline
\end{tabular}

- Centrality \& Rapidity dependence

Anomalous increase in pheripheral collisions

Suppression of the peak in forward $(y>0)$ : less or no peak

No measurement in the backward $(y<0)$ : ???? 


\section{What have we learnt about Cronin so far?}

- Overall properties of MB case:

CM energy dependence: Higher the sqrt(s) lower the peak

No relevant change in the place of the peak

Shape is similar, but hard to say anything on width

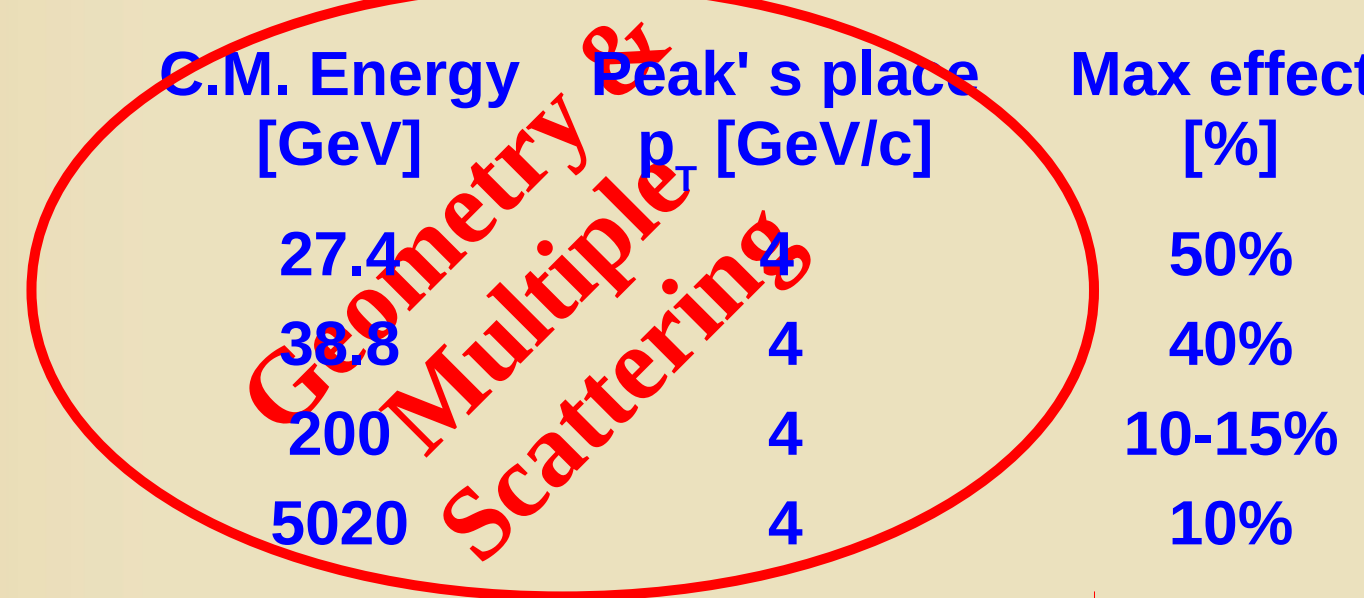

Type

Peak in $\mathrm{x}$

- Centrality \& Rapidity dependence

Anomalous increase in pheripheral collisions

Suppression of the peak in forward $(y>0)$ : less or no peak

No measurement in the backward $(y<0)$ : ???? 


\section{Models \& Parameters}

HIJING B/B 2.0

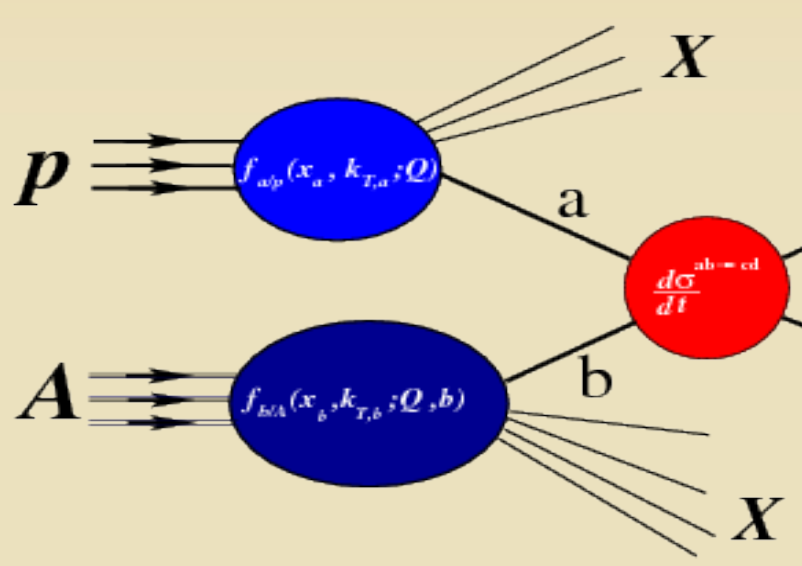

PRC83 024902, PRC84 022002 (2010)

Modified version of HIJING 2.0

Minijet cutoff: $p_{0}=3.1 \mathrm{GeV} / \mathrm{c}$

$$
p_{0}(s, A)=0.416 \sqrt{s}^{0.191} A^{0.128} \mathrm{GeV} / c
$$

String tension: $\mathrm{k}=2.9 \mathrm{GeV} / \mathrm{fm}$

$$
\kappa(s, A)=\kappa_{0}(s / s 0)^{0.06} A^{0.167} \mathrm{GeV} / \mathrm{fm} .
$$

PDF: GRV+ HIJING shadowing

FF: PYTHIA + minijet

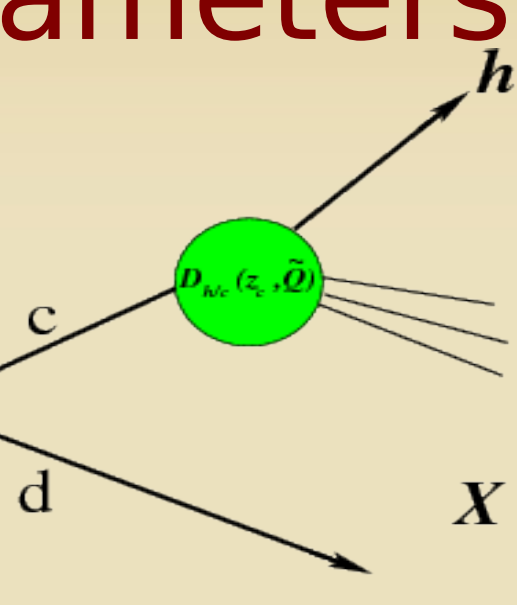

kTpQCD_v2.0 PRC65 (2002)034903

NLO pQCD based parton model with intrinsic- $\mathrm{k}_{\mathrm{T}}, \mathrm{k}_{\mathrm{T}}-$ broadening, and various shadowing parametrization.

$$
\begin{aligned}
E_{\pi} \frac{\mathrm{d} \sigma_{\pi}^{d A u}}{\mathrm{~d}^{3} p_{\pi}} & =f_{a / d}\left(x_{a}, Q^{2} ; \mathbf{k}_{T a}\right) \otimes f_{b / A u}\left(x_{b}, Q^{2} ; \mathbf{k}_{T b}\right) \otimes \\
& \otimes \frac{\mathrm{d} \sigma^{a b \rightarrow c d}}{\mathrm{~d} \hat{t}} \otimes \frac{D_{\pi / c}\left(z_{c}, \widehat{Q}^{2}\right)}{\pi z_{c}^{2}}
\end{aligned}
$$

PDF:GRV/MRST+Shad, FF:KKP 


\section{on the Shadowing}

\section{aka suppression}




\section{Multiple Scattering in kTpQCD_v2.0}

- What experiment gives... :

No relevant change in the place of the peak

Shape is similar, but hard to say anything on width

Anomalous increase in pheripheral collisions

Suppression of the peak in forward $(y>0)$ : less or no peak

No measurement in the backward $(y<0)$ : ????

- you just take into account in a theory...

Peak does not move or very slowly: assume no or log(s) dependence, and not follows $x$.

MB agrees with models: integrated values are constraints Geometry matters: shadowing, nuclear size/density, rapidity, asymmetry, correlations, etc 


\section{The Spectra and $R_{p P b}\left(p_{T}\right)$ for $|\eta|<0.35 \& 0.3$}

\section{Predictions by kTpQCD_2.0 @ 5.02 ATeV \& 0.2 AGeV}

\section{Shadowing:}

$$
f_{a / A}\left(x, Q^{2}\right)=S_{a / A}\left(x, Q^{2}\right) f_{a / N}\left(x, Q^{2}\right)
$$

\section{b-dependent part}

$$
s_{a}(b)=s_{a} \frac{5}{3}\left(1-\frac{b^{2}}{R_{A}^{2}}\right)
$$

\section{with}

$$
R_{A}=1.12 A^{1 / 3}
$$

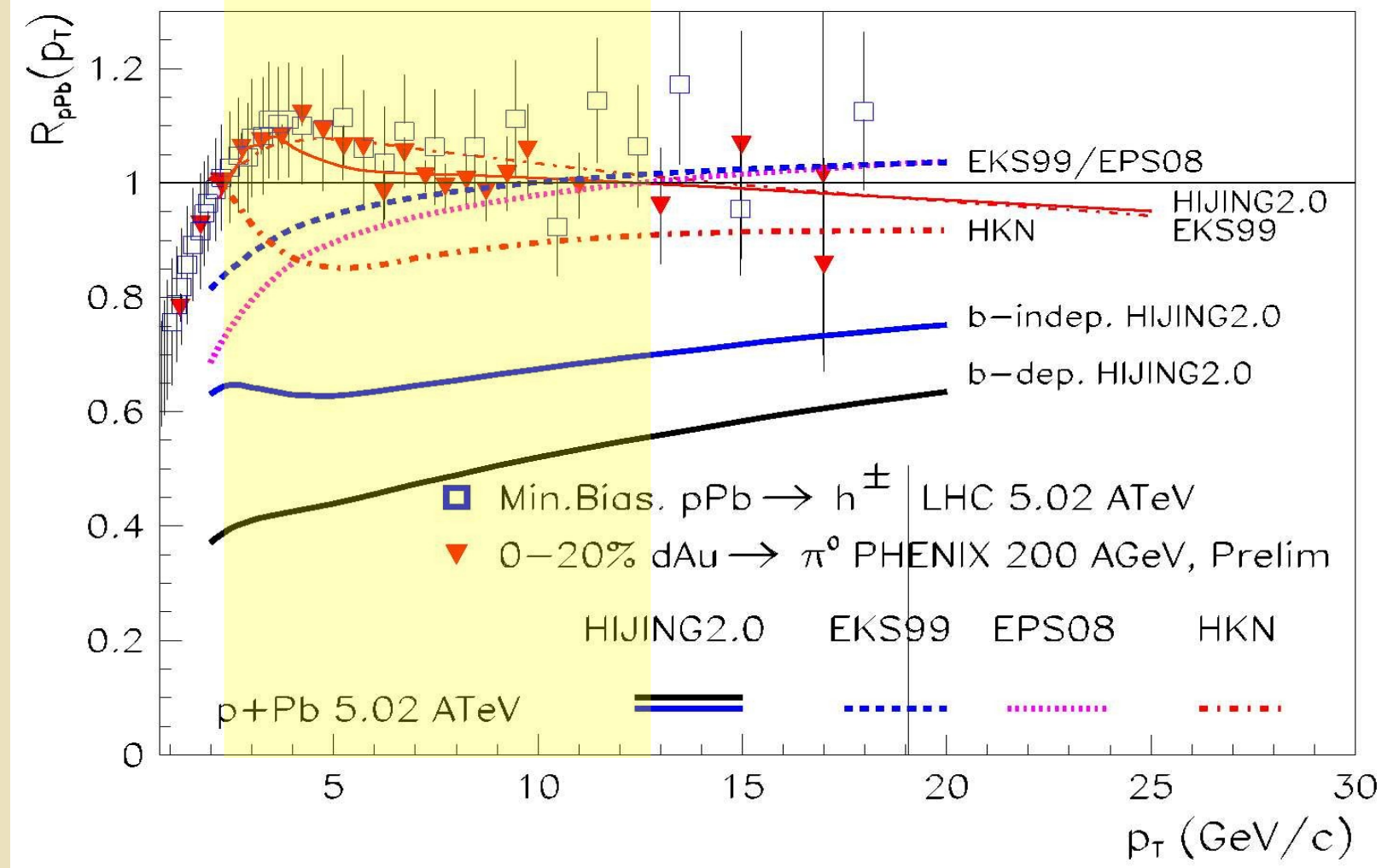

GGB, J. Barrette, M. Gyulassy, P. Lévai, V. Topor Pop: PRC 024903 (2012), arXiv:1211.2256, Int. Mod. Phys E22 1330007 (2013) G.G. Barnaföldi: Cronin \& Shadowing in pA 


\section{The Spectra and $R_{p P b}\left(p_{T}\right)$ for $|\eta|<0.35 \& 0.3$}

\section{Predictions by kTpQCD_2.0 @ 5.02 ATeV}

\section{Shadowing:}

$$
f_{a / A}\left(x, Q^{2}\right)=S_{a / A}\left(x, Q^{2}\right) f_{a / N}\left(x, Q^{2}\right)
$$

\section{b-dependent part}

$$
s_{a}(b)=s_{a} \frac{5}{3}\left(1-\frac{b^{2}}{R_{A}^{2}}\right)
$$

\section{with}

$$
R_{A}=1.12 A^{1 / 3}
$$

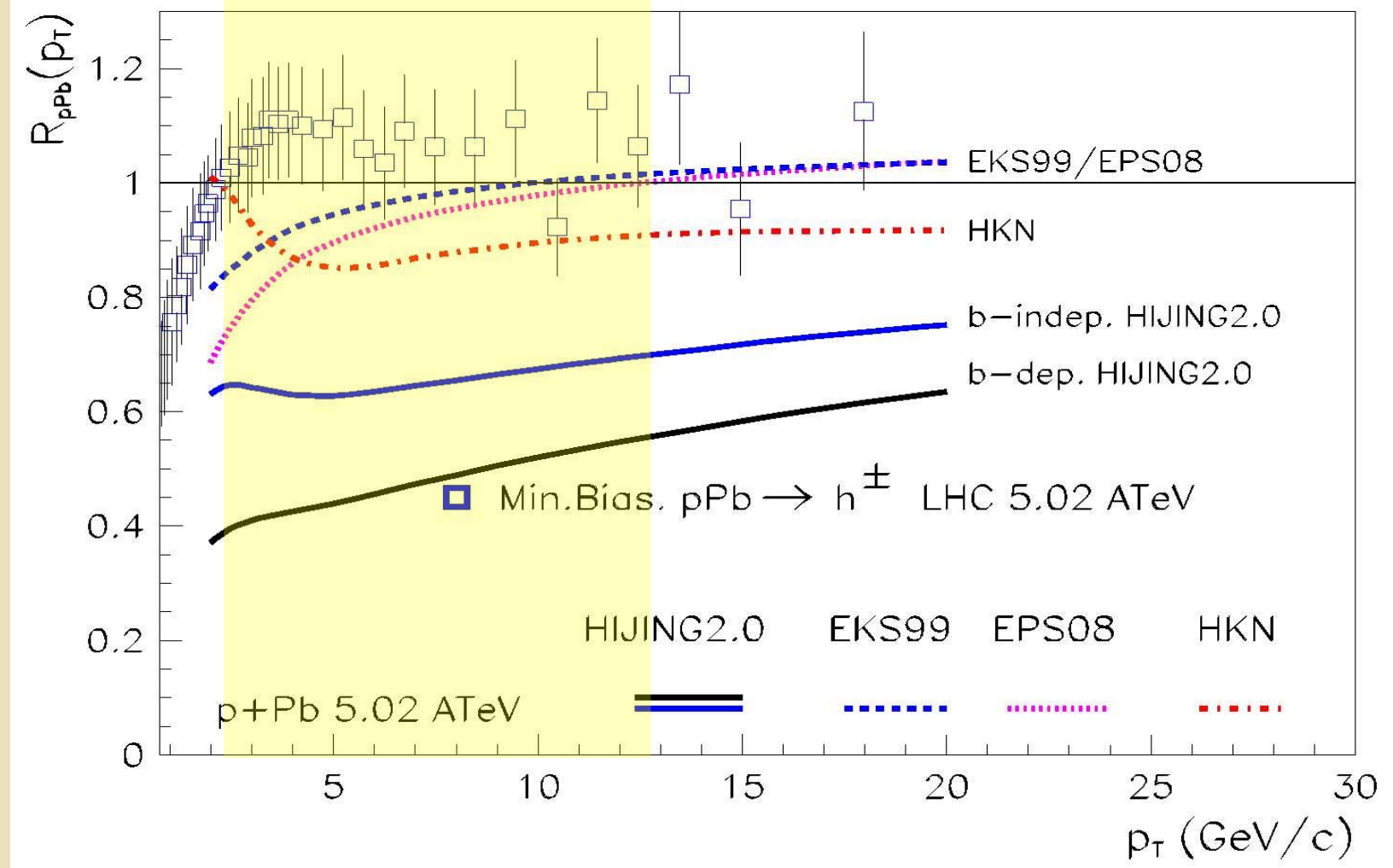

GGB, J. Barrette, M. Gyulassy, P. Lévai, V. Topor Pop: PRC 024903 (2012), arXiv:1211.2256, Int. Mod. Phys E22 1330007 (2013) G.G. Barnaföldi: Cronin \& Shadowing in pA 


\section{The Spectra and $R_{p P b}\left(p_{T}\right)$ for $|\eta|<0.8$}

Charged hadron production with HIJING $2.0 @ 4.4 \mathrm{ATeV} \quad \mathbf{R}_{\mathrm{pA}} \sim 0.7$
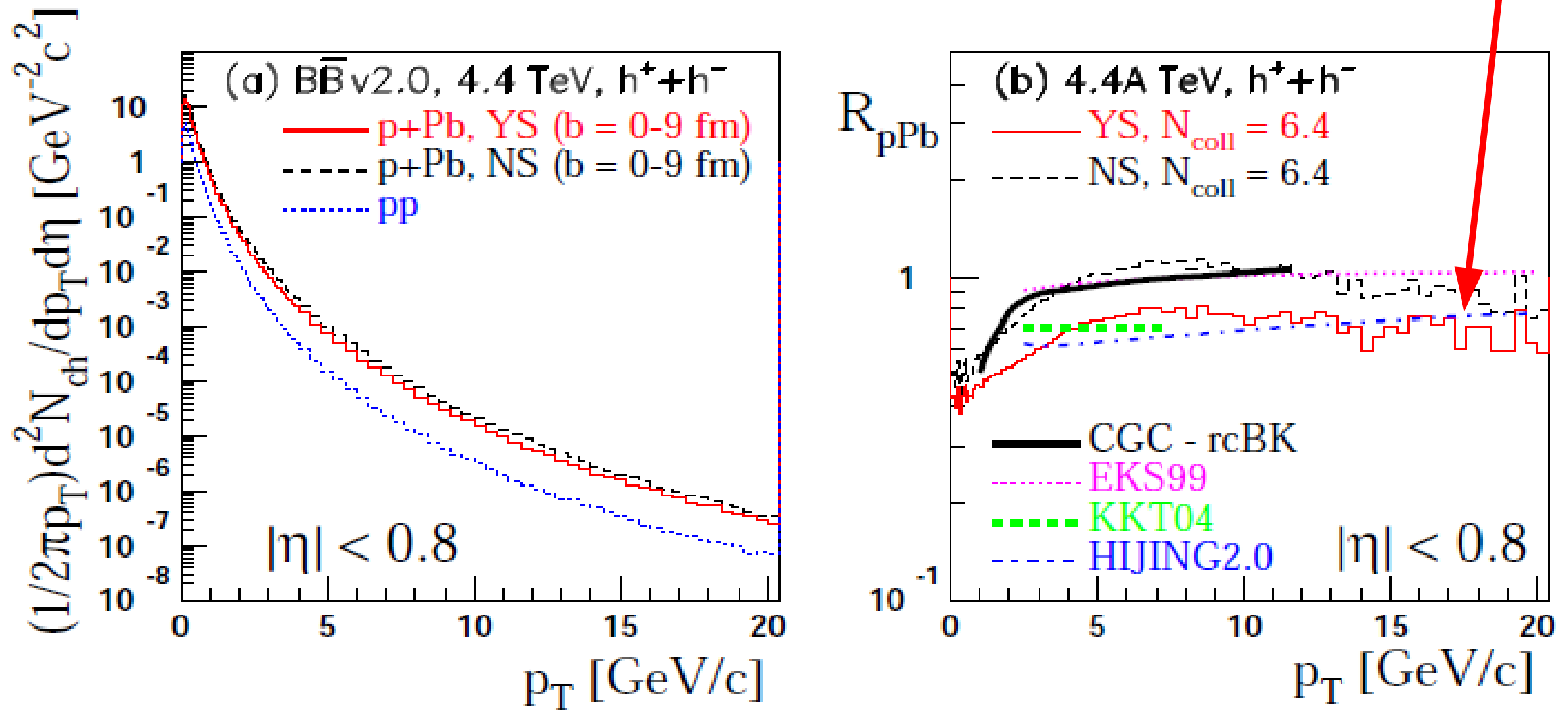

GGB, J. Barrette, M. Gyulassy, P. Lévai, V. Topor Pop PRC85 024903 (2012) 


\section{Forward $R_{p P b}\left(p_{T}\right)$ at $|\eta|<0.8 \& \eta=6.0$}

Charged hadron production with HIJING $2.0 @ 4.4 \mathrm{ATeV} \quad \mathbf{R} \sim 0.35$
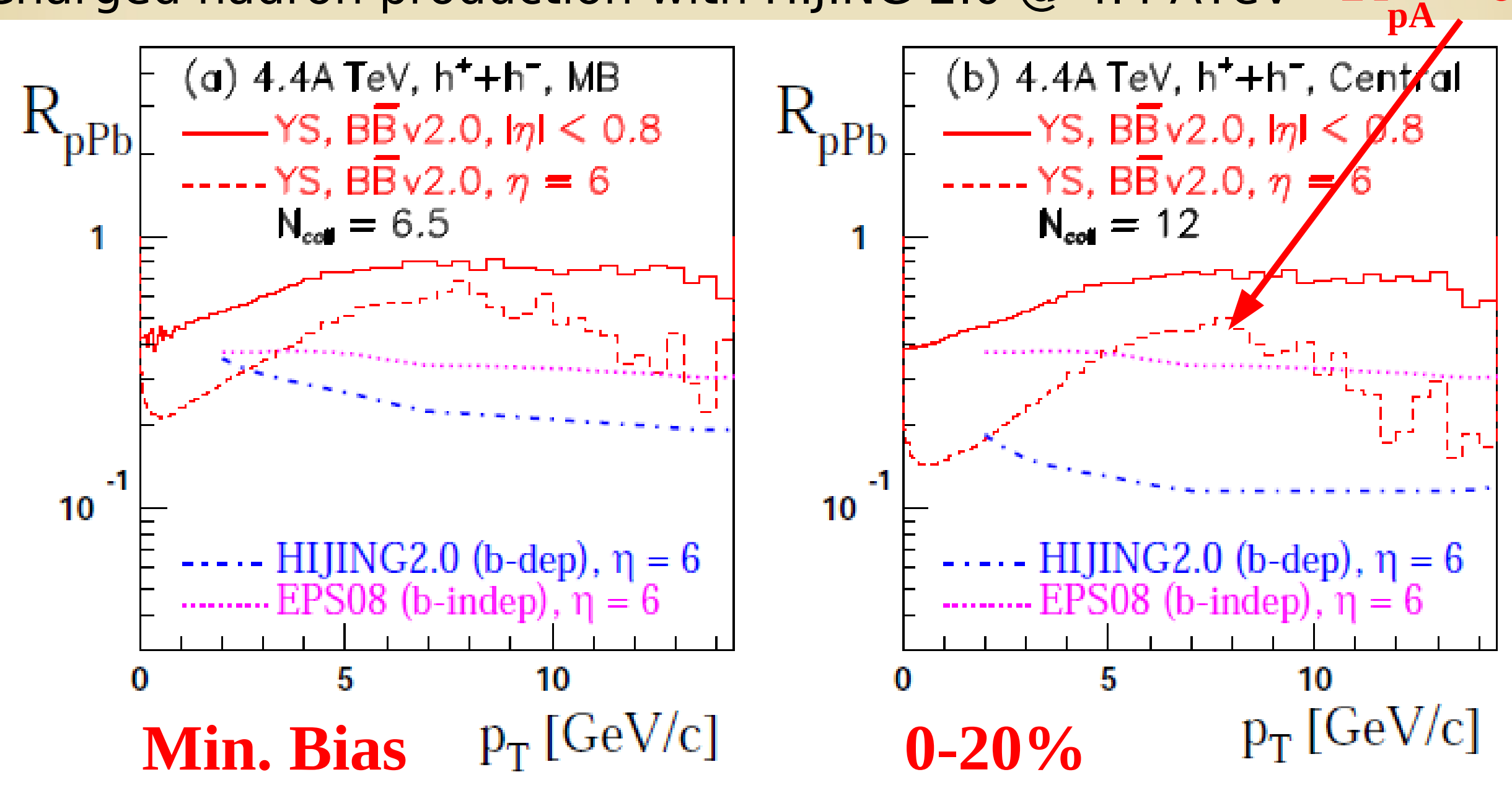

GGB, J. Barrette, M. Gyulassy, P. Lévai, V. Topor Pop PRC85 024903 (2012) 


\section{HIJINGB/B 2.0: Rapidity distribution for $p p \& p P b$}

Charged hadron production with HIJING 2.0 @ 5.02 ATeV (0-20\%)

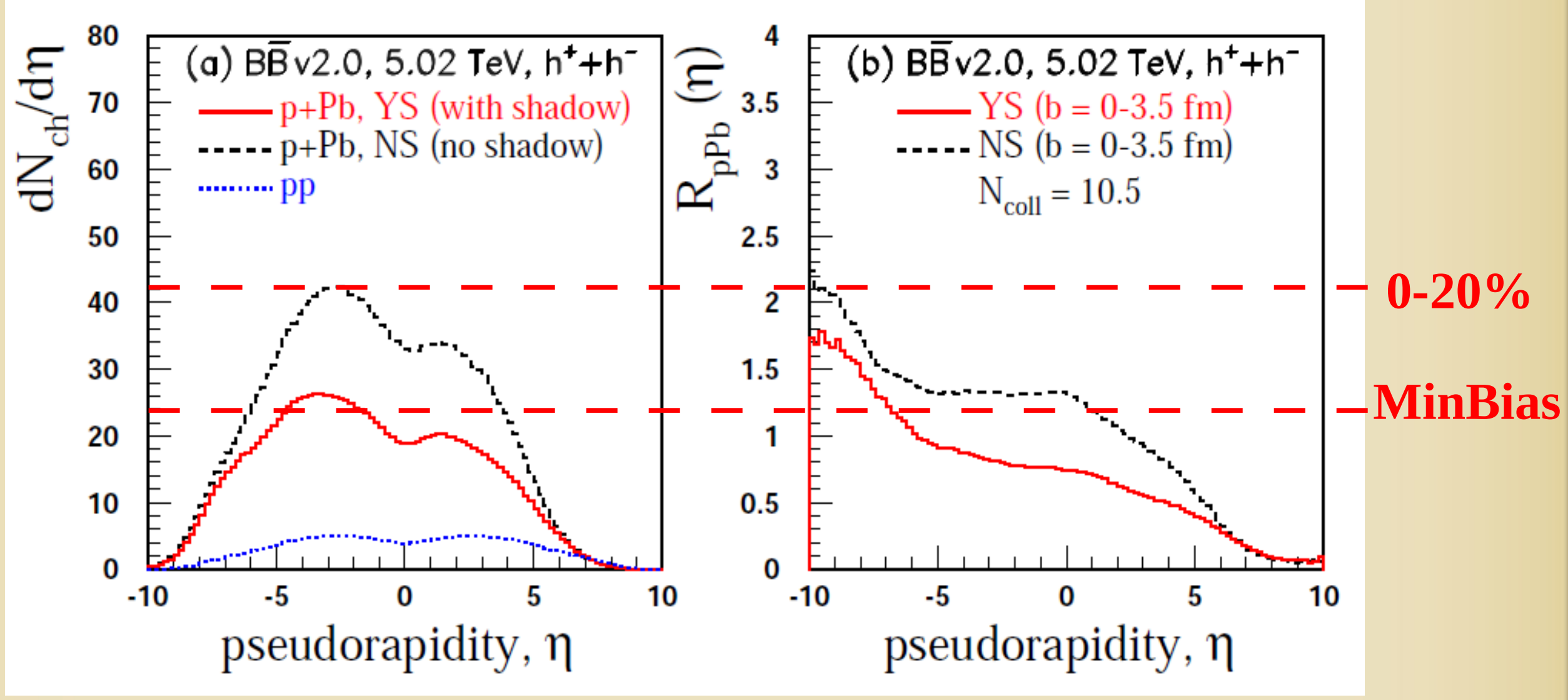

GGB, J. Barret, M. Gyulassy, P. Lévai, V. Topor Pop (in preparation 2012) 


\section{Shadowing effects on $R_{d A u}\left(p_{T}\right)$ for $|\eta|<0.35$}

Extreme high- $\mathrm{p}_{\mathrm{T}}$ Pion production with kTpQCD @ $200 \mathrm{AGeV}$

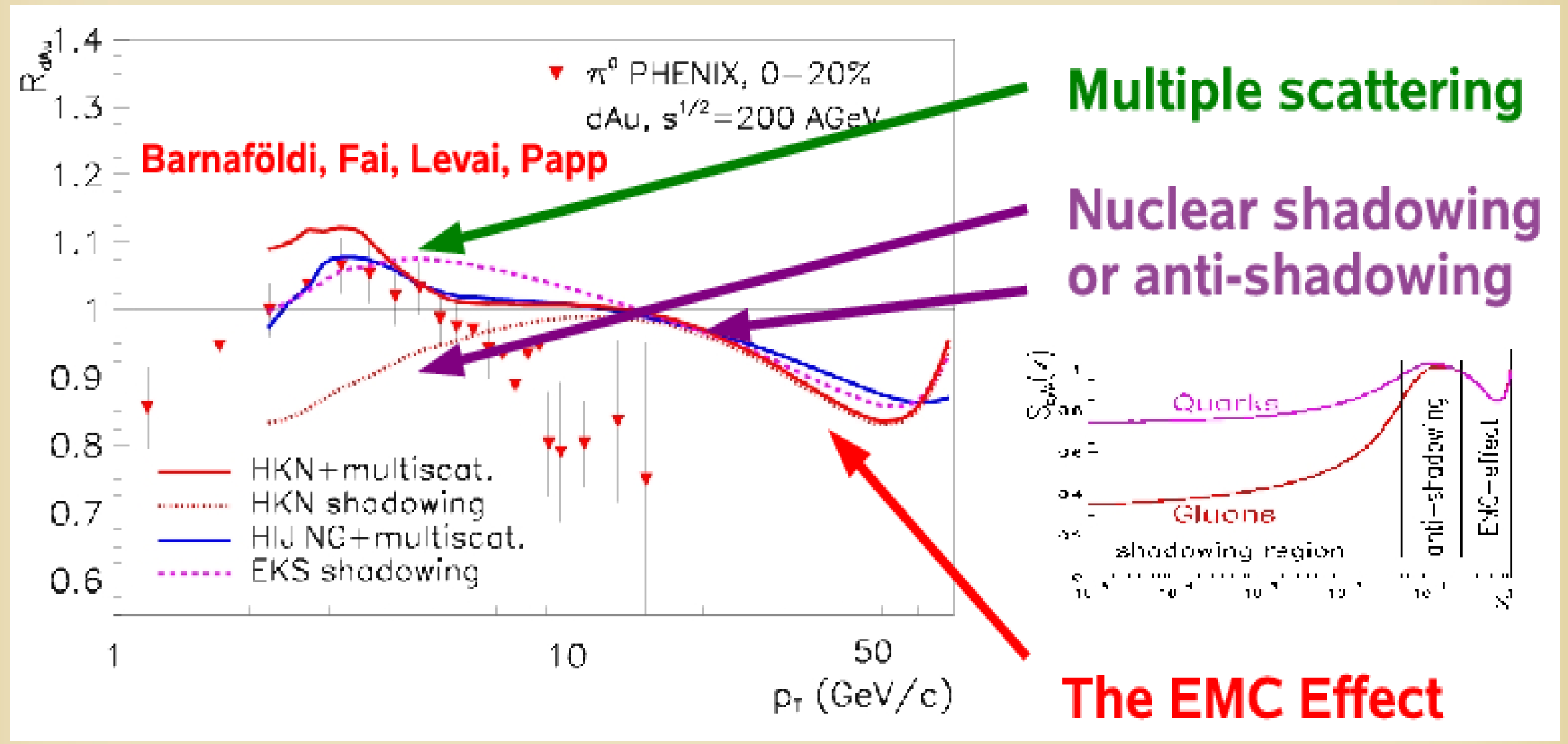

BA Cole, GGB, G. Fai, P. Lévai, G. Papp, arXiv:08073384 (2007) 


\section{Shadowing effects on $R_{d A U}\left(p_{T}\right)$ for $|\eta|<0.35$}

Slopes \& values are OK with new dAu 0-20\% data @ 200 AGeV

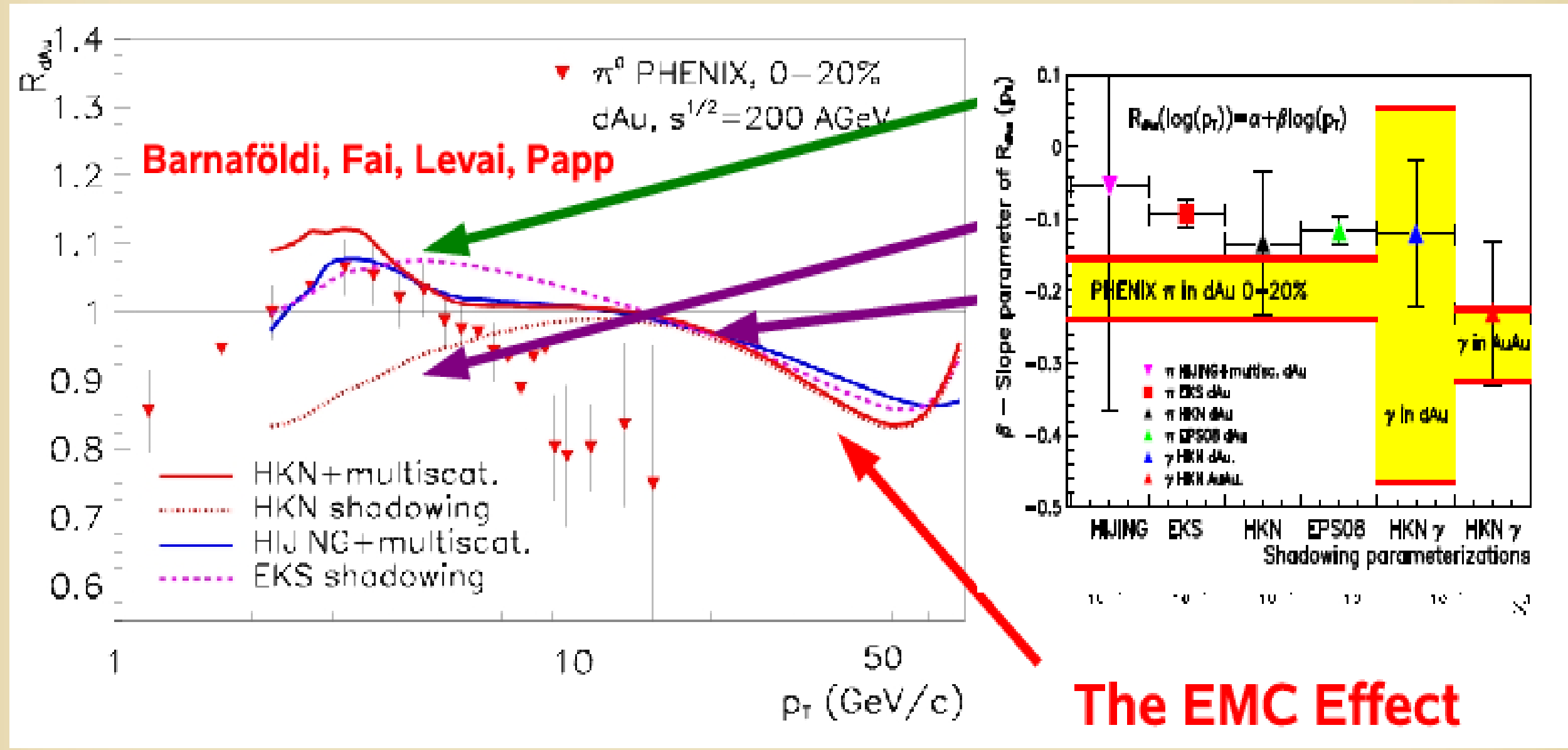

BA Cole, GGB, G. Fai, P. Lévai, G. Papp, arXiv:08073384 (2007) 


\section{on the Multiple Scattering}

aka the enhacement 


\section{Multiple Scattering in KTpQCD_v2.0}

Intrinsic $\mathrm{k}_{\mathrm{T}}$ from theory $\& \mathrm{pp}$ experiments

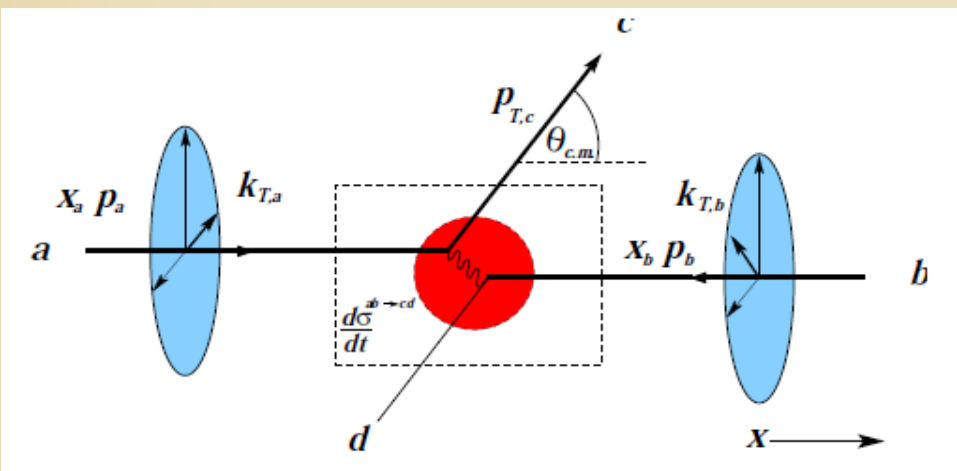

\section{$\mathrm{k}_{\mathrm{T}}$-broadening:}

\section{$f_{a / p}\left(x_{a}, \mathbf{k}_{T a}, Q^{2}\right)=f_{a / p}\left(x_{a}, Q^{2}\right) \cdot g_{a / p}\left(\mathbf{k}_{T a}\right)$}

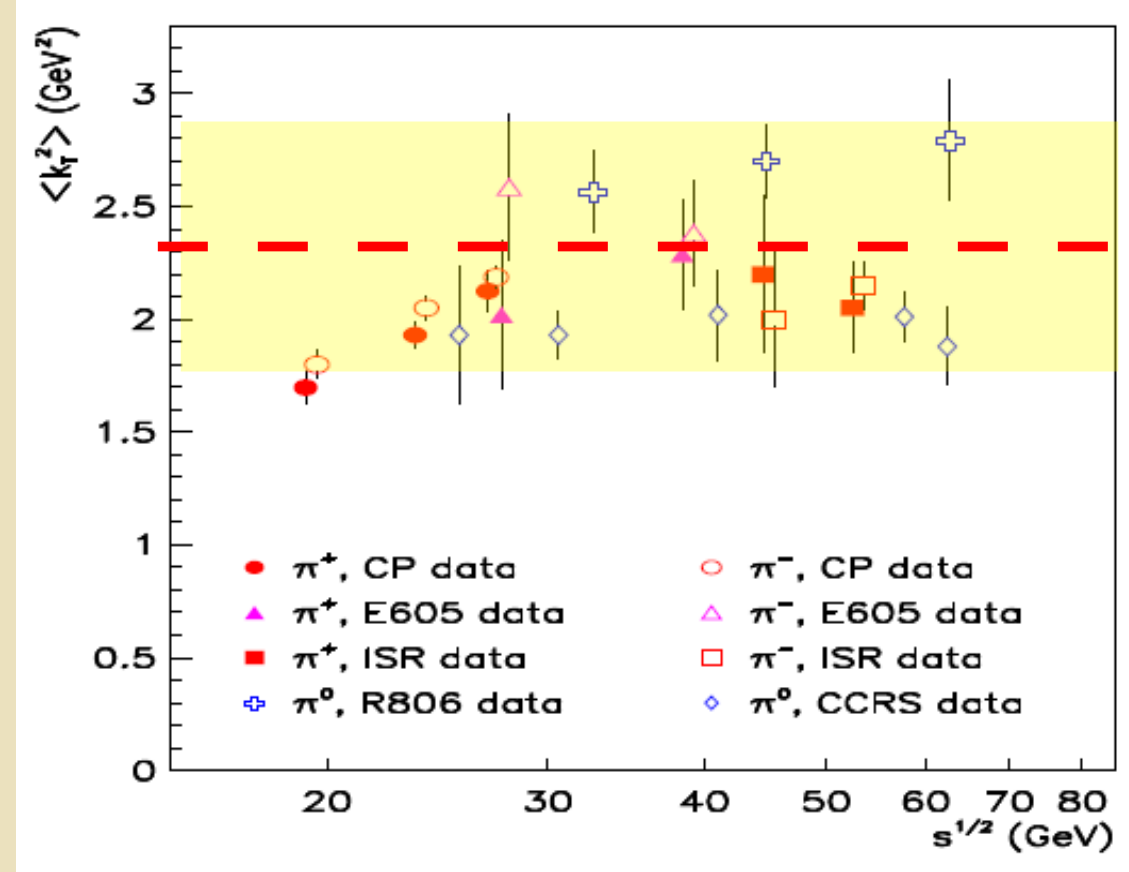

$g_{a / n}\left(\mathbf{k}_{T}\right):=\frac{1}{\pi\left\langle k_{T}^{2}\right\rangle} e^{-\mathbf{k}_{T}^{2} /\left\langle k_{T}^{2}\right\rangle}$

$\left\langle k_{T}^{2}\right\rangle_{p p}=\left\langle p_{T}\right\rangle_{\text {pair }}^{2} / \pi$

Y. Zhang, GGB, G. Fai, P. Lévai, G. Papp, PRC65 034903 (2002) 


\section{Multiple Scattering in KTpQCD_v2.0}

Intrinsic $\mathrm{k}_{\mathrm{T}}$ from theory $\& \mathrm{pp}$ experiments

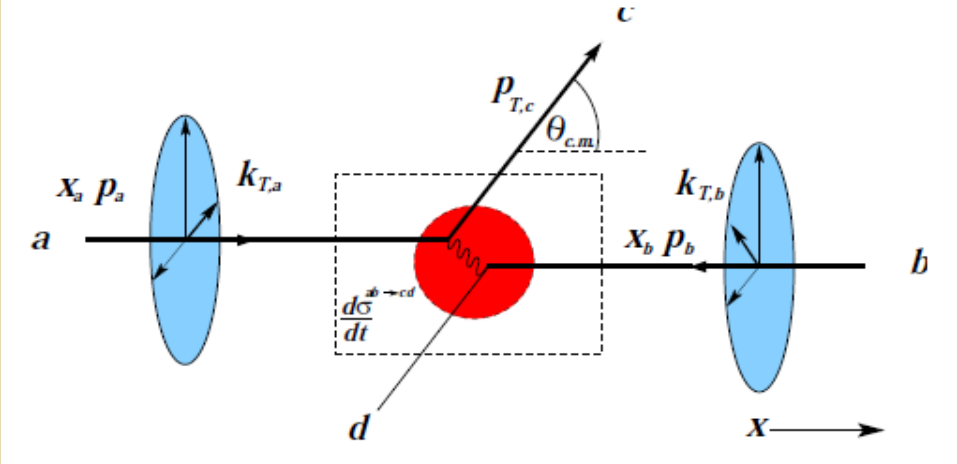

\section{$\mathrm{k}_{\mathrm{T}}$-broadening:}

$$
f_{a / p}\left(x_{a}, \mathrm{k}_{T a}, Q^{2}\right)=f_{a / p}\left(x_{a}, Q^{2}\right) \cdot g_{a / p}\left(\mathbf{k}_{T a}\right)
$$

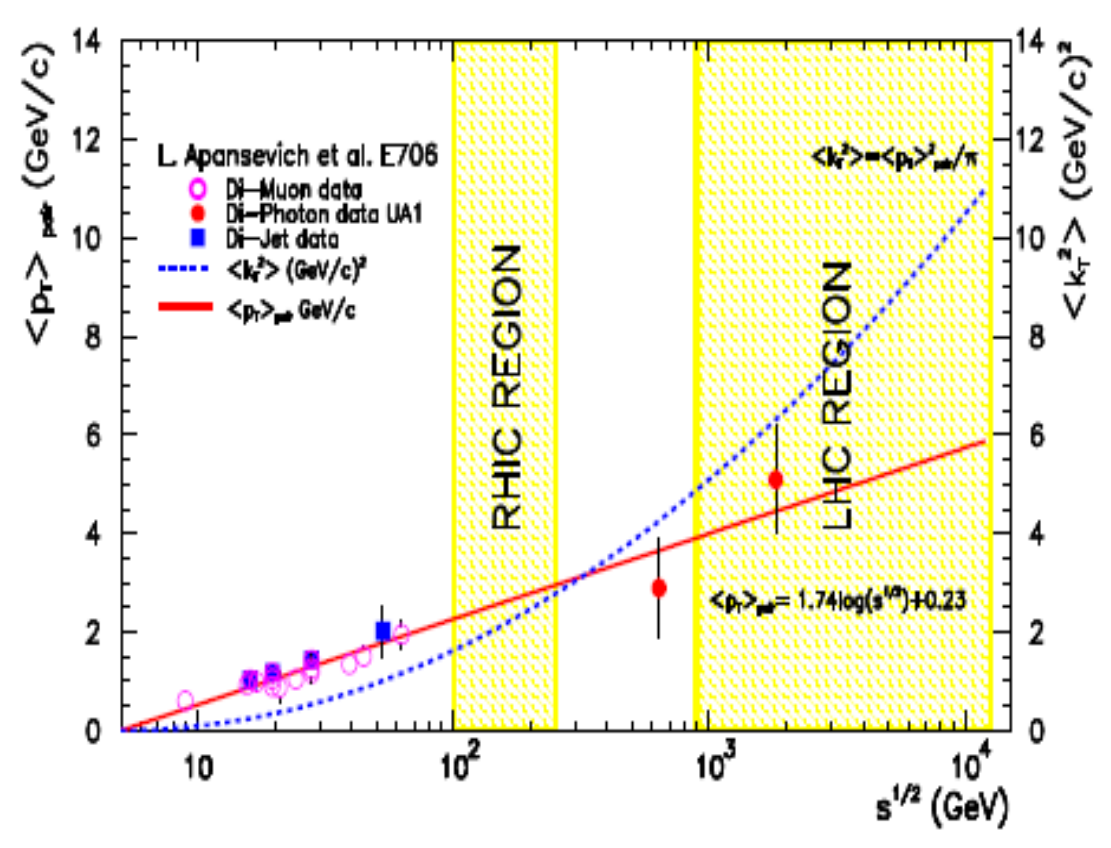

$g_{a / n}\left(\mathbf{k}_{T}\right):=\frac{1}{\pi\left\langle k_{T}^{2}\right\rangle} e^{-\mathbf{k}_{T}^{2} /\left\langle k_{T}^{2}\right\rangle}$

$\left\langle k_{T}^{2}\right\rangle_{p p}=\left\langle p_{T}\right\rangle_{\text {pair }}^{2} / \pi$

BA Cole, GGB, G. Fai, P. Lévai, G. Papp, arXiv:08073384 (2007) 


\section{Multiple Scattering in KTpQCD_v2.0}

Intrinsic $\mathrm{k}_{\mathrm{T}}$ from theory $\& \mathrm{pp}$ experiments

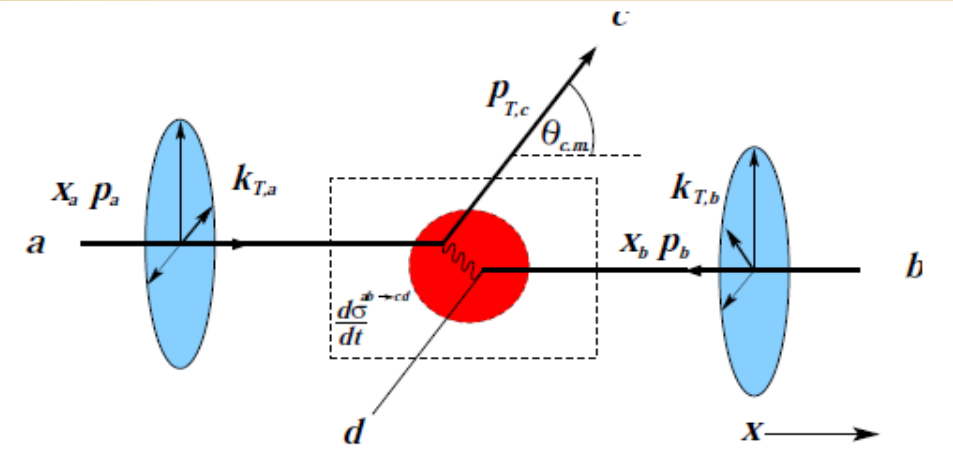

\section{$\mathrm{k}_{\mathrm{T}}$-broadening:}

$$
f_{a / p}\left(x_{a}, \mathbf{k}_{T a}, Q^{2}\right)=f_{a / p}\left(x_{a}, Q^{2}\right) \cdot g_{a / p}\left(\mathbf{k}_{T a}\right)
$$

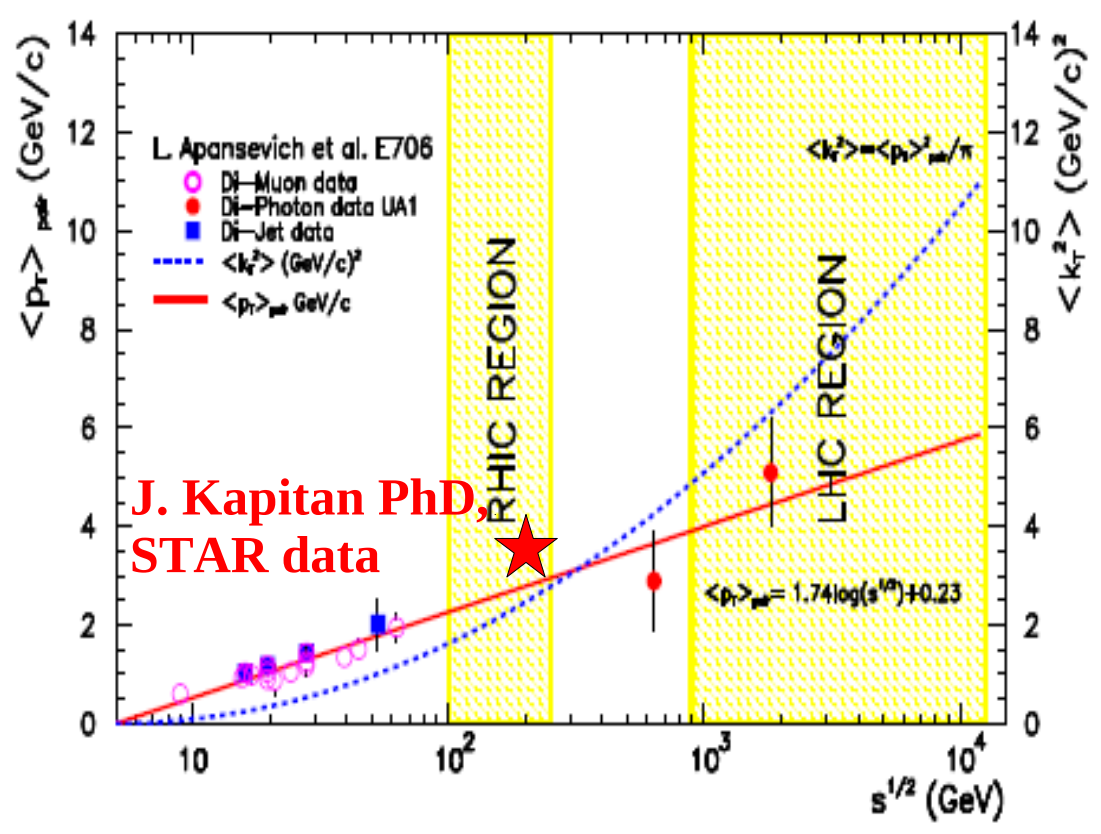

$g_{a / n}\left(\mathbf{k}_{T}\right):=\frac{1}{\pi\left\langle k_{T}^{2}\right\rangle} e^{-\mathbf{k}_{T}^{2} /\left\langle k_{T}^{2}\right\rangle}$

$\left\langle k_{T}^{2}\right\rangle_{p p}=\left\langle p_{T}\right\rangle_{\text {pair }}^{2} / \pi$

BA Cole, GGB, G. Fai, P. Lévai, G. Papp, arXiv:08073384 (2007)

J. Kapitan, PhD Thesis, 201 I.G. Barnaföldi: Cronin \& Shadowing in pA 


\section{Multiple Scattering in KTpQCD_v2.0}

Glauber model

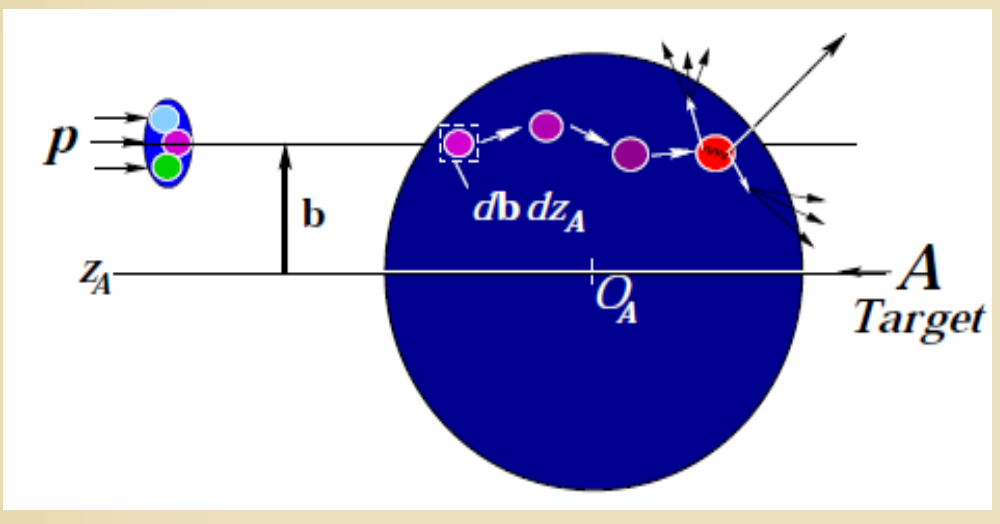

$$
\int_{0}^{\mathbf{b}_{\max }} t_{A}(\mathbf{b}) \mathrm{d}^{2} \mathbf{b}=\int_{0}^{\mathbf{b}_{\max }} \mathrm{d}^{2} \mathbf{b} \int \mathrm{d} z \rho(\mathbf{b}, z)=A
$$

with nuclear density:

$$
\rho(r)=\frac{\rho_{0}}{1+\mathrm{e}^{\frac{r-r_{0}}{c}}}
$$

There must be 1 hard and, further semi-hard collisions (number of NN collisions):

$$
\nu_{A}(b)=\sigma_{N N}^{i n} \cdot t_{A}(b)
$$

$\mathrm{NN}$-like collisions followed by enhancement of intrinsic $\mathrm{k}_{\mathrm{T}}$

PA: Gaussian broadening

$$
g_{a / p}\left(\mathbf{k}_{T, a}, b\right):=\frac{1}{\pi\left\langle k_{T a}^{2}(b)\right\rangle_{p A}} \mathrm{e}^{-\frac{\mathbf{k}_{T, a}^{2}}{\left\langle k_{T, a}^{2}(b)\right\rangle_{p A}}}
$$

BA Cole, GGB, G. Fai, P. Lévai, G. Papp, arXiv:08073384 (2007) 


\section{Multiple Scattering in KTpQCD_v2.0}

Phenomenological determination of the broadening
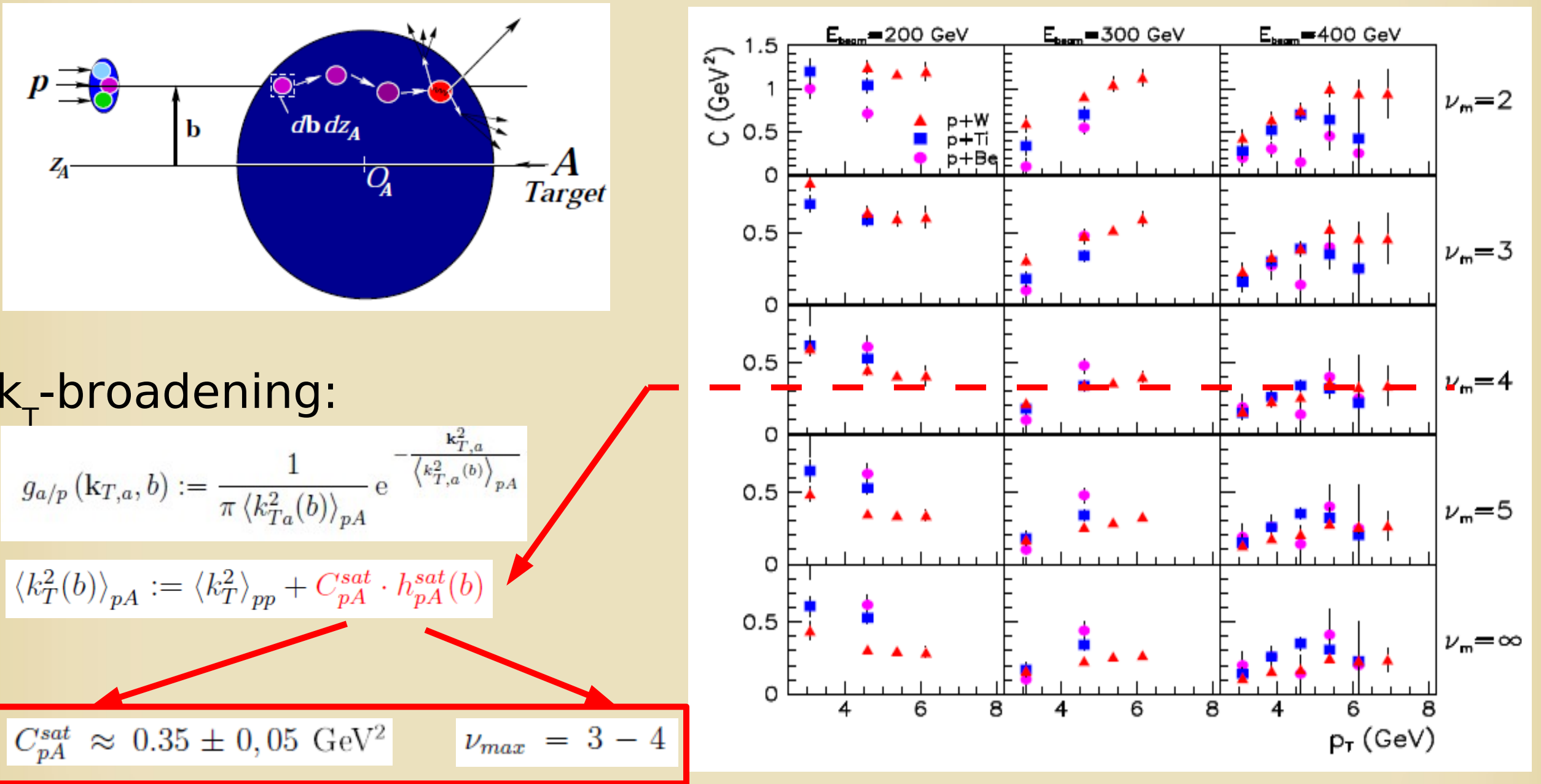

$\mathrm{k}_{\mathrm{T}}$-broadening:

$g_{a / p}\left(k_{T, a}, b\right):=\frac{1}{\pi\left\langle k_{T a}^{2}(b)\right\rangle_{p A}} e^{-\frac{k_{2}^{2}, a}{\left\langle k_{T}^{2}, a(b)\right\rangle_{p A}}}$ $\left\langle k_{T}^{2}(b)\right\rangle_{p A}:=\left\langle k_{T}^{2}\right\rangle_{p p}+C_{p A}^{s a t} \cdot h_{p A}^{s a t}(b)$

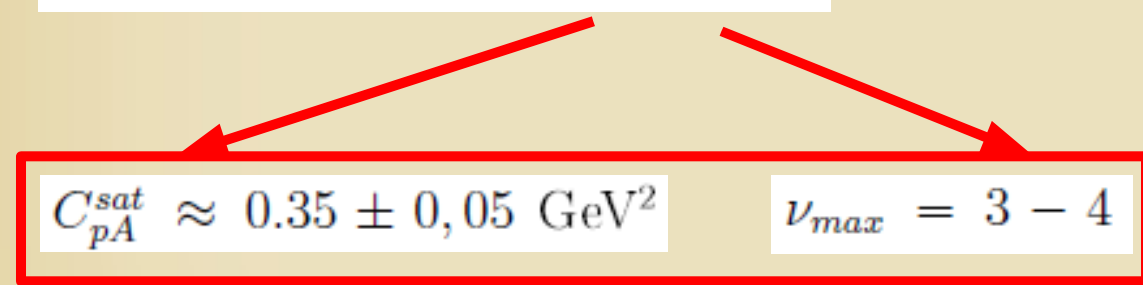

BA Cole, GGB, G. Fai, P. Lévai, G. Papp, arXiv:08073384 (2007) 


\section{Multiple Scattering in KTpQCD_v2.0}

Intrinsic $\mathrm{k}_{\mathrm{T}}$ from theory $\& \mathrm{pA}$ experiment
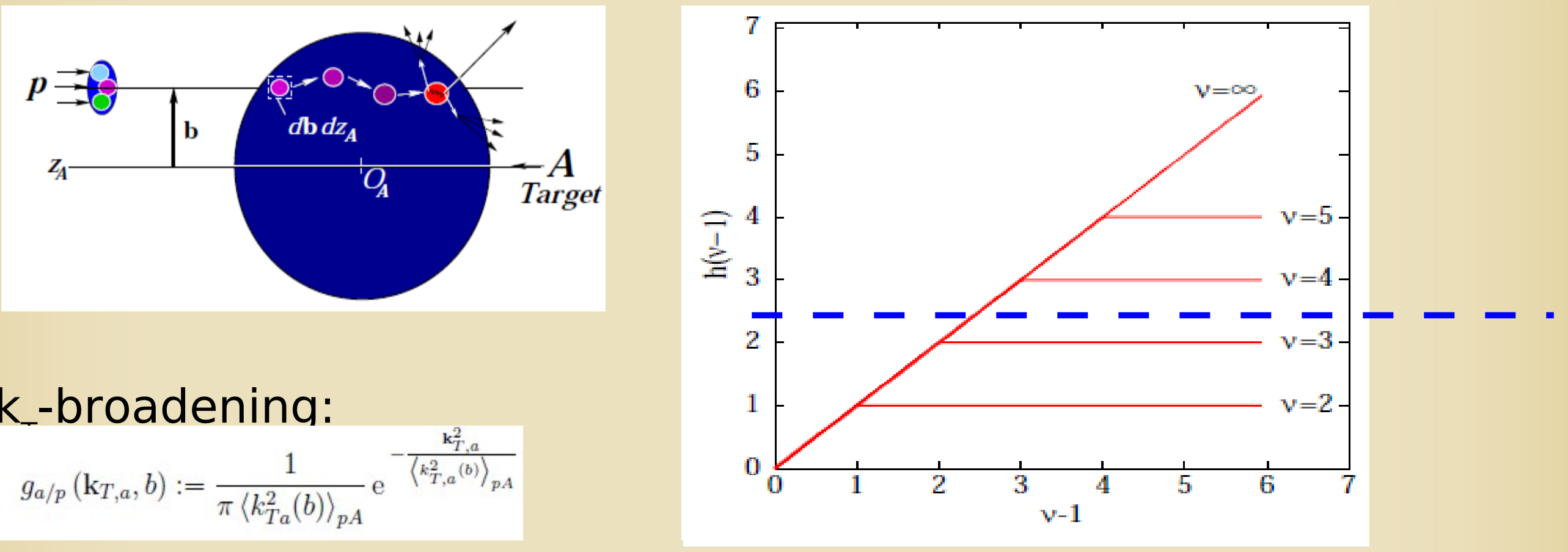

k_-broadenina:

$g_{a / p}\left(\mathbf{k}_{T, a}, b\right):=\frac{1}{\pi\left\langle k_{T a}^{2}(b)\right\rangle_{p A}} \mathrm{e}^{-\frac{k_{T}^{2}, a}{\left\langle k_{T}^{2},(a)\right\rangle_{p A}}}$

model: $h_{p A}^{\text {sat }}(b)=\left\{\begin{array}{lll}0 & \text { ha } & \nu_{A}(b)<1 \\ \nu_{A}(b)-1 & \text { ha } & 1 \leq \nu_{A}(b)<4 \\ 3 & \text { ha } & \nu_{A}(b) \geq 4\end{array}\right.$

$\left\langle k_{T}^{2}(b)\right\rangle_{p A}:=\left\langle k_{T}^{2}\right\rangle_{p p}+C_{p A}^{s a t} \cdot h_{p A}^{s a t}(b)$

Staurated Glauber model:

BA Cole, GGB, G. Fai, P. Lévai, G. Papp, arXiv:08073384 (2007) 


\section{Multiple Scattering in KTpQCD_v2.0}

Intrinsic $\mathrm{k}_{\mathrm{T}}$ from theory $\& \mathrm{pp} / \mathrm{dAu}$ experiments

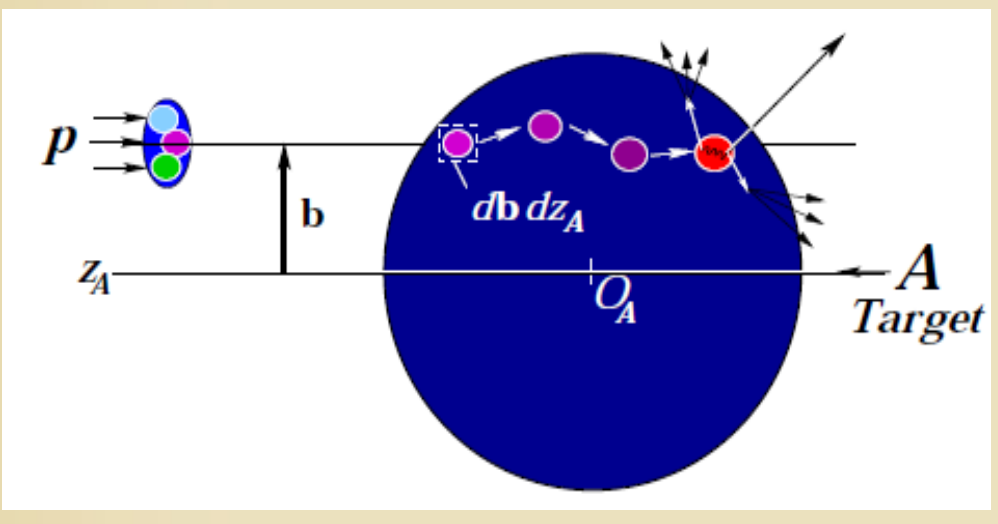

k_-broadening:

$$
\begin{aligned}
& g_{a / p}\left(\mathbf{k}_{T, a,}, b\right):=\frac{1}{\pi\left\langle k_{T a}^{2}(b)\right\rangle_{p A}} \mathrm{e}^{-\frac{k_{T}^{2}, a}{\left\langle k_{T, a}^{2}(b)\right\rangle_{p A}}} \\
& \left\langle k_{T}^{2}(b)\right\rangle_{p A}:=\left\langle k_{T}^{2}\right\rangle_{p p}+C_{p A}^{s a t} \cdot h_{p A}^{s a t}(b) \\
& \left\langle k_{T}^{2}\right\rangle_{p p}=\left\langle p_{T}\right\rangle_{p a i r}^{2} / \pi
\end{aligned}
$$

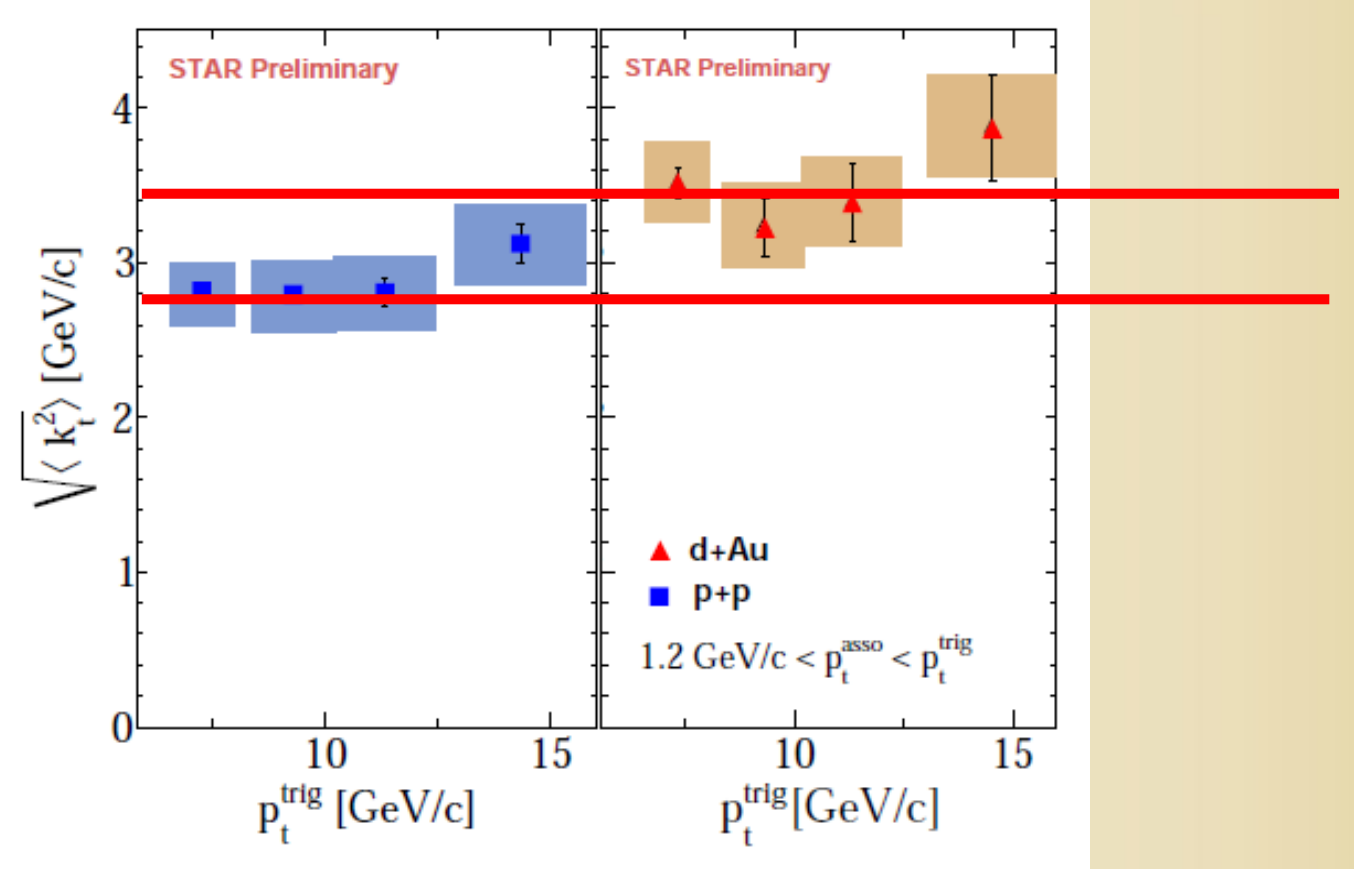

BA Cole, GGB, G. Fai, P. Lévai, G. Papp, arXiv:08073384 (2007) 


\section{Multiple Scattering in KTpQCD_v2.0}

A proposed effect of saturated Cronin effect on NMF

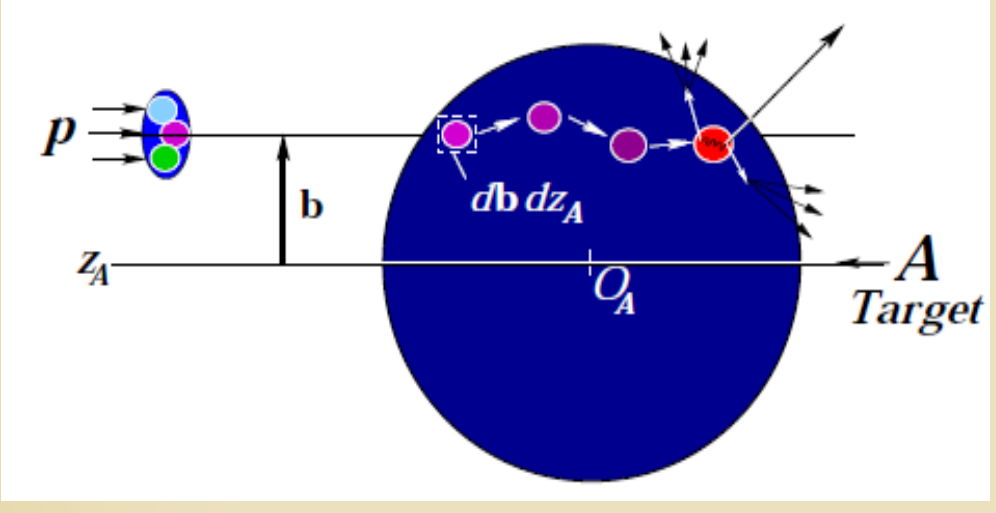

NMF as we know:

$$
R_{p A}^{h}\left(p_{T}\right):=\frac{1}{\left\langle N_{b i n}\right\rangle_{A}} \cdot \frac{\mathrm{d}^{3} \sigma_{p A}^{h} / \mathrm{d}^{3} p_{T}}{\mathrm{~d}^{3} \sigma_{p p}^{h} / \mathrm{d}^{3} p_{T}}
$$

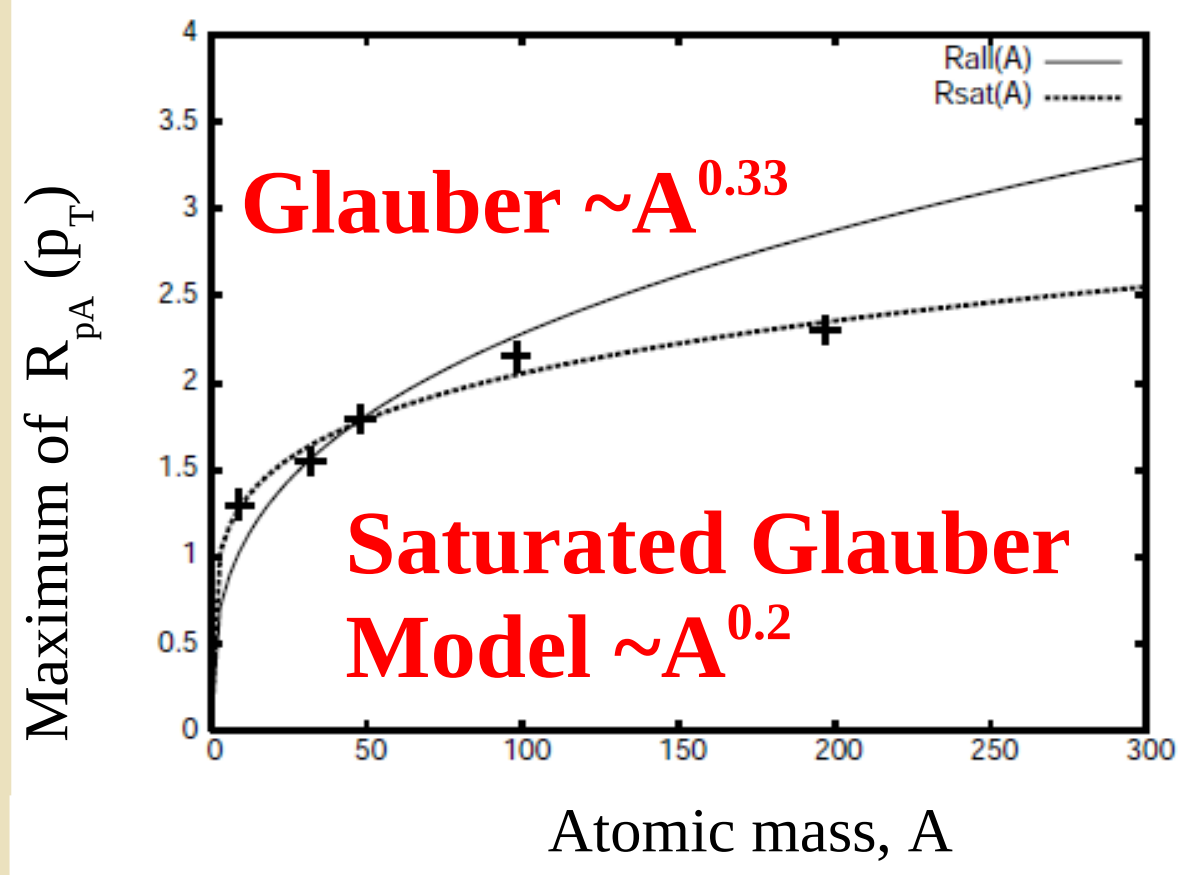

As it was in the beginning, for MB (see e.g. W. Busza's talk)

$$
\frac{\mathrm{d}^{3} \sigma_{p A}^{h} / \mathrm{d}^{3} p_{T}}{\mathrm{~d}^{3} \sigma_{p p}^{h} / \mathrm{d}^{3} p_{T}} \sim A^{\alpha\left(p_{T}\right)} \longrightarrow R_{p A}^{h} \sim A^{\alpha\left(p_{T}\right)-1}
$$

G. Fai, P. Lévai, G. Papp, PRC61 (2000) 021902,

GGB PhD Thesis, 2006 


\section{Multiple Scattering in KTpQCD_v2.0}

A proposed effect of saturated Cronin effect on NMF

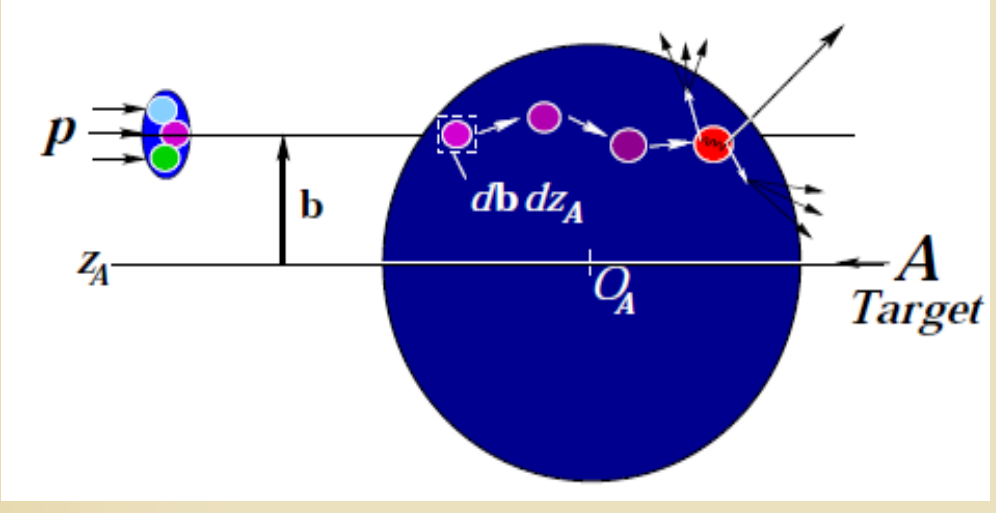

NMF as we know:

$$
R_{p A}^{h}\left(p_{T}\right):=\frac{1}{\left\langle N_{b i n}\right\rangle_{A}} \cdot \frac{\mathrm{d}^{3} \sigma_{p A}^{h} / \mathrm{d}^{3} p_{T}}{\mathrm{~d}^{3} \sigma_{p p}^{h} / \mathrm{d}^{3} p_{T}}
$$

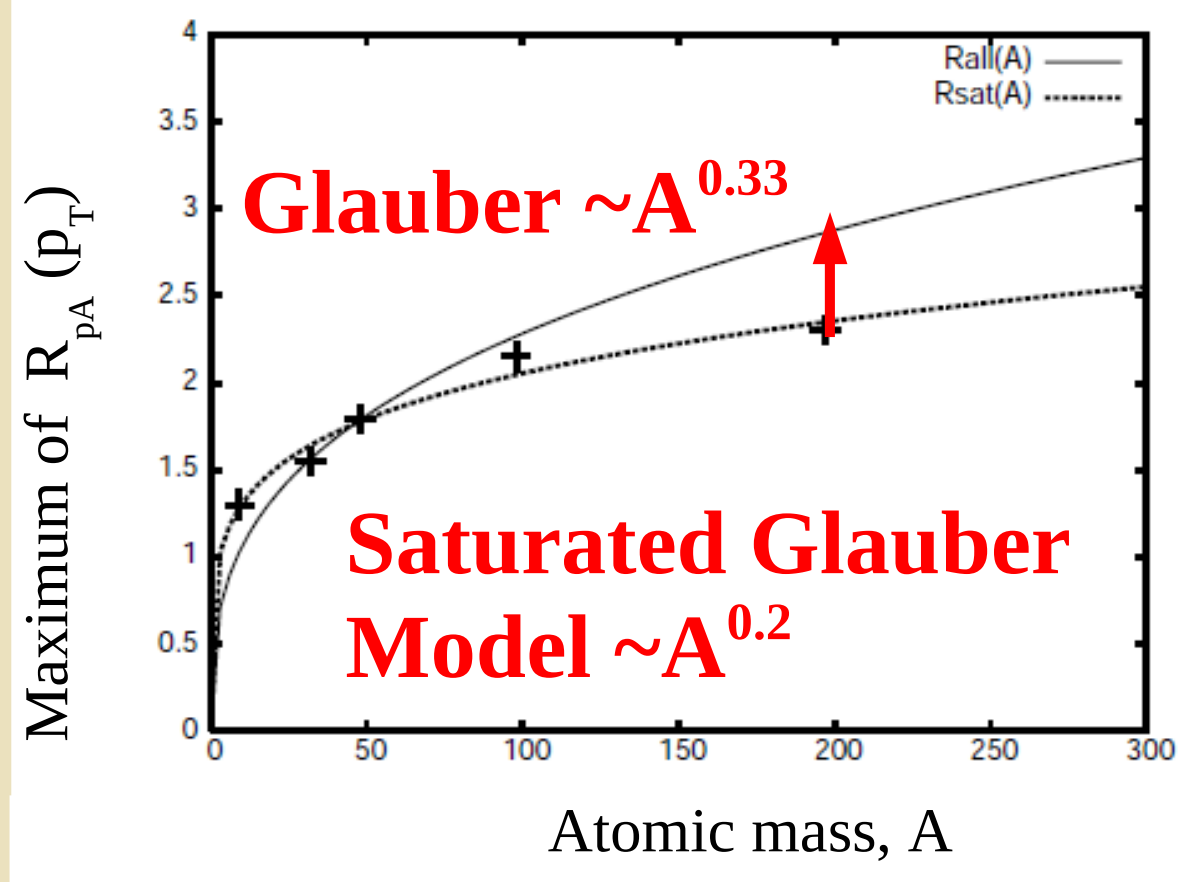

As it was in the beginning, for MB (see e.g. W. Busza's talk)

$$
\frac{\mathrm{d}^{3} \sigma_{p A}^{h} / \mathrm{d}^{3} p_{T}}{\mathrm{~d}^{3} \sigma_{p p}^{h} / \mathrm{d}^{3} p_{T}} \sim A^{\alpha\left(p_{T}\right)} \longrightarrow R_{p A}^{h} \sim A^{\alpha\left(p_{T}\right)-1} \quad 20 \% \text { effect for large } \mathbf{A}
$$

G. Fai, P. Lévai, G. Papp, PRC61 (2000) 021902,

GGB PhD Thesis, 2006 


\section{Suggestion for take away...}

It is high time to understand the Cronin effect!

- High precision experimental data are available!

- Handle with care

- Make consistent physical picture (10 AGeV to $10 \mathrm{ATeV}$ )

-We have tools to test it (energy, geometry, etc.)

- Universal description requires solid baseline :-)

- Suggest a pA study experiment....

- Can we test the Saturated Glauber picture?

- Test of geometry and scalings.

- Large rapidities (forward \& backward)

- Correlations 
B A C KU P 


\section{Rapidity asymmetry for $p B e$ at FNAL}

\section{Pion production with kTpQCD for pPb@ 5.02 ATeV $|n|$ [0.3:0.8]}

$$
Y_{\text {Asym }}^{h}\left(p_{T}\right)=\left.E_{h} \frac{\mathrm{d}^{3} \sigma_{A B}^{h}}{\mathrm{~d}^{3} p_{T}}\right|_{\eta<0} /\left.E_{h} \frac{\mathrm{d}^{3} \sigma_{A B}^{h}}{\mathrm{~d}^{3} p_{T}}\right|_{\eta>0}
$$
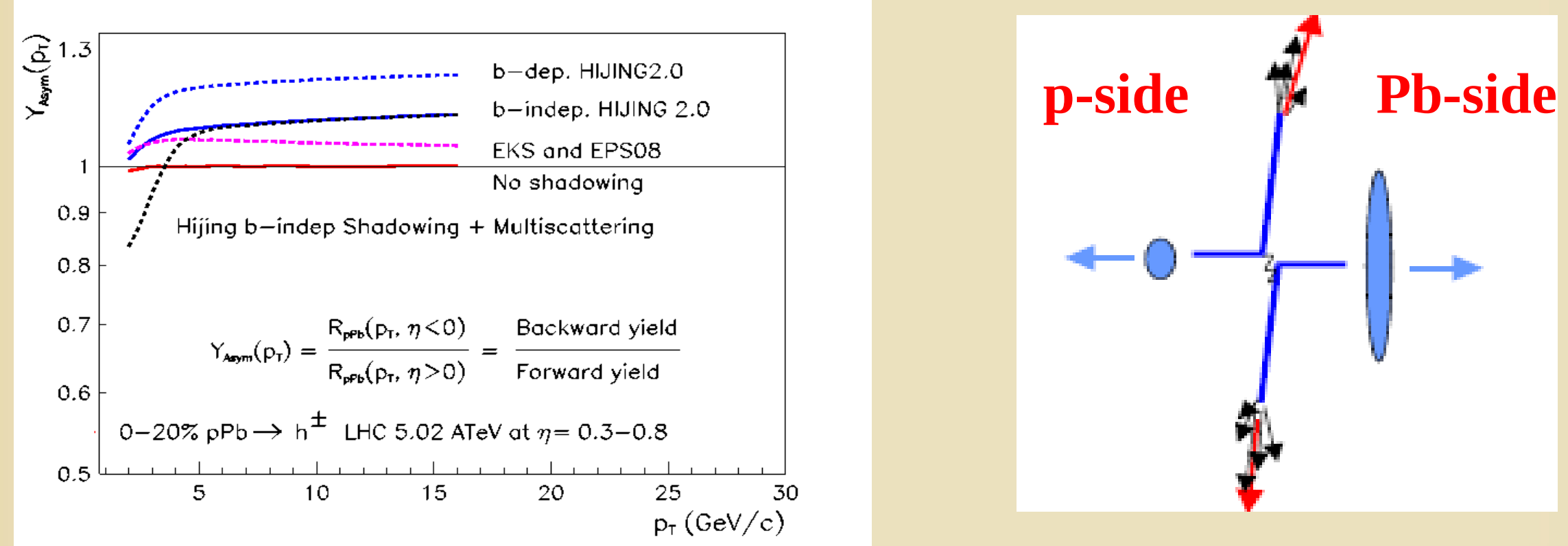

GGB, J. Barret, M. Gyulassy, P. Lévai, V. Topor Pop (in preparation 2012) 


\section{Rapidity asymmetry for $d P b$ at LHC}

\section{Pion production with kTpQCD @ 8.8 ATeV}

$$
Y_{\text {Asym }}^{h}\left(p_{T}\right)=\left.E_{h} \frac{\mathrm{d}^{3} \sigma_{A B}^{h}}{\mathrm{~d}^{3} p_{T}}\right|_{\eta<0} /\left.E_{h} \frac{\mathrm{d}^{3} \sigma_{A B}^{h}}{\mathrm{~d}^{3} p_{T}}\right|_{\eta>0}
$$
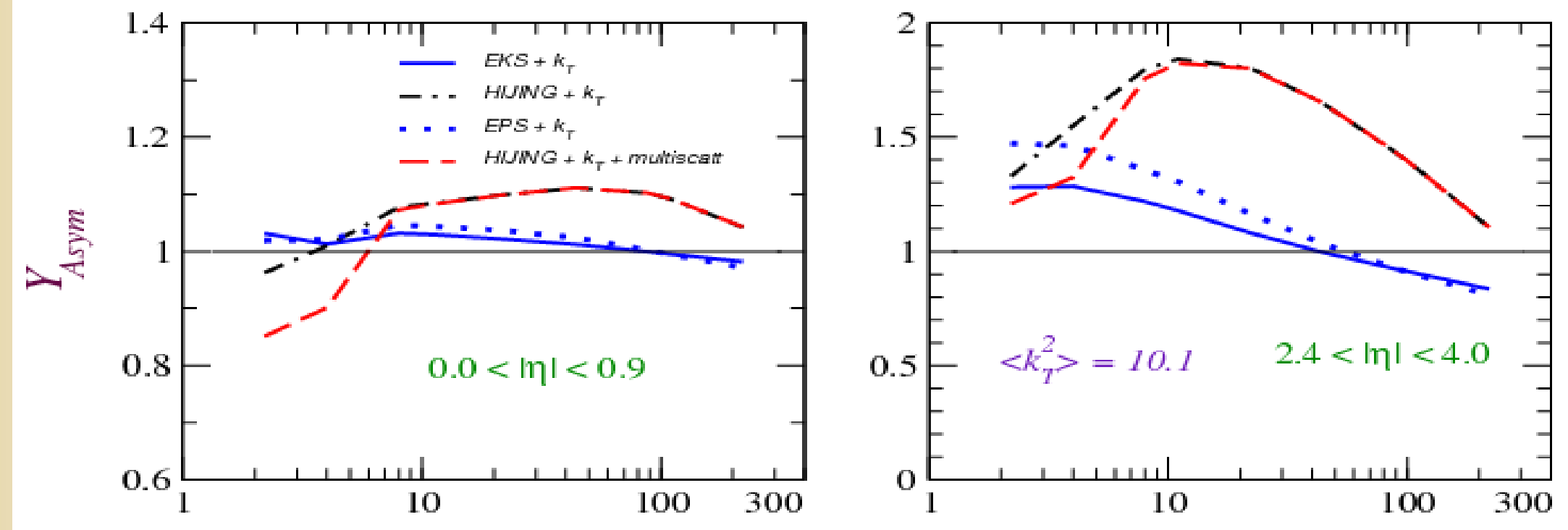

$p_{T}[\mathrm{GeV} / \mathrm{c}]$

A. Adeluy, GGB, G. Fai, P. Lévai, PRC80 (2009) 014903 


\section{Rapidity asymmetry for $d A u$ at RHIC}

\section{Pion production with kTpQCD @ 200 AGeV}

Rapidity asymmatry

$$
Y_{\text {Asym }}^{h}\left(p_{T}\right)=\left.E_{h} \frac{\mathrm{d}^{3} \sigma_{A B}^{h}}{\mathrm{~d}^{3} p_{T}}\right|_{\eta<0} /\left.E_{h} \frac{\mathrm{d}^{3} \sigma_{A B}^{h}}{\mathrm{~d}^{3} p_{T}}\right|_{\eta>0}
$$

\section{Relation to NMF}

$$
Y_{\text {Asym }}^{h}\left(p_{T}\right)=R_{\eta}^{h}\left(p_{T}\right)=\frac{R_{d A u}^{h}\left(p_{T}, \eta<0\right)}{R_{d A u}^{h}\left(p_{T}, \eta>0\right)}
$$

\section{X-scaling is OK,}

Multiscattering changes $Y(\eta)$

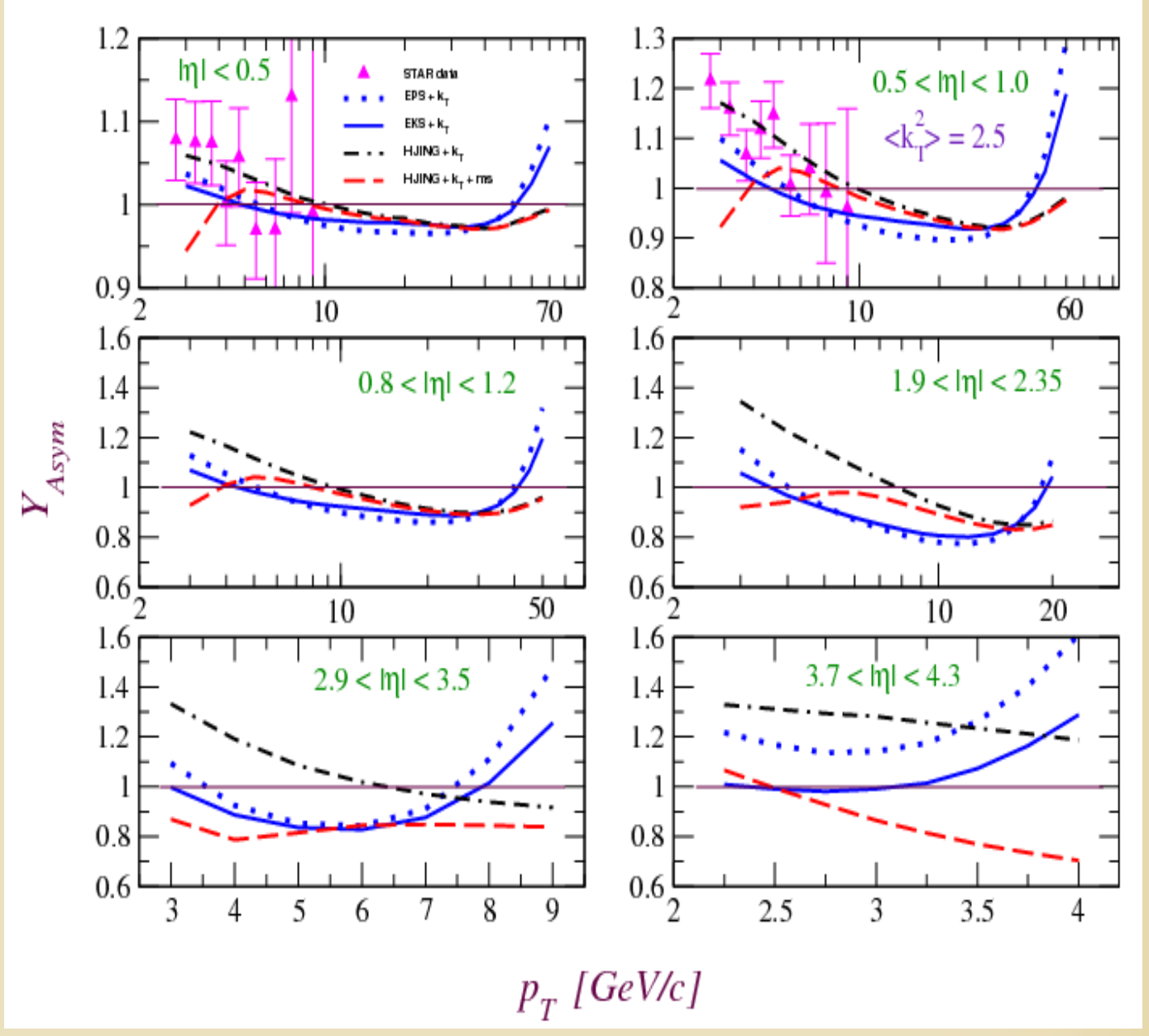

A. Adeluy, GGB, G. Fai, P. Lévai, PRC80 (2009) 014903 


\section{Rapidity asymmetry for $p B e$ at FNAL}

\section{Pion production with kTpQCD @ 30.7 GeV}

$$
Y_{\text {Asym }}^{h}\left(p_{T}\right)=\left.E_{h} \frac{\mathrm{d}^{3} \sigma_{A B}^{h}}{\mathrm{~d}^{3} p_{T}}\right|_{\eta<0} /\left.E_{h} \frac{\mathrm{d}^{3} \sigma_{A B}^{h}}{\mathrm{~d}^{3} p_{T}}\right|_{\eta>0}
$$
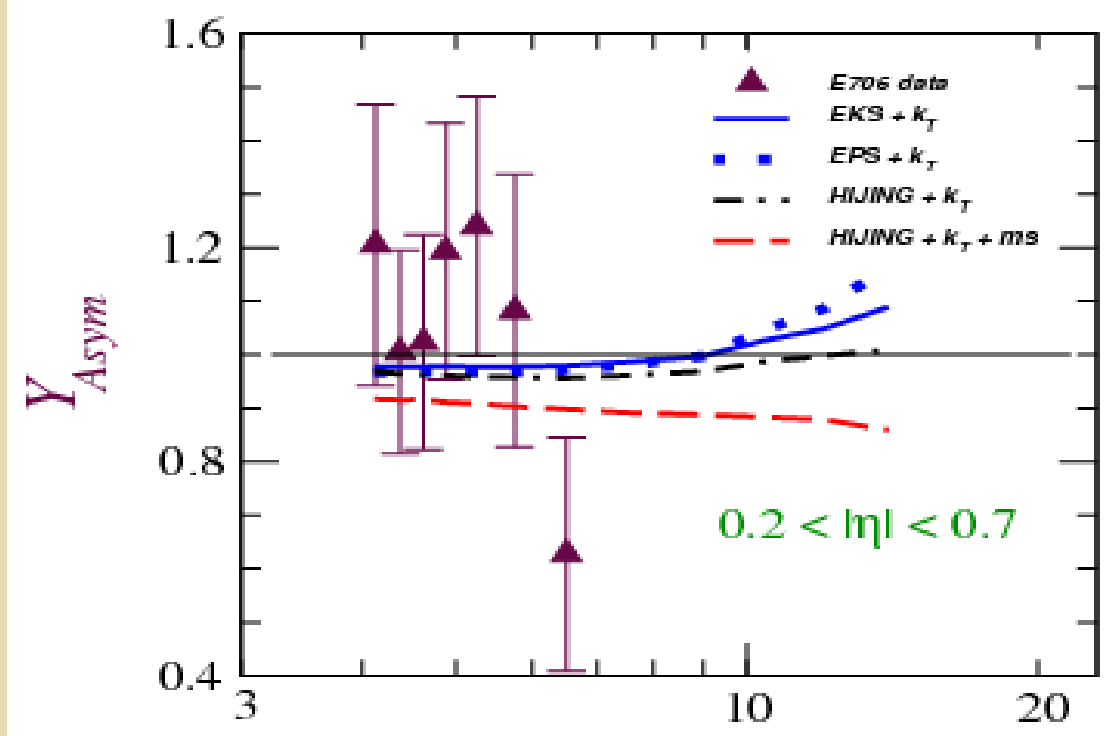

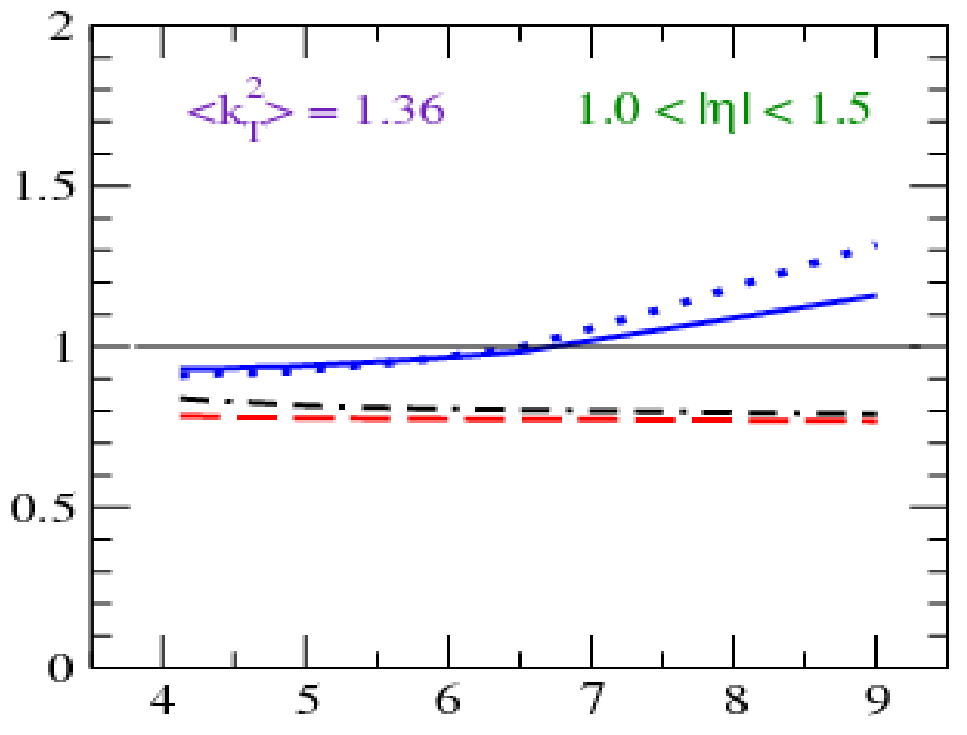

$p_{T}[\mathrm{GeV} / \mathrm{c}]$

A. Adeluy, GGB, G. Fai, P. Lévai, PRC80 (2009) 014903 


\section{Midrapidity $R_{d A}\left(x_{T}\right)$ for $|\eta|<0.35$}

Pion production with HIJING shadowing kTpQCD @ $0.2 \& 8.8 \mathrm{ATeV}$

HIJING Shadowing

x-scaling

DGLAP evolution

Need for additional multiple scattering

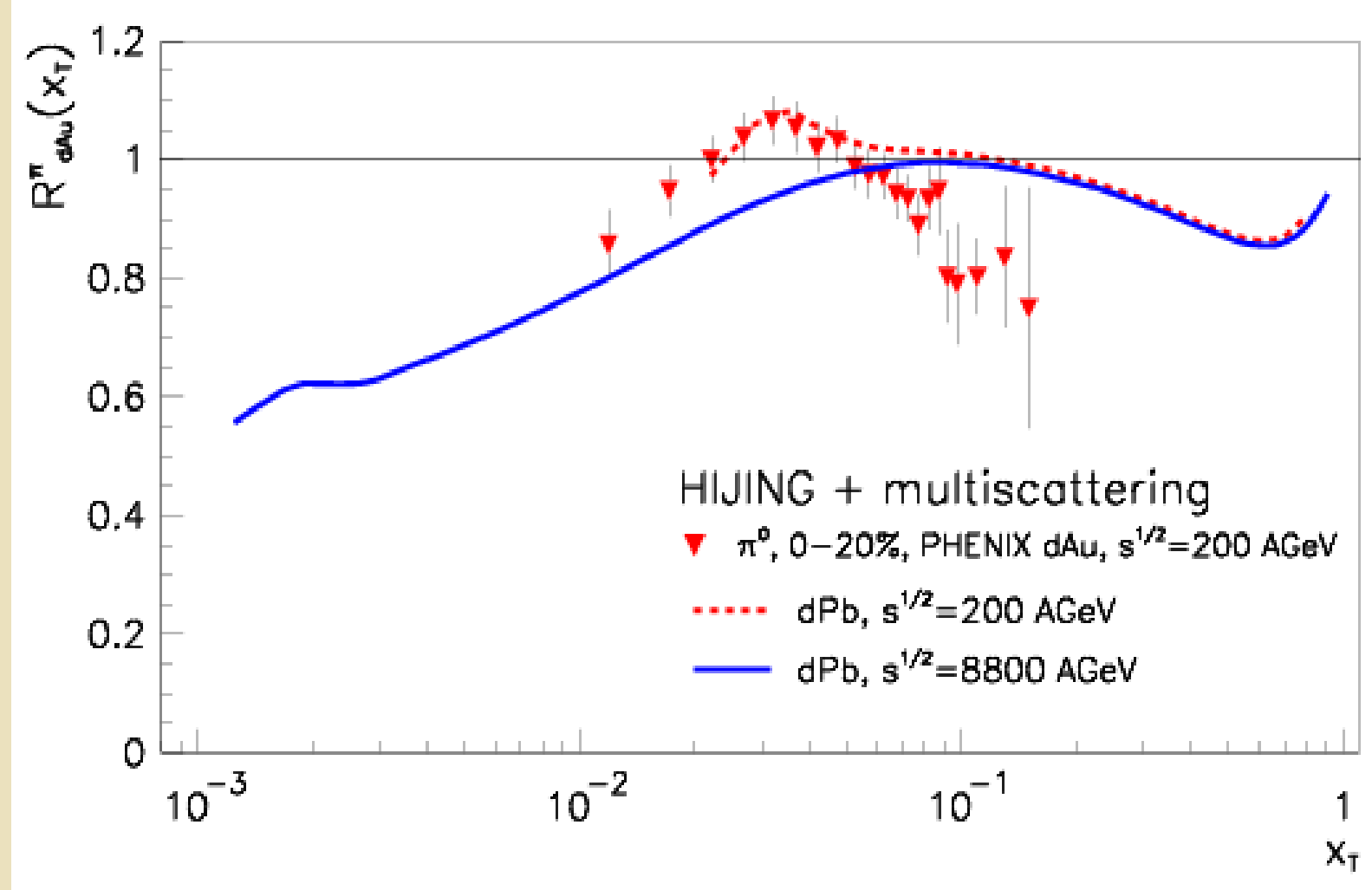

GGB, G. Fai, P. Lévai, BA Cole, G. Papp, Indian J.Phys. 84 (2010) 1721-1725 


\section{Midrapidity $R_{d A}\left(x_{T}\right)$ for $|\eta|<0.35$}

Pion production with HKN shadowing kTpQCD @ $0.2 \& 8.8$ ATeV

HKN Shadowing

x-scaling

DGLAP evolution

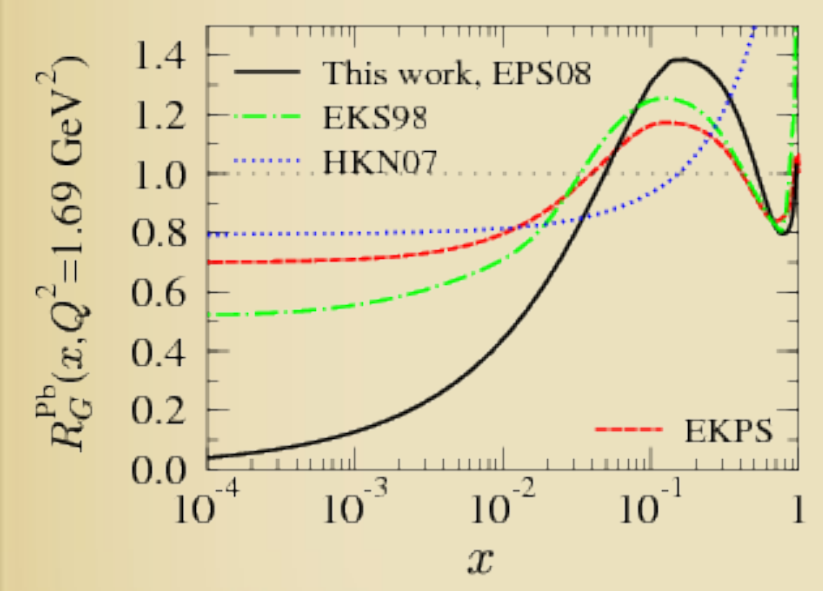

BA Cole, GGB, G. Fai, P. Lévai, G. Papp, arXiv:08073384 (2007)

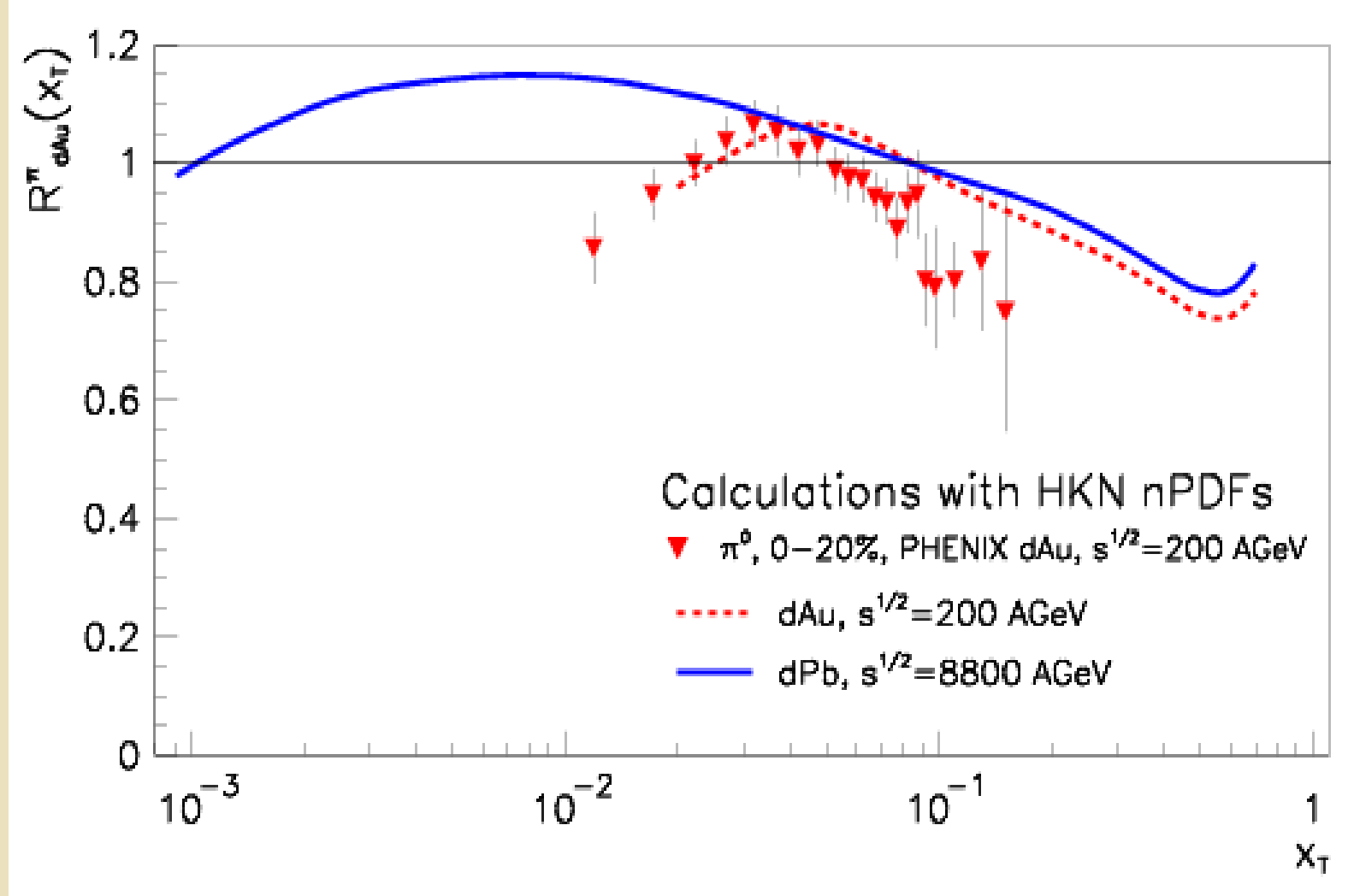

1
$X_{T}$ 


\section{The Nuclear Modification Factor, $\mathrm{R}$}

- Measuring nuclear effects 'precisely'

ratio of the

$$
R_{d \mathrm{Au}} \equiv \frac{1}{\left\langle N_{\text {coll }}\right\rangle} \frac{d^{2} N^{d+\mathrm{Au}} / d p_{T} d \eta}{d^{2} N_{\text {inel }}^{p+p} / d p_{T} d \eta} .
$$

hadron spectra

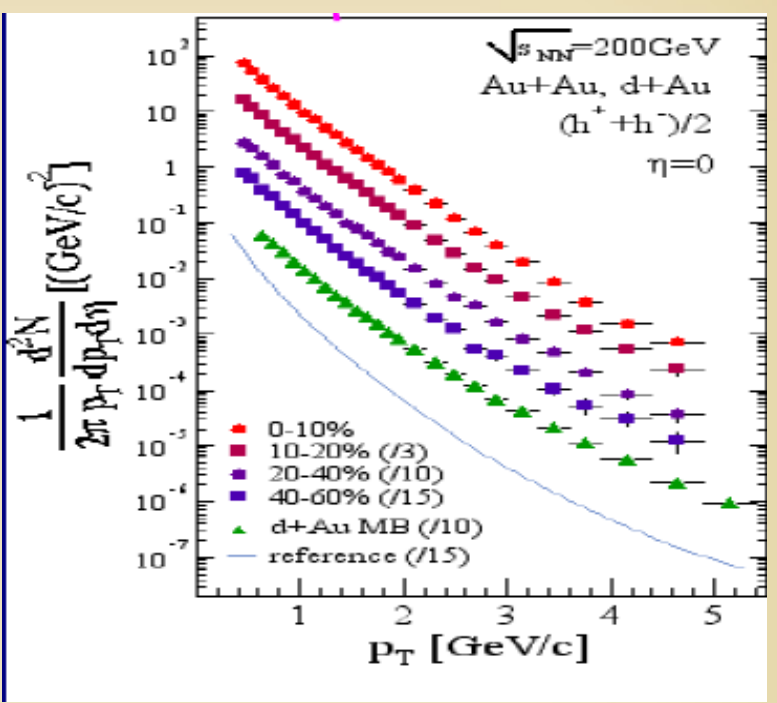

- Collisions:

$$
R_{\mathrm{AA}}\left(p_{\mathrm{T}}\right)=\frac{d^{2} N_{\mathrm{ch}}^{\mathrm{AA}} / d p_{\mathrm{T}} d \eta}{\left\langle T_{\mathrm{AA}}\right\rangle d^{2} \sigma_{\mathrm{ch}}^{\mathrm{NN}} / d p_{\mathrm{T}} d \eta}
$$

\section{proton-nucleus (pA)}

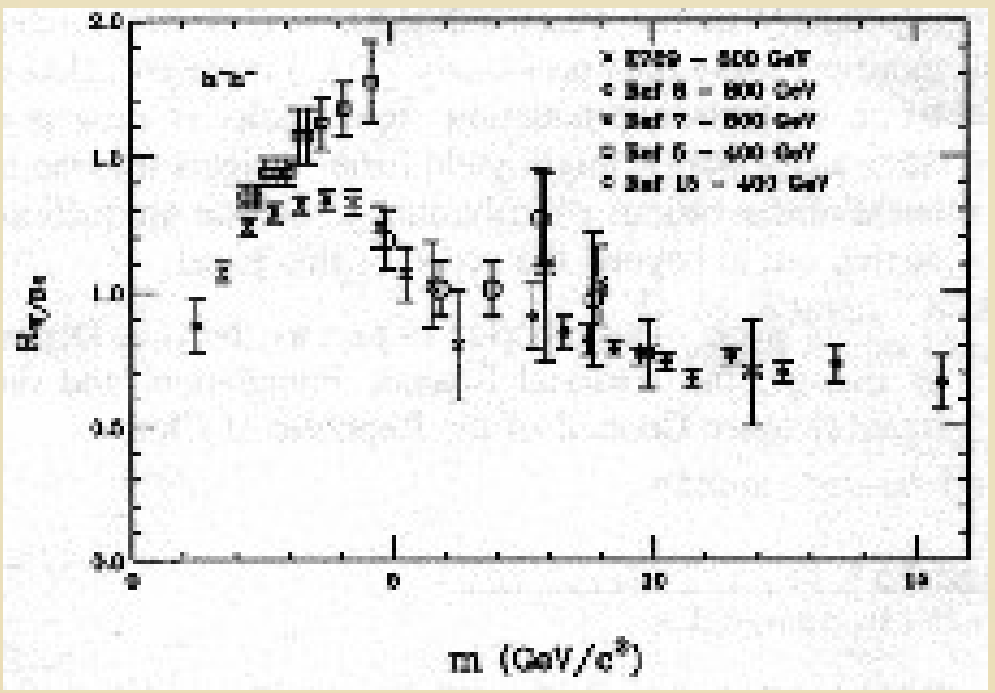

or nucleus-nucleus (AA, $A A^{\prime}$ )

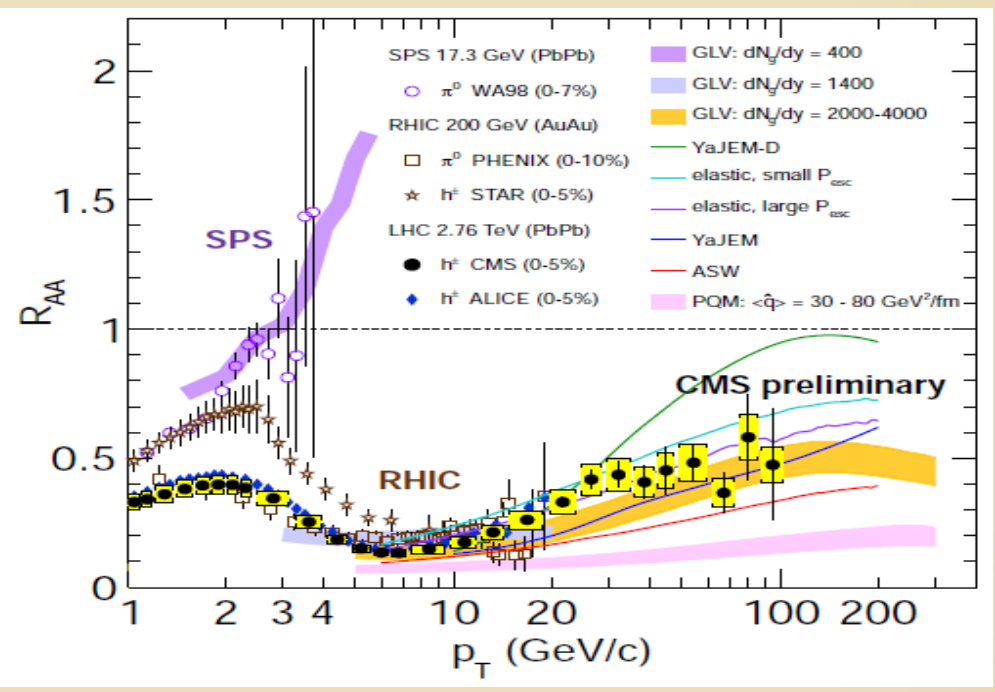




\section{Midrapidity $R_{d A}\left(p_{T}\right)$ fot LHC - Summary}

Extreme high- $\mathrm{p}_{\mathrm{T}}$ Pion production kTpQCD @ 0.2, 0.9, \& 8.8 ATeV
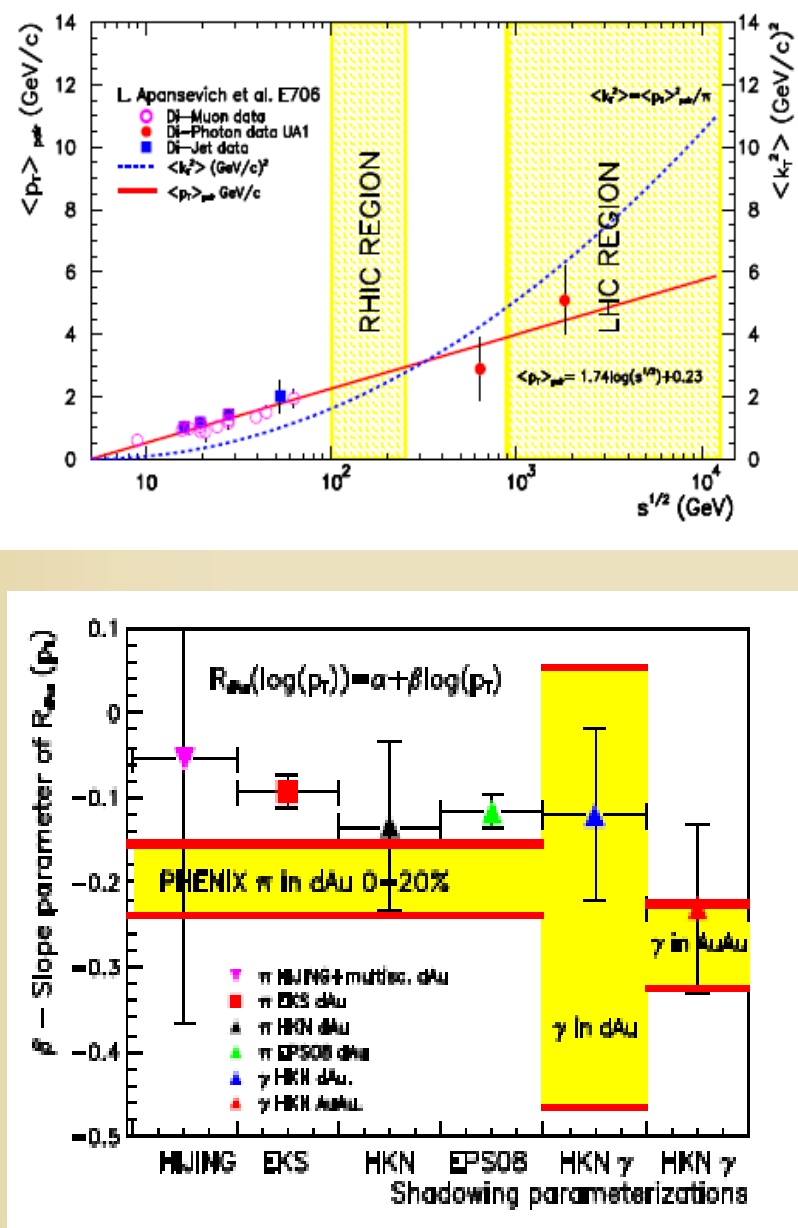

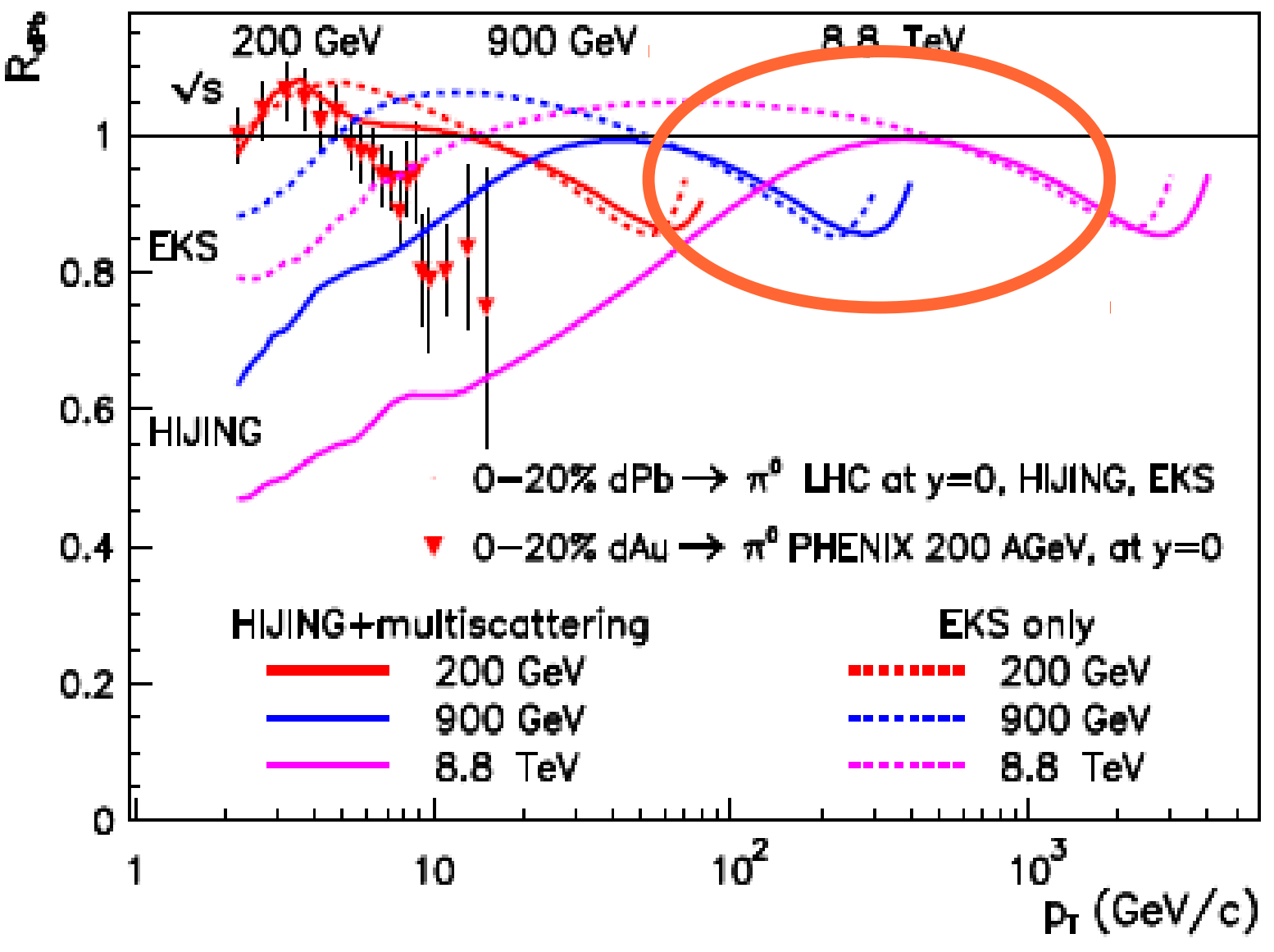

BA Cole, GGB, G. Fai, P. Lévai, G. Papp, arXiv:08073384 (2007) 


\section{MOTIVATION}

\section{- Motivation for $p P b / d A u$ collisions}

\section{Preliminary dAu data from PHENIX@QM12 (B. Sahlmueller)}
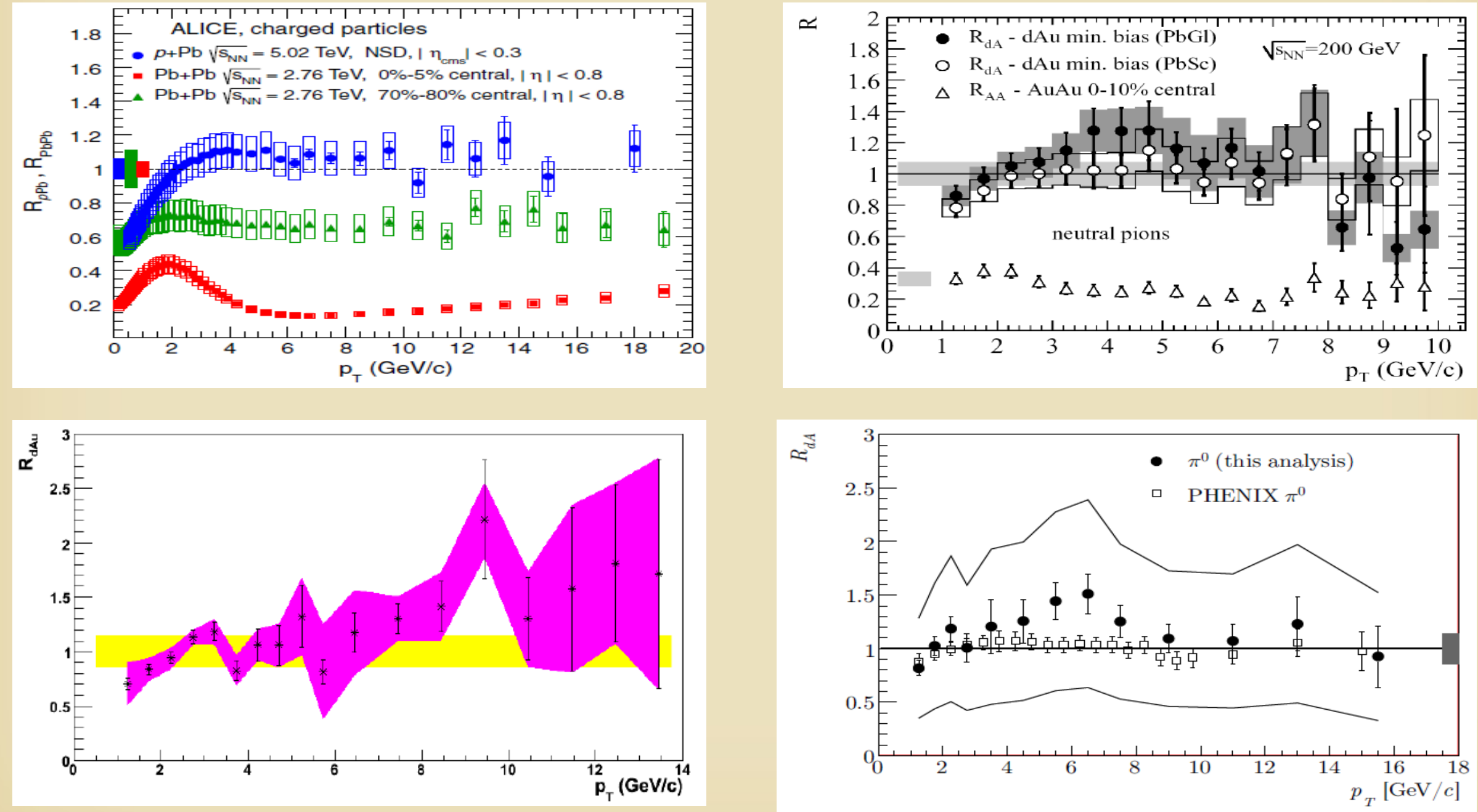

G.G. Barnaföldi: Cronin \& Shadowing in pA 


\section{HIJINGB/B 2.0: Rapidity distribution for $p p \& p P b$}

Charged hadron production with HIJING 2.0 @ 5.02 ATeV MinBias
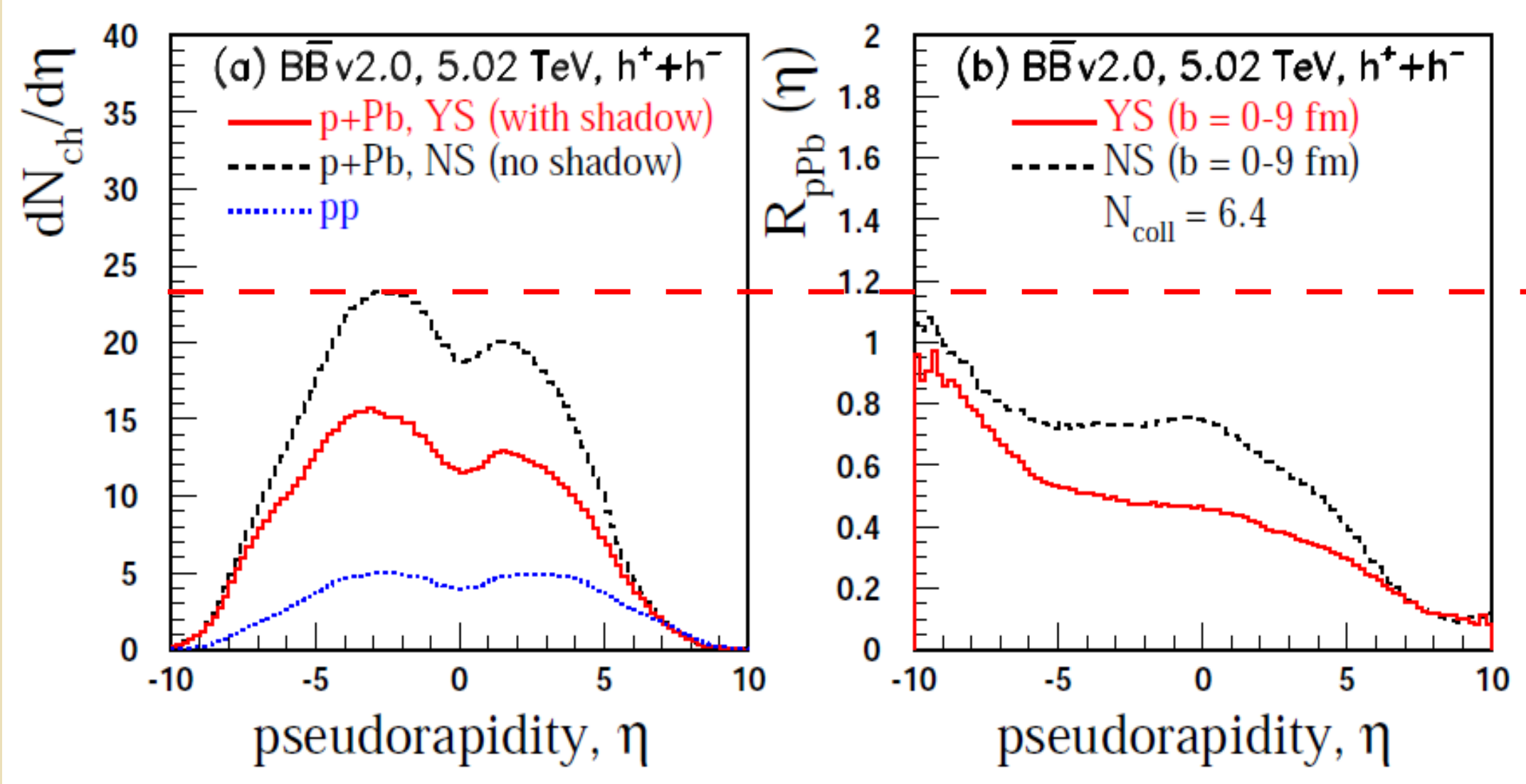

GGB, J. Barret, M. Gyulassy, P. Lévai, V. Topor Pop (in preparation 2012) 


\section{The Collection of $\mathrm{pA} / \mathrm{dAu}$ Data}

\begin{tabular}{|l||c|c|c|c|c|c|}
\hline \hline CP $|23,24|$ & $p$ & $d, B e, T i, W$ & 19,$4 ; 23,7 ; 27,4$ & $\approx 0$ & {$[0,77 ; 6,91]$} & $\pi^{ \pm}, K^{ \pm}, p^{ \pm}, d^{ \pm}$ \\
\hline ITA $|115|$ & $p$ & $C, W$ & $p_{\text {inc }}=50-275$ & {$[0,7 ; 1,0]$} & {$[0,2 ; 2,35]$} & $\pi^{ \pm}, K^{ \pm}, p^{ \pm}$ \\
\hline FNAL $|119|$ & $n$ & $B e, A l, C, S n, P b$ & 27,4 & {$[4,0 ; 8,0]$} & {$[0,1 ; 1,7]$} & $h^{ \pm}, \pi^{+}, p$ \\
\hline FNAL $|116|$ & $p$ & $B e, W$ & 19,$4 ; 23,7 ; 27,4$ & $\approx 0$ & {$[0,2 ; 4,5]$} & $\pi^{ \pm}, K^{ \pm}, p^{ \pm}, h^{ \pm}$ \\
\hline CP $|118|$ & $\pi^{-}$ & $p, B e, C u, W$ & 19,$4 ; 23,7$ & $\approx 0$ & {$[0,8 ; 5,78]$} & $\pi^{ \pm}, K^{ \pm}, p^{ \pm}$ \\
\hline E577/E672 $|117|$ & $p$ & $p, B e, C, A l, C u, P b$ & 38,8 & {$[-0,75 ; 0,75]$} & {$[0,6 ; 11,5]$} & $h^{ \pm}$ \\
\hline E605/E789 $|114|$ & $p$ & $B e, W$ & 38,8 & $\approx 0$ & {$[0,5 ; 11,5]$} & $h^{ \pm}$ \\
\hline E605 $|69,70|$ & $p$ & $d, B e, W$ & 38,8 & $\approx 0$ & {$[0,5 ; 11,0]$} & $\pi^{ \pm}, K^{ \pm}, p^{ \pm}$ \\
\hline E706 $|59|$ & $p$ & $B e$ & 31,$6 ; 38,8$ & {$[-0,75 ; 0,75]$} & {$[1,0 ; 12,0]$} & $\pi^{0}, \eta$ \\
\hline E706 $|113|$ & $p, \pi^{-}$ & $B e, C u$ & 30,7 & {$[-0,7 ; 0,7]$} & {$[3,5 ; 10,0]$} & $\pi^{0}, \gamma$ \\
\hline WA80 $|144|$ & $S$ & $S, A u$ & 19,4 & {$[2,1 ; 2,9]$} & {$[0,3 ; 3,9]$} & $\pi^{0}, \gamma$ \\
\hline WA98 $|140|$ & $P b$ & $P b, N b$ & 17,3 & {$[2,3 ; 4,4]$} & {$[0,3 ; 3,7]$} & $\pi^{0}, \gamma$ \\
\hline CERES $|126|$ & $P b$ & $A u$ & 17,3 & {$[2,1 ; 2,6]$} & {$[1,5 ; 3,3]$} & $\pi^{ \pm}$ \\
\hline PHENIX $|122,123|$ & $p, A u$ & $p, d, A u$ & 130,200 & $|\eta| \leq 0,35 ; 2,0$ & {$[0,5 ; 10,0]$} & $\pi^{0}, h^{ \pm}, \gamma$ \\
\hline BRAHMS $|127,128|$ & $p, A u$ & $p, d, A u$ & 130,200 & 0,$0 ; 1,0 ; 2,2 ; 3,2$ & {$[0,5 ; 6,0]$} & $\pi^{0}, h^{ \pm}$ \\
\hline STAR $|129,130|$ & $p, A u$ & $p, d, A u$ & 130,200 & $|\eta| \leq 0,5 ; 2,0$ & {$[0,2 ; 5,5]$} & $\pi^{0}, h^{ \pm}, \Lambda, K, \gamma$ \\
\hline PHOBOS $|132,133|$ & $p, A u$ & $p, d, A u$ & 62,$4 ; 130 ; 200$ & 0,$4 ; 0,8 ; 1,2$ & {$[0,5 ; 3,5]$} & $K^{ \pm}, p^{ \pm} \pi^{0}, h^{ \pm}$ \\
\hline
\end{tabular}




\section{Raa and High pT azimuthal anisotropy}

\section{(in light of recent $\mathrm{p}+\mathrm{A}$ and $\mathrm{D}+\mathrm{A}($ un)Control)}

Mklz Gyulassy, alias Barbara Betz

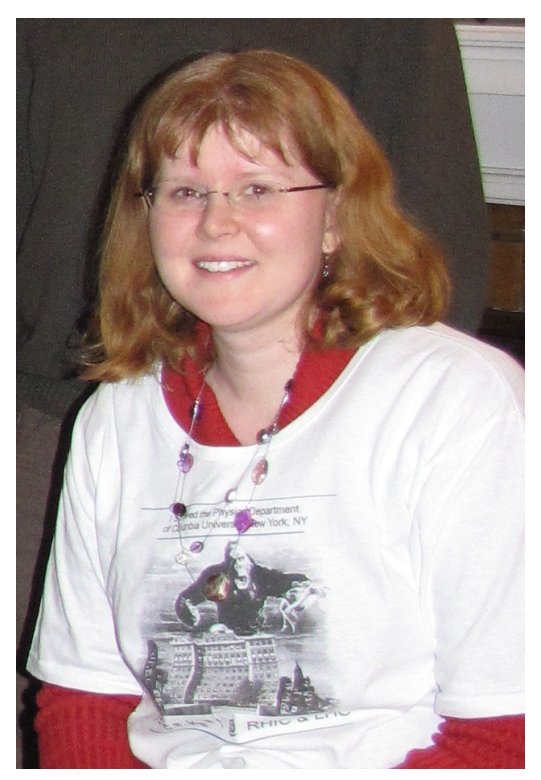

High pT nuclear modification of jets in and out of reaction plane at RHIC and LHC B.Betz, MG, in preparation

Examining a reduced jet-medium coupling in $\mathrm{Pb}+\mathrm{Pb}$ collisions at the $\mathrm{LHC}$, B.Betz, MG PRC86 (2012)

Fourier Harmonics of High-pT Particles Probing the Fluctuating Intitial Condition Geometries in Heavy-Ion Collisions, B.Betz, MG, G.Torrieri, PRC84 (2011) 
Part 1: Jet Quenching prior to 2012

Part 2: D+Au and p+Pb Shock Waves QM12 and RBRC13

Part 3: Azimuthal Tomography (ignoring part 2) 


\section{High- $p_{T}$ Tomography of $d+$ Au and Au + Au at SPS, RHIC, and LHC}

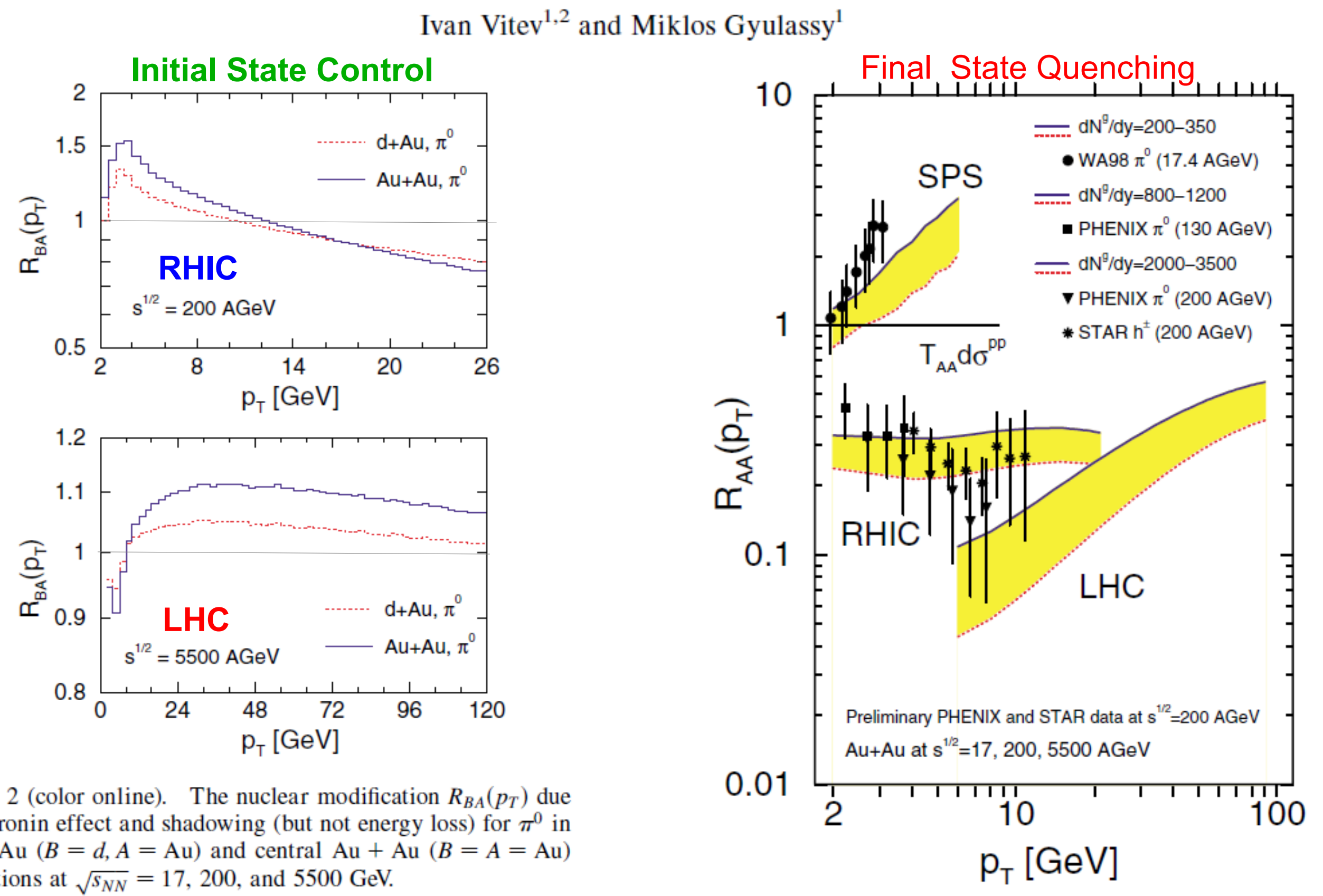

FIG. 2 (color online). The nuclear modification $R_{B A}\left(p_{T}\right)$ due to Cronin effect and shadowing (but not energy loss) for $\pi^{0}$ in $d+\mathrm{Au}(B=d, A=\mathrm{Au})$ and central $\mathrm{Au}+\mathrm{Au}(B=A=\mathrm{Au})$ $\mathrm{M}$ reactions at $\sqrt{s_{N N}}=17,200$, and $5500 \mathrm{GeV}$. 


\section{In the beginning... Gabor David RBRC13}

STAR, PRL 91 (2003) 072304

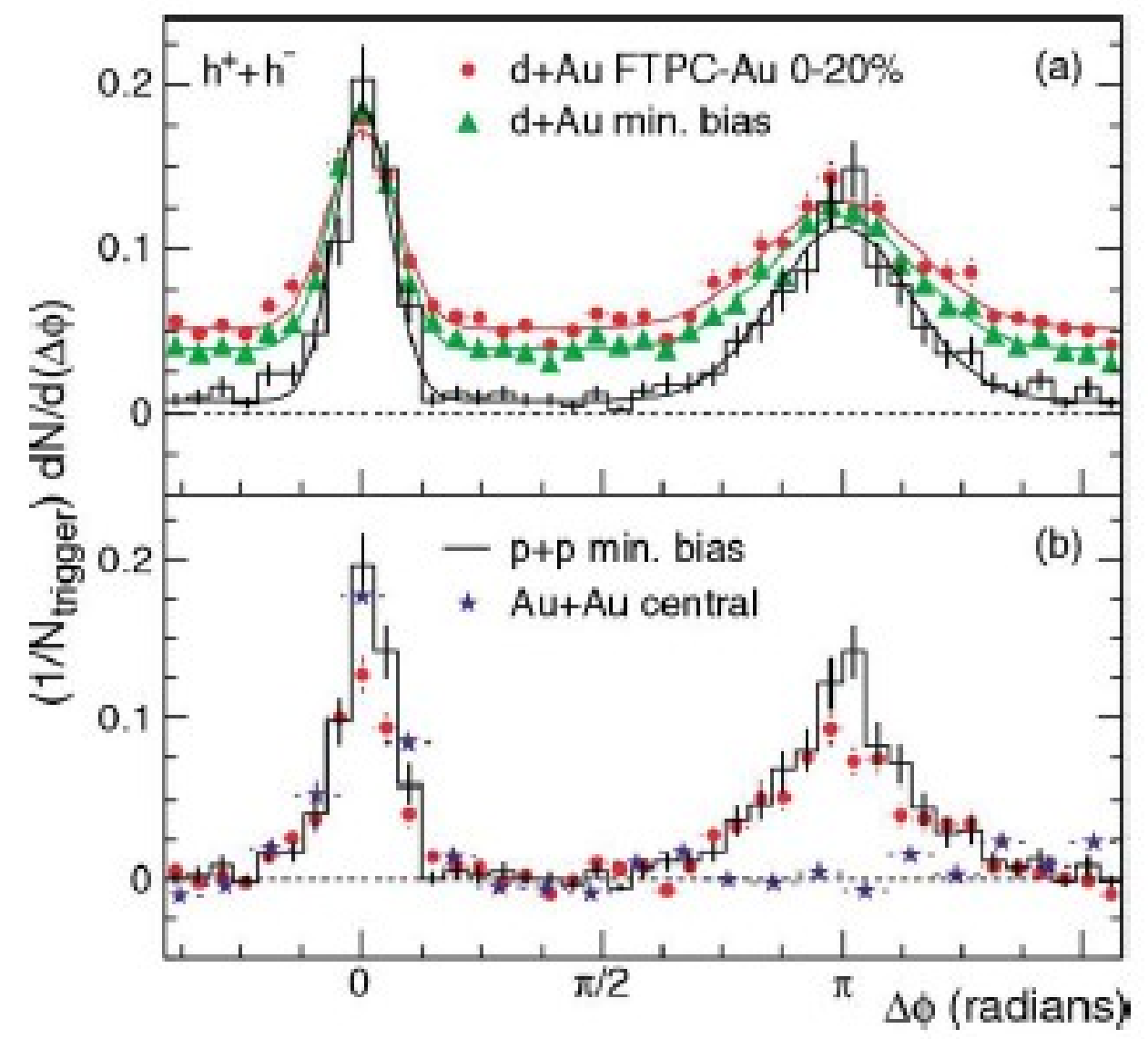

STAR: back-to-back jets reappear in $d+A u$ $\mathrm{PHENIX}$ : large suppression in $\mathrm{Au}+\mathrm{Au}$, no suppression in $\mathrm{d}+\mathrm{Au}$

$\rightarrow$ final state effect (as of 2003)
PHENIX, PRL 91 (2003) 072303

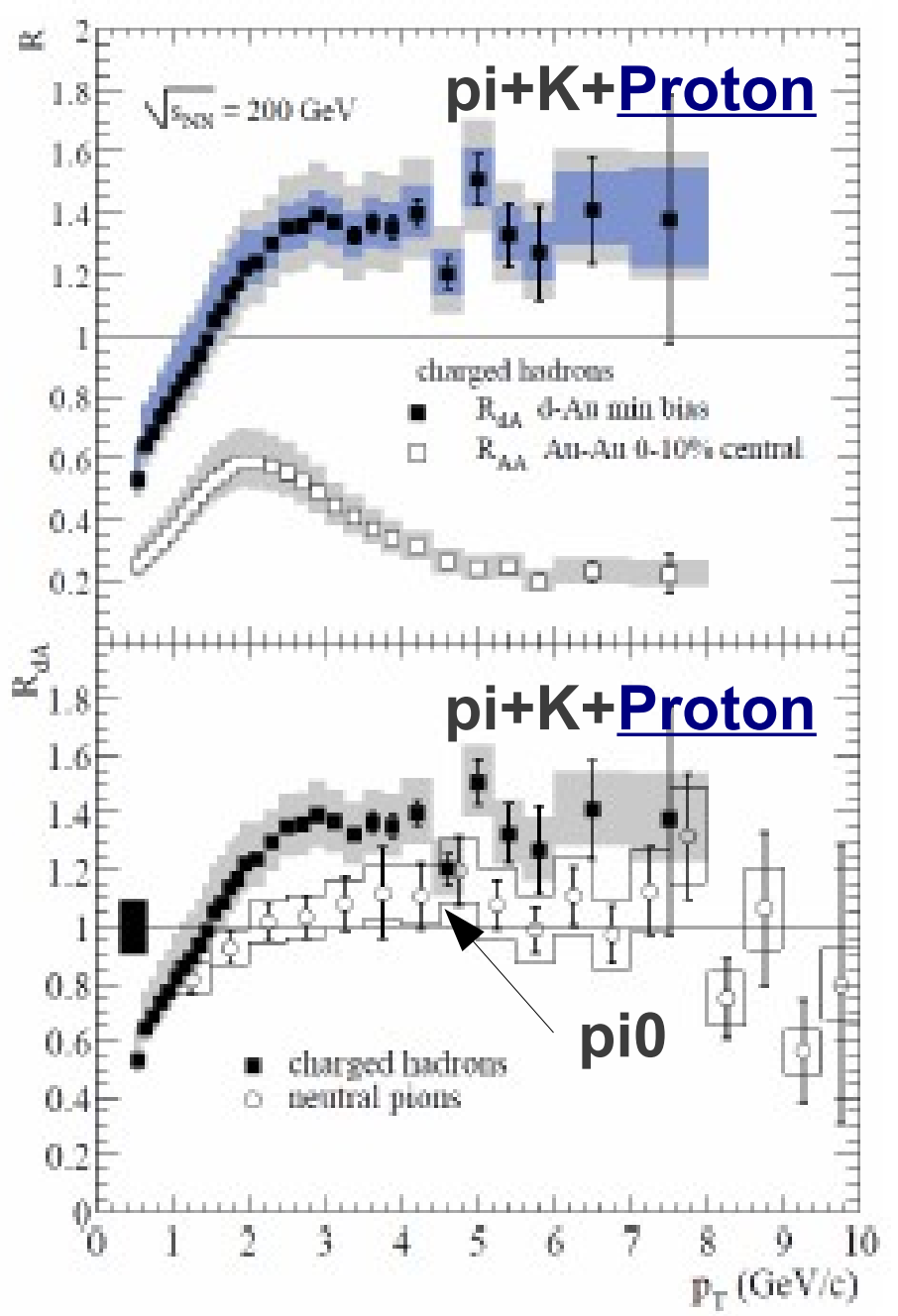


Gabor David RBRC13

\section{Null-Control $D+A u \rightarrow$ Direct Photon $\mathbf{N}_{\text {coll }}$ scaling works!}

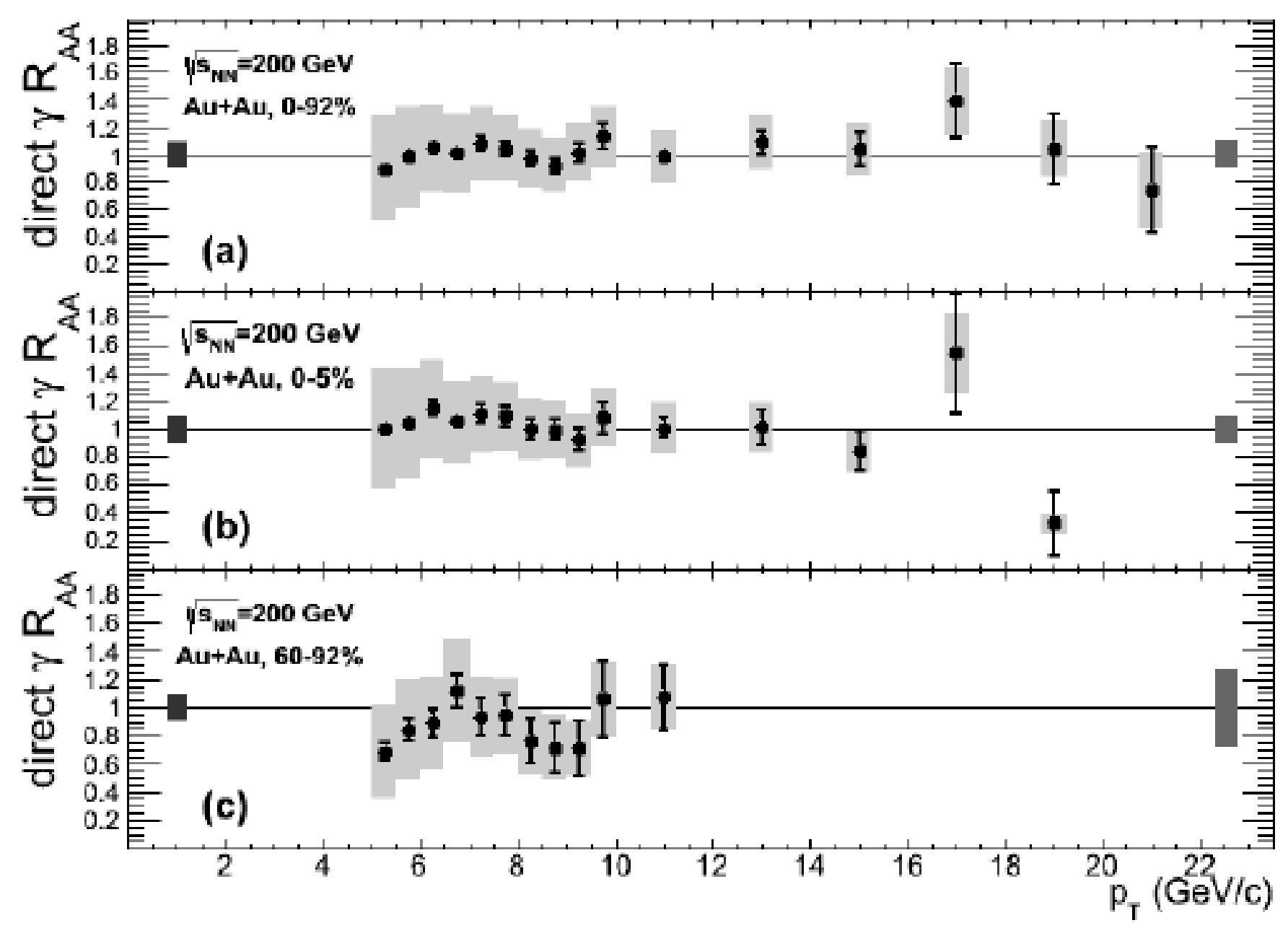


High- $p_{T}$ Tomography of $d+$ Au and Au + Au at SPS, RHIC, and LHC Ivan Vitev $^{1,2}$ and Miklos Gyulassy ${ }^{1}$

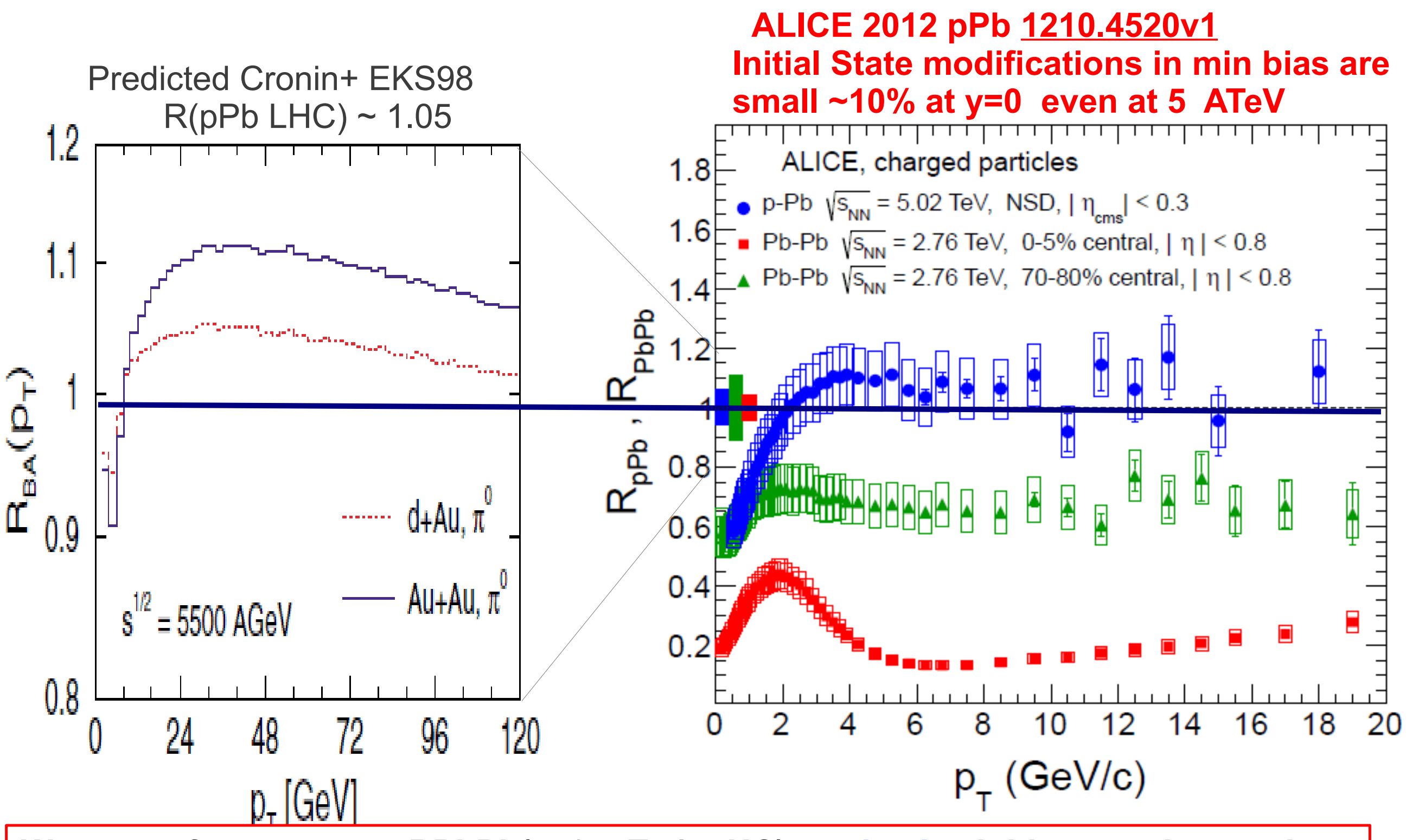

We can safely calculate $\mathrm{RPbPb}(\mathrm{y}=0, \mathrm{pT}>4, \mathrm{LHC})$ neglecting initial state interactions 


\section{$\mathbf{N}_{\text {part }}$ scaling for asymmetric collisions:}

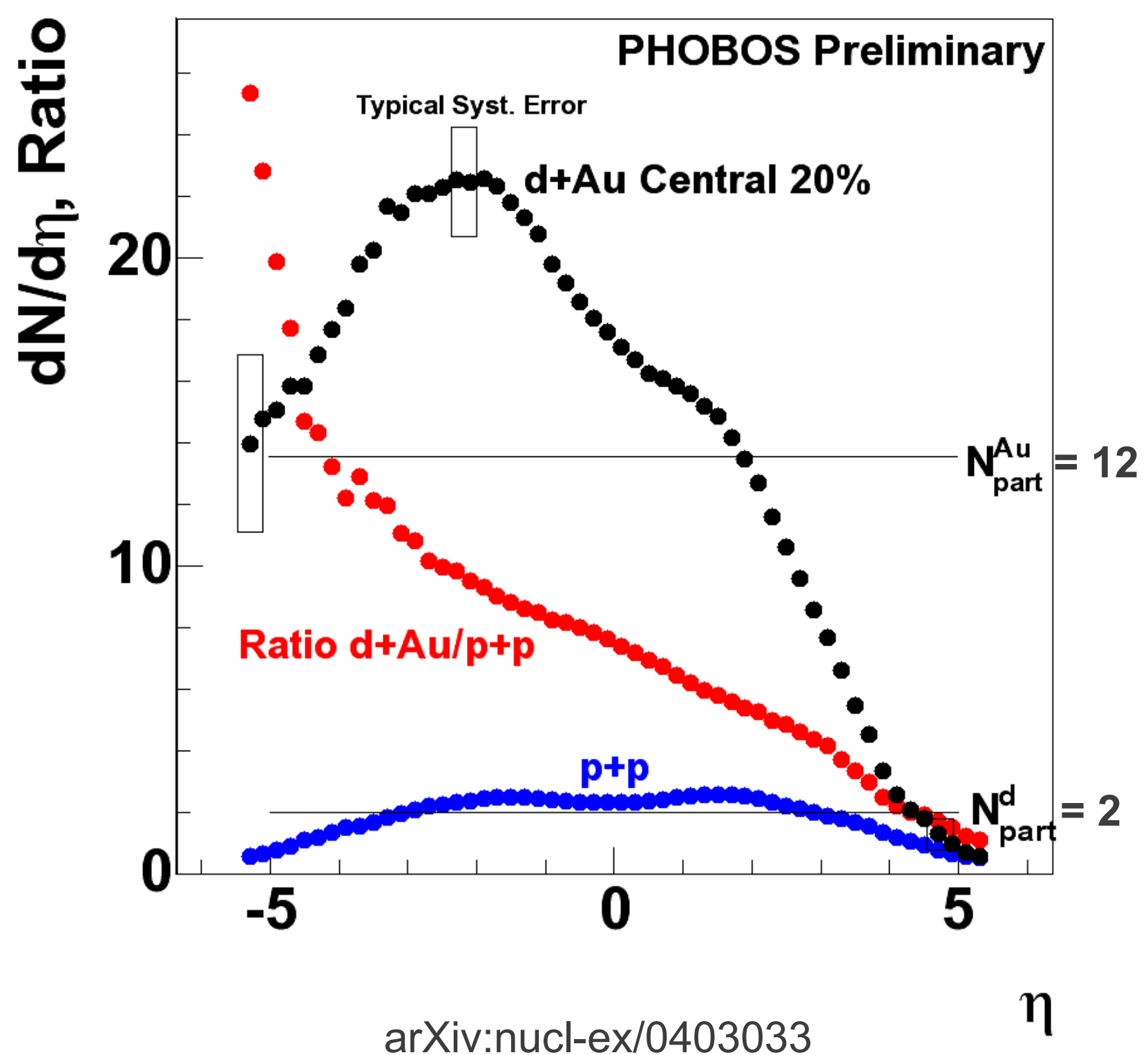




\section{Busza $p+A / p+p d N d y$ Triangle well described at RHIC via HIJING}

- Lund model beam jet flux tubes (as encoded in HIJING) account well for RHIC data and reproduced the basic BGK77 triangle form of the ratio $p+A / p+p, \quad$ as well as the absolute magnitudes dNch/deta

- Except in target frag region $\mathrm{y}<-3$ where $\mathrm{FSI}$ enhance nch yields
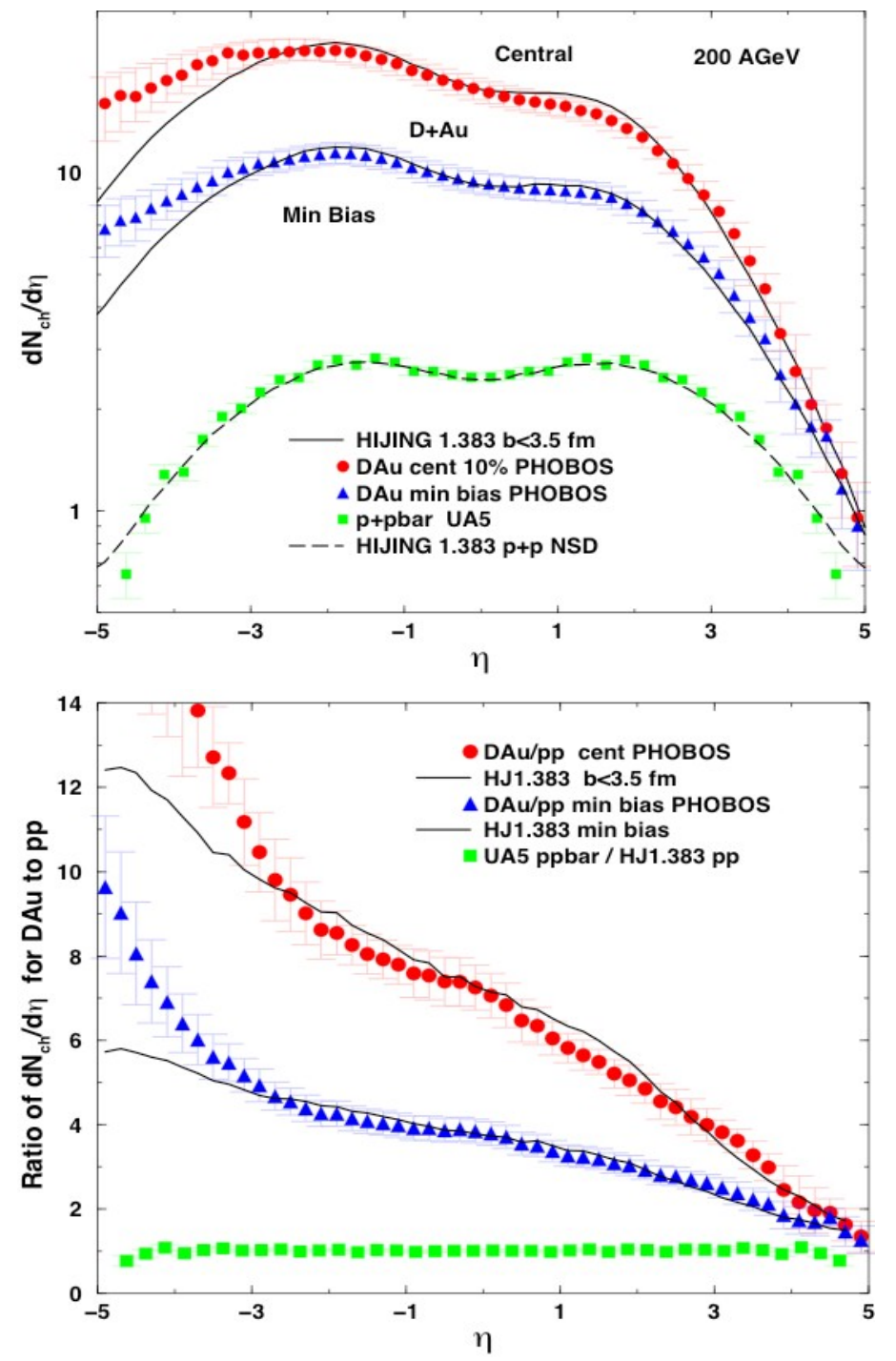
In 2003 Three Lines of Data Seemed to Converged to QGP at RHIC

\section{Null Control}

Three Legs Stable even if unequal

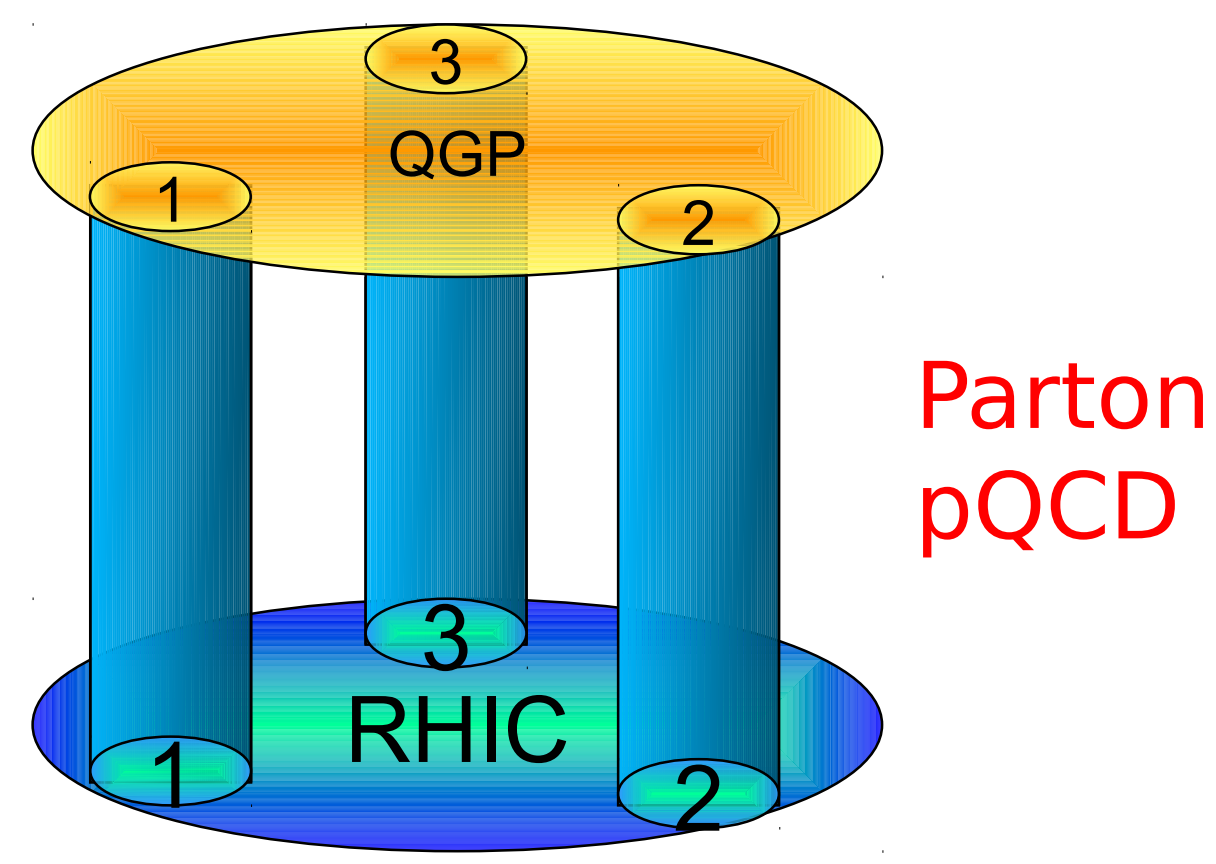

1. Bulk $P_{\mathrm{QCD}}$ Collective Elliptic Flow v2

2. Parton $\mathrm{pQCD}$ Jet Quenching RAA

3. $p+p$ Calibration and $\underline{d+A}$ Null Control

$Q G P=P_{Q C D}+p Q C D+d A=v_{2}+\left(R_{A A}+I_{A A}\right)+R_{d A}$ 
Part 1: Jet Quenching prior to 2012

Part 2: $\mathrm{D}+\mathrm{Au}$ and $\mathrm{p}+\mathrm{Pb}$ Shock Waves $\mathrm{QM12}$ and RBRC13

Part 3: Azimuthal Tomography (ignoring part 2) 


\section{My Summary of QM12}

M Gyulassy RBRC 4

Mklaz

\section{Energy Scan}

\section{$\mathrm{D}+\mathrm{Au}$}




\section{$\underline{\ln }$ \\ I \\ QM12 PHENIX story: (same as STAR BES story) \\ Ecm dependence of $\mathrm{vn}\{\Psi \mathrm{n}\}$ for charged hadrons}

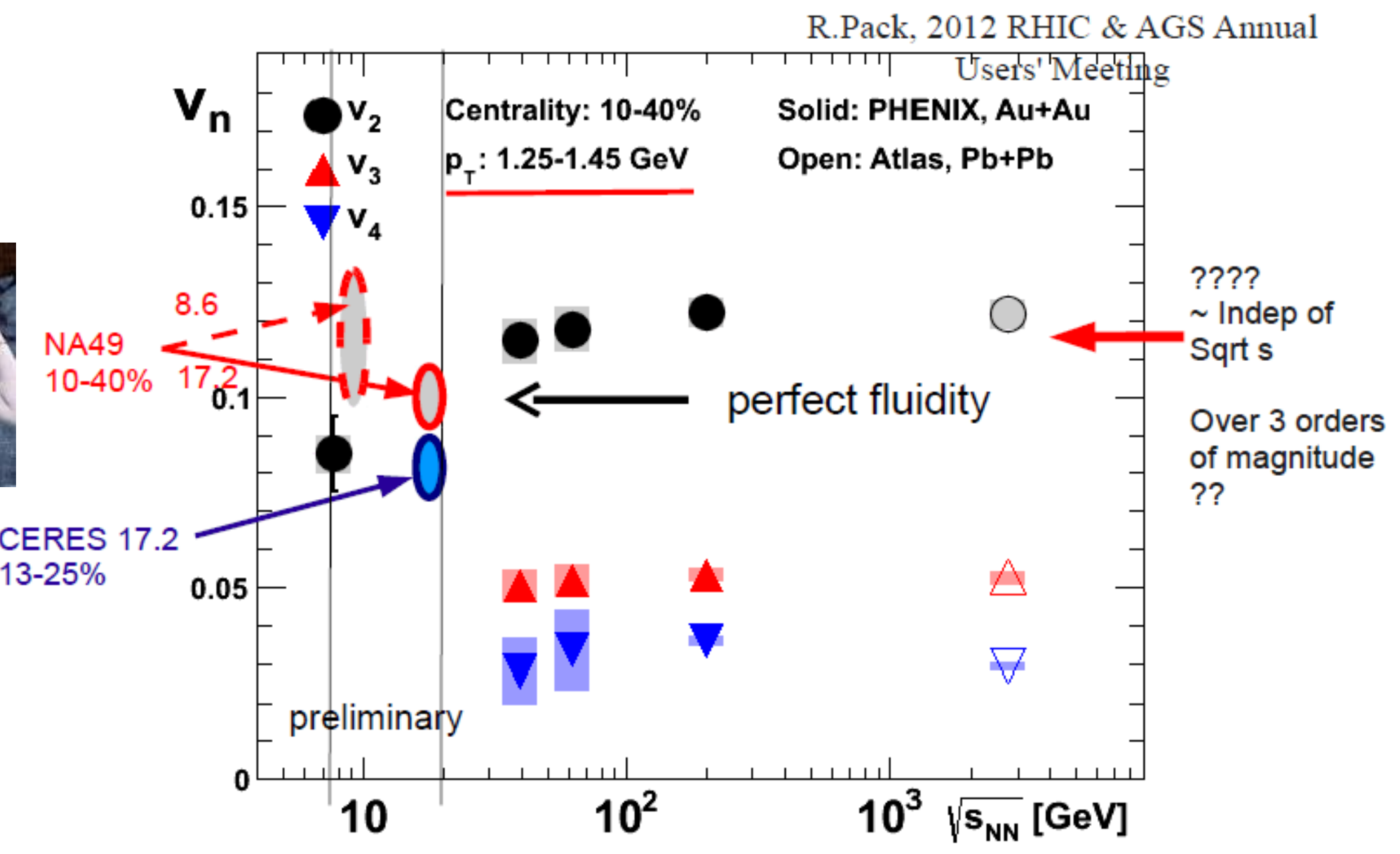

The (Bevalac, AGS, SPS, RHIC 200) Perfect Fluid QGP Core + Lousy HRG Corona Was Crushed: vn(pt) shows no sign of LOUSY HRG Corona down to AGS!!! 


\section{more}

\section{Now sometresults to lose sleep over}

\section{PHENIX preliminary, QM'12}

2008 (high) statistics d+Au data, nuclear modification factors vs centrality
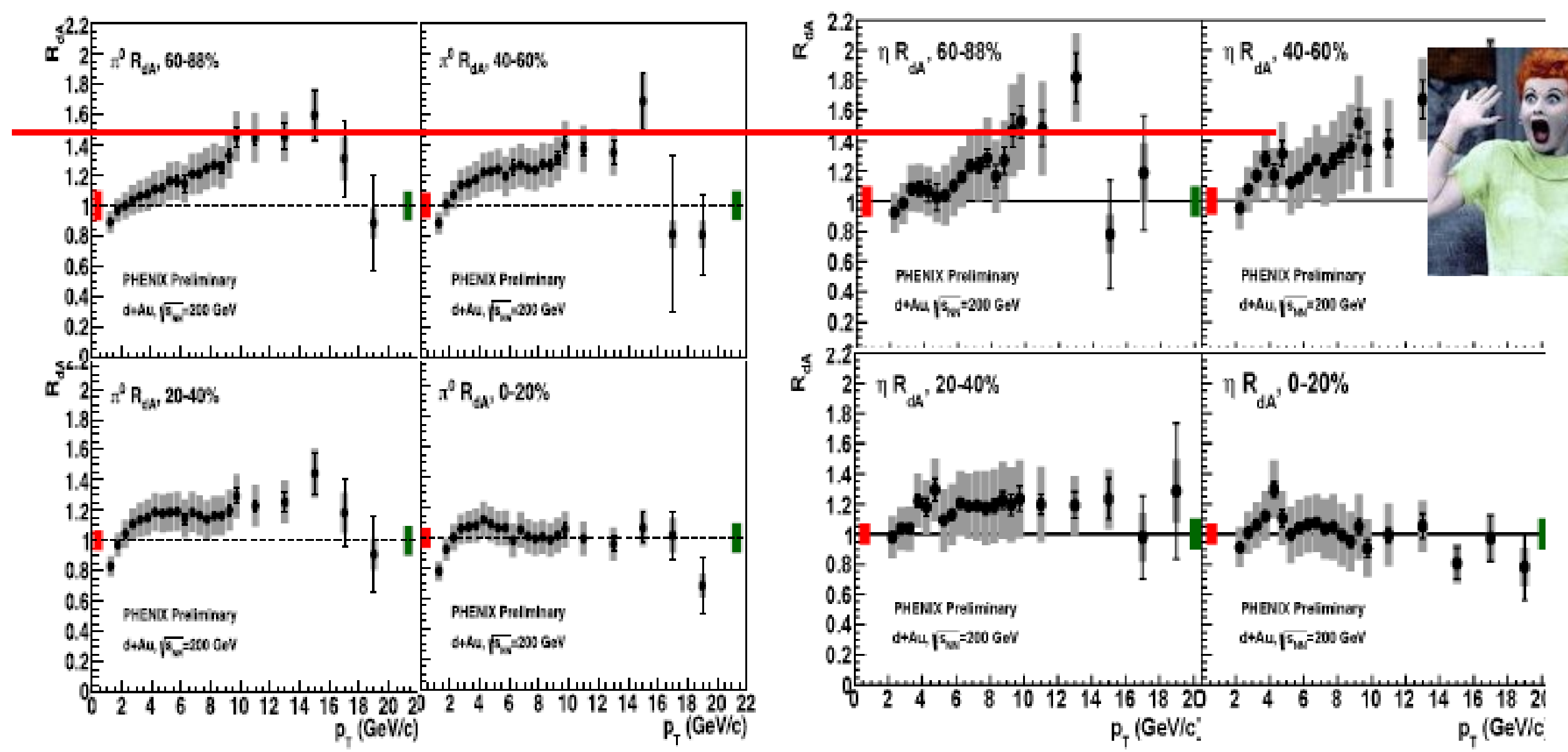

Is it possible that $\pi_{0}, \eta$ production at high $\mathrm{p}_{\mathrm{T}}$ in peripherals is enhanced??? 


\section{$\mathrm{V}_{3}$ in $\mathrm{pPb}$ and $\mathrm{PbPb}$}

PbPb RBRC13 CMS SHOCK waves from super central pPb @ LHC

$\mathrm{PbPb}$

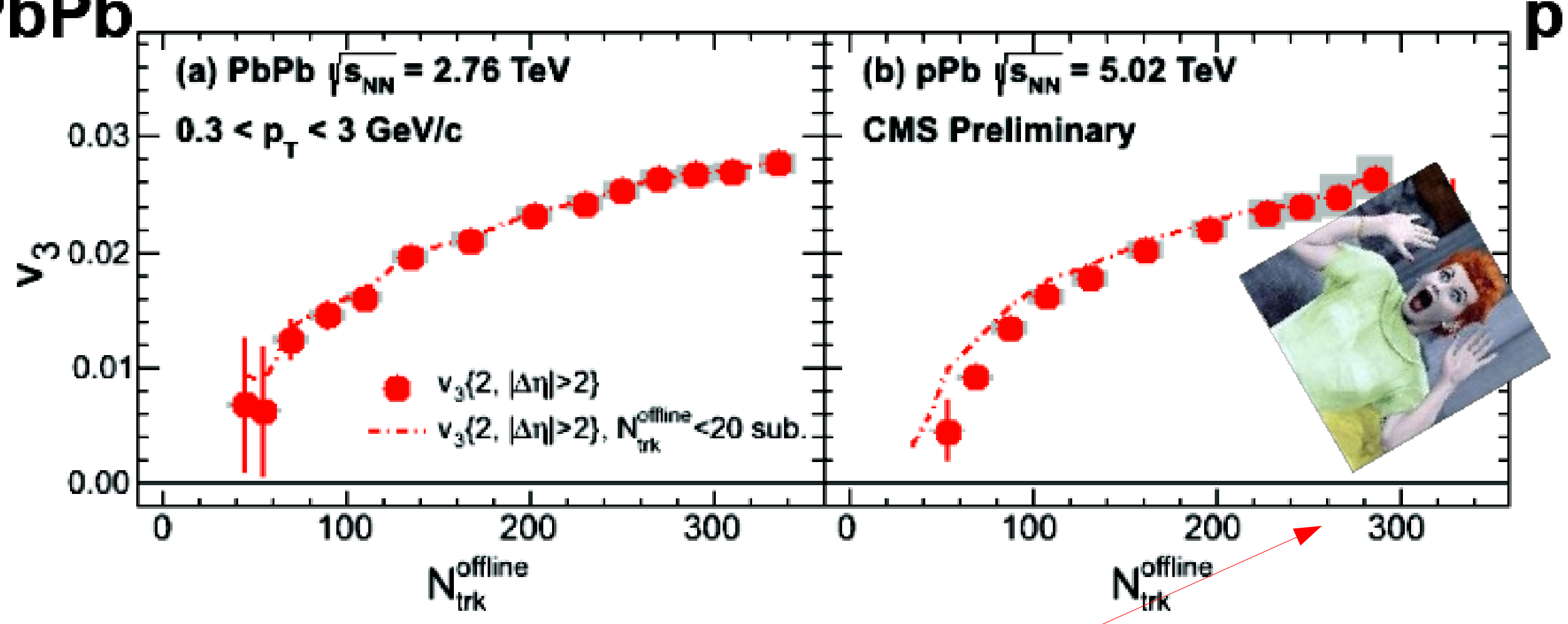

$\mathrm{pPb}$

$\mathrm{v}_{3}$ shows similar shape in $\mathrm{pPb}$ and $\mathrm{PbPb}$; magnitude comparable

very rare events

$1 / 1,000,000$ 


\section{$\mathrm{V}_{3}$ in $\mathrm{pPb}$ and $\mathrm{PbPb}$}

\section{$\mathrm{PbPb}$}

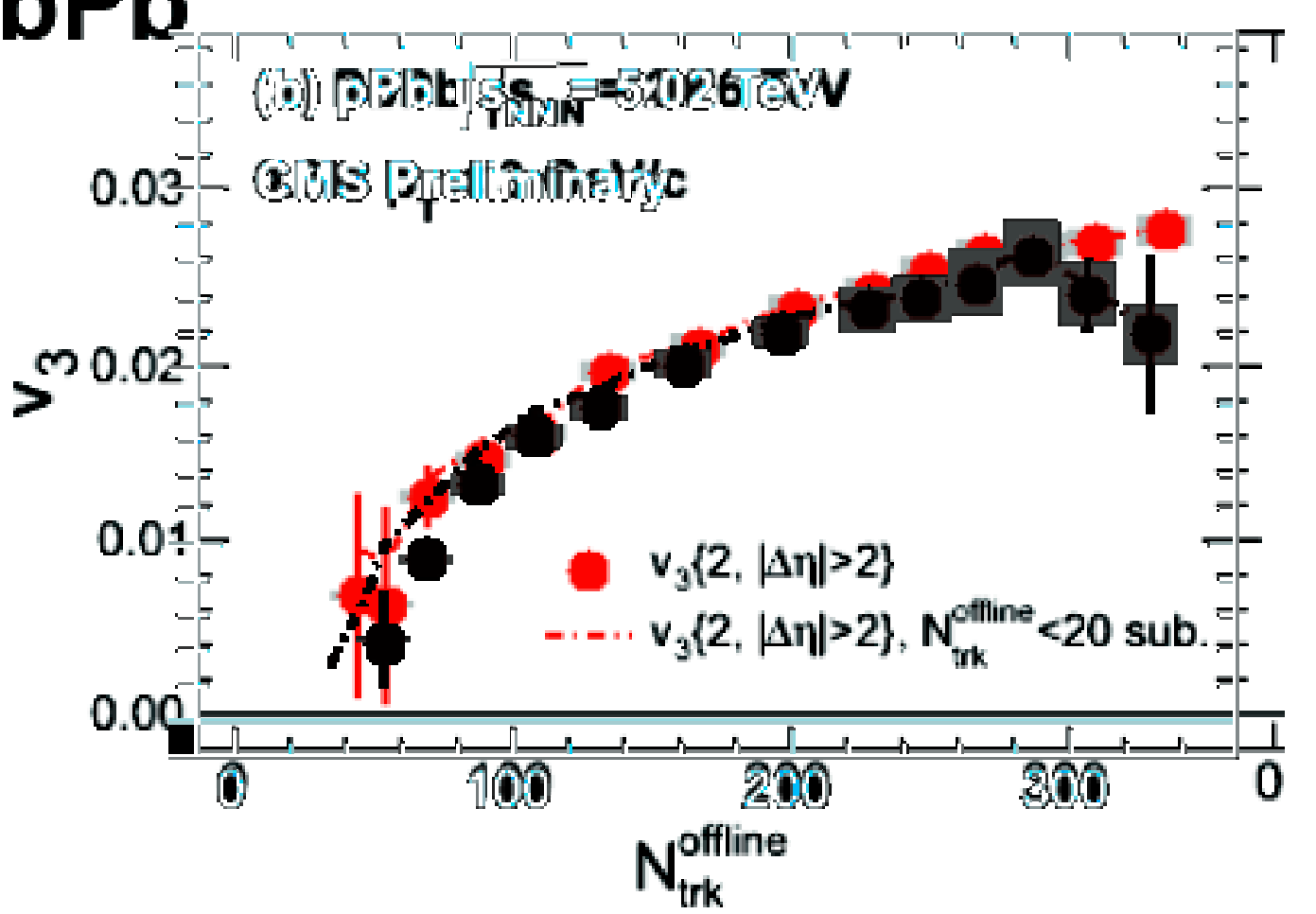

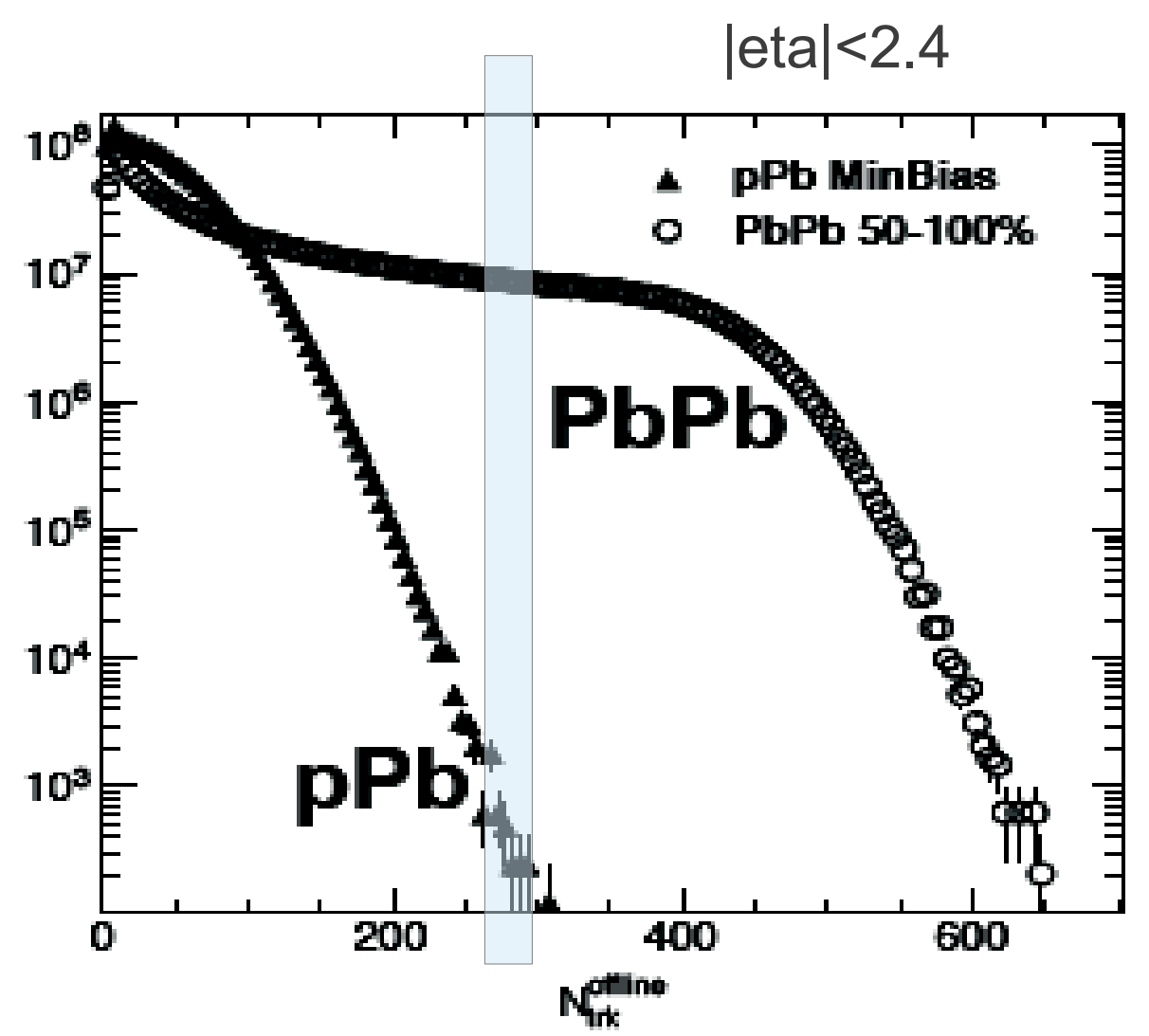

$\mathrm{v}_{3}$ shows similar shape in $\mathrm{pPb}$ and $\mathrm{PbPb}$; magnitude comparable same

same

Data seem to falsify "Apples = Oranges" theorem

But dN/deta shapes could differ a lot (Or it could be multi jetty)
$\mathrm{PbPb}$ 


\section{Recall old BGK p+A "Rapidity Triangle"}

- Multiple independent wee parton $\mathrm{dx} / \mathrm{x}$ collisions produce uniform in rapidity color charges between valence $p$ and valence wounded $A$.

- Color neutralizes via pair production between wee and valence partons

- Leaving a stack in $10 \%$ of

- $\mathrm{A}^{1 / 3} \sim 10$ Target beam jets

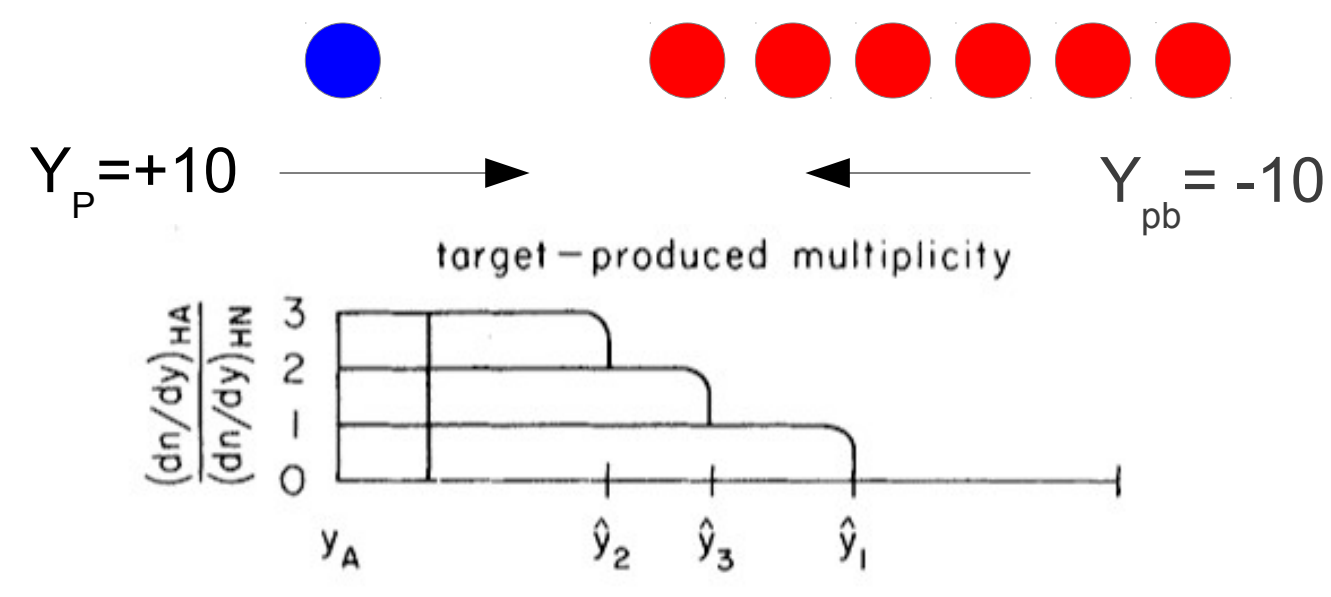

projectile - produced multiplicity

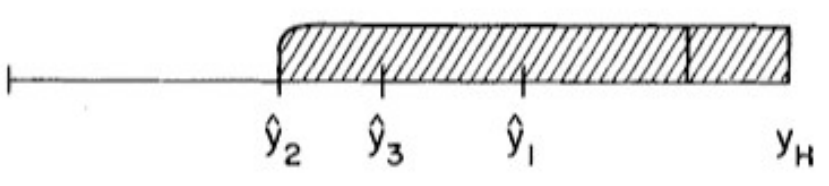

- For rare Ntr 300 maybe $30 \mathrm{~Pb}$ nucleons line up

- There is 1 Projectile beam jet

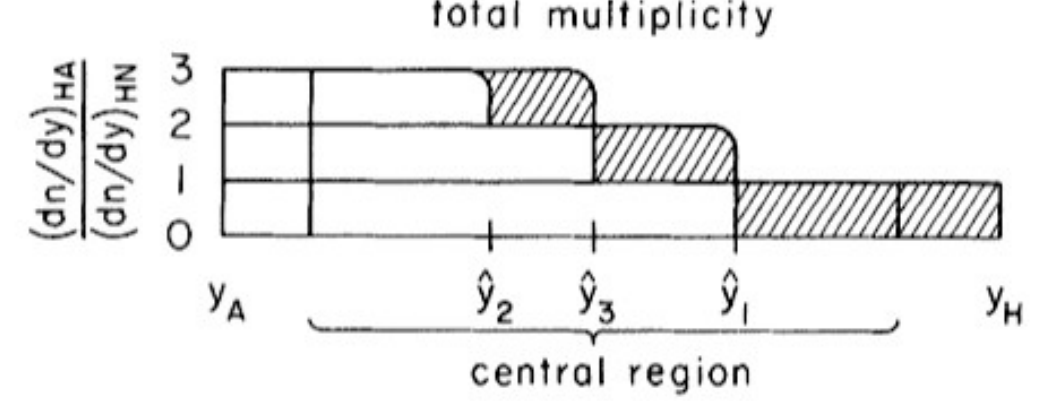

- Y Slope $\delta=N$ tr / log(s)

Figure from Brodsky, Gunion, Kuhn 1977

- $\mathrm{RHIC} \delta \sim 2 \times$ LHC $\delta$ 
Can sQGP survive the RBRC $2013 D+A u$ and $p+P b$ tsunami?

BES, $\mathrm{D}+\mathrm{Au}$ and $\mathrm{p}+\mathrm{Pb}$ uncalibrated our v2 Barometer of sQGP $\mathrm{D}+\mathrm{Au}$ (and ? soon $\mathrm{p}+\mathrm{Pb}$ ) uncalibrated our RAA opacity meter

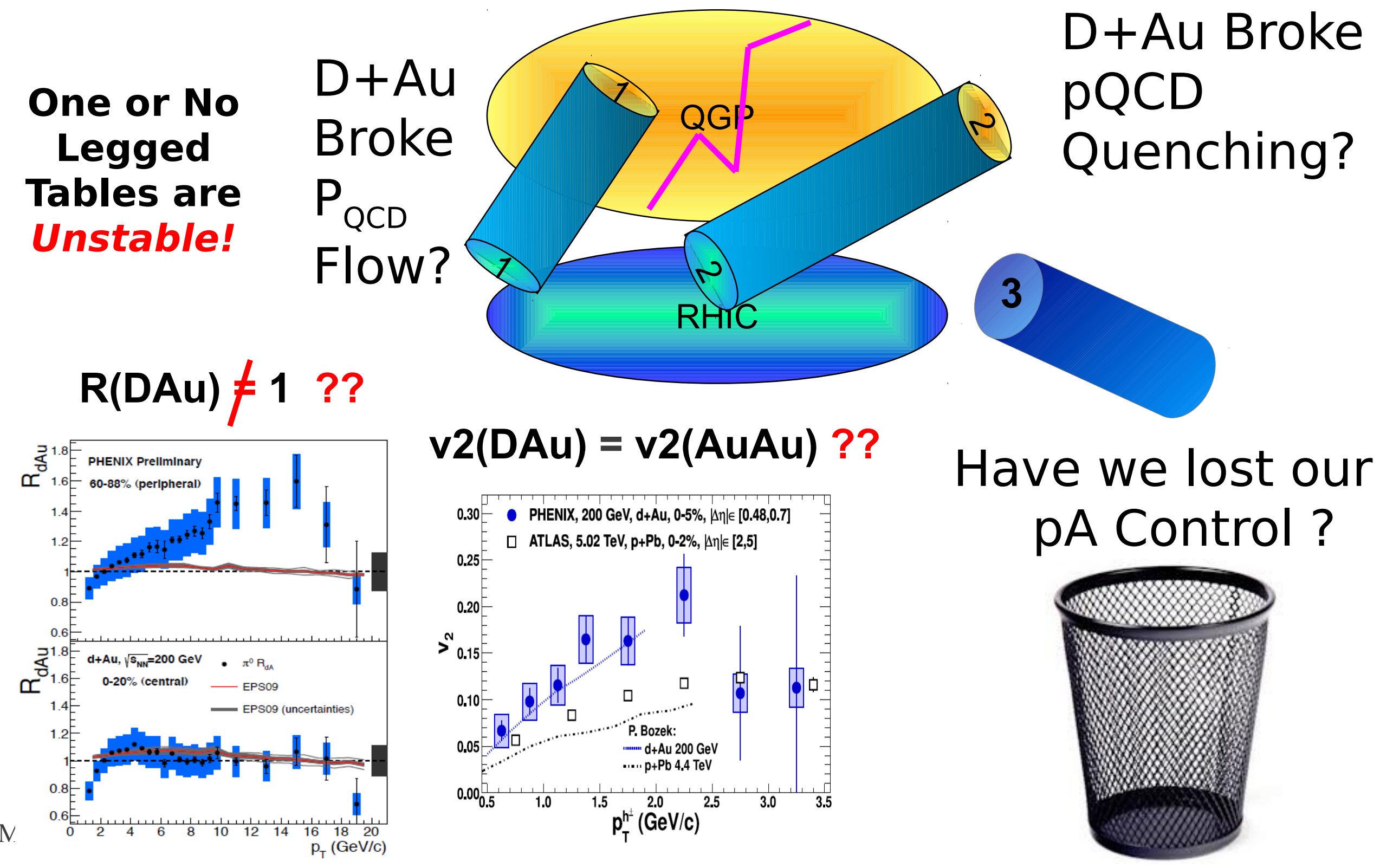


Maybe v2(DAu , $5 \%)=v 2(\mathrm{AuAu}, 30 \%)$ is just coincidence bad luck ??

Maybe QM12 BES is also just coincidence ?

v2(AuAu, 7 AGeV) v2(AuAu,200 AGeV) v2(PbPb,2800 AGeV) ??

Should we ignore the "peripheral" $R_{\mathrm{DAu}}(15 \mathrm{GeV}) \sim 1.5$ anomaly

and rely instead only on Null Control direct gammas

$R_{\mathrm{DA}}($ gamma $) \sim R_{\mathrm{AuAu}}($ gamma $) \sim 1$

We need global data on dN/dy, dET/dy, and dN/dydpT at both RHIC and LHC on rare $p+A$ processes

Entropy has been produced (Clausius is happy) but we need to step back and reassess which of our (many) Geomtric and dynamical assumptions may fail in very rare pA and DA processes. 
Maybe v2(DAu , $5 \%)=v 2(\mathrm{AuAu}, 30 \%)$ is just coincidence bad luck ??

Maybe QM12 BES is also just coincidence ?

v2(AuAu, 7 AGeV) v2(AuAu,200 AGeV) v2(PbPb,2800 AGeV) ??

Should we ignore the "peripheral" $R_{\mathrm{DAu}}(15 \mathrm{GeV}) \sim 1.5$ anomaly

and rely instead only on Null Control direct gammas

$R_{\mathrm{DA}}($ gamma $) \sim R_{\mathrm{AuAu}}($ gamma $) \sim 1$

We need global data on dN/dy, dET/dy, and dN/dydpT at both RHIC and LHC on rare $p+A$ processes

Entropy has been produced (Clausius is happy) but we need to step back and reassess which of our (many) Geomtric and dynamical assumptions may fail in very rare pA and DA processes. 
Part 3:

The High pT azimuthal asymmetry v2( pT, Ecm)

In the $\underline{\mathrm{IM} 2<\mathrm{pT}<10}$ and the deep UV $\mathrm{pT}>10$

\author{
Puzzling Status update
}


The Deep UV Band pT>10 GeV seemed to agree with pQCD tomography @LHC Unlike the intermediate IM Band $2<$ pT $<10$ interpolating between IR and UV bands
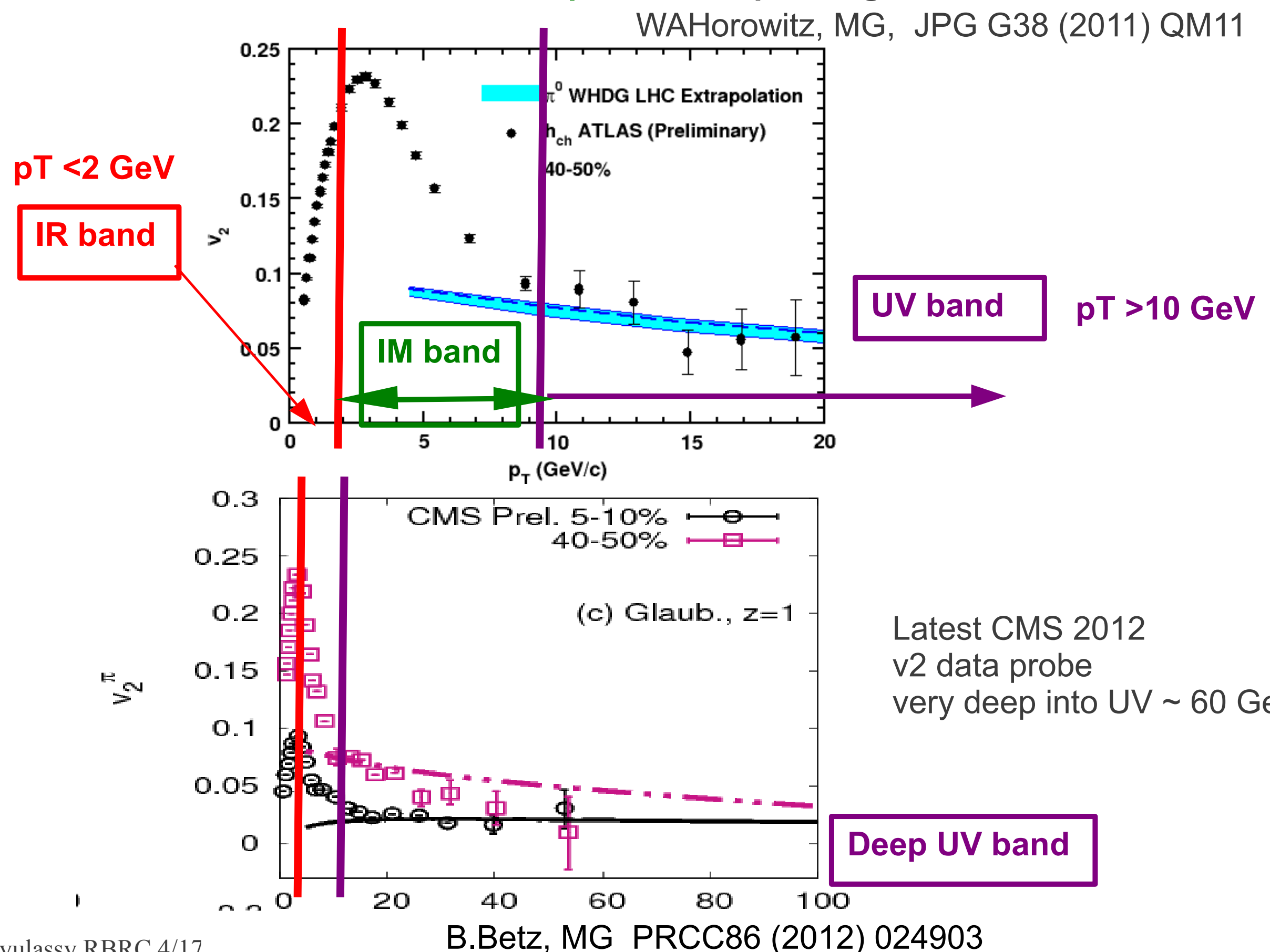
Denes Molnar, Deke Sun, QM12 used MPC bulk evolution to evaluate GLV En Loss

high-pT pion v2, LHC ( $\alpha_{s}$ scaled to RAA at pT $\left.=6 \mathrm{GeV}\right)$
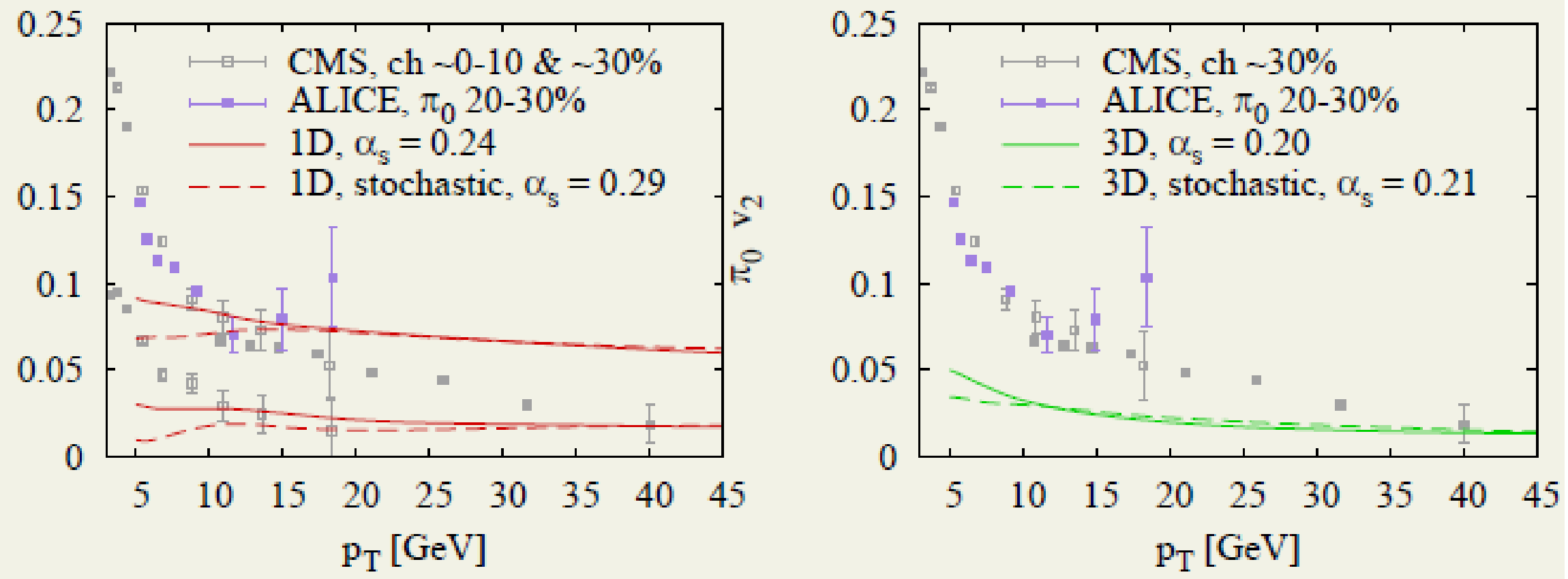

They concluded that transverse expansion reduced $\mathrm{pQCD} v 2(\mathrm{pT})$ by factor $\sim 2$ below data 
Denes Molnar, Deke Sun, QM12 used MPC bulk evolution to evaluate GLV En Loss

average $A u+A u, b=8 \mathrm{fm}$

$$
\tau d N / d^{2} x_{T}[\mathrm{fm}]
$$

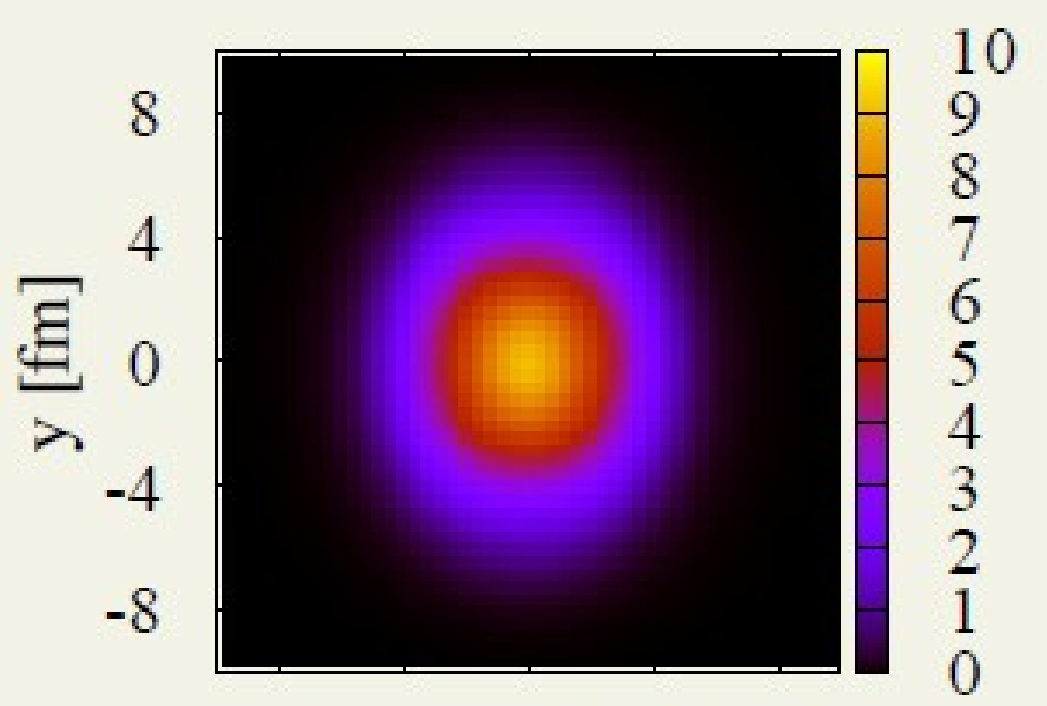

$\begin{array}{lllll}-8 & -4 & 0 & 4 & 8\end{array}$

$\mathrm{x}[\mathrm{fm}]$ single MC Glauber

$$
\tau d N / d^{2} x_{T}[\mathrm{fm}]
$$

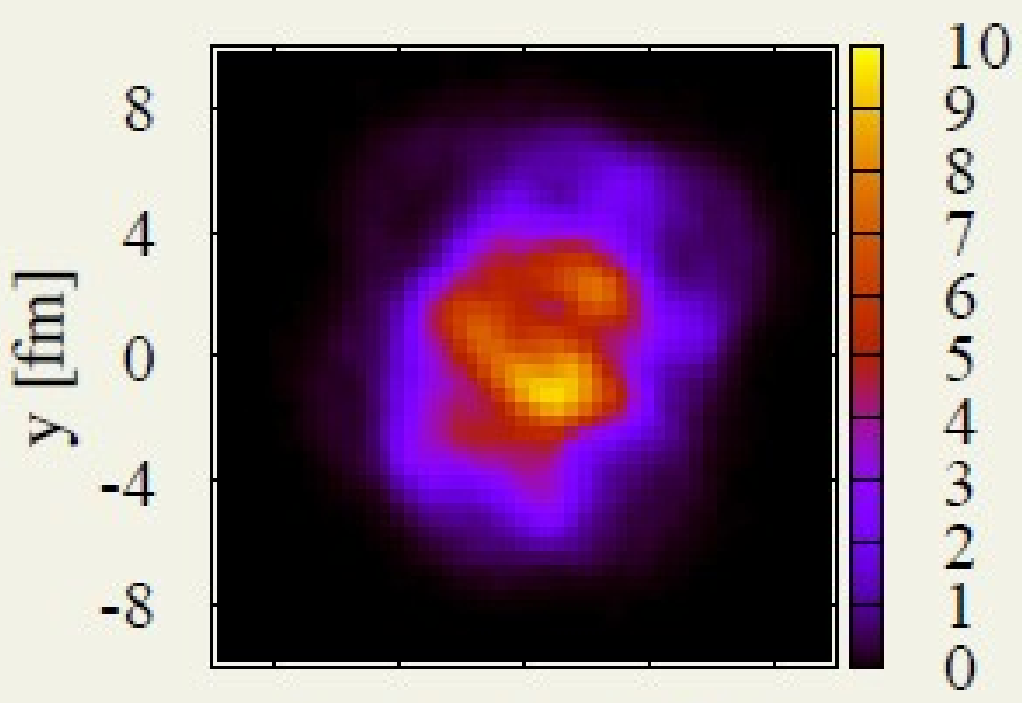

$\begin{array}{lllll}-8 & -4 & 0 & 4 & 8\end{array}$

$\mathrm{x}[\mathrm{fm}]$ 
Denes Molnar, Deke Sun, QM12 used MPC bulk evolution to evaluate GLV En Loss

Similar to the Ed's 1999 "Nutcracker" scenario bulk hydro like evolution

With rapid decrease of eccentricity to negative values for $t>R$

average $A u+A u, b=8 \mathrm{fm}$

single MC Glauber

$\tau d N / d^{2} x_{T}[\mathrm{fm}]$

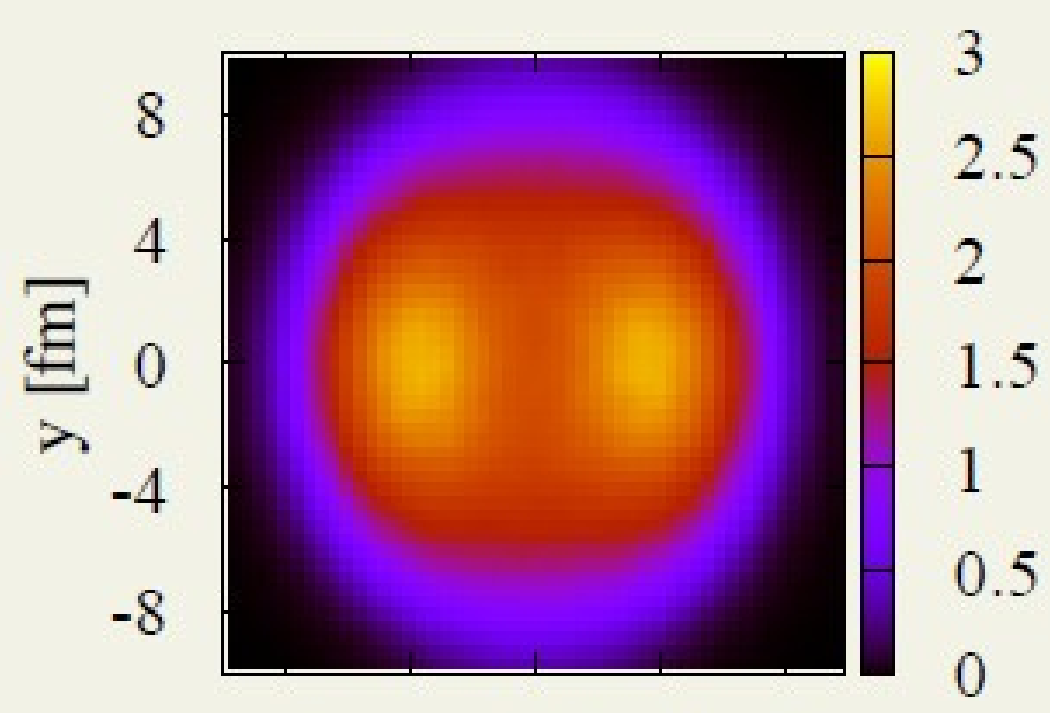

$\begin{array}{lllll}-8 & -4 & 0 & 4 & 8\end{array}$

$\mathrm{x}[\mathrm{fm}]$ $\tau d N / d^{2} x_{T}[\mathrm{fm}]$

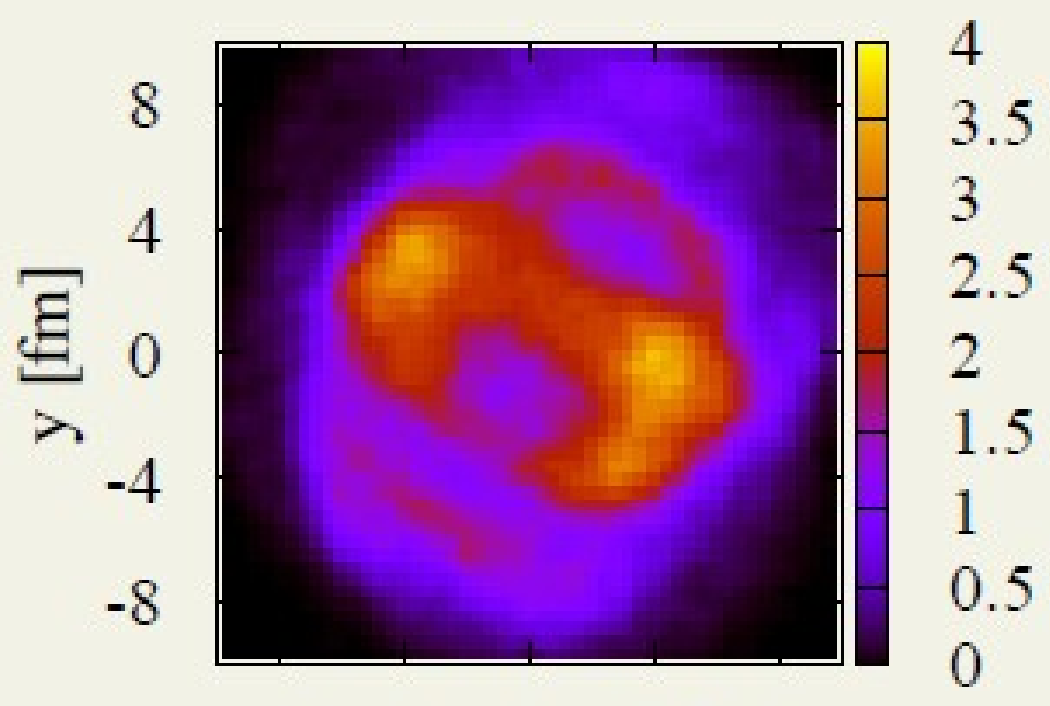

$\begin{array}{lllll}-8 & -4 & 0 & 4 & 8\end{array}$

$\mathrm{x}[\mathrm{fm}]$

$$
\tau=6.6 \mathrm{fm}
$$




\section{PHENIX $R_{\mathrm{AA}}^{\text {in }}=R_{\mathrm{AA}}\left(1+2 \mathrm{v}_{2}\right) \quad$ vs $\quad R_{\mathrm{AA}}^{\text {out }}=\mathrm{R}_{\mathrm{AA}}\left(1-2 \mathrm{v}_{2}\right)$}

A. Adare et al., arXiv:1208.2254

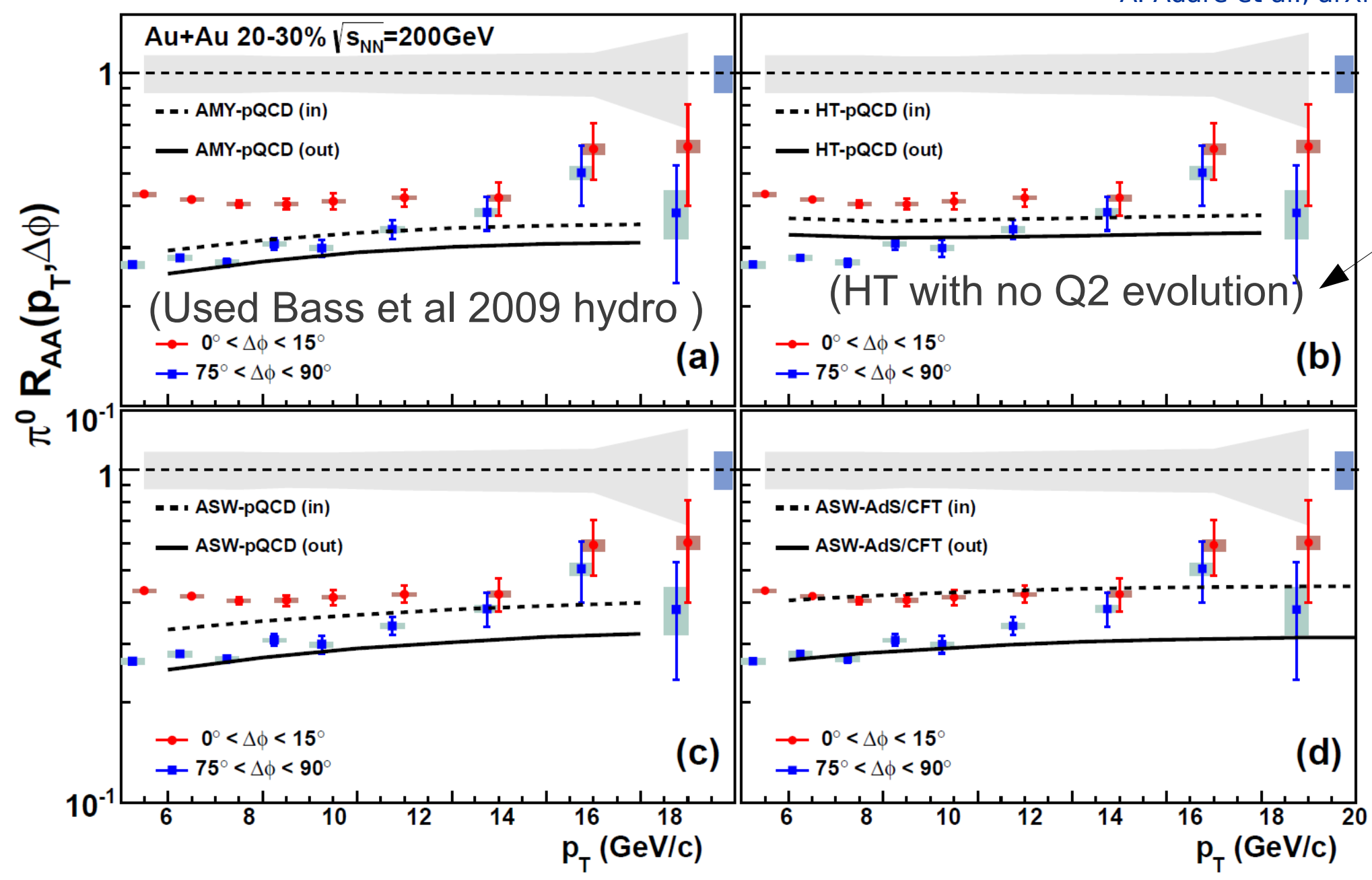

See A.

Majumder

RBRC13

update

$E^{\wedge} 0 L^{\wedge} 2$

En loss

$\rightarrow$ PHENIX Concluded: Only Schematic ASW-AdS/CFT can fit both $\mathrm{R}_{\mathrm{AA} \text { in/out }}$ for a $3 \mathrm{~d}$ expanding medium. PQCD based dedx underestimate

Provide information about both $\mathrm{R}_{\mathrm{AA}}$ and $\mathrm{v}_{2}$ 
abc Model of Jet Energy Loss and RAA

$\begin{aligned} & \text { For } \mathrm{pQCD} \\ & \mathrm{a} \sim 1 / 3, \mathrm{~b} \sim 1\end{aligned} \quad \frac{d P}{d \tau}=-\kappa P^{a} \tau^{b} T^{2-a+b}(x(\tau), \tau)$

$$
P_{0}\left(P_{f}\right)=\left[P_{f}^{1-a}+K \int_{\tau_{0}}^{\tau_{f}} \tau^{z} T^{c}\left[\vec{x}_{\perp}(\tau), \tau\right] d \tau\right]^{\frac{1}{1-a}}
$$

$R_{A A}\left(p_{f} ; s, A\right)=\frac{\partial p_{0}}{\partial p_{f}} \frac{d \sigma\left(p_{0}\left(p_{f}\right)\right) / d p}{d \sigma\left(p_{f}\right) / d p}$

For Bj Brick $\approx\left(1+\kappa^{\prime} \frac{(d N / d y)^{(2-a+b) / 3}}{\left(L p_{f}\right)^{1-a}}\right)^{\frac{a-n\left(p_{f}\right)}{1-a}}$

Fix k' by fit to one RHIC $\mathrm{R}(\mathrm{pf}=10 \mathrm{GeV}, \mathrm{dNdy}=1000)$ reference point. Horowitz,MG, NPA872(2011); $\quad$ B.Betz, MG, Torrieri PRC84(11), PRC86(12) 
„Magnetic Monopole“ scenario of Liao,Shuryak

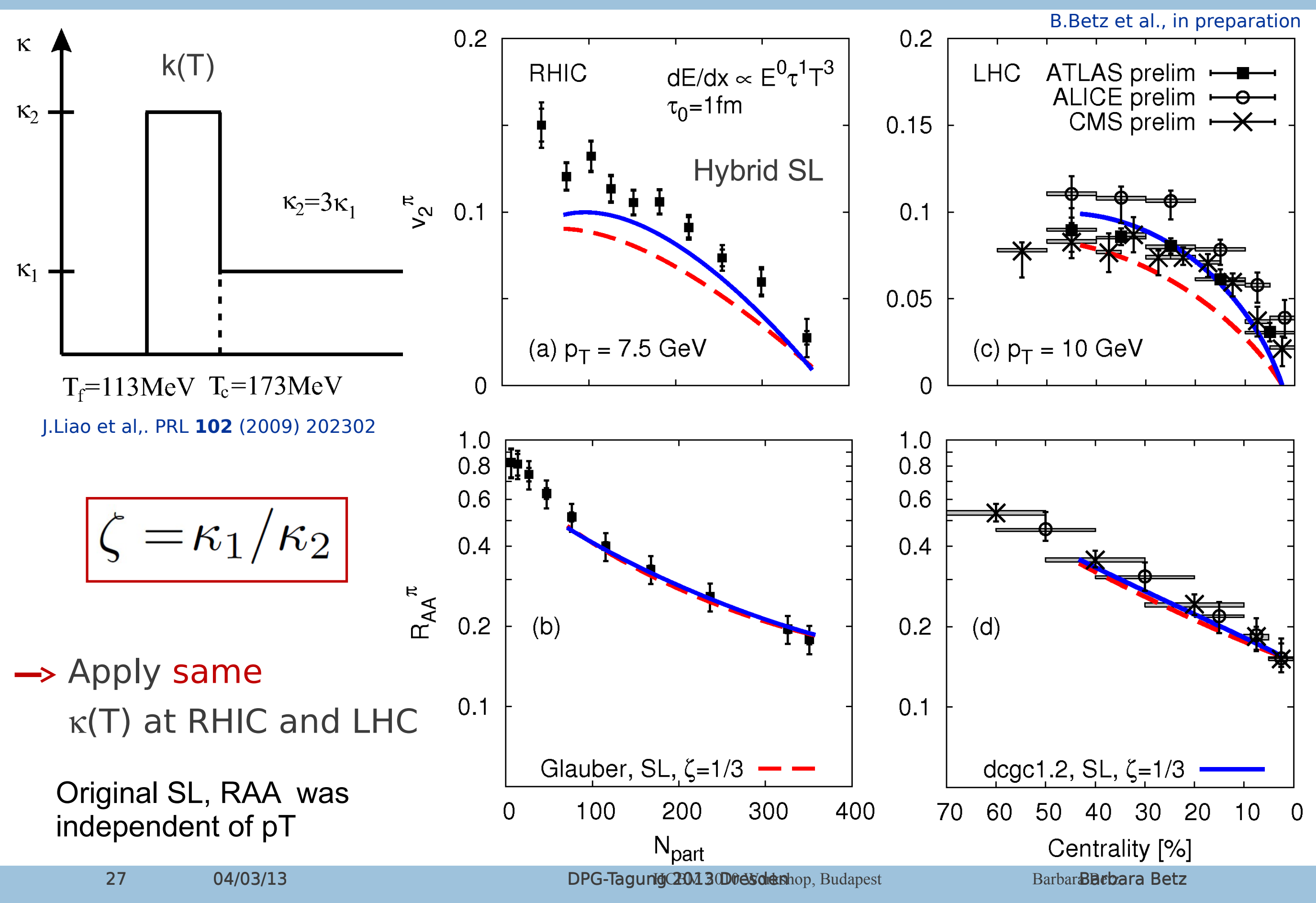


Compare $4 \mathrm{dEdx}$ models using Romatschke hydro or $\mathrm{Bj}+$ radial flow $\mathrm{v}=\mathbf{0 . 6}$ bulk models
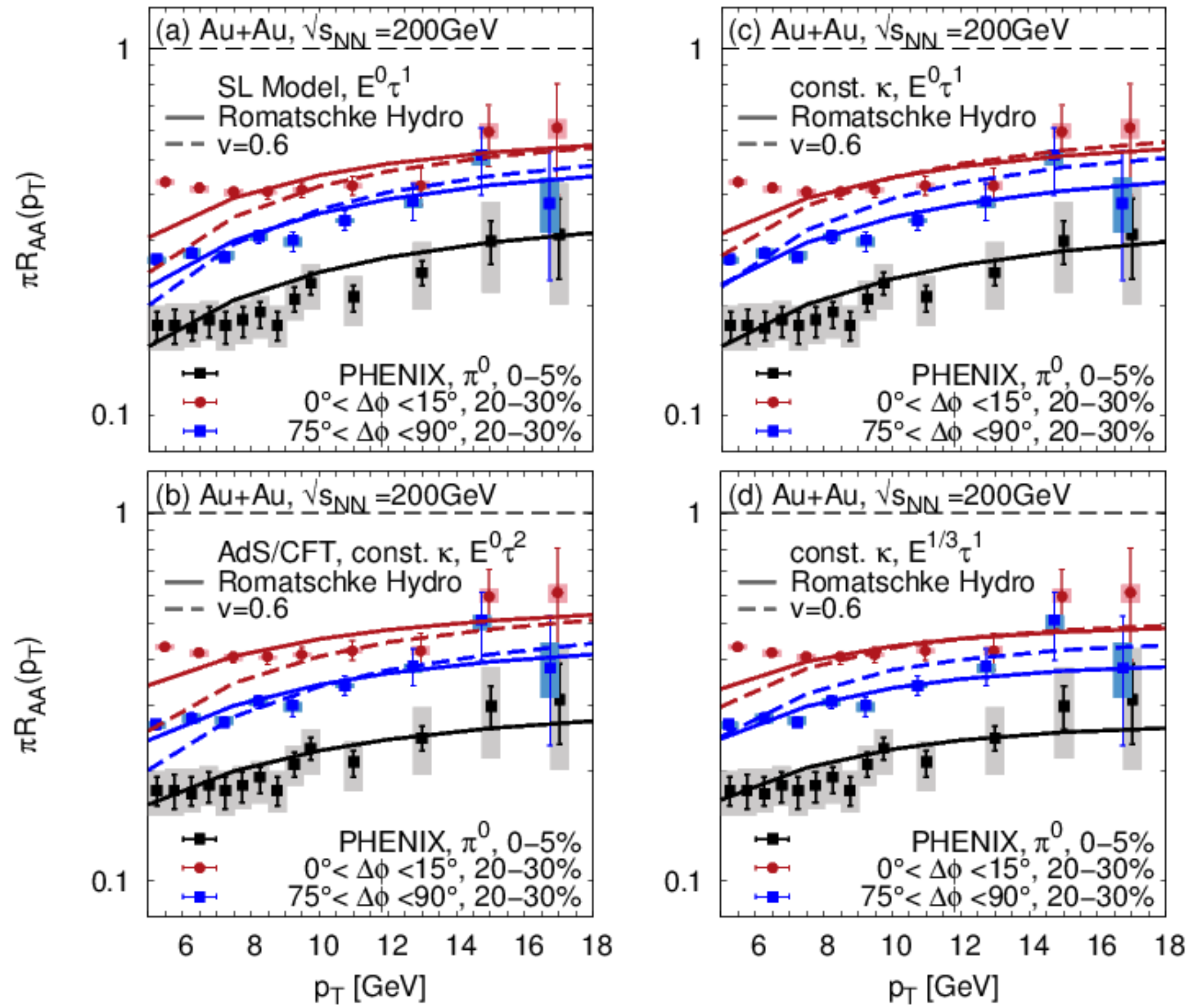

None of 8 scenarios appears preferred within current errors at RHIC

B.Betz, MG (13) 
8 Scenarios Extrapolated to LHC

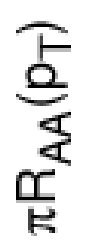

Sensitivity To dEdx (abc) and kappa(T) is weaker than at RHIC because spectral shapes are harder

\section{Large}

Sensitivity of RAAin/out To bulk flow Evolution (as per Renk 11)

点

$\frac{\text { 竞 }}{\frac{\pi}{4}}$

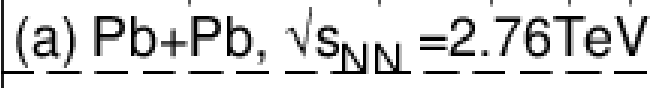
SL Model, $E^{0} \tau^{1}$

0.1

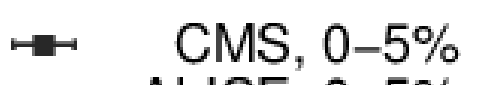

$-\quad$ ALICE, $0-5 \%$

1

(b) $\underline{P b}+\underline{P b}, \underline{\sqrt{ }} \mathrm{s}_{\mathrm{NN}}=2.7 \underline{6} \underline{\mathrm{TeV}}$ AdS/CFT, const. $\kappa, \mathrm{E}^{0} \tau^{2}$ $-\mathrm{v}=0.0$

$-\mathrm{v}=0.6$

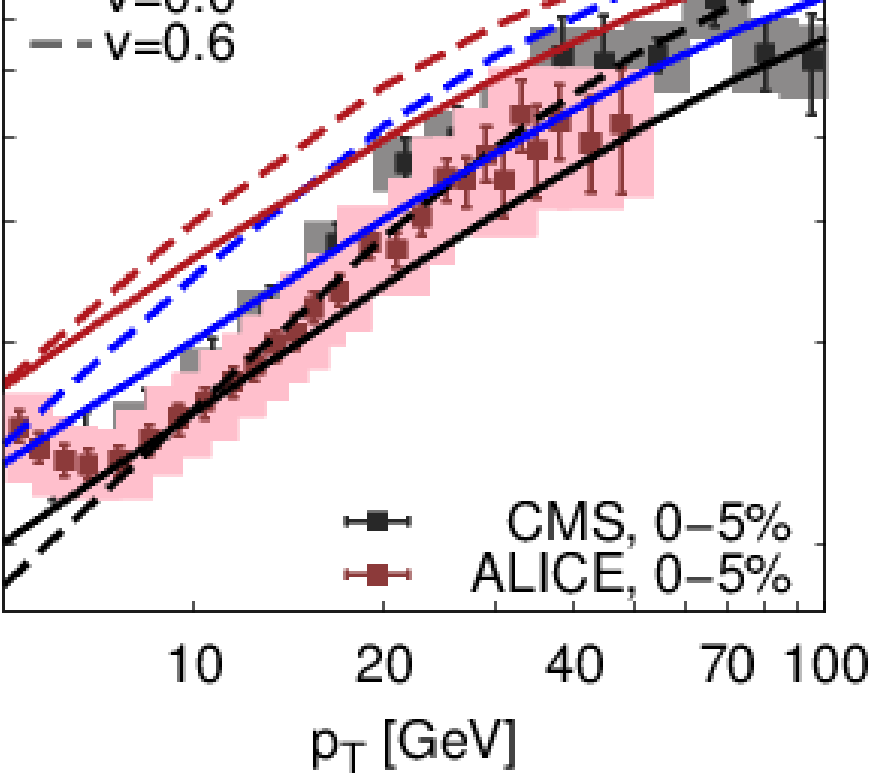

1

0.1

(c) $\underline{\mathrm{Pb}}+\underline{\mathrm{Pb}}, \underline{\sqrt{ } \mathrm{s}_{\mathrm{NN}}=2.76 \mathrm{TeV}}$ const. $\kappa, E^{0} \tau^{1}$

$-v=0.0$

$-v=0.6$

1

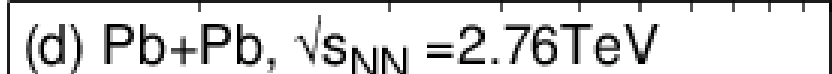

$\rightarrow \quad$ CMS, $0-5 \%$

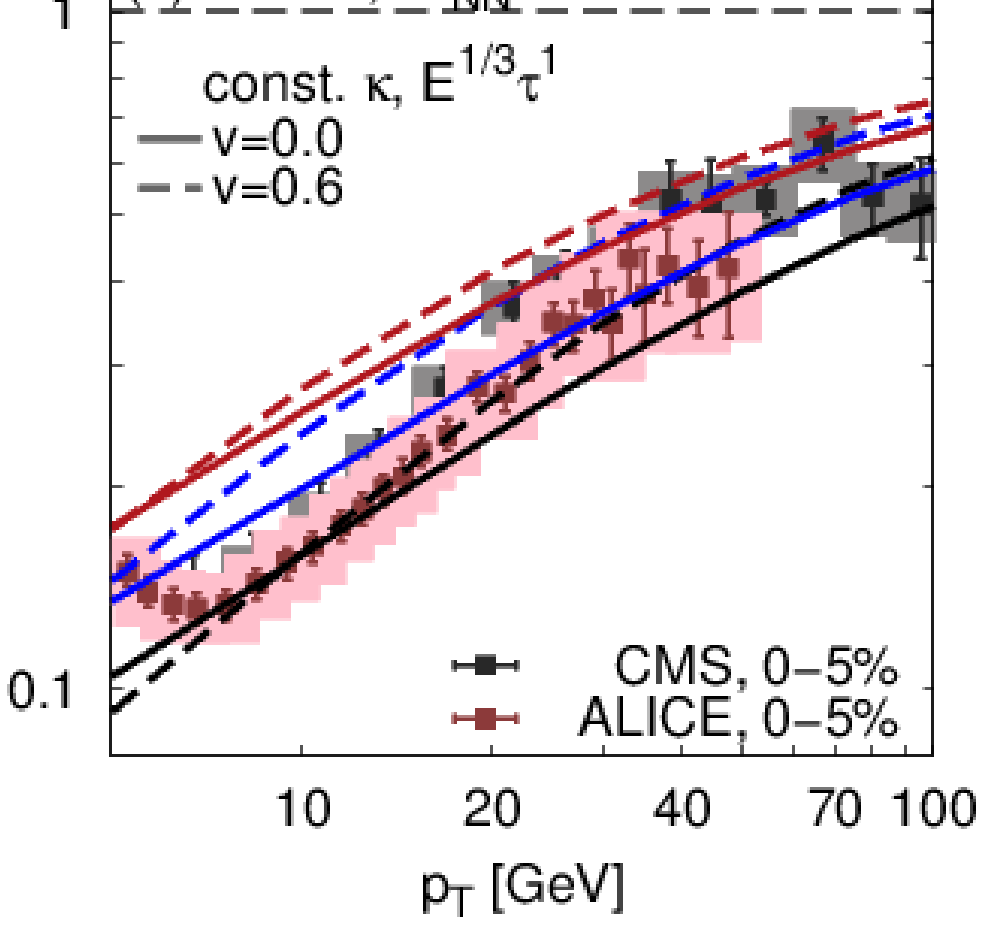


Final remarks :

1) Azimuthal tomography is sensitive to details of the bulk flow $T(x, t)$ and $u(x, t)$ field evolutions that is complementary to low pT vn systematics. This is the good news.

2) However sensitivity is weak more precise RAAin/out(pT) needed to resolve current array of ( $p Q C D, S L$, and AdS dEdx models) times the array bulk flow models (Bass, Heinz, Romat, MPC)

3) Different jet asymmetry using Romat hydro and Bass hydro flow fields needs to be clarified.

4) Meanwhile, the new QM12 and RBRC13 p+A and D+A data on bulk vn and anomalous jet modification "discoveries" severely challenge our entire framework of A+A analysis.

Our homework now is to try to regain the lost Null-Controls ! 


\section{The $p-A / p+p$ "Rapidity Triangle"- BGK Model}

- Multiple independent wee parton $\mathrm{dx} / \mathrm{x}$ collisions produce uniform in rapidity color charges between valence $p$ and and valence wounded $A$.

- Color neutralizes via pair production between wee and valence partons

- Leaving a stack of

- $\mathrm{A}^{1 / 3}$ Target beam jets

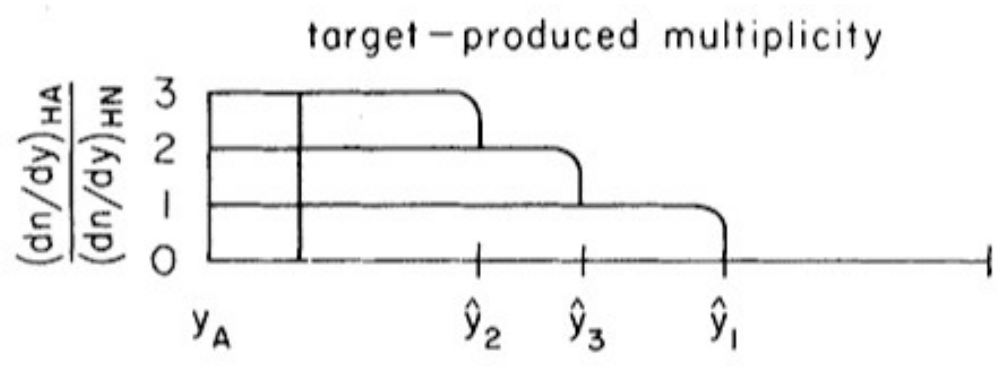

projectile - produced multiplicity

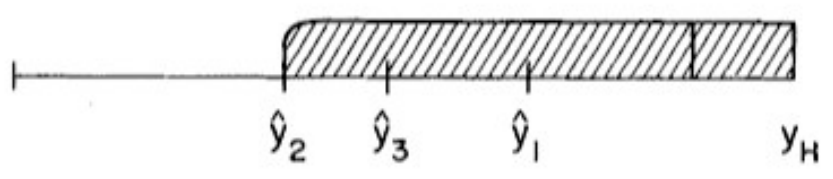

- and 1 Projectile beam jet

- $\nu_{A}(b) \approx \sigma_{i n} T_{A}(b)$

- Y Slope $\delta=O\left(\mathrm{~A}^{13} / \log (\mathrm{s})\right)$

Figure from Brodsky, Gunion, Kuhn 1977

- RHIC $\delta \sim 0.45$, LHC $\sim \delta 0.28$ 


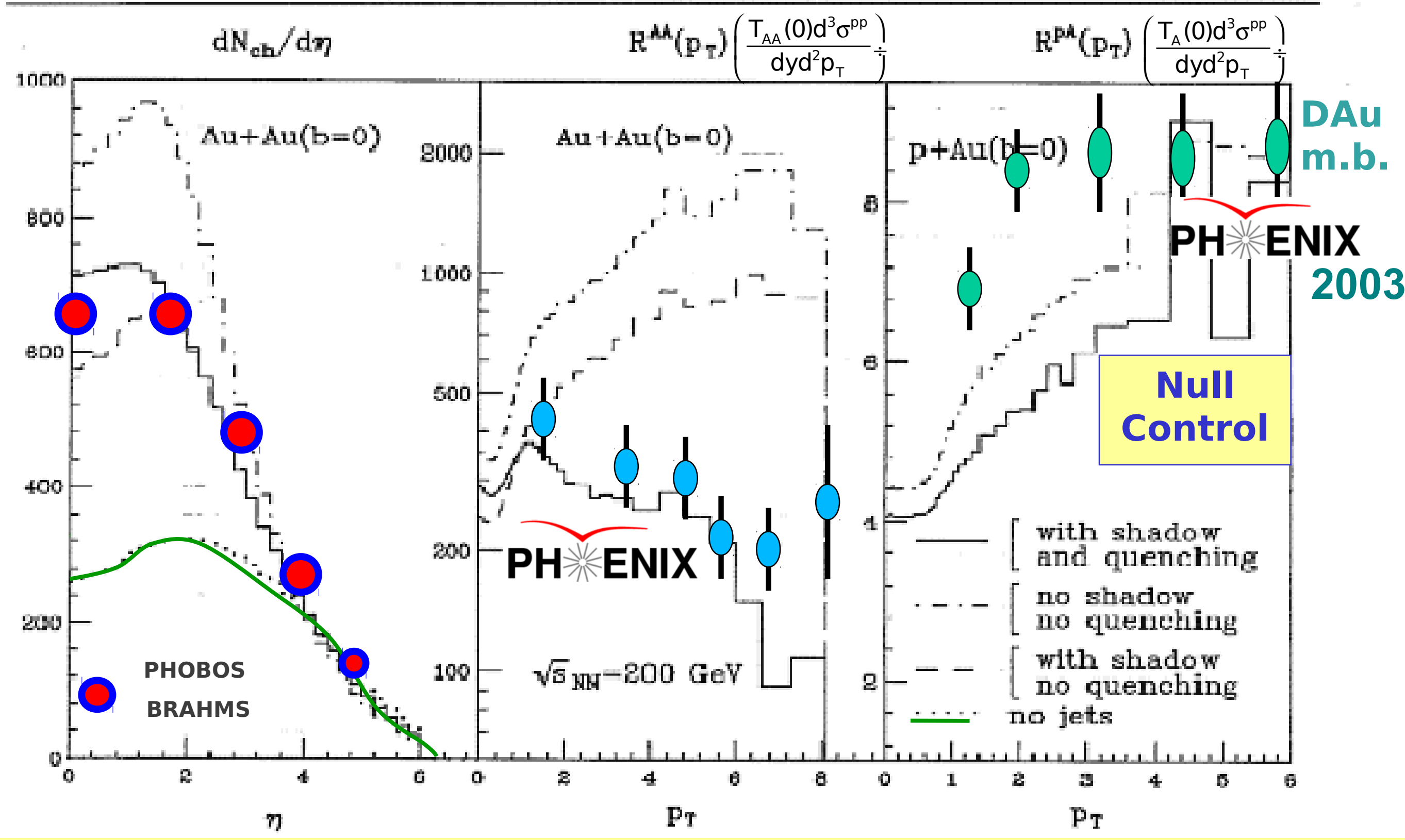

Null Control with p+A @ RHIC was critical to prove that RAA 0.2 Was due to final state jet quenching (not to initial state shadow) 


\section{Evidence from the suppression of high- $p_{T}$ particles:}

[in central AA but not in D+A]

PHOBOS dAu $200 \mathrm{GeV}$

W.Busza, RBRC 4/15/2004

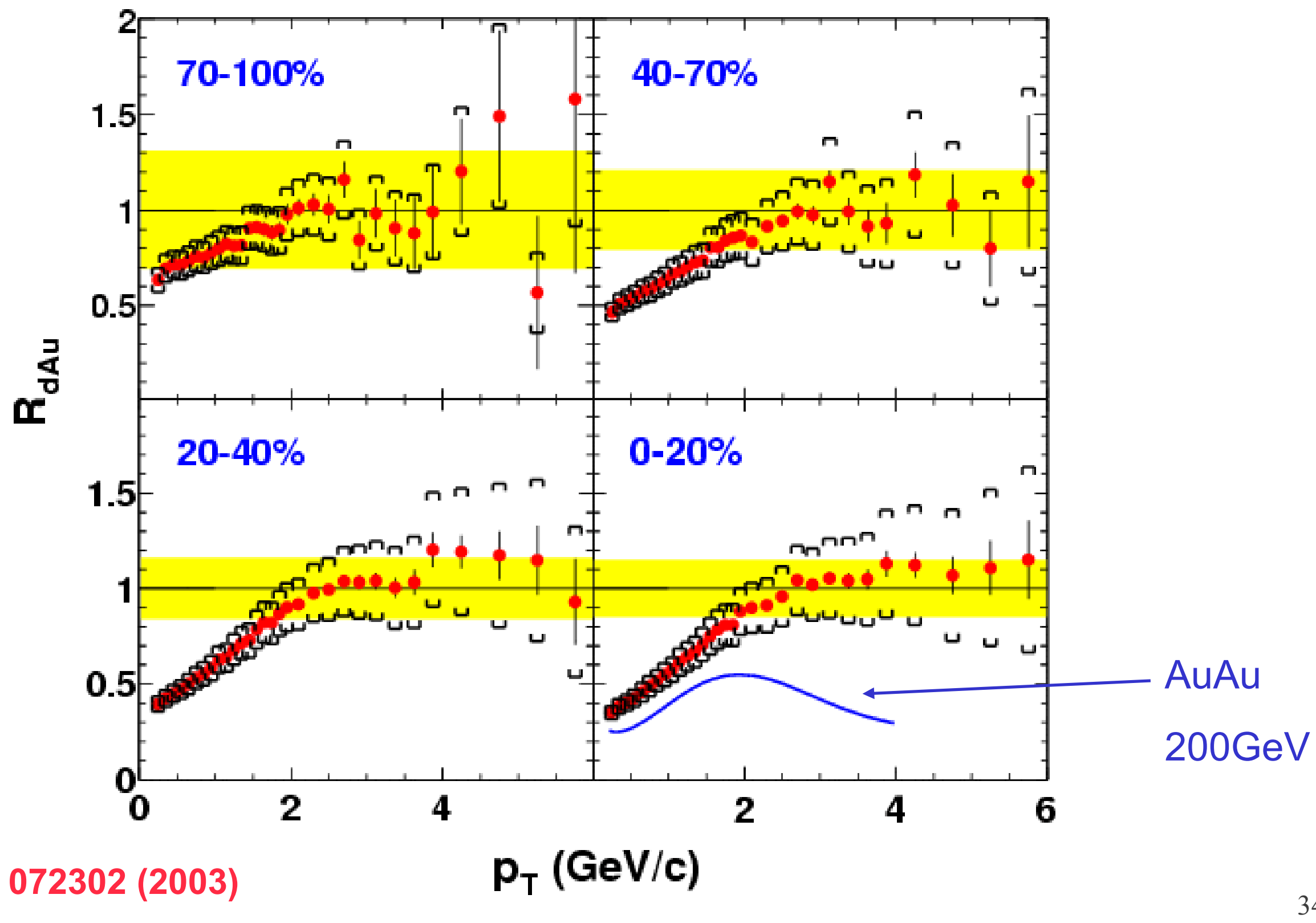




\section{In-medium QCD cascade: democratic branching and wave turbulence}

Jet Quenching at RHIC Vs LHC BNL

April 17, 2013 


\section{outline}

- Phenomenological motivations

- In-medium gluon branching (BDMPSZ mechanism)

- Multiple branching, (de)coherence, in-medium cascade

- In-medium cascade, turbulent flow

- Relevance to di-jet asymmetry

- Conclusion

Work done in collaboration with F. Dominguez, E. Iancu and Y. Mehtar-Tani (arXiv:1209.4585, 1301.6102) 
Phenomenological motivations 


\section{Di-jet asymmetry}

\section{there is more to it than just 'jet quenching'...}
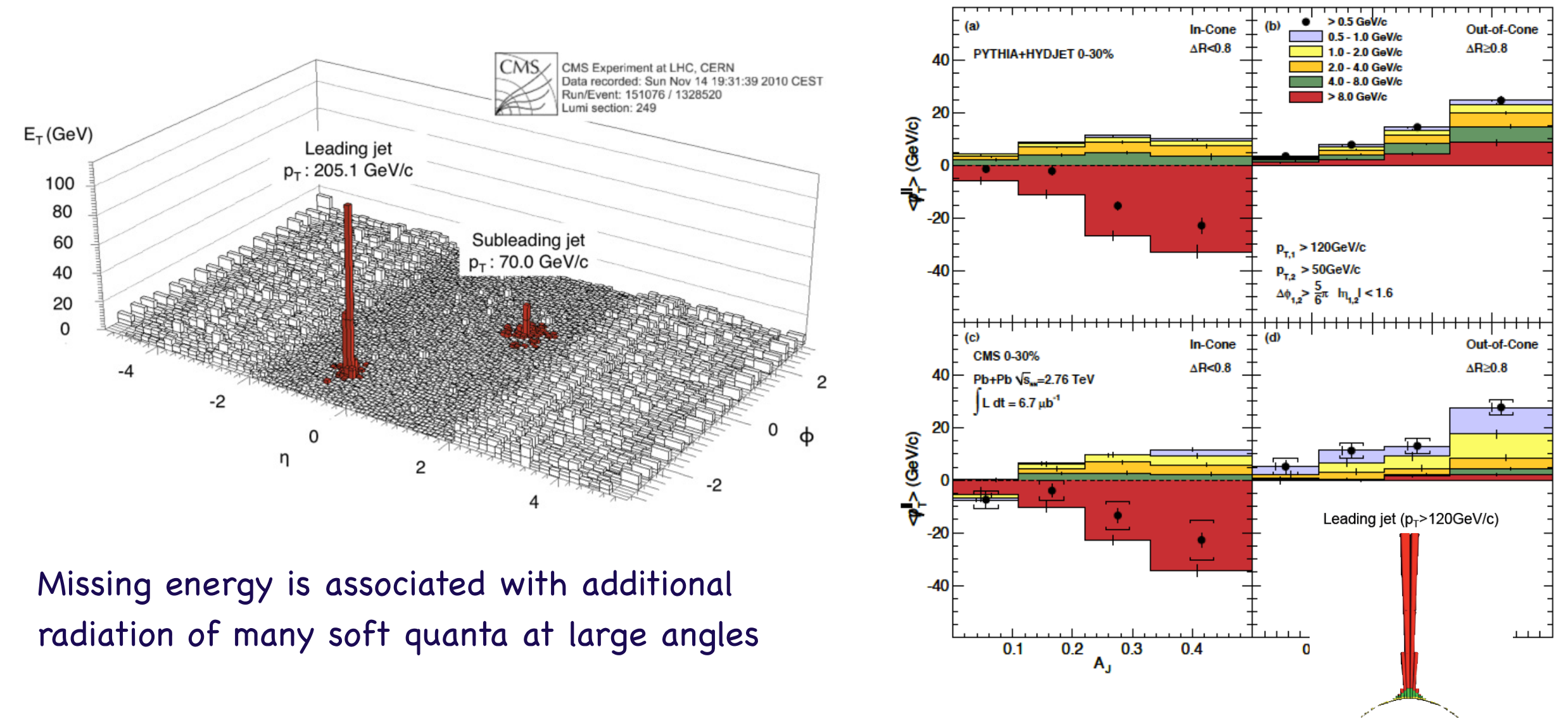

Perhaps reflecting a genuine feature of the in-medium QCD cascade (JPB, E. Iancu and Y. Mehtar-Tani, arXiv: 1301.6102)

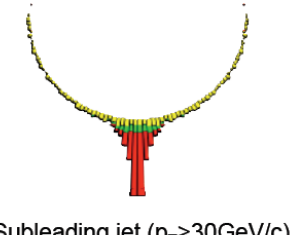

Missing energy is associated with additional radiation of many soft quanta at large angles 


\section{In-medium parton branching BDMPSZ mechanism}

(Baier, Dokshitzer, Mueller, Peigné, Schiff; Zakharov 1996) 


\section{The BDMPSZ mechanism for in-medium branching}

Gluon emission is linked to momentum broadening

$$
\frac{1}{\tau_{\mathrm{f}}} \sim \frac{k_{\perp}^{2}}{2 \omega} \quad \Delta k_{\perp}^{2}=\hat{q} \Delta t
$$

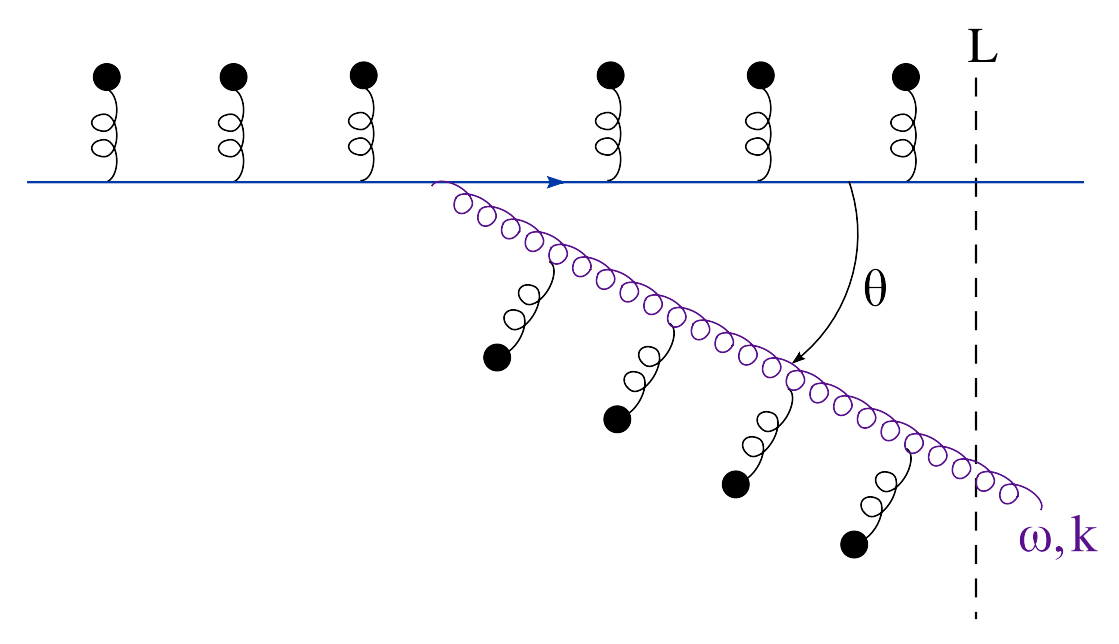

Time scale for the branching process $\tau_{\mathrm{br}}(\omega) \sim \sqrt{\frac{2 \omega}{\hat{q}}}$

Medium of finite extent $\quad \tau_{\text {br }} \lesssim L \Rightarrow \omega \lesssim \omega_{c} \quad \omega_{c} \sim \hat{q} L^{2}$ 
Formation time and emission angle

Typical branching $k T$ and angle

$$
\begin{aligned}
& k_{\mathrm{br}}^{2}=\hat{q} \tau_{\mathrm{br}} \\
& \theta_{\mathrm{br}} \sim k_{\mathrm{br}} / \omega \sim\left(\hat{q} / \omega^{3}\right)^{1 / 4}
\end{aligned}
$$

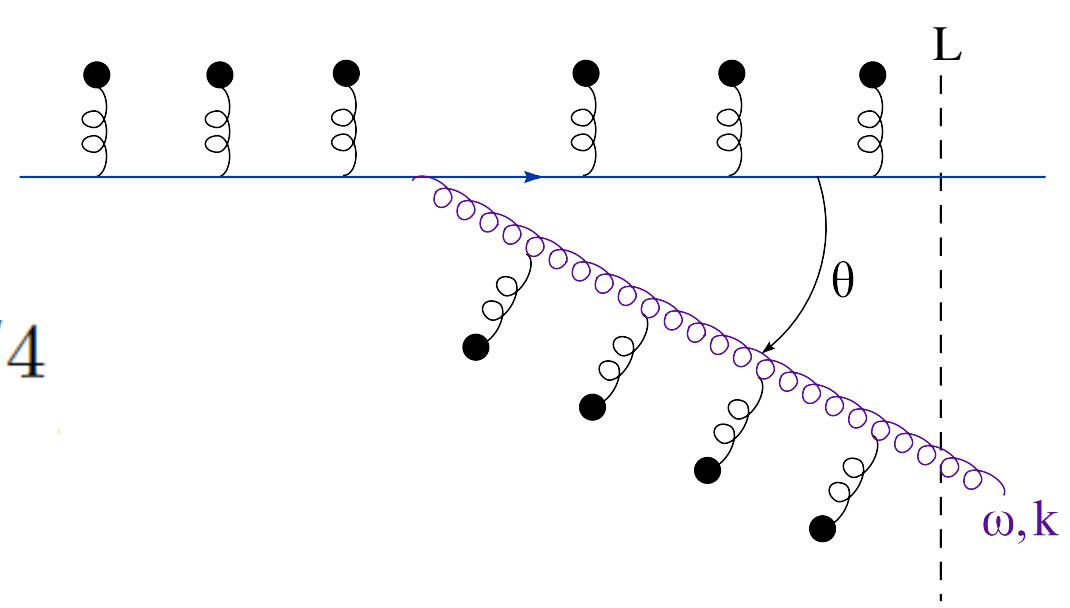

Hard gluon: small angle, long time

$$
\tau_{\mathrm{br}} \lesssim L \quad \omega \lesssim \omega_{c} \quad \theta_{\mathrm{br}} \gtrsim \theta_{c}
$$

Soft gluon: large angle, short time

$$
\tau_{\text {br }} \ll L \quad \omega \ll \omega_{c} \quad \theta_{\text {br }} \gg \theta_{c}
$$




\section{BDMPSZ spectrum}

$$
\omega \frac{\mathrm{d} N}{\mathrm{~d} \omega} \simeq \frac{\alpha_{s} N_{c}}{\pi} \sqrt{\frac{\omega_{c}}{\omega}} \equiv \bar{\alpha} \sqrt{\frac{\omega_{c}}{\omega}}=\bar{\alpha} \frac{L}{\tau_{\mathrm{br}}(\omega)}
$$

\section{Hard emissions}

- rare events, with probability $\sim \mathcal{O}\left(\alpha_{s}\right)$

- dominate energy loss: $E_{\text {hard }} \sim \alpha_{s} \omega_{c}$

- small angle, not important for di-jet asymmetry

\section{Soft emissions}

- frequent, with probability $\sim \mathcal{O}(1)$

- weaker energy loss: $E_{\text {soft }} \sim \alpha_{s}^{2} \omega_{c}$

- but arbitrary large angles: control di-jet asymmetry

large angles emissions are dominated by soft multiple branchings 
Multiple branchings

(de)-coherence

in-medium cascade

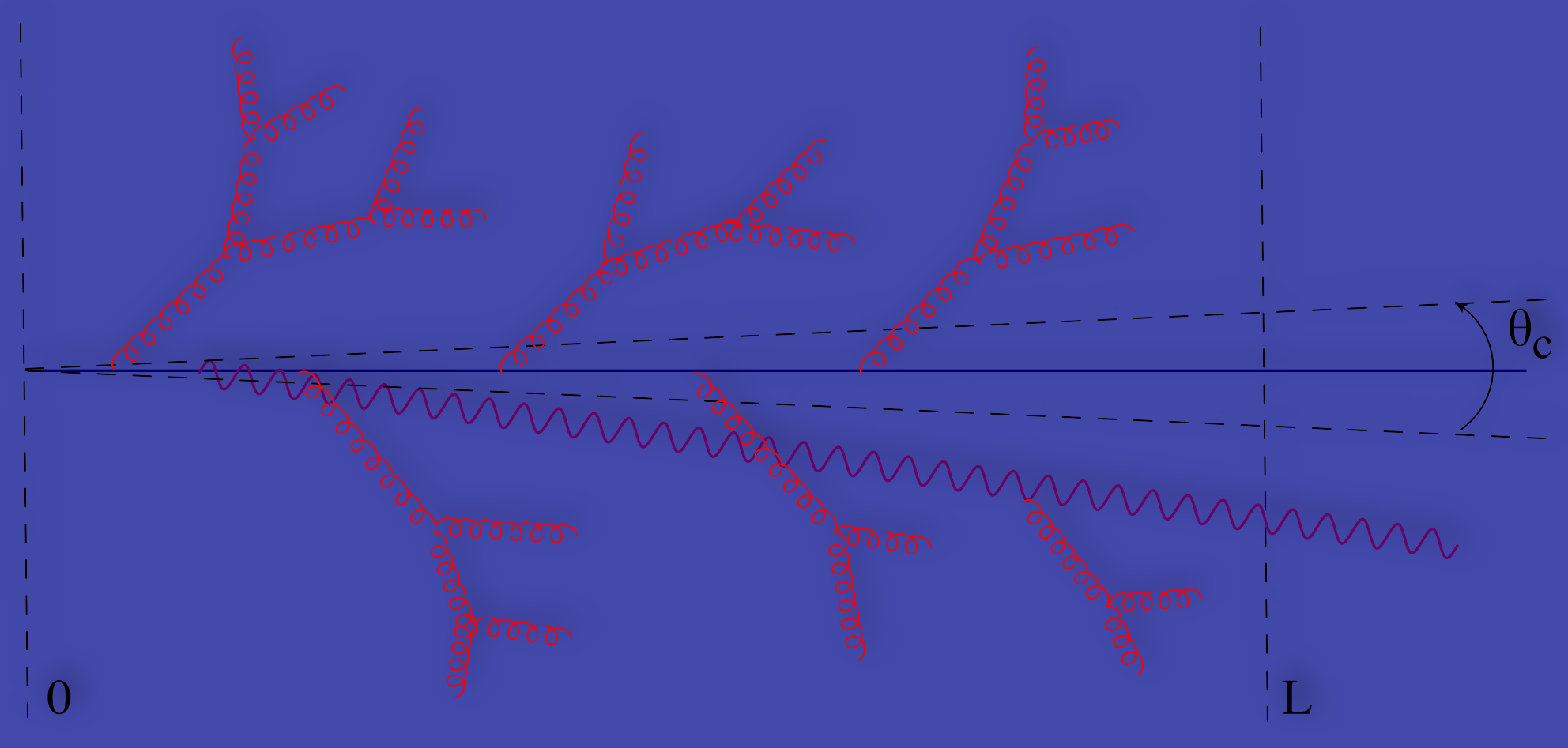




\section{Multiple emissions}

A priori complicated by interferences

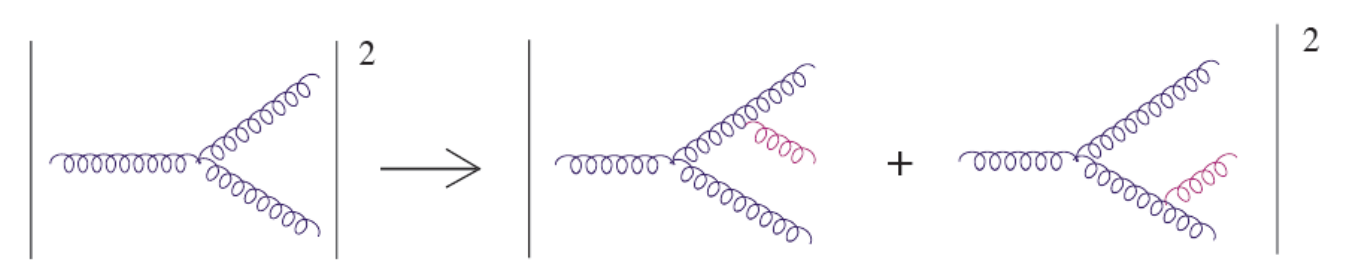

In vacuum, these interferences lead to angular ordering

In medium color coherence is rapidly lost via rescattering

Mehtar-Tani, Salgado, Tywoniuk (1009.2965; 1102.4317)

Iancu, Casalderey-Solana (1106.3864)

In medium, interference effects are subleading

Independent emissions are enhanced by a factor $L / \tau_{f}$

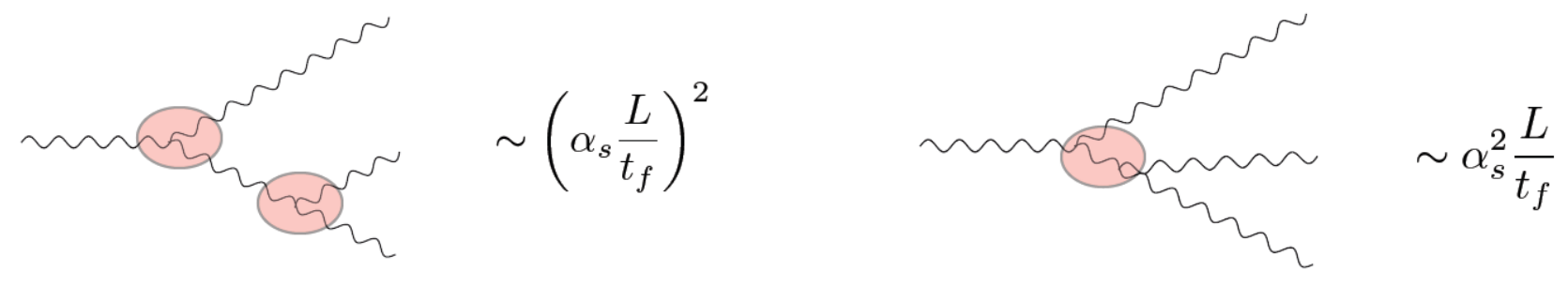

JPB, F. Dominguez, E. Iancu, Y. Mehtar-Tani, arXiv: 1209.4585 


\section{Resumming the leading terms}

When $\bar{\alpha} L / \tau_{b r} \sim 1$ all powers of $\bar{\alpha} L / \tau_{b r} \sim 1$ need to be resummed.

Since independent emissions dominate, the leading order resummation is equivalent to a probabilistic cascade, with nearly local branchings

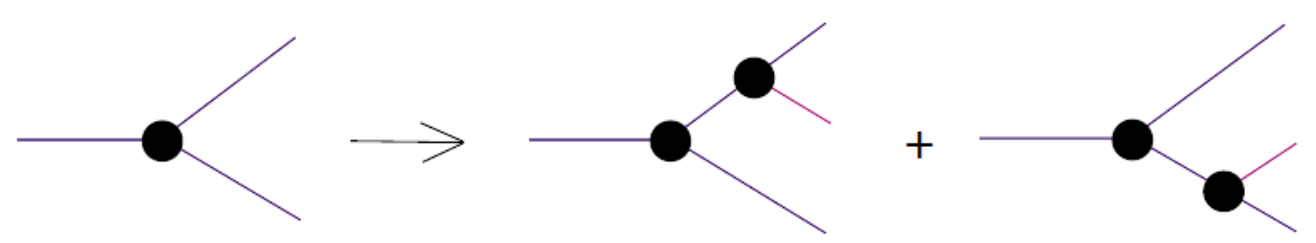

Blob: BDMPSZ spectrum

Line: momentum broadening

JPB, Dominguez, Iancu and Mehtar-Tani (arXiv:1209.4585)

Note: already implemented in Monte Carlo codes

MARTINI (Jeon, Gale, Schenke)

Q Pythia (Armesto, Salgado et al)

Stachel, Wiedemann, Zapp 
Evolution equation for the gluon spectrum (after integration over $k T$ )

$$
D(x, \tau)=x \frac{d N}{d x} \quad x=\frac{\omega}{E}
$$

Probabilistic equation ('gain-loss')

$$
\frac{\partial D(x, \tau)}{\partial \tau}=\int \mathrm{d} z \mathcal{K}(z)\left[\sqrt{\frac{z}{x}} D\left(\frac{x}{z}, \tau\right)-\frac{z}{\sqrt{x}} D(x, \tau)\right]
$$

Formally analogous to DGLAP. But very different kernel... and physics.

$\Longrightarrow A Q C D$ cascade of a new type 


\section{In-medium QCD cascade \\ Turbulent flow at small $x$}

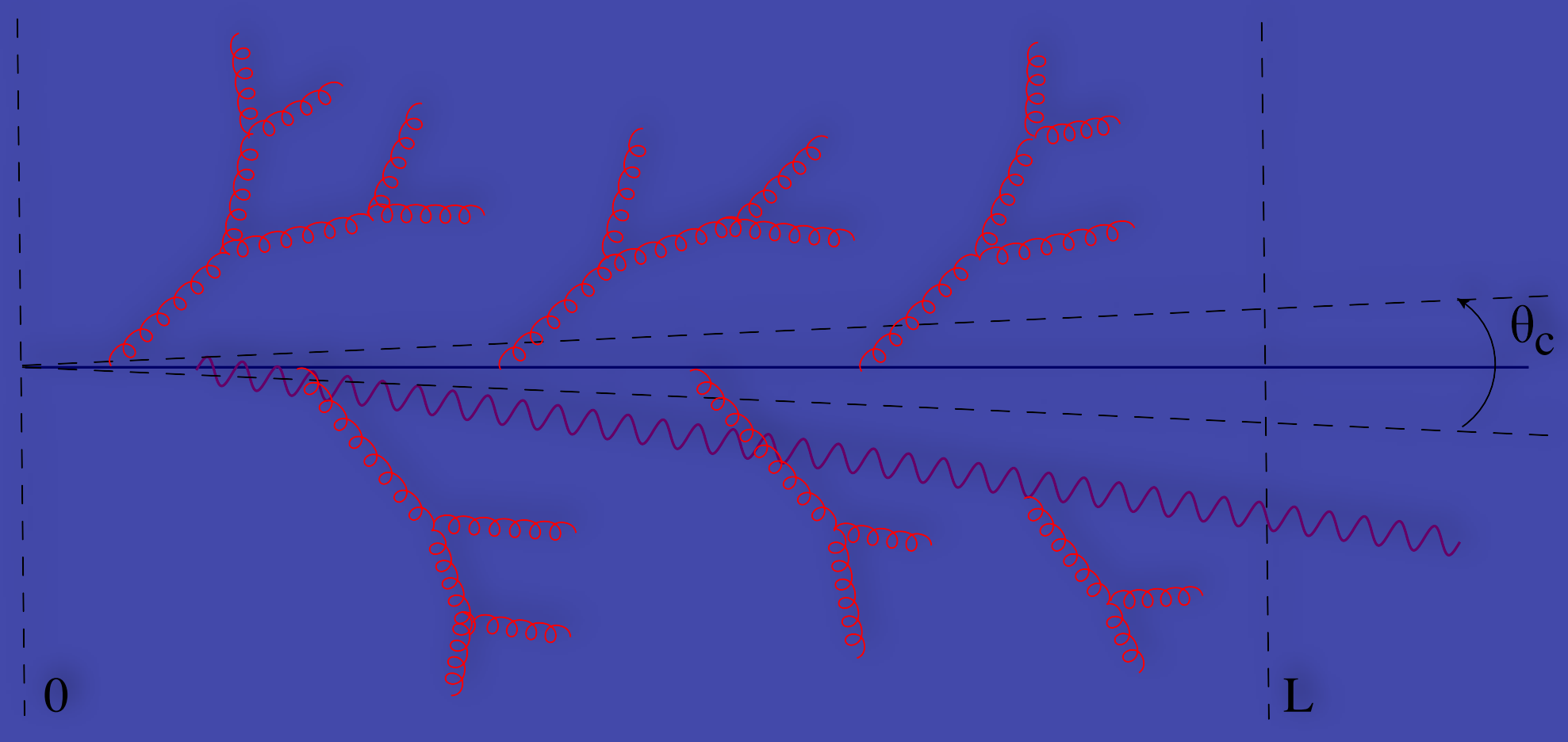




\section{short times}

$$
\frac{\partial D(x, \tau)}{\partial \tau}=\int \mathrm{d} z \mathcal{K}(z)\left[\sqrt{\frac{z}{x}} D\left(\frac{x}{z}, \tau\right)-\frac{z}{\sqrt{x}} D(x, \tau)\right]
$$

At short time, single emission by the leading particle $\left(D_{0}(\tau=0, x)=\delta(x-1)\right)$ $D$ is the BDMSZ spectrum

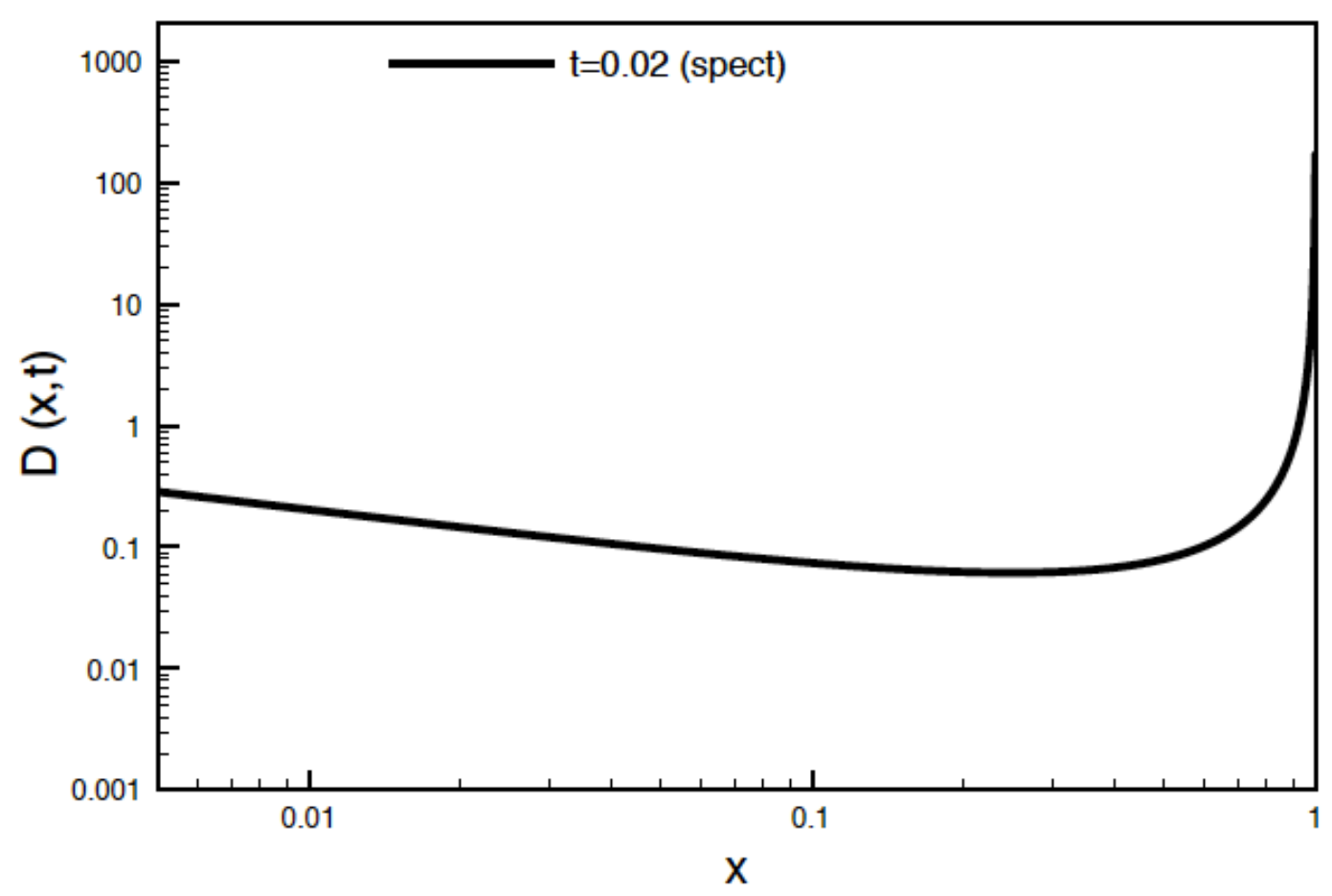

How do multiple branchings affect this spectrum? 
Naively, we could expect the spectrum to be depleted at large $x$ and to increase rapidly at small $x$, so as to keep the total energy constant

$$
\int_{0}^{1} \mathrm{~d} x D(\tau, x)=1
$$

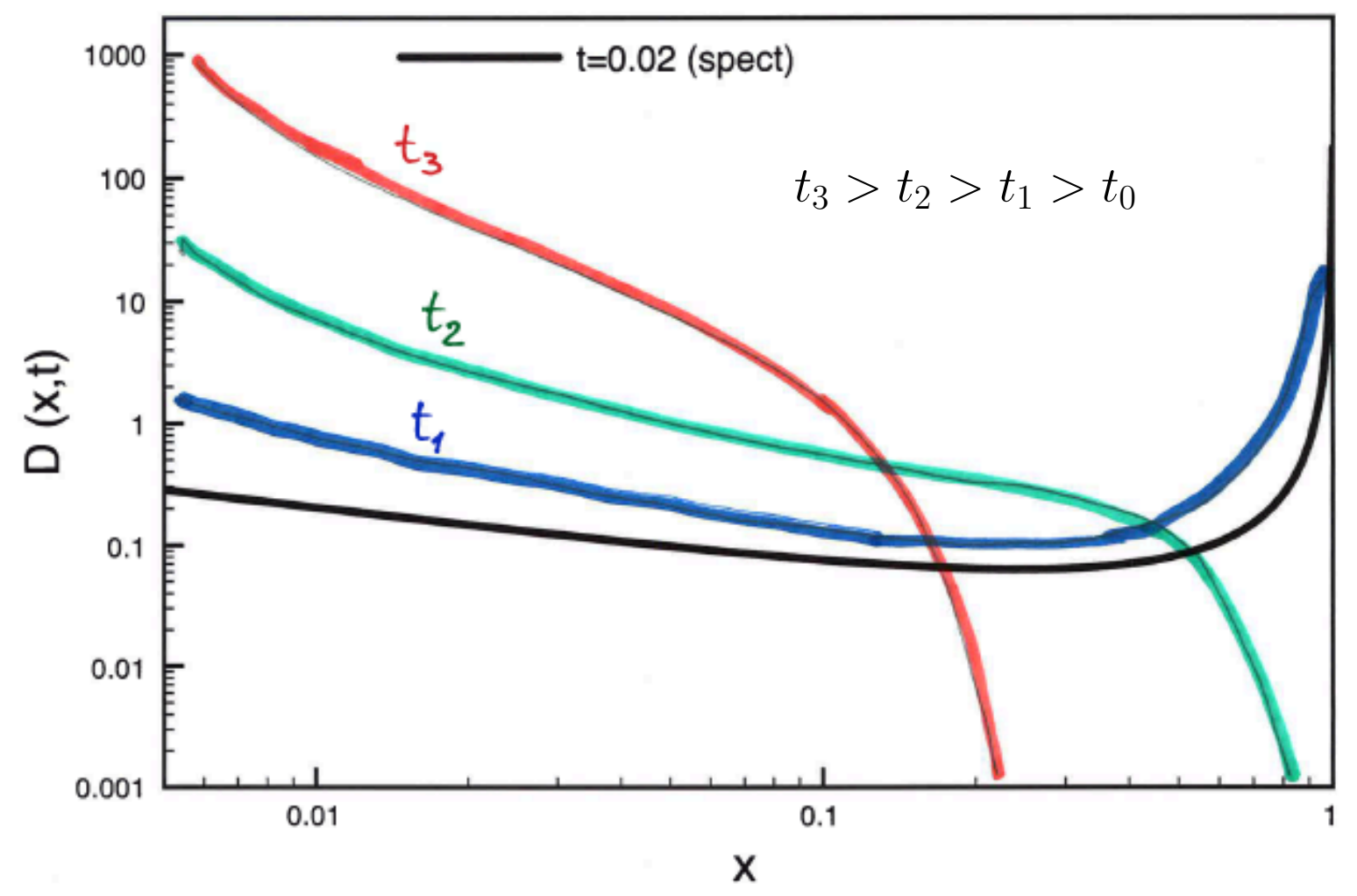


But this is not what happens!

One finds (exact result) $\quad D(x, t) \simeq \frac{t}{\sqrt{x}} \mathrm{e}^{-\pi t^{2}} \quad$ for $x \ll 1$

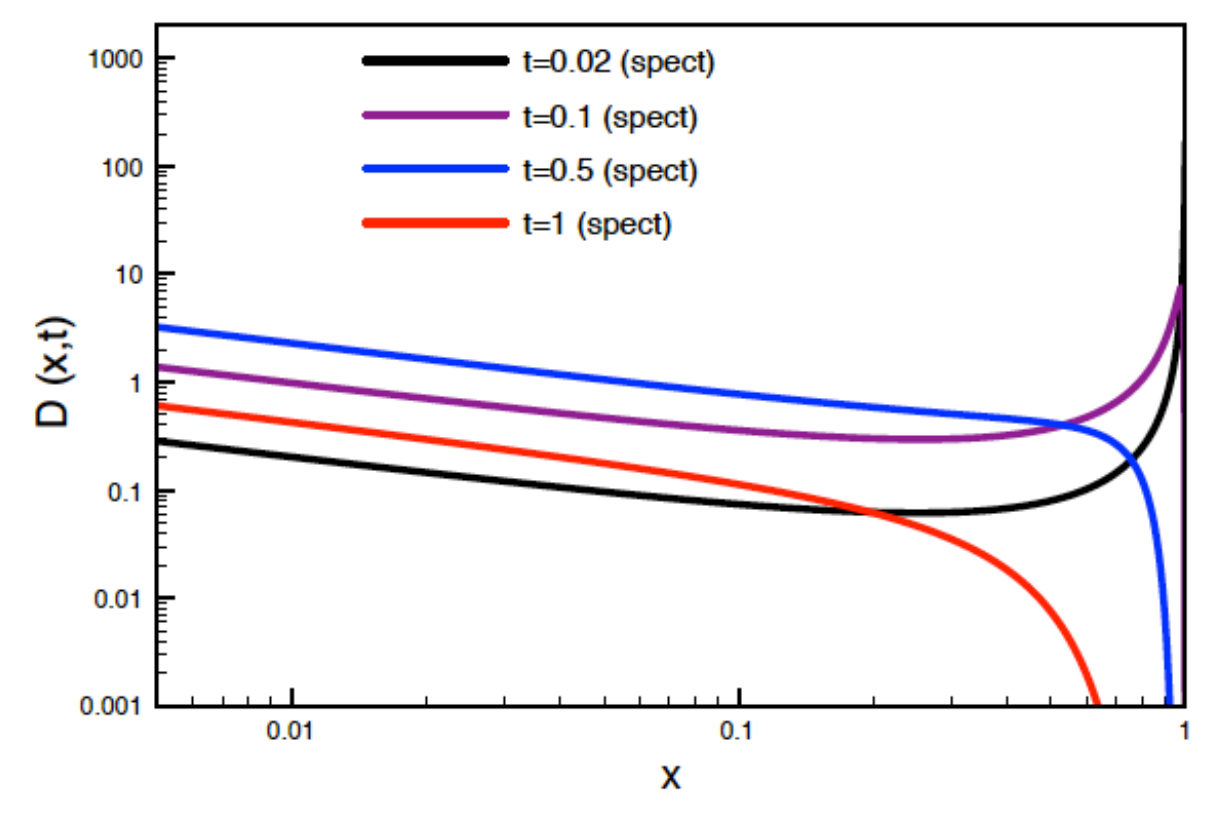

Fine (local) cancellations between gain and loss terms BDMPS spectrum emerges as a fixed point, scaling, spectrum Characteristic features of wave turbulence (Kolmogoroz, Zakharov) 


\section{The source problem}

At this (fixed) point

ALL the energy flows

through the whole

system

Energy is injected at $x=1$, at a

constant rate

The population of

the various $x$-modes

grows, keeping the

shape of the

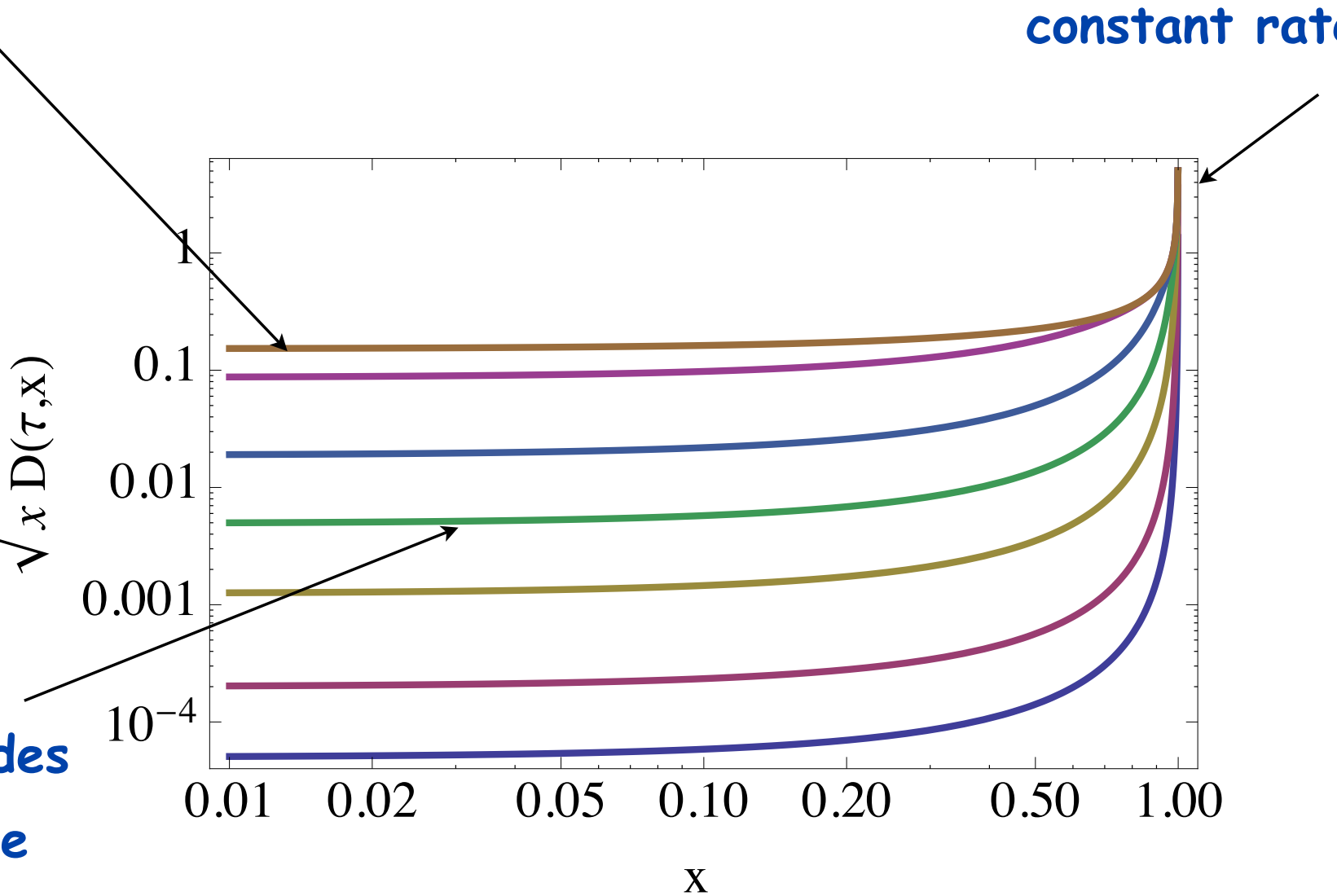

spectrum at small $x$ 
Relevance to di-jet asymmetry

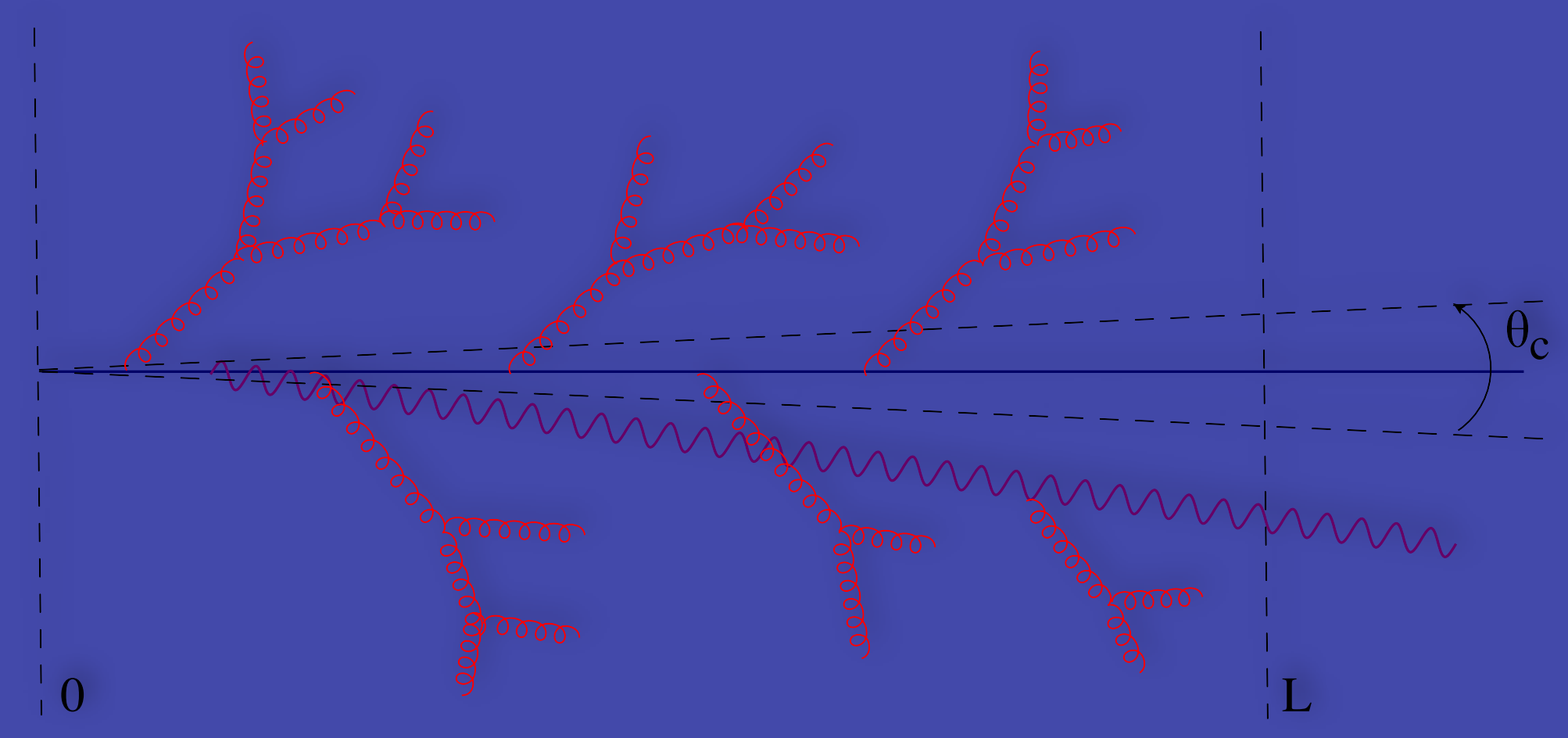




\section{Evolution of the inclusive spectrum}

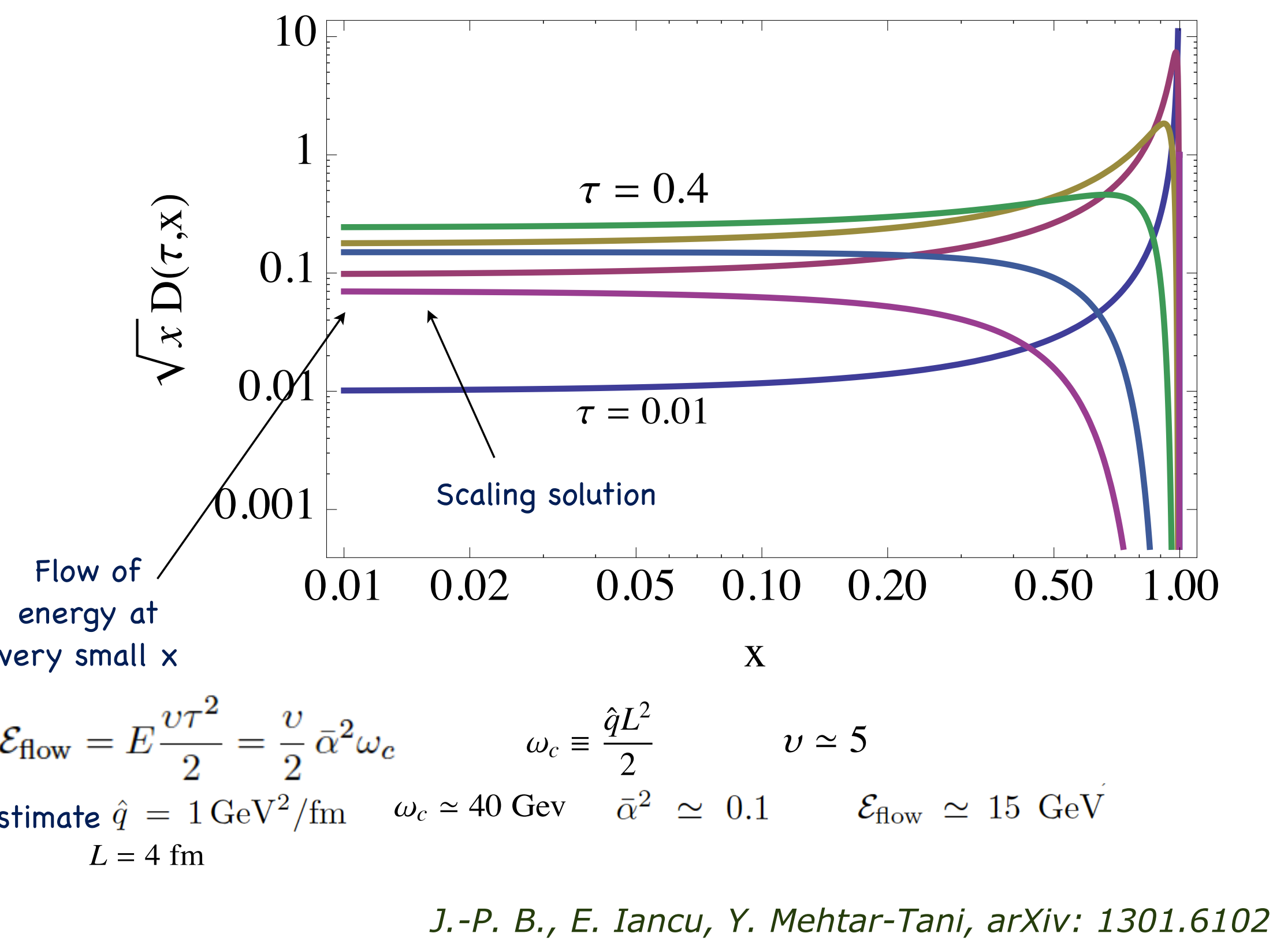




\section{Energy flow at large angle}

$E_{\text {in }}$ energy in the jet with $x>x 0$

$E_{\text {out }}$ energy in the spectrum with $x<x 0$

$E_{\text {out }}+E_{\text {flow }}$ energy out of the jet cone
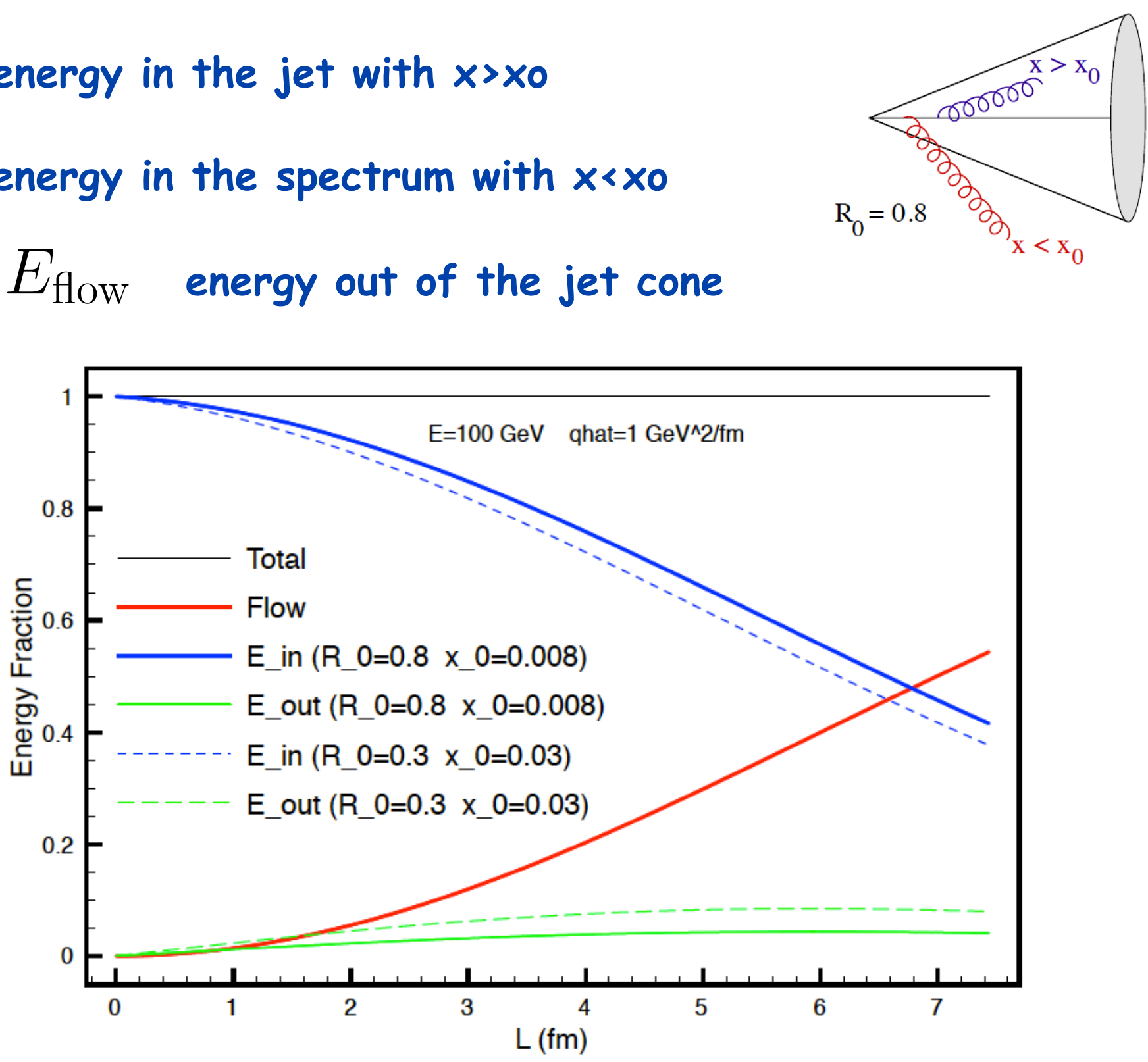


\section{conclusions}

In-medium cascade is very different from the in-vacum cascade (no angular ordering, turbulent flow)

Provides a simple and natural mechanism for transfer of jet energy towards very small angles 


\title{
Ridge correlations in $\mathrm{pp} / \mathrm{pPb}$ : hydro perspective
}

\author{
Piotr Bożek
}

PB Phys. Rev. C85 (2012) 014911

PB, W. Broniowski Phys. Lett. B718 (2013) 1557

PB, W. Broniowski Phys. Lett. B720 (2013) 250

PB, W. Broniowski arXiv:1304.3044 

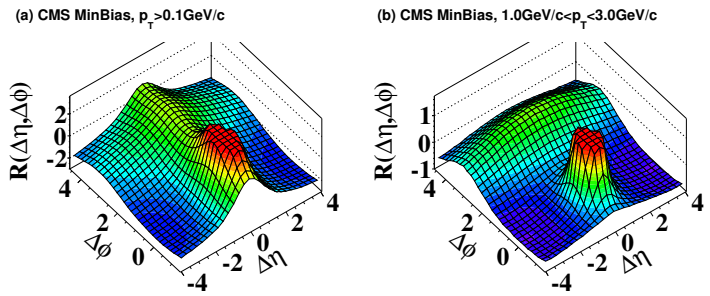

(c) CMS N $\geq 110, \mathrm{p}_{\mathrm{T}}>0.1 \mathrm{GeV} / \mathrm{c}$

(d) $\mathrm{CMS} \mathrm{N} \geq 110,1.0 \mathrm{GeV} / \mathrm{c}<\mathrm{p}_{\mathrm{T}}<3.0 \mathrm{GeV} / \mathrm{c}$
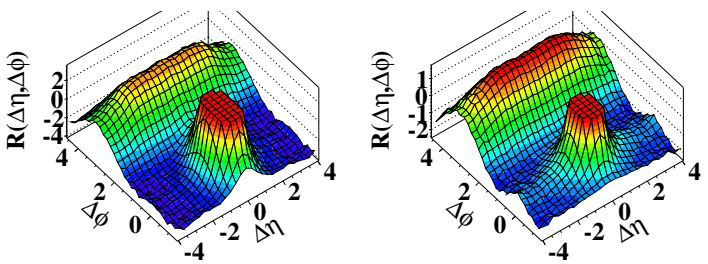

intriguing ridge seen in $p-p$ 


\section{Flow in $\mathrm{A}-\mathrm{A}$}
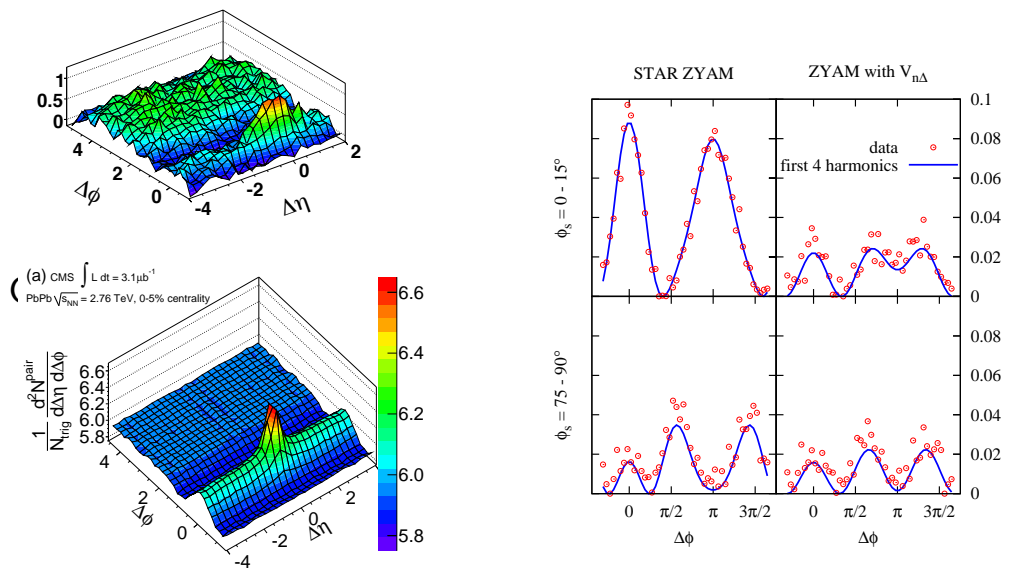

Luzum arXiv: 1011.5773 - flow harmonics

\section{Piotr Bożek}

Flow in $\mathrm{p}-\mathrm{p}$ and $\mathrm{p}-\mathrm{Pb}$ ? 
Collective elliptic flow in $\mathrm{p}-\mathrm{Pb}$ ?

- Large enough density? yes

- Large enough eccentricity? yes?

- Large enough size? (?) but should and can be tested in pA

- Small enough gradients? no - beyond viscous hydro 


\section{$\mathrm{p}-\mathrm{Pb}, \mathrm{d}-\mathrm{Pb} @ \mathrm{LHC}$}

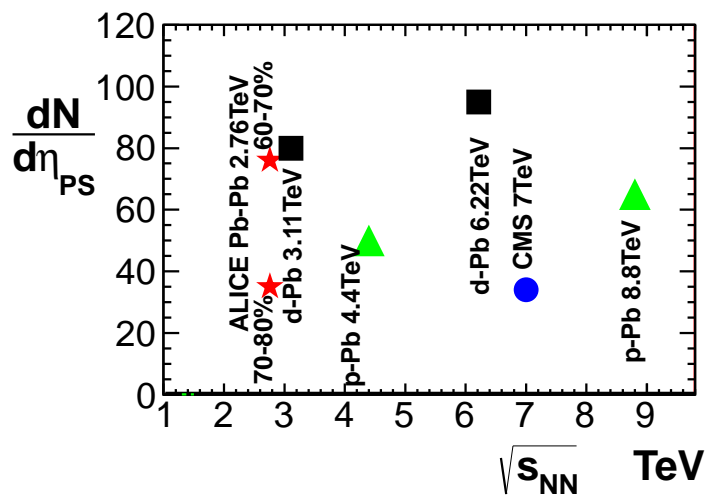

large multiplicity - large fireball - collective expansion? 


\section{Fireball in $\mathrm{p}-\mathrm{Pb}$}
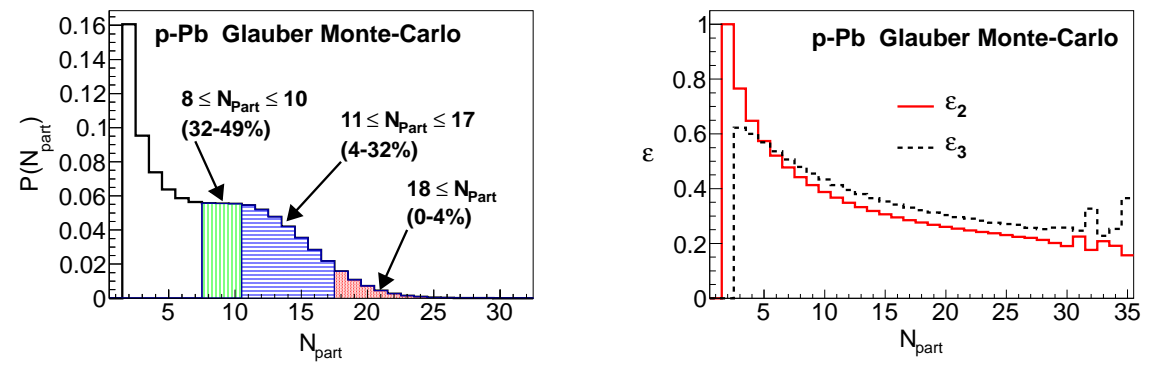


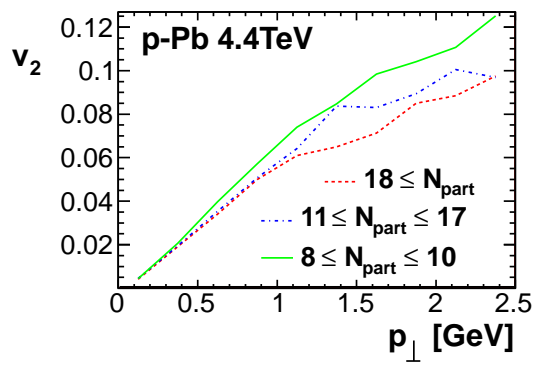

elliptic flow in $\mathrm{p}-\mathrm{Pb}$

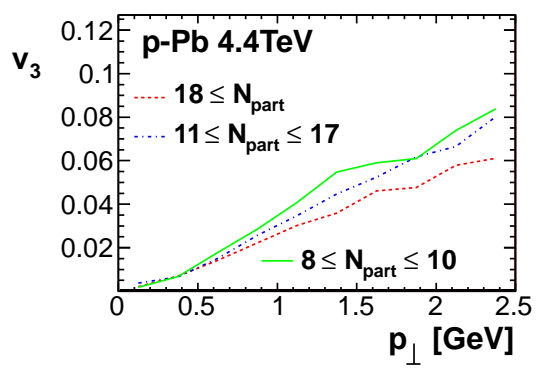

triangular flow 


\section{$\mathrm{d}-\mathrm{Pb}$}

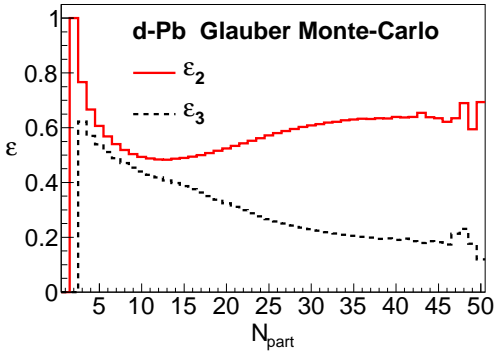

large elliptic flow
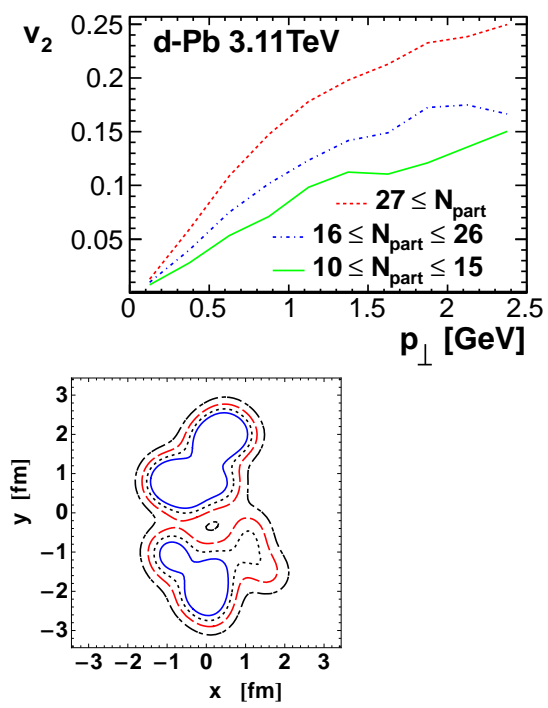
prediction 11.2011

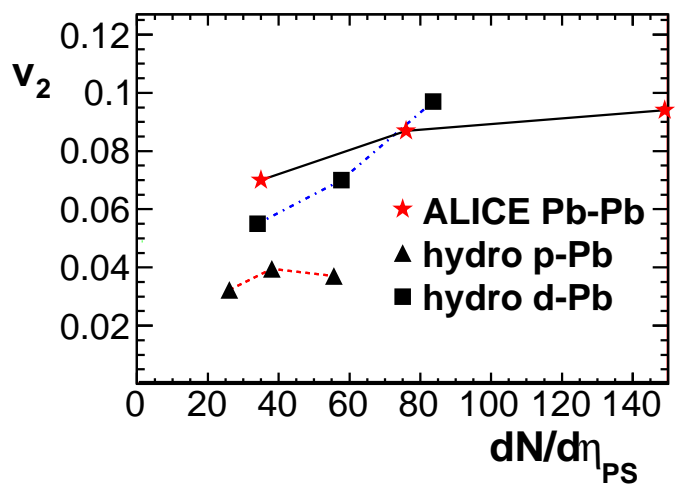

- collective flow effects $\simeq$ peripheral $\mathrm{Pb}-\mathrm{Pb}$

- can be observed

- $\mathrm{p}-\mathrm{Pb}(\mathrm{d}-\mathrm{Pb})$ is not $\mathrm{p}-\mathrm{p}$ superposition

- only p-p as baseline 
First results on pPb@5.02TeV - ALICE, CMS, ATLAS

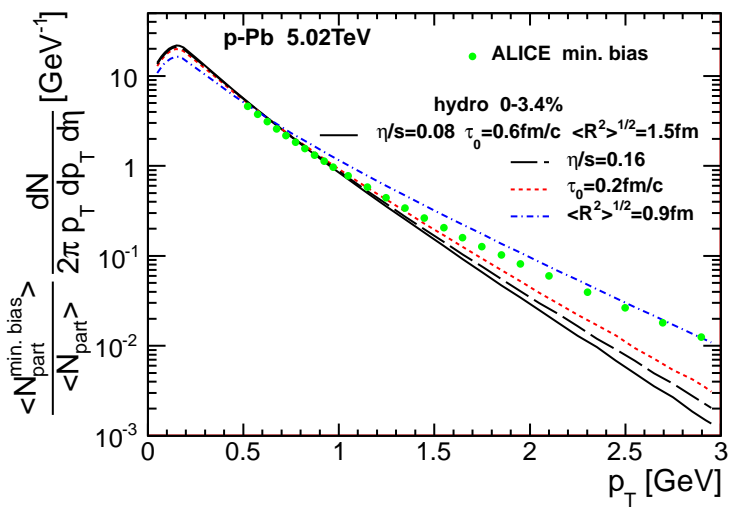

- statistical for $p_{\perp}<1.5-2 \mathrm{Gev}$

- early flow, viscosity, density profile? 


\section{Ridge in $\mathrm{p}-\mathrm{Pb}$}

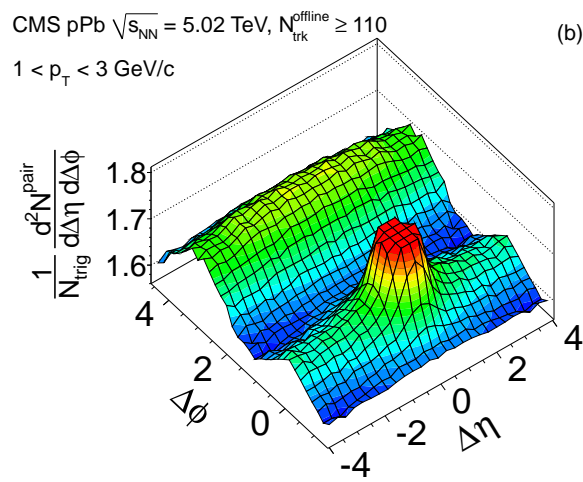
(a) $\mathrm{N}_{\text {trk }} \geq 110,1<\mathrm{p}_{\mathrm{T}}<3 \mathrm{GeV}$

(b)

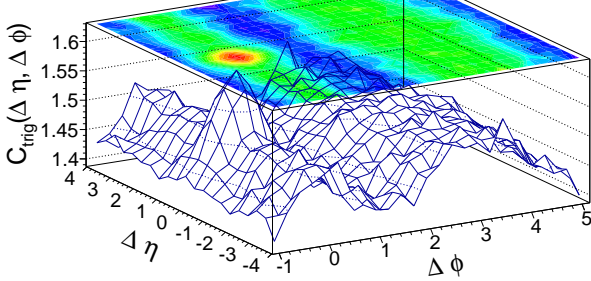




\section{Charge balancing}

local charge conservation

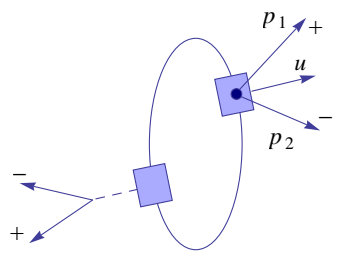

charge balance function

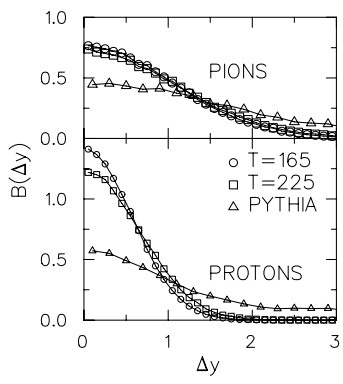

Bass et al. (2000) 


\section{Correlation function per pair}

\section{ATLAS}

ATLAS $\mathrm{p}+\mathrm{Pb} \sqrt{\mathrm{S}_{\mathrm{NN}}}=5.02 \mathrm{TeV}$ $\int \mathrm{L} \approx 1 \mu \mathrm{b}^{-1} \quad 0.5<\mathrm{p}_{\mathrm{T}}^{\mathrm{a}, \mathrm{b}}<4 \mathrm{GeV}$

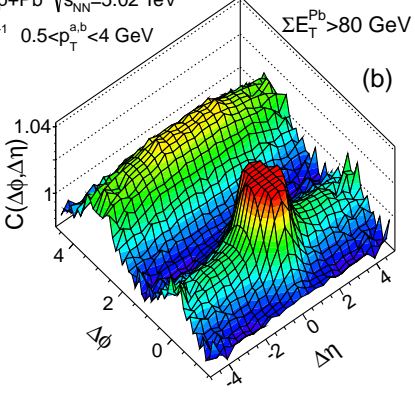

\section{hydro}

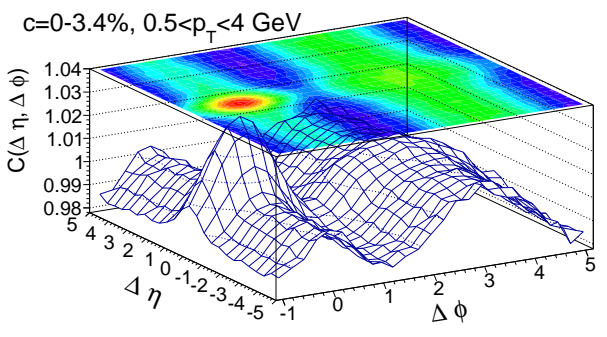


Glauber+NB

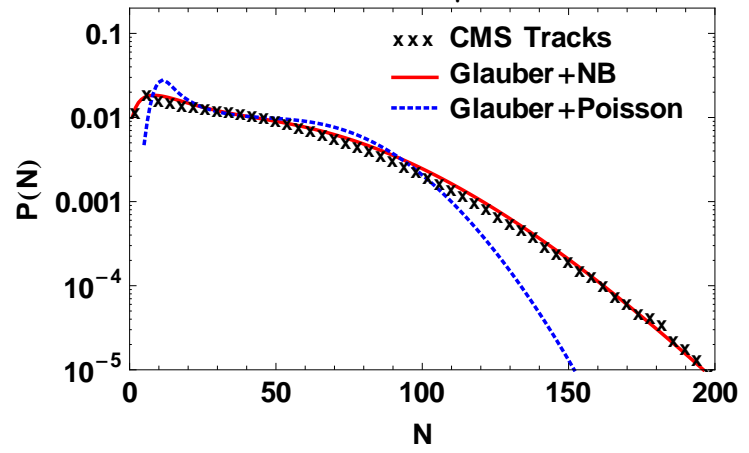

$P(n)=\sum_{i} P_{p a r t}(i) N_{p \lambda i, \kappa i}(n)$

Additional fluctuations of density ( compared to Glauber ) 


\section{Very different fireball size}

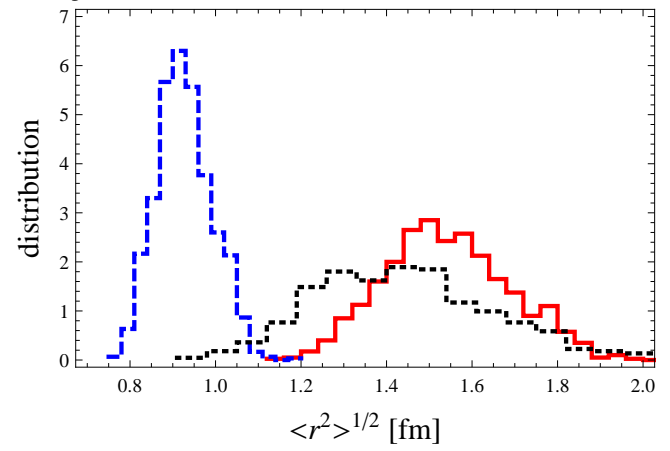

Compact $(0.9 \mathrm{fm})$, Glauber+NB $(1.4 \mathrm{fm})$, Standard $(1.5 \mathrm{fm})$ 


\section{Expansion of the fireball}

Compact source

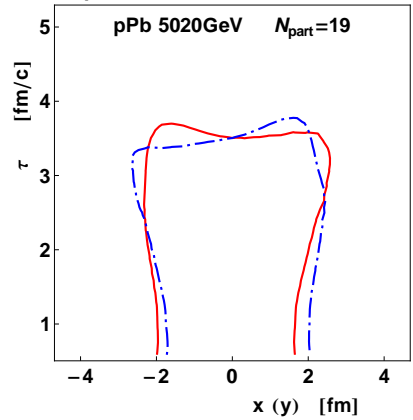

\section{Standard (large) source}

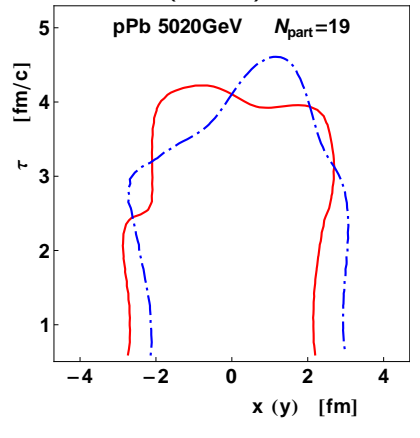




\section{Azimuthal correlations}

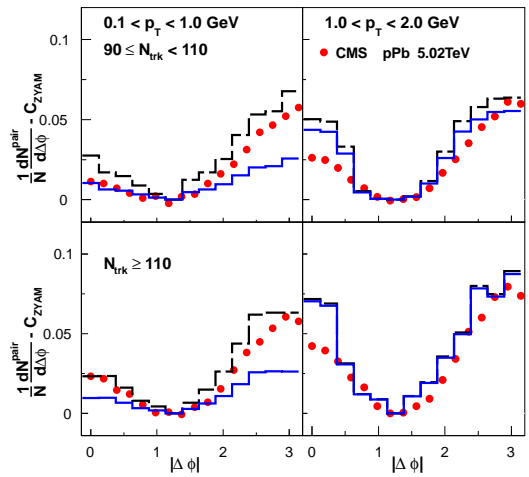

- collective flow 


\section{Azimuthal correlations ATLAS}

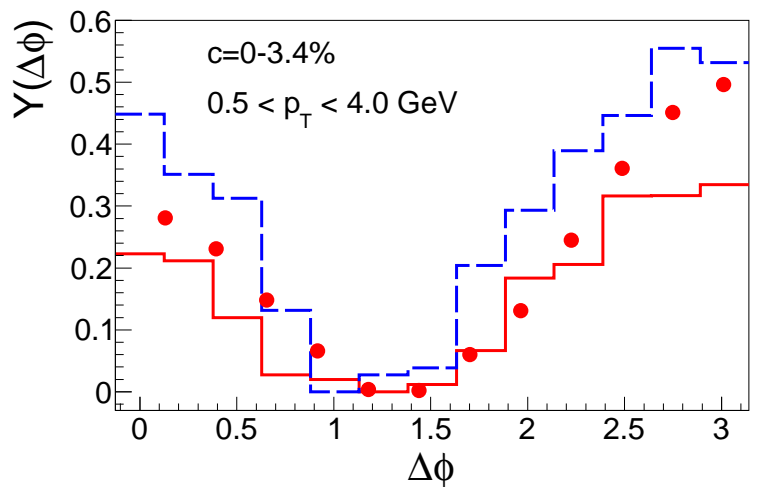


HBT systematics

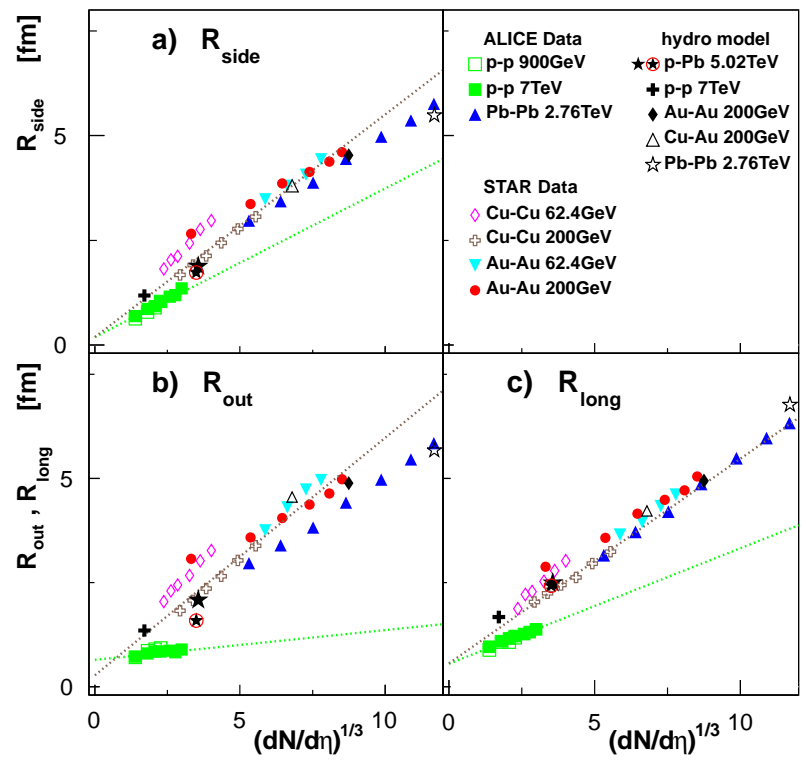

Piotr Bożek

Flow in $\mathrm{p}-\mathrm{p}$ and $\mathrm{p}-\mathrm{Pb}$ ? 


\section{d-Au $200 \mathrm{GeV}$}

- large eccentricity

- large $v_{2}$

- small $v_{3}$

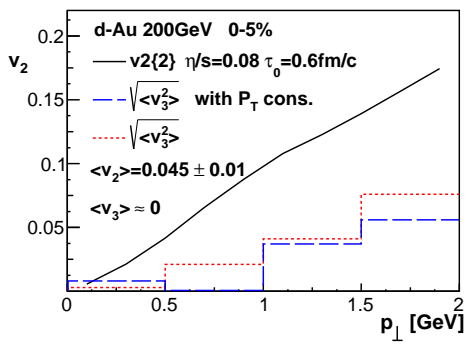

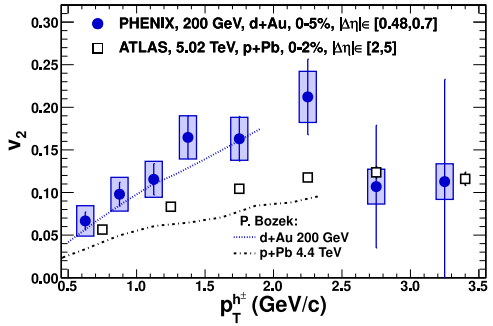

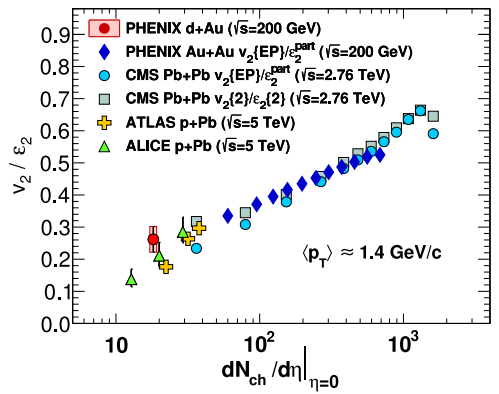


Hydro in pA, dA compares well with data

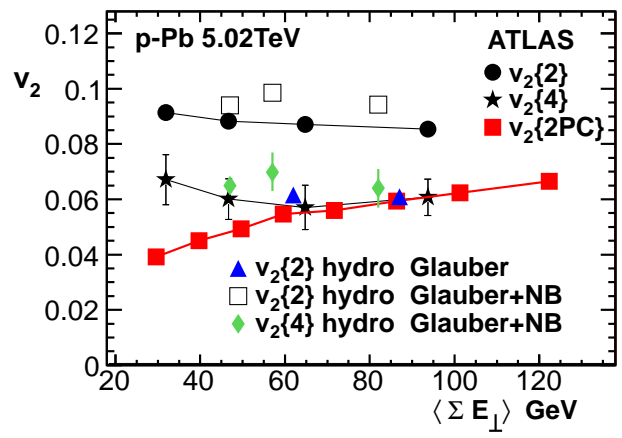

Initial conditions matter a lot! 


\section{Hydrodynamic flow in p-p?}

- Romatschke, Luzum - arXiv: 0901.4588 (overlap)

- Bozek - arXiv: 0911.2392 (flux-tubes)

- Chaudhuri - arXiv: 0912.2578 (hot-spots)

- Werner, Karpenko, Pierog - arXiv: 1011.0375 (EPOS)

- Bzdak, Schenke, Tribedy, Venugopalan - arXiv: 1304.3403 (IP-Glasma)

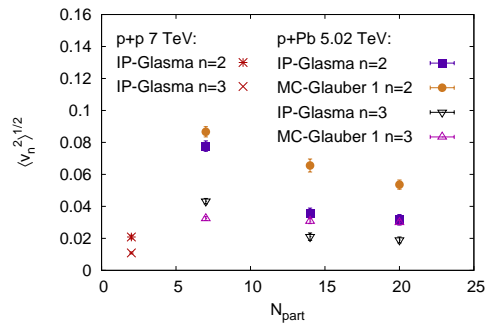

- Is hydrodynamics valid?

- What is the initial eccentricity ? 


\section{Fireball shape in pp}
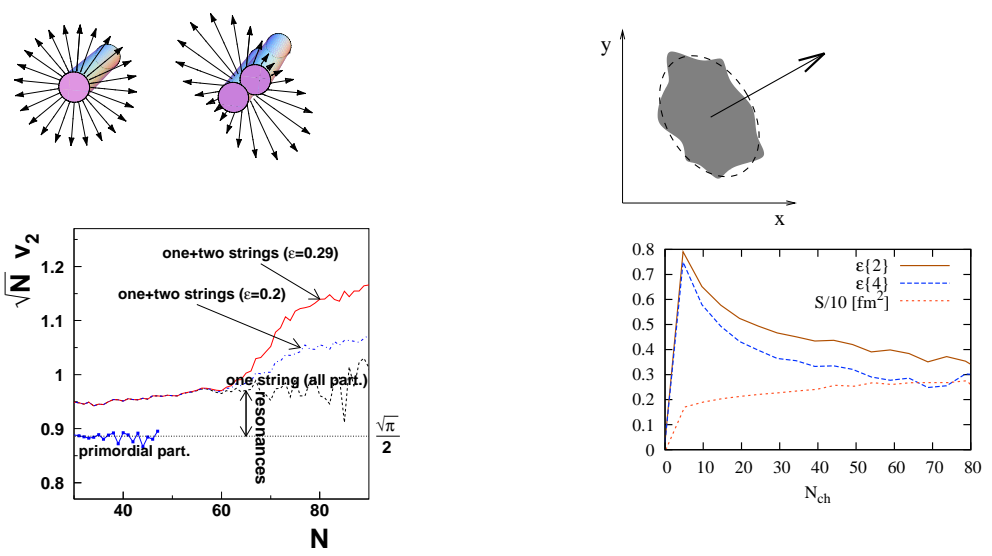

E.Asar et al., 1009.5643

PB, 0911.2397

EbE $v_{2}$ deconvolution? (ATLAS, Jia, Mohapatra) 


\section{Hydro ridge in p-p}

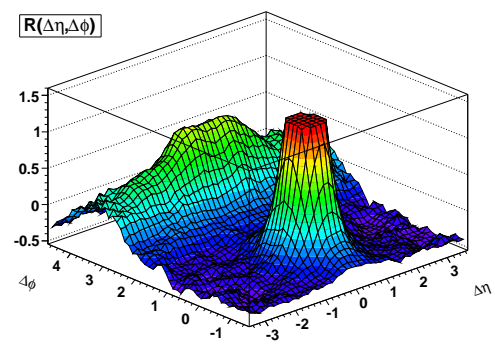

No collectivity

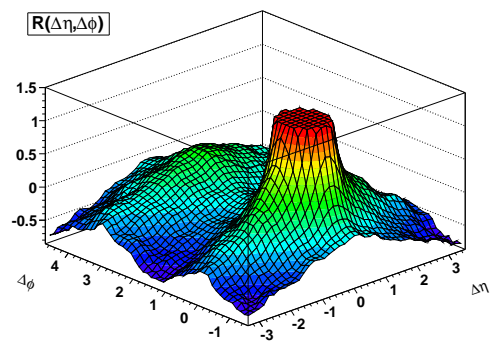

EPOS+hydro

Werner, Karpenko, Pierog arXiv: 1011.0375 


\section{Can we reduce the uncertainties}

go back to very peripheral A-A

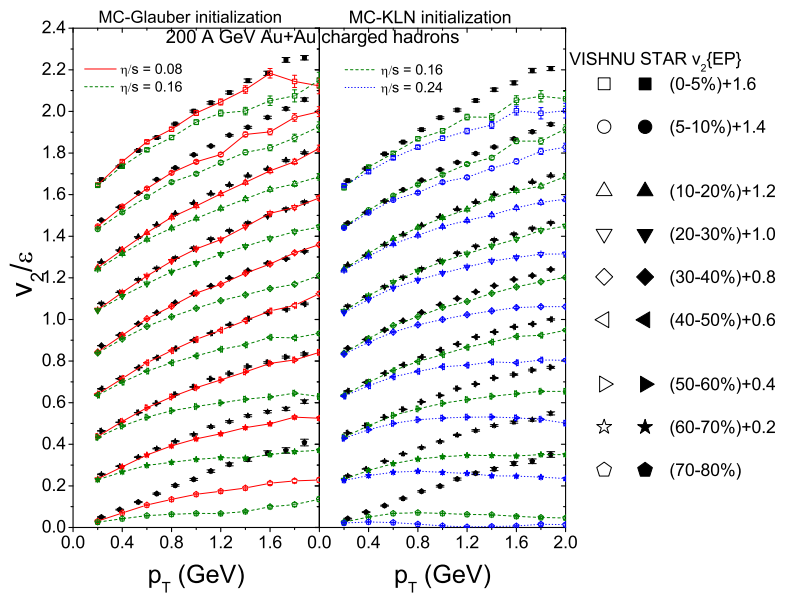

Song, Bass, Heinz, Hirano, Shen arXiv: 1101.4638 


\section{Summary}

- Ev-by-ev hydro for pA, dA

- Collectivity (FSI) in pPb@LHC, explains observed ridge and $v_{2}$

- d-Au data consistent with collective picture

- HBT radii in $\mathrm{p}-\mathrm{Pb}$ ?

- Is it collective flow ? - In manys ascpects consistent

- Other sources of correlations!

- Limits of hydro!

- Why hydrodynamics would work?

- Prospects: Experiment

... and initial state, peripheral A-A, p-p??, core-corona, disantengling: collectivity, CGC, jets ... 


\section{Pressure anisotropy}
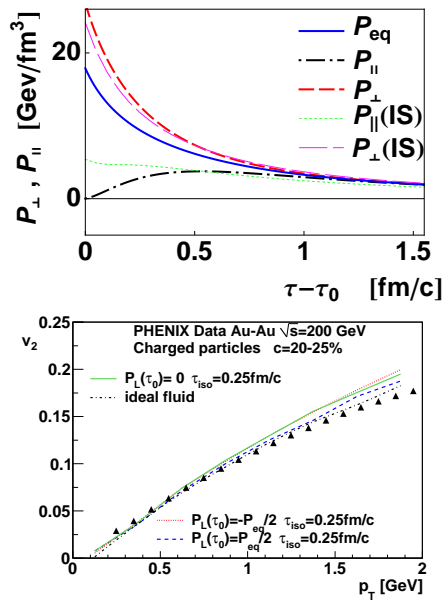

PB, I.Wyskiel, 2010
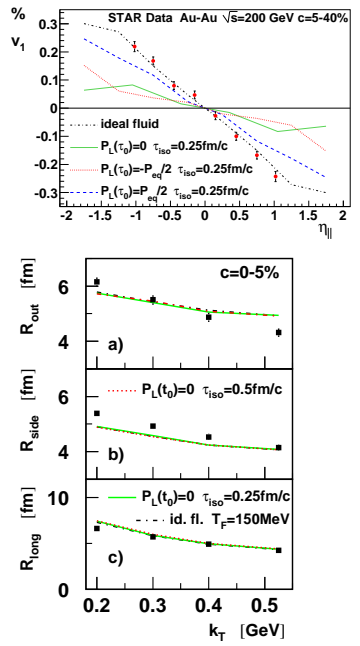

early pressure anisotropy irrelevant - Vredevoogd, Pratt, 2009 other higher gradients could be important? 


\section{Fireball anisotropy - flow asymmetry}
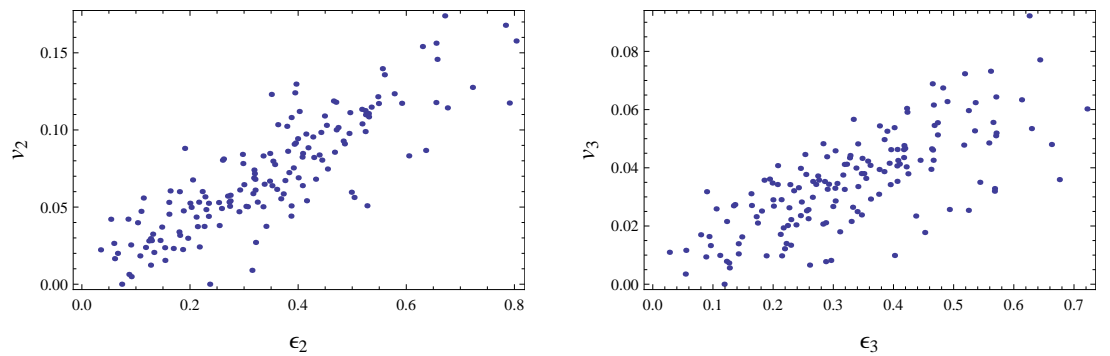

- Ev-by-Ev hydro response to geometry valid

- response strength depends on details 


\section{Eccentricity fluctuations, central collisions}

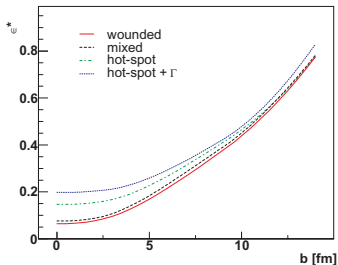

$$
\frac{\Delta \epsilon}{\epsilon}=\sqrt{\frac{4}{\pi}-1}
$$

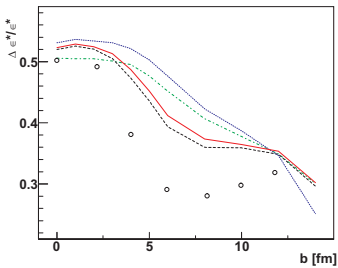

Broniowski, Bozek, Rybczynski arXiv: 0706.4266

central A-A, Gaussian limit $\longrightarrow v_{2}\{4\} \simeq 0$

central p-A (Glauber+NB) $\frac{\Delta \epsilon}{\epsilon}<\sqrt{\frac{4}{\pi}-1}$ and $v_{2}\{4\}>0$ 


\section{pA studies 1972-2013}

reminiscences 
The pA play

as seen through the eyes of one of the actors

Act 1

before the early 1970's

Act 2

The 1970's

Act 3

late 1970's, early 1980's

Act 4

Late 1980's, 1990's \& 2000's

Act 5

To-day
The " $A$ " of " $p A$ " is more of a nuisance than a help!

Is there too much or too little cascading?

Is there too much or too little quenching in the forward direction?

Who cares about the details of "pA" ? After all, it's only a reference!

Who is helping whom?

$p p \& p A$ the understanding of $A A$ or

$A A$ the understanding of $p p \& p A$ ? 
Cosmic ray interactions in nuclear emulsions $\approx 1950$

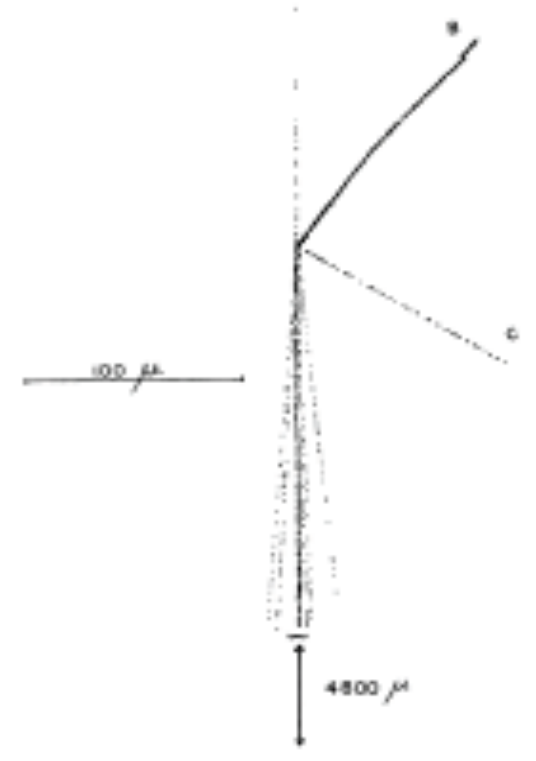

p-p collision

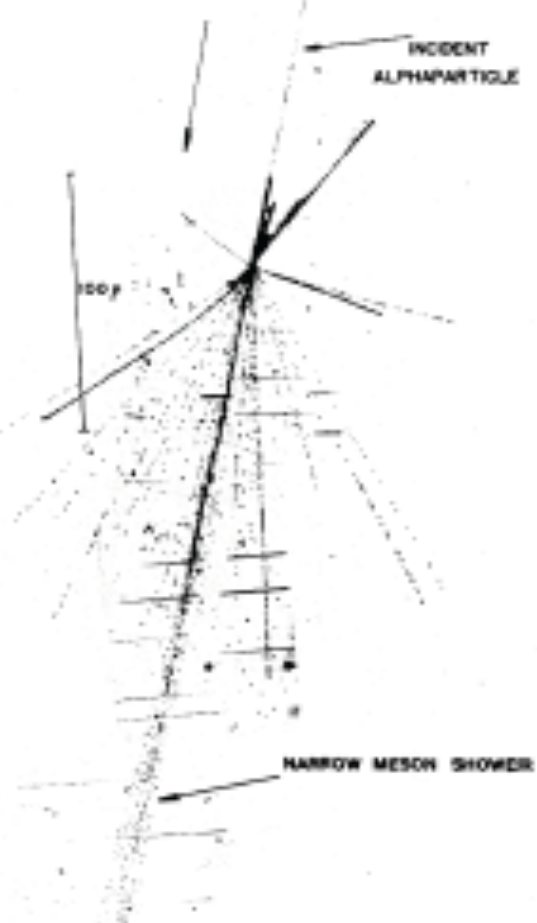

$\alpha$-emulsion collision

\section{visualph'ofos.com}

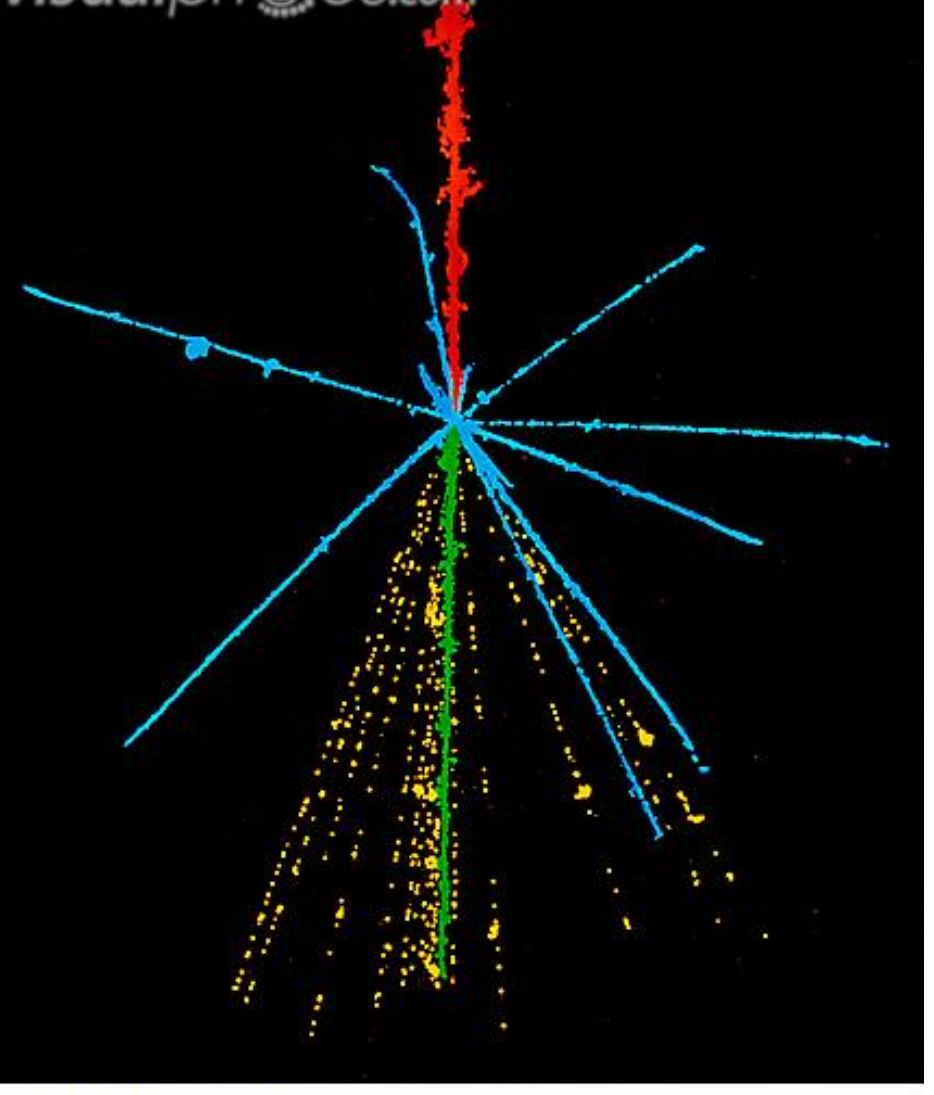

a134005 [RM] $\odot$ www.visualphotos.com

Composition of nuclear emulsion by weight: $83 \%(\mathrm{Ag}+\mathrm{Br}+\mathrm{l}), 16 \%(\mathrm{C}+\mathrm{N}+\mathrm{O}), 1 \% \mathrm{H}$ 
Echo Lake Calorimeter-Spark Chamber ( L.Jones et al. Preprint UM HE 74-23)

$$
\sqrt{s_{N N}}=13-31 G e V
$$
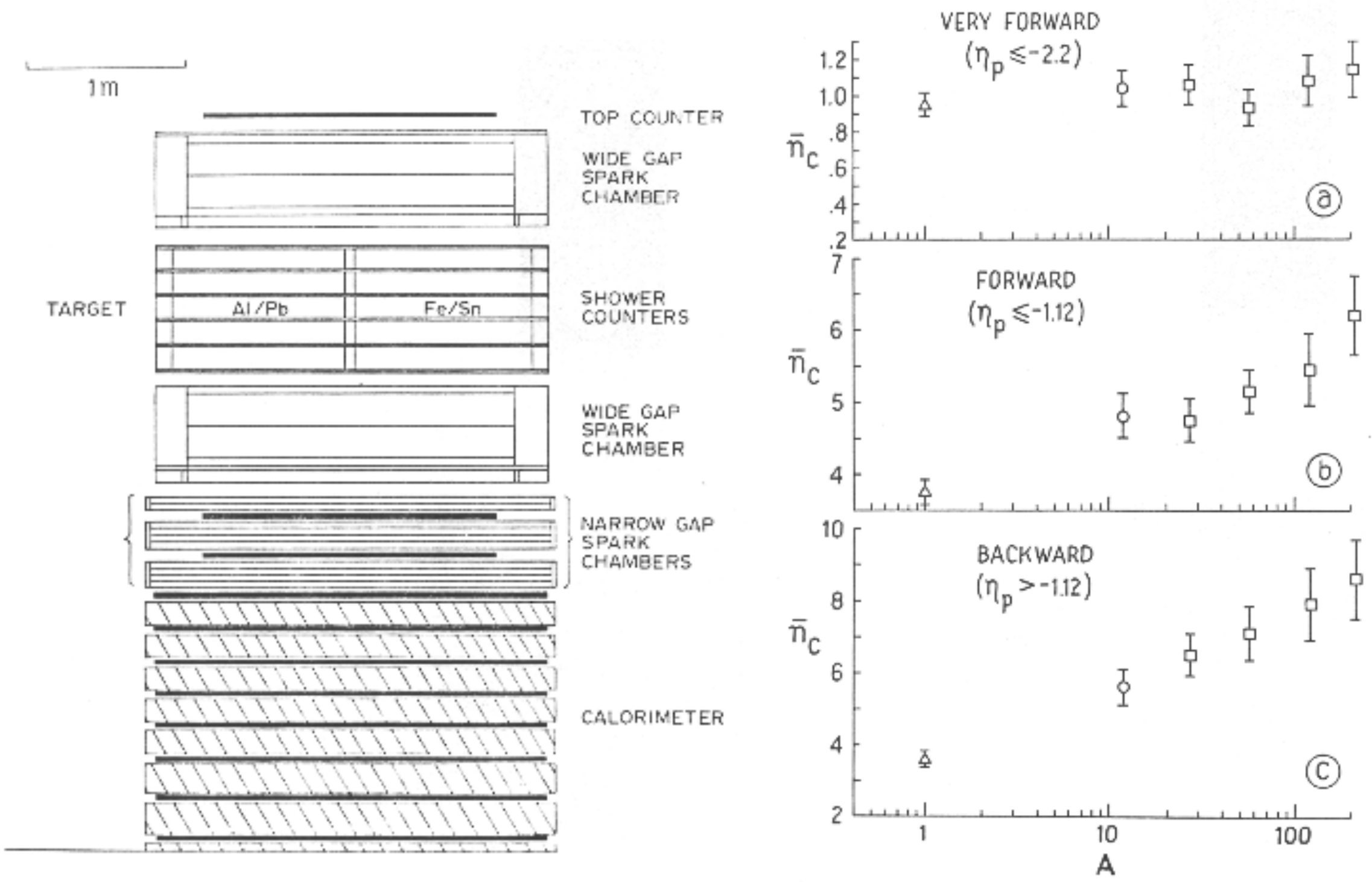
The pA play

as seen through the eyes of one of the actors

Act 1

before the early 1970's

Act 2

The 1970's

Act 3

late 1970 's, early 1980 's

Act 4

Late 1980's, 1990's \& 2000's

Act 5

To-day
The " $A$ " of " $p A$ " is more of a nuisance than a help!

Is there too much or too little cascading?

Is there too much or too little quenching in the forward direction?

Who cares about the details of "pA" ? After all, it's only a reference!

Who is helping whom?

$\mathrm{pp} \& \mathrm{pA}$ the understanding of $\mathrm{AA}$ or

$A A$ the understanding of $p p \& p A$ ? 


\section{Questions from the early 1970's}

- Mechanism of particle production in pp collisions?

- Space-Time evolution of the production process?

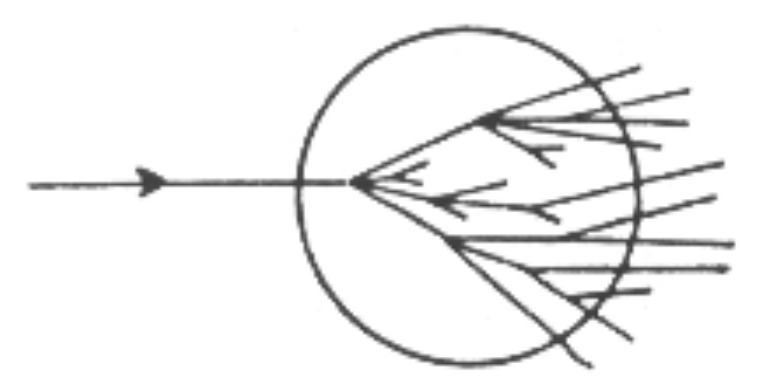

$$
\langle n\rangle_{A} \sim\langle n\rangle_{p}^{A_{p}^{1 / 3}}
$$

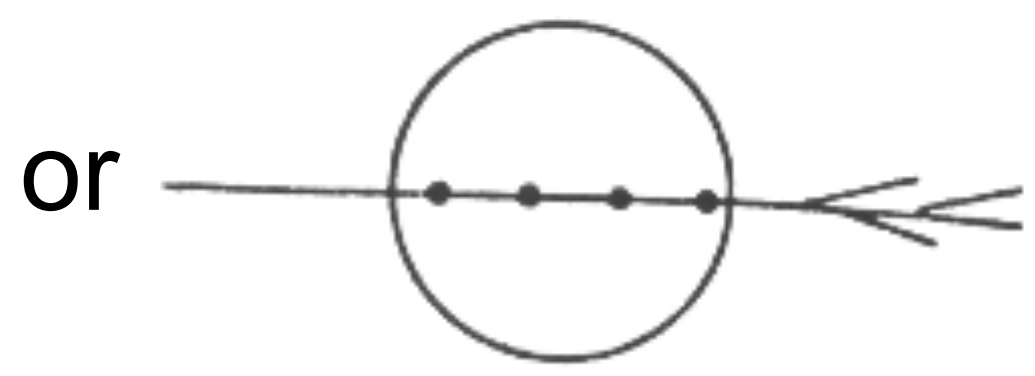

$$
\begin{gathered}
\langle n\rangle_{A} \sim\langle n\rangle_{p} \text { or } \\
\langle n(A, s)\rangle \sim\langle n(p, \bar{v})\rangle
\end{gathered}
$$

From Fermilab E178 proposal 


\section{Fashions in theory in 1970's}

Godfried's energy cascade model
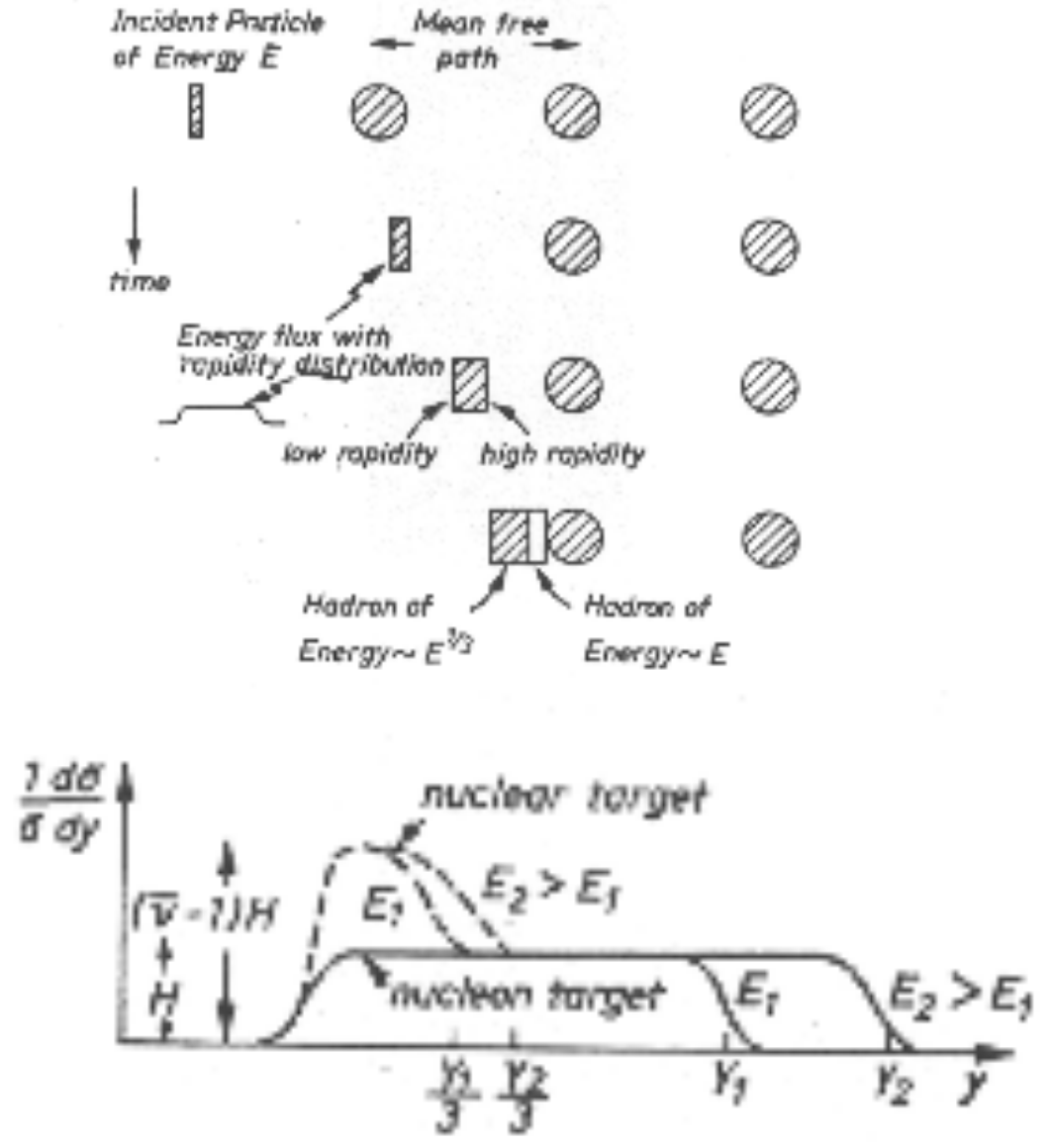

Parton and single chain multiperipheral type models

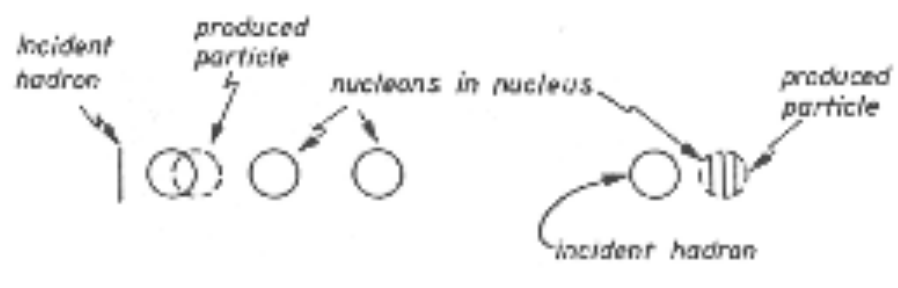

in rest frome of particles araducar with low rapidity io rest reame af porticies

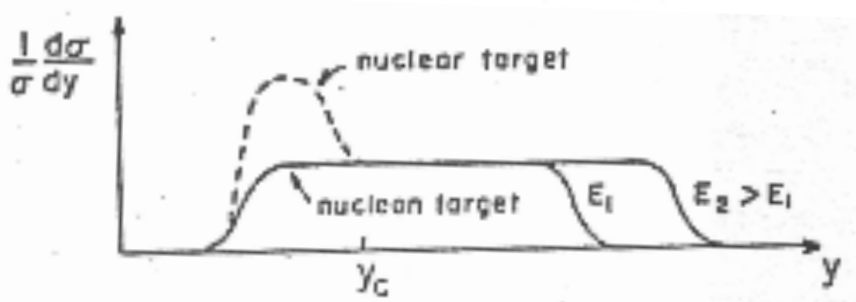

$\mathrm{pA}=\mathrm{pp}$ at higher energy

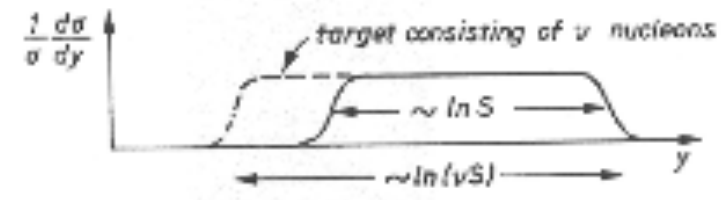

$R_{A} \sim \frac{\ln (v S)}{\ln S} \sim 1+\frac{\ln v}{\ln S} \rightarrow 1$ as $E \rightarrow \infty$
None of these lead to the observed rapidity distributions, long range correlations, $\mathrm{N}_{\text {part }}$-scaling or extended longitudinal scaling
From WB review,

Acta Phys. Pol. B8(1977) 


\section{Fermilab E178 (PHOBOS - 1)}
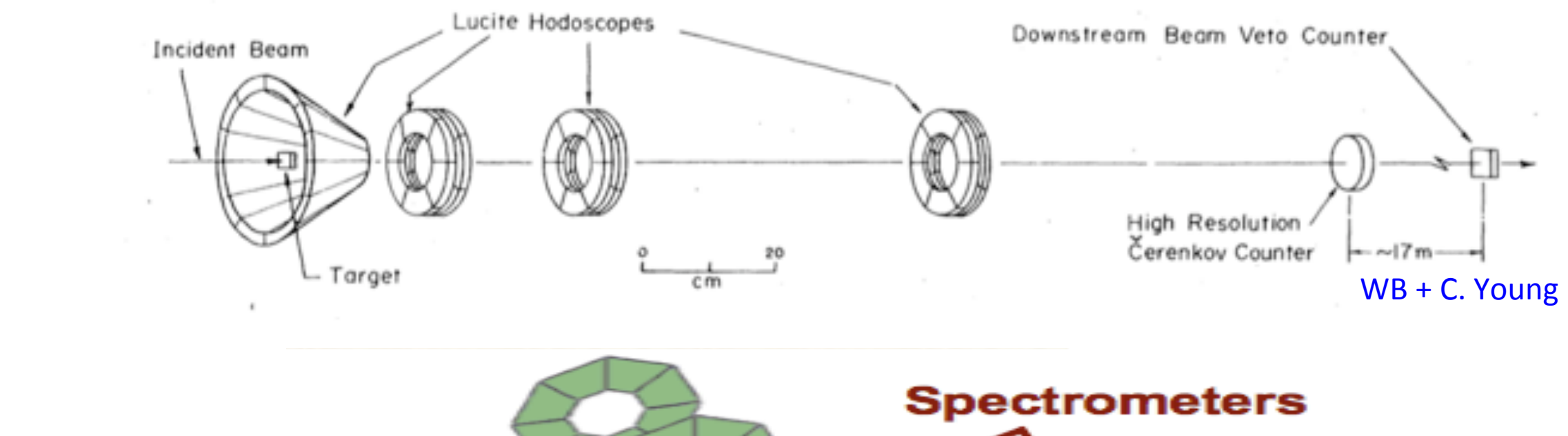

1991 PHOBOS 
Surprise: participant scaling

$$
R_{A}=N_{p A} / N_{p p}=1 / 2+1 / 2 \bar{v}=1 / 2+1 / 2 N_{\text {coll }}=1 / 2 \text { wounded nucleons }=1 / 2 N_{\text {part }}
$$
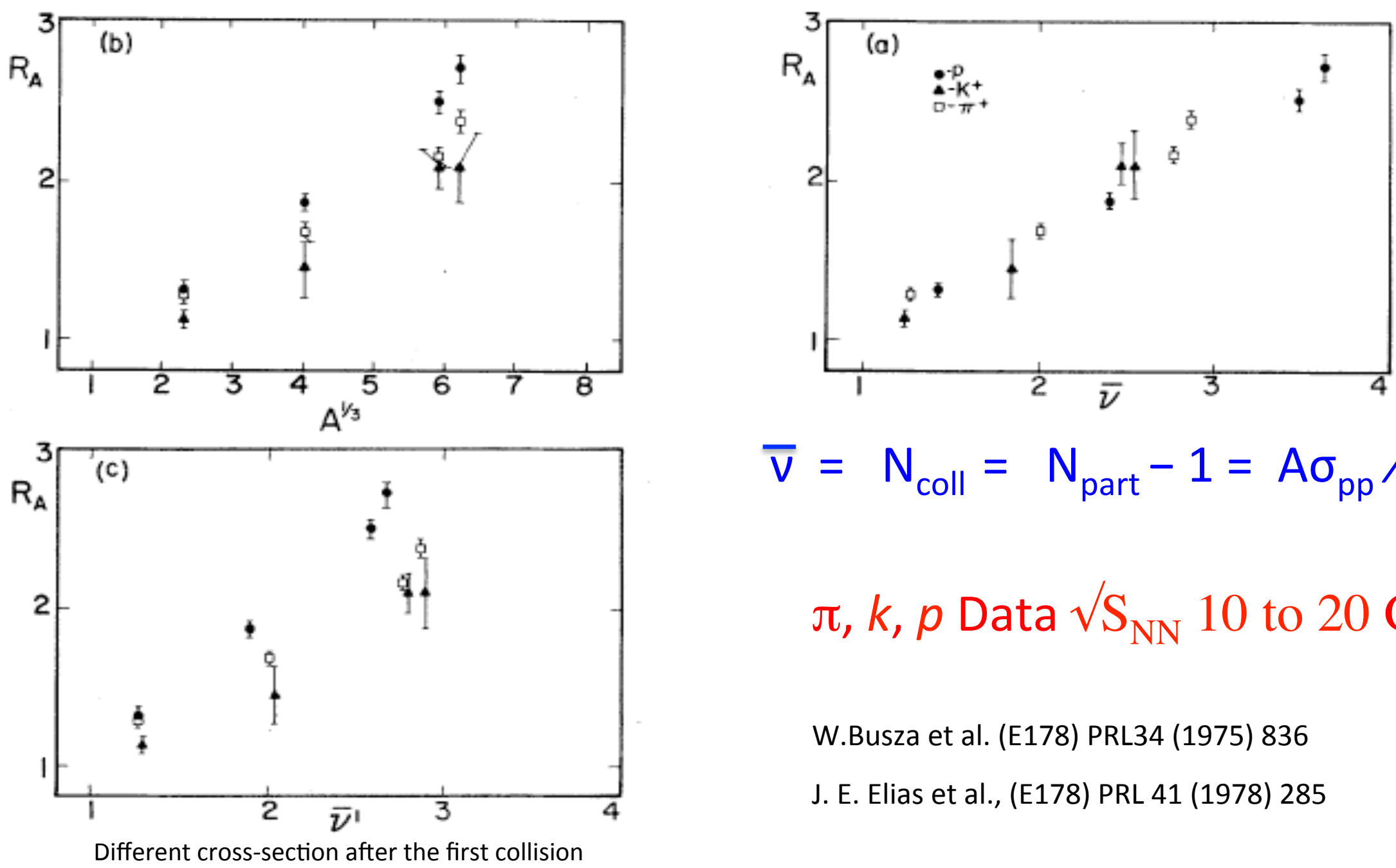

$\bar{v}=N_{\text {coll }}=N_{\text {part }}-1=A \sigma_{p p} / \sigma_{p A}$

$\pi, k, p$ Data $\sqrt{ } \mathrm{S}_{\mathrm{NN}} 10$ to $20 \mathrm{GeV}$

W.Busza et al. (E178) PRL34 (1975) 836

J. E. Elias et al., (E178) PRL 41 (1978) 285 


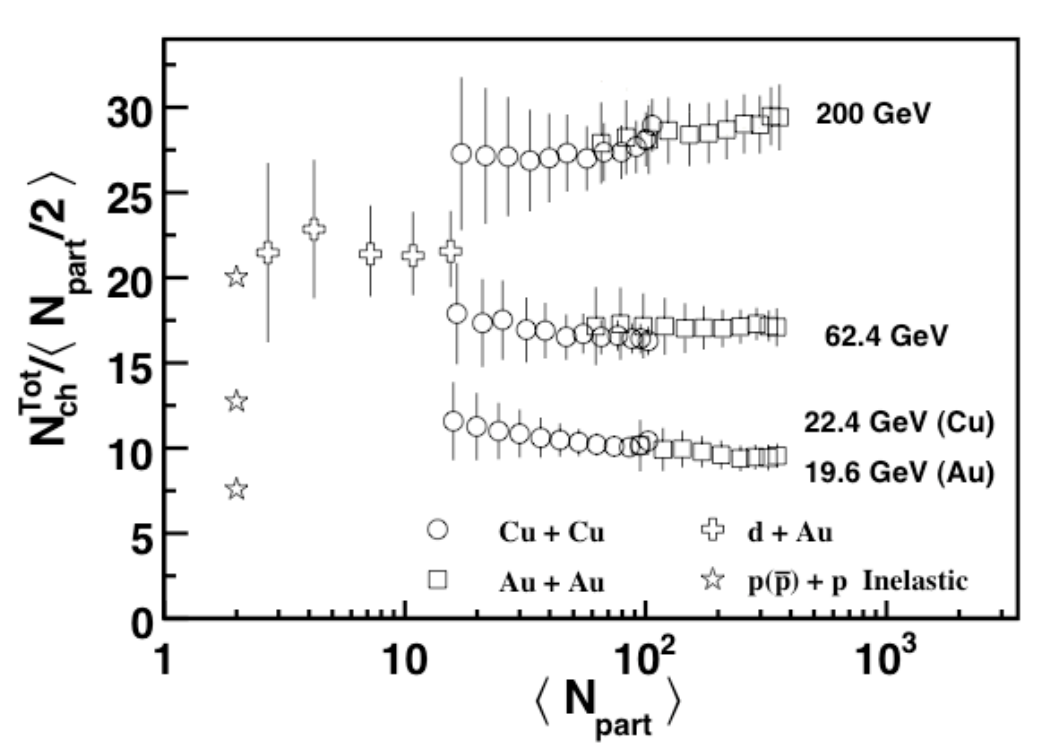

PHOBOS, arXiv:0709.4008 [nucl-ex]

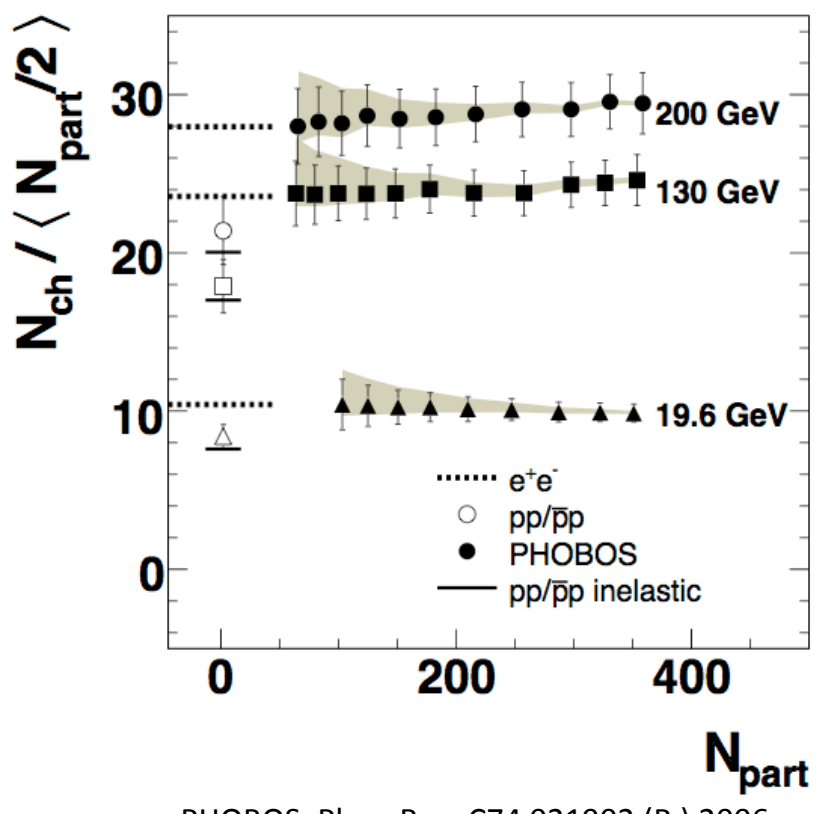

PHOBOS, Phys. Rev. C74 021902 (R ) 2006 Wit Busza

\section{AA collisions consistent with $\mathrm{N}_{\text {part }}$ - Scaling}

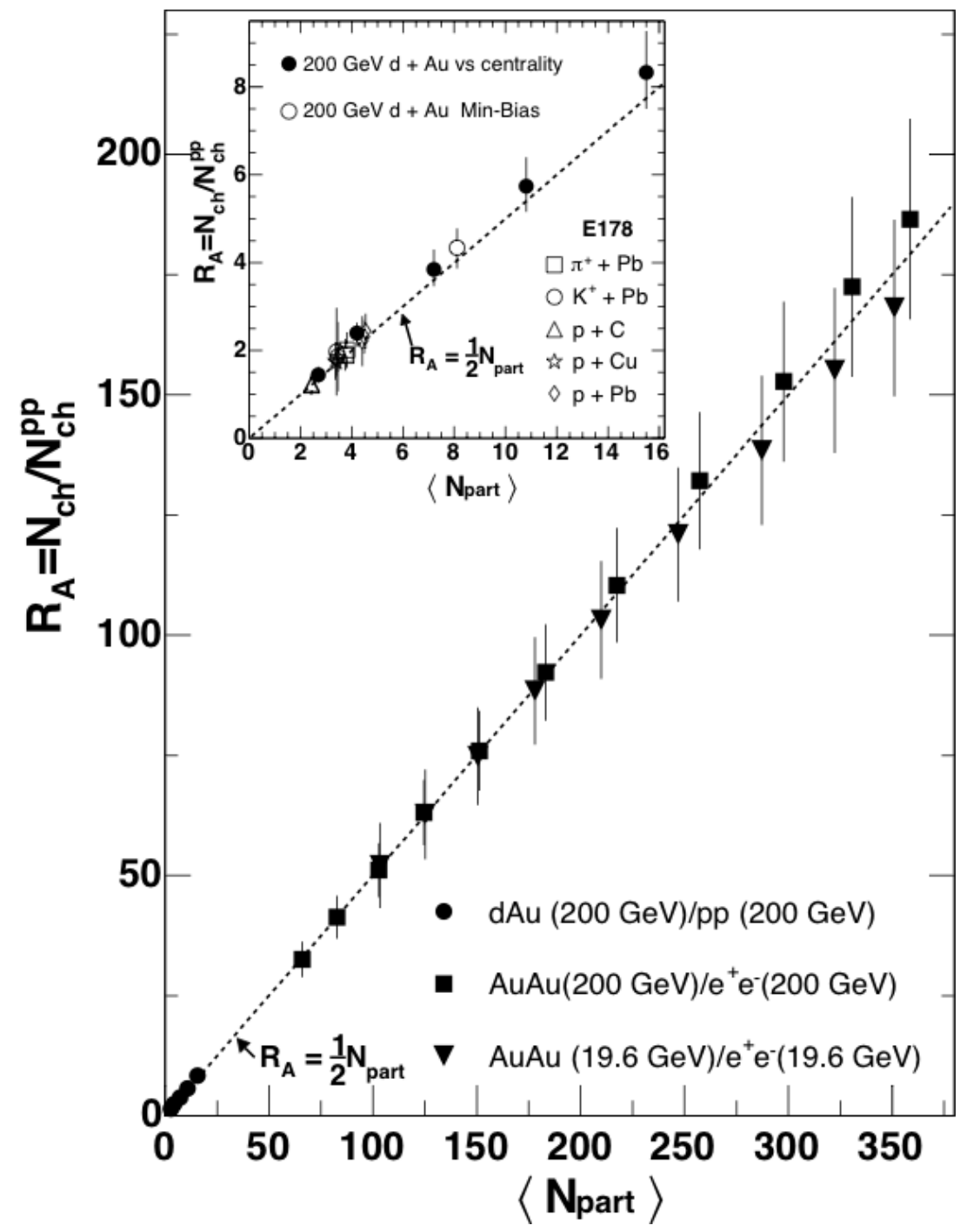

WB, Acta Phys. Pol. B35 (2004)2873

W.Busza et al. (E178) PRL34 (1975) 836 


\section{Extended Longitudinal Scaling in E178 Data for $\sqrt{ } \mathrm{s}_{\mathrm{NN}} 10-20 \mathrm{GeV}$}

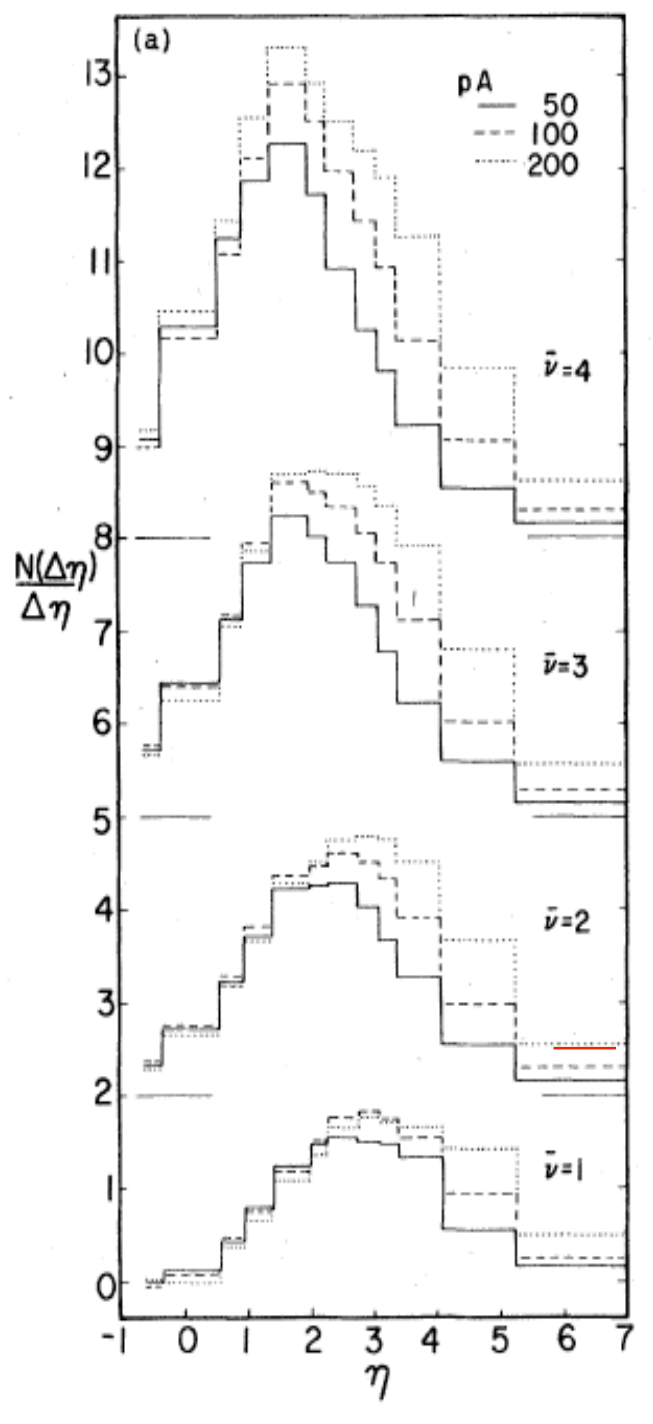

Nucleus rest frame

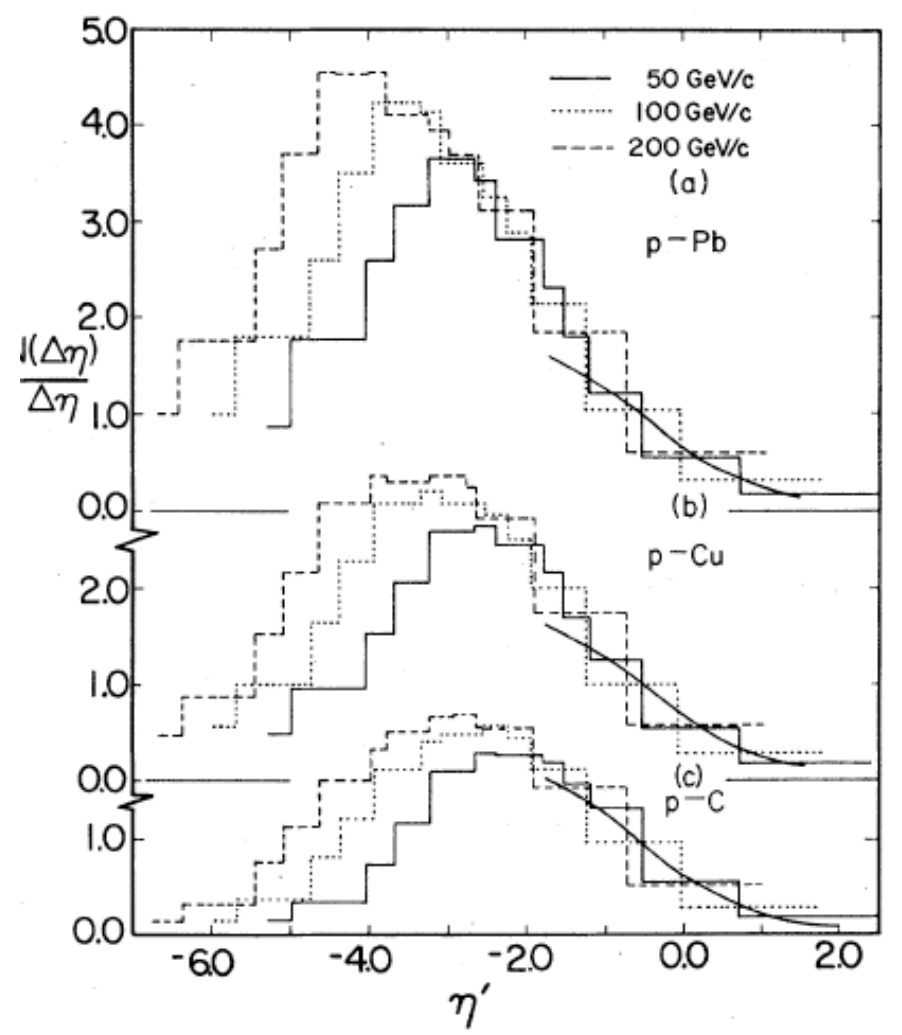

Projectile rest frame

J. Elias et al., (E178) PR D22 (1980)13
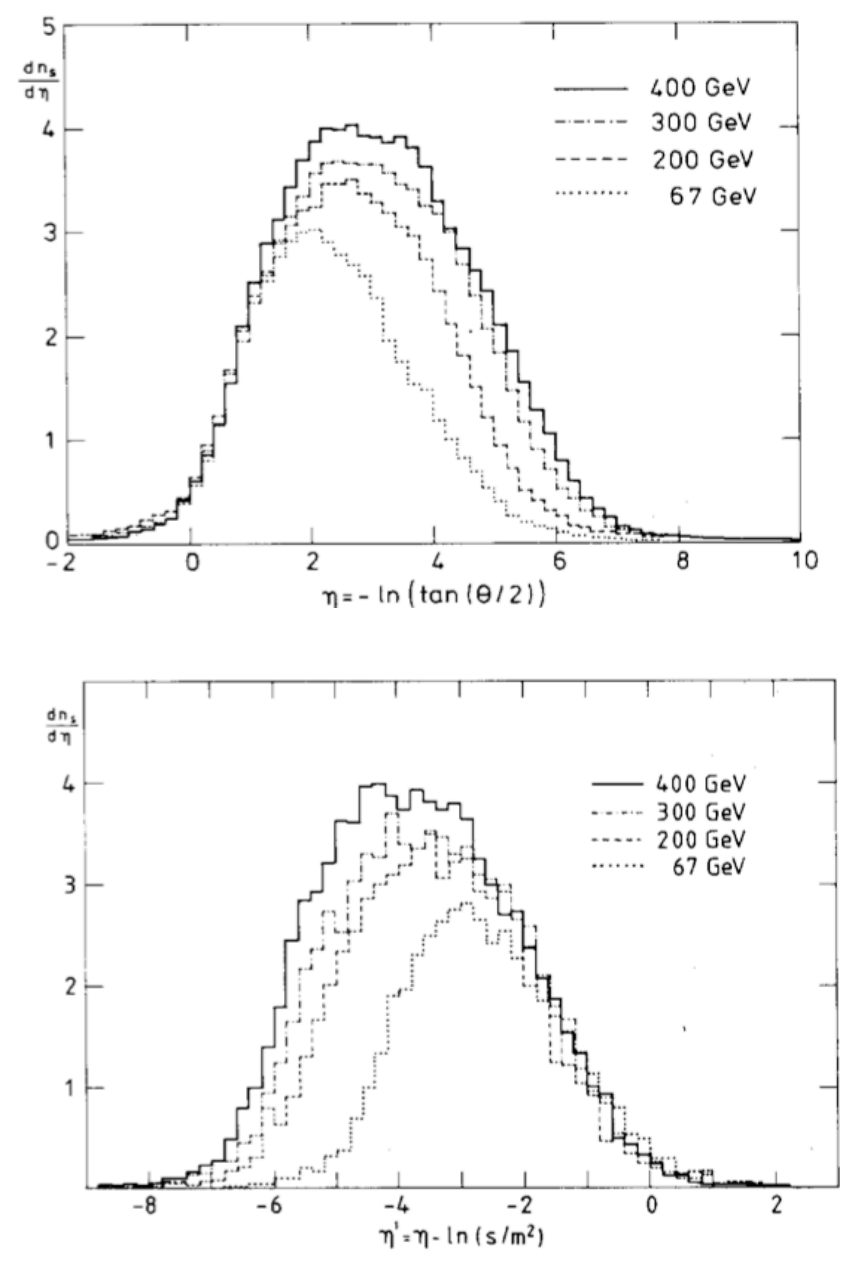

pEmulsion data (Otterlund et al., compilation NP B142 (1978) 445 


\section{Universality of extended longitudinal scaling}

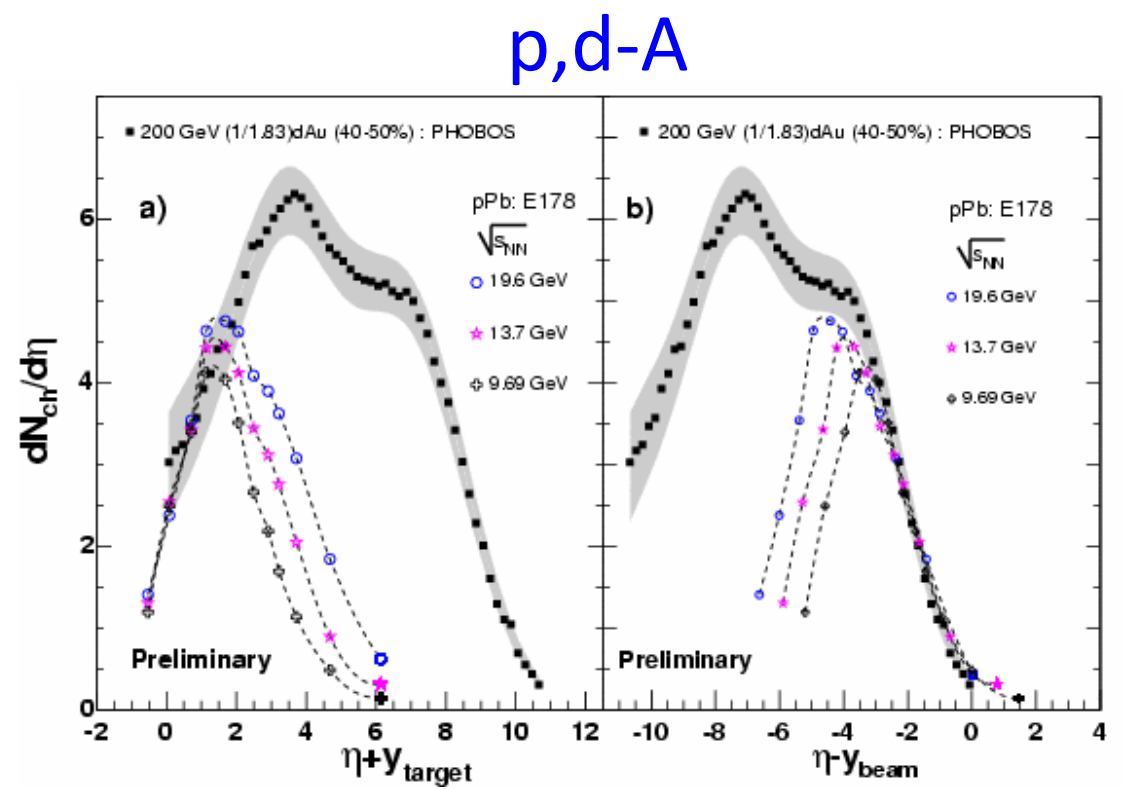

PHOBOS, Nucl. Phys. A 757 (2005) 28.

E178: PRD 22 (1980) 13
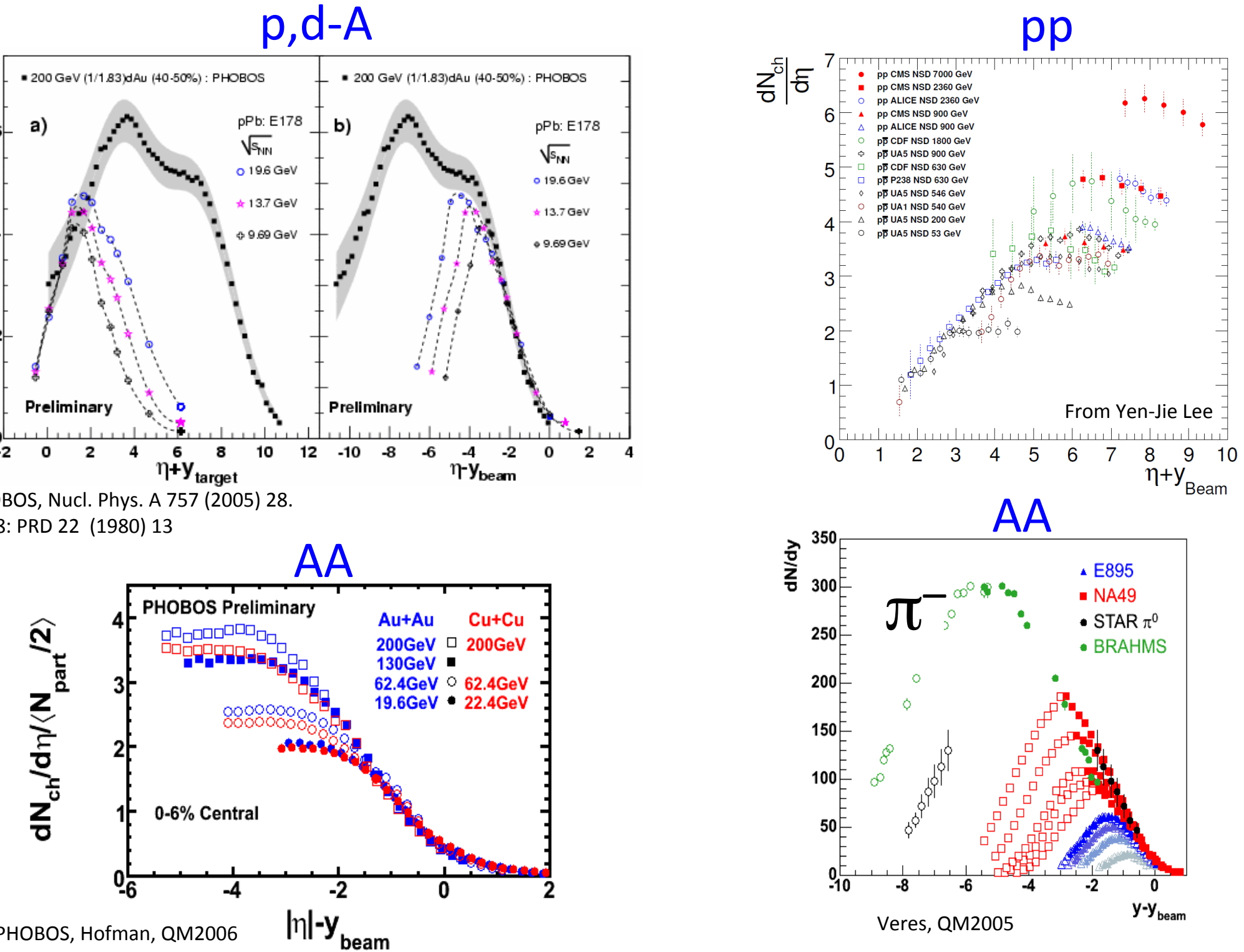

PHOBOS, Hofman, QM2006 $\quad$ ml-y beam 


\section{First Reference to RHIC}

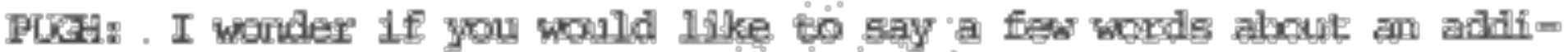

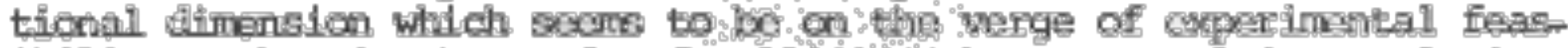
ibility. That is the staxy of sollisions-betwern muclei at relativistic speeds. I an, referring to the prosent feasibility or soarreasibility of acoelerating partilcies:ip to carbon or so In tho Intersecting storage rings at trav and of: concelvaloly desigrang now

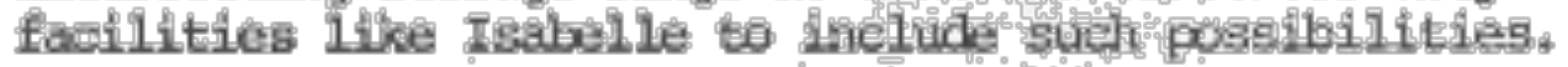

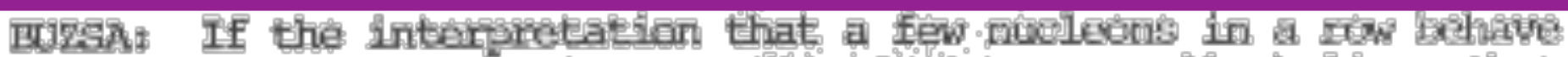

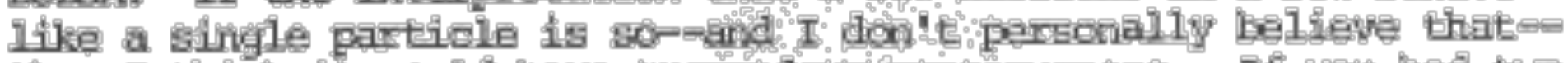
then I think it sould have teremendous cursoxuncus. If you hat tho

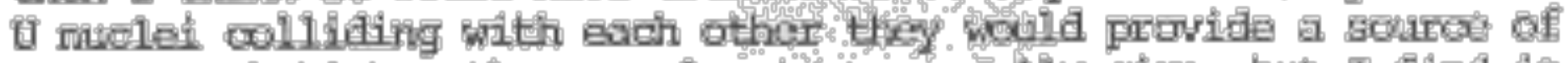

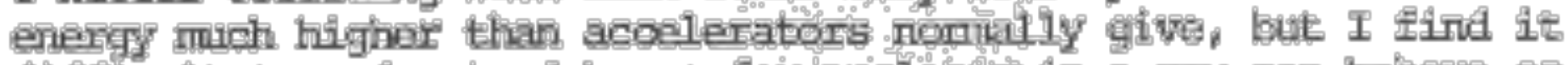

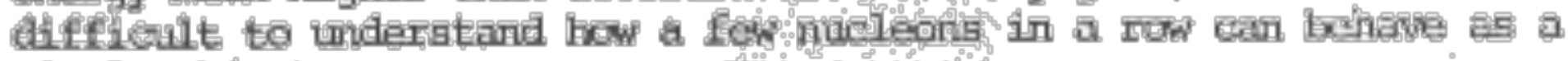
ingle object.

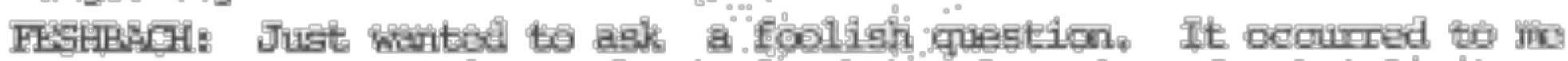

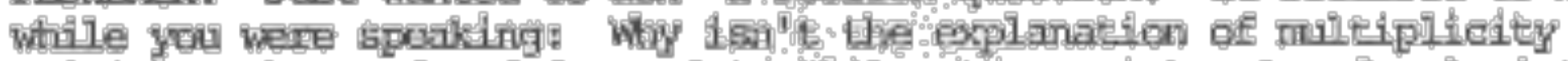

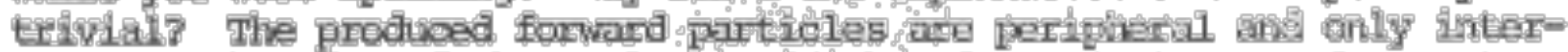

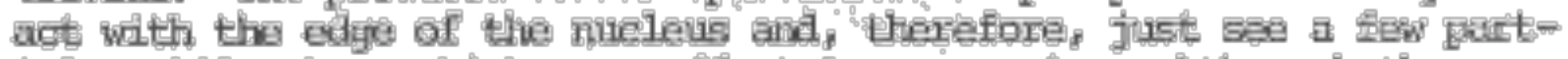

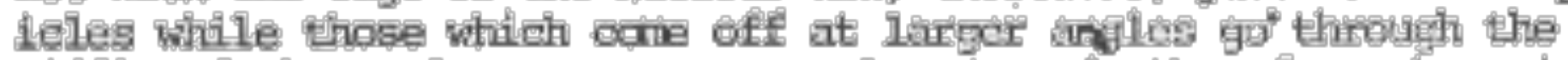

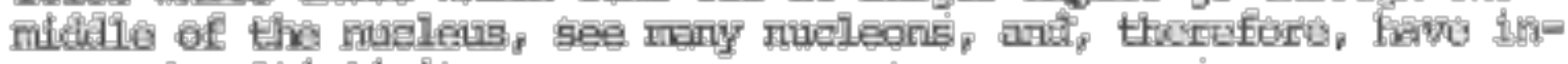

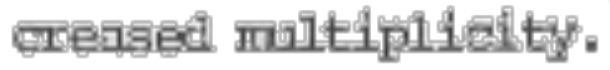

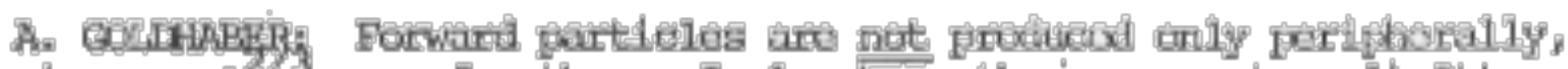
sinoe

Questions and Answers at "HE Physics and Nuclear Structure - 1975", page 237 @ Santa Fe \& Los Alamos 
The "Cronin effect": that's another play! 
The pA play

as seen through the eyes of one of the actors

Act 1

before the early 1970's

Act 2

The 1970's

Act 3

late 1970 's, early 1980 's

Act 4

Late 1980's, 1990's \& 2000's

Act 5

To-day
The " $A$ " of " $p A$ " is more of a nuisance than a help!

Is there too much or too little cascading in $\mathrm{pA}$ ?

Is there too much or too little quenching in the forward direction?

Who cares about the details of "pA" ? After all, it's only a reference!

Who is helping whom?

$\mathrm{pp} \& \mathrm{pA}$ the understanding of $A A$ or $A A$ the understanding of $p p \& p A$ ? 
Forward production of particles in pA collsions ( $\pi$ A results are similar)

Various final states: $\phi, \pi^{+}, \pi^{-}, \mathrm{p}, \mathrm{p}, \mathrm{n}, \Lambda, \mathrm{K}^{0}, \Xi, \mathrm{K}^{+}, \mathrm{K}^{-}$ Various beam energies: $24,100,300,400 \mathrm{GeV}$

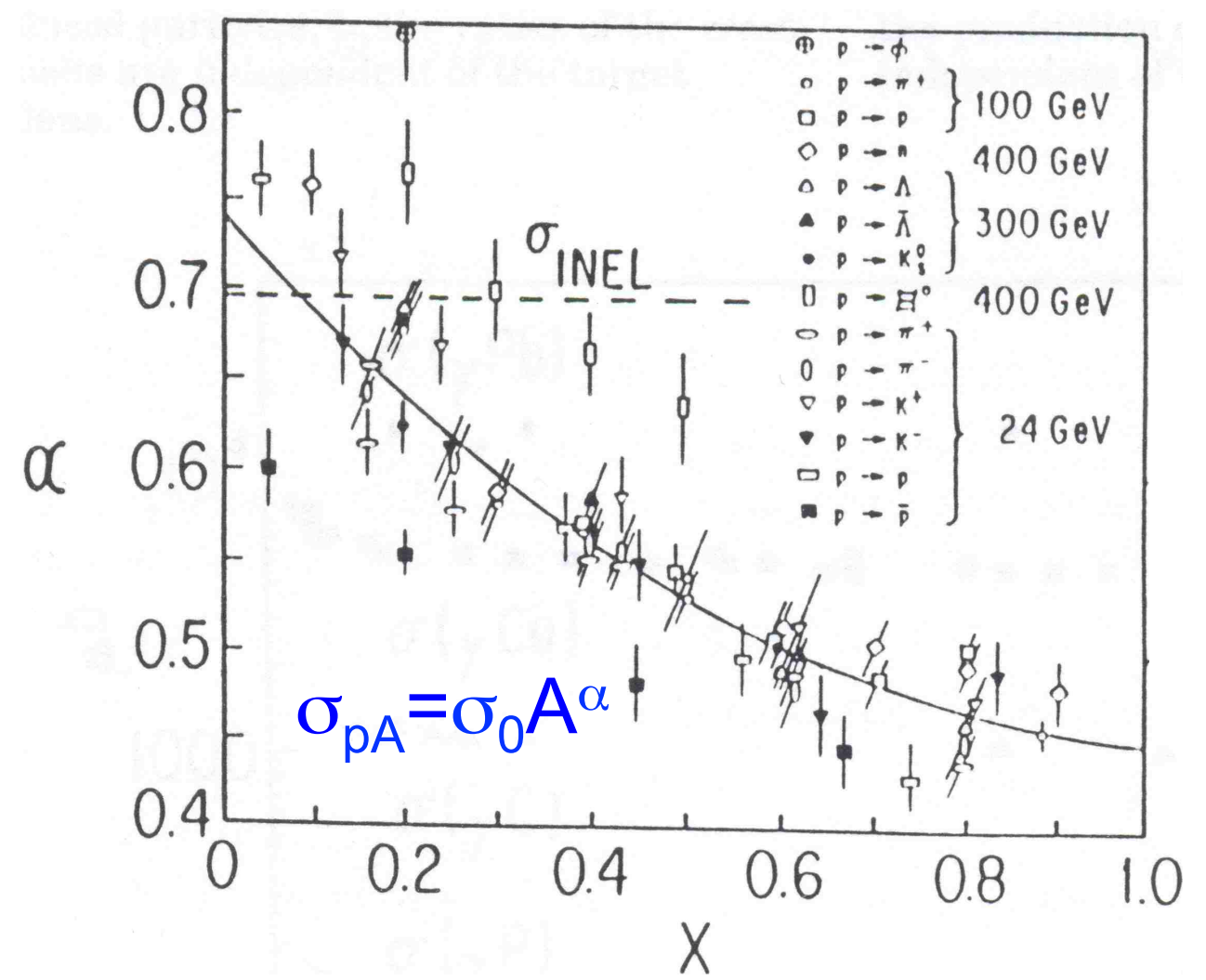

D.Barton et al. (E451) PRD27 (1983) 2580 WB, Nucl. Phys. A544:49 (1992)

$800 \mathrm{GeV}$ p-A (FNAL) $\sigma_{\mathrm{A}}=\sigma_{\mathrm{p}}{ }^{*} \mathbf{A}^{\alpha}$ PRL 84, 3256 (2000); PRL 72, 2542 (1994)
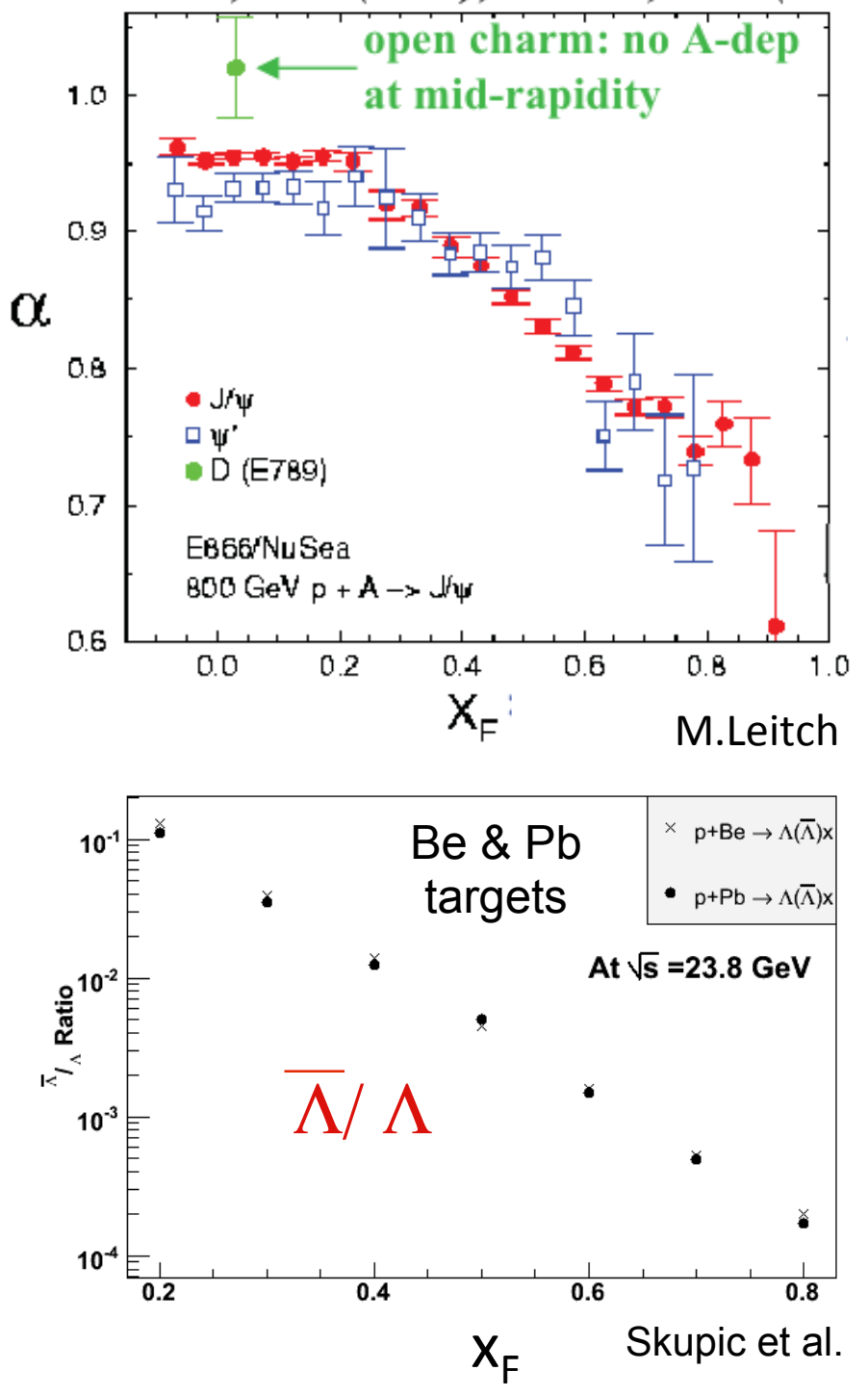


\section{By-product of forward quenching studies:}

estimate that, on average at RHIC, baryon will lose $85 \%$ of its energy as it goes through the center of the Au nucleus
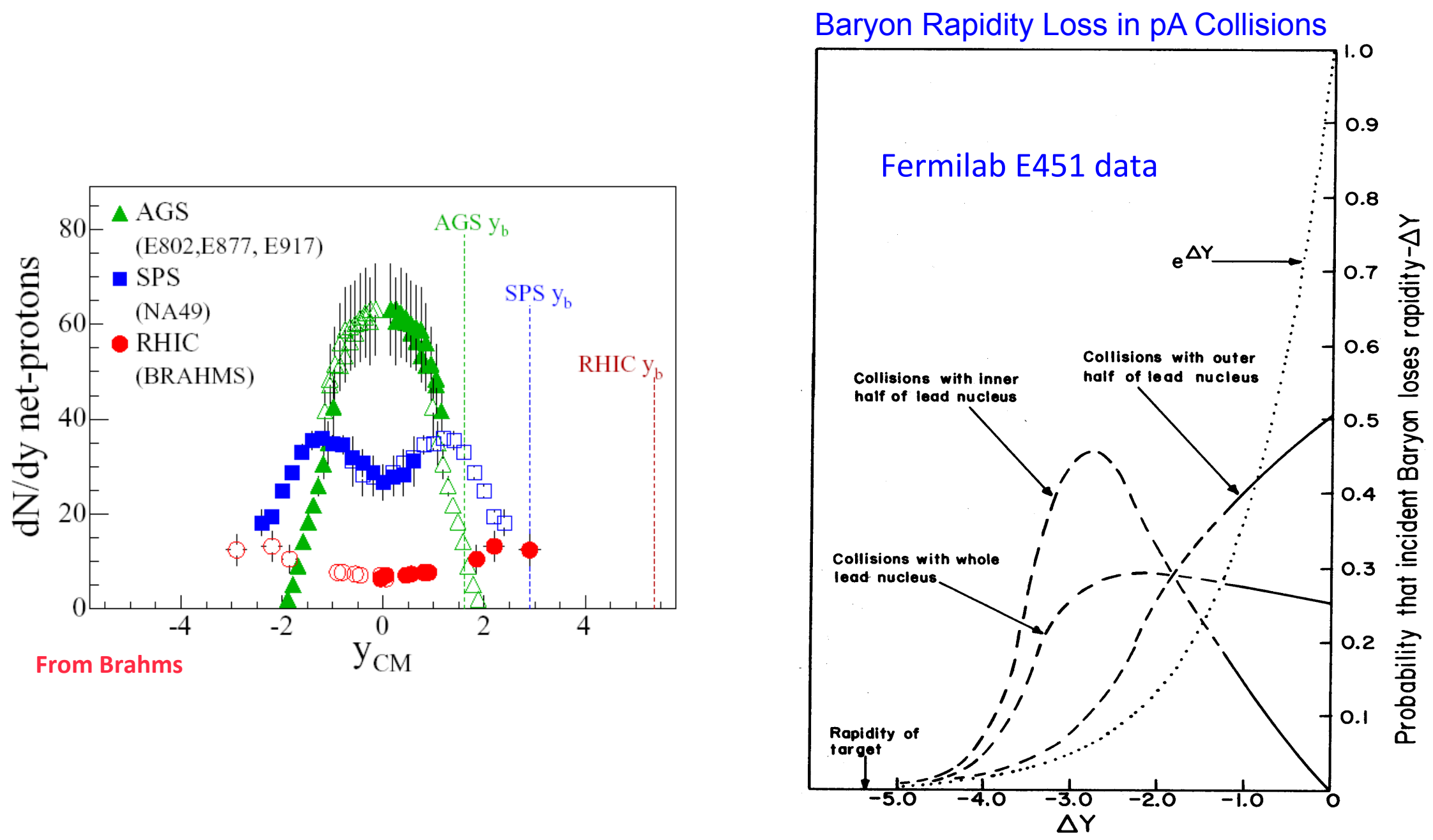

From WB and A.S. Goldhaber 
The pA play

as seen through the eyes of one of the actors

Act 1

before the early 1970's

Act 2

The 1970's

Act 3

late 1970 's, early 1980 's

Act 4

Late 1980's, 1990's \& 2000's

Act 5

To-day
The " $A$ " of " $p A$ " is more of a nuisance than a help!

Is there too much or too little cascading in $\mathrm{pA}$ ?

Is there too much or too little quenching in the forward direction?

Who cares about the details of "pA" ? After all, it's only a reference!

Who is helping whom?

$\mathrm{pp} \& \mathrm{pA}$ the understanding of $A A$ or $A A$ the understanding of $p p \& p A$ ? 


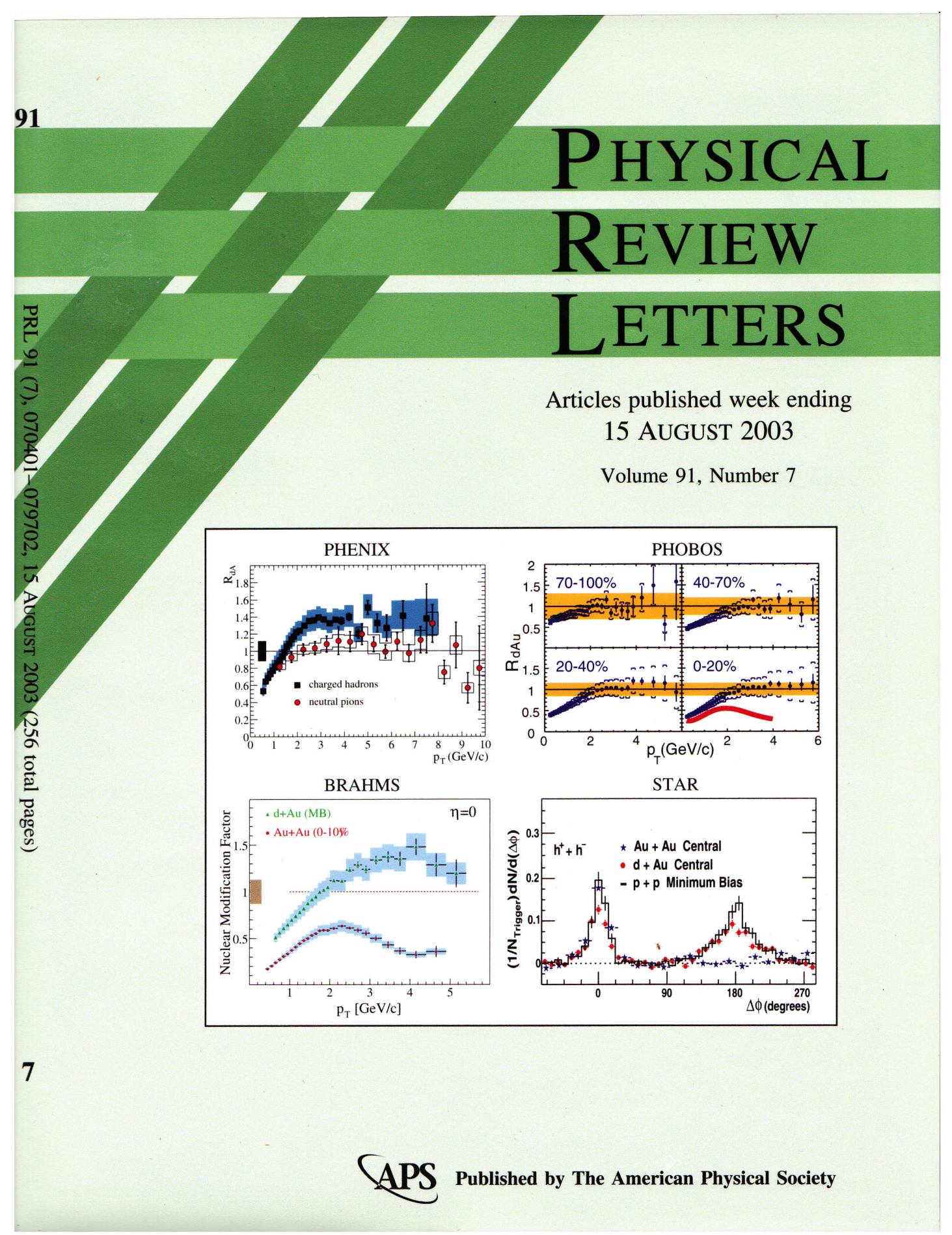


The pA play

as seen through the eyes of one of the actors

Act 1

before the early 1970's

Act 2

The 1970's

Act 3

late 1970 's, early 1980 's

Act 4

Late 1980's, 1990's \& 2000's

Act 5

To-day
The " $A$ " of " $p A$ " is more of a nuisance than a help!

Is there too much or too little cascading in $\mathrm{pA}$ ?

Is there too much or too little quenching in the forward direction?

Who cares about the details of " $p A$ " ? After all, it's only a reference!

Who is helping whom?

$\mathrm{pp} \& \mathrm{pA}$ the understanding of $A A$ or $A A$ the understanding of $p p \& p A$ ? 


\section{Remarkable similarity of mass dependence of average $P_{t}$ in AA and $p p$}
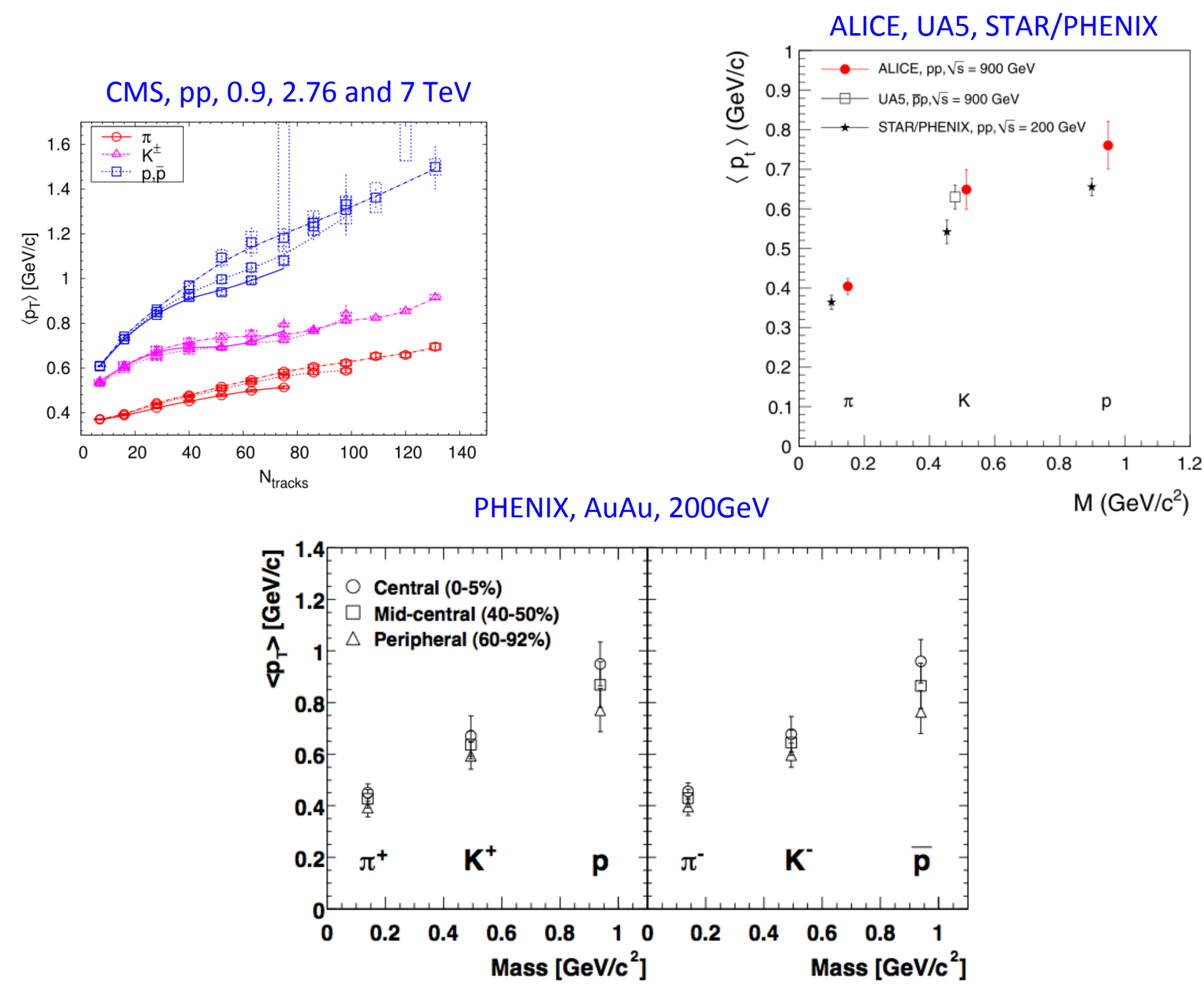
The ridge phenomenon

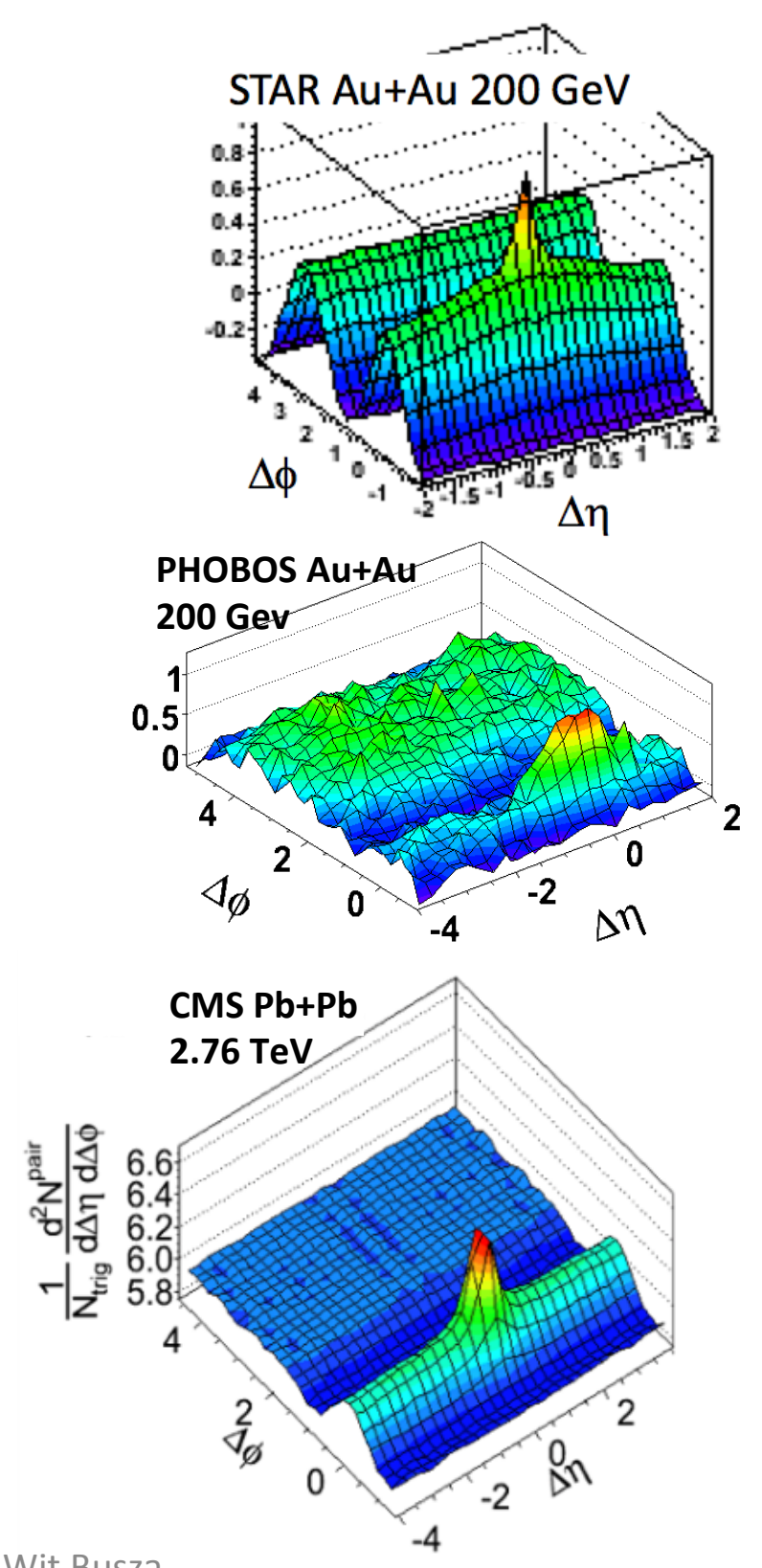

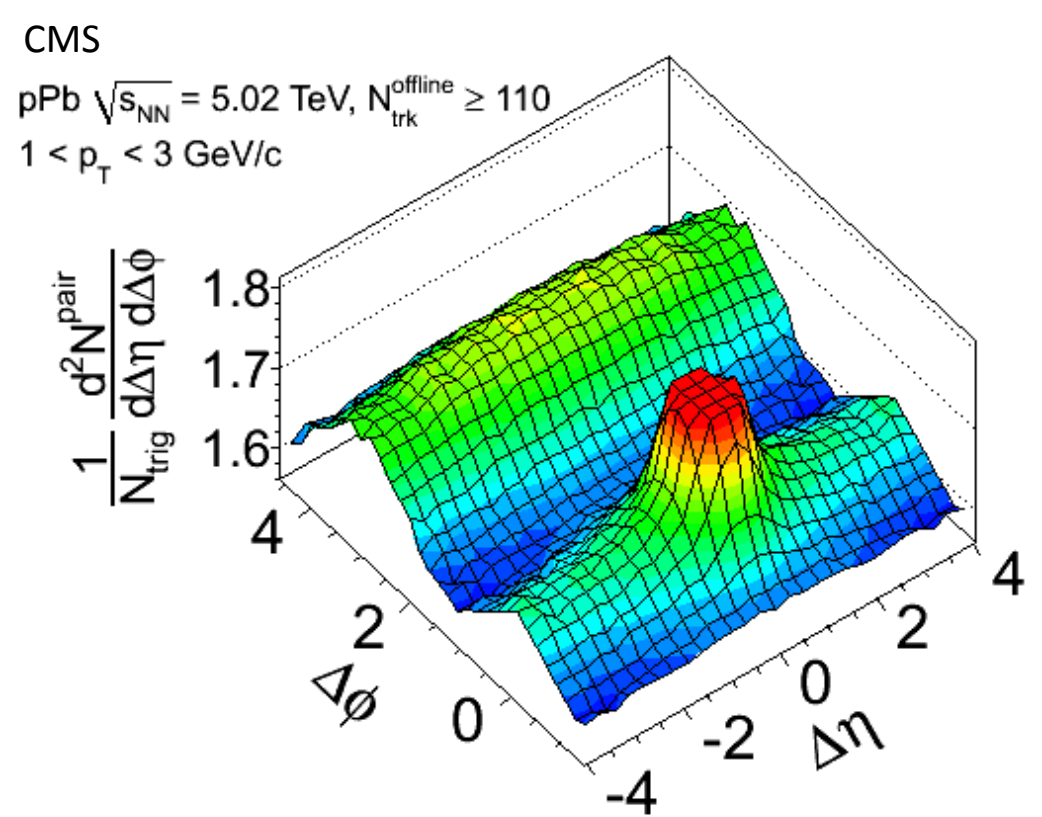

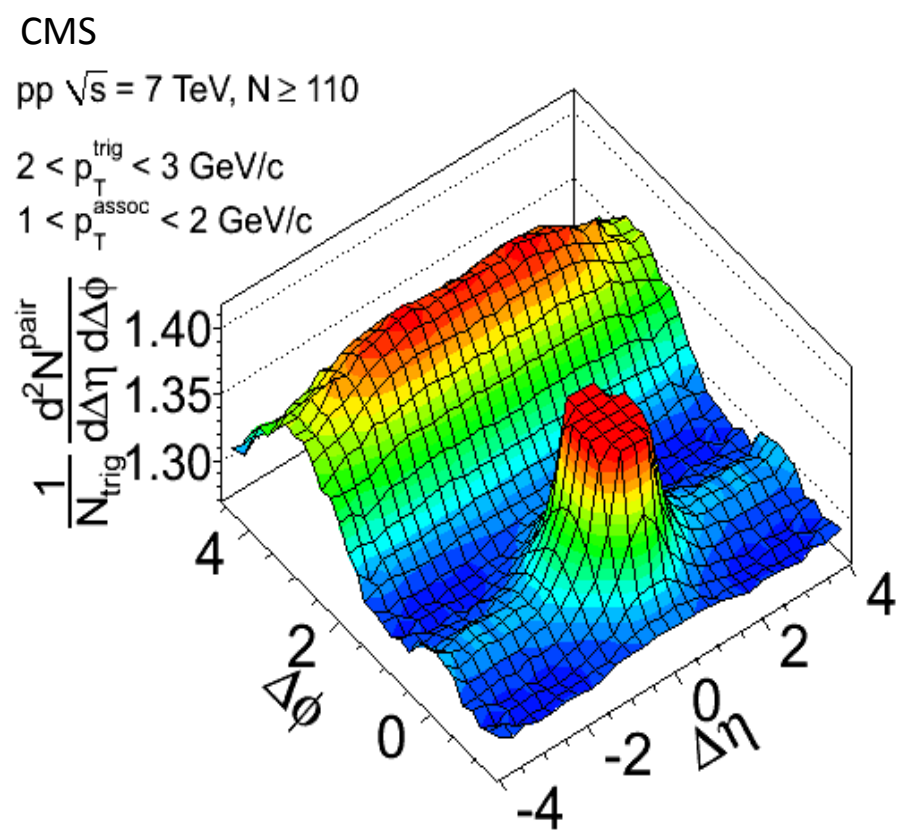


The pA play

as seen through the eyes of one of the actors

Act 1

before the early 1970's

Act 2

The 1970's

Act 3

late 1970 's, early 1980 's

Act 4

Late 1980's, 1990's \& 2000's

Act 5

To-day
The " $A$ " of " $p A$ " is more of a nuisance than a help!

Is there too much or too little cascading in $\mathrm{pA}$ ?

Is there too much or too little quenching in the forward direction?

Who cares about the details of "pA" ? After all, it's only a reference!

Who is helping whom?

$p p \& p A$ the understanding of $A A$ or $A A$ the understanding of $p p \& p A$ ? 


\section{Concluding remarks}

pA data has, and continues to surprise us

- lack of cascading in the 1950' and 1960's

- long range correlations and simplicity of participant scaling in the 1970's

- "Cronin effect" in the the 1970's

- strong quenching of forward particles in the 1970's and 1980's

- "flow-like" behavior in the 2010's

$p A$ is like a litmus test. Until we understand $p A$ from our understanding of $p p$ and $A A$, we cannot claim to have a deep understanding of $p p$ and $A A$.

I take this opportunity to thank Andrzej BiaŁas, Bj, Kurt Godfried, Freddie Goldhaber, Larry Jones, Miklos Gyulassy and Al Mueller, for helping make pA so much fun for me ! 


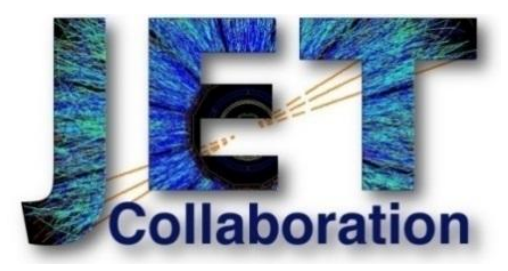

\title{
Flavor Tomography
}

Alessandro Buzzatti

\author{
Miklos Gyulassy
}
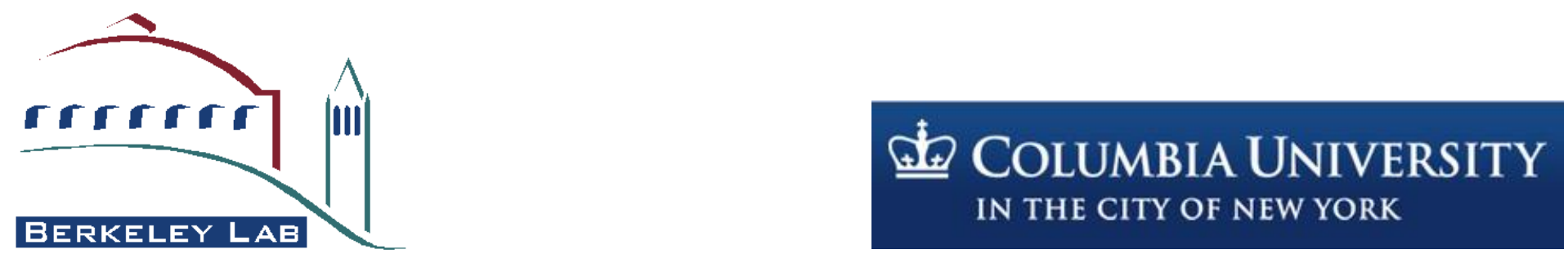


\section{Outline}

- Introduction

- Jet tomography and jet quenching

- CUJET

- Analysis of the model

- Geometry, path integral, partonic spectra

- Jet tomography at RHIC and LHC

- Heavy quark puzzle (RHIC)

- Surprising transparency (LHC)

- Future developments

- Elliptic flow

- Conclusions 


\section{Quark Gluon Plasma}

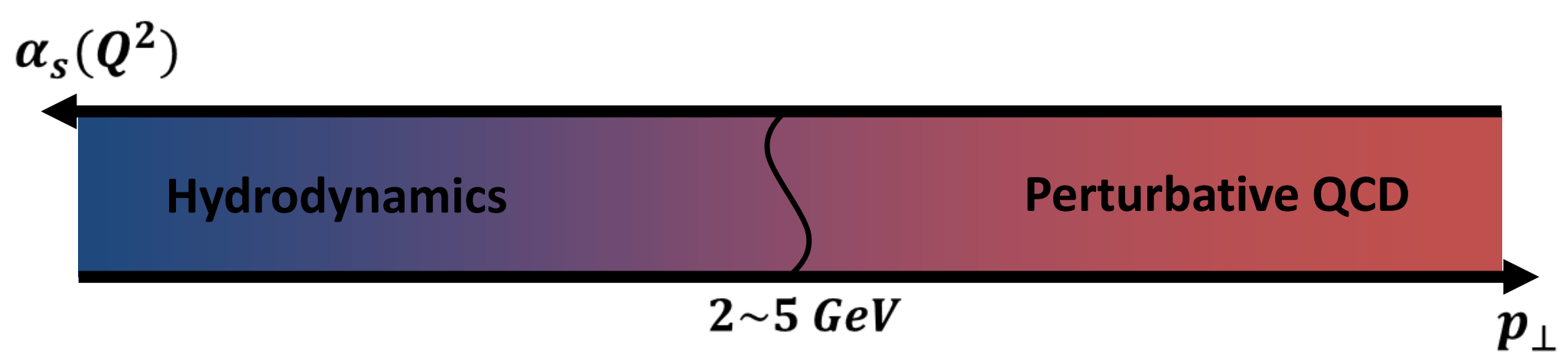

- Medium is strongly coupled

- Local thermal equilibrium is maintained

- Hydrodynamic expansion and collective flow

- Measurement of 'bulk' properties of QGP
- High- $p_{\perp}$ probes are weakly coupled with medium

- Perturbative QCD methods are applicable

- Indication of jet-medium coupling mechanism

- Indirect but 'cleaner' measurements of QGP properties 


\section{Jet Tomography}

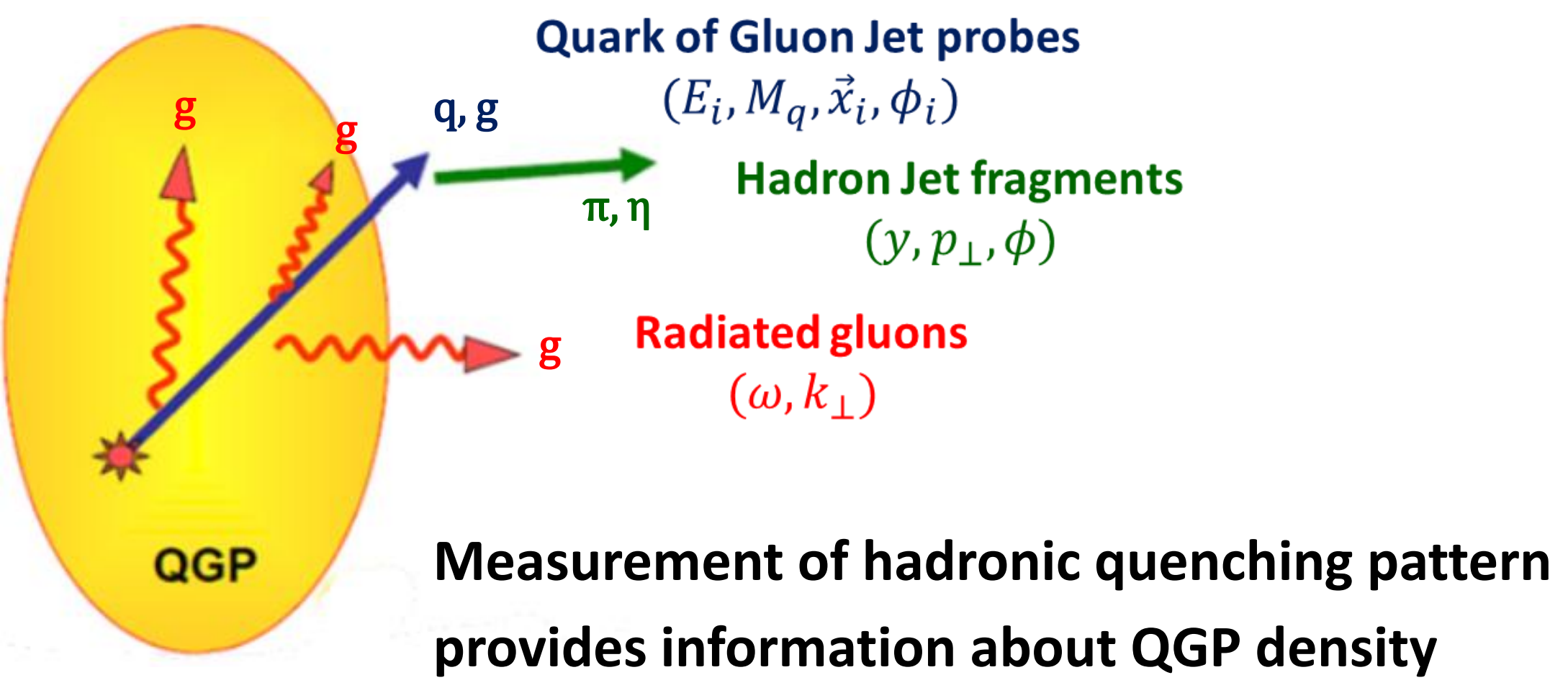

- Requires detailed knowledge of jet-medium interaction mechanism and jet transverse diffusion

- Knowledge of initial conditions, 'cold' nuclear matter effects and hadronization process 


\section{$\mathrm{R}_{\mathrm{AA}}$ measurements}
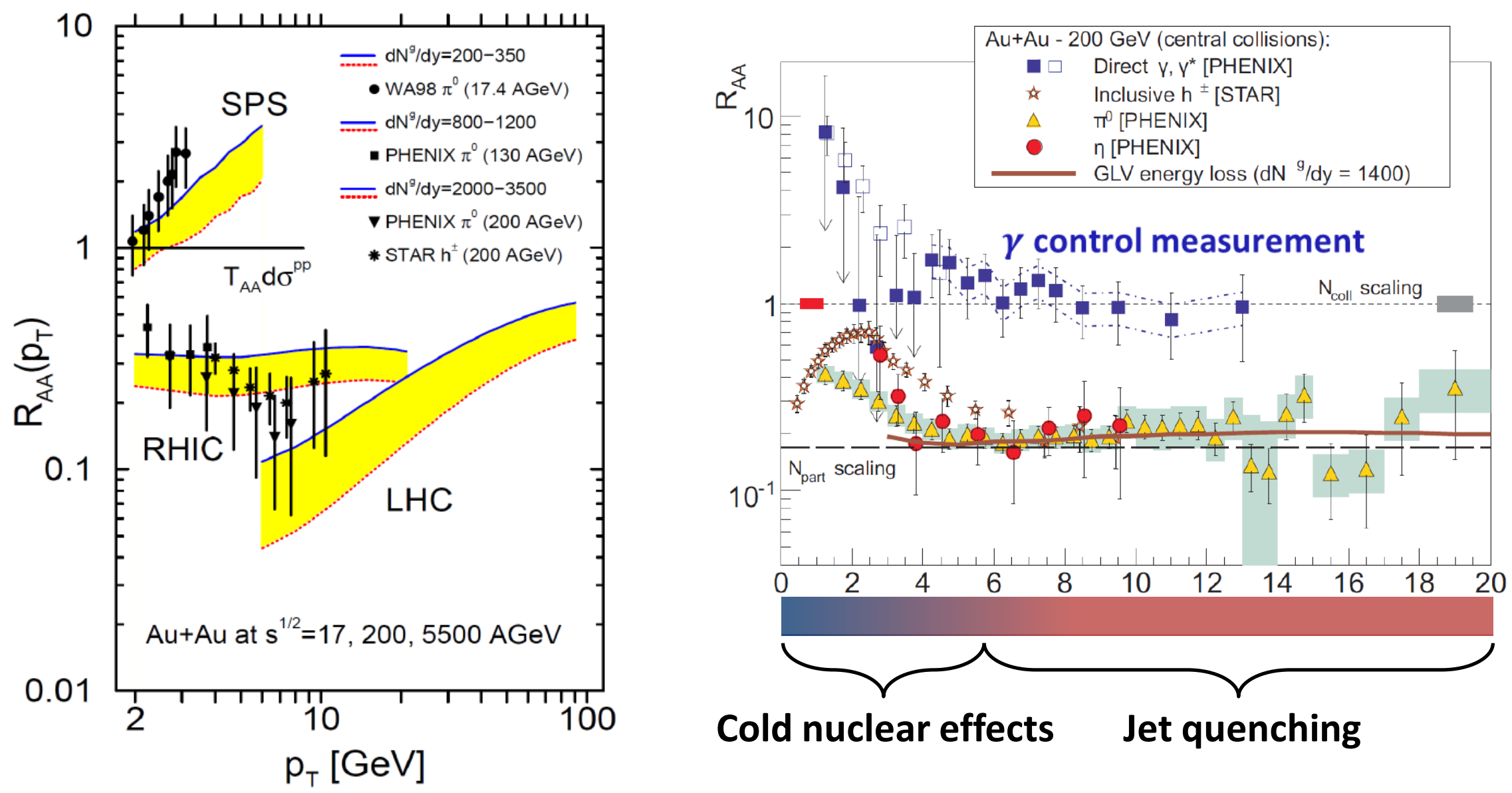

Relevance of 'cold' nuclear matter effects depends on $\sqrt{s}$ and $p_{\perp}$

$>$ Use $\mathrm{p}+\mathrm{A}$ collisions as control experiments 


\section{Jet quenching}

- Radiative $\Delta E^{\text {raditive }} \propto \alpha_{s}^{3} \int d \tau \tau \rho_{Q G P}(\tau, \vec{r}(\tau)) \log \left(\frac{E_{j e t}}{\mu_{D}}\right)$

(Soft eikonal approximation, incoherent multiple gluon emission)

- Opacity expansion

- Thin plasma approximation

- Includes the hard tails of the transferred momentum distribution

- Medium described by density $\rho_{Q G P}$ and Debye screening mass $\mu_{D}=g T$

- Multiple soft scattering (MSS)

- Thick plasma approximation

- Gaussian transverse diffusion

- Medium described by transport coefficient $\widehat{q}$

- Elastic $\Delta E^{\text {elastic }} \propto \alpha_{s}^{2} \int d \tau \rho_{Q G P}^{2 / 3}(\tau, \vec{r}(\tau)) \log \left(\frac{E_{j e t}}{T}\right)$

- Bjorken collisional model 


\section{Model comparison}

Characterize the medium by $\widehat{q}$ :

$>$ mean of the squared transverse momentum exchanged per unit path length

$>$ In the opacity expansion, $\widehat{\boldsymbol{q}} \sim \frac{\mu_{D}^{2}}{\lambda}$

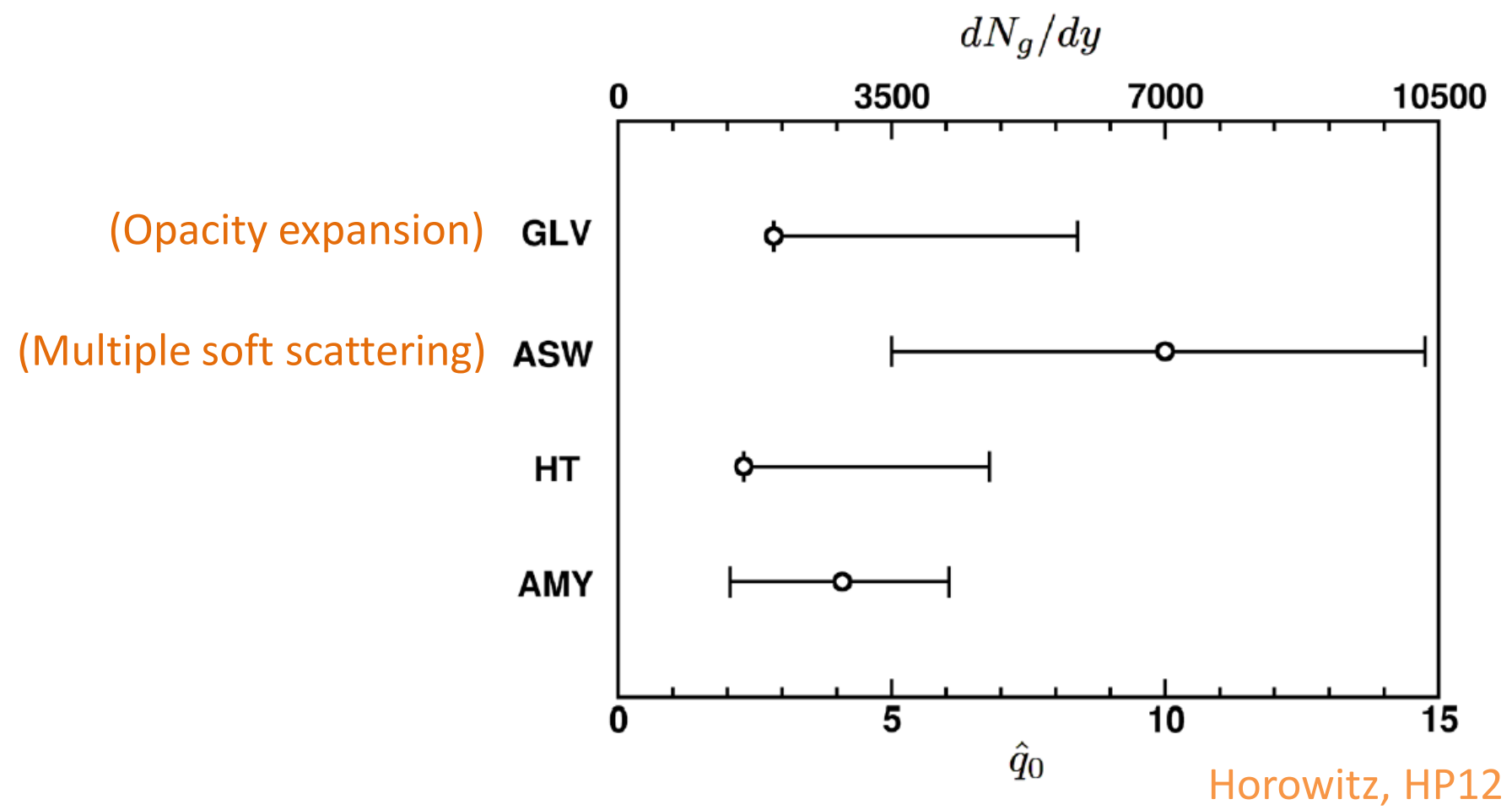

$>$ Need to reduce systematic uncertainties! 


\section{Outline}

- Introduction

- CUJET

- Analysis of the model

- Geometry, path integral, partonic spectra

- Jet tomography at RHIC and LHC

- Heavy quark puzzle (RHIC)

- Surprising transparency (LHC)

- Future developments

- Elliptic flow

- Conclusions 


\section{History}

- Genealogy of Jet tomography at Columbia University: Vitev, Molnar, Djordjevic, Adil, Wicks, Horowitz, Ficnar.

- Past efforts culminated with the construction of the WHDG model

- Radiative (static potential) + Elastic energy loss

- Static medium (approximated at half the time of the expansion)

- Static jet-medium coupling parameters

- Simplified convolution over partonic spectra (spectral index approximation)

- Applications of WHDG to RHIC and LHC phenomenology got mixed response

- Correct predictions of flat pion $\mathrm{R}_{\mathrm{AA}}$ behavior at RHIC

- Under-quenching of non-photonic electrons at RHIC

- Over-quenching of pion $\mathrm{R}_{\mathrm{AA}}$ at $\mathrm{LHC}$ 


\section{CUJET}

- Geometry

- Glauber model

- Bjorken longitudinal expansion

- Energy loss

- Full jet path length integration

$$
\begin{aligned}
\frac{d N_{g}}{d x}\left(x_{\perp}, \phi\right) & =\frac{C_{R}}{\pi} \int d \tau \alpha_{S}(\tau) \frac{d^{2} k}{\pi} \frac{d^{2} q}{\pi} \frac{1}{x} \frac{\frac{9}{2} \pi \alpha_{S}^{2}(\tau)}{q^{2}\left(q^{2}+\mu^{2}(\tau)\right)} \times \\
& \times \frac{2(k+q)}{(k+q)^{2}+\chi(\tau)}\left(\frac{(k+q)}{(k+q)^{2}+\chi(\tau)}-\frac{k}{k^{2}+\chi(\tau)}\right) \times \\
& \times\left(1-\cos \left[\frac{(k+q)^{2}+\chi(\tau)}{2 x E} \tau\right]\right) \rho_{Q G P}\left(x_{\perp}+\widehat{\phi} \tau, \tau\right)
\end{aligned}
$$

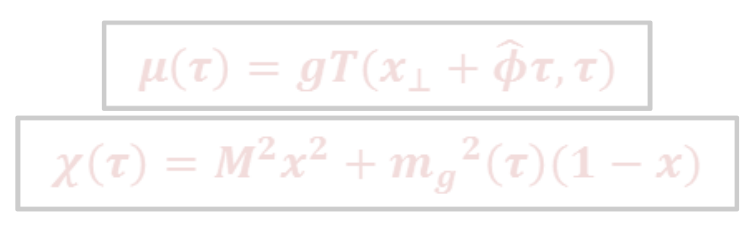

- Collisional energy losses

- Gluon emission fluctuation effects

- Detailed convolution over initial production spectra

- In vacuum Fragmentation Functions 


\section{Bjorken expansion}

- The local thermal equilibrium is established at $\tau_{0}$

$$
S(\tau)=s_{0} \frac{\tau_{0}}{\tau} \quad \text { (entropy density) }
$$$$
s_{0} \approx 3.6 \rho_{0}=3.6 \frac{1}{\pi R^{2} \tau_{0}} \frac{d N}{d y} \quad\left(\frac{d N}{d y} \text { is the observed rapidity density }\right)
$$

MONOTONIC density dependence

$$
\rho_{Q G P}\left(x_{\perp}, \tau\right)=\frac{1}{\tau_{0}} \frac{\rho_{\text {part }}\left(x_{\perp}\right)}{N_{\text {part }}} \frac{d N}{d y} f\left(\frac{\tau}{\tau_{0}}\right)
$$

\section{- Before equilibrium}

Temporal envelopes: linear, divergent, free streaming

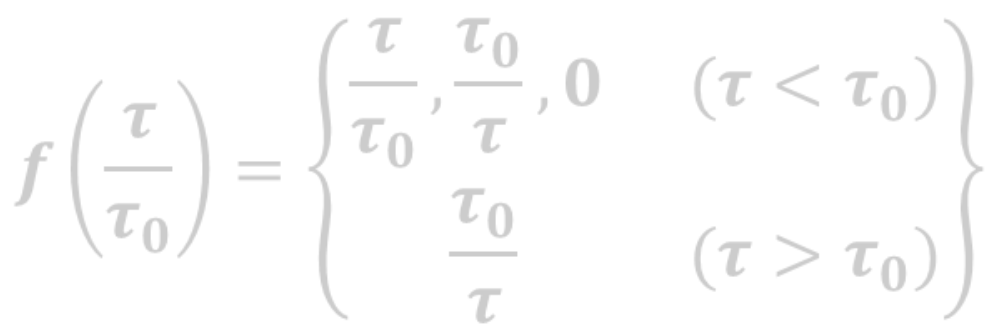




\section{JET \\ CUJET}

- Geometry

- Glauber model

- Bjorken longitudinal expansion

- Energy loss

- Full jet path length integration

Possibility to evaluate systematic theoretical uncertainties such as sensitivity to formation and decoupling phases of the QGP evolution, local running coupling and screening scale variations, and other effects out of reach with analytic approximations.

- Detailed convolution over initial production spectra - In vacuum Fragmentation Functions 


\section{Strong running coupling}

- Introduce one-loop alpha running

$$
\boldsymbol{\alpha}_{s} \rightarrow \alpha_{s}\left(\boldsymbol{Q}^{2}\right)\left\{\begin{array}{cc}
\boldsymbol{\alpha}_{\mathbf{0}} & \text { if } \boldsymbol{Q} \leq \boldsymbol{Q}_{\mathbf{0}} \\
\frac{2 \pi}{9 \log (\boldsymbol{Q} / \Lambda)} & \text { if } \boldsymbol{Q}>\boldsymbol{Q}_{\mathbf{0}}
\end{array}\right\}
$$

B. G. Zakharov, JETP Lett. 88 (2008) 781-786

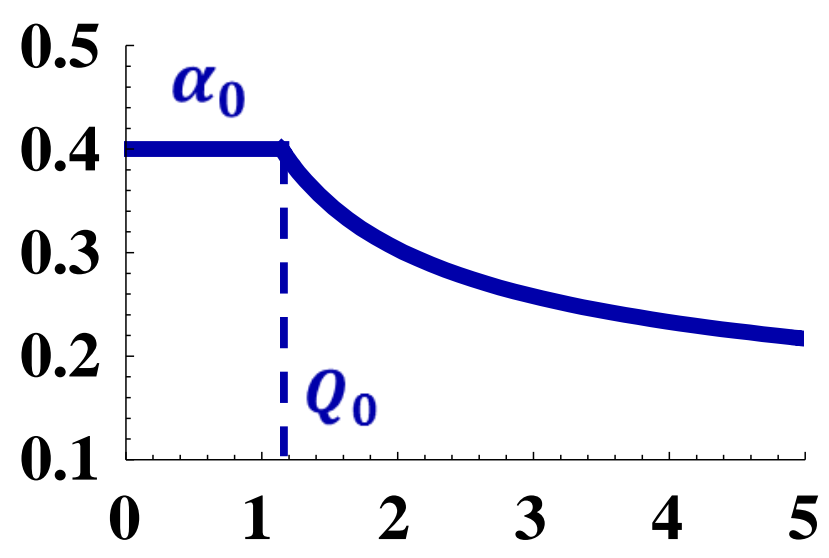

$-\quad$ Radiative $=\left\{\begin{array}{c}\alpha\left(q^{2}\right)^{2} \\ \alpha\left(\frac{k_{\perp}^{2}}{x(1-x)}\right) \\ \mu=g\left(\alpha\left(2 T^{2}\right)\right) T\end{array}\right.$

$-\quad$ Elastic $=\left\{\begin{array}{c}\alpha(E T) \\ \alpha\left(\mu^{2}\right)\end{array}\right.$

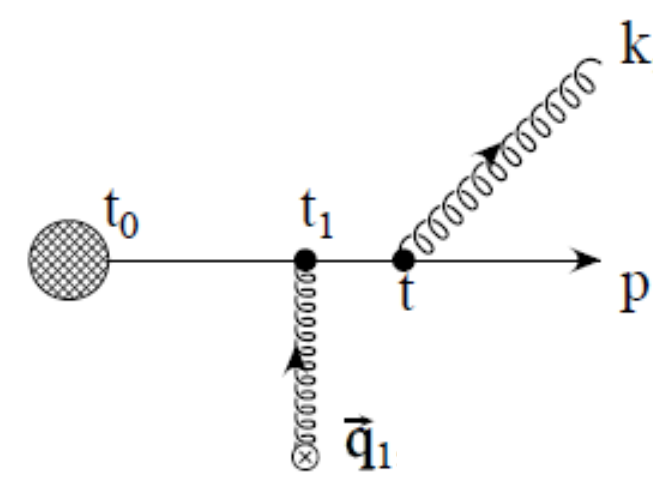

S. Peigne and A. Peshier, Phys.Rev. D77 (2008) 114017 


\section{JET \\ CUJET}

- Geometry

- Glauber model

- Bjorken longitudinal expansion

- Energy loss

- Full jet path length integration

Possibility to evaluate systematic theoretical uncertainties such as sensitivity to formation and decoupling phases of the QGP evolution, local running coupling and screening scale variations, and other effects out of reach with analytic approximations.

- Detailed convolution over initial production spectra - In vacuum Fragmentation Functions 


\section{Production spectra}

- Convolute over $\sqrt{s}$ and flavor dependent pQCD invariant jet spectra

$\frac{d \sigma^{A A}}{d p_{f}}=\int \frac{d \phi}{2 \pi} d x_{\perp} \overline{\boldsymbol{\rho}}_{J e t}\left(x_{\perp}\right) \int d p_{i} P\left(p_{f}, p_{i} ; x_{\perp}, \varphi ;\right) \frac{d \sigma^{p p}}{d p_{i}}$

RHIC

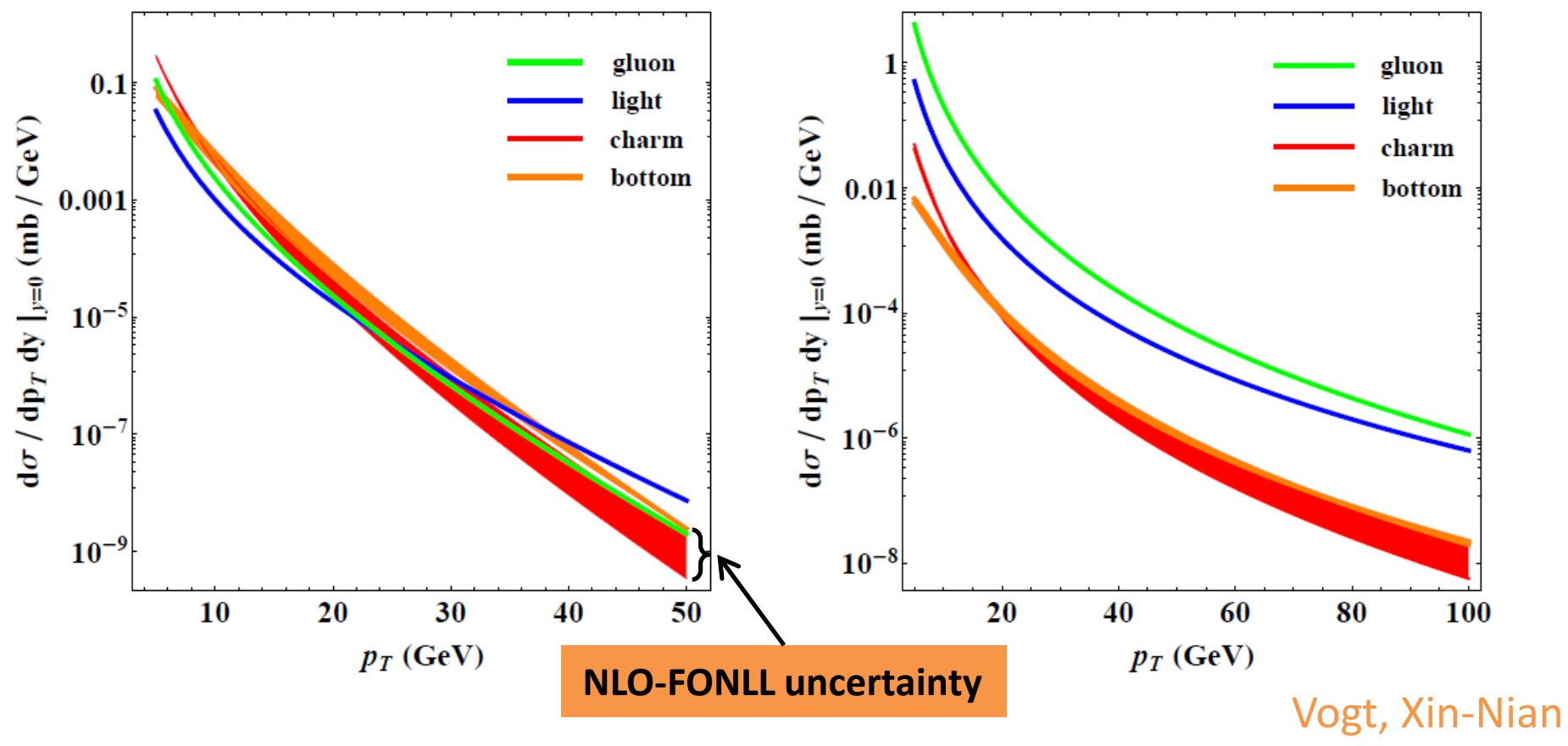




\section{JET \\ CUJET}

- Geometry

- Glauber model

- Bjorken longitudinal expansion

- Energy loss

- Full jet path length integration

Possibility to evaluate systematic theoretical uncertainties such as sensitivity to formation and decoupling phases of the QGP evolution, local running coupling and screening scale variations, and other effects out of reach with analytic approximations.

- Collisional energy losses

- Gluon emission fluctuation effects

- Detailed convolution over initial production spectra - In vacuum Fragmentation Functions 


\section{Systematic errors}

- Initial conditions and plasma evolution

- Thermalization phase, plasma density profile and fluctuations, plasma constituents, hydrodynamic expansion, freeze-out temperature

- $p p$ reference spectra

- Jet-medium coupling

- Interaction potential, order of opacity expansion, running coupling scales, multiple gluon emission, large angle radiation

1. One free parameter in the model: $\alpha_{s}$ (if fixed) or $\alpha_{0}$ (if running)

2. Constraint $\alpha_{s}$ or $\alpha_{0}$ to one $R_{A A}^{h}\left(p_{\perp}, \sqrt{s}, y, \mathcal{C}\right)$ reference point

3. Extrapolations to different $\left(h ; p_{\perp}, \sqrt{S}, y, \mathcal{C}\right)$ are parameter free 


\section{Running coupling}

- a-b-c model

Gyulassy, Horowitz

$\stackrel{\mathrm{GLV}}{\longrightarrow} \quad a=\frac{1}{3} \sim \frac{1}{4}$

$>$ Contribution from hard $\boldsymbol{k}_{\perp}$ tails

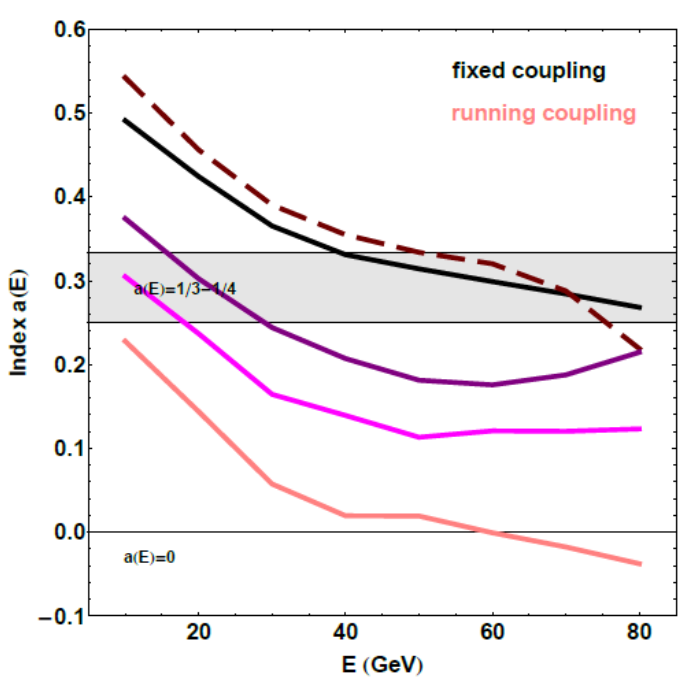

$\frac{d P}{d \tau}=-\kappa P^{a} \tau^{b} T^{2-a+b}$

$\left(\Delta E \propto \log (E / T) L^{2} T^{3}\right)$

E (GeV)
Running scales

fixed

only $T$

only $\boldsymbol{q}_{\perp}$

only $\boldsymbol{k}_{\perp}$

all

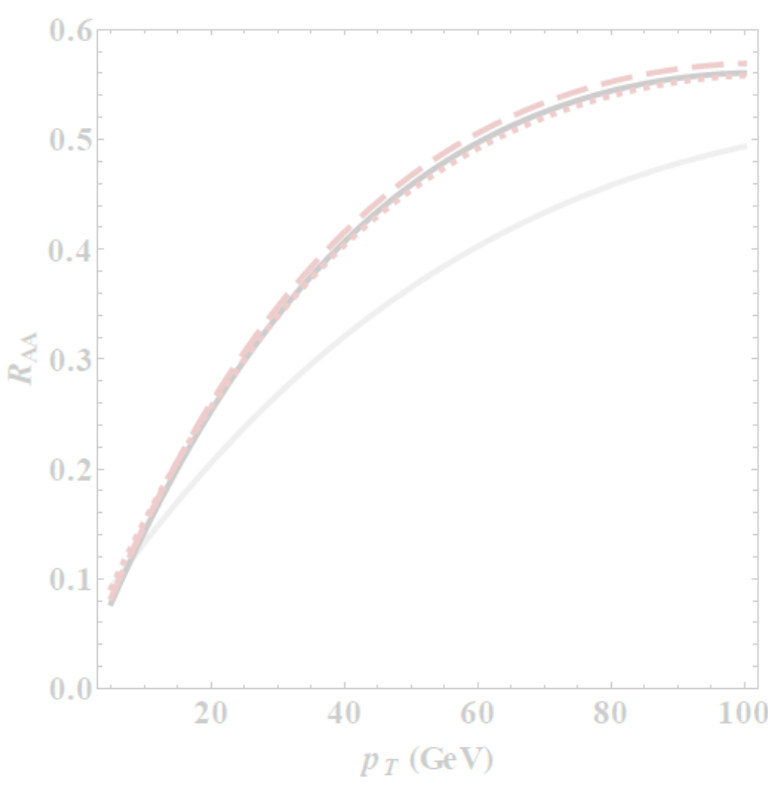

Solid: $\alpha_{S}(Q)$ with $\alpha_{0}=0.4$

Red Dotted:

Red Dashed: $\alpha_{S}(0.5 \times Q)$ with $\alpha_{0}=0.3$

Opaque: Fixed $\alpha_{s}=0.3$ 


\section{Initial pQCD spectra}

Initial quark production spectra

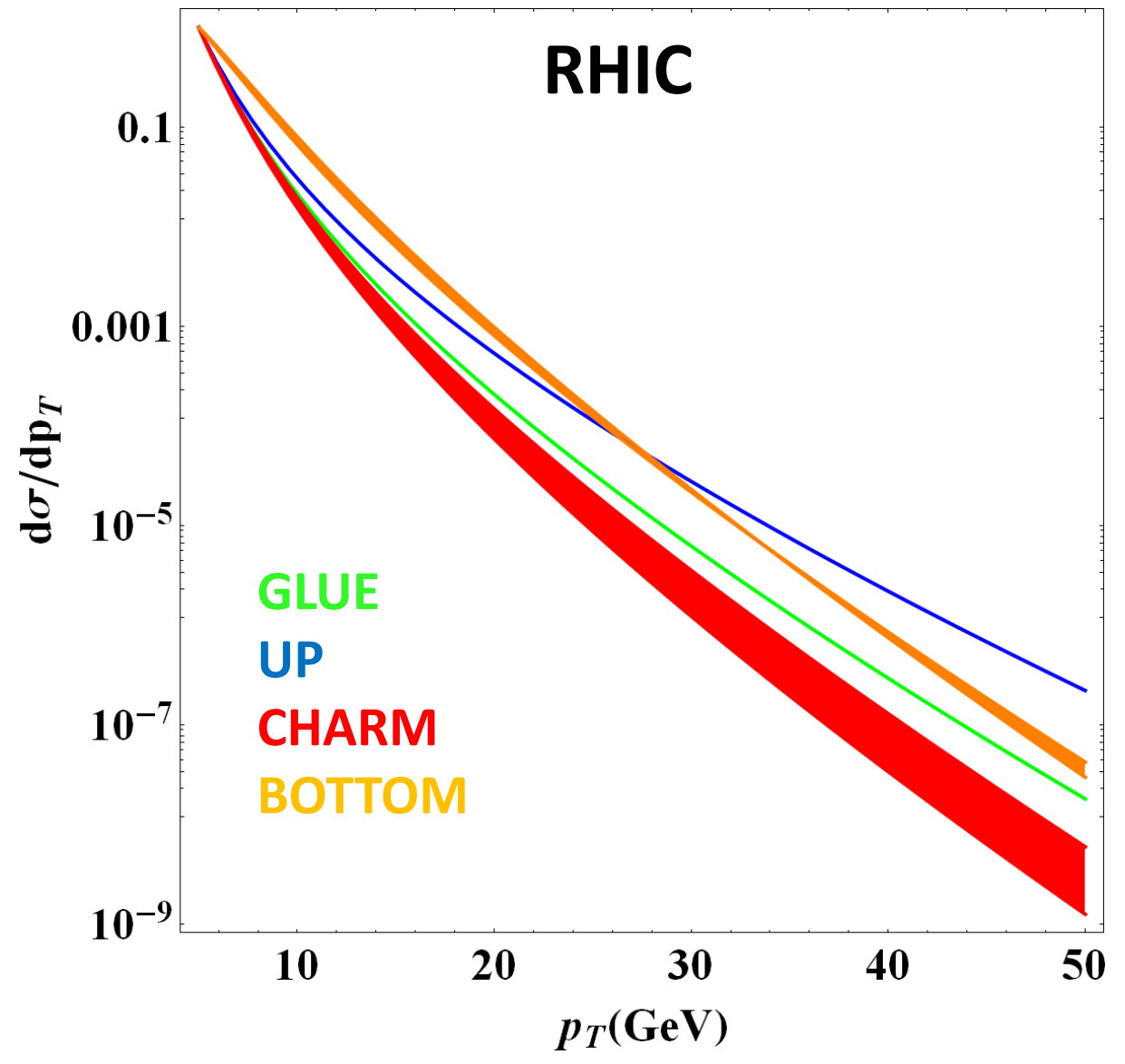

Competing effects between increased density and harder production spectra

- RHIC density and spectra

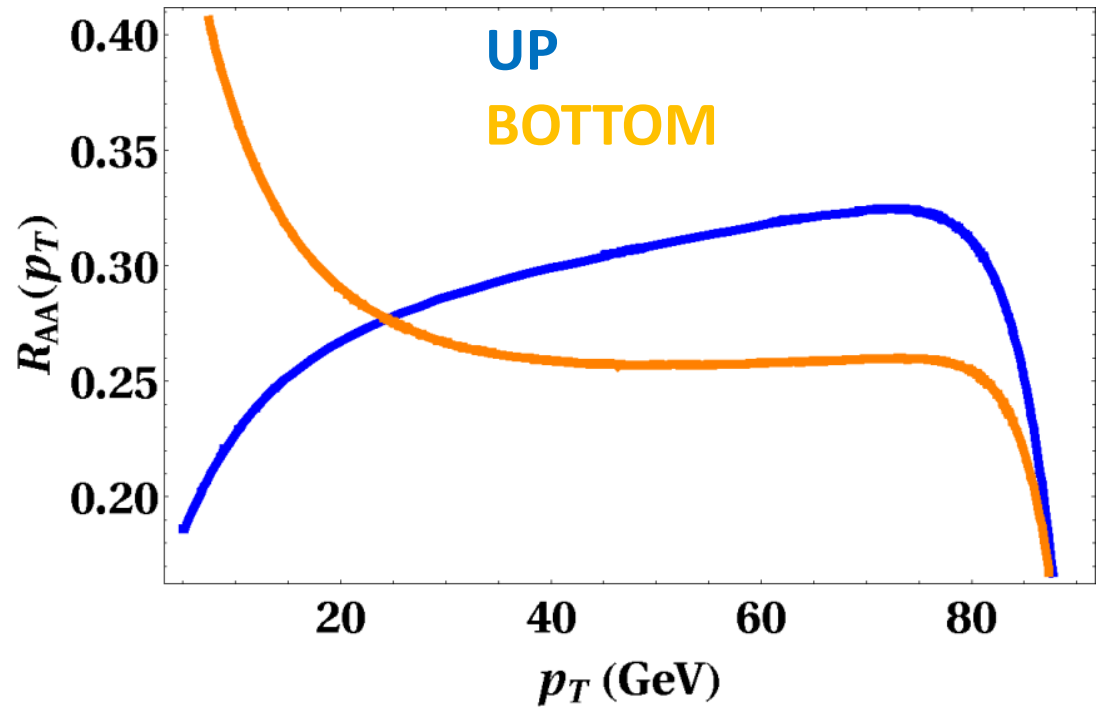




\section{Initial pQCD spectra}

Initial quark production spectra

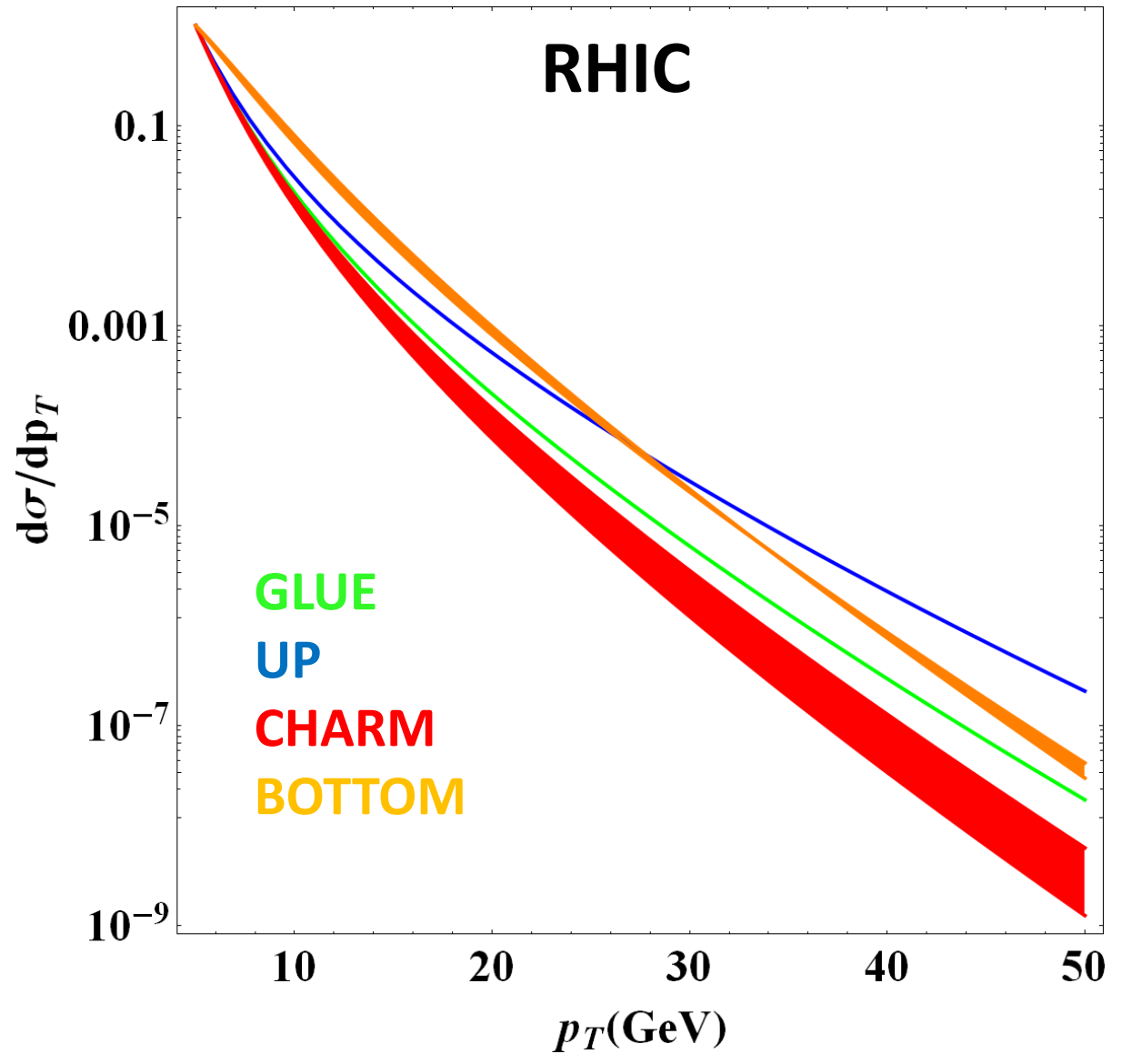

Competing effects between increased density and harder production spectra

- LHC density, RHIC spectra

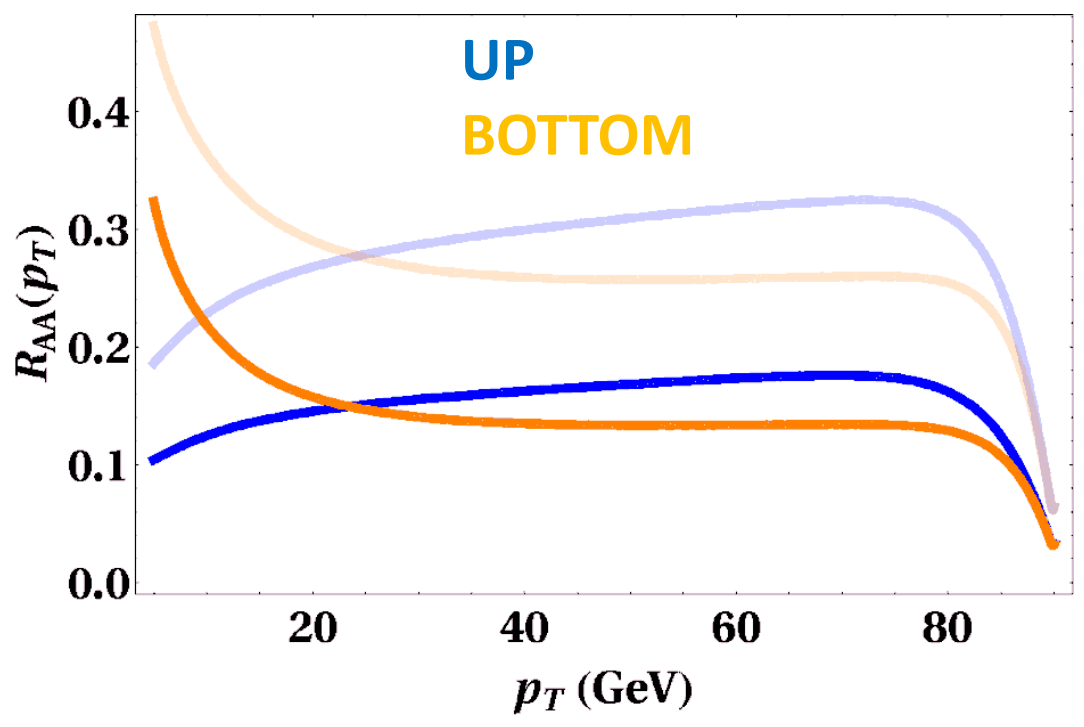




\section{Initial pQCD spectra}

Initial quark production spectra

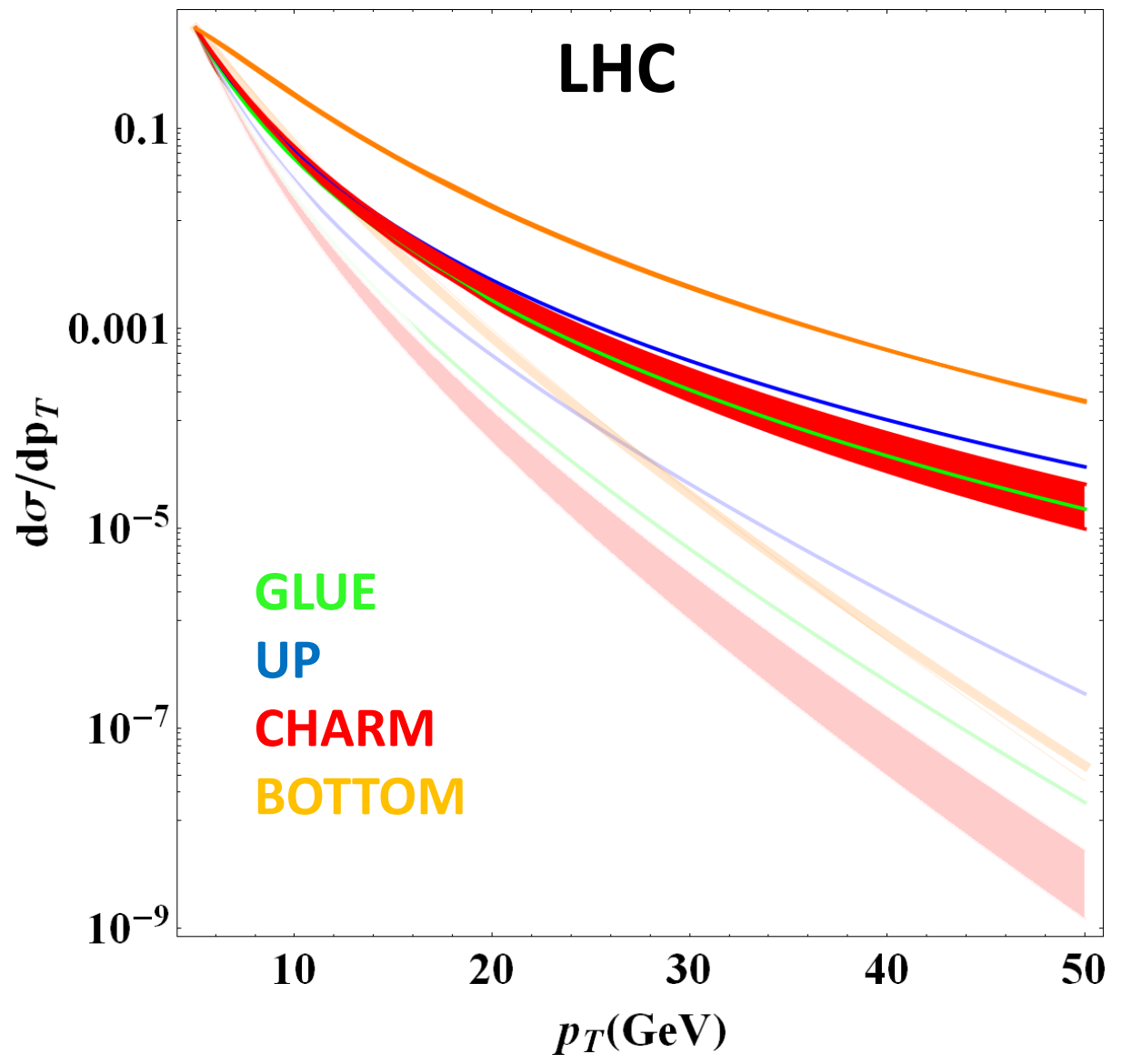

Competing effects between increased density and harder production spectra

- LHC density and spectra

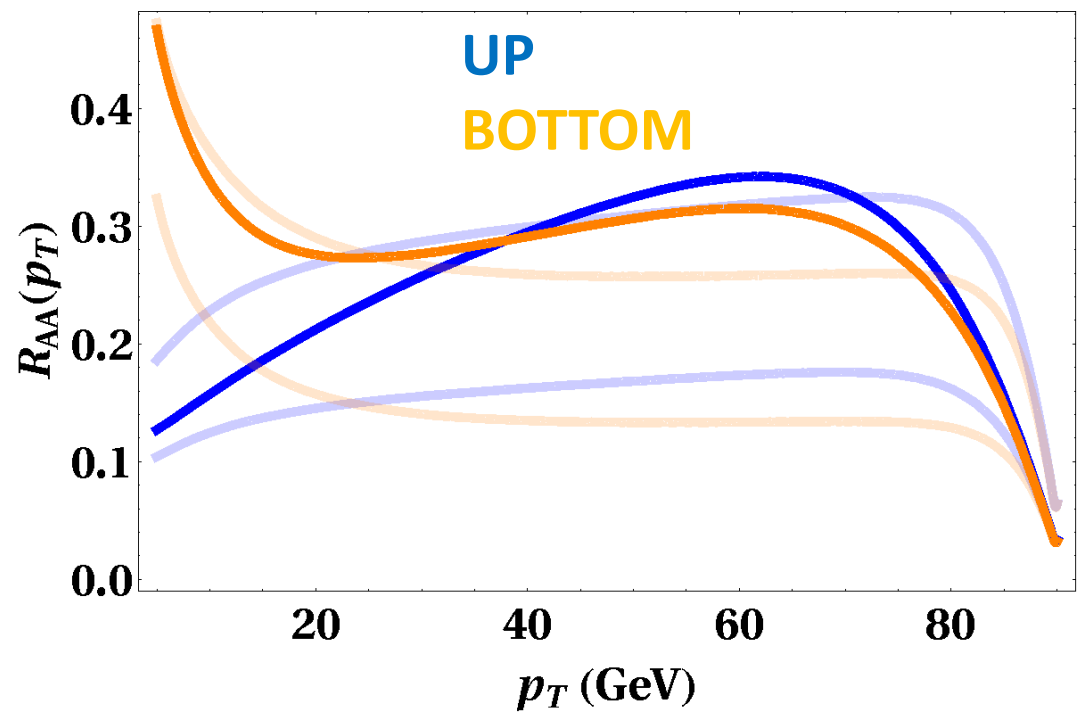




\section{Outline}

- Introduction

- Jet tomography and jet quenching

- CUJET

- Analysis of the model

- Geometry, path integral, partonic spectra

- Jet tomography at RHIC and LHC

- Heavy quark puzzle (RHIC)

- Surprising transparency (LHC)

- Future developments

- Elliptic flow

- Conclusions 


\section{Explained issues}

\section{Heavy quark puzzle (RHIC)}

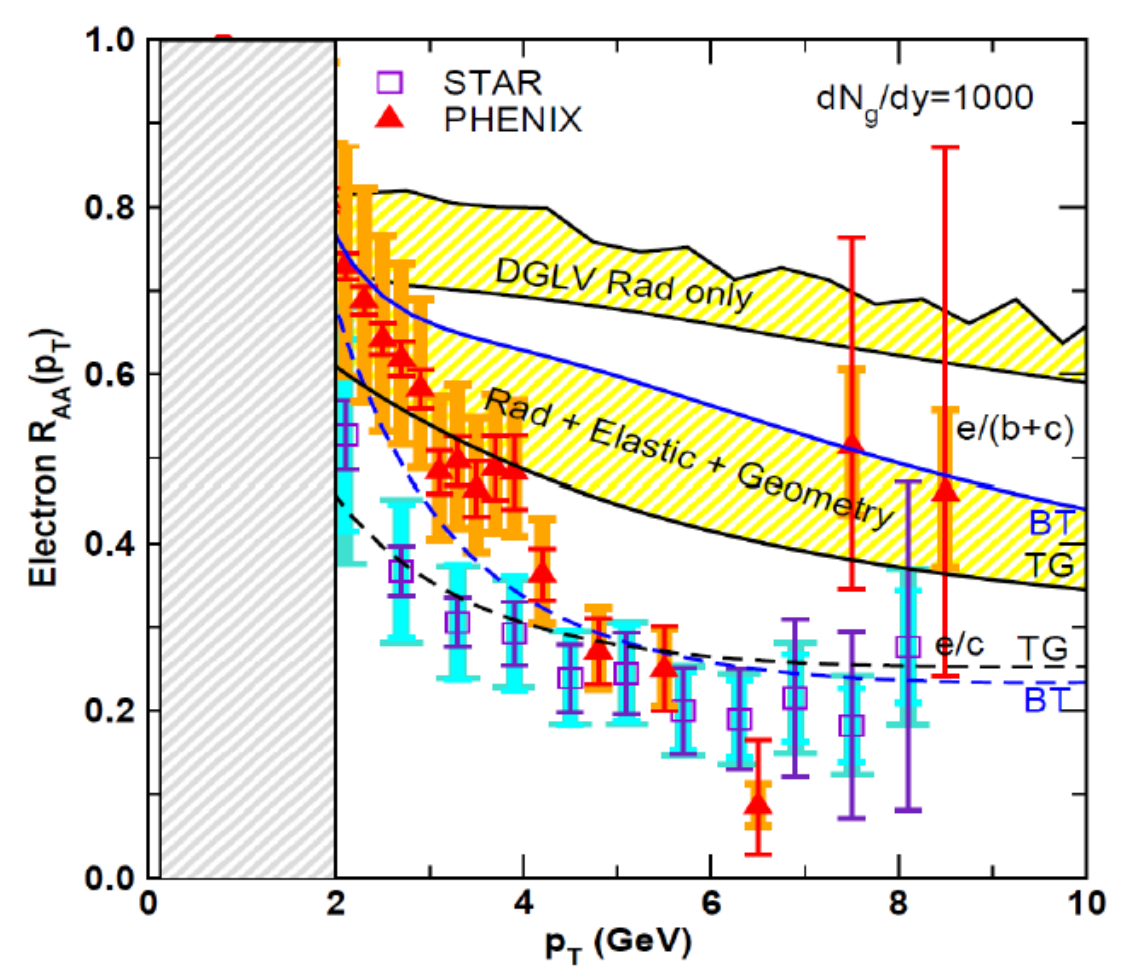

\section{Surprising transparency (LHC)}

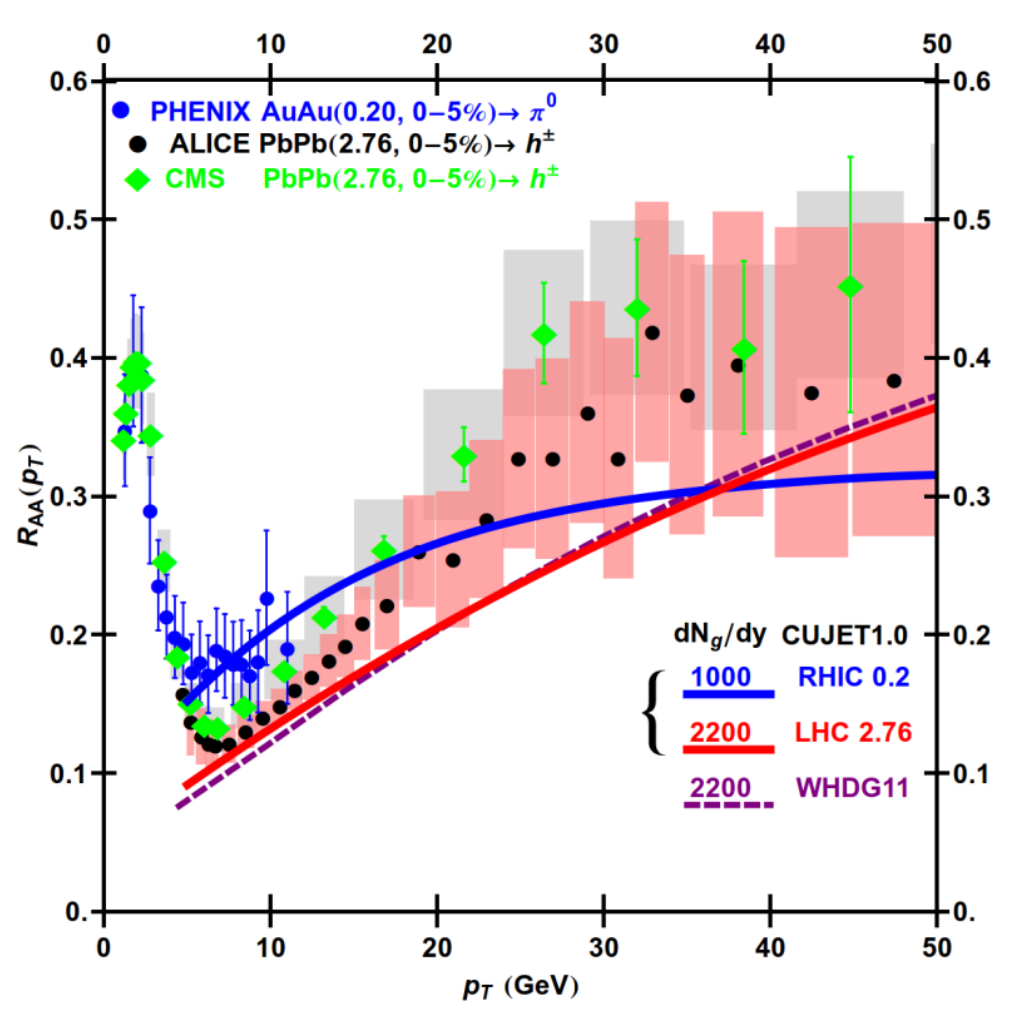




\section{Level crossing at RHIC}

\section{RHIC fit}

Glauber+Bjorken, dynamical, rad+col central collision

$\mathrm{dN} / \mathrm{dy}=1000$

fixed coupling, $\alpha_{\mathrm{s}}=0.3$

$\tau_{0}=1 \mathrm{fm} / \mathrm{c}$

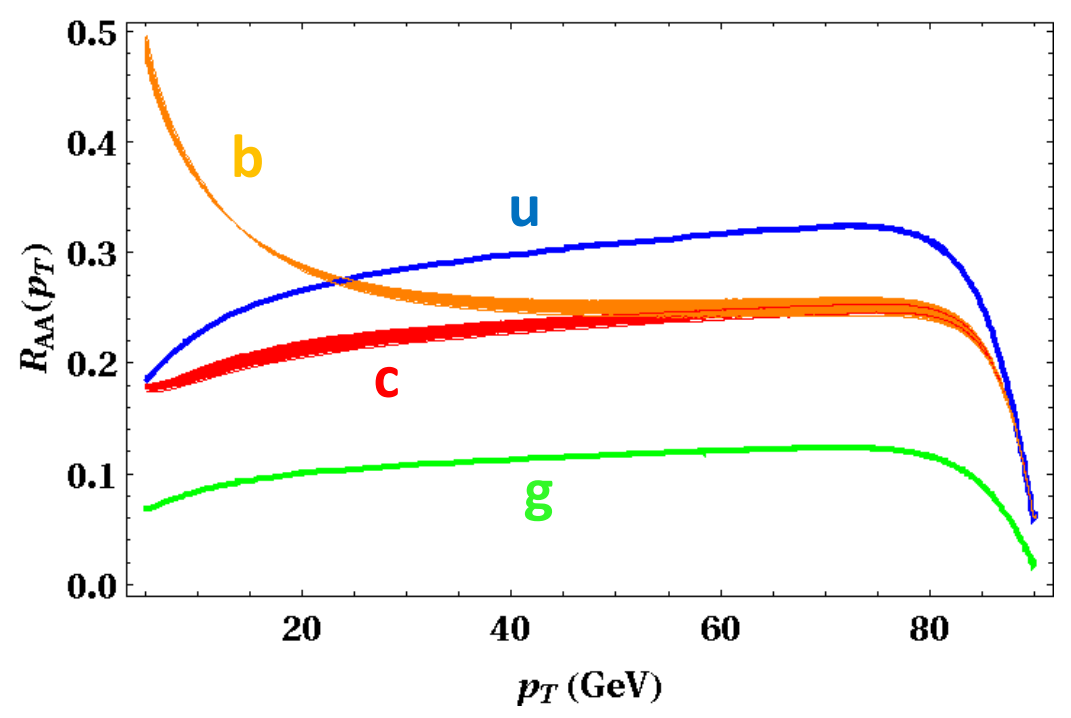

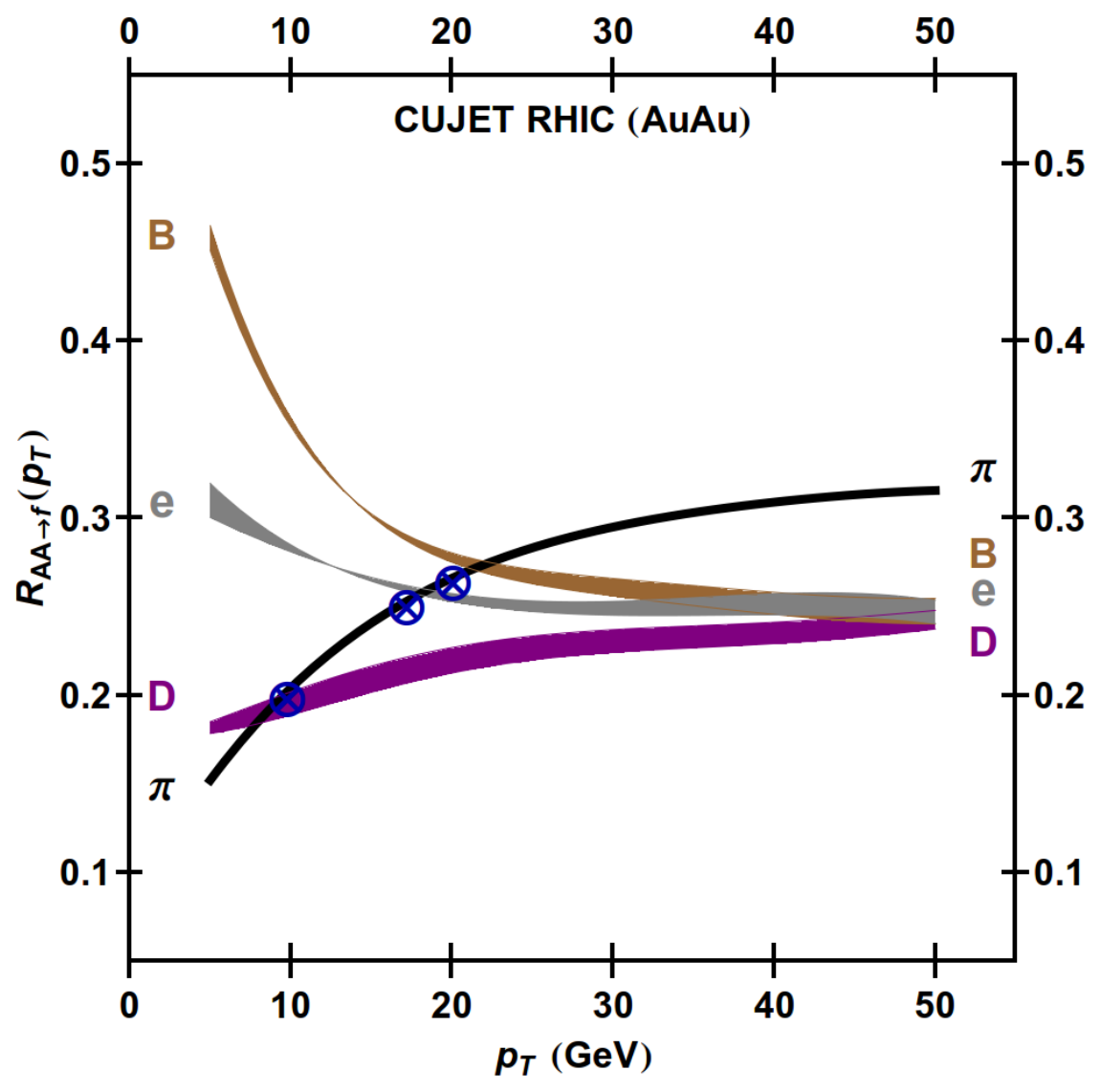

$>$ Inversion of the expected hierarchy order $\pi<D<e<B$ at sufficiently high $p_{\perp}$

$>$ Interplay between energy loss, initial partonic spectra and fragmentation functions 


\section{RHIC data}

\section{RHIC fit}

Glauber+Bjorken, dynamical, rad+col central collision

$\mathrm{dN} / \mathrm{dy}=1000$

fixed coupling, $\alpha_{s}=0.3$

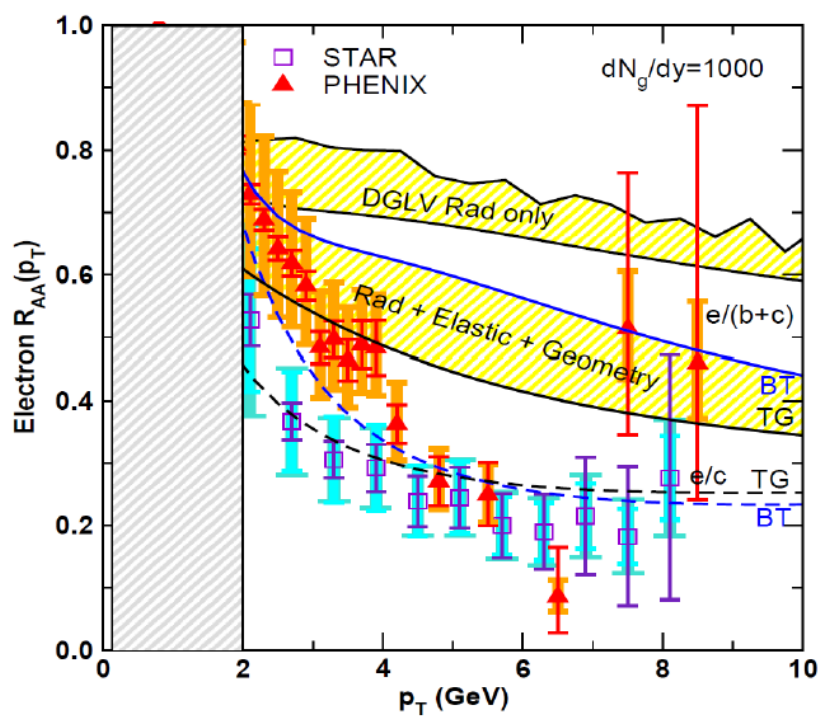

CUJET @ RHIC

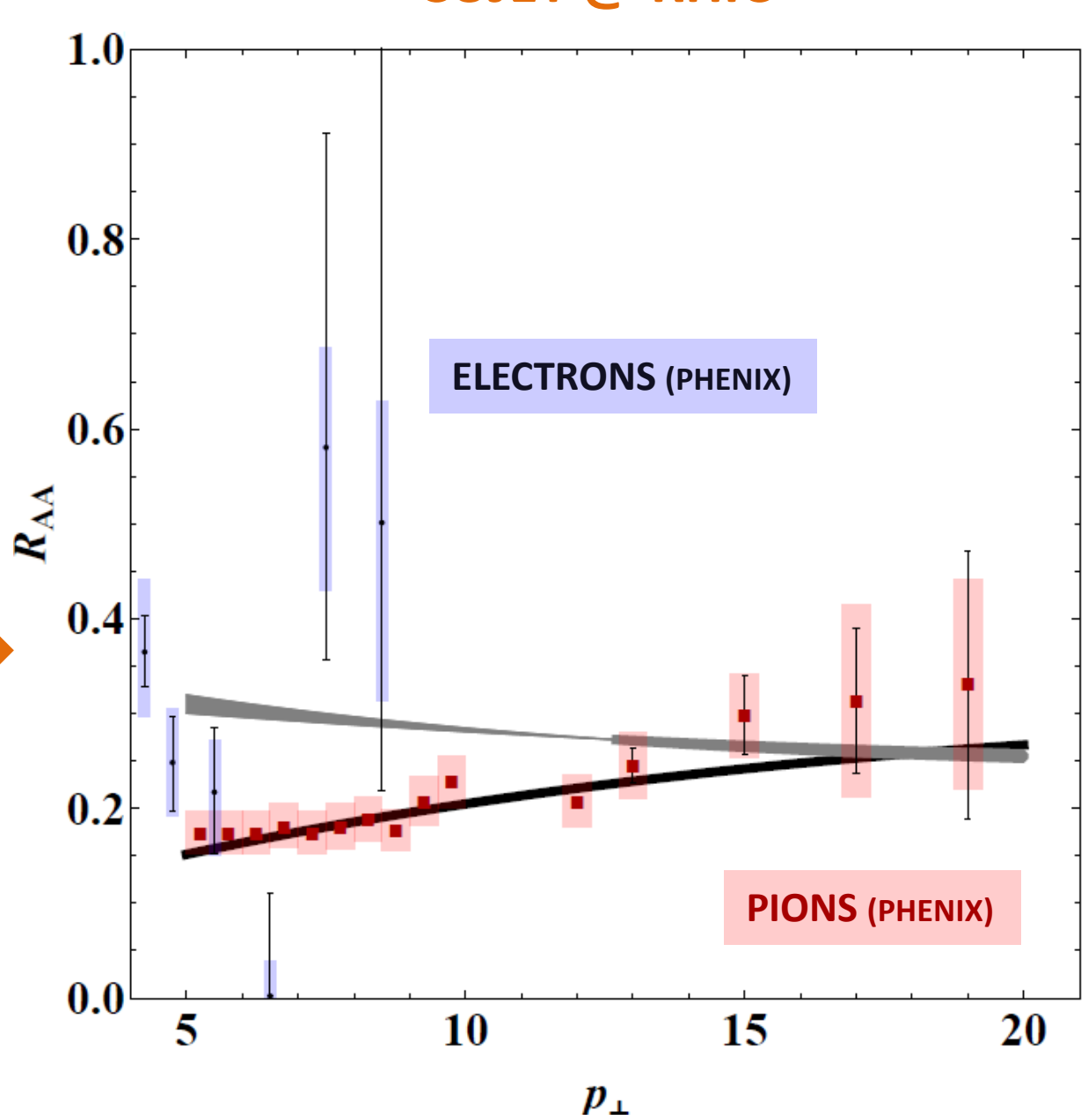

Reduced discrepancy between pions and non-photonic electrons 


\section{Level crossing at LHC}
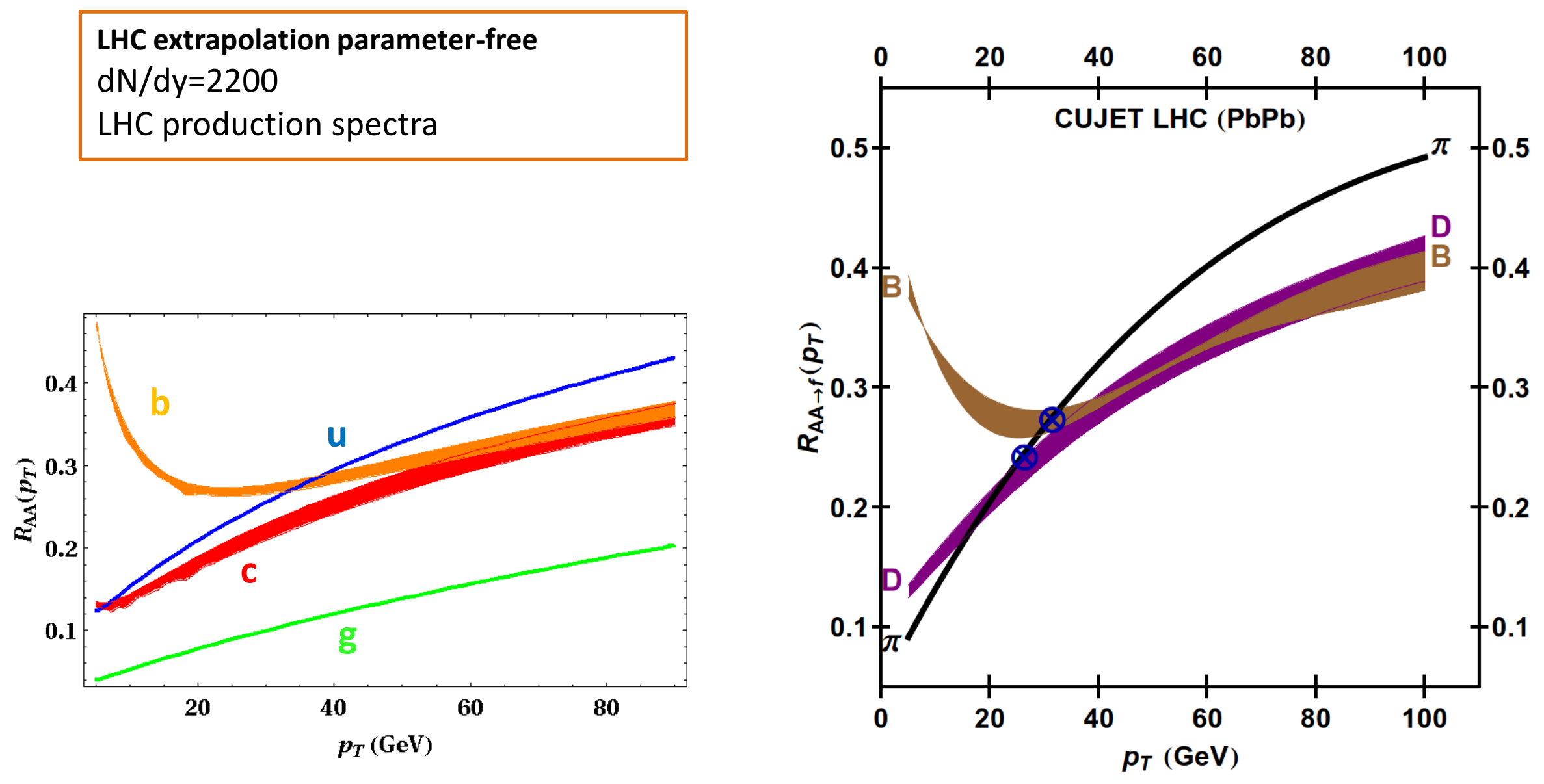

$>$ Inversion of the expected hierarchy order $\pi<D<B$ at energies accessible experimentally

$>$ Steep rise of $\mathrm{R}_{\mathrm{AA}}$ with $p_{\perp}$ 


\section{LHC data}

\section{LHC extrapolation parameter-free}

(fixed coupling, $\alpha_{s}=0.3$ )

$\mathrm{dN} / \mathrm{dy}=2200$

LHC production spectra

D Predictions are over-quenched: the QGP at LHC is surprisingly transparent to hard probes

$>$ Possibility of reduced coupling?

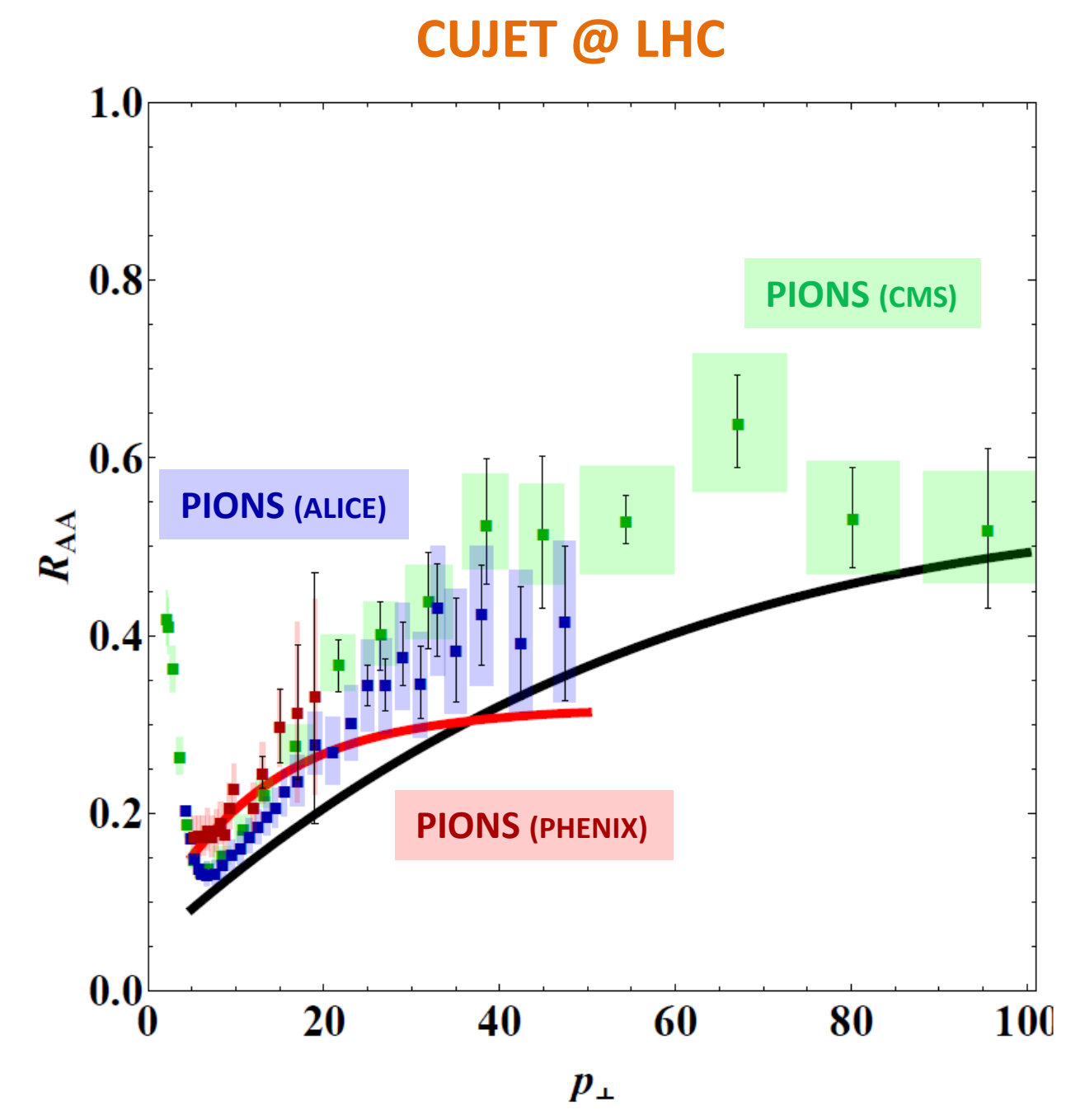




\section{LHC data with running coupling}

LHC fit, RHIC extrapolation parameter-free
Glauber+Bjorken, dynamical, rad+col
central collision
$d N / d y=2200$
running coupling, $\boldsymbol{\alpha}_{0}=\mathbf{0 . 4}$

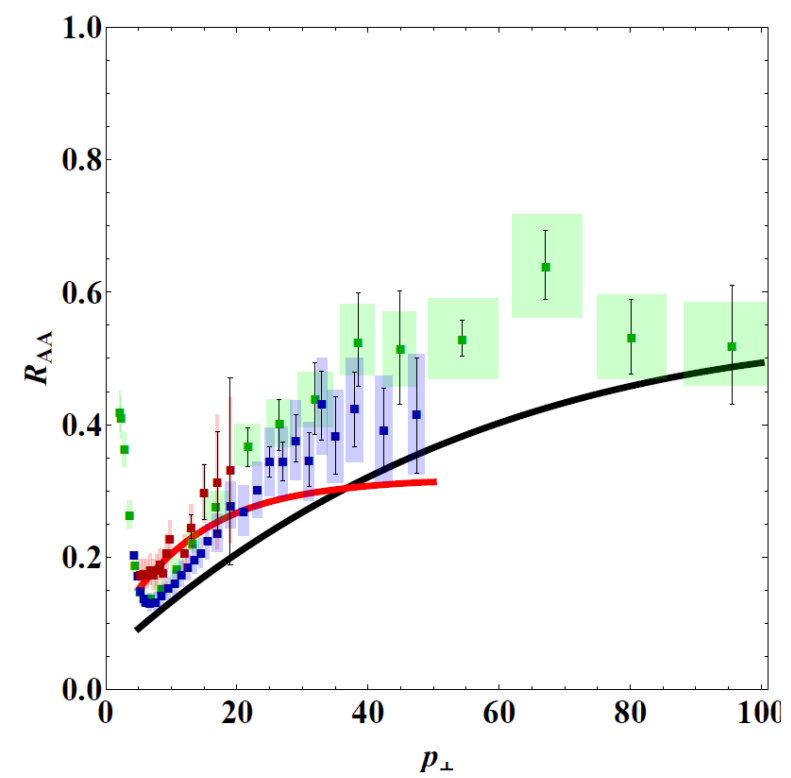

$>$ Surprising agreement with data

\section{CUJET @ LHC}

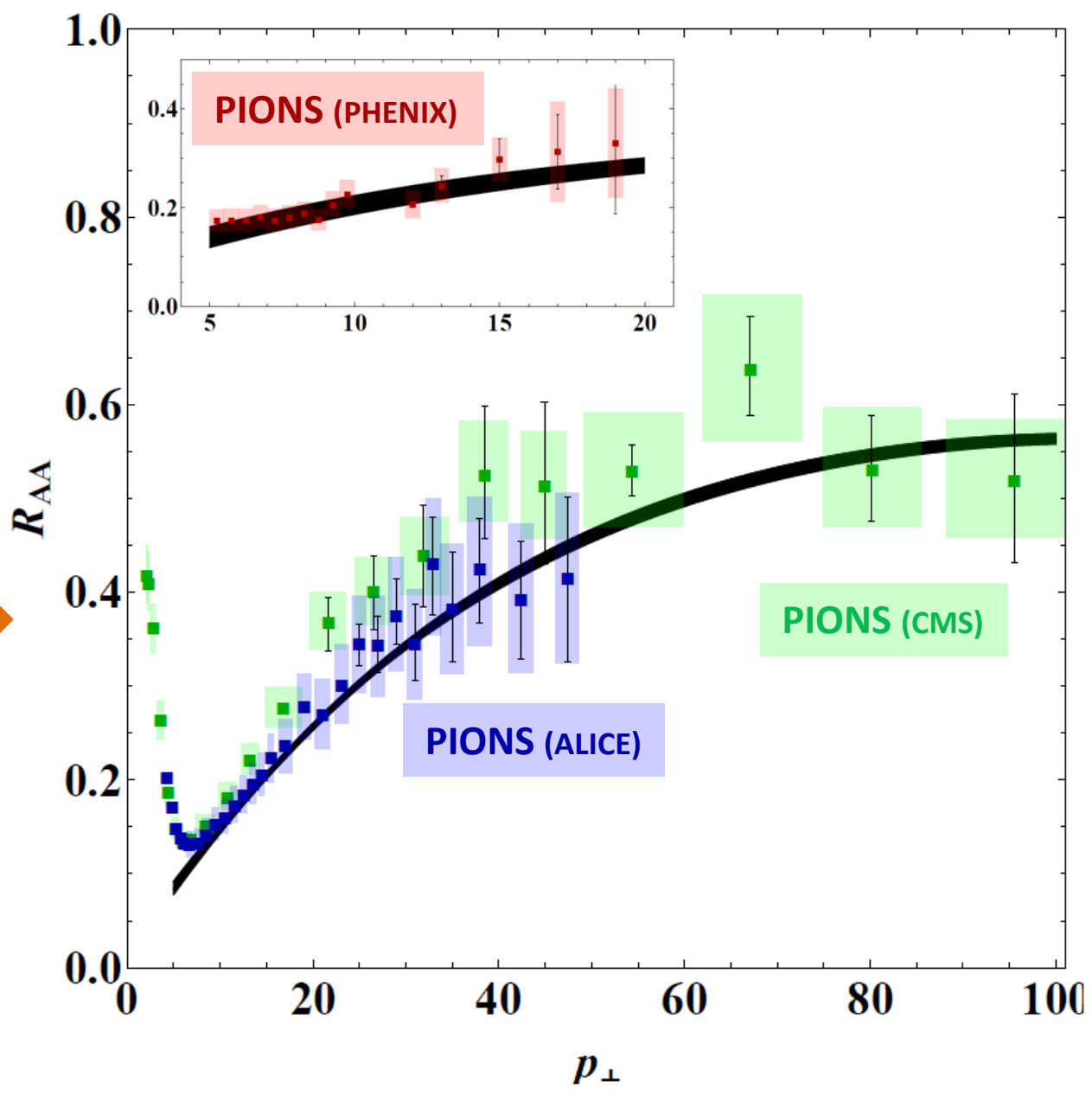

> Consistent with backward extrapolated RHIC predictions 


\section{Open heavy flavor data}

CUJET @ LHC
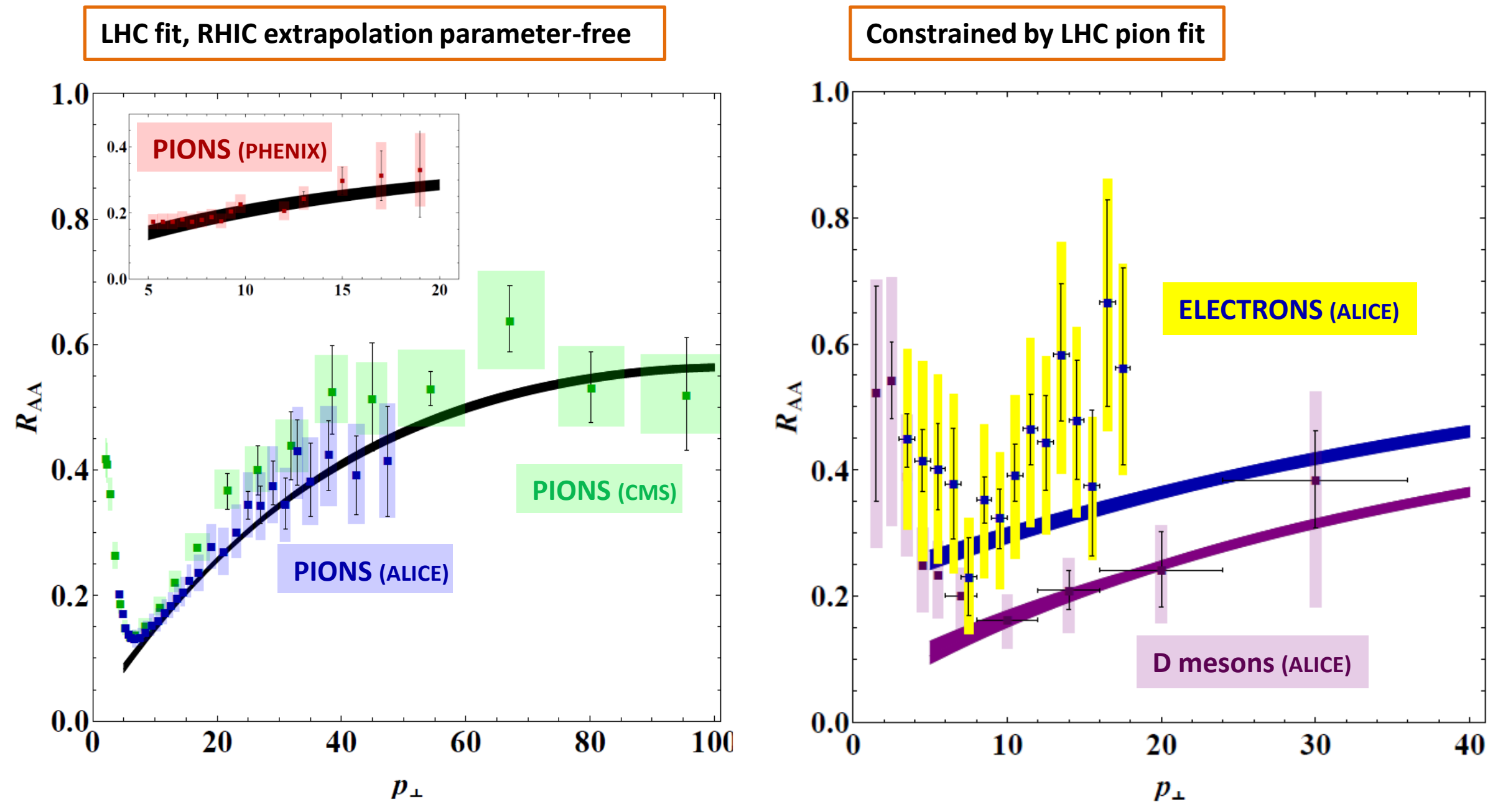


\section{Open heavy flavor data}

\section{CUJET @ LHC}
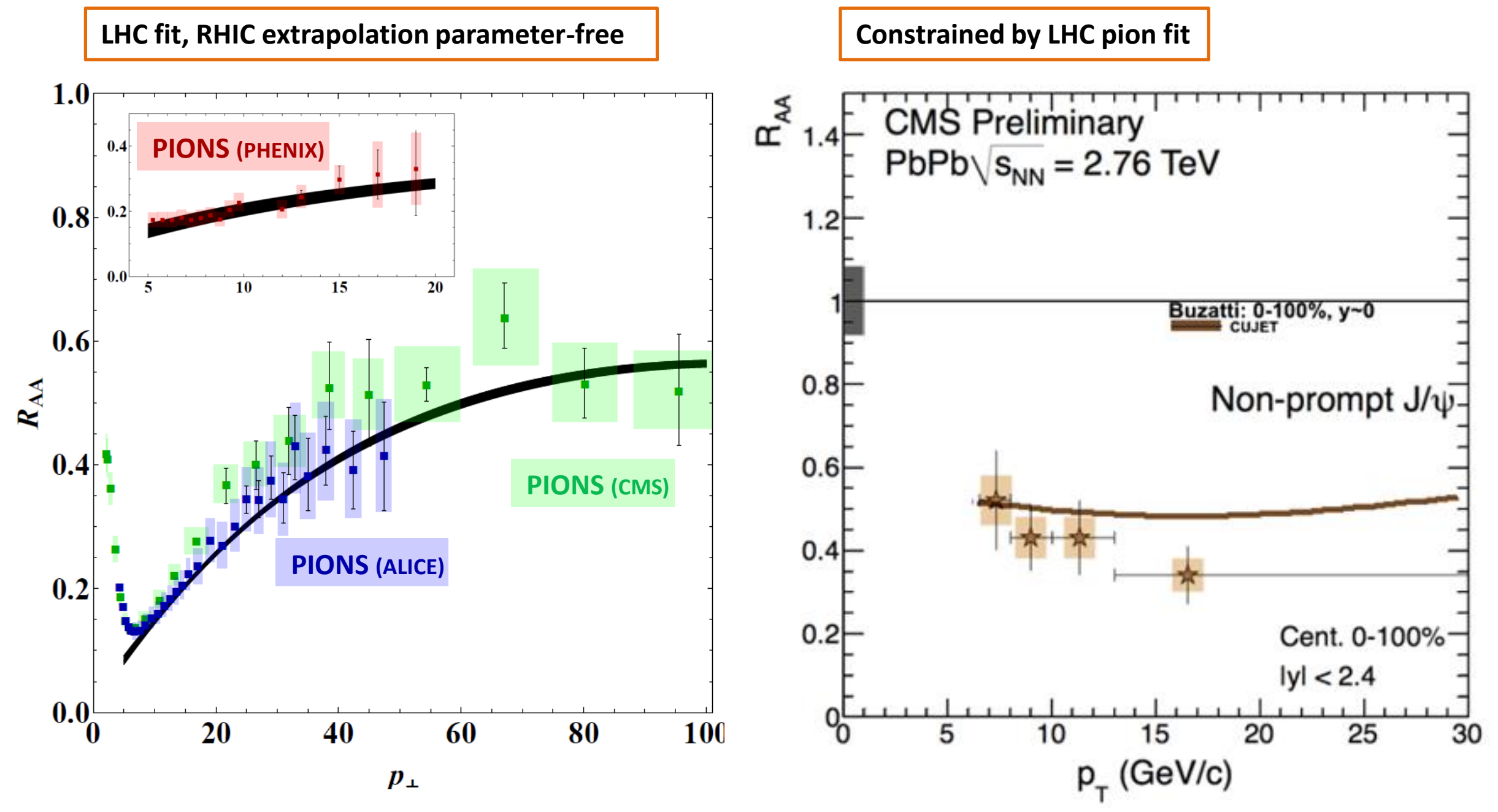


\section{Outline}

- Introduction

- Jet to mography and jet quenching

- CUJET

- Analysis of the model

- Geometry, path integral, partonic spectra

- Jet tomography at RHIC and LHC

- Heavy quark puzzle (RHIC)

- Surprising transparency (LHC)

- Future developments

- Elliptic flow

- Conclusions 


\section{Elliptic flow}

Recent presliminary PHENIX/RHIC 2012 azimuthal tomography

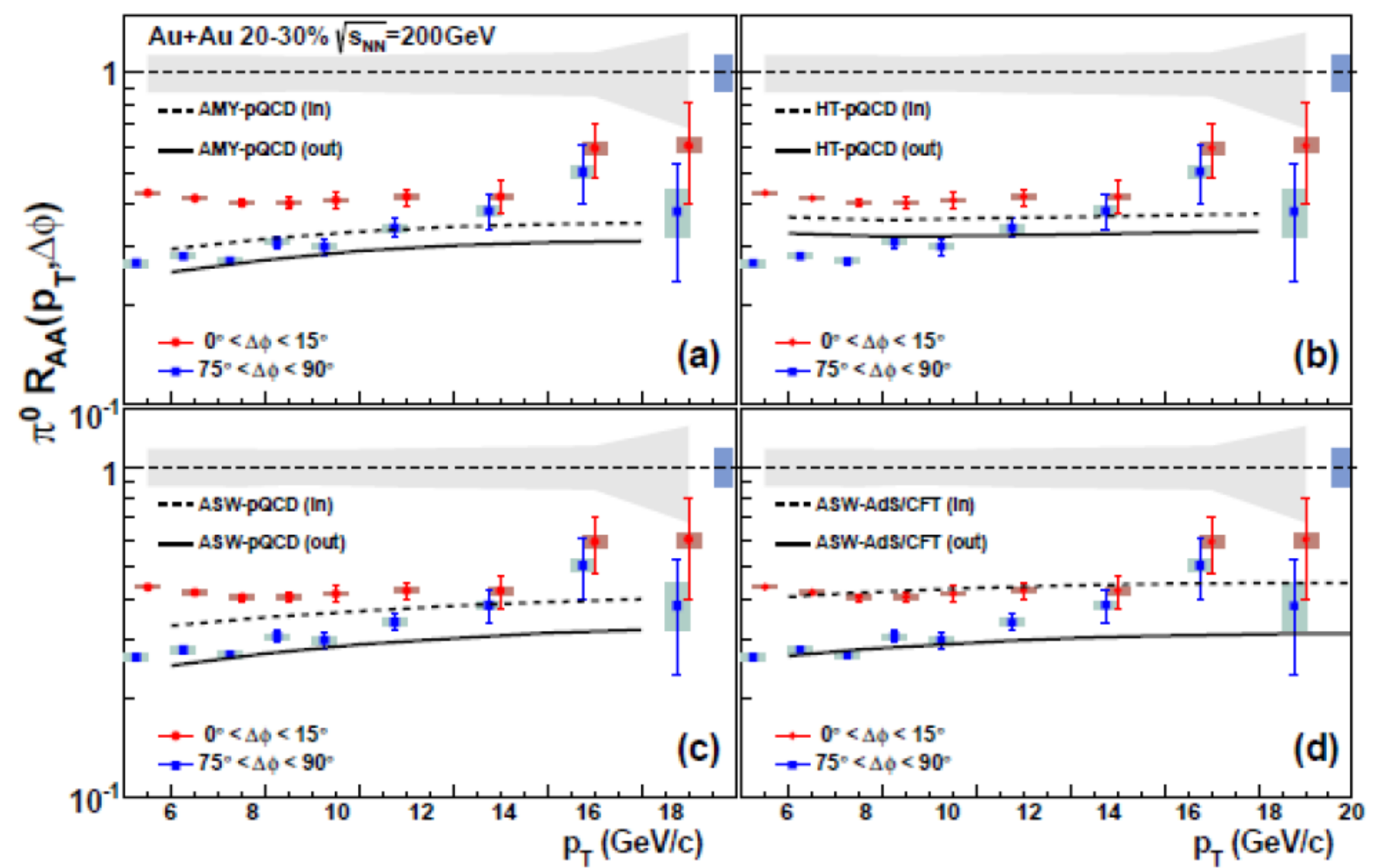

FIG. 22: (Color online) The data points are $R_{A A}(\Delta \phi)$ in 20-30\% centrality as a function of $p_{T}$ for in-plane (red, upper points) and out-of-plane $\pi^{0}$ (blue, lower points), compared to four model calculations (see text for description and references). (a) pQCD-based AMY [9, 42], (b) HT [1], (c) pQCD-based ASW [1]], (d) ASW using AdS/CFT correspondence [12]. The curves on panels (a)-(c) are taken from[3i]. The dotted and solid lines are the in-plane and out-of-plane predictions, respectively. The definition of the gray bands and blue boxes is same as in Fig. 207] 


\section{Elliptic flow}

At LHC Deep UV Band $p T>10 \mathrm{GeV}$ seems to agree finally with pQCD tomography An intermediate IM Band $2<\mathrm{pT}<10$ interpolates between IR and UV bands

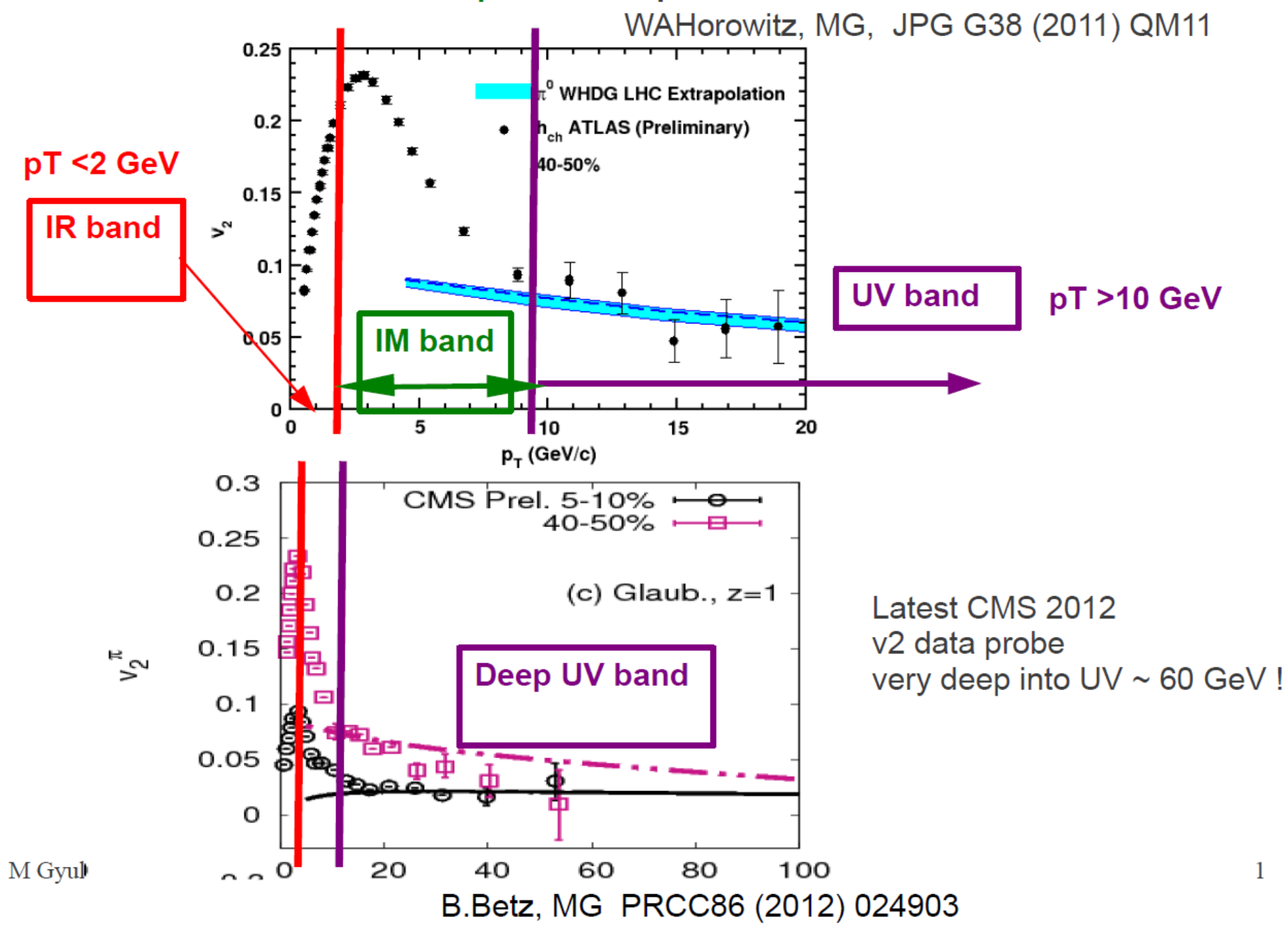




\section{Hydro expansion}

Denes Molnar, Deke Sun, QM12 used MPC bulk evolution to evaluate GLV En Loss

high-pT pion v2, LHC ( $\alpha_{s}$ scaled to RAA at pT $\left.=6 \mathrm{GeV}\right)$
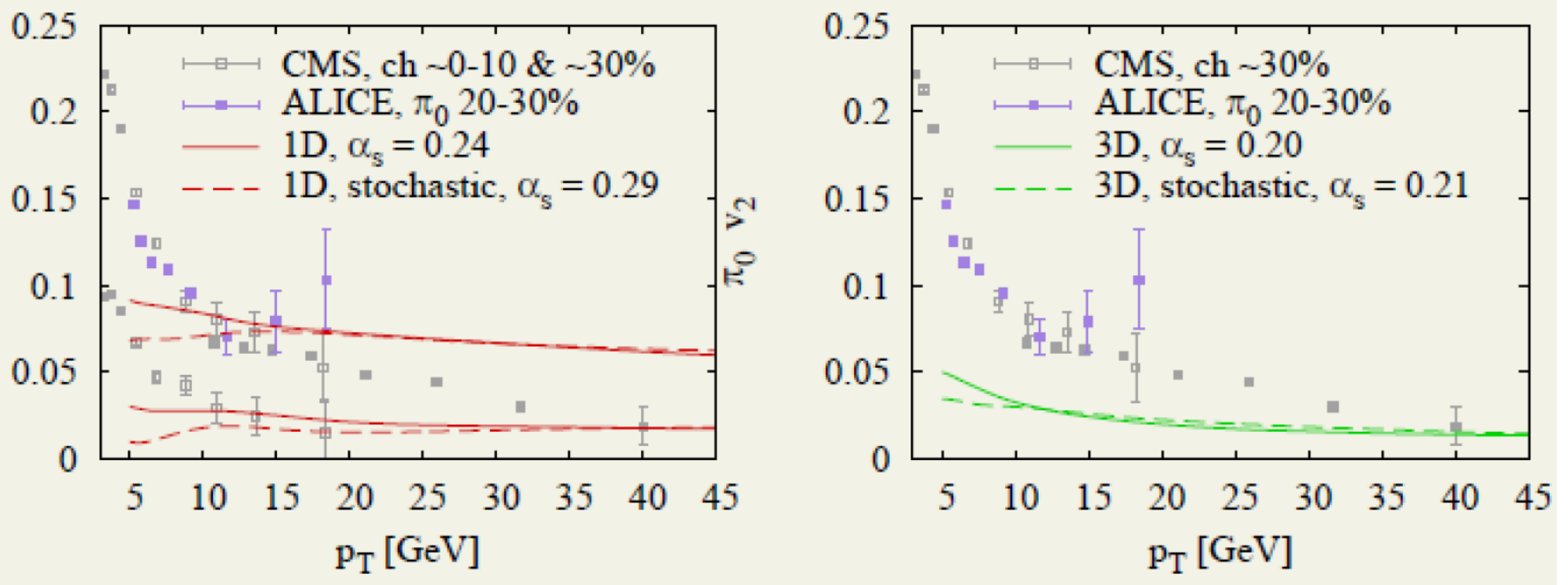

They concluded that transverse expansion reduced $\mathrm{pQCD} v 2(\mathrm{pT})$ by factor $\sim 2$ below data 


\section{More on hydro expansion}
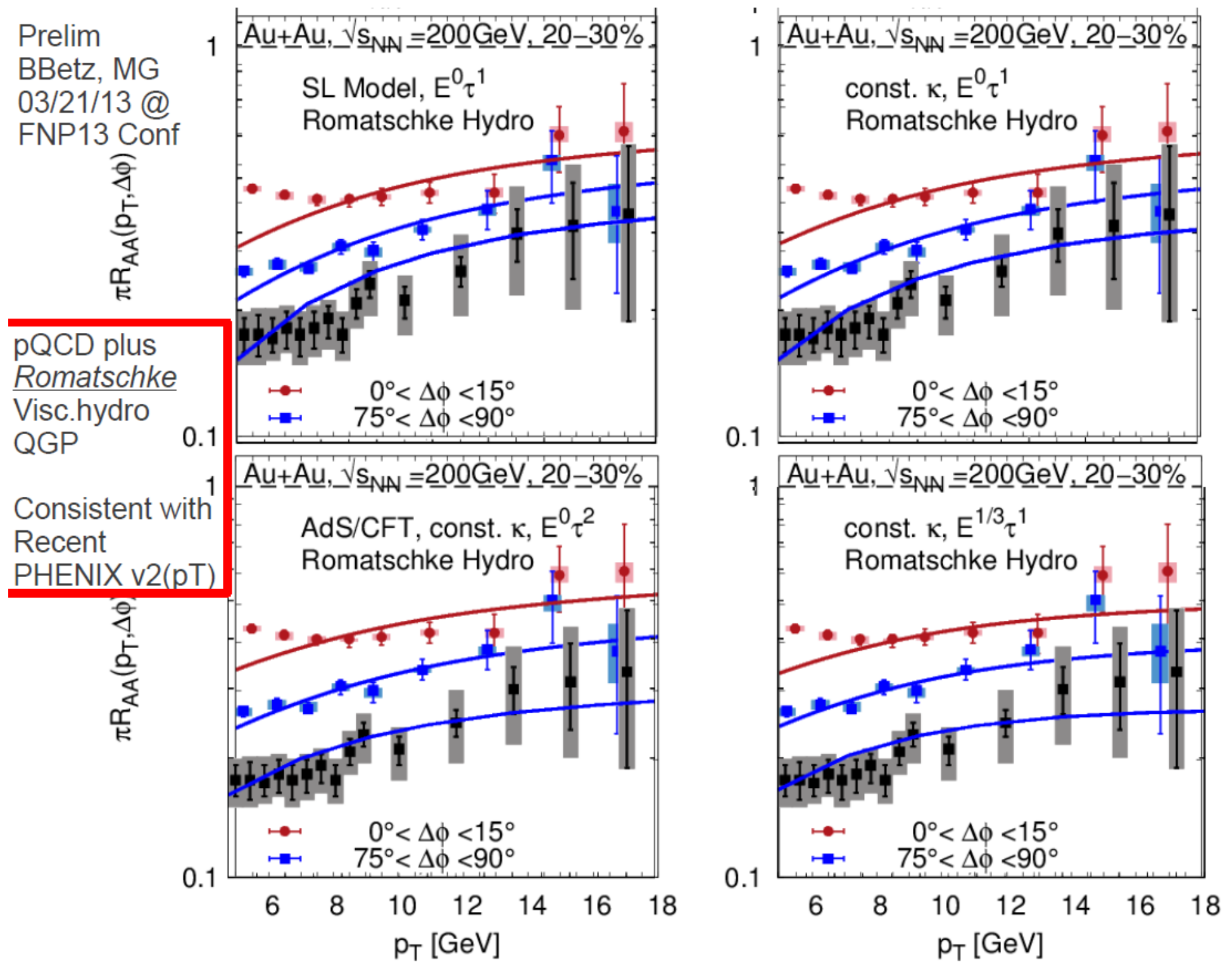


\section{Conclusions}

- Quark Gluon Plasma

- Low- hydro vs. high- pQCD

- QGP properties can be studied measuring high- Jets

- Jet tomography requires knowledge of: (1) initial conditions (2) plasma evolution (3) energy loss (4) jet fragmentation

- CUJET

- Quantitative predictions insensitive to many of the systematic theoretical uncertainties

- Evolution over WHDG

" Geometry, Jet path integration, Partonic spectra, Dynamical potential

- Predicted level crossing pattern, reduced light-bottom quark jets discrepancy, steep rise of $\mathrm{R}_{A A}$ at $\mathrm{LHC}$

- Future

- Address azimuthal asymmetry puzzle

» Include viscous hydrodynamics

- Full jet measurements 


\section{WHDG}

- Elliptic flow $\frac{d N}{d p_{\perp} d \phi}=\frac{d N}{d p_{\perp}} \frac{1}{2 \pi}\left(1+2 v_{1} \cos \phi+2 v_{2} \cos 2 \phi+\cdots\right)$
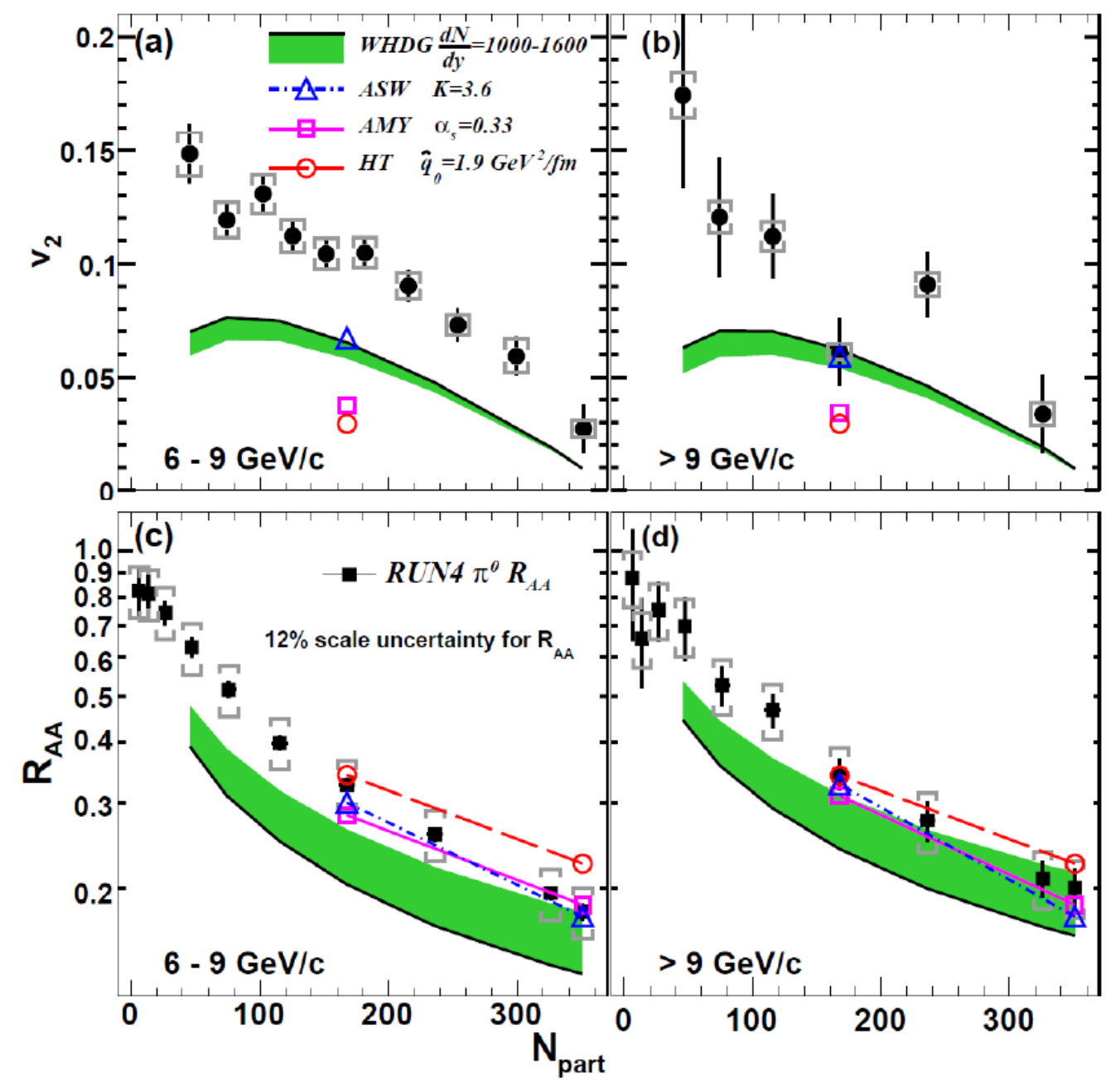

$>$ Need stronger coupling between jet and medium, but $R_{A A}$ will be over-quenched

$>$ The agreement between data and theoretical curves worsen for higher centralities and lower transverse momenta. 


\section{Elliptic flow}

\section{BBMG 032112 FNP13}

Azimuthal Tomography over full range RHIC pT=0-20 GeV , RAAin out

Sensistive to both $\mathrm{dEdX}$ as well as detailed spacetime QGP expansion

Results below couple pQCD with RL (Romatsch.Luzum) min visc hydro QGP

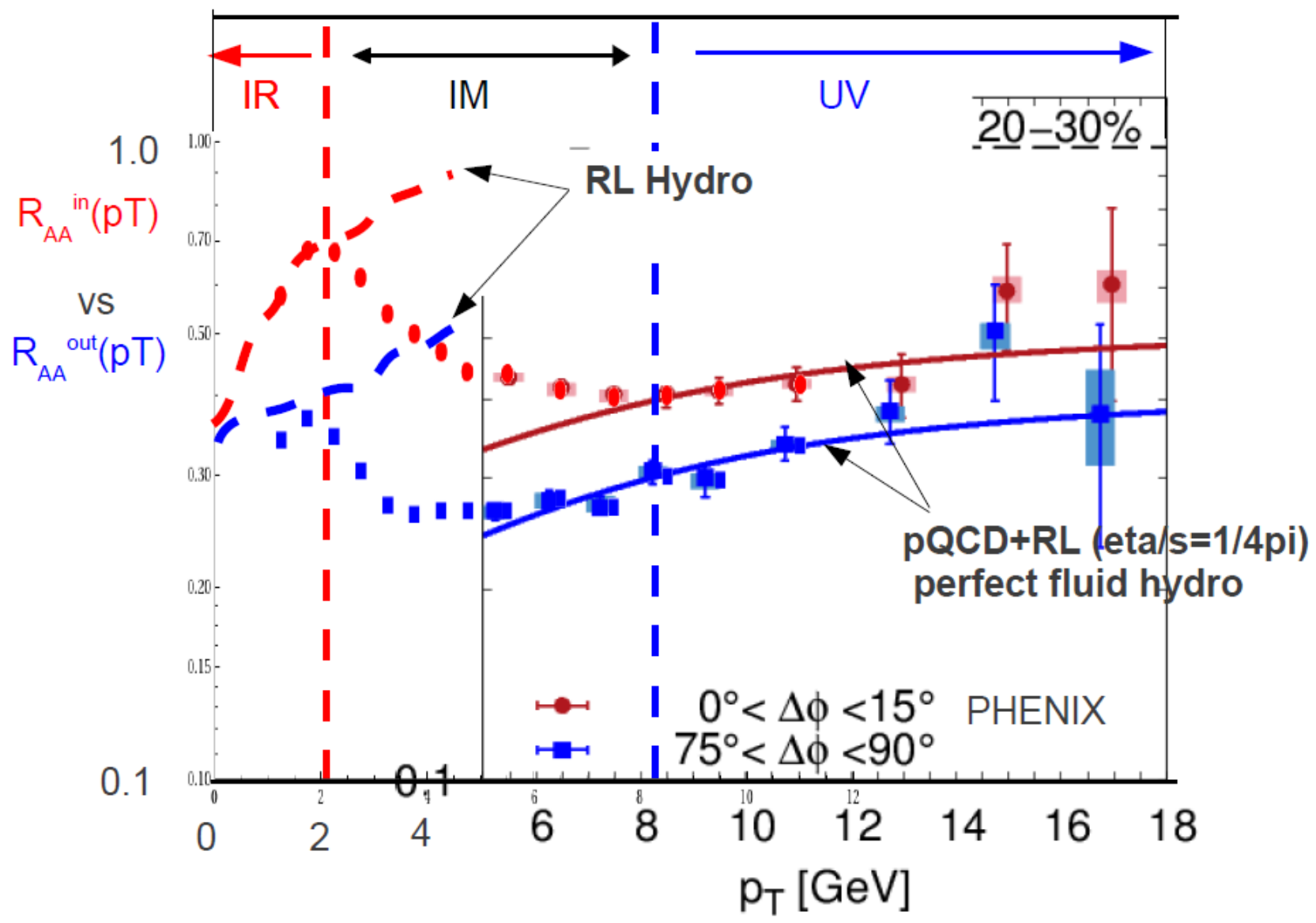




\section{Radiative energy loss}

\section{Incoherent limit: Gunion-Bertsch}

- $\frac{d N}{d x d k_{\perp}}=\frac{1}{x} \frac{\alpha_{s} C_{A}}{\pi^{2}} \frac{q_{\perp}^{2}}{k_{\perp}^{2}\left(q_{\perp}-k_{\perp}\right)^{2}}$

- Non-abelian equivalent of Bethe-Heitler limit

- Flat distribution at mid-rapidity

- Multiple scattering amplitudes are summed incoherently

- Incoming quark is on-shell and massless

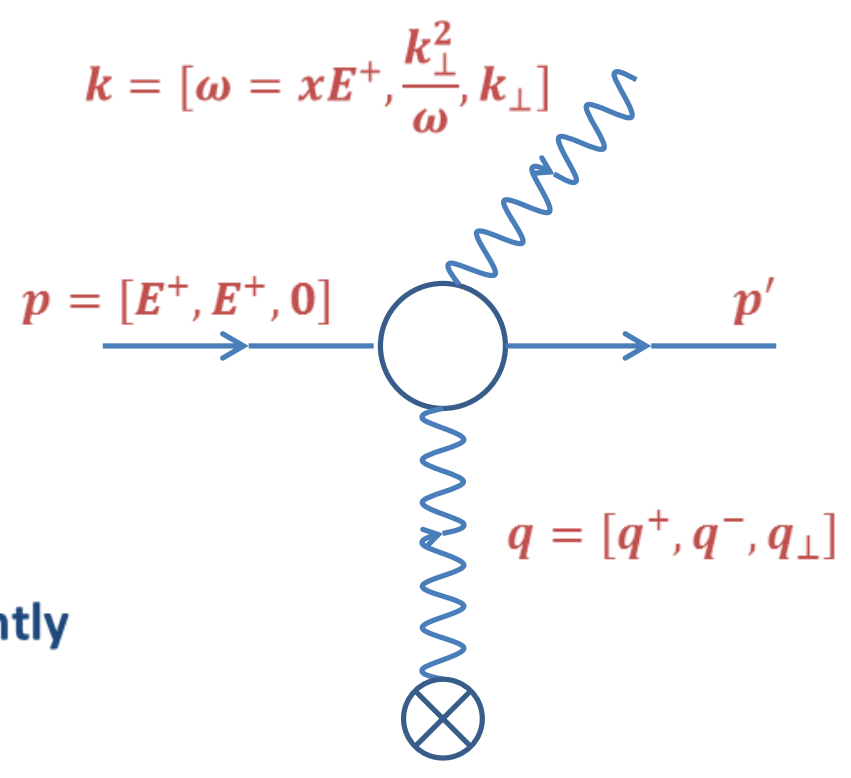

\section{Formation time physics}

$\bullet$

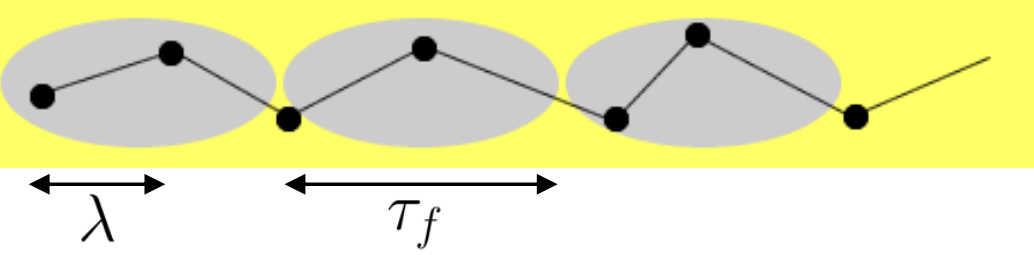

$$
\tau_{f} \sim \frac{2 \omega}{k_{\perp}^{2}}
$$

- $\tau_{f}<\lambda<L$ Incoherent multiple collisions

$-\lambda<\tau_{f}<L$ LPM effect (radiation suppressed by multiple scatterings within one coherence length)

- $\lambda<L<\boldsymbol{\tau}_{f}$ Factorization limit (acts as one single scatterer) 


\section{GLV interference effects}

- Energy loss LPM interference effects with hard production vertex (jet created at finite time)
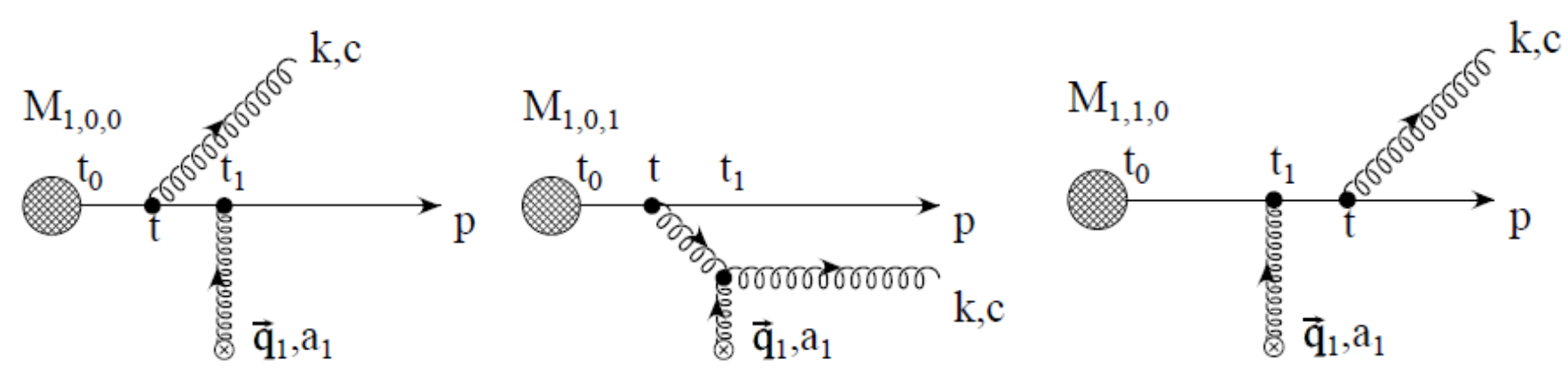

- Transverse diffusion LPM interference effects with successive scatterings (gluon cascading)

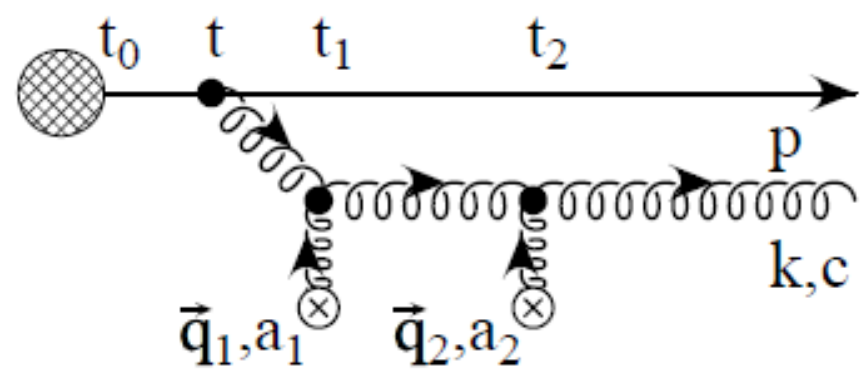




\section{DGLV mass effects}

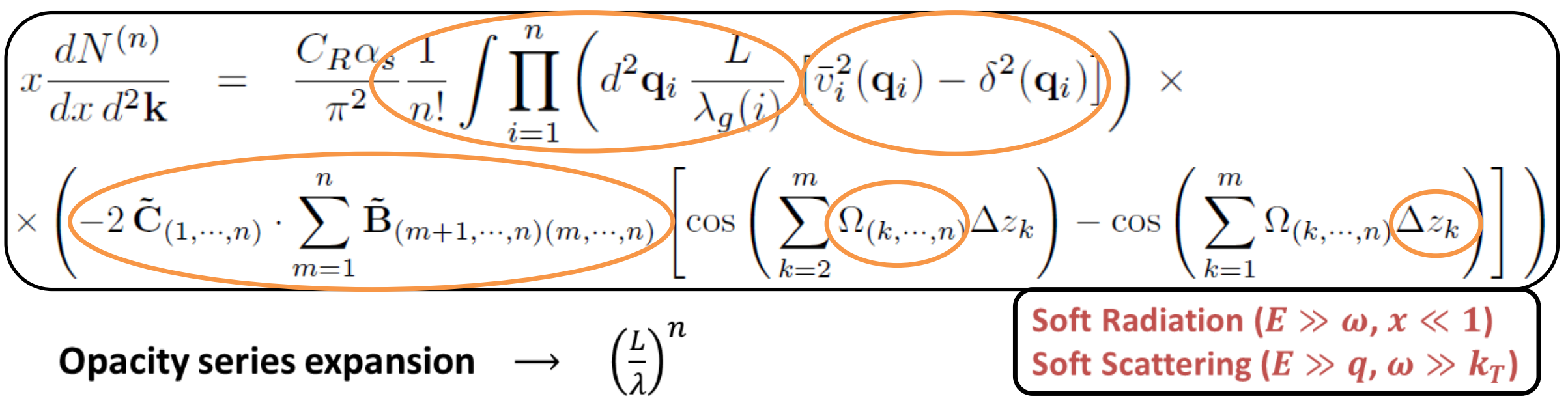

$\begin{array}{ll}\text { Radiation antenna } \rightarrow \text { Cascade terms } & \tilde{\mathbf{C}}_{\left(i_{1} i_{2} \cdots i_{m}\right)}=\frac{\left(\mathbf{k}-\mathbf{q}_{i_{1}}-\mathbf{q}_{i_{2}}-\cdots-\mathbf{q}_{i_{m}}\right)}{\left(\mathbf{k}-\mathbf{q}_{i_{1}}-\mathbf{q}_{i_{2}}-\cdots-\mathbf{q}_{i_{m}}\right)^{2}+m_{g}^{2}+M^{2} x^{2}}, \\ & \tilde{\mathbf{B}}_{\left(i_{1} i_{2} \cdots i_{m}\right)\left(j_{1} j_{2} \cdots i_{n}\right)}=\tilde{\mathbf{C}}_{\left(i_{1} i_{2} \cdots j_{m}\right)}-\tilde{\mathbf{C}}_{\left(j_{1} j_{2} \cdots j_{n}\right)} .\end{array}$

Gunion-Bertsch $\quad \tilde{\mathbf{B}}_{i}=\tilde{\mathbf{H}}-\tilde{\mathbf{C}}_{i}$,

Hard

$\tilde{\mathbf{H}}=\frac{\mathbf{k}}{\mathbf{k}^{2}+m_{g}^{2}+M^{2} x^{2}}$,

LPM effect $\rightarrow \quad \Omega_{(m, \cdots, n)}=\frac{\left(\mathbf{k}-\mathbf{q}_{m}-\cdots-\mathbf{q}_{n}\right)^{2}}{2 x E}+\frac{m_{g}^{2}+M^{2} x^{2}}{2 x E}$

Inverse formation time Mass effects

Scattering center distribution $\rightarrow \Delta z_{k}=z_{k}-z_{k-1} \sim L /(n+1)$ 


\section{Effective Potential}

Static potential (DGLV)

$\left|\bar{v}_{i}\left(q_{i}\right)\right|^{2}=\frac{1}{\pi} \frac{\mu\left(z_{i}\right)^{2}}{\left(q^{2}+\mu\left(z_{i}\right)^{2}\right)^{2}}$

- Static scattering centers

- Color-electric screened Yukawa potential (Debye mass)

- Full opacity series

\section{Dynamical potential (MD)}

$$
\left|\bar{v}_{i}\left(q_{i}\right)\right|^{2}=\frac{1}{\pi} \frac{\mu\left(z_{i}\right)^{2}}{q^{2}\left(q^{2}+\mu\left(z_{i}\right)^{2}\right)}
$$

- Dynamical scattering centers

- Includes screened color-magnetic effects (HTL gluon propagators)

- Only first order in opacity

Interpolating potential (CUJET)

$$
\left|\bar{v}_{i}\left(q_{i}\right)\right|^{2}=\frac{\mathcal{N}\left(\mu_{m}\right)}{\pi} \frac{\mu_{e}\left(z_{i}\right)^{2}}{\left(q^{2}+\mu_{e}\left(z_{i}\right)^{2}\right)\left(q^{2}+\mu_{m}\left(z_{i}\right)^{2}\right)}
$$

- Introduces effective Debye magnetic mass

- Interpolates between the static and dynamical limits

- Magnetic screening allows full opacity series 


\section{Outline}

- Introduction

\section{- QCD phase diagrams and equation of state \\ - Heavy ion collisions and formation of Quark Gluon Plasma \\ - Jet tomography and jet quenching \\ - Energy loss models}

- Opacity expansion series

- Convergence of the series

- Radiated gluon transverse momentum distribution

- CUJET

- Analysis of the model

- Geometry, path integral, partonic spectra

- Jet tomography at RHIC and LHC

- Heavy quark puzzle (RHIC)

- Surprising transparency (LHC)

- Conclusions 


\section{Convergence of the series}

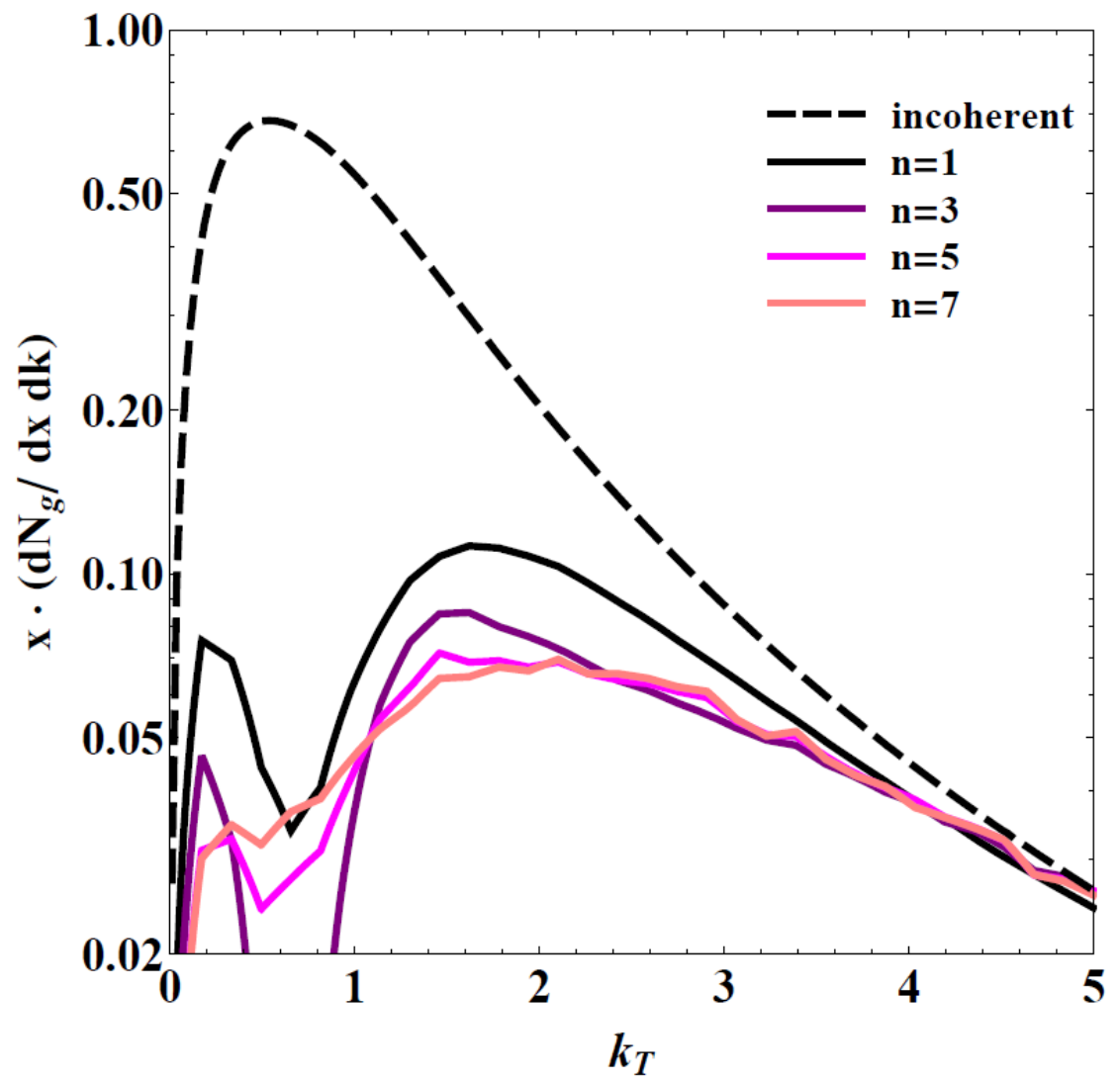

\section{Static brick}

$\mathrm{E}=50 \mathrm{GeV}$

$\omega=5 \mathrm{GeV}(\mathrm{x}=0.1)$

$\mathrm{L}=5 \mathrm{fm}$

$\mathrm{T}=250 \mathrm{MeV}$

$>$ Interfering effects dominated by $\mathrm{n}=1$ order in opacity

$>$ Convergence already at $n=5$ 


\section{Light-charm quark similarity}

Light (dashed) and charm (solid)
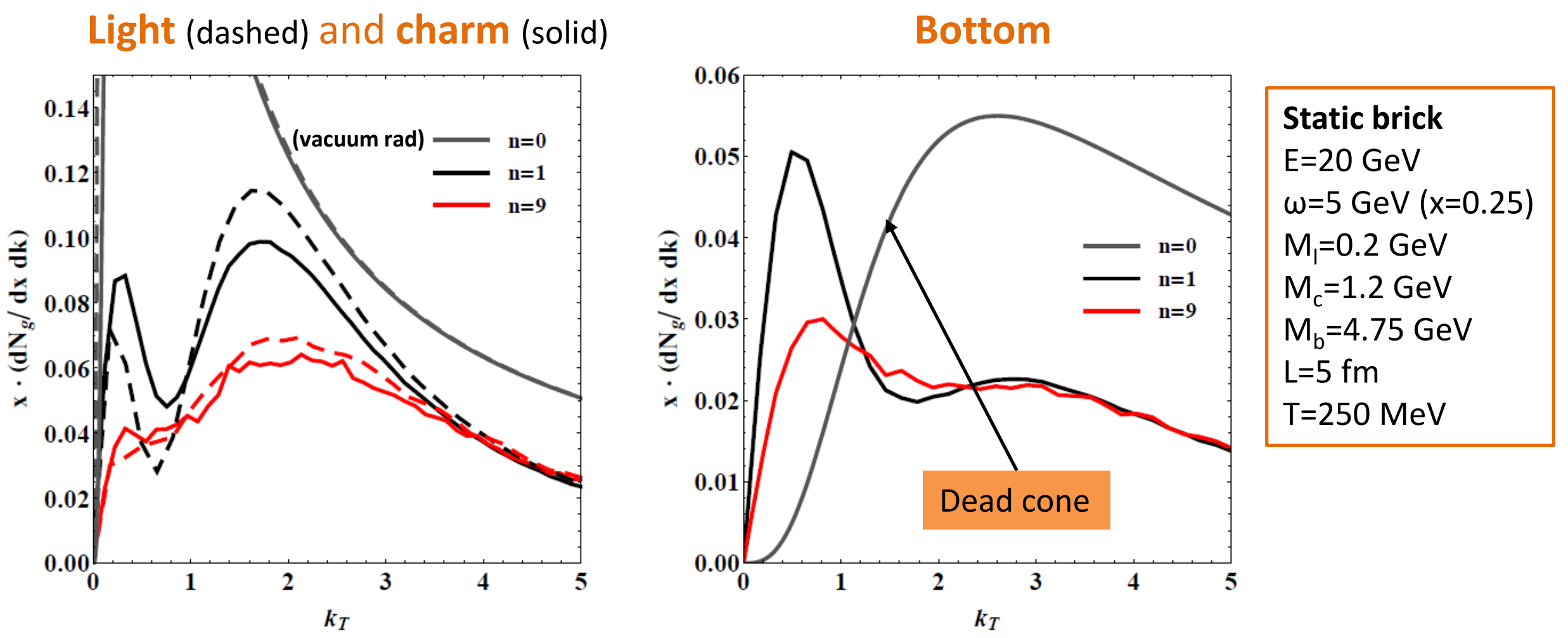

$>$ Charm and light quark transverse momentum spectra are almost identical

$>$ Bottom quark radiation is suppressed by heavy mass

$>$ Dead cone filled by induced radiation 


\section{'Thin'-'thick' plasma approximation}

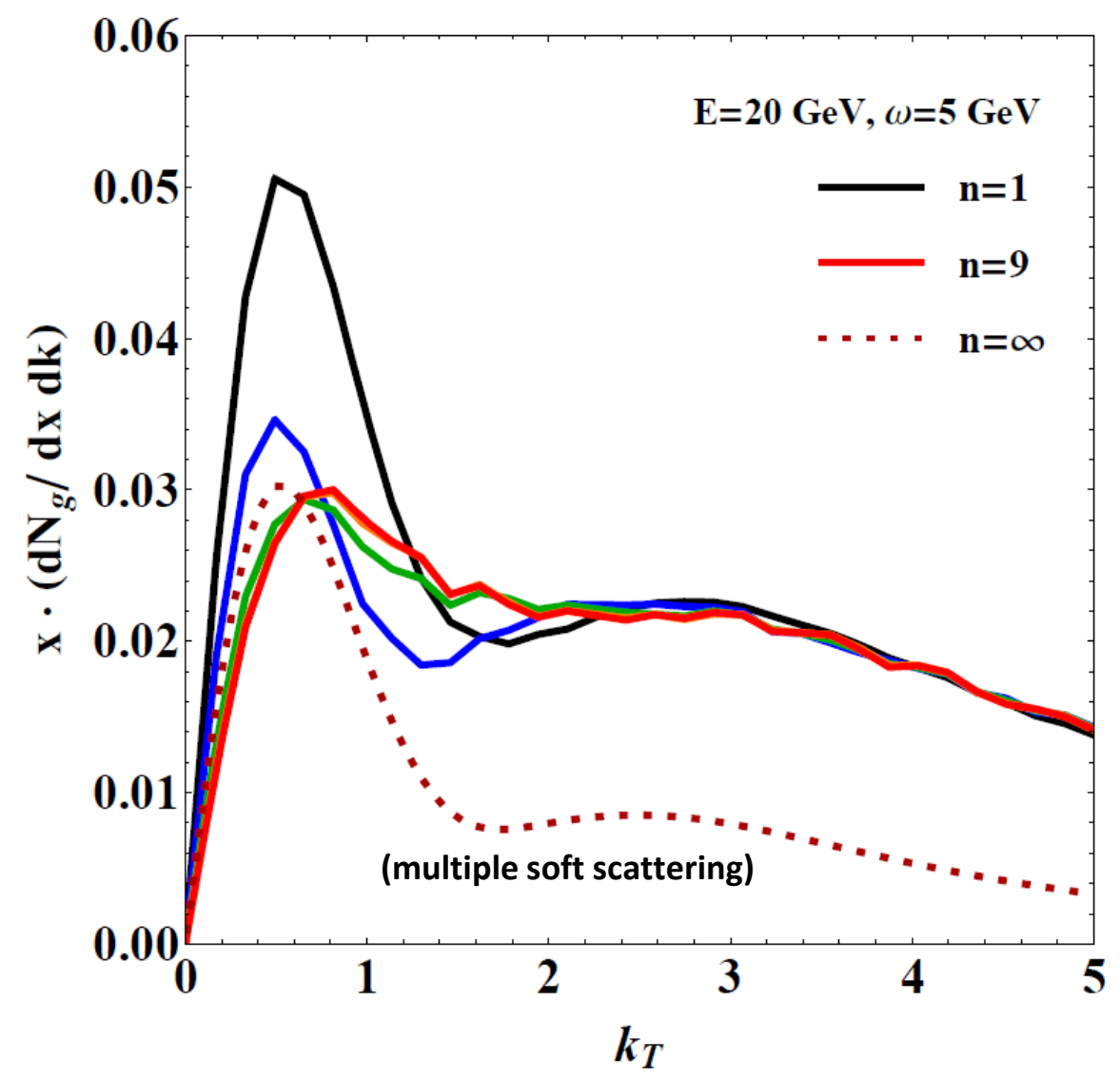

Static brick

$\mathrm{E}=20 \mathrm{GeV}$

$\omega=5 \mathrm{GeV}(x=0.25)$

$\mathrm{L}=5 \mathrm{fm}$

$\mathrm{T}=250 \mathrm{MeV}$

D At small emission angles, the opacity series converges to the m.s.s. limit

$>$ At large $k_{\perp}$, the hard power-law tail of the distribution dominates 


\section{$\mathrm{n}=1$ approximation}

\section{Light to heavy quark energy loss ratio}
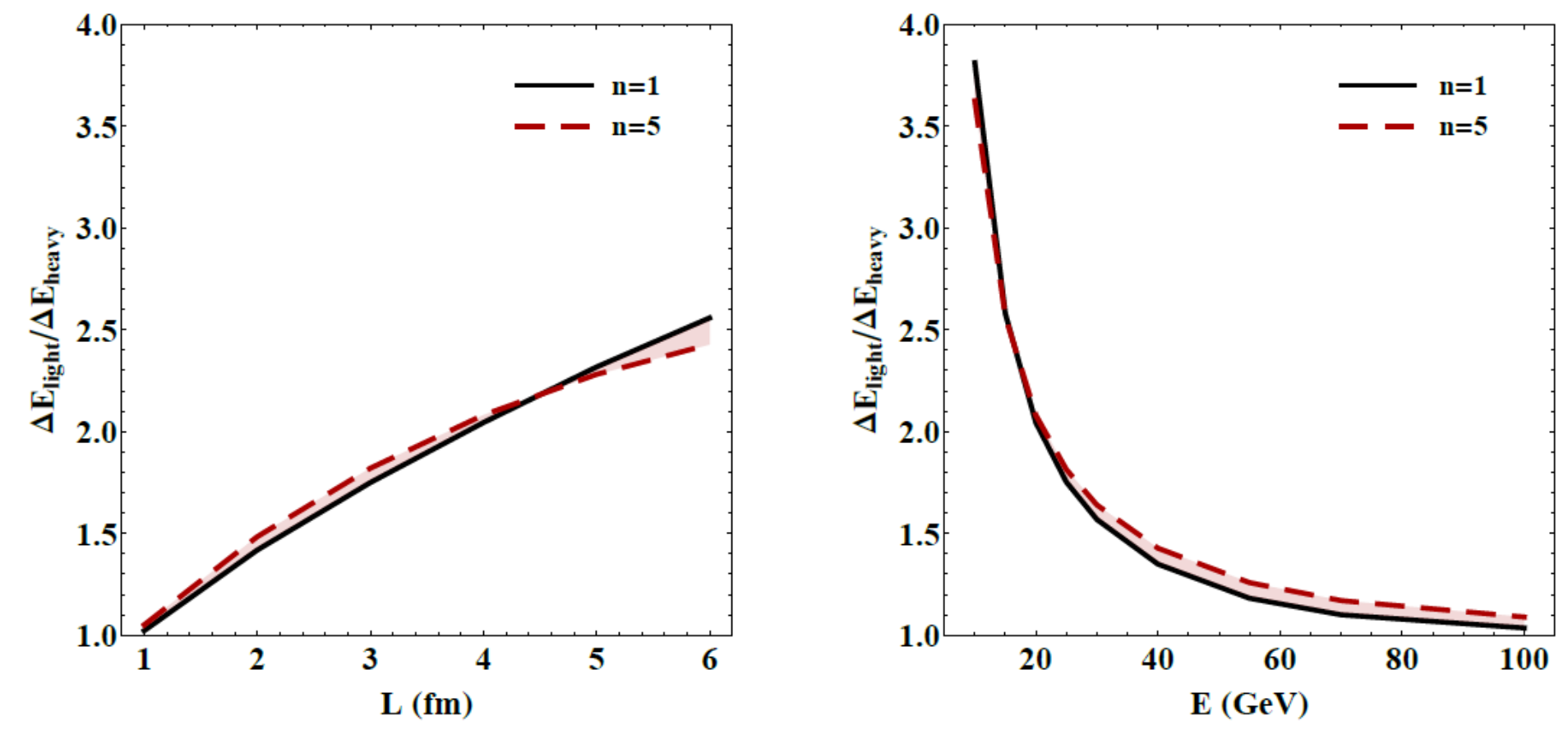

$$
\begin{aligned}
& \text { Static brick } \\
& E=20 \mathrm{GeV} \\
& \omega=[\text { integrated }] \\
& M_{1}=0.2 \mathrm{GeV} \\
& M_{\mathrm{h}}=4.75 \mathrm{GeV} \\
& L=5 \mathrm{fm} \\
& T=250 \mathrm{MeV}
\end{aligned}
$$

The energy loss ratio between light and heavy quark jets is insensitive to higher order in opacity corrections

$>$ Flavor tomography can be studied at $n=1$

$>$ Jet measurements require full transverse radiative spectrum at high orders 


\section{Interpolating potential}

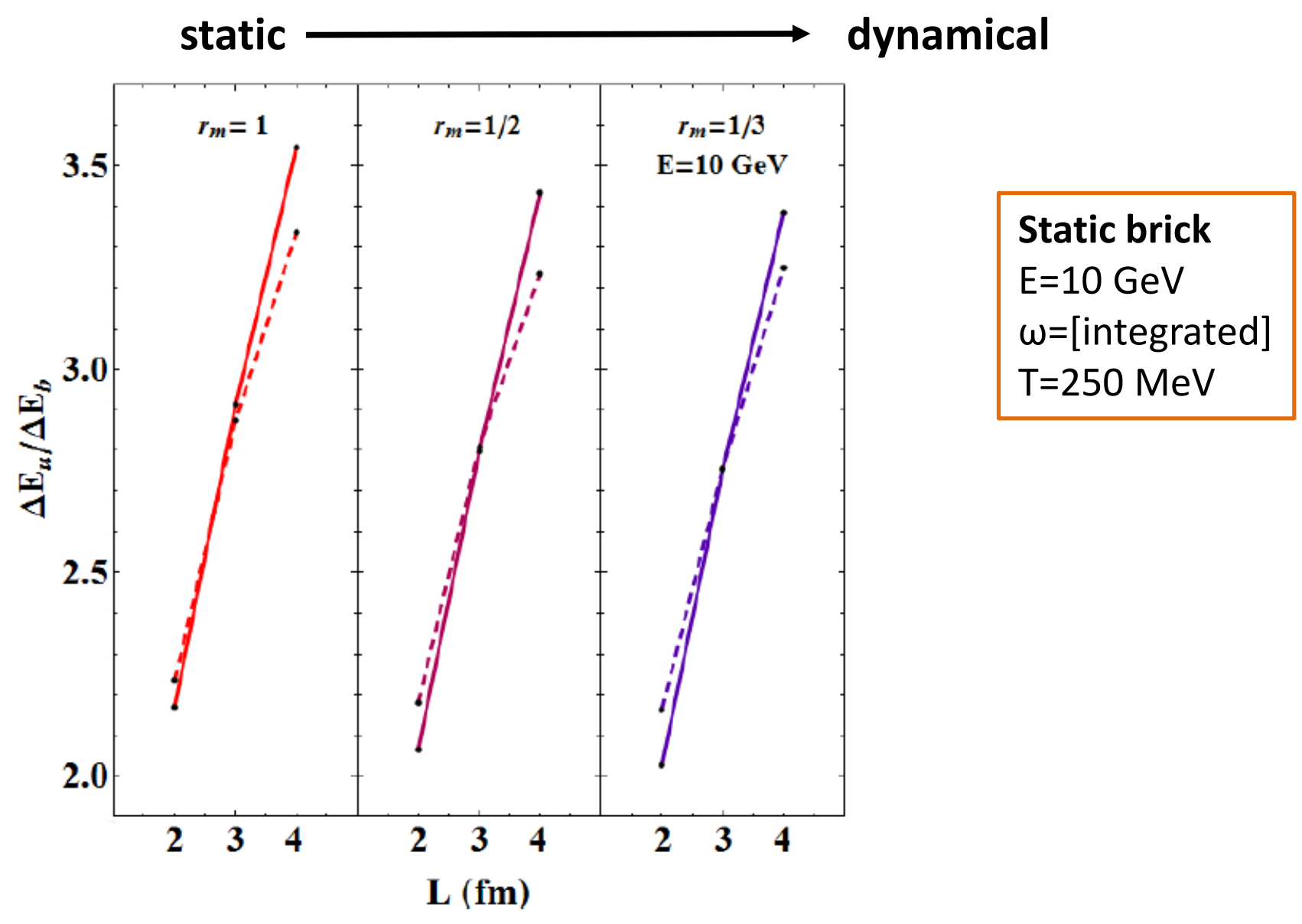

$>$ Strong indication of similar convergence for static and dynamical potentials 


\section{Static and dynamical limits}

Static (dashed) and dynamical (solid) energy loss $\Delta E / E$
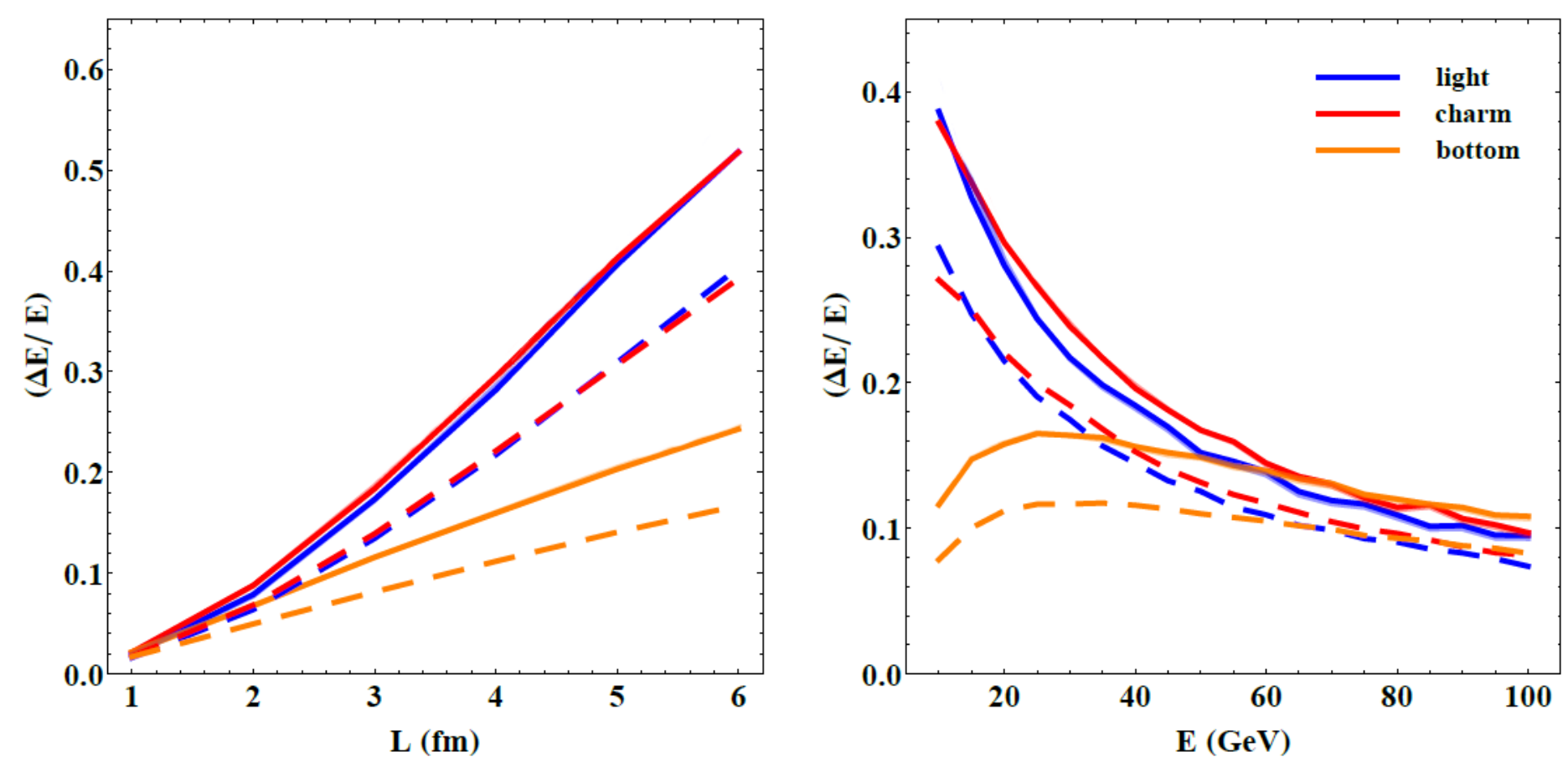

Static brick
$E=20 \mathrm{GeV}$
$M_{1}=0.2 \mathrm{GeV}$
$M_{c}=1.2 \mathrm{GeV}$
$M_{b}=4.75 \mathrm{GeV}$
$L=5 \mathrm{fm}$
$T=250 \mathrm{MeV}$

$>$ The dynamical potential increases the energy loss

$>$ Quadratic L-dependence for light and charm (LPM effect) vs. linear for bottom

$>$ Remarkably different dependence on E for bottom 


\section{Thermalization}

RHIC

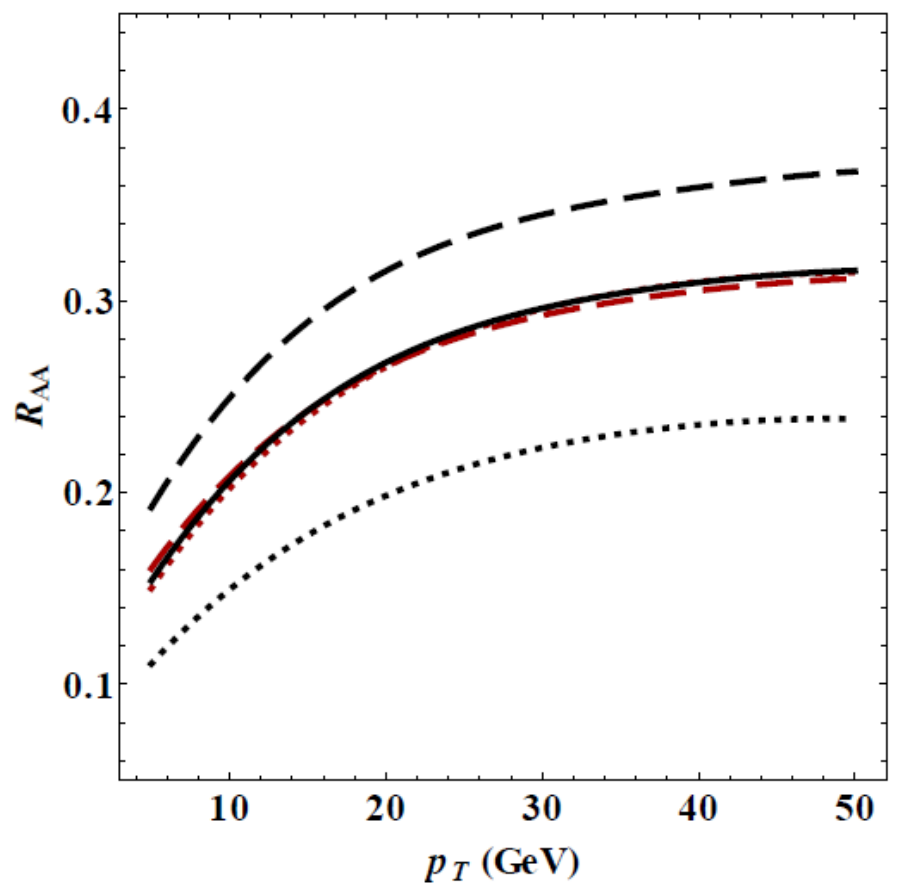

LHC

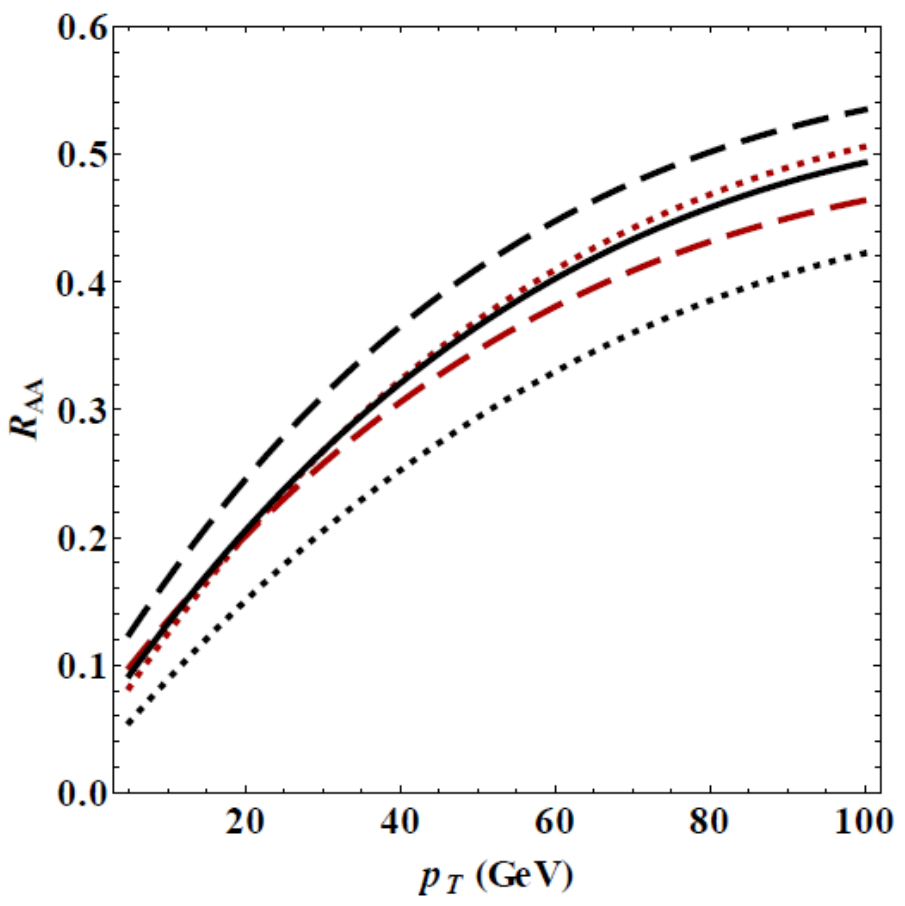

\section{Glauber+Bjorken}

$\mathrm{dN} / \mathrm{dy}=1000(\mathrm{RHIC})$

2200 (LHC)

$\tau_{0}=1 \mathrm{fm} / \mathrm{c}$

dynamical potential fixed coupling radiative+collisional

Solid: Linear with $\boldsymbol{\alpha}_{s}=\mathbf{0 . 3}$

Dotted/Dashed: Divergent or Free streaming with $\alpha_{s}=0.3$

Red: Divergent with $\alpha_{s}=0.27$ or Free streaming with $\alpha_{s}=0.325$

$>$ Large sensitivity to pre-thermalization phase, canceled by a small $( \pm 10 \%)$ rescaling of the coupling constant 


\section{BDMPS}

Baier, Dokshitzer, Mueller, Peigne, Schiff, Nucl.Phys.B483:291,1997

- Multiple soft scattering approximation $(\mathbf{N} \sim \infty)$

- The quark performs a Brownian motion in transverse momentum

$$
\begin{aligned}
& \text { START FROM PATH INTEGRAL FORMULATION } \\
& \begin{aligned}
\omega \frac{d I}{d \omega}= & \frac{\alpha_{s} C_{R}}{(2 \pi)^{2} \omega^{2}} 2 \operatorname{Re} \int_{\xi_{0}}^{\infty} d y_{l} \int_{y_{l}}^{\infty} d \bar{y}_{l} \int d \mathbf{u} \int_{0}^{\chi \omega} d \mathbf{k}_{\perp} e^{-i \mathbf{k}_{\perp} \cdot \mathbf{u}} e^{-\frac{1}{2} \int_{\bar{y}_{l}}^{\infty} d \xi n(\xi) \sigma(\mathbf{u})} \\
& \times \frac{\partial}{\partial \mathbf{y}} \cdot \frac{\partial}{\partial \mathbf{u}} \int_{\mathbf{y}=0}^{\mathbf{u}=\mathbf{r}\left(\bar{y}_{y}\right)} \mathcal{D} \mathbf{r} \exp \left[i \int_{y_{l}}^{\bar{y}_{l}} d \xi \frac{\omega}{2}\left(\dot{\mathbf{r}}^{2}-\frac{n(\xi) \sigma(\mathbf{r})}{i \omega}\right)\right] \\
& n(\xi) \sigma(\mathbf{r}) \simeq \frac{1}{2} \hat{q}(\xi) \mathbf{r}^{2} \\
- & \hat{q}=\frac{\left\langle q_{\perp}^{2}\right\rangle_{\text {med }}}{\lambda} \text { is the transport coefficient that characterize the } \\
& \text { transverse momentum squared per unit path length } \\
& \text { transferred to the parton }
\end{aligned}
\end{aligned}
$$




\section{ASW}

- Without fixing kinematic limits

$$
\begin{aligned}
& \omega \frac{d}{d \omega}\left(I-I_{\text {vac }}\right)=\frac{\alpha}{\pi} x P_{s \rightarrow \mathrm{g}}(x) \ln \left|\cos \left(\omega_{0} L\right)\right| \\
& \text { - Scaling with } \mathrm{z}: \quad z \equiv\left|\omega_{0}^{2}\right| L^{2} \\
& \text { - Taylor expansion in } \mathrm{z} \sim \mathrm{O}(\mathrm{L} / \lambda)^{1:} \\
& \quad \ln \left[\cos \left(e^{i \pi / 4} z^{1 / 2}\right) \cos \left(e^{-i \pi / 4} z^{1 / 2}\right)\right]=\frac{1}{6} z^{2}-\frac{17}{1260} z^{4}+\frac{691}{467775} z^{6}-\cdots \\
& \omega_{\mathrm{q} \rightarrow \mathrm{g}}(x)=C_{\mathrm{F}} \frac{\left[1+(1-x)^{2}\right]}{x}
\end{aligned}
$$

$>$ Z scaling cannot hold at intermediate opacity, i.e. qhat alone is insufficient to describe radiative processes at intermediate opacity

- Consider kinematic limits on $\mathbf{k}_{\mathbf{T}}$

Armesto, Salgado, Wiedemann, arXiv:hep-ph/0312106v1

$$
\left.\omega \frac{d I}{d \omega d \kappa^{2}}=\omega \frac{d I_{4}}{d \omega d \kappa^{2}}+\omega \frac{d I_{5}}{d \omega d \kappa^{2}}+\omega \frac{d I_{6}}{d \omega d \kappa^{2}}\right]\left[\begin{array}{l}
\text { OUR dN(0) } \\
\text { ORIGIN OF } \\
\text { DEAD CONE }
\end{array}\right.
$$

- $I_{5}$ and $I_{6}$ terms fill the dead cone

- They depend on qhat, which is adjusted to obtain quenching 


\section{Dynamical potential}

\section{A.1.9 Recovering Djordjevic's 'dynamical medium'}

We have just seen that the leading contribution to the collisional energy loss comes from the first term odd in $\omega$ in the $(\omega, q) / T$ expansion. But the leading contribution to the $q_{p e r p}$ distribution for small momentum and energy exchange comes from the first term in the series. Taking the leading term in the $q_{p e r p}$ integral, evaluating the $d \phi$ integral

$$
\begin{gathered}
\Gamma(E)=4 \pi C_{J} \alpha^{2} T^{3}\left(1+\frac{n_{f}}{6}\right) \int \frac{d^{2} q_{\perp}}{2 \pi} \int \frac{d \omega}{q}\left(1-\frac{\omega^{2}}{q^{2}}\right)^{2} \times \\
\left(\left|\Delta_{L}\right|^{2}+\frac{1}{2}\left(\frac{v^{2}-\frac{\omega^{2}}{q^{2}}}{1-\frac{\omega^{2}}{q^{2}}}\right)\left|\Delta_{T}\right|^{2}\right)
\end{gathered}
$$

with $q_{z} \approx \frac{q^{0}}{v}$ and $q=\sqrt{q_{\perp}^{2}+q_{z}^{2}} \approx \sqrt{q_{\perp}^{2}+\omega^{2} / v^{2}}$. Note that the coefficient of the magnetic term has an explicit $v$ dependence (not seen in Djordjevic). Also, if we had kept a mass for the medium particles, there would be a $v_{k}$ dependence as well. Using the properties

$$
\begin{aligned}
\operatorname{Im} \Delta_{L}(Q) & =\frac{\omega \pi \mu^{2}}{2 q}\left(1-\frac{\omega^{2}}{q^{2}}\right)\left|\Delta_{L}\right|^{2} \\
\operatorname{Im} \Delta_{T}(Q) & =-\frac{1}{2} \frac{\omega \pi \mu^{2}}{2 q}\left(1-\frac{\omega^{2}}{q^{2}}\right)\left|\Delta_{T}\right|^{2}
\end{aligned}
$$

and the change of variables

$$
x=\frac{\omega}{q}, \frac{d x}{x}=\frac{d \omega}{\omega}\left(1-\frac{\omega^{2}}{q^{2}}\right)
$$

produces, for $v=1$,

$$
\begin{aligned}
\Gamma(E)= & 4 \pi C_{J} \alpha^{2} T^{3}\left(1+\frac{n_{f}}{6}\right) \int \frac{d^{2} q_{\perp}}{2 \pi} \frac{2}{\pi \mu^{2}} \int \frac{d x}{x} \times \\
& \left(\frac{\operatorname{Im} \Delta_{L}}{\left(q_{\perp}^{2}+\operatorname{Re} \Delta_{L}\right)^{2}+\left(\operatorname{Im} \Delta_{L}\right)^{2}}-\frac{\operatorname{Im} \Delta_{T}}{\left(q_{\perp}^{2}+\operatorname{Re} \Delta_{T}\right)^{2}+\left(\operatorname{Im} \Delta_{T}\right)^{2}}\right)
\end{aligned}
$$

$$
\begin{aligned}
& \text { Using the sum rule, } \quad \text { Aurenche, Gelis, et al, } 2002 \\
& \qquad \begin{array}{l}
\int_{-1}^{1} \frac{d x}{x} \frac{1}{2 \pi} \frac{2 \operatorname{Im} \Delta_{L, T}}{\left(q_{\perp}^{2}+\operatorname{Re} \Delta_{L, T}\right)^{2}+\left(\operatorname{Im} \Delta_{L, T}\right)^{2}}= \\
\frac{1}{q_{\perp}^{2}+\operatorname{Re} \Pi_{L, T}(x=\infty)}-\frac{1}{q_{\perp}^{2}+\operatorname{Re} \Pi_{L, T}(x=0)}
\end{array}
\end{aligned}
$$

This is an integral in the $\omega-q$ plane, with $q_{\perp}$ at a fixed value. This traces out a hyperbola. The asymptotic Пs satisfy:

$$
\begin{gathered}
\operatorname{Re} \Pi_{L}(x=\infty)=\frac{\mu^{2}}{3}, \quad \operatorname{Re} \Pi_{T}(x=\infty)=\frac{\mu^{2}}{3} \\
\operatorname{Re} \Pi_{L}(x=0)=\mu^{2}, \quad \operatorname{Re} \Pi_{T}(x=0) \\
\text { Djordjevic, Heinz, } \\
=4 \pi C_{J} \alpha^{2} T^{3}\left(1+\frac{n_{f}}{6}\right) \int \frac{d^{2} q_{\perp}}{2 \pi} \frac{2}{\mu^{2}} \\
\left(\frac{1}{q_{\perp}^{2}+\frac{\mu^{2}}{3}}-\frac{1}{q_{\perp}^{2}+\mu^{2}}-\frac{1}{q_{\perp}^{2}+\frac{\mu^{2}}{3}+\mu_{\text {mag }}^{2}}+\frac{1}{q_{\perp}^{2}+\mu_{\text {mag }}^{2}}\right)
\end{gathered}
$$

Pisarski, 1980

Hence,

For the case of vanishing magnetic screening mass, this becomes

$$
\begin{aligned}
\Gamma(E) & =4 \pi C_{J} \alpha^{2} T^{3}\left(1+\frac{n_{f}}{6}\right) \int \frac{d^{2} q_{\perp}}{2 \pi} \frac{2}{\mu^{2}}\left(\frac{1}{q_{\perp}^{2}}-\frac{1}{q_{\perp}^{2}+\mu^{2}}\right) \\
& =8 \pi C_{j} \alpha^{2} T^{3}\left(1+\frac{n_{f}}{6}\right) \int \frac{d^{2} q_{\perp}}{2 \pi} \frac{1}{q_{\perp}^{2}\left(q_{\perp}^{2}+\mu^{2}\right)}
\end{aligned}
$$




\section{Gunion-Bertsch}

situation. The multiplicity distribution is then given by the differential of this expression, i.e.,

$$
\frac{d n_{g}}{d x d^{2} q_{\perp}}=\frac{C_{F} \alpha_{s}}{\pi^{2}} \frac{1}{x q_{\perp}^{2}} .
$$

This distribution is uniform in rapidity,

$$
\frac{d n_{g}}{d \eta d^{2} q_{\perp}}=\frac{C_{F} \alpha_{s}}{\pi^{2}} \frac{1}{q_{\perp}^{2}} .
$$

To relate this gluon distribution to an observable quantity, assume that $f_{\text {ch }}$ charged particles are produced in the final state for each perturbative gluon. We also integrate over $q_{\perp}$, with a lower limit $q_{\perp} \sim \Lambda$ determined by confinement, and an upper limit $q_{\perp}{ }^{2} \sim s$ determined by kinematics. The charged-particle distribution is then

$$
\frac{d n_{\mathrm{ch}}}{d \eta} \sim \frac{C_{F} f_{\mathrm{ch}} \alpha_{s}}{\pi} \ln \left(s / \Lambda^{2}\right)
$$




\section{Elastic energy loss and Fluctuations}

Bjorken elastic collisions

$$
\frac{d E}{d x}=-C_{R} \pi \alpha^{2} T^{2} \log [B]
$$

- Soft scattering

- Thoma-Gyulassy model $\rightarrow B_{T G}=\frac{4 p T}{E-p+4 T} / \mu$

\section{Energy loss fluctuations}

- The probability of losing a fractional energy $\varepsilon=\frac{\Delta E}{E}$ is the convolution of Radiative and Elastic contributions

$$
P(\varepsilon)=\int d x P_{r a d}(\varepsilon) P_{e l}(x-\varepsilon)
$$

Poisson expansion of the number of INCOHERENTLY emitted gluons

- Radiative: $\boldsymbol{P}_{\text {rad }}(\varepsilon)=\boldsymbol{P}_{0} \delta(\varepsilon)+\left.\widetilde{P}(\varepsilon)\right|_{0} ^{1}+\boldsymbol{P}_{\text {stop }} \delta(1-\varepsilon)$ 


\section{Geometry of the collision}

\section{Glauber model is used to compute}

number of binary collisions and participant nucleons

- Start from Wood-Saxon nuclear density profile...

$-\rho_{A}(r) \propto[1+\operatorname{Exp}[(r-R) / a]]^{-1}$

$-T_{A}(x)=\int d z \rho_{A}(z, x)$

- ...to obtain:

$-\rho_{\text {binary }}(x, b)$

$-\rho_{\text {participants }}(x, b)$

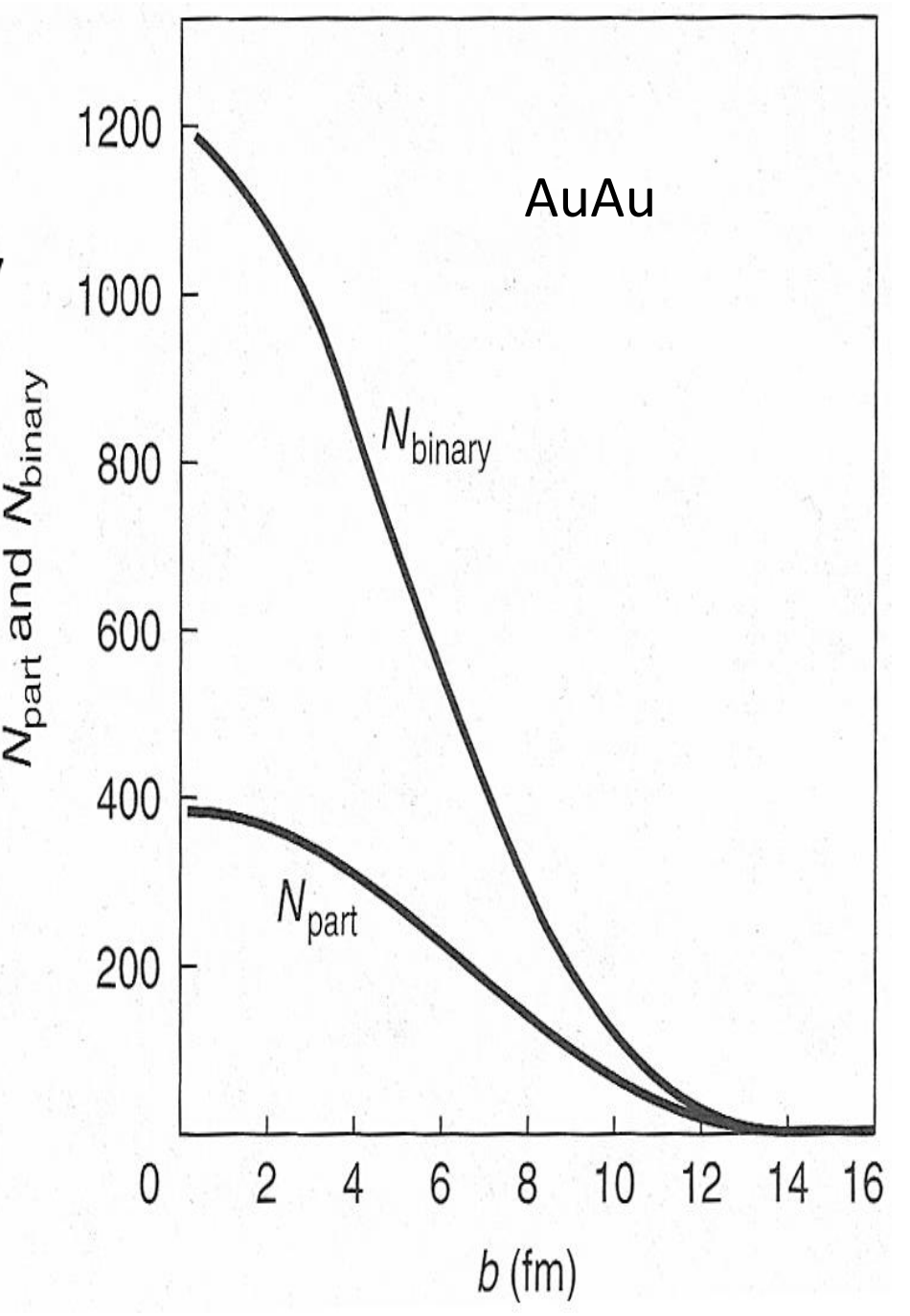




\section{$\mathrm{k}_{\mathrm{T}}$ sensitivity}

- Collinear approximation: $x_{E}=x_{+}\left(1+O\left(\frac{k_{T}}{x_{+} E^{+}}\right)^{2}\right)$

- DGLV formula has the same functional form for $x_{E}$ or $x_{+}$

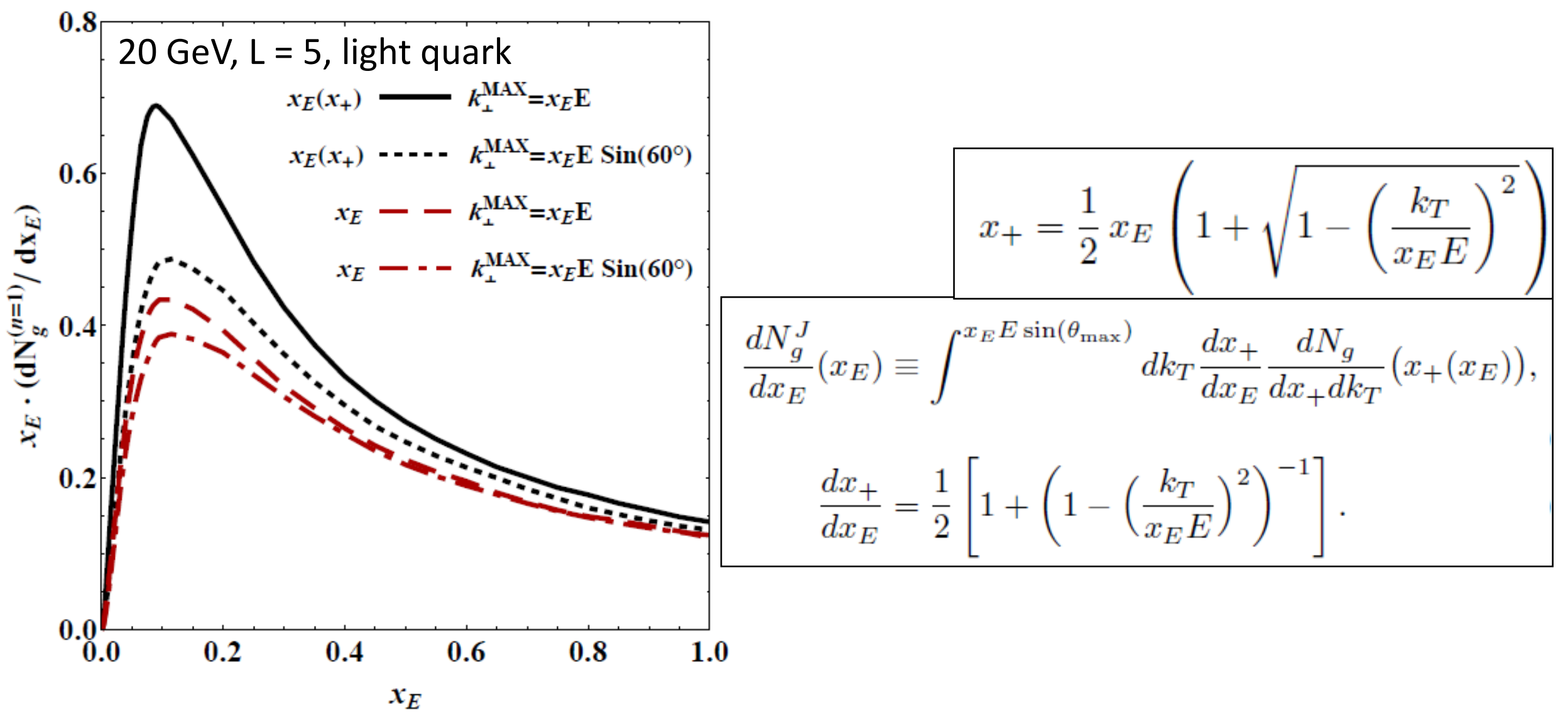




\section{Holographic models}

- AdS/CFT correspondence: strong coupling between jet and medium

- The energy loss of a parton quenched in a thermal plasma is described by the dynamics of its dual classical string moving in a five-dimensional AdS space with a black hole

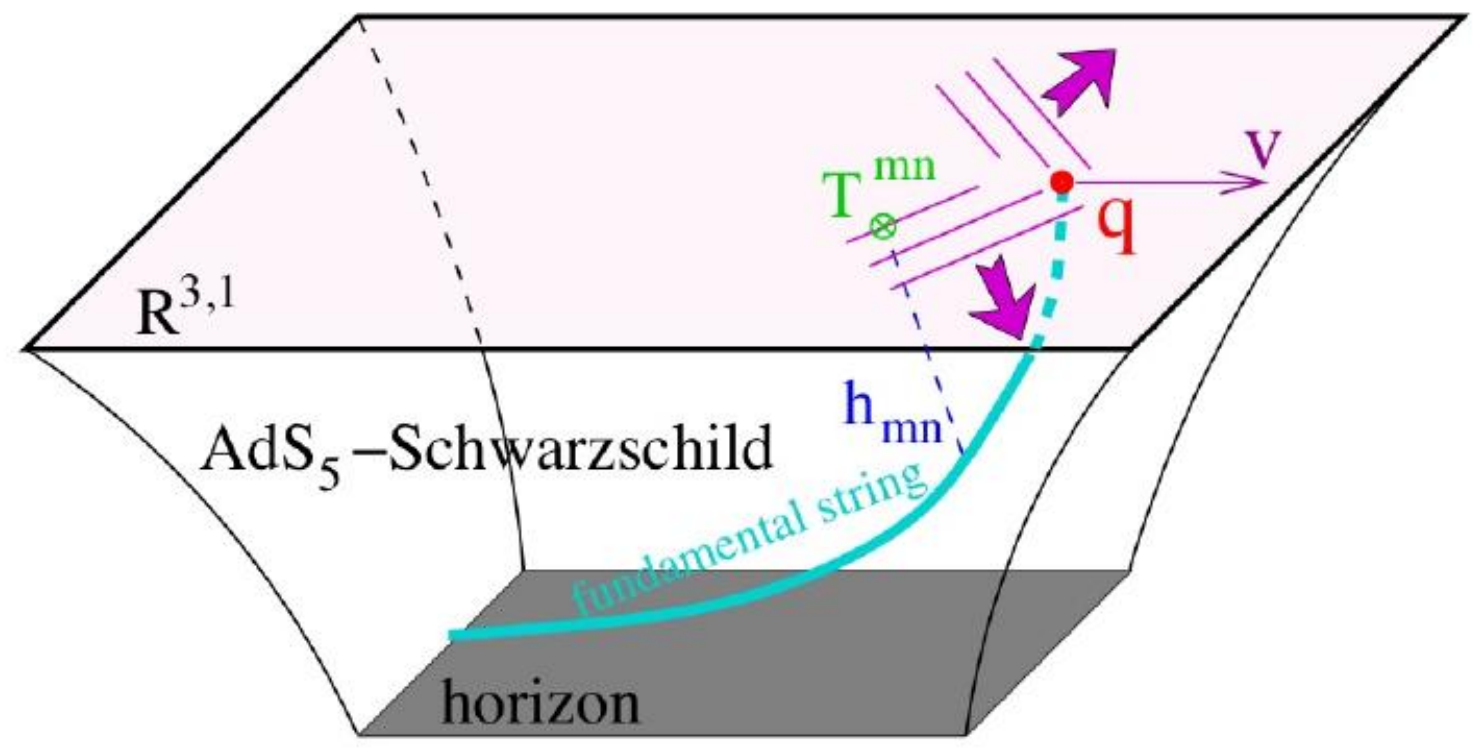




\section{Jet Quenching and Initial Geometry/Conditions from the RHIC BES}

Helen Caines - Yale University 


\section{RHIC data available}

$\mathrm{Au}-\mathrm{Au}$

200, 130, 62.4, 39, 27, 19.6, 11.5(STAR only), 7.7, 5(test run)

$\mathrm{Cu}-\mathrm{Cu}$

$200,62.4,22.5$

$\mathrm{U}-\mathrm{U}$

193

$\mathrm{C}-\mathrm{Au}$

200

$\mathrm{p}-\mathrm{p}$

200,500

$\mathrm{d}-\mathrm{Au}$

200

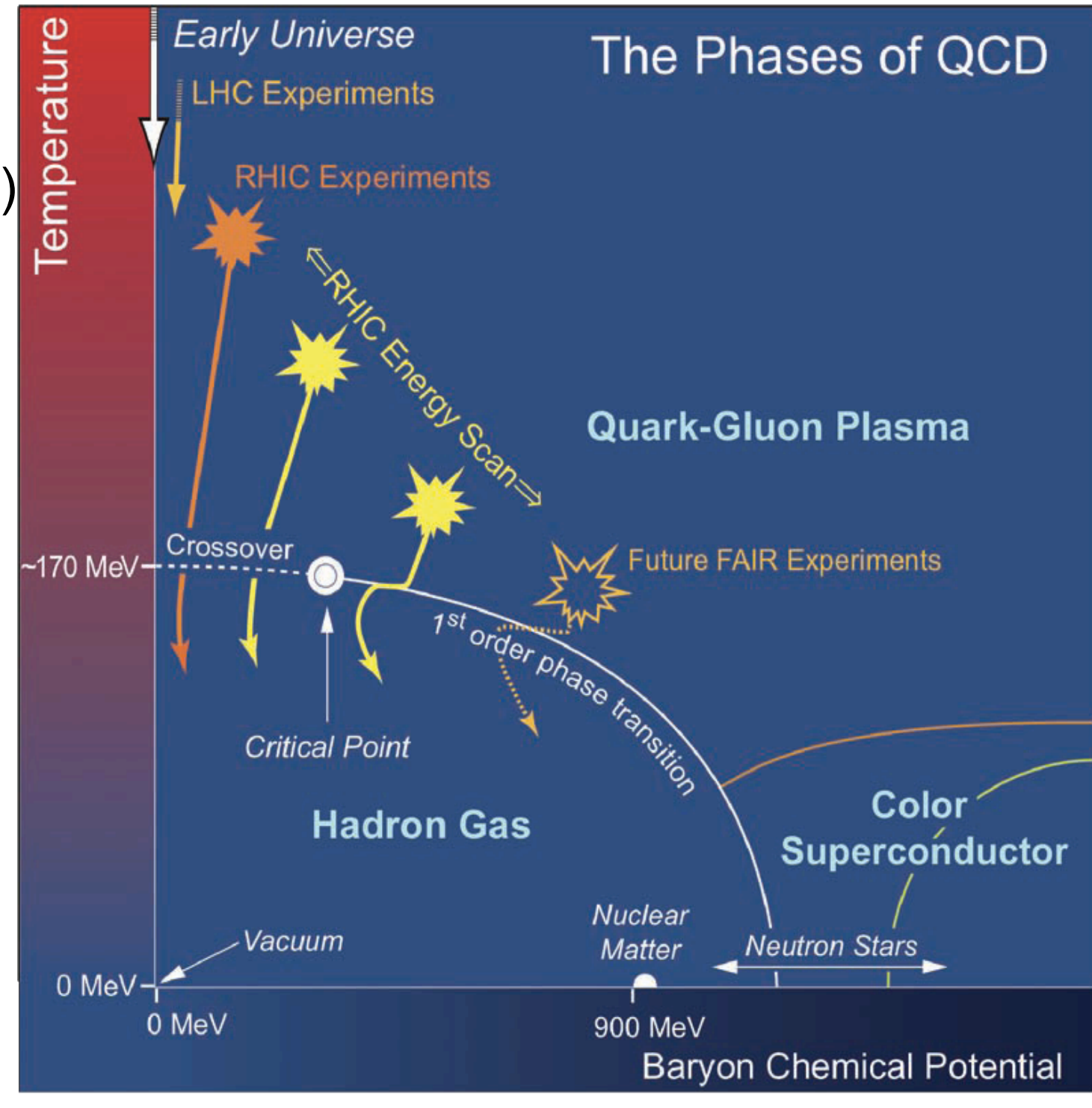




\section{Generic features of $A-A$ collisions - I}

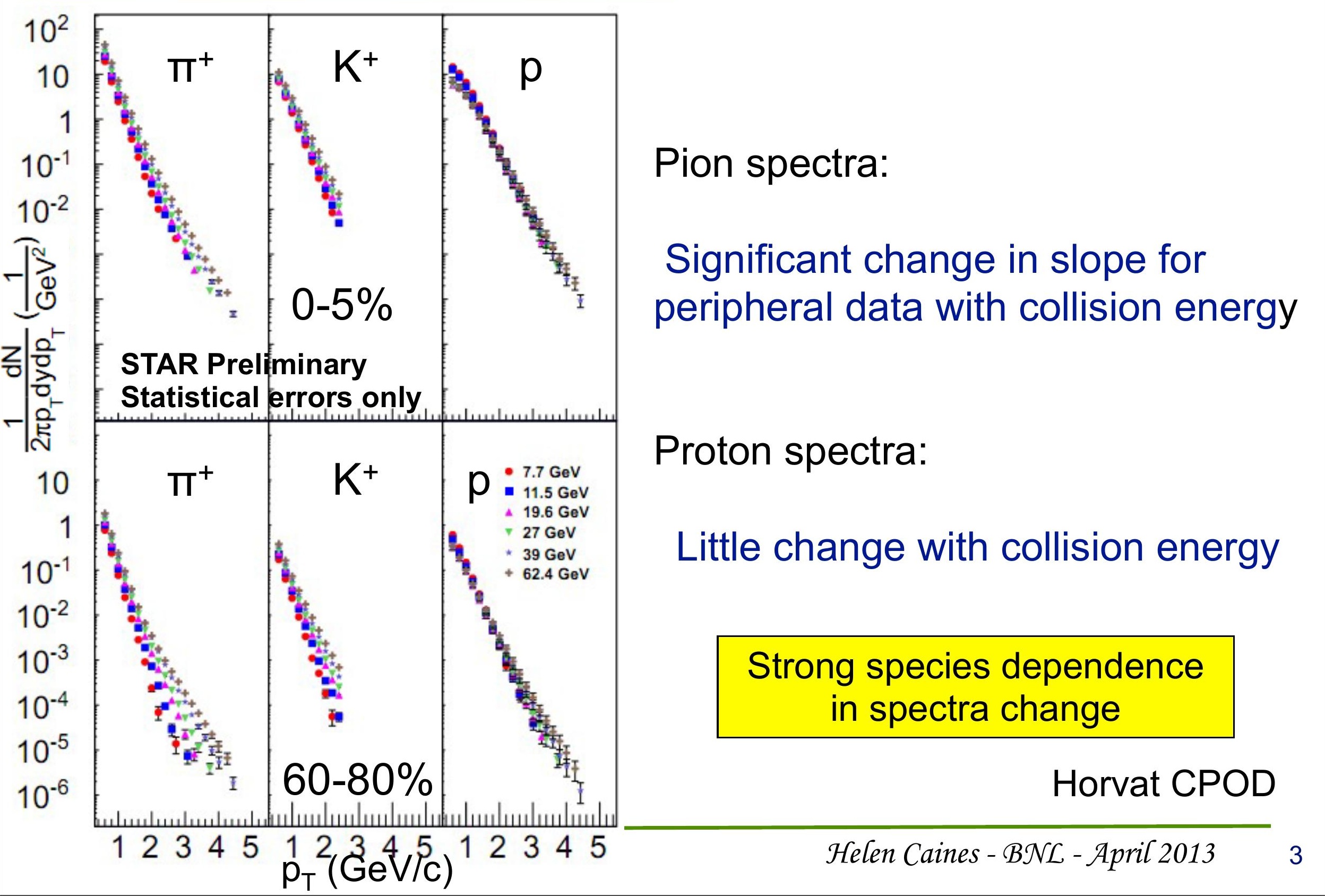




\section{Generic features of A-A collisions - III}
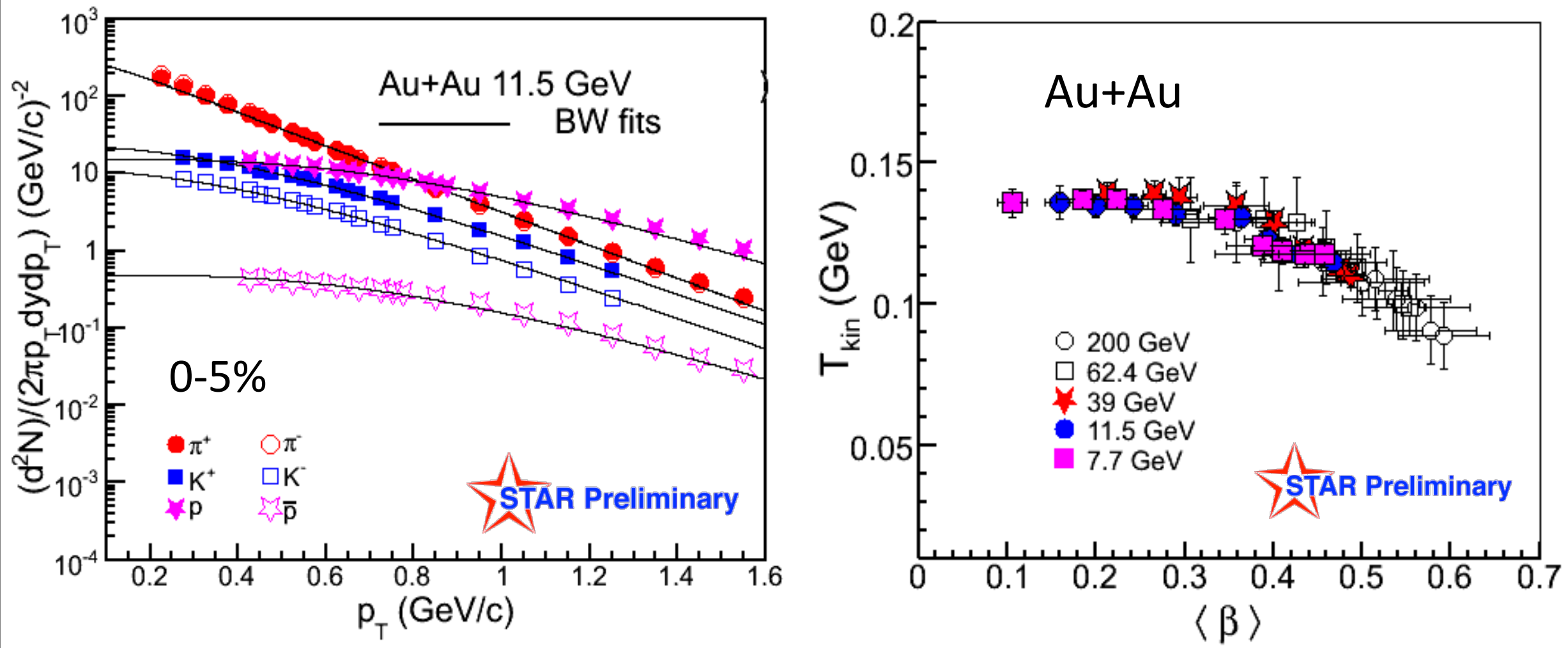

$\pi, K, p$ used in fits

Blastwave fits seem OK even at lowest collision energies

Expansion gets less violent and kinetic freeze-out occurs "earlier" ( or at least higher T) 


\section{Generic features of A-A collisions - III}

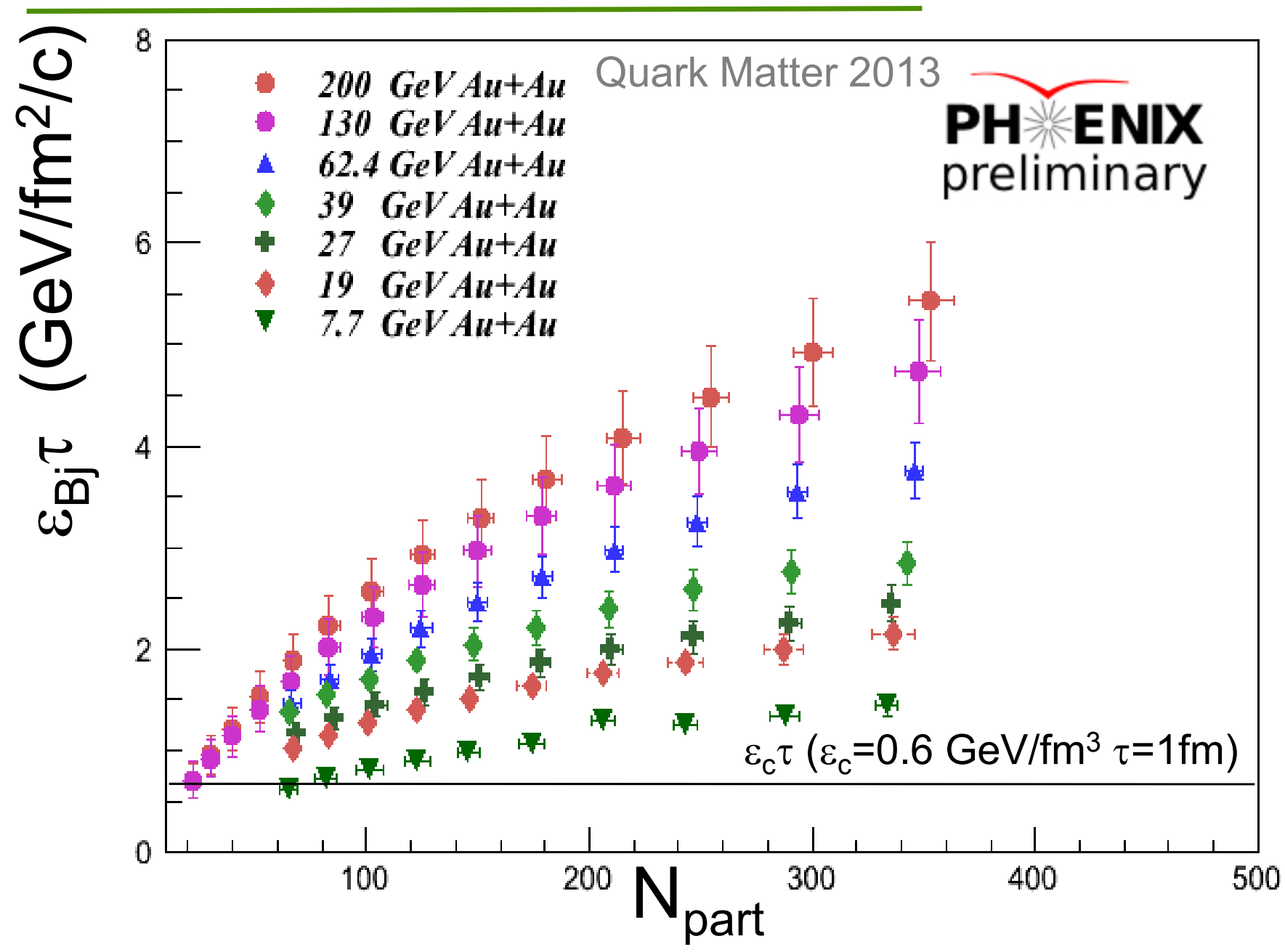

Lattice:

$\varepsilon_{c} \sim 0.6 \mathrm{GeV} / \mathrm{fm}^{3}$

Above critical density for all collision energies and centralities

QGP at all energies? 


\section{Au-Au vs Cu-Cu}

$$
\pi^{0}
$$

\section{$\mathrm{Cu}-\mathrm{Cu}$}

$\mathrm{R}_{\mathrm{AA}}{ }^{22.4}>\mathrm{R}_{\mathrm{AA}}{ }^{62} \sim \mathrm{R}_{\mathrm{AA}}{ }^{200}$
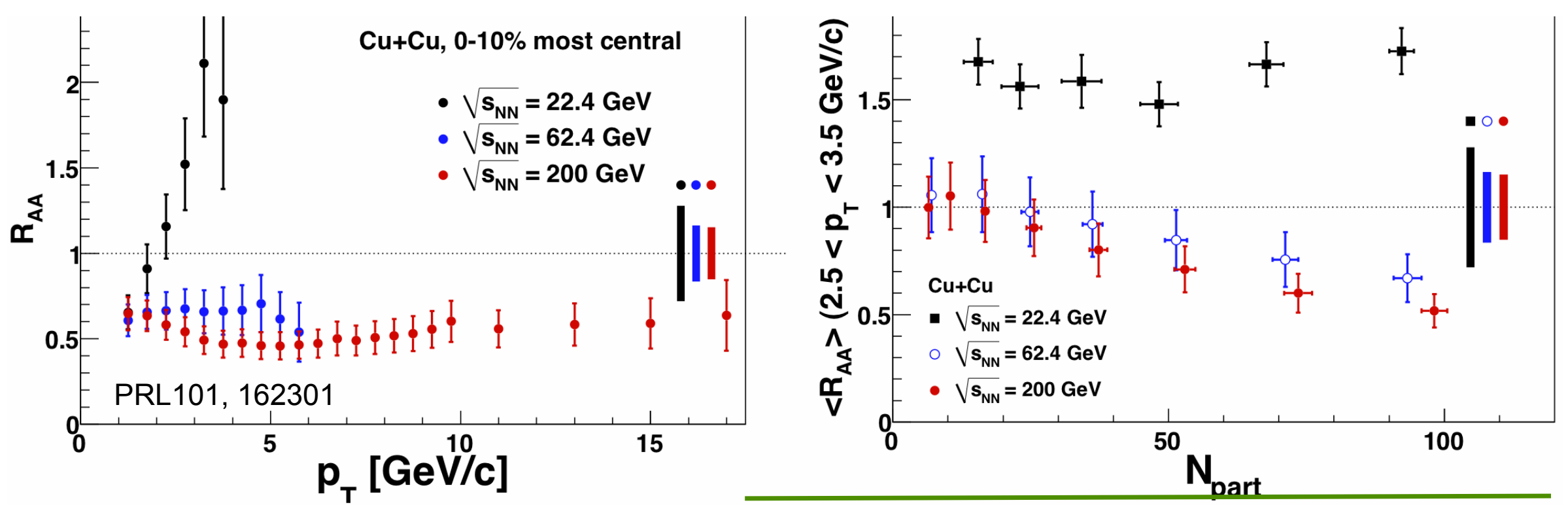
$\pi^{0}$

Au-Au

$\mathrm{R}_{\mathrm{AA}}{ }^{39}>\mathrm{R}_{\mathrm{AA}}{ }^{62} \sim \mathrm{R}_{\mathrm{AA}} 200$

$\mathrm{Cu}-\mathrm{Cu}$

$\mathrm{R}_{\mathrm{AA}} 22.4>\mathrm{R}_{\mathrm{AA}}{ }^{62} \sim \mathrm{R}_{\mathrm{AA}}{ }^{200}$
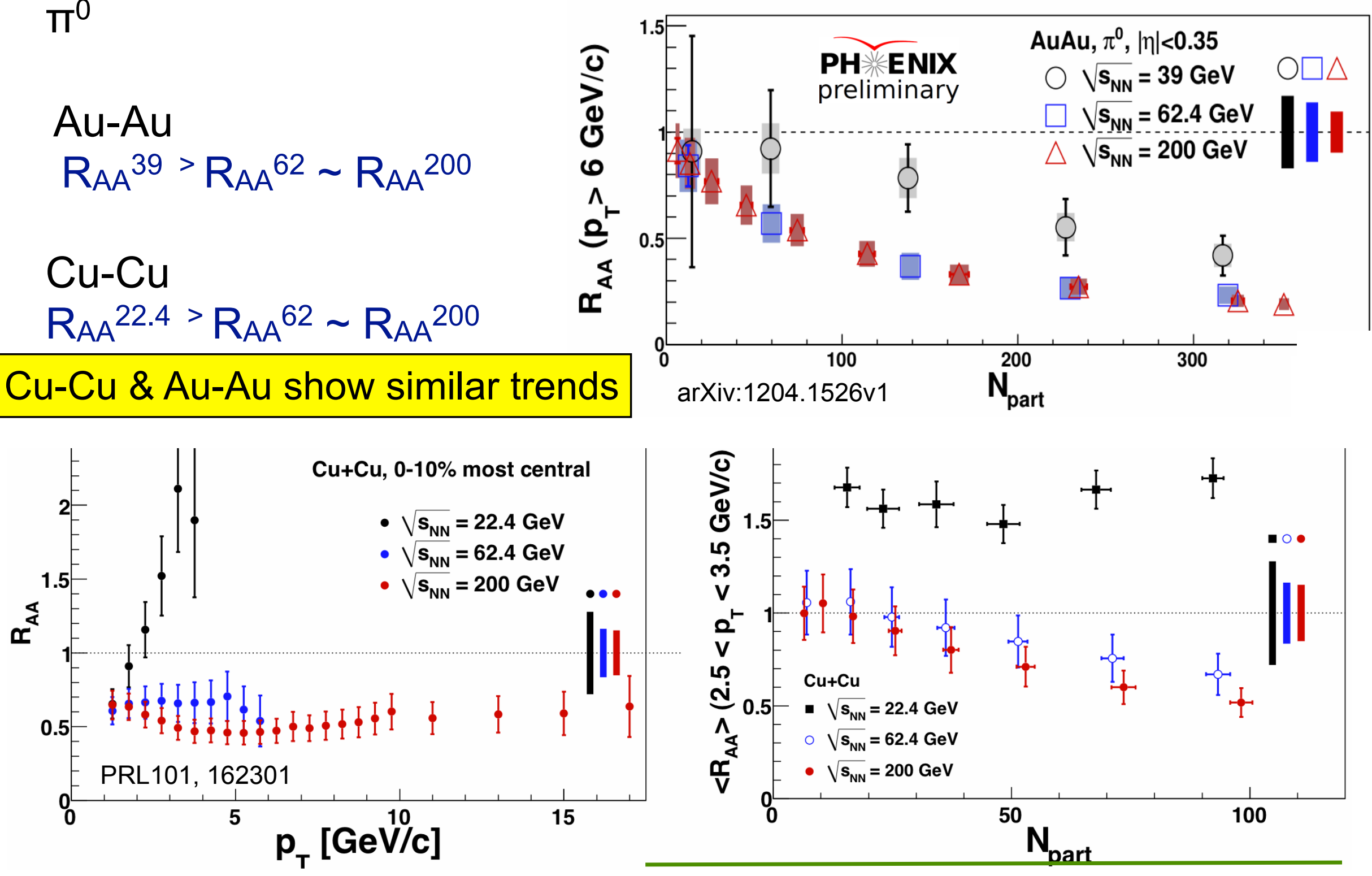

Helen Caines - BNL - April 2013 


\section{Parton recombination ending}
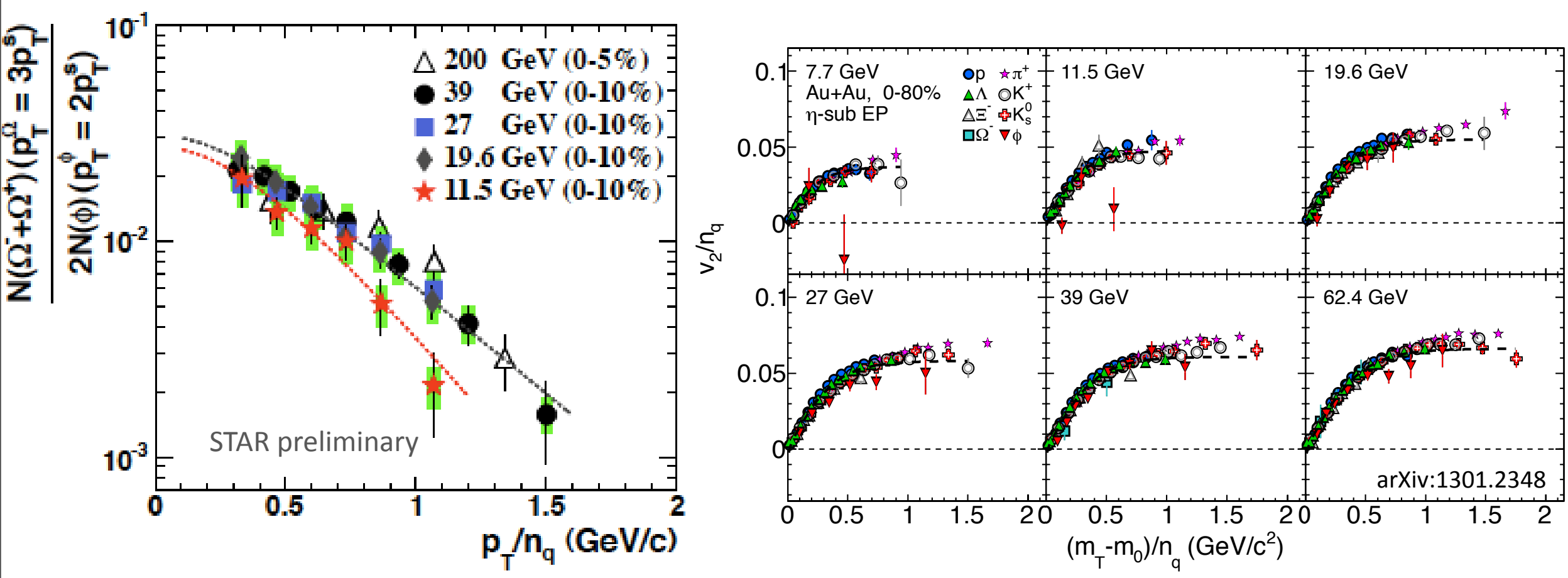

Between 19.6 and $11 \mathrm{GeV}$ :

Derived s quark pт distribution changes

Change in dominant particle production mechanisms?

$\phi v_{2}$ no longer follows meson distribution

- low $\phi v_{2}$ could imply hadronic dominance

(Better statistics would be nice) 


\section{$R_{A A}$ vs $R_{C P}$}

Peripheral

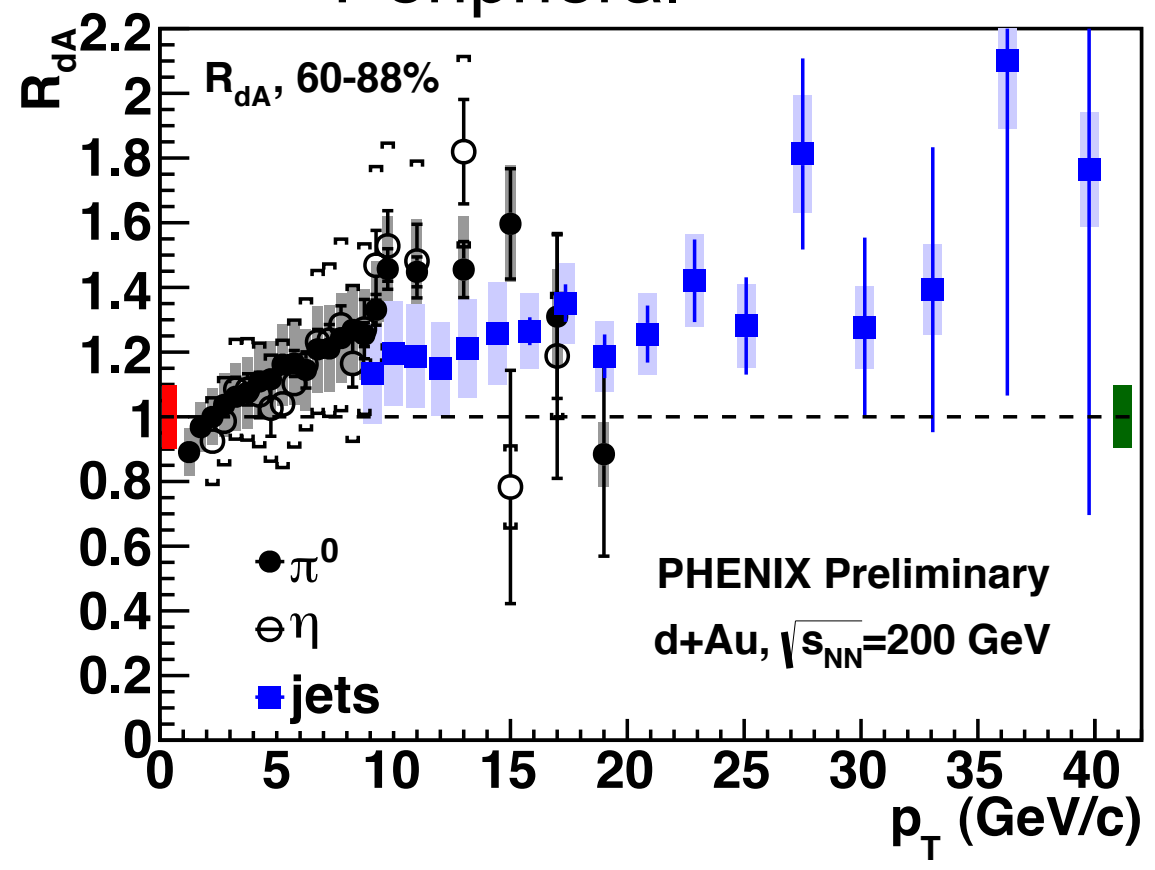

Central

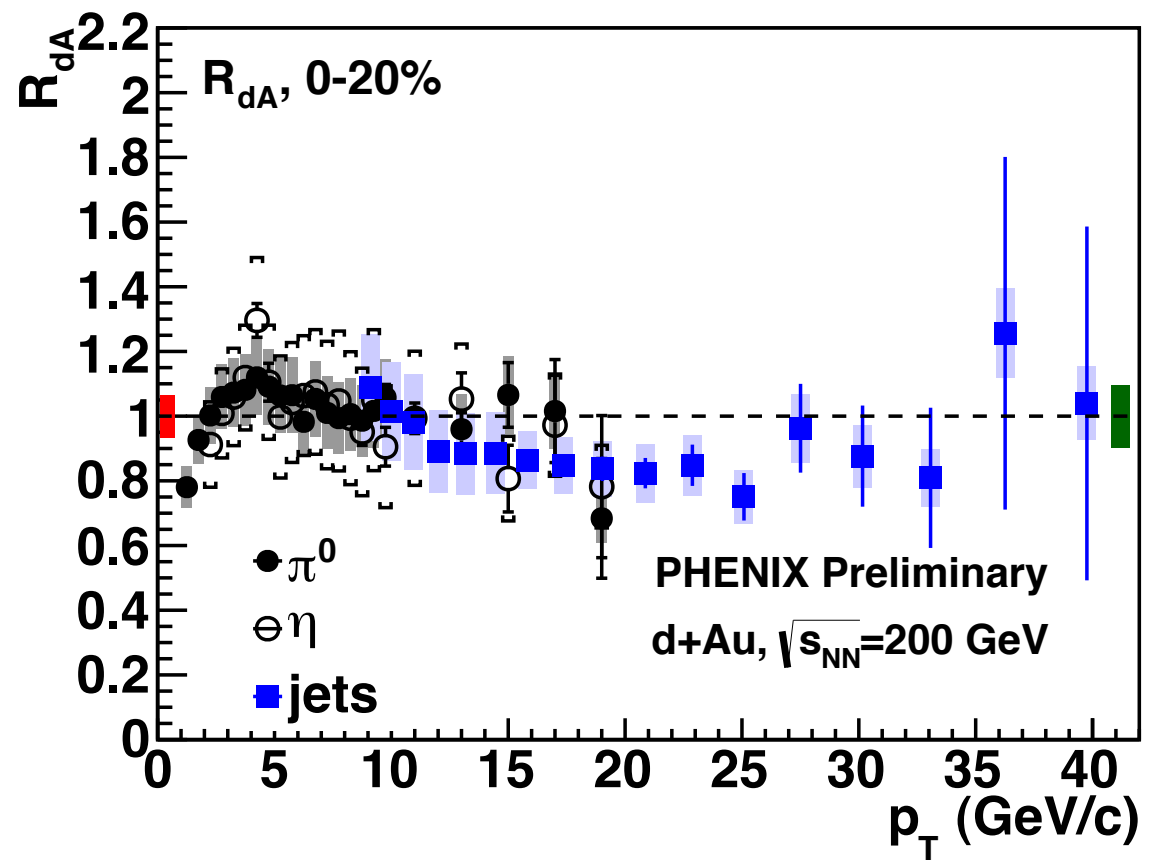

Centrality dependence to the Cronin Effect?

Au-Au 200

d-Au: enhancement larger in peripheral collisions

- were expected to be more "p-p" like 


\section{$R_{A A}$ vs $R_{C P}$}

Peripheral

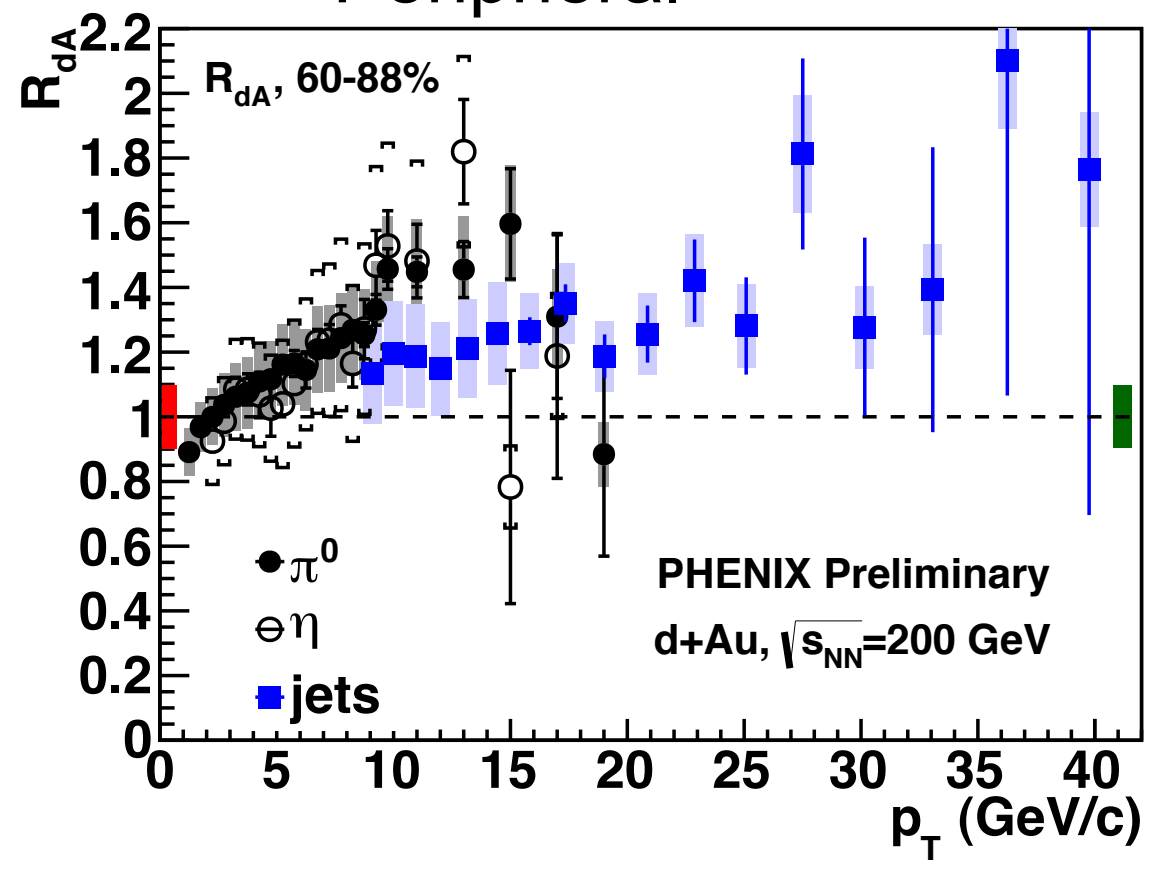

Central

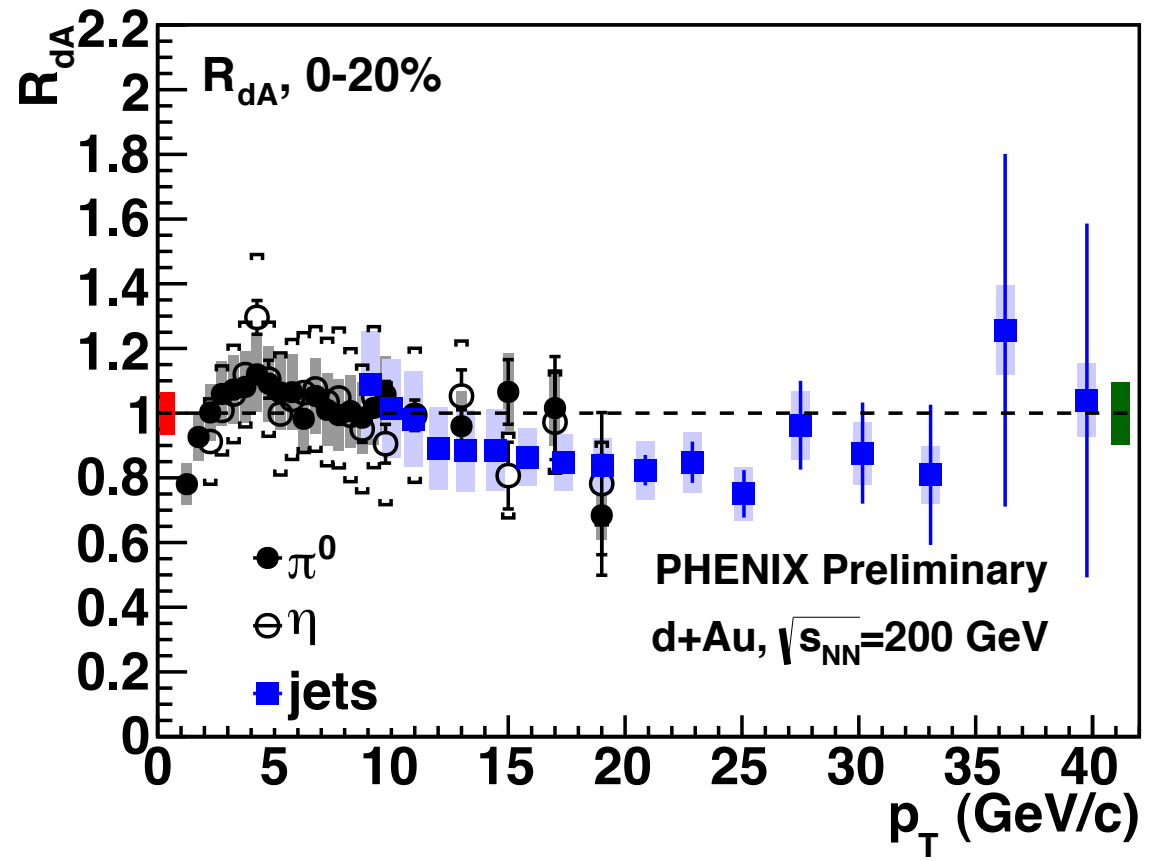

Centrality dependence to the Cronin Effect?

d-Au: enhancement larger in peripheral collisions

- were expected to be more "p-p" like

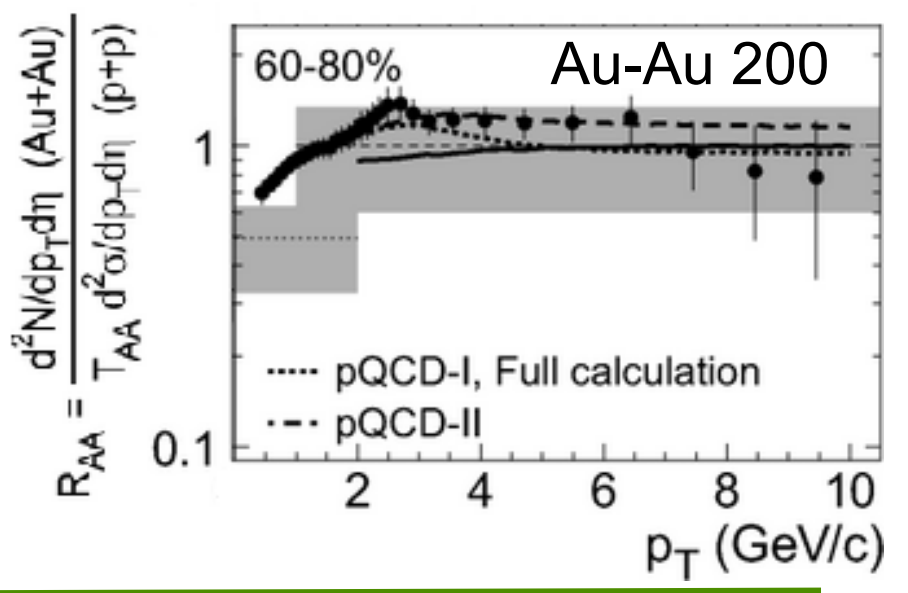




\section{$R_{A A}$ vs $R_{C P}$}

Peripheral

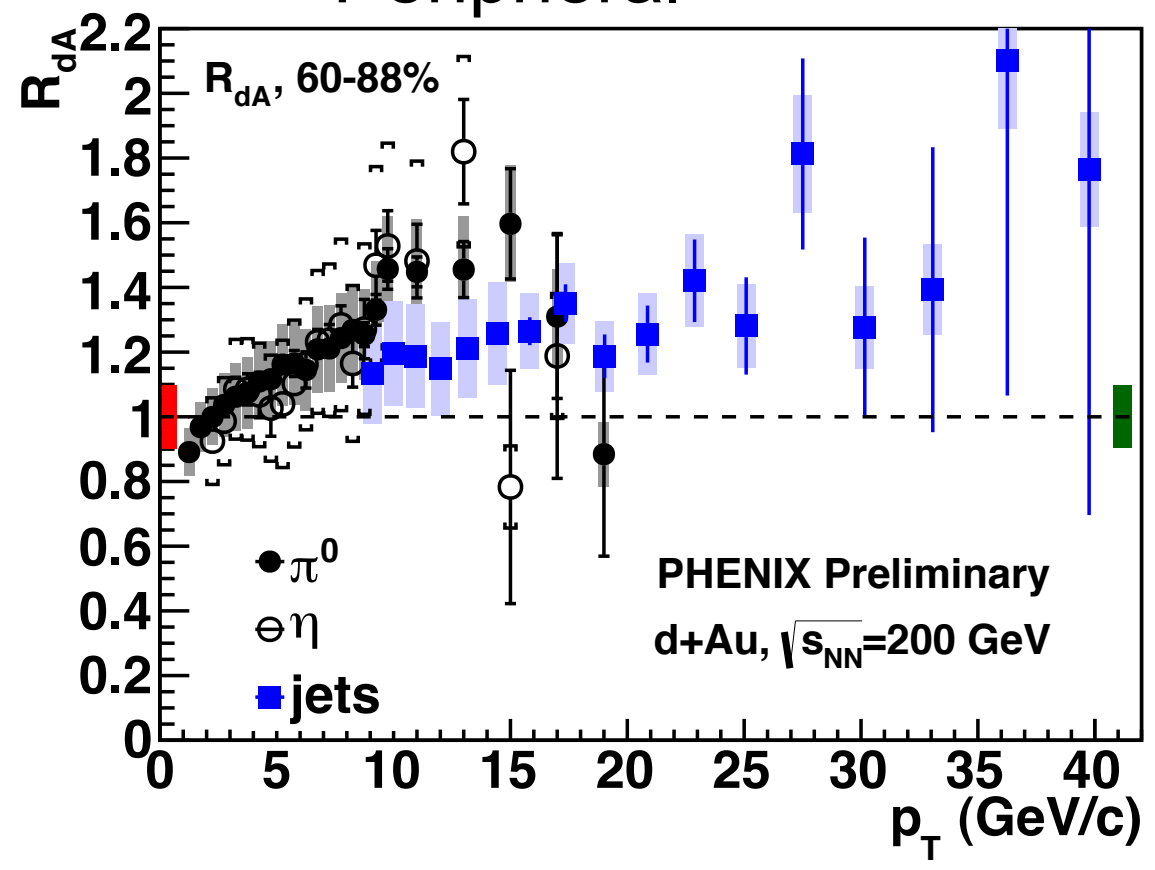

Central

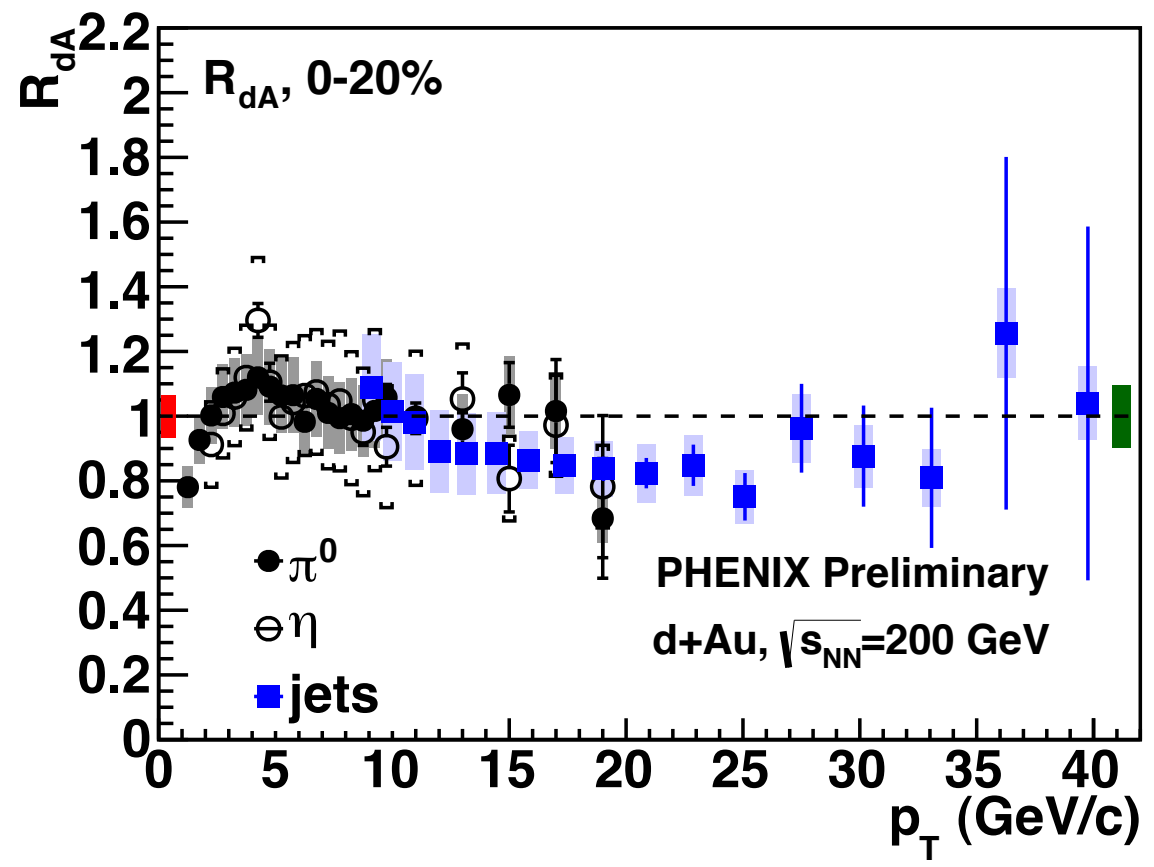

Centrality dependence to the Cronin Effect?

d-Au: enhancement larger in peripheral collisions

- were expected to be more "p-p" like

$\mathrm{R}_{\mathrm{CP}}$ cancels some of the Cronin - maybe too much

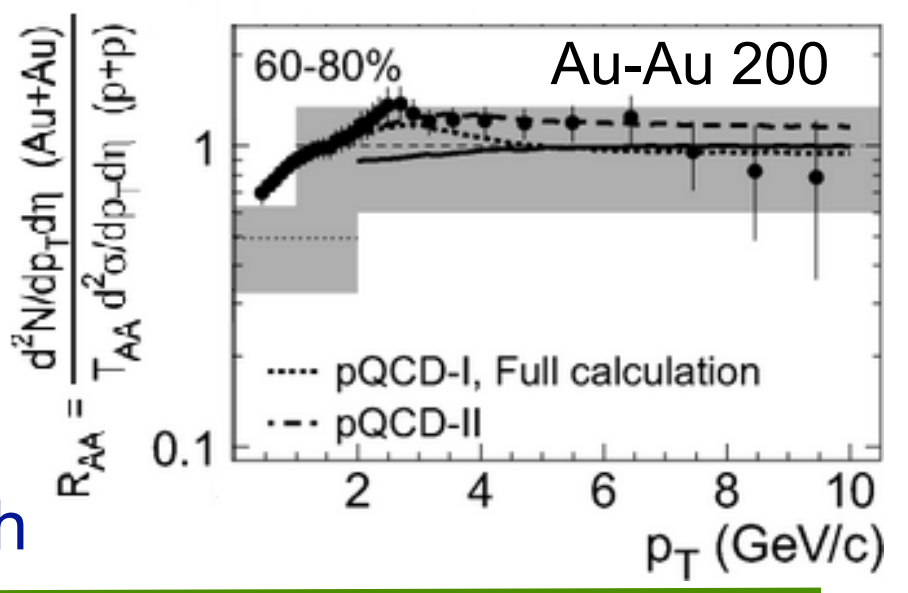




\section{Is $x_{T}$ better variable than $p_{T}$ ?}

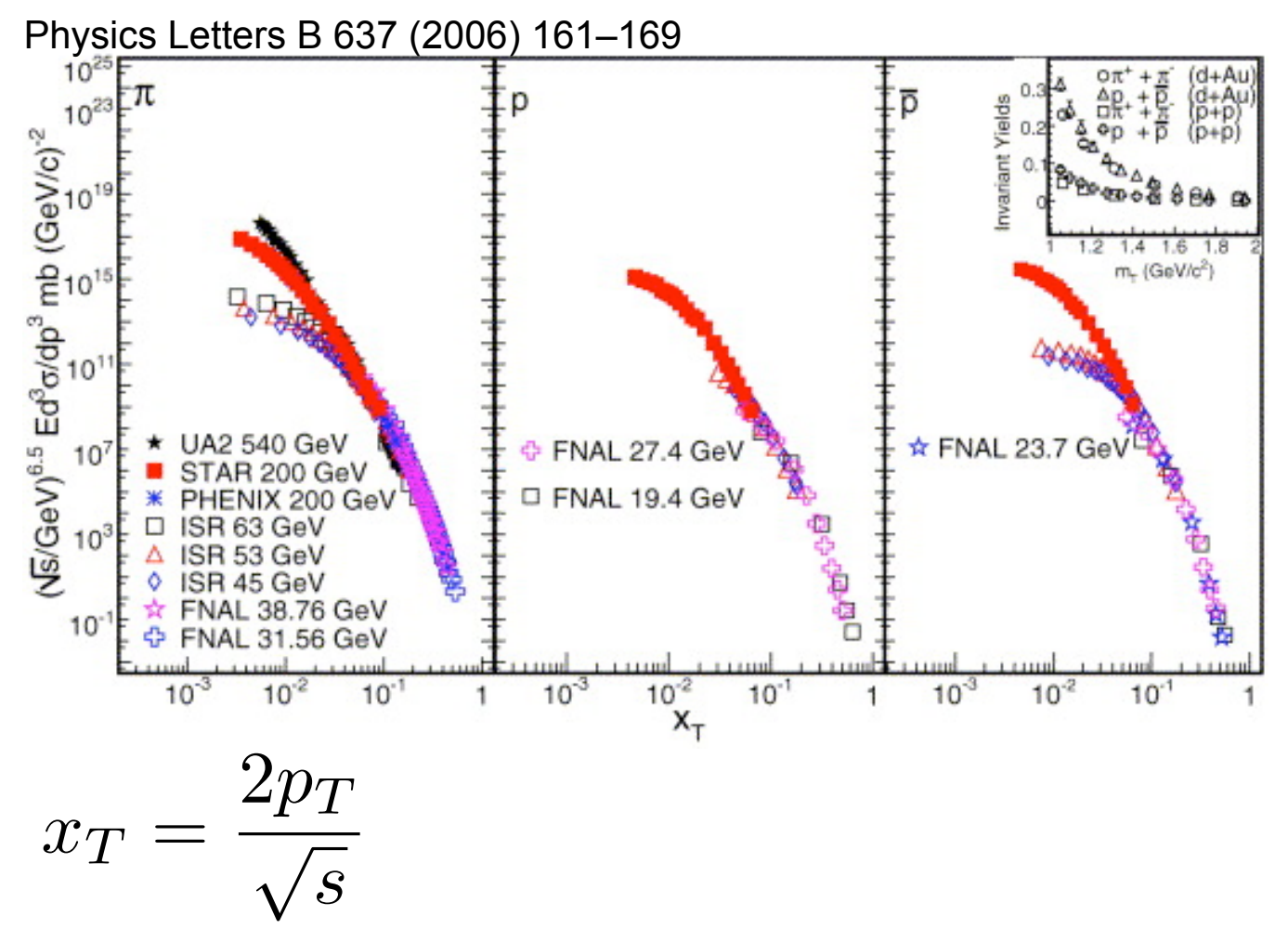

p-p spectra converge at large $\mathrm{x}_{\mathrm{T}}$ over large range in $\sqrt{\mathrm{S}_{\mathrm{NN}}}$ when yield scaled by $(\sqrt{s} / G e V)^{6.5 \pm 0.8}$

Does this scaling persist in A-A collisions? 


\section{Is $x_{T}$ better variable than $p_{T}$ ?}

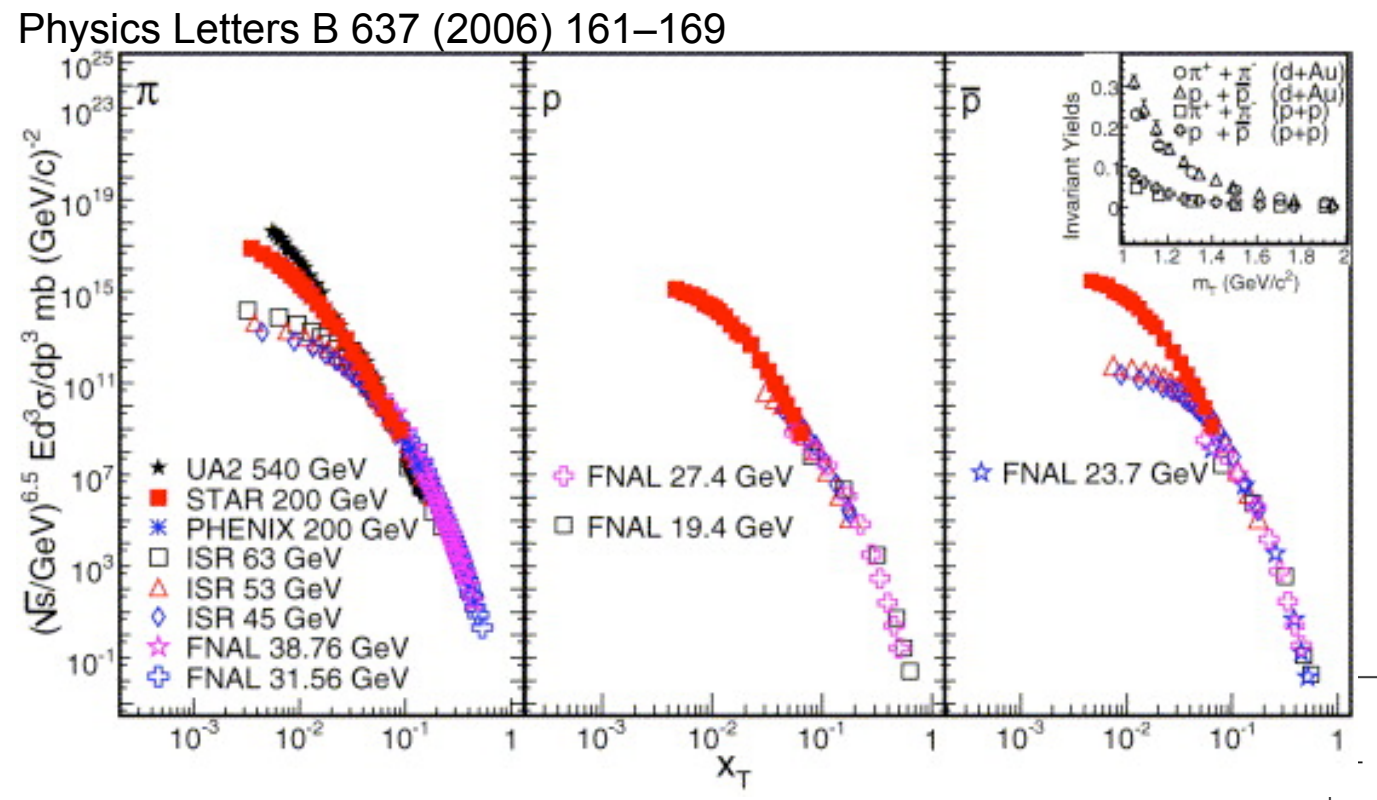

$$
x_{T}=\frac{2 p_{T}}{\sqrt{s}}
$$

$\mathrm{R}_{\mathrm{CP}} \sqrt{\mathrm{S}_{\mathrm{NN}}}$ dependence remains

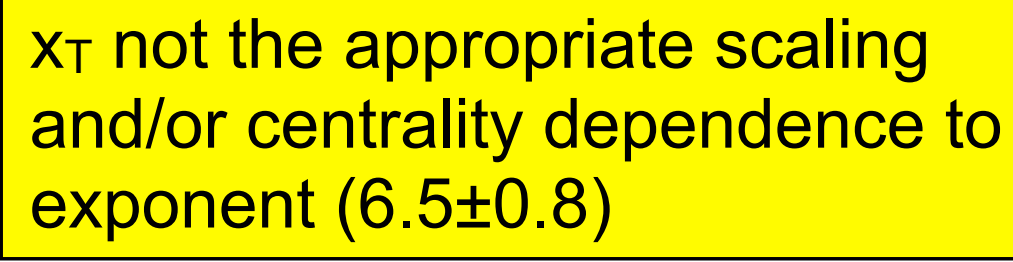

p-p spectra converge at large $\mathrm{XT}_{\mathrm{T}}$ over large range in $\sqrt{S_{N N}}$ when yield scaled by $(\sqrt{s} / G e V)^{6.5 \pm 0.8}$

Does this scaling persist in A-A collisions?

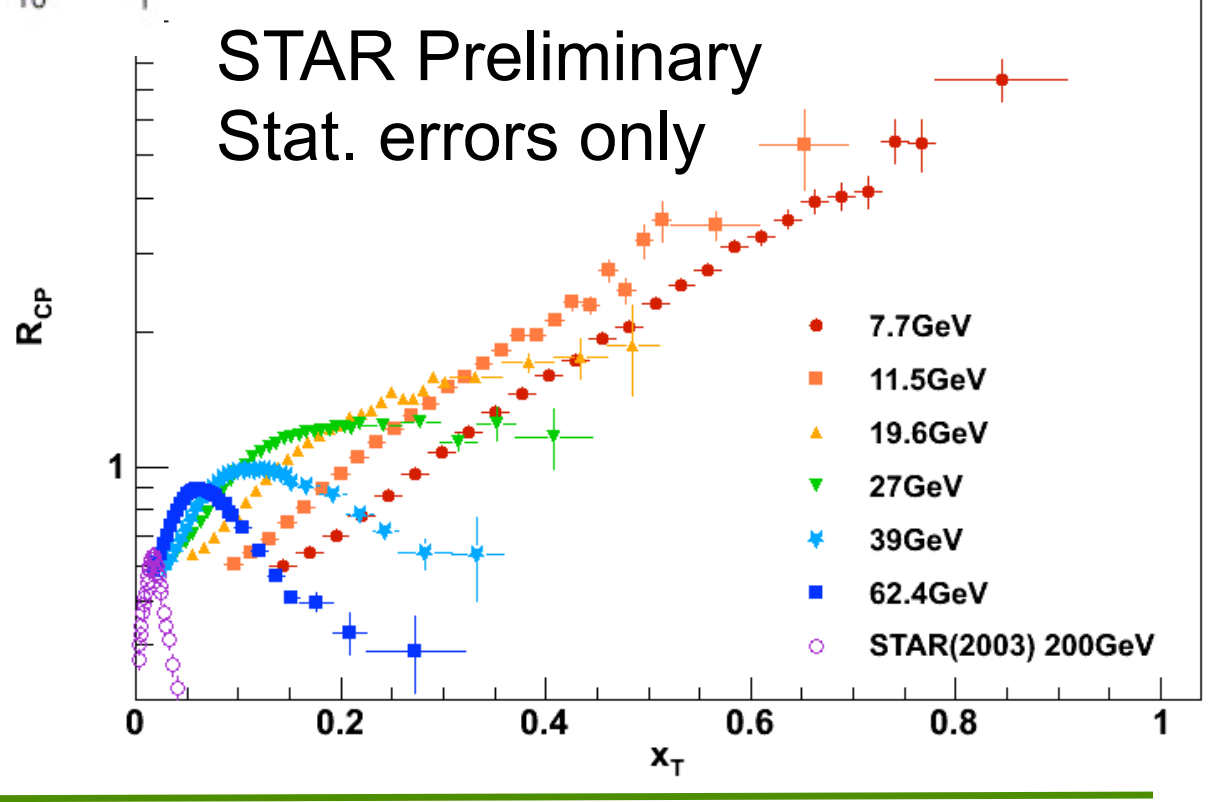

Horvat CPOD 


\section{Can we extract Cronin from models}

- HIJING 1.383

- jet quenching on or off

- modeled as $-\mathrm{dE} / \mathrm{dx}$ within the medium

- default Lund splitting parameters $a=0.5, b=0.9$

- AMPT v1.21/v2.21(uses HIJING 1.383)

- string melting (SM) off uses Lund string fragmentation for hadronization (v1.21)

- SM on uses quark coalescence for hadronization (v2.21)

- default Lund splitting parameters $a=2.2, b=0.5$

Lund fragmentation formula: $f(z) \propto \frac{(1-z)^{a}}{z} e^{-b m_{\perp}^{2} / z}$ 


\section{HIJING with differing Lund parameters}

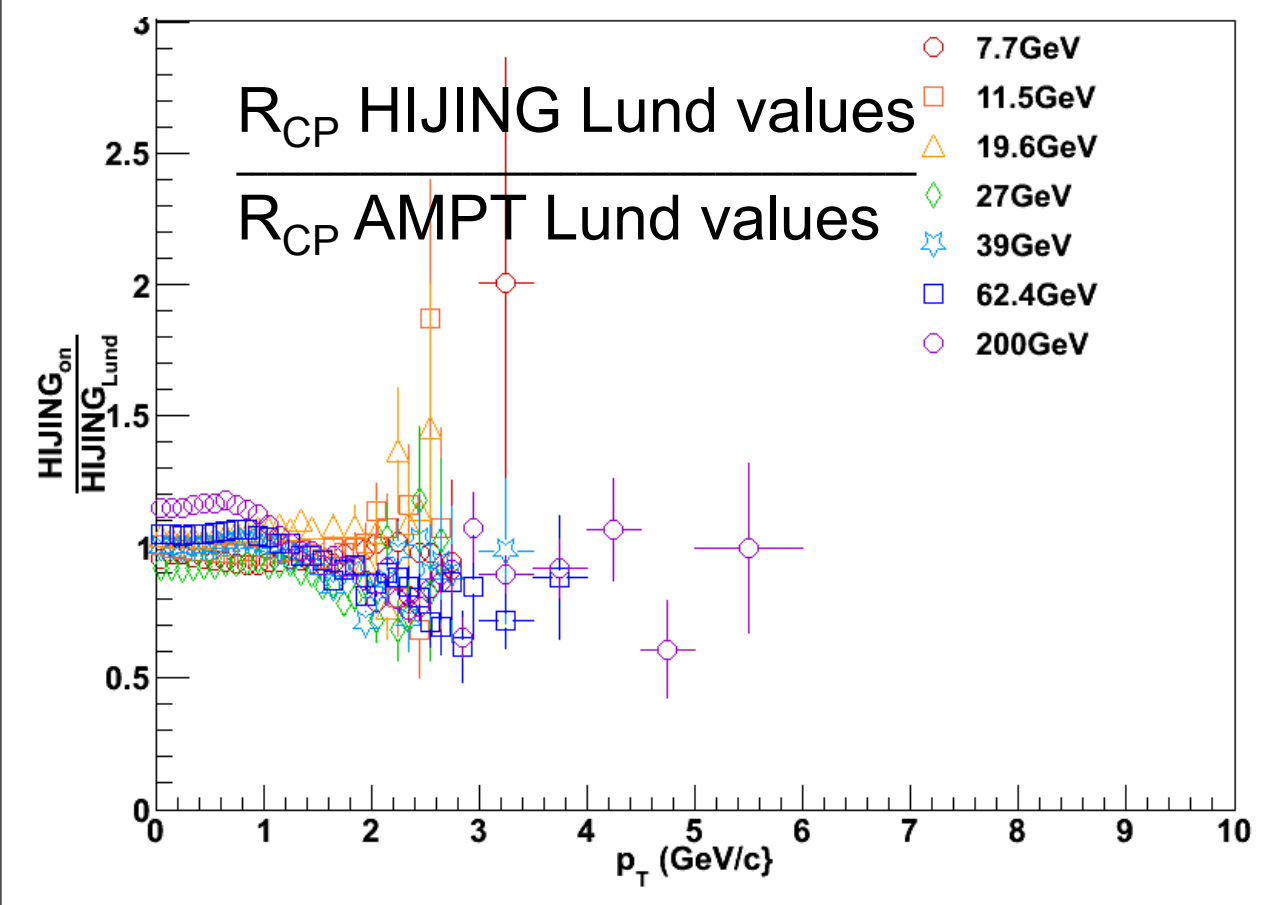

$R_{C P}$ essentially unchanged 


\section{HIJING with differing Lund parameters}
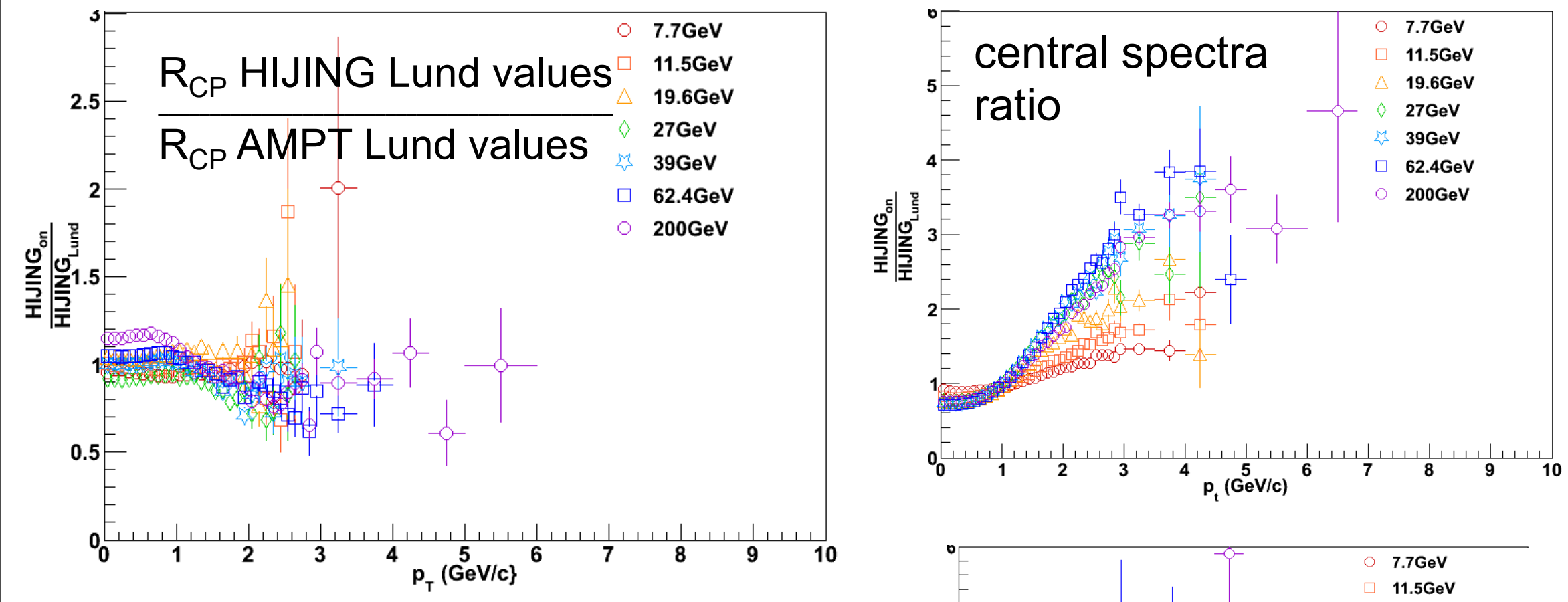

$R_{C P}$ essentially unchanged

Peripheral and central spectra vary in same manner

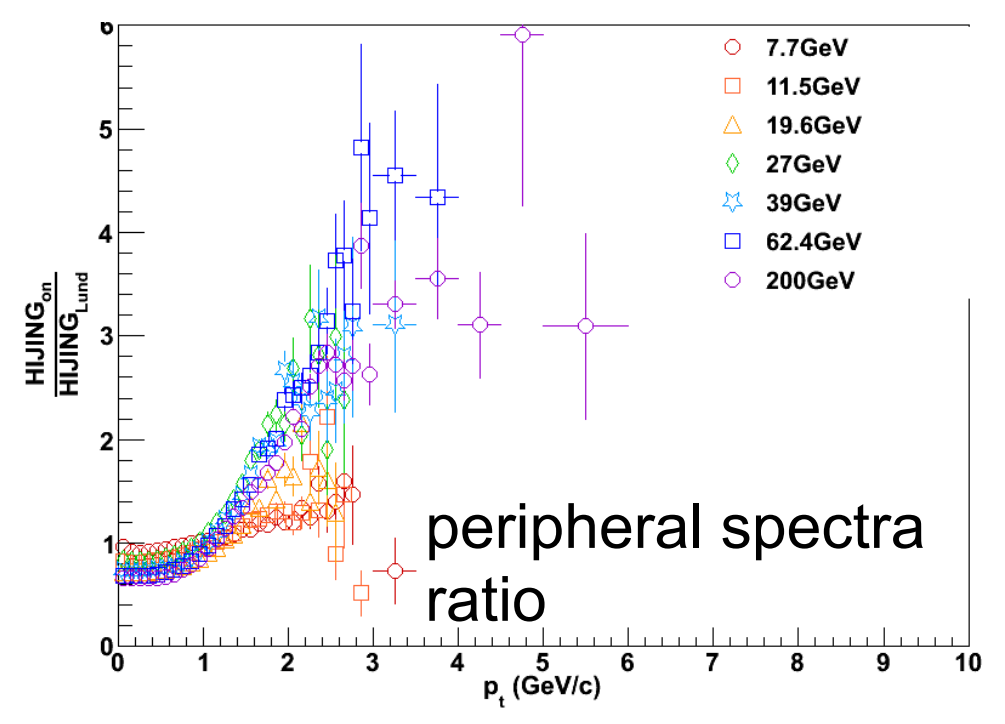




\section{Conclusions on quenching}

- Results taken at face value suggest QGP down to lowest beam energy

- Disentangling initial from final state close to impossible without control data

- (some) Models get trends OK but none get details

- Need to compare to numerous measurements to gain understanding

- BES data confusing, and more interesting than folks thought 


\section{Initial Conditions}

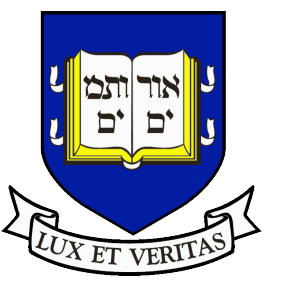




\section{Importance of initial conditions}

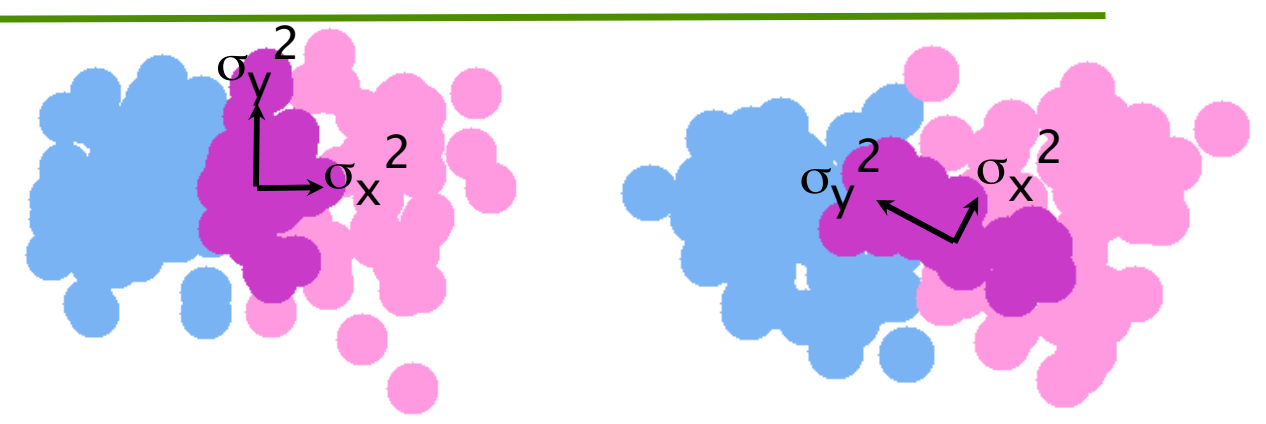

PHOBOS - Cu-Cu importance of principle axis

Alver and Roland $-v_{3}$ exists and plays key role

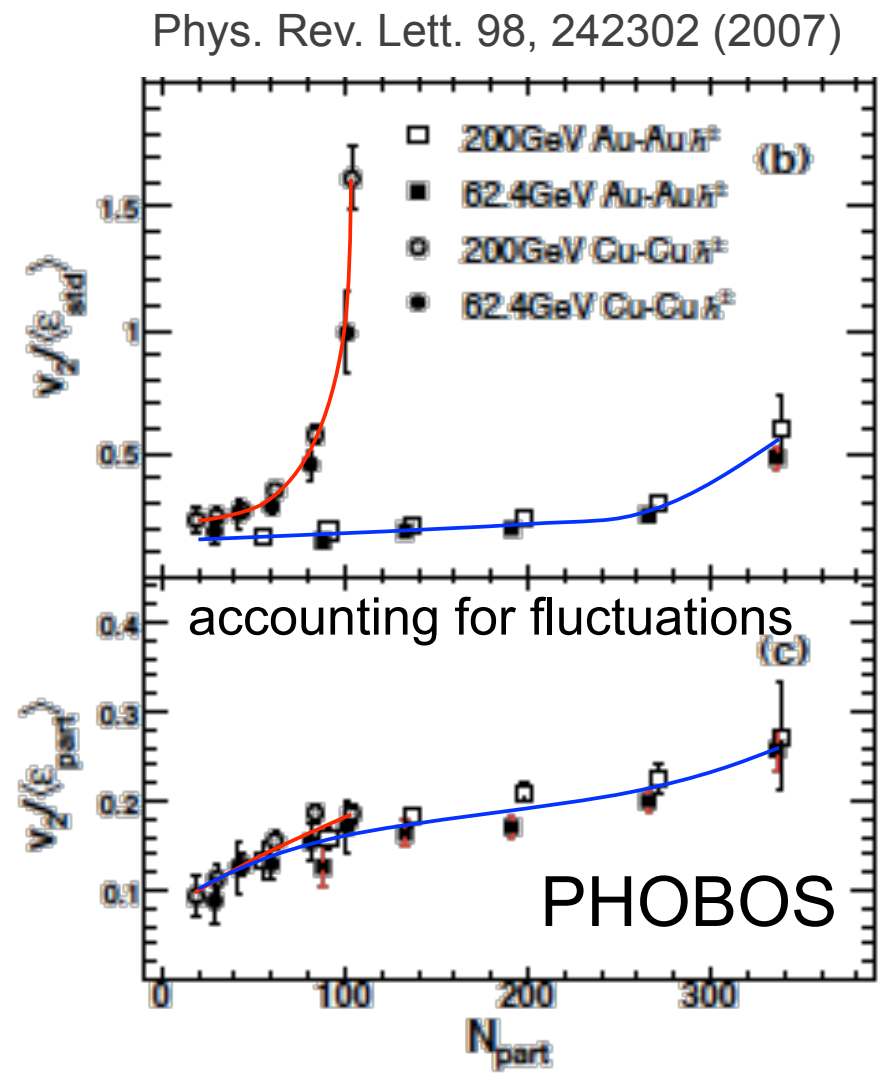




\section{Importance of initial conditions}

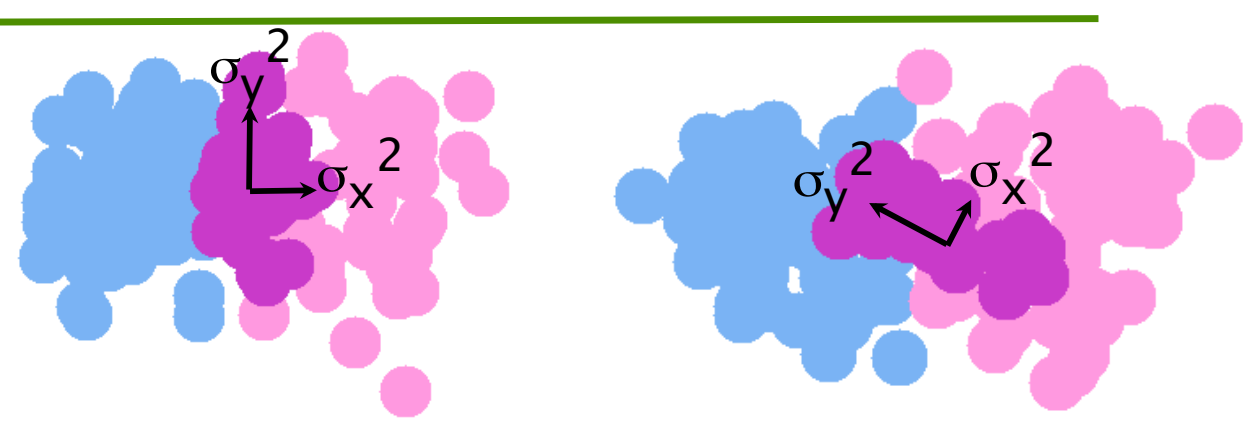

PHOBOS - Cu-Cu importance of principle axis

Alver and Roland - $v_{3}$ exists and plays key role
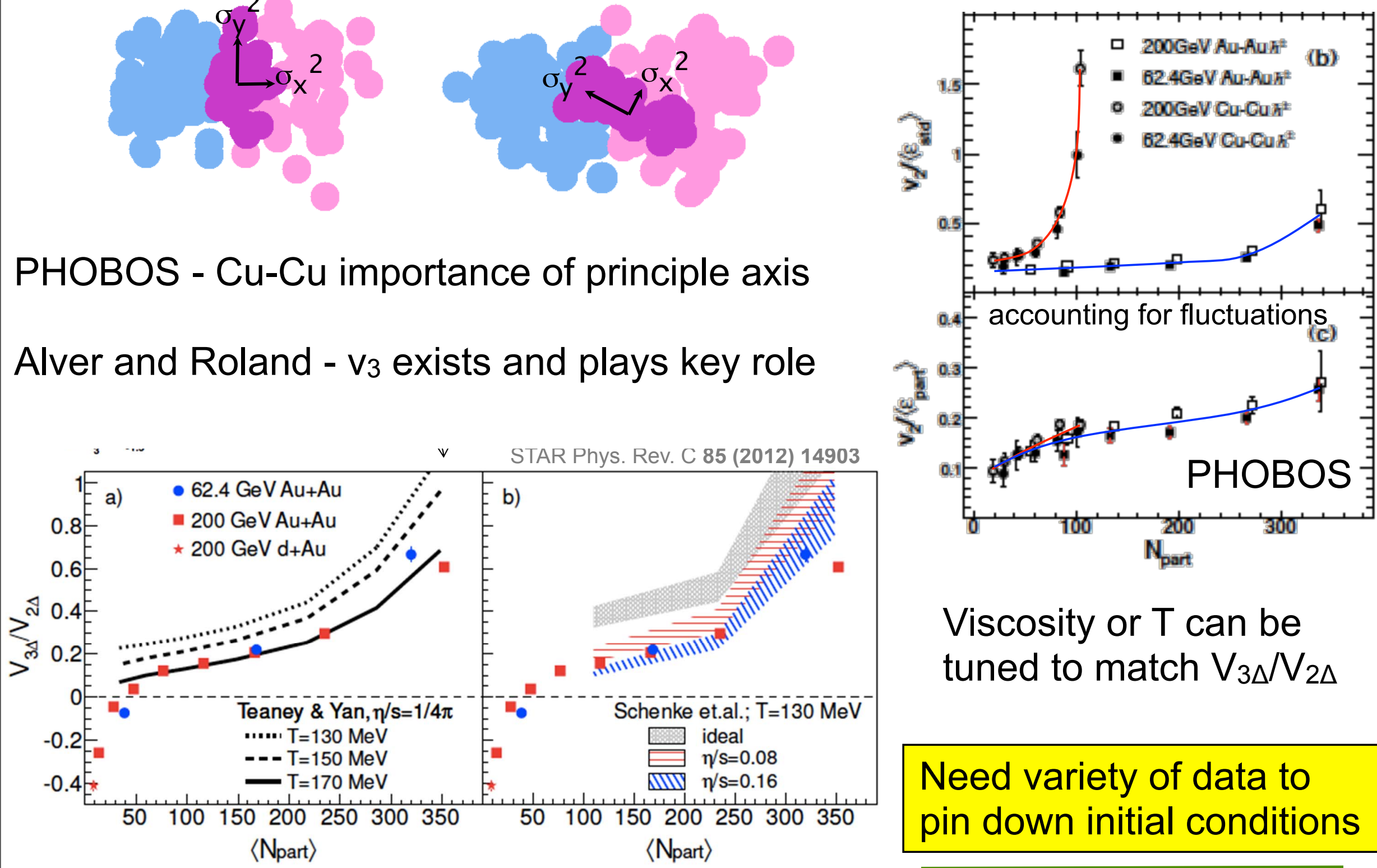

Viscosity or T can be tuned to match $\mathrm{V}_{3 \Delta} / \mathrm{V}_{2 \Delta}$

Need variety of data to pin down initial conditions

Helen Caines - BNNL - April 2013 


\section{Importance of Initial conditions - III}

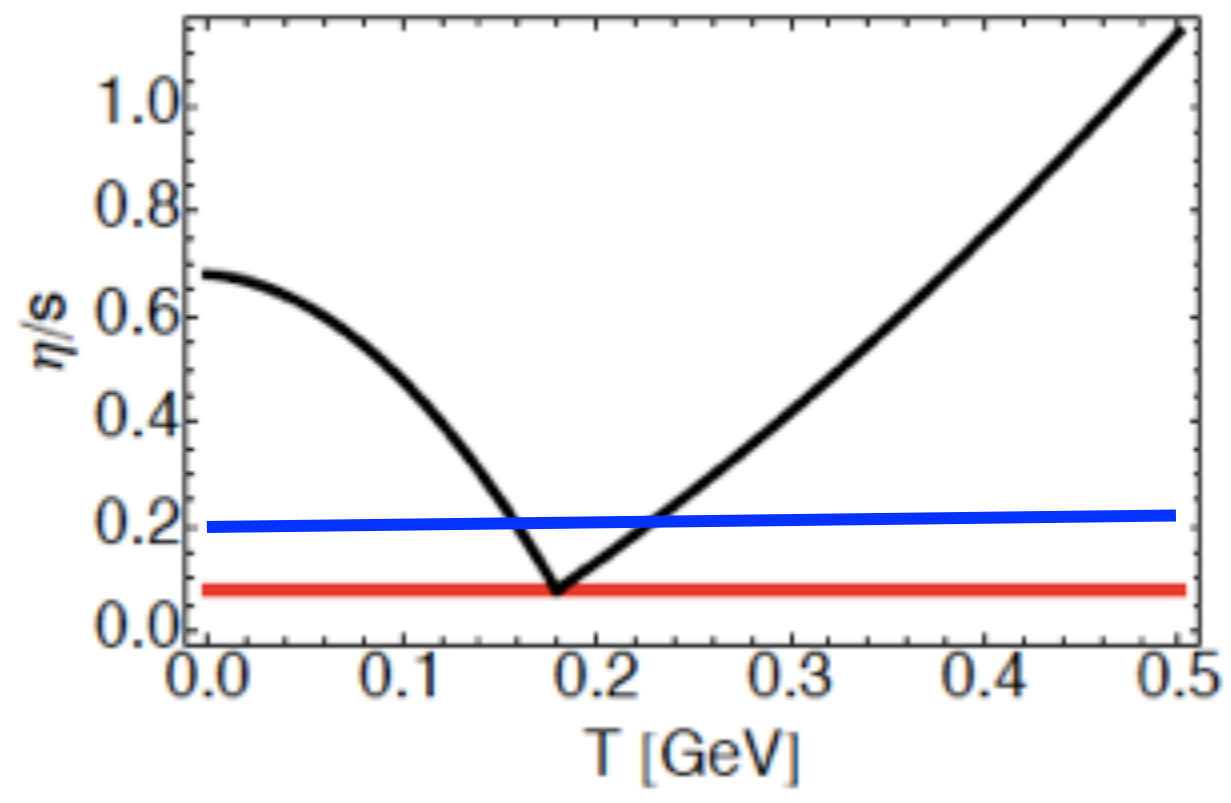

Gale, Jeon, Schenke, Tribedy, Venugopalan

Different functional forms for $\eta / s(T)$ can result in similar mean $\eta / s$ values

Can't distinguish with models between constant $\eta / s$ or temperature dependent $\eta / s$ with minimum at $T_{c}$ with only one collision energy

Variety of collision energies needed to disentangle 


\section{$\eta /$ s as a function of collision energy}

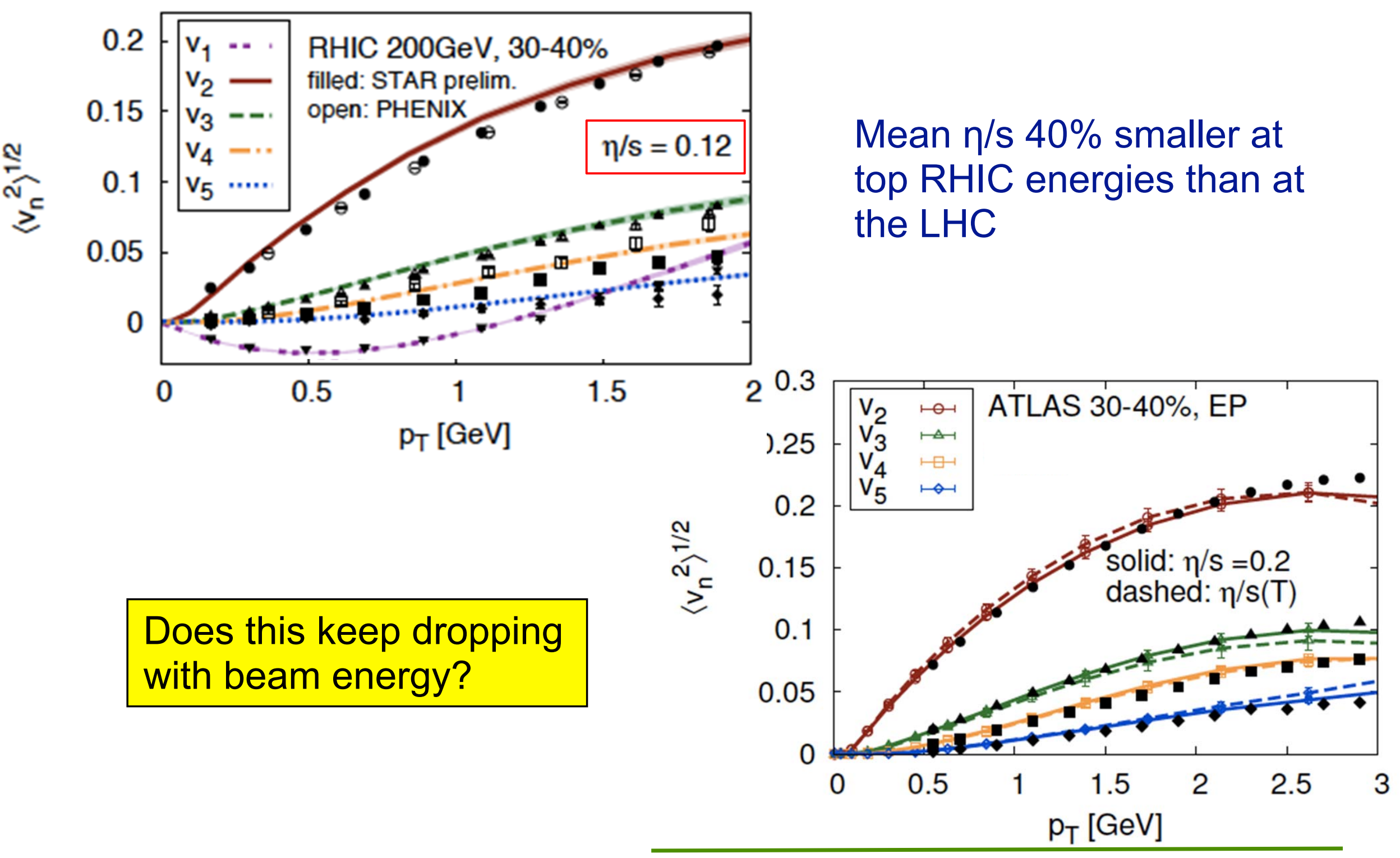




\section{$V_{n}$ at top beam energies}

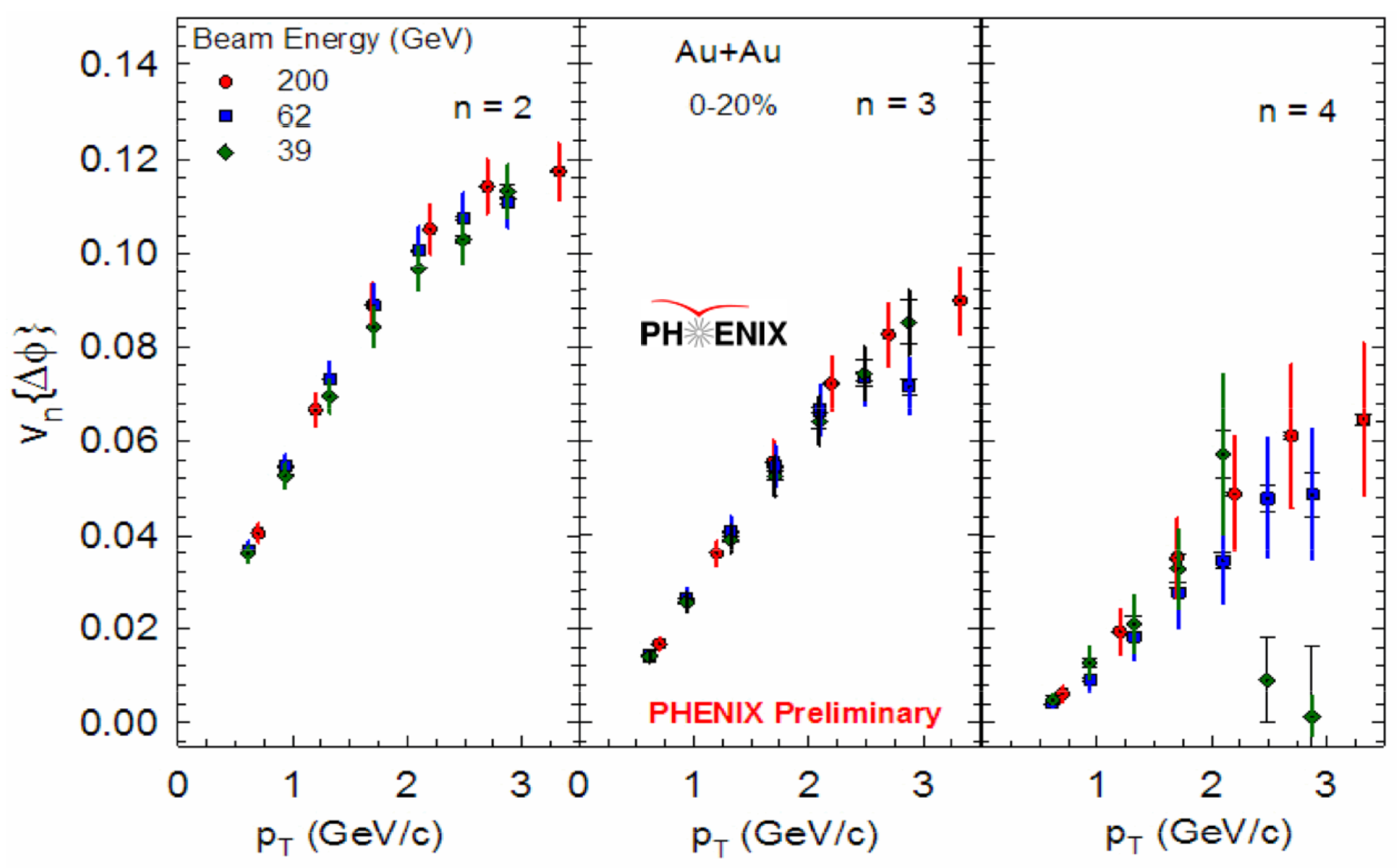

Similar $p_{\mathrm{T}}$ distributions for all $v_{\mathrm{n}}$ for $\sqrt{\mathrm{S}_{\mathrm{NN}}}=39-200 \mathrm{GeV}$

Likely numerous things changing that happen to cancel out 


\section{$v_{3}$ and sensitivity to EoS}

Y. Pandit, STAR, Quark Matter 2013

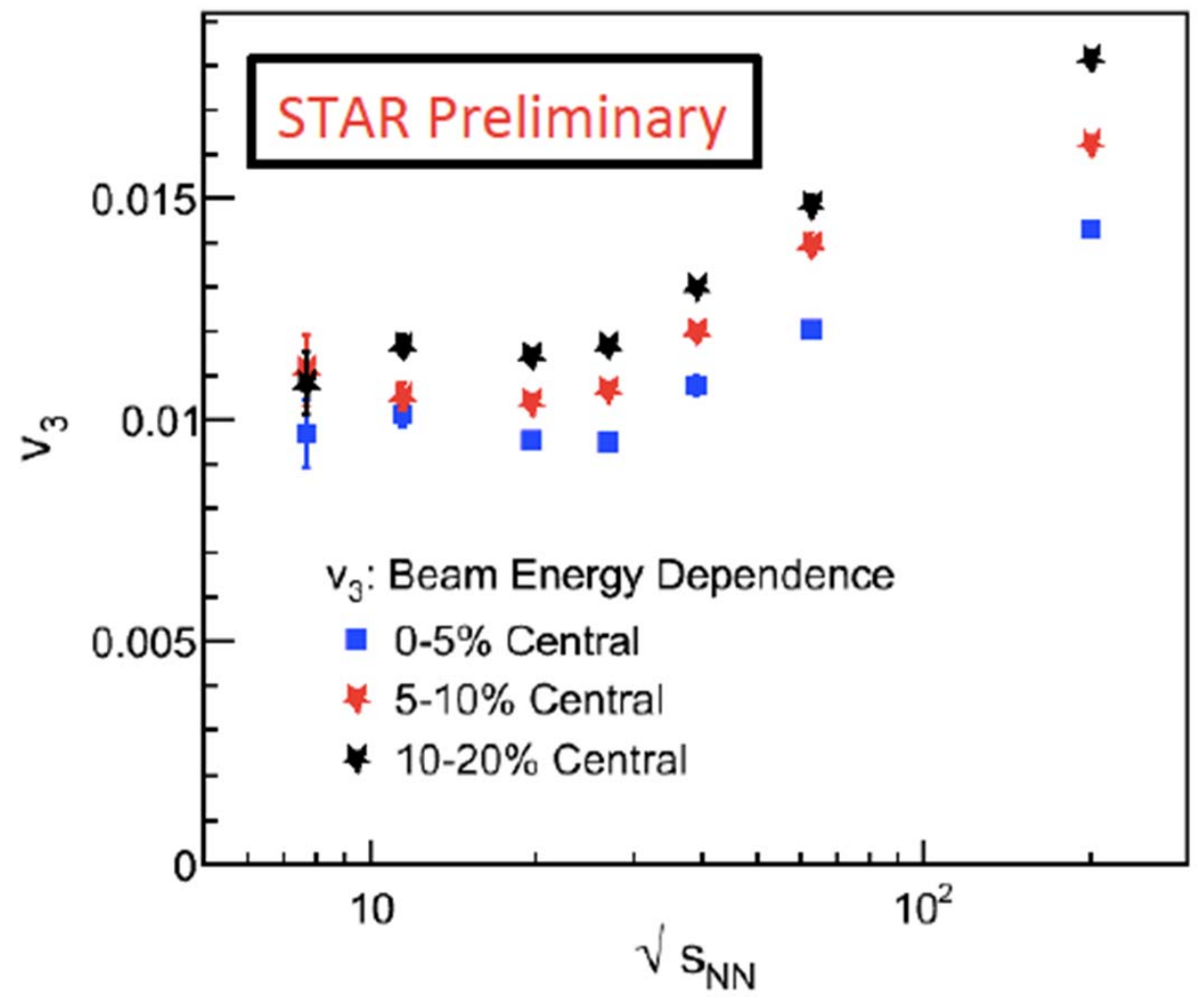

V3 sizable even at $7.7 \mathrm{GeV}$

- jet contribution essentially zero 


\section{$v_{3}$ and sensitivity to EoS}

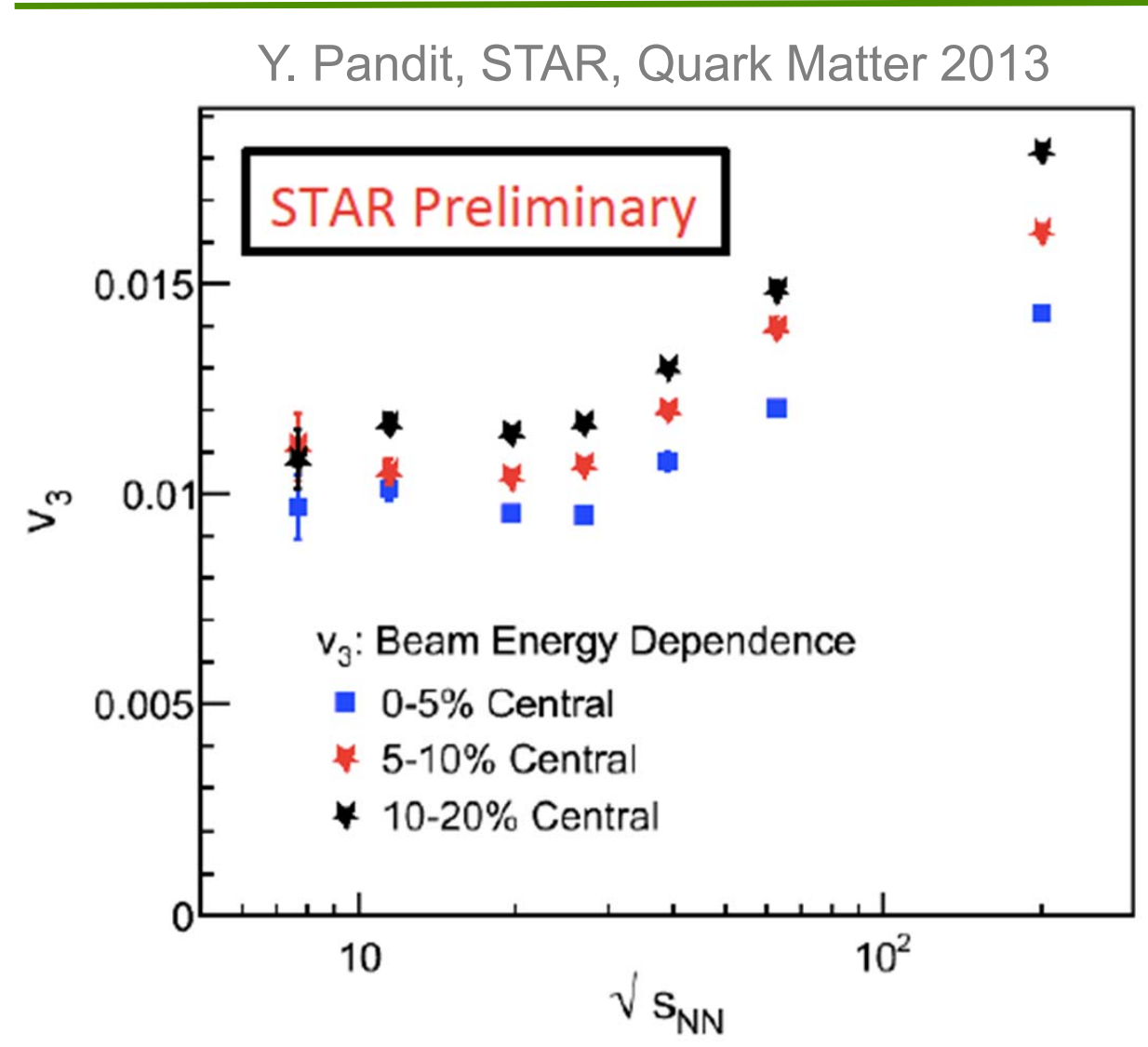

V3 sizable even at $7.7 \mathrm{GeV}$

- jet contribution essentially zero

STAR data in reasonable agreement with QGP version of AMPT for all $\sqrt{S_{N N}}$

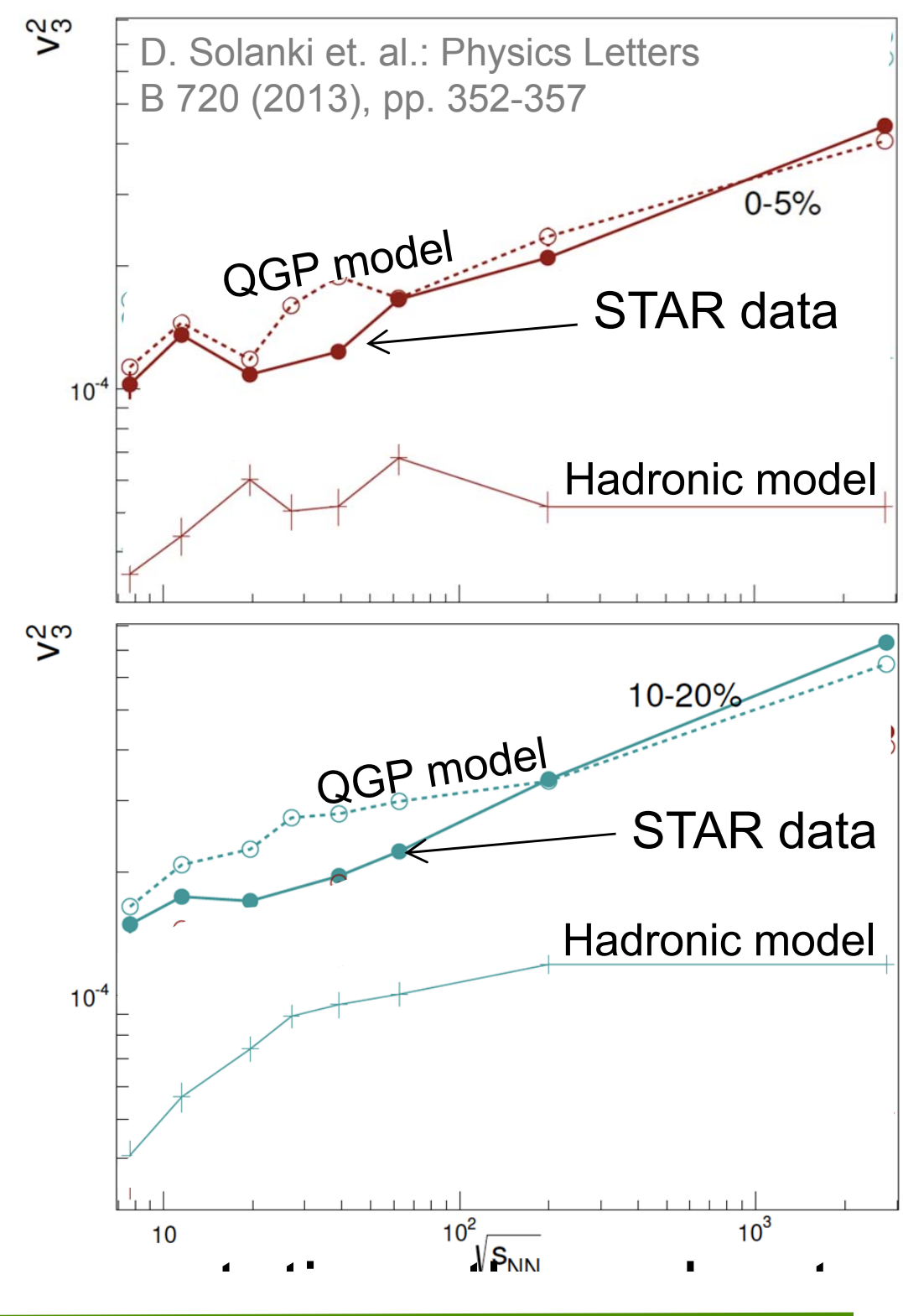




\section{Pre BES - Azimuthally sensitive HBT}

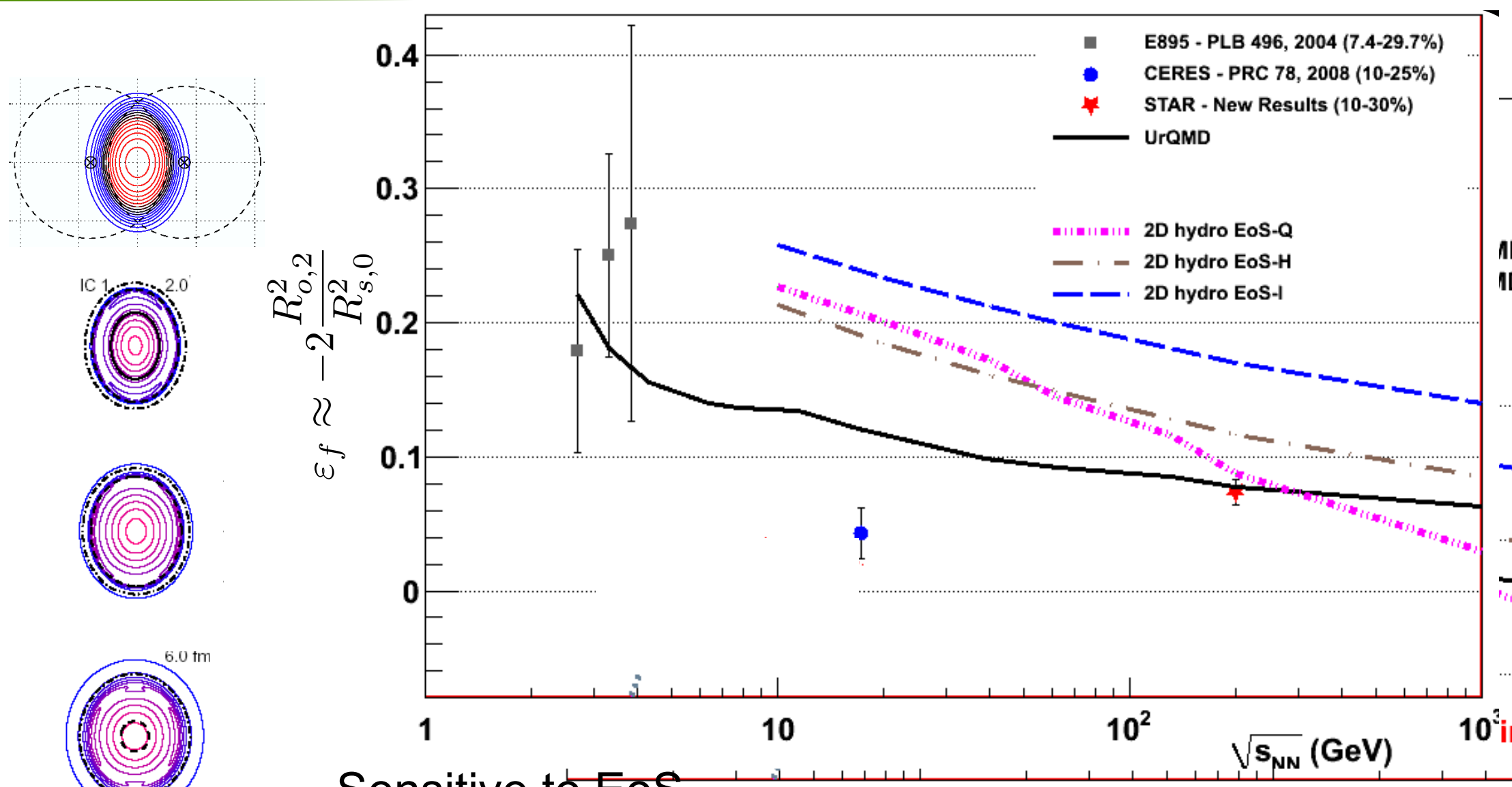

Sensitive to EoS

Naively:

higher $\sqrt{\mathrm{S}_{\mathrm{NN}}} \rightarrow$ higher pressure $\rightarrow$ evolve to smaller $\varepsilon_{f}$ higher $\sqrt{\mathrm{S}_{\mathrm{NN}}} \rightarrow$ longer lifetime $\rightarrow$ evolve to smaller $\varepsilon_{f}$

CERES data hint at something interesting 


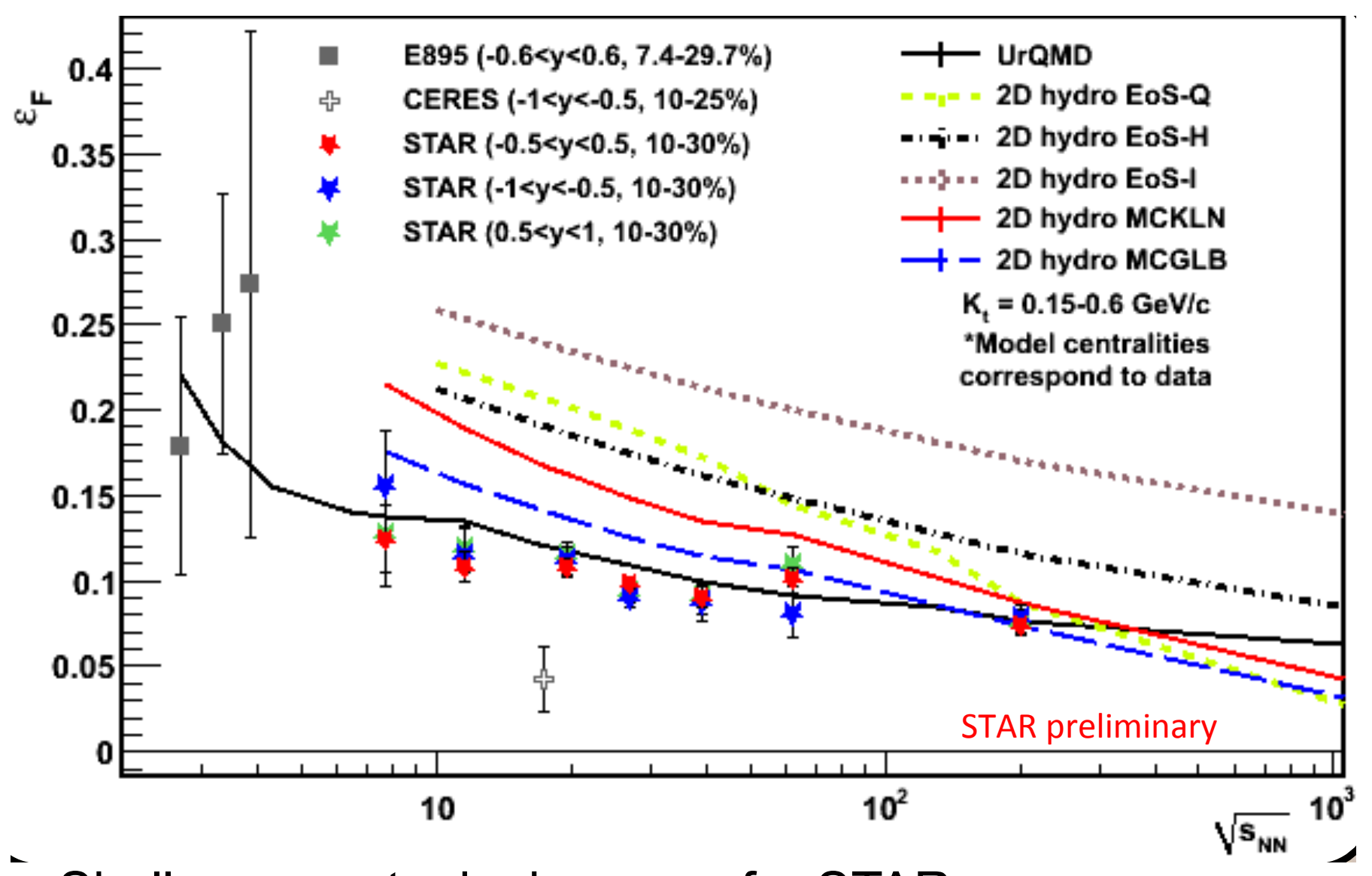

Shallow monotonic decrease for STAR data - even for CERES acceptance

Sensitivity to initial-state/viscosity 


\section{Future prospects - BES-II and fixed target}

\begin{tabular}{|c|c|c|c|c|c|}
\hline $\begin{array}{c}\sqrt{\boldsymbol{s}_{N N}} \\
(\mathrm{GeV})\end{array}$ & $\begin{array}{c}\mu_{B} \\
(\mathrm{MeV})\end{array}$ & BES-I & BES-II & Physics Motivation & Weeks** \\
\hline 200 & 24 & & $0.5-2(B)$ & Heavy flavor hadron $v_{2} \& R_{A A}$ & \\
\hline 39 & 112 & $130(\mathrm{M})$ & & & \\
\hline 27 & 156 & $70(\mathrm{M})$ & & & \\
\hline 19.6 & 206 & $36(\mathrm{M})$ & $400(M)$ & LMR di-electron ${ }^{*}$, net-p k>5 $\sigma$ & 2 \\
\hline 15 & 250 & & $100(\mathrm{M})$ & $\Omega$ yield, $\phi$-meson $v_{2}(\leq 3 \mathrm{GeV} / \mathrm{c})$ & 2 \\
\hline 11.5 & 316 & $12(\mathrm{M})$ & $120(\mathrm{M})$ & net-p k & 3.5 \\
\hline 7.7 & 420 & $5(\mathrm{M})$ & $80(M)$ & net-p k & 10 \\
\hline
\end{tabular}

* Di-electron measurements below $19.6 \mathrm{GeV}$ are not planned

** Estimates are based on electron cooling upgrade currently under development and are approximate without electron cooling, the program would require $\sim 150$ weeks

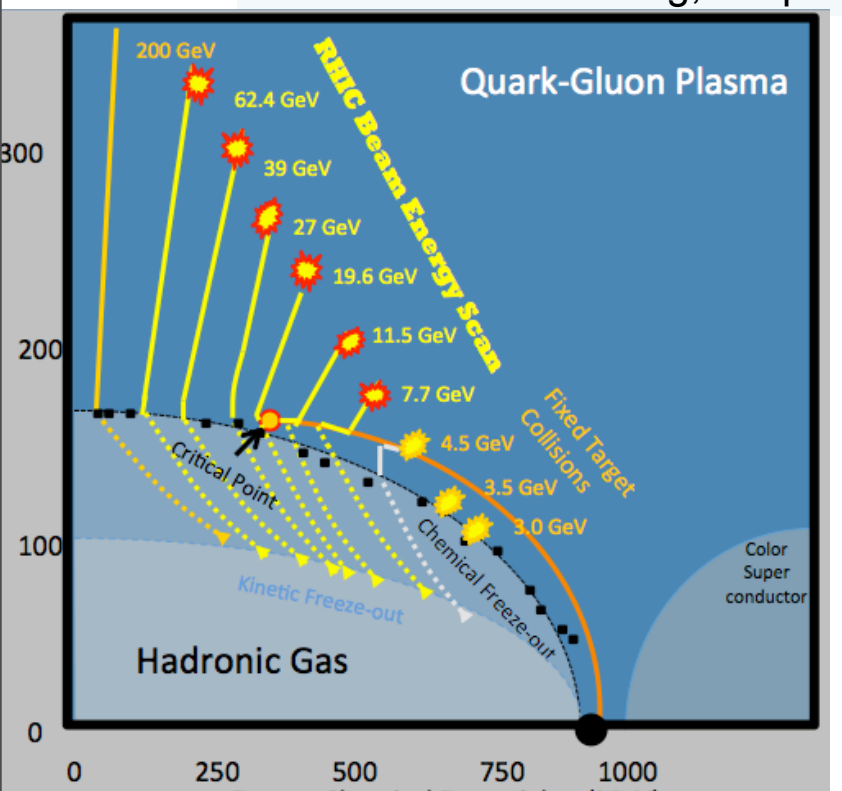

Fixed target experiment at STAR will push down to even lower $\mu_{B}$

\begin{tabular}{|c|c|c|}
\hline $\begin{array}{c}\text { Collider mode } \\
{\sqrt{S_{N N}}}(\mathrm{GeV})\end{array}$ & $\begin{array}{c}\text { Fixed-target mode } \\
{\sqrt{S_{N N}}}(\mathrm{GeV})\end{array}$ & $\begin{array}{c}\text { Fixed-target mode } \\
\mu_{\mathrm{B}}(\mathrm{MeV})\end{array}$ \\
\hline 19.6 & 4.5 & 585 \\
\hline 15 & 4.0 & 625 \\
\hline 11.5 & 3.5 & 670 \\
\hline 7.7 & 3.0 & 720 \\
\hline 5 & 2.5 & 775 \\
\hline
\end{tabular}




\section{Conclusions}

- I'm in the minority but I think pA BES would be very interesting

- Hopeful can disentangle contributions to initial state via variety of measurements - HBT making a comeback?!?!!

- Lots of puzzles from the BES

- even if hints for Critical Point and 1st order transitions are not leaping out 

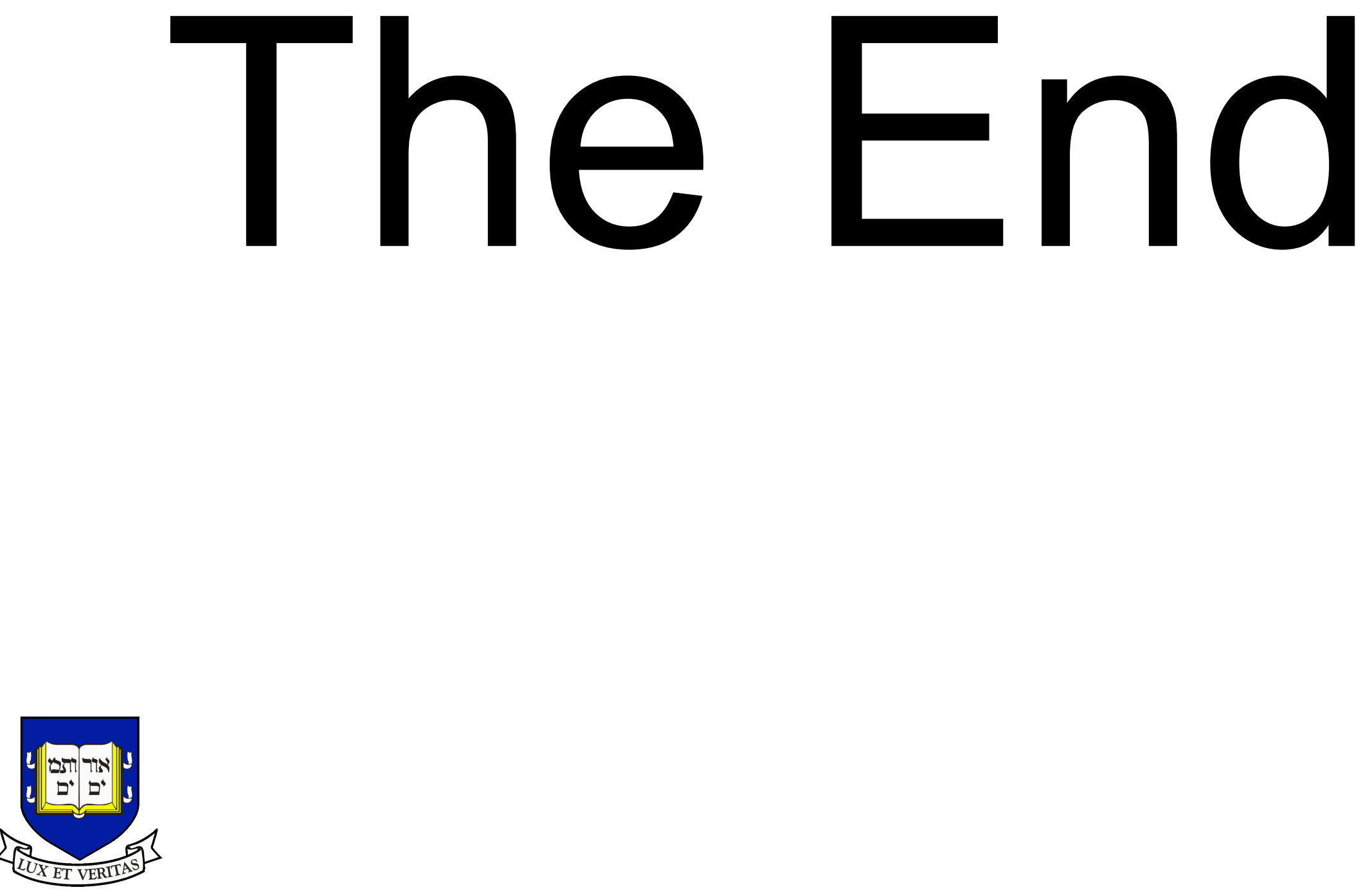


\title{
Jet quenching II: or
}

\section{What do we know, and how well do we know it?}

\author{
Brian. A Cole \\ April 16, 2013
}
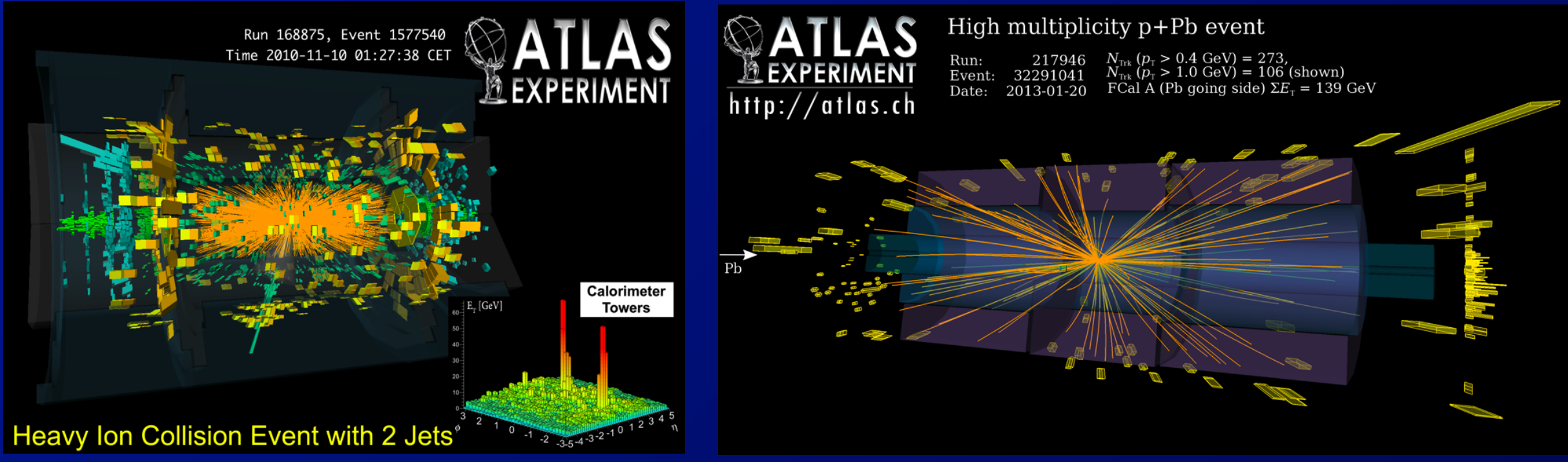


\section{Overview}

- From workshop web page:

- Specific topics we plan to address include:

To what extent do we achieve control over initial state effects in light of the new dAu and $\mathrm{pPb}$ data?

How do the medium properties change from RHIC to LHC?

Can we achieve precision by combining the vast amount of data from RHIC and LHC?

How does the jet quenching response to fluctuating initial geometry (jet tomography) evolve from RHIC to LHC?

- Discuss:

- Theory, $\mathrm{p}+\mathrm{Pb}, \mathrm{Pb}+\mathrm{Pb}$ constraints on nuclear modifications on hard scattering rates at high $p_{\text {T. }}$

$\Rightarrow$ Single hadron, jet and heavy flavor suppression

- Jet quenching probes insensitive to PDFs/rates

- Tomographic measurements 
Constraints on initial-state effects 


\section{Initial-state effects (not saturation)}

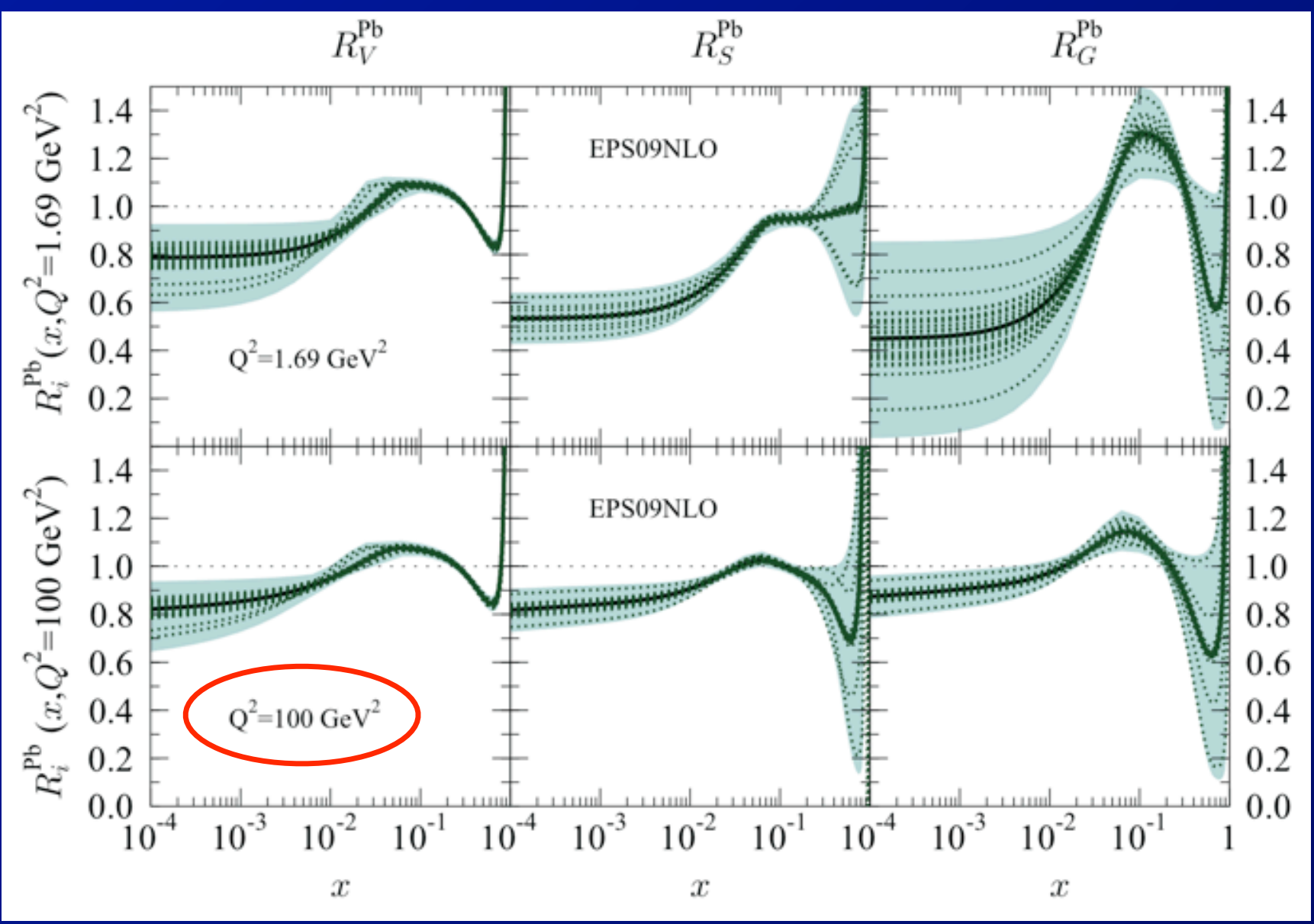

- For $Q^{2}=100 \mathrm{GeV}^{2} \Rightarrow$ jet $p_{T} \sim 10 \mathrm{GeV}$

- Expect modest low $x$ effects (b dependence?) for high-pt processes

$\Rightarrow \mathrm{p}+\mathrm{Pb}$ measurements will provide stringent test 


\section{$\mathrm{p}+\mathrm{Pb}$ inclusive spectra}
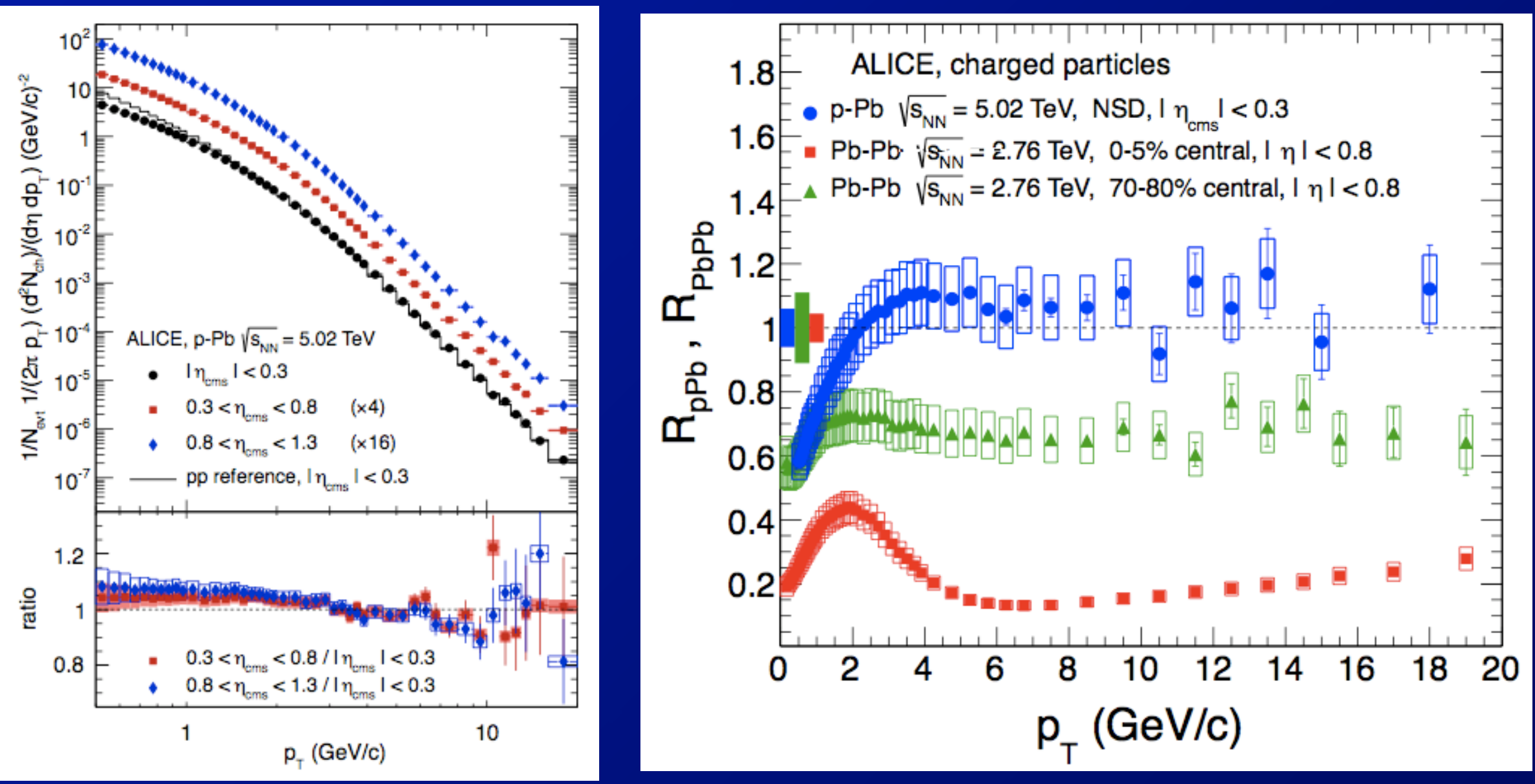

- $1^{\text {st }}$ look at charged particle spectra - arXiv:1210.4520, ALICE inclusive, NSD

$\Rightarrow R_{\mathrm{pPb}}$ consistent with 1, no suppression at midrapidity, also little or no "Cronin" 


\section{$\mathrm{Pb}+\mathrm{Pb}$ : Prompt photon production}
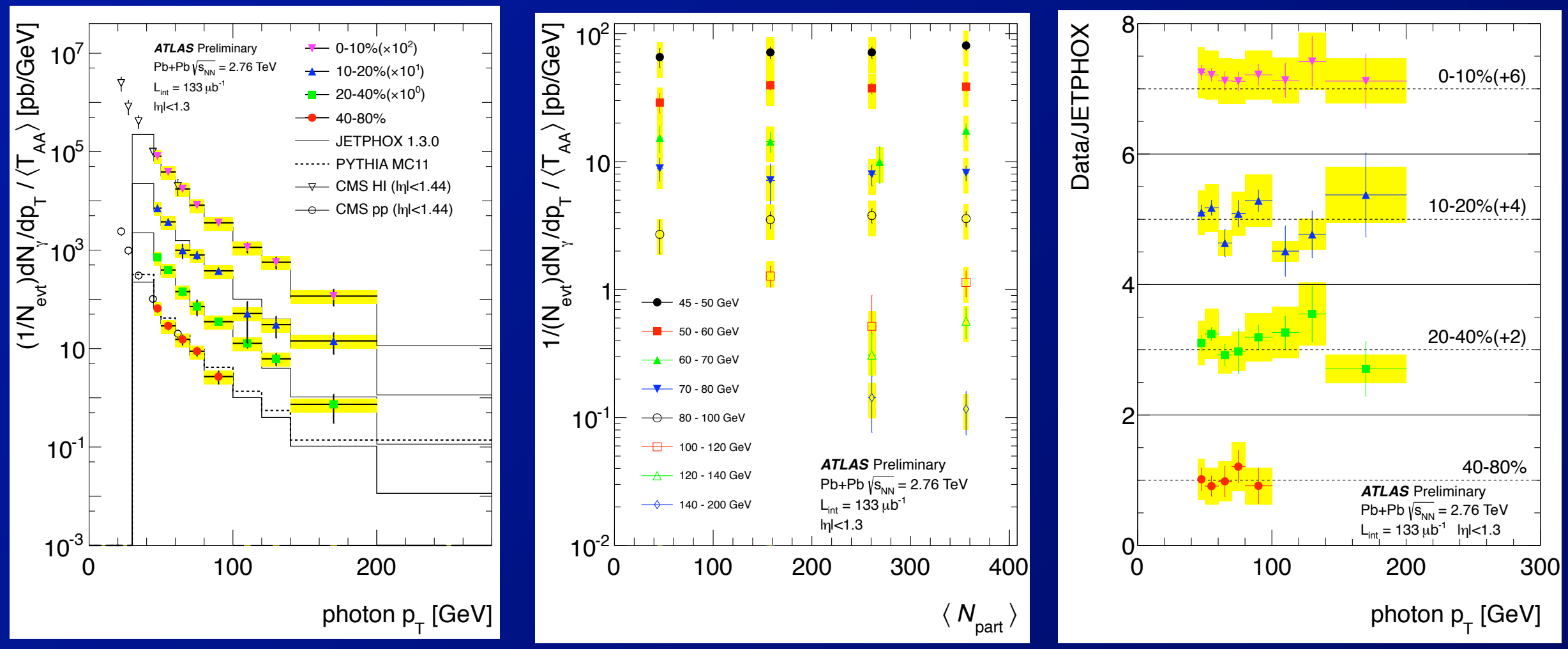

- Photon spectra over $40<p_{T}<200 \mathrm{GeV}$

- well described by JETPHOX multiplied by $T_{A A}$

- Yield / $T_{A A} \sim$ independent of centrality

$\Rightarrow$ Hard QCD photon production varies with $\mathrm{Pb}+\mathrm{Pb}$ centrality as expected 


\section{Pb+Pb: Z production (3)}

Phys. Rev. Lett. 110, 022301 (2013)

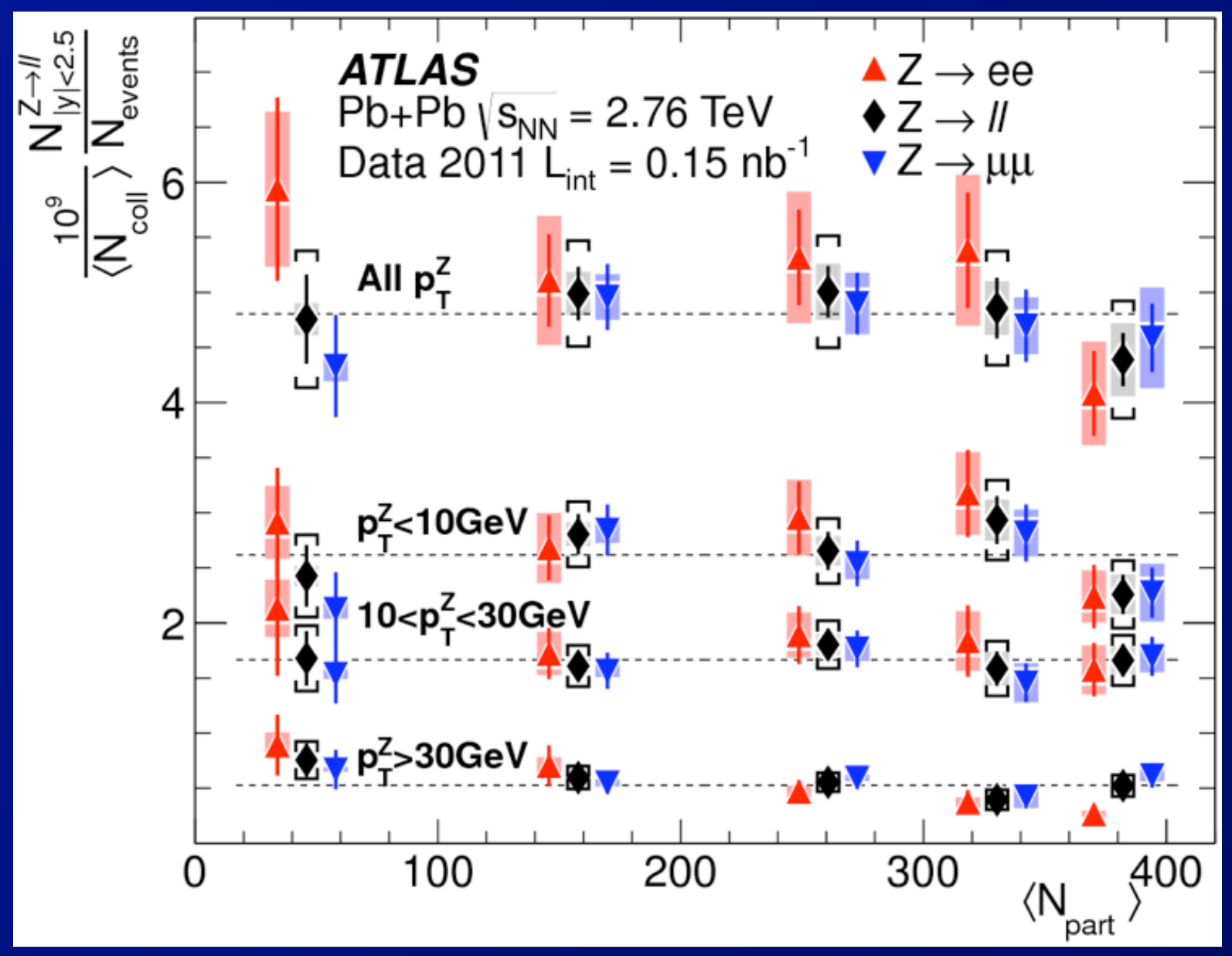

- Check Ncoll scaling of $Z$ yield in different $p_{T}$ intervals

- Slight drop in central yield/Ncoll for $p_{T}<10 \mathrm{GeV}$

$\Rightarrow$ But not significant given errors. Expected? 


\section{$\mathrm{Pb}+\mathrm{Pb}$ : Z production (4)}

Guzey et al, Eur. Phys. J. A49 (2013) 35
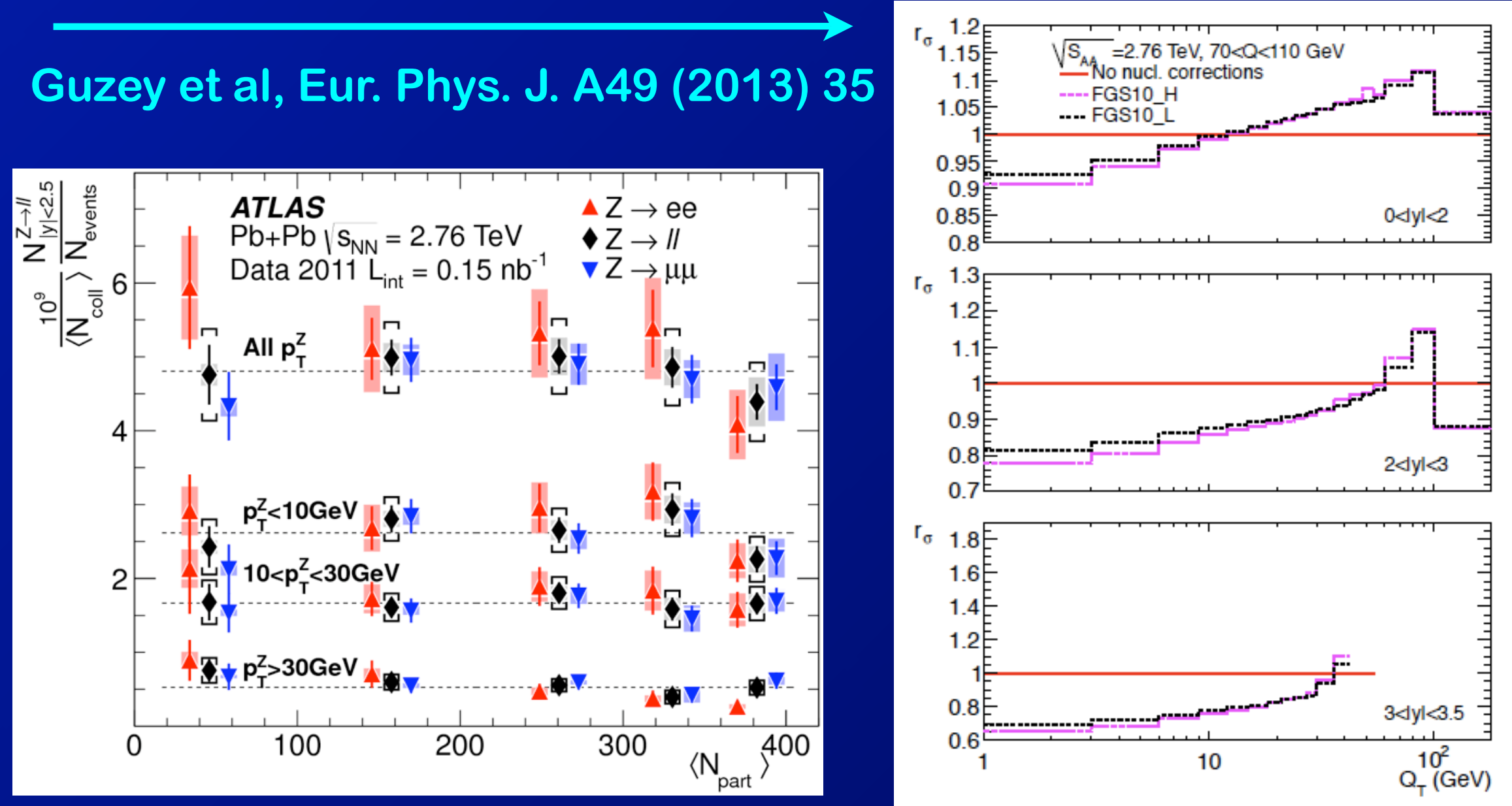

- Predictions of $\sim 10 \%$ nuclear modifications in $\mathrm{Pb}+\mathrm{Pb}$ at both low $Q_{T}$ and high $Q_{T}$

$\Rightarrow$ Measurements not precise enough to test yet

$\Rightarrow$ But maybe with 20132.76 TeV p-p data 


\section{Single hadron, heavy flavor (muon), jet suppression}




\section{Charged particle $\mathbf{R}_{\mathrm{cp}}$}

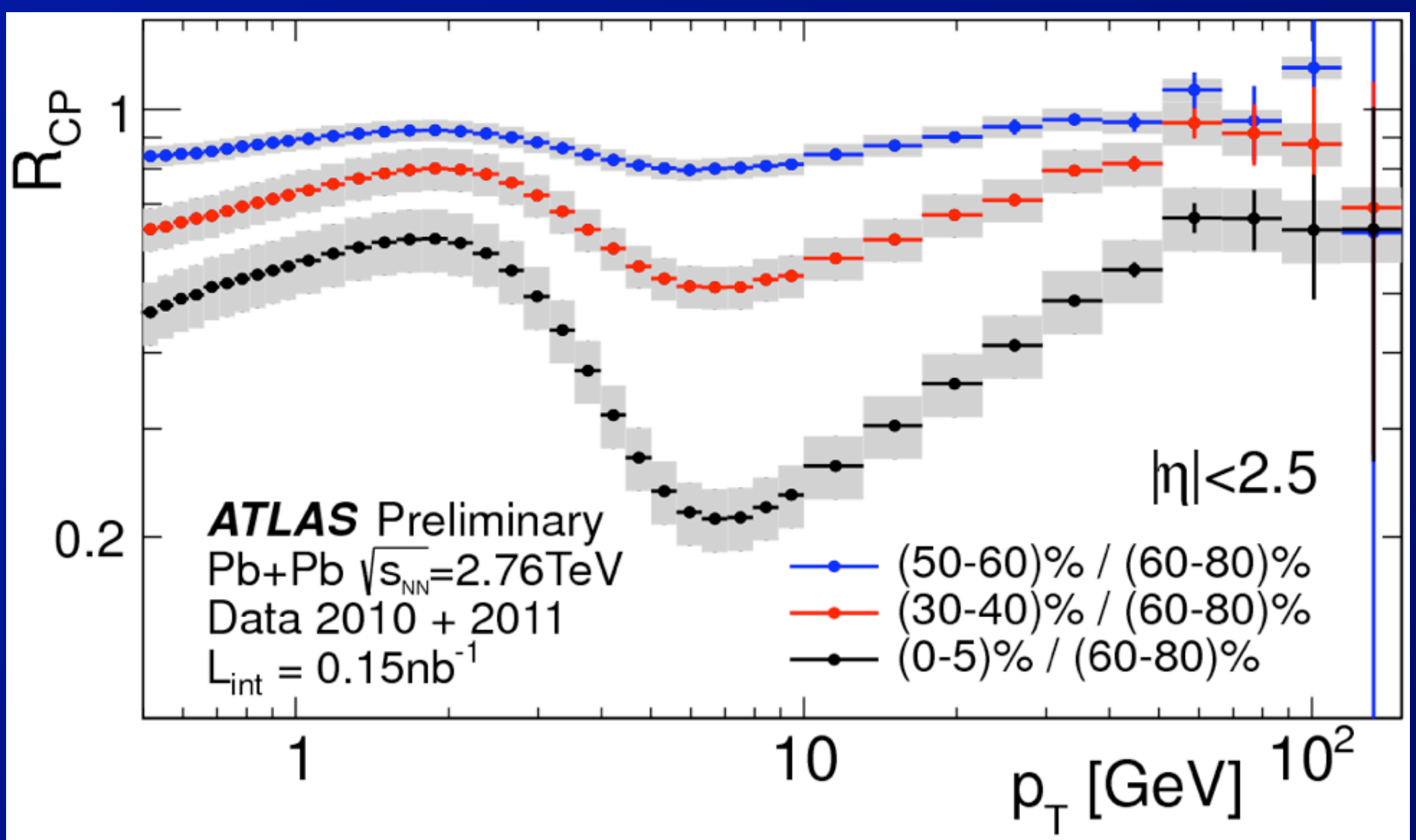

- Similar to ALICE, CMS results

$-R_{c p} \sim 0.6$ for $p_{T}>60 \mathrm{GeV}$

$\Rightarrow$ (change in) slope for $\mathrm{pT}>50 \mathrm{GeV}$ important 


\section{Single muons from heavy quark decays}
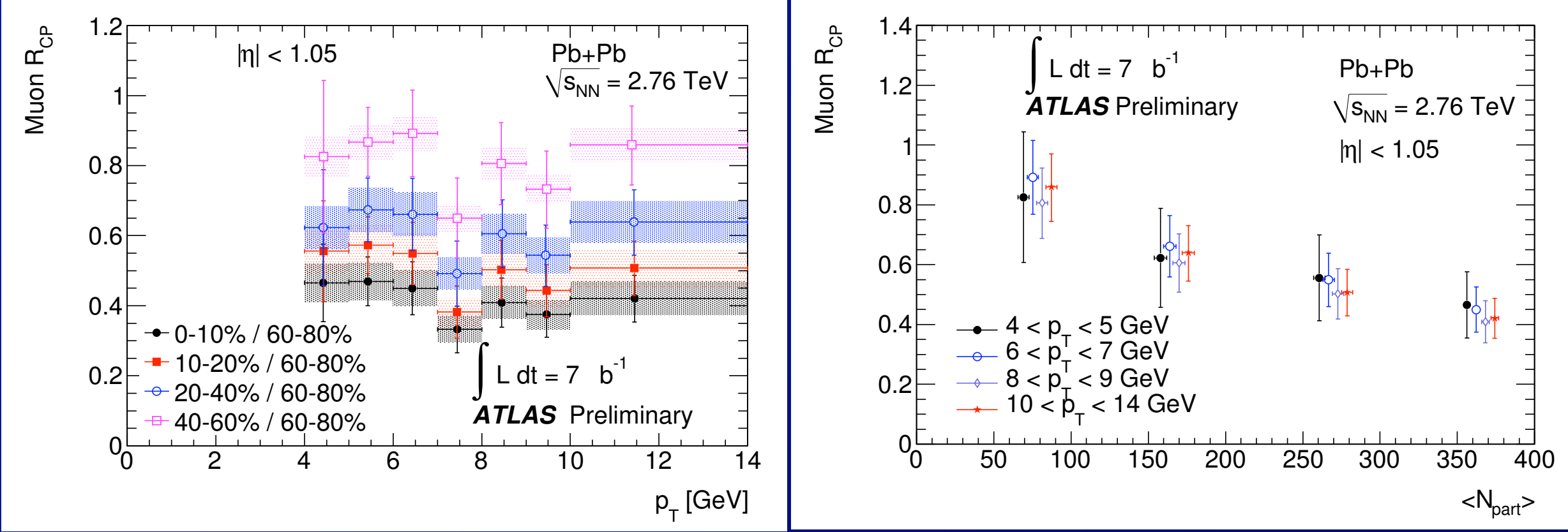

- In measured pт range, muons primarily from charm and bottom decays.

$-\mathrm{J} / \Psi$ contribution $\sim 1 \%$

- Evaluate $R_{c p}$ using 60-80\% peripheral reference

$\Rightarrow$ Factor of 2.5 suppression in $0-10 \%$ relative to $60-80 \%$

$\Rightarrow$ Independent of muon pi within errors

$\Rightarrow$ Evolution with $\mathbf{N}_{\text {part }}$ consistent between $p_{\text {T }}$ bins 


\section{Single muons, charged comparison}
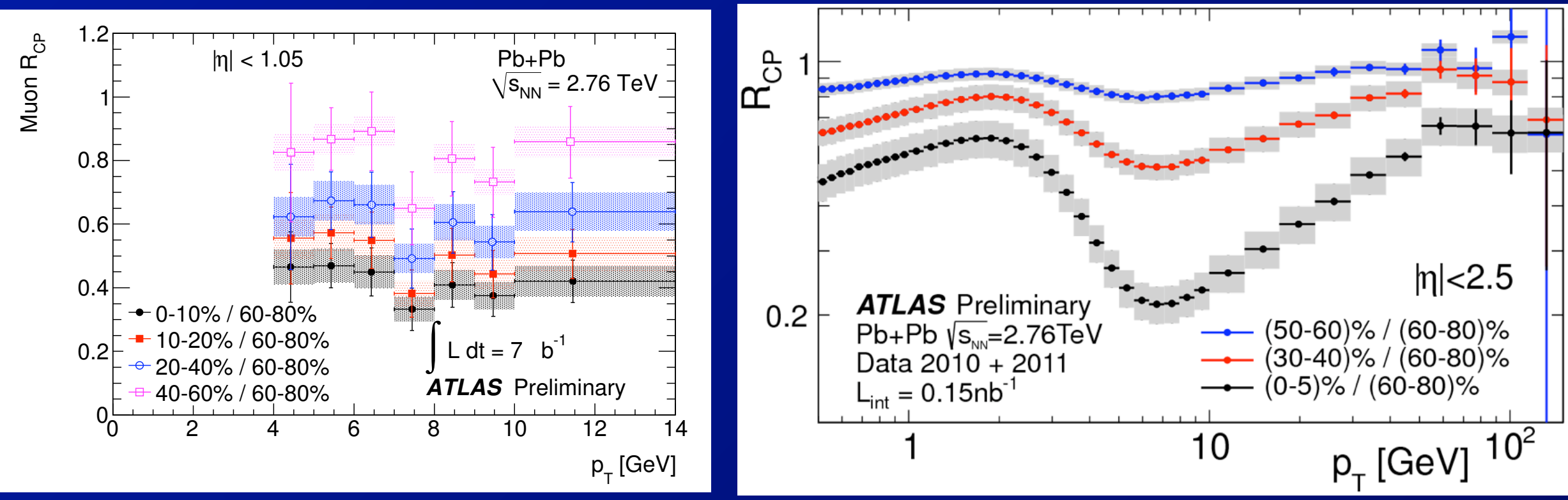

- See less suppression of heavy flavor decay muons than single hadrons @ lower pt

-But, both muon and hadron pt poorly correlated with jet momentum.

$\Rightarrow$ Single jets (below)

$\Rightarrow$ b-tagged jets, especially at lower $p_{T}$ (to come) 


\section{$\mathrm{Pb}+\mathrm{Pb}$ Jet Spectra}
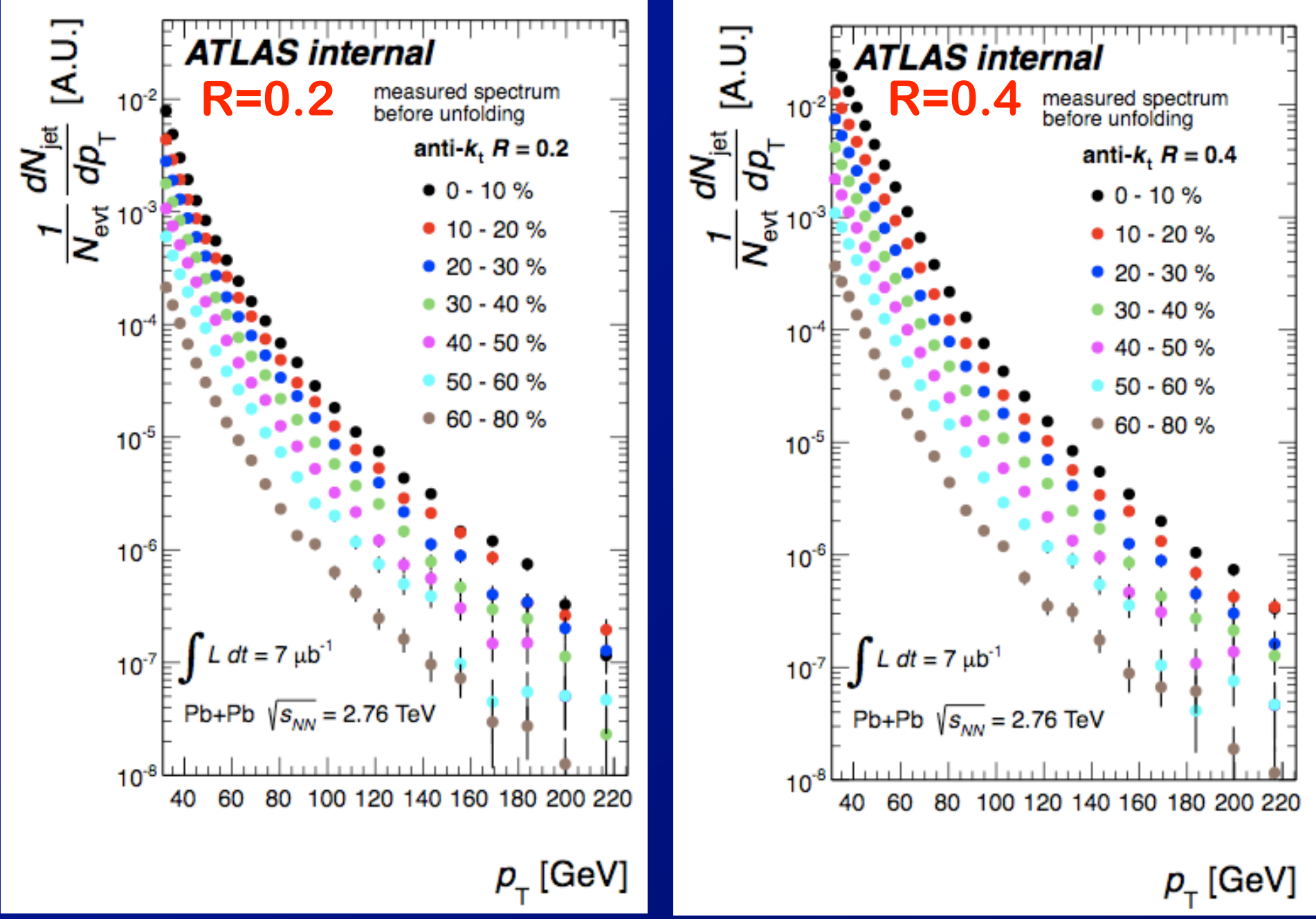

Unfolded (SVD) and efficiency corrected

$p_{\mathrm{T}}[\mathrm{GeV}]$ $p_{\mathrm{T}}[\mathrm{GeV}]$

- For these results, no absolute normalization - awaiting absolute jet energy scale uncertainty $\Rightarrow \mathrm{RSN}$ 


\section{$\mathbf{R}=0.4$ Jet $R_{\mathrm{cp}}$}

\section{Phys. Lett. B 719 (2013) 220-241}

- Systematic errors

- Black band: fully correlated systematics

- all points move up/down together

- JES, JER, efficiency, $x_{\text {ini }}, \mathbf{R}_{\text {coll }}$

- Red boxes: partially correlated systematics

- unfolding

- Error bars: square root of diagonal elements of covariance matrix

- No significance to horizontal width of error bars

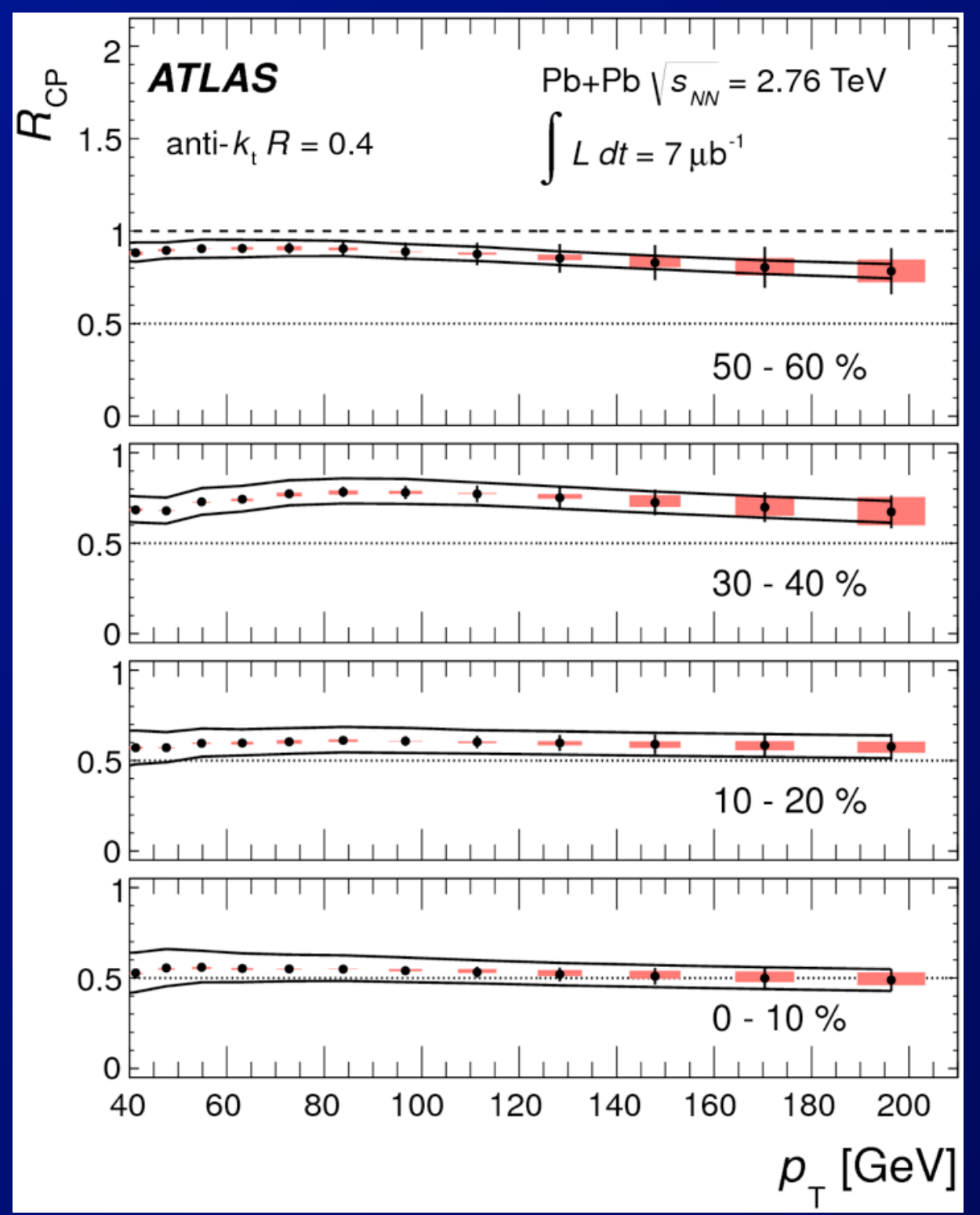




\section{$\mathbf{R}=0.4$ Jet $R_{\mathrm{cp}}$ Uncertainties}
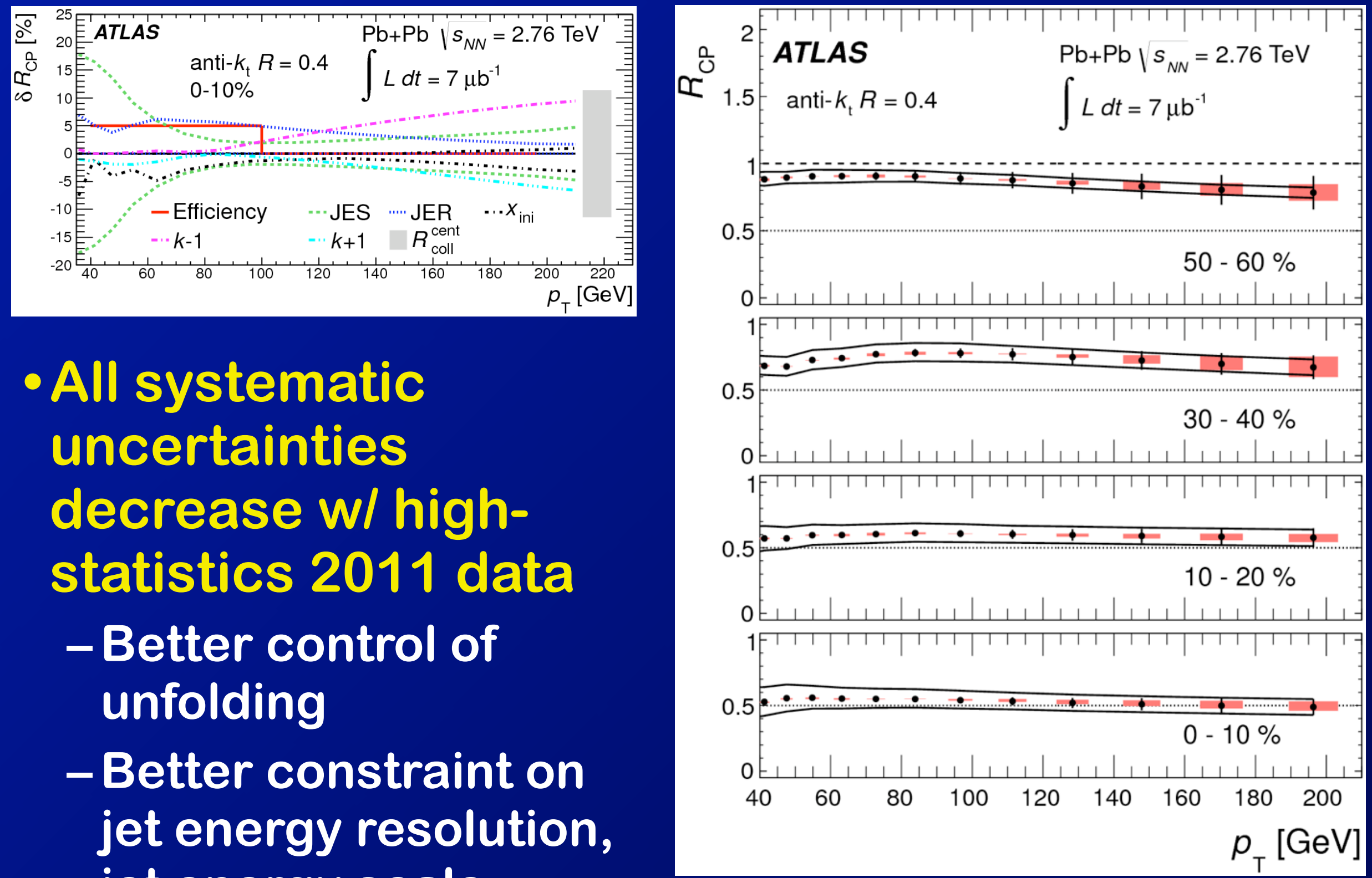

- All systematic uncertainties

decrease w/ highstatistics 2011 data

-Better control of unfolding

- Better constraint on jet energy resolution, jet energy scale $\Rightarrow \mathrm{MC}$, cross-checks 


\section{Jet yields: centrality dependence}

Phys. Lett. B 719 (2013) 220-241

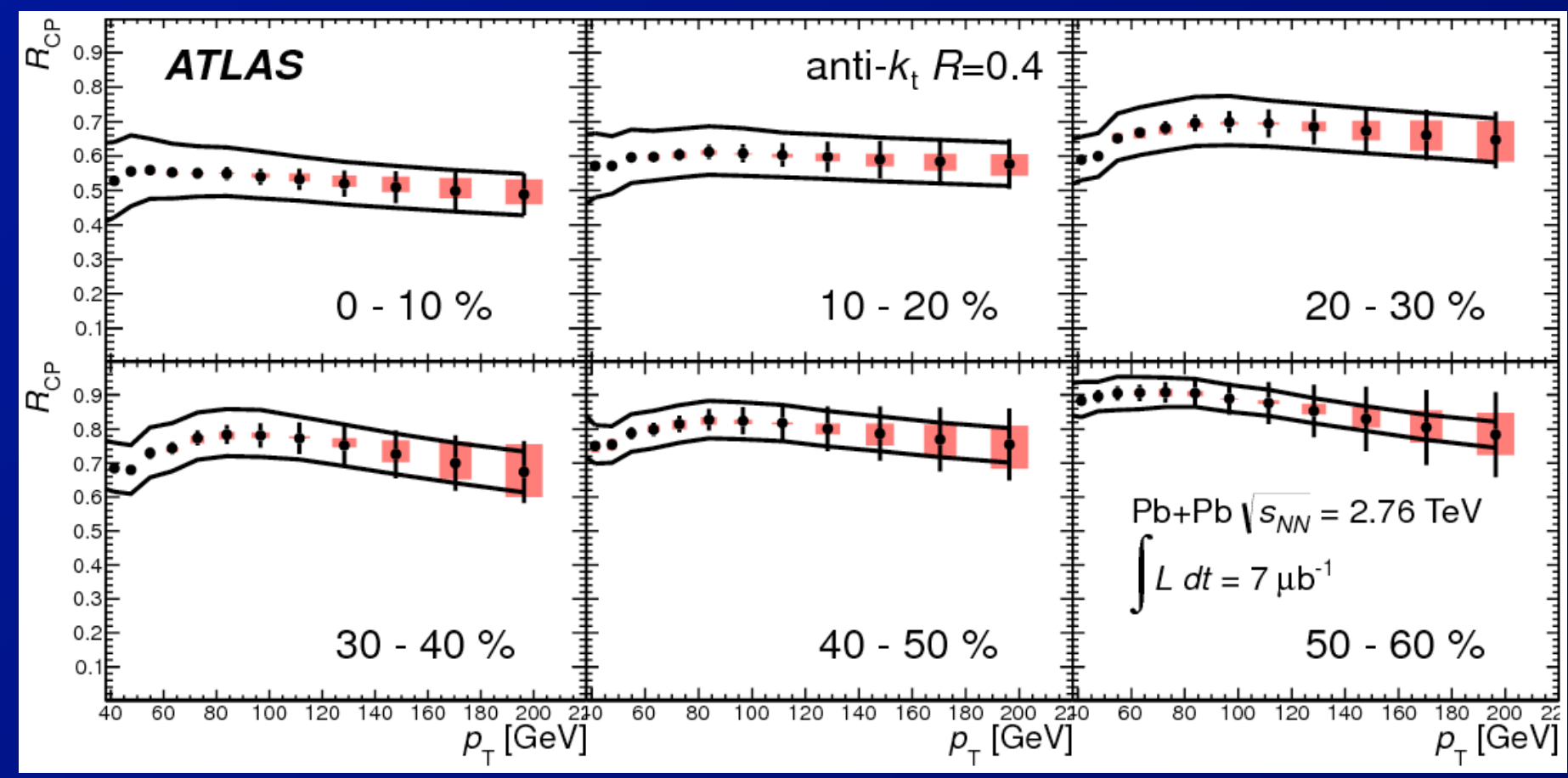

- Consider systematics:

- Based on previous slides, $Y, Z$ measurements constrain initial conditions to $<\sim 20 \%$

$\Rightarrow$ Will be reduced by $\mathrm{p}$ - $\mathrm{Pb}$ measurements

- $15 \%$ systematic uncertainties in $R_{\mathrm{cp}}$

$\Rightarrow$ Reduced in 2011 data, in $R_{A A}$ 


\section{Jet radius dependence of $\boldsymbol{R}_{\mathbf{c p}}$}

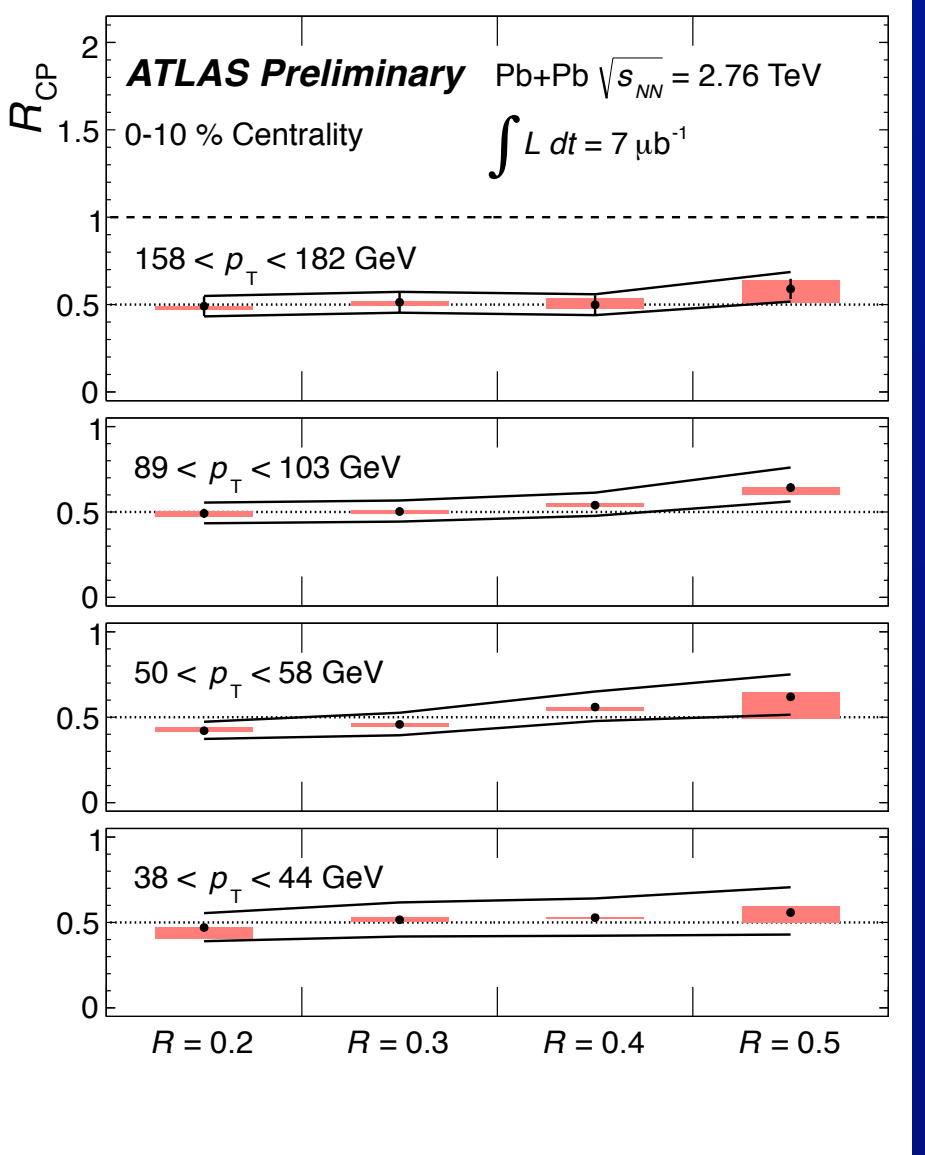

\section{Significant cancellation of correlated errors}

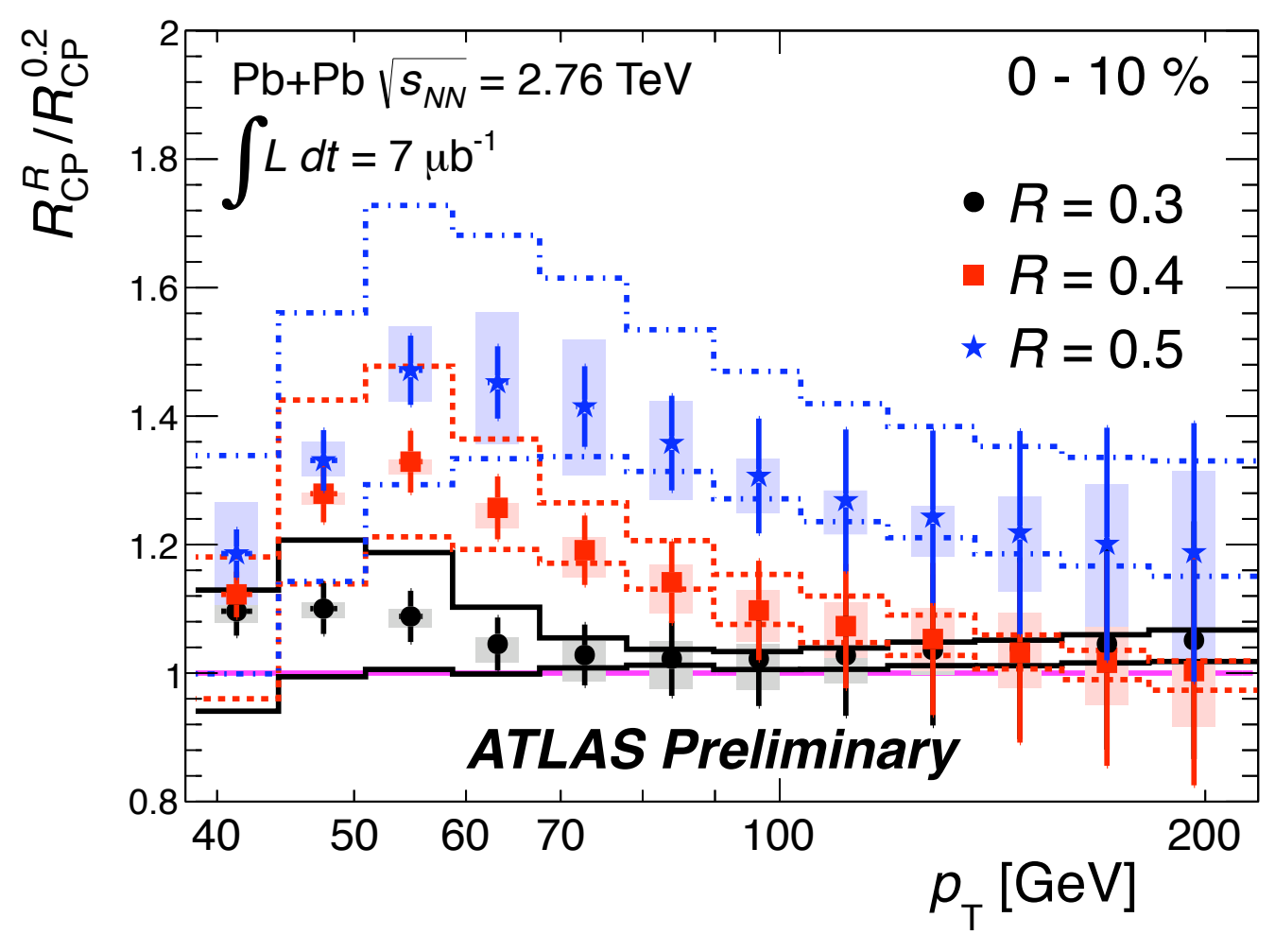

- Evaluate jet radius dependence of $\boldsymbol{R}_{\mathrm{cp}}$

- Modest but significant variation of $R_{c p}$

- Less suppression for larger $\mathbf{R}$

$\Rightarrow$ An indication of jet broadening? 


\section{Probes insensitive to nuclear PDFs, hard-scattering rates}




\section{Inclusive jet fragmentation}
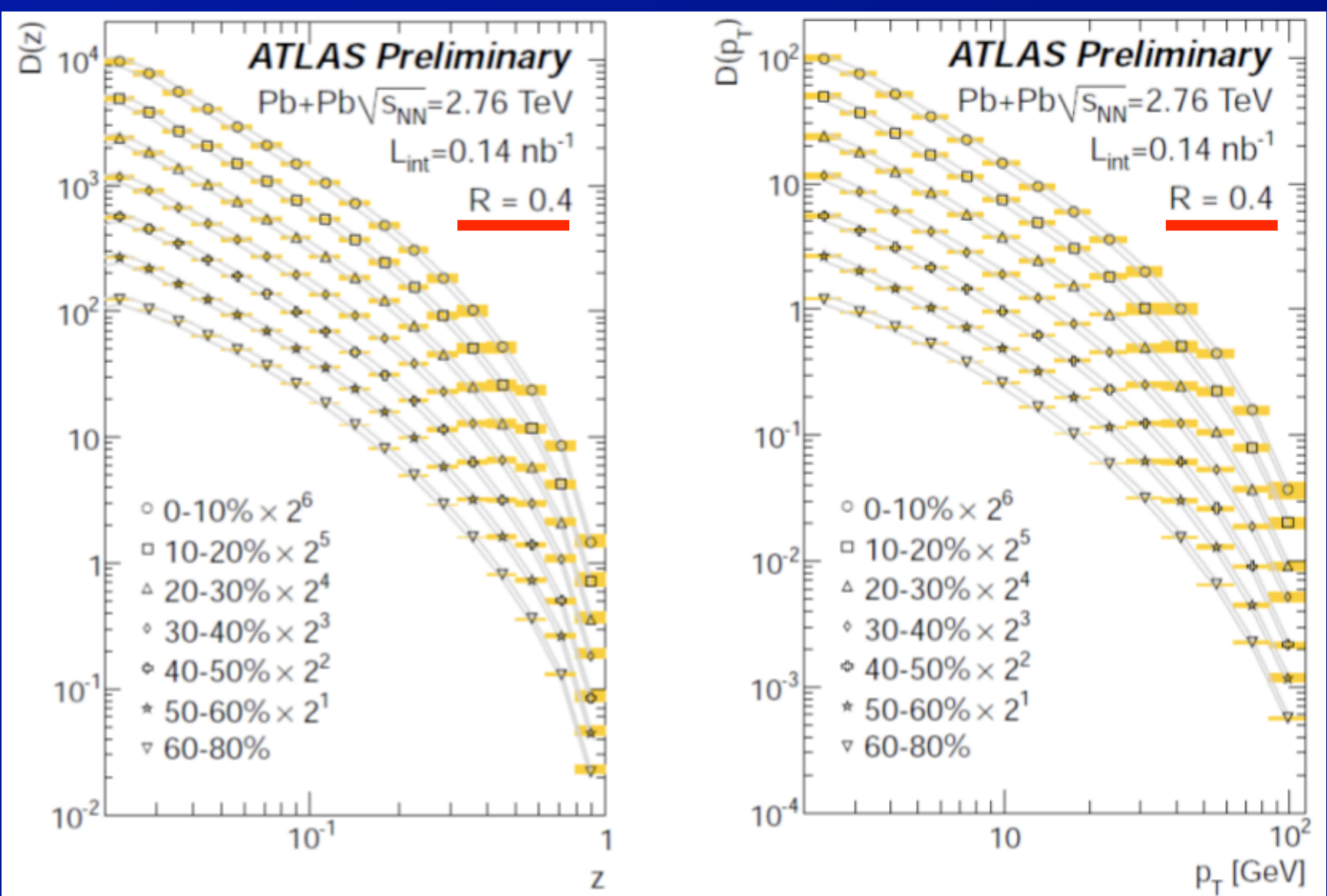

Unfolded for jet and charged particle resolution

$$
\begin{aligned}
& D(z)=\frac{1}{N_{j e t}} \frac{d N_{c h g}}{d z}, z=\vec{p}_{c h g} \cdot \vec{p}_{j e t} /\left|\vec{p}_{j e t}\right| \\
& D\left(p_{T}\right)=\frac{1}{N_{j e t}} \frac{d N_{c h g}}{d p_{T}}
\end{aligned}
$$




\section{Inclusive jet fragmentation (2)}
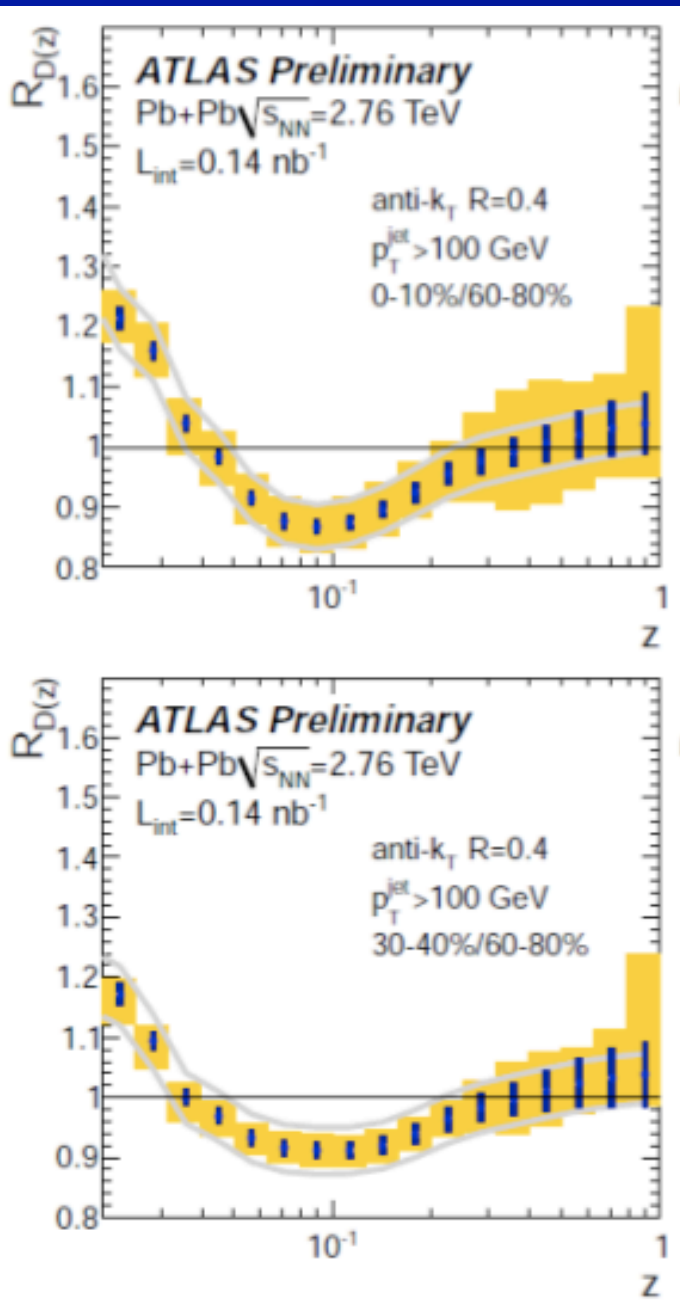
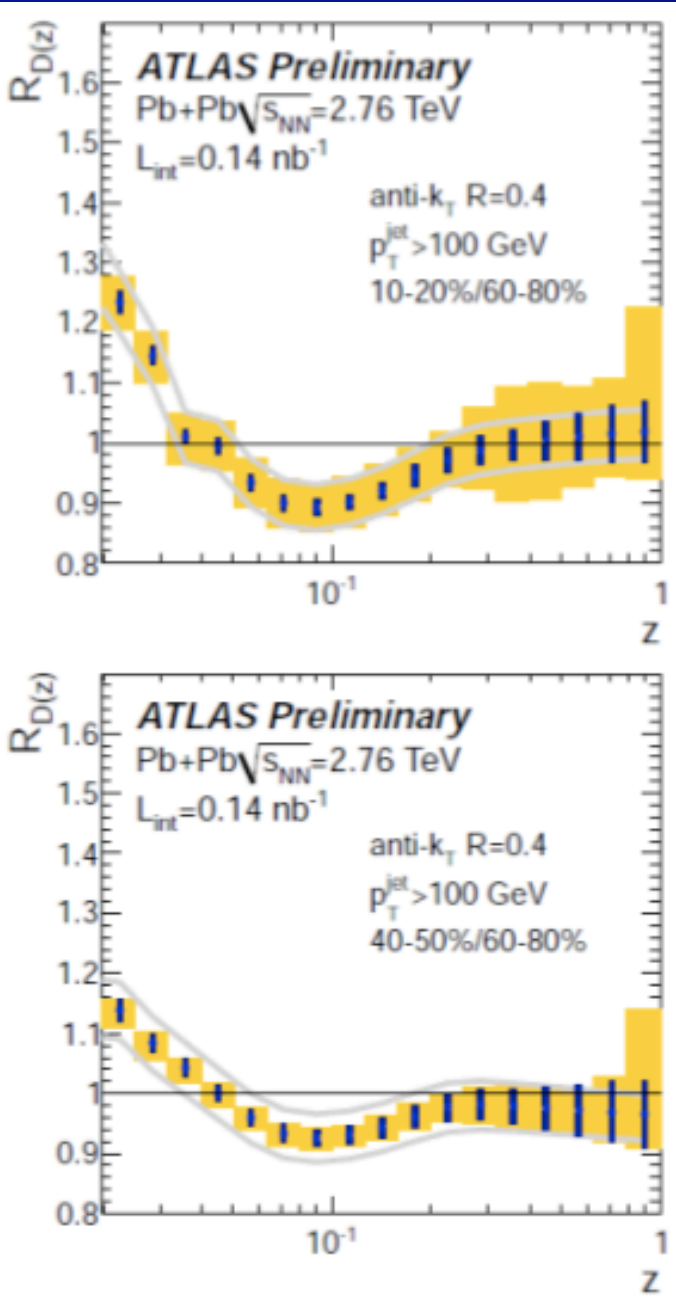

$R=0.4$
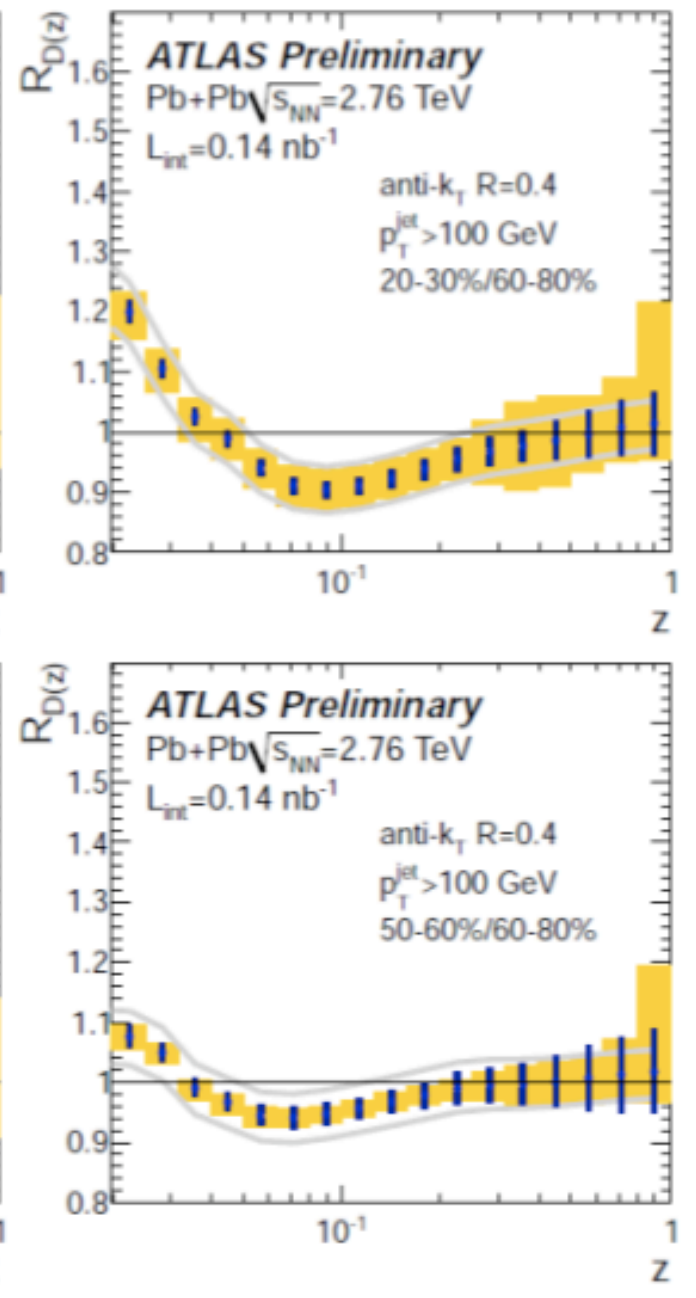

- First observation of modified parton shower in inclusive jets

$\Rightarrow$ Not only seeing "left over" unquenched jets. 


\section{Inclusive jet fragmentation (3)}
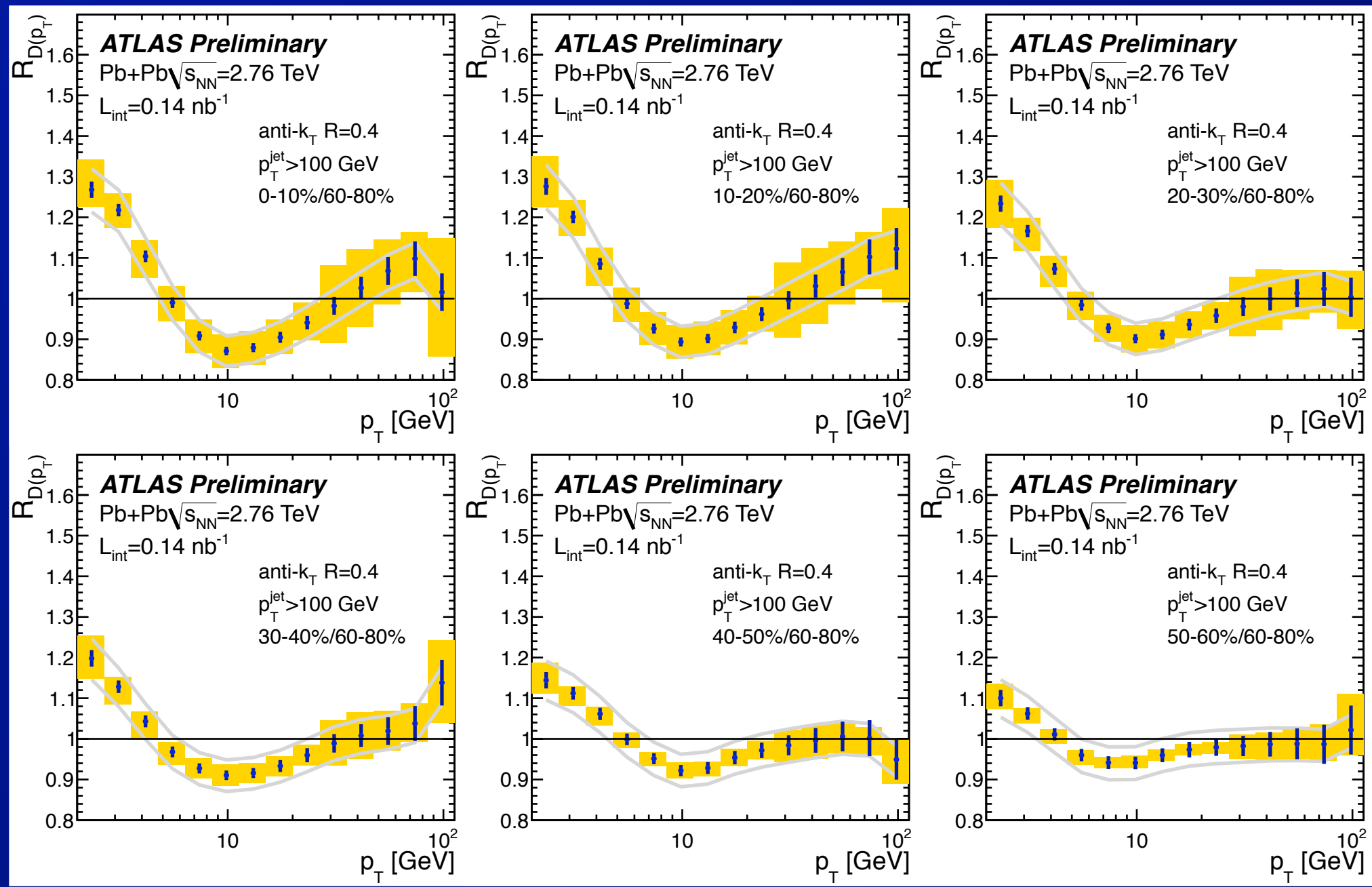

- Check that the modification is not due to the measurement of jet $p_{T} \Rightarrow D\left(p_{T}\right)$

$\Rightarrow \mathrm{D}\left(\mathrm{p}_{\mathrm{T}}\right)$ shows similar modifications 


\section{Jet fragmentation: $\mathbf{R}$ dependence}

- Check that the modification is not due to underlying event fluctuations

-Use different jet sizes:

$R=0.2,0.3$

- Obtain the same results as $R=0.4$
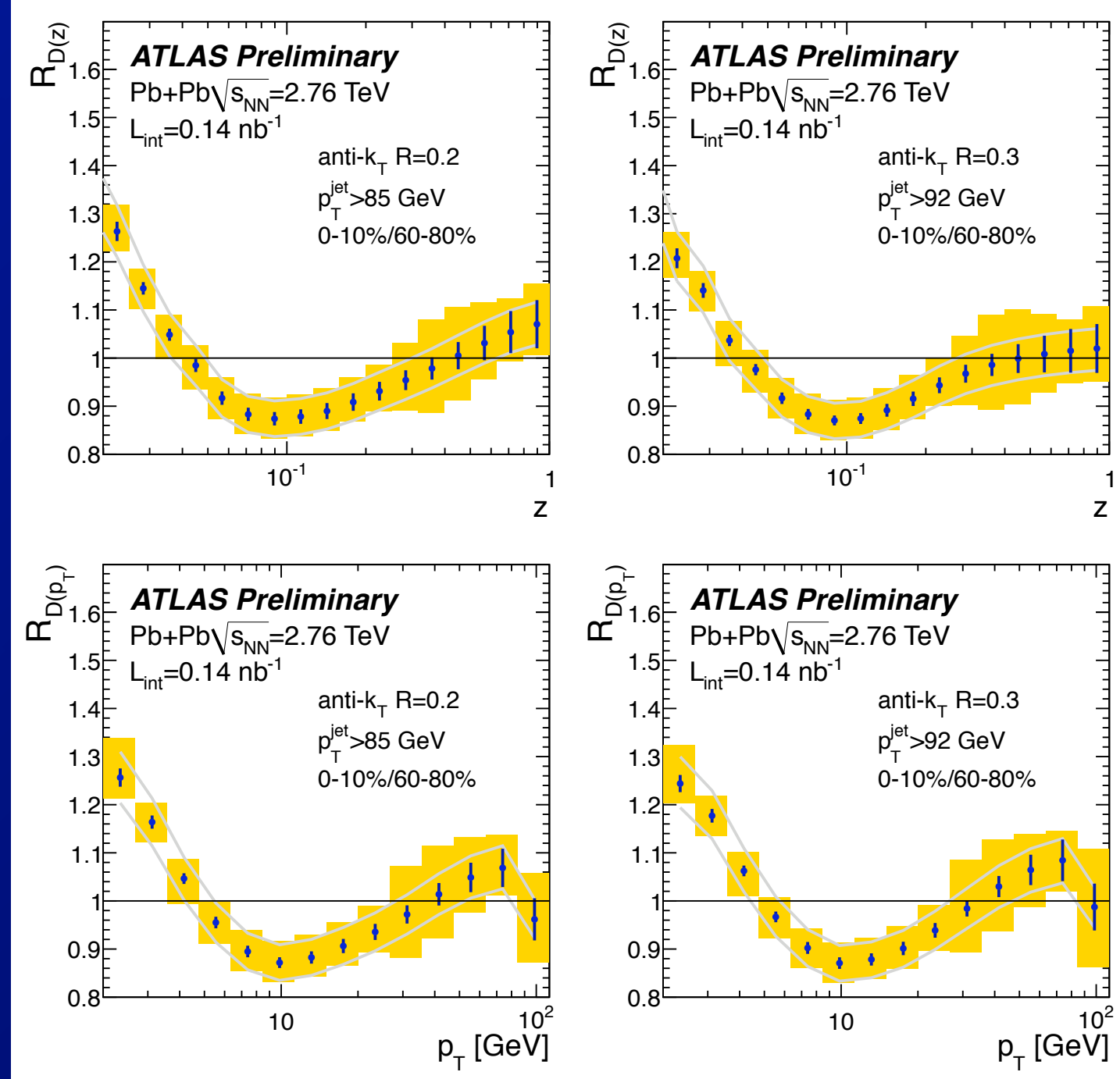

$\Rightarrow$ Observed modifications are robust 


\section{Di-jet asymmetry \& acoplanarity}
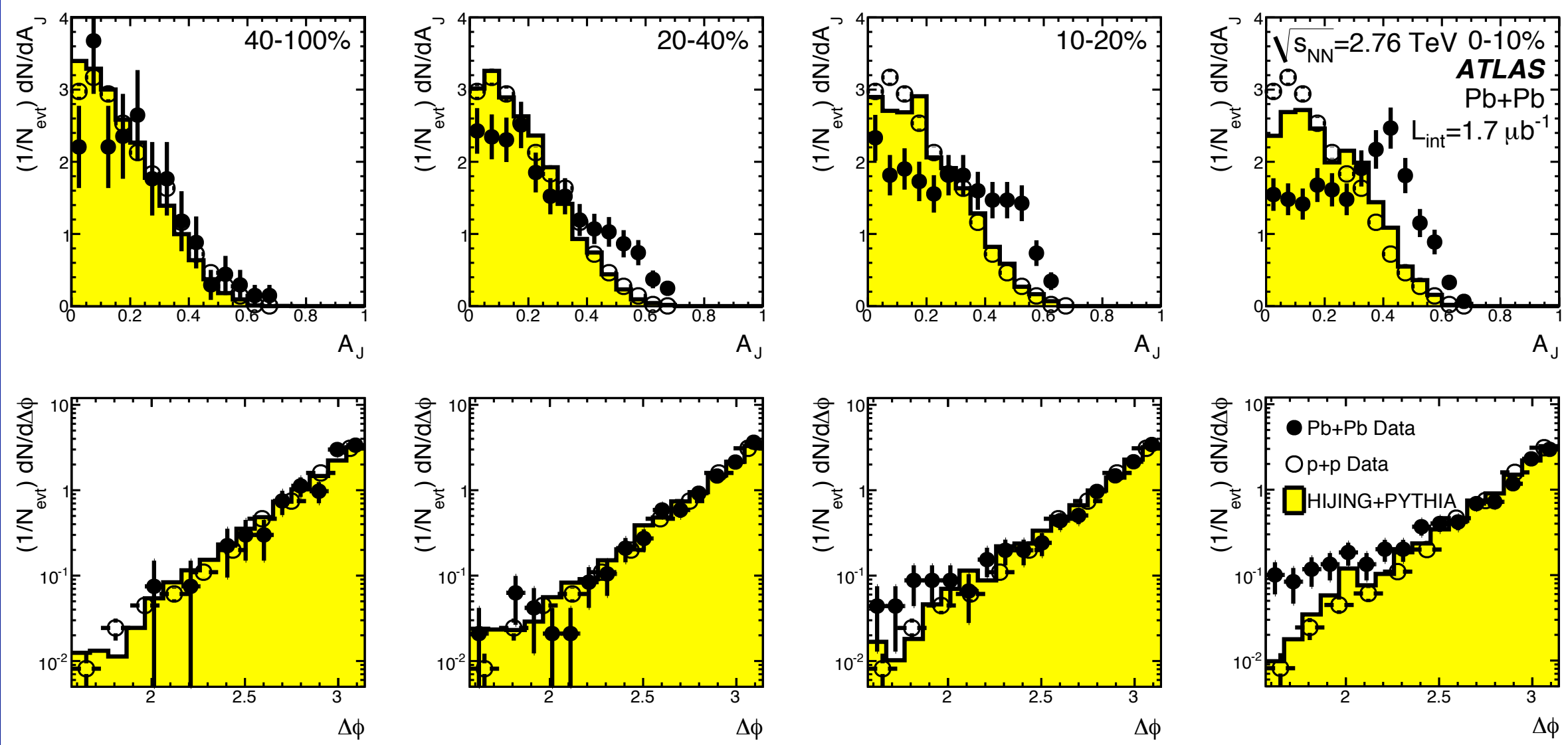

- For more central collisions, see:

- Change in distribution of dijet asymmetry

-While no change in the distribution of $\Delta \varphi$

$\Rightarrow$ Except for combinatoric pairs in central 


\section{Dijet acoplanarity, $\mathbf{R}=0.2$}

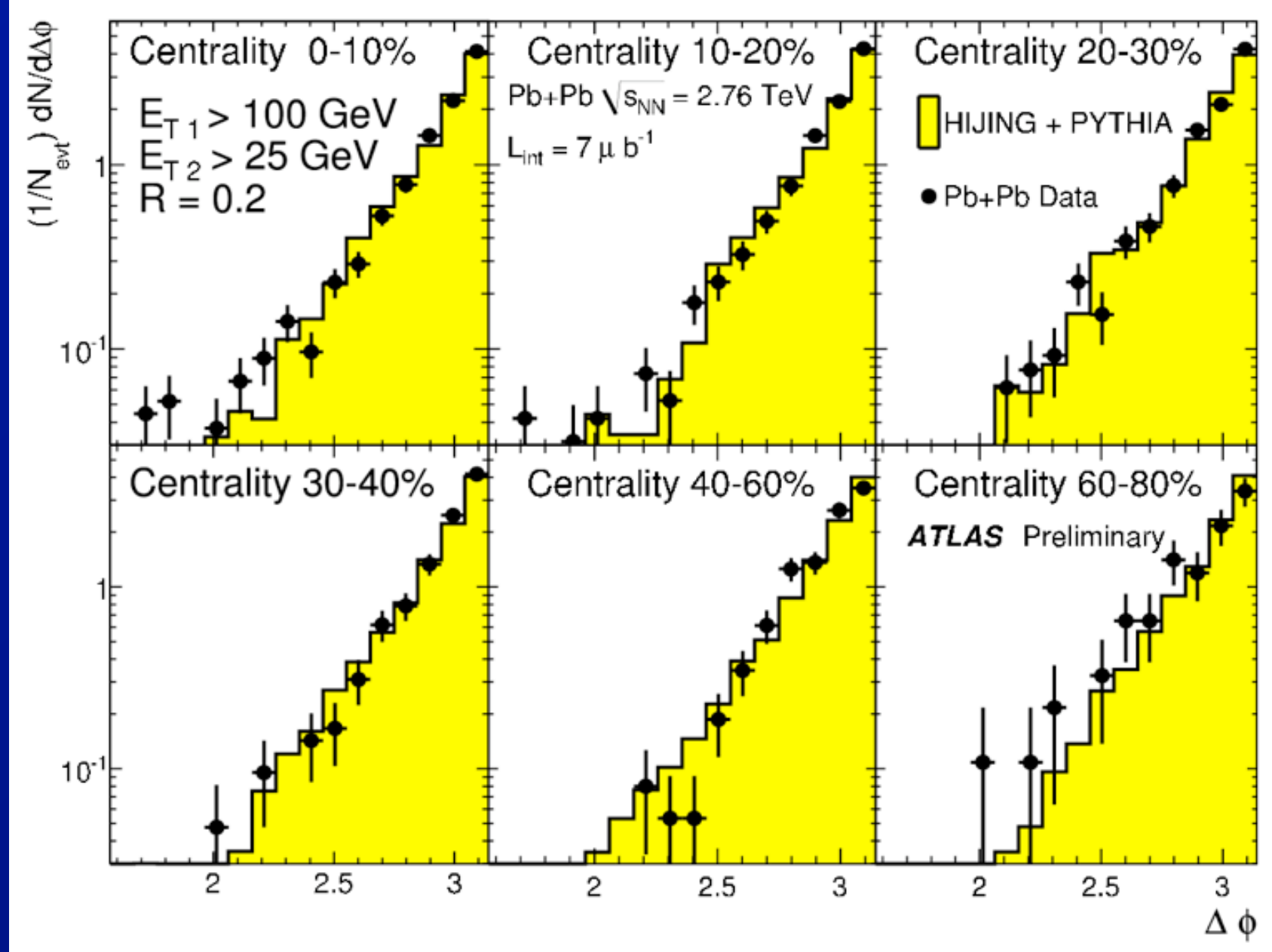

- Look at dijet acoplanarity $W / R=0.2$ jets

$\Rightarrow$ small UE effects

$\Rightarrow<\mathrm{NO}\rangle$ broadening 


\section{$\gamma$-jet angular distribution}

\section{Peripheral}
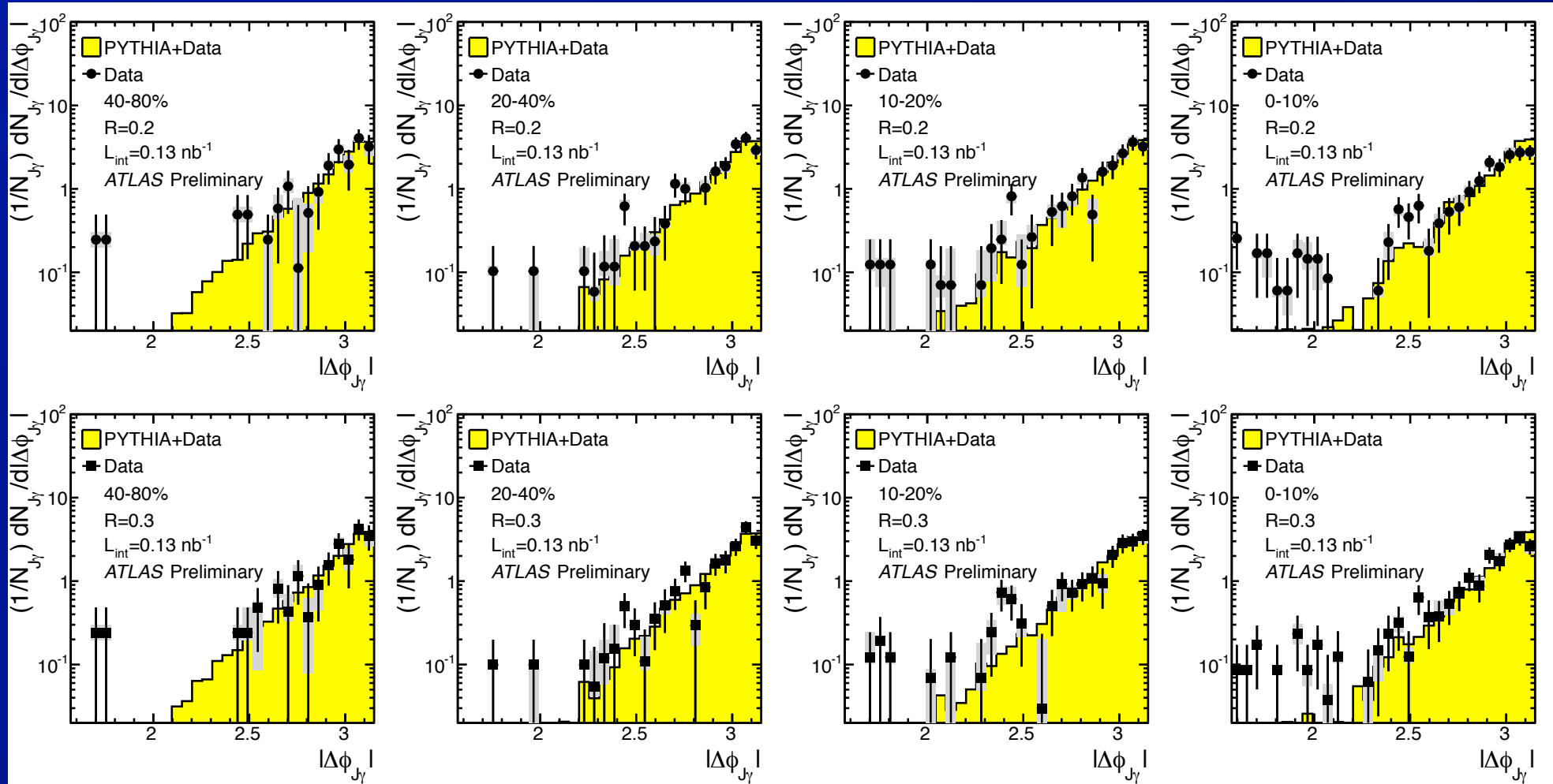

$R=0.2$

$R=0.3$

- Take leading jet in hemisphere opposite photons with $60<p_{T}<90 \mathrm{GeV}$

- Jets with $p_{T}>25 \mathrm{GeV}, \boldsymbol{R}=0.2$ and 0.3

$\Rightarrow$ Distribution of $\Delta \phi$ peaked at $\pi$

$\Rightarrow$ For following, apply cut $|\Delta \phi-\pi|<7 \pi / 8$ 


\section{y-jet momentum balance}

\section{Peripheral}
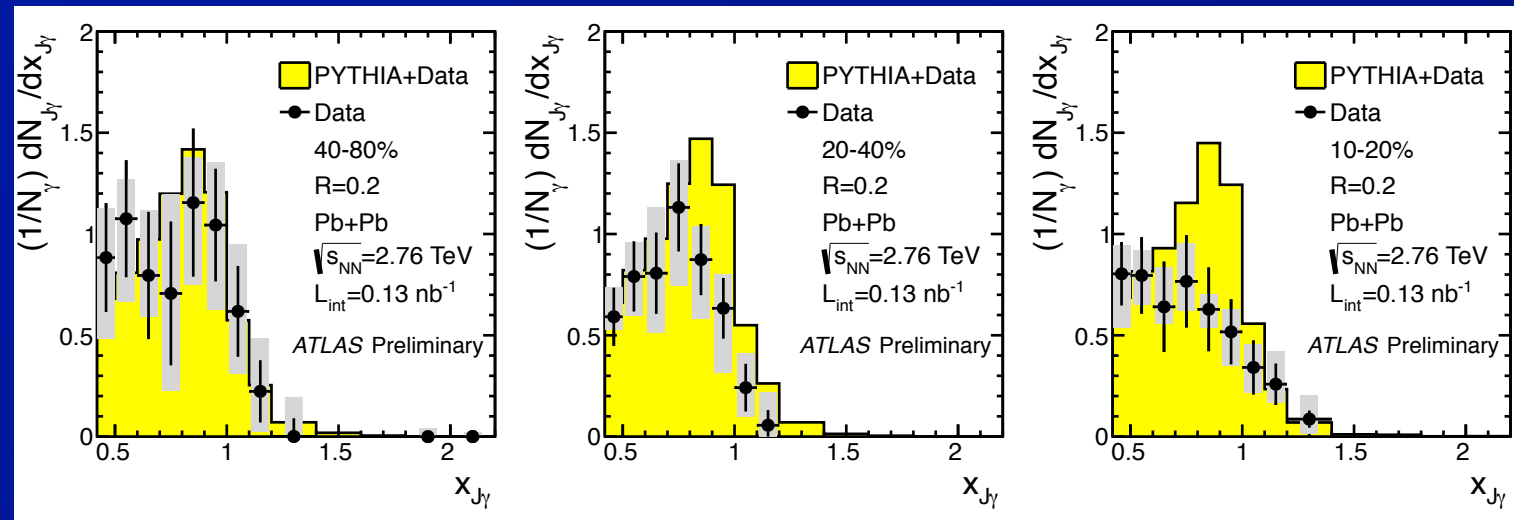

$\rightarrow$ central
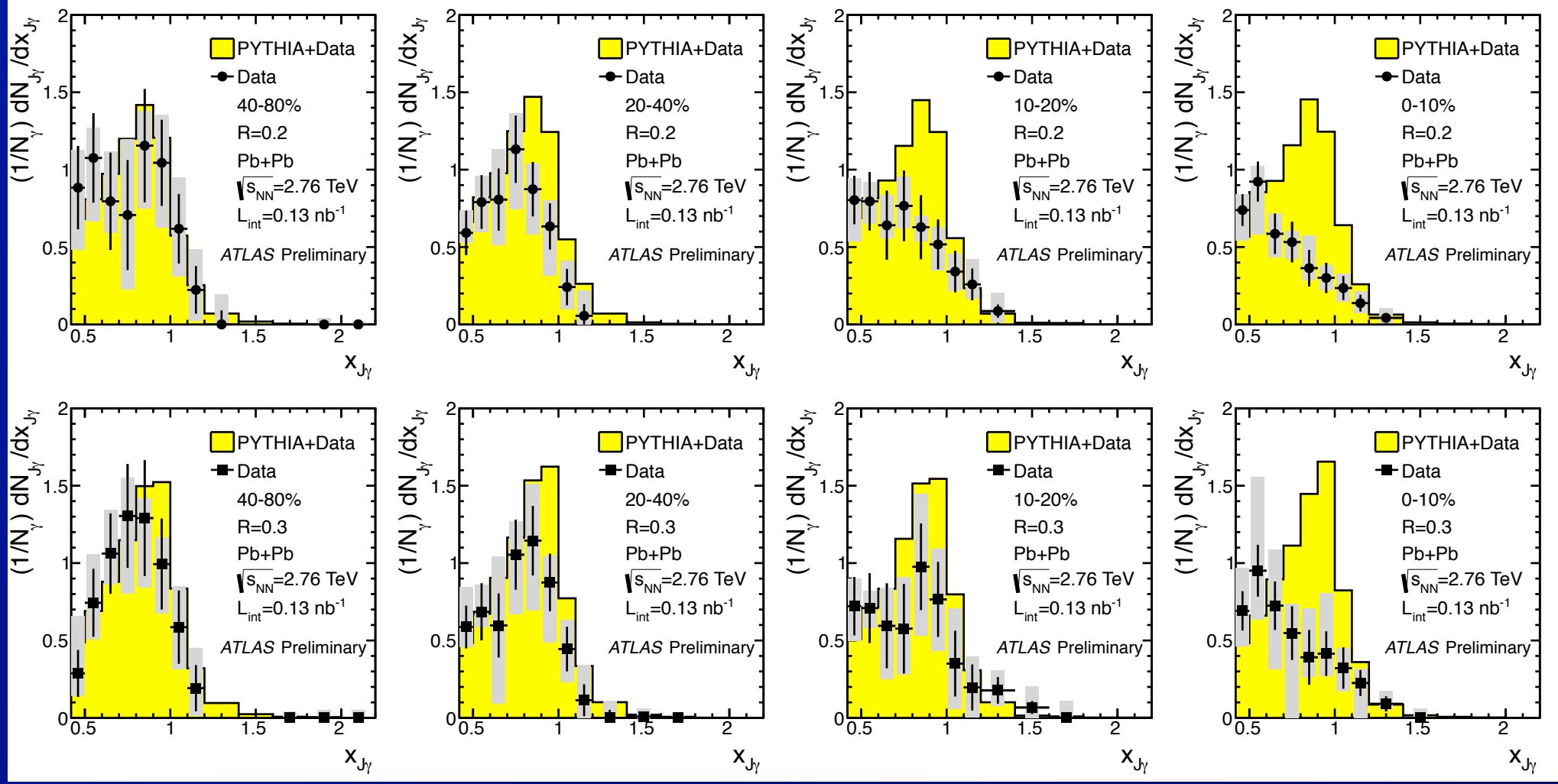

$R=0.2$

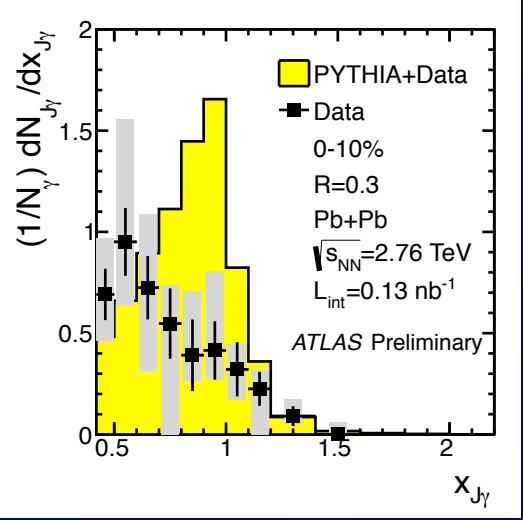

$R=0.3$

- Plot distribution of $x_{J}=p_{T}^{\mathrm{jet}} / p_{T}^{\gamma}$

- photon background pairs subtracted

- unfolded for jet energy resolution

$\Rightarrow$ Substantial change in $\mathrm{Y}$-jet balance 


\section{$\mathrm{Pb}+\mathrm{Pb} \mathrm{Z}$-jet measurement}
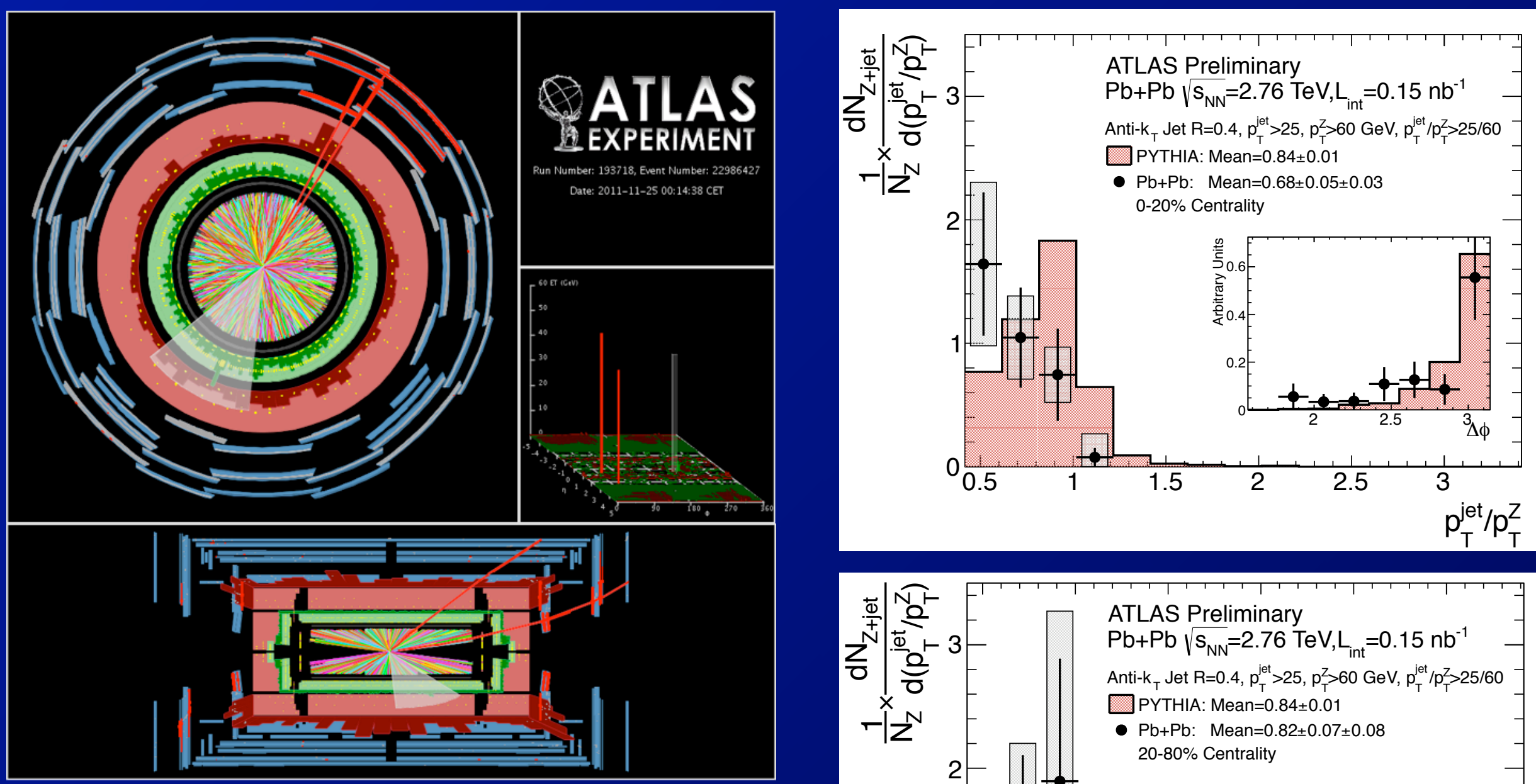

- Z-jet measurements have less background than $\gamma-$ jet, but smaller rate

$\Rightarrow 1^{\text {st }}$ results

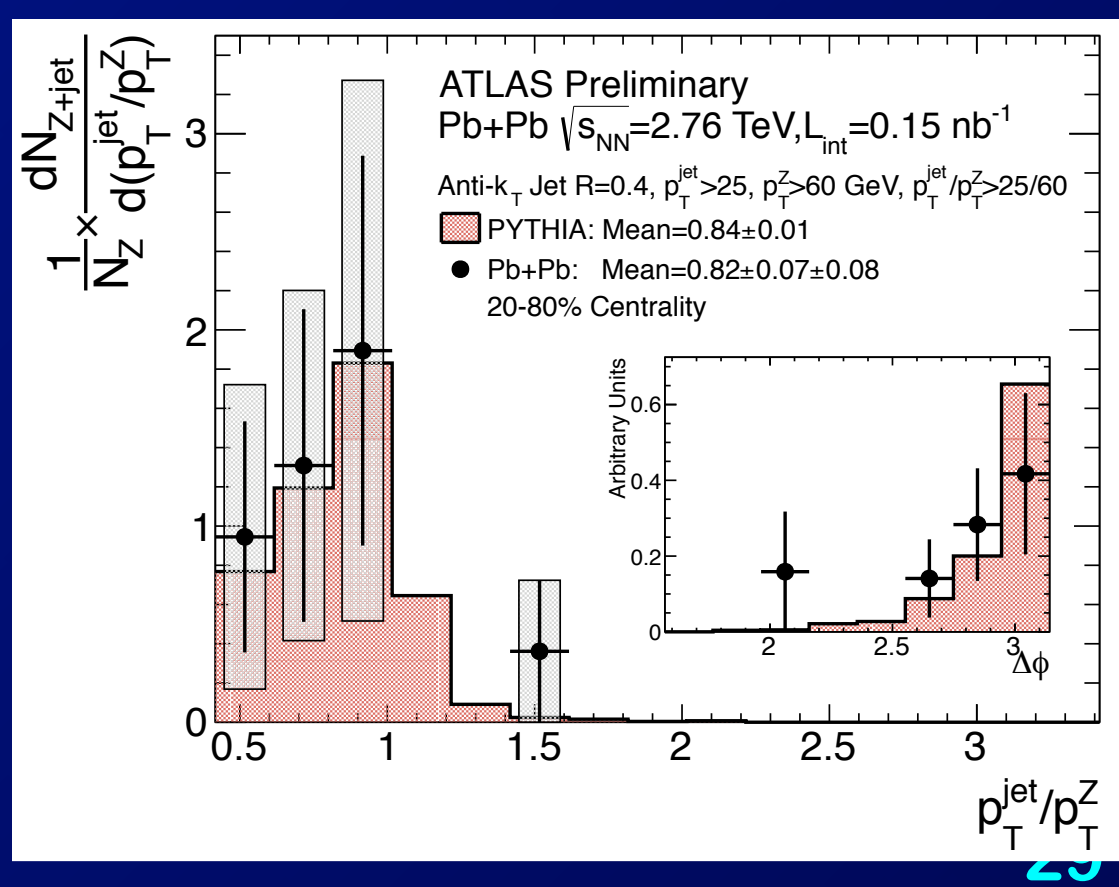




\section{Dijet (and gamma-jet) acoplanarity}
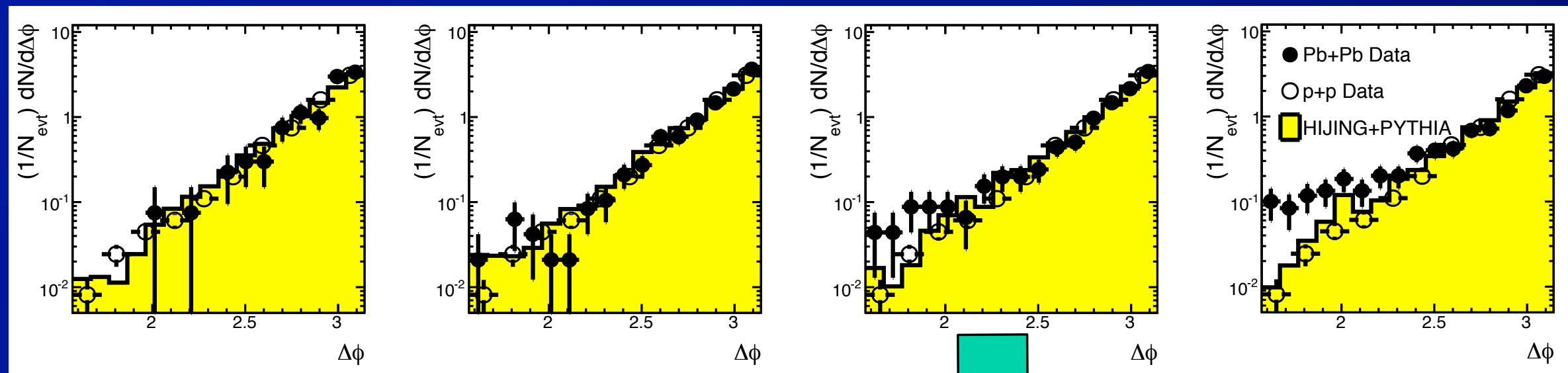

\section{Virtuality matters}

Virtuality $\mathrm{Q}^{2}$ of the parton in the medium controls physics of radiative energy loss:

Weak coupling scenario

RHIC: $20 \mathrm{GeV}$ parton, $L=3 \mathrm{fm}$

$\hat{q} L \approx 4.5 \mathrm{GeV}^{2} \gg \frac{E}{L} \approx 1.5 \mathrm{GeV}^{2}$

Virtuality of primary parton is medium dominated and small enough to "experience" the strongly coupled medium

$$
Q^{2}(L) \approx \max \left(\hat{q} L, \frac{E}{L}\right)
$$

LHC: $200 \mathrm{GeV}$ parton, $L=3 \mathrm{fm}$

$\hat{q} L \approx 9 \mathrm{GeV}^{2}<\frac{E}{L} \approx 13 \mathrm{GeV}^{2}$

Virtuality of primary parton is vacuum dominated and only its gluon cloud "experiences" the strongly coupled medium
Is the lack of $k_{T}$ broadening in the presence of significant quenching a death knell for "leading parton" models of energy loss? 
Tomography 


\section{ATLAS: Charged particle $v_{2}\left(p_{T}\right)$}

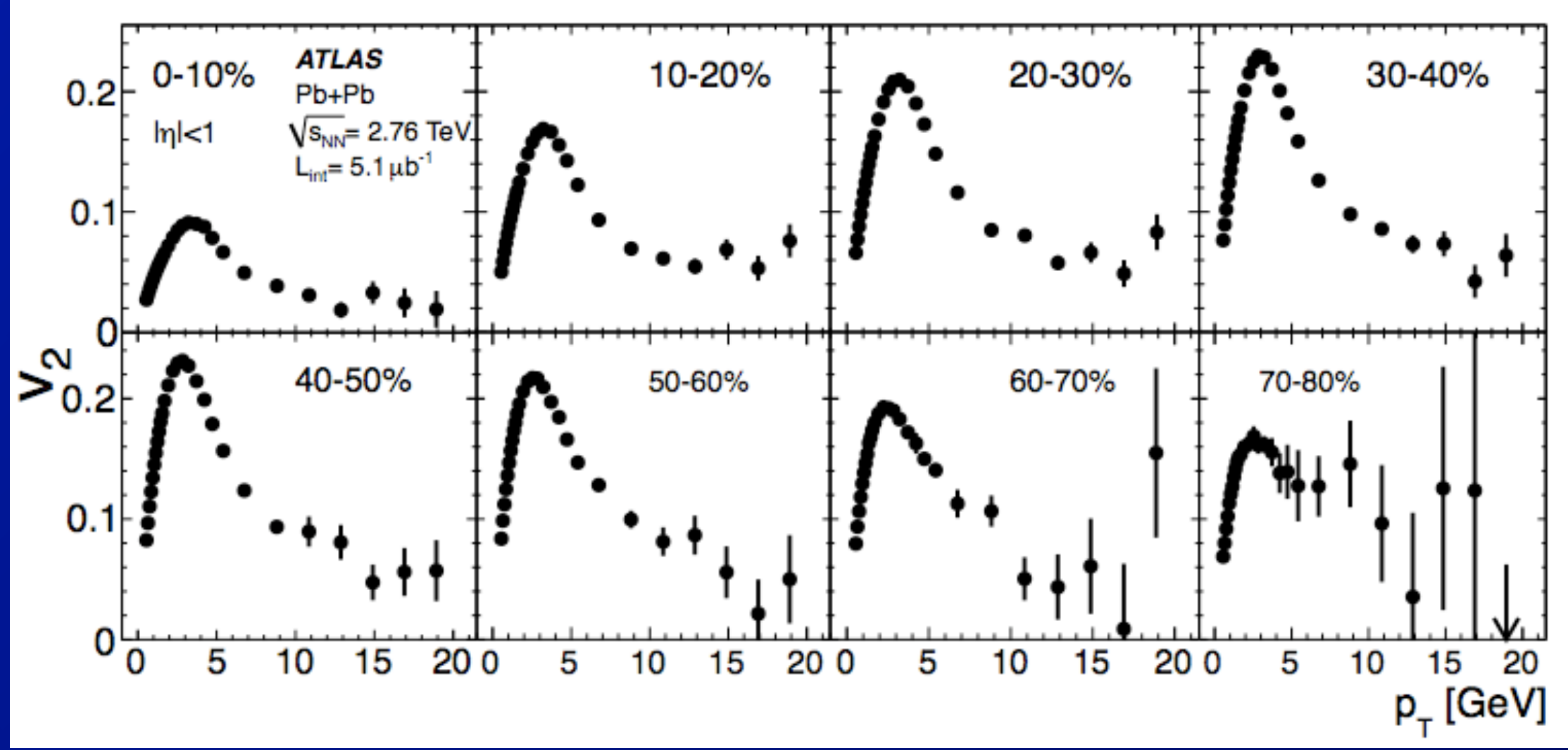

- Single hadron $v_{2}\left(p_{T}\right)$ :

-Evolution from flow ( $p_{T}<6-7 \mathrm{GeV}$ )

-to quenching ( $p_{\mathrm{T}}>\sim 10 \mathrm{GeV}$ )

$\Rightarrow$ Consistent with conclusions from similar analyses in PHENIX 


\section{ATLAS: Charged particle $v_{2}\left(p_{T}\right)$}
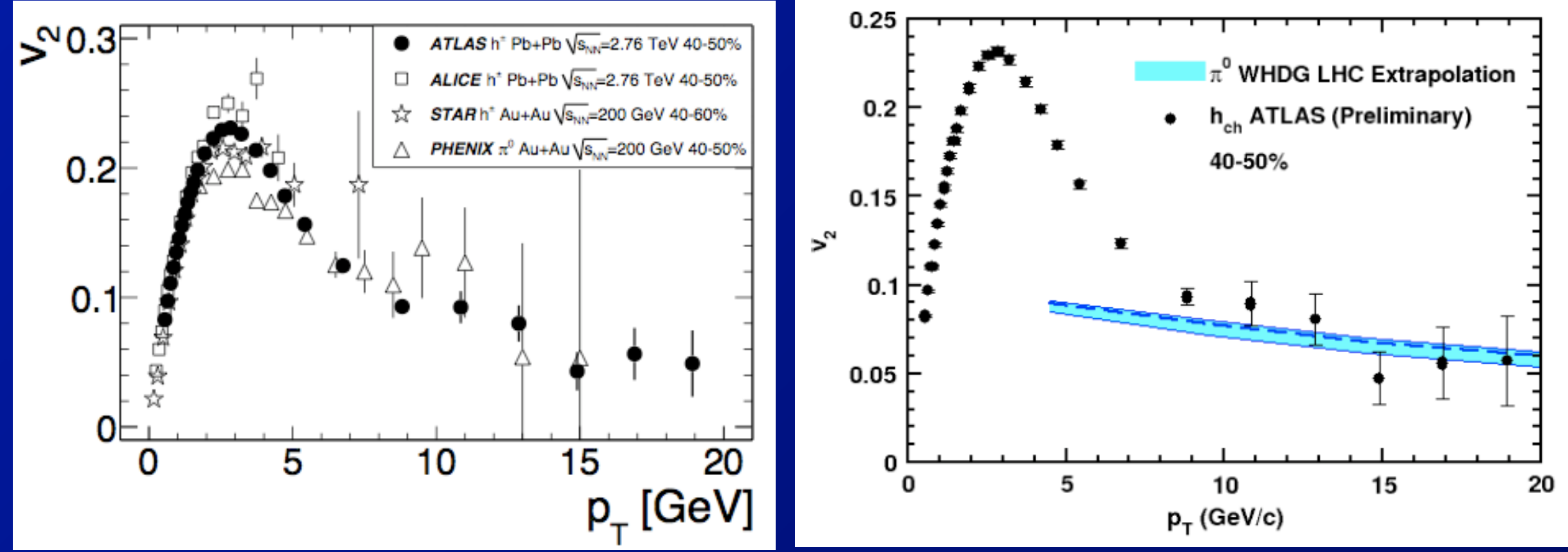

- Surprising agreement between RHIC and LHC $v_{2}\left(p_{T}\right)$, but beware "apples and oranges"

- Charged (ATLAS, STAR), $\pi^{0}$ (PHENIX)

-WHDG energy loss describes $\mathrm{V}_{2}\left(\mathrm{p}_{\mathrm{T}}\right)$ for $\mathrm{p}_{\mathrm{T}}>10$ $\Rightarrow$ Flow dominates for $\mathrm{p}_{\mathrm{T}}<\sim 8 \mathrm{GeV}$ 


\section{Differential jet suppression}

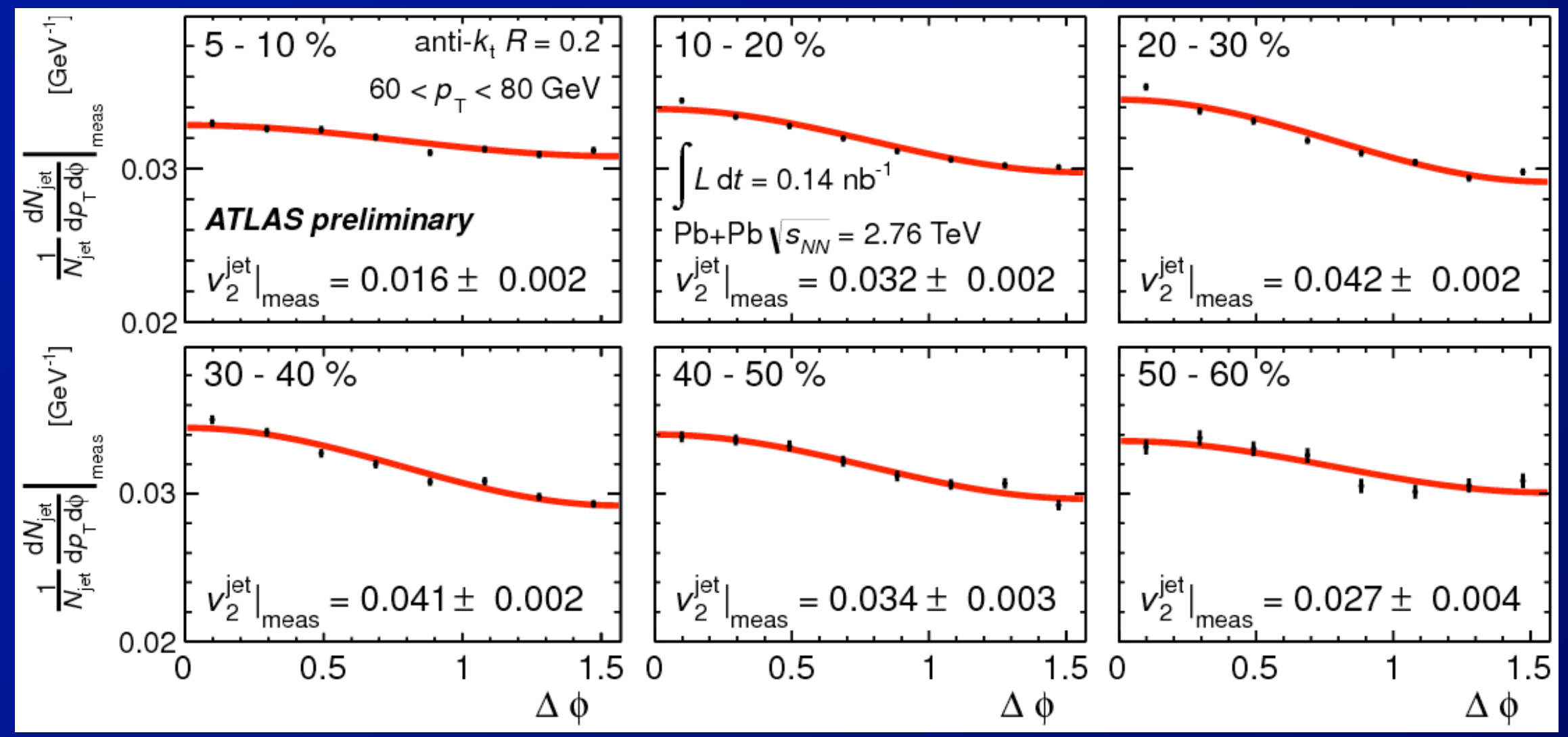

- Measure jet yields in 8 bins of $\Delta \phi$ with respect to the elliptic event plane

- Here for $R=0.2$ jets, $60<p_{T}<80 \mathrm{GeV}$

$\Rightarrow$ UE subtraction corrected for elliptic flow modulation in calorimeter 


\section{Differential jet suppression}

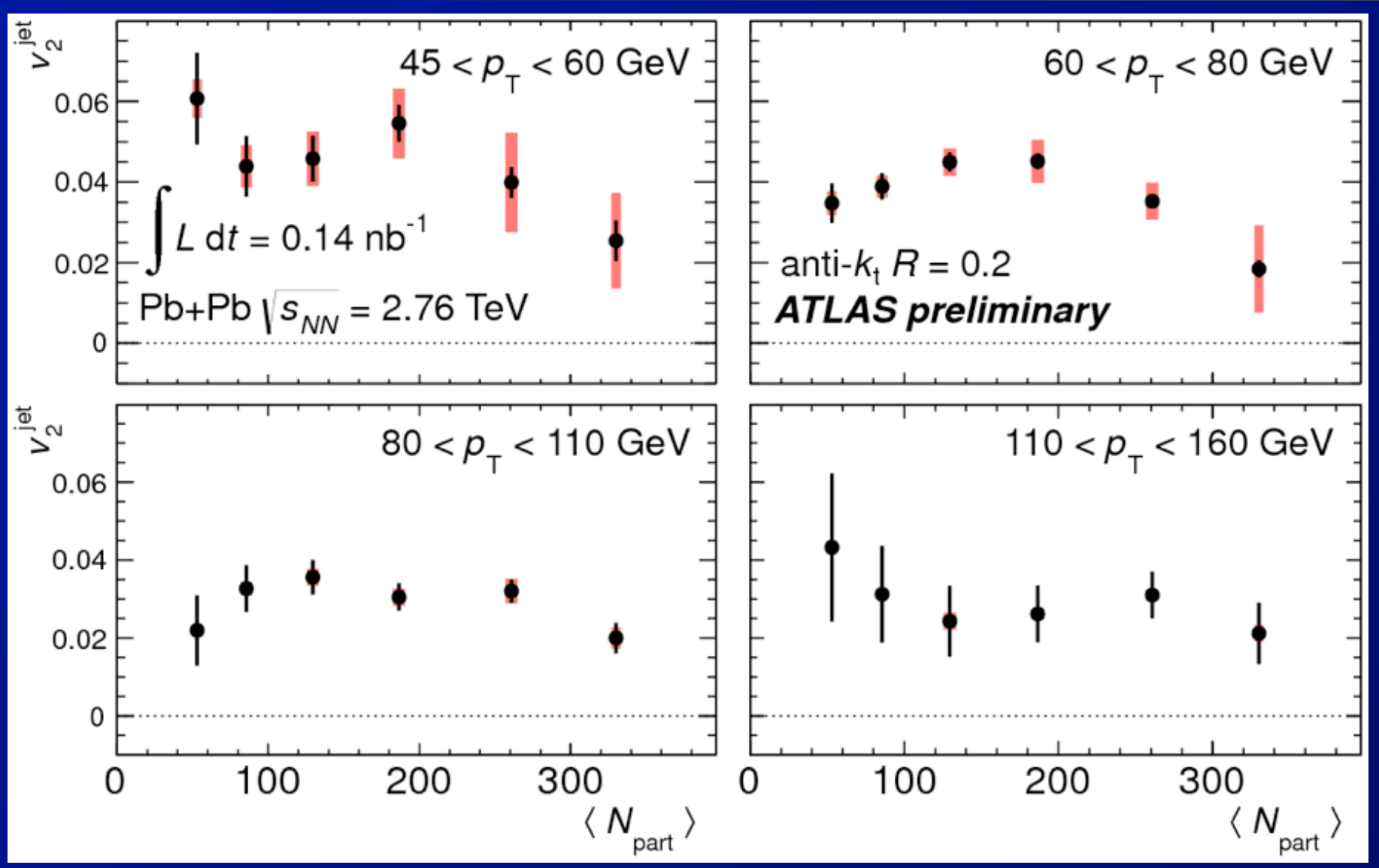

- Observe non-zero jet $V_{2}$ for $(R=0.2) p_{T}$ values $>100 \mathrm{GeV}$

$\Rightarrow$ jet quenching clearly sensitive to initial geometry out to very high pT 


\section{Jet $v_{2}\left(p_{T}\right)$}
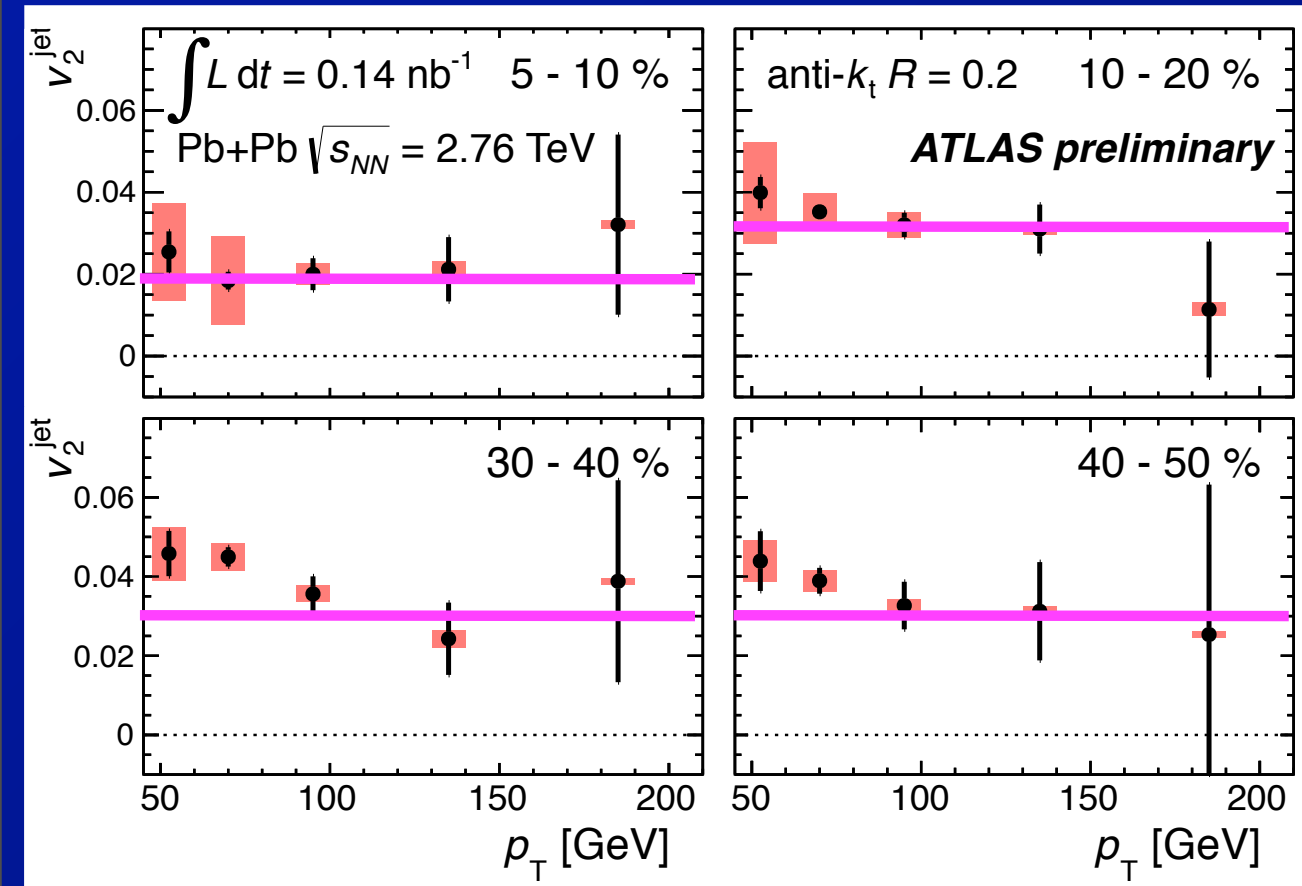

- Do rough comparison of jet, charged v2 at high pT

- plot 0.02 for $0 / 5-10 \%$ - plot 0.03 for $>10 \%$

$\Rightarrow$ As good as could be expected
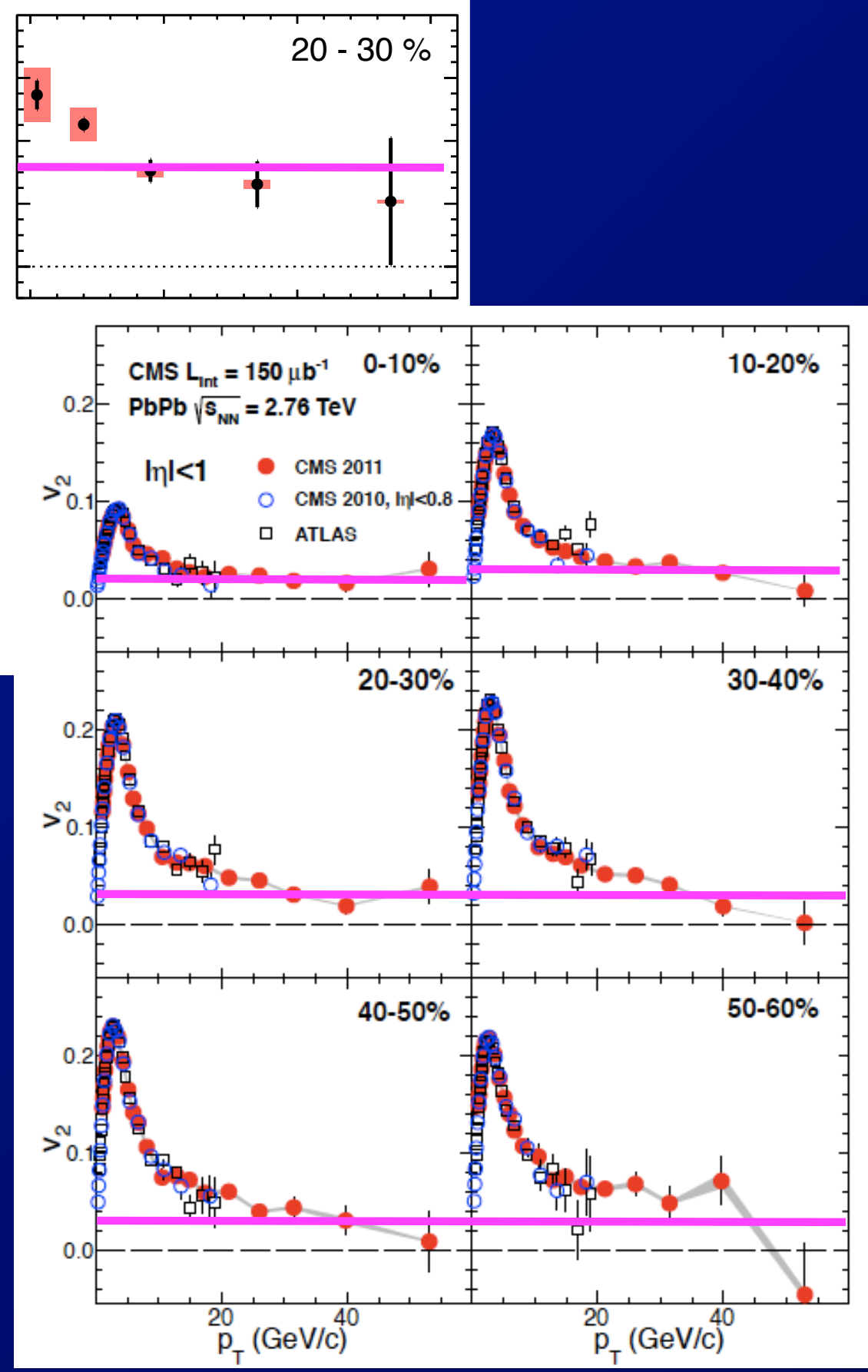


\section{Differential jet suppression}
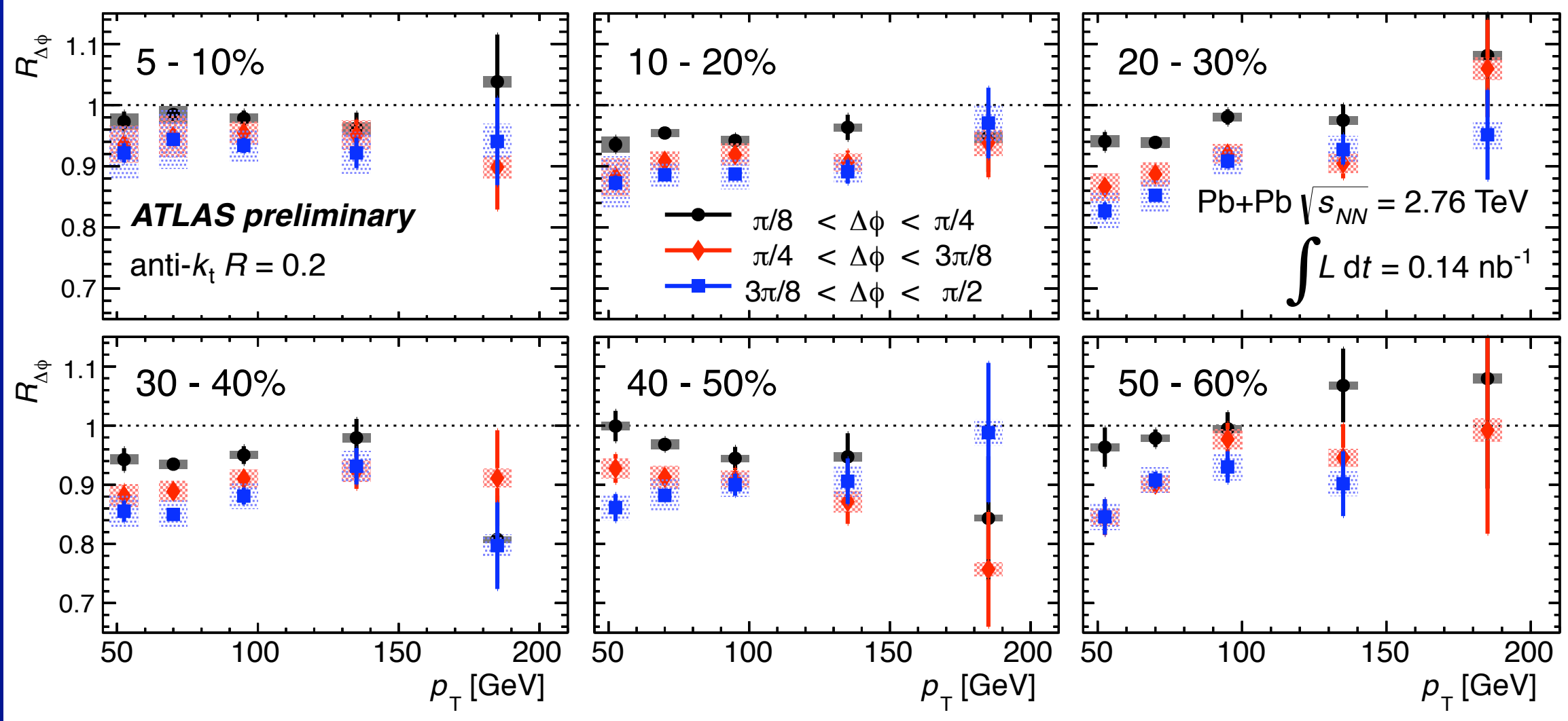

- Evaluate ratio of jet yields in different $\Delta \phi$ bins to the yield in $0<\Delta \phi<\pi / 8$.

$-\mathbf{R}_{\mathrm{AA}}(\Delta \boldsymbol{\phi}) / \mathbf{R}_{\mathrm{AA}}(\mathbf{0}-\pi / \mathbf{8})$

$\Rightarrow \sim 15 \%$ change in single jet suppression between in-plane, out-of-plane @ high pt 


\section{$n>2$ collective flow @ high(er) pt}

Phys. Rev. C86 (2012) 014907

- Full set of event plane $v_{n}\left(p_{T}\right)$ results

- Observe non-zero $v_{n}$ for $p_{T}>10 \mathrm{GeV}$ in central collisions

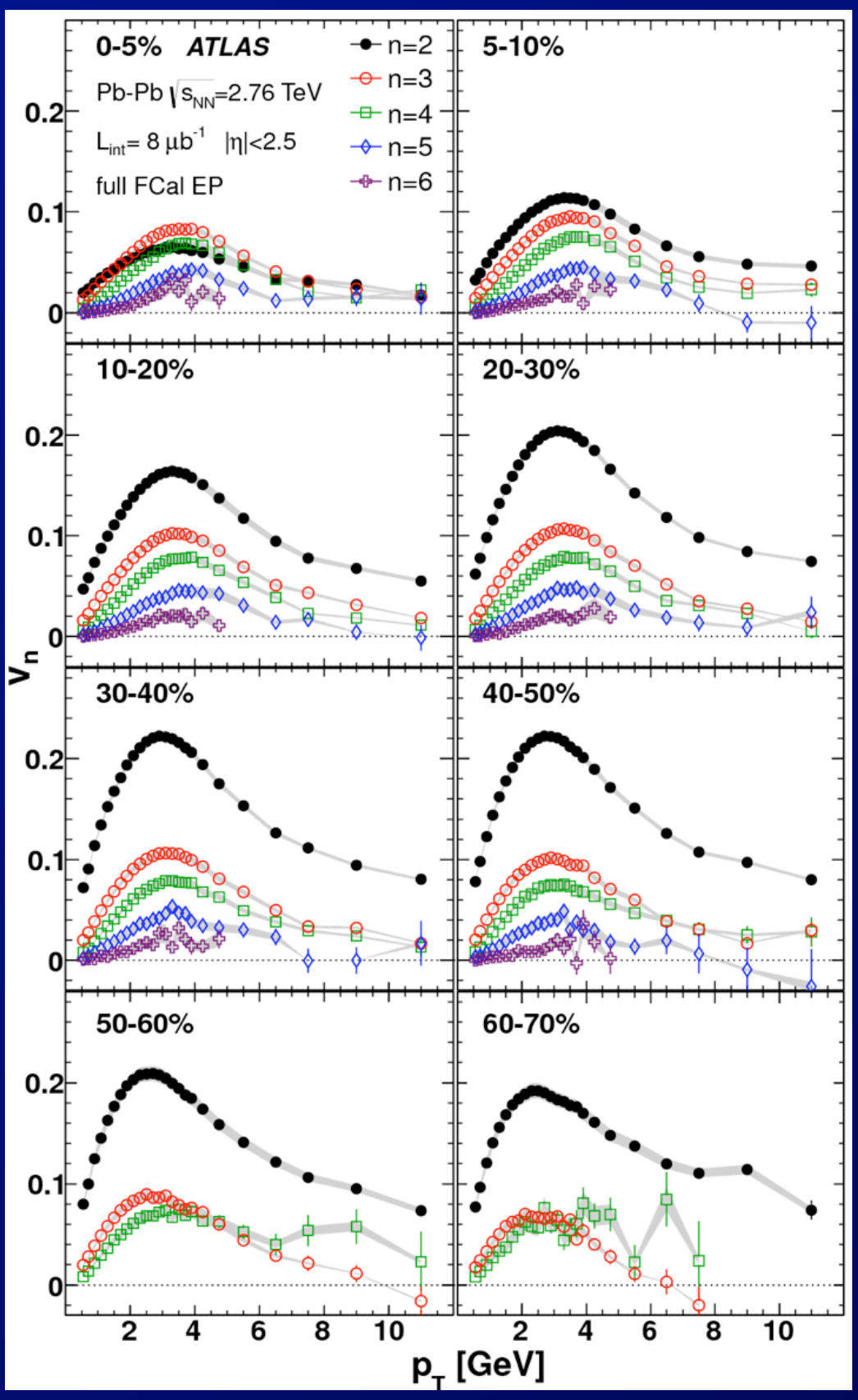




\section{$n>2$ collective flow @ high(er) pt}

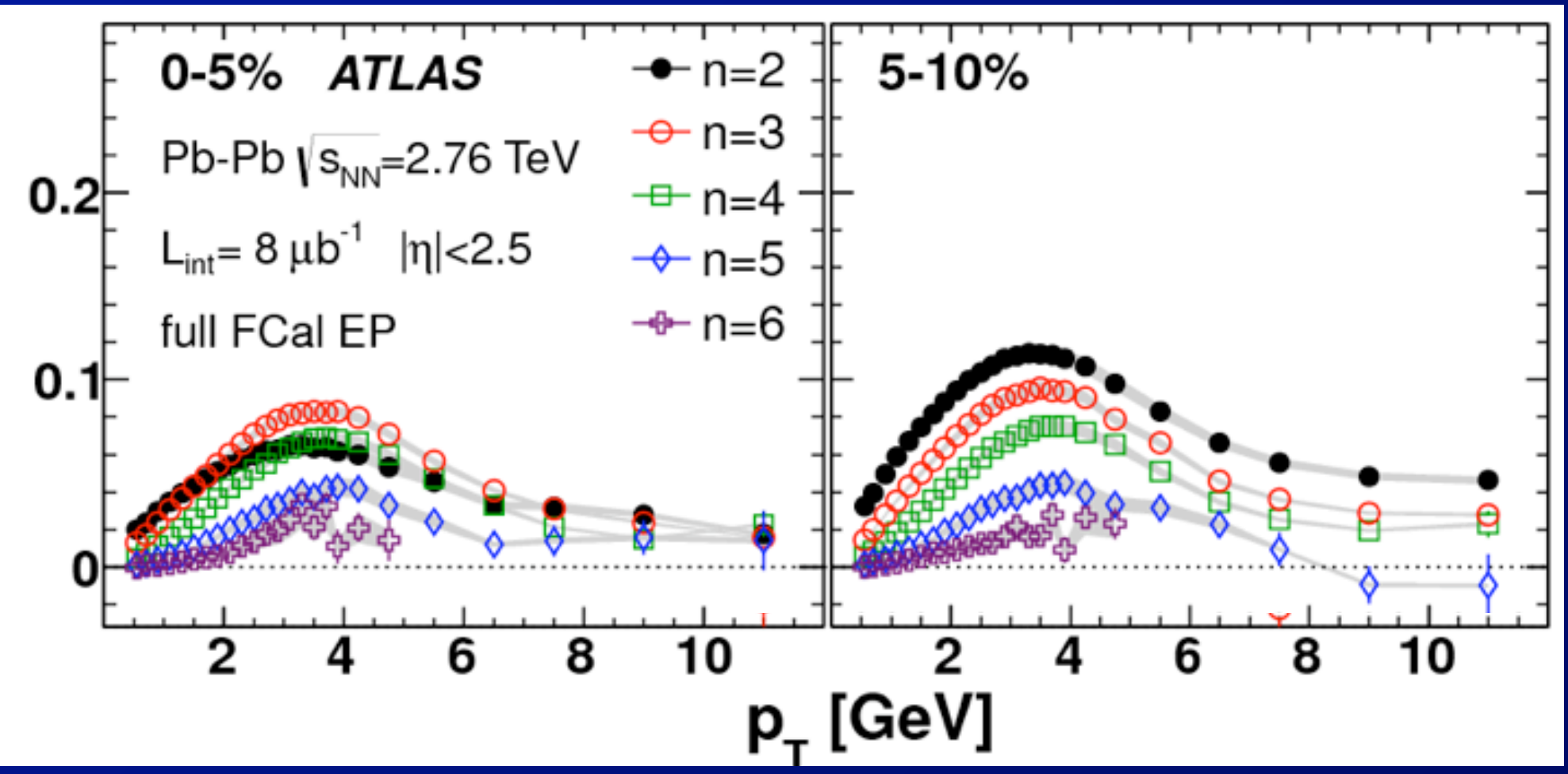

- For $0-5 \%, 5-10 \%$

- get non-zero, flat $v_{n}$ 's in region where $v_{2}$ is due to quenching?

$\Rightarrow$ Suggestive, but not conclusive.

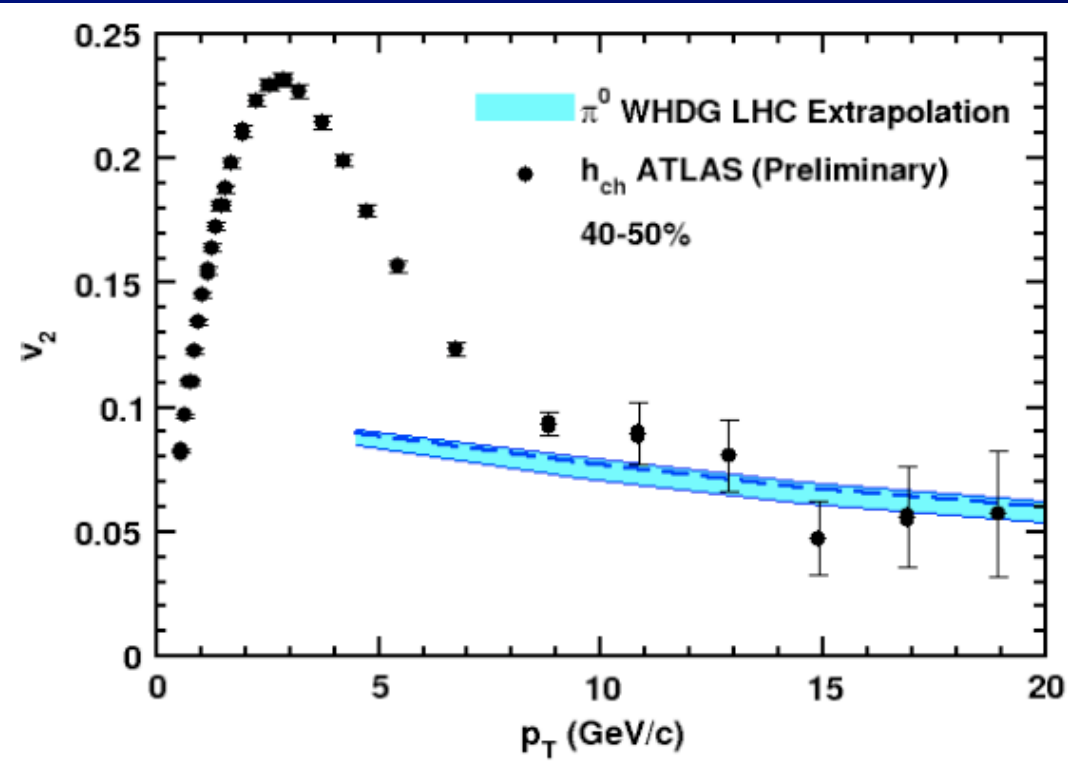




\section{Summary, ruminations}




\section{Jet probes of the quark gluon plasma}

Jet - QGP interactions schematically

From Quark Matter 2011 talk by Muller, Qiu

\section{A partonic jet shower in medium}

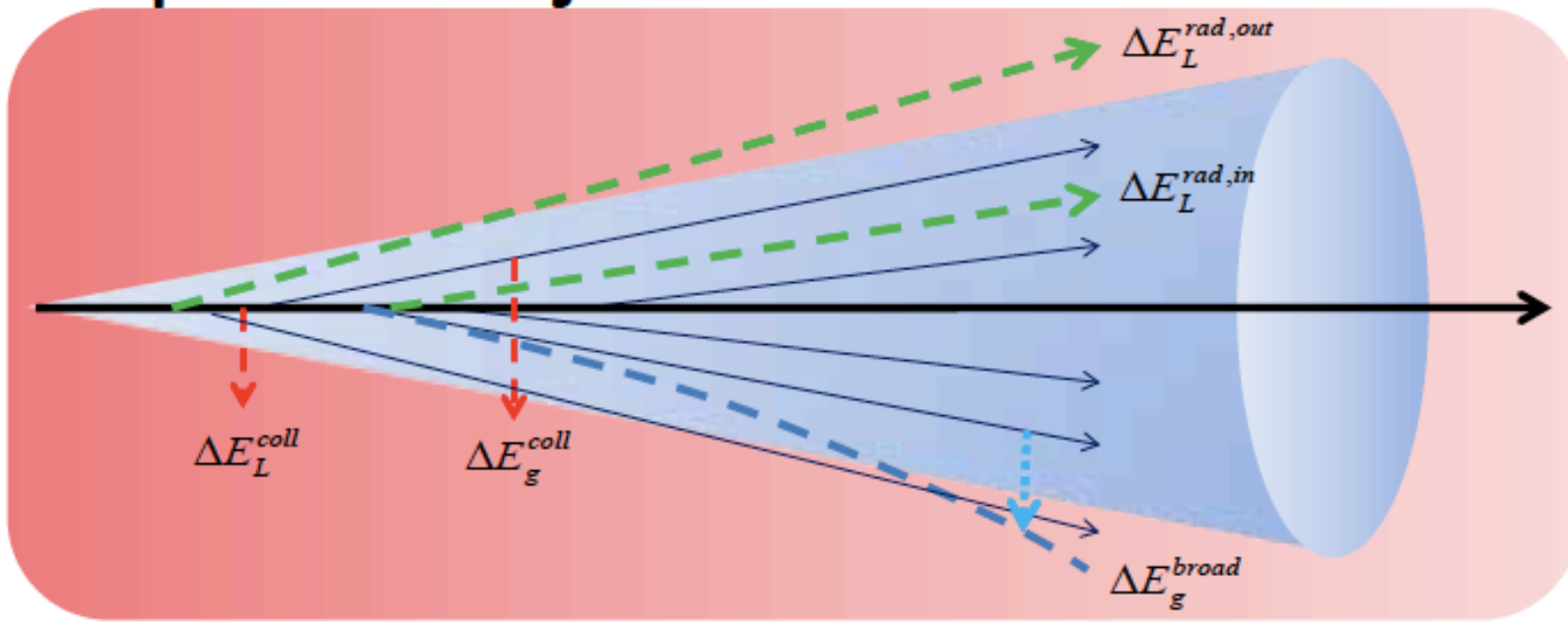

Leading parton:

Transfers energy to medium by elastic collisions

Radiates gluons due to scatterings in the medium (inside and outside jet cone)

Radiated gluons (vacuum \& medium-induced):

Transfer energy to medium by elastic collisions

Be kicked out of the jet cone by multiple scatterings after emission

- Complicated theoretical problem

- Addressing complicated measurements

- Need to be patient (but not too patient), but:

$\Rightarrow$ Are we asking, answering right questions? 


\section{Jet probes of the quark gluon plasma}

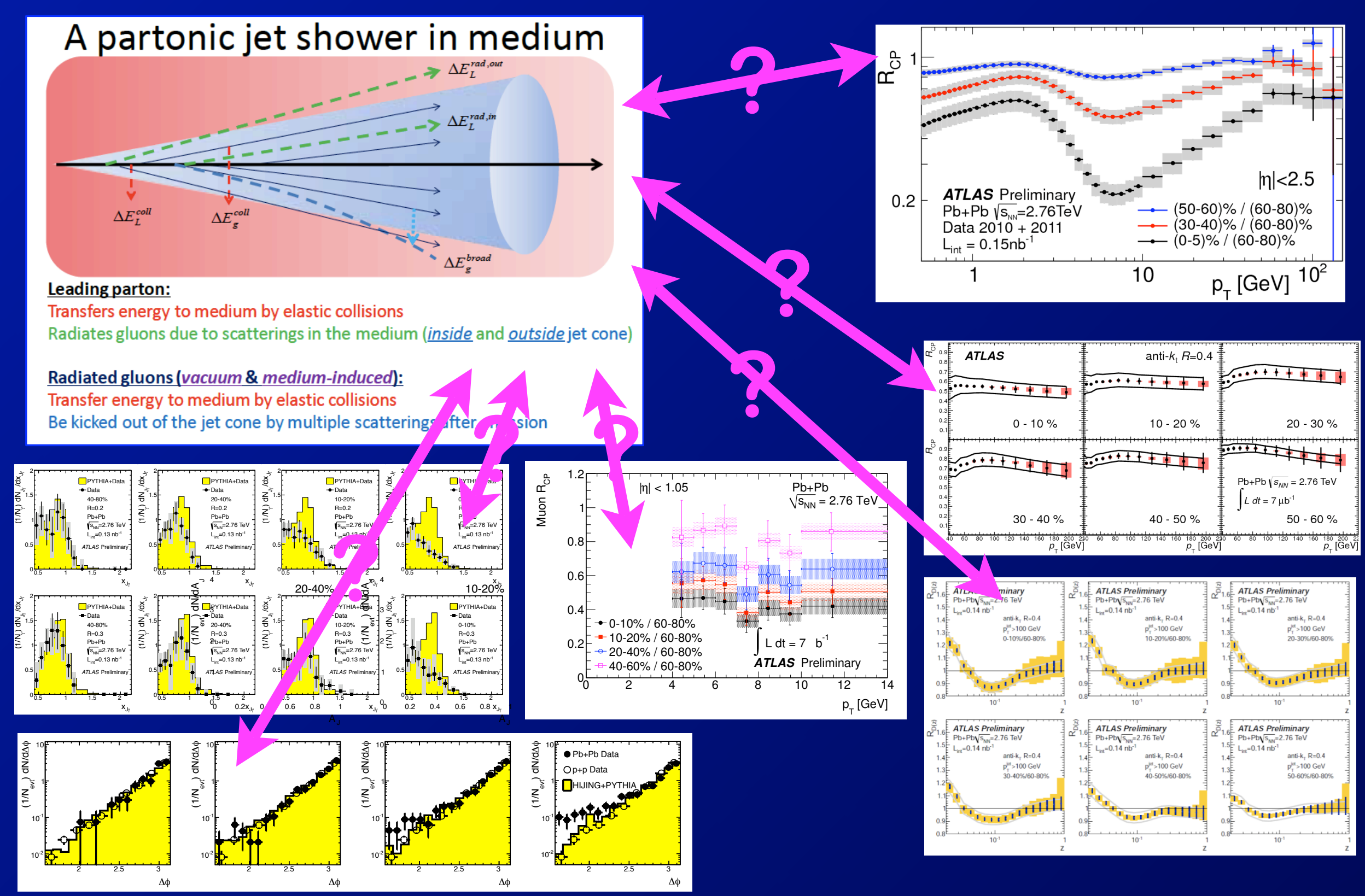




\section{$\mathrm{p}+\mathrm{Pb}$ ridges, who ordered that?}

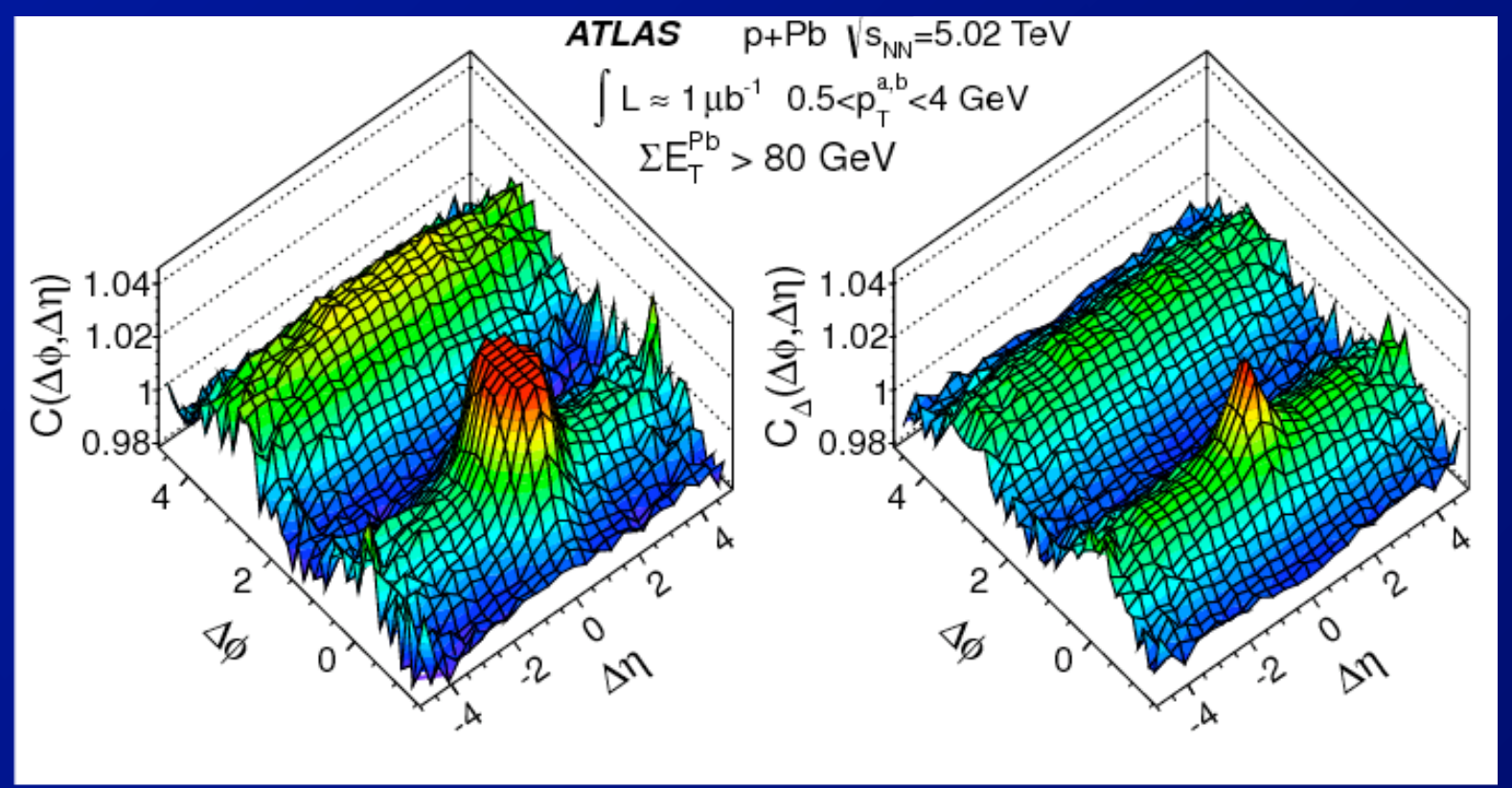

- What are the implications of ridge(s) for understanding jet quenching in $\mathrm{Pb}+\mathrm{Pb}$ ?

- Improved understanding of initial conditions?

- Yet more evidence of strong coupling?

- Potential pre-equilibrium effects that we don't know (yet) know how to control?

$\Rightarrow$ Need to understand connection between ridges \& hard scattering (if any) in $\mathrm{p}+\mathrm{Pb}$. 


\section{Wither the Glauber model ...}

Frankfurt, Strikman, Weiss, Phys. Rev. D83:054012,2011

(a)
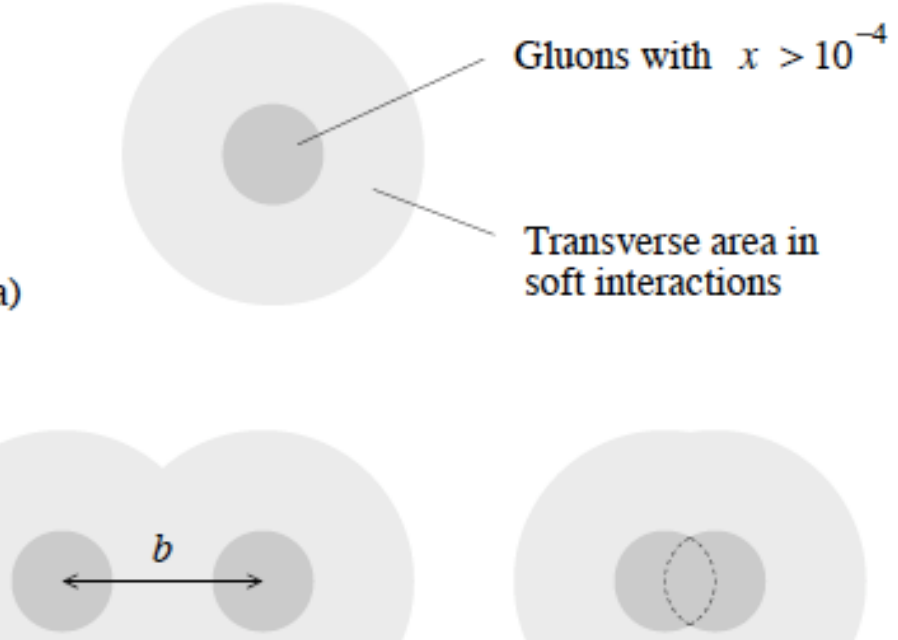

(b) peripheral collision

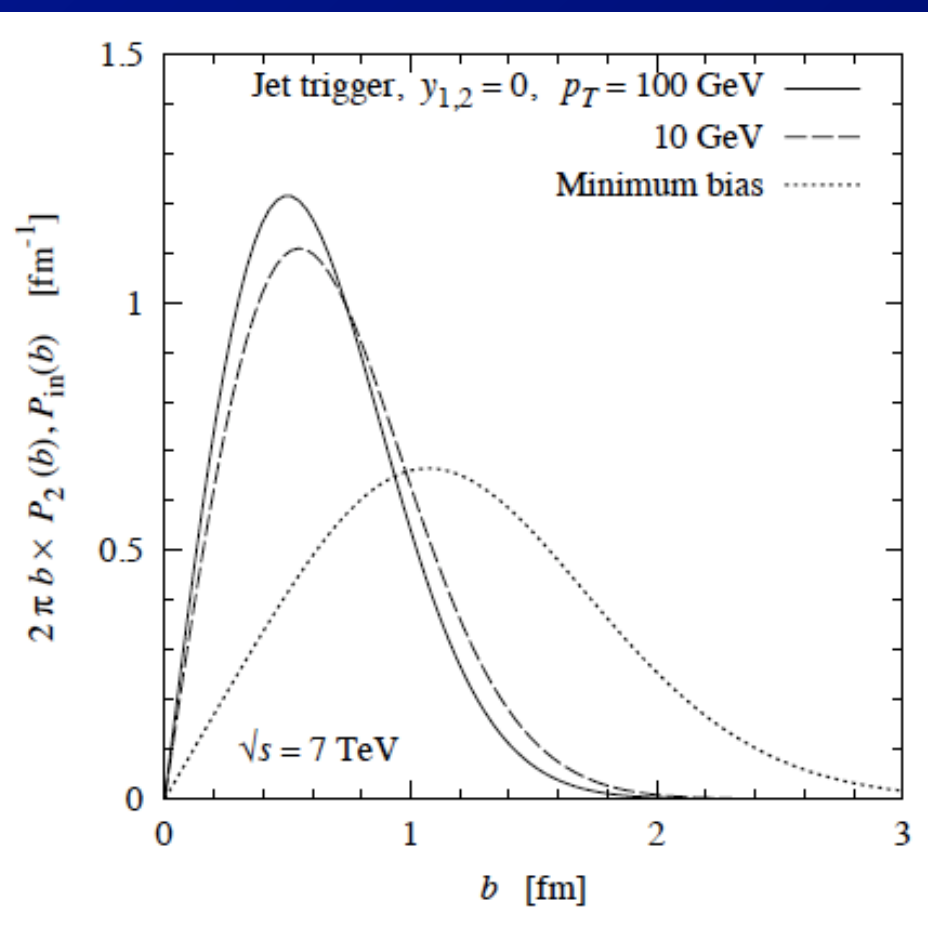

- Impact parameter dependence to physics in p-p collisions?

$\Rightarrow$ Implications for $d+A, p+A$ 


\section{Wither the Glauber model ... (2)}

\section{Alvioli, M. Strikman, arXiv:1301.0728}
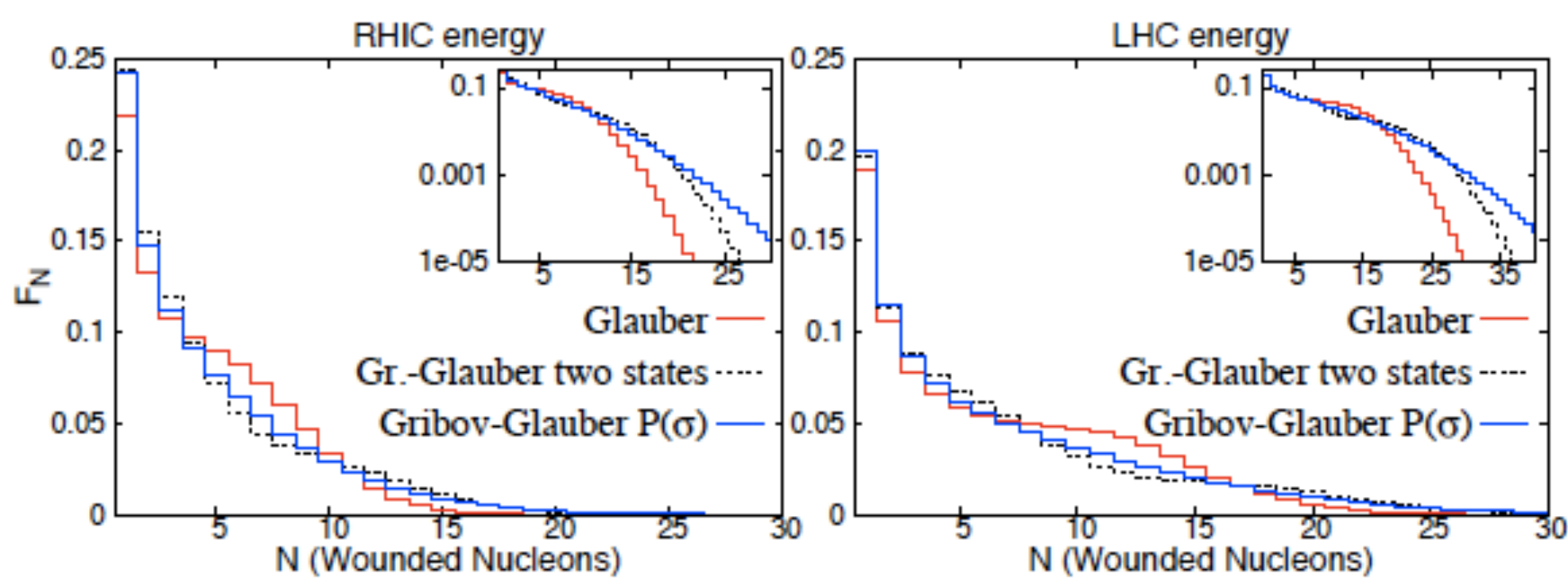

Figure 3: Effect of the event-by-event fluctuating values of $\sigma_{\text {tot }}$, for RHIC (left panel) and LHC energies (right panel) on the number of wounded nucleons, calculated as $F_{N}=$ $\int d b P_{N}(b) / \sigma_{i n}^{h A}$. Red curves show the results obtained with the usual Glauber calculation with fixed cross section, black curves correspond to calculations with the two-component $\sigma_{\text {tot }}$ model and blue curves correspond to calculations with fluctuating cross section with $P_{h}\left(\sigma_{\text {tot }}\right)$ distribution. The insets show the same quantities in logarithmic scale.

\section{- The Glauber model works well ... until it doesn't $\Rightarrow \mathrm{d} / \mathrm{p}$-A may force us to go beyond Glauber.}




\section{Backup}




\section{1th century view of jet quenching}

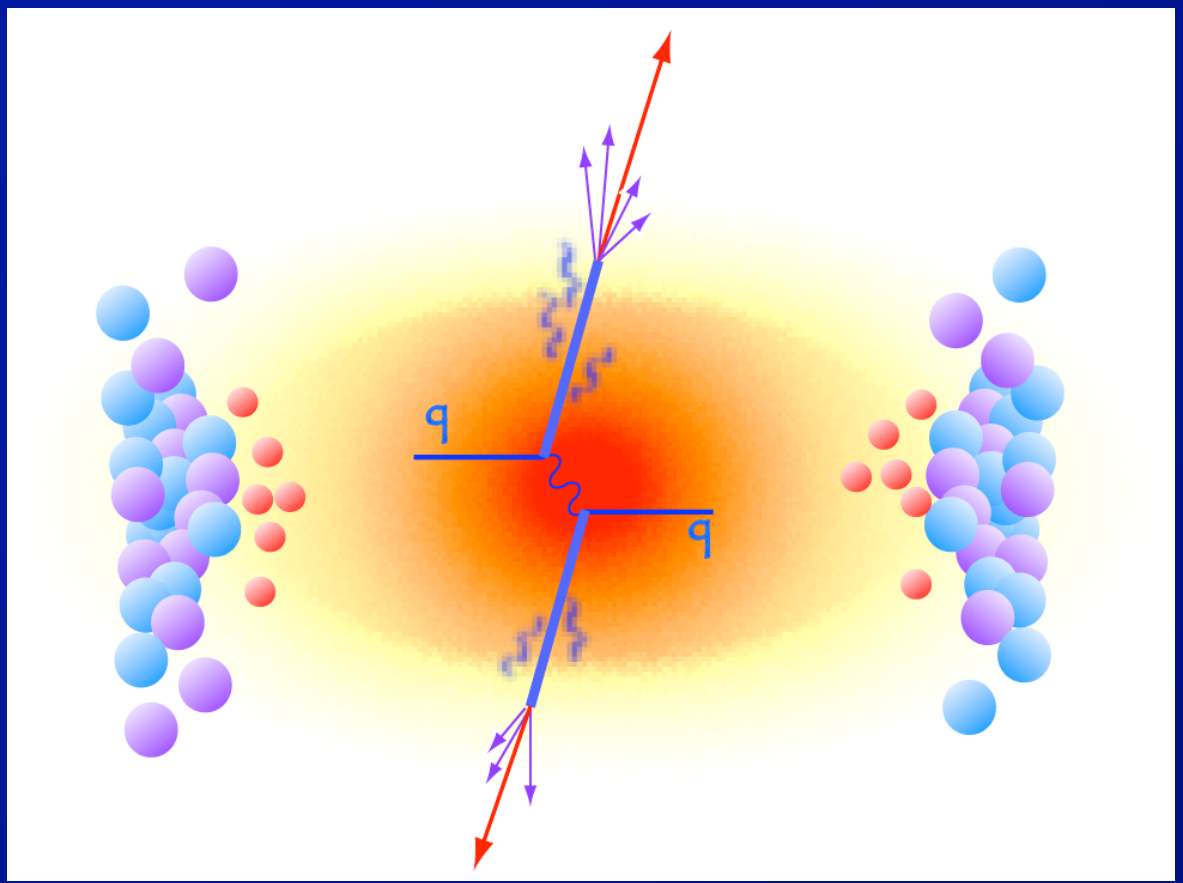

e.g. opacity expansion

a la GLV

- High-pт quarks or gluons propagate through and scatter in the QGP

- with collisional and radiative energy loss

- interference between vacuum and medium-induced radiation + LPM interference of multiple emissions

-Fragmentation in vacuum 


\section{1th century view of jet quenching}

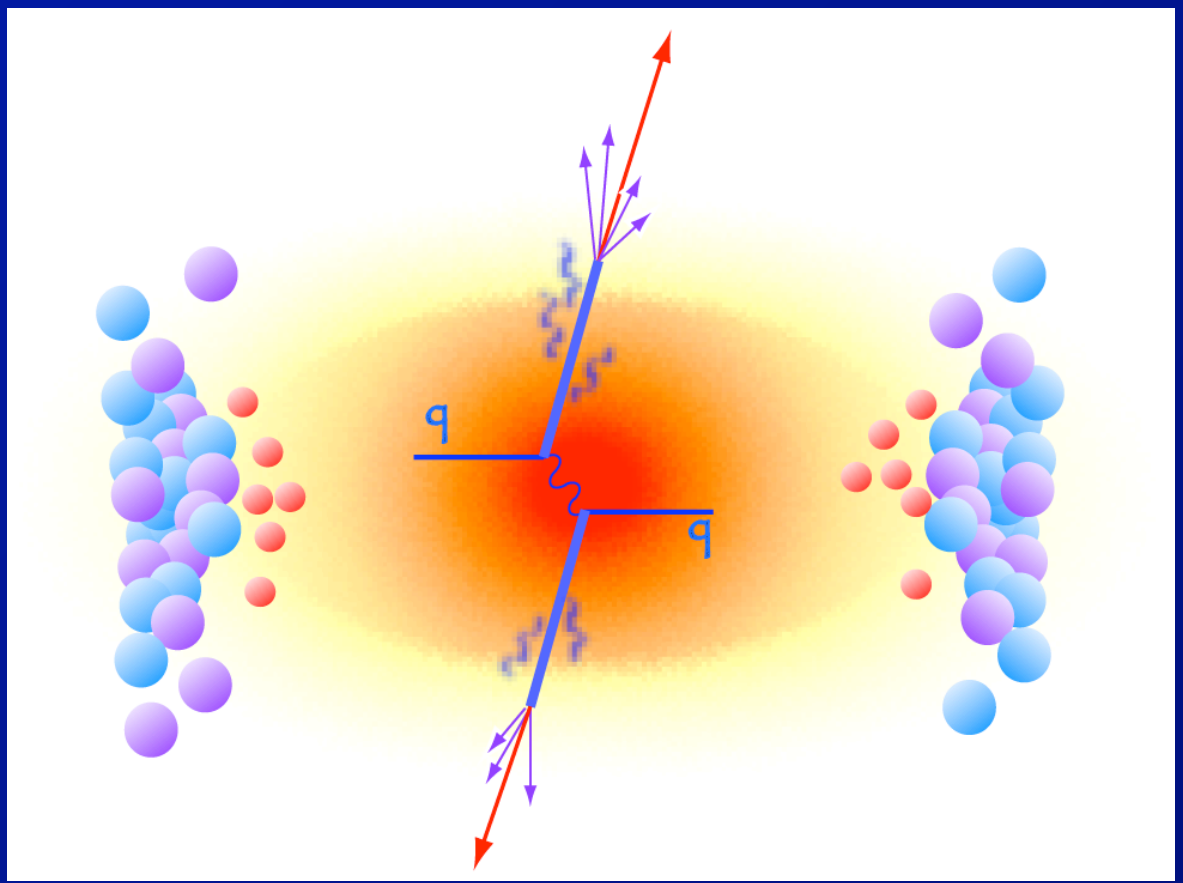

e.g. opacity expansion a la GLV

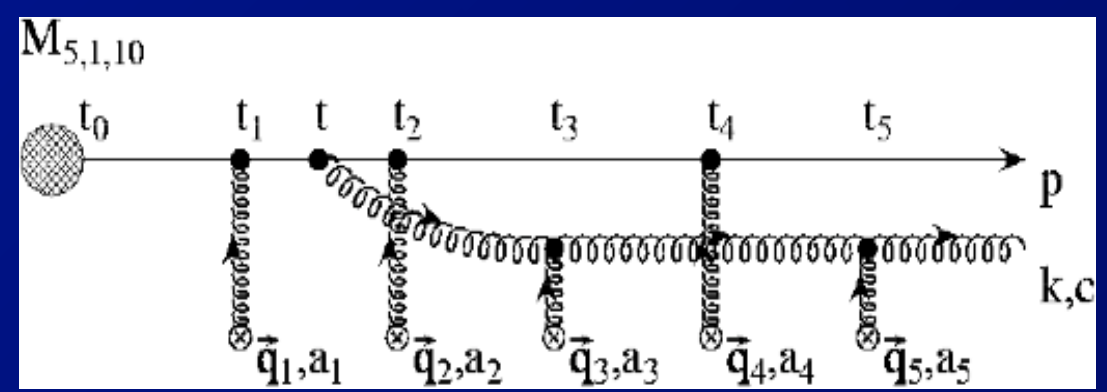

- High-pт quarks or gluons propagate through and scatter in the QGP

- with collisional and radiative energy loss

- interference between vacuum and medium-induced radiation + LPM interference of multiple emissions

-Fragmentation in vacuum 


\section{ATLAS: Pb+Pb centrality}

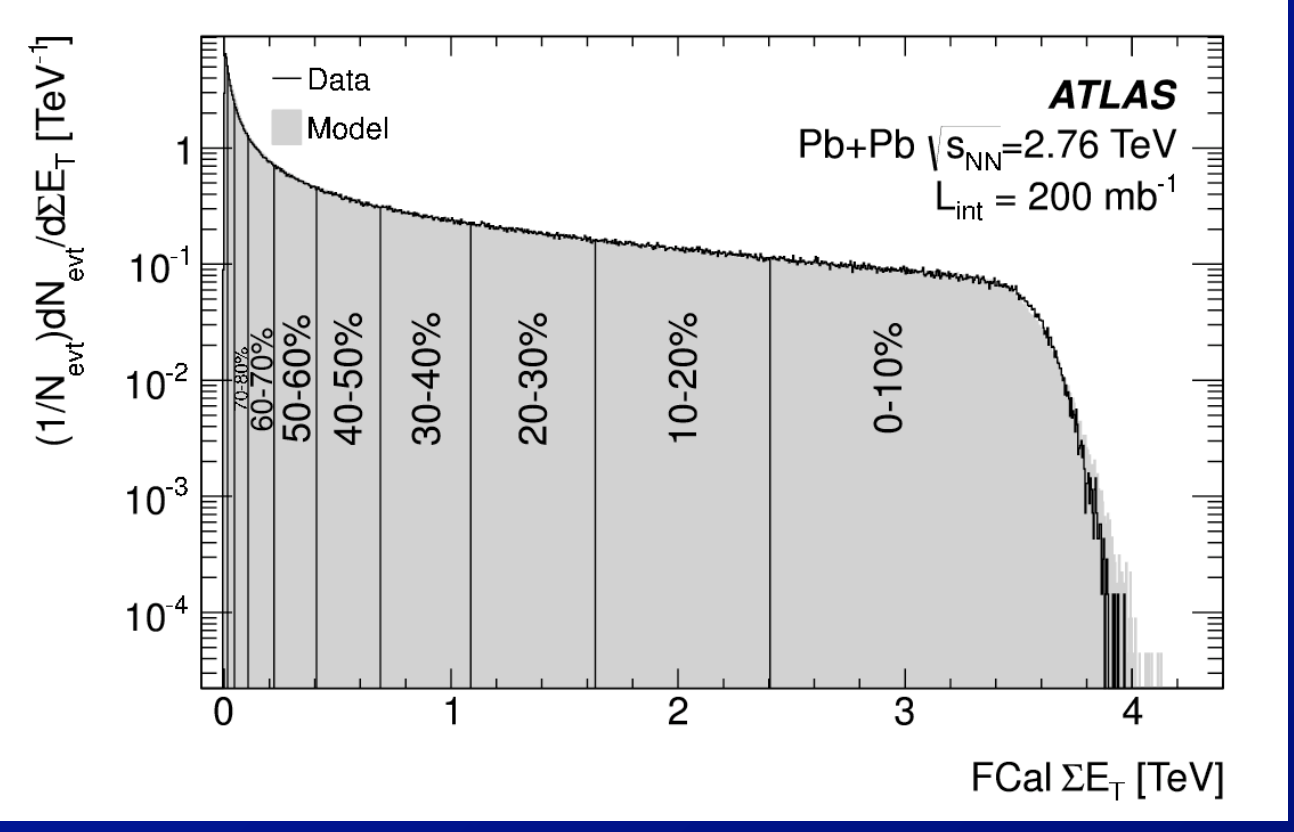

\begin{tabular}{|r|c|c|}
\hline Centrality & $\left\langle N_{\text {part }}\right\rangle$ & $\left\langle N_{\text {coll }}\right\rangle$ \\
\hline $0-10 \%$ & $356 \pm 2$ & $1500 \pm 115$ \\
\hline $10-20 \%$ & $261 \pm 4$ & $923 \pm 68$ \\
\hline $20-30 \%$ & $186 \pm 4$ & $559 \pm 41$ \\
\hline $30-40 \%$ & $129 \pm 4$ & $322 \pm 24$ \\
\hline $40-50 \%$ & $86 \pm 4$ & $173 \pm 14$ \\
\hline $50-60 \%$ & $53 \pm 3$ & $85 \pm 8$ \\
\hline $60-80 \%$ & $23 \pm 2$ & $27 \pm 4$ \\
\hline
\end{tabular}

- $\mathrm{Pb}+\mathrm{Pb}$ collision centrality characterized by $\Sigma E_{T}$ in forward calorimeters $(3.2<|\eta|<4.9)$.

- Also quantified using number of participants ( $\left.N_{\text {part }}\right)$

$-\mathrm{Pb}+\mathrm{Pb}$ partonic luminosity expressed in terms of "number of nucleon-nucleon collisions" ( $\left.N_{\text {coll }}\right)$ or $T_{A A}$ $\Rightarrow$ Calculated using standard Glauber Monte Carlo. 


\section{Centrality dependence of jet $R_{c p}$}

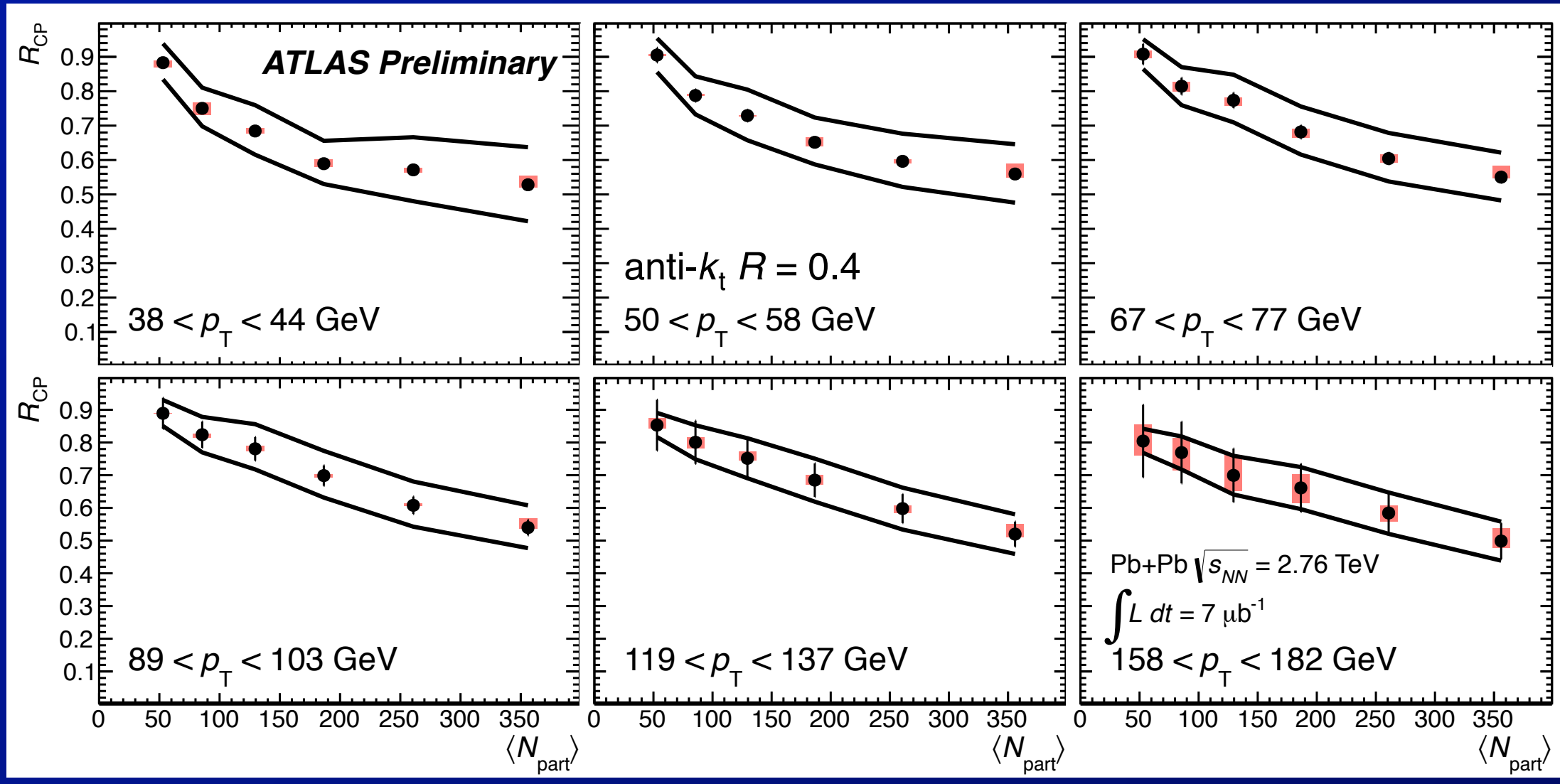

- Study centrality evolution for fixed jet $p_{T}$

- $R_{c p}$ vs $N_{\text {part }}$

$\Rightarrow$ Smooth turn on of jet suppression between peripheral and central collisions. 
Jet Quenching at RHIC vs LHC in Light of Recent dAu vs pPb Controls

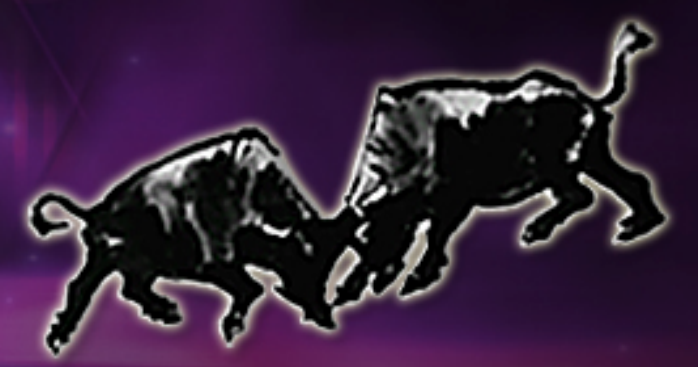

\section{Experimental status \\ of flavour tomography}

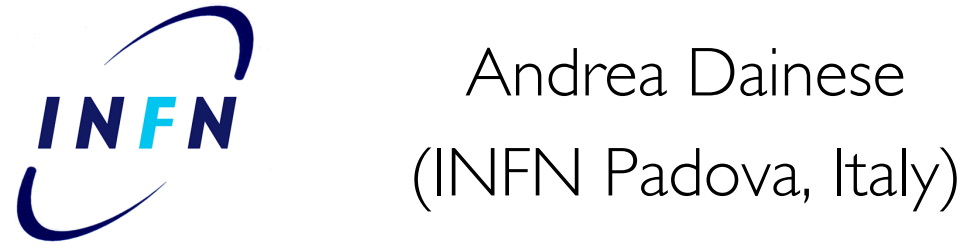




\section{Outline of the Talk}

$\rightarrow$ Introduction: HF probes of the medium ( $\rightarrow$ see A.Buzzatti)

Calibrating HF probes: pp results

$\checkmark \mathrm{HF}$ production in nucleus-nucleus:

$>$ Semi-leptonic decays $\underline{U}$ PHENIX, STAR

$>\mathrm{D}$ mesons

$>B$ and b-jets
U ALICE, ATLAS

STAR
ALICE

CMS

- Proton-nucleus control data

- HF azimuthal anisotropy

- Outlook: detector upgrades at RHIC and LHC 


\section{Outline of the Talk}

- Introduction: HF probes of the medium

- Calibrating HF probes: pp results

- HF production in nucleus-nucleus:

$>$ Semi-leptonic decays

$>\mathrm{D}$ mesons

$>\mathrm{B}$ and $\mathrm{b}$-jets

- Proton-nucleus control data

- HF azimuthal anisotropy

- Outlook: detector upgrades at RHIC and LHC 


\section{Heavy flavour production in pp}

- State-of-the-art pQCD calculation: Fixed Order Next-to-Leading Log

$$
\begin{aligned}
& \frac{d \sigma}{d p_{T}}=A(m) \alpha_{s}^{2}+B(m) \alpha_{s}^{3}+G\left(m, p_{T}\right)\left[\alpha_{s}^{2} \sum_{i=2}^{\infty} a_{i}\left[\alpha_{s} \log (\mu / m)\right]^{i}+\alpha_{s}^{3} \sum_{i=1}^{\infty} b_{i}\left[\alpha_{s} \log (\mu / m)\right]^{i}\right] \quad \mu \approx \vec{p}_{T} \\
& \text { FONLL: Cacciari, Frixione, Mangano, Nason and Ridolfi, JHEP0407 (2004) } 033 \quad
\end{aligned}
$$

[coincides with NLO for low $p_{\mathrm{T}}$ (total cross section); more accurate at high $p_{\mathrm{T}}$ ]
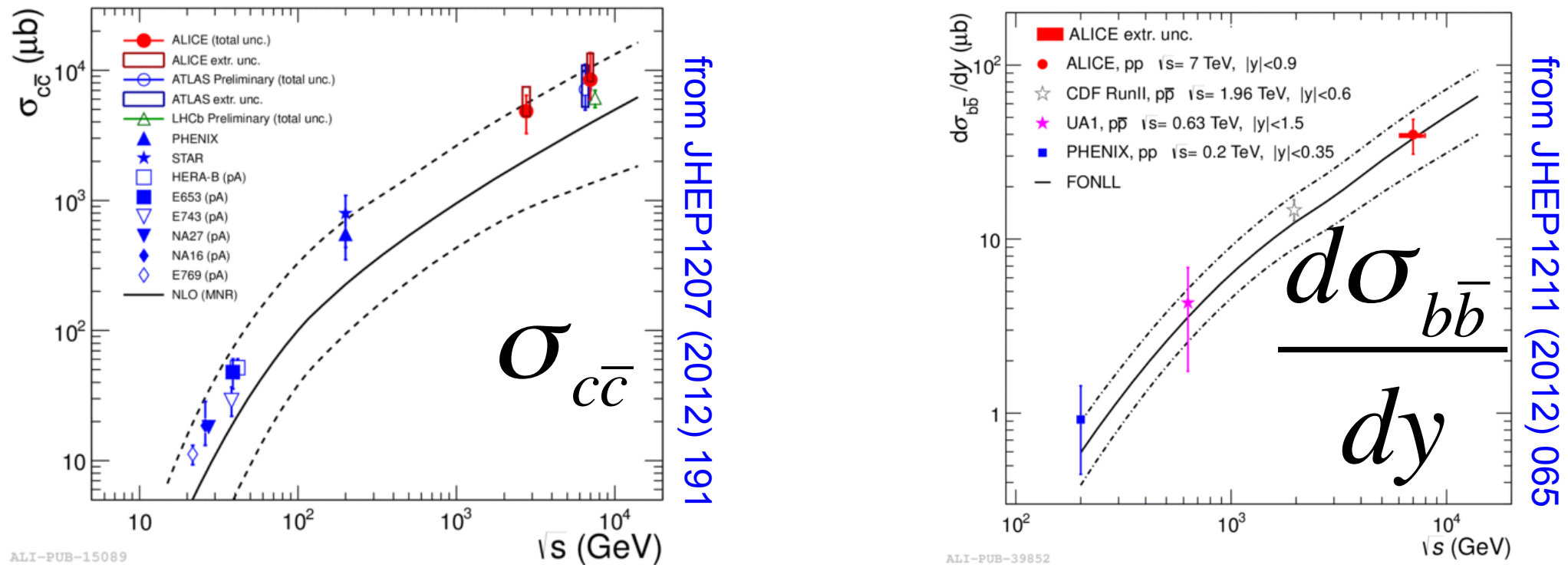

$\checkmark$ Describes consistently energy dependence of total cross sections

$\checkmark$ Charm (beauty) x10 (100) from 0.2 to $2.76 \mathrm{TeV}$ 
pp: pQCD calculations vs data

Charm $p_{\text {T-differential cross section }}$

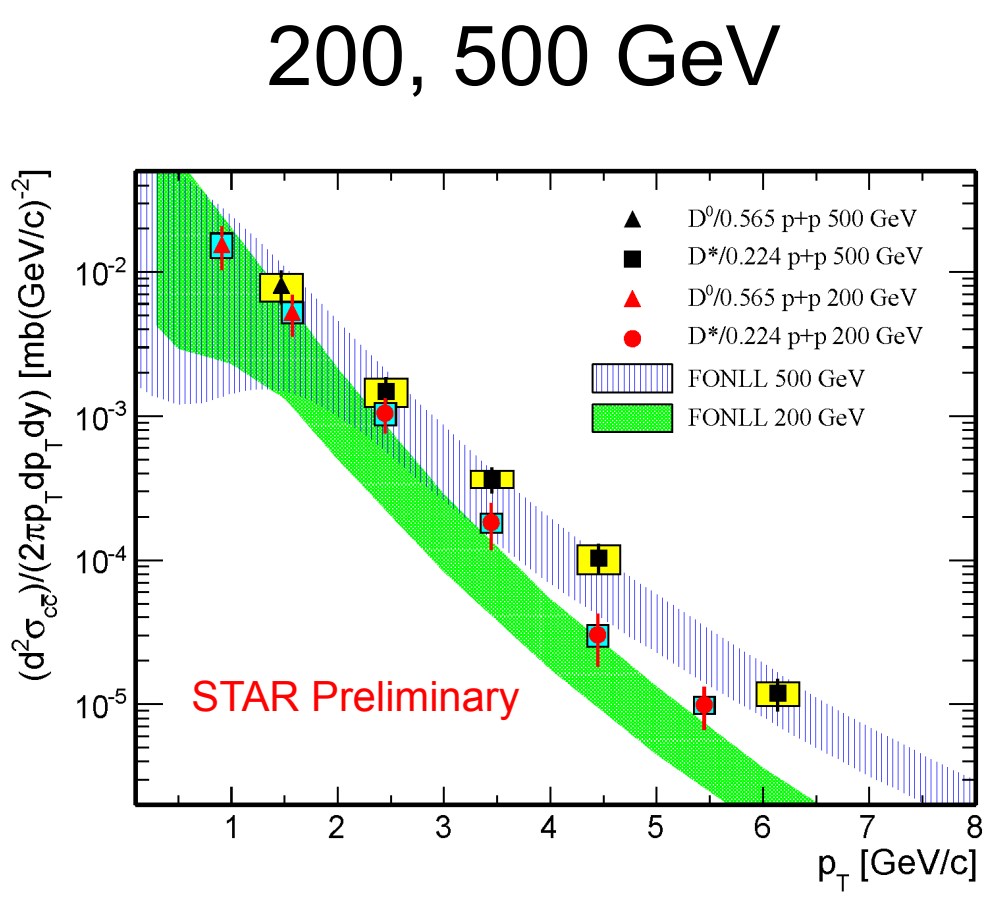

STAR, PRD 86 (2012) 72013 (200 GeV) J. Bielcik (Moriond2013)

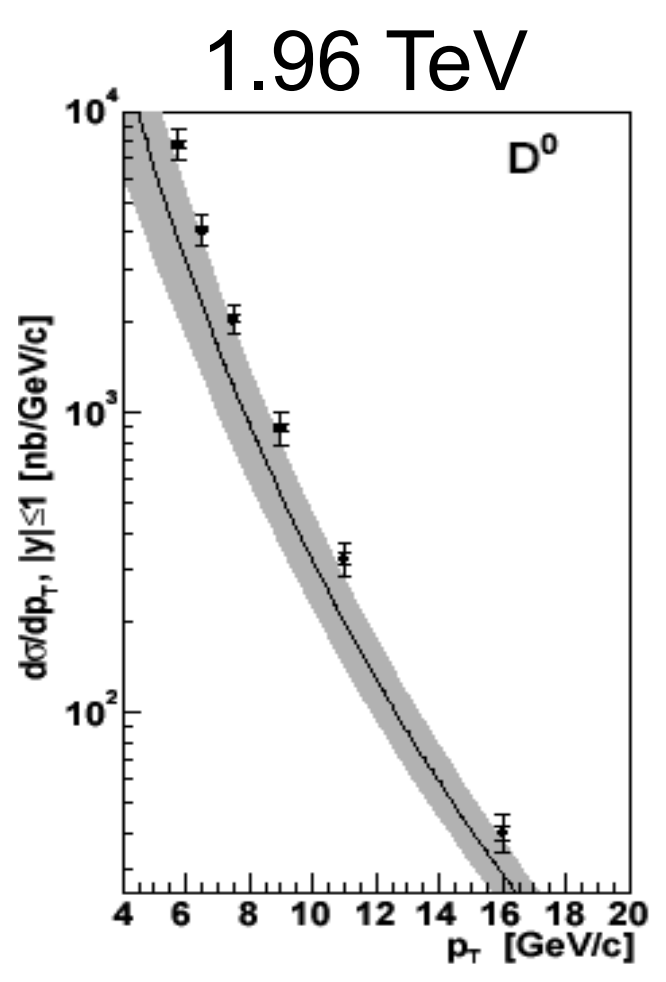

CDF, PRL91 (2003) 241804

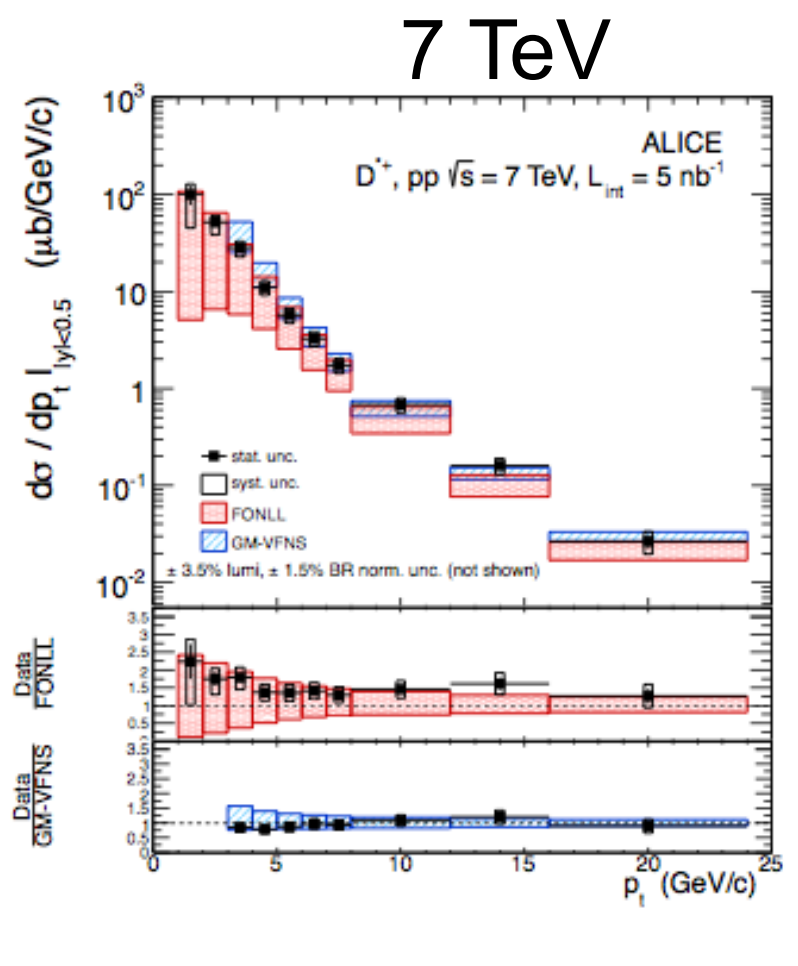

ALICE, JHEP01 (2012) 128

- Charm production described within uncertainties

- Consistently at upper limit of theoretical band from 0.2 to $7 \mathrm{TeV}$ 


\section{pp: pQCD calculations vs data Beauty $p_{T}$-differential cross section}

$1.96 \mathrm{TeV}$

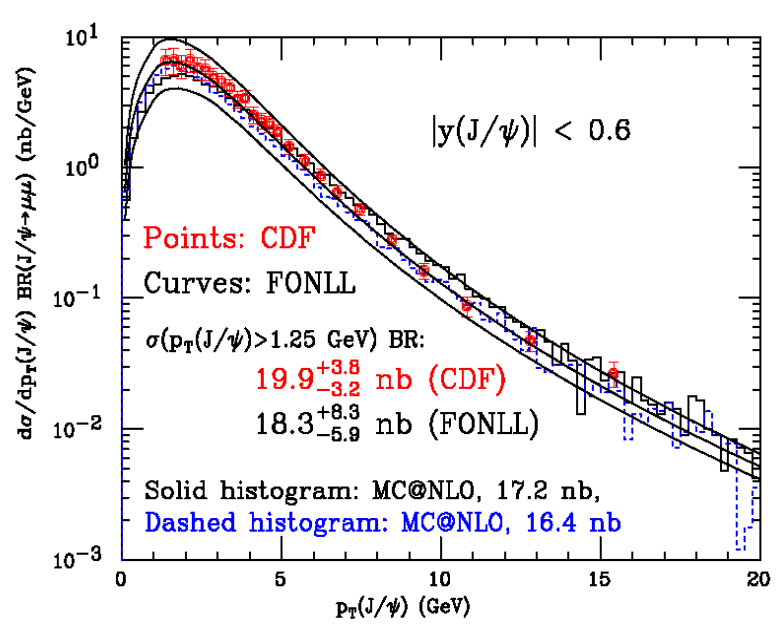

CDF, PRD71 (2005) 032001

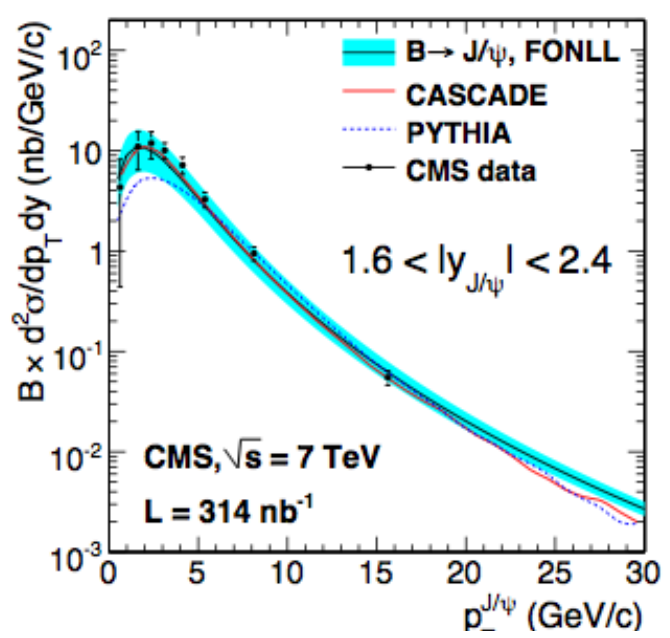

CMS, EPJC71 (2011) 1575

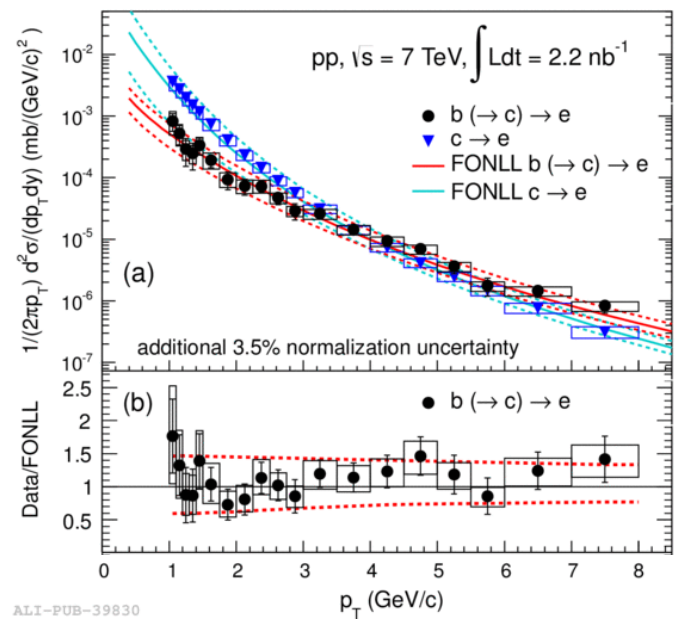

ALICE, PLB721 (2013) 13

- Beauty production described very well by central value of calculation 


\section{pp: pQCD calculations vs data HF-lepton $p_{T}$-differential cross section}

$200 \mathrm{GeV}$

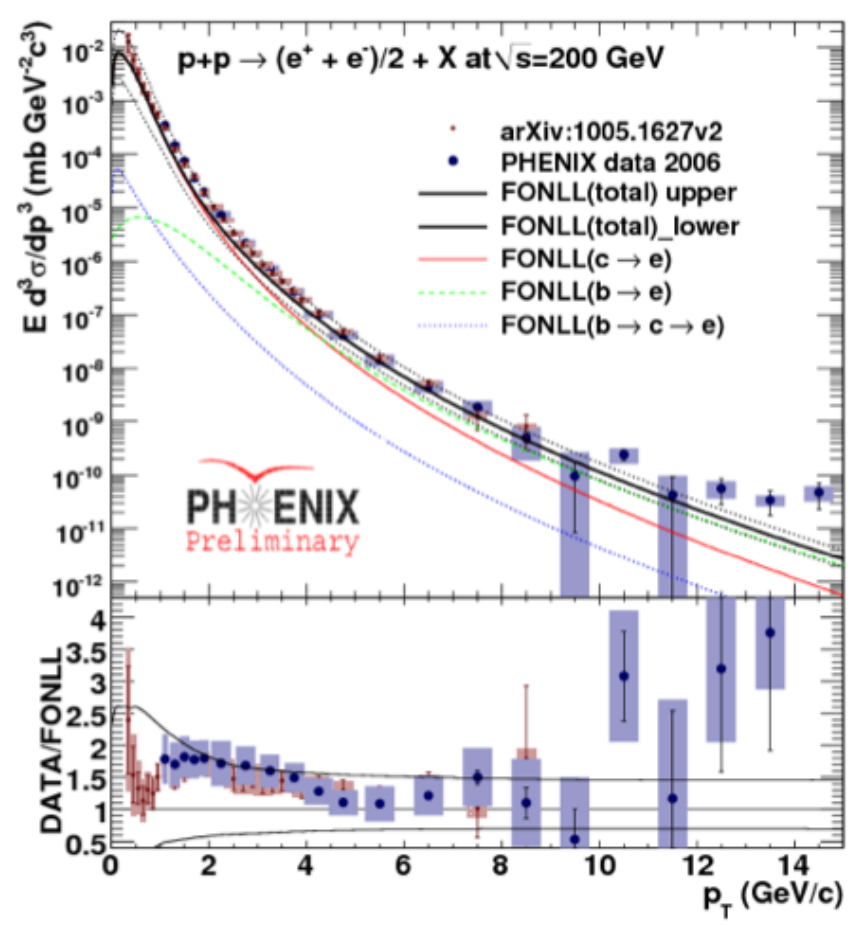

PHENIX, PRC84 (2011) 044905

N.Apadula (WWND2013)
$2.76 \mathrm{TeV}$

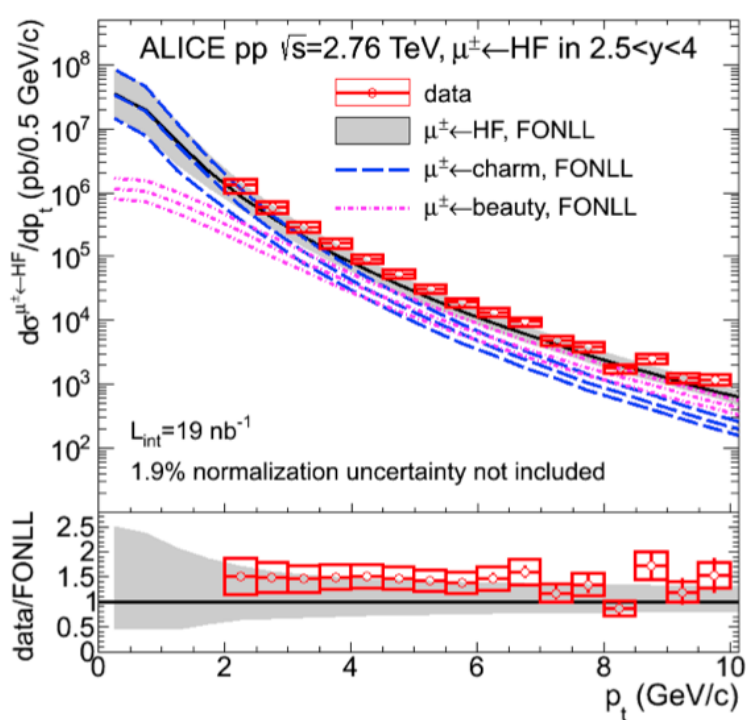

ALICE, PRL 109 (2012) 112301

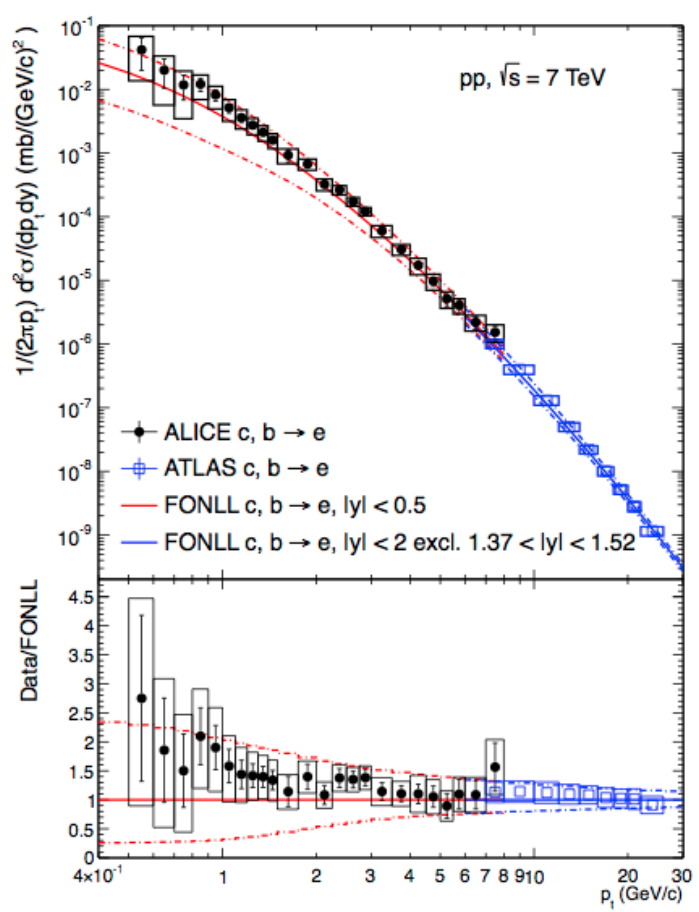

ALICE, PRD86 (2012) 112007 ATLAS, PLB707 (2012) 438

- HF-decay electrons and muons at central and forward y

- FONLL: "b > c" for $p_{\mathrm{T}}>4$ (5) GeV/c at RHIC (LHC) 


\section{Outline of the Talk}

- Introduction: HF probes of the medium

- Calibrating HF probes: pp results

- HF production in nucleus-nucleus:

$>$ Semi-leptonic decays U PHENIX, STAR

$>$ D mesons

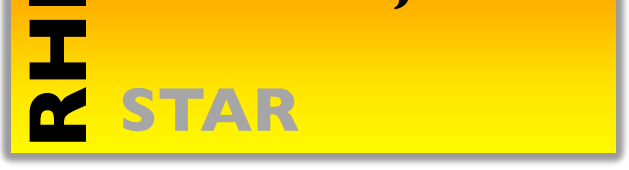

$>\mathrm{B}$ and b-jets

u ALICE, ATLAS

I

CMS

- Proton-nucleus control data

- HF azimuthal anisotropy

- Outlook: detector upgrades at RHIC and LHC 


\section{HF-decay electrons at RHIC (Au-Au)}

- Inclusive measurement $(\mathrm{c}+\mathrm{b})$ using non-photonic electrons

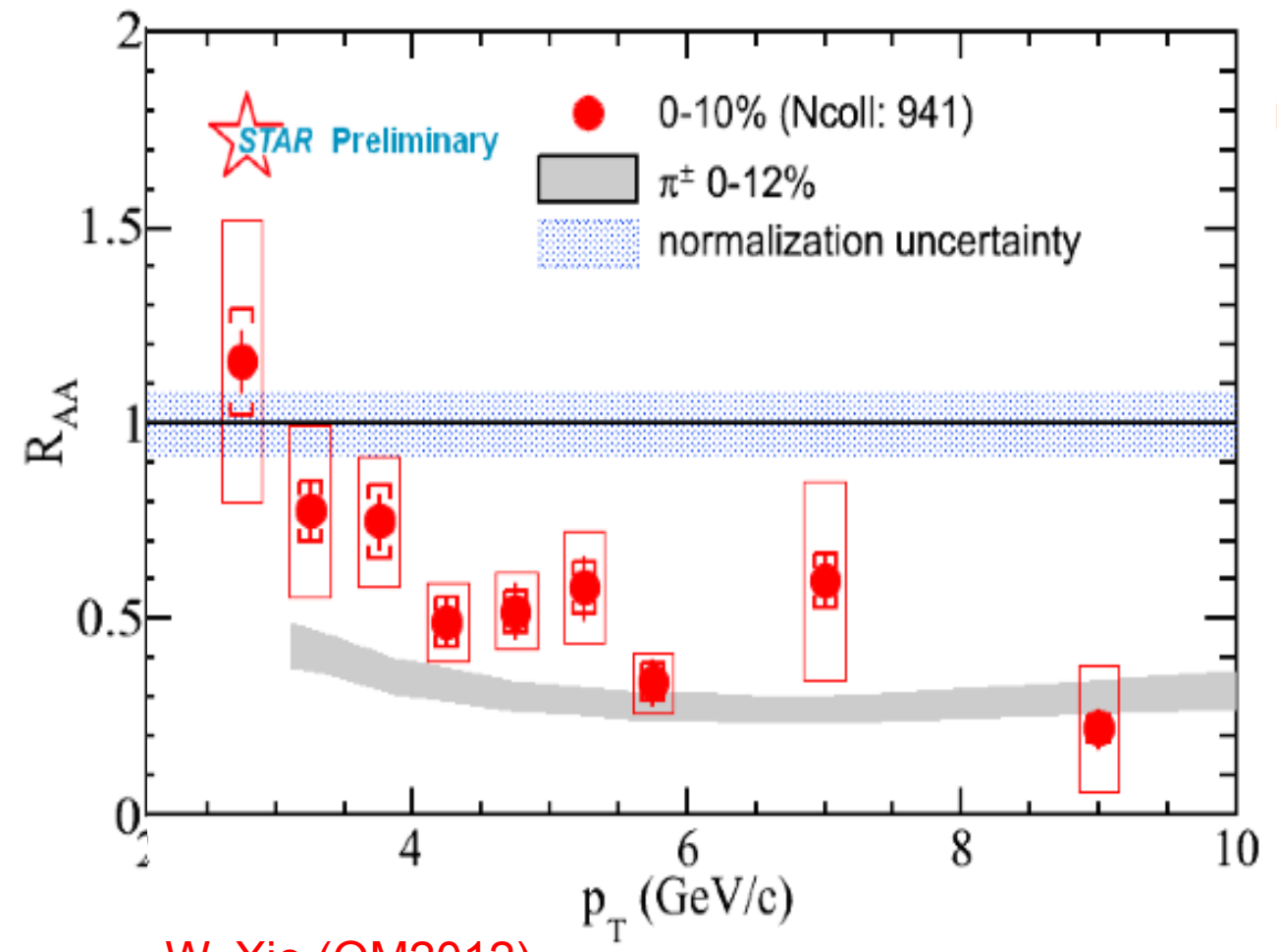

W. Xie (QM2012)

see also Phys. Rev. Lett. 98, 192301 (2007)

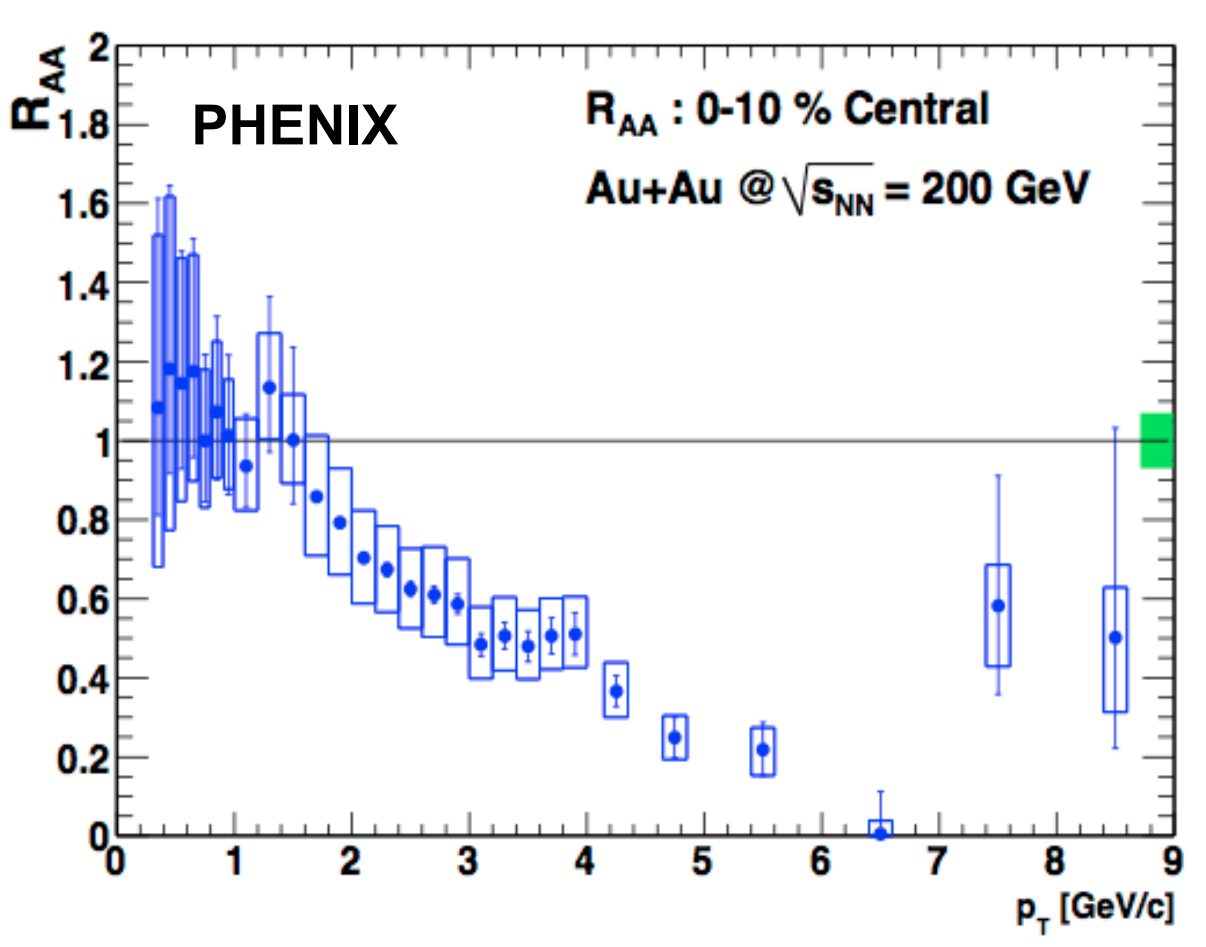

Phys. Rev. C 84, 044905 (2011)

- Same suppression as for light-flavour hadrons above $5 \mathrm{GeV} / \mathrm{c}$

$>$ Mass already irrelevant?

$\checkmark$ Smaller suppression at 2-3 GeV/c: is this the dead cone?? 


\section{HF-decay e at RHIC (Cu-Cu vs. Au-Au) INFN}
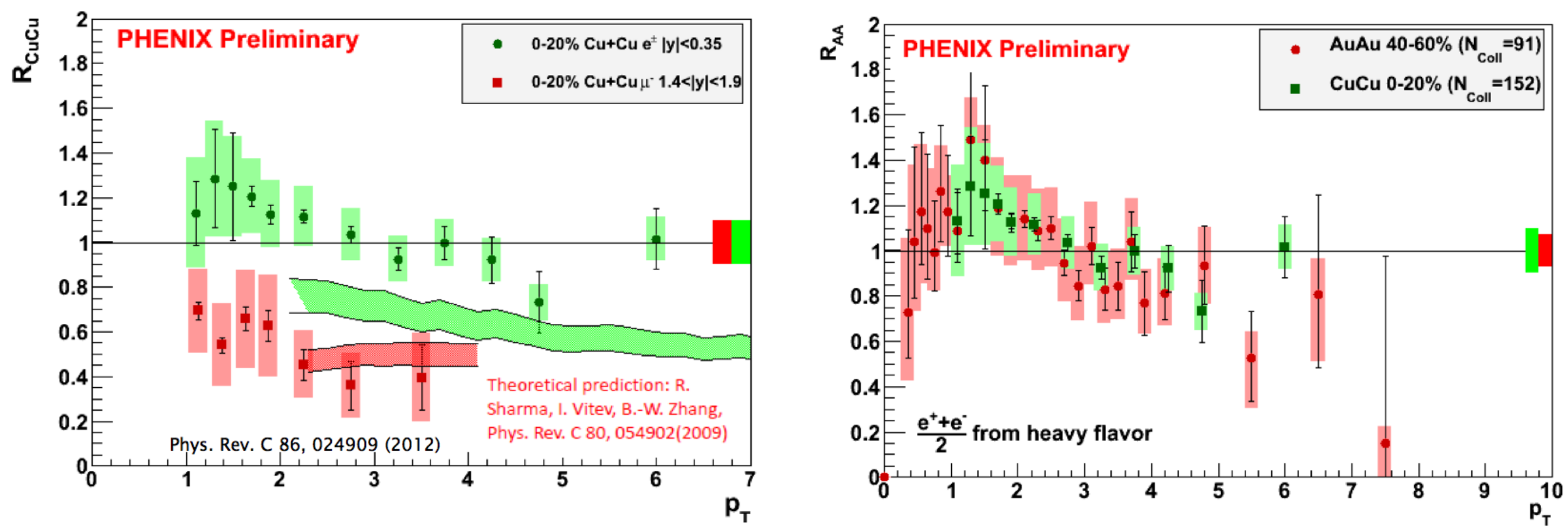

$\diamond \mathrm{Cu}-\mathrm{Cu}:$

$>$ low $\mathrm{p}_{\mathrm{T}}$ enhancement in central collisions?

$>$ no suppression even at $6 \mathrm{GeV} / \mathrm{c}$ : not expected in models!?

- Similar $\mathrm{R}_{\mathrm{AA}}$ as in $\mathrm{Au}$-Au for similar $\mathrm{N}_{\text {coll }}$ values 


\section{HF-decay e and $\mu$ at $\mathrm{RHIC}(\mathrm{Cu}-\mathrm{Cu})$}

- Mid-rapidity (e): $\mathrm{R}_{\mathrm{AA}} \sim 1 \rightarrow$ enhancement (from centr. to periph.)

$\rightarrow$ Forward rapidity $(\mu)$ : suppression $\rightarrow \mathrm{R}_{\mathrm{AA}} \sim 1$
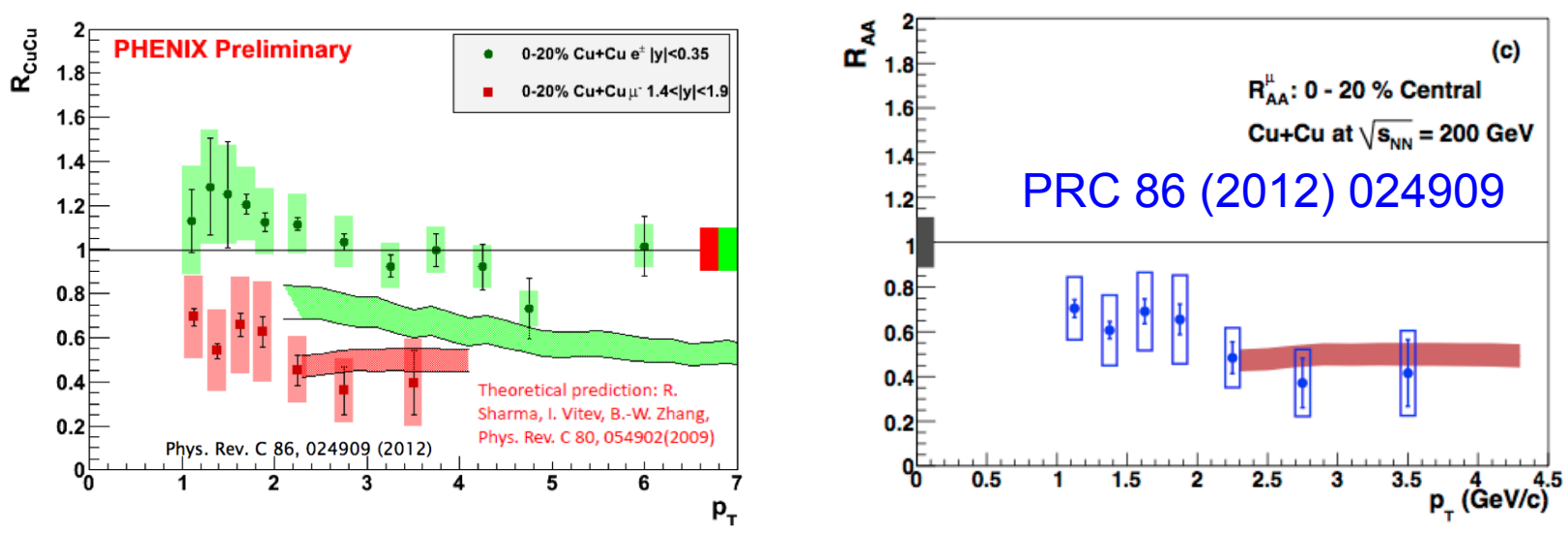

Theoretical prediction: $\mathrm{R}$.

Sharma, I. Vitev, B.-W. Zhang,

Phys. Rev. C 80, 054902(2009)
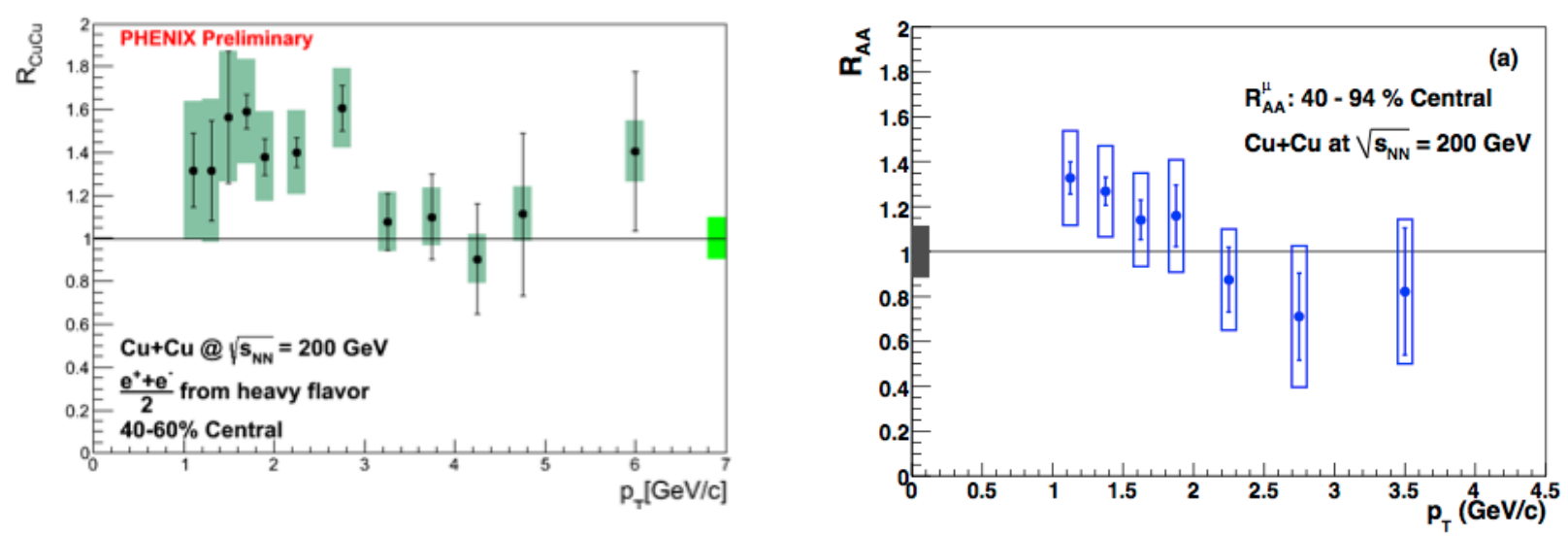

N.Apadula (WWND2013) 


\section{HF-decay e and $\mu$ at LHC: $R_{A A}$ vs $P_{T}$}

- Electrons and muons from $D+B \rightarrow e, \mu$ decays

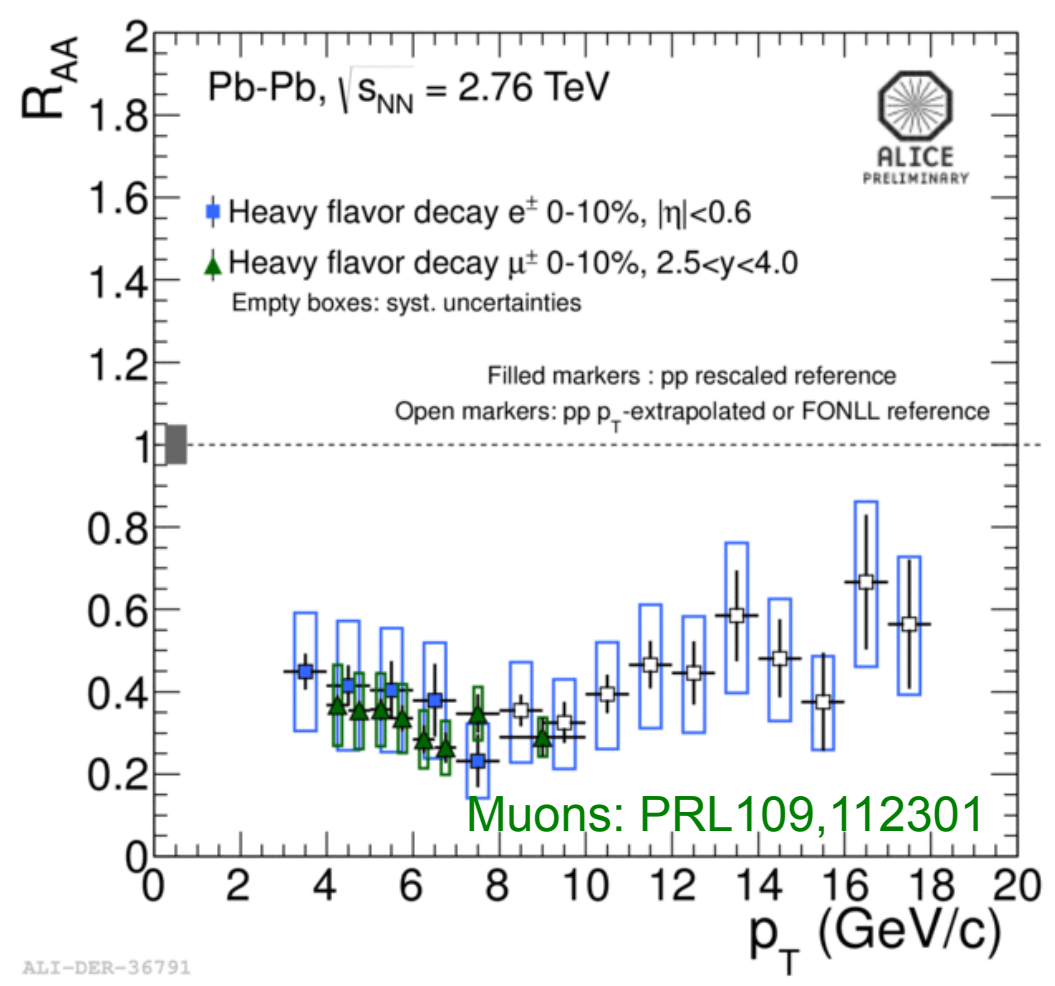

- Comparable suppression at central $(|y|<0.6)$ and forward $(2.5<y<4)$ rapidity

- Suppression by a factor about 2 up to $18 \mathrm{GeV} / \mathrm{c}$

$>$ Dominated by beauty at such high $p_{\mathrm{T}}$ 


\section{HF-decay $\mu$ at LHC vs. centrality}
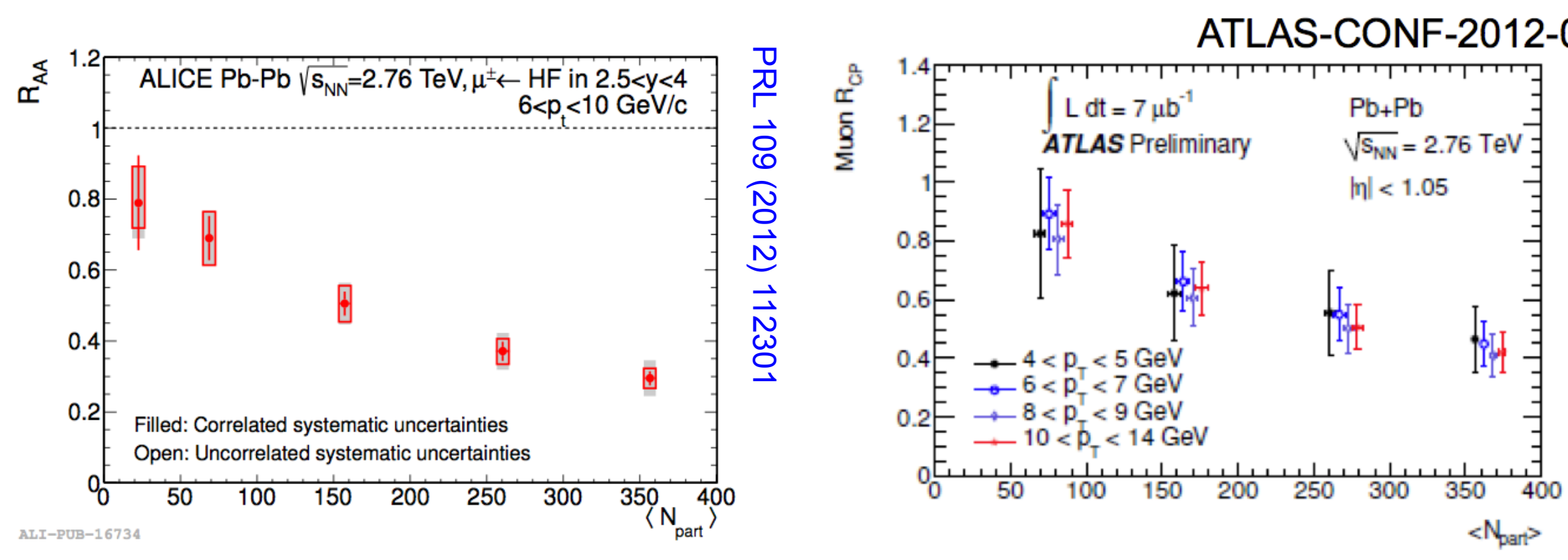

- Clear and consistent centrality dependence for

$>\mathrm{R}_{\mathrm{AA}}$ of muons at forward rapidity (ALICE)

$>\mathrm{R}_{\mathrm{CP}}$ of muons at central rapidity (ATLAS)

No sign of $p_{\mathrm{T}}$ dependence from 4 to $12 \mathrm{GeV} / \mathrm{c}$ 


\section{Outline of the Talk}

- Introduction: HF probes of the medium

- Calibrating HF probes: pp results

- HF production in nucleus-nucleus:

$>$ Semi-leptonic decays

$>\mathrm{D}$ mesons

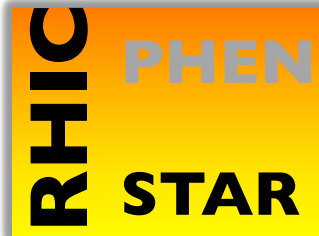

$>B$ and b-jets

I ALICE

CMS

- Proton-nucleus control data

- HF azimuthal anisotropy

- Outlook: detector upgrades at RHIC and LHC 


\section{Charm: D mesons at RHIC}

- STAR: first D $\mathrm{R}_{\mathrm{AA}}$ in central Au-Au at RHIC
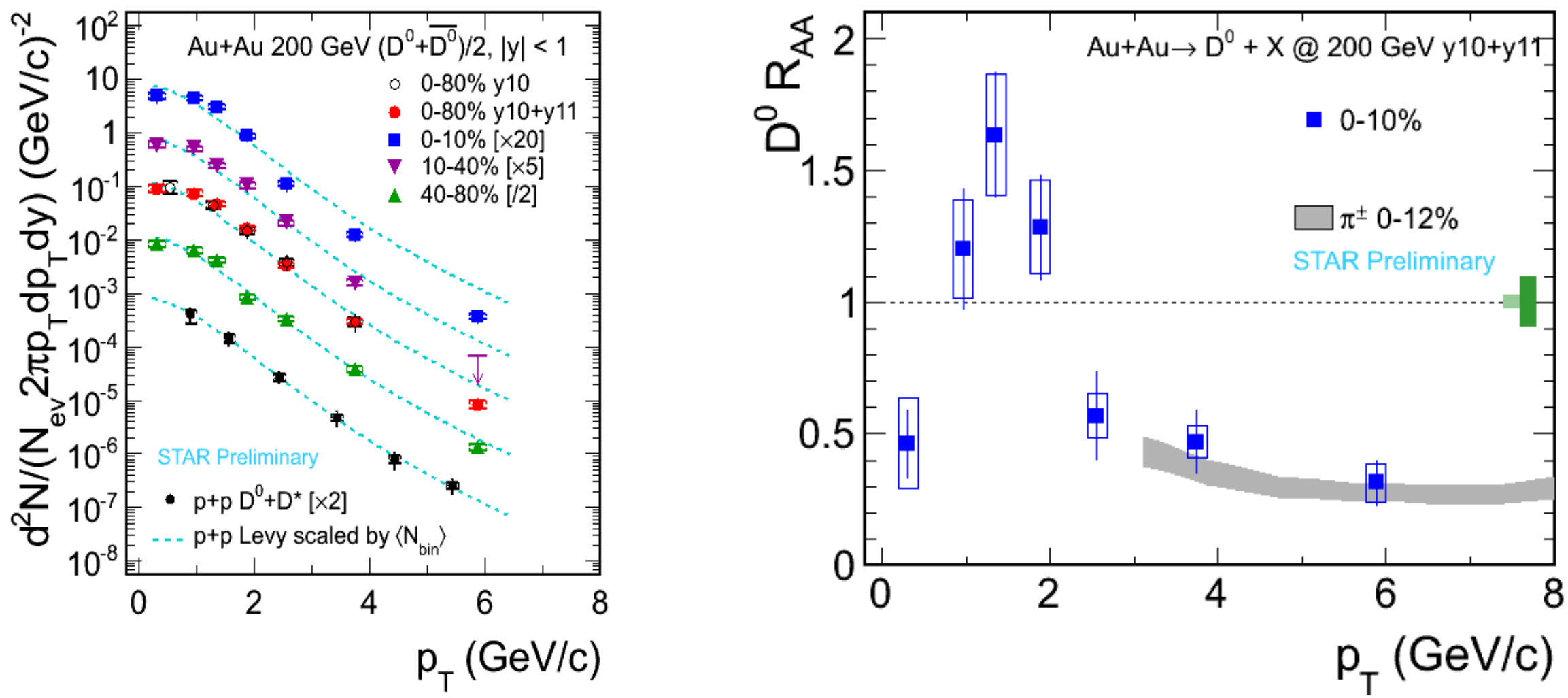

$>$ Suppressed as much as pions at high $p_{\mathrm{T}}$ : no mass effect?

$>$ Large enhancement at $1.5 \mathrm{GeV} / \mathrm{c}$ : radial flow + coalescence? 


\section{Charm: D mesons at LHC}

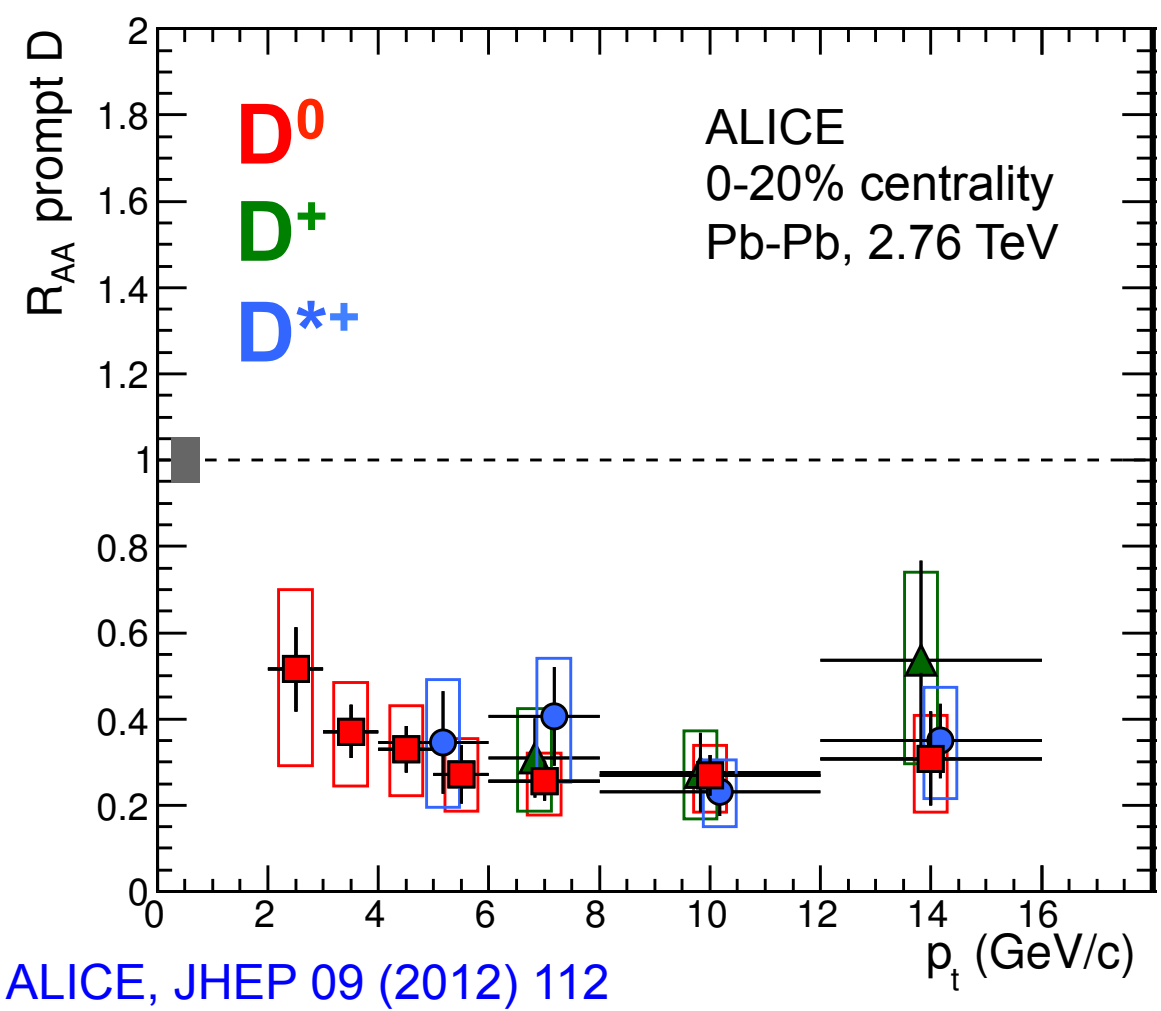

Secondary vertex reconstruction (ALICE) ${ }^{\pi}$

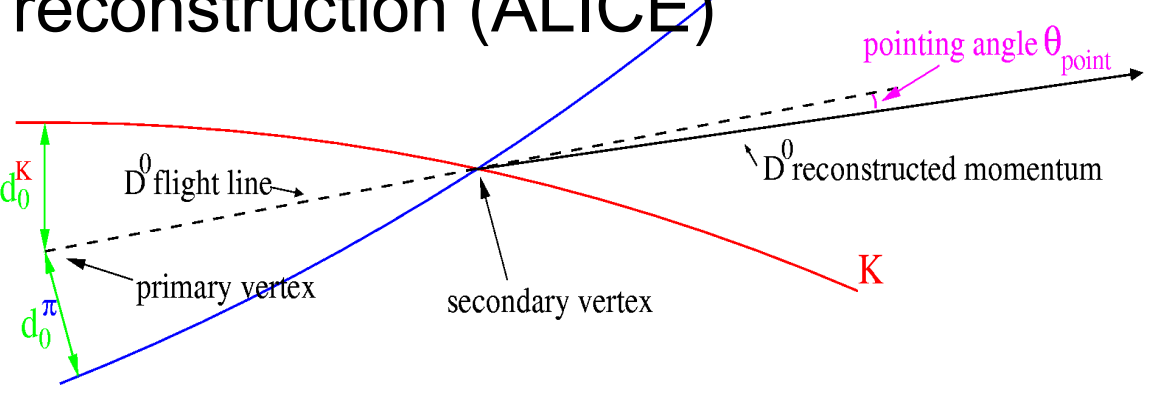

impact parameters $\sim 100 \mu \mathrm{m}$

- First $D \mathrm{R}_{\mathrm{AA}}$ measurement in heavy-ion collisions, presented by ALICE at QM2011 (LHC run 2010)

$>$ Strong suppression observed 


\section{Charm: D mesons at LHC}
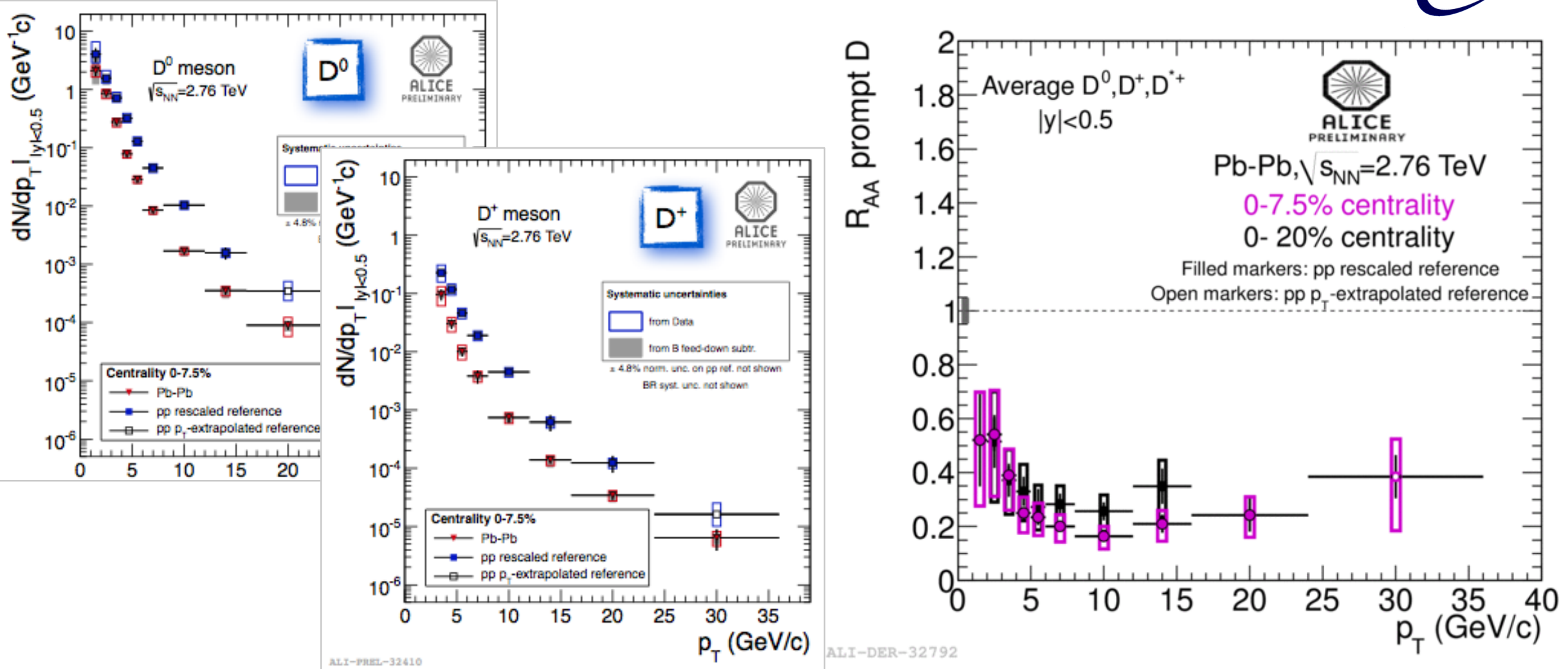

- First $D R_{A A}$ measurement in heavy-ion collisions, presented by ALICE at QM2011 (LHC run 2010)

$>$ Strong suppression observed

- Measurement extended with LHC run 2011, from 1 to $30 \mathrm{GeV} / \mathrm{c}$ 


\section{Charm: D mesons vs. pions}

\section{at $\mathrm{RHIC}$ and $\mathrm{LHC}$}
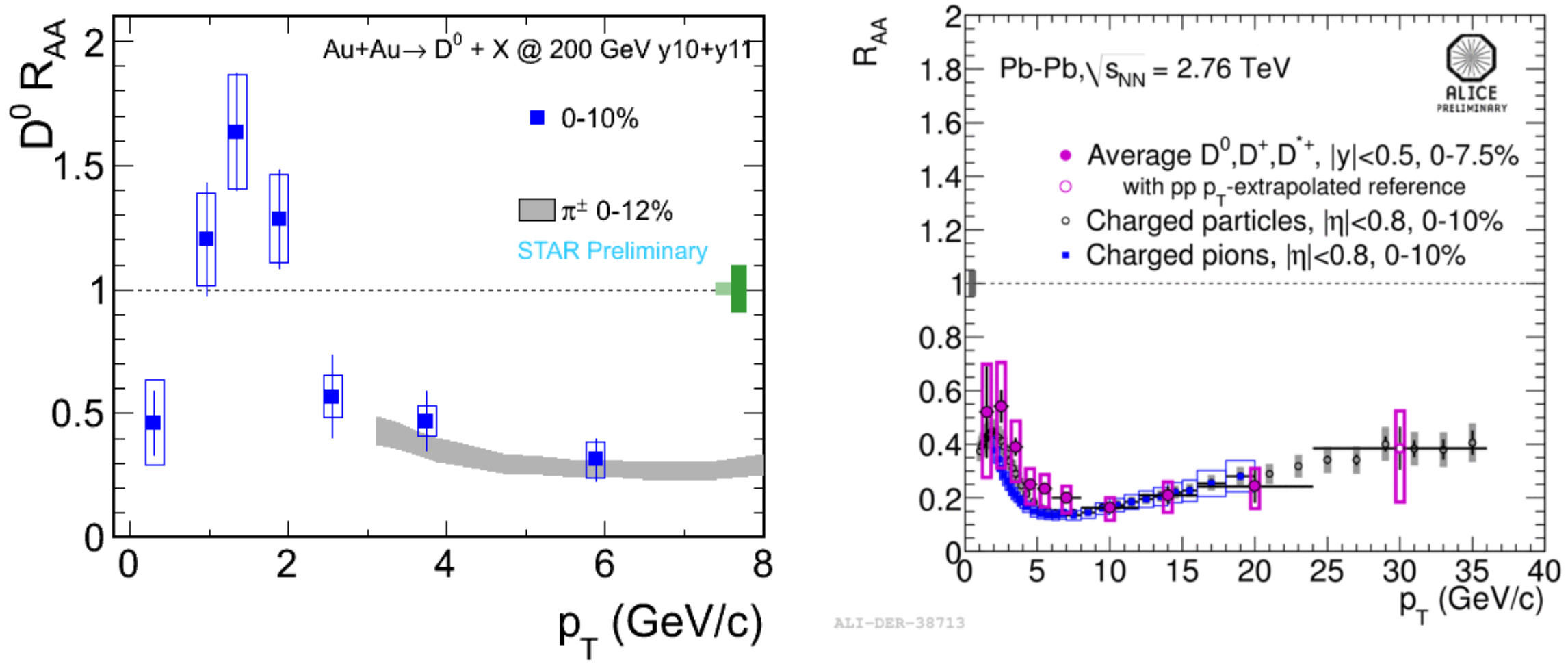

- D consistent with pions at both energies

$>$ Hint for $D>\pi$ in 3-6 GeV/c at LHC?

- D meson $\mathrm{R}_{\mathrm{AA}}$ similar at RHIC and LHC at $6 \mathrm{GeV} / \mathrm{c}$

- Strikingly different at 1-2 GeV/c: stronger effect from coalescence at RHIC due to steeper spectra? 


\section{$\mathrm{D}_{\mathrm{s}}$ meson $\mathrm{R}_{\mathrm{AA}}$ at $\mathrm{LHC}$}

- First measurement of $D_{s}$ in heavy ions

$>$ Large $\mathrm{D}_{\mathrm{s}}$ enhancement expected, if $c$ quarks recombine in the QGP
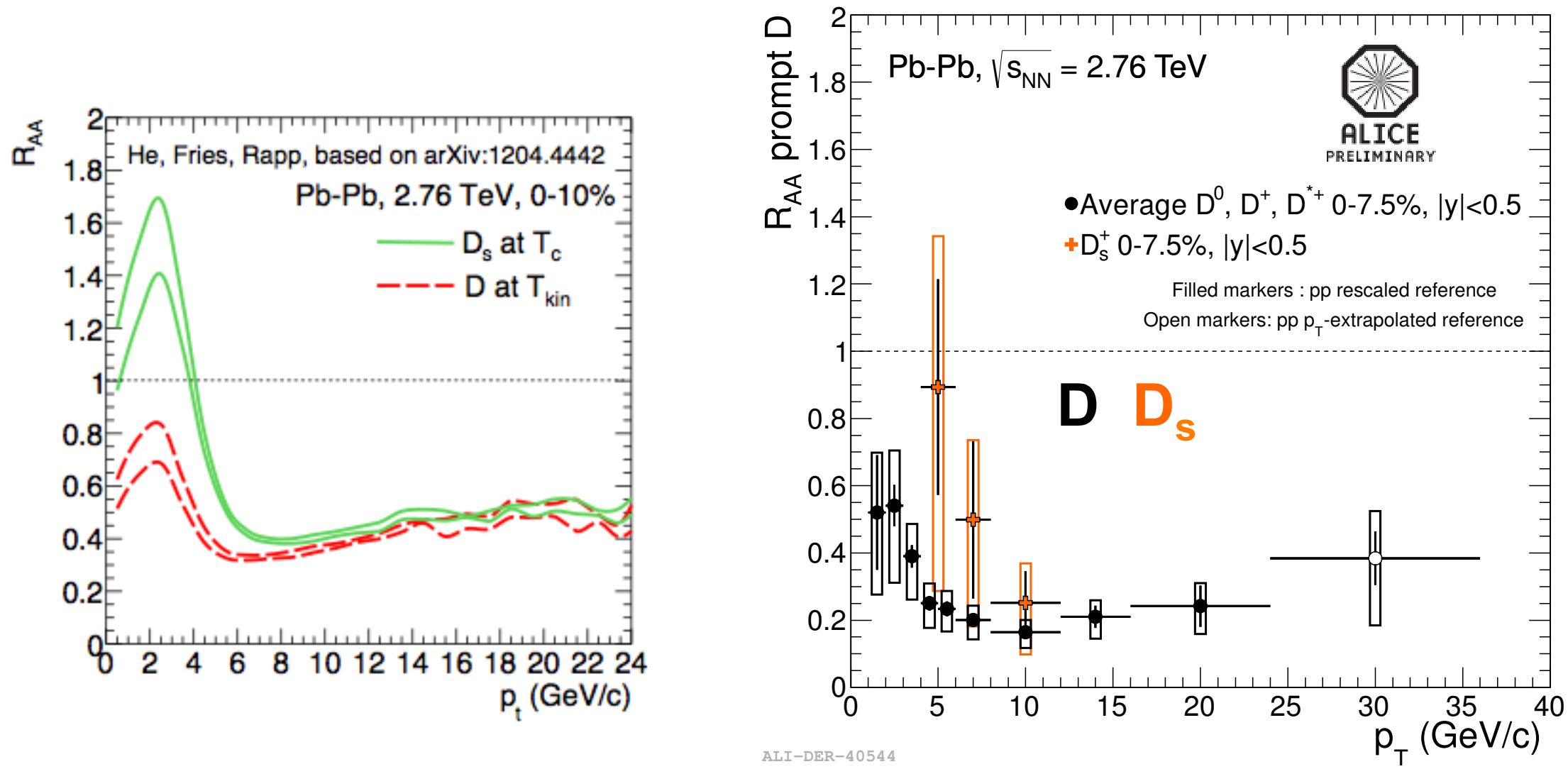

$>$ Data very intriguing, but not conclusive ( $\rightarrow$ next LHC run, upgrades) 


\section{Outline of the Talk}

- Introduction: HF probes of the medium

- Calibrating HF probes: pp results

- HF production in nucleus-nucleus:

$>$ Semi-leptonic decays

$>$ D mesons

$>B$ and $b$-jets

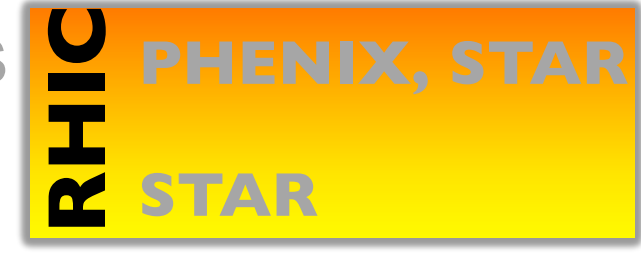

U

CMS

- Proton-nucleus control data

- HF azimuthal anisotropy

- Outlook: detector upgrades at RHIC and LHC 


\section{Beauty suppression at LHC}

$\checkmark$ First measurement of beauty $\mathrm{R}_{\mathrm{AA}}$ by CMS (CMS-PAS-HIN-12-014)
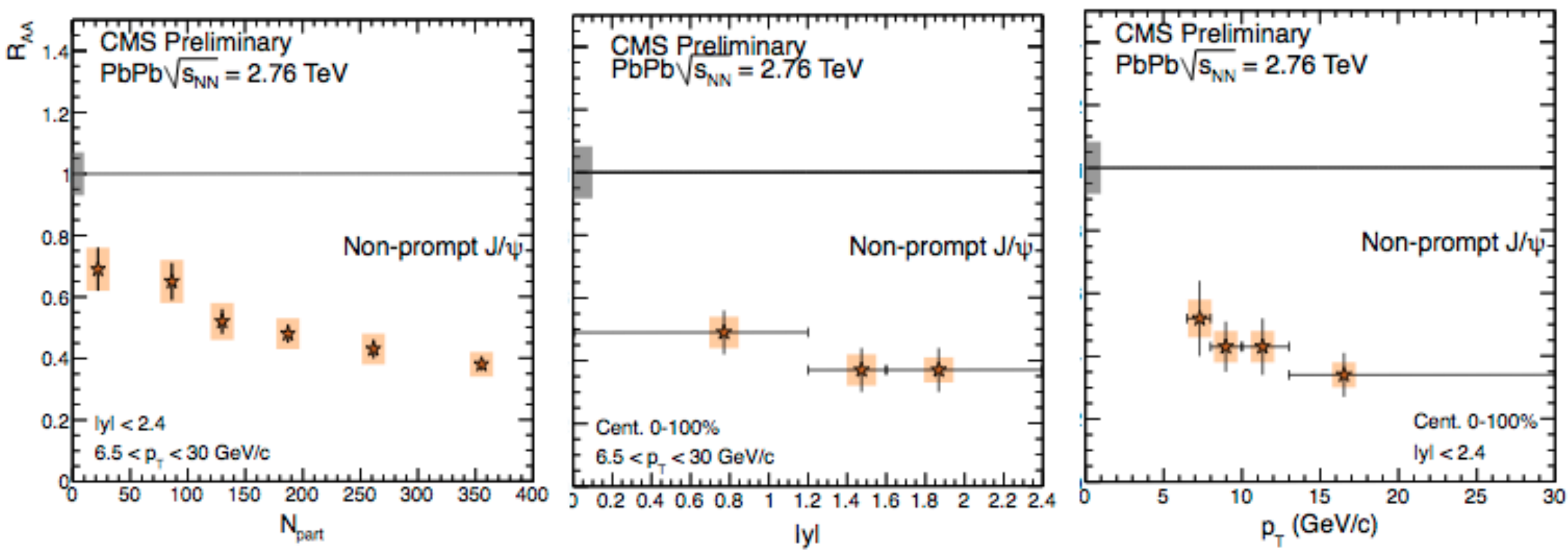

$>$ Centrality dependence of $\mathrm{B} \rightarrow \mathrm{J} / \Psi \mathrm{R}_{\mathrm{AA}}$

- $50-100 \%$ : factor $\sim 1.4 \rightarrow 0-5 \%$ : factor $\sim 2.5$

$>$ Hint of less suppression at mid-rapidity

$>$ Hint of larger suppression at higher $\mathrm{p}_{\mathrm{T}}$

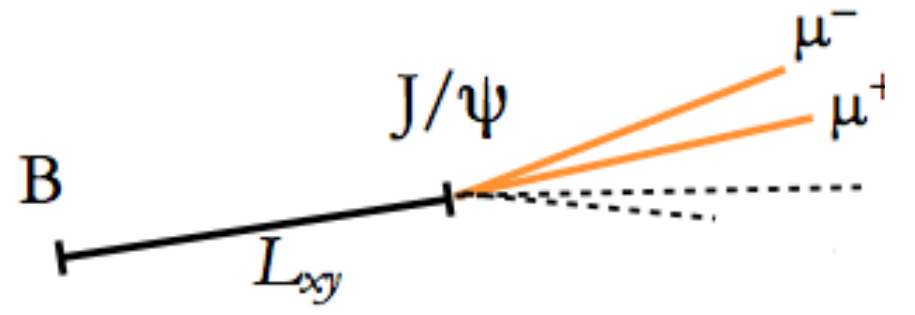




\section{Is this the dead cone?}

- Comparison of charmed mesons (ALICE) with $\mathrm{J} / \psi$ from beauty decays (CMS)

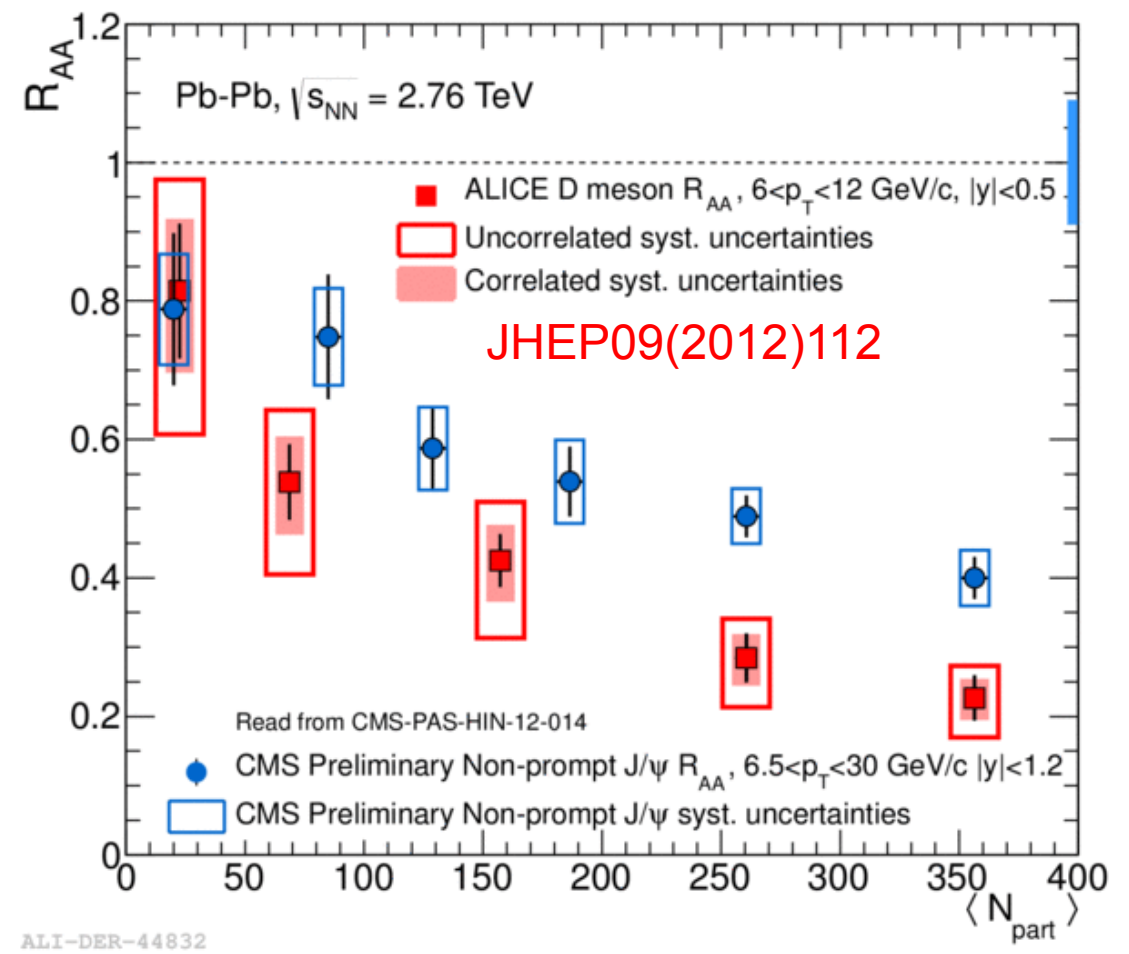

- First indication of a dependence on heavy quark mass:

$$
R_{A A}{ }^{B}>R_{A A}{ }^{D}
$$

$>$ However, kinematical ranges are not exactly the same 


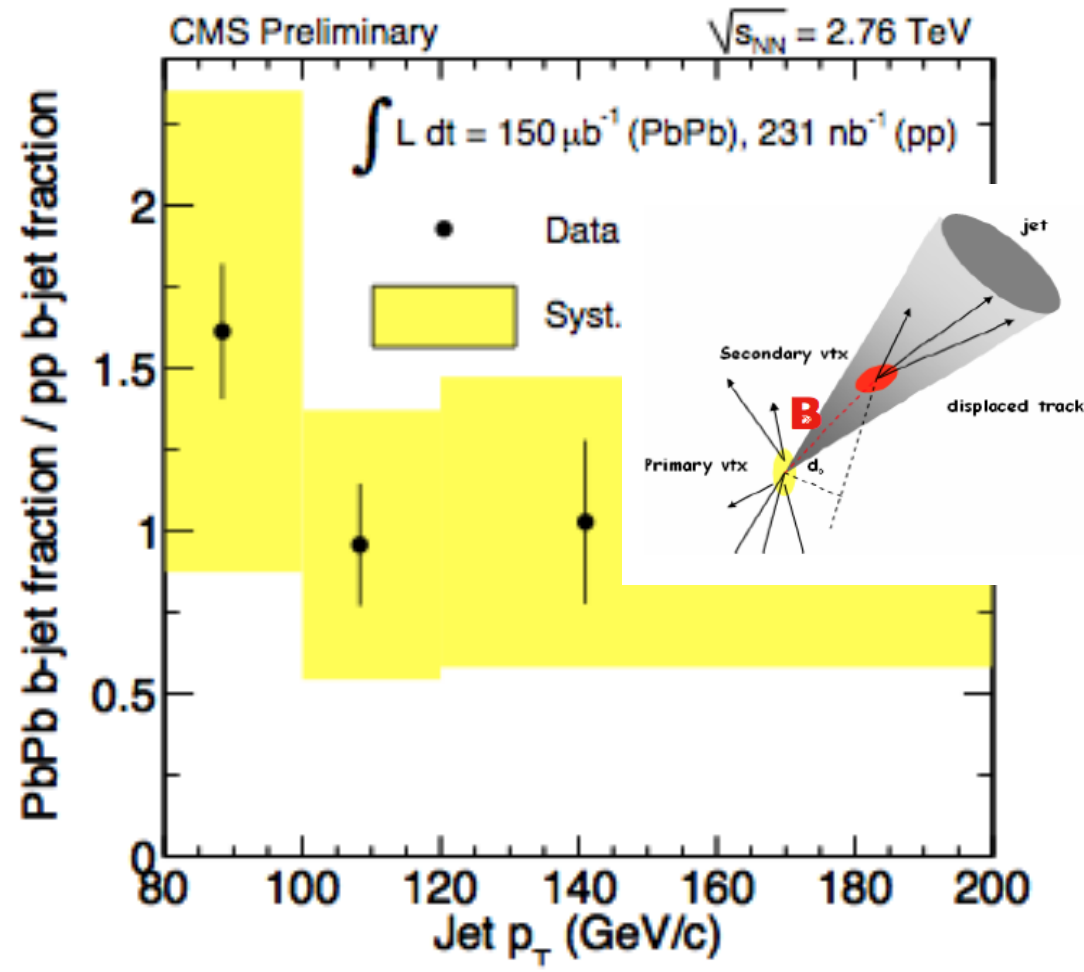

b-jet double_ratio $=$

b-fraction in $\mathrm{PbPb} /$ b-fraction in $\mathrm{PP}$

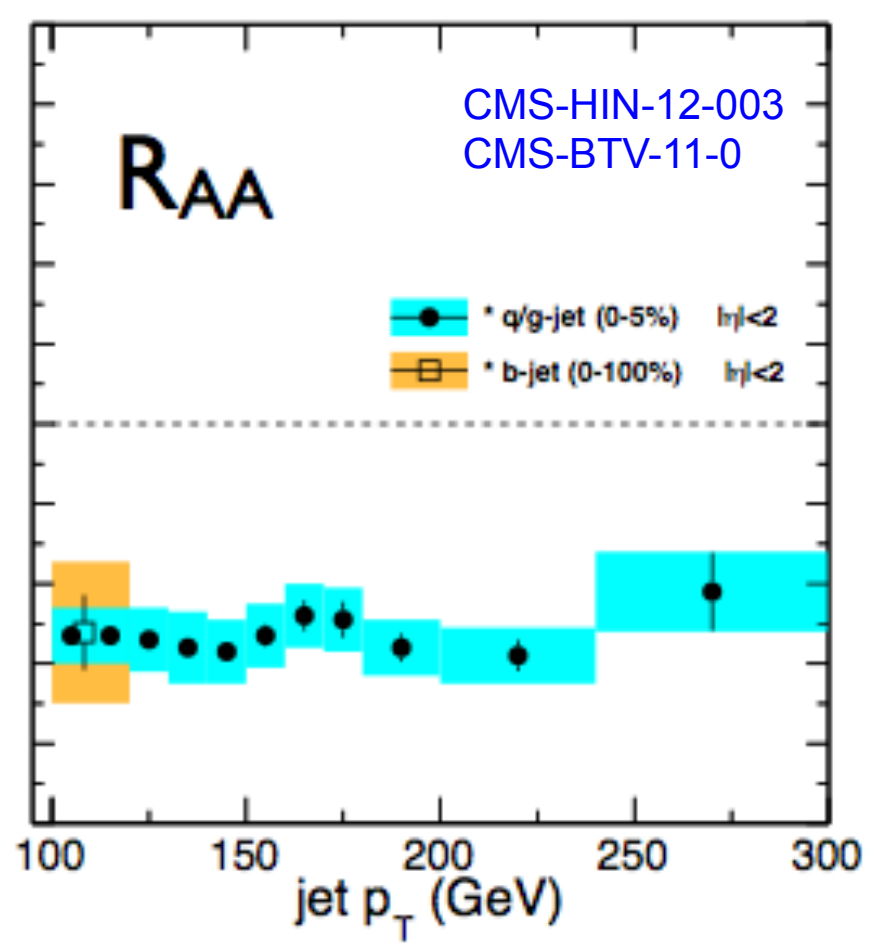

b-jet $\mathbf{R}_{\mathrm{AA}}=$

inclusive-jet $\mathrm{R}_{\mathrm{AA}}$ * b-jet double_ratio

$-\mathrm{CMS}$ finds the same $\mathrm{R}_{\mathrm{AA}}$ for b-jets as for q/g-jets, as expected at this $p_{T}$ 


\section{Outline of the Talk}

- Introduction: HF probes of the medium

- Calibrating HF probes: pp results

- HF production in nucleus-nucleus:

$>$ Semi-leptonic decays

$>\mathrm{D}$ mesons

$>\mathrm{B}$ and $\mathrm{b}$-jets

- Proton-nucleus control data

- HF azimuthal anisotropy

- Outlook: detector upgrades at RHIC and LHC 


\section{Initial state effects on heavy quarks}

- Charm production at low $p_{\mathrm{T}}: \mathrm{Q}^{2}\left(4 \mathrm{~m}_{\mathrm{c}}{ }^{2} \sim 5-10 \mathrm{GeV}^{2}\right)$

\begin{tabular}{|c|cccc|}
\hline Machine & SPS & RHIC & LHC & LHC \\
System & $\mathrm{Pb}-\mathrm{Pb}$ & $\mathrm{Au}-\mathrm{Au}$ & $\mathrm{Pb}-\mathrm{Pb}$ & pp \\
$\sqrt{s_{\mathrm{NN}}}$ & $17 \mathrm{GeV}$ & $200 \mathrm{GeV}$ & $5.5 \mathrm{TeV}$ & $14 \mathrm{TeV}$ \\
\hline \hline $\mathrm{c} \overline{\mathrm{c}}$ & $x \simeq 10^{-1}$ & $x \simeq 10^{-2}$ & $x \simeq 4 \times 10^{-4}$ & $x \simeq 2 \times 10^{-4}$ \\
$\mathrm{~b} \overline{\mathrm{b}}$ & - & - & $x \simeq 2 \times 10^{-3}$ & $x \simeq 6 \times 10^{-4}$ \\
\hline
\end{tabular}

$\rightarrow$ interpretation of HF (charm) measurements in AA requires $\mathrm{pA}$ reference

$\rightarrow$ charm in $\mathrm{pA}$ : access to small- $x$ gluons with perturbative probes

\section{Nuclear modification of PDFs}

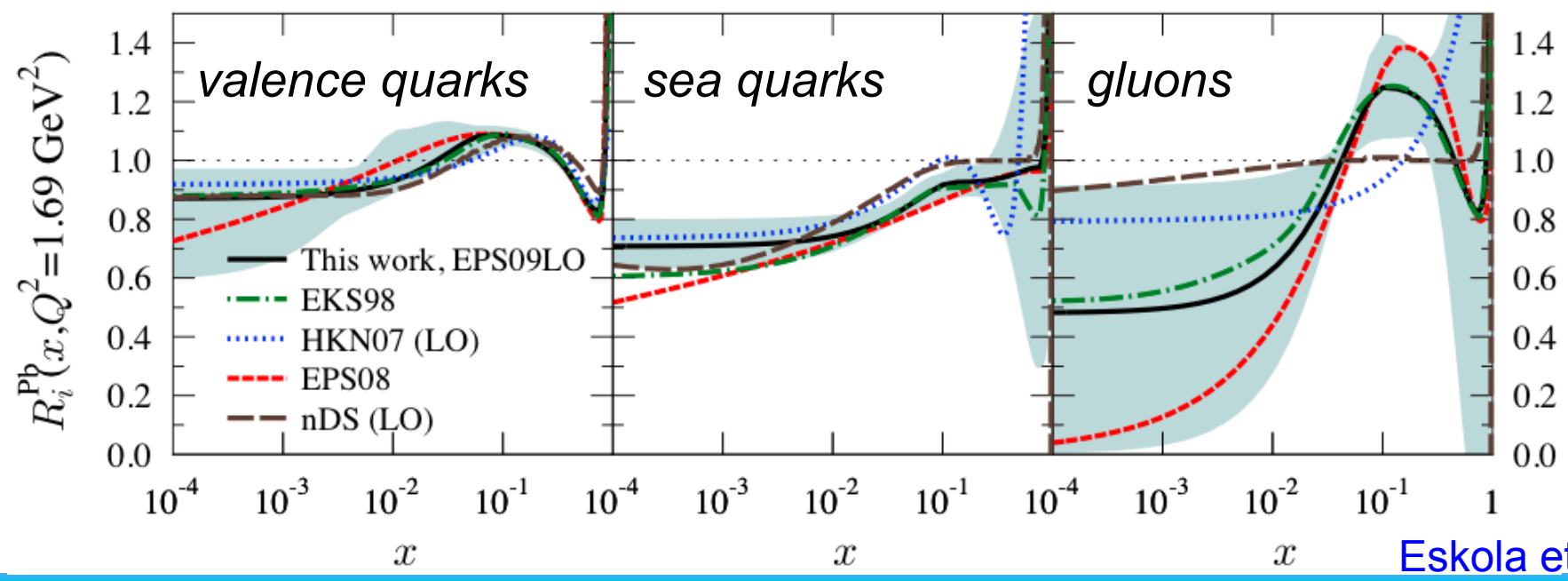




\section{Only a QCD medium effect? RHIC}

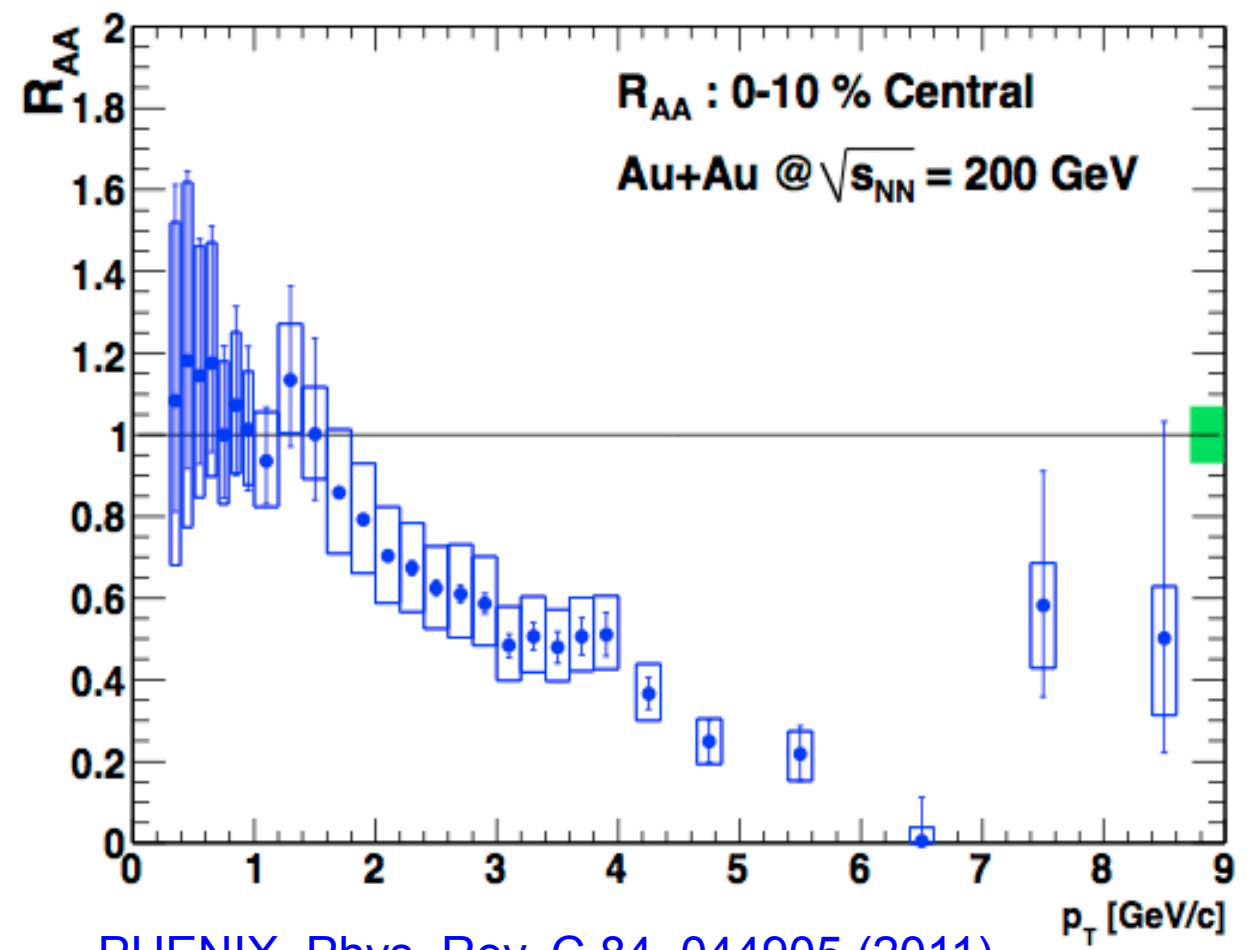

PHENIX, Phys. Rev. C 84, 044905 (2011)

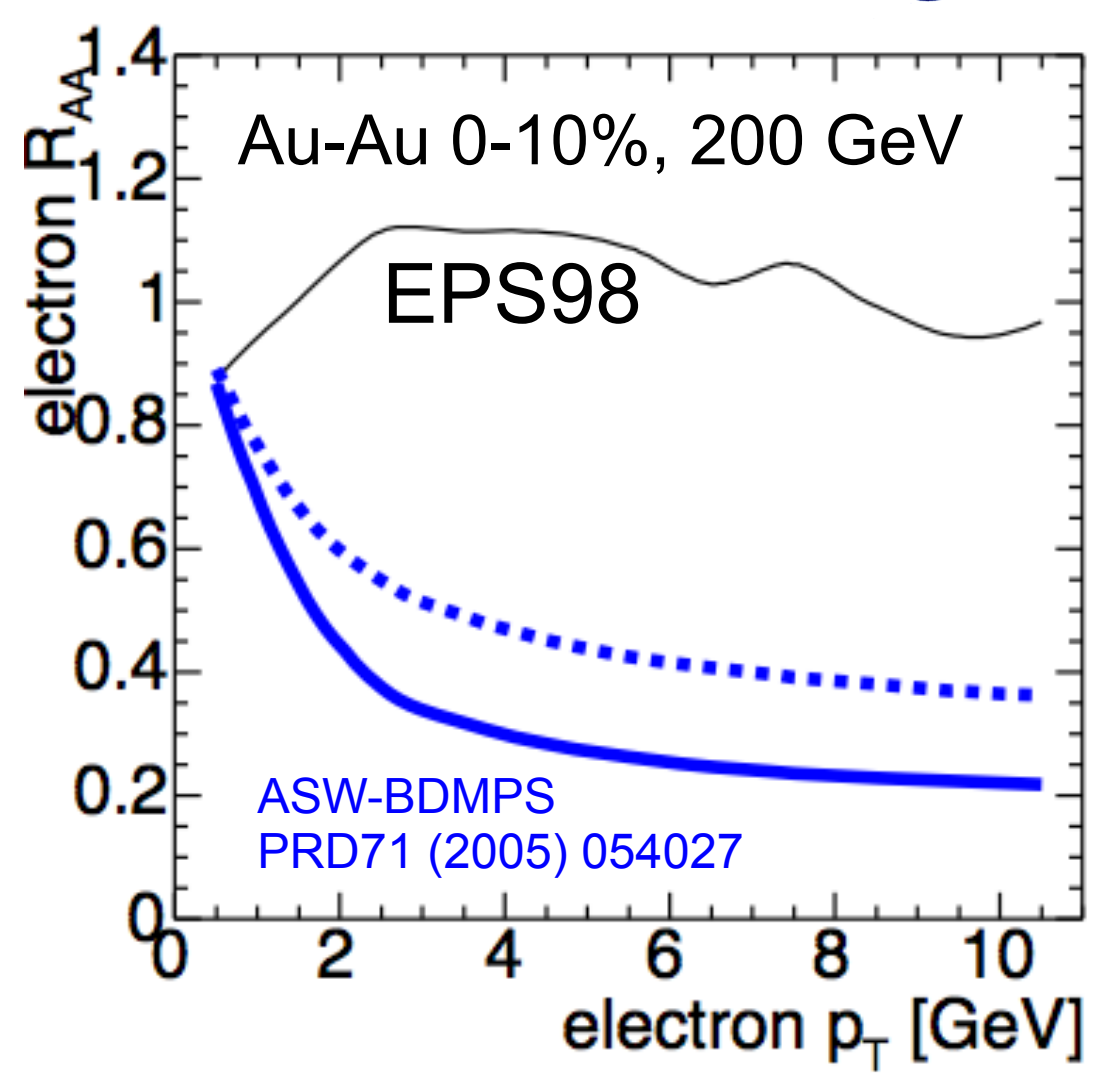

- Small effect expected from PDFs shadowing ( $<10 \%)$

- Mostly a hot medium effect?

p/d-A control crucial to quantify initial-state effects 


\section{Only a QCD medium effect? LHC}
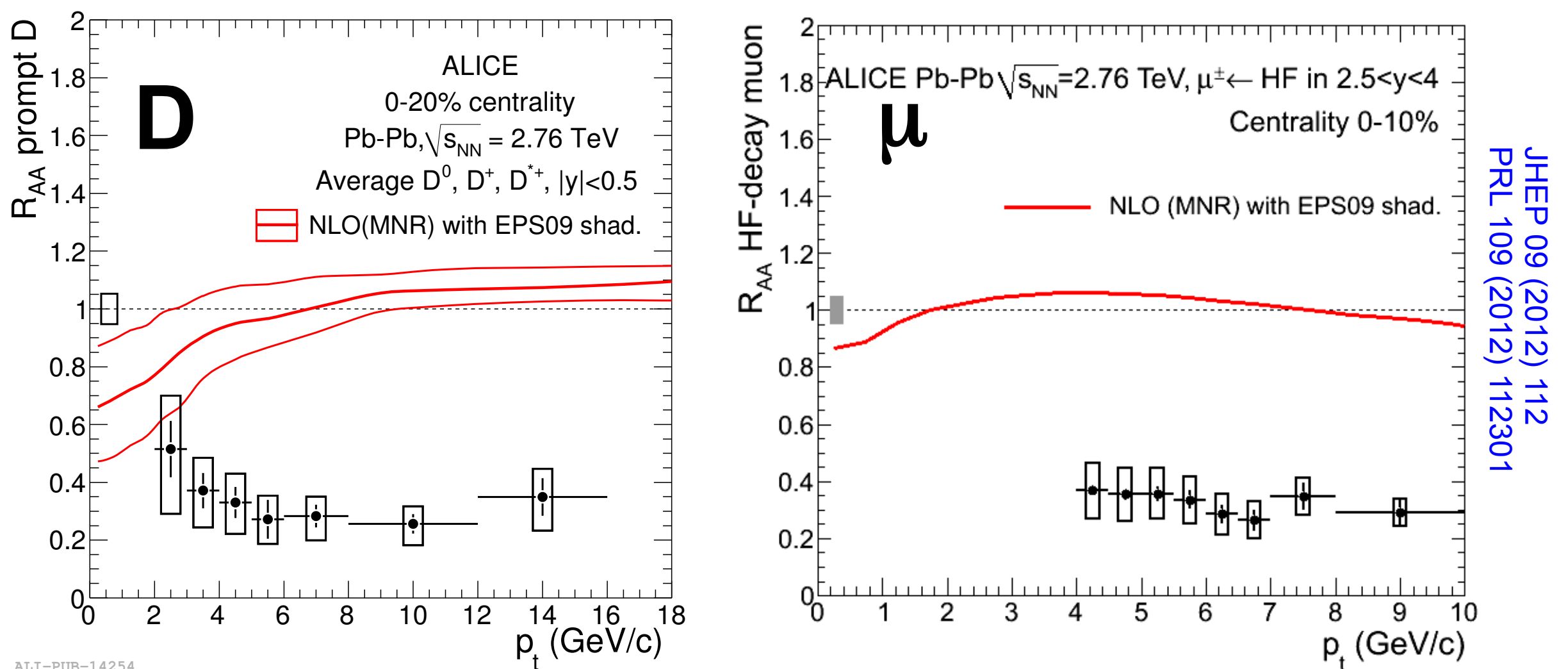

- Small effect expected from PDFs shadowing above $5 \mathrm{GeV} / \mathrm{c}$

- Mostly a hot medium effect above $5 \mathrm{GeV} / \mathrm{c}$ ?

$\checkmark$ p/d-A control crucial to quantify initial-state effects 


\section{$H F$ e in d-Au: $R H I C$ results}

Phys. Rev. Lett. 109, 242301 (2012)
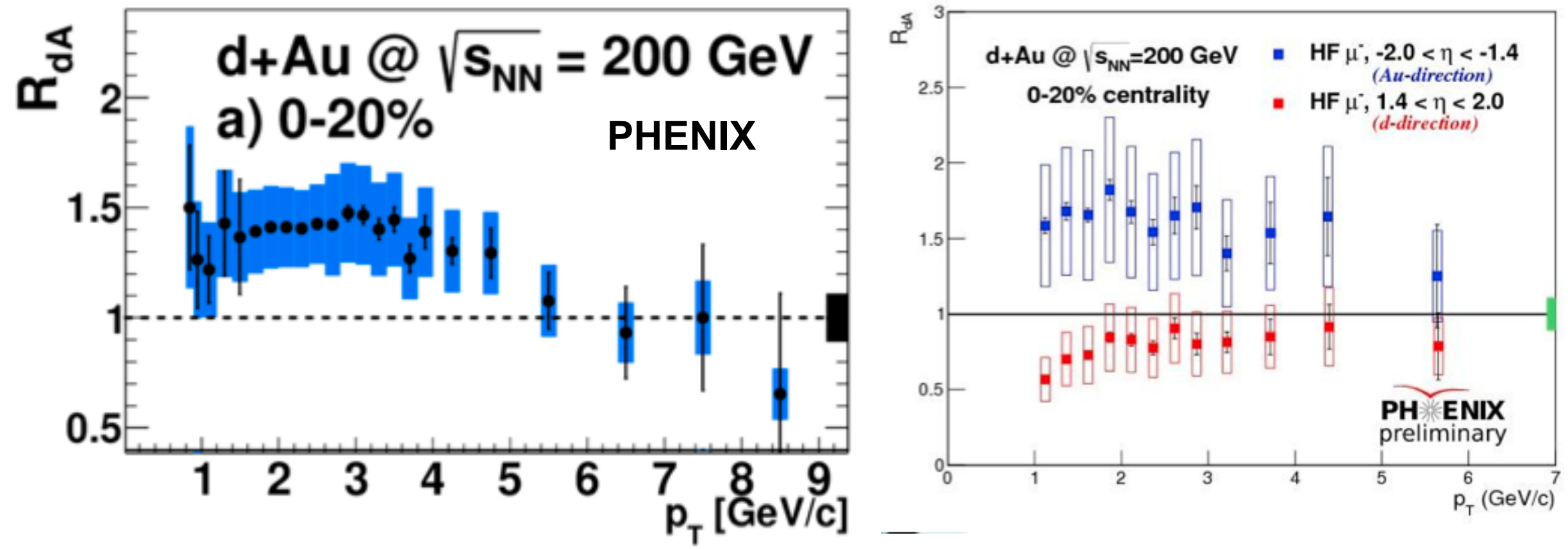

- Low- $p_{\mathrm{T}}$ electrons (mid-y) and muons (forward y) largely enhanced

More than expected from anti-shadowing? 


\section{$H F$ e in d-Au: $R H I C$ results}

Phys. Rev. Lett. 109, 242301 (2012)
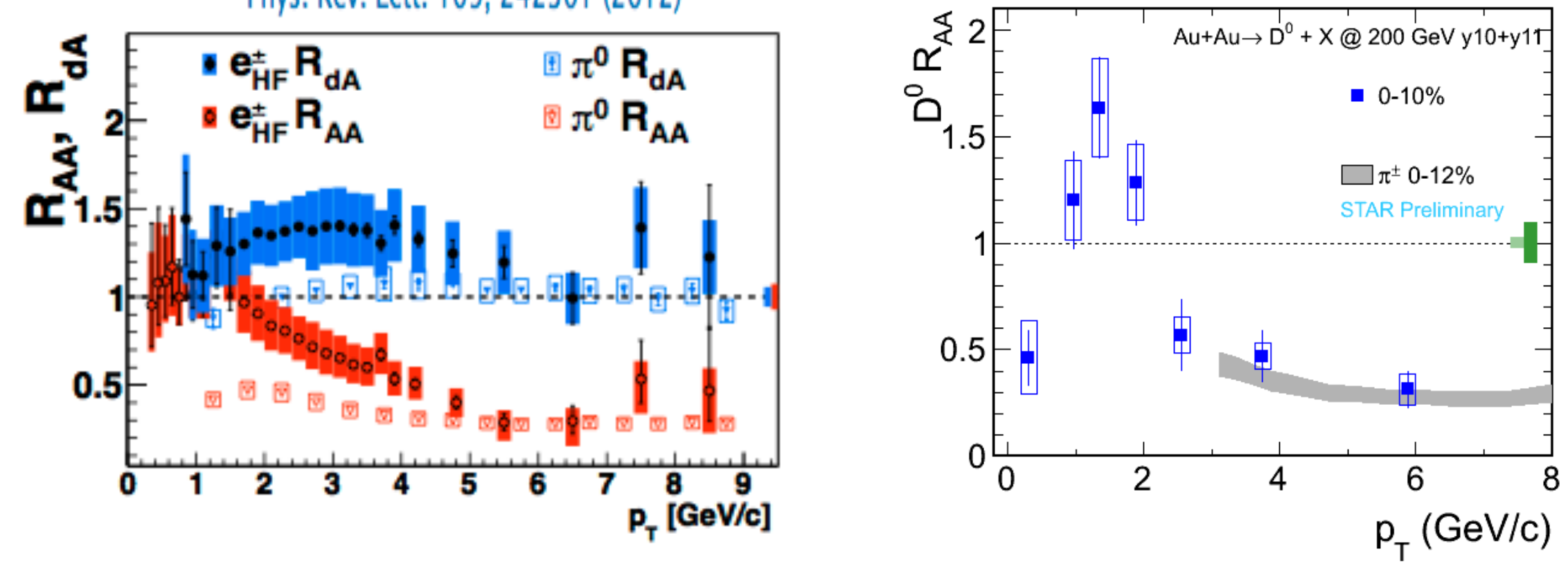

- Low- $p_{\mathrm{T}}$ electrons (mid-y) and muons (forward y) largely enhanced

More than expected from anti-shadowing?

- Significant role of (mass-dependent?) $\mathrm{k}_{\mathrm{T}}$ broadening / initialstate partonic scattering?

- Or is there some final-state "activity"? $\rightarrow$ need D mesons!!! 


\section{$\mathrm{HF}$ in $\mathrm{p}-\mathrm{Pb}$ at $\mathrm{LHC}$ : analyses ongoing ...}

- Large integrated lumi of the $\mathrm{p}-\mathrm{Pb}$ run $(\sim 30 / \mathrm{nb})$ will allow the experiments (including $\mathrm{LHCb}$ !) to measure, with precision PbPb2011:

$>$ HF-decay electrons and muons

$>D$ mesons, including $D_{s}$

$>\mathrm{B} \rightarrow \mathrm{J} / \psi$, b-jets

- Example: D meson signals in ALICE
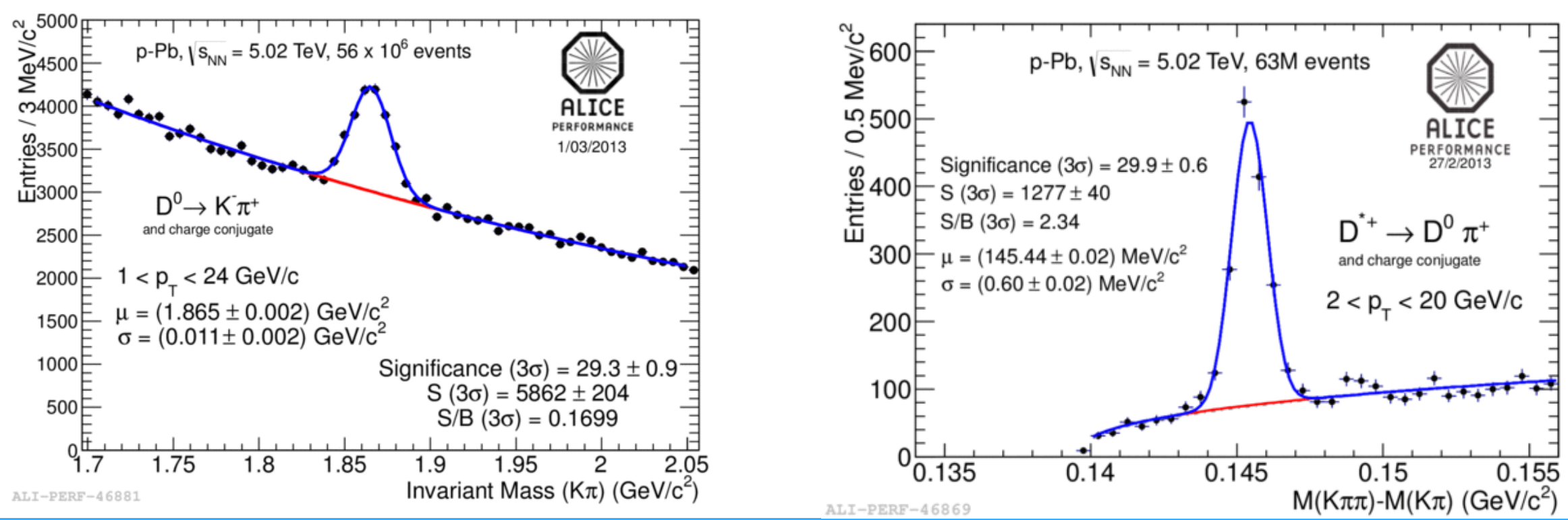


\section{Outline of the Talk}

- Introduction: HF probes of the medium

- Calibrating HF probes: pp results

- HF production in nucleus-nucleus:

$>$ Semi-leptonic decays

$>$ D mesons

$>\mathrm{B}$ and $\mathrm{b}$-jets

- Proton-nucleus control data

$\checkmark$ HF azimuthal anisotropy

- Outlook: detector upgrades at RHIC and LHC 


\section{Heavy-flavour azimuthal anisotropy,}

\section{from $\mathrm{RHIC}$ to LHC}

- Electrons from HF show a $v_{2}$ of up to 0.15 at RHIC (PHENIX, STAR)

$>$ Charm does flow!

$>v_{2}$ significantly smaller than for pions above $2 \mathrm{GeV} / \mathrm{c}$ (might be decay kinematics, rather than a difference heavy vs. light)

- First measurements at the LHC (ALICE): electron $v_{2}$ comparable to RHIC
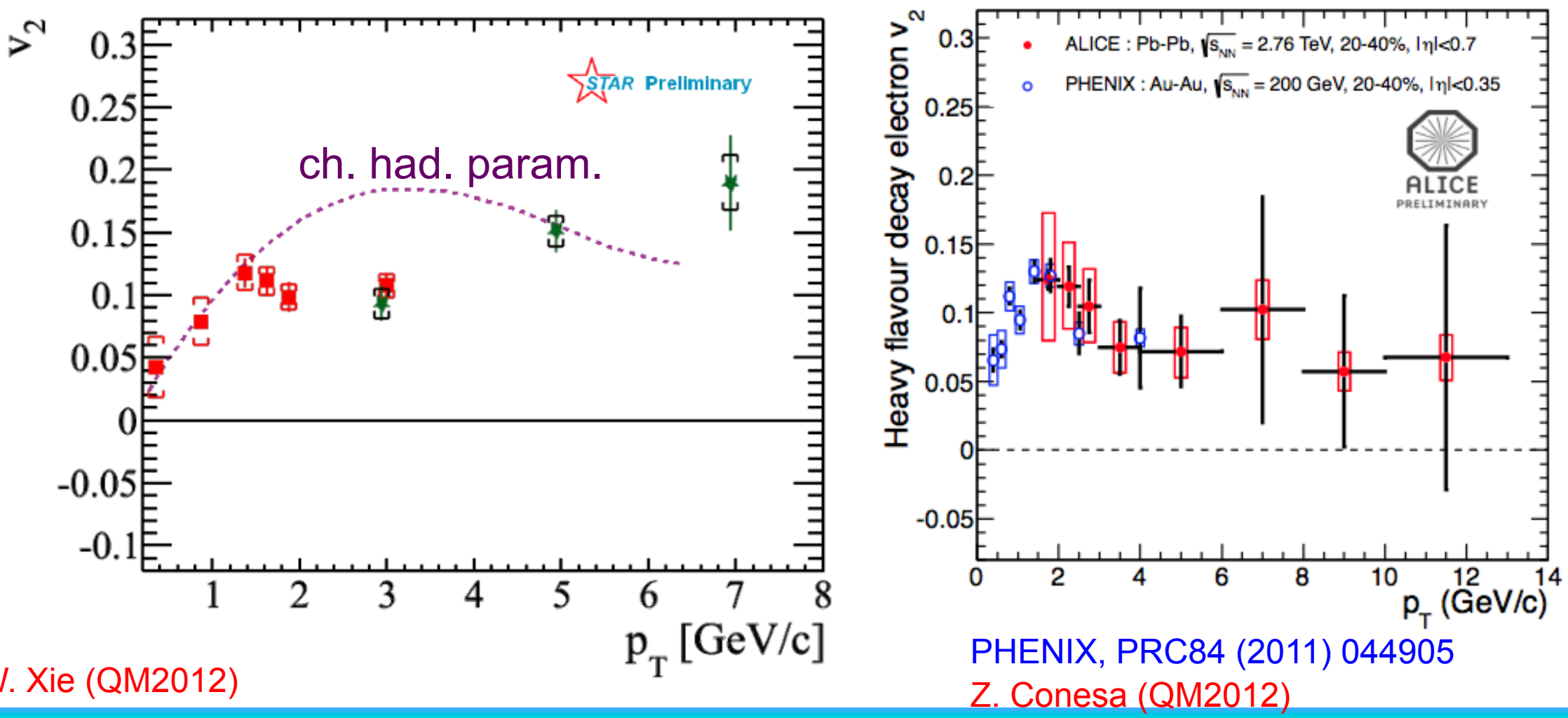


\section{Heavy-flavour azimuthal anisotropy,}

\section{from $\mathrm{RHIC}$ to LHC}

- First D measurements at the LHC (ALICE): D meson $v_{2}>0$ in $30-50 \%$ class

$>\mathrm{D}$ meson $\mathrm{v}_{2}>0$ in $30-50 \%$ class \& Comparable to $\mathrm{v}_{2}$ of charged hadrons

$>$ Suggests flow at low $p_{T}$ and path-length dependent suppression at high $p_{T}$

- First $D v_{2}$ measurement at RHIC presented at QM by STAR (not shown)
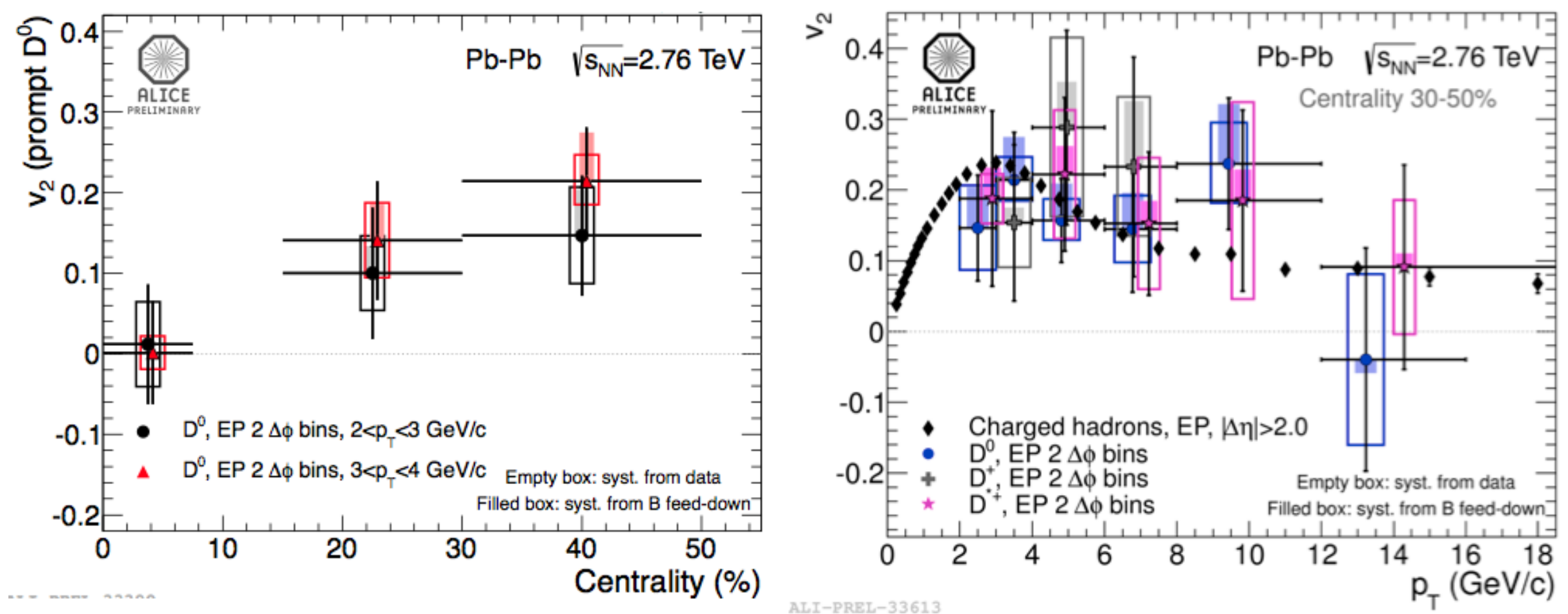

Z. Conesa (QM2012) 


\section{LHC: comparison with models $\left(R_{A A}\right)$}

- Several models based on E-loss and heavy-quark transport describe qualitatively the measured light, charm, and beauty $R_{A A}$

\section{charm}

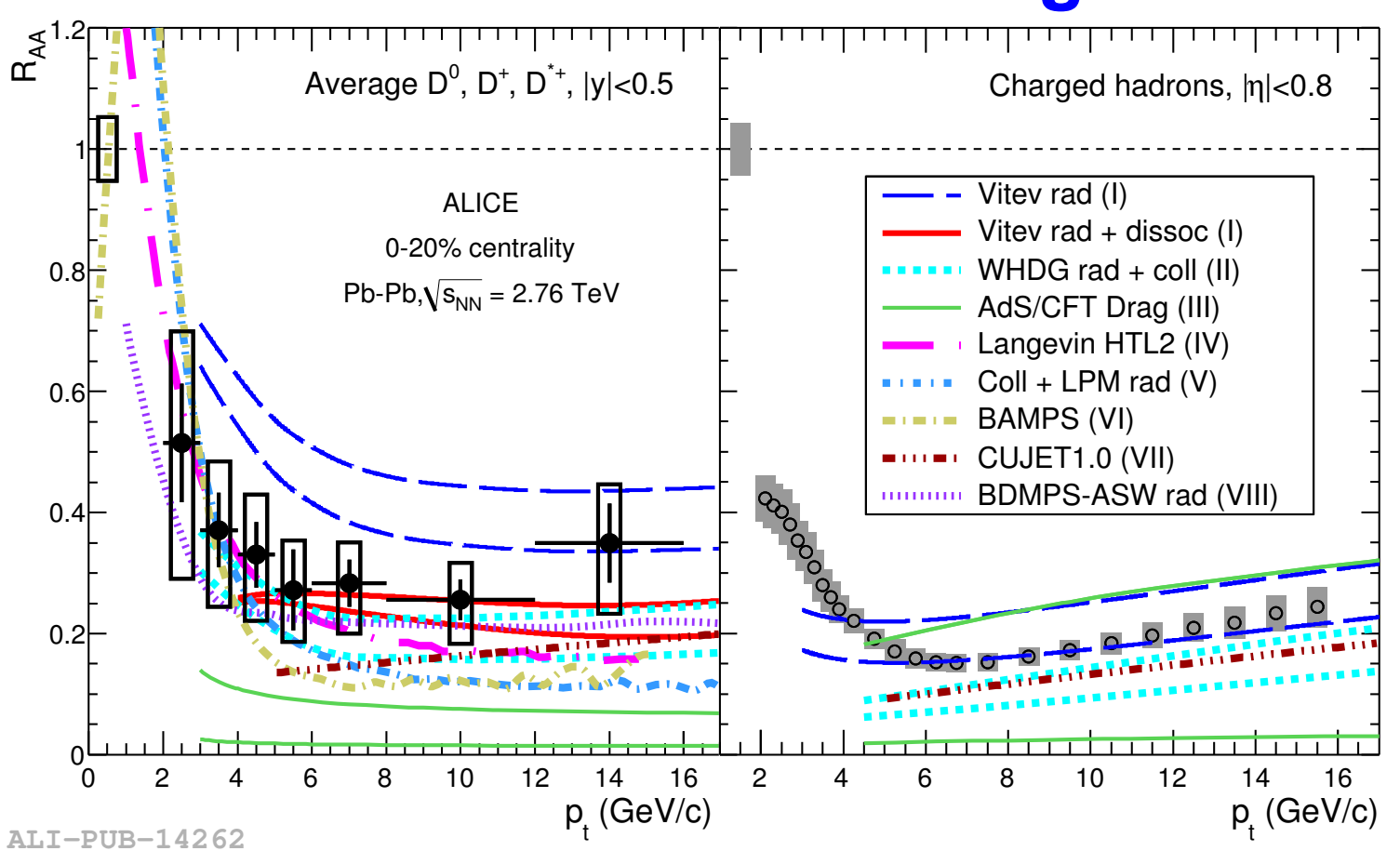

ALICE, JHEP 09 (2012) 112

\section{"light"}

Charged hadrons, $|\eta|<0.8$

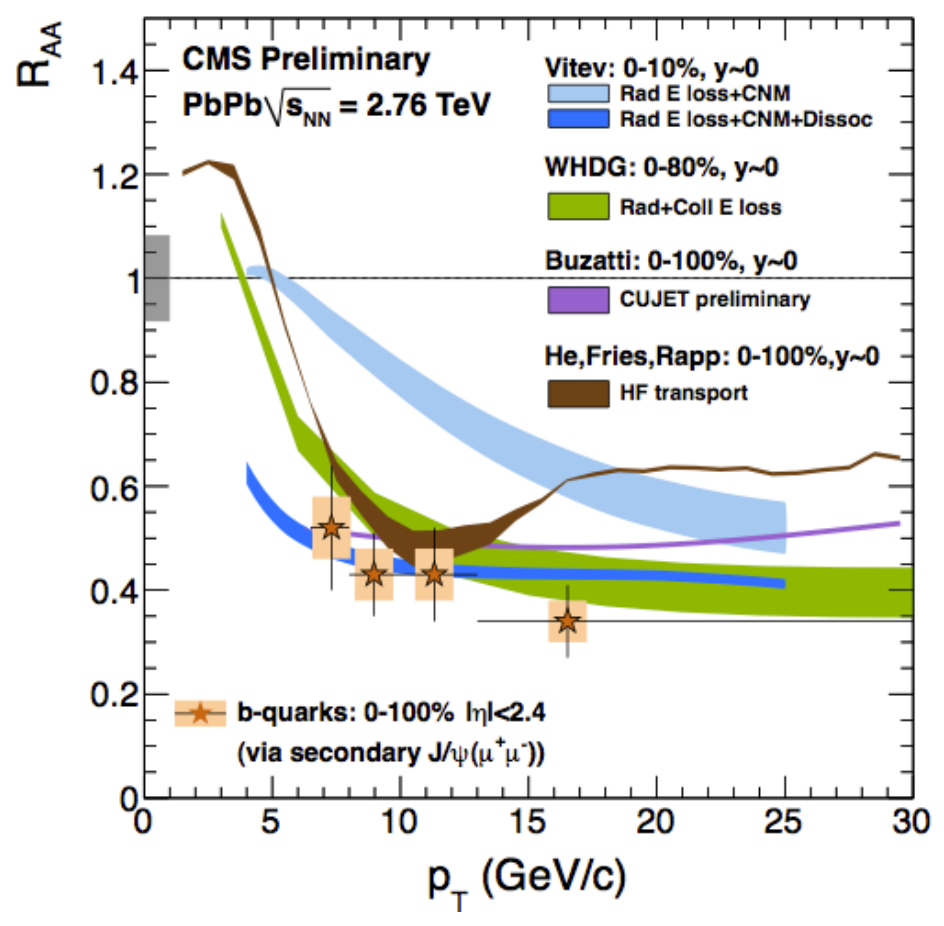

CMS-PAS-HIN-12-014

\section{beauty}




\section{LHC: comparison with models $\left(R_{A A}\right.$ and $\left.v_{2}\right) \iota^{\text {INFN }}$}
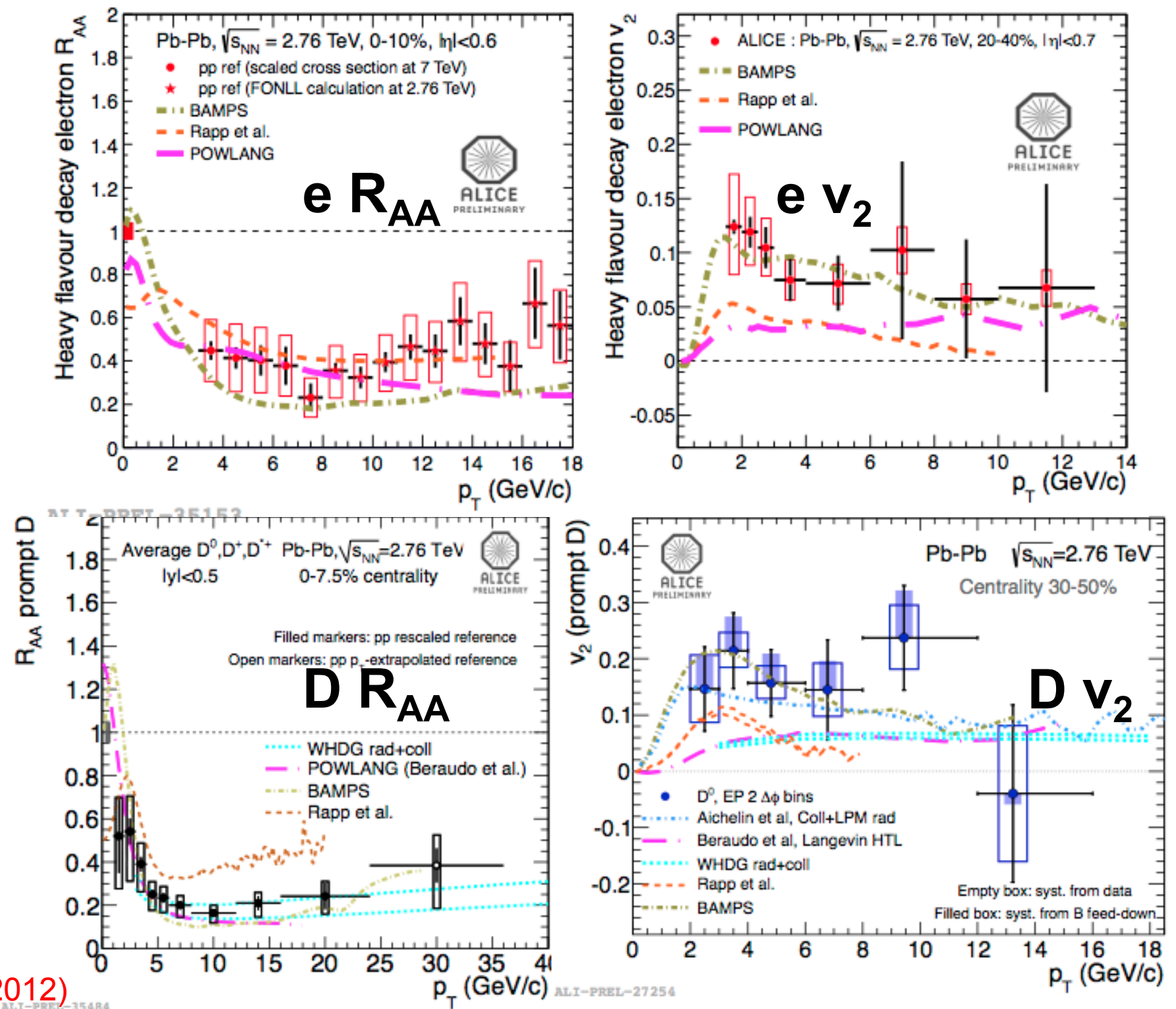

Z. Conesa (QM2012) 
LHC: comparison with models $\left(R_{A A}\right.$ and $\left.v_{2}\right) \bigcup^{\text {INFN }}$

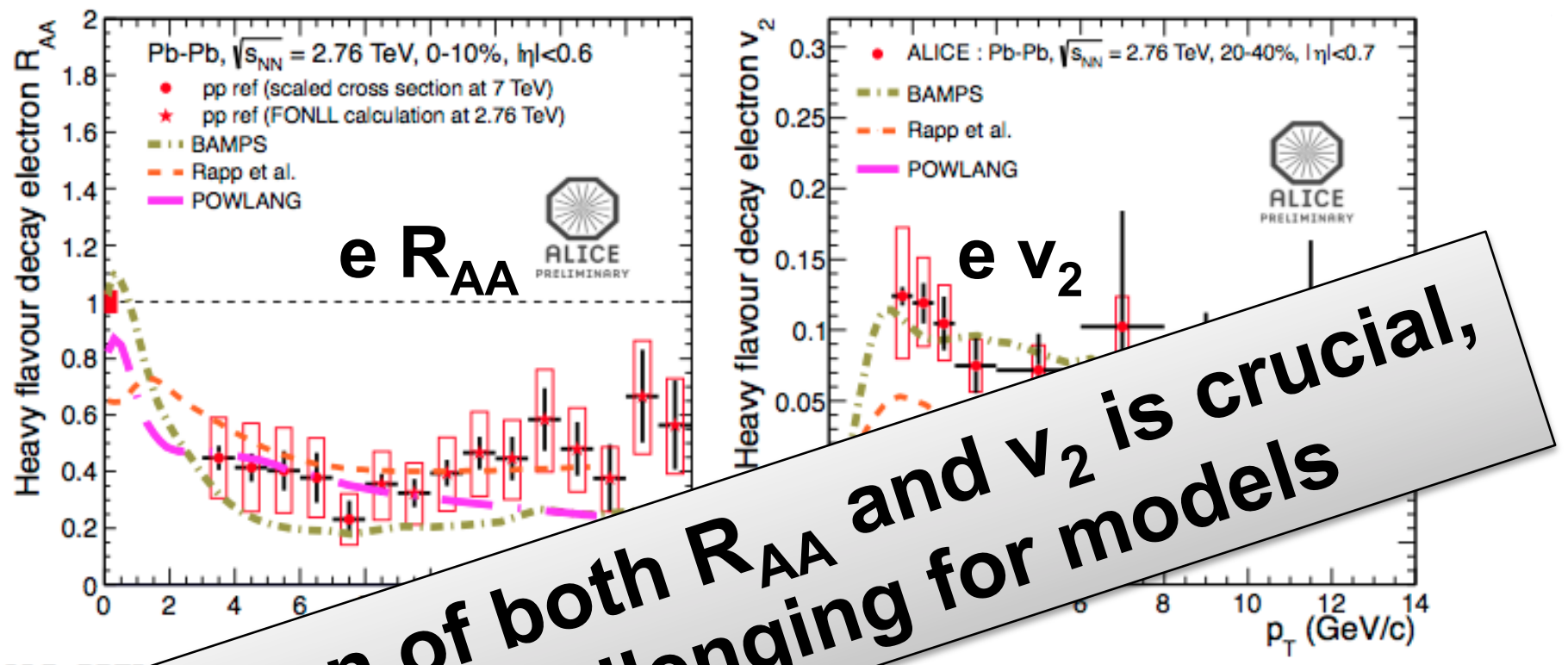

Z. Conesa (QM2012)

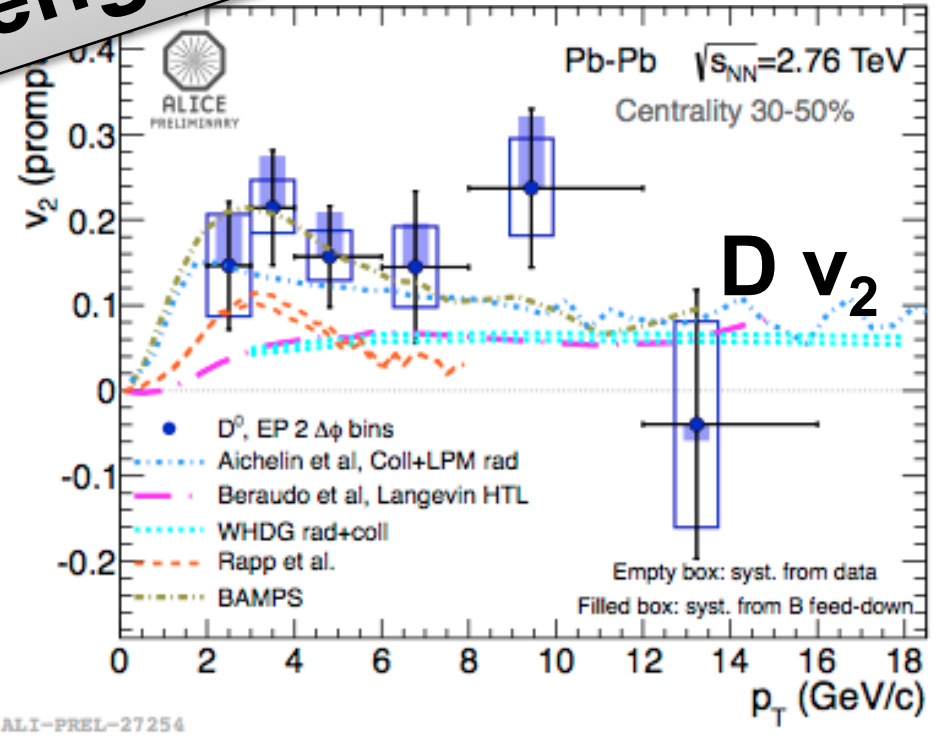




\section{Towards HF tomography}

\section{- D suppression in different azimuthal directions}

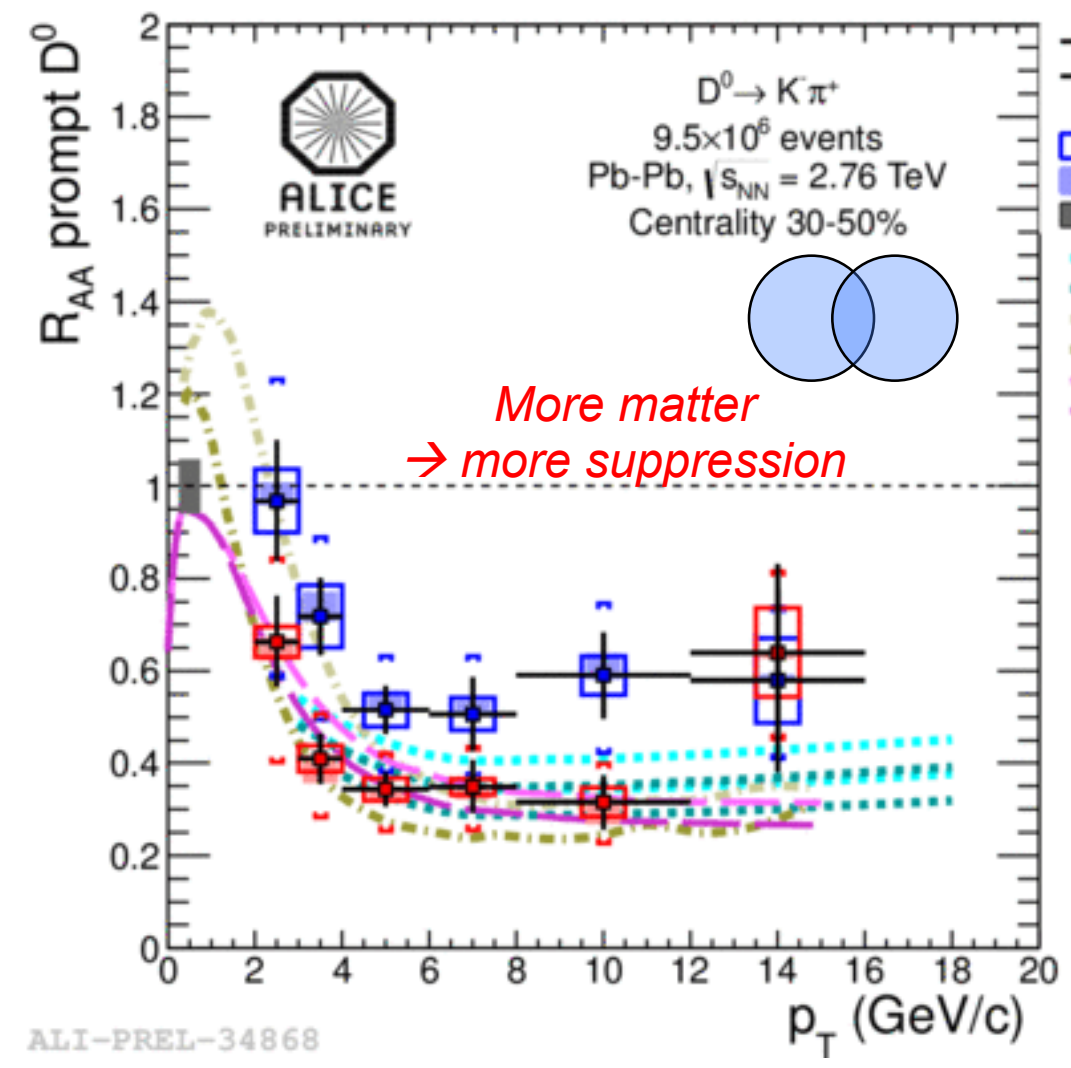

much more of this with next

LHC run, then upgrades
$D^{0} R_{A A}$ In Plane

$D^{0} R_{A A}$ Out Of Plane

Correlated syst. uncenaintles

Uncorrelated syst. uncertainties

Anticorrelated syst. uncertainties

Global norm uncertainty

WHDG rad+coll (In)

WHDG rad+coll (Out)

BAMPS (In)

BAMPS (Out)

POWLANG (Beraudo et

POWLANG (Beraudo et

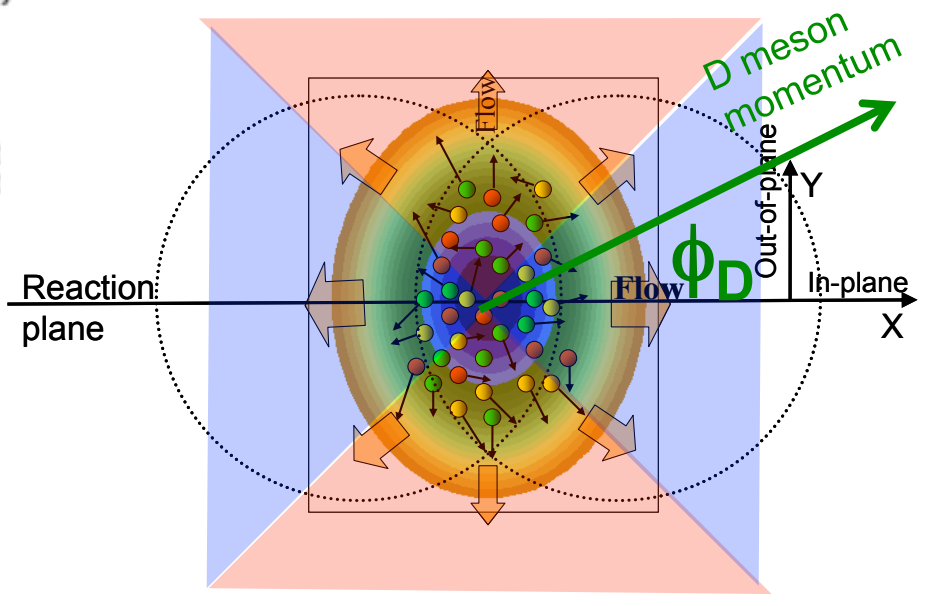

- Models tend to describe $\mathrm{R}_{\mathrm{AA}}$ (out-of-plane) better than $R_{A A}$ (in-plane) 


\section{Outline of the Talk}

- Introduction: HF probes of the medium

- Calibrating HF probes: pp results

- HF production in nucleus-nucleus:

$>$ Semi-leptonic decays

$>\mathrm{D}$ mesons

$>\mathrm{B}$ and $\mathrm{b}$-jets

- Proton-nucleus control data

- HF azimuthal anisotropy

- Outlook: detector upgrades at RHIC and LHC

$>$ Heavy flavour: a central topic for upgrades of all the HI experiments! 


\section{STAR: Heavy Flavour Tracker}

Projections 500M evts
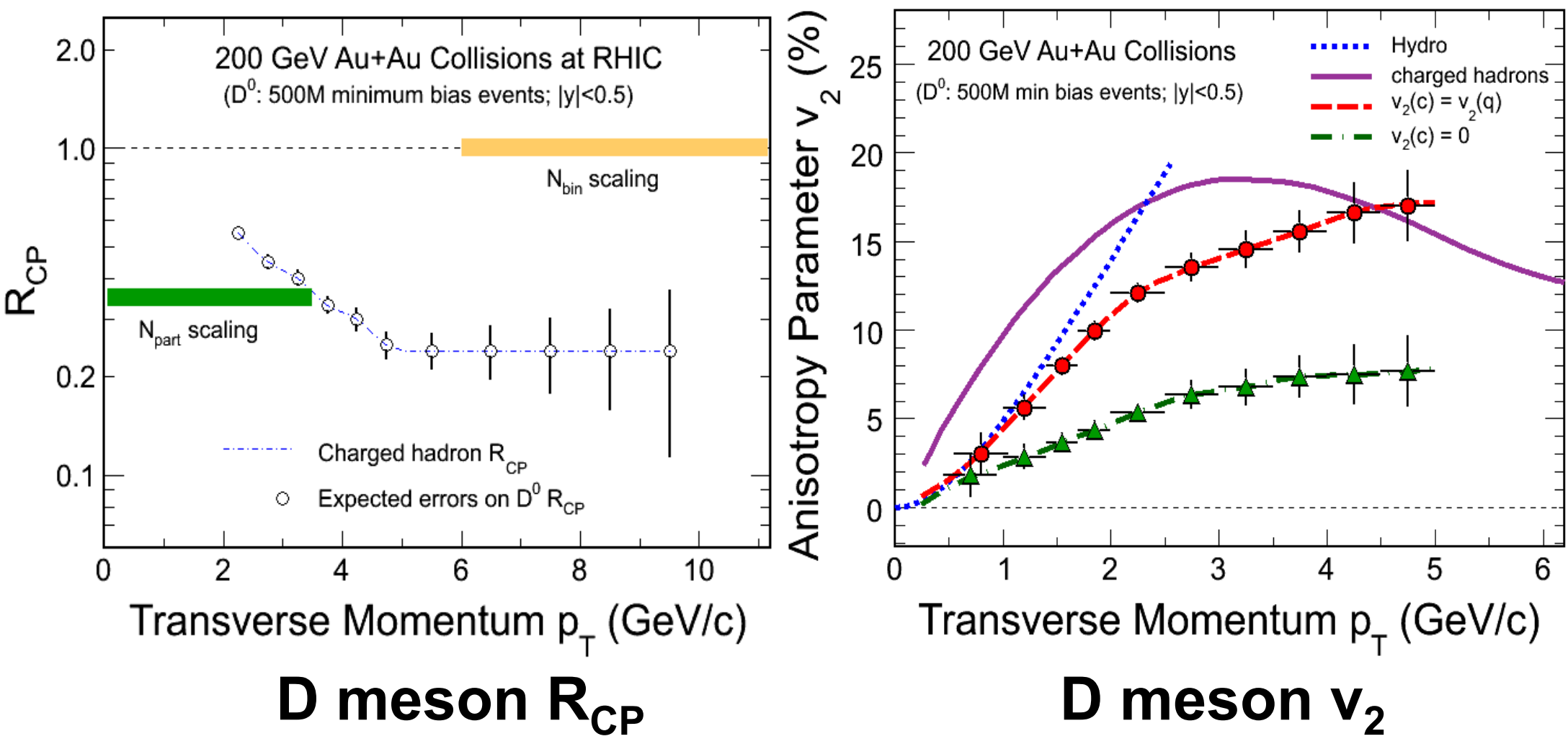

J. Bielcik, Moriond2013 


\section{ALICE: new Inner Tracking System}

\section{Projections LHC Run3 (10/nb)}
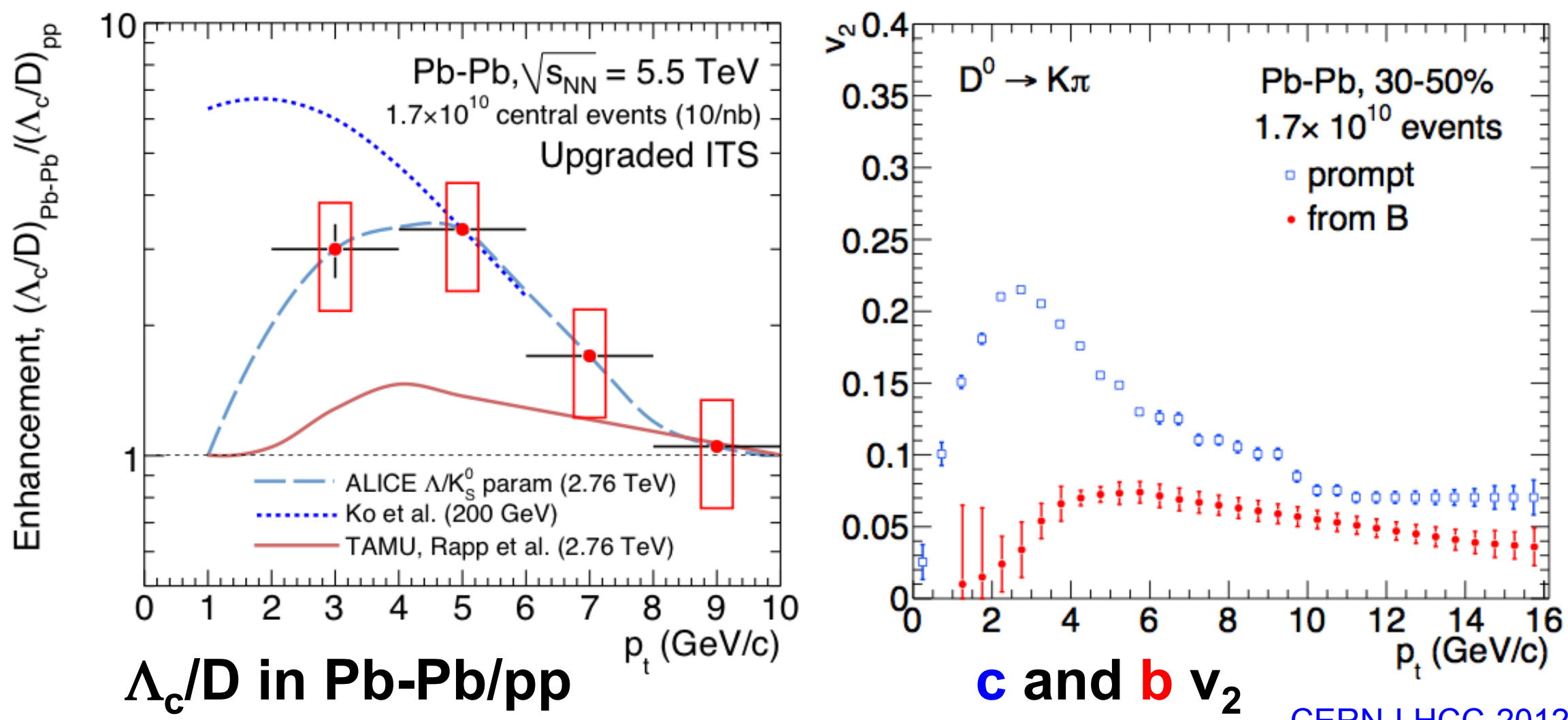

CERN-LHCC-2012-013 


\section{Conclusions}

- From the experimental point of view, we have just entered the "golden age" for heavy-flavour observables in $\mathrm{HI}$ collisions

$>$ Thanks to the LHC detectors and RHIC upgrades

\section{Whom and What (in AA, as of today)}

\begin{tabular}{|c|c|c|c|c|c|}
\hline & PHENIX & STAR & ALICE & ATLAS & CMS \\
\hline HF electrons & $\boldsymbol{v}$ & $\boldsymbol{v}$ & & \\
\hline HF muons & $\boldsymbol{V}$ & & $\boldsymbol{v}$ & \\
\hline $\mathrm{D}^{0}, \mathrm{D}^{+}, \mathrm{D}^{*+}$ & & $\boldsymbol{v}$ & $\boldsymbol{v}$ & \\
\hline $\mathrm{D}_{\mathrm{s}}^{+}$ & & & $\boldsymbol{v}$ & \\
\hline $\mathrm{B} \rightarrow \mathrm{J} / \psi$ & & & \\
\hline $\mathrm{B}$ jets & & & & $\boldsymbol{V}$ \\
\hline
\end{tabular}




\section{Conclusions}

- From the experimental point of view, we have just entered the "golden age" for heavy-flavour observables in $\mathrm{HI}$ collisions

$>$ Thanks to the LHC detectors and RHIC upgrades

\section{Whom and What (in AA, as of today)}

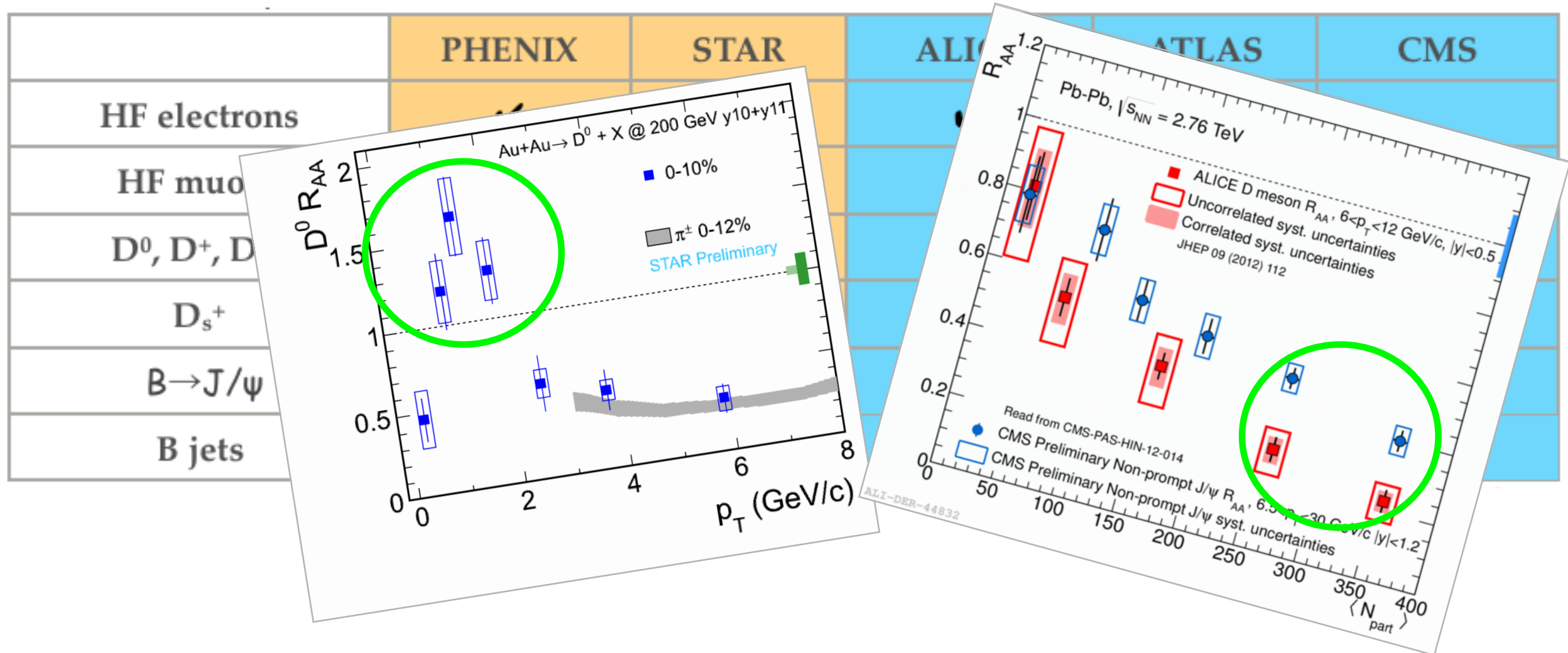


EXTRA SLIDES 


\section{What's special about heavy quarks: probes through the full system history}

Large mass $\left(\mathrm{m}_{\mathrm{c}} \sim 1.5 \mathrm{GeV}, \mathrm{m}_{\mathrm{b}} \sim 5 \mathrm{GeV}\right) \rightarrow$ produced in large virtuality $Q^{2}$ processes at the initial stage of the collision with short formation time $\Delta \mathrm{t}>1 / 2 \mathrm{~m} \sim 0.1 \mathrm{fm} \ll \tau_{\mathrm{QGP}} \sim 5-10 \mathrm{fm}$

$\checkmark$ Characteristic flavour, conserved in strong interactions

Production in the QGP is subdominant

$>$ Interactions with QGP don't change flavour identity

- Uniqueness of heavy quarks: cannot be "destroyed/created" in the medium $\rightarrow$ transported through the full system evolution

$\rightarrow$ "Brownian motion markers of the medium" $\left.{ }^{*}\right)$ 
What's special about heavy quarks: probes through the full system history

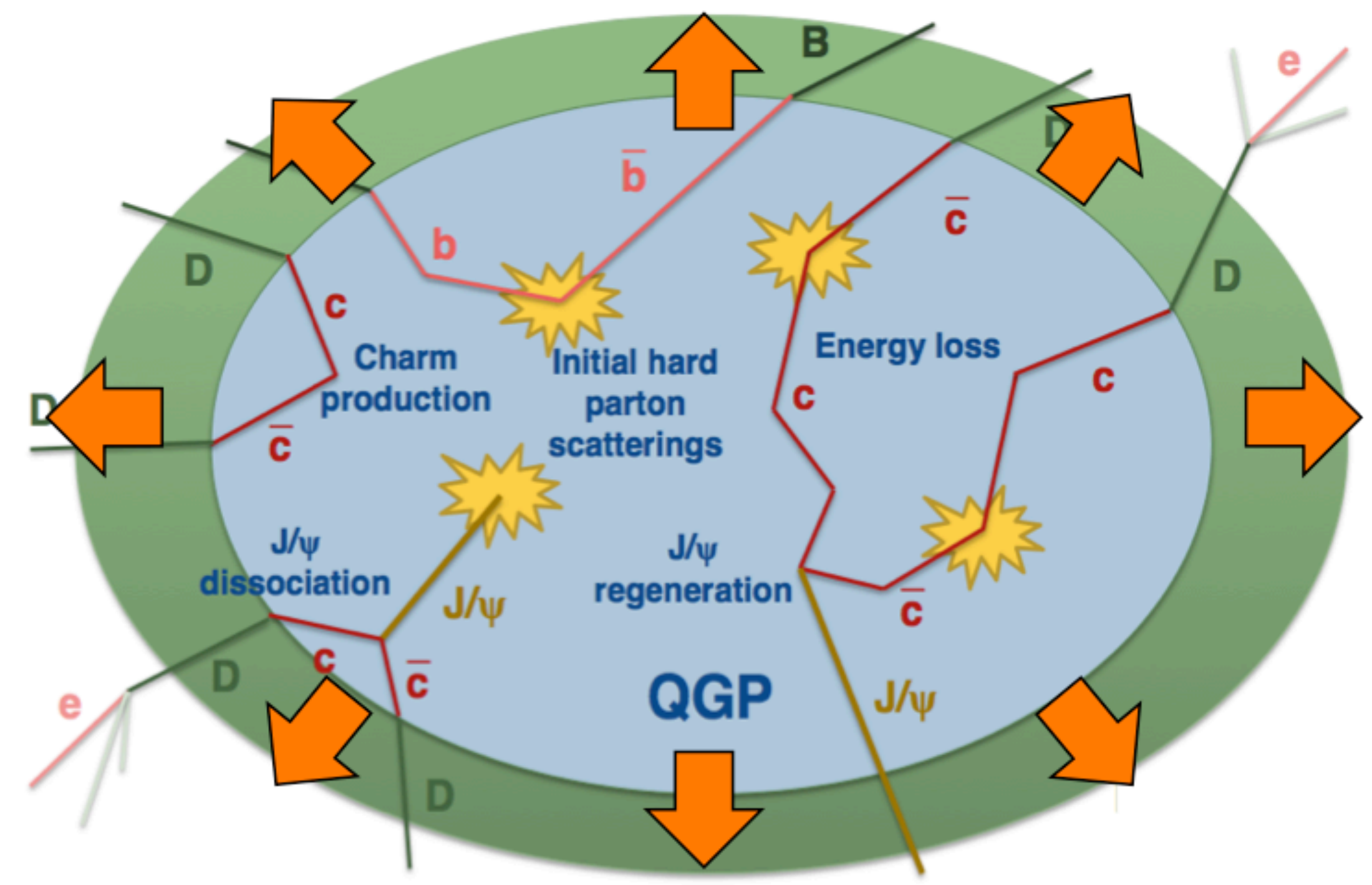

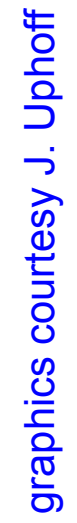




\section{What's special about heavy quarks: probing the properties of QCD E-loss}

q: colour triplet u,d,s: $m \sim 0, C_{R}=4 / 3$

g: colour octet g: $\quad m=0, C_{R}=3$

\section{Q: colour triplet}

c: $m \sim 1.5 \mathrm{GeV}, \mathrm{C}_{\mathrm{R}}=4 / 3$

b: $m \sim 5 \mathrm{GeV}, \quad \mathrm{C}_{\mathrm{R}}=4 / 3$

Parton Energy Loss by

- medium-induced gluon radiation

- collisions with medium gluons

$$
\Delta E\left(\varepsilon_{\text {medium }} ; C_{R}, m, L\right)
$$

$C_{R}$ : colour charge dep.

$m$ : mass dep. (dead cone, ...)

$$
\begin{array}{ll}
\rightarrow & \Delta E_{g}>\Delta E_{c \approx q}>\Delta E_{b} \\
\text { pred: } & R_{A A}^{\pi}<R_{A A}^{D}<R_{A A}^{B}
\end{array}
$$

Much more on this in the talk of A. Buzzatti 
HF in heavy ion collisions: Who and What

\begin{tabular}{|c|c|c|c|c|c|}
\hline & PHENIX & STAR & ALICE & ATLAS & CMS \\
\hline HF electrons & $\boldsymbol{V}$ & $\boldsymbol{v}$ & & \\
\hline HF muons & $\boldsymbol{V}$ & & $\boldsymbol{v}$ & \\
\hline $\mathrm{D}^{0}, \mathrm{D}^{+}, \mathrm{D}^{*+}$ & & $\boldsymbol{v}$ & & \\
\hline $\mathrm{D}_{\mathrm{s}}^{+}$ & & & $\boldsymbol{v}$ & \\
\hline $\mathrm{B} \rightarrow \mathrm{J} / \Psi$ & & & \\
\hline $\mathrm{B}$ jets & & & & $\boldsymbol{V}$ \\
\hline
\end{tabular}




\section{Less gluon radiation for heavy quarks?}

- In vacuum, gluon radiation suppressed at $\theta<m_{\mathrm{Q}} / E_{\mathrm{Q}}$

$\rightarrow$ "dead cone" effect

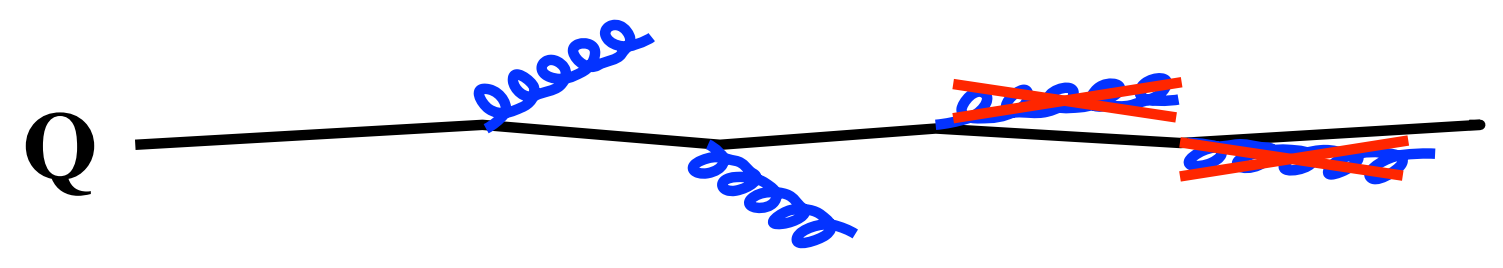

Gluonsstrahlung probability

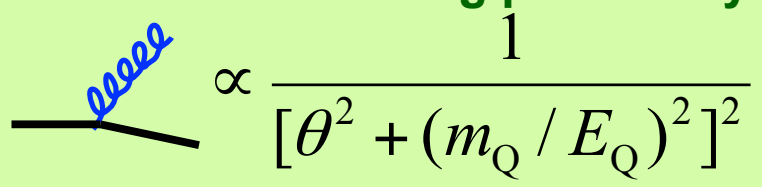

- Dead cone implies lower energy loss (Dokshitzer-Kharzeev, 2001):

* energy distribution $\omega d I / d \omega$ of radiated gluons suppressed by angle-dependent factor

* suppresses high- $\omega$ tail

$$
\left.\omega \frac{\mathrm{d} I}{\mathrm{~d} \omega}\right|_{\text {HEAVY }}=\left.\omega \frac{\mathrm{d} I}{\mathrm{~d} \omega}\right|_{\text {LIGHT }} \times\left(1+\left(\frac{m_{\mathrm{Q}}}{E_{\mathrm{Q}}}\right)^{2} \frac{1}{\theta^{2}}\right)^{-2}
$$

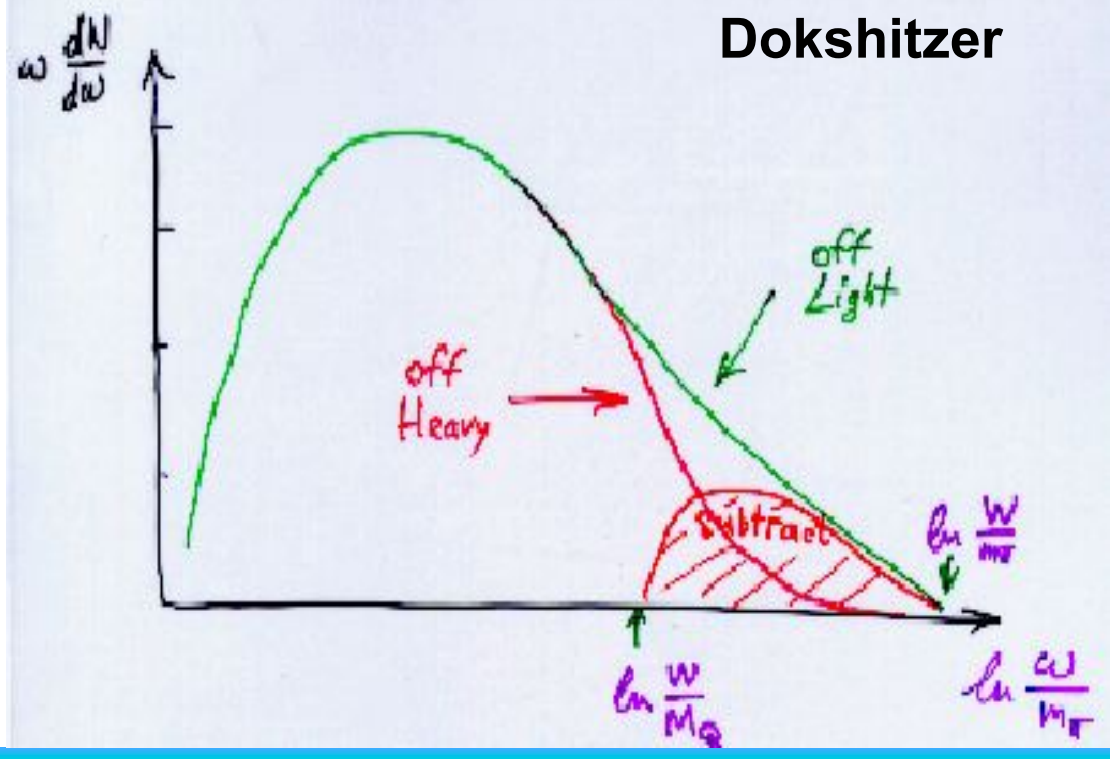




\section{HQs E loss: some expectations ...}

- Energy loss based predictions: factor 3-5 suppression for D mesons

- Significantly smaller suppression for B

$$
R_{A A}^{D}\left(p_{T}\right) \text { and } R_{A A}^{B}\left(p_{T}\right)
$$

Radiative E loss

$$
\begin{aligned}
& B_{\left(m_{b} \sim 5 \mathrm{GeV}\right)} \\
& \mathbf{D}_{\left(\mathrm{m}_{\mathrm{c}} \sim 1.5 \mathrm{GeV}\right)}
\end{aligned}
$$

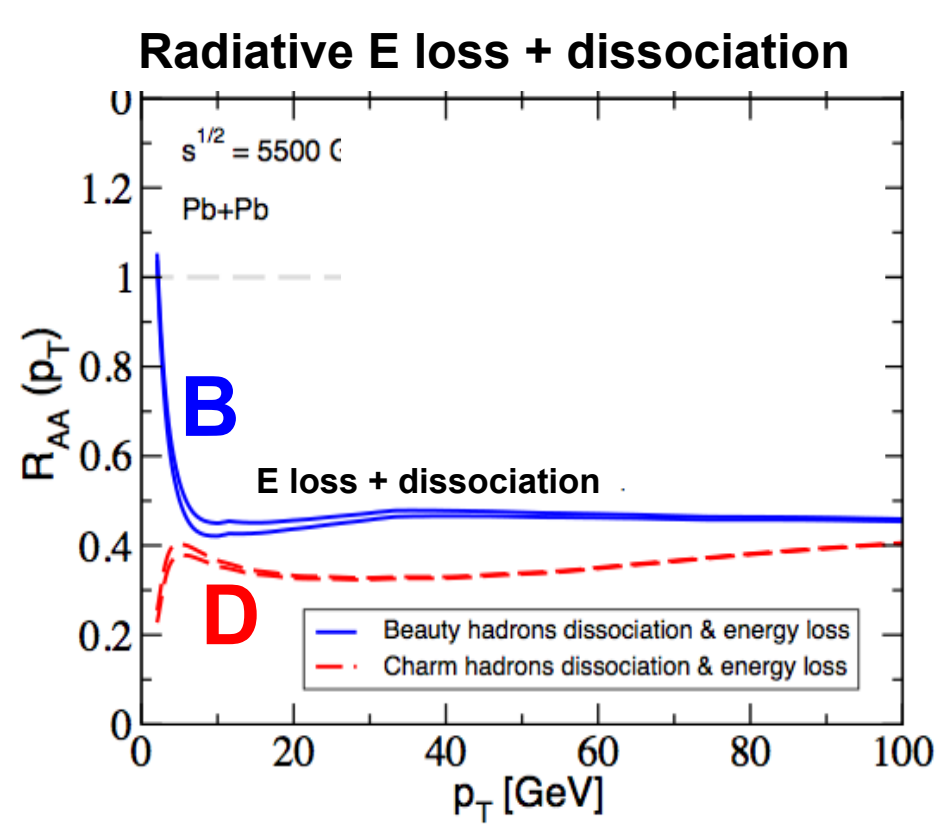

Shorter formation time of heavy hadrons $\rightarrow$ additional $\mathrm{R}_{\mathrm{AA}}$ suppression due to inmedium dissociation?

$$
\tau_{\text {form }}\left(p_{T}=10 \mathrm{GeV}\right)
$$

$\pi \quad D \quad B$

$25 \mathrm{fm} \quad 1.6 \mathrm{fm} \quad 0.4 \mathrm{fm}$

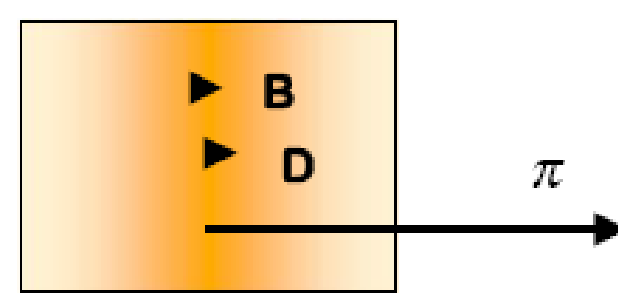




\section{HQs E loss: the AdS/CFT way...?}

- Maldacena conjecture: correspondence between super-gravity (Super Yang Mills) and QCD

$\rightarrow$ calculate strongly-coupled QCD in SUGRA

- Model energy loss by embedding a string in AdS space

- One distinctive prediction:

$>$ Very strong suppression for charm

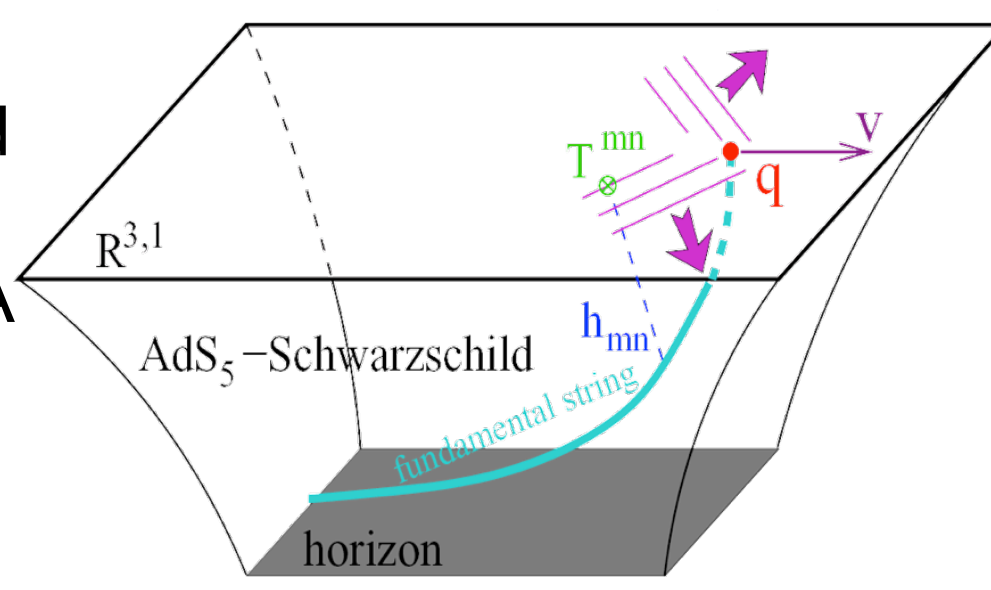

$>$ Small suppression for beauty up to very large $p_{T}$
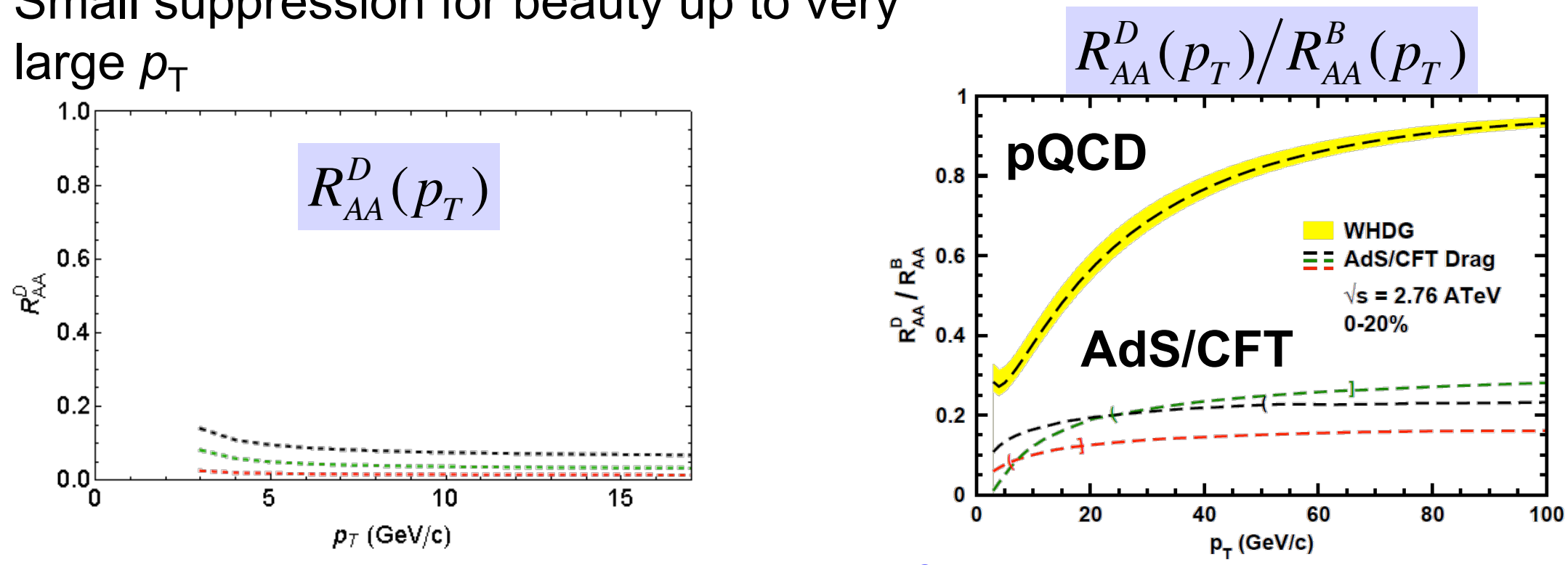

Horowitz, Gyulassy, PLB666 (2008), Horowitz, arXiv:1108.5876 


\section{Pp reference at $2.76 \mathrm{TeV}$ via $\sqrt{\mathrm{s} \text { scaling IN FN }}$ (ALICE D mesons and electrons)}

- Scale the $7 \mathrm{TeV}$ cross sections by the 2.76/7 factor from FONLL, with full theoretical uncertainty

$$
R_{A A}\left(p_{t}\right)=\frac{1}{\left\langle T_{A A}\right\rangle} \frac{d N_{A A} / d p_{t}}{d \sigma_{p p} / d p_{t}}
$$

$>$ relative scaling uncertainty: $30 \% \rightarrow 5 \%$ in the $p_{\mathrm{t}}$ range $2 \rightarrow 16 \mathrm{GeV} / c$

- Validated by comparing to measured cross section at 2.76 TeV (fewer $p_{t}$ bins)

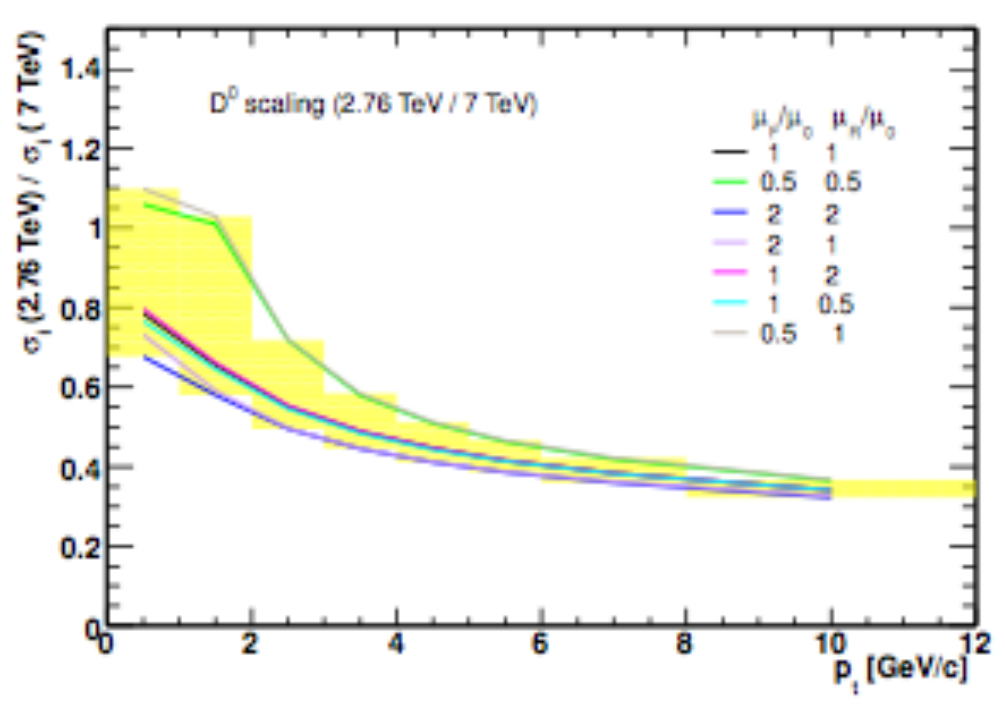

Averbeck et al., arXiv:1107.3243

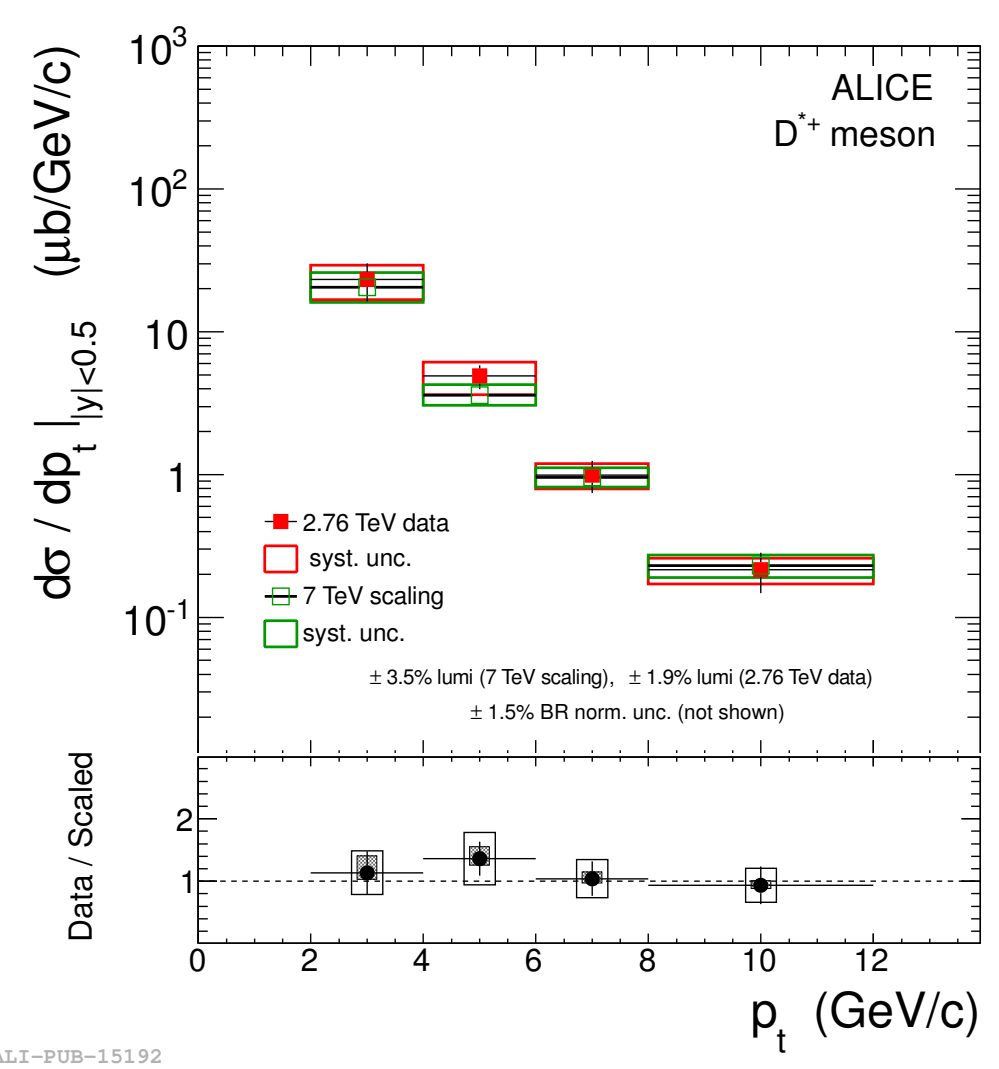

JHEP1207 (2012) 191 


\section{$H F$ e in d-Au: $R H I C$ results}

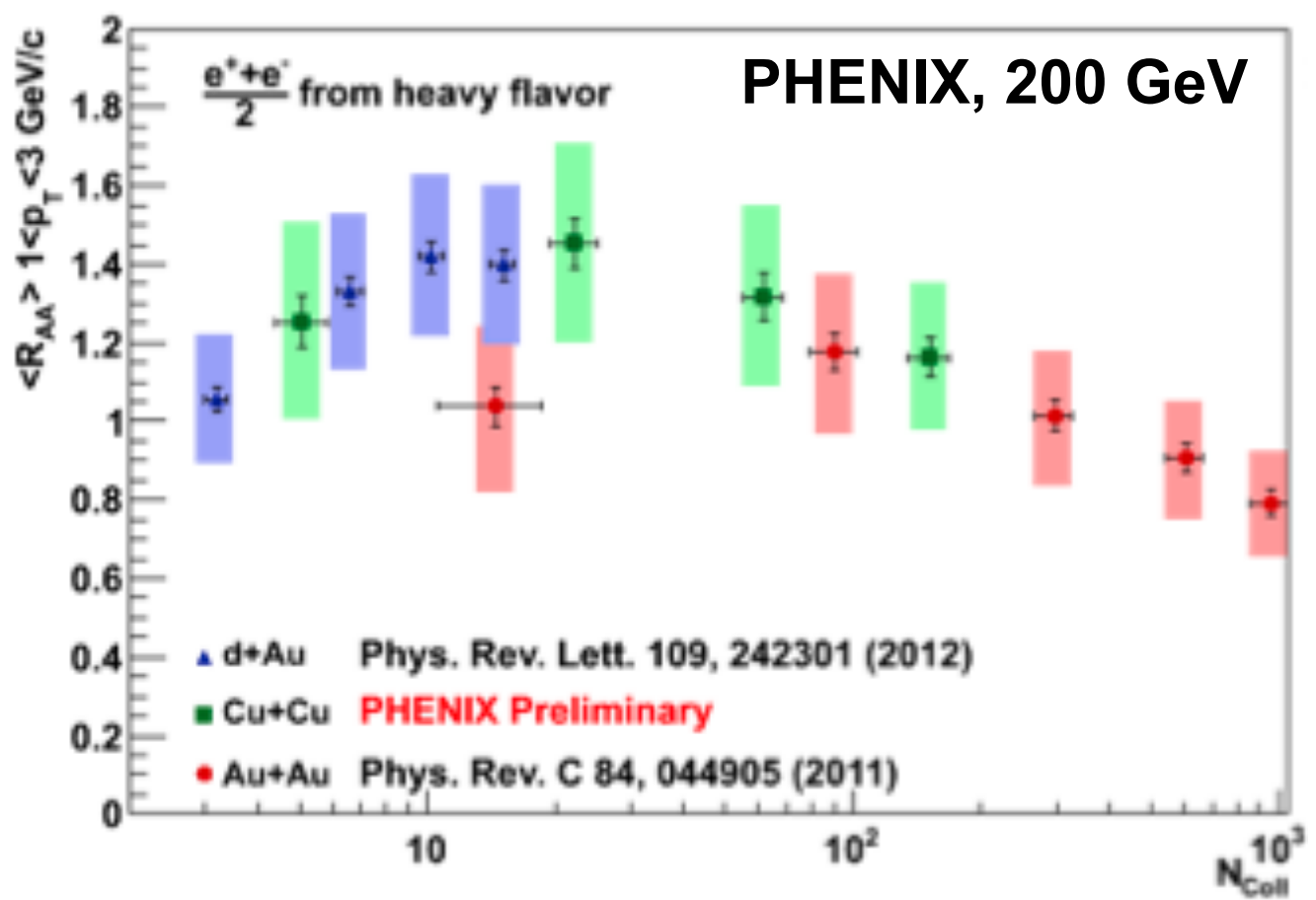

- Consistent $\mathrm{N}_{\text {coll }}$-dependence in d-Au, Cu-Cu, Au-Au

- Move from initial-state effects in d-Au/peripheral Cu-Cu to where the hot medium takes over as system size increases in $\mathrm{Cu}-\mathrm{Cu}$ and $\mathrm{Au}-\mathrm{Au}$ 


\title{
Updates/puzzles of dAu at RHIC
}

\author{
G. David, BNL
}

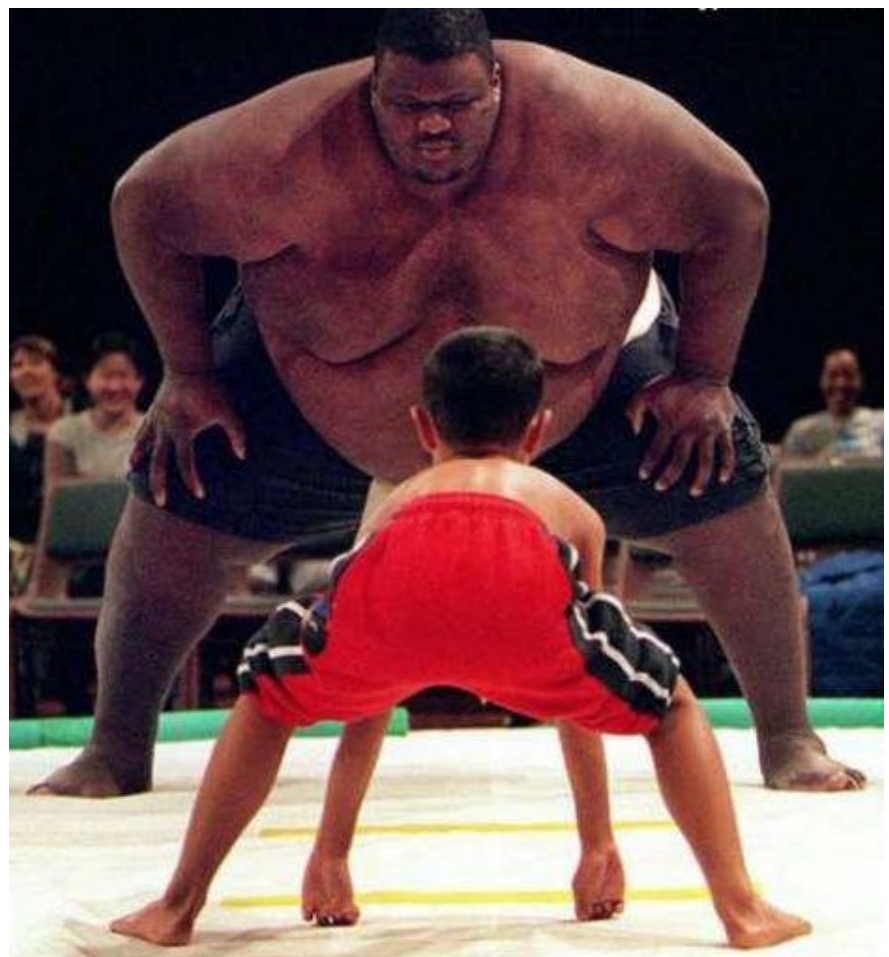

(Picture shamelessly stolen from Kwangbok Lee)

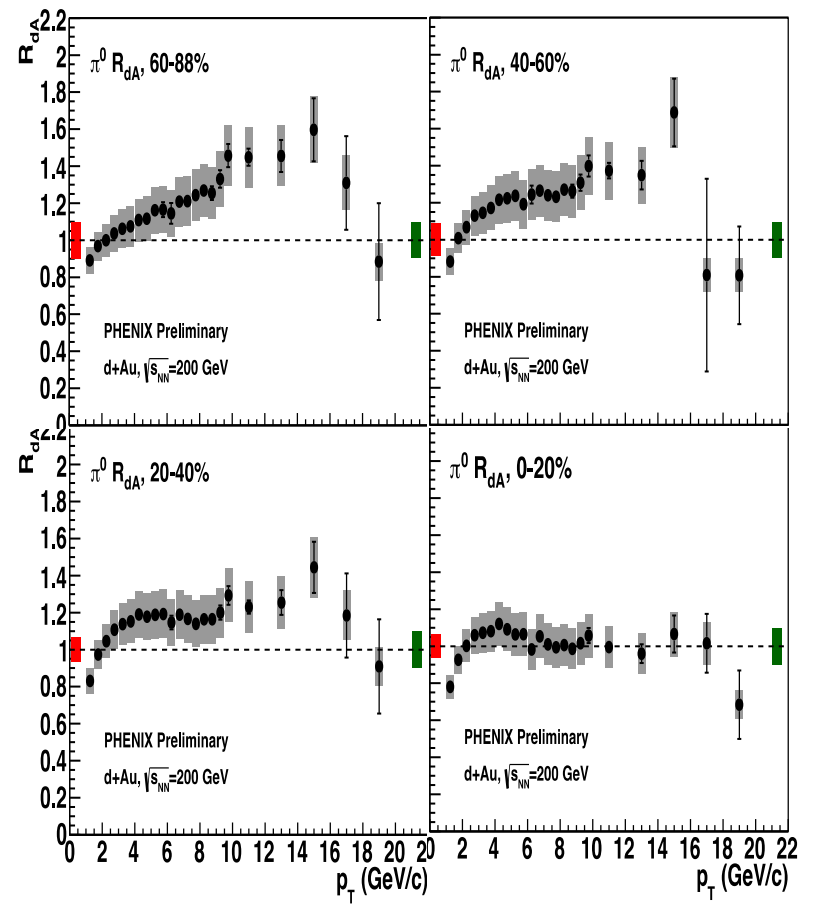

(Will focus on results at $\eta \sim 0$ and higher $p_{T}$ ) 


\section{In the beginning...}

STAR, PRL 91 (2003) 072304

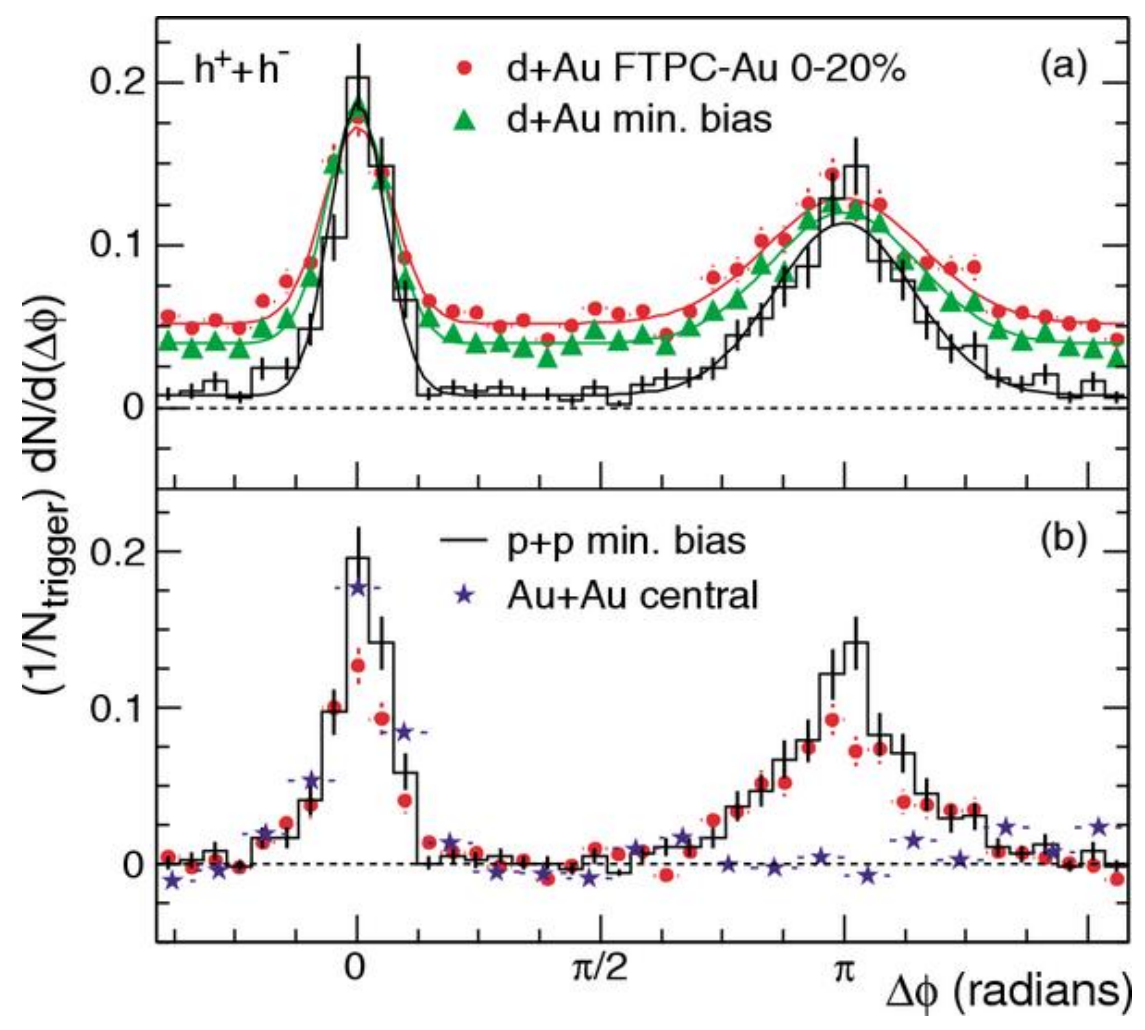

STAR: back-to-back jets reappear in $d+A u$ PHENIX: large suppression in $\mathrm{Au}+\mathrm{Au}$, no suppression in $\mathrm{d}+\mathrm{Au}$

$\rightarrow$ final state effect (as of 2003)
PHENIX, PRL 91 (2003) 072303

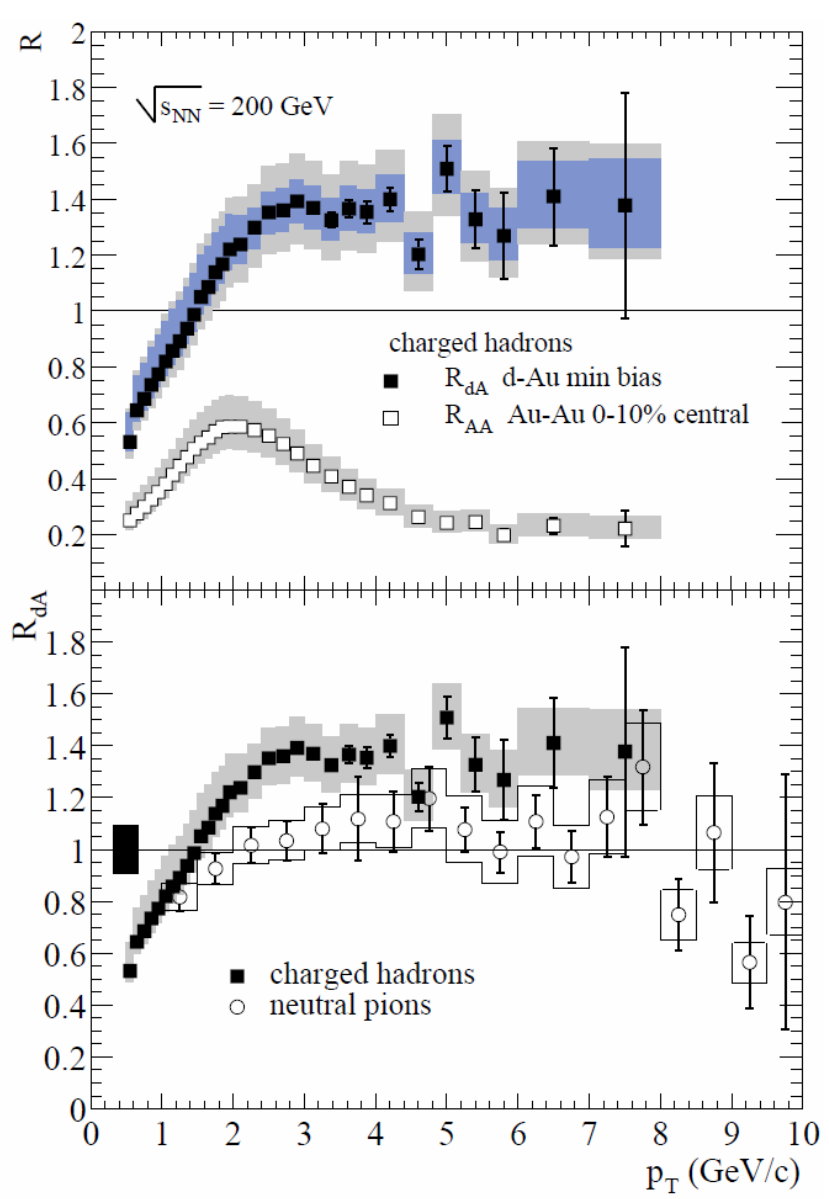




\section{What the photons tell}

STAR, d+Au, PRC 81 (2010) 064904

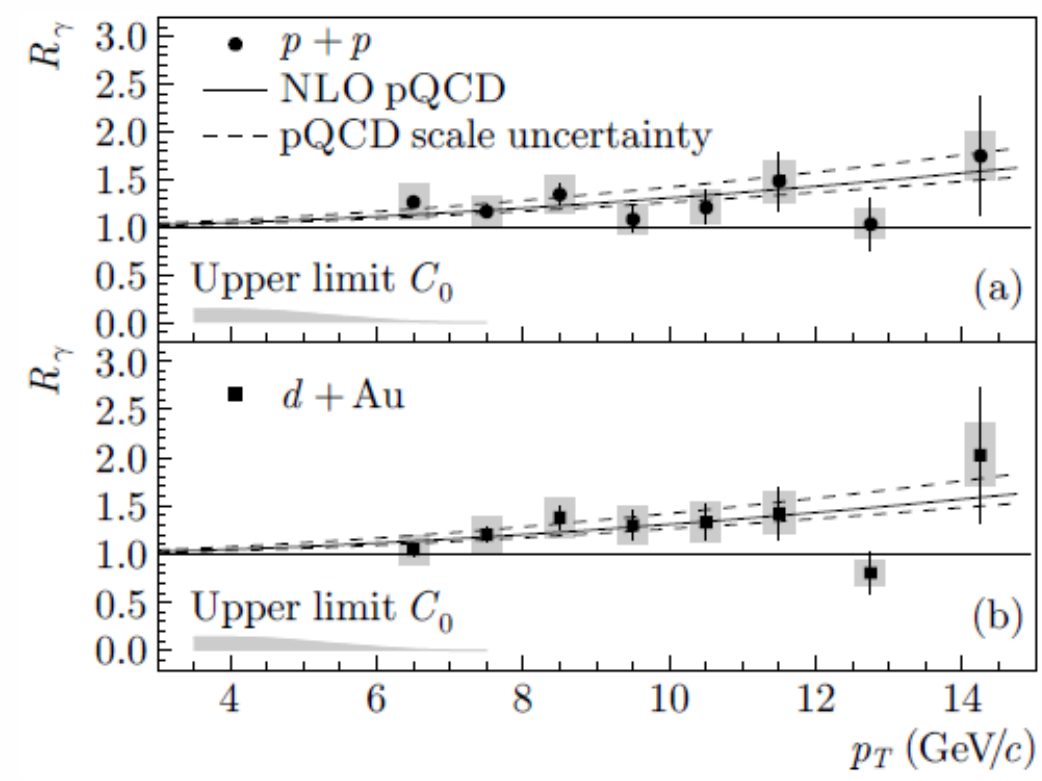

First published direct photons in $\mathrm{d}+\mathrm{Au}$ at high $\mathrm{p}_{\mathbf{T}}$ (shown here as $\mathrm{R}_{\gamma}$ )

No hint of medium formation so far
PHENIX, arXiv:1208.1234

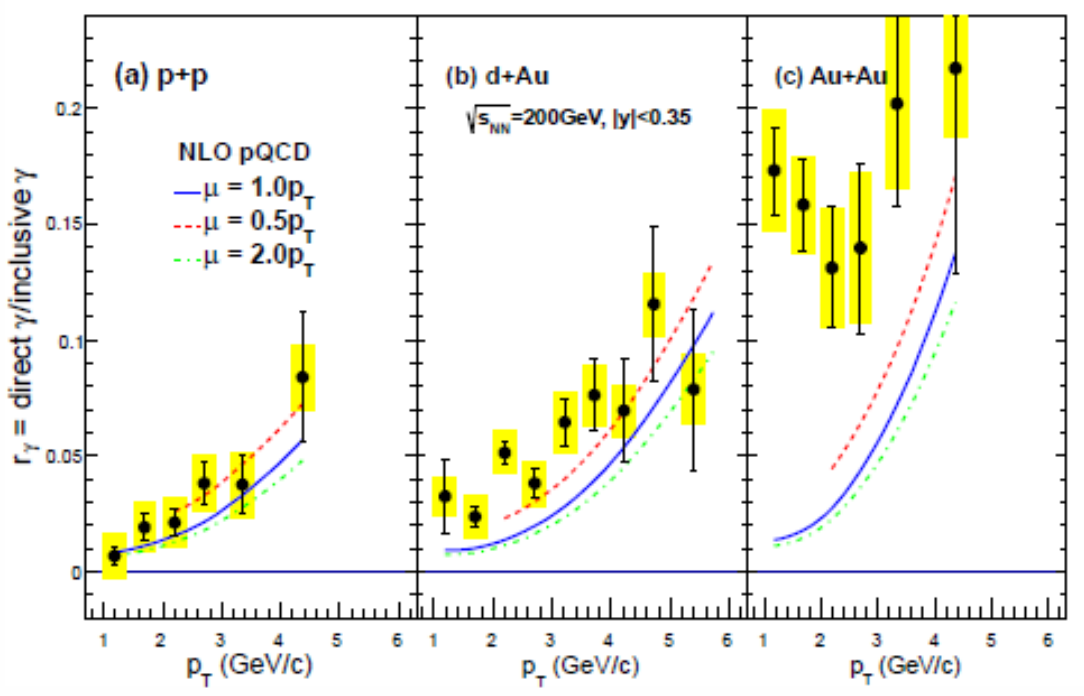

No (thermal) radiation at low $\mathrm{p}_{\mathbf{T}}$

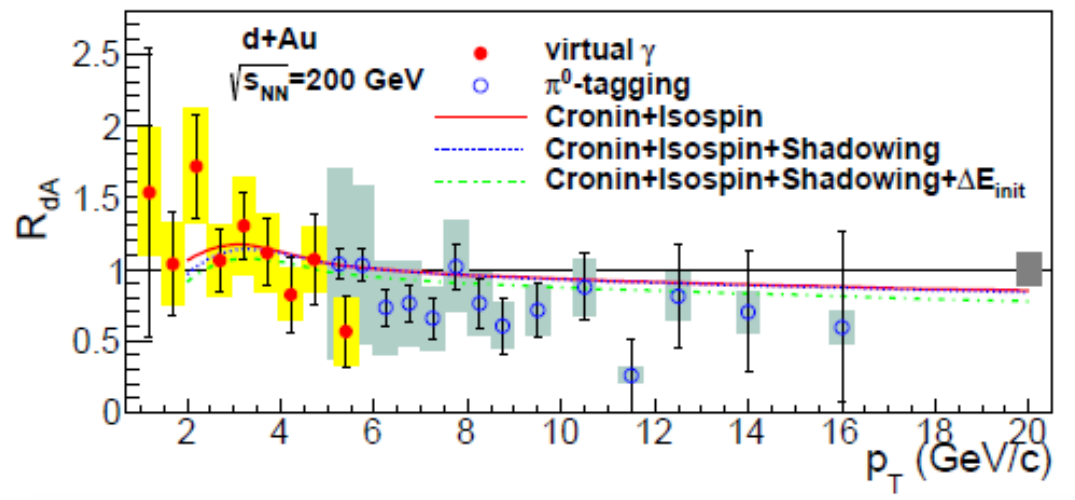

No nuclear modification (modulo isospin effect) 


\section{Low to medium $p_{T}-$ Cronin-effect}

For unidentified charged particles $R_{A A}$ enhanced at $\mathrm{RHIC}$, but consistent with one at $\mathrm{LHC}$

The Cronin-effect appears to fade away at higher energies (why would it?) $\rightarrow$ but maybe it is because of some $\mathrm{E}_{\text {loss }}$ ?

More pieces to the puzzle on the next slides

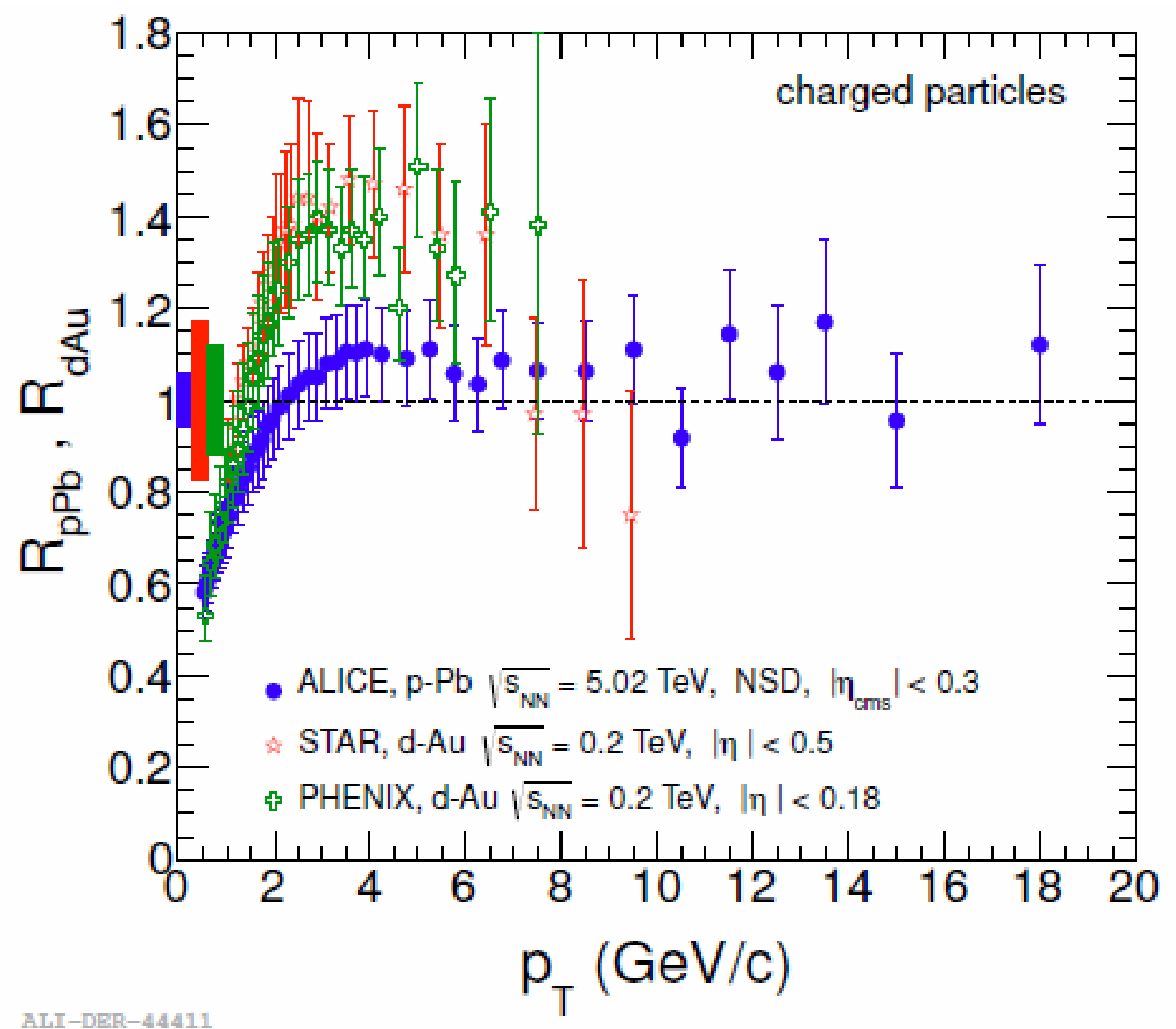




\section{Identified hadron $\mathbf{R}_{\mathrm{AA}}$ in $\mathrm{Au}+\mathrm{Au}\left(\right.$ medium $\mathrm{p}_{\mathrm{T}}$ )}

PHENIX, arXiv:1304.3410

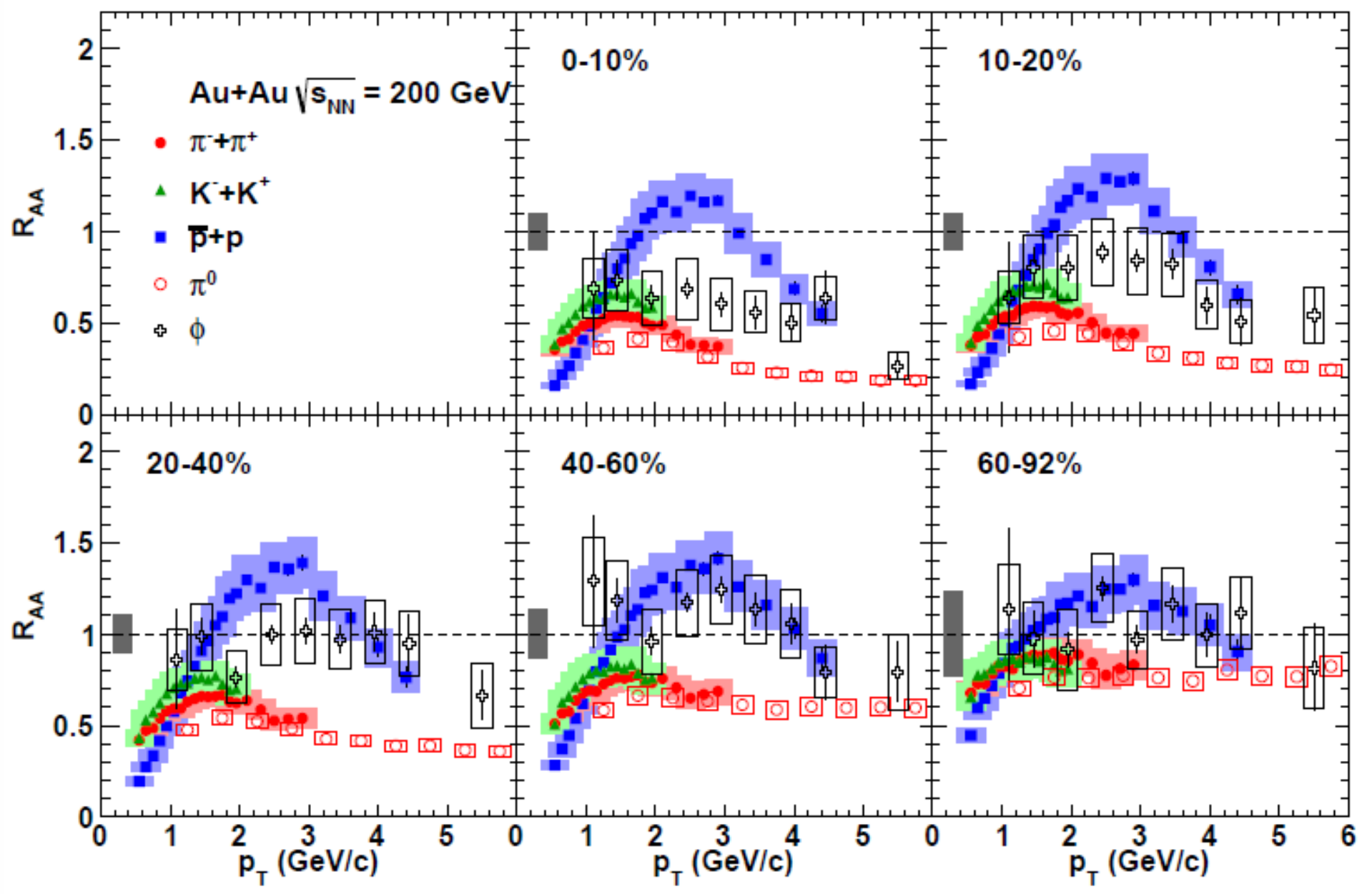

Proton: small variation with centrality.

$\mathrm{K}, \pi$ very similar (although kaons slightly higher): large variation with centrality

$\Phi$ : intermediate

Baryon vs meson; radial flow would order by mass 


\section{Identified hadron $\mathbf{R}_{\mathrm{dA}}$ in $\mathbf{d}+\mathrm{Au}\left(\right.$ medium $\mathrm{p}_{\mathrm{T}}$ )}

PHENIX, arXiv:1304.3410

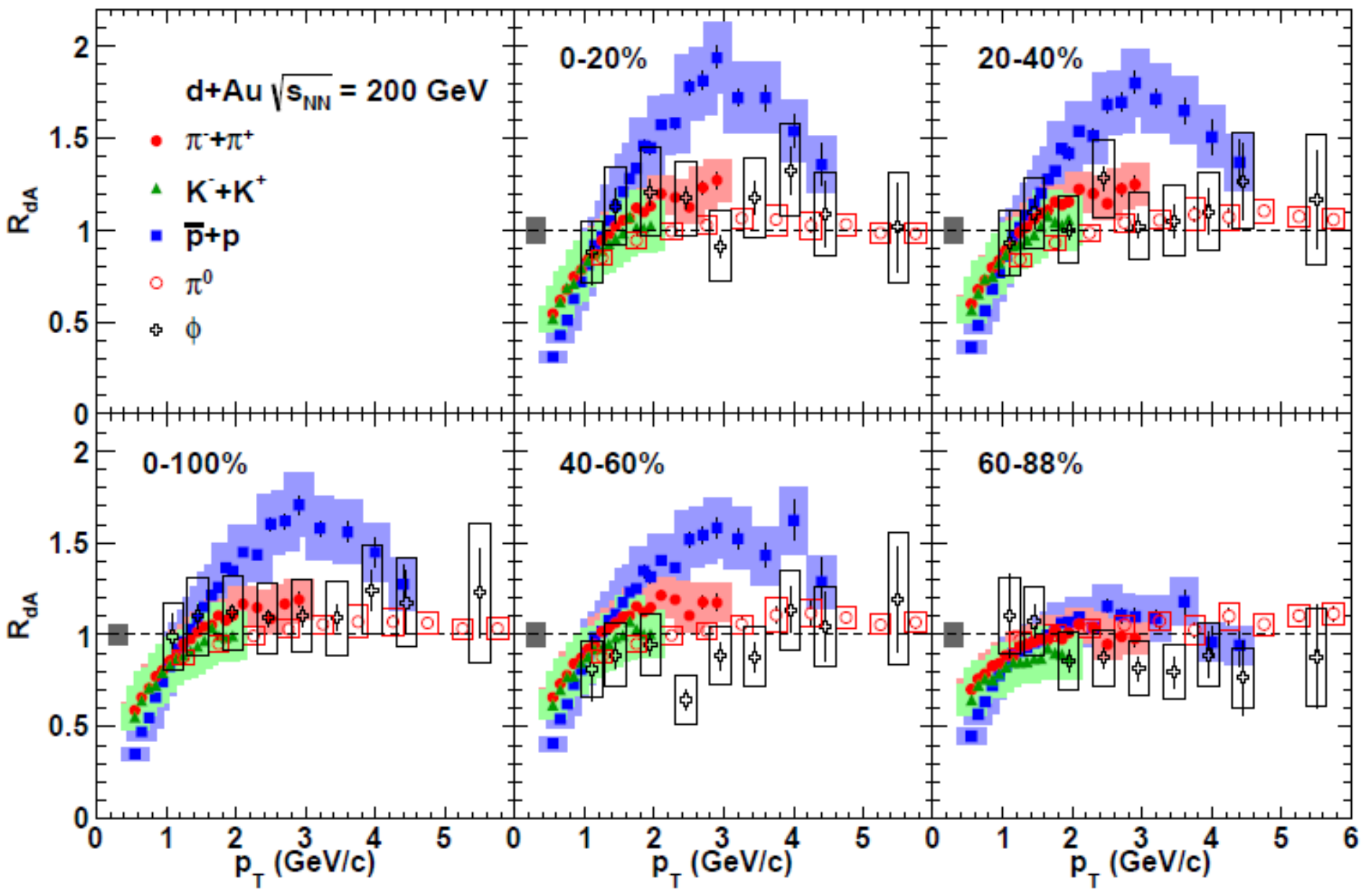

Proton: large variation $\rightarrow$ Cronin larger for baryons, and strongly depends on centrality $\Phi, \mathrm{K}, \pi$ very similar: little if any change with centrality Mass irrelevant, valence quarks count - recombination? 


\section{Corollary: particle ratios}

PHENIX, arXiv:1304.3410

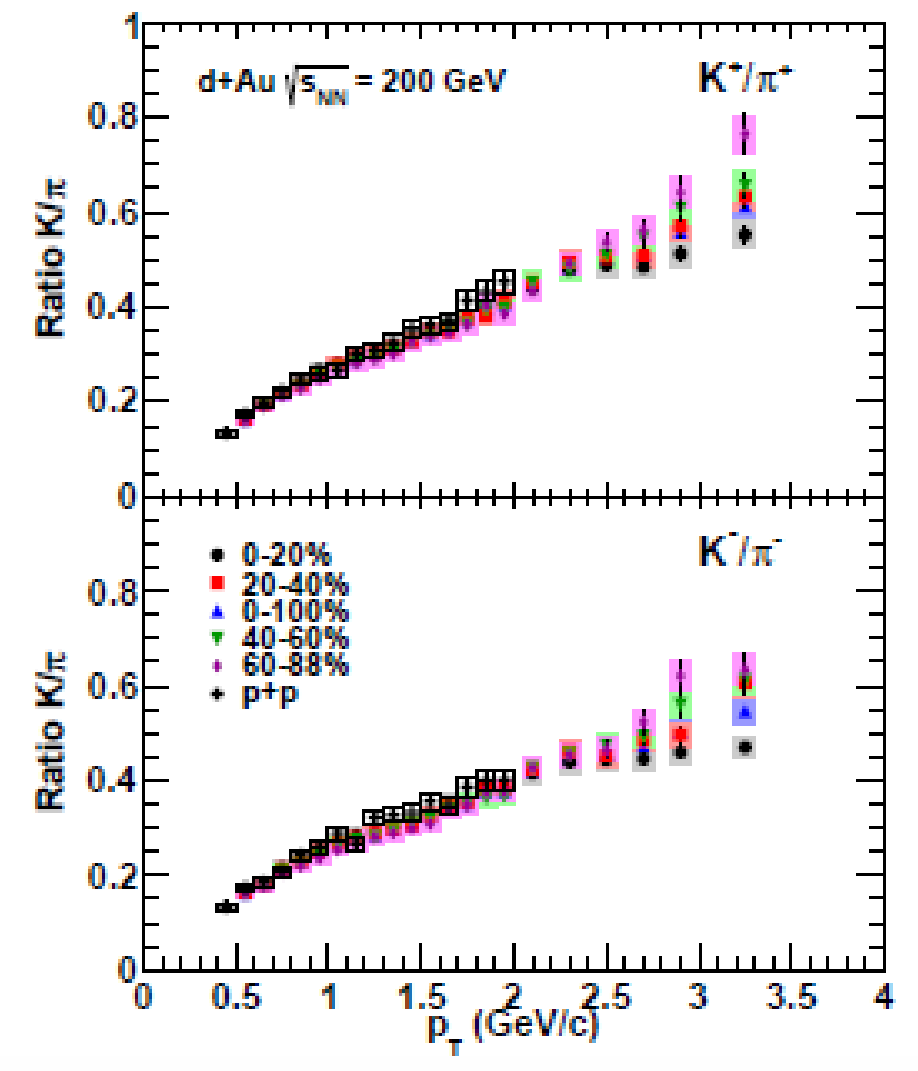

All very similar: strangeness production is the same for all centrality classes and consistent with $p+p$

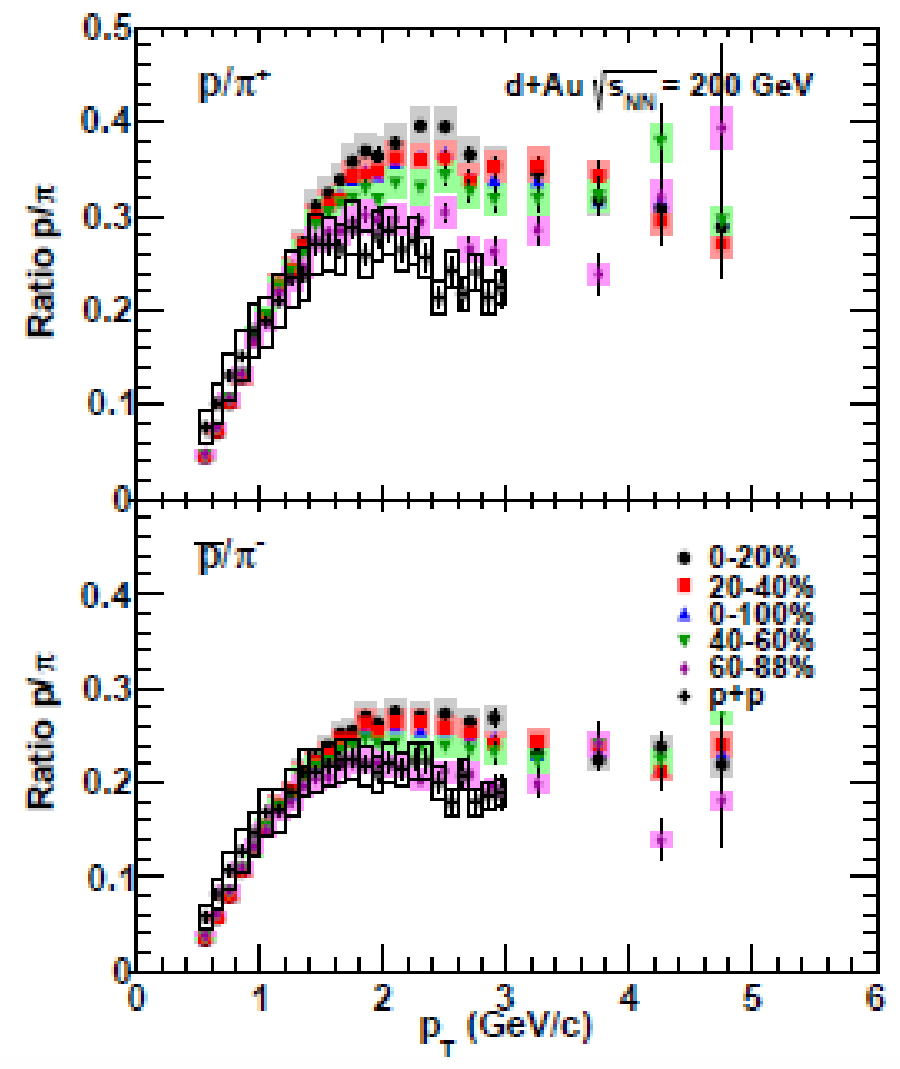

Enhanced with centrality (similar trend as in $\mathrm{Au}+\mathrm{Au}$ ): recombination? (Which would favor protons.) 


\section{Peripheral $\mathbf{A u}+\mathbf{A u}-$ central $\mathrm{d}+\mathrm{Au}$ Why are they similar?}

PHENIX, arXiv:1304.3410

$A u+A u$ 60-92\% and d+Au 0-20\% have similar $\mathrm{N}_{\text {part }}, \mathrm{N}_{\text {coll }}$.

The ratio of all ID'd hadron spectra are on the same curve, and go to a constant $\sim 0.65$

Common production mechanism?

Baryon enhancement the same?

If all CNM scales with $\mathrm{N}_{\text {part }}$, does this ratio (non-unity) mean $\mathrm{E}_{\text {loss }}$ in the medium in peripheral $\mathrm{Au}+\mathrm{Au}$ (even for protons?)

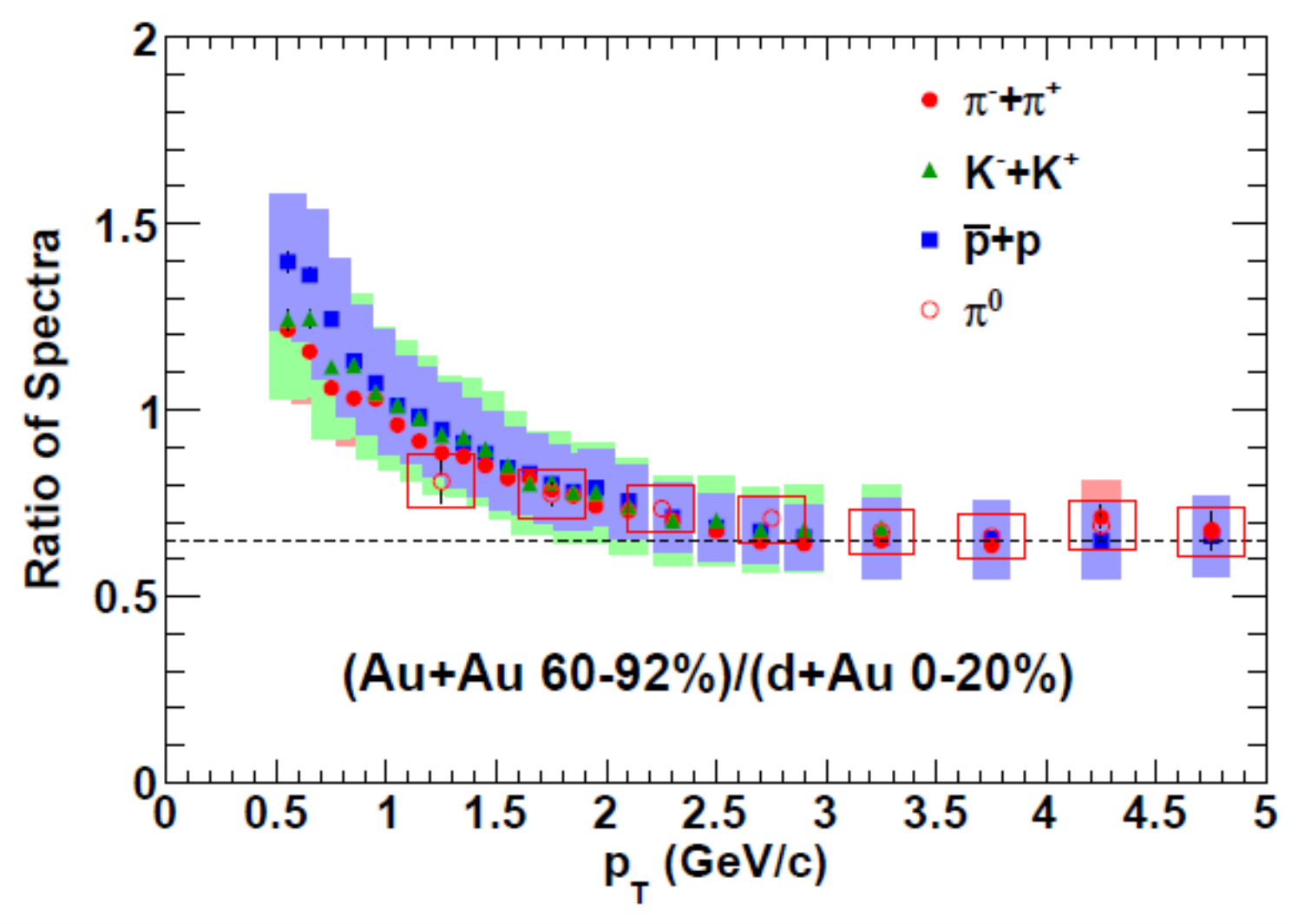

Rapidity shift in $d+A u$ causing low $p_{\mathrm{T}}$ increase?

nPDF's modified? 


\section{Reminder: $\mathbf{N}_{\text {coll }}$ scaling works!}

Remember: direct photons yields in Au+Au (presumed to be blind to any medium formed) should be proportional to the (" $\mathrm{N}_{\text {coll }}$ " times) increased, but still very-very small probability of photon production in $p+p$. And this is exactly what is seen here.

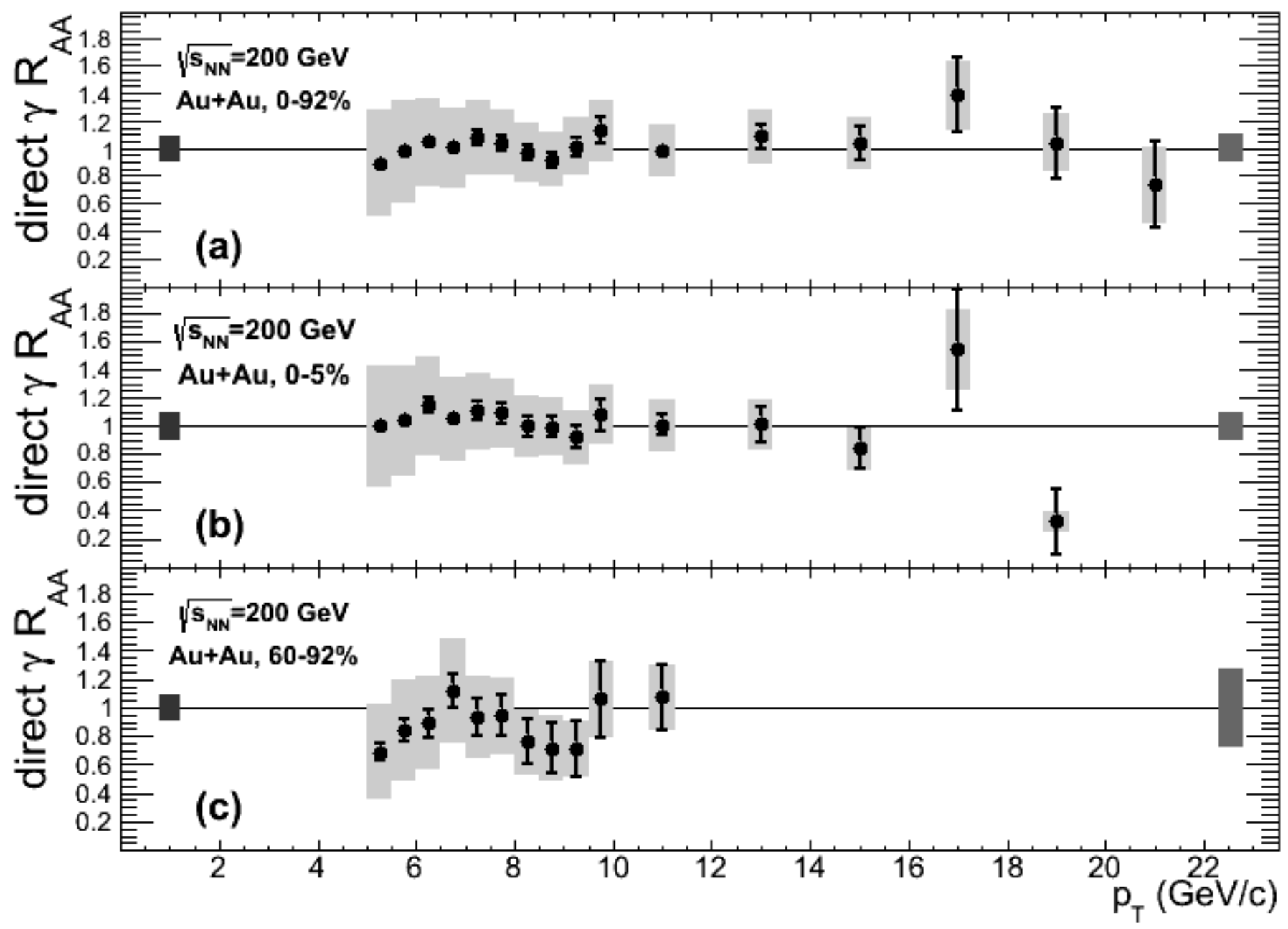




\section{Minimum bias $R_{d A}$ is unity as expected everywhere}

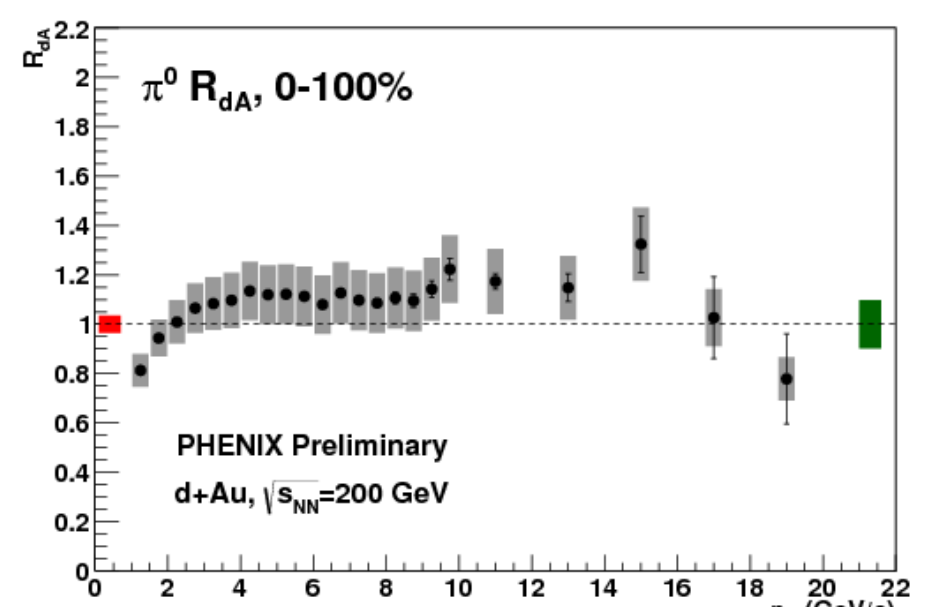

STAR, PRC 81 064904 (2010)
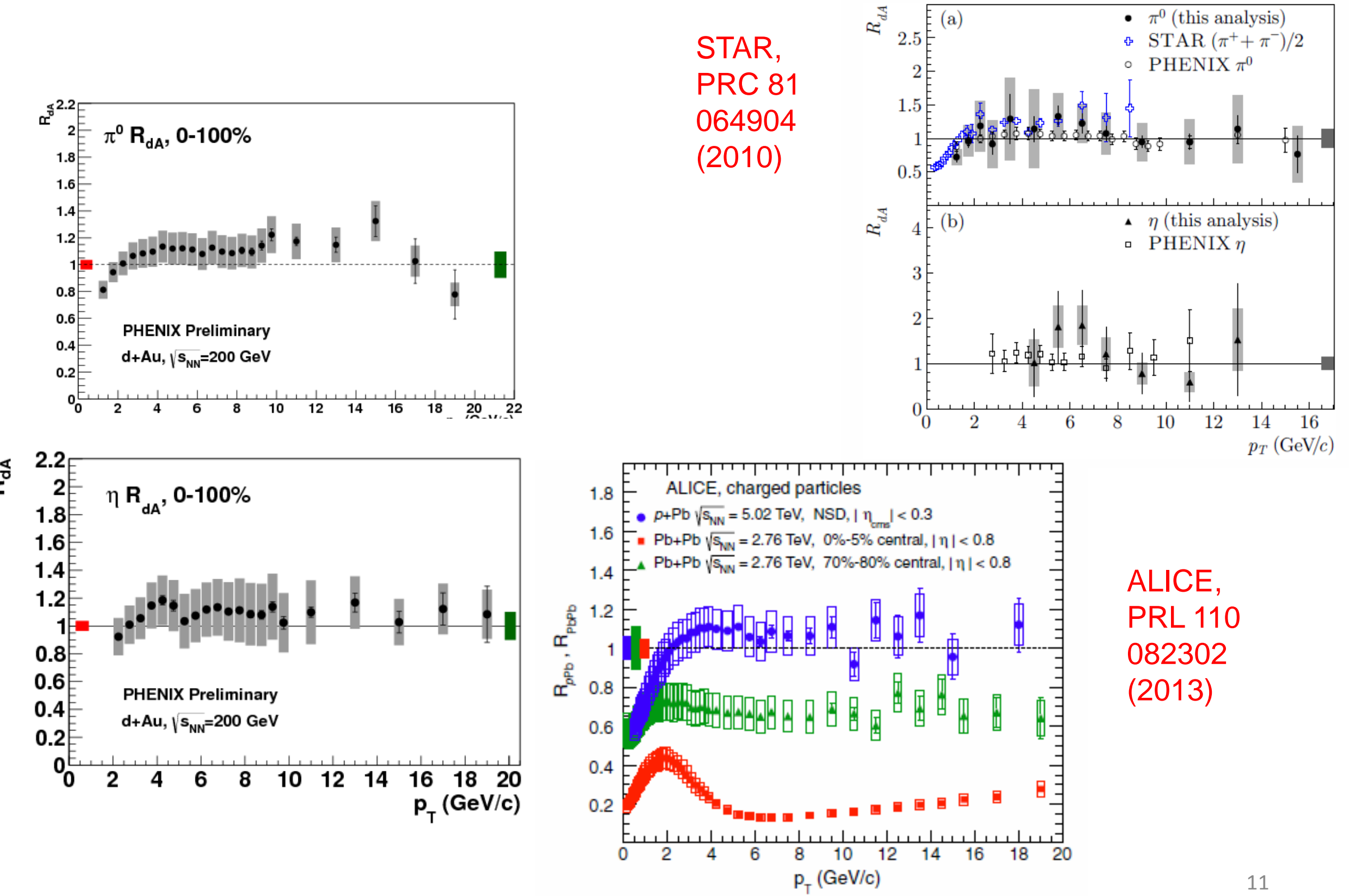

ALICE, PRL 110 082302 (2013)

КВКС jet quenchıng workshop, Aprı 1b-17, 2013 -- G. David, BNL 


\section{Jets - a very different kind of analysis}

Inclusive (leading) particle

vs jet reconstruction

(related, yet quite different

observable, analyzed differently)

Gaussian filter method 0.57
0.5
20
$\frac{0}{2}$
$10 \frac{0}{5}$
$\frac{5}{2}$
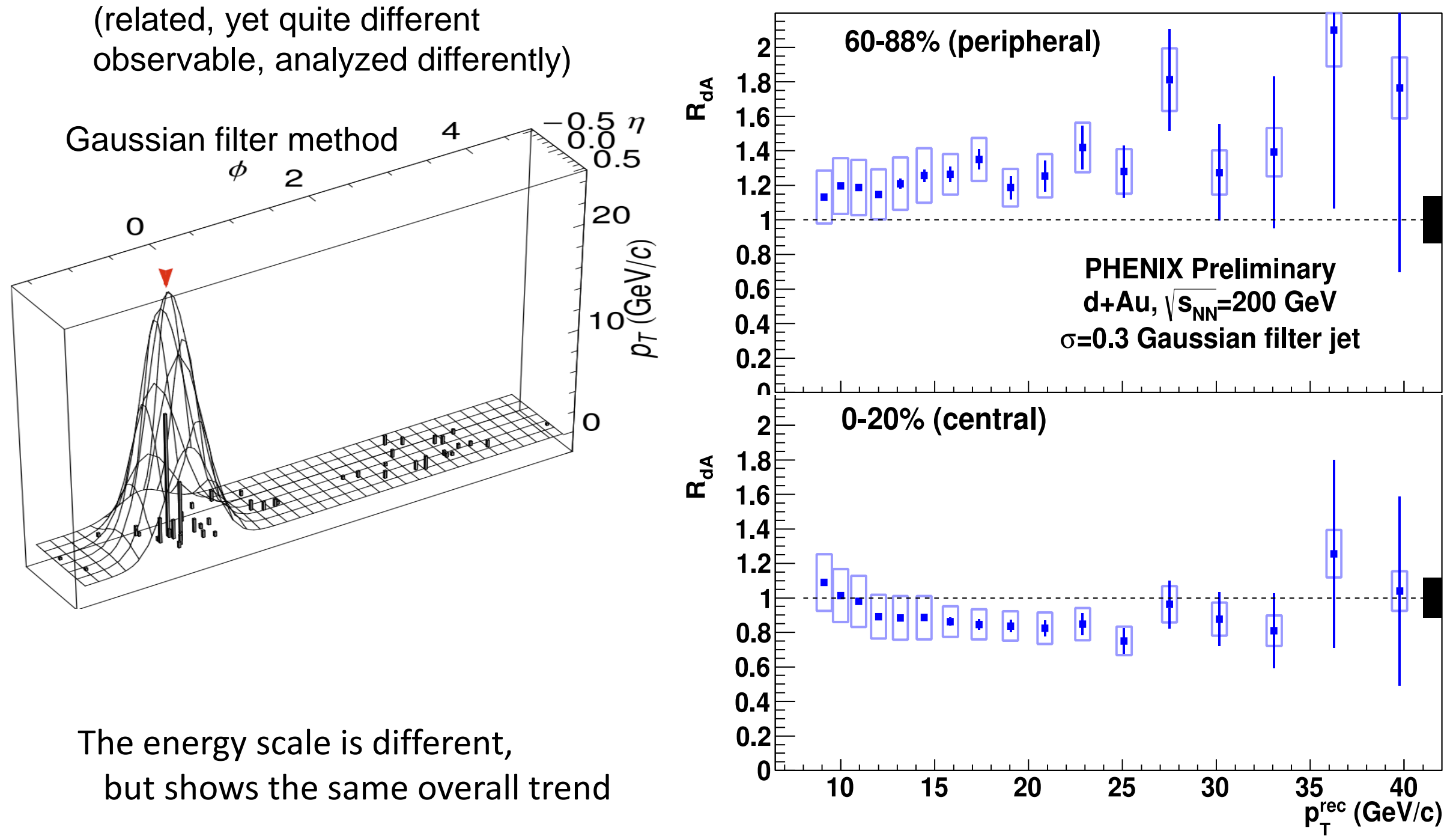

The energy scale is different, but shows the same overall trend 


\section{Jets, $\pi^{0}, \eta$-central to peripheral}

For $\pi^{0}, \eta$ this is true $p_{T}$, for jets it is total jet energy.

There is no unambiguous transformation, but 1./0.7 is a reasonable compromise, and would put the points almost on top of each other.

Important: $R_{C P}$ is independent of any $p+p$ reference!

The only "external" quantity here is the $N_{\text {coll }}$ value attributed to the individual centrality classes

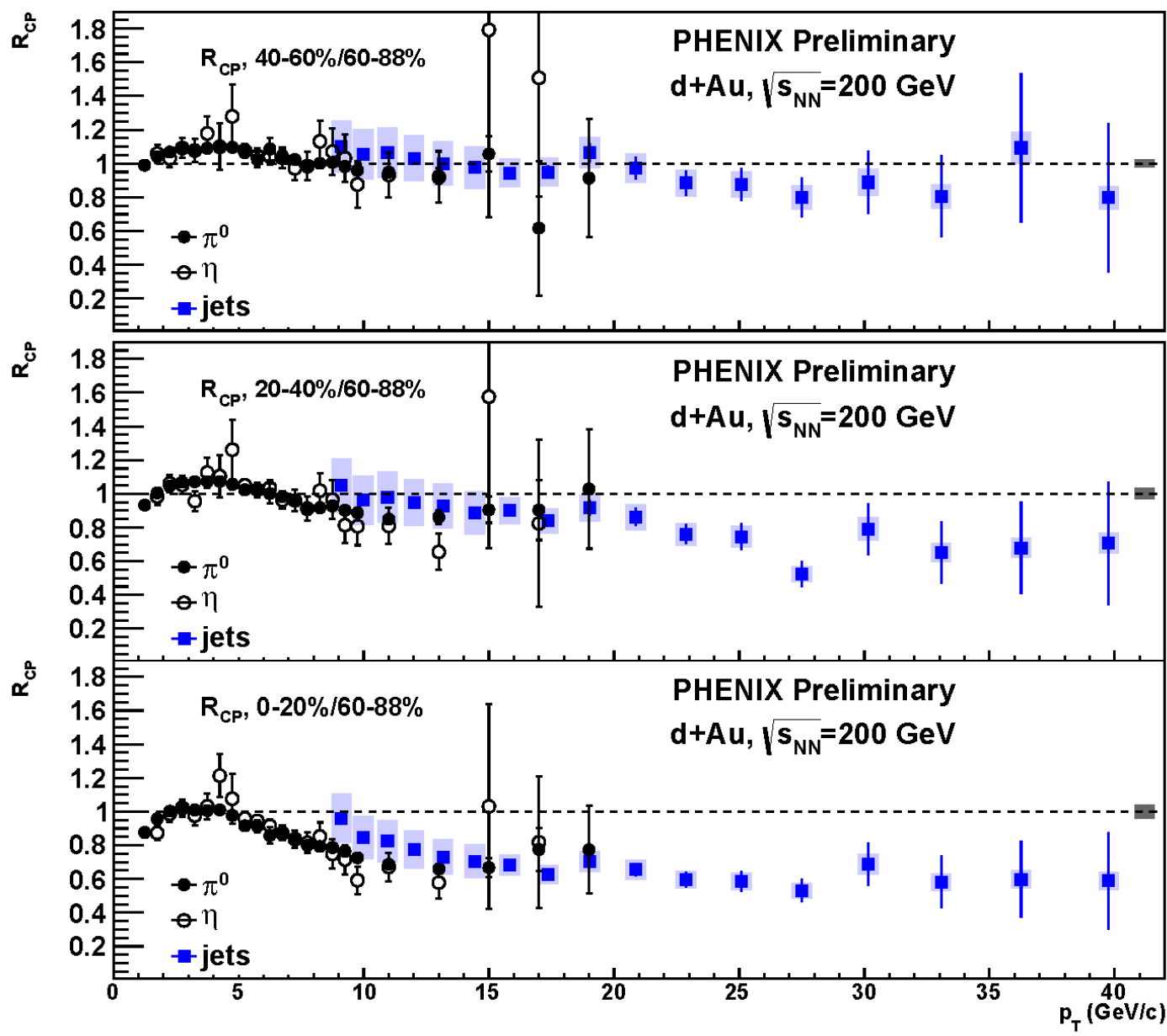

Note that $\boldsymbol{R}_{C P}$ drops sharply, indicating major shape change from peripheral to central 


\section{Something to be made VERY clear}

I am not speaking for PHENIX this time - only and exclusively for myself

Of course l'm aware that there is very intense work going on in PHENIX to verify this result (there's a reason why it is called preliminary!) The result, if it survives scrutiny, is extraordinary - extraordinary care is warranted.

However, even if I knew the answer, I will not comment on this specific work, checks, discussion in PHENIX related to this issue. Progress will be reported by the experiment in due time.

What I will do is to grab the opportunity to give a close look how centrality, a crucial quantity in heavy ion collisions, is interpreted and connected between experiment and theory, deliberately avoiding (almost always) any numbers, since nothing here is specific to PHENIX.

"Anybody thinking otherwise is itching for a fight" :) 


\section{Centrality: thinking out loud}

The theorist tends to think in terms of impact parameter (b), or $N_{\text {part }}, N_{\text {coll }}, T_{A B}, \varepsilon, \ldots$ none of which is directly accessible in the experiment

The experimenter is concerned whether a/ the event is taken at all (trigger bias/efficiency)

$\mathrm{b} /$ there are some global observables that can be tied to the theorists' quantities and while they are correlated to those quantities, they are as uncorrelated as possible to the specific features of the event (like presence of jets, flow, etc.)

Assuming such observable(s) exist, a model is agreed upon that makes the translation between experimental observables and theoretical quantities

Since you want to avoid introducing biases as much as possible, the model is tuned with a large number of (more or less) average events, in regions preferably "far" from the regions with the "specific features" studied (like a large $\eta$ gap)

The correlation between the global observable and the theoretical quantity is typically wide: events on the average will be properly classified - but not necessarily individually. 


\section{Connecting theoretical and experimental quantitites}

$3.1 \quad$ Methodology

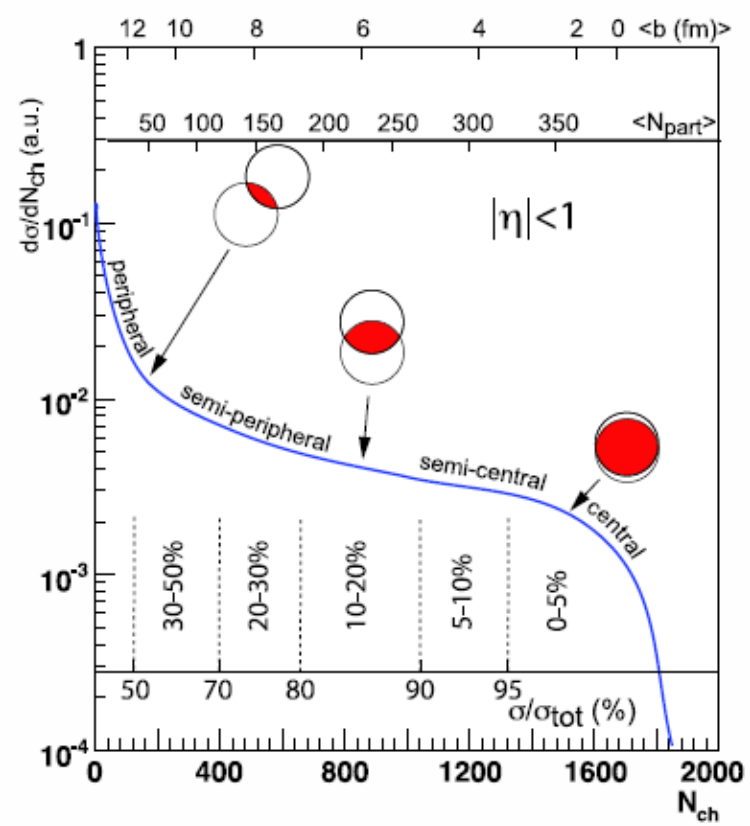

Figure 8: A cartoon example of the correlation of the final state observable $N_{\text {ch }}$ with Glauber calculated quantities $\left(b, N_{\text {part }}\right)$. The plotted distribution and various values are illustrative and not actual measurements (T. Ullrich, private communication).

\section{Ann.Rev.Nucl.Part.Sci.57:205-243,2007 (arXiv:nucl-ex/0701025)}

Glauber Modeling in Nuclear Collisions

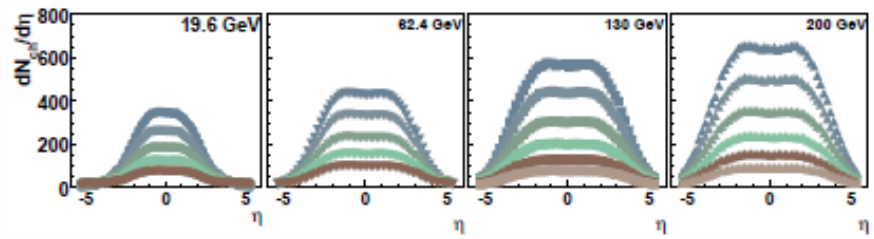

Figure 10: Charged particle multiplicity (PHOBOS) in $\mathrm{Au}+\mathrm{Au}$ collisions at various center-of-mass energies for $|\eta|<5(57,58)$. The different colors represent different centrality selections.

tion (41). However, the multiplicity is also known to scale with the hardness $\left(q^{2}\right)$ of the collision - the multiplicity for hard jet events is significantly higher than that of MB collisions. In heavy ion collisions, we manipulate the fact that the majority of the initial state nucleon-nucleon collisions will be analogous to minimum bias $\mathrm{p}+\mathrm{p}$ collisions, with a small perturbation from much rarer hard interactions. The final integrated multiplicity of heavy ion events is then roughly described as a superposition of many negative binomial distributions, which quickly approaches the Gaussian limit.

"In heavy ion collisions, we manipulate the fact that the majority of the initial state nucleon-nucleon collisions will be analogous to minimum bias $p+p$ collisions..." 


\section{The verifiable case: $p+p$}

Triggering and event characterization:

looking for activity (e.g. charged particle production $\mathrm{N}_{\text {ch }}$, transverse energy $\mathrm{E}_{\mathrm{T}}$ )

preferably close to the beam and far from the region of interest (mid-rapidity)

Now study those distributions as a function of the activity observed at $\eta \sim 0$ "Activity" here is the highest $p_{T}$ for any particle seen around $\eta \sim 0$; could be jet energy, etc. Can be done both in simulation and in data!

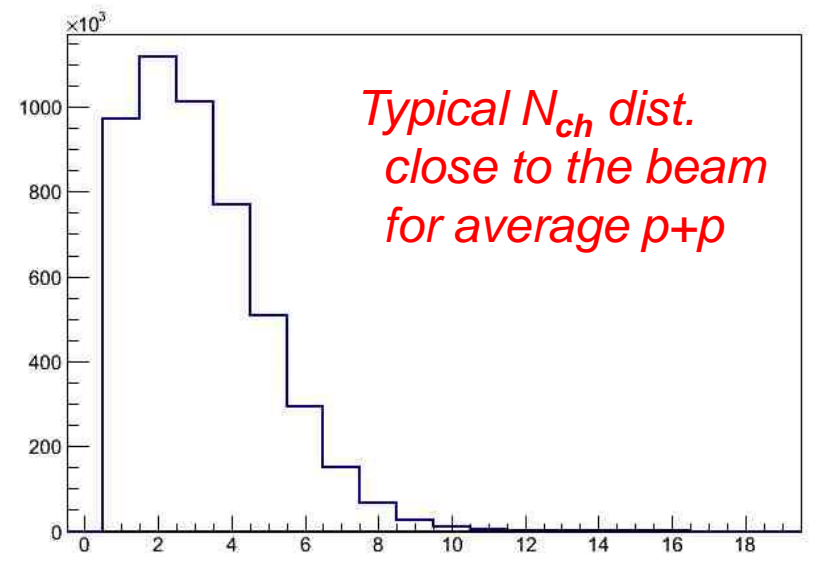

Mean and RMS of the $N_{c h}$ dist. vs $\max p_{T}$ in the center

Note the characteristic rise initially (well-known: higher activity when hard scattering occurs) However, at higher pT they start to drop slowly. They have to, at least asymptotically, for simple kinematic reasons.

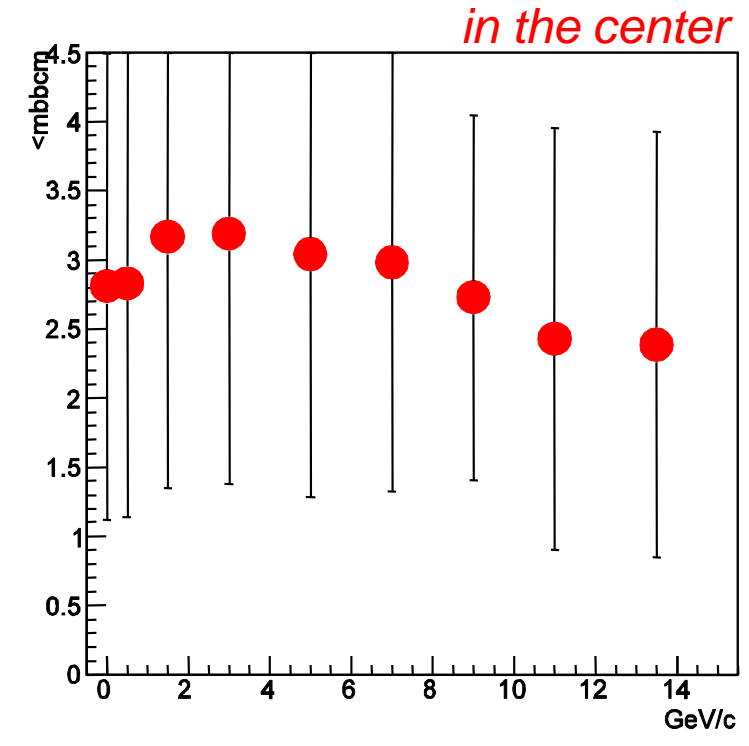

Trigger efficiency vs $\max p_{T}$ in the center

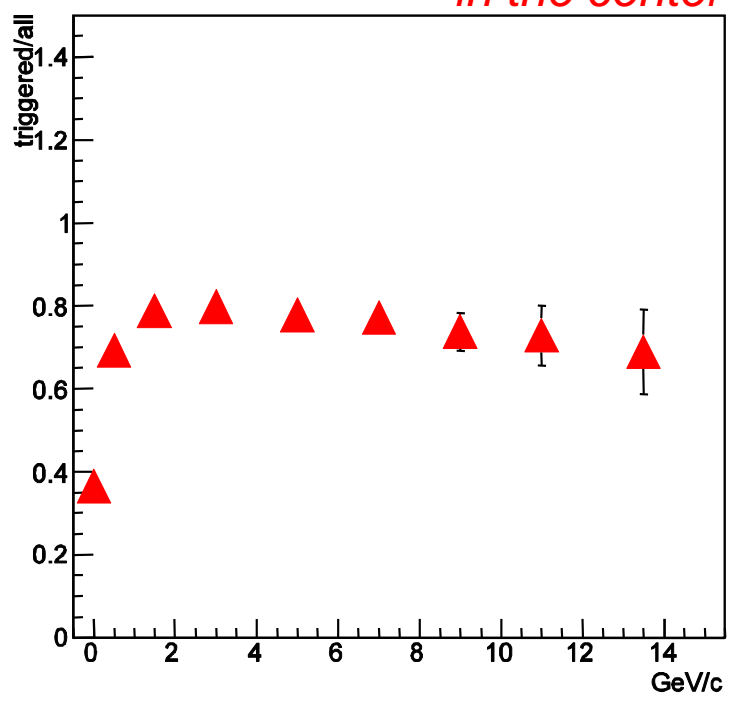

Of course other mechanisms can deplete forward activity way before kinematics does! 


\section{Glauber-model and centrality in $p+A, d+A, \ldots$}

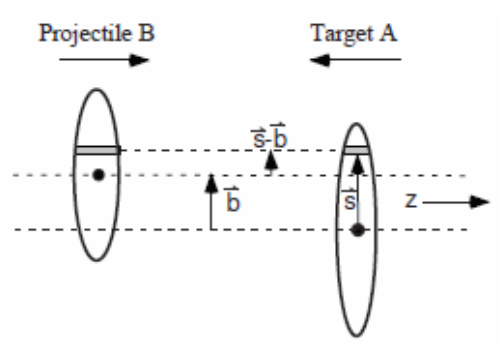

a) Side View

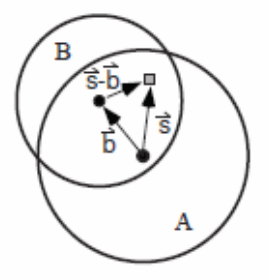

b) Beam-line View

Straight path, independent collisions with the same probability (cross section) $\rightarrow \mathrm{N}_{\text {coll }}, \mathrm{N}_{\text {part }}$ Folding with the average response observed in $\mathrm{p}+\mathrm{p}$ can tie $\mathrm{N}_{\text {coll }}$, $\mathrm{N}_{\text {part }}$ to observed $\mathrm{N}_{\text {ch }}$ statistically Weather or not fluctuations are taken into account is irrelevant here

For instance:

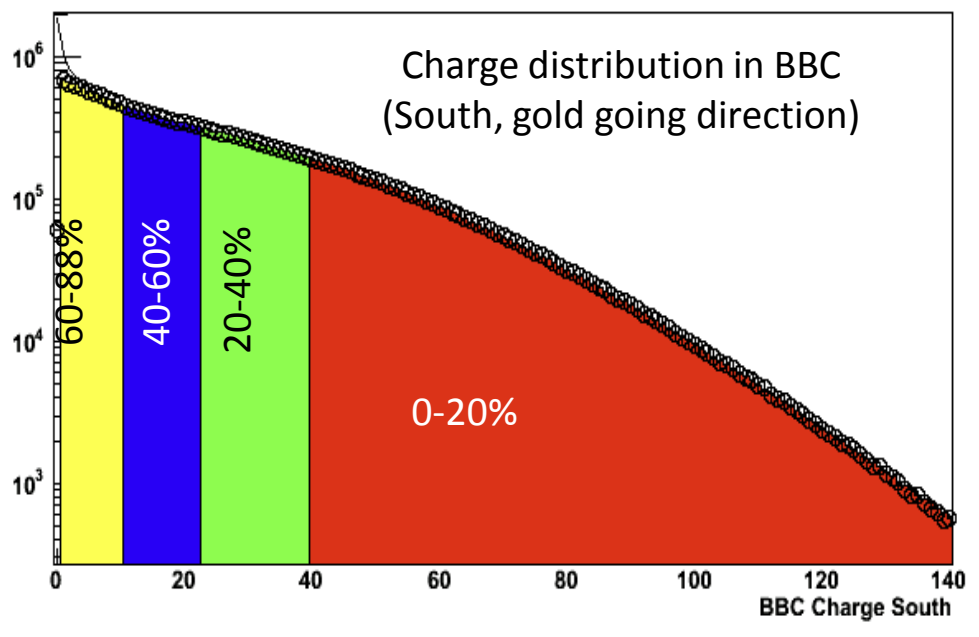

Experimentally defined centrality classes

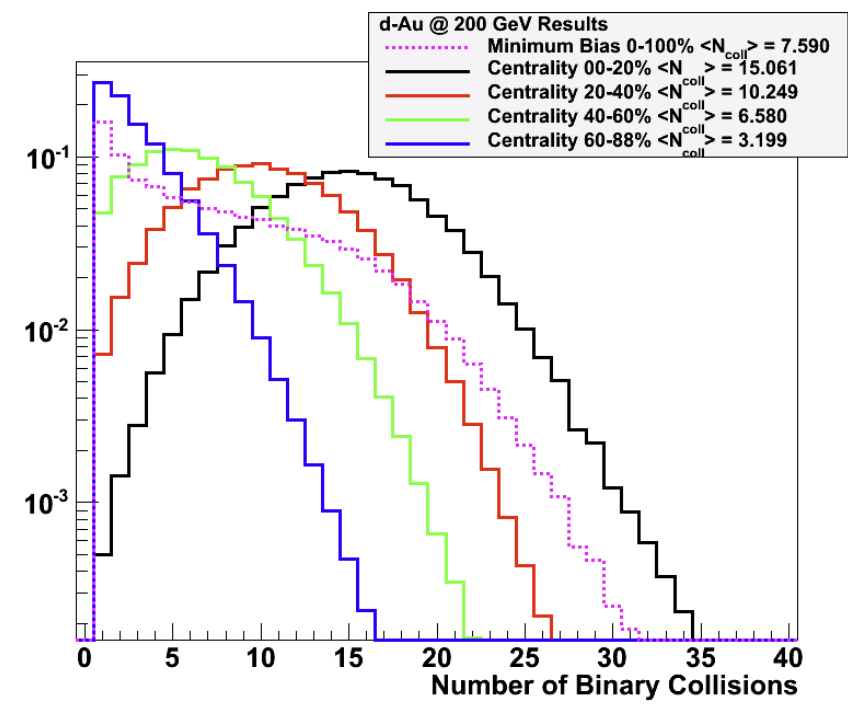

$\mathrm{N}_{\text {coll }}$ distribution for each class from the model

Based on average responses, does not take into account possible special features of rare events (like high $p_{T}$ particle or jet in the central region) 


\section{Will this always work without further corrections?}

Not necessarily.

For instance, as we have seen for $p+p$, the trigger efficiency decreases with increasing energy in the center. Since the trigger requires coincidence on both sides and in $\mathrm{pA}, \mathrm{dA}$ on one side there are at most two nucleons, a similar drop in efficiency is expected. This is well known and usually taken into account.

Centrality is usually defined in the direction where the large ion goes. Assume the projectile makes $\mathbf{N}$ collisions, one of them with very high $\mathrm{p}_{\mathbf{T}}$. Then the expected multiplicity forward is only ( $\mathbf{N}-1)$ times the average plus one reduced response

$\rightarrow$ the multiplicity observed by the experimenter (forward) is smaller than it would be for an event that is identical except that no high $\mathrm{p}_{\mathrm{T}}$ is present

If centrality is defined with fixed multiplicity thresholds based on the average events but applied to the rare, special ones, those rare events may be (mistakenly) classified as lower centrality (lower average $\mathrm{N}_{\text {coll }}$ ) than they really are.

At higher $p_{\mathrm{T}}$ this effect typically shifts to lower multiplicity (i.e. lower centrality) classes events that $\mathrm{N}_{\text {coll }}$-wise - i.e. from the point of view of how probable a rare, hard collision is - would belong in a higher centrality class. 


\section{Illustration: shift between multiplicity classes / 1}

Here is your average,

higher centrality event
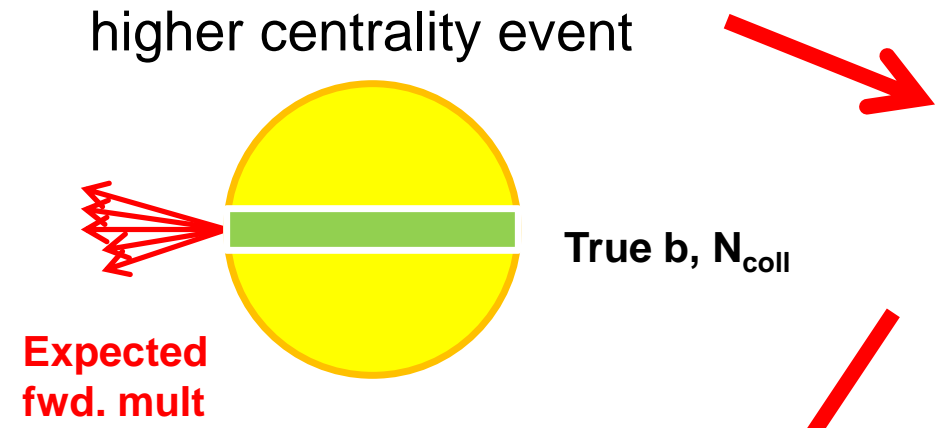

But now a very hard scattering happened (one in a True $\mathbf{b}, \mathbf{N}_{\text {coll }}$

...this is how you classify

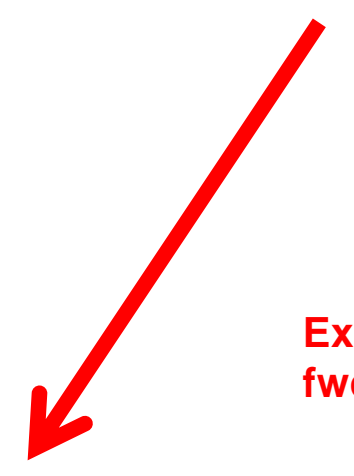
million!), with reduced fwd. response, therefore... the event...

\section{Observed}

fwd. mult

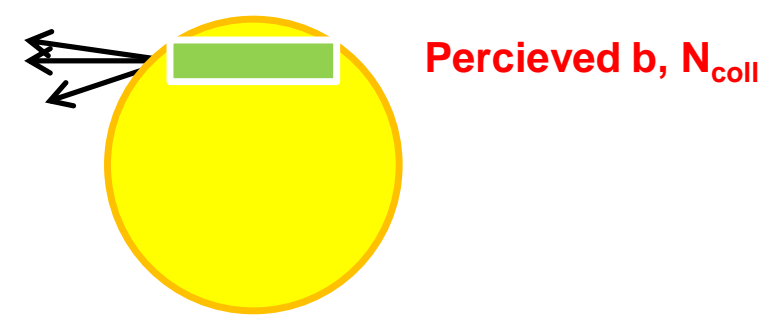

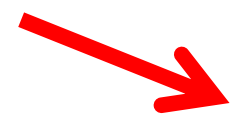

... and when you calculate $R_{A A}$, the denumerator $\left(N_{\text {coll }}{ }^{*} \sigma_{p p}\right)$ will be smaller than it should be $\rightarrow \mathrm{R}_{\mathrm{AA}}$ increases

(There can be other, even more serious effects, as we'll theorize later) 


\section{Illustration: shift between multiplicity classes / 2}

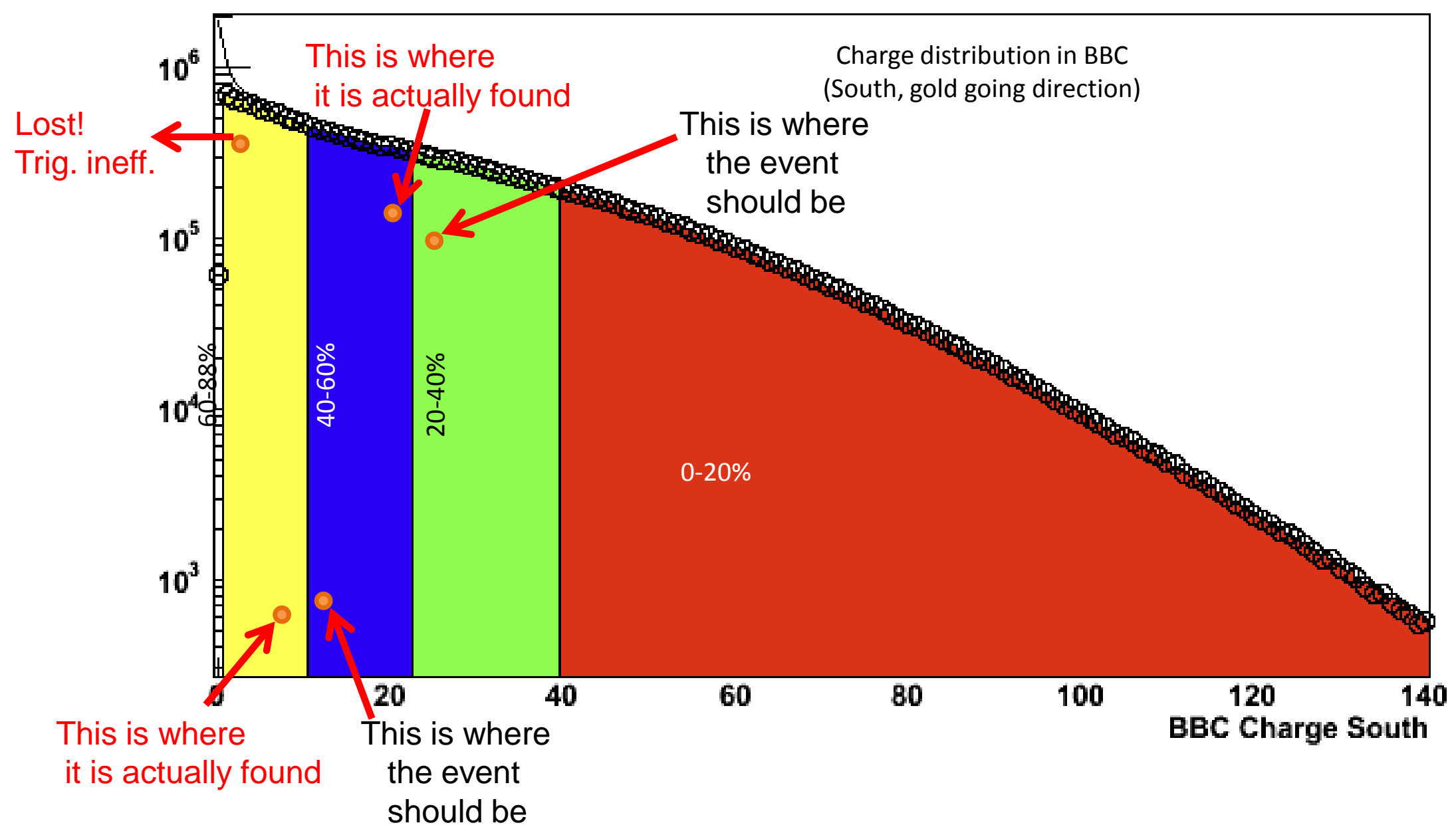

If (experimental) centrality is determined with fixed (forward) multiplicity thresholds, irrespective of what happened at $\boldsymbol{\eta} \sim 0$, events may end up in the wrong centrality class - and attributed an incorrect $\left\langle N_{\text {coll }}>\right.$ 


\section{More exotic possibilities}

Confusion from the dual use of $\mathrm{N}_{\text {coll }}$ (???)

We use it both to estimate the average soft response by folding the $p+p$ distribution (which assumes that the likes of $\mathrm{N}_{\text {coll }}$ average $\mathrm{p}+\mathrm{p}$ collisions in fact do happen in the event)

but then we also use $N_{\text {coll }}$ to estimate how much an extremely rare $p+p$ process (hard scattering) is enhanced in $p / d+A$, where it is still very-very rare $(<<1 /$ event $)$

But in those very rare instances when hard scattering did in fact happen, will the $d / p$ nucleon for the rest of its path interact with the remaining A nucleons and original, intact nucleon (i.e. with the same $\sigma_{\mathrm{pp}}$ a la Glauber?)

If not, what will happen?

Will it keep interacting, but with reduced cross-section (like $\left.\sigma_{\pi \mathfrak{p}}\right)$ ?

Will it be completely out of the pool (no more soft production whatsoever?)

Something in between? If so, what? 


\title{
Can this be tested?
}

\section{Reduced/vanishing cross-section in a different context:}

\author{
Papp, Levai, Barnafoldi, Zhang, Fai -- nucl-th/0203075
}

A possible candidate for the reduction of the cross section in large systems is the proton dissociation mentioned in the introduction. Our $p A$ collision study showed that each $p p$ inelastic collision adds $\sim 400 \mathrm{MeV}$ transverse momentum to the partons inside the proton (on the average). After a few such collisions the partons gain high enough transverse momenta to become free of the proton and during this transition process they do not interact (dead time). We assume that such a proton is "lost" for the reaction and does not participate in particle production anymore. We note that such a picture corresponds to a modification of the original Glauber model. It re-

\section{High $\mathrm{p}_{\mathrm{T}}$ biases}

Renk, arXiv:1212.0646

However, it has to be realized that almost all current high $P_{T}$ observables measure conditional probabilities of events, not probabilities. Thus, the correct starting point for their theoretical understanding is Bayes' formula, and the biases introduced by the conditioning are crucial to understanding the outcome. Once this is introduced properly into the modelling process, the counter-intuitive results are seen to find a natural explanation in terms of various biases and the puzzles largely disappear.

Would comparison to LHC help?

If (with similar centrality determination) LHC would see no effect in our $\mathrm{p}_{\mathrm{T}}$ range, but similar effect at higher $\mathrm{p}_{\mathrm{T}}$, the "kinematic" effect (depletion of available energy forward) could be the culprit (or dominant)

If LHC would see a similar effect already in our $\mathrm{p}_{\mathrm{T}}$ range, the "dynamic" effect (reduced or vanishing cross section) could be the dominant contributor 


\section{Summary}

In $d+A u$ thermal radiation is not there, but identified hadrons up to $6 \mathrm{GeV} / \mathrm{c}$ show features consistent with some medium formation

Specifically, all hadron (baryon and meson) spectra are similar in peripheral $A u+A u$ and central $d+A u$

Preliminary $\pi^{0}, \eta$ and jet nuclear modification factors show a very unexpected rise in peripheral collisions, while for minimum bias $R_{A A} \sim 1$

Very large drop in $R_{C p}$, too - i.e. the reference $p+p$ can not be the source of such effect

Different observables, different groups, codes, systematic uncertainties unlikely that the spectra have a substantial problem

Is it some new physics - or can there be some problem with the way centrality is inferred for these very rare events?

One thing is certain: just redefining centrality such that $R_{A A}=1$ is not a good idea (:) 


\section{Alfonso the Xth ("Alfonse the Wise")}

1221-1284

Monarch of Castilia

One of the best scientists of his age

(and big time supporter of science)

"Alfonsine tables" used even by

Copernicus, superseded only

by Kepler in 1627

So he knew what he was talking about, when sighed (and we all should agree...):

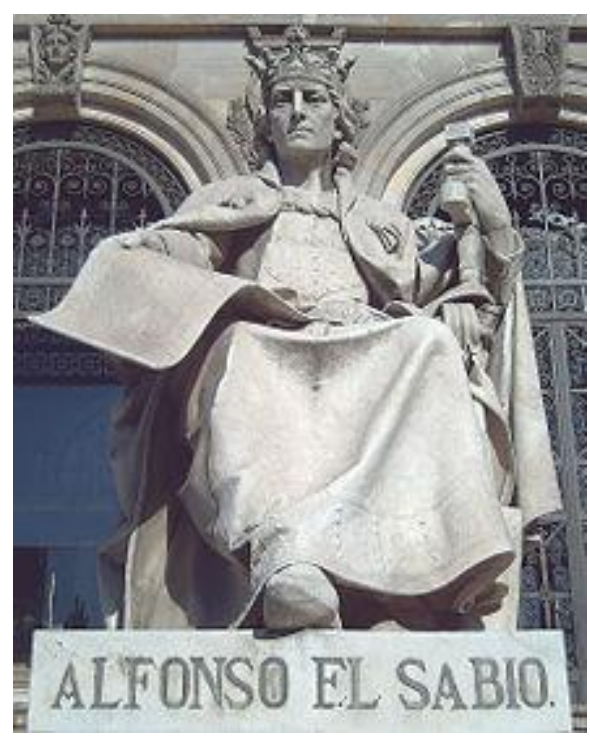

If the Lord Almighty had consulted me before embarking upon creation, I should have recommended something simpler. 


\section{... or on a more serene note}

Even if all these speculations turn out to be wrong, the measurement that prompted them is very tantalizing and certainly consequential - in one way or another. No matter what the outcome, the process itself has been fun. Or to quote...

\section{Albert Szent-Györgyi}

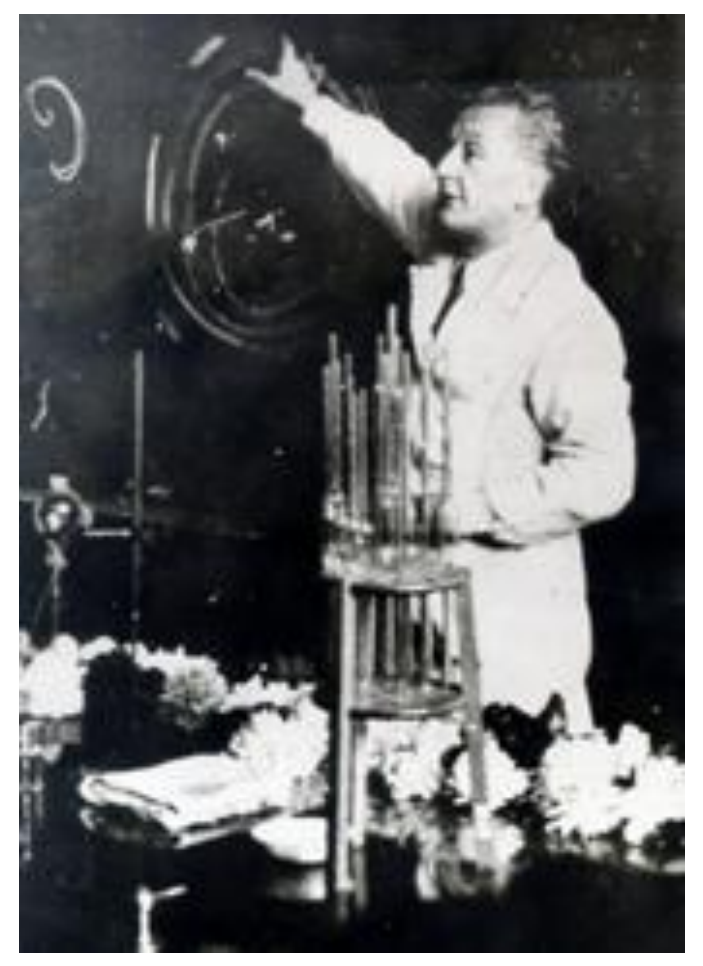

In later years, - he worked in his newly organized Muscle Research Laboratory at the Marine Biological Laboratory in Woods Hole, Mass., USA, - when asked why, he passionately used such a big hook for his beloved fishing, he used to say, "I think it is more exciting not to catch a big fish, than not to catch a small one." He really made some big catches in his lifetime: the Nobel-Prize for discovering the vitamin- $C$ and the biochemical steps of catalysis of the fumaric acid in the tricarboxylic acid cycle. 


\section{$\infty$}




\section{Small-x physics and pA control measurements}

Adrian Dumitru

RIKEN BNL and Baruch College, CUNY

Jet Quenching 2013

RBRC workshop 03/2013

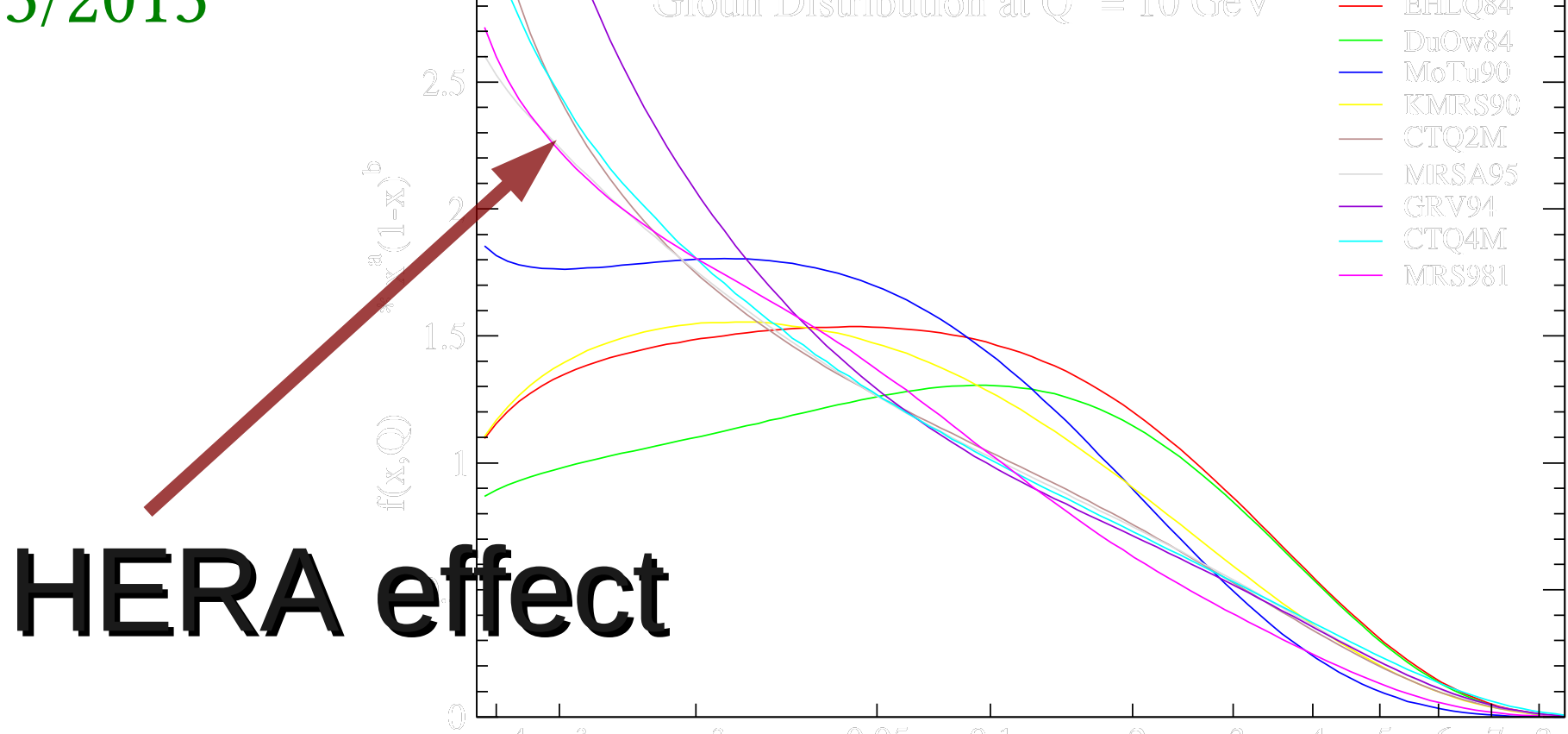




\section{rcBK evolution:}

basic "degrees of freedom": dipole scattering amplitude in fund. rep. (2-point fct)

$$
\begin{aligned}
& \mathcal{N}_{F}(r, Y ; b, A) \equiv \frac{1}{N_{c}} \operatorname{tr}\left\langle 1-V^{\dagger}(y) V(z)\right\rangle_{Y} \\
& \mathbf{r}=\mathbf{y}-\mathbf{z}
\end{aligned}
$$

BK equation (incl. non-linear terms $\rightarrow$ saturation of scattering amplitude!)

$$
\begin{array}{r}
\frac{\partial \mathcal{N}(r, Y)}{\partial Y}=\int d^{2} r_{1} K\left(r, r_{1}, r_{2}\right)\left[\mathcal{N}\left(r_{1}, Y\right)+\mathcal{N}\left(r_{2}, Y\right)-\mathcal{N}(r, Y)-\mathcal{N}\left(r_{1}, Y\right) \mathcal{N}\left(r_{2}, Y\right)\right] \\
\mathbf{r}=\mathbf{r}_{1}-\mathbf{r}_{\mathbf{2}}
\end{array}
$$

running-coupling kernel (Balitsky prescription)

$$
\begin{gathered}
K\left(\mathbf{r}, \mathbf{r}_{\mathbf{1}}, \mathbf{r}_{\mathbf{2}}\right)=\frac{N_{c} \alpha_{s}\left(r^{2}\right)}{2 \pi^{2}}\left[\frac{1}{r_{1}^{2}}\left(\frac{\alpha_{s}\left(r_{1}^{2}\right)}{\alpha_{s}\left(r_{2}^{2}\right)}-1\right)+\frac{r^{2}}{r_{1}^{2} r_{2}^{2}}+\frac{1}{r_{2}^{2}}\left(\frac{\alpha_{s}\left(r_{2}^{2}\right)}{\alpha_{s}\left(r_{1}^{2}\right)}-1\right)\right] \\
\alpha_{s}\left(r^{2}\right)=\frac{4 \pi}{\beta \log \left(4 \frac{C^{2}}{r^{2} \Lambda^{2}}+\mu\right)} \\
\quad \text { dipole scattering amplitude in adj. rep. } \quad \mathcal{N}_{A}=2 \mathcal{N}_{F}-\mathcal{N}_{F}^{2}
\end{gathered}
$$


uGD at $x=3 \times 10^{-4}$ (e.g. $p t=2 G e V, y=0, v s=7 T e V$ )

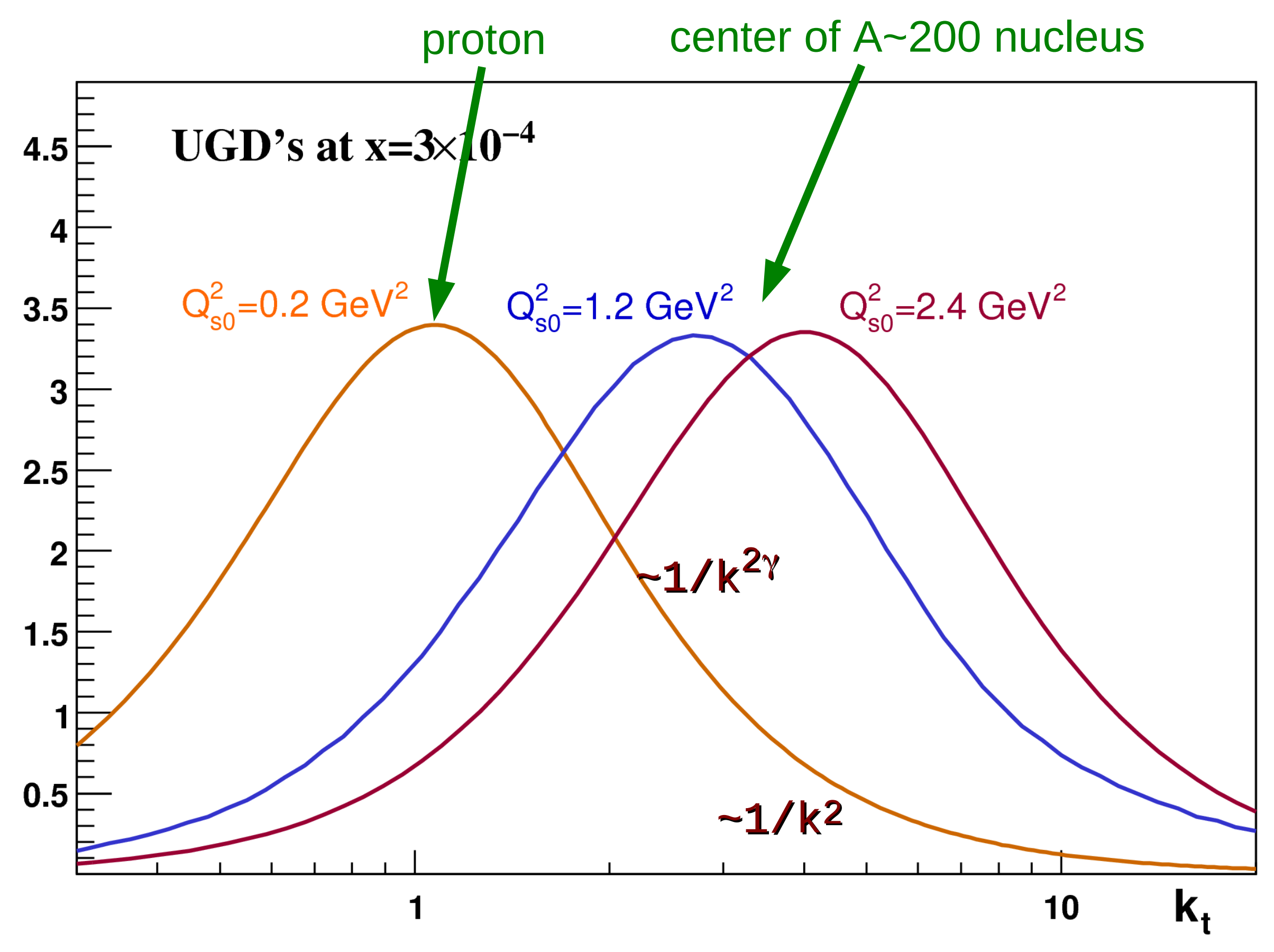




\section{what is the initial condition for rcBK evolution?}

- don't really know, small-x doesn't tell

- needs to be set at "sufficiently" small $x_{0}$ so that rcBK can take it from there; in practice, $x_{0}=0.01$ ?

- for large A, MV model may provide a decent ini. cond. :

$$
\mathcal{N}_{F}(r, Y=0 ; b)=1-\exp \left[-\frac{r^{2} Q_{s 0}^{2}(b)}{4} \ln \left(\frac{1}{\Lambda r}+e\right)\right]
$$

- alternative I.C. (AAMQS 2011), also denoted by $\mathrm{MV}^{\gamma}$ or Set $2(\gamma>1$ !):

$$
\mathcal{N}_{F}(r, Y=0 ; b)=1-\exp \left[-\frac{\left[r^{2} Q_{s 0}^{2}(b)\right]^{\gamma}}{4} \ln \left(\frac{1}{\Lambda r}+e\right)\right]
$$


$\gamma^{*} \mathrm{p} \quad$ Fit with only light quarks

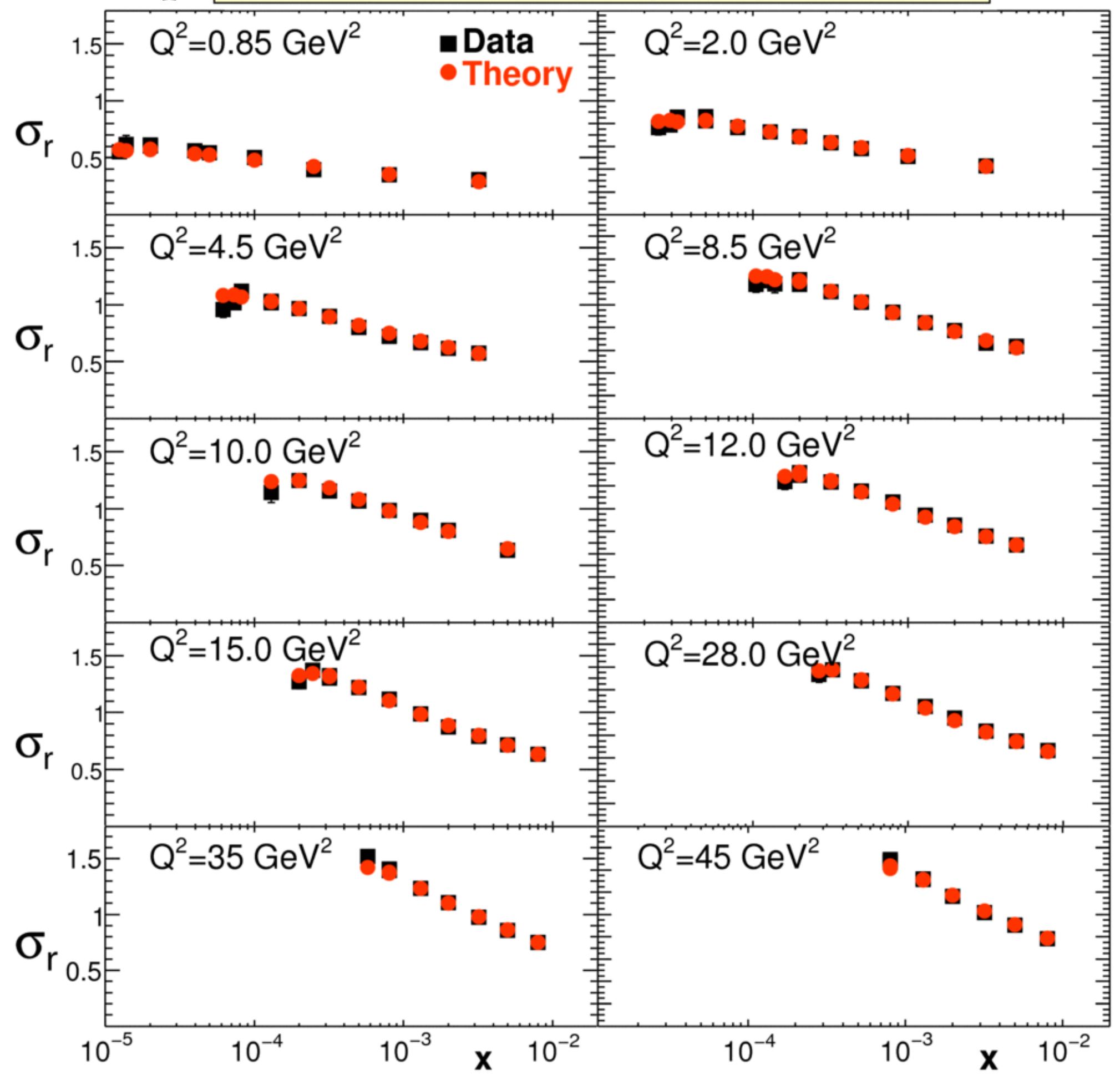

AAMQS:

EPJ C71 (2011) 


\section{J. Albacete et al ("AAMQS"): arXiv:1012.4408}

\begin{tabular}{|l||c||c||c|c|c|c|c|}
\hline & fit & $\frac{\chi^{2}}{\text { d.o.f }}$ & $Q_{s 0}^{2}$ & $\sigma_{0}$ & $\gamma$ & $C$ & $\mathrm{~m}_{l}^{2}$ \\
\hline \hline & GBW & & & & & & \\
\hline a & $\alpha_{f r}=0.7$ & 1.226 & 0.241 & 32.357 & 0.971 & 2.46 & fixed \\
\hline a' & $\alpha_{f r}=0.7\left(\Lambda_{m_{\tau}}\right)$ & 1.235 & 0.240 & 32.569 & 0.959 & 2.507 & fixed \\
\hline b & $\alpha_{f r}=0.7$ & 1.264 & 0.2633 & 30.325 & 0.968 & 2.246 & $1.74 \mathrm{E}-2$ \\
\hline c & $\alpha_{f r}=1$ & 1.279 & 0.254 & 31.906 & 0.981 & 2.378 & fixed \\
\hline c' & $\alpha_{f r}=1\left(\Lambda_{m_{\tau}}\right)$ & 1.244 & 0.2329 & 33.608 & 0.9612 & 2.451 & fixed \\
\hline d & $\alpha_{f r}=1$ & 1.248 & 0.239 & 33.761 & 0.980 & 2.656 & $2.212 \mathrm{E}-2$ \\
\hline \hline & MV & & & & & & \\
\hline e & $\alpha_{f r}=0.7$ & 1.171 & 0.165 & 32.895 & 1.135 & 2.52 & fixed \\
\hline f & $\alpha_{f r}=0.7$ & 1.161 & 0.164 & 32.324 & 1.123 & 2.48 & $1.823 \mathrm{E}-2$ \\
\hline g & $\alpha_{f r}=1$ & 1.140 & 0.1557 & 33.696 & 1.113 & 2.56 & fixed \\
\hline h & $\alpha_{f r}=1$ & 1.117 & 0.1597 & 33.105 & 1.118 & 2.47 & $1.845 \mathrm{E}-2$ \\
\hline h' & $\alpha_{f r}=1\left(\Lambda_{m_{\tau}}\right)$ & 1.104 & 0.168 & 30.265 & 1.119 & 1.715 & $1.463 \mathrm{E}-2$ \\
\hline
\end{tabular}

Table 1: Parameters from fits with only light quarks to data with $x \leq 10^{-2}$ and for all available values of $Q^{2} \leq 50 \mathrm{GeV}^{2}$ for different initial conditions, fixed values of the coupling in the infrared $\alpha_{f r}=0.7$ and 1 and light quark masses either taken fixed $m_{l}=0.14 \mathrm{GeV}$ or left as a free parameter. Fits a', c' and h' correspond to taking the $\tau$ mass as reference scale for the running of the coupling. Units: $Q_{s 0}^{2}$ and $m_{l}^{2}$ are in $\mathrm{GeV}^{2}$ and $\sigma_{0}$ in $\mathrm{mb}$. 
- in what follows, this I.C. is used even for a nucleon, with

$$
\mathrm{MV}: Q_{s 0, N}^{2}=0.2 \mathrm{GeV}^{2} \quad \mathrm{MV}^{\gamma}: Q_{s 0, N}^{2}=0.168 \mathrm{GeV}^{2}
$$

- for nucleus, at transv. position b:

$$
\mathrm{Q}^{2}{ }_{\mathrm{sO}}(\mathrm{b})=(\# \text { nucleons at } \mathrm{b}) \times \mathrm{Q}^{2} \mathrm{~s} \mathrm{~s}, \mathrm{~N}
$$

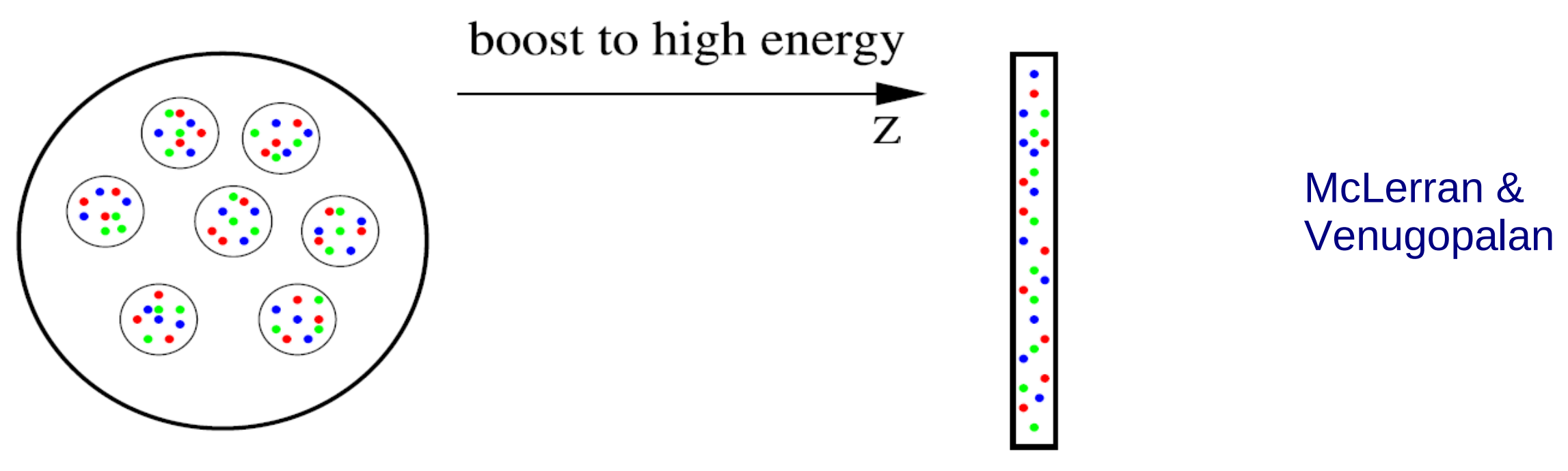

side view 


\section{fluctuations of valence partons in $\perp$ plane}
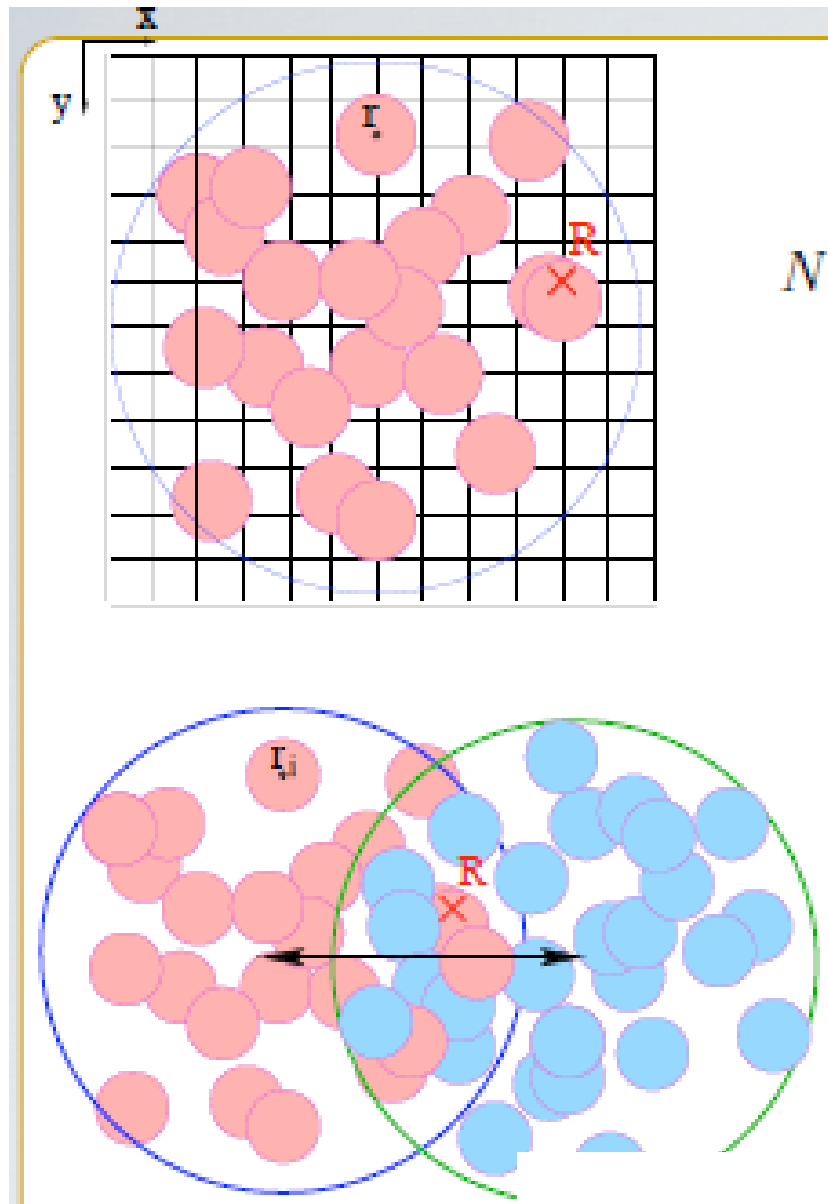

$N_{\text {part,A }}$

$(\vec{b})=$

1. Initial conditions for the evolution ( $\mathrm{x}=0.01)$

$$
\begin{aligned}
N(\mathbf{R})=\sum_{i=1}^{A} \Theta\left(\sqrt{\frac{\sigma_{0}}{\pi}}-\left|\mathbf{R}-\mathbf{r}_{\mathbf{i}}\right|\right) \longrightarrow & Q_{s 0}^{2}(\mathbf{R})=N(\mathbf{R}) Q_{s 0, \text { nucl }}^{2} \\
\downarrow & \varphi\left(x_{0}=0.01, k_{t}, R\right)
\end{aligned}
$$

2. Solve local running coupling BK evolution at each transverse point rcBK equation or KLN model

$$
\varphi\left(x, k_{t}, R\right)
$$

3 Calculate gluon production at each transverse point according to kt-factorization

INPUT: $\varphi\left(\mathrm{x}=0.01, \mathrm{k}_{\mathbf{t}}\right)$ FOR A SINGLE NUCLEON:

$$
\sum_{i=1 \cdots A} \Theta\left(P\left(\vec{b}-\vec{r}_{i}\right)-\nu_{i}\right)
$$

$$
P(b)=1-\exp \left[-\sigma_{g} T_{p p}(b)\right],
$$$$
T_{p p}(b)=\int d^{2} s T_{p}(s) T_{p}(s-b)
$$

$T_{p}(r)=\frac{1}{2 \pi B} \exp \left[-r^{2} /(2 B)\right]$

$$
\sigma_{N N}(\sqrt{s})=\int d^{2} b\left(1-\exp \left[-\sigma_{g} T_{p p}(b)\right]\right)
$$




\section{thickness fluctuations:}

(average of 1 / 9 nucleon target compared to 5-nucl. target)

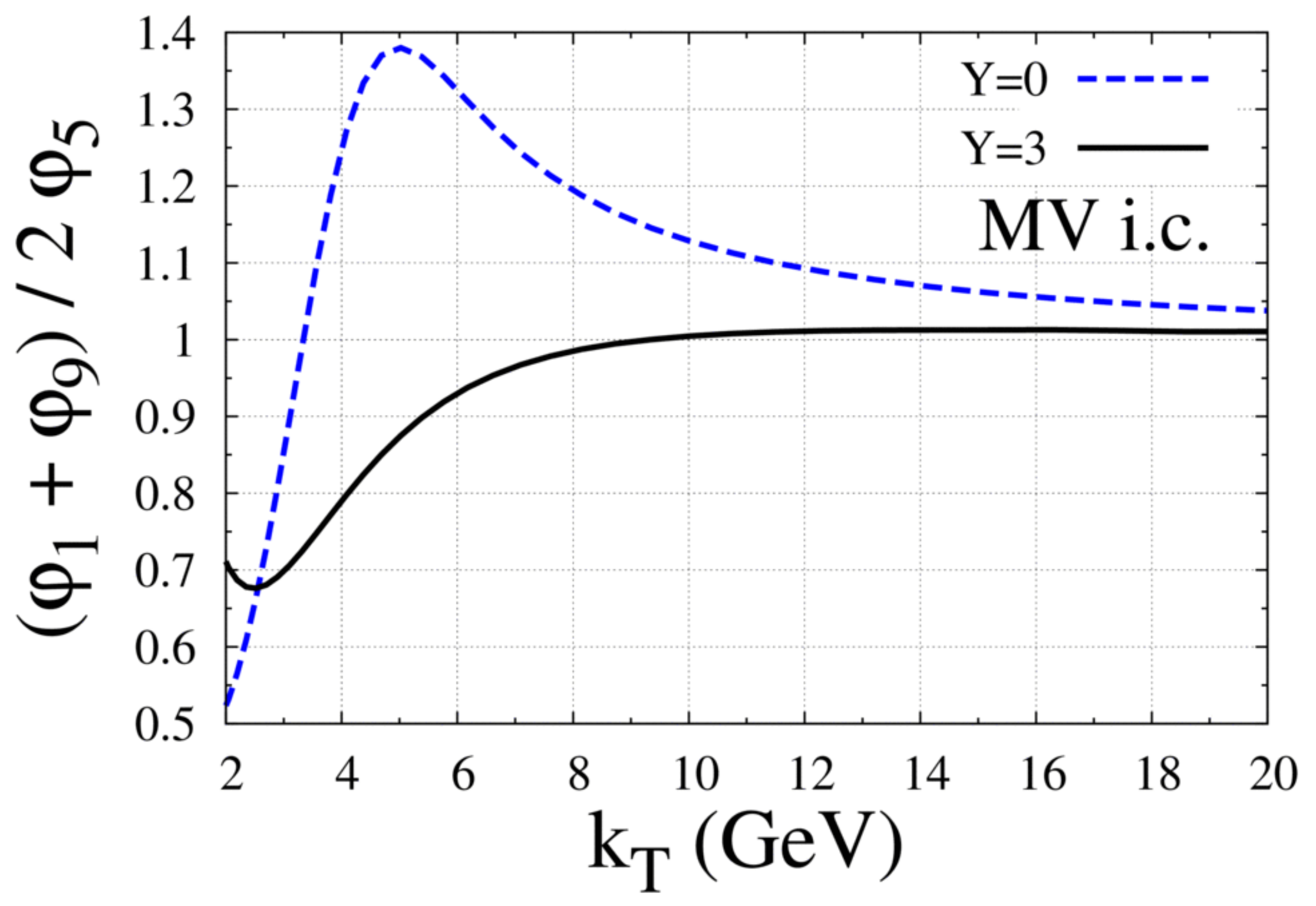




\section{$k_{\perp}$-factorization, multiplicity in $A+B-->g+X$}

unintegrated gluon distribution:

$$
\varphi(k, Y ; b, A)=\frac{C_{F} k^{2}}{\alpha_{s}(k)} \int \frac{d^{2} \mathbf{r}}{(2 \pi)^{3}} e^{-i \mathbf{k} \cdot \mathbf{r}} \mathcal{N}_{A}(r, Y ; b, A)
$$

multiplicity:

$$
\frac{d N^{A+B \rightarrow g}}{d y d^{2} p_{t} d^{2} b}=K \frac{2}{C_{F}} \frac{1}{p_{t}^{2}} \int^{p_{t}} \frac{d^{2} k_{t}}{4} \alpha_{s}(Q) \varphi\left(\frac{\left|p_{t}+k_{t}\right|}{2}, x_{1}\right) \varphi\left(\frac{\left|p_{t}-k_{t}\right|}{2}, x_{2}\right)
$$

Notes:

- finite as $p_{t} \rightarrow 0$ if UGD does not blow up

- $x_{1,2}=\left(p_{t} / \sqrt{ } s\right) \exp ( \pm y) ; \quad Y_{1,2}=\log \left(x_{0} / x_{1,2}\right)$ where $x_{0}=0.01$ is assumed onset of rcBK evol.

- $\mathrm{K}=1.5-2$, appears reasonable 
high-pt hadron production in pp @ LHC requires steeper I.C.

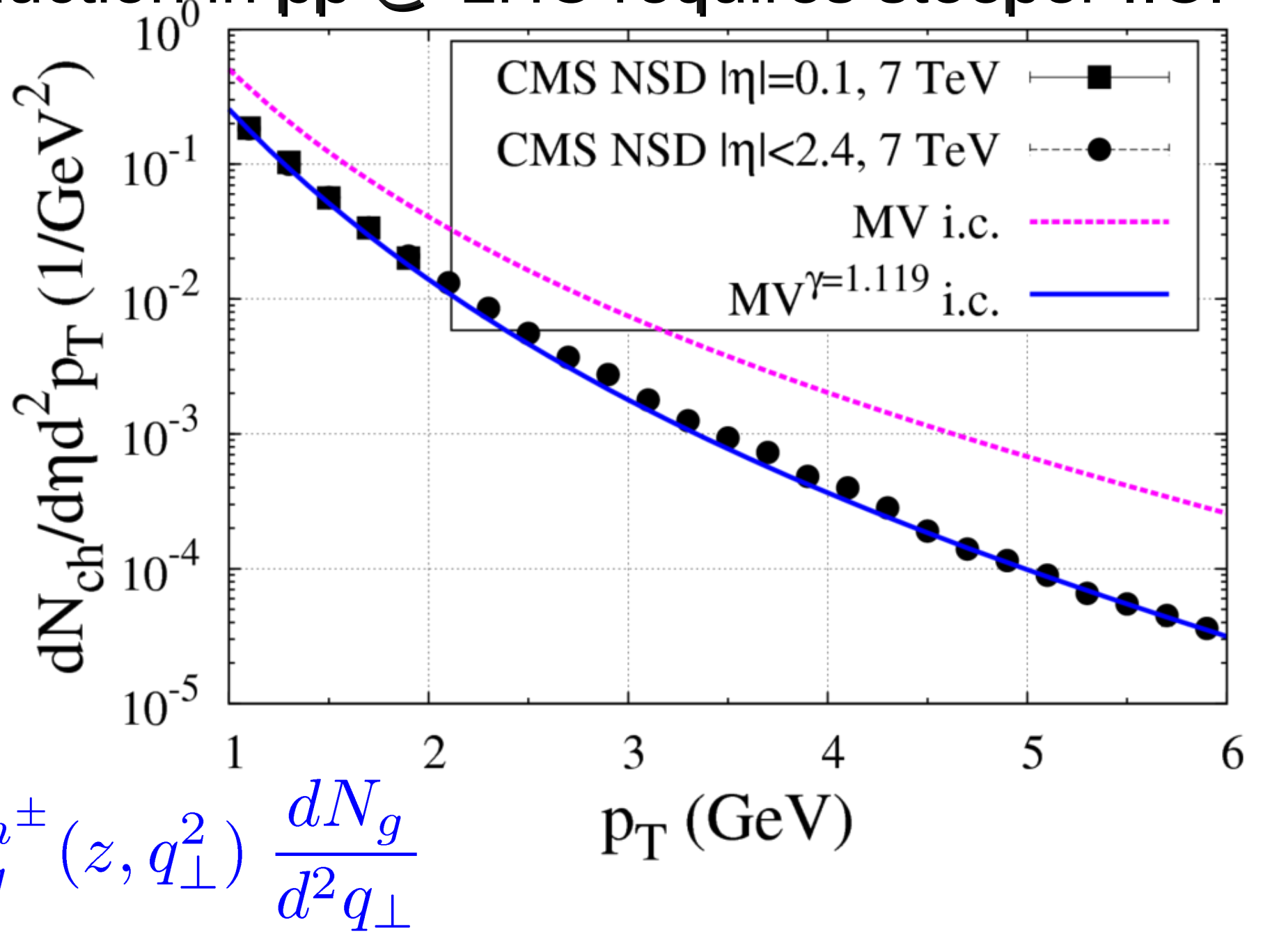

- no $\mathrm{g} \rightarrow \mathrm{h}$ multiplication factor $\kappa$ here!

(normalization set by Fragm. Func.)

- rcBK in dilute regime

- LHC constrains initial condition 


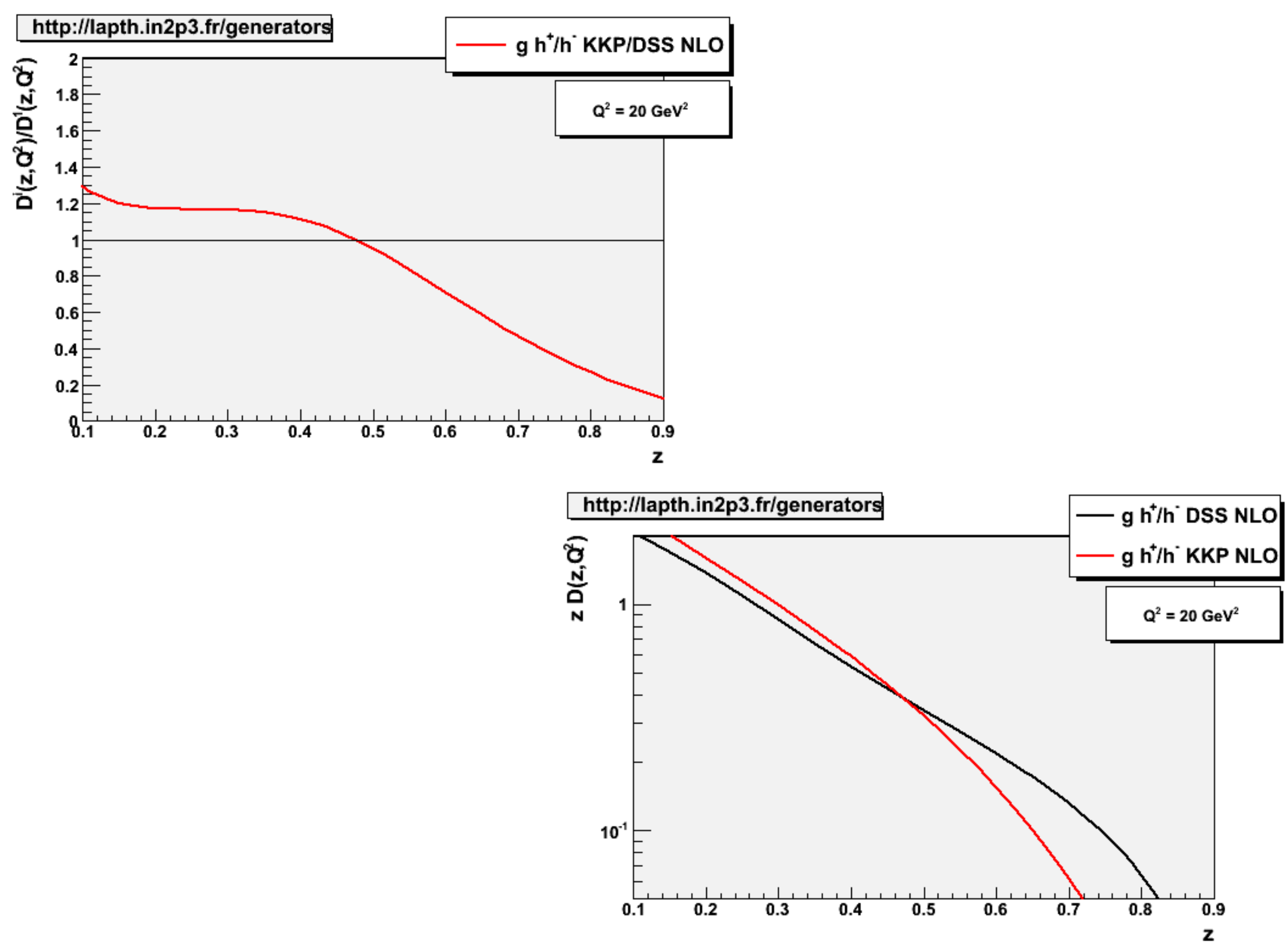




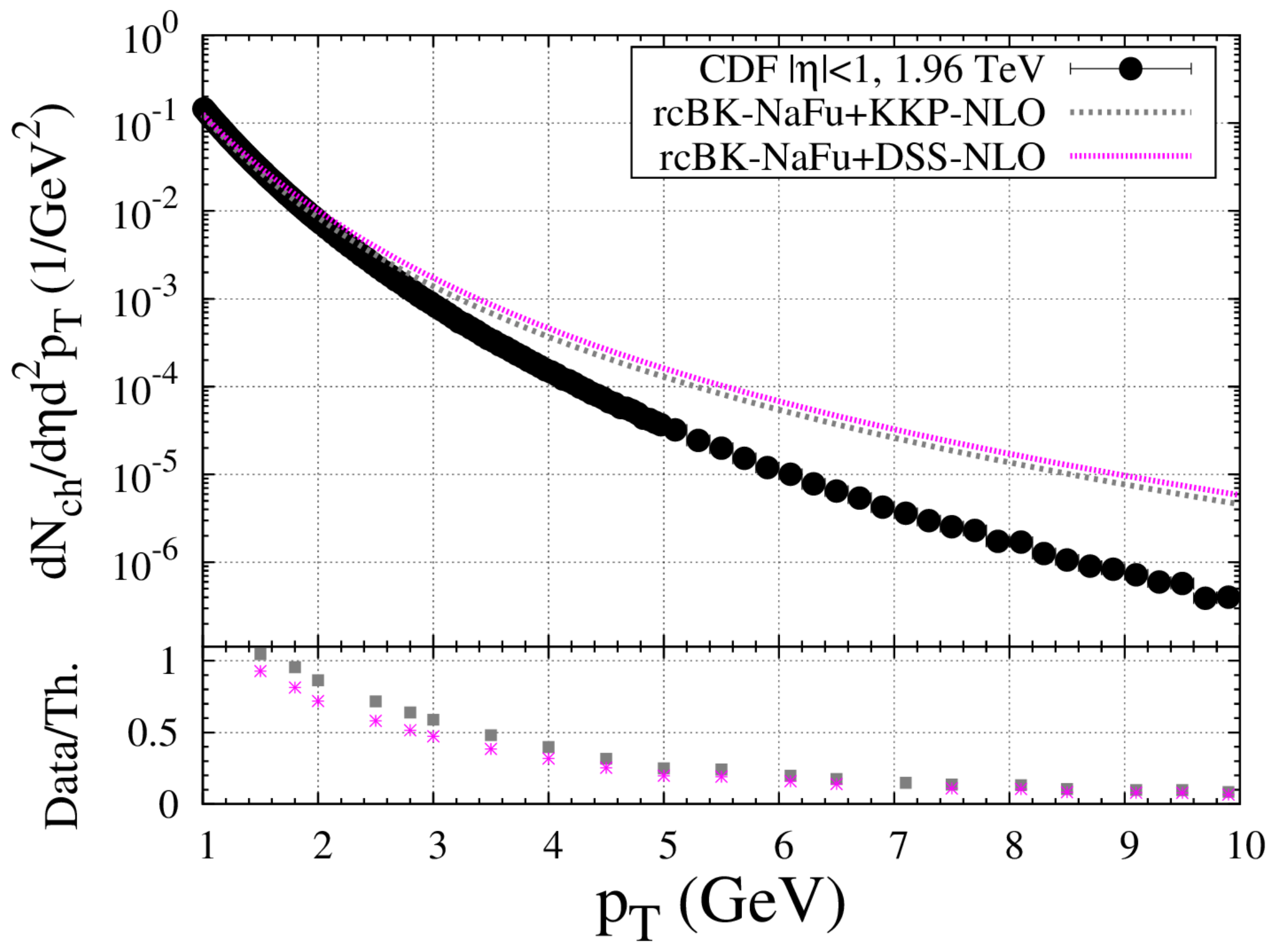


pt distributions in $\mathrm{pp} \rightarrow$ ch at TEVATRON / LHC energies

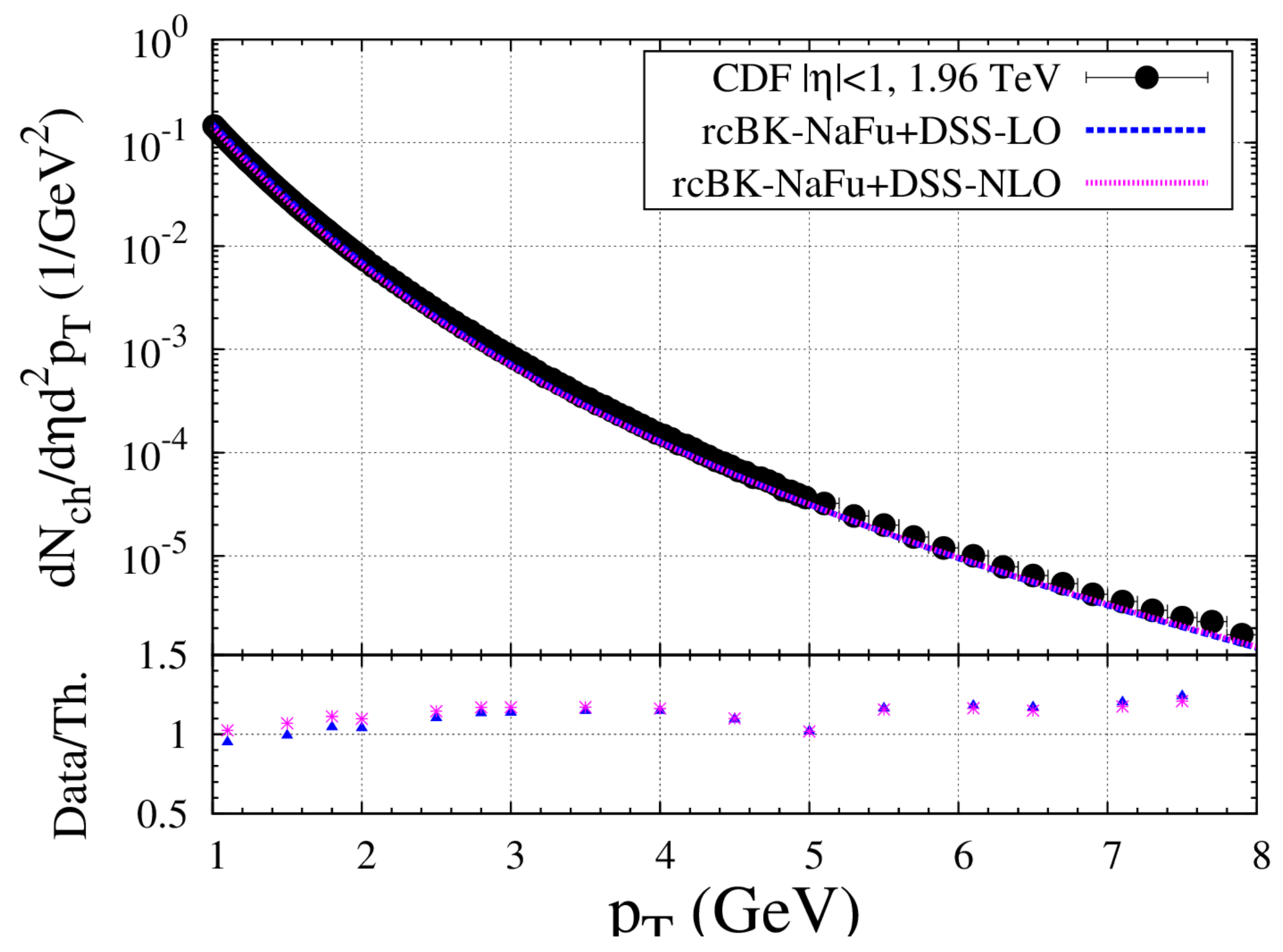




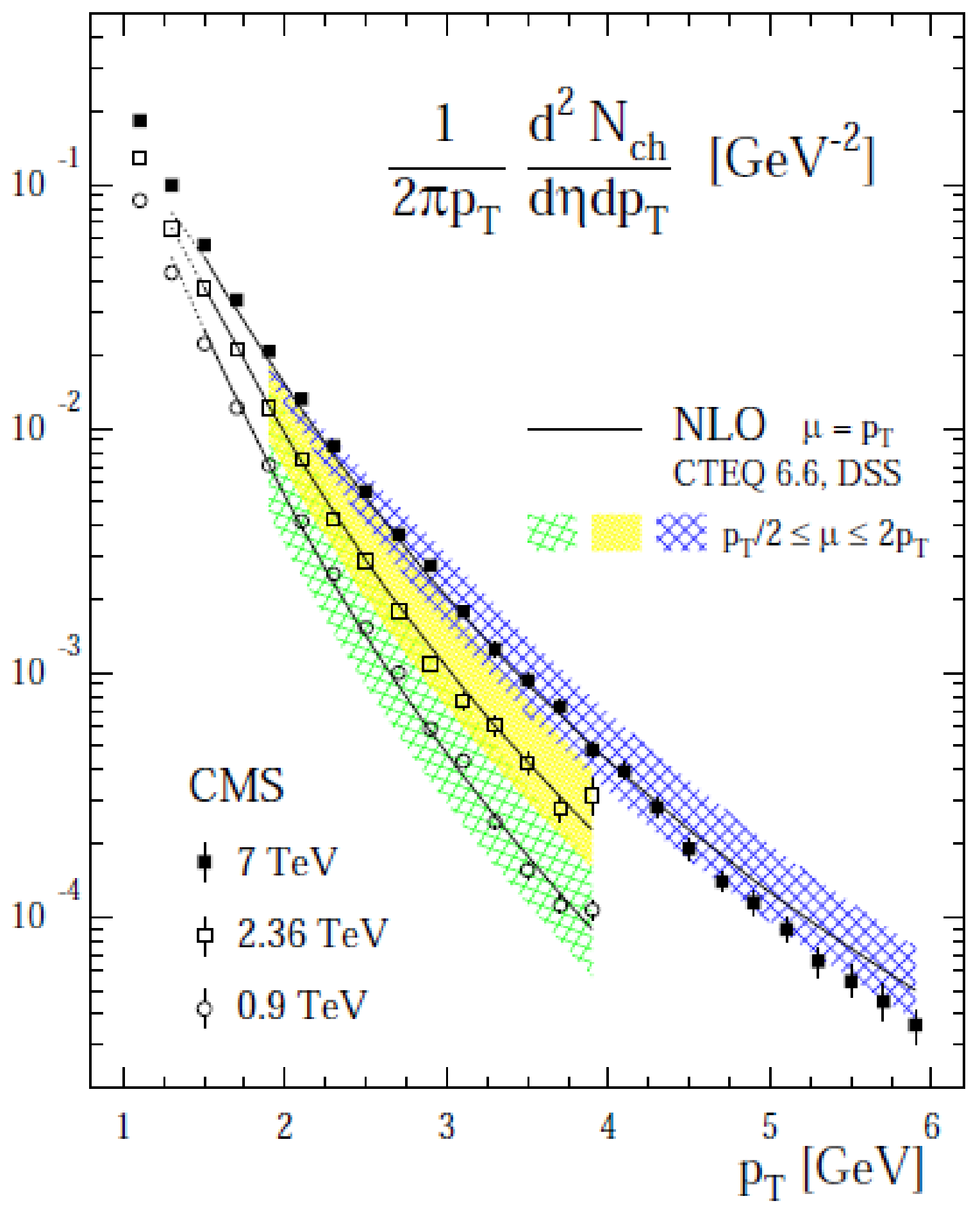

Collinear pQCD fact. Sassot, Zurita, Stratmann PRD 82 (2010) 
$\mathcal{N}_{F}(r, Y=0 ; b)=1-\exp \left[-\frac{\left[r^{2} Q_{s 0}^{2}(b)\right]^{\gamma}}{4} \ln \left(\frac{1}{\Lambda r}+e\right)\right]$

for nuclei:

- do we use same $\gamma$ as for a proton?

- do we assume $Q_{s 0}^{2} \sim T_{A}$ or $Q_{s 0}^{2} \sim T_{A}^{1 / \gamma}$

$\rightarrow \mathrm{w} / \mathrm{o}$ a better idea of where the AAMQS parameter comes from, we need to factor this into uncertainties... 


\section{$\mathrm{A}^{1 / 3}$ dependence of initial conditions for BK/JIMWLK beyond MV action}

$S=\int d^{2} x_{\perp}\left\{\frac{1}{2 \mu^{2}} \rho^{a} \rho^{a}-\frac{1}{\kappa_{3}} d^{a b c} \rho^{a} \rho^{b} \rho^{c}+\right.$
$\left[\frac{1}{\kappa_{4}}\left(\delta^{a b} \delta^{c d}+\delta^{a c} \delta^{b d}+\delta^{a d} \delta^{b c}\right)+\right.$
$\left.\left.\frac{1}{\kappa_{4}^{\prime}}\left(d^{a b e} d^{c d e}+d^{a c e} d^{b d e}+d^{a d e} d^{b c e}\right)\right] \rho^{a} \rho^{b} \rho^{c} \rho^{d}\right\}$

+ soft YM fields + coupling of soft $\leftrightarrow$ hard

- $\mu^{2} \sim g^{2} A^{1 / 3} ; \quad \kappa_{3} \sim g^{3} A^{2 / 3} ; \quad \kappa_{4} \sim g^{4} A$ 
E. Petreska + A.D., NPA 2012

$1-\mathcal{N}\left(r, x_{0}\right)=\frac{1}{4} r^{2} Q_{s 0}^{2} \log \frac{1}{r \Lambda}-\frac{1}{6 \pi^{3}} \frac{g^{8}}{\kappa_{4}}\left(\int d x^{-} \mu^{4}\left(x^{-}\right)\right)^{2} r^{2} \log ^{3} \frac{1}{r \Lambda}$

(in $\log (1 / \mathrm{r} \wedge) » 1$ limit)
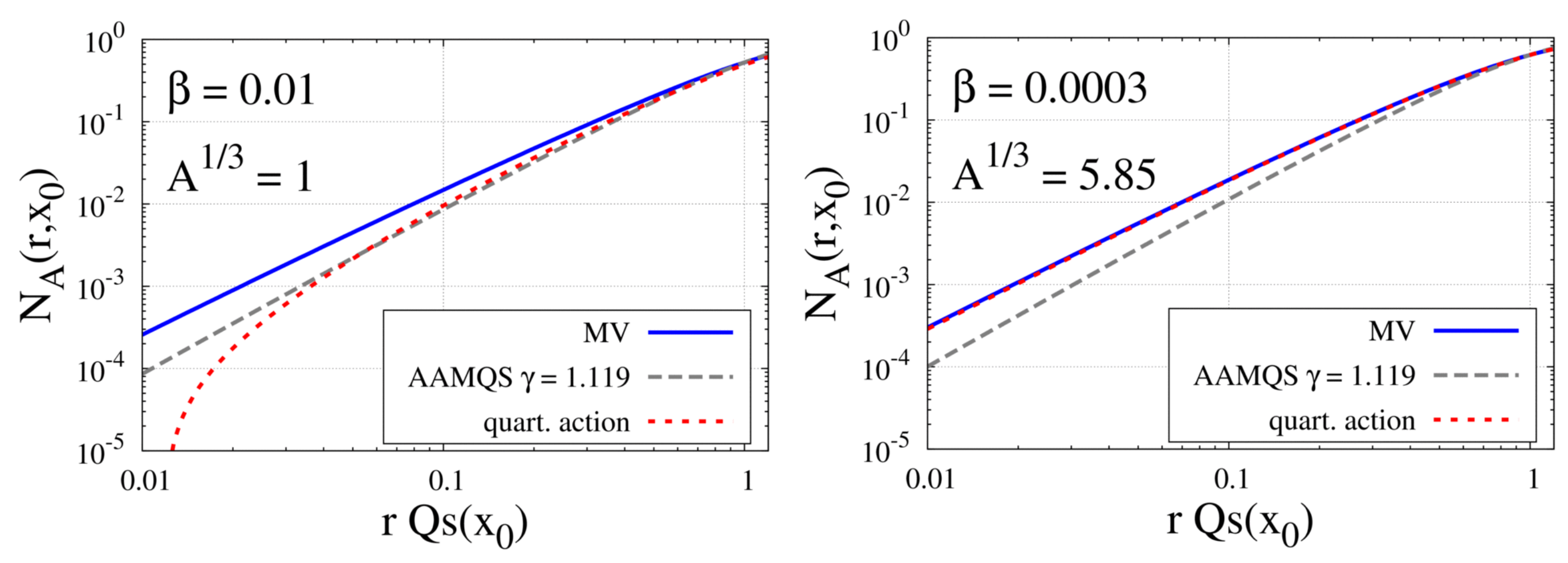


\section{Let's start with AA : centrality and energy dependence of multiplicities; (confirms KLN idea)}

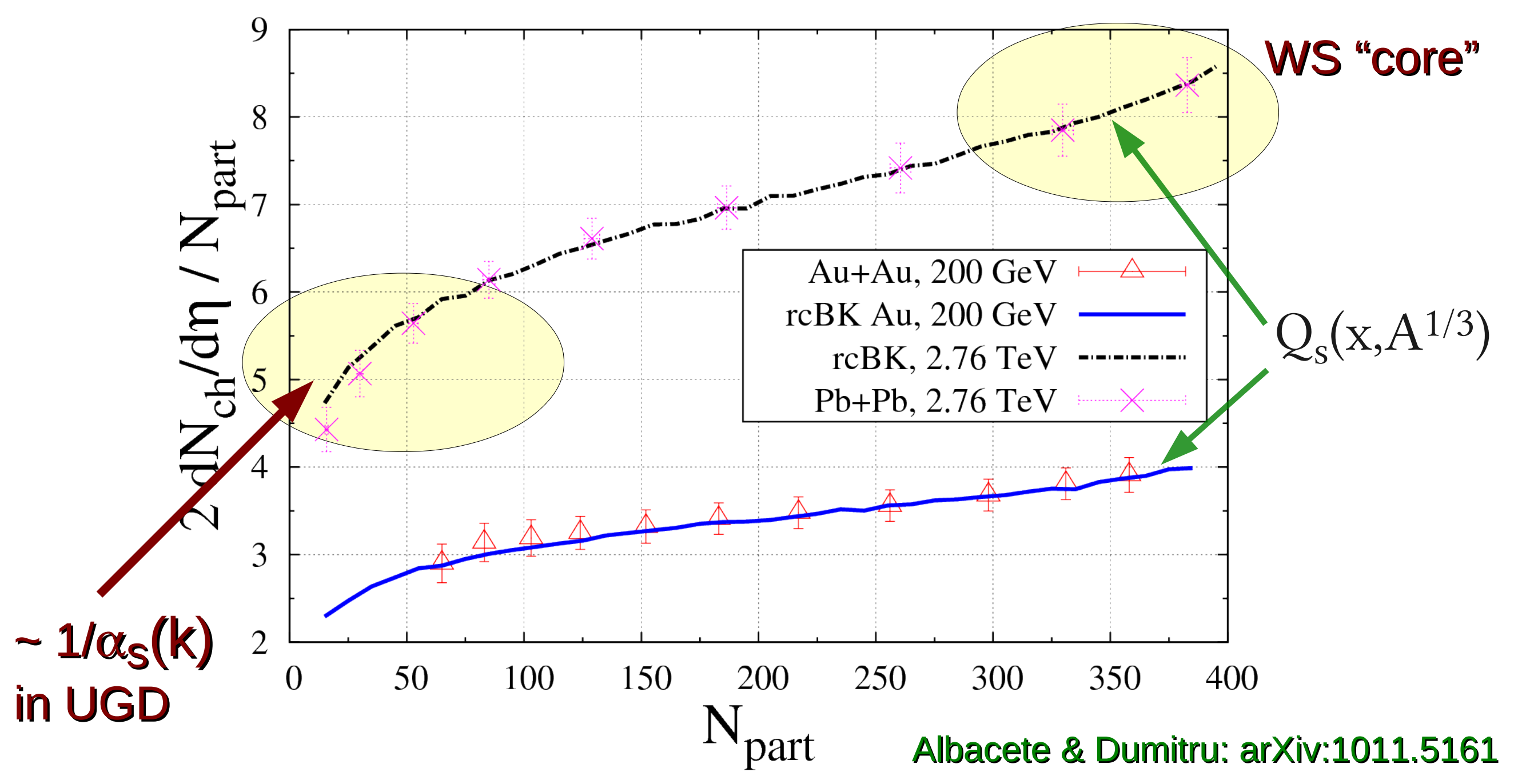

- assumes $N_{\text {hadr }}=\kappa \cdot N_{\text {glue }}$ with $\kappa \cong 5$ 


\section{Back to AA : centrality and energy dependence of $E_{\perp}$}
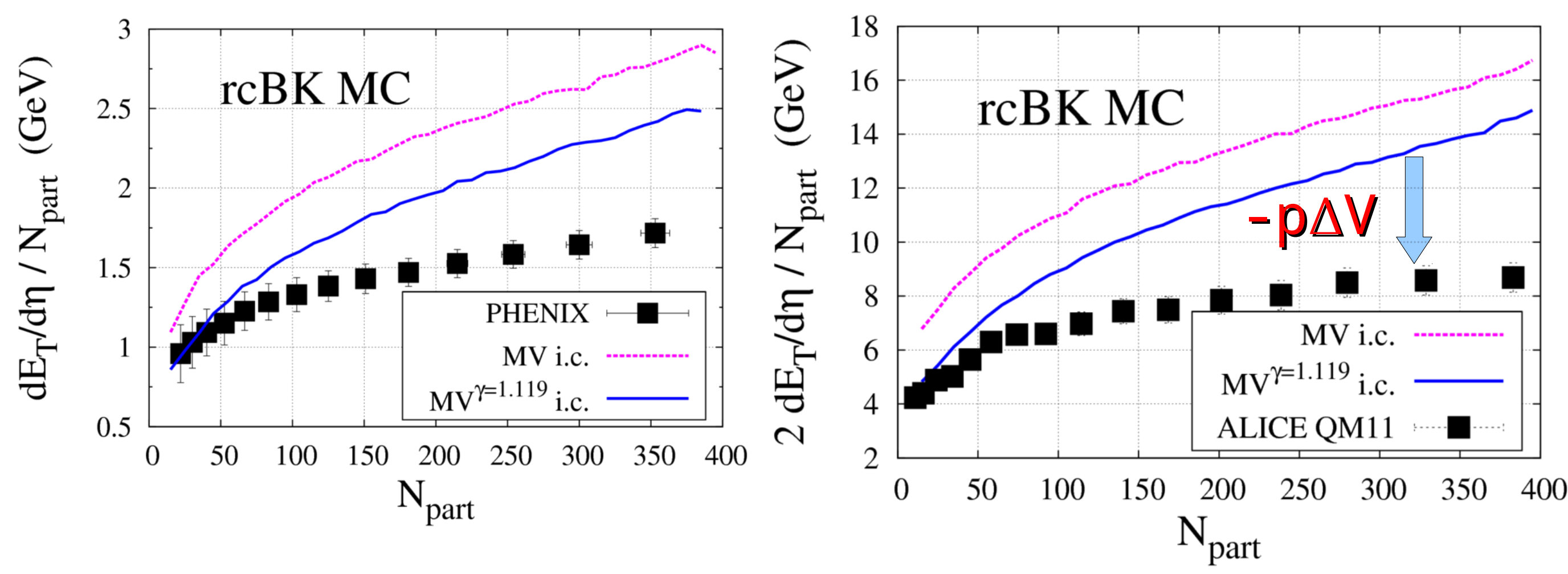

- (again, no $\mathrm{g} \rightarrow \mathrm{h}$ multiplication factor $\kappa$ here)

- 1d ideal hydro: $E_{\perp}^{f} / E_{\perp}^{i} \approx T_{f} / T_{i} \approx 1 / 2$

- interesting: $\left(\mathrm{dE}_{\perp} / \mathrm{d} \eta\right) /(\mathrm{A} \sqrt{\mathrm{s}}) \approx 0.5 \%$ at $\mathrm{LHC}_{2760}$, centrl $\mathrm{Pb}+\mathrm{Pb}$ ! 
$\mathrm{R}_{\mathrm{pA}}$ for $\mathrm{p}+\mathrm{Pb}$ at $5 \mathrm{TeV}$ :

$$
R_{p A}\left(p_{T}\right)=\frac{d N_{\mathrm{ch}}^{p A} / d^{2} p_{T} d \eta}{N_{\mathrm{coll}} d N_{\mathrm{ch}}^{p p} / d^{2} p_{T} d \eta}
$$

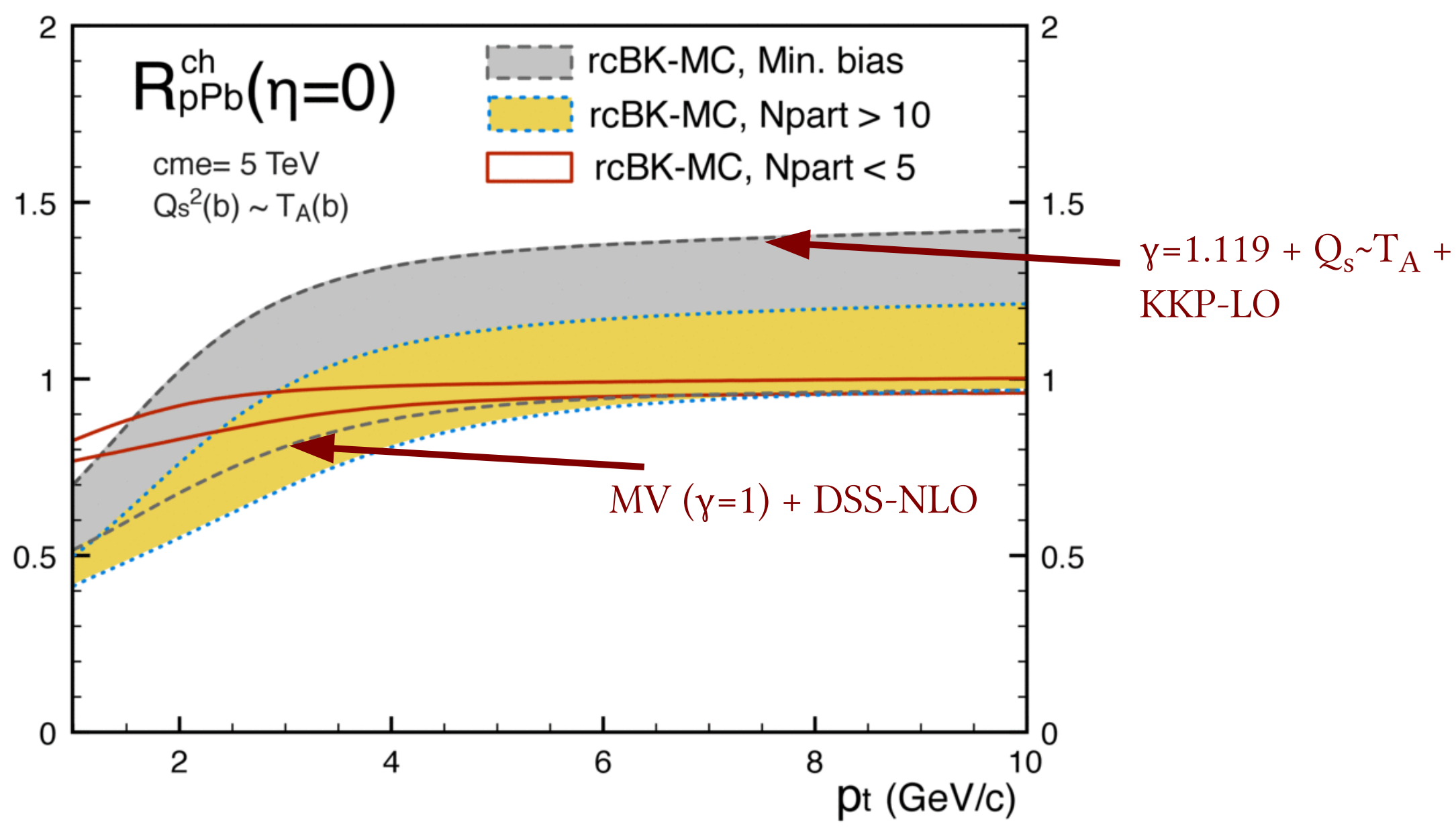

- $\mathrm{R}_{\mathrm{pA}}<1$ at $\mathrm{p}_{\mathrm{T} \text { (hadron) }} \sim 1-2 \mathrm{GeV}$

- $\mathrm{R}_{\mathrm{pA}}$ decreases (slightly) with rapidity

- generically $\mathrm{R}_{\mathrm{pA}}$ (central) $<\mathrm{R}_{\mathrm{pA}}(\mathrm{mb})$

- Cronin peak washed out by evolution 


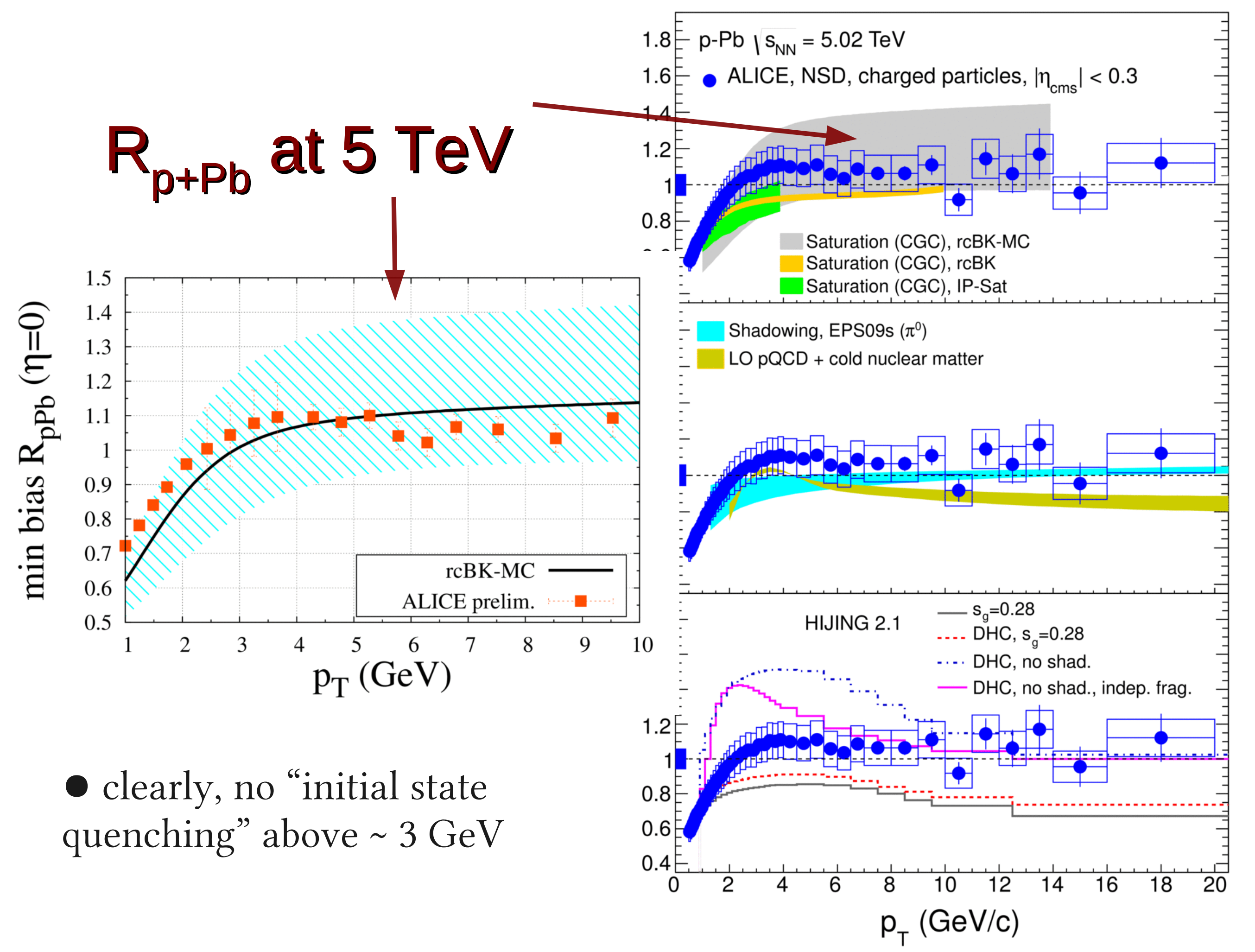




\section{forward rapidity :}
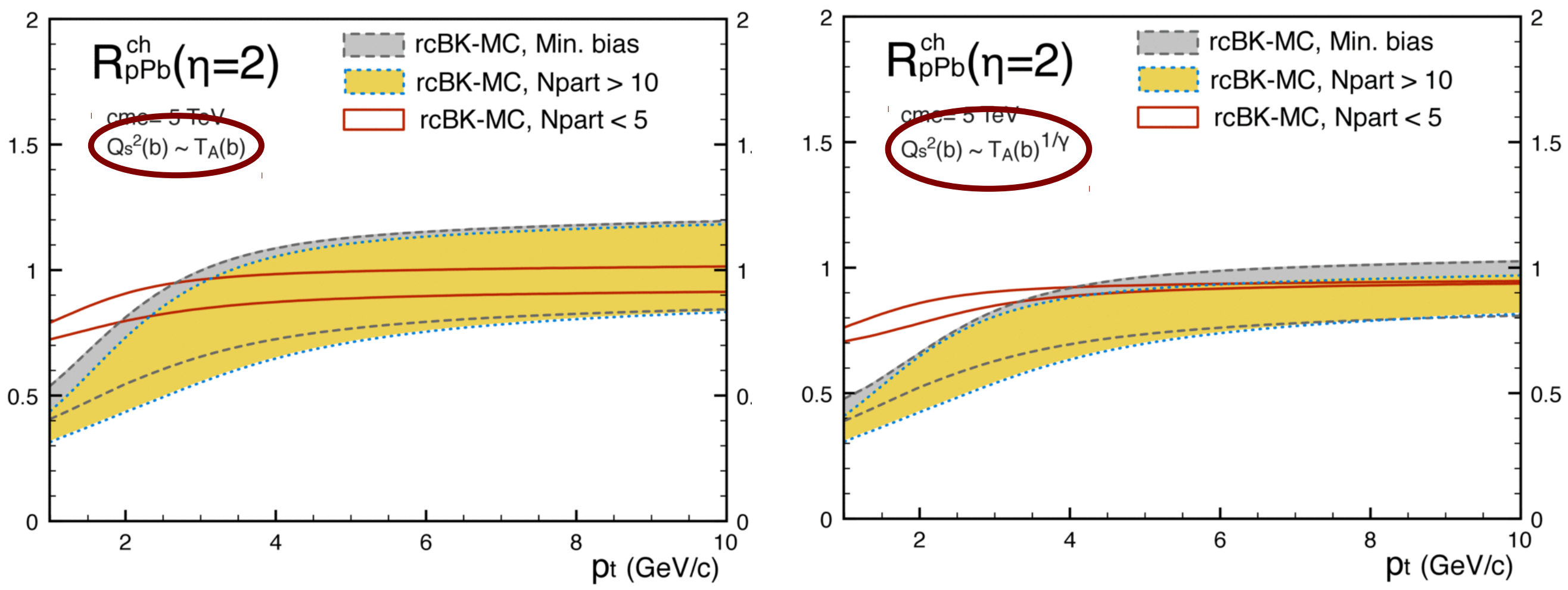

- more suppression due to small-x evolution

- $\mathrm{R}_{\mathrm{pA}}$ goes down by some $0.1-0.2$ 


\section{More detailed view of the "medium" in AA?}

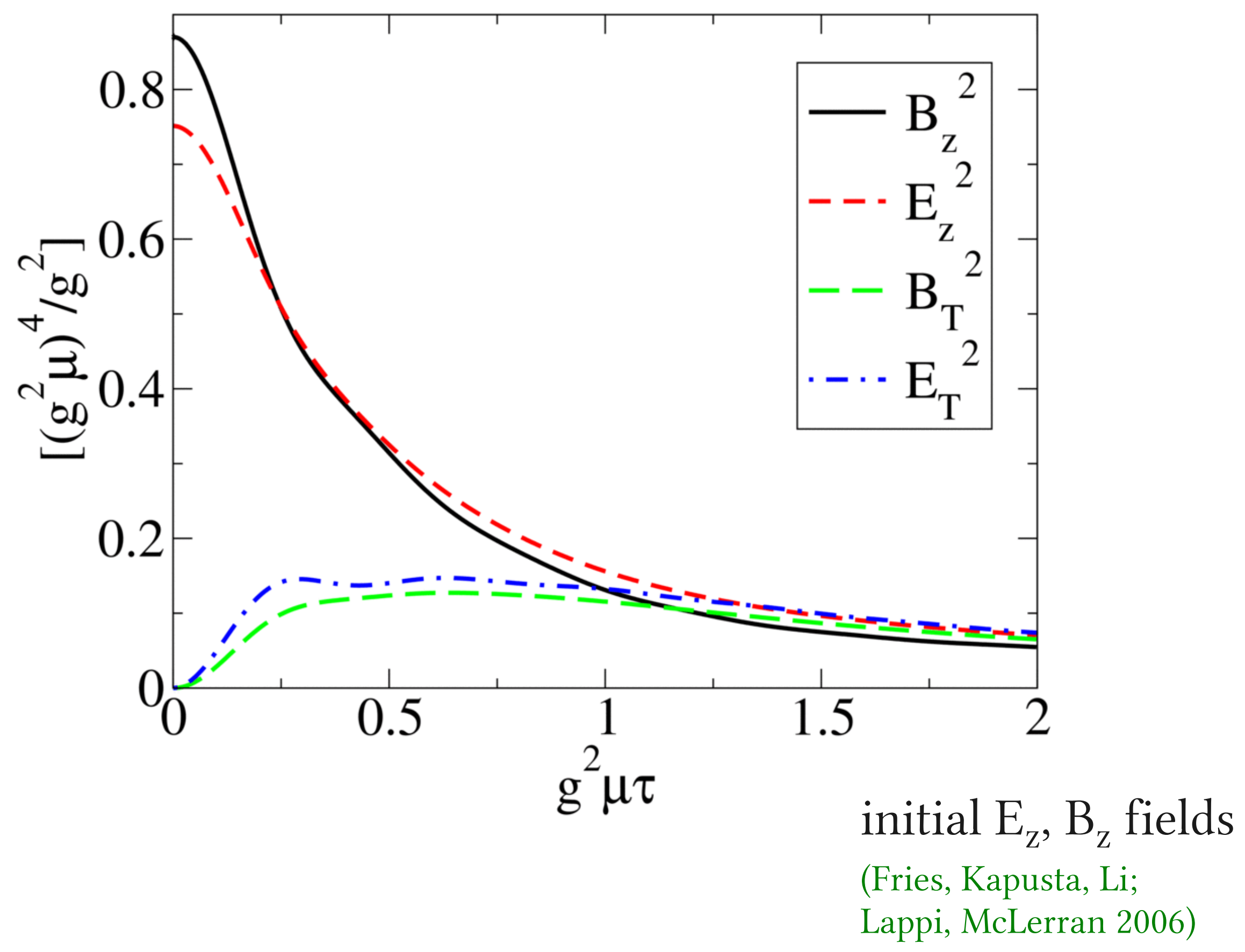




\section{Analyze classical field configurations at midrapidity: $\eta=0,2 D$}

\section{what is structure of $\mathrm{B}_{\underline{\underline{z}}}$ field?}

magnetic flux loop in $x^{-} y$ plane:

$$
M(R)=\mathcal{P} \exp \left(i g \oint d x^{i} A^{i}\right)
$$

$W_{M}(R)=\frac{1}{N_{c}}\langle\operatorname{tr} M(R)\rangle$

$W_{M}^{Z(2)}(R)=\langle\operatorname{sgn} \operatorname{tr} M(R)\rangle$

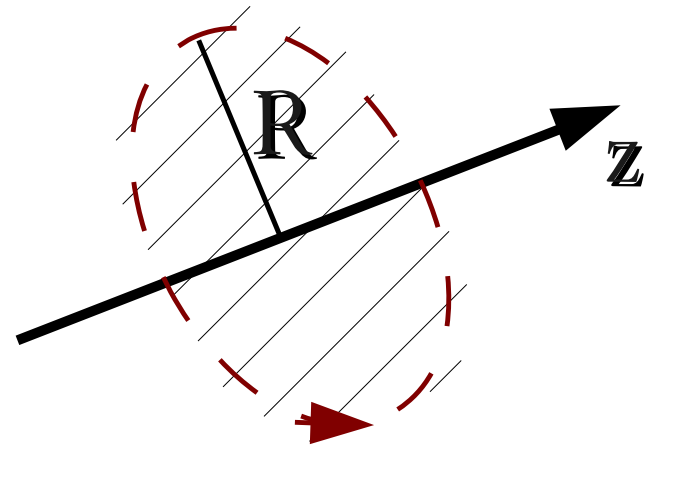




\section{Magnetic field domains $\left(\mathrm{B}_{\mathrm{z}}{ }^{3}\right.$ at $\left.\tau=0\right)$}

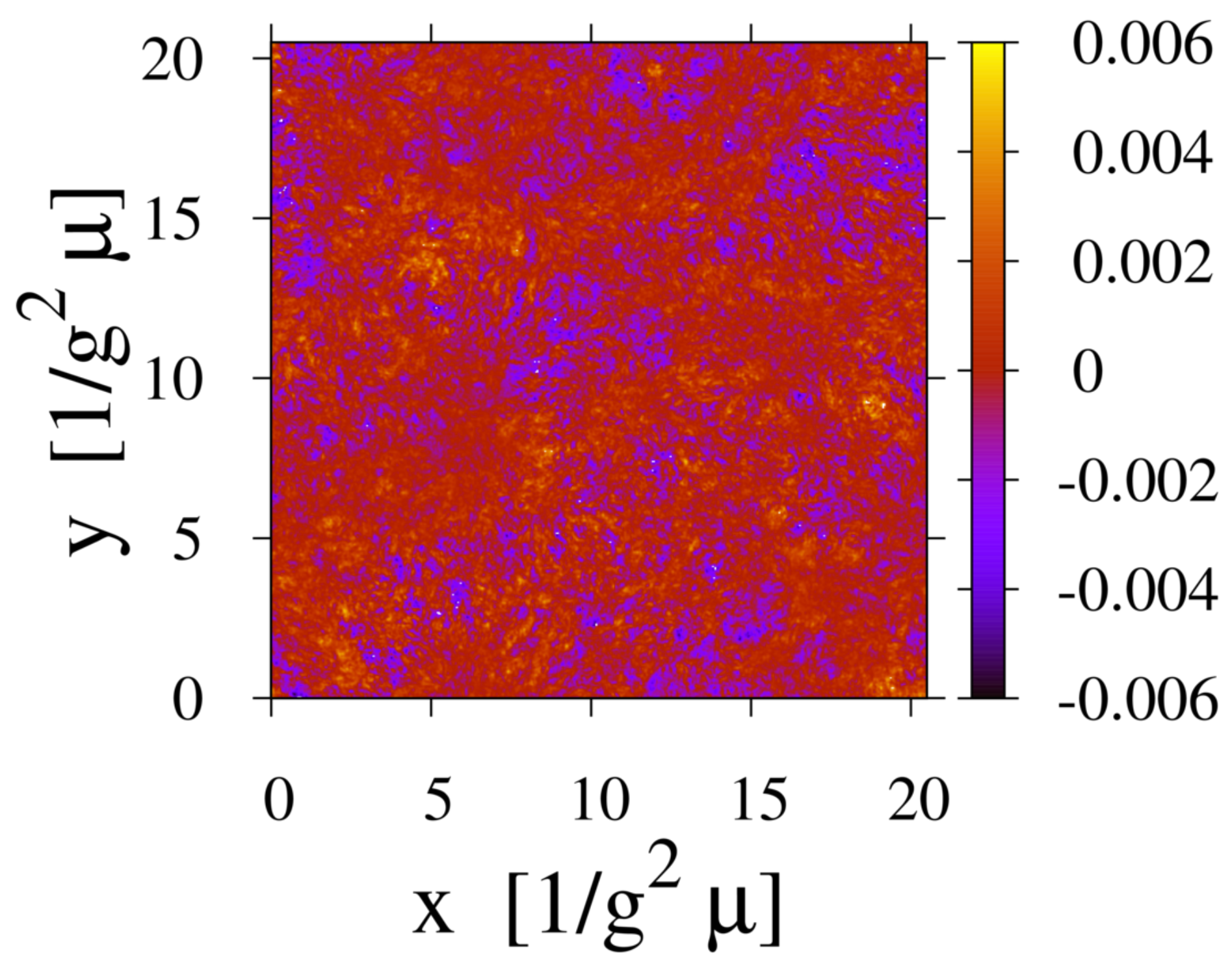




\section{SU(2) solution :}

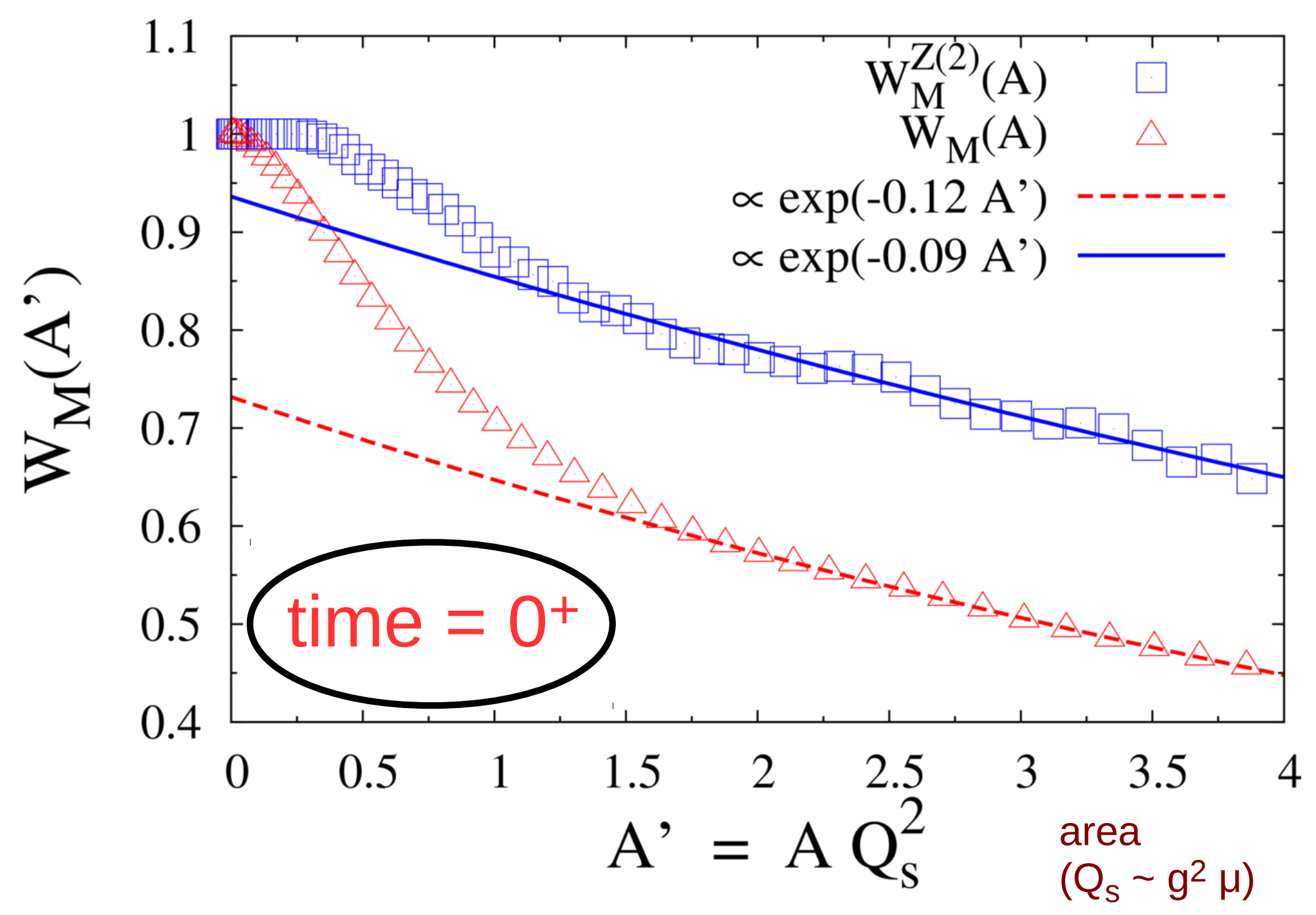

- area law for loops with area $\mathrm{A} \geq 1.5-2$

- $\sigma_{\mathrm{M}} \sim 0.12 \mathrm{Q}_{\mathrm{s}}^{2}$; thermal SU(N): $\sigma_{M} \sim g_{3 \mathrm{D}}^{2} \sim\left(g^{2} T\right)^{2}$

- small loops $\notin \mathrm{Z}(2)$ but roughly ok for large ones!

- structure of $\mathrm{B}_{\mathrm{z}} \sim$ uncorrelated vortices?!

- $\mathrm{R}_{\mathrm{vtx}} \sim 1 / \mathrm{Q}_{\mathrm{s}}$ from onset of area law 


\section{Propagation of hard particles in background of magnetic $\mathrm{Z}(\mathrm{N})$ vortices}

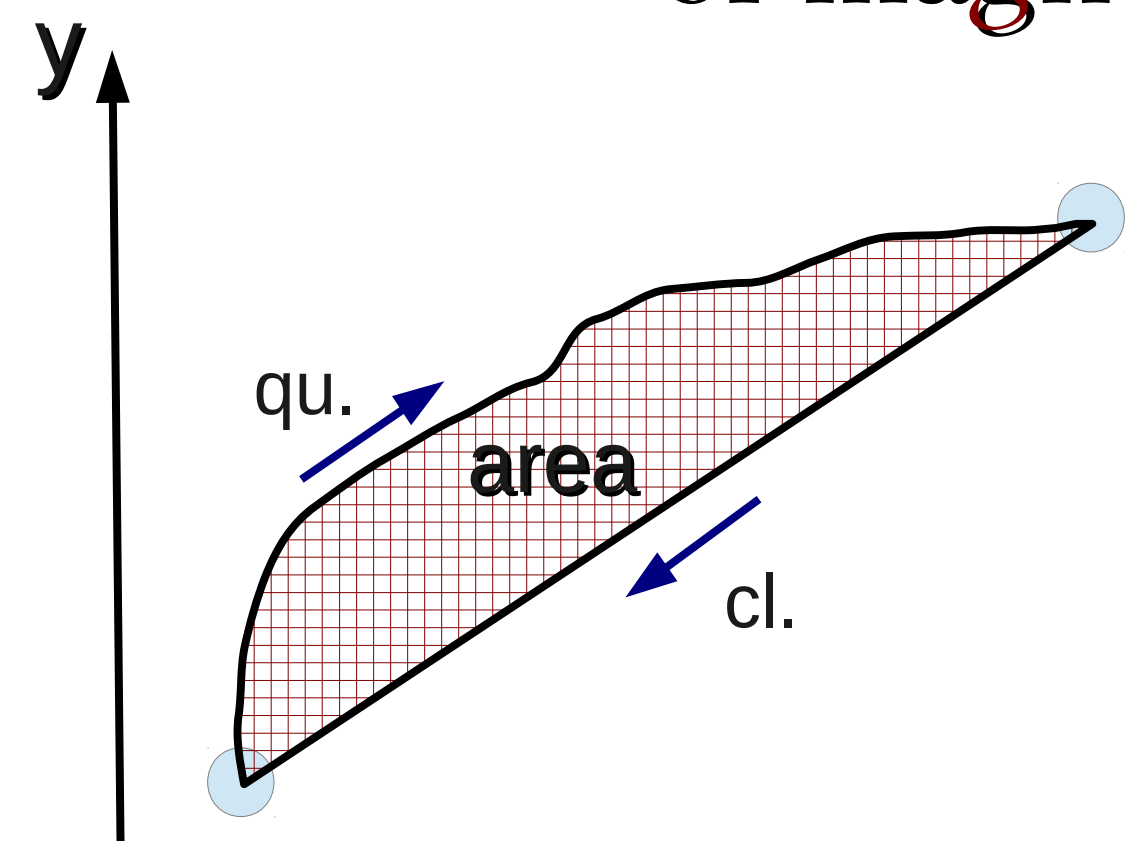

classical trajectory?

- only if paths within one de Broglie length $\left(1 / p_{T}\right)$ have same Aharonov-Bohm phase

- destructive interference leads to Anderson localization

$\int_{0}^{\infty} d s \int \mathcal{D} x^{\mu}\left\langle\exp i \int_{0}^{s} d \tau\left(m \dot{x}^{2}+g A_{\mu} \dot{x}^{\mu}\right)\right\rangle \sim$

$\int_{0}^{\infty} d s \int \mathcal{D} x^{\mu} \exp \left(i \int_{0}^{s} d \tau m \dot{x}^{2}\right) \exp \left(-\sigma_{M} A\right)=\frac{i}{p^{2}+i \sigma_{M} \frac{m}{p_{T}}}$ 


\section{Summary}

- p+Pb @ LHC control shows no "initial state jet quench"; (wasn't expected by more recent/realistic CGC predictions) suppression was predicted and seen at semi-hard pt $\sim$ a few $\mathrm{GeV}$

- forward rapidity data from LHC very important

- the "medium" appears much more interesting than featureless homogenous soup: density fluctuations, long. fields, vortices, ... 


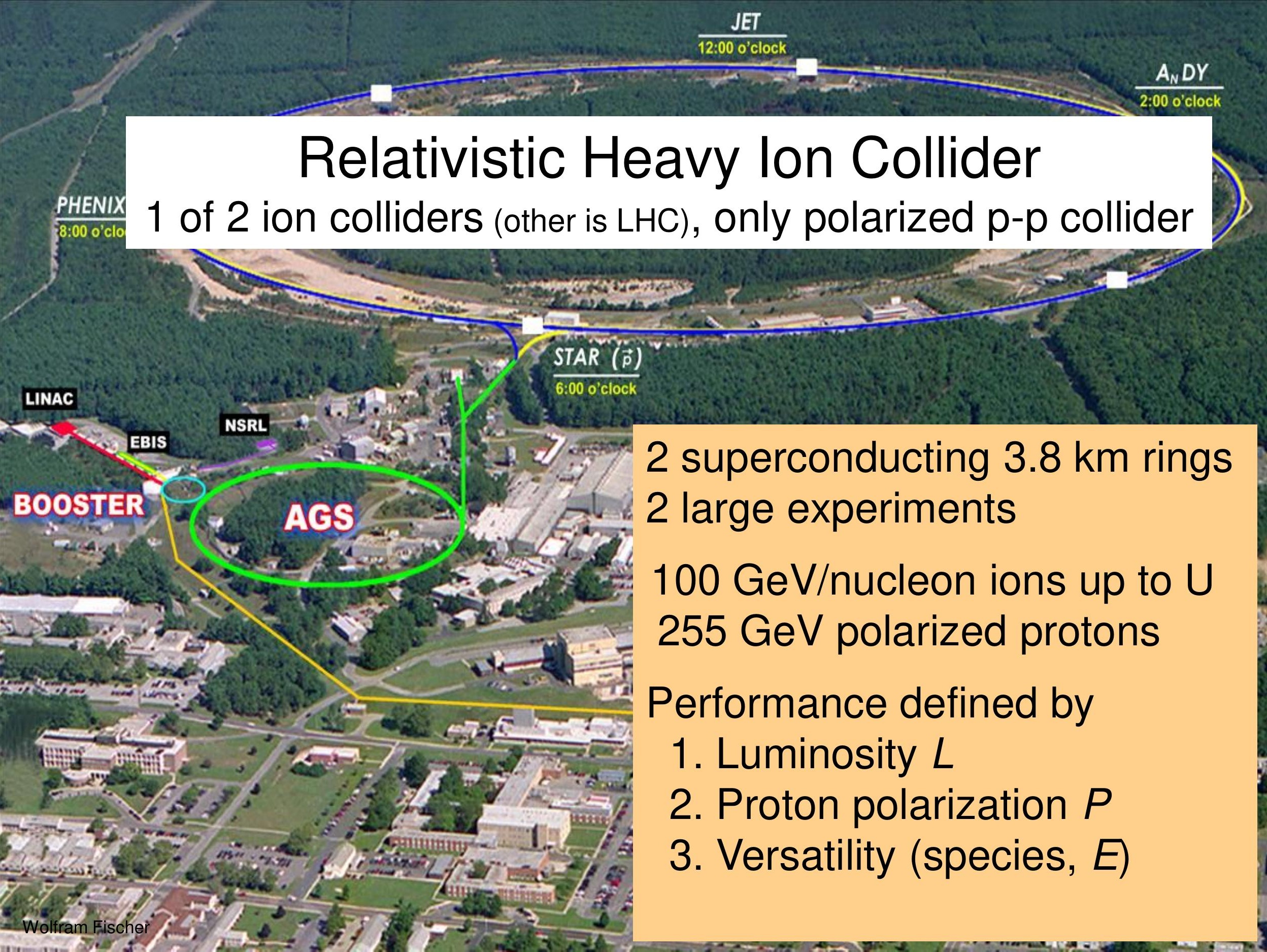




\section{Content}

- What do we have for $p+A u$ collisions?

- What do we still need for $p+A u$ collisions?

- Luminosity projection 


\section{Delivered Integrated Luminosity and Polarization}

--- RHIC-II performance is here ---
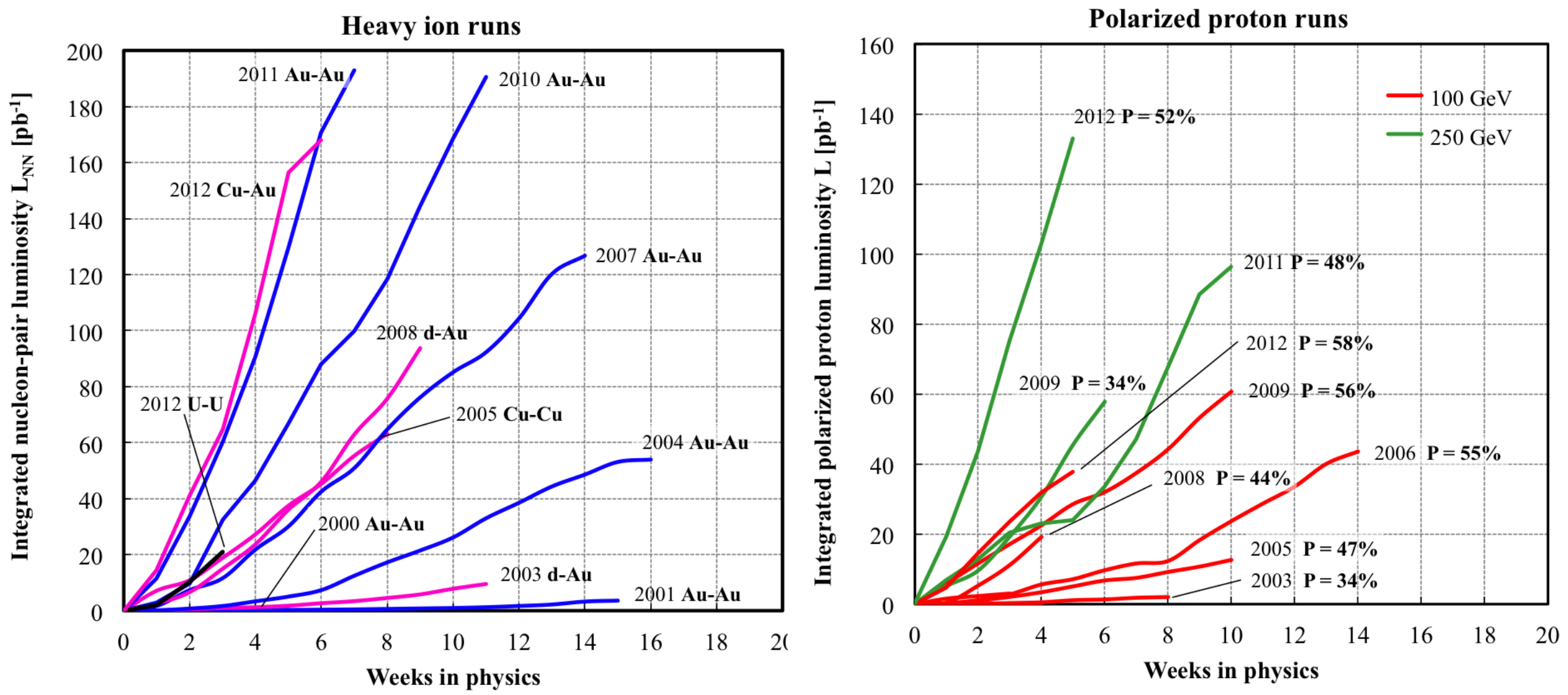

Nucleon-pair luminosity: luminosity calculated with nucleons of nuclei treated independently; allows comparison of luminosities of different species; appropriate quantity for comparison runs. 


\section{RHIC luminosity and polarization goals}

\begin{tabular}{|c|c|c|c|c|c|c|c|}
\hline parameter & unit & \multicolumn{2}{|c|}{ achieved } & \multicolumn{4}{|c|}{ goals } \\
\hline Au-Au operation & & \multicolumn{2}{|c|}{2011} & \multicolumn{4}{|c|}{$\begin{array}{c}\geq 2014 \\
\text { 3D stochastic cooling }+56 \mathrm{MHz} \text { SRF }\end{array}$} \\
\hline energy & GeV/nucleon & \multicolumn{2}{|c|}{100} & \multicolumn{4}{|c|}{100} \\
\hline no colliding bunches & $\cdots$ & \multicolumn{2}{|c|}{111} & \multicolumn{4}{|c|}{111} \\
\hline bunch intensity & $10^{9}$ & \multicolumn{2}{|c|}{1.3} & \multicolumn{4}{|c|}{1.3} \\
\hline avg. Iuminosity & $10^{26} \mathrm{~cm}^{-2} \mathrm{~s}^{-1}$ & \multicolumn{2}{|c|}{30} & \multicolumn{4}{|c|}{40} \\
\hline$p \uparrow-p \uparrow$ operation & & \multicolumn{2}{|c|}{2012} & \multicolumn{2}{|c|}{$\begin{array}{l}\geq 2013 \\
\text { source }\end{array}$} & \multicolumn{2}{|c|}{$\begin{array}{c}\geq 2014 \\
\text { source + e-lenses }\end{array}$} \\
\hline energy & $\mathrm{GeV}$ & 100 & 255 & 100 & 250 & 100 & 250 \\
\hline no colliding bunches & $\ldots$ & \multicolumn{2}{|c|}{$-107-$} & \multicolumn{2}{|c|}{$-107-$} & \multicolumn{2}{|c|}{$-107-$} \\
\hline bunch intensity & $10^{11}$ & 1.6 & 1.7 & 1.6 & 2.0 & 1.8 & 2.5 \\
\hline avg. Iuminosity & $10^{30} \mathrm{~cm}^{-2} \mathrm{~s}^{-1}$ & 33 & 105 & 30 & 150 & 60 & 300 \\
\hline avg. polarization* & $\%$ & 59 & 52 & \multicolumn{2}{|c|}{$-60-$} & \multicolumn{2}{|c|}{$-65-$} \\
\hline
\end{tabular}

* Intensity and time-averaged polarization as measured by the $\mathrm{H}$-jet. Luminosity-averaged polarizations, relevant in single-spin colliding beam experiments, are higher. For example, for intensity-averaged $P=48 \%$ and $R_{\mathrm{x}}=R_{\mathrm{y}}=0.2(250 \mathrm{GeV}, 2011)$, the luminosity-averaged polarization is $P=52 \%$. 


\section{Asymmetric ion species under consideration in RHIC (to date)}

proton-gold ( $\mathrm{p}-\mathrm{Au})$

- not yet done, part of the design

- requires moving IR6 and IR8 DX magnets horizontally by $\sim 1 \mathrm{~cm}$

deuteron-gold (d-Au)

- operated in 2003 and 2008

copper-gold (Cu-Au)

- operated in 2012

Polarized proton-polarized ${ }^{3} \mathrm{He}\left(\mathrm{p} \mathrm{m}^{3}{ }^{3} \mathrm{He} \mathrm{m}_{\mathrm{m}}\right)$

- likely request when ${ }^{3} \mathrm{He} m$ is available

- RBRC Workshop on ${ }^{3} \mathrm{He} m$ at BNL, 28-30 September 2011 


\section{What do we have for $p+A u$ collisions?}

- RHIC was designed for $p+A u$ collisions

Independent rings except DX magnets

DX magnets movable ( 1 shift)

- With stochastic cooling initial Au beam size is at its maximum

Allows for DX move in IR6 and IR8 only ( 1 shift)

- Solutions for lattice, injection and acceleration

Lattice takes advantage of stochastic cooling

- Machine with fast setup (beam-based feedbacks)

- Experience with asymmetric collisions $(\mathrm{d}+\mathrm{Au}, \mathrm{Cu}+\mathrm{Au})$

- Proton beam with $N_{\mathrm{b}}=2 \times 10^{11}, P=55 \%$

with upgrades (OPPIS): $N_{\mathrm{b}}=3 \times 10^{11}, P=65 \%$

- Au beam with $N_{b}=1.3 \times 10^{9}$

with upgrades (EBIS/Booster/AGS/RHIC): 2.0×109 (emittance?) 


$$
\text { Run-11: } L_{\text {avg }}=3010^{26} \mathrm{~cm}^{-2} \mathrm{~s}^{-1}
$$

Luminosity. The collider is designed for a Au-Au luminosity of about $2 \times 10^{26} \mathrm{~cm}^{-2}$ $\mathrm{sec}^{-1}$ at top energy, while maintaining the potential for future upgrades by an order of magnitude. Operation with the heaviest ions imposes the most demanding requirements on the collider design, and gold-on-gold is taken as the prototypical example. The luminosity is energy dependent and decreases in first approximation propor Run-12: $L_{\text {avg }}=10.510^{31} \mathrm{~cm}^{-2} \mathrm{~s}^{-1}\left(\right.$ with $P_{\text {avg }}=53 \%$ ) will be higher, with $\sim 1 \times 10^{31} \mathrm{~cm}^{-2} \mathrm{sec}^{-1}$ for pp collisions.

Range of ion masses. The expectations for interesting physics phenomena require a broad range of nuclei from the heaviest to the lightest, including protons. Asymmetric operation with heavy ions colliding on protons is considered to be crucial for the experimental program. The collider will allow collisions of beams of equal ion species from $\mathrm{Au}-\mathrm{Au}$ all the way down to p-p. It will also allow operation of unequal species such as protons on gold ions.

Uranium is a viable species and can be considered as a future upgrade. However, at the present, an adequate source for uranium does not exist at Brookhaven and further R\&D will be needed to achieve this goal.

Intersection Regions. The existing tunnel and the magnet lattice configuration provides for six experimental areas where the circulating beams cross. Three of the experimental areas presently 


\section{RHIC Design Manual (July 1998)}

\section{IR design with beam splitting DX dipoles first}

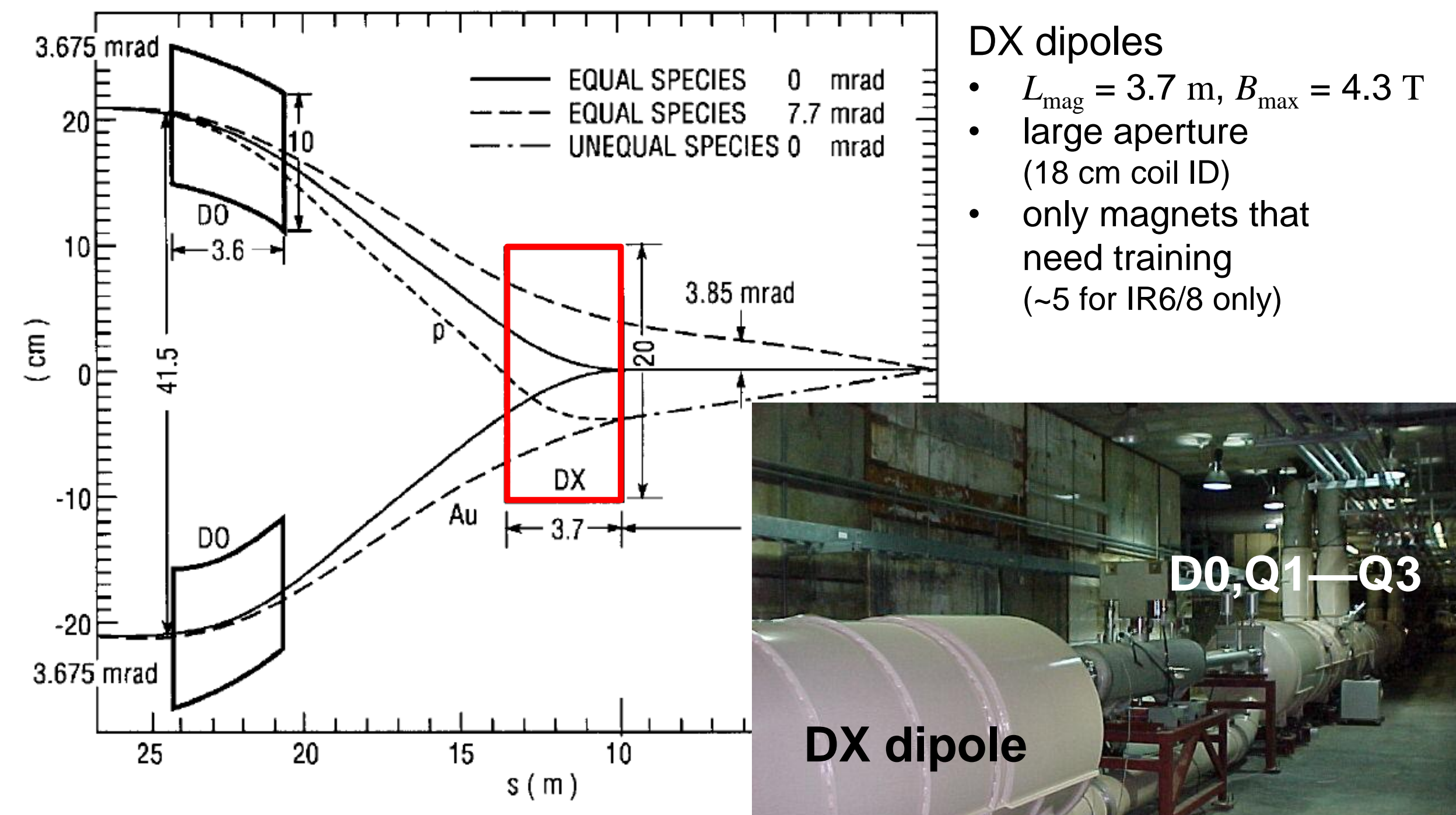




\section{Now have full 3D stochastic cooling for heavy ions}

longitudinal pickup

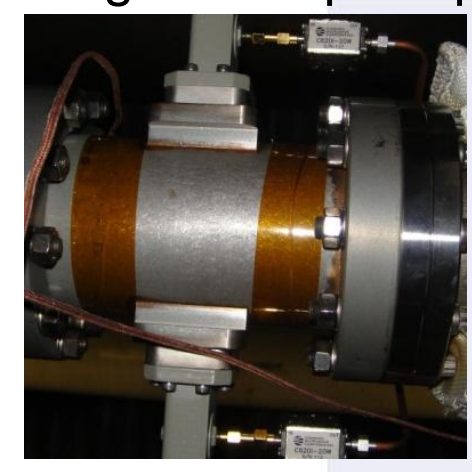

horizontal and vertical pickups

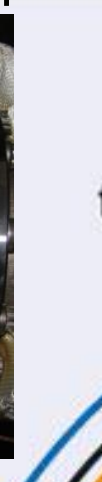

longitudinal kicker

(closed)
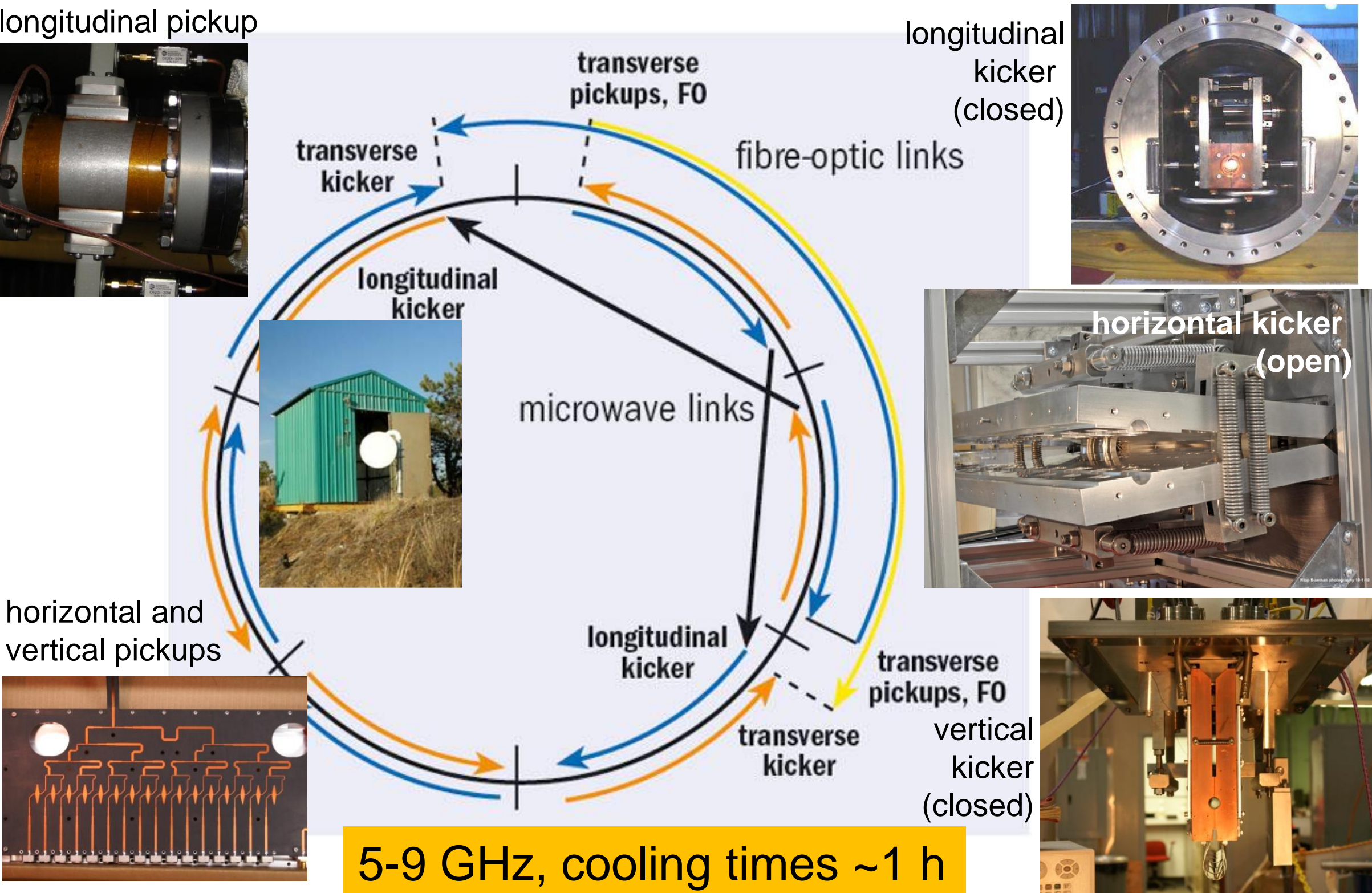

pickups, F0

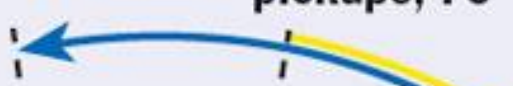

fibre-optic links transverse
Iongitudinal kicker

kicker
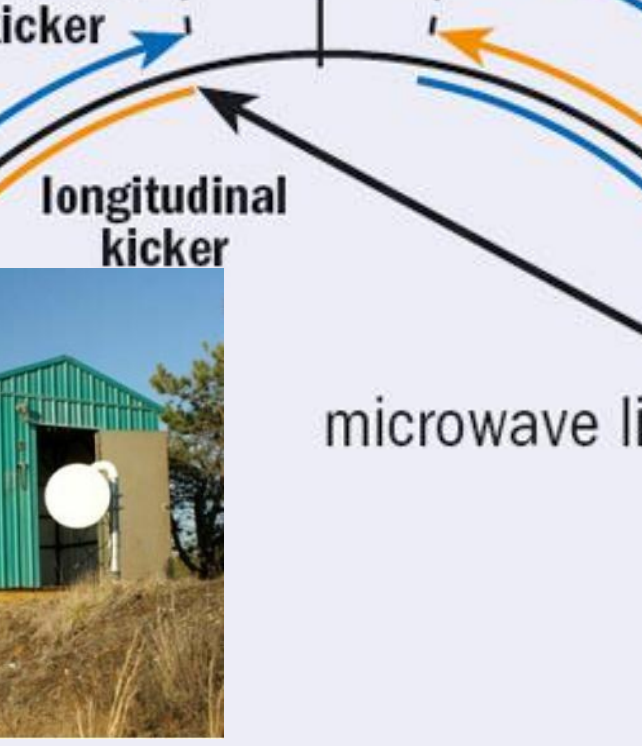

microwave links

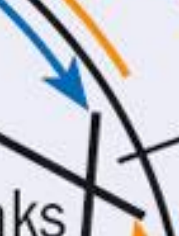

t

longitudinal kicker

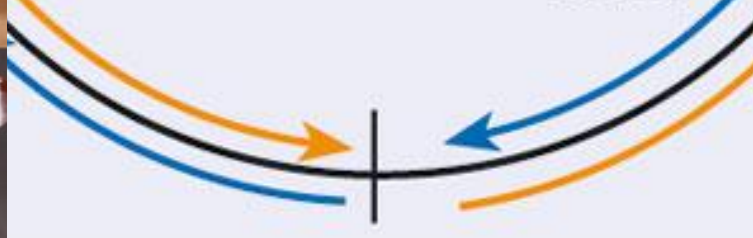

tran

5-9 GHz, cooling times $\sim 1 \mathrm{~h}$

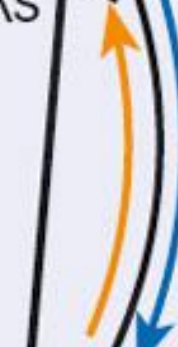

transverse pickups, F0 vertical kicker (closed)

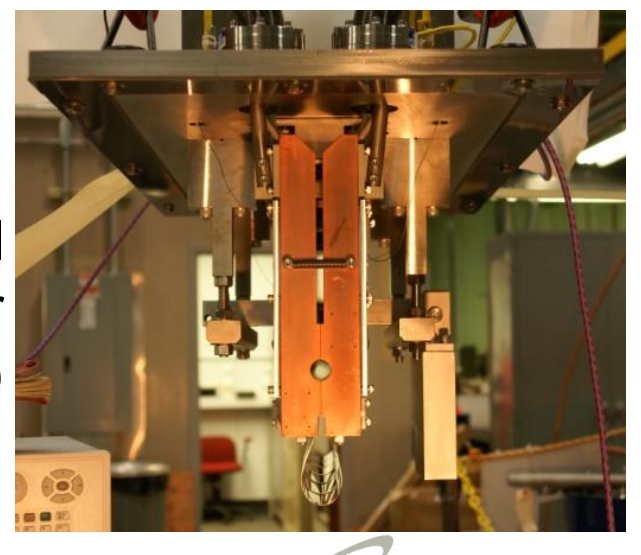

M. Brennan, M. Blaskiewicz, F. Severino, PRL 100174803 (2008); PRSTAB, PAC, EPAC 


\section{Stochastic cooling in $U-U$ operation}

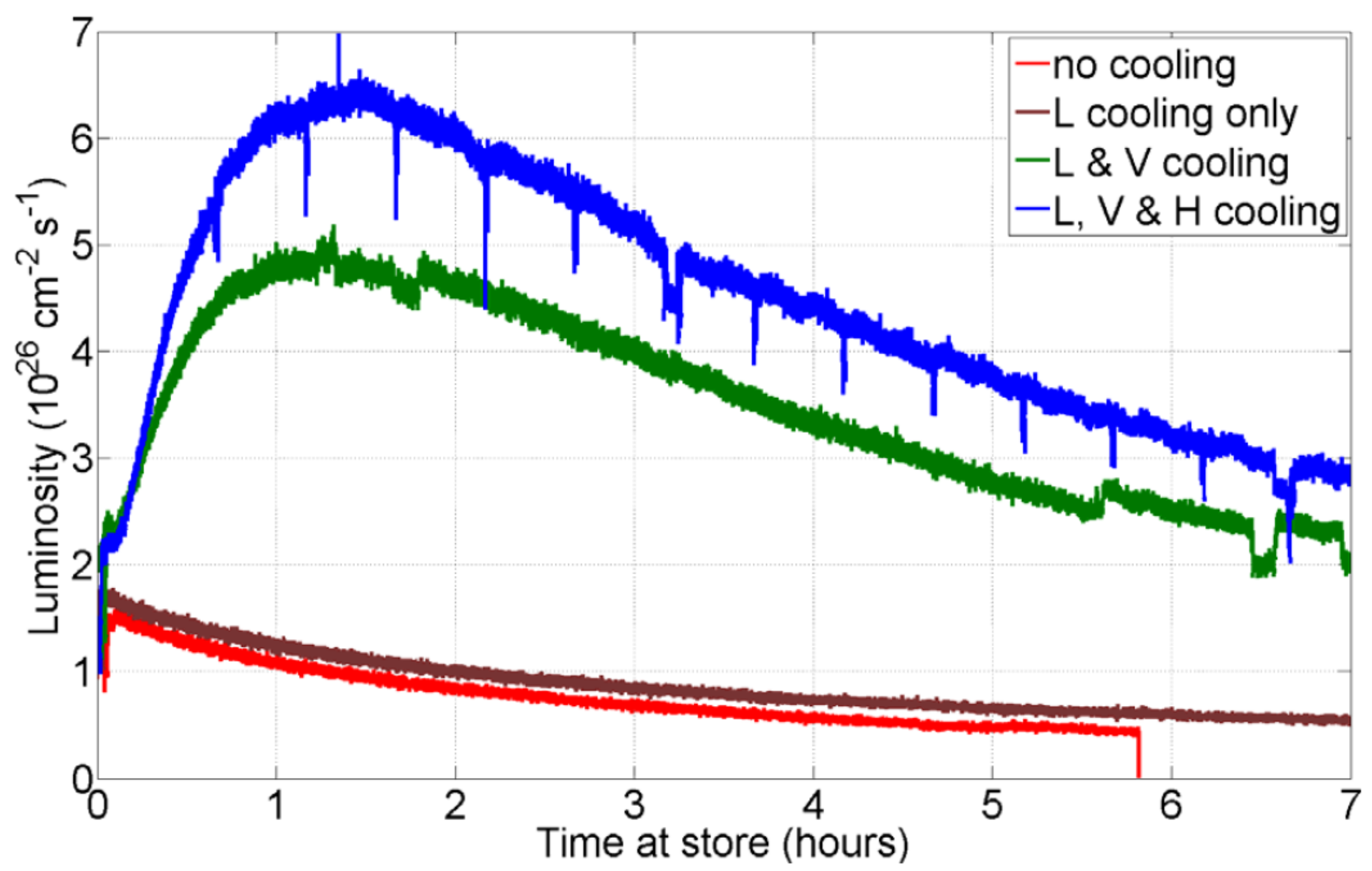




\section{Cu-Au store - new mode in 2012}

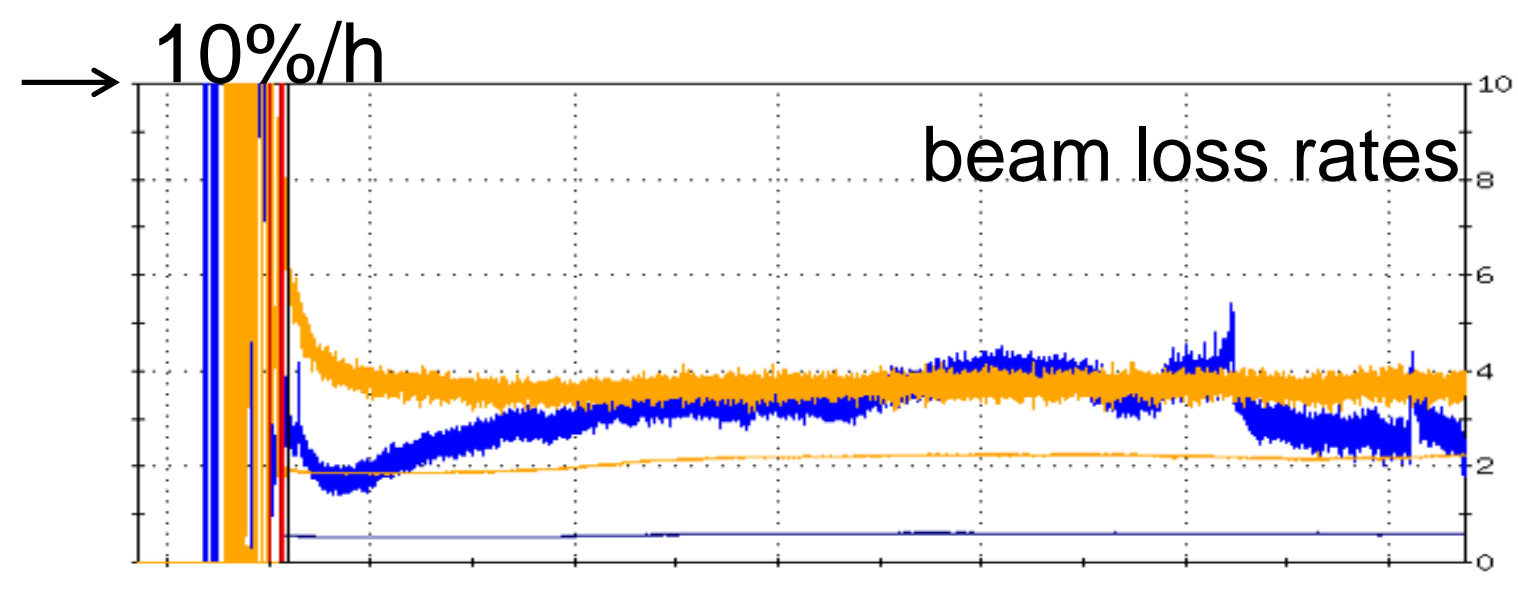

$\mathrm{Cu}$ and Au have different

- intrabeam scattering growth rates

$\left(\sim Z^{4} N_{\mathrm{b}} / A^{2}\right) \quad r_{\mathrm{IBS}, \mathrm{Au}} \approx 2 \mathrm{x} r_{\mathrm{IBS}, \mathrm{Cu}}$

- cooling rates

$\left(\sim 1 / N_{\mathrm{b}}\right) r_{\mathrm{SC}, \mathrm{Au}} \approx 3 \mathrm{x} r_{\mathrm{SC}, \mathrm{Cu}}$

\section{$25 \mathrm{~mm} . \mathrm{mrad}$}

\section{transverse emittances}

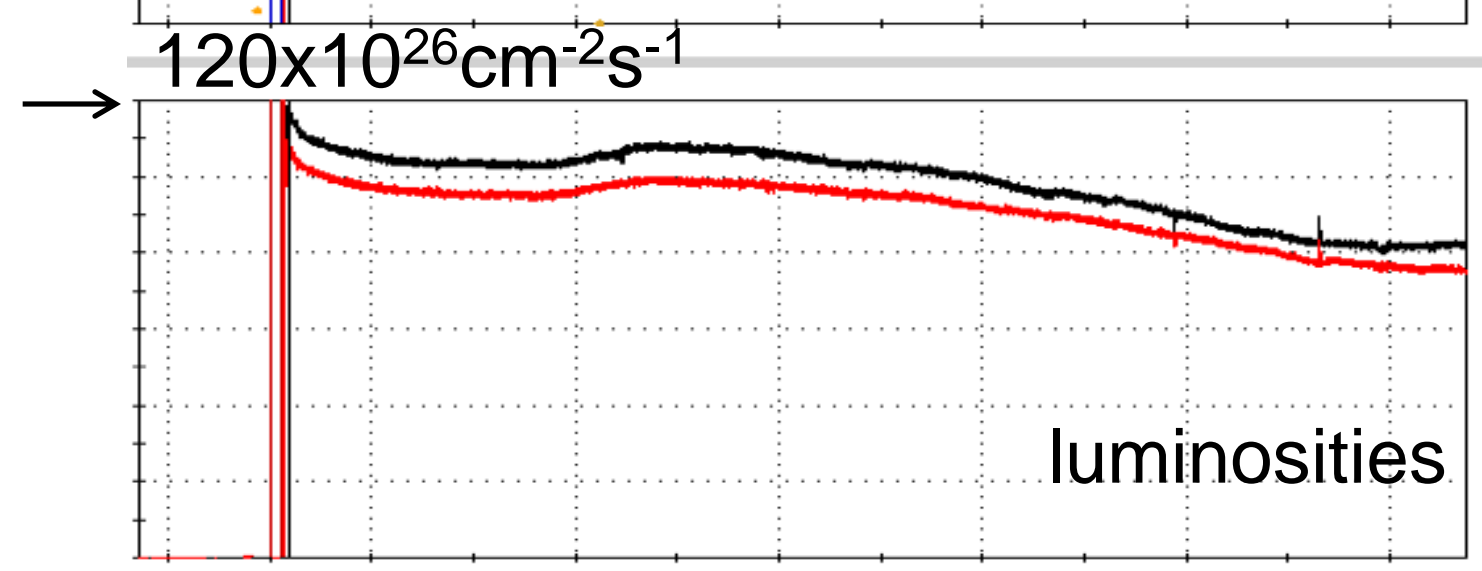

Stores start with large $\varepsilon$ after undergoing instability at transition

Possible with stochastic cooling Increase bunch intensity until loss at transition 


\section{p+Au easier with stochastic cooling}

- only need to accommodate initial Au emittances

- sufficient to move IR6 and IR8 DX magnets

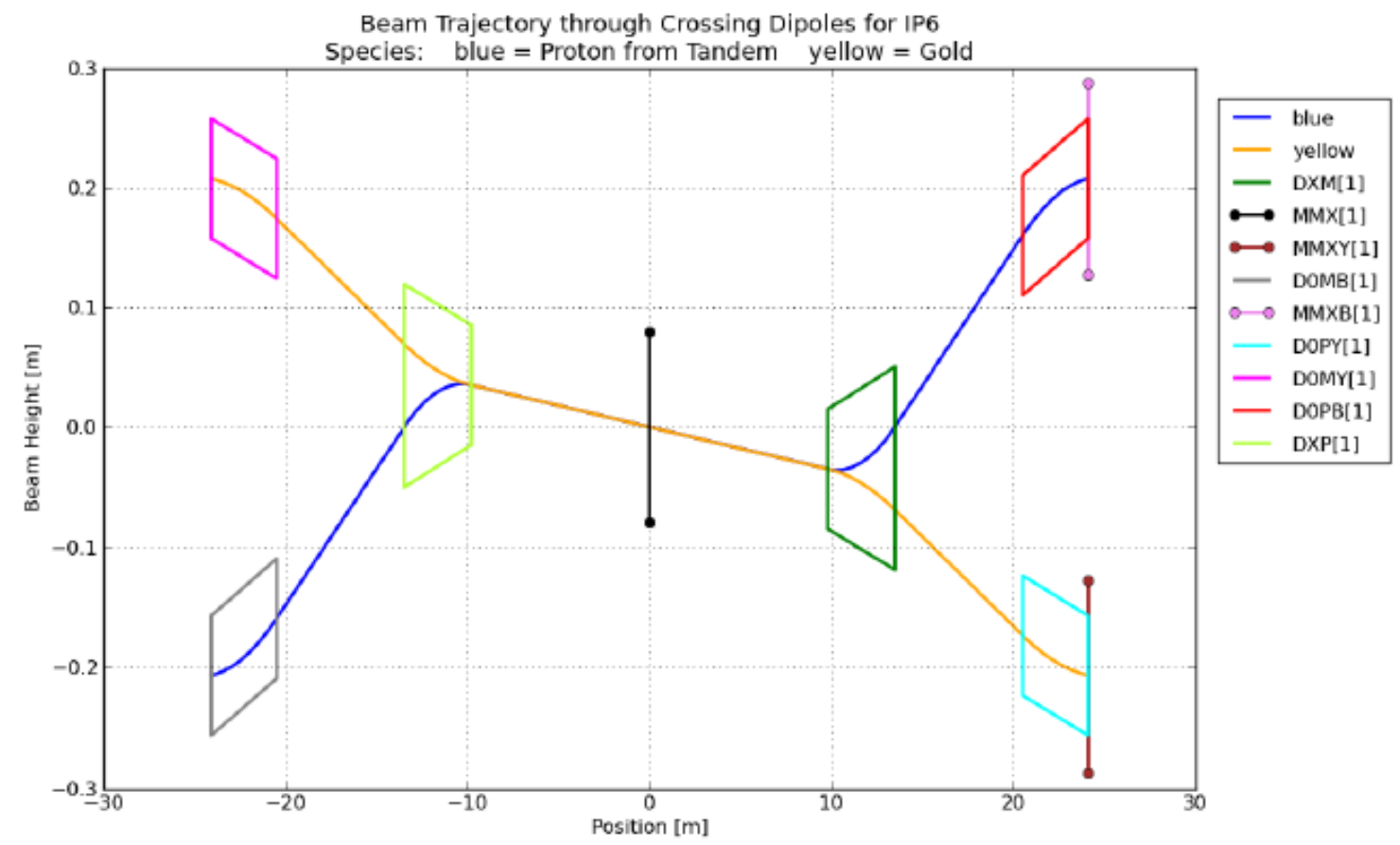

colliding IR move DX by $1 \mathrm{~cm}$

Figure 1. The beam trajectory through the crossing dipoles D0 and DX. The Au beam is $69.4 \mathrm{~mm}$ from the central line in the DX magnet in the worst case. Additional room for beam size must also be taken into account.

S. Tepikian, D. Trbojevic, C-A/AP/447 (Jan. 2012) 


\section{p+Au easier with stochastic cooling}

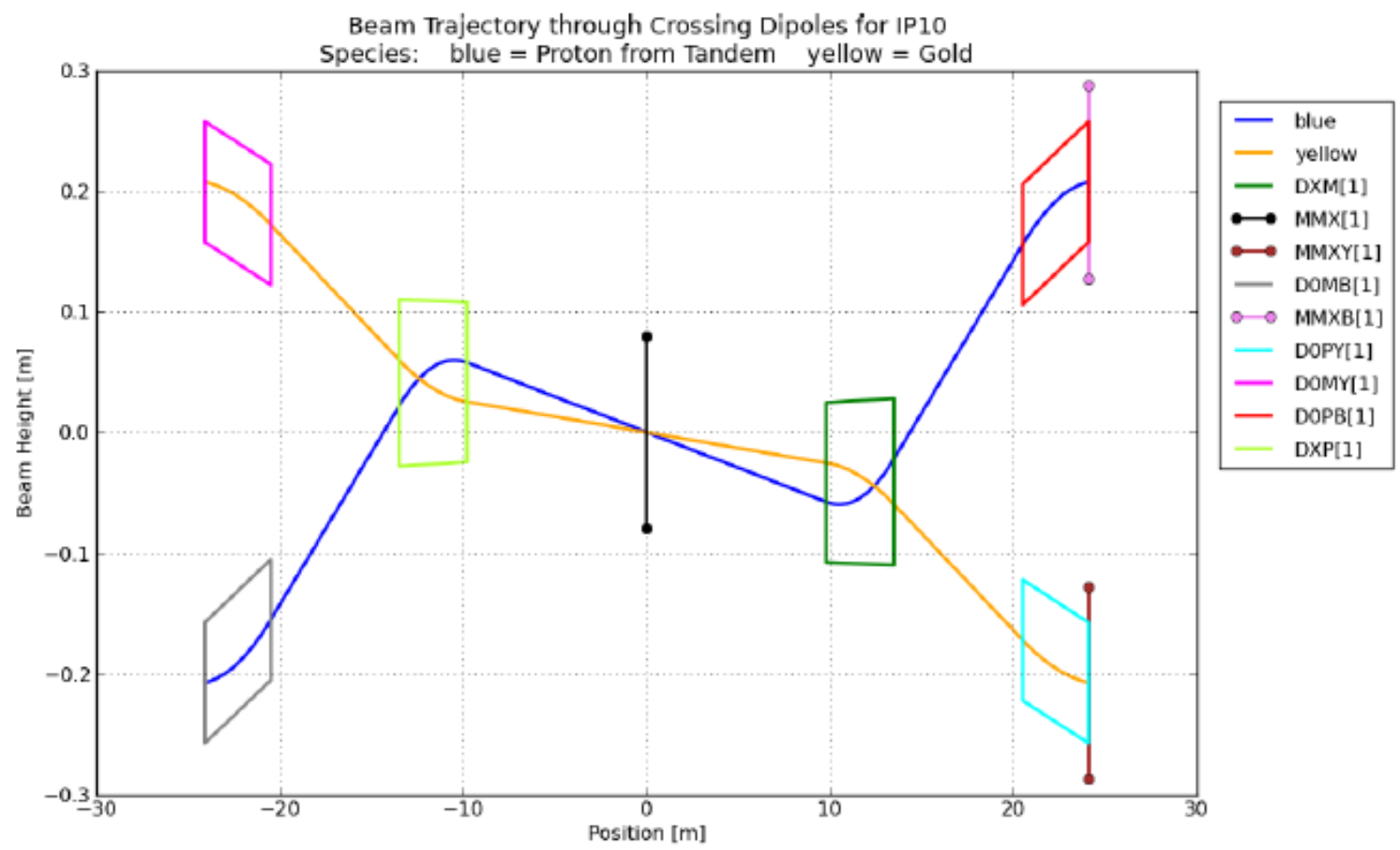

non-colliding IR no DX move

Figure 2. A non-colliding insertion. The crossing angle is $-0.3305 \mathrm{mrad}$. The beam trajectory is $59.8 \mathrm{~mm}$ from the central axises for both beams in the DX magnet. The Blue beam reaches its peak at $10.5 \mathrm{~m}$ from the IP, while the Yellow beam reaches its peak at $13.5 \mathrm{~m}$ from the IP.

S. Tepikian, D. Trbojevic, C-A/AP/447 (Jan. 2012) 


\section{Beam control improvement - feedbacks on ramp}
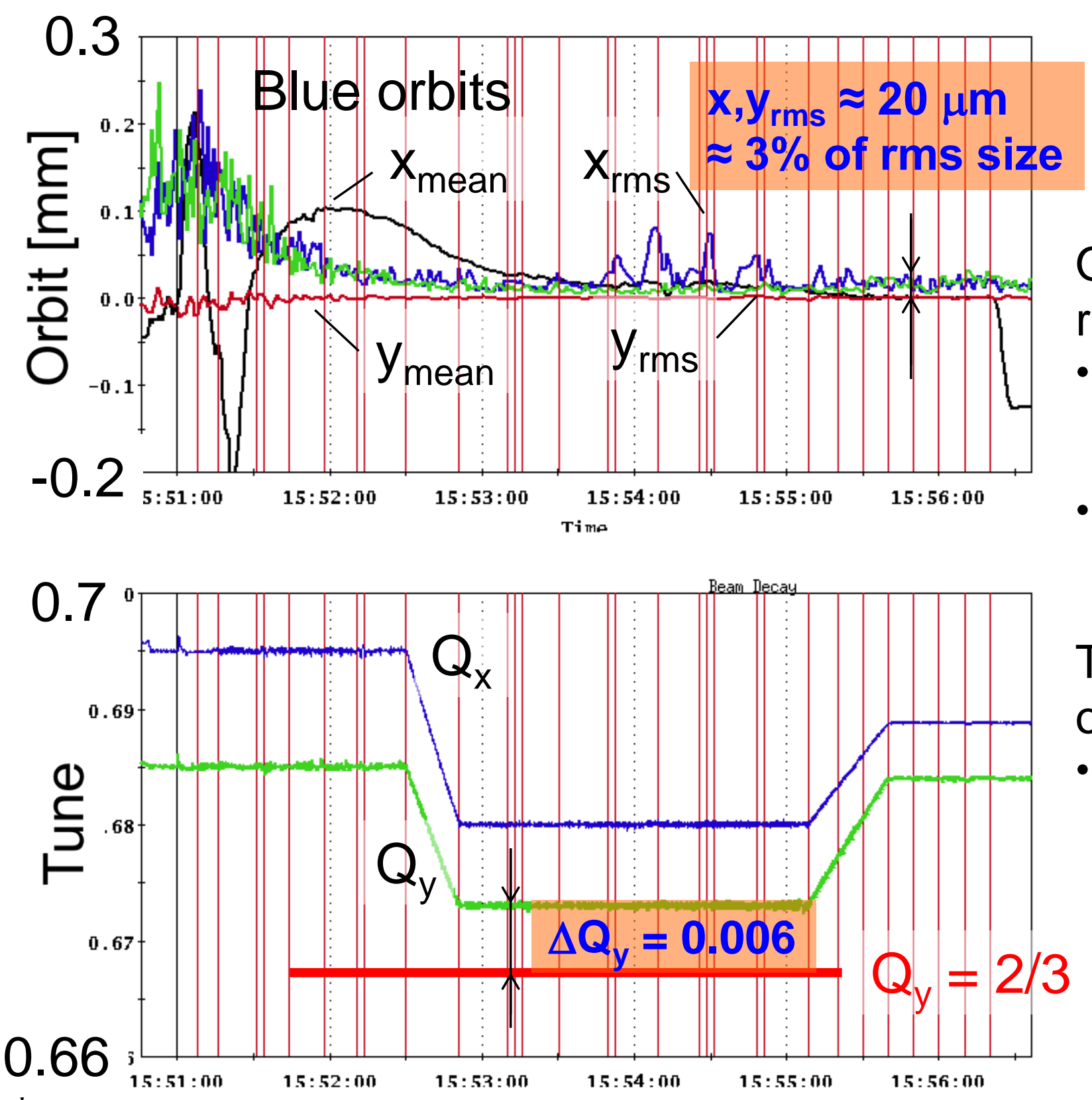

M. Minty, A. Marusic et al.

Orbit feedback on every ramp allows for

- Smaller $y_{\text {rms }}$ (smaller imperfection resonance strength)

- Ramp reproducibility (have $24 \mathrm{~h}$ orbit variation)

Tune/coupling feedback on every ramp allows for

- Acceleration near $Q_{y}=2 / 3$ (better $\mathrm{P}$ transmission compared to higher tune) 


\section{Time-in-store as fraction of calendar time}

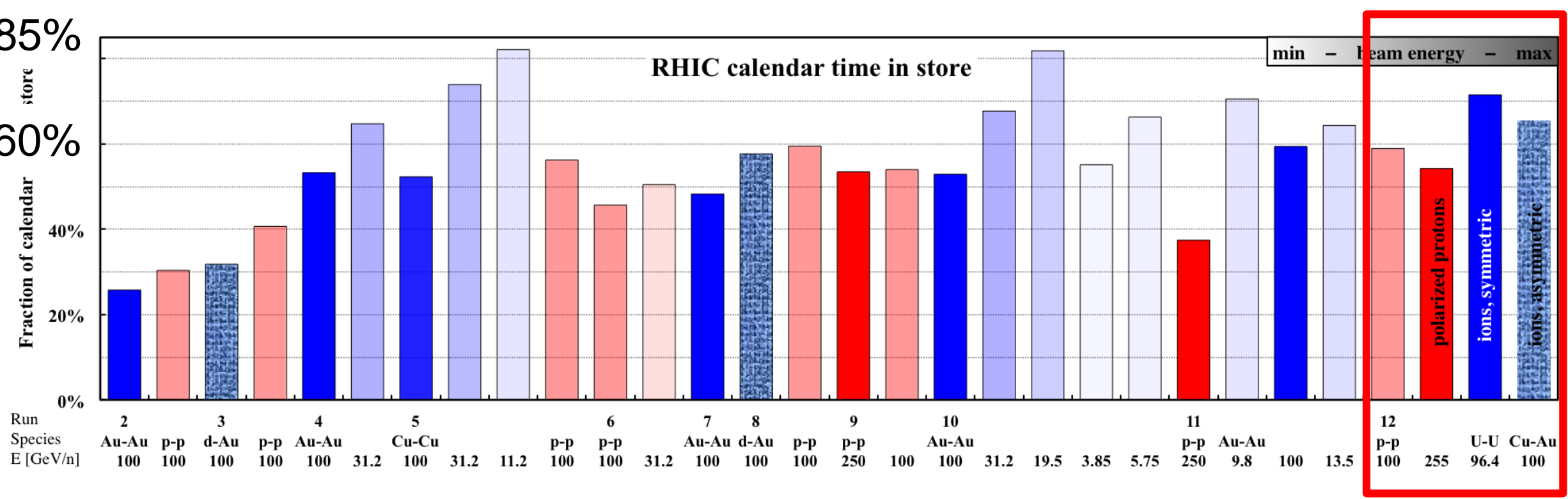

- Run-12 with low failure rates in all systems

- Highest time-in-store ratios to date

even with increased APEX time during $255 \mathrm{GeV}$ protons, and few weeks per species

- Have higher failure rates in Run-13 so far 


\section{What do we still need for $p+A u$ ?}

- Demonstrate DX move by $1 \mathrm{~cm}$

Can be done at end of Run-13

Move for $p$ beam in Yellow, Au beam in Blue (different from d-Au)

- If $p$ beam in Blue is needed (and Au beam in Yellow)

Modify vacuum pump stands in IR6 and IR8

Modify shielding IR6 and IR8

- Operate with new injection and acceleration scheme

Inject and accelerate Au to intermediate level above transition

Then inject $p$ and accelerate both beams

- Ensure that luminosity can be measured

- Study DX aperture and possible experimental background 


\section{Moving IR6 and IR8 DX magnets by $1 \mathrm{~cm}$}

- Bellows allow for $1 \mathrm{~cm}$ movement

- Installed shielding creates tight spaces but acceptable "6:00 the bellows and ion pump stand were swapped to make space for the shielding, ... cannot be moved because of the ion pump stand" (M. Mapes)

- Easier to have $p$ in Yellow, Au in Blue (different from d-Au!)

$\Rightarrow$ Clear preference for Au beam in Yellow ring. (Workshop on Physics of p+A Collisioins in RHIC, 7-9 Jan 2013)

Can be done in 1 shifts (i.e. during a run) when properly prepared in previous shut-down 


\section{p+Au injection and acceleration}

maintains same $f_{\text {rev }}$ for both beams

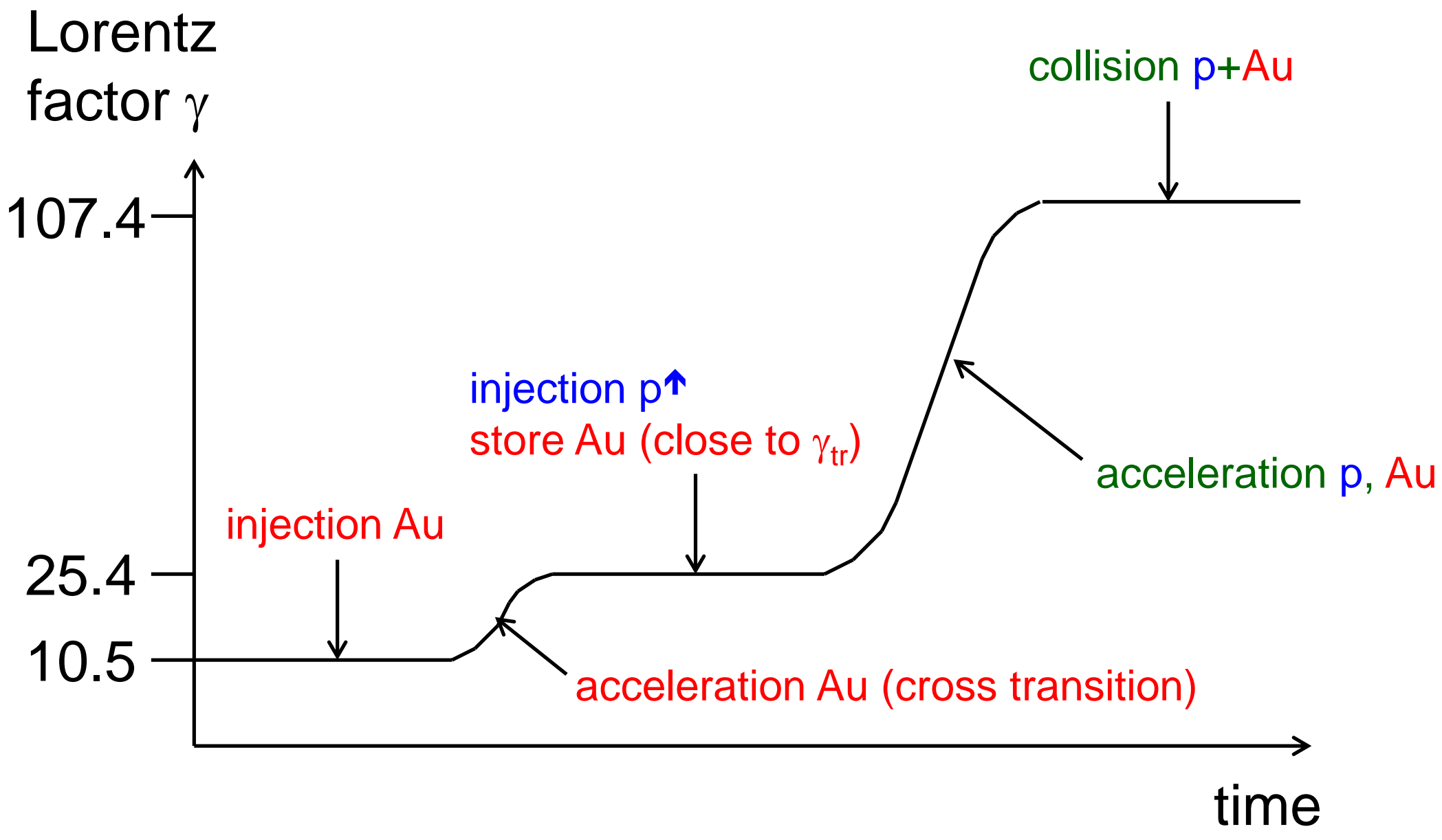

Note: now tolerate ion beam instabilities at transition to obtain higher intensity (can be cooled down again), not possible with $p+A u$ since have smaller aperture available 


\section{ZDC Lumi Monitor Acceptance (M. Bai)}

The $3.58 \mathrm{mrad}$ common angle through IP for head-on collisions can blind the ZDC detector right behind DX magnet

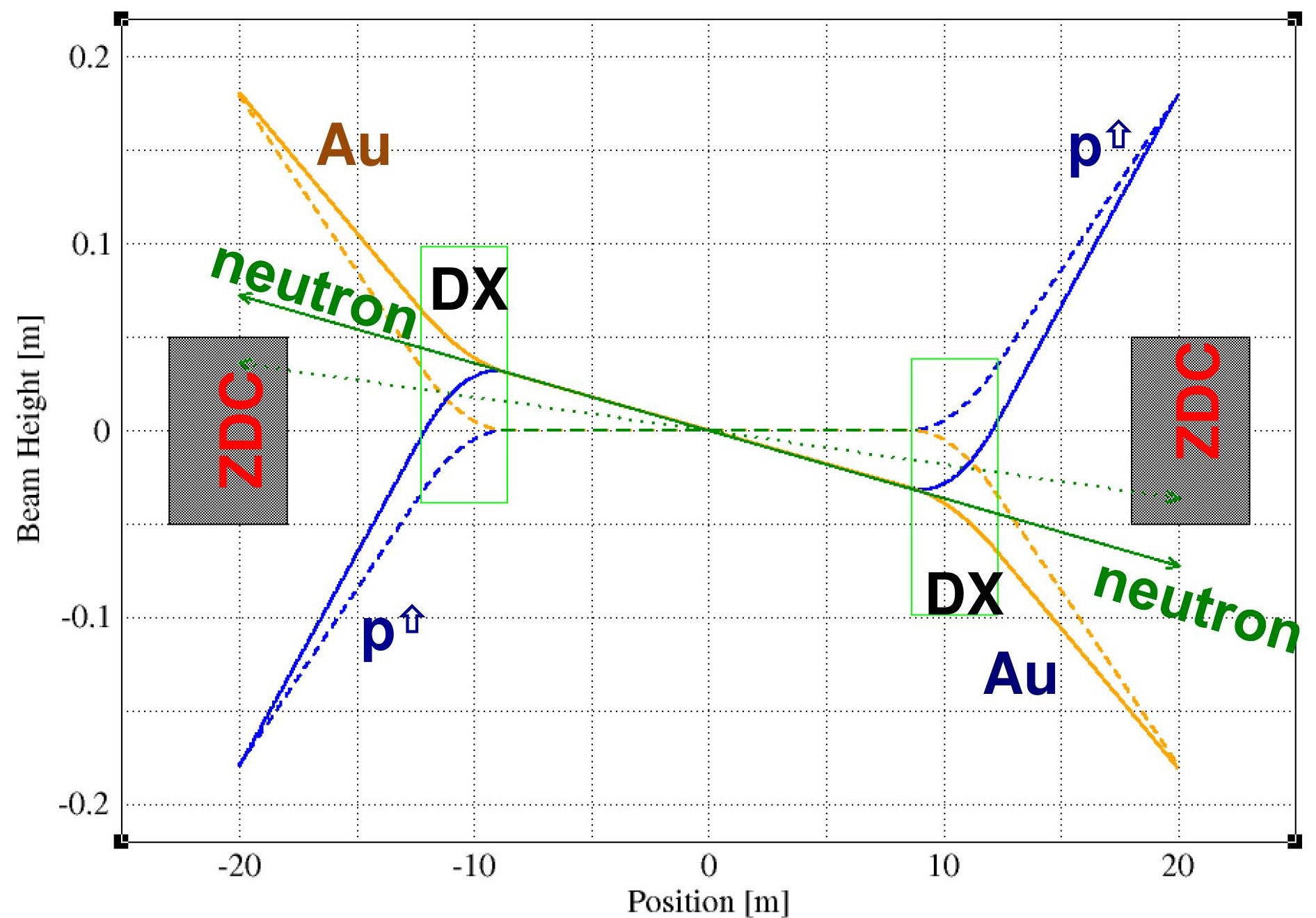




\section{ZDC Lumi Monitor Acceptance (M. Bai)}

\section{Layout between DX and D0}

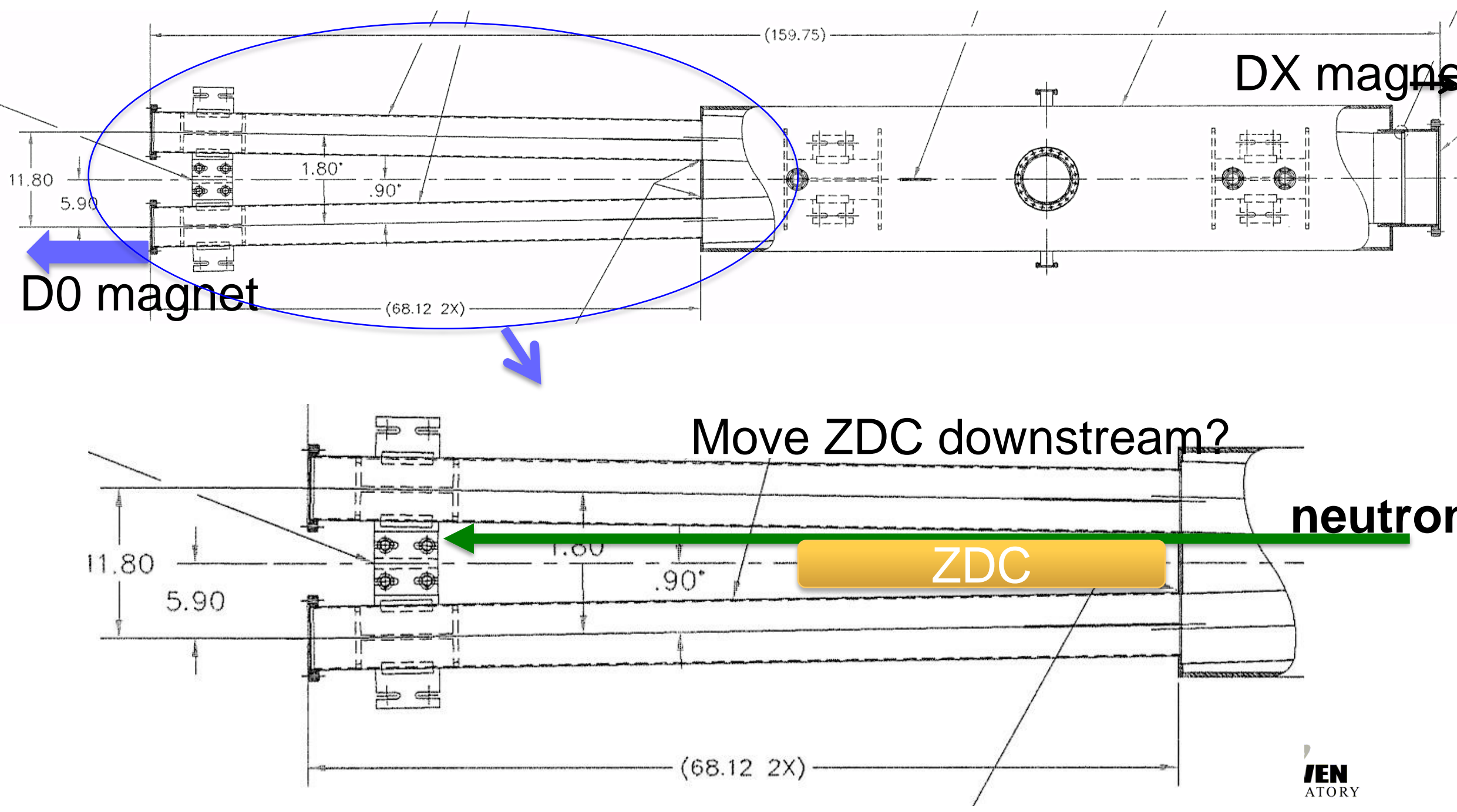




\section{ZDC Lumi Monitor Acceptance (M. Bai)}

If not enough room to move ZDC downstream, need to find a way to reposition ZDC move not only DX magnet but also the beam pipe between DX and D0

Difficult but could be explored

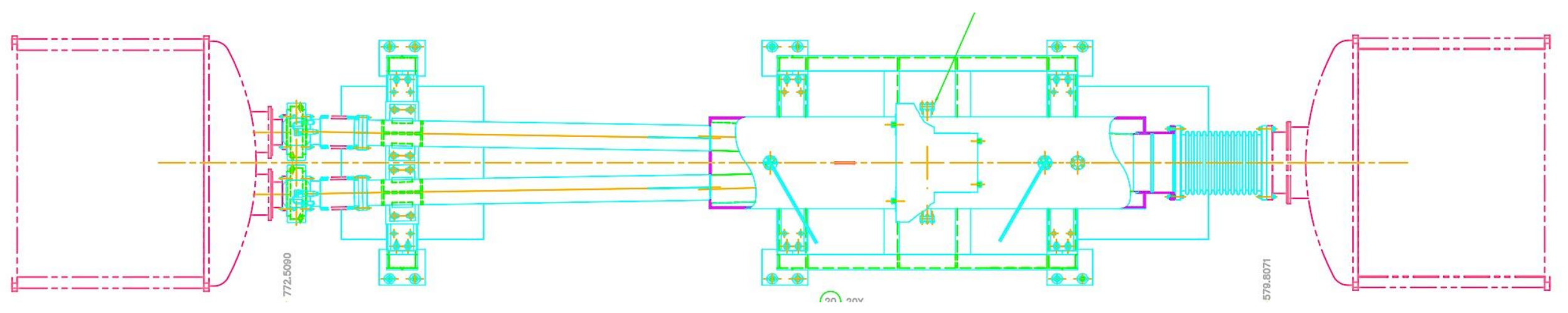


Y. Luo, A. Marusic, S. Tepikian

04/10/13

$$
\sigma=\sqrt{\frac{\left(\frac{\epsilon_{N}}{\pi}\right)\left(\beta^{*}+\frac{s^{2}}{\beta^{*}}\right)}{6(\beta \gamma)}}
$$

Store conditions:

Gold at $B \rho=831.763 \mathrm{Tm}$ or $(\beta Y)=107.391$ Proton at $B \rho=358.647 \mathrm{Tm}$ or $(\beta Y)=114.593$

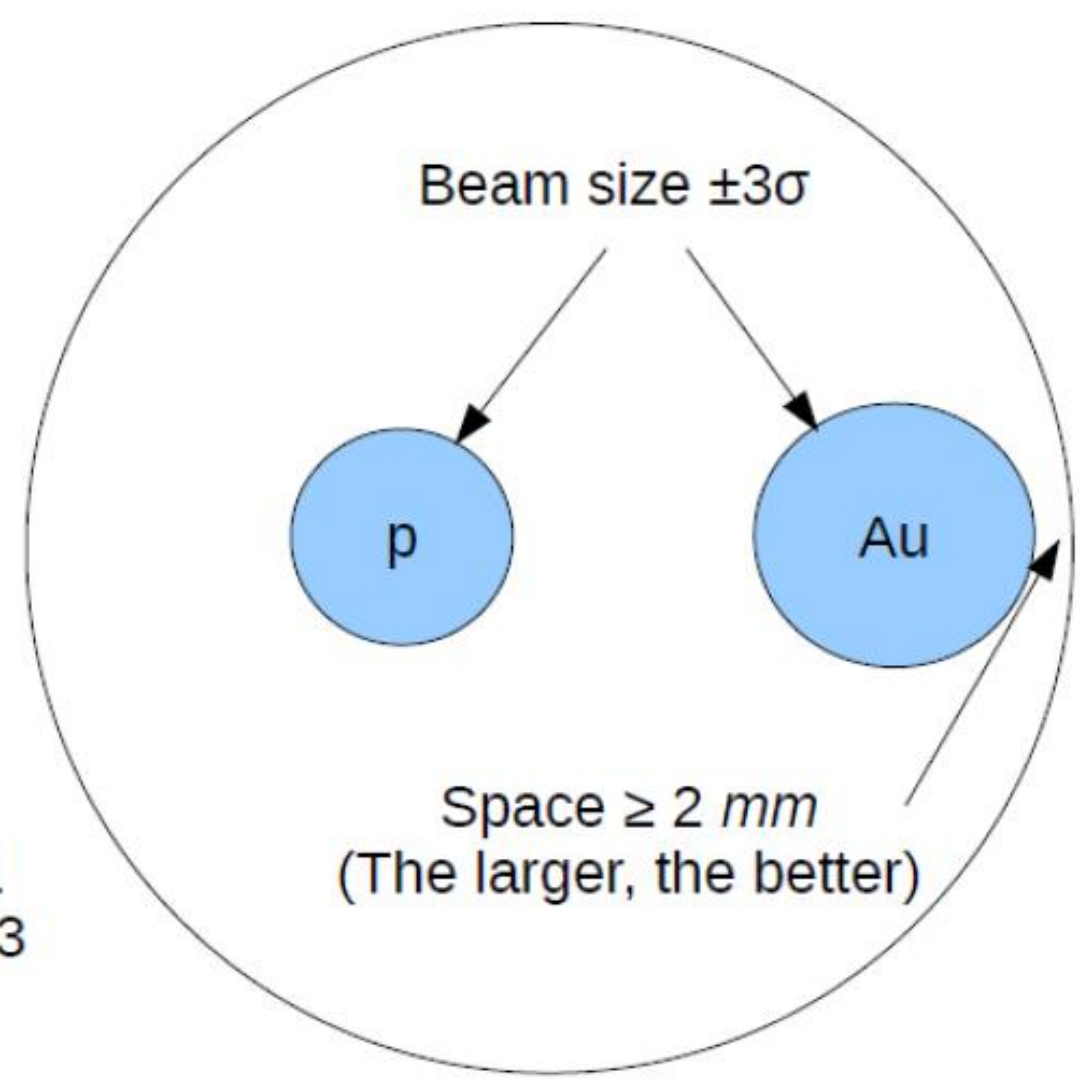

DX magnet $($ radius $=68.326 \mathrm{~mm})$ 


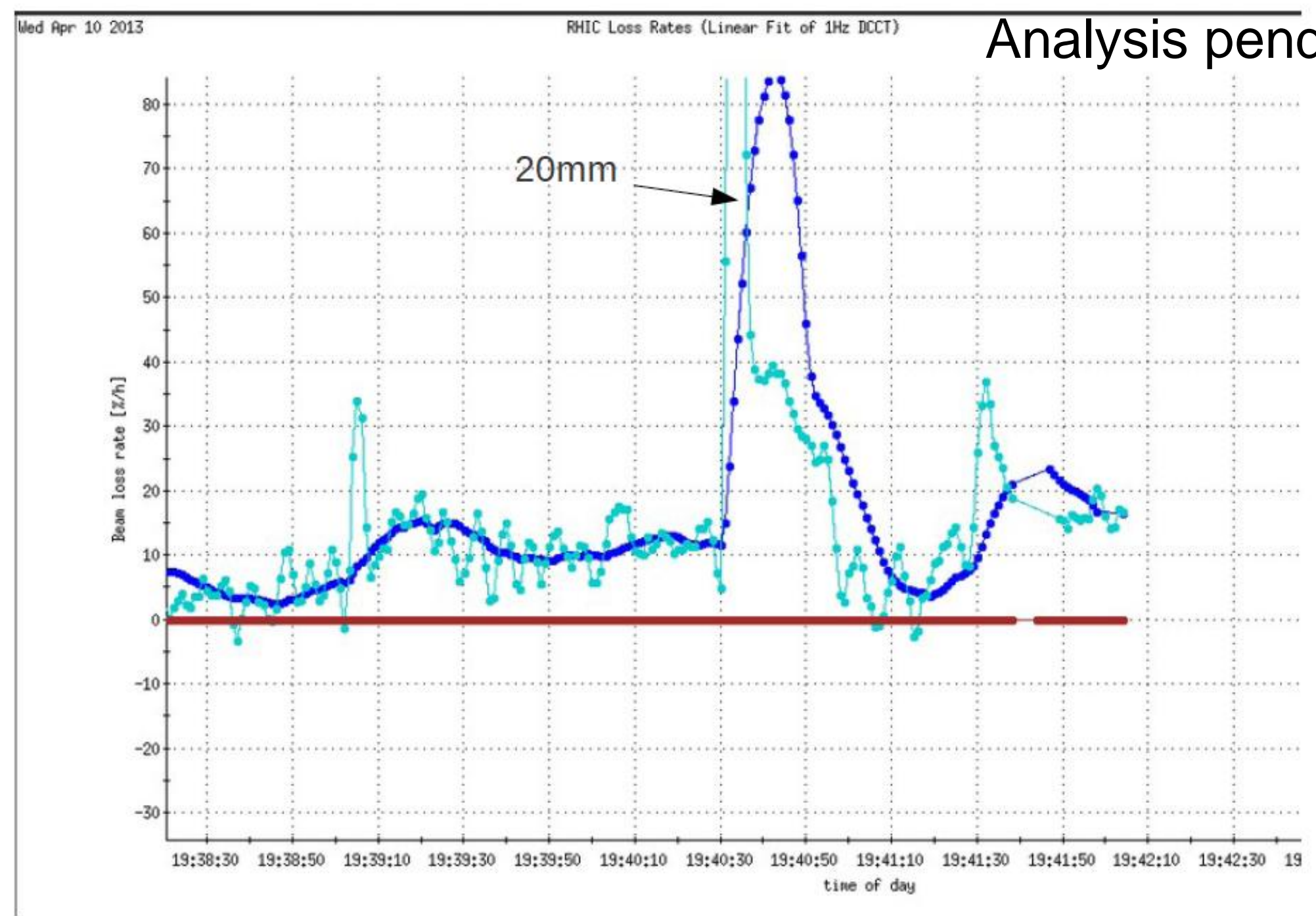

IR 12 Horizontal bump size $20 \mathrm{~mm}$

Zero crossing angle at IR2 ( $33 \mathrm{~mm}$ offset at DX exit )

Vertical bump at IR2 removed during this measurement 


\section{Asymmetric collisions $(p+A u)$}

- $\mathrm{p}+\mathrm{Au}$ energies:

100.8 GeV p on $100.0 \mathrm{GeV} /$ nucleon $\mathrm{Au}\left(\gamma_{\mathrm{p}}=\gamma_{\mathrm{Au}}=107.4\right)$

- For energy scan need to match Lorentz factor $\gamma$ of both beams

\begin{tabular}{|c|c|c|c|c|c|}
\hline $\begin{array}{l}\text { Au-Au history and project } \\
\text { Parameter }\end{array}$ & ${ }_{\text {unit }}$ & $\underset{2013 E}{p}$ & Au & $\frac{p}{2013 E}$ & Au \\
\hline no of colliding bunches & & 111 & 111 & 111 & 111 \\
\hline ions/bunch, initial & $10^{9}$ & 140 & 1.0 & 180 & 1.4 \\
\hline charges per bunch & $10^{\circ} \mathrm{e}$ & 140 & 79 & 180 & 107 \\
\hline average beam current/ring $I_{\mathrm{IM}}$ & $\mathrm{mA}$ & 194 & 110 & 250 & 148 \\
\hline stored energy per beam & MJ & 0.25 & 0.35 & 0.32 & 0.47 \\
\hline transverse rms emittance $E_{x y}$ & mm.mrad & 2.9 & 2.9 & 2.8 & 2.8 \\
\hline rms bunch length $\sigma_{s}$ & $\mathrm{~m}$ & 0.5 & 0.3 & 0.5 & 0.3 \\
\hline envelope function at IP $\beta^{*}$ & $\mathrm{~m}$ & 0.9 & 0.9 & 0.7 & 0.7 \\
\hline beam-beam parameter $g / \mathrm{IP}$ & $10^{-5}$ & 4.3 & 2.4 & 5.9 & 3.1 \\
\hline initial luminosity $L / I P$ & $10^{26} \mathrm{~cm}^{-1} \mathrm{~s}^{-1}$ & 39 & & 82 & \\
\hline events per bunch-bunch crossing & $\ldots$ & & & & \\
\hline average / initial luminosity & $\%$ & 60 & & 70 & \\
\hline average store luminosity $L_{\mathrm{avg}}$ & $10^{26} \mathrm{~cm}^{-1} \mathrm{~s}^{-1}$ & 24 & & 57 & \\
\hline time in store & $\%$ & 55 & & 55 & \\
\hline $\begin{array}{l}\max \text { luminosity/week } \\
\text { min luminosity/week }\end{array}$ & $\begin{array}{l}\mu \mathrm{b}^{-1} \\
\mu \mathrm{b}^{-1}\end{array}$ & 78 & & 190 & \\
\hline$L_{\mathrm{NN}} /$ week, $\min / \max$ & $\mathrm{pb}^{-1}$ & 15 & & 37 & \\
\hline
\end{tabular}




\section{Summary}

- $p+A u$ is possible

max energy $100 \mathrm{GeV} /$ nucleon for both beams

- Stochastic cooling for ion beams helps:

DX move only in IR6 \& IR8, no Au beam growth

- DX move in $\sim 1$ shifts

possible upgrade for pp2pp in IR6 requires change of DX bellows

will reduce flexibility, cannot move DX magnets during run

- New injection/acceleration scheme

store Au beam above transition for $\sim 15 \mathrm{~min}$

- Luminosity estimate based on $\mathrm{p} \uparrow$ beam available (anticipated), and $\mathrm{Au}$ beam available (anticipated)

$L_{\mathrm{NN}}=15 \mathrm{pb} /$ week min (now)

$L_{\mathrm{NN}}=37 \mathrm{pb} / \mathrm{week} \max$ (few years) 


\section{Fluctuations, flow and viscosity in the Little Bang*}

\section{Ulrich Heinz (The Ohio State University)}

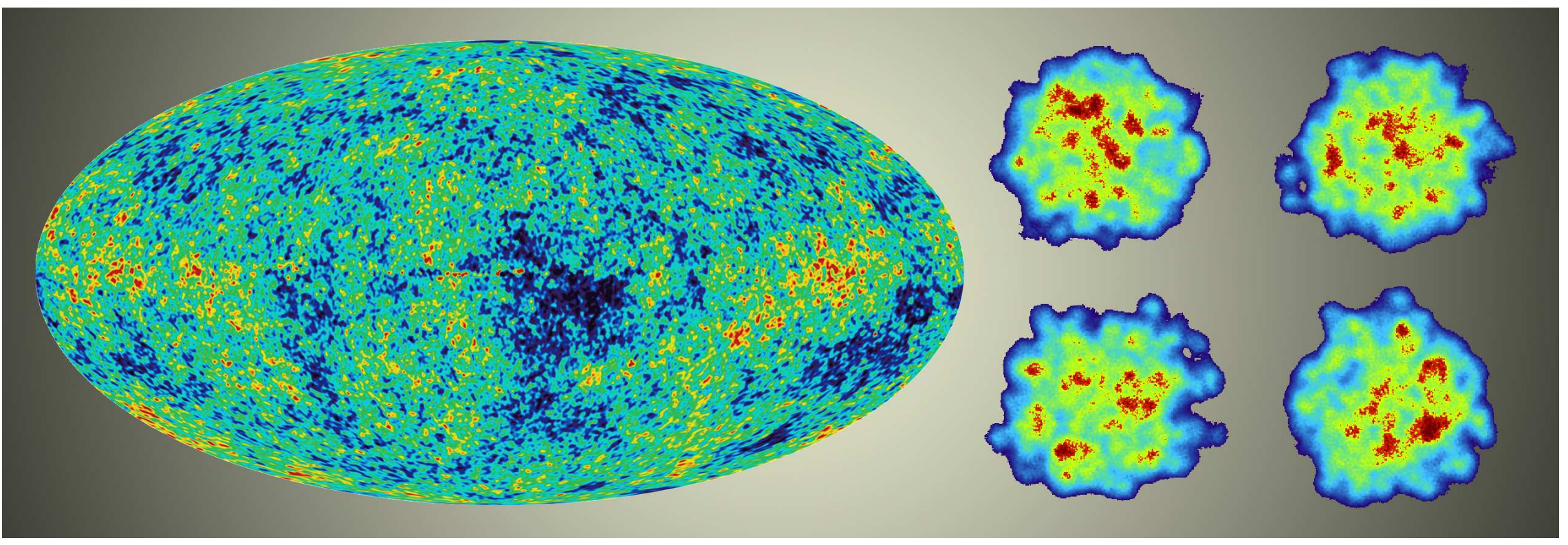

presented at:

Jet quenching at RHIC vs. LHC in Light of Recent dAu vs. pPb Controls Brookhaven National Laboratory, 15-17 April 2013

\section{OHH H' ${ }^{\mathrm{R}}$ DEPARTMENT OF STAIE PHYSICS}

*Supported by the U.S. Department of Energy

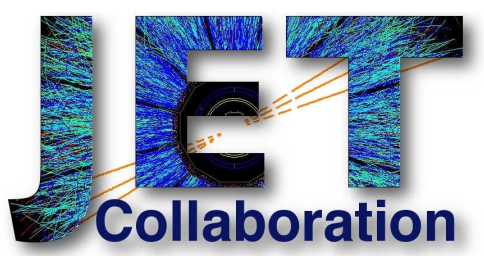

References: Heinz, Qiu, Shen, PRC 87 (2013) 034913

Qiu, Heinz, PLB 717 (2012) 261 


\section{Big Bang vs. Little Bang: The fluctuation power spectrum}
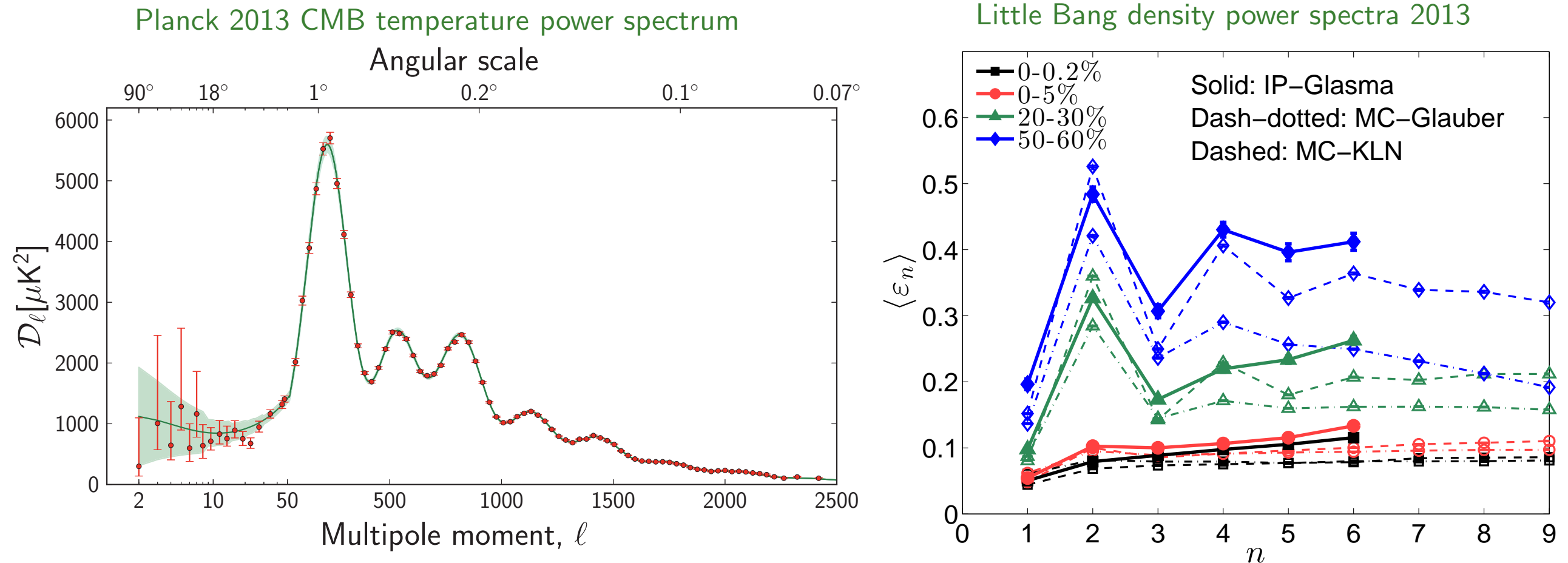

Initial eccentricities $\varepsilon_{n}$ and associated participant-plane angles $\Phi_{n}$ of the Little Bang:

$$
\begin{array}{r}
\varepsilon_{1} e^{i \Phi_{1}} \equiv-\frac{\int r d r d \varphi r^{3} e^{i \varphi} e(r, \varphi)}{\int r d r d \varphi r^{3} e(r, \varphi)}, \quad \varepsilon_{n} e^{i n \Phi_{n}} \equiv-\frac{\int r d r d \varphi r^{n} e^{i n \varphi} e(r, \varphi)}{\int r d r d \varphi r^{n} e(r, \varphi)}(n) \\
\text { A detailed study of fluctuations is a powerful } \\
\text { discriminator between models! }
\end{array}
$$




\section{The fluctuation power spectrum: initial vs. final}

Flow power spectrum for ultracentral pPb Little Bangs

Little Bang density power spectra

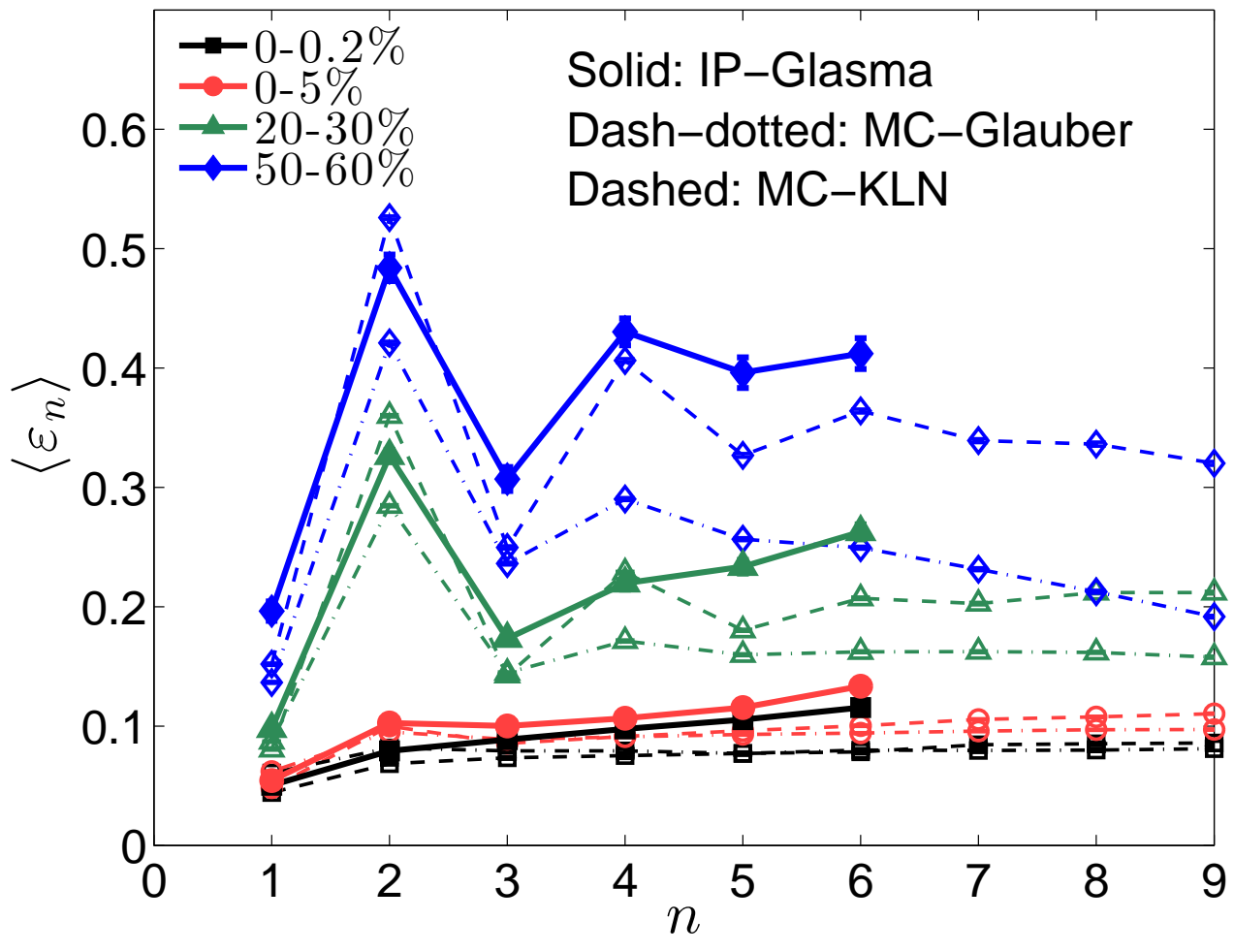

(CMS, Quark Matter 2012)

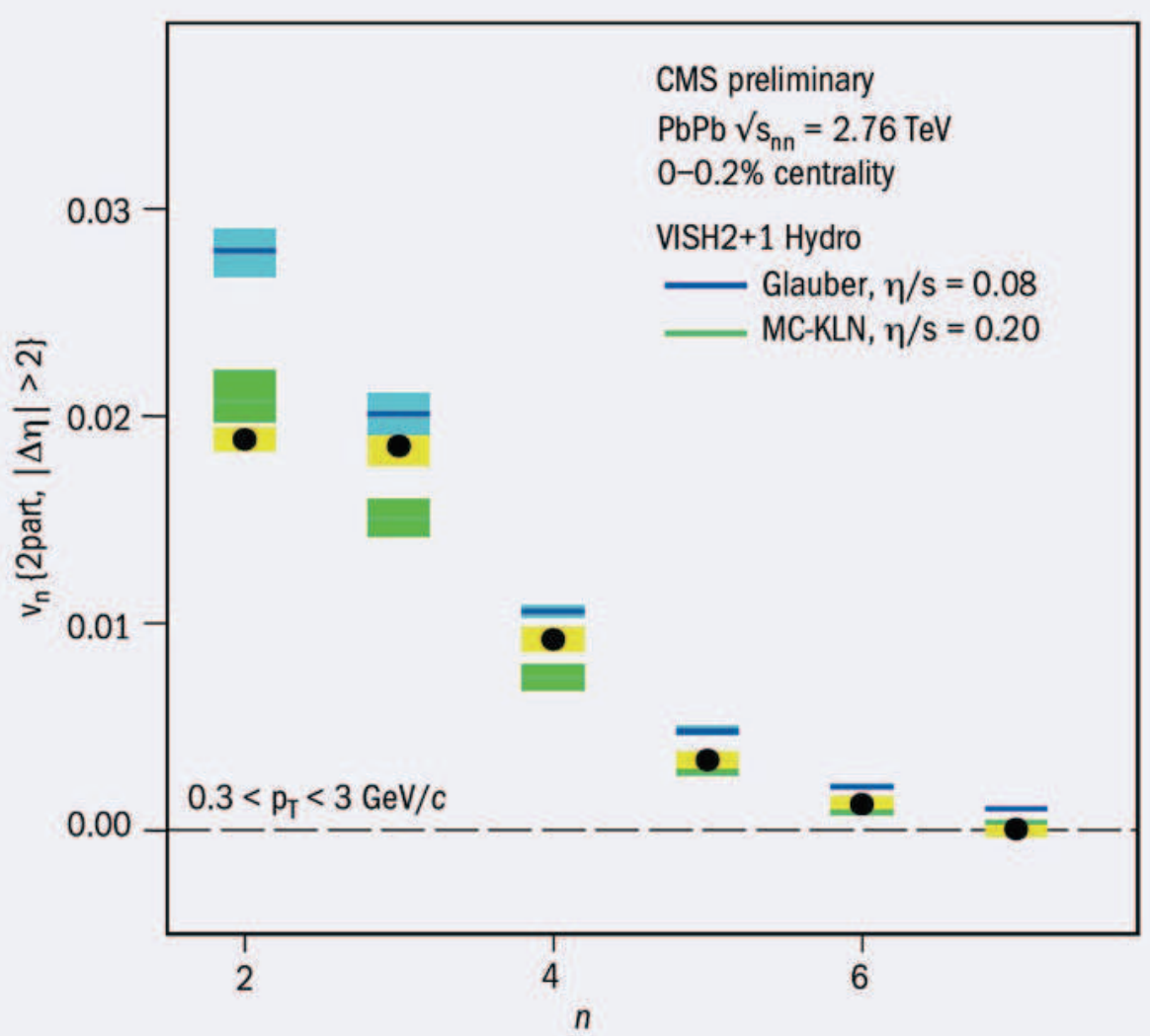

Higher flow harmonics get suppressed by shear viscosity

A detailed study of fluctuations is a powerful discriminator between models! 


\section{Each Little Bang evolves differently!}

Density evolution of a single $b=8 \mathrm{fm} \mathrm{Au}+\mathrm{Au}$ collision at RHIC, with IP-Glasma initial conditions, Glasma evolution to $\tau=0.2 \mathrm{fm} / c$ followed by $(3+1)$-d viscous hydrodynamic evolution with MUSIC using $\eta / s=0.12=1.5 /(4 \pi)$

Schenke, Tribedy, Venugopalan, PRL 108 (2012) 252301:
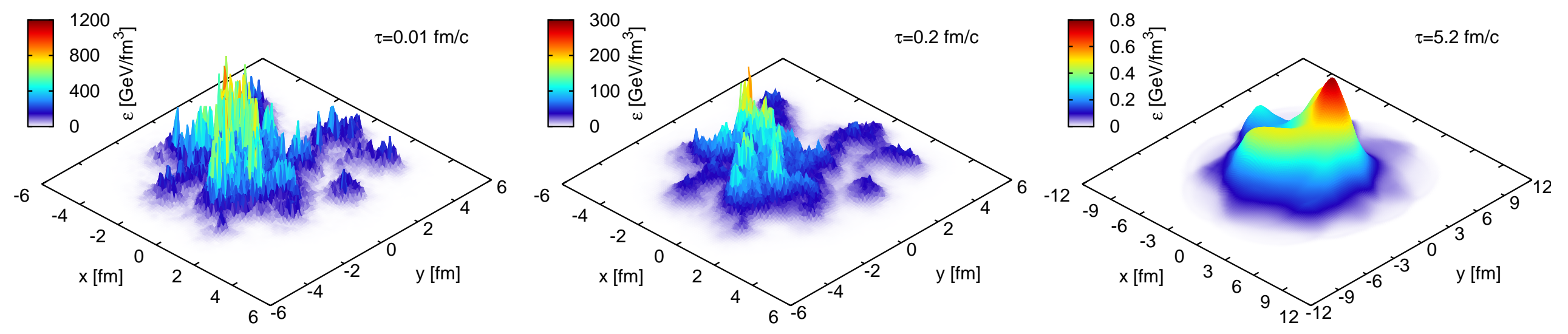


\section{Single event anisotropic flow coefficients}

In a single event, the specific initial density profile results in a set of complex, $y$ - and $p_{T}$-dependent flow coefficients (we'll suppress the $y$-dependence):

$$
\begin{gathered}
V_{n}=v_{n} e^{i n \Psi n}:=\frac{\int p_{T} d p_{T} d \phi e^{i n \phi} \frac{d N}{d y p_{T} d p_{T} d \phi}}{\int p_{T} d p_{T} d \phi \frac{d N}{d y p_{T} d p_{T} d \phi}} \equiv\left\{e^{i n \phi}\right\}, \\
V_{n}\left(p_{T}\right)=v_{n}\left(p_{T}\right) e^{i n \Psi n\left(p_{T}\right)}:=\frac{\int d \phi e^{i n \phi} \frac{d N}{d y p_{T} d p_{T} d \phi}}{\int d \phi \frac{d N}{d y p_{T} d p_{T} d \phi}} \equiv\left\{e^{i n \phi}\right\}_{p_{T}} .
\end{gathered}
$$

Together with the azimuthally averaged spectrum, these completely characterize the measurable singleparticle information for that event:

$$
\begin{gathered}
\frac{d N}{d y d \phi}=\frac{1}{2 \pi} \frac{d N}{d y}\left(1+2 \sum_{n=1}^{\infty} v_{n} \cos \left[n\left(\phi-\Psi_{n}\right)\right]\right) \\
\frac{d N}{d y p_{T} d p_{T} d \phi}=\frac{1}{2 \pi} \frac{d N}{d y p_{T} d p_{T}}\left(1+2 \sum_{n=1}^{\infty} v_{n}\left(p_{T}\right) \cos \left[n\left(\phi-\Psi_{n}\left(p_{T}\right)\right)\right]\right)
\end{gathered}
$$

- Both the magnitude $v_{n}$ and the direction $\Psi_{n}$ ("flow angle") depend on $p_{T}$.

- $v_{n}, \Psi_{n}, v_{n}\left(p_{T}\right), \Psi_{n}\left(p_{T}\right)$ all fluctuate from event to event.

- $\Psi_{n}\left(p_{T}\right)-\Psi_{n}$ fluctuates from event to event. 


\section{Initial participant plane correlations in $\mathrm{PbPb} Q \mathrm{LHC}$}

Zhi Qiu, UH, PLB 717 (2012) 261
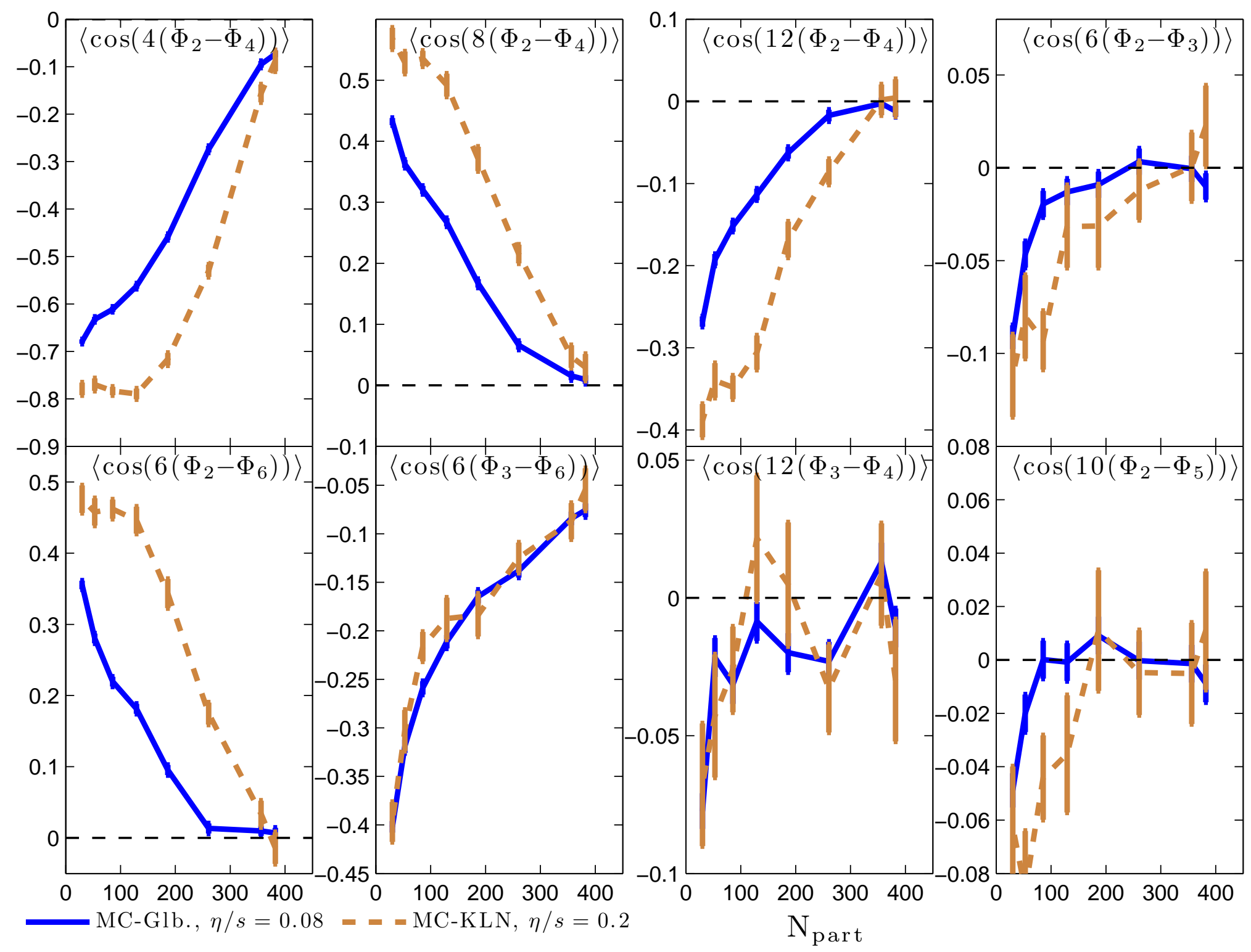

Qualitatively similar, but quantitative differences between models 


\section{Final flow angle correlations in $\mathrm{PbPb} @ \mathrm{LHC}$}

Data: ATLAS Coll., J. Jia et al., Hard Probes 2012

Event-by-event hydrodynamics: Zhi Qiu, UH, PLB 717 (2012) 261 (VISH2+1)
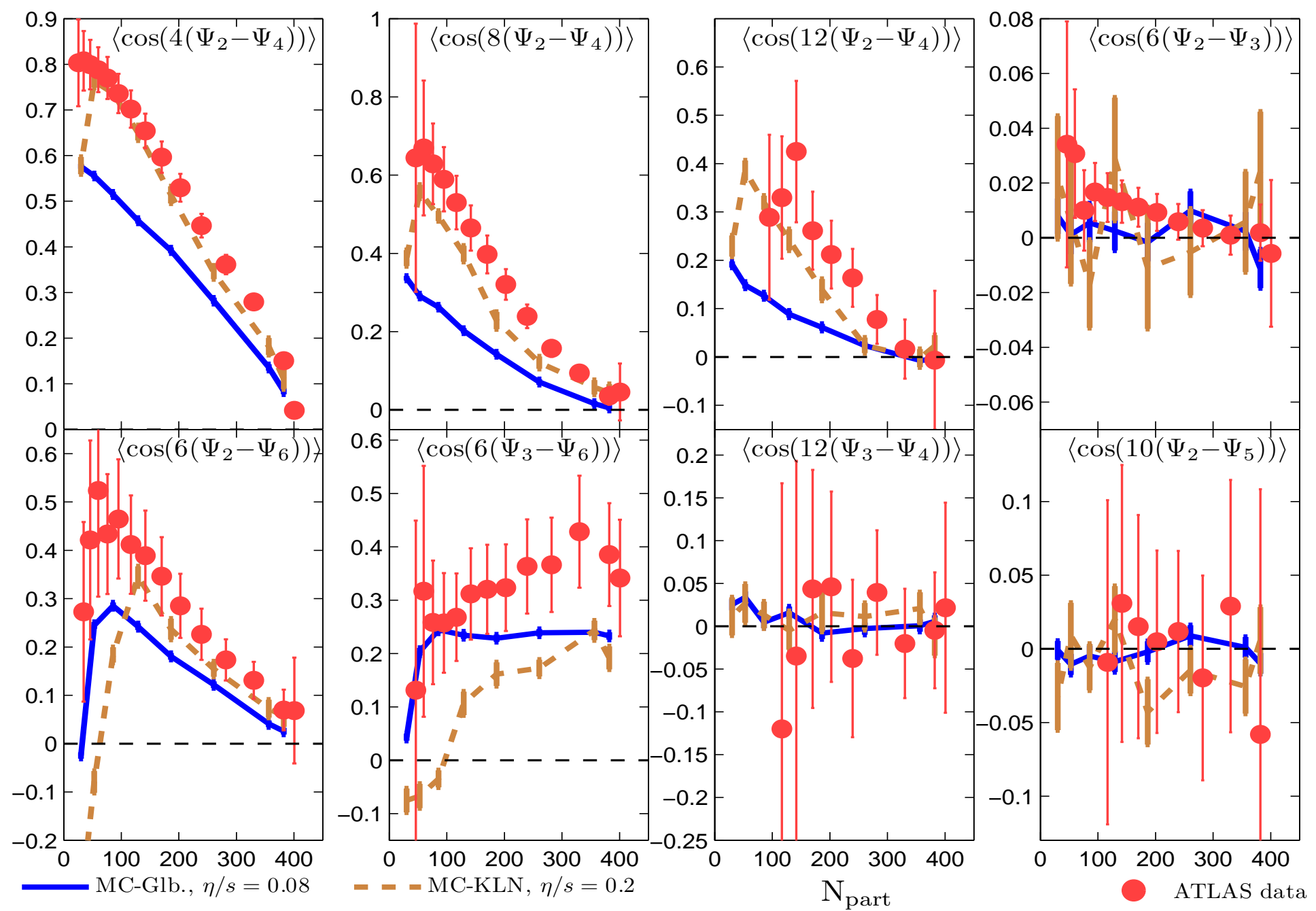

$\mathrm{VISH} 2+1$ reproduces qualitatively the centrality dependence of all measured event-plane correlations Initial part.-plane correlations disagree qualitatively with the measured final-state flow-plane correlations $\Longrightarrow$ Nonlinear mode coupling through hydrodynamic evolution essential to describe the data!

Larger viscosity appears to yield stronger flow-angle correlations 


\section{Initial three-plane correlations in PbPb@LHC}

Zhi Qiu, UH, PLB 717 (2012) 261

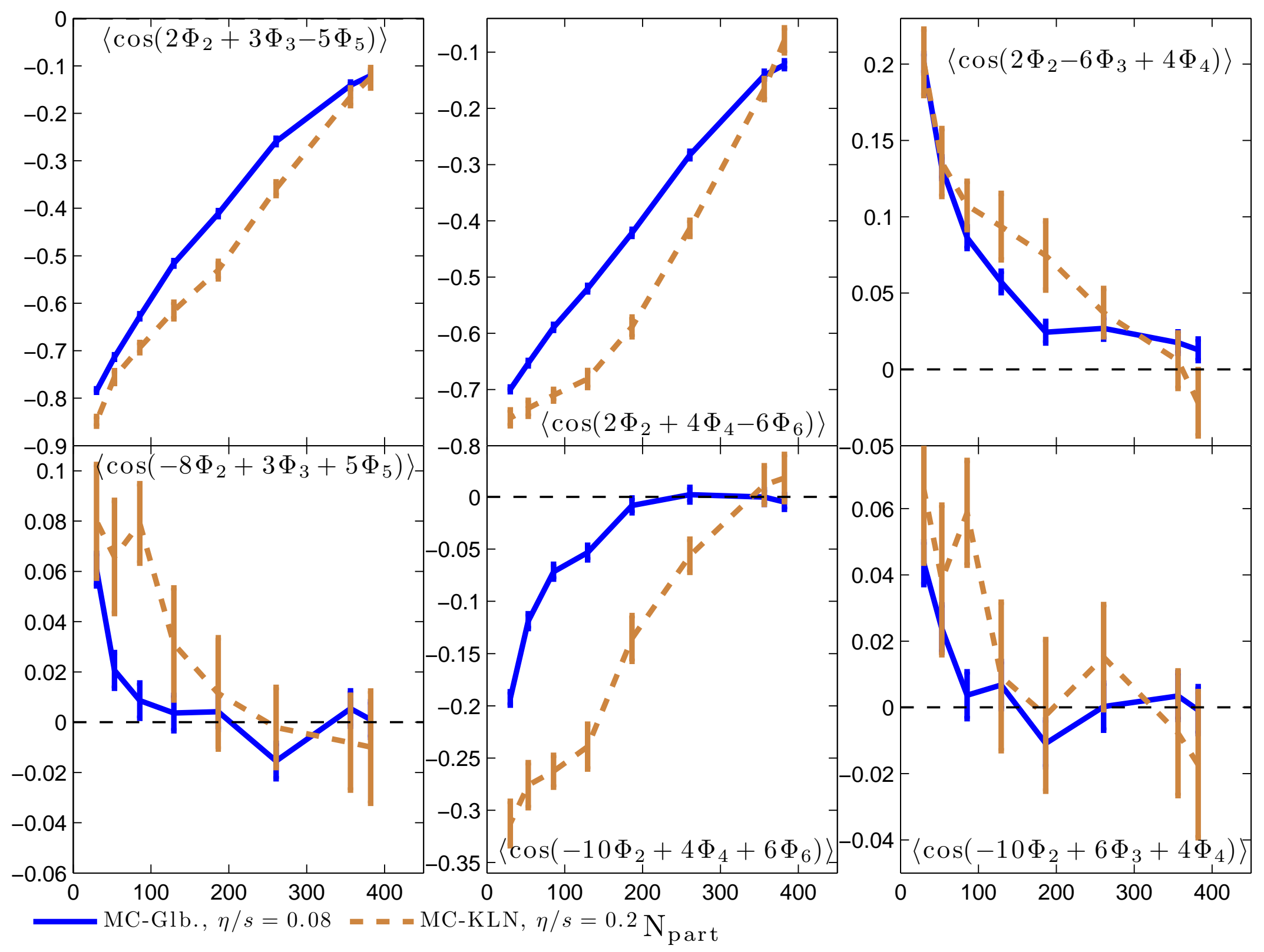




\section{Final three-plane flow correlations in PbPb@LHC}

Data: ATLAS Coll., J. Jia et al., Hard Probes 2012

Event-by-event hydrodynamics: Zhi Qiu, UH, PLB 717 (2012) 261 (VISH2+1)
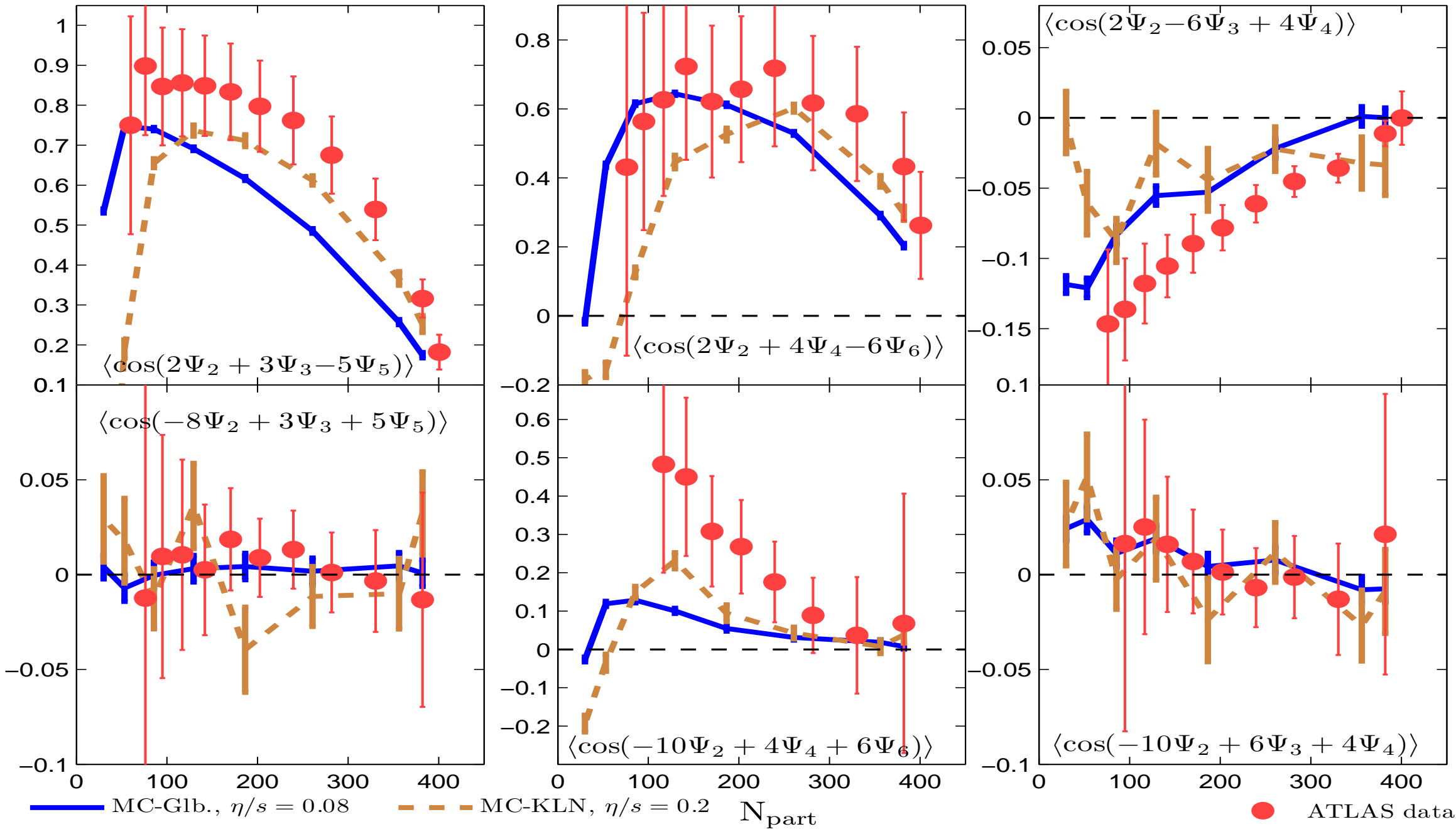

Nonlinear mode coupling through hydrodynamic evolution essential to describe the data! 


\section{Single event anisotropic flow coefficients}

In a single event, the specific initial density profile results in a set of complex, $y$ - and $p_{T}$-dependent flow coefficients (we'll suppress the $y$-dependence):

$$
\begin{gathered}
V_{n}=v_{n} e^{i n \Psi n}:=\frac{\int p_{T} d p_{T} d \phi e^{i n \phi} \frac{d N}{d y p_{T} d p_{T} d \phi}}{\int p_{T} d p_{T} d \phi \frac{d N}{d y p_{T} d p_{T} d \phi}} \equiv\left\{e^{i n \phi}\right\}, \\
V_{n}\left(p_{T}\right)=v_{n}\left(p_{T}\right) e^{i n \Psi n\left(p_{T}\right)}:=\frac{\int d \phi e^{i n \phi} \frac{d N}{d y p_{T} d p_{T} d \phi}}{\int d \phi \frac{d N}{d y p_{T} d p_{T} d \phi}} \equiv\left\{e^{i n \phi}\right\}_{p_{T}} .
\end{gathered}
$$

Together with the azimuthally averaged spectrum, these completely characterize the measurable singleparticle information for that event:

$$
\begin{gathered}
\frac{d N}{d y d \phi}=\frac{1}{2 \pi} \frac{d N}{d y}\left(1+2 \sum_{n=1}^{\infty} v_{n} \cos \left[n\left(\phi-\Psi_{n}\right)\right]\right) \\
\frac{d N}{d y p_{T} d p_{T} d \phi}=\frac{1}{2 \pi} \frac{d N}{d y p_{T} d p_{T}}\left(1+2 \sum_{n=1}^{\infty} v_{n}\left(p_{T}\right) \cos \left[n\left(\phi-\Psi_{n}\left(p_{T}\right)\right)\right]\right)
\end{gathered}
$$

- Both the magnitude $v_{n}$ and the direction $\Psi_{n}$ ("flow angle") depend on $p_{T}$.

- $v_{n}, \Psi_{n}, v_{n}\left(p_{T}\right), \Psi_{n}\left(p_{T}\right)$ all fluctuate from event to event.

- $\Psi_{n}\left(p_{T}\right)-\Psi_{n}$ fluctuates from event to event. 


\section{$p_{T^{-}}$-dependent flow angles and their fluctuations}
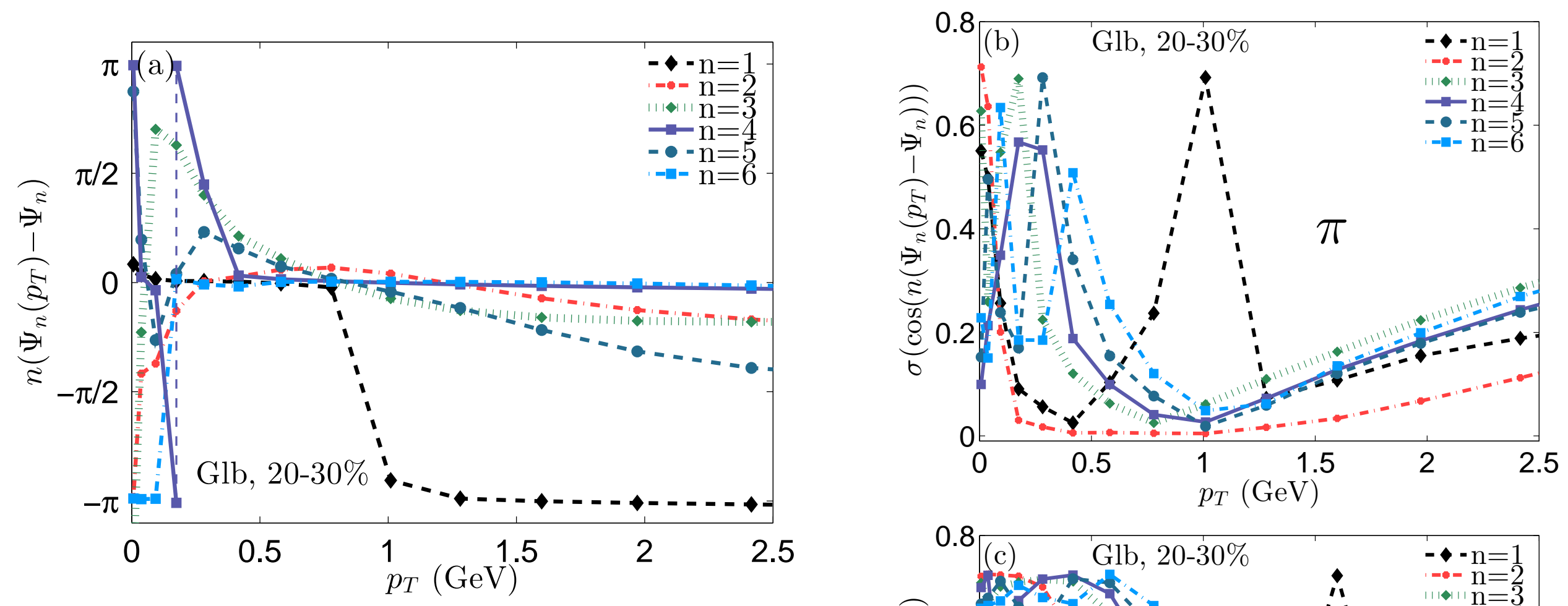

- Except for directed flow $(n=1)$, $\Psi_{n}\left(p_{T}\right)-\Psi_{n}$ fluctuates most strongly at low $p_{T}$

- Directed flow angle $\Psi_{1}\left(p_{T}\right)$ flips by $180^{\circ}$ at $p_{T} \sim 1 \mathrm{GeV}$ for charged hadrons (pions) and at $p_{T} \sim 1.5 \mathrm{GeV}$ for protons (momentum conservation)

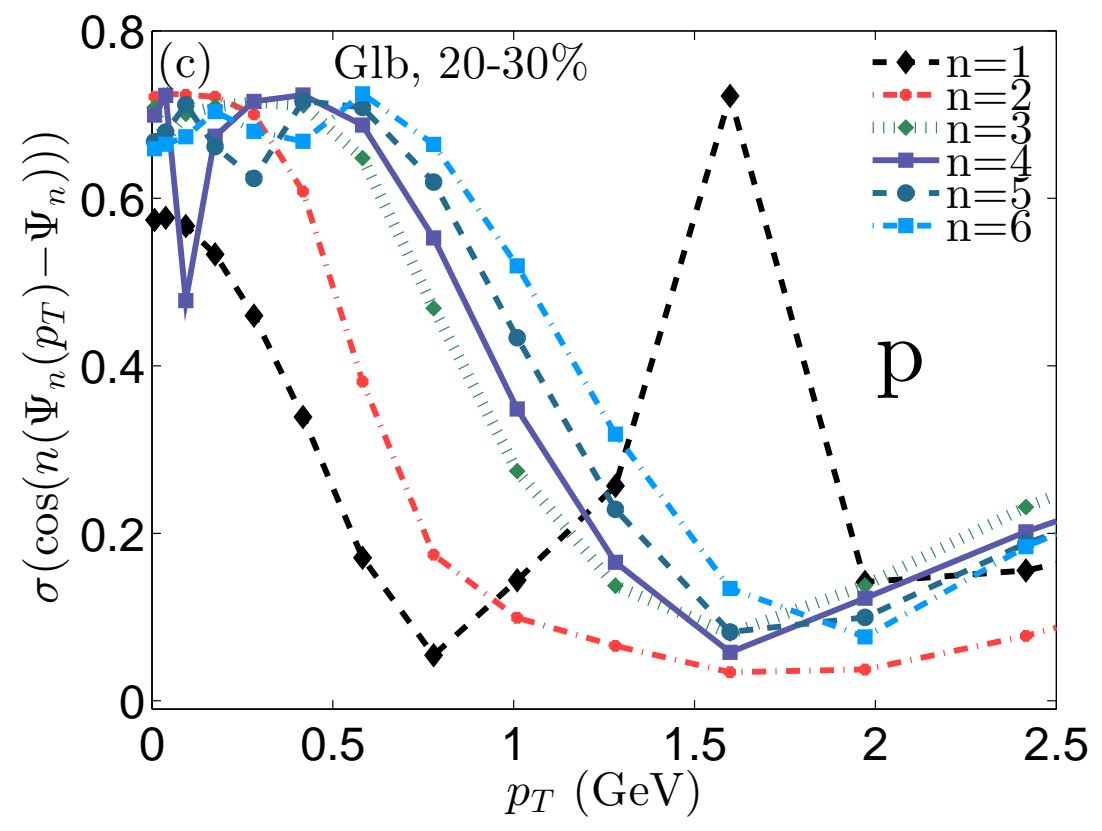




\section{Flow measures from two-particle correlations $\left\langle\left\{e^{i n\left(\phi_{1}-\phi_{2}\right)}\right\}\right\rangle$}

"rms flow":

$$
\begin{aligned}
& v_{n}^{2}[2]:=\left\langle\left\{e^{i n \phi_{1}}\right\}\left\{e^{-i n \phi_{2}}\right\}\right\rangle=\left\langle v_{n}^{2}\right\rangle \equiv v_{n}\{2\} \\
& v_{n}^{2}[2]\left(p_{T}\right):=\left\langle\left\{e^{i n \phi_{1}}\right\}_{p_{T}}\left\{e^{-i n \phi_{2}}\right\}_{p_{T}}\right\rangle=\left\langle v_{n}^{2}\left(p_{T}\right)\right\rangle \quad\left(\neq v_{n}^{2}\{2\}\left(p_{T}\right) !\right) .
\end{aligned}
$$

\section{"differential 2-particle cumulant flow":}

$$
v_{n}\{2\}\left(p_{T}\right):=\left\langle\left\{e^{i n \phi_{1}}\right\}_{p_{T}}\left\{e^{-i n \phi_{2}}\right\}\right\rangle / v_{n}\{2\}=\left\langle v_{n}\left(p_{T}\right) v_{n} \cos \left[n\left(\Psi_{n}\left(p_{T}\right)-\Psi_{n}\right)\right]\right\rangle / v_{n}[2] .
$$

“event plane flow":

\section{"mean flow":}

$$
v_{n}\{\operatorname{EP}\}\left(p_{T}\right):=\left\langle\left\{e^{i n \phi}\right\}_{p_{T}} e^{-i n \Psi n}\right\rangle=\left\langle v_{n}\left(p_{T}\right) \cos \left[n\left(\Psi_{n}\left(p_{T}\right)-\Psi_{n}\right)\right]\right\rangle .
$$

$$
\left\langle v_{n}\left(p_{T}\right)\right\rangle:=\left\langle\left|\left\{e^{i n \phi}\right\}_{p_{T}} e^{-i n \Psi n}\right|\right\rangle=\left\langle\sqrt{\{\cos (n \phi)\}_{p_{T}}^{2}+\{\sin (n \phi)\}_{p_{T}}^{2}}\right\rangle .
$$

\section{"two-particle flows":}

$$
\begin{gathered}
\tilde{V}_{n \Delta}\left(p_{T 1}, p_{T 2}\right):=\left\langle\left\{e^{i n\left(\phi_{1}-\phi_{2}\right)}\right\}_{p_{T 1} p_{T 2}}\right\rangle=\left\langle v_{n}\left(p_{T 1}\right) v_{n}\left(p_{T 2}\right) \cos \left[n\left(\Psi_{n}\left(p_{T 1}\right)-\Psi_{n}\left(p_{T 2}\right)\right)\right]\right\rangle \\
\left\langle v_{n}\left(p_{T 1}\right) v_{n}\left(p_{T 2}\right)\right\rangle:=\left\langle\sqrt{\{\cos (n \Delta \phi)\}_{p_{T 1}, p_{T 2}}^{2}+\{\sin (n \Delta \phi)\}_{p_{T 1}, p_{T 2}}^{2}}\right\rangle
\end{gathered}
$$

Here: both particles taken from same species (but this is not necessary).

Fluctation effects related to finite number of particles in the observed final state are ignored. 


\section{Elliptic and triangular flow comparison (I)}
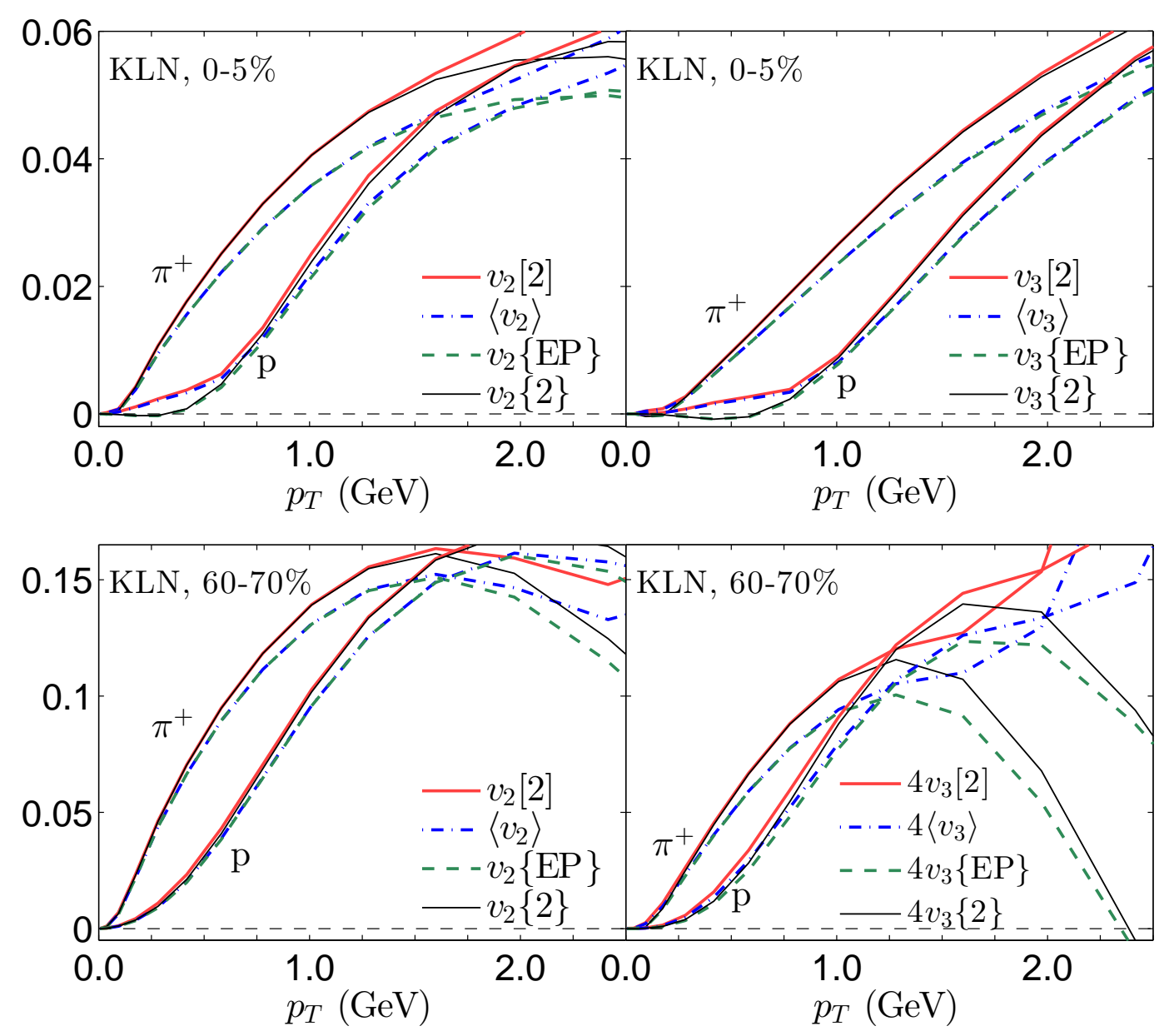

In central collisions, angular fluctuations suppress $v_{n}\{\mathrm{EP}\}\left(p_{T}\right)$ and $v_{n}\{2\}\left(p_{T}\right)$ below the mean and rms flows at low $p_{T}$ (clearly visible for protons)

This effect disappears in peripheral collisions, but a similar effect then takes over at higher $p_{T}$, for both pions and protons. 


\section{Elliptic and triangular flow comparison (II): $v_{n}$ ratios}

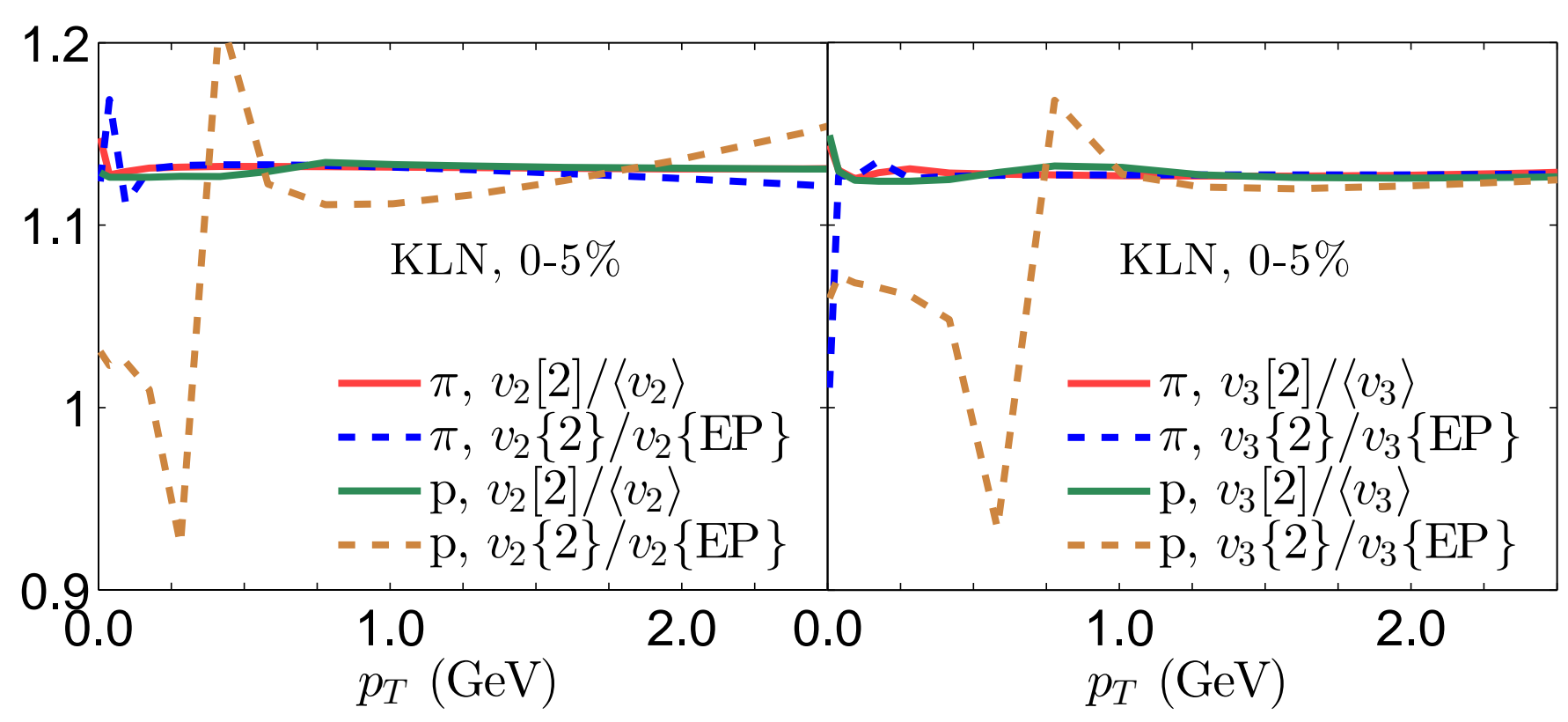

Except for where the numerator or denominator goes through zero, for central collisions these ratios are equal to $2 / \sqrt{\pi} \approx 1.13$, independent of $p_{T}$. Expected if flow angles are randomly oriented (BesselGaussian distribution for $v_{n}$, see Voloshin et al., PLB 659, 537 (2008)).

Not true in peripheral collisions, especially not for $v_{2}$ (Gardim et al., 1209.2323)

That this works even for $v_{n}\{2\} / v_{n}\{\mathrm{EP}\}$ suggests an approximate factorization of angular fluctuation effects! 


\section{Elliptic and triangular flow comparison (III): $v_{n}$ ratios}

Central collisions:

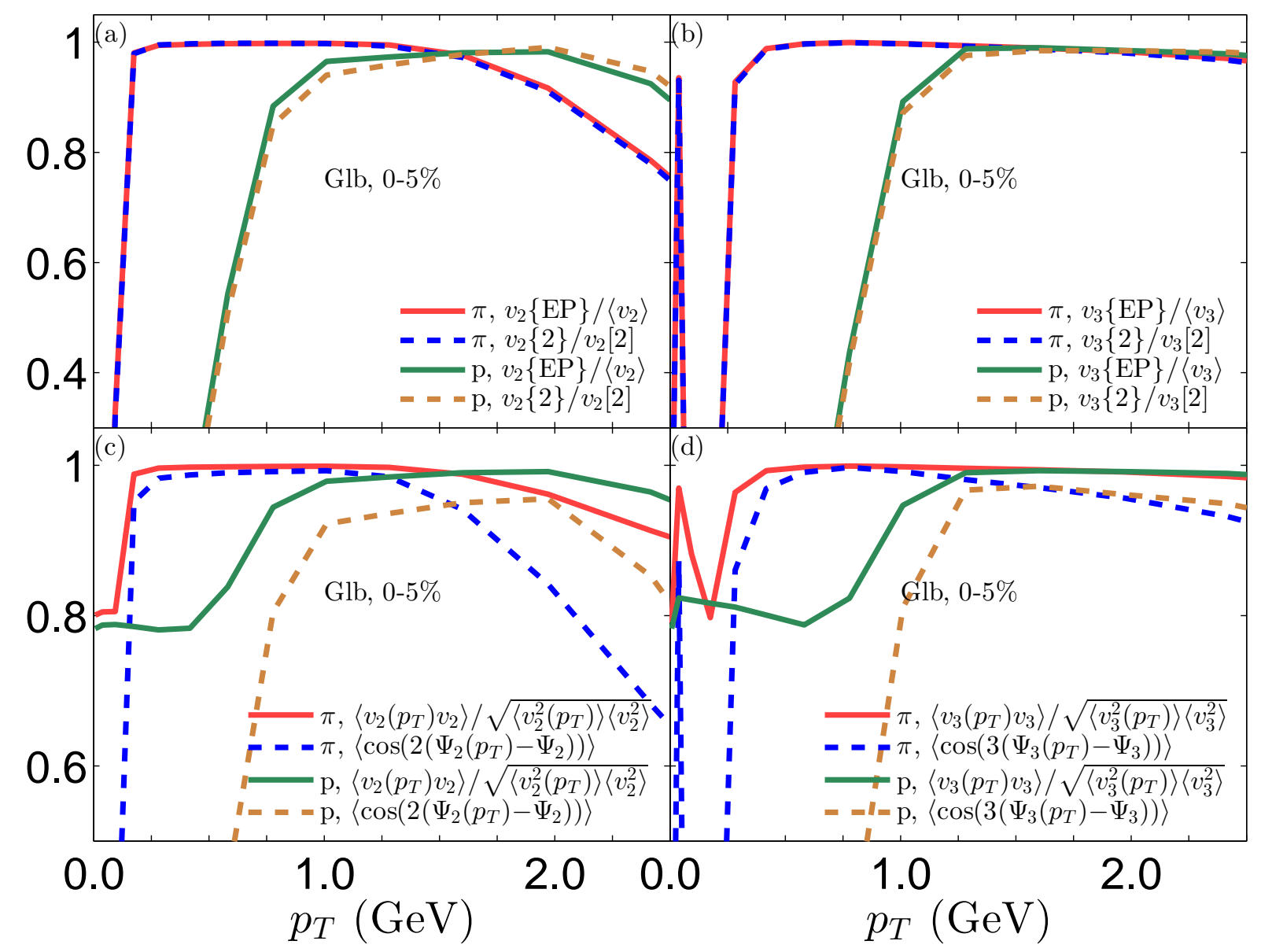

- The angular fluctuation factor $\left\langle\cos \left[n\left(\Psi_{n}\left(p_{T}\right)-\Psi_{n}\right)\right]\right\rangle$ completely dominates the $p_{T}$-dependence of these ratios!

- Angular fluctuations have similar effect as poor event-plane resolution: they reduce $v_{n}$.

- Angular fluctuations are effective both at low and high $p_{T}$, but not at intermediate $p_{T}$.

- The window for seeing flow angle fluctuation effects at low $p_{T}$ is smaller for pions than for protons. 


\section{Elliptic and triangular flow comparison (IV): $v_{n}$ ratios}

Peripheral collisions:

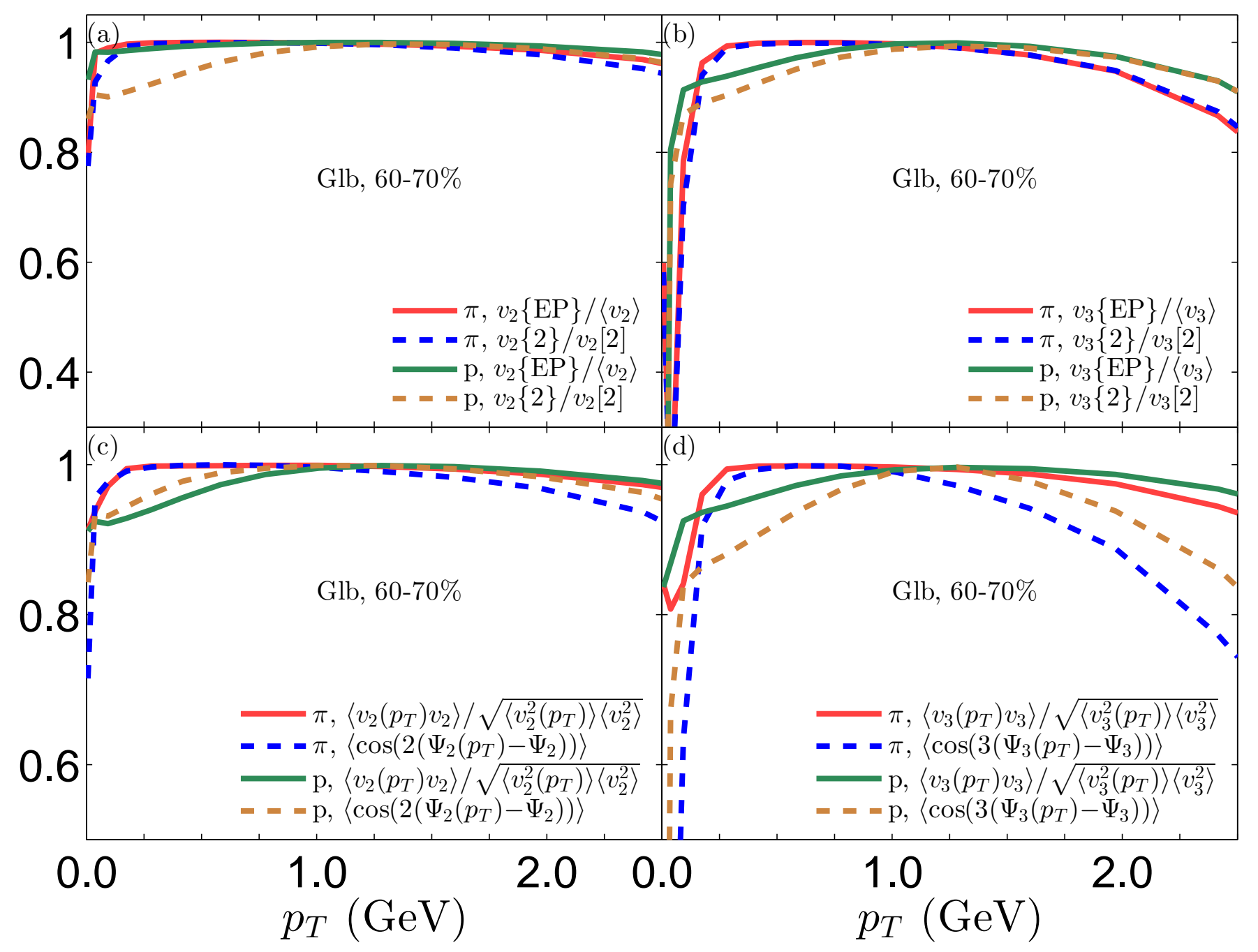

The window for seeing flow angle fluctuation effects at low $p_{T}$ closes in peripheral collisions. 


\section{Flow angle fluctuation effects for higher order $v_{n}\left(p_{T}\right)$}

Central collisions; solid: $\left\langle v_{n}\left(p_{T}\right)\right\rangle$; dashed: $v_{n}\{\operatorname{EP}\}\left(p_{T}\right)$ :

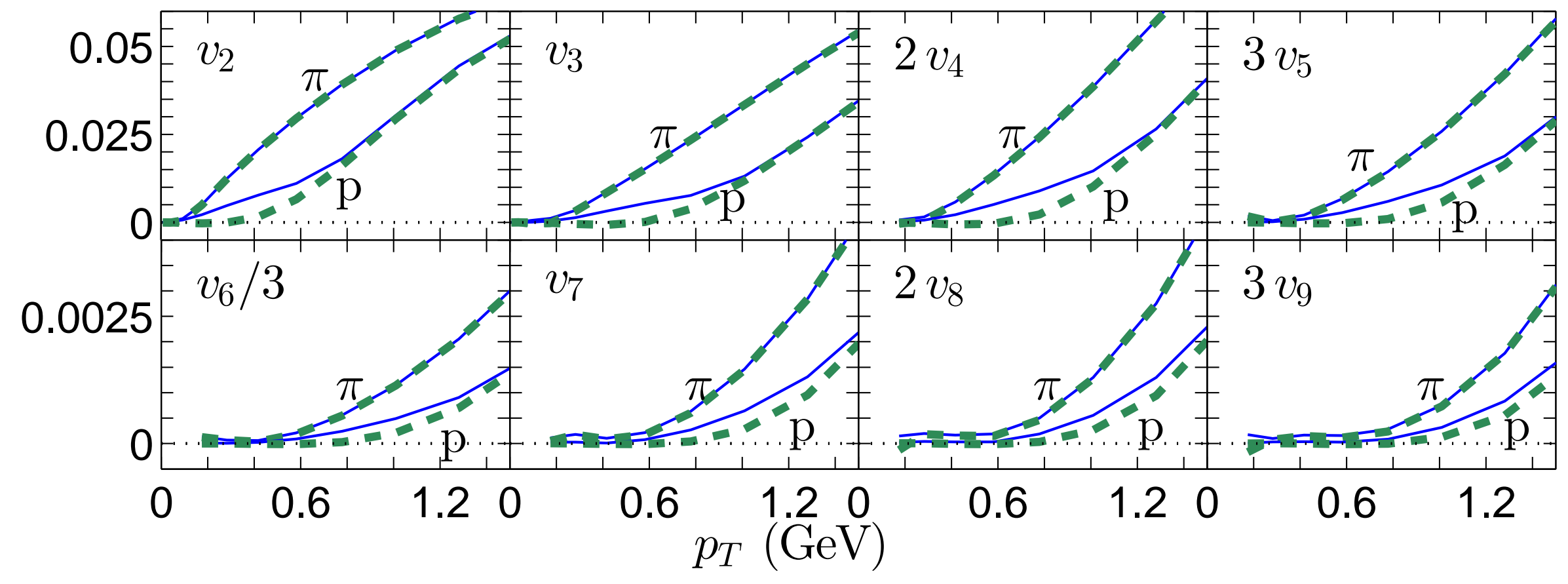

As harmonic order $n$ increases, suppression of $v_{n}\{\mathrm{EP}\}\left(p_{T}\right)$ (or $v_{n}\{2\}\left(p_{T}\right)$ ) from flow angle fluctuations for protons gets somewhat weaker but persists to larger $p_{T}$. 


\section{Test of factorization of two-particle spectra}

Factorization $V_{n \Delta}\left(p_{T 1}, p_{T 2}\right):=\left\langle\left\{\cos \left[n\left(\phi_{1}-\phi_{2}\right)\right]\right\}_{p_{T 1} p_{T 2}}\right\rangle \approx{ }^{\prime} v_{n}\left(p_{T 1}\right) \times v_{n}\left(p_{T 2}\right)$ " was checked experimentally as a test of hydrodynamic behavior, and found to hold to good approximation.

Gardim et al. (1211.0989) pointed out that event-by-event fluctuations break this factorization even if 2-particle correlations are exclusively due to flow.

They proposed to study the following ratio:

$r_{n}\left(p_{T 1}, p_{T 2}\right):=\frac{V_{n \Delta}\left(p_{T 1}, p_{T 2}\right)}{\sqrt{V_{n \Delta}\left(p_{T 1}, p_{T 1}\right) V_{n \Delta}\left(p_{T 2}, p_{T 2}\right)}}=\frac{\left\langle v_{n}\left(p_{T 1}\right) v_{n}\left(p_{T 2}\right) \cos \left[n\left(\Psi_{n}\left(p_{T 1}\right)-\Psi_{n}\left(p_{T 2}\right)\right)\right]\right\rangle}{v_{n}[2]\left(p_{T 1}\right) v_{n}[2]\left(p_{T 2}\right)}$.

Even in the absence of flow angle fluctuations, this ratio is $<1$ due to $v_{n}$ fluctuations (Schwarz inequality), except for $p_{T 1}=p_{T 2}$.

But it additionally depends on flow angle fluctuations.

To assess what share of the deviation from 1 is due to flow angle fluctuations, we can compare with

$$
\tilde{r}_{n}\left(p_{T 1}, p_{T 2}\right):=\frac{\left\langle v_{n}\left(p_{T 1}\right) v_{n}\left(p_{T 2}\right) \cos \left[n\left(\Psi_{n}\left(p_{T 1}\right)-\Psi_{n}\left(p_{T 2}\right)\right)\right]\right\rangle}{\left\langle v_{n}\left(p_{T 1}\right) v_{n}\left(p_{T 2}\right)\right\rangle}
$$

which deviates from 1 only due to flow angle fluctuations. Again, this ratio approaches 1 for $p_{T 1}=p_{T 2}$. Gardim et al. studied $r_{n}$ for ideal hydro; we have studied $r_{n}$ and $\tilde{r}_{n}$ for viscous hydro. 


\section{Breaking of factorization by e-by-e fluctuations (I)}

Monte Carlo Glauber initial conditions, $\eta / s=0.08=1 /(4 \pi)$ :

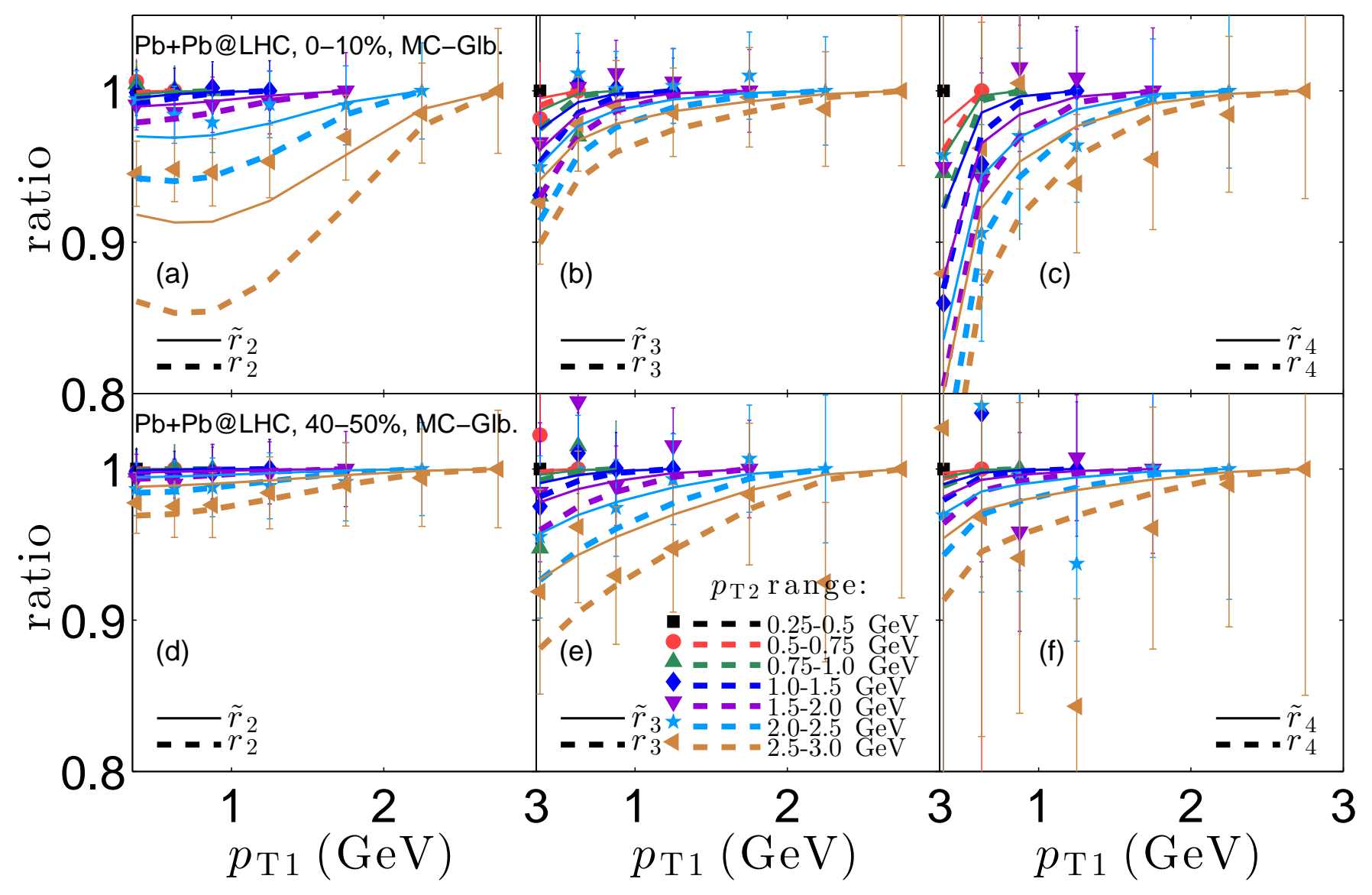

More than half of the factorization breaking effects are due to flow angle fluctuations.

In central collisions, $\eta / s=0.08$ appears to overpredict the breaking of factorization (consistent with Gardim et al. who saw still larger effects for ideal hydro).

Factorization breaking effects appear to be larger for fluctuation-dominated flow harmonics. 


\section{Breaking of factorization by e-by-e fluctuations (II)}

Monte Carlo KLN initial conditions, $\eta / s=0.2=2.5 /(4 \pi)$ :

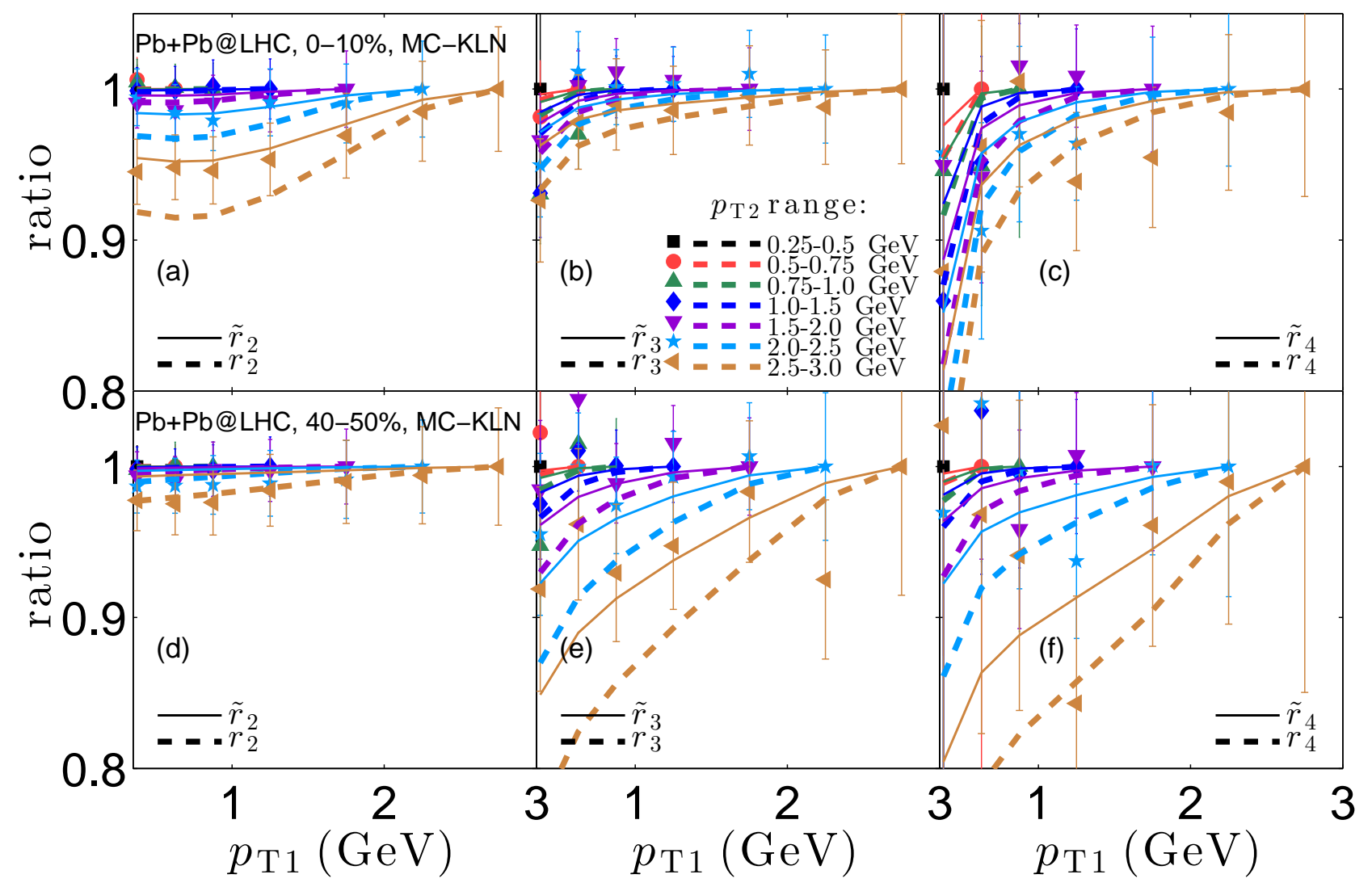

In central collisions, factorization-breaking effects decrease with increasing $\eta / s$.

In peripheral collisions, larger $\eta / s$ appears to cause a larger breaking of factorization, mostly due to flow angle fluctuations.

Data may indicate slight preference for larger $\eta / s$ value, but more experimental precision and more detailed theoretical studies are needed to settle this. Analysis of ATLAS data in progress. 


\section{Conclusions}

- Both the magnitudes $v_{n}$ and the flow angles $\Psi_{n}$ depend on $p_{T}$ and fluctuate from event to event.

- In each event, the " $p_{T}$-averaged" (total-event) flow angles $\Psi_{n}$ are identical for all particle species, but their $p_{T}$ distribution differs from species to species.

- The mean $v_{n}$ values and their $p_{T}$-dependence at RHIC and LHC have already been shown to put useful constraints on the QGP shear viscosity and its temperature dependence (see next talk by B. Schenke)

- The effects of $v_{n}$ and $\Psi_{n}$ fluctuations can be separated experimentally by studying different $V_{n}$ measures based on two-particle correlations.

- Flow angle correlations are a powerful test of the hydrodynamic paradigm and will help to further constrain the spectrum of initial-state fluctuations and QGP transport coefficients.

- Studying event-by-event fluctuations of the anisotropic flows $v_{n}$ and their flow angles $\Psi_{n}$ as functions of $p_{T}$, as well as the correlations between different harmonic flows (both their magnitudes and angles), provides a rich data base for identifying the "Standard Model of the Little Bang", by pinning down its initial fluctuation spectrum and its transport coefficients. 


\section{Nurcleaur efffects un pA}

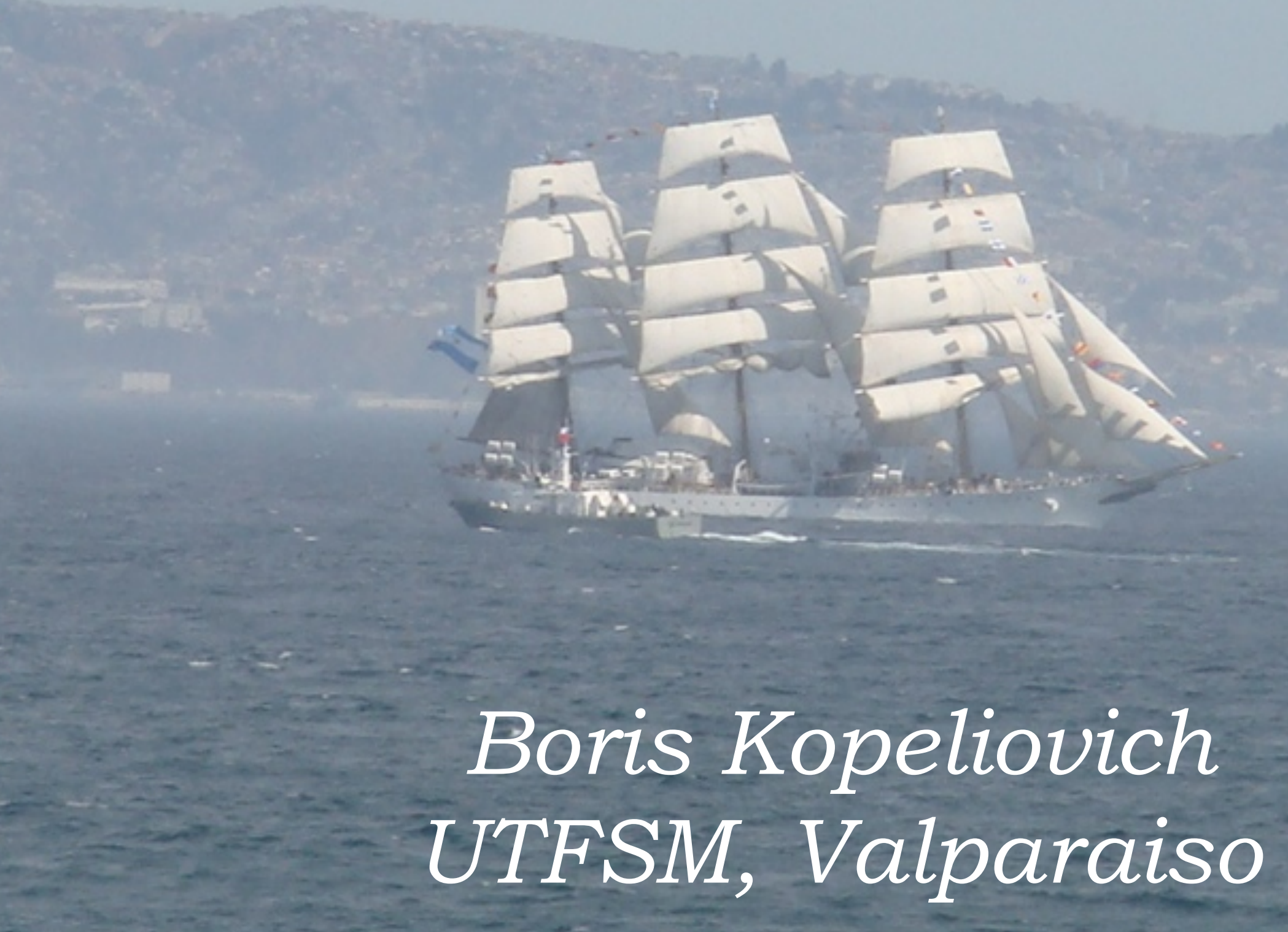




\title{
In collaboration with
}

\author{
Jan Nemchilk \\ Irina Potashnikova \\ Ivan Schmidt
}
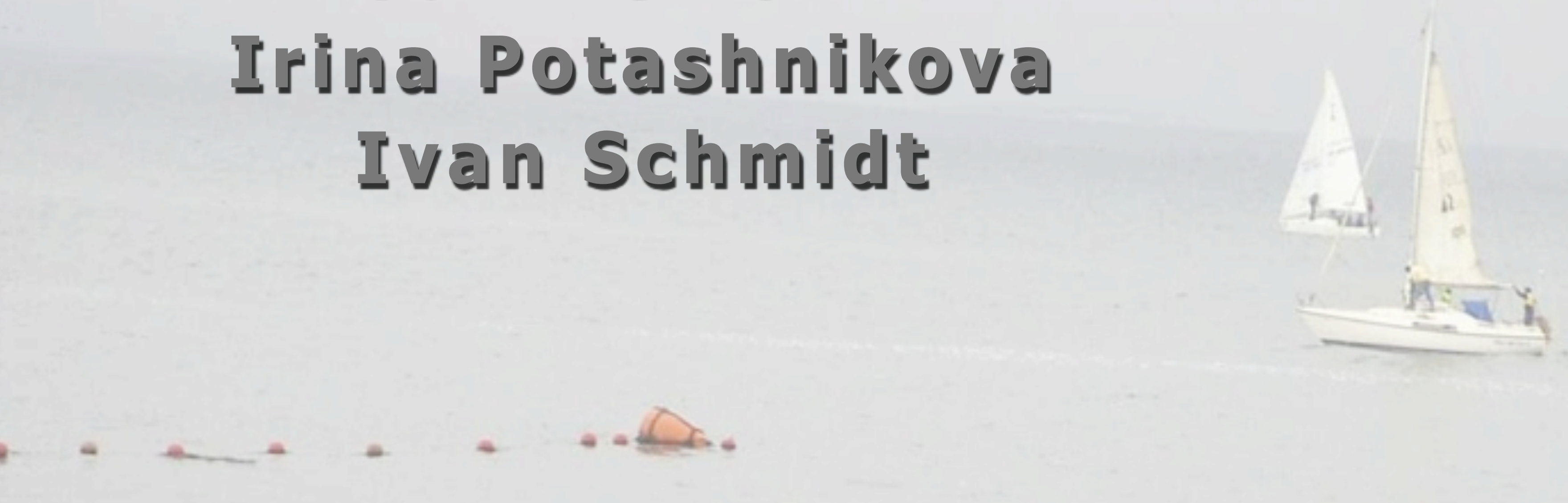


\section{Cronin effect}

High-pT hadrons can be produced coherently from multiple interactions in nuclei at very high energies (LHC), but not at low energies of fixed target experiments. Correspondingly, the mechanisms for the Cronin enhancement are different.

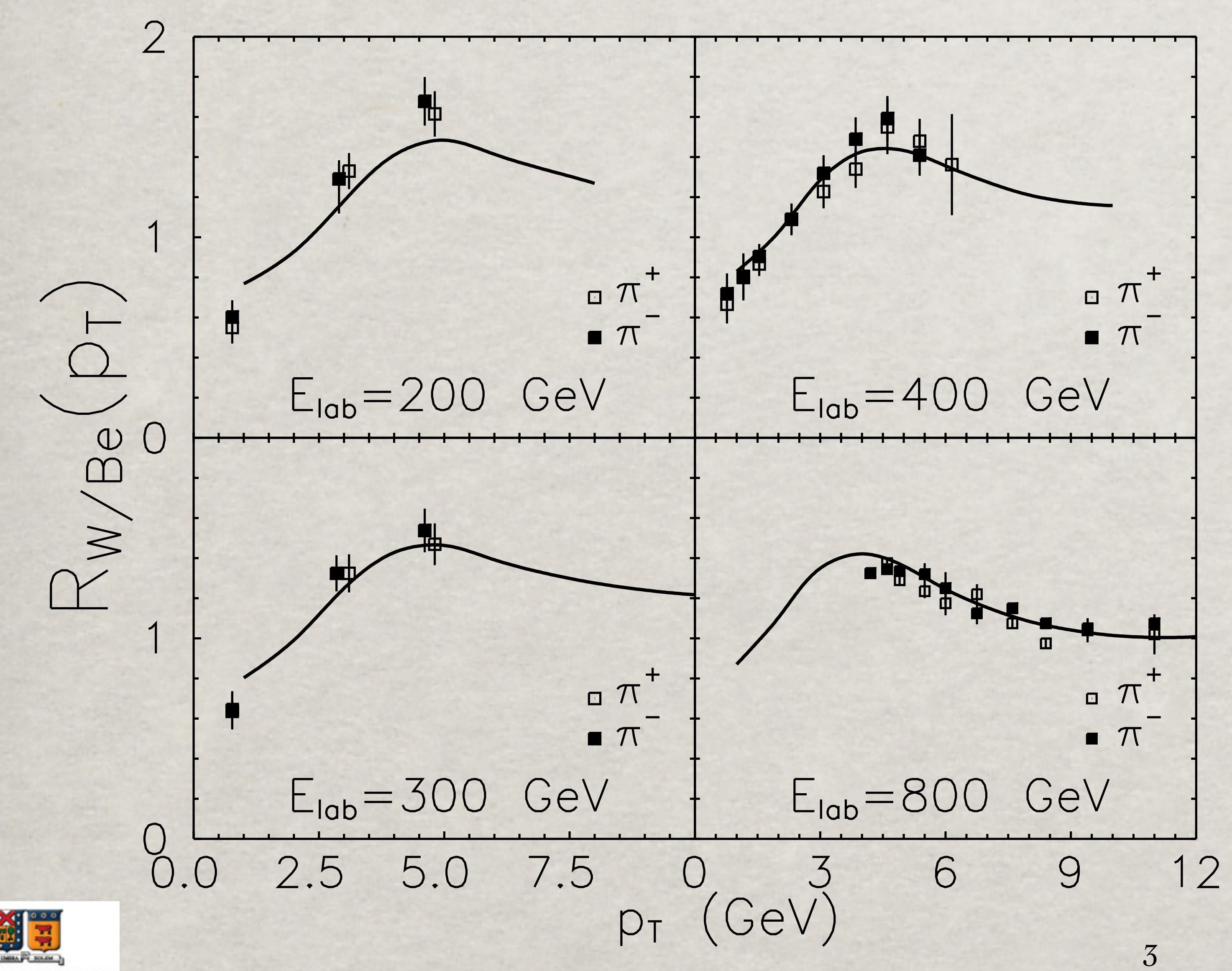

B.K., J.Nemchik, A.Schafer, A.Tarasov, PRL 88(2002)232303
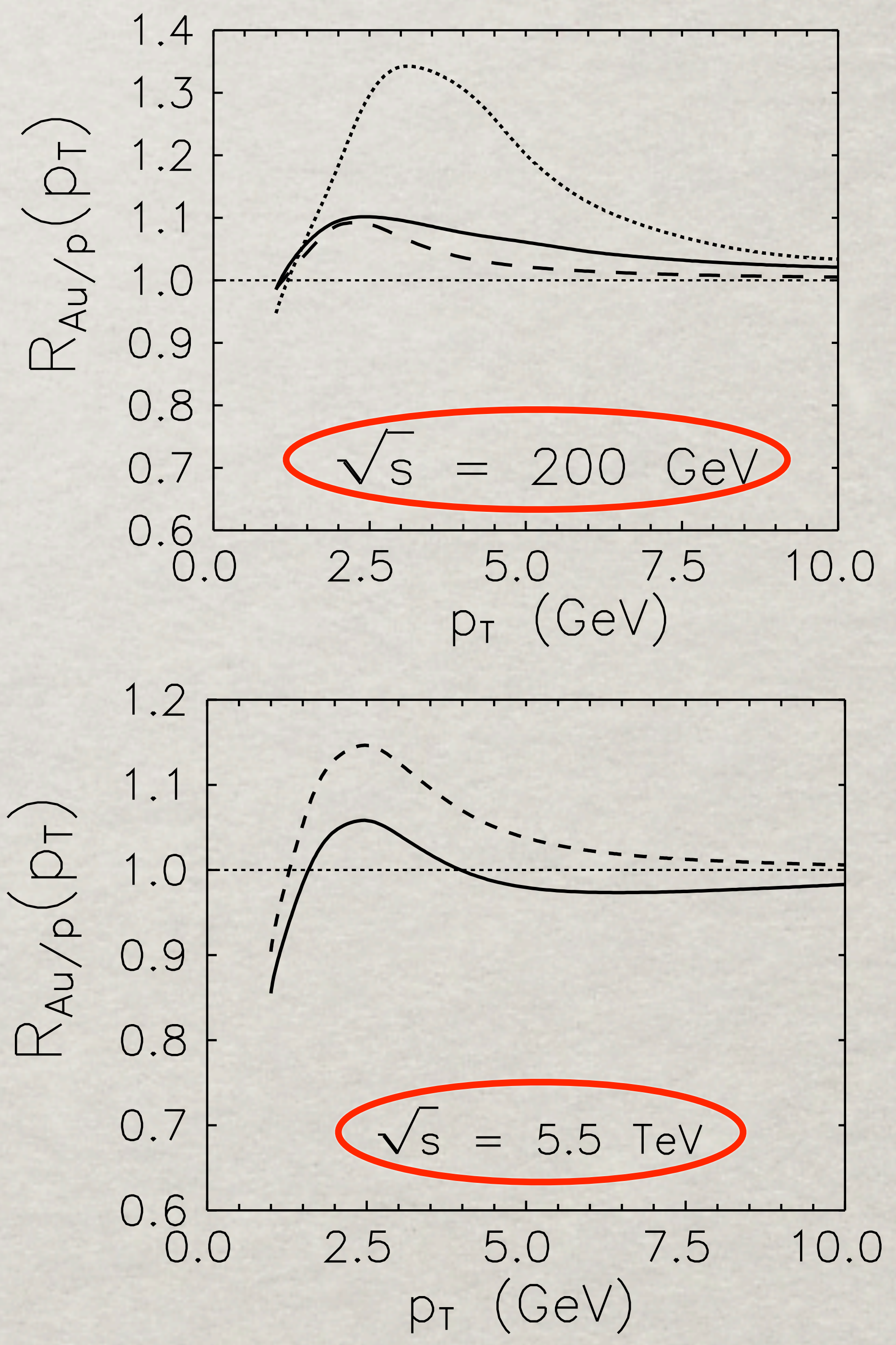

B. Kopeliovich, Jet Quenching, BNL, April 15, 2013 


\section{Cronin effect at RHIC: predicted and observed}

The predicted magnitude was OK, but the shape was not. The employed unintegrated gluon density of K.J.Golec-Biernat \& M.Wustoff, 1999 (GBW) peakes at too small pT.

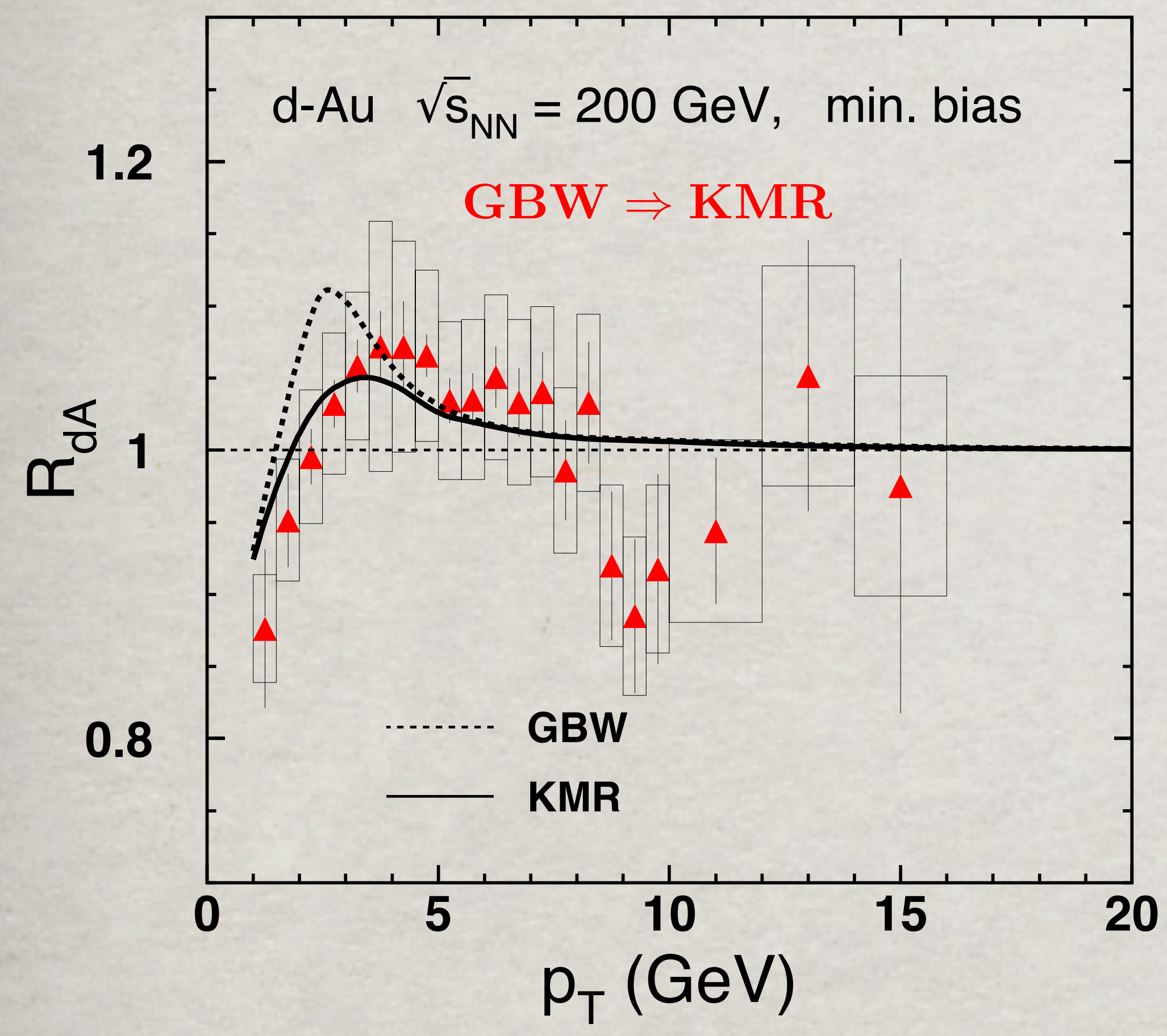

More realistic parametrization for the unintegrated gluon distribution proposed later. M.Kimber, A.Martin \& M.Ryskin, 2001 (KMR)

A.Martin, M.Ryskin \& G.Watt, 2010

improves the shape (with no other modifications in the computing code).

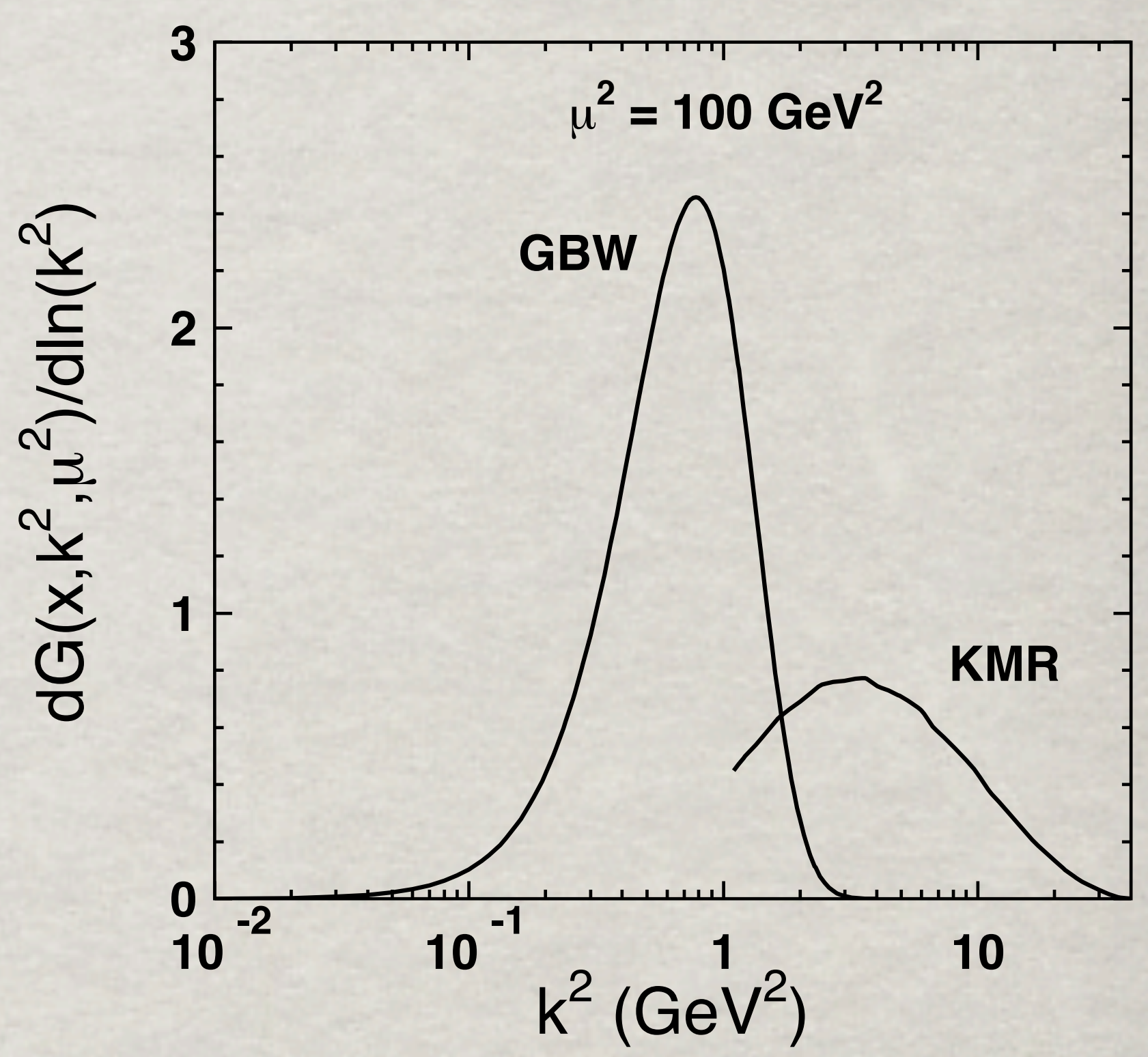

D.Kharzeev, E.Levin,L.McLerran, PL B561(2003)93:

Color Glass Condensate models exaggerated the magnitude of the coherence effects predicting a sizable suppression $R_{\mathrm{dA}}=\mathbf{0 . 7 5}$ 
Cronin effect at LHC: predicted and observed

R.Vogt et al, arXiv: 1301.3395

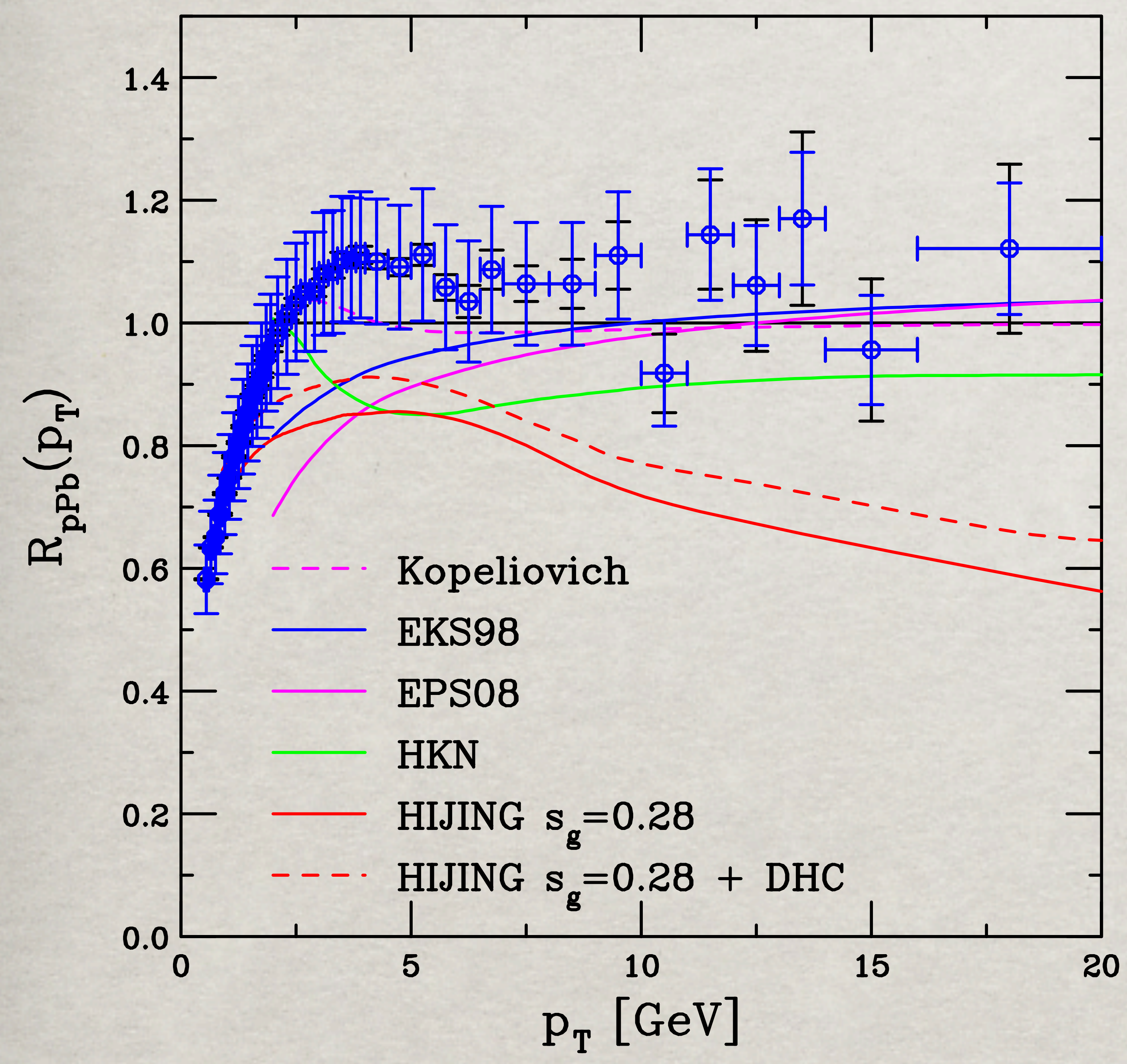

The first successful prediction

B.K., J.Nemchik, A.Schafer, A.Tarasov (2002)

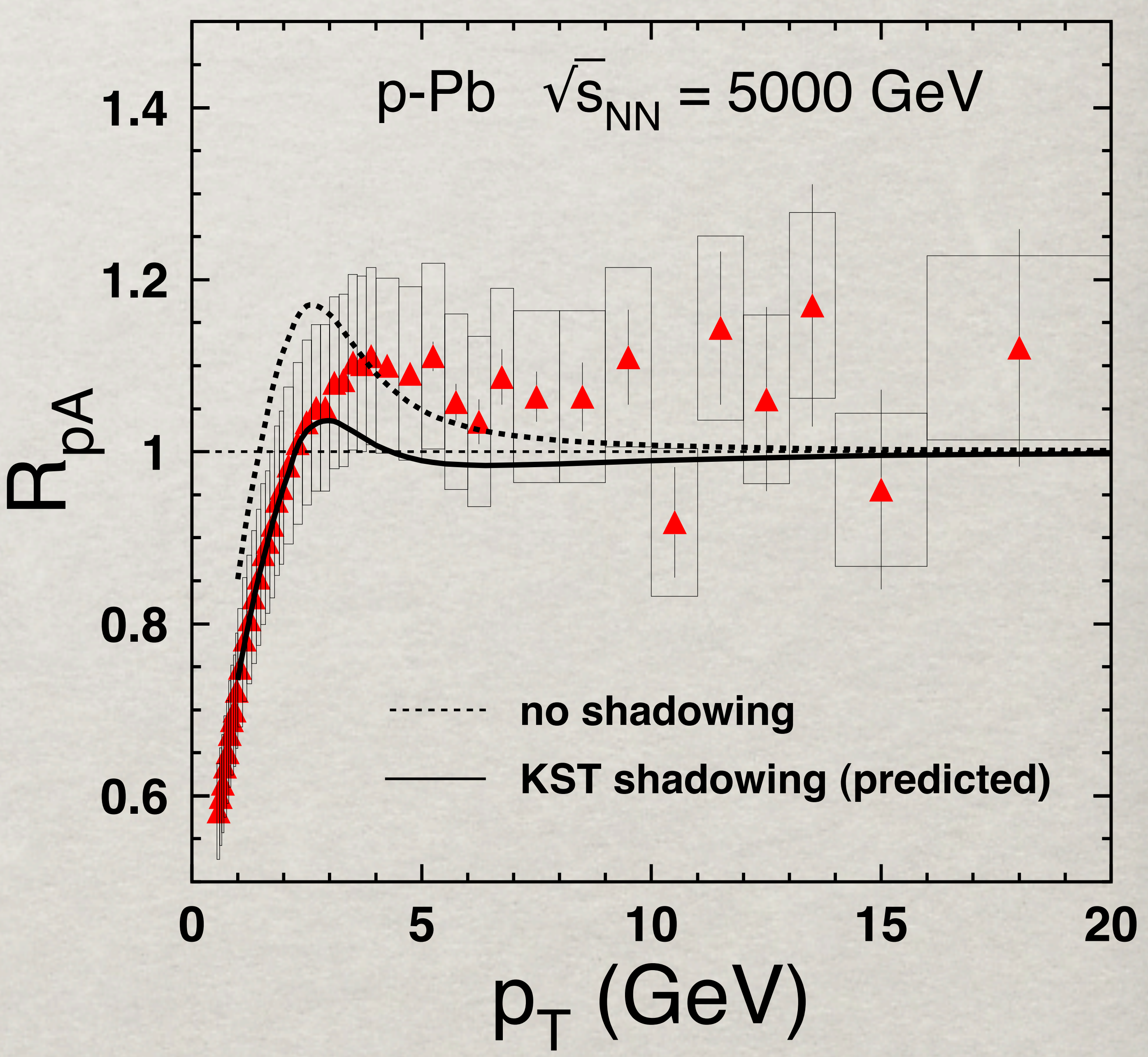

B. Kopeliovich, Jet Quenching, BNL, April 15, 2013 

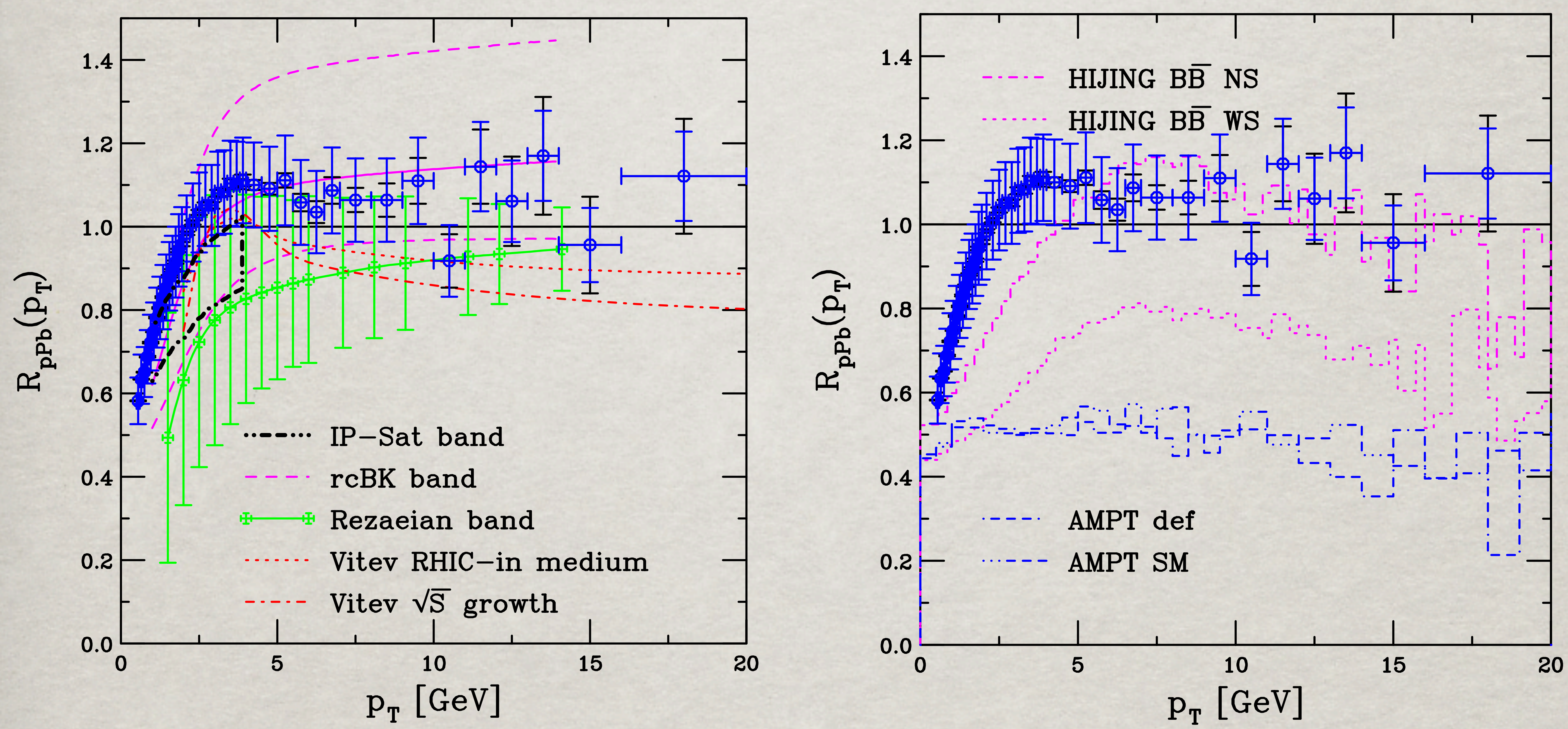

Not successful either 


\section{Details}

\section{parton model}

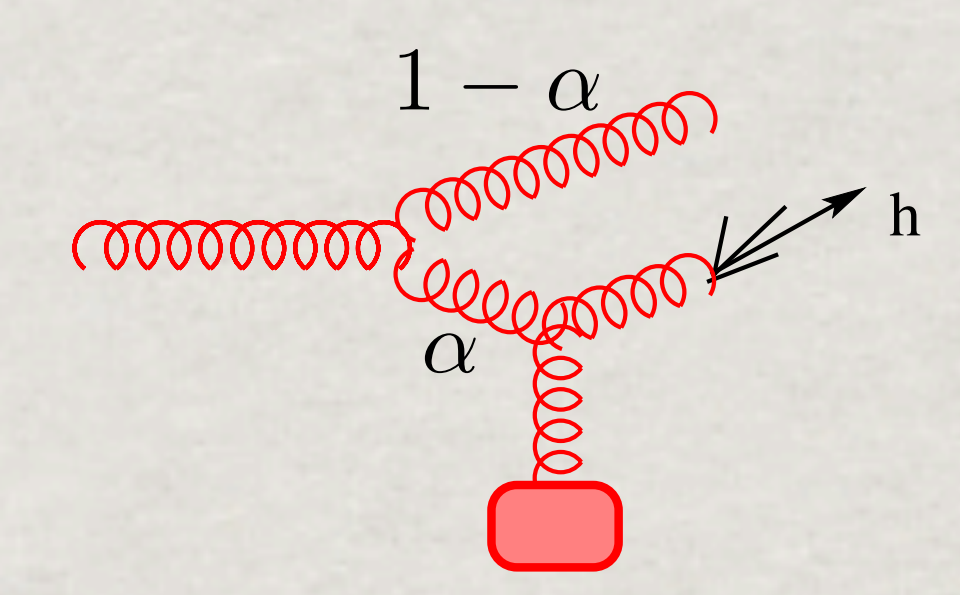

\section{dipole description}

$$
\frac{\mathbf{d} \sigma_{\mathrm{g} \rightarrow \mathbf{2 g}}^{\mathbf{N}(\mathbf{A})}\left(\alpha, \mathbf{x}_{\mathbf{2}}\right)}{\mathbf{d}^{\mathbf{2}} \mathbf{k}_{\mathbf{T}} \mathbf{d y}}=\int \mathbf{d}^{2} \mathbf{r d}^{2} \mathbf{r}^{\prime} \mathbf{e}^{\mathrm{i} \mathbf{k}_{\mathbf{T}}\left(\mathbf{r}-\mathbf{r}^{\prime}\right)}\left\langle\boldsymbol{\Psi}_{\mathrm{gg}}^{\dagger}(\mathbf{r}, \alpha) \Psi_{\mathrm{gg}}\left(\mathbf{r}^{\prime}, \alpha\right)\right\rangle \boldsymbol{\Sigma}_{\mathbf{3 g}}^{\mathbf{N}(\mathbf{A})}\left(\mathbf{r}, \mathbf{r}^{\prime}, \alpha, \mathbf{x}_{\mathbf{2}}\right)
$$

$$
\begin{aligned}
& \Sigma_{3 g}^{N(A)}\left(\vec{r}, \vec{r}^{\prime}, \alpha, x_{2}\right)=\left\{\begin{array}{l}
\sigma_{3 g}^{N}(r, \alpha)+\sigma_{3 g}^{N}\left(r^{\prime}, \alpha\right)-\sigma_{3 g}^{N}\left(\vec{r}-\vec{r}^{\prime}, \alpha\right) ; \quad(\mathrm{pp}) \\
2 \int d^{2} b\left[1-e^{-\frac{1}{2} \sigma_{3 g}^{N}(r, \alpha) T_{A}(b)} e^{-\frac{1}{2} \sigma_{3 g}^{N}\left(r^{\prime}, \alpha\right) T_{A}(b)}+e^{-\frac{1}{2} \sigma_{3 g}^{N}\left(\vec{r}-\vec{r}^{\prime}, \alpha\right) T_{A}(b)}\right]
\end{array}\right. \\
& \sigma_{3 g}^{N}(r, \alpha)=\frac{9}{8}\left\{\sigma_{\bar{q} q}(r)+\sigma_{\bar{q} q}(\alpha r)+\sigma_{\bar{q} q}[(1-\alpha) r]\right\}
\end{aligned}
$$

$\Psi_{g g}(\vec{r}, \alpha)=\frac{\sqrt{8 \alpha_{s}}}{\pi r^{2}} \exp \left[-\frac{r^{2}}{2 r_{0}^{2}}\right]\left[\alpha\left(\vec{e}_{1}^{*} \cdot \vec{e}\right)\left(\vec{e}_{2}^{*} \cdot \vec{r}\right)(1-\alpha)\left(\vec{e}_{2}^{*} \cdot \vec{e}\right)\left(\vec{e}_{1}^{*} \cdot \vec{r}\right)-\alpha(1-\alpha)\left(\vec{e}_{1}^{*} \cdot \vec{e}_{2}^{*}\right)(\vec{e} \cdot \vec{r})\right]$ $\mathrm{r}_{0} \approx 0.3 \mathrm{fm}$

$$
\left\langle\Psi_{g g}^{\dagger}(\vec{r}) \Psi_{g g}\left(\vec{r}^{\prime}\right)\right\rangle=\frac{4 \alpha_{s}}{\pi^{2}} \frac{\vec{r} \cdot \vec{r}^{\prime}}{r^{2}{r^{\prime}}^{2}} \exp \left[-\frac{r^{2}+{r^{\prime}}^{2}}{2 r_{0}^{2}}\right]\left[1+\alpha^{4}+(1-\alpha)^{4}\right]
$$




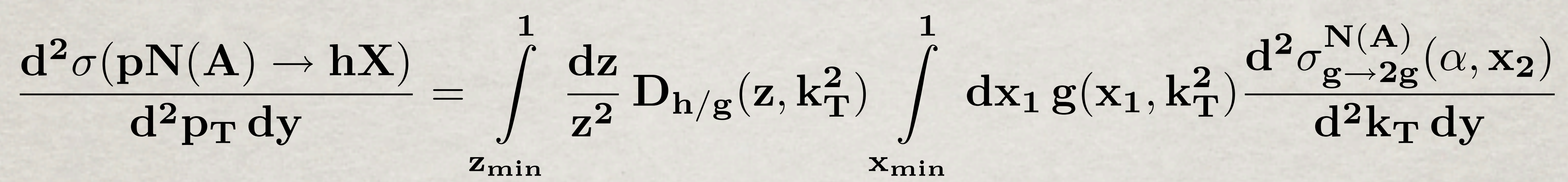

$$
\begin{aligned}
& \mathbf{k}_{\mathbf{T}}=\mathbf{p}_{\mathbf{T}} / \mathbf{z} \\
& \mathbf{z}_{\min }=\frac{\mathbf{p}_{\mathbf{T}}}{\sqrt{\mathrm{s}}} \mathbf{e}^{\mathrm{y}} ; \quad \mathbf{x}_{\min }=\mathbf{z}_{\min } / \mathbf{z} \\
& \alpha=\frac{\mathbf{p}_{\mathbf{T}} \mathbf{e}^{\mathbf{y}}}{\mathbf{x}_{1} \mathbf{z} \sqrt{\mathbf{s}}} \\
& \mathbf{x}_{\mathbf{2}}=\frac{\mathbf{p}_{\mathbf{T}}^{\mathbf{2}}}{\mathbf{x}_{1} \mathbf{z}^{2} \alpha(\mathbf{1}-\alpha) \mathbf{s}}
\end{aligned}
$$

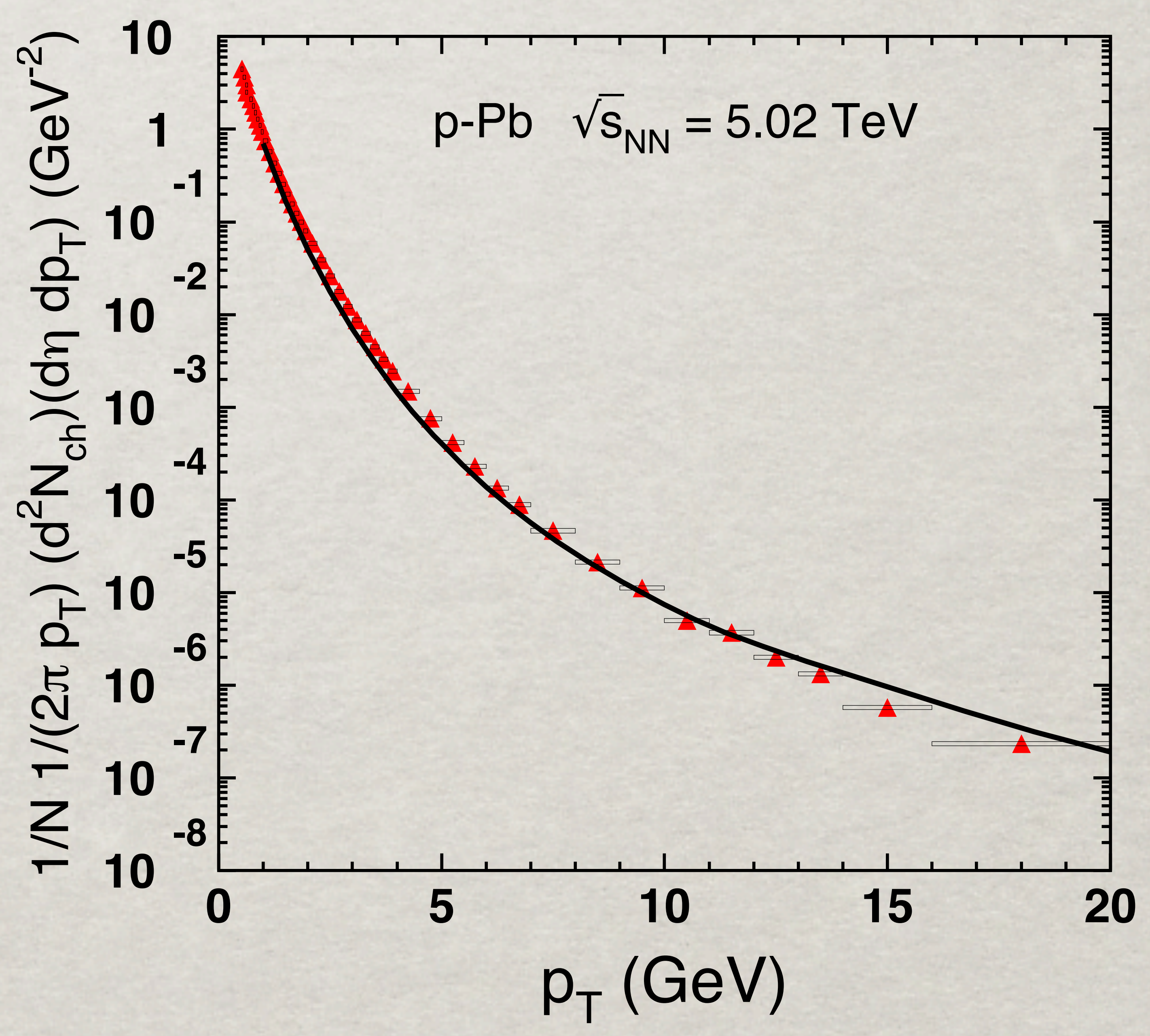


Gluon shadowing is a part of the Gribov inelastic corrections related to the triple-Pomeron term in diffraction.
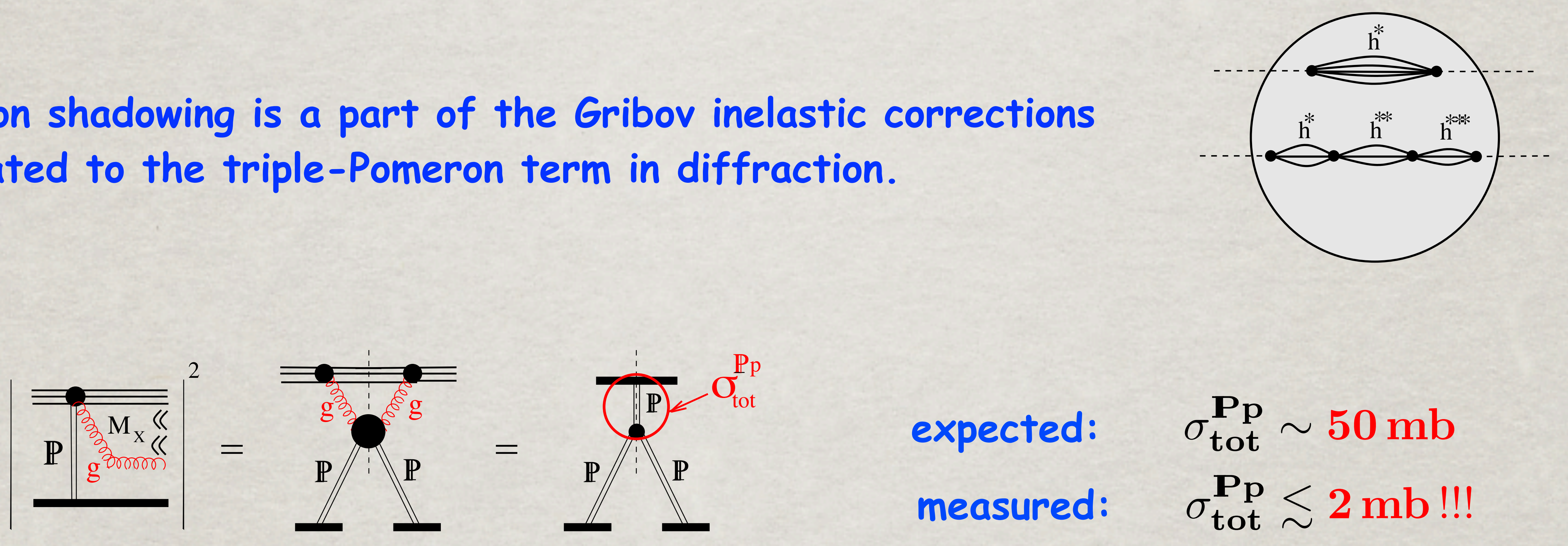

Smallness of the diffractive cross section means weakness of gluon shadowing.

In terms of $P Q C D$ this shows a suppression of diffractive gluon radiation, which can only be related to smallness of gluonic dipoles. 


\section{Gluon shadowing from DIS}

Gluon shadowing in DIS correspond to inclusion of the higher Fock components of the photon, $\gamma^{*} \rightarrow \overline{\mathbf{q}} \mathbf{q}+\mathrm{g}$, B.K., A.Schaefer, A.Tarasov, 1999 .

Gluon PDFs in DIS are probed via the DGLAP evolution from the $Q^{2}$ dependence of $F_{2}\left(x, Q^{2}\right)$ So far only the NMC experiment managed to detect a variation of the nuclear PDF with $Q^{2}$

B.K., A.Schaefer, A.Tarasov, 1999 (KST)

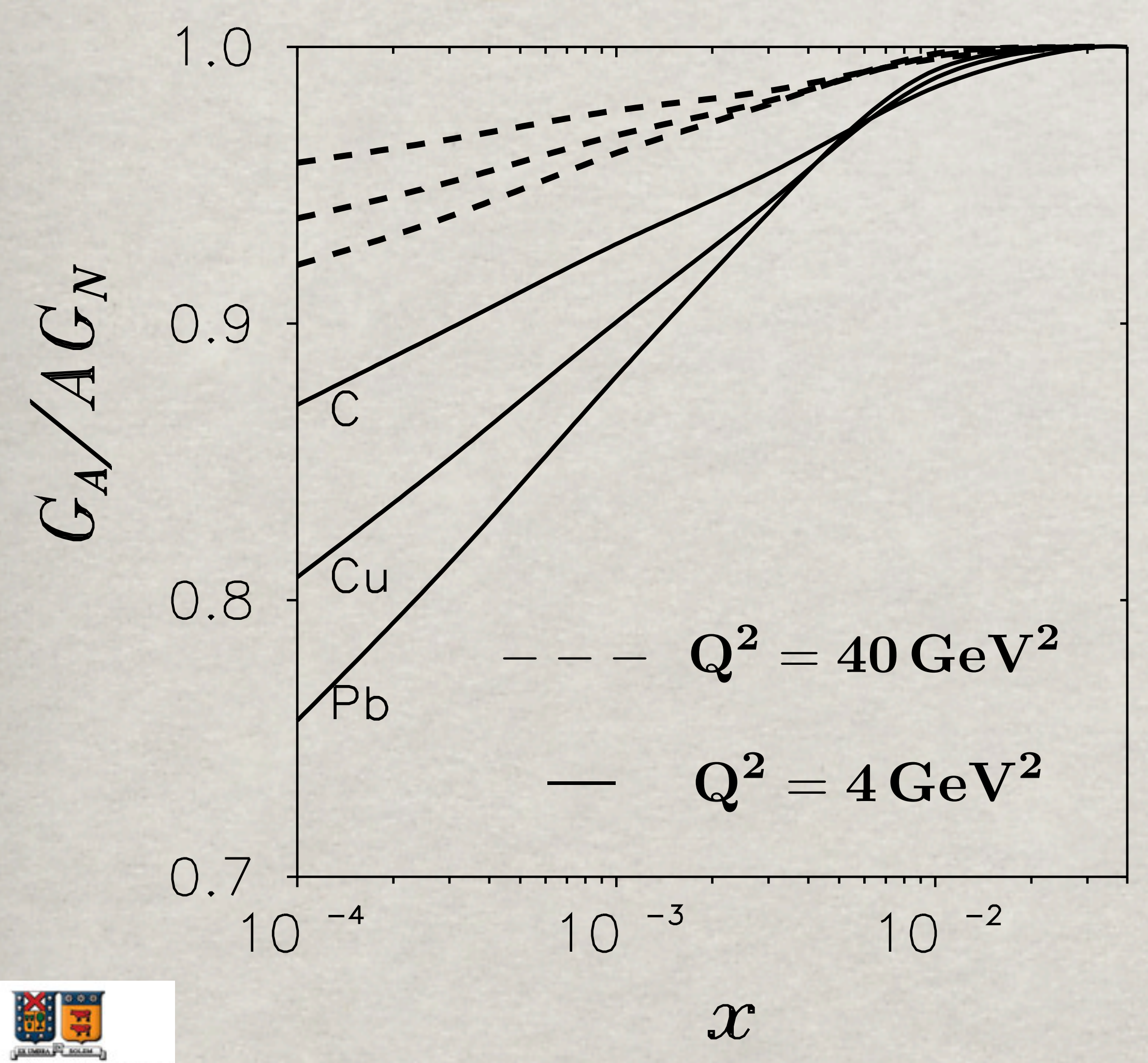

D.de Florian, R.Sassot, 2004 (DS)

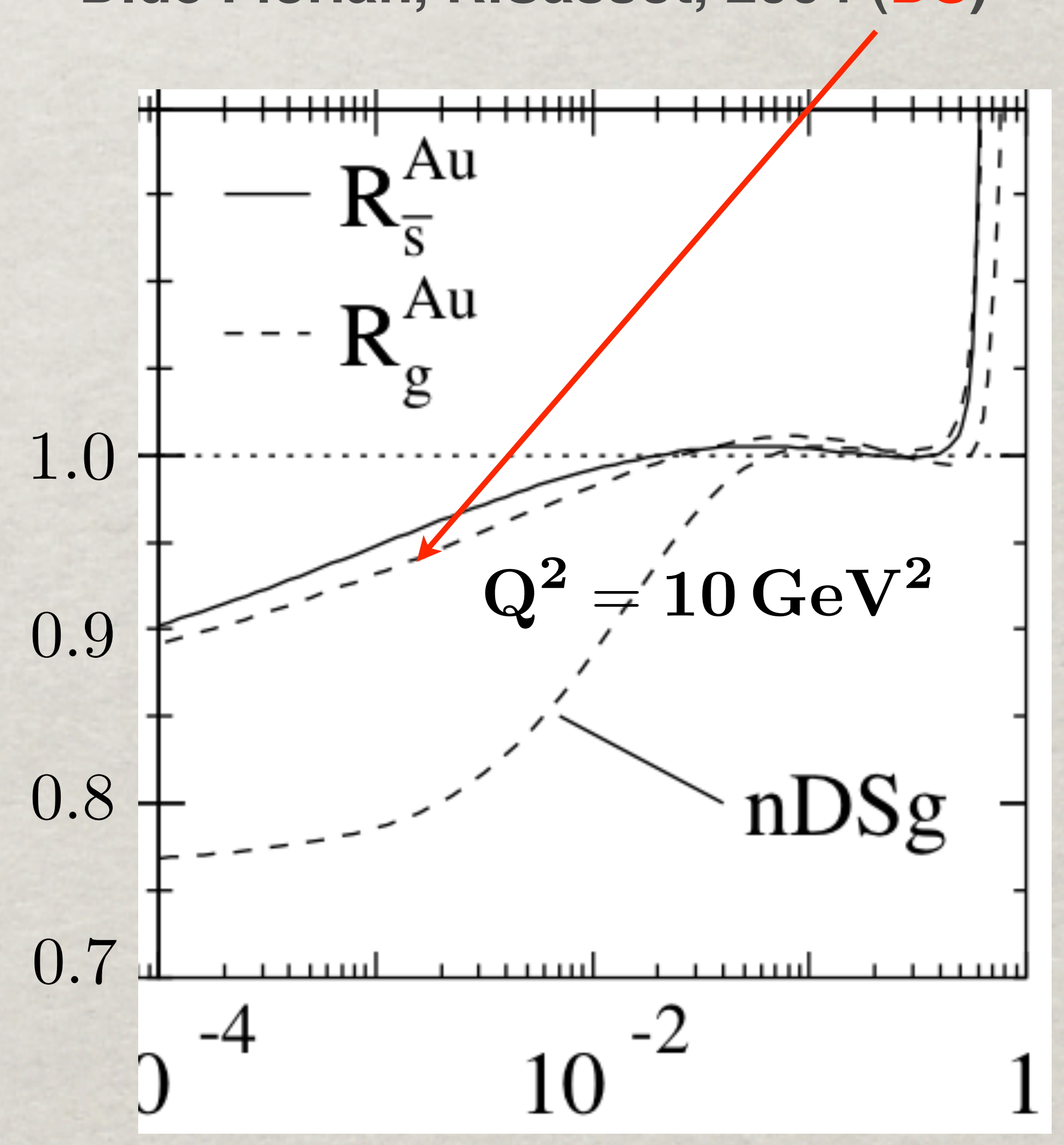

B. Kopeliovich, Jet Quenching, BNL, April 15, 2013 


\section{Two-scale hadronic structure}

B.K., A.Schafer, A.Tarasov(1999): the valence quarks carry small size gluon clouds, $r_{0} \approx 0.3 \mathrm{fm}$
Shuryak \& Zakhed (2004): gluonic spots of small size, $r_{0} \approx 0.3 \mathrm{fm}$ are floating in the proton.

Small gluonic spots $==>$ weak gluon shadowing:

$\left.\frac{\mathbf{G}_{\mathbf{A}}(\mathbf{x})}{\mathbf{A G}_{\mathbf{N}}(\mathbf{x})}\right|_{\mathbf{x} \ll \mathbf{1}}=\frac{\mathbf{2}}{\left\langle\sigma_{\mathbf{G G}}(\mathbf{r})\right\rangle} \int \mathbf{d}^{2} \mathbf{b}\left[\mathbf{1}-\left\langle\mathbf{e}^{-\frac{1}{2} \sigma_{\mathrm{GG}}(\mathbf{r}) \mathbf{T}_{\mathbf{A}}(\mathbf{b})}\right\rangle\right]=1-\frac{3 \mathbf{C}}{\mathbf{8}} \mathbf{r}_{\mathbf{0}}^{2} \rho_{\mathbf{A}} \mathbf{R}_{\mathrm{A}}+\ldots \approx 0.8$

Even if small-x gluons overlap in the longitudinal direction, they can miss each other in transverse plane, if they are located within small spots. Indeed, for a heavy nucleus (lead) the mean number of gluonic spots overlapping with a given one is,

$$
\langle\mathbf{n}\rangle=\frac{3 \pi}{4} \mathbf{r}_{\mathbf{0}}^{2}\left\langle\mathbf{T}_{\mathbf{A}}\right\rangle=\pi \mathbf{r}_{\mathbf{0}}^{2} \rho_{\mathbf{A}} \mathbf{R}_{\mathbf{A}}=0.3
$$




\section{Evidences for two-scales}

As far as gluon radiation is suppressed, hadronic cross sections should rise slowly with energy. Indeed, the observed energy dependence of the total pp cross section is well described [B.K., I.Potashnikova, E.Predazzi, B.Povh, PRL 85(2000)507]

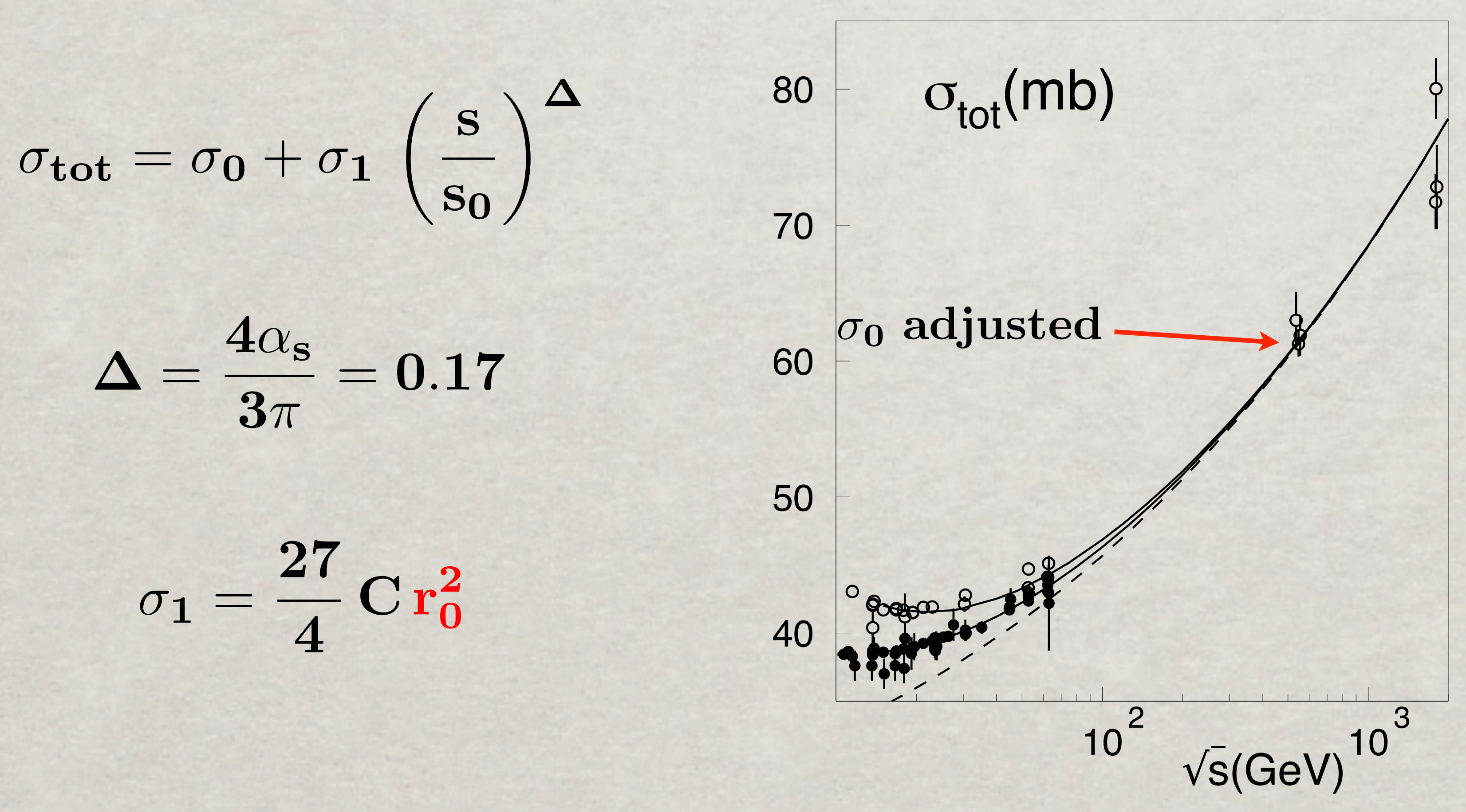

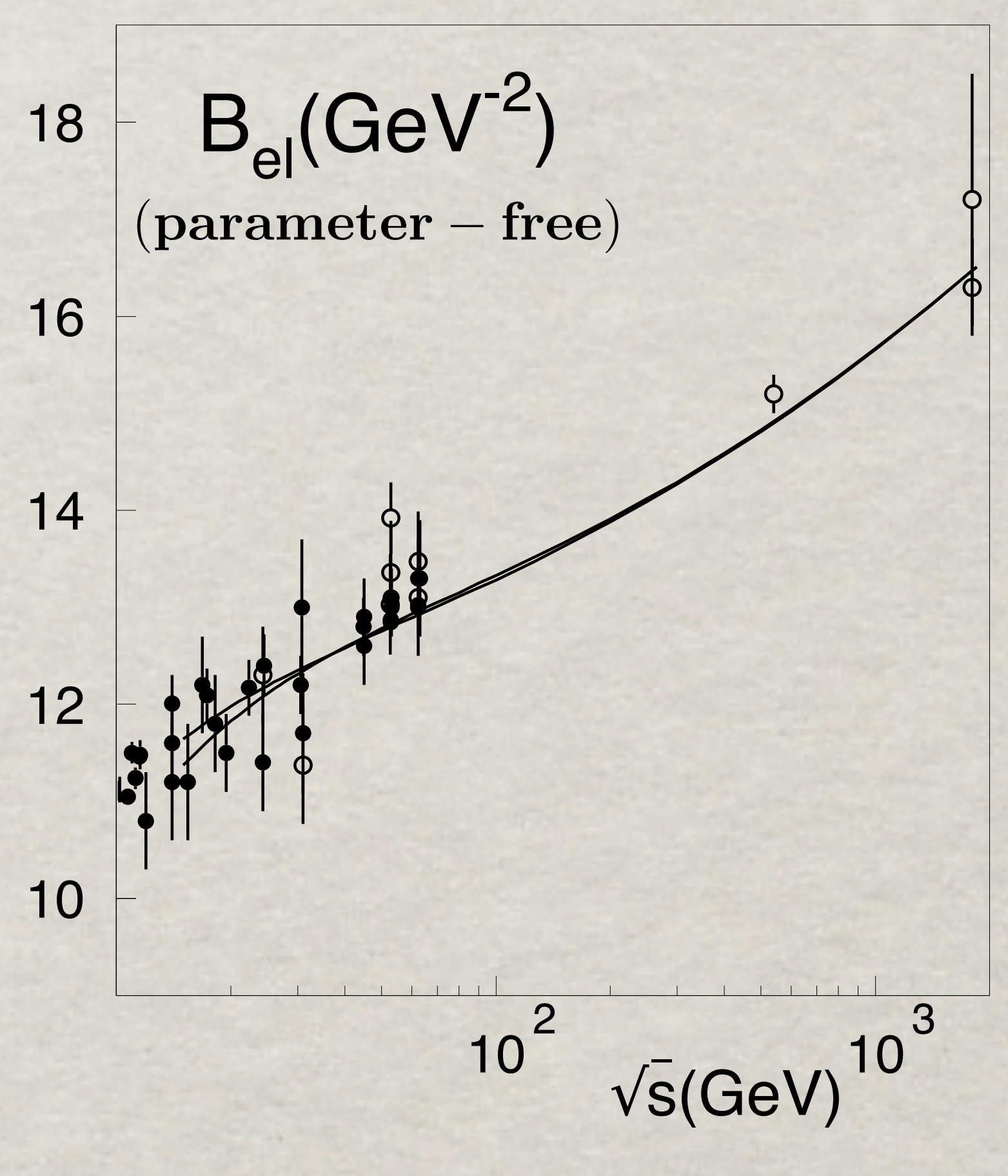

B. Kopeliovich, Jet Quenching, BNL, April 15, 2013 


\section{Evidences for two-scales}

\section{B.K., I.Potashnikova, B.Povh, E.Predazzi (2000)}
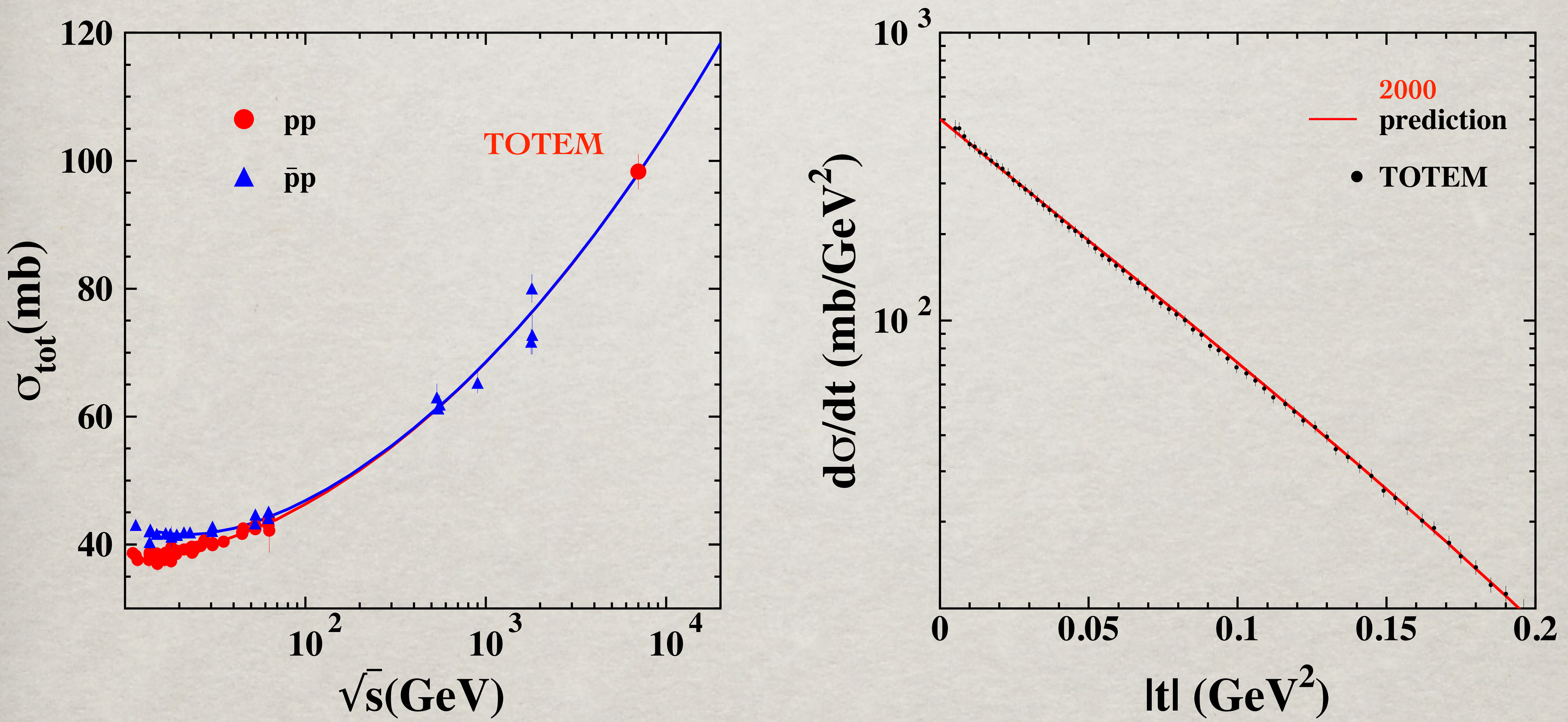

B.K., I.Potashnikova, B.Povh (2012) 


\section{Improved predictions}

With the same 2002 computer code, but using a contemporary versions of the unintegrated gluon distribution (KMR) one can improve the shape of pT-dependence.
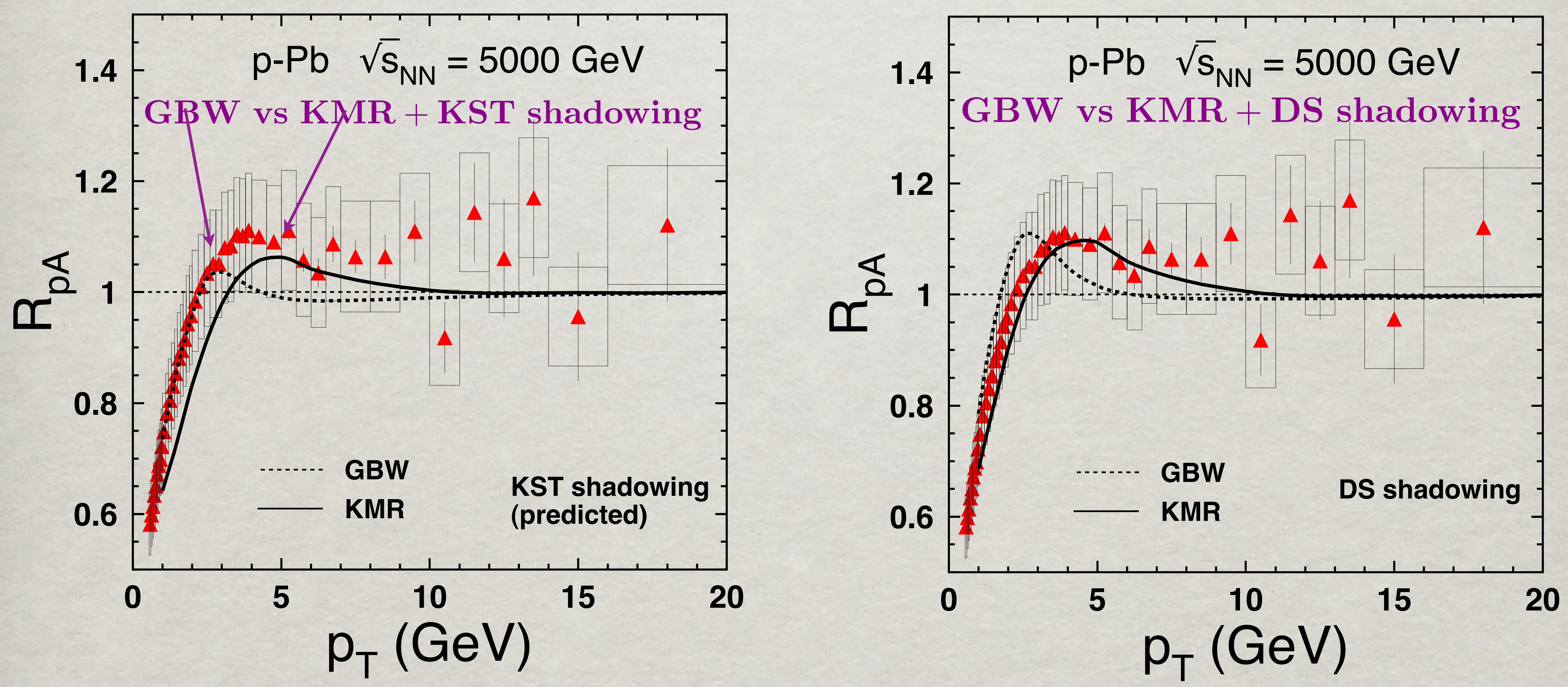


\section{POSTDICTIONS: Further improvements}

A better choice of the scale for gluon shadowung.

$\mathrm{Q}^{2}=\frac{4}{\mathrm{xg}\left(\mathrm{x}, \mathrm{k}_{\mathrm{T}}^{2}\right)} \int \mathrm{dq}^{2} \mathcal{F}_{\mathrm{g}}\left(\mathrm{x}, \mathrm{q}, \mathrm{k}_{\mathrm{T}}^{2}\right)$

Alternative parametrizations for the dipole cross section:

J.Bartels, K.J.Golec-Biernat \& H.Kowalsky, 2002 (BGBK)

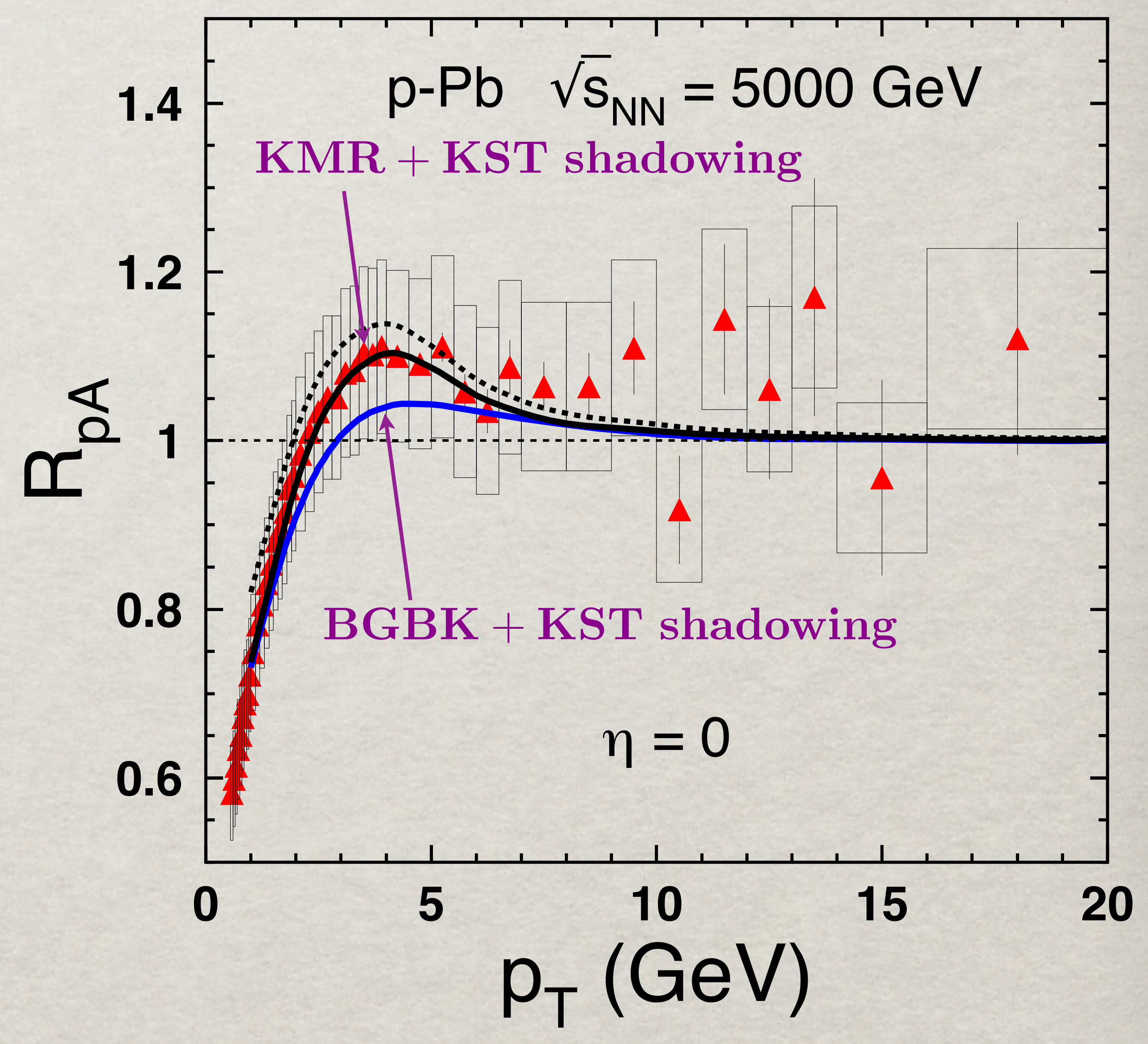


Smallest $x 2$ are reached at forward rapidities. This is why it was tempting to interpret the suppression observed at forward rapidities by BRAHMS and STAR, as a result of coherence, CGC [D.Kharzeev, Yu.Kovchegov, K.Tuchin (2003)]

Initial-state energy loss suppresses particle production toward the kinematic limit $x 1->1$ [B.K., J.Nemchik, I.Potashnikova, I.Schmidt (2005)]

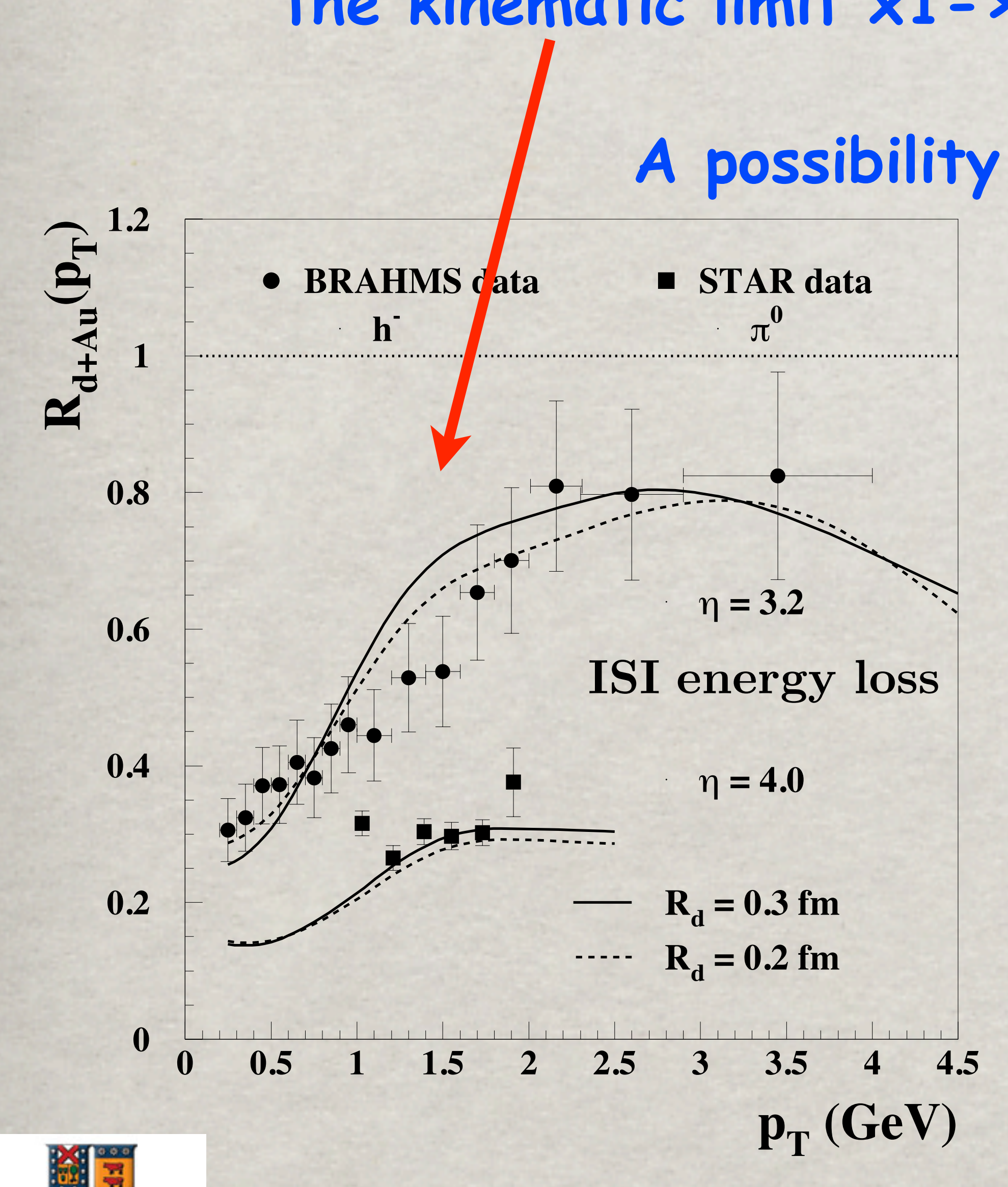

One also approaches the kinematic limit at the mid rapidity, but high $\mathrm{pT}$. go to higher energies and check with the nuclear effects at the same $\times 2$. but further away from the kinematic limit (see LHC data below).

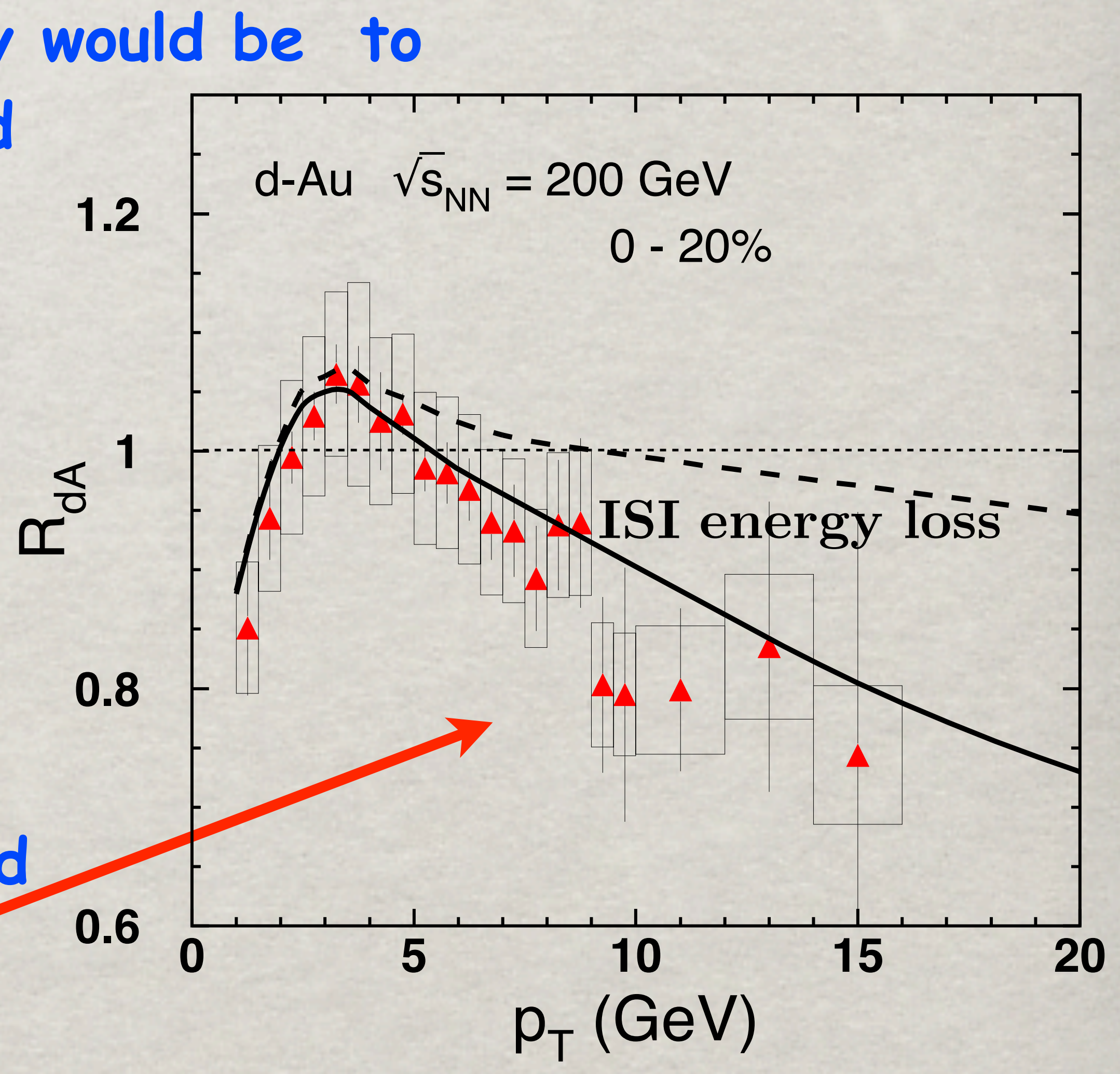

B. Kopeliovich, Jet Quenching, BNL, April 15, 2013 
Suppression of high-pT hadrons by ISI E-loss in AA collisions at RHIC B.K., J.Nemchik, I.Potashnikova, I.Schmidt, PRC86(20012)054904
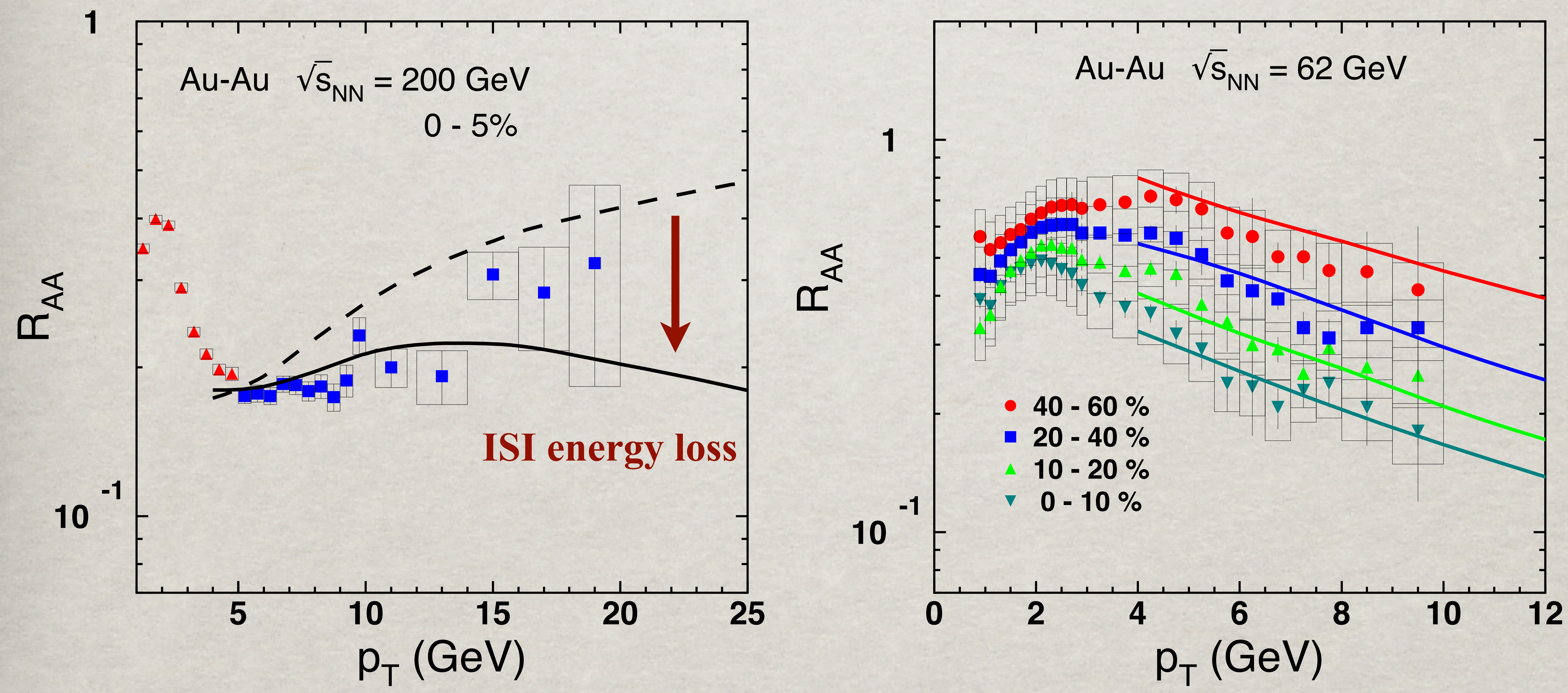
One can enhance the role of ISI energy loss either moving to forward rapidities, or higher PT, or both

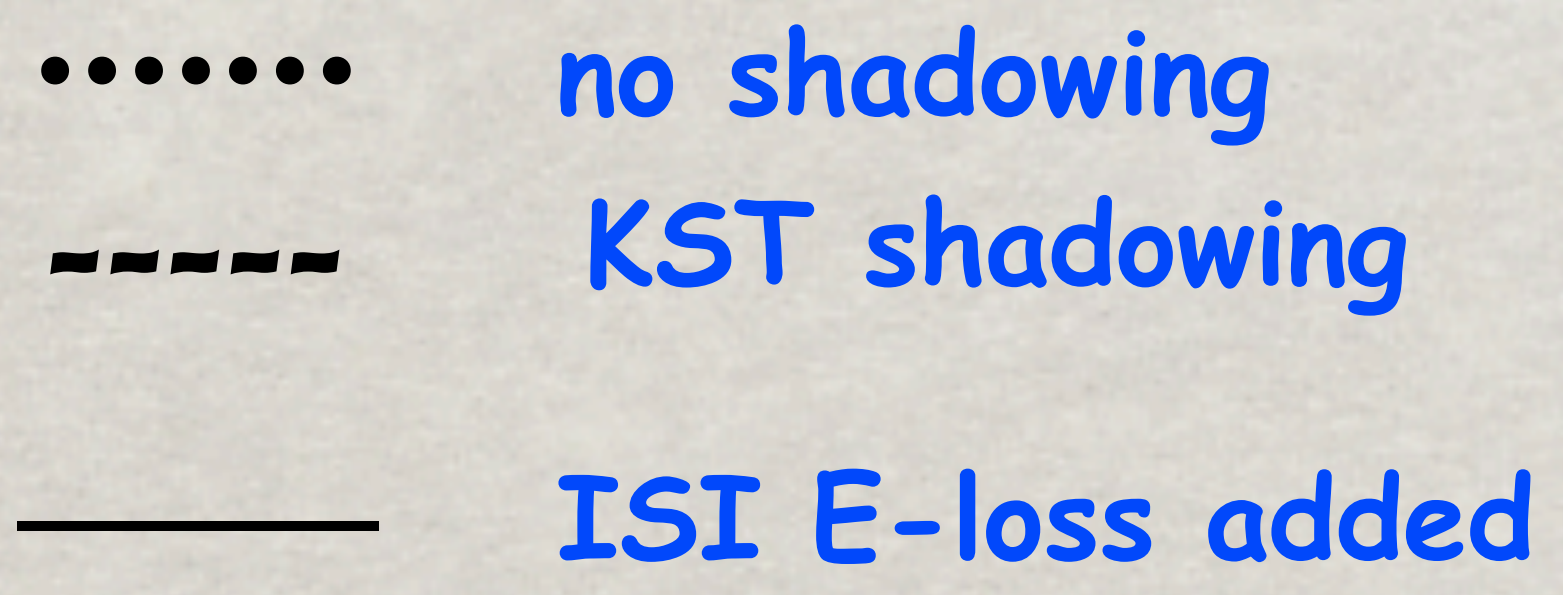

KMR unintegrated gluon density
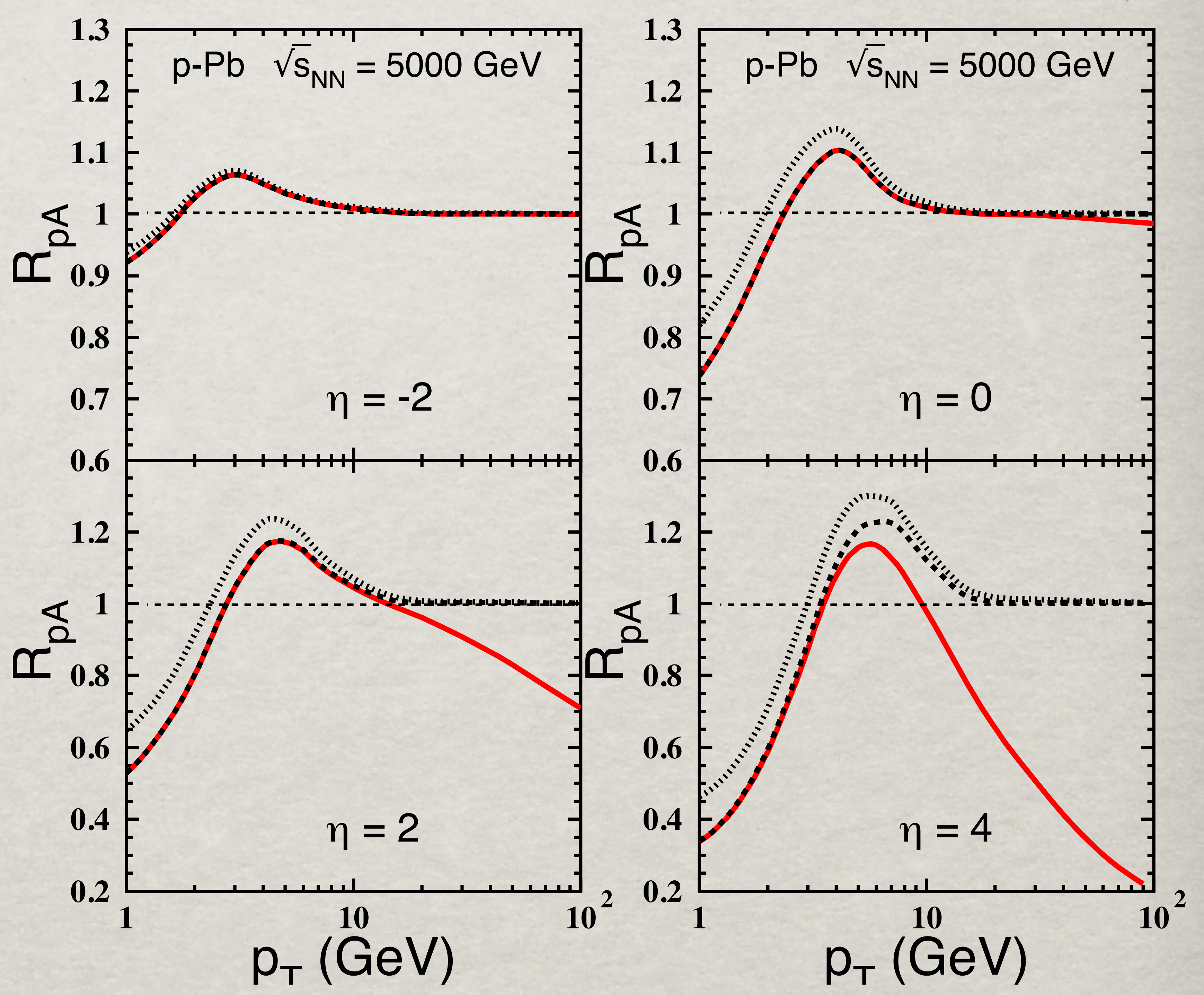

B. Kopeliovich, Jet Quenching, BNL, April 15, 2013 


\section{Summarizing,}

For the first time high-pT hadrons can be produced in $\mathrm{pA}$ coherently. Data for the Cronin effect at LHC provide a strong support for the two-scale hadronic structure and weak gluon shadowing. Many popular models are "ruled out".

The magnitude of the Cronin enhancement predicted in 2002 was correct, while the shape of the $\mathrm{PT}$ dependence can be improved applying more realistic up-to-date phenomenology.

Initial state energy loss is expected to suppress significantly inclusive hadron production at large $\mathrm{PT}$ and/or at forward rapidities in PA, as well as in AA collisions. 


\section{BACKUPS}

In order to study the sensitivity of different observables on the amount of shadowing in the nuclear gluon distribution, we have performed an alternative extraction of nPDF from the same data set but constraining the gluon density in heavy nuclei to show a stronger shadowing effect at small $x_{N}$. We provide the result in set called $\mathrm{nDSg}$, constrained to satisfy $R_{g}^{A u}=0.75$ at $x_{N}=0.001$ and $Q^{2}=5$. The $\chi^{2}$ value of this analysis is around 550 , considerably larger than the unconstrained fit, and should be considered only as a mean to study variations on, mainly, the gluon nuclear distribution. An example 


\title{
Long-Range Rapidity Correlations in Heavy-Light Ion Collisions
}

\author{
Yuri V. Kovchegov \\ The Ohio State University
}

based on arXiv:1212.1195 [hep-ph]

with Douglas Wertepny 


\section{Outline}

- Long-range rapidity correlations in hadronic and nuclear collisions: general discussion

- Two-gluon production in heavy-light ion collisions:

- Geometric correlations

- Long-range rapidity correlations

- Perturbative HBT correlations

- Conclusions 


\section{Introduction to saturation physics}

\section{Quantum Chromodynamics}

at High Energy

veki \&, kOVCargov

AND EUOKNE LRVIN

Published in September 2012 by Cambridge U Press 


\section{Long-range rapidity correlations}




\section{Ridge in heavy ion collisions}

- Heavy ion collisions, along with high-multiplicity $p+p$ and $p+A$ collisions, are known to have long-range rapidity correlations, known as 'the ridge':

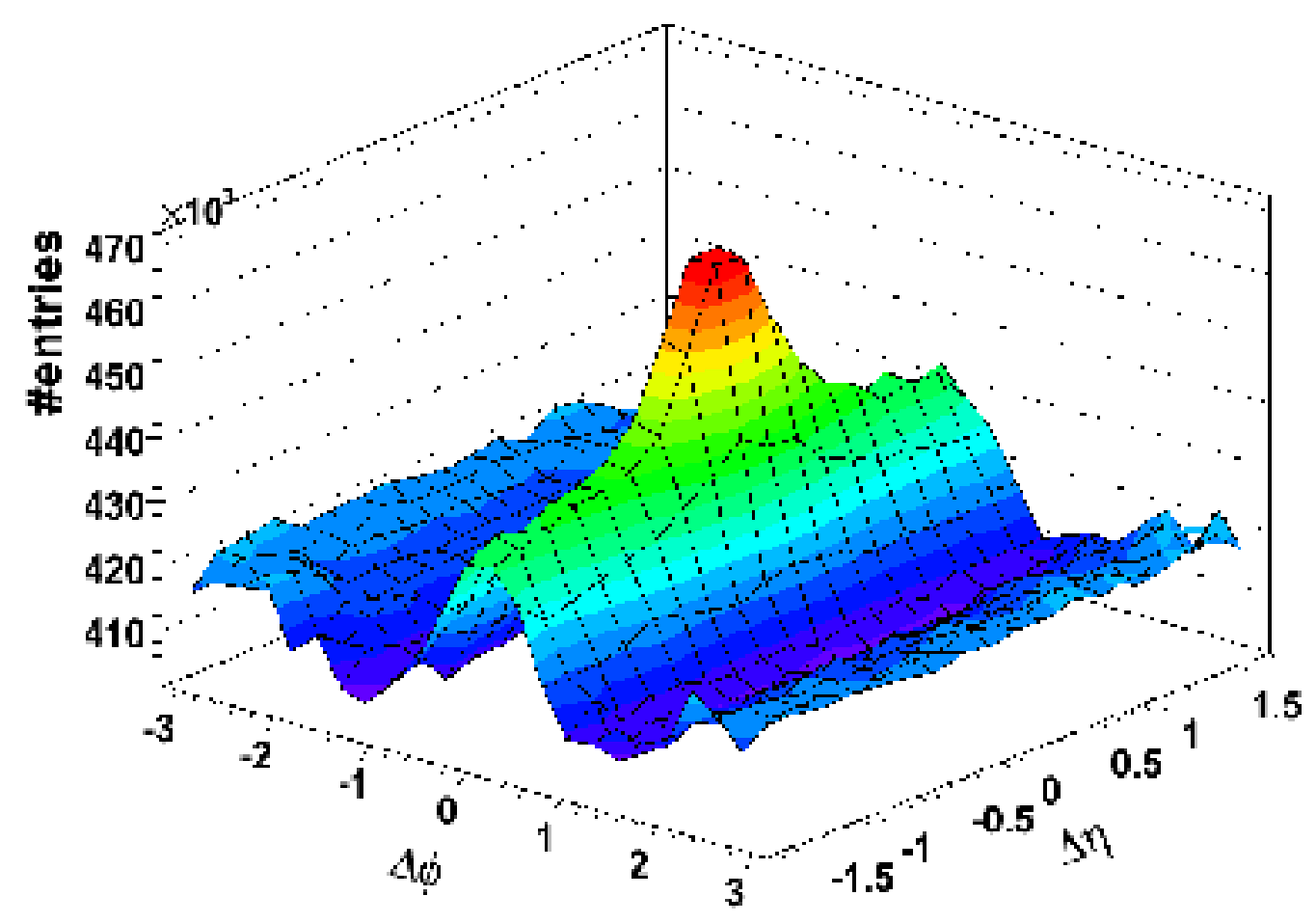




\section{Origin of rapidity correlations}

Causality demands that long-range rapidity correlations originate at very early times (cf. explanation of the CMB homogeneity in the Universe)

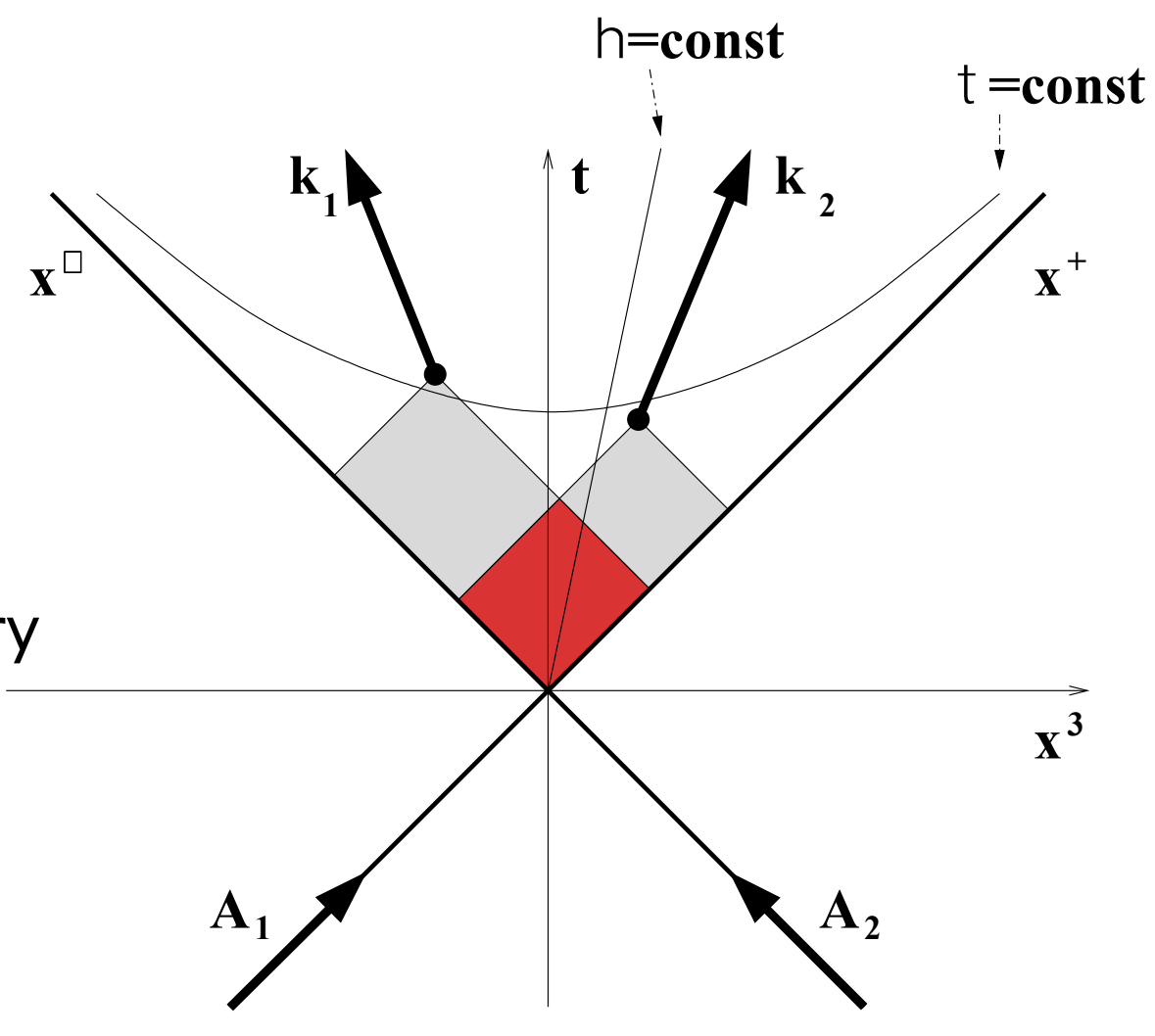

Gavin, McLerran, Moschelli '08; Dumitru, Gelis, McLerran, Venugopalan '08. 


\section{Correlations in AdS shock wave collisions}

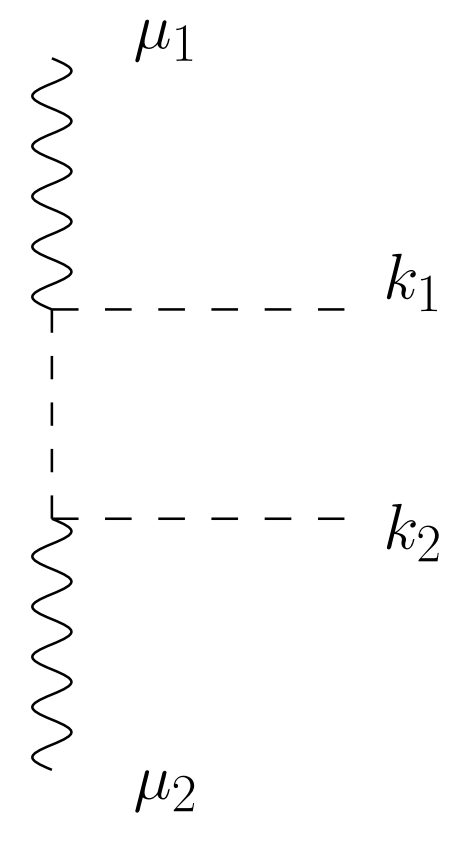

I

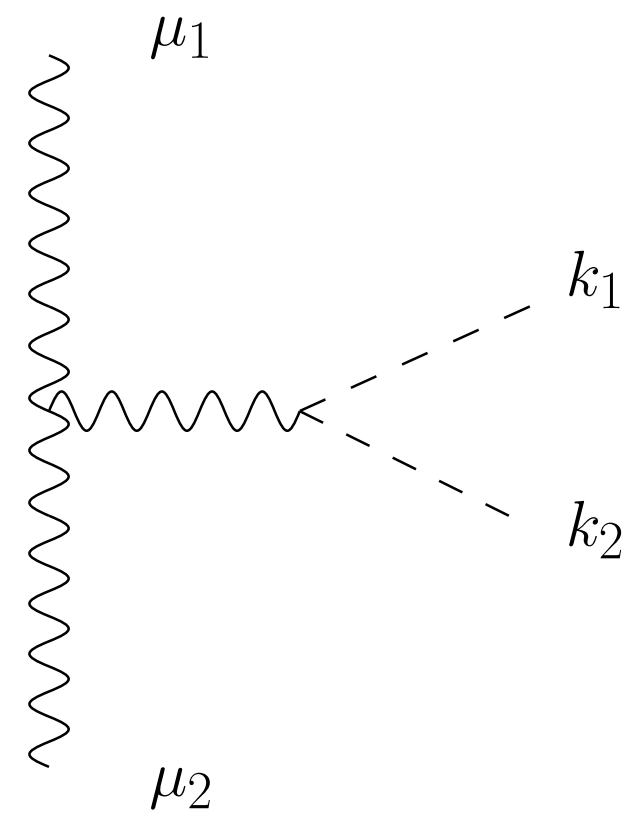

II

$C\left(k_{1}, k_{2}\right) \sim \cosh (4 \Delta y)$

H. Grigoryan, Yu.K. '10 


\section{Correlations in AdS shock wave collisions}

$$
C\left(k_{1}, k_{2}\right) \sim \cosh (4 \Delta y)
$$

- Correlations grow with rapidity interval???

- It is possible that higher-order corrections in shock wave strengths will modify this result, making it closer to real life.

- However, such corrections are important at later times, and are less likely to affect the long-range rapidity correlation...

- This could be another argument in favor of weakly-coupled dynamics in the early stages of heavy ion collisions. 


\section{Ridge in CGC}

- There are two explanations of the ridge in CGC:

- Long-range rapidity-independent fields are created at early times, with correlations generated soon after and with azimuthal collimation produced by radial hydro flow. (Gavin, McLerran, Moschelli '08)

- Both long-range rapidity correlations and the azimuthal correlations are created in the collision due to a particular class of diagrams referred to as the "Glasma graphs". 


\section{Glasma graphs}

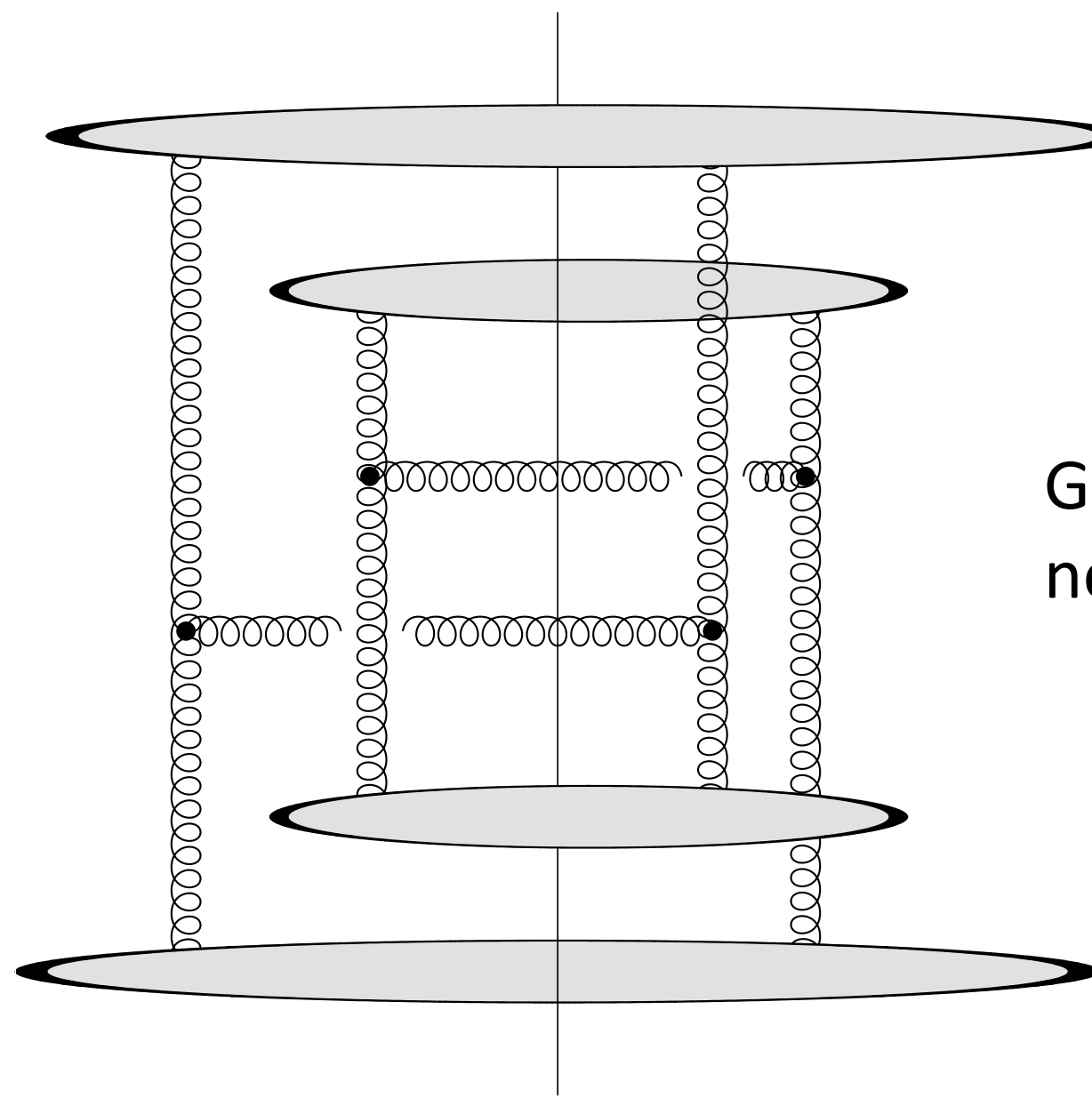

Generate back-to-back and near-side azimuthal correlations.

Dumitru, Gelis, McLerran, Venugopalan ‘08. 


\section{What to calculate?}

- To systematically include Glasma graphs in the CGC formalism it would be great to solve the two-gluon inclusive production problem in the MV model, that is, including multiple rescatterings in both nuclei to all orders (the two produced gluons only talk to each other through sources):

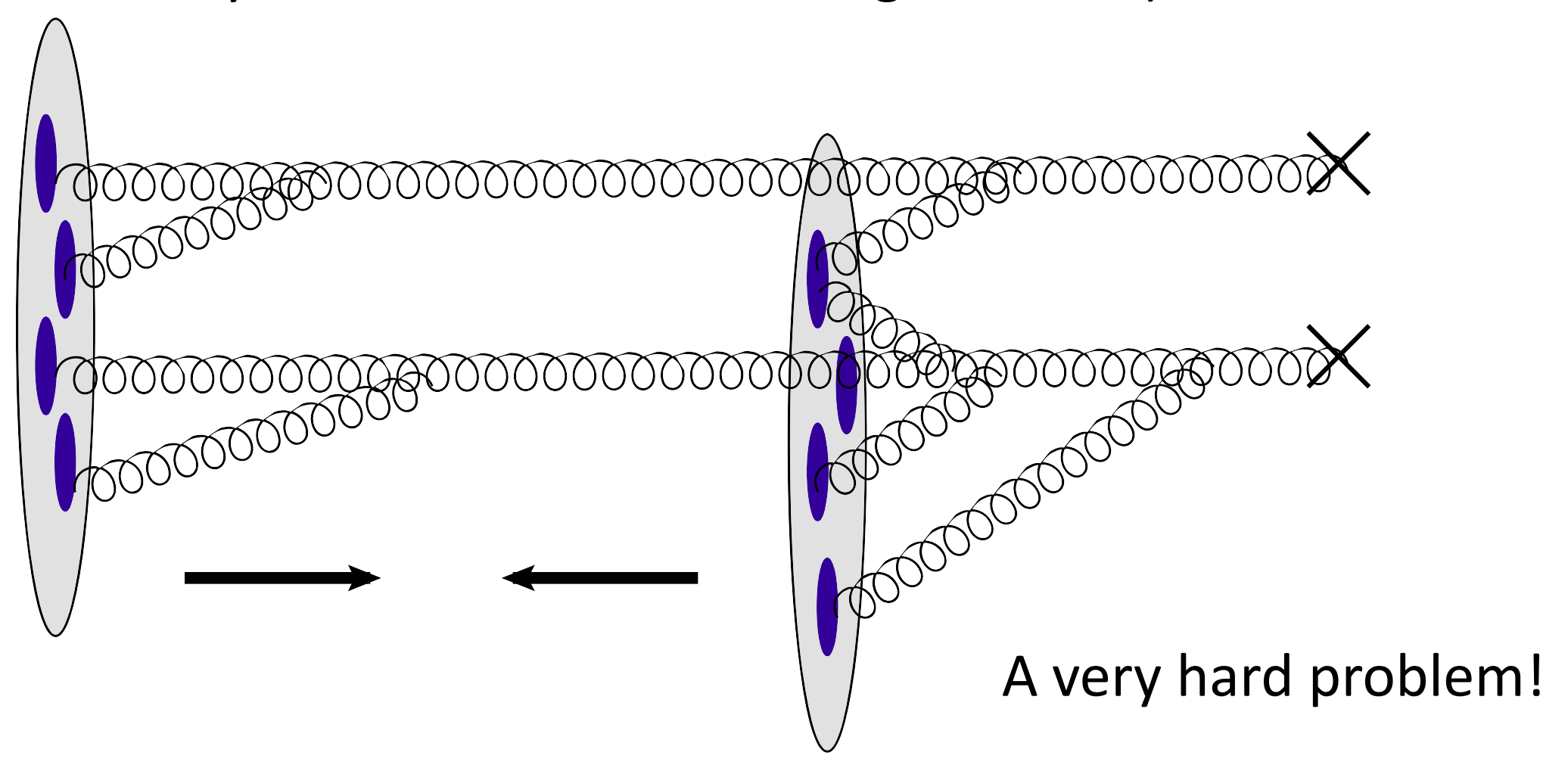




\section{Heavy-Light Ion Collisions}

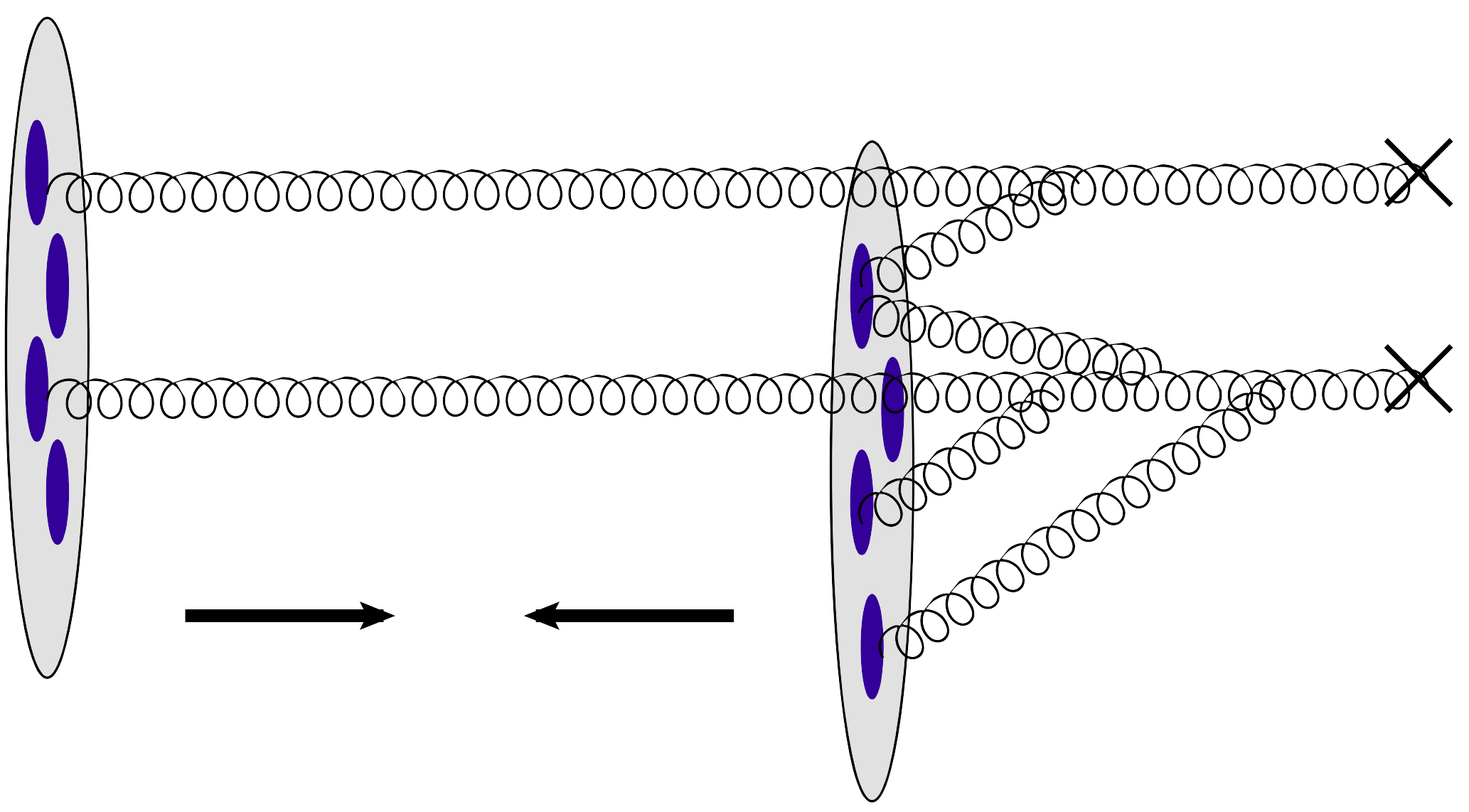

Little steps for the little feet: consider multiple rescatterings only in one of the two nuclei. 


\section{Double gluon production in heavy-light ion collisions}




\section{A. Setting up the problem: geometric correlations}




\section{Two-gluon production}

- We want to calculate two gluon production in $A_{1}+A_{2}$ collisions with $1 \ll A_{1} \ll A_{2}$ resumming all powers of

$$
\alpha_{s}^{2} A_{2}^{1 / 3} \sim 1
$$

(multiple rescatterings in the target nucleus)

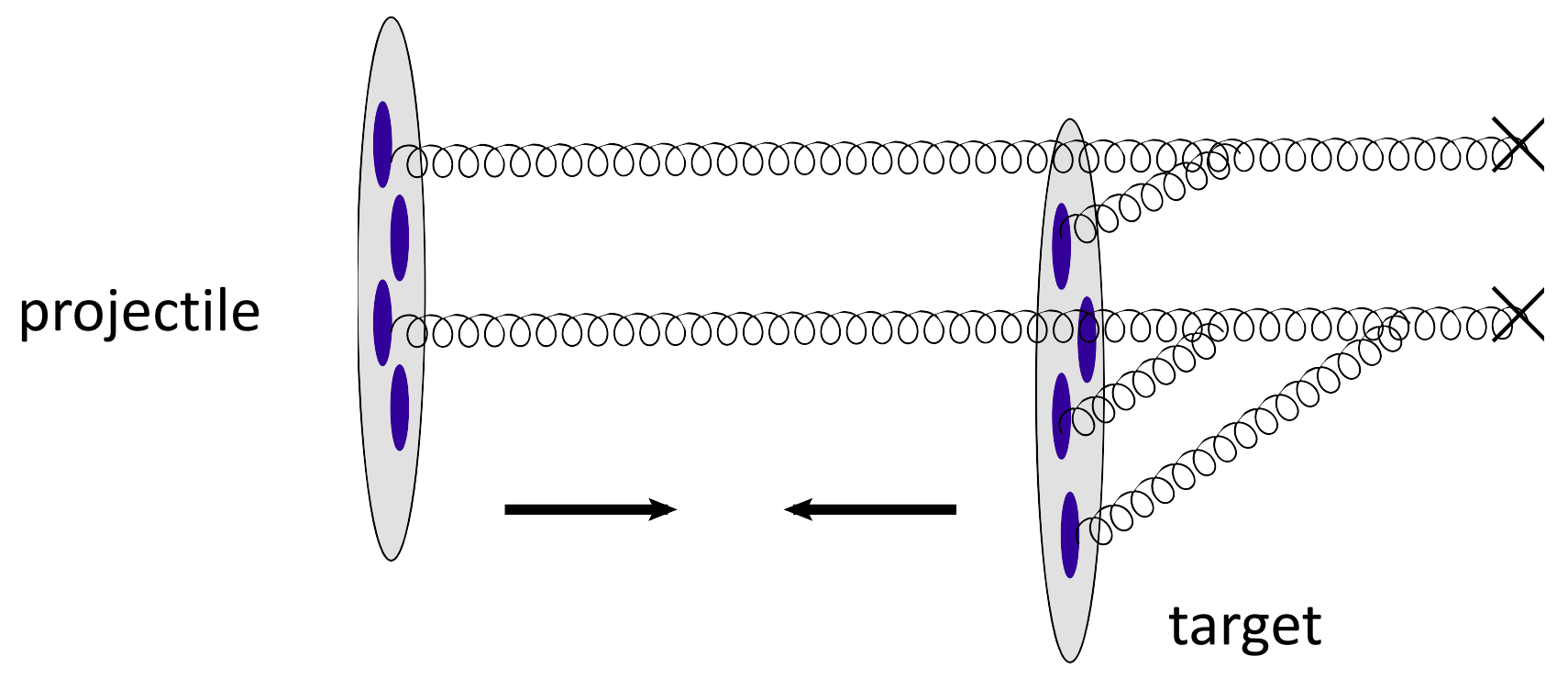

- The gluons come from different nucleons in the projectile nucleus as $A_{1} \gg 1$ and this is enhanced compared to emission from the same nucleon. 


\section{Applicability region}

- The saturation scales of the two nuclei are very different:

$$
\Lambda_{Q C D} \ll Q_{s 1} \ll Q_{s 2}
$$

- We are working above the saturation scale of the smaller nucleus:

$$
k_{T} \gg Q_{s 1}
$$

- We thus sum all multiple rescatterings in the larger nucleus, $\mathrm{Q}_{\mathrm{s} 2} / \mathrm{k}_{\mathrm{T}} \sim 1$, staying at the lowest non-trivial order in $\mathrm{Q}_{\mathrm{s} 1} / \mathrm{k}_{\mathrm{T}} \ll<1$.

- Multiple interactions with the same nucleon in either nucleus are suppressed by $\Lambda_{\mathrm{QCD}} / \mathrm{k}_{\mathrm{T}} \ll<1$. 


\section{Production cross-section}

- The single- and double-inclusive cross sections can be written as

$$
\begin{gathered}
\frac{d \sigma}{d^{2} k d y}=\int d^{2} B d^{2} b\left|\Psi_{I}(\mathbf{B}-\mathbf{b})\right|^{2}\left\langle\frac{d \sigma^{p A_{2}}}{d^{2} k d y d^{2} b}\right\rangle \\
\frac{d \sigma}{d^{2} k_{1} d y_{1} d^{2} k_{2} d y_{2}}=\int d^{2} B d^{2} b_{1} d^{2} b_{2}\left|\Psi_{I I}\left(\mathbf{B}-\mathbf{b}_{1}, \mathbf{B}-\mathbf{b}_{2}\right)\right|^{2}\left\langle\frac{d \sigma^{p A_{2}}}{d^{2} k_{1} d y_{1} d^{2} b_{1}} \frac{d \sigma^{p A_{2}}}{d^{2} k_{2} d y_{2} d^{2} b_{2}}\right\rangle
\end{gathered}
$$

- Assume a large nucleus with uncorrelated nucleons (MV/Glauber model). Then the single- and double-nucleon wave functions are (with $T_{1}$ the nuclear profile function)

$$
\begin{gathered}
\left|\Psi_{I}(\mathbf{b})\right|^{2}=T_{1}(\mathbf{b}) \\
\left|\Psi_{I I}\left(\mathbf{b}_{1}, \mathbf{b}_{2}\right)\right|^{2}=T_{1}\left(\mathbf{b}_{1}\right) T_{1}\left(\mathbf{b}_{2}\right)
\end{gathered}
$$

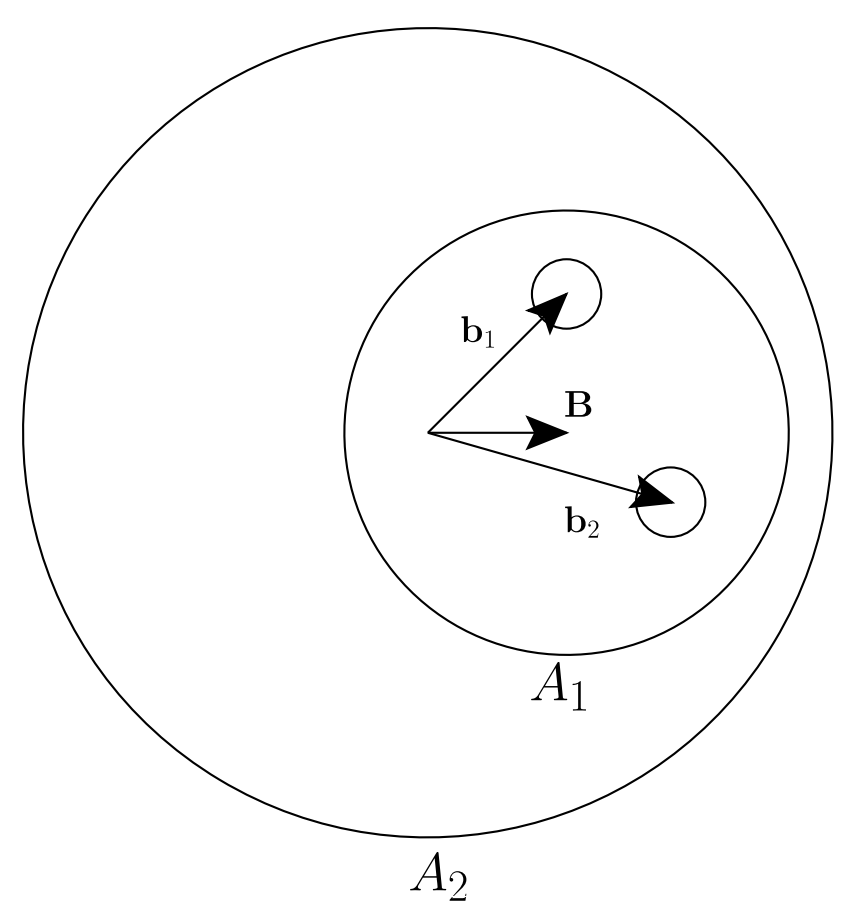




\section{Geometric Correlations}

- Assume uncorrelated interaction with the target:

$$
\left\langle\frac{d \sigma^{p A_{2}}}{d^{2} k_{1} d y_{1} d^{2} b_{1}} \frac{d \sigma^{p A_{2}}}{d^{2} k_{2} d y_{2} d^{2} b_{2}}\right\rangle \approx\left\langle\frac{d \sigma^{p A_{2}}}{d^{2} k_{1} d y_{1} d^{2} b_{1}}\right\rangle\left\langle\frac{d \sigma^{p A_{2}}}{d^{2} k_{2} d y_{2} d^{2} b_{2}}\right\rangle
$$

- For cross sections we have

$$
\begin{gathered}
\frac{d \sigma}{d^{2} k d y}=\int d^{2} B d^{2} b T_{1}(\mathbf{B}-\mathbf{b})\left\langle\frac{d \sigma^{p A_{2}}}{d^{2} k d y d^{2} b}\right\rangle \\
\frac{d \sigma}{d^{2} k_{1} d y_{1} d^{2} k_{2} d y_{2}}=\int d^{2} B d^{2} b_{1} d^{2} b_{2} T_{1}\left(\mathbf{B}-\mathbf{b}_{\mathbf{1}}\right) T_{1}\left(\mathbf{B}-\mathbf{b}_{\mathbf{2}}\right)\left\langle\frac{d \sigma^{p A_{2}}}{d^{2} k_{1} d y_{1} d^{2} b_{1}}\right\rangle\left\langle\frac{d \sigma^{p A_{2}}}{d^{2} k_{2} d y_{2} d^{2} b_{2}}\right\rangle \\
\text { • Clearly } \\
\frac{d \sigma}{d^{2} k_{1} d y_{1} d^{2} k_{2} d y_{2}} \nsim \frac{d \sigma}{d^{2} k_{1} d y_{1}} \frac{d \sigma}{d^{2} k_{2} d y_{2}}
\end{gathered}
$$

- Correlations due to the integration over the impact parameter B -> Geometric correlations! 


\section{Geometric correlations: physical meaning}

- In the same even the two nucleons are always within the smaller nucleus radius from each other (in transverse plane). In different events they can be anywhere in the larger nucleus.

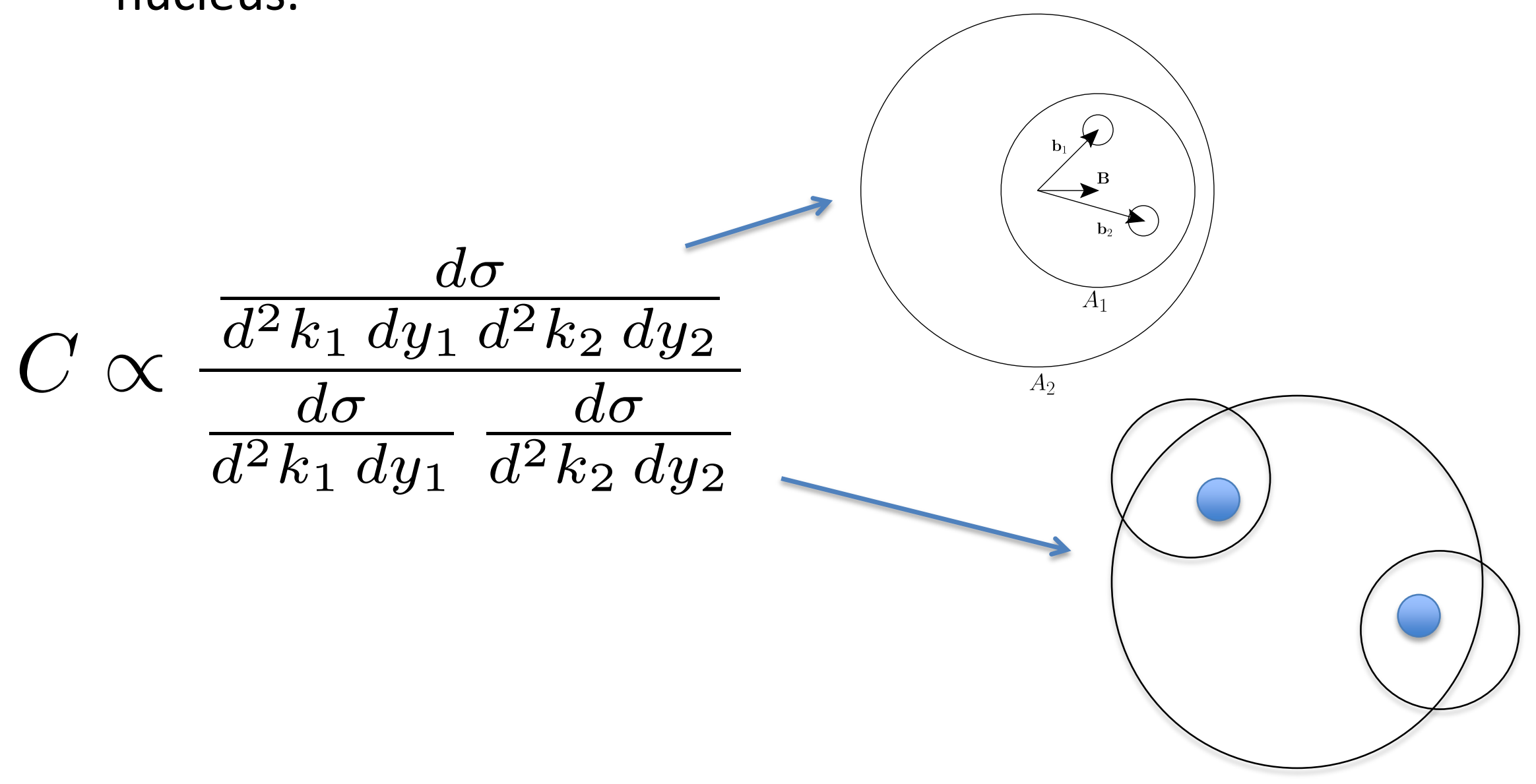




\section{Geometric correlations at fixed B}

- are zero as

$\frac{d \sigma}{d^{2} k_{1} d y_{1} d^{2} k_{2} d y_{2} d^{2} B}=\frac{d \sigma}{d^{2} k_{1} d y_{1} d^{2} B} \frac{d \sigma}{d^{2} k_{2} d y_{2} d^{2} B}$

which is clear from

$$
\frac{d \sigma}{d^{2} k d y}=\int d^{2} B d^{2} b T_{1}(\mathbf{B}-\mathbf{b})\left\langle\frac{d \sigma^{p A_{2}}}{d^{2} k d y d^{2} b}\right\rangle
$$

$\frac{d \sigma}{d^{2} k_{1} d y_{1} d^{2} k_{2} d y_{2}}=\int d^{2} B d^{2} b_{1} d^{2} b_{2} T_{1}\left(\mathbf{B}-\mathbf{b}_{\mathbf{1}}\right) T_{1}\left(\mathbf{B}-\mathbf{b}_{\mathbf{2}}\right)\left\langle\frac{d \sigma^{p A_{2}}}{d^{2} k_{1} d y_{1} d^{2} b_{1}}\right\rangle\left\langle\frac{d \sigma^{p A_{2}}}{d^{2} k_{2} d y_{2} d^{2} b_{2}}\right\rangle$

- Note that direction of $B$ has to be fixed (to remove the geometric correlations), in other words the vector $B$ should be fixed with respect to vectors $k_{1}$ and $k_{2}$. Maybe hard to do, but perhaps possible. 
B. Two-gluon production 


\section{(i) Single gluon production in $\mathrm{pA}$}




\section{Single gluon production in $\mathrm{pA}$}

Model the proton by a single quark (can be easily improved upon). The diagrams are shown below (Yu.K., A. Mueller '97):
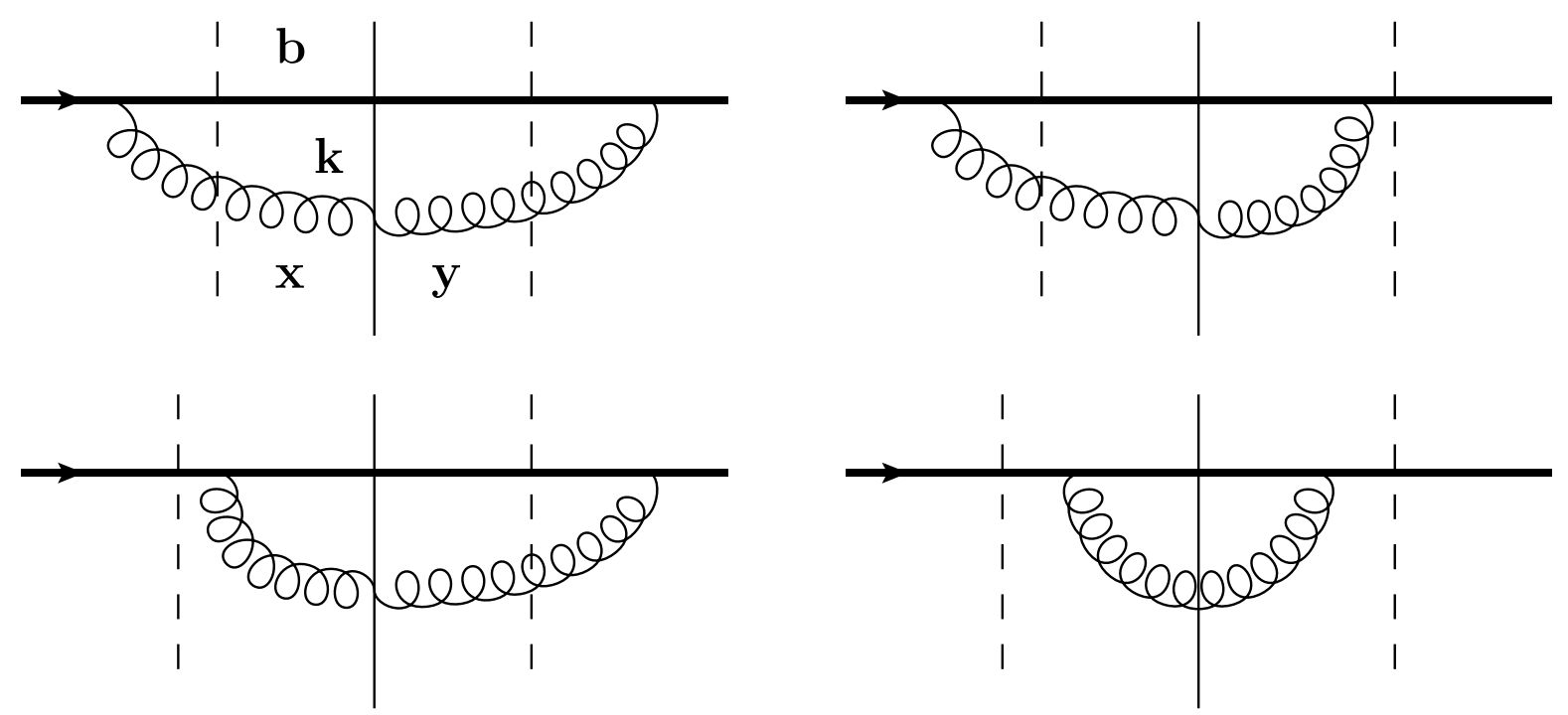

Multiple rescatterings are denoted by a single dashed line:
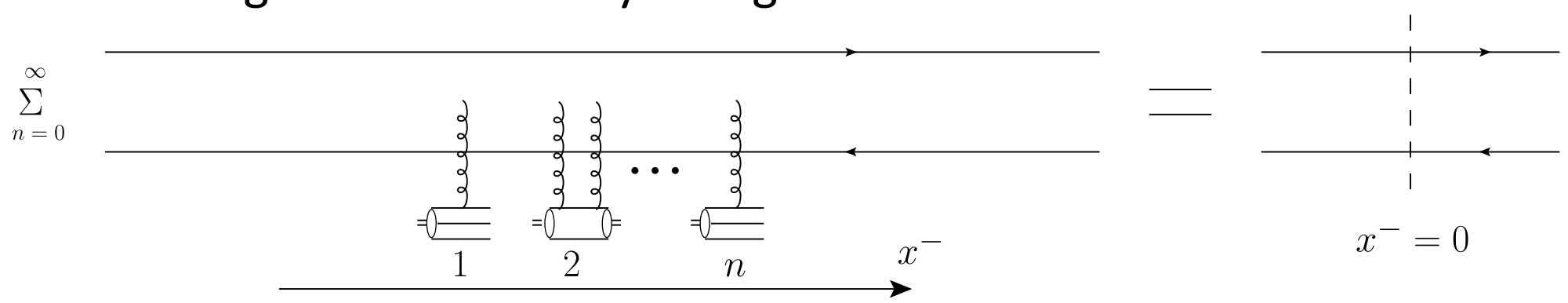


\section{Single gluon production in $\mathrm{pA}$}
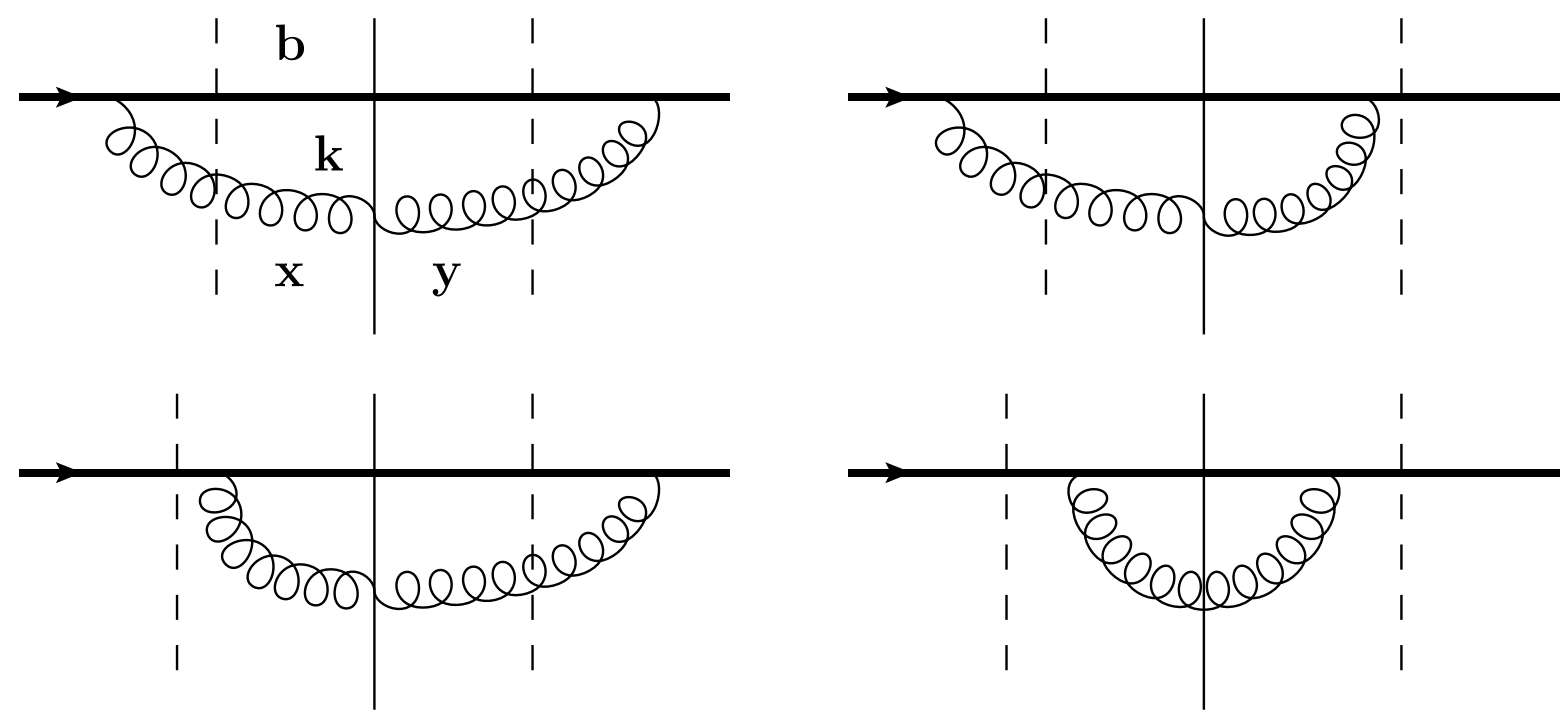

The gluon production cross section can be readily written as $(U=$ Wilson line in adjoint representation, represents gluon interactions)

$$
\begin{aligned}
& \left\langle\frac{d \sigma^{p A_{2}}}{d^{2} k d y d^{2} b}\right\rangle=\frac{\alpha_{s} C_{F}}{4 \pi^{4}} \int d^{2} x d^{2} y e^{-i \mathbf{k} \cdot(\mathbf{x}-\mathbf{y})} \frac{\mathbf{x}-\mathbf{b}}{|\mathbf{x}-\mathbf{b}|^{2}} \cdot \frac{\mathbf{y}-\mathbf{b}}{|\mathbf{y}-\mathbf{b}|^{2}} \\
& \times\left\langle\frac{1}{N_{c}^{2}-1} \operatorname{Tr}\left[U_{\mathbf{x}} U_{\mathbf{y}}^{\dagger}\right]-\frac{1}{N_{c}^{2}-1} \operatorname{Tr}\left[U_{\mathbf{x}} U_{\mathbf{b}}^{\dagger}\right]-\frac{1}{N_{c}^{2}-1} \operatorname{Tr}\left[U_{\mathbf{b}} U_{\mathbf{y}}^{\dagger}\right]+1\right\rangle
\end{aligned}
$$




\section{Forward dipole amplitude}

- The eikonal quark propagator is given by the Wilson line

$$
V(\underline{x})=\mathrm{P} \exp \left[i g \int_{-\infty}^{\infty} d x^{+} A^{-}\left(x^{+}, x^{-}=0, \underline{x}\right)\right]
$$

with the light cone coordinates

$$
x^{ \pm}=\frac{t \pm z}{\sqrt{2}}
$$

- The quark dipole scattering amplitude is

$$
N\left(\underline{x}_{1}, \underline{x}_{2}\right)=1-\frac{1}{N_{c}}\left\langle\operatorname{tr}\left[V\left(\underline{x}_{1}\right) V^{\dagger}\left(\underline{x}_{2}\right)\right]\right\rangle
$$

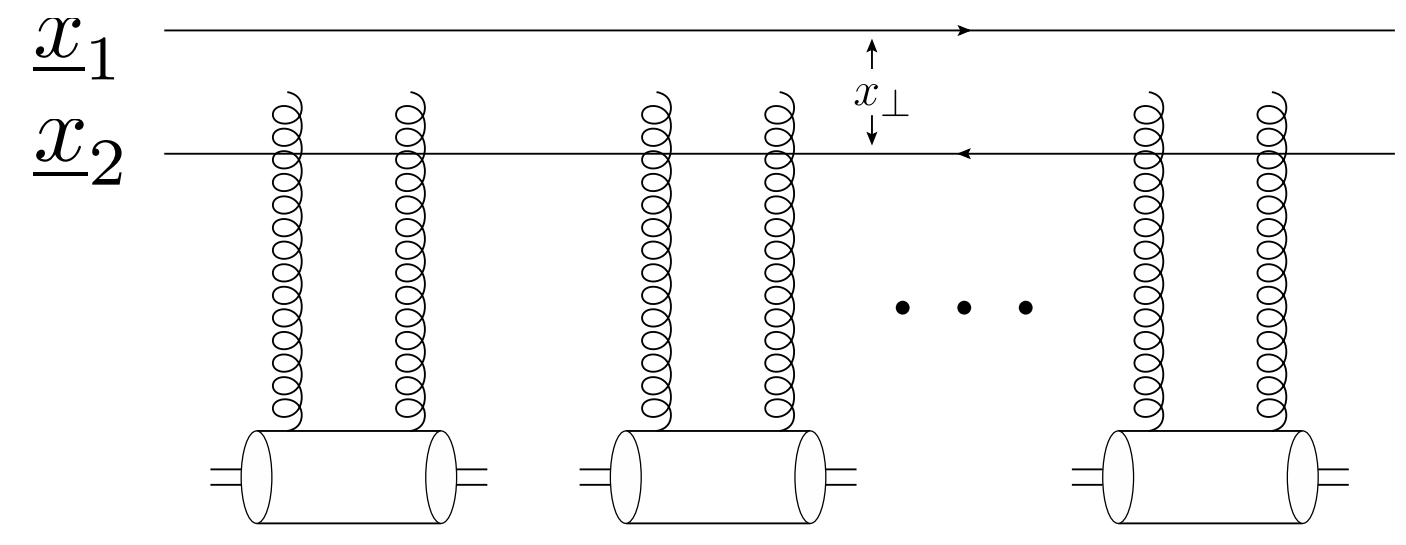




\section{(ii) Two-gluon production in heavy-light ion collisions}




\section{The process}

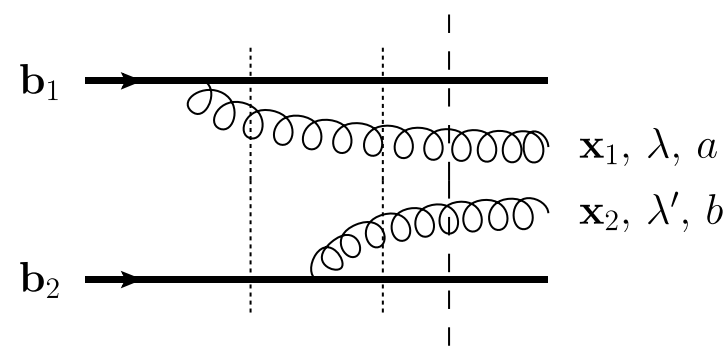

A

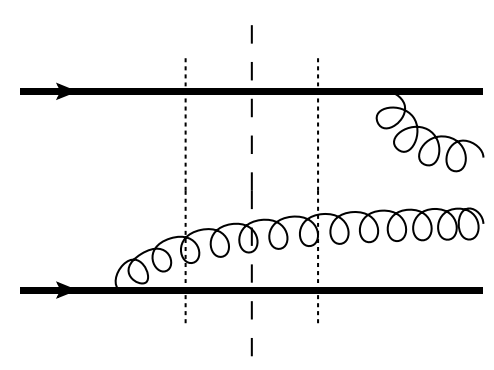

D

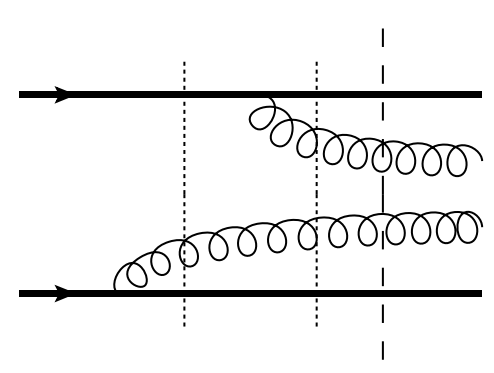

B

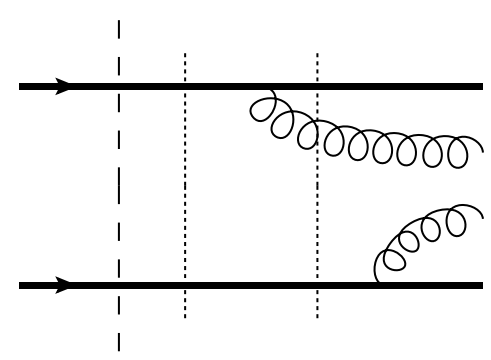

E

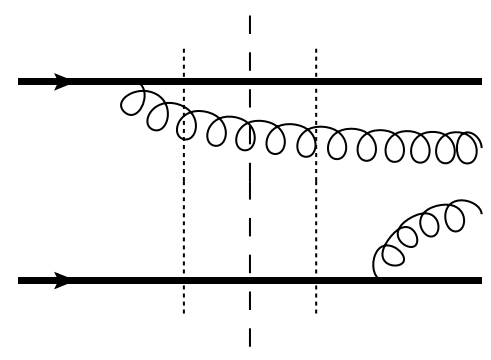

C

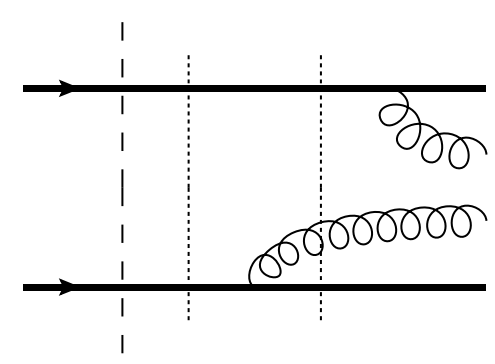

F

Solid horizontal lines = quarks in the incoming nucleons.

Dashed vertical line $=$ interaction with the target.

Dotted vertical lines = energy denominators. 


\section{Amplitude squared}

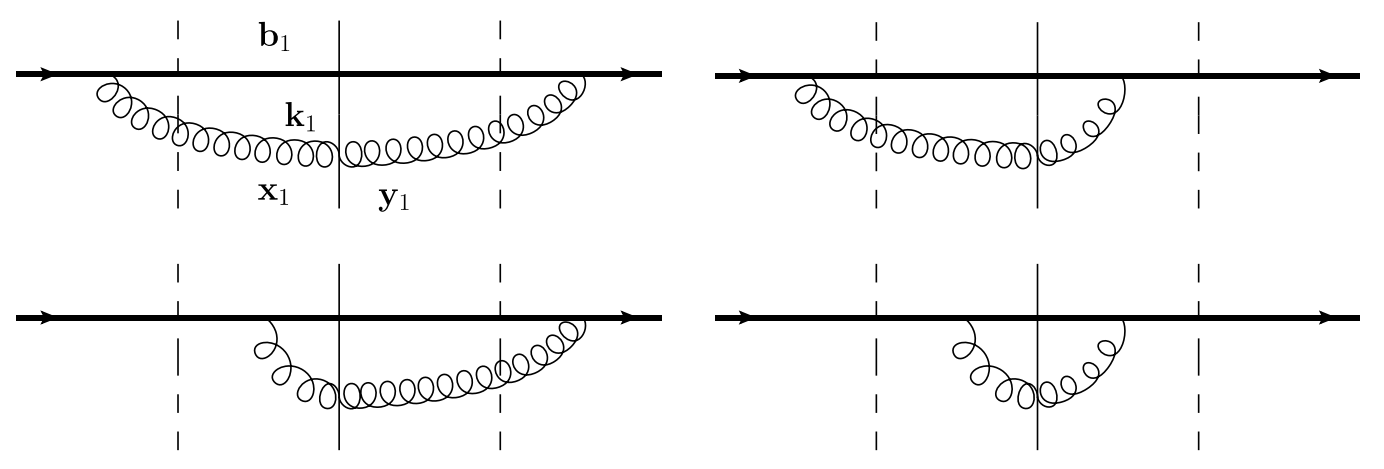

$\bigcirc$
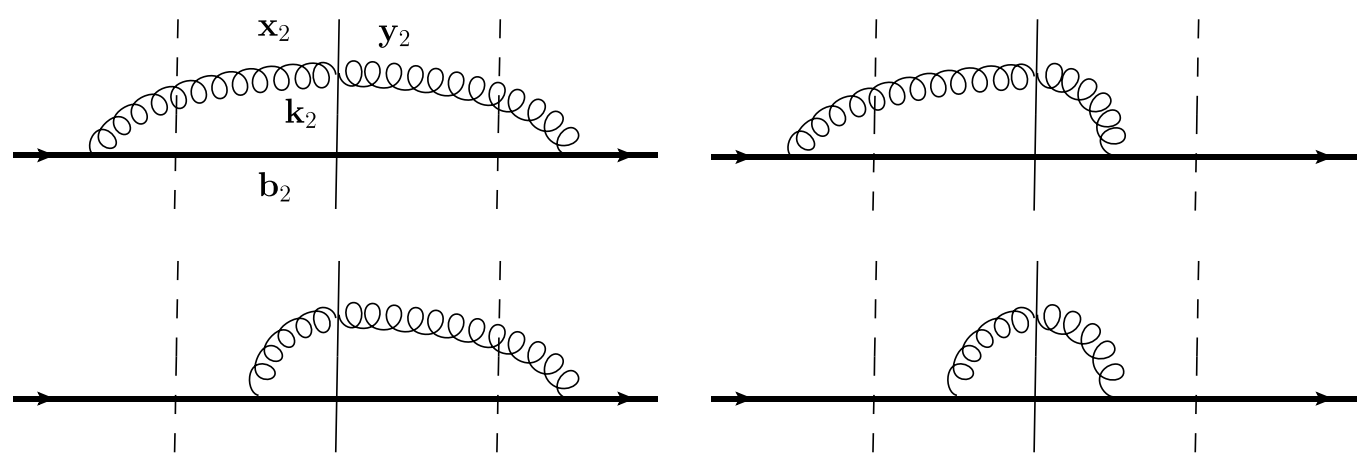

This contribution to two-gluon production looks like one-gluon production squared, with the target averaging applied to both. 


\section{Amplitude squared}

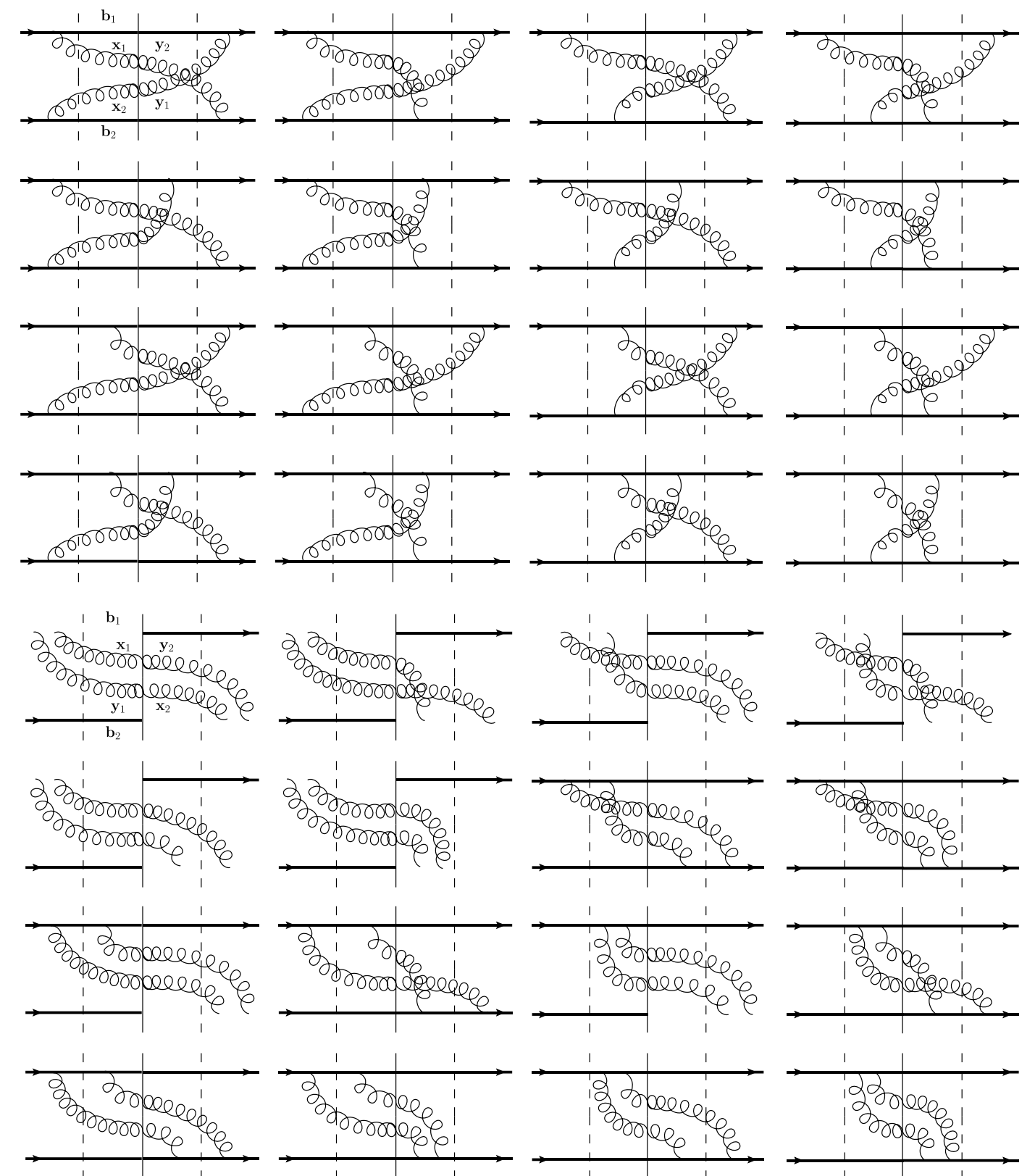

These contributions to two-gluon production contain cross-talk between the emissions from different nucleons. 


\section{Two-gluon production cross section}

- "Squaring" the single gluon production cross section yields

$$
\begin{aligned}
\frac{d \sigma}{d^{2} k_{1} d y_{1} d^{2} k_{2} d y_{2}} & =\frac{\alpha_{s}^{2} C_{F}^{2}}{16 \pi^{8}} \int d^{2} B d^{2} b_{1} d^{2} b_{2} T_{1}\left(\mathbf{B}-\mathbf{b}_{1}\right) T_{1}\left(\mathbf{B}-\mathbf{b}_{2}\right) d^{2} x_{1} d^{2} y_{1} d^{2} x_{2} d^{2} y_{2} e^{-i \mathbf{k}_{1} \cdot\left(\mathbf{x}_{1}-\mathbf{y}_{1}\right)-i \mathbf{k}_{2} \cdot\left(\mathbf{x}_{2}-\mathbf{y}_{2}\right)} \\
& \times \frac{\mathbf{x}_{1}-\mathbf{b}_{1}}{\left|\mathbf{x}_{1}-\mathbf{b}_{1}\right|^{2}} \cdot \frac{\mathbf{y}_{1}-\mathbf{b}_{1}}{\left|\mathbf{y}_{1}-\mathbf{b}_{1}\right|^{2}} \frac{\mathbf{x}_{2}-\mathbf{b}_{2}}{\left|\mathbf{x}_{2}-\mathbf{b}_{2}\right|^{2}} \cdot \frac{\mathbf{y}_{2}-\mathbf{b}_{2}}{\left|\mathbf{y}_{2}-\mathbf{b}_{2}\right|^{2}} \\
& \times\left\langle\left(\frac{1}{N_{c}^{2}-1} \operatorname{Tr}\left[U_{\mathbf{x}_{1}} U_{\mathbf{y}_{1}}^{\dagger}\right]-\frac{1}{N_{c}^{2}-1} \operatorname{Tr}\left[U_{\mathbf{x}_{1}} U_{\mathbf{b}_{1}}^{\dagger}\right]-\frac{1}{N_{c}^{2}-1} \operatorname{Tr}\left[U_{\mathbf{b}_{1}} U_{\mathbf{y}_{1}}^{\dagger}\right]+1\right)\right. \\
& \left.\times\left(\frac{1}{N_{c}^{2}-1} \operatorname{Tr}\left[U_{\mathbf{x}_{2}} U_{\mathbf{y}_{2}}^{\dagger}\right]-\frac{1}{N_{c}^{2}-1} \operatorname{Tr}\left[U_{\mathbf{x}_{2}} U_{\mathbf{b}_{2}}^{\dagger}\right]-\frac{1}{N_{c}^{2}-1} \operatorname{Tr}\left[U_{\mathbf{b}_{2}} U_{\mathbf{y}_{2}}^{\dagger}\right]+1\right)\right\rangle
\end{aligned}
$$
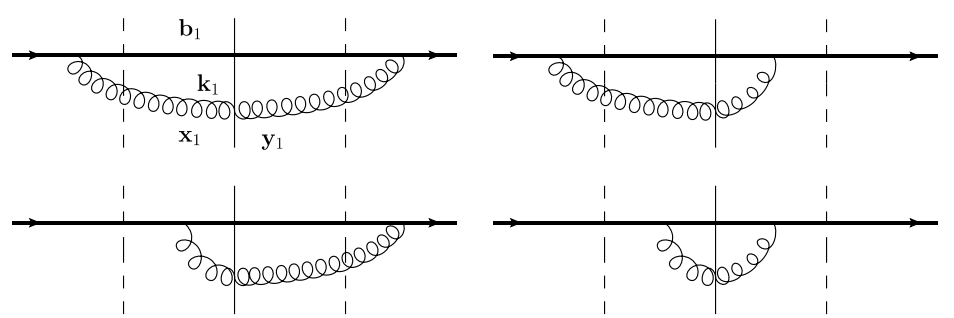

(cf. Kovner \& Lublinsky, '12)

$\bigcirc$

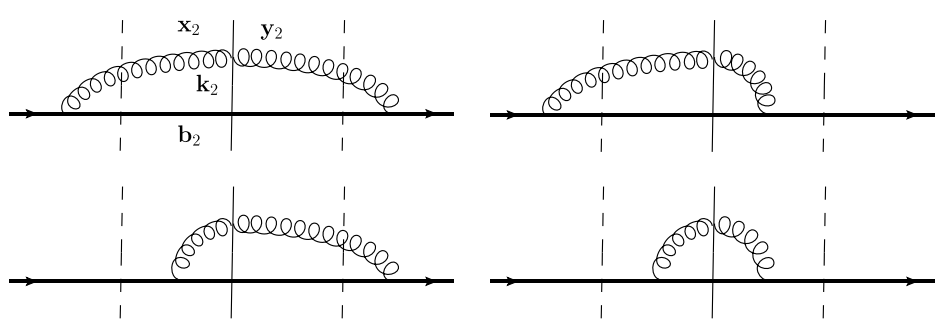




\section{Two-gluon production cross section}

- The "crossed" diagrams give

$$
\begin{aligned}
& \frac{d \sigma_{\text {crossed }}}{d^{2} k_{1} d y_{1} d^{2} k_{2} d y_{2}}=\frac{1}{\left[2(2 \pi)^{3}\right]^{2}} \int d^{2} B d^{2} b_{1} d^{2} b_{2} T_{1}\left(\mathbf{B}-\mathbf{b}_{1}\right) T_{1}\left(\mathbf{B}-\mathbf{b}_{2}\right) d^{2} x_{1} d^{2} y_{1} d^{2} x_{2} d^{2} y_{2} \\
& \times\left[e^{-i \mathbf{k}_{1} \cdot\left(\mathbf{x}_{1}-\mathbf{y}_{2}\right)-i \mathbf{k}_{2} \cdot\left(\mathbf{x}_{2}-\mathbf{y}_{1}\right)}+e^{-i \mathbf{k}_{1} \cdot\left(\mathbf{x}_{1}-\mathbf{y}_{2}\right)+i \mathbf{k}_{2} \cdot\left(\mathbf{x}_{2}-\mathbf{y}_{1}\right)}\right] \\
& \times \frac{16 \alpha_{s}^{2}}{\pi^{2}} \frac{C_{F}}{2 N_{c}} \frac{\mathbf{x}_{1}-\mathbf{b}_{1}}{\left|\mathbf{x}_{1}-\mathbf{b}_{1}\right|^{2}} \cdot \frac{\mathbf{y}_{2}-\mathbf{b}_{2}}{\left|\mathbf{y}_{2}-\mathbf{b}_{2}\right|^{2}} \frac{\mathbf{x}_{2}-\mathbf{b}_{2}}{\left|\mathbf{x}_{2}-\mathbf{b}_{2}\right|^{2}} \cdot \frac{\mathbf{y}_{1}-\mathbf{b}_{1}}{\left|\mathbf{y}_{1}-\mathbf{b}_{1}\right|^{2}} \\
& \times\left[Q\left(\mathbf{x}_{1}, \mathbf{y}_{1}, \mathbf{x}_{2}, \mathbf{y}_{2}\right)-Q\left(\mathbf{x}_{1}, \mathbf{y}_{1}, \mathbf{x}_{2}, \mathbf{b}_{2}\right)-Q\left(\mathbf{x}_{1}, \mathbf{y}_{1}, \mathbf{b}_{2}, \mathbf{y}_{2}\right)+S_{G}\left(\mathbf{x}_{1}, \mathbf{y}_{1}\right)-Q\left(\mathbf{x}_{1}, \mathbf{b}_{1}, \mathbf{x}_{2}, \mathbf{y}_{2}\right)\right. \\
& +Q\left(\mathbf{x}_{1}, \mathbf{b}_{1}, \mathbf{x}_{2}, \mathbf{b}_{2}\right)+Q\left(\mathbf{x}_{1}, \mathbf{b}_{1}, \mathbf{b}_{2}, \mathbf{y}_{2}\right)-S_{G}\left(\mathbf{x}_{1}, \mathbf{b}_{1}\right)-Q\left(\mathbf{b}_{1}, \mathbf{y}_{1}, \mathbf{x}_{2}, \mathbf{y}_{2}\right)+Q\left(\mathbf{b}_{1}, \mathbf{y}_{1}, \mathbf{x}_{2}, \mathbf{b}_{2}\right) \\
& \left.+Q\left(\mathbf{b}_{1}, \mathbf{y}_{1}, \mathbf{b}_{2}, \mathbf{y}_{2}\right)-S_{G}\left(\mathbf{b}_{1}, \mathbf{y}_{1}\right)+S_{G}\left(\mathbf{x}_{2}, \mathbf{y}_{2}\right)-S_{G}\left(\mathbf{x}_{2}, \mathbf{b}_{2}\right)-S_{G}\left(\mathbf{b}_{2}, \mathbf{y}_{2}\right)+1\right]
\end{aligned}
$$
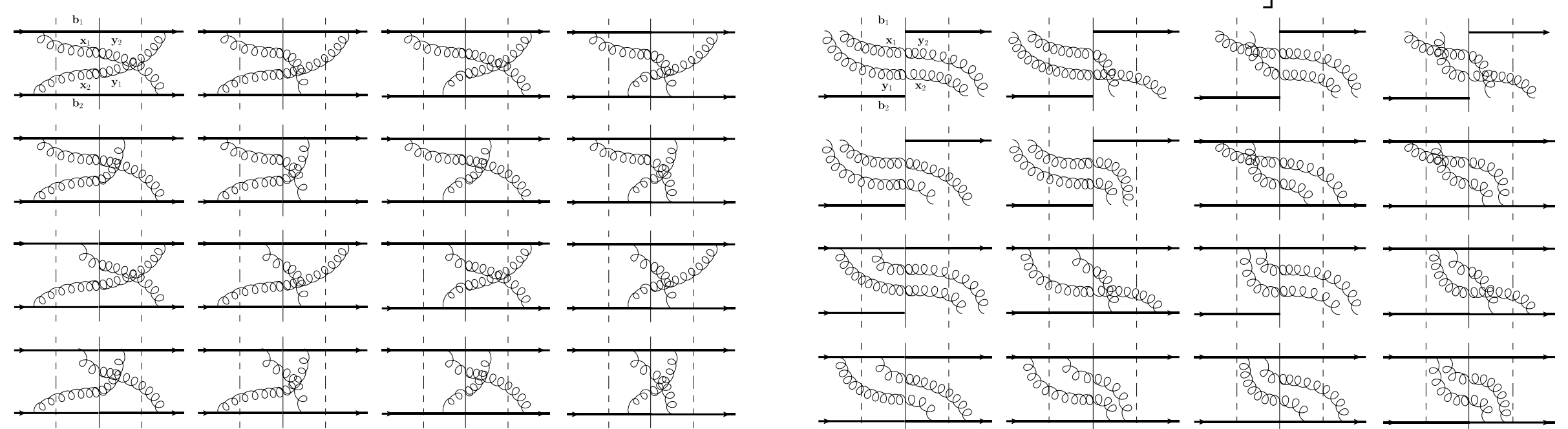


\section{Two-gluon production cross section}

- The "crossed" diagrams give

$$
\begin{aligned}
& \frac{d \sigma_{\text {crossed }}}{d^{2} k_{1} d y_{1} d^{2} k_{2} d y_{2}}=\frac{1}{\left[2(2 \pi)^{3}\right]^{2}} \int d^{2} B d^{2} b_{1} d^{2} b_{2} T_{1}\left(\mathbf{B}-\mathbf{b}_{1}\right) T_{1}\left(\mathbf{B}-\mathbf{b}_{2}\right) d^{2} x_{1} d^{2} y_{1} d^{2} x_{2} d^{2} y_{2} \\
& \times\left[e^{-i \mathbf{k}_{1} \cdot\left(\mathbf{x}_{1}-\mathbf{y}_{2}\right)-i \mathbf{k}_{2} \cdot\left(\mathbf{x}_{2}-\mathbf{y}_{1}\right)}+e^{-i \mathbf{k}_{1} \cdot\left(\mathbf{x}_{1}-\mathbf{y}_{2}\right)+i \mathbf{k}_{2} \cdot\left(\mathbf{x}_{2}-\mathbf{y}_{1}\right)}\right] \\
& \times \frac{16 \alpha_{s}^{2}}{\pi^{2}} \frac{C_{F}}{2 N_{c}} \frac{\mathbf{x}_{1}-\mathbf{b}_{1}}{\left|\mathbf{x}_{1}-\mathbf{b}_{1}\right|^{2}} \cdot \frac{\mathbf{y}_{2}-\mathbf{b}_{2}}{\left|\mathbf{y}_{2}-\mathbf{b}_{2}\right|^{2}} \frac{\mathbf{x}_{2}-\mathbf{b}_{2}}{\left|\mathbf{x}_{2}-\mathbf{b}_{2}\right|^{2}} \cdot \frac{\mathbf{y}_{1}-\mathbf{b}_{1}}{\left|\mathbf{y}_{1}-\mathbf{b}_{1}\right|^{2}} \\
& \times\left[Q\left(\mathbf{x}_{1}, \mathbf{y}_{1}, \mathbf{x}_{2}, \mathbf{y}_{2}\right)-Q\left(\mathbf{x}_{1}, \mathbf{y}_{1}, \mathbf{x}_{2}, \mathbf{b}_{2}\right)-Q\left(\mathbf{x}_{1}, \mathbf{y}_{1}, \mathbf{b}_{2}, \mathbf{y}_{2}\right)+S_{G}\left(\mathbf{x}_{1}, \mathbf{y}_{1}\right)-Q\left(\mathbf{x}_{1}, \mathbf{b}_{1}, \mathbf{x}_{2}, \mathbf{y}_{2}\right)\right. \\
& +Q\left(\mathbf{x}_{1}, \mathbf{b}_{1}, \mathbf{x}_{2}, \mathbf{b}_{2}\right)+Q\left(\mathbf{x}_{1}, \mathbf{b}_{1}, \mathbf{b}_{2}, \mathbf{y}_{2}\right)-S_{G}\left(\mathbf{x}_{1}, \mathbf{b}_{1}\right)-Q\left(\mathbf{b}_{1}, \mathbf{y}_{1}, \mathbf{x}_{2}, \mathbf{y}_{2}\right)+Q\left(\mathbf{b}_{1}, \mathbf{y}_{1}, \mathbf{x}_{2}, \mathbf{b}_{2}\right) \\
& \left.+Q\left(\mathbf{b}_{1}, \mathbf{y}_{1}, \mathbf{b}_{2}, \mathbf{y}_{2}\right)-S_{G}\left(\mathbf{b}_{1}, \mathbf{y}_{1}\right)+S_{G}\left(\mathbf{x}_{2}, \mathbf{y}_{2}\right)-S_{G}\left(\mathbf{x}_{2}, \mathbf{b}_{2}\right)-S_{G}\left(\mathbf{b}_{2}, \mathbf{y}_{2}\right)+1\right]
\end{aligned}
$$

- We introduced the adjoint color-dipole and color quadrupole amplitudes:

$$
\begin{aligned}
& S_{G}\left(\mathbf{x}_{1}, \mathbf{x}_{2}, y\right) \equiv \frac{1}{N_{c}^{2}-1}\left\langle\operatorname{Tr}\left[U_{\mathbf{x}_{1}} U_{\mathbf{x}_{2}}^{\dagger}\right]\right\rangle \\
& Q\left(\mathbf{x}_{1}, \mathbf{x}_{2}, \mathbf{x}_{3}, \mathbf{x}_{4}\right) \equiv \frac{1}{N_{c}^{2}-1}\left\langle\operatorname{Tr}\left[U_{\mathbf{x}_{1}} U_{\mathbf{x}_{2}}^{\dagger} U_{\mathbf{x}_{3}} U_{\mathbf{x}_{4}}^{\dagger}\right]\right\rangle
\end{aligned}
$$




\section{Two-gluon production: properties}

$$
\begin{aligned}
\frac{d \sigma}{d^{2} k_{1} d y_{1} d^{2} k_{2} d y_{2}} & =\frac{\alpha_{s}^{2} C_{F}^{2}}{16 \pi^{8}} \int d^{2} B d^{2} b_{1} d^{2} b_{2} T_{1}\left(\mathbf{B}-\mathbf{b}_{1}\right) T_{1}\left(\mathbf{B}-\mathbf{b}_{2}\right) d^{2} x_{1} d^{2} y_{1} d^{2} x_{2} d^{2} y_{2} e^{-i \mathbf{k}_{1} \cdot\left(\mathbf{x}_{1}-\mathbf{y}_{1}\right)-i \mathbf{k}_{2} \cdot\left(\mathbf{x}_{2}-\mathbf{y}_{2}\right)} \\
& \times \frac{\mathbf{x}_{1}-\mathbf{b}_{1}}{\left|\mathbf{x}_{1}-\mathbf{b}_{1}\right|^{2}} \cdot \frac{\mathbf{y}_{1}-\mathbf{b}_{1}}{\left|\mathbf{y}_{1}-\mathbf{b}_{1}\right|^{2}} \frac{\mathbf{x}_{2}-\mathbf{b}_{2}}{\left|\mathbf{x}_{2}-\mathbf{b}_{2}\right|^{2}} \cdot \frac{\mathbf{y}_{2}-\mathbf{b}_{2}}{\left|\mathbf{y}_{2}-\mathbf{b}_{2}\right|^{2}} \\
& \times\left\langle\left(\frac{1}{N_{c}^{2}-1} \operatorname{Tr}\left[U_{\mathbf{x}_{1}} U_{\mathbf{y}_{1}}^{\dagger}\right]-\frac{1}{N_{c}^{2}-1} \operatorname{Tr}\left[U_{\mathbf{x}_{1}} U_{\mathbf{b}_{1}}^{\dagger}\right]-\frac{1}{N_{c}^{2}-1} \operatorname{Tr}\left[U_{\mathbf{b}_{1}} U_{\mathbf{y}_{1}}^{\dagger}\right]+1\right)\right. \\
& \left.\times\left(\frac{1}{N_{c}^{2}-1} \operatorname{Tr}\left[U_{\mathbf{x}_{2}} U_{\mathbf{y}_{2}}^{\dagger}\right]-\frac{1}{N_{c}^{2}-1} \operatorname{Tr}\left[U_{\mathbf{x}_{2}} U_{\mathbf{b}_{2}}^{\dagger}\right]-\frac{1}{N_{c}^{2}-1} \operatorname{Tr}\left[U_{\mathbf{b}_{2}} U_{\mathbf{y}_{2}}^{\dagger}\right]+1\right)\right\rangle
\end{aligned}
$$

- Note that if the interaction with the target factorizes,

$$
\left.\left\langle\operatorname{Tr}\left[U_{\mathbf{x}_{1}} U_{\mathbf{y}_{1}}^{\dagger}\right] \operatorname{Tr}\left[U_{\mathbf{x}_{2}} U_{\mathbf{y}_{2}}^{\dagger}\right]\right\rangle\right|_{\text {large }-N_{c}, \operatorname{large}-A_{2}} \approx\left\langle\operatorname{Tr}\left[U_{\mathbf{x}_{1}} U_{\mathbf{y}_{1}}^{\dagger}\right]\right\rangle\left\langle\operatorname{Tr}\left[U_{\mathbf{x}_{2}} U_{\mathbf{y}_{2}}^{\dagger}\right]\right\rangle
$$

we still have the geometric correlations.

- The geometric correlations lead to non-zero cumulants! (Like everything that depends on geometry.) 


\section{Two-gluon production: properties}

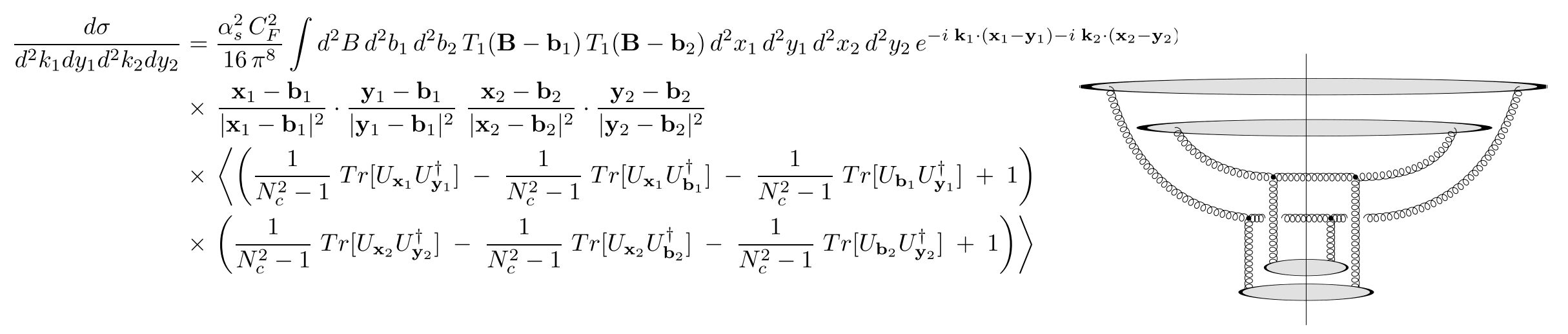

- If we expand the interaction with the target to the lowest non-trivial order, one reproduced the contribution of the 'glasma' graphs:

$$
\begin{aligned}
& \left.\frac{d \sigma_{c o r r}}{d^{2} k_{1} d y_{1} d^{2} k_{2} d y_{2}}\right|_{L O}=\frac{\alpha_{s}^{2}}{4 \pi^{4}} \int d^{2} B d^{2} b\left[T_{1}(\mathbf{B}-\mathbf{b})\right]^{2} \frac{Q_{s 0}^{4}(\mathbf{b})}{\mathbf{k}_{1}^{2} \mathbf{k}_{2}^{2}} \int_{\Lambda} \frac{d^{2} l}{\left(\mathbf{l}^{2}\right)^{2}}\left[\frac{1}{\left(\mathbf{k}_{1}-\mathbf{l}\right)^{2}\left(\mathbf{k}_{2}+\mathbf{l}\right)^{2}}+\frac{1}{\left(\mathbf{k}_{1}-\mathbf{l}\right)^{2}\left(\mathbf{k}_{2}-\mathbf{l}\right)^{2}}\right] \\
& \sim \frac{1}{\left(\mathbf{k}_{1}+\mathbf{k}_{2}\right)^{2}} \\
& \sim \frac{1}{\left(\mathbf{k}_{1}-\mathbf{k}_{2}\right)^{2}}
\end{aligned}
$$

(cf. Dumitru, Gelis, McLerran, Venugopalan '08) 


\section{Two-gluon production: properties}

- Crossed diagrams at lowest nontrivial order
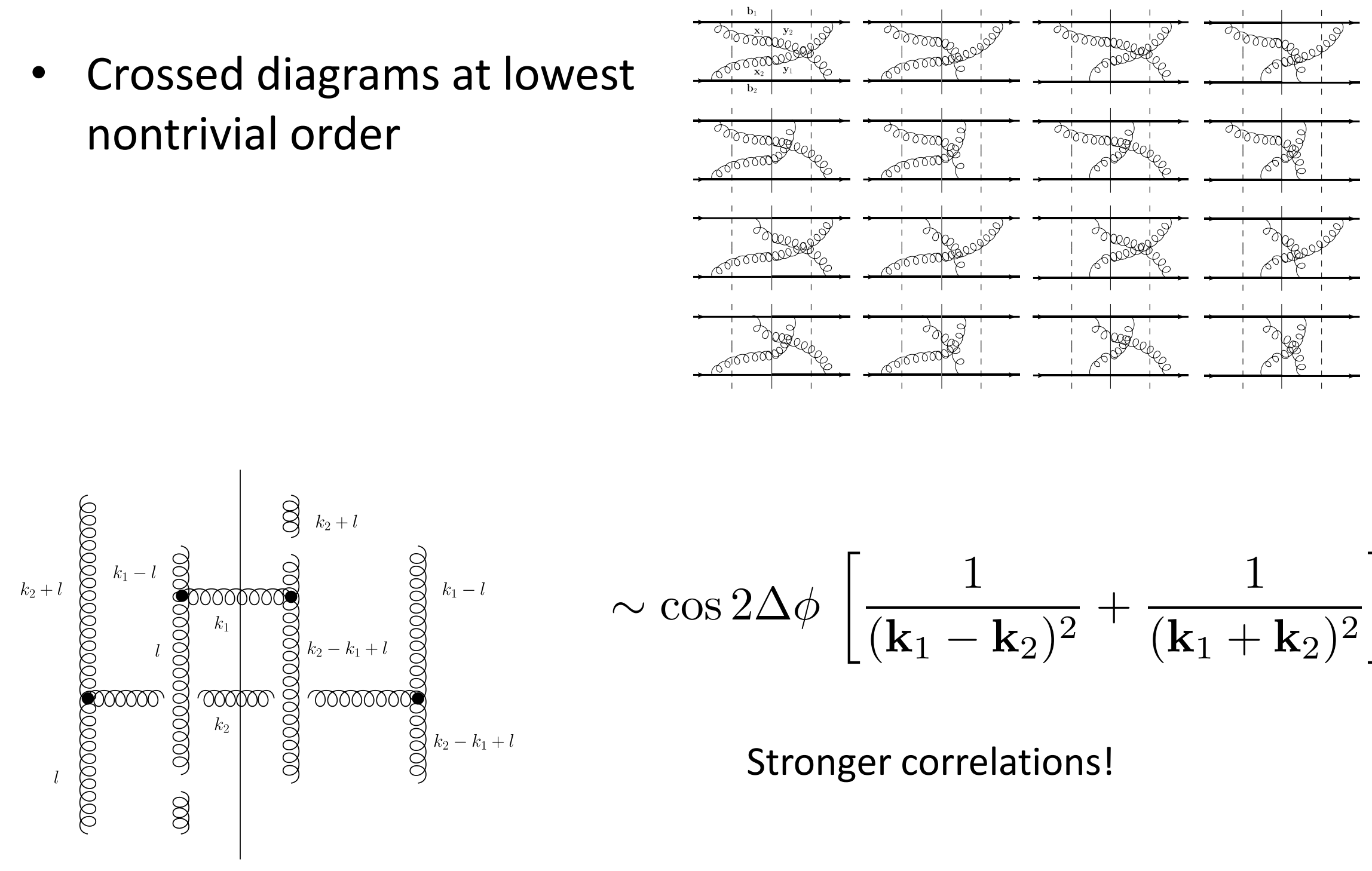

$$
\sim \cos 2 \Delta \phi\left[\frac{1}{\left(\mathbf{k}_{1}-\mathbf{k}_{2}\right)^{2}}+\frac{1}{\left(\mathbf{k}_{1}+\mathbf{k}_{2}\right)^{2}}\right]
$$

Stronger correlations! 


\section{Two-gluon production: properties}

$$
\begin{aligned}
\frac{d \sigma}{d^{2} k_{1} d y_{1} d^{2} k_{2} d y_{2}} & =\frac{\alpha_{s}^{2} C_{F}^{2}}{16 \pi^{8}} \int d^{2} B d^{2} b_{1} d^{2} b_{2} T_{1}\left(\mathbf{B}-\mathbf{b}_{1}\right) T_{1}\left(\mathbf{B}-\mathbf{b}_{2}\right) d^{2} x_{1} d^{2} y_{1} d^{2} x_{2} d^{2} y_{2} e^{-i \mathbf{k}_{1} \cdot\left(\mathbf{x}_{1}-\mathbf{y}_{1}\right)-i \mathbf{k}_{2} \cdot\left(\mathbf{x}_{2}-\mathbf{y}_{2}\right)} \\
& \times \frac{\mathbf{x}_{1}-\mathbf{b}_{1}}{\left|\mathbf{x}_{1}-\mathbf{b}_{1}\right|^{2}} \cdot \frac{\mathbf{y}_{1}-\mathbf{b}_{1}}{\left|\mathbf{y}_{1}-\mathbf{b}_{1}\right|^{2}} \frac{\mathbf{x}_{2}-\mathbf{b}_{2}}{\left|\mathbf{x}_{2}-\mathbf{b}_{2}\right|^{2}} \cdot \frac{\mathbf{y}_{2}-\mathbf{b}_{2}}{\left|\mathbf{y}_{2}-\mathbf{b}_{2}\right|^{2}} \\
& \times\left\langle\left(\frac{1}{N_{c}^{2}-1} \operatorname{Tr}\left[U_{\mathbf{x}_{1}} U_{\mathbf{y}_{1}}^{\dagger}\right]-\frac{1}{N_{c}^{2}-1} \operatorname{Tr}\left[U_{\mathbf{x}_{1}} U_{\mathbf{b}_{1}}^{\dagger}\right]-\frac{1}{N_{c}^{2}-1} \operatorname{Tr}\left[U_{\mathbf{b}_{1}} U_{\mathbf{y}_{1}}^{\dagger}\right]+1\right)\right. \\
& \left.\times\left(\frac{1}{N_{c}^{2}-1} \operatorname{Tr}\left[U_{\mathbf{x}_{2}} U_{\mathbf{y}_{2}}^{\dagger}\right]-\frac{1}{N_{c}^{2}-1} \operatorname{Tr}\left[U_{\mathbf{x}_{2}} U_{\mathbf{b}_{2}}^{\dagger}\right]-\frac{1}{N_{c}^{2}-1} \operatorname{Tr}\left[U_{\mathbf{b}_{2}} U_{\mathbf{y}_{2}}^{\dagger}\right]+1\right)\right\rangle
\end{aligned}
$$

- The cross section is symmetric under (ditto for the "crossed" term)

$$
\begin{aligned}
& \mathbf{k}_{1} \leftrightarrow \mathbf{k}_{2} \quad \text { (just coordinate relabeling) } \\
& \mathbf{k}_{2} \rightarrow-\mathbf{k}_{2} \quad \text { as } \quad \operatorname{Tr}\left[U_{\mathbf{x}} U_{\mathbf{y}}^{\dagger}\right]=\operatorname{Tr}\left[U_{\mathbf{y}} U_{\mathbf{x}}^{\dagger}\right]
\end{aligned}
$$

- Hence the correlations generate only even azimuthal harmonics

$$
\sim \cos 2 n\left(\phi_{1}-\phi_{2}\right)
$$




\section{Correlation function}

May look like this (a toy model; two particles far separated in rapidity, jets subtracted, $p A$ and $A A$ ):

$\mathrm{C}(\Delta \phi)$
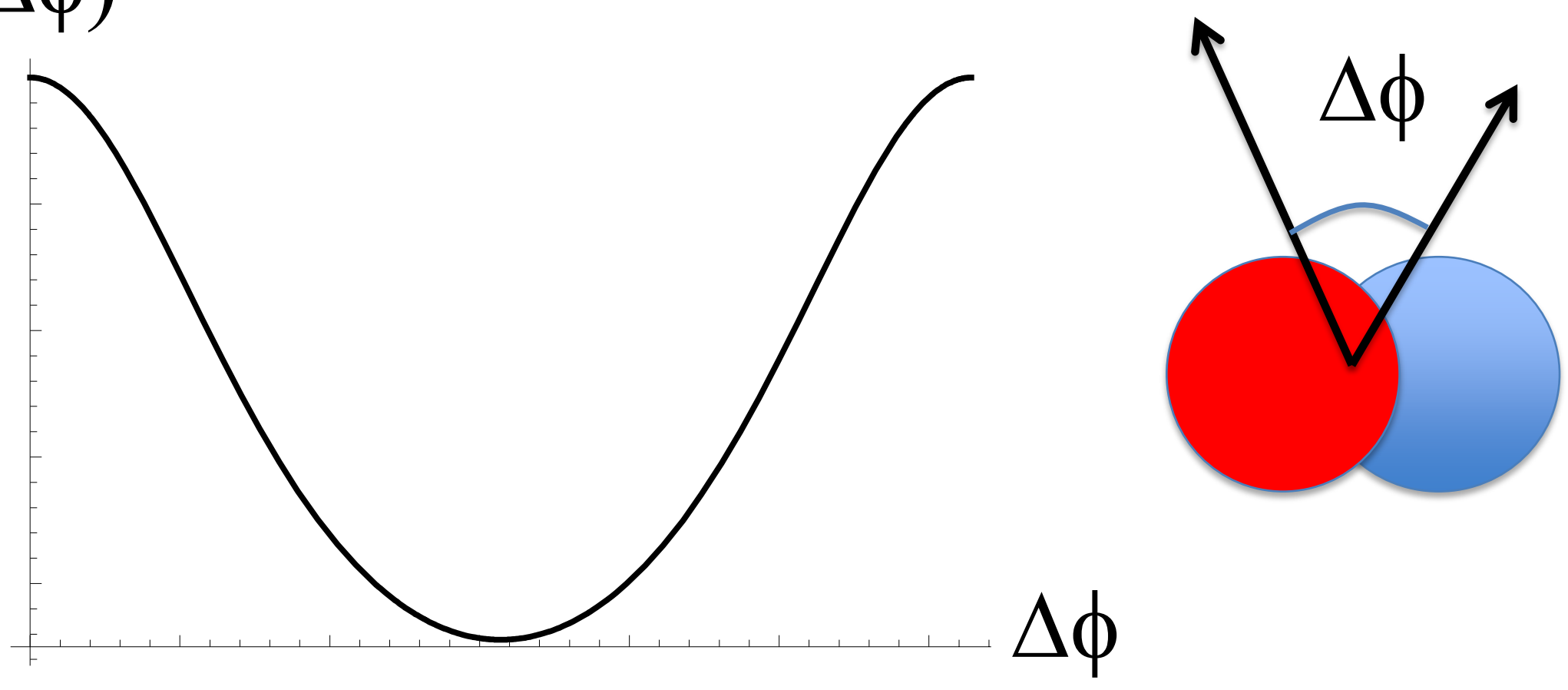

Dumitru, Gelis, McLerran, Venugopalan '08; Yu.K., D. Wertepny '12; Lappi, Srednyak, and Venugopalan '09 


\section{This conclusion is consistent with the data}

\section{Long-Range Rapidity Correlations in Heavy-Light Ion Collisions}

Yuri V. Kovchegov, Douglas E. Wertepny

(Submitted on 5 Dec 2012)

We study two-particle long-range rapidity correlations arising in the early stages of heavy ion collisions in the saturation/Color Glass Condensate framework, assuming for simplicity that one colliding nucleus is much larger than the other. We calculate the two-gluon production cross section while including all-order saturation effects in the heavy nucleus with the lowest-order rescattering in the lighter nucleus. We find four types of correlations in the two-gluon production cross section: (i) geometric correlations, (ii) HBT correlations, (iii) back-to-back correlations, and (iv) near-side azimuthal correlations which are long-range in rapidity. The geometric correlations (i) are due to the fact that nucleons are correlated by simply being confined within the same nucleus and may lead to long-range rapidity correlations for the produced particles without strong azimuthal angle dependence. Somewhat surprisingly, long-range rapidity correlations (iii) and (iv) have exactly the same amplitudes along with azimuthal and rapidity shapes: one centered around $\backslash$ Delta $\backslash$ phi $=\backslash \mathrm{pi} \backslash$ with the other one centered around $\backslash$ Delta $\backslash \mathrm{phi}=0$ (here $\backslash$ Delta $\backslash \mathrm{phi} \backslash$ is the azimuthal angle between the two produced gluons).

We thus observe that the early-time CGC dynamics in nucleus-nucleus collisions generates azimuthal non-flow correlations which are qualitatively different from jet correlations by being long-range in rapidity. If strong enough, they have the potential of mimicking the elliptic (and higher-order even-harmonic) flow in the di-hadron correlators: one may need to take them into account in the experimental determination of the flow observables. 


\section{This conclusion is consistent with the data}

\section{Long-range angular correlations on the near and away side in $\mathrm{p}-\mathrm{Pb}$ collisions at sqrt $(\mathrm{sNN})=5.02 \mathrm{TeV}$}

\section{ALICE Collaboration}

\section{(Submitted on 10 Dec 2012)}

Angular correlations between charged trigger and associated particles are measured by the ALICE detector in $\mathrm{p}-\mathrm{Pb}$ collisions at a nucleon-nucleon centre-of-mass energy of $5.02 \mathrm{TeV}$ for transverse momentum ranges within $0.5<\mathrm{pT}$, assoc $<\mathrm{pT}$, trig $<4 \mathrm{GeV} / \mathrm{c}$. The correlations are measured over two units of pseudorapidity and full azimuthal angle in different intervals of event multiplicity, and expressed as associated yield per trigger particle. Two long-range ridge-like structures, one on the near side and one on the away side, are observed when the per-trigger yield obtained in low-multiplicity events is subtracted from the one in high-multiplicity events. The excess on the near-side is qualitatively similar to that recently reported by the CMS collaboration, while the excess on the away-side is reported for the first time. The two-ridge structure projected onto azimuthal angle is quantified with the second and third Fourier coefficients as well as by near-side and away-side yields and widths. The yields on the near side and on the away side are equal within the uncertainties for all studied event multiplicity and PT bins, and the widths show no significant evolution with event multiplicity or pT. These findings suggest that the near-side ridge is accompanied by an essentially identical away-side ridge. 


\section{LHC $p+P b$ data from ALICE}

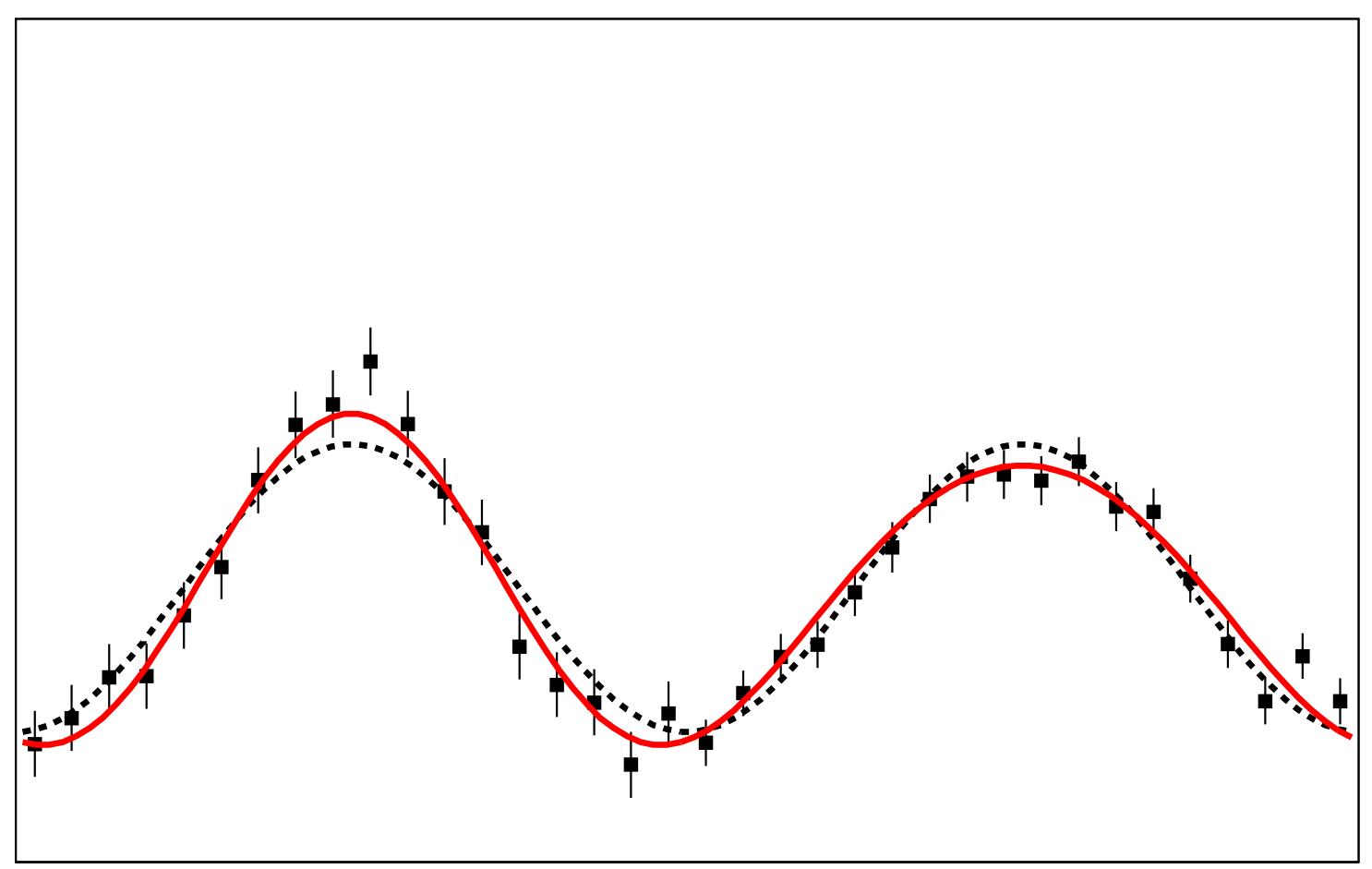

- These are high-multiplicity collisions: it is possible that quark-gluon plasma is created in those, with the hydrodynamics contributing to these correlations.

- Saturation approach is lacking the odd harmonics, like $\cos (3 \Delta \phi)$, etc. Can they be generated in a pure CGC approach EBE? 
C. HBT correlations 


\section{HBT diagrams}

- There is another contribution coming from the "crossed" diagrams
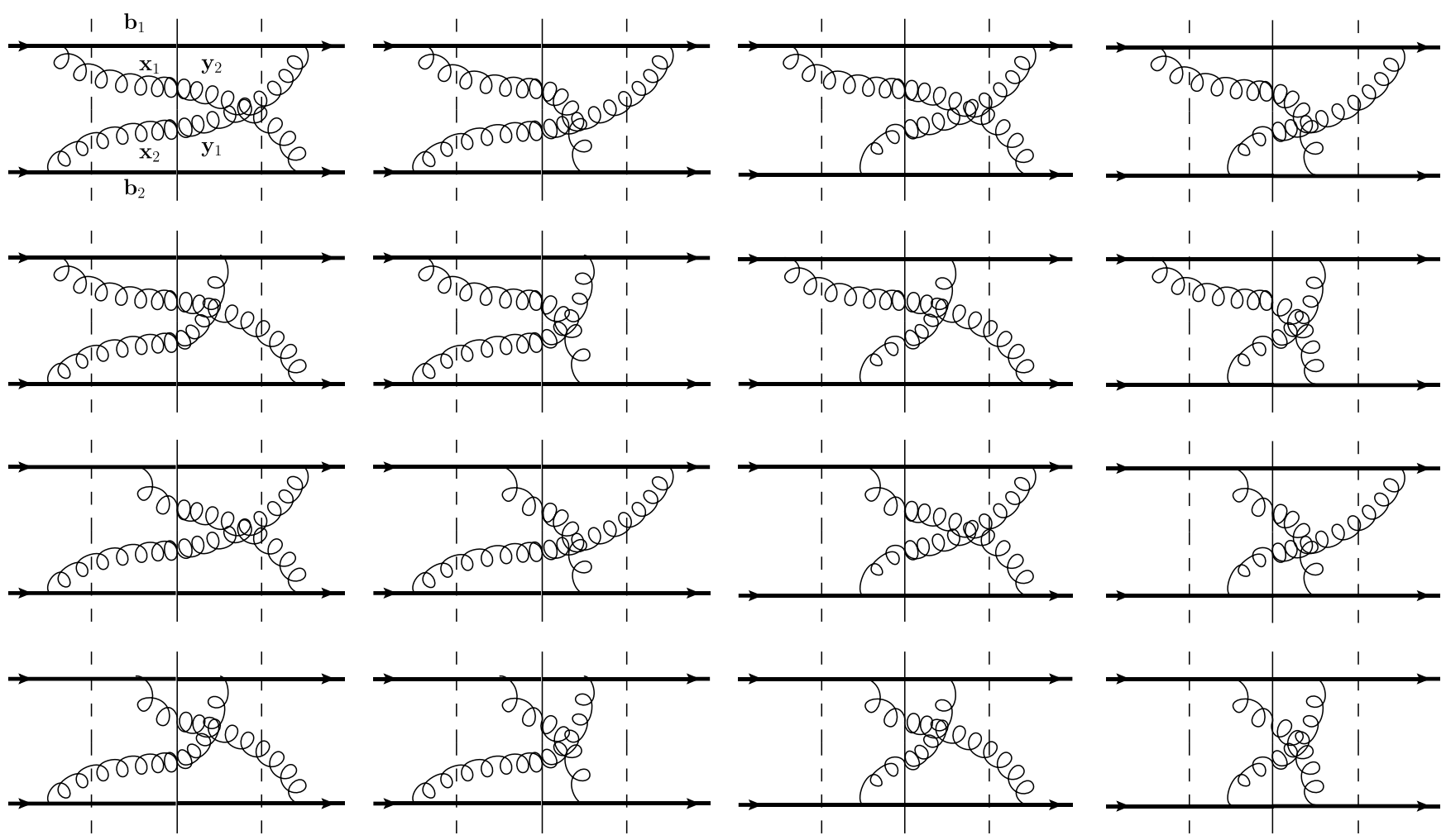


\section{HBT diagrams}

- They give HBT correlations (with $\mathrm{R}_{\text {long }}=0$ due to Lorentz contraction)

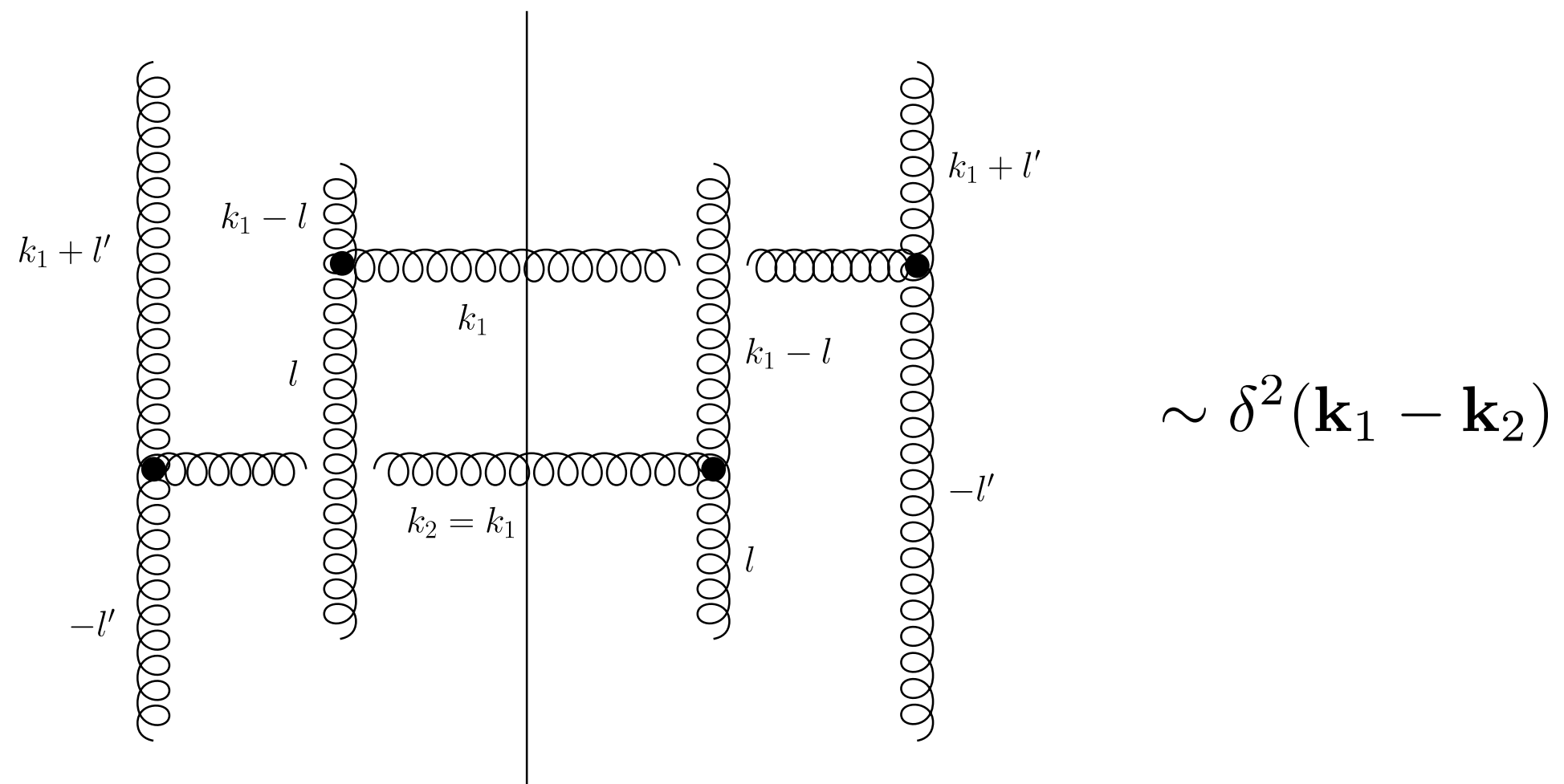

- Just like the standard HBT correlations

$\left|\Psi_{1}\left(\mathbf{k}_{1}\right) \Psi_{2}\left(\mathbf{k}_{2}\right)+\Psi_{1}\left(\mathbf{k}_{2}\right) \Psi_{2}\left(\mathbf{k}_{1}\right)\right|^{2} \rightarrow \Psi_{1}\left(\mathbf{k}_{1}\right) \Psi_{2}\left(\mathbf{k}_{2}\right) \Psi_{1}^{*}\left(\mathbf{k}_{2}\right) \Psi_{2}^{*}\left(\mathbf{k}_{1}\right)+$ c.c. $+\ldots$

- Possibly fragmentation would break phase coherence making these perturbative HBT correlations not observable. 


\section{Back-to-back HBT?}

- Note that all our formulas are symmetric under

$$
\mathbf{k}_{2} \rightarrow-\mathbf{k}_{2}
$$

- Therefore, the HBT correlation is accompanied by the identical back-to-back HBT correlation

$$
\sim \delta^{2}\left(\mathbf{k}_{1}+\mathbf{k}_{2}\right)
$$

- Note again that this correlation may be destroyed in hadronization. 


\section{Conclusions}

- In this talk I have shown a calculation of the two-gluon production cross section in nuclear collisions including saturation effects in one nucleus to all orders (heavy-light ion collisions).

- Correlations we see:

- Geometric correlations $\checkmark$

- HBT correlations (along with b2b HBT ones)

- Away-side correlations $\checkmark$

- Near-side long-range rapidity correlations $\checkmark$ ( $\checkmark$ = long-range in rapidity)

- Near- and away-side correlations are identical to all orders in saturation effects: this is mainly consistent with the recent $\mathrm{p}+\mathrm{Pb}$ data from LHC.

- Seems like geometric correlations are hard to remove by cumulants! 


\section{Backup Slides}




\section{Ridge in heavy ion collisions}

- Heavy ion collisions, along with high-multiplicity pp collisions, are known to have long-range rapidity correlations, known as 'the ridge':

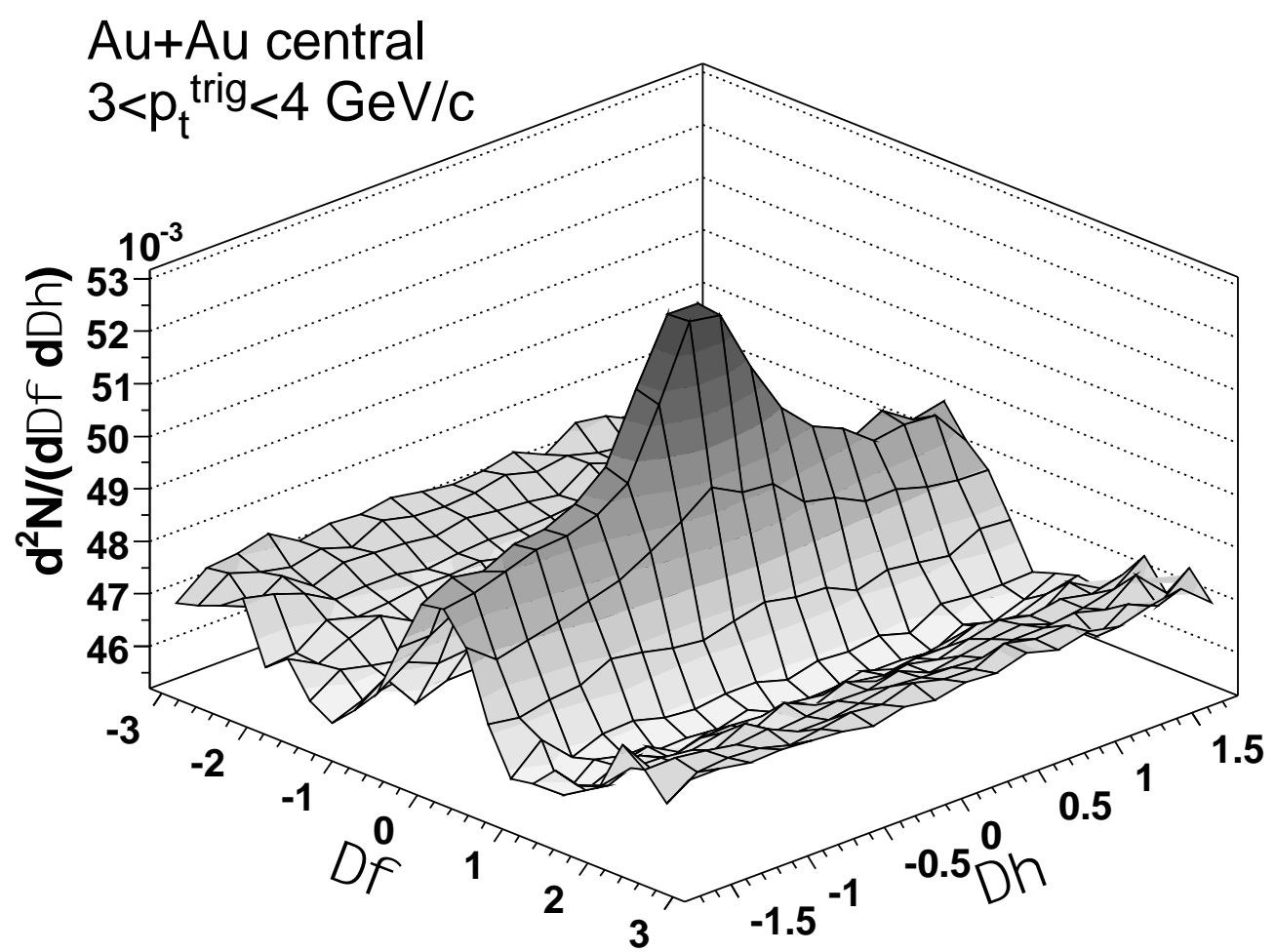




\section{What to calculate?}

- To systematically include glasma graphs in the CGC formalism it would be great to solve the two-gluon inclusive production problem in the MV model, that is, including multiple rescatterings in both nuclei to all orders:

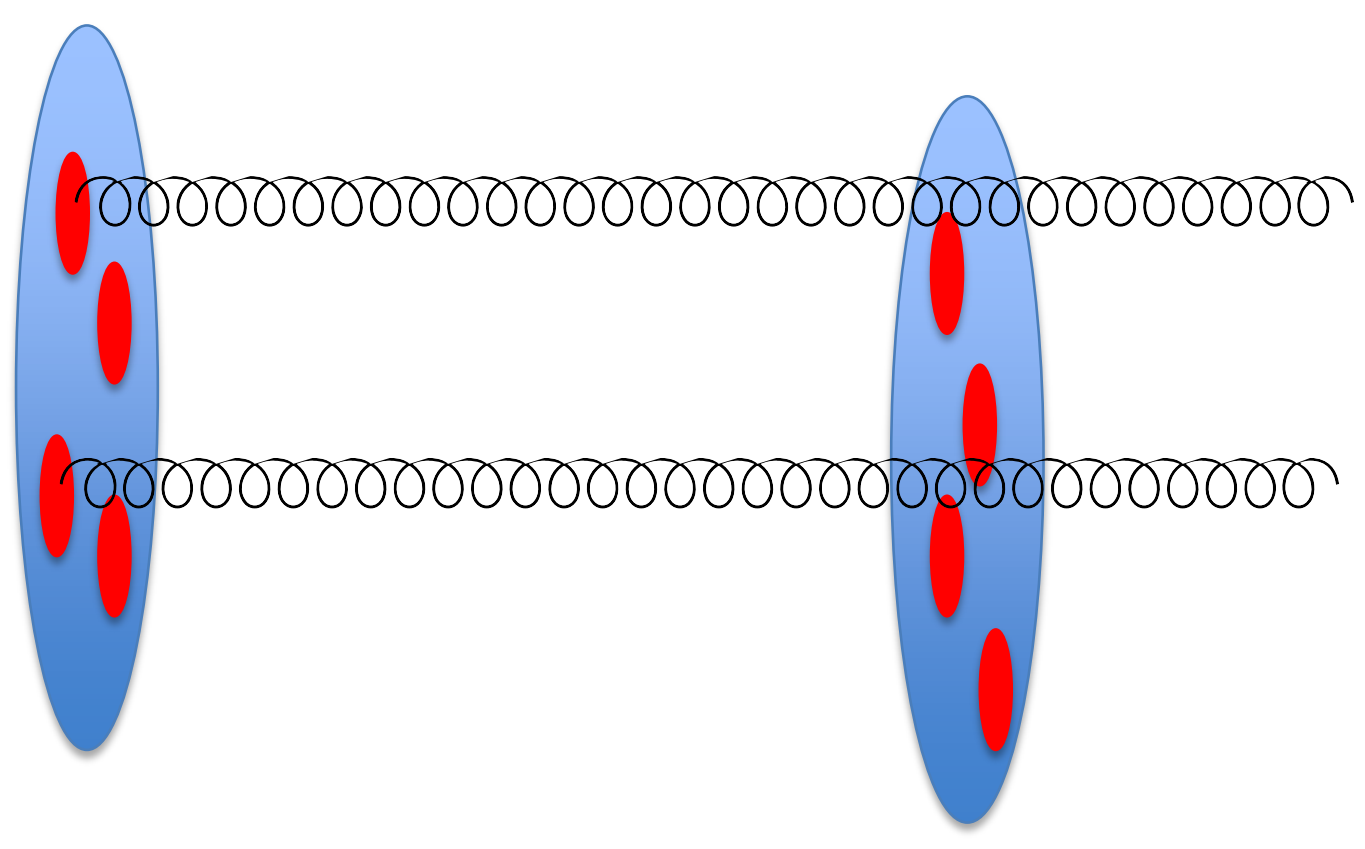




\section{Forward dipole amplitude}

$$
N\left(\underline{x}_{1}, \underline{x}_{2}\right)=1-\frac{1}{N_{c}}\left\langle\operatorname{tr}\left[V\left(\underline{x}_{1}\right) V^{\dagger}\left(\underline{x}_{2}\right)\right]\right\rangle
$$

- Valid both in the quasi-classical Glauber-Mueller/McLerranVenugopalan multiple-rescattering approximation and for the LLA small-x evolution (BFKL/BK/JIMWLK). 


\section{Two-gluon production}

- The approximation can also be formulated as resumming all powers of target color charge density $\rho_{2}$ while staying at the order- $\rho_{1}^{2}$ in the projectile charge density.

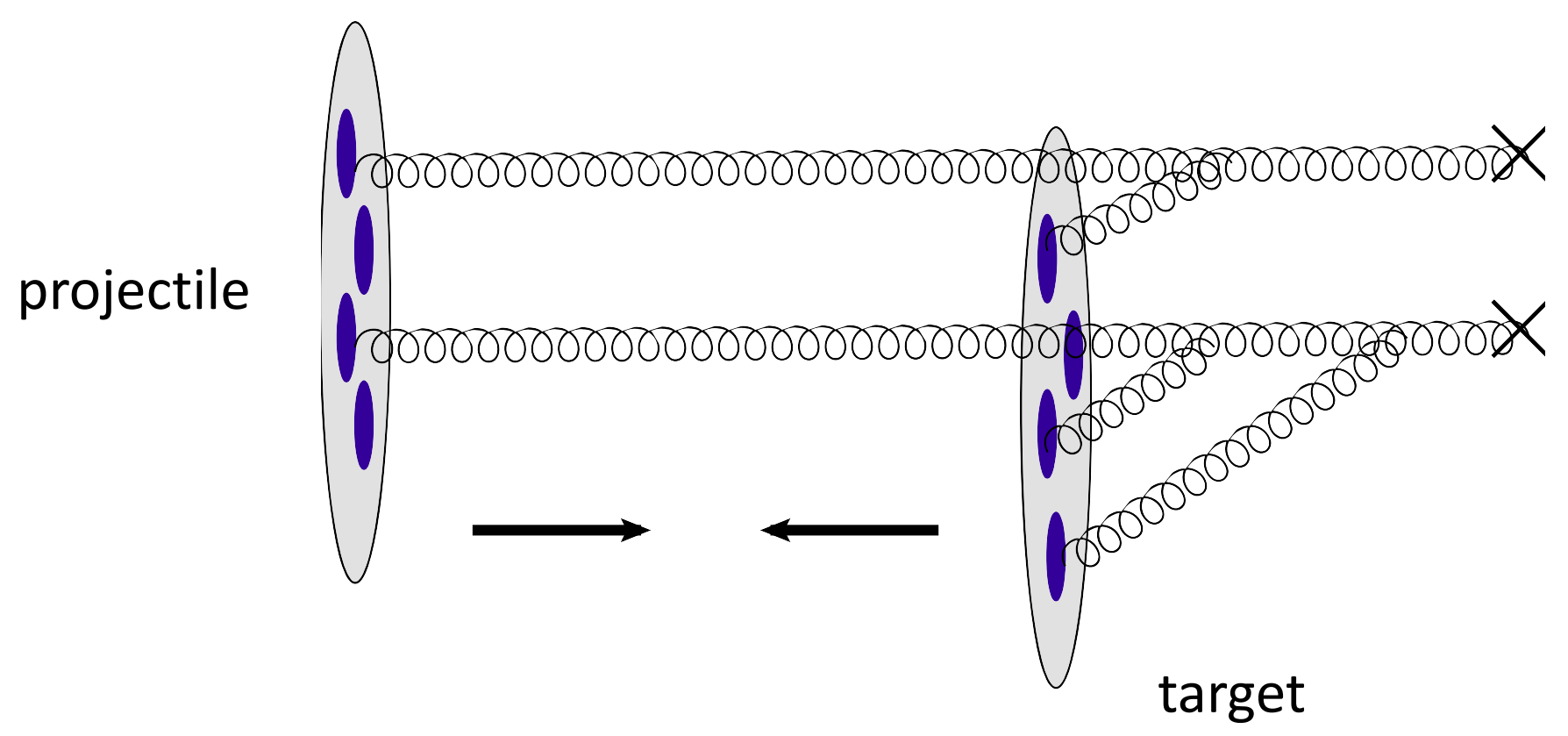




\section{Origin of correlations}

- It appears that the correlation is in the connected part $\Delta$ of the double-trace correlator

$\frac{1}{\left(N_{c}^{2}-1\right)^{2}}\left\langle\operatorname{Tr}\left[U_{\mathbf{x}_{1}} U_{\mathbf{x}_{2}}^{\dagger}\right] \operatorname{Tr}\left[U_{\mathbf{x}_{3}} U_{\mathbf{x}_{4}}^{\dagger}\right]\right\rangle=\frac{1}{\left(N_{c}^{2}-1\right)^{2}}\left\langle\operatorname{Tr}\left[U_{\mathbf{x}_{1}} U_{\mathbf{x}_{2}}^{\dagger}\right]\right\rangle\left\langle\operatorname{Tr}\left[U_{\mathbf{x}_{3}} U_{\mathbf{x}_{4}}^{\dagger}\right]\right\rangle+\Delta\left(\mathbf{x}_{1}, \mathbf{x}_{2}, \mathbf{x}_{3}, \mathbf{x}_{4}\right)$

- We evaluated the correlator in the Gaussian (MV) approximation in the large- $\mathrm{N}_{\mathrm{c}}$ limit.

- Its evolution is not straightforwards: JIMWLK gives a pretty hard to work with expression. 


\section{Correlations in AdS shock wave collisions}

$$
C\left(k_{1}, k_{2}\right) \sim \cosh (4 \Delta y)
$$

- Correlations grow with rapidity interval???

- It is possible that higher-order corrections in shock wave strengths will modify this result, making it closer to real life.

- However, such corrections are important at later times, and are less likely to affect the long-range rapidity correlation...

- This could be another argument in favor of weakly-coupled dynamics in the early stages of heavy ion collisions. 


\title{
CGC 2013 - A PERSPECTIVE.
}

\author{
Alex Kovner \\ University of Connecticut
}

p-A COLLISIONS A NATURAL HABITAT FOR SATURATION.

SO IS IT THERE? IS IT NOT THERE? CAN WE EVEN TELL IF IT IS THERE? 


\section{A WONDERFUL AND SIMPLE IDEA}

IN A DENSE SYSTEM (HADRON OR NUCLEUS) THE AVERAGE DENSITY OF PARTONS (OR FIELDS OR WHAT NOT...) DEFINES A TRANSVERSE SCALE $Q_{S}^{2} \propto \rho$.

AT DISTANCE SCALES $\Delta x^{2}<Q_{S}^{-2}$ THE SYSTEM IS DILUTE PARTONIC: GLUON DENSITY AT HIGH MOMENTUM $\Phi(k) \propto 1 / k^{2}$ (up to logarithmic corrections)

AT LOW RESOLUTION SCALES $\Delta x^{2}>Q_{S}^{-2}$ THE SYSTEM IS SATURATED. THERE ARE MUCH LESS GLUONS THAN PERTURBATIVE EXPECTATION: AT $k<$ $Q_{S}, \Phi(k) \propto$ const, OR MAYBE EVEN $\Phi(k) \propto k^{2}$.

$Q_{S}$ IS ALWAYS THERE - EVEN FOR A PROTON AT REST, BUT $Q_{s}^{2} \sim 0.04 G e v^{2}$ - NONPERTURBATIVELY SMALL AND SATURATED REGIME IS INTRACTABLE.

BUT IT GROWS WITH ATOMIC NUMBER AND WITH ENERGY $Q_{S}^{2} \propto A^{1 / 3} s^{\# \alpha_{s}}$

SO NATURALLY THE BEST WAY TO GET INTO " 'PERTURBATIVE SATURATION REGIME"' (CGC ?) IS TO PROBE HEAVY NUCLEUS AT HIGH ENERGY.

TO MINIMIZE STRONG FINAL STATE EFFECTS THE PROBE BETTER BE SMALL, SO IN THE ABSENCE OF e-A MACHINE, THE BEST REACTION IS p-A. 
WHAT DO WE EXPECT FORM CGC?

WITH WHAT LEVEL OF ACCURACY CAN WE CALCULATE INTERESTING OBSERVABLES?

MULTIPLICITES

$k_{T}$ SPECTRUM AND $R_{p A}$ TWO PARTICLE CORRELATIONS - "THE RIDGE" 


\section{MULTIPLICITIES}

THE SIMPLEST OBSERVABLE. ROUGHLY SPEAKING PROPORTIONAL TO THE SATURATION MOMENTUM

$$
\frac{d N}{d y} \propto \frac{1}{\alpha_{S}\left(Q_{S}^{2}\right)} S Q_{S}^{2} \times \ln \frac{Q_{S}^{2}(1)}{Q_{S}^{2}(2)}
$$

ASSUMES $\phi(k) \sim$ const; $\quad k<Q_{S}$ AND $k_{T}$ FACTORISED GLUON PRODUCTION, AND LOCAL GLUON-HADRON DUALITY - KLN MODEL

$Q_{S}$ DEPENDS ON ENERGY, RAPIDITY AND CENTRALITY

$$
Q_{S}^{2}=Q_{0}^{2} N_{\text {part }}\left[x_{0} \frac{W}{Q_{0}} e^{ \pm y}\right]^{\lambda} ; \quad Q_{0}=0.6 \mathrm{Gev} ; \quad x_{0}=0.01 ; \quad \lambda=0.205
$$

THIS SIMPLE INPUT PLUS SOME MODELLING AT $x \rightarrow 1$ PLUS GLAUBER MONTE CARLO FOR FLUCTUATIONS OF $N_{\text {part }}$ AND THEREFORE $Q_{S}$ OF $\mathrm{Pb}$ PRODUCES VERY COMPELLING RESULTS. 


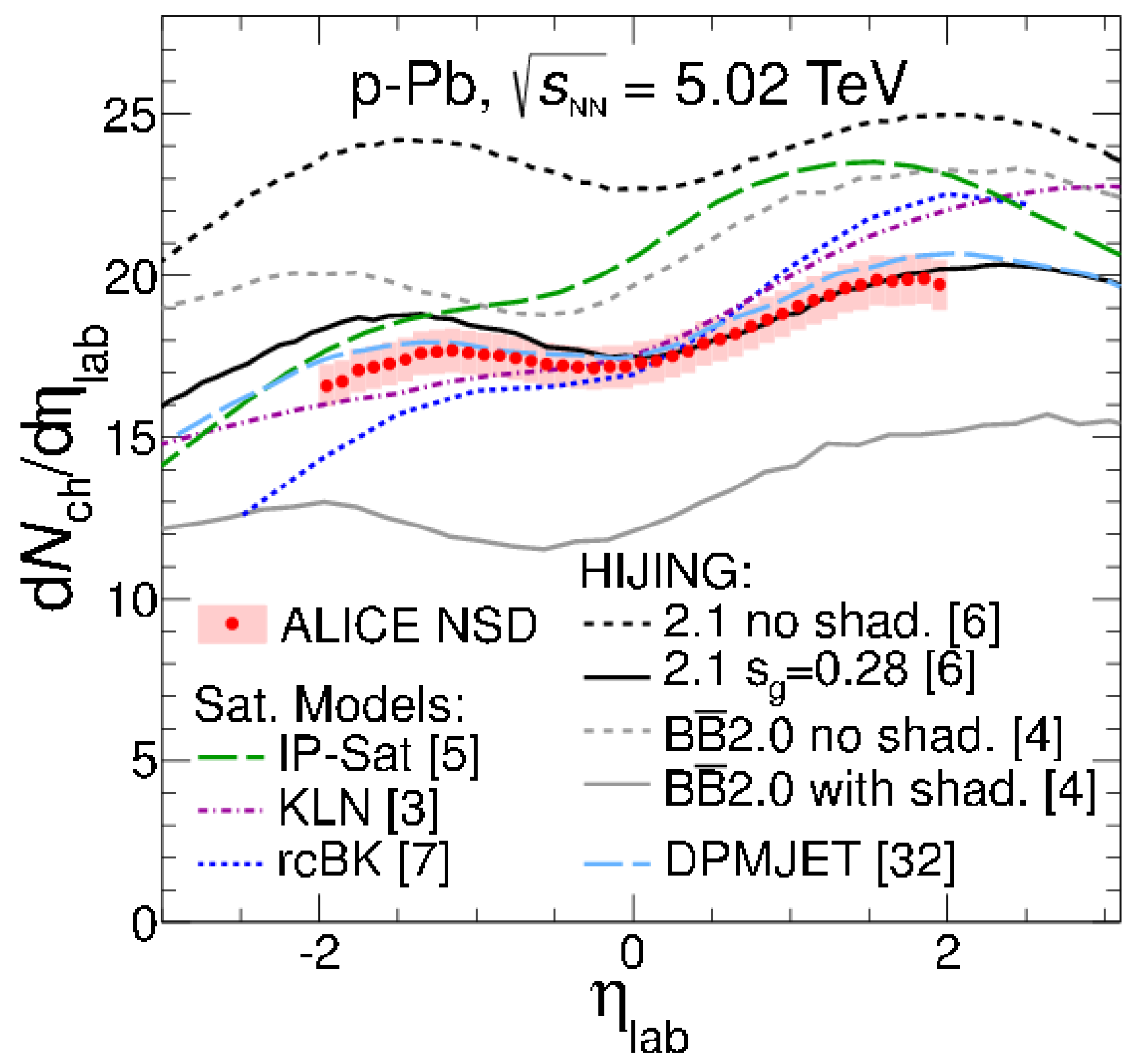




\section{rc BK AND FRIENDS}

\section{BUT ONE WANTS TO DO MORE AND TO DO IT BETTER.}

IN PRINCIPLE WE DO NOT HAVE TO MODEL THE DEPENDENCE OF $Q_{S}$ ON $W$ AND $y$. THIS DEPENDENCE SHOULD FOLLLOW FROM THE QCD EVOLUTION: BK/JIMWLK EQUATION.

LAST SEVERAL YEARS "rCBK" APPROACH HAS BEEN DEVELOPED AND VIGOROUSLY APPLIED.

ONE CHOOSES A "REASONABLE" INITIAL CONDITION FOR $\Phi(k)$ AT INITIAL $x_{0}$ AND EVOLVES IT IN RAPIDITY WITH THE BALITSKY-KOVCHEGOV EQUATION, WHERE THE COUPLING CONSTANT RUNS AT THE SCALE CHOSEN TO MINIMIZE SOME NEXT TO LEADING ORDER CONTRIBUTIONS. 
NEED TO CHOOSE INITIAL CONDITIONS.

$N\left(r, x_{0}\right)=1-\exp \left[-\frac{\left(r^{2} Q_{0 S, \text { proton }}^{2}\right)^{\gamma}}{4} \ln \left(\frac{1}{\Lambda r}\right)\right] ; \quad \Phi(k) \propto \frac{1}{\alpha_{s}(k)} k^{2} N(k)$

PARAMETERS $Q_{0 s}$ AND $\gamma$ ARE CONSTRAINED TO A DEGREE BY FITS TO DIS HERA DATA (ANOTHER ADJUSTABLE PARAMETER OR TWO IN $\alpha_{S}$ )

NEED TO MODEL THE $Q_{S}$ OF NUCLEUS.

TWO BASIC APPROACHES:

1. THE SIMPLEST IS FIX ONE $Q_{0 S}$, nucleus AND SOLVE BK EQUATION WITH THIS INITIAL CONDITION (REZAEIAN also TRIBEDY-VENUGOPALAN).

2. MORE ELABORATE: GLAUBER MONTE CARLO FOR DISTRIBUTION OF NUCLEONS IN THE NUCLEUS YIELDS TRANSVERSE POSITION DEPENDENT $Q_{S}$. CALCULATE PARTICLE PRODUCTION LOCALLY IN $b$ AND INTEGRATE OVER $b$ IN THE END (ALBACETE, DUMITRU, FUJII, NARA) 
FINALLY: HOW DO WE CALCULATE HADRON PRODUCTION?

1. $k_{T}$ FACTORIZATION:

$$
\frac{d N}{d y d^{2} p_{T} d^{2} R} \propto \frac{1}{p_{T}^{2}} \int d^{2} k_{T} \int d^{2} b \alpha_{S}(Q) \Phi_{P}\left(k_{T}, x_{1}, b\right) \Phi_{T}\left(p_{T}-k_{T}, x_{2}, R-b\right)
$$

$R$-NUCLEAR IMPACT PARAMTER, $b$ TRANSVERSE COORDINATE IN THE PROTON. AT HIGH $P_{T}$ CONVOLUTED WITH GLUON FRAGMENTATION FUNCTION. THIS IS DERIVED FOR PRODUCTION OF SOFT GLUONS - FAR IN RAPIDITY FROM VALENCE PARTONS.

2. FOR MORE FORWARD RAPIDITIES - HYBRID FORMALIZM:

$$
\begin{gathered}
\frac{d N}{d \eta d^{2} k}=\left[\frac{d N}{d \eta d^{2} k}\right]_{\text {elastic }}+\left[\frac{d N}{d \eta d^{2} k}\right]_{\text {inelastic }} \\
{\left[\frac{d N}{d \eta d^{2} k}\right]_{\text {elastic }} \propto \Sigma_{q, g} x_{1} f_{q, g / p}\left(x_{1}\right) N_{q, g}\left(x_{2}, k_{T}\right)} \\
{\left[\frac{d N}{d \eta d^{2} k}\right]_{\text {inelastic }} \propto \alpha_{S}(Q) \frac{1}{k^{4}} \int d^{2} p p^{2} N\left(x_{2}, p\right) \int \frac{d \zeta}{\zeta} \omega_{j}(\zeta) f_{j}\left(\frac{x_{1}}{\zeta}, Q^{2}\right)}
\end{gathered}
$$




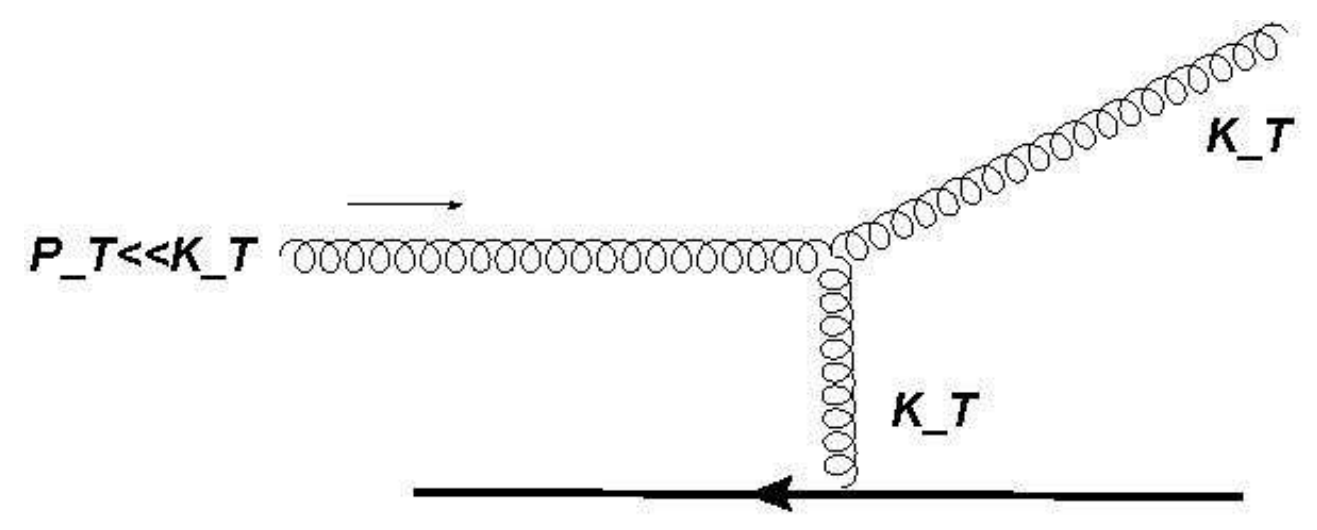

Figure 1: Elastic contribution to particle production.

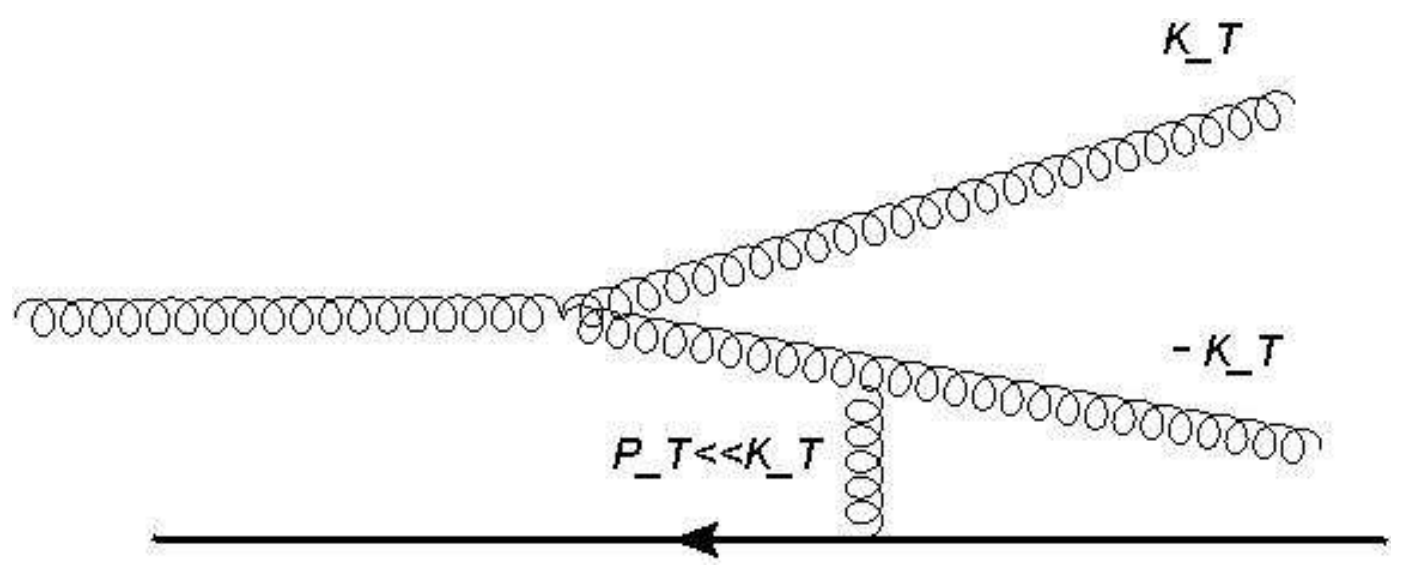

Figure 2: Inelastic contribution to particle production.

INELASTIC CONTRIBUTION FORMALLY A PERTURBATIVE CORRECTION, BUT IS OF THE SAME ORDER AS DGLAP EVOLUTION OF PARTONIC DISTRIBUTIONS IN THE ELASTIC PART. 


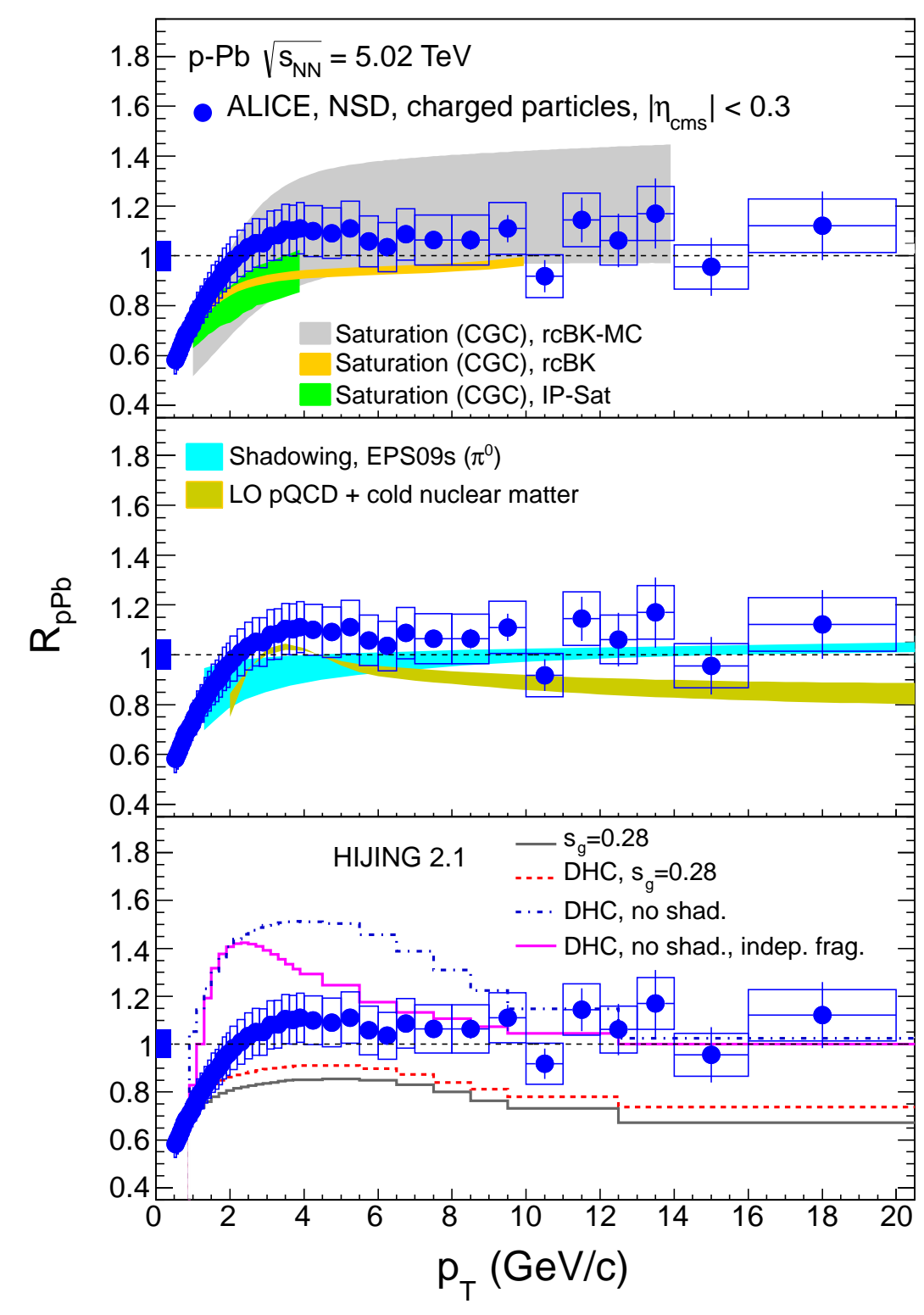

Figure 3: ALICE data: NO CRONIN PEAK (check), SUPPRESSION UP TO $2 \mathrm{Gev}$ (check), VERY FLAT $R_{p A} \sim 1$ ABOVE 2-3 Gev (kh-m, check) 

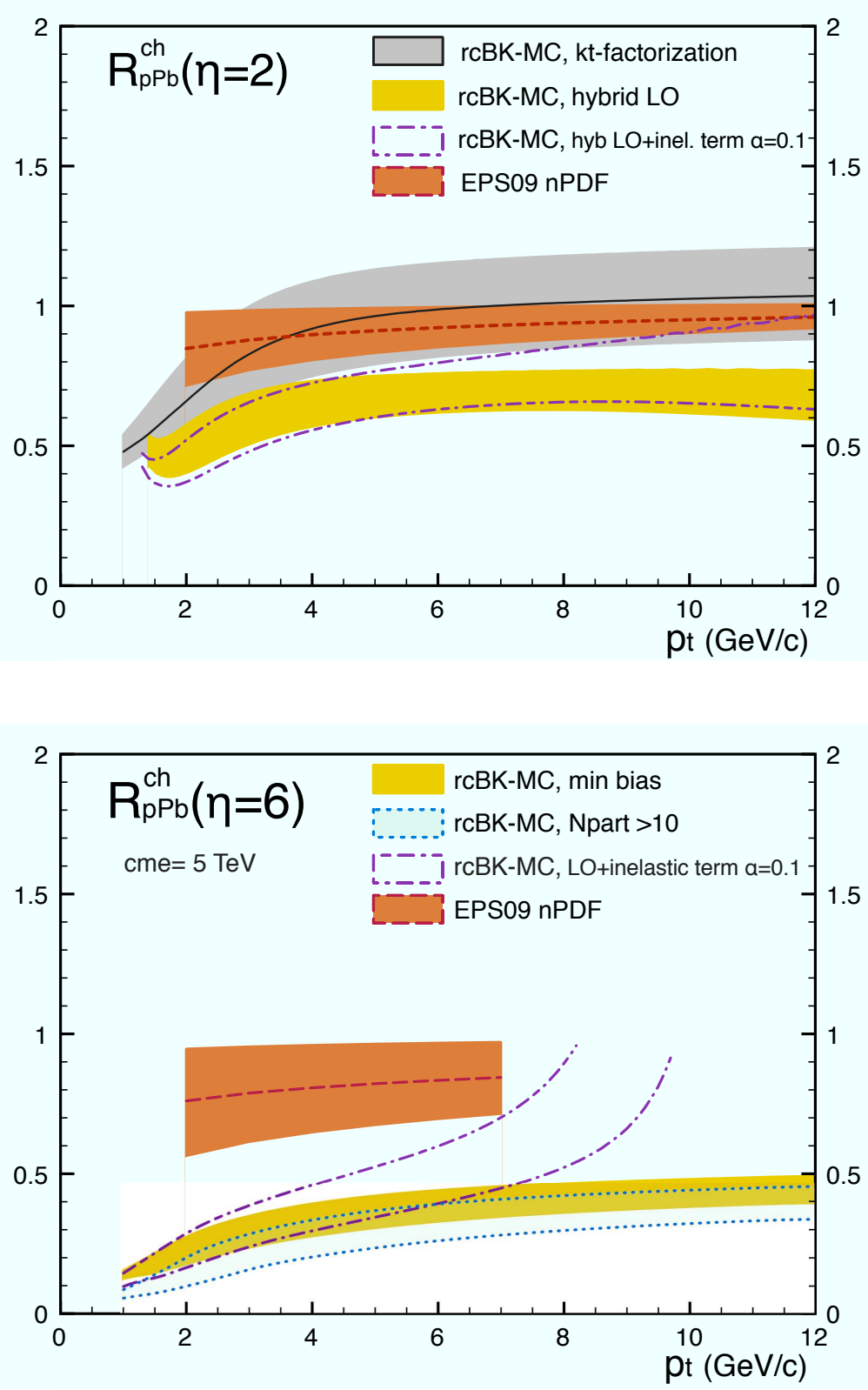

Figure 4: Albacete et.al. - predictions for $\mathrm{p}-\mathrm{Pb}$ at $5 \mathrm{Tev}$ 


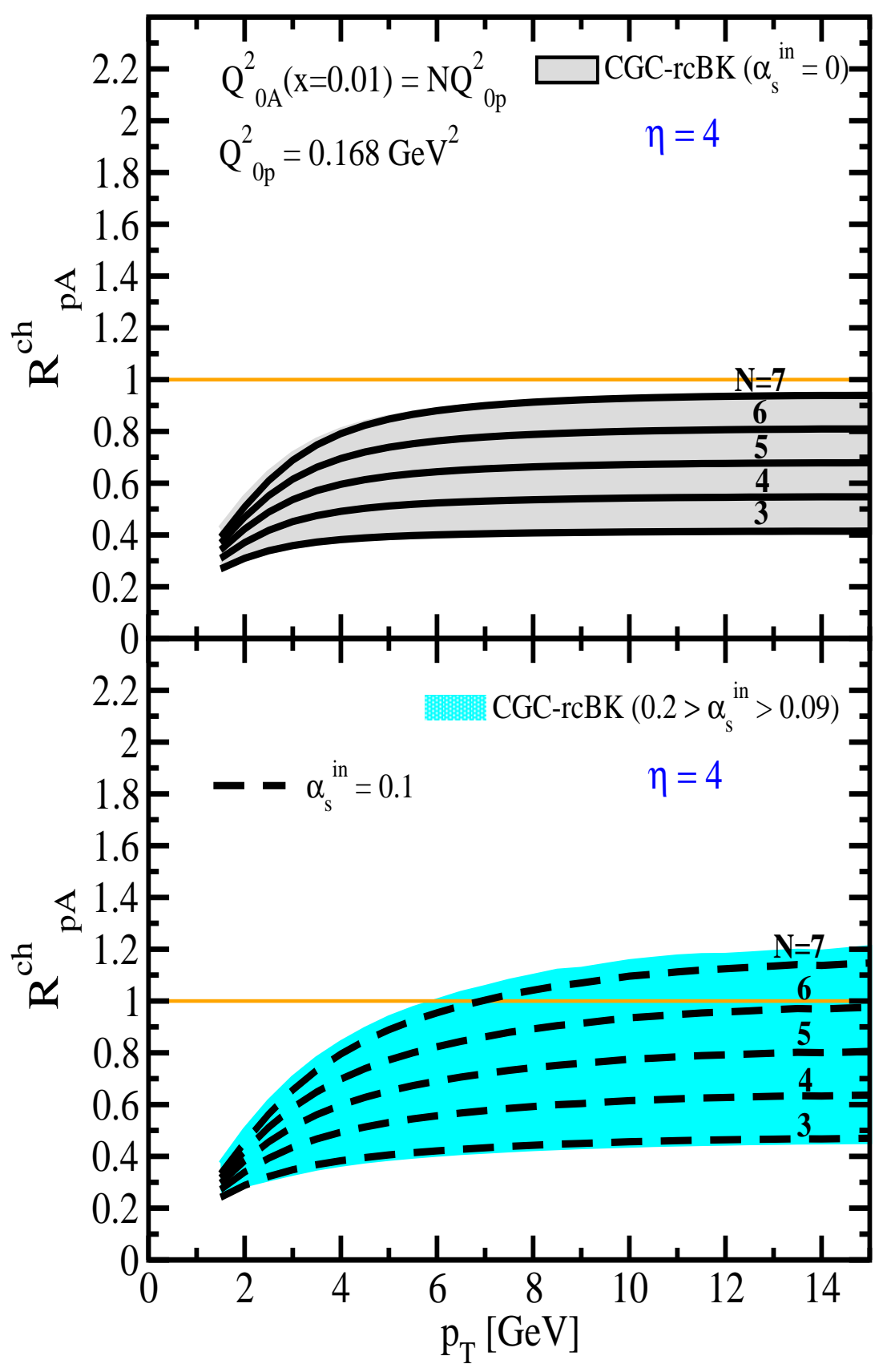

Figure 5: Rezaeian - predictions for $\mathrm{p}-\mathrm{Pb}$ at $5 \mathrm{Tev}$ 


\section{CLEARLY THE PREDICTION BAND IS VERY WIDE}

CLEARLY THE EFFECT OF THE PERTURBATIVE CORRECTION - THE INELASTIC TERM IS VERY LARGE.

\section{SO WE NEED TO UNDERSTAND THINGS BETTER.}

IS PERTURBATION THEORY UNSTABLE? POSSIBLY. THEN WE ARE IN TROUBLE.

MORE LIKELY, I THINK, THE INELASTIC CORRECTION IS NOT AS BIG AS IT LOOKS. AT FORWARD RAPIDITIES AND LARGE TRANSVERSE MOMENTA THE PARTON PAIR ONLY EXIST FOR A SHORT TIME AND SHOULD NOT BE ABLE TO SCATTER COHERENTLY OFF THE TARGET. COHERENT SCATTERING APPROXIMATION MAY WELL BE AT FAULT.

MORE COMPLETE PERTURBATIVE CORRECTION EXISTS: CHIRILLI, XIAO, YUAN - BUT THE SCATTERING IS STILL TREATED THERE AS COHERENT. 
rCBK ALSO PRODUCES PARTICLE MULTIPLICITIES. SO DO OTHER PHENOMENOLOGICAL SATURATION ANSATZE FOR THE GLUON DENSITY (IPSAT, b-CGC)

IRONICALLY THE "OF THE SHELF" KLN MODEL STILL DOES THE BEST JOB.

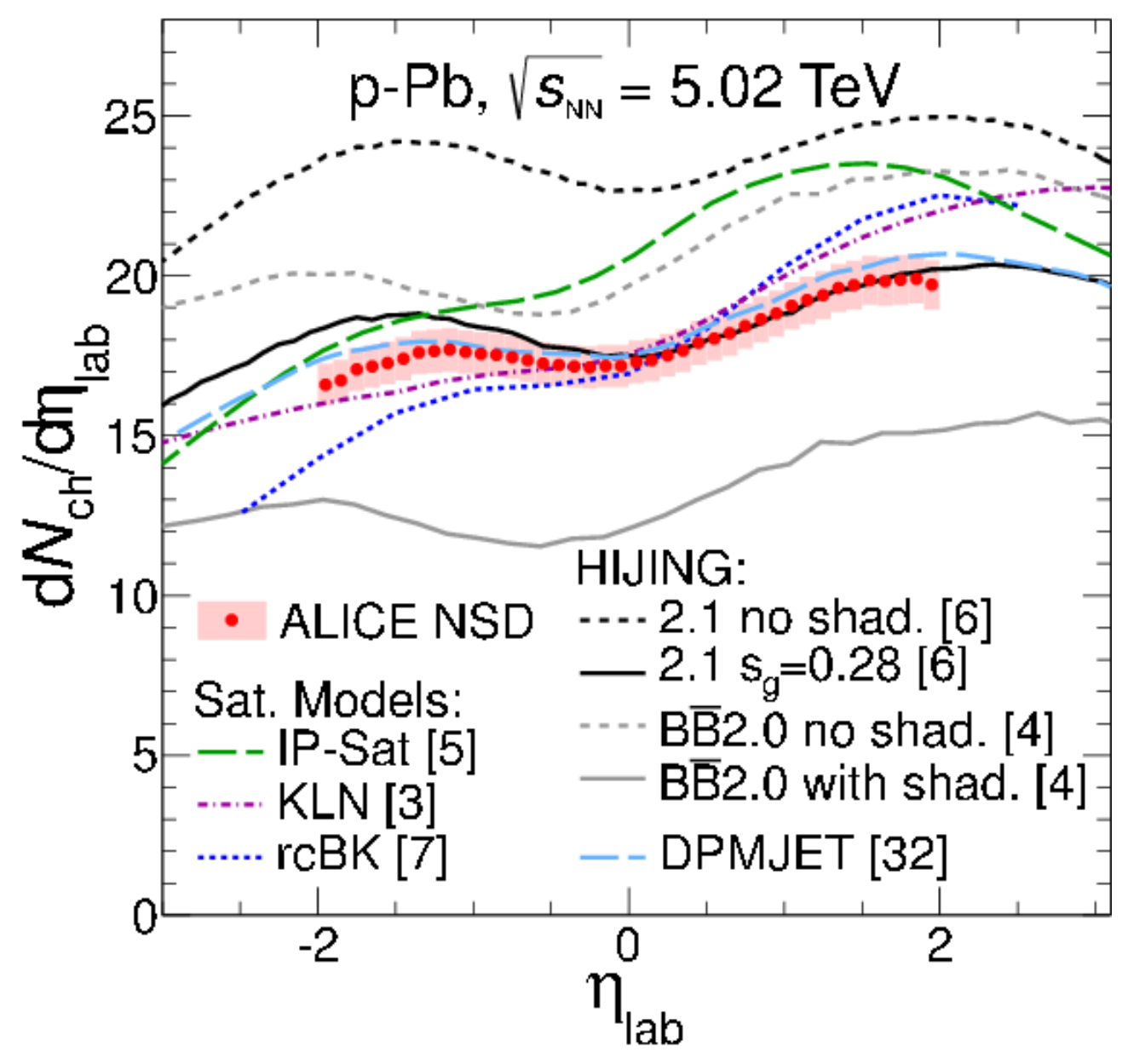




\section{THE RIDGE}

CMS OBSERVATION OF RIDGE IN p-p FOLLOWED BY ALICE AND ATLAS OBSERVATION IN p-Pb

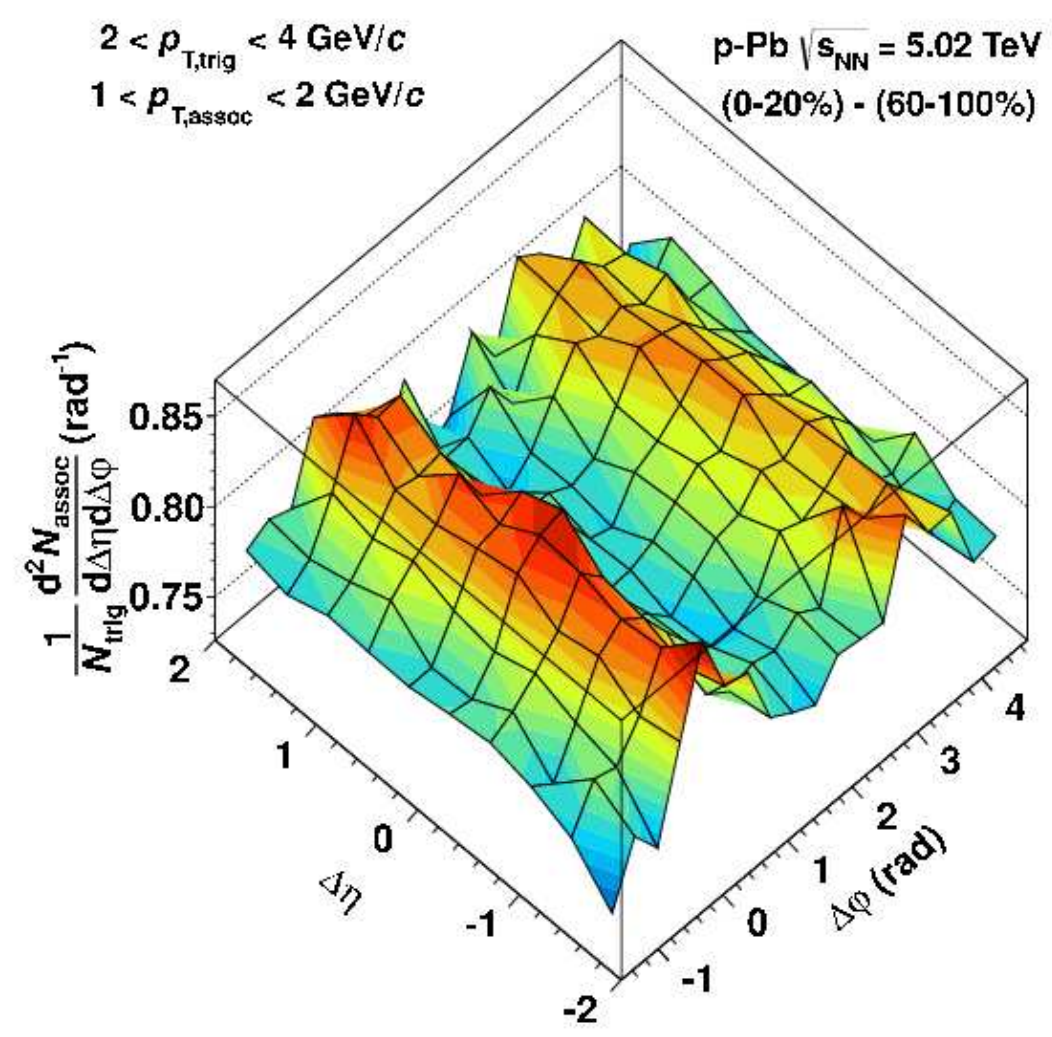

Figure 6: ALICE RIDGE 


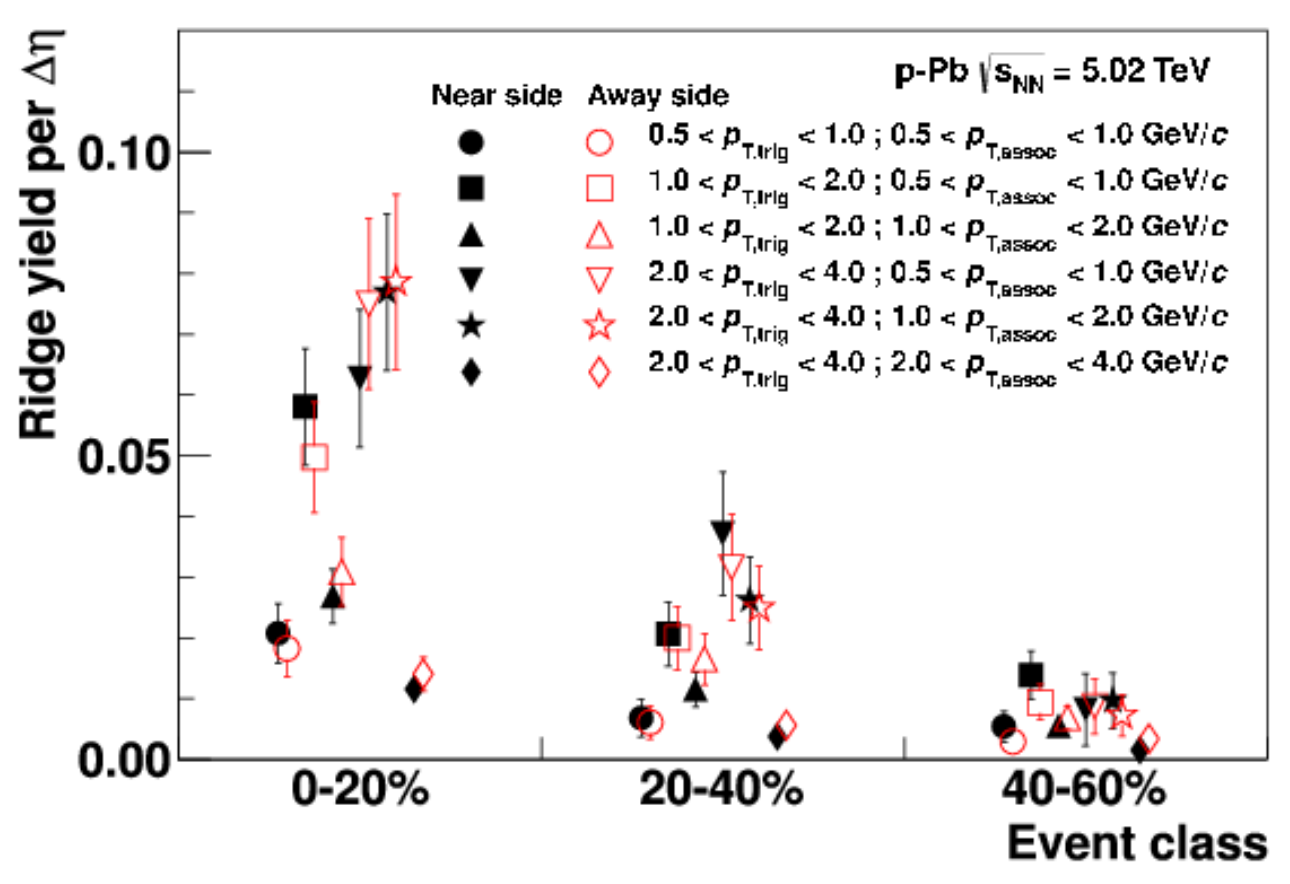

Figure 7: ALICE ridge at different $p_{T}$

QUALITATIVELY SIMILAR STRUCTURE: TWO PARTICLE CORRELATIONS LONG RANGE IN RAPIDITY ( $>4$ UNITS); AND COLLIMATED IN AZYMUTHAL ANGLE. $\mathrm{p}-\mathrm{Pb}$ SIGNAL IS STRONGER (ABOUT A FACTOR OF 2.5-3 FOR CORRELATED YIELD). THE PLOTS ARE FOR ASSOCIATED YIED $R(\Delta \eta) \sim \frac{N_{\text {correlated pairs }}}{N_{\text {trigger particles }}}$ 


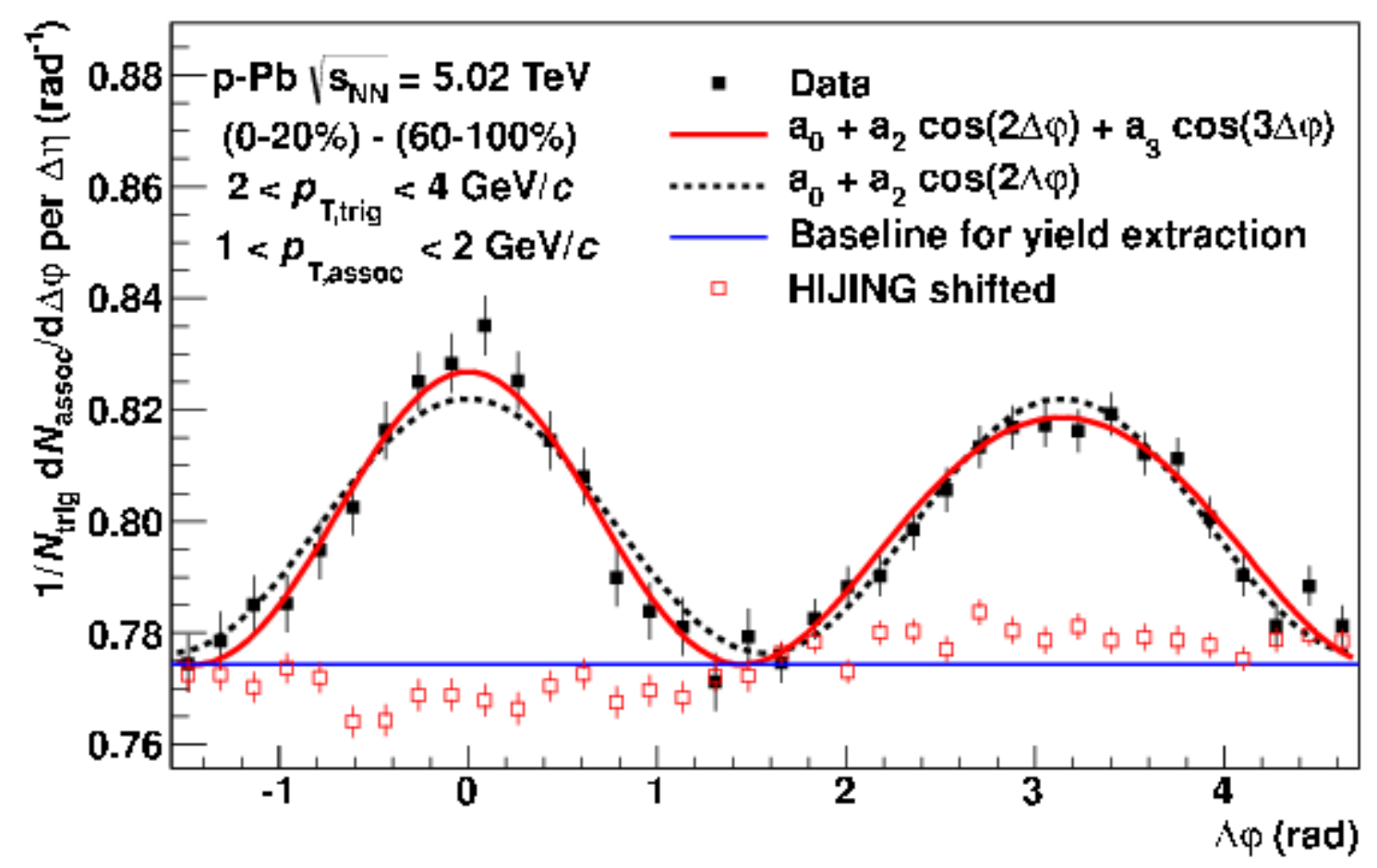

Figure 8: ALICE RIDGE IS SYMMETRIC

ALSO THE $\mathrm{p}$-Pb SIGNAL IS SYMMETRIC IN $\Delta \phi \rightarrow \Delta \phi+\pi$. 
VIGOROUS ACTIVITY TO DESCRIBE THE RIDGE WITHIN CGC APPROACH DUSLING AND VENUGOPALAN

IT IS CERTAINLY CGC INSPIRED.

INVOLVES QUITE A BIT OF MODELING. E.G. REQUIRES A CHOICE OF $Q_{S}$ FOR EVERY MULTIPICITY CLASS (NOT UNREASONABLE).

MOST IMPORTANT LIMITATION IN MY VIEW IS THAT IT TAKES AN ANSATZ FOR TWO PARTICLE PRODUCTION PROBABILITY FROM DILUTE LIMIT ( $\left.\mathrm{HIGH} p_{T}\right)$, AND APPLIES IT TO ALL MOMENTA.

THE VALIDITY IS NOT UNDER THEORETICAL CONTROL.

SOME IMPORTANT LEADING IN $N_{C}$ EFFECTS ARE NOT INCLUDED. THE RESULTS OF DV ARE BASED ON A SUBLEADING IN $N_{C}$ CONTRIBUTION.

\section{A SHORT RUNDOWN OF EFFECTS THAT LEAD TO COLLIMATION.}

1. DIRECTED COLOR FIELDS IN THE TARGET (KOVNER, LUBLINSKY). COLOR FIELDS IN THE TARGET HAVE FINITE CORRELATION LENGTH GIVEN

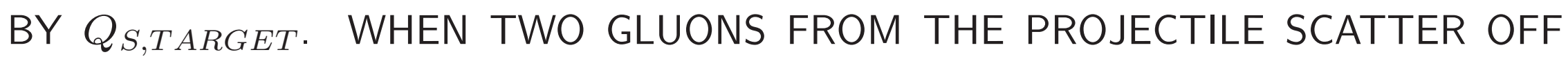
THE SAME FIELD, THEY PICK UP THE SAME MOMENTUM TRANSFER. THIS LEADS TO POSITIVE ANGULAR CORRELATIONS. THIS IS LEADING $N_{C}$ EFFECT. AT PRESENT WE DO NOT UNDERSTAND ITS ENERGY DEPENDENCE.

THIS EFFCT IS NOT INCLUDED IN DV 
2. VARIATION OF COLOR FILED DENSITY IN THE TARGET (LEVIN AND REZAEIAN). IF THE SIZE OF THE INCOMING TWO GLUON STATE IS COMPARABLE TO THE SIZE OF THE REGION OVER WHICH $Q_{S}$ VARIES, THERE IS A PREFERRED DIRECTION FOR SCATTERING - ALONG THE GRADIENT OF $Q_{S}$. THIS IS ALSO A LEADING $N_{C}$ EFFECT AND HAS LEADING BEHAVIOR AT HIGH ENERGY. THE RELEVANT MOMENTUM RANGE HOWEVER IS NOT $Q_{S}$, BUT "THE CORRELATION LENGTH OF $Q_{S}$ ". STILL, GIVEN THAT $Q_{S}$ FLUCTUATES IN IMPACT PARAMETER, IT MAY BE QUITE IMPORTANT.

THIS EFFCT IS NOT INCLUDED IN DV

3. BOSE CORRELATIONS BETWEEN THE GLUONS OF PROJECTILE. (DUMITRU, DUSLING, GELIS, JALILIAN MARIAN, LAPPI, MCLERRAN, VENUGOPALAN) DENSITY OF PROJECTILE GLUONS WITH THE SAME QUANTUM NUMBERS (TRANSVERSE MOMENTUM AND COLOR) IS ENHANCED DUE TO BOSE CORRELATIONS. THE GLUONS IN THIS CORRELATED CONFIGURATION PREFER TO SCATTER IN THE SAME DIRECTION. SINCE THE INCOMING GLUONS HAVE TO BE IN THE SAME COLOR STATE, THIS IS A SUBLEADING IN $N_{C}$ CONTRIBUTION RELATIVE TO GENERIC SITUATION WHERE THE COLOR OF INCOMING GLUONS IS INDEPENDENT.

THIS EFFCT IS INCLUDED IN DV AND GIVES ALL THE CORRELATED CONTRIBUTION. 


\section{ALL THE ABOVE MECHANISMS LEAD TO SYMMETRIC CONTRIBUTIONS}

AT $\phi$ AND $\phi+\pi$.

DV CALCULATION IS AN INTERESTING MODEL, BUT IT IS HARD TO SEE HOW IT CAN BE THE LAST WORD. TANTALIZING THAT IT GIVES SUCH GOOD QUANTITIVE AGREEMENT WITH THE DATA. COULD BE AN ACCIDENT, OR COULD BE THAT THE OTHER EFFECTS ARE FOR SOME REASON SUPPRESSED.

IT IS IMPORTANT THAT THIS CALCULATION IS OUT THERE AS A BASELINE FOR FUTURE, HOPEFULLY MORE COMPLETE ONES

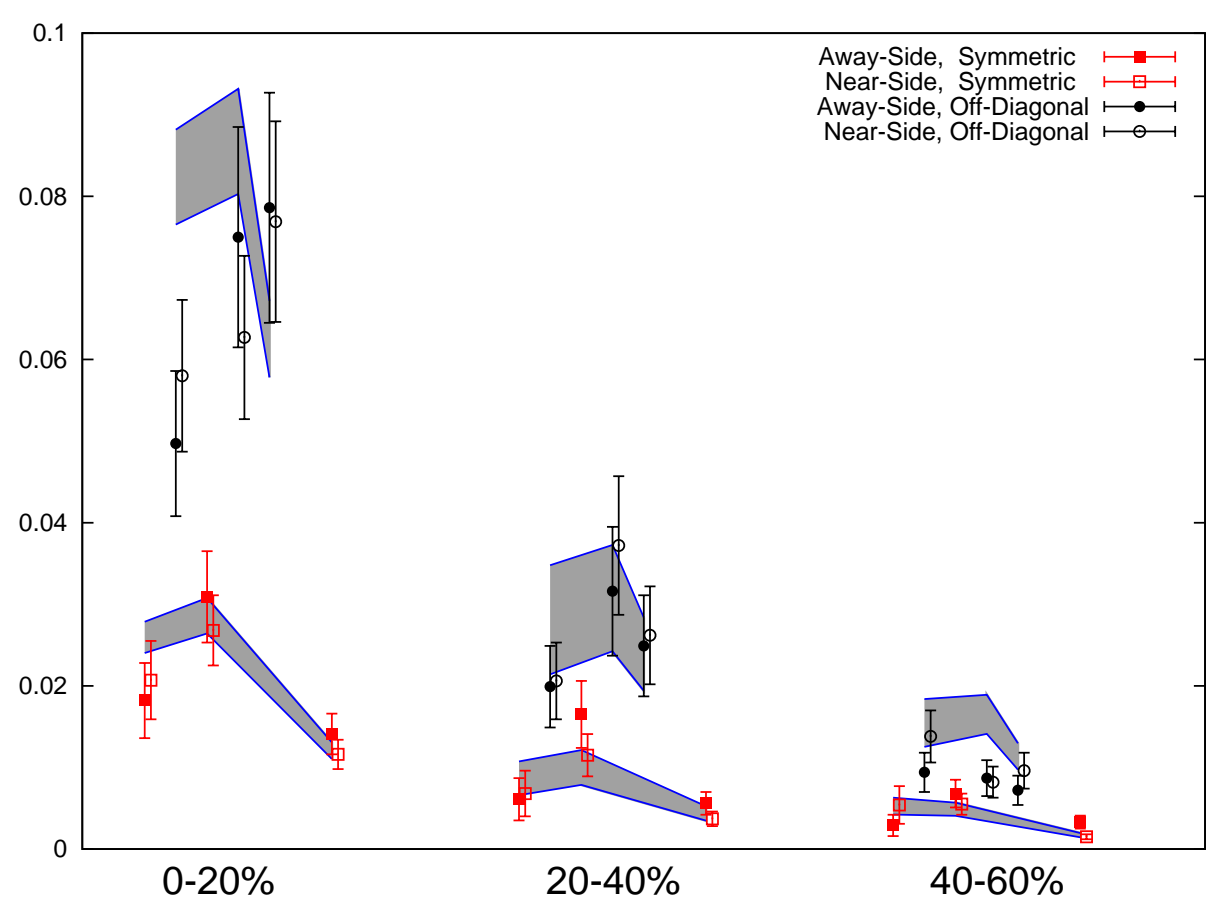

Figure 9: DUSLING-VENUGOPALAN FIT TO ALICE (LARGE FLUCTUATIONS IN $Q_{S}$ ?) 


\section{CONCLUSIONS}

A MIXED BAG: QUALITATIVELY THINGS WORK QUITE WELL - A LOT OF FEATURES OF THE DATA ARE REPRODUCED.

IS IT A THEORY AS OPPOSED TO MODEL? NOT YET. STILL MANY AD HOC APPROXIMATIONS WITH NO OBVIOUS CONTROL ON THEIR VALIDITY PLUS A LOT OF FREEDOM IN FITTING (CHANGING) PARAMETERS.

THE USUAL ANNOYING THING. HERE IS A GOOD, SIMPLE IDEA. YOU MAKE THE SIMPLEST POSSIBLE ESTIMATE INSPIRED BY IT, AND IT WORKS BETTER THAN IT HAS THE RIGHT TO WORK. YOU IMPROVE IT BY MAKING REAL CALCULATION (INCLUDING PERTURBATIVE CORRECTIONS AND SUCH) AND THINGS GET ONLY WORSE. USUALLY ONE HAS TO WORK REALLY HARD TO GET BACK THE ACCURACY OF THE ORIGINAL BACK-OF-THE ENVELOPE ESTIMATE FROM THE REAL CONTROLLABLE CALCULATION.

TYPICAL TEENAGER: SHOOTS UP A FOOT IN HIGHT IN A YEAR, BUT DOES NOT QUITE KNOW WHAT TO DO WITH HIS LONG ARMS, PLUS HAS TONS OF PIMPLES. IT WILL TAKE TIME AND EFFORT TO GROW HIM INTO A PROFESSIONAL ATHLETE WITH SMOOTH SKIN (OR ALTERNATIVELY INTO A RESPECTED UNIVERSITY PROFESSOR). BUT IN THE END IT MAY HAPPEN. 
SO I HOPE THAT WE ARE AT THIS STAGE IN THE CHAIN - MOVING FROM BACK OF THE ENVELOPE STUFF TO REAL CALCULATIONS, ALTHOUGH NOT QUITE THERE YET. 


\title{
Combined analysis of RHIC to LHC suppression \& Geometry
}

\author{
Roy A. Lacey \\ Chemistry Depta, \\ Stony Brook University
}

Primary focus:

Scaling properties of flow \& Jet quenching 


\section{Flow \& Jet quenching are important probes of the QGP!}

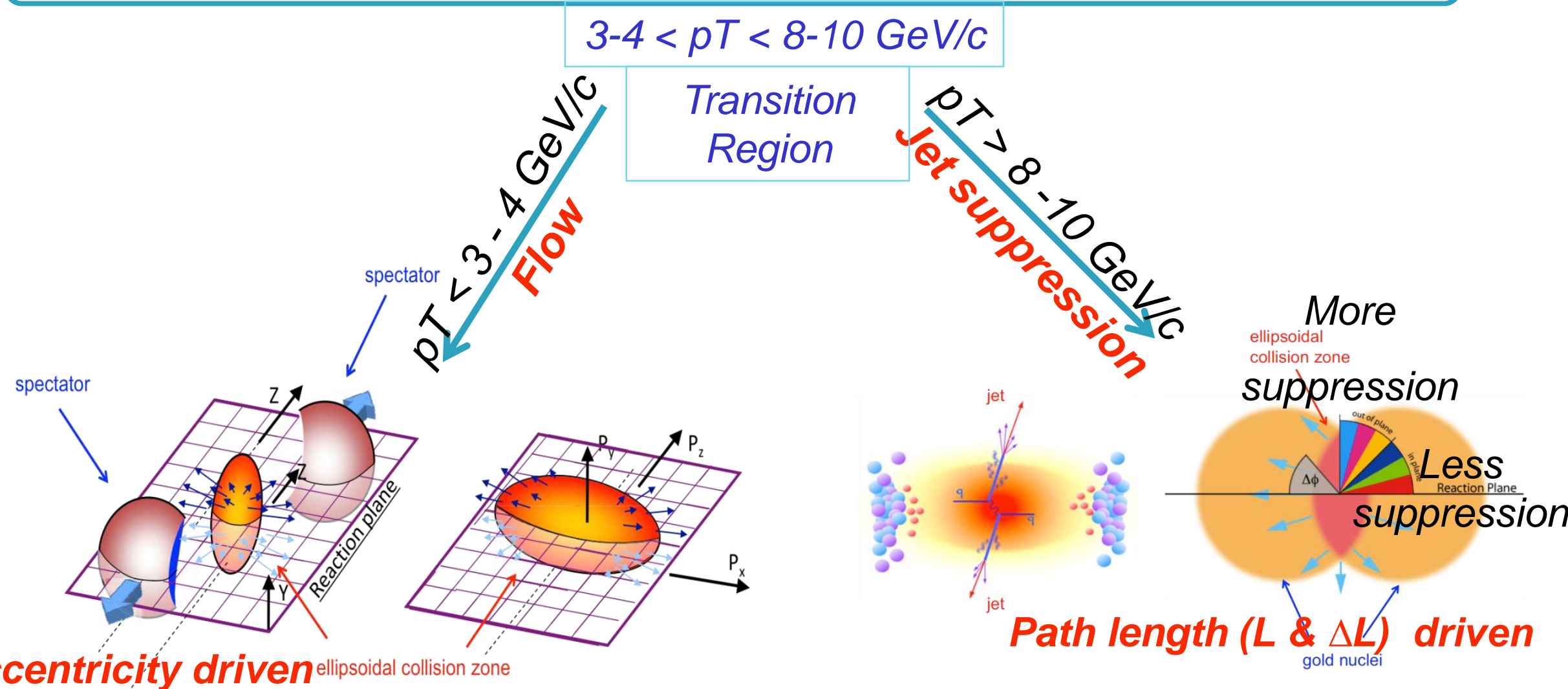

\& acoustic

Flow and Jet suppression are linked to

Geometry \& the interactions in the QGP

$>$ This implies very specific scaling properties for flow and jet suppression (respectively), which can be tested experimentally

$\checkmark$ Scaling validation provide important insights, as well as straightforward probes of transport coefficients 


\section{Geometric Quantities for scaling}

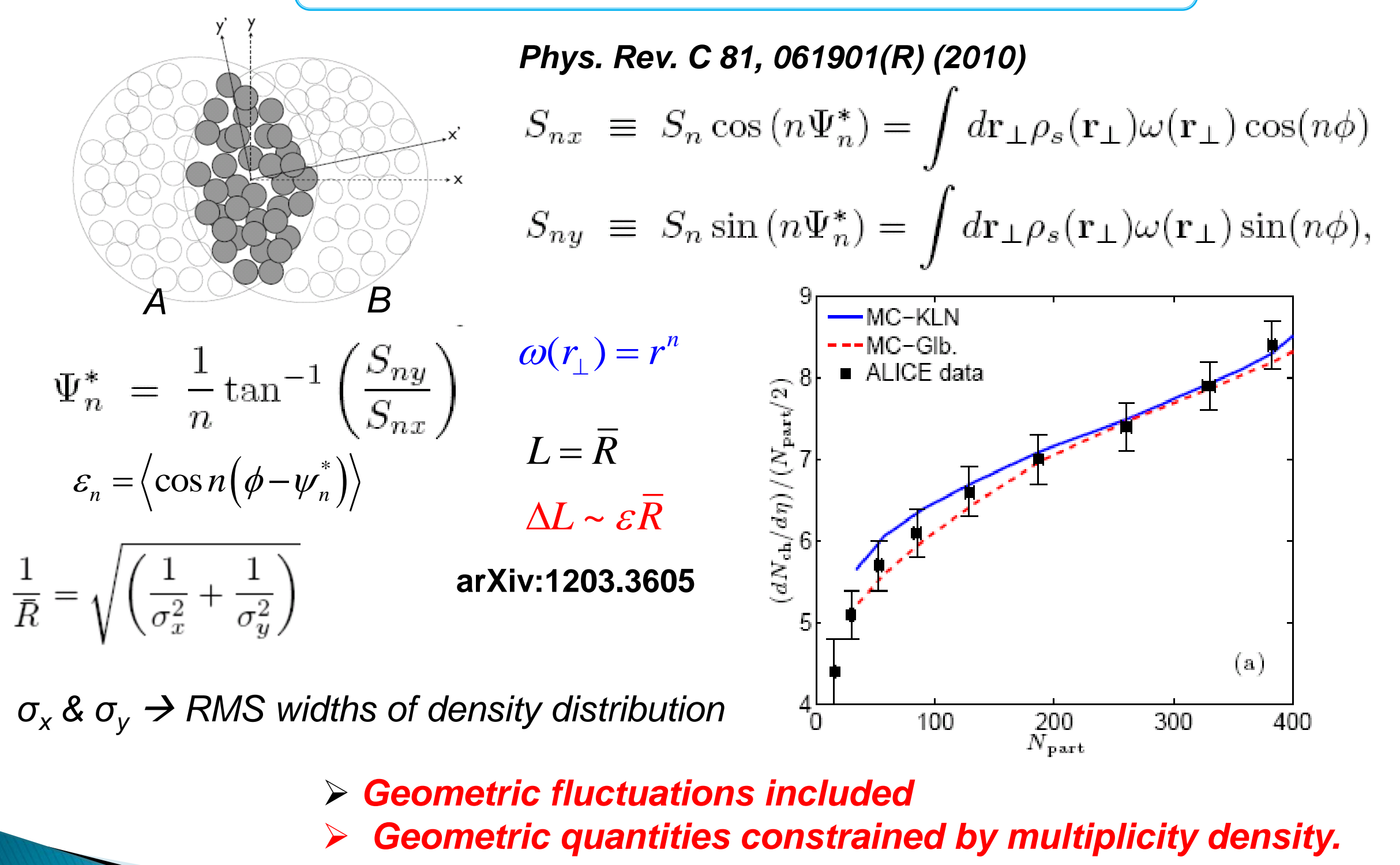




\section{The Flow Probe}

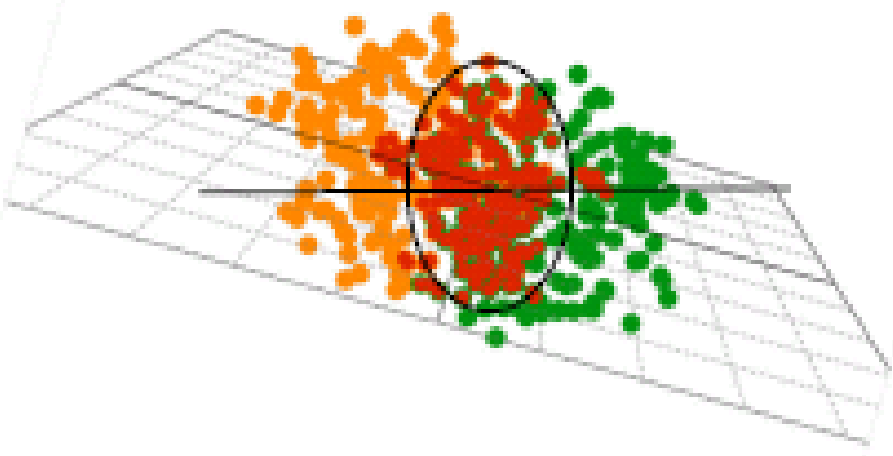

Acoustic viscous modulation of $v_{n}$ $\delta T_{\mu \nu}(t, k)=\exp \left(-\frac{2}{3} \frac{\eta}{s} k^{2} \frac{t}{T}\right) \delta T_{\mu v}(0)$ Staig \& Shuryak arXiv:1008.3139

Scaling expectations

Particle Distribution
Initial Geometry characterized by many shape harmonics $\left(\varepsilon_{n}\right) \rightarrow$ drive $v_{n}$

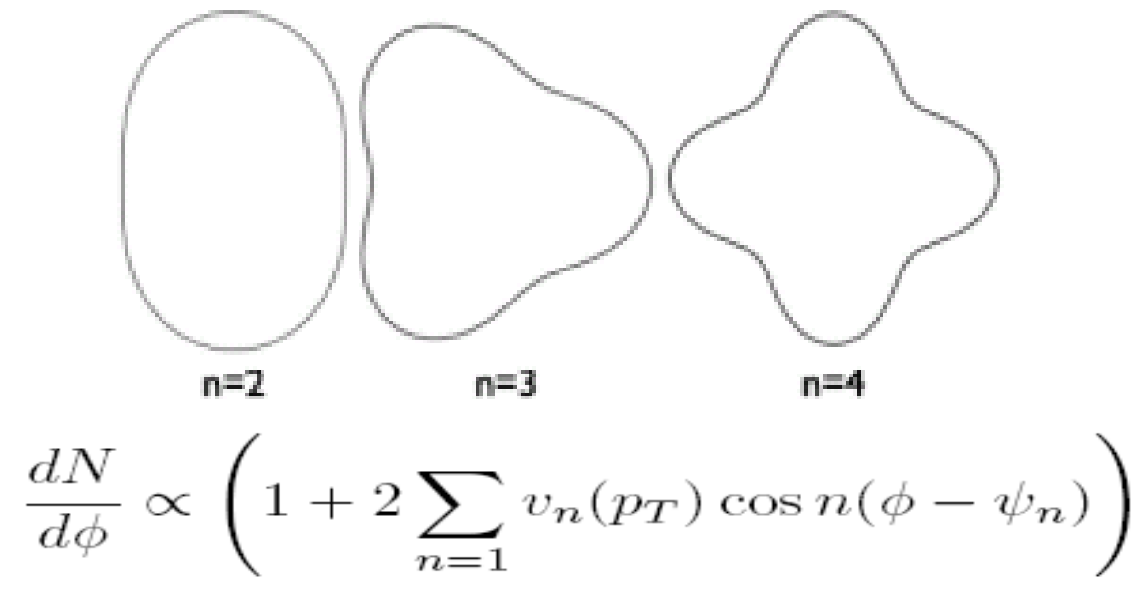

$\frac{v_{n}\left(p_{T}\right)}{\varepsilon_{n}} \propto \exp \left(-\beta^{\prime} n^{2}\right)$

$$
\frac{\mid \frac{v_{n}\left(p_{T}\right)}{v_{2}\left(p_{T}\right)}=\frac{\varepsilon_{n}}{\varepsilon_{2}} \cdot \exp \left(-\beta^{\prime}\left(n^{2}-4\right)\right)}{\text { Note that } v_{n} \text { is related to } v_{2}}
$$$$
\begin{aligned}
& \ln \left(\frac{v_{n}}{\varepsilon_{n}}\right) \propto \frac{-\beta^{\prime \prime}}{\bar{R}} \\
& \beta^{\prime \prime}\left(\tilde{p}_{T}\right) \propto p_{T}^{\alpha^{\prime}}
\end{aligned}
$$ 


\section{$v_{n}\left(\Psi_{n}\right)$ Measurements - ATLAS}

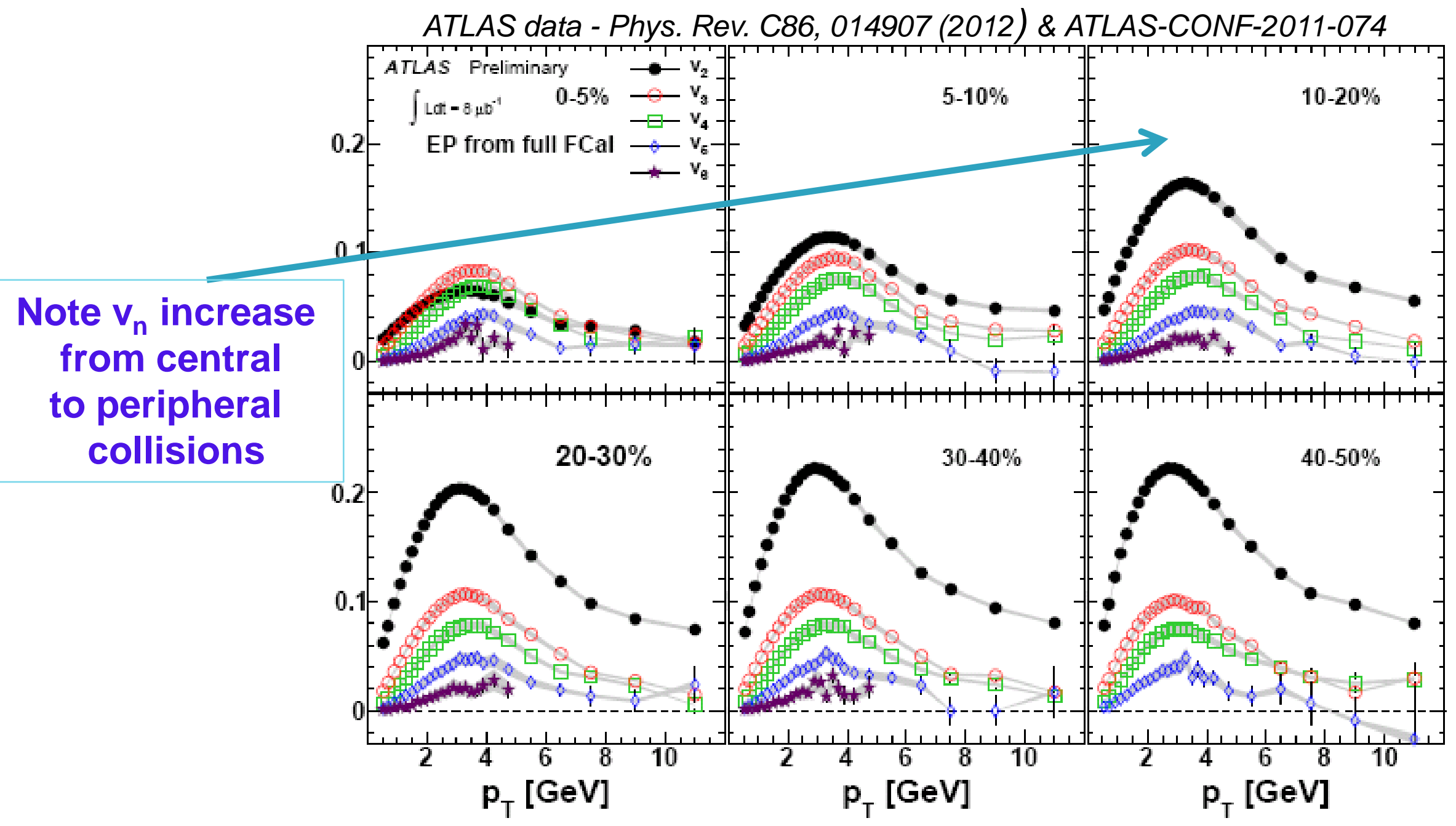

High precision double differential Measurements for $\mathrm{Pb}+\mathrm{Pb}$ are pervasive $\rightarrow$ Do they scale? 


\section{$v_{n}\left(\Psi_{n}\right)$ Measurements - LHC}

ATLAS Data - arXiv:1212.5198.

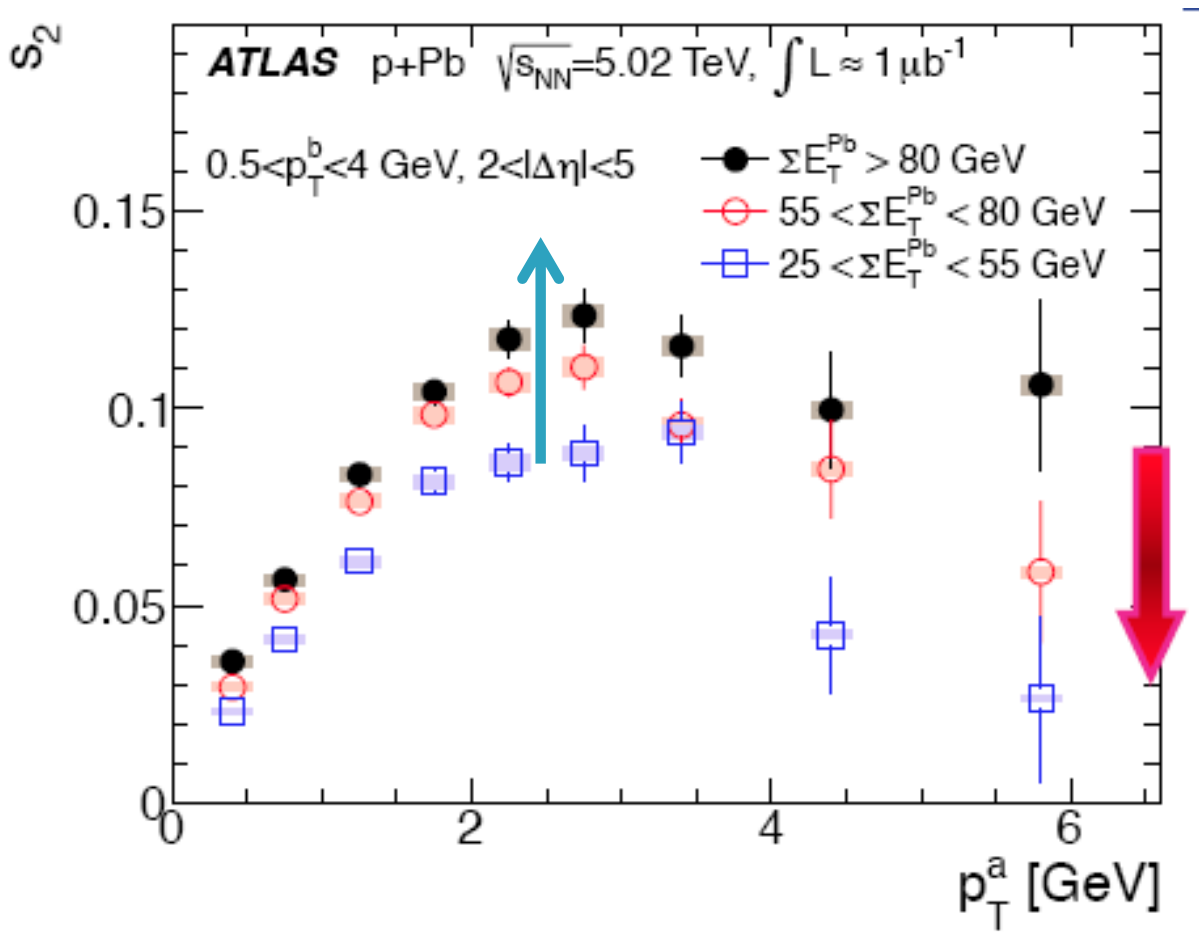

Recent $\mathrm{p}+\mathrm{Pb}$ measurements

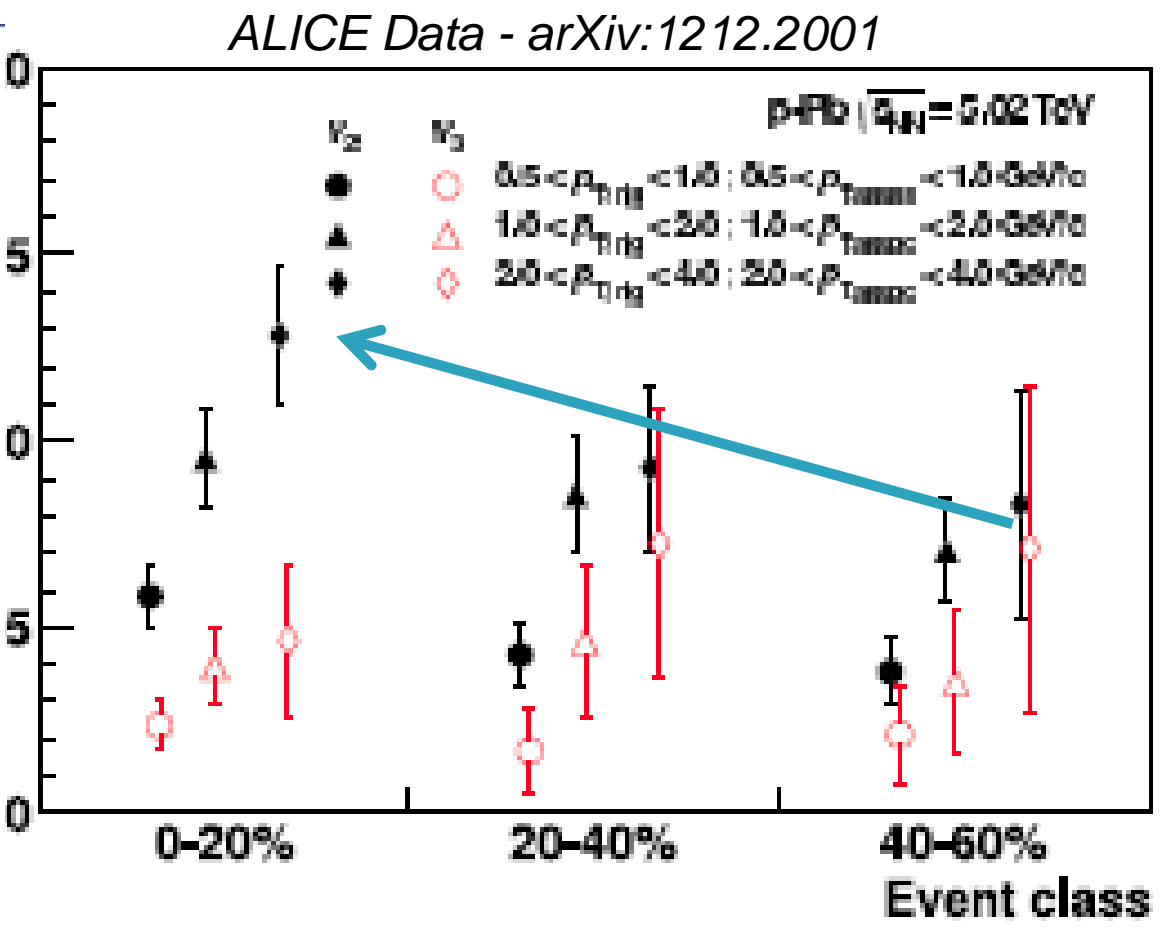

$\checkmark$ Note increase of $v_{2}\left(\right.$ or $\left.s_{2}\right)$ from peripheral to central collisions

Double differential Measurements for $p+P b$ are pervasive $\rightarrow$ Do they scale? 


\section{Acoustic Scaling $-n^{2}$}

ATLAS data - Phys. Rev. C86, 014907 (2012)

$$
\frac{v_{n}\left(p_{T}\right)}{\varepsilon_{n}} \propto \cdot \exp \left(-\beta^{\prime} n^{2}\right)
$$

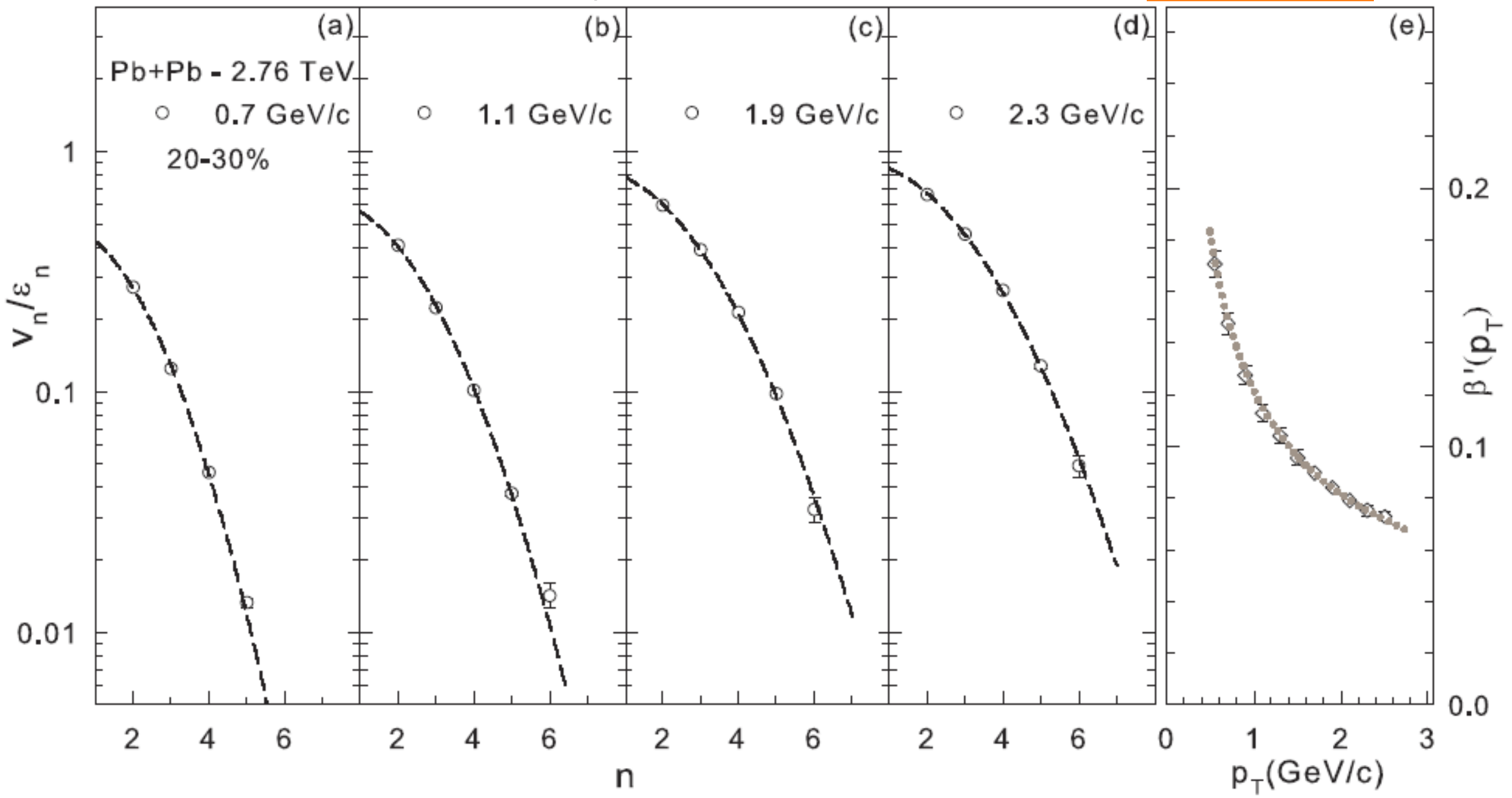

$\checkmark$ Characteristic $\mathrm{n}^{2}$ viscous damping validated

$\checkmark$ Characteristic $1 /\left(p_{T}\right)^{\alpha}$ dependence of extracted $\beta$ values validated Constraint for $\eta / s$ and $\delta f$ 


\section{Acoustic Scaling - Ratios}

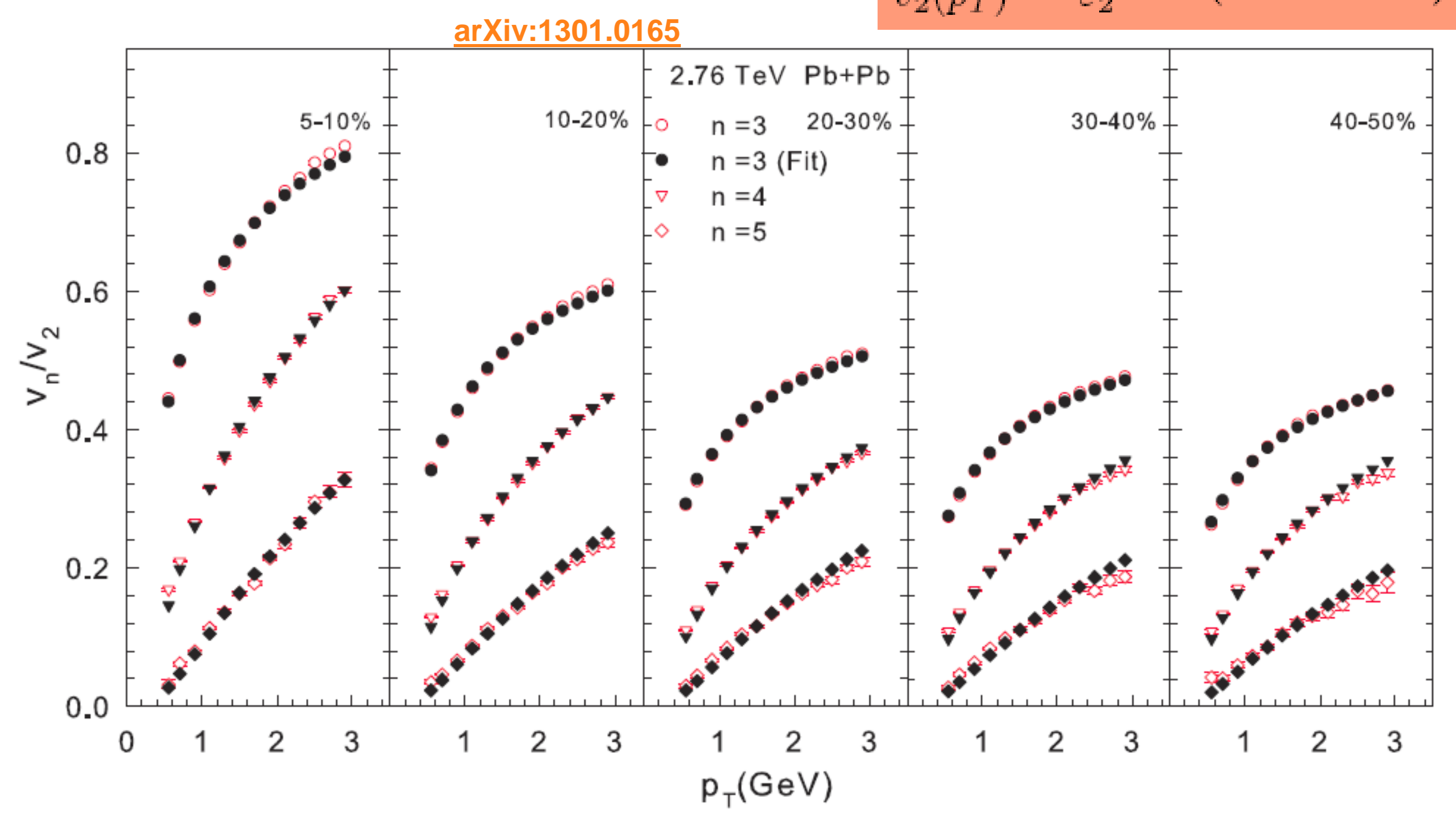

$$
\frac{v_{n}\left(p_{T}\right)}{v_{2}\left(p_{T}\right)}=\frac{\varepsilon_{n}}{\varepsilon_{2}} \exp \left(-\beta^{\prime}\left(n^{2}-4\right)\right)
$$

$\checkmark$ Characteristic $\left(\mathrm{n}^{2}-4\right)$ viscous damping validated

$\checkmark$ Characteristic $1 /\left(p_{T}\right)^{\alpha}$ dependence of $\beta$ validated

Constraint for $\eta / s$ 


\section{Acoustic Scaling - Ratios}

$$
\frac{v_{n}\left(p_{T}\right)}{v_{2}\left(p_{T}\right)}=\frac{\varepsilon_{n}}{\varepsilon_{2}} \exp \left(-\beta^{\prime}\left(n^{2}-4\right)\right)
$$

Fits performed for each centrality

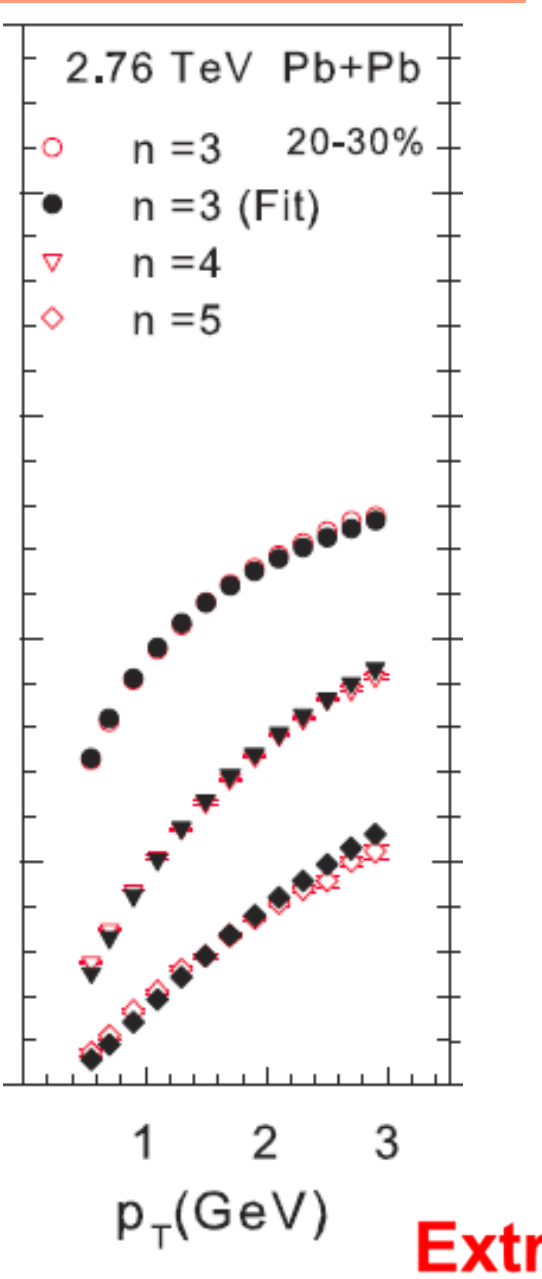

arXiv:1301.0165
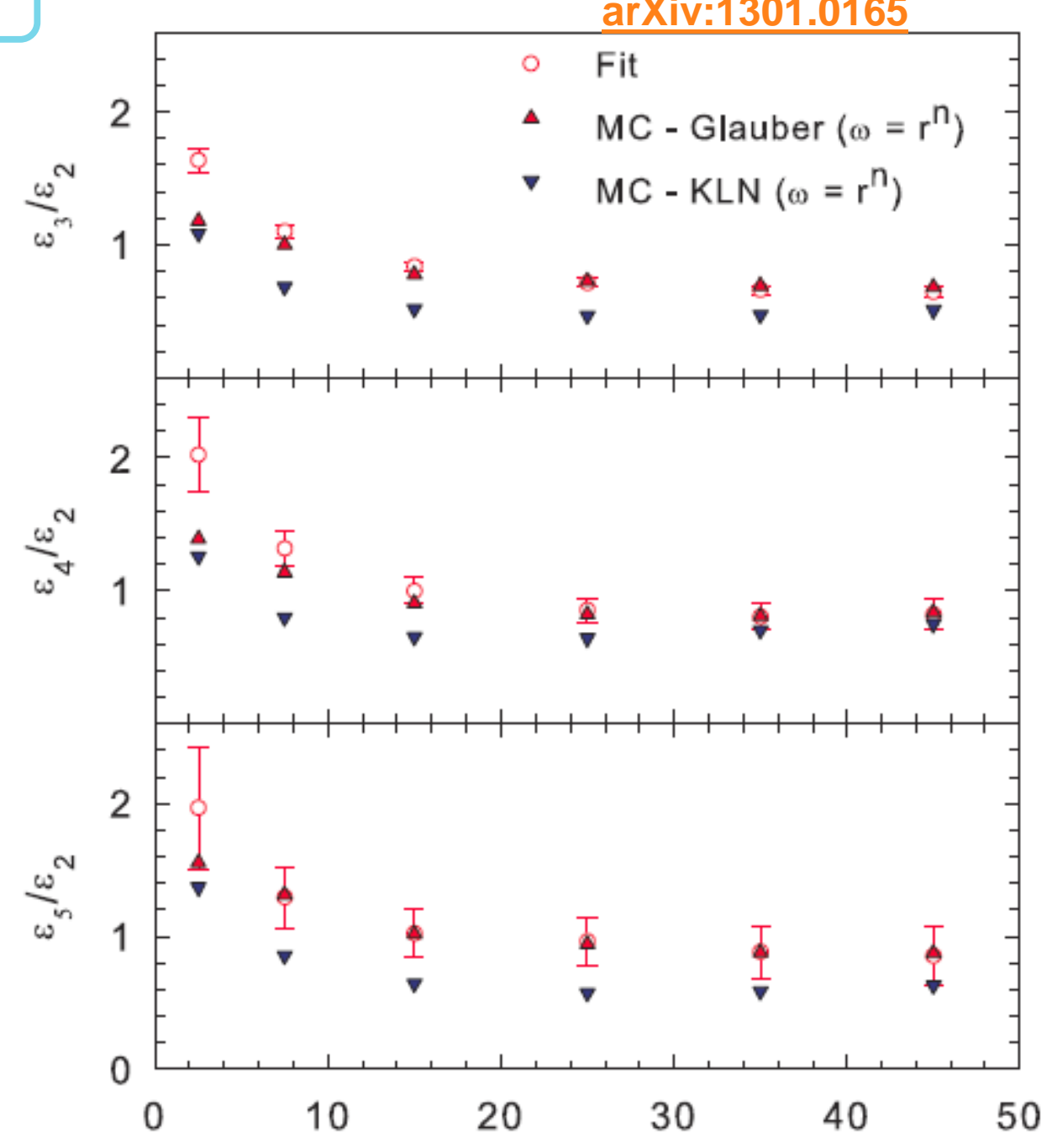

Centrality (\%)

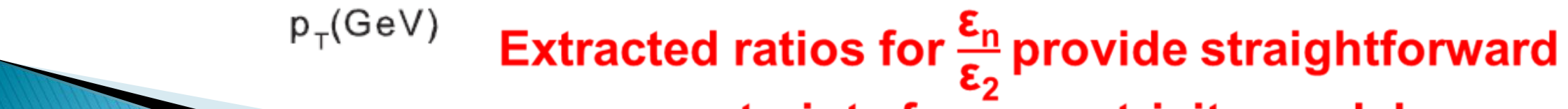

constraints for eccentricity models 


\section{Acoustic Scaling - Ratios}

$$
\frac{v_{n}\left(p_{T}\right)}{v_{2}\left(p_{T}\right)}=\frac{\varepsilon_{n}}{\varepsilon_{2}} \exp \left(-\beta^{\prime}\left(n^{2}-4\right)\right)
$$

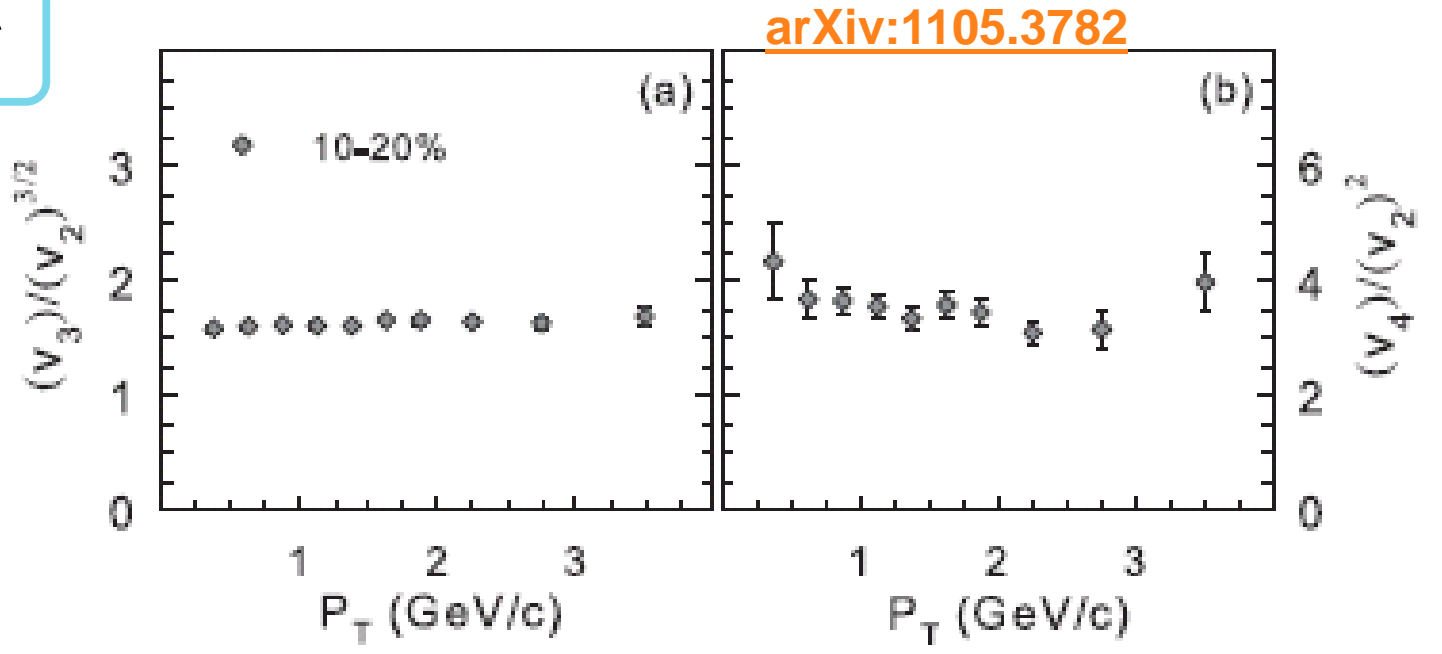

$$
v_{n}\left(p_{T}\right) \propto\left(v_{2}\right)^{n / 2}
$$

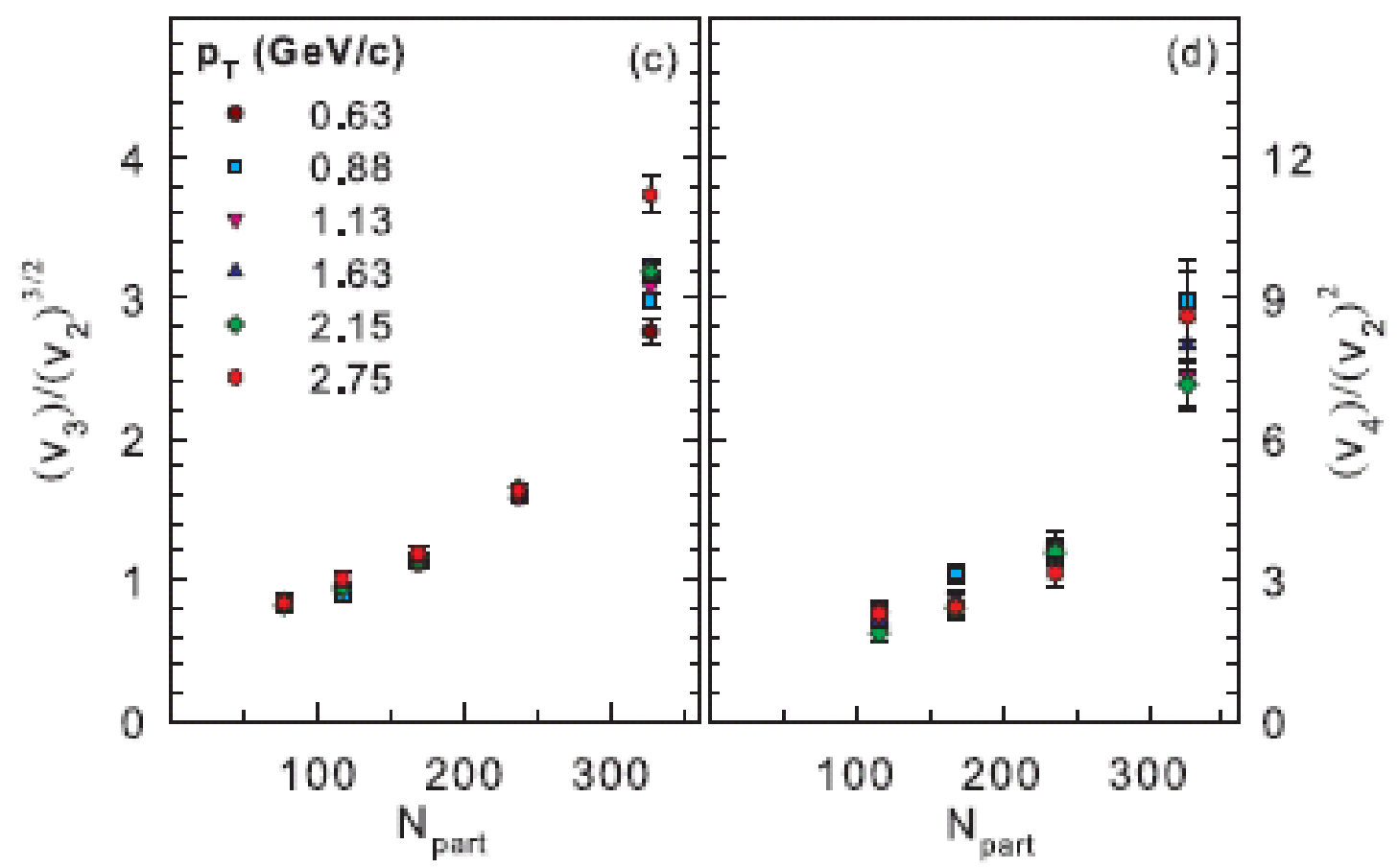

The expected relation between $v_{n}$ and $v_{2}$ is validated 


\section{Flow is partonic \& Acoustic?}

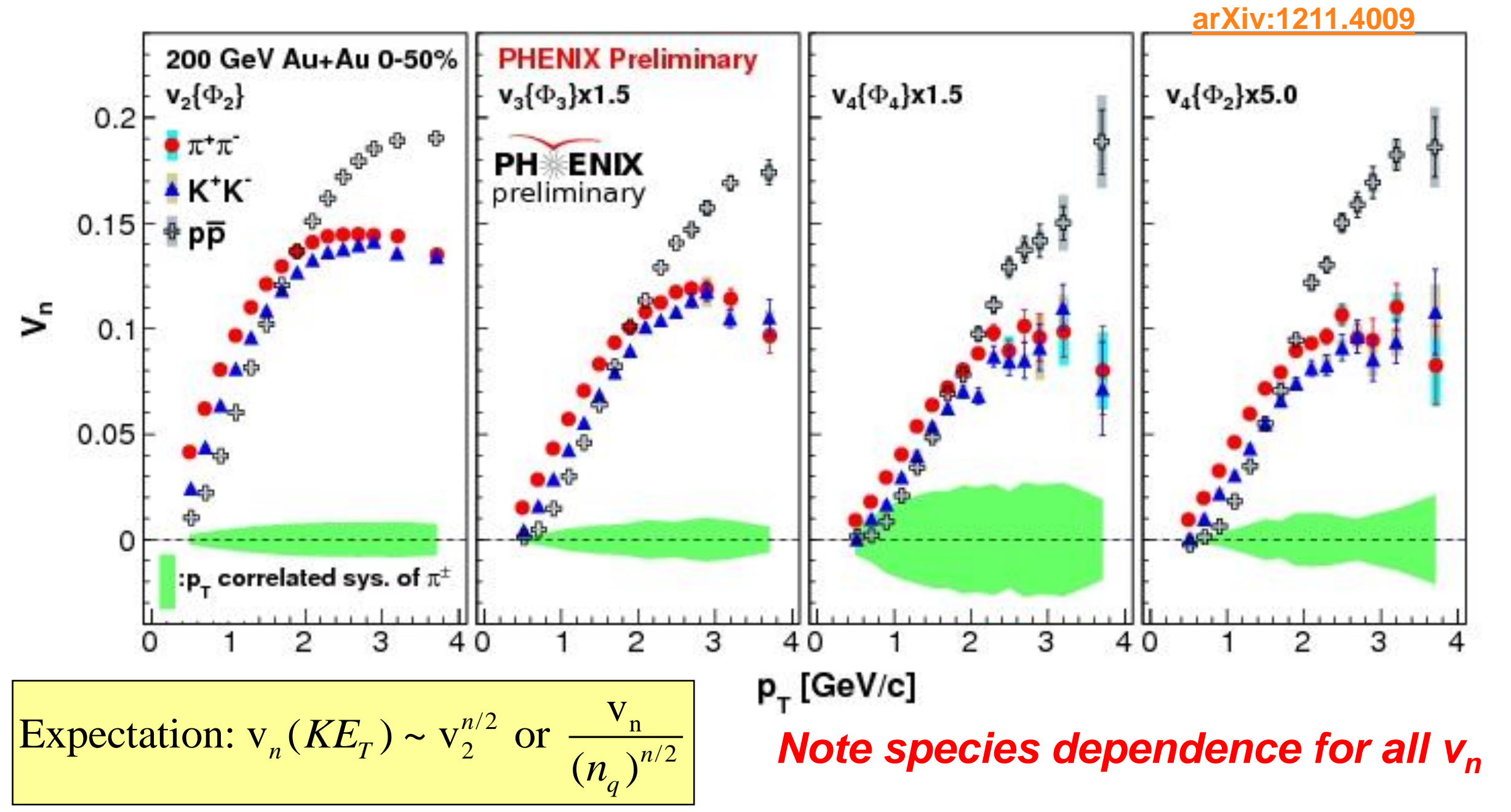

For partonic flow, quark number scaling expected $\rightarrow$ single curve for identified particle species $v_{n}$ 


\section{Flow is partonic \& acoustic}

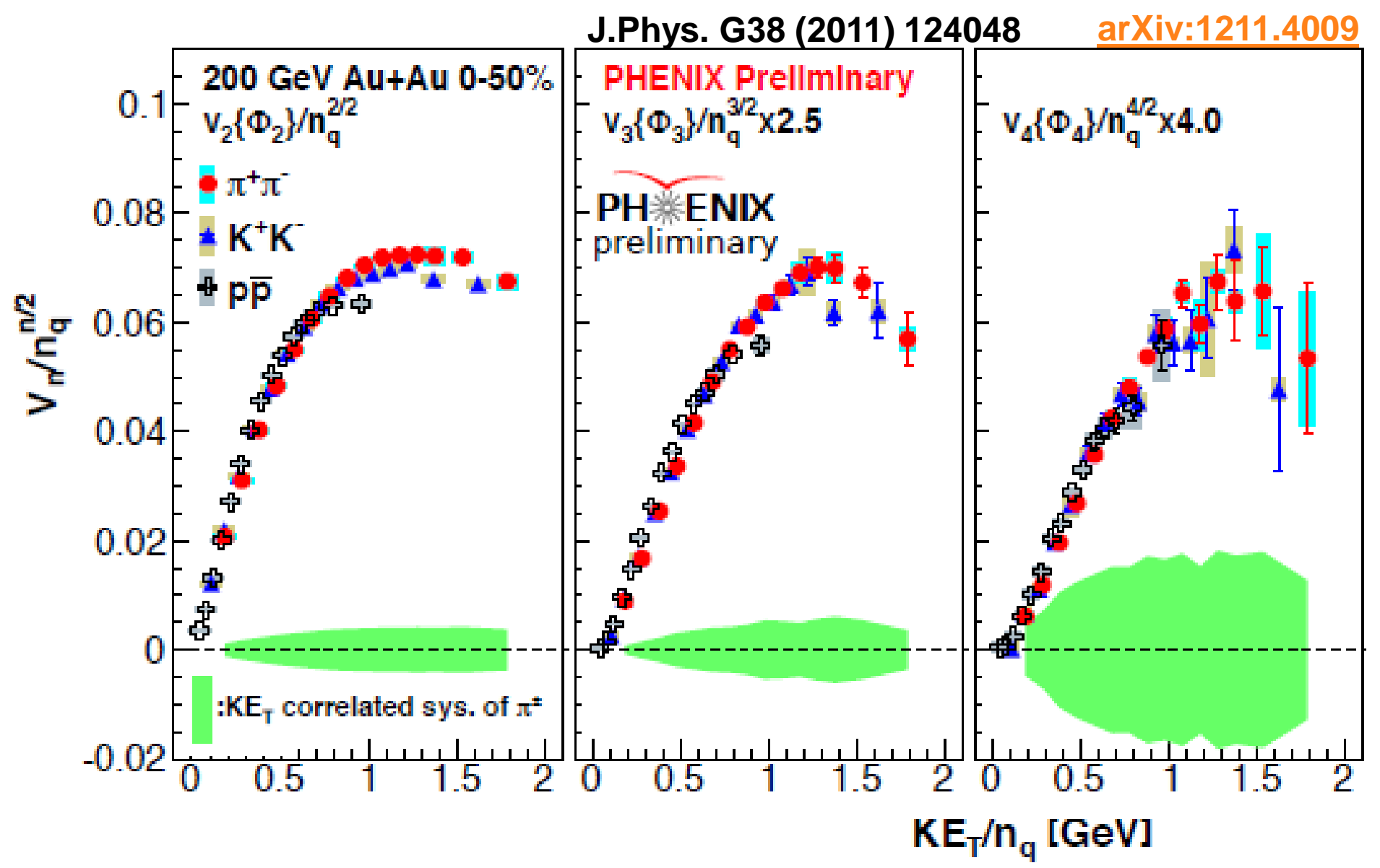

$K E_{T} \&\left(n_{q}\right)^{n / 2}$ scaling validated for $v_{n}$

$\rightarrow$ Partonic flow 


\section{Acoustic Scaling - 1/R}

Specific dependence dictated by sound attenuation

$$
\ln \left(\frac{v_{n}}{\varepsilon_{n}}\right) \propto \frac{-\beta^{\prime \prime}}{\bar{R}}
$$

ATLAS data - Phys. Rev. C86, 014907 (2012)

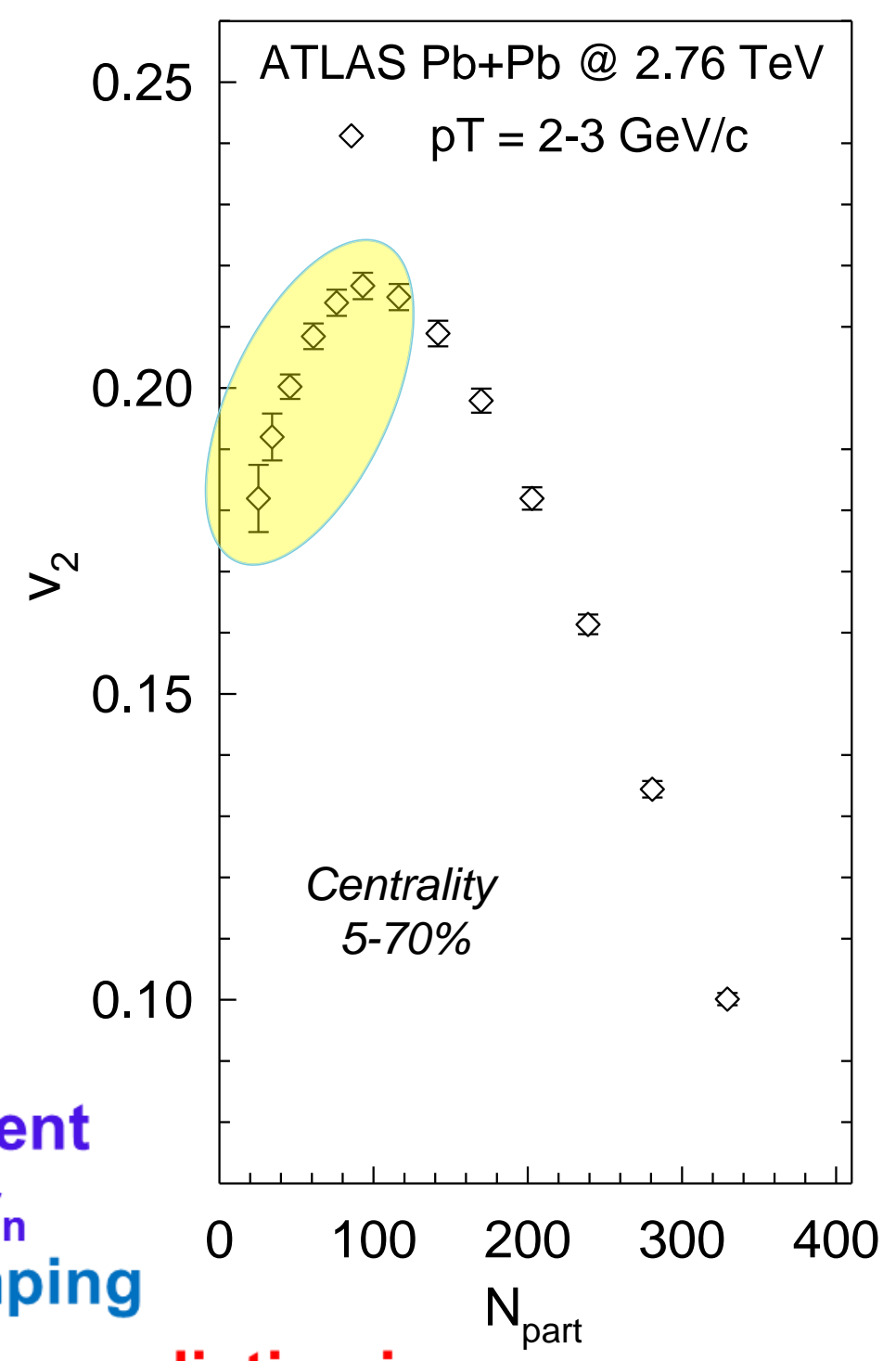

$\checkmark$ Characteristic $1 / \bar{R}$ scaling prediction is non-trivial 


\section{Acoustic Scaling - 1/R}

$\ln \left(\frac{v_{n}}{\varepsilon_{n}}\right) \propto \frac{-\beta^{\prime \prime}}{\bar{R}}$

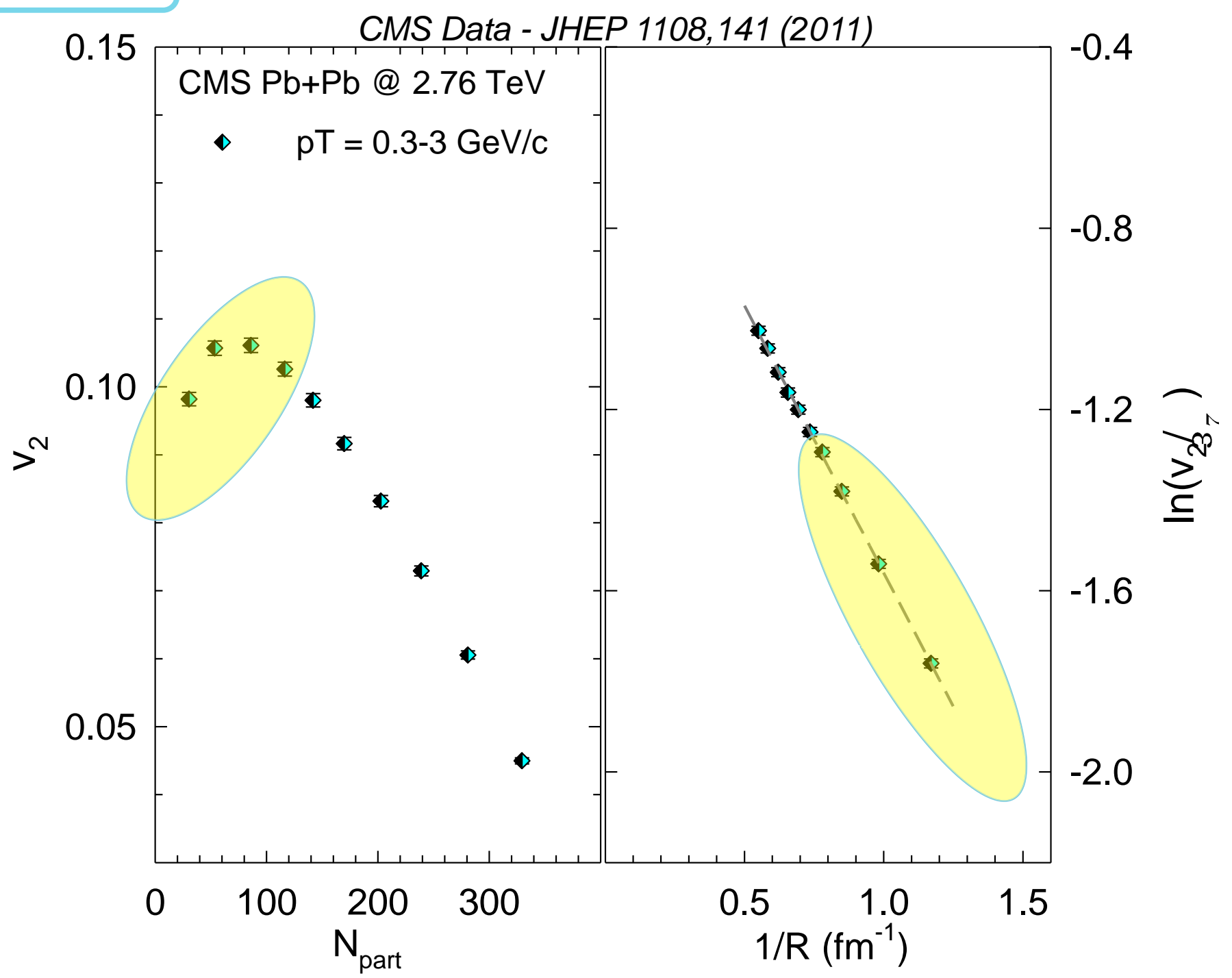

$\checkmark$ Characteristic $1 / \bar{R}$ viscous damping validated $\checkmark \quad$ A further constraint for $\eta / s$ of 


\section{Acoustic Scaling - 1/R}

$\ln \left(\frac{v_{n}}{\varepsilon_{n}}\right) \propto \frac{-\beta^{\prime \prime}}{\bar{R}}$

slope $\rightarrow$

$\beta$ constraint

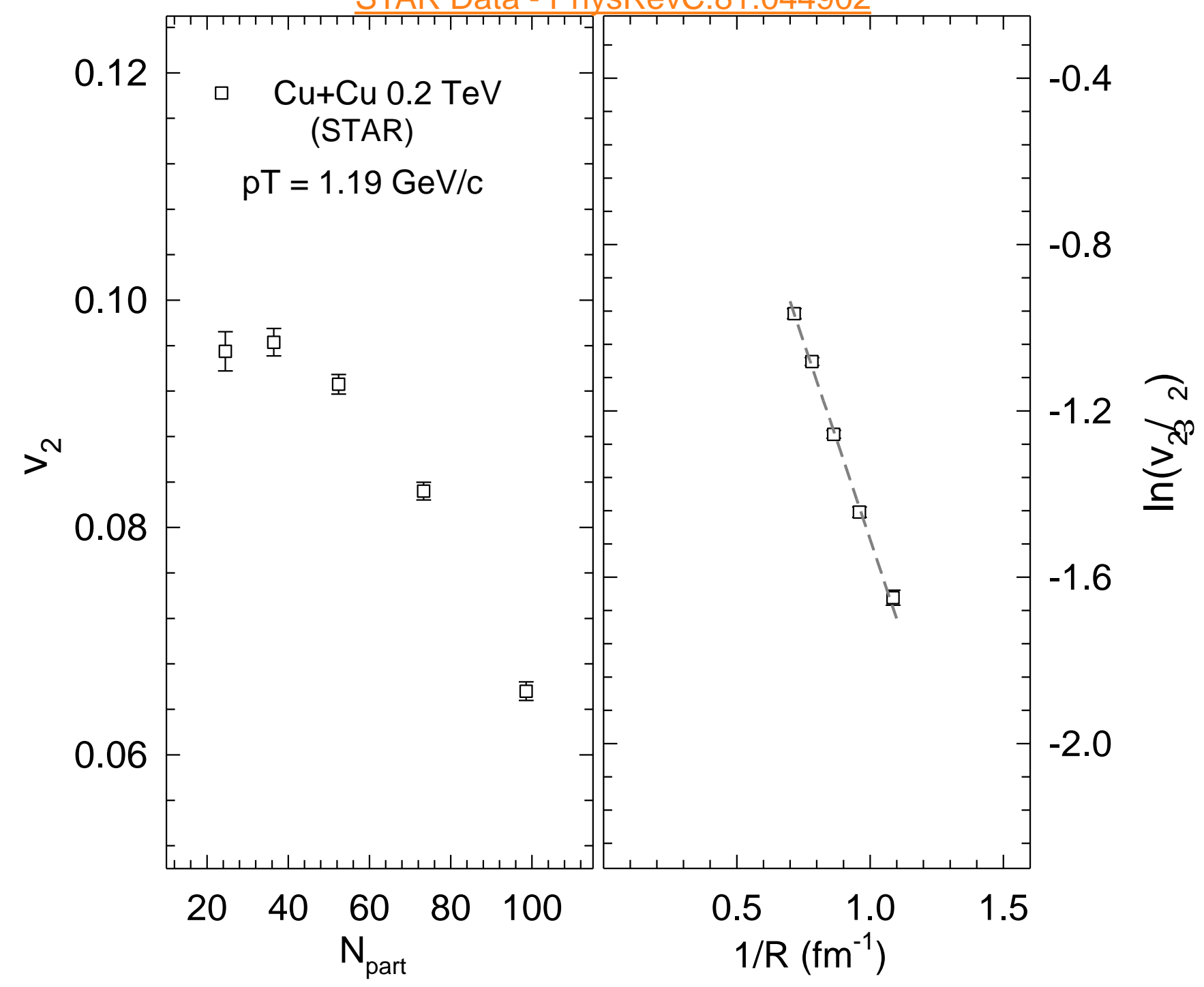

$\checkmark$ Characteristic $1 / \bar{R}$ viscous damping validated for light system A further constraint for $\eta / s$ 


\section{Acoustic Scaling - 1/R}

ATLAS data - Phys. Rev. C86, 014907 (2012)

$$
\ln \left(\frac{v_{n}}{\varepsilon_{n}}\right) \propto \frac{-\beta^{\prime \prime}}{\bar{R}}
$$

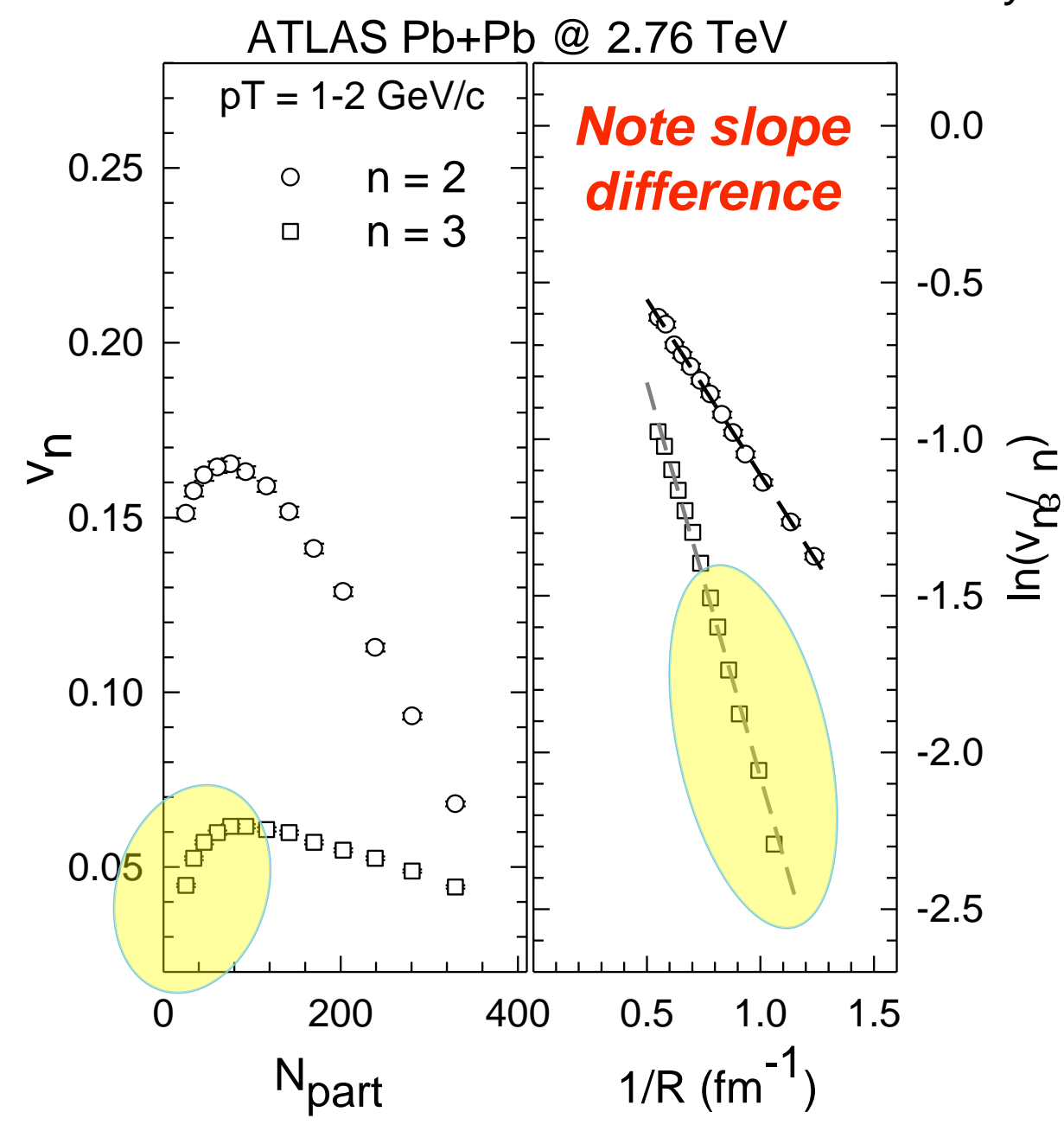

ATLAS Pb+Pb @ 2.76 TeV

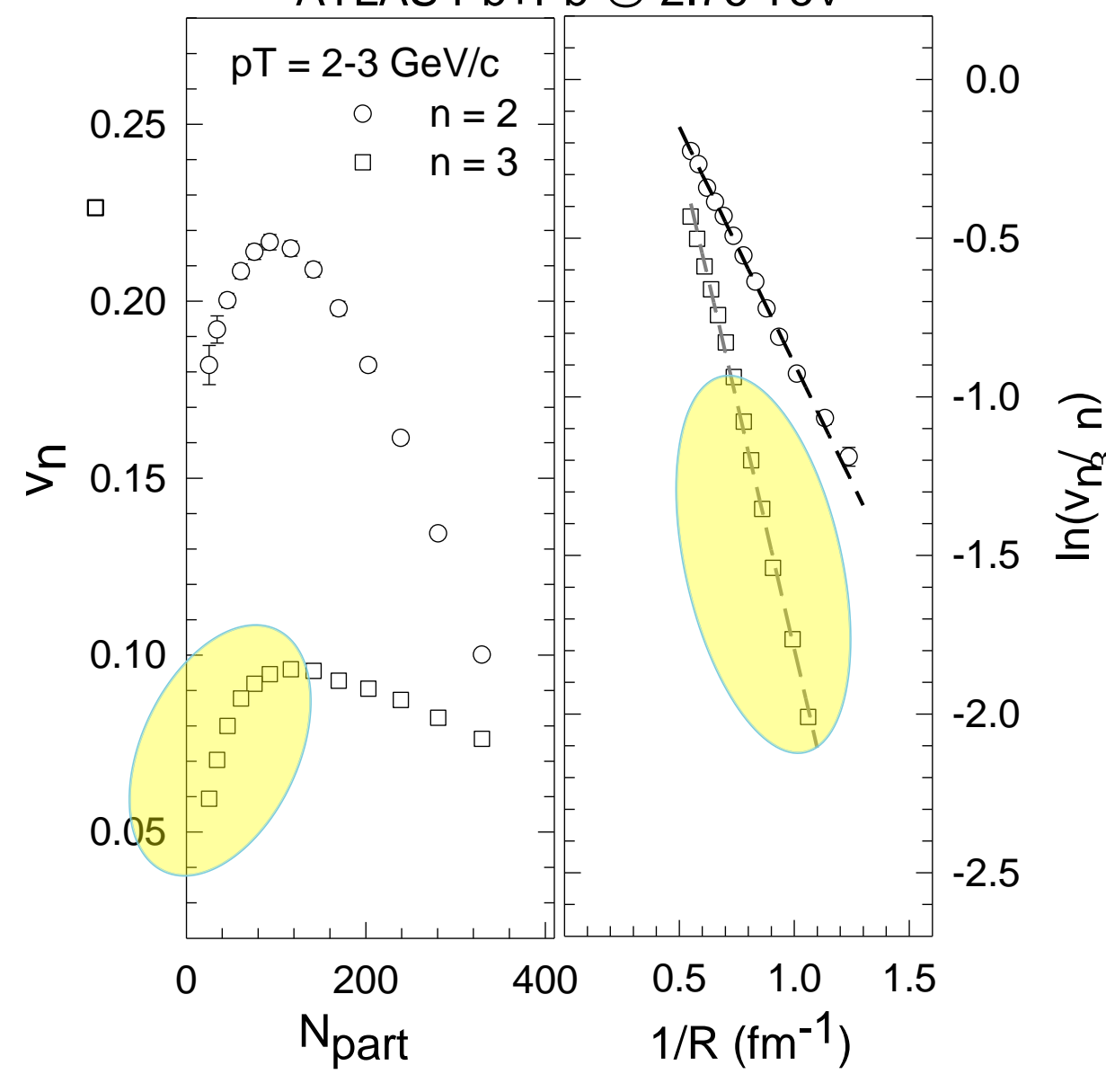

$\checkmark$ Characteristic $1 / \bar{R}$ viscous damping validated $\checkmark \quad$ A further constraint for $\eta / s$ and $\delta f$ 


\section{Acoustic Scaling - 1/R}

$\ln \left(\frac{v_{n}}{\varepsilon_{n}}\right) \propto \frac{-\beta^{\prime \prime}}{\bar{R}}$

ATLAS Data - arXiv:1212.5198.

$\omega^{N}$

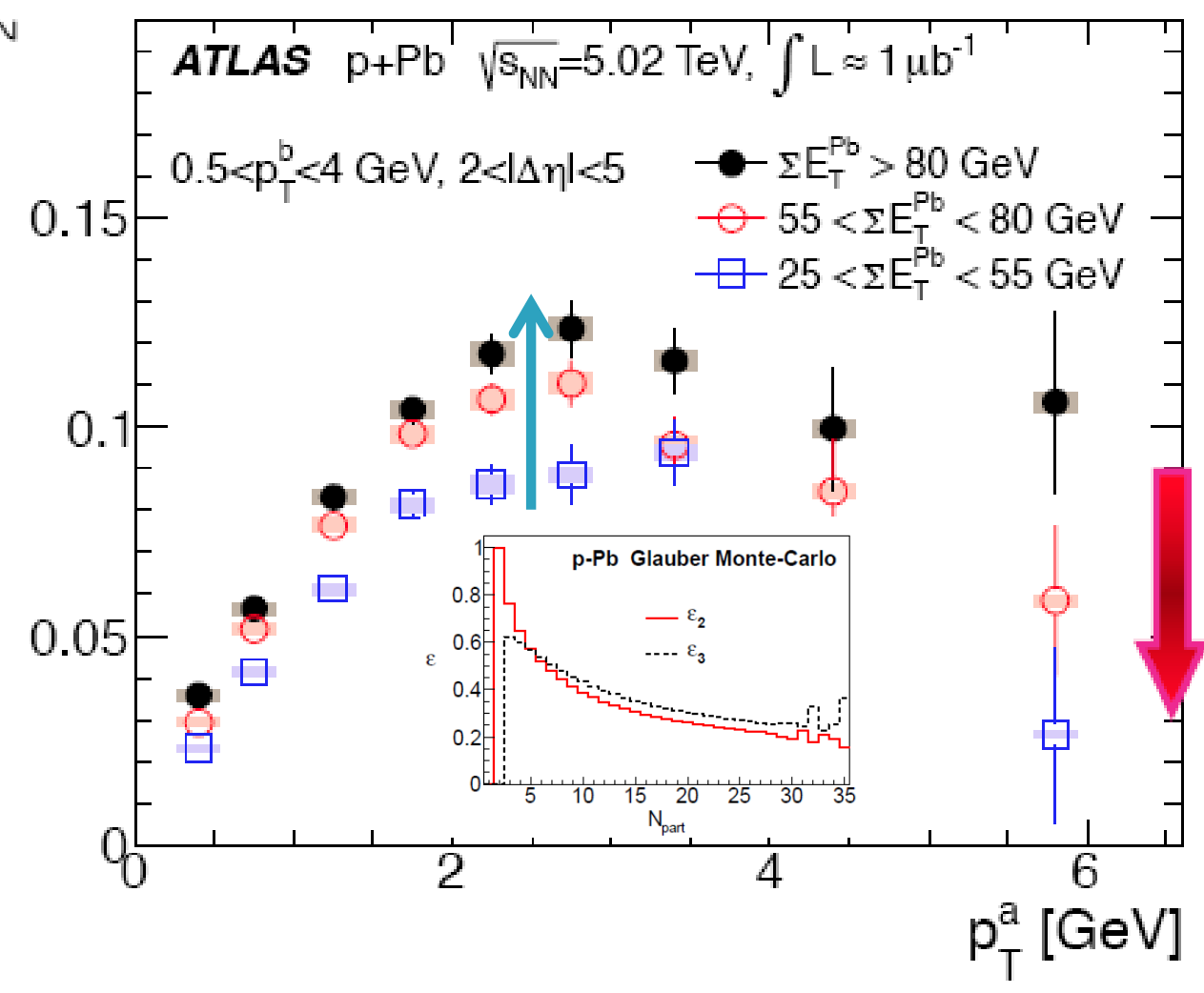

$\checkmark$ Increase of $s_{2}\left(v_{2}\right)$ with centrality for $\mathrm{p}+\mathrm{Pb}$ collisions is to be expected If the mechanism is similar!

$\rightarrow$ Effects of system size $(R)$ dominates
0.25

$>$

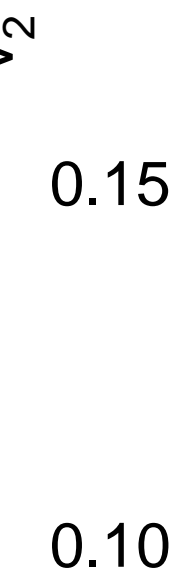

$\mathbf{v}_{2}$ increases with centrality $\lesssim$

$\bar{\Sigma}$

$\bar{\Sigma}$
ATLAS Pb+Pb @ 2.76 TeV

$\diamond \quad \mathrm{pT}=2-3 \mathrm{GeV} / \mathrm{c}$

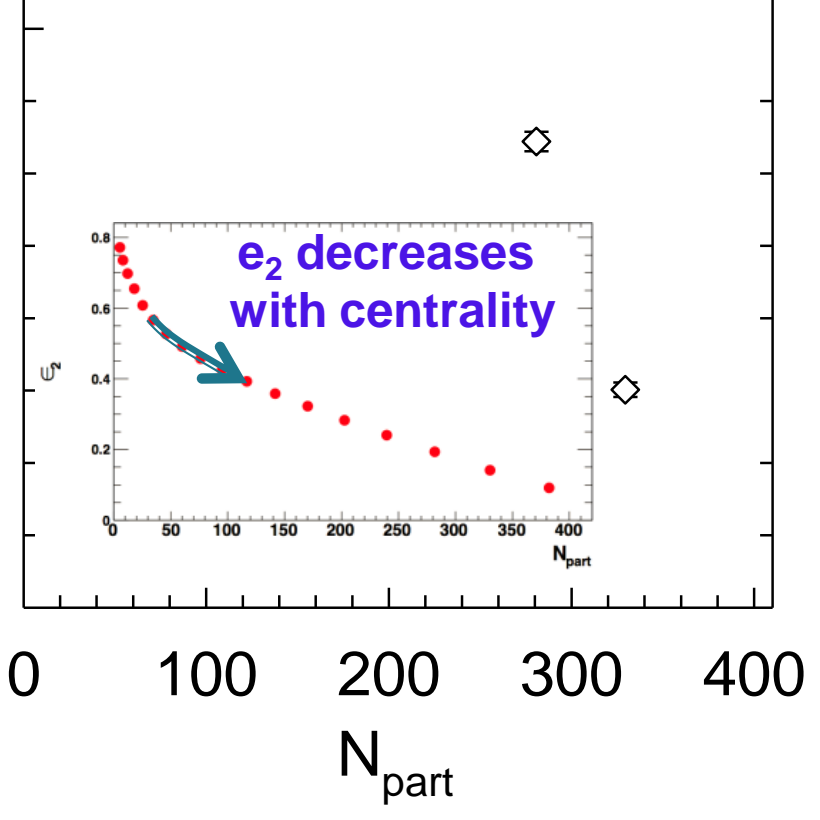




\section{Acoustic Scaling - 1/R}

Compare system size@RHIC

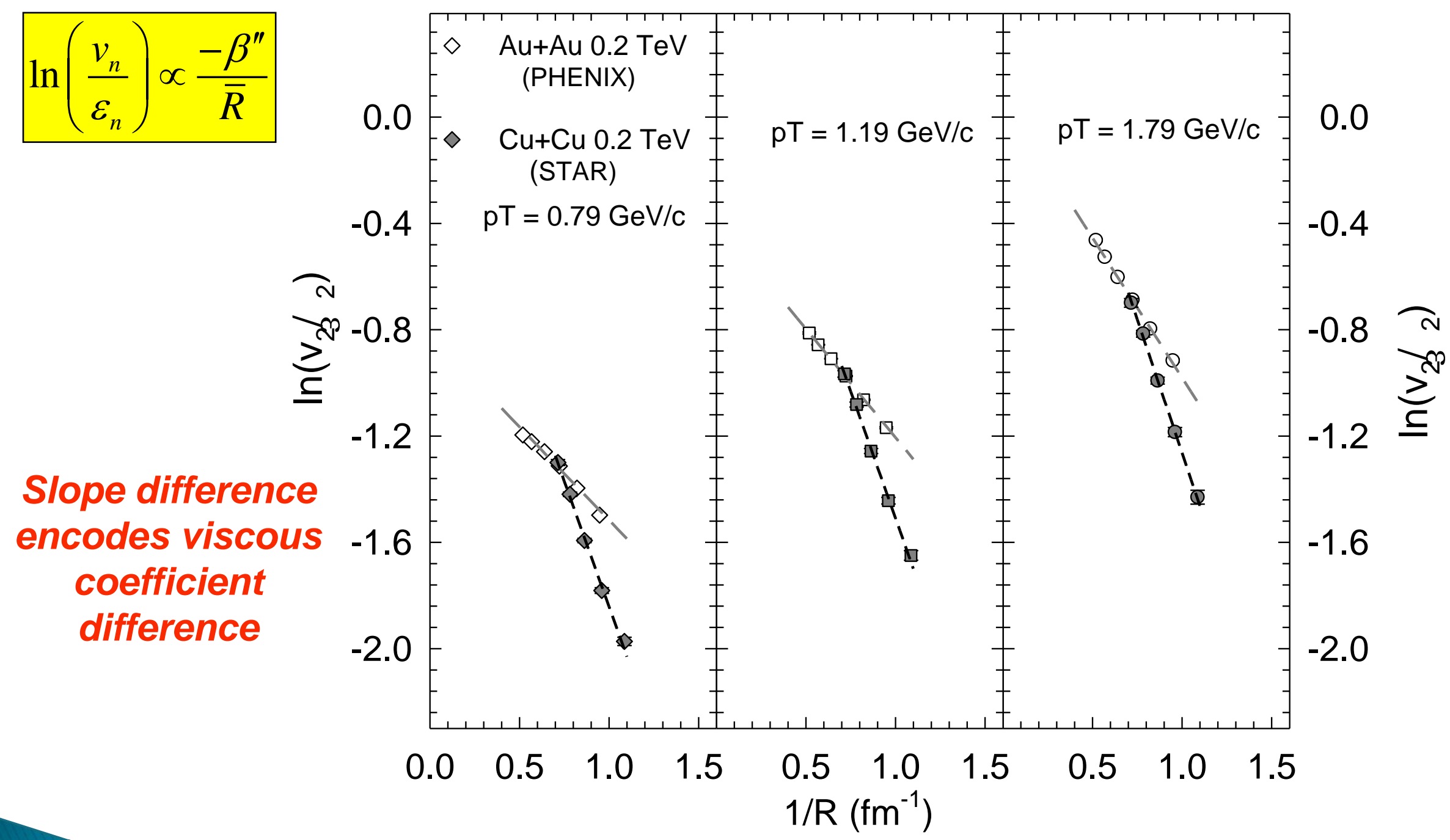

$\checkmark$ Viscous coefficient larger for more dilute system 


\section{Acoustic Scaling - 1/R}

$$
\ln \left(\frac{v_{n}}{\varepsilon_{n}}\right) \propto \frac{-\beta^{\prime \prime}}{\bar{R}}
$$

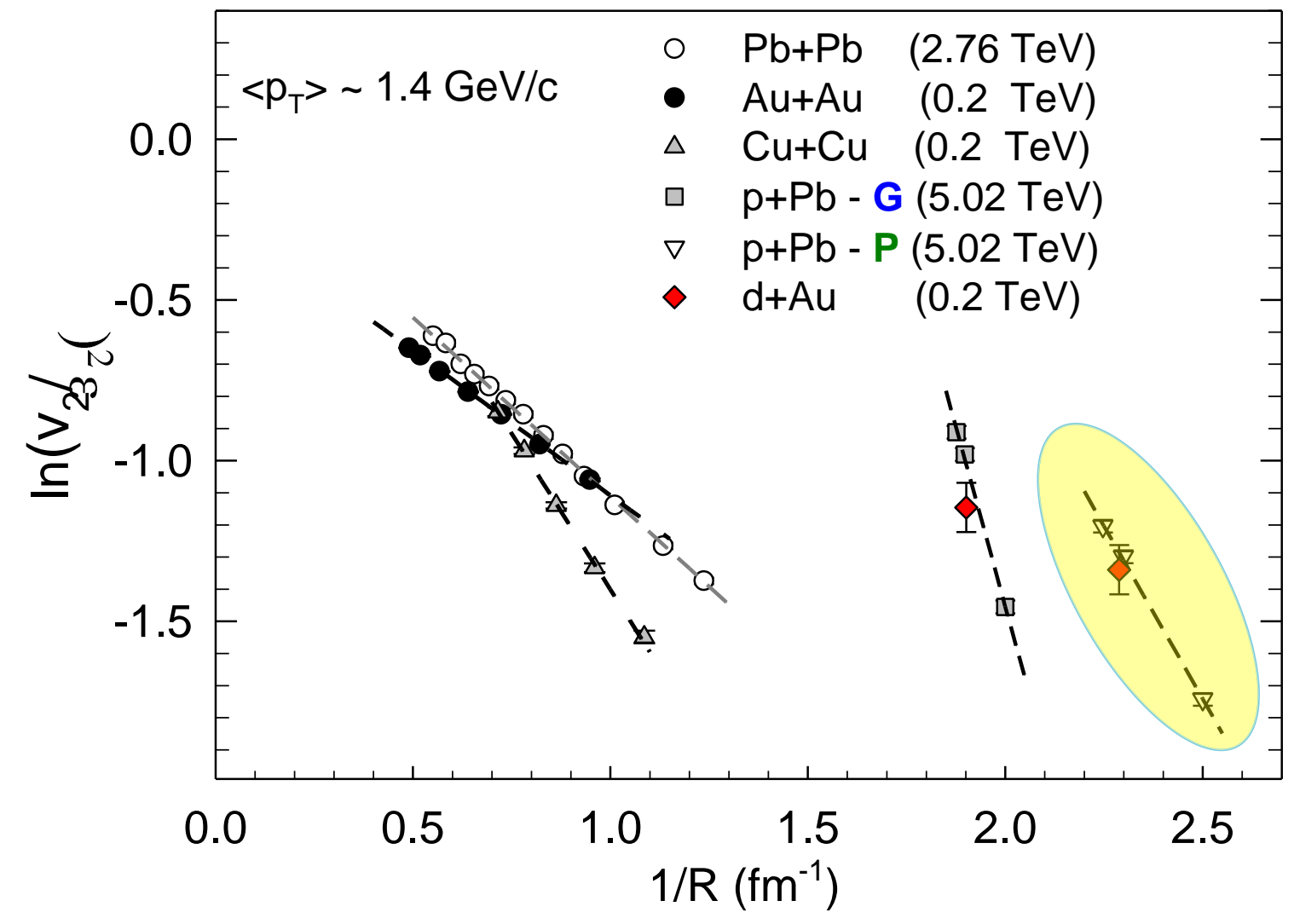

$\checkmark$ Characteristic $1 / \bar{R}$ viscous damping validated across systems $\rightarrow$ Similar mechanism

$\checkmark \quad$ Clear system size dependence of $\beta \rightarrow$ signature of dilute fluid? This is very important 


\section{Jet suppression Probe}

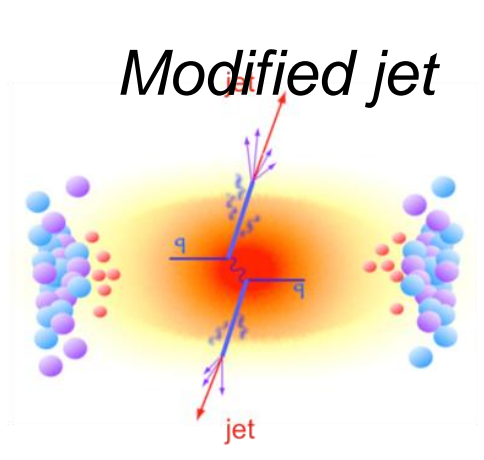

Suppression (L) $\mathrm{R}_{\mathrm{AA}}\left(p_{T}, L\right)=\frac{\text { Yield }_{\mathrm{AA}}}{\left\langle\mathrm{N}_{\text {binary }}\right\rangle_{\mathrm{AA}} \text { Yield }_{\mathrm{pp}}}$

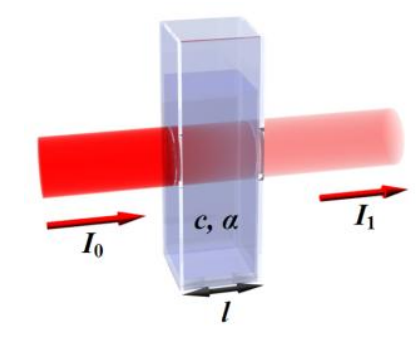

$$
T=\frac{I}{I_{0}}=e^{-\varepsilon L c}
$$

Fixed Geometry

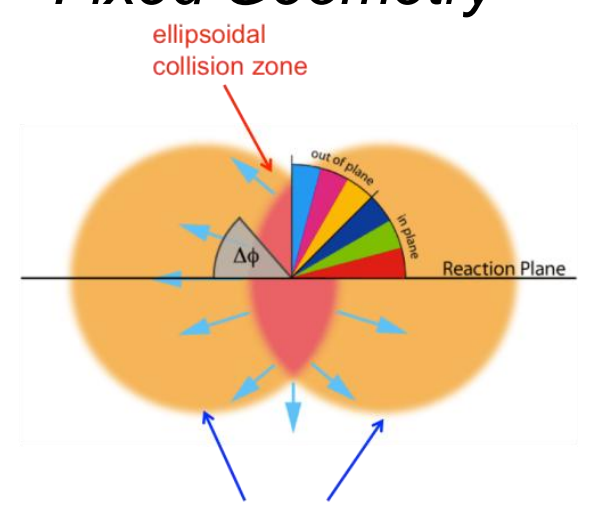

More

suppression

Less

suppression

$$
\operatorname{Yield}\left(\Delta \phi, p_{T}\right) \propto\left[1+2 v_{2}\left(p_{T}\right) \cos (2 \Delta \phi)\right]
$$

Suppression $(\Delta L)$ - pp yield unnecessary

$$
R_{2}\left(p_{T}, \Delta L\right)=\frac{R_{\mathrm{AA}}\left(90^{\circ}, p_{T}\right)}{R_{\mathrm{AA}}\left(0^{0}, p_{T}\right)}=\frac{1-2 v_{2}\left(p_{T}\right)}{1+2 v_{2}\left(p_{T}\right)}
$$

\section{For Radiative Energy loss:}

Path length (related to collision centrality)

$$
\begin{aligned}
R_{\mathrm{AA}}\left(p_{T}, L\right) \simeq \exp \left[-\frac{2 \alpha_{s} C_{F}}{\sqrt{\pi}} L \sqrt{\hat{q} \frac{\mathcal{L}}{p_{\perp}}}+\frac{16 \alpha_{s} C_{F}}{9 \sqrt{3}} L\left(\frac{\hat{q} M^{2}}{M^{2}+p_{\perp}^{2}}\right)^{1 / 3}\right] \\
\mathcal{L} \equiv \frac{d}{d \ln p_{\perp}} \ln \left[\frac{d \sigma^{v a c}}{d p_{\perp}^{2}}\left(p_{\perp}\right)\right]
\end{aligned}
$$

Jet suppression drives $R_{A A}$ \& azimuthal anisotropy with specific scaling properties 


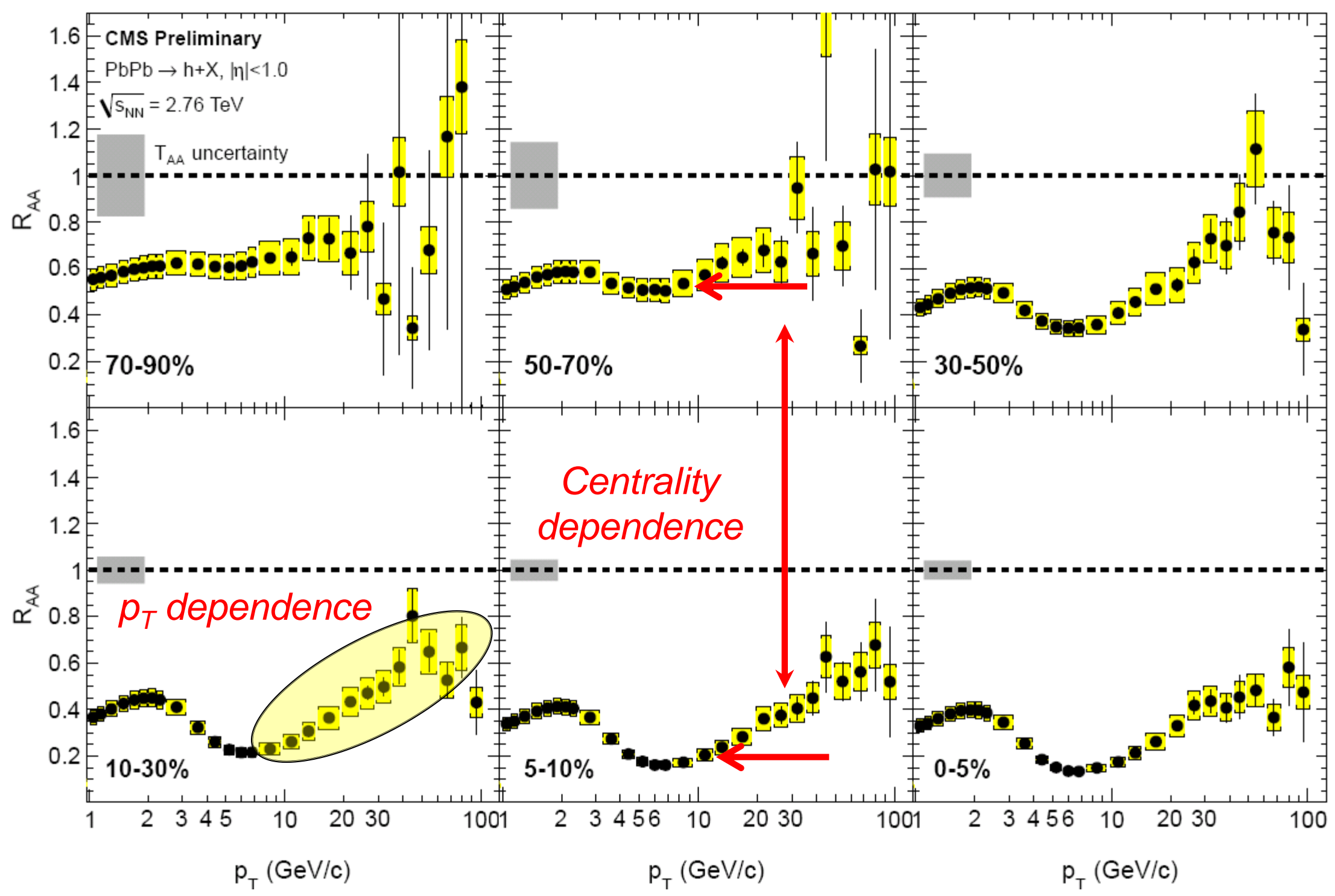

Specific $p_{T}$ and centrality dependencies - Do they scale? 


\section{L scaling of Jet Quenching - LHC}

Dokshitzer and D. E. Kharzeev, Phys.Lett.B519:199-206,2001

$$
R_{\mathrm{AA}}^{l}\left(p_{T}, L\right) \simeq \exp \left[-\frac{2 \alpha_{s} C_{F}}{\sqrt{\pi}} L \sqrt{\hat{q}} \frac{\overline{\mathcal{L}_{l}}}{p_{T}}\right]
$$

arXiv:1202.5537

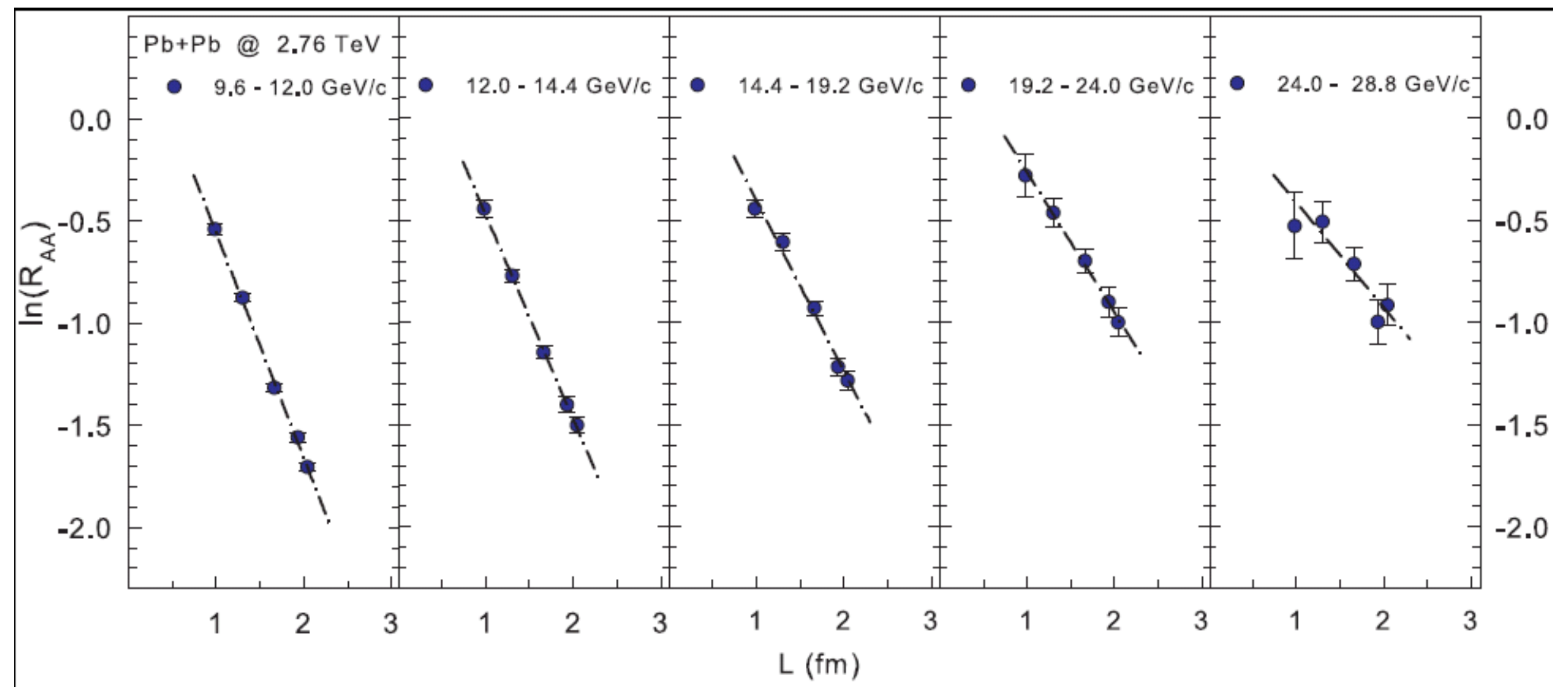

$R_{A A}$ scales with $L$, slopes $\left(S_{L}\right)$ encodes info on $\alpha_{s}$ and $\hat{q}$ $\checkmark$ Compatible with the dominance of radiative energy loss 


\section{L scaling of Jet Quenching - RHIC}

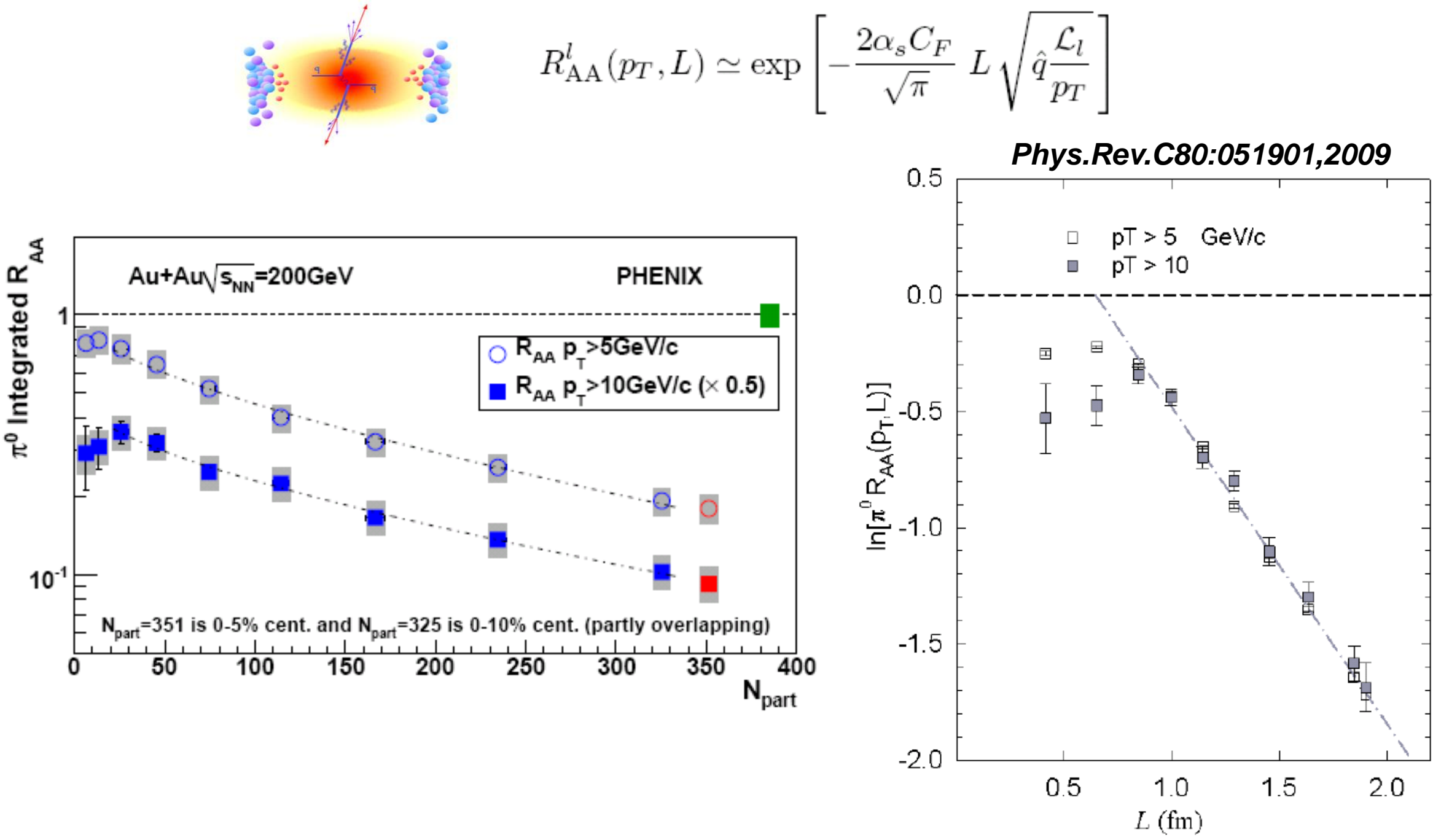

$R_{A A}$ scales with $L$, slopes $\left(S_{L}\right)$ encodes info on $\alpha_{s}$ and $\hat{q}$ $\checkmark$ Compatible with the dominance of radiative energy loss 


\section{$p_{T}$ scaling of Jet Quenching}

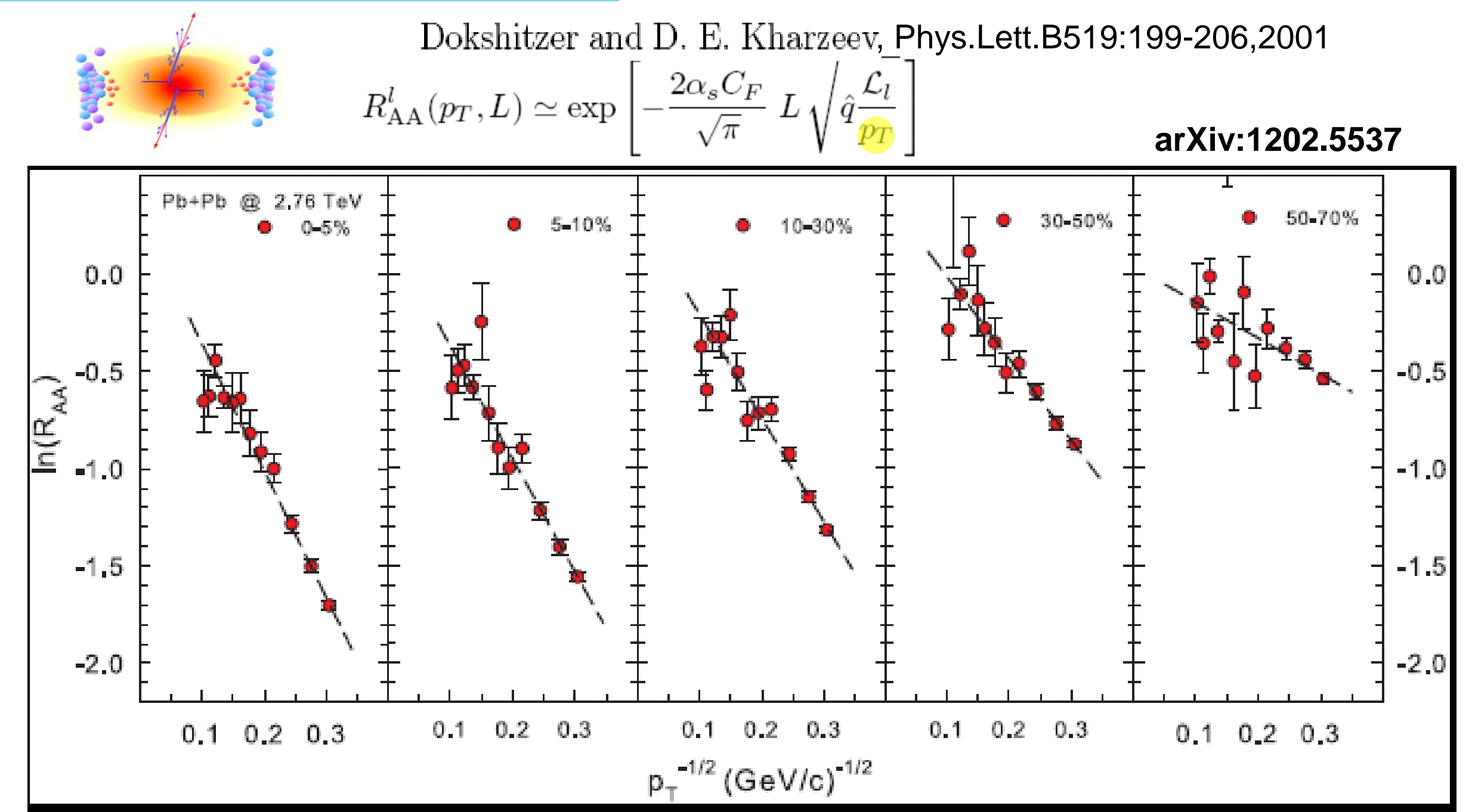

$R_{A A}$ scales as $1 / N p_{T}$; slopes $\left(S_{p T}\right)$ encode info on $\alpha_{s}$ and $\hat{q}$

$\checkmark L$ and $1 / N p_{T}$ scaling $\rightarrow$ single universal curve

$\checkmark$ Compatible with the dominance of radiative energy loss 


\section{Heavy quark suppression}

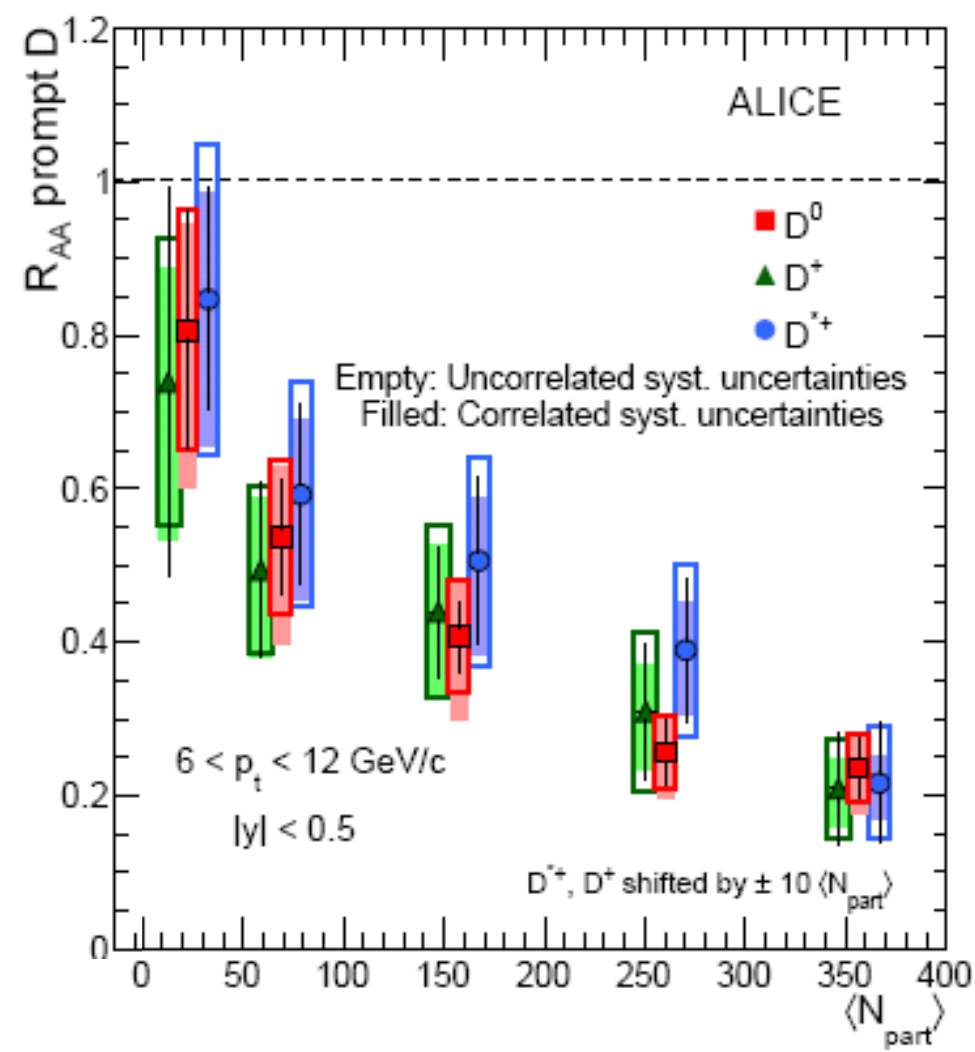

$R_{\mathrm{AA}}\left(p_{T}, L\right) \simeq \exp \left\lfloor-\frac{2 \alpha_{s} C_{F}}{\sqrt{\pi}} L \sqrt{\hat{q} \frac{\mathcal{L}}{p_{\perp}}}+\right.$.

$$
\begin{aligned}
& \left.\frac{16 \alpha_{s} C_{F}}{9 \sqrt{3}} L\left(\frac{\hat{q} M^{2}}{M^{2}+p_{\perp}^{2}}\right)^{1 / 3}\right] \\
& \mathcal{L} \equiv \frac{d}{d \ln p_{\perp}} \ln \left[\frac{d \sigma^{v a c}}{d p_{\perp}^{2}}\left(p_{\perp}\right)\right]
\end{aligned}
$$

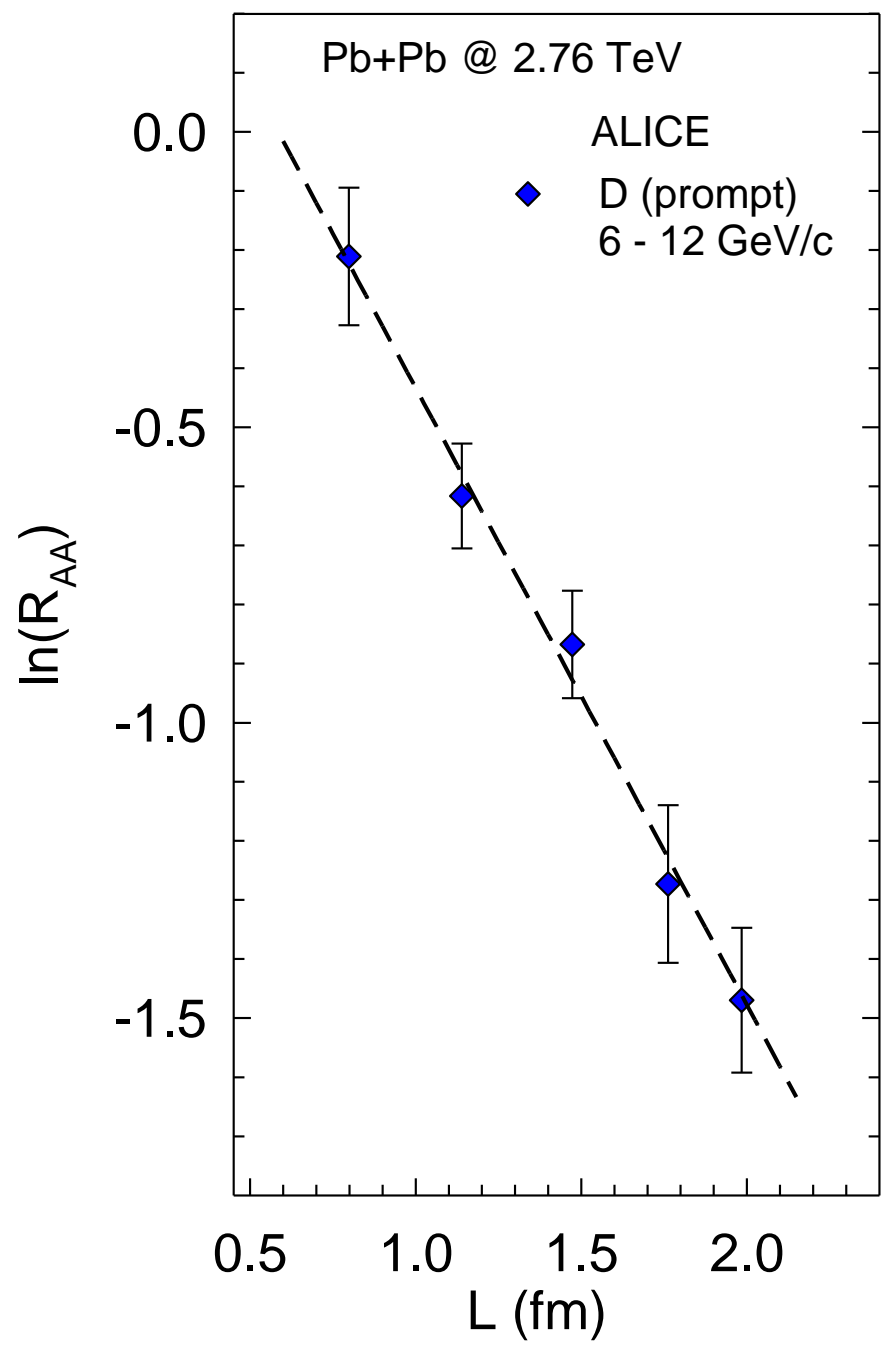

Consistent $\hat{q}_{\text {LHC }}$ obtained 


\section{High-pT $v_{2}$ measurements - PHENIX}

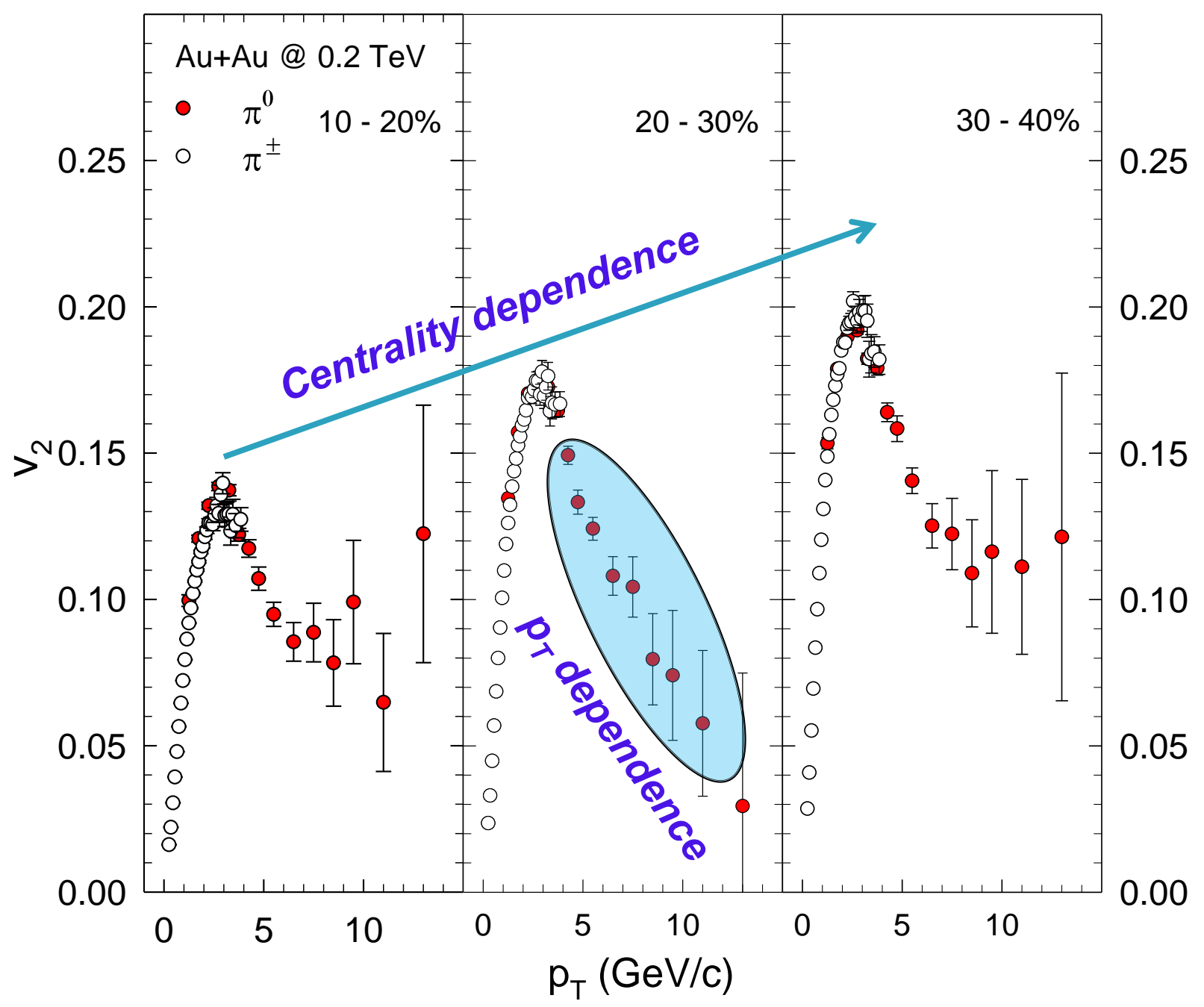

Specific $p_{T}$ and centrality dependencies - Do they scale? 


\section{High-pT $v_{2}$ measurements - CMS}

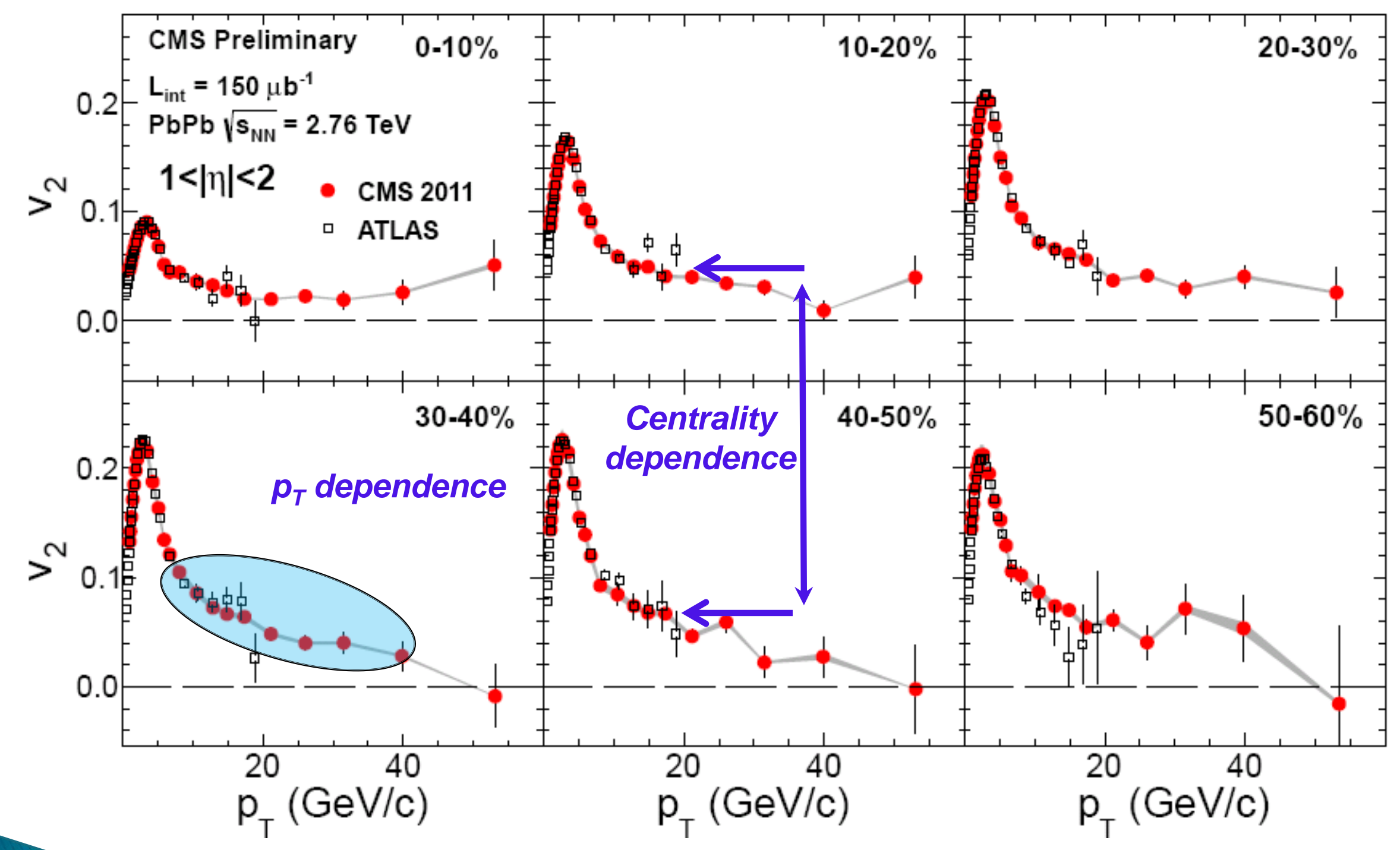

Specific $p_{T}$ and centrality dependencies - Do they scale? 


\section{High-pT $v_{2}$ scaling - LHC}

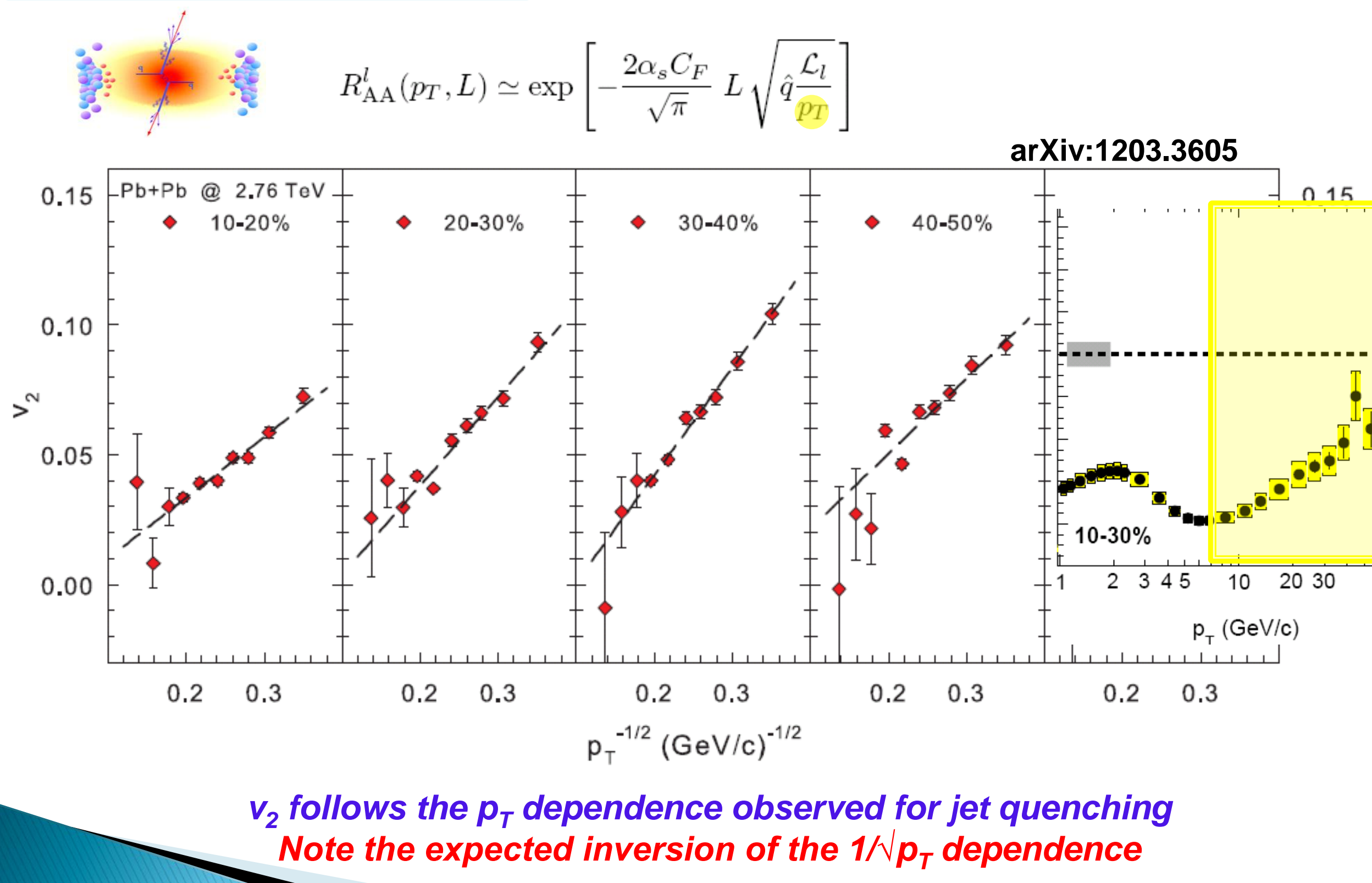




\section{$\Delta L$ Scaling of high-pT $v_{2}-L H C \& R H I C$}

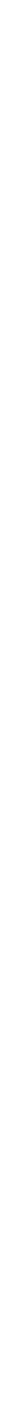

Combined $\Delta L$ and $1 / N p_{T}$ scaling $\rightarrow$ single universal curve for $v_{2}$ 


\section{$\Delta L$ Scaling of high-pT $v_{2}-R H I C$}

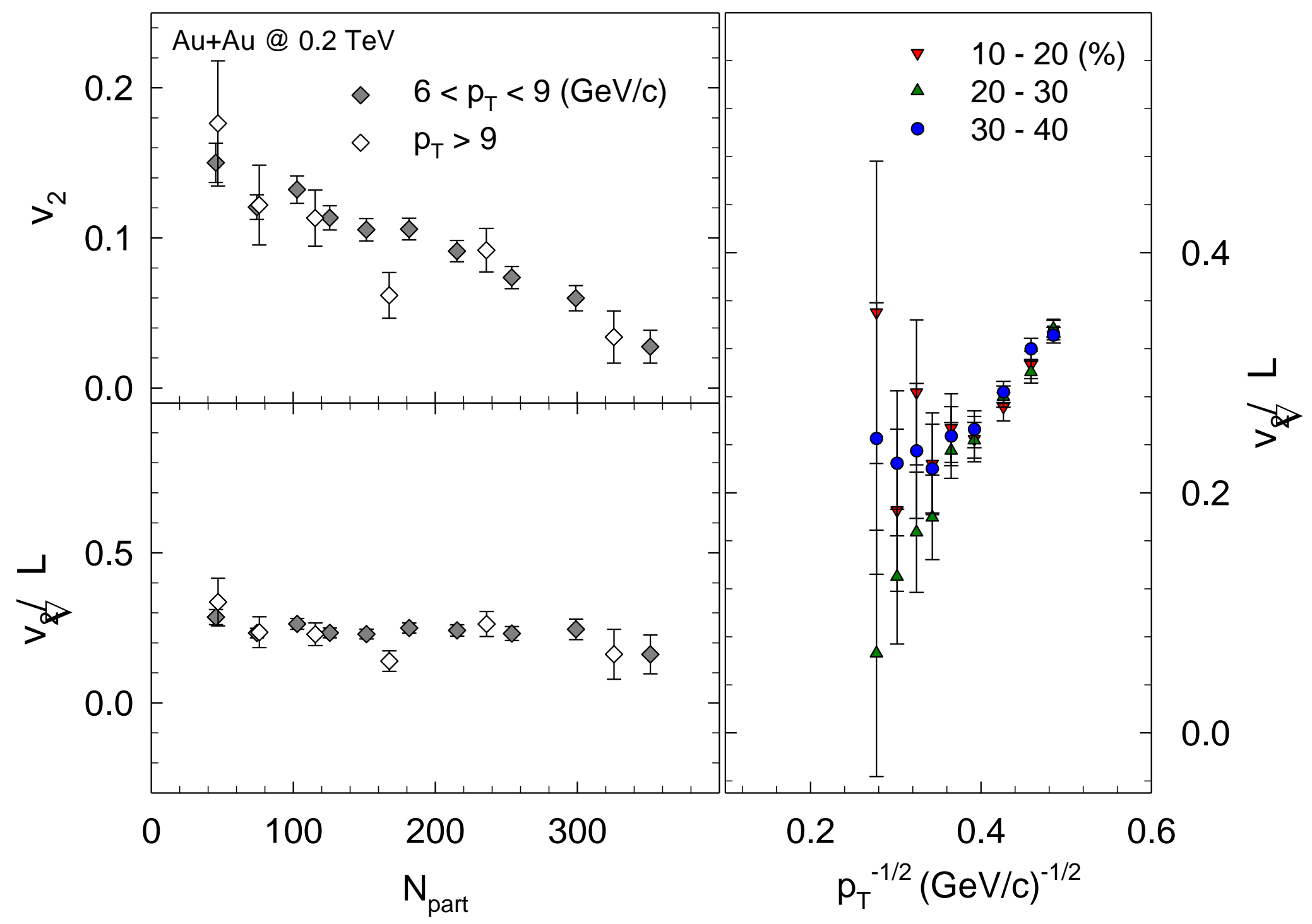

Combined $\Delta L$ and $1 / N p_{T}$ scaling $\rightarrow$ single universal curve for $v_{2}$ 
Jet $v_{2}$ scaling - LHC

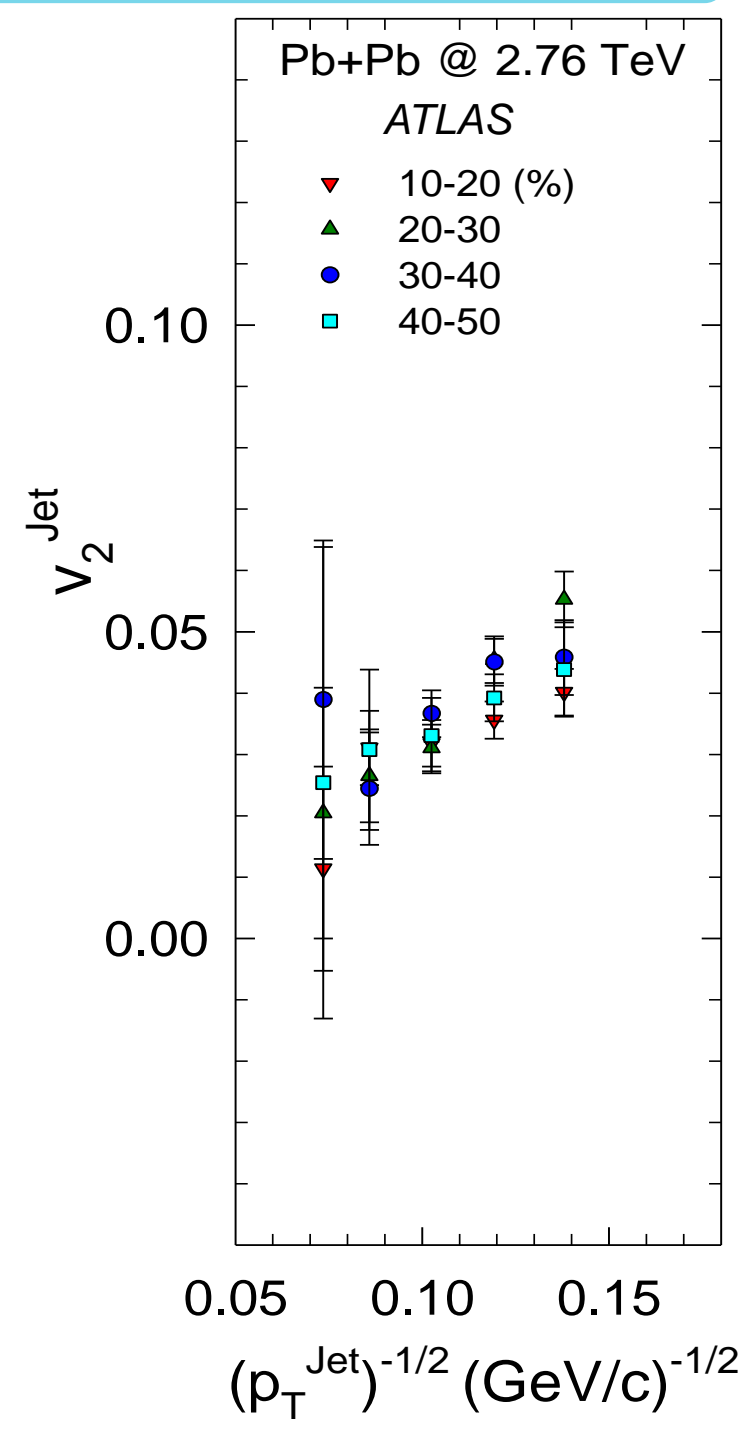

$$
R_{\mathrm{AA}}^{l}\left(p_{T}, L\right) \simeq \exp \left[-\frac{2 \alpha_{s} C_{F}}{\sqrt{\pi}} L \sqrt{\hat{q} \frac{\mathcal{L}_{l}}{p_{T}}}\right]
$$

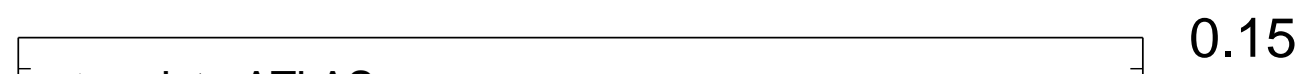

$\diamond \quad$ Jet - ATLAS

$\square$ Hadrons - CMS

- Hadrons - scaled $(z \sim .62)$

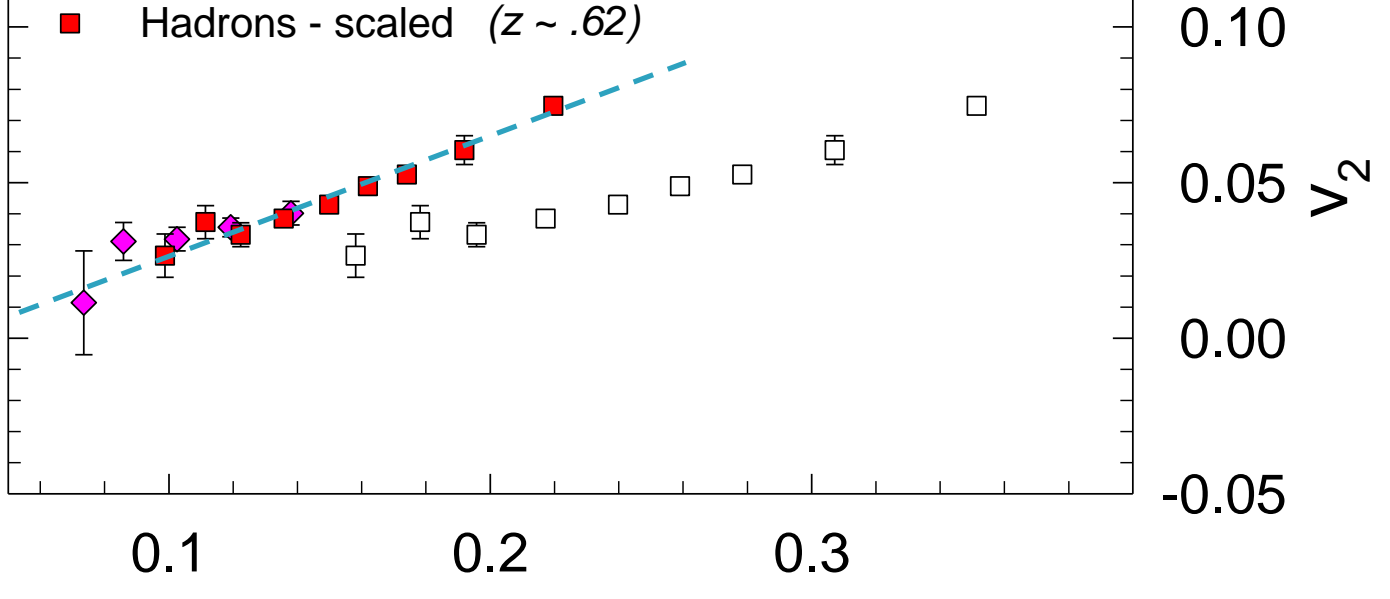

$\mathrm{p}_{\mathrm{T}}^{-1 / 2}(\mathrm{GeV} / \mathrm{c})^{-1 / 2}$

$v_{2}$ for reconstructed Jets follows the $p_{T}$ dependence for jet quenching Similar magnitude and trend for Jet and hadron $v_{2}$ after scaling 


\section{Jet suppression from high-pT $v_{2}$}

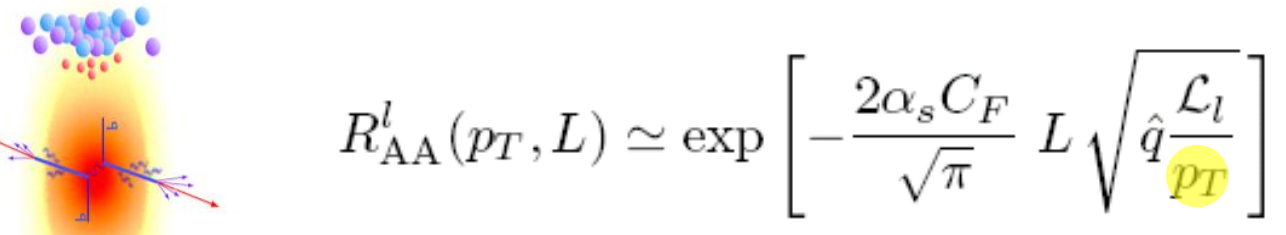

$$
\begin{array}{r}
N\left(\Delta \phi, p_{T}\right) \propto\left[1+2 v_{2}\left(p_{T}\right) \cos (2 \Delta \phi)\right] \\
R_{v_{2}}\left(p_{T}, \Delta L\right)=\frac{R_{\mathrm{AA}}\left(90^{o}, p_{T}\right)}{R_{\mathrm{AA}}\left(0^{0}, p_{T}\right)}=\frac{1-2 v_{2}\left(p_{T}\right)}{1+2 v_{2}\left(p_{T}\right)}
\end{array}
$$

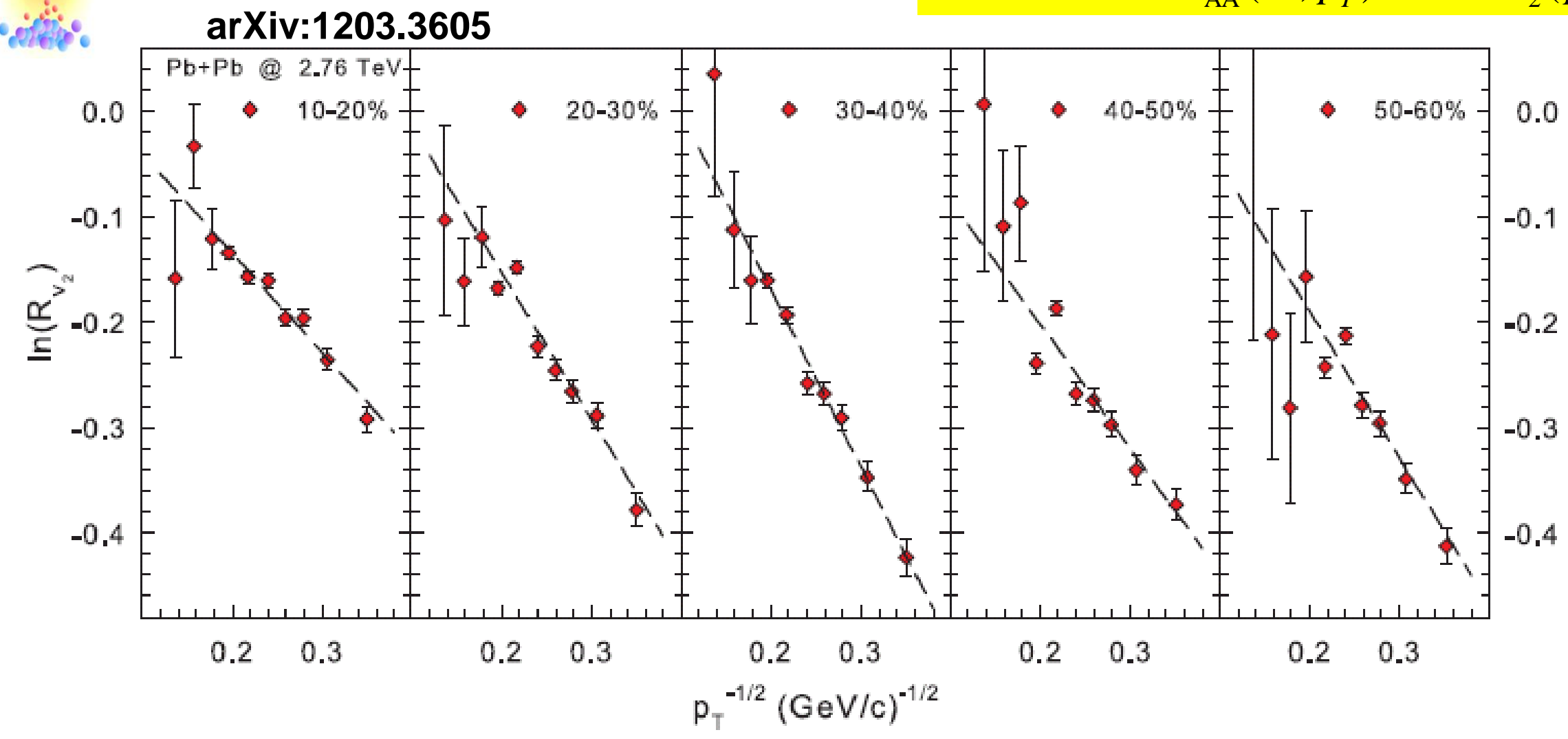

Jet suppression obtained directly from pT dependence of $v_{2}$ $R_{v 2}$ scales as $1 / N p_{T}$, slopes encodes info on $\alpha_{s}$ and $\hat{q}$ 


\section{Extracted stopping power}
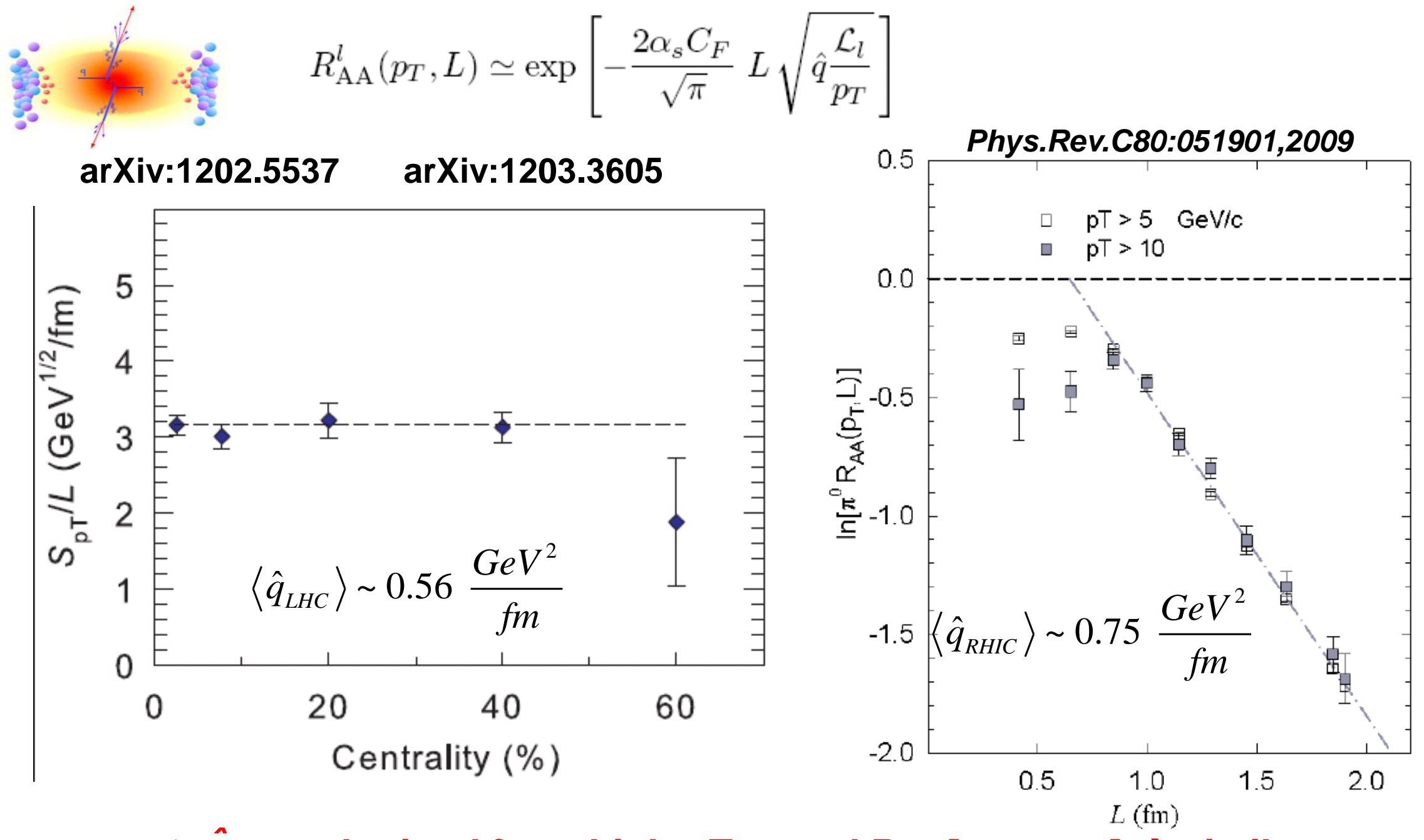

$>\hat{q}_{L H C}$ obtained from high-pT $v_{2}$ and $R_{A A}$ [same $\alpha_{s}$ ] $\rightarrow$ similar

$>\hat{q}_{R H I C}>q_{L H C}$ - medium produced in LHC collisions less opaque!

Conclusion similar to those of Liao, Betz, Horowitz,

$\rightarrow$ Stronger coupling near $T_{c}$ ? 


\section{Summary}

Robust scaling observed for both Flow and Jet Quenching

They lend profound mechanistic insights, as well as New constraints for estimates of the transport and thermodynamic coefficients!

\section{What do we learn?}

$>\mathbf{R}_{\mathrm{AA}}$ and high-pT azimuthal anisotropy stem from the same energy loss mechanism

$>$ Energy loss is dominantly radiative

$>\mathbf{R}_{\mathrm{AA}}$ and anisotropy measurements give consistent estimates for $\langle\dot{q}\rangle$ $>R_{A A}$ for D's give consistent estimates for $\langle\hat{q}\rangle$
$>$ Flow is acoustic

$\checkmark$ Flow is pressure driven

$\checkmark$ Obeys the dispersion relation for sound propagation

$\checkmark$ clear system size dependence

$>$ The QGP created in LHC collisions > Constraints for:

is less opaque than that produced at RHIC - Note density increase from $>$ Flow is partonic

$\checkmark$ exhibits scaling

$$
\mathrm{v}_{n, q}\left(K E_{T}\right) \sim \mathrm{v}_{2, q}^{n / 2} \text { or } \frac{\mathrm{v}_{\mathrm{n}}}{\left(n_{q}\right)^{n / 2}}
$$

$\checkmark \varepsilon, \beta$, and $\delta f$

$\checkmark \eta / s @ R H I C(\sim 1 / 4 \pi)$

$\checkmark \eta / s @$ LHC a bit larger than at RHIC 


\section{End}




\section{Acoustic Scaling - 1/R}

$\ln \left(\frac{v_{n}}{\varepsilon_{n}}\right) \propto \frac{-\beta^{\prime \prime}}{\bar{R}}$

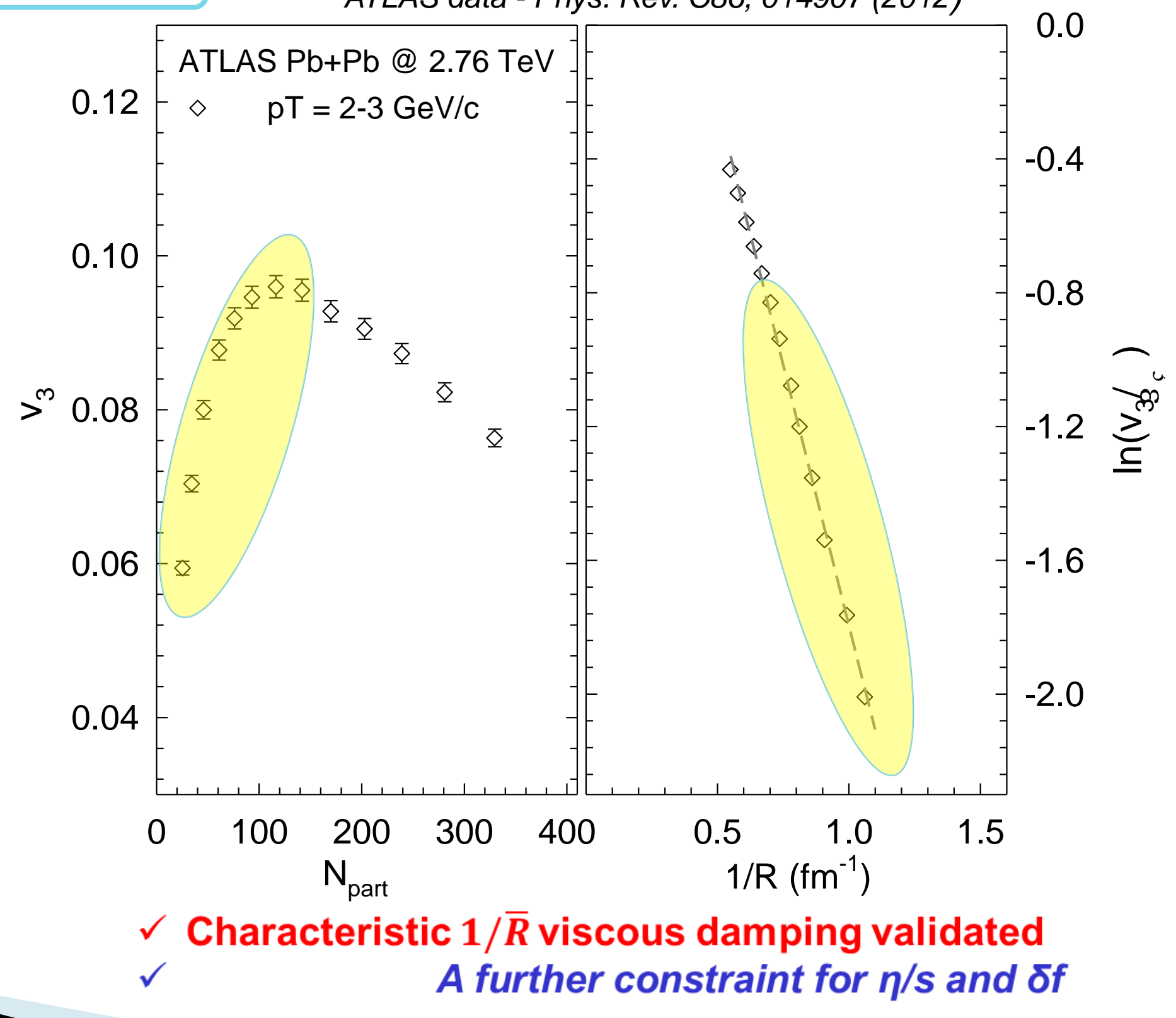

ATLAS data - Phys. Rev. C86, 014907 (2012)

Centrality $-5-70 \%$ 


\section{Acoustic Scaling - 1/R}

STAR Data - PhysRevC.81.044902

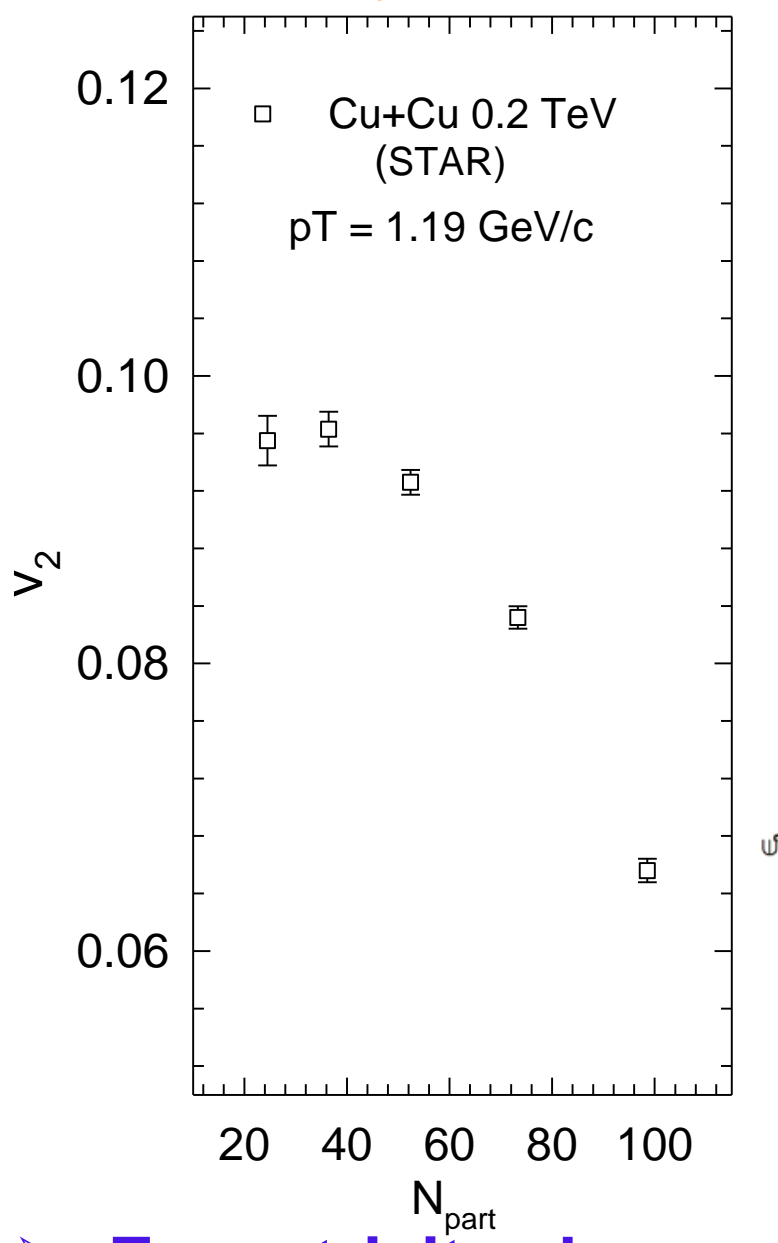

$>$ Eccentricity change alone is not sufficient To account for $\mathrm{N}_{\text {part }}$ dependence of $\mathrm{v}_{2}$ In light \& heavy systems

$\ln \left(\frac{v_{n}}{\varepsilon_{n}}\right) \propto \frac{-\beta^{\prime \prime}}{\bar{R}}$
ATLAS data - Phys. Rev. C86, 014907 (2012) ATLAS Pb+Pb @ 2.76 TeV

$\diamond \quad \mathrm{pT}=2-3 \mathrm{GeV} / \mathrm{c}$

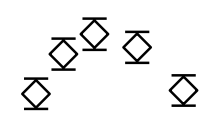

$0.20-\frac{\bar{\sigma}}{\bar{\sigma}} \bar{\Omega}$

$\overline{1}$

$\checkmark$

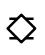

$\diamond$

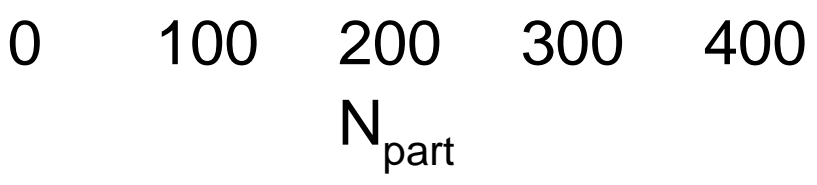




\section{OPAQUENESS EVOLUTION FROM COLOR LIBERATION}

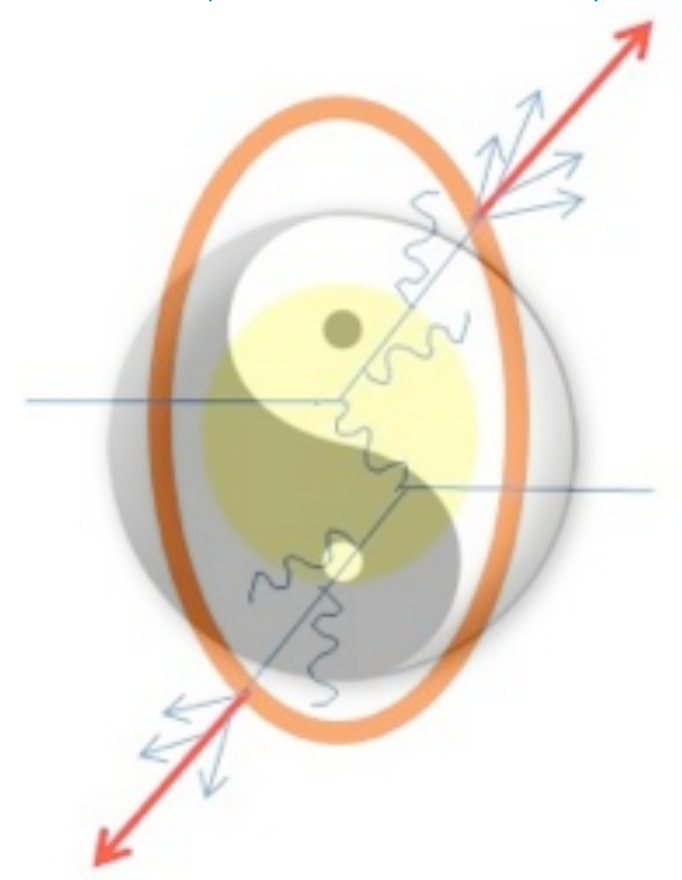

\section{Jinfeng Liao}

Indiana University, Physics Dept. \& CEEM RIKEN BNL Research Center 


\section{Outline}

- Introduction: An Opaque QCD Plasma

- What We Learn From Geometry of Jet Quenching

- Opaqueness Evolution from RHIC to LHC

- Discussions \& Summary

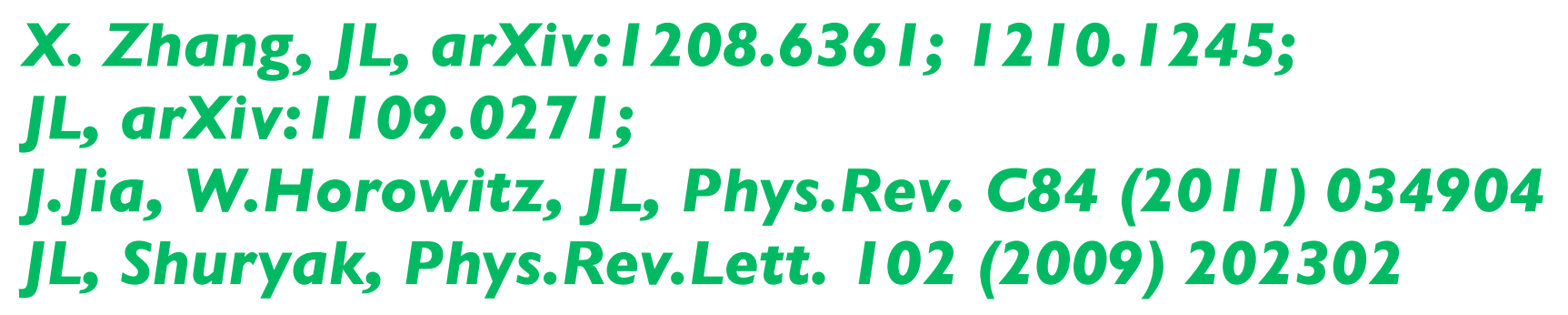




\section{Years of Asymptotic Freedom}

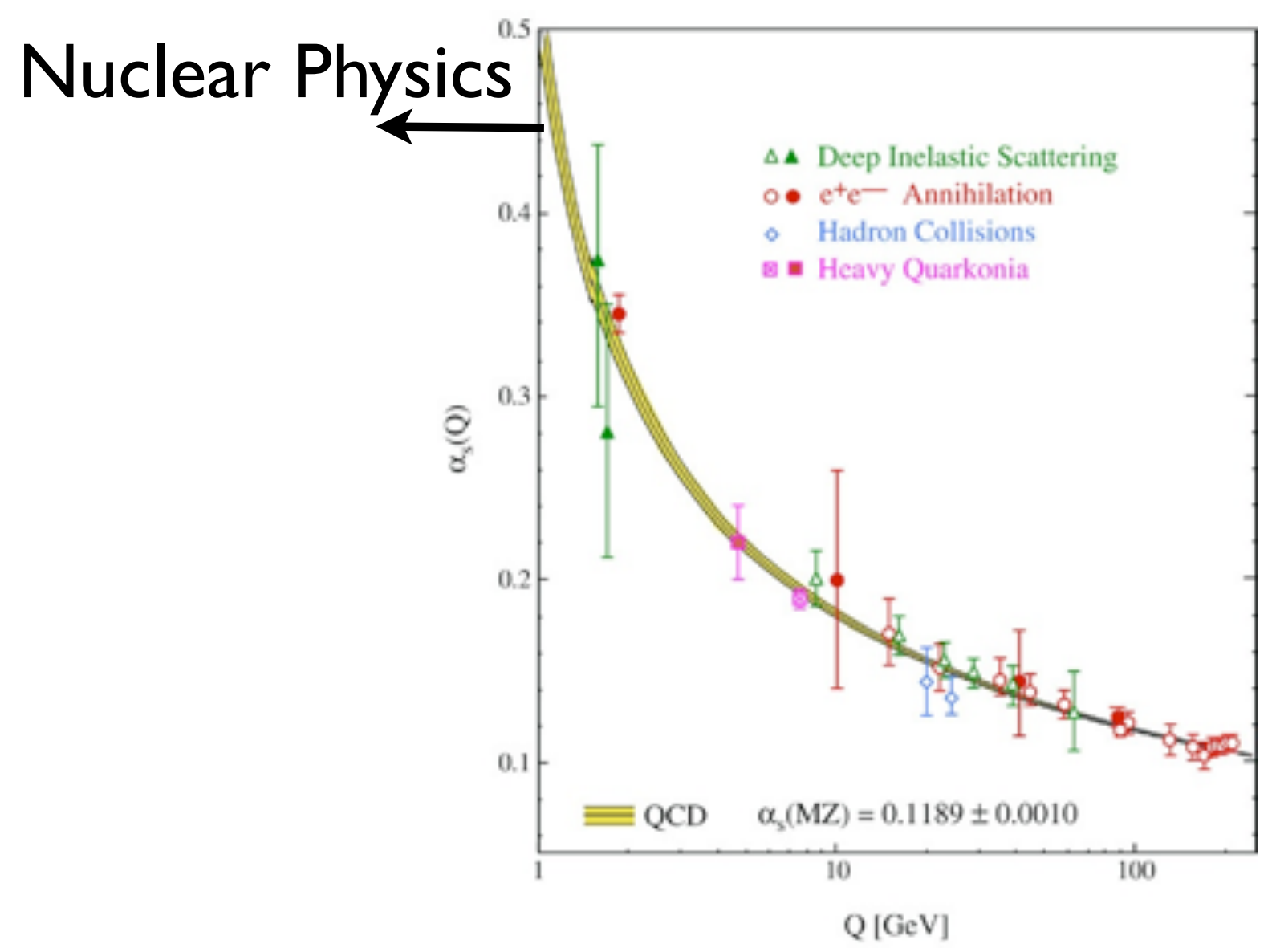




\section{Asymptotically Free Matter}

T

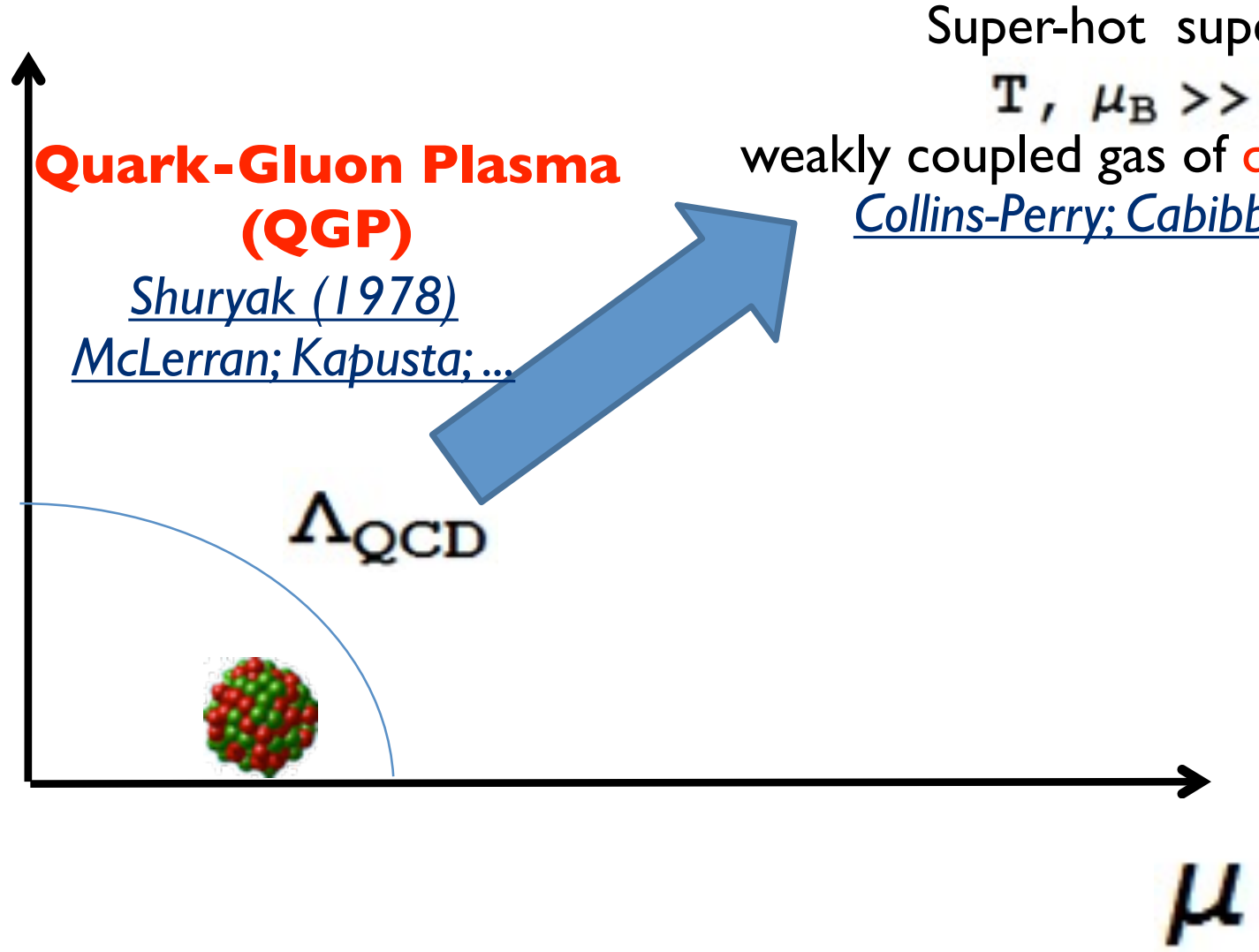

color confined world --> color (fully) liberated world 


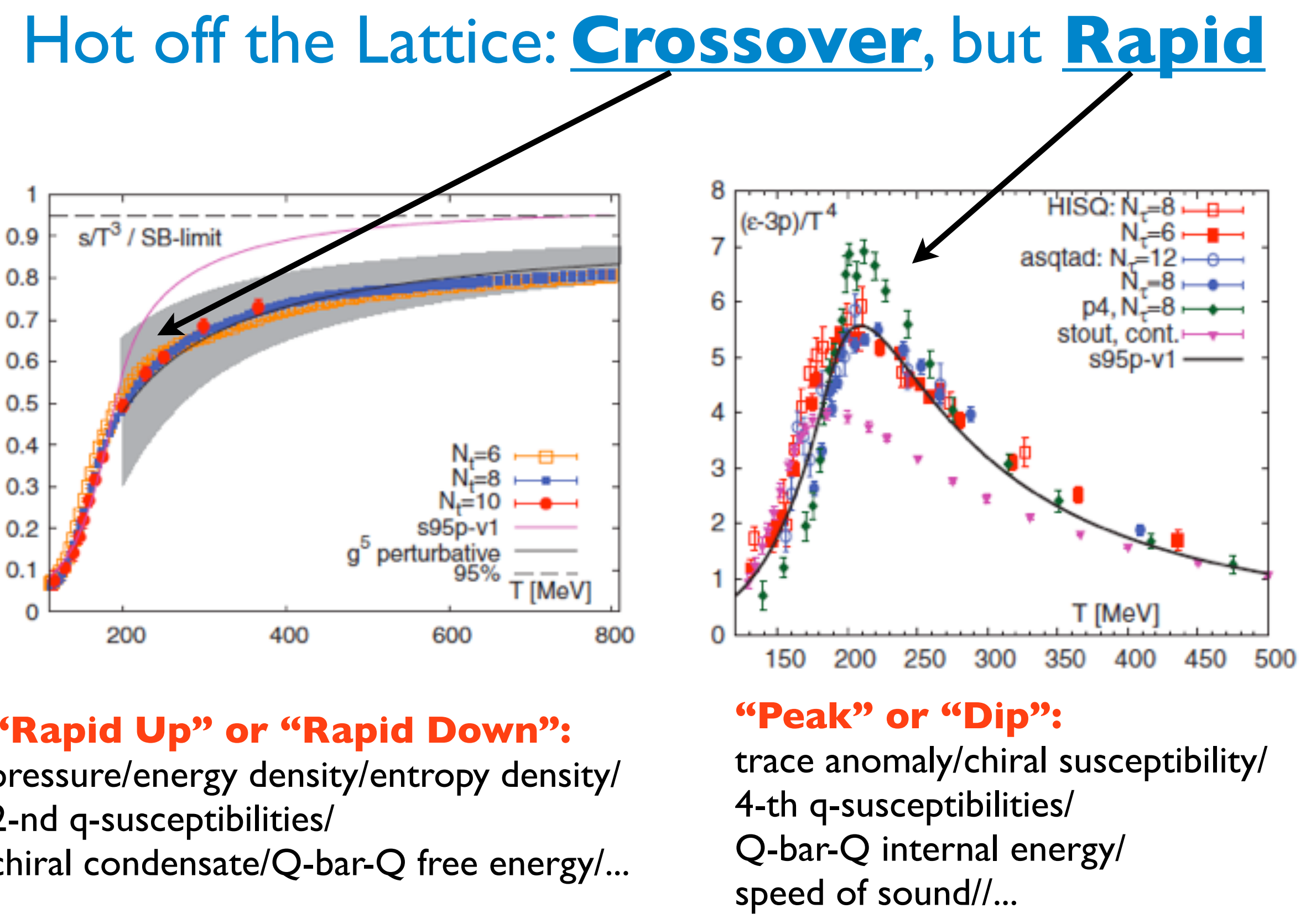




\section{Liberation of Color?}

\section{Degrees of freedom}

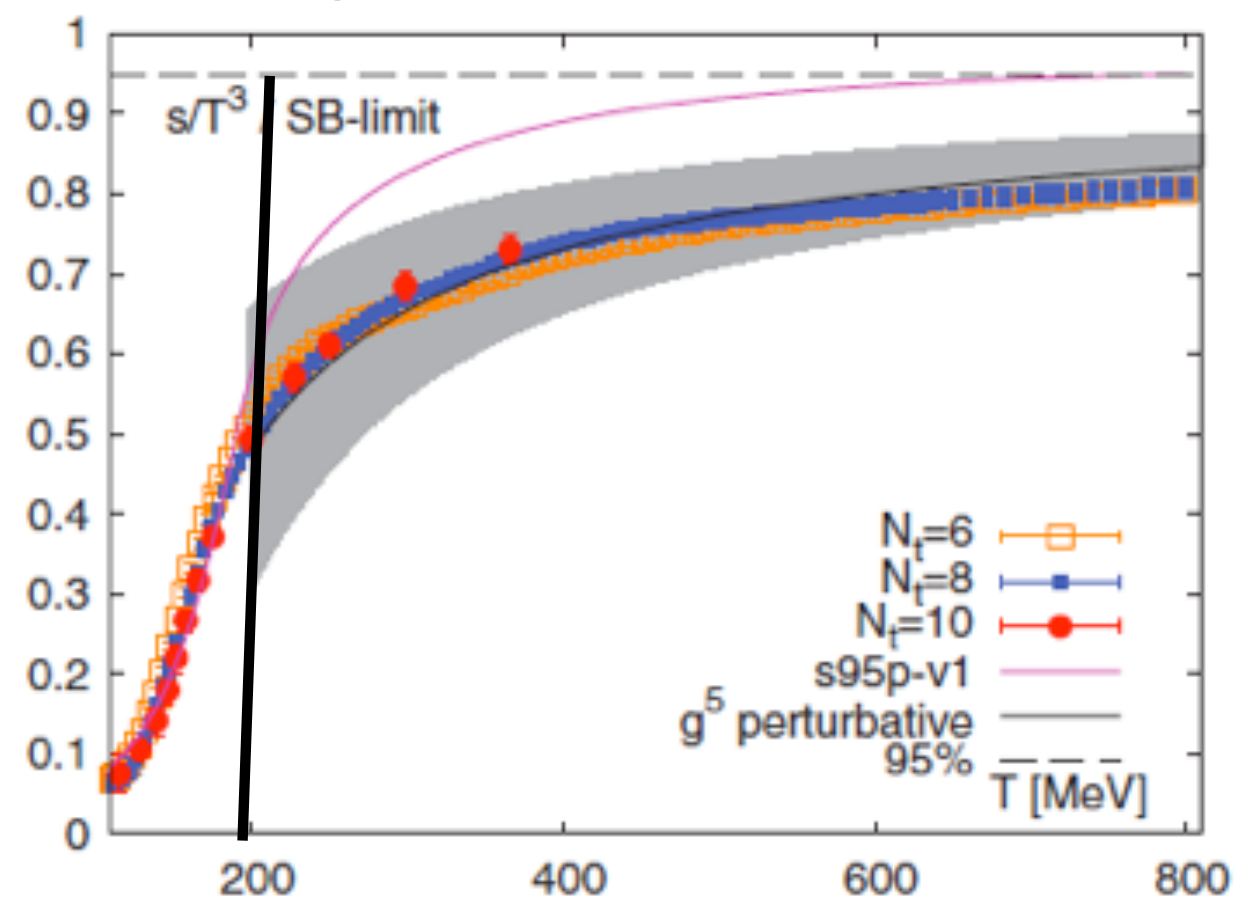

Degree of color liberation

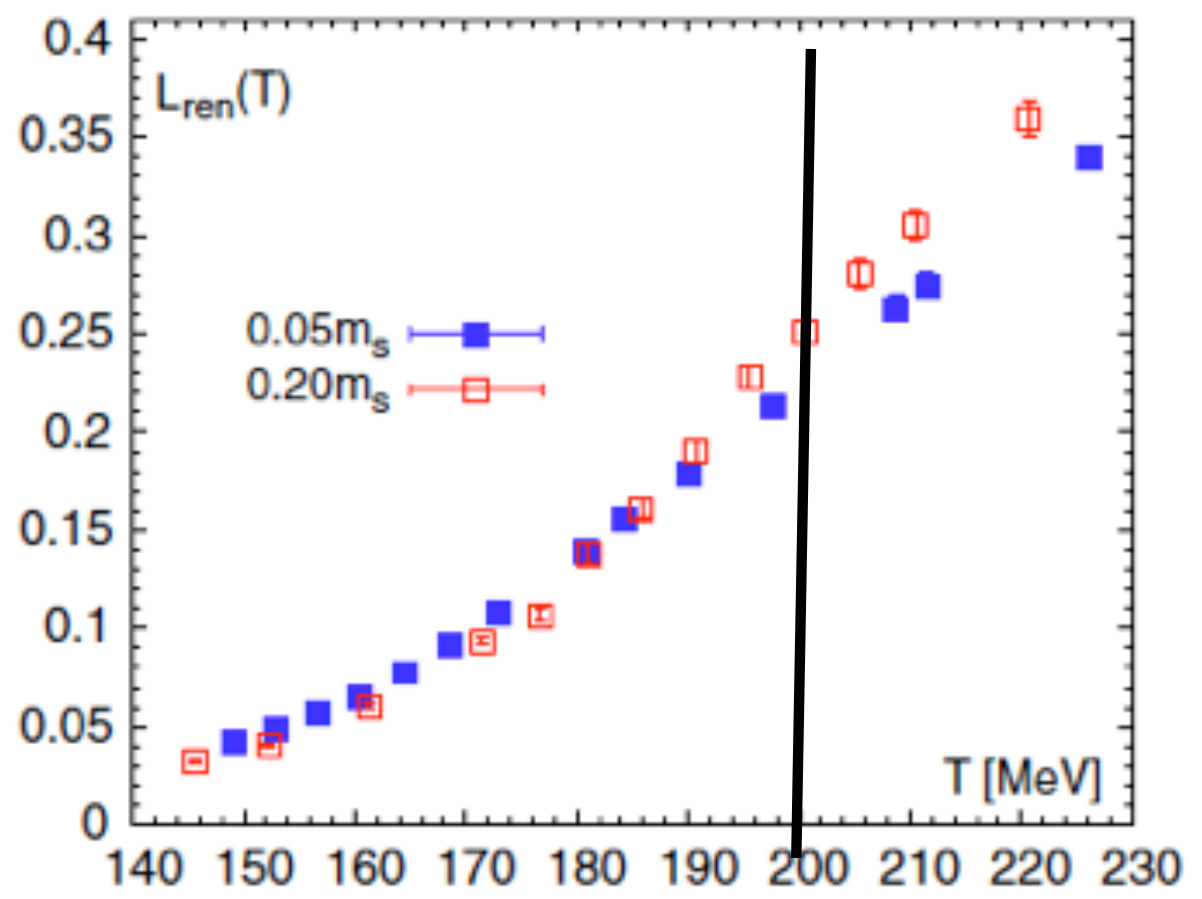

A region around Tc with liberated degrees of freedom but only partially liberated color-electric objects. (Pisarski \& collaborators: semi-QGP --- see Skokov's talk) Then what are the "extra" dominant DoF here??? Let's come to this later, for the moment: sth. special NearTc, not yet the AFM 


\section{QGP from the "Little Bangs"}

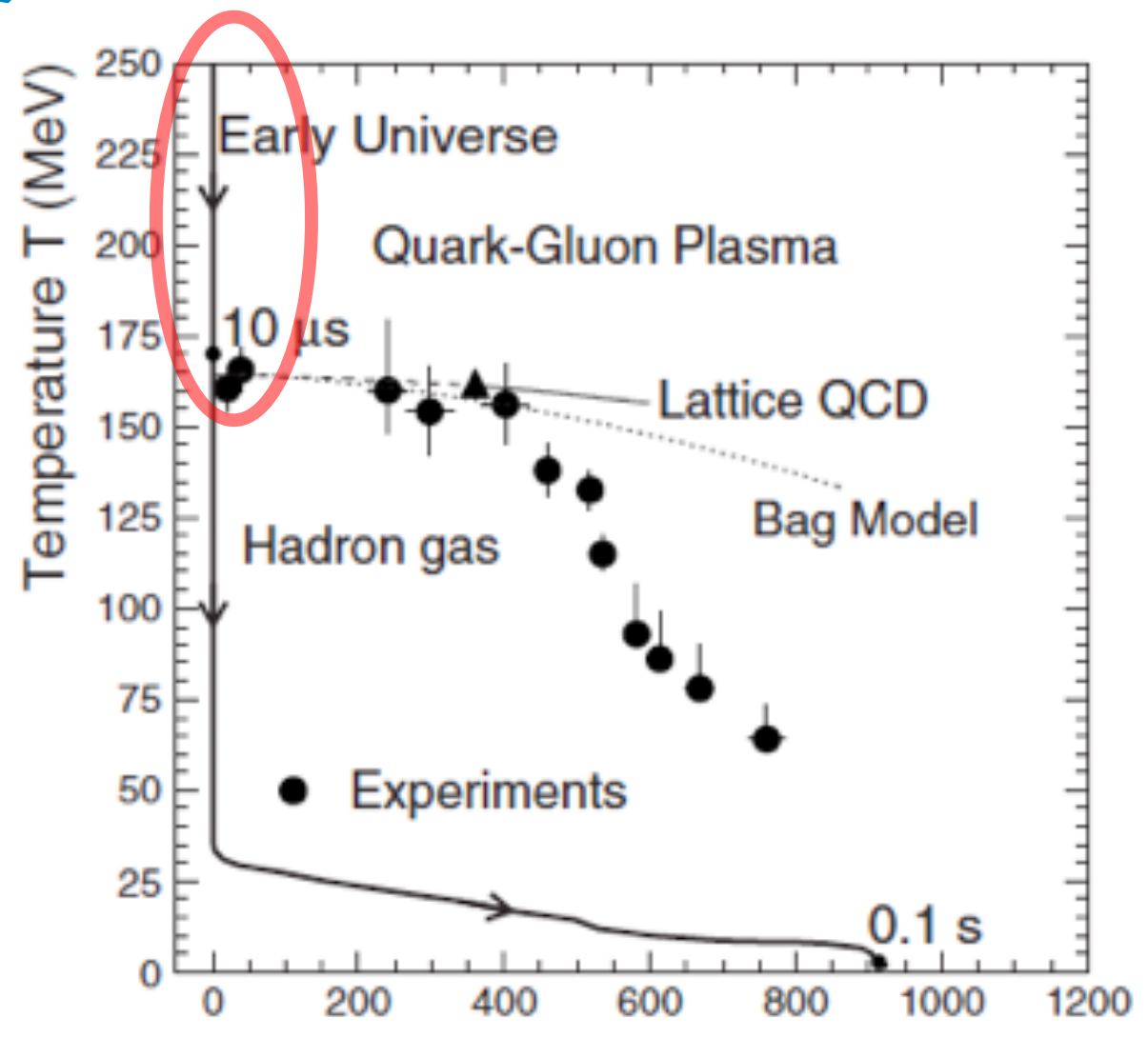

Baryochemical potential $\mu_{\mathrm{b}}(\mathrm{MeV})$

Surprising QGP: nearly perfect fluid; color opaque; .......

It is not the Asymptotically Free Matter yet! (not unrelated to the partial liberation of color) 


\section{A Color-Opaque Plasma}

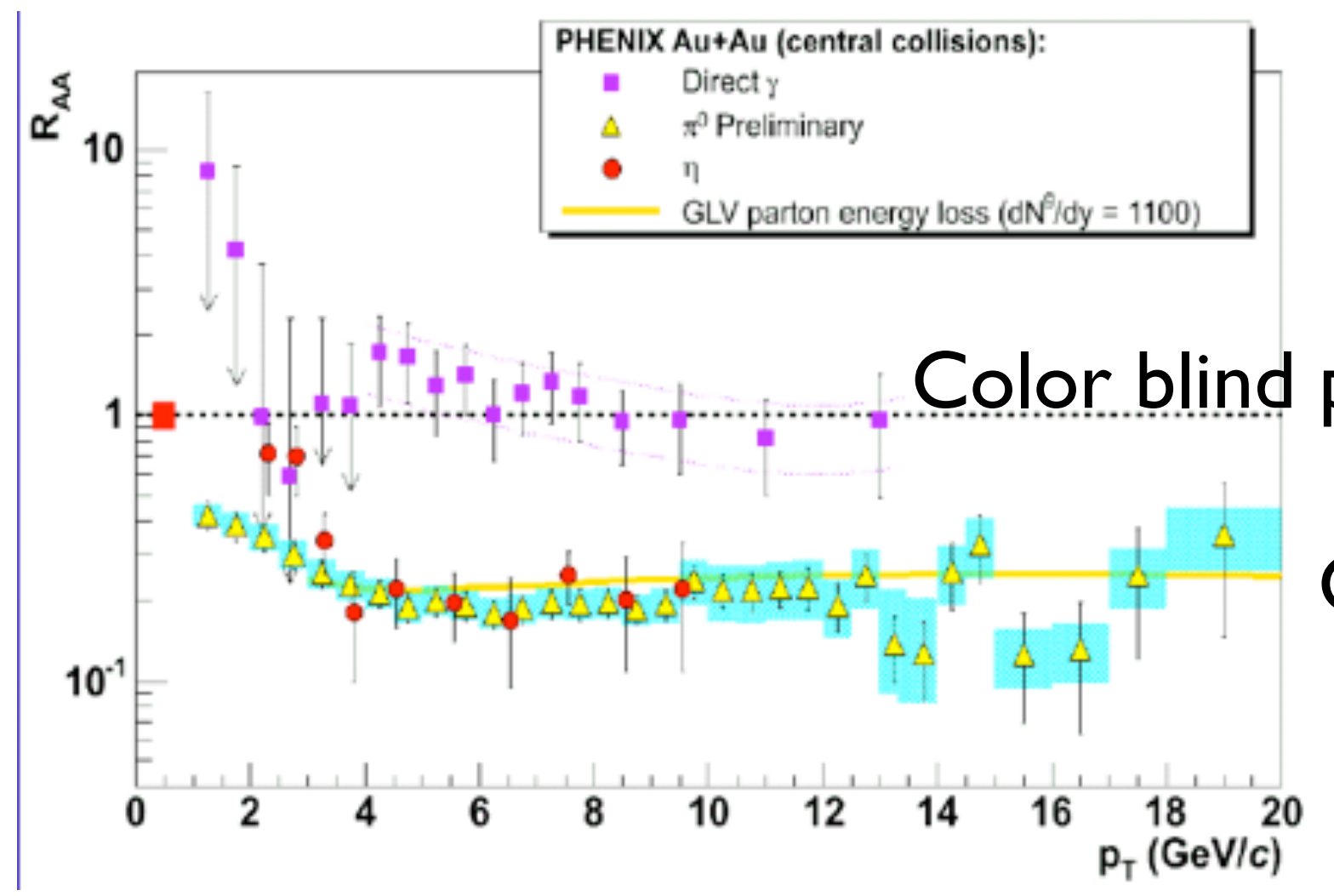

Colorful probe

A qualitatively different medium Jet-Medium
Coupling

\section{High (liberated)}

\section{Zero/Low \\ (Confined)}

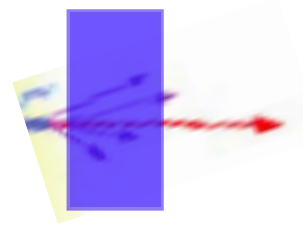




\section{From Transparency to Opaqueness}

Jet-Medium

Coupling

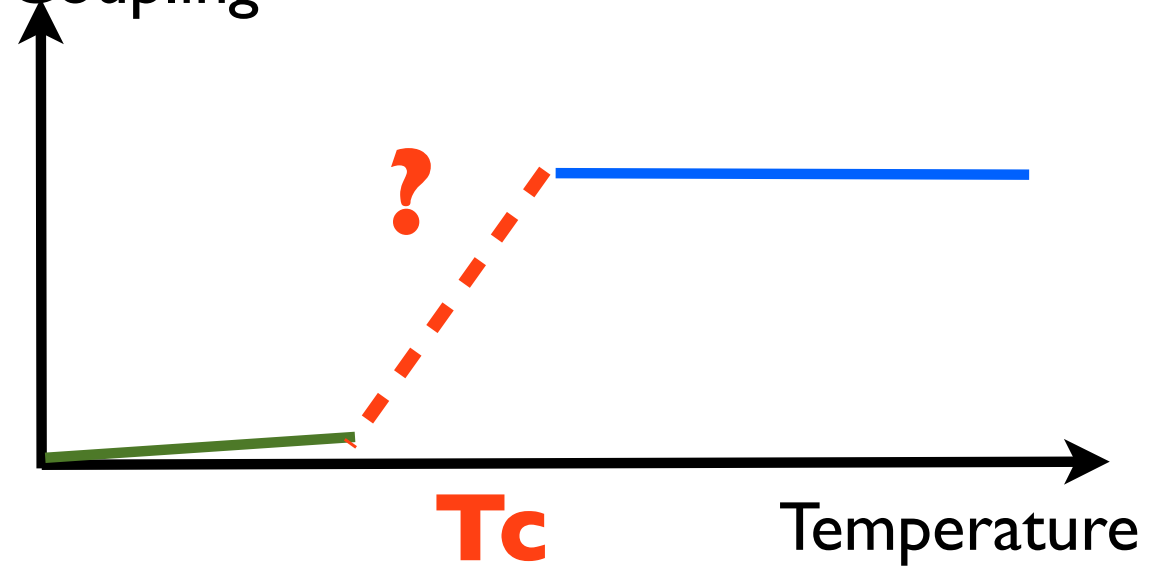

"Waterfall" scenario

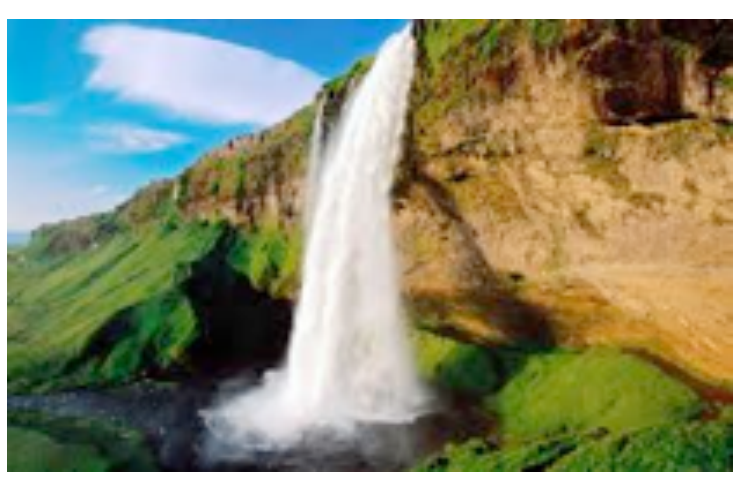

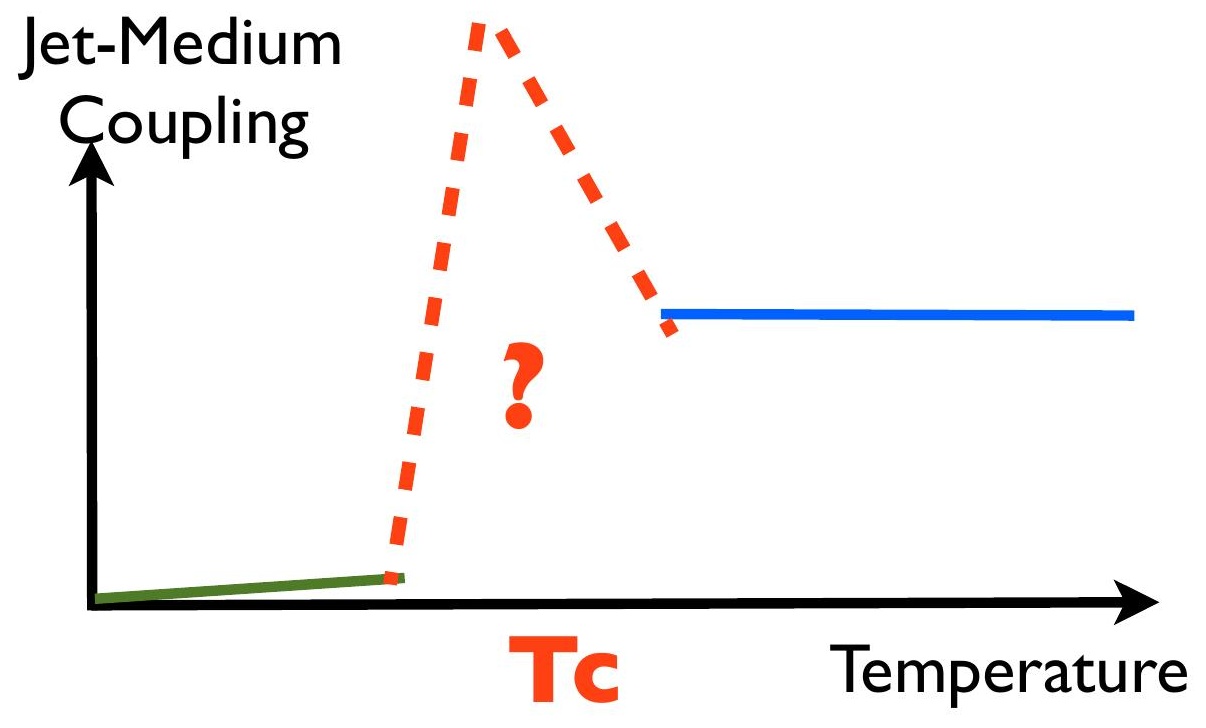

"Volcano" scenario

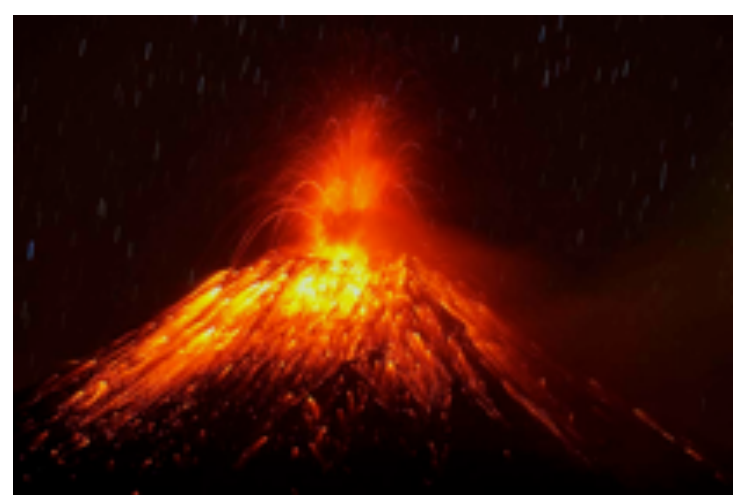

To me, this is a question of fundamental interest, and one we must answer for understanding of jet-quenching \& of the medium itself. 


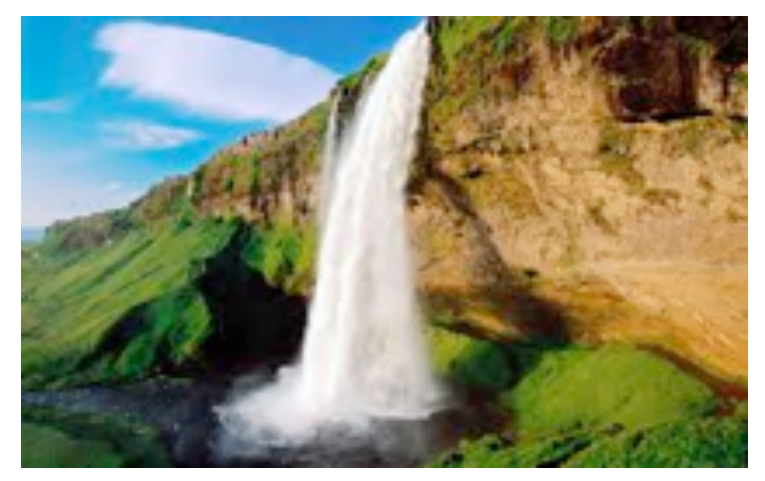

V.S.

How can we get the answer?

Do we even have a chance to find out the answer?

Luckily, we seem to be able to:

Geometry; evolution RHIC --> LHC 


\section{Geometric Anisotropy of Jet Quenching}

Geometric tomography( 200I): Gyulassy,Vitev,Wang,... Geometric limit of high-pt v2: Shuryak; Drees,Feng,Jia;... Till 2008: clear discrepancy between data / any model

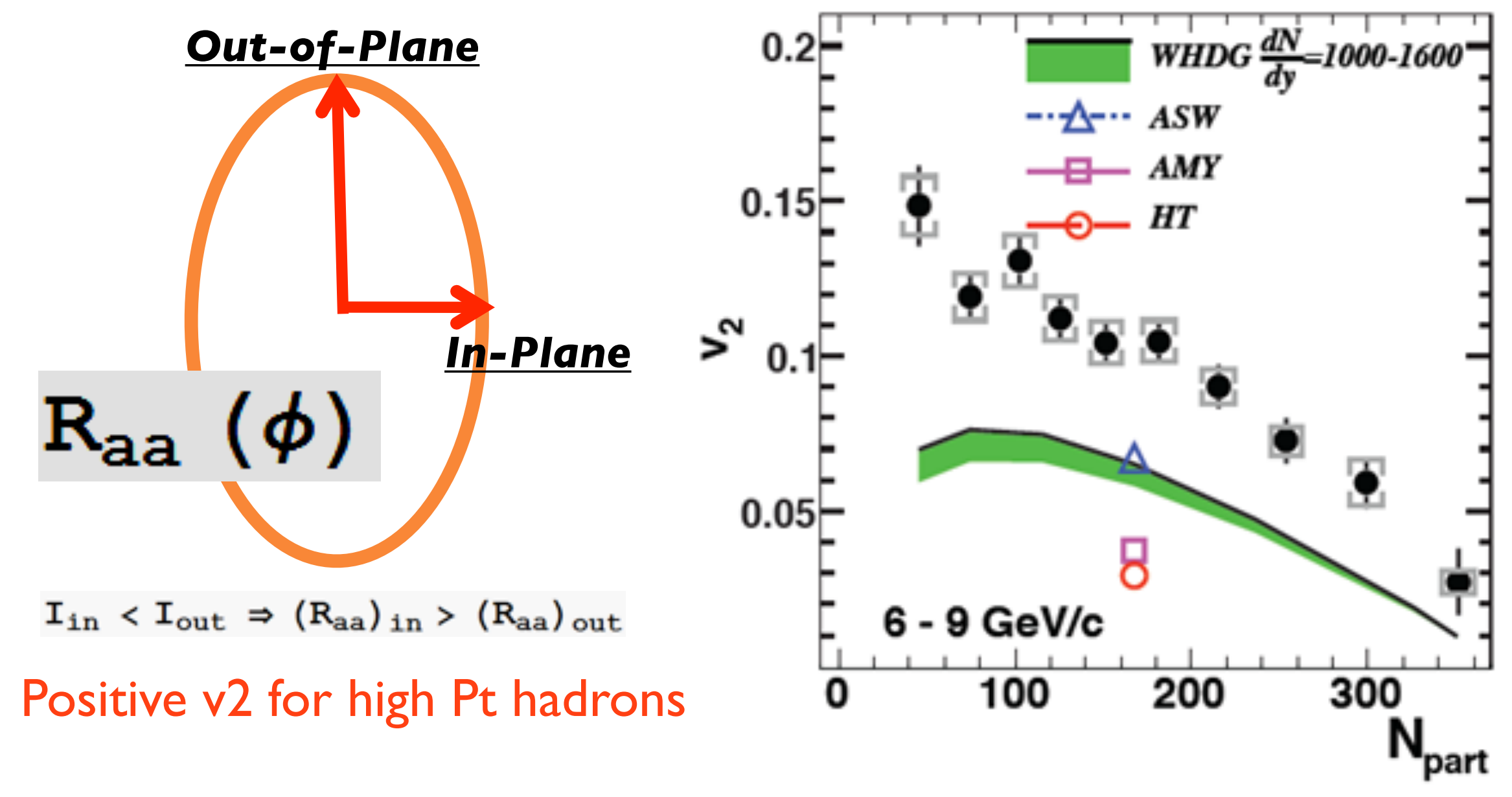




\section{Where Are Jets Quenched (More Strongly)?}

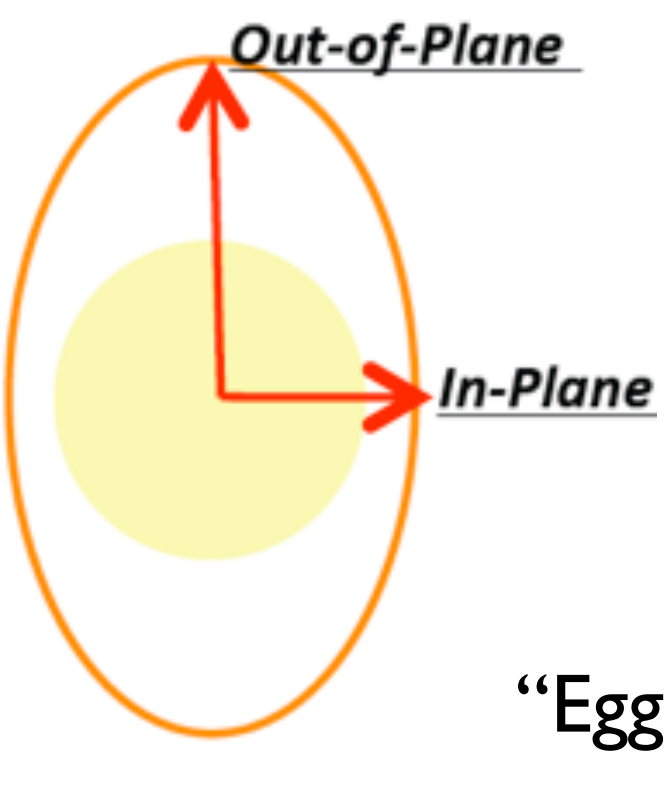

PRL 102, 202302 (2009)
Taken for granted in all previous models: "waterfall" scenario.

We realized the puzzle may concern more radical questions:

Where are jets quenched (more strongly)?

Geometry is a sensitive feature: "Egg yolk" has one geometry,"Egg white" has another.

Angular Dependence of Jet Quenching Indicates Its Strong Enhancement near the QCD Phase Transition

Jinfeng Liao ${ }^{1,2, *}$ and Edward Shuryak ${ }^{1, \uparrow}$

${ }^{1}$ Department of Physics and Astronomy, State University of New York, Stony Brook, New York 11794, USA

${ }^{2}$ Nuclear Science Division, Lawrence Berkeley National Laboratory, Berkeley, California 94720, USA

(Received 22 October 2008; revised manuscript received 19 February 2009; published 22 May 2009) 


\section{Layer-wise Jet Quenching}
$f_{P}=\exp \left\{-\int_{P} \kappa[s(l)] s(l) l^{m} d l\right\}$
scan the jet quenching geometry
layer by layer in density
$R_{A A}(\phi)=<\left(f_{P}\right)^{n-2}>_{P(\phi)}$
$\kappa[s]=\kappa_{c} * \theta\left[s-s_{a}\right] * \theta\left[s_{b}-s\right]$

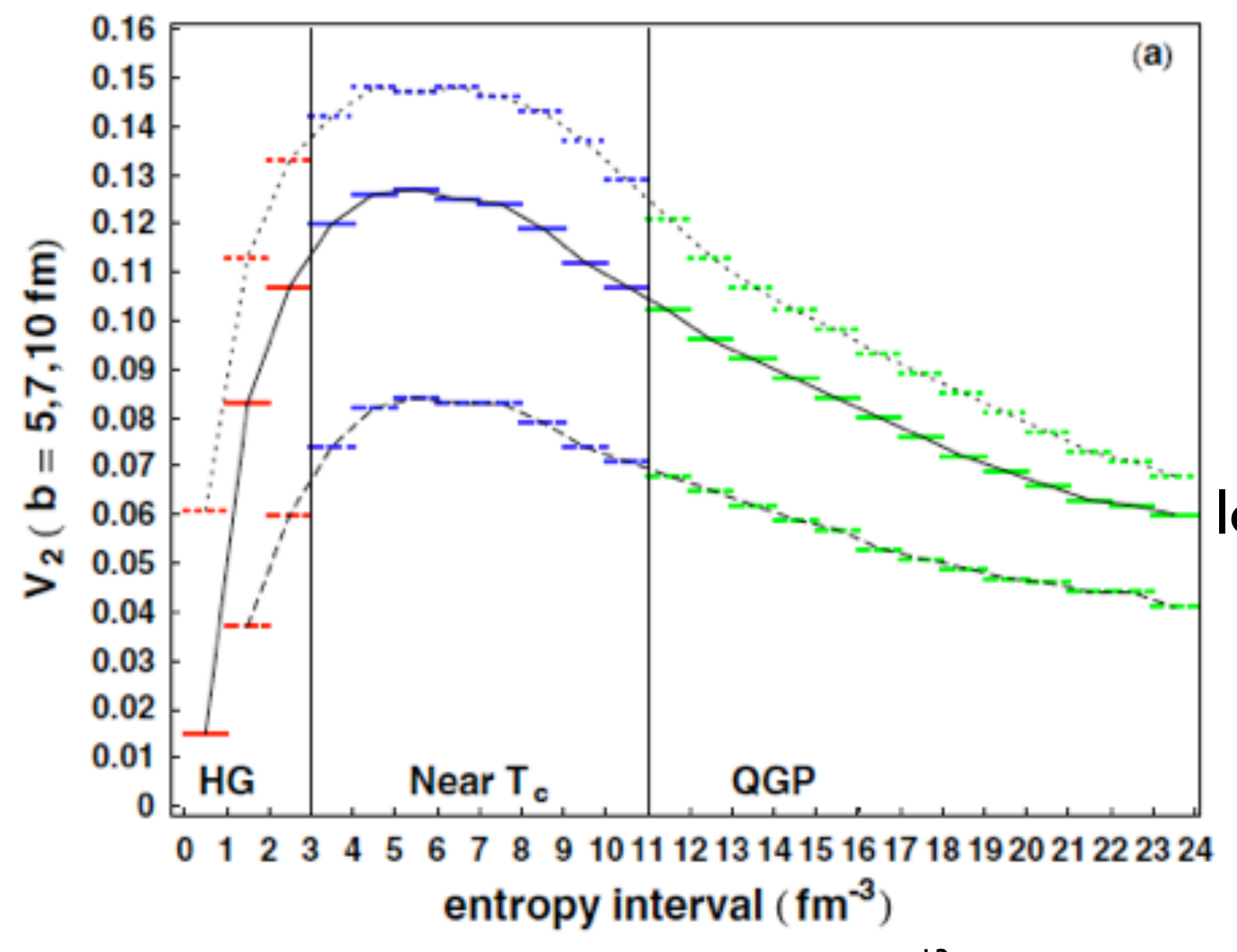

Assume jet quenching occurs only in a specific density interval with constraint from overall Raa $-->$

look at v2 from that layer:

Near-Tc layers give the strongest anisotropy! 


\section{Near-Tc Enhancement (NTcE)}
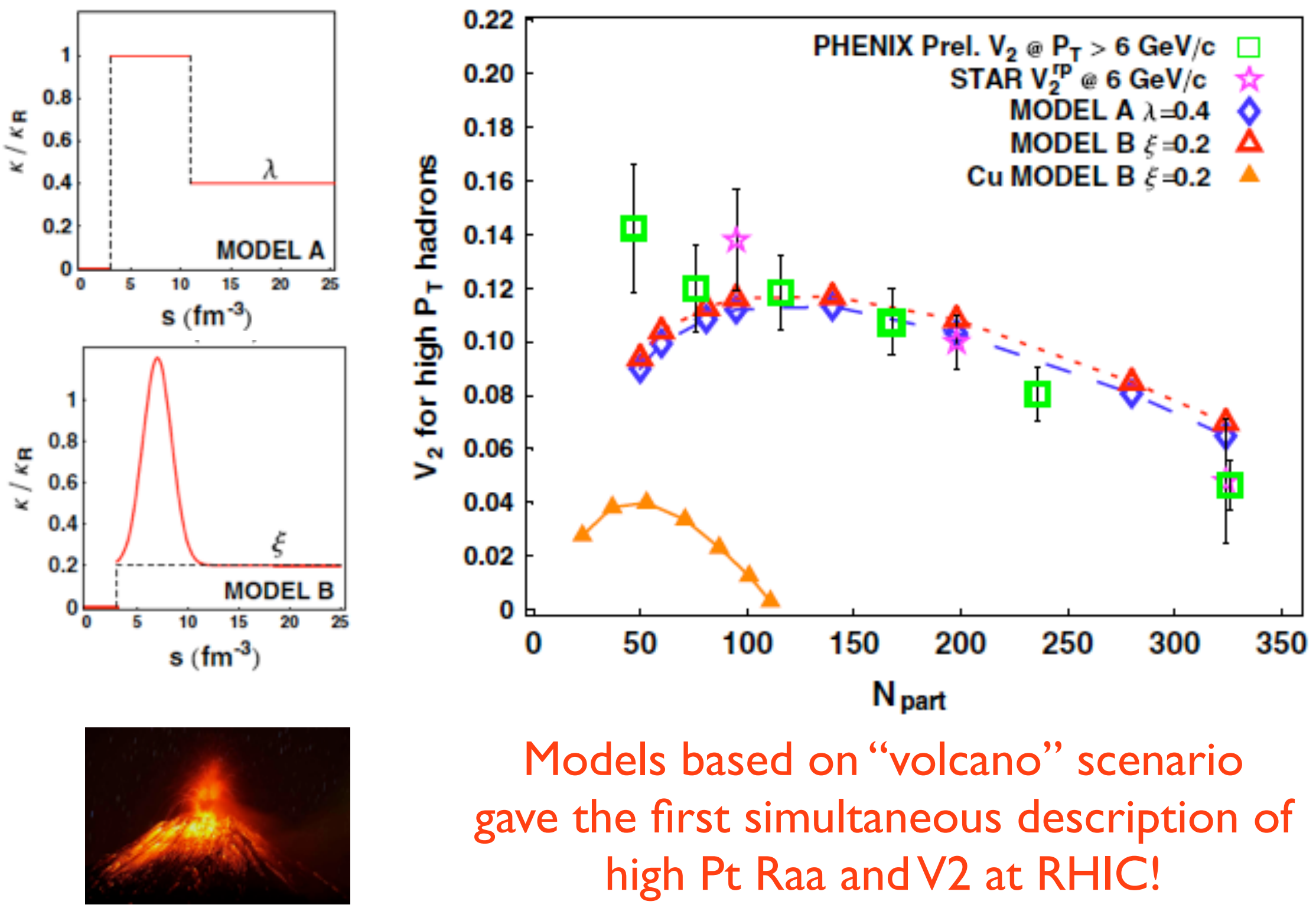

Models based on "volcano" scenario gave the first simultaneous description of high Pt Raa and V2 at RHIC! 


\section{NTcE as a Generic Mechanism}

Near Tc Enhancement (the "volcano") generically increases the contribution to jet quenching from later stage and outer layer of the fireball, and "picks" up more anisotropy.

* relatively insensitive to detailed shape of "volcano"

* works in jet quenching modelings with varied implementations (e.g. geometric models, or GLV/WHDG/ CUJET, or ASW, with/without fluctuations/transverse expansions)

Francesco-Di Toro-Greco

Renk-Holopainen-Heinz-Shen

Gyulassy,Buzzatti,Bezt

Fries \& students

Marquet \& Renk

Jia \&Wei 


\section{The RHIC+LHC Era}

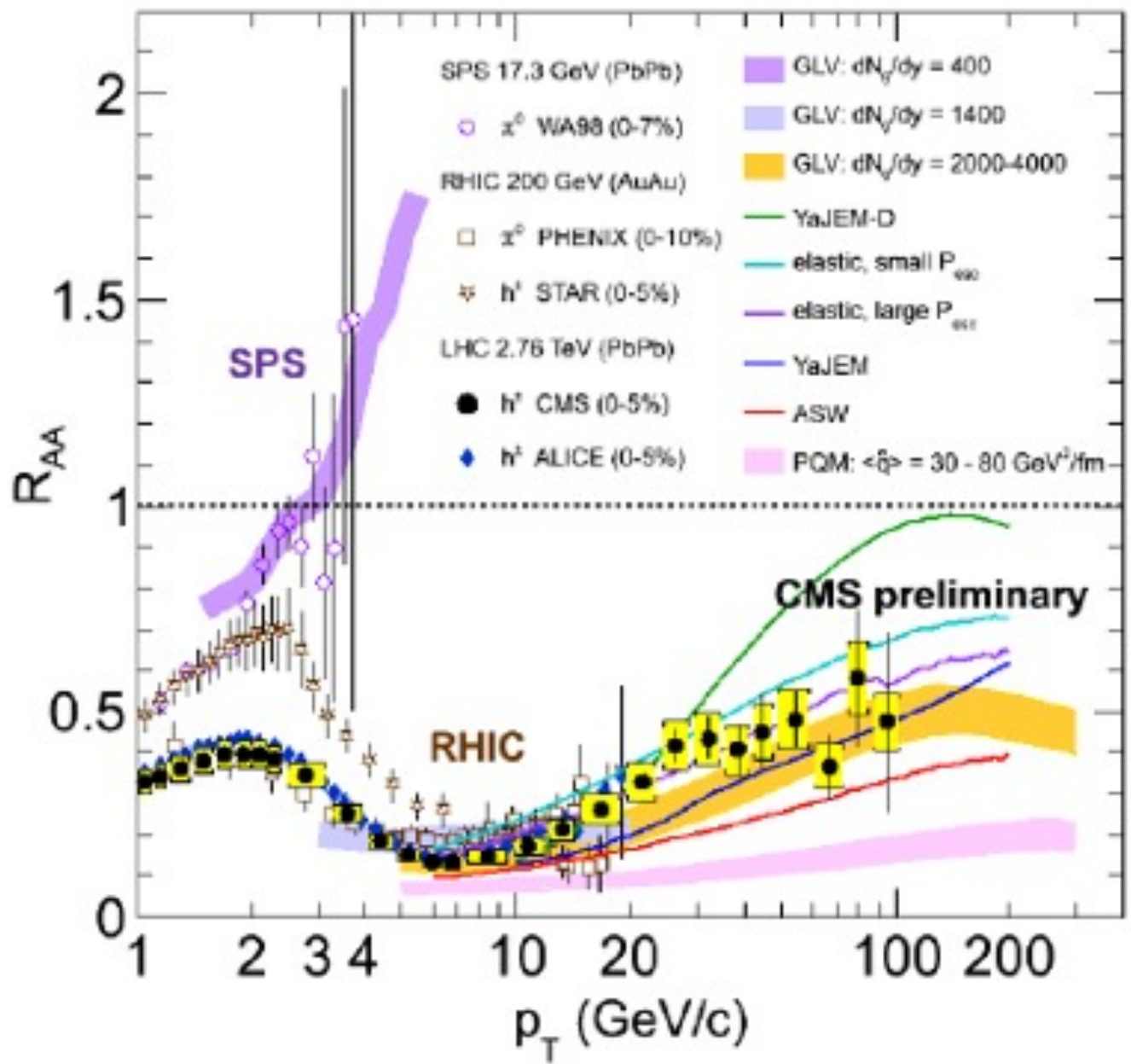

Beautiful jet quenching measurements from ALICE,ATLAS,CMS 
NTcE: Shift to Less Opaque Medium at LHC

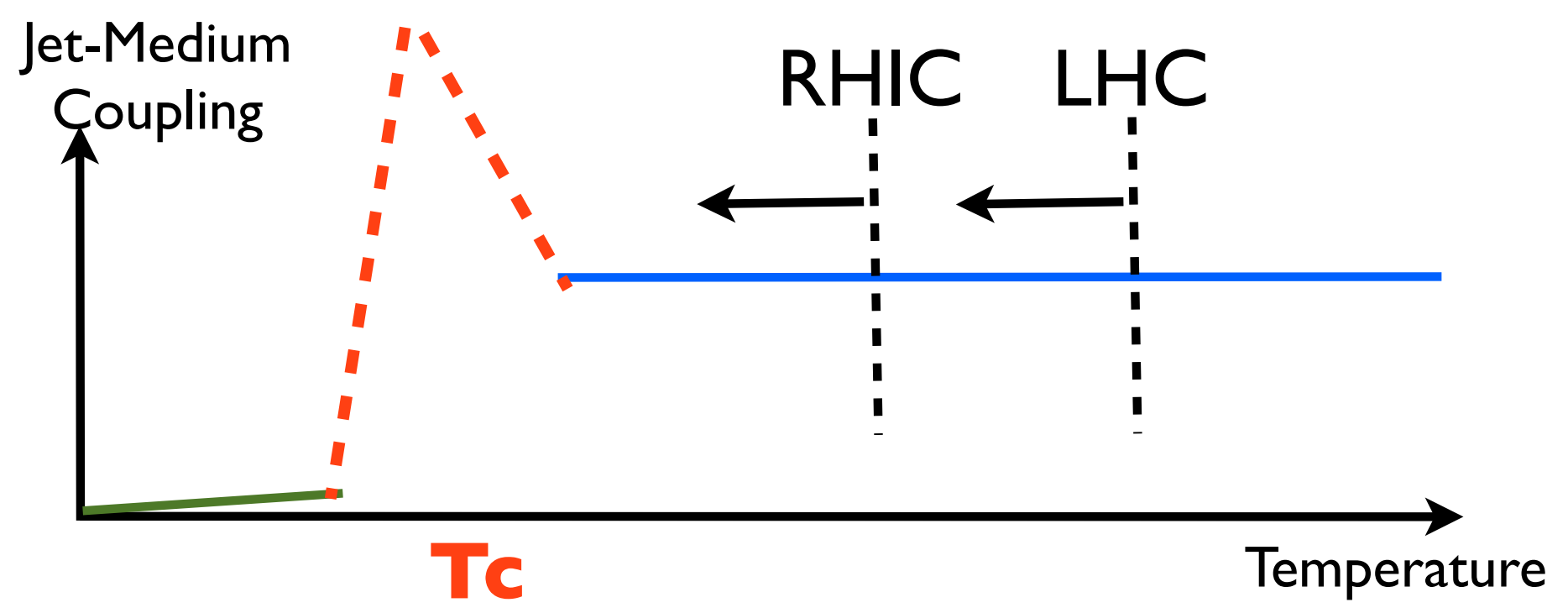

LHC compared with RHIC:

* high T QGP occupies more space-time evolution

* the near-Tc will weigh less, with "volcano" effect reduced $->>$

* Naturally predicts a less opaque (on average) medium seen by jets (note however density doubles)

* Anisotropy from the "volcano" and "waterfall" scenarios will become closer 


\section{Raa from RHIC to LHC}
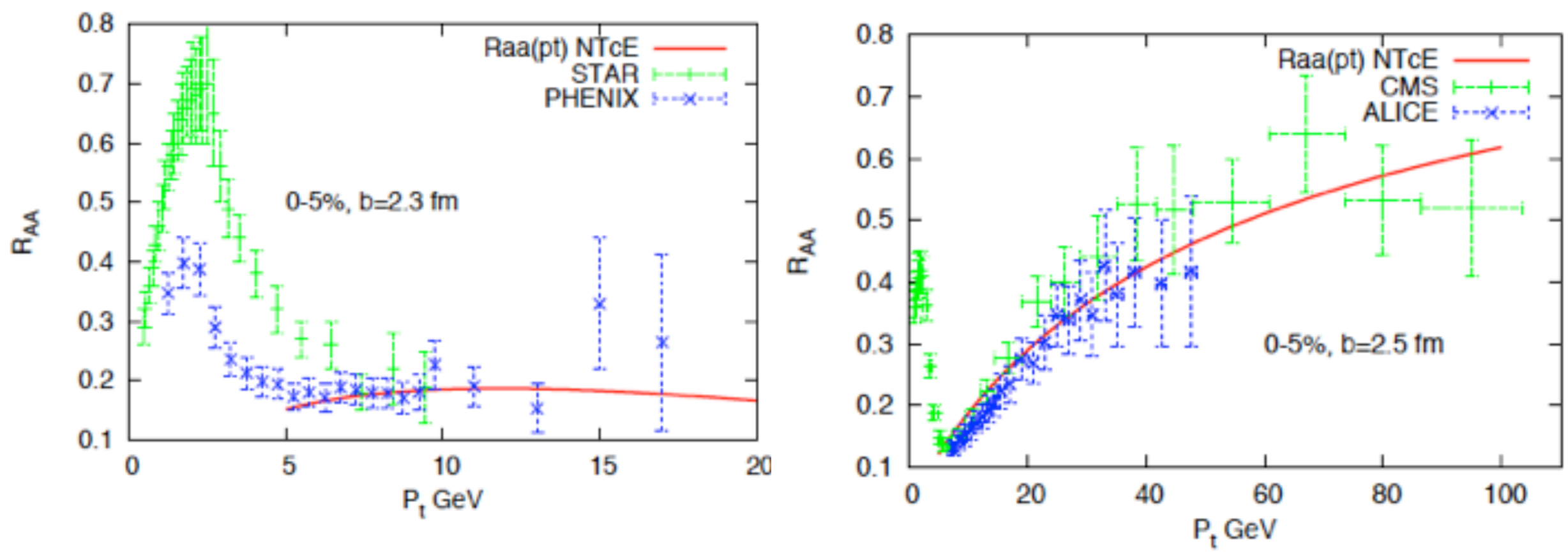

\section{Average jet-medium coupling from RHIC to LHC: reduced by $\sim 30 \%$}

$<\kappa[s(l)]>_{P}=\frac{\int_{P} \kappa[s(l)] s(l) l d l}{\int_{P} s(l) l d l}$

$<\kappa>_{\mathrm{RHIC}}:<\kappa>_{\mathrm{LHC}} \approx 1: 0.72$ 


\section{V2 from RHIC to LHC}
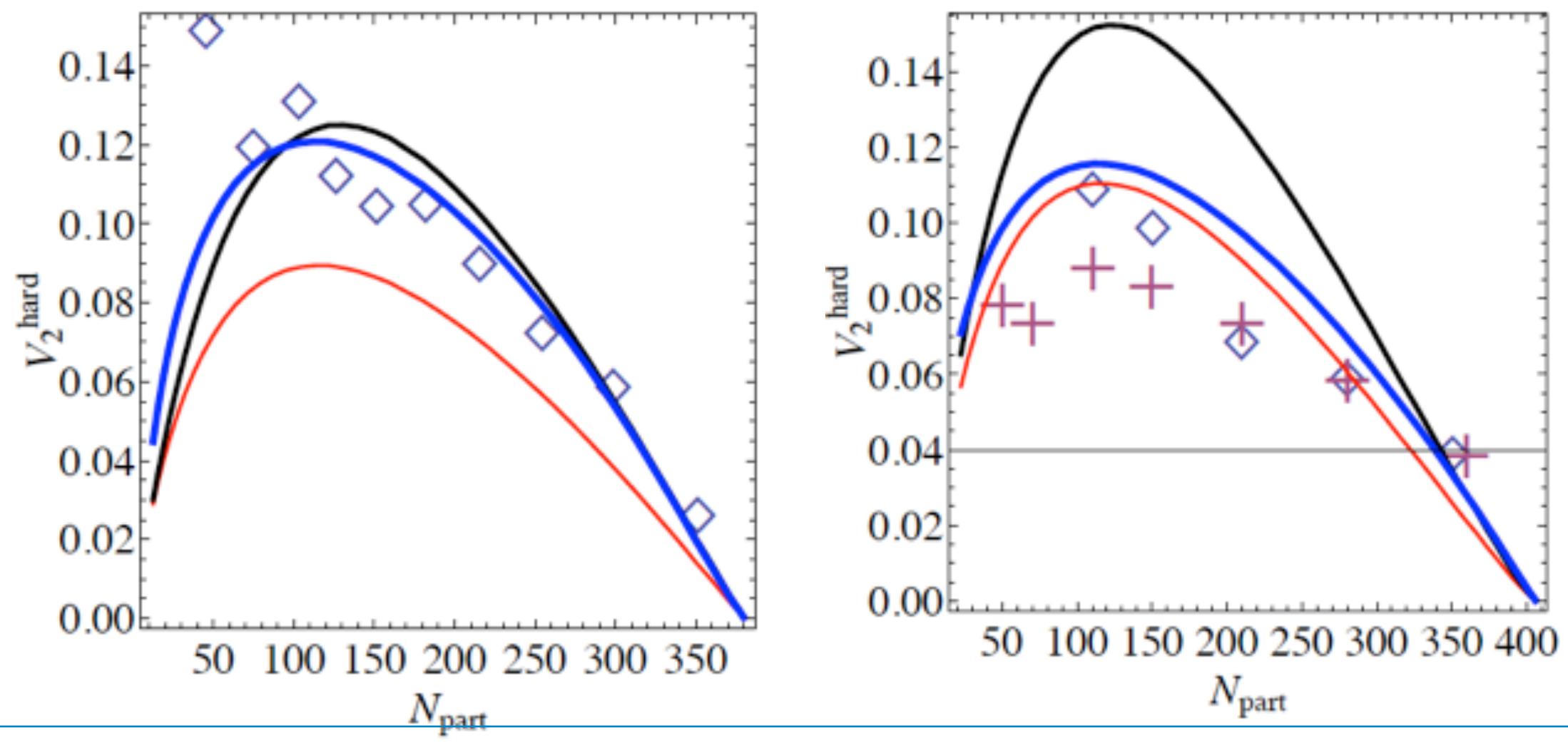

RED: L^2 model+waterfall

BLUE: L^2+volcano

BLACK: L^3+waterfall

* We do see big difference between waterfall/volcano at RHIC, and this difference becomes much smaller at LHC

$*$ RHIC + LHC data are in favor of the $L^{\wedge} 2+$ Volcano scenario

(See Xilin Zhang talk tomorrow for newest results with initial fluctuations and higher harmonics) 


\section{"Volcano" Seen from "Different Angles"}

* Horowitz \& Gyulassy:

"surprising transparency" when

simply extrapolating RHIC to LHC

* Betz \& Gyulassy:

10 30\% reduction in "polytrope" model

* Buzzatti \& Gyulassy:

Strong running coupling at T --> Tc

(also in Zakharov calculation)
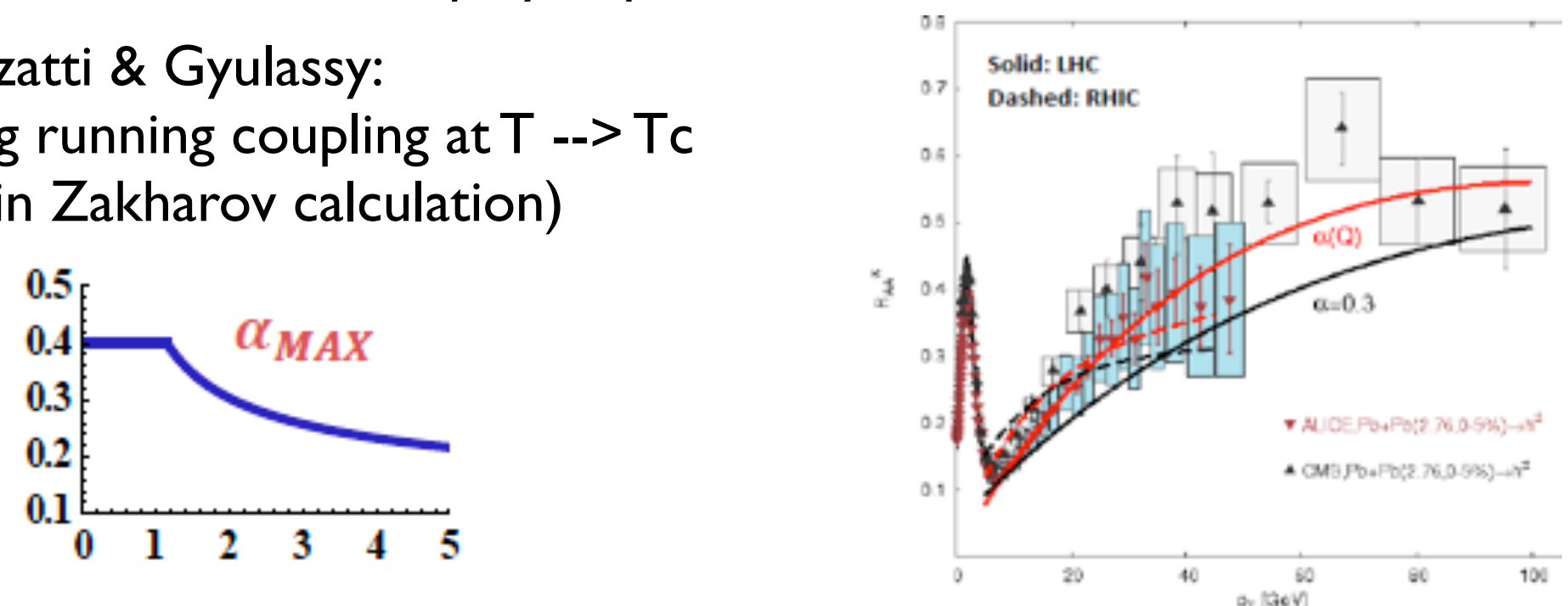

* Lacey et al, scaling analysis: q-hat $(\mathrm{LHC}) \sim \mathrm{q}-\mathrm{hat}(\mathrm{RHIC})$ despite twice the density

* Lattice QCD: Q-bar-Q internal energy shows strong peak at Tc

* Muller-Majumder-Wang, Dusling-Moore-Teaney:

peak in q-hat/density related with dip in leta/s ?

* Majumder: lattice attempt -->q-hat/density showing peak? 


\section{What are Underlying the "Volcano"?}

T $<<$ Lambda_QCD

Vacuum: confined

4

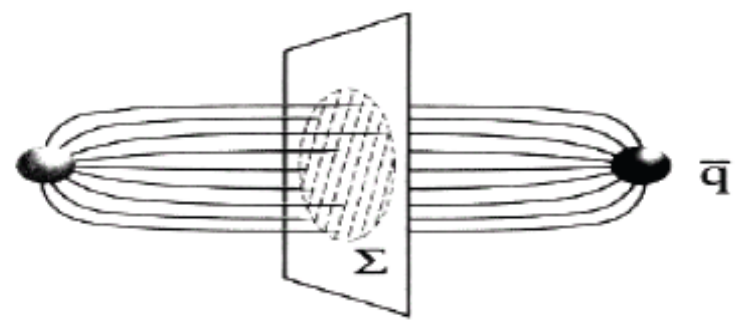

Electric Flux Tube:

Magnetic Condensate

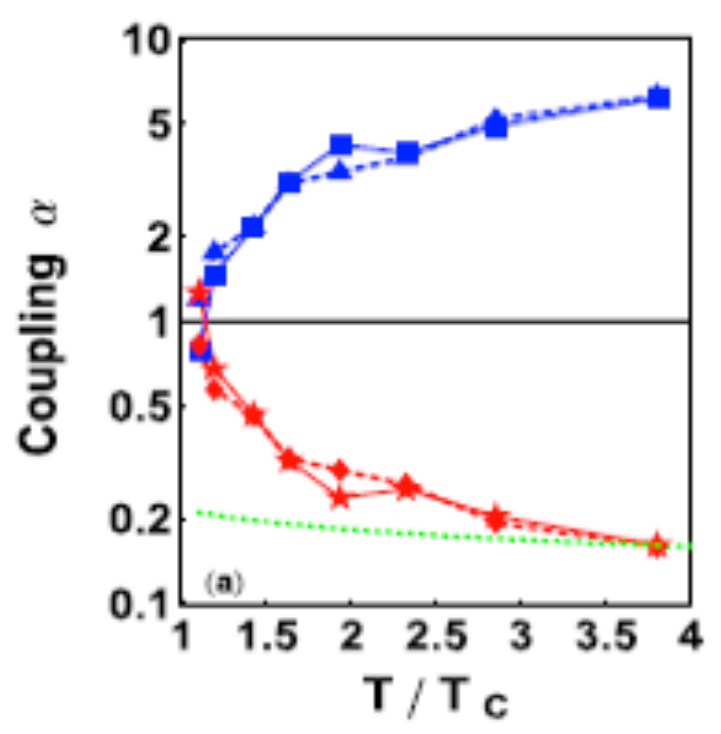

$\mathrm{T} \sim$ Lambda_QCD

Tc

sQGP
T>> Lambda_QCD

WQGP: screening

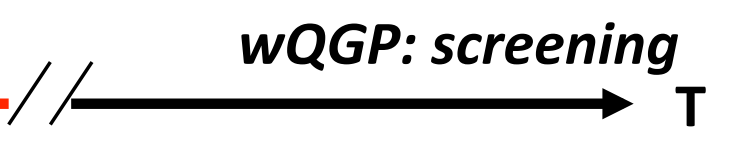

Plasma of E-charges

E-screening: $g \boldsymbol{T}$

M-screening: $g^{\wedge} \mathbf{2} T$

$$
\alpha_{E} * \alpha_{M}=1
$$

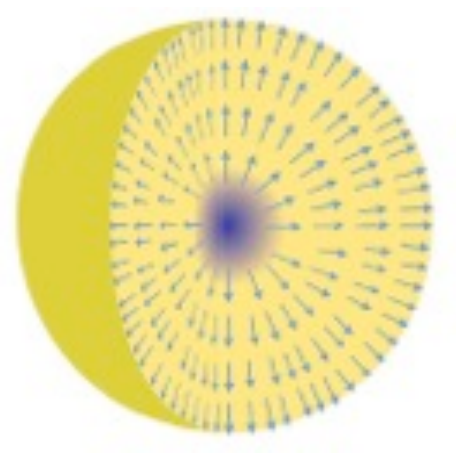

$\kappa \sim \frac{\alpha_{E}(T) \alpha_{M}(T) n_{m}(T)}{s(T)}=\frac{n_{m} / T^{3}}{s / T^{3}}$

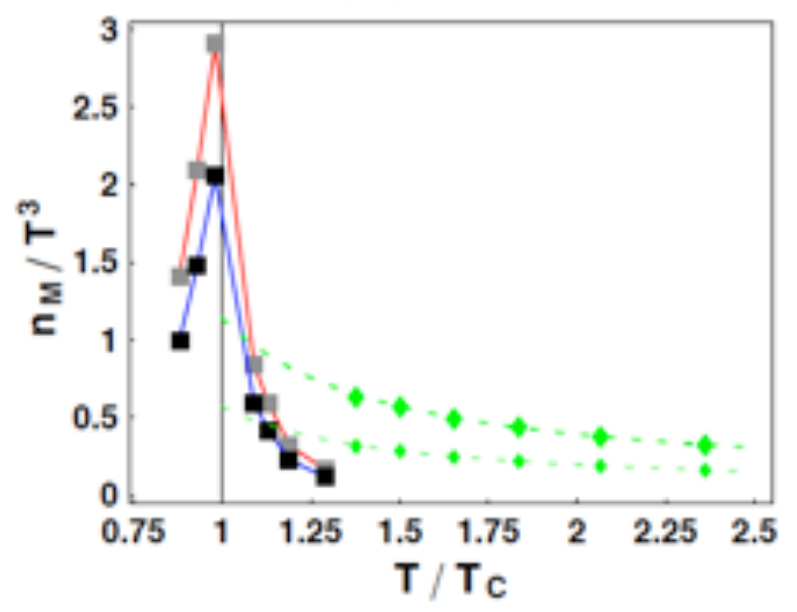

JL \& Shuryak:

Phys.Rev.C75:054907,2007; Phys.Rev.Lett. I 0 I : I 62302,2008; Phys.Rev.C77:064905,2008; Phys.Rev.D82:094007,20 I 0;

21 Phys.Rev.Lett. I09:I5200 I,20 I 2. 


\section{Summary}

* An exciting problem: determine and understand the temperature dependence of jet-medium coupling

* Geometry + Evolution from RHIC to LHC: strong evidences for Near-Nc Enhancement

* RHIC + LHC together provide unique opportunities for mapping out the detailed shape of the "volcano" and for probing the fascinating midland between the confined world and the asymptotically free matter.

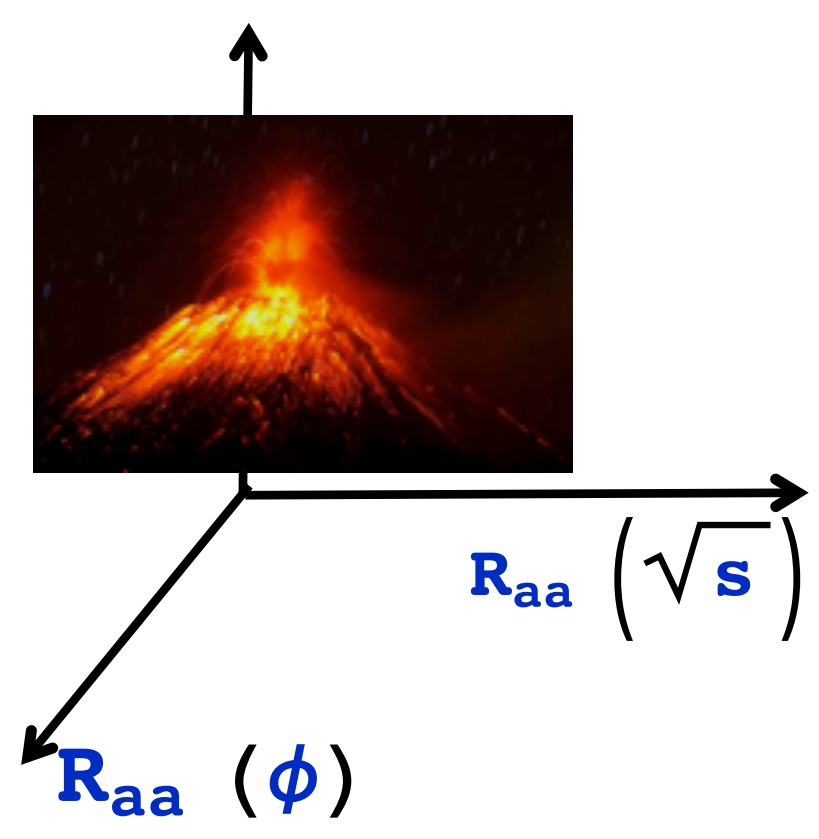




\section{BACKUP SLIDES}




\section{Near-Tc Matter:Thermodynamics}

Near Tc: a wide window in terms of entropy density !

What is the nature of confinement transition?

Can H.I.C. help us understand the matter just about to confine?

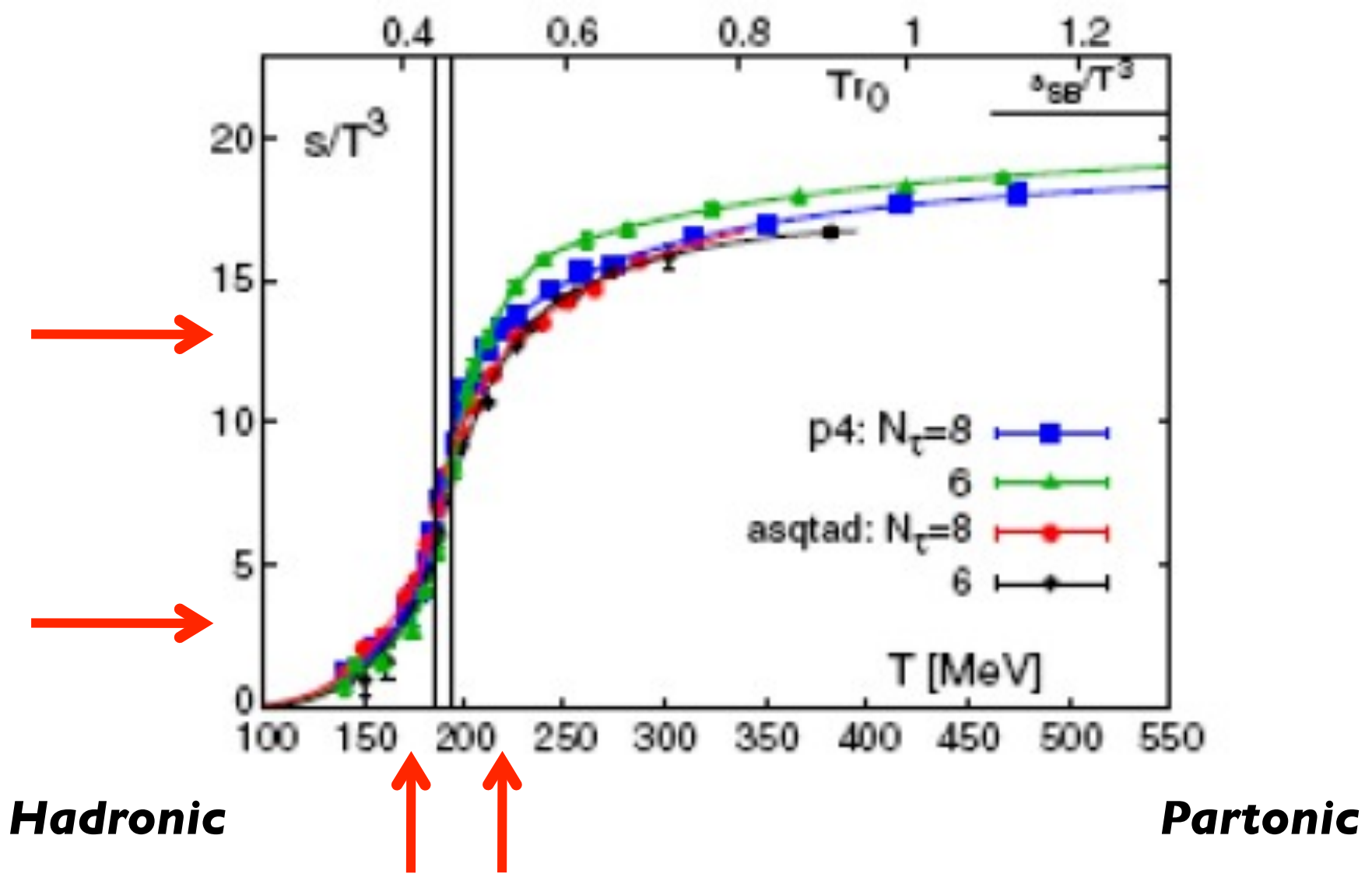

The world is much richer than just a HRG and a Stefan-Boltzmann QGP! 


\section{Near-Tc Matter: Hydrodynamics}

Near Tc Matter (between HRG and QGP) occupies large space time volume ( / /3) during the fireball evolution.

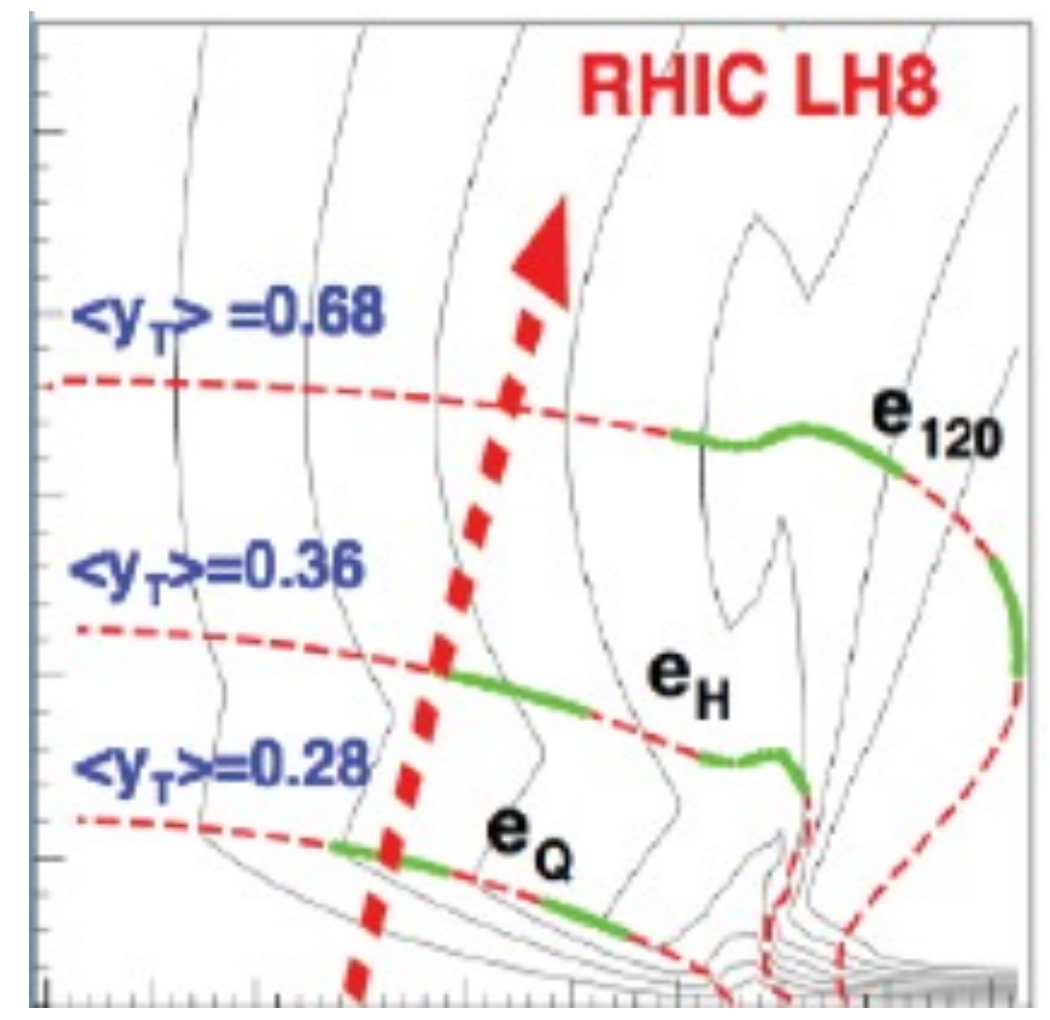

Teaney \& Shuryak

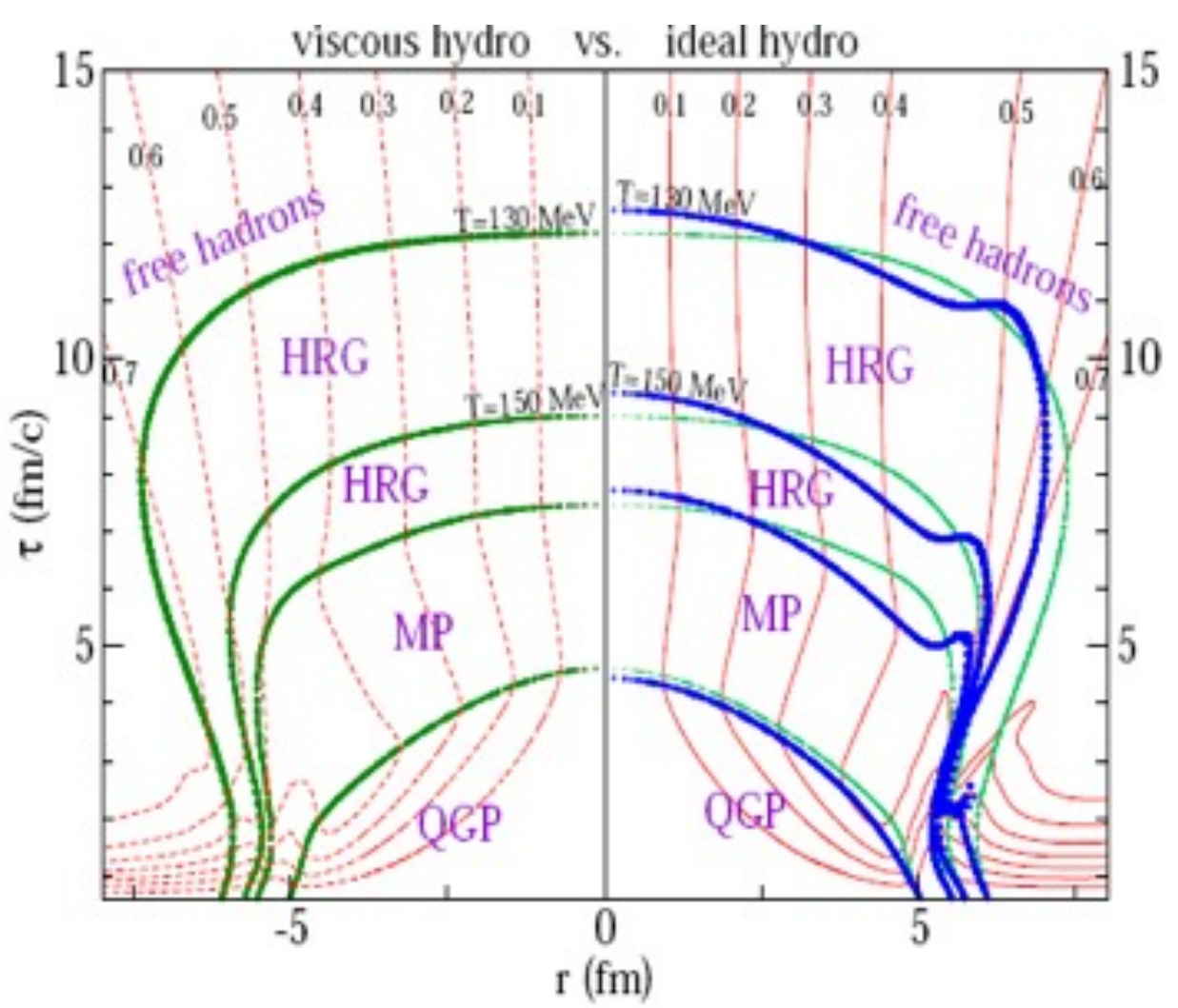

25

Heinz \& Song 


\section{Sensitivity to T-dependence of Energy Loss}

\section{Francesco-Di Toro-Greco (arXiv: 1009.1 26I)}
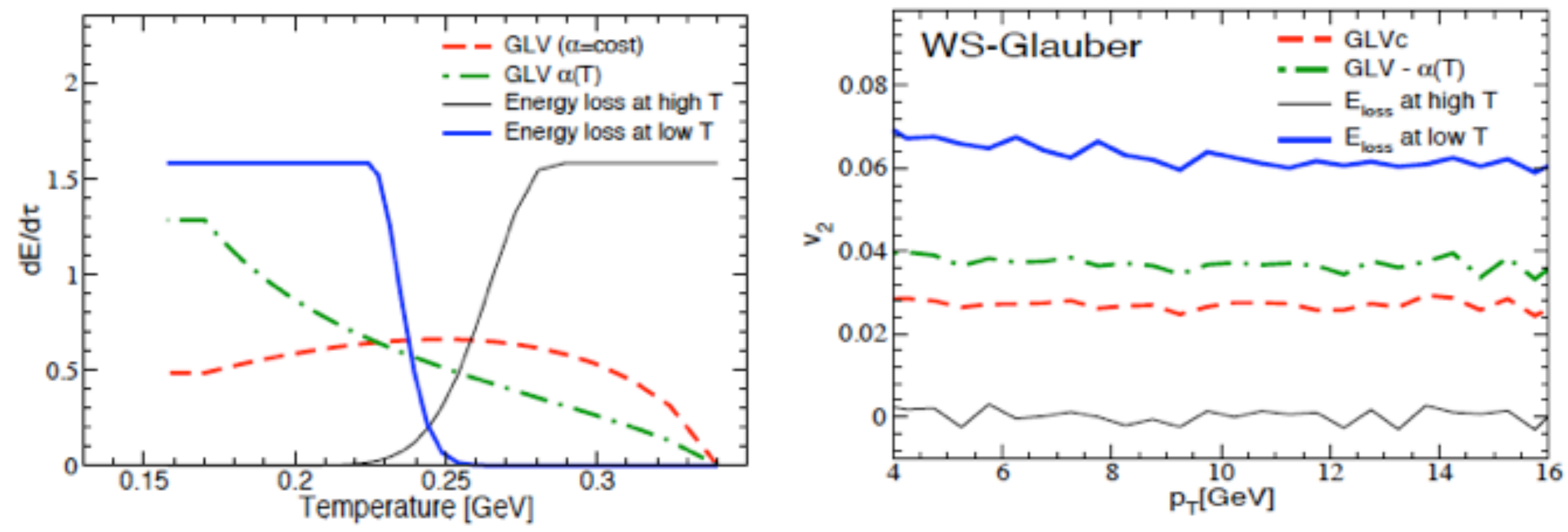


\section{Magnetic Monopoles \& E-M Duality}

$$
\text { 't Hooft-Polyakov (1974): }
$$

monopoles naturally arise as topological solutions to classical EoM in non-Abelian gauge theories; Dirac Quantization obeyed, mass \& size $\sim \mathrm{I} / \mathrm{g}$

$$
\text { Dirac : } e^{\star} g=1
$$

What happens if the gauge theory with monopoles is in strongly coupled regime?

E-M Duality: (Motonen, Olive, I 977) strong coupling $\rightarrow$ change of D.o.F. toward emergent ones;

Dirac condition $\rightarrow \quad E$ and $M$ couplings inversely related

E weakly coupled $\rightarrow$ theory in terms of E language E strongly coupled $\rightarrow$ theory better described by ${ }_{27}$ Magnetic. 


\title{
Recent results from $\mathrm{pPb}$ collisions at the LHC
}

\author{
Constantin Loizides \\ (LBNL/EMMI)
}

15.04 .2013

RBRC Workshop on "Jet quenching at RHIC vs LHC in light of recent dAu vs pPb controls" 


\section{LHC p+Pb runs at 5.02 TeV}

- LHC operated with

- $4 \mathrm{TeV}$ proton beam and 1.57 TeV / nucleon $\mathrm{Pb}$ beam

- Center of mass energy 5.02 per nucleon pair

- Center of mass rapidity shift $\mathrm{dY}=-0.465$ in direction of proton

- 2012 pilot run (4 hours of data taking)

- About $1 / \mu b$ per experiment with very low pileup

- 2013 long run (3 weeks of data taking)

- Delivered about 30/nb to ATLAS, CMS and ALICE

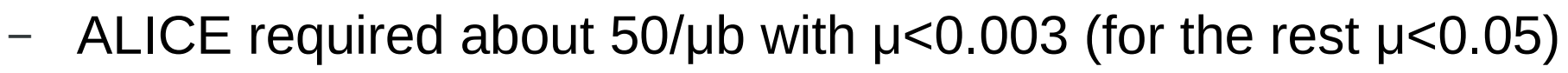

- Few $1 /$ nb for LHCb (new to heavy-ion operation)

- Beam reversal (relevant for ALICE and LHCb) for about half of statistics

- Van der Meer scans in both beam configuations

- No pp run at $5.02 \mathrm{TeV}$ until 2015

- Instead a run at 2.76 TeV with 0.1/pb for ALICE and 5/pb for the rest 


\section{Acceptance of LHC experiments}

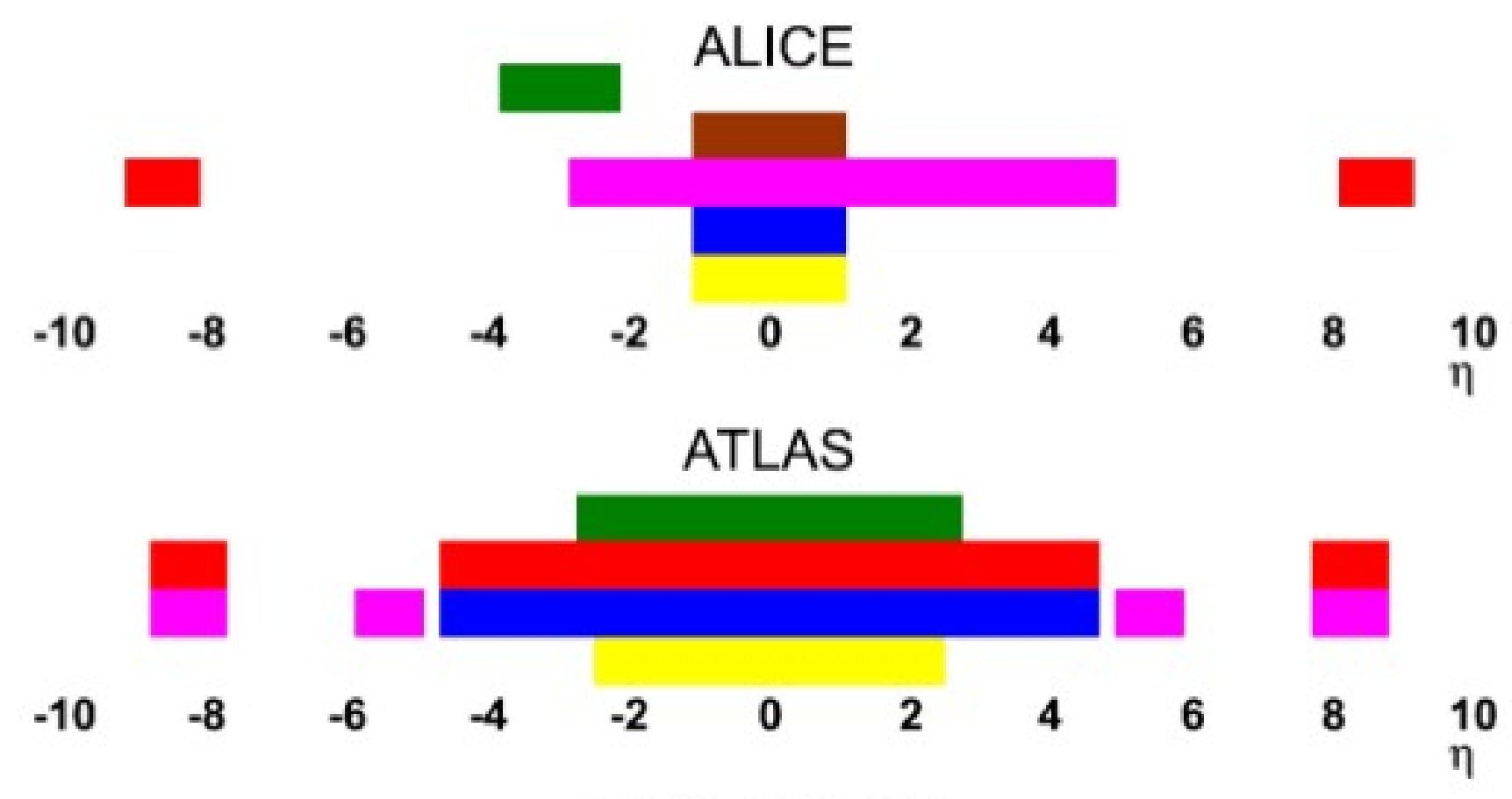

CMS+TOTEM

$\begin{array}{lllllllllll}-10 & -8 & -6 & -4 & -2 & 0 & 2 & 4 & 6 & 8 & 10 \\ & & & & & & & & & & \eta\end{array}$

$\begin{array}{lllllllllll}-10 & -8 & -6 & -4 & -2 & 0 & 2 & 4 & 6 & 8 & 10 \\ & & & & & & & & \end{array}$




\section{Physics results from pilot run}

ALICE, PRL 110 (2013) 032301
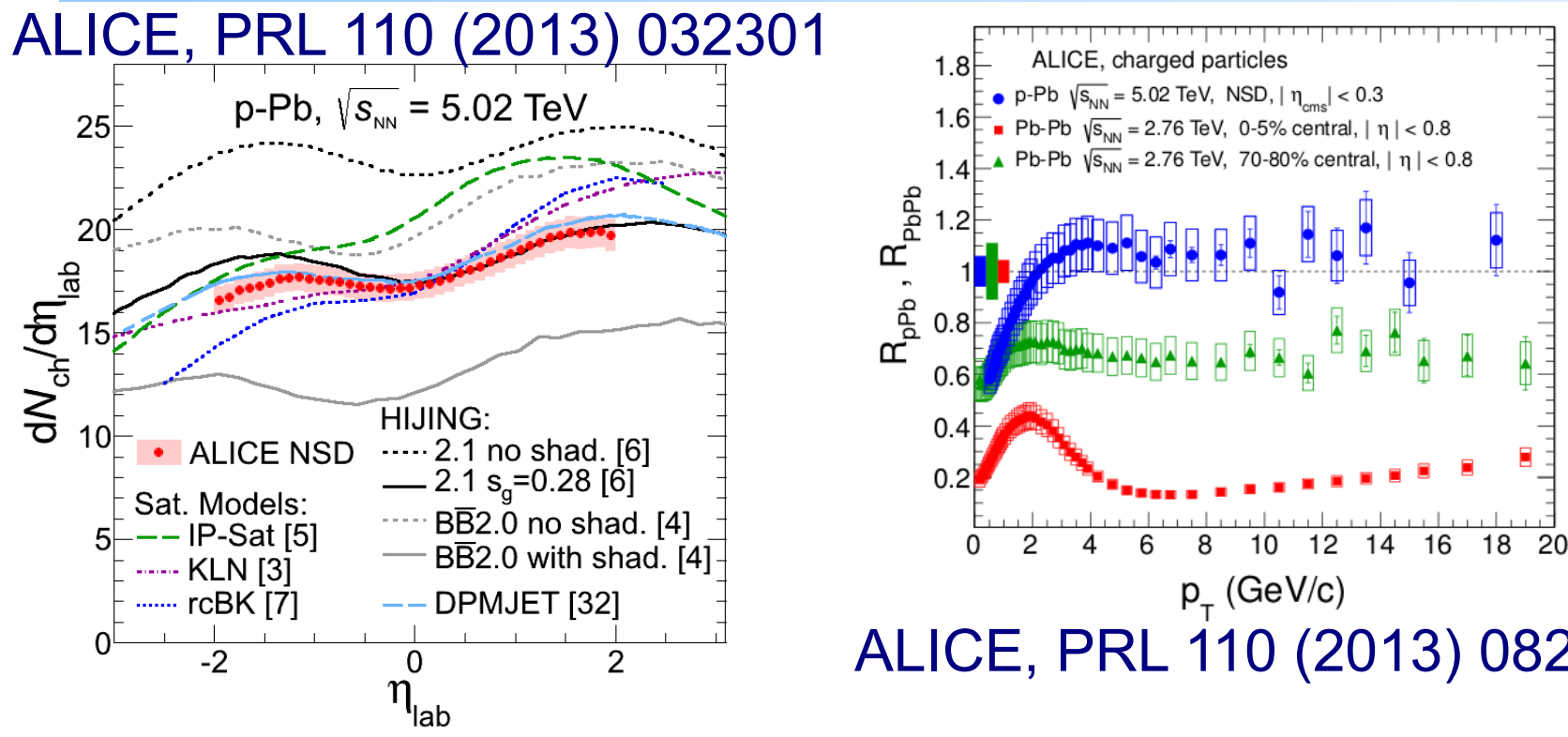

ALICE, PRL 110 (2013) 082302
LHCb, CERN-LHCb-CONF-2012-034

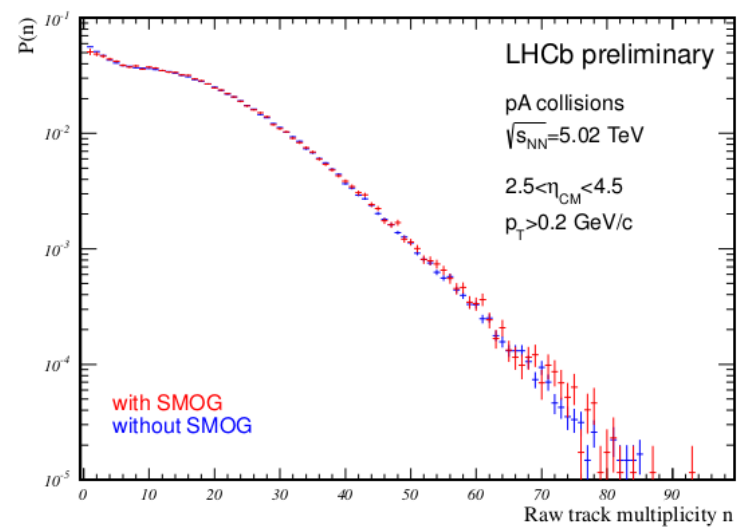

ATLAS, arXiv: 1212.5198

CMS, PLB 718 (2012) 章 955

CMS pPb $\sqrt{\mathrm{s}_{\mathrm{NN}}}=5.02 \mathrm{TeV}, \mathrm{N}_{\text {trik }}^{\text {offline }} \geq 110$
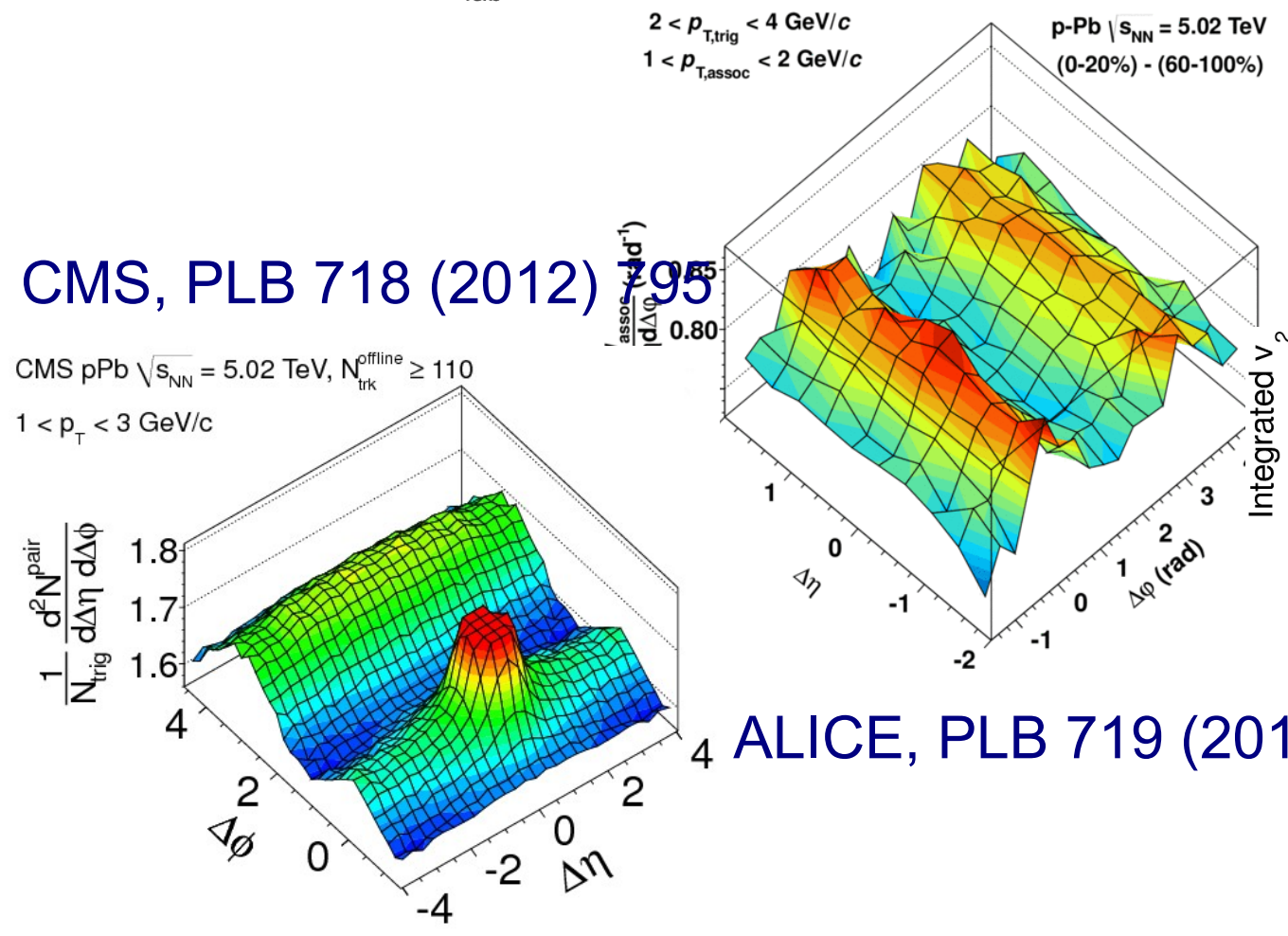

${ }_{4}$ ALICE, PLB 719 (2013) $29<\mathrm{p}_{\mathrm{T}}<5 \mathrm{GeV}$

ATLAS, arXiv:1303.2084

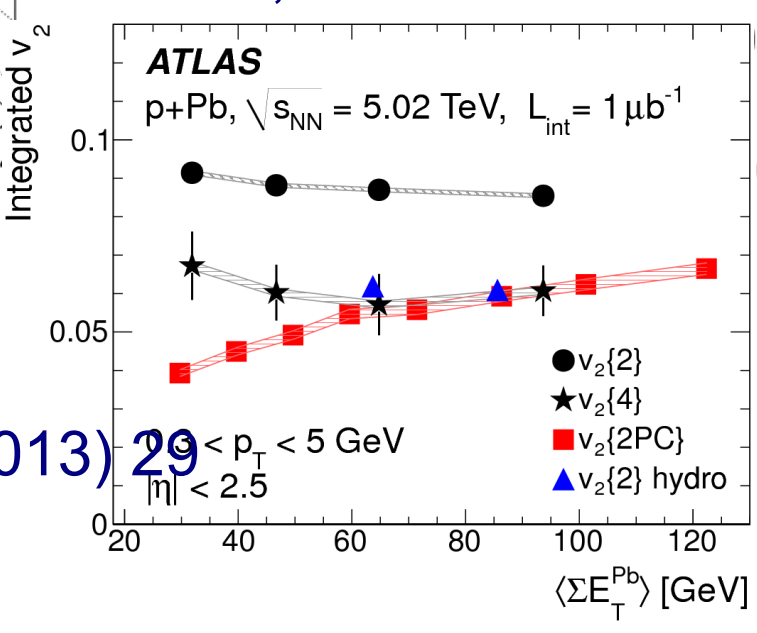




\section{LHCb: Normal and SMOG running}

- Two running scenarios:

- Normal background conditions

- Running with "SMOG" (System for Measuring Overlap with Gas)

- Ne injection to measure beam-profile + luminosity (JINST 7 P01010)

- Increase beam-gas factor by $\sim 100$

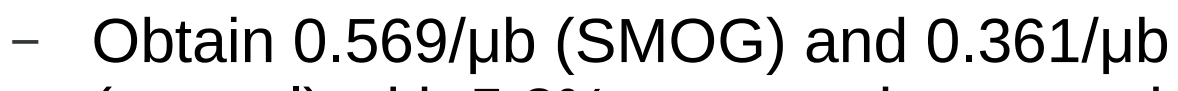
(normal) with $5.2 \%$ systematic uncertainty

- Need to perform beam-gas subtraction

- Measure observables with BX1 (beam1), BX2 (beam2) and BX3 (coll.beams)

- Calculate BX3 - $a_{1} B X 1-a_{2} B X 2$

- Determine $a_{1}$ and $a_{2}$ from primary vertex distribution for $|z|>300 \mathrm{~mm}$

- Common for w and w/o SMOG

- Same weights apply for other observables
LHCb, CERN-LHCb-CONF-2012-034
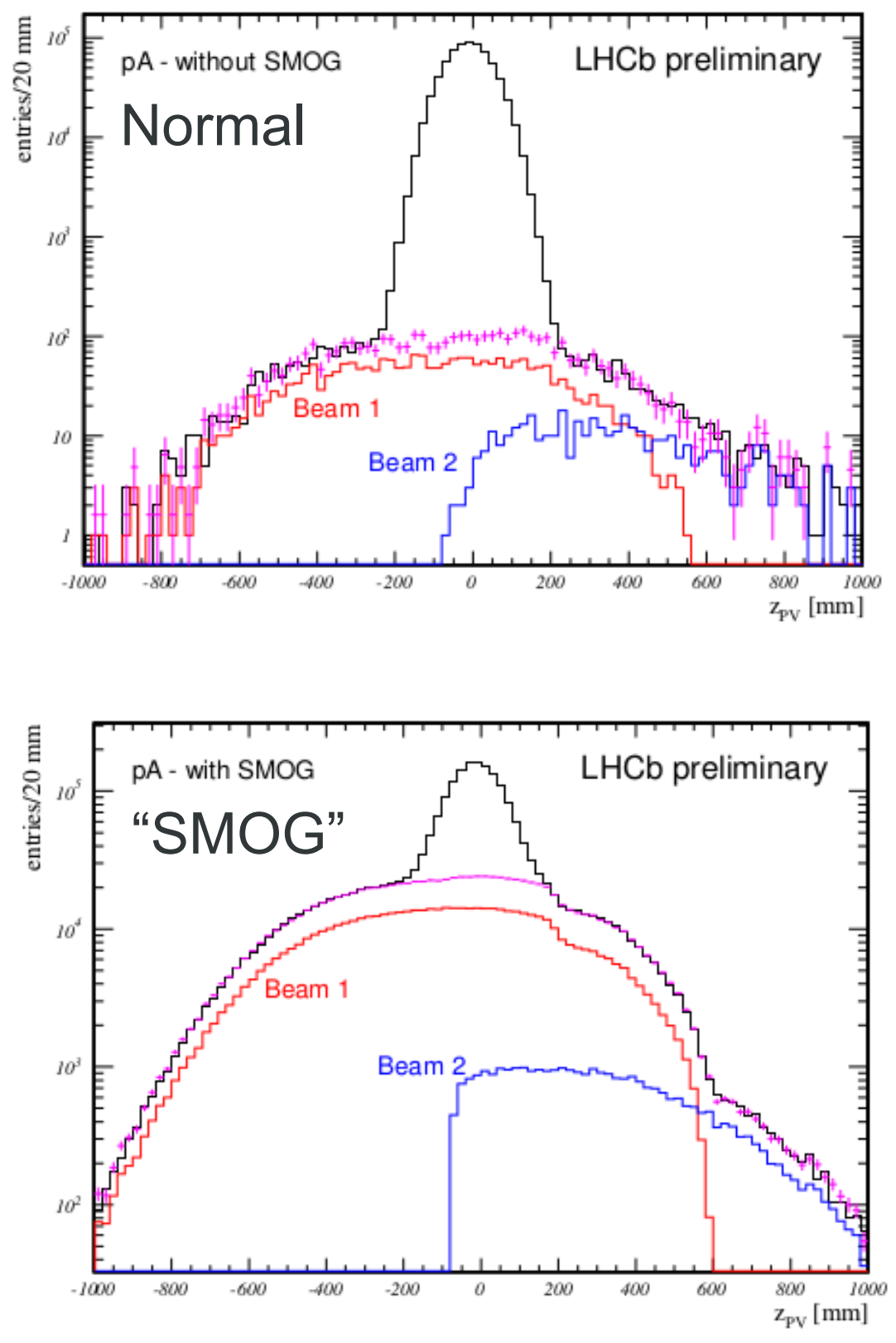


\section{LHCb: Beam gas subtraction}

z-vertex positions of primary vertices in $\mathrm{pPb}$ and $\mathrm{pp}$

LHCb, CERN-LHCb-CONF-2012-034
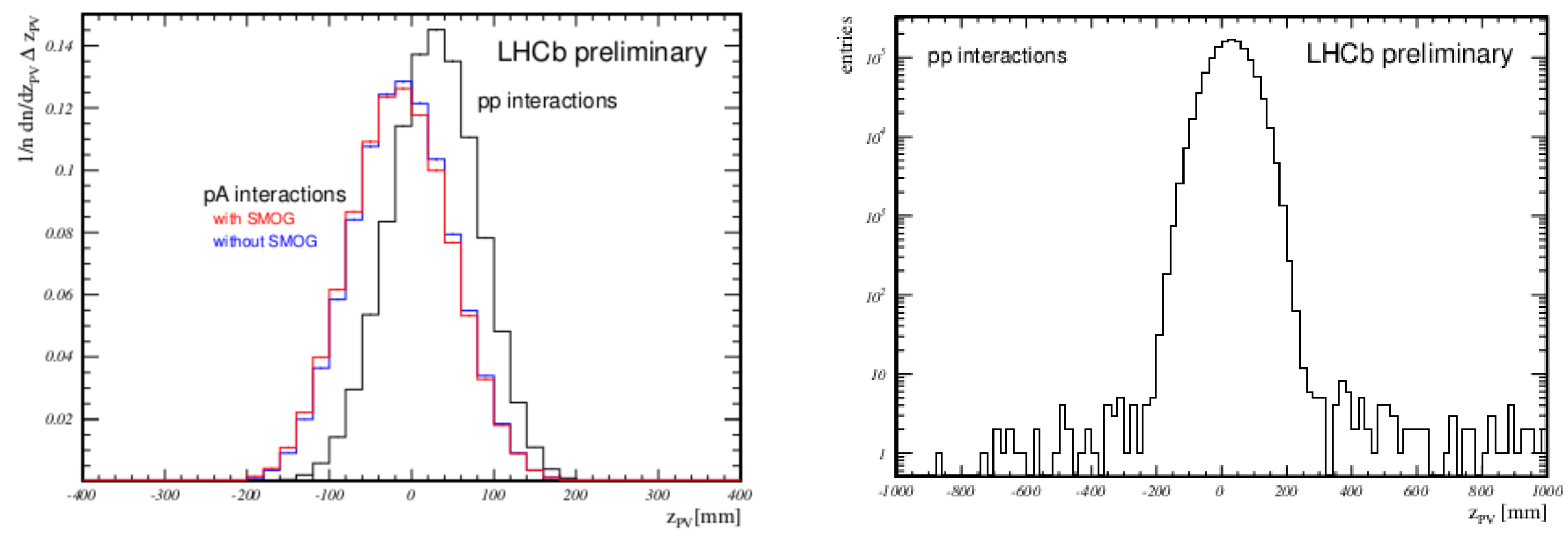

- After beam-gas subtraction vertex distributions in $\mathrm{pPb}$ are very similar for collisions taken with and without SMOG

- Differences in luminous region between $\mathrm{pPb}$ and $\mathrm{pp}$

- Luminous region in $\left|z_{P V}\right|<200 m m$ for $\mathrm{pPb}$ and $\mathrm{pp}$ 


\section{Inelastic $\mathrm{pPb}$ cross section}

- Count collisions which produce at least one track in $2.5<\eta<4.5$ (proton side) with $p_{\mathrm{T}}>0.2 \mathrm{GeV} / \mathrm{c}$

- In HIJING/DPMJET only 1-2\% events without a charged particle

- Analysis steps

- Beam gas subtraction

- Pileup below permille level ignored

- Trigger efficiency $100 \% \pm 1 \%$

- Correction for finite single track finding efficiency: $98 \% \pm 2 \%$

- Convert using integrated LHCb, CERN-LHCb-CONF-2012-034 luminosity measured with SMOG

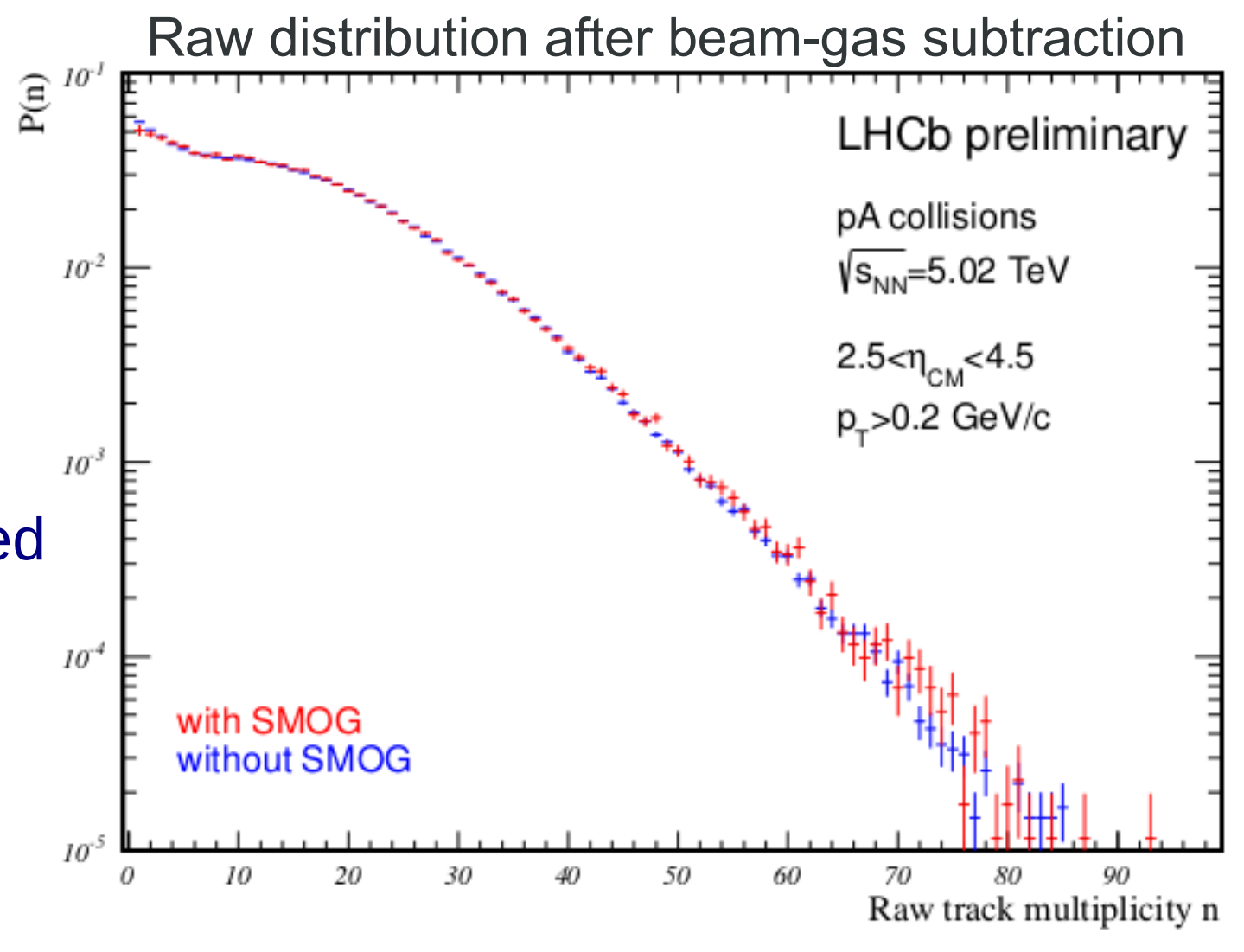

- Systematic uncertainty dominated by $5.2 \%$ error on luminosity

$$
\sigma_{\text {inel }}\left(2.5<\eta_{c m}<4.5, p_{T}>0.2 \mathrm{GeV} / c\right)=2.09 \pm 0.12 \mathrm{~b}
$$

(consistent with HIJING, DPMJET and Glauber with $\sigma_{\mathrm{NN}}=70 \mathrm{mb}$ ) 


\section{NSD pPb normalization}

- Event selection

ALICE, PRL 110 (2013) 032301

- VZERO-A $(2.8<\eta<5.1)$ and VZERO-C $(-3.7<\eta<-1.7)$ incl. time cuts

- Systematic variation using ZDC on nucleus side (ZNA)

- Resulting event sample

- Non single-diffractive (NSD)

- At least one binary N+N interaction is NSD (Glauber picture)

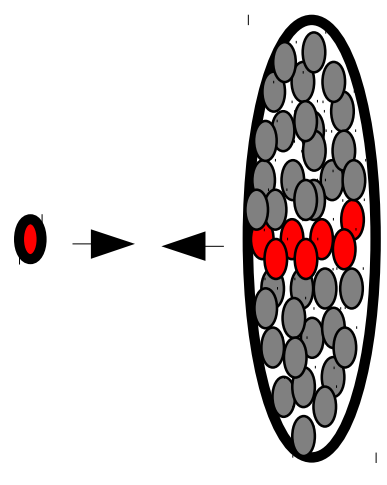

- Inspired from DPMJET, which includes incoherent SD of the projectile with target nucleons that are mainly concentrated on the surface of the nucleus

- SD about 4\% from HIJING, DPMJET or standalone Glauber

- Negligible contamination from SD and EM processes

- Validated with a cocktail of generators

- DPMJET for NSD (2b)

- PHOJET + Glauber for incoherent SD part (0.1b)

- SD/INEL $=0.2$ in pp at $7 \mathrm{TeV}$ ( arXiv:1208.4968)

- EM with STARLIGHT (0.1-0.2b) 


\section{Pseudorapidity density}

ALICE, PRL 110 (2013) 032301

- Tracklet analysis using SPD hits

- Dominant systematic uncertainty from NSD normalization of $3.1 \%$

- Reach of SPD extended to $|\eta|<2$ by extending the $z$-vertex range

- Results in ALICE laboratory system

- $\mathrm{y}_{\mathrm{cms}}=-0.465$

- Comparison

- Most models within 20\%

- Saturation models have to steep rise between $\mathrm{p}$ and $\mathrm{Pb}$ region

NB: HJING calculations for NSD expected to

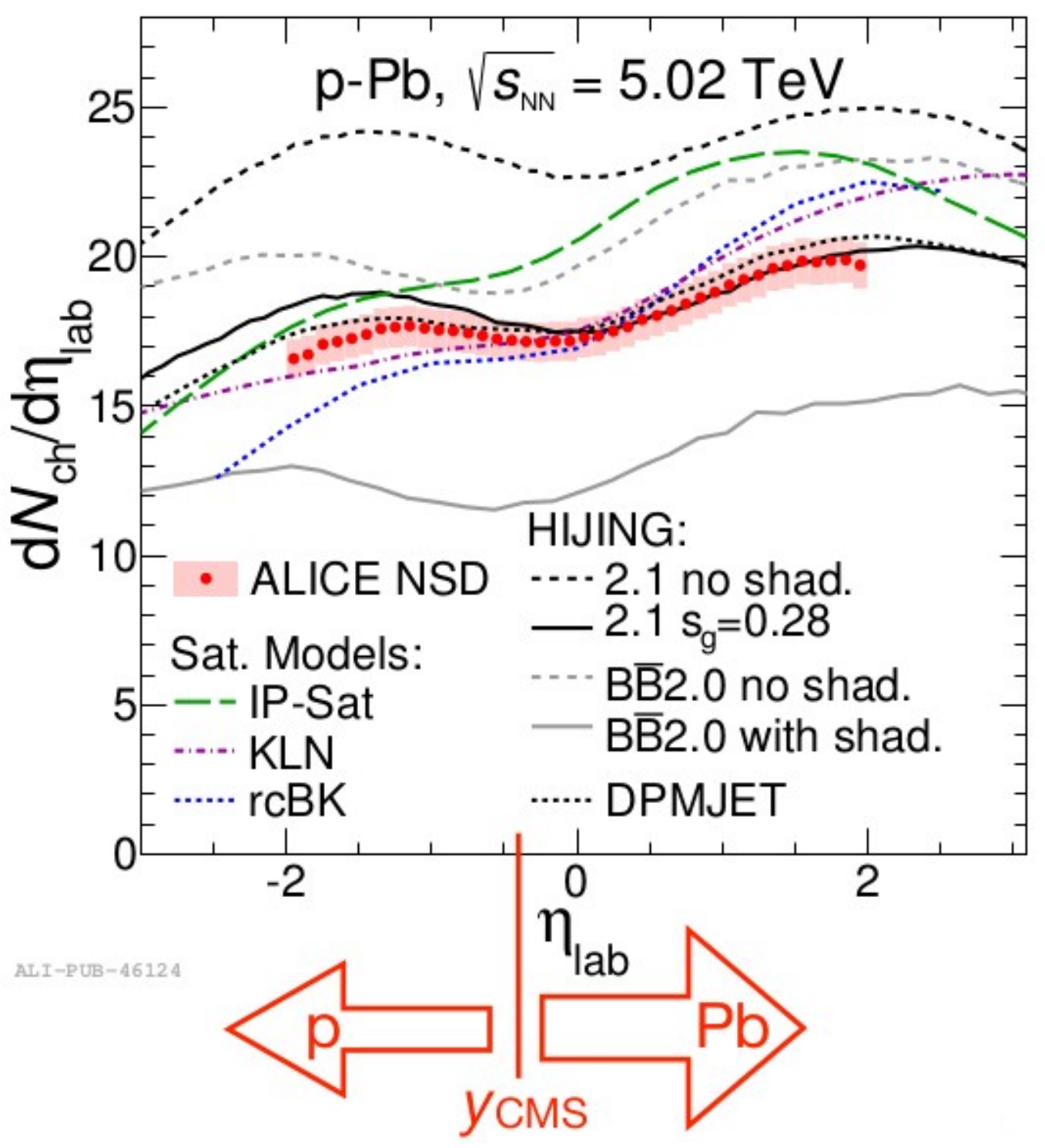
increase by $\sim 4 \%$ 


\section{Pseudorapidity density at midrapidity}

- Measurement (tracklet based)

ALICE, PRL 110 (2013) 032301

- $\mathrm{dN} / \mathrm{d} \eta=16.81 \pm 0.71$ (syst)

- Converted into centre-of-mass system using HIJING

- Dominant uncertainty from NSD normalization of $3.1 \%$

- Glauber model for pPb

- With $\sigma_{\text {INEL }}=70 \pm 5 \mathrm{mb}$

- $<$ Npart $>=7.9 \pm 0.6$ (syst)

- Participant scaled value

- $(\mathrm{dN} / \mathrm{d \eta}) /<$ Npart $>=2.14 \pm 0.17$ (syst)

- About 15\% below NSD pp

- Similar to pp INEL

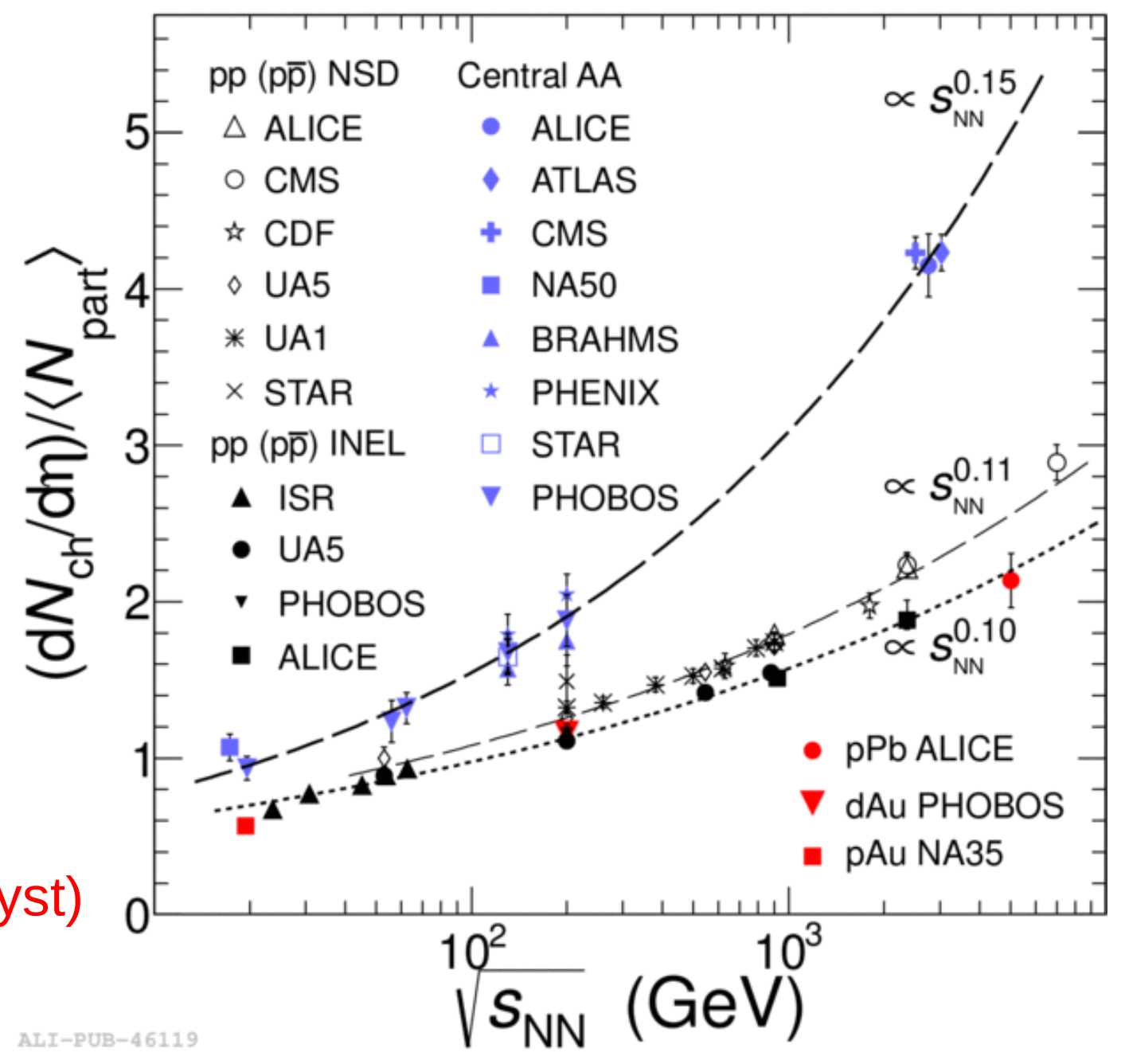

- Inelastic pPb would be 4\% lower (estimate from models) 


\section{Charged particle spectrum}

- Primary charged tracks ( $3 \eta$ bins)

- Reconstructed in ITS+TPC $(|\eta|<0.8)$ 褰

- Assume $\eta_{\mathrm{cms}}=\eta_{\mathrm{lab}-} \mathrm{y}_{\mathrm{cms}}$, then correct

- Systematic uncertainty: 5.2-7.1\%

- NSD normalization: $3.1 \%$

- Hint for slightly softer spectrum at higher $\eta(\mathrm{Pb}$ side)?

- Reference constructed from pp (INEL) data at 2.76 and $7 \mathrm{TeV}$

- Interpolation below $5 \mathrm{GeV} / \mathrm{c}$, and above scaled by factor obtained from NLO calculation

- Systematic uncertainty: 8\%

- Normalization uncertainty: $3.6 \%$

- $\left\langle\mathrm{T}_{\mathrm{pPb}}>=0.0983 \pm 0.0035 \mathrm{mb}^{-1}\right.$ from Glauber model

ALICE, PRL $110(2013) 082302$

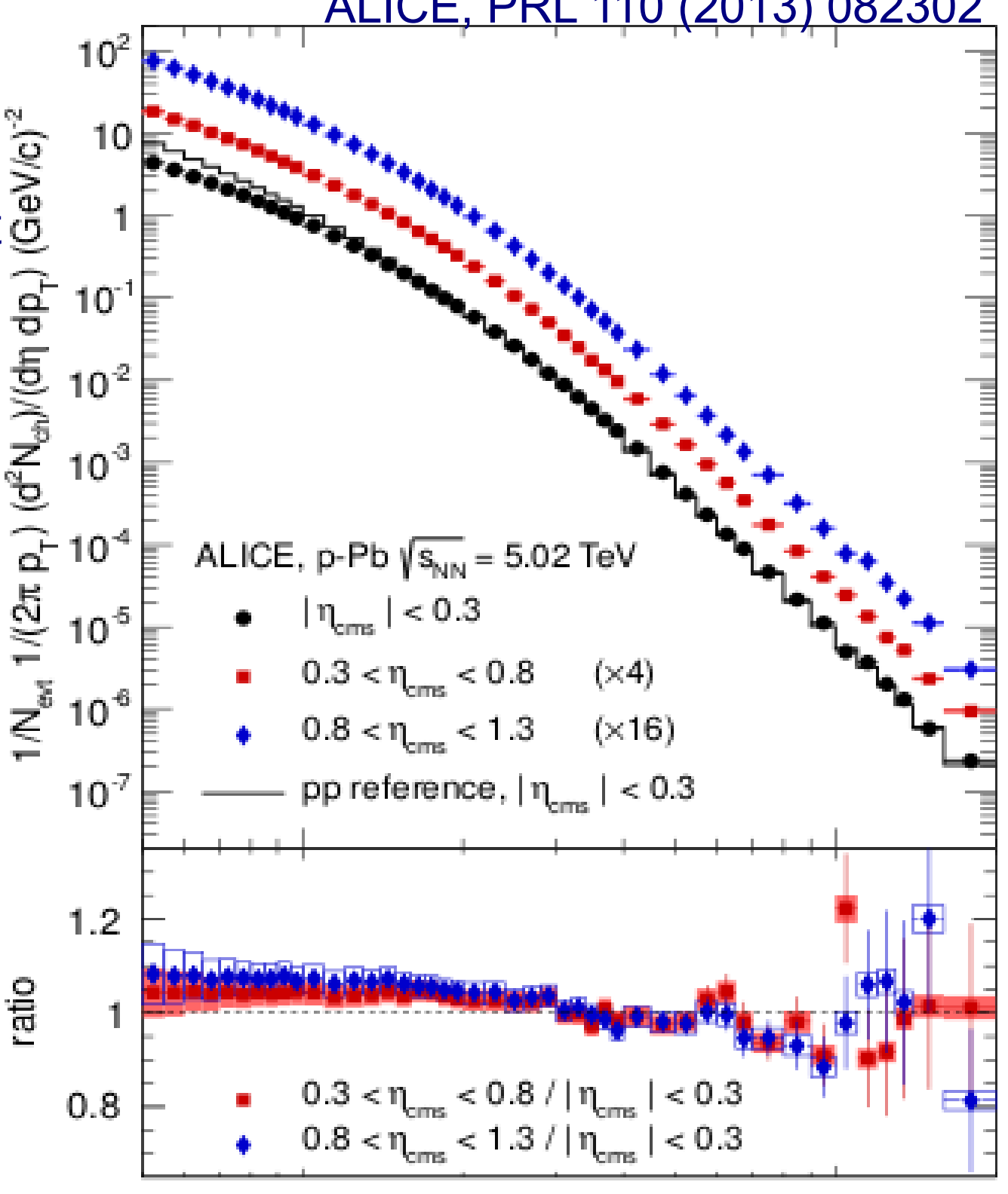

1

$p_{T}(\mathrm{GeV} / \mathrm{c})$ 


\section{Nuclear modification factor}

ALICE, PRL 110 (2013) 082302

$R_{A B}=\frac{\mathrm{d} N_{A B} / \mathrm{d} p_{\mathrm{T}}}{\left\langle N_{\text {coll }}\right\rangle \mathrm{d} N_{\mathrm{pp}} / \mathrm{d} p_{\mathrm{T}}}$

- $\mathrm{R}_{\mathrm{pPb}}$ (at mid-rapidity) consistent with unity for $p_{T}>2 \mathrm{GeV} / \mathrm{c}$

- High- $p_{T}$ charged particles exhibit binary scaling

- Unlike in $\mathrm{PbPb}$, no suppression is observed

- Suppression in $\mathrm{PbPb}$ is not an initial state effect

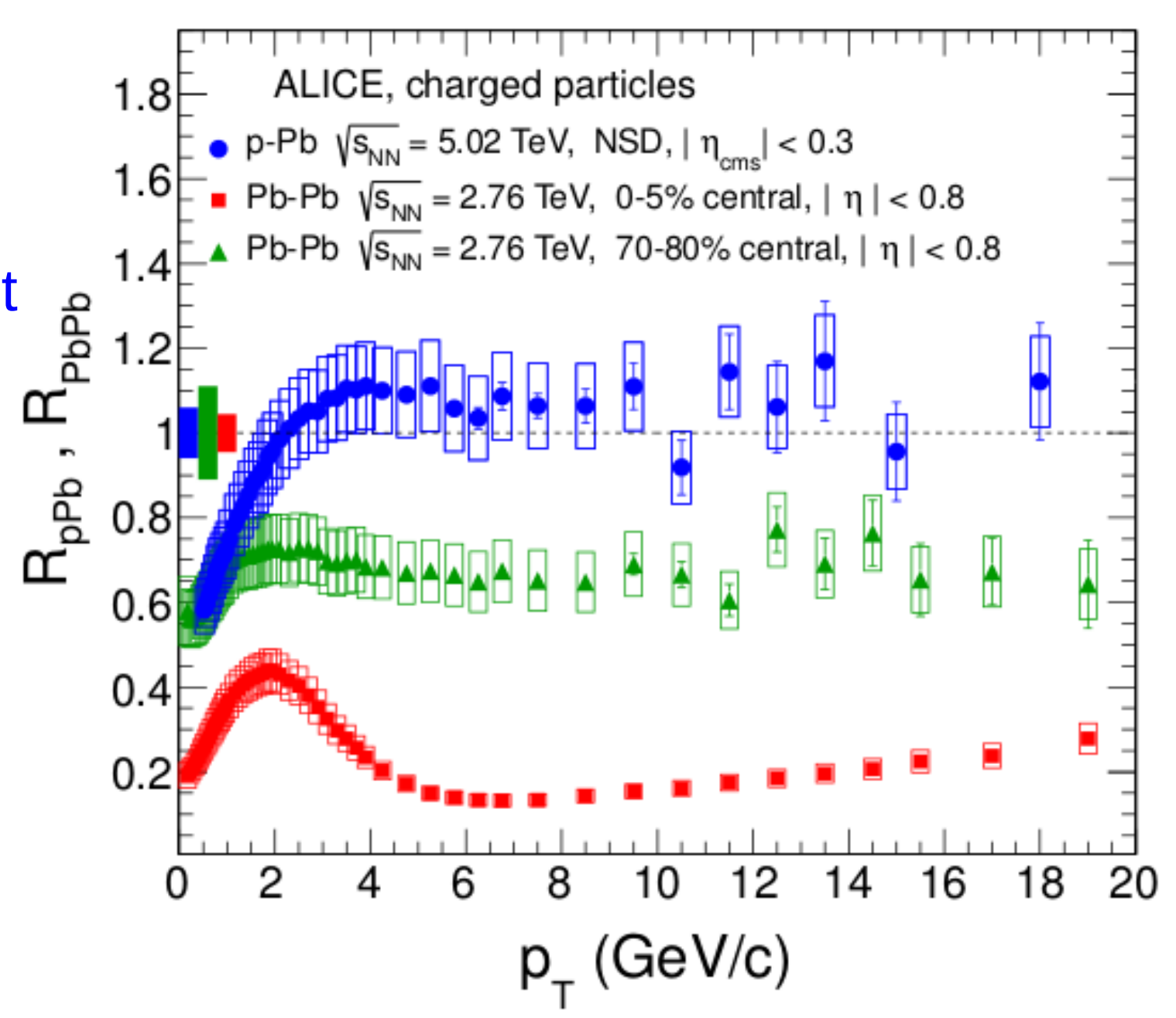




\section{Cronin effect at RHIC and LHC}

ALICE, PRL 110 (2013) 082302

STAR, PRL 91 (2003) 072304

$R_{A B}=\frac{\mathrm{d} N_{A B} / \mathrm{d} p_{\mathrm{T}}}{\left\langle N_{\text {coll }}\right\rangle \mathrm{d} N_{\mathrm{pp}} / \mathrm{d} p_{\mathrm{T}}}$

- $\mathrm{R}_{\mathrm{AB}}>1$ at intermediate $\mathrm{p}_{\mathrm{T}}$ observed in dAu collisions at RHIC typically attributed to Cronin effect

- No enhancement seen in $\mathrm{pPb}$ at the LHC

- No Cronin effect?

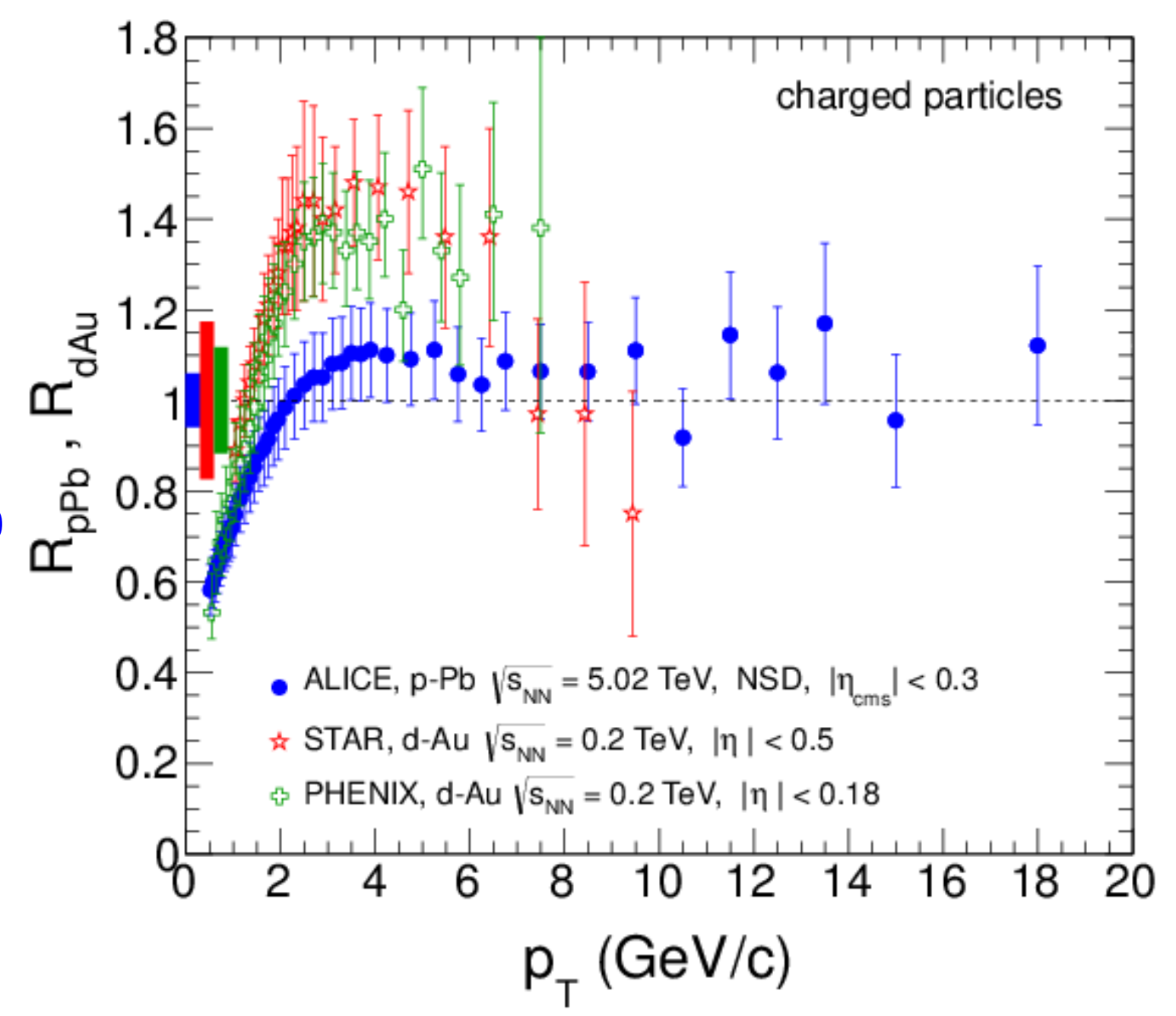




\section{Cronin effect at SPS}

- Reminder from SPS energies: $\mathrm{RAB} \approx 1$ does not necessarily imply absence of effects

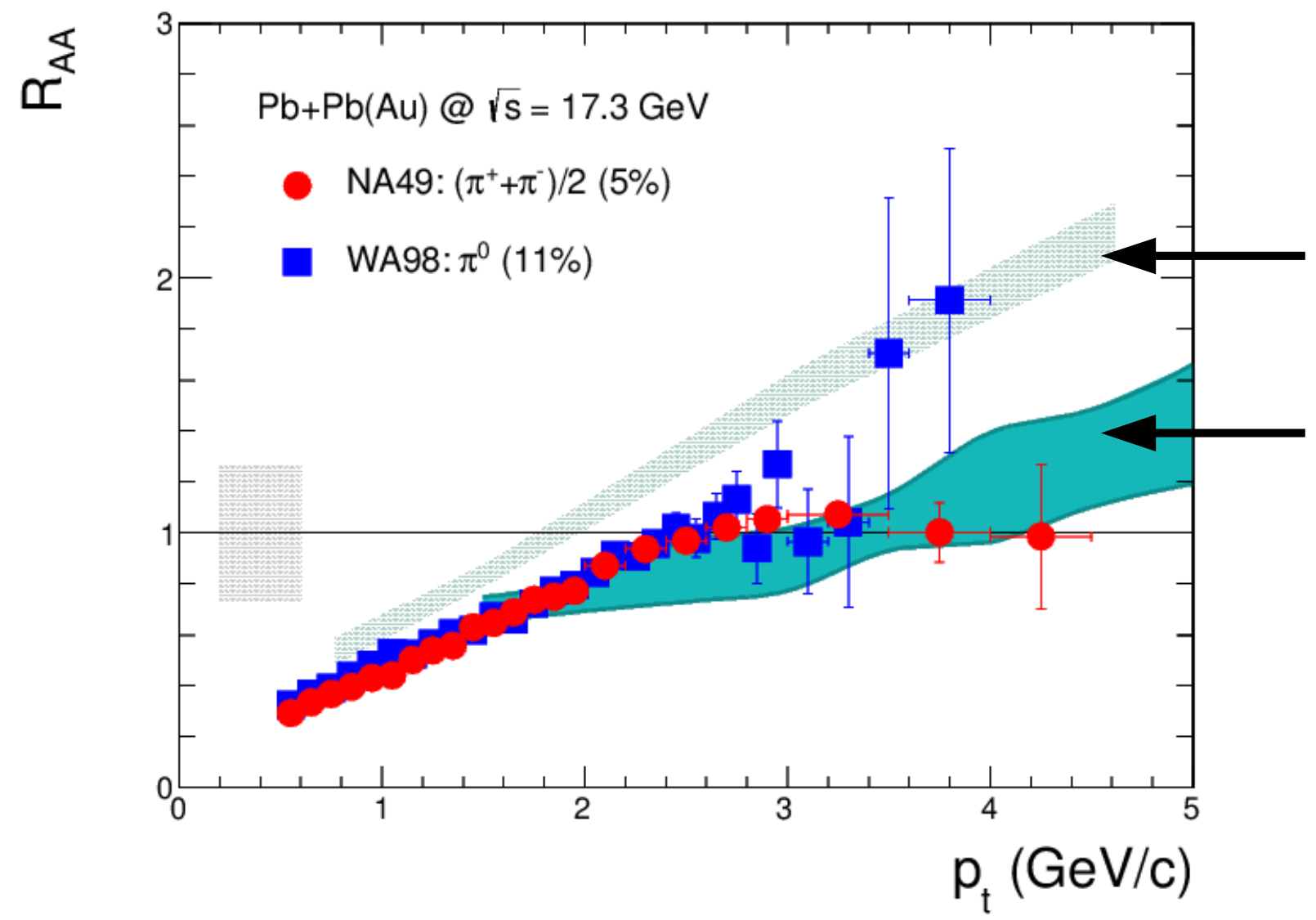

NA49, NPA 783 (2007) 65

WA98, PRL 89 (2002) 252301

Calculation

taking into account:

Cronin effect + shadowing

Cronin effect, shadowing plus partonic energy loss

- Model comparisons are required to understand $\mathrm{R}_{\mathrm{pPb}}$ at the LHC 


\section{Nuclear modification factor vs models}

- Saturation (CGC) models:

- Consistent with the data

- Large uncertainties

- pQCD models with shadowing

- Consistent at low $\mathrm{p}_{\mathrm{T}}$

- Discrepancies at high $\mathrm{p}_{\mathrm{T}}$

- HIJING

- With shadowing describes $\eta$ and low $\mathrm{p}_{\mathrm{T}}$ better

- No shadowing better at high $\mathrm{p}_{\mathrm{T}}$

- Spectrum on its own interesting

- Neither HIJING nor DPMJET do describe the $\mathrm{p}-\mathrm{Pb} \mathrm{p}_{\mathrm{T}}$ spectra

NB: HJING calculations for NSD expected to increase by $\sim 4 \%$

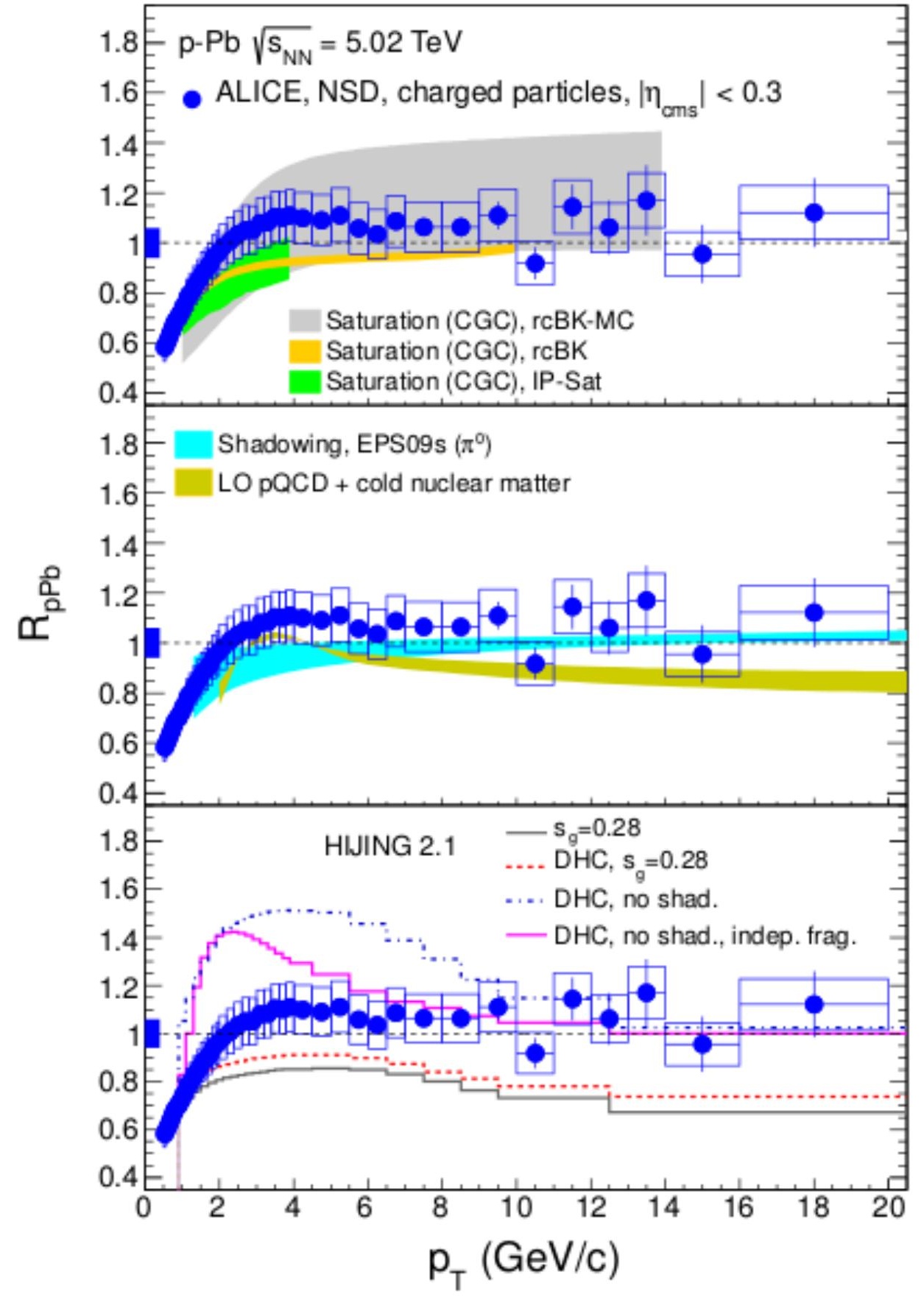




\section{Di-Hadron Correlations (DHC)}

- CMS: pp, pPb at LHC

- Long-range near-side correlations (ridge) appear at high-multiplicity

- Collective effects in $\mathrm{pp}$ and $\mathrm{pPb}$ ?

- CGC initial state effects?
CMS, JHEP 1009 (2010) 91

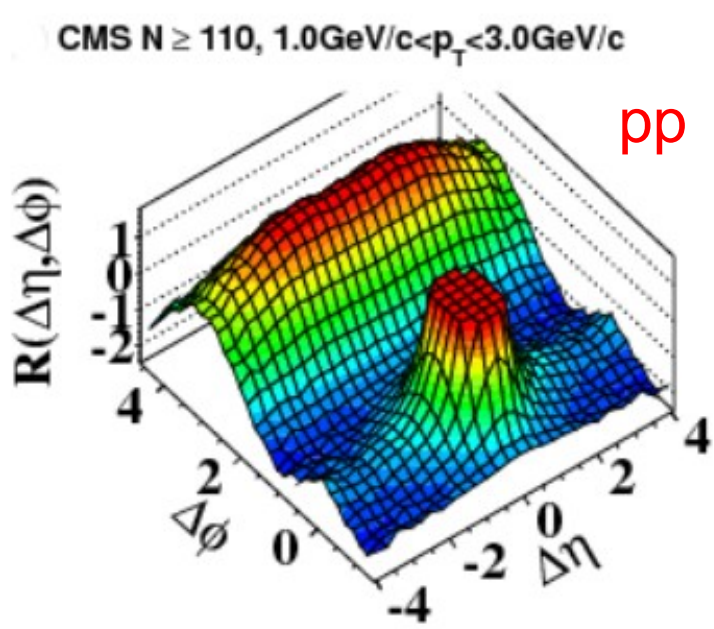

CMS, PLB 718 (2012) 795

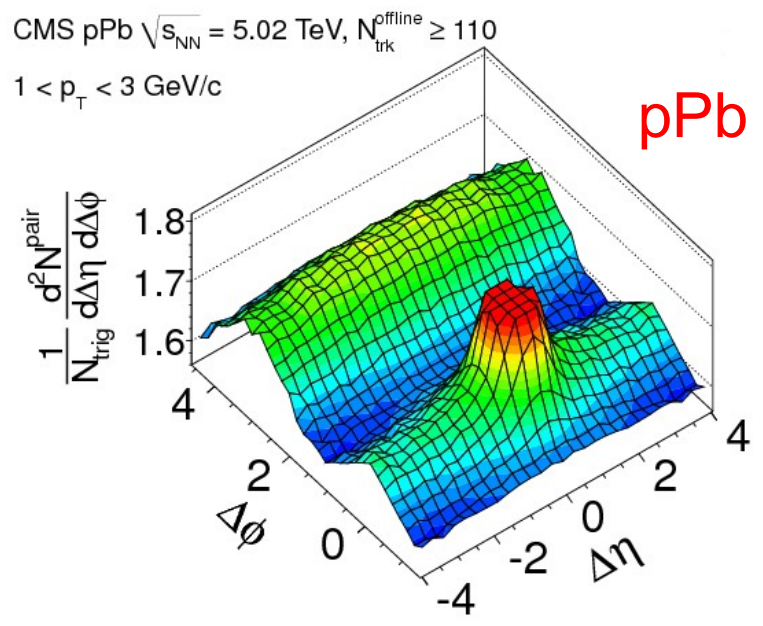

- STAR: dAu at RHIC

- Back-to-back (jet-like) correlations in forward $\pi^{0}$ correlations disappear in high-multiplicity events

- Compatible with CGC predictions

- LHC mid- and RHIC forward- $\eta$ probe a similar $\mathrm{x}$ regime
STAR, arXiv:1005.2378

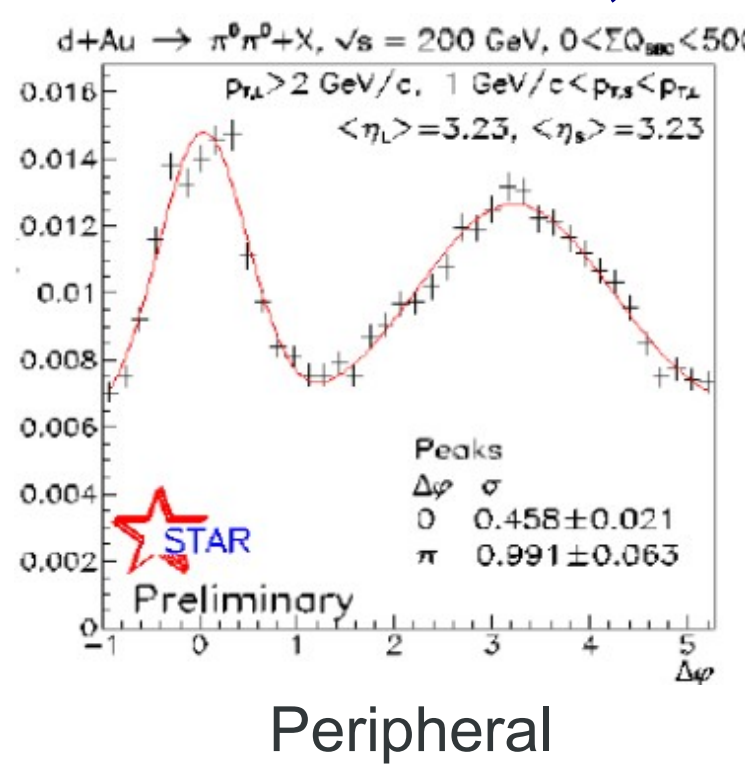




\section{DHC: Multiplicity classes}

ALICE, PLB 719 (2013) 29

- Correlation between geometry and multiplicity in pA is not as strong as in $A A$

- System also shows features of biased pp (NN) collisions in the low and high multiplicity tails

- Define multiplicity classes

- Use charge in VZERO to avoid correlation with tracks in barrel

- VOM: sum of amplitudes from

- VZERO-A $(2.8<\eta<5.1)$

- VZERO-C $(-3.7<\eta<-1.7)$

- Systematic checks using

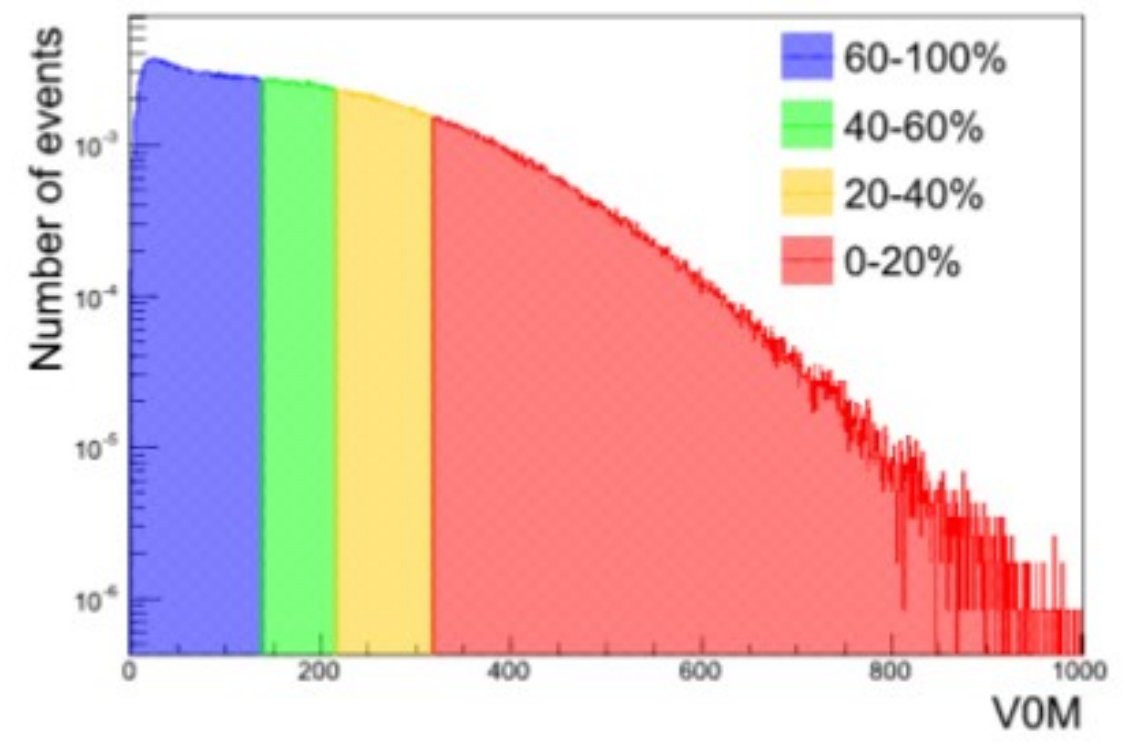

\begin{tabular}{cccc}
\hline $\begin{array}{c}\text { Event } \\
\text { class }\end{array}$ & $\begin{array}{c}\text { V0M range } \\
\text { (a.u.) }\end{array}$ & $\begin{array}{c}\left.\left\langle\mathrm{d} N_{\text {ch }} / \mathrm{d} \eta\right\rangle\right|_{|\eta|<0.5} \\
p_{\mathrm{T}}>0 \mathrm{GeV} / c\end{array}$ & $\begin{array}{c}\left.\left\langle N_{\text {trk }}\right\rangle\right|_{|\eta|<1.2} \\
p_{\mathrm{T}}>0.5 \mathrm{GeV} / c\end{array}$ \\
\hline $60-100 \%$ & $<138$ & $6.6 \pm 0.2$ & $6.4 \pm 0.2$ \\
$40-60 \%$ & $138-216$ & $16.2 \pm 0.4$ & $16.9 \pm 0.6$ \\
$20-40 \%$ & $216-318$ & $23.7 \pm 0.5$ & $26.1 \pm 0.9$ \\
$0-20 \%$ & $>318$ & $34.9 \pm 0.5$ & $42.5 \pm 1.5$ \\
\hline
\end{tabular}

- $\operatorname{SPD}(|\eta|<1.4)$

- ZNA (beam neutron on Pb side) 


\section{DHC: Correlation measure}

ALICE, PLB 719 (2013) 29

- Associated yield per trigger particle (with $p_{T}^{\text {trig }}>p_{T}^{\text {assoc }}$ )

$$
\frac{1}{N_{\text {trig }}} \frac{\mathrm{d}^{2} N_{\text {assoc }}}{\mathrm{d} \Delta \eta \mathrm{d} \Delta \varphi}=\frac{S(\Delta \eta, \Delta \varphi)}{B(\Delta \eta, \Delta \varphi)}
$$

- Signal (same event) pair yield

$$
S(\Delta \eta, \Delta \varphi)=\frac{1}{N_{\text {trig }}} \frac{\mathrm{d}^{2} N_{\text {same }}}{\mathrm{d} \Delta \eta \mathrm{d} \Delta \varphi}
$$

- Definition as ratio of sums is multiplicity independent

$$
\begin{aligned}
& \frac{N_{\text {pair }}}{N_{\text {trig }}}=\frac{\sum_{i=1}^{N_{\text {evt }}} \sum_{j=1}^{N_{\text {source }}^{i}} \frac{1}{2} n_{i j}\left(n_{i j}-1\right)}{\sum_{i=1}^{N_{\text {evt }}} \sum_{j=1}^{N_{\text {iource }}^{2}} n_{i j}} \\
& =\frac{N_{\text {evt }}\left\langle N_{\text {source }}\right\rangle \frac{1}{2}\langle n(n-1)\rangle}{N_{\text {evt }}\left\langle N_{\text {source }}\right\rangle\langle n\rangle} \\
& =\frac{1}{2} \frac{\langle n(n-1)\rangle}{\langle n\rangle}
\end{aligned}
$$

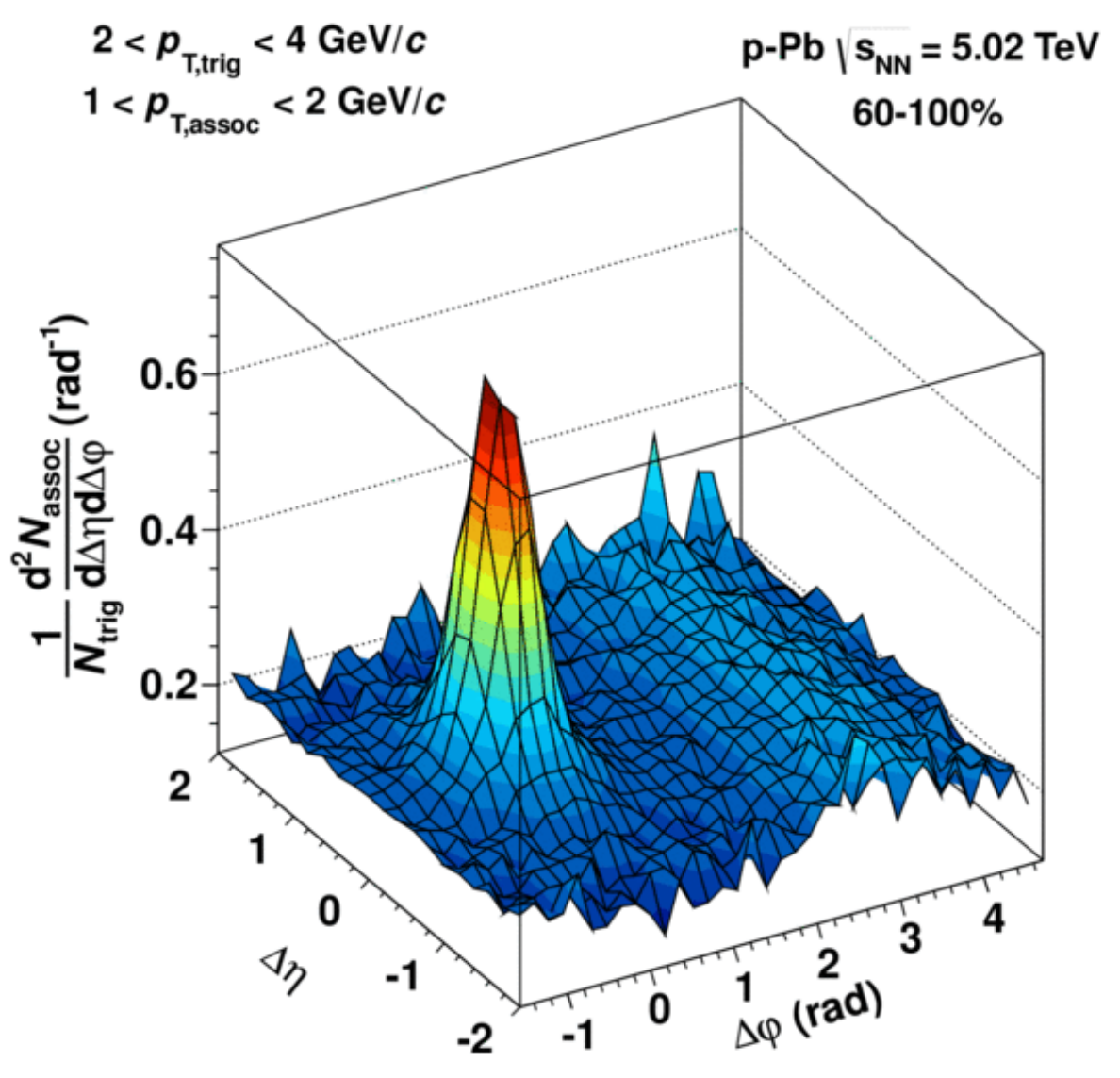

- Background (mixed event) pair yield

$$
B(\Delta \eta, \Delta \varphi)=\frac{1}{B(0,0)} \frac{\mathrm{d}^{2} N_{\text {mixed }}}{\mathrm{d} \Delta \eta \mathrm{d} \Delta \varphi}
$$




\section{DHC: Multiplicity dependence}

ALICE, PLB 719 (2013) 29

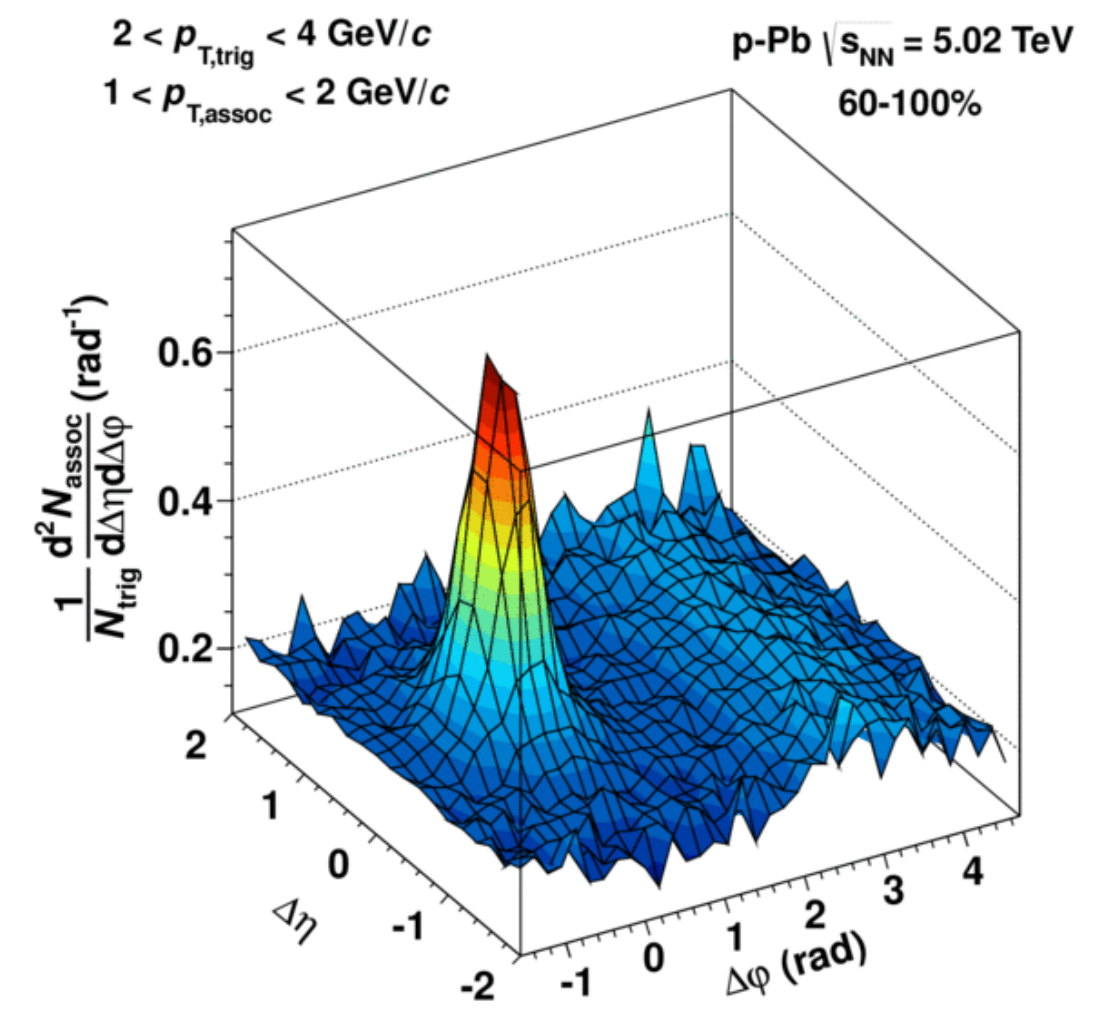

- Low-multiplicity p-Pb (60-100\%)

- pp-like (jet-like) correlation structures

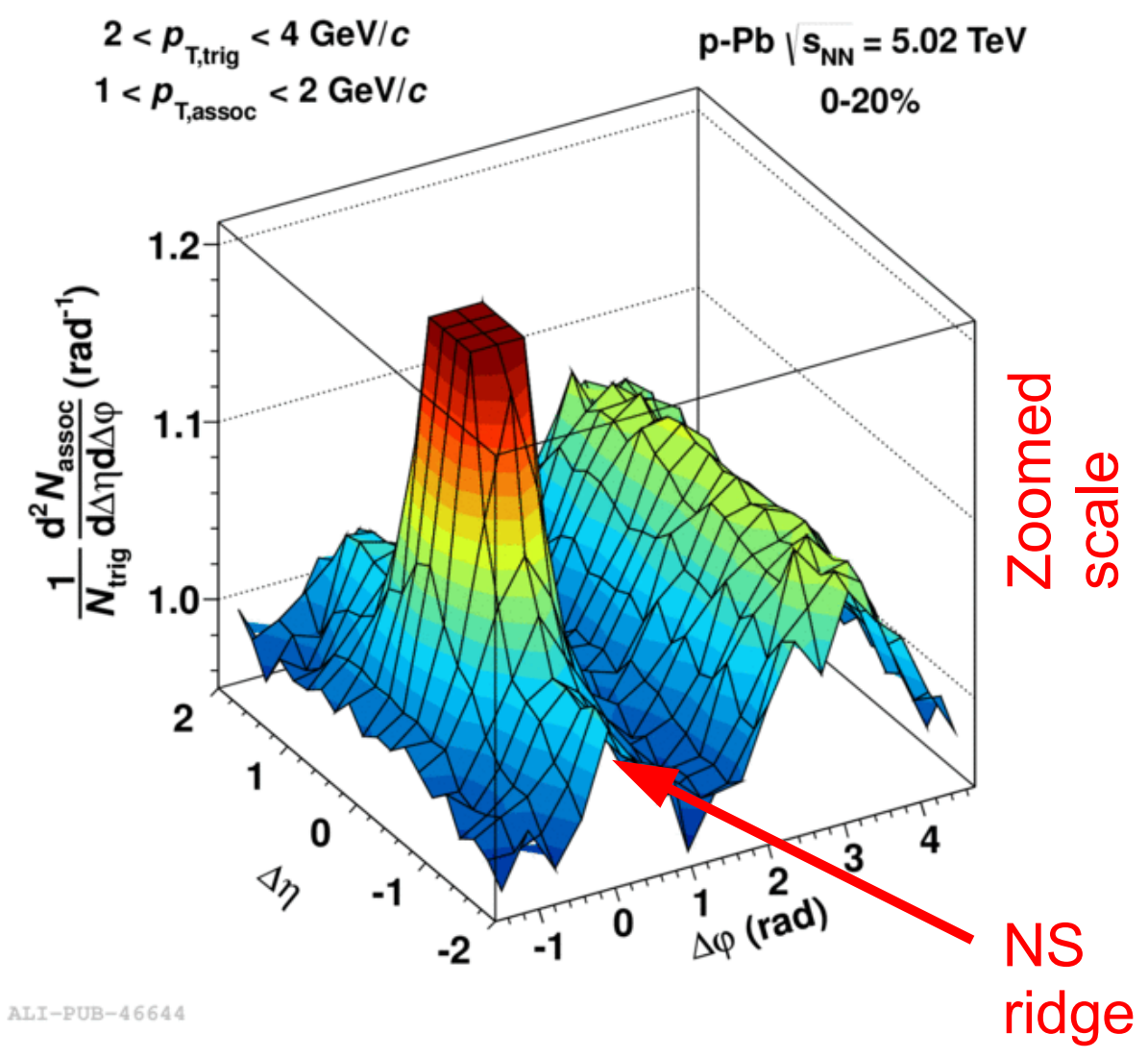

- High-multiplicity p-Pb (0-20\%)

- Near-side ridge appears (first seen in CMS)

- Higher yields on near- and away-side 


\section{DHC: Multiplicity dependence}

ALICE, PLB 719 (2013) 29

- Compare associated yield in $\mathrm{pPb}$ multiplicity classes and $\mathrm{pp}$

- Project to $\Delta \varphi$ over $|\Delta \eta|<1.8$

- Subtract baseline at $\Delta \varphi \sim 1.3$

- Low multiplicity pPb is similar to pp (at $7 \mathrm{TeV}$ )

- Yield rises on near and away side with increasing multiplicity

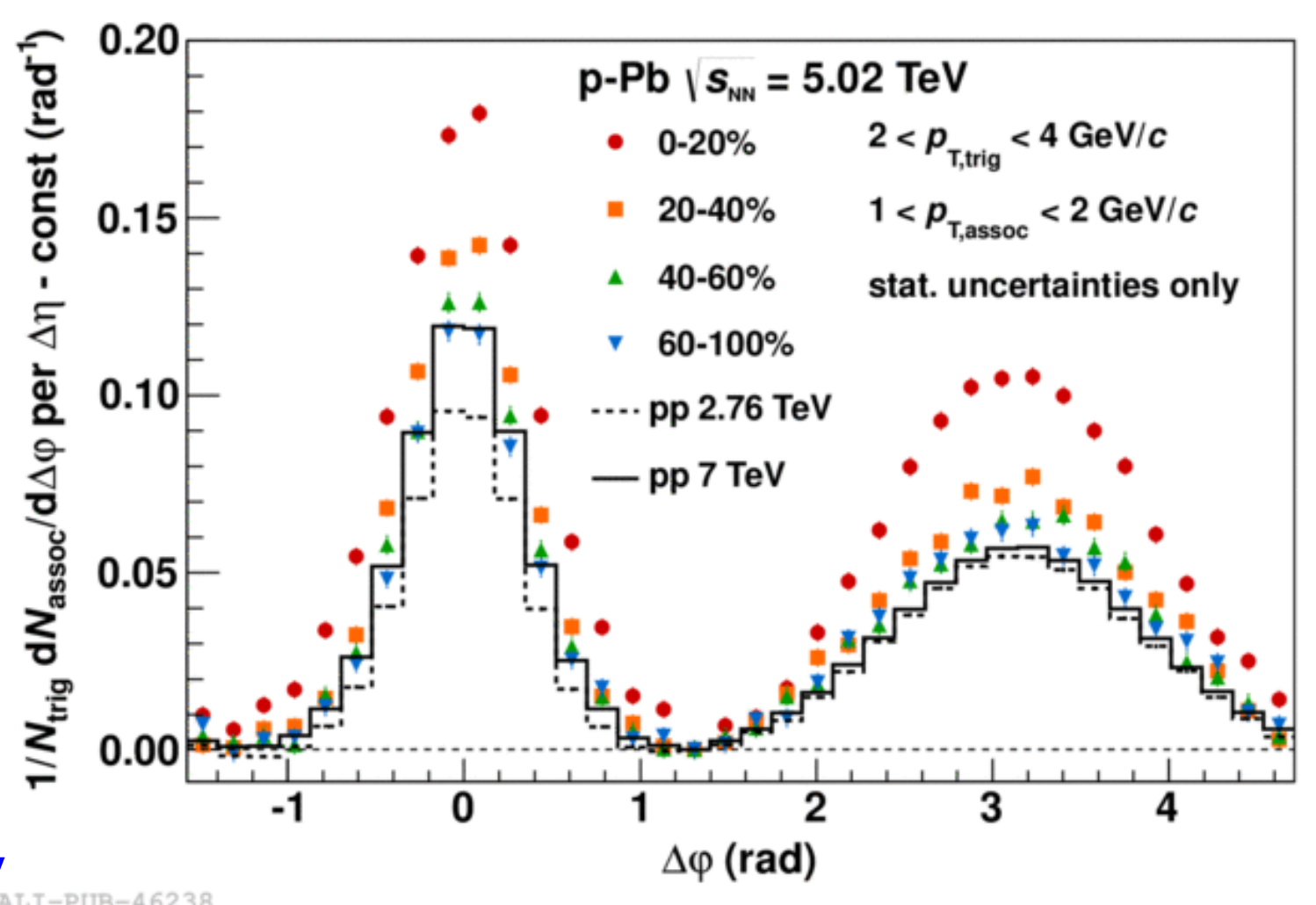

- In contrast with away-side suppression observed in dAu at $\mathrm{RHIC}$ at forward $\eta$ (similar $\mathrm{x}$ ) 


\section{DHC: Two ridges}

- Quantify the excess in high-multiplicity

ALICE, PLB 719 (2013) 29 $\mathrm{pPb}$ by subtracting the jet-like correlations:
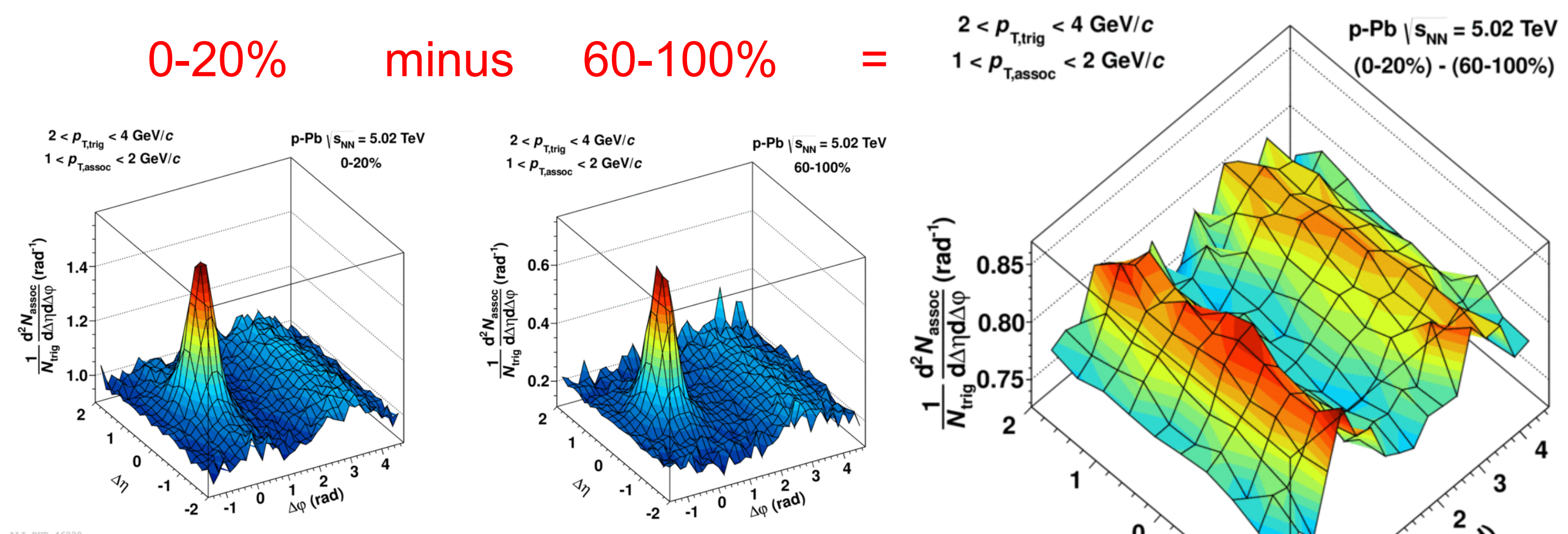

- The near-side is accompanied by an almost identical ridge structure on the away-side 


\section{DHC: Two ridges}

ALICE, PLB 719 (2013) 29

- A residual jet peak at $(0,0)$ remains even after subtraction of $60-100 \%$ from the $0-20 \%$ multiplicity class

- Compare effects using different event class definition
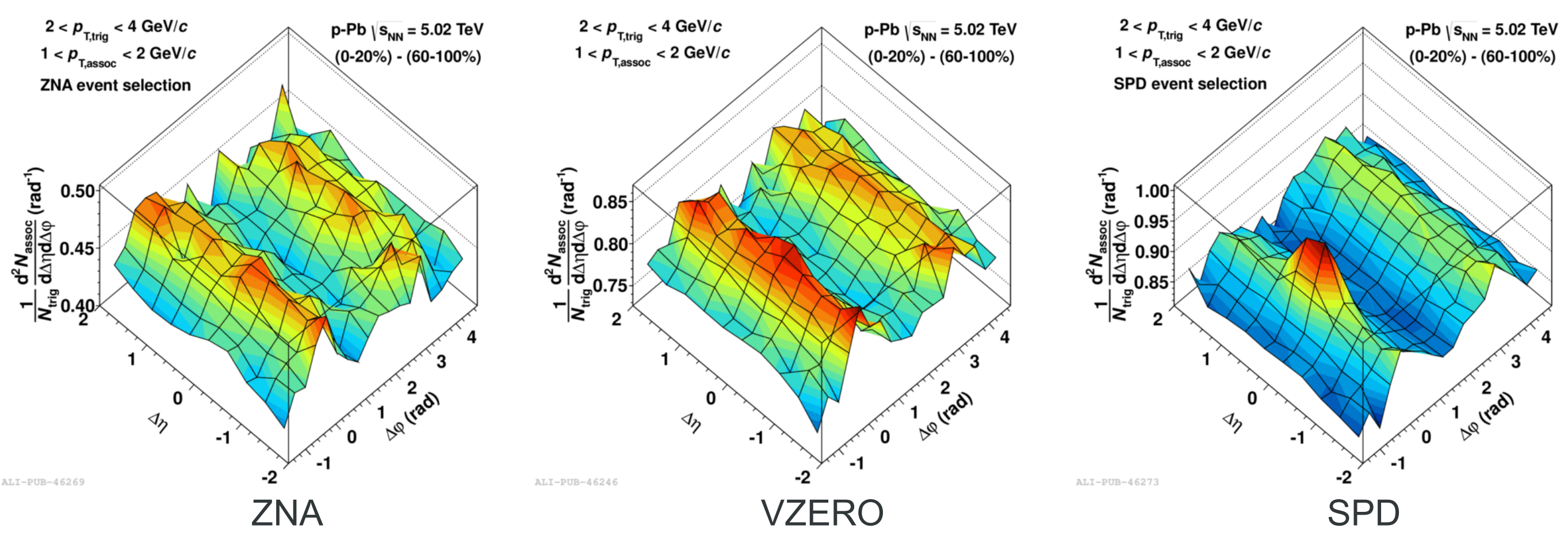

auto-correlation 


\section{DHC: Selection bias on fragmentation (pp) 30}

Per trigger near-side pair yield in $\mathrm{pp}$
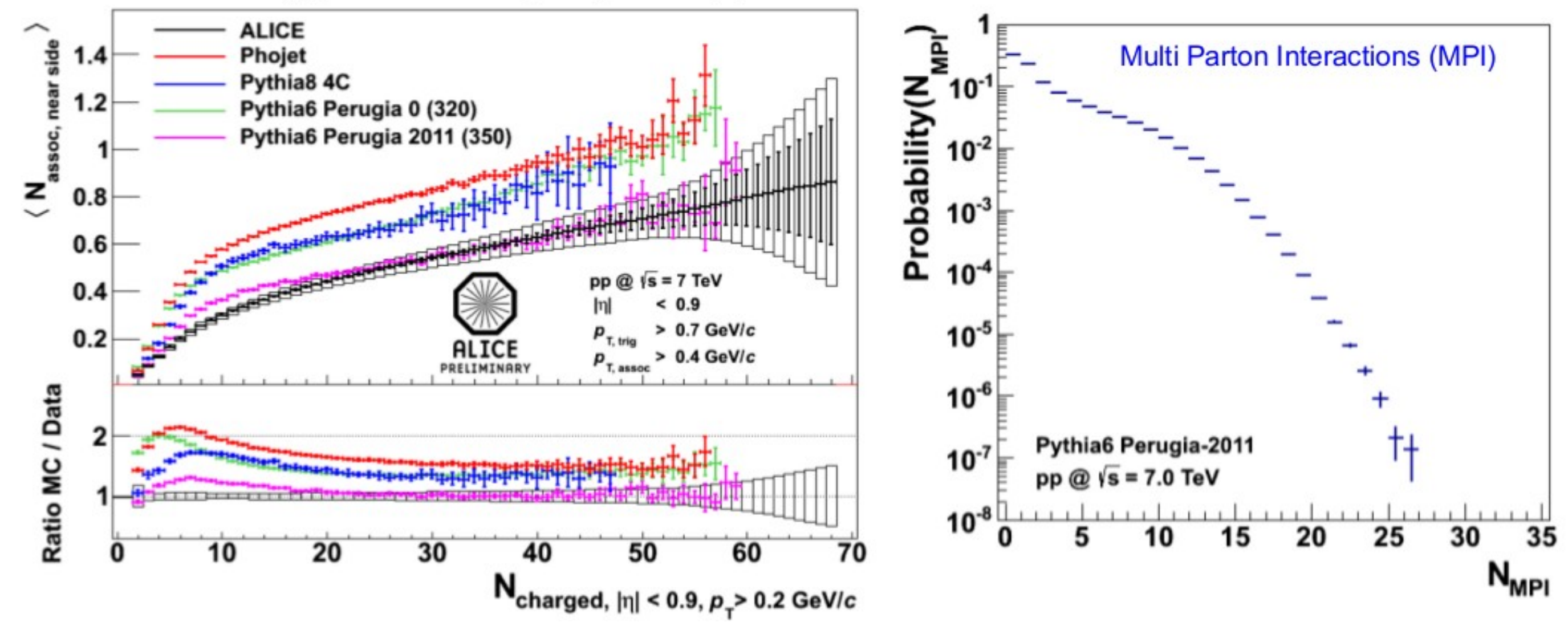

- By selecting on multiplicity, jet fragmentation is biased towards higher number of fragmenting products

- Competition between higher number of MPI and fragmentation 


\section{DHC: Two ridges (ATLAS)}

ATLAS, arXiv:1212.5198

- Similar two ridge structures also observed by ATLAS

- Event multiplicity classes defined by sum of transverse energy $(3.1<\eta<4.9)$ on the $\mathrm{Pb}$ nucleus side

- Also here, the jet peak at $(0,0)$ remains even after subtraction of $50-100 \%$ from the $0-2 \%$ multiplicity class

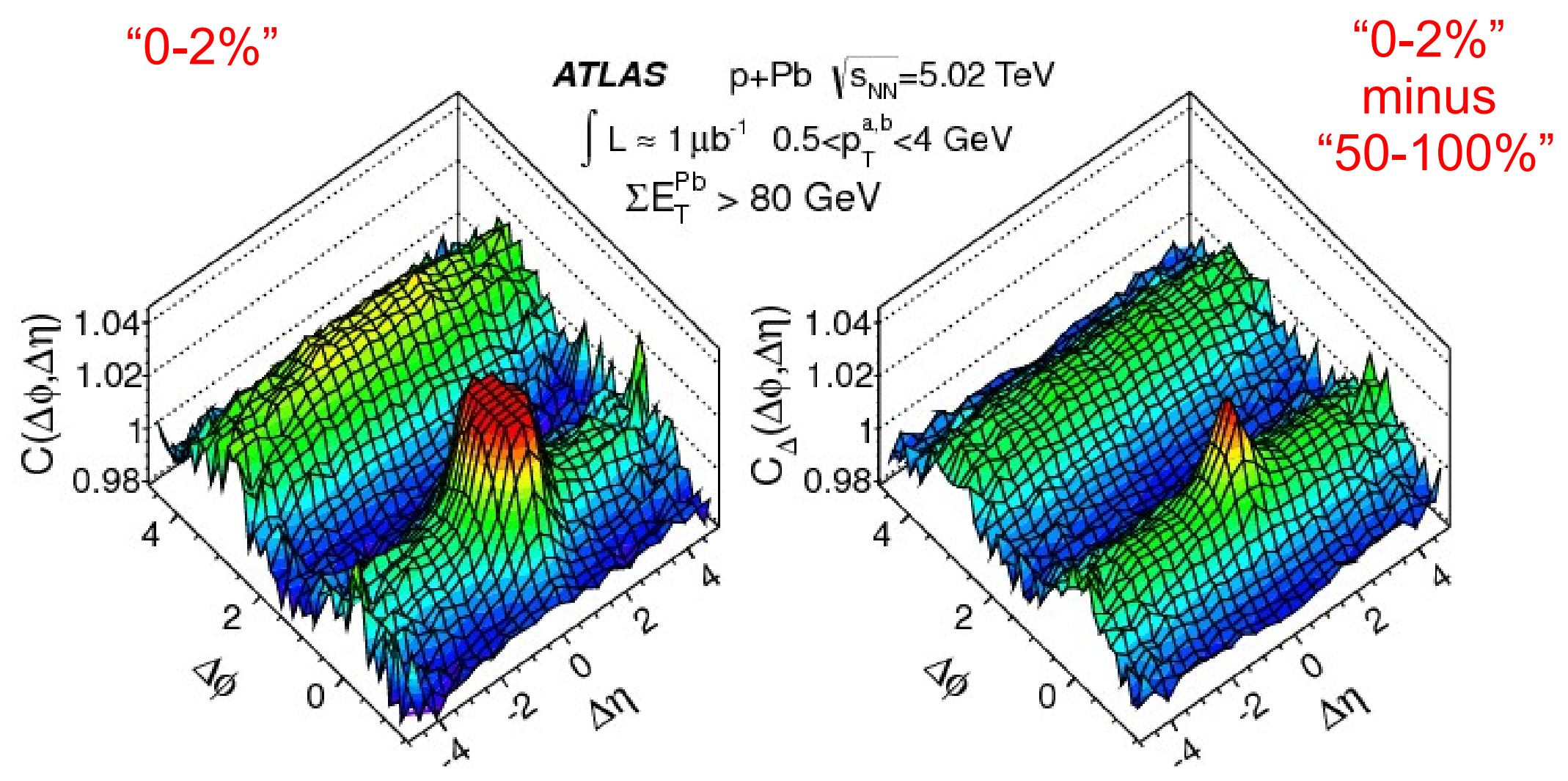




\section{DHC: Two ridges}

ALICE, PLB 719 (2013) 29

- A closer look at the two ridges: the near- and away-side ridges

- Are essentially flat in $\Delta \eta$

- Slight excess on near side due to small residual jet peak

- Have the same magnitude

- Projection to $\Delta \varphi$

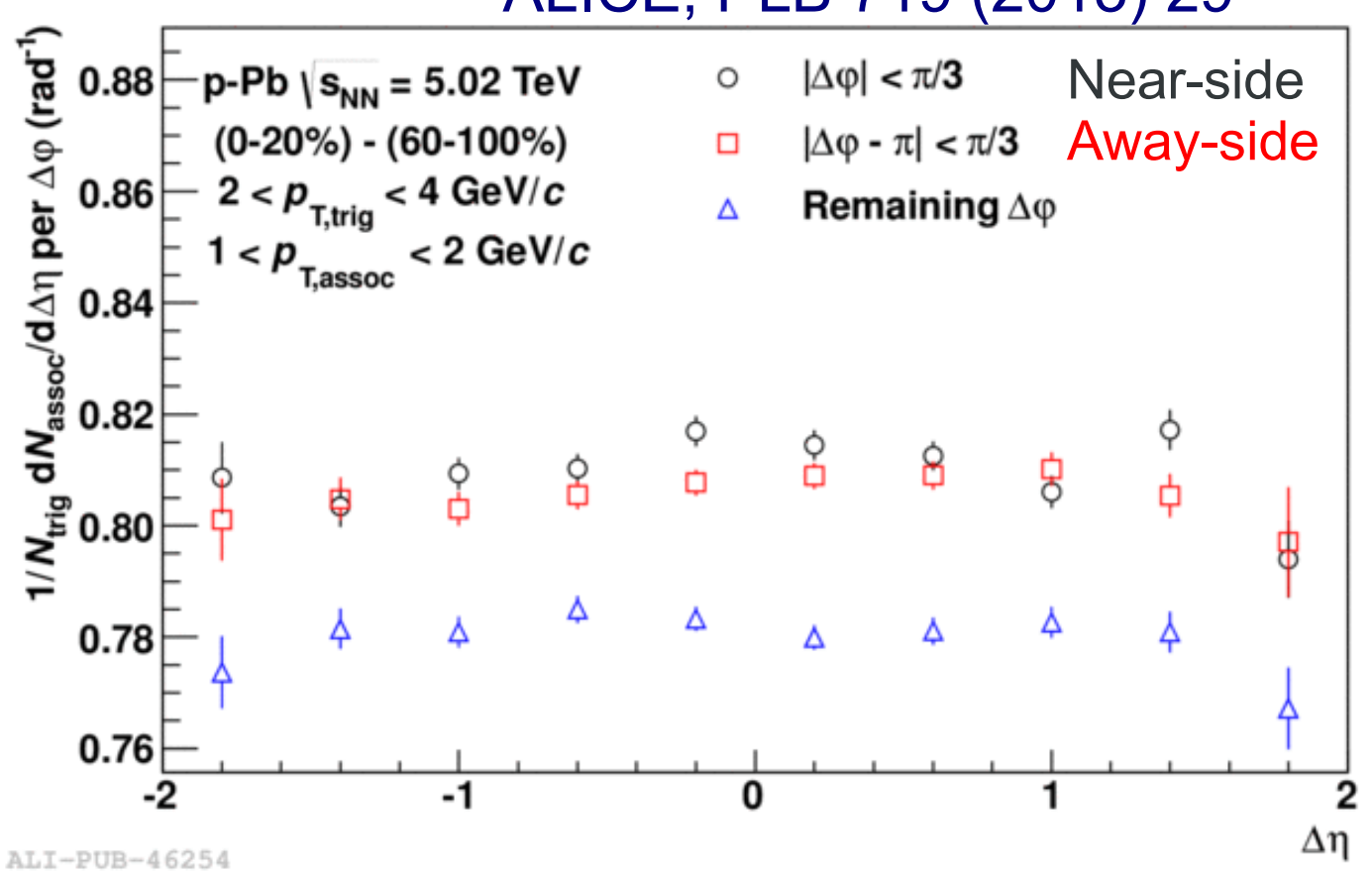

- Exclude residual peak $(|\Delta \eta<0.8|$ on near-side $)$ exhibits a modulation

- In HIJING, the correlation shows no qualitative changes with multiplicity

- Quantify the ridges

- Ridge yields

- Fourier coefficients

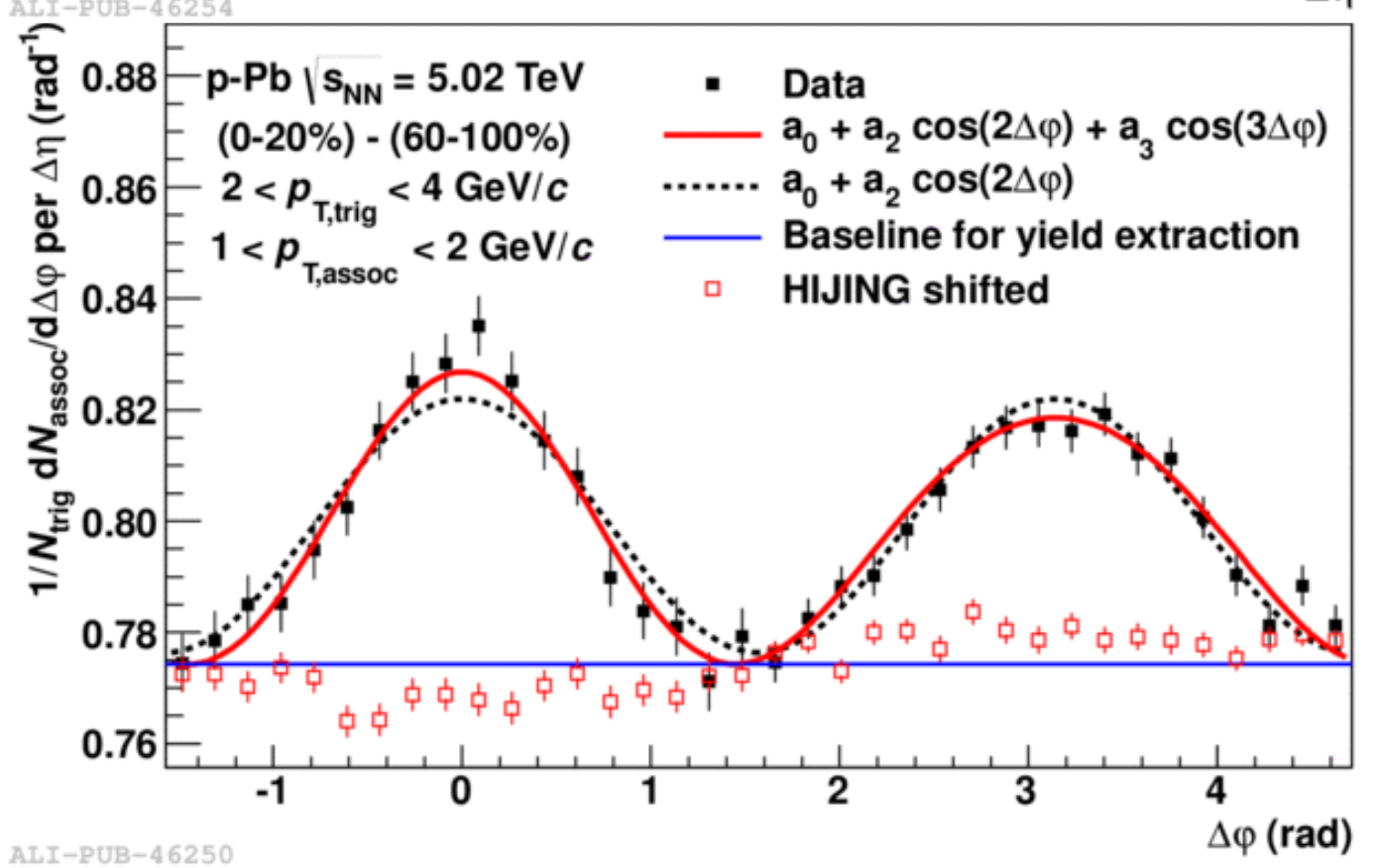




\section{DHC: Ridge yields}

- Integrate two ridges above baseline on the

- Near side $(|\Delta|<\pi / 2)$

- Away side $(\pi / 2<|\Delta|<3 \pi / 2)$

- Near and away-side ridge yields

- Change significantly

- Agree for all $p_{T}$ and multiplicity ranges

- Increase with trigger $p_{T}$ and multiplicity

- Widths are approximately the same (not shown)

- The correlation between nearand away-side yields suggests a common underlying origin
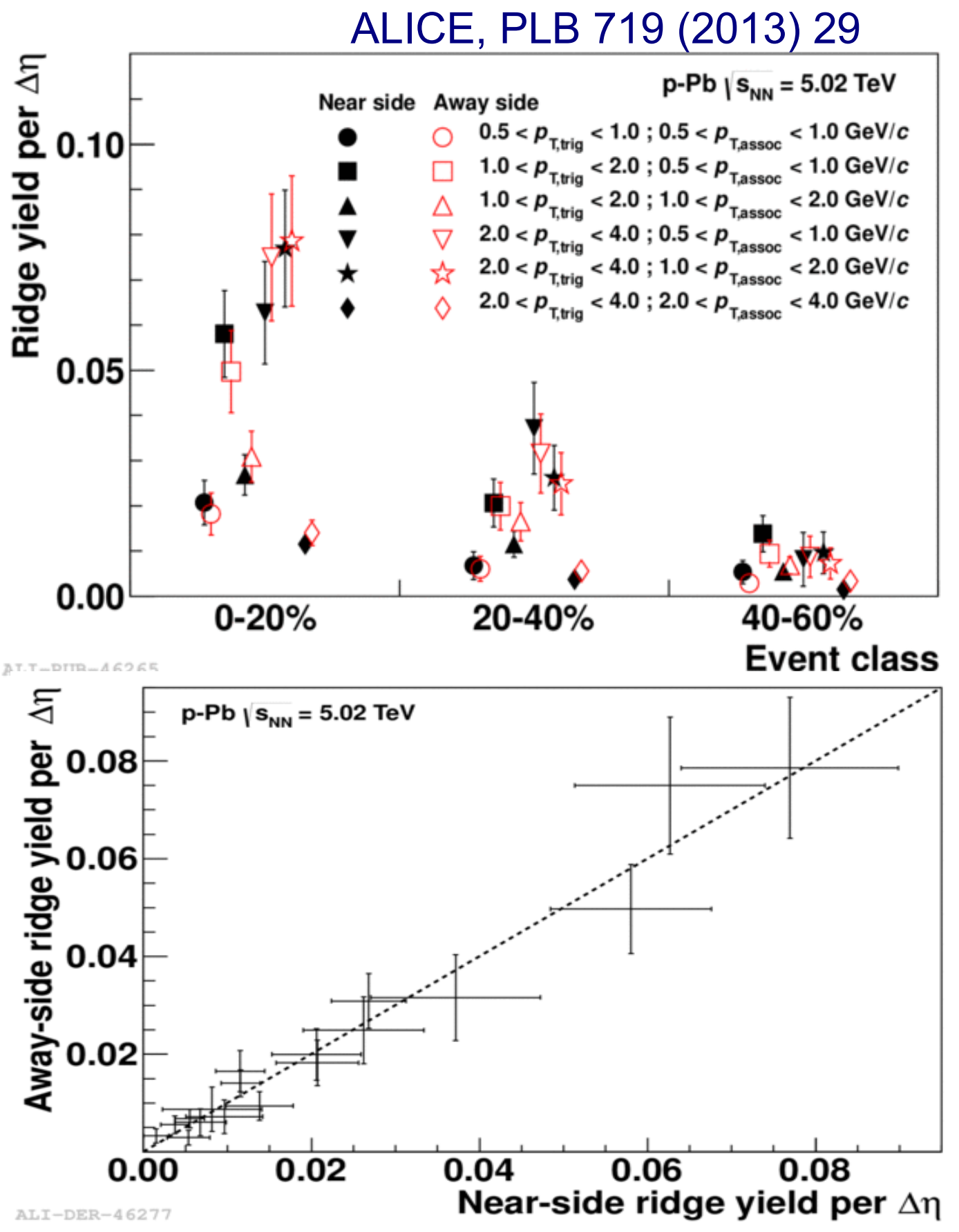


\section{DHC: Ridge modulation}

- Obtain $v_{n}=\sqrt{ }\left(a_{n} / b\right)$ from $a_{0}+2 a_{2} \cos (2 \Delta \varphi)+2 a_{3} \cos (3 \Delta \varphi)$ fit where $b$ is baseline in higher multiplicity class

- $v_{2}$ increases strongly with $p_{T}$ and mildly with multiplicity

- $v_{3}$ increases with $p_{T}$ within large uncertainties

- These trends are in qualitative agreement with expectations from viscous hydrodynamical predictions

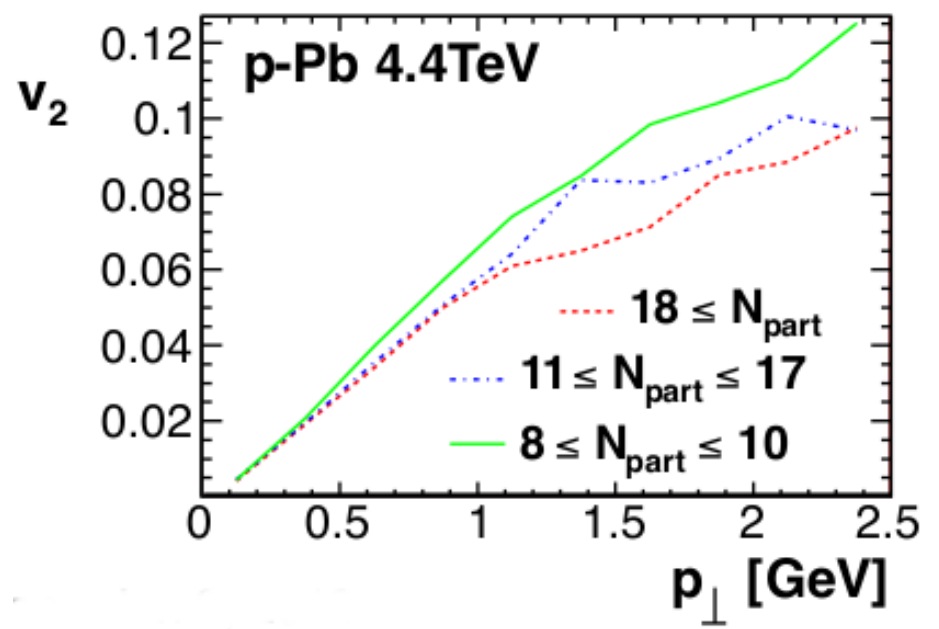

ALICE, PLB 719 (2013) 29

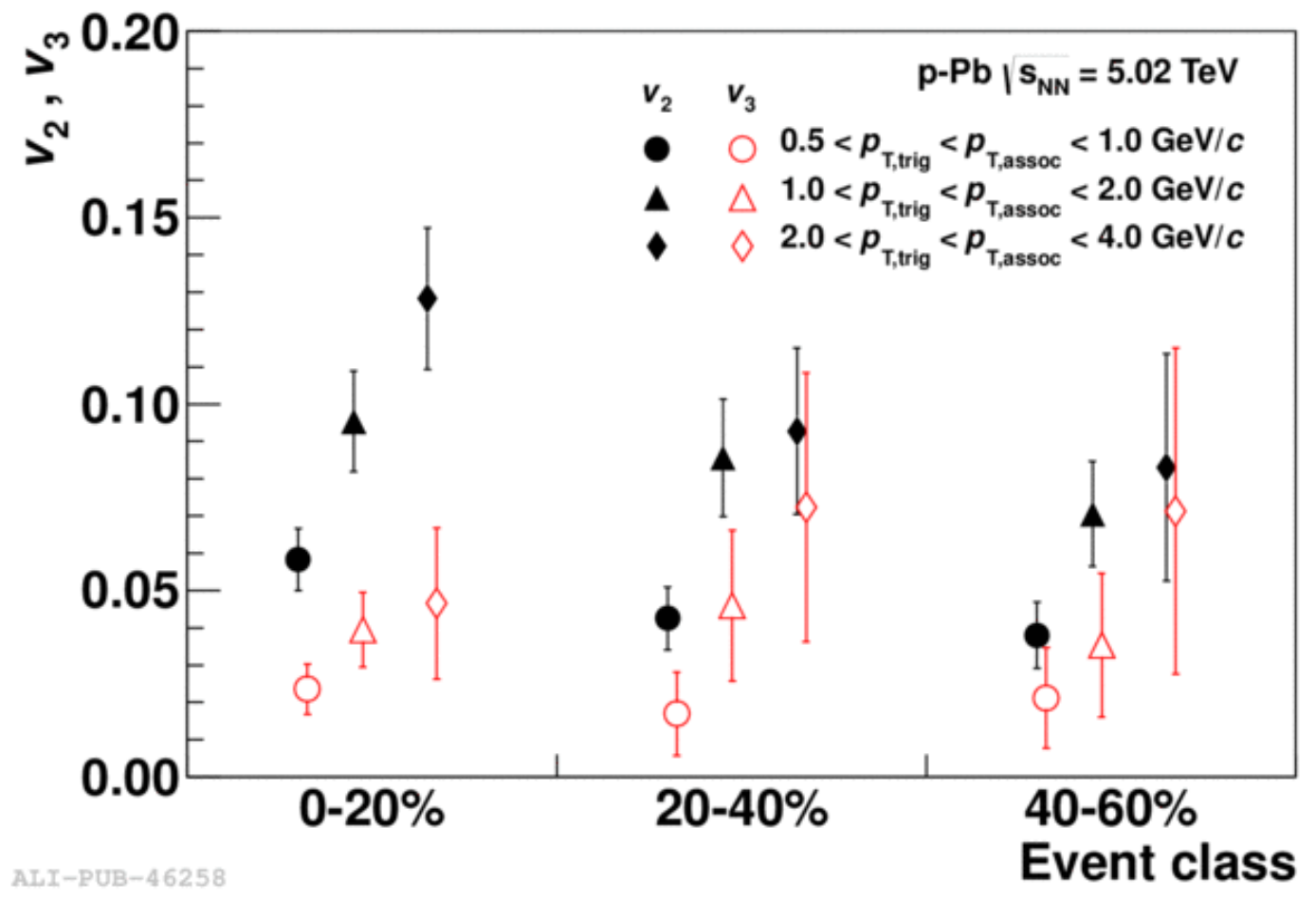

Bozek, PRC 85 (2012) 014911

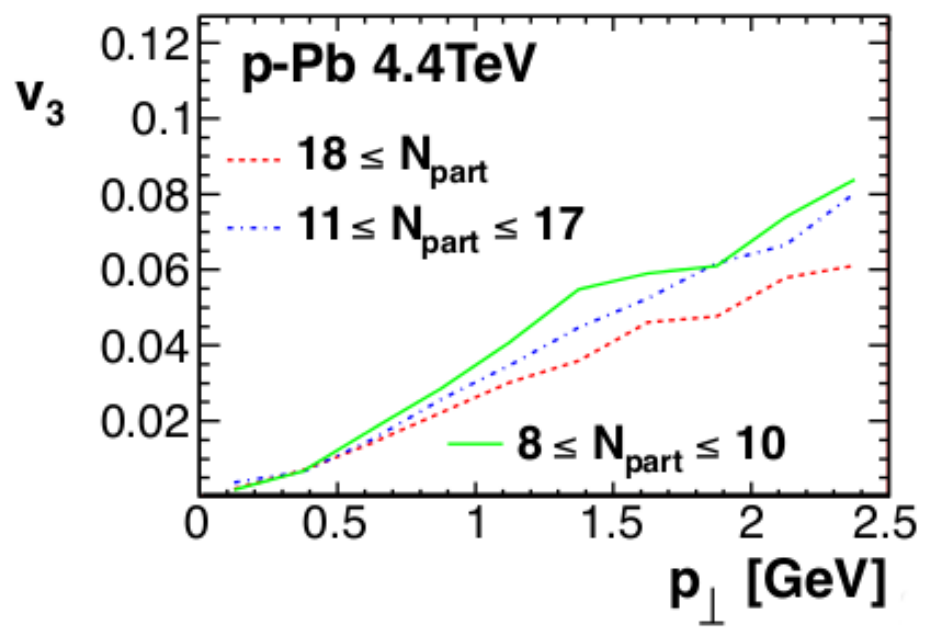




\section{DHC: Ridge modulation}

- Two ridges are also predicted by CGC

$\frac{1}{N_{\text {Trig }}} \frac{d^{2} N}{d \Delta \phi}$

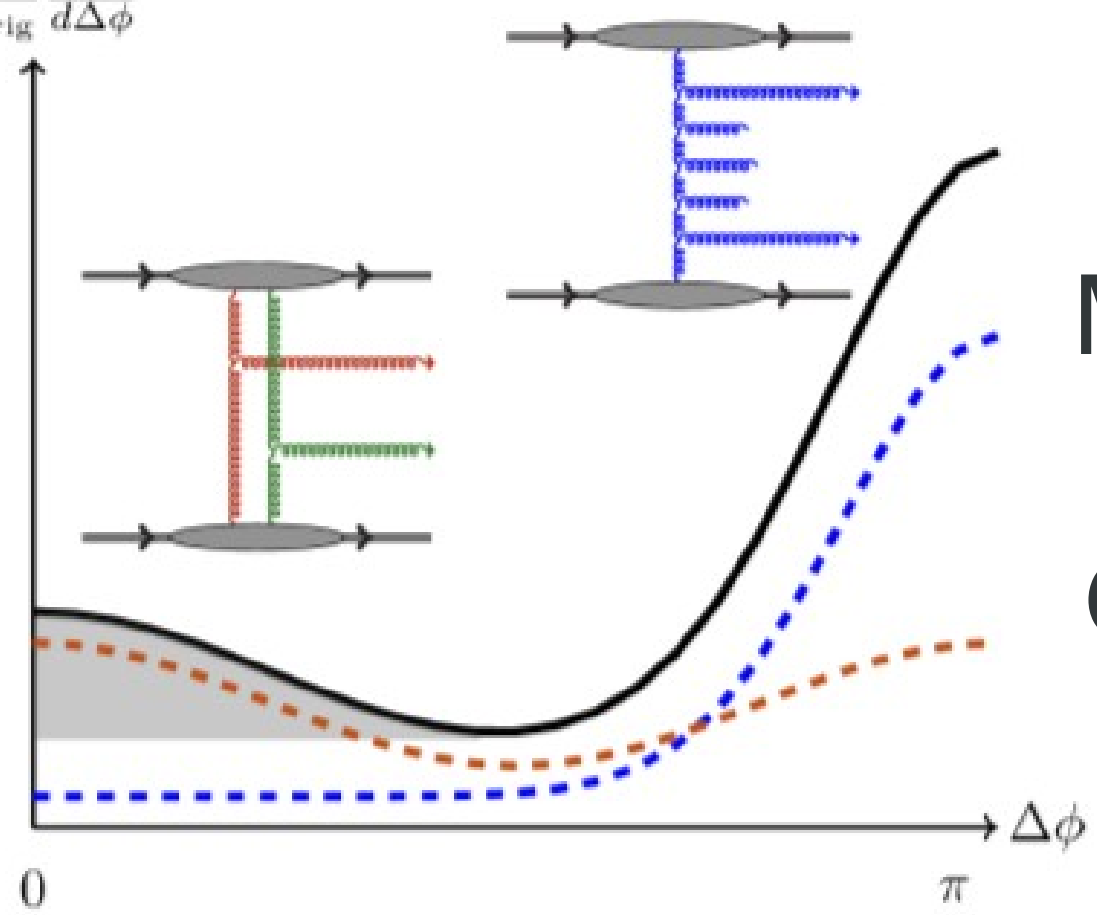

Dusling and Venugopalan, arXiv:1302.7018
Minijets

Glasma

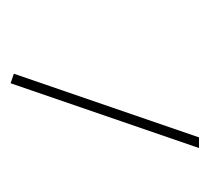

$2<p_{\text {T,trig }}<4 \mathrm{GeV} / c$
$1<p<2 \mathrm{GeV} / c$ $\mathrm{p}-\mathrm{Pb} \mid \mathrm{s}_{\mathrm{NN}}=5.02 \mathrm{TeV}$ $0-20 \%$
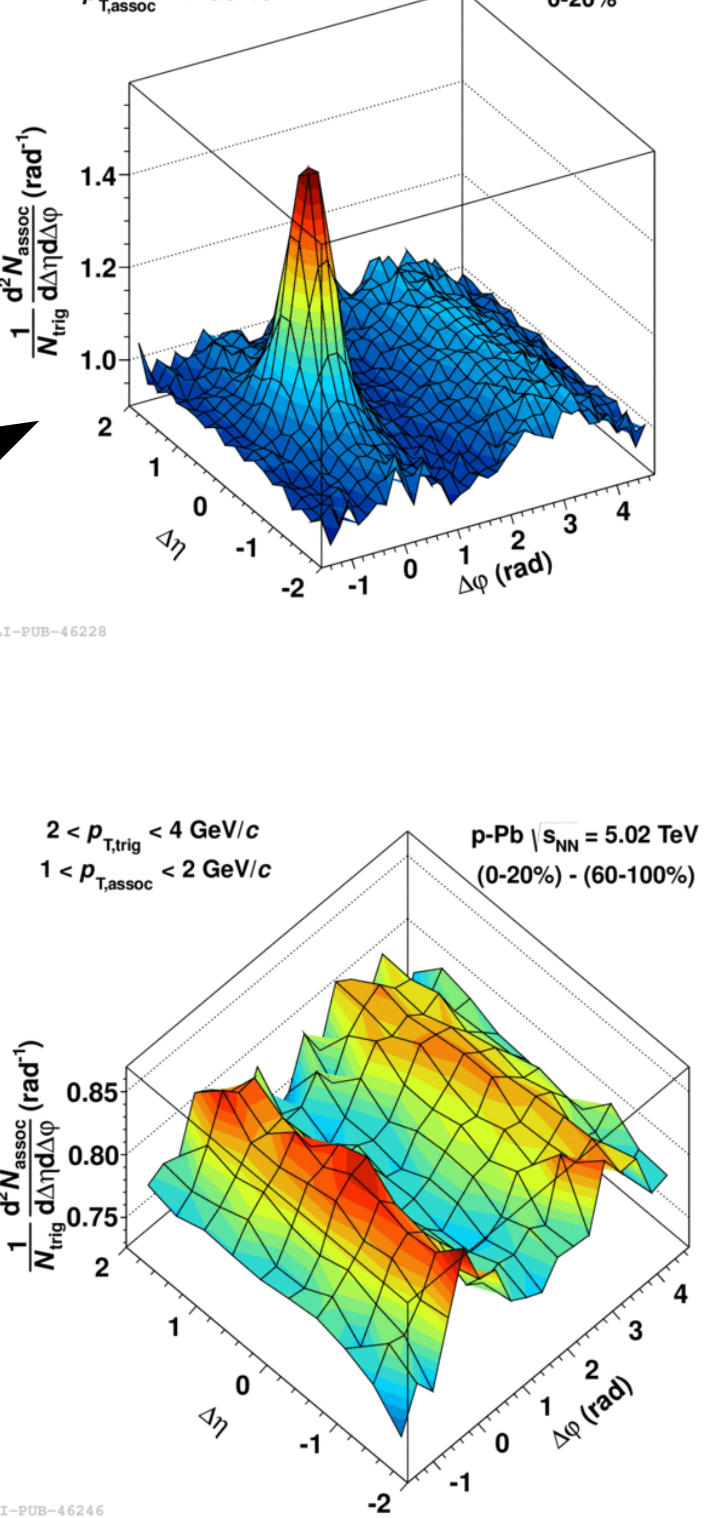


\section{DHC: Symmetric ridge}

ALICE, PLB 719 (2013) 29

- What would the assumption of a symmetric ridge give?

- Determine the near-side ridge in $1.2<|\Delta \eta|<1.8$

- Mirror to away-side and subtract

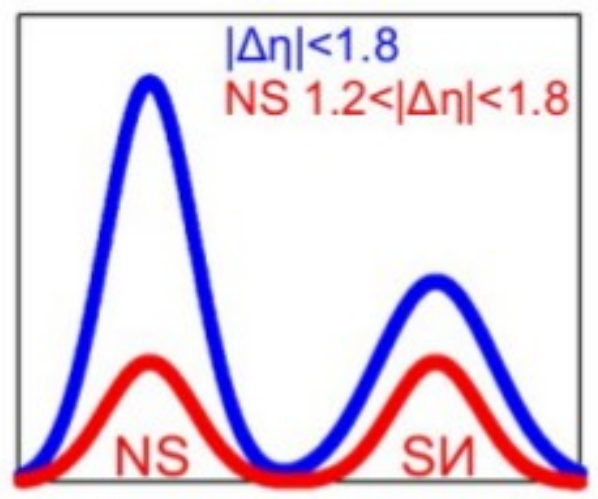

$\Delta \phi$
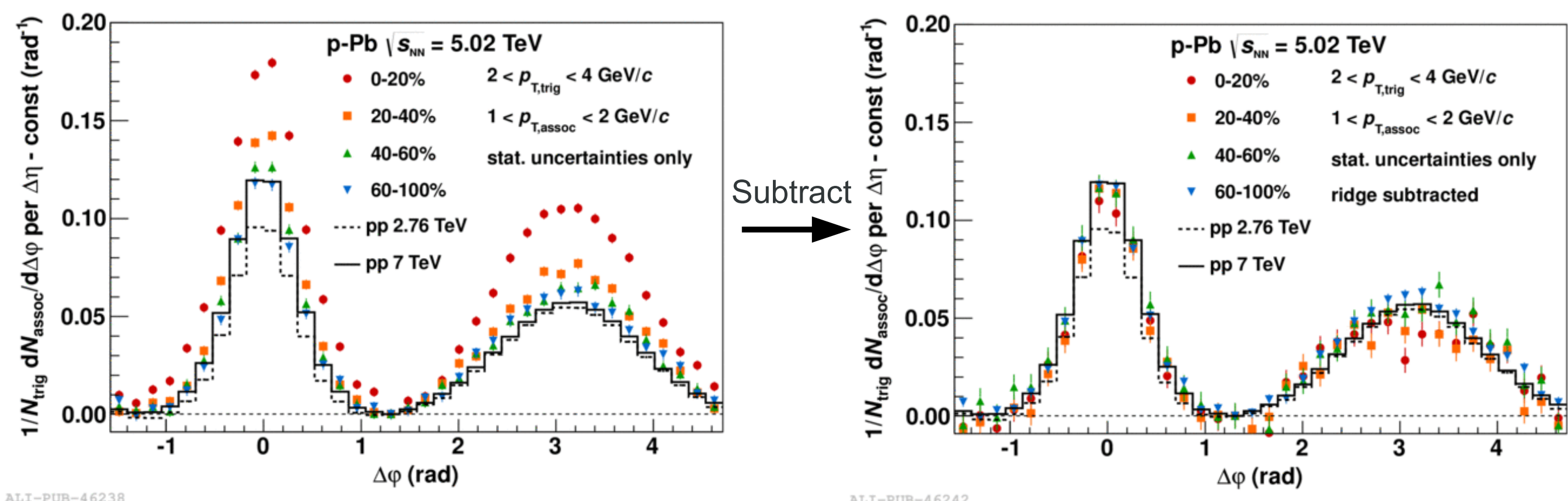

- No significant other multiplicity dependent structures left over 


\section{DHC: CMS and ATLAS}

- CMS

- Reported near-side ridge

- ATLAS

- Confirms two-ridge structure

- Larger acceptance

- More $\mathrm{p}_{\mathrm{T}}$ and multiplicity bins

- Results by the 3 collaborations are qualitatively similar

- Differences in event selection, normalization, acceptance and $\mathrm{p}_{\mathrm{T}}$ ranges, as well as in the per-trigger yield definition make direct comparisons difficult

- A few cases were checked "together" and found to be consistent

- See appendix: arXiv:1302.7018
CMS, PLB 718 (2012) 795

Low mult.

(Ntrack<35)

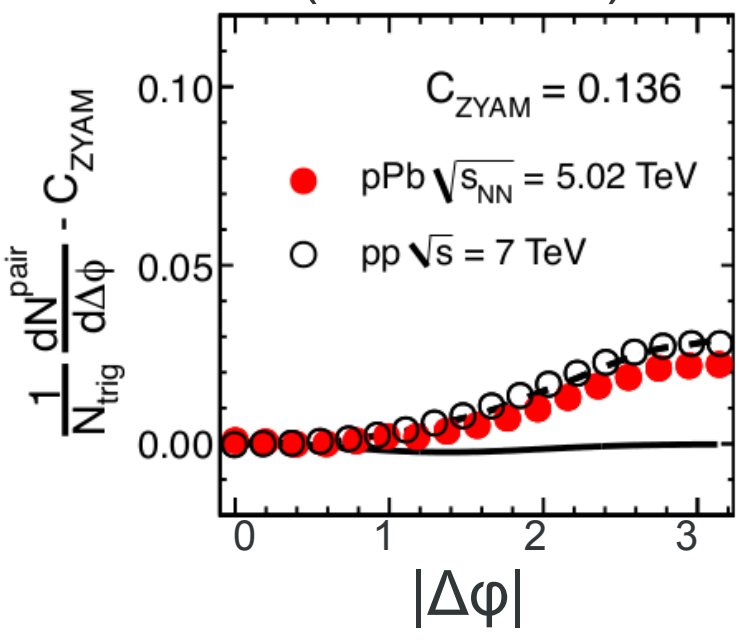

High mult.

(Ntrack $\geq 110)$

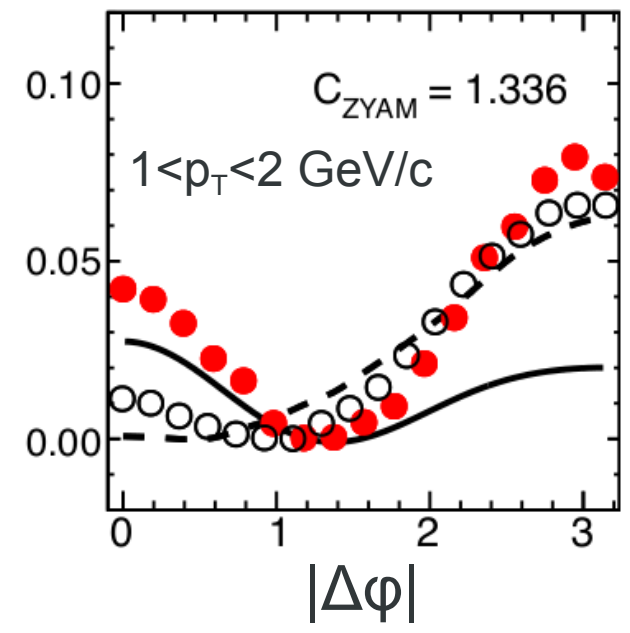

ATLAS, arXiv:1212.5198

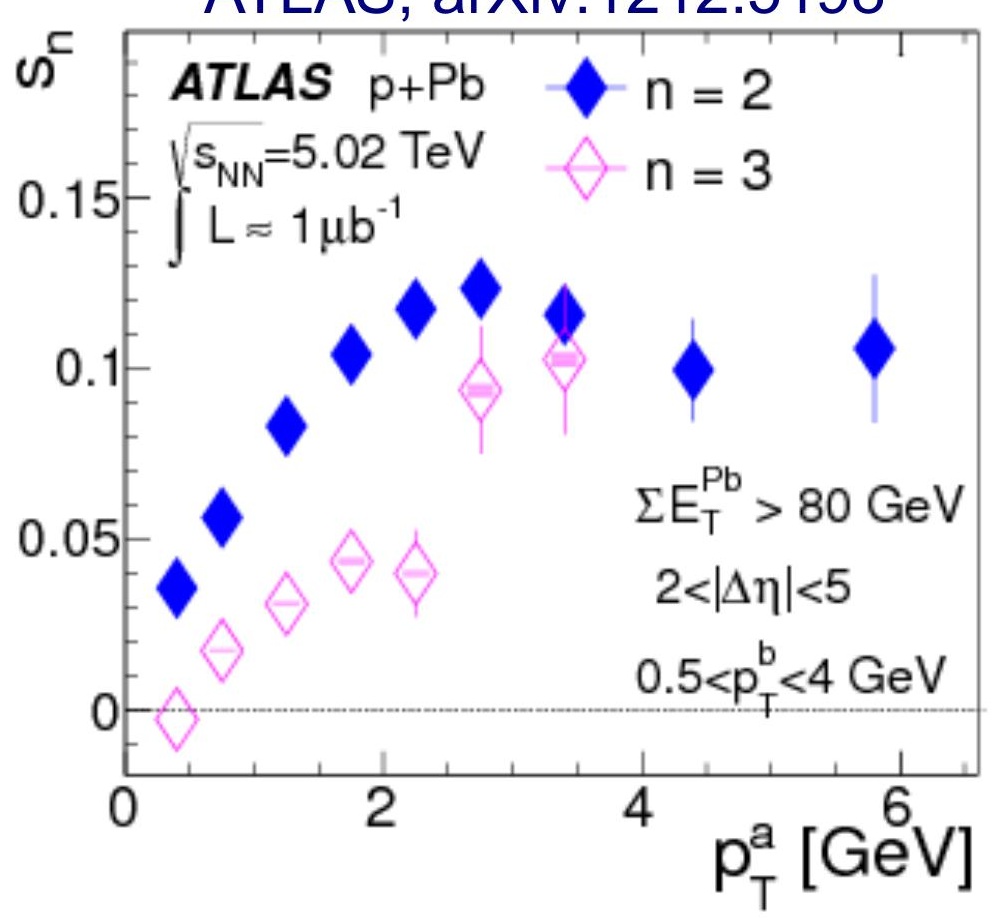




\section{Multi-particle correlations in $\mathrm{PbPb}$}

38

- Cumulants to extract genuine k-particle correlations excluding those from k-1 particles

- Higher order cumulant nicely work out in $\mathrm{PbPb}$, where multiplicity is large

- Definitions

- $\mathrm{v}_{2}\{2\}^{2}=\left\langle\mathrm{v}_{2}\right\rangle^{2}+\sigma_{\mathrm{v}_{2}}^{2}+\delta$ $v_{2} \gg 1 / \sqrt{M}$

- $\mathrm{v}_{2}\{4\}^{2}=\left\langle\mathrm{v}_{2}\right\rangle^{2}-\sigma_{\mathrm{v}_{2}}^{2}$

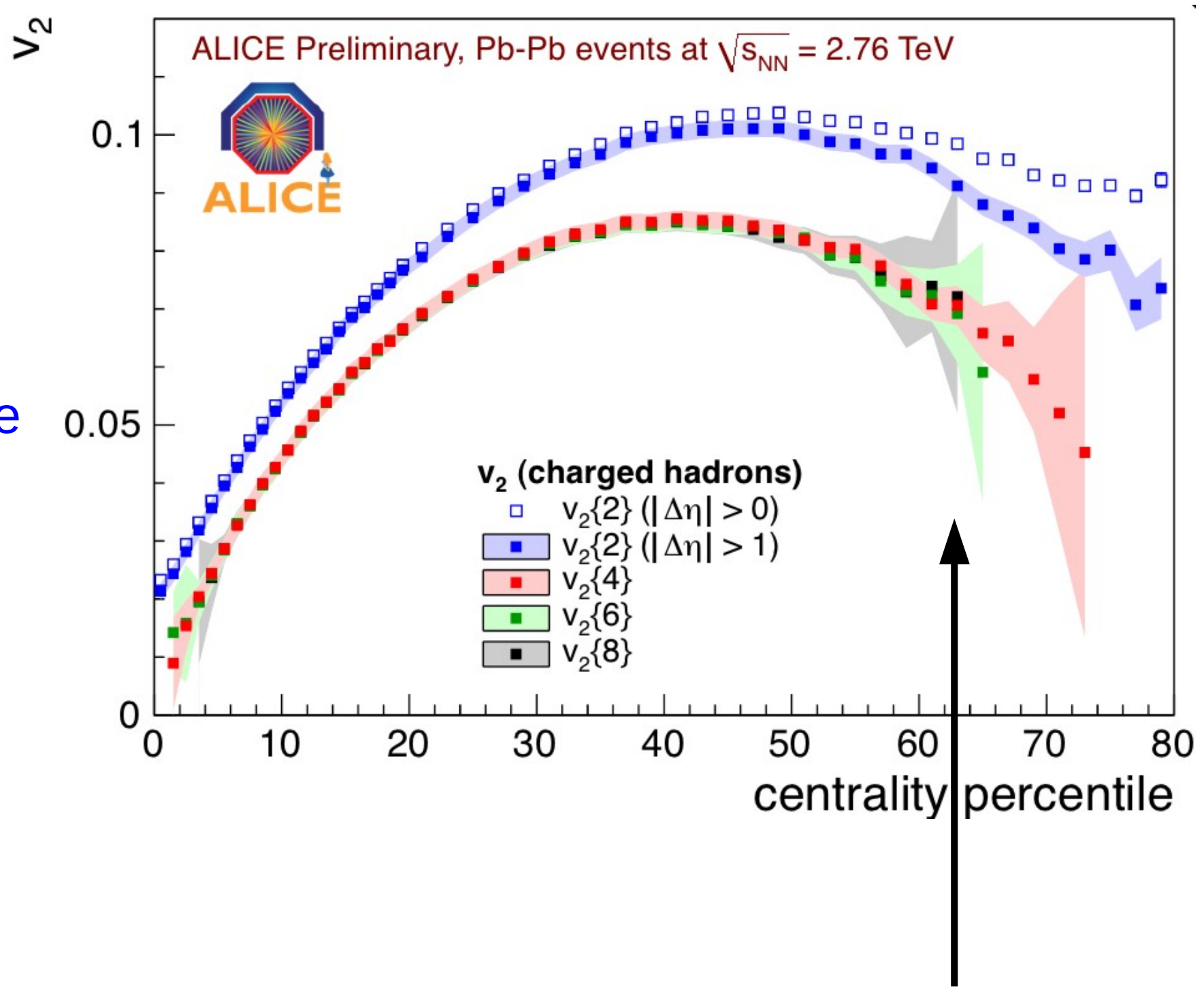

- Care is needed when averaging $<\mathrm{N}_{\mathrm{ch}}>\approx 100$ over $\mathrm{M}$, as cumulants are also sensitive to multiplicity fluctuations 


\section{Multi-particle correlations in pPb}

ATLAS, arXiv:1303.2084
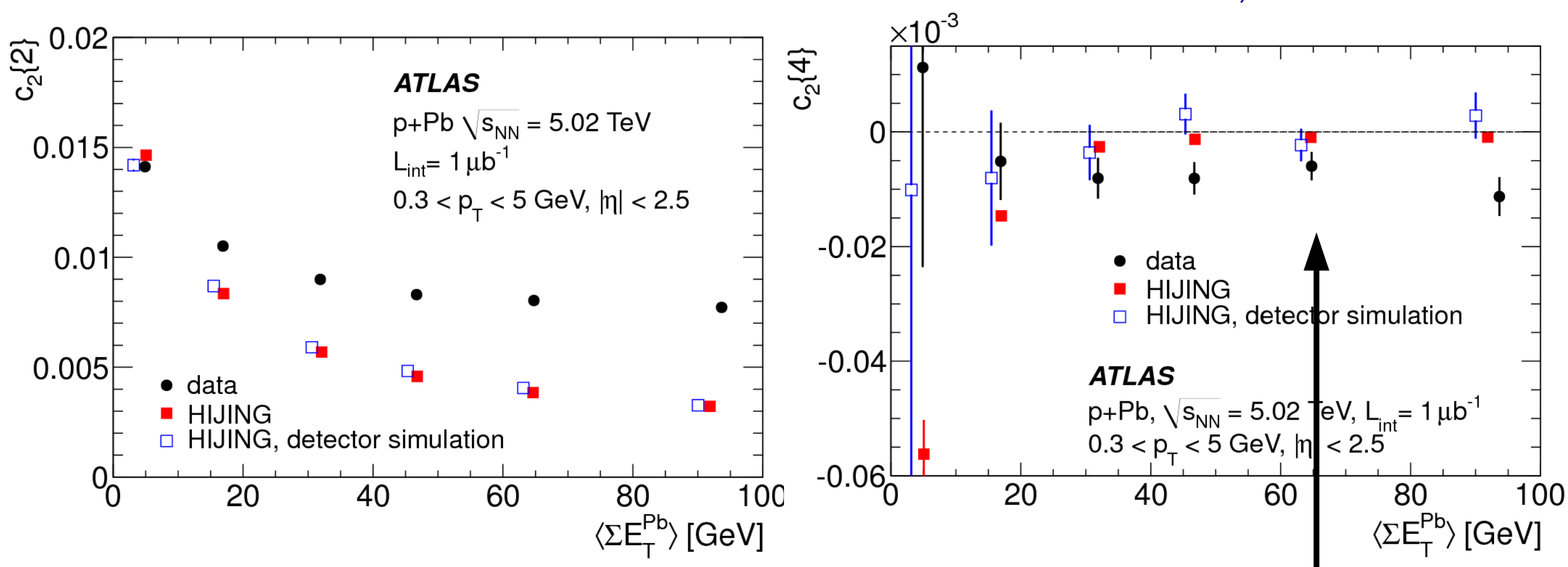

- Second and fourth order cumulant extracted

- Second order above HIJING as expected if additional correlations present

- Fourth order has different trend than HIJING

- In high-multiplicity region there are four or higher particle correlations not present in HIJING 


\section{Integrated $v_{2}$ for $\mathrm{pPb}$}

ATLAS, arXiv:1303.2084

$$
\begin{aligned}
& \text { In PbPb one would expect } \\
& \mathrm{v}_{2}\{2\}^{2}=\left\langle\mathrm{v}_{2}\right\rangle^{2}+\sigma_{\mathrm{v}_{2}}^{2}+\delta
\end{aligned}
$$

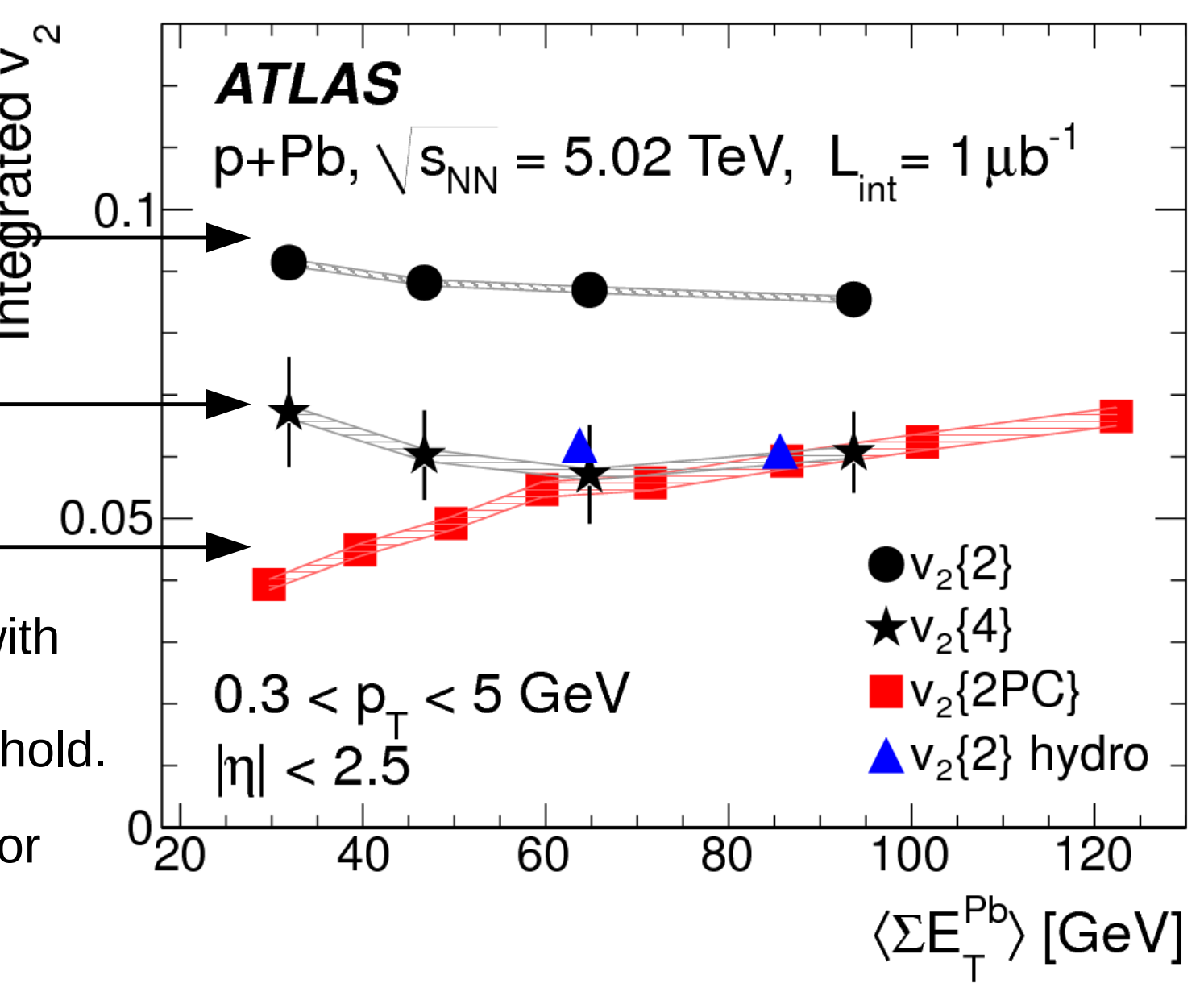

$v_{2}\{4\}$ compatible with $v_{2}\{P C\}$ supports the importance of final state effects, even in $\mathrm{pPb}$. Or else, are there other Glasma contributions (or different theory) which predict four azimuthally correlated particles? 


\section{ALICE configuration (pilot run)}

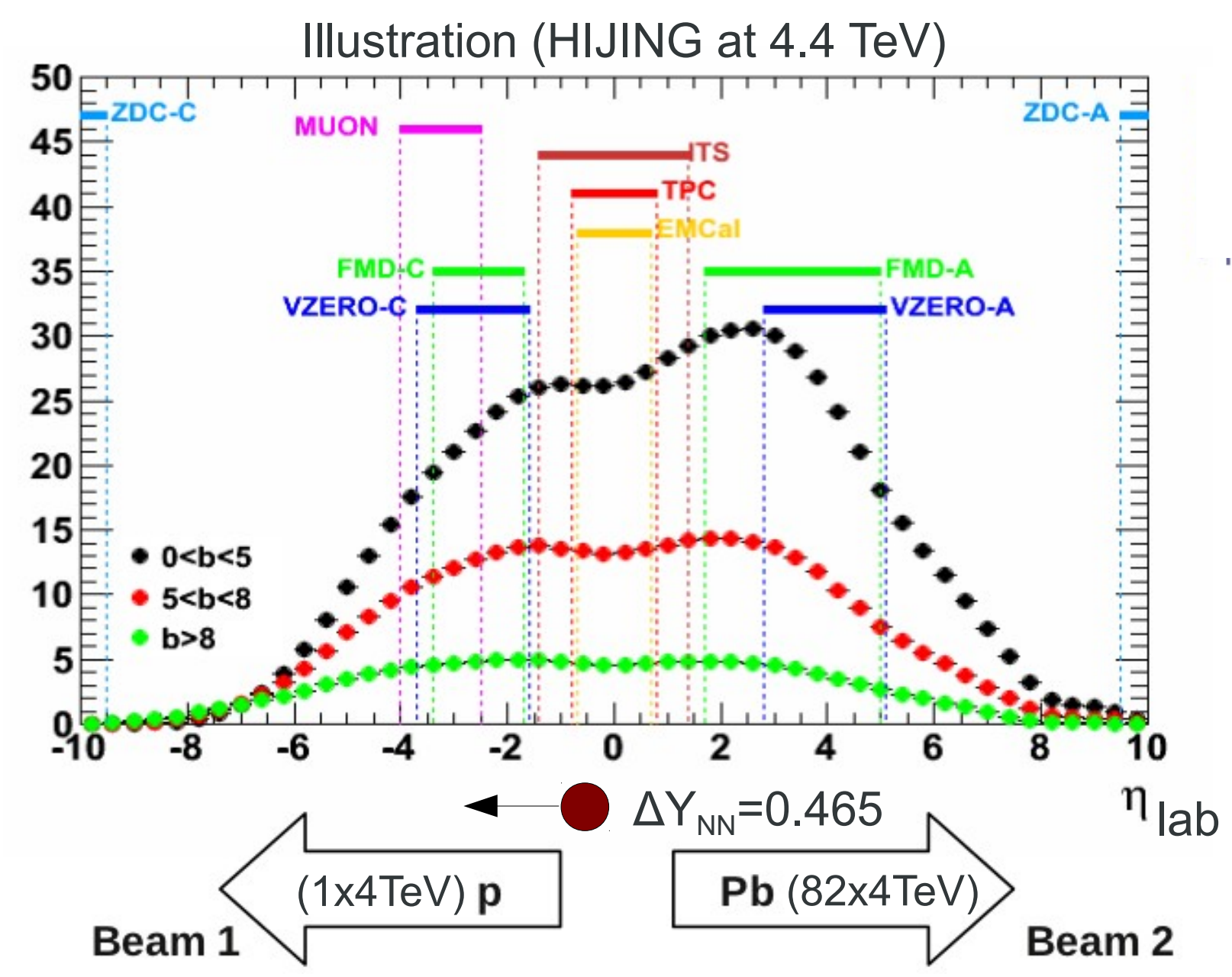

- Trigger

- VZERO-A or C

- ZDC-A or ZDC-C
- Dataset

- One fill (a few millions triggers)

- A part with displaced vertex to have ITS coverage over 6 units in $\eta$ 


\section{Nuclear modification factor}

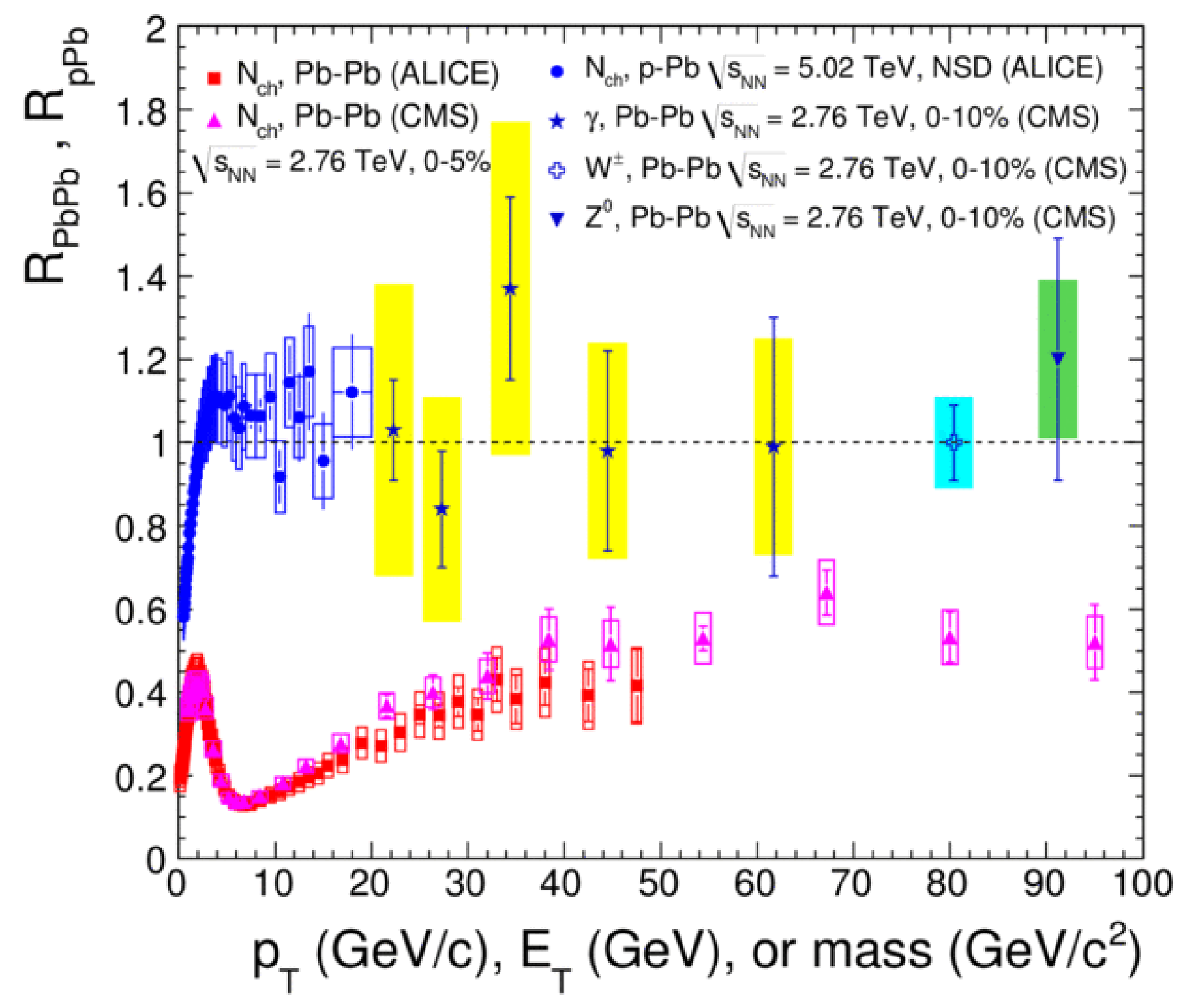


Jet Quenching at RHIC vs LHC in Light of Recent dAu vs pPb Controls RIKEN BNL Research Center Workshop

April 15-17, 2013 at Brookhaven National Laboratory

\section{Jet quenching and fragmentation from an exactly soluble model}

\section{Frashër Loshaj}

Department of Physics \& Astronomy

Stony Brook University

16 April 2013

based on F.L. and D. E. Kharzeev, Int.J.Mod.Phys. E21 (2012) 1250088, arXiv:1111.0493 [hep-ph]; D. E. Kharzeev and F.L., to appear in Phys. Rev. D, arXiv:1212.5857 [hep-ph] 


\section{Outline}

(1) Introduction and motivation

(2) Overview of the model

(3) Jet fragmentation in vacuum

(4) In-medium fragmentation

(5) Summary and Outlook 


\section{Jet quenching - General introduction}

- It is observed at RHIC and LHC that large $p_{T}$ hadron yields are suppressed in heavy ion collisions.

- This suppression is attributed to jet quenching.

- Traditional approaches to jet quenching are:

- Perturbative QCD ( $p Q C D)$

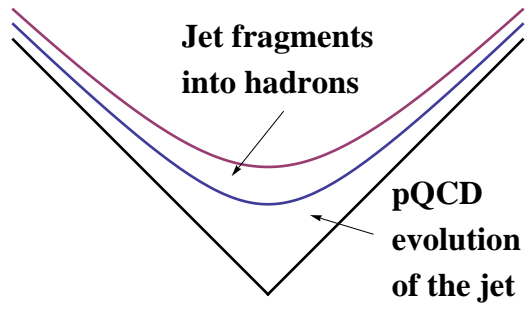

- At some scale $Q_{0}^{2} \sim 1-3 \mathrm{GeV}^{2}, p Q C D$ is not valid anymore.

- Fragmentation functions are used to describe hadronization phenomenologically.

- Gauge/Gravity duality 
General properties of the model

We want to study nonperturbative effects on jet fragmentation. Properties we would like to have:

- Confinement in the presence of light quarks - screening of color charge.

- Chiral symmetry breaking, topology, anomalies.

Other assumptions

- We study very energetic jets, therefore we assume effective dimensional reduction - namely a $1+1$ dimensional theory, where the spatial direction is along jet axis.

- The picture of confinement we adopt is one based on the Abelian projection - we project the non Abelian theory to $N_{c}$ (number of colors) Abelian sectors (e.g. condensation of magnetic monopoles). 
In the large $N_{c}$ approximation we can neglect interference between individual Abelian sectors. We then have $N_{c}$ independent Abelian sectors in the theory. We assume that the dynamics in these sectors is described by the Schwinger model or $Q E D$ in $1+1$ dimensions.

$$
\mathcal{L}=-\frac{1}{4} F_{\mu \nu} F^{\mu \nu}+\bar{\psi}\left(i \gamma^{\mu} \partial_{\mu}-g \gamma^{\mu} A_{\mu}-m_{q}\right) \psi
$$

It is very well known that this theory is exactly soluble when $m_{q}=0$. Dimensional analysis:

$$
[A]=0, \quad[\psi]=1 / 2 \Rightarrow[g]=1
$$

Vector current

$$
J_{V}^{\mu}(x)=\bar{\psi}(x) \gamma^{\mu} \psi(x)
$$

Is conserved. For the massless case, $m_{q}=0$, the axial current

$$
J_{A}^{\mu}(x)=\bar{\psi}(x) \gamma^{\mu} \gamma^{5} \psi(x)
$$

is conserved classically. Quantum corrections give rise to axial anomaly. We will illustrate some of the properties in the following slides. 
The theta angle [Coleman, 1975]

- In $1+1$ dimensions, in the $A_{1}=0$ gauge, we can write

$$
F_{01}=g \partial_{1}^{-1} j_{0}+F
$$

- the constant electric field is allowed in $1+1$ dimensions

$\mathbf{L}$

$$
\begin{aligned}
& F \bigodot F+g \quad \bigcirc F \\
& \boldsymbol{F} \bigodot \boldsymbol{F}-\boldsymbol{g} \odot \boldsymbol{F}
\end{aligned}
$$

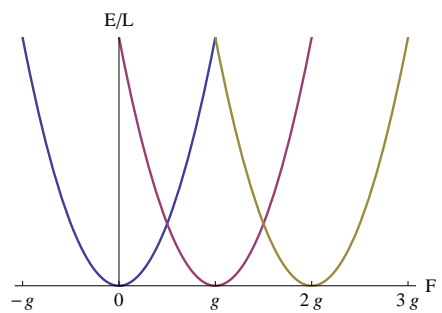

Ground state energy per unit length.
- Energy difference

$$
\Delta E=\frac{1}{2} \int d x\left[F_{01}^{2}-F^{2}\right]=\frac{1}{2} L\left[(F \pm g)^{2}-F^{2}\right]
$$

- Pair creation favorable for $|F|>\frac{1}{2} g$, until $|F| \leq \frac{1}{2} g$
Physics is periodic with period $g$.

$$
\theta=\frac{2 \pi F}{g}
$$




\section{Confinement and chiral symmetry breaking}

Let's consider two infinitely heavy quarks with charges $\pm g$, separated by $2 L$

$$
J_{\text {ext }}^{0}(x)=\delta(z+L)-\delta(z-L)
$$

The potential can be computed to be

$$
\begin{aligned}
V(L) & =2 \pi^{2} M^{2} 2 L+\frac{g \sqrt{\pi}}{2}\left(1-e^{-\frac{g}{\sqrt{\pi}} 2 L}\right) \\
M^{2} & \propto m_{q} g
\end{aligned}
$$

$m_{q}=0$ - Charge screening.

$m_{q} \neq 0$ - Linear confinement.

Axial anomaly in $1+1$ dimensions has the form

$$
\partial_{\mu} J_{A}^{\mu}=\frac{g}{2 \pi} \epsilon^{\mu \nu} F_{\mu \nu}=\frac{g}{\pi} F_{01}
$$

Using Gauss' theorem

$$
\int d z d t \partial_{\mu} J_{A}^{\mu}=Q_{A}(t=\infty)-Q_{A}(t=-\infty)=N_{R}-N_{L}=\frac{g}{2 \pi} \int d z d t \epsilon^{\mu \nu} F_{\mu \nu}
$$

If we have a nonzero background electric field $F$

$$
N_{R}-N_{L}=\frac{g}{\pi} \int d z d t F
$$

Chiral symmetry is not spontaneously broken, but explicitly through anemaly: 


\section{Abelian bosonization}

In $1+1$ dimensions we can use bosonization

$$
J_{V}^{\mu}(x)=\bar{\psi}(x) \gamma^{\mu} \psi(x)=-\frac{1}{\sqrt{\pi}} \epsilon^{\mu \nu} \partial_{\nu} \phi(x)
$$

where $\phi$ is a real scalar field. Using the identity $\gamma^{\mu} \gamma^{5}=-\epsilon^{\mu \nu} \gamma_{\nu}$, we have

$$
J_{A}^{\mu}(x)=\bar{\psi}(x) \gamma^{\mu} \gamma^{5} \psi(x)=\frac{1}{\sqrt{\pi}} \partial^{\mu} \phi(x)
$$

Bosonized Lagrangian can be written as $\left(m_{q}=0\right)$

$$
\begin{aligned}
\mathcal{L} & =-\frac{1}{4} F_{\mu \nu} F^{\mu \nu}+\frac{1}{2} \partial_{\mu} \phi \partial^{\mu} \phi+\frac{g}{\sqrt{\pi}} \epsilon^{\mu \nu} \partial_{\nu} \phi A_{\mu} \\
& =\frac{1}{2} F_{01}^{2}+\frac{1}{2} \partial_{\mu} \phi \partial^{\mu} \phi-\frac{g}{\sqrt{\pi}} \epsilon^{\mu \nu} \partial_{\nu} A_{\mu} \\
& =\frac{1}{2} F_{01}^{2}+\frac{1}{2} \partial_{\mu} \phi \partial^{\mu} \phi+\frac{g}{\sqrt{\pi}} \phi F_{01}
\end{aligned}
$$

We can integrate $F_{01}$ (e.g. choose the gauge $A_{0}=0$, Jacobian of $\int \mathcal{D} A_{1} \rightarrow \int \mathcal{D} F_{01}$ doesn't depend on $\left.F_{01}\right)$ to get

$$
\mathcal{L}_{\text {eff }}=\frac{1}{2} \partial_{\mu} \phi \partial^{\mu} \phi-\frac{1}{2} \frac{g^{2}}{\pi} \phi^{2}
$$

This is just a free massive scalar field, with mass $m=\frac{g}{\sqrt{\pi}}$. 


\section{Anomaly equation}

From Maxwell's equations

$$
\partial_{1} F^{10}=-\frac{g}{\sqrt{\pi}} \partial_{1} \phi \Rightarrow F_{01}=\frac{-g}{\sqrt{\pi}} \phi
$$

We have assumed that fields vanish at infinity.

Equation of motion for $\phi$ is just the Klein-Gordon equation

$$
\left(\square+\frac{g^{2}}{\pi}\right) \phi=0
$$

Using bosonization relations

$$
\partial_{\mu} J_{A}^{\mu}=\partial_{\mu}\left(\frac{1}{\sqrt{\pi}} \partial^{\mu} \phi\right)=\frac{1}{\sqrt{\pi}} \square \phi
$$

Using EOM for $\phi$ and relation between $F_{01}$ and $\phi$, we get

$$
\partial_{\mu} J_{A}^{\mu}=\frac{g}{\pi} F_{01}=\frac{g}{2 \pi} \epsilon^{\mu \nu} F_{\mu \nu}
$$

This is the same expression we saw before for the axial anomaly for QED in $1+1$ dimensions. 


\section{Adding an external source}

Consider a general external source $J_{\text {ext }}^{\mu}(x)=j_{\text {ext }}^{\mu}(z, t)$. We use the parametrization

$$
J_{e x t}^{\mu}(x)=-\frac{1}{\sqrt{\pi}} \epsilon^{\mu \nu} \partial_{\nu} \phi_{\text {ext }}(x)
$$

In the same way as before, we get the effective Lagrangian

$$
\mathcal{L}_{\text {eff }}=\frac{1}{2}\left(\partial_{\mu} \phi\right)^{2}-\frac{1}{2} \frac{g^{2}}{\pi}\left(\phi+\phi_{e x t}\right)^{2}
$$

Which gives

$$
\left(\square+m^{2}\right) \phi(x)=-m^{2} \phi_{e x t}(x)
$$

- Corresponds to a massive scalar field, coupled to a classical source.

- Coherent particle creation. 


\section{Jet fragmentation by quark-antiquark pair production}

We consider the source (Casher, Kogut and Susskind,1974)

$$
\underbrace{J_{\text {ext }}^{0}(x)=\delta(z-t) \theta(z)-\delta(z+t) \theta(-z)}_{\text {Quark anti quark pair, moving back to back. }}
$$

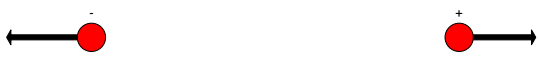

Using bosonization relations we have

$$
\phi_{\text {ext }}(x)=-\theta(t-z) \theta(t+z)
$$

We therefore have to solve

$$
\left(\square+m^{2}\right) \phi=m^{2} \theta(t-z) \theta(t+z)
$$

The solution to the equation of motion is (F.L. and D. E. Kharzeev, arXiv:1111.0493 [hep-ph])

$$
\phi(x)=\theta(t+z) \theta(t-z)\left(1-J_{0}\left(m \sqrt{x^{2}}\right)\right)
$$

where $x^{2}=t^{2}-z^{2}$. 


\section{Jet fragmentation (cont'd)}

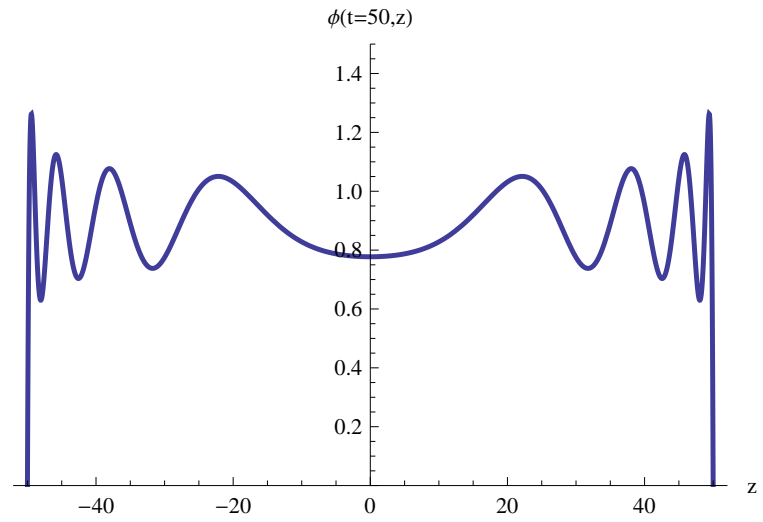

Quark anti quark pair, moving back to back.

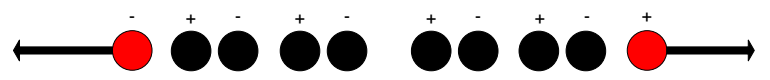

Quark anti quark pair, moving back to back.

[D. E. Kharzeev and F.L., arXiv:1212.5857 [hep-ph]]

(Anti-)Kinks correpond to (anti-)fermions. Quark fragmentation in the $\theta$-vacuum. 


\section{Particle creation by a general source}

Let's consider

$$
\left(\square+m^{2}\right) \phi(x)=f(x)
$$

It is known that

$$
\begin{aligned}
\frac{d N}{d p} & =\frac{|\tilde{f}(p)|^{2}}{2 E_{p}} \\
\tilde{f}(p) & =\int d^{2} x e^{i p \cdot x} f(x), \quad E_{p}=\sqrt{p^{2}+m^{2}}
\end{aligned}
$$

For jets with finite energy - quarks move with velocity $v$

$$
j_{\text {ext }}^{0}(x)=\delta(z-v t) \theta(z)-\delta(z+v t) \theta(-z)
$$

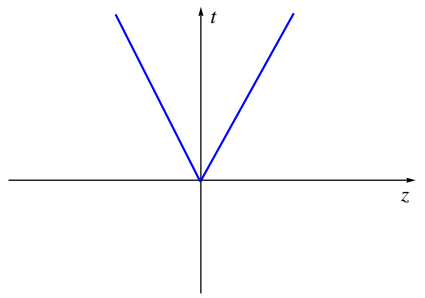

Velocity is calculated from

$$
\begin{gathered}
v=\frac{p_{q}}{E_{q}}=\frac{p_{\text {jet }}}{\sqrt{p_{j e t}^{2}+Q_{0}^{2}}} \\
\frac{d N}{d p}=2 \pi \frac{v^{2} m^{4}}{E_{p}\left(E_{p}^{2}-v^{2} p^{2}\right)^{2}}
\end{gathered}
$$

We fix $Q_{0}$ by comparing our result to experimental data. 


\section{Rapidity distribution}

We now change variables to $y=\frac{1}{2} \ln \frac{E_{p}+p}{E_{p}-p}$

$$
\frac{d N}{d y}=2 \pi \frac{v^{2}}{\left(\cosh ^{2} y-v^{2} \sinh ^{2} y\right)^{2}}
$$

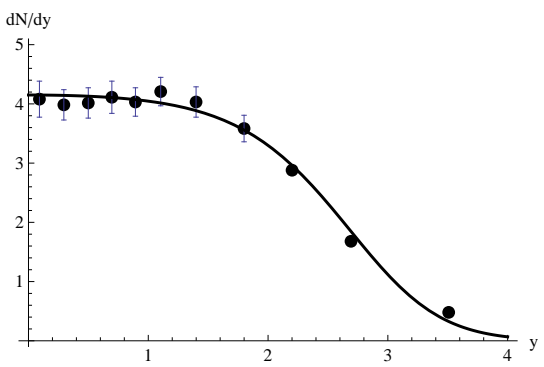

Comparison to experimental data [Aiphara (TPC/Two Gamma Collaboration), 1988], for $\sqrt{s}=29 \mathrm{GeV}$

- $Q_{0}$ is fixed by above fit. We get $Q_{0} \approx 1.8 \mathrm{GeV}$.

- Since $\sqrt{s}$ is small, the effect of jet evolution is small and our model fits the data well. 


\section{Fragmentation functions}

Fragmentation function for $e^{+} e^{-}$annihilation

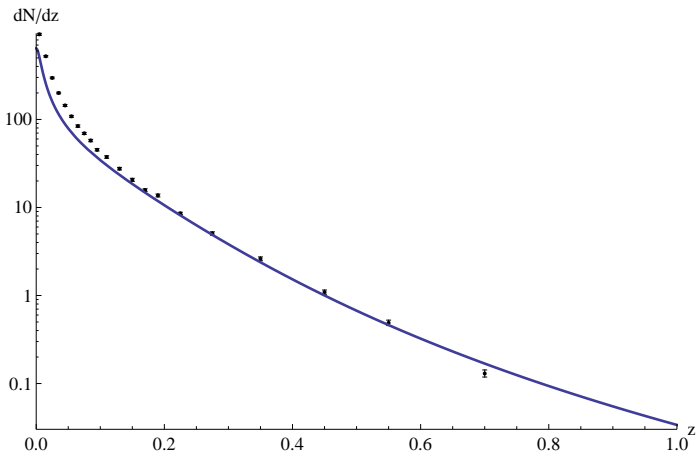

Charged particle distribution for $\sqrt{s}=201.7 \mathrm{GeV}$, [Abbiendi (OPAL Collaboration), 2003].

- Reasonable agreement with the data for $\frac{p}{p_{j e t}}=z>0.1$.

- By fitting to data at different center of mass energies $m \simeq 0.6 \mathrm{GeV}$.

- Enhancement at small $z$ and suppression at large $z$ are attributed to $p Q C D$ evolution of the jet, which is not included in our model. 
Medium modified fragmentation functions [CMS Collaboration, 2012], [PHENIX Collaboration, 2012]
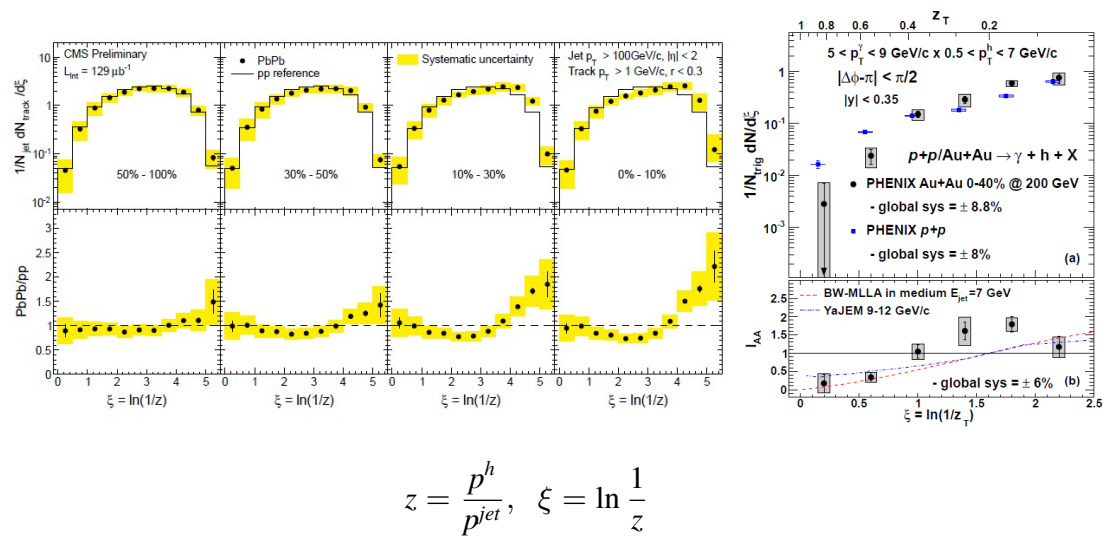

- There is enhancement of soft particles for central collisions.

- For the most central collisions, we also see a depletion of particles for intermediate $\xi$. 


\section{In-medium scattering}

We consider a very simple model: static scatterers, no expansion of the medium. [D. E. Kharzeev and F.L., arXiv:1212.5857 [hep-ph]]

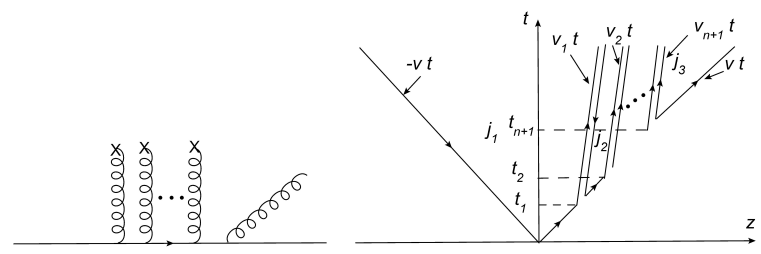

We have three types of currents and their Fourier transform is

$$
\begin{aligned}
& \tilde{j}_{1}^{0}(p)=\frac{i p}{E_{p}-v p}\left[\frac{2 v}{E_{p}+v p}-\frac{v-v_{1}}{E_{p}-v_{1} p} e^{i\left(E_{p}-v p\right) t_{1}}\right] \\
& \tilde{j}_{2}^{0}(p)=\frac{-i p}{E_{p}-v p}\left[\frac{v-v_{2}}{E_{p}-v_{2} p} e^{i\left(E_{p}-v p\right) t_{2}}-\frac{v-v_{1}}{E_{p}-v_{1} p} e^{i\left(E_{p}-v p\right) t_{1}}\right] \\
& \tilde{j}_{3}^{0}(p)=\frac{i p}{E_{p}-v p} \frac{v-v_{n+1}}{E_{p}-v_{n+1} p} e^{i\left(E_{p}-v p\right) t_{n+1}}
\end{aligned}
$$




\section{Nonperturbative LPM effect}

Total momentum distribution is given by

$$
\frac{d N^{\text {med }}}{d p}=\frac{1}{2 E_{p}}|\tilde{f}(p)|^{2}=\frac{1}{2 E_{p}}\left(\left|\tilde{f}_{1}(p)\right|^{2}+\sum_{i=1}^{N-1}\left|\tilde{f}_{2}(p)\right|^{2}+\left|\tilde{f}_{3}(p)\right|^{2}\right)
$$

where as before we construct $f_{i}$ 's from $j_{i}^{0}$ 's.

$\tilde{j}_{2}^{0}(p)$ responsible for soft radiation. We can define the formation time

$$
t_{f}=\frac{1}{E_{p}-v p} \simeq \frac{2 p}{m^{2}}
$$

Similar to the perturbative result $t_{f}=\frac{2 \omega}{k^{2}}$. Radiation is suppressed when mean free path $\lambda=t_{2}-t_{1}<<t_{f}$ - LPM effect. 


\section{Medium fragmentation functions - results}

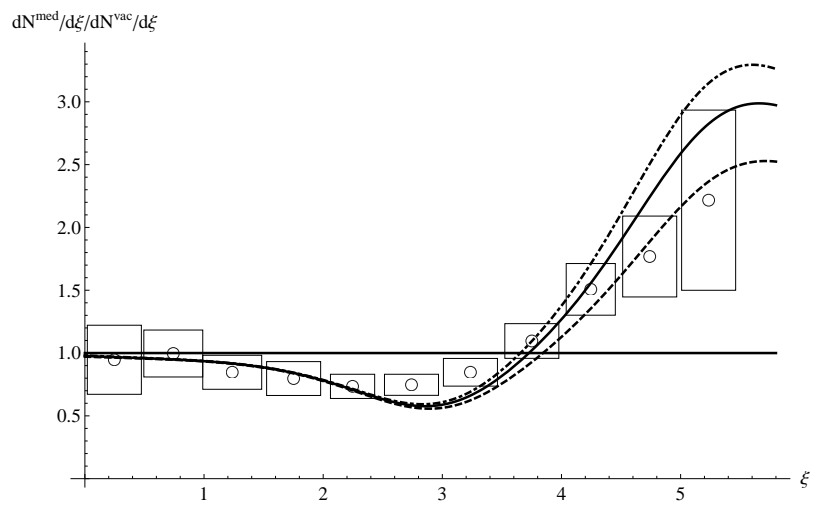

The ratio of in-medium and vacuum fragmentation functions for $p_{j e t}=120 \mathrm{GeV}$. The first scattering occurs at $t_{1} \simeq 1 \mathrm{fm}$, which is the assumed thermalization time. The length of the medium is $L=5 \mathrm{fm}$. The curves correspond to mean free paths of $\lambda=0.57,0.4$ and $0.2 \mathrm{fm}$ from top to bottom respectively.

- Scaling for small $\xi$ - Nonperturbative LPM effect.

- Suppression at intermediate $\xi$ is a result of the partial screening of the color charge of the jet by a comoving medium-induced gluon. 


\section{Transverse-momentum difference - $D_{A A}$ (Talk by J. Putschke)}

We study jet modification using another observable proposed in [STAR Collaboration, arXiv:1302.6184 [nucl-ex]]

$$
D_{A A}(p)=Y_{A u-A u}(p)\langle p\rangle_{A u-A u}-Y_{p-p}(p)\langle p\rangle_{p-p}
$$

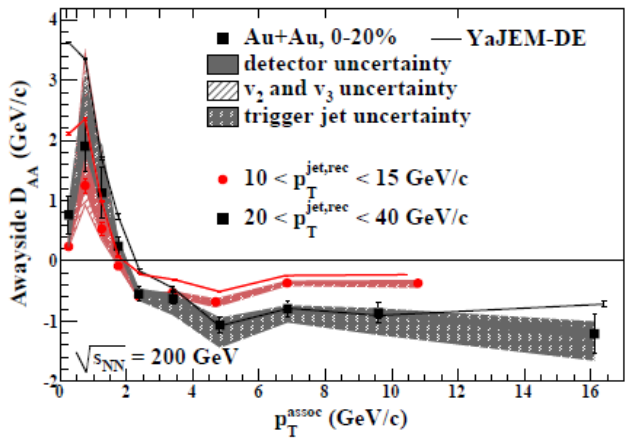

$Y$ are yields in $A u-A u$ and $p-p$ and $\langle p\rangle$ is the average momentum inside a bin.

In our case

$$
D_{A A}(p)=\langle p\rangle \int_{\text {bin with average }\langle p\rangle} d p^{\prime} \frac{d N^{\text {med }}}{d p^{\prime}}-\langle p\rangle \int_{\text {bin with average }\langle p\rangle} d p^{\prime} \frac{d N^{\text {vac }}}{d p^{\prime}}
$$




\section{Transverse-momentum difference - $D_{A A}$ - Results}

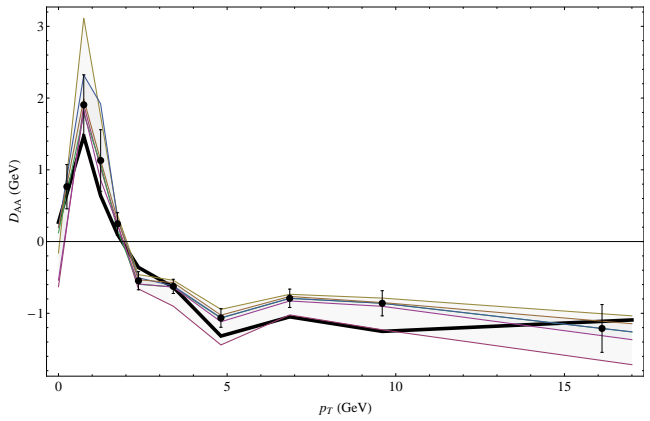

Circles show the data for the awayside momentum difference for $20<p_{T}^{j e t, r e c}<40 \mathrm{GeV}$ and shaded areas show jet energy scale, v2/v3 and detector uncertainties (taken from [STAR Collaboration, arXiv:1302.6184 [nucl-ex]]). Calculations are done using $\lambda=0.4$ fm (solid black line). 


\section{Transverse-momentum difference - $D_{A A}$ - Results}

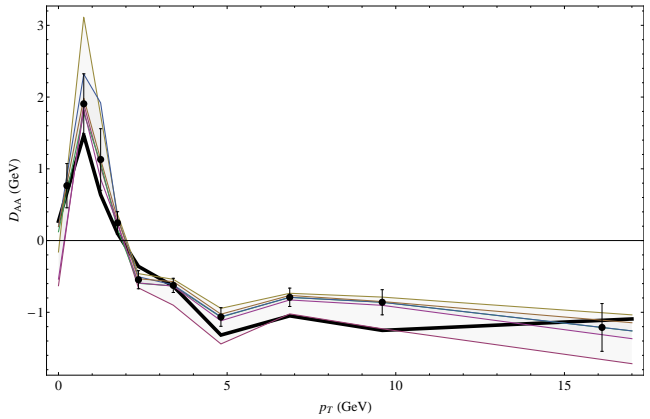

Circles show the data for the awayside momentum difference for $20<p_{T}^{j e t, r e c}<40 \mathrm{GeV}$ and shaded areas show jet energy scale, v2/v3 and detector uncertainties (taken from [STAR Collaboration, arXiv:1302.6184 [nucl-ex]]). Calculations are done using $\lambda=0.4$ fm (solid black line).

$D_{A A}$ was also addressed in [T. Renk, Phys.Rev. C87 (2013) 024905] In this approach jet broadening contributes to jet modification and energy loss.

In our $1+1$ model we only have energy redistribution in the longitudinal (along jet axis) direction.

Two models could be distinguished experimentally by looking for the modification of the jet cone. 


\section{Summary and Outlook}

- We considered an effective theory of jet fragmentation based on an exactly soluble model

- This model incorporated confinement in the presence of light quarks, topology and chiral symmetry breaking.

- Topology was shown to be responsible for particle production.

- Would be interesting to investigate further the effects of topology in high energy processes.

- A systematic derivation of the dimensionally reduced theory for high energy processes is needed. 


\title{
Jet modification in
}

$A-A, P-A$ and $D-A$

at RHIC and LHC

(within HT scheme)

\author{
Abhijit Majumder \\ Wayne State University
}

RIKEN BNL workshop on Jet Quenching at RHIC and LHC ... , BNL, April 15-17 


\section{Outline}

- The present status of HT jet modification

- Some background on HT E-loss + MC routines

- Observables that are easily understood

- Observables less easily understood

- Really difficult observables!

- What else are we doing... 


\section{What goes into HT-E-loss}
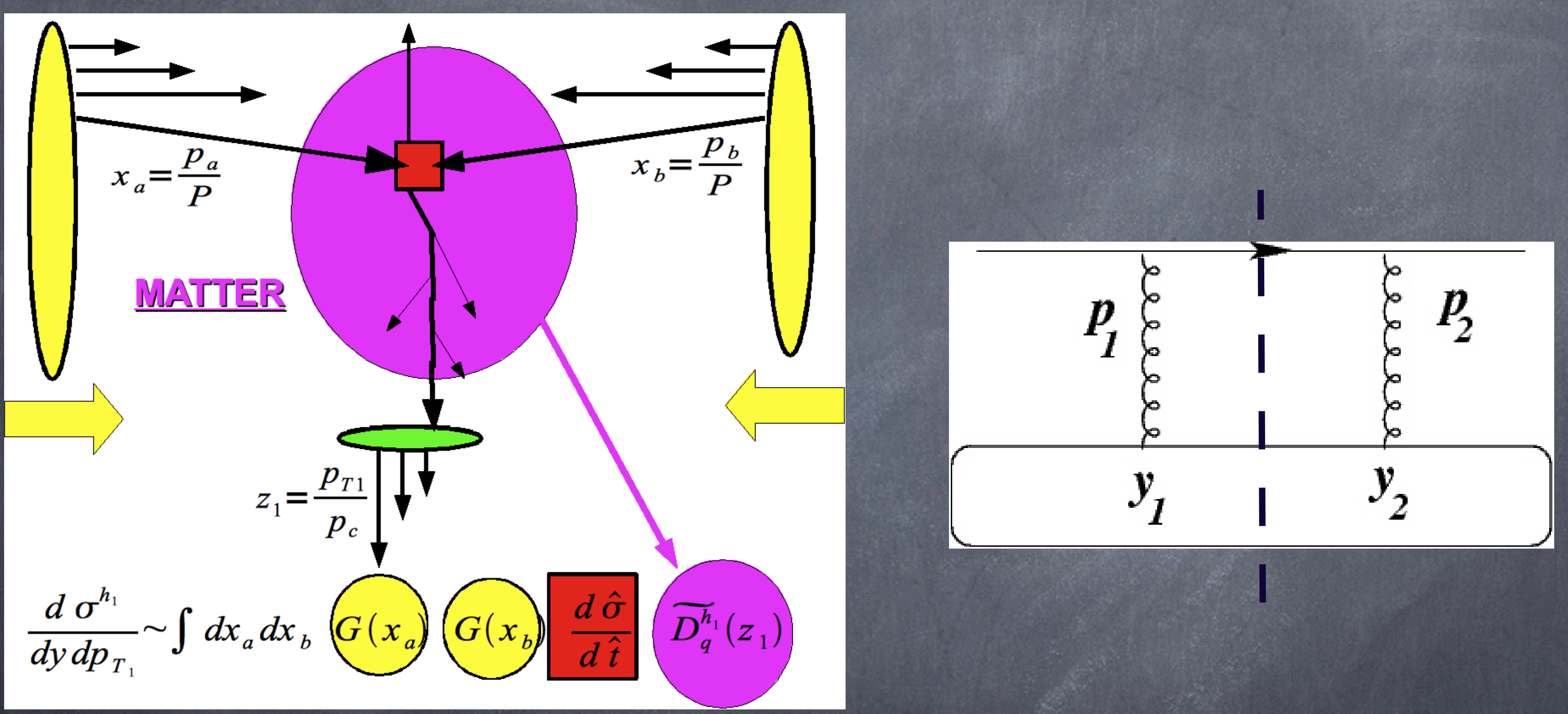

Double factorized perturbative approach 


\section{What goes into this calculation}

Modification derived in A-DIS and applied to HIC (implied factorization of hard scattering)

Jet scale assumed much harder than medium scale (factorization of jet from soft matrix element)

Multiple scatterings resummed in single gluon emission

Expansion in powers of $\Lambda^{2} / Q^{2}$

DGLAP $\mathrm{K}_{\mathrm{T}}^{2}$ systematics assumed for multiple emissions Fluid dynamical simulation of medium and trans. coeffs. 


\section{How the medium affects the parton.}

A parton in a jet shower, has momentum components $q=\left(q^{-}, q^{+}, q_{T}\right)=\left(1, \lambda^{2}, \lambda\right) Q, Q:$ Hard scale, $\lambda \ll 1, \lambda Q \gg \Lambda_{Q C D}$

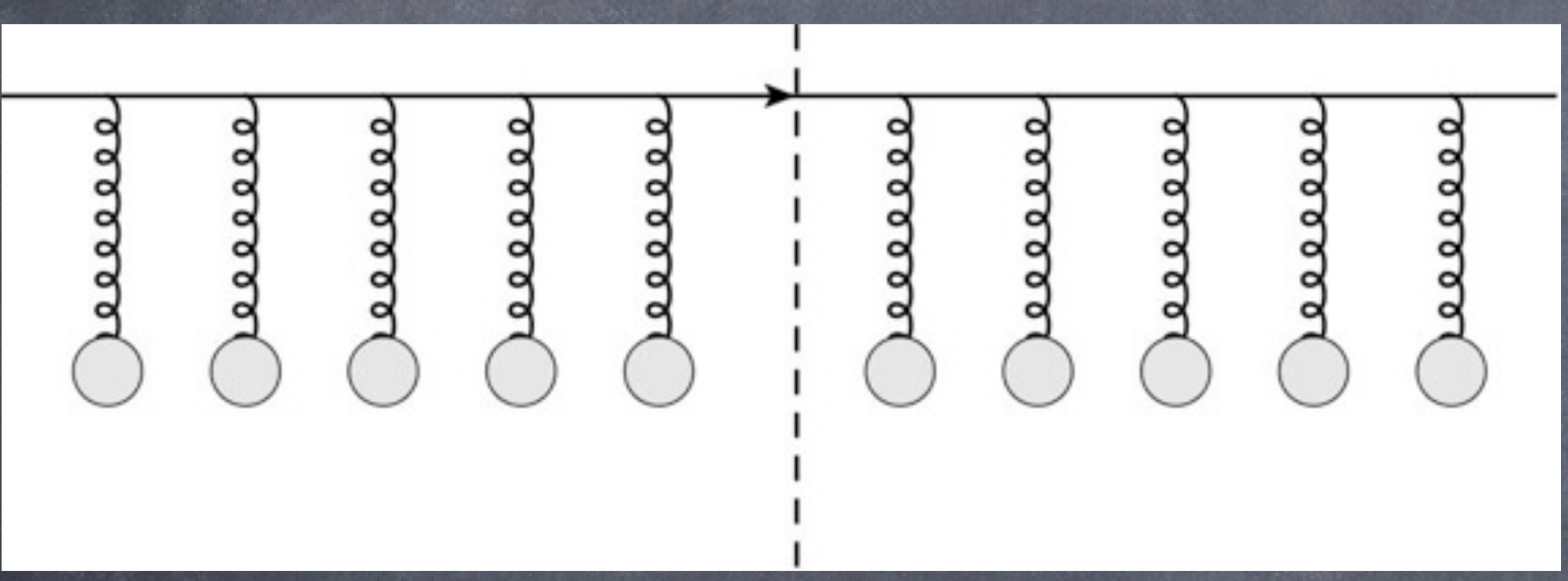

$$
\begin{aligned}
& p^{+}=\frac{p^{0}+p_{z}}{\sqrt{2}} \\
& p^{-}=\frac{p^{0}-p_{z}}{\sqrt{2}}
\end{aligned}
$$

hence, gluons have

$$
k_{\perp} \sim \lambda Q, \quad k^{+} \sim \lambda^{2} Q
$$

could also have $k^{-} \sim \lambda Q$

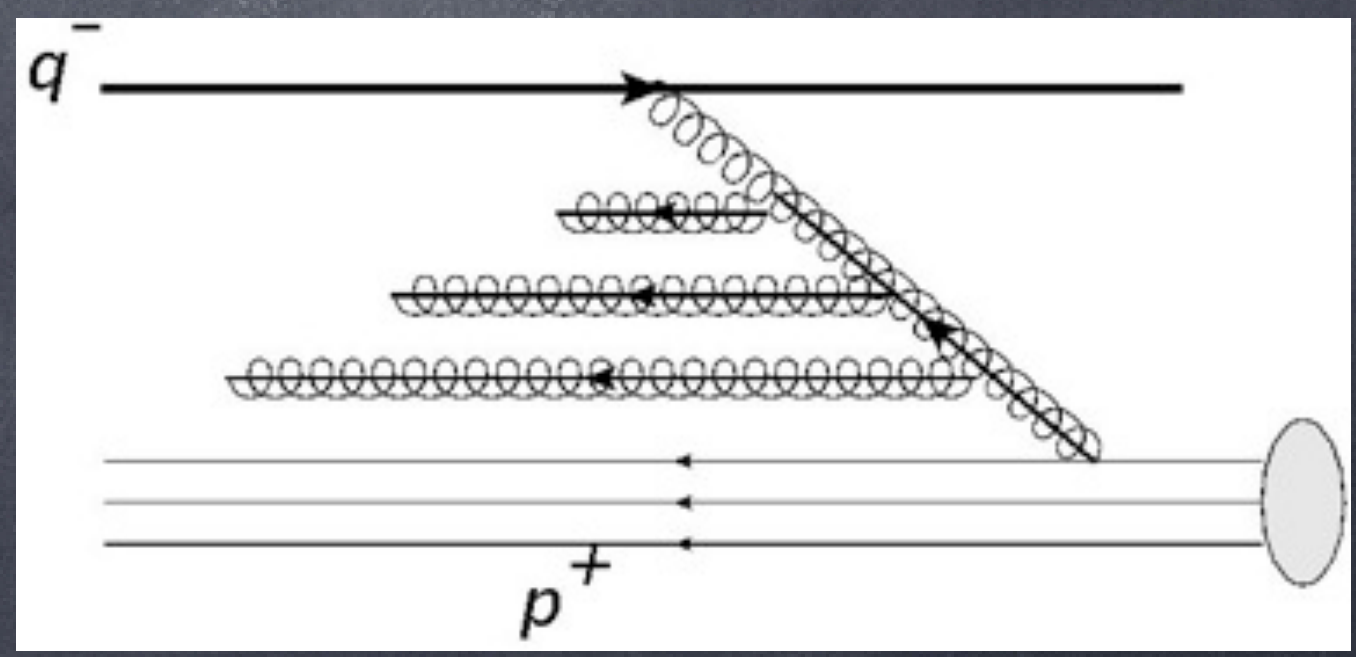




\section{So what do we get from resumming?} transverse broadening
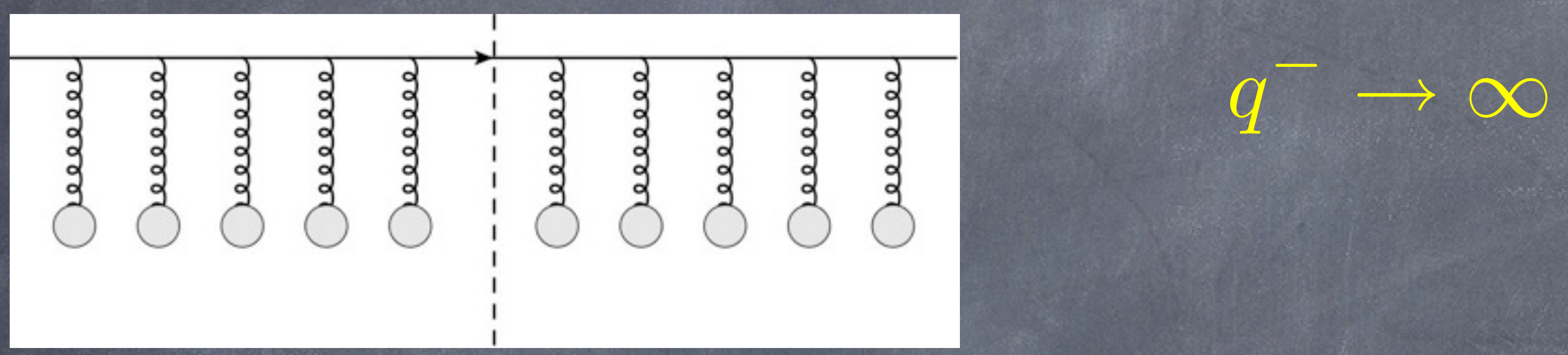

Assuming independent scattering of nucleons gives a diff. equation These cannot be soft, they must have transverse momentum, Glauber gluons.

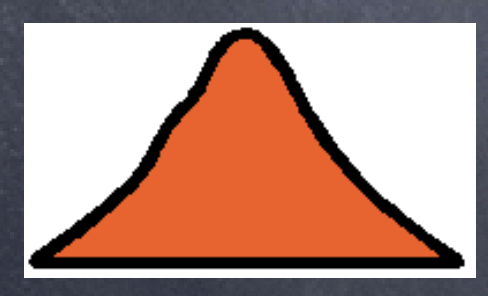

$$
\frac{\partial f\left(p_{\perp}, t\right)}{\partial t}=\nabla_{p_{\perp}} \cdot D \cdot \nabla_{p_{\perp}} f\left(p_{\perp}, t\right)
$$

$$
\left\langle p_{\perp}^{2}\right\rangle=4 D t \quad \hat{q}=\frac{p_{\perp}^{2}}{t}=\frac{2 \pi^{2} \alpha_{s} C_{R}}{N_{c}^{2}-1} \int d \tilde{t}\left\langle F^{\mu \alpha}(\tilde{t}) v_{\alpha} F_{\mu}^{\beta}(0) v_{\beta}\right\rangle
$$


There are a bunch of medium properties which modify the parton and frag. func.

$$
\hat{q}, \hat{e}=d E / d L \text { and } f=d N / d L
$$

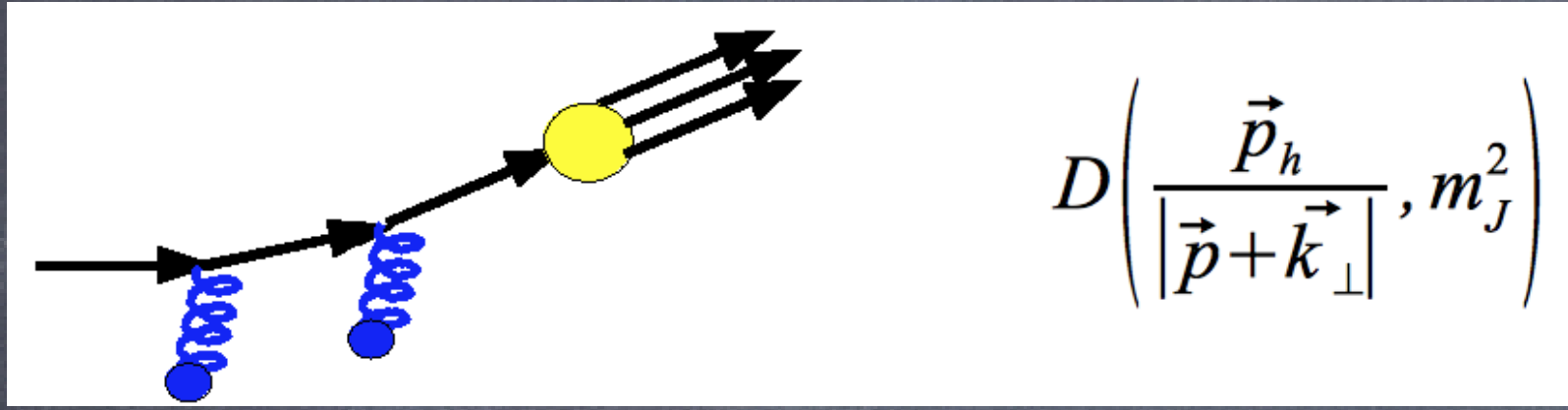

$\hat{q}=\frac{\left\langle p_{T}^{2}\right\rangle_{L}}{L} \quad \begin{aligned} & \text { Transverse momemtum } \\ & \text { diffusion rate }\end{aligned}$

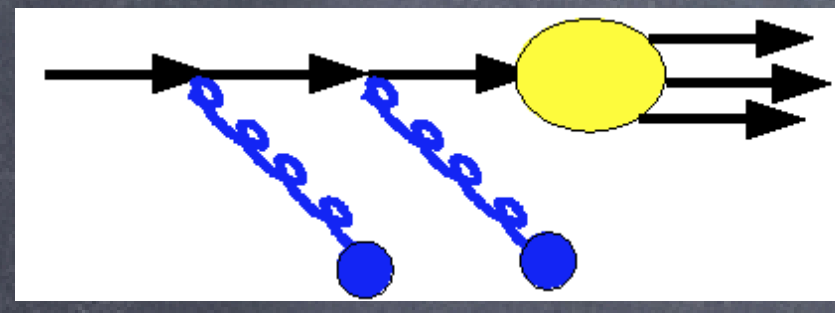

$$
D\left(\frac{p_{h}}{p-k}, m_{J}^{2}\right)
$$

$\hat{e}=\frac{\langle\Delta E\rangle_{L}}{L}$

Elastic energy loss rate also diffusion rate $e_{2}$
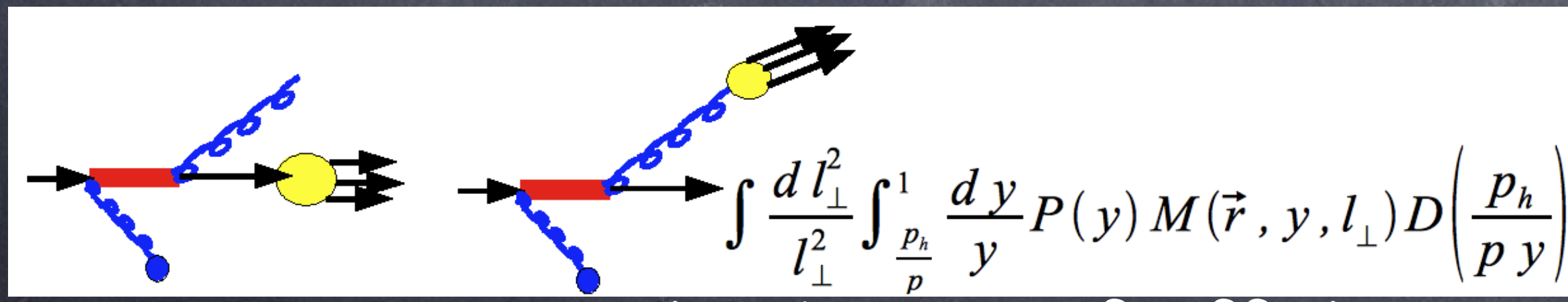

Gluon radiation is sensitive to all these transport coefficients

And a bunch of off diagonal and higher order transport coefficients 
Need to repeat the kernel

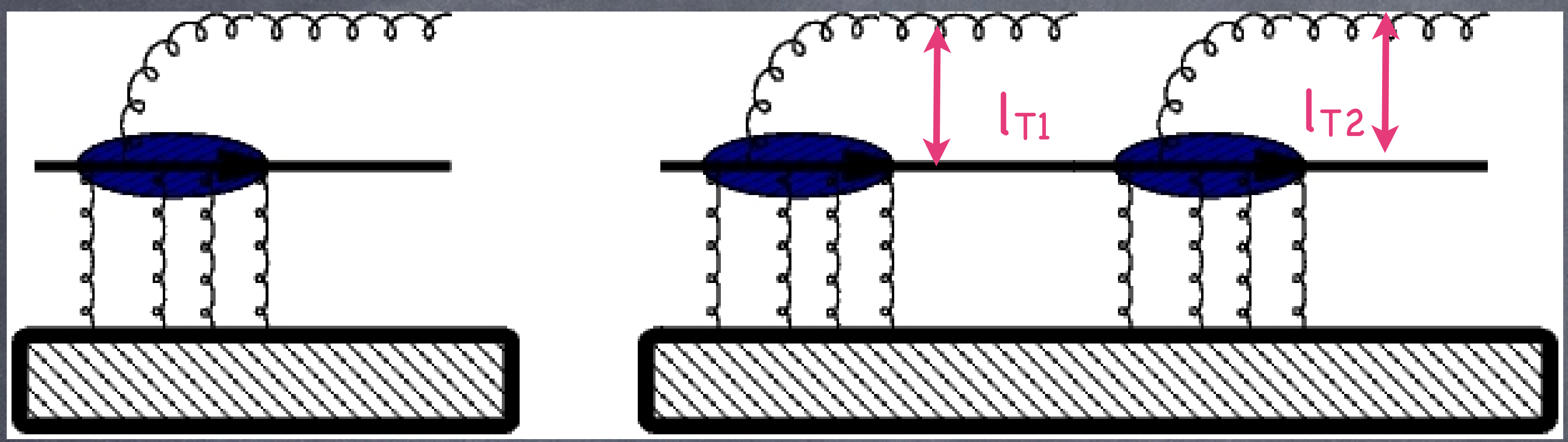

What is the relation between subsequent radiations?

In the large $Q^{2}$ we can argue that there should be ordering of $I_{T}$.

$$
\begin{aligned}
\text { if } \hat{q} L & <Q^{2} \\
\text { then } & \frac{d Q^{2}}{Q^{2}}\left[1+c_{1} \frac{\hat{q} L}{Q^{2}}\right] \leq \frac{d Q^{2}}{Q^{2}}\left[1+c_{1}\right]
\end{aligned}
$$


A DGLAP formalism requires an upper scale and a lower scale

Upper scale is $p_{T}^{2}$, same as in vacuum What is the lower scale?

what is the virtuality of a parton on exit?

Natural choice

$Q^{2}{ }_{\min }=E / L$

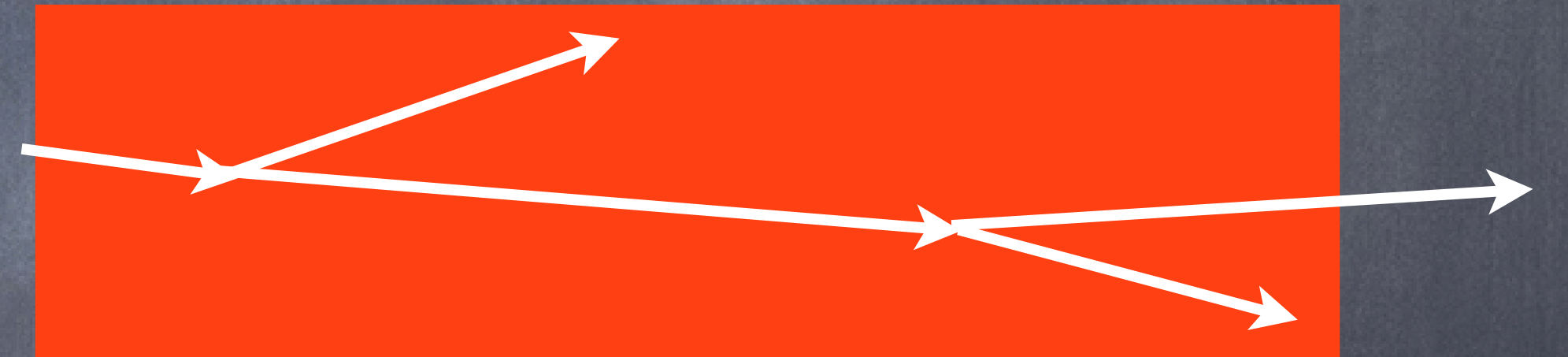

Realistically, this should be done for each path In reality we average kernel over many paths and calculate a mean distance based on the maximum length that the jet can travel in the representative brick 


\section{Gaussian distribution/temperature dependence/fit parameter !!!}

Multiple scattering off any

distribution samples a Gaussian

$\hat{q} \sim T^{3}, s, \epsilon^{3 / 4}$

is basically a model

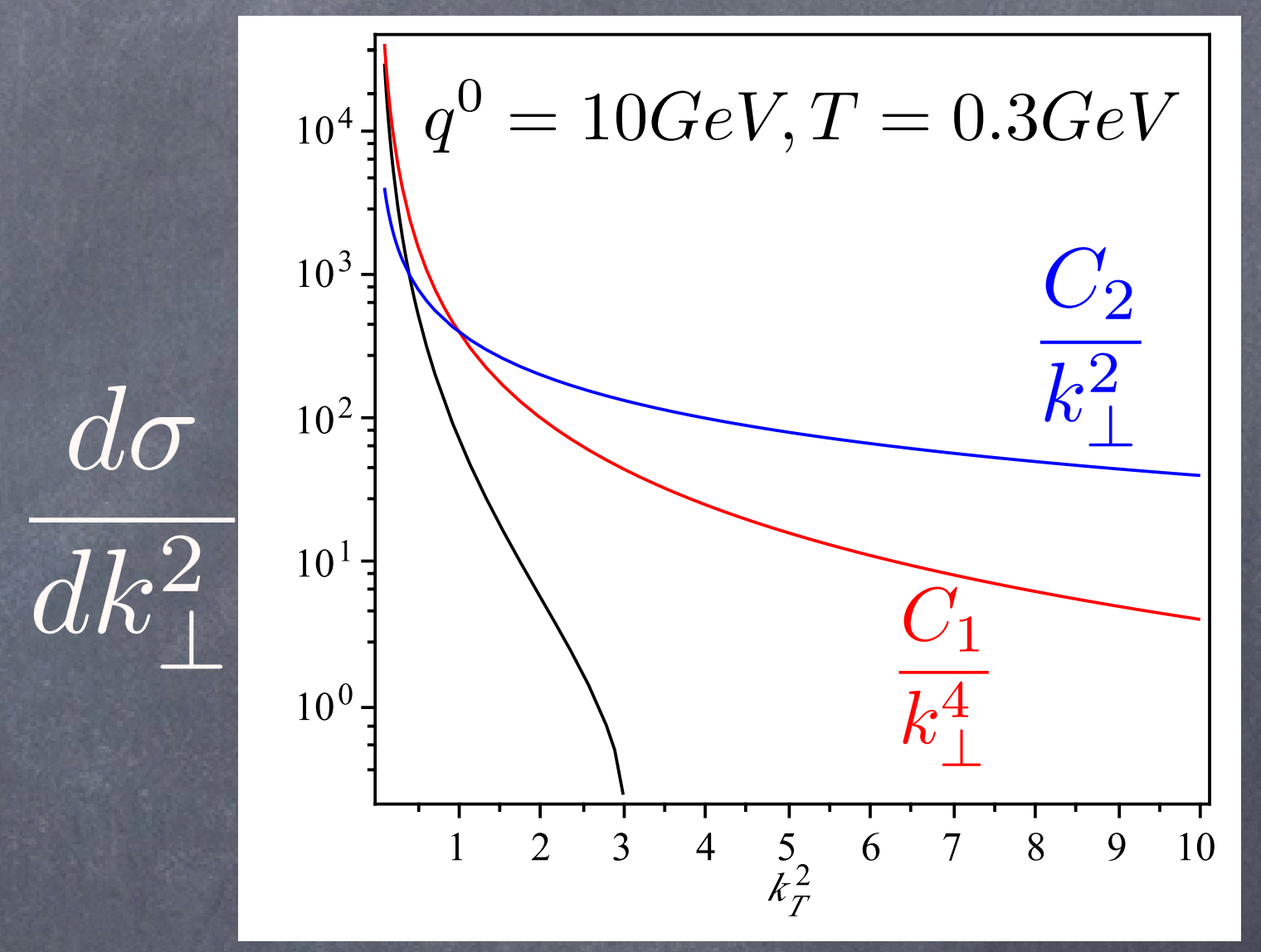

Ultimately you have to fit the normalization to 1 data point at one centrality, one value of $\mathrm{P}_{\mathrm{T}}$, one HIC energy 


\section{Bulk medium described by viscous fluid dynamics}

Medium evolves hydro-dynamically as the jet moves through it

Fit the $q$ for the initial $T$ in the hydro in central coll.
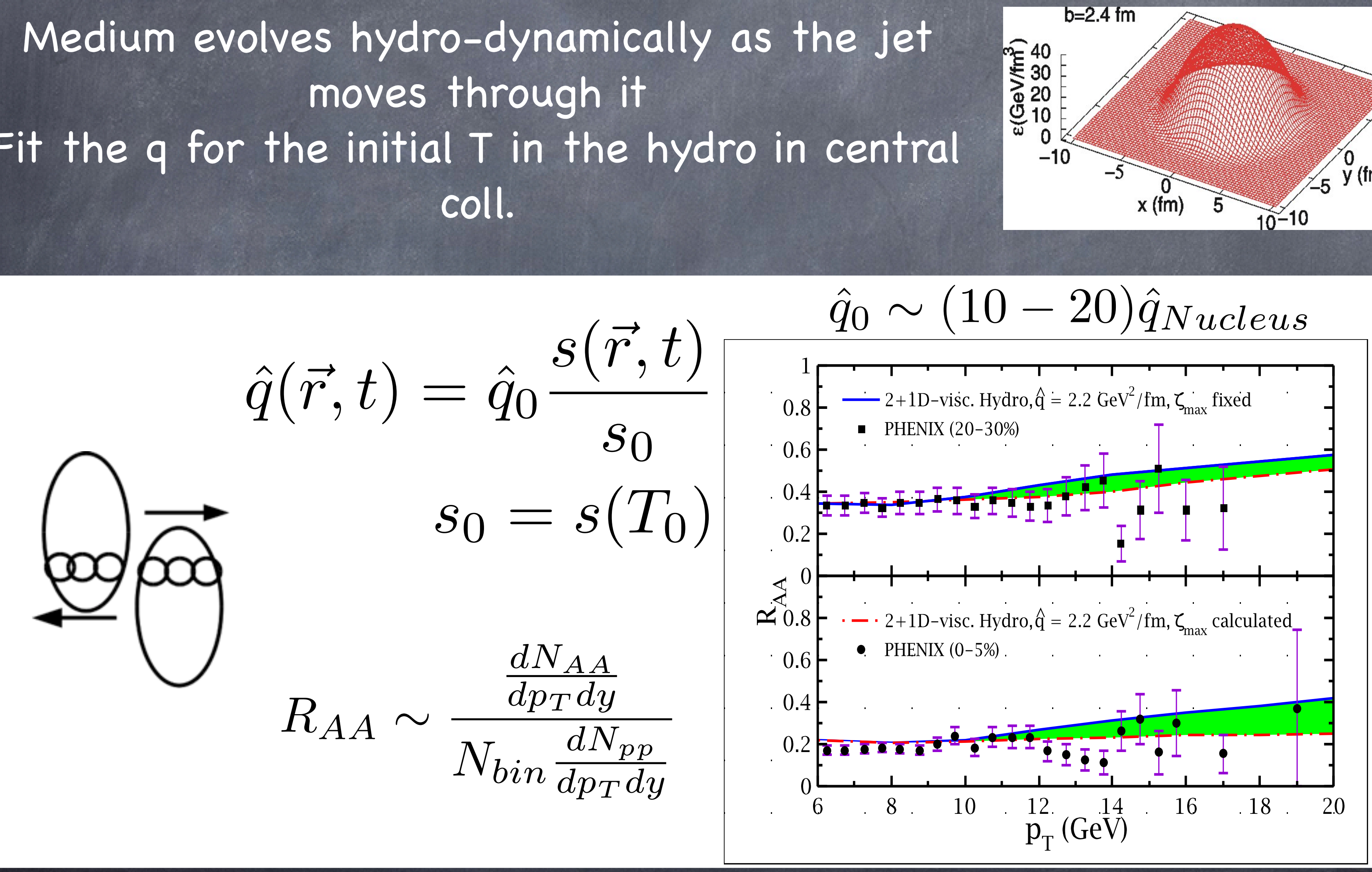
Note: no refitting between RHIC and LHC.

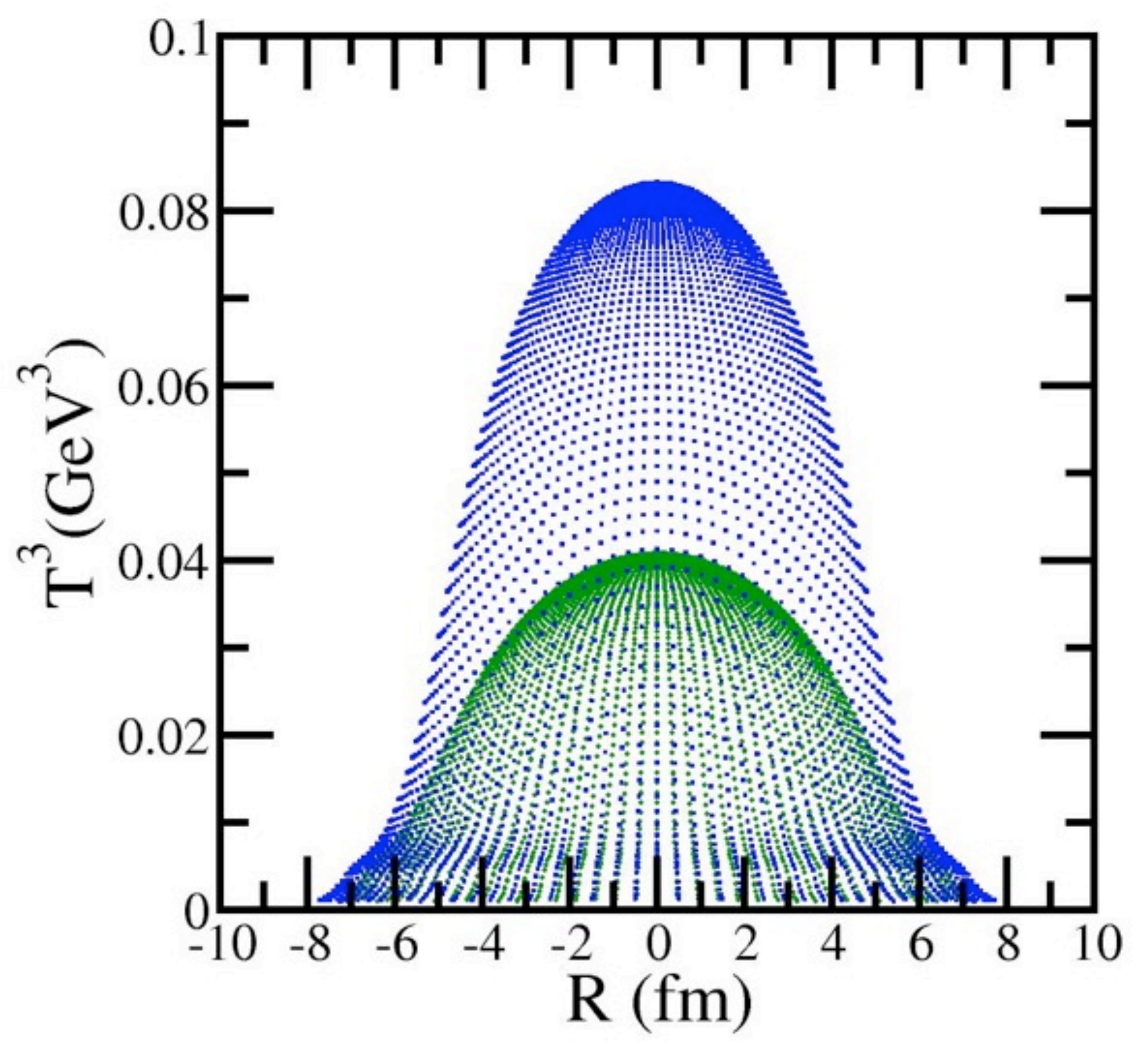




\section{Versus reaction plane, versus energy}
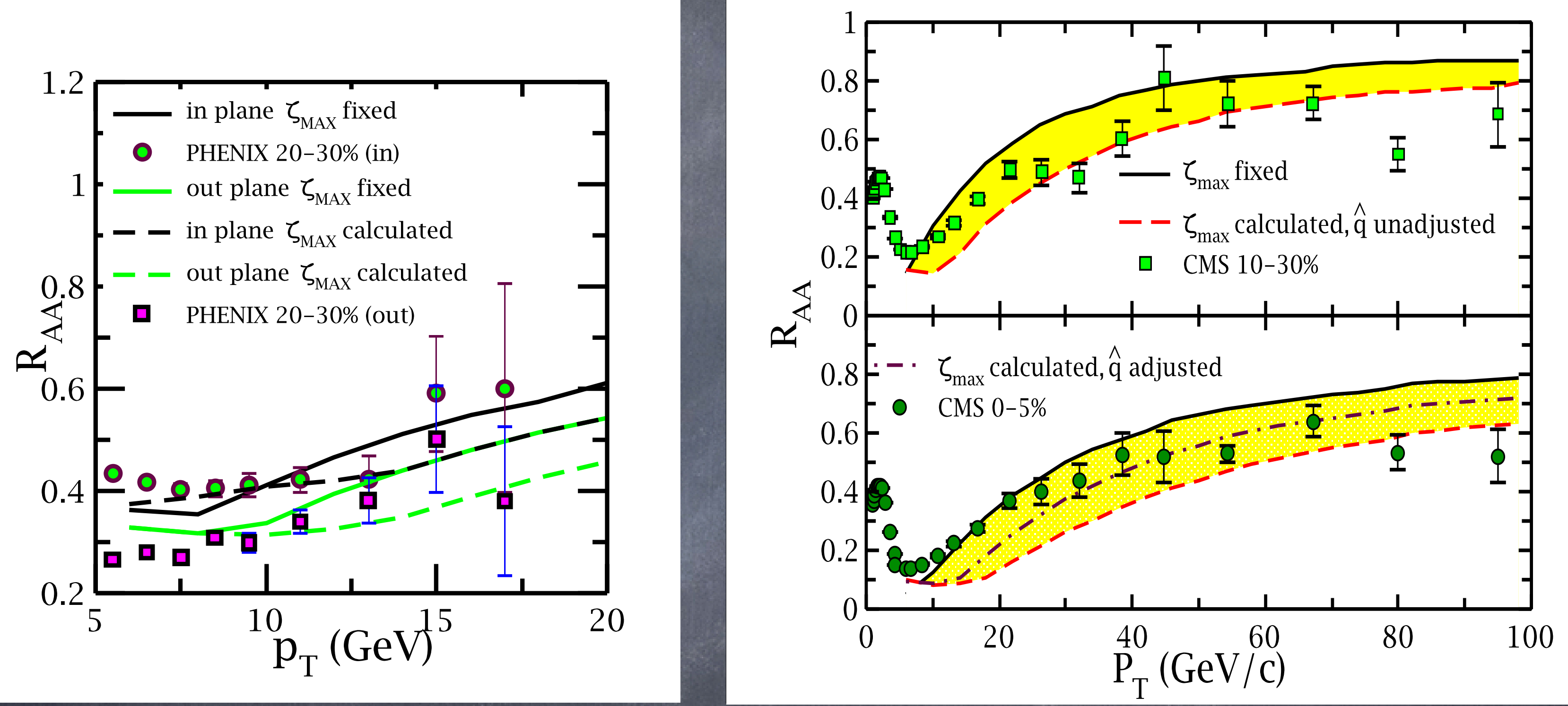

AM, C. Shen, PRL 1092023012012

Reasonable agreement with data

The band is from the uncertainty in $Q_{\min }$ 


\section{Predictions for $\mathrm{p}-\mathrm{Pb} \mathrm{R}_{\mathrm{AA}}$ at mid-rapidity}

\section{No surprises here!}
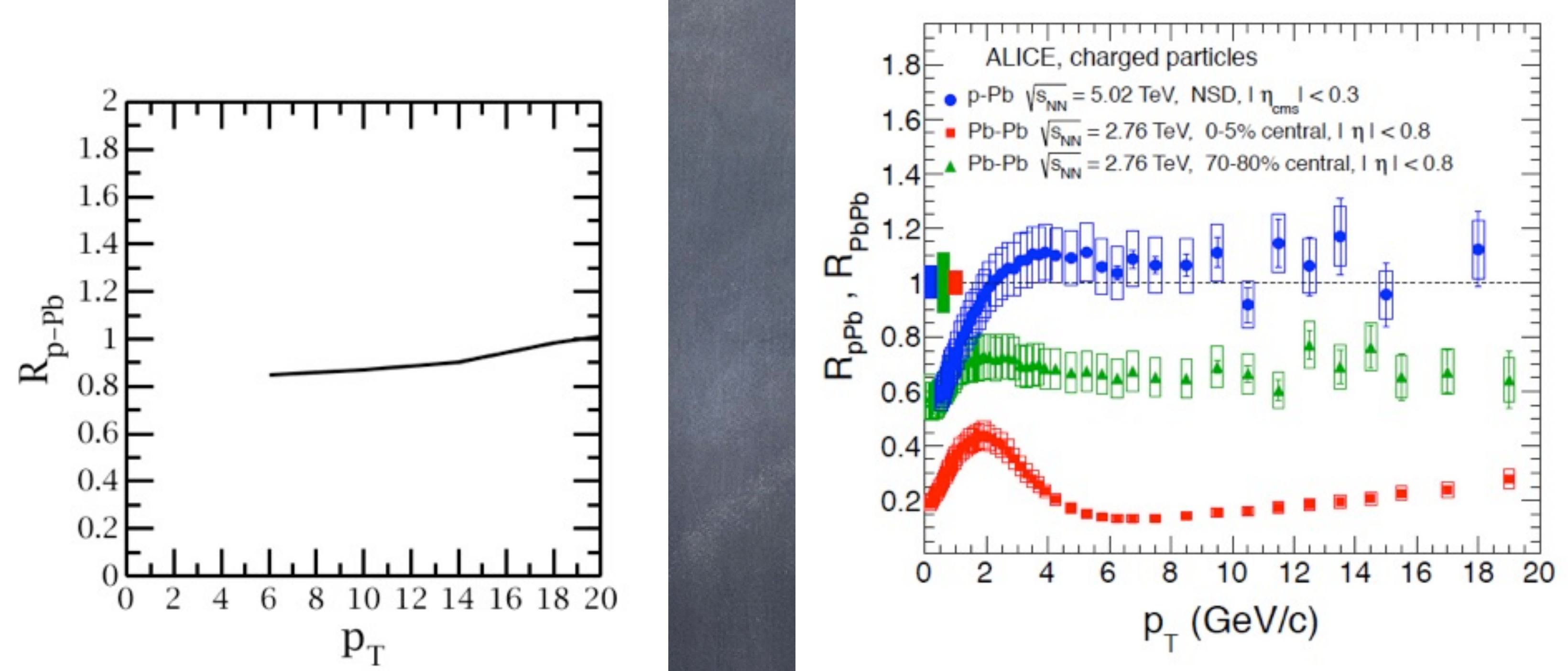

This means that our baseline is in control We can do more detailed analysis 


\section{Predictions for $\mathrm{p}-\mathrm{Pb} \mathrm{R}_{\mathrm{AA}}$ at mid-rapidity}

\section{No surprises here!}

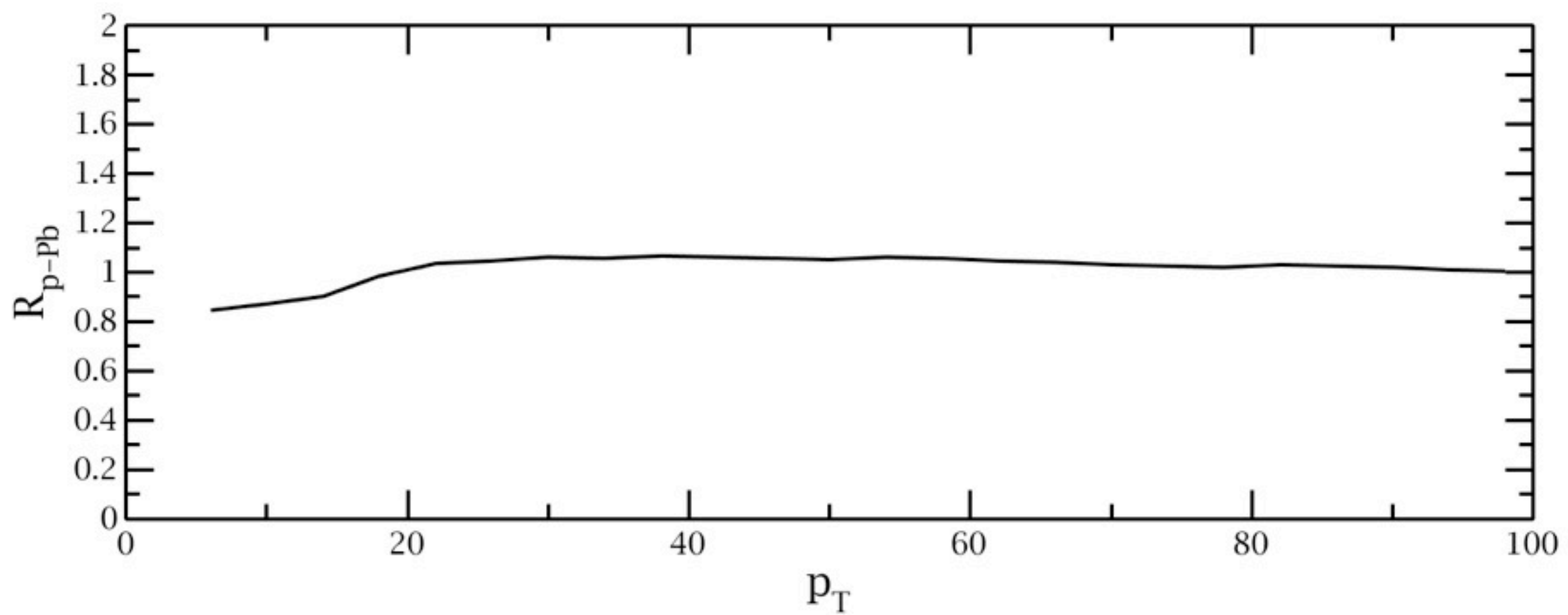

This means that our baseline is in control We can do more detailed analysis 
Four ways to go from here 


\section{Four ways to go from here}

Move from a few particles to full jets... Need MC 


\section{Four ways to go from here}

Move from a few particles to full jets... Need MC Progress towards a first principles calculation... Lattice QCD 


\section{Four ways to go from here}

Move from a few particles to full jets... Need MC Progress towards a first principles calculation... Lattice QCD Improve the error in current calculations... NLO 


\section{Four ways to go from here}

Move from a few particles to full jets... Need MC

Progress towards a first principles calculation... Lattice QCD

Improve the error in current calculations... NLO

Study flavor/mass dependence... more transport coefficients 


\section{MATTER++ a HT based MC event generator}

Main problem: Introducing distance into a DGLAP shower No space-time in the usual Monte-Carlo showers

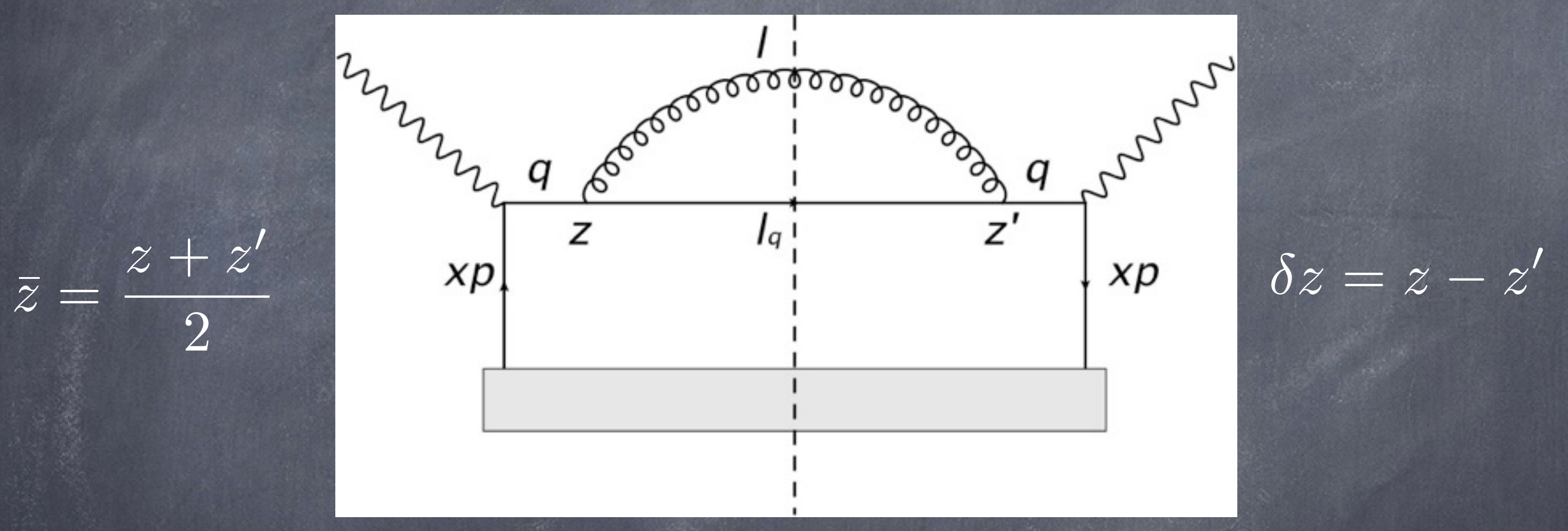

what is the role of $z$ and $z^{\prime}$ ?

$$
\int_{0}^{\infty} d^{4} \bar{z} \exp [i(\delta q) \bar{z}] \quad \int d^{4} \delta z \exp \left[i \delta z\left(l+l_{q}-q\right)\right]
$$

$\delta q$ is the uncertainty in $q$, 


\section{How much uncertainty can there be?}

\section{To be sensible: $\delta q \ll q$}

we assume a Gaussian distribution around $\mathrm{q}^{+}$

And try different functional forms of the width

We set the form by insisting $\langle T\rangle=2 q^{-} /\left(Q^{2}\right)$

to obtain the $\mathrm{z}^{-}$distribution only need to assume a $\delta q^{+}$distribution

$\rho\left(\delta q^{+}\right)=\frac{e^{-\frac{\left(\delta q^{+}\right)^{2}}{2\left[2\left(q^{+}\right)^{2} / \pi\right]}}}{\sqrt{2 \pi\left[2\left(q^{+}\right)^{2} / \pi\right]}}$

A normalized Gaussian with a variance $2 q^{+} / \pi$

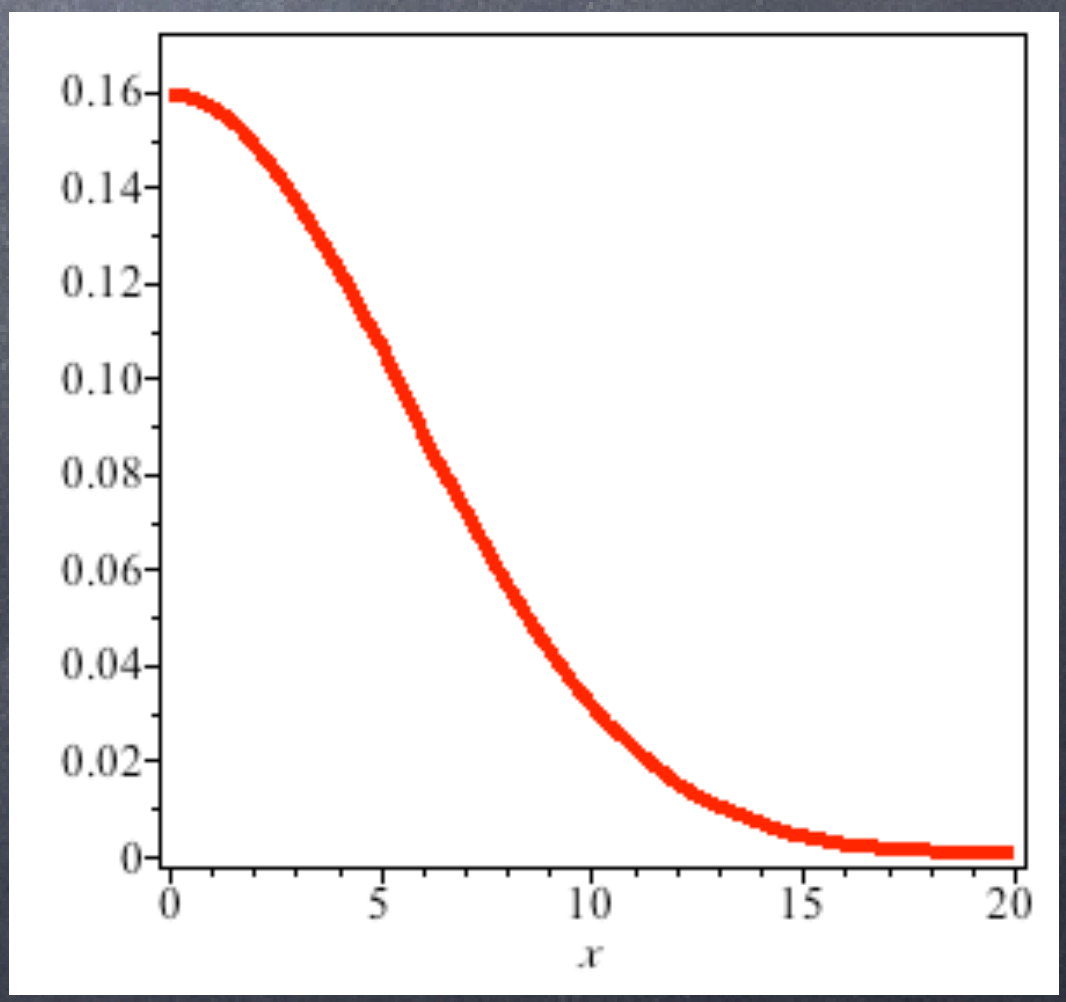




\section{Consider a jet moving through a QGP Brick}

We now construct a

Sudakov with the constraint

$$
\frac{Q_{0}^{2}}{Q^{2}}<z<1-\frac{Q_{0}^{2}}{Q^{2}}
$$

Have a distribution of locations of splittings

length dependent transverse broadening

Partons whose virtuality drops below $1 \mathrm{GeV}$ are removed form cascade
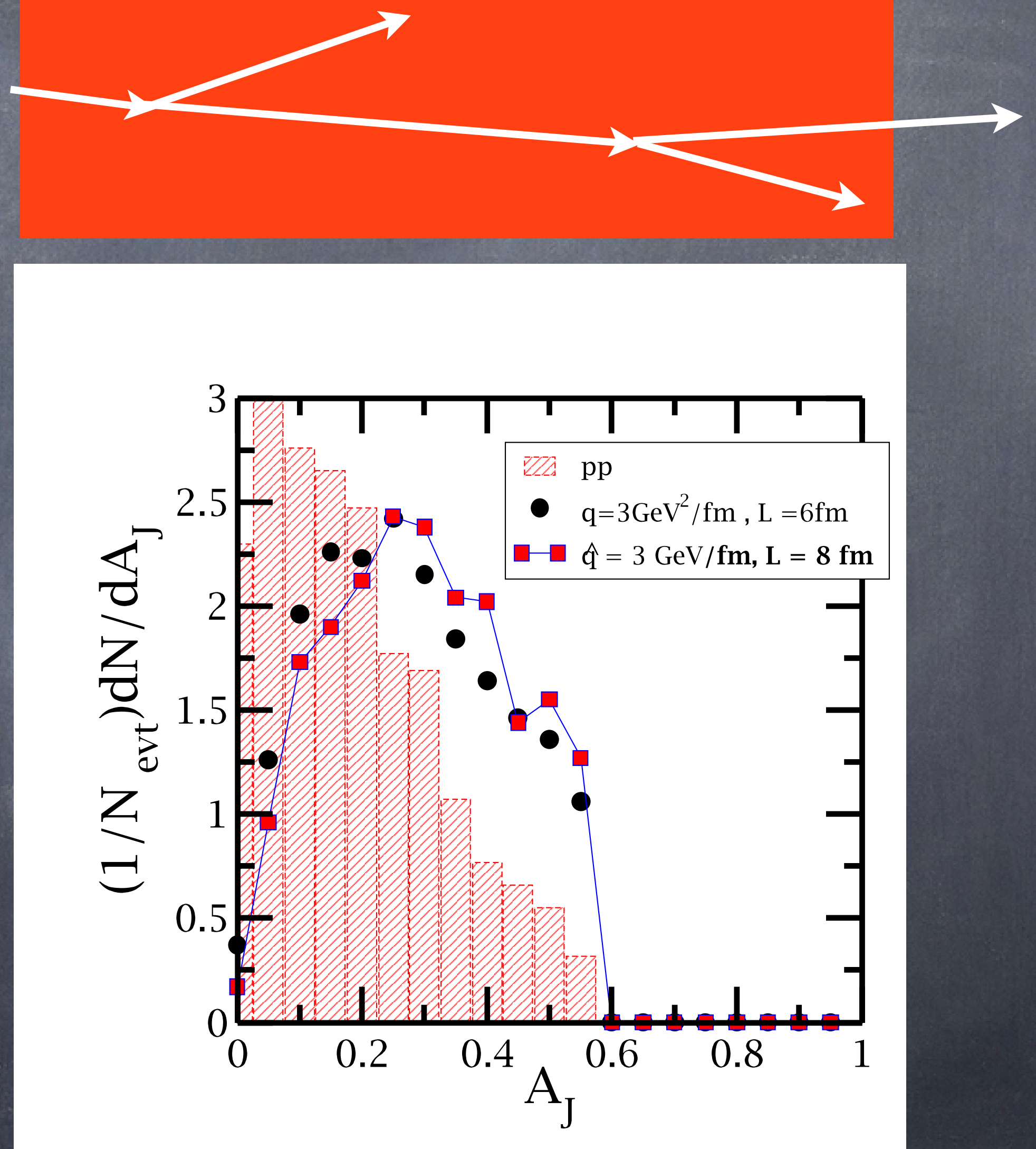


\section{Getting around to the CMS/ATLAS Fragmentation function}

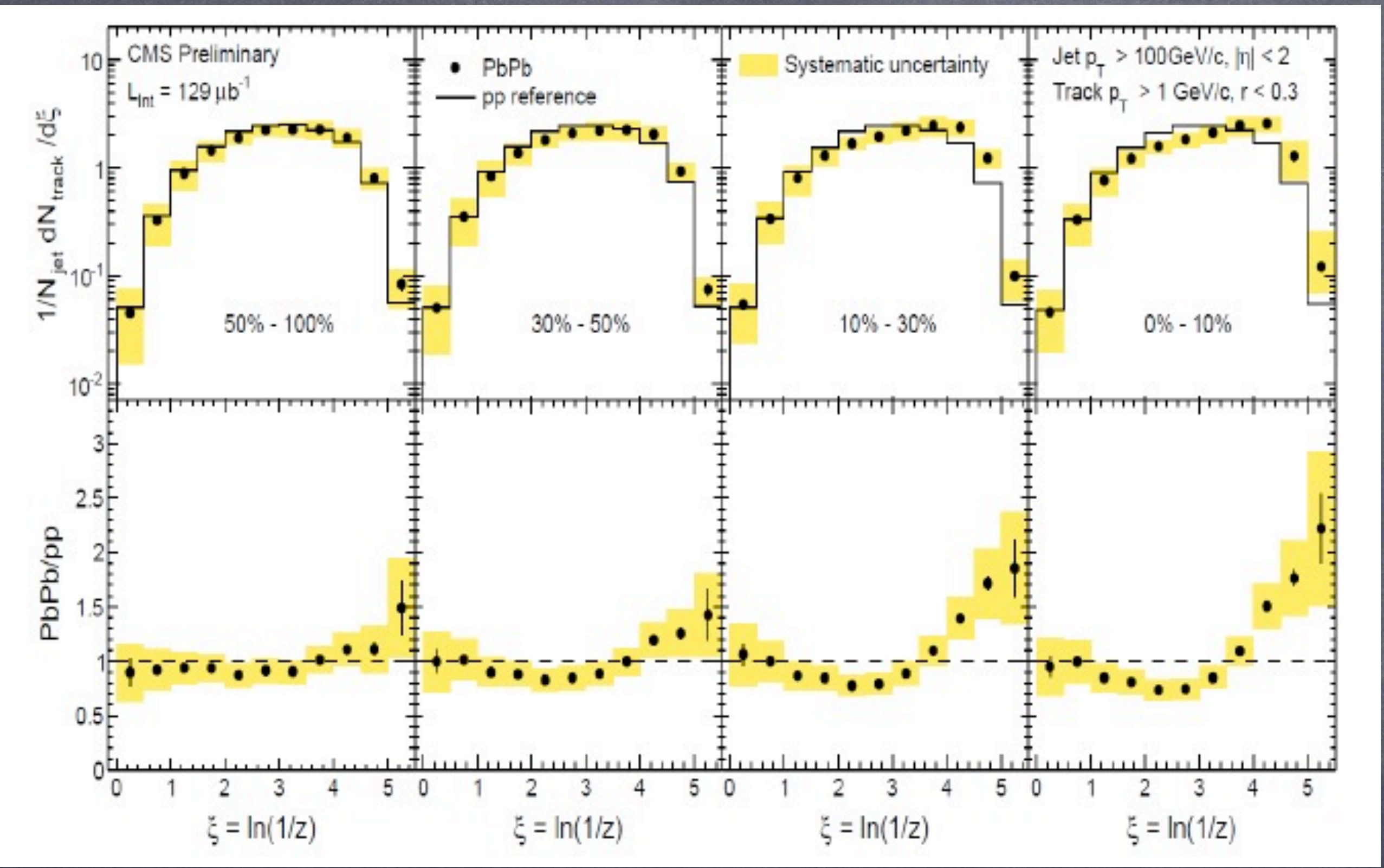

AM, arXiv:1301.5323 [nucl-th]

Brick MC, need E-by-E hydro and realistic hadronization 


\section{Getting around to the CMS/ATLAS Fragmentation function}
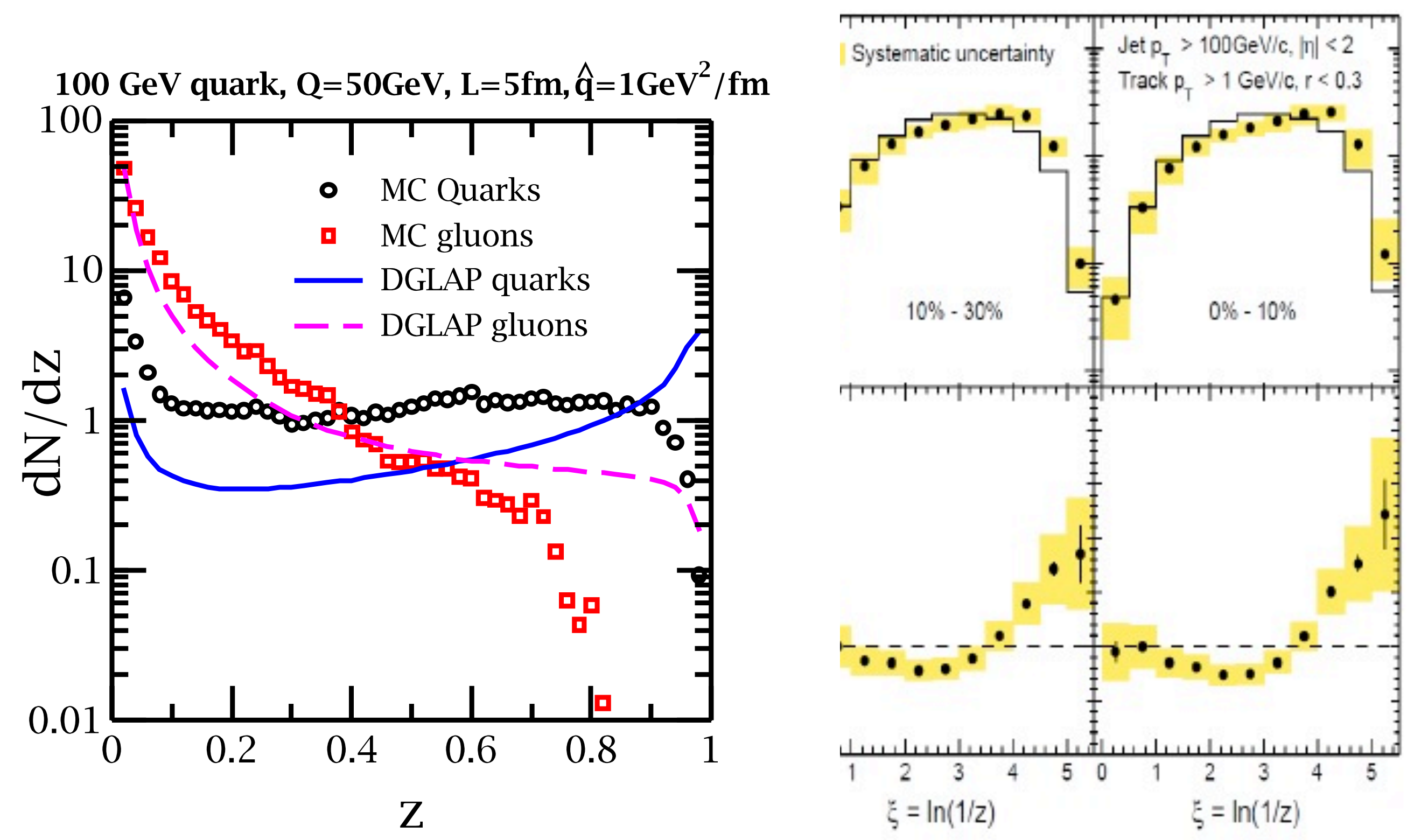

AM, arXiv:1301.5323 [nucl-th]

Brick MC, need E-by-E hydro and realistic hadronization 


\section{Getting around to the CMS/ATLAS Fragmentation function}
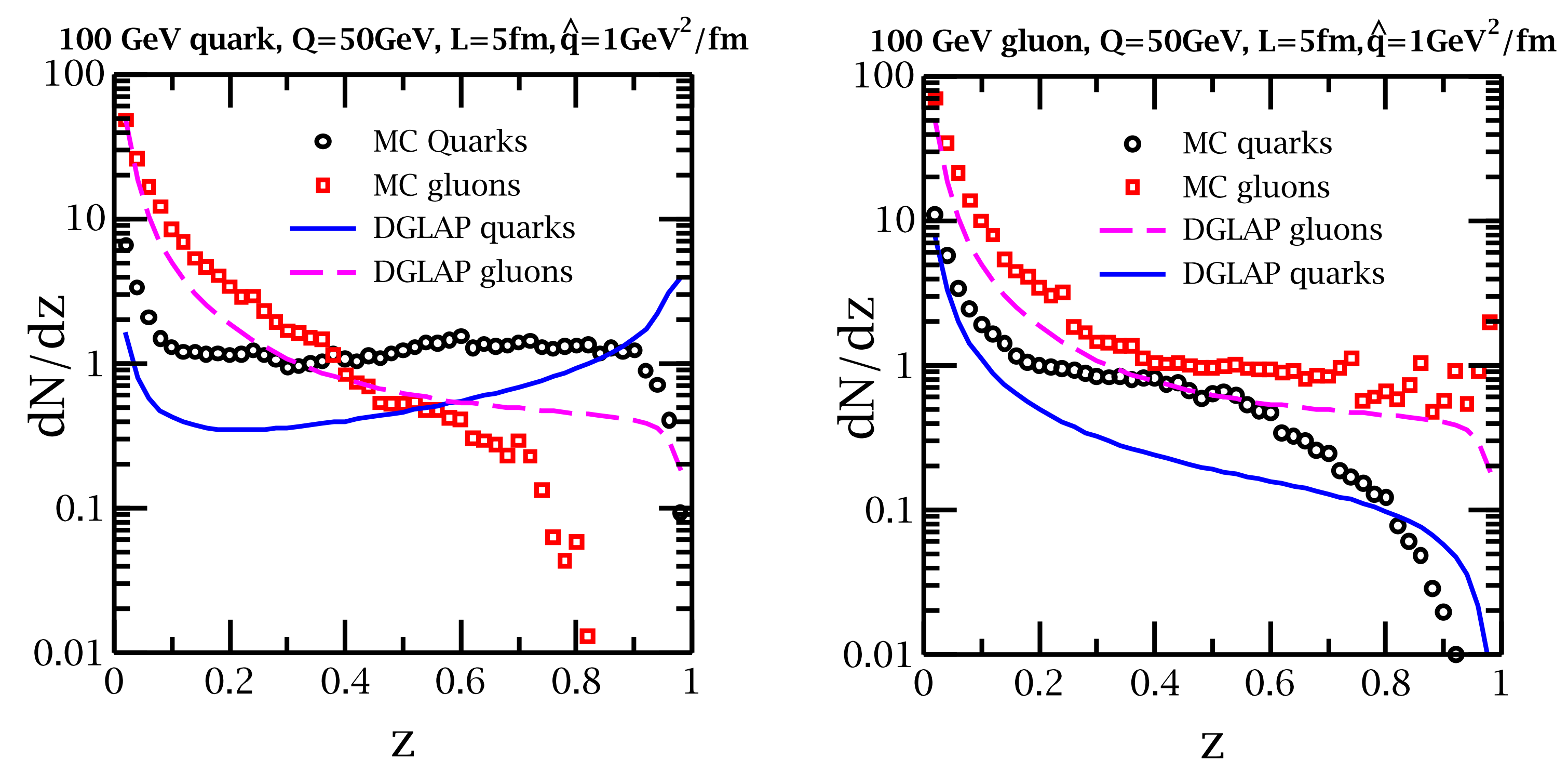

AM, arXiv:1301.5323 [nucl-th]

Brick MC, need E-by-E hydro and realistic hadronization 


\section{Getting around to the CMS/ATLAS Fragmentation function}
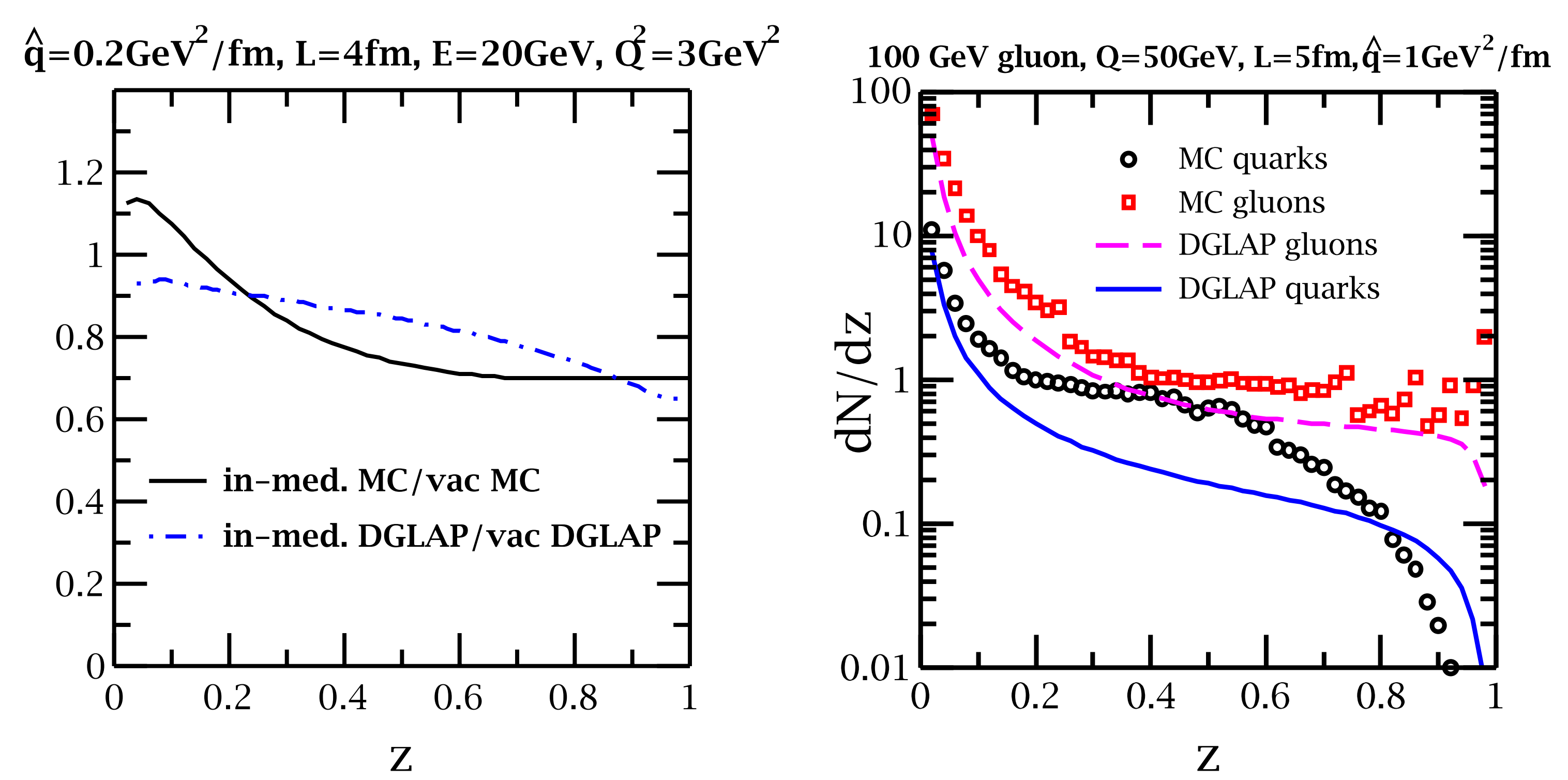

AM, arXiv:1301.5323 [nucl-th]

Brick MC, need E-by-E hydro and realistic hadronization 


\section{Getting around to the CMS/ATLAS Fragmentation function}
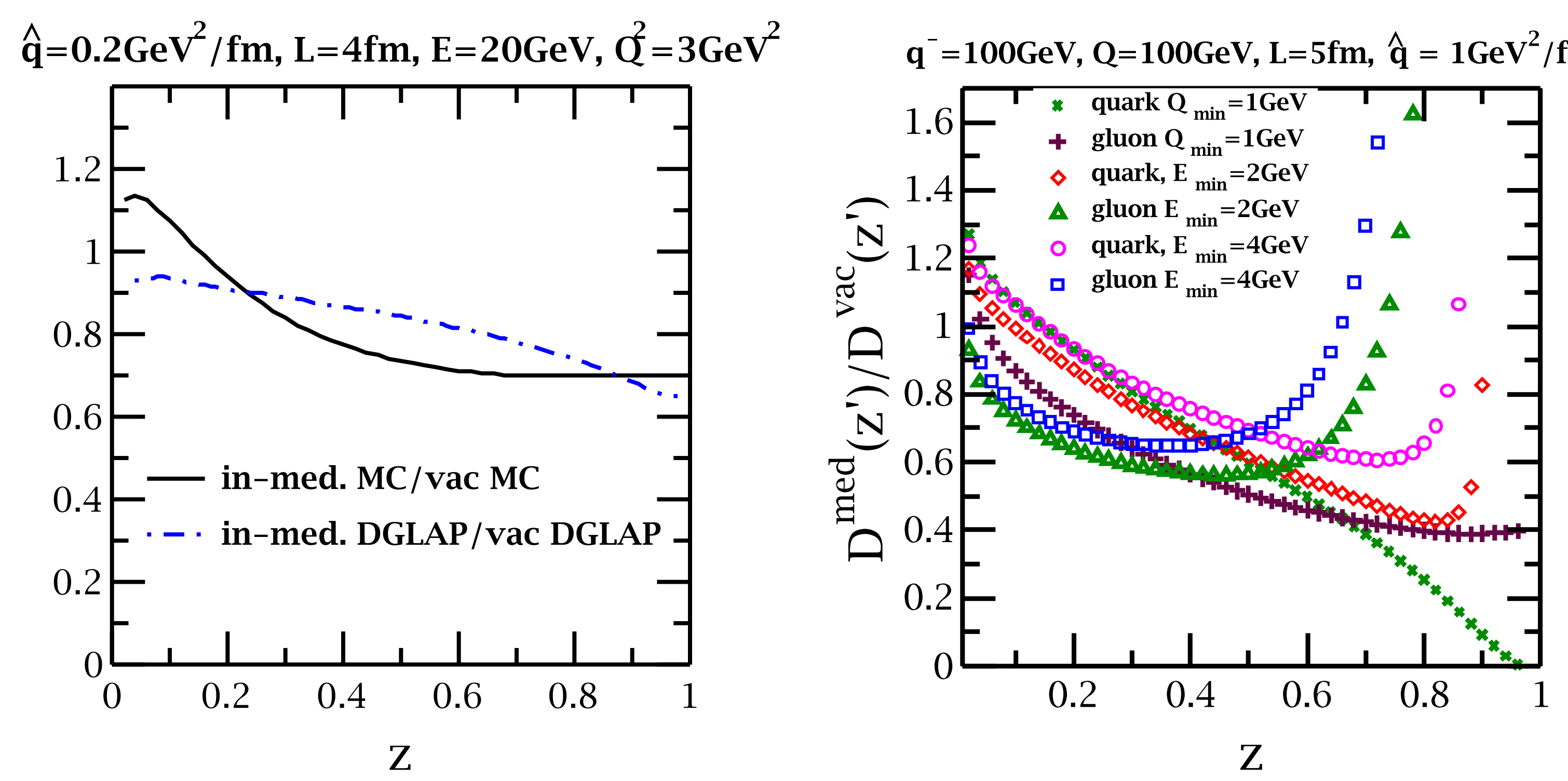

AM, arXiv:1301.5323 [nucl-th]

Brick MC, need E-by-E hydro and realistic hadronization 


\section{Energy in and out of cone!}

\section{answering this question is not trivial!}

From the surviving hard partons, very little energy $\sim 5 \%$ is outside $R=1$ cone

A lot of the energy is lost as particles become less energetic or less virtual than $1 \mathrm{GeV}$, thus strongly interacting with the medium

So far cannot estimate in MC, no Sudakov, which readjusts formation time by removing deposited energy from shower

No single gluon emission with coherent drag calculation 


\section{However, can be analytically estimated}

\section{At lower momentum need some no-pert. input}

A single parton deposits energy and transverse momentum in medium.

This just $q$ and $e$

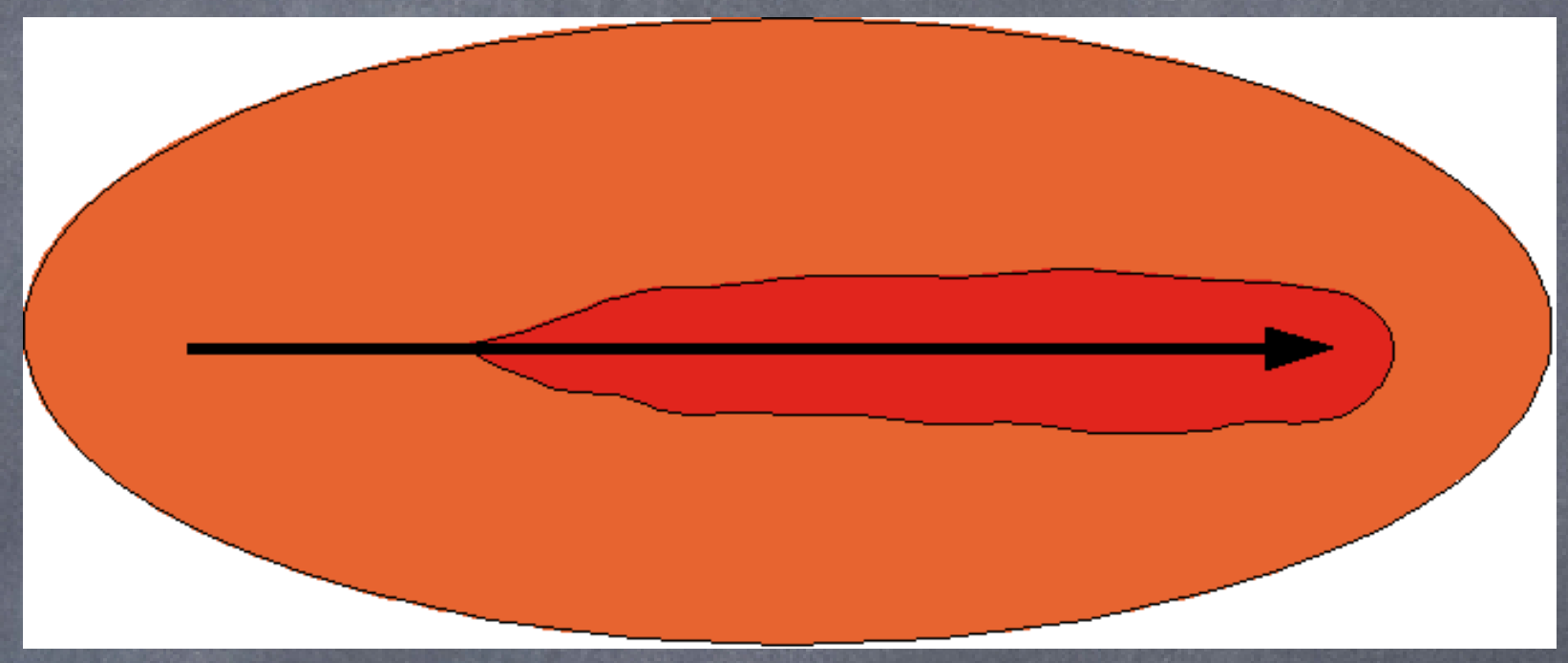

multiple radiation increases the sources of mom. dep.

We know how much radiation as we already calculated it for energy loss

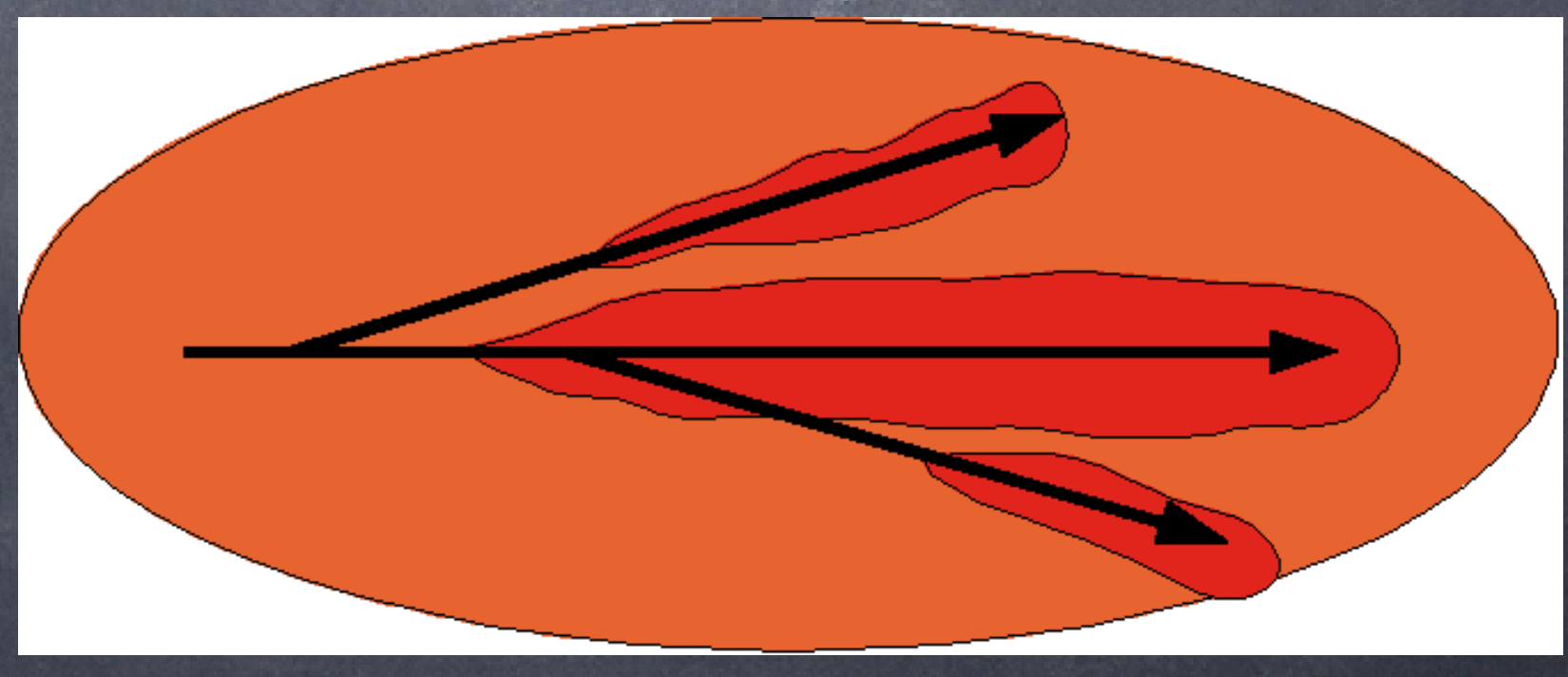




\section{Radiative loss enhances the deposited energy}

Do a DGLAP with $\triangle E=\hat{e} L$ as input

partons with $E=4 T$ and $Q<1 G e V$ are added to medium

\section{Energy}

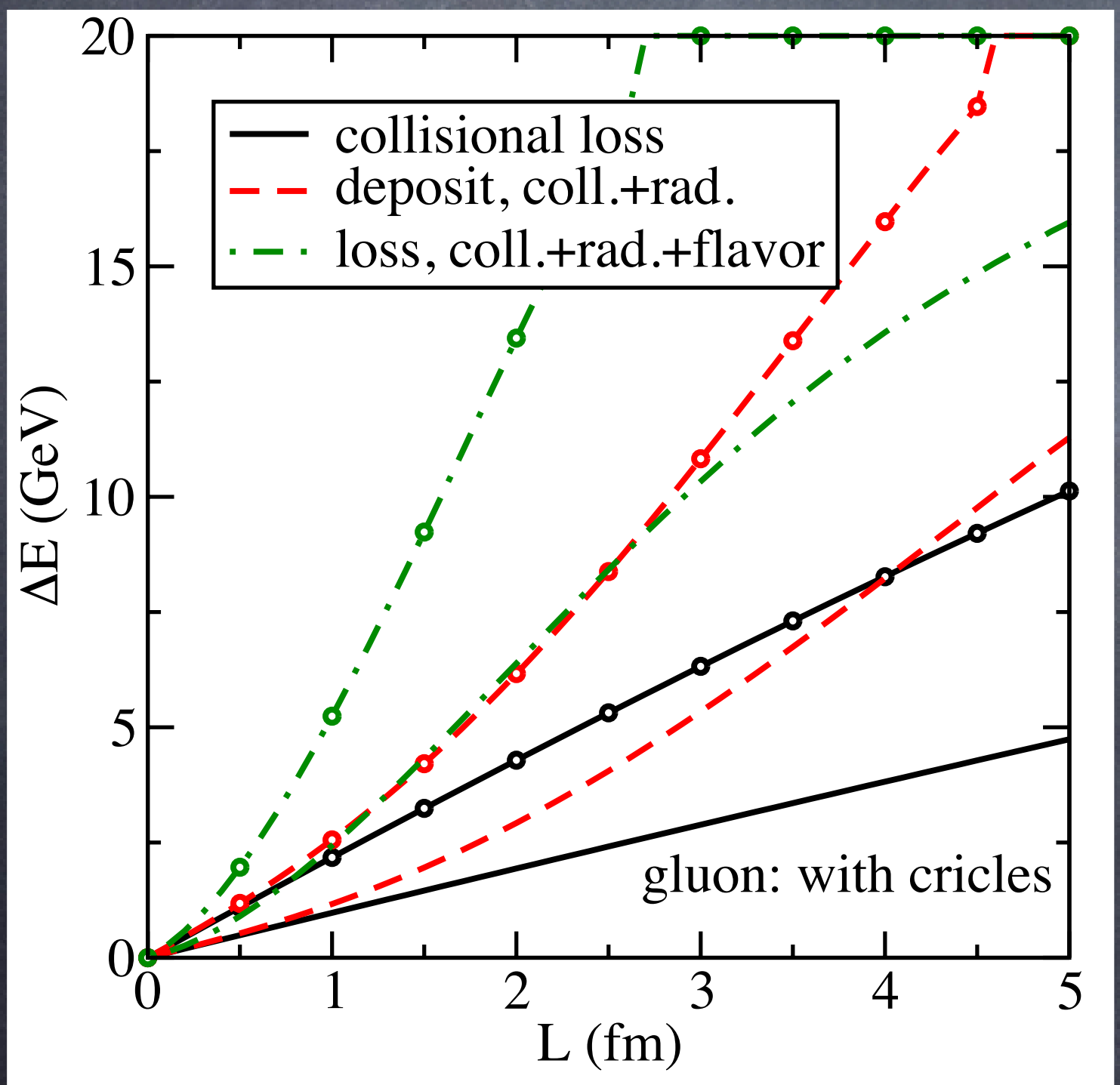

Deposited energy will thermalize and spread out

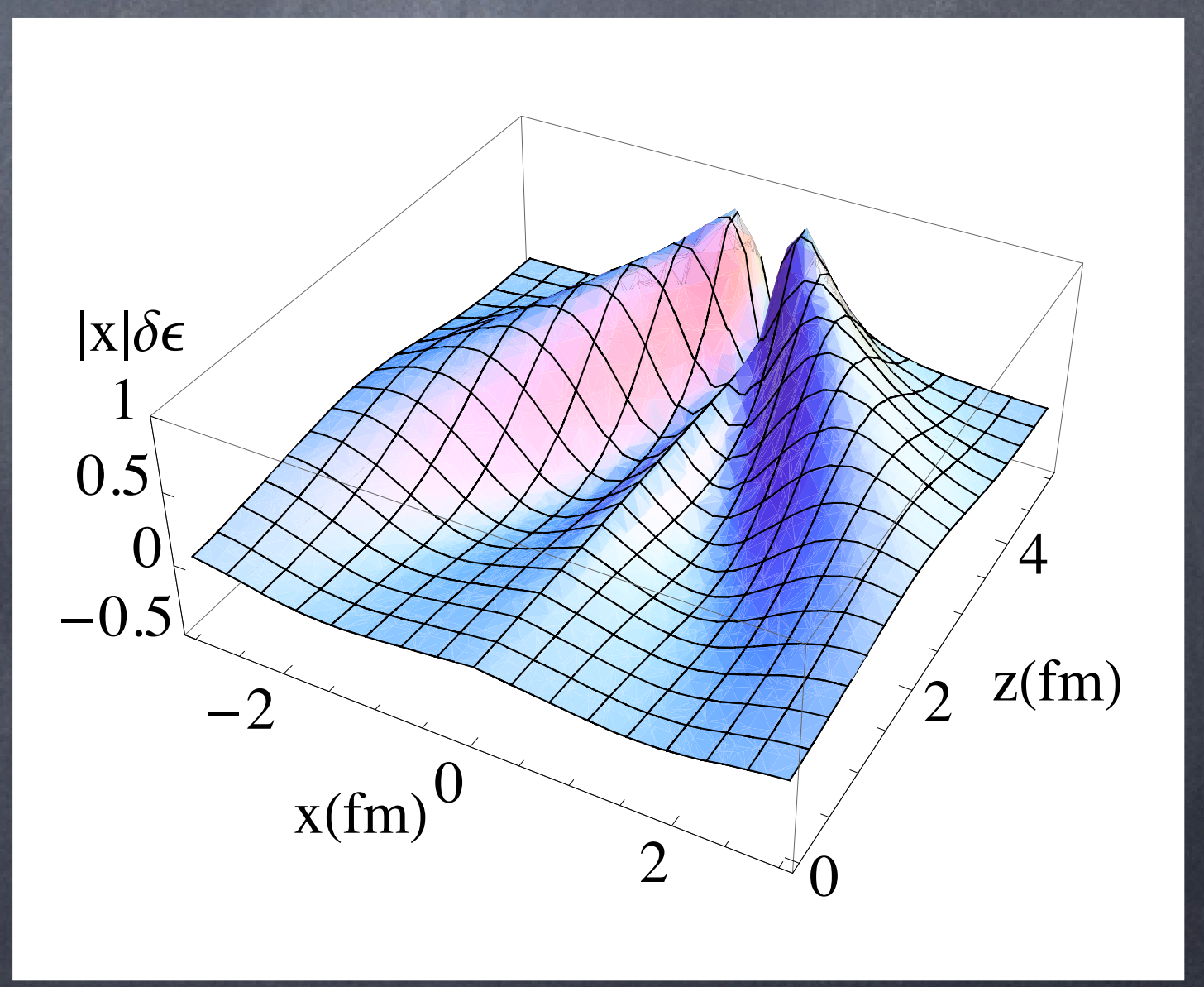




\section{A simple picture}

Assign E deposited before $L / 2$ as early deposition.

$$
\mathrm{L}=4 \mathrm{fm}(\mathrm{RHIC}) \text { and } \mathrm{L}=6 \mathrm{fm}(\mathrm{LHC})
$$

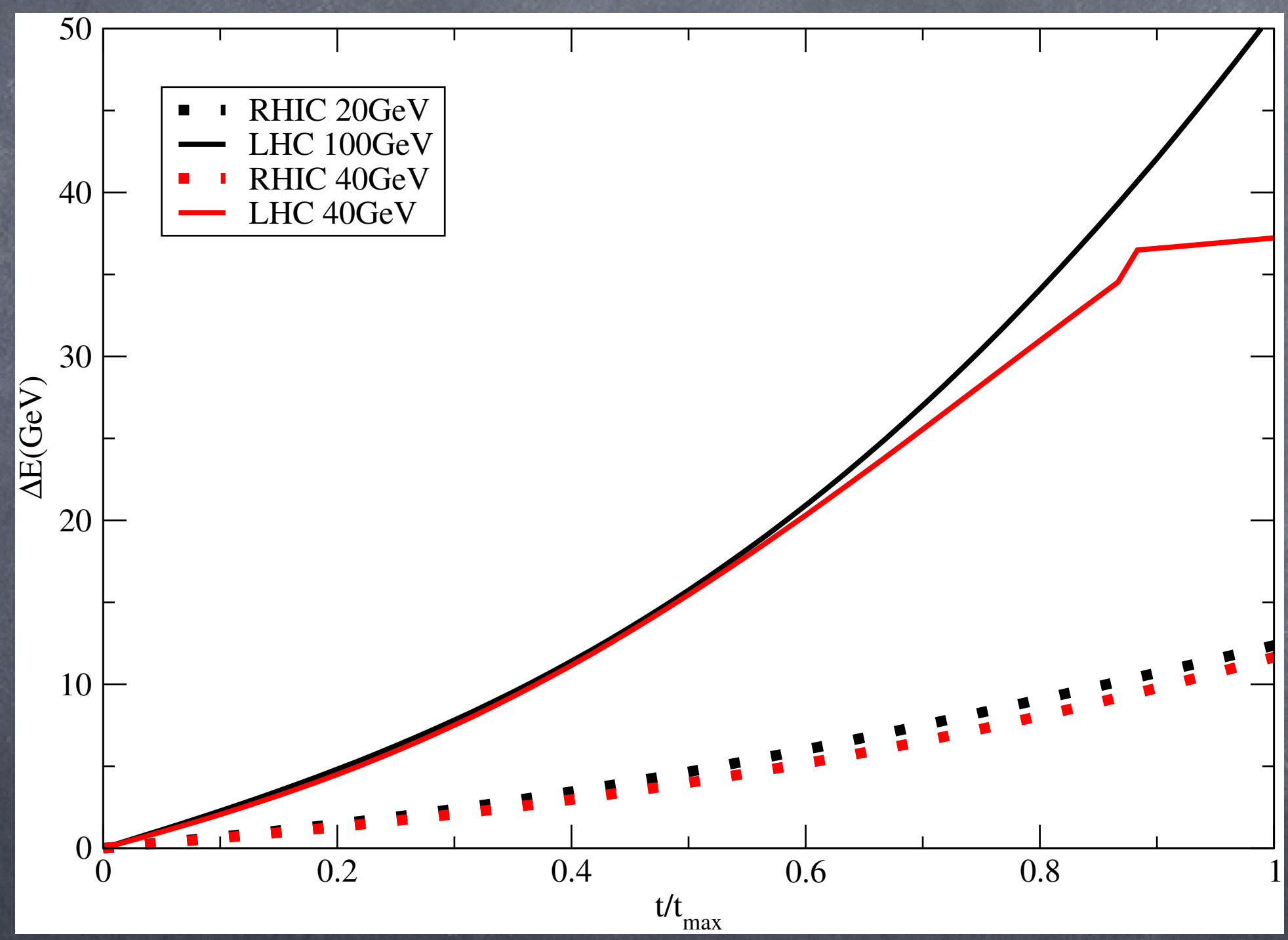

G.Y. Qin, AM, J. Putschke

A complete understanding requires a $M C$ and a new soft-hard transport coefficient 


\section{A simple picture}

Assign $E$ deposited before $L / 2$ as early deposition.

$$
\mathrm{L}=4 \mathrm{fm}(\mathrm{RHIC}) \text { and } \mathrm{L}=6 \mathrm{fm}(\mathrm{LHC})
$$

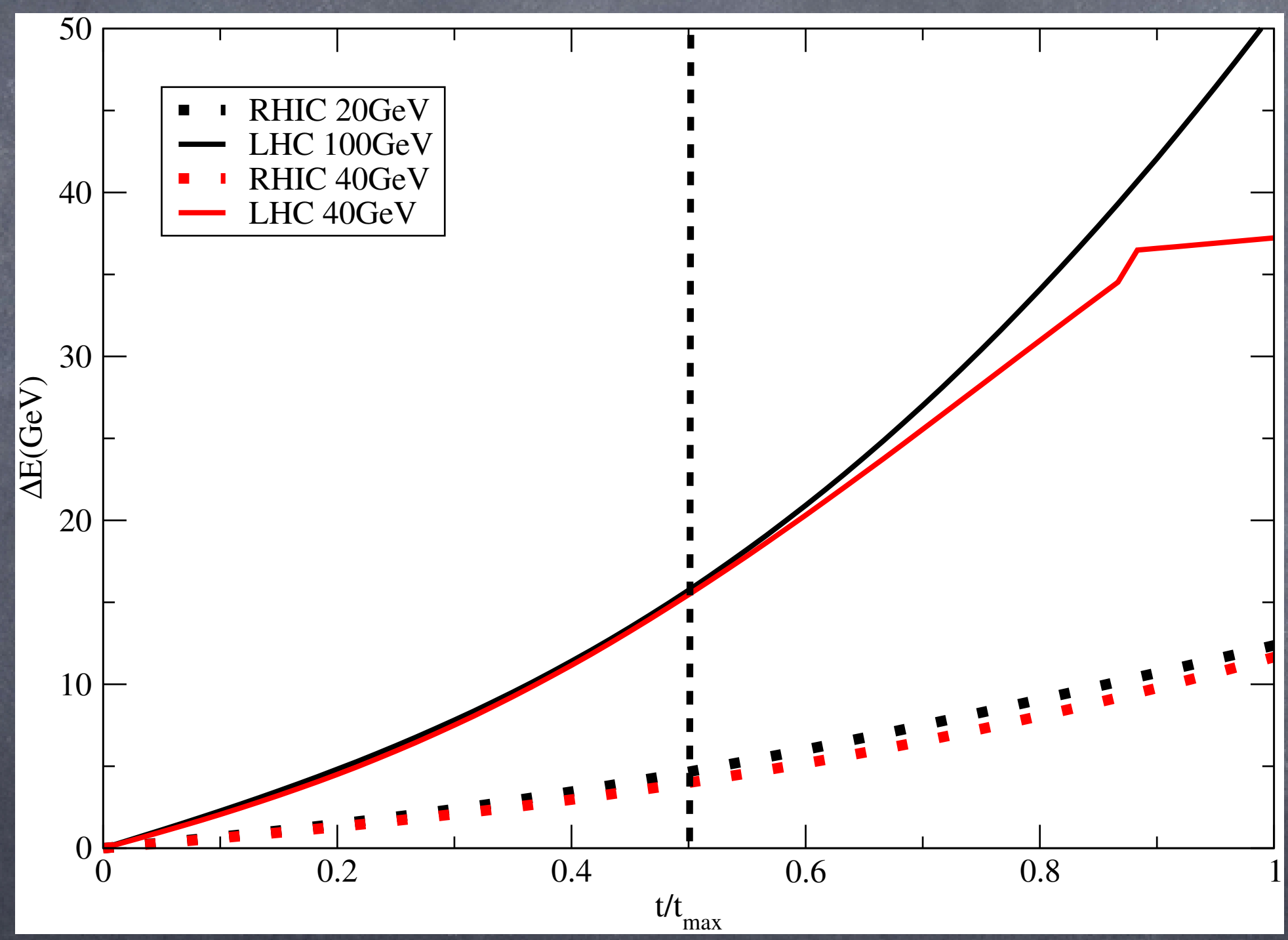

G.Y. Qin, AM, J. Putschke

A complete understanding requires a $M C$ and a new soft-hard transport coefficient 
Even with the best MC energy loss routine on the best E-by-E hydro The exchange momentum distribution of

$\hat{q}, \hat{e}$ and their normalization are assumptions! 


\section{A first principles method to calculate $\hat{q}$}

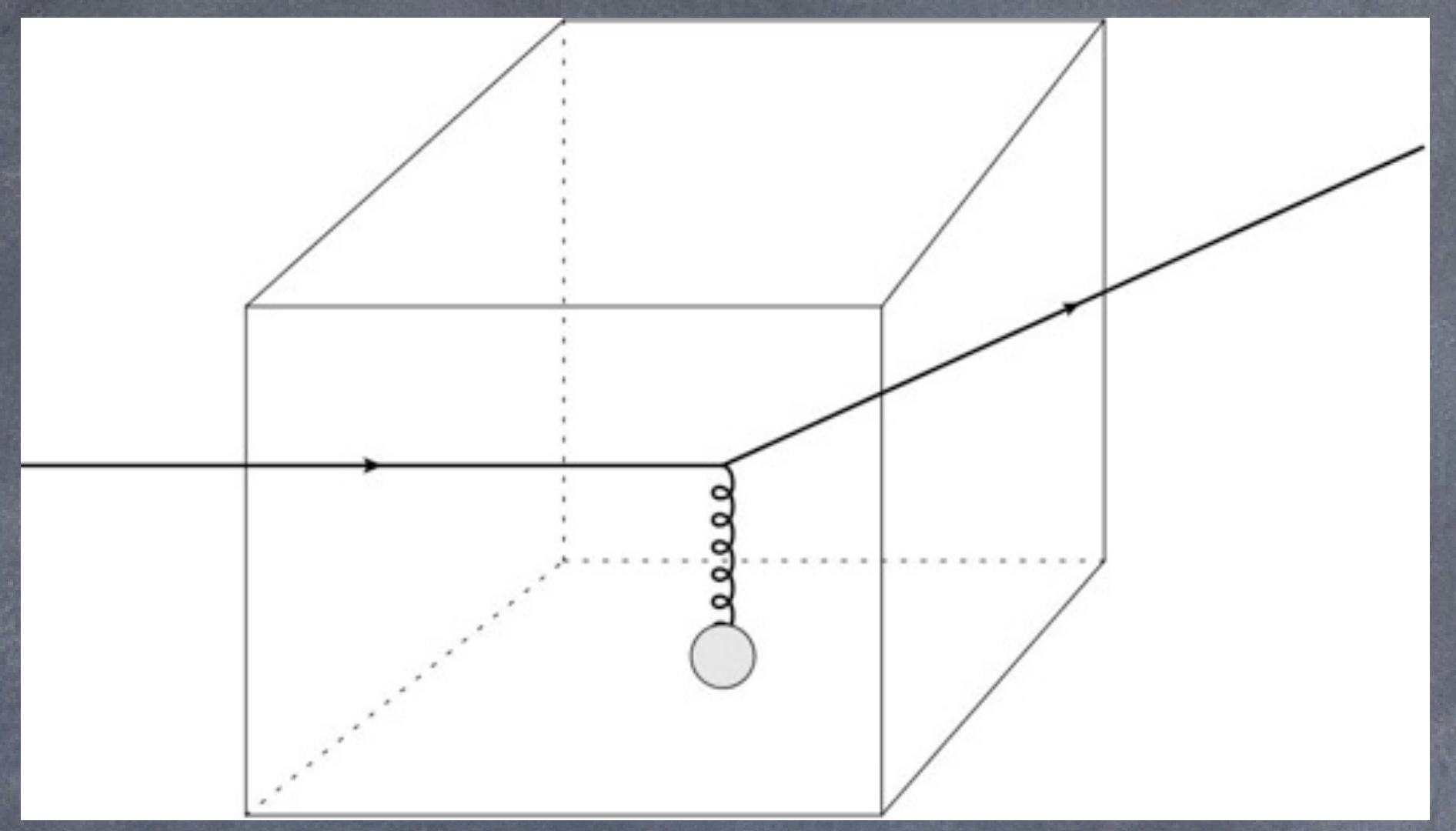

$$
\begin{aligned}
W(k) & =\frac{g^{2}}{2 N_{c}}\left\langle q^{-} ; M\right| \int d^{4} x d^{4} y \bar{\psi}(y) A(y) \psi(y) \\
& \times\left|q^{-}+k_{\perp} ; X\right\rangle\left\langle q^{-}+k_{\perp} ; X\right| \\
& \times \bar{\psi}(x) A(x) \psi(x)\left|q^{-} ; M\right\rangle
\end{aligned}
$$

in terms of W, we get

$$
\hat{q}=\sum_{k} k_{\perp}^{2} \frac{W(k)}{t}
$$


Final state is "on-shell"

$$
\delta\left[(q+k)^{2}\right] \simeq \frac{1}{2 q^{-}} \delta\left(k^{+}-\frac{k_{\perp}^{2}}{2 q^{-}}\right)
$$

Also we are calculating in a finite temperature heat bath

$$
\begin{array}{r}
\hat{q}=\frac{4 \pi^{2} \alpha_{s}}{N_{c}} \int \frac{d y^{-} d^{2} y_{\perp}}{(2 \pi)^{3}} d^{2} k_{\perp} e^{-i \frac{k_{\perp}^{2}}{2 q-} \cdot y^{-}+i \vec{k}_{\perp} \cdot \overrightarrow{y_{\perp}}} \\
\left\langle n\left|\frac{e^{-\beta E_{n}}}{Z} F^{+,}{ }_{\perp}\left(y^{-}\right) F_{\perp}^{+}(0)\right| n\right\rangle
\end{array}
$$

physical $\hat{q}\left(q^{-}, q^{+}\right)$where $q^{+} \sim \lambda^{2} Q$ 
Consider a more general object

$\hat{Q}=\frac{4 \pi^{2} \alpha_{s}}{N_{c}} \int \frac{d^{4} y d^{4} k}{(2 \pi)^{4}} e^{i k \cdot y} \frac{2\left(q^{-}\right)^{2}}{\sqrt{2} q^{-}} \frac{\left\langle M\left|F^{+\perp}(0) F_{\perp,}^{+}(y)\right| M\right\rangle}{(q+k)^{2}+i \epsilon}$

Consider $q^{-}$large $(\sim Q)$ and fixed

Consider $q^{+}$to be a variable

$\frac{d^{2} \hat{Q}}{d k_{\perp}^{2}}$ has a pole at $q^{+}=\frac{k_{\perp}^{2}}{2 q^{-}}$

$\hat{Q}$ has a branch cut on the real axis at $\mathrm{q}^{+} \sim \lambda^{2} Q$
$\hat{q}=\operatorname{Im}(\hat{Q})$
$q^{+}$complex plain 
Consider a more general object

$\hat{Q}=\frac{4 \pi^{2} \alpha_{s}}{N_{c}} \int \frac{d^{4} y d^{4} k}{(2 \pi)^{4}} e^{i k \cdot y} \frac{2\left(q^{-}\right)^{2}}{\sqrt{2} q^{-}} \frac{\left\langle M\left|F^{+\perp}(0) F_{\perp,}^{+}(y)\right| M\right\rangle}{(q+k)^{2}+i \epsilon}$

Consider $q^{-}$large $(\sim Q)$ and fixed

Consider $q^{+}$to be a variable

$\frac{d^{2} \hat{Q}}{d k_{\perp}^{2}}$ has a pole at $q^{+}=\frac{k_{\perp}^{2}}{2 q^{-}}$

$\hat{Q}$ has a branch cut on the real axis at $\mathrm{q}^{+} \sim \lambda^{2} Q$
$\hat{q}=\operatorname{Im}(\hat{Q})$
$q^{+}$complex plain 
Consider the following integral

$$
I_{1}=\oint \frac{d q^{+}}{2 \pi i} \frac{\hat{Q}\left(q^{+}\right)}{\left(q^{+}+Q_{0}\right)}
$$

$q^{+}$complex plain

$Q_{0}$

For $Q_{0} \sim-Q$, can Taylor expand $\hat{Q}$ in terms of local operators

$$
=\frac{4 \sqrt{2} \pi^{2} \alpha_{s}\left\langle M\left|F_{\perp}^{+\mu} \sum_{n=0}^{\infty}\left(\frac{-q \cdot i \mathcal{D}-\mathcal{D}_{\perp}^{2}}{2 q^{-} Q_{0}}\right)^{n} F_{\perp, \mu}^{+}\right| M\right.}{N_{c} 2 Q_{0}}
$$


Consider the following integral

$$
I_{1}=\oint \frac{d q^{+}}{2 \pi i} \frac{\hat{Q}\left(q^{+}\right)}{\left(q^{+}+Q_{0}\right)}
$$

$q^{+}$complex plain

$Q_{0}$

For $Q_{0} \sim-Q$, can Taylor expand $\hat{Q}$ in terms of local operators

$$
=\frac{4 \sqrt{2} \pi^{2} \alpha_{s}\left\langle M\left|F_{\perp}^{+\mu} \sum_{n=0}^{\infty}\left(\frac{-q \cdot i \mathcal{D}-\mathcal{D}_{\perp}^{2}}{2 q^{-} Q_{0}}\right)^{n} F_{\perp, \mu}^{+}\right| M\right.}{N_{c} 2 Q_{0}}
$$


Consider the following integral

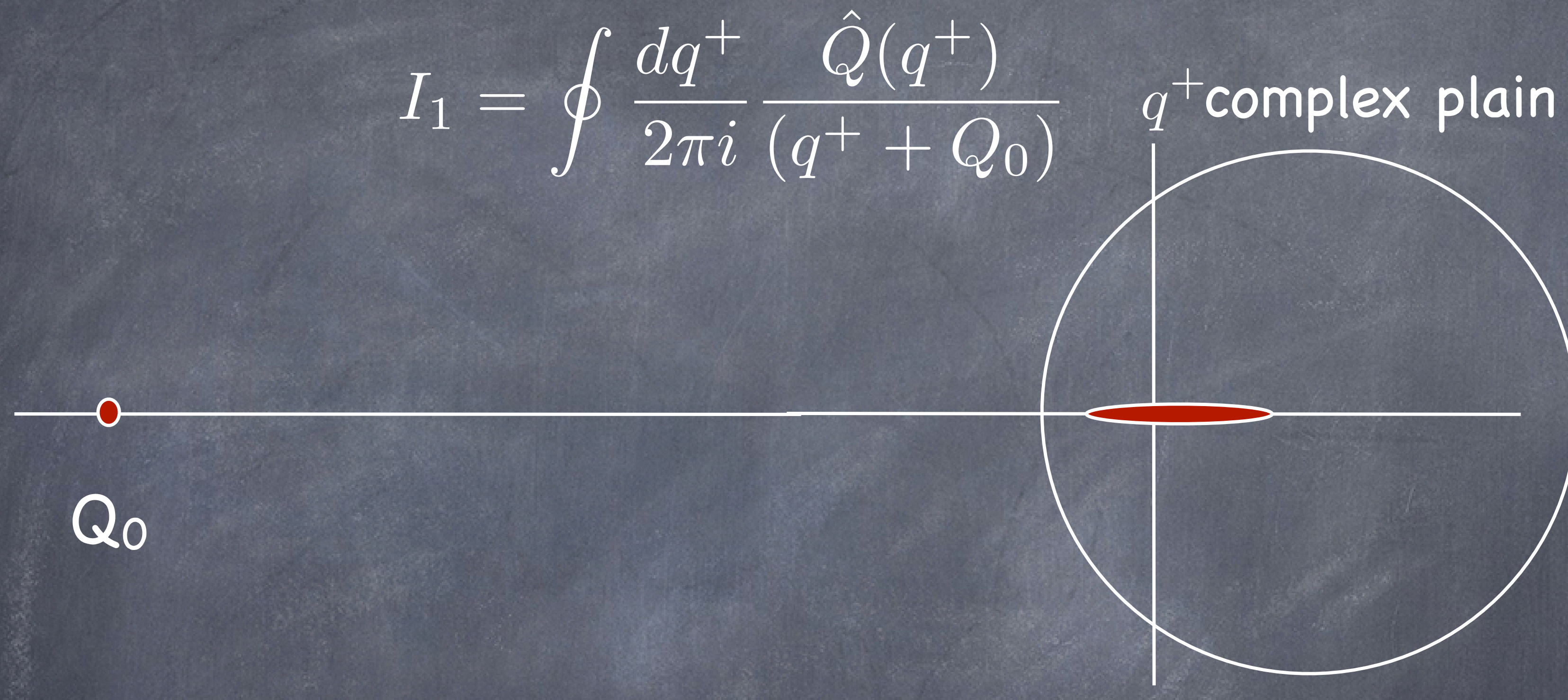

For $Q_{0} \sim-Q$, can Taylor expand $\hat{Q}$ in terms of local operators

$$
=\frac{4 \sqrt{2} \pi^{2} \alpha_{s}\left\langle M\left|F_{\perp}^{+\mu} \sum_{n=0}^{\infty}\left(\frac{-q \cdot i \mathcal{D}-\mathcal{D}_{\perp}^{2}}{2 q-Q_{0}}\right)^{n} F_{\perp, \mu}^{+}\right| M\right.}{N_{c} 2 Q_{0}}
$$


Consider the following integral

$$
I_{1}=\oint \frac{d q^{+}}{2 \pi i} \frac{\hat{Q}\left(q^{+}\right)}{\left(q^{+}+Q_{0}\right)}
$$

$q^{+}$complex plain

$Q_{0}$

For $Q_{0} \sim-Q$, can Taylor expand $\hat{Q}$ in terms of local operators

$$
=\frac{4 \sqrt{2} \pi^{2} \alpha_{s}\left\langle M\left|F_{\perp}^{+\mu} \sum_{n=0}^{\infty}\left(\frac{-q \cdot i \mathcal{D}-\mathcal{D}_{\perp}^{2}}{2 q^{-} Q_{0}}\right)^{n} F_{\perp, \mu}^{+}\right| M\right.}{N_{c} 2 Q_{0}}
$$


Consider the following integral

$$
I_{1}=\oint \frac{d q^{+}}{2 \pi i} \frac{\hat{Q}\left(q^{+}\right)}{\left(q^{+}+Q_{0}\right)}
$$

$q^{+}$complex plain

$Q_{0}$

For $Q_{0} \sim-Q$, can Taylor expand $\hat{Q}$ in terms of local operators

$$
=\frac{4 \sqrt{2} \pi^{2} \alpha_{s}\left\langle M\left|F_{\perp}^{+\mu} \sum_{n=0}^{\infty}\left(\frac{-q \cdot i \mathcal{D}-\mathcal{D}_{\perp}^{2}}{2 q^{-} Q_{0}}\right)^{n} F_{\perp, \mu}^{+}\right| M\right.}{N_{c} 2 Q_{0}}
$$




\section{Rotating everything to Euclidean space and calculating \\ $x^{0} \rightarrow-i x^{4}$ and $A^{0} \rightarrow i A^{4}$ $\rightarrow F^{0 i} \rightarrow i F^{4 i}$}

Calculate in quark less SU(2) gauge theory
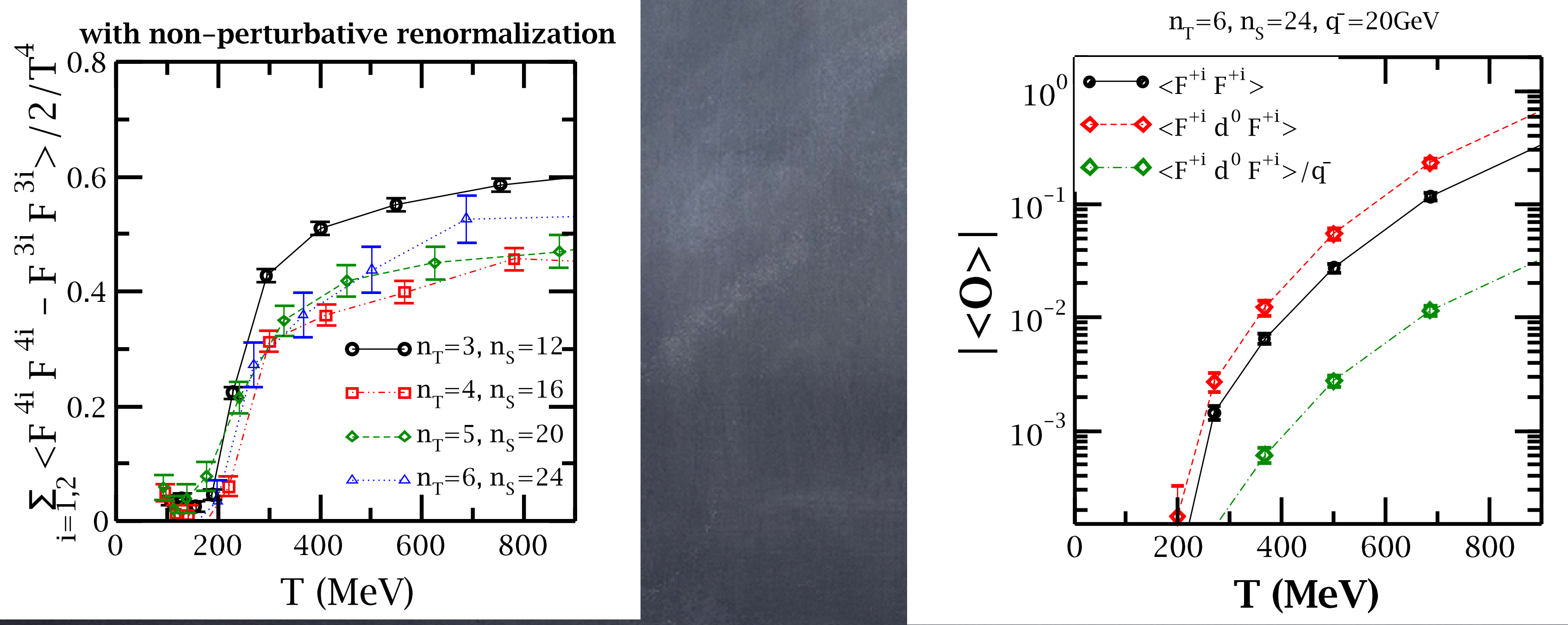


\section{Concluding and Extrapolating !}

Need to calculate in SU(3)

Better renormalization prescription

More complicated processes on the lattice

Need to do a higher order perturbative calculation But lets estimate anyways

at $\mathrm{T}=400, \mathrm{FF}=0.01 \mathrm{GeV}^{4}$

Lattice size $\sim 2 \mathrm{fm}, \mathrm{E}=20 \mathrm{GeV}, \mu^{2}=1.3 \mathrm{GeV}^{2}$

Gluon $\hat{q}$ is $C_{A} / C_{F}$ of quark $\hat{q}$

SU(2) has 3 gluons, SU(3) has 8, and 6 quarks + antiquarks

$\hat{q}(T=400 \mathrm{MeV})=1 \mathrm{GeV}^{2} / \mathrm{fm}-2 \mathrm{GeV}^{2} / \mathrm{fm}$ 


\section{A lingering problem with D-Au}
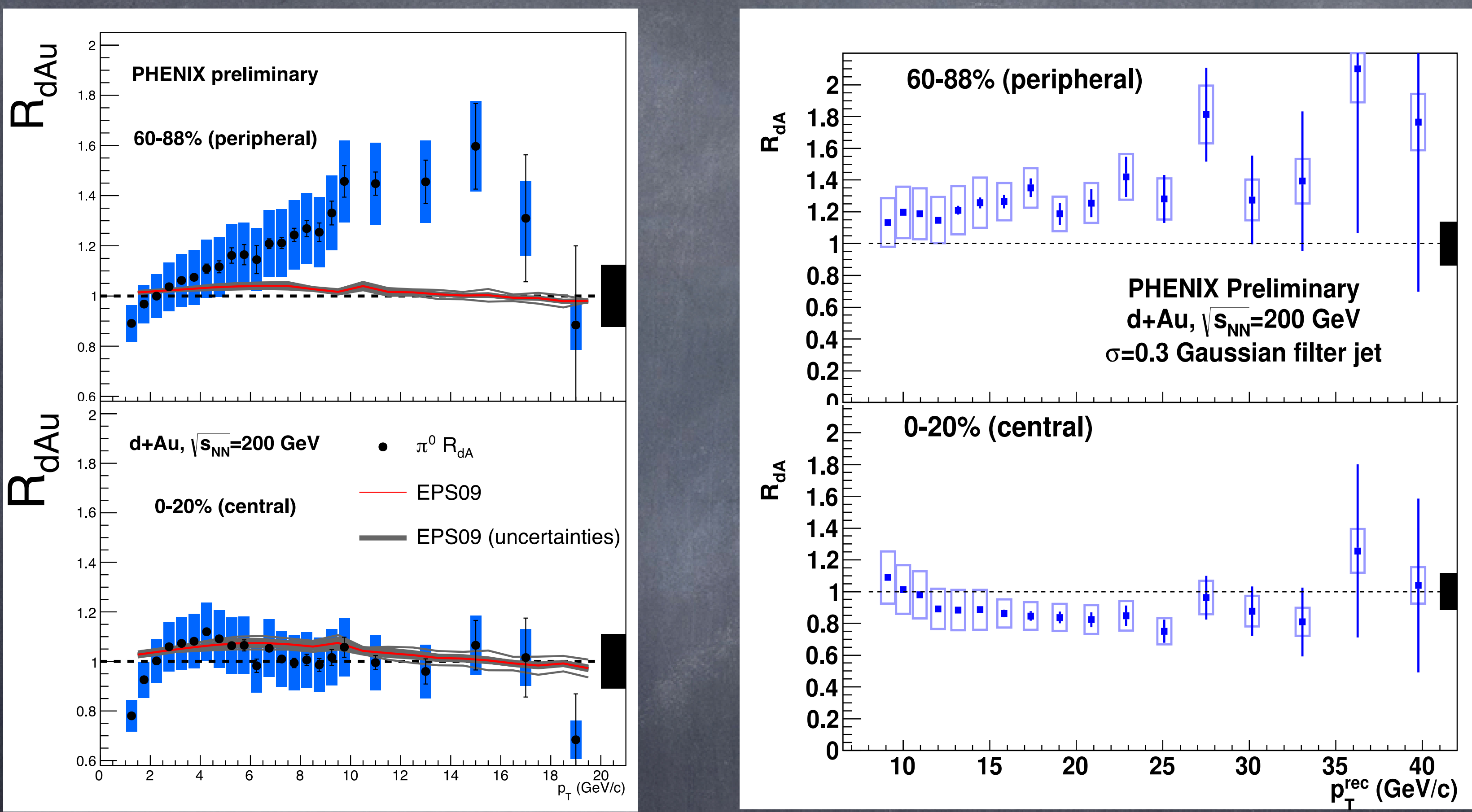

Why does this happen? 


\section{Are there more scatterings per nucleon in peripheral vs. central D-Au}
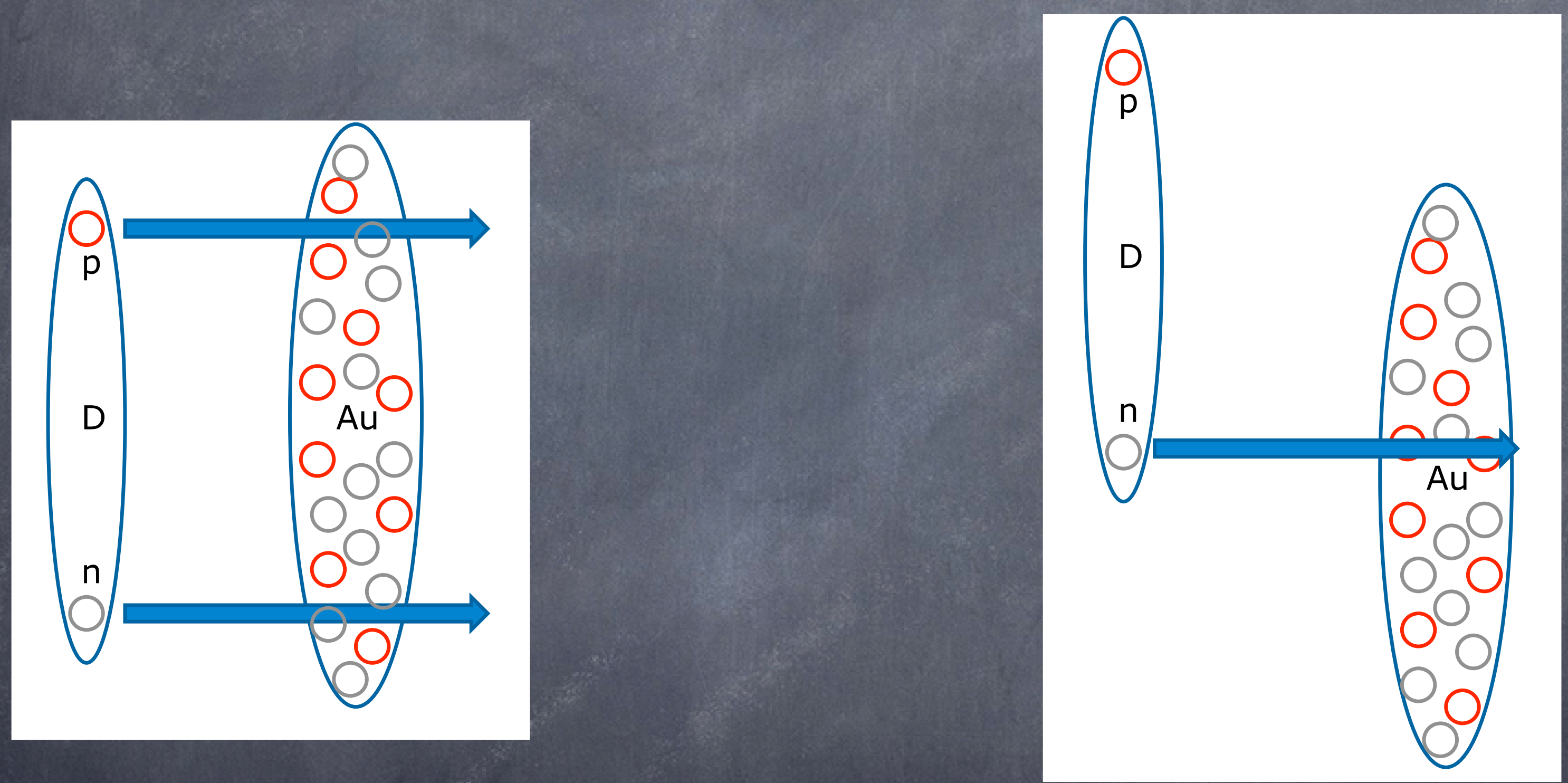

No, but the numbers are very close.

Testing to see if correlated initial state and final state scattering can explain the data. 


\section{Done more carefully than you would think!}
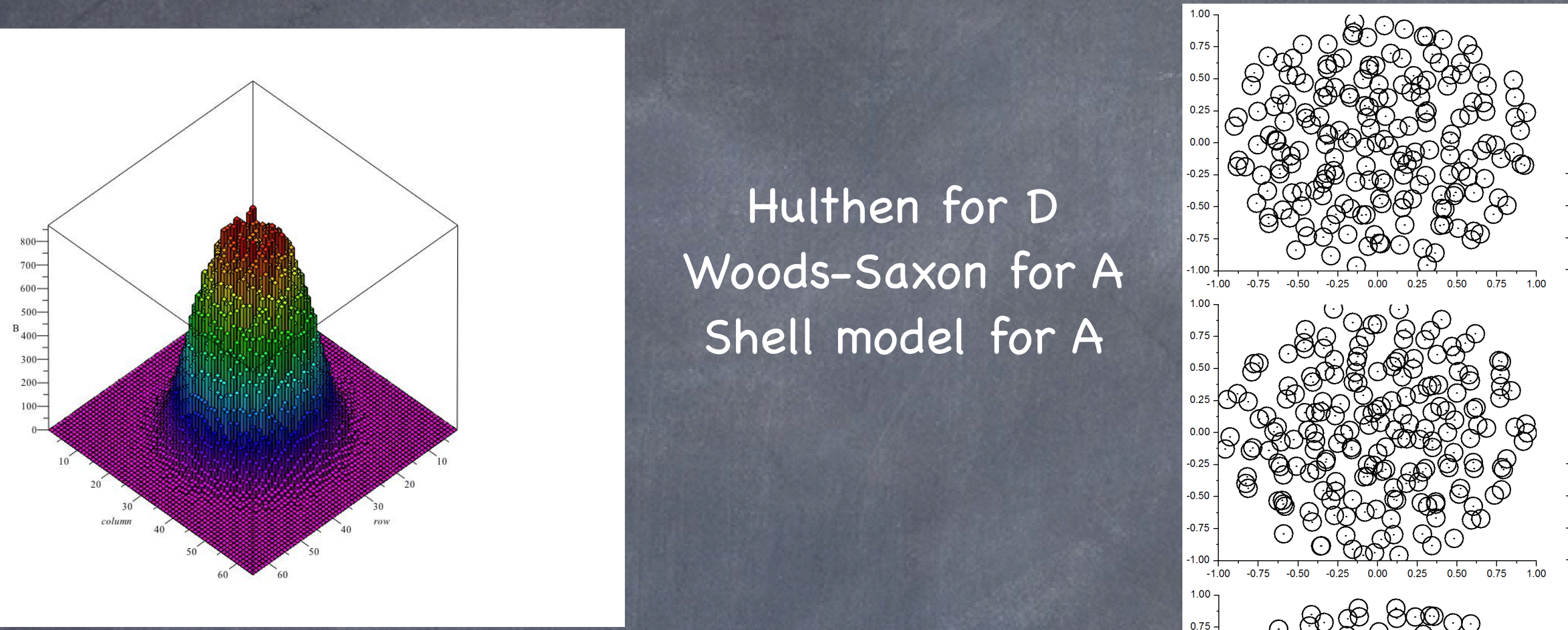

Woods-Saxon for A Shell model for $A$

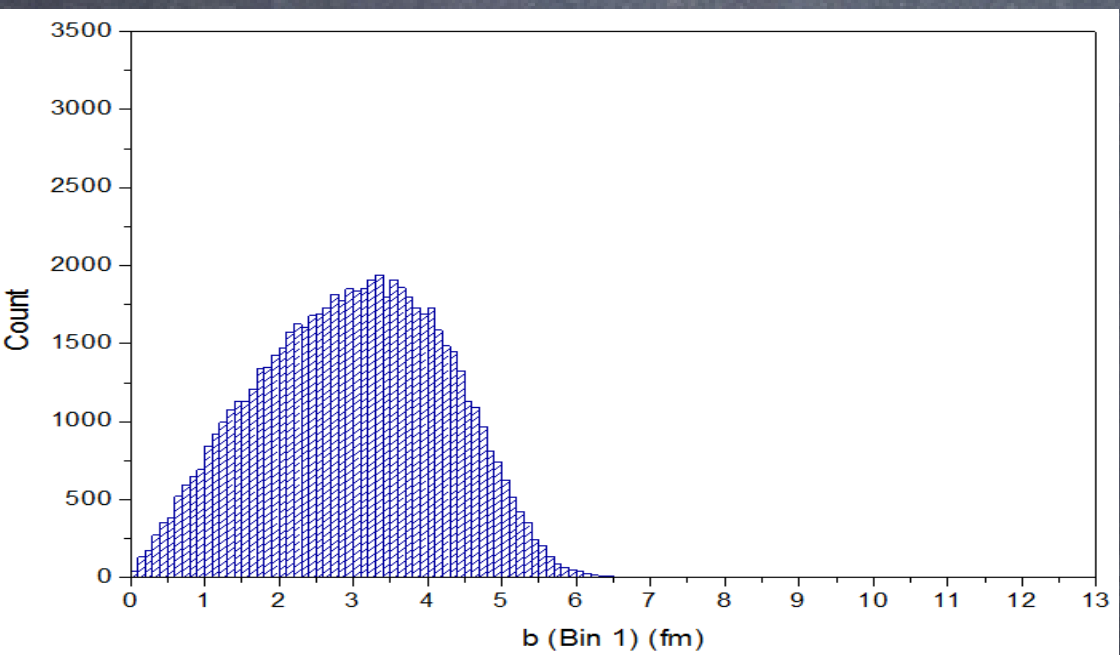

Sum of $1 M$ events
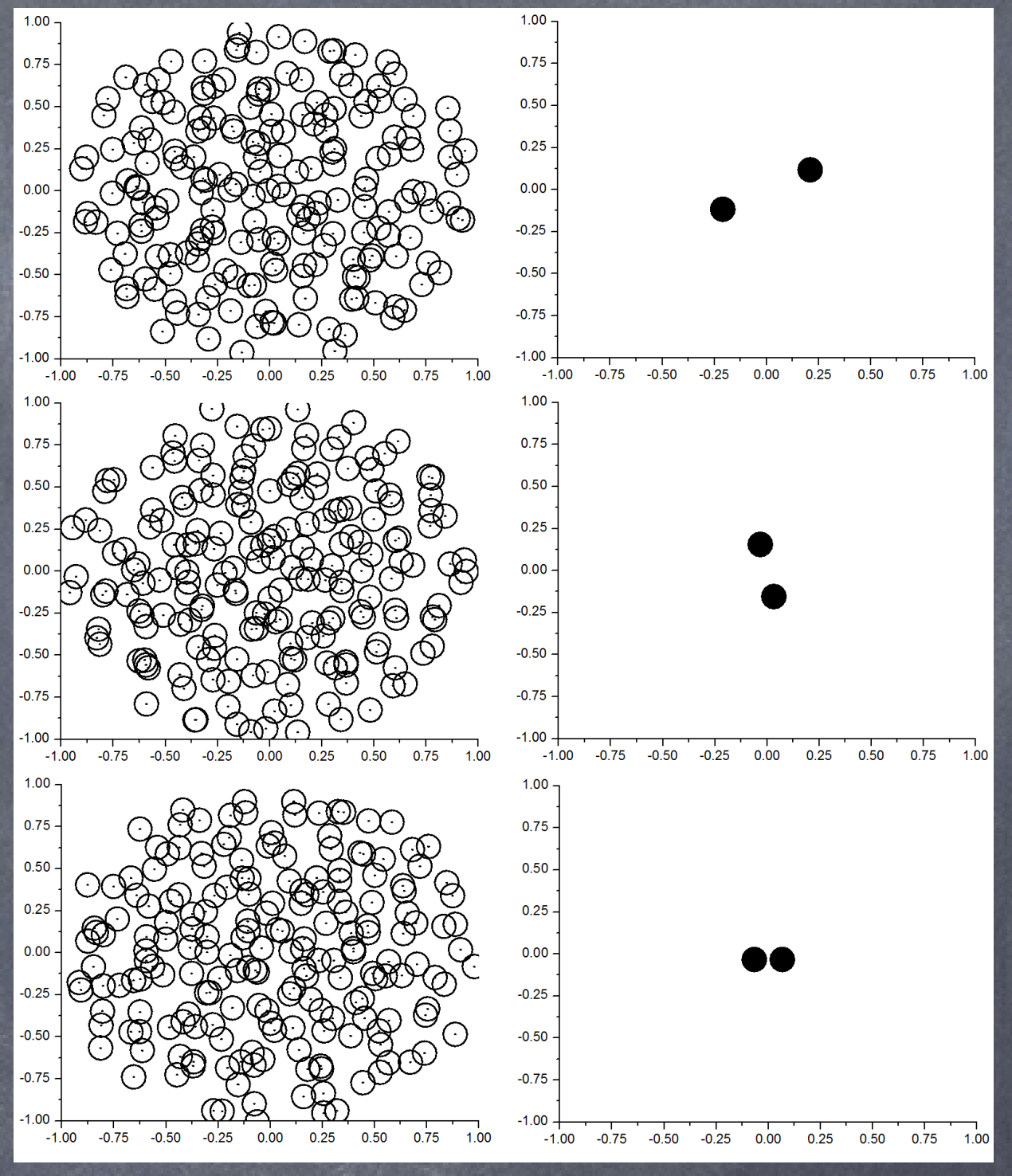
Distribution of $b$ for each $N_{\text {bin }}$

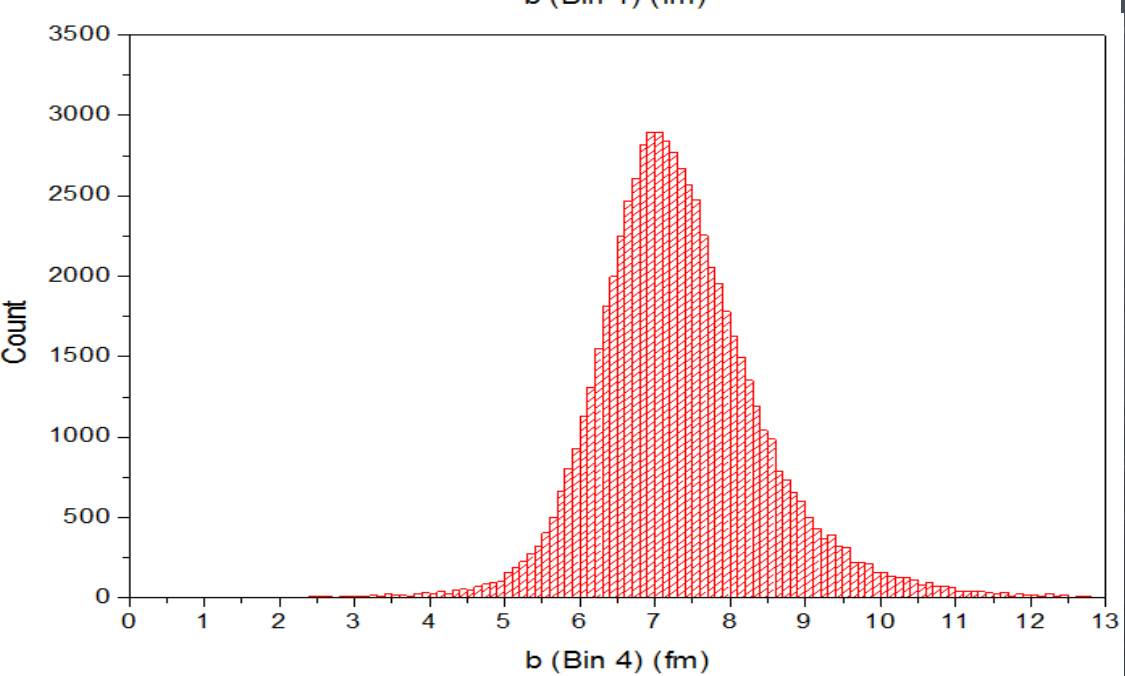

A new e-by-e shadowing 


\section{Summary}

After $R_{A A}$ test HT energy loss scheme on the expansion

Full Jet MC being incorporated with E-by-E hydro

Energy deposition calculations being developed

Lattice QCD calculations of transport parameters

Detailed phenomenology for D-A and AA being set up

Interesting issues with heavy-quarks and NLO 


\section{Jets at Detroit, part II}

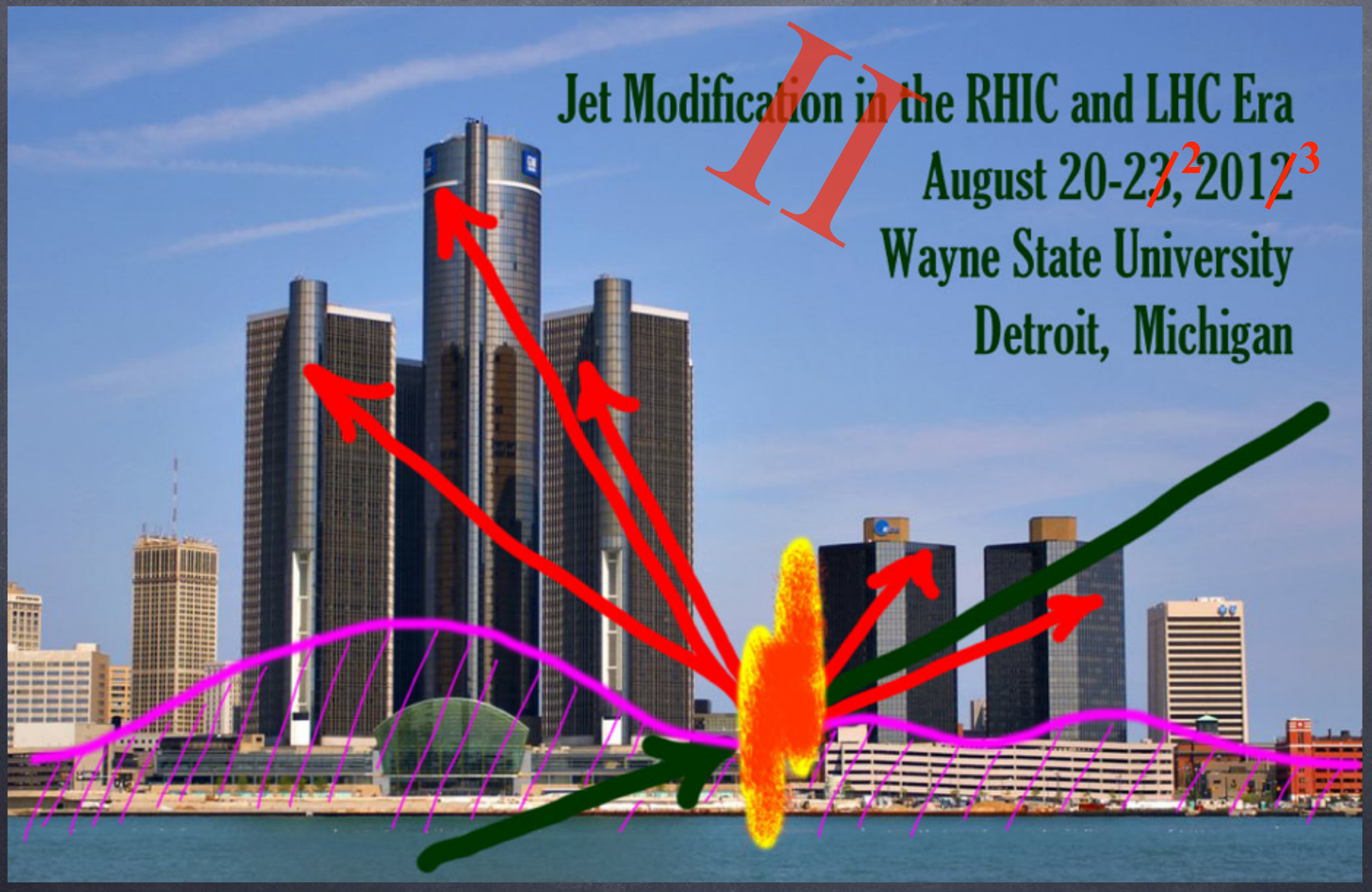




\section{Event-by-event flow and initial geometry from LHC}

Soumya Mohapatra

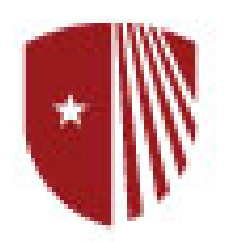

Stony Brook

University

Jet Quenching Workshop, BNL

16 $6^{\text {th }}$ April 2013 


\section{Importance of fluctuations}

- Initial spatial fluctuations of nucleons lead to higher moments of deformations in the fireball, each with its own orientation. Alver, Roland (arXiv:1003.0194)

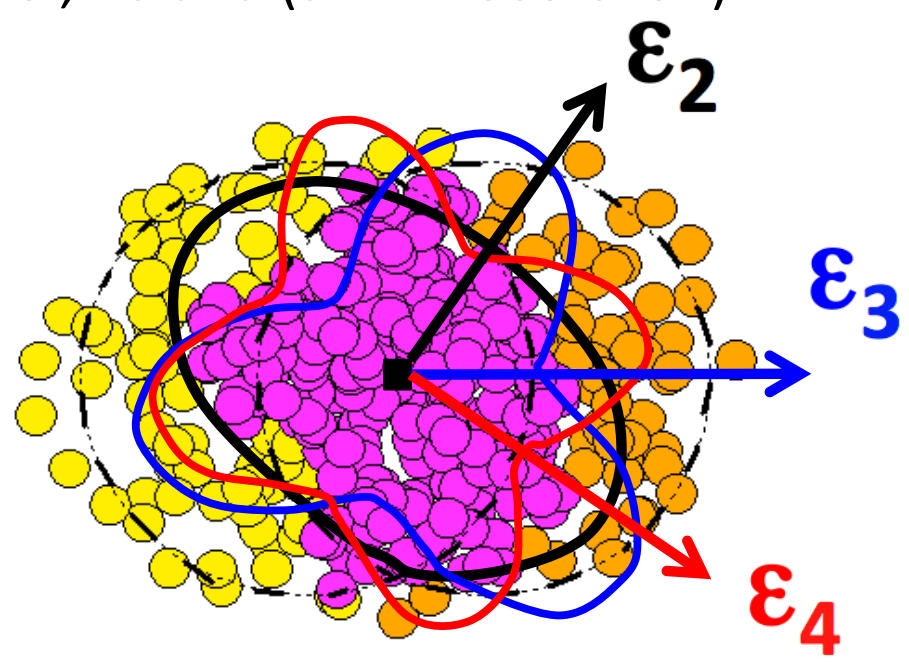

1. Odd harmonics present

2. $v_{n}$ is a distribution, can be characterized by mean and width

3. Each harmonic has a separate phase (phases may be correlated)

$\varepsilon_{n}=\sqrt{\frac{\left\langle r^{n} \cos (n \phi)\right)+\left\langle\left\langle r^{n} \sin (n \phi)\right)\right\rangle}{r^{n}}} \quad \tan \left(n{ }_{n}^{n}\right)=\frac{\left\langle r^{n} \sin (n)\right\rangle}{\left\langle r^{n} \cos (n)\right\rangle}$

Large acceptance of the LHC experiments coupled with the increased multiplicity has allowed for great precision is studying the nature of these fluctuations

Understanding the initial geometry is critical for understanding jetsuppression 


\section{OUTLINE}

- Multi-particle correlation measurements

- Cumulants, 2PC, LYZ

- Event by Event $v_{n}$ measurements

- Event-plane correlations

Emphasis on

- Removing non-flow

- Comparison between experiments and methods

- Theory interpretation 


\section{Gaussian model of flow fluctuations}

$$
\vec{n}_{n}=\left({ }_{x},{ }_{y}\right)
$$

$p\left(\vec{n}_{n}\right) \mu \exp \frac{\left(\begin{array}{cc}\vec{n}_{n} & \rightarrow R P \\ 2_{n}^{2}\end{array}\right)^{2}}{\dot{\div}}$

$p\left(\vec{v}_{n}\right) \mu \exp \frac{\left(\vec{v}_{n} \vec{v}_{n}^{R P}\right)^{2}}{2_{n}^{2}} \div$

$\underset{n}{\rightarrow R P} \rightarrow$ Mean Geometry

$\delta_{n} \rightarrow$ Fluctuations

$p\left(v_{n}\right) \mu v_{n} \exp \frac{\left(v_{n}^{2}+\left(v_{n}^{R P}\right)^{2}\right.}{2_{n}^{2}} \div I_{0}\left(\frac{v_{n} v_{n}^{R P}}{2_{n}}\right)$

arXiv: 0708.0800

For pure fluctuations $\mathrm{v}_{\mathrm{n}}{ }^{\mathrm{RP}}=0$

arXiv:0809.2949

$$
p\left(v_{n}\right) \propto v_{n} \exp \left(\frac{-\left(v_{n}^{2}\right)}{2 \delta_{n}^{2}}\right)
$$




\section{Multi-particle correlations}

$v_{n}\{2\}=\sqrt{\left(v_{n}^{R P}\right)^{2}+2_{n}^{2}} \quad$ Sensitive to mean geometry and fluctuations

arXiv: 0708.0800

0809.2949

$v_{n}\{4\}=v_{n}\{6\}=v_{n}\{8\}=v_{n}^{R P}$ Mean geometry only

$\frac{n}{\left\langle v_{n}\right\rangle} \sqrt{\frac{v_{n}\{2\} \quad v_{n}\{4\}}{v_{n}\{2\}+v_{n}\{4\}}} \quad \begin{aligned} & \text { Limit when } v_{n}{ }^{\mathrm{RP}}>>\delta_{n} \text { (i.e. Average geo } \\ & \text { dominates over fluctuations) } \\ & \text { Expected for } v_{2} \text { in mid-central events }\end{aligned}$

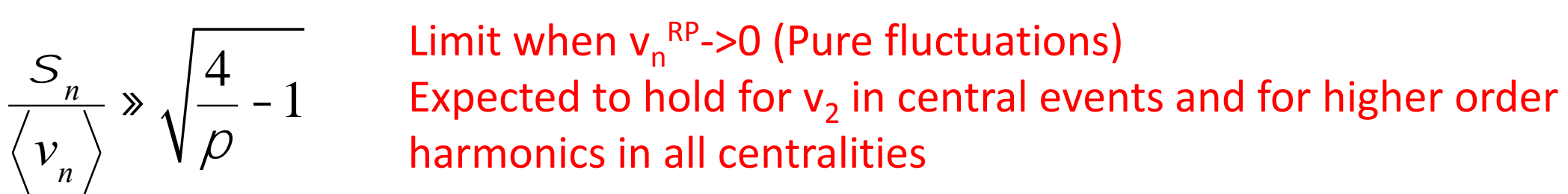

Lee-Yang Zeros : Multi-particle correlations involving all particles in the event. suppresses non-flow

Two particle correlations: similar to $v_{n}\{2\}$, but often done with dh gap to suppress non-flow. Measures $\left\langle\mathrm{v}_{\mathrm{n}}{ }^{2}\right\rangle$

Event Plane (EP) Method : Returns a value in between $\left\langle v_{n}\right\rangle$ and $\left\langle v_{n}{ }^{2}\right\rangle$ 


\section{$v_{2}$ from multi-particle correlations}

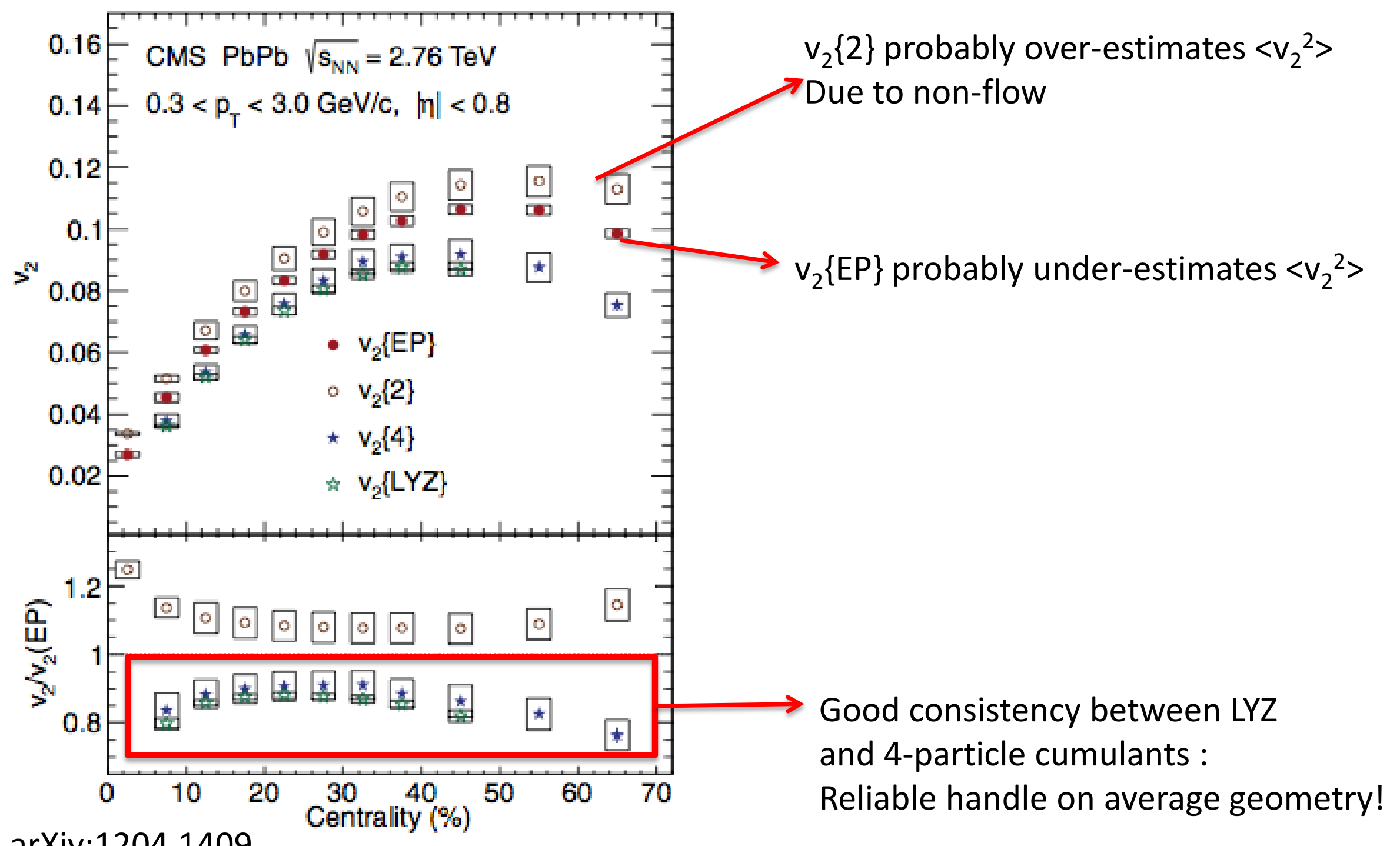

arXiv:1204.1409 


\section{Comparison across experiments}

ATLAS-CONF-2012-118

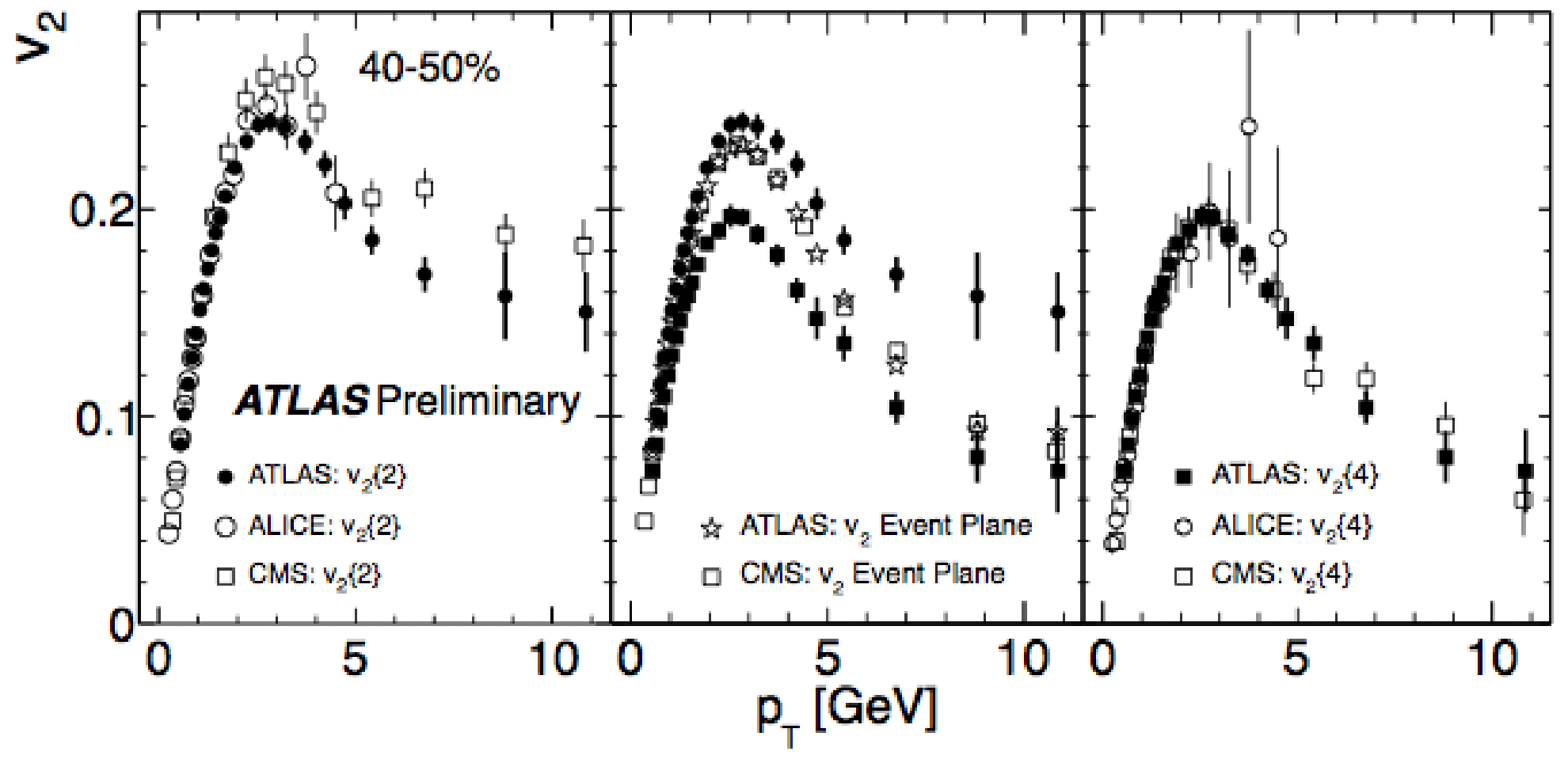

Good agreement among experiments for cumulants and even $v_{2}\{E P\}$ 


\section{pT dependence of EbE fluctuations}

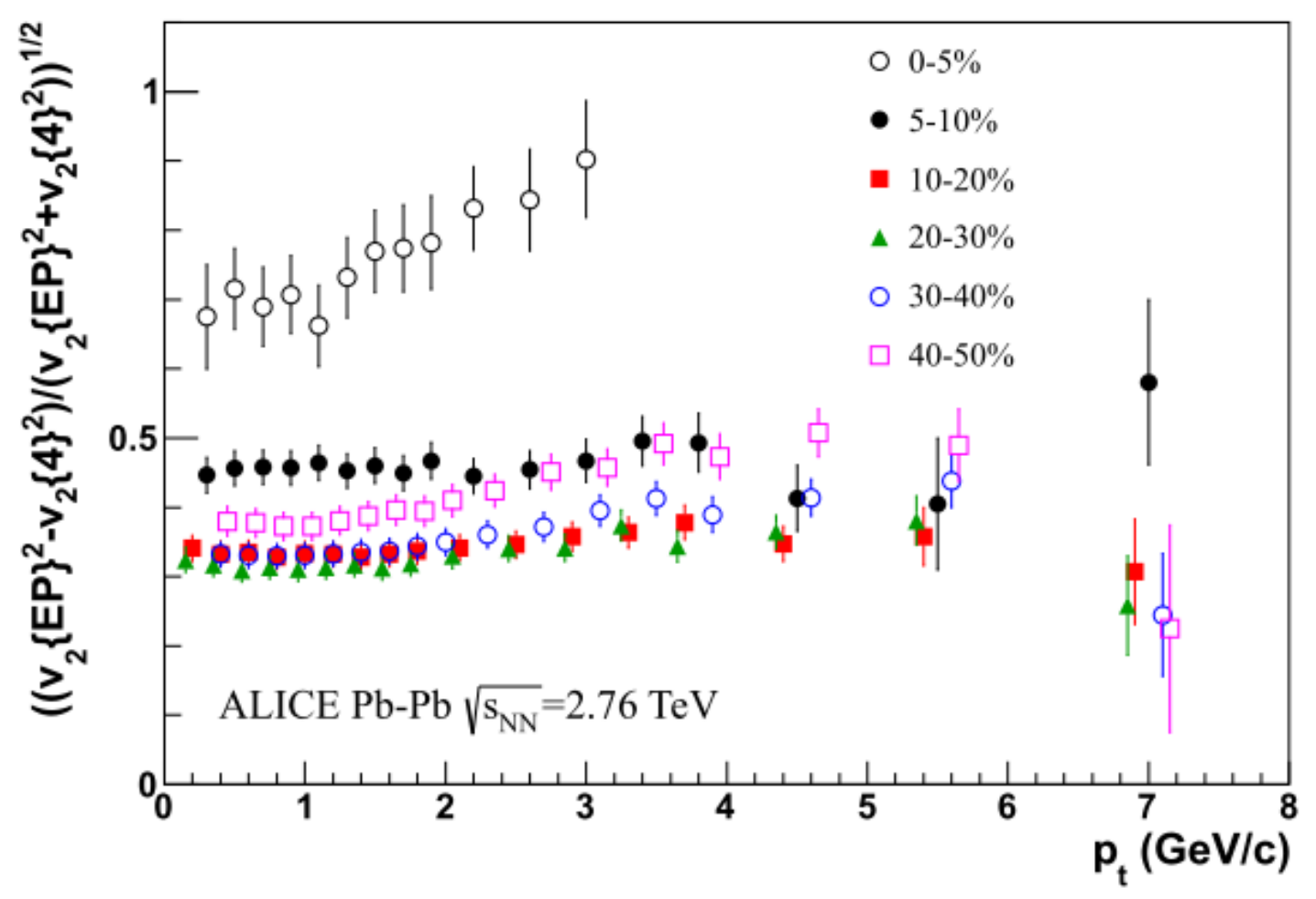

arXiv:1205.5761

$$
\frac{2}{\left\langle v_{2}\right\rangle} \sqrt{\frac{v_{2}\{E P\} \quad v_{2}\{4\}}{v_{2}\{E P\}+v_{2}\{4\}}}
$$

Ratio of fluctuations in $v_{2}$ to mean $v_{2}$ is relatively independent of $p_{T}$

Note that $v_{2}\{E P\}$ changes by an order of magnitude over this $p_{T}$ range but ratio is remarkably stable

Hydro response factorizes of function of $\mathrm{p}_{\mathrm{T}}$ and initial geometry! 


\section{Higher order cumulants for $\mathrm{v}_{2}$}

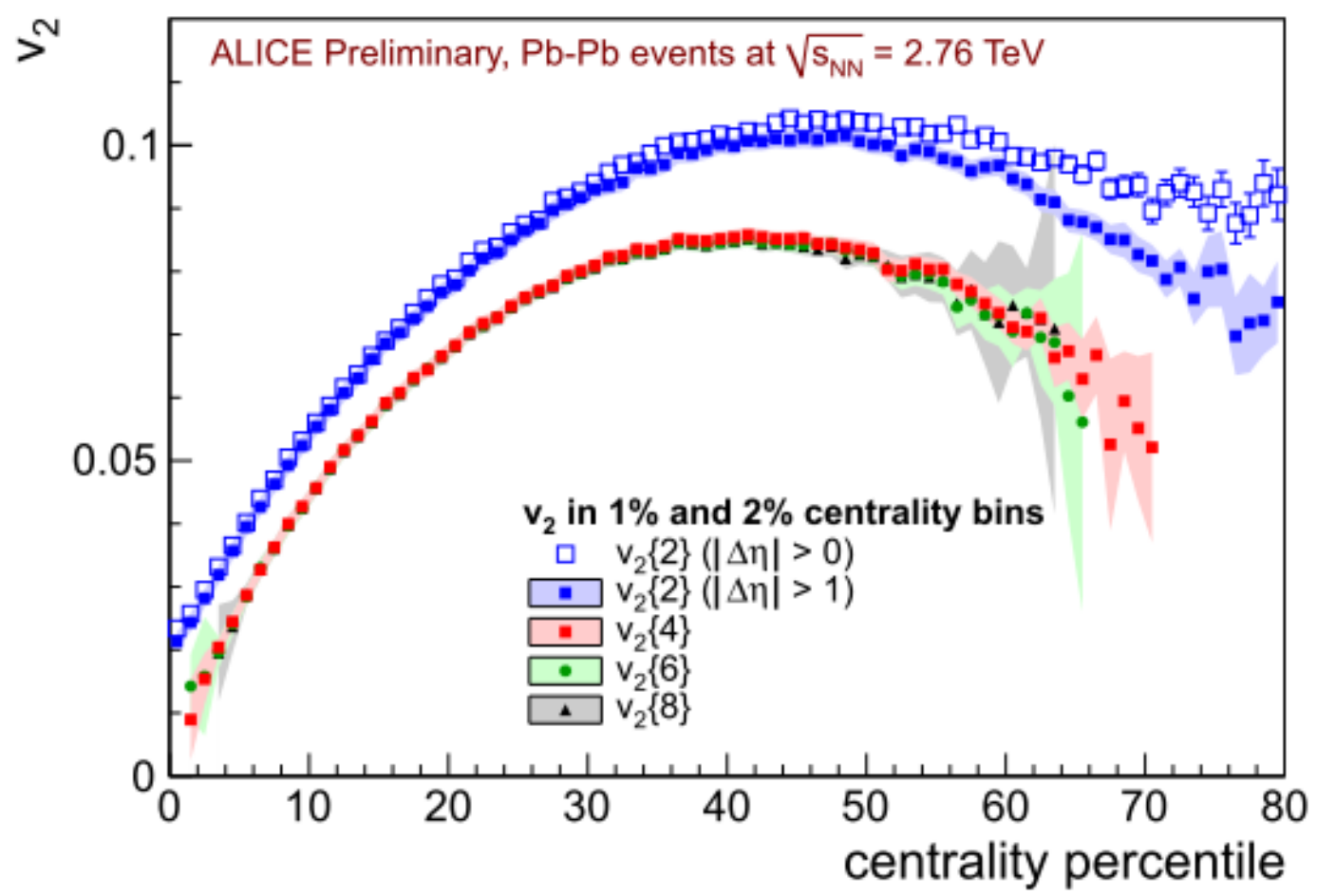

- Higher order cumulants such as $v_{n}\{6\}, v_{n}\{8\}$ all measure $v_{n}{ }^{R P}$

- $v_{n}{ }^{R P}$ is less susceptible to non-flow and so are $v_{n}\{4\}, v_{n}\{6\}, v_{n}\{8\}$.

- ALICE results show consistency among them

- Note these measurements are done in $1 \%$ bins (Good!) 


\section{Cumulant results v3}

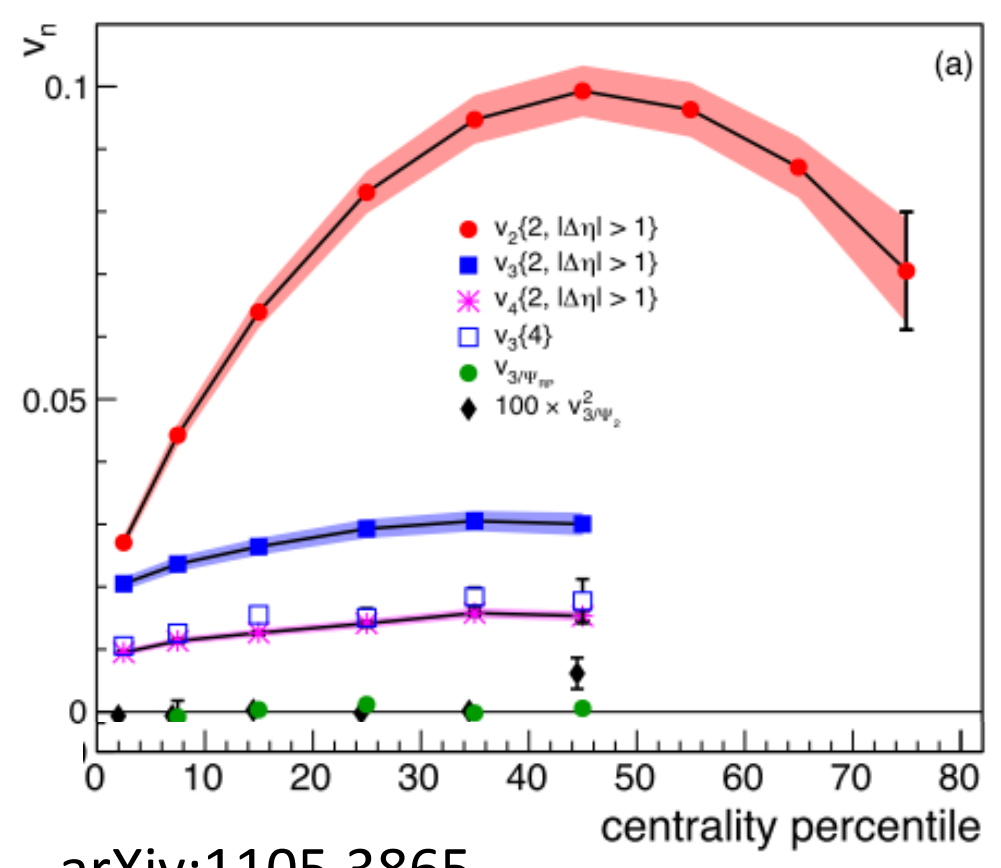

arXiv:1105.3865

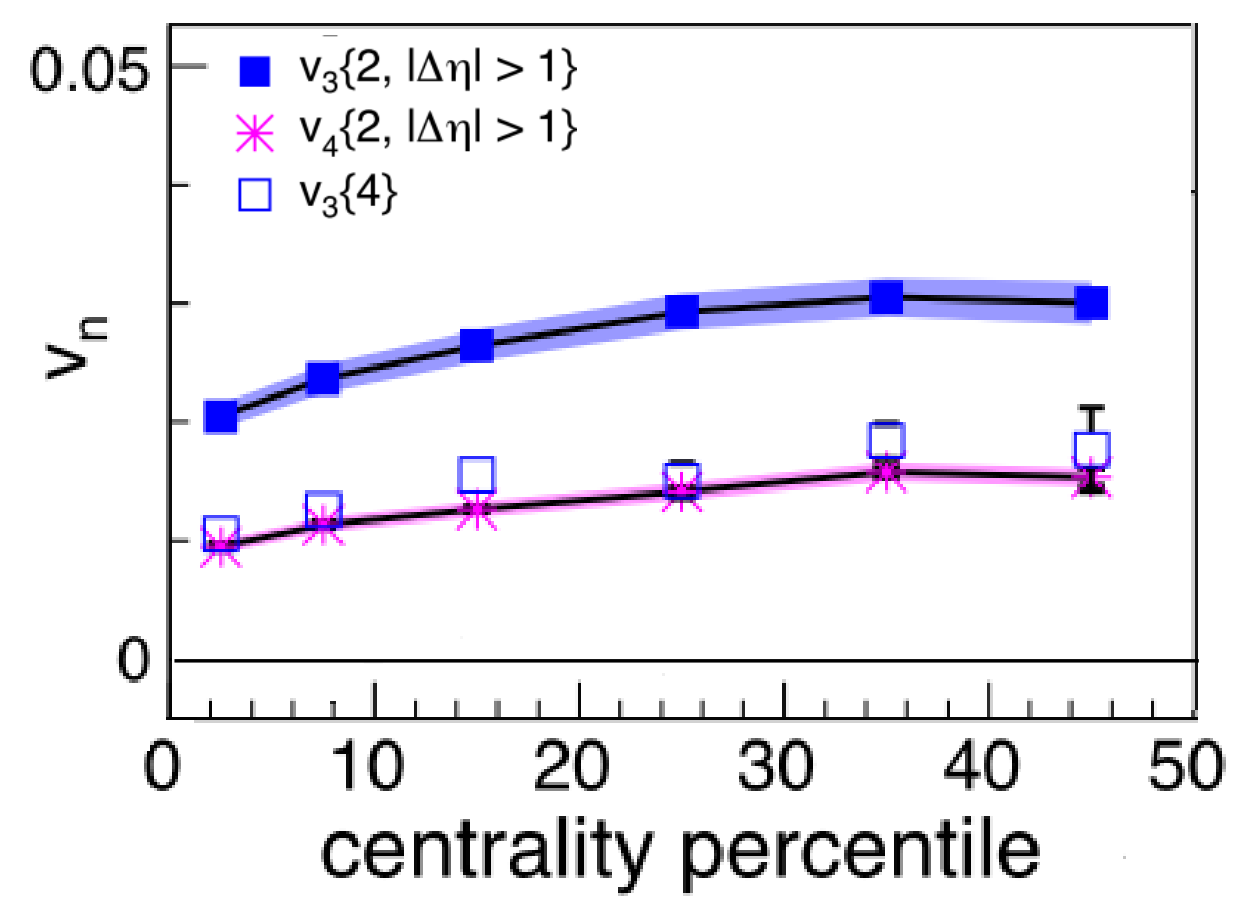

- Relatively weak centrality dependence as compared to $v_{2}$

- Sizable $v_{3}\{4\}$ is seen $\sim 50 \%$ of $v_{3}\{2\}$

- Implies mean geometry effects for $v_{3}$ !

- $v_{3}\{4\} / v_{3}\{2\}=0.5 \Rightarrow v_{3}{ }^{R P} / \delta_{3}=0.8$ 


\section{$v_{3}\{4\}$ and average geometry}
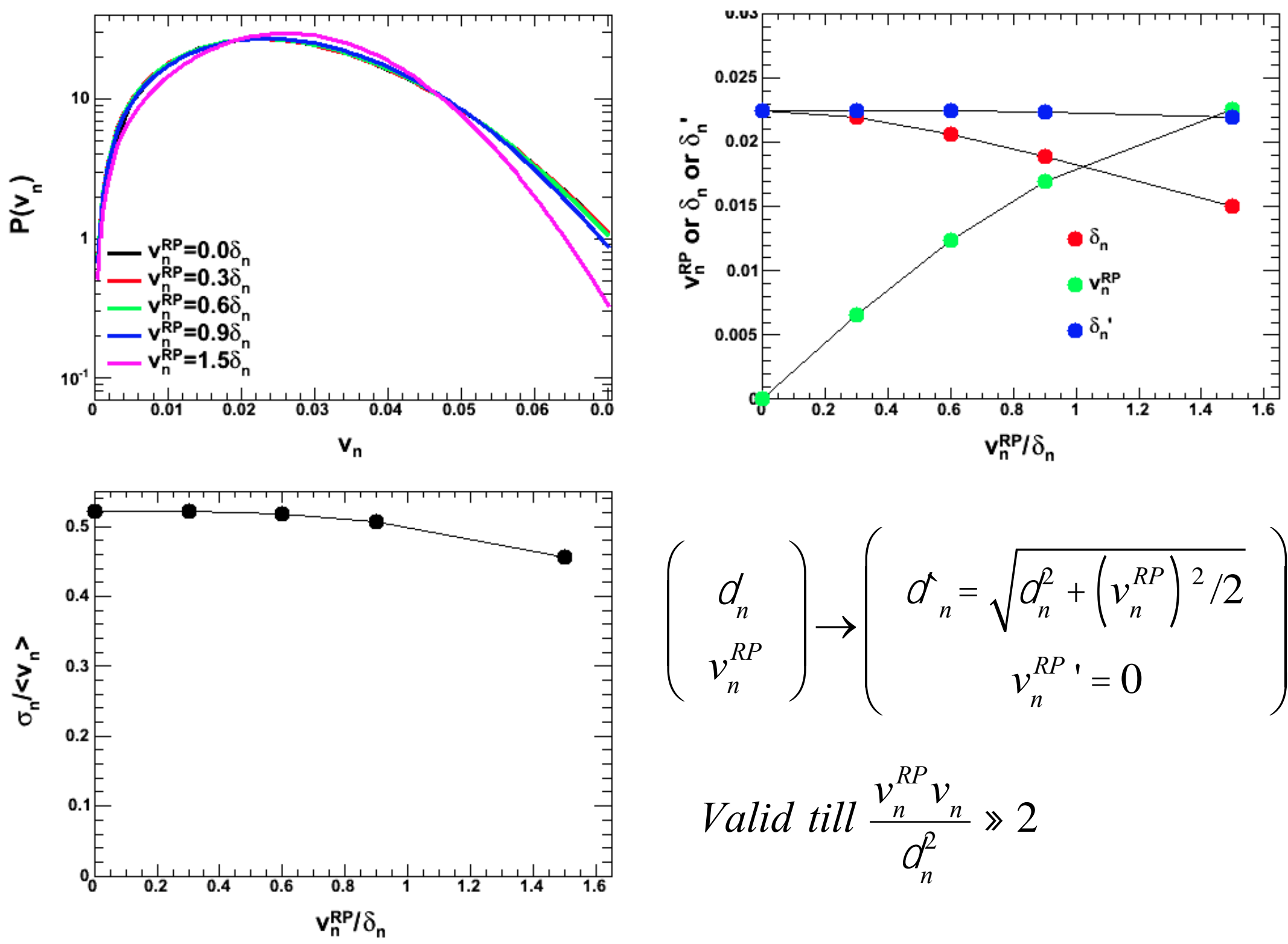

$$
\begin{gathered}
\left(\begin{array}{c}
n \\
v_{n}^{R P}
\end{array}\right) \rightarrow\left(\begin{array}{c}
\bar{n}_{n}=\sqrt{{ }_{n}^{2}+\left(v_{n}^{R P}\right)^{2} / 2} \\
v_{n}^{R P}{ }^{\prime}=0
\end{array}\right) \\
\text { Valid till } \frac{v_{n}^{R P} v_{n}}{2} \\
n_{n}
\end{gathered}
$$




\section{Event by Event flow measurements}

Track distribution in three central events
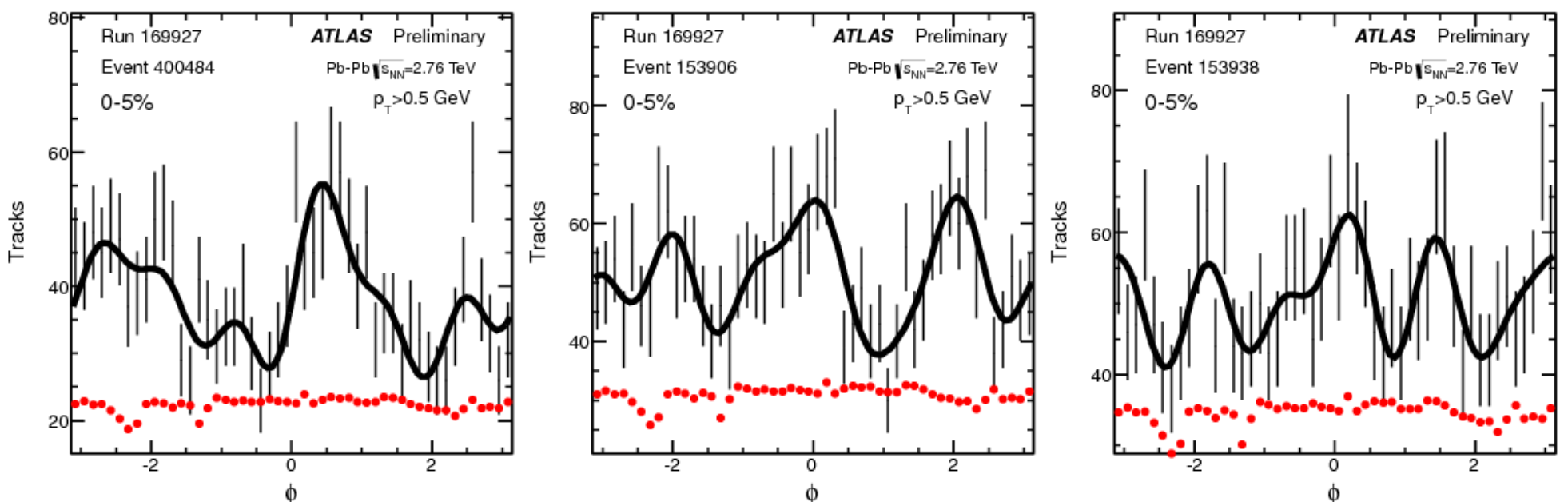

Corresponding twoparticle correlations
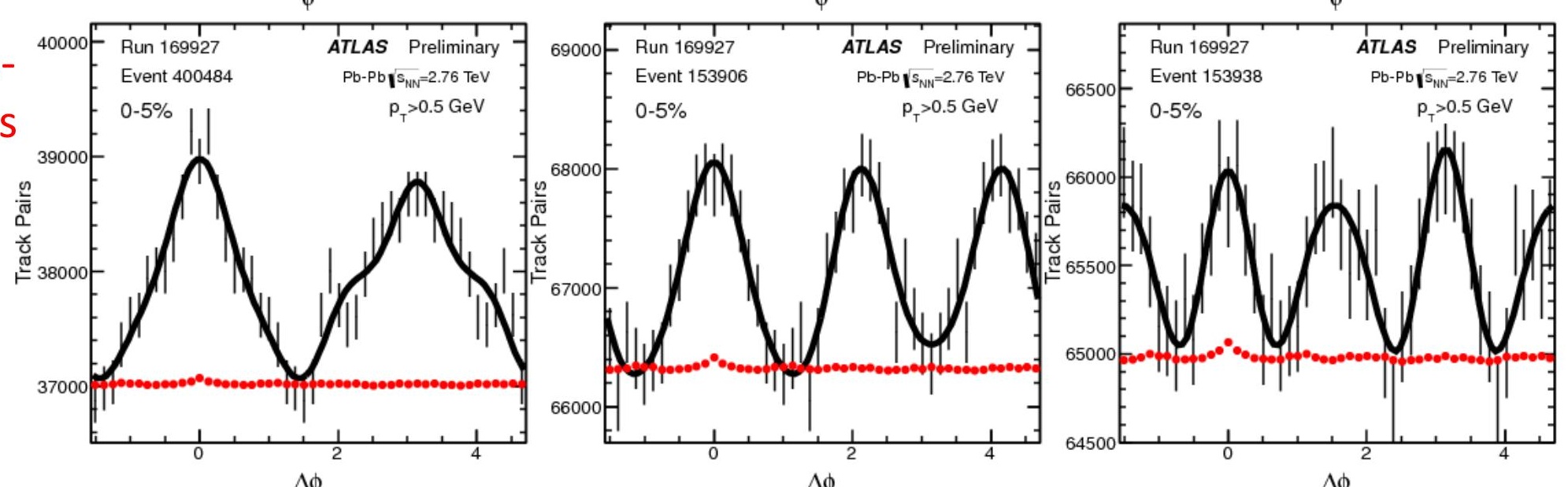

The large acceptance of the ATLAS/ALICE detectors and large multiplicity at LHC makes EbE $v_{n}$ measurements possible for the first time. 


\section{$v_{2}-v_{3}$ probability distributions}
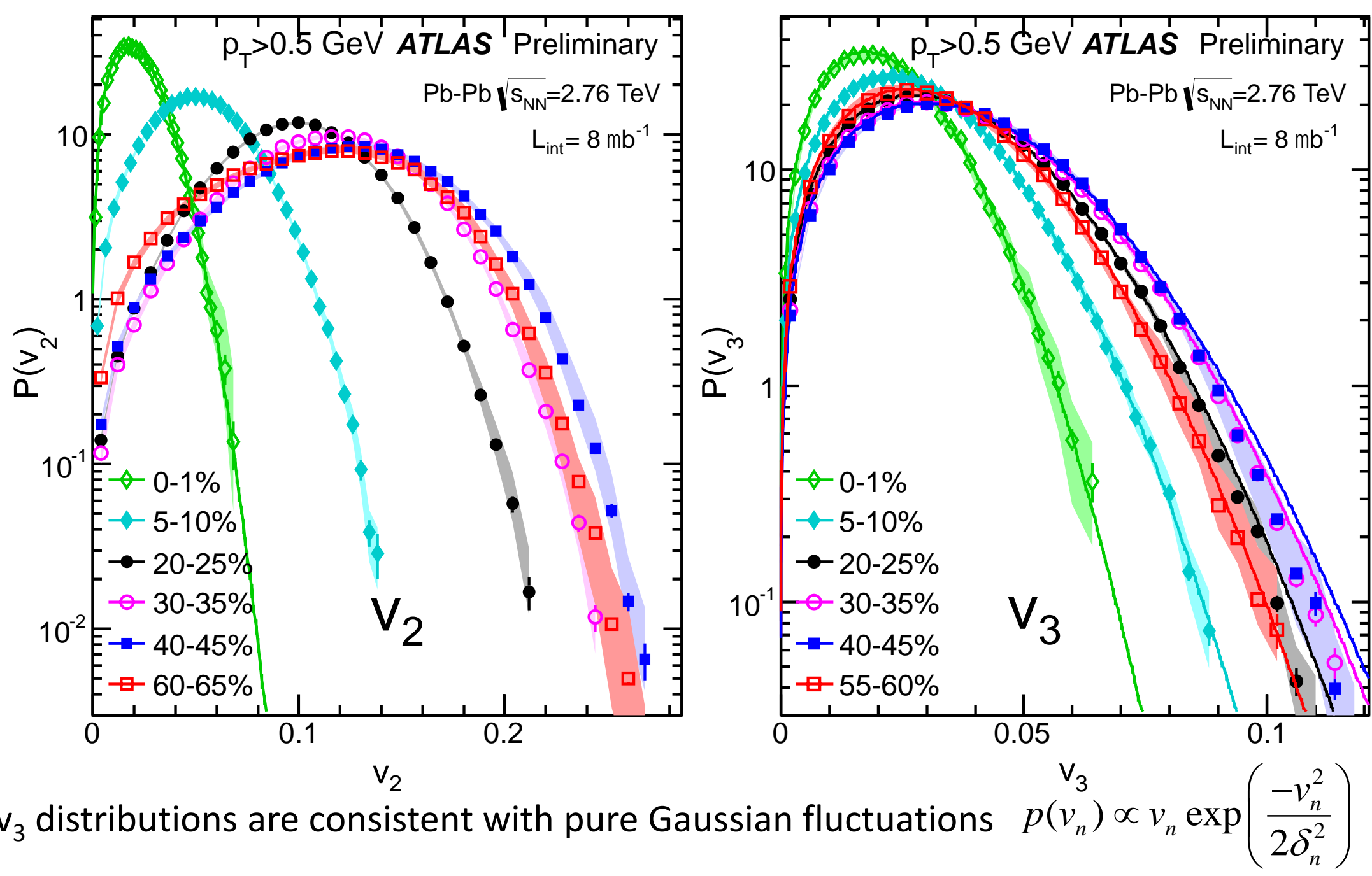

deviations in the tail (increases central->midcentral), Also see caveat in slide 11

For $v_{2}$ pure Gaussian fits only work for most central (2\%) events 


\section{$v_{2}$ probability distributions via $2 \mathrm{PC}$}

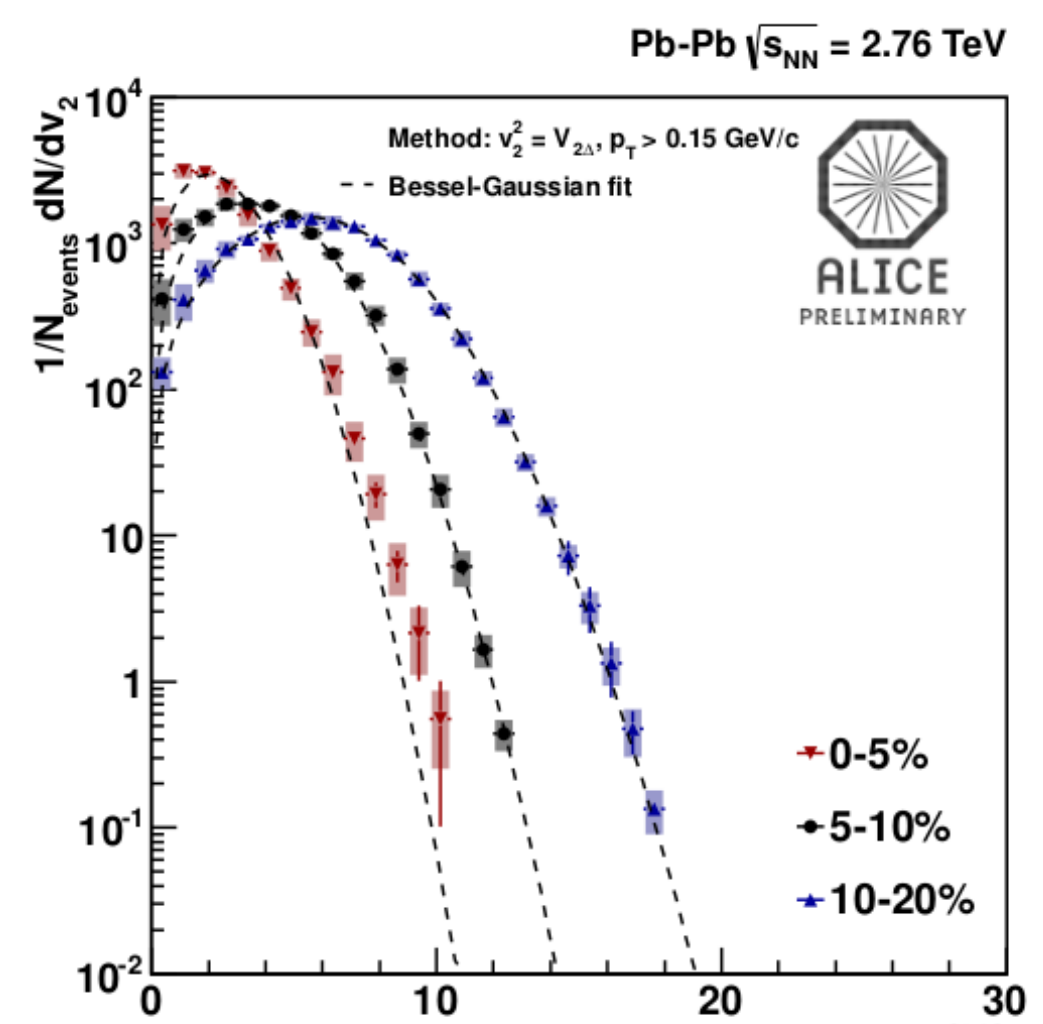

A. Timmins, Hot Quarks $2012^{\mathbf{v}_{2}(\%)}$

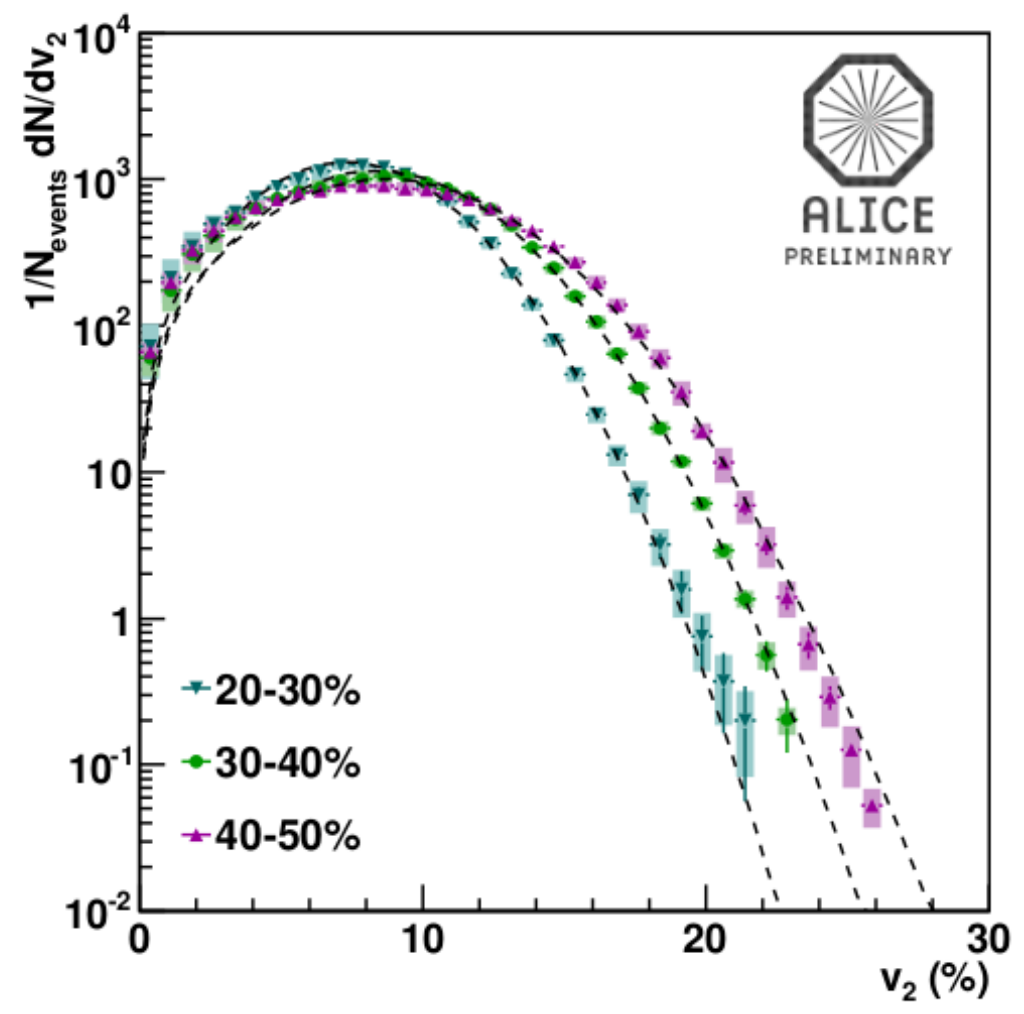

ALICE EbE $v_{2}$ measurements obtained via $2 \mathrm{PC}$ followed by unfolding.

$v_{2}$ described by Bessel-Gaussian distribution: Contribution from mean geometry+fluctuations.

$$
p\left(v_{n}\right) \mu v_{n} \exp \frac{\left(v_{n}^{2}+\left(v_{n}^{R P}\right)^{2}\right)}{2_{n}^{2}} \div I_{0}\left(\frac{v_{n} v_{n}^{R P}}{2}\right)
$$




\section{Relative fluctuations of $v_{3}$}
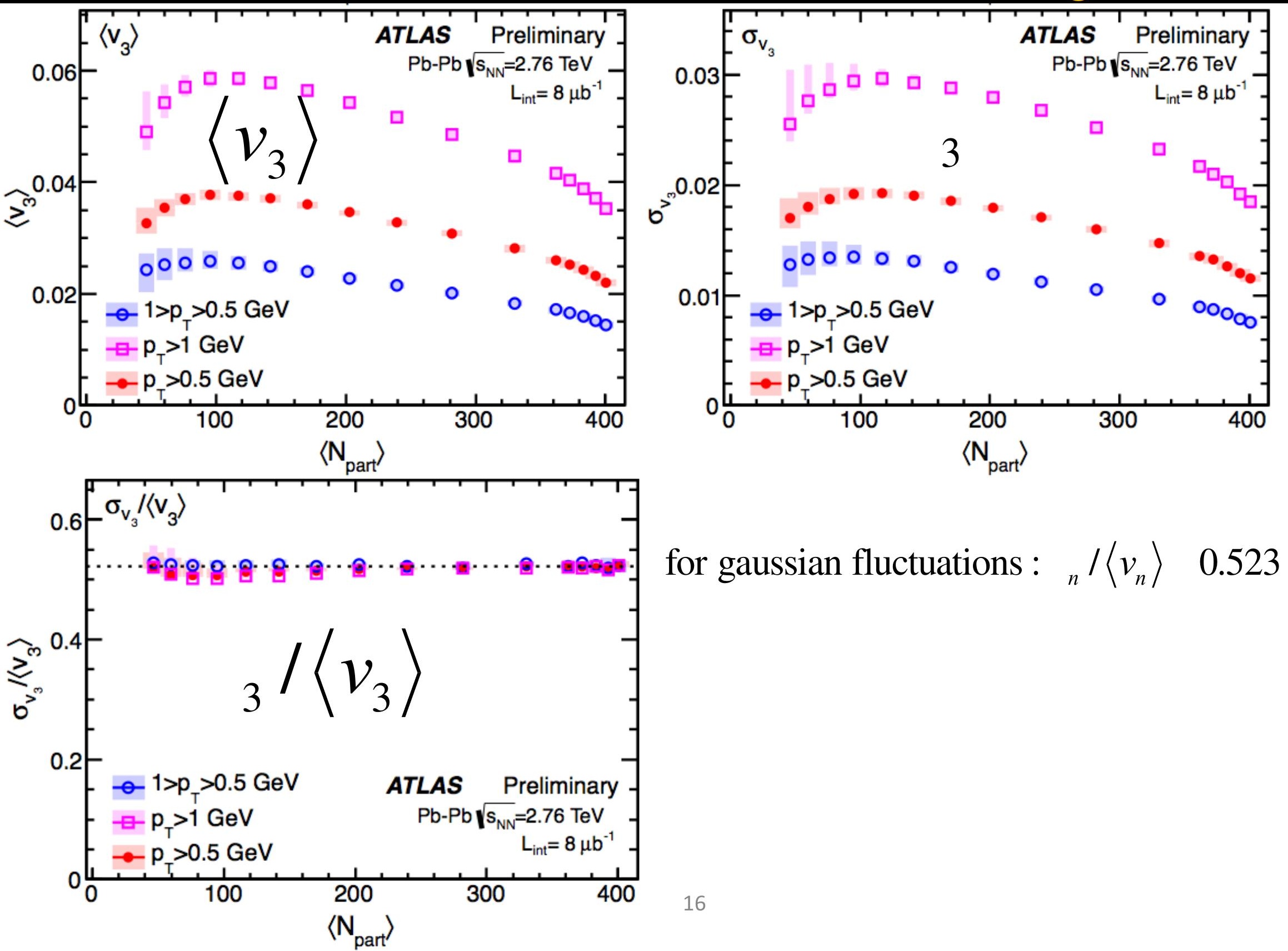

for gaussian fluctuations : ${ }_{n} /\left\langle v_{n}\right\rangle \quad 0.523$ 


\section{Comparison to cumulant results}
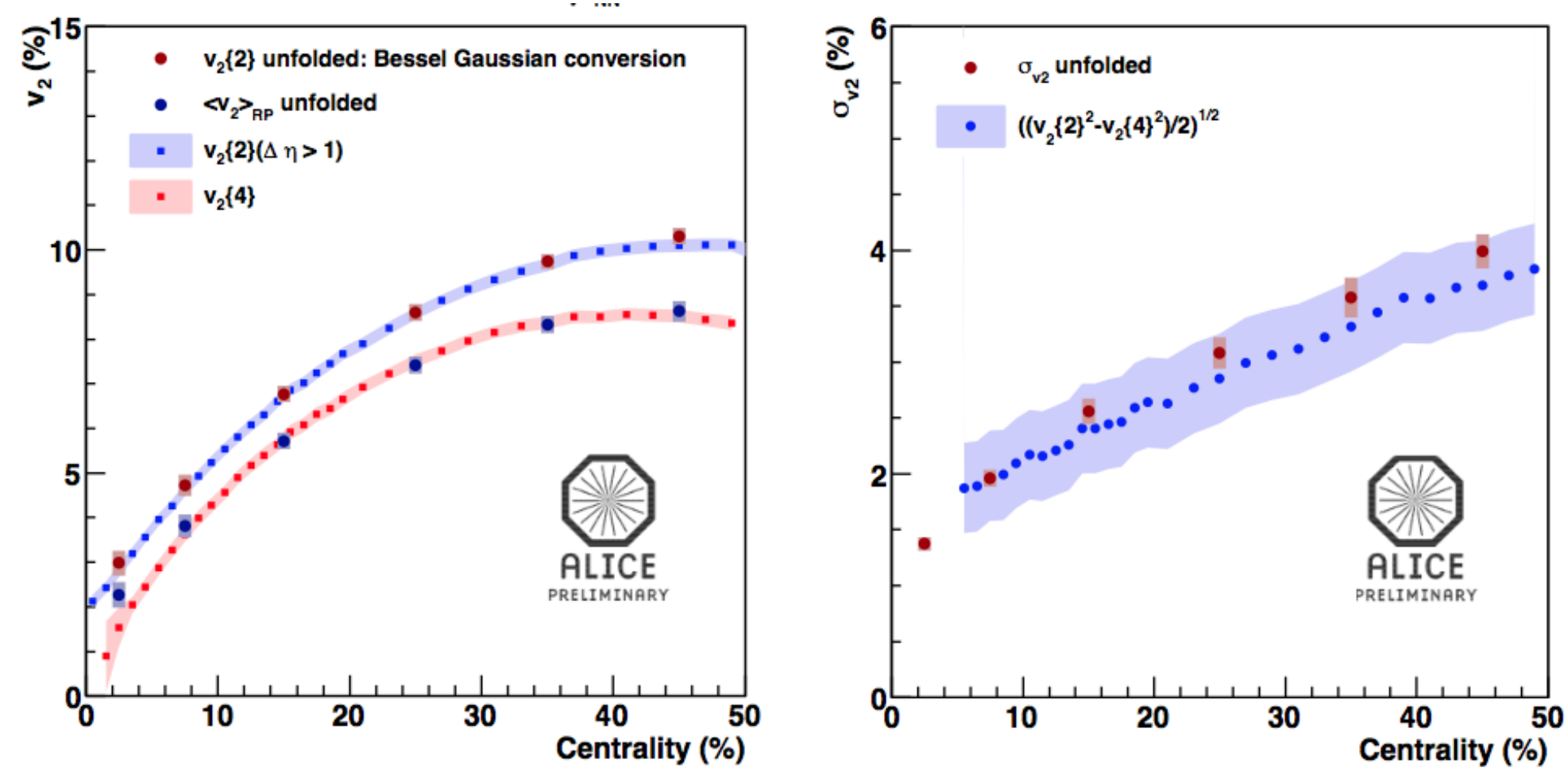

A. Timmins Hot Quarks 2012

Extracted $v_{2}\{2\}, v_{2}\{4\}$ and sigma from EbE distributions are consistent with cumulant measurements 


\section{Non-flow bias on fluctuation measurements ${ }^{18}$}

- Non-flow effects can bias the cumulant and EbE results

- For cumulant the main effect is to enhance $v_{n}\{2\}$

- Can use $v_{n}\{2\}$ with $\Delta n$ gap as substitute

- $v_{n}\{4\}$ and higher cumulants relatively unaffected by non-flow

- Can estimate non-flow from MC (ALICE EbE Measurements)

- Not data driven

- For EbE $v_{n}$ measurement the unfolding procedure can be used to remove non-flow (ATLAS Measurements)

- Data driven procedure 


\section{Non-flow effects : ATLAS EbE}

- Non-flow effects are mostly uncorrelated between sub-events

- They are removed during unfolding

- HIJING+Flow afterburner test demonstrates this

- Get response function by dividing tracks with $\eta>0$ and $\eta<0$ into sub-events

- Get response function by randomly dividing tracks into sub-events

- Do unfolding with both response functions and compare to input vn distribution
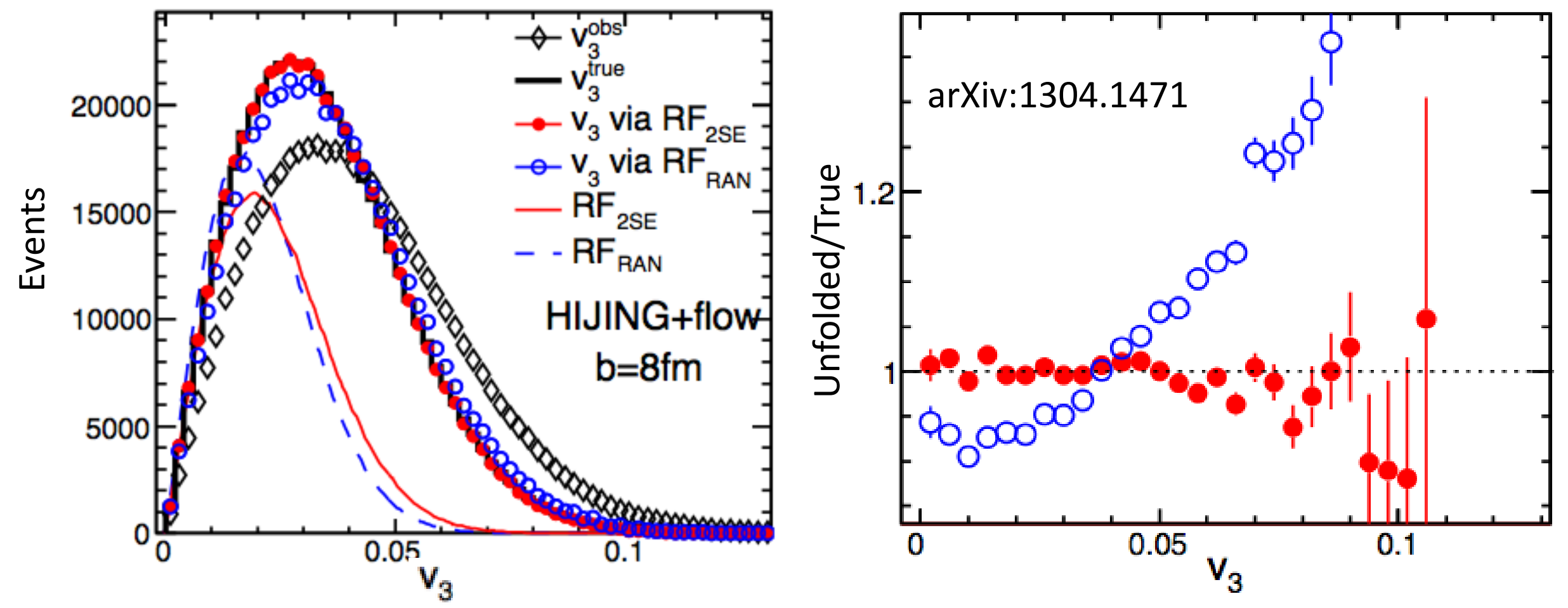


\section{Comparison to initial geometry: $v_{2}$}

$$
v_{n} \propto \epsilon_{n}=\frac{\sqrt{\left\langle r^{n} \cos n \phi\right\rangle^{2}+\left\langle r^{n} \sin n \phi\right\rangle^{2}}}{\left\langle r^{n}\right\rangle} \quad \text { For Glauber and CGC mckln }
$$

Rescale $\varepsilon_{\mathrm{n}}$ distribution to the mean of data
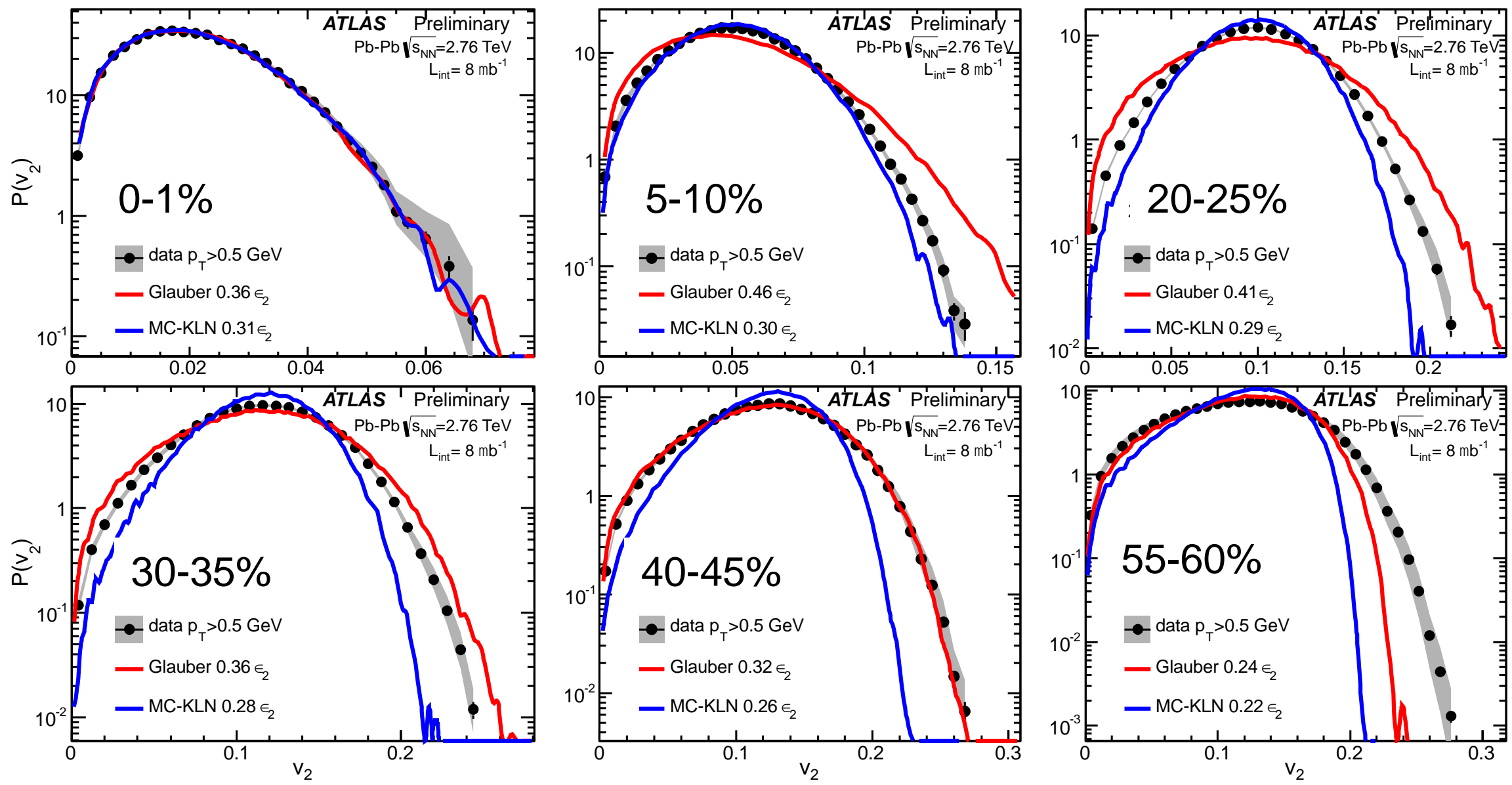

Both models fail describing $p\left(v_{2}\right)$ across the full centrality range 


\section{Comparison to IP-Glasma model}
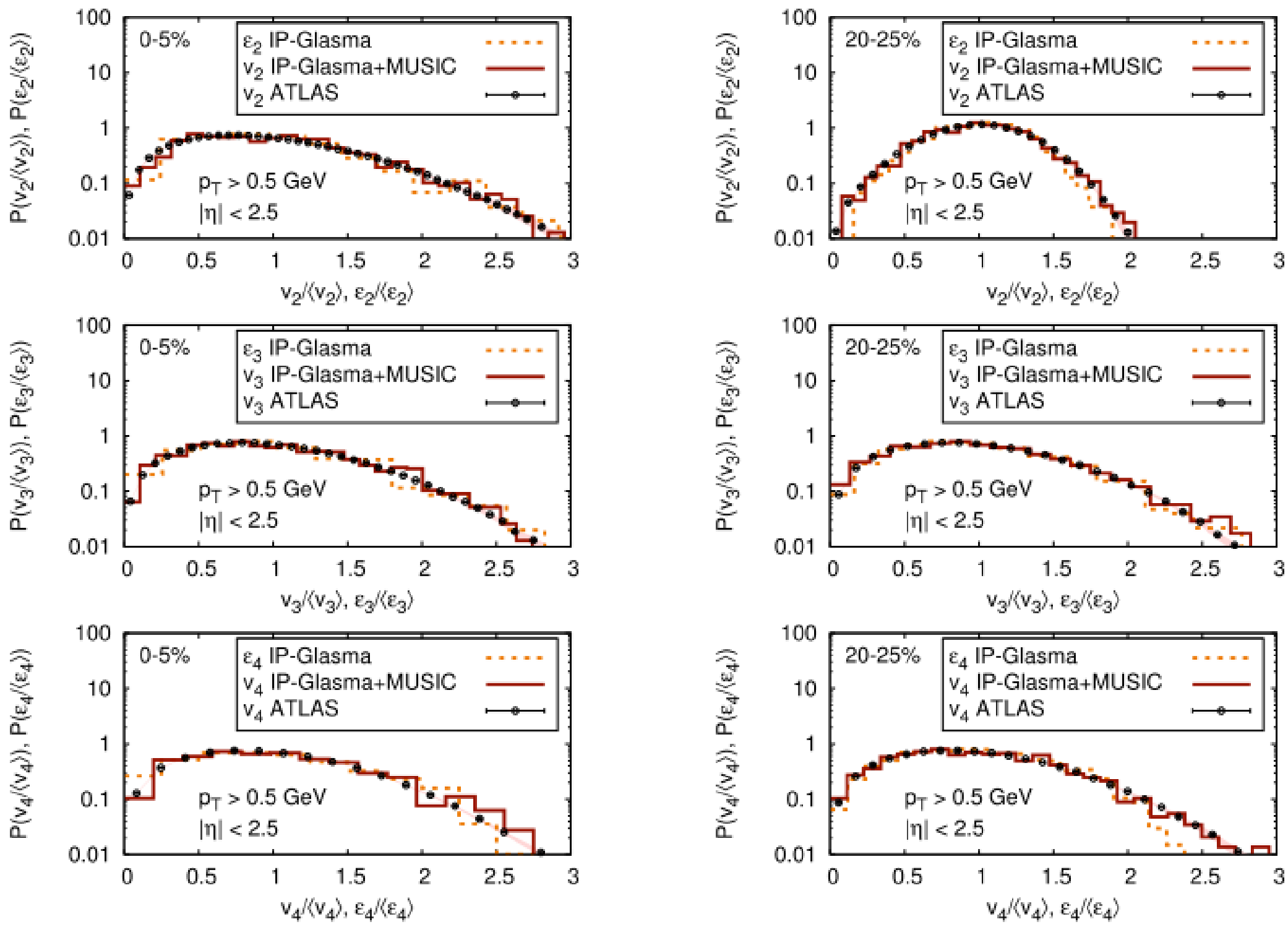

arXiv:1301.5893

Talk tomorrow by Bjorn Schenke 1209.6330 (Gale, Jeon, Schenke, Tribedi, Venugopalan) 


\section{Correlation between phases of harmonic flow ${ }^{22}$}

- Complementary observables to $v_{n}$

- Correlation can exist in the initial geometry and also generated during hydro evolution

- The correlation can be quantified via a set of correlators

$$
\begin{aligned}
& \frac{d N_{\text {events }}}{\left.d\left(\begin{array}{ll}
\text { n }_{n} &
\end{array}\right)\right)}=1+2 V_{j=1}^{j} \cos \left(j k\left(\begin{array}{ll}
n, m &
\end{array}\right)\right) \\
& V_{n, m}^{j}=\left\langle\cos \left(j k\left(\begin{array}{ll}
{ }_{n} &
\end{array}\right)\right)\right\rangle \quad \text { arXiv:1203.5095 }
\end{aligned}
$$

- This can be generalized into multi-plane correlations

$$
\left.\left\langle\cos \left(c_{1}{ }_{1}+2 c_{2} \quad 2 \ldots l c_{l} \quad l\right)\right)\right\rangle c_{1}+2 c_{2}+\ldots l c_{l}=0
$$

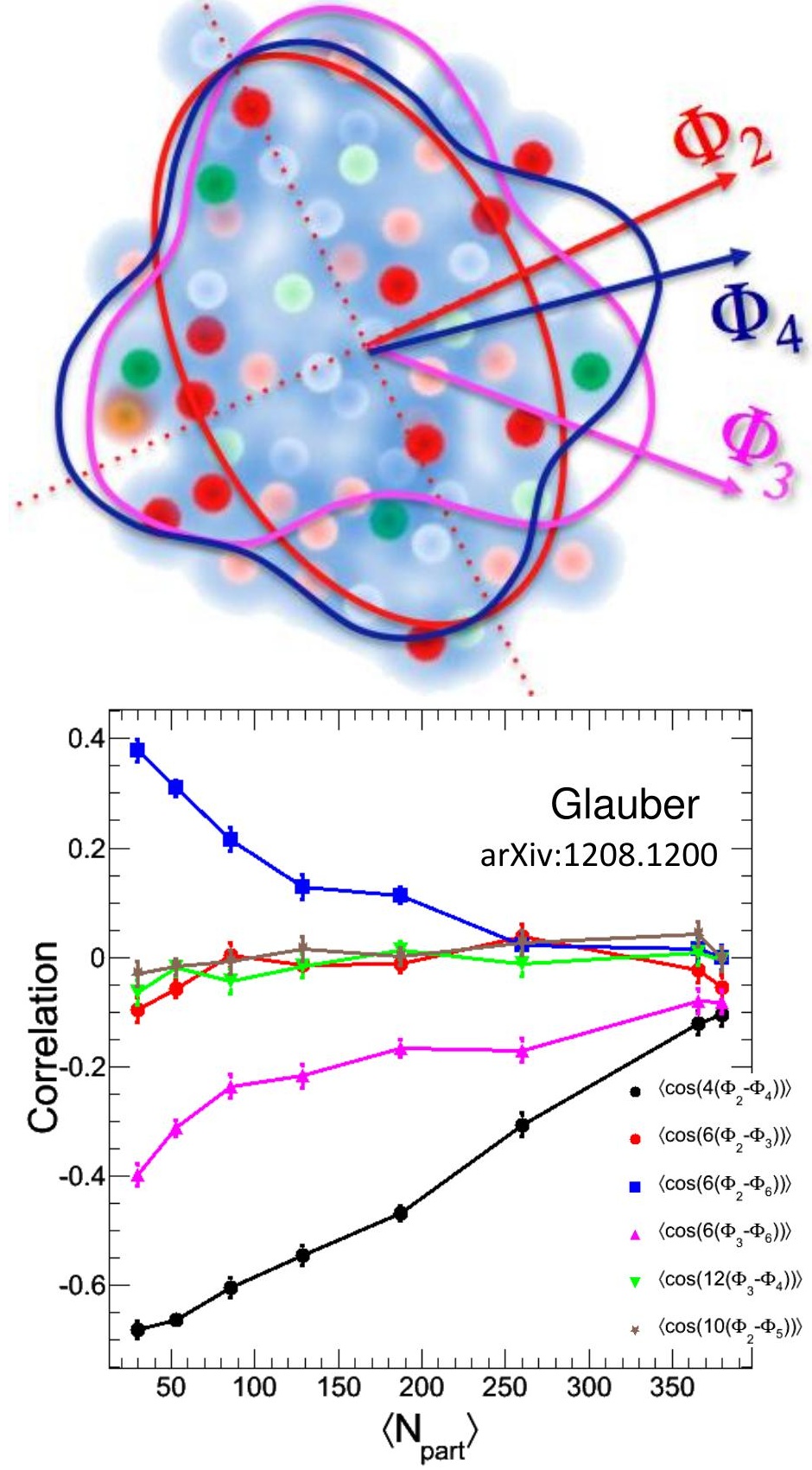




\section{Event plane correlations}

Initial geometry + hydrodynamic

ATLAS data

MC-Glb., $\eta / s=0.08$
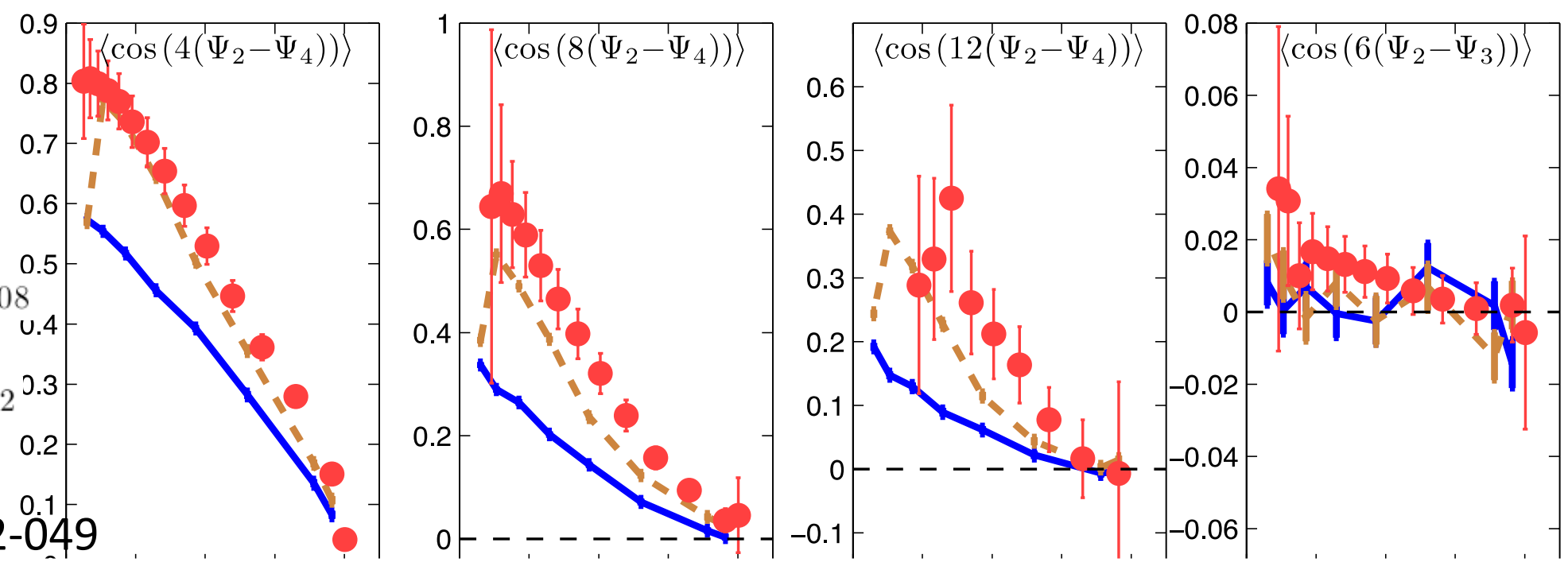

ATLAS-CONF-2012-049

arXiv:1208.1200 Heinz \& Qui geometry only

MC-Glb.

- = = MC-KLN
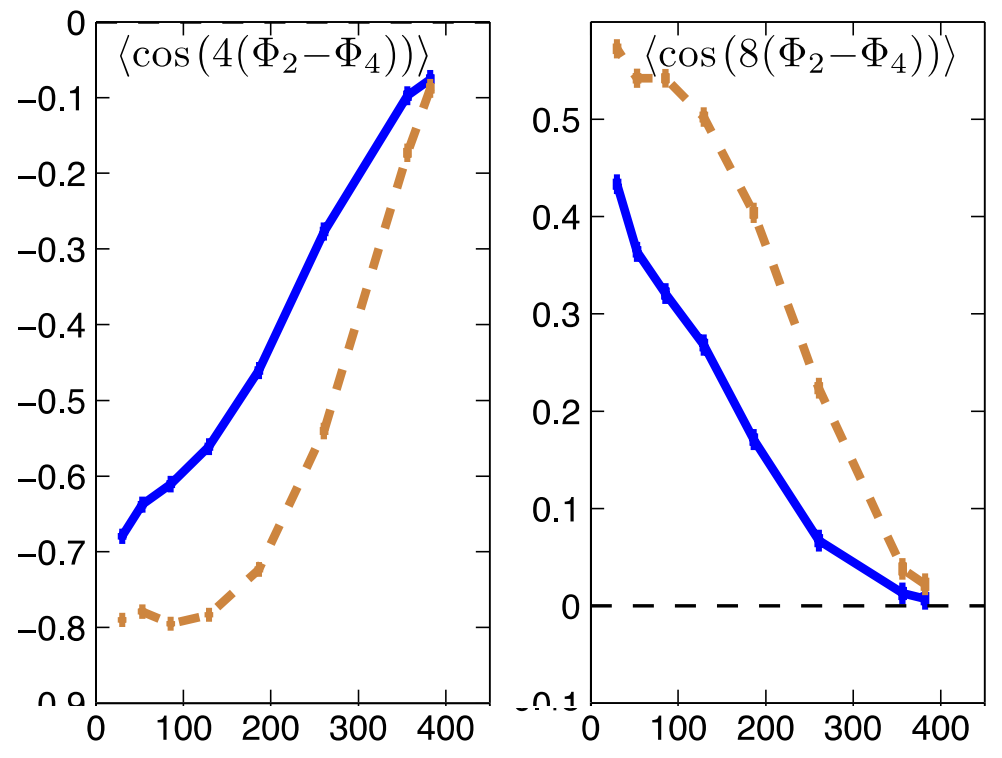

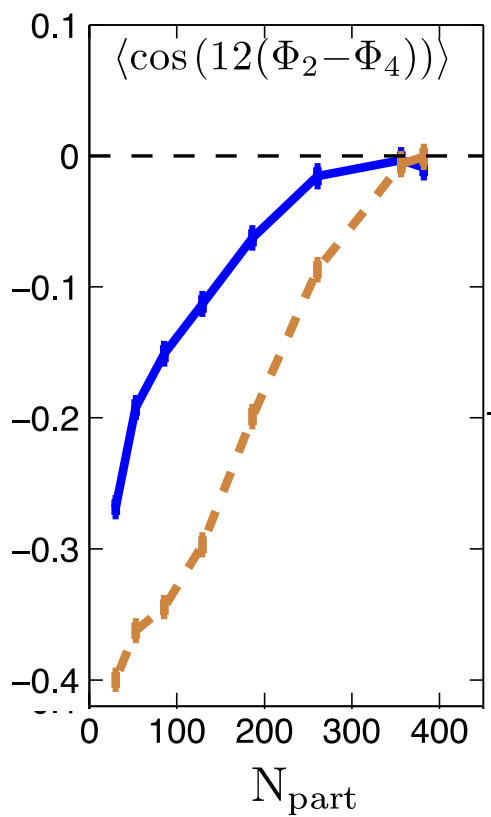

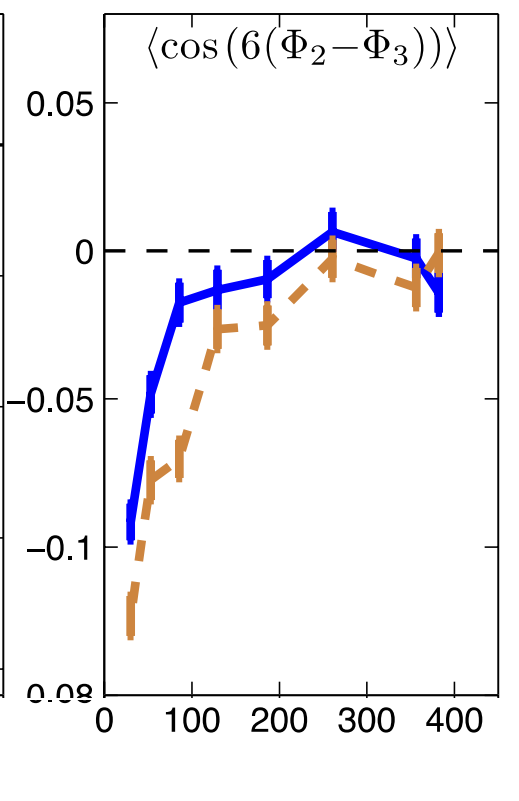

EbE hydro qualitatively reproduces features in the data 


\section{Summary}

- Cumulants provide overview into nature of fluctuations

- $v_{2}\{2\}$ used to probe average geometry+fluctuations.

- $\mathrm{v}_{2}\{4\}=\mathrm{v}_{2}\{6\}=\mathrm{v}_{2}\{8\}=\mathrm{v}_{2}{ }^{\mathrm{RP}}$ and LYZ probe average geometry.

- Dependence of $v_{n}$ on $p_{T}$ and initial geometry factorizes.

- EBE measurements of $v_{2}, v_{3}$ and $v_{4}$ distributions done by ATLAS and $\operatorname{ALICE}\left(v_{2}\right)$.

- EbE measurement handles non-flow.

- Does not assume a particular form of the EbE distributions.

- Distributions look Bessel-Gaussian like (deviations in the tail).

- Distributions for $v_{2}, v_{3}$ and $v_{4}$ well reproduced by IP-Glasma+MUSIC, but not by Glauber.

- EP Corrs give further insight into initial geometry as well as hydroevolution

- Can differentiate hydro-effects from initial geometry effects.

- Also gives information on initial geometry. 


\section{Hard and soft responses from parton transport}

Denes Molnar, Purdue University

Workshop on Jet Quenching at RHIC vs LHC in Light of Recent dAu vs $\mathrm{pPb}$ controls

Apr 15-17, 2013, Brookhaven National Laboratory, Upton, NY

in collaboration with Deke Sun 


\section{(parton) transport can give you:}

- bulk medium evolution

- nonequilibrium effects

- fluctuations 


\section{Covariant transport}

(on-shell) phase-space density $f(x, \vec{p}) \equiv \frac{d N(\vec{x}, \vec{p}, t)}{d^{3} x d^{3} p}$

transport equation:

$$
p^{\mu} \partial_{\mu} f_{i}(x, p)=C_{2 \rightarrow 2}^{i}\left[\left\{f_{j}\right\}\right](x, p)+C_{2 \leftrightarrow 3}^{i}\left[\left\{f_{j}\right\}\right](x, p)+\cdots
$$

with, e.g.,

$C_{2 \rightarrow 2}^{i}=\frac{1}{2} \sum_{j k l} \int_{234}\left(f_{3}^{k} f_{4}^{l}-f_{1}^{i} f_{2}^{j}\right) W_{12 \rightarrow 34}^{i j \rightarrow k l}$

$$
\left(\int_{j} \equiv \int \frac{d^{3} p_{j}}{2 E_{j}}, \quad f_{a}^{k} \equiv f^{k}\left(x, p_{a}\right)\right)
$$

fully causal and stable, can handle large gradients

near hydrodynamic limit, transport coefficients and relaxation times:

$$
\eta \approx 1.2 T / \sigma_{t r}, \quad \tau_{\pi} \approx 1.2 \lambda_{t r}
$$




\section{Freezeout}

Cooper-Frye: assumed sudden transition to a gas on a 3D hypersurface

$$
E d N=p^{\mu} d \sigma_{\mu}(x) d^{3} p f_{g a s}(x, \vec{p})
$$

Huovinen \& Holopainen (QM2012): $\boldsymbol{T}_{F O}=$ const vs $\tau_{\text {scatt }} / \tau_{\text {exp }}=$ const ?!
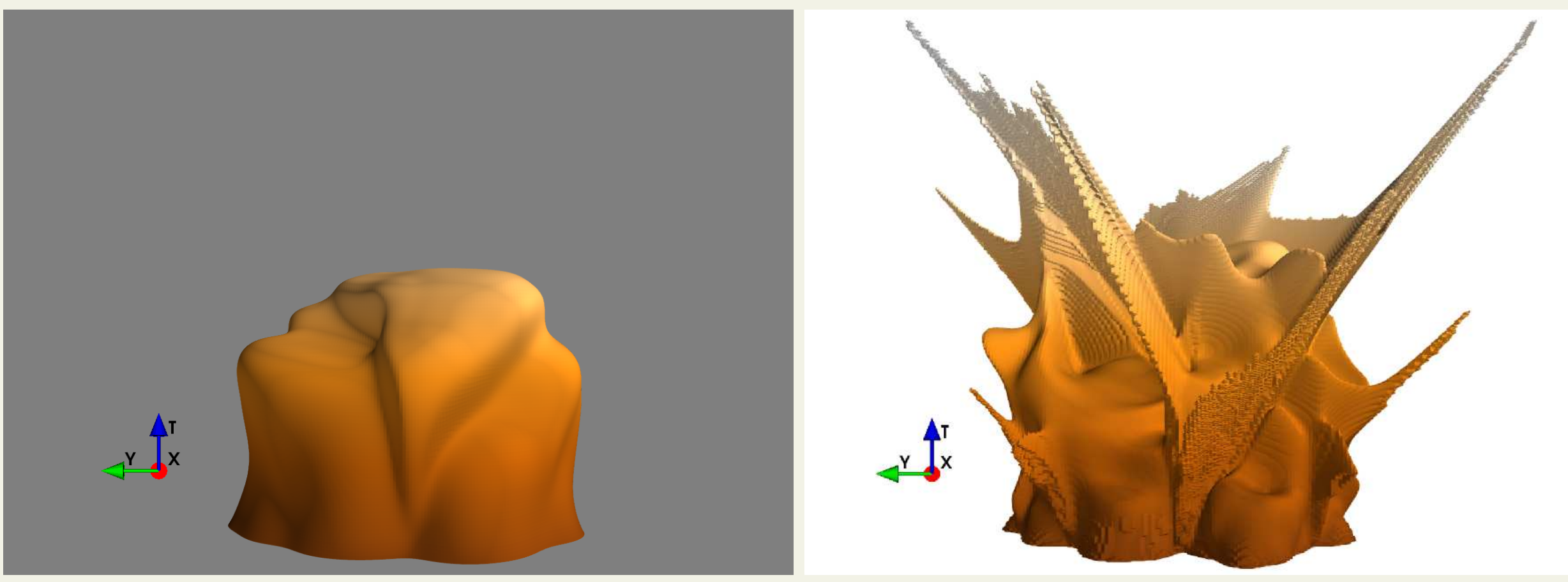

also need viscous corrections $f_{\text {gas }}=f_{\text {equil }}+\delta f$ (e.g., Wolff \& Molnar @ QM2012) 


\section{Collectivity with suitable rates}

Zhang, Gyulassy \& Ko ('99):

$2 \rightarrow 2$ transport ZPC

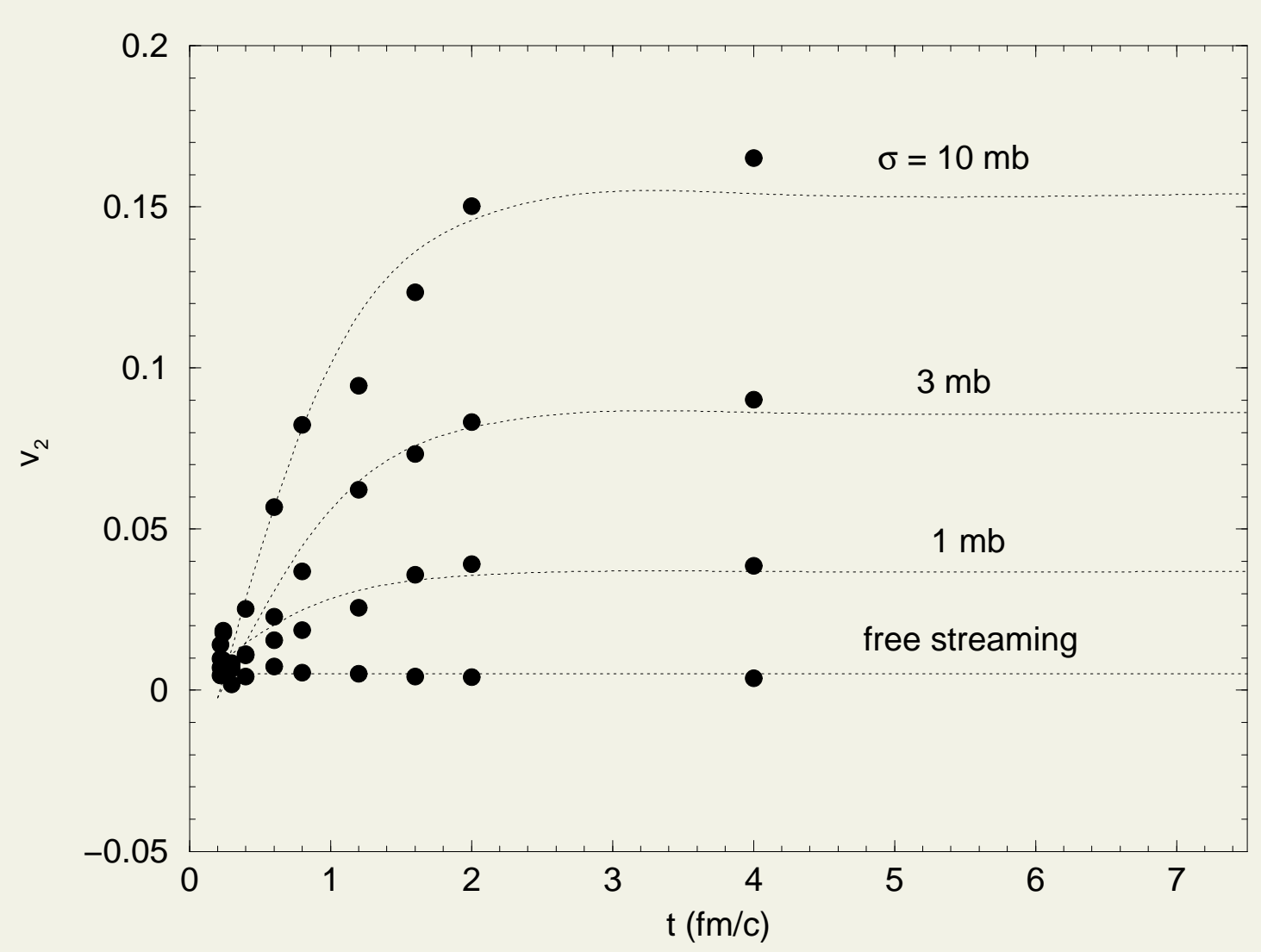

DM \& Gyulassy, NPA 697 ('02):

$2 \rightarrow 2$ transport MPC

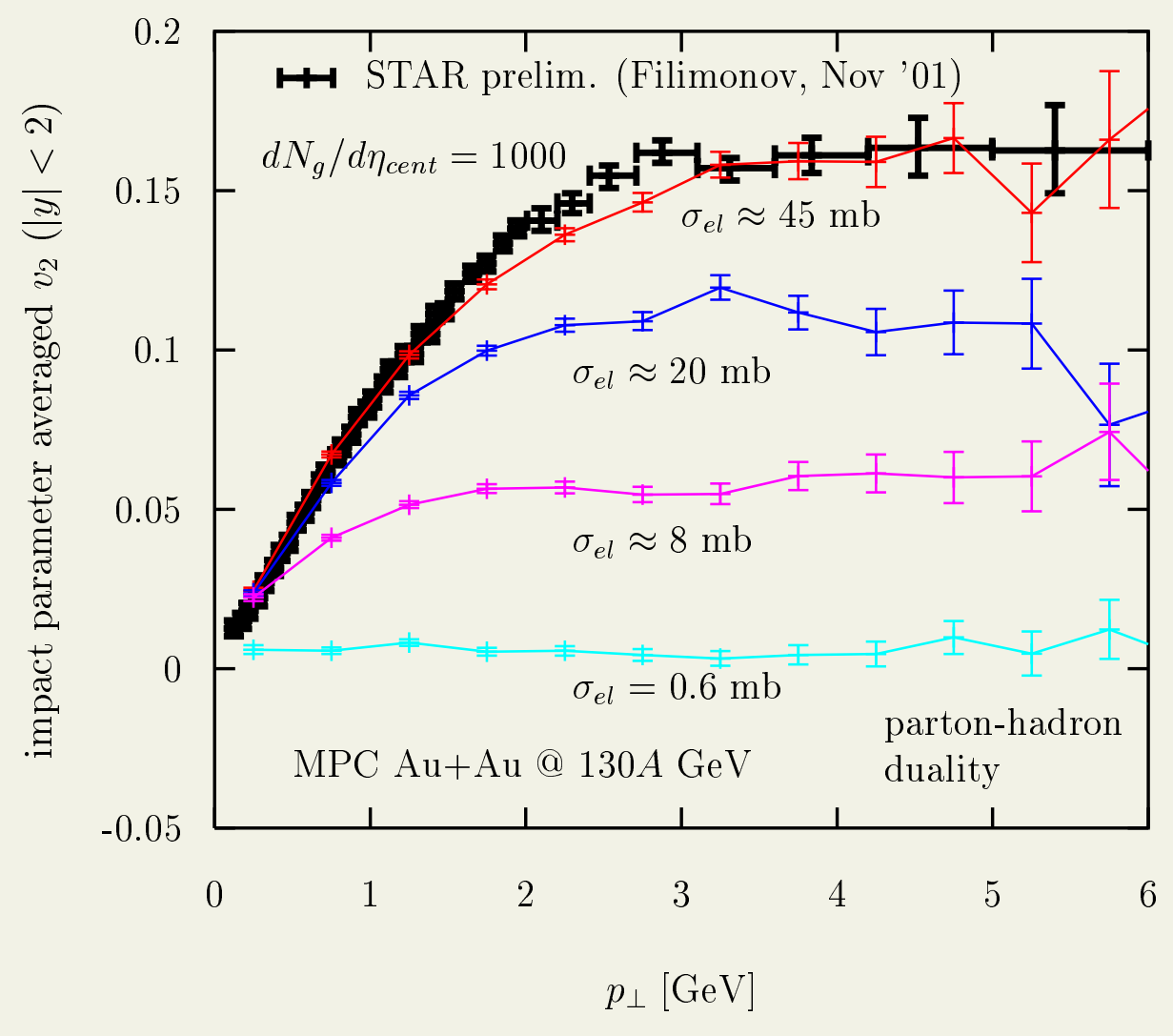


Xu \& Greiner, ('08) BAMPS claim: perturbative $3 \leftrightarrow 2$ rates thermalize

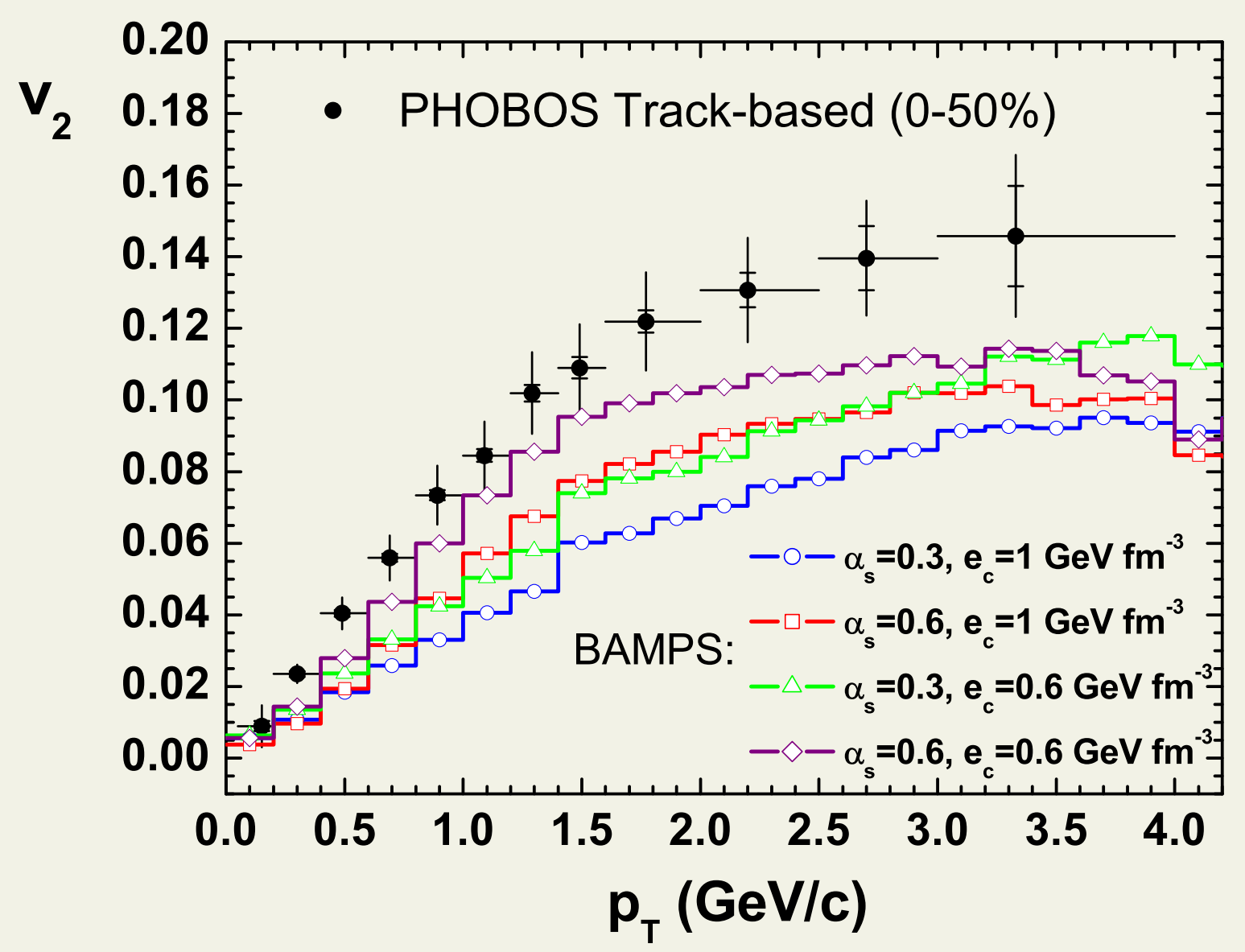

Still to be confirmed...

matrix elements? 


\section{Application: bulk medium for jet quenching}

E.g., line integrals

$$
\begin{aligned}
\Delta E_{G L V}^{(1)} & \approx \frac{9 \pi C_{R} \alpha_{s}^{3}}{4} \int d \tau \tau \rho\left(z_{0}+v \tau, \tau\right) \ln \frac{2 E}{\mu^{2} \tau} \\
\frac{d E}{d L} & =\kappa[s(L)] s(L) L \\
\frac{d E}{d L} & =\text { const } \times E^{\alpha} L^{\beta} T^{2-\alpha+\beta}(L)
\end{aligned}
$$

Gyulassy, Vitev, et al...

Shuryak \& Liao

Betz et al

or stochastic E-loss

$\Delta E$ depends on the medium. E.g, GLV needs scattering center information

$\rightarrow$ natural to combine (D)GLV with a parton transport model (MPC) 


\section{GLV - opacity expansion}

Gyulassy, Levai, Vitev NPB594 ('00)
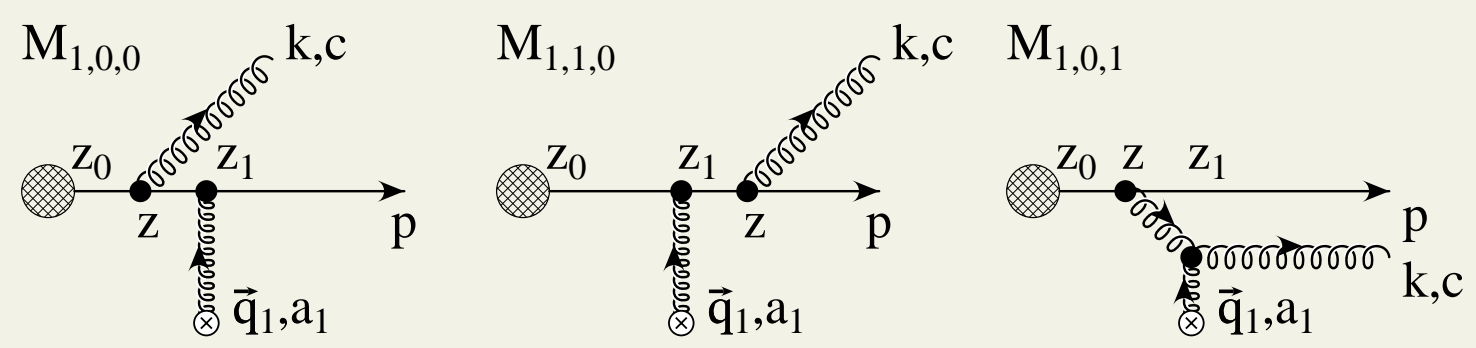

$$
\begin{aligned}
x \frac{d N^{(n)}}{d x d^{2} \mathbf{k}}= & \frac{C_{R} \alpha_{s}}{\pi^{2}} \frac{\chi^{n}}{n !} \int \prod_{i=1}^{n}\left\{d \mathbf{q}_{i}\left(\frac{d z_{i} \rho_{i} \sigma_{i}}{\chi}\right)\left(\bar{v}_{i}^{2}\left(\mathbf{q}_{i}\right)-\delta^{2}\left(\mathbf{q}_{i}\right)\right)\right\} \\
\times & {\left[-2 \mathbf{C}_{(1, \cdots, n)} \cdot \sum_{m=1}^{n} \mathbf{B}_{(m+1, \cdots, n)(m, \cdots, n)}\right.} \\
& \left.\left(\cos \left(\sum_{k=2}^{m} \omega_{(k, \cdots, n)} \Delta z_{k}\right)-\cos \left(\sum_{k=1}^{m} \omega_{(k, \cdots, n)} \Delta z_{k}\right)\right)\right]
\end{aligned}
$$

formation time $\omega_{n \cdots m}=\frac{2 x E}{\left(\mathbf{k}-\mathbf{q}_{n}-\cdots \mathbf{q}_{m}\right)^{2}}$

key assumptions: static Yukawa scatterers, soft emission, $\lambda_{M F P} \gg 1 / \mu_{D}$

as usual, in the end interpret $\rho_{i}(\vec{x}) \rightarrow \rho_{i}(\vec{x}, t)$ along jet trajectory 


\section{Setup:}

- Bulk dynamics evolution computed from covariant transport (MPC code)

- Here: only use the density $\rho\left(x_{\perp}, \tau\right)$

- in principle, we could use spacetime location of scatterers for external jets embedded in transport (no jet recoil, forward scattering)

- Keep energy loss stochastic (no averaging over scattering location)

- Radiated glue considered "lost" and feedback on medium ignored - focus on high $p_{T}$

After E-loss, fragment as in vacuum (LO pQCD) to get some hadronic observables: $R_{A A}$ and $v_{2}$ 
Medium evolution from kinetic theory (MPC transport code):

$-2 \rightarrow 2$ with massless gluons

- opacity set to generate sufficient $v_{2}\left(p_{T}\right) \sim 0.2$ at RHIC / LHC

- $\eta / s \approx 0.1$ dynamics via $\sigma_{g g} \sim 1 / T^{2} \sim \tau^{2 / 3} \quad$ DM, arXiv:0806.0026

- boost-invariant initial conditions in $|\eta|<5$ window

- LO pQCD jet production \& fragmentation (CTEQ5L, BKK95, $K_{N L O} \approx 2.5$ )

- jet and bulk transverse profiles $\propto \rho^{\text {binary }}\left(x_{\perp}\right)$, with $d N^{b u l k} / d y \propto N_{\text {part }}$

- $T$ set by $\rho(T)$ for massless gluon gas, $\mu_{D}=g T$

- $\tau_{0}=0.6$ fm formation, and LINEAR density buildup $\rho(\tau) \propto \tau$ for $\tau<\tau_{0}$

Two centralities: $0-10 \%(b \approx 3 \mathbf{f m})$ and $25-35 \%(b \approx 8 \mathbf{f m})$ 
4 scenarios:

1D = longitudinal Bjorken expansion, $\langle\Delta E\rangle$

$1 \mathrm{D}$, stochastic $=$ longitudinal Bjorken expansion, $\Delta E(z)$

3D $=$ Bjorken AND transverse expansion, $\langle\Delta E\rangle$

3D, stochastic $=$ Bjorken AND transverse expansion, $\Delta E(z)$

not the most sophisticated E-loss treatment

but we did include transverse expansion (unlike Buzzatti et al, Horowitz et al, Betz et al) 
Finite energy \& kinematics crucial: $\quad|k|<\sim x E$

$$
\begin{aligned}
& |q|<\sim \sqrt{s} \sim \sqrt{6 E T} \\
& x E>\sim \mu \quad \text { (plasma) } \\
& \Delta E_{G L V}^{(1)}(z)=\frac{C_{R} \alpha_{s}}{\pi^{2}} \chi \int d x d \mathbf{k} d \mathbf{q} \frac{\mu^{2}}{\pi\left(\mathbf{q}^{2}+\mu^{2}\right)^{2}} \frac{2 \mathbf{k} \cdot \mathbf{q}}{\mathbf{k}^{2}(\mathbf{k}-\mathbf{q})^{2}}(1-\cos \omega \Delta z) \\
& \equiv \frac{2 C_{R} \alpha_{s}}{\pi} E \chi I(\Delta z / \tau(z), E / \mu(z)) \\
& \omega \equiv(\mathbf{k}-\mathbf{q})^{2} /(2 E x) \\
& \tau(z) \equiv \frac{2 E}{\mu^{2}(z)} \\
& \left\langle\Delta E_{G L V}^{(1)}\right\rangle \sim \int \rho\left(z_{0}+v \tau, \tau\right) \ln \frac{2 E}{\mu^{2} \tau}
\end{aligned}
$$

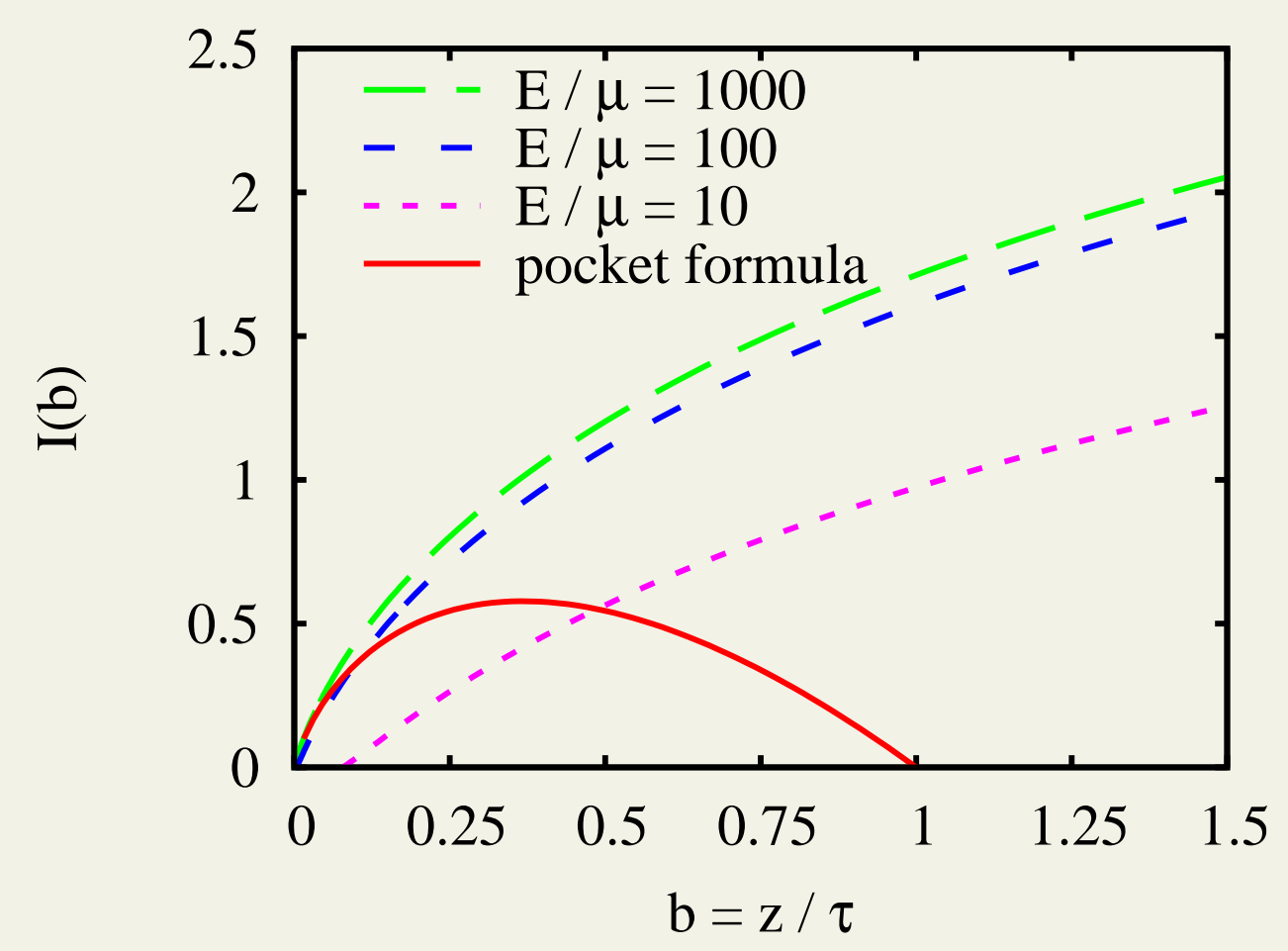




\section{pion RAA, RHIC}
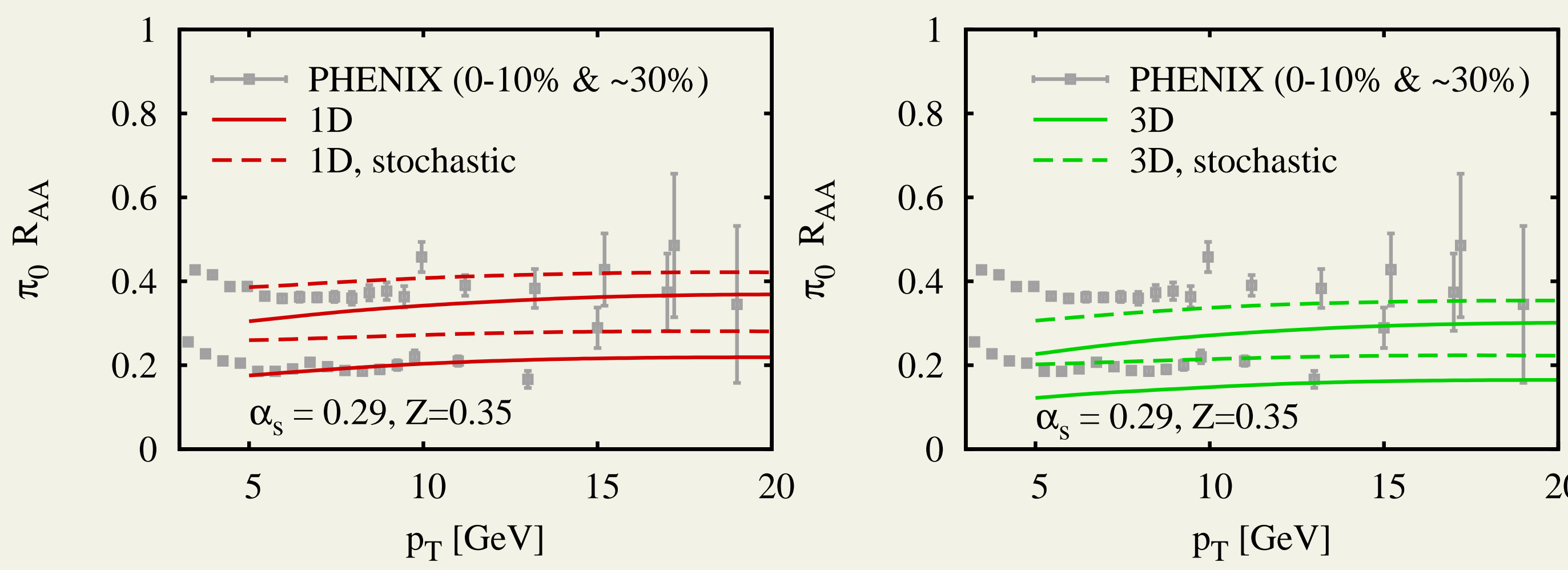

fluctuations and transverse expansion matter

more energy loss with transverse expansion because GLV favors large $\Delta z / \tau_{f}$ 
pion RAA, RHIC - $\alpha_{s}$ scaled to RAA for central
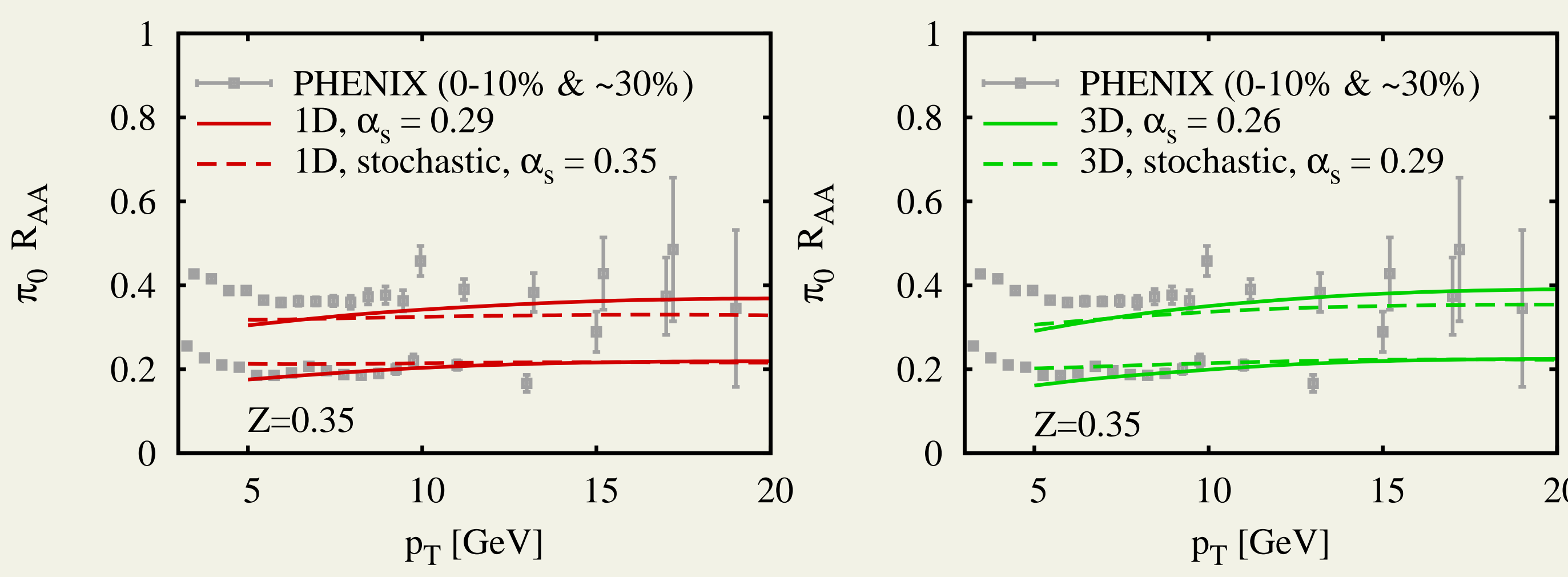

only small $10-15 \%$ differences after tuning parameters to central data 


\section{pion v2, RHIC}
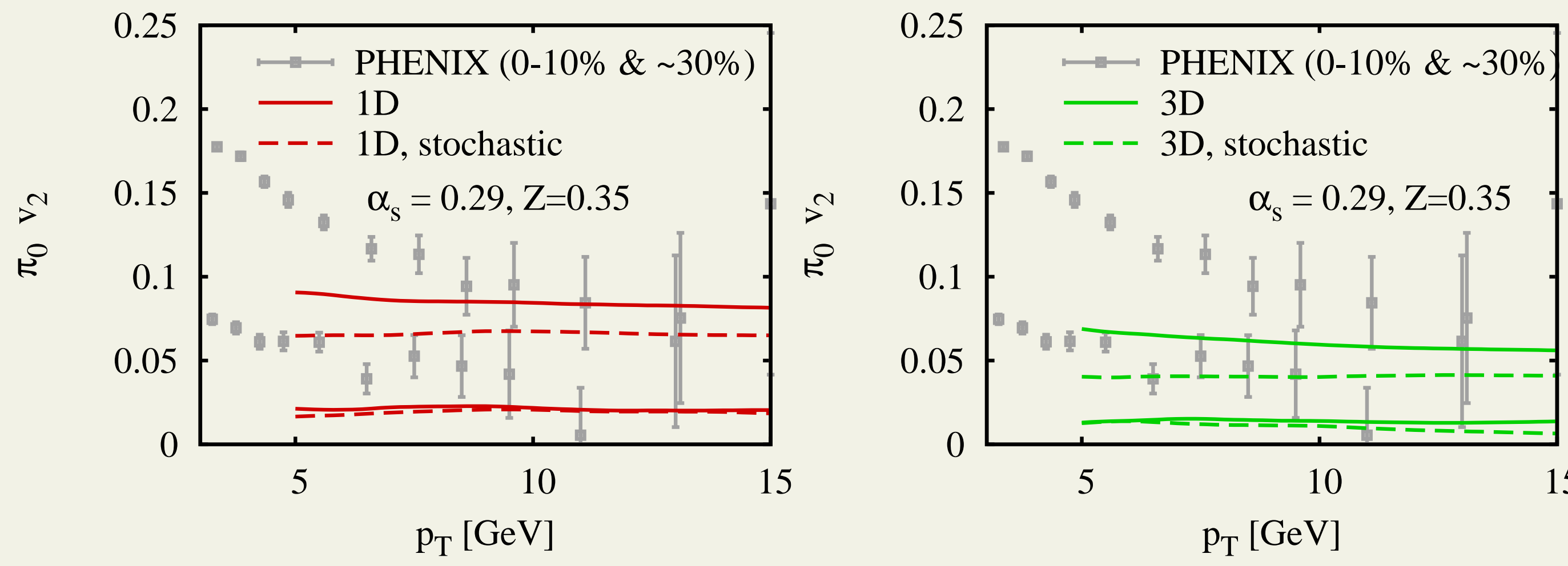
pion v2, RHIC $-\alpha_{s}$ scaled to RAA for central
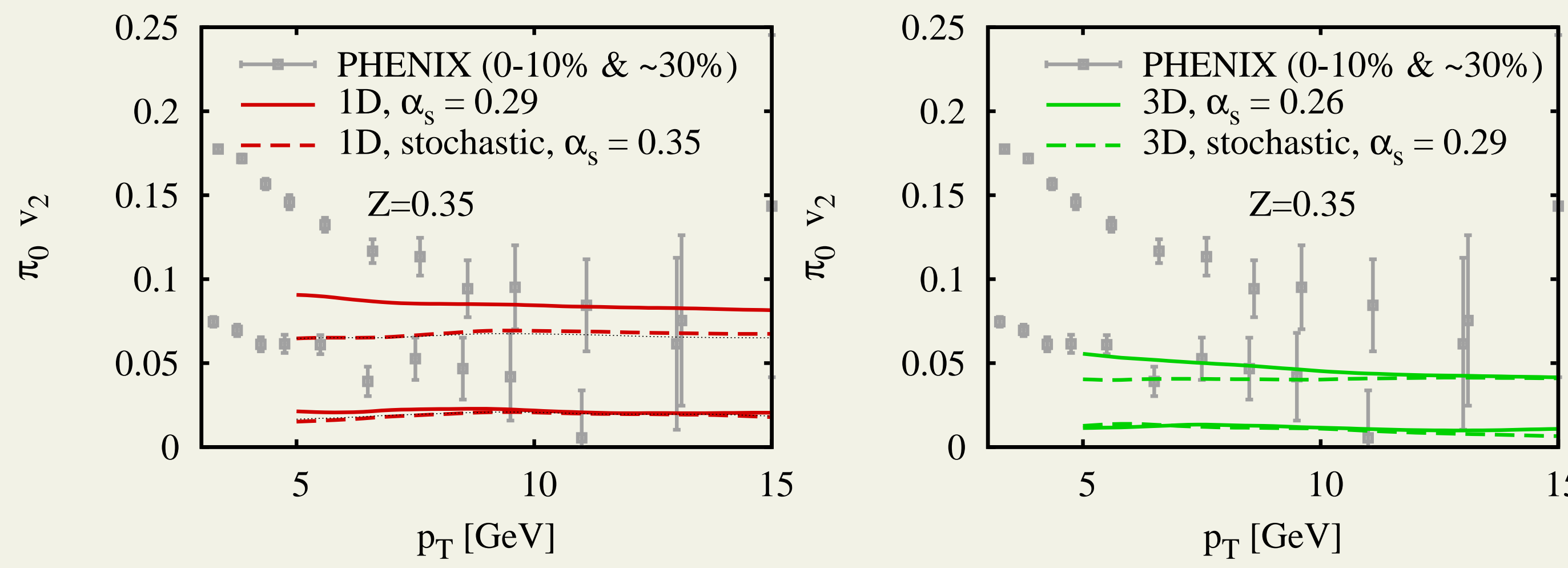

challenging to get enough $v_{2}>4-5 \%$ with expanding medium smaller $v_{2}$ with transverse expansion - GLV favors later times (large $\Delta z / \tau_{f}$ ) 
Now move to LHC energies. Simple assumption:

- higher $d N / d \eta=2400$ in central collisions $(b=0)$

- all other ingredients stay same

at present we only have mid-peripheral results in the 3D case 


\section{pion RAA, LHC}
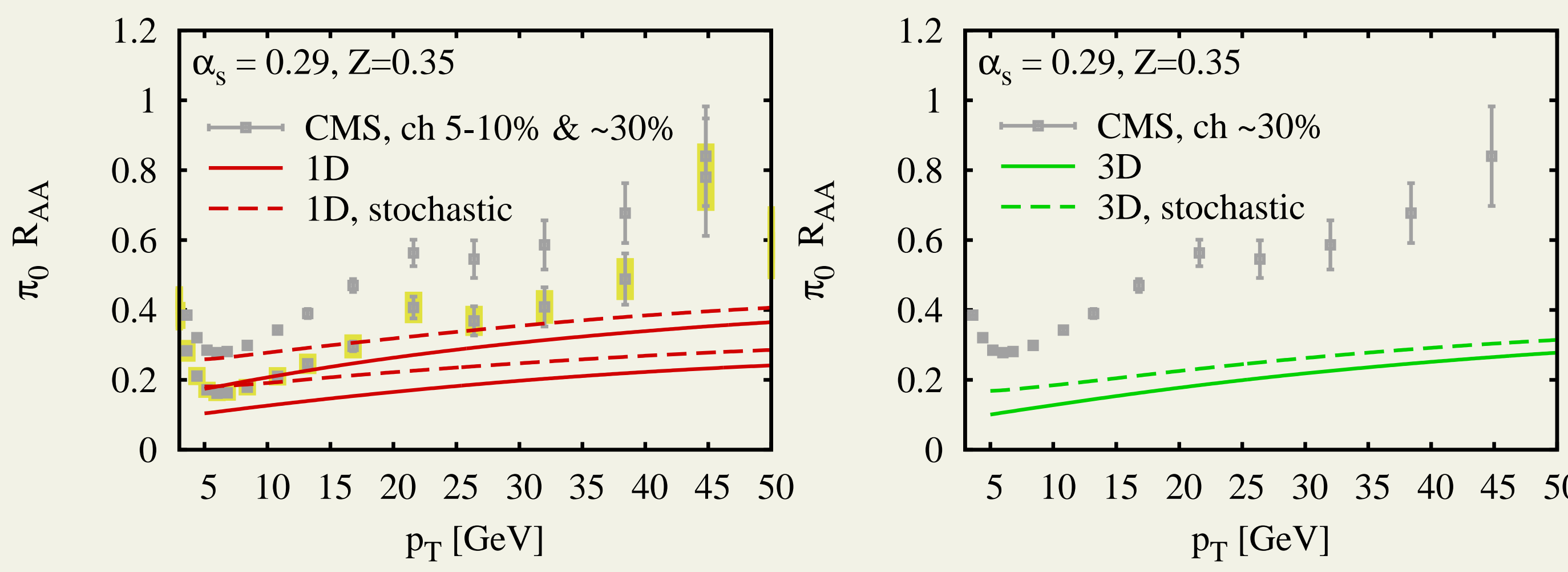
pion RAA, LHC - $\alpha_{s}$ scaled to RAA for central at $\mathrm{pT}=6 \mathrm{GeV}$
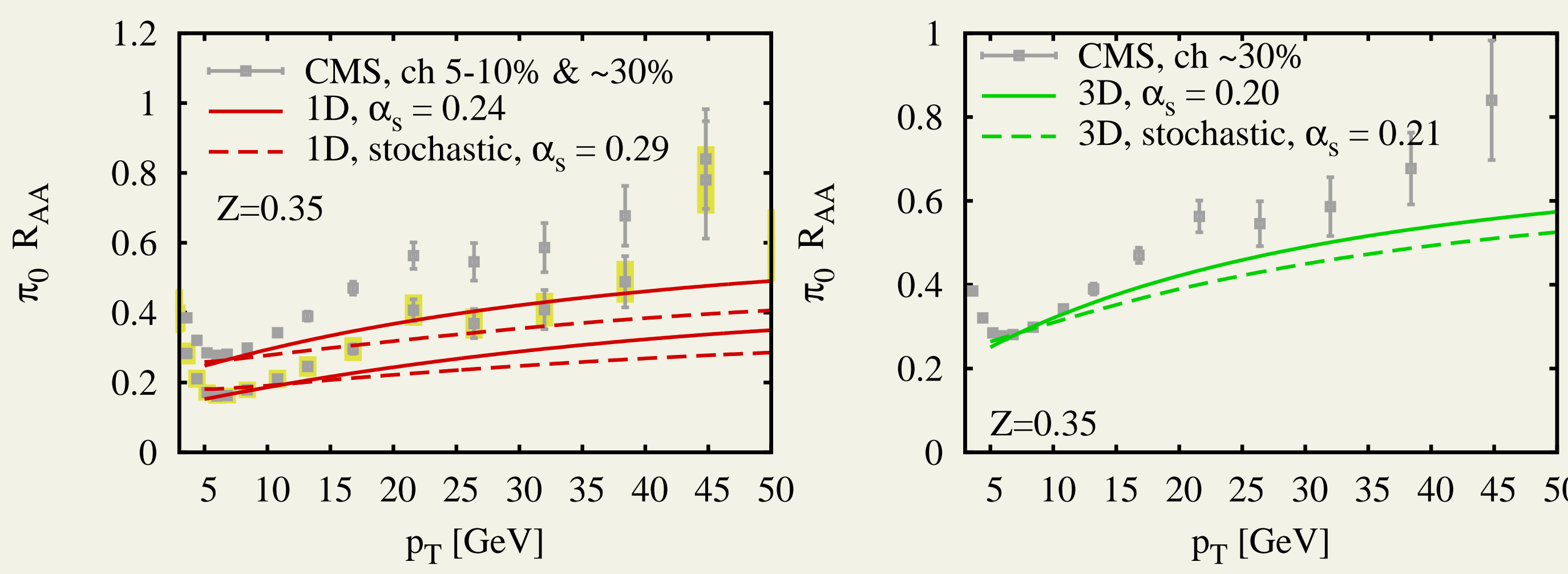

GLV implementation here is simplified, also $\alpha_{s}=$ const (no running) 

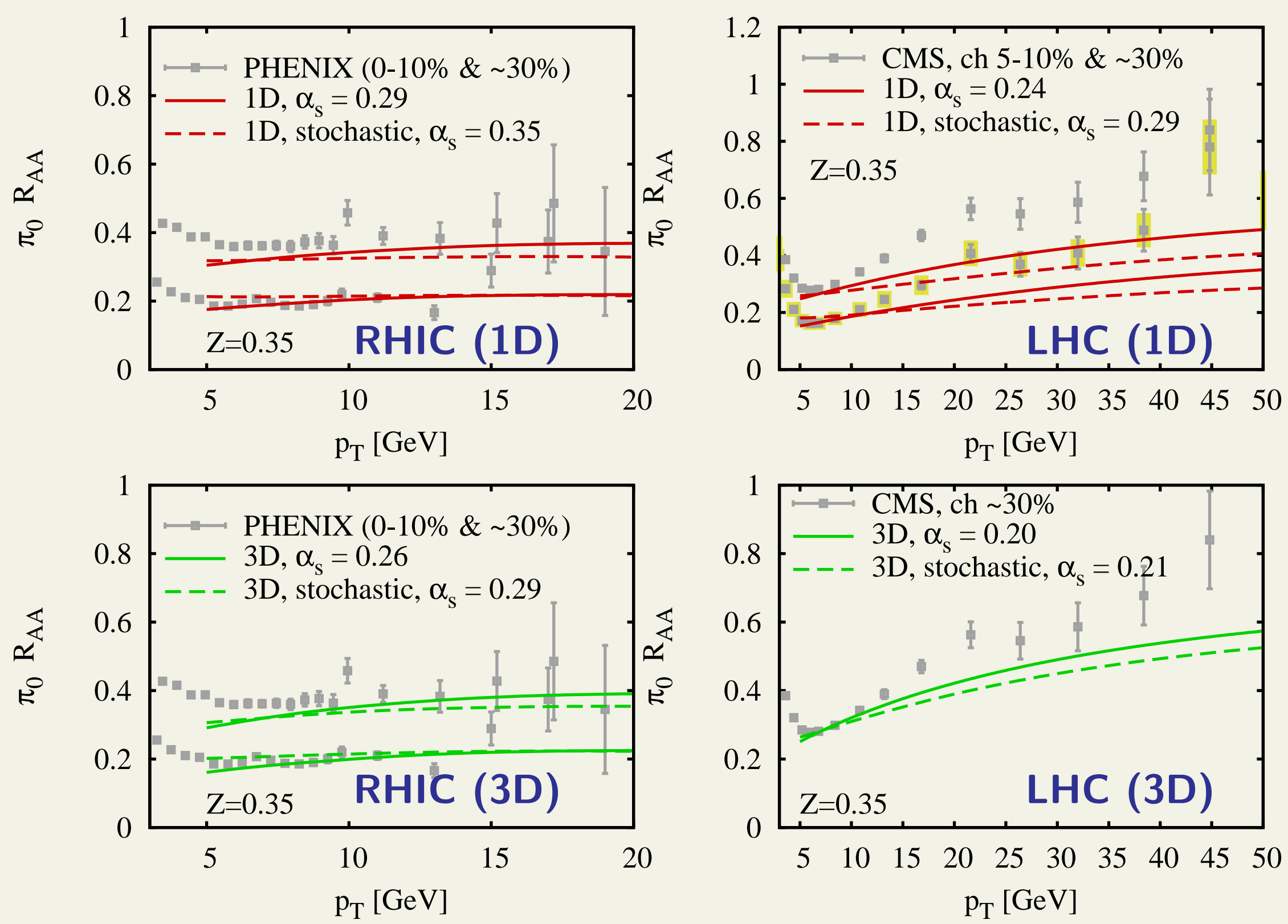

need $20-25 \%$ smaller $\alpha_{s}$ at LHC than at RHIC - similar to Betz et al, 1201.0281 


\section{pion v2, LHC}
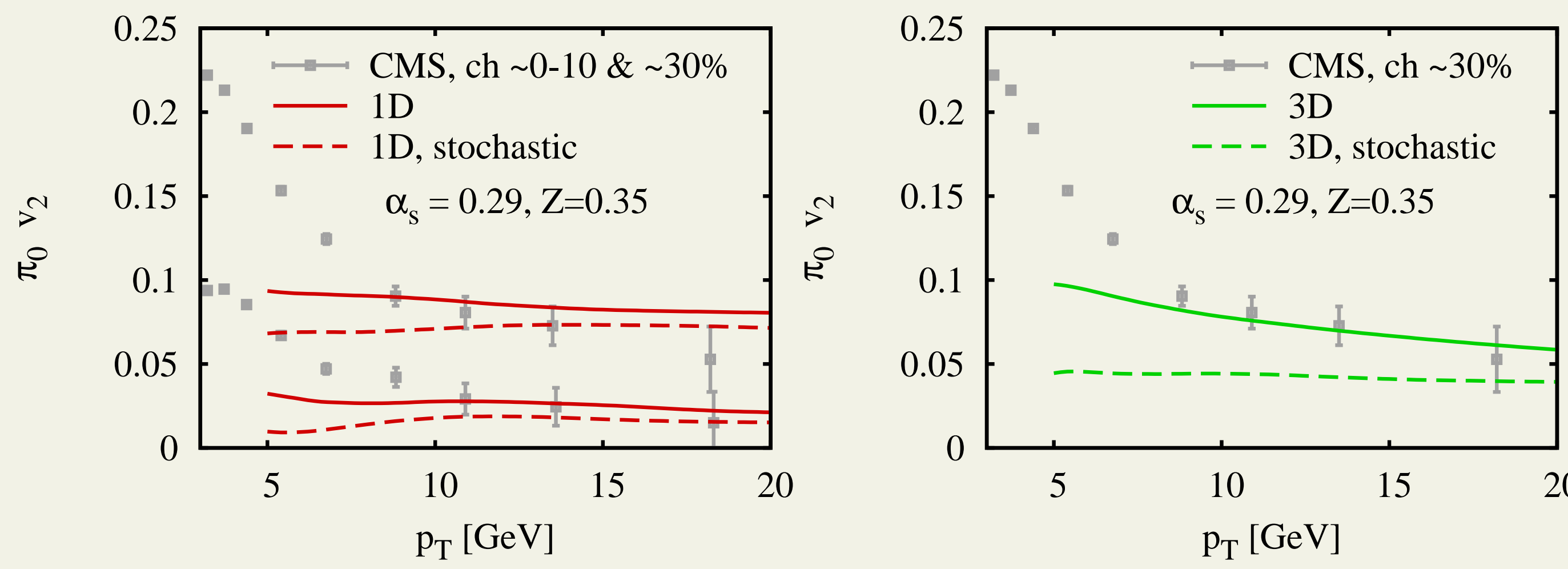
pion v2, LHC $-\alpha_{s}$ scaled to RAA at $\mathbf{p T}=6 \mathrm{GeV}$
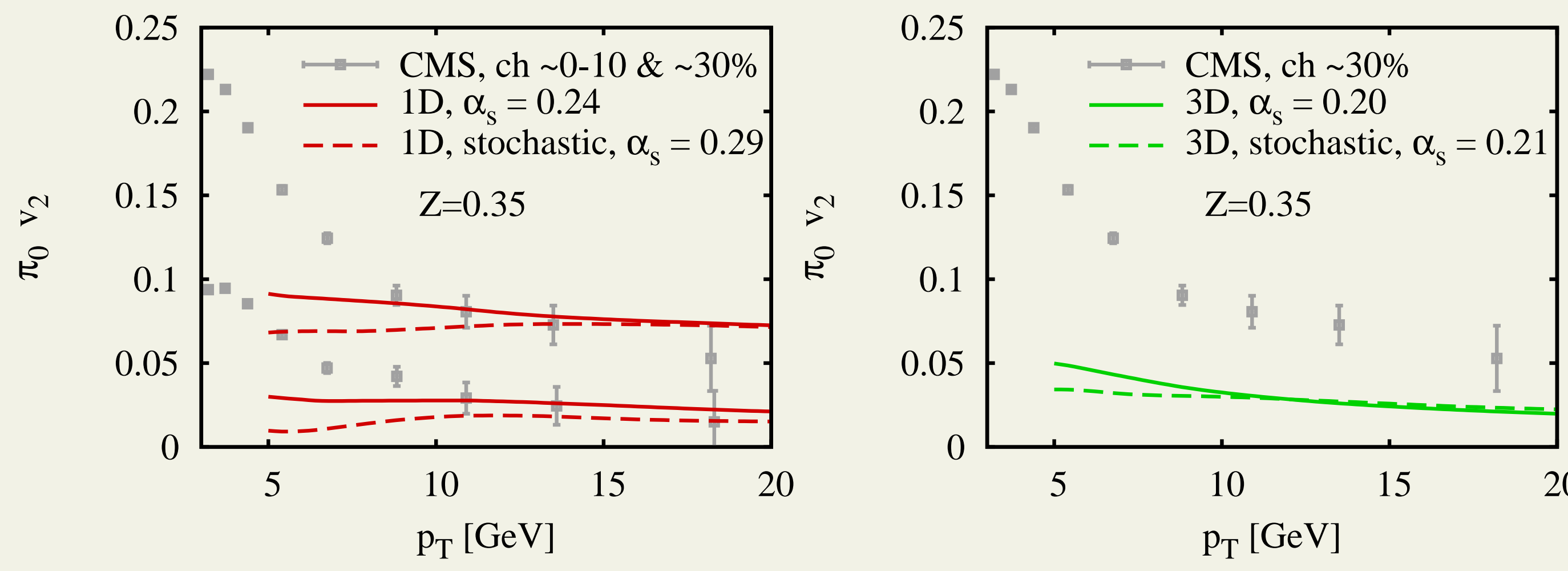

similar reduction in 3D case as at RHIC energies 


\section{high-pT pion v2, LHC ( $\alpha_{s}$ scaled to RAA at $\left.\mathbf{p T}=6 \mathrm{GeV}\right)$}
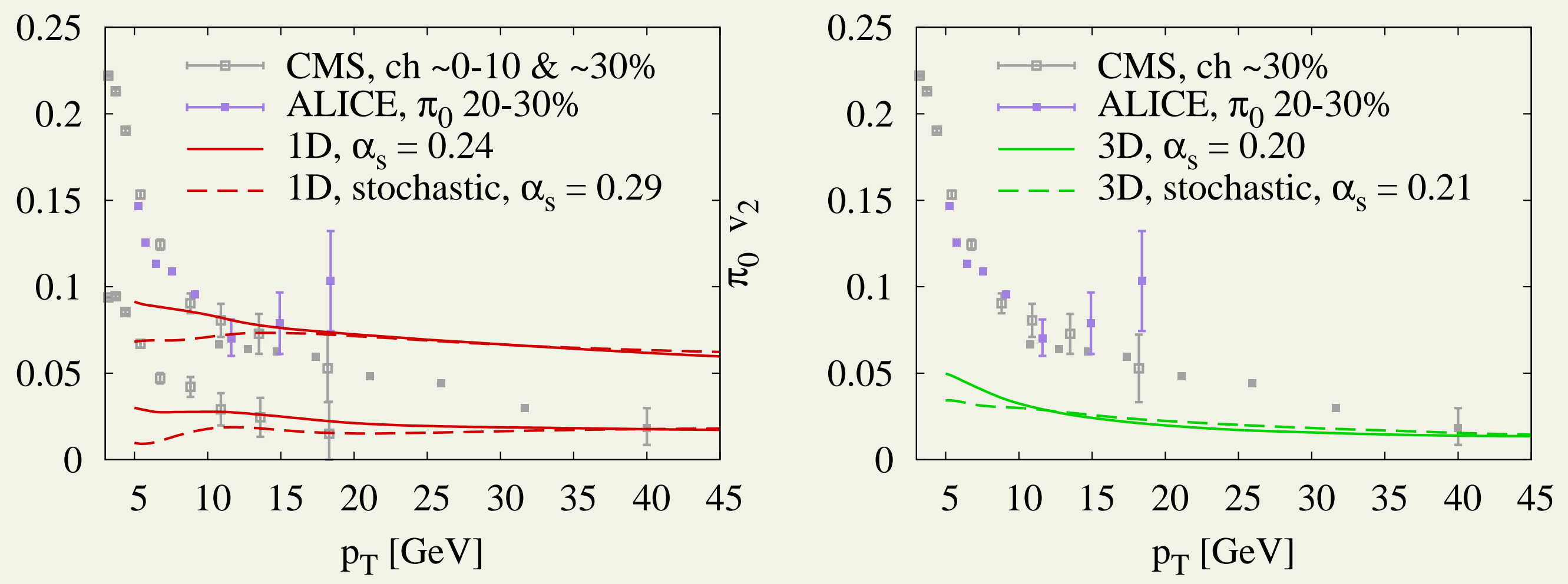
In short: for transversely expanding medium, both $R_{A A}$ and $v_{2}$ are smaller with GLV energy loss. LPM interference favors late scattering.

$\Delta E_{G L V}^{(1)}(z) \propto \int d x d \mathbf{k} d \mathbf{q} \frac{\mu^{2}}{\pi\left(\mathbf{q}^{2}+\mu^{2}\right)^{2}} \frac{2 \mathbf{k} \cdot \mathbf{q}}{\mathbf{k}^{2}(\mathbf{k}-\mathbf{q})^{2}}(1-\cos \omega \Delta z) \propto I(\Delta z / \tau(z), E / \mu(z)$

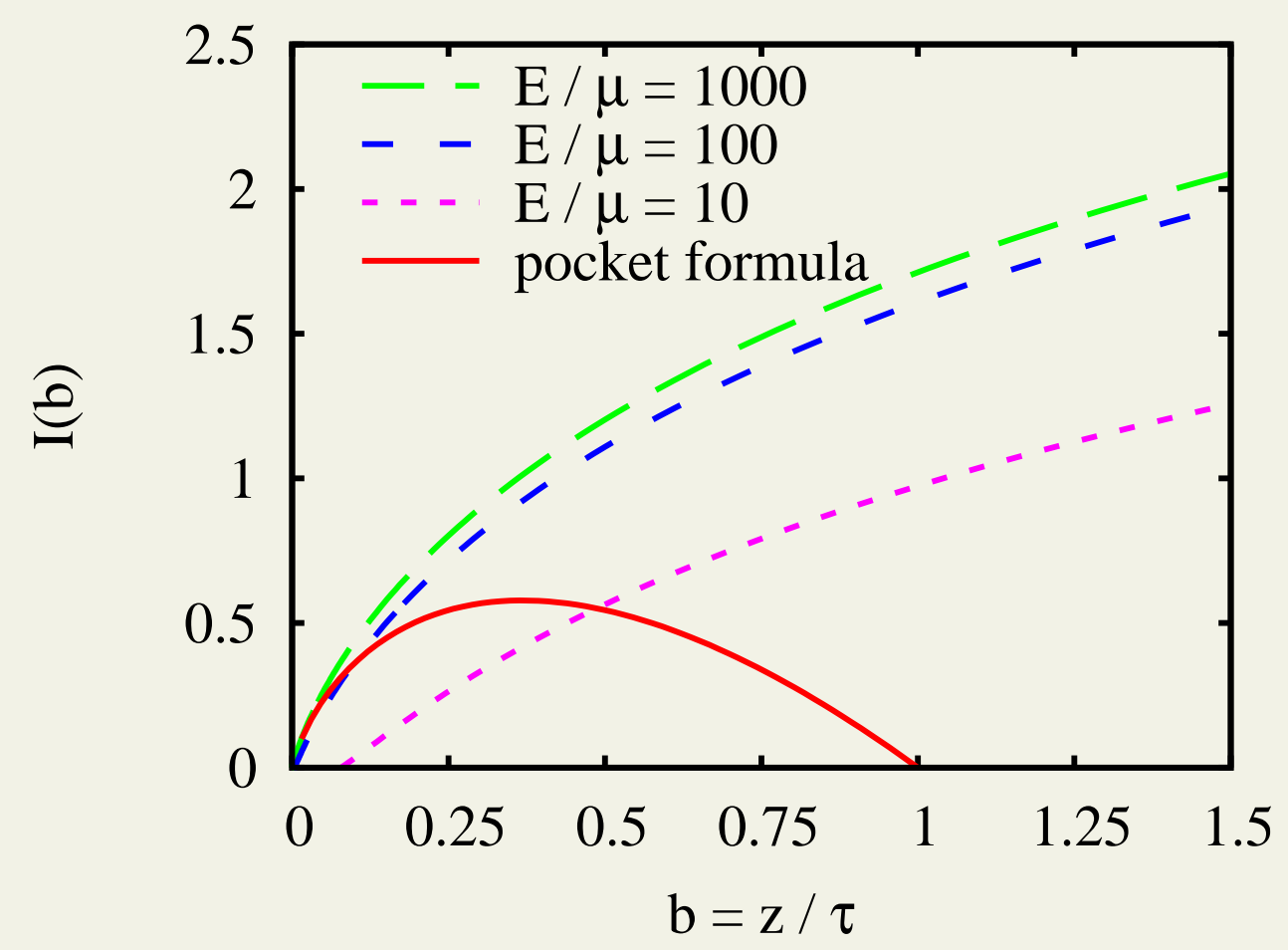

$$
\tau(z) \equiv \frac{2 E}{\mu^{2}(z)}
$$




\section{Could fluctuations help?}

- MC Glauber initconds (binary)

- RHIC Au+Au at $b=8 \mathbf{f m}, 200 A \mathbf{G e V}$

- same $2 \rightarrow 2$ transport (MPC) 
average $A u+A u, b=8 \mathrm{fm}$

$$
\tau d N / d^{2} x_{T}[\mathrm{fm}]
$$

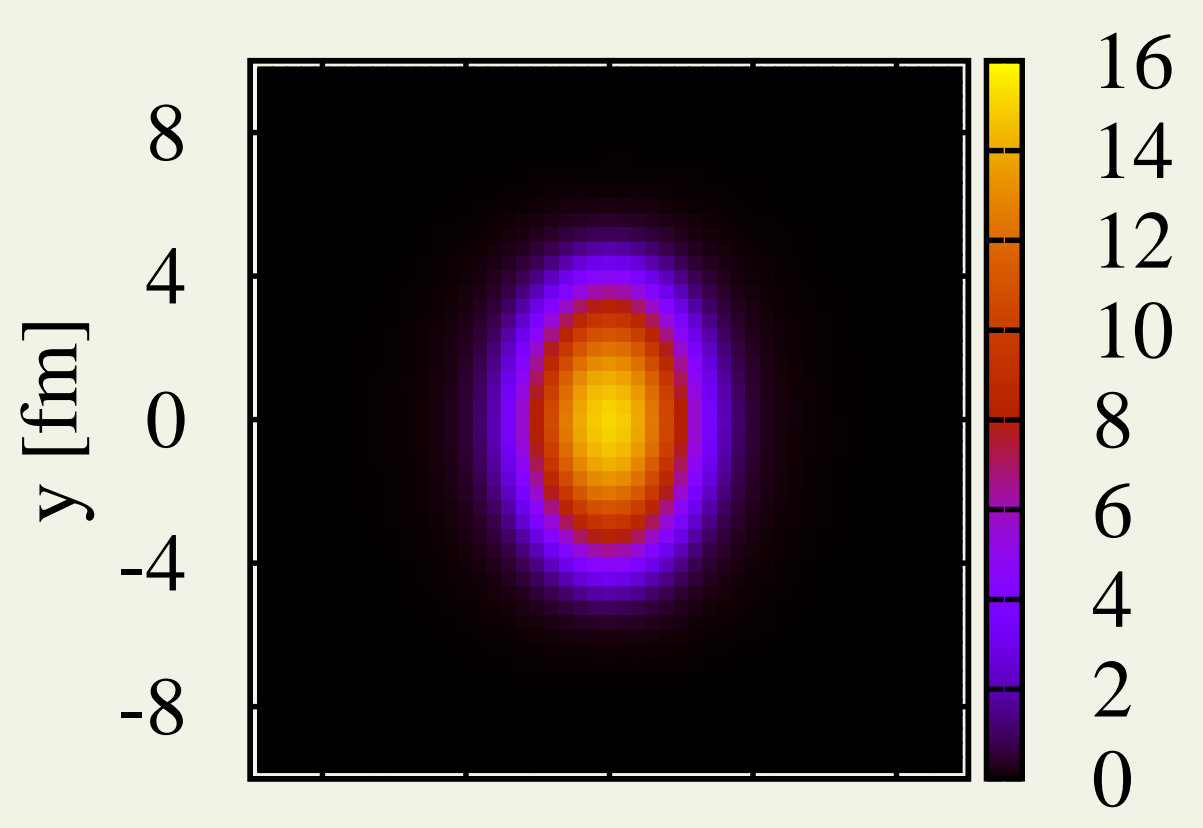

$\begin{array}{lllll}-8 & -4 & 0 & 4 & 8\end{array}$

$\mathrm{x}[\mathrm{fm}]$

\section{single MC Glauber}

$$
\tau d N / d^{2} x_{T}[\mathrm{fm}]
$$

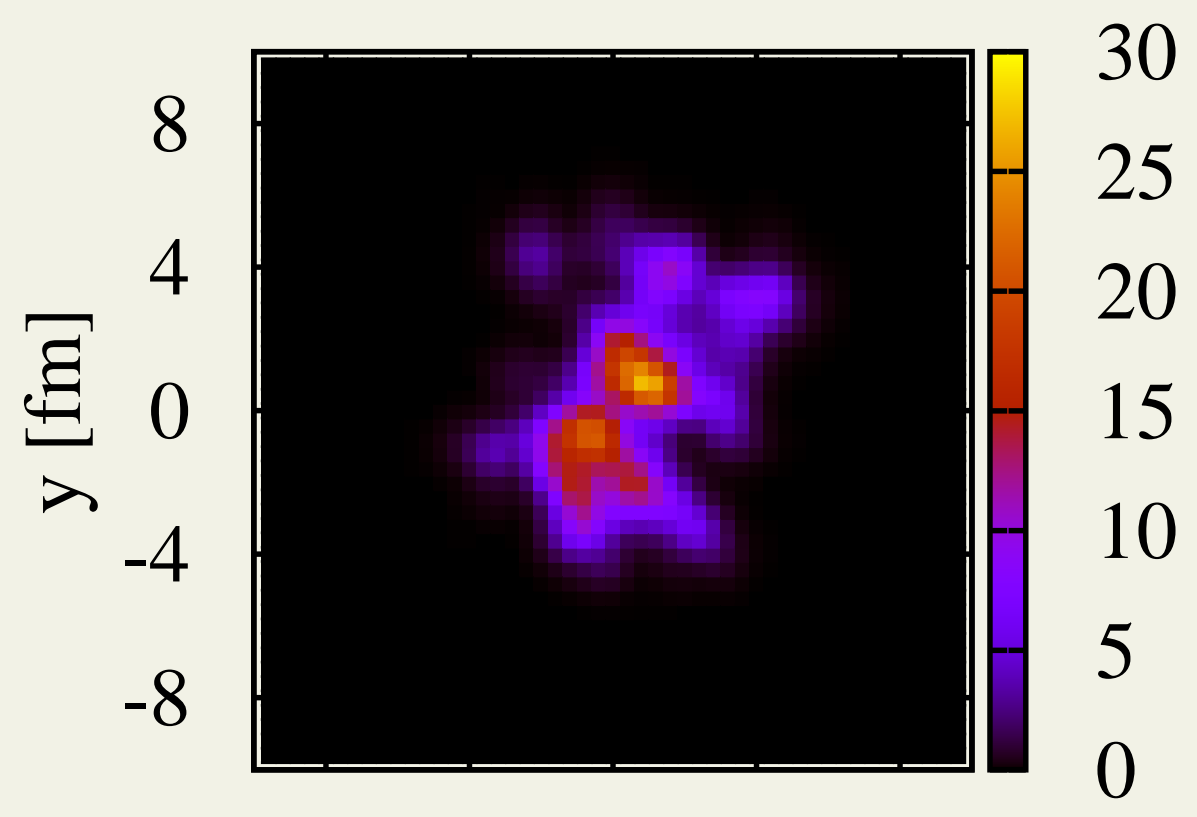

$$
\begin{array}{lllll}
-8 & -4 & 0 & 4 & 8 \\
& \\
& & \\
& & {[\mathrm{fm}]}
\end{array}
$$

$$
\tau=0.6 \mathrm{fm}
$$


average $A u+A u, b=8 \mathrm{fm}$

$$
\tau d N / d^{2} x_{T}[\mathrm{fm}]
$$

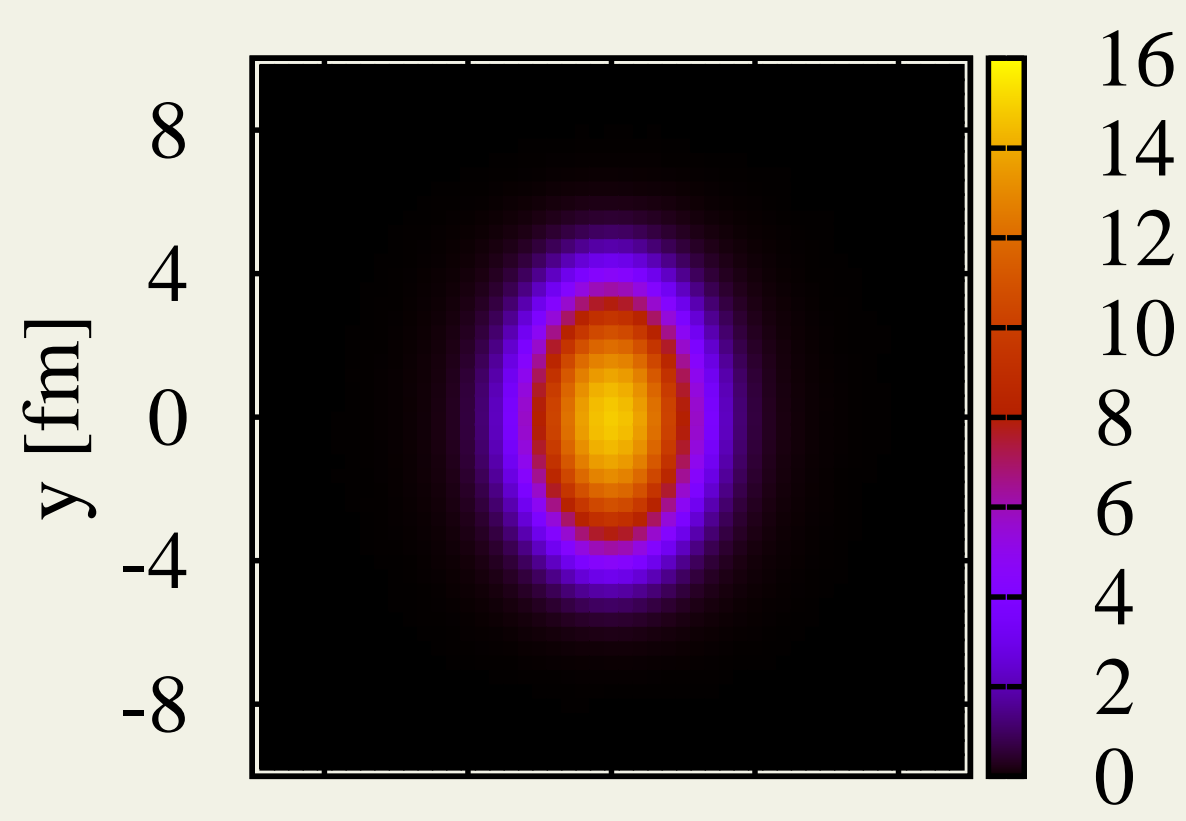

$$
\begin{array}{ccccc}
-8 & -4 & 0 & 4 & 8 \\
\\
\end{array}
$$

single MC Glauber

$$
\tau d N / d^{2} x_{T}[\mathrm{fm}]
$$

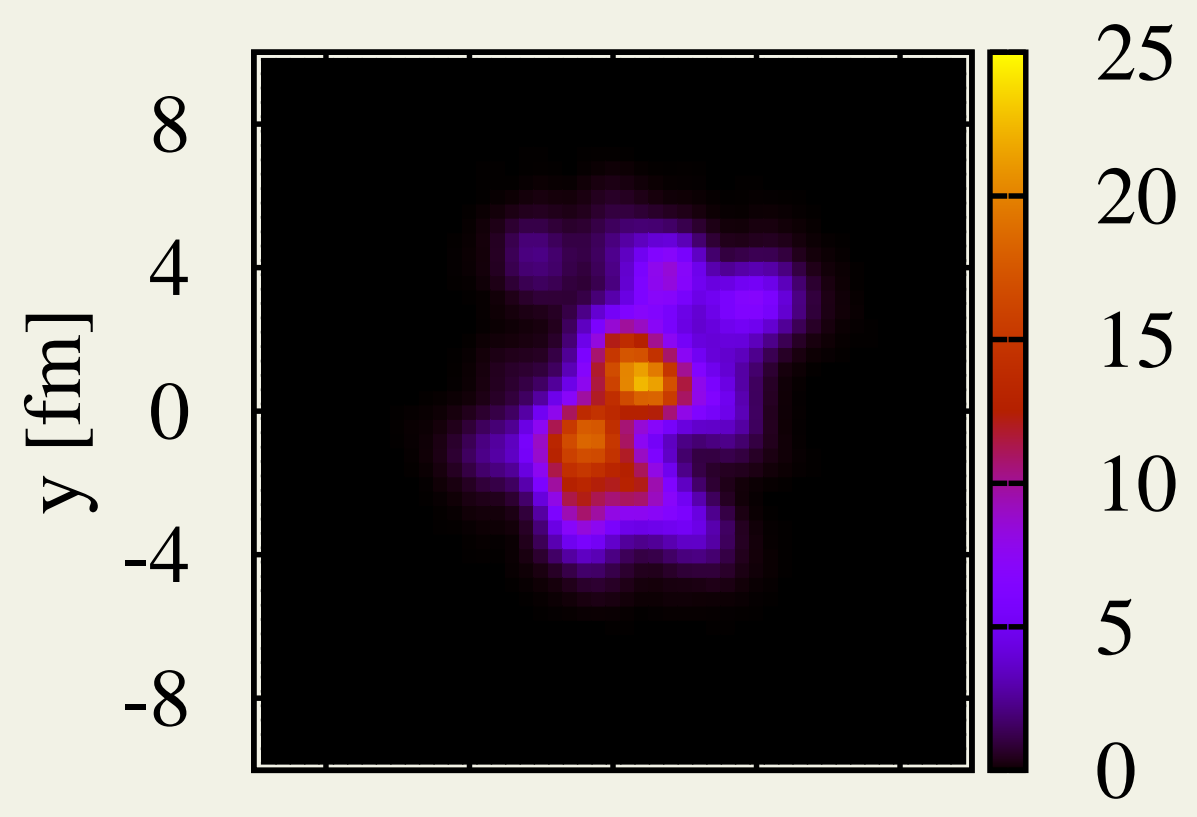

$$
\begin{array}{ccccc}
-8 & -4 & 0 & 4 & 8 \\
& \\
& & & & {[\mathrm{fm}]}
\end{array}
$$


average $A u+A u, b=8 \mathrm{fm}$

$$
\tau d N / d^{2} x_{T}[\mathrm{fm}]
$$

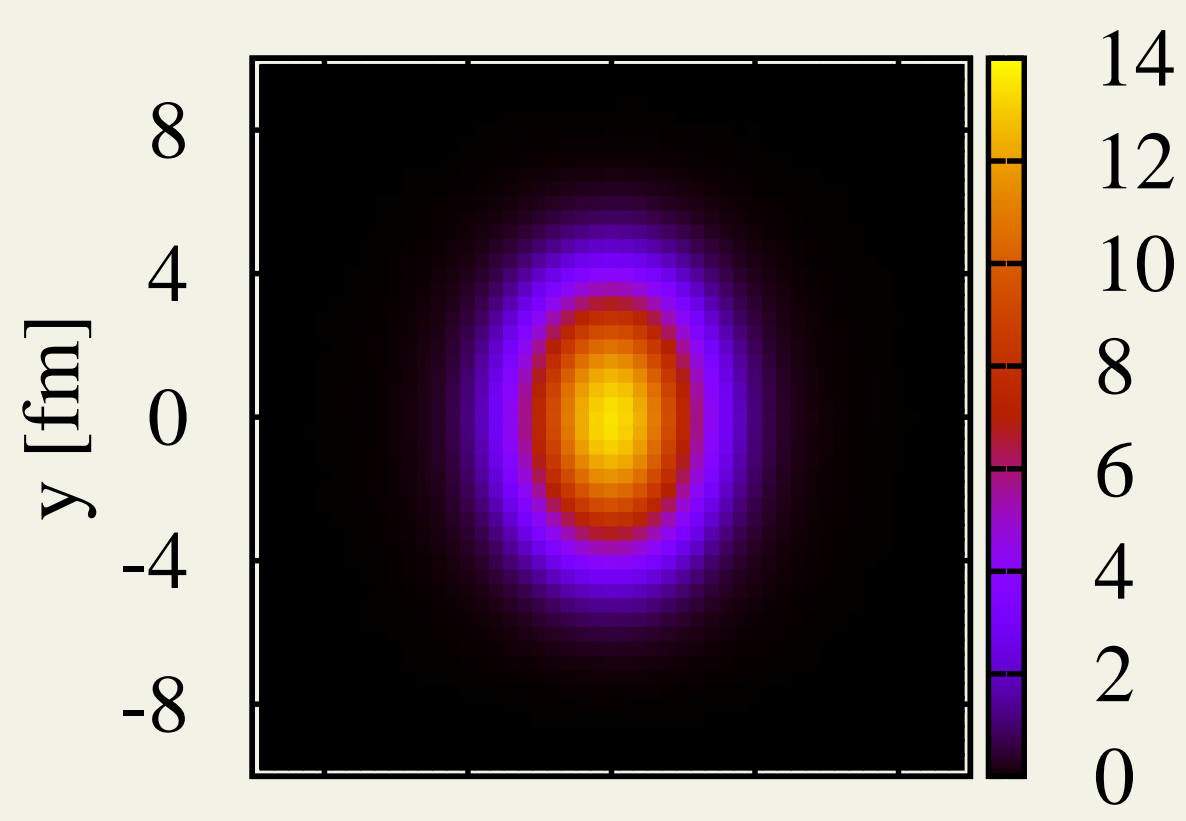

$\begin{array}{lllll}-8 & -4 & 0 & 4 & 8\end{array}$

$\mathrm{x}[\mathrm{fm}]$

\section{single MC Glauber}

$$
\tau d N / d^{2} x_{T}[\mathrm{fm}]
$$

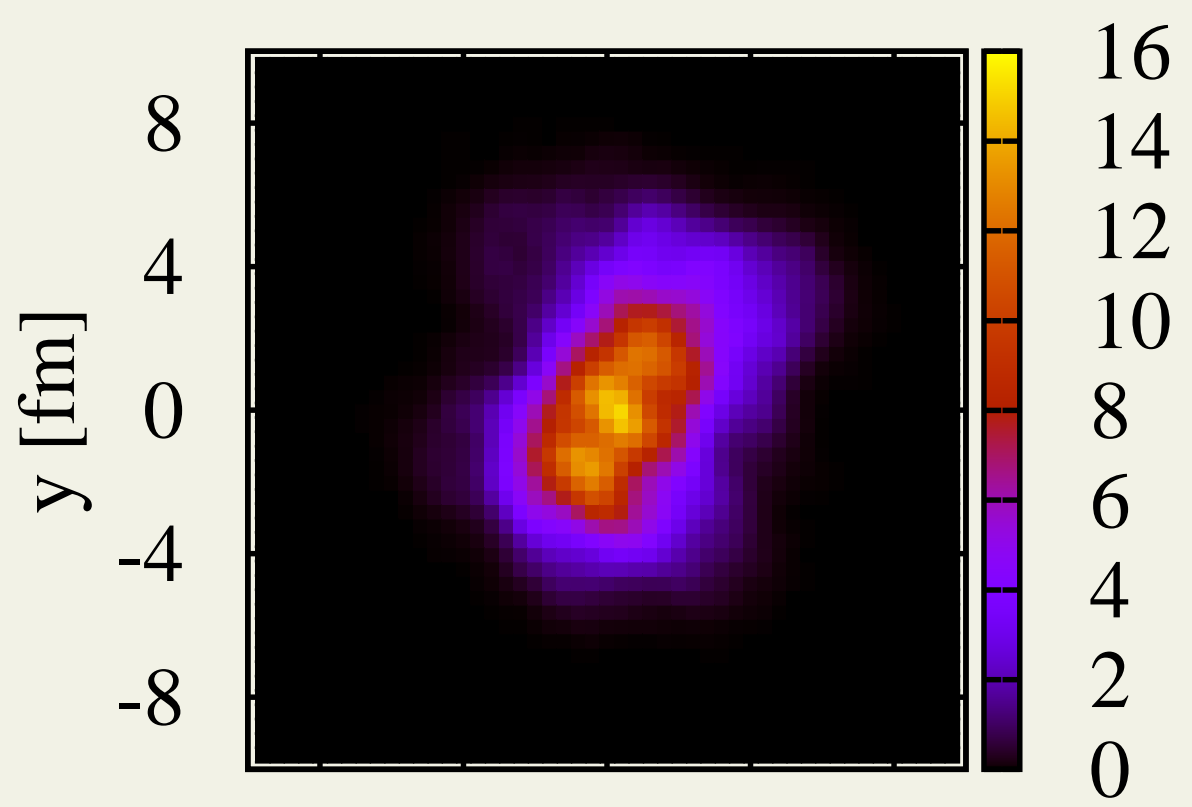

$$
\begin{array}{cccccc}
-8 & -4 & 0 & 4 & 8 \\
\\
\end{array}
$$


average $A u+A u, b=8 \mathrm{fm}$

$$
\tau d N / d^{2} x_{T}[\mathrm{fm}]
$$

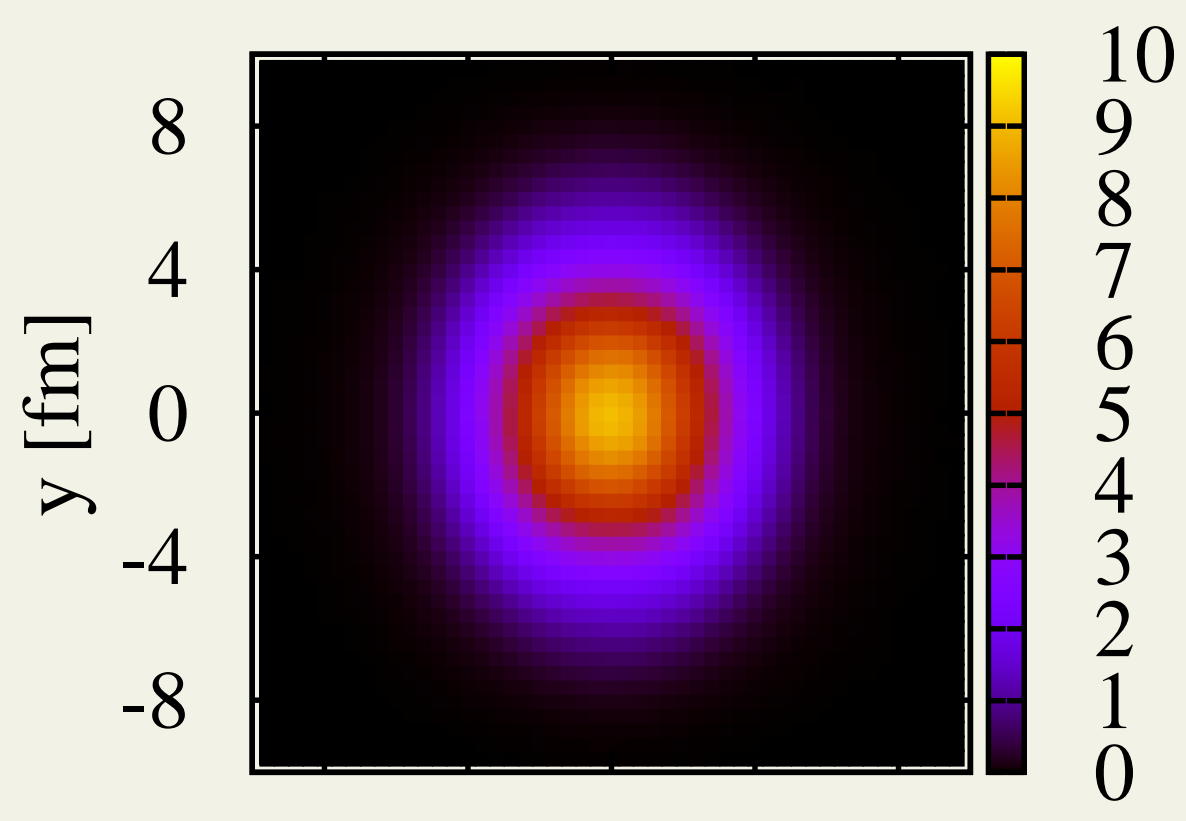

$$
\begin{array}{ccccc}
-8 & -4 & 0 & 4 & 8 \\
\\
\end{array}
$$

\section{single MC Glauber}

$$
\tau d N / d^{2} x_{T}[\mathrm{fm}]
$$

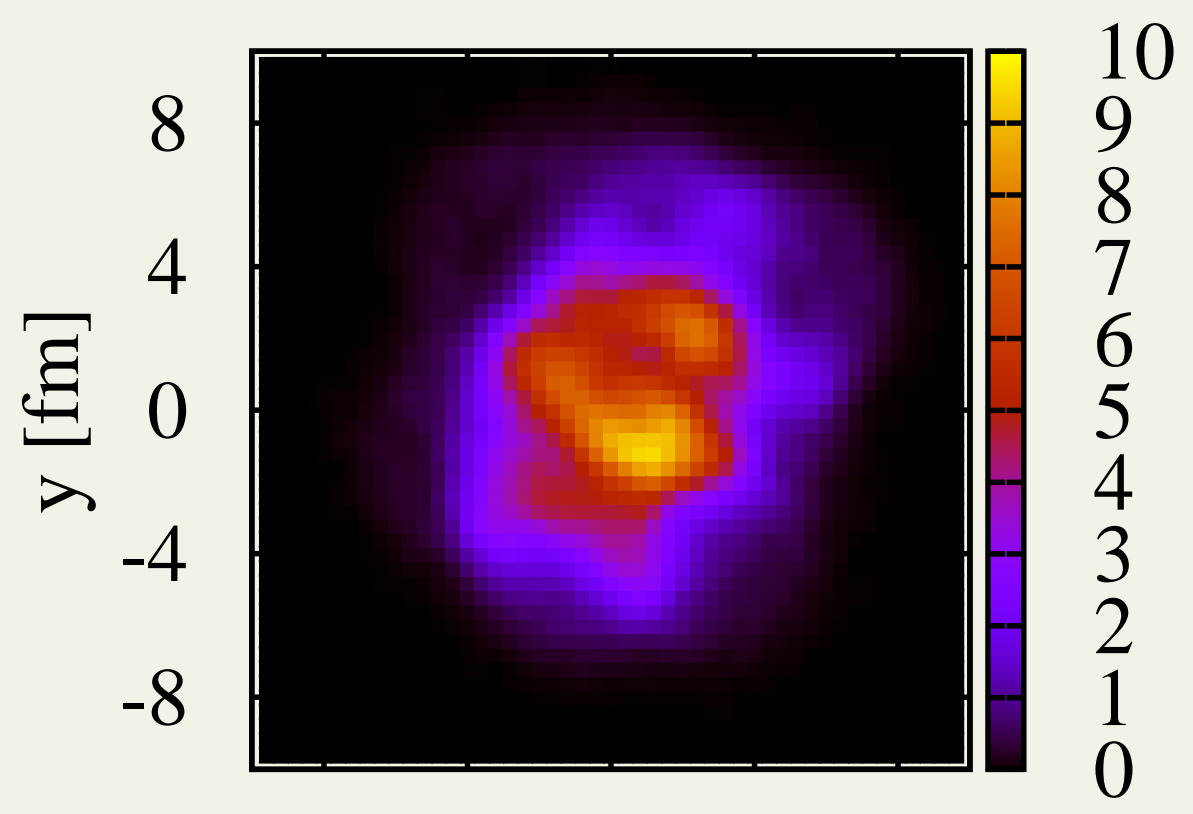

$$
\begin{array}{ccccc}
-8 & -4 & 0 & 4 & 8 \\
& \\
& & & & {[\mathrm{fm}]}
\end{array}
$$


average $A u+A u, b=8 \mathrm{fm}$

$$
\tau d N / d^{2} x_{T}[\mathrm{fm}]
$$

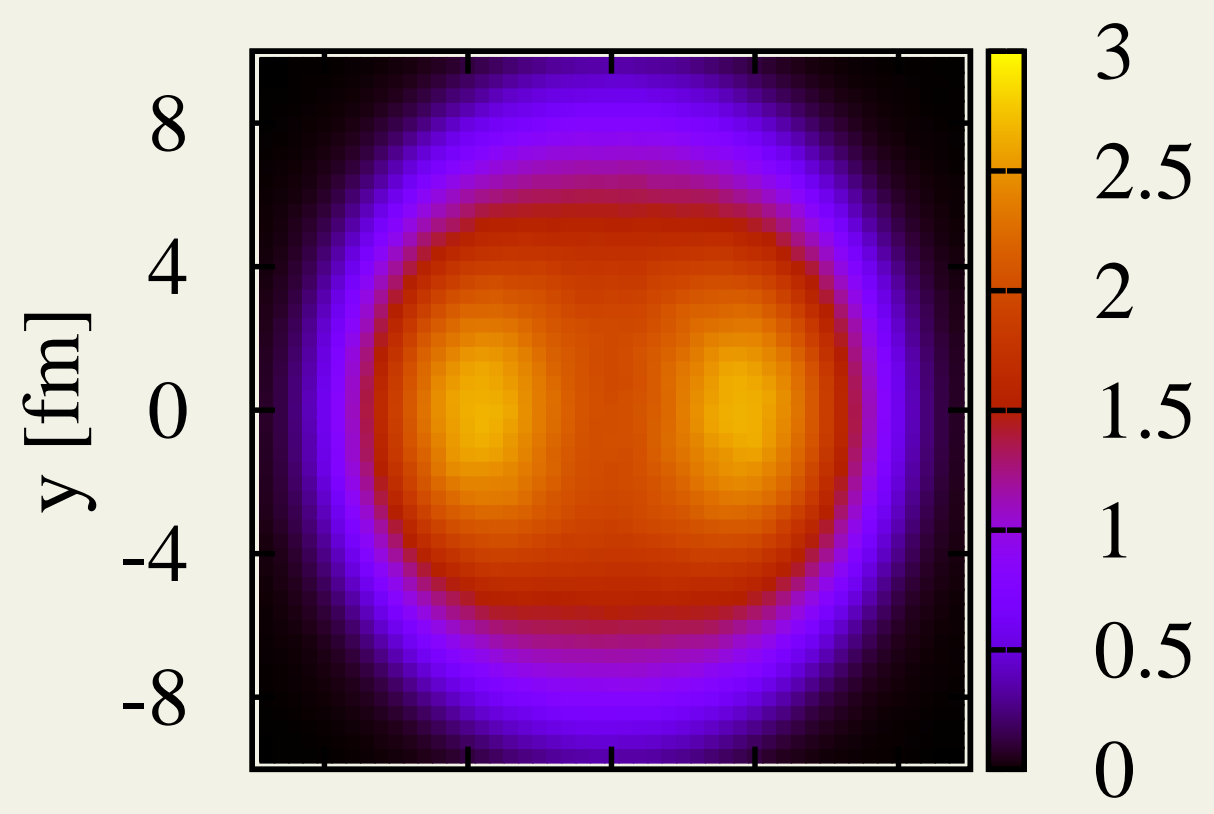

$$
\begin{array}{ccccc}
-8 & -4 & 0 & 4 & 8 \\
\\
\end{array}
$$

\section{single MC Glauber}

$$
\tau d N / d^{2} x_{T}[\mathrm{fm}]
$$

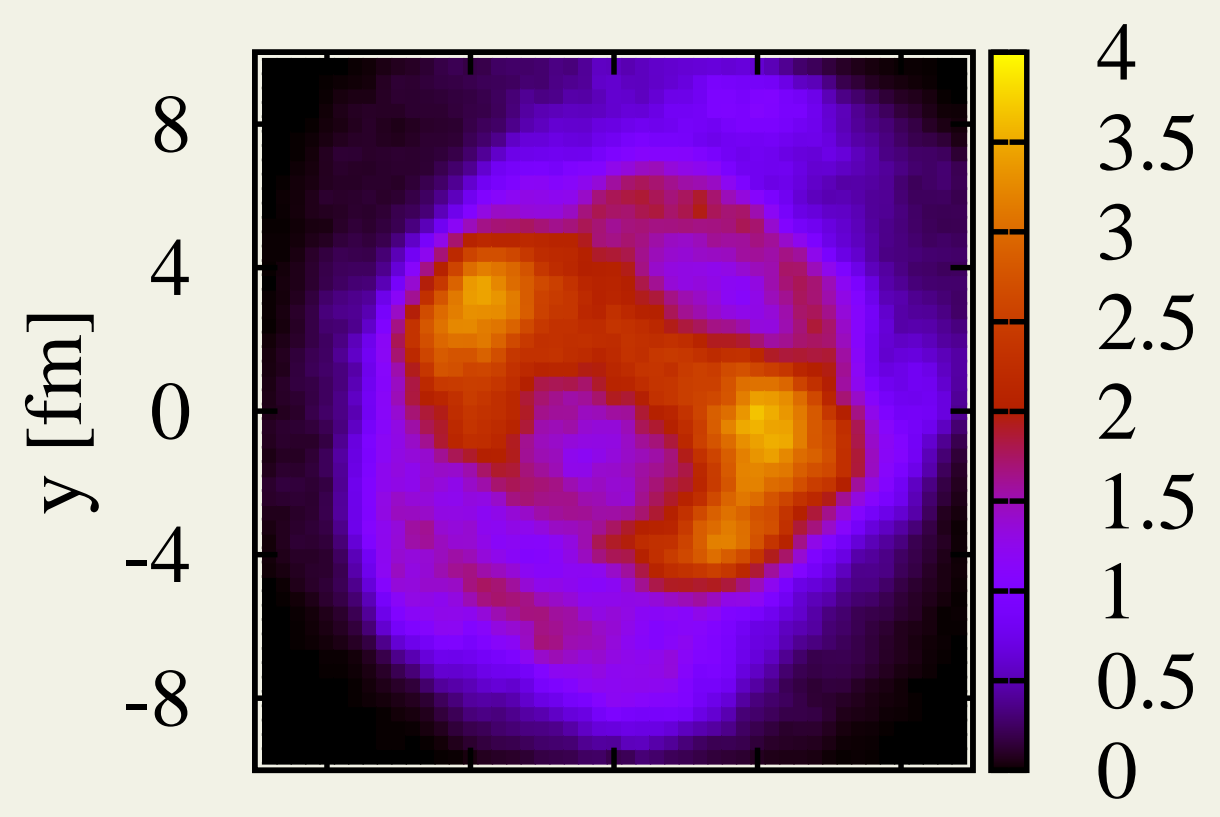

$$
\begin{array}{lllll}
-8 & -4 & 0 & 4 & 8 \\
& \\
& & & {\left[\begin{array}{lll}
\mathrm{fm}
\end{array}\right]}
\end{array}
$$


average $A u+A u, b=8 \mathrm{fm}$

$$
\tau d N / d^{2} x_{T}[\mathrm{fm}]
$$

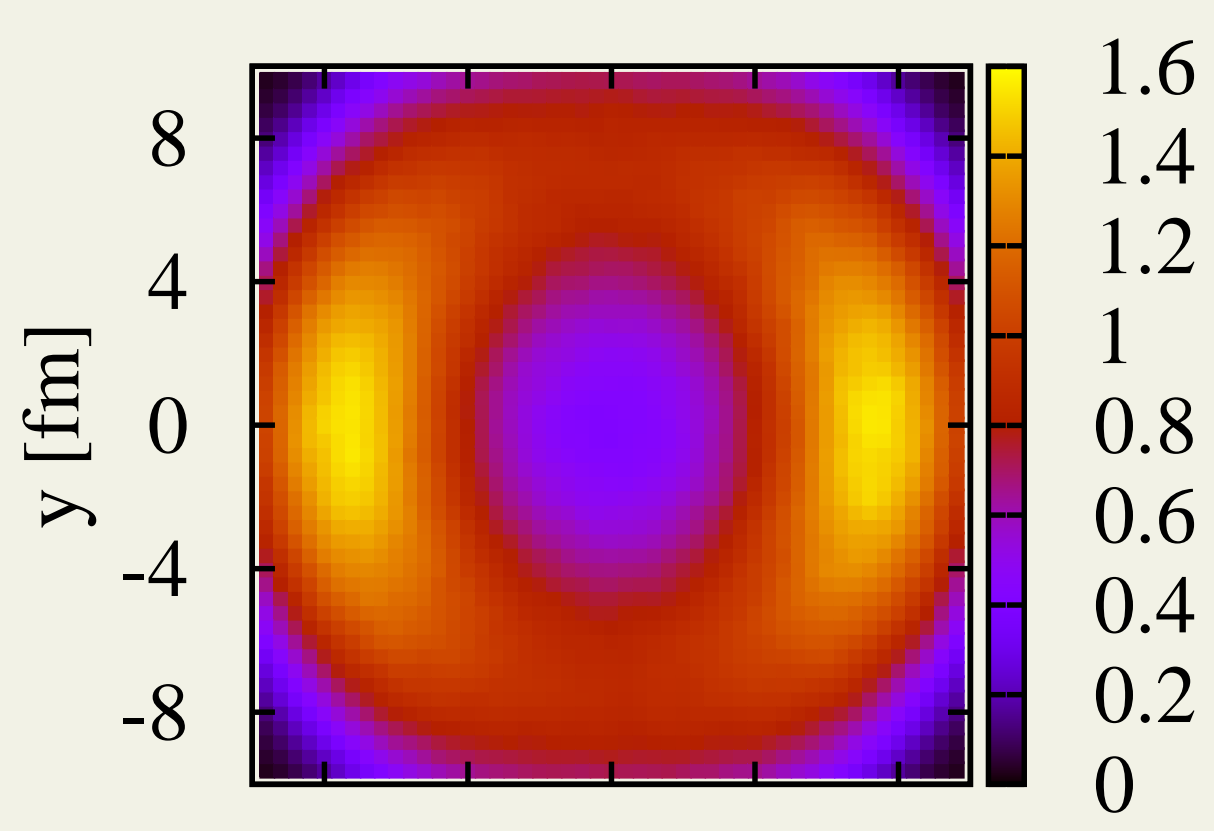

$$
\begin{array}{ccccc}
-8 & -4 & 0 & 4 & 8 \\
\\
\end{array}
$$

\section{single MC Glauber}

$$
\tau d N / d^{2} x_{T}[\mathrm{fm}]
$$

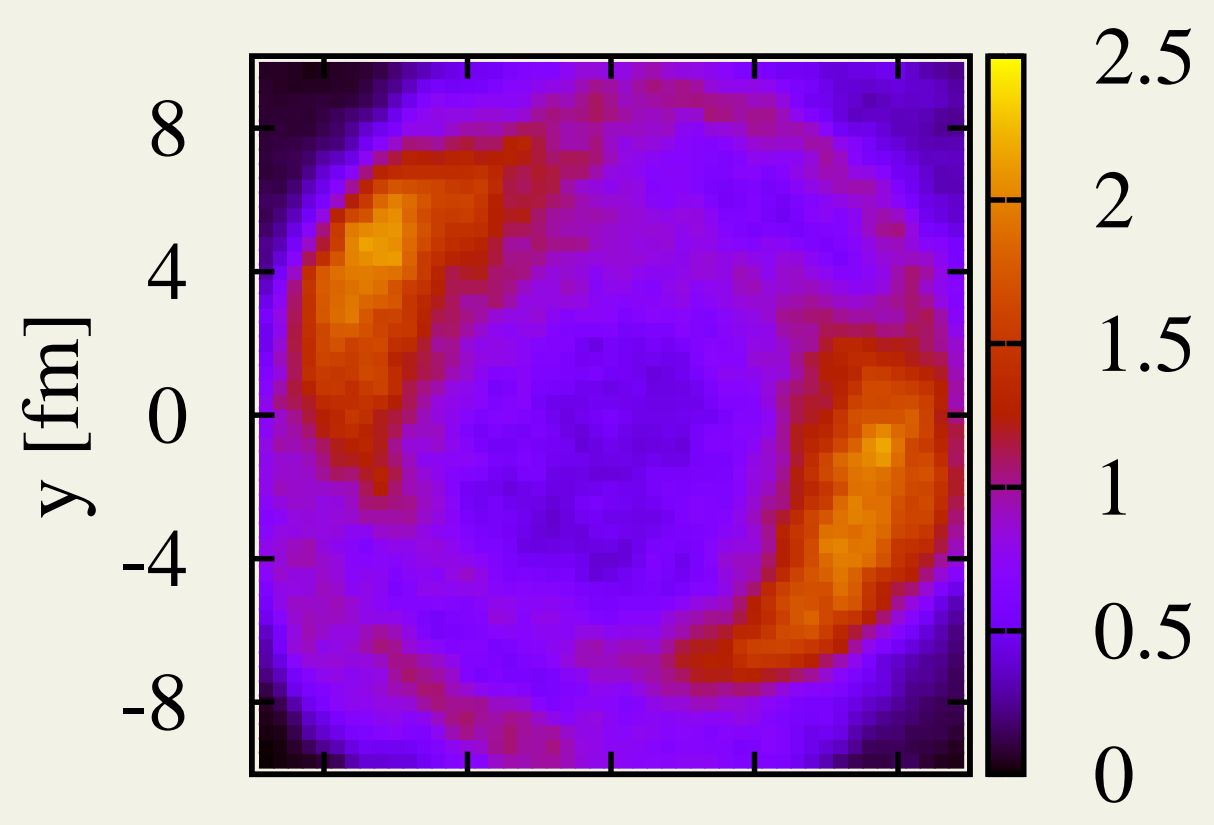

$$
\begin{array}{ccccc}
-8 & -4 & 0 & 4 & 8 \\
& \\
& & & {[\mathrm{fm}]}
\end{array}
$$

$$
\tau=9.6 \mathrm{fm}
$$


$\tau \mathrm{n}\left[\mathrm{fm}^{2}\right]$

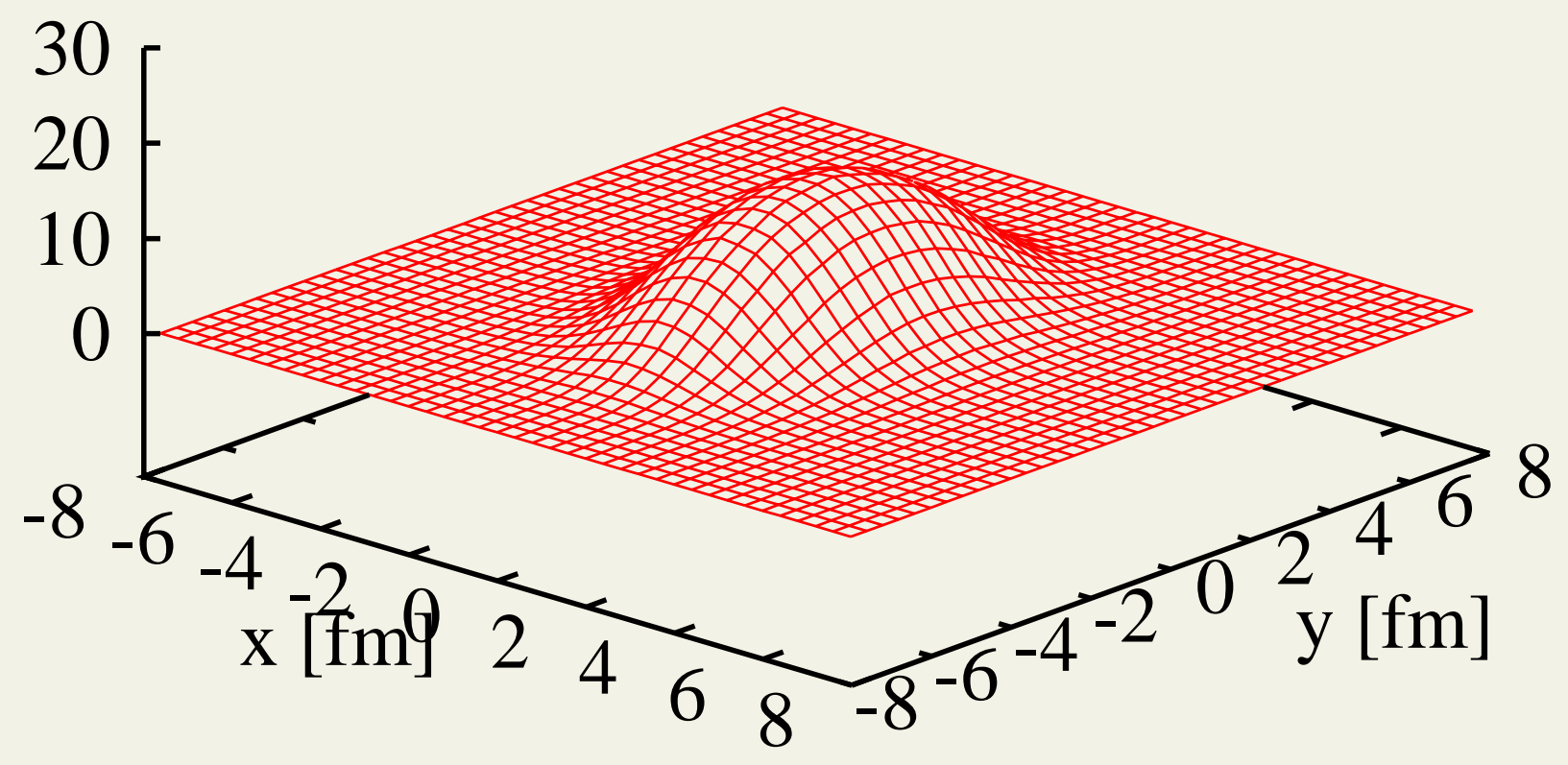

$\tau \mathrm{n}\left[\mathrm{fm}^{2}\right]$

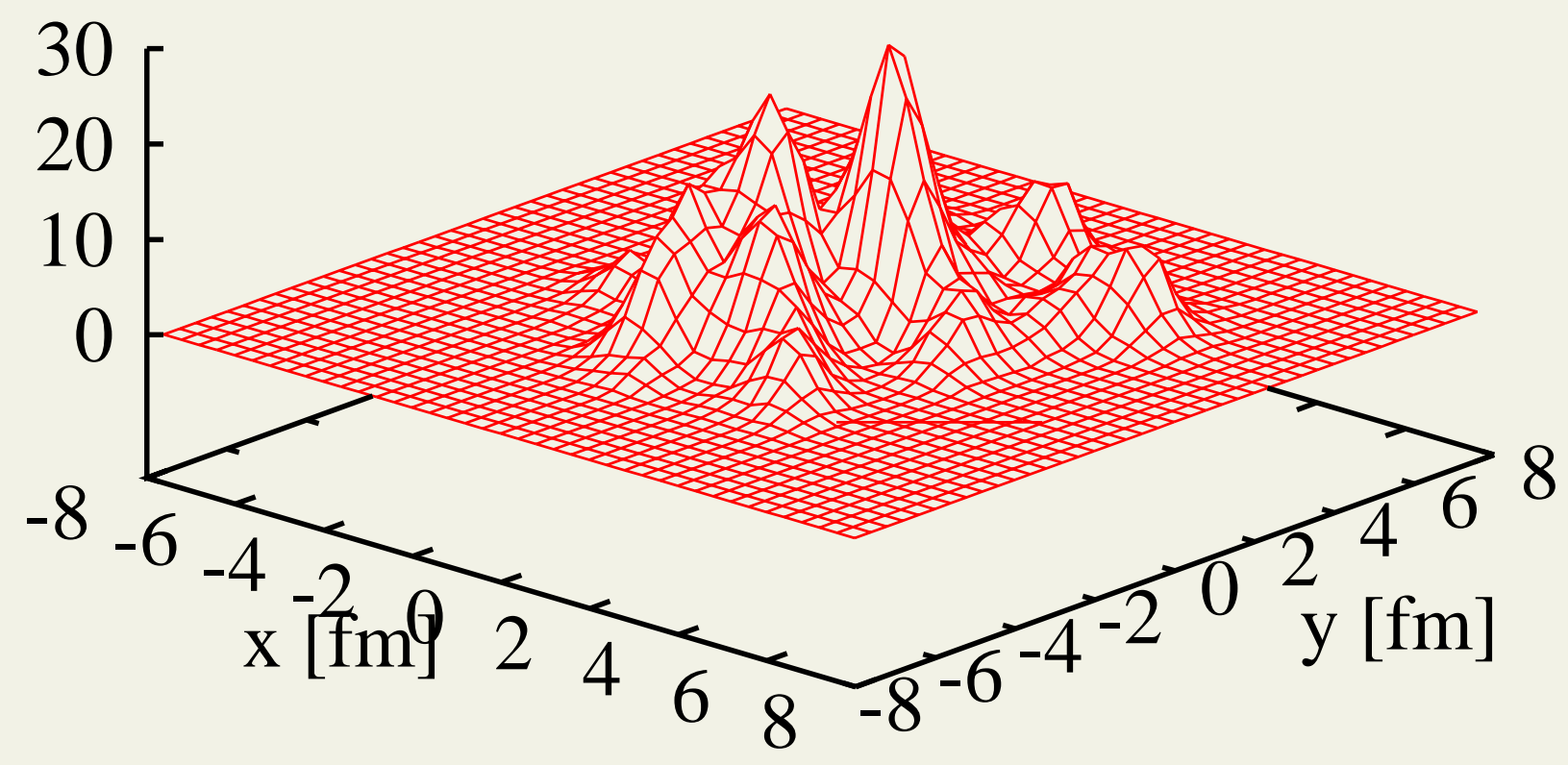

"./flı 
Analyze MEDIUM and JET response using eccentricity coefficients

$$
\begin{aligned}
\vec{\epsilon}_{n, k} & \equiv \frac{1}{\left\langle r^{k}\right\rangle}\left(\left\langle r^{k} \cos \left(n \phi_{x}\right)\right\rangle,\left\langle r^{k} \sin \left(n \phi_{x}\right)\right\rangle\right) \\
& =\epsilon_{n, k}^{*}\left(\cos \Phi_{n, k}, \sin \Phi_{n, k}\right)
\end{aligned}
$$

and similar decomposition for transverse momenta $\left(v_{n}, \Phi_{n}\right)$

$$
\frac{d N}{d \varphi} \propto 1+\sum_{n=1}^{\infty} 2 v_{n} \cos n\left(\varphi-\Phi_{n}\right)
$$



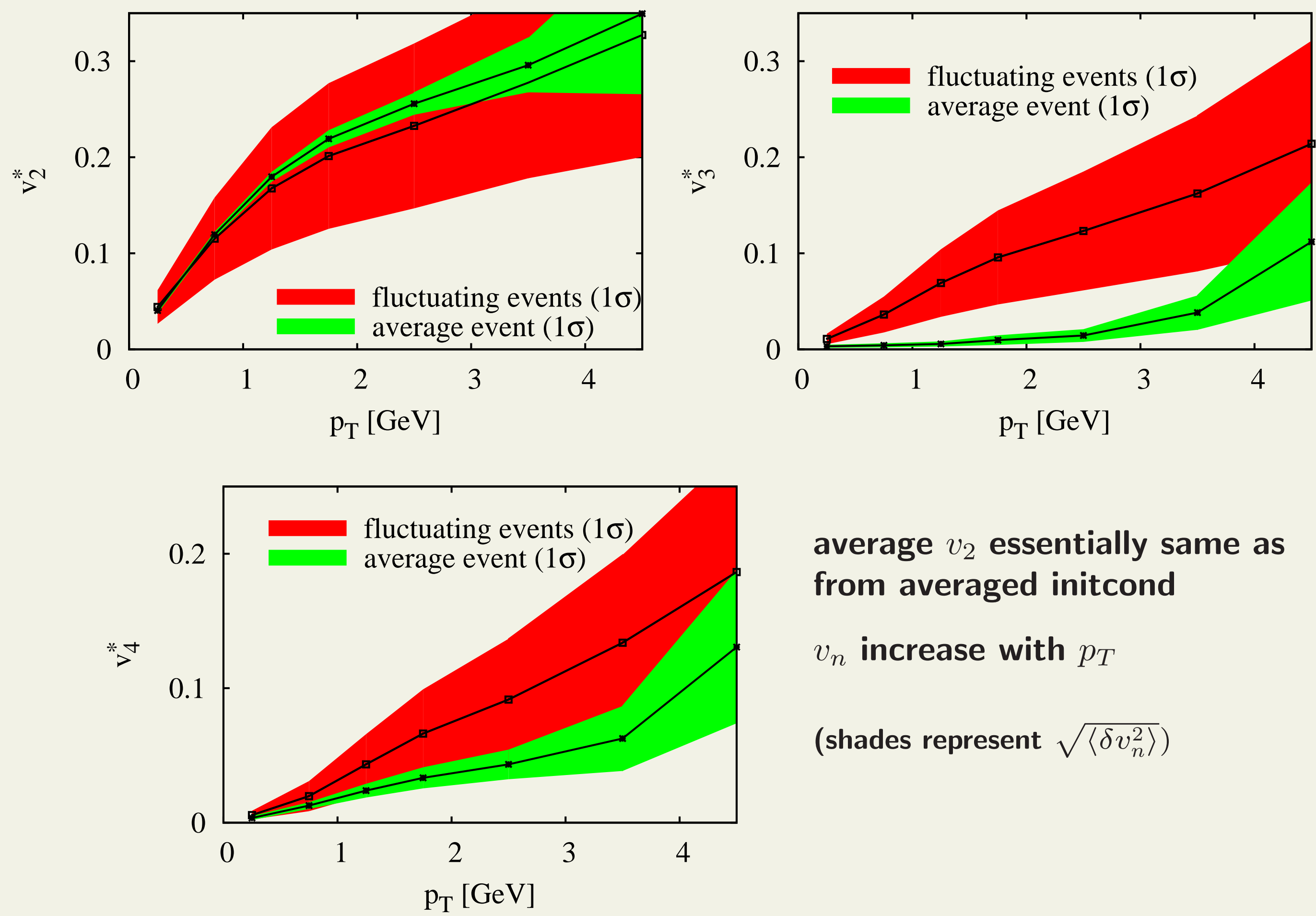

average $v_{2}$ essentially same as from averaged initcond

$v_{n}$ increase with $p_{T}$

(shades represent $\sqrt{\left\langle\delta v_{n}^{2}\right\rangle}$ )

- Typeset by FoilTEX - D. Molnar @ BNL JET 2013, Apr 15-17, 2013 

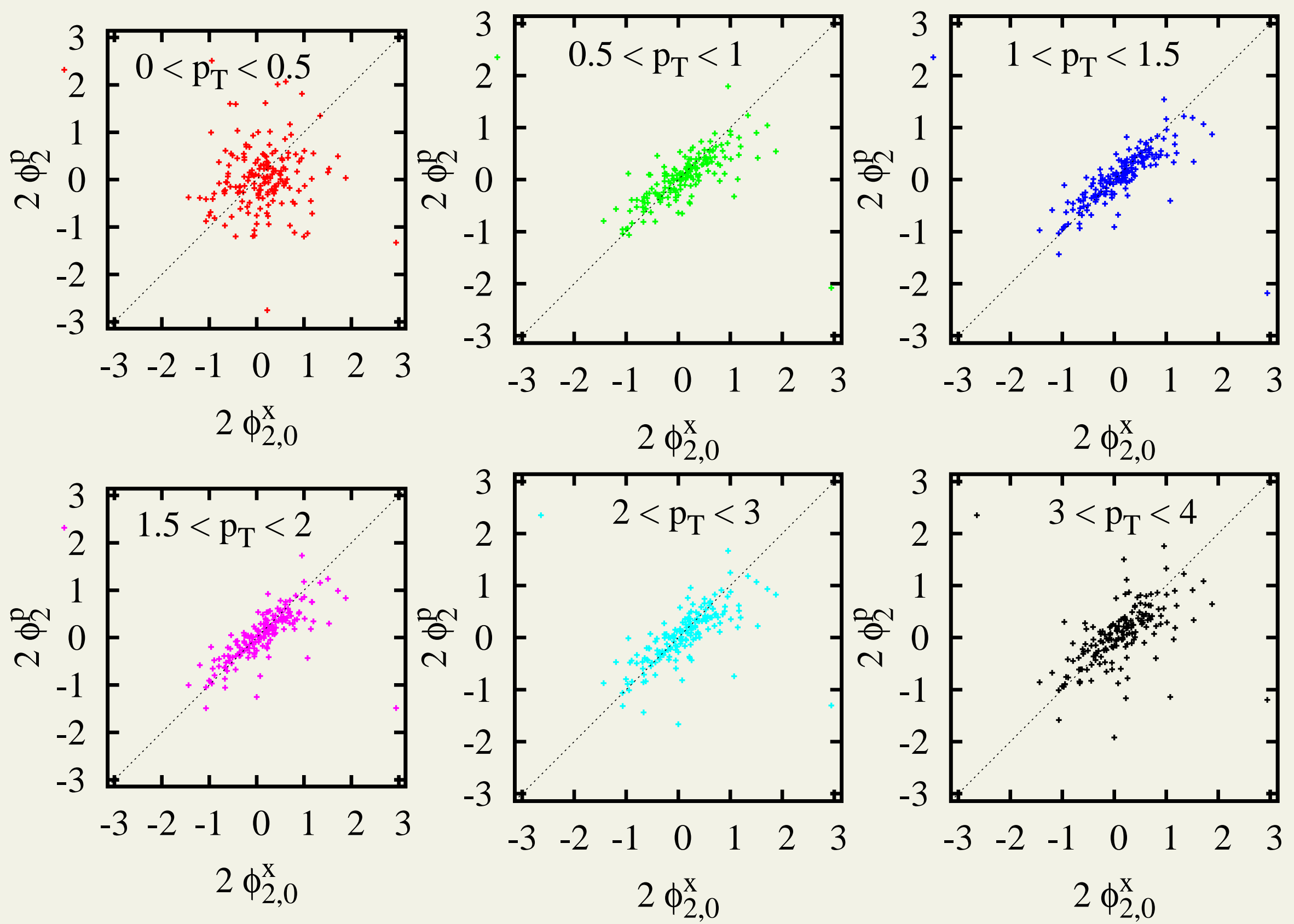

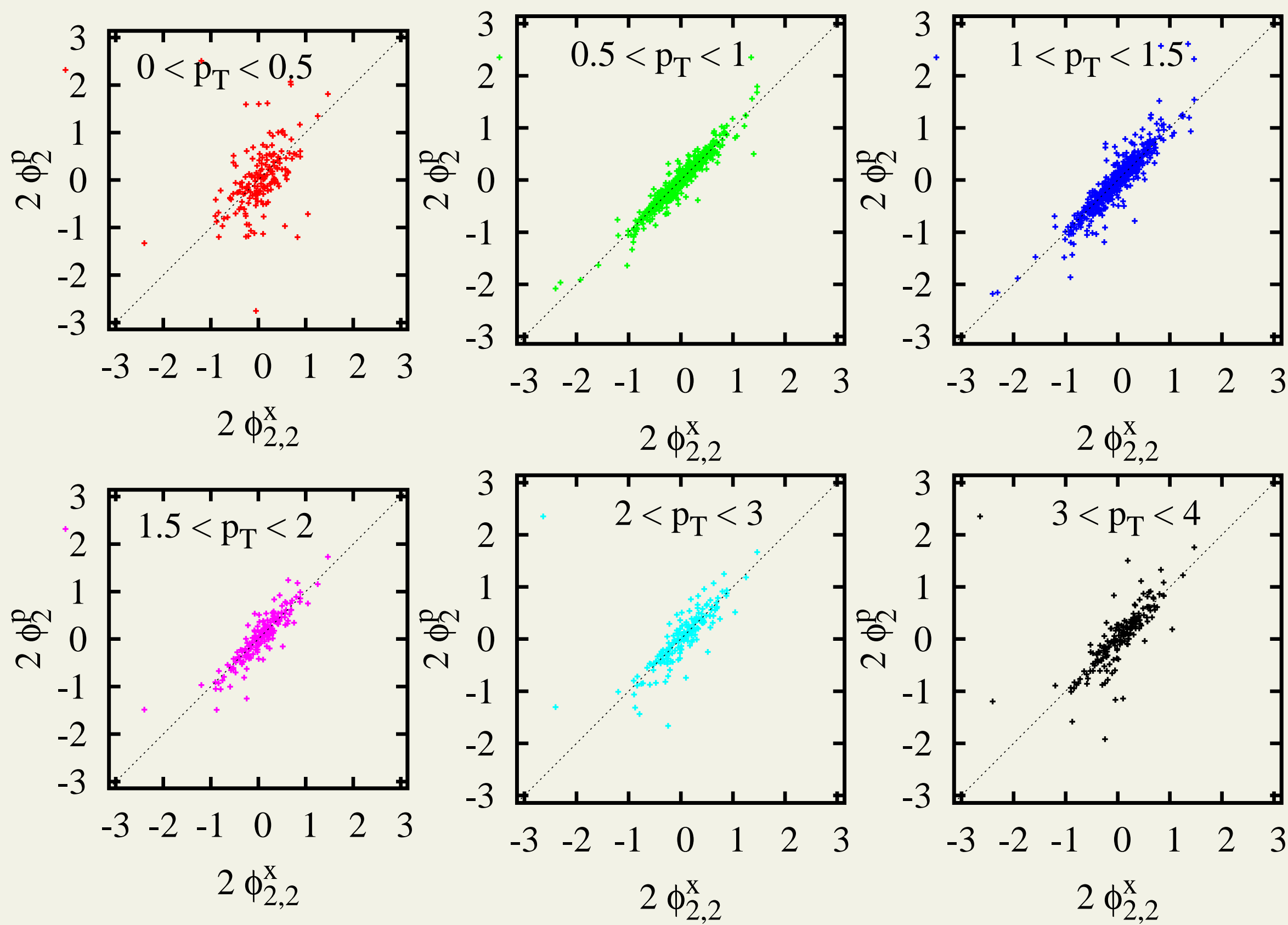

$\epsilon_{2,2}$ orientation correlates best with $v_{2}$ orientation 

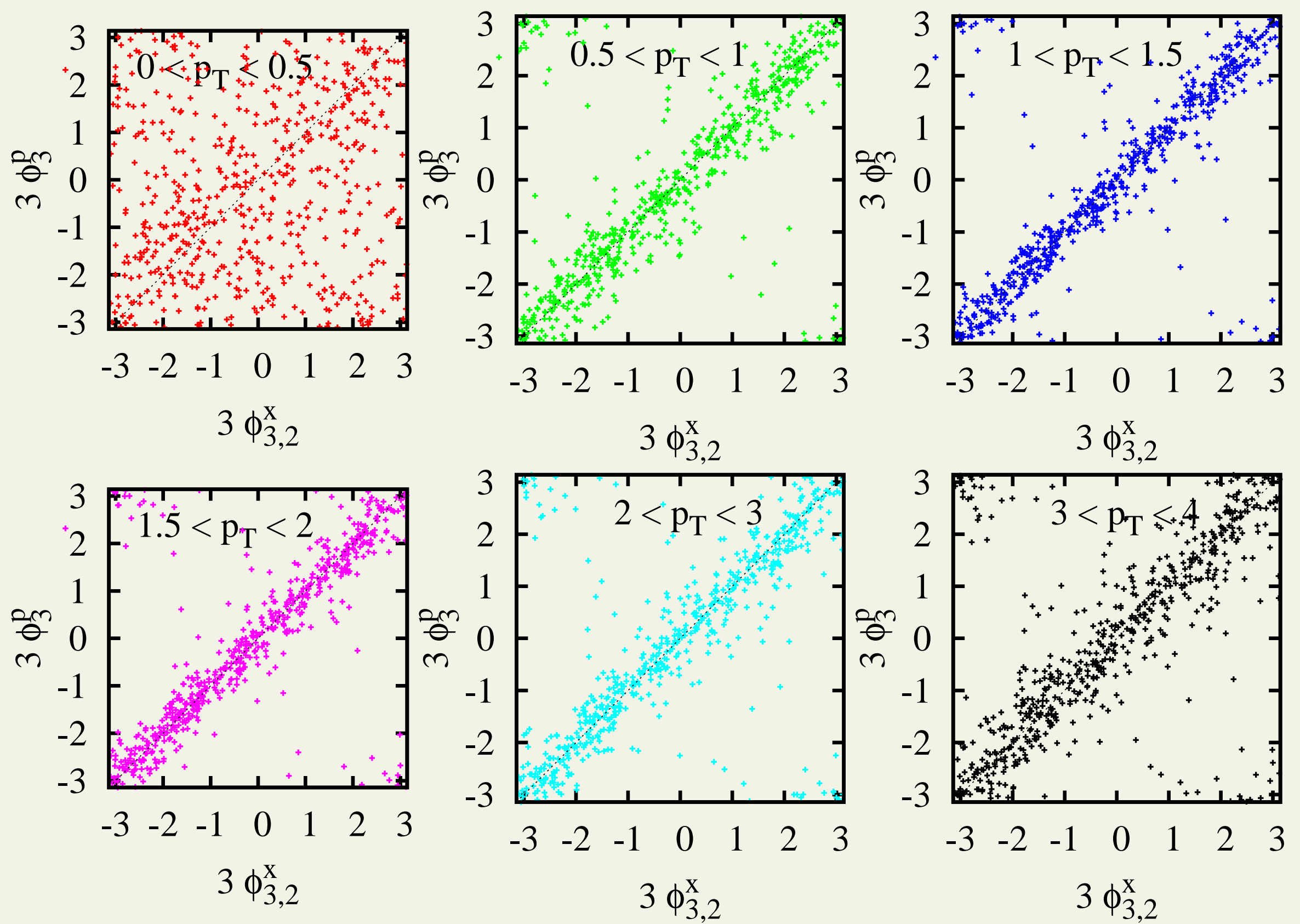

$\epsilon_{3,2}$ correlates strongly with $v_{3}$ orientation 


\section{Bulk medium response qualitatively similar to hydrodynamics.}

What about the jets? 

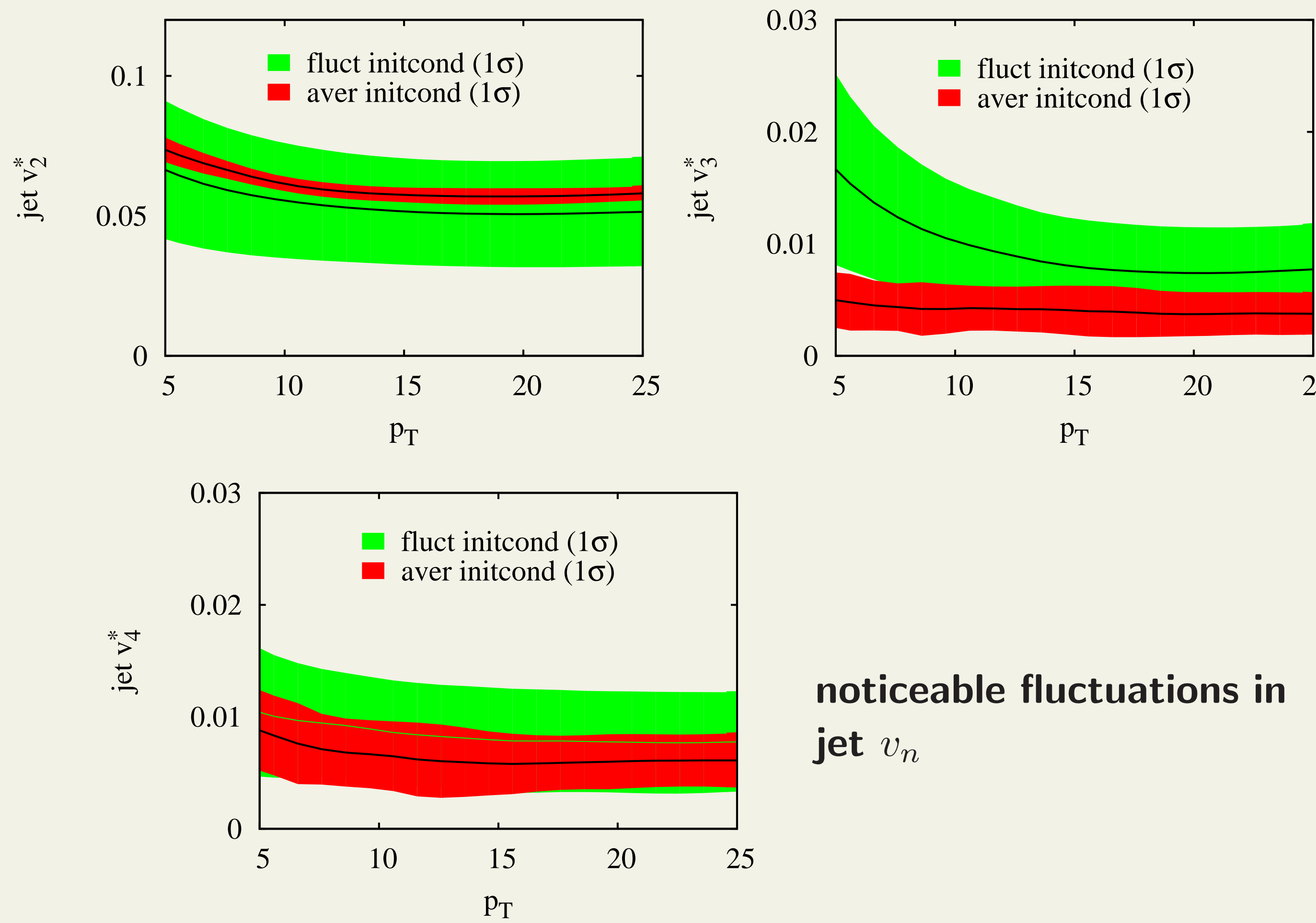

noticeable fluctuations in jet $v_{n}$ 

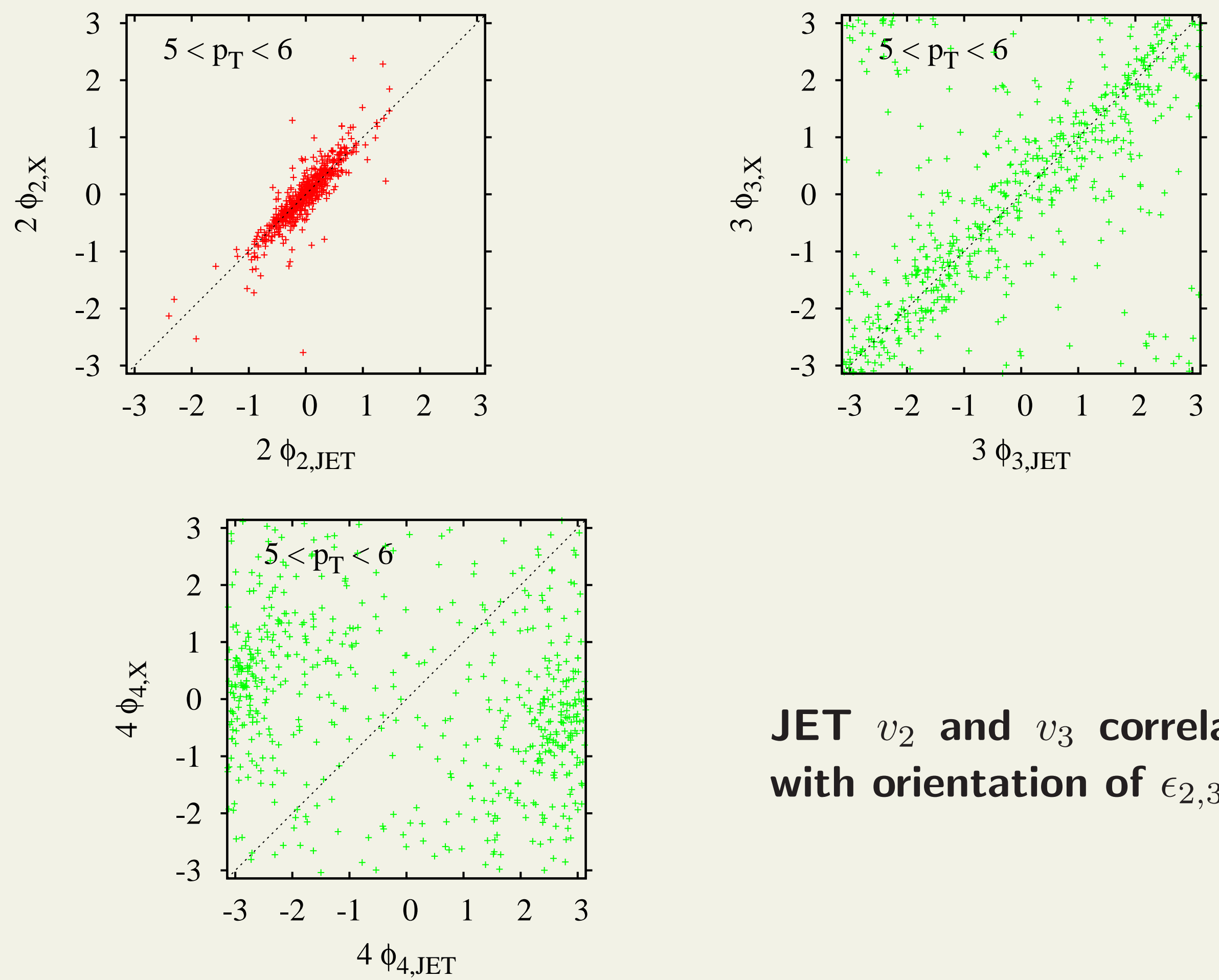

JET $v_{2}$ and $v_{3}$ correlate with orientation of $\epsilon_{2,3}$ 


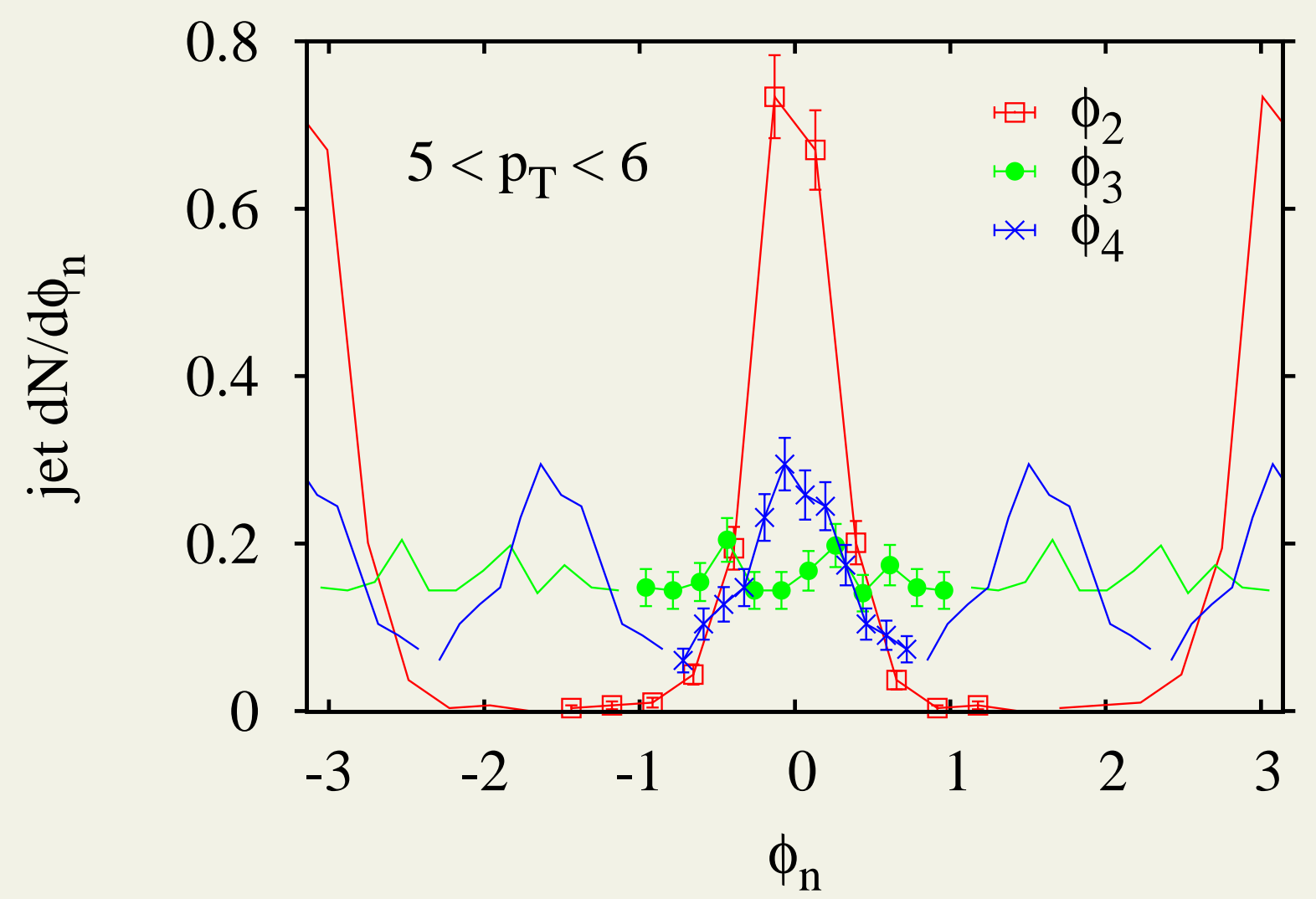

angles relative to theorist's reaction plane $\rightarrow$ positive $v_{4}$ 


\section{Summary}

Parton transport can serve as bulk medium model. It responds to fluctuating initial conditions similarly to hydrodynamics.

Though initial fluctuations NOT small perturbations, jet and medium $v_{2,3,4}$ correlate well with initial $\vec{\epsilon}_{2,3,4}$. For basic observables averaging even appears to nearly commute with evolution. (Where is the nonlinearity??)

We also find that, with GLV energy loss, transverse expansion reduces both $R_{A A}$ and $v_{2}$ at high $p_{T}$.

Some interesting avenues to investigate:

- dynamical fluctuations in transport

- migration in $p_{T}$ ("plasma push" DM nucl-th/0503051)

- corona 
DM ('05):
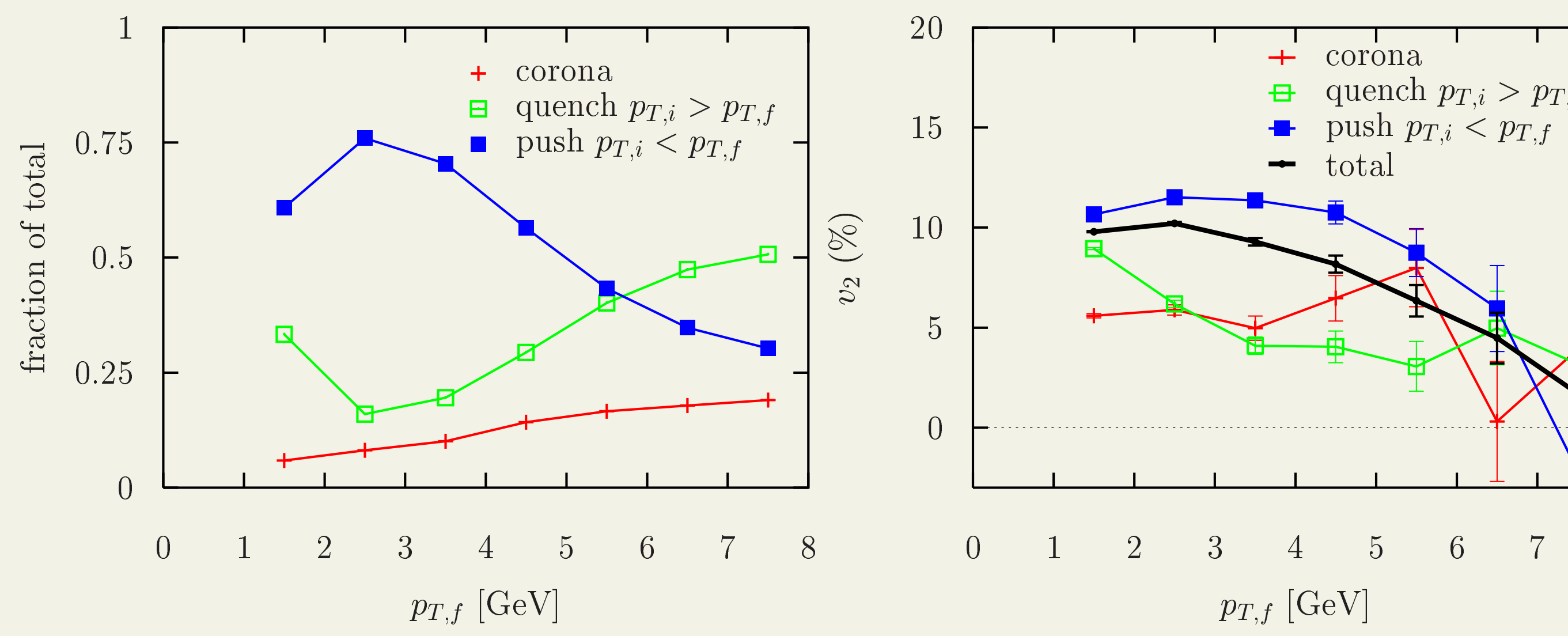
average $A u+A u, b=8 \mathrm{fm}$

$$
\tau d N / d^{2} x_{T}[\mathrm{fm}]
$$

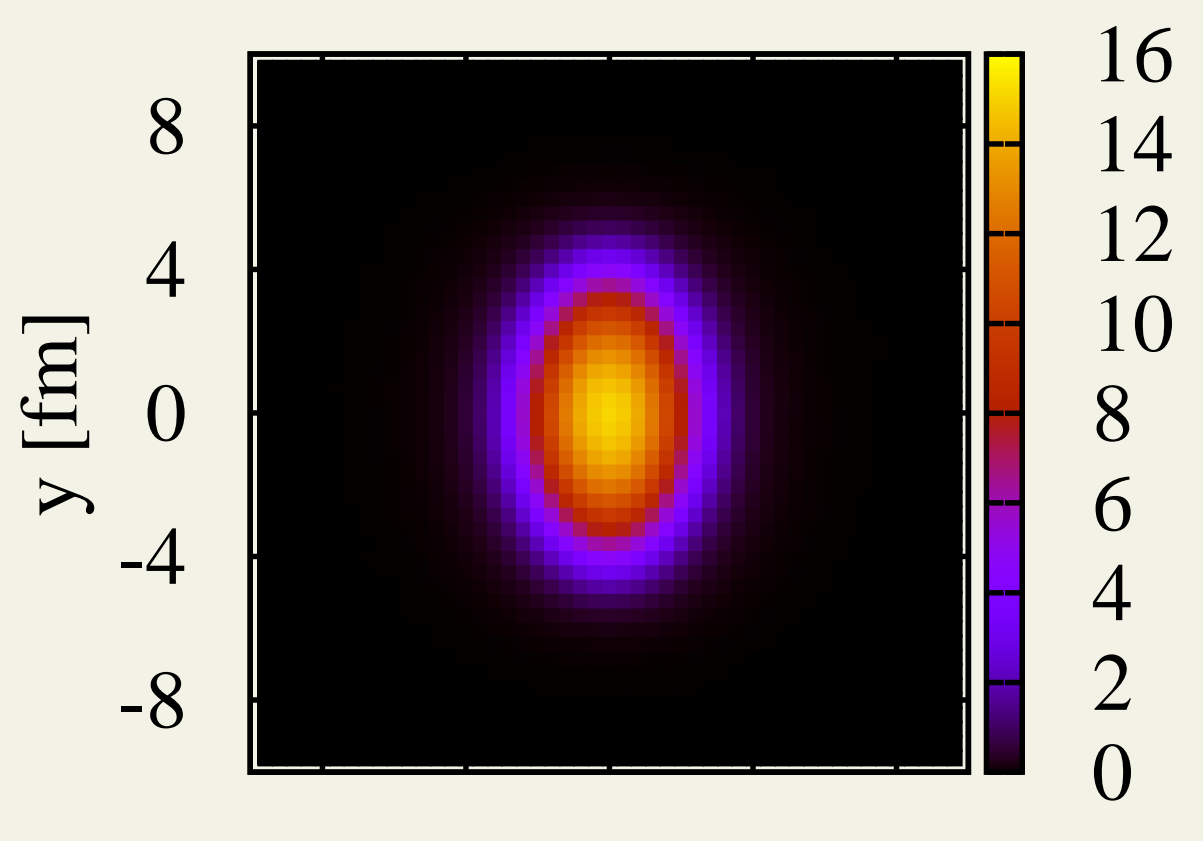

\section{0 averaged MC Glauber runs}

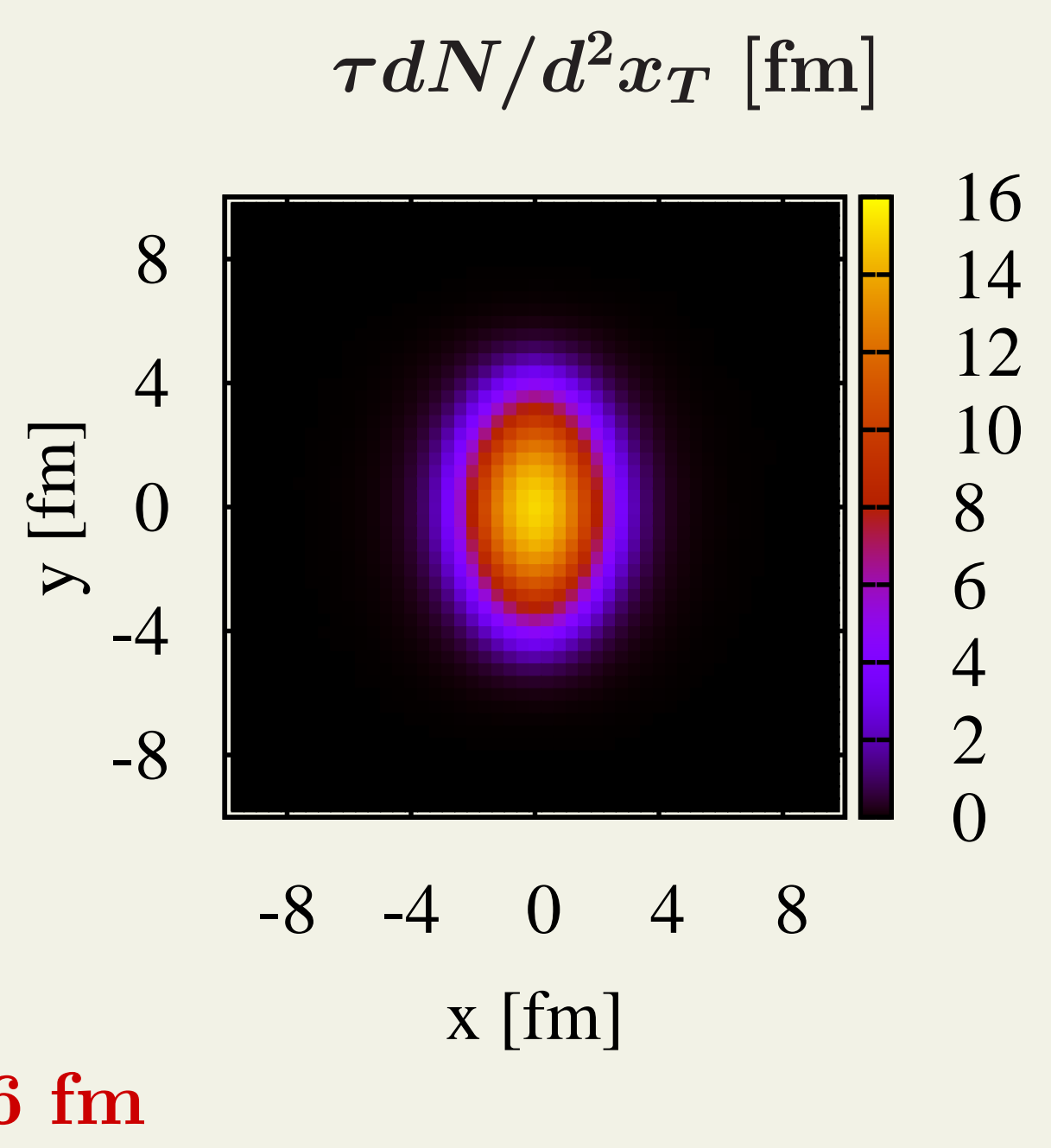


average $A u+A u, b=8 \mathrm{fm}$

$$
\tau d N / d^{2} x_{T}[\mathrm{fm}]
$$

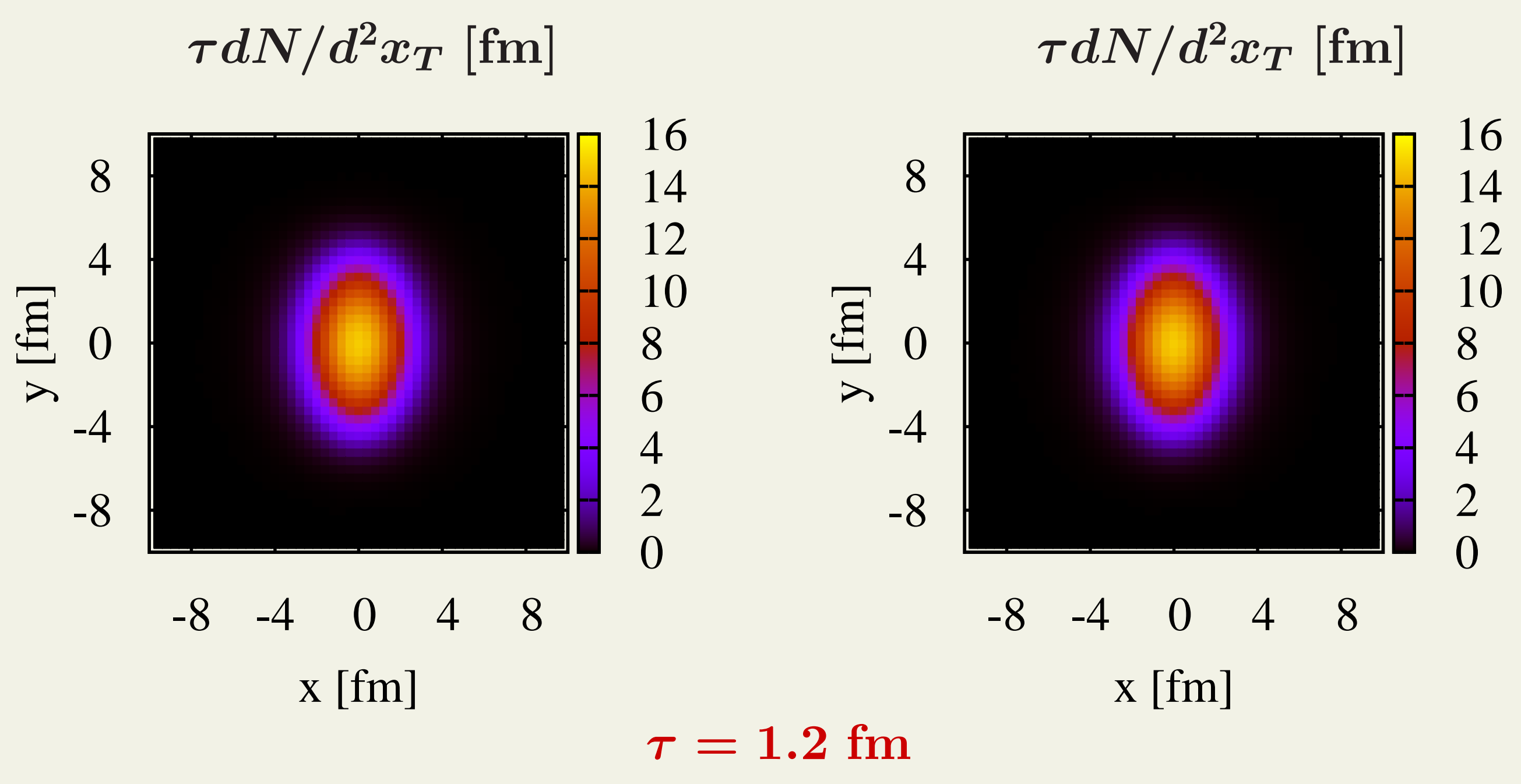

\section{0 averaged MC Glauber}


average $A u+A u, b=8 \mathrm{fm}$

$$
\tau d N / d^{2} x_{T}[\mathrm{fm}]
$$

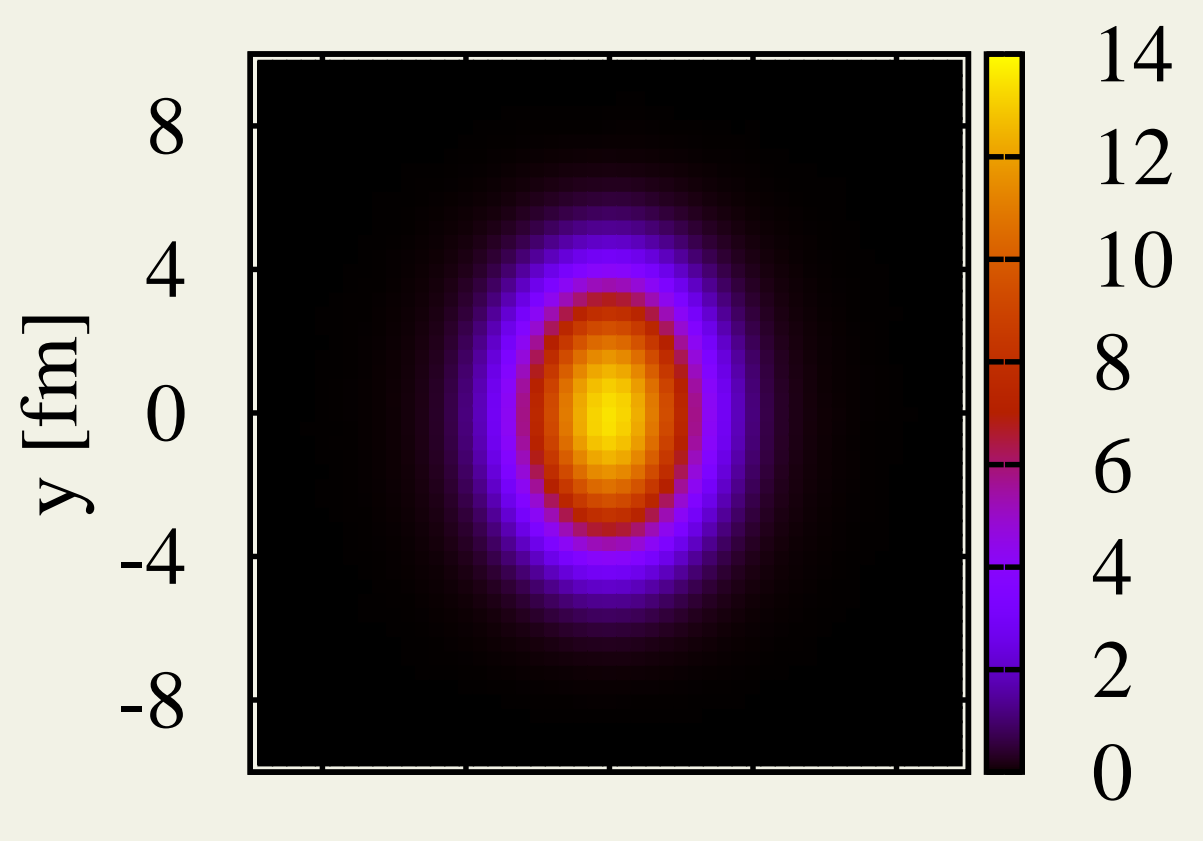

\section{0 averaged MC Glauber}

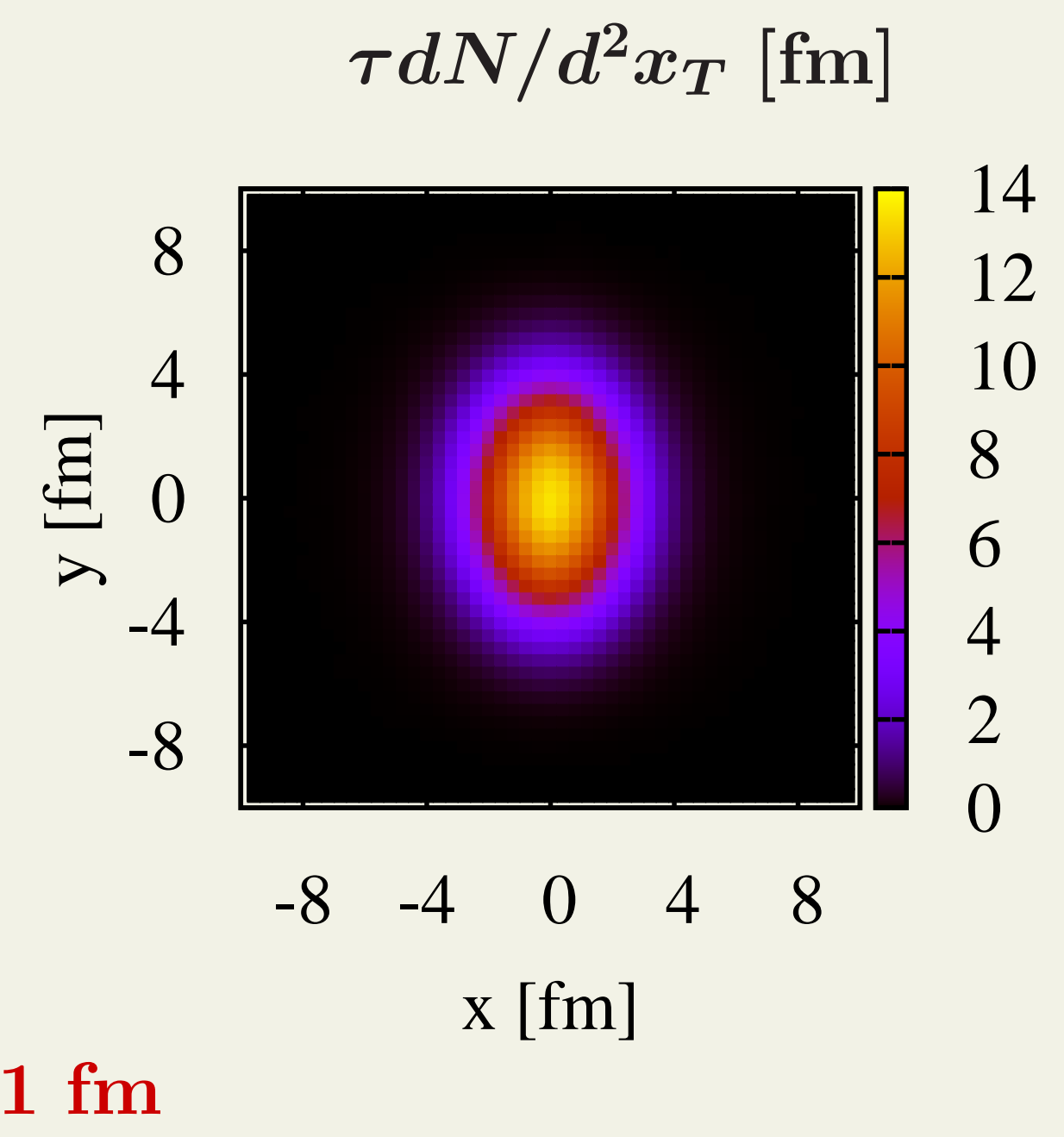


average $A u+A u, b=8 \mathrm{fm}$

$$
\tau d N / d^{2} x_{T}[\mathrm{fm}]
$$

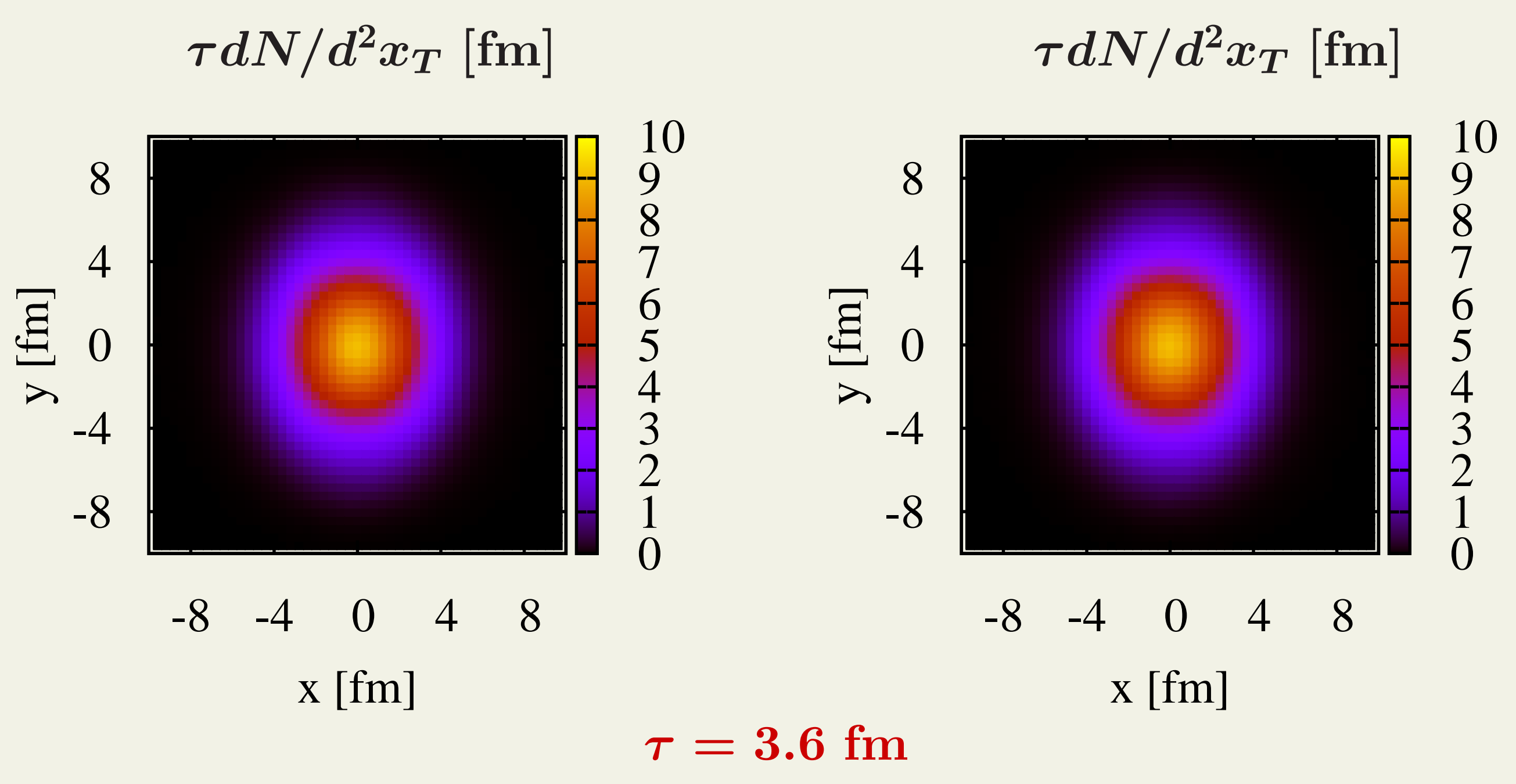

\section{0 averaged MC Glauber}


average $A u+A u, b=8 \mathrm{fm}$

$$
\tau d N / d^{2} x_{T}[\mathrm{fm}]
$$

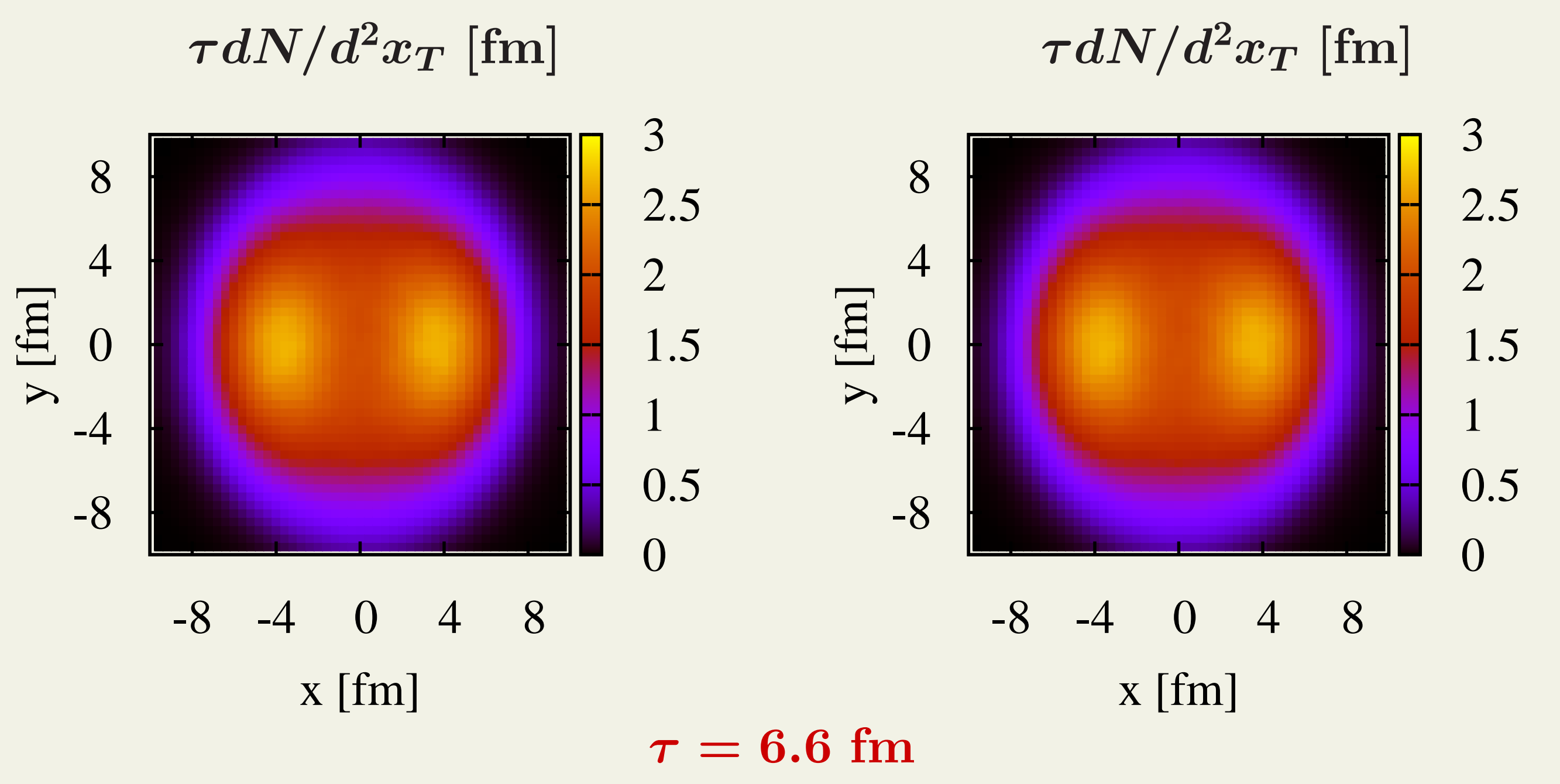

\section{0 averaged MC Glauber}


average $A u+A u, b=8 \mathrm{fm}$

$$
\tau d N / d^{2} x_{T}[\mathrm{fm}]
$$

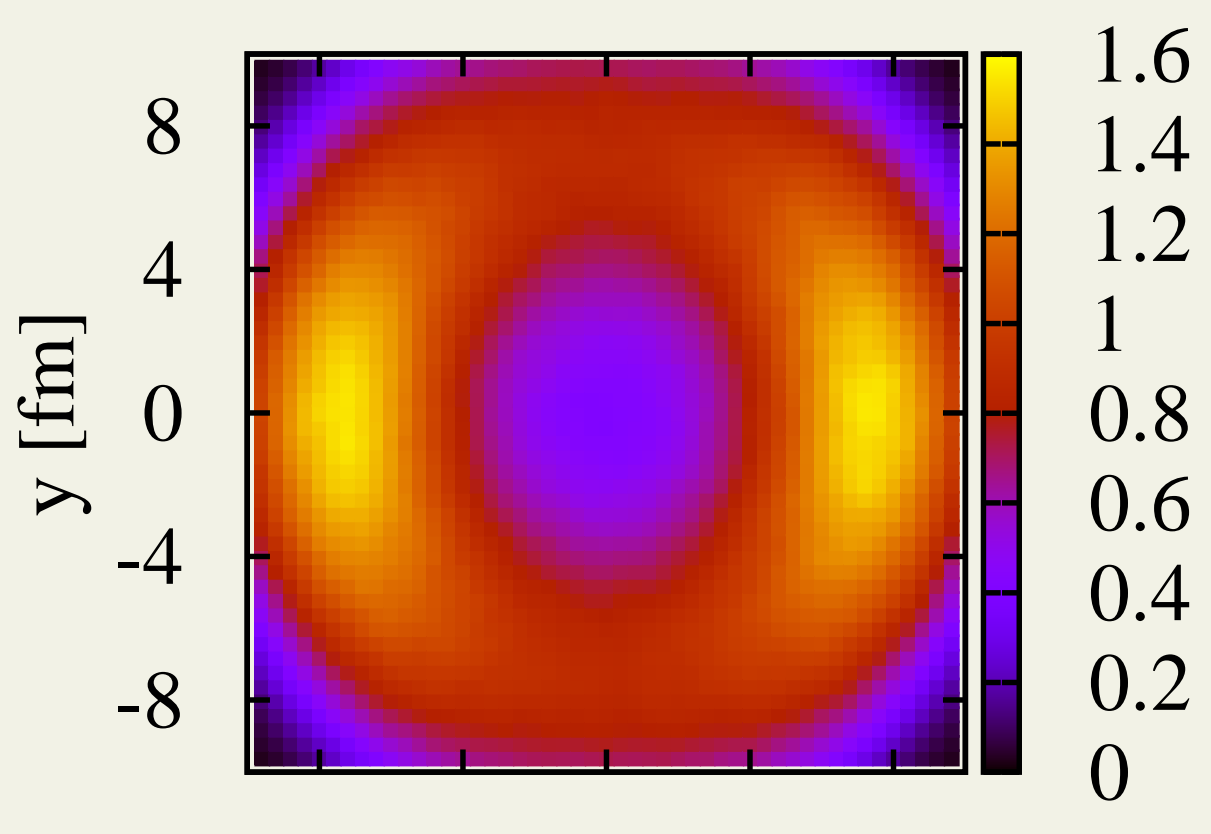

$$
\begin{array}{lllll}
-8 & -4 & 0 & 4 & 8 \\
& \\
& & \\
& & {[\mathrm{fm}]}
\end{array}
$$

\section{0 averaged MC Glauber}

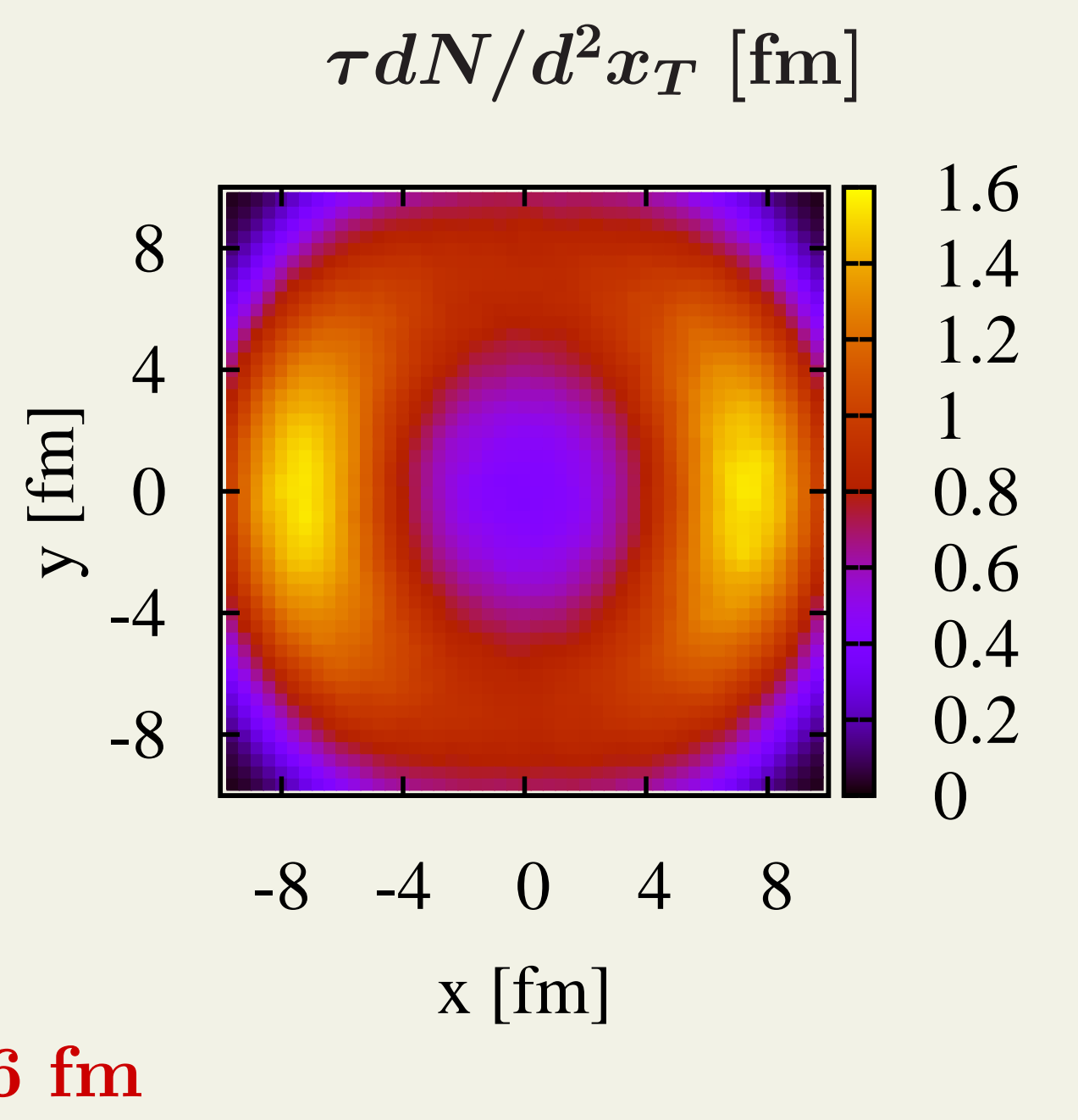




\section{Backup slides}


CUJET 1.0 Buzzatti et al ('11)

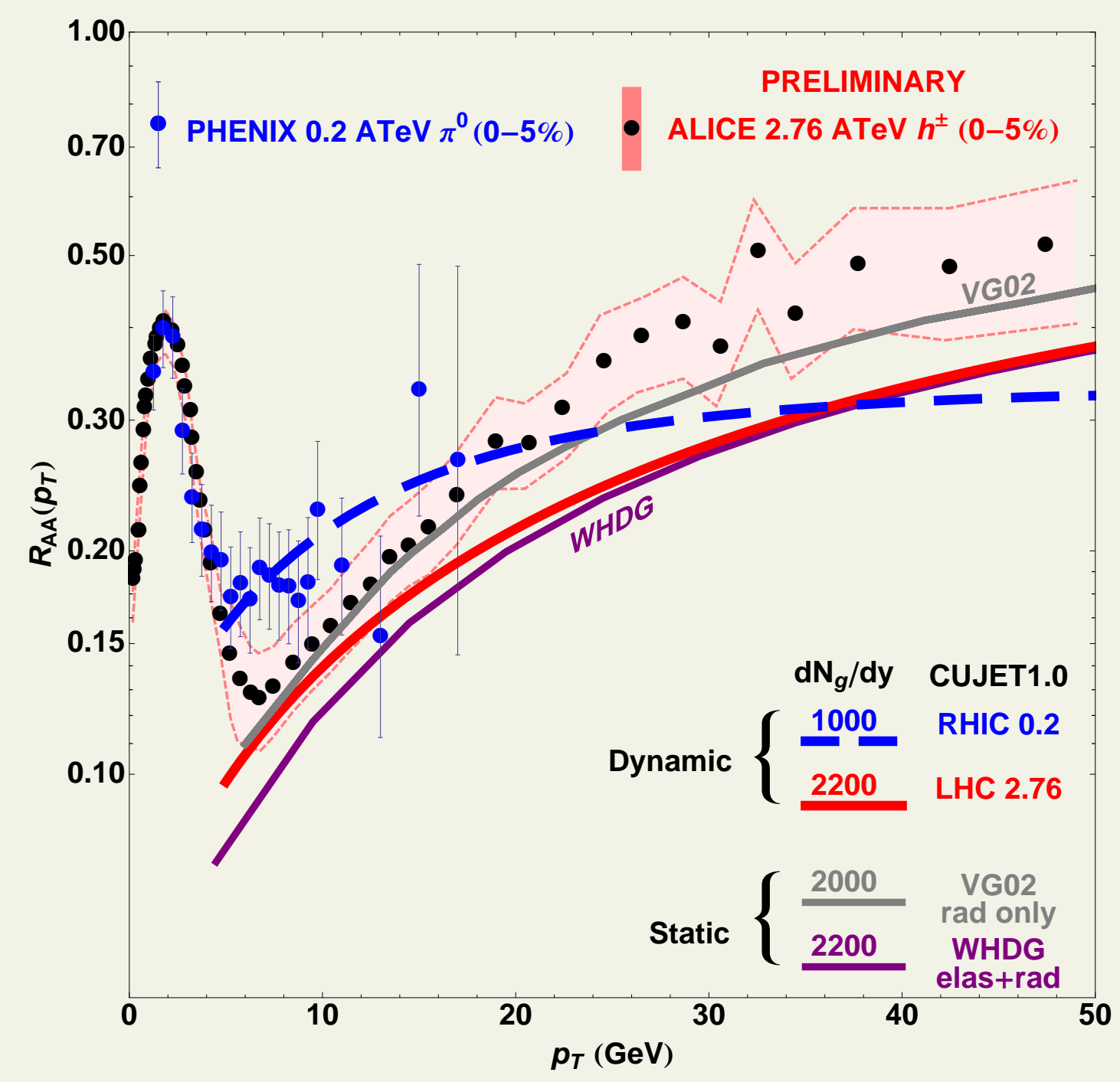


Betz \& Gyulassy, arXiv:1201.0281

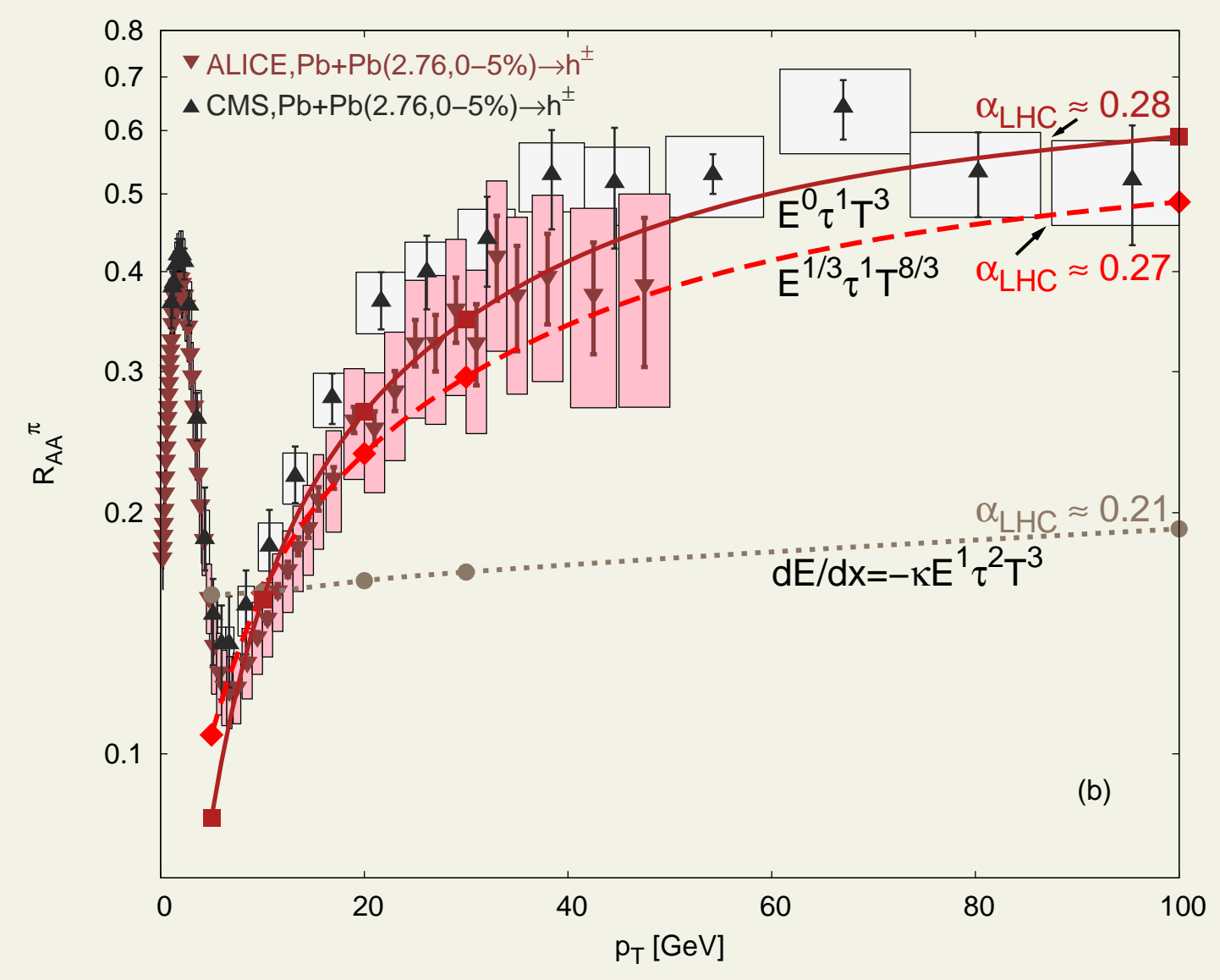

$$
\alpha_{s}^{R H I C}=0.3
$$


CYM eccentricity Venugopalan \& Lappi, PRC74 ('06):

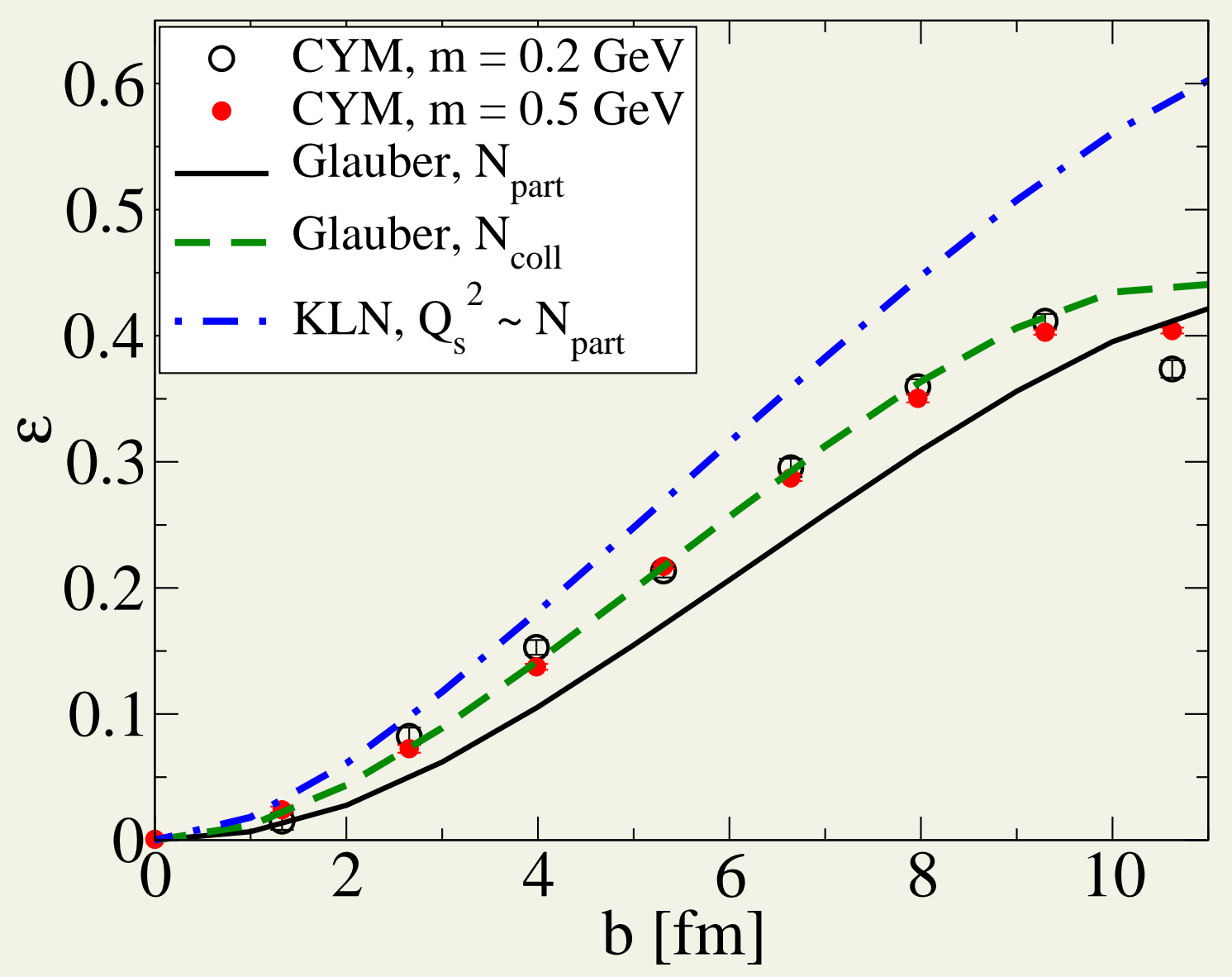


MARTINI $R_{A A}(\phi)$ Schenke et al, PRC80 ('09):

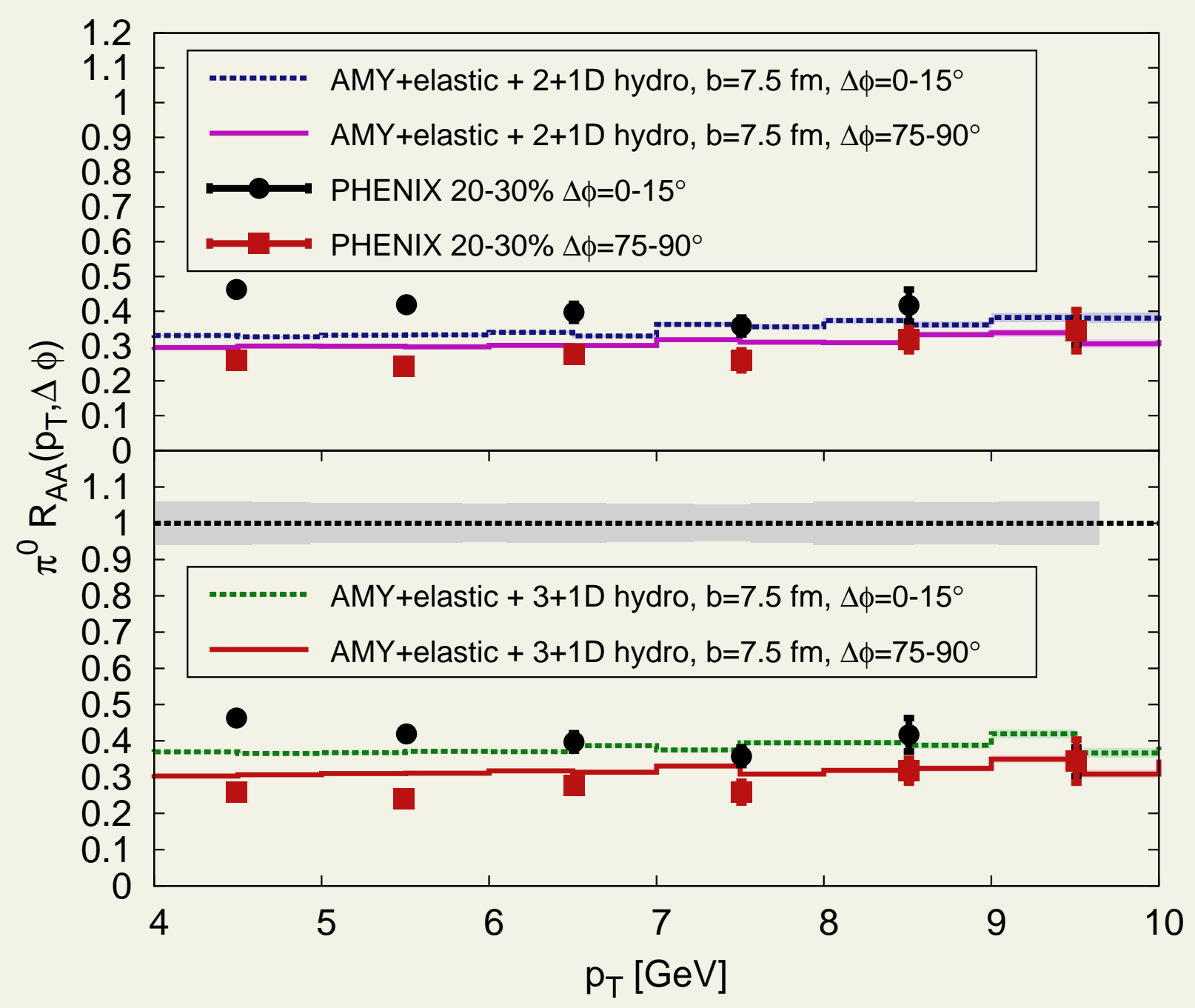




\section{Parton subdivision}

Naive $2 \rightarrow 2$ cascade nonlocal - action at distance $d<\sqrt{\frac{\sigma}{\pi}}$ 
subdivision: rescale $f \rightarrow f \cdot \ell, \sigma \rightarrow \sigma / \ell \Rightarrow d \propto \ell^{-1 / 2}$ local as $\ell \rightarrow \infty$
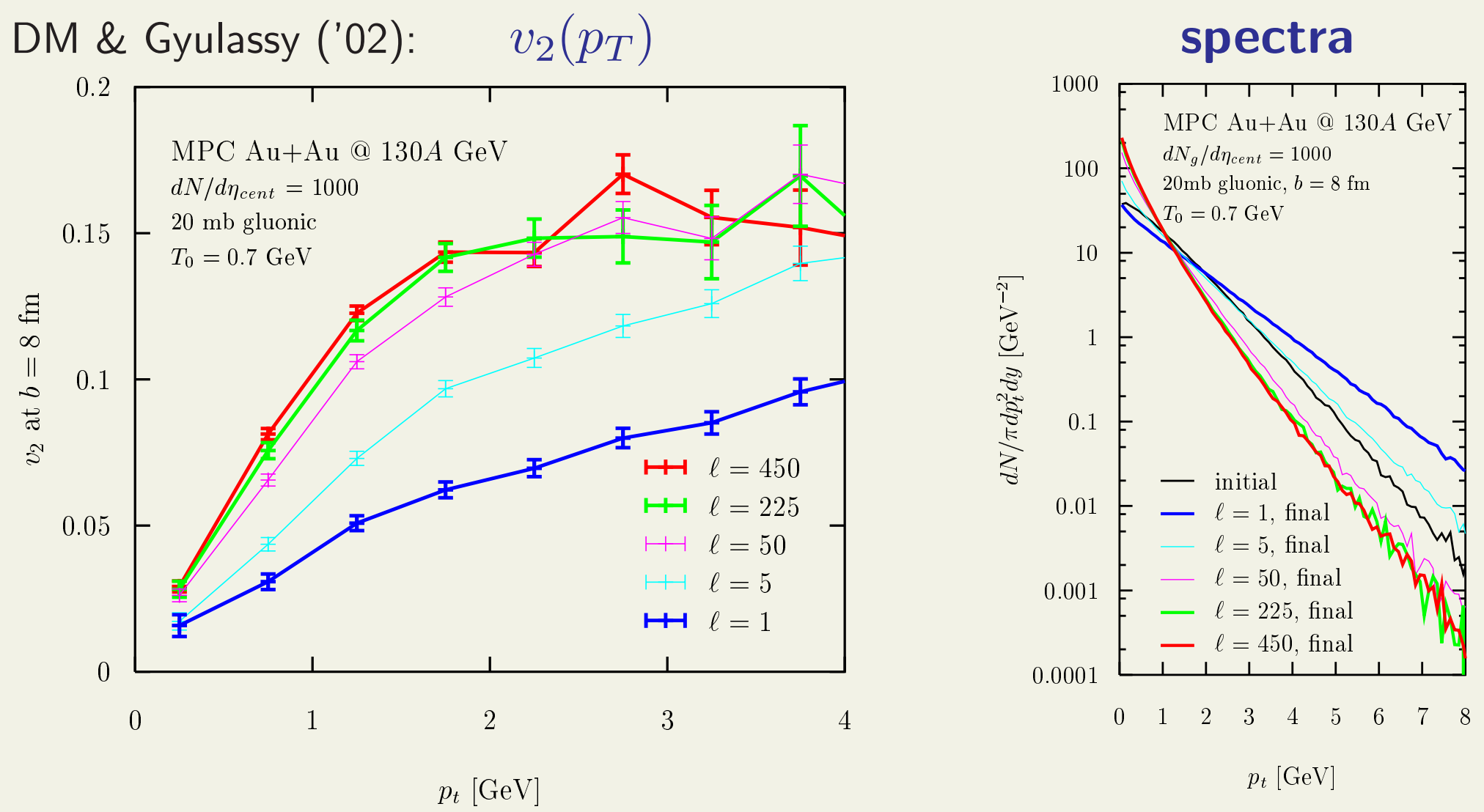

at RHIC: need subdivision $\ell \sim 200$ to eliminate large artifacts

$\rightarrow$ computational challenge - CPU time scales as $\ell^{\sim 3 / 2}$ per run $\rightarrow$ barely fits on PC 


\section{RHIC/LHC Complementarity}

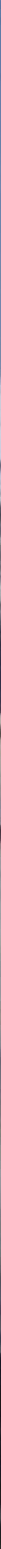




\section{Used as a guideline}

RHIC White Paper: 
Used as a guideline 


\section{RHIC and LHC "Landscape"}

\section{The QGP at the LHC:}

- fireball hotter ( 20\%) and denser $(\sim \times 2)$ and longer lifetime wrt RHIC

- bulk dynamics, $\mathrm{v}_{\mathrm{n}}\left(\mathrm{p}_{\mathrm{T}}\right)$, similar at RHIC and LHC, mainly driven by initial state "geometry"

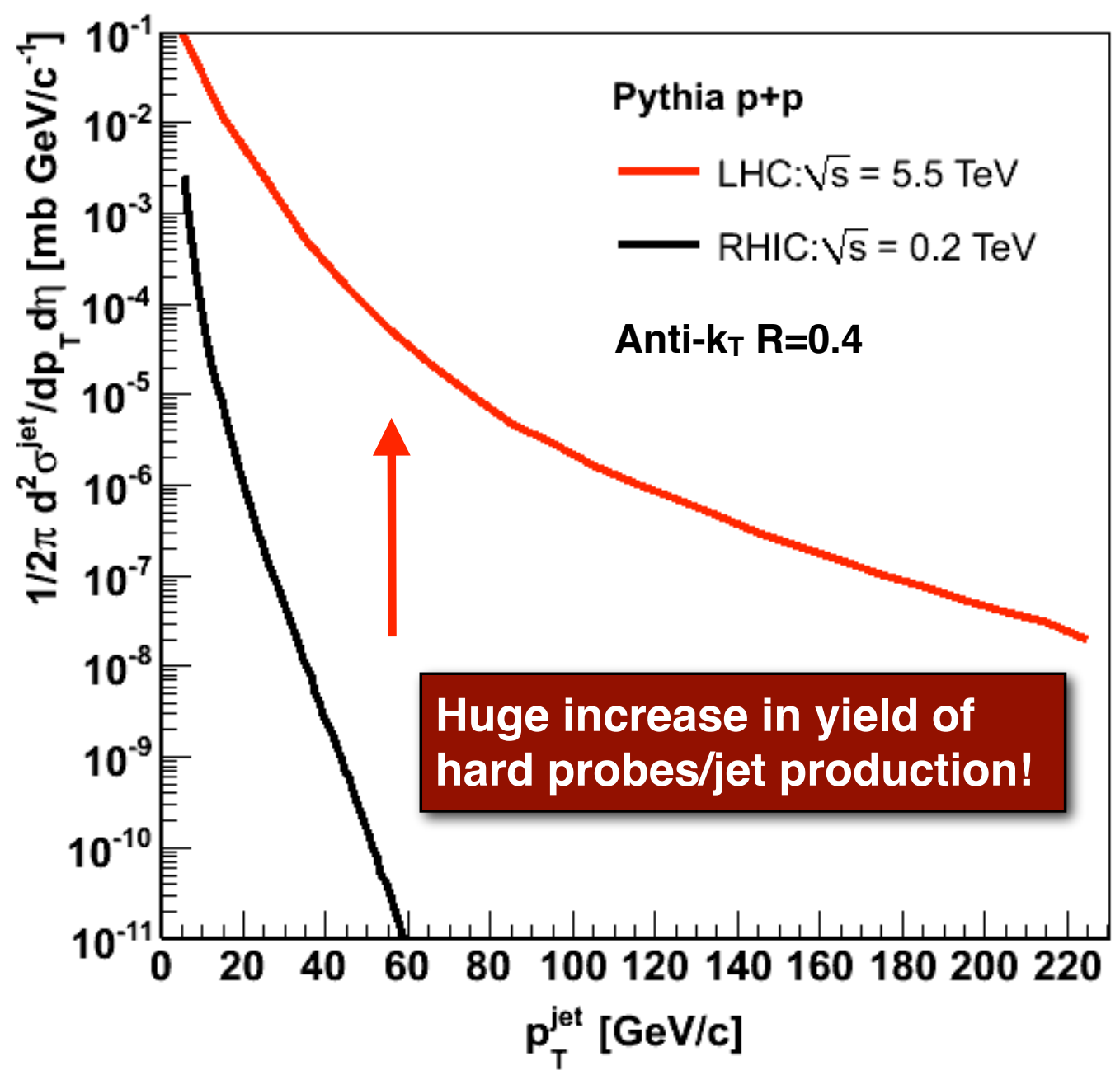




\section{RHIC and LHC "Landscape"}

\section{The QGP at the LHC:}

- fireball hotter ( 20\%) and denser $(\sim \mathrm{x})$ and longer lifetime wrt RHIC

- bulk dynamics, $\mathrm{v}_{\mathrm{n}}\left(\mathrm{p}_{\mathrm{T}}\right)$, similar at RHIC and LHC, mainly driven by initial state "geometry"

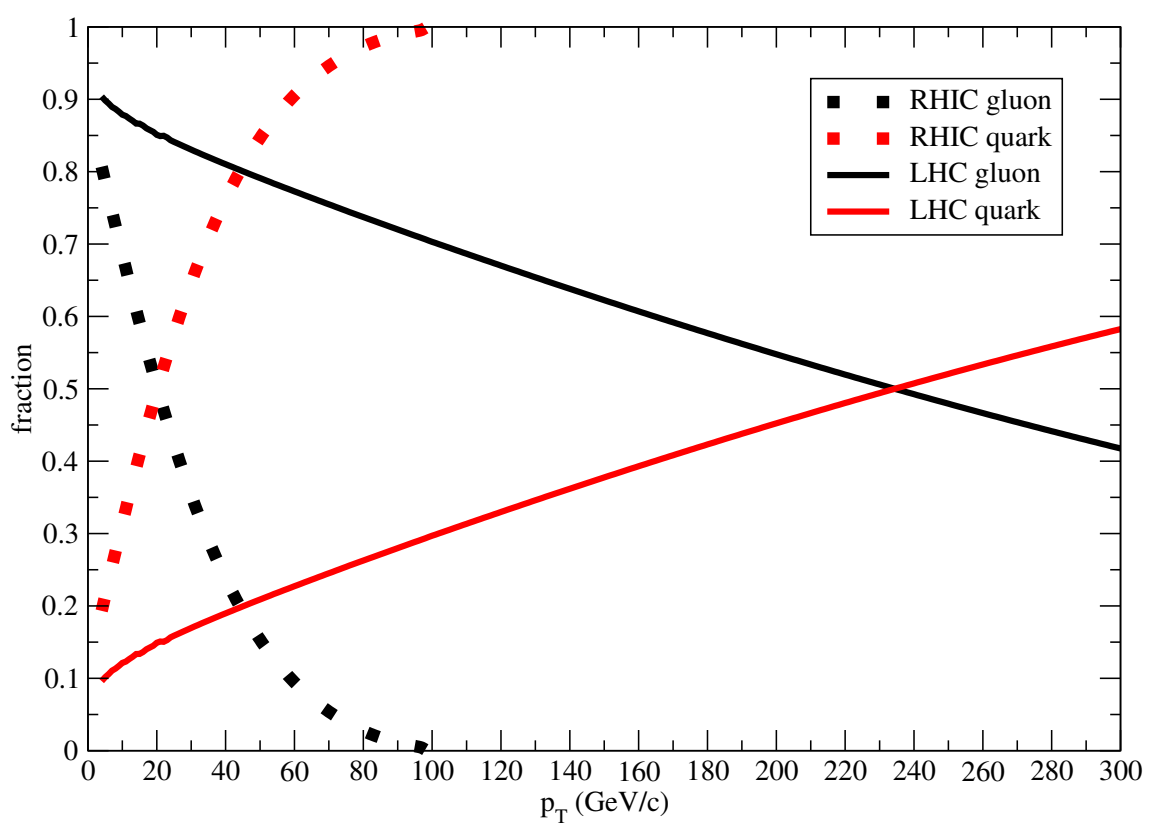

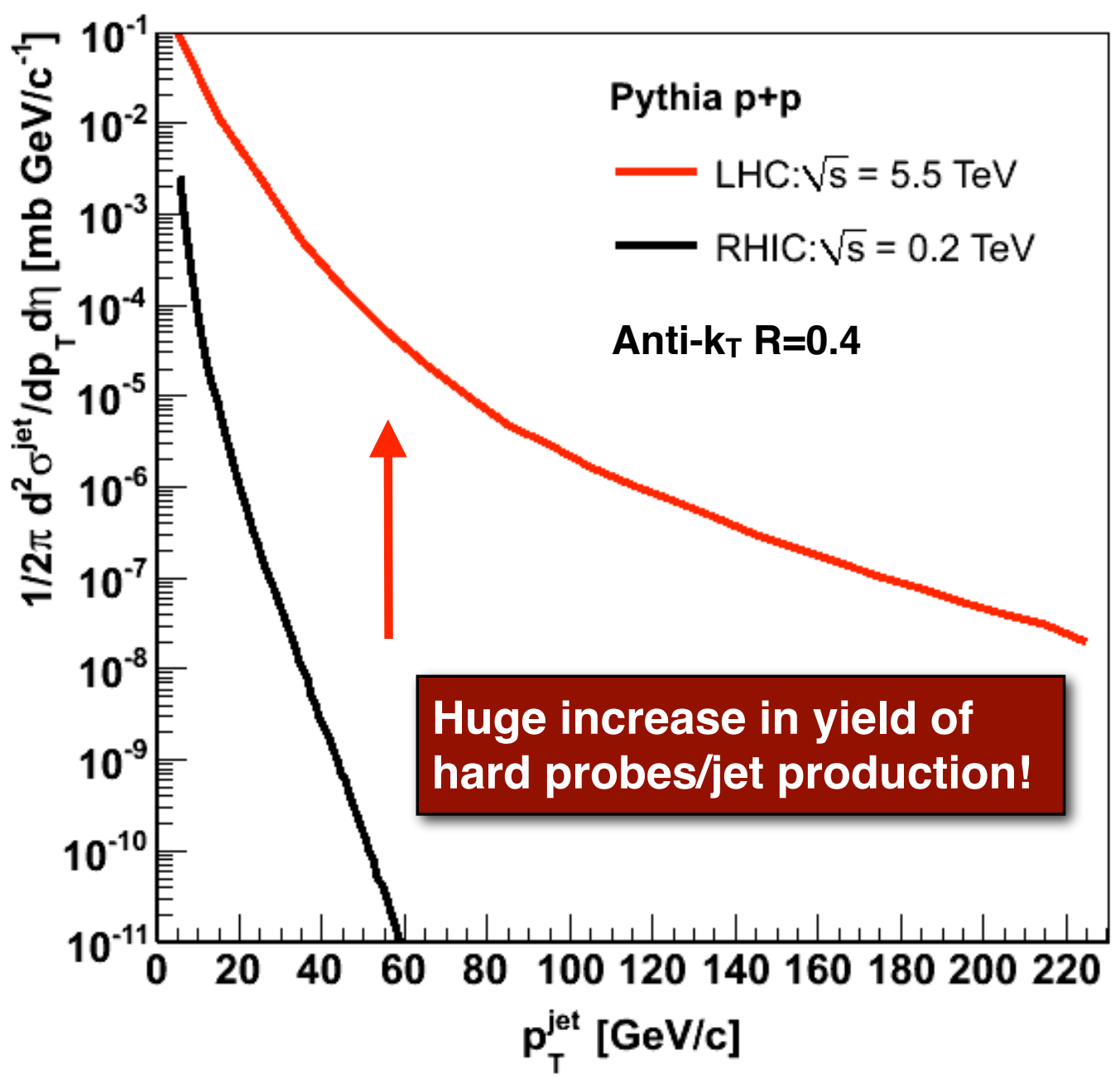

Mainly gluon jets $\left(p_{T}<200 \mathrm{GeV}\right)$ at the LHC. Quark jets at RHIC $p_{T}>40 \mathrm{GeV}$. 


\section{The issue: Background in $\mathrm{HI}$ collisions}
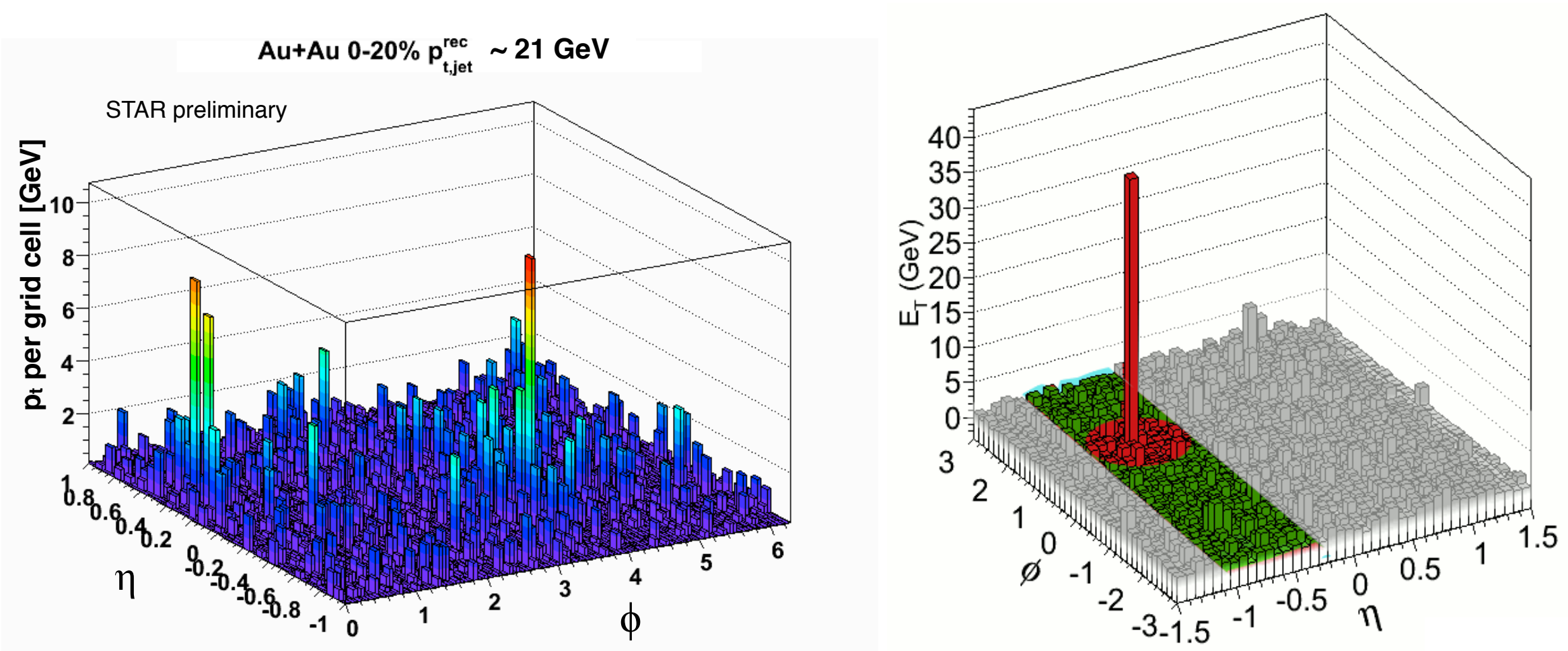

Full jet reconstruction in $\mathrm{HI}$ collisions is a challenge due to the underlying background

- Overall background pedestal

- Region-to-region background fluctuations and $v_{n}$ contributions

- Multiple independent hard scattering in HI collisions

\section{Different contributions depending on coincidence vs. inclusive measurements!}

Remark: I will not talk about this in detail, a comprehensive summary concerning the different approaches currently used can be found in a talk by $\mathrm{G}$. Roland: $\mathrm{https}$ ://indico.cern.ch/getFile.py/access? contribld=3\&sessionld=0\&resld=0\&materialld=slides\&confld=198761 


\section{LHC and RHIC RAA}

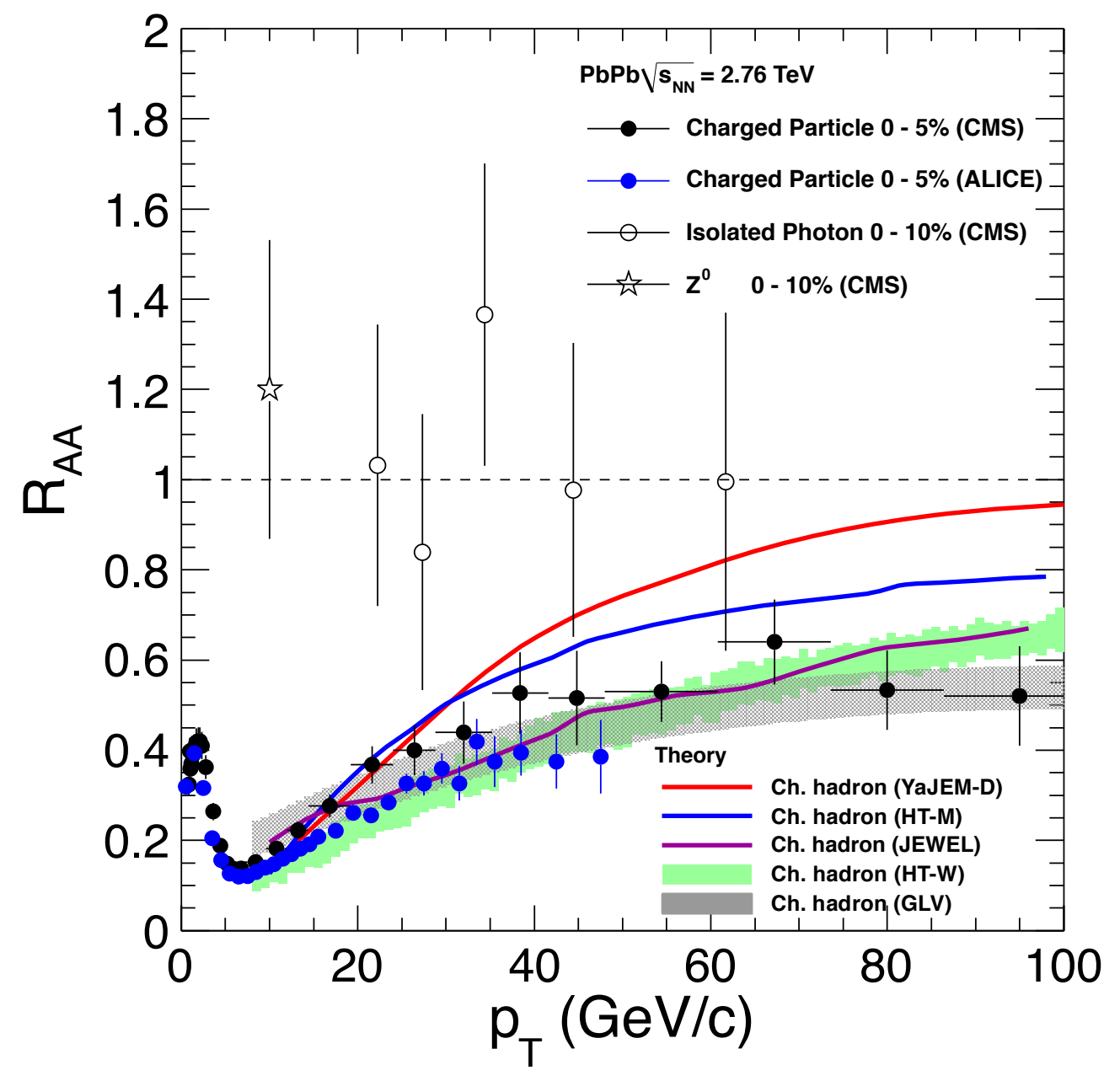

$R_{A A}$ rising as function of $p_{T}$; constant for $p_{T}>50 \mathrm{GeV}$ ? 


\section{LHC and RHIC $R_{A A}$}

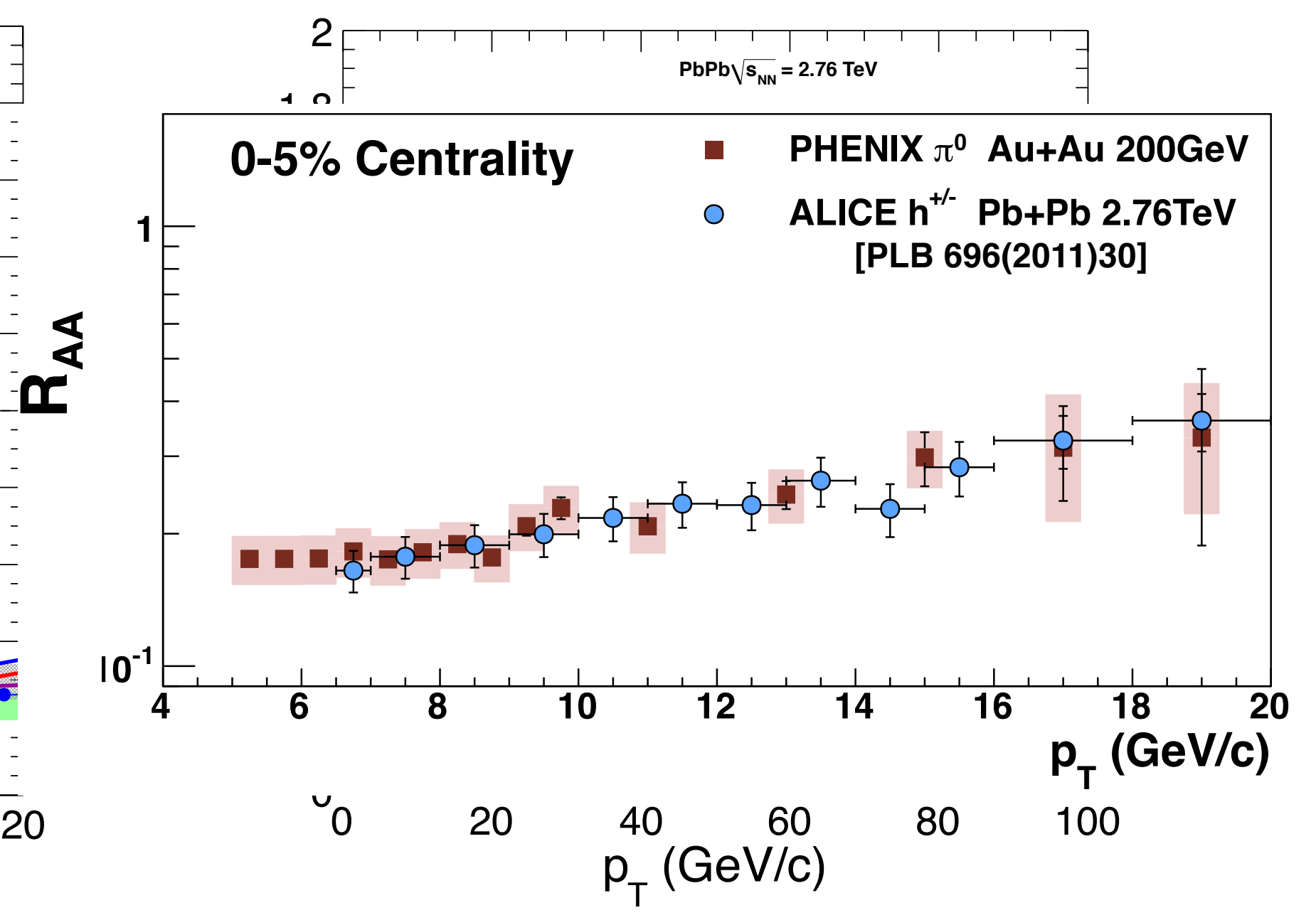

$R_{A A}$ rising as function of $p_{T}$; constant for $p_{T}>50 \mathrm{GeV}$ ? RHIC $R_{A A} \sim$ LHC $R_{A A}$ up to $p_{T} \sim 20 \mathrm{GeV}$ 


\section{Jet $\mathbf{R}_{\mathrm{AA}} / \mathbf{R}_{\mathrm{CP}}$ at the LHC}
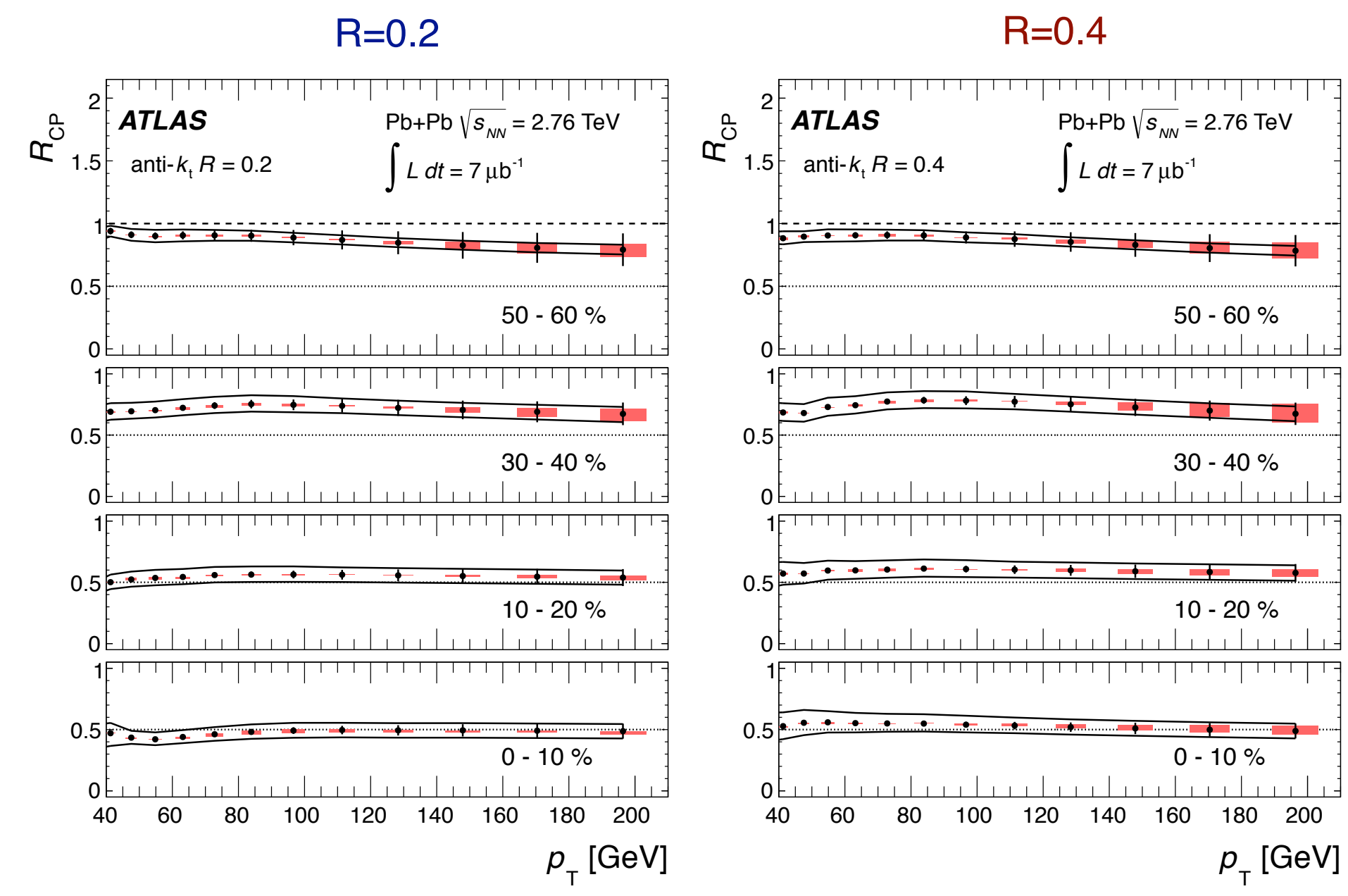

$\mathbf{R}_{\text {CP }}{ }^{\text {Jet }} \sim R_{\text {AA }} \sim 0.5$ (>50 GeV)

No significant $p_{T}$ and $R$ dependence of $R_{C P}$ for $p_{T}>100 \mathrm{GeV}$ 


\section{Jet $R_{A A} / R_{C P}$ at the $L H C$}
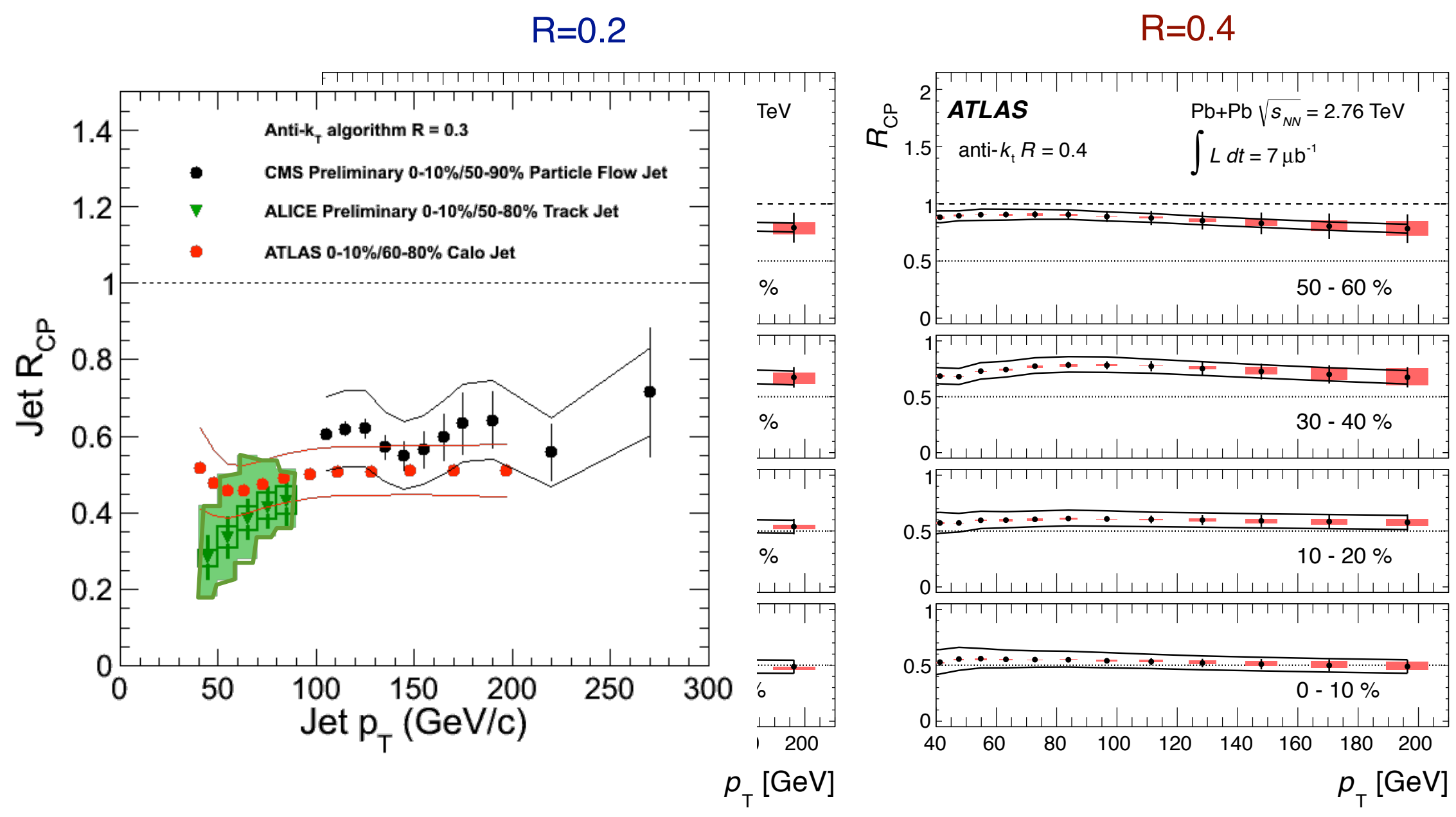

$\mathbf{R}_{\mathrm{CP}} \mathrm{Jet}^{\sim} \mathbf{R}_{\mathrm{AA}} \sim 0.5$ (>50 GeV)

No significant $p_{T}$ and $R$ dependence of $R_{C P}$ for $p_{T}>100 \mathrm{GeV}$ 


\section{Jet $\mathbf{R}_{\mathrm{AA}} / \mathbf{R}_{\mathrm{CP}}$ at the LHC}
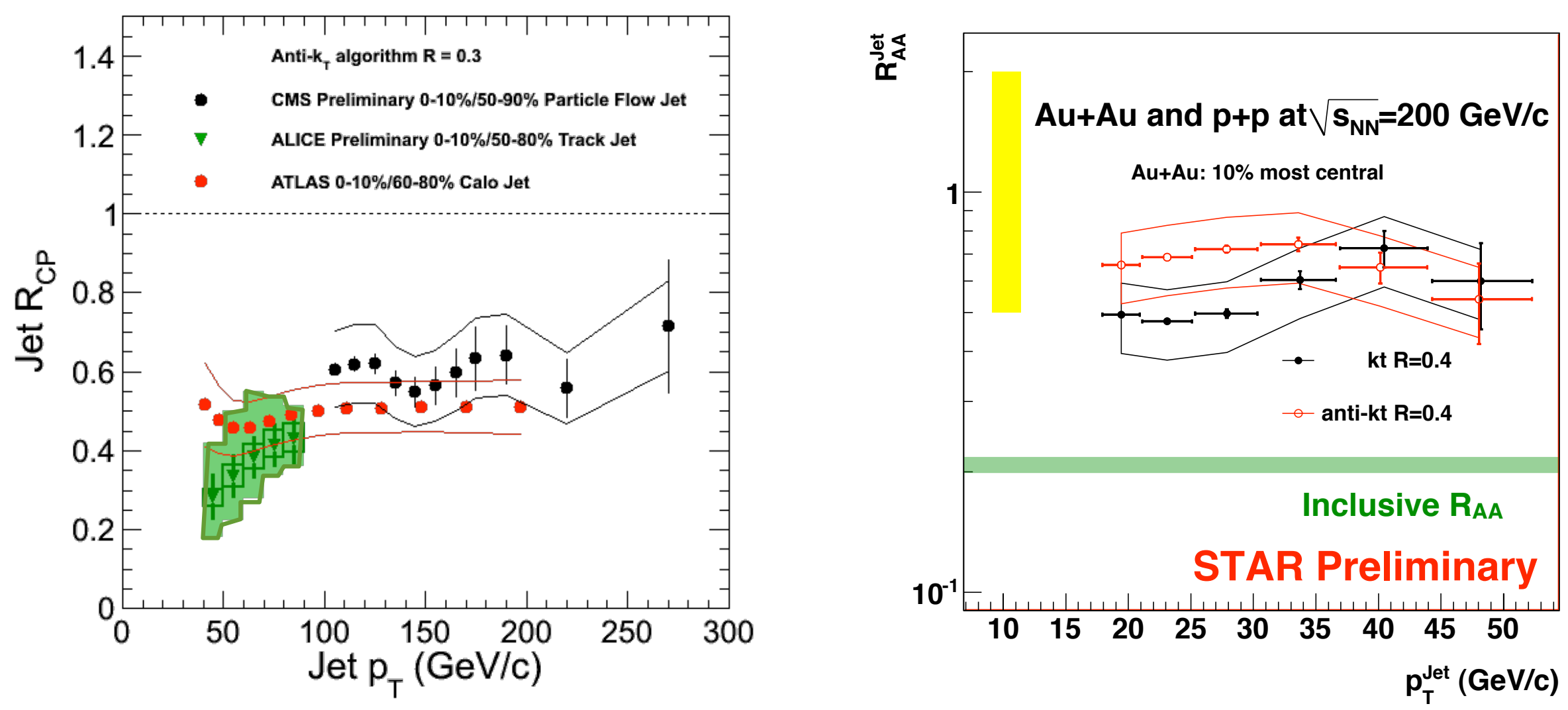

$\mathbf{R}_{\mathrm{CP}} \mathrm{Jet}^{\sim} \mathbf{R}_{\mathrm{AA}} \sim 0.5$ (>50 GeV)

No significant $p_{T}$ and $R$ dependence of $R_{C P}$ for $p_{T}>100 \mathrm{GeV}$

$R H I C$ : Jet $R_{\text {AA }}$ less suppressed than hadrons!

Caveat: Large systematic uncertainties 


\section{Di-jet asymmetry/imbalance as function of leading jet $\mathrm{p}_{\mathrm{T}}$}

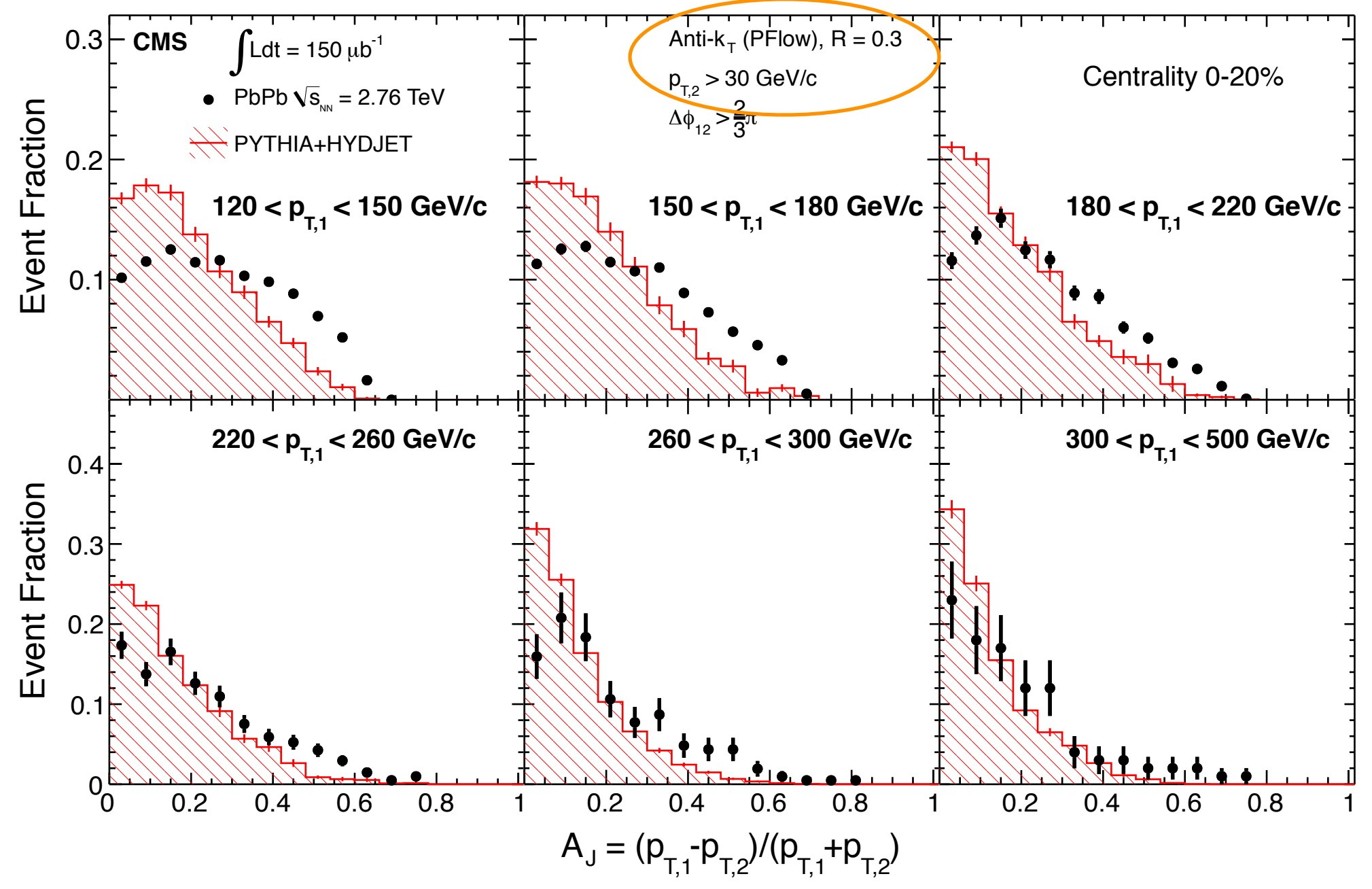




\section{Di-jet asymmetry/imbalance as function of leading jet $\mathrm{p}_{\mathrm{T}}$}

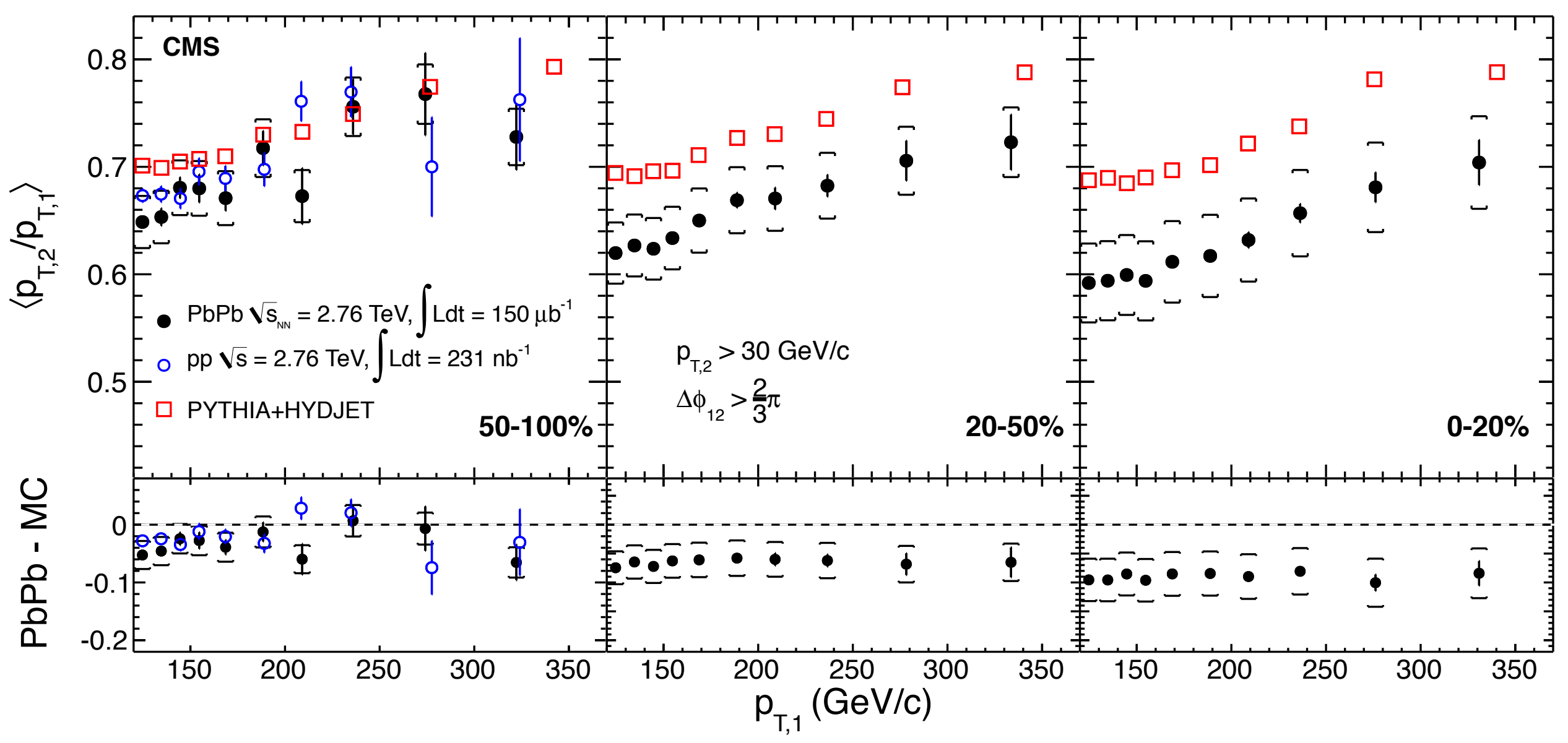

\section{Di-Jet imbalance decreasing with increasing jet energy!}

"Can be explained in terms of essentially known physics, i.e. the increased collimation of jets

due to kinematics and a transition to a less gluon- dominated regime." : T.Renk, arXiv:1204.5572 


\section{Direct Photon-Jet Measurements}
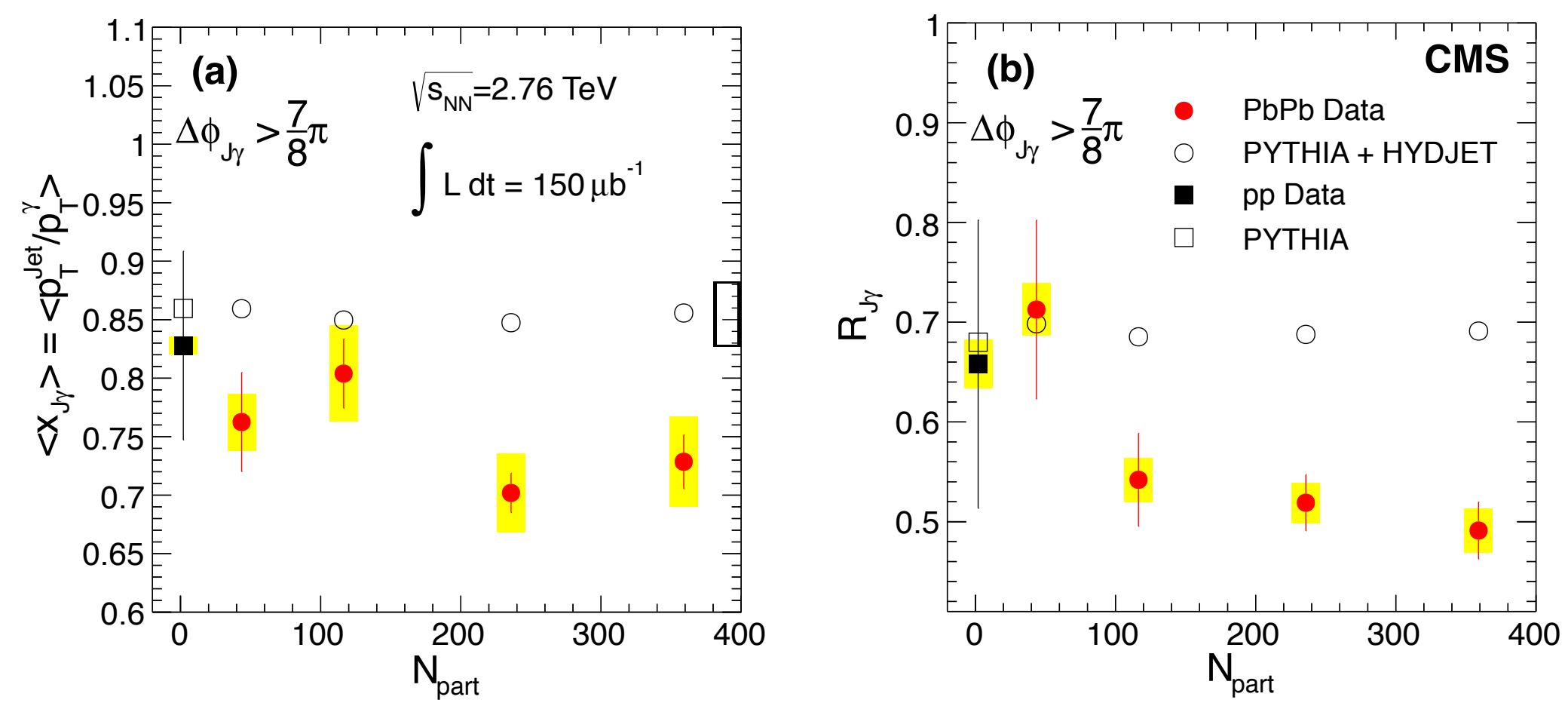

$\mathrm{p}_{\mathrm{T}} \mathrm{\gamma}>60 \mathrm{GeV}$

$\mathrm{p}^{\mathrm{Jet}}>30 \mathrm{GeV}$
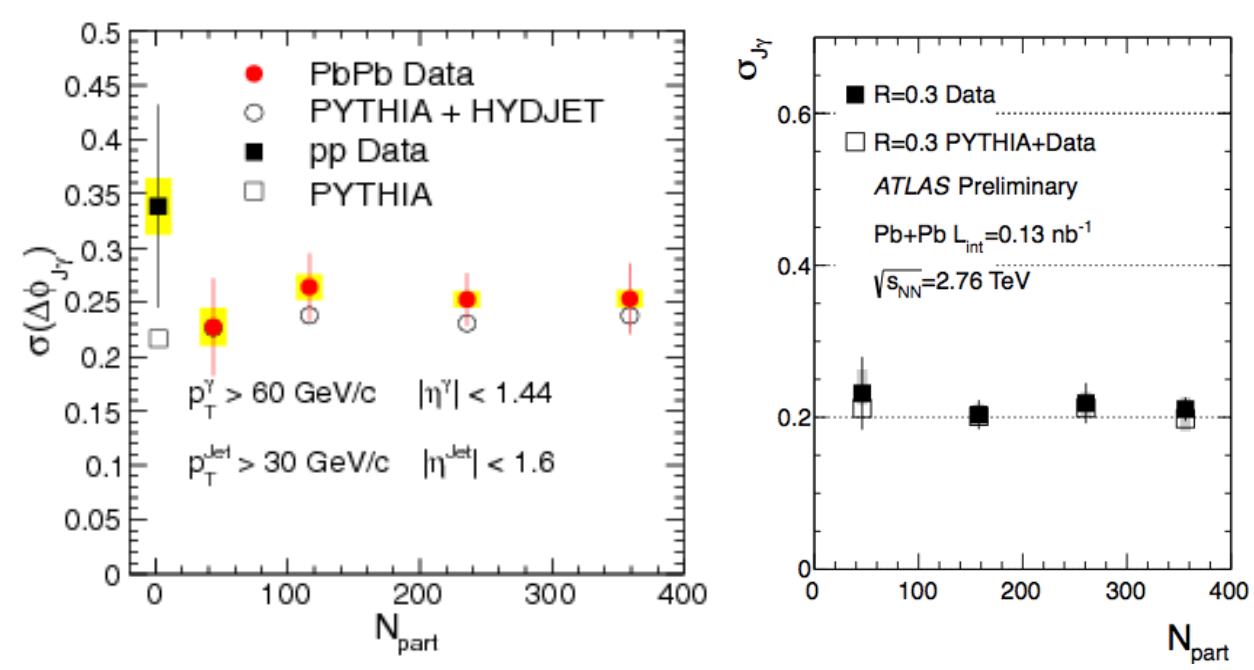

Large quenching effects seen in direct photon measurements

(Consistent with jets measurements?

Quark vs. gluon energy loss?)

No angular de-correlation (also seen in di-jets @RHIC) 


\section{Fragmentation Functions in $\mathrm{Pb}+\mathrm{Pb}$ at the $\mathrm{LHC}$}
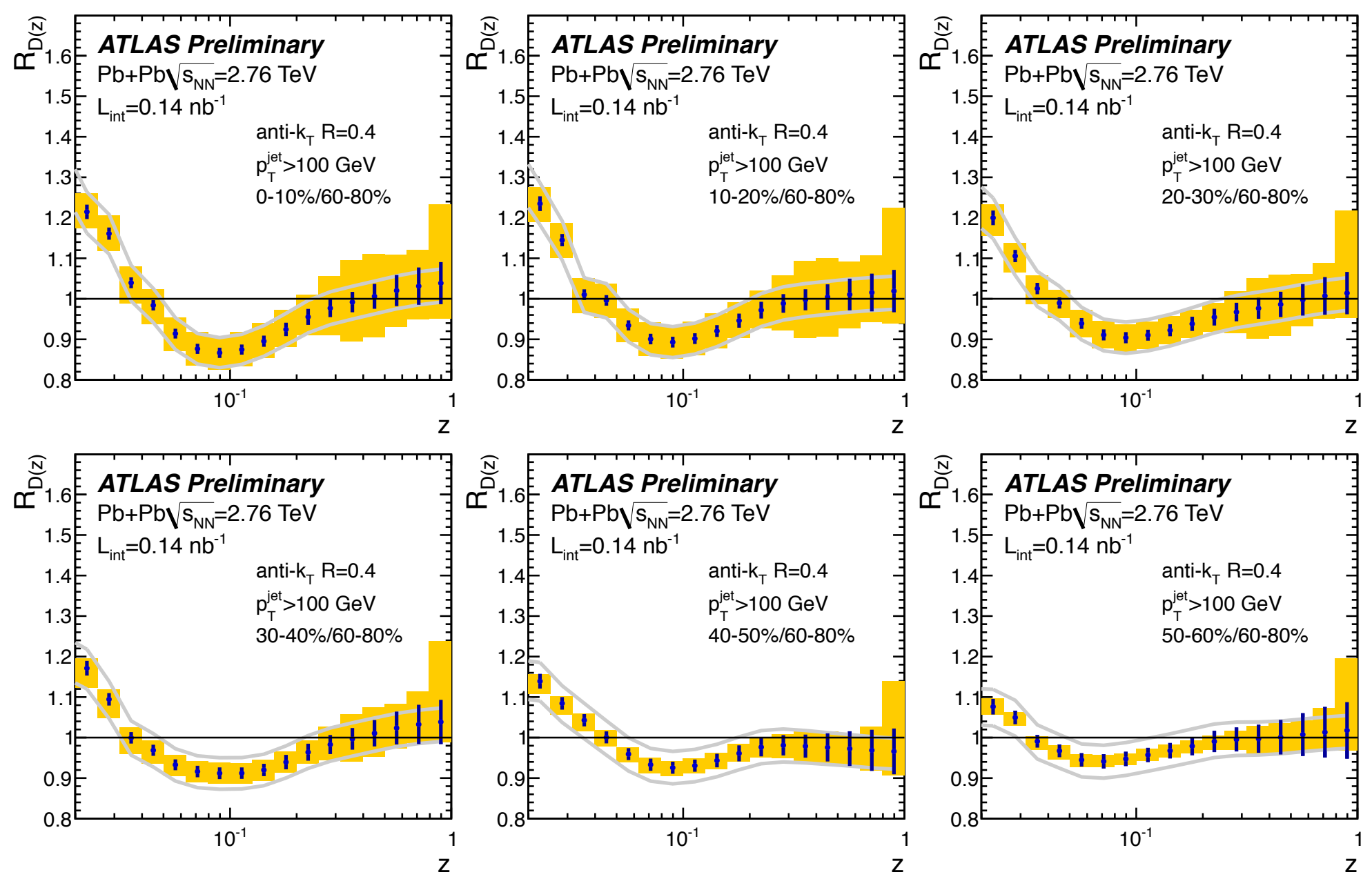

\section{Enhancement at low z Suppression at intermediate $\mathbf{z}$ No suppression at high z}




\section{Fragmentation Functions in $\mathrm{Pb}+\mathrm{Pb}$ at the $\mathrm{LHC}$}

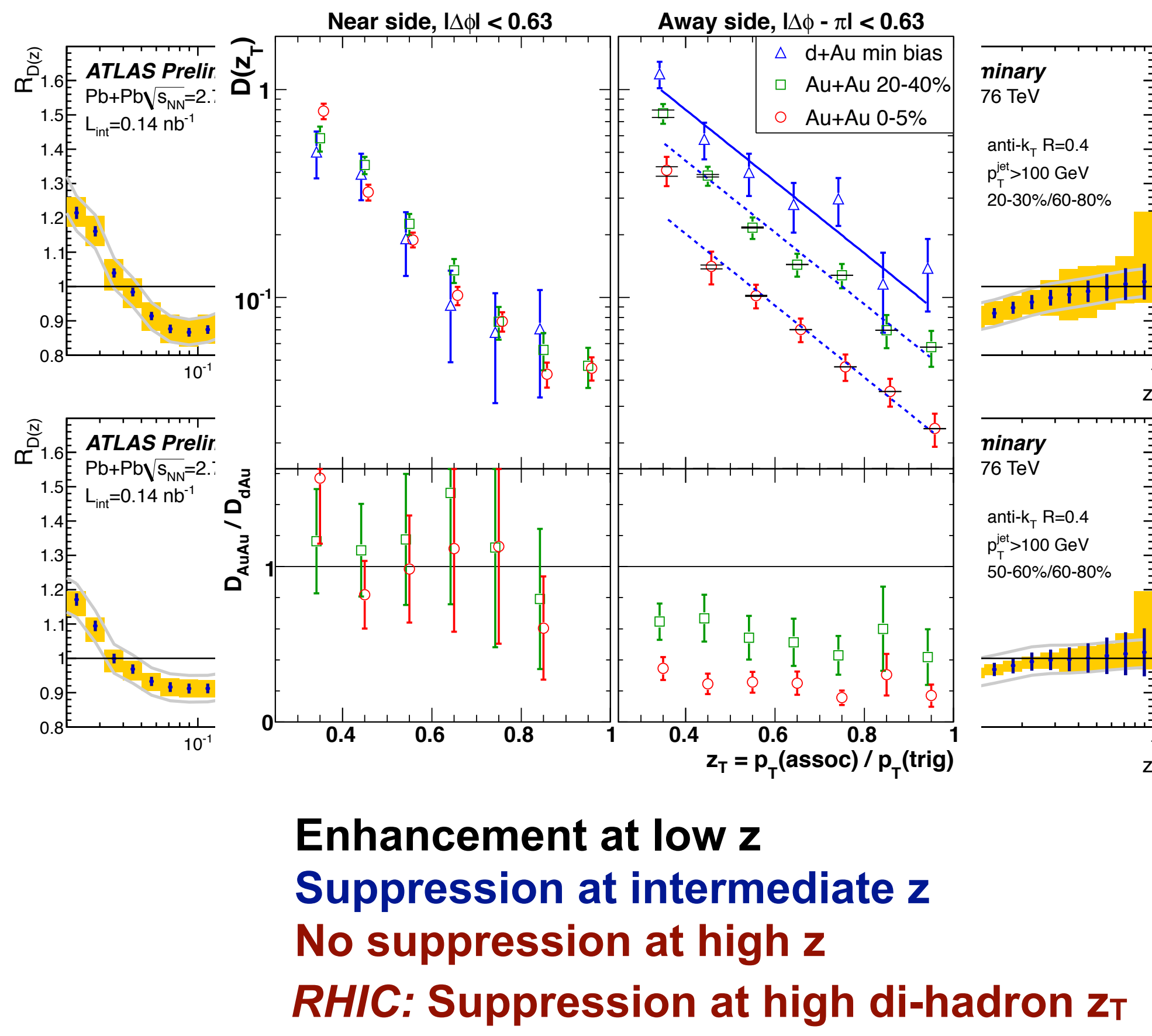




\section{Jet Shape Observables}
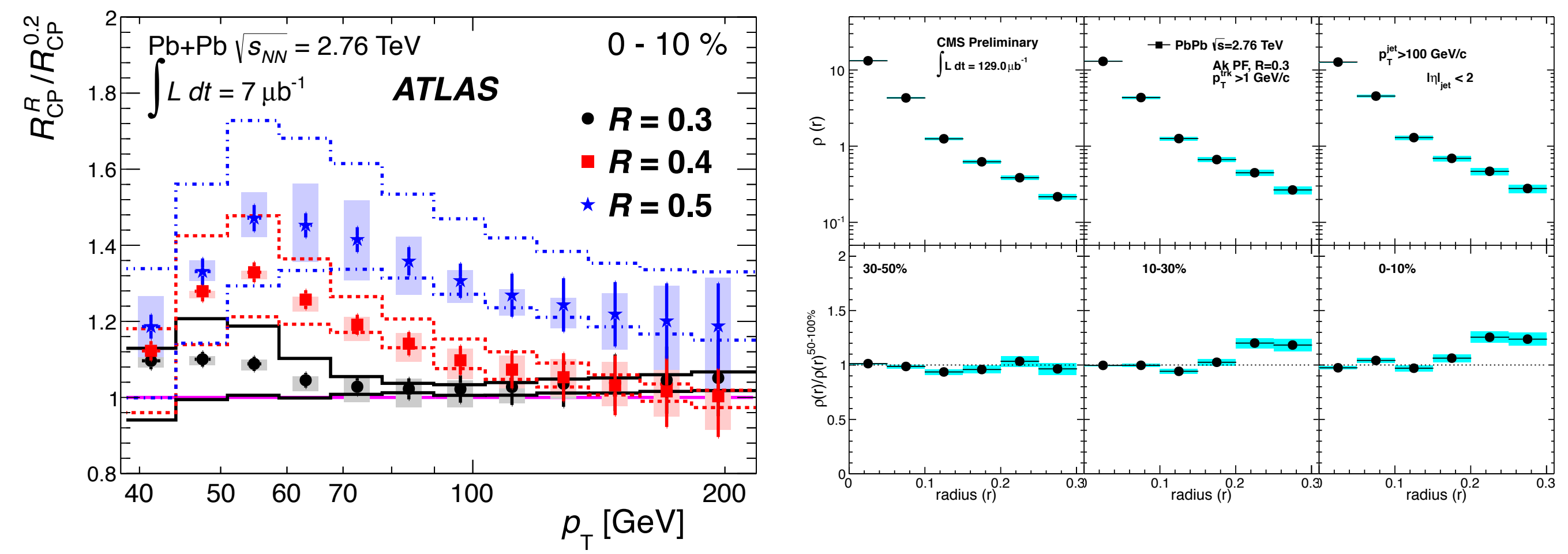

Jet broadening at the LHC: Seen in differential jet shape and $R$ dependence of jet $R_{C P}$ (especially at lower jet $\mathrm{p}_{\mathrm{T}}$ ) 


\section{Where does the lost energy go? Missing $\mathrm{p}_{\mathrm{T}}{ }^{\prime \prime}$}

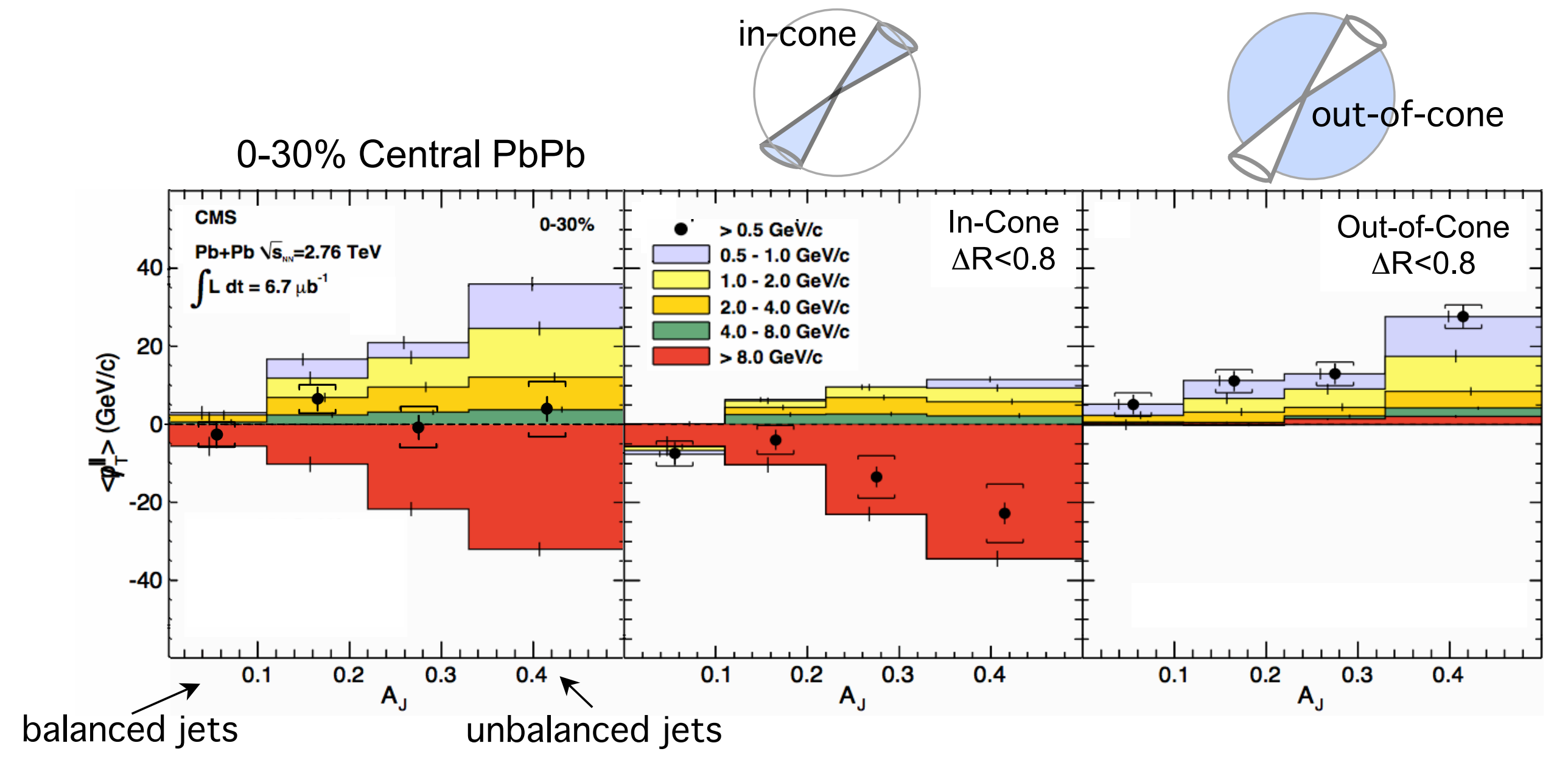

The momentum difference in the di-jet is balanced by low $\mathrm{p}_{\mathrm{T}}$ particles at large angles relative to the away side jet axis 


\section{RHIC: Direct Photon - Hadron Correlations}

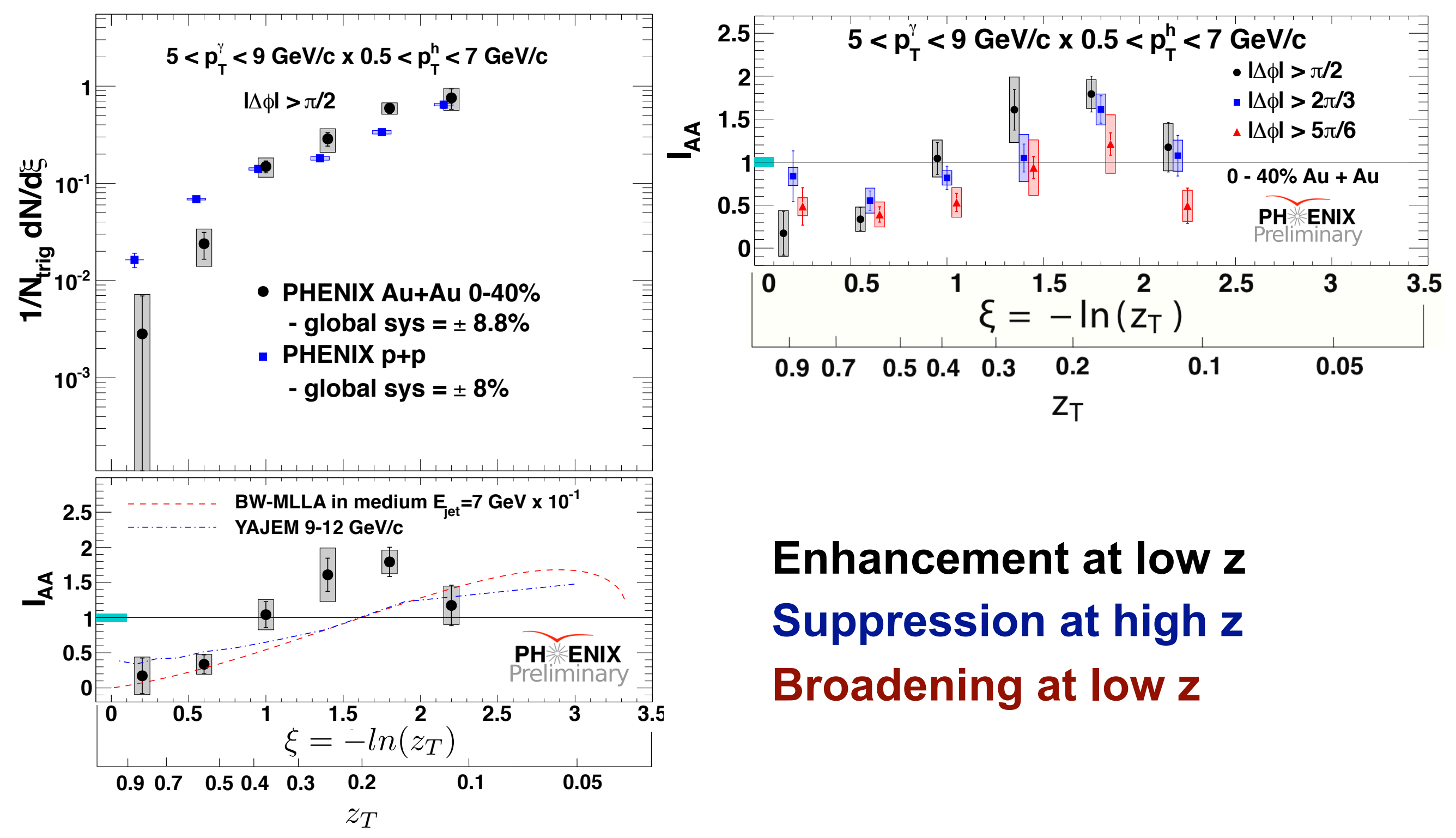




\section{RHIC: Jet-Hadron Correlations}

Trigger Jet: $\mathbf{R}=0.4, \mathrm{p}_{\mathrm{T}, \mathrm{cut}}=2 \mathrm{GeV} / \mathrm{c}$ and EMCal Tower $>6 \mathrm{GeV}$

Energy difference: AuAu-pp
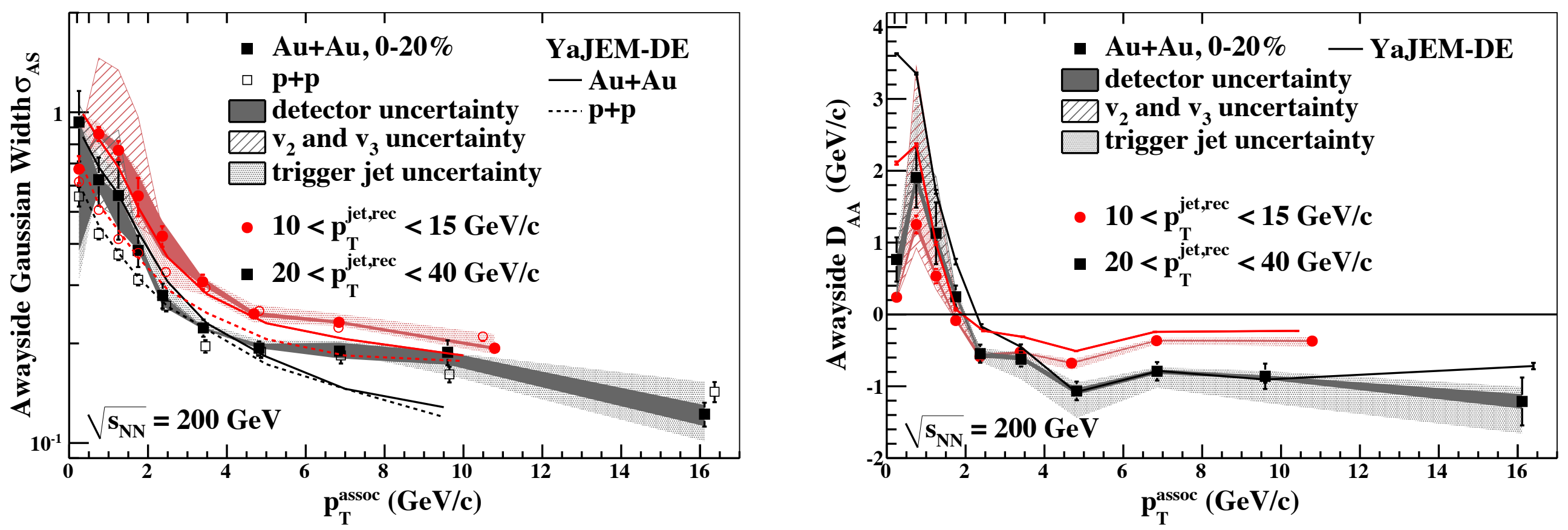

Hint of Jet Broadening at low $\mathrm{p}_{\mathrm{T}}$ (large uncertainties due to potential jet $\mathrm{v}_{2} / \mathrm{v}_{3}$ )

Quenched energy at high $p_{T}$ balanced by low $p_{T}$ enhancement

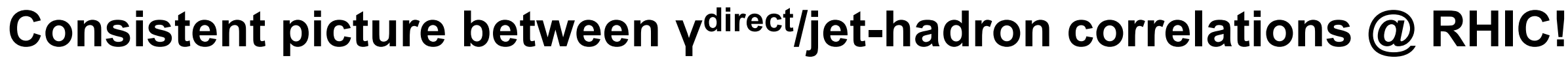




\section{RHIC: Jet-Hadron Correlations}

Trigger Jet: $\mathrm{R}=0.4, \mathrm{p}_{\mathrm{T}, \mathrm{cut}}=2 \mathrm{GeV} / \mathrm{c}$ and EMCal Tower $>6 \mathrm{GeV}$

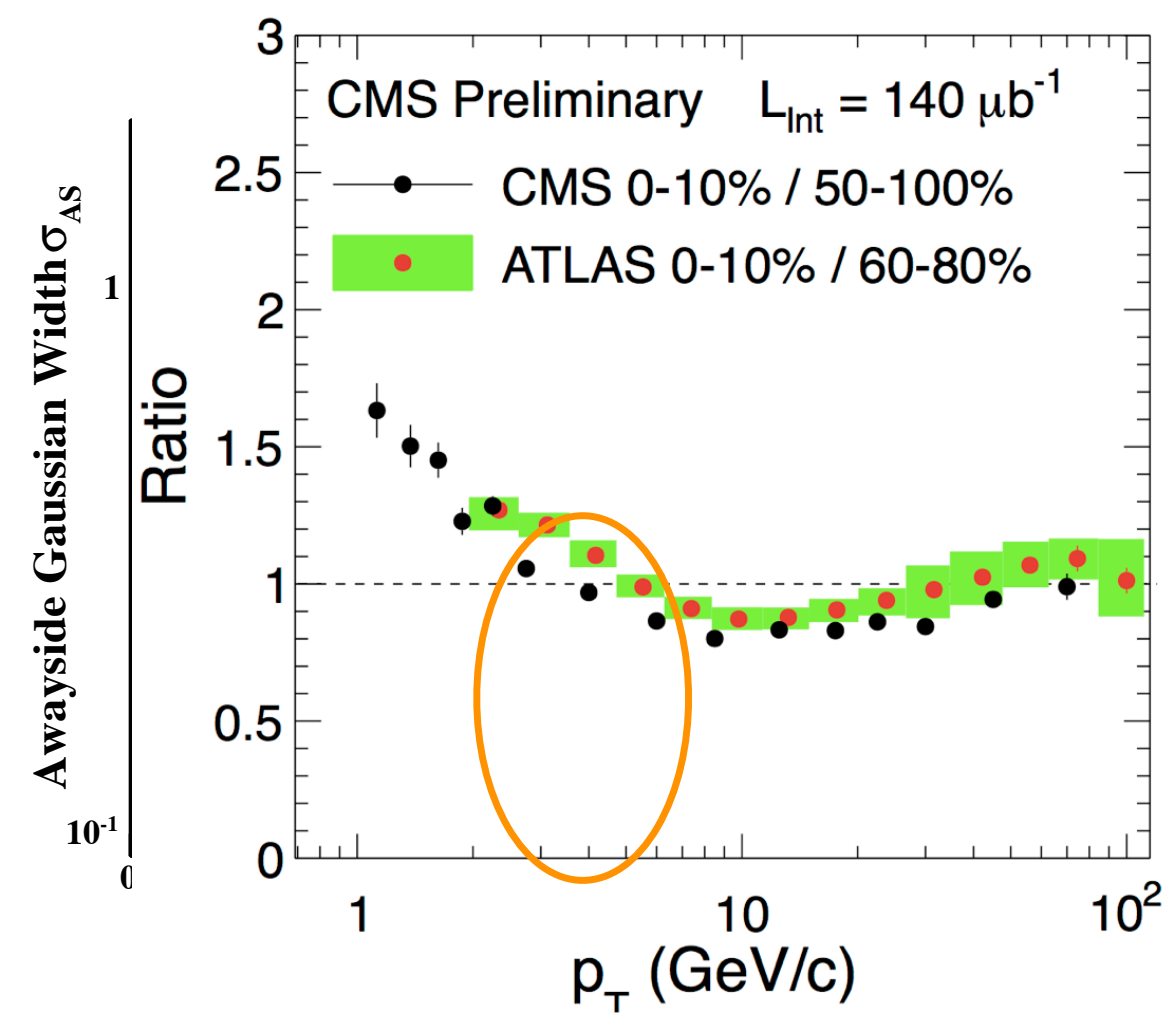

Energy difference: AuAu-pp

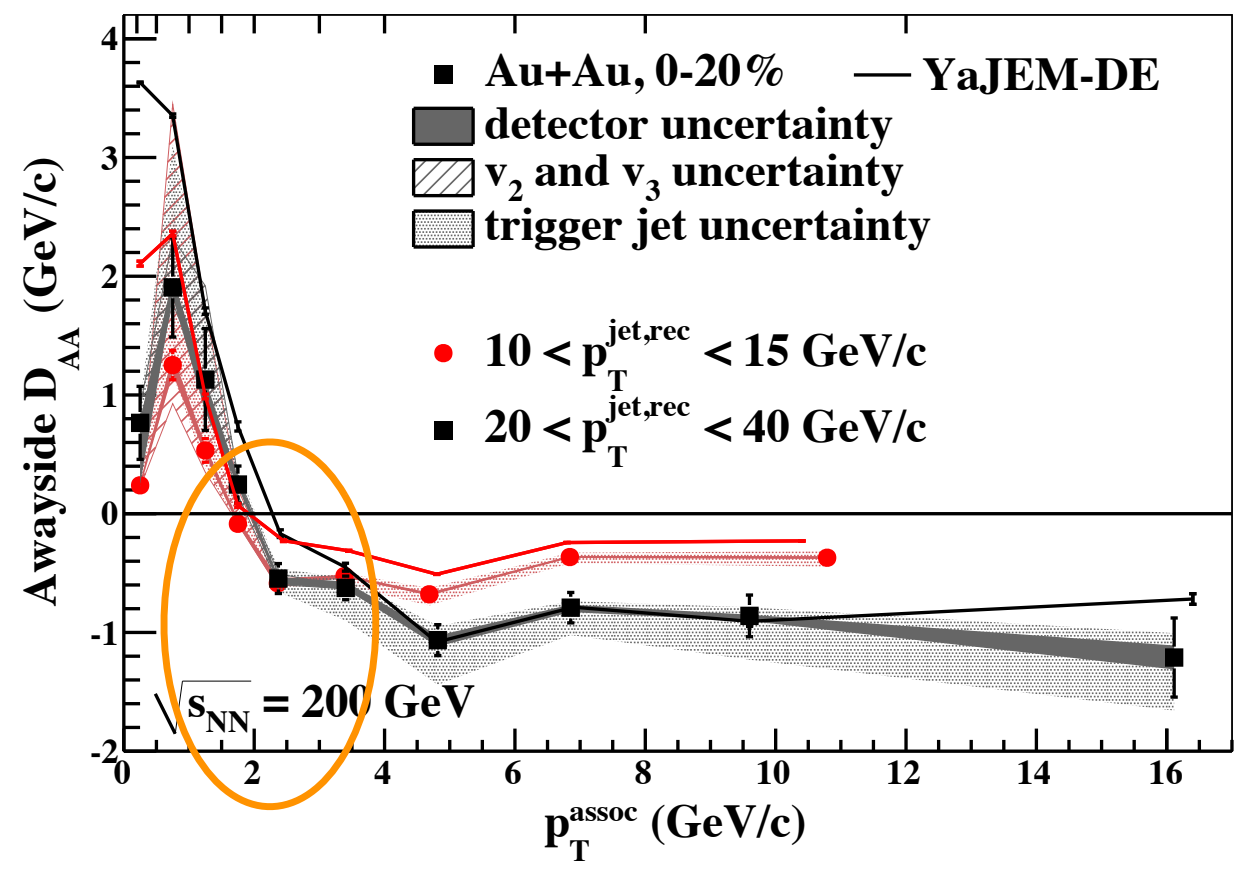

Hint of Jet Broadening at low $\mathrm{p}_{\mathrm{T}}$ (large uncertainties due to potential jet $\mathrm{v}_{2} / \mathrm{v}_{3}$ )

Quenched energy at high $p_{T}$ balanced by low $p_{T}$ enhancement

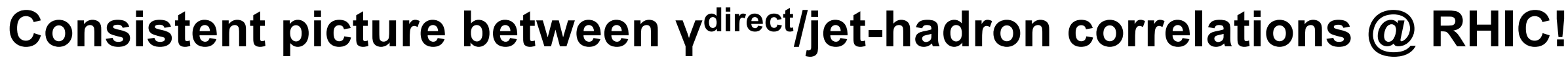
pт scale of low pт enhancement: $\sim 2$ GeV RHIC, 3-4 GeV LHC

Caveat: RHIC measurement: Statistical. Need per jet quantities $\left(A_{j}, F F\right)$ to allow one-to-one comparison to LHC. 


\section{Consistency or a way too simplistic explanation?}

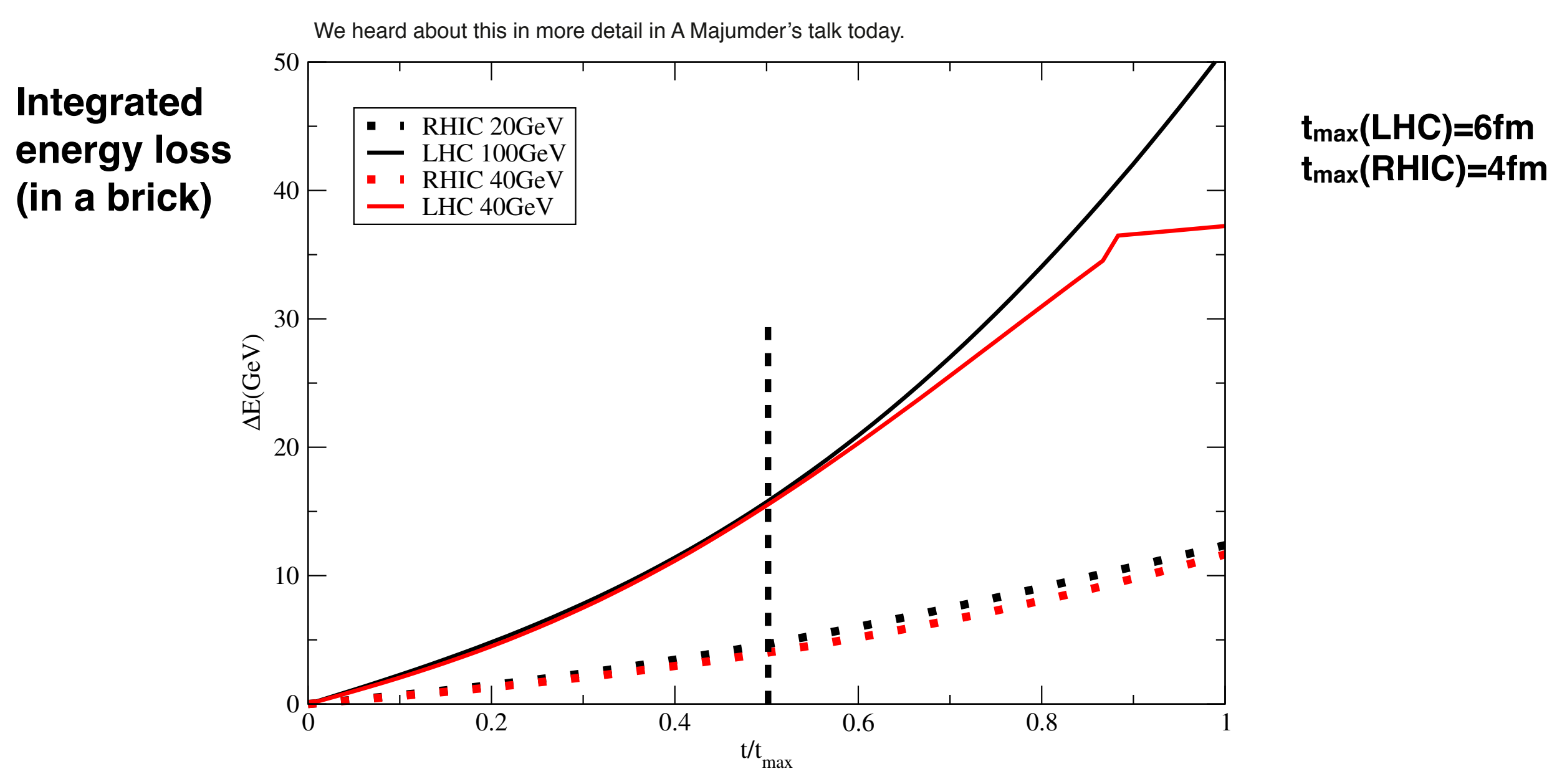

LHC larger energy loss at early times $\rightarrow$ diffusion in medium $\rightarrow$ larger angles

RHIC smaller energy loss at early times $\rightarrow$ less diffusion in the medium $\rightarrow$ closer to jet axis $\rightarrow$ can qualitatively explain the differences RHIC/LHC (!?)

Easier to study details of soft gluon radiation at RHIC!?

Caveat: Realistic calculation needed? Can current MC models explain RHIC and LHC at the same time? 


\section{Biases are not always bad ...}

T. Renk, Phys.Rev. C87 (2013) 024905

$\operatorname{Tr} 20-40 \mathrm{GeV}$

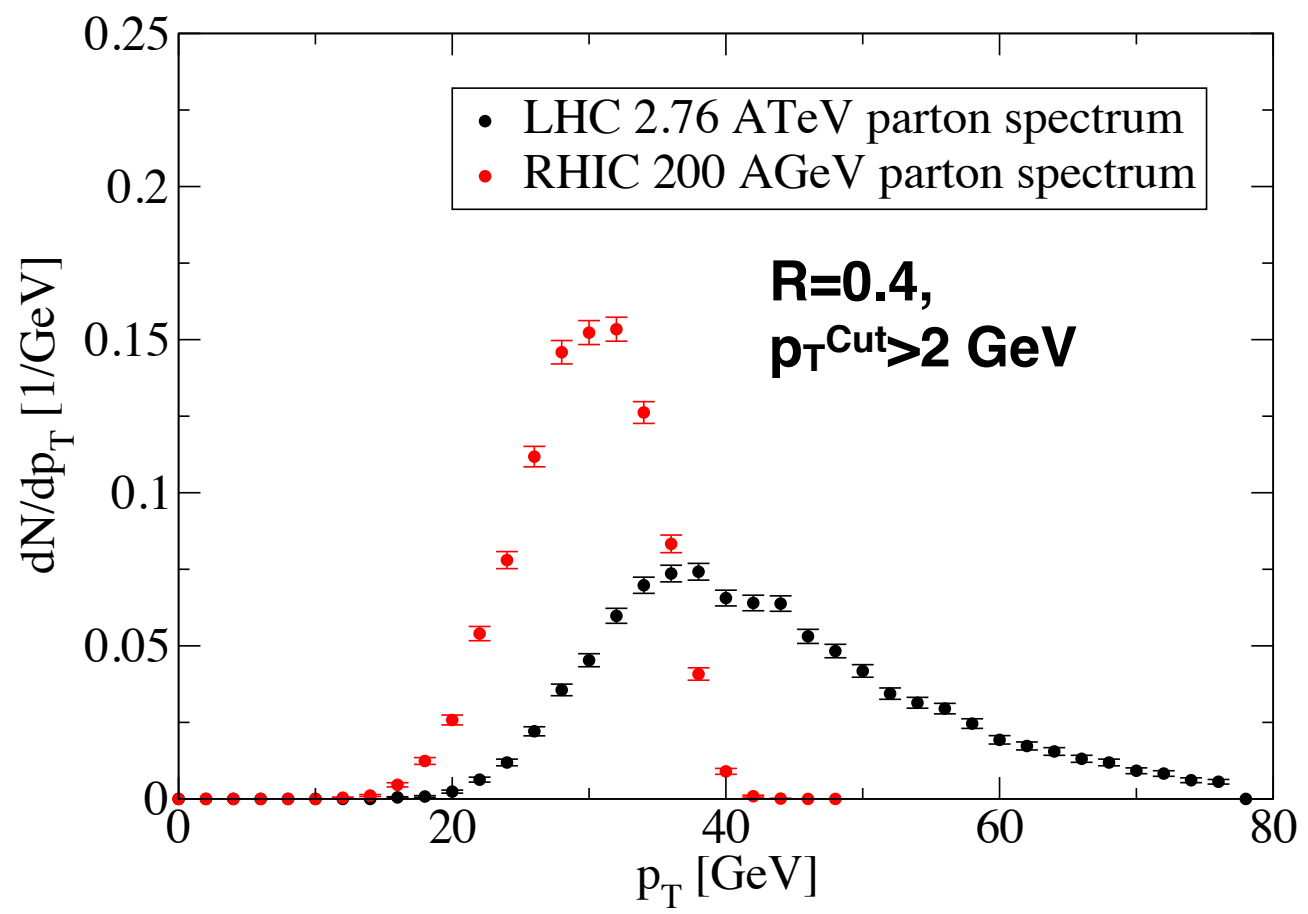

Due to the steeply falling spectrum at RHIC, even with imposing biases $\left(p_{T}{ }^{C u t}, \ldots\right)$, a good correlation to the initial parton energy is preserved 


\section{Biases are not always bad ...}

$\operatorname{Tr} 20-40 \mathrm{GeV}$

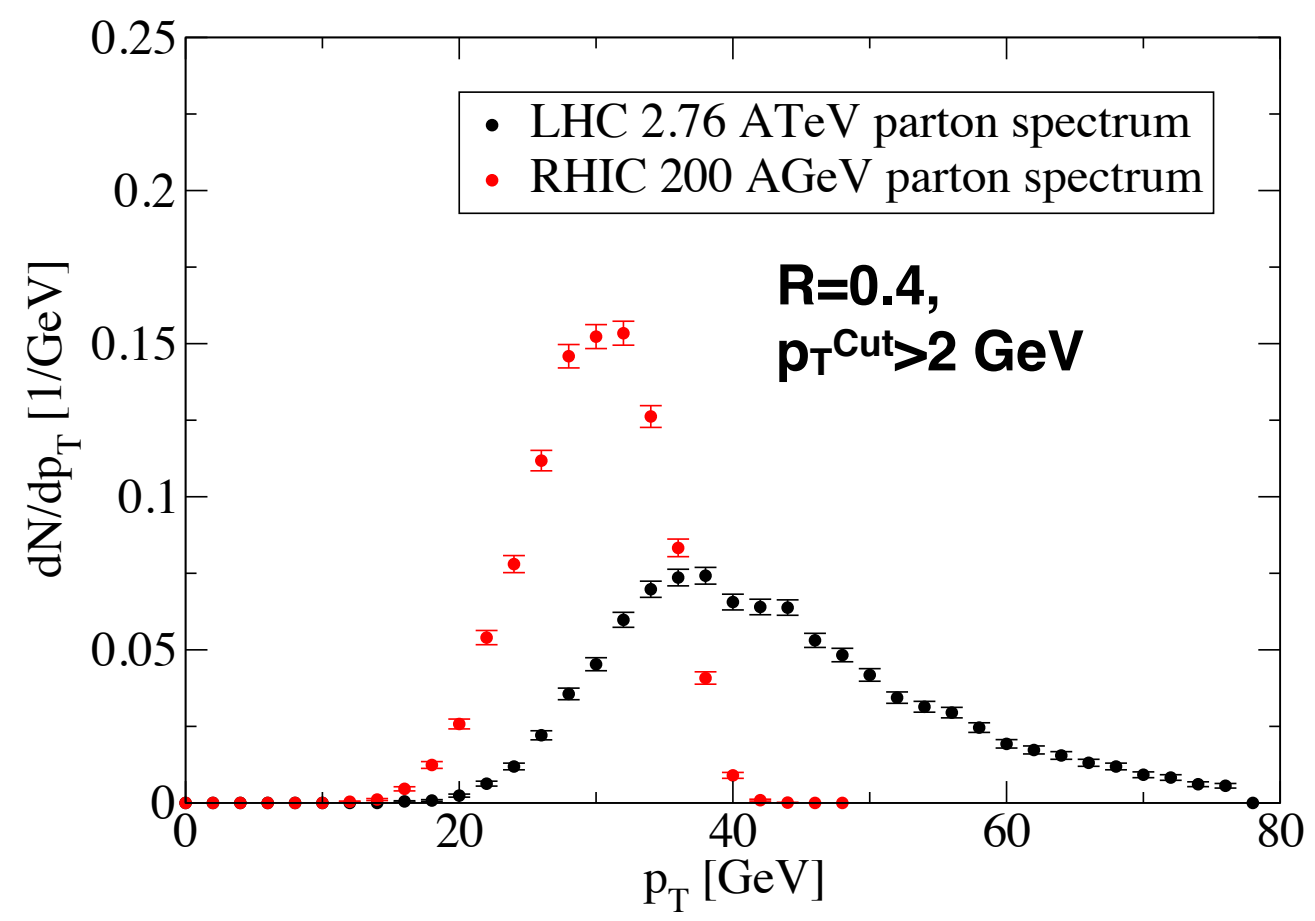

Due to the steeply falling spectrum at RHIC, even with imposing biases ( $p_{T}$ Cut, ...), a good correlation to the initial parton energy is preserved

Biases $\left(p_{T}{ }^{C u t}, \ldots\right)$ can be used to change

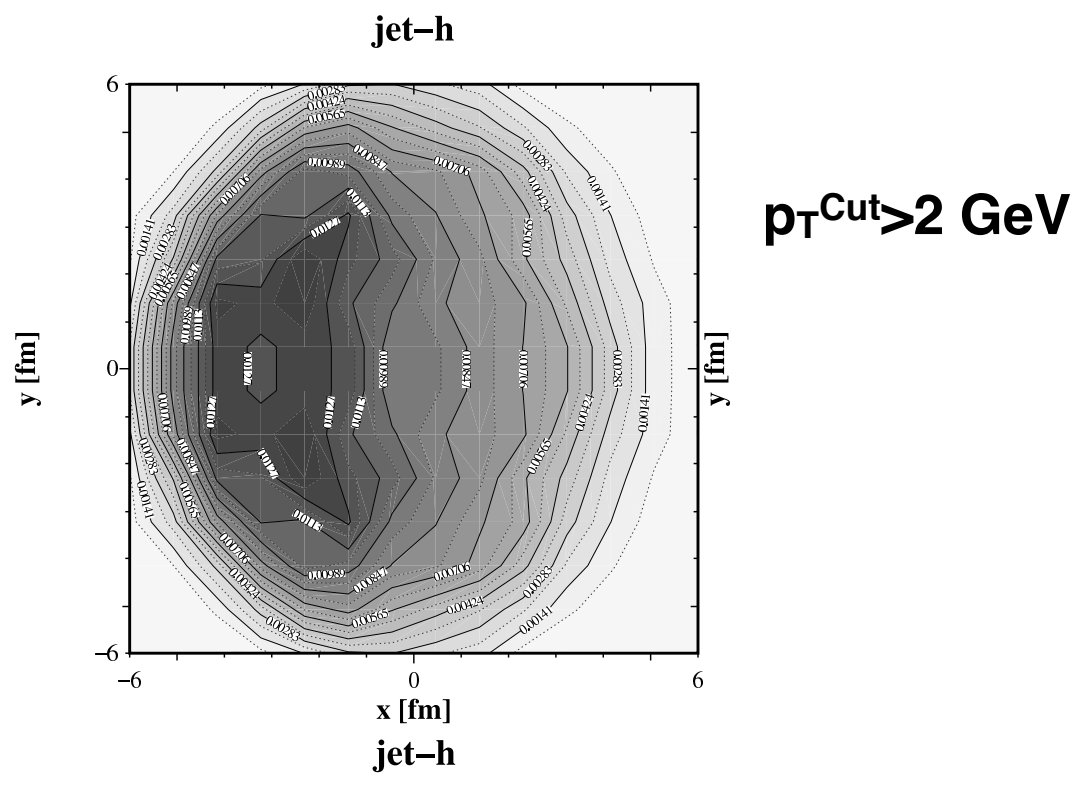
systematically the pathlength of the recoil jet

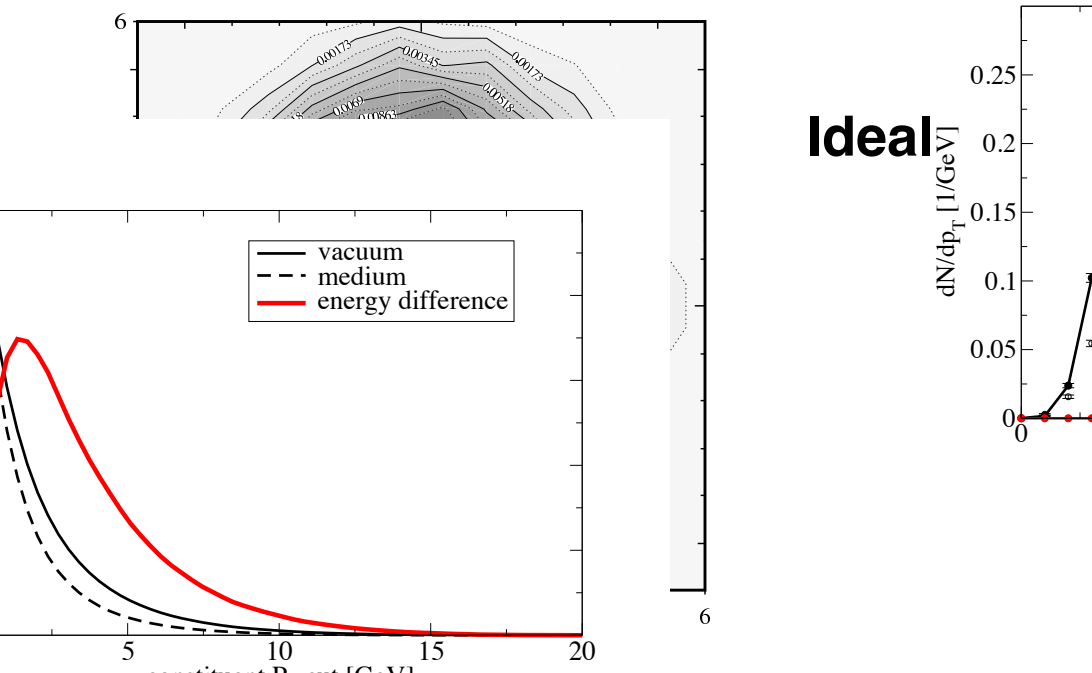

Biases ( $p_{T} \mathrm{Cut}, \ldots$ ) can be further utilized to favor gluon recoil jets 


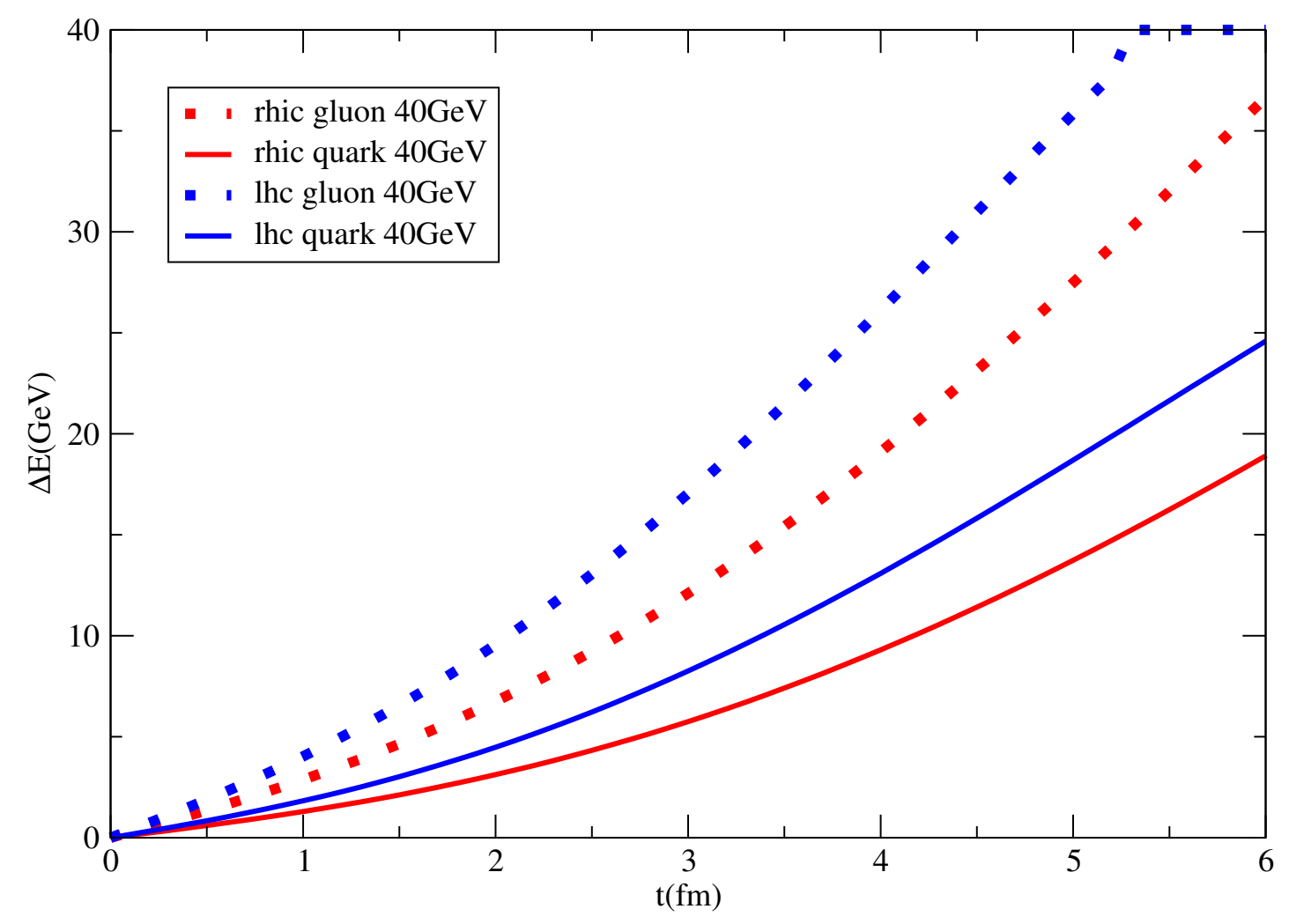

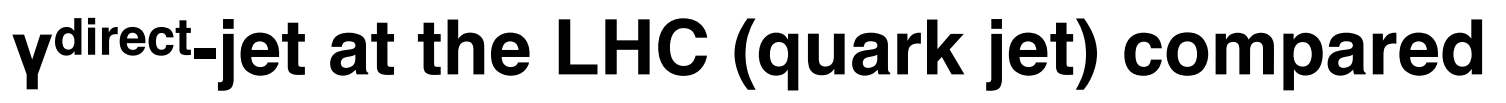
to di-jets at RHIC (quark jets) @ 40-50 GeV

Caveat: To remove geometric biases one needs an unbiased jet measurement at RHIC! 


\section{Temperature dependence of energy loss}

We had talks this morning discussing this in more detail!

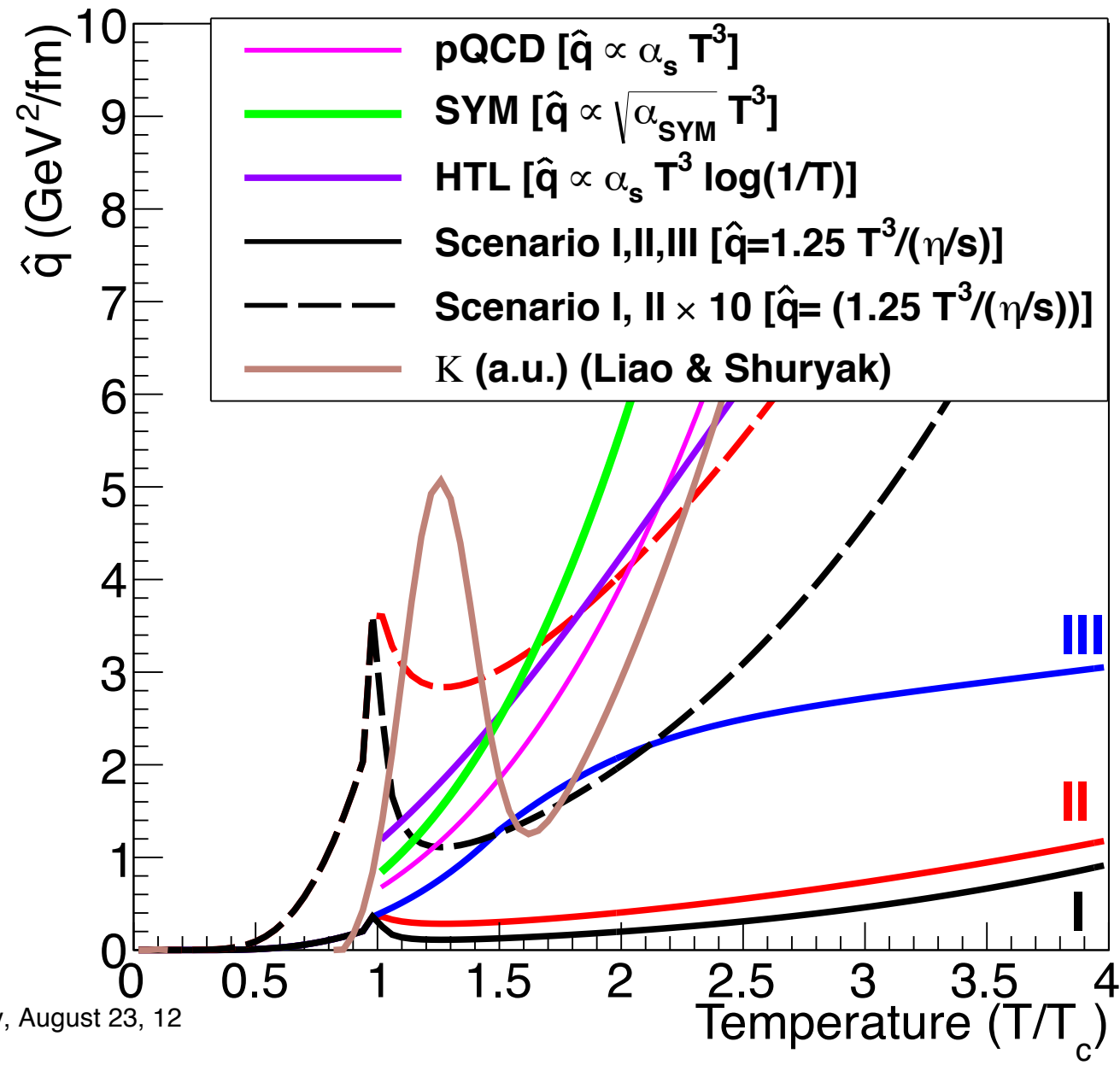

$\eta / s=$ const $\times \mathrm{T}^{3} / \hat{q}$

for weak coupling (PRL 99, 192301, 2007)

Differential measurements of transport properties of the QGP: Temperature dependence of $q(\hat{\mathbf{e}}, \eta / \mathbf{s}, .$.

Sensitivity of q to 1-2 $T_{c}$ requires RHIC measurements for different colliding systems and smaller $\sqrt{ } \mathbf{s}$ (LHC larger initial T) 


\section{Testing the quasi-particle nature of the QGP}
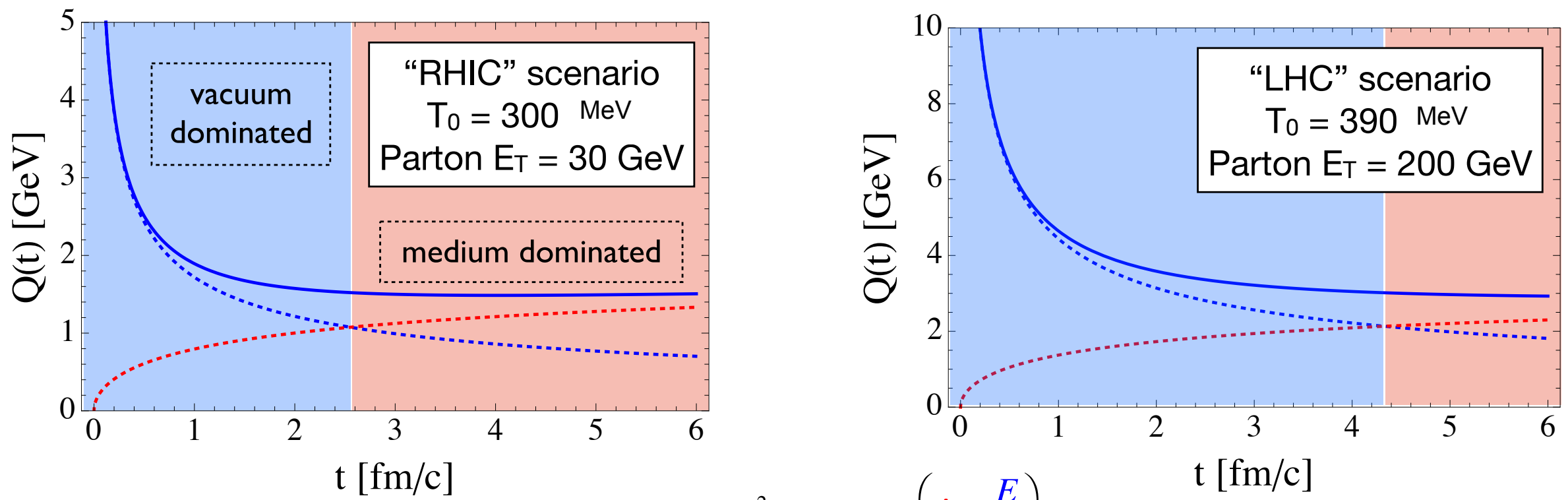

\section{Jet Virtuality: Controls the Physics of Radiative Energy Loss}

RHIC: $20 \mathrm{GeV}$ parton, $L=3 \mathrm{fm}$

$\hat{q} L \approx 1.5 \mathrm{GeV}^{2} \approx \frac{E}{L} \approx 1.5 \mathrm{GeV}^{2}$

Virtuality of primary parton is medium influenced and small enough to "experience" the strongly coupled medium
LHC: $200 \mathrm{GeV}$ parton, $L=3 \mathrm{fm}$

$$
Q^{2}(L) \approx \max \left(\underset{\uparrow}{\hat{q} L, \frac{E}{L}}\right)
$$

$\hat{q} L \approx 3.5 \mathrm{GeV}^{2}<\frac{E}{L} \approx 13 \mathrm{GeV}^{2}$

Virtuality of primary parton is vacuum dominated and only its gluon cloud "experiences" the strongly coupled medium
RHIC can explore the region between the weak and strong coupling limits! 


\section{"QCD Analog of Bethe-Bloch"}

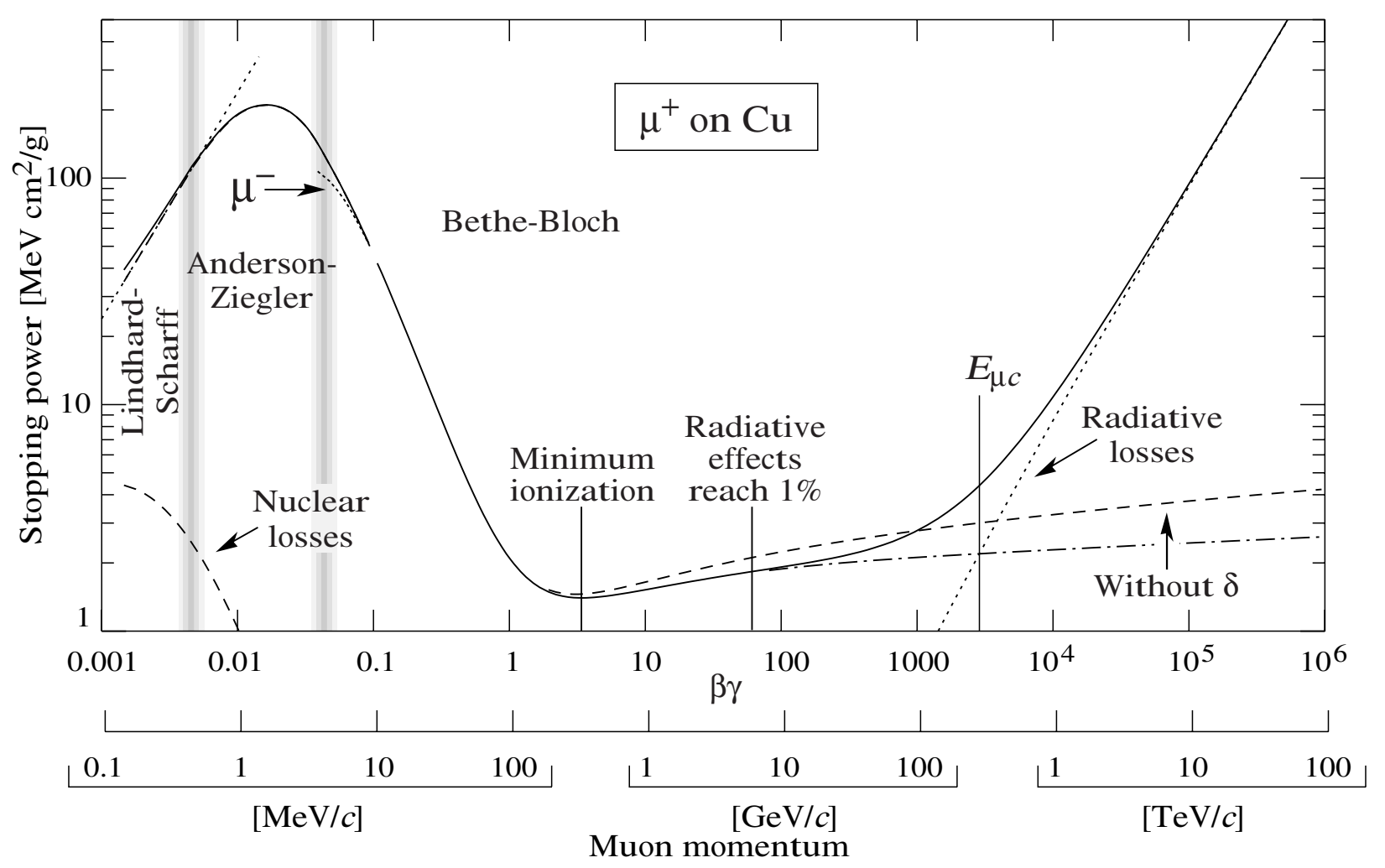

At the LHC/at large jet energies, jet modification dominated by radiative energy loss

At lower jet energies balance/interplay between radiative energy and collisional energy loss

RHIC and LHC combined will map out the stopping power $-\mathrm{dE} / \mathrm{dx}$ of hot and dense QGP for colored patrons 


\section{RHIC is always good for surprises: $d+A_{u} R_{A A}$}

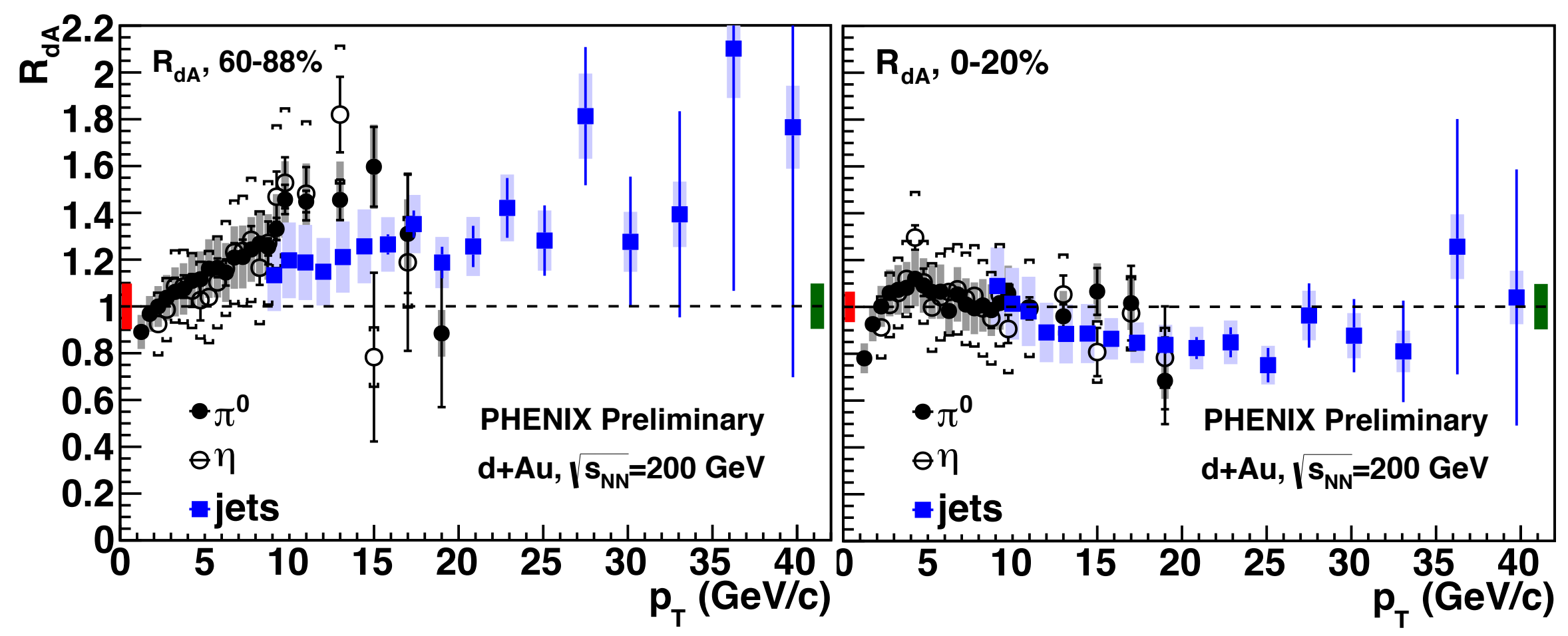

Enhancement of Jet $\mathbf{R}_{\mathrm{AA}}$ in peripheral $\mathbf{d}+\mathrm{Au}$ collision?

Caveat: We saw yesterday (G. David) that centrality determination in $d+A u$ is not trivial ... 


\section{Future: Precision Jet Measurements @ RHIC / sPhenix}
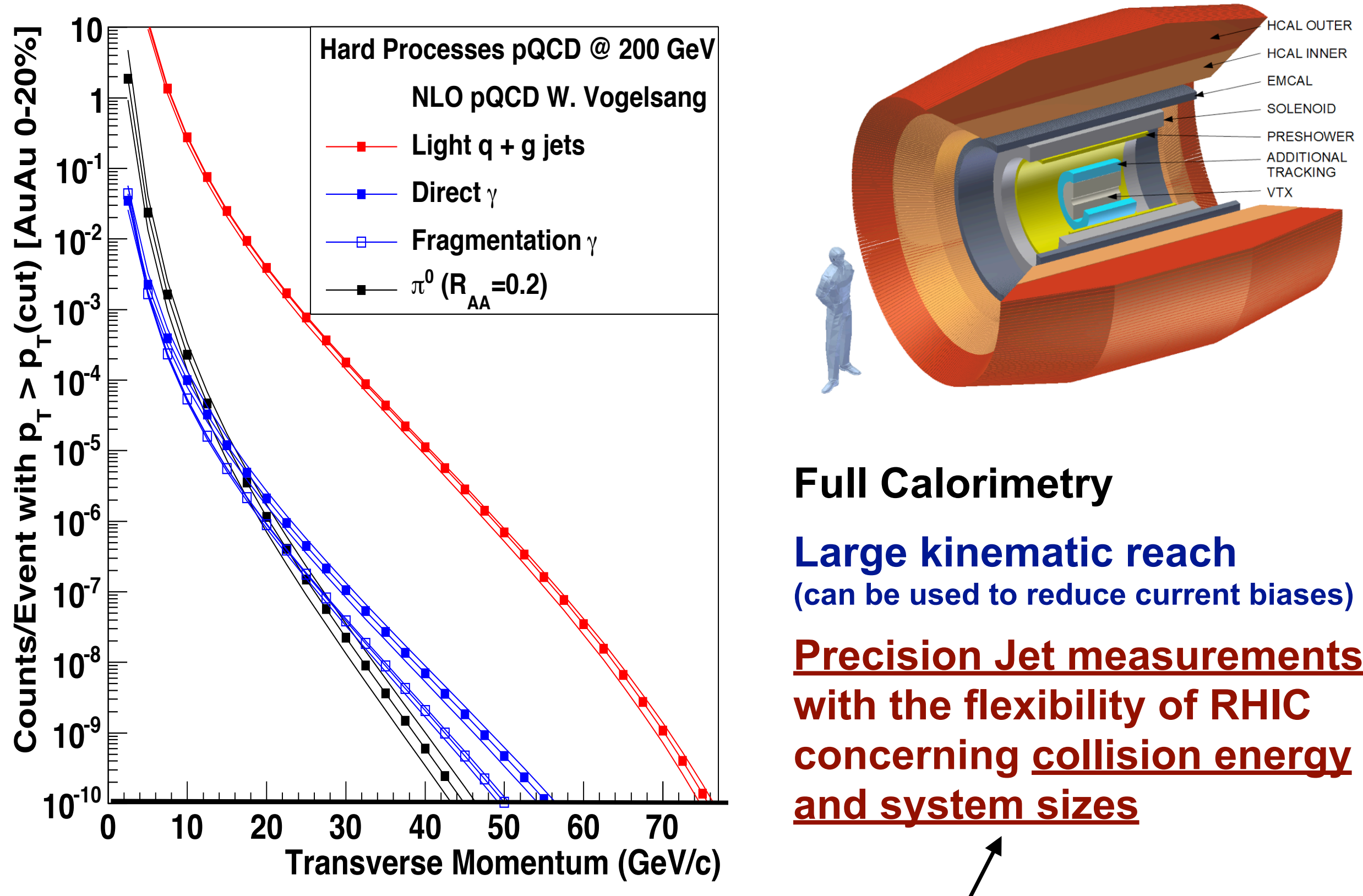

Full Calorimetry

Large kinematic reach (can be used to reduce current biases)

Precision Jet measurements with the flexibility of RHIC concerning collision energy and system sizes

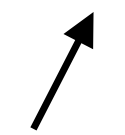

Can this be utilized to study pre-equilibrium effects? 


\section{Summary}

Consistent (qualitative) jet quenching picture at RHIC emerging: suppression at high $z$, enhancement at low $z$. Jet broadening has to be quantified.

Can current LHC and RHIC quenching measurements be explained in a consistent picture?

In many respects RHIC and LHC a complementary and an active jet program at RHIC is essential to further and quantify our understanding of partonic energy loss in the future!

Important experimental and theoretical developments
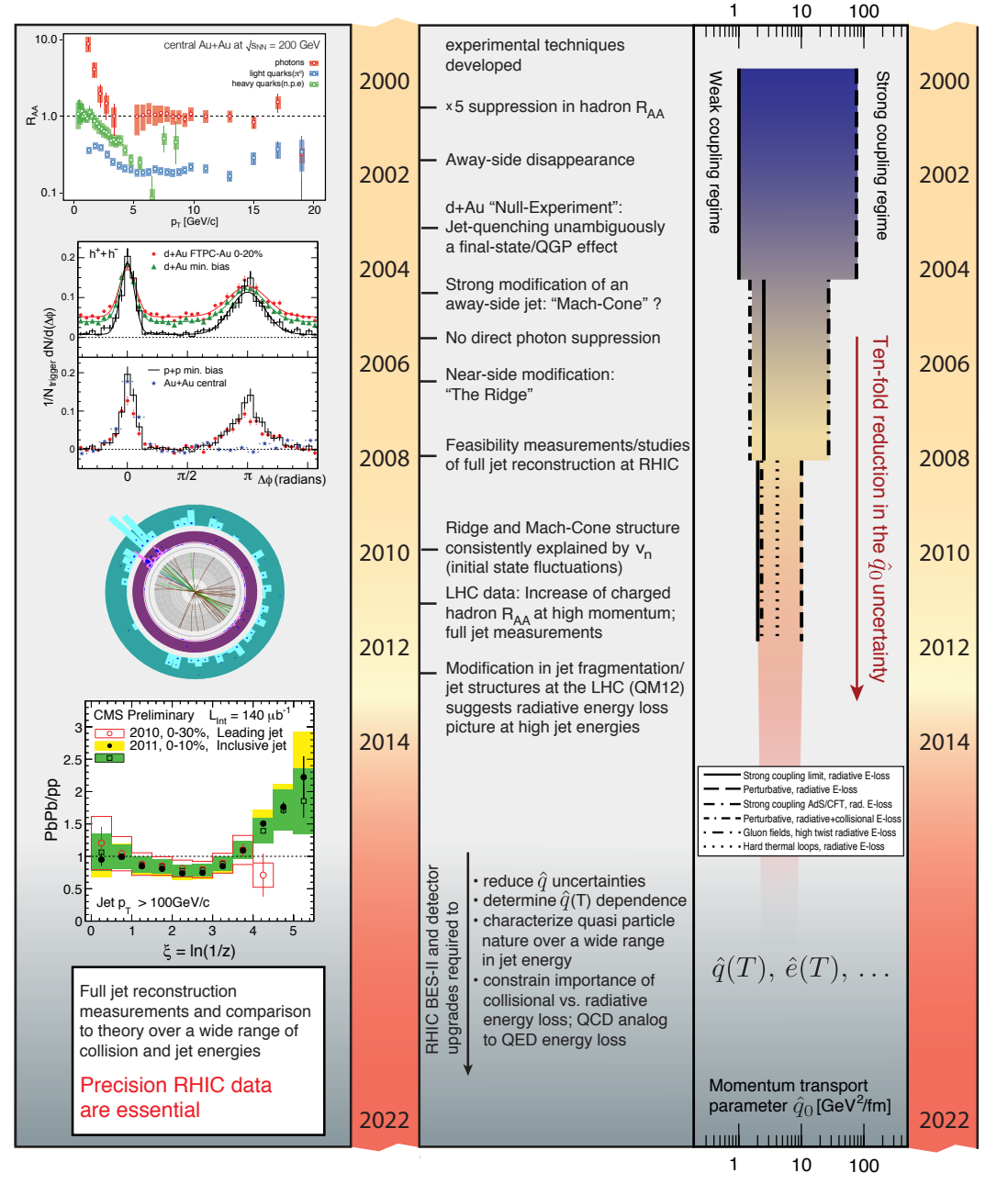


\section{Near Future: RJE(T)T ?}

\section{RJE(T)T=RHIC Jet Experiments (\& Theory) Taskforce}
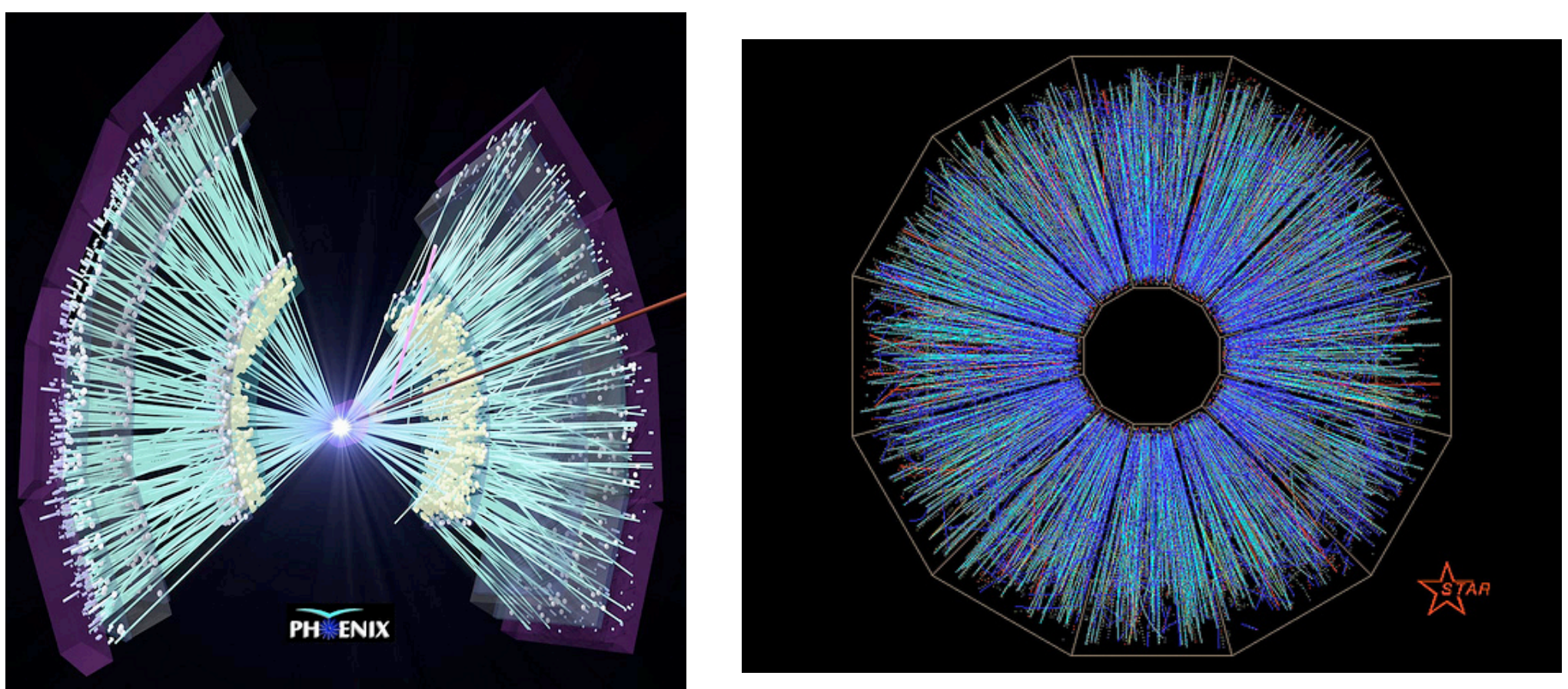

Can something like this be realized at $\mathrm{RHIC}$ ? 


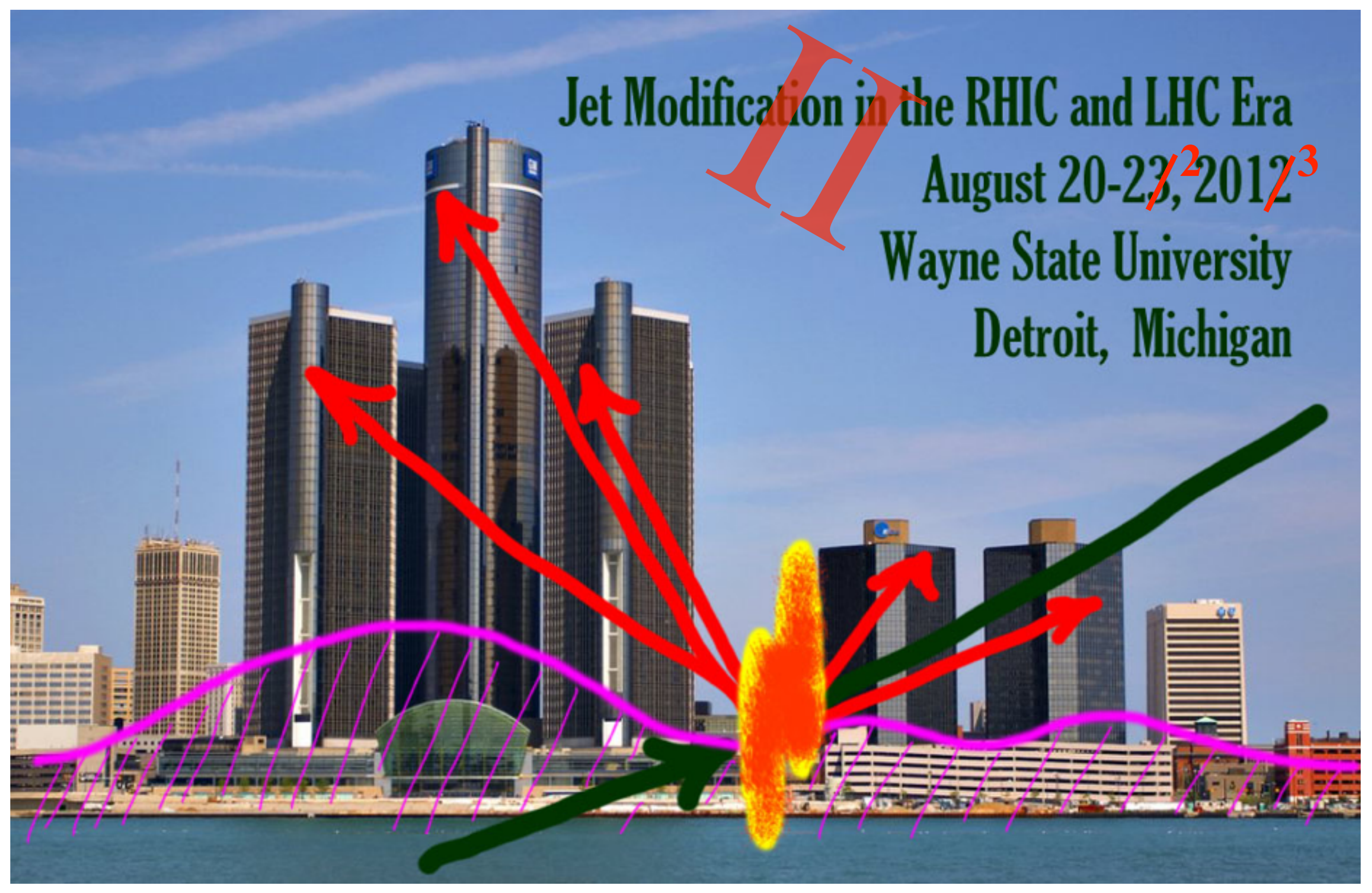




\section{Backup}




\section{Temperature dependence of energy loss}

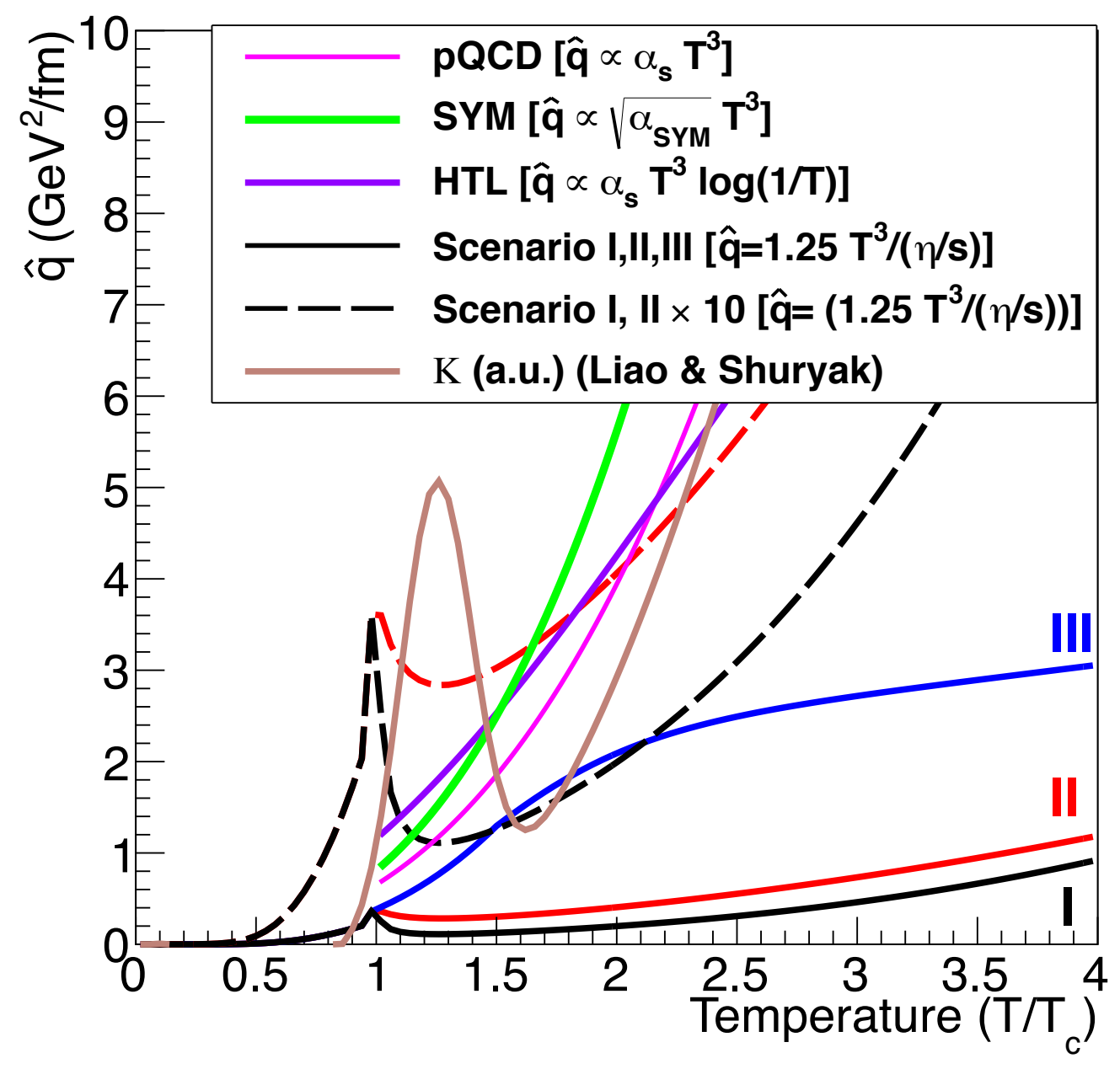

$\eta / s=$ const $\times \mathrm{T}^{3} / \hat{q}$ for weak coupling (PRL 99, 192301, 2007)

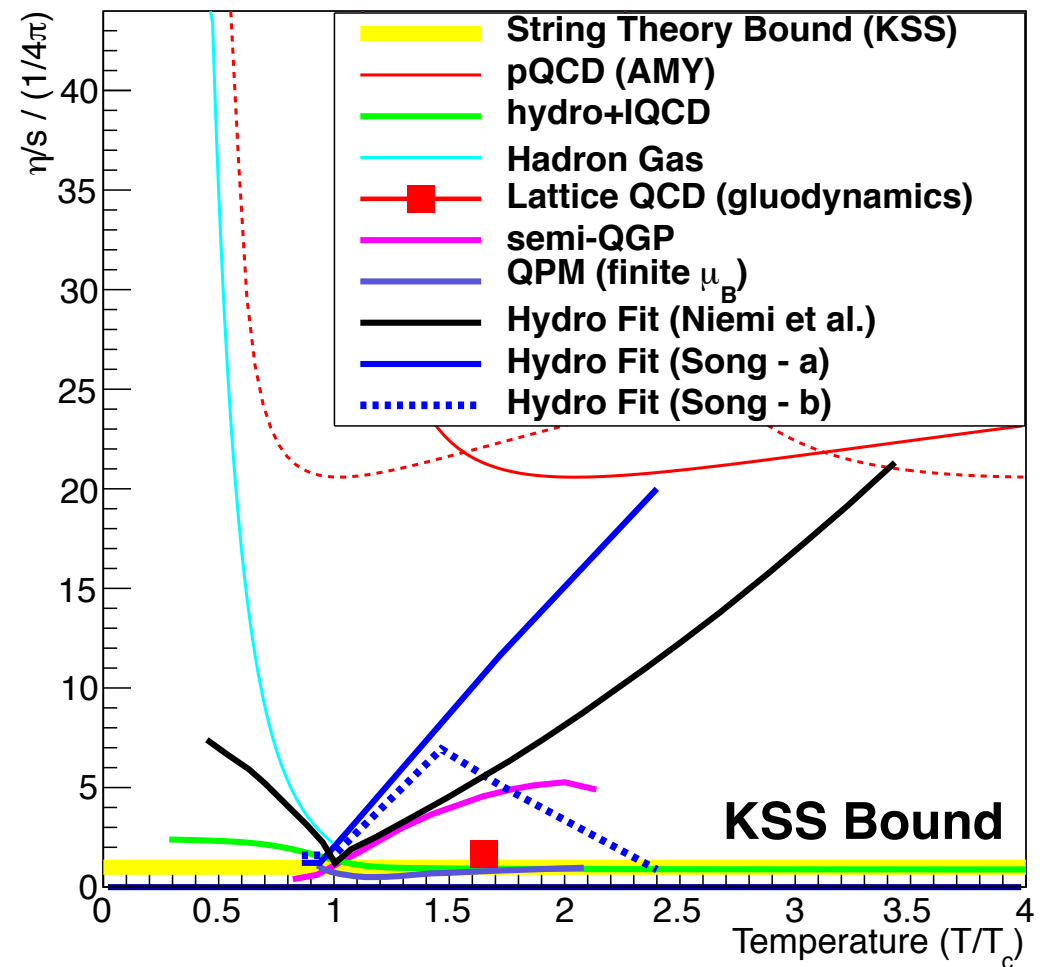

Majumder, BM, V that $\eta / s$ and $\hat{q}$ are weak coupling in [PRL 99, 192301

$$
\eta / s=\text { const }>
$$

$\eta / s$ saturates in strong coupling, but energy loss increases w/o limpit at $1 / 4 \pi$, but $\hat{q}$ incr limit. Unambiguo weak vs. strong $c$ 


\section{Where does the lost energy go? Missing $\mathrm{p}_{\mathrm{T}}{ }^{\prime \prime}$}

Taken from C. Roland (CMS), QM11

Missing $\mathrm{p}_{\mathrm{T}} \|: \quad \phi_{\mathrm{T}}^{\|}=\sum_{\text {Tracks }}-p_{\mathrm{T}}^{\text {Track }} \cos \left(\phi_{\text {Track }}-\phi_{\text {Leading Jet }}\right) \quad|\eta|<2.4$

Calculate projection of $p_{T}$ on leading jet axis and average over selected tracks with

$\mathrm{p}_{\mathrm{T}}>0.5 \mathrm{GeV} / \mathrm{c}$ and

$|\eta|<2.4$
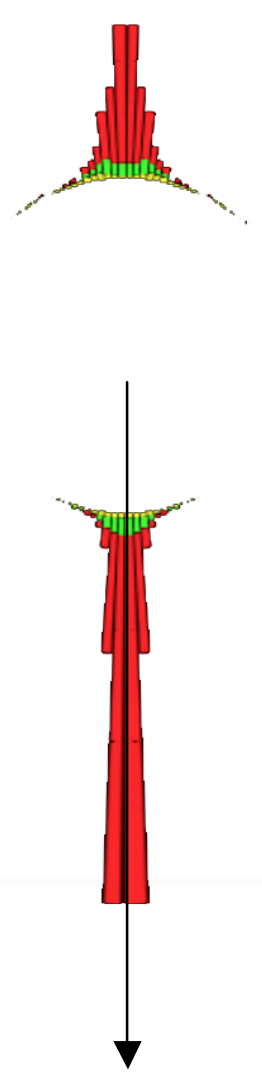

Leading Jet defines direction 


\section{Where does the lost energy go? Missing $\mathrm{p}_{\mathrm{T}}{ }^{\prime \prime}$}

Taken from C. Roland (CMS), QM11

Missing $\mathrm{p}_{\mathrm{T}} \|: \quad \phi_{\mathrm{T}}^{\|}=\sum_{\text {Tracks }}-p_{\mathrm{T}}^{\text {Track }} \cos \left(\phi_{\text {Track }}-\phi_{\text {Leading Jet }}\right) \quad|\eta|<2.4$

Calculate projection of $p_{T}$ on leading jet axis and average over selected tracks with

$\mathrm{p}_{\mathrm{T}}>0.5 \mathrm{GeV} / \mathrm{c}$ and

$|\eta|<2.4$

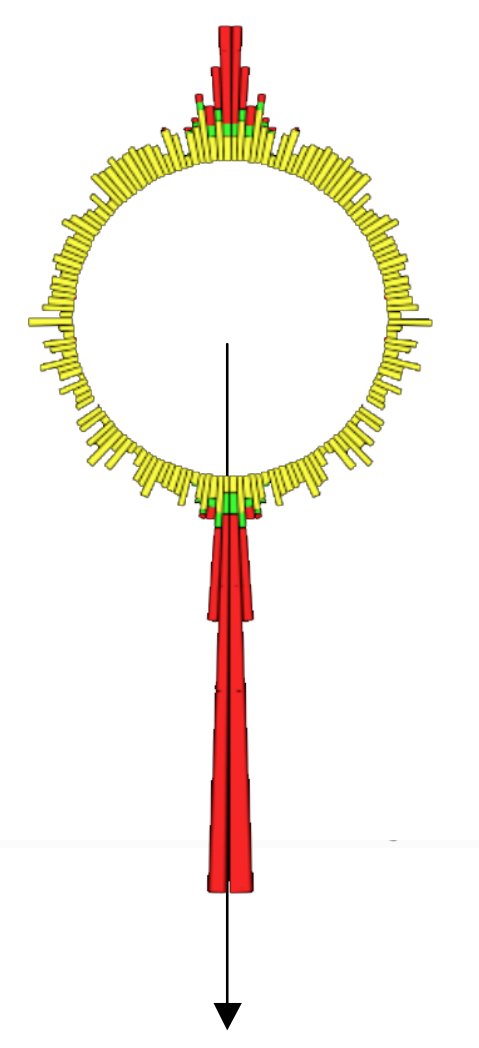

Sum all tracks in the event 


\section{Where does the lost energy go? Missing $\mathrm{p}_{\mathrm{T}}$ "}

Taken from C. Roland (CMS), QM11

Missing $\mathrm{p}_{\mathrm{T}} \|: \quad \phi_{\mathrm{T}}^{\|}=\sum_{\text {Tracks }}-p_{\mathrm{T}}^{\text {Track }} \cos \left(\phi_{\text {Track }}-\phi_{\text {Leading Jet }}\right) \quad|\eta|<2.4$
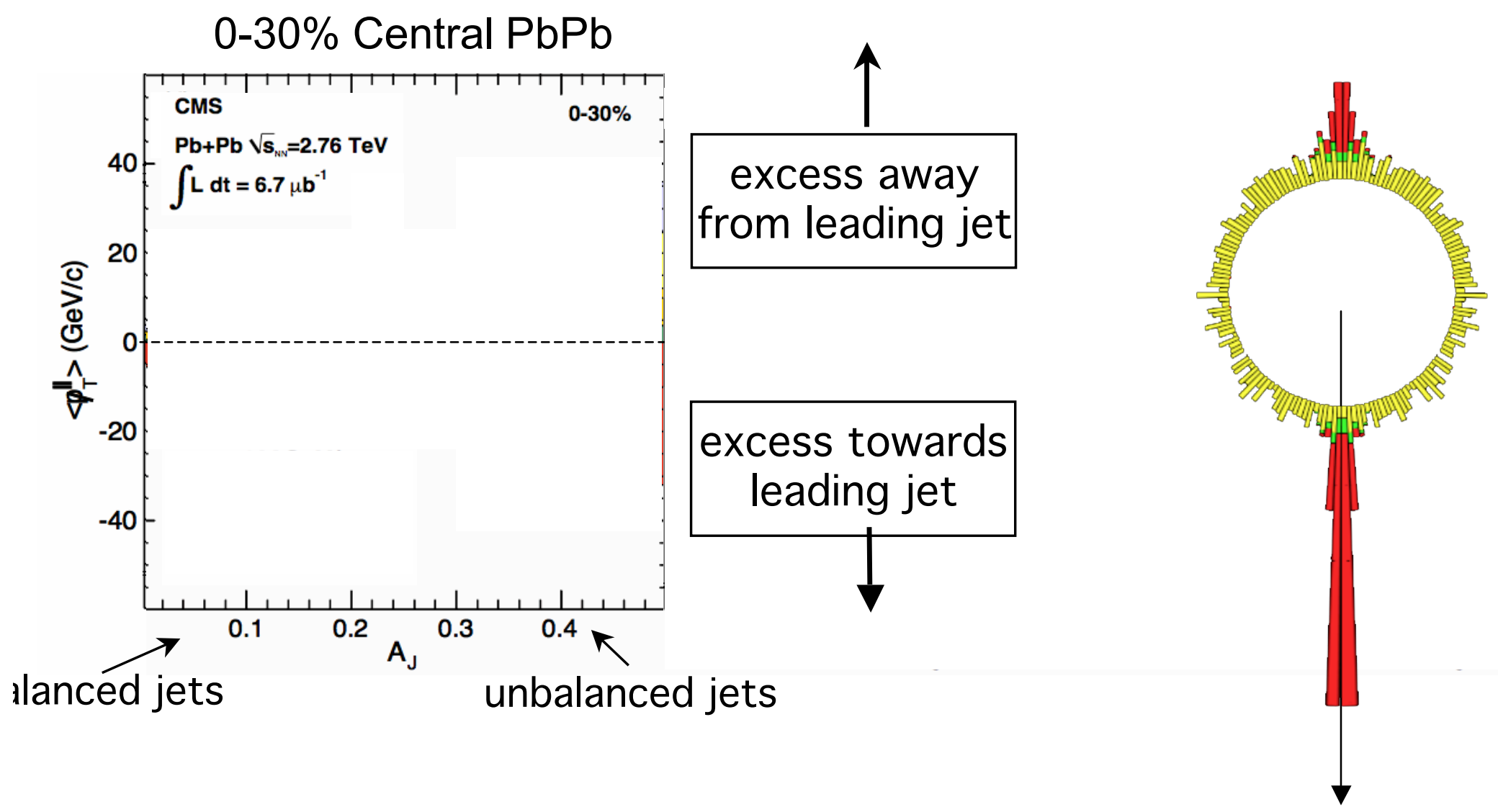


\section{Where does the lost energy go? Missing $\mathrm{p}_{\mathrm{T}}{ }^{\prime \prime}$}

Taken from C. Roland (CMS), QM11

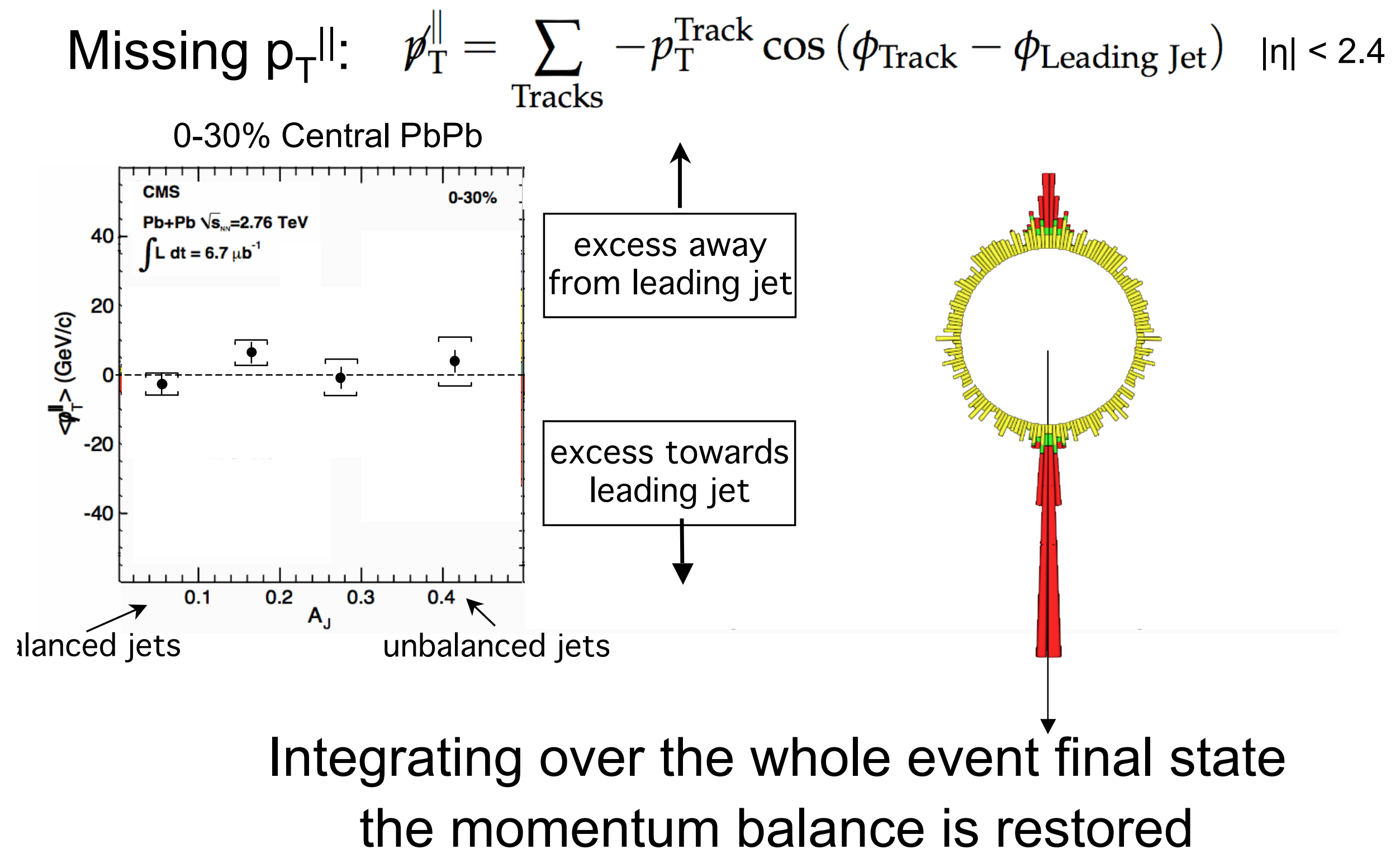




\section{Where does the lost energy go? Missing $\mathrm{p}_{\mathrm{T}}{ }^{\prime \prime}$}

Taken from C. Roland (CMS), QM11

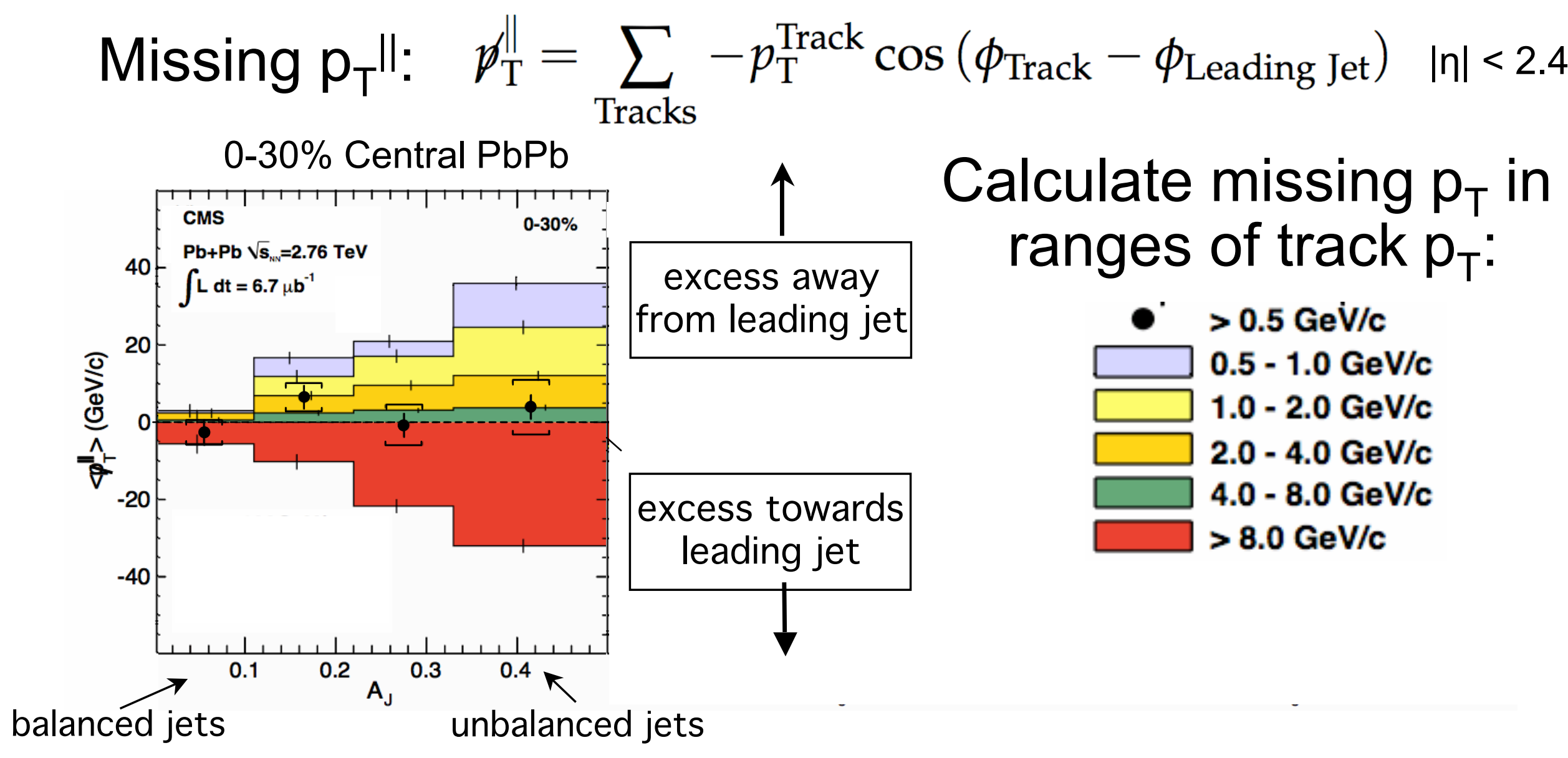

The momentum difference in the dijet is balanced by low $p_{T}$ particles 


\section{Jet $R_{A A}$ in central $A u+A u$ and $C u+C u$}
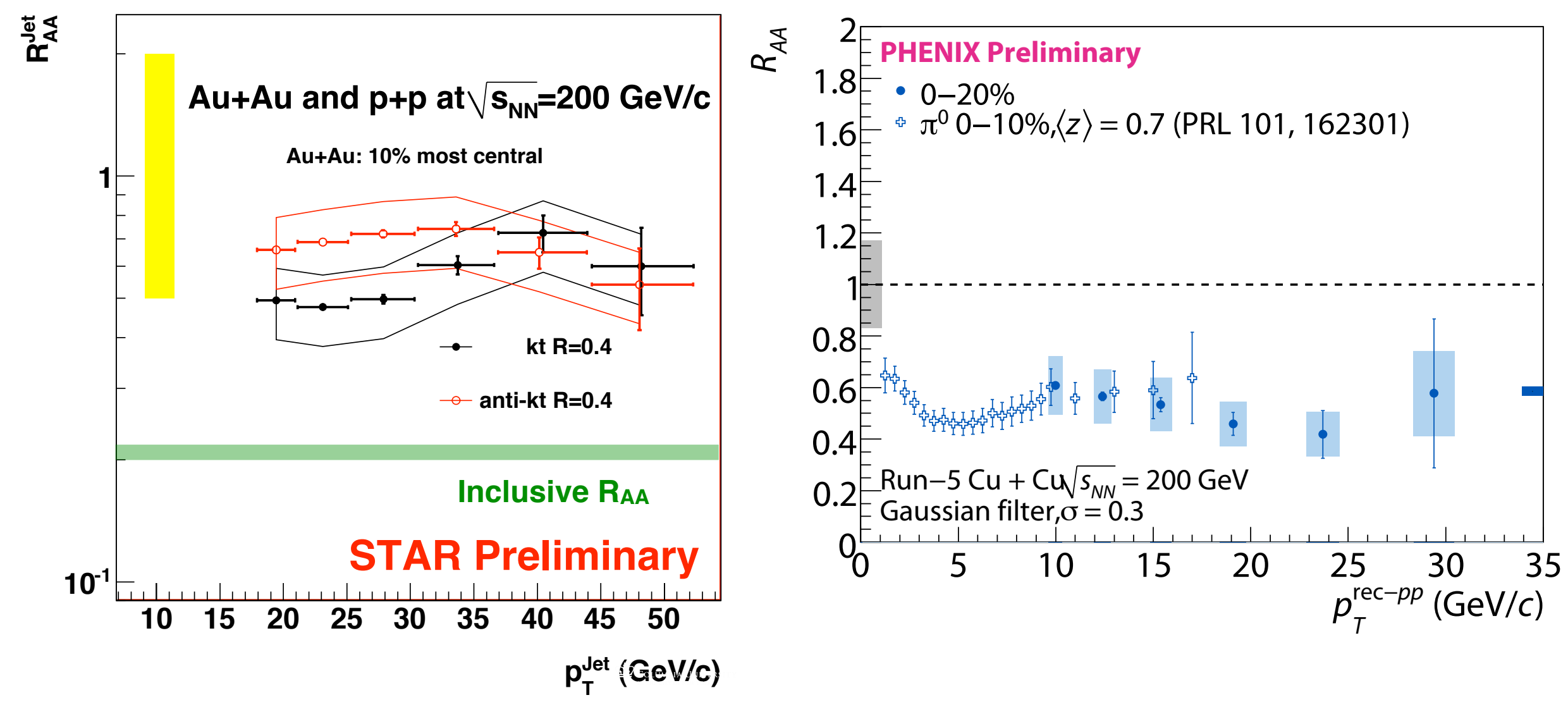

STAR sees a substantial fraction of jets in Au+Au

- in contrast to $\times 5$ suppression for light hadron $R_{A A}$

Strong suppression (similar to single particle) in $\mathrm{Cu}+\mathrm{Cu}$ measured by PHENIX 


\section{Recoil jet spectrum $R_{A A}$}
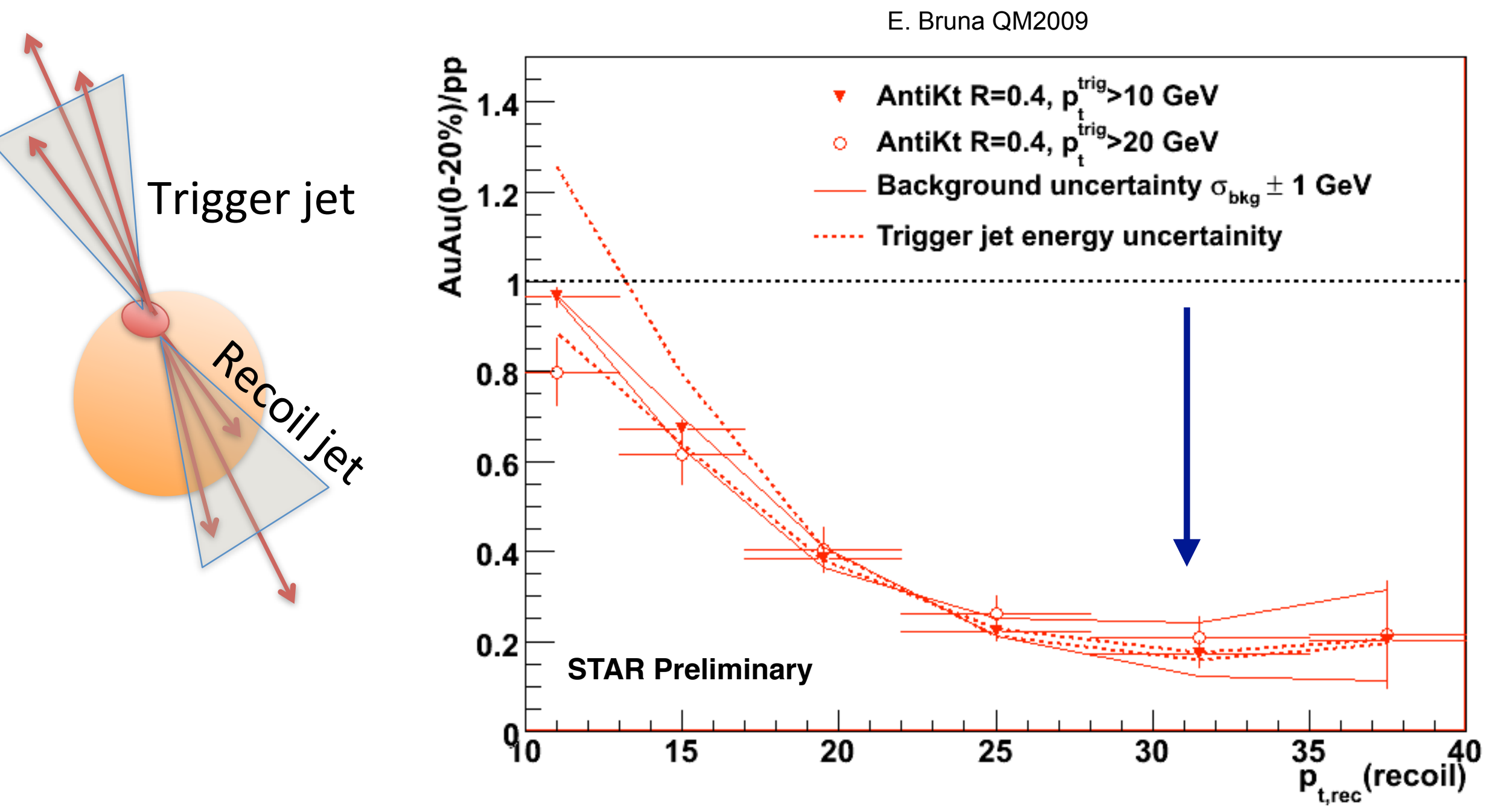

- Selecting biased trigger jet maximizes pathlength for the back-to-back jets: extreme selection of jet population

- Significant suppression in di-jet coincidence measurements! 


\section{CMS results on jet quenching and $\mathrm{pPb}$ collisions}

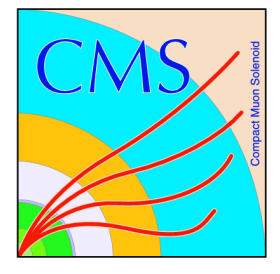

\section{Gunther Roland (MIT) \\ for the CMS Collaboration}

\section{RBRC Workshop}

"Jet Quenching at RHIC vs LHC in Light of Recent dAu vs pPb Controls"

BNL Apr 15-17, 2013 


\title{
CMS results on jet quenching and $\mathrm{pPb}$ collisions
}

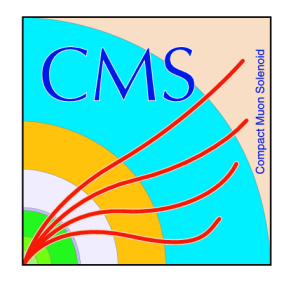

\section{Gunther Roland (MIT) \\ for the CMS Collaboration}

\author{
RBRC Workshop
}

"Jet Quenching at RHIC vs LHC in Light of Recent dAu vs pPb Controls"

BNL Apr 15-17, 2013 


\section{CMS Detector}

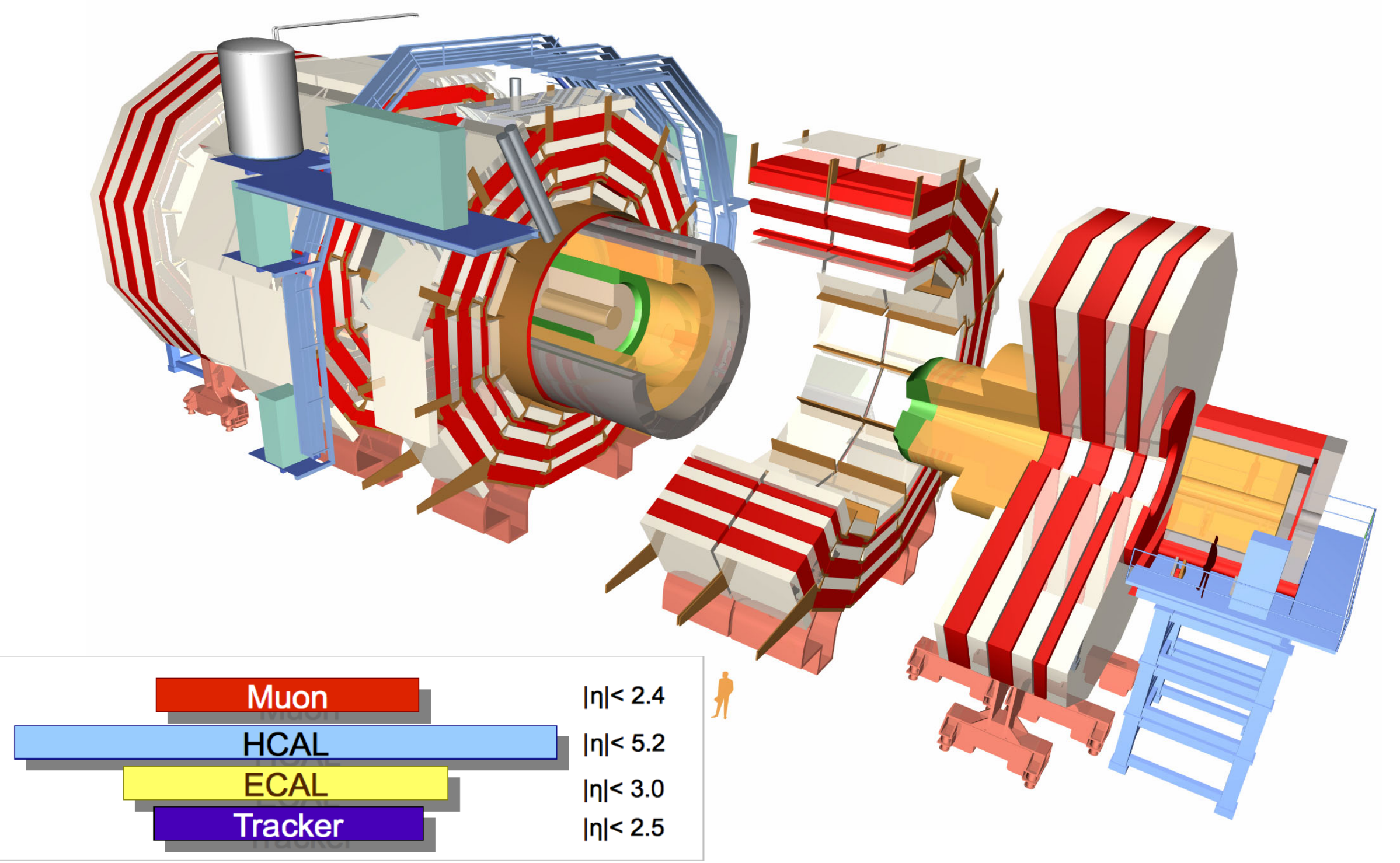




\section{$\mathrm{PbPb}$ dijet phenomenology}

\section{Leading jet $\left(p_{T}>120 \mathrm{GeV} / \mathrm{c}\right)$}
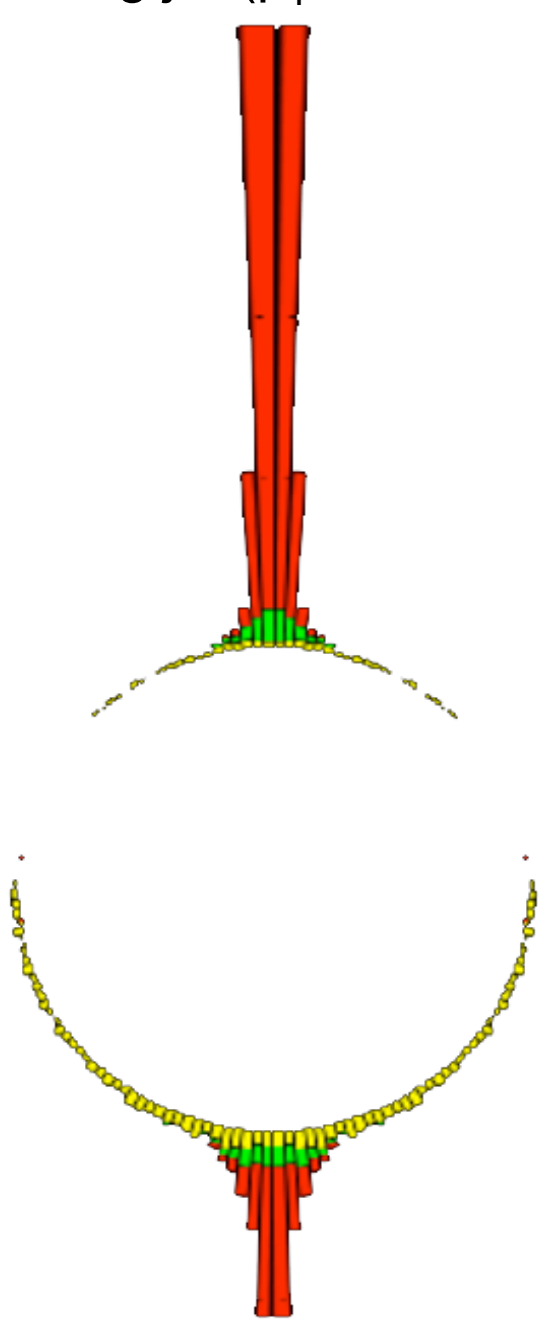

Subleading jet $\left(p_{T}>30 \mathrm{GeV} / \mathrm{c}\right)$ 


\section{$\mathrm{PbPb}$ dijet phenomenology}
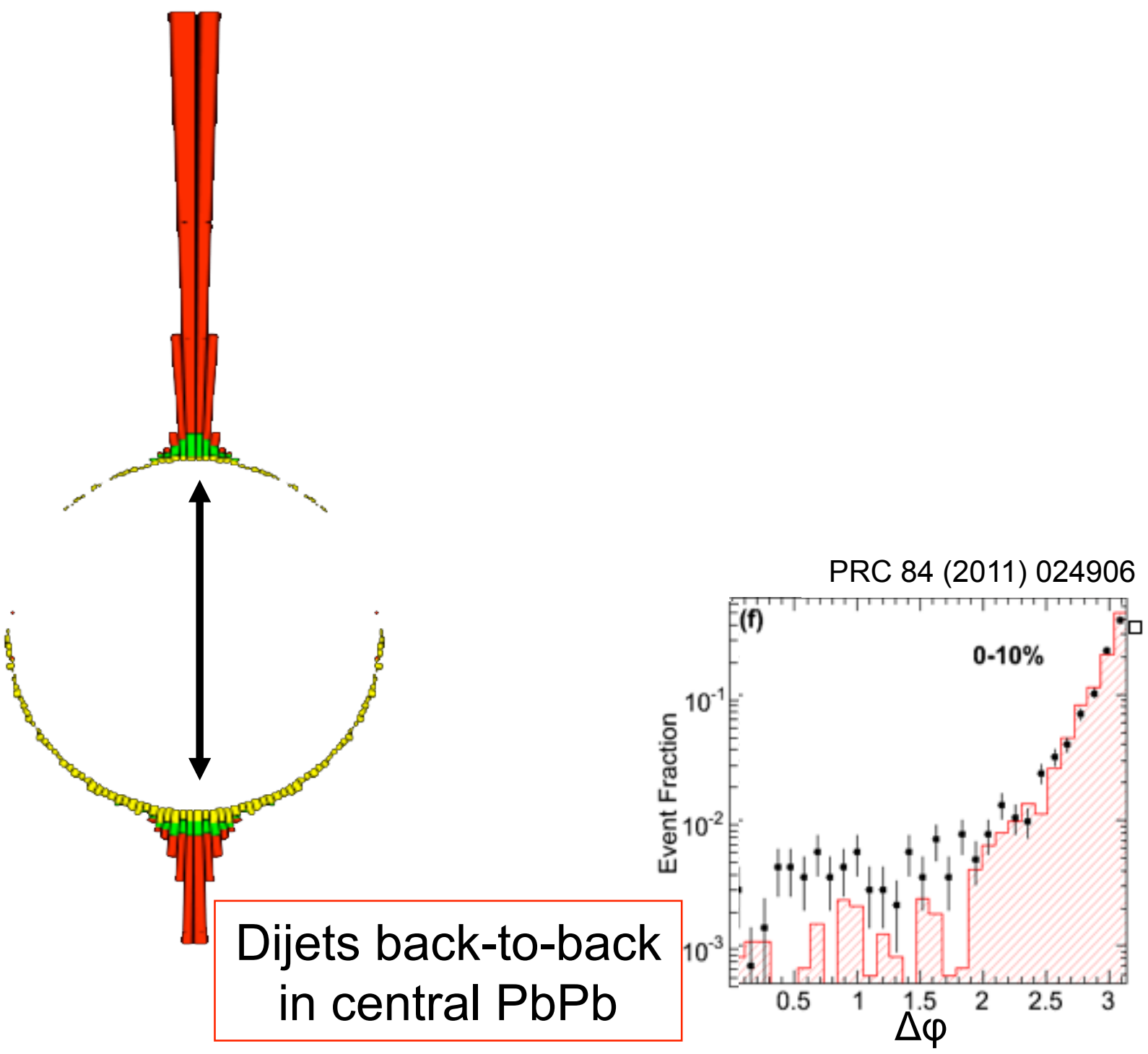


\section{$\mathrm{PbPb}$ dijet phenomenology}

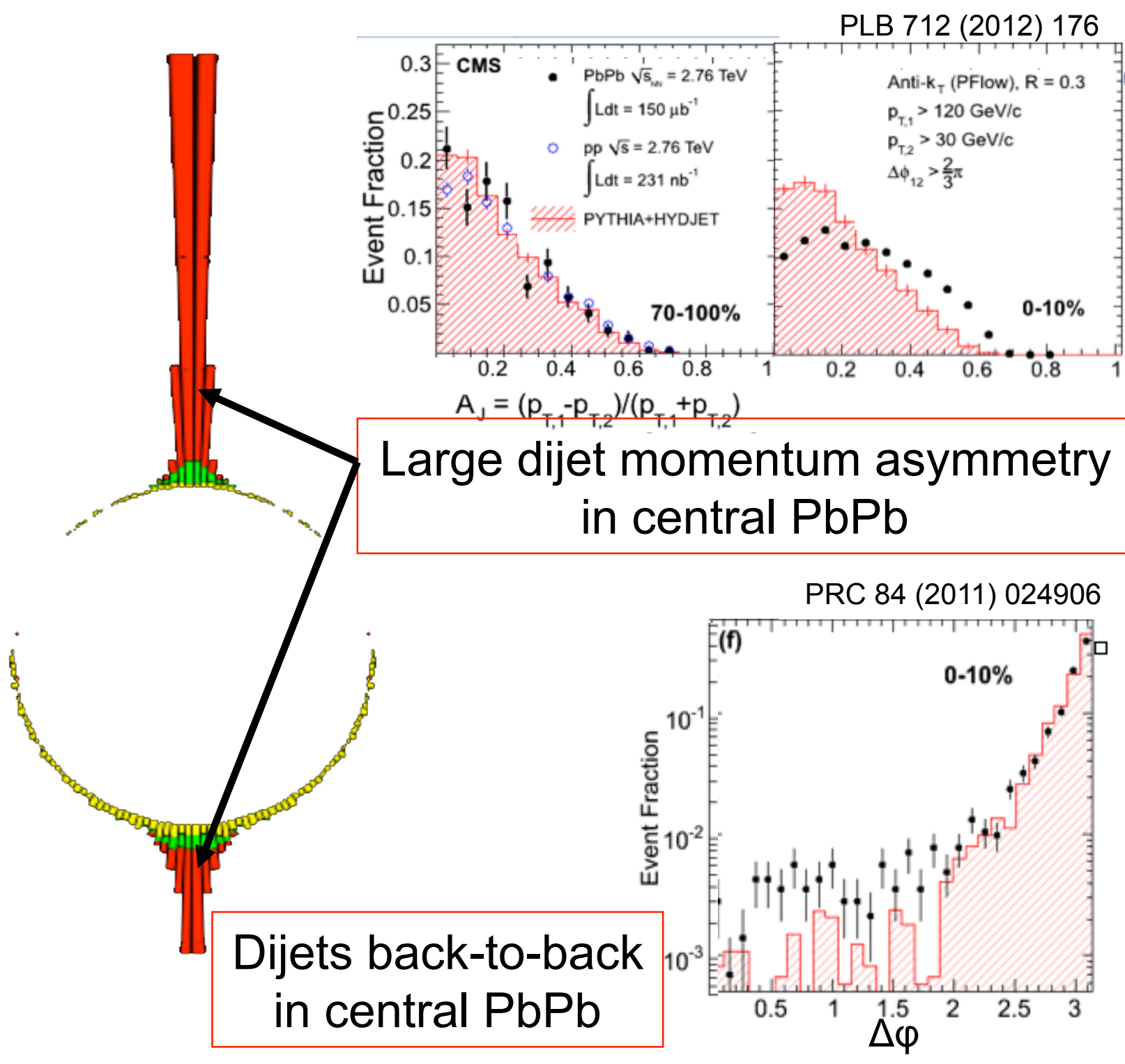




\section{$\mathrm{PbPb}$ dijet phenomenology}

$p_{\mathrm{T}}^{\|}=\sum_{\text {Tracks }}-p_{\mathrm{T}}^{\text {Track }} \cos \left(\phi_{\text {Track }}-\phi_{\text {Leading Jet }}\right)$

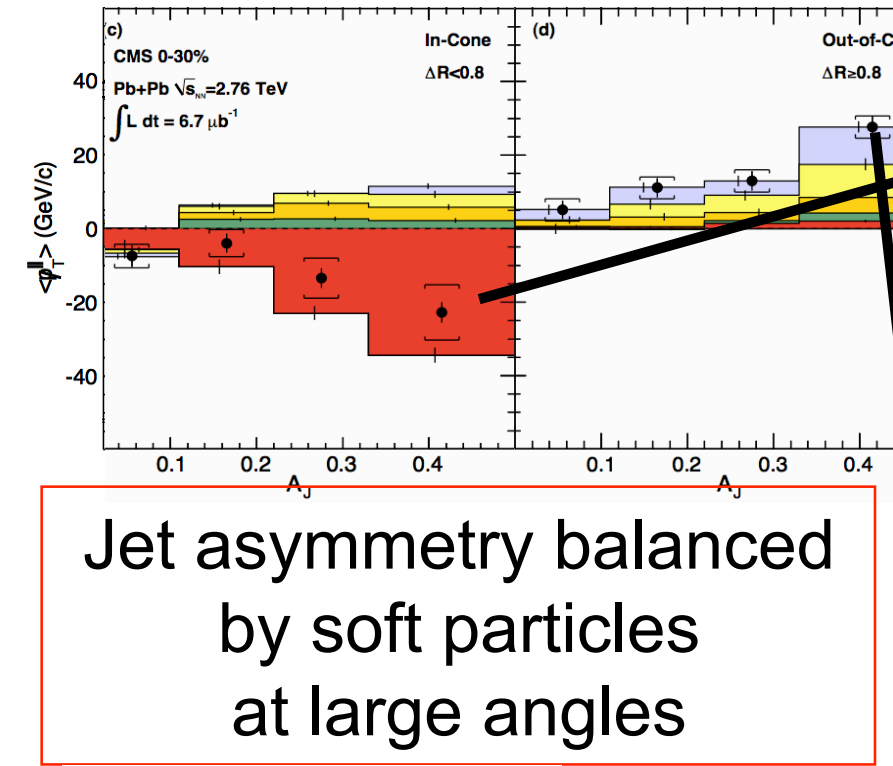

PRC 84 (2011) 024906

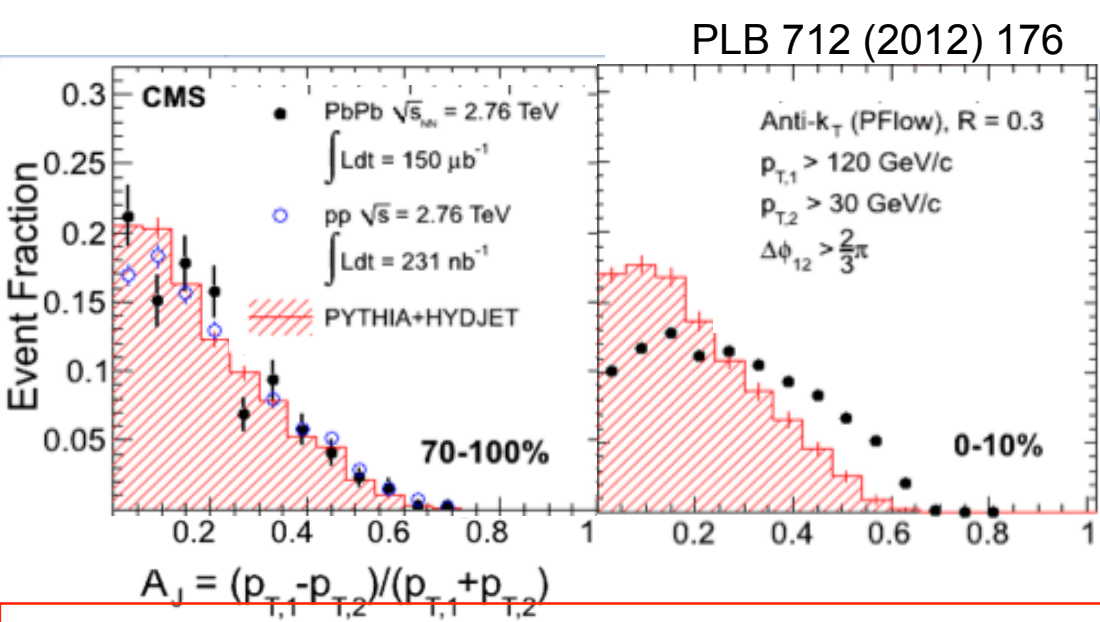

Large dijet momentum asymmetry in central $\mathrm{PbPb}$
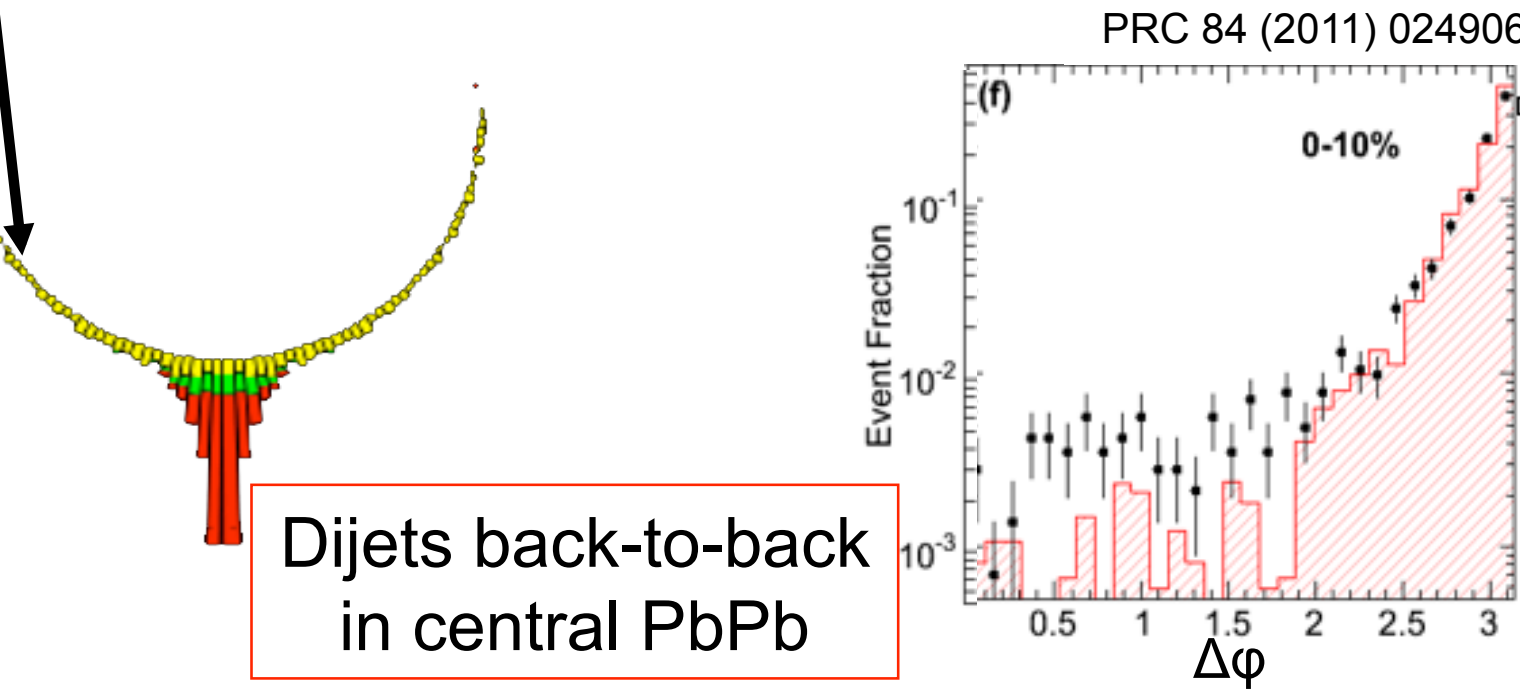


\section{Jet anatomy}

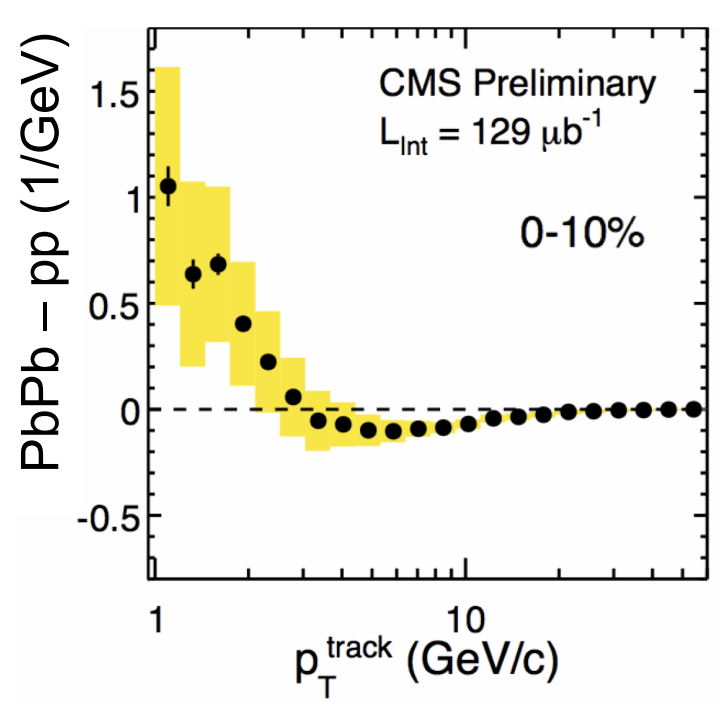

Fragmentation function difference $(\mathrm{PbPb}-\mathrm{pp})$ : Shows redistribution of particles in $p_{T}$
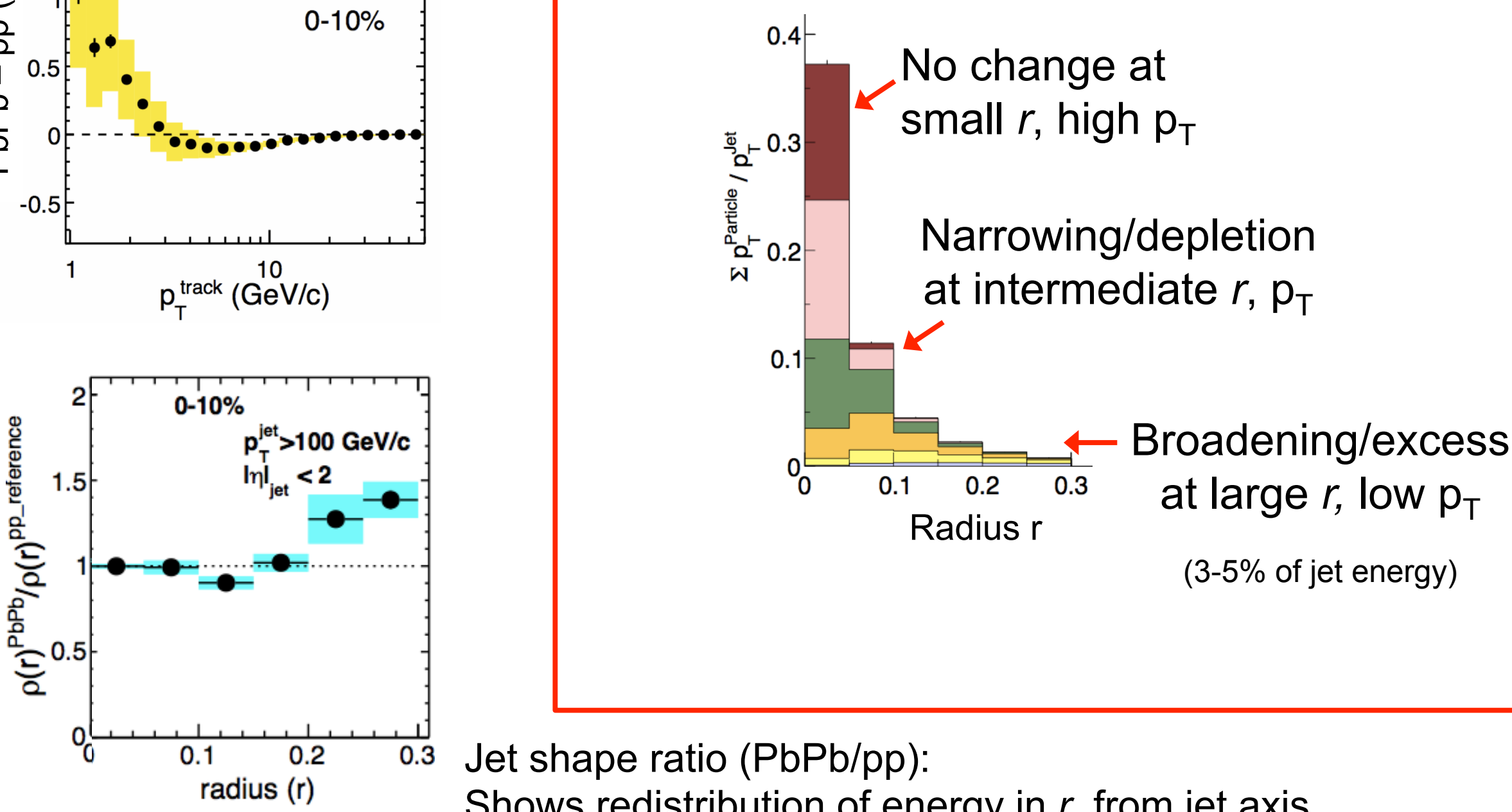

(3-5\% of jet energy)

Jet shape ratio $(\mathrm{PbPb} / \mathrm{pp})$ :

Shows redistribution of energy in $r$ from jet axis 


\section{$\gamma+$ jet: $u, d$ quark energy loss}

PLB 718 (2013) 773

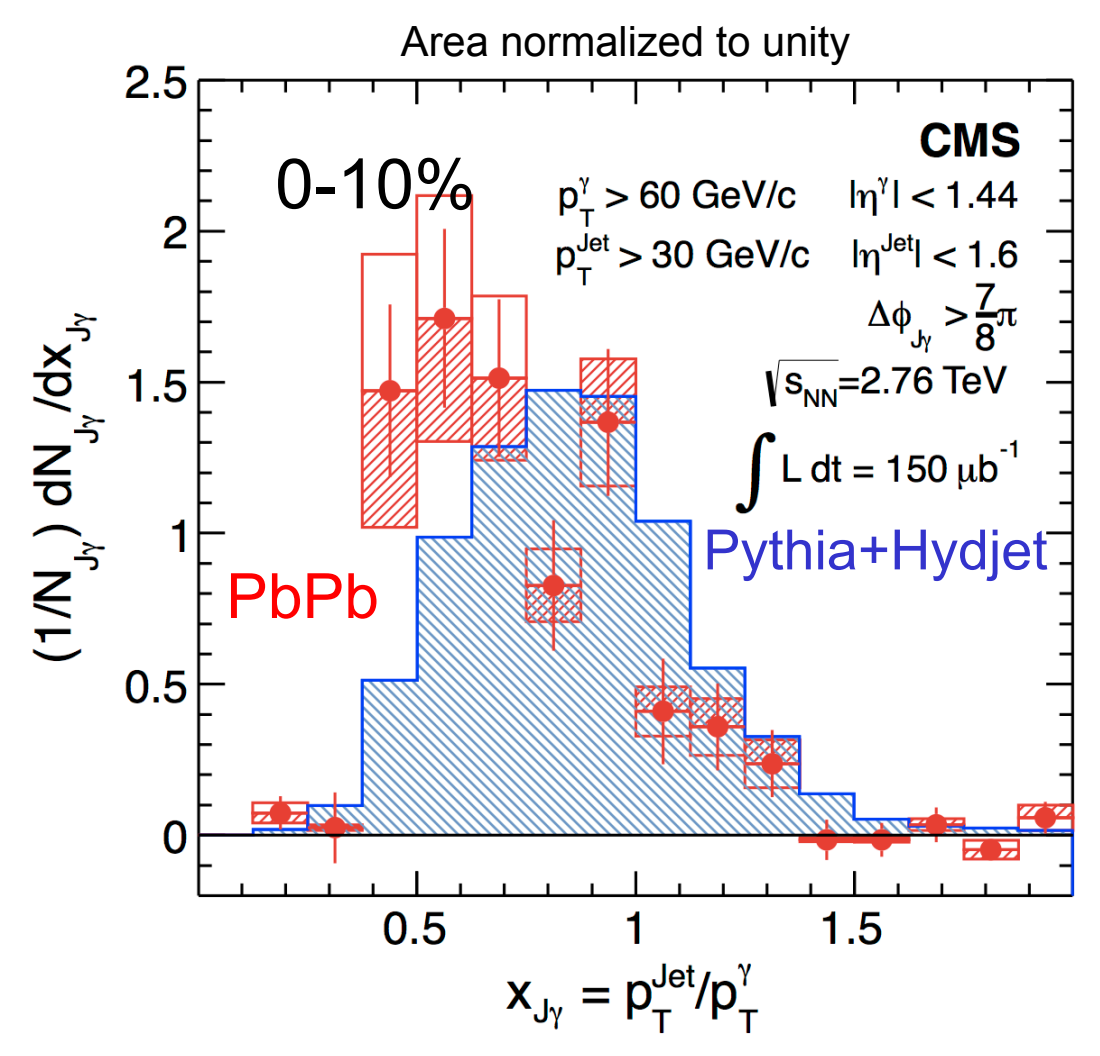

Photon-jet momentum balance

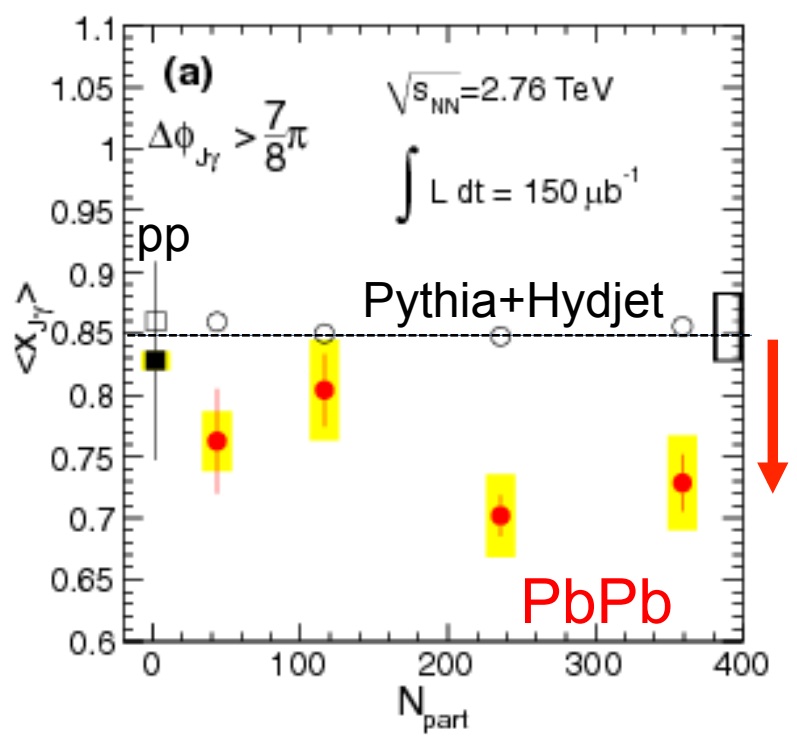

Jet-photon $\mathrm{p}_{\mathrm{T}}$ balance drops by $14 \%$

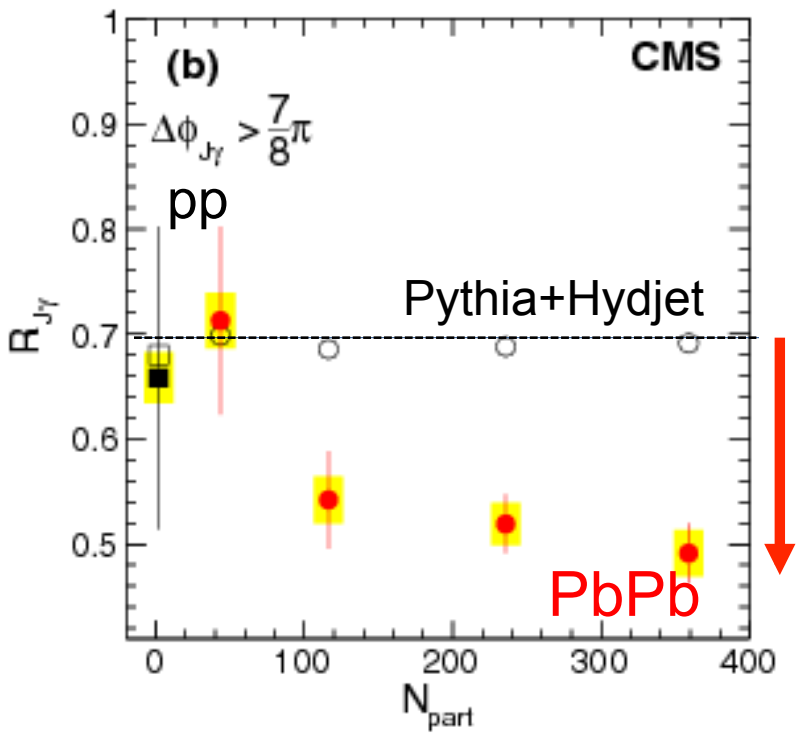

$20 \%$ of photons lose jet partner 


\section{$\mathrm{R}_{\mathrm{AA}}$ Zoo: Signal and Control}
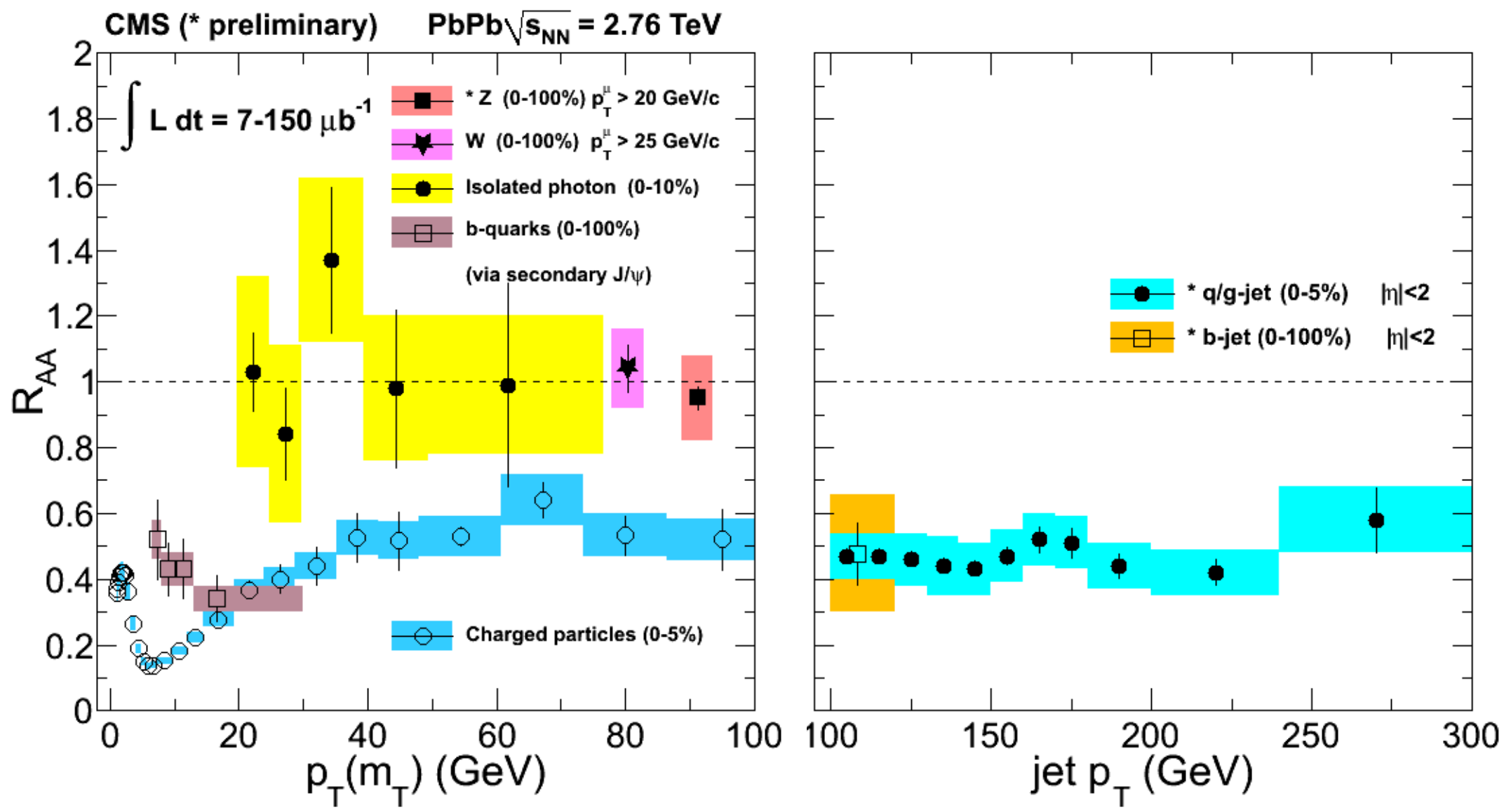

Vector-bosons show that hard scattering rates are under good control 


\section{Long-range correlations in $\mathrm{pp}$ and $\mathrm{pPb}$}

PUBlished FOR SISSA BY SPRINGER

RECEIVED: September 22, 2010

ACCEPTED: September 23, 2010

Published: September 27, 2010

Observation of long-range, near-side angular

correlations in proton-proton collisions at the LHC

Paysis Lettes \& 718 [2013) 395-814

Contents lists available at SciVerse ScienceDirect

Physics Letters B

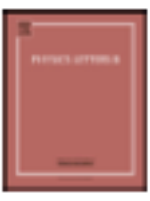

Observation of long-range, near-side angular correlations in $\mathrm{pPb}$ collisions at the $\mathrm{LHC}^{\text {मे }}$

CMS Collaboration *

cres suntoretind

Observed "flow-like" effects raise possibility of "final state" effects in pPb for many observables, incl. e.g. jets (but this is going to be subject of a talk at a different meeting...) 


\section{Correlations in $7 \mathrm{TeV}$ pp collisions}

Results based on $1 \mathrm{fb}^{-1}$, i.e. sampling 50billion pp events with high multiplicity trigger

\section{Intermediate $p_{\mathrm{T}}: 1-3 \mathrm{GeV} / \mathrm{c}$}

(b) MinBias, $1.0 \mathrm{GeV} / \mathrm{c}<\mathrm{p}_{\mathrm{T}}<3.0 \mathrm{GeV} / \mathrm{c}$

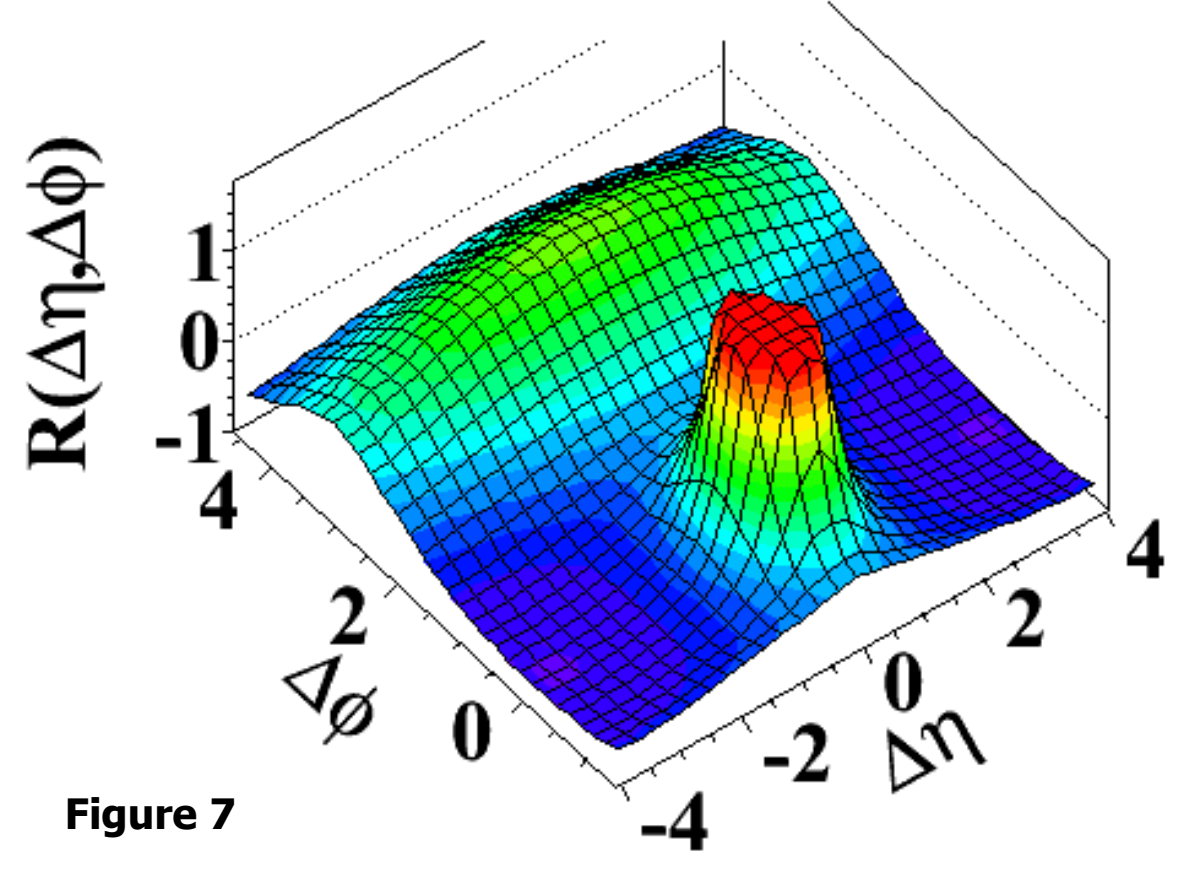

(d) $\mathrm{N}>110,1.0 \mathrm{GeV} / \mathrm{c}<\mathrm{p}_{\mathrm{T}}<3.0 \mathrm{GeV} / \mathrm{c}$

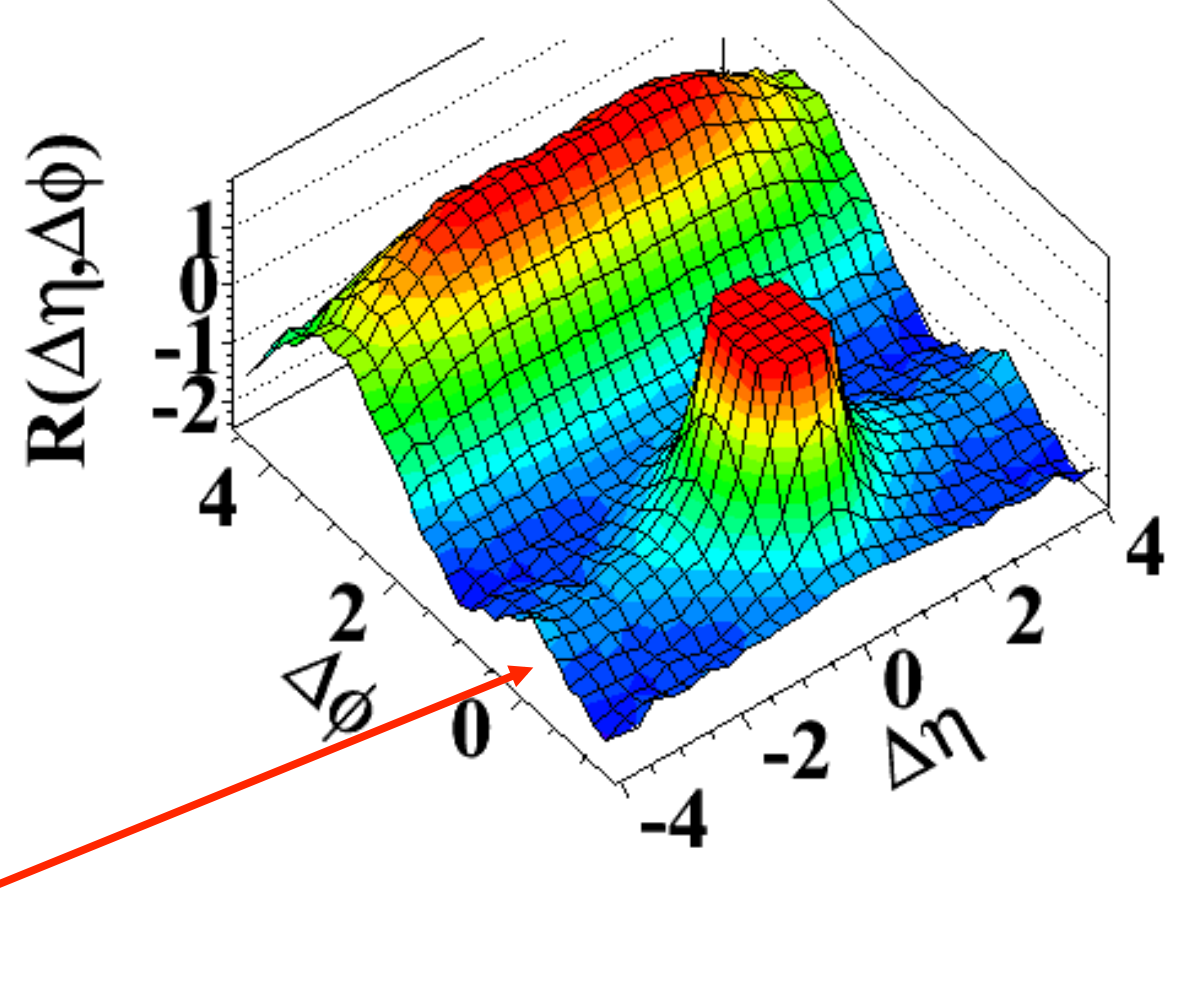

Pronounced structure at large $\delta \eta$ around $\delta \phi \sim 0$ ! 


\section{Multiplicity Evolution in pPb}

\section{Low multiplicity}

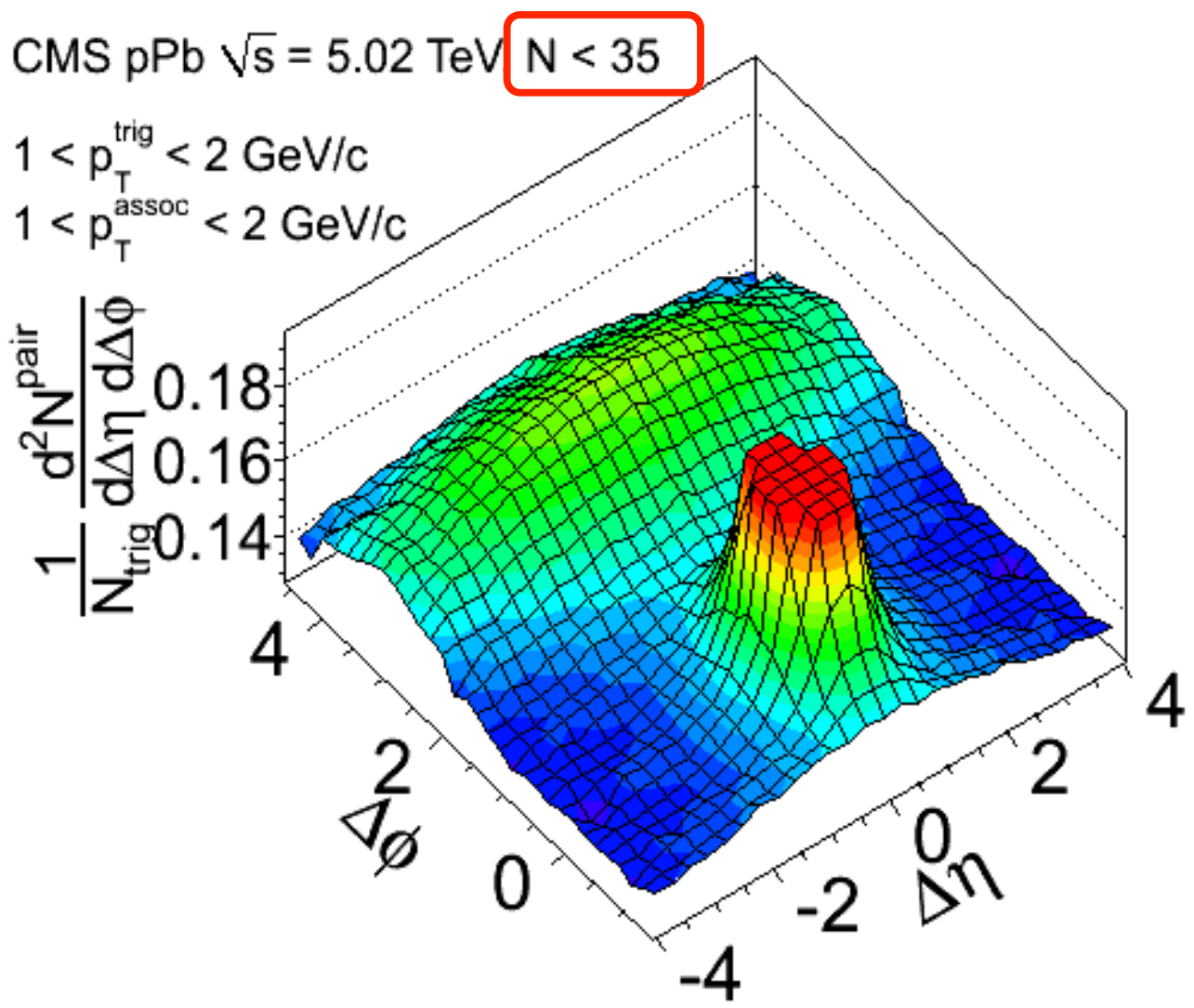

Divide into 4 multiplicity bins:

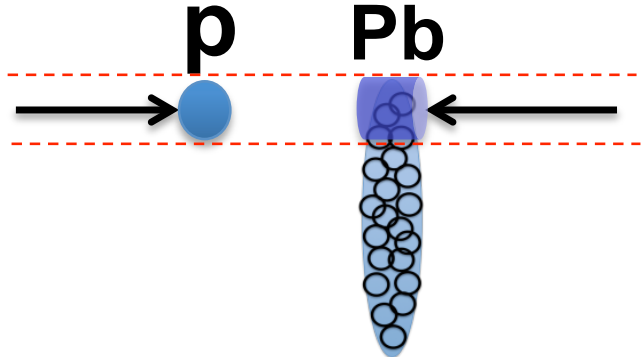

4

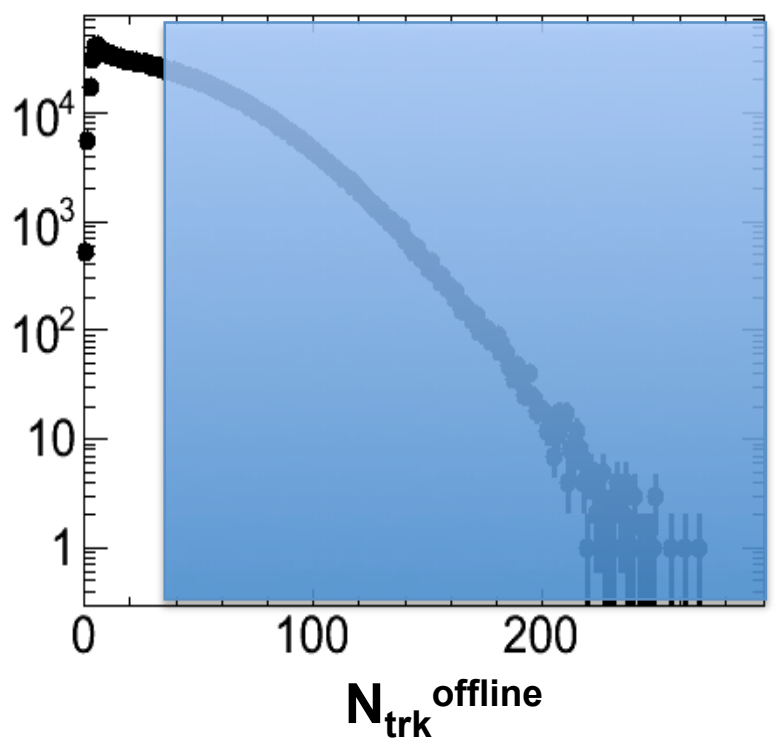




\section{Multiplicity Evolution in pPb}

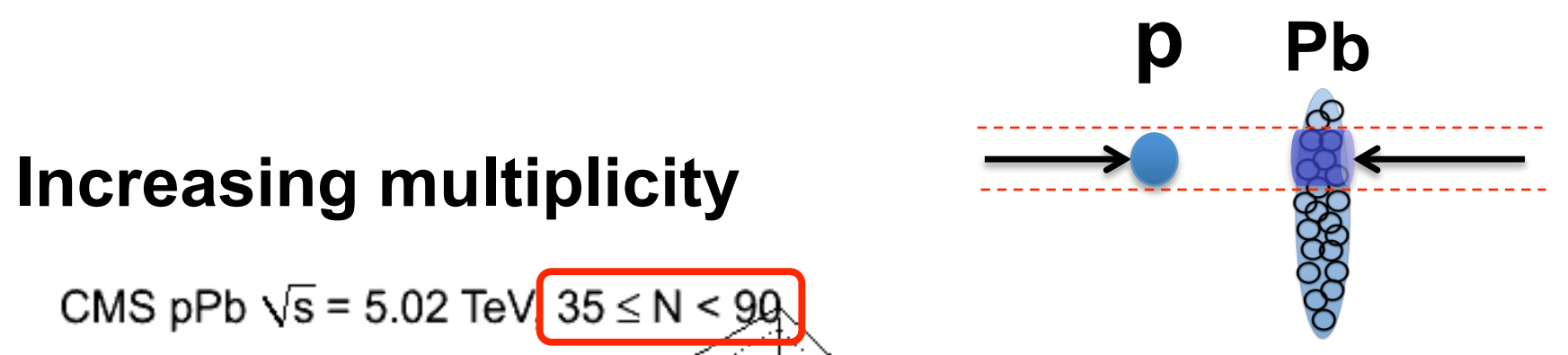

$1<\mathrm{p}_{\mathrm{T}}^{\text {trig }}<2 \mathrm{GeV} / \mathrm{c}$

$1<\mathrm{p}_{\mathrm{T}}^{\text {assoc }}<2 \mathrm{GeV} / \mathrm{c}$

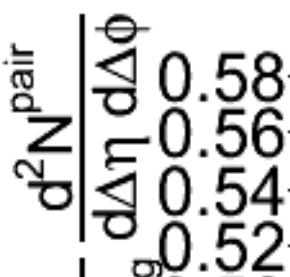

$-z_{z}=0.50=$

4

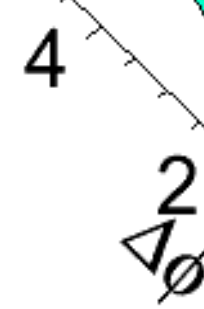

Divide into 4 multiplicity bins:

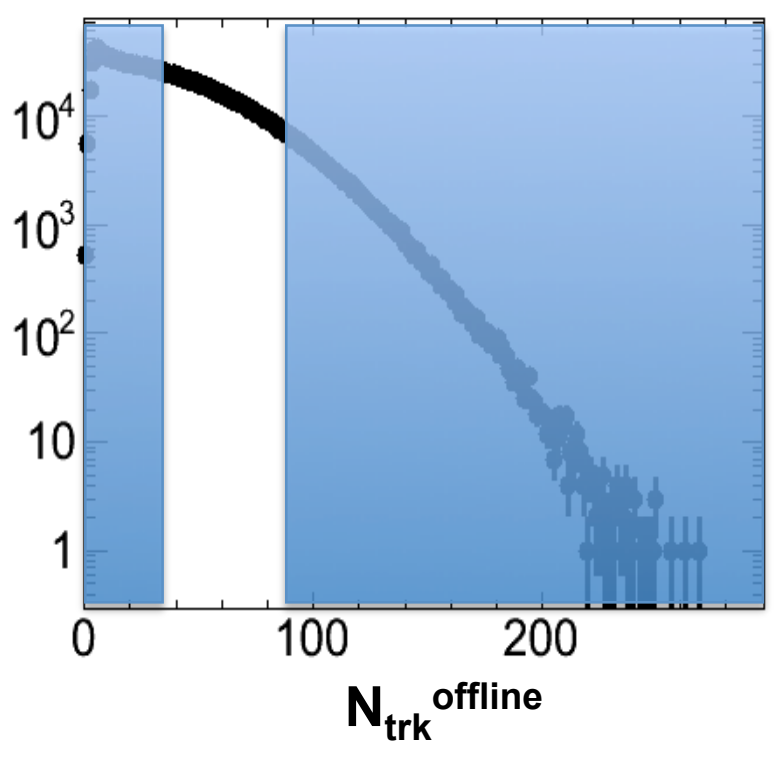




\section{Multiplicity Evolution in pPb}

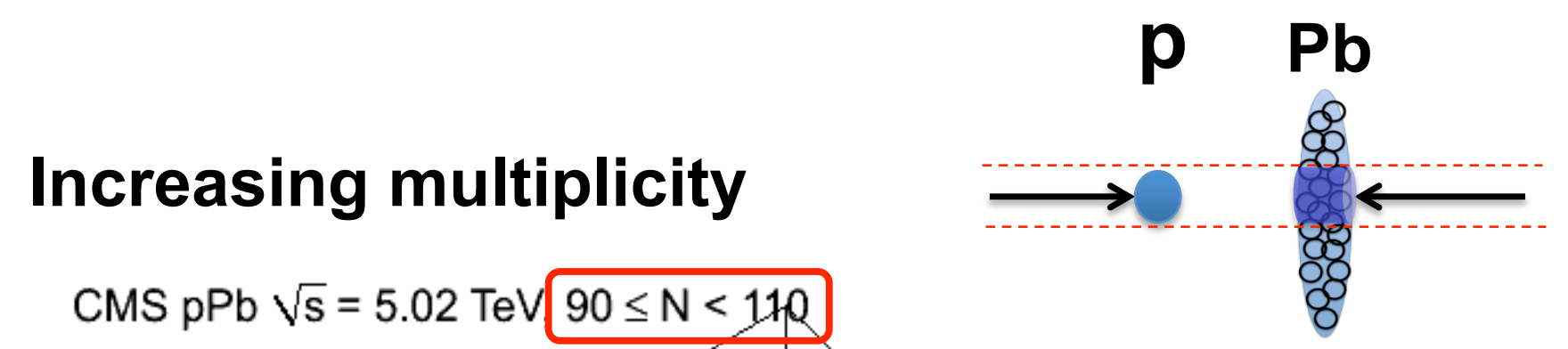

$1<\mathrm{p}_{\mathrm{T}}^{\text {trig }}<2 \mathrm{GeV} / \mathrm{c}$

$1<p_{T}^{\text {assoc }}<2 \mathrm{GeV} / \mathrm{c}$

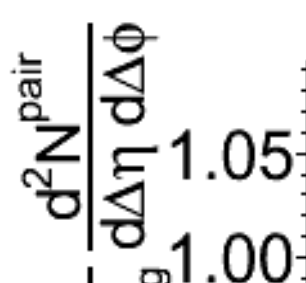

$-z^{1.00}$

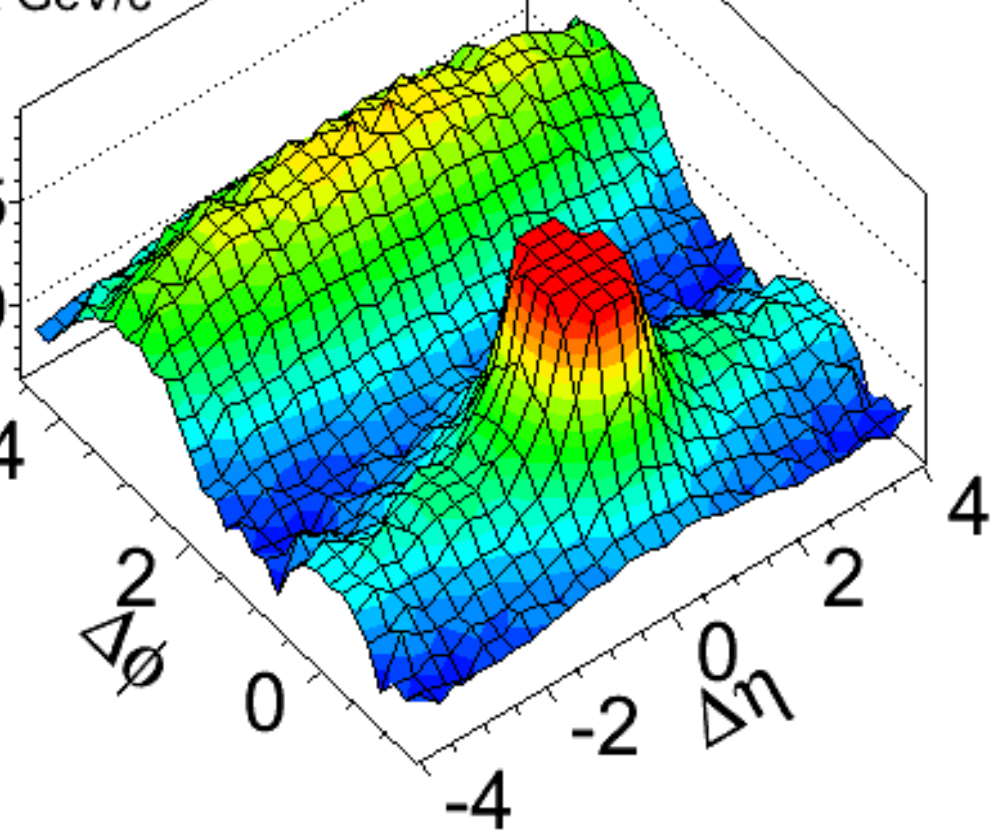

Divide into 4 multiplicity bins:

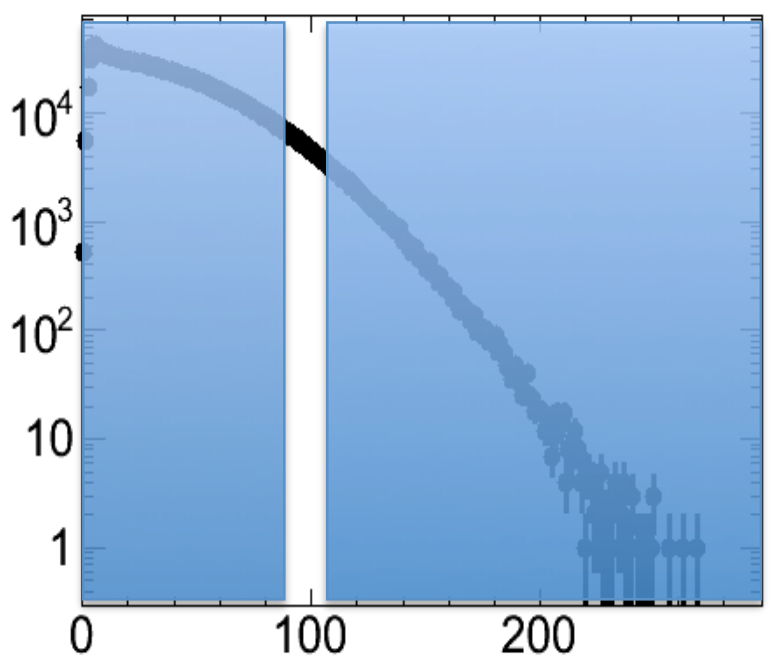




\section{Multiplicity Evolution in pPb}

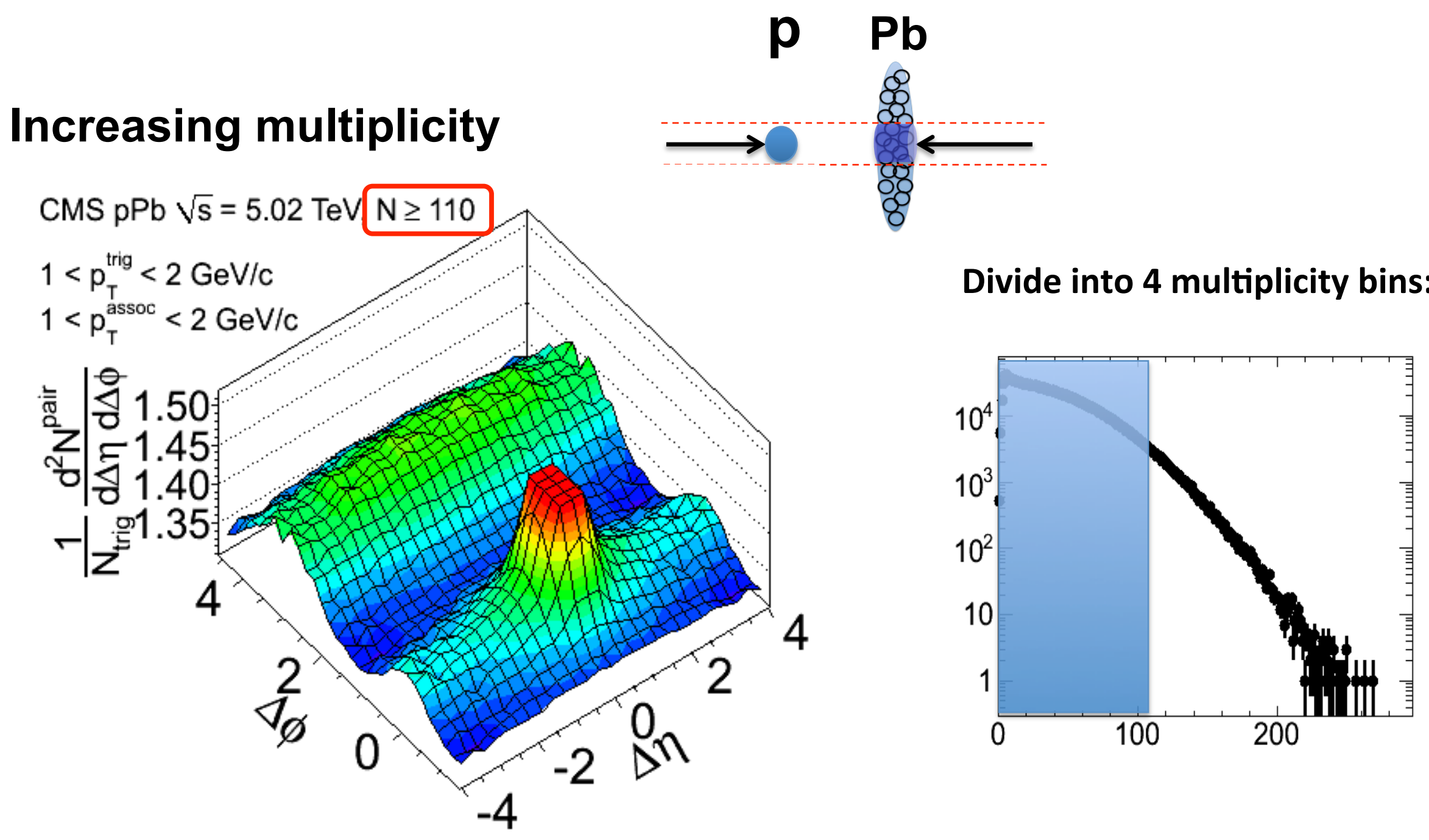




\section{Ridge Associated Yield}

ZYAM example

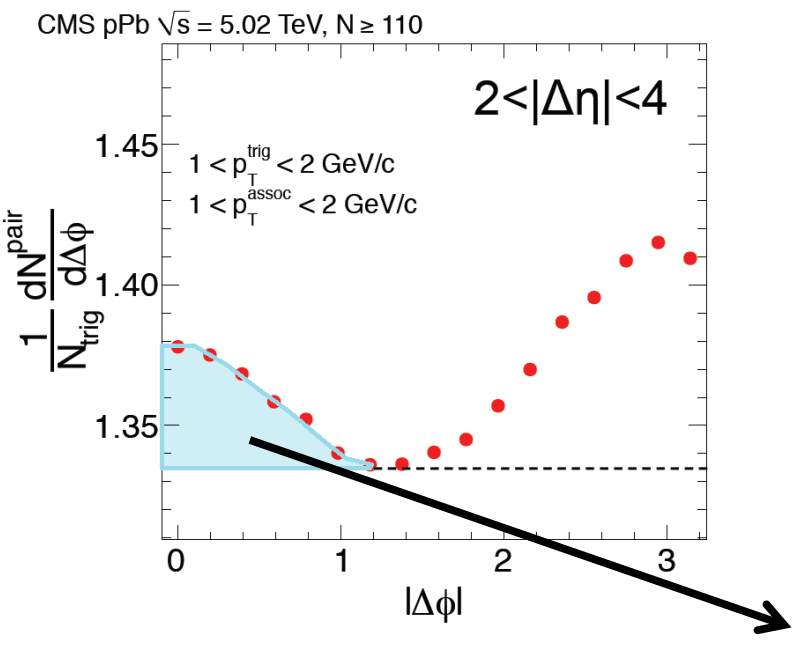

In the signal $(\mathrm{N}>110)$ region, the strength of the effect rises and falls with $p_{T}$
In the $p_{T}$ range where the yield is the strongest, the ridge turns on at $\mathrm{N} \approx 40$

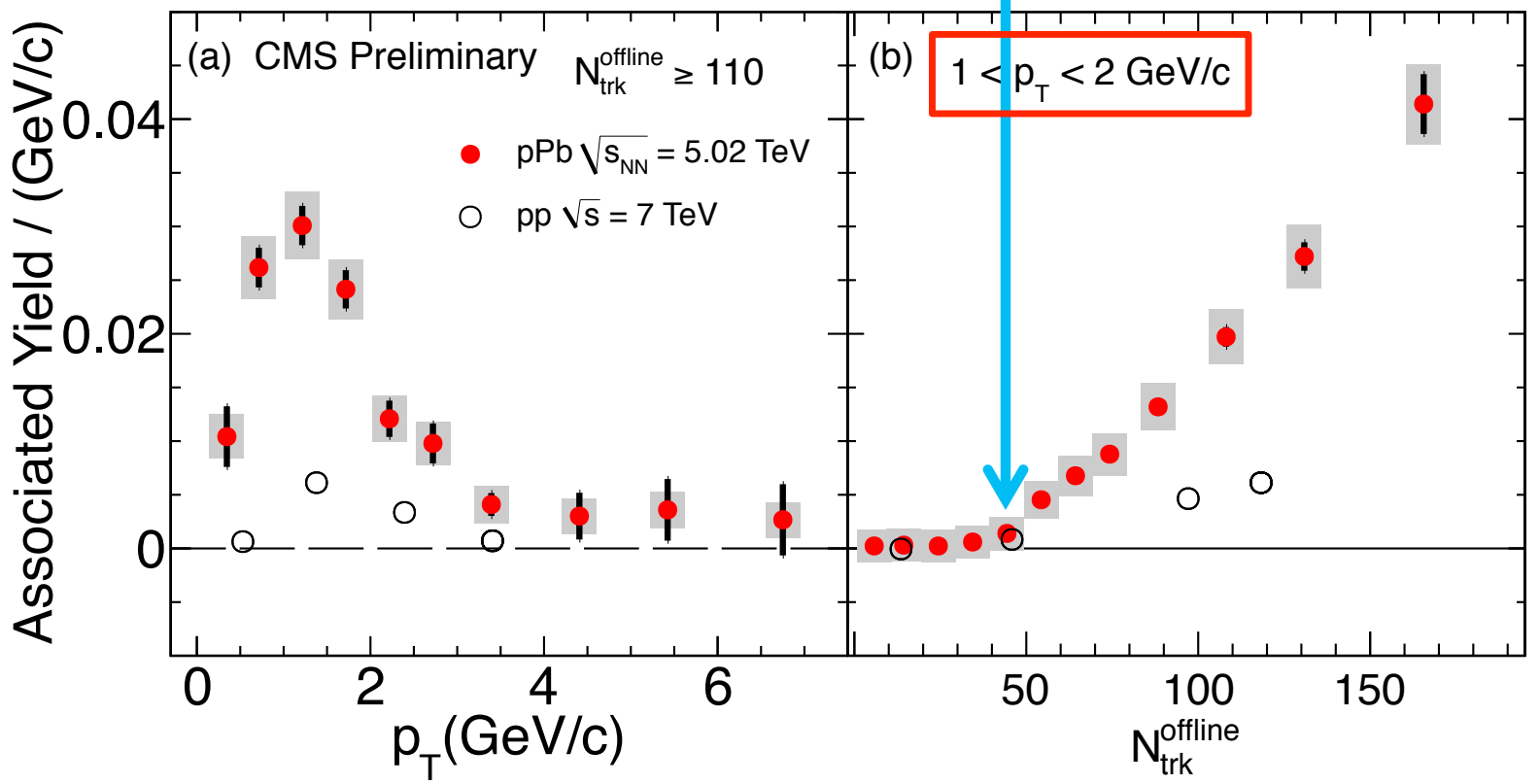




\section{New results on $\mathrm{pPb}$ correlations}

- Analysis of $2013 \mathrm{pPb}$ data set

- Including high multiplicity triggers sampling full luminosity of $31 / \mathrm{nb}$ (30000x pilot run stat's)

- Re-analysis of peripheral $\mathrm{PbPb}$ using identical reconstruction, event selection, analysis code, ...

- Comprehensive set of observables

- Associate yields (as in previous CMS pp and pPb analyses)

- $v_{2}$ and $v_{3}$ from two particle correlations ( $w / \eta$ gap)

- "Peripheral subtraction" a la ALICE, ATLAS

- $v_{2}\{4\}$ four-particle cumulants 


\section{"Centrality" (multiplicity density) binning}

n.b., particles are counted for $p_{T}>0.4 \mathrm{GeV} / \mathrm{c},|\eta|<2.4$

\begin{tabular}{l||l|l|l||l|l|l}
\hline \multicolumn{1}{c||}{} & \multicolumn{3}{c||}{ pPb data } & \multicolumn{3}{c}{ PbP data } \\
\hline$N_{\text {trk }}^{\text {offline }}$ bin & Fraction & $\left\langle N_{\text {trk }}^{\text {offline }}\right\rangle$ & $\left\langle N_{\text {trk }}^{\text {corrected }}\right\rangle$ & $\begin{array}{l}\langle\text { Centrality }\rangle \\
\pm \text { RMS }(\%)\end{array}$ & $\left\langle N_{\text {trk }}^{\text {offline }}\right\rangle$ & $\left\langle N_{\text {trk }}^{\text {corrected }}\right\rangle$ \\
\hline$[0, \infty)$ & 1.00 & 40 & $50 \pm 2$ & & & \\
{$[0,20)$} & 0.31 & 10 & $12 \pm 1$ & $92 \pm 4$ & 10 & $13 \pm 1$ \\
{$[20,30)$} & 0.14 & 25 & $30 \pm 1$ & $86 \pm 4$ & 24 & $30 \pm 1$ \\
{$[30,40)$} & 0.12 & 35 & $42 \pm 2$ & $83 \pm 4$ & 34 & $43 \pm 2$ \\
{$[40,50)$} & 0.10 & 45 & $54 \pm 2$ & $80 \pm 4$ & 44 & $55 \pm 2$ \\
{$[50,60)$} & 0.09 & 54 & $66 \pm 3$ & $78 \pm 3$ & 54 & $68 \pm 3$ \\
{$[60,80)$} & 0.12 & 69 & $84 \pm 4$ & $75 \pm 3$ & 69 & $87 \pm 4$ \\
{$[80,100)$} & 0.07 & 89 & $108 \pm 5$ & $72 \pm 3$ & 89 & $112 \pm 5$ \\
{$[100,120)$} & 0.03 & 109 & $132 \pm 6$ & $70 \pm 3$ & 109 & $137 \pm 6$ \\
{$[120,150)$} & 0.02 & 132 & $159 \pm 7$ & $67 \pm 3$ & 134 & $168 \pm 7$ \\
{$[150,185)$} & $4 \times 10^{-3}$ & 162 & $195 \pm 9$ & $64 \pm 3$ & 167 & $210 \pm 9$ \\
{$[185,220)$} & $5 \times 10^{-4}$ & 196 & $236 \pm 10$ & $62 \pm 2$ & 202 & $253 \pm 11$ \\
{$[220,260)$} & $6 \times 10^{-5}$ & 232 & $280 \pm 12$ & $59 \pm 2$ & 239 & $299 \pm 13$ \\
{$[260,300)$} & $3 \times 10^{-6}$ & 271 & $328 \pm 14$ & $57 \pm 2$ & 279 & $350 \pm 15$ \\
{$[300,350)$} & $1 \times 10^{-7}$ & 311 & $374 \pm 16$ & $55 \pm 2$ & 324 & $405 \pm 18$ \\
\hline
\end{tabular}
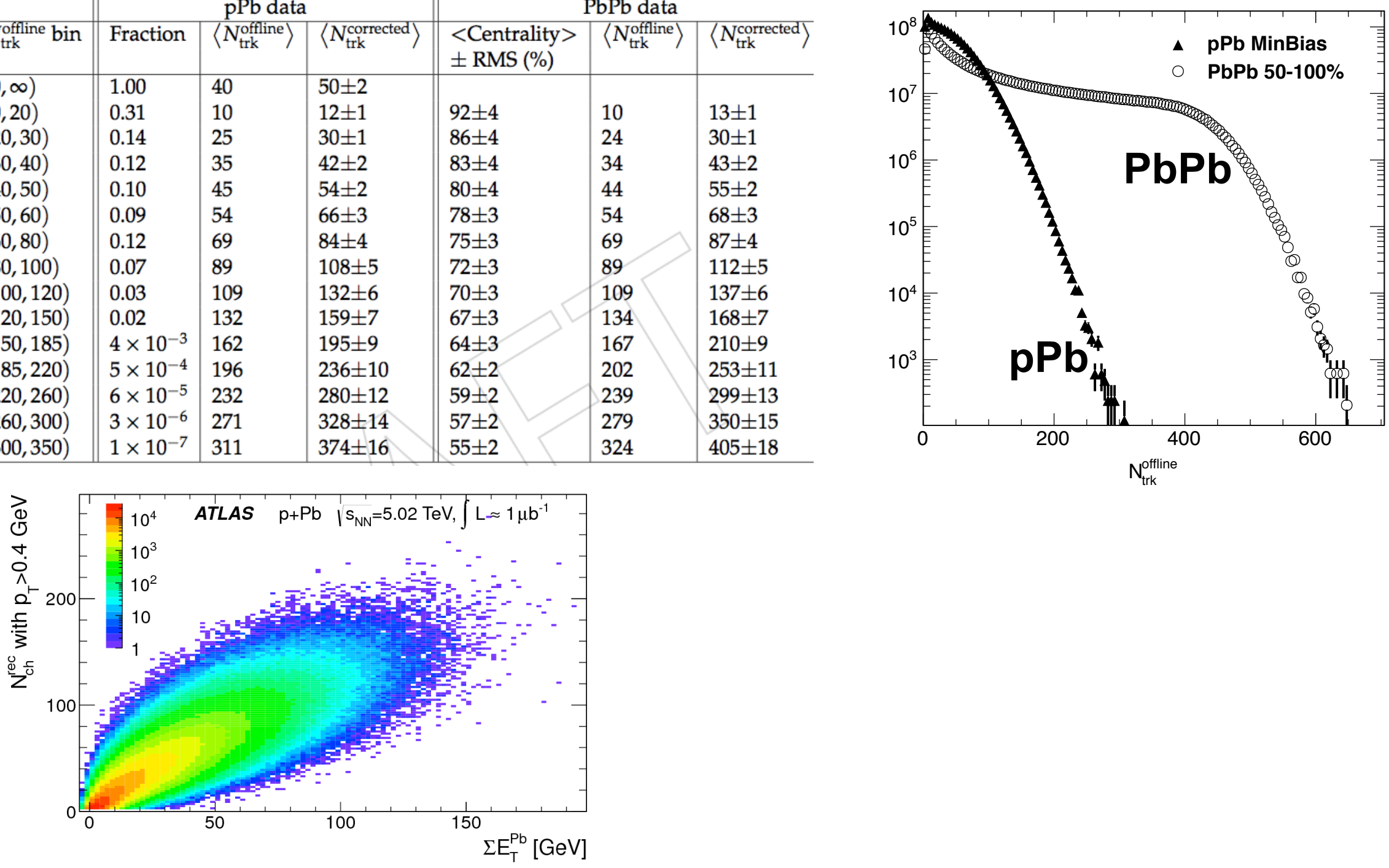


\section{$\mathrm{pPb}$ vs $\mathrm{PbPb}$}

CMS Preliminary PbPb $\sqrt{\mathrm{s}_{\mathrm{NN}}}=2.76 \mathrm{TeV}, 220 \leq \mathrm{N}<260$

$\mathbf{P b P b}$
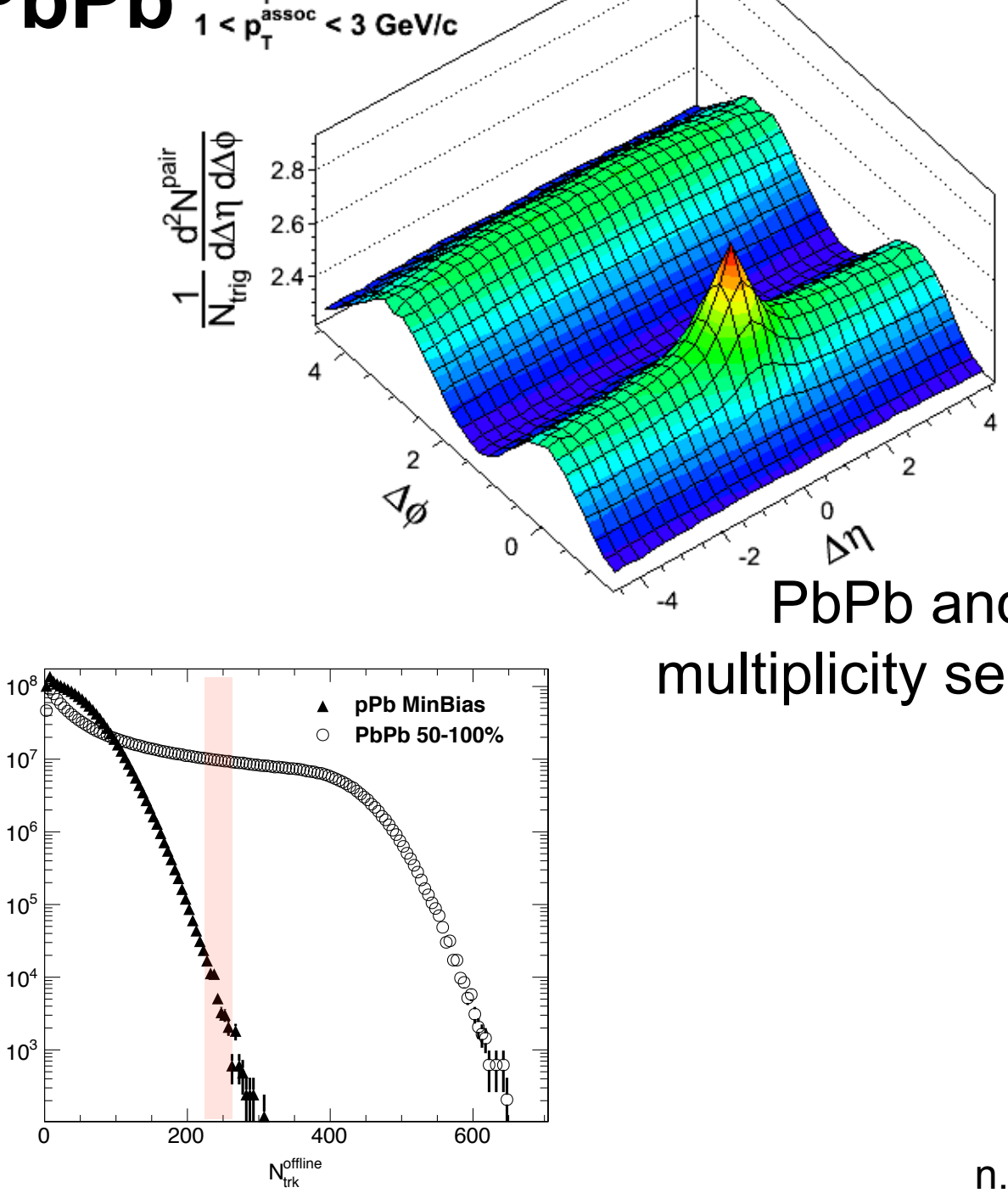

CMS Preliminary pPb $\sqrt{\mathrm{S}_{\mathrm{NN}}}=5.02 \mathrm{TeV}, 220 \leq \mathrm{N}<260$

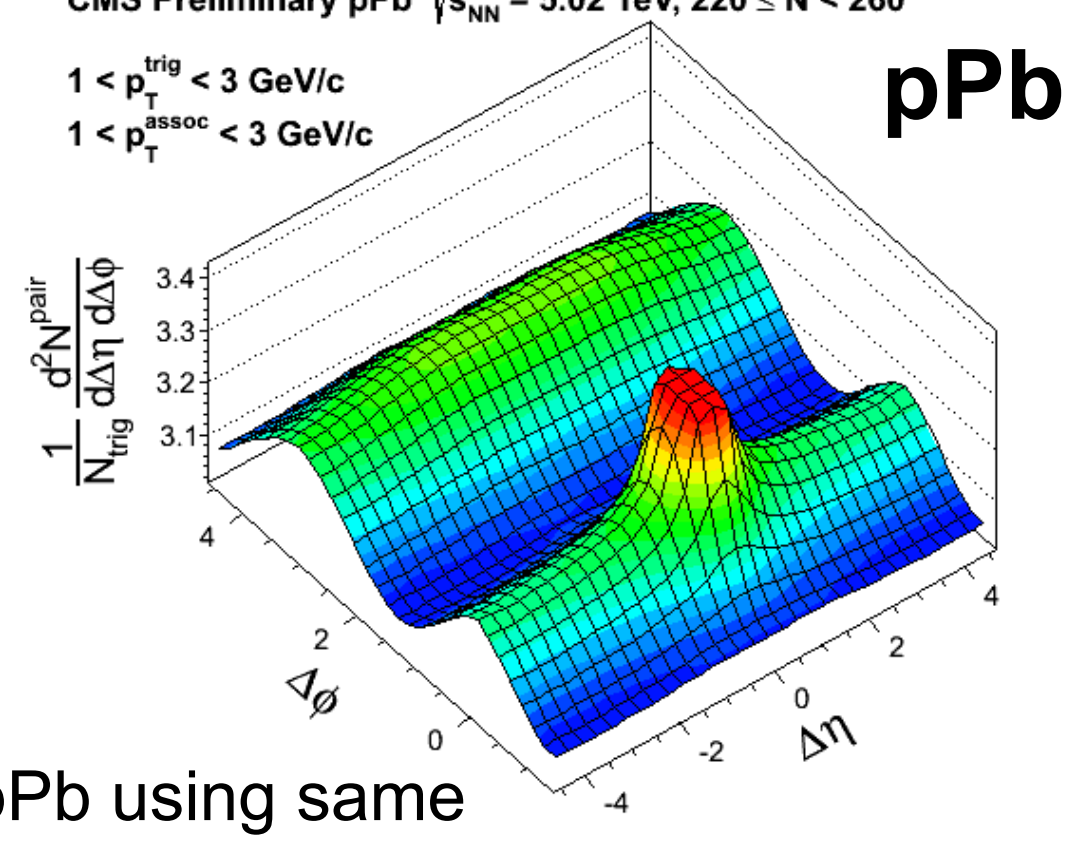
multiplicity selection, $220<\mathrm{N}<260$ 


\section{Multiplicity dependence}

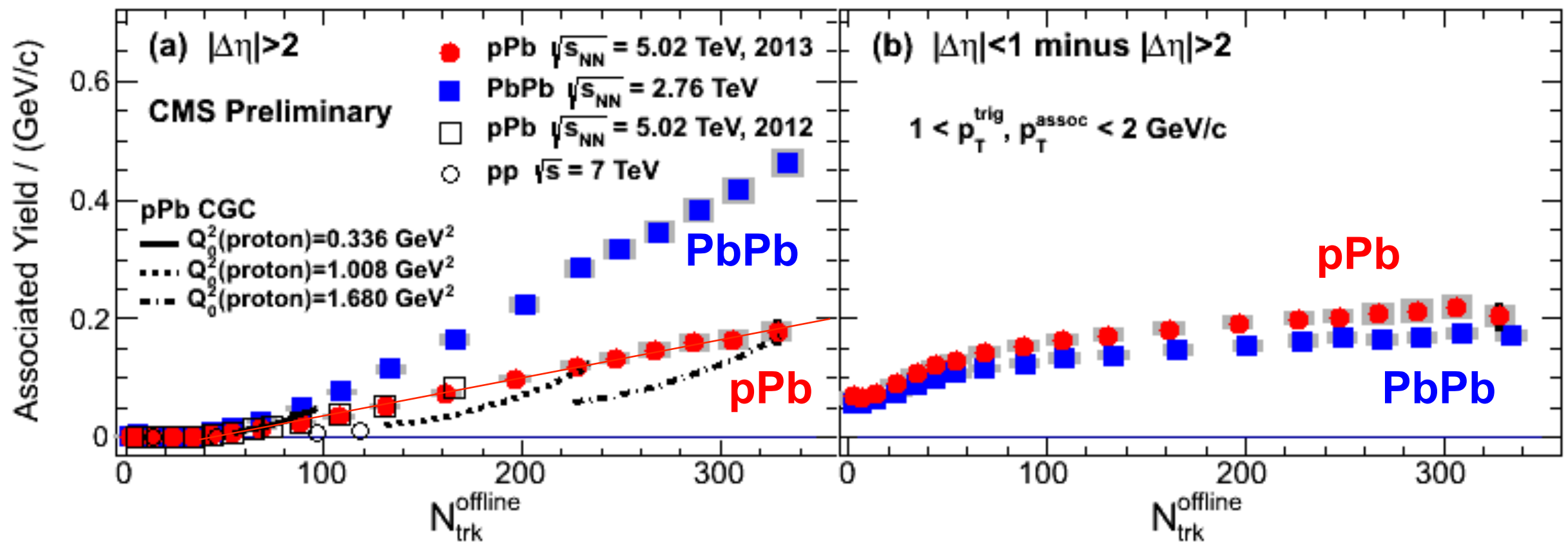

"Long-range" associated yield: "Jet" yield: Similar multiplicity dependence for $\mathrm{pPb}$ and $\mathrm{PbPb}$

Difference in absolute yield 


\section{$4^{\text {th }}$ order cumulants in multiplicity bins}

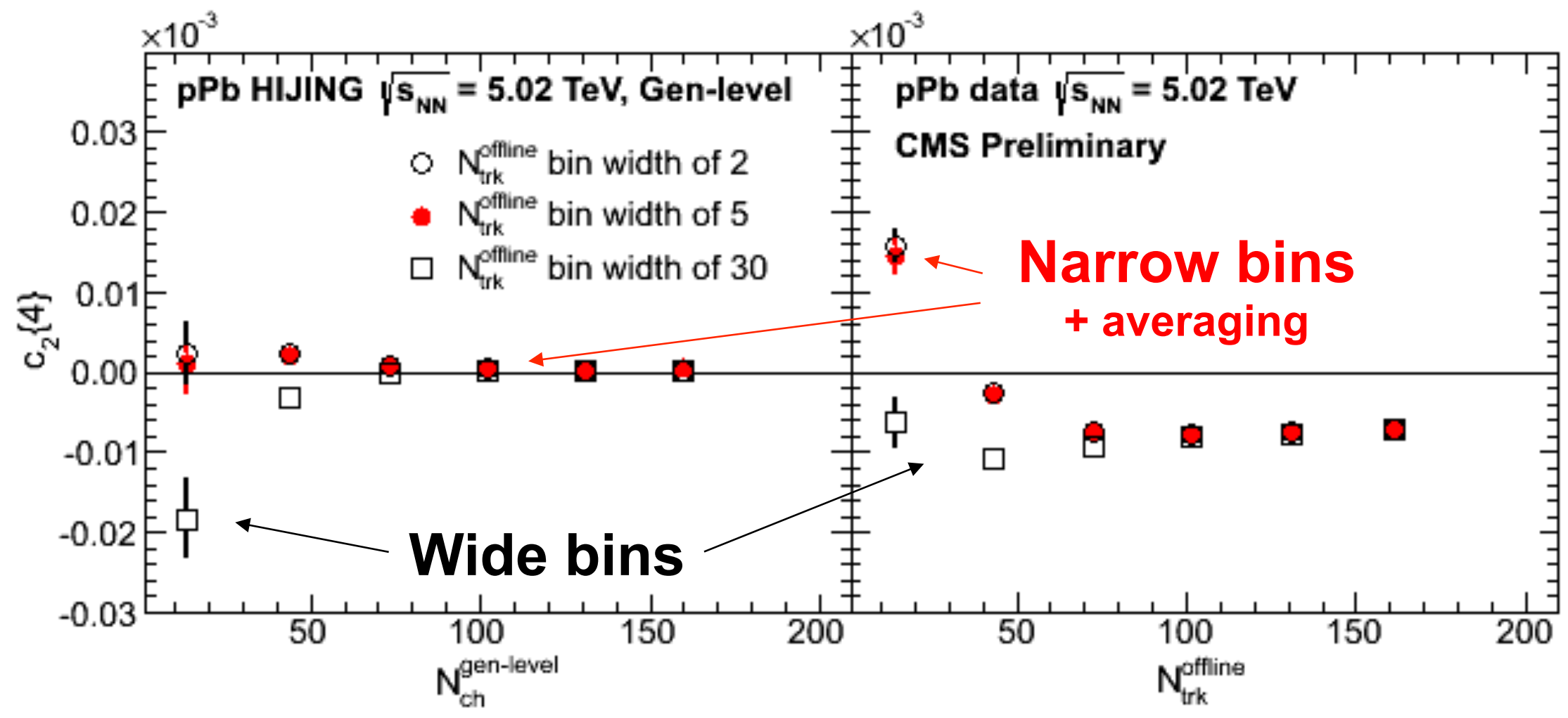

Cumulant analysis in bins of multiplicity Need to consider bin widths carefully CMS analysis: (narrow bins + averaging) 


\section{$\mathrm{v}_{2}$ in $\mathrm{pPb}$ and $\mathrm{PbPb}$}

Dash-dot line: peripheral subtracted

multiplicity

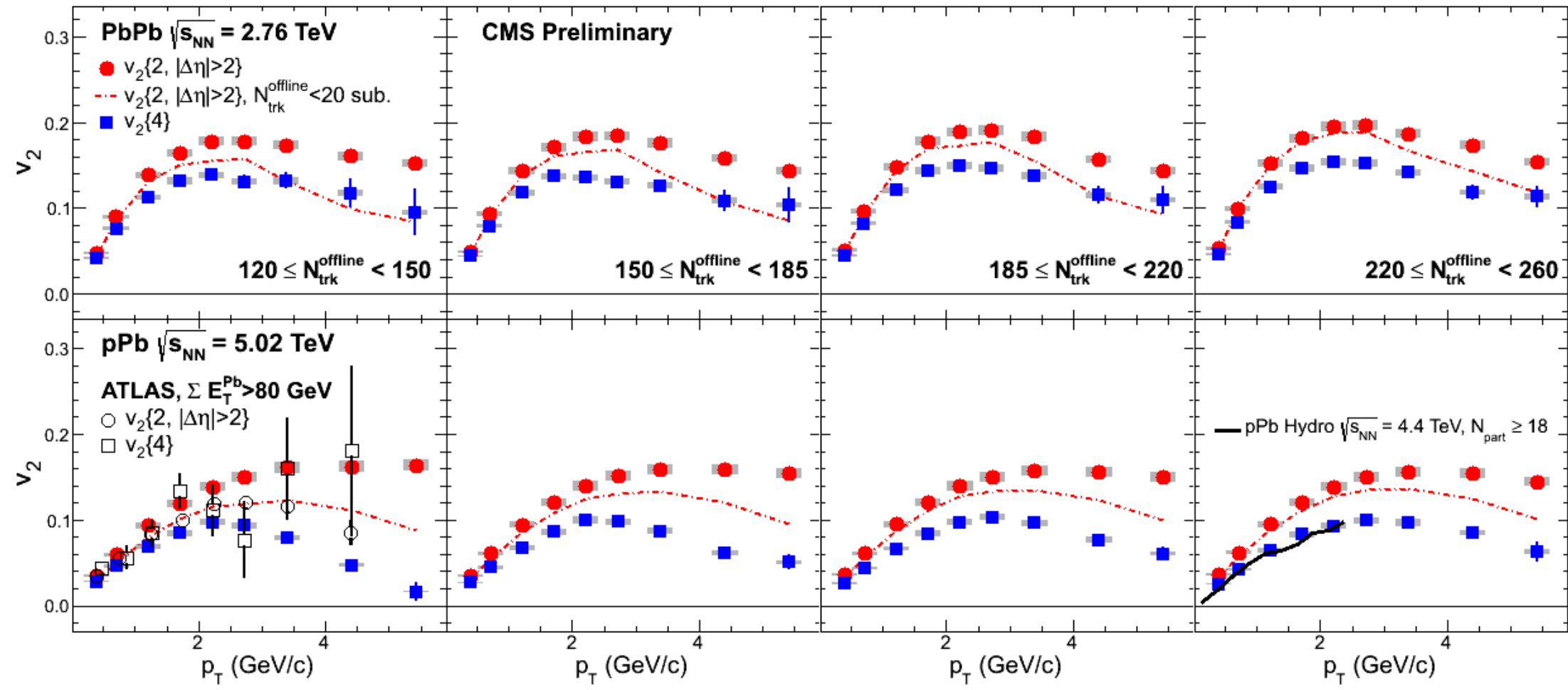

$\mathrm{PbPb}$

$\mathrm{pPb}$

$\mathrm{v}_{2}$ shows similar shape in $\mathrm{pPb}$ and $\mathrm{PbPb}$, but is smaller in $\mathrm{pPb}$ $v_{2}\{4\}$ is only $20 \%$ smaller than $v_{2}\{2\}$ below $2 \mathrm{GeV} / \mathrm{c}$ "Peripheral subtraction" has small effect at high multiplicity 


\section{$\mathrm{v}_{2}$ in $\mathrm{pPb}$ and $\mathrm{PbPb}$}

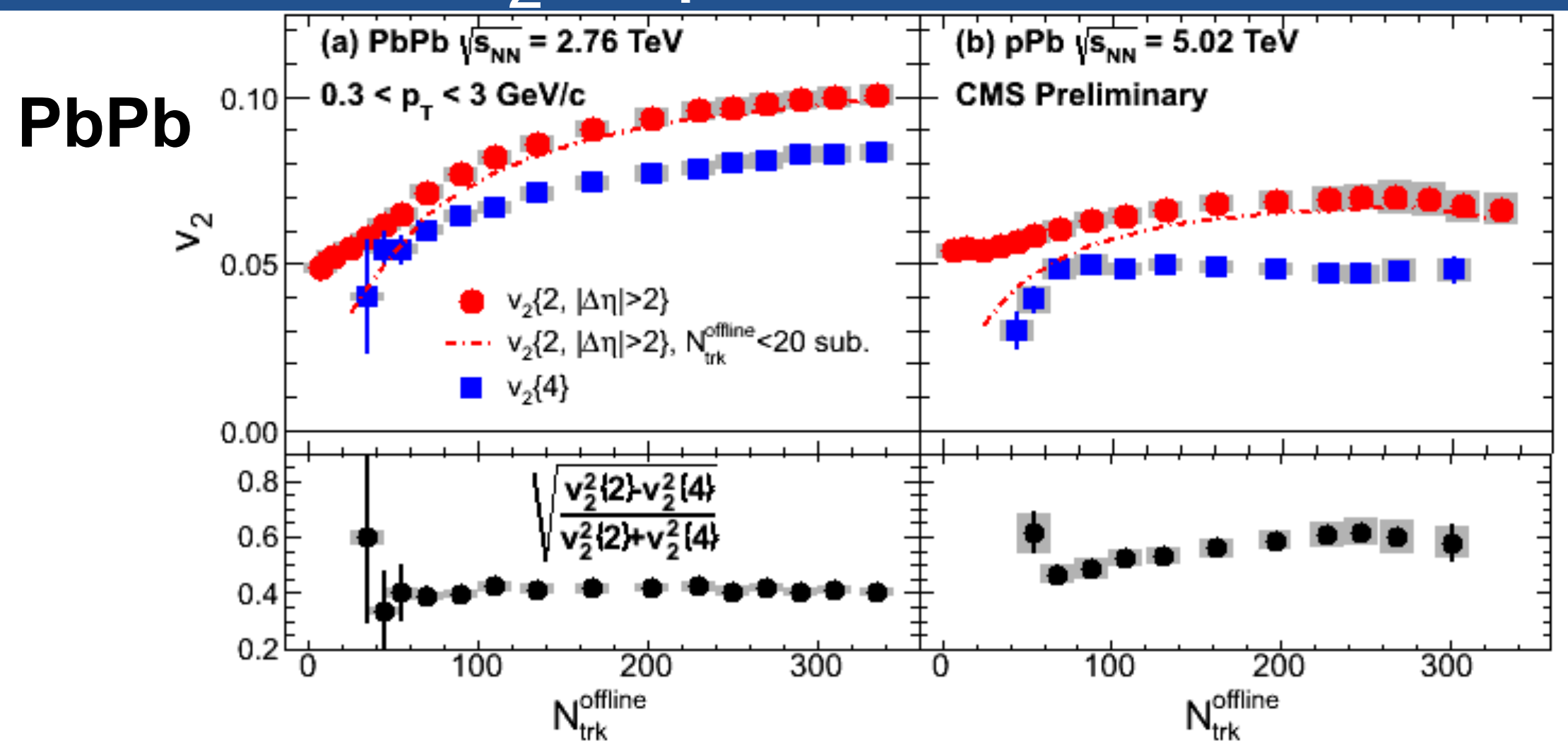

$\mathrm{pPb}$

$\mathrm{v}_{2}$ smaller in $\mathrm{pPb}$ than $\mathrm{PbPb}$

$\mathrm{v}_{2}\{4\}$ drops at low multiplicity

"Peripheral subtraction" has small effect at high multiplicity 


\section{$\mathrm{v}_{2}$ in $\mathrm{pPb}$ and $\mathrm{PbPb}$}

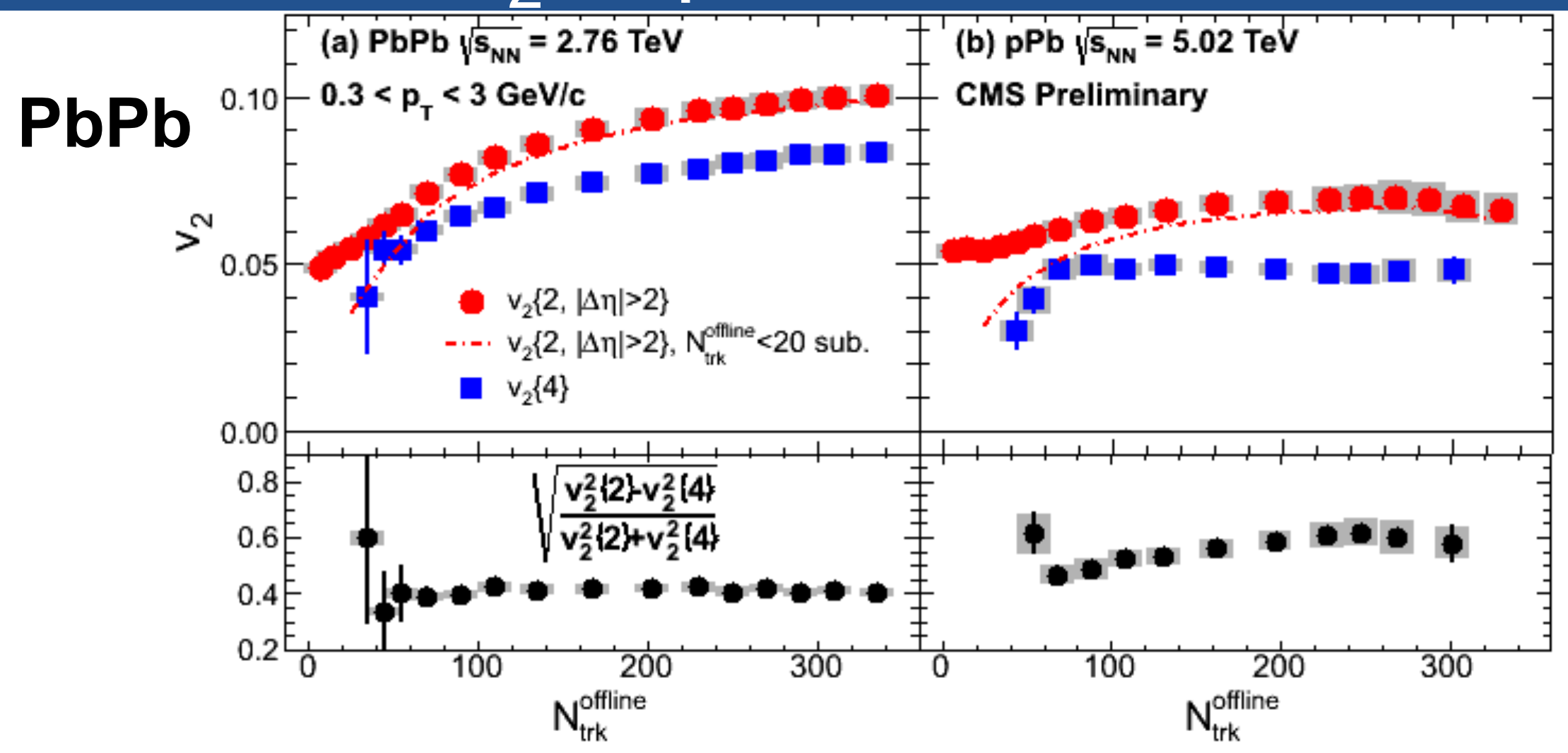

$\mathrm{pPb}$

"Fluctuations" larger in pPb, $v_{2}\{2\}=\sqrt{\left\langle v_{2}>^{2}+\sigma_{v_{2}}^{2}\right.}$
$v_{2}\{4\}=\sqrt{\left\langle v_{2}>^{2}-\sigma_{v_{2}}^{2}\right.}, \quad \frac{\sigma_{v_{2}}}{v_{2}}=\sqrt{\frac{v_{2}^{2}\{2\}-v_{2}^{2}\{4\}}{v_{2}^{2}\{2\}+v_{2}^{2}\{4\}}}$. with moderate multiplicity dependence 


\section{$\mathrm{v}_{3}$ in $\mathrm{pPb}$ and $\mathrm{PbPb}$}

Dash-dot line: peripheral subtracted

\section{multiplicity}

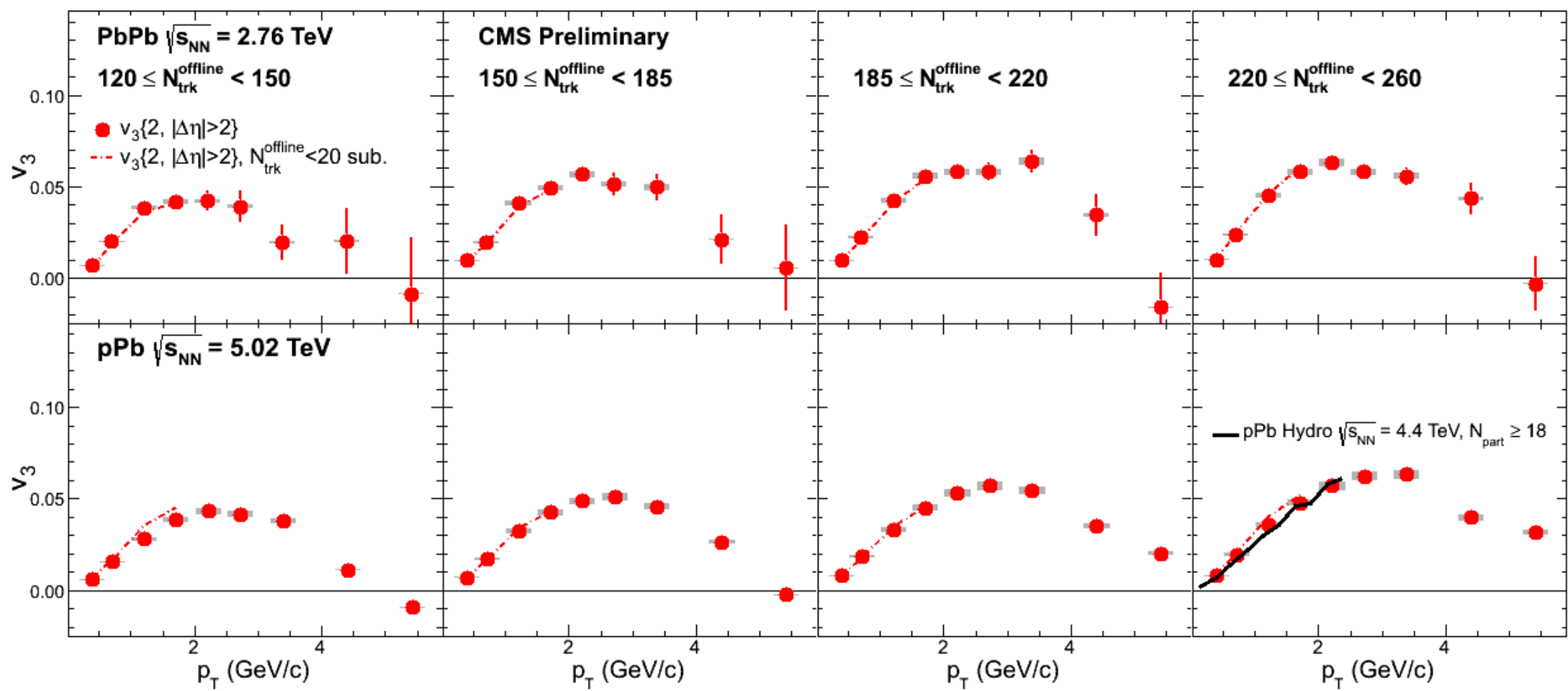

$\mathrm{PbPb}$

$\mathrm{pPb}$

$\mathrm{v}_{3}$ has similar shape in $\mathrm{pPb}$ and $\mathrm{PbPb}$; magnitude comparable "Peripheral subtraction" makes essentially no difference

Hydro prediction: Bozek, $v_{3}\{P P\}$, not including fluctuations 


\section{$\mathrm{V}_{3}$ in $\mathrm{pPb}$ and $\mathrm{PbPb}$}

$\mathrm{PbPb}$

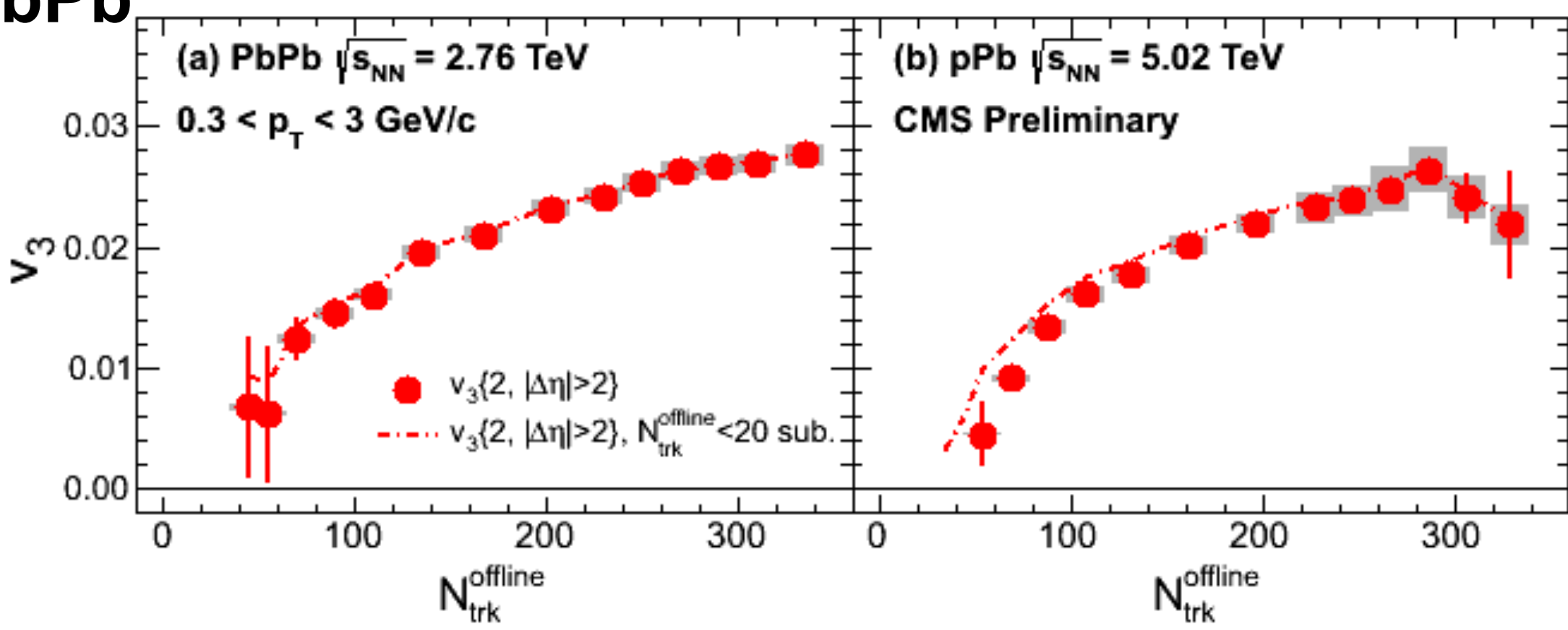

$\mathrm{pPb}$

$\mathrm{v}_{3}$ shows similar shape in $\mathrm{pPb}$ and $\mathrm{PbPb}$; magnitude comparable

"Peripheral subtraction" makes essentially no difference 


\section{$\mathrm{v}_{2}$ comparison with ATLAS in $\mathrm{pPb}$}

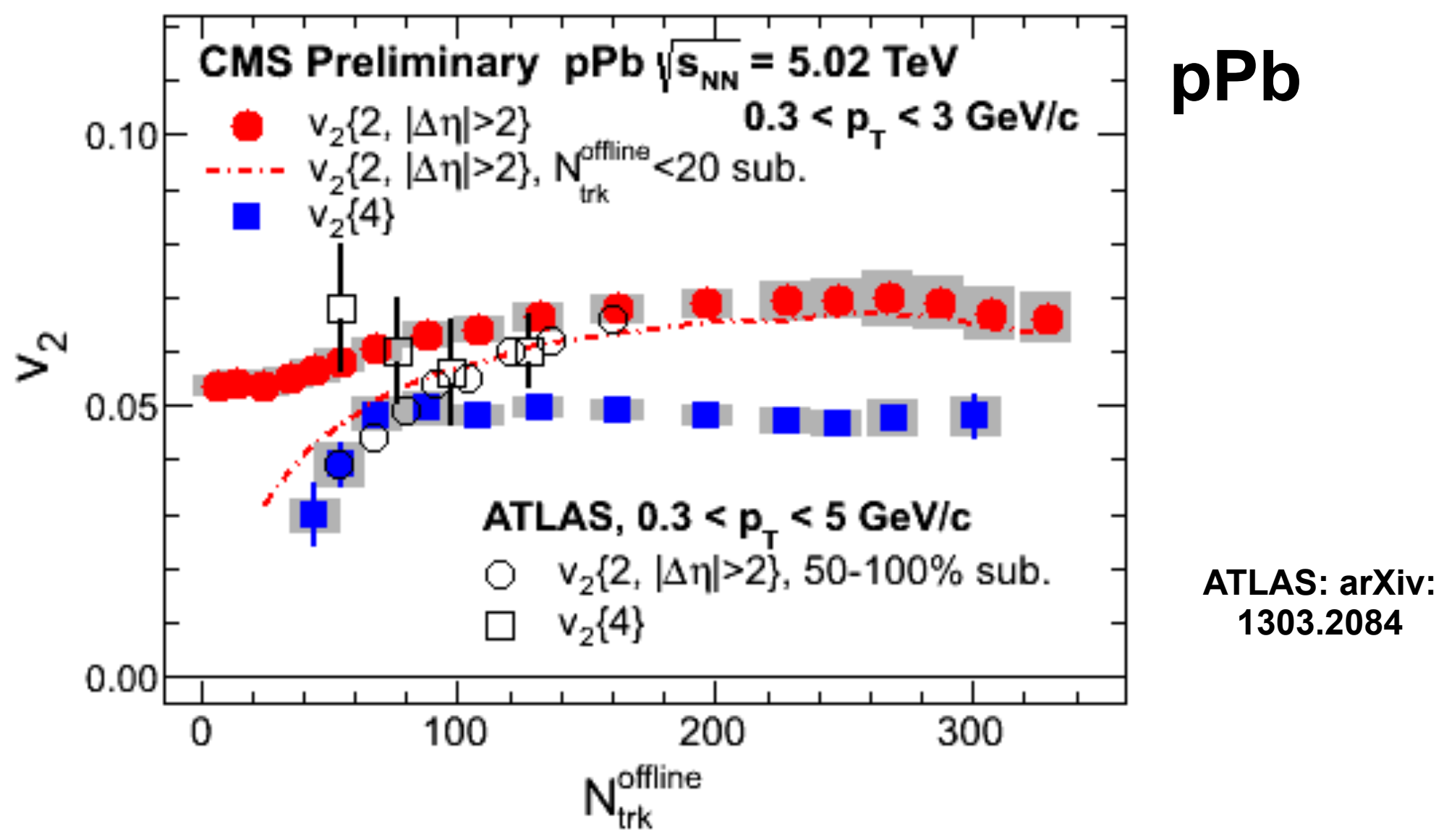

Subtract $\mathrm{N}_{\text {trk }}$ ottline $<20(70-100 \%)$ to avoid removing signal - ATLAS subtract $50-100 \%$; forward-calorimeter centrality Some difference vs ATLAS in $\mathrm{v}_{2}\{4\}$ : multiplicity fluctuations? 


\section{Summary}

- Direct comparison of high statistics, high multiplicity $\mathrm{pPb}$ and $\mathrm{PbPb}$ data

- Studied $\mathrm{v}_{2}$ multiplicity dependence in $\mathrm{pPb}$ and $\mathrm{PbPb}$

- Somewhat smaller magnitude of $\mathrm{v}_{2}$ in $\mathrm{pPb}$

- Large $v_{2}\{4\}$ in $\mathrm{pPb}$

- Large $\mathrm{v}_{3}\{2\}$ in $\mathrm{pPb}$ (comparable to $\mathrm{PbPb}$ )

- Ready to use full arsenal of jet studies in $\mathrm{pPb}$ 


\section{No ridge anywhere in $\mathrm{pPb} \mathrm{MC}$}

\section{Compare to AMPT and HIJING pPb}

HIJING pPb, N>=120

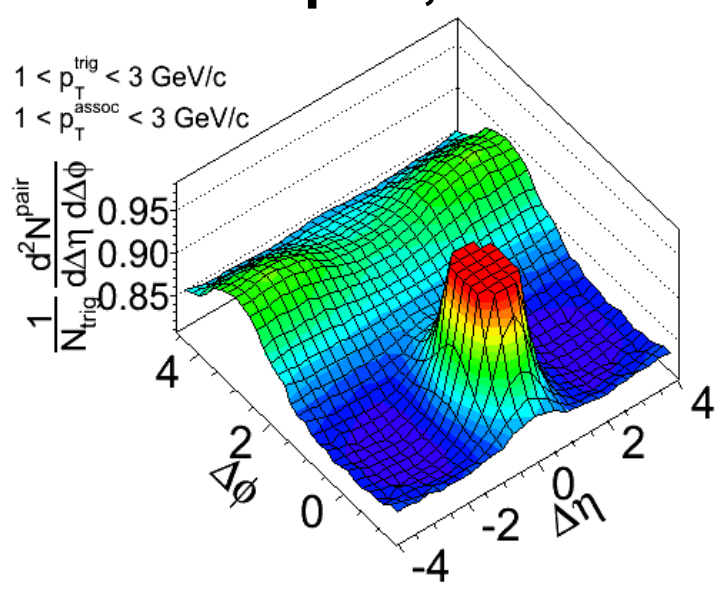

AMPT $p P b, N>=100$
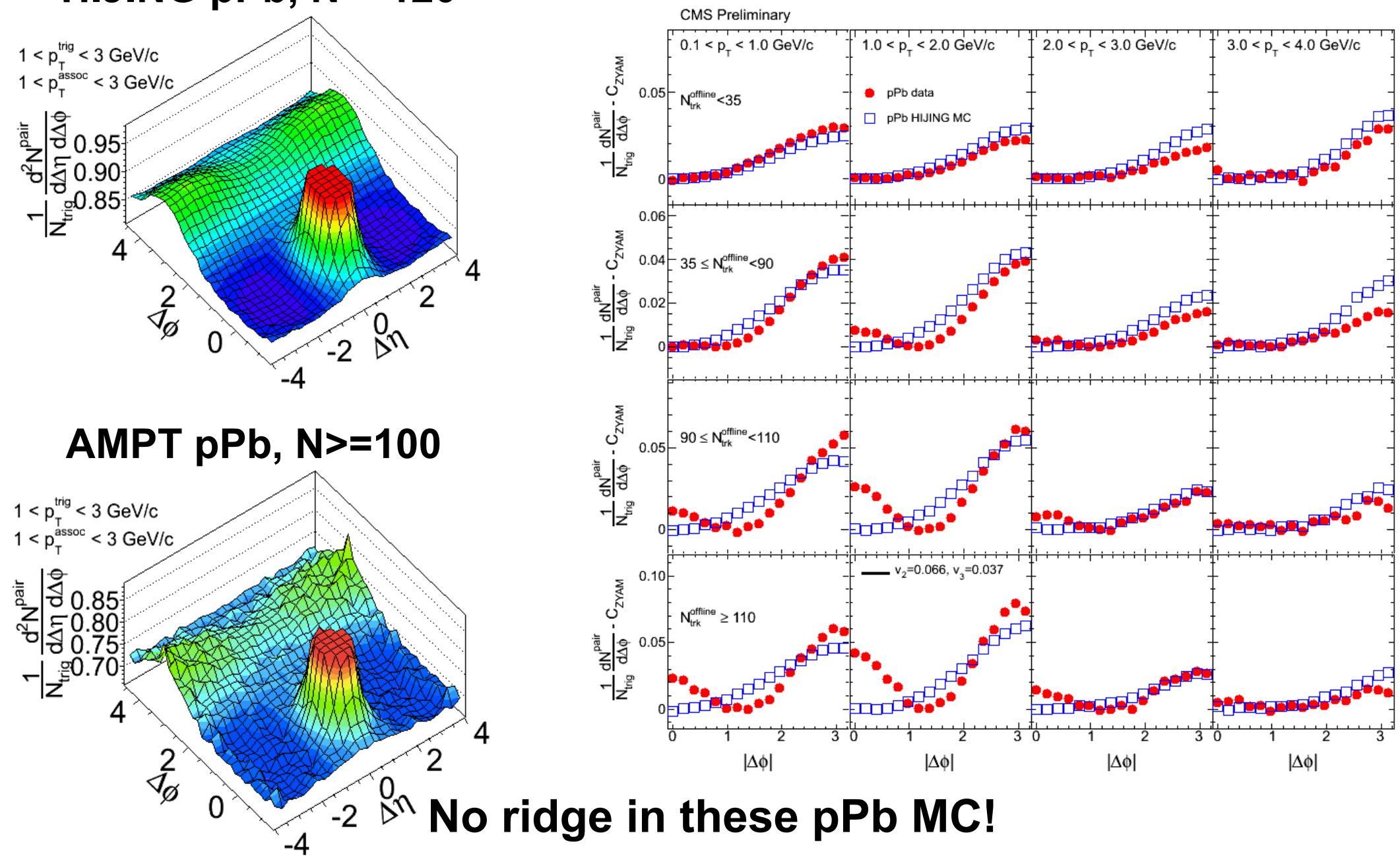

No ridge in these $\mathrm{pPb} \mathrm{MC}$ ! 


\section{Triggers and event selection}

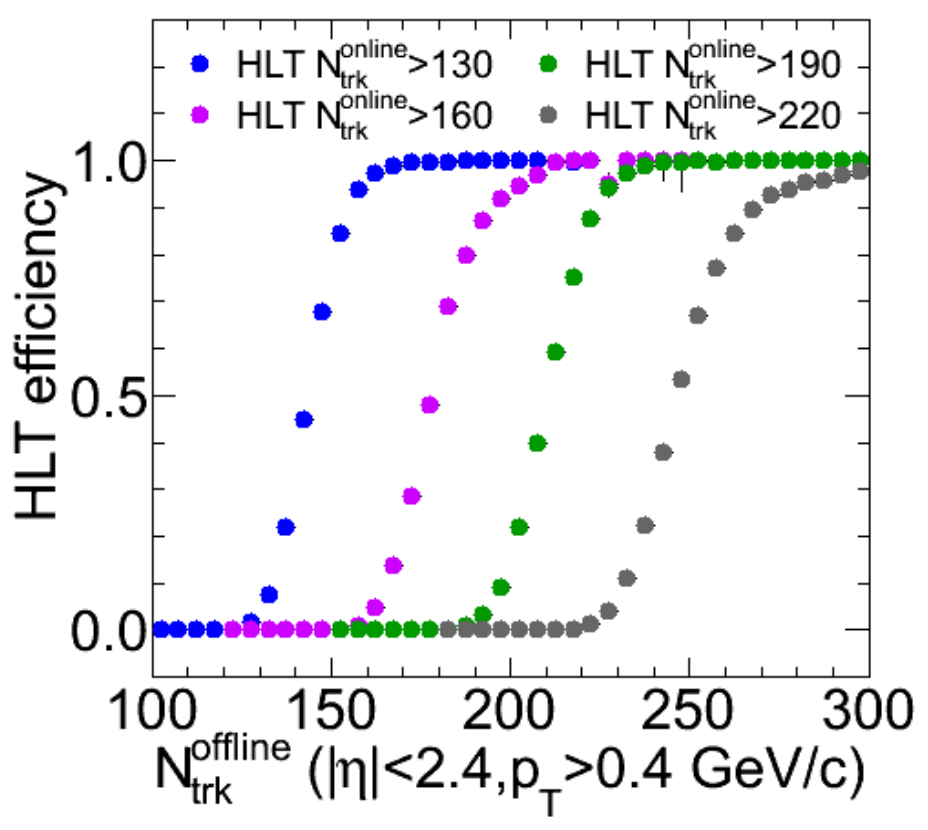

Event selection:

- HF coincidence of at least 1 tower above $3 \mathrm{GeV}$

- Vertex requirement: !isFake \& $\left|\mathrm{v}_{\mathrm{z}}\right|<15 \mathrm{~cm} \&$ position. Rho $<0.15 \mathrm{~cm}$ $\& \mathrm{nTracks}>=2$

- Fraction of highPurity tracks $>25 \%$ to remove beam scraping events 


\section{Initial conditions \\ in $A+A, p+A, d+A$ and $p+p$ collisions}

\section{Björn Schenke}

Physics Department, Brookhaven National Laboratory, Upton, NY, USA

April 172013

Jet Quenching at RHIC vs LHC in Light of Recent $\mathrm{dAu}$ and $\mathrm{pPb}$ Controls BNL, Upton, NY

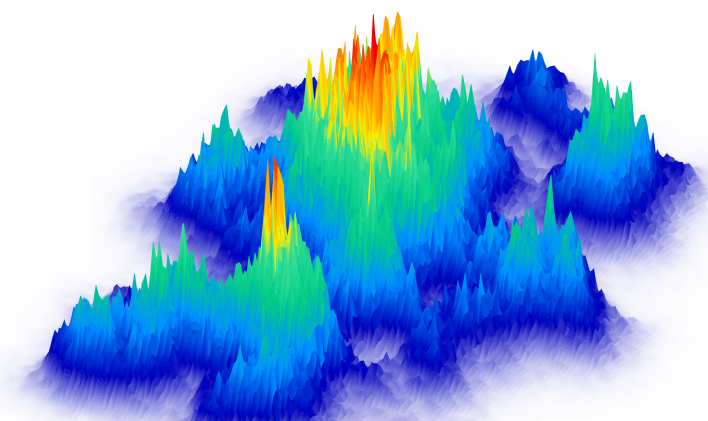




\section{Introduction - Initial state fluctuations and flow}

- Large elliptic flow has indicated fluid behavior of matter created at RHIC in early 2000'S BNL announces "perfect liquid" in 2005 press release

- The importance of fluctuations in the initial state was realized later and analysis of odd flow harmonics began in 2010

- Measured anisotropic flow is largely due to the response to the initial fluctuating geometry

- Geometry of the medium is relevant for jet evolution

- I will

- present different models for the initial state in $A+A$

- show some results of harmonic flow from these models

- apply the models to $p+p, p+A$ and $d+A u$ collisions 


\section{Modeling the initial state}

Flow is driven by the initial geometry

Final result depends on what we start with

Hard partons are affected by the geometry of the background

We need a rigorous understanding

of the initial state and its fluctuations

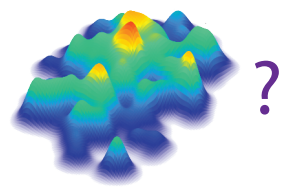

MC-Glauber

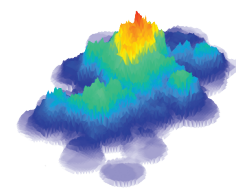

MC-KLN

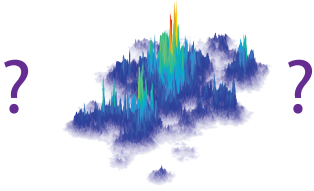

IP-Glasma 


\section{MC-Glauber}

- Sample (un-)correlated nucleons in nucleus $A$ and $B$, then overlap the two distributions with impact parameter $b$

- Interaction when the distance $d$ between a nucleon from nucleus $A$ and one from nucleus $B$ fulfills (black disk):

$$
d \leq \sqrt{\sigma_{\text {inel }}^{N N} / \pi}
$$

(alternatively use a Gaussian overlap function)

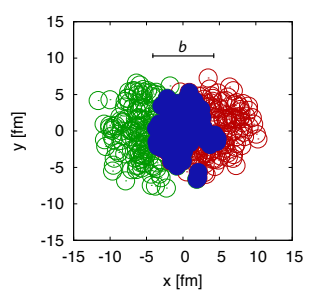

- Add Gaussian energy density with width $\sigma_{0}$ for every wounded nucleon, binary collision, or combination

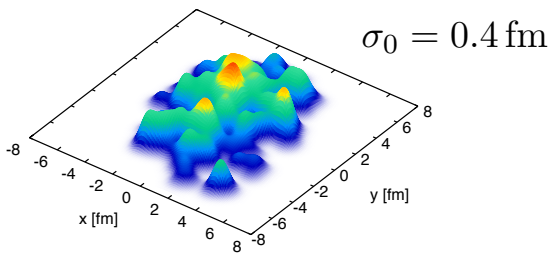


- Sample nucleon positions from Woods-Saxon distribution

- Local nucleon density $T_{A, B}\left(\mathbf{x}_{\perp}\right)$ from counting nucleons in tube of transverse area $S=\sigma_{\text {inel }}^{N N}$

- Kharzeev-Levin-Nardi (KLN) model for uGDF $\phi$ :

$$
\phi_{A, B}\left(x, k_{\perp}^{2}, \mathbf{r}_{\perp}\right) \sim \frac{1}{\alpha_{s}\left(Q_{s}^{2}\right)} \frac{Q_{s}^{2}}{\max \left(Q_{s}^{2}, k_{\perp}^{2}\right)}
$$

with $Q_{s, A}^{2}\left(x, \mathbf{r}_{\perp}\right)=2 \mathrm{GeV}^{2} \frac{T_{A}\left(\mathbf{x}_{\perp}\right)}{1.53 \mathrm{fm}^{-2}}\left(\frac{0.01}{x}\right)^{\lambda}, \lambda=0.28$

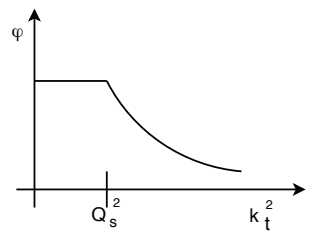

- Determine gluon production using $k_{T}$-factorization:

$$
\frac{d N}{d r_{\perp}^{2} d y} \sim \int \frac{d^{2} p_{\perp}}{p_{\perp}^{2}} \int d^{2} k_{\perp} \alpha_{s} \phi_{A}\left(x_{1}, k_{\perp}^{2}\right) \phi_{B}\left(x_{2},\left(p_{\perp}-k_{\perp}\right)^{2}\right)
$$

- Energy density analogously

Drescher, Nara, Phys.Rev. C75 (2007) 034905

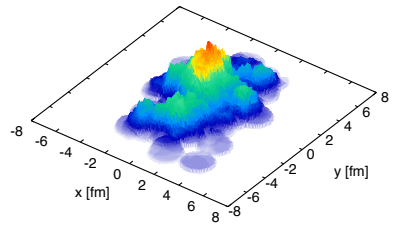




\section{IP-Glasma: before the collision}

- Energy and impact parameter $b$ dependence of $Q_{s}(x, \mathbf{b})$ can be modeled in the IP-Sat model Kowalski, Teaney, Phys.Rev. D68 (2003) 114005

- Parametrize cross sections for DIS on protons and fit to HERA diffractive data

A. Rezaeian, M. Siddikov, M. Van de Klundert, R. Venugopalan Phys.Rev. D87 (2013) 034002

- Sample nucleon positions from Woods-Saxon distribution

- Sum Gaussian thickness functions $T_{p}$ of $A$ nucleons

$$
\frac{\mathrm{d} \sigma_{\mathrm{dip}}^{\mathrm{p}}}{\mathrm{d}^{2} \mathbf{b}_{\perp}}\left(\mathbf{r}_{\perp}, x, \mathbf{b}_{\perp}\right)=2 \mathcal{N}\left(\mathbf{r}_{\perp}, x, \mathbf{b}_{\perp}\right)=2\left[1-\exp \left(-\frac{\pi^{2}}{2 N_{c}} \mathbf{r}_{\perp}{ }^{2} \alpha_{s}\left(Q^{2}\right) x g\left(x, Q^{2}\right) \sum_{i=1}^{A} T_{p}\left(\mathbf{b}_{\perp}-\mathbf{b}_{T}^{i}\right)\right)\right]
$$

then determine local $Q_{s}\left(\mathbf{x}_{\perp}\right)\left(\mathcal{N}\left(1 / Q_{s}\left(x, \mathbf{x}_{\perp}\right), x, \mathbf{x}_{\perp}\right)=1-e^{-1 / 2}\right)$

- Color charge density $g \mu\left(\mathbf{x}_{\perp}\right)$ is proportional to $Q_{s}\left(\mathbf{x}_{\perp}\right)$ 


\section{IP-Glasma: before the collision}

- Self-consistently solve $x=Q_{s}\left(\mathbf{x}_{\perp}, x\right) / \sqrt{s}$

- Run the coupling with $Q_{s}\left(\mathbf{x}_{\perp}\right)$

- Sample color charges $\rho^{a}$ from local Gaussian

$$
\left\langle\rho^{a}\left(\mathbf{x}_{\perp}\right) \rho^{b}\left(\mathbf{y}_{\perp}\right)\right\rangle=\delta^{a b} \delta^{2}\left(\mathbf{x}_{\perp}-\mathbf{y}_{\perp}\right) g^{2} \mu^{2}\left(\mathbf{x}_{\perp}\right)
$$

- Color charges determine incoming color currents:

$$
\begin{aligned}
& J_{1}^{\nu}=\delta^{\mu+} \rho_{1}\left(x^{-}, \mathbf{x}_{\perp}\right) \\
& {\left[D_{\mu}, F^{\mu \nu}\right]=J_{1}^{\nu}}
\end{aligned}
$$

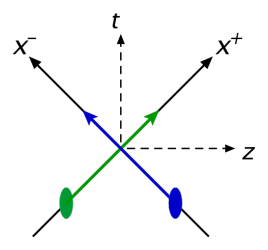

$$
\begin{aligned}
& J_{2}^{\nu}=\delta^{\mu-} \rho_{2}\left(x^{+}, \mathbf{x}_{\perp}\right) \\
& {\left[D_{\mu}, F^{\mu \nu}\right]=J_{2}^{\nu}}
\end{aligned}
$$

- Solve Yang-Mills equations for the gauge fields

$$
A^{+}\left(x^{-}, \mathbf{x}_{\perp}\right)=-\frac{g \rho\left(x^{-}, \mathbf{x}_{\perp}\right)}{\nabla_{\perp}^{2}+m^{2}}
$$




\section{IP-Glasma: after the collision}

Initial condition on the lightcone:

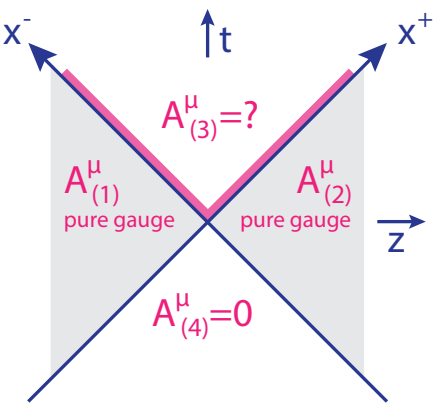

Solution:

Kovner, McLerran, Weigert, Phys. Rev. D52, 3809 (1995)

$$
\begin{aligned}
& \left.A_{(3)}^{i}\right|_{\tau=0}=A_{(1)}^{i}+A_{(2)}^{i} \\
& \left.A_{(3)}^{\eta}\right|_{\tau=0}=\frac{i g}{2}\left[A_{(1)}^{i}, A_{(2)}^{i}\right]
\end{aligned}
$$

\section{Numerical solution}

Krasnitz, Venugopalan, Nucl.Phys. B557 (1999) 237

Configuration in Schwinger gauge $A^{\tau}=0$
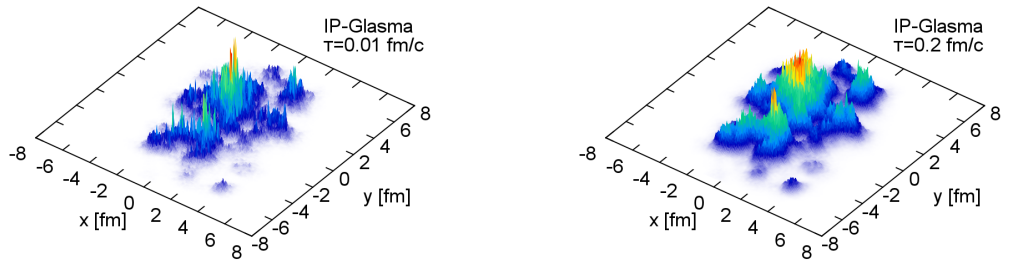


\section{Eccentricities в.Scchenke, P.T.ibedy, R.Venugopalan, Phys.Rev.Let. 108, 252301 (2012)}

Characterize the initial distribution by its ellipticity, triangularity, etc...

$$
\varepsilon_{n}=\sqrt{\left\langle r^{n} \cos (n \phi)\right\rangle^{2}+\left\langle r^{n} \sin (n \phi)\right\rangle^{2}} /\left\langle r^{n}\right\rangle
$$
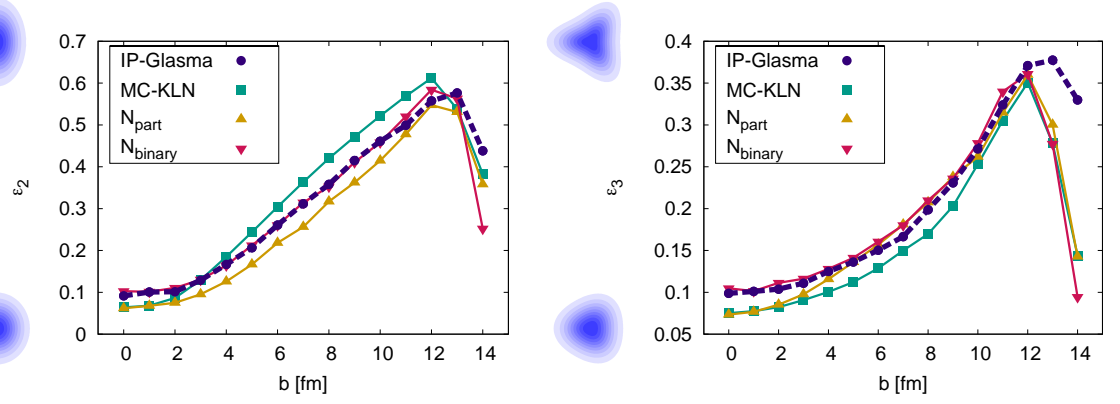

- $\varepsilon_{2}$ smaller in IP-Glasma than MC-KLN for (for $b>3 \mathrm{fm}$ )

- $\varepsilon_{2}$ and $\varepsilon_{3}$ very similar between IP-Glasma and MC-Glauber with binary collision scaling 


\section{Multiplicity в.Scchenke, P.T.ribedy, R. venugopalan, Phys. Rev. C88, 034908 (2012)}

$P\left(d N_{\mathrm{g}} / d y\right)$ at time $\tau=0.4 \mathrm{fm}$ with $P(b)$ from a Glauber model Experimental data: STAR, Phys. Rev. C79, 034909 (2009)

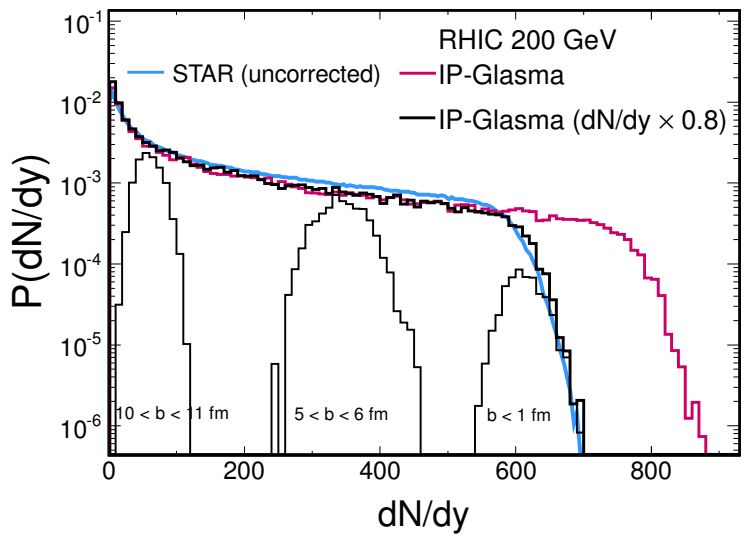

Glasma model gives a convolution of negative binomial distributions No need to put them in by hand 


\section{Negative binomial fluctuations}

B.Schenke, P.Tribedy, R.Venugopalan, Phys.Rev.Lett.108, 252301 (2012)

Fluctuations in the total energy per unit rapidity produce negative binomial distribution (NBD).

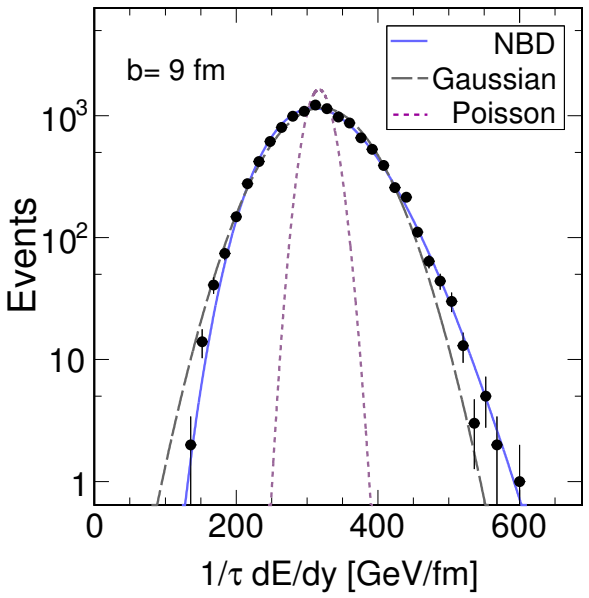

$$
P(n)=\frac{\Gamma(k+n)}{\Gamma(k) \Gamma(n+1)} \frac{\bar{n}^{n} k^{k}}{(\bar{n}+k)^{n+k}}
$$

Good, since multiplicity in pp collisions can be described well with NBD.

In AA, convolution of NBDs at all impact parameters describes data well too.

P. Tribedy and R. Venugopalan

Nucl.Phys. A850 (2011) 136-156

MC-KLN does not do that - these fluctuations need to be added by hand.

see Dumitru and Nara, Phys. Rev. C85 (2012) 034907 


\section{Viscous flow at RHIC and LHC}

$$
\mathrm{RHIC} \eta / s=0.12
$$

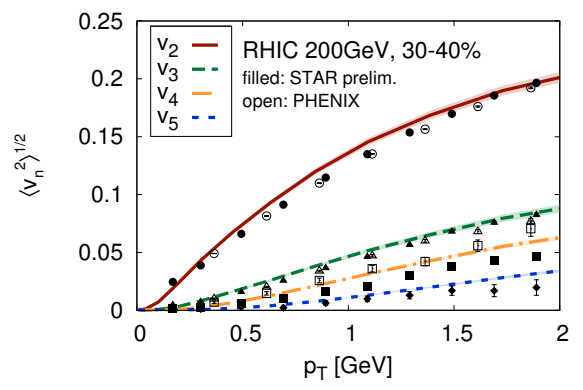

$$
\mathrm{LHC} \eta / s=0.2
$$

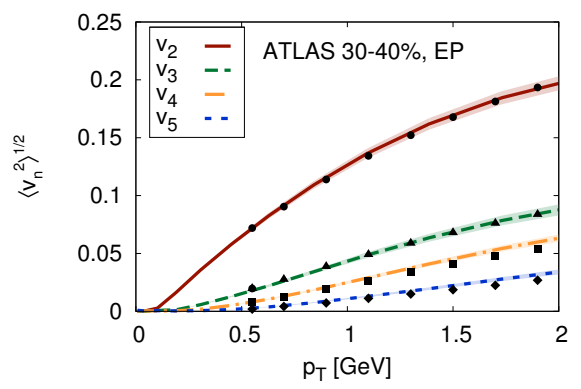

Initial state fluctuations + flow work to describe $v_{n}$ in $\mathrm{A}+\mathrm{A}$ Lower effective $\eta / s$ at RHIC than at LHC needed to describe data Hints at increasing $\eta / s$ with increasing temperature

\section{Experimental data:}

A. Adare et al. (PHENIX Collaboration), Phys.Rev.Lett. 107, 252301 (2011)

Y. Pandit (STAR Collaboration), Quark Matter 2012, (2012)

ATLAS collaboration, Phys. Rev. C 86, 014907 (2012) 


\section{Event-by-event distributions of $v_{n}$}

\section{Experimenatal data:}

ATLAS collaboration https://atlas.web.cern.ch/Atlas/GROUPS/PHYSICS/CONENOTES/ATLAS-CONF-2012-114/ J. Jia, S. Mohapatra, arXiv:1304.1471

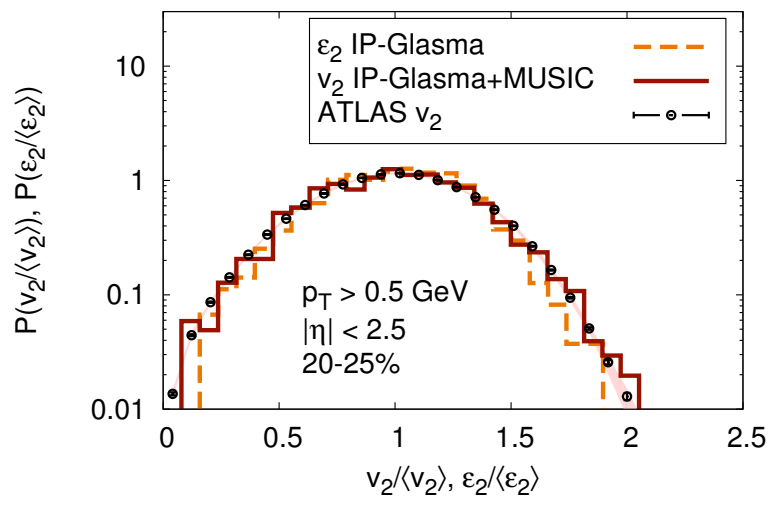

C. Gale, S. Jeon, B.Schenke, P.Tribedy, R.Venugopalan, PRL110, 012302 (2013) 


\section{Event-by-event distributions of $v_{n}$}

\section{Experimenatal data:}

ATLAS collaboration https://atlas.web.cern.ch/Atlas/GROUPS/PHYSICS/CONFNOTES/ATLAS-CONF-2012-114/ J. Jia, S. Mohapatra, arXiv:1304.1471

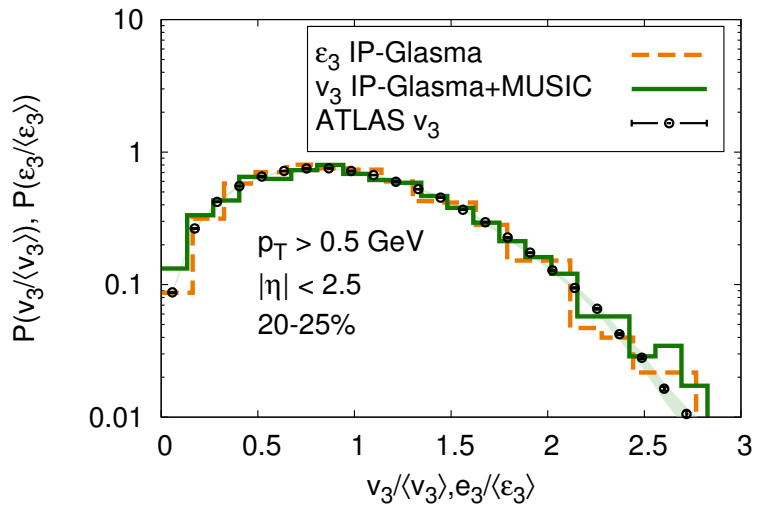

C. Gale, S. Jeon, B.Schenke, P.Tribedy, R.Venugopalan, PRL110, 012302 (2013) 


\section{Event-by-event distributions of $v_{n}$}

\section{Experimenatal data:}

ATLAS collaboration https://atlas.web.cern.ch/Atlas/GROUPS/PHYSICS/CONFNOTES/ATLAS-CONF-2012-114/ J. Jia, S. Mohapatra, arXiv:1304.1471

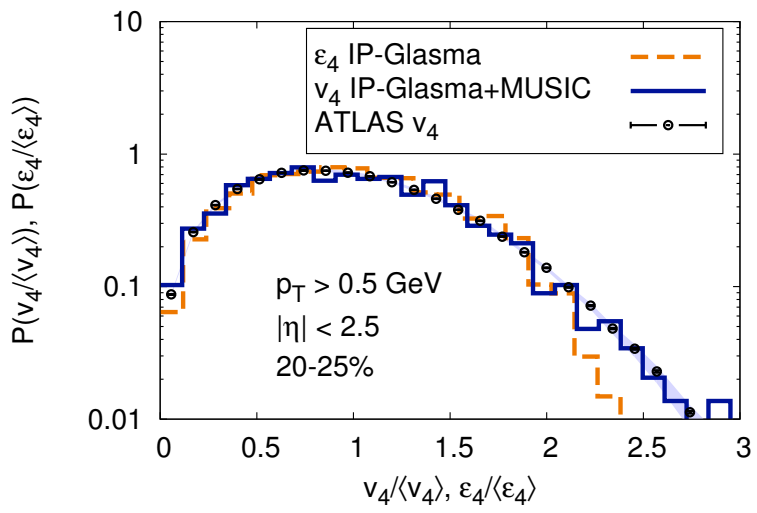

C. Gale, S. Jeon, B.Schenke, P.Tribedy, R.Venugopalan, PRL110, 012302 (2013) 


\section{Event-by-event distributions of $v_{n}$}

\section{Experimenatal data:}

ATLAS collaboration https: / /atlas.web.cern.ch/Atlas/GROUPS/PHYSICS/CONFNOTES/ATLAS-CONF-2012-114/ J. Jia, S. Mohapatra, arXiv:1304.1471
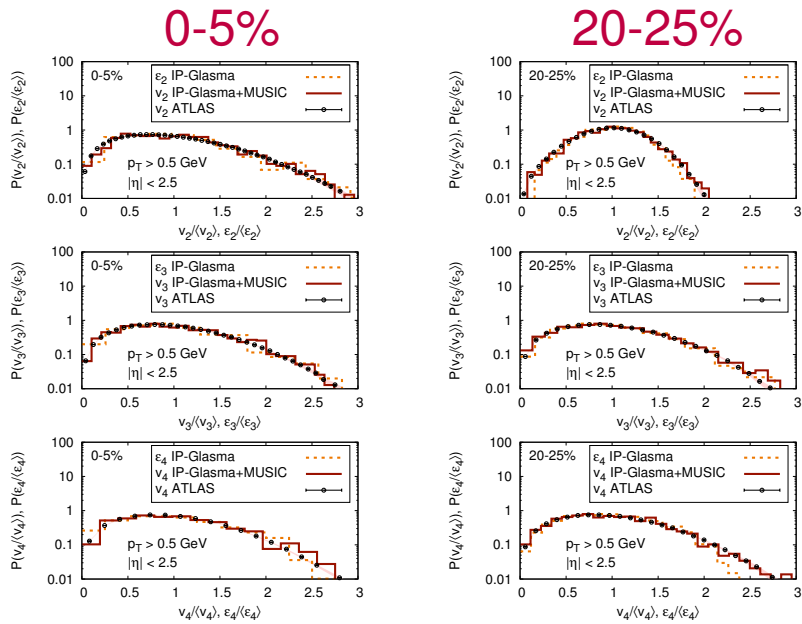

C. Gale, S. Jeon, B.Schenke, P.Tribedy, R.Venugopalan, PRL110, 012302 (2013) 


\section{Event-by-event distributions of $v_{n}$ - other models}

Showing eccentricity distributions (yellow on the right)
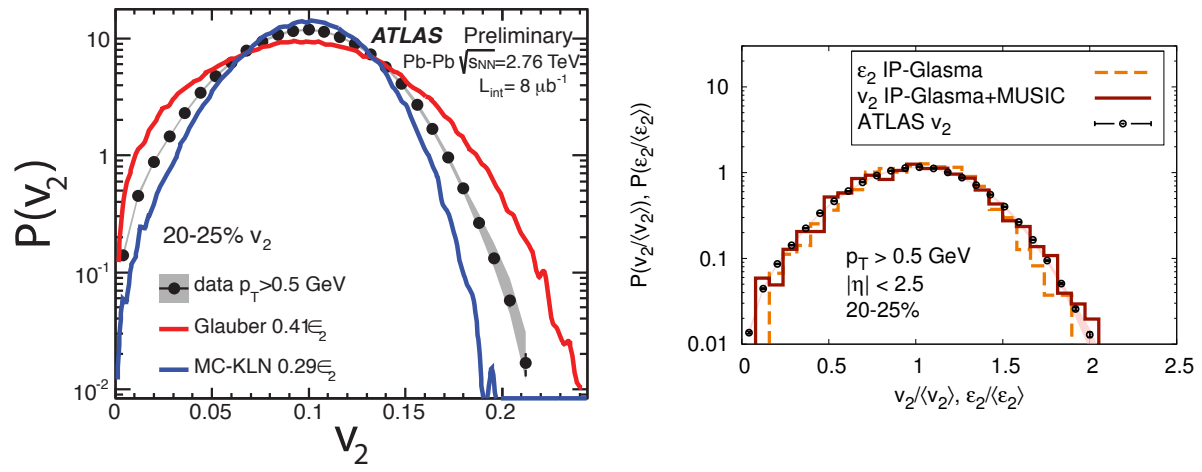

Event-by-event distributions can potentially distinguish between different initial state models 


\section{$\mathrm{d}+\mathrm{Au}$ collisions}

In small systems the different models lead to very different intial states

Energy density for the same nucleon positions:
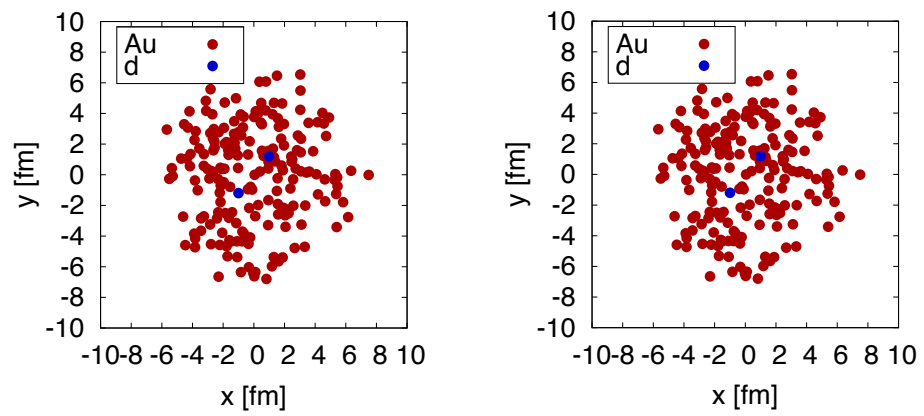

In MC-Glauber all nucleons that are barely 'touched' contribute fully to the energy density

an MC-Glauber implementation is used in e.g. P. Bozek, Phys.Rev. C85 (2012) 014911 


\section{$\mathrm{d}+\mathrm{Au}$ collisions}

In small systems the different models lead to very different intial states

Energy density for the same nucleon positions:
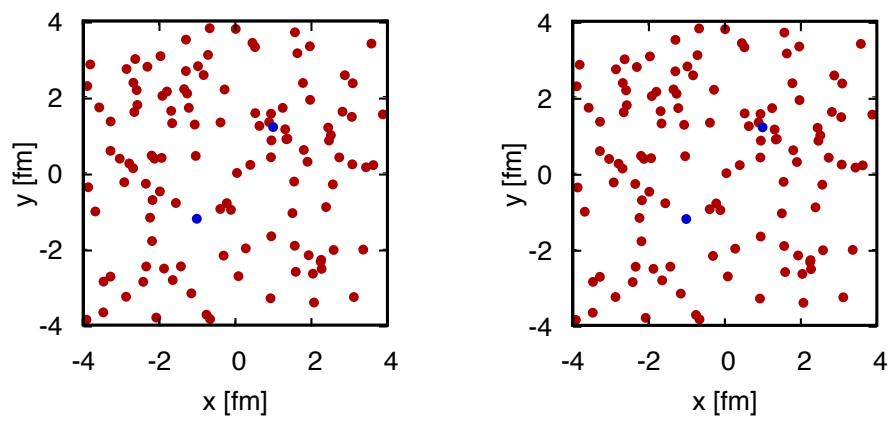

In MC-Glauber all nucleons that are barely 'touched' contribute fully to the energy density an MC-Glauber implementation is used in e.g. P. Bozek, Phys.Rev. C85 (2012) 014911 


\section{$\mathrm{d}+\mathrm{Au}$ collisions}

In small systems the different models lead to very different intial states

Energy density for the same nucleon positions:
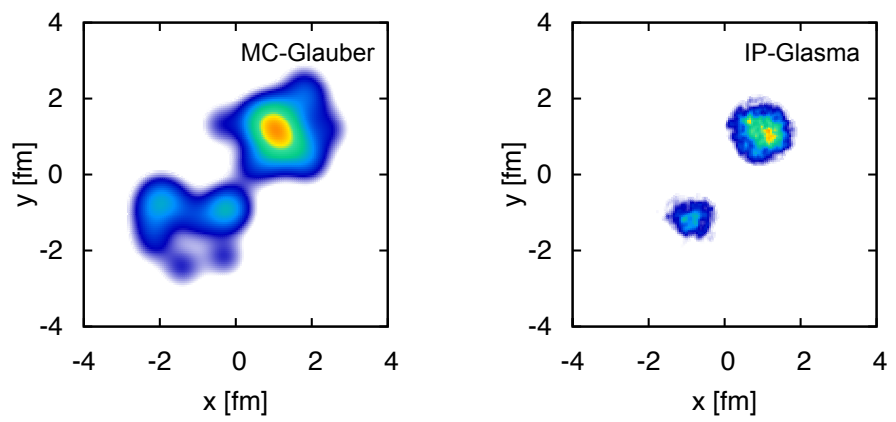

In MC-Glauber all nucleons that are barely 'touched' contribute fully to the energy density

an MC-Glauber implementation is used in e.g. P. Bozek, Phys.Rev. C85 (2012) 014911 


\section{$\mathrm{d}+\mathrm{Au}$ collisions}

In small systems the different models lead to very different intial states

Energy density for the same nucleon positions:
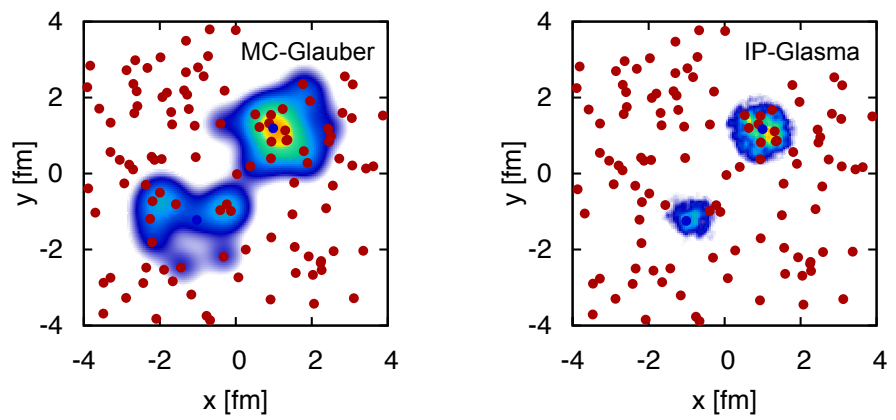

In MC-Glauber all nucleons that are barely 'touched' contribute fully to the energy density an MC-Glauber implementation is used in e.g. P. Bozek, Phys.Rev. C85 (2012) 014911 


\section{Evolution in $d+A u$}

Energy density and initial flow velocity from $u_{\mu} T^{\mu \nu}=\varepsilon u^{\nu}$ as input for hydrodynamic simulation

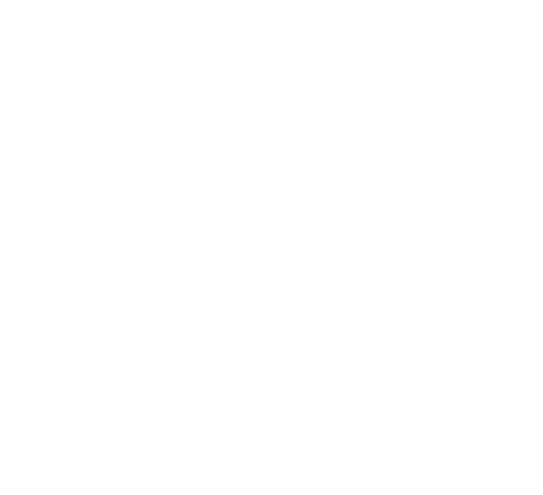




\section{Evolution in $d+A u$}

Energy density and initial flow velocity from $u_{\mu} T^{\mu \nu}=\varepsilon u^{\nu}$ as input for hydrodynamic simulation

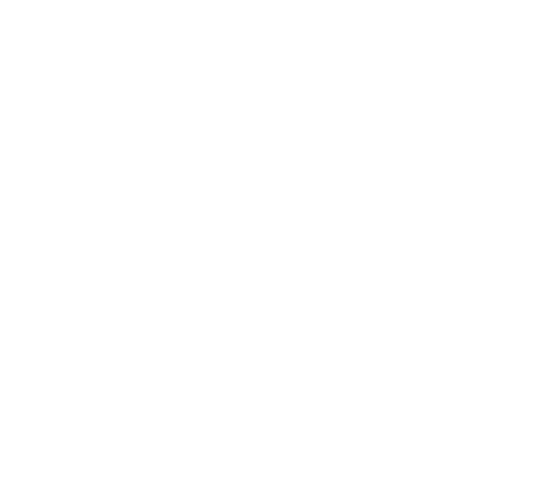




\section{$p+p$ and $p+P b$ collisions $A$. Bzdak, B. Schenke, P. Tribedy, R. Venugopalan, axtivi:1304.3403}

Initial system size (vs $d N / d y$ ) in $\mathrm{p}+\mathrm{p}$ and $\mathrm{p}+\mathrm{Pb}$ collisions is very similar in the IP-Glasma model

$r_{\max }=$ maximal radius at which energy density is above $\sim \Lambda_{\mathrm{QCD}}^{4}$

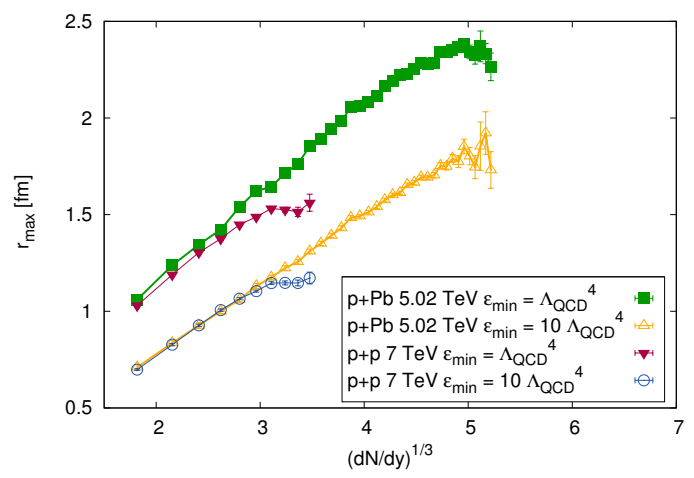

If there is no flow in either system, HBT radii will be similar 


\section{$p+p$ and $p+P b$ collisions $A$. Bzdak, B. Schenke, P. Tribedy, R. Venugopalan, axtivi:1304.3403}

Initial eccentricities (vs $d N / d y$ ) in $\mathrm{p}+\mathrm{p}$ and $\mathrm{p}+\mathrm{Pb}$ collisions are quite different in the IP-Glasma model

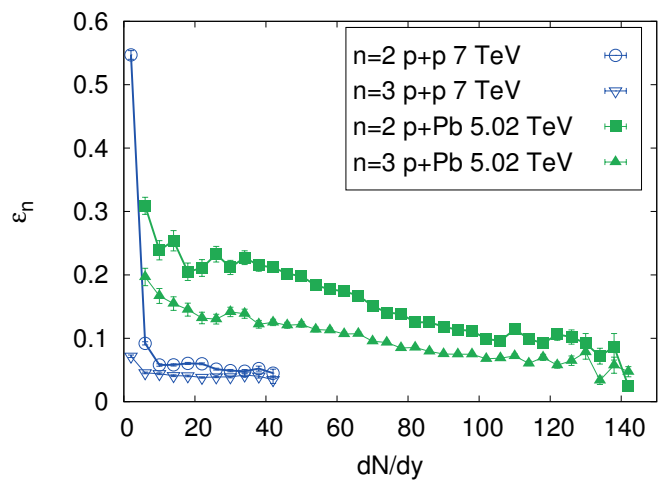

How well do eccentricities represent flow in $p+p$ and $p+P b$ collisions? 


\section{$\mathrm{p}+\mathrm{Pb}$ collisions}

Eccentricities from different models can differ significantly

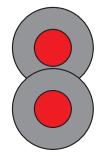

MC-Glauber 1

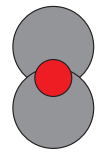

MC-Glauber 2
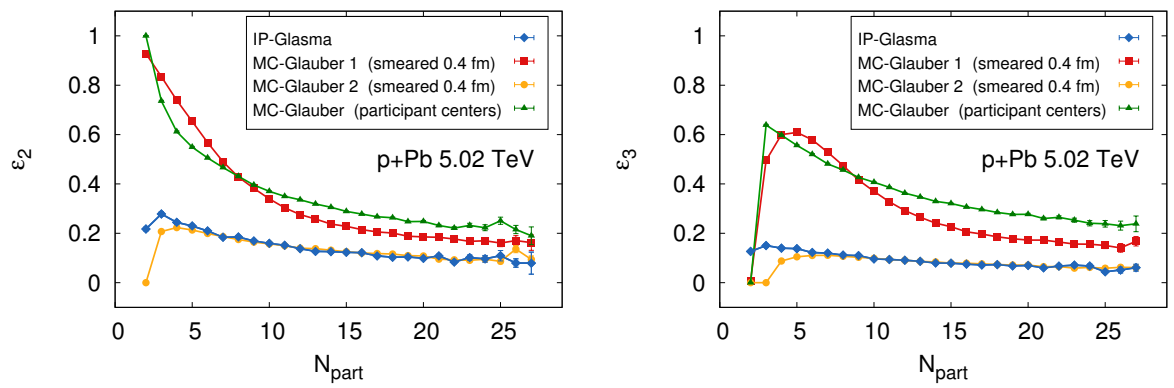

Lesson: Do not divide $v_{n}$ by $\varepsilon_{n}$ in small systems 


\section{Flow in $p+p, p+P b$ and $d+A u$ collisions}

Only qualitative scaling between flow and eccentricities
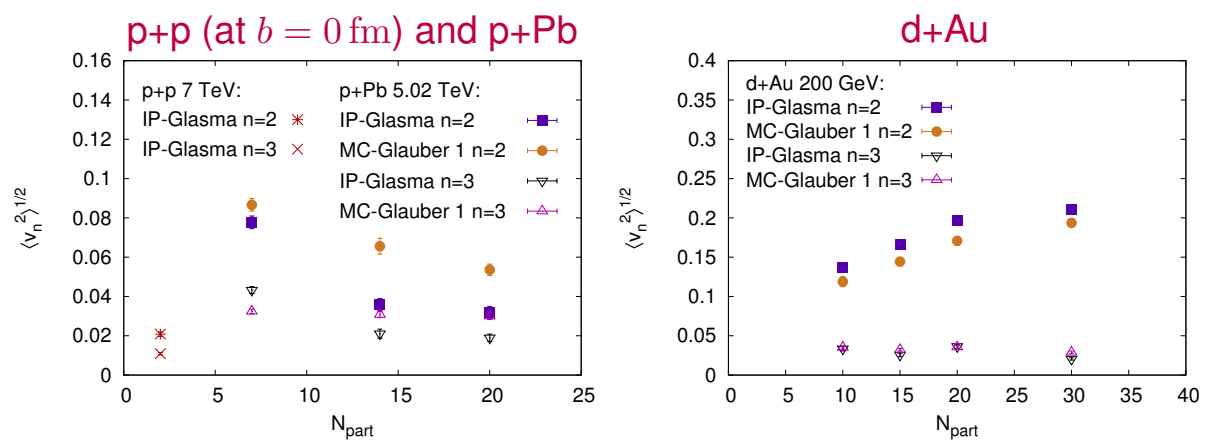

$\mathrm{p}+\mathrm{Pb}$ : Ellipitc flow decreases with $N_{\text {part }}$ d+Au: Elliptic flow increases with $N_{\text {part }}$ $\mathrm{p}+\mathrm{p}$ : Elliptic flow small, but not as small as expected from eccentricity Need sophisticated centrality selection to compare with experiments

A. Bzdak, B. Schenke, P. Tribedy, R. Venugopalan, arXiv:1304.3403 


\section{$\mathrm{p}+\mathrm{A}$ collisions - is viscous hydro valid?}

How "crazy" is it to use hydrodynamics in $p+A$ ?

Initial $\pi_{0}^{\mu \nu}=0, b=0 \mathrm{fm}$, IP-Glasma. Cells within f.o. surface that have $>25 \%$ viscous correction in $\mathrm{p}+\mathrm{Pb}$ and $\mathrm{Pb}+\mathrm{Pb}$ :

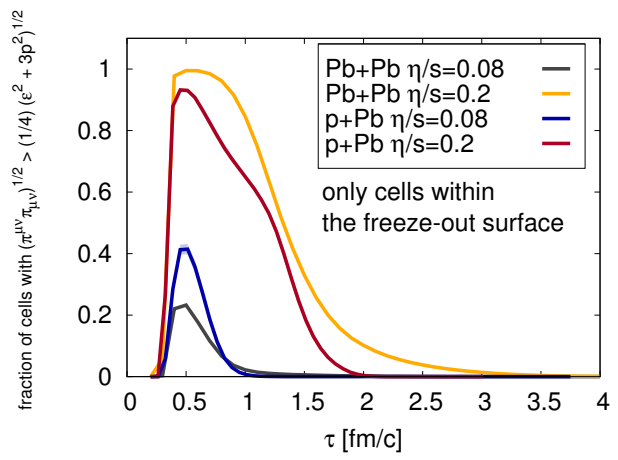

Important: Lifetime in $\mathrm{Pb}+\mathrm{Pb}$ is about 6 times longer than in $\mathrm{p}+\mathrm{Pb}$ Also see Dumitru, Molnar, Nara, Phys.Rev. C76 (2007) 024910 


\section{$\mathrm{p}+\mathrm{A}$ collisions - is viscous hydro valid?}

same with Navier-Stokes $\pi_{0}^{\mu \nu}$, count cells within f.o. surface that have more than a $25 \%$ viscous correction in $\mathrm{p}+\mathrm{Pb}$ and $\mathrm{Pb}+\mathrm{Pb}$ :

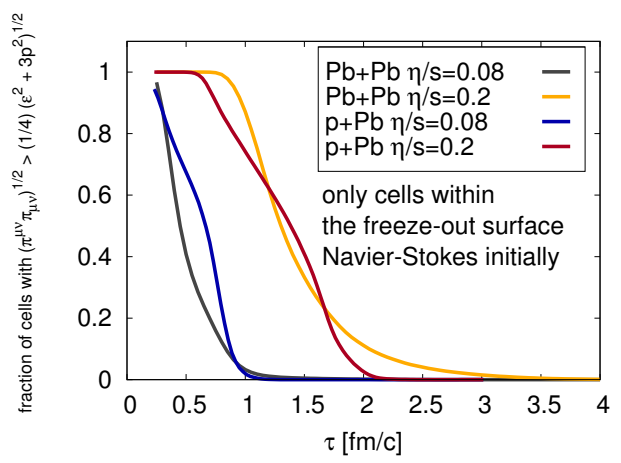

Important: Lifetime in $\mathrm{Pb}+\mathrm{Pb}$ is about 6 times longer than in $\mathrm{p}+\mathrm{Pb}$ 


\section{$\mathrm{p}+\mathrm{A}$ collisions - is viscous hydro valid?}

Initial Navier-Stokes $\pi_{0}^{\mu \nu}$, count cells within f.o. surface that have more than a $50 \%$ viscous correction in $\mathrm{p}+\mathrm{Pb}$ and $\mathrm{Pb}+\mathrm{Pb}$ :

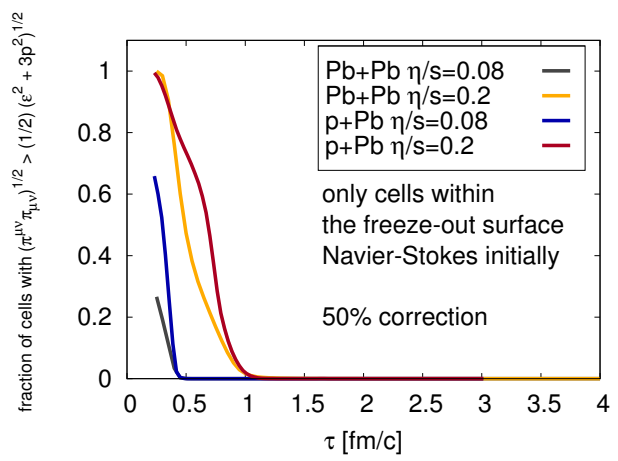

Important: Lifetime in $\mathrm{Pb}+\mathrm{Pb}$ is about 6 times longer than in $\mathrm{p}+\mathrm{Pb}$ 


\section{Summary and conclusions}

- Different initial state models produce different geometries

- Differences are not very large in $A+A$, details still relevant for flow and jet energy loss studies (e.g. jet $v_{2}$ )

- Differences are much larger in small systems $(p+p, p+A, d+A)$

- Eccentricites not the only relevant measure/predictor of flow

- Correct centrality selection in the model important

- Viscous corrections: Large at early times - affect larger fraction of total evolution time in $\mathrm{p}+\mathrm{A}$ than $\mathrm{A}+\mathrm{A}$ 


\section{BACKUP}




\section{Flow in $\mathrm{p}+\mathrm{Pb}$}
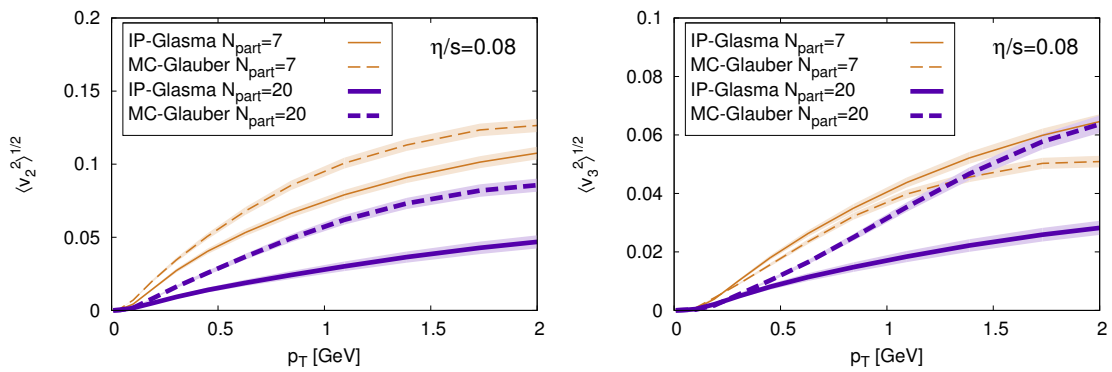

A. Bzdak, B. Schenke, P. Tribedy, R. Venugopalan, arXiv:1304.3403 


\section{Flow in $d+A u$}
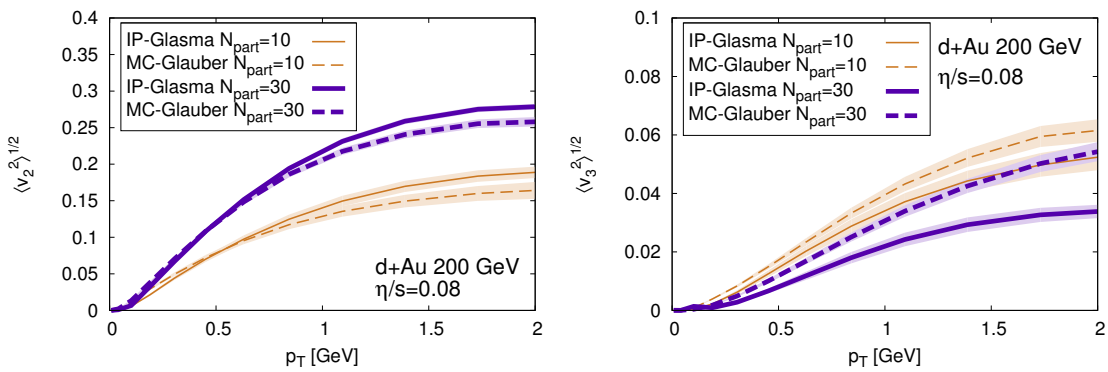

A. Bzdak, B. Schenke, P. Tribedy, R. Venugopalan, arXiv:1304.3403 


\section{Learning about QCD}

Example: extraction of $(\eta / s)(T)$
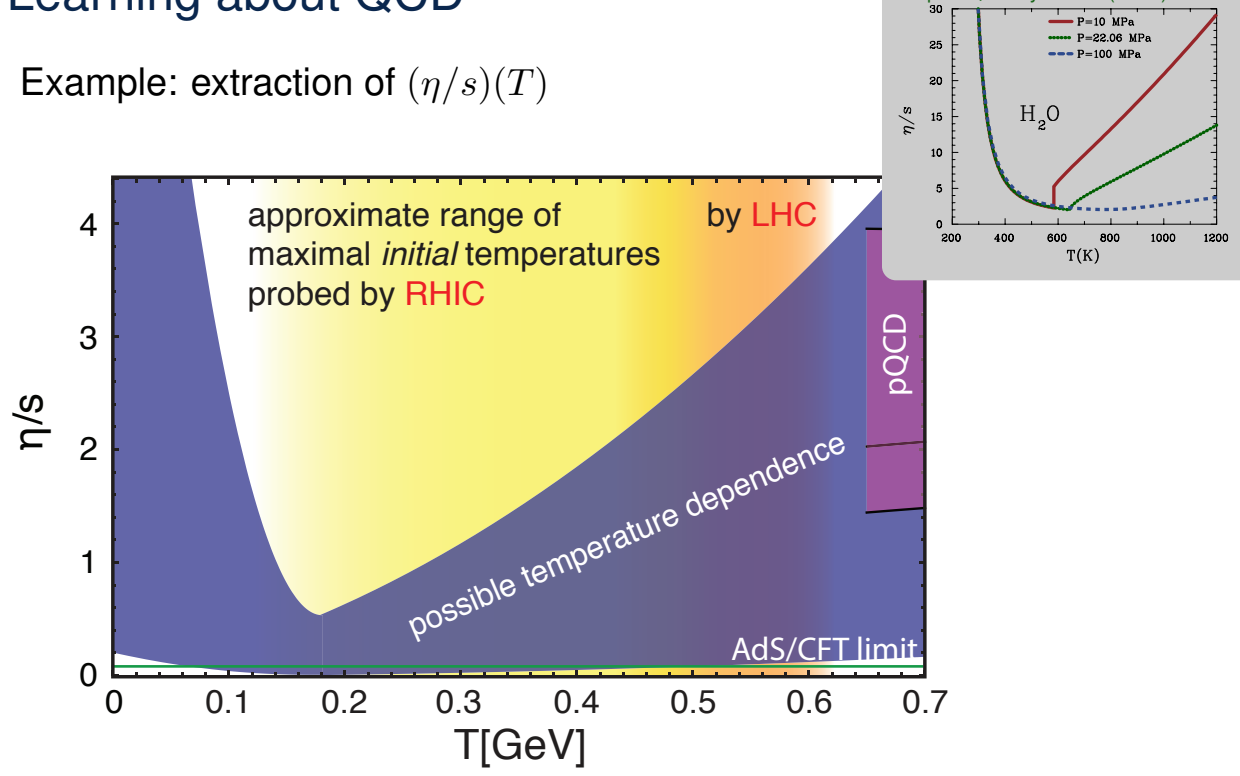

maximal initial temperatures 


\section{Temperature dependent $\eta / s$}

C. Gale, S. Jeon, B.Schenke,

P.Tribedy, R.Venugopalan, PRL110, 012302 (2013)

Use $\eta / s(T)$ as in Niemi et al., Phys.Rev.Lett. 106 (2011) 212302

Experimental data: A. Adare et al. (PHENIX), Phys.Rev.Lett. 107, 252301 (2011)

Y. Pandit (STAR), Quark Matter 2012, (2012)

ATLAS collaboration, Phys. Rev. C 86, 014907 (2012)
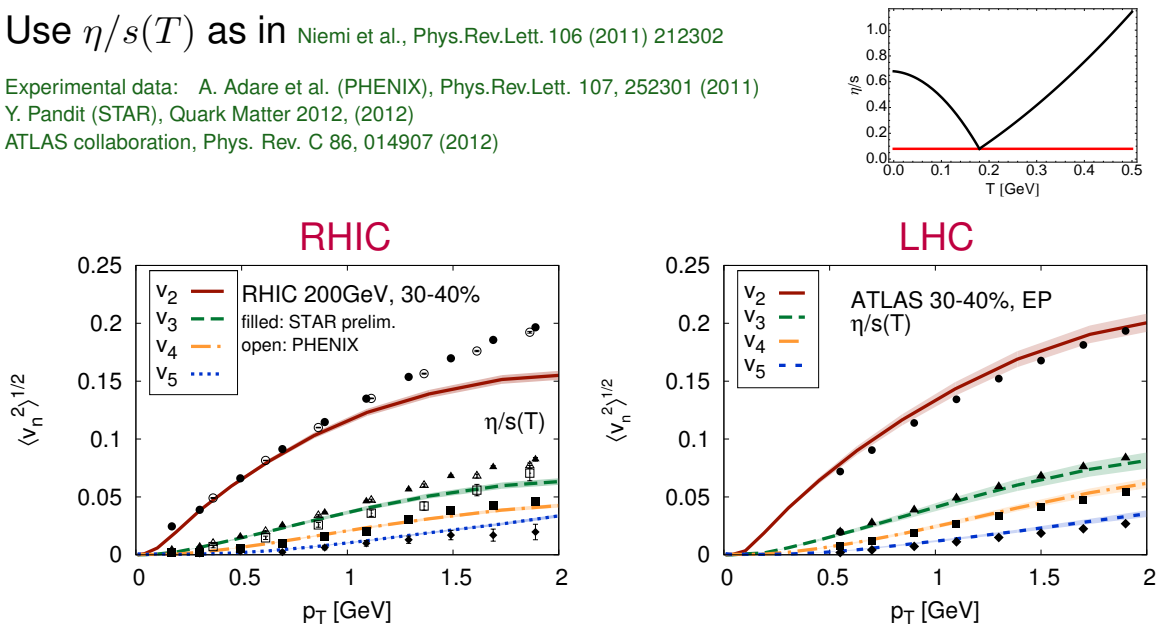

One $(\eta / s)(T)$ will be able to describe both RHIC and LHC data Used parametrization not yet perfect: no surprise

More detailed study needed - include different RHIC energies and LHC 


\section{Eccentricities}

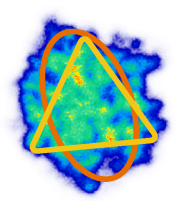

Characterize the initial distribution by its ellipticity, triangularity, etc...

$$
\varepsilon_{n}=\sqrt{\left\langle r^{n} \cos (n \phi)\right\rangle^{2}+\left\langle r^{n} \sin (n \phi)\right\rangle^{2}} /\left\langle r^{n}\right\rangle
$$
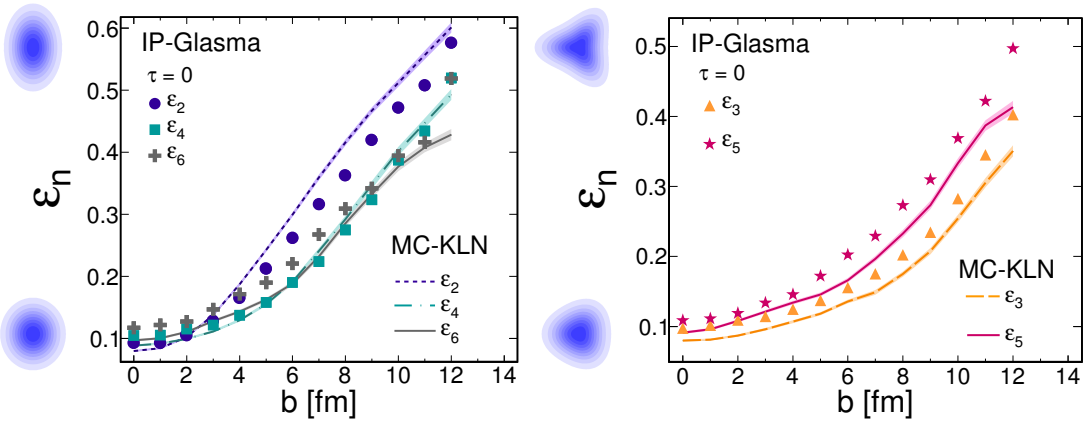

- $\varepsilon_{n}$ larger in Glasma model for odd $n$

- $\varepsilon_{n}$ smaller in Glasma model for $n=2$ (for $b>3 \mathrm{fm}$ ) about equal for $n=4$, larger for $n=6$ 


\section{Viscous flow at LHC}

C. Gale, S. Jeon, B.Schenke, P.Tribedy, R.Venugopalan, PRL110, 012302 (2013)

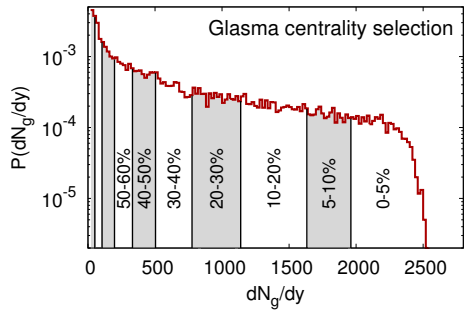



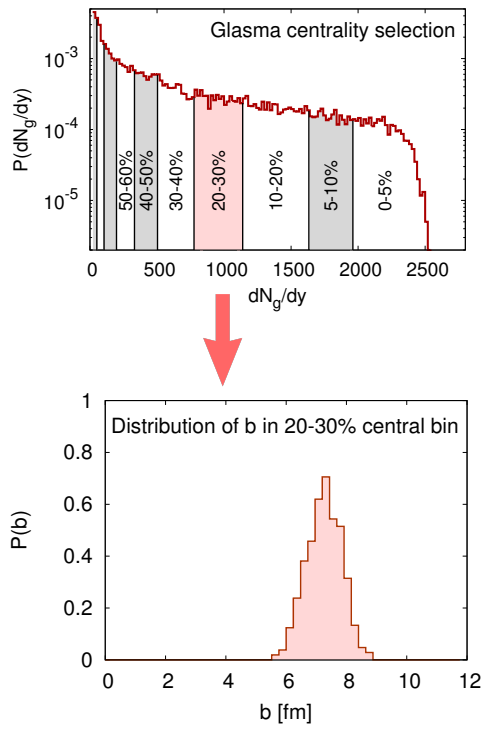

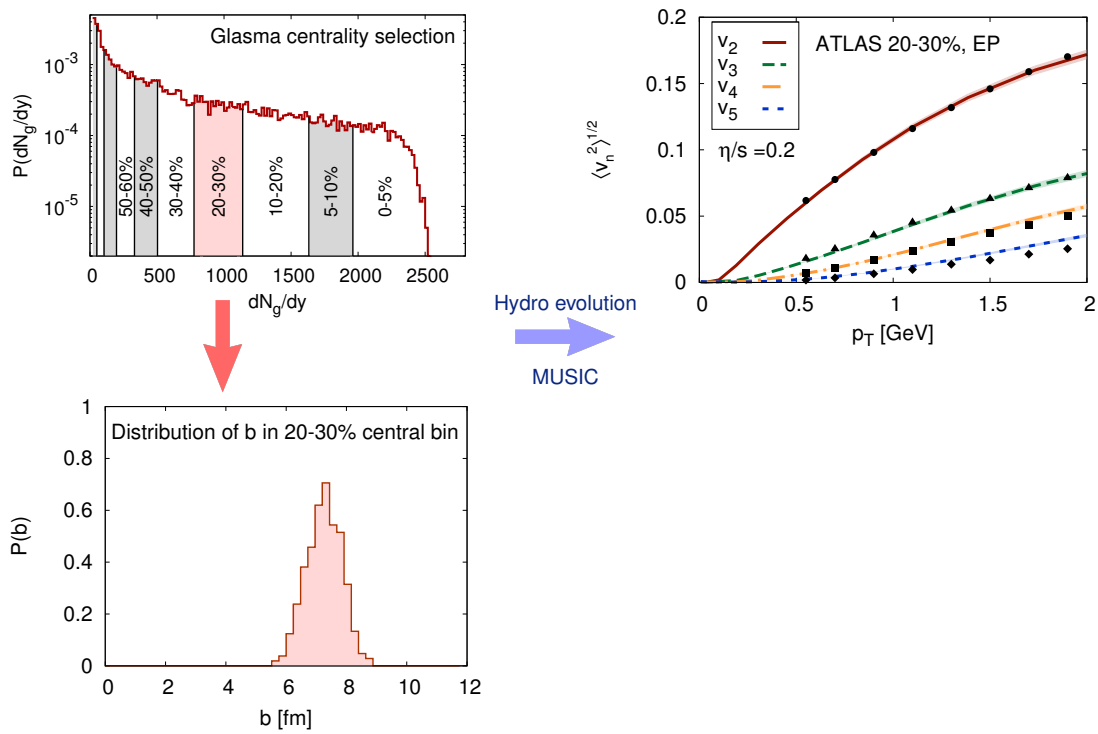

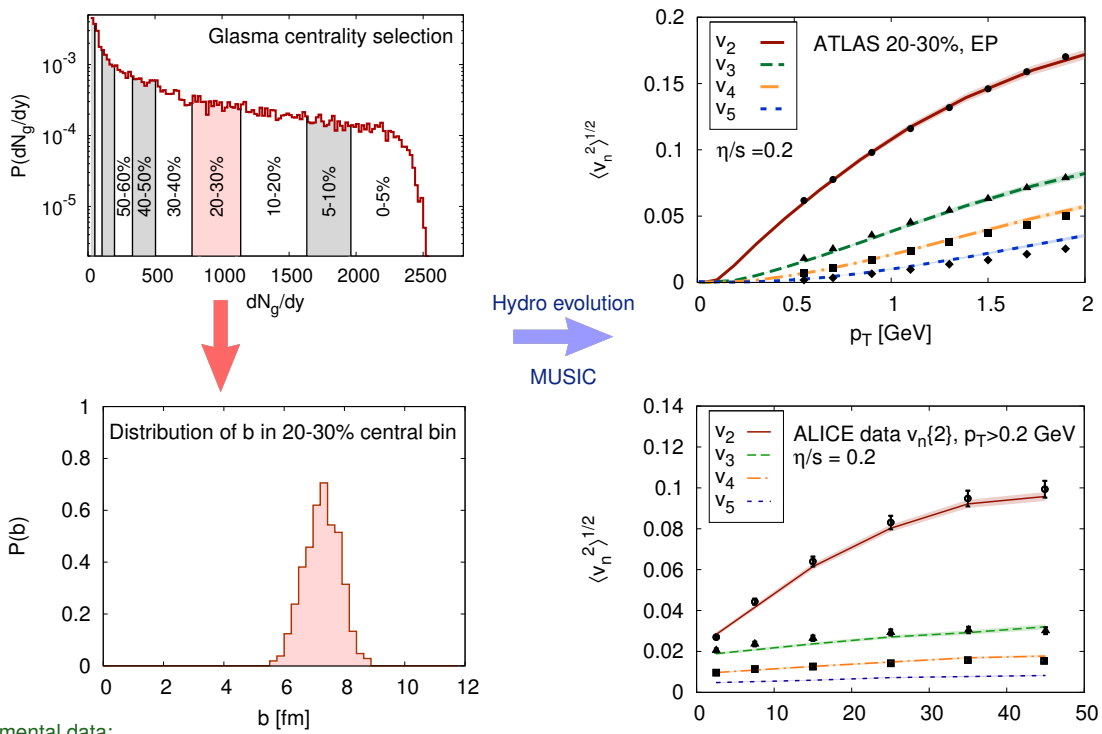

Experimental data:

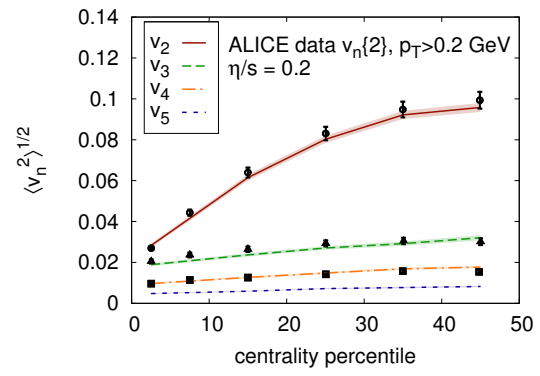

ATLAS collaboration, Phys. Rev. C 86, 014907 (2012) ALICE collaboration, Phys. Rev. Lett. 107, 032301 (2011) 


\section{Relativistic fluid-dynamics}

Bulk of the matter produced in heavy-ion collisions is well described by fluid-dynamics...

... and that is just energy and momentum conservation in a system with small mean free path (compared to the system size)

$$
\begin{gathered}
\partial_{\mu} T^{\mu \nu}=0 \\
T^{\mu \nu}=(\epsilon+P) u^{\mu} u^{\nu}-P g^{\mu \nu}+\pi^{\mu \nu}
\end{gathered}
$$

need additional equation to close the set:

$$
\text { Equation of state: } P=P(\epsilon)
$$

(comes e.g. from lattice QCD / hadron gas model)

$\pi^{\mu \nu}$ contains dissipative effects 


\section{Event-by-event fluctuations}

- Fluctuations are important

Affect all harmonics $v_{1}, v_{2}, v_{3}$, etc.

$$
\frac{d N}{d \phi}=\frac{N}{2 \pi}\left(1+\sum_{n} 2 v_{n} \cos \left(n\left(\phi-\psi_{n}\right)\right)\right)
$$

In particular: odd harmonics are not zero

Mishra et al., Phys.Rev. C77, 064902 (2008), Takahashi et al., Phys. Rev. Lett. 103, 242301 (2009)

Alver and Roland, Phys. Rev. C81, 054905 (2010)

- Axes and eccentricities determined by fluctuating geometry

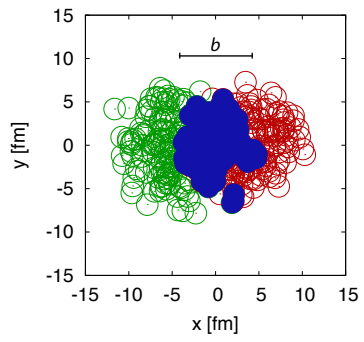




\section{Event-by-event fluctuations}

- Fluctuations are important Affect all harmonics $v_{1}, v_{2}, v_{3}$, etc.

$$
\frac{d N}{d \phi}=\frac{N}{2 \pi}\left(1+\sum_{n} 2 v_{n} \cos \left(n\left(\phi-\psi_{n}\right)\right)\right)
$$

In particular: odd harmonics are not zero

Mishra et al., Phys.Rev. C77, 064902 (2008), Takahashi et al., Phys. Rev. Lett. 103, 242301 (2009)

Alver and Roland, Phys. Rev. C81, 054905 (2010)

- Axes and eccentricities determined by fluctuating geometry

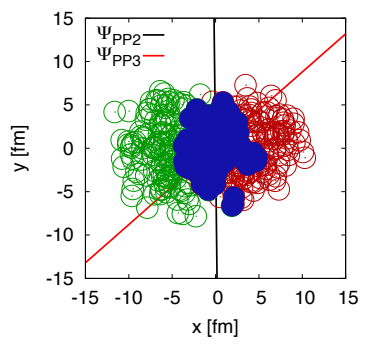

Participant plane angle (spatial):

$\psi_{P P n}=\frac{1}{n} \arctan \frac{\left\langle r^{n} \sin (n \phi)\right\rangle}{\left\langle r^{n} \cos (n \phi)\right\rangle}$

average over nucleon positions

Event plane angle (momentum):

$\psi_{E P n}=\frac{1}{n} \arctan \frac{\left\langle p_{T}^{\alpha} \sin (n \psi)\right\rangle}{\left\langle p_{T}^{\alpha} \cos (n \psi)\right\rangle}$

average over produced particles, $\alpha=0,1, \ldots$ 


\section{Event-by-event fluctuations}

- Fluctuations are important Affect all harmonics $v_{1}, v_{2}, v_{3}$, etc.

$$
\frac{d N}{d \phi}=\frac{N}{2 \pi}\left(1+\sum_{n} 2 v_{n} \cos \left(n\left(\phi-\psi_{n}\right)\right)\right)
$$

In particular: odd harmonics are not zero

Mishra et al., Phys.Rev. C77, 064902 (2008), Takahashi et al., Phys. Rev. Lett. 103, 242301 (2009)

Alver and Roland, Phys. Rev. C81, 054905 (2010)

- Axes and eccentricities determined by fluctuating geometry

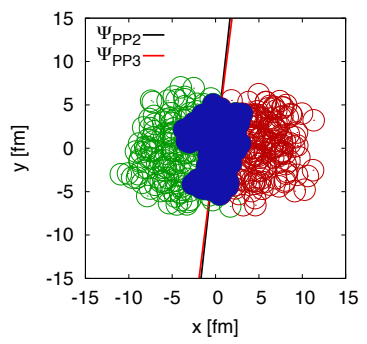

Participant plane angle (spatial):

$\psi_{P P n}=\frac{1}{n} \arctan \frac{\left\langle r^{n} \sin (n \phi)\right\rangle}{\left\langle r^{n} \cos (n \phi)\right\rangle}$

average over nucleon positions

Event plane angle (momentum):

$\psi_{E P n}=\frac{1}{n} \arctan \frac{\left\langle p_{T}^{\alpha} \sin (n \psi)\right\rangle}{\left\langle p_{T}^{\alpha} \cos (n \psi)\right\rangle}$

average over produced particles, $\alpha=0,1, \ldots$ 


\section{Event-by-event fluctuations}

- Fluctuations are important Affect all harmonics $v_{1}, v_{2}, v_{3}$, etc.

$$
\frac{d N}{d \phi}=\frac{N}{2 \pi}\left(1+\sum_{n} 2 v_{n} \cos \left(n\left(\phi-\psi_{n}\right)\right)\right)
$$

In particular: odd harmonics are not zero

Mishra et al., Phys.Rev. C77, 064902 (2008), Takahashi et al., Phys. Rev. Lett. 103, 242301 (2009)

Alver and Roland, Phys. Rev. C81, 054905 (2010)

- Axes and eccentricities determined by fluctuating geometry

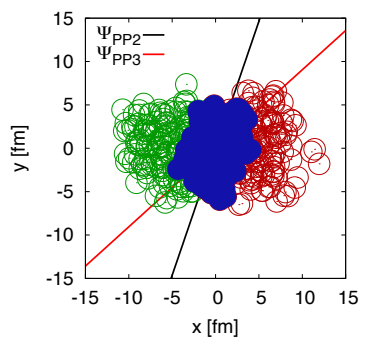

Participant plane angle (spatial):

$\psi_{P P n}=\frac{1}{n} \arctan \frac{\left\langle r^{n} \sin (n \phi)\right\rangle}{\left\langle r^{n} \cos (n \phi)\right\rangle}$

average over nucleon positions

Event plane angle (momentum):

$\psi_{E P n}=\frac{1}{n} \arctan \frac{\left\langle p_{T}^{\alpha} \sin (n \psi)\right\rangle}{\left\langle p_{T}^{\alpha} \cos (n \psi)\right\rangle}$

average over produced particles, $\alpha=0,1, \ldots$ 


\section{Event-by-event fluctuations}

- Fluctuations are important Affect all harmonics $v_{1}, v_{2}, v_{3}$, etc.

$$
\frac{d N}{d \phi}=\frac{N}{2 \pi}\left(1+\sum_{n} 2 v_{n} \cos \left(n\left(\phi-\psi_{n}\right)\right)\right)
$$

In particular: odd harmonics are not zero

Mishra et al., Phys.Rev. C77, 064902 (2008), Takahashi et al., Phys. Rev. Lett. 103, 242301 (2009)

Alver and Roland, Phys. Rev. C81, 054905 (2010)

- Axes and eccentricities determined by fluctuating geometry

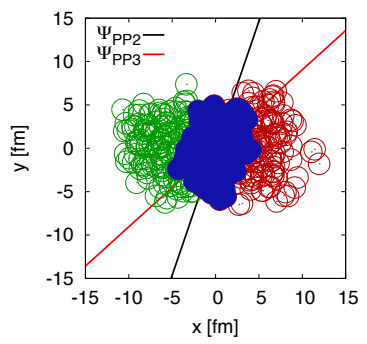

Eccentricities:

$\varepsilon_{n}=\frac{\sqrt{\left\langle r^{n} \cos (n \phi)\right\rangle^{2}+\left\langle r^{n} \sin (n \phi)\right\rangle^{2}}}{\left\langle r^{n}\right\rangle}$

All $\varepsilon_{n}=0 \rightarrow$ perfect circle

$\varepsilon_{n} \neq 0$ : shape modulation

in the $n^{\text {th }}$ harmonic 


\section{$3+1 \mathrm{D}$ event-by-event viscous fluid-dynamics}

The first relativistic hydrodynamic simulation to include viscosity, fluctuations and 3+1 dimensions:

\section{MUSIC: MUScl for lon Collisions}

MUSCL = Monotonic Upstream Centered Scheme for Conservation Laws

B. Schenke, S. Jeon, and C. Gale, Phys.Rev.Lett.106, 042301 (2011)

- 3+1 dimensions

- Kurganov-Tadmor algorithm

A. Kurganov, E. Tadmor, Journal of Computational Physics 160, 241-282 (2000)

- expanding geometry

- viscous effects ( $2^{\text {nd }}$ order Israel-Stewart formalism)

- fluctuating initial conditions

- equation of state from lattice QCD and hadron resonance gas 


\section{MUSIC: studying the effect of viscosity and fluctuations}

- Setup:

energy density

Wounded nucleons are assigned

a Gaussian energy density distribution

width $\sigma_{0}$ is a free parameter

- Evolution:

Hydrodynamic evolution with shear viscous effects

System expands, becomes dilute, freezes out

Initial spatial anisotropy is transformed into momentum anisotropy

- Energy density $\rightarrow$ Particle spectra:

Cooper-Frye formula:

$$
E \frac{d N}{d^{3} p}=\int_{\Sigma} d \Sigma_{\mu} p^{\mu} f\left(T, p_{\mu} u^{\mu}, \pi^{\mu \nu}\right)
$$

followed by resonance decays

$\Sigma=$ freeze-out surface (surface of constant temperature)

$f=$ particle distribution

Cooper and Frye, Phys.Rev.D10, 186 (1974) 


\section{Sensitivity of $v_{n}$ on viscosity and fluctuations}

B. Schenke, S. Jeon, C. Gale, Phys.Rev.C85, 024901 (2012)

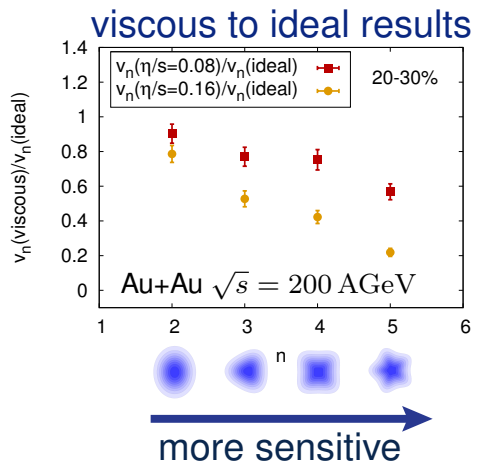

smoother to more granular results

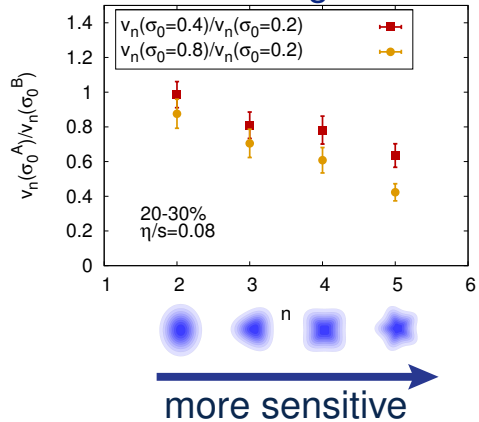

Viscosity decreases anisotropic flow (it's friction)

Smoother initial conditions decrease anisotropic flow

Sensitivity to viscosity and initial state structure increases with $n$ 


\section{Nuclei at high energy: Gluon saturation}

As we go to higher energy / smaller $x$, gluons split, number increases:

BFKL (Balitsky,Fadin,Kuraev,Lipatov) equation describes $x$-evolution but violates unitarity: cross-sections grow without bound

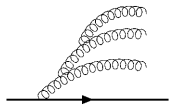

JIMWLK (Jalilian-Marian, lancu, McLerran, Weigert, Leonidov, Kovner) and BK (Balitsky, Kovchegov) equations include non-linear evolution $\rightarrow$ saturation

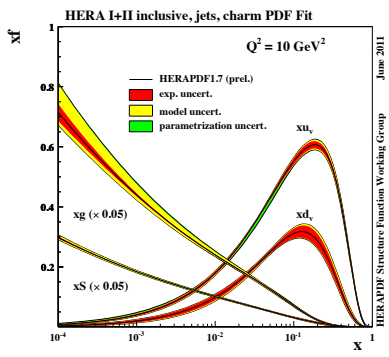

- Transverse gluon density:

$$
\rho \sim \frac{x g_{A}\left(x, Q^{2}\right)}{S_{\perp}} \sim \frac{A x g\left(x, Q^{2}\right)}{A^{2 / 3}} \sim A^{1 / 3} x g\left(x, Q^{2}\right)
$$

- Recombination cross section:

$$
\sigma_{g g \rightarrow g} \sim \frac{\alpha_{s}}{Q^{2}}
$$

- Saturation when

$$
\rho \sigma_{g g \rightarrow g} \gtrsim 1
$$

$\Rightarrow$ Saturation at scale $Q_{s}^{2}=\alpha_{s} A^{1 / 3} x g\left(x, Q_{s}^{2}\right)$

\section{$x=$ longitudinal momentum fraction of partons in a hadron or nucleus}




\section{Nuclei at high energy: Gluon saturation}

As we go to higher energy / smaller $x$, gluons split, number increases:

BFKL (Balitsky,Fadin,Kuraev,Lipatov) equation describes $x$-evolution but violates unitarity: cross-sections grow without bound

JIMWLK (Jalilian-Marian, lancu, McLerran, Weigert, Leonidov, Kovner) and BK (Balitsky, Kovchegov) equations include non-linear evolution $\rightarrow$ saturation

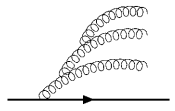

$\mathbf{p}_{T} \lesssim Q_{s}(x):$

- strong saturated fields $A_{\mu} \sim 1 / g$

- occupation numbers $\sim 1 / \alpha_{s}$

- $\Rightarrow$ classical field approximation 


\section{Energy density в.Schenke, P.Tibedy, R. Renugopalan, Phys. Rev.Lett. 108, 252301 (2012)}

Compute energy density in the fields at $\tau=0$ and later times with CYM evolution

arbitrary units

same nucleon positions

in all events, $b=4 \mathrm{fm}$
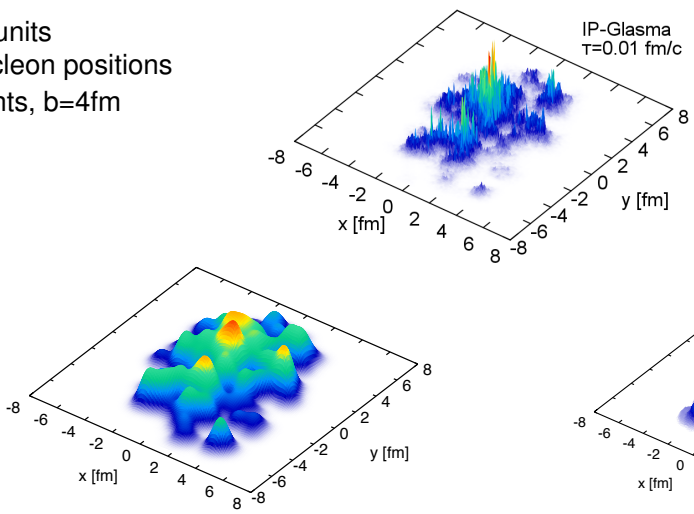

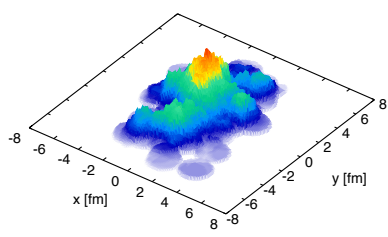

MC-KLN: Drescher, Nara, nucl-th/0611017

mckln-3.52 from http://physics.baruch. cuny.edu/files/CGC/CGC_IC.html with defaults, energy density scaling 


\section{Energy density в.Schenke, P.Tibedy, R. Renugopalan, Phys. Rev.Lett. 108, 252301 (2012)}

Compute energy density in the fields at $\tau=0$ and later times with CYM evolution

arbitrary units

same nucleon positions

in all events, $b=4 \mathrm{fm}$
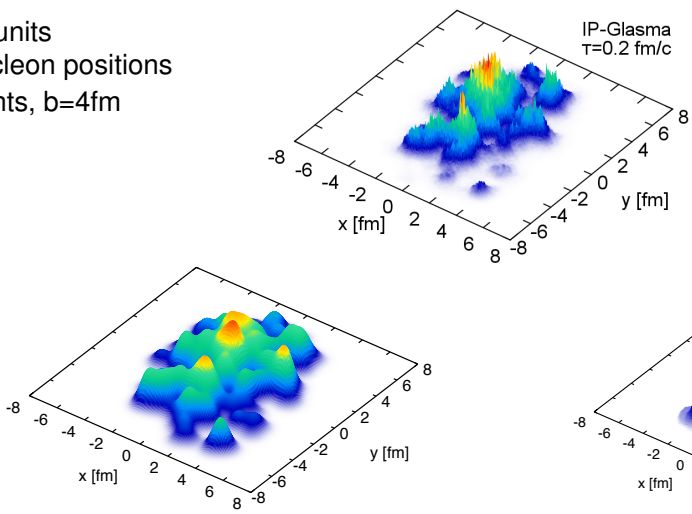

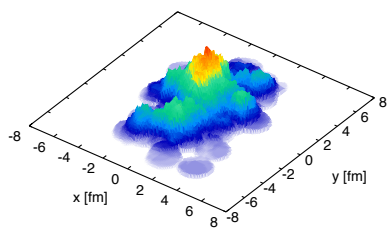

MC-KLN: Drescher, Nara, nucl-th/0611017

mckln-3.52 from http://physics.baruch. cuny.edu/files/CGC/CGC_IC.html with defaults, energy density scaling 


\section{Multiplicity в.Scchenke, P.T.ribedy, R.V.enugopalan, Phys. Rev. C86, 034908 (2012)}

$d N_{\mathrm{g}} / d y$ in transverse Coulomb gauge $\partial_{i} A^{i}=0$

$N_{\text {part }}$ from MC-Glauber with $\sigma_{N N}=42 \mathrm{mb}$ and $64 \mathrm{mb}$ respectively

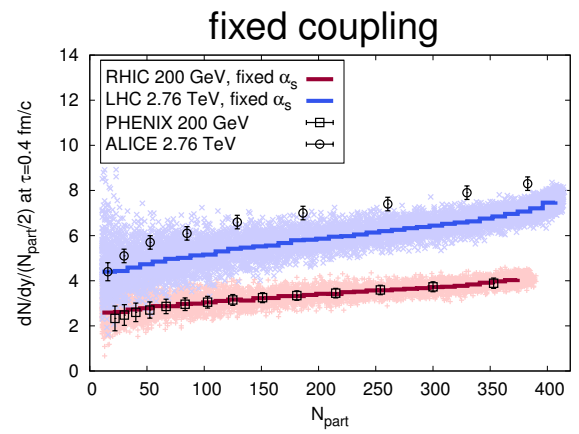

Experimental data: PHENIX, Phys.Rev.C71 034908 (2004) and ALICE, Phys.Rev.Lett. 106, 032301 (2011)

Scaled by $2 / 3$ to compare to charged particles.

Original version. Nuclear 'oomph' added by hand. 


\section{Multiplicity в.Scchenke, P.T.ribedy, R.V.enugopalan, Phys. Rev. C86, 034908 (2012)}

$d N_{\mathrm{g}} / d y$ in transverse Coulomb gauge $\partial_{i} A^{i}=0$

$N_{\text {part }}$ from MC-Glauber with $\sigma_{N N}=42 \mathrm{mb}$ and $64 \mathrm{mb}$ respectively

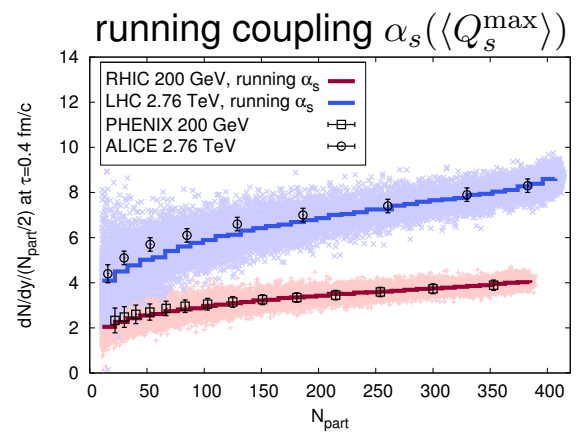

Experimental data: PHENIX, Phys.Rev.C71 034908 (2004) and ALICE, Phys.Rev.Lett. 106, 032301 (2011)

Scaled by $2 / 3$ to compare to charged particles.

Original version. Nuclear 'oomph' added by hand. 


\section{Multiplicity в.Scchenke, P.T.ribedy, R.V.enugopalan, Phys. Rev. C86, 034908 (2012)}

New version. Using IP-Sat for nuclei. No adjustments by hand anymore.

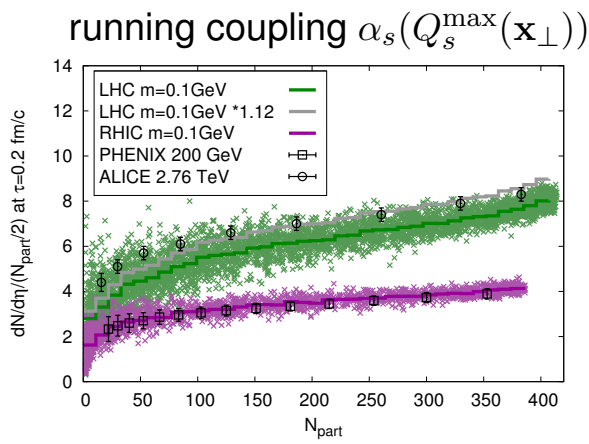

Experimental data: PHENIX, Phys.Rev.C71 034908 (2004) and ALICE, Phys.Rev.Lett. 106, 032301 (2011)

Expect more entropy production at LHC - need hydro results to check 


\section{Negative binomial fluctuations}

B.Schenke, P.Tribedy, R.Venugopalan, Phys.Rev.C86, 034908 (2012)

Extract $k$ and $\bar{n}$ using a fit with

$$
P(n)=\frac{\Gamma(k+n)}{\Gamma(k) \Gamma(n+1)} \frac{\bar{n}^{n} k^{k}}{(\bar{n}+k)^{n+k}}
$$

at fixed impact parameters

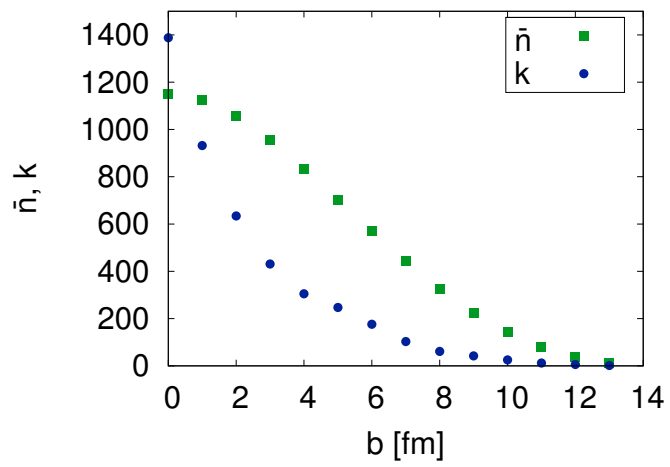

Ratio of $k / \bar{n}$ is $>1$ for small $b$ and becomes small $\sim 0.14$ for large $b$. That is close to the value extracted for $p+p$ collisions: Dumitru and Nara arXiv:1201.6382 


\section{NBDs and Glasma flux tubes}

B.Schenke, P.Tribedy, R.Venugopalan, Phys.Rev.C86, 034908 (2012)

Glasma flux tube picture:

$$
k=\zeta \frac{N_{c}^{2}-1}{2 \pi} Q_{s}^{2} S_{\perp}
$$

Gelis, Lappi, Mclerran, arXiv:0905.3234

Width of NBD is inversely proportional to the number of flux tubes $Q_{s}^{2} S_{\perp}$. $S_{\perp}=$ interaction area

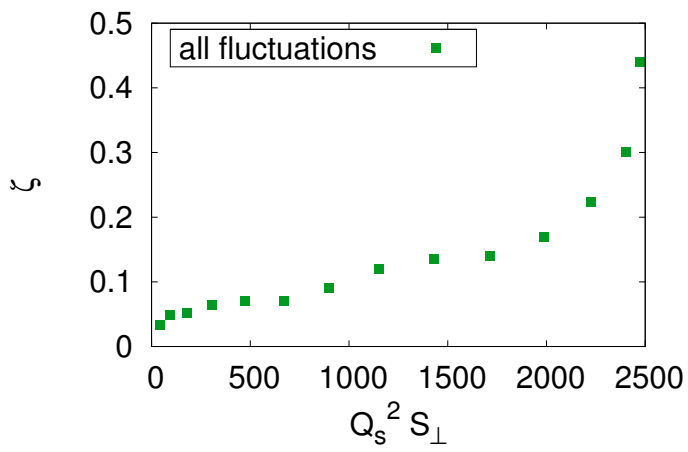

$\zeta$ should be close to constant in the flux tube picture 


\section{NBDs and Glasma flux tubes}

B.Schenke, P.Tribedy, R.Venugopalan, Phys.Rev.C86, 034908 (2012)

$\zeta$ is not constant because geometric fluctuations are very important Were not considered in the derivation of

$$
k=\zeta \frac{N_{c}^{2}-1}{2 \pi} Q_{s}^{2} S_{\perp}
$$

Eliminate by using smooth nucleon distributions:

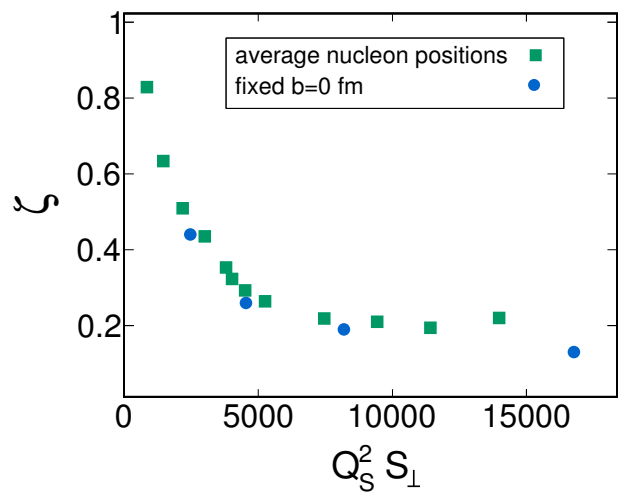




\section{Color charge densities of incoming nuclei}

- Sample positions of nucleons from Woods-Saxon distributions in nucleus A and B.

- IP-Sat provides $Q_{s}^{2}\left(x, \mathbf{b}_{\perp}\right)$ for each nucleon.

The color charge density squared $g^{2} \mu^{2}$ is proportional to $Q_{s}^{2}$.

(proportionality factor depends on details of calculation - see Lappi, arXiv:0711.3039)

- We add all $g^{2} \mu^{2}\left(\mathbf{x}_{\perp}\right)$ in each nucleus to obtain $g^{2} \mu_{1}^{2}\left(\mathbf{x}_{\perp}\right)$ and $g^{2} \mu_{2}^{2}\left(\mathbf{x}_{\perp}\right)$.

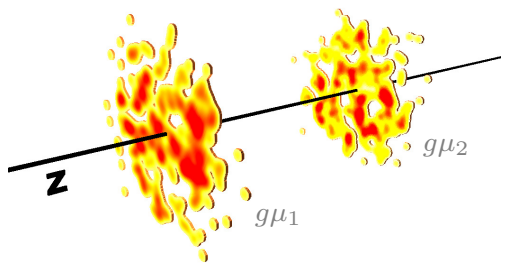

- Sample $\rho^{a}$ from local Gaussian distribution for each nucleus

$$
\left\langle\rho^{a}\left(\mathbf{x}_{\perp}\right) \rho^{b}\left(\mathbf{y}_{\perp}\right)\right\rangle=\delta^{a b} \delta^{2}\left(\mathbf{x}_{\perp}-\mathbf{y}_{\perp}\right) g^{2} \mu^{2}\left(\mathbf{x}_{\perp}\right)
$$




\section{Gauge fields before the collision}

Color currents:

$$
\begin{aligned}
& J_{1}^{\nu}=\delta^{\mu+} \rho_{1}\left(x^{-}, \mathbf{x}_{\perp}\right) \\
& {\left[D_{\mu}, F^{\mu \nu}\right]=J_{1}^{\nu}}
\end{aligned}
$$

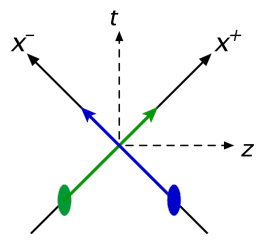

$$
\begin{aligned}
& J_{2}^{\nu}=\delta^{\mu-} \rho_{2}\left(x^{+}, \mathbf{x}_{\perp}\right) \\
& {\left[D_{\mu}, F^{\mu \nu}\right]=J_{2}^{\nu}}
\end{aligned}
$$

Solution in covariant gauge:

$$
A_{\operatorname{cov}(1,2)}^{+}\left(x^{-}, \mathbf{x}_{\perp}\right)=-\frac{g \rho_{(1,2)}\left(x^{-}, \mathbf{x}_{\perp}\right)}{\nabla_{\perp}^{2}+m^{2}}
$$

with infrared cutoff $m$ of order $\Lambda_{\mathrm{QCD}}$.

Solution in light cone gauge:

$V$ is the path-ordered exponential of $A_{\mathrm{Cov}(1,2)}^{+}$

$$
\begin{aligned}
& A_{(1,2)}^{+}\left(\mathbf{x}_{\perp}\right)=A_{(1,2)}^{-}\left(\mathbf{x}_{\perp}\right)=0 \\
& A_{(1,2)}^{i}\left(\mathbf{x}_{\perp}\right)=\frac{i}{g} V_{(1,2)}\left(\mathbf{x}_{\perp}\right) \partial_{i} V_{(1,2)}^{\dagger}\left(\mathbf{x}_{\perp}\right)
\end{aligned}
$$




\section{Gauge fields before the collision}

The correlator of the Wilson lines (same one we looked at before)

$$
C_{(1,2)}\left(\mathbf{x}_{\perp}\right)=\frac{1}{N_{c}} \operatorname{Re}\left[\operatorname{tr}\left(V(1,2)^{\dagger}(0,0) V(1,2)(x, y)\right)\right]
$$

with

$$
V_{(1,2)}\left(\mathbf{x}_{\perp}\right)=P \exp \left(-i g \int d x^{-} \frac{\rho_{(1,2)}\left(x^{-}, \mathbf{x}_{\perp}\right)}{\nabla_{\perp}^{2}+m^{2}}\right)
$$

shows the degree of correlations and fluctuations in the gluon fields.

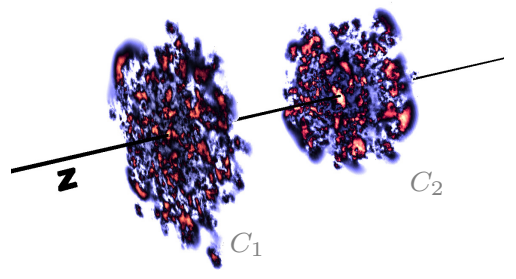

The length scale of fluctuations is $1 / Q_{s}$. Not the nucleon size. 


\section{IP-Glasma: Gauge fields after the collision (Glasma)}

Initial condition on the lightcone:

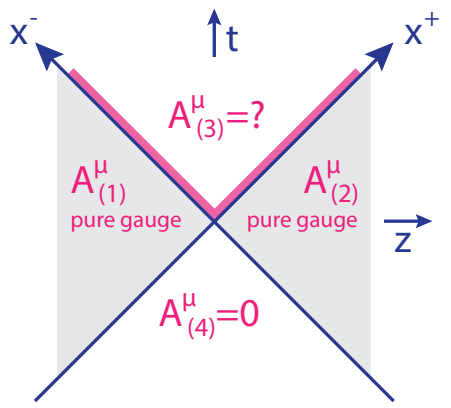

\section{Solution:}

$$
\begin{aligned}
& \left.A_{(3)}^{i}\right|_{\tau=0}=A_{(1)}^{i}+A_{(2)}^{i} \\
& \left.A_{(3)}^{\eta}\right|_{\tau=0}=\frac{i g}{2}\left[A_{(1)}^{i}, A_{(2)}^{i}\right]
\end{aligned}
$$

Configuration in Schwinger gauge $A^{\tau}=0$

We solve for the gauge fields numerically

Krasnitz, Venugopalan, Nucl.Phys. B557 (1999) 237

Time evolution follows Yang-Mills equations 


\section{Modeling $x$ and $b$ dependence: IP-Sat model}

Want to determine color charge distribution in a nucleus. Proton first: Use IP-Sat model to parametrize

- $x$-dependence

- Impact parameter dependence (IP)

Kowalski, Teaney, Phys.Rev. D68 (2003) 114005

$x$-evolution can be computed using JIMWLK,

but parametrization is easier for now 


\section{Modeling $x$ and $b$ dependence: IP-Sat model}

Want to determine color charge distribution in a nucleus. Proton first: Use IP-Sat model to parametrize

- $x$-dependence

- Impact parameter dependence (IP)

Kowalski, Teaney, Phys.Rev. D68 (2003) 114005

$x$-evolution can be computed using JIMWLK,

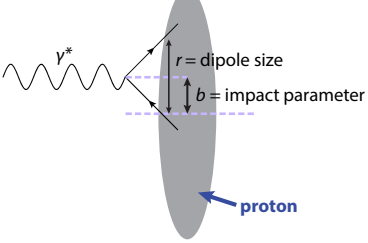

but parametrization is easier for now

IP-Sat proton dipole cross section in deeply inealstic scattering (DIS):

$$
\frac{\mathrm{d} \sigma_{\mathrm{dip}}^{\mathrm{p}}}{\mathrm{d}^{2} \mathbf{b}_{\perp}}\left(\mathbf{r}_{\perp}, x, \mathbf{b}_{\perp}\right)=2 \mathcal{N}\left(\mathbf{r}_{\perp}, x, \mathbf{b}_{\perp}\right)=2\left[1-\exp \left(-\frac{\pi^{2}}{2 N_{c}} \mathbf{r}_{\perp}{ }^{2} \alpha_{s}\left(Q^{2}\right) x g\left(x, Q^{2}\right) T_{p}\left(\mathbf{b}_{\perp}\right)\right)\right]
$$

with $Q^{2}$ an energy scale related to the dipole size $\mathbf{r}_{\perp}$

Parameters fit to HERA diffractive data

gluon density

\section{sity}

$Q_{s}$ is defined by the scale $r$ where $\mathcal{N}$ reaches the saturated regime

$\mathcal{N}\left(R_{s}, x, \mathbf{b}_{\perp}\right)=1-e^{-1 / 2}$, with $Q_{s}^{2}=\frac{2}{R_{s}^{2}}$ 


\section{Gauge fields after the collision (Glasma)}

Initial condition on the lightcone: require that fields match smoothly on the lightcone.

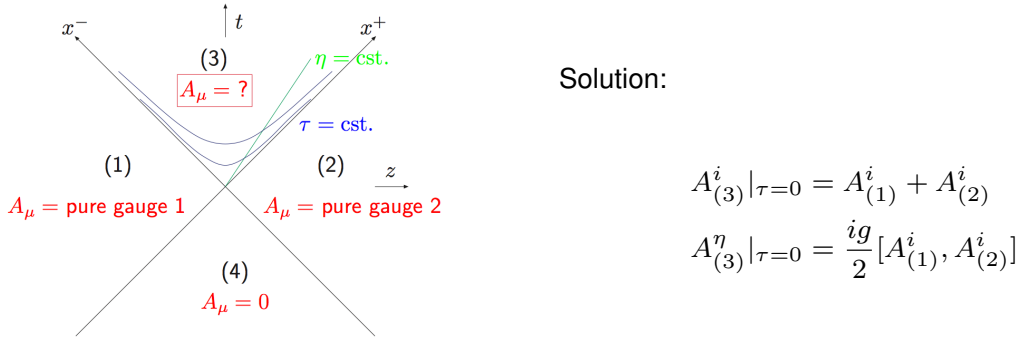

figure from Lappi, arXiv:1003.1852

On the lattice the Wilson lines in the future lightcone are obtained from the condition:

$$
\operatorname{tr}\left\{t^{a}\left[\left(U_{(1)}^{i}+U_{(2)}^{i}\right)\left(1+U_{(3)}^{i \dagger}\right)-\left(1+U_{(3)}^{i}\right)\left(U_{(1)}^{i \dagger}+U_{(2)}^{i \dagger}\right)\right]\right\}=0
$$

where $t^{a}$ are the generators of $S U\left(N_{c}\right)$ in the fundamental representation. Solve iteratively. Krasnitz, Venugopalan, Nucl.Phys. B557 (1999) 237

$$
U_{(1,2), j}^{i}=V_{(1,2), j} V_{(1,2), j+\hat{e_{i}}}^{\dagger} \quad \text { (gauge transform of 1: pure gauge) }
$$

$E_{\eta}$ can be obtained from $U_{(1,2,3)}^{i}$ 


\section{Modeling $x$ and $b$ dependence of $Q_{s}$ : IP-Sat model}

After fitting parameters to HERA DIS data the model provides a distribution of $Q_{s}^{2}\left(x, \mathbf{b}_{\perp}\right)$ of the proton, which will be our input It is determined self consistently from the requirement that

$$
\mathcal{N}\left(R_{s}, x, \mathbf{b}_{\perp}\right)=1-e^{-1 / 2}, \text { with } Q_{s}^{2}=\frac{2}{R_{s}^{2}}
$$

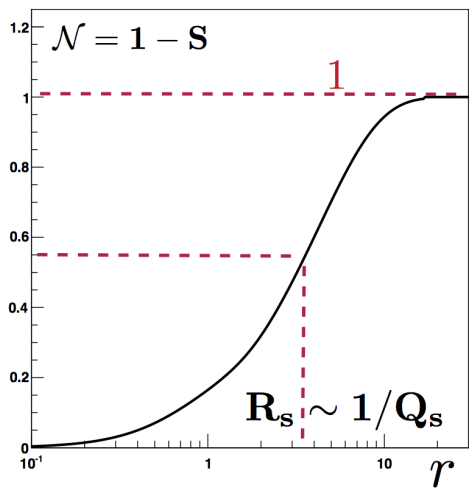




\section{Multiplicity в.Scchenke, P.T.ribedy, R.V.enugopalan, Phys. Rev. C86, 034908 (2012)}

$d N_{\mathrm{g}} / d y$ in transverse Coulomb gauge $\partial_{i} A^{i}=0$

$N_{\text {part }}$ from MC-Glauber with $\sigma_{N N}=42 \mathrm{mb}$ and $64 \mathrm{mb}$ respectively

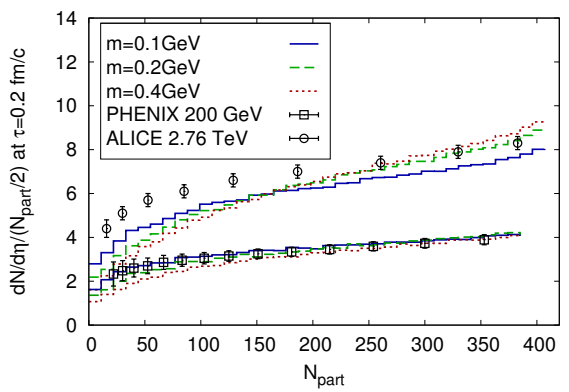

Experimental data: PHENIX, Phys.Rev.C71 034908 (2004) and ALICE, Phys.Rev.Lett. 106, 032301 (2011)

Scaled by $2 / 3$ to compare to charged particles.

New version. Using IP-Sat for nuclei. $m$-dependence. 


\section{Equations to be solved}

Explicit version of $\partial_{\mu} T^{\mu \nu}=0$ in an appropriate coordinate system that expands longitudinally

proper time $\tau=\sqrt{t^{2}-z^{2}}$, space-time rapidity $\eta_{s}=\frac{1}{2} \ln \frac{t+z}{t-z}$

$$
\begin{aligned}
& \partial_{\tau}\left(\tau T^{\tau \tau}\right)+\partial_{v}\left(\tau T^{v \tau}\right)+\partial_{\eta_{s}}\left(T^{\eta_{s} \tau}\right)+T^{\eta_{s} \eta_{s}} \\
& +\partial_{\tau}\left(\tau \pi^{\tau \tau}\right)+\partial_{v}\left(\tau \pi^{v \tau}\right)+\partial_{\eta_{s}}\left(\pi^{\eta_{s} \tau}\right)+\pi^{\eta_{s} \eta_{s}}=0 \\
& \partial_{\tau}\left(\tau T^{\tau \eta_{s}}\right)+\partial_{v}\left(\tau T^{v \eta_{s}}\right)+\partial_{\eta_{s}}\left(T^{\eta_{s} \eta_{s}}\right)+T^{\tau \eta_{s}} \\
& +\partial_{\tau}\left(\tau \pi^{\tau \eta_{s}}\right)+\partial_{v}\left(\tau \pi^{v \eta_{s}}\right)+\partial_{\eta_{s}}\left(\pi^{\eta_{s} \eta_{s}}\right)+\pi^{\tau \eta_{s}}=0 \\
& \quad \partial_{\tau}\left(\tau T^{\tau v}\right)+\partial_{w}\left(\tau T^{w v}\right)+\partial_{\eta_{s}}\left(T^{\eta_{s} v}\right) \\
& \quad+\partial_{\tau}\left(\tau \pi^{\tau v}\right)+\partial_{w}\left(\tau \pi^{w v}\right)+\partial_{\eta_{s}}\left(\pi^{\eta_{s} v}\right)=0
\end{aligned}
$$

spatial derivatives (computed using Kurganov-Tadmor method) treated as sources 


\section{Equations to be solved}

In addition, we have to solve the equation for $\pi^{\mu \nu}$ ( $2^{\text {nd }}$ order Israel-Stewart viscous fluid-dynamics)

$$
\begin{aligned}
\partial_{c}\left(u^{c} \pi^{a b}\right)= & -\frac{1}{2 \tau} u^{\tau} \pi^{a b}+\frac{1}{\tau} \Delta^{a \eta} u^{\eta} \pi^{b \tau}-\frac{1}{\tau} \Delta^{a \tau} u^{\eta} \pi^{b \eta} \\
& -g_{c f} \pi^{c b} u^{a} D u^{f}-\frac{\pi^{a b}}{2 \tau_{\pi}}-\frac{1}{6} \pi^{a b} \partial_{c} u^{c} \\
& +\frac{\eta}{\tau_{\pi}}\left(-\frac{1}{\tau} \Delta^{a \eta} g^{b \eta} u^{\tau}+\frac{1}{\tau} \Delta^{a \eta} g^{b \tau} u^{\tau}\right. \\
& \left.+g^{a c} \partial_{c} u^{b}-u^{a} D u^{b}-\frac{1}{3} \Delta^{a b} \partial_{c} u^{c}\right) \\
& +(a \leftrightarrow b),
\end{aligned}
$$

relaxation time $\tau_{\pi}$

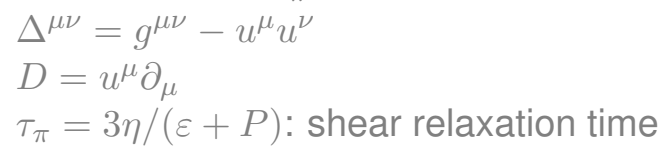




\section{Yang-Mills evolution}

The equations of motion are formulated on the lattice in $2+1 \mathrm{D}\left(\phi=A_{\eta}\right)$

$$
\begin{aligned}
\dot{U}_{i} & \left.=i \frac{g^{2}}{\tau} E^{i} U_{i} \text { (no sum over } i\right) \\
\dot{\phi} & =\tau E^{\eta} \\
\dot{E}^{1} & =\frac{i \tau}{2 g^{2}}\left[U_{1,2}+U_{1,-2}-U_{1,2}^{\dagger}-U_{1,-2}^{\dagger}-T_{1}\right]+\frac{i}{\tau}\left[\tilde{\phi}_{1}, \phi\right] \\
\dot{E}^{2} & =\frac{i \tau}{2 g^{2}}\left[U_{2,1}+U_{2,-1}-U_{2,1}^{\dagger}-U_{2,-1}^{\dagger}-T_{2}\right]+\frac{i}{\tau}\left[\tilde{\phi}_{1}, \phi\right] \\
\dot{E}^{\eta} & =\frac{1}{\tau} \sum_{i}\left[\tilde{\phi}_{i}+\tilde{\phi}_{-i}-2 \phi\right]
\end{aligned}
$$

where $T_{1}=\frac{1}{N_{c}} \operatorname{tr}\left[U_{1,2}+U_{1,-2}-U_{1,2}^{\dagger}-U_{1,-2}^{\dagger}\right] \mathbf{1}$, and $T_{2}=\frac{1}{N_{c}} \operatorname{tr}\left[\left[U_{2,1}+U_{2,-1}-U_{2,1}^{\dagger}-U_{2,-1}^{\dagger}\right] \mathbf{1}\right.$ with the $N_{c} \times N_{c}$ unit matrix $\mathbf{1}$ $\tilde{\phi}_{i}^{j}=U_{j}^{i} \phi_{j+\hat{e}_{i}} U_{j}^{i \dagger}$ and $U_{1,2}^{j}=U_{j}^{1} U_{j+\hat{e}_{1}}^{2} U_{j+\hat{e}_{2}}^{1 \dagger} U_{j}^{2 \dagger}$ 


\section{Event plane}

To get non-zero odd moments, we rotate the event plane in each event. Event plane is defined by the angle:

$$
\psi_{n}=\frac{1}{n} \arctan \frac{\langle w \sin (n \phi)\rangle}{\langle w \cos (n \phi)\rangle}
$$

using particle momenta.

$\left(w=p_{T}\right.$ (first results) or 1 (later results))

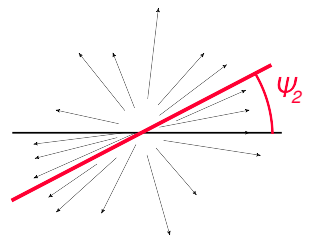

A.Poskanzer and S.Voloshin, Phys.Rev.C58:1671-1678 (1998)

$$
v_{n}=\left\langle\cos \left(n\left(\phi-\psi_{n}\right)\right)\right\rangle
$$

... different angle for every flow coefficient.

In the simulation we know the true event-plane (no correction factor). 


\section{Effect of initial flow}

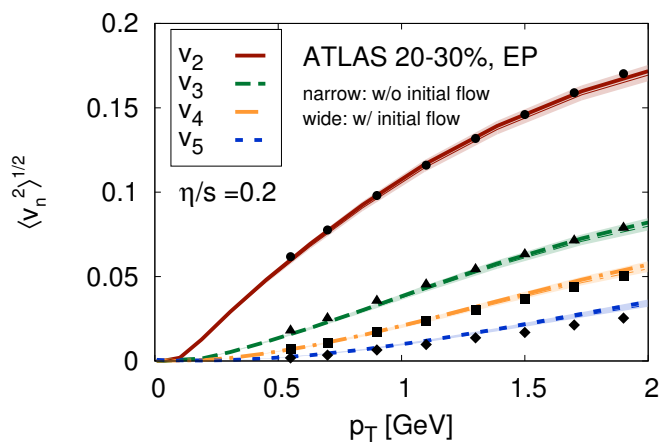

Weak effect of initial flow on hadron $v_{n}\left(p_{T}\right)$

Expect stronger effect for photon $v_{n}$ :

Photons are mostly produced early at high temperatures

Effect of different switching time $0.4 \mathrm{fm} / c$ is very weak

\section{Experimental data:}

ATLAS collaboration, Phys. Rev. C 86, 014907 (2012) 


\section{Directed flow $v_{1}$}
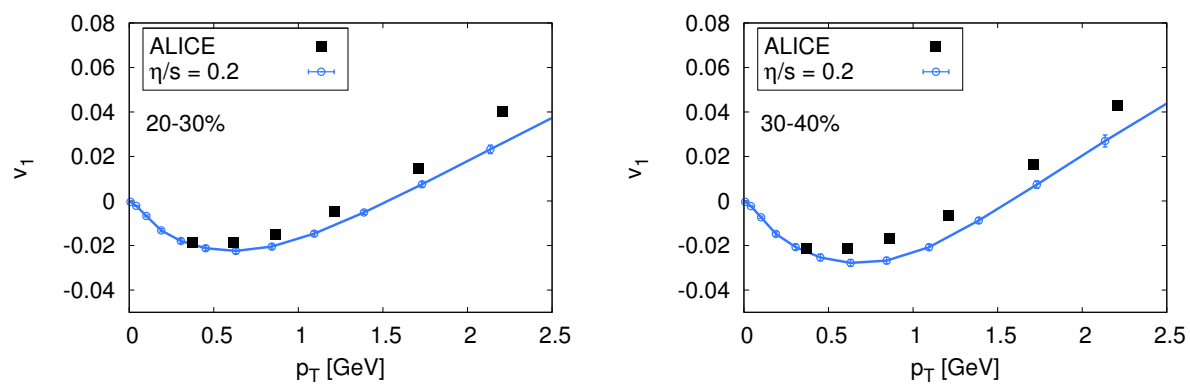

\section{Experimental data:}

extracted in Retinskaya et al., Phys.Rev.Lett. 108 (2012) 252302

from ALICE data in K. Aamodt et al., Phys. Lett. B 708, 249 (2012) 


\section{More centrality classes: IP-Glasma + MUSIC}
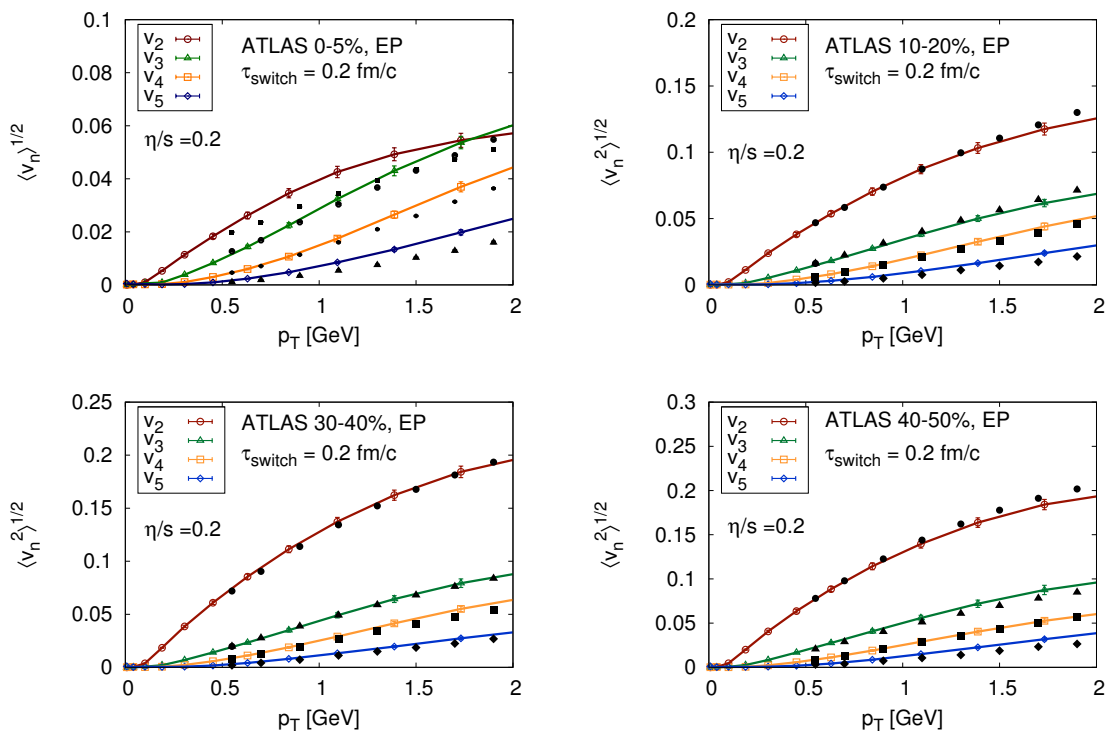


\section{Smaller average $\eta / s$}

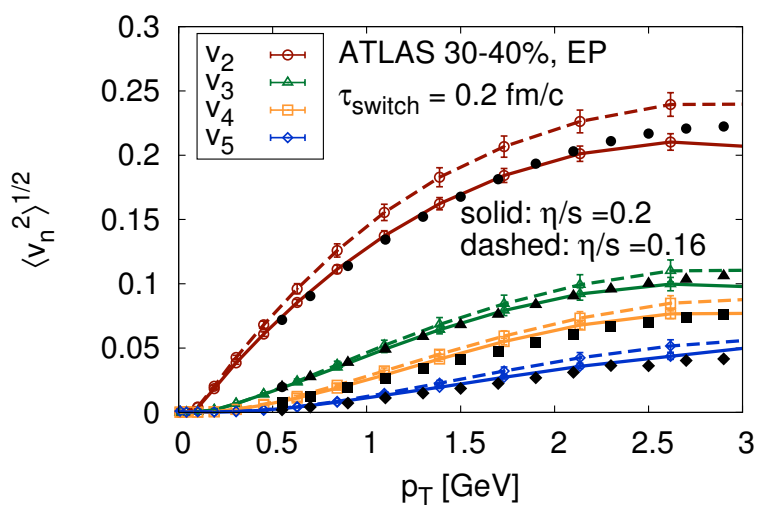

Using $\eta / s=0.16$ overestimates all $v_{n}$

Experimental data:

ATLAS collaboration, Phys. Rev. C 86, 014907 (2012) 


\section{High Multiplicity pp and pA: explosive Stringy Pomeron and Hydrodynamics at its Edge}

\section{arXiv:1301.4470}

Edward Shuryak and Ismail Zahed

Stony Brook

Talk at RBRC workshop,April 2013

2 parts:

micro $=>$ macro

macro $=>$ micro 


\section{micro-to-macro: excitation of stringy Pomeron}

- stringy Pomeron: Stoffers-Zahed model

- $\mathrm{T}=>\mathrm{T}$ (Hagedorn) in bulk and for one string

- transition to explosive regime

- black hole connection 


\section{stringy Pomeron stringy Pomeron}

At very high energies the rapidity interval parameter is large

$$
\chi=\ln \left(s / s_{0}\right) \gg 1
$$

and will play the role of the effective time in

sion. Two longitudinal directions - time and the beam direction, also often used as light cone variables $x_{ \pm}$- are complemented by two transverse coordinates plus a "scale coordinate" $z$. Its initial value corresponds to a physical size of the colliding dipoles and diffusion means the production of small size closed strings. The zcoordinate is not flat. We will model its metric by an $\mathrm{AdS}_{5}$ with a wall. The number of transverse coordinates, which will play an important role in the following, is thus

$$
D_{\perp}=3
$$

$\frac{1}{-2 i s} \mathcal{T}(s, t ; k) \approx g_{s}^{2} \int d^{2} \mathbf{b} e^{i q \cdot \mathbf{b}} \mathbf{K}_{T}(\beta, \mathbf{b} ; k)(50)$

where $\mathbf{K}_{T}$ is the pomeron propagator for dipole sources of color $N_{c}$-ality $k$ describing the string flux. $k$ runs over all integers till $N_{c} / 2$ for even $N_{c}$ and $N_{c} / 2+1 / 2$ for odd ones. In the real world with the $\mathrm{SU}(3)$ color group, $k=1$ is the usual string between fundamental charges (quarks) and the largest tension $k=2$ is the one between two baryon junctions. The first argu-

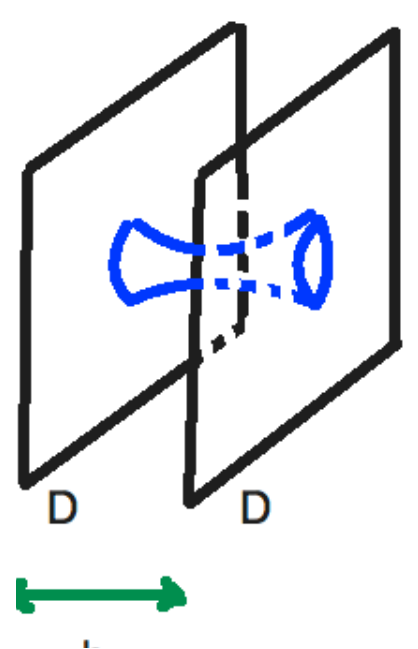

$$
\mathcal{T}(s, t ; k) \approx i g_{s}^{2}\left(\frac{s}{s_{0}}\right)^{1+\frac{k D \perp}{12}+\frac{\alpha^{\prime}}{2 k} t}
$$

Thus the resulting pomeron is supercritical, with the intercept above 1

$$
\alpha_{\mathbf{P}, k}(0)=1+\frac{k D_{\perp}}{12} \rightarrow 1+\frac{k D_{\perp}}{12}-\frac{\left(D_{\perp}-1\right)^{2}}{8 \sqrt{\lambda}}
$$

where the first term is due to Casimir-Luscher contribution and the $1 / \sqrt{\lambda}$ correction follows from the tachyonic correction (58) as discussed in $[1]$.

$D_{k} \chi$. A more precise bound follows from the inclusion of the $1 / \lambda$ corrections in the tachyon mass (58) or

$$
\beta>\sqrt{2\left(\alpha_{\mathbf{P}}-1\right)} \beta_{H}
$$

This leads to the bound $\chi<10$ for the corrected phenomenological value of the pomeron intercept $\alpha_{\mathbf{P}}-1=0.08$ in (60), which roughly corresponds to energies below the LHC. This 
now our paper

a stringy cylinder generates temperature, entropy and even viscosity (Kubo)

$\mathrm{I} / \mathrm{T}$ is circle's sircumference, so $T$ is highest at the middle

$$
\begin{aligned}
\mathbf{S}= & -D_{\perp} \sum_{n=1}^{\infty}\left(\ln \left(1-e^{-\beta_{k} n}\right)+\frac{\beta_{k} n}{e^{\beta_{k} n}-1}\right) \\
& +D_{\perp}\left(\frac{\beta_{k}}{12}-\frac{1}{2}\left(1+\ln \left(\frac{\beta_{k}}{2 \pi}\right)\right)\right)
\end{aligned}
$$

\section{Pure glue thermodynamics}

Near-Hagedorn regime: a string gets excited into a string ball, with growing entropy,energy but not free energy (=p)!

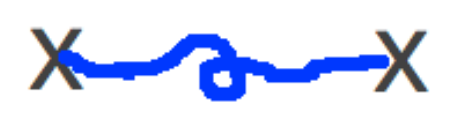

a

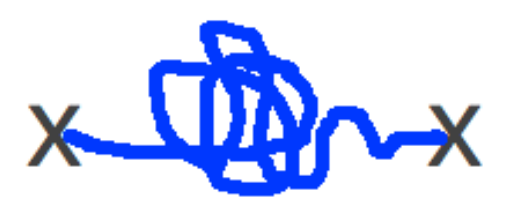

b

Polyakov, Susskind 1978

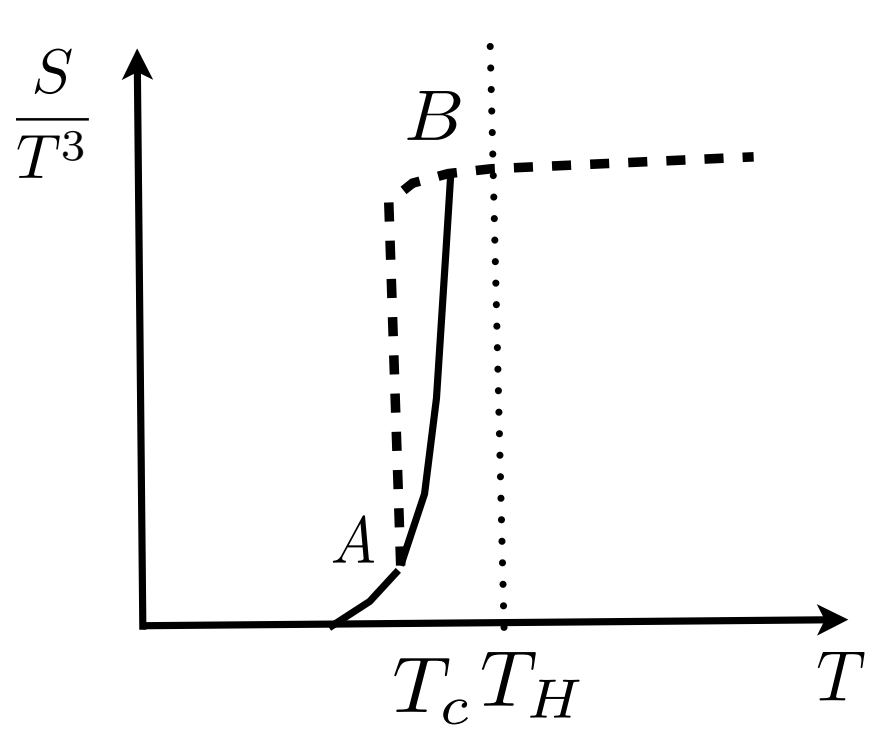

FIG. 2: Schematic temperature dependence of the entropy density. The dashed line represents equilibrium gluodynamics with a first order transition at $T=T_{c}$. The solid line between points $\mathrm{A}$ and $\mathrm{B}$ represents the expected behavior of a single string approaching its Hagedorn temperature $T_{H}$. 


\section{but when $T$ is too close to $\mathbf{T}$ (Hagedorn), transition to QGP happens, pressure grows and the ball can explode!}

\section{$\mathrm{T}=>\mathrm{T}_{\mathrm{H}}$}

\section{Hagedorn regime}

string makes a ball

small F,p large E,S

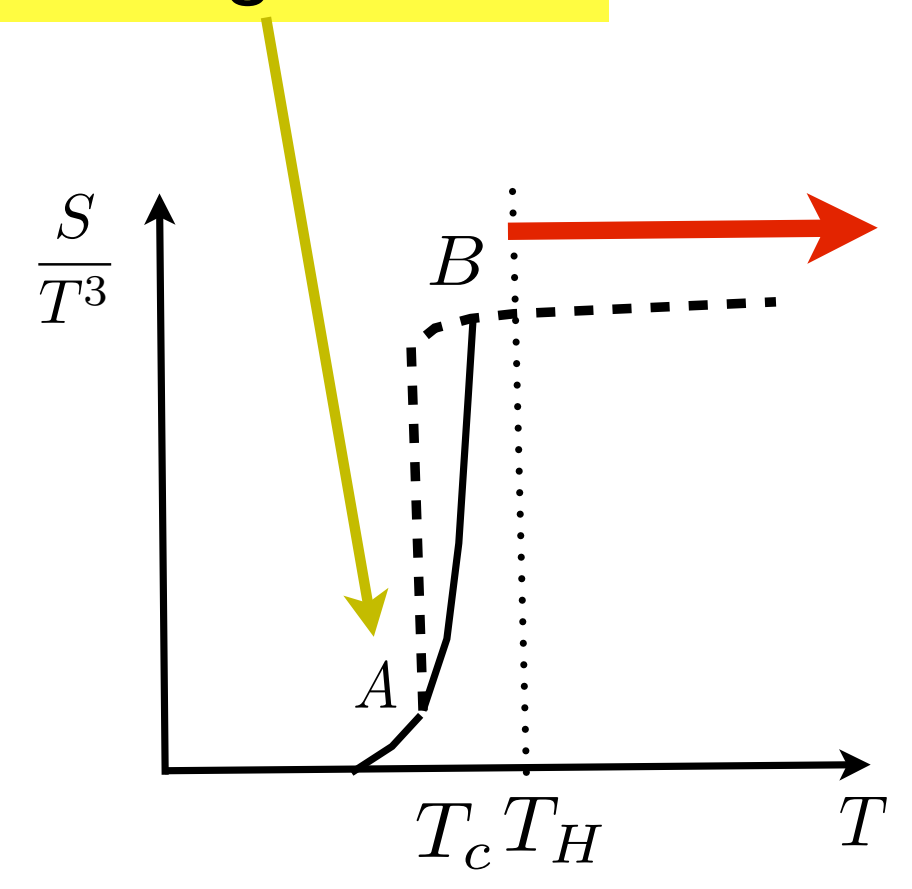

FIG. 2: Schematic temperature dependence of the entropy density. The dashed line represents equilibrium gluodynamics with a first order transition at $T=T_{c}$. The solid line between points $\mathrm{A}$ and $\mathrm{B}$ represents the expected behavior of a single string approaching its Hagedorn temperature $T_{H}$.

$$
\frac{\Delta \beta}{\beta_{H}}=\frac{T_{H}}{T}-1=\mathcal{O}\left(\frac{1}{N_{c}}\right)
$$

The ratio of the high multiplicity events to the minimum bias events can be estimated as

$$
\frac{\sigma_{H M}}{\sigma_{M B}} \approx g_{s}^{2} \frac{1}{\chi^{D_{\perp} / 2}}\left(\frac{\Delta \beta}{\beta_{H}}\right) e^{-\chi \sqrt{\Delta \beta / 2 \beta_{H}}}
$$

where we have dropped an overall number of order 1 and $D_{\perp}=3$. For near critical strings we have $(70)$ and $g_{s} \approx 1 / N_{c}$. At LHC, $\chi \approx 10$ so that

$$
\frac{\sigma_{H M}}{\sigma_{M B}} \approx 10^{-5}
$$

This estimate is comparable to the probability of the high multiplicity trigger used by the CMS collaboration for events displaying the ridge in pp collisions at LHC. 


\section{a string ball is dual to a black hole}

The

correspondence is

usually derived via

the entropy

(=Hawking-Bekenstein)

\section{But one can also}

calculate viscosity,

which gives I/4pi

although the calculation is stringy

not $\mathrm{BH}$. (And even if $\mathrm{BH}$ it is

very different from that in AdS/

CFT, not located in 5-th

dimension, so were surprised) follow [2] and write the needed expression on the streched horizon for the excited string

Kubo

$$
\eta_{R}=\lim _{\omega_{R} \rightarrow 0} \frac{A_{R}}{2 \omega_{R}} \int_{0}^{\infty} d \tau e^{i \omega_{R} \tau} \mathbf{R}_{23,23}(\tau)
$$

with $A_{R}$ the area of the black-hole and $\tau$ a dimensionless Rindler time. The retarded commutator of the normal ordered transverse stress tensor for the Polyakov string on the Rindler horizon reads

$$
\mathbf{R}_{23,23}(\tau)=\left\langle\left[T_{\perp}^{23}(\tau), T_{\perp}^{23}(0)\right]\right\rangle
$$

with

$$
T_{\perp}^{23}(\tau)=\frac{1}{2 A_{R}} \sum_{n \neq 0}: a_{n}^{2} a_{n}^{3}: e^{-2 i n \tau}
$$

and the canonical rules $\left[a_{m}^{i}, a_{n}^{j}\right]=m \delta_{m+n, 0} \delta^{i j}$. The averaging in (88) is carried using the blackbody spectrum as in (84). The result is

$$
\eta_{R}=\lim _{\omega_{R} \rightarrow 0} \frac{A_{R}}{2 \omega_{R}} \frac{\pi}{2 A_{R}^{2}} \frac{\left(\omega_{R} / 2\right)^{2}}{e^{\beta_{R} \omega_{R} / 2}-1}=\frac{1}{A_{R}} \frac{\pi}{8 \beta_{R}}
$$

We note the occurence of the BekensteinHawking or Rindler temperature $\beta_{B H}=\beta_{R}$ in the thermal factor.

Combining (86) for the entropy to (90) yields the viscosity on the streched horizon

$$
\frac{\eta_{R}}{\mathbf{S}_{\mathbf{R}} / A_{R}}=\frac{1}{4 \pi}\left(\frac{3}{D_{\perp}}\right) \equiv \frac{1}{4 \pi}
$$




\section{summary micro $=>$ macro}

- at very high energy the strings of the Pomeron gets longer and effectively hotter

- as its T approach Haderon temperature, a string ball regime appears $=>$ high $S, E$ but not $P$

- as T grows too close to T(Hagedorn), string ball saturates the space and transition to sQGP happens. $=>$ p grows $=>$ hydro explosion 


\section{Macro to micro: Outline}




\section{Macro to micro: Outline}

- history before LHC 


\section{Macro to micro: Outline}

- history before LHC

- 4 papers from LHC experiments 


\section{Macro to micro: Outline}

- history before LHC

- 4 papers from LHC experiments

- two small parameters of hydro 


\section{Macro to micro: Outline}

- history before LHC

- 4 papers from LHC experiments

- two small parameters of hydro

- the radial flow, gradients and viscosity 


\section{Macro to micro: Outline}

- history before LHC

- 4 papers from LHC experiments

- two small parameters of hydro

- the radial flow, gradients and viscosity

- higher harmonics, sound damping 


\section{Macro to micro: Outline}

- history before LHC

- 4 papers from LHC experiments

- two small parameters of hydro

- the radial flow, gradients and viscosity

- higher harmonics, sound damping

- higher harmonics for PP and PA 


\section{Macro to micro: Outline}

- history before LHC

- 4 papers from LHC experiments

- two small parameters of hydro

- the radial flow, gradients and viscosity

- higher harmonics, sound damping

- higher harmonics for PP and PA

- higher gradients and LS resummation 


\section{prehistory:}

Can there be hydro in PP? 


\section{prehistory:}

Can there be hydro in PP?

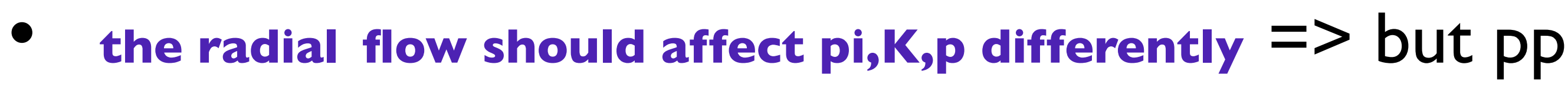
ISR data showed no effect (excellent Mt scaling)

E. V. Shuryak and O. V. Zhirov, Phys. Lett. B 89, 253 (1979). 


\section{prehistory:}

\section{Can there be hydro in PP?}

- $\quad$ the radial flow should affect $p i, K, p$ differently $=>$ but $P P$ ISR data showed no effect (excellent Mt scaling)

E. V. Shuryak and O. V. Zhirov, Phys. Lett. B 89, 253 (1979).

- 20 years later Bjorken and co [MiniMax Collaboration], T. C. Brooks et al. Phys. Rev. D 61, 032003 (2000) [hep-ex/9906026] took data at TEVATRON: minor effect, inconclusive 


\section{prehistory:}

\section{Can there be hydro in PP?}

- the radial flow should affect $p i, K, p$ differently $=>$ but $P P$ ISR data showed no effect (excellent Mt scaling)

E. V. Shuryak and O. V. Zhirov, Phys. Lett. B 89, 253 (1979).

- 20 years later Bjorken and co [MiniMax Collaboration], T. C. Brooks et al. Phys. Rev. D 61, 032003 (2000) [hep-ex/9906026] took data at TEVATRON: minor effect, inconclusive

- the radial flow was seen in AA at AGS/SPS

Good pre-RHIC hydro description C.Hung.E.Shuryak Phys.Rev.C57 (1998) 1891 -1906 


\section{prehistory:}

\section{Can there be hydro in PP?}

- the radial flow should affect $p i, K, p$ differently $=>$ but $P P$ ISR data showed no effect (excellent Mt scaling)

E. V. Shuryak and O. V. Zhirov, Phys. Lett. B 89, 253 (1979).

- 20 years later Bjorken and co [MiniMax Collaboration], T. C. Brooks et al. Phys. Rev. D 61, 032003 (2000) [hep-ex/9906026] took data at TEVATRON: minor effect, inconclusive

- the radial flow was seen in AA at AGS/SPS Good pre-RHIC hydro description C.Hung.E.Shuryak Phys.Rev.C57 (1998) 1891 -1906

- and everyone knows what happened at RHIC, $v \_n$ etc 


\section{LHC era begins}

Observation of long-range, near-side angular correlations in $\mathrm{pPb}$ collisions at the LHC

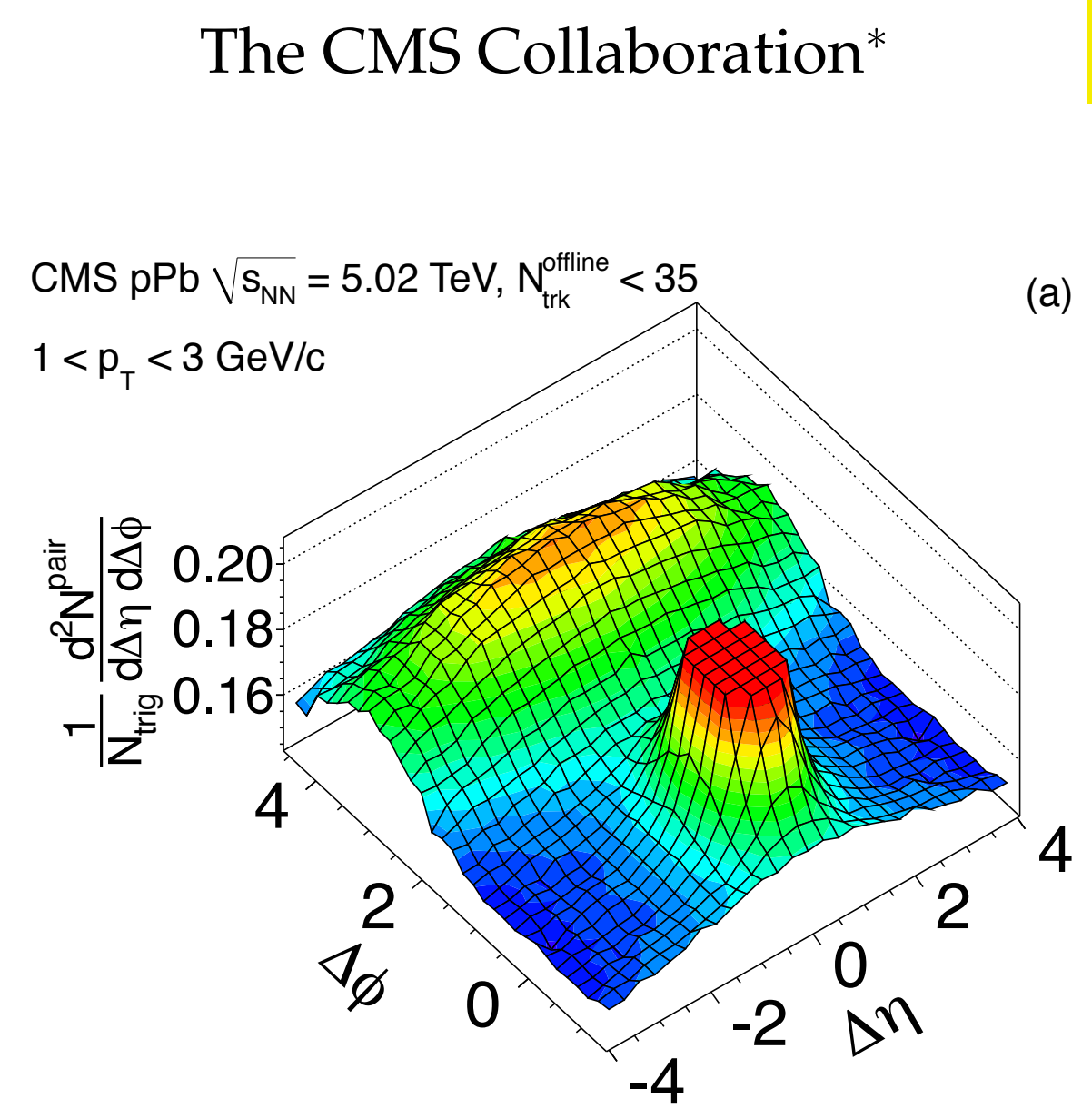

high multiplicity trigger in PP reveals a "ridge"

(a) which is also there in $\mathrm{pPb}$

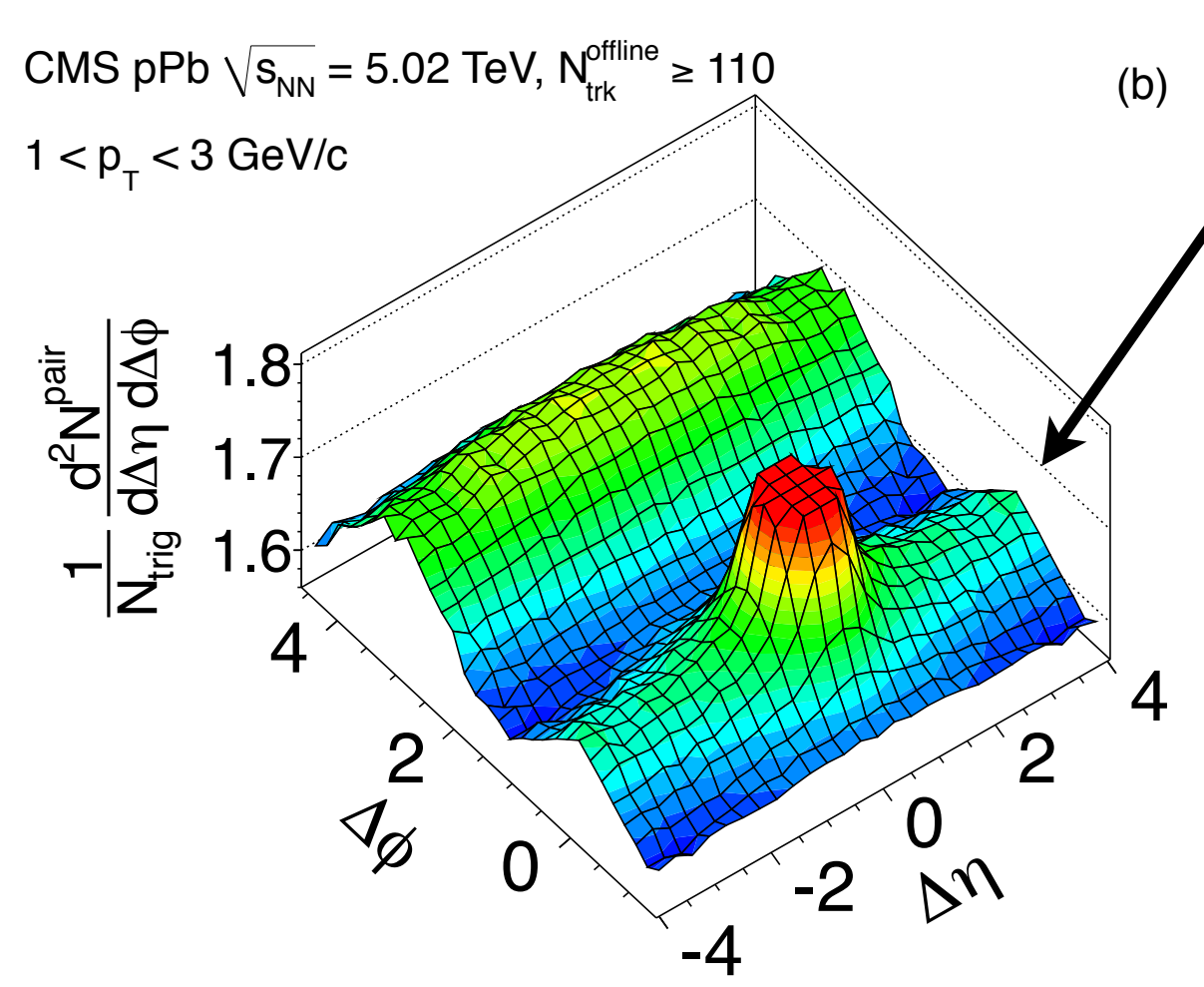

Figure 1: 2-D two-particle correlation functions for $5.02 \mathrm{TeV} \mathrm{pPb}$ collisions for pairs of charged particles with $1<p_{\mathrm{T}}<3 \mathrm{GeV} / c$. Results are shown (a) for low-multiplicity events $\left(N_{\text {trk }}^{\text {offline }}<\right.$ $35)$ and (b) for a high-multiplicity selection ( $\left.N_{\text {trk }}^{\text {offline }} \geq 110\right)$. The sharp near-side peaks from jet correlations have been truncated to better illustrate the structure outside that region. 
- all bins show backto-back correlation well described by "minijet" models like HIJING

- Ridge is a peak at the same azimuth, seen at highest multiplicity only and at $p r=1-2 \mathrm{GeV}$

- it is stronger in $\mathrm{pA}$ than at Pp, yet the same multiplicity is needed

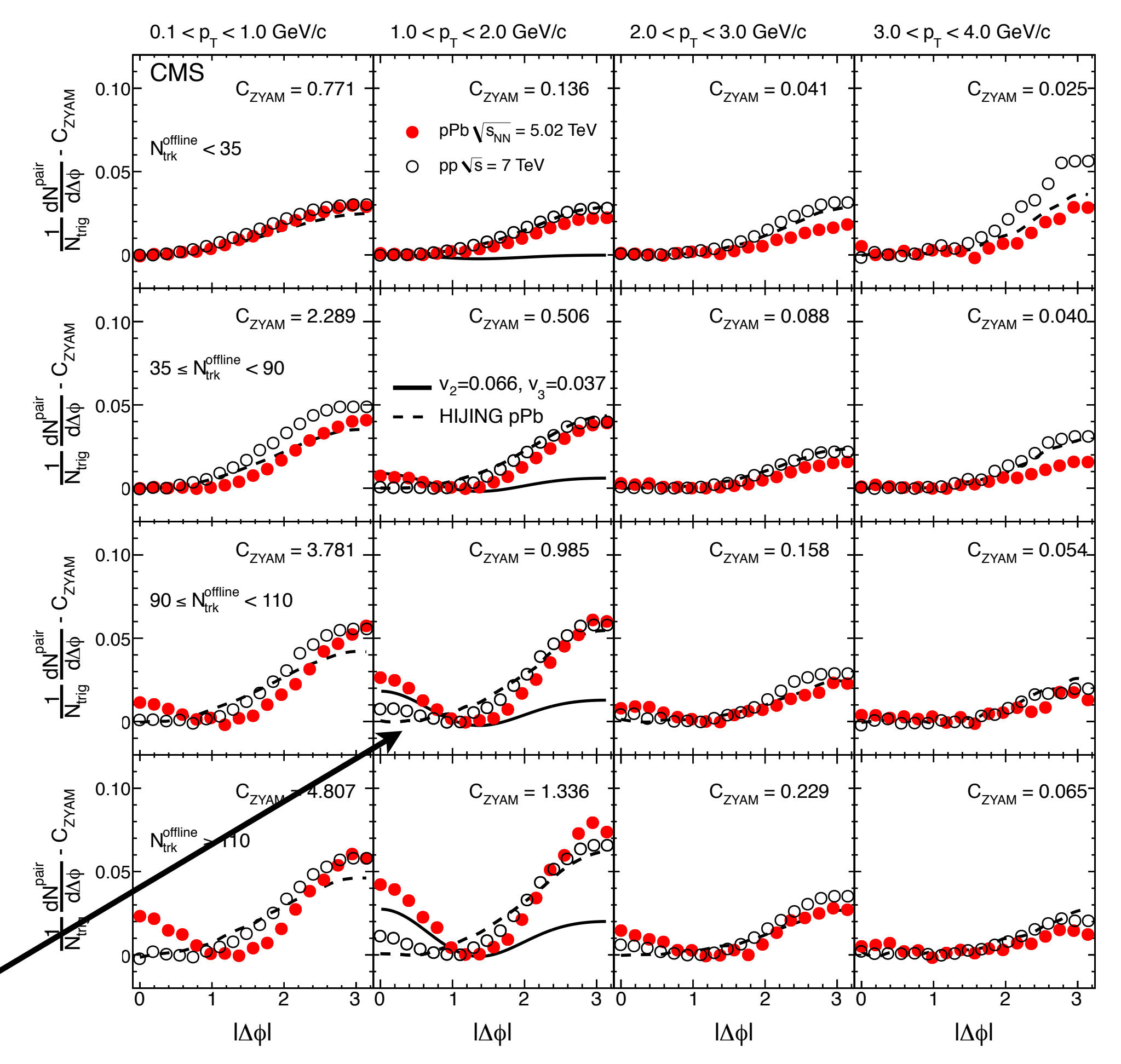

Figure 2: Correlated yield obtained from the ZYAM procedure as a function of $|\Delta \phi|$ averaged over $2<|\Delta \eta|<4$ in different $p_{\mathrm{T}}$ and multiplicity bins for $5.02 \mathrm{TeV} \mathrm{pPb}$ data (solid circles) and $7 \mathrm{TeV}$ pp data (open circles). The $p_{\mathrm{T}}$ selection applies to both particles in each pair. Statistical uncertainties are smaller than the marker size. The subtracted ZYAM constant is listed in each panel. Also shown are $\mathrm{pPb}$ predictions for HIJING [24] (dashed curves) and a hydrodynamic model [25] (solid curves shown for $1<p_{\mathrm{T}}<2 \mathrm{GeV} / \mathrm{c}$ ). 


\section{- a "double ridge" in ALICE and ATLAS}

\section{Long-range angular correlations on the near and away side}

in $\mathrm{p}-\mathrm{Pb}$ collisions at $\sqrt{\mathrm{s}_{\mathrm{NN}}}=\mathbf{5 . 0 2} \mathrm{TeV}$
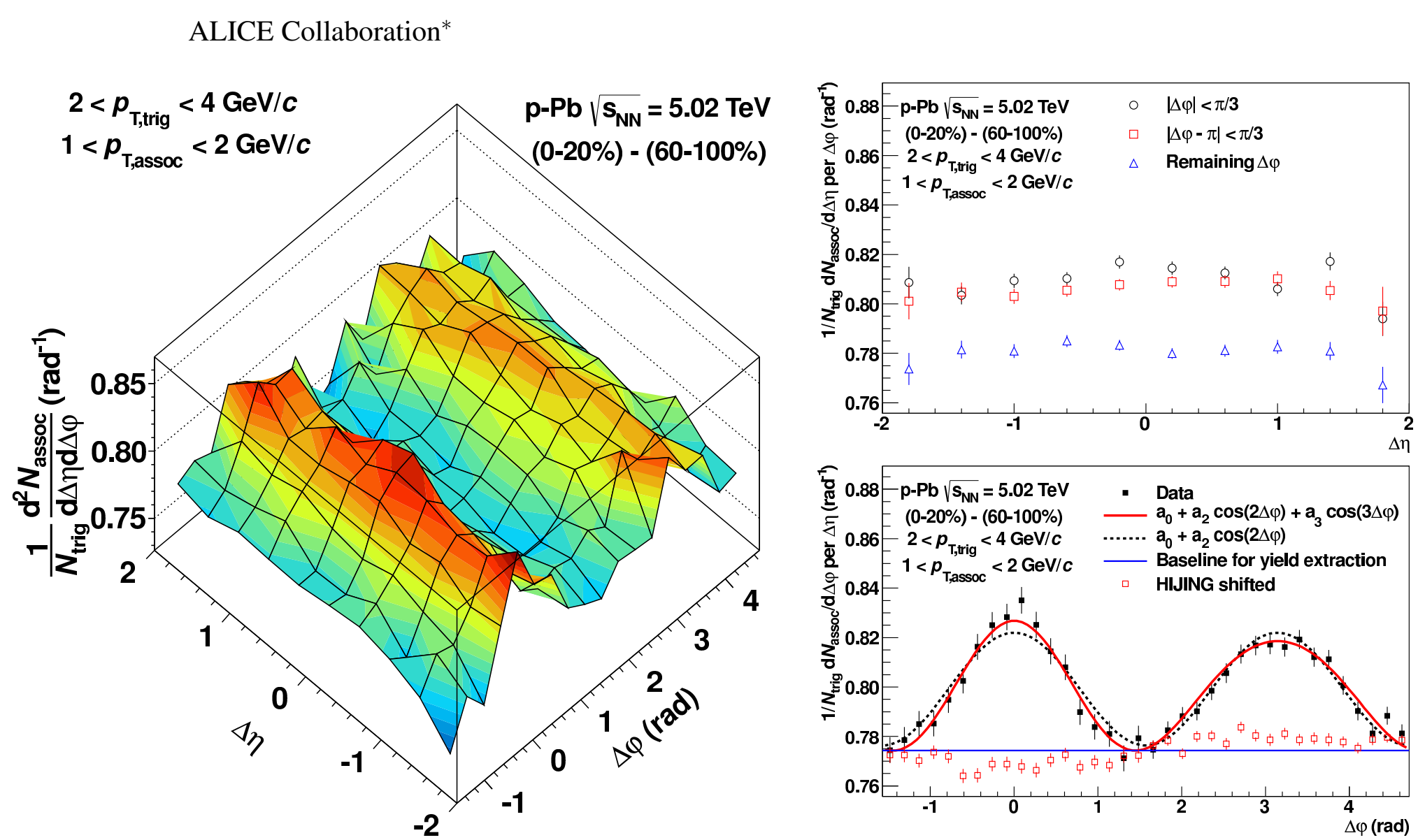

Fig. 3: Left: Associated yield per trigger particle in $\Delta \varphi$ and $\Delta \eta$ for pairs of charged particles with $2<p_{\text {T,trig }}<4 \mathrm{GeV} / c$ and $1<p_{\text {T,assoc }}<2 \mathrm{GeV} / c$ in $\mathrm{p}-\mathrm{Pb}$ collisions at $\sqrt{s_{\mathrm{NN}}}=5.02 \mathrm{TeV}$ for the $0-20 \%$ multiplicity class, after subtraction of the associated yield obtained in the $60-100 \%$ event class. Top right: the associated per-trigger yield after subtraction (as shown on the left) projected onto $\Delta \eta$ averaged over $|\Delta \varphi|<\pi / 3$ (black circles), $|\Delta \varphi-\pi|<\pi / 3$ (red squares), and the remaining area (blue triangles, $\Delta \varphi<-\pi / 3, \pi / 3<\Delta \varphi<2 \pi / 3$ and $\Delta \varphi>4 \pi / 3$ ). Bottom right: as above but projected onto $\Delta \varphi$ averaged over $0.8<|\Delta \eta|<1.8$ on the near side and $|\Delta \eta|<1.8$ on the away side. Superimposed are fits containing a $\cos (2 \Delta \varphi)$ shape alone (black dashed line) and a combination of $\cos (2 \Delta \varphi)$ and $\cos (3 \Delta \varphi)$ shapes (red solid line). The blue horizontal line shows the baseline obtained from the latter fit which is used for the yield calculation. Also shown for comparison is the subtracted associated yield when the same procedure is applied on HIJING shifted to the same baseline. The figure shows only statisti- 


\section{- a "double ridge" in ALICE and ATLAS}

\section{Long-range angular correlations on the near and away side}

in $\mathrm{p}-\mathrm{Pb}$ collisions at $\sqrt{\mathrm{s}_{\mathrm{NN}}}=\mathbf{5 . 0 2} \mathrm{TeV}$

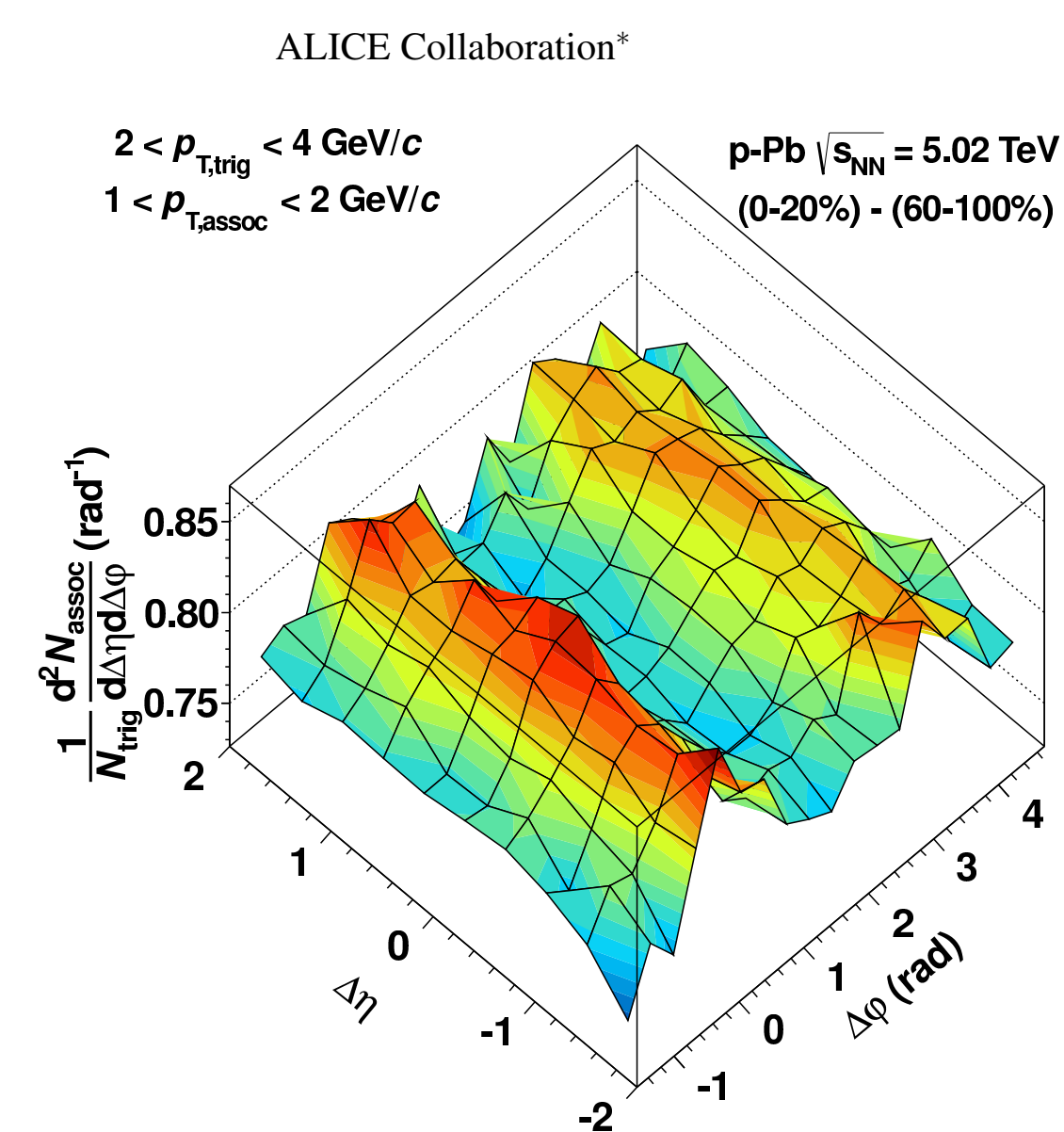

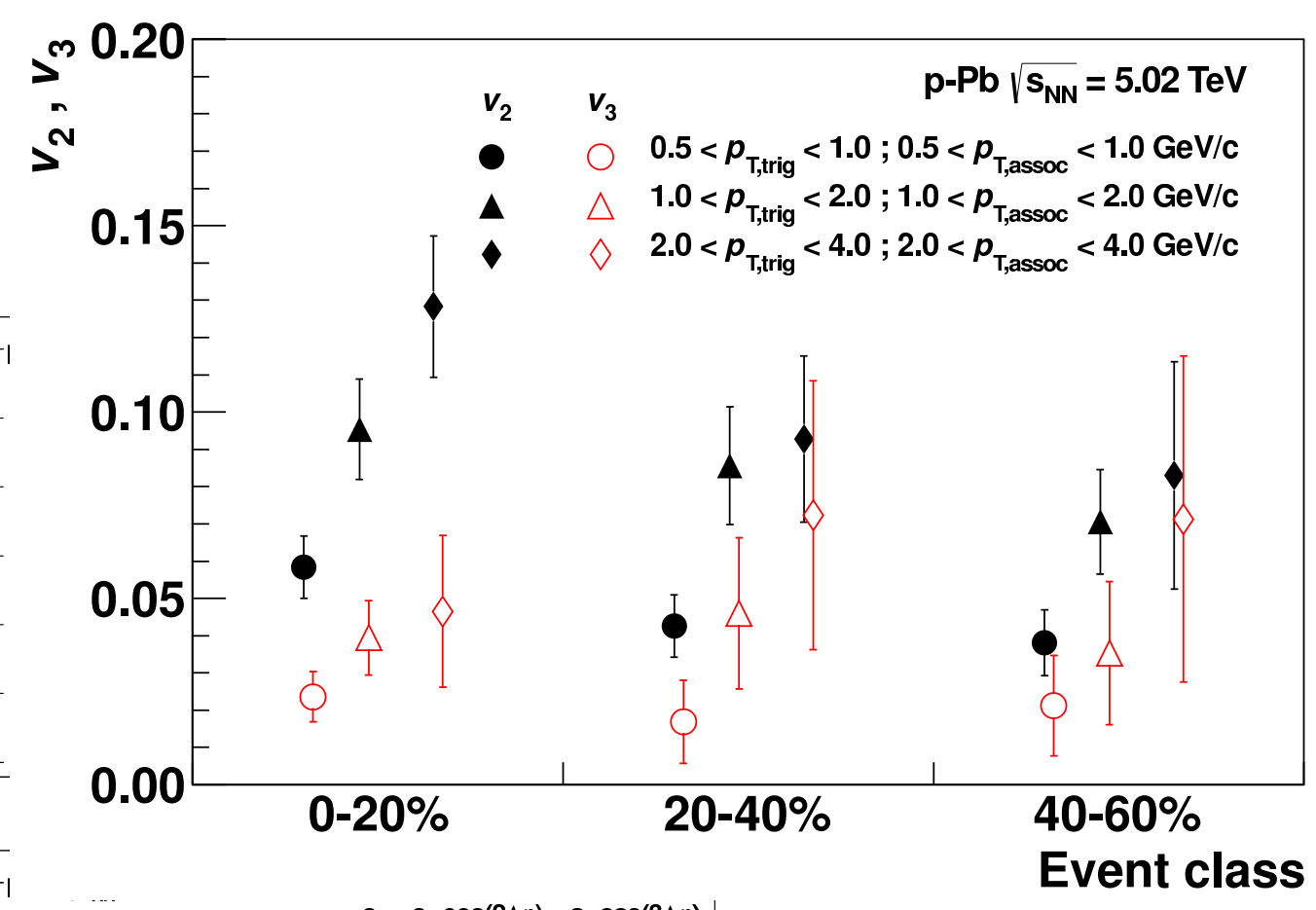

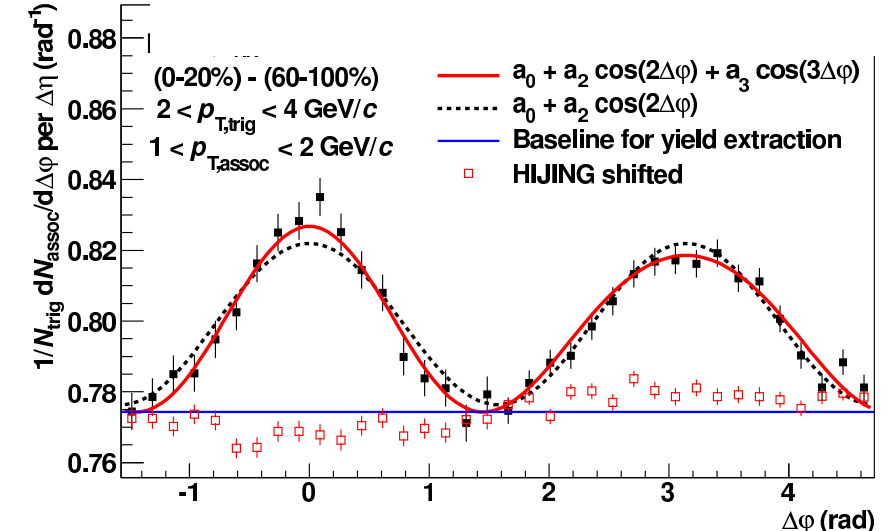

Fig. 3: Left: Associated yield per trigger particle in $\Delta \varphi$ and $\Delta \eta$ for pairs of charged particles with $2<p_{\text {T,trig }}<4 \mathrm{GeV} / c$ and $1<p_{\text {T,assoc }}<2 \mathrm{GeV} / c$ in $\mathrm{p}-\mathrm{Pb}$ collisions at $\sqrt{s_{\mathrm{NN}}}=5.02 \mathrm{TeV}$ for the $0-20 \%$ multiplicity class, after subtraction of the associated yield obtained in the $60-100 \%$ event class. Top right: the associated per-trigger yield after subtraction (as shown on the left) projected onto $\Delta \eta$ averaged over $|\Delta \varphi|<\pi / 3$ (black circles), $|\Delta \varphi-\pi|<\pi / 3$ (red squares), and the remaining area (blue triangles, $\Delta \varphi<-\pi / 3, \pi / 3<\Delta \varphi<2 \pi / 3$ and $\Delta \varphi>4 \pi / 3$ ). Bottom right: as above but projected onto $\Delta \varphi$ averaged over $0.8<|\Delta \eta|<1.8$ on the near side and $|\Delta \eta|<1.8$ on the away side. Superimposed are fits containing a $\cos (2 \Delta \varphi)$ shape alone (black dashed line) and a combination of $\cos (2 \Delta \varphi)$ and $\cos (3 \Delta \varphi)$ shapes (red solid line). The blue horizontal line shows the baseline obtained from the latter fit which is used for the yield calculation. Also shown for comparison is the subtracted associated yield when the same procedure is applied on HIJING shifted to the same baseline. The figure shows only statisti- 


\section{- a "double ridge" in ALICE and ATLAS}

\section{Long-range angular correlations on the near and away side}

in $\mathrm{p}-\mathrm{Pb}$ collisions at $\sqrt{\mathrm{s}_{\mathrm{NN}}}=\mathbf{5 . 0 2} \mathrm{TeV}$

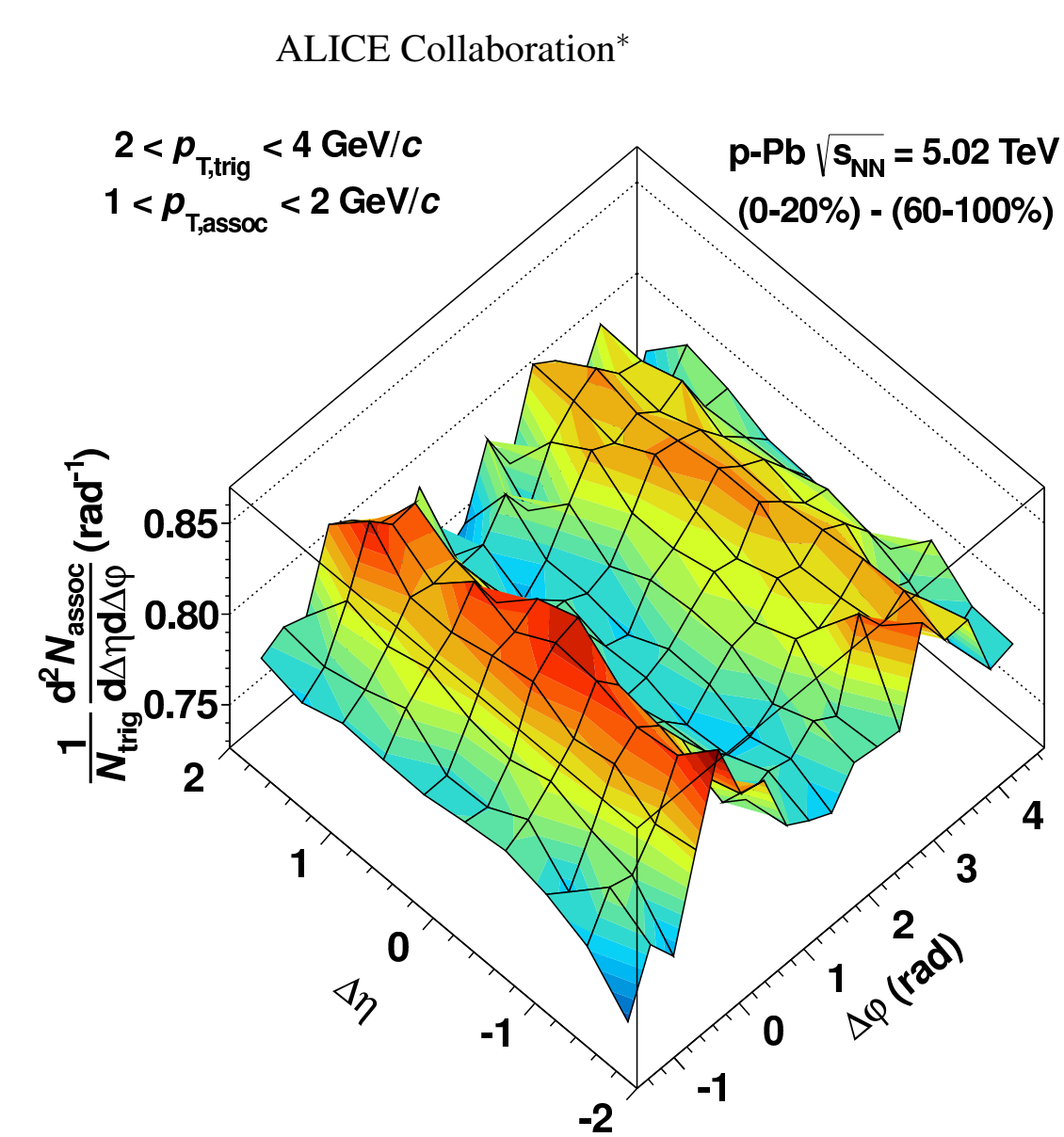

Fig. 3: Left: Associated yield per trigger particle in $\Delta \varphi$ and $\Delta \eta$ for pairs of charged particles with $2<p_{\mathrm{T}, \text { trig }}<4 \mathrm{GeV} / c$ and $1<p_{\mathrm{T} \text {,assoc }}<2 \mathrm{GeV} / c$ in $\mathrm{p}-\mathrm{Pb}$ collisions at $\sqrt{s_{\mathrm{NN}}}=5.02 \mathrm{TeV}$ for the $0-20 \%$ multiplicity class, after subtraction of the associated yield obtained in the $60-100 \%$ event class. Top right: the associated per-trigger yield after subtraction (as shown on the left) projected onto $\Delta \eta$ averaged over $|\Delta \varphi|<\pi / 3$ (black circles), $|\Delta \varphi-\pi|<\pi / 3$ (red squares), and the remaining area (blue triangles, $\Delta \varphi<-\pi / 3, \pi / 3<\Delta \varphi<2 \pi / 3$ and $\Delta \varphi>4 \pi / 3$ ). Bottom right: as above but projected onto $\Delta \varphi$ averaged over $0.8<|\Delta \eta|<1.8$ on the near side and $|\Delta \eta|<1.8$ on the away side. Superimposed are fits containing a $\cos (2 \Delta \varphi)$ shape alone (black dashed line) and a combination of $\cos (2 \Delta \varphi)$ and $\cos (3 \Delta \varphi)$ shapes (red solid line). The blue horizontal line shows the baseline obtained from the latter fit which is used for the yield calculation. Also shown for comparison is the subtracted associated yield when the same procedure is applied on HIJING shifted to the same baseline. The figure shows only statisti- 


\section{Observation of Associated Near-side and Away-side Long-range Correlations in $\sqrt{s_{\mathrm{NN}}}=5.02 \mathrm{TeV}$ Proton-lead Collisions with the ATLAS Detector}

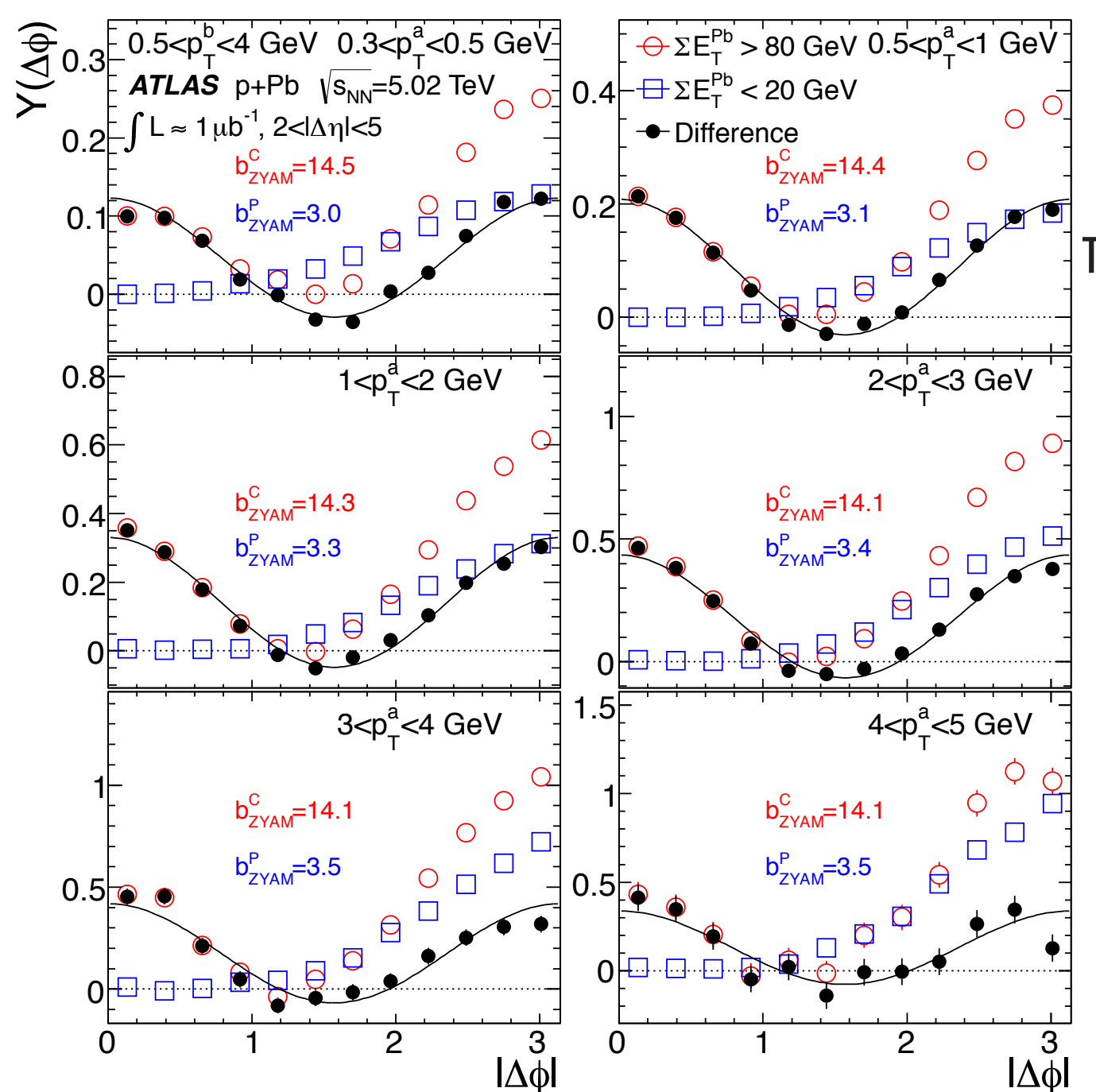

FIG. 3. Distributions of per-trigger yield in the peripheral and the central event activity classes and their differences (solid symbols), for different ranges of $p_{\mathrm{T}}^{\mathrm{a}}$ and $0.5<p_{\mathrm{T}}^{\mathrm{b}}<4 \mathrm{GeV}$, together with functions $a_{0}+2 a_{2} \cos 2 \Delta \phi$ obtained via a Fourier decomposition (see text). The values for the ZYAMdetermined pedestal levels are indicated on each panel for peripheral $\left(b_{\mathrm{ZYAM}}^{\mathrm{P}}\right)$ and central $\left(b_{\mathrm{ZYAM}}^{\mathrm{C}}\right) \Sigma E_{\mathrm{T}}^{\mathrm{Pb}}$ bins.
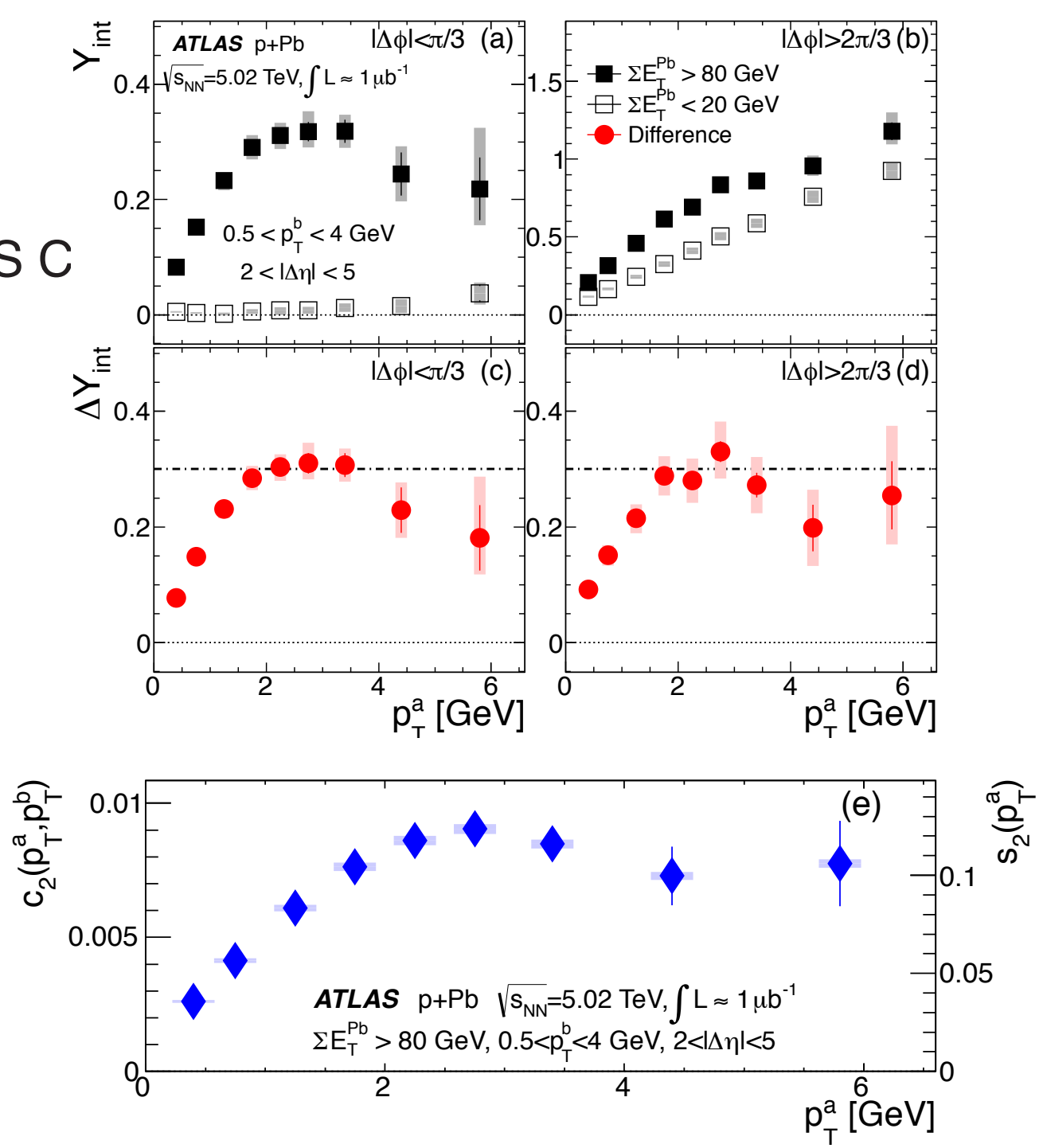

FIG. 4. Integrated per-trigger yields, $Y_{\text {int }}$, (see text) vs $p_{\mathrm{T}}^{\mathrm{a}}$ for $0.5<p_{\mathrm{T}}^{\mathrm{b}}<4 \mathrm{GeV}$ in peripheral and central events, on the (a) near-side and (b) away-side. The panels (c) and (d) show the difference, $\Delta Y_{\text {int }}$. Panel (e) shows the $p_{\mathrm{T}}$ dependence of $c_{2}$ (left axis) and $s_{2}$ (right axis). The right axis of (e) differs from the left only by a multiplicative factor $1 / \sqrt{5.4 \times 10^{-3}}$ (see text). The error bars and shaded boxes represent the statistical and systematic uncertainties, respectively. 


\section{Observation of Associated Near-side and Away-side Long-range Correlations in $\sqrt{s_{\mathrm{NN}}}=5.02 \mathrm{TeV}$ Proton-lead Collisions with the ATLAS Detector}

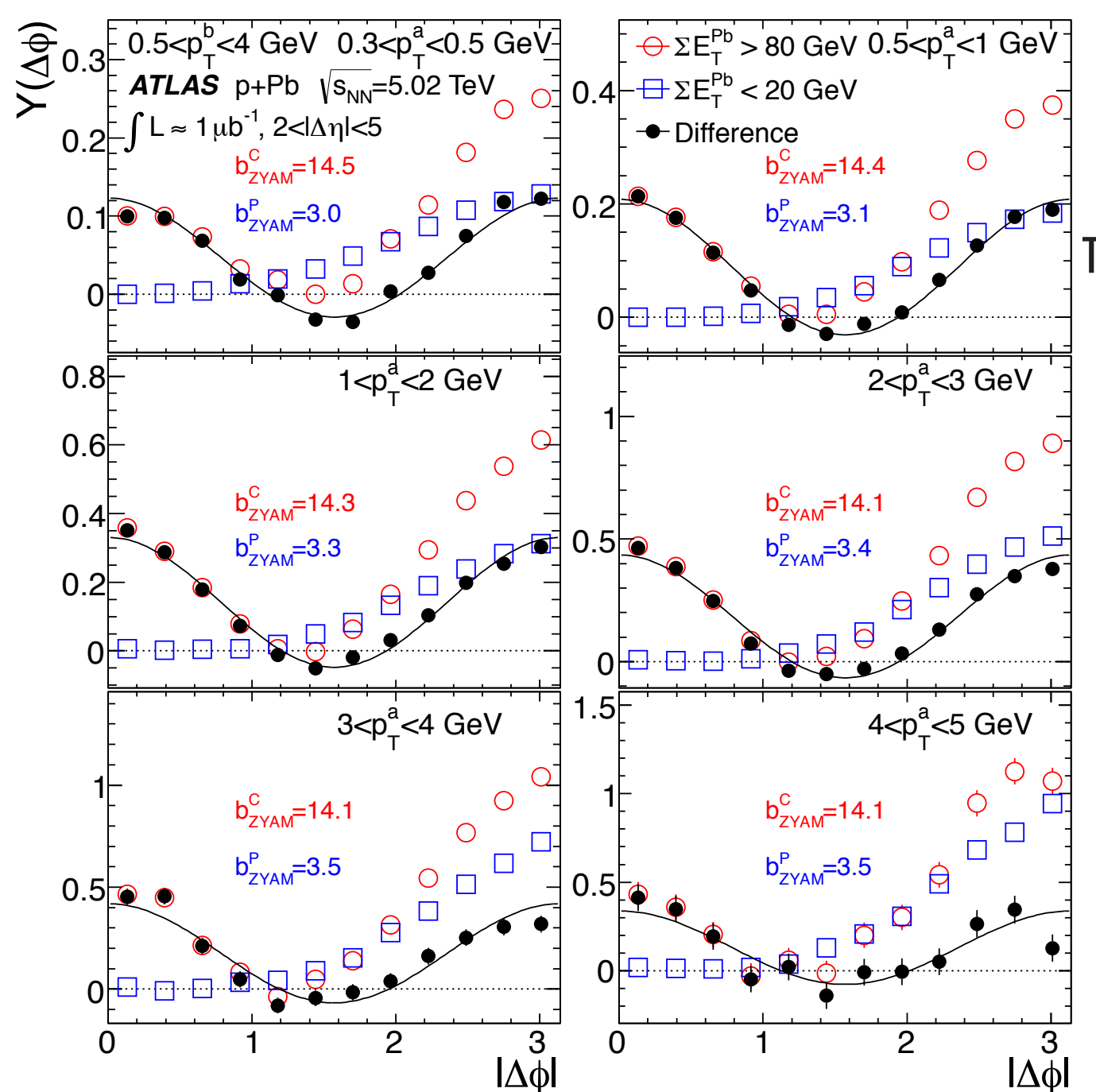

FIG. 3. Distributions of per-trigger yield in the peripheral and the central event activity classes and their differences (solid symbols), for different ranges of $p_{\mathrm{T}}^{\mathrm{a}}$ and $0.5<p_{\mathrm{T}}^{\mathrm{b}}<4 \mathrm{GeV}$, together with functions $a_{0}+2 a_{2} \cos 2 \Delta \phi$ obtained via a Fourier decomposition (see text). The values for the ZYAMdetermined pedestal levels are indicated on each panel for peripheral $\left(b_{\mathrm{ZYAM}}^{\mathrm{P}}\right)$ and central $\left(b_{\mathrm{ZYAM}}^{\mathrm{C}}\right) \Sigma E_{\mathrm{T}}^{\mathrm{Pb}}$ bins.
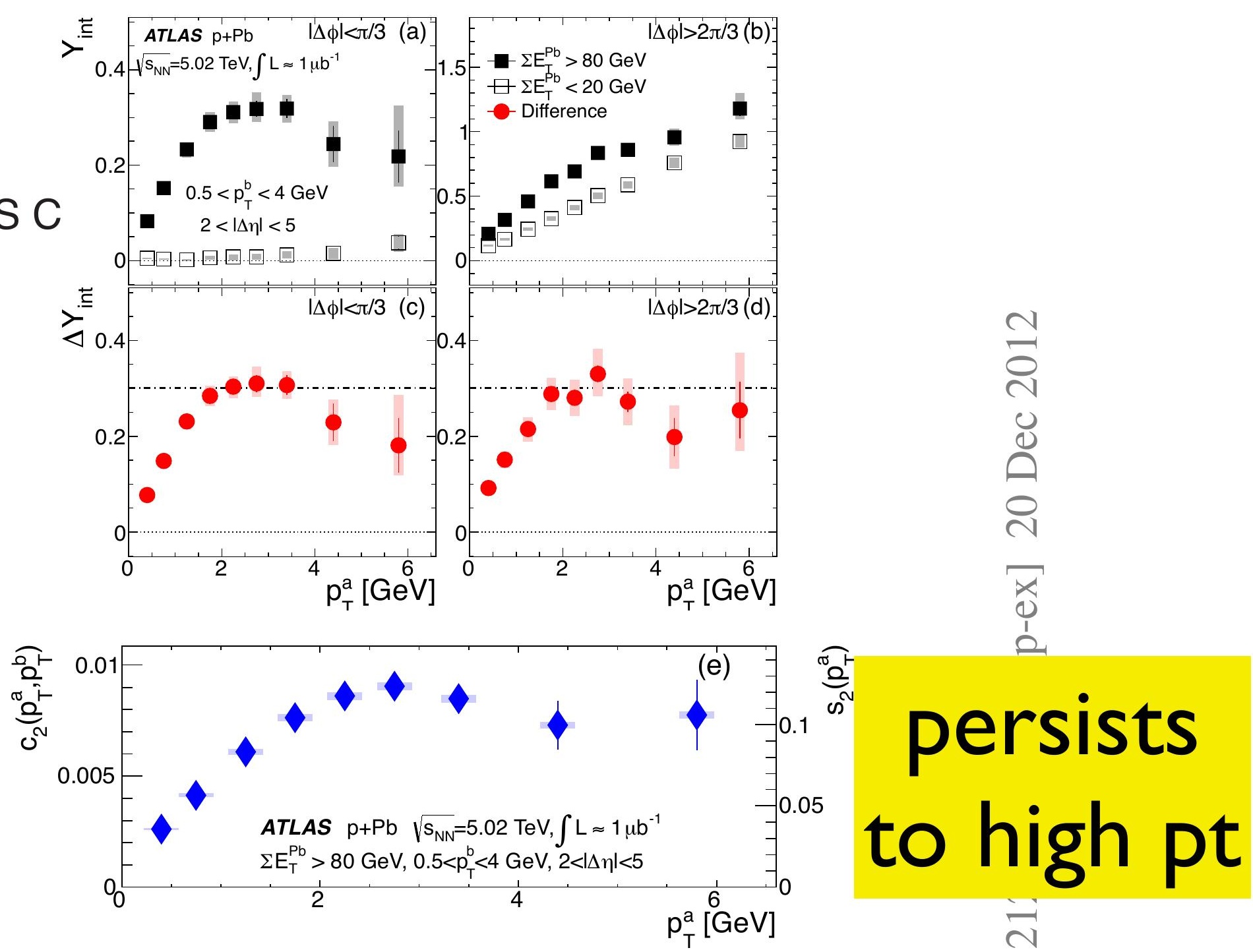

FIG. 4. Integrated per-trigger yields, $Y_{\text {int }}$, (see text) vs $p_{\mathrm{T}}^{\mathrm{a}}$ for $0.5<p_{\mathrm{T}}^{\mathrm{b}}<4 \mathrm{GeV}$ in peripheral and central events, on the (a) near-side and (b) away-side. The panels (c) and (d) show the difference, $\Delta Y_{\text {int }}$. Panel (e) shows the $p_{\mathrm{T}}$ dependence of $c_{2}$ (left axis) and $s_{2}$ (right axis). The right axis of (e) differs from the left only by a multiplicative factor $1 / \sqrt{5.4 \times 10^{-3}}$ (see text). The error bars and shaded boxes represent the statistical and systematic uncertainties, respectively. 


\section{High Multiplicity pp and pA Collisions: Hydrodynamics at its Edge and Stringy Black Hole}

- What is the smallest system size which still undergoes a hydrodynamical explosion?

- How do all hydrodynamical observables scale with the system size $R$ and viscosityto-entropy ratio $\eta / s$, for such systems? In particular, how large are the viscous corrections for radial and elliptic flows?

- How do amplitudes of higher angular harmonics $v_{n}$ scale with $n, R$ and $\eta / s ?$ In which $p_{t}$ region do we expect hydrodynamics to work, and for each $v_{n}$ ?

- Do high multiplicity pp and pA collisions in which the (double) "ridge" has been recently observed at LHC [7-9] fit into such a hydrodynamical scaling?

$$
\begin{aligned}
& \text { two small parameters } \\
& \text { of hydro } \\
& \mathcal{O}(1) \approx \frac{1}{T R^{\text {pp,high mutiplicity }}} \\
& >\frac{1}{T R^{\text {central } A A}}>\frac{1}{T R^{\text {central } A A}} \approx \mathcal{O}(1 / 10)
\end{aligned}
$$

Another important small parameter which we seem to have for strongly coupled Quark-Gluon Plasma (sQGP) is the viscosity-to-entropydensity ratio

$$
\frac{\eta}{s} \approx \mathcal{O}(1 / 10) \ll 1
$$

Roughly speaking, it tells us that viscous effects - or the mean free path - is additionally suppressed compared to the micro scale $1 / T$. 


\section{the radial (Gubser's) flow}

Gubser's solution of ideal relativistic hydrodynamics, for the transverse velocity and the energy density reads

$$
\begin{gathered}
v_{\perp}(t, r)=\frac{2 t r}{1+t^{2}+r^{2}} \quad(10) \\
\frac{\epsilon}{q^{4}}=\frac{\hat{\epsilon}_{0} 2^{8 / 3}}{t^{4 / 3}\left[1+2\left(t^{2}+r^{2}\right)+\left(t^{2}-r^{2}\right)^{2}\right]^{4 / 3}} \\
t=q \bar{\tau}, \quad r=q \bar{r} \\
q_{A A}^{-1}=4.3, q^{-1}=1, q^{-1}=0.5(\mathrm{fm}) \\
\text { pA } \\
\text { my guesses of the } \\
\text { system's size } \\
\text { central PbPb 400 } \\
\text { pA: I 5-20 participants } \\
\text { Pp 2 }
\end{gathered}
$$

$$
v_{\perp}^{\max }[A A, p A, p p]=[0.69,0.83,0.95]
$$

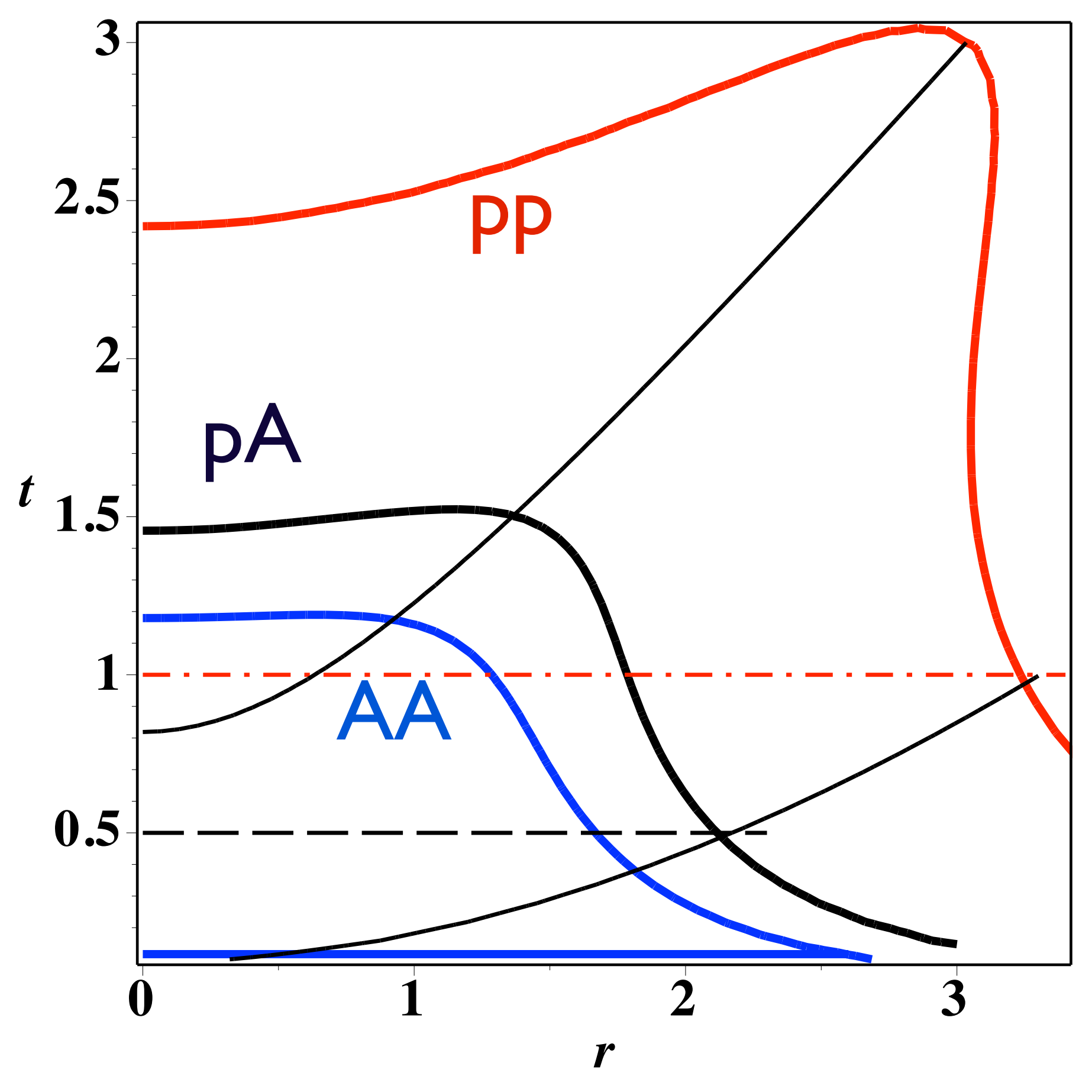




\section{$H_{0}=\frac{\eta}{\epsilon^{3 / 4}}=\frac{\eta}{s} \frac{4}{3} f_{*}^{1 / 4}$ \\ the role of viscosity}

for $\eta / s=0.134$ we will use as representative number $H_{0}=0.33$.

nonzero viscosity the solution is

$$
\begin{aligned}
\hat{T} & =\frac{\hat{T}_{0}}{(\cosh \rho)^{2 / 3}}+\frac{H_{0} \sinh ^{3} \rho}{9(\cosh \rho)^{2 / 3}} \\
& \times{ }_{2} F_{1}\left(\frac{3}{2}, \frac{7}{6} ; \frac{5}{2},-\sinh ^{2} \rho\right)
\end{aligned}
$$

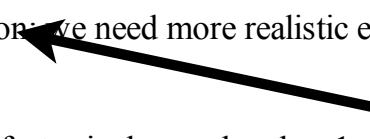

with $\hat{T}=\tau f_{*}^{1 / 4} T$ and $f_{*}=\epsilon / T^{4}=11$ as in [18].
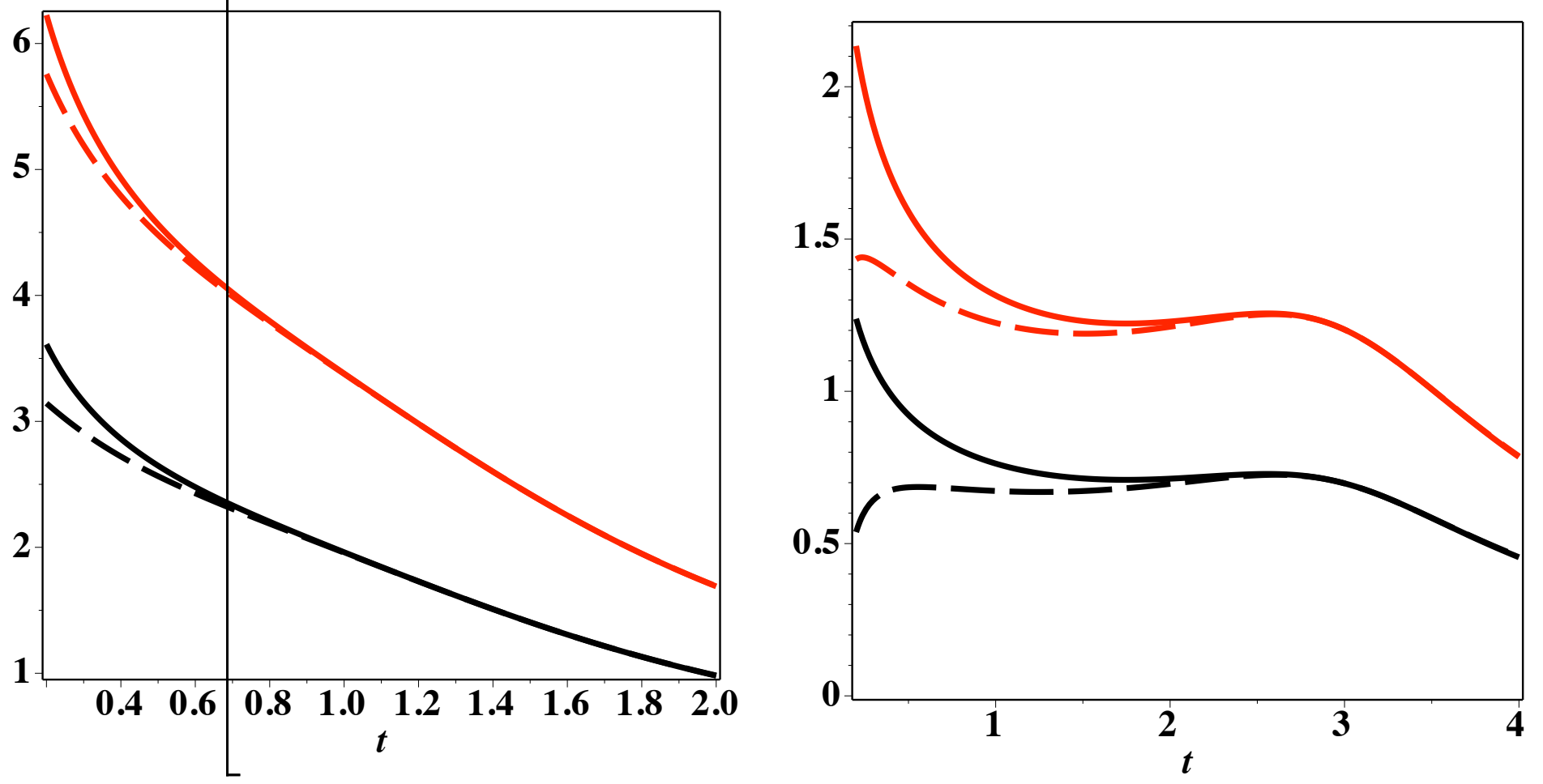

In [19] Gubser and Yarom re-derived the radial solution by going into the co-moving frame via a coordinate transformation from the $\tau, r$ to a new set $\rho, \theta$ given by:

$$
\begin{aligned}
\sinh \rho & =-\frac{1-\tau^{2}+r^{2}}{2 \tau} \\
\tan \theta & =\frac{2 r}{1+\tau^{2}-r^{2}}
\end{aligned}
$$

\section{The radial flow in $\mathrm{pA}, \mathrm{pp}$ has moderate corrections!}

FIG. 3: (color online) The temperature versus dimensionless time $t$, for ideal hydrodynamics (solid) and viscous hydrodynamics with $\eta / s=0.132$ (dashed) lines. The upper pair of (red) curves are for $\mathrm{pp}$, the lower (black) ones for $\mathrm{pA}$ collisions. The upper plot is for $r=1$, the lower plot for $r=3$. 


\section{viscosity for higher harmonics}

Staig+ES (2010) suggested to use "acoustic" damping expression

\section{$t=O(R)$}

$P_{m}=\exp \left[-m^{2} \frac{4}{3}\left(\frac{\eta}{s}\right)\left(\frac{1}{T R}\right)\right]$

AA 


\section{viscosity for higher harmonics}

Staig+ES (20I0) suggested to use "acoustic" damping expression

$\mathrm{t}=\mathrm{O}(\mathrm{R})$

$P_{m}=\exp \left[-m^{2} \frac{4}{3}\left(\frac{\eta}{s}\right)\left(\frac{1}{T R}\right)\right]$

AA

$\ln A A:(m / 10)^{\wedge} 2$

in $P A / P P(m / 3)^{\wedge} 2$

PP 


\section{viscosity for higher harmonics}

Staig+ES (2010) suggested to use "acoustic" damping expression

\section{$\mathrm{t}=\mathrm{O}(\mathrm{R})$}

$P_{m}=\exp \left[-m^{2} \frac{4}{3}\left(\frac{\eta}{s}\right)\left(\frac{1}{T R}\right)\right]$

\section{$\ln A A:(\mathrm{m} / \mathrm{lo})^{\wedge} 2$ in $\mathrm{PA} / \mathrm{pP}(\mathrm{m} / 3)^{\wedge} 2$}

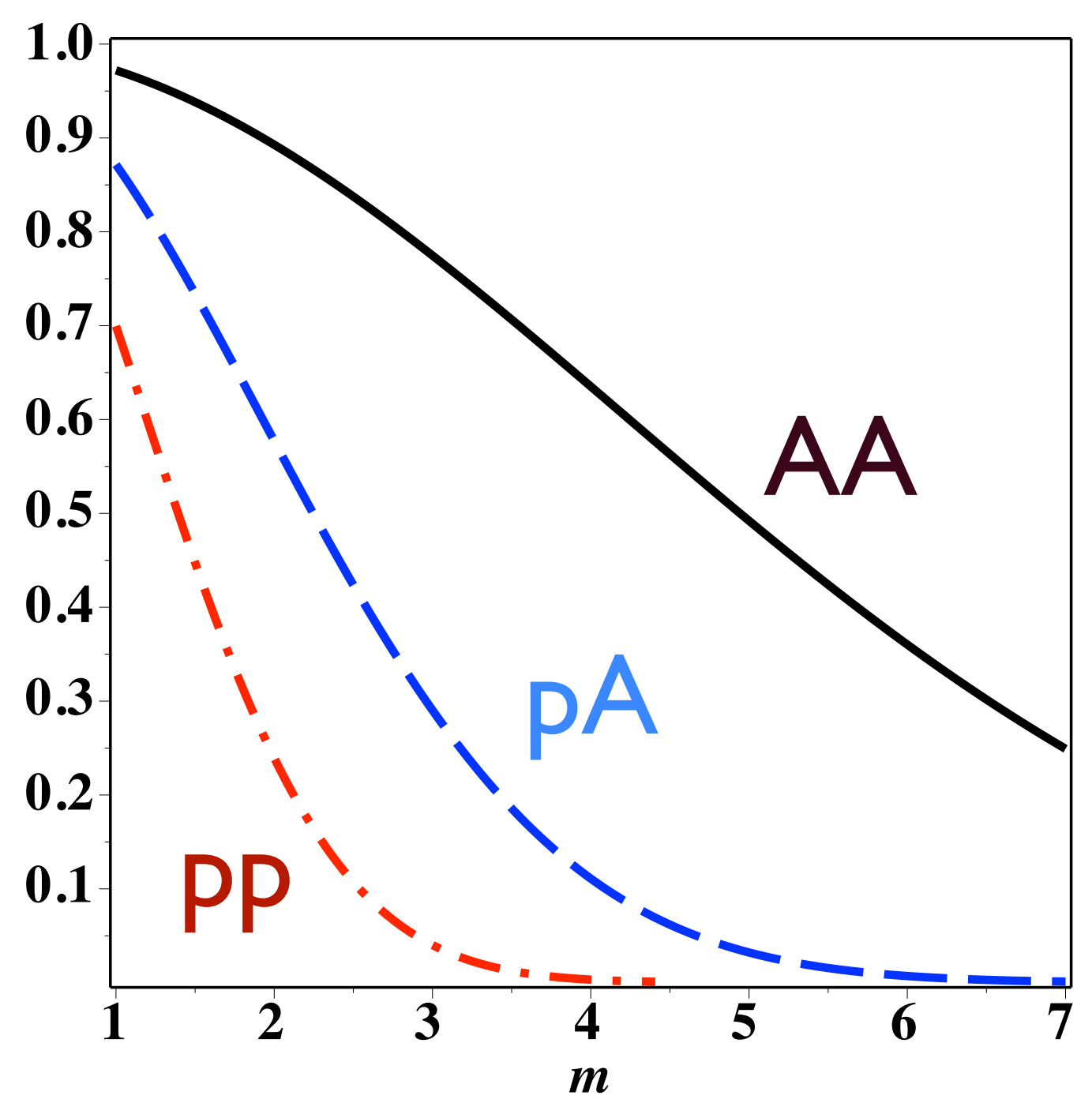

FIG. 4: (color online) Squared amplitude dissipation factor $P_{m}^{2}$ (as it appears in 2-particle correlators) for $\eta / s=.134$ as a function of the azimuthal harmonics $m$ for AA (black) solid, pA (blue) dash and pp (red) dash-dot. 


\section{Is anisotropic flow really acoustic?}

Roy A. Lacey, ${ }^{1}, *$ Yi Gu, ${ }^{1}$ X. Gong, ${ }^{1}$ D. Reynolds, ${ }^{1}$ N. N. Ajitanand,${ }^{1}$ J. M. Alexander, ${ }^{1}$ A. Mawi, ${ }^{1}$ and A. Taranenko ${ }^{1}$

${ }^{1}$ Department of Chemistry, Stony Brook University, Stony Brook, NY, 11794-3400, USA

(Dated: January 3, 2013)

$$
P_{m}=\exp \left[-m^{2} \frac{4}{3}\left(\frac{\eta}{s}\right)\left(\frac{1}{T R}\right)\right]
$$

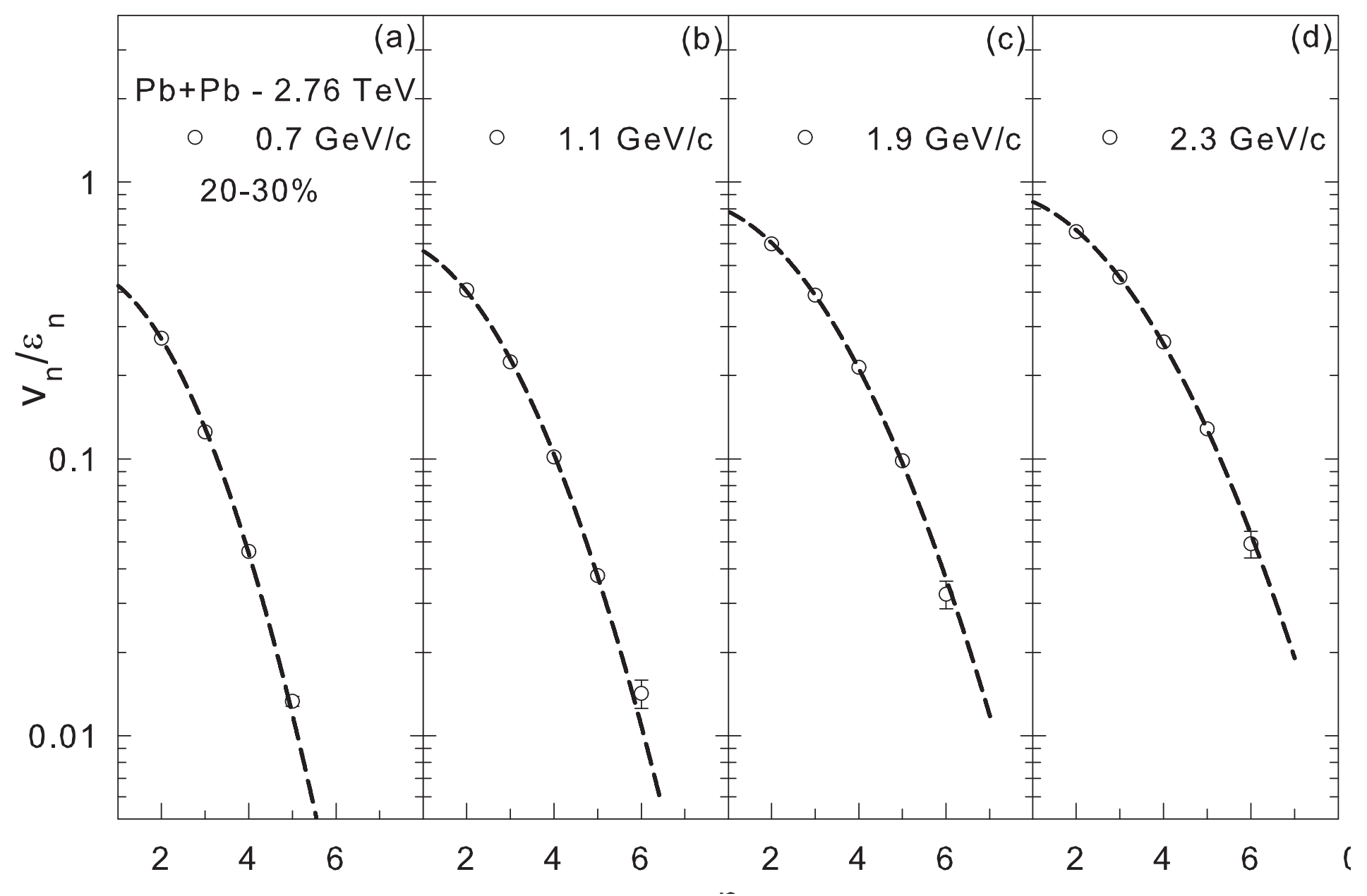




\section{resummation of higher gradients a la Lublinsky-Shuryak}

An approximate PADE-like re-summation of the higher order terms has been suggested by Lublinsky and Shuryak (LS) [20]. The main point is to notice the alternating signs of the series, which calls for an resummation a la geometrical series. Here we discuss only the single pole resummation model or LS2 in [20] in which the Navier-Stokes viscosity or NS is subtituted by an effective viscosity

$\eta_{L S 2}(\omega, k)=\frac{\eta_{N S}}{1-\eta_{2,0} k^{2} /(2 \pi T)^{2}-i \omega \eta_{0,1} /(2 \pi T)}$

Note that (40) involves only two dimensionless coefficients

$$
\eta_{2,0}=-\frac{1}{2} \eta_{0,1}=2-\ln 2=1.30
$$

Schematically the resummed hydro equations look as

$$
(\text { Euler })=\eta \mathbf{O}_{L S}(\text { Navier }- \text { Stokes })
$$

where $\mathbf{O}_{L S}$ is an integral operator. However, one can act with its inverse on the hydrodynamical equation as a whole, acting on the Euler part but canceling it in the viscous term

$$
\mathbf{O}_{L S}^{-1}(\text { Euler })=\eta(\text { Navier }- \text { Stokes })
$$

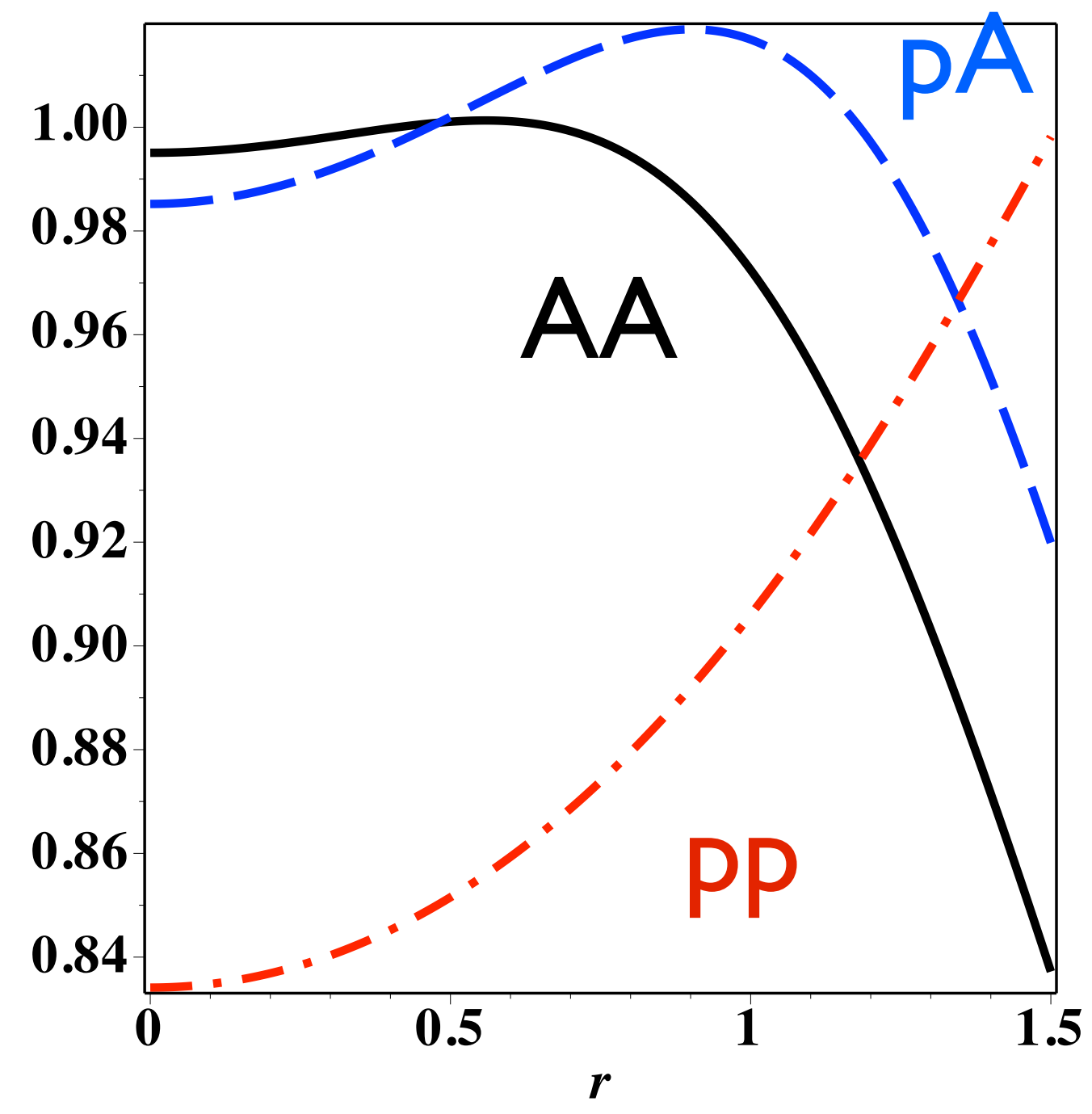

$$
\begin{aligned}
\mathbf{O}_{L S}^{-1}(f)= & 1+\frac{q^{2}}{2(2 \pi T)^{2}}\left(\frac{\partial^{2} f}{\partial r^{2}}+\frac{1}{r} \frac{\partial f}{\partial r}\right) \frac{1}{f} \\
& +(2-\ln 2) \frac{q}{2 \pi T} \frac{\partial f}{\partial t} \frac{1}{f}
\end{aligned}
$$




\section{summary of the hydro}

- the applicability of hydrodynamics rests on two small parameters:

(i) the micro-to- macro ratio $1 / \mathrm{TR}$, (ii) the viscosity-to-entropy ratio $\eta / \mathrm{s}$. For central AA collisions, both are $\mathrm{O}(1 / 10)$. For high multiplicity $\mathrm{pA}$ and $\mathrm{pp}$ collisions, the first parameter is no longer small $1 / \mathrm{TR}=\mathrm{O}(1)$, prompting us to ask which hydrodynamical predictions are preserved by the smallness of only the second parameter $\eta / s$.

- After solving the hydrodynamical equations we found that the radial (axially symmetric) flow is little modified by viscosity and is in fact enhanced by higher transverse gradients. Thus our main prediction is an enhanced radial flow $=>$ a change in the observed pt spectra on the particle mass, or growing proton-to-pion-ratio with pt. The magnitude of the effect should be even larger (=> ALICE ?)

- Higher harmonics are penalized by larger viscous corrections. We obtained explicit solution for Gubser flow for $m=2,3,4$ as shown in Fig.5. We have found a small $v 3 / v 2 \approx 1 / 3$ ratio for $P A$ in agreement with the reported ALICE data (in contrast to $v 3 / v 2>1$ in central AA). The value of $v 2$ itself is also suppressed by viscosity, and the relative suppression we have found between the PP and PA collisions agree reasonably with the CMS data. 


\section{Staig+ES 20II prediction vs ATLAS data}

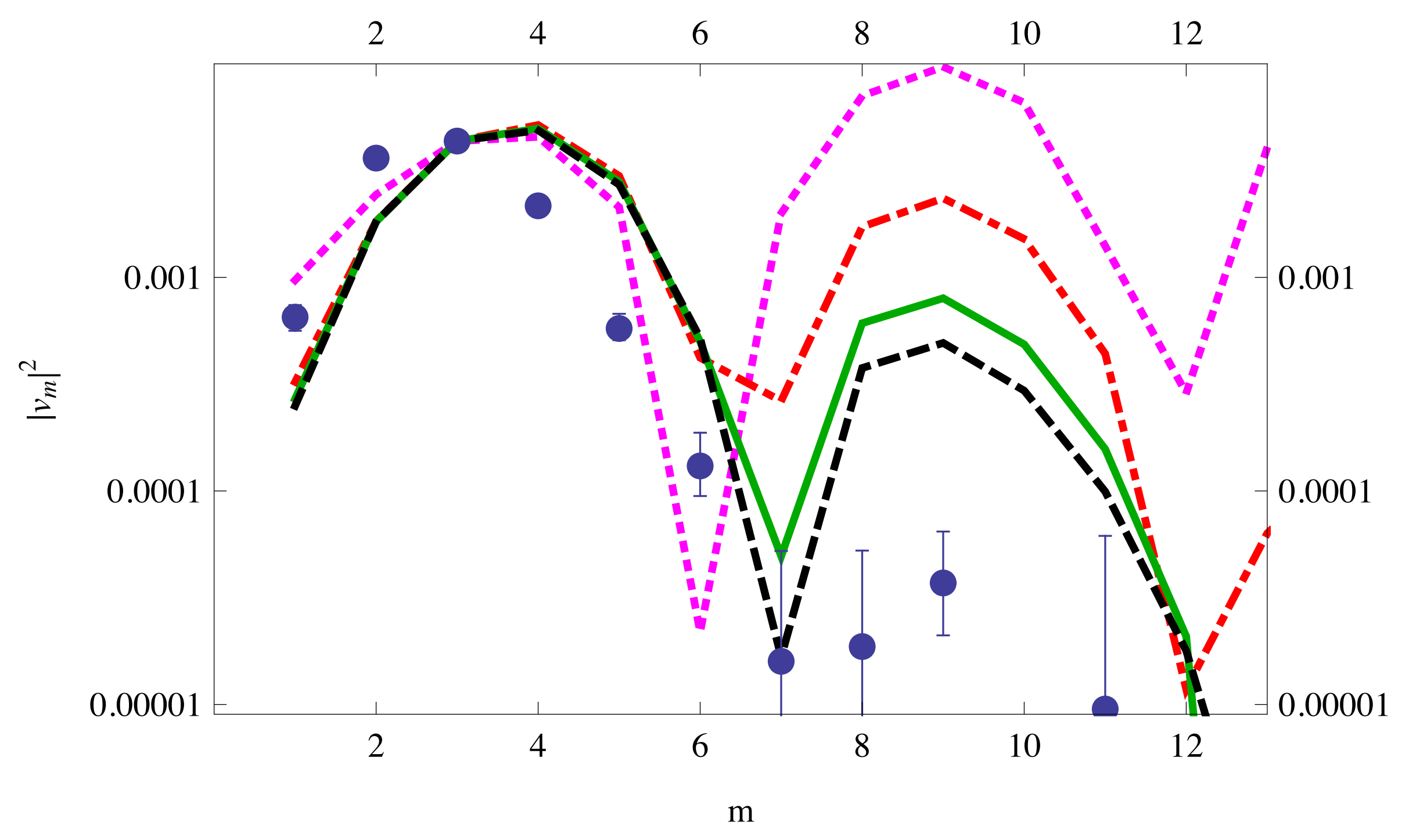





\section{Jet Quenching at RHIC}

vs LHC in Light of Recent dAu vs pPb Controls

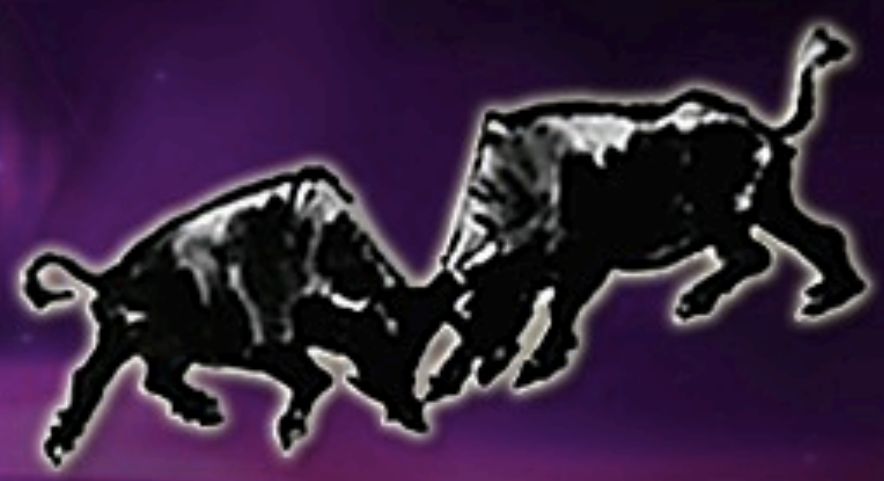

\section{Jets Quenching at RHIC: Present and Future}

Anne Sickles

Brookhaven National Lab 
- LHC has brought the field fantastic new jet measurements

- high jet rates, huge detectors, new system, large kinematic reach
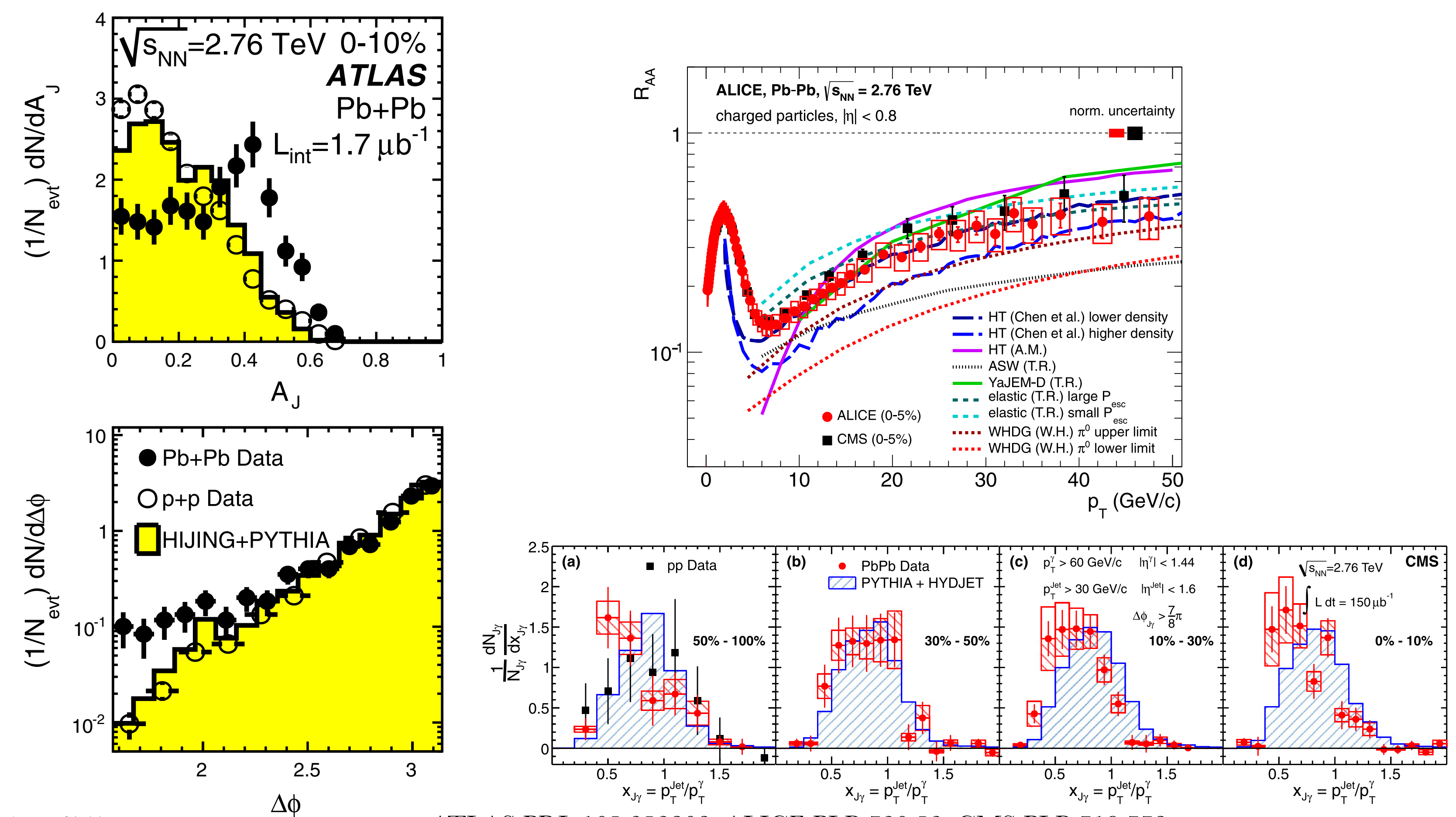


\section{what do we want from jets@RHIC?}

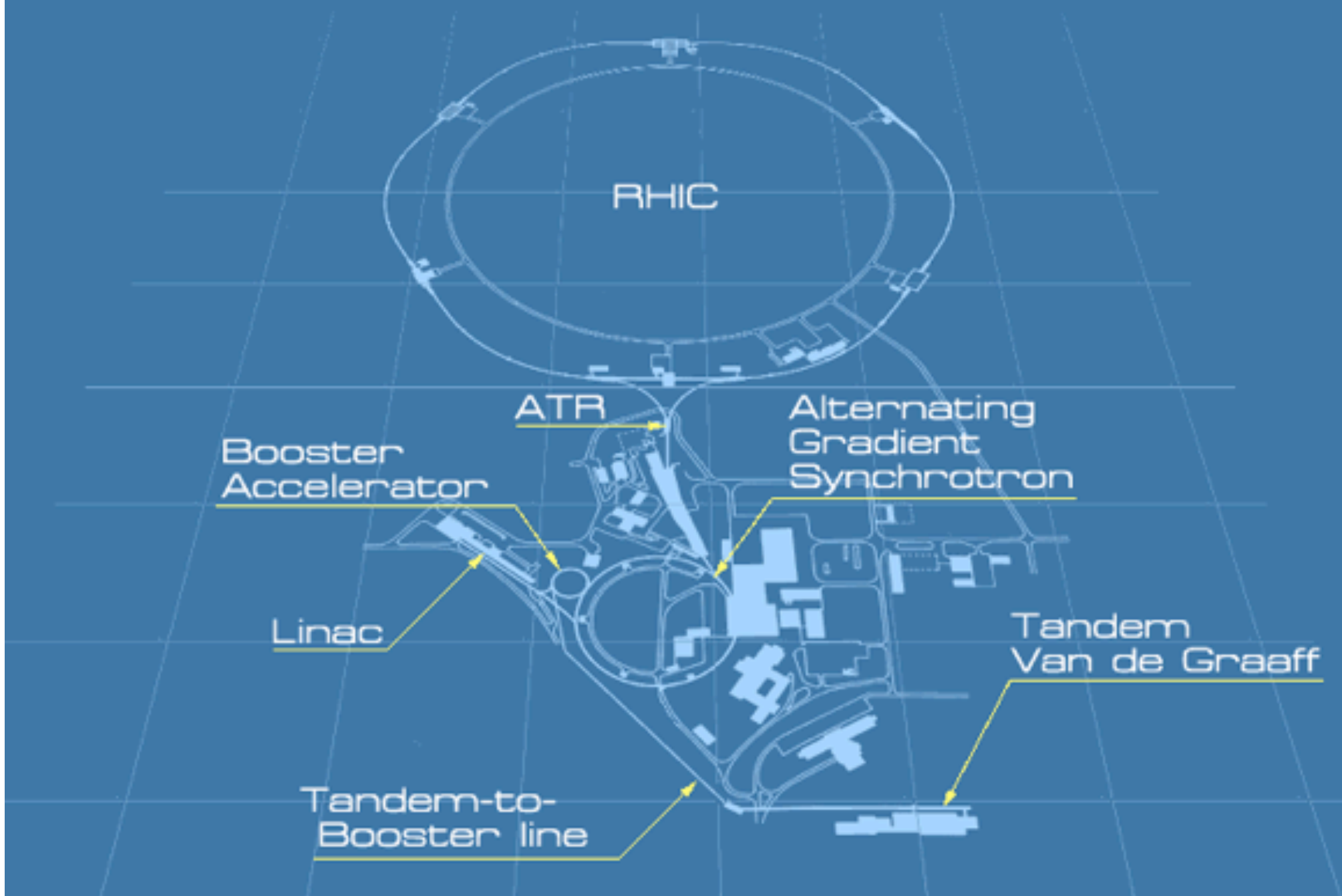

- probe the properties of jets near Tc

- exploit the large collision energy difference between RHIC and LHC to understand the physics of quenching

- exploit RHIC's flexibility to study different systems, small and large:

- geometry, initial state effects... 


\section{quenching vs temperatue}

strongest quenching at the highest temperature in the collision
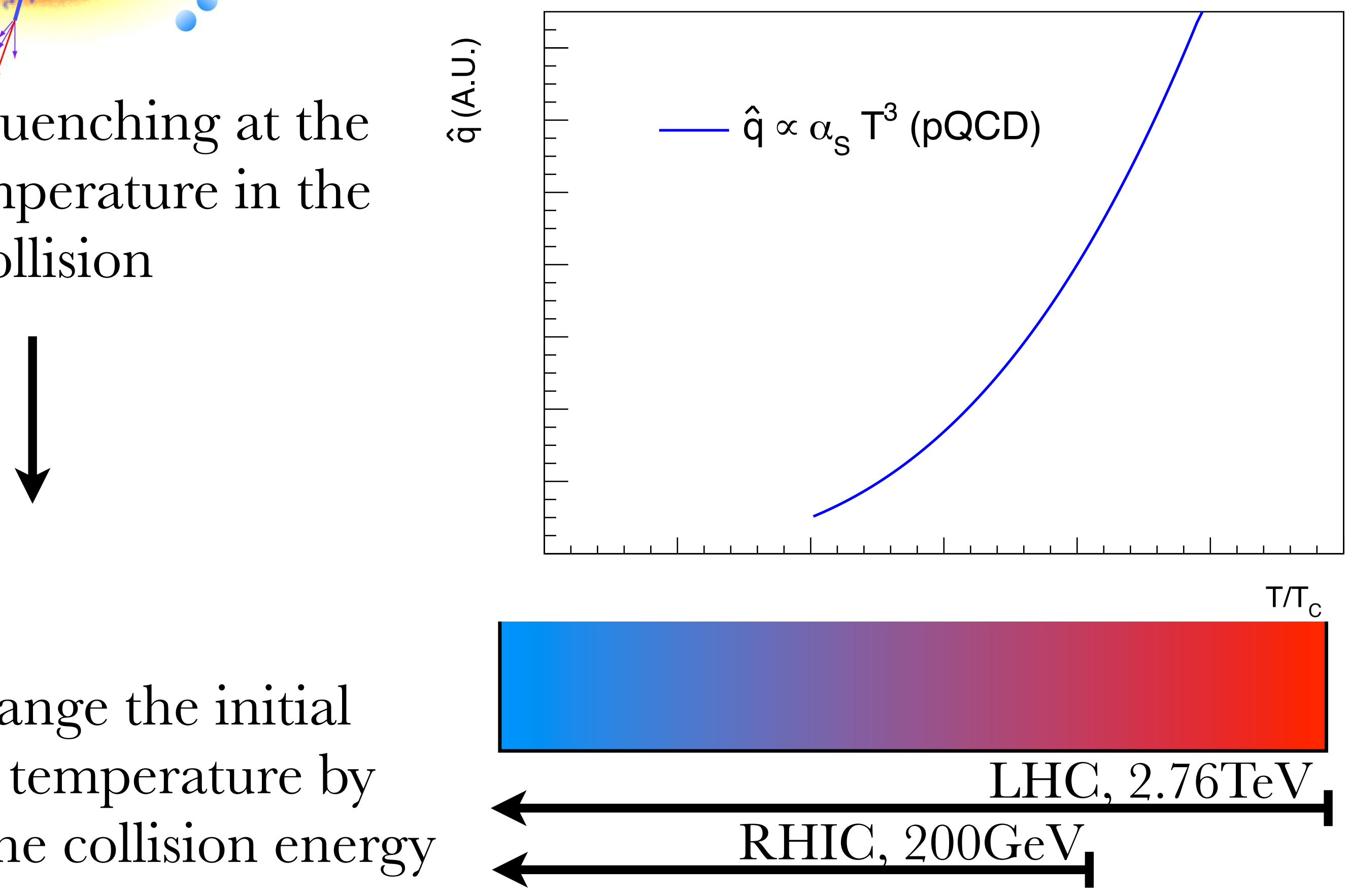

$\mathrm{q}(\mathrm{T})$ change the initial collision temperature by changing the collision energy 


\section{modified fragmentation patterns}

CMS: balancing energy at low $\mathrm{p}$ T and large angles

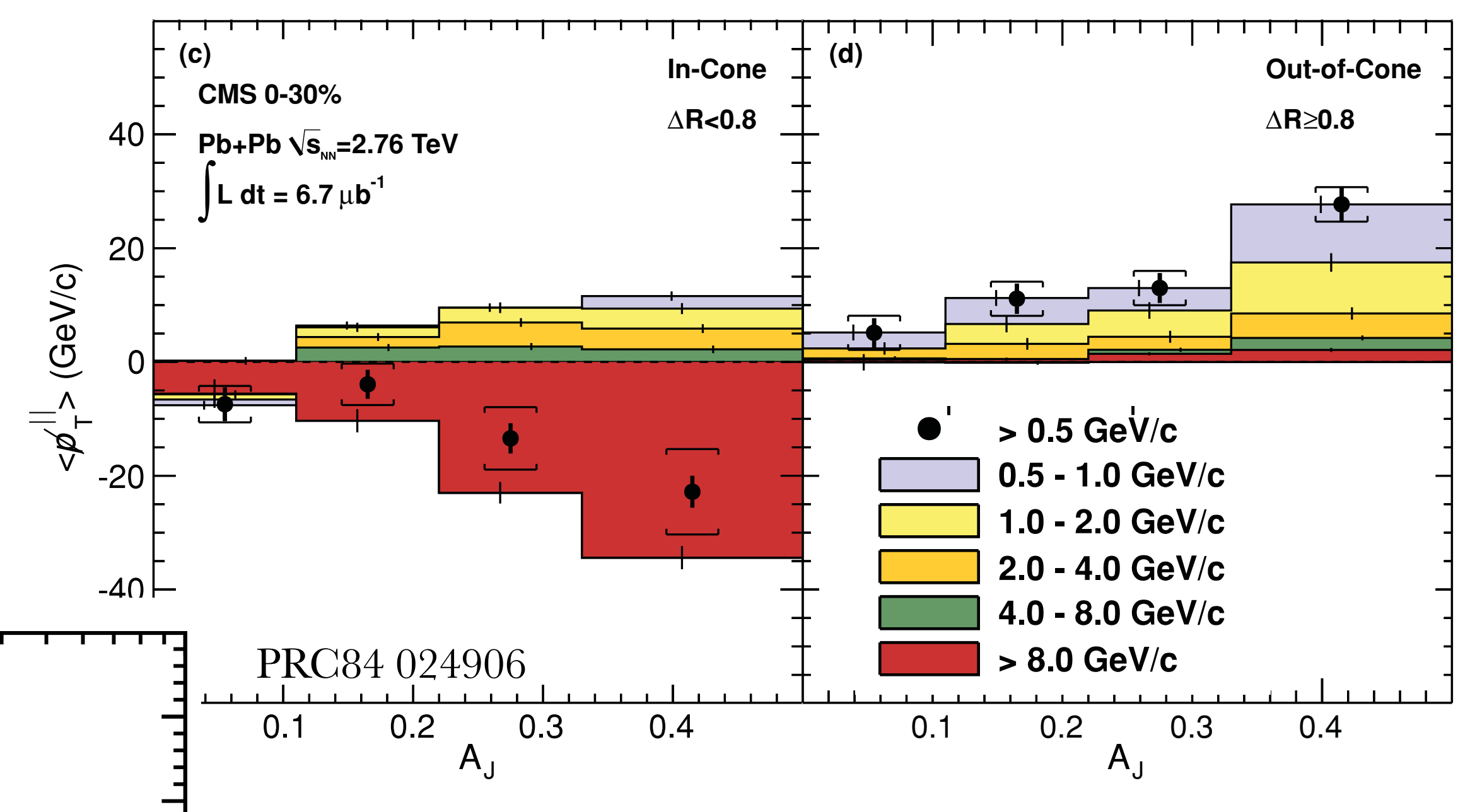

ATLAS: softening of jet fragmentation functions anti-k $\mathrm{T}=0.4$ $p_{\mathrm{T}}^{\text {jet }}>100 \mathrm{GeV}$

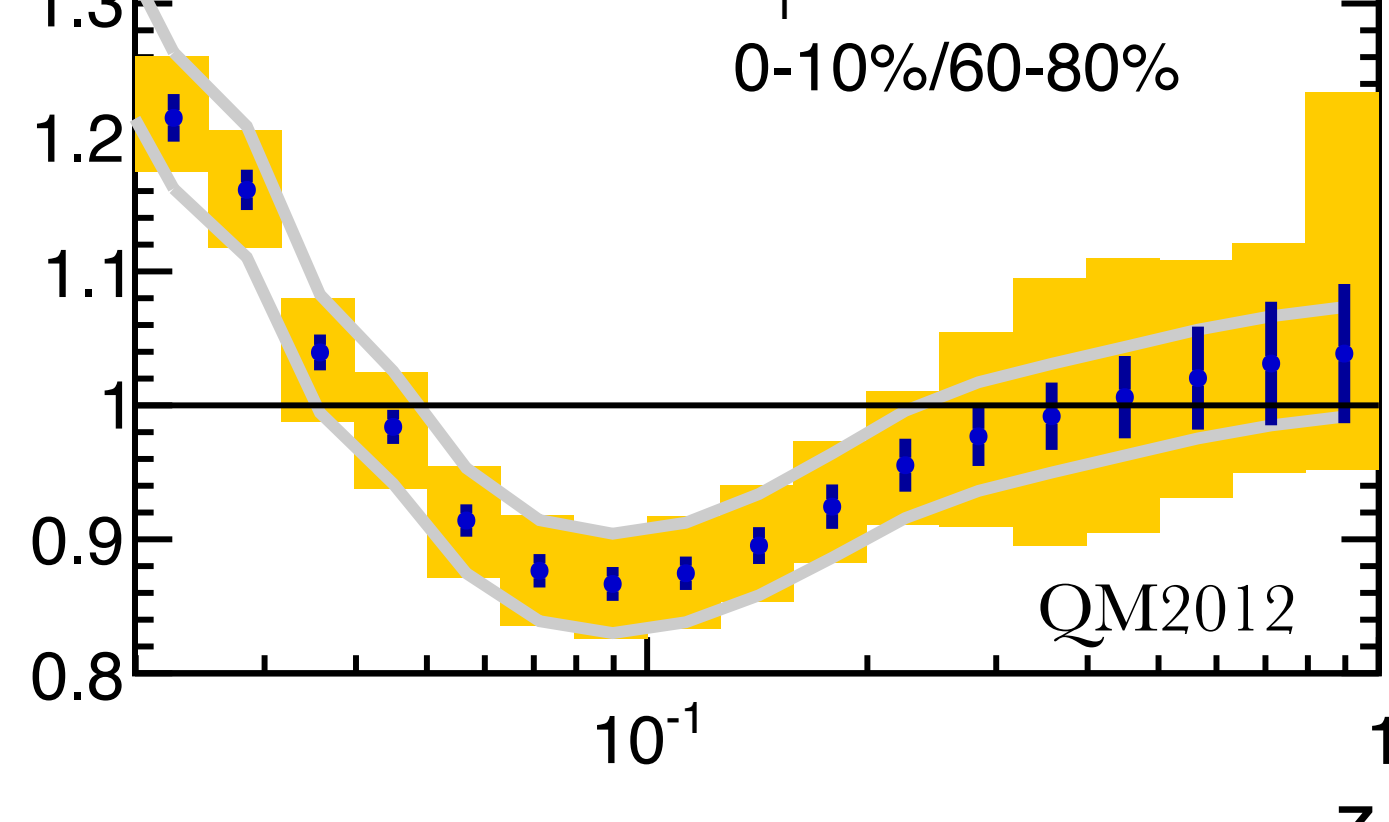




\section{modifying fragmentation patterns}

$\begin{array}{llllll}1 & 0.8 & 0.6 & 0.4 & Z_{T} & 0.2\end{array}$

\section{$\gamma$-hadron correlations}

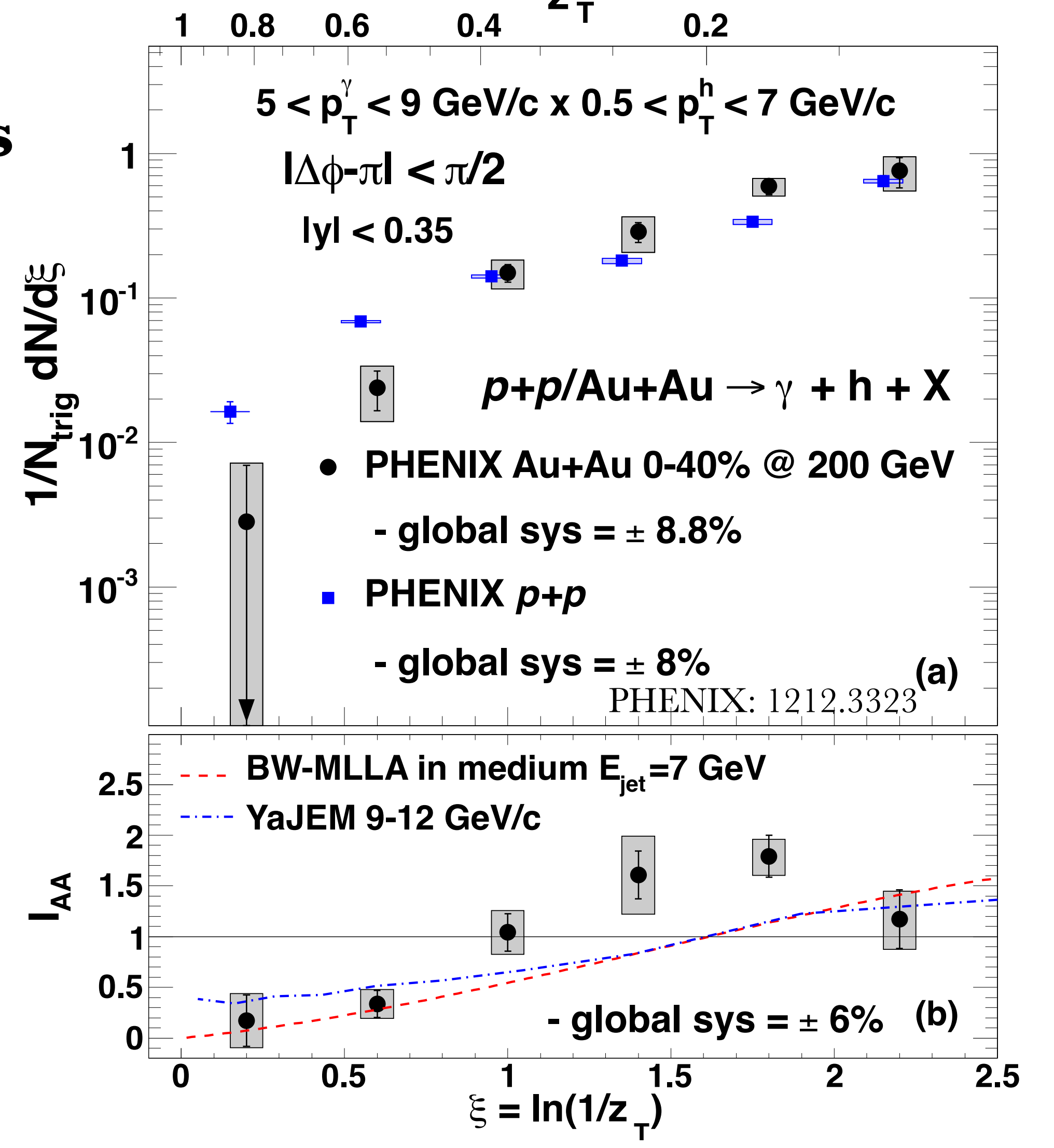




\section{broadening of angular distribution}
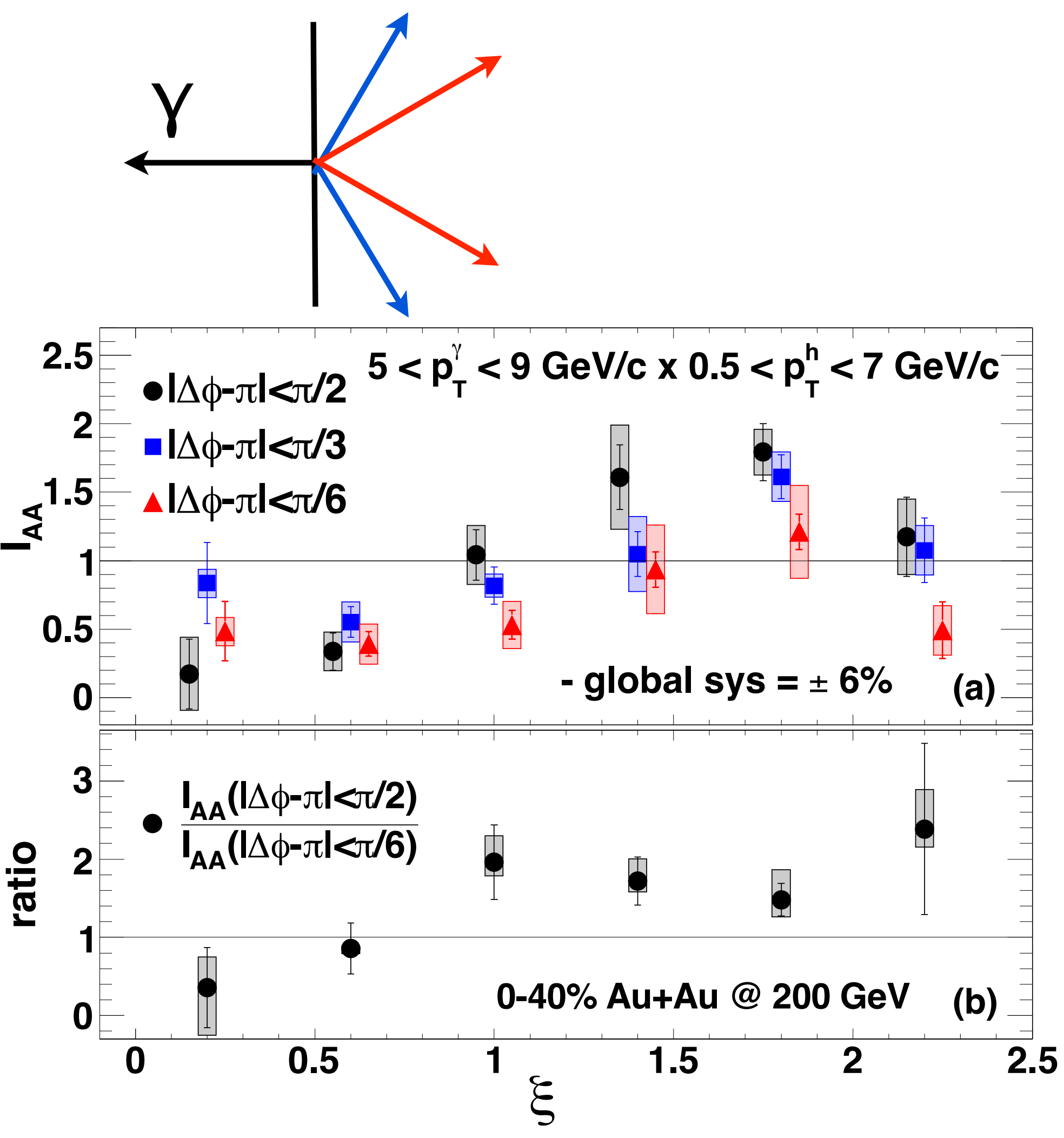


\section{broadening of angular distribution}

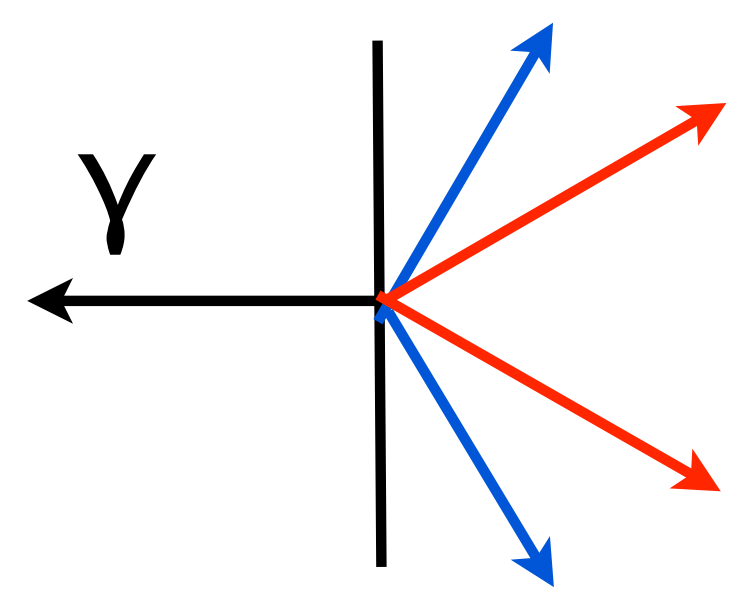

$2.5-|\Delta \phi-\pi|<\pi / 25<p_{\mathrm{T}}^{\gamma}<9 \mathrm{GeV} / \mathrm{c} \times 0.5<\mathrm{p}_{\mathrm{T}}^{\mathrm{h}}<7 \mathrm{GeV} / \mathrm{c}$ $2=-|\Delta \phi-\pi|<\pi / 3$ $\varangle^{1.5} \Delta|\Delta \phi-\pi|<\pi / 6$ $-$
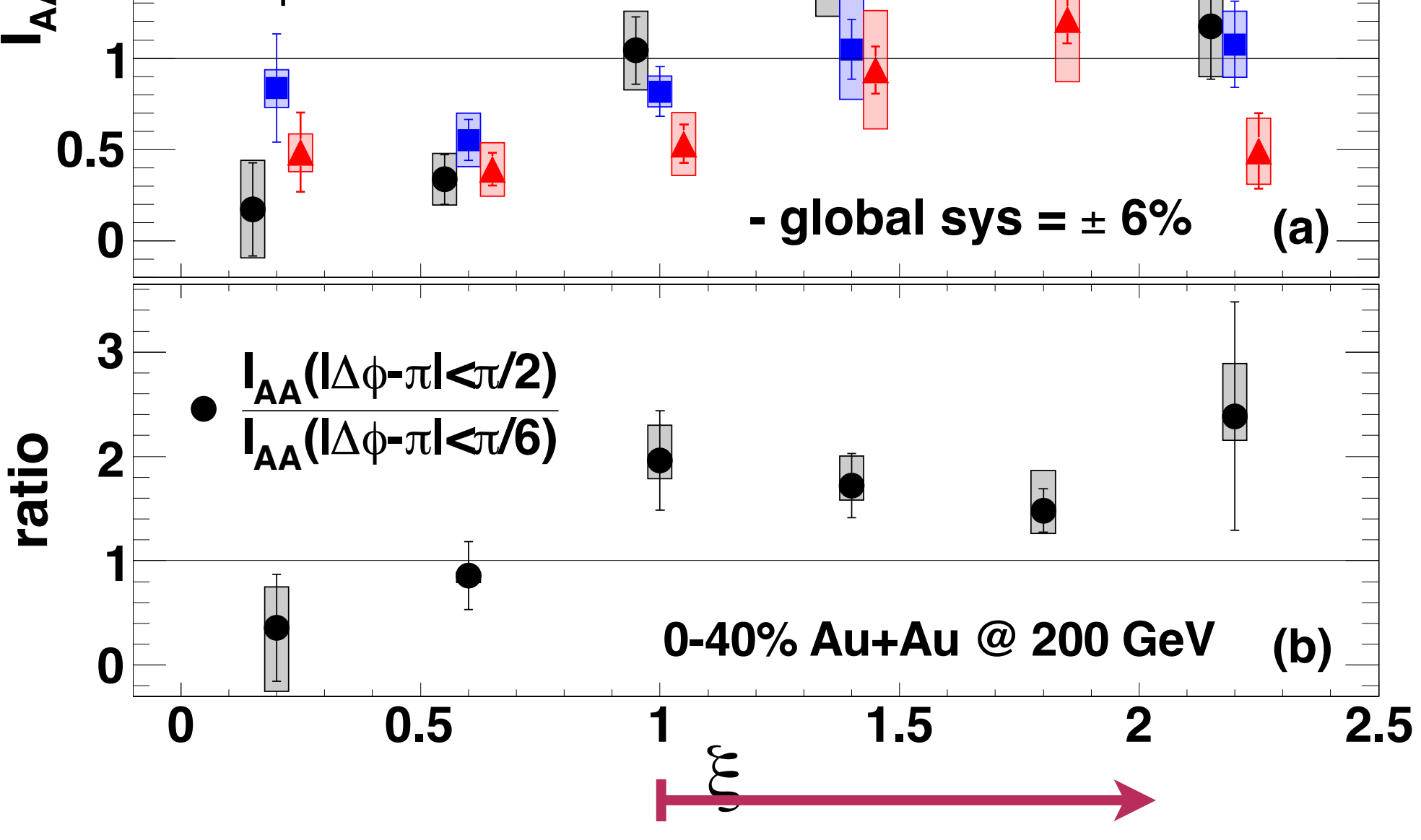

broadening of hadron distribution 


\section{broadening of angular distribution}

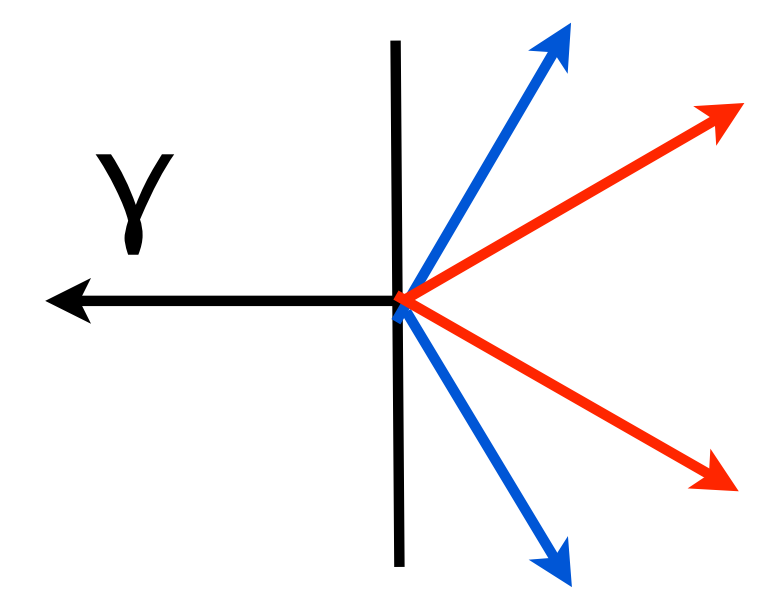

$2.5 \quad|\Delta \phi-\pi|<\pi / 25<p_{\mathrm{T}}^{\gamma}<9 \mathrm{GeV} / \mathrm{c} \times 0.5<p_{\mathrm{T}}^{\mathrm{h}}<7 \mathrm{GeV} / \mathrm{c}$

\section{$2=-|\Delta \phi-\pi|<\pi / 3$}

$\varangle^{1.5} \Delta|\Delta \phi-\pi|<\pi / 6$

$<$

0.5

$$
0
$$$$
\frac{0}{\frac{0}{2}}
$$

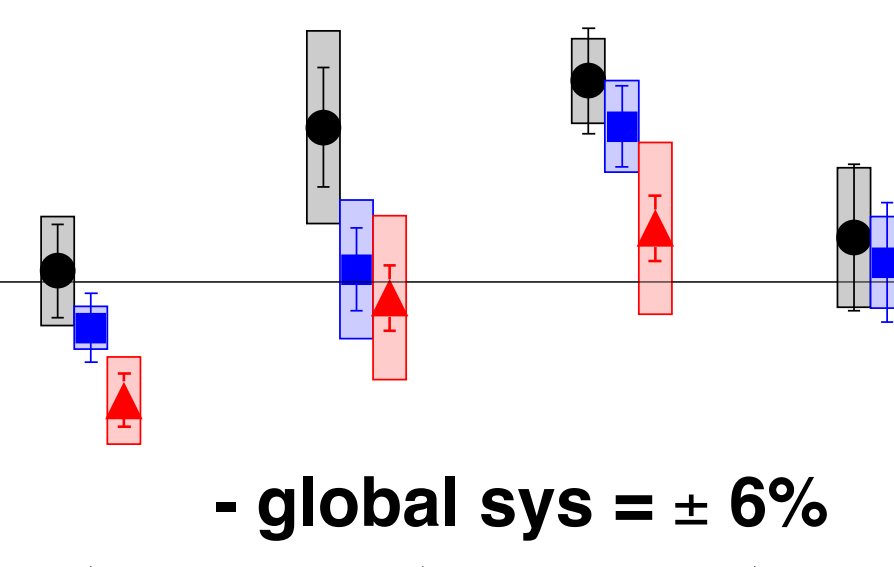

(a)

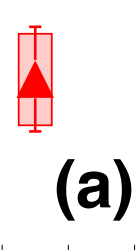

운 


\section{quenching vs $\sqrt{S N N}_{\text {SN }}$}
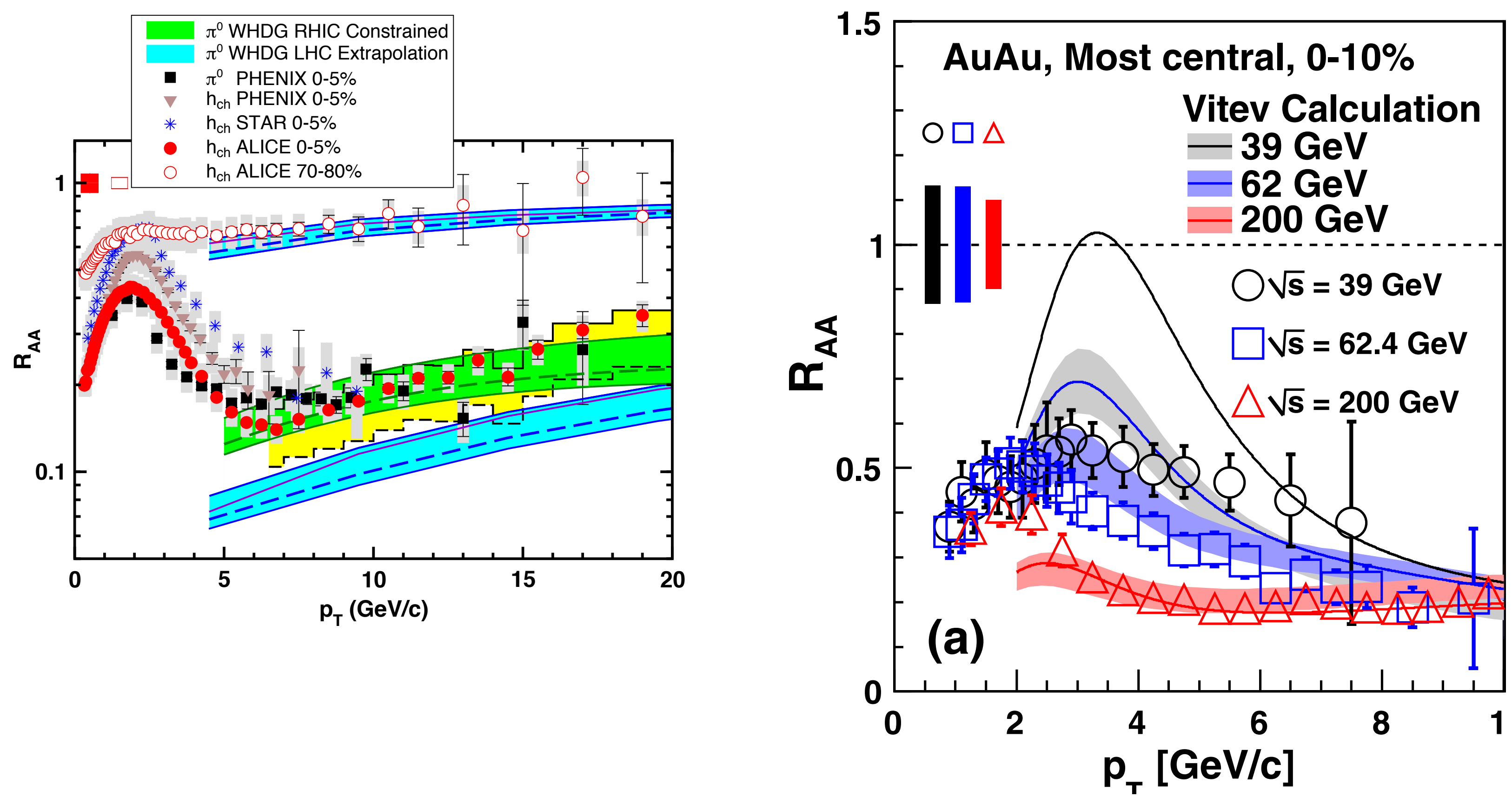

- $\mathrm{R}_{\mathrm{AA}} \sim 0.5$ at $39 \mathrm{GeV}$

- pT $_{\text {reach }} \sim 10 \mathrm{GeV}$ at $62.4 \mathrm{GeV}, \mathrm{R}_{\mathrm{AA}}$ consistent with $200 \mathrm{GeV}$

- *pA measurements needed to understand initial state effect 


\section{a peak into the future of hard probes...}

using RHIC and LHC to constrain temperature dependence of $\eta$ /s within hydro
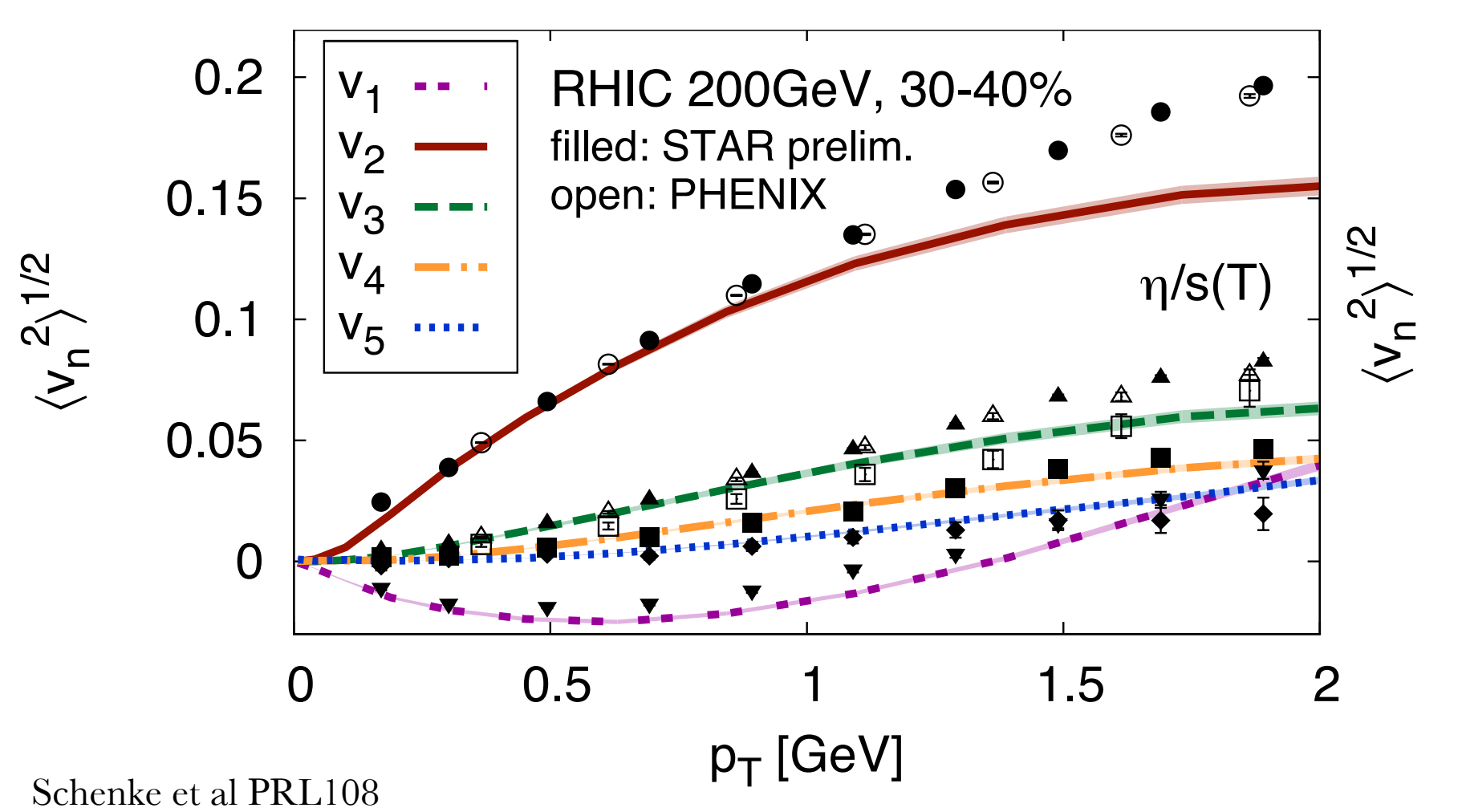
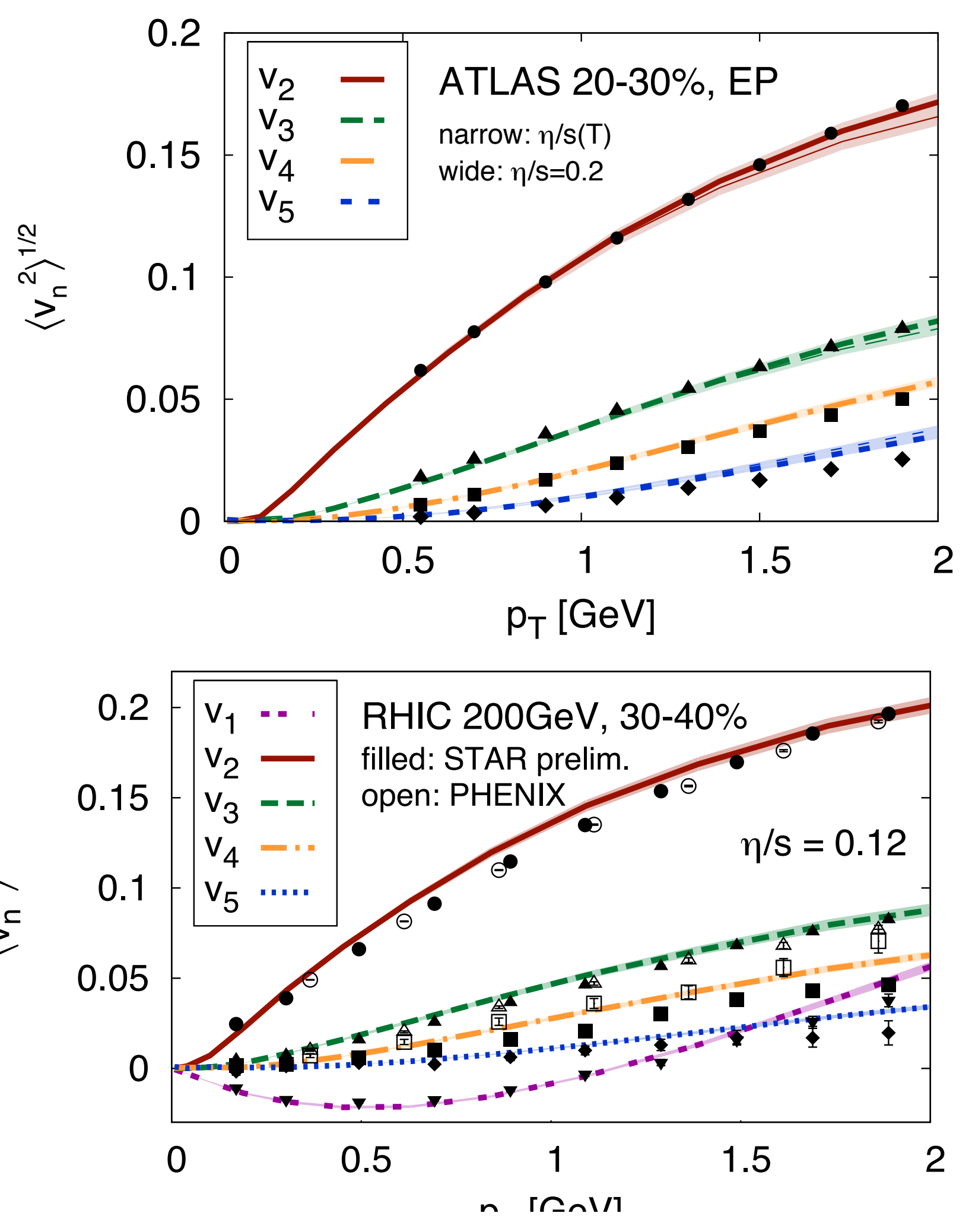


\section{leveraging RHIC/LHC differences}

1303.1794 initial entropy densitiy $0.30=$
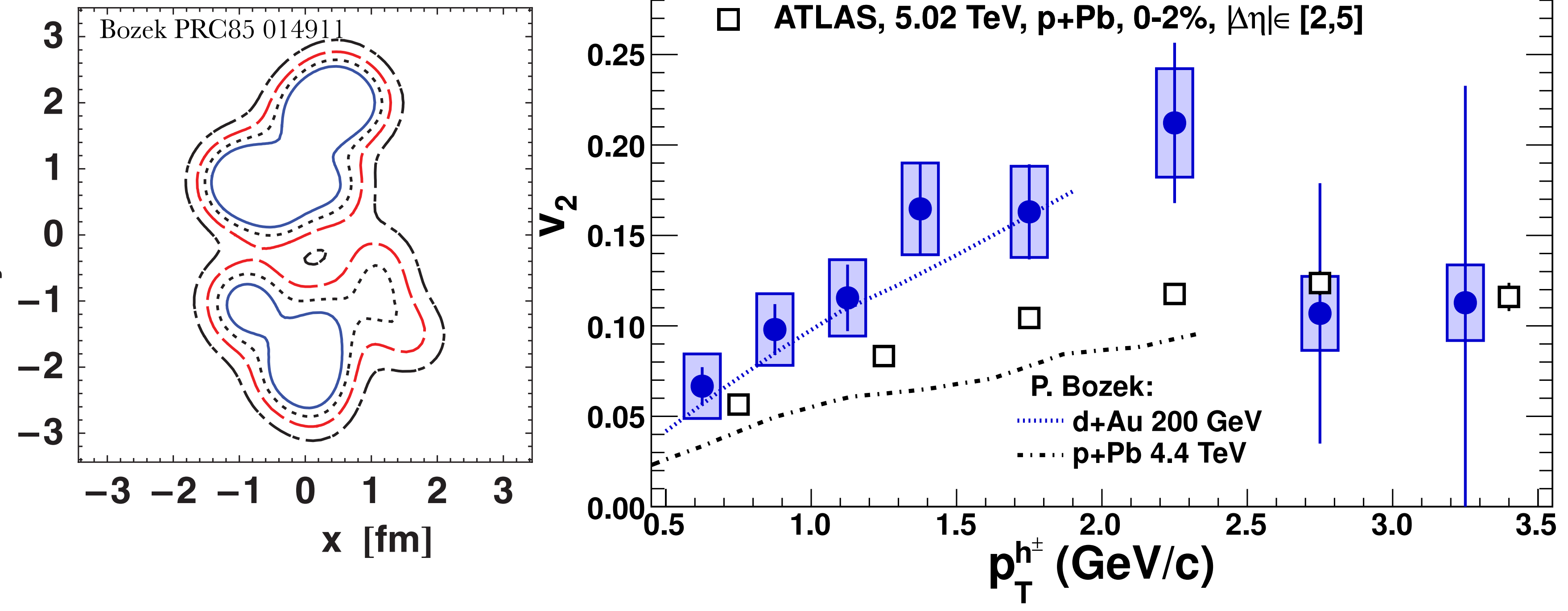

- changing both the collision energy and the shape of the initial system as compared to the ridge seen in $\mathrm{pPb}$ 
- upgrade optimized around jet/di-jet/photon measurements

- high rate, large uniform acceptance over $|\eta|<1$, hadronic calorimetry

- submitted last month to DOE for CD-0 review

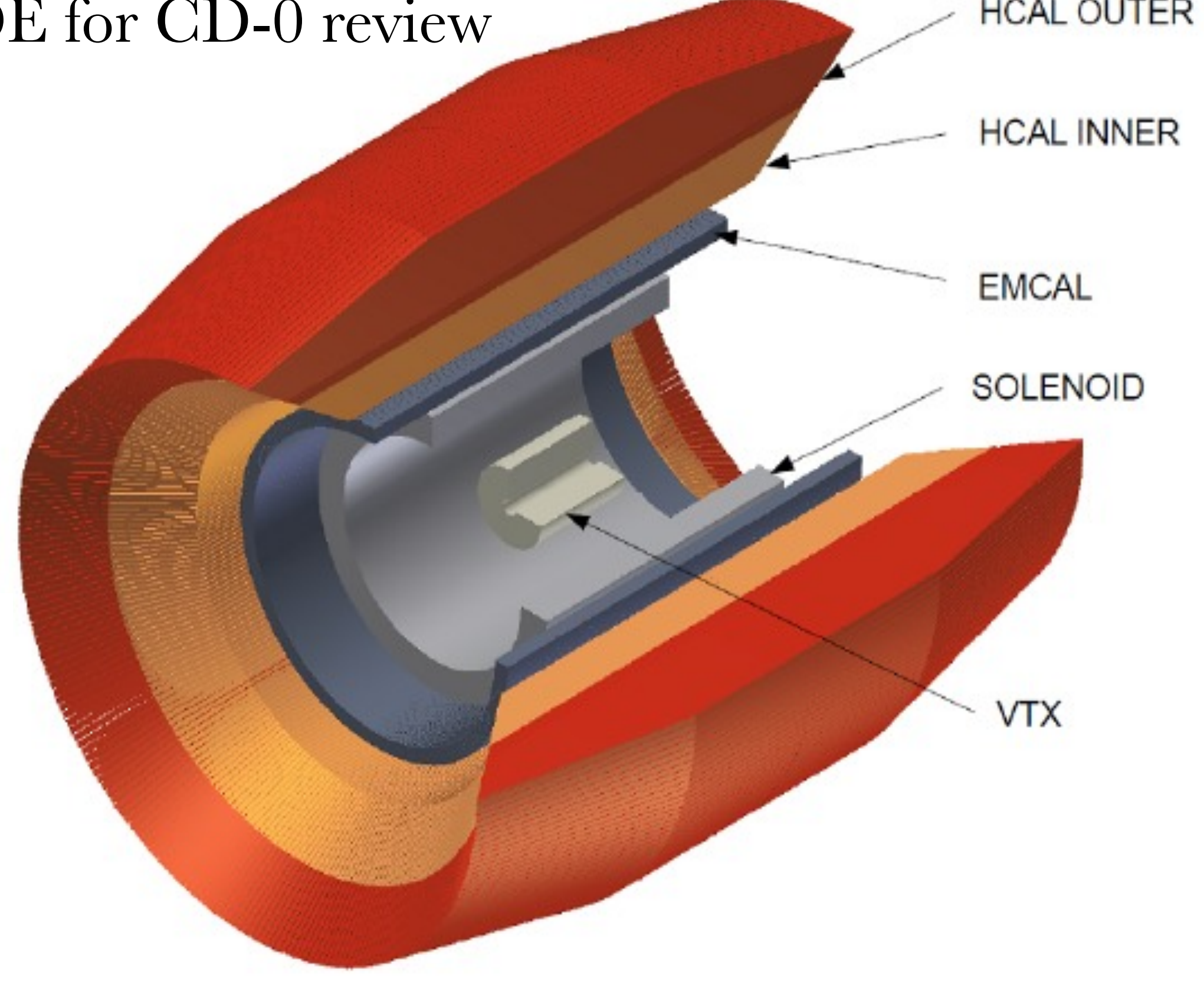



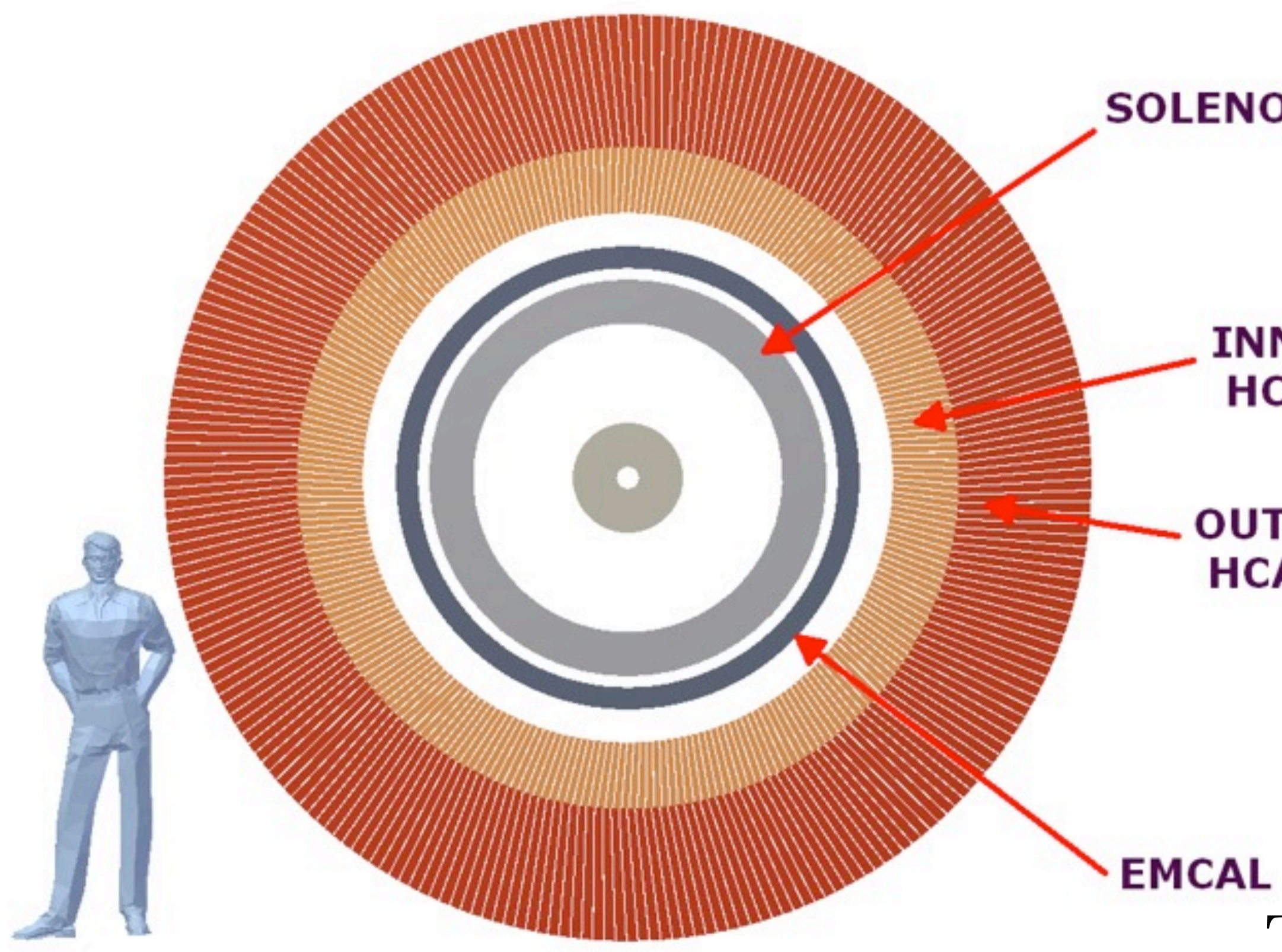

$\operatorname{Rin}=70 \mathrm{~cm}$

$2 \mathrm{~T}, 1 \mathrm{X}_{0}$

INNER

HCAL

Fe-Scintillator

HCal

\section{Tungsten-scintillator \\ $10 \mathrm{~cm}$ thick}




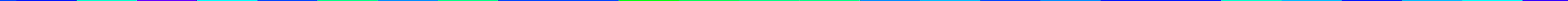




\section{RHIC Jet Rates}

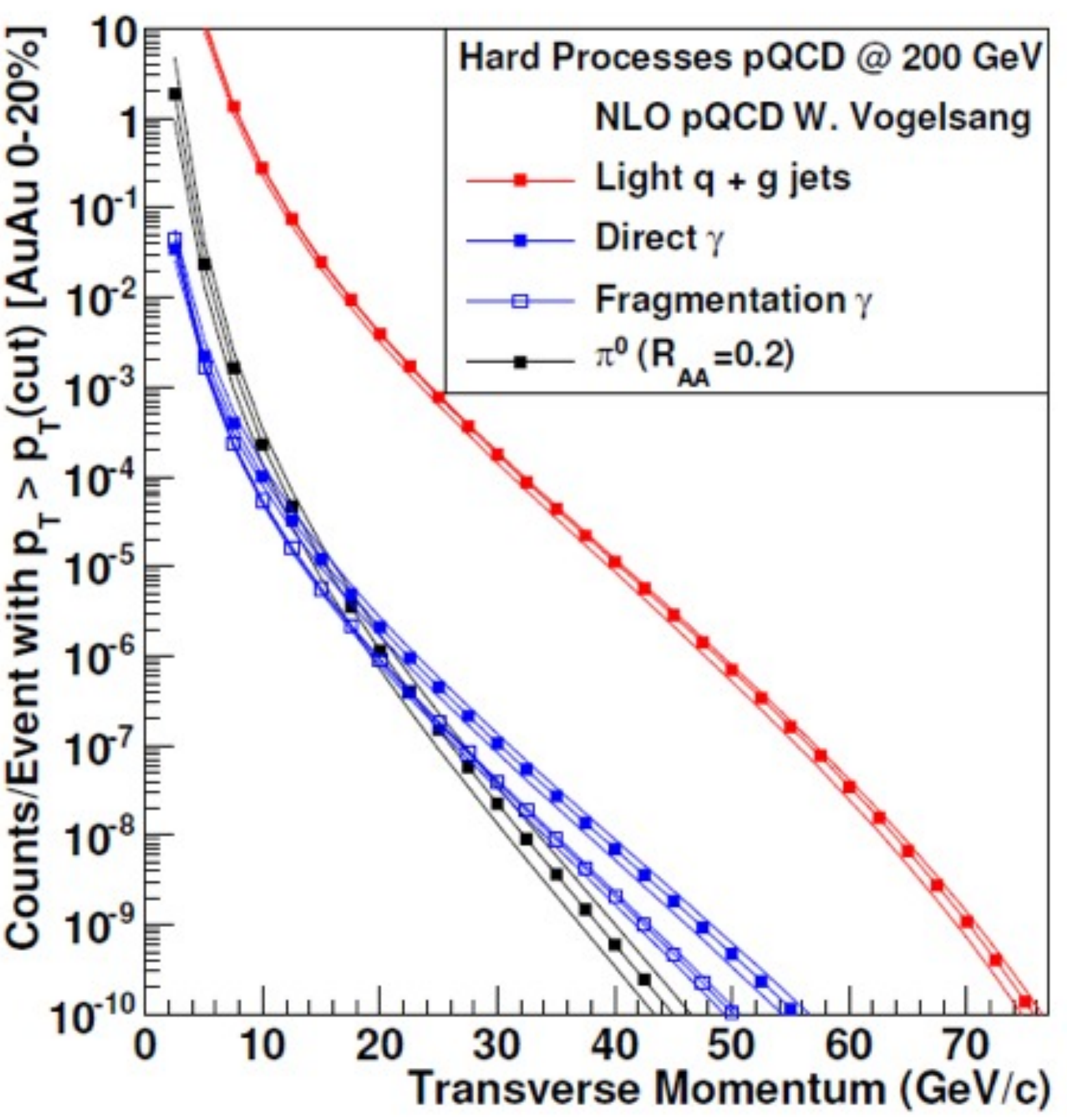

rates based on full stochastic cooling, but no additional accelerator upgrades

\begin{tabular}{l|l|l|l} 
& $\begin{array}{l}\text { Au+Au } \\
\text { (central 20\%) }\end{array}$ & $p+p$ & $d+$ Au \\
\hline > $20 \mathrm{GeV}$ & $\begin{array}{l}10^{7} \text { jets } \\
10^{4} \text { photons }\end{array}$ & $\begin{array}{l}10^{6} \text { jets } \\
10^{3} \text { photons }\end{array}$ & $\begin{array}{l}10^{7} \text { jets } \\
10^{4} \text { photons }\end{array}$ \\
\hline >30GeV & $\begin{array}{l}10^{6} \text { jets } \\
10^{3} \text { photons }\end{array}$ & $\begin{array}{l}10^{5} \text { jets } \\
10^{2} \text { photons }\end{array}$ & $\begin{array}{l}10^{6} \text { jets } \\
10^{3} \text { photons }\end{array}$ \\
\hline >40GeV & $10^{5}$ jets & $10^{4}$ jets & $10^{5}$ jets \\
\hline >50GeV & $10^{4}$ jets & $10^{3}$ jets & $10^{4}$ jets
\end{tabular}

Huge rates allow differential measurements with geometry $\left(\mathbf{v}_{2}, \mathbf{v}_{3}, \mathbf{A}+\mathbf{B}, \mathbf{U}+\mathbf{U}, \ldots\right) \&$ precise control measurements (dAu \& pp) over $80 \%$ as dijets! 
- how well can we measure real jets?

- jet energy scale, jet energy resolution

- how are the jet measurements impacted by background fluctuations masquerading as jets--fakes

- large HIJING study

- embedding PYTHIA jets into HIJING events to evaluate jet reconstruction performance

- 750M minimum bias HIJING events to study relative rates of fake and real jets in HI background

- iterative background subtraction as in ATLAS

detailed study in: Hanks, Sickles et al: PRC86 024908 


\section{reconstruction performance}
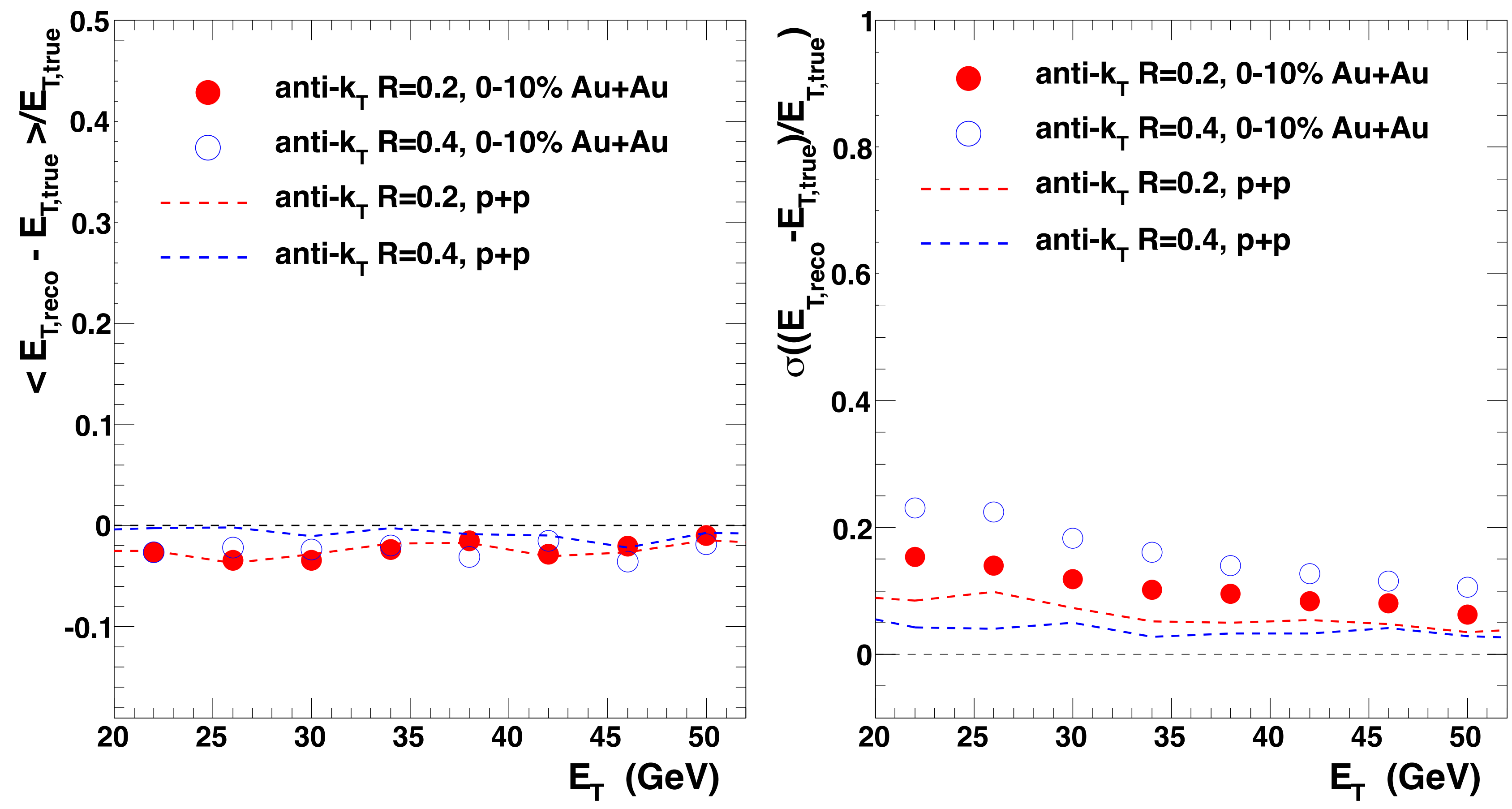

- good performance in heavy ion background

- resolution only from the underlying event, no detector resolution included 


\section{reconstructed jets}

\section{reconstructed jets}

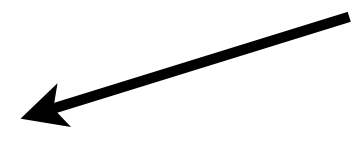

\begin{tabular}{|c|}
\hline matched jets: \\
within $\Delta \mathrm{R}<0.25$ of a HIJING \\
truth jet $(>5 \mathrm{GeV})$
\end{tabular}

not matched jets:

no nearby HIJING jets

"fakes"

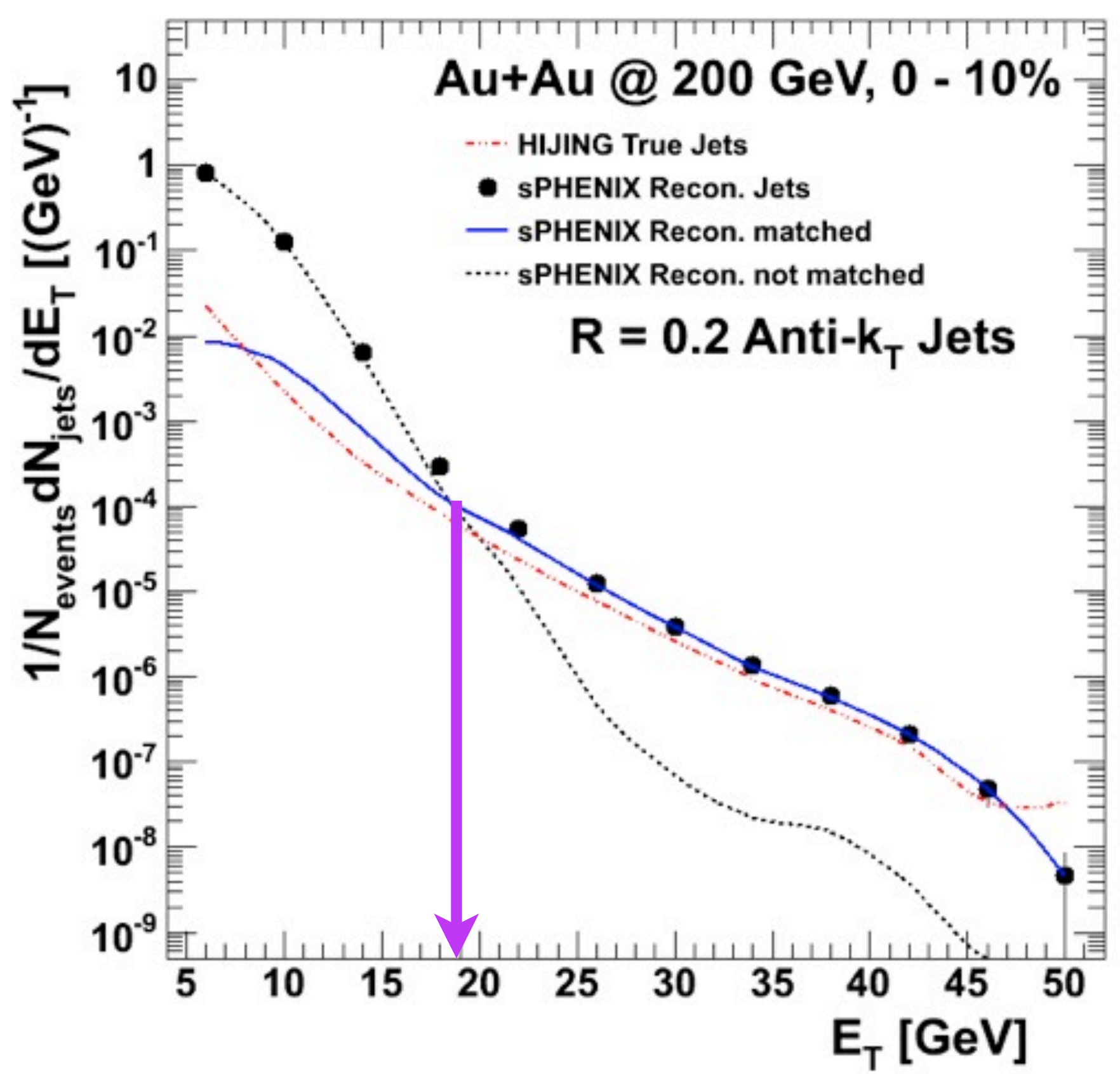




\section{Fake Jets at RHIC $(\mathrm{R}=0.2)$}

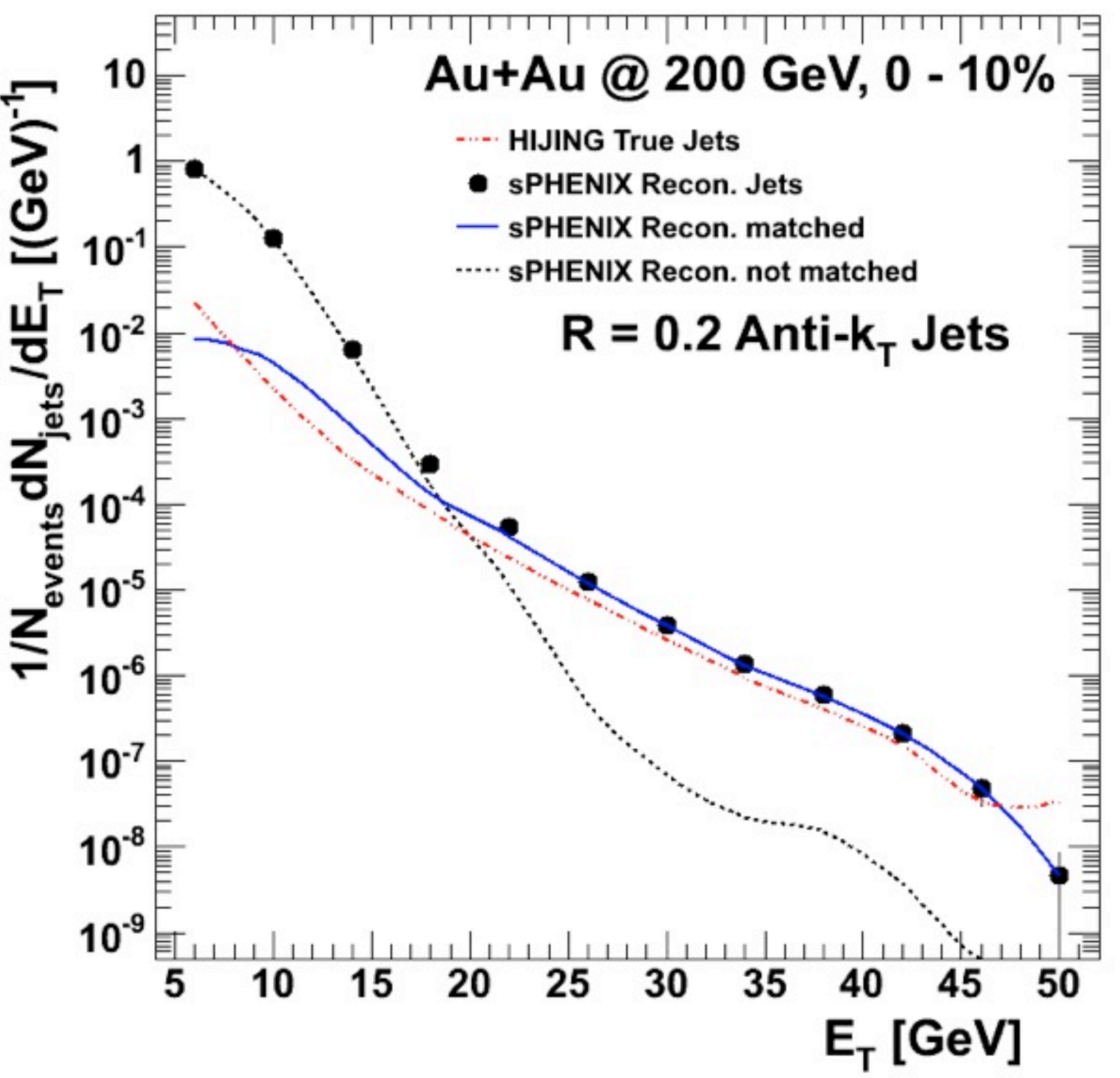




\section{Fake Jets at RHIC $(\mathrm{R}=0.2)$}

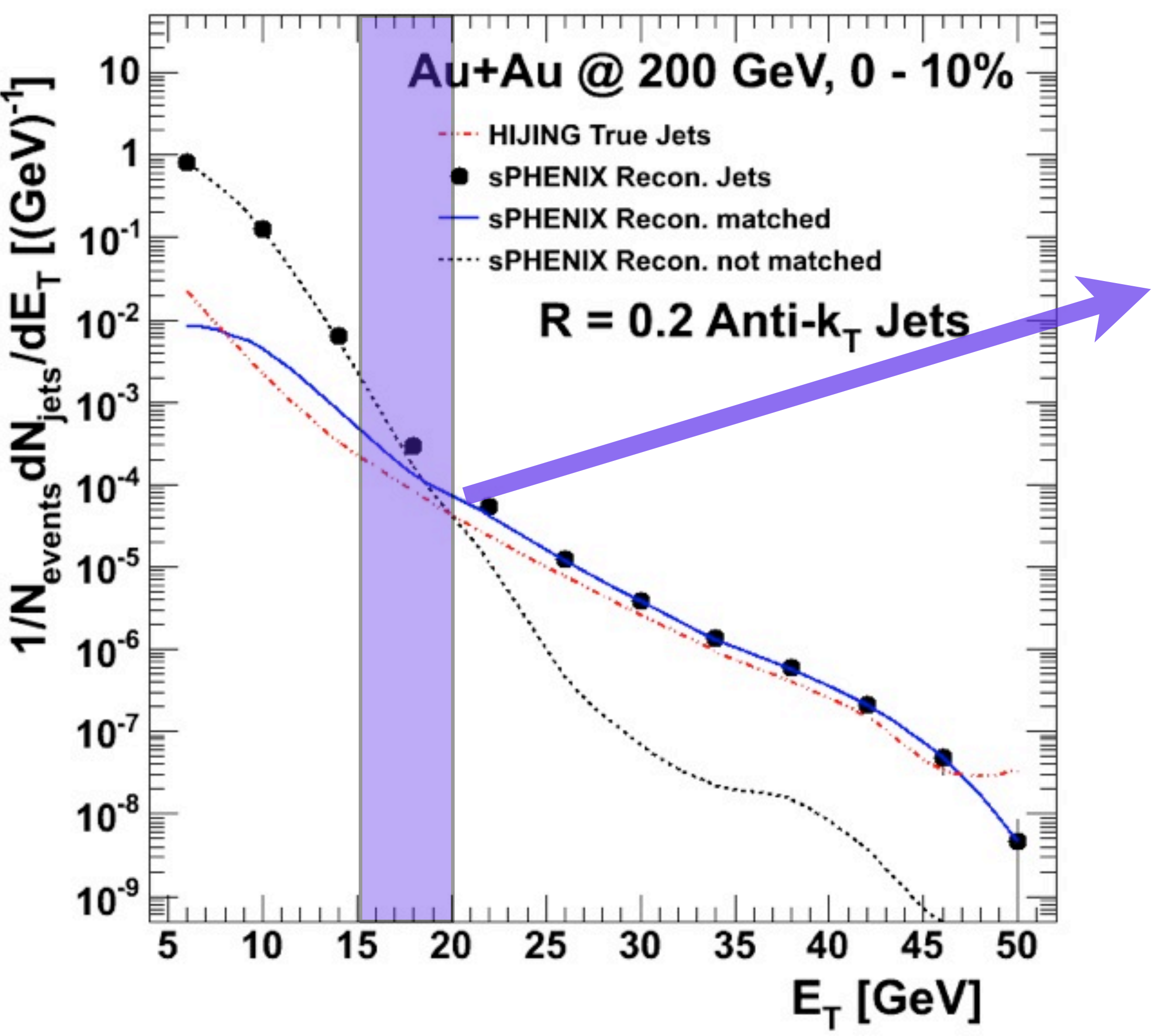




\section{Fake Jets at RHIC $(\mathrm{R}=0.2)$}

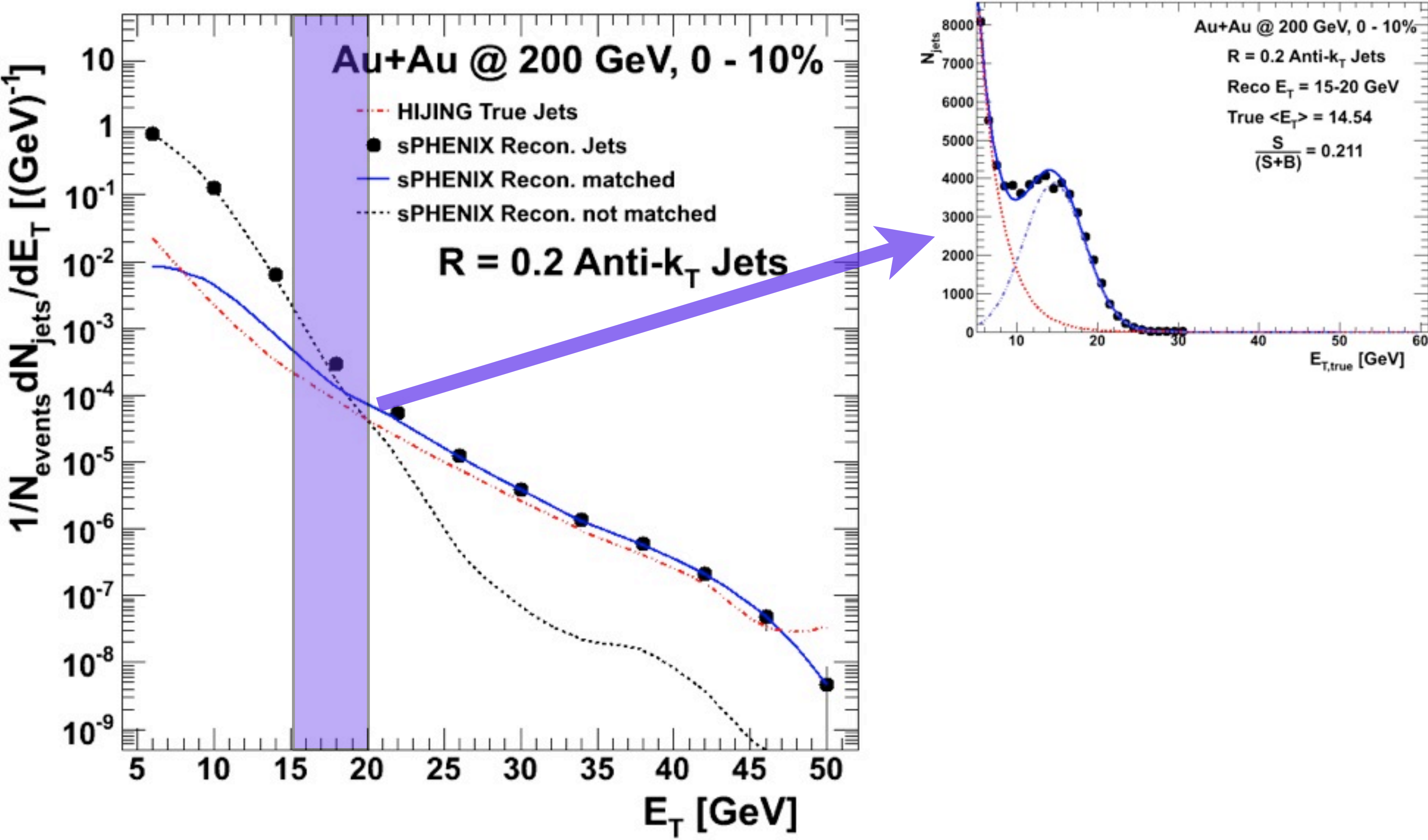




\section{Fake Jets at RHIC $(\mathrm{R}=0.2)$}

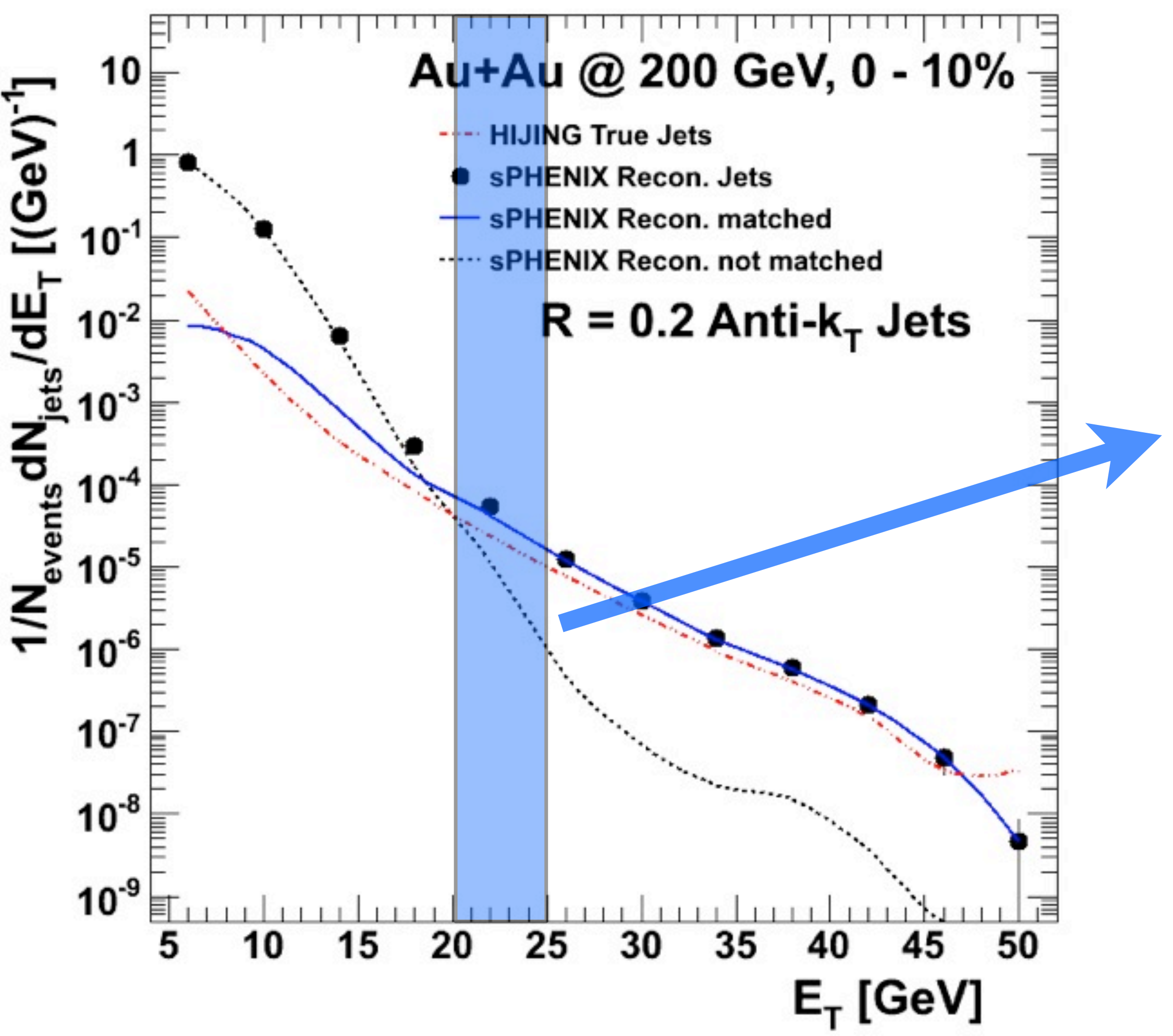




\section{Fake Jets at RHIC $(\mathrm{R}=0.2)$}

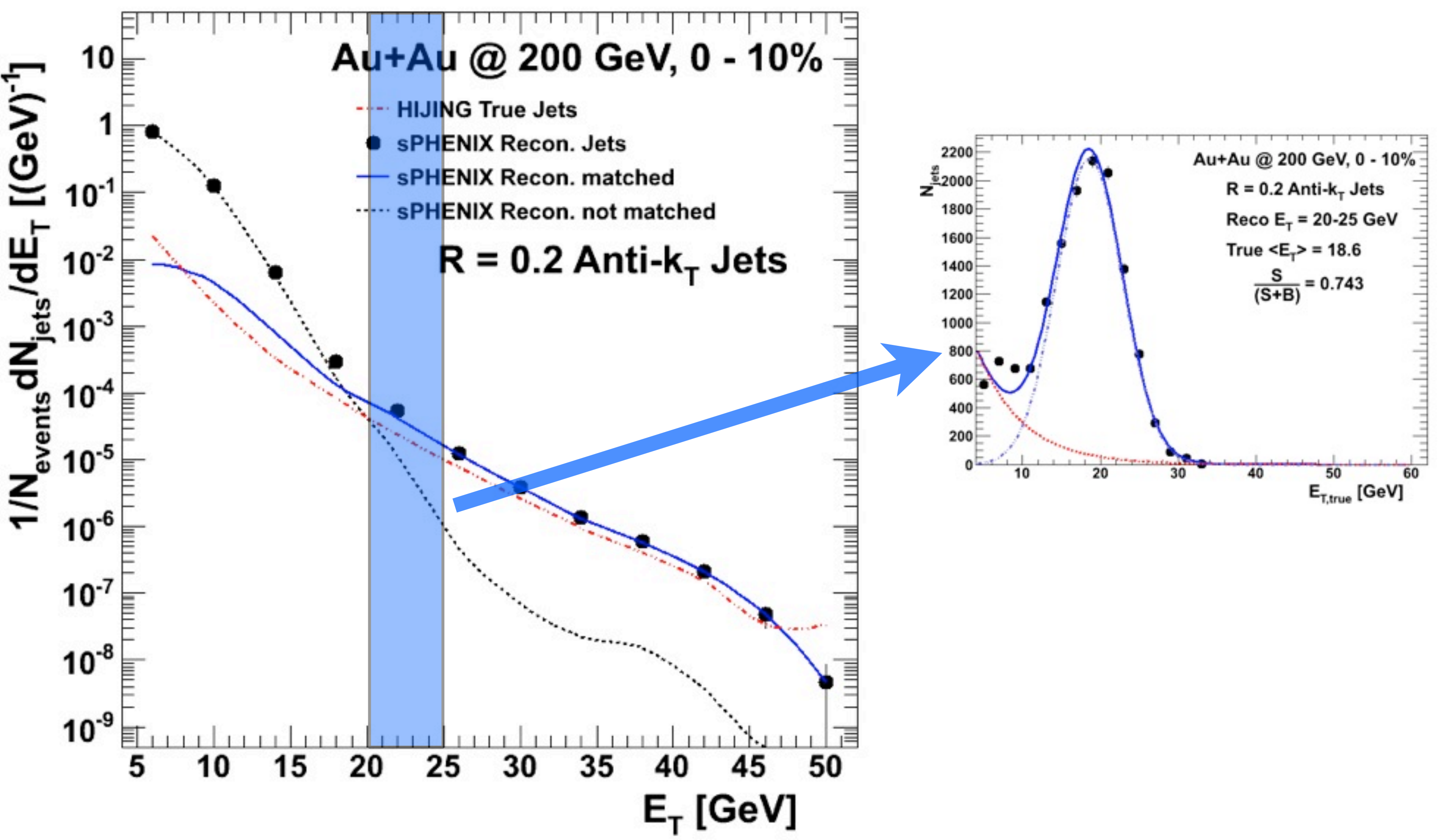




\section{Fake Jets at RHIC $(\mathrm{R}=0.2)$}

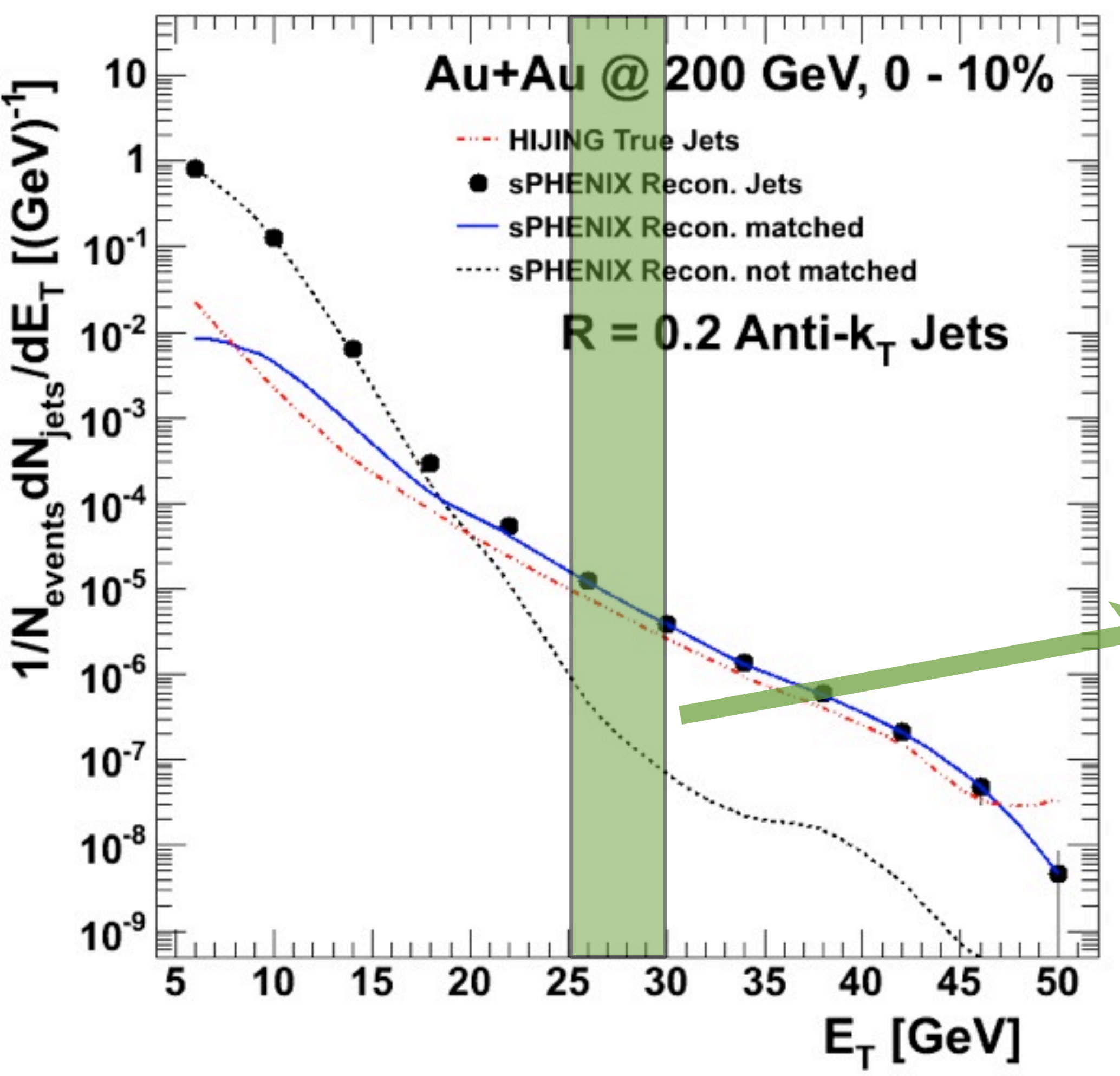




\section{Fake Jets at RHIC $(\mathrm{R}=0.2)$}

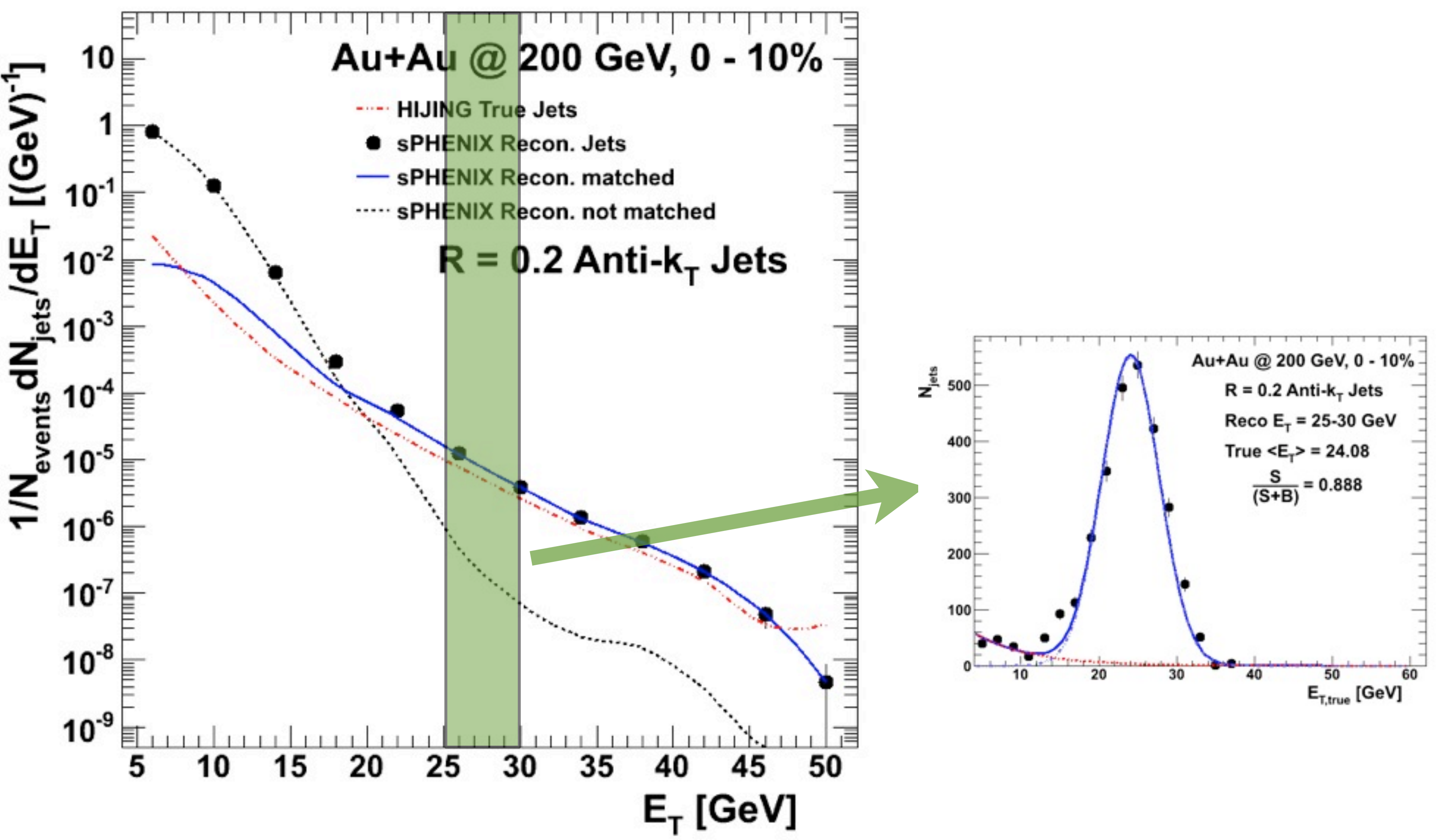




\section{Fake Jets at RHIC $(\mathrm{R}=0.2)$}

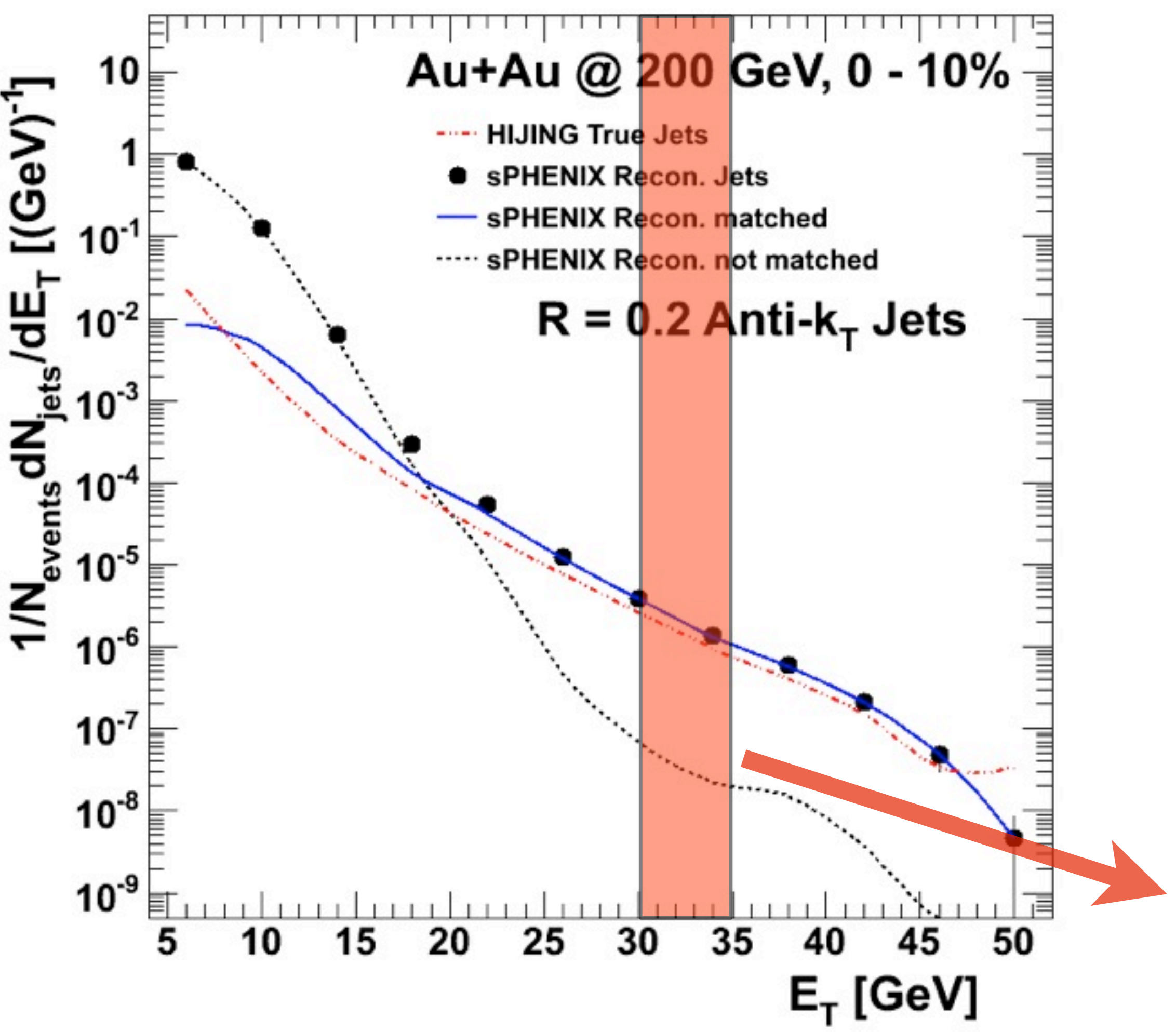




\section{Fake Jets at RHIC $(\mathrm{R}=0.2)$}

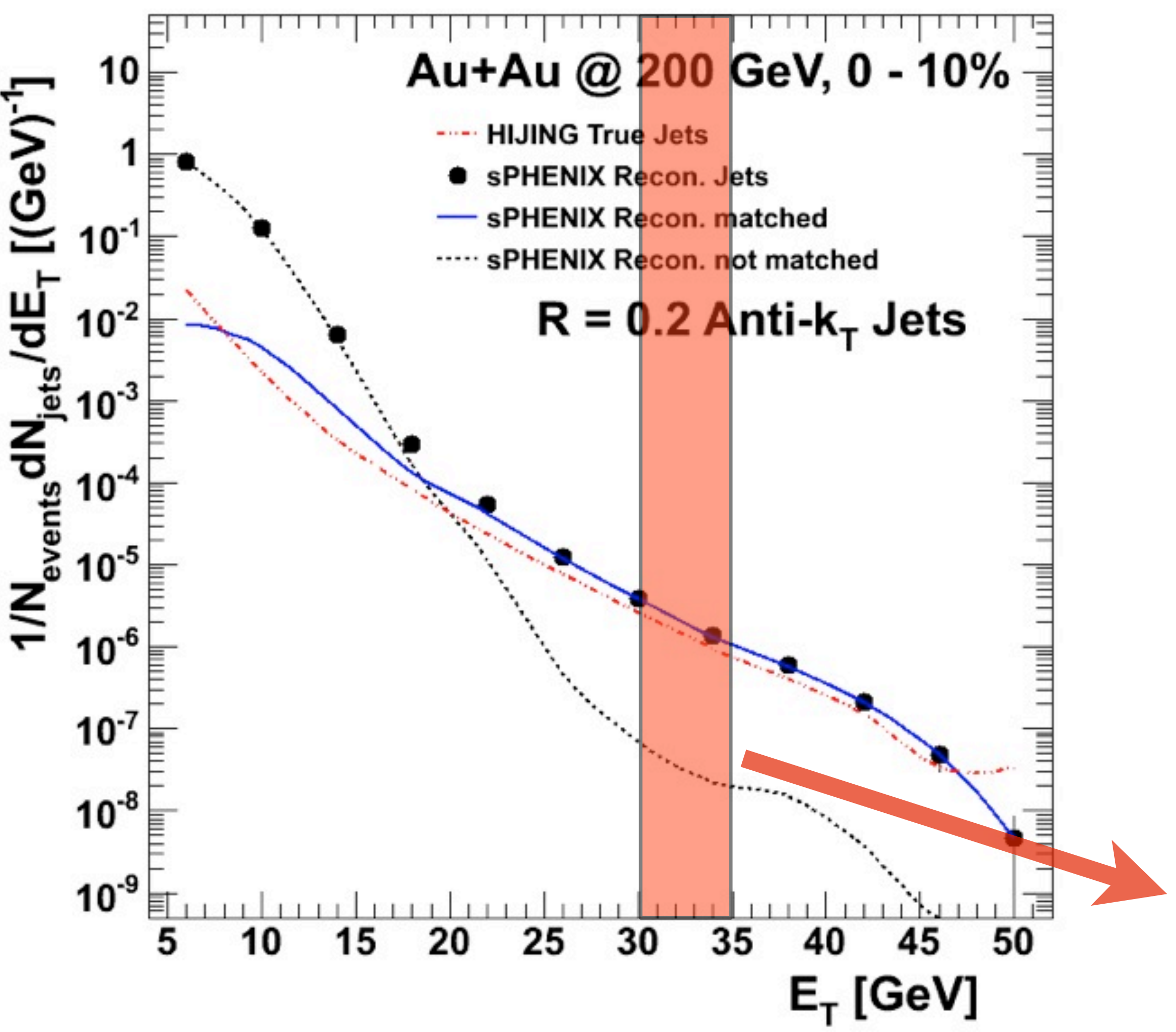




\section{Fake Jets at RHIC $(\mathrm{R}=0.4)$}

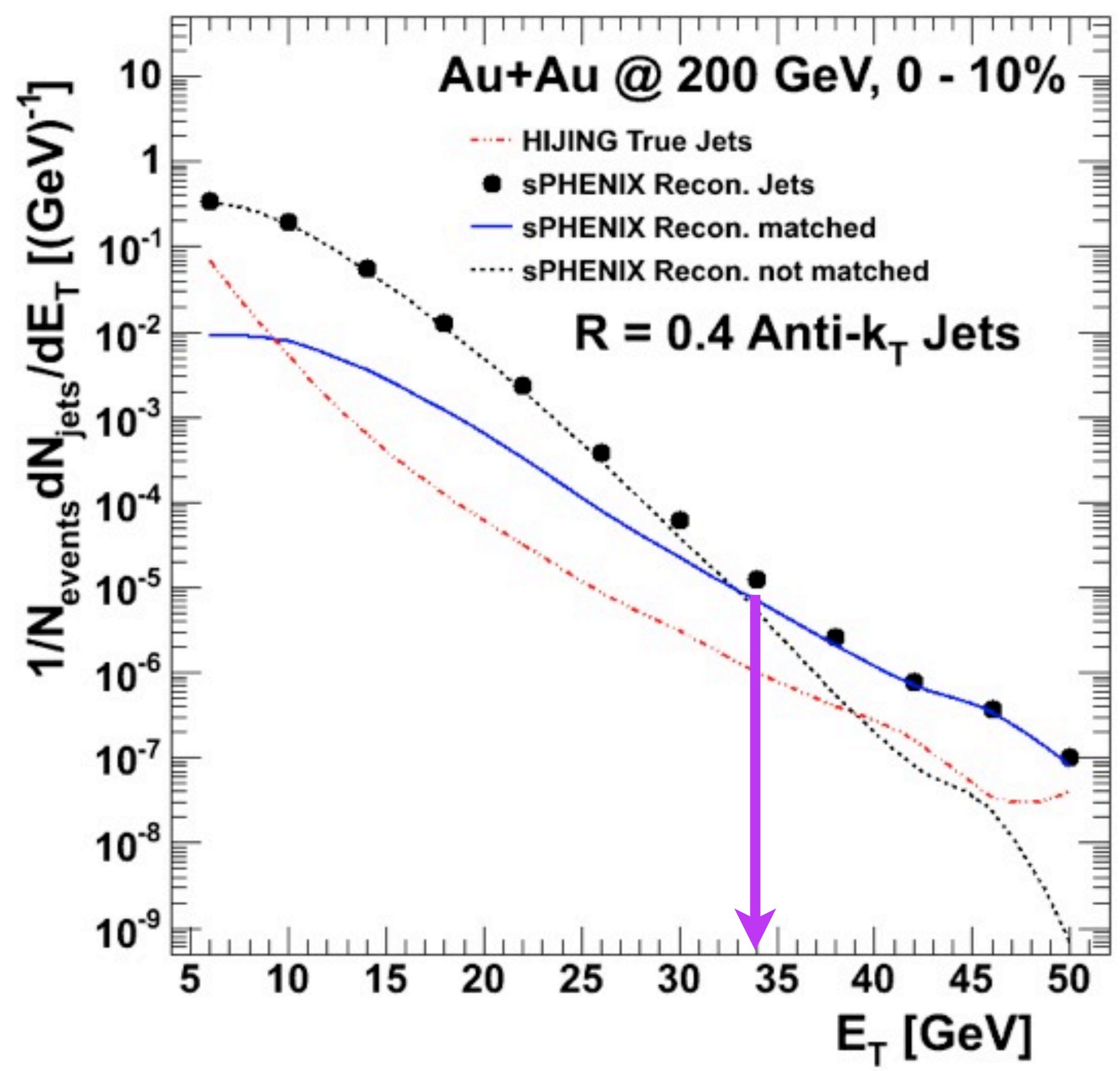

real jets outnumber fakes by $\sim 35 \mathrm{GeV}$ however, no fake jet rejection done yet 


\section{why are large jets so interesting?}

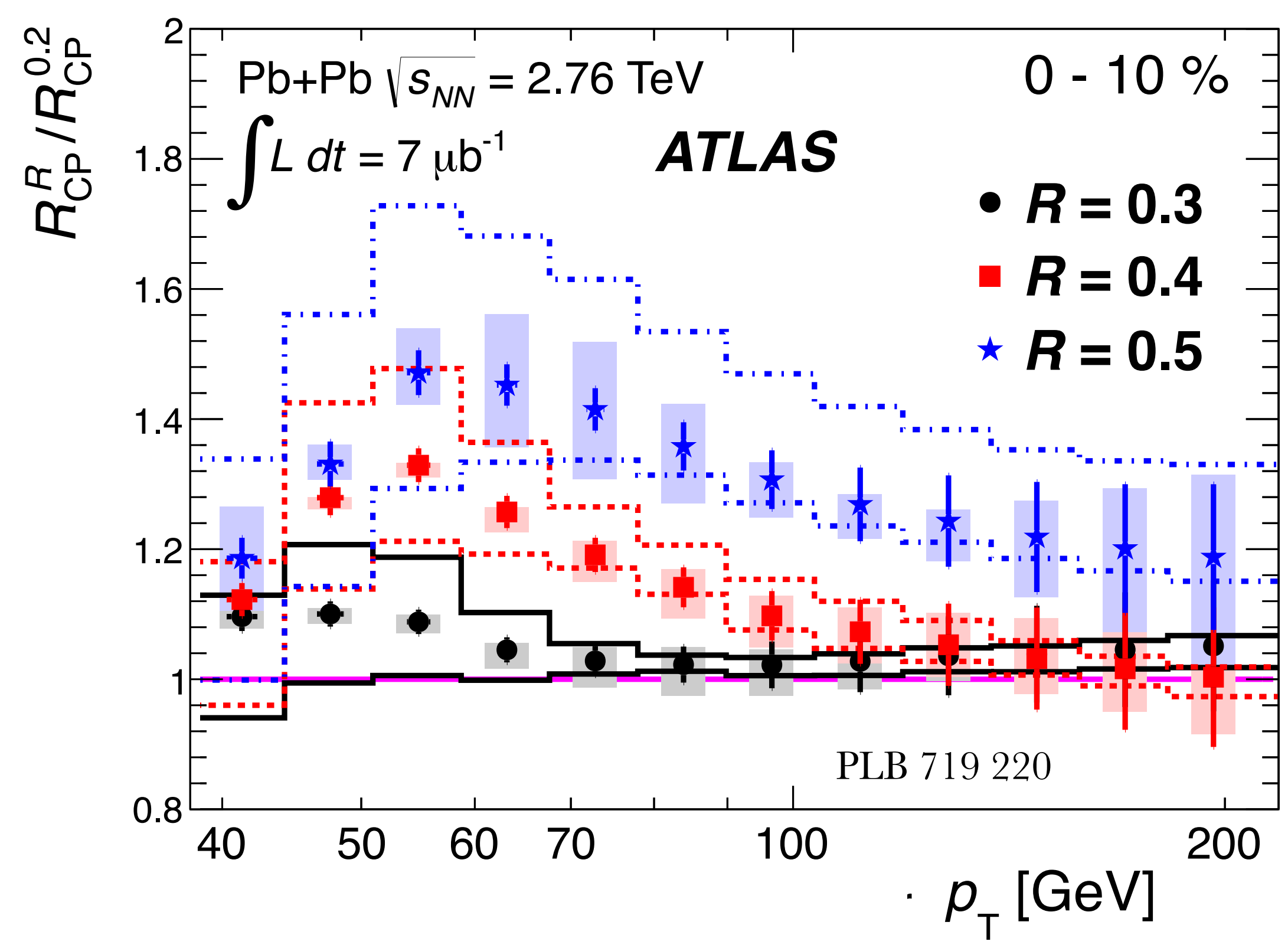

- evidence for large shape modifications in the region accessible at RHIG

- experimentally: require high statistics \& good control of systematics 


\section{dijet measurements}

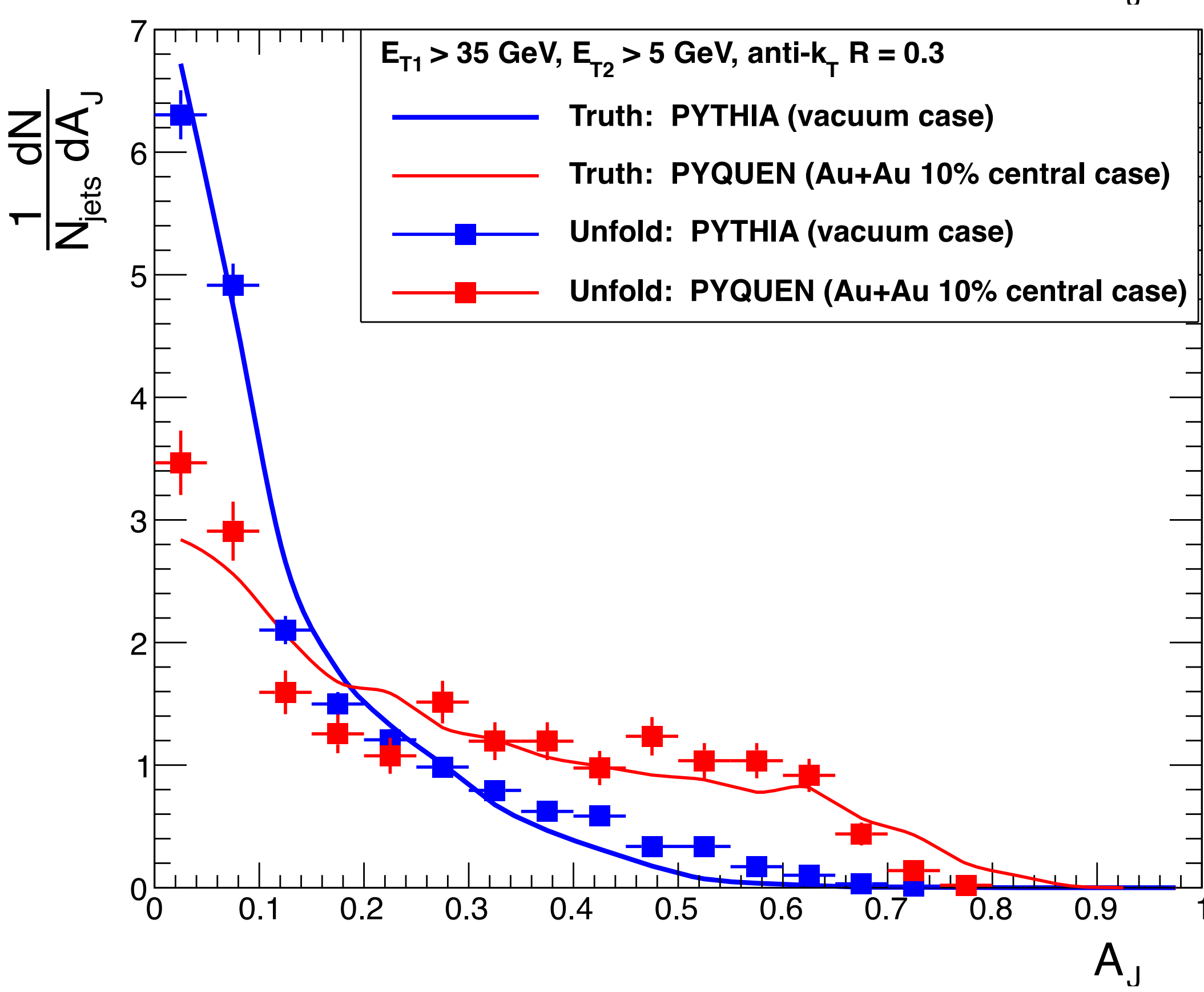

- full Geant 4 detector simulation of dijets embedded in $0-10 \%$ central HIJING

- unfolding recovers the truth distribution very well 


\section{$\gamma$-jet measurements}
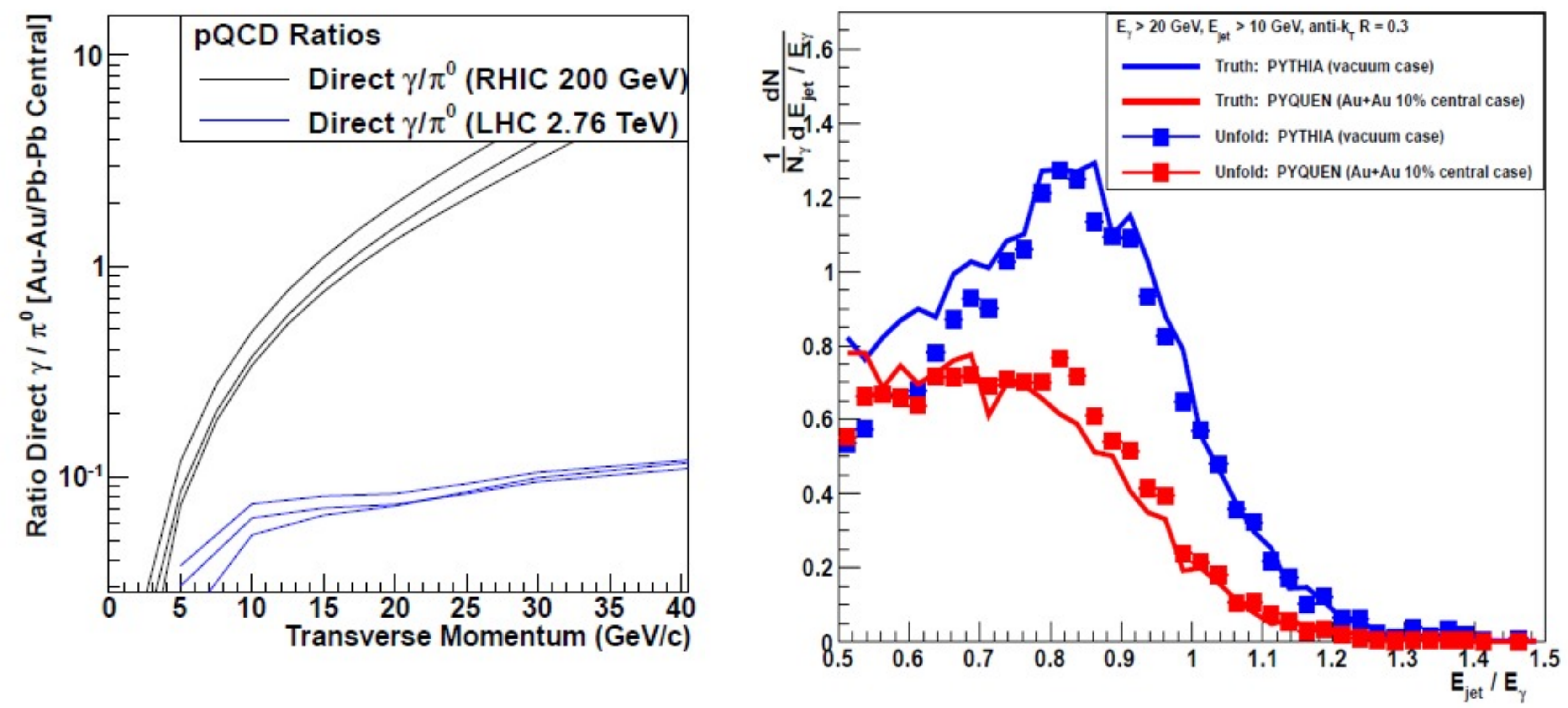

- $\gamma / \pi 0$ very large at $\mathrm{RHIC}$, large numbers out $>30 \mathrm{GeV}$

- unfolding the jet energy recovers the truth distributions from Geant4 simulation into central HIJING 


\section{heavy flavor at RHIC}

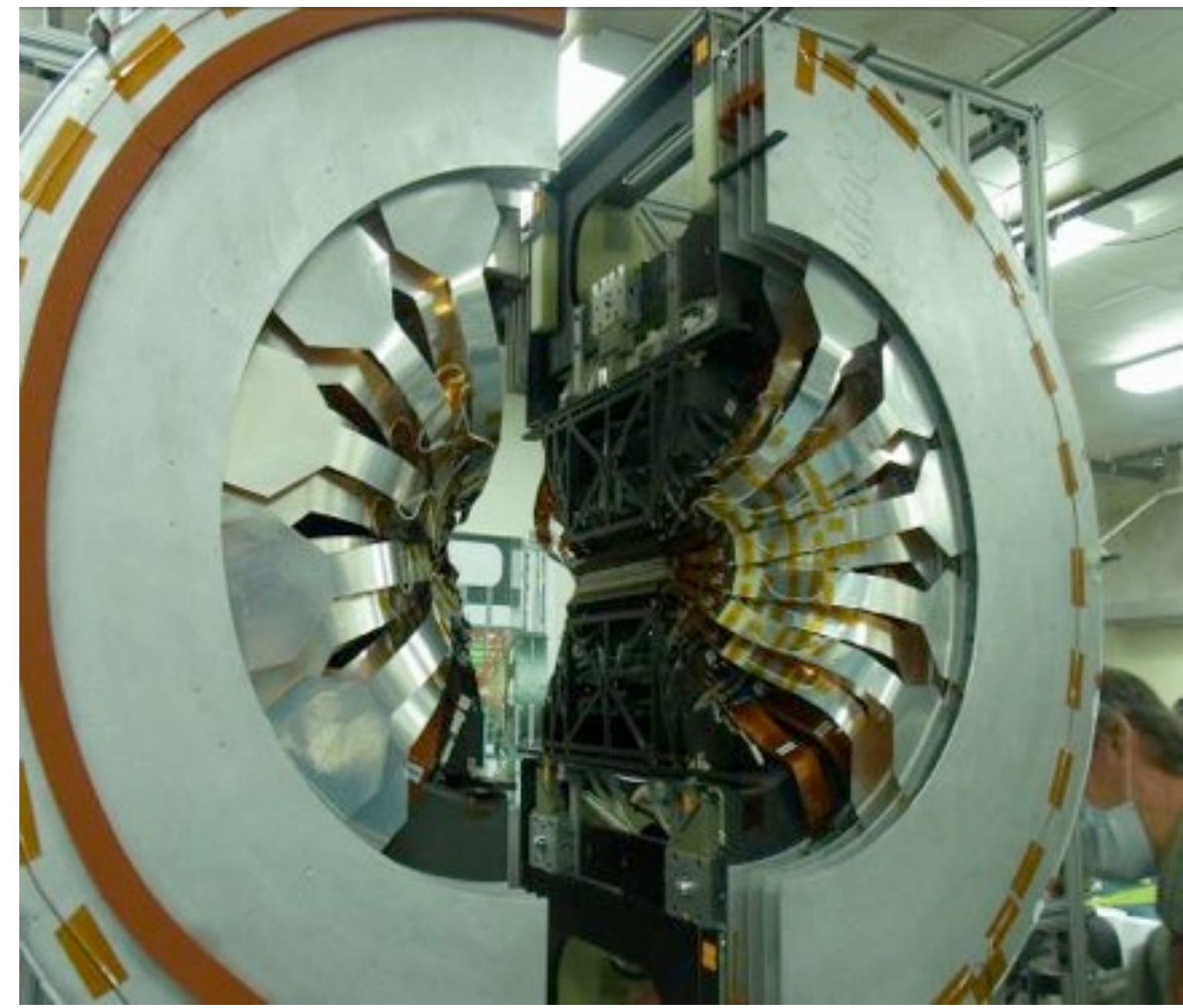

PHENIX (F)VTX

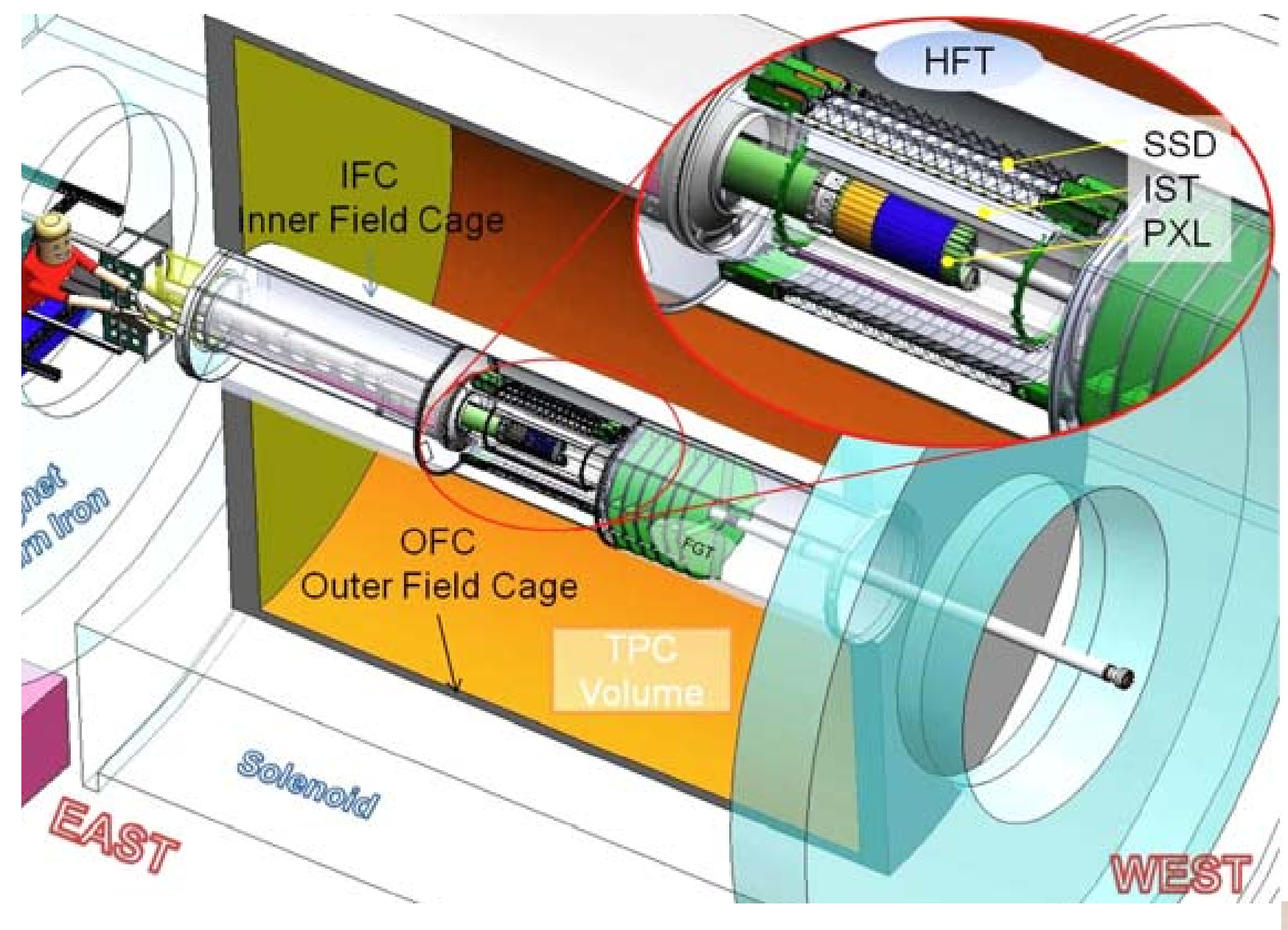

STAR HFT

- upgrades coming online to address charm and bottom separation at RHIC

- essential piece of the jet quenching puzzle 


\section{jet quenching at RHIC}

current measurements show evidence for softening of fragmentation and broadening of angular correlations

ATLAS measurements show $R$ dependence of jet suppression at jet energies accessible at RHIC

together with LHC constrain T dependence of jet quenching

extremely interesting physics accessible at RHIC

sPHENIX: full calorimeter coverage at mid-rapidity

- take advantage of full RHIC luminosity: large acceptance, high rate

- becomes central to ePHENIX

- proposal submitted to DOE for CD-0 review last month!

silicon detectors at STAR and PHENIX will provide handle on heavy flavor

- detector upgrades critical to the success of hard probes at RHIC 


\section{backups}




\section{further exploration of $\mathrm{T}$ dependence}

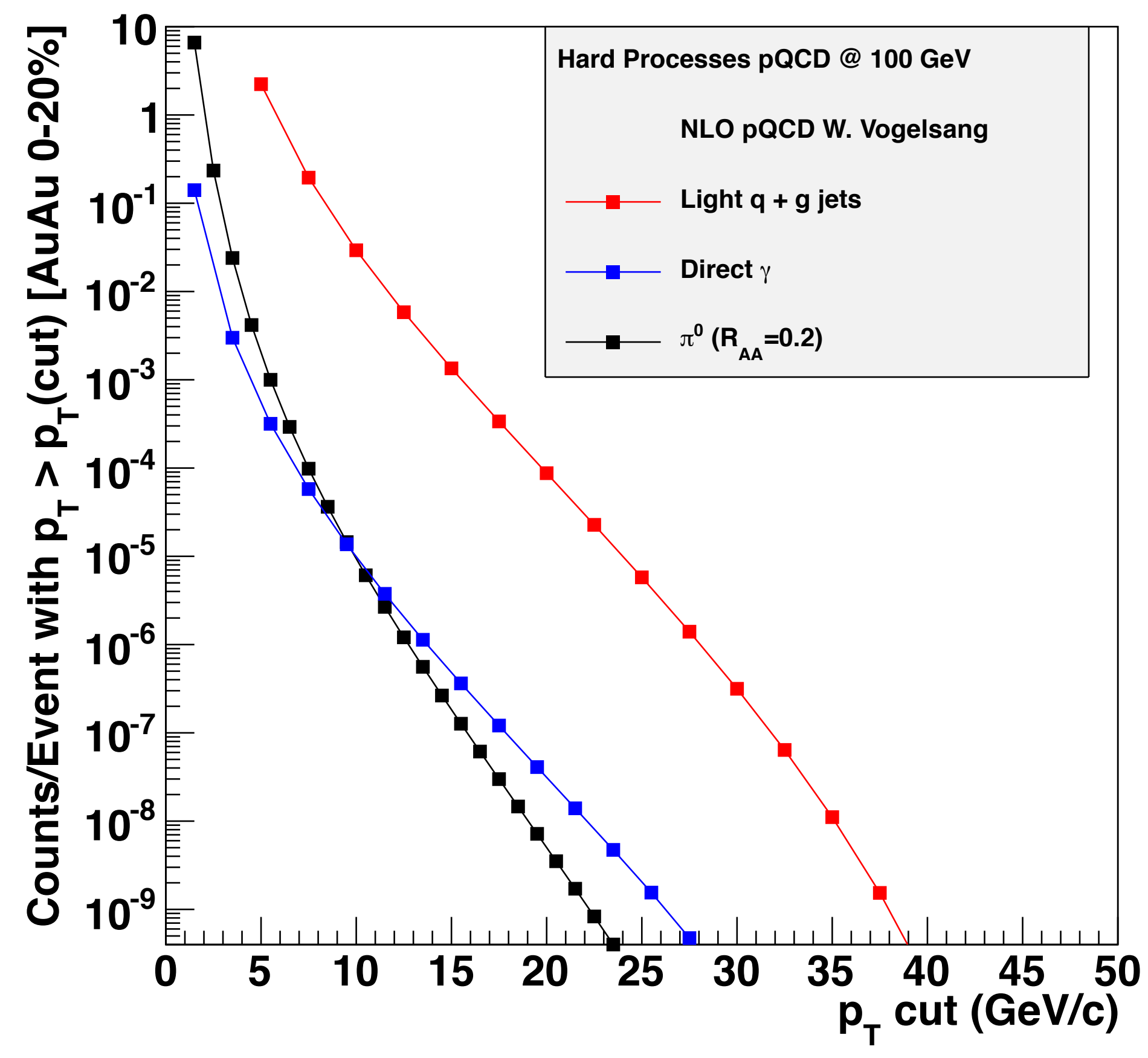




\section{heavy quarks}

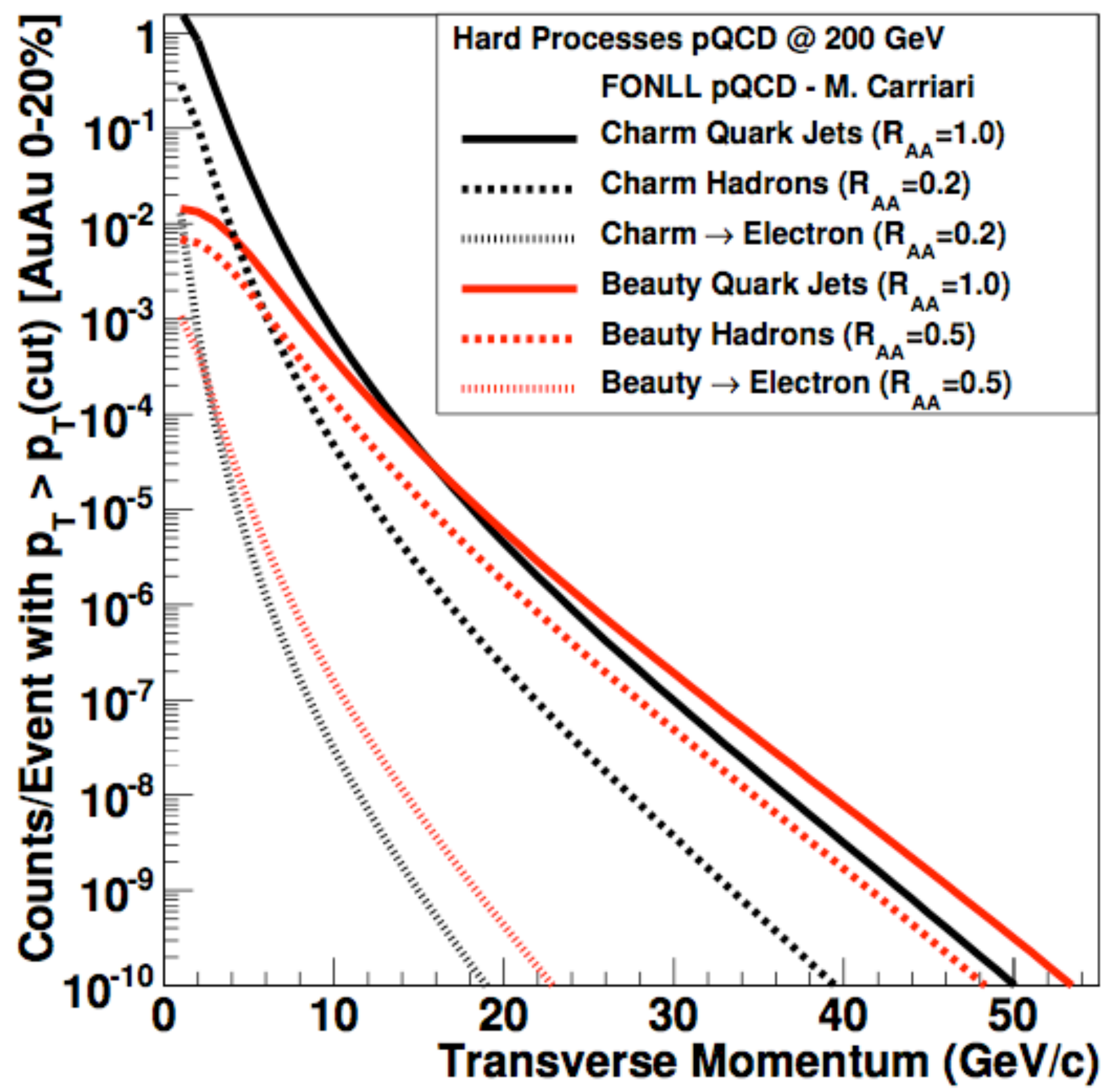




\section{identifying truth jets}




\section{identifying truth jets}

deep within the HIJING Event Generation... 


\section{identifying truth jets}

deep within the HIJING Event Generation...

parton 1 


\section{identifying truth jets}

deep within the HIJING Event Generation...

parton 1

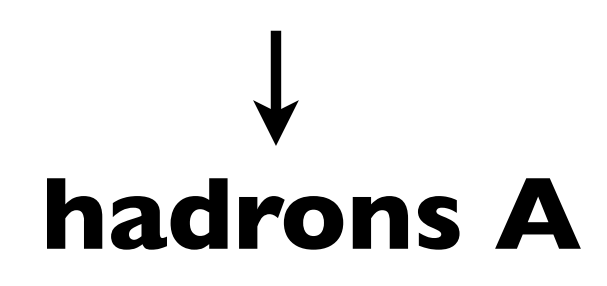




\section{identifying truth jets}

deep within the HIJING Event Generation...

parton 1

$\underset{\downarrow}{\downarrow}$ hadrons $A$

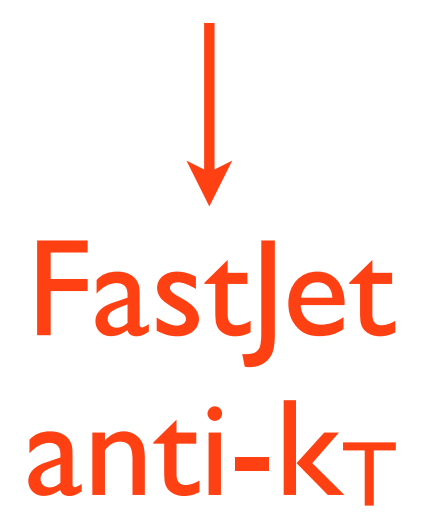




\section{identifying truth jets}

deep within the HIJING Event Generation...

parton 1

$\downarrow$

hadrons $A$

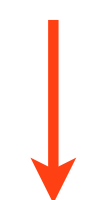

Fastjet

anti-kT

jet $A(R=0.2)$

jet $A(R=0.3)$

jet $A(R=0.4)$

A. M. Striles 


\section{identifying truth jets}

deep within the HIJING Event Generation...

parton 1

$\downarrow$

hadrons $A$

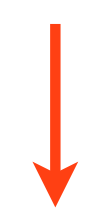

Fastjet

anti-kT

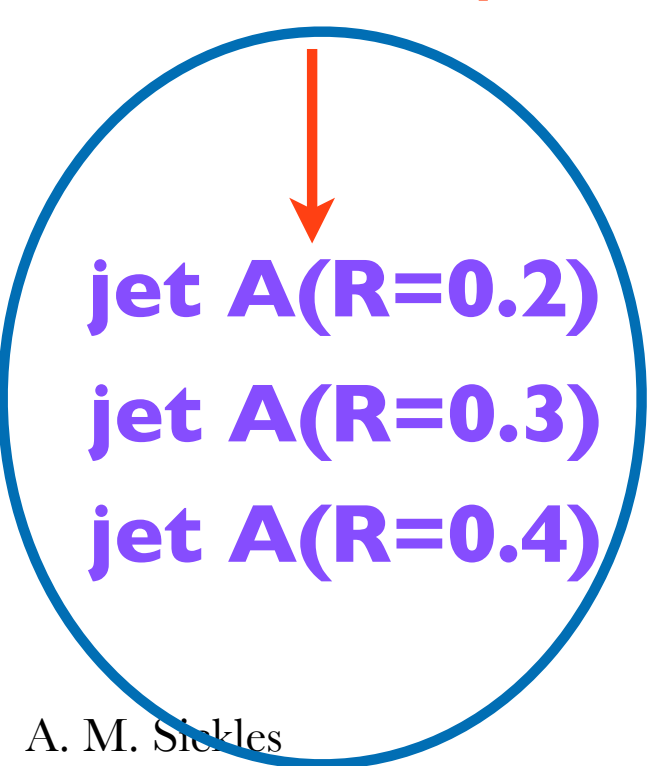

parton 2

$\downarrow$

hadrons B

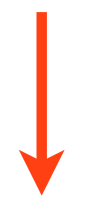

Fastjet

anti-kT

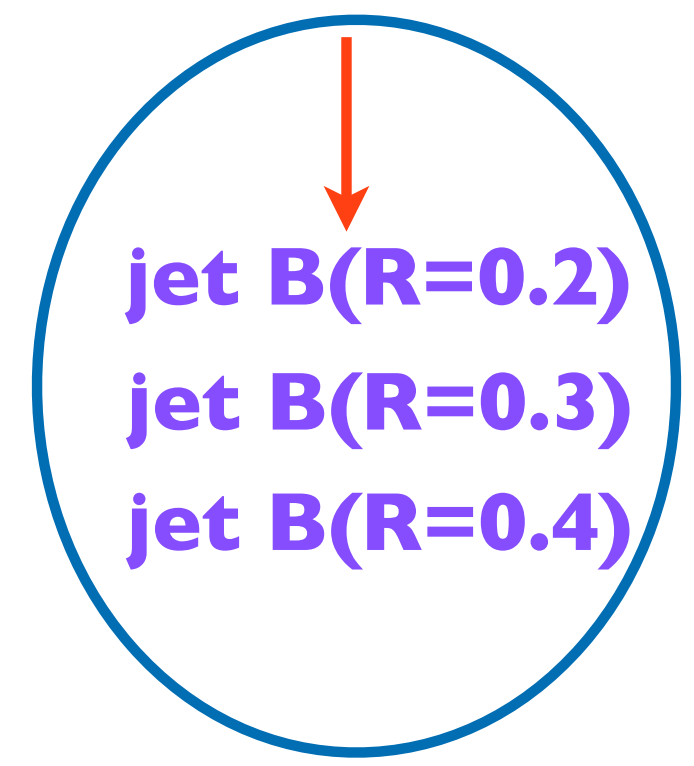


deep within the HIJING Event Generation...

parton 1

$\downarrow$

hadrons A

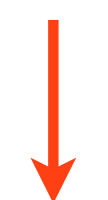

Fastjet

anti-kT

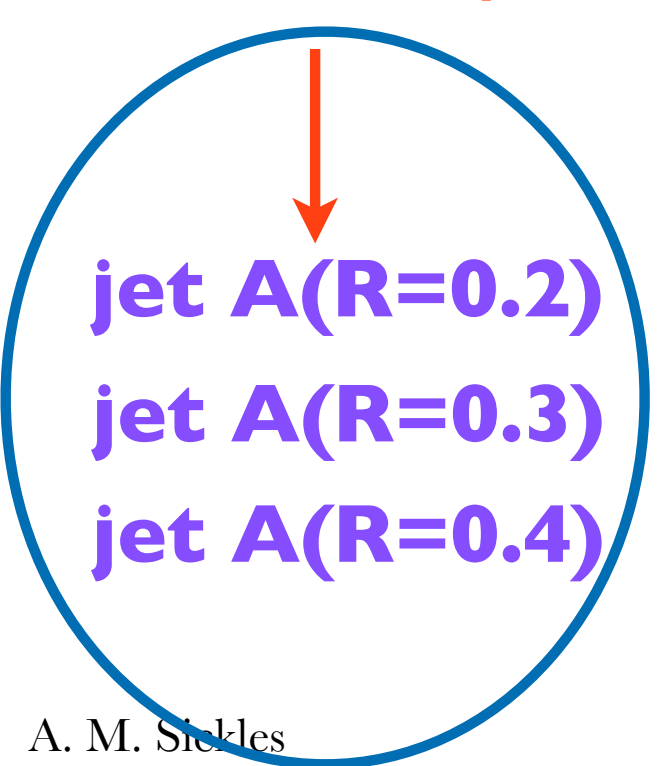

parton 2 parton 3

$\downarrow$

hadrons B hadrons C

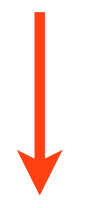

Fastjet

anti-kT

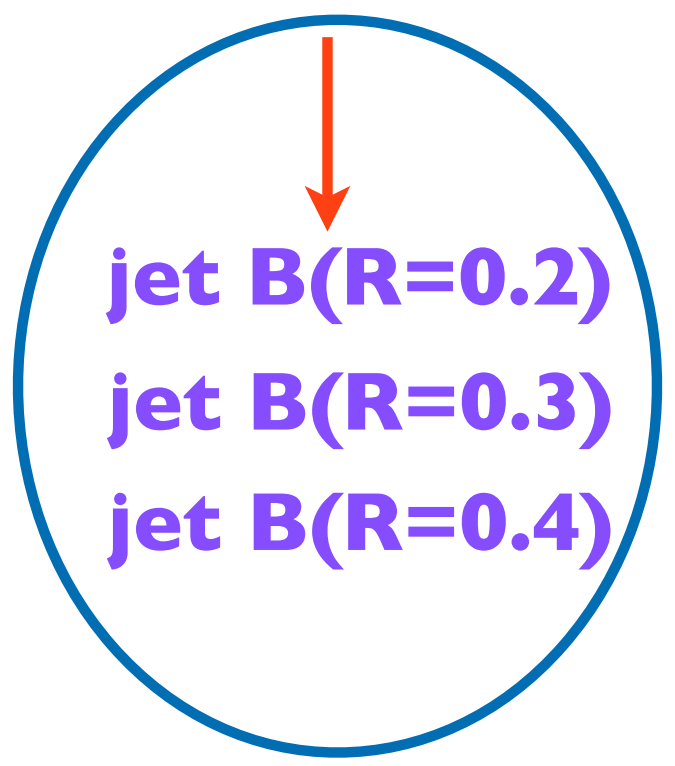

Fastjet

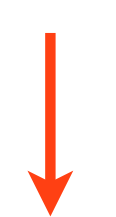

anti-kT

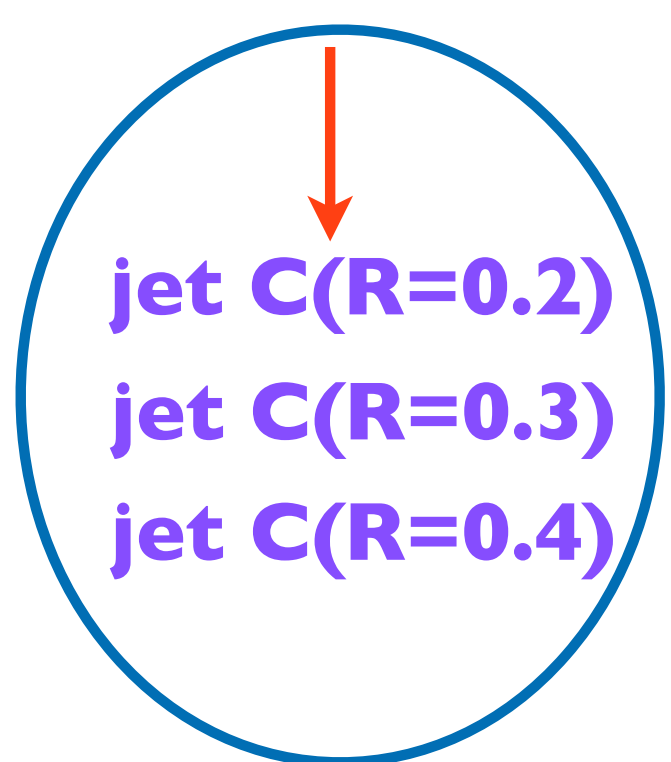




\section{identifying truth jets}

deep within the HIJING Event Generation...

parton 1

$\downarrow$

hadrons A

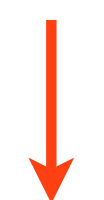

Fastjet

anti-kT

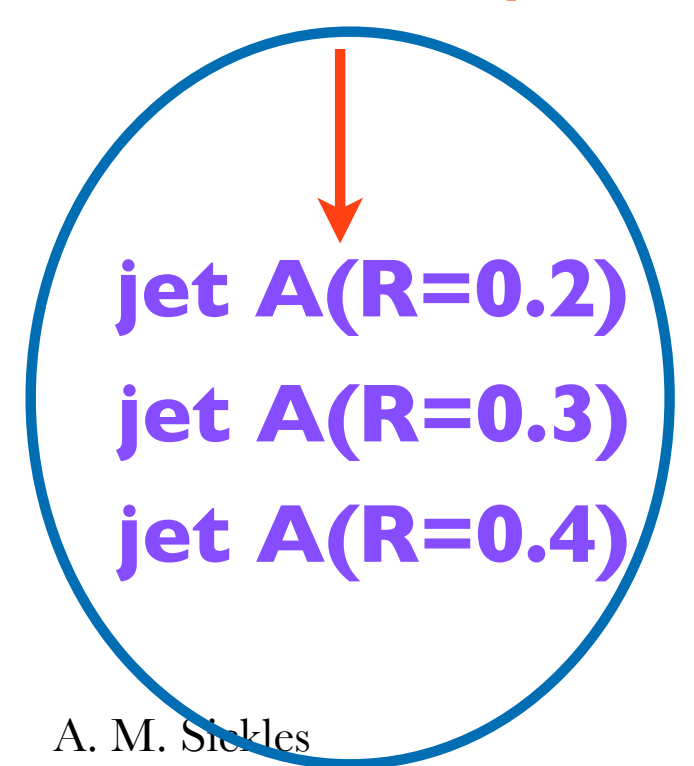

hadrons B hadrons C

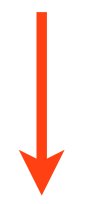

Fastjet

anti-kT

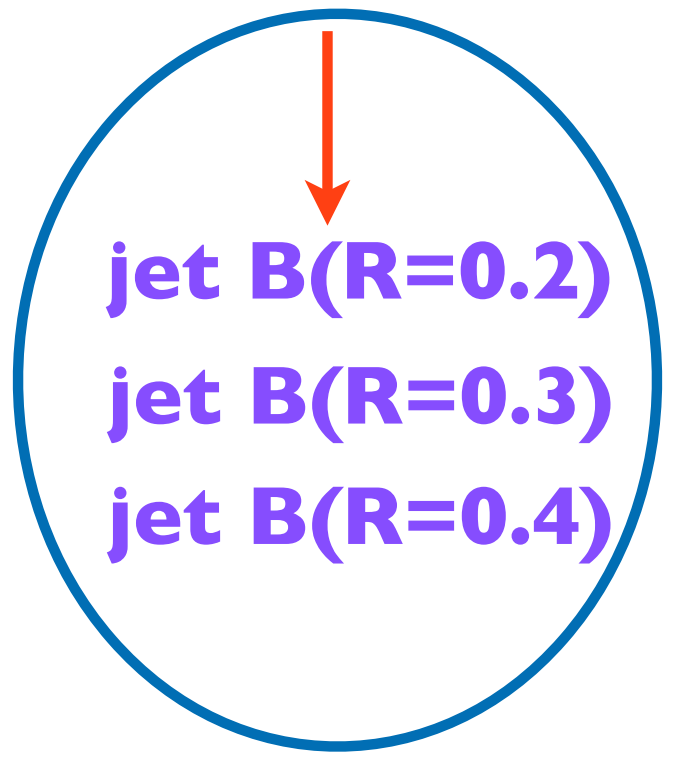

parton 3

$\downarrow$
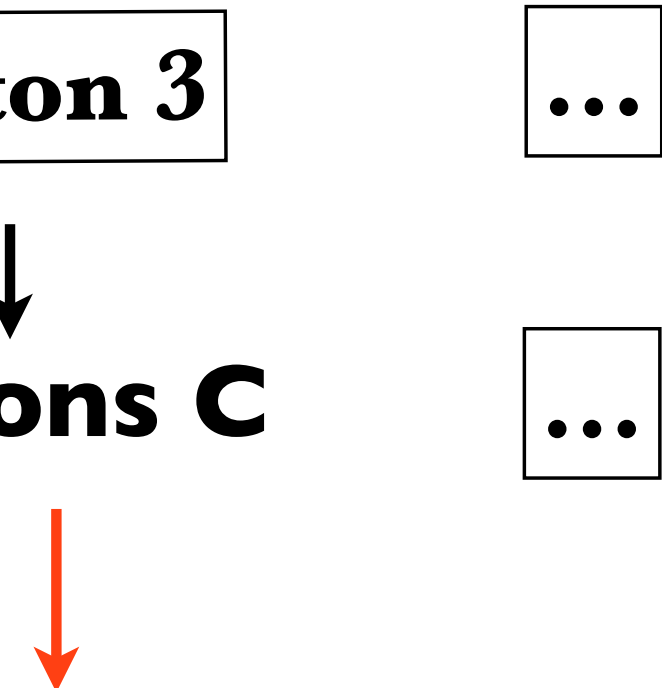

Fastjet

anti-kT

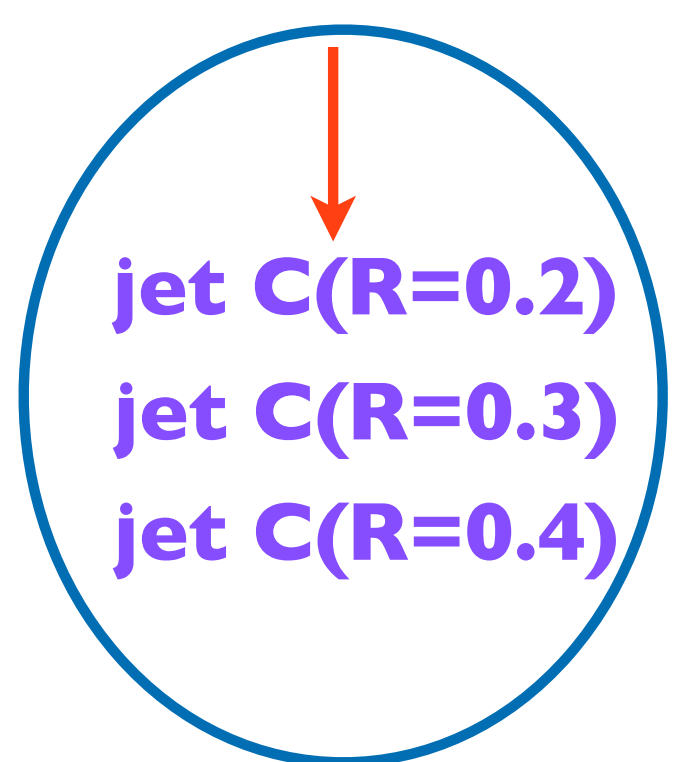

parton n

$\downarrow$

hadrons z

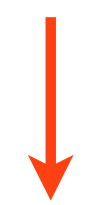

Fastjet

anti-kT

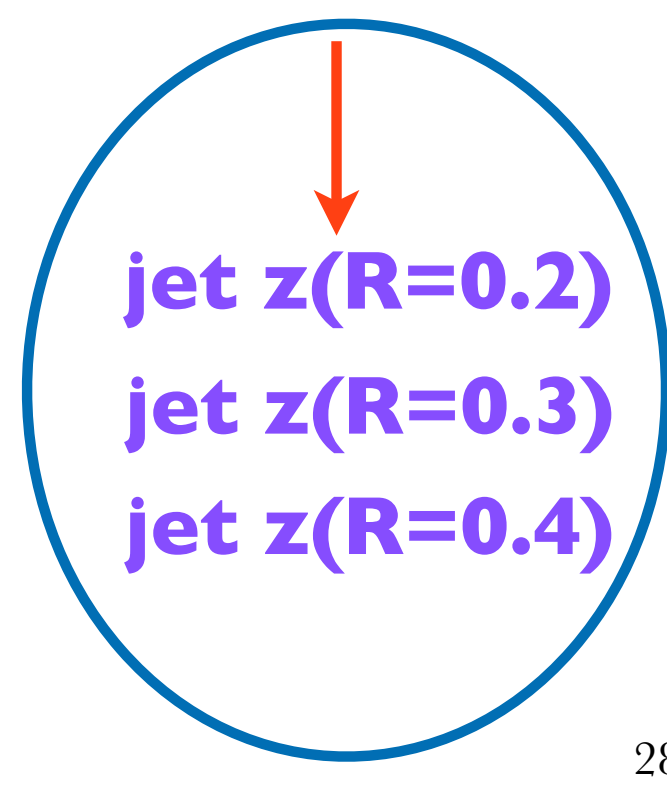




\section{well reconstructed jets}

- $\mathrm{b}=1.8 \mathrm{fm}$ HIJING dijet event

- well reconstructed with anti-k $\mathrm{k}_{\mathrm{T}} \mathrm{R}=0.2$

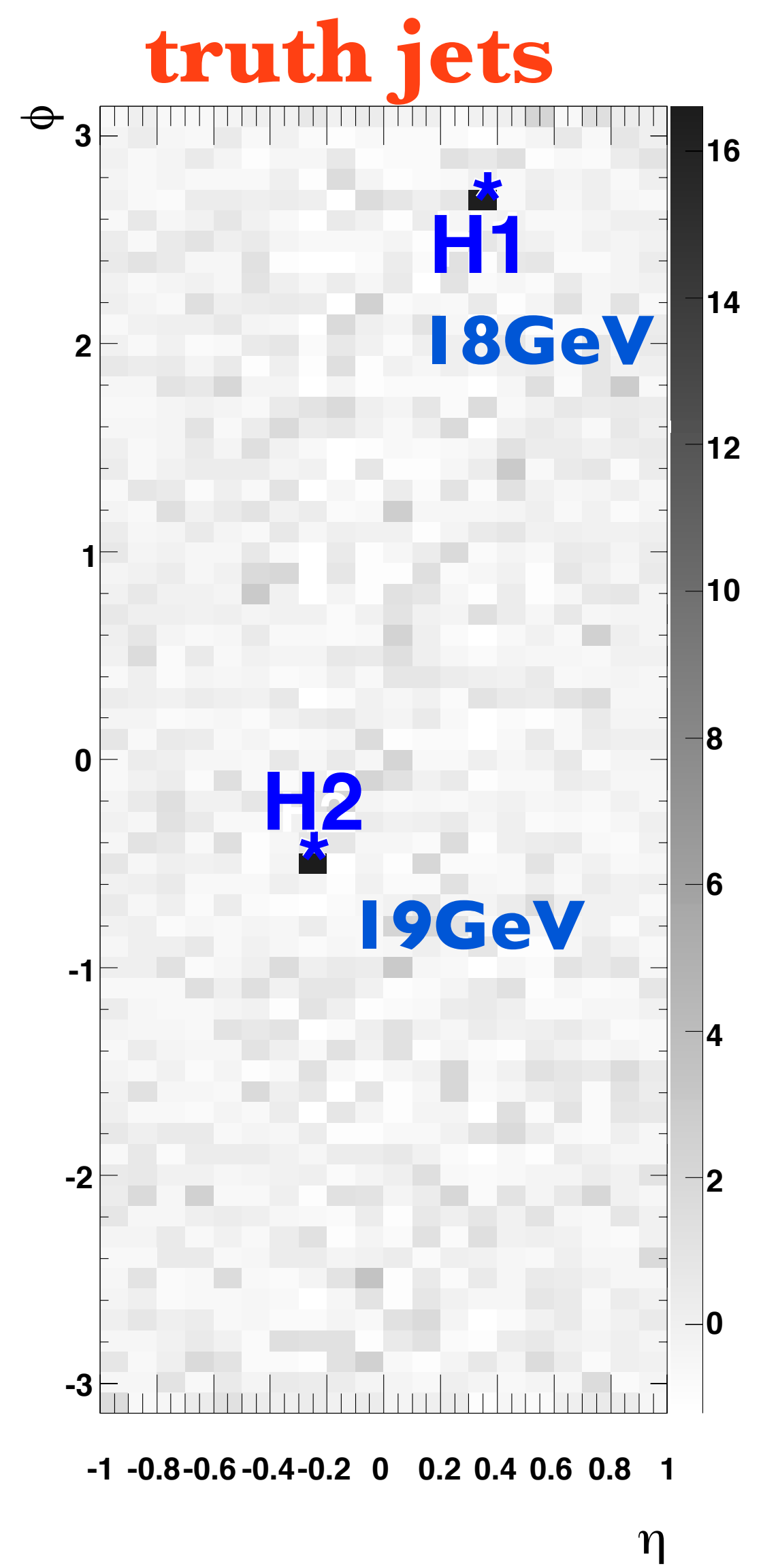
reconstructed jets

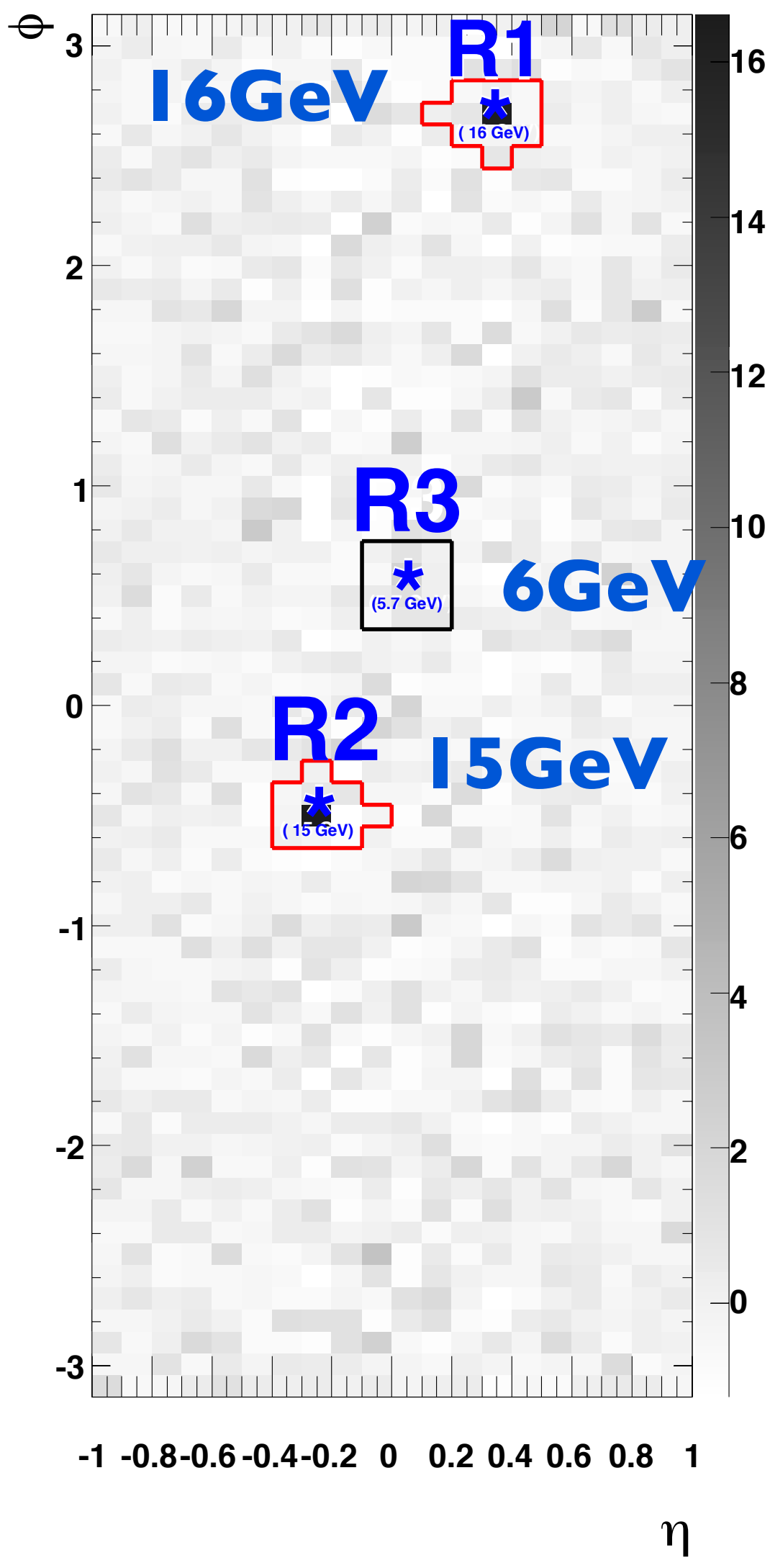




\section{fake jets}

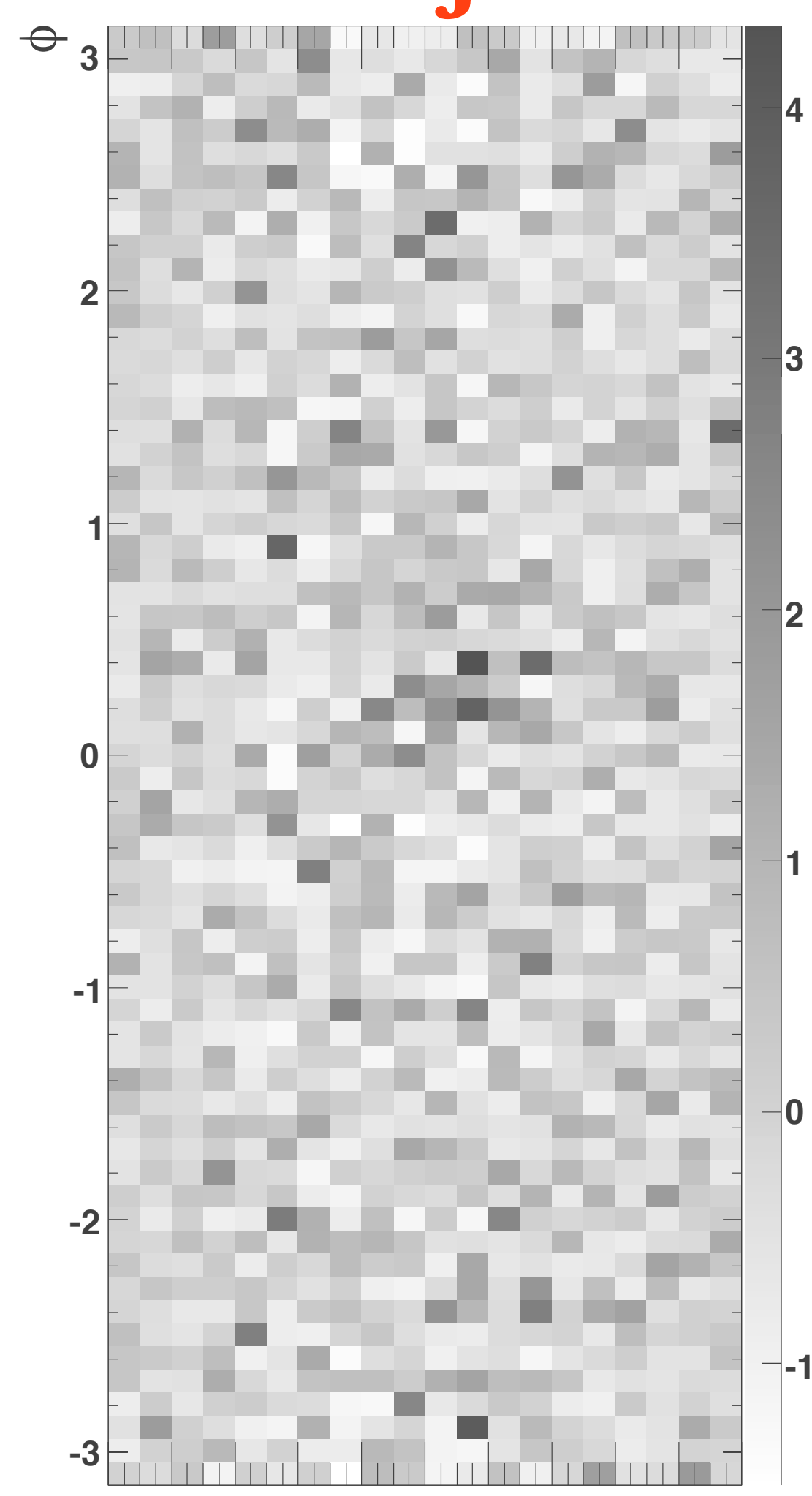

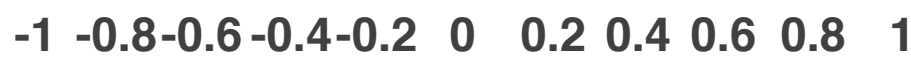

reconstructed jets

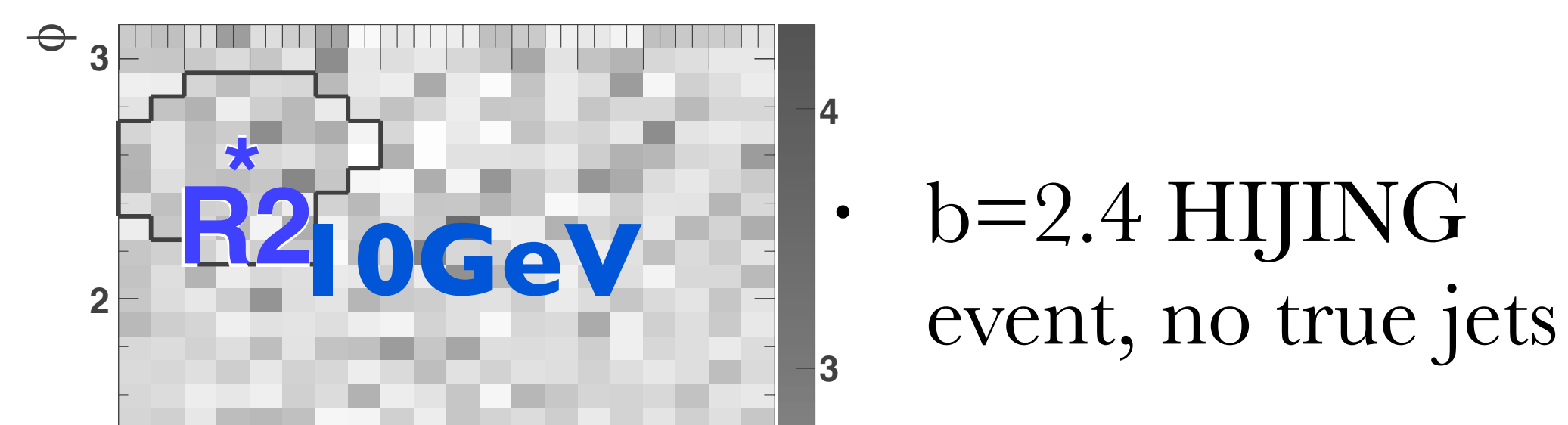

- 30 \& 10GeV fake jets with anti-kT $\mathrm{R}=0.4$

however, we looked at 750M+ events! need quantitative rate assessment 


\section{iterative jet finding algorithm}

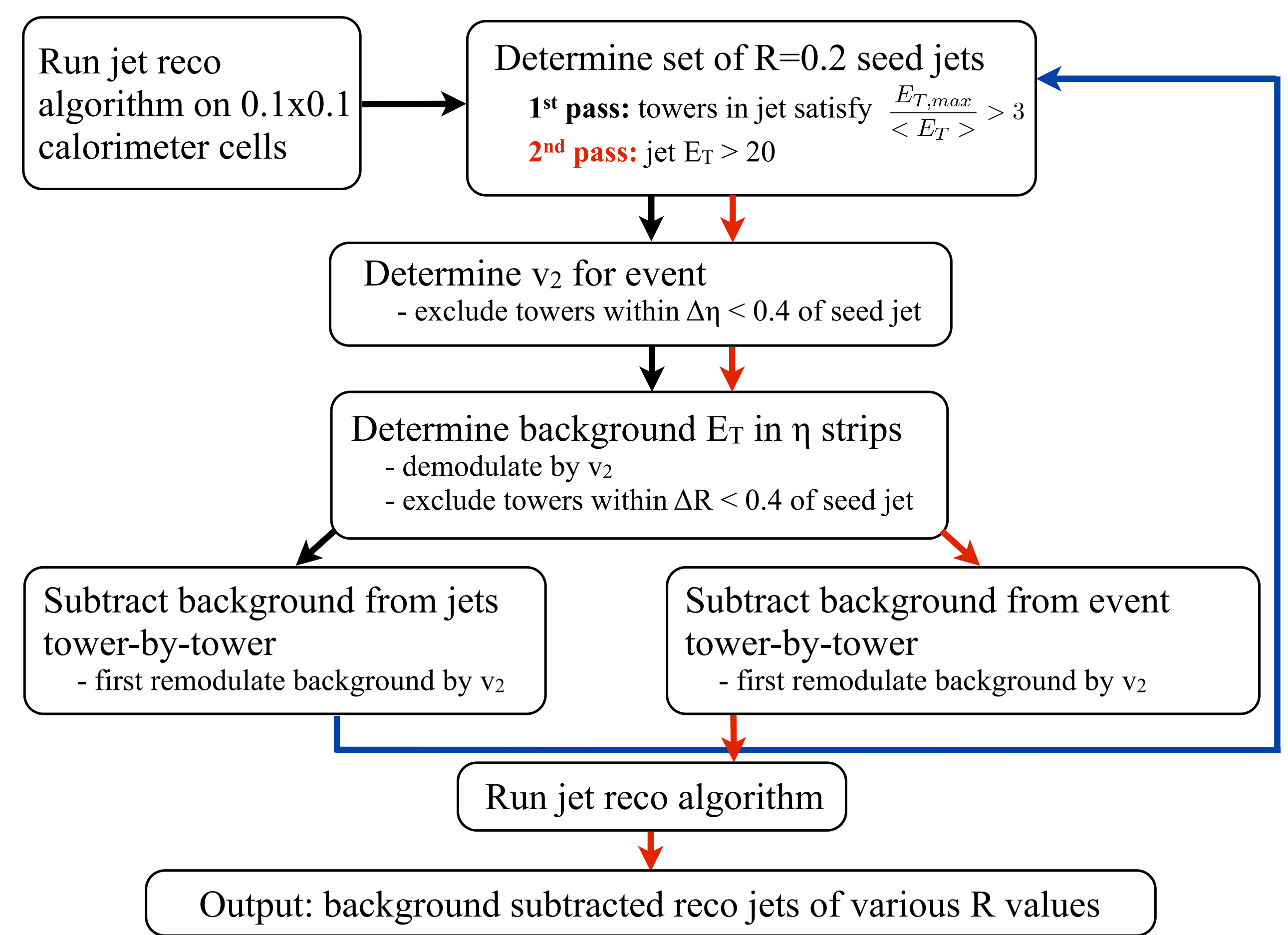

- uses anti-k $\mathrm{k}_{\mathrm{T}}$ algorithm

- $\quad$ inspired by ATLAS algorithm 


\title{
SUPPRESSION OF ENERGY LOSS FROM PARTIALLY IONIZED COLOR
}

\author{
Vladimir Skokov
}

Y. Hidaka, S. Lin, R. Pisarski

\section{BROOKHSWEN}

NATIONAL LABORATORY

pA workshop BNL 2013 


\section{Outline}

- Introduction: ionization in QED/QCD plasmas 


\section{Outline}

- Introduction: ionization in QED/QCD plasmas

- Collisional energy loss in partially ionized plasma 


\section{Outline}

- Introduction: ionization in QED/QCD plasmas

- Collisional energy loss in partially ionized plasma

- Conclusions 


\section{IONIZATION IN QED PLASMA}

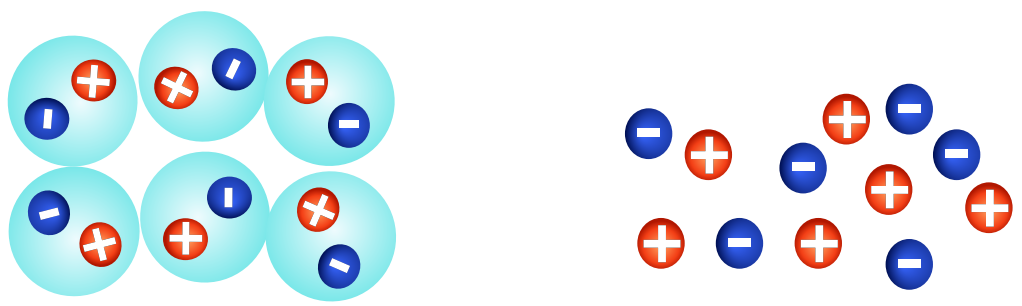

Neutral state $\sim$ atoms, electric neutrality $>$ atomic scales
Completely ionized plasma $\sim$ plasma with freely moving electric charges

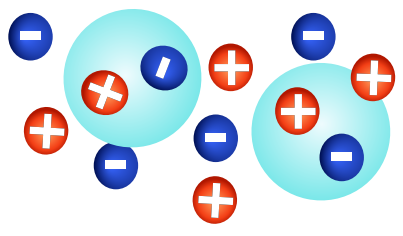

Partially ionized plasma $\leadsto$ partially ionized plasma with atoms and electric charges 


\section{IONIZATION IN QCD PLASMA}

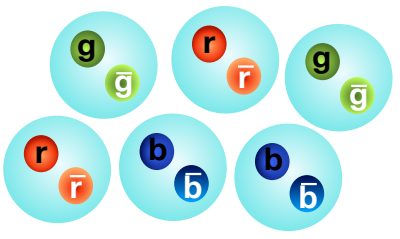

Neutral state $\sim$ confined phase, color neutrality $>$ hadronic scale

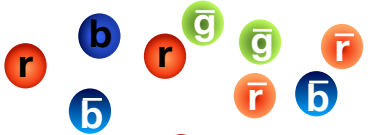 (r) 9}

Completely ionized plasma $\leadsto$ perturbative QGP with freely moving charges

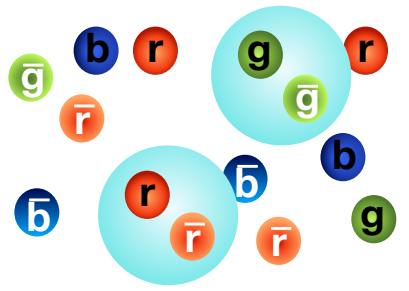

Partially ionized plasma $\sim$ partial ionization of color: hadrons and color charges; semi-QGP, nontrivial holonomy 


\section{POLYAKOV LOOP AS A MEASURE OF PARTIAL IONIZATION: PURE GLUE}

Polyakov loop: $\langle L\rangle \sim e^{-F_{\text {test qk }} / T}$

Confined: $F_{\text {test qk }} \rightarrow \infty, \quad$ Semi QGP: $0<\langle L\rangle<1 \quad$ Perturbative QGP:

$\langle L\rangle \rightarrow 0$

$\langle L\rangle$ measures degree of ionization

$F_{\text {test qk }} / T \rightarrow 0,\langle L\rangle \rightarrow 1$

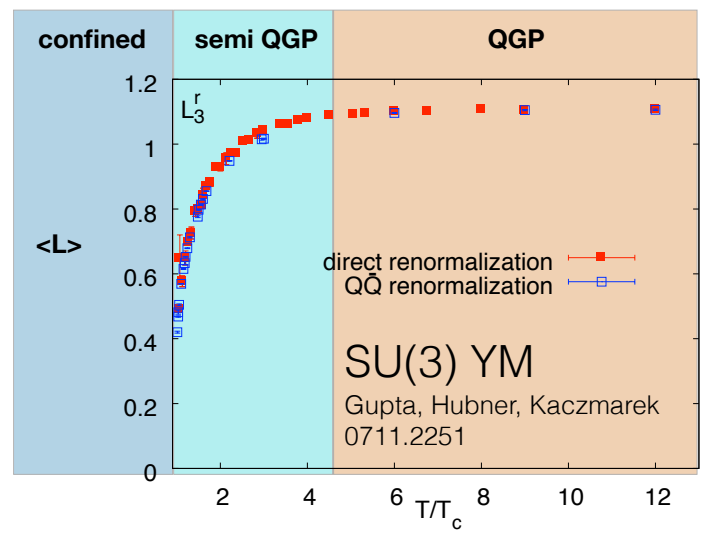




\section{Polyakov loOP AS A MEASURE OF PARTIAL IONIZATION: LQCD}

$$
\text { Polyakov loop: }\langle L\rangle \sim e^{-F_{\text {test qk }} / T}
$$

Confined: $F_{\text {test qk }} \rightarrow \infty, \quad$ Intermediate regime: $0<\langle L\rangle<1 \quad$ Deconfined:

$\langle L\rangle \rightarrow 0$

$\langle L\rangle$ measures degree of ionization $\quad F_{\text {test qk }} / T \rightarrow 0,\langle L\rangle \rightarrow 1$

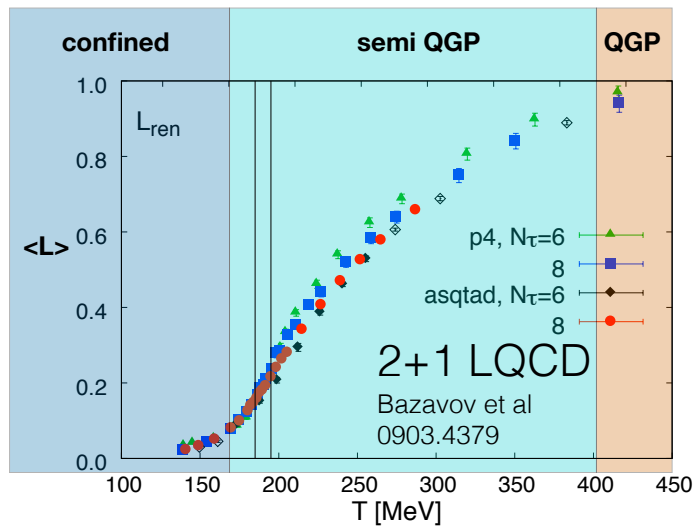




\section{POLYAKOV LOOP: MATRIX MODEL}
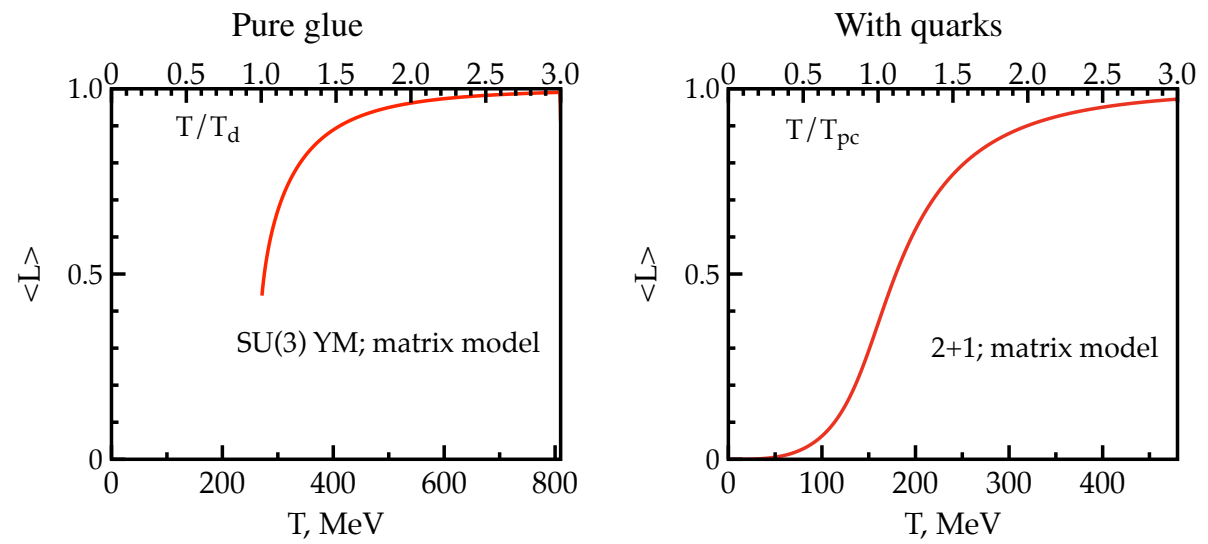


\section{Punchline: transition region ("semi"-QGP): must exhibit partial ionization of color}

shear viscosity, energy loss... must depend upon the degree of ionization

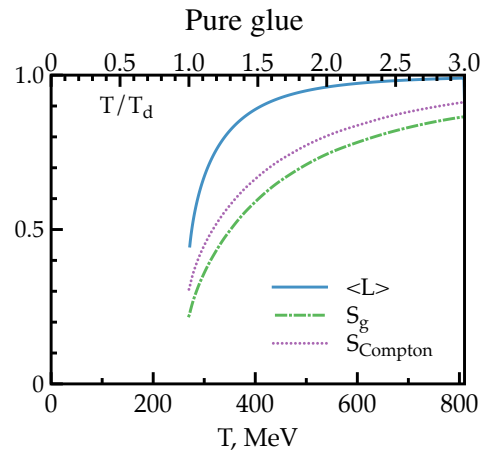

$S_{i}=\frac{\text { energy loss in semi-QGP }}{\text { energy loss inperturbative QGP }}$

$S_{i}$ increases as color is ionized
With quarks

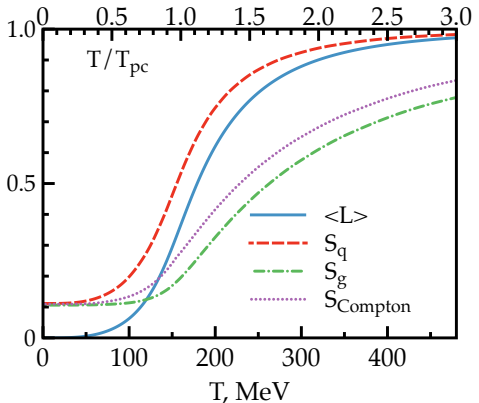

- $i=q$ scattering on light quark $(t$ channel)

- $i=g$ scattering on gluons ( $t$ channel)

- $i=$ Compton scattering on gluons, Compton scattering ( $u$ channel) 


\section{Perturbative vs Semi-QGP}

Usual argument of kinetic theory

Majumder, Muller and Wang, hep-ph/0703082

Liao and Shuryak, 0810.4116

Asakawa, Bass, and Muller, hep-ph/0603092, 1208.2426

- Viscosity $\eta \sim \rho^{2} / \sigma$

- $\rho$ - density of color charges $\rho \sim 1$

- $\sigma$ - crossection: $\sigma \sim g^{4}$, $g$ - coupling

- large $g \leadsto$ small $\eta$

\section{Semi-QGP}

Y. Hidaka, R, Pisarski 0912.0940

R. Pisarksi, V. Skokov proceedings of QM2013

- Viscosity $\eta \sim \rho^{2} / \sigma$

- $\rho$ - density of color charges, $\rho \sim\langle L\rangle^{2}$

- $\sigma$ - crossection: $\sigma \sim\langle L\rangle^{2}$

- $\eta \sim\langle L\rangle^{2}$, small in semi-QGP
- Energy loss $\frac{d E}{d x} \sim g^{2} \rho^{2}$

- large $g \leadsto$ large $\frac{d E}{d x}$
- Energy loss (large $N_{c}$ )

- $\frac{\frac{d E}{d x} \sim\langle L\rangle \cdot \frac{d E}{d x} \text { on light quarks }}{+\langle L\rangle^{2} \cdot \frac{d E}{d x} \text { on gluons }}$ 


\section{Details}

- Matrix model

- Collisional energy loss in large $N_{c}$ limit

- Collisional energy loss due to scattering on light quark, $N_{c}=3$

- Collisional energy loss due to scattering on gluons, $N_{c}=3$

- Outlook: radiative?! 


\section{NoN-ZERO POLYAKOV LOOP $\leadsto$ NON-TRIVIAL HOLONOMY}

- Polyakov loop $L=\operatorname{Tr} \mathcal{P} \exp \left(i g \int_{0}^{1 / T} A_{0} d \tau\right)$

- Anzatz for $\left[A_{0}\right]_{a b}=\delta_{a b} \frac{Q^{a}}{g}$, for the sake of simplicity $Q^{a}=2 \pi T \cdot q^{a}$

- Tracelessness tr $\mathrm{A}_{0}=0 \leadsto \sum_{a} Q^{a}=\sum_{a} q^{a}=0$

- Classical approximation: zero action for $A_{0}$

- One loop about $A_{0}$ : Gross, Pisraski, Yaffe '81:

$$
U_{\text {pert }}=-2 \pi^{2} T^{4}\left[\frac{N^{2}-1}{45}-\frac{1}{3} \sum_{a, b}\left(q_{a}-q_{b}\right)^{2}\left(1-\left|q_{a}-q_{b}\right|\right)^{2}\right]
$$

Gives only trivial $A_{0}$

- Non-perturbative contribution are modeled by (R. Pisarski et al)

$$
U_{\text {non-pert }}=T^{2} T_{d}^{2}\left[c_{1} \sum_{a, b}^{N}\left|q_{a}-q_{b}\right|\left(1-\left|q_{a}-q_{b}\right|\right)+c_{2} \sum_{a, b}^{N}\left(q_{a}-q_{b}\right)^{2}\left(1-\left|q_{a}-q_{b}\right|\right)^{2}+c_{3}\right]
$$

- $c_{i}$ are fixed to get transition at $T=T_{d}$, and describe lattice data

- three colors: $q_{1}=-q_{2}=q, q_{3}=0$. Confining at $q=1 / 3$ and perturbative $q=0$. 


\section{Model vs LATTICE}

Pressure for pure glue

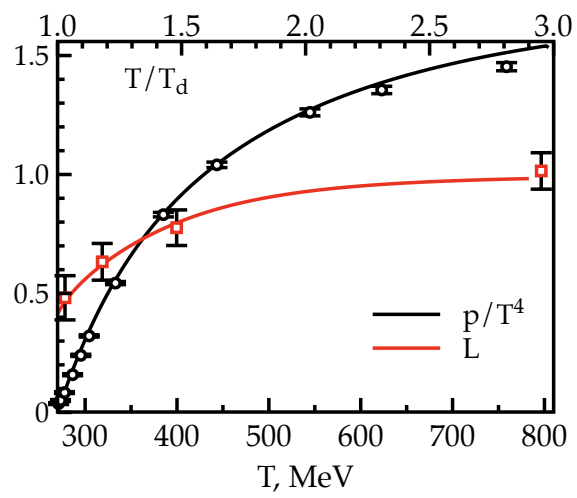

t'Hooft loop

(interface between different $Z(N)$ sectors)

Dumitru et al, 1011.3820

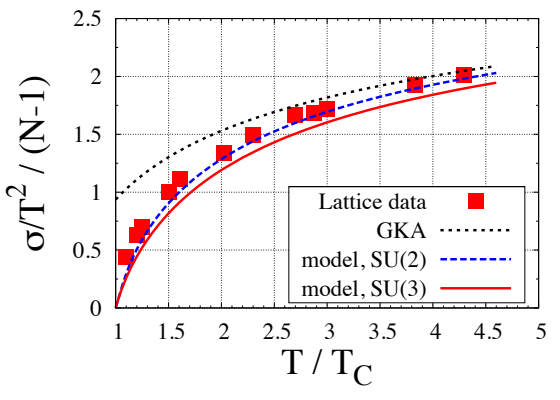

$V \rightarrow V+\frac{1}{g^{2}} \sum_{a}^{N}\left(\frac{\partial Q_{a}}{\partial z}\right)^{2}$ 
Quark and gluon propagator in a background $A_{0}$ field: Hidaka, Pisarski 0906.1751

- Distribution function for gluons

$$
n_{a, b}^{g}(p, Q)=\left[\exp \left(\frac{E-i\left(Q_{a}-Q_{b}\right)}{T}\right)-1\right]^{-1}
$$

- Distribution function for quarks

$$
n_{a}^{q}(p, Q)=\left[\exp \left(\frac{E-i Q_{a}}{T}\right)+1\right]^{-1}
$$

Limits:

- Trivial holonomy or perturbative QGP, $Q=0$

$$
\begin{aligned}
& n^{g}(p, Q=0)=\left[\exp \left(\frac{E}{T}\right)-1\right]^{-1} \\
& n^{q}(p, Q=0)=\left[\exp \left(\frac{E}{T}\right)+1\right]^{-1}
\end{aligned}
$$

- Confining limit, large $N$ :

$$
\begin{aligned}
& n^{q}=0 \\
& n^{g}=0
\end{aligned}
$$




\section{LARGE N LIMIT FOR SCATTERING OFF LIGHT QUARK: BIRDTRACKS}

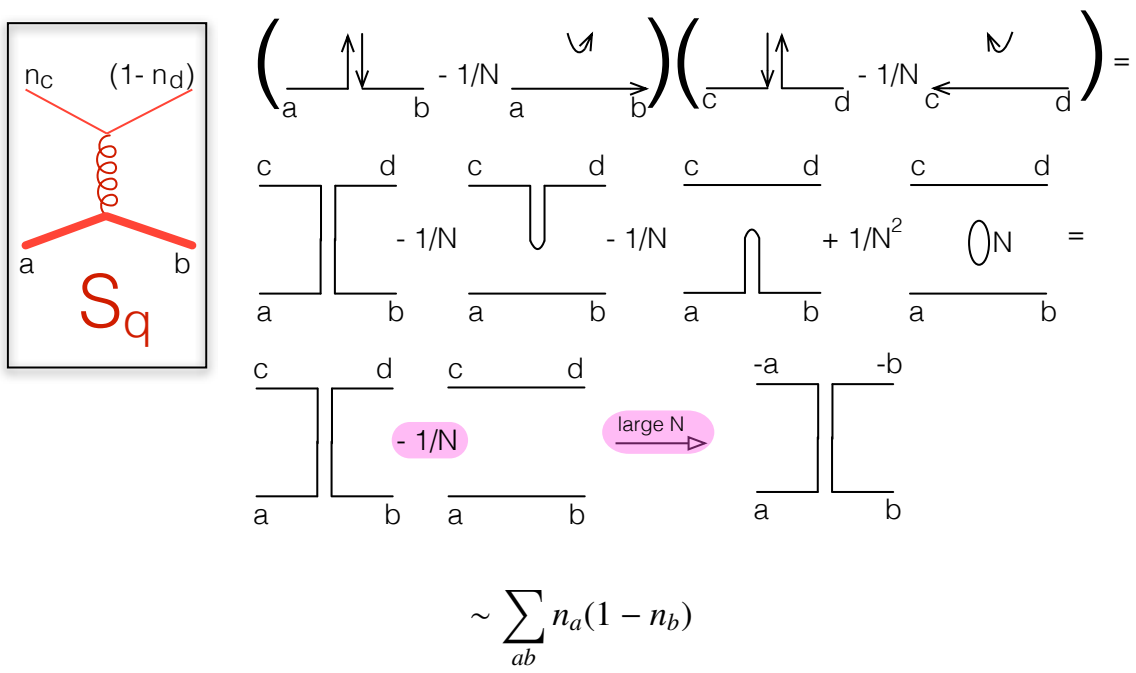




\section{LARGE N LIMIT}

$$
\begin{gathered}
\frac{d E}{d x} \propto \sum_{a, b}^{N} \int[d k]\left[d k^{\prime}\right]\left[d p^{\prime}\right] f\left(p, k, k^{\prime}, p^{\prime}\right) n\left(E_{k}+i Q_{a}\right)\left[1-n\left(E_{k^{\prime}}+i Q_{b}\right)\right] \\
\sum_{a} n\left(E_{k}+i Q_{a}\right)=\sum_{a}\left[\exp \left(\beta E_{k}+i \beta Q_{a}\right)+1\right]^{-1} \\
=\sum_{n=1}^{\infty}(-1)^{n} \exp \left(-\beta E_{k}\right) \sum_{a} \exp \left(i n \beta Q_{a}\right)=\sum_{n=1}^{\infty}(-1)^{n} \exp \left(-\beta E_{k}\right) \operatorname{tr} L^{a}
\end{gathered}
$$

for small tr $L$ scattering off light quarks

$$
\frac{d E}{d x} \propto \operatorname{tr} L \cdot\left(\frac{d E}{d x}\right)_{\text {pert. }}
$$

Similar argument for scattering off gluons gives

$$
\frac{d E}{d x} \propto(\operatorname{tr} L)^{2} \cdot\left(\frac{d E}{d x}\right)_{\text {pert. }}
$$

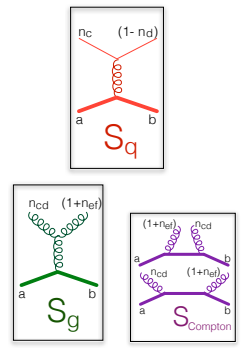




\section{$\mathrm{N}=3$}

As in $\mathrm{PQCD}$, but taking into account modification of distribution function in final/initial state. 


\section{$\mathrm{N}=3$ : ScATTERING OFF LIGHT QUARKS}

$$
S_{i}=\left(\frac{d E}{d x}\right)_{i} /\left(\frac{d E}{d x}\right)_{i, \text { pert. }}
$$

- Scattering off light quark

$$
S_{q}=\frac{12}{\pi^{2}\left(N^{2}-1\right)} \sum_{l=1}^{\infty} \sum_{m=0}^{l-1}(-1)^{l+1} \frac{l-2 m}{l^{3}}\left(\operatorname{tr} L^{l-m} \operatorname{tr} L^{m}-\frac{1}{N} \operatorname{tr} L^{l}\right)
$$

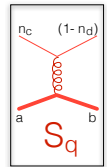

- Perturbative limit $Q \rightarrow 0$, so $\forall i$ tr $L^{i} \rightarrow 1$ :

$$
S_{q}(Q=0)=\frac{12}{\pi^{2}\left(N^{2}-1\right)} \sum_{l=1}^{\infty} \sum_{m=0}^{l-1}(-1)^{l+1} \frac{l-2 m}{l^{3}}\left(N^{2}-1\right)=1
$$

- Confining limit $q \rightarrow 1 / 3$, so $\forall i=k N$, where $k$ is integer, tr $L^{i} \rightarrow N$, otherwise $\operatorname{tr} L^{j \neq k N} \rightarrow 0$ :

$$
\begin{gathered}
S_{q}(q=1 / 3)=\frac{12}{\pi^{2}\left(N^{2}-1\right)} \sum_{k=1}^{\infty} \sum_{m=0}^{N k-1}(-1)^{k N+1} \delta_{m} j N \frac{k N-2 m}{k^{3} N^{3}}\left(N^{2}-1\right) \\
=\frac{1}{N^{2}} \text { for odd } N, \frac{2}{N^{2}} \text { otherwize }
\end{gathered}
$$




\section{$\mathrm{N}=3:$ ScATTERING OFF GLUONS}

$$
S_{i}=\left(\frac{d E}{d x}\right)_{i} /\left(\frac{d E}{d x}\right)_{i, \text { pert. }}
$$

- Scattering off gluons ( $t$ channel)

$$
S_{g}=\frac{6}{\pi^{2}\left(N^{2}-1\right)} \sum_{l=1}^{\infty} \sum_{m=0}^{l-1} \frac{l-2 m}{l^{3}}\left(\operatorname{tr} L^{l-m} \cdot \operatorname{tr} L^{l} \cdot \operatorname{tr} L^{m}-\operatorname{tr} L^{2 l}\right)
$$

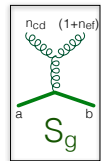

- Compton scattering off gluons ( $u$ channel), only Polyakov loop terms

$$
\begin{gathered}
S_{\text {Compton }}=\cdots\left(\operatorname{tr} L^{l} \operatorname{tr} L^{l-m} \operatorname{tr} L^{m}\right. \\
-\frac{2}{N} \operatorname{tr} L^{m} \operatorname{tr} L^{2 l-m}-\frac{2}{N} \operatorname{tr} L^{l-m} \operatorname{tr} L^{l+m}+\frac{4}{N} \operatorname{tr} L^{2 l} \\
+\frac{1}{N^{2}}\left(\operatorname{tr} L^{m}\right)^{2} \operatorname{tr} L^{2(l-m)}+\frac{1}{N^{2}}\left(\operatorname{tr} L^{l-m}\right)^{2} \operatorname{tr} L^{2 m}-\frac{4}{N^{2}} \operatorname{tr} L^{2(l-m)} \operatorname{tr} L^{2 m} \\
\left.+\frac{1}{N^{3}}\left(\operatorname{tr} L^{2 m}\right)^{2} \operatorname{tr} L^{2(l-m)}\right)
\end{gathered}
$$

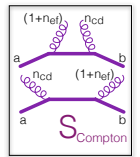




\section{NumERICAL RESUlts}

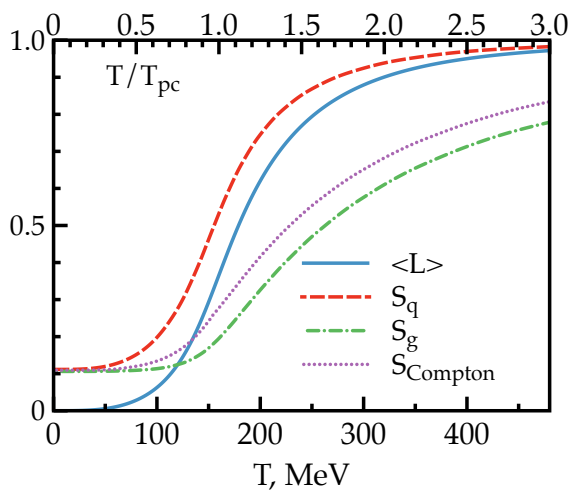

$$
S_{i}=\left(\frac{d E}{d x}\right)_{i} /\left(\frac{d E}{d x}\right)_{i, \text { pert. }}
$$
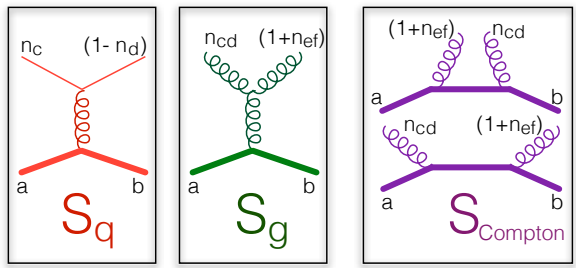

- Different processes are supressed dufferently

- Processes with gluons are supressed stronger then thouse with quarks

- $\forall i ; S_{i} \rightarrow 1 / N^{2}$ at low temperatures 


\section{RADIATIVE ENERGY LOSS: PRELIMINARY LARGE N RESULT}
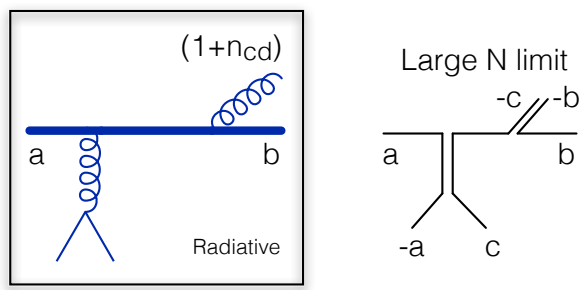

Suppressed by

$$
n_{-a}\left(1-n_{c}\right)\left(1+n_{-c,-b}\right) \sim \operatorname{tr} L
$$


- LQCD: shallow dependence of $\langle L\rangle$ on $T$

- This suggest that semi-QGP region (region with partial ionization of color) is broad and has to be taken into account when computing energy loss viscosity and etc

- Collisional energy loss is supressed in semi-QGP either linearly (for scattering off light quarks) or quadratically (for scattering off gluons) by Polyakov loop

- Radiative energy loss is harder to cumpute, but, at least, in large $N$ limit it also gets supressed at least quadratically by Polyakov loop 


\section{Thank you!}




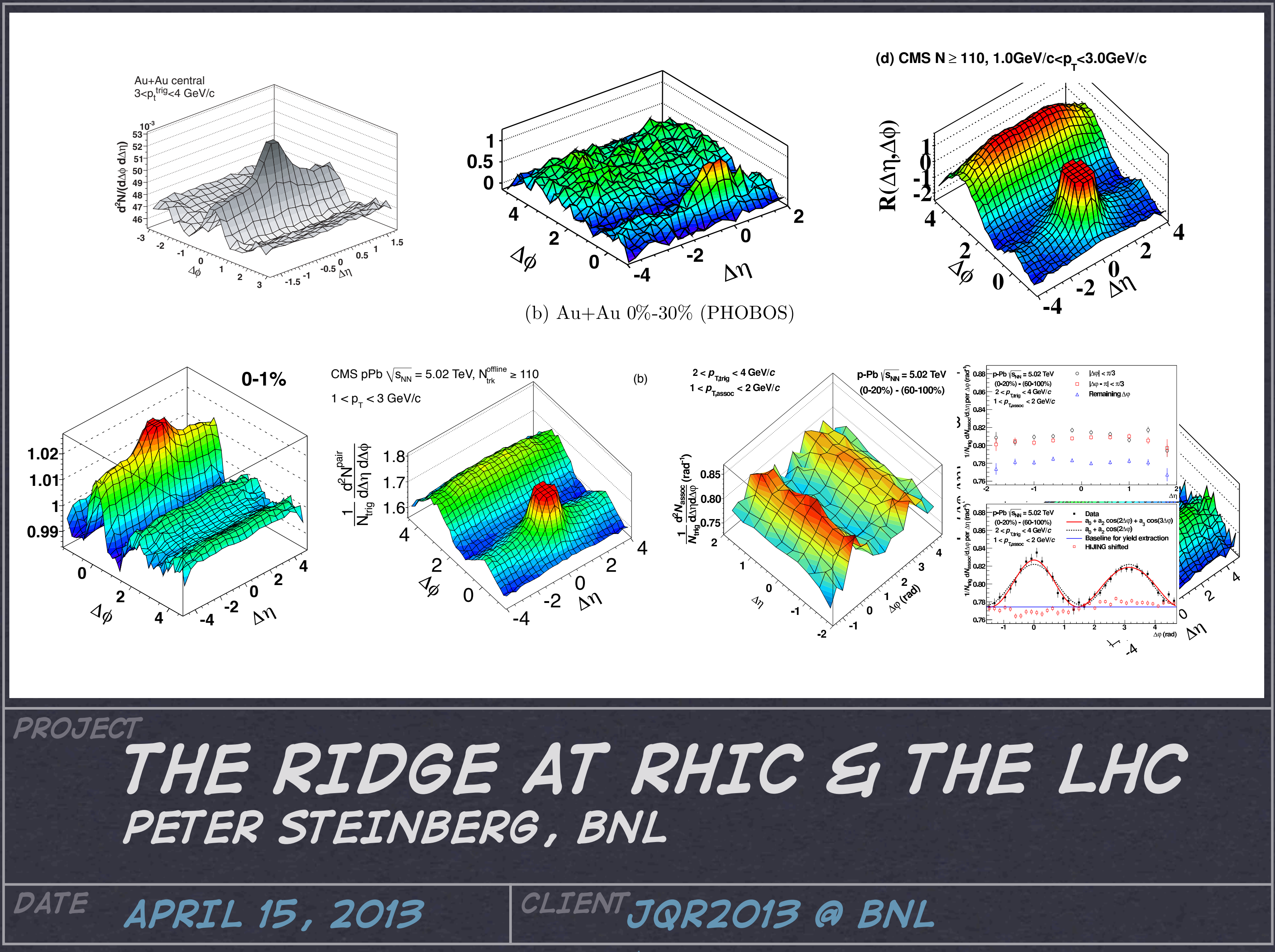




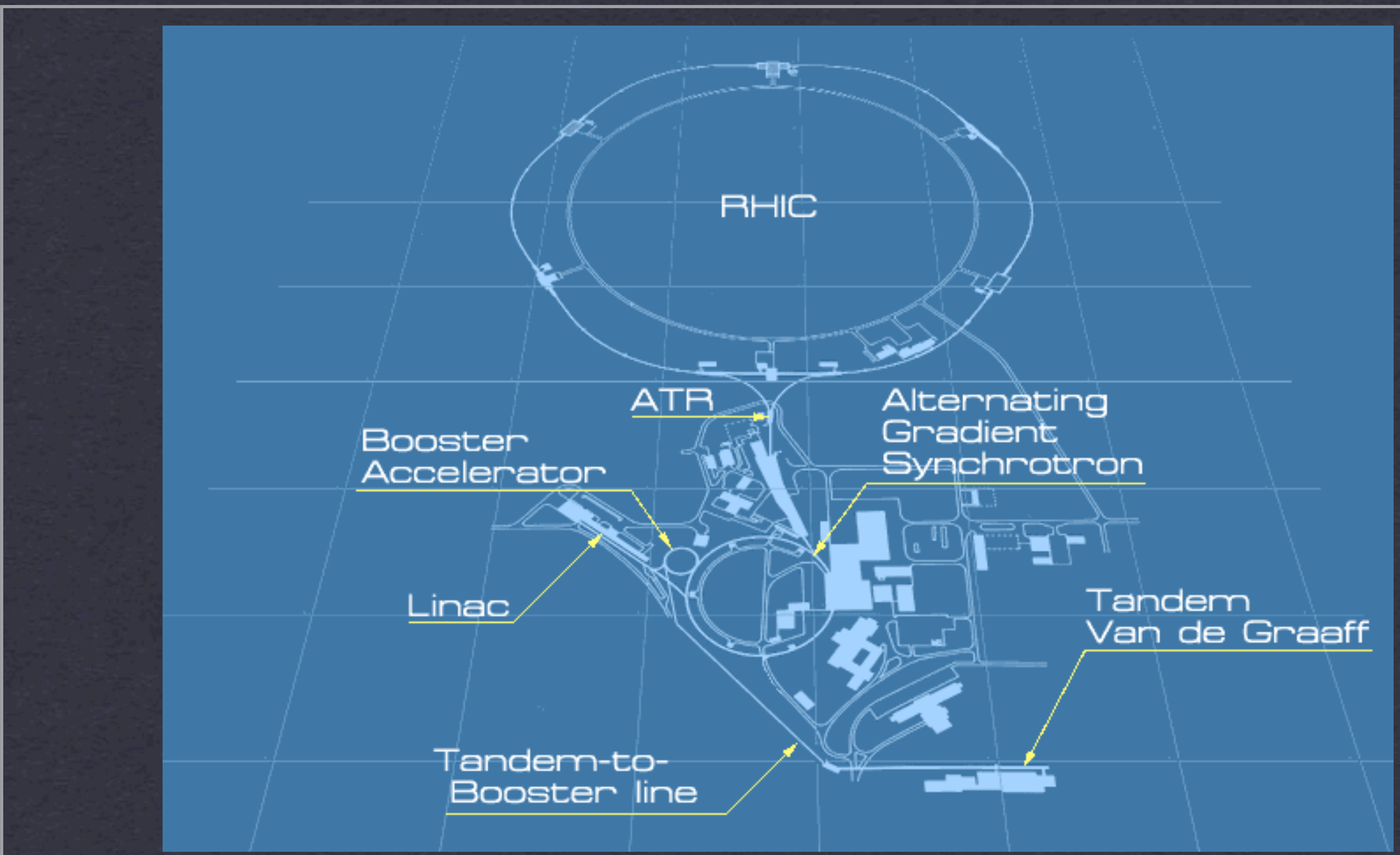

\section{PROJECT}

\section{RELATIVISTIC HEAVY ION COLLIDER BROOKHAVEN NATIONAL LABORATORY, LISA}




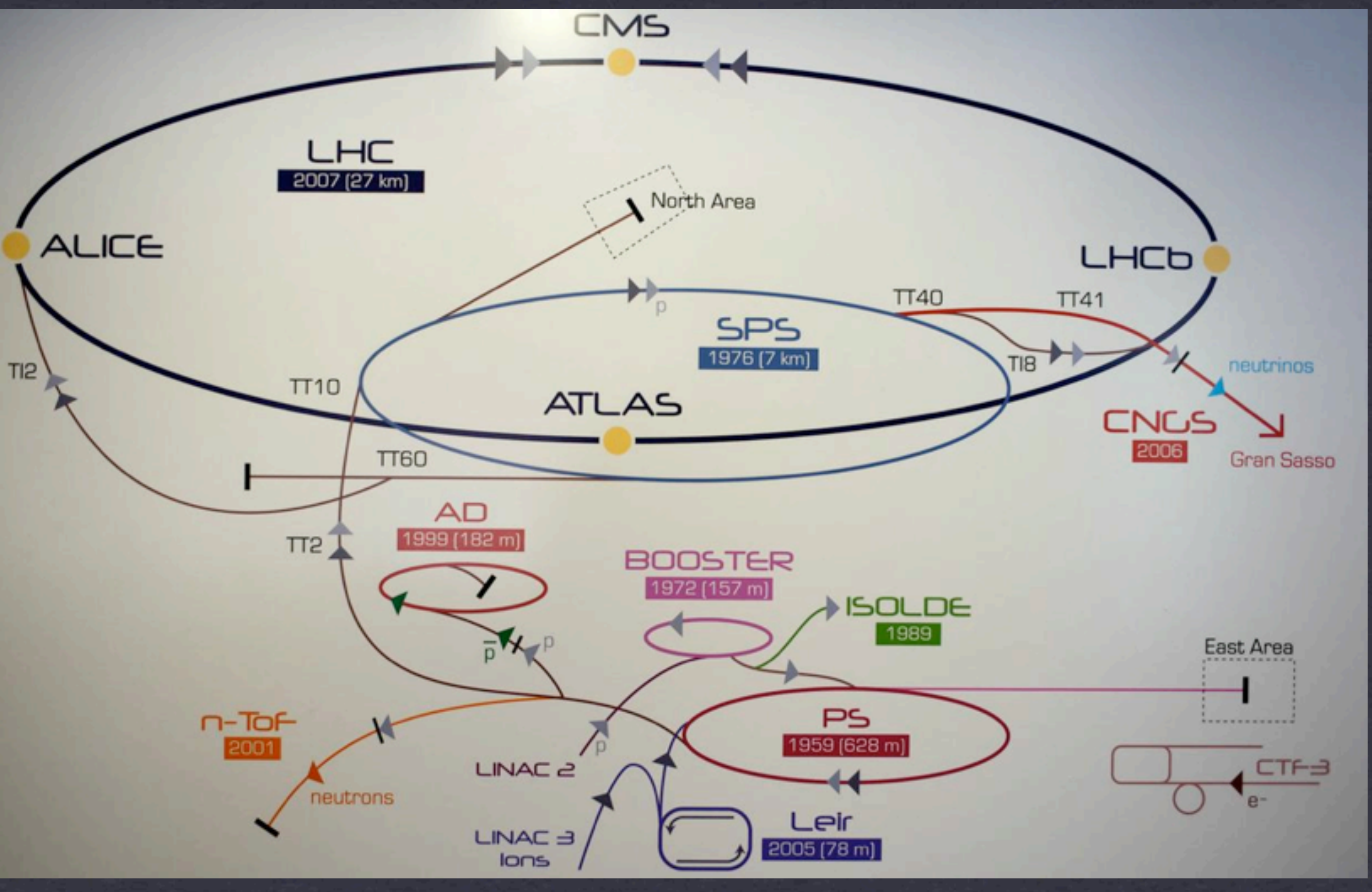

PROJECT

LARGE HADRON COLLIDER CERN, SWITZERLAND 

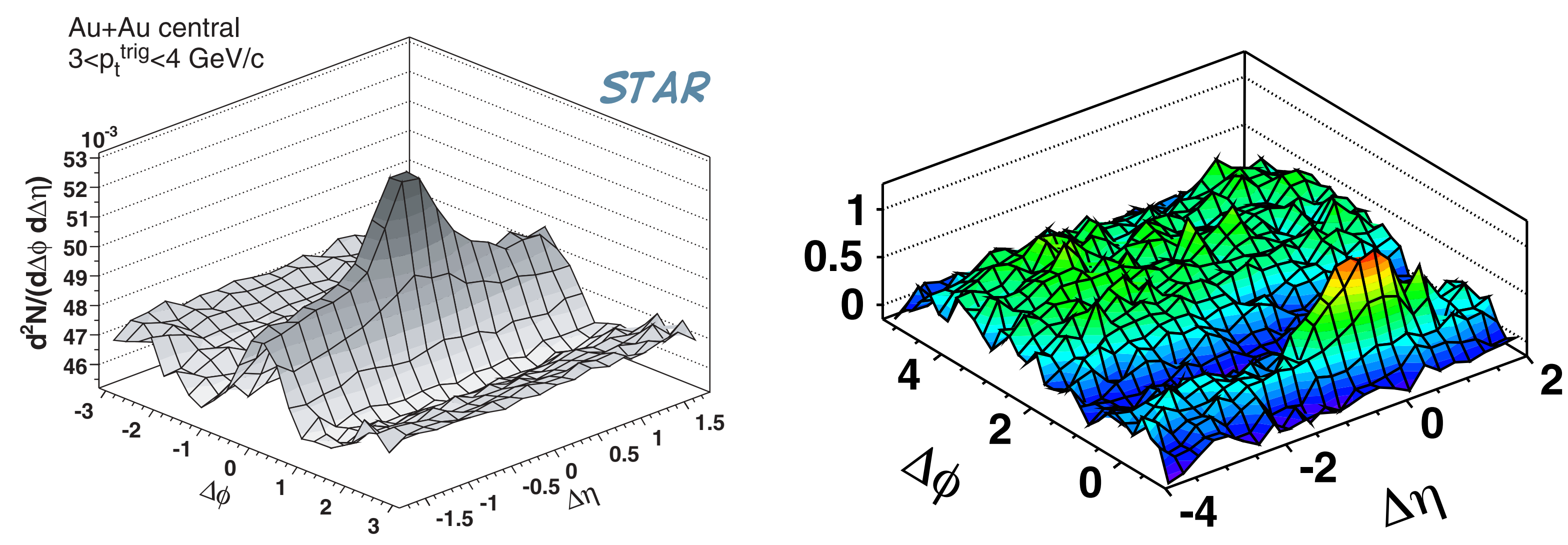

(b) $\mathrm{Au}+\mathrm{Au} \mathrm{0 \% -30 \%} \mathrm{(PHOBOS)}$

\section{PROJECT}

THE RHIC RIDGE
PHENIX, PHOBOS, AND STAR 


\section{STAR AL+AU RIDGE}

* RIDGE FIRST OBSERVED AT RHIC IN AL+AL

* BY-PRODLCT OF STLDY OF HIGH PT CORRELATIONS

* clear strLICTLRE EXTENDED IN ETA, BUT LOCALIZED IN PHI

CLEARLY SEEN IN AL+AL ...BLT NOT OBSERVED IN D+AL COLLISIONS!
STAR, PRC 80064912 (2009)
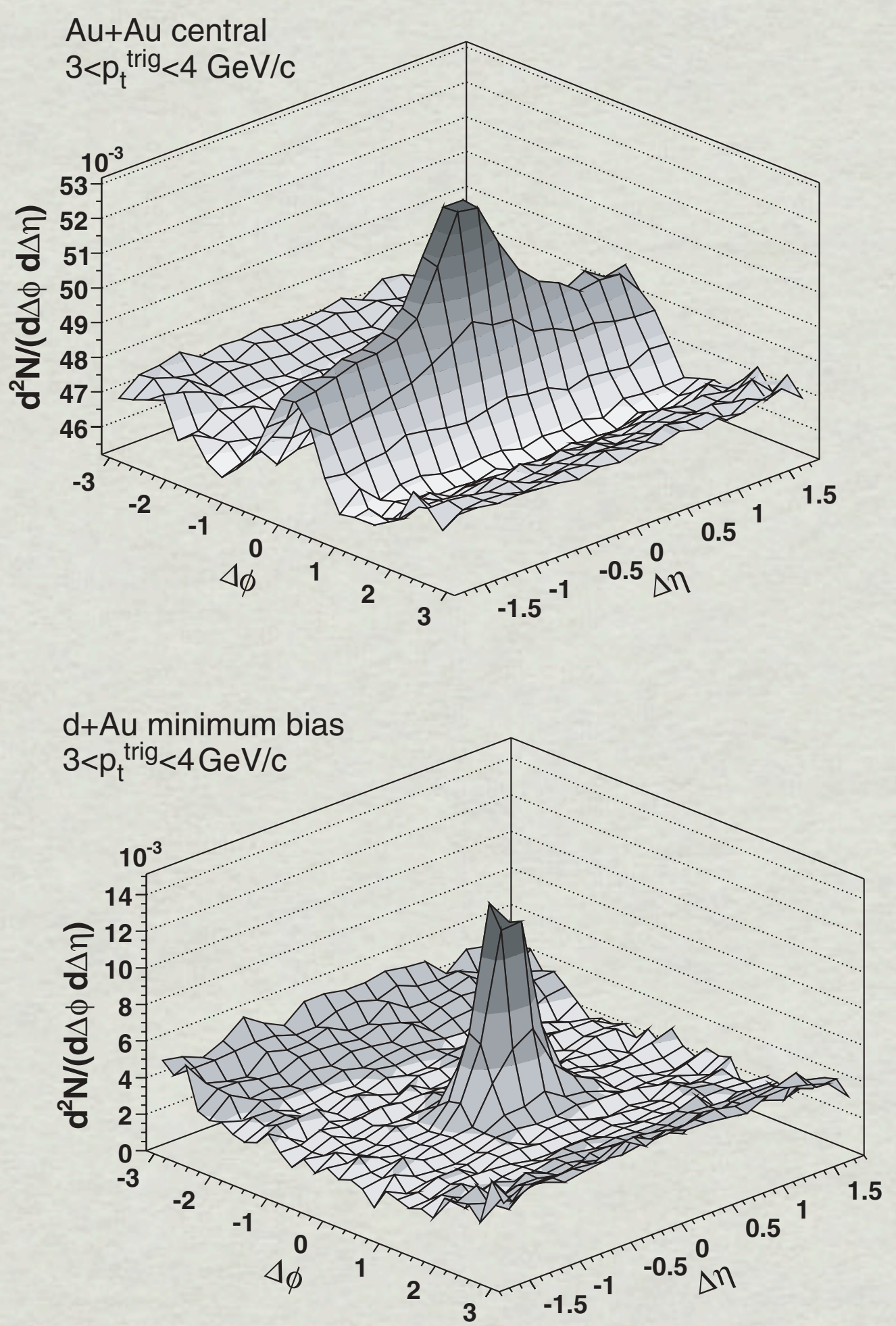


\section{PHOBOS ALI+AL RIDGE}

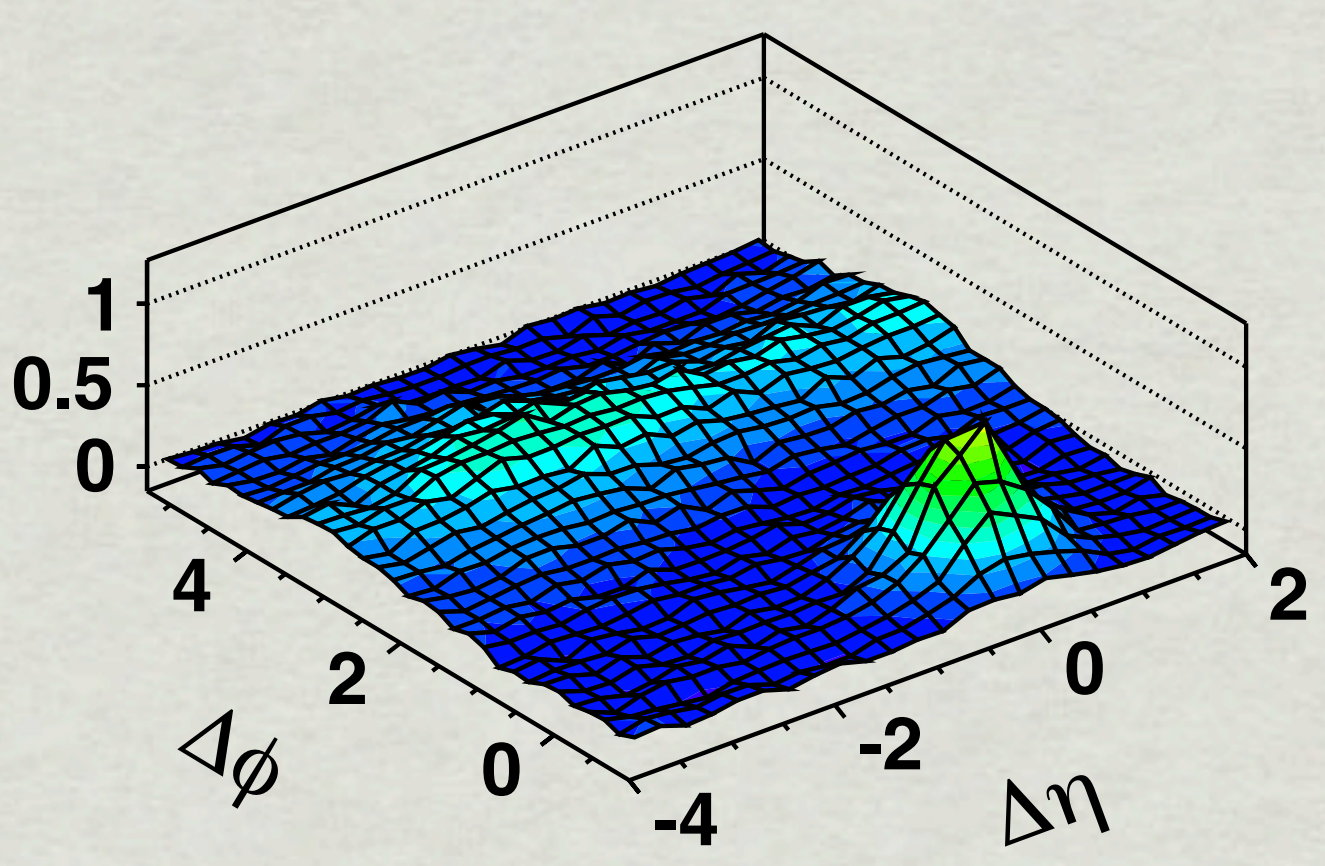

(a) $\mathrm{p}+\mathrm{p}$ PYTHIA (version 6.325)

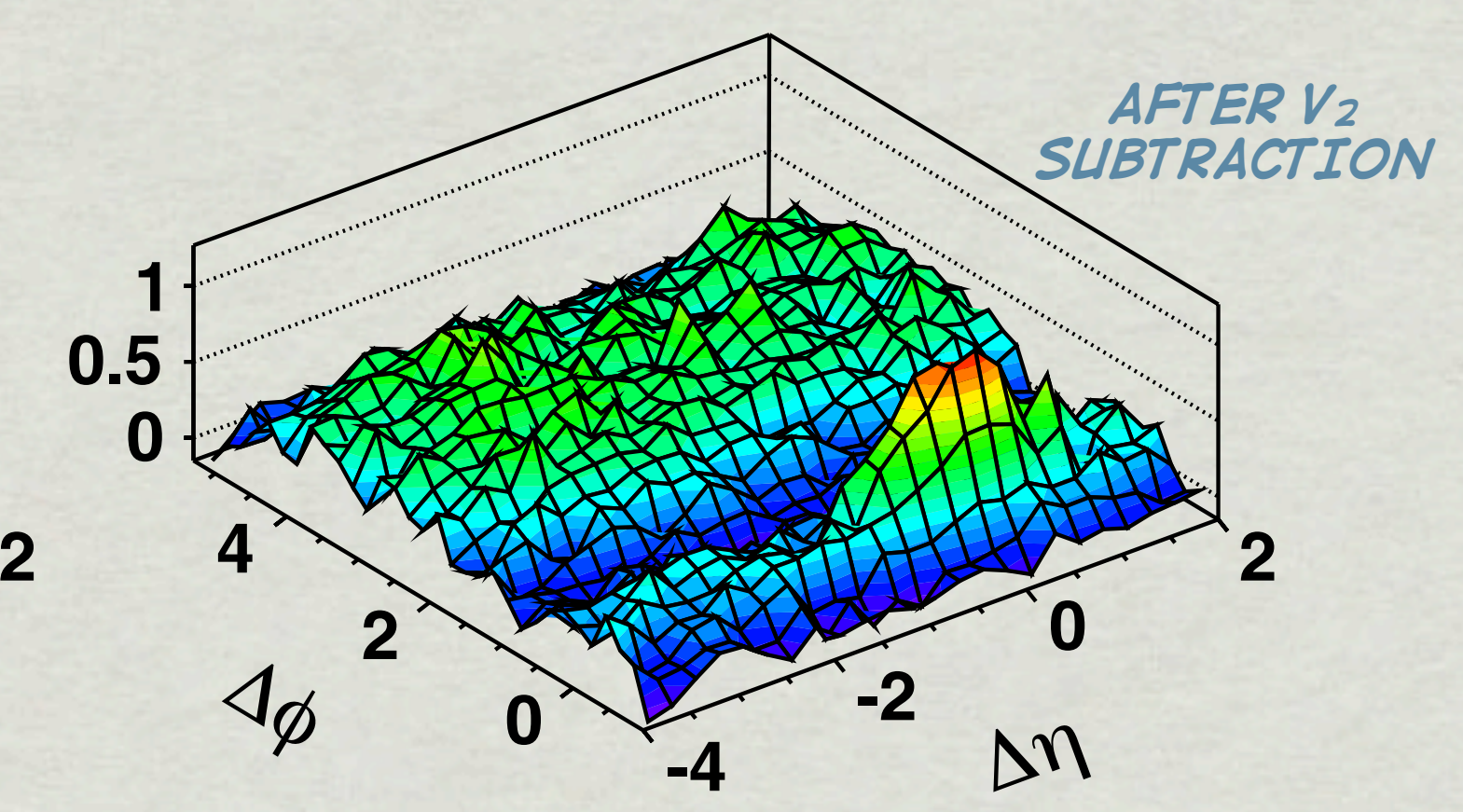

(b) $\mathrm{Au}+\mathrm{Au} 0 \%-30 \%$ (PHOBOS)

WHILE PHOBOS COLILD NOT PROVIDE PT DEPENDENCE, THE LARGE ETA COVERAGE GAVE FIRST LOOK AT THE RIDGE AT VERY LARGE $\triangle E T A$ SEPARATIONS... ...AND THERE WAS NO END IN SIGHT! 


\section{MANY

...FROM A 2008 TALK BY ED WENGER (PHOBOS)

- Coupling of induced radiation to longitudinal flow Armesto et al., PRL 93,242301

- Recombination of shower + thermal partons Hwa, arXiv:nucl-th/0609017v1

- Anisotropic plasma Romatschke, PRC 75, 014901

- Turbulent color fields

Shuryak, arXiv:0706.3531v1

- Bremsstrahlung + transverse flow + jet-quenching Majumder, Muller, Bass, axXivinep-phoo611135v2

- Splashback from away-side shock Pantuev, arXiv:0710.1882v1

- Momentum kick imparted on medium partons Wong, arXiv:0707.2385v2

- Glasma Flux Tubes

Dumitru, Gelis, McLerran, Venugopalan, arXiv:0804.3858; Gavin, McLerran, Moscelli, arXiv:0806.4718 
a

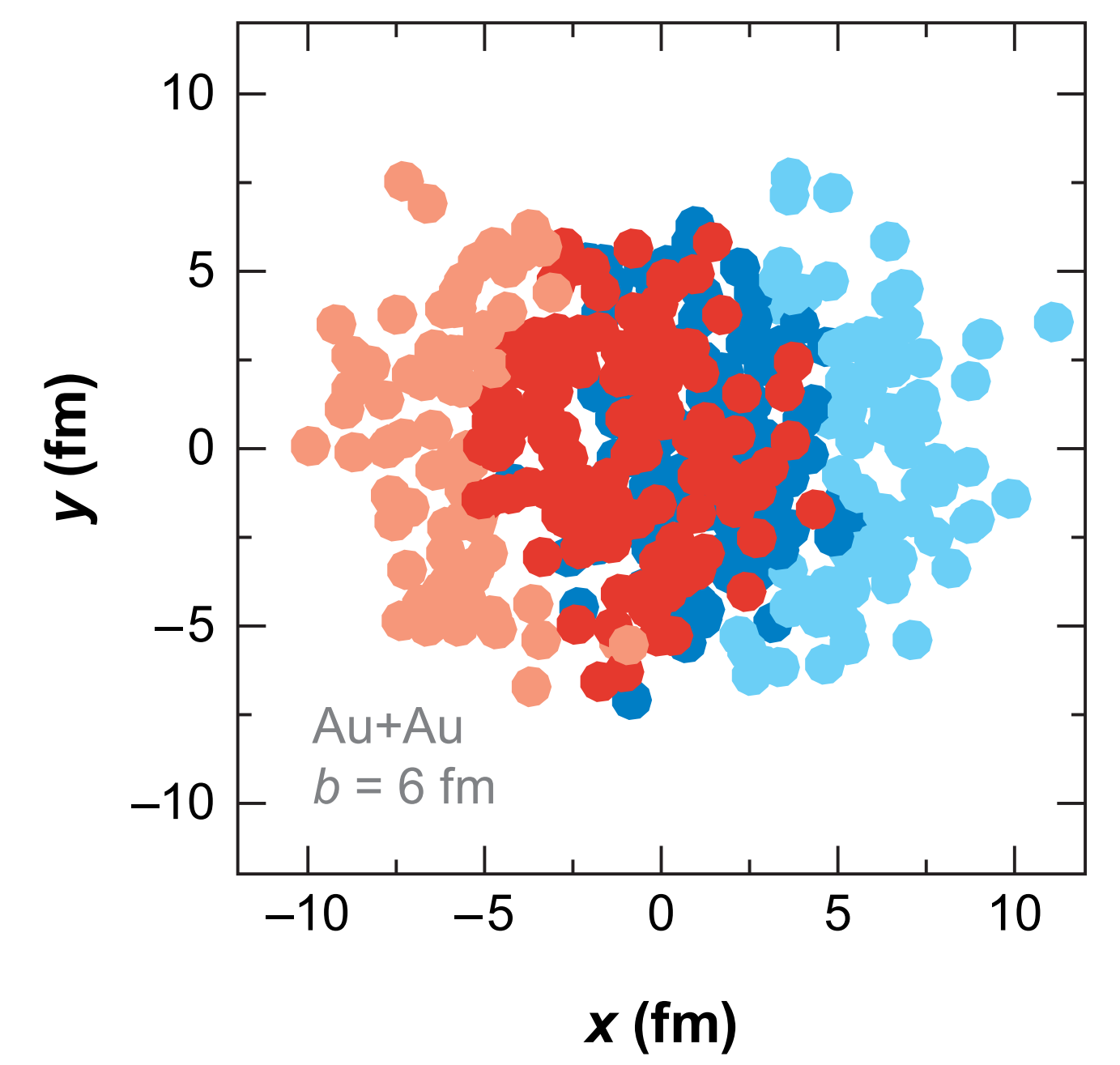

b

Ann.Rev.Nucl.Part.Sci.57:205-243,2007

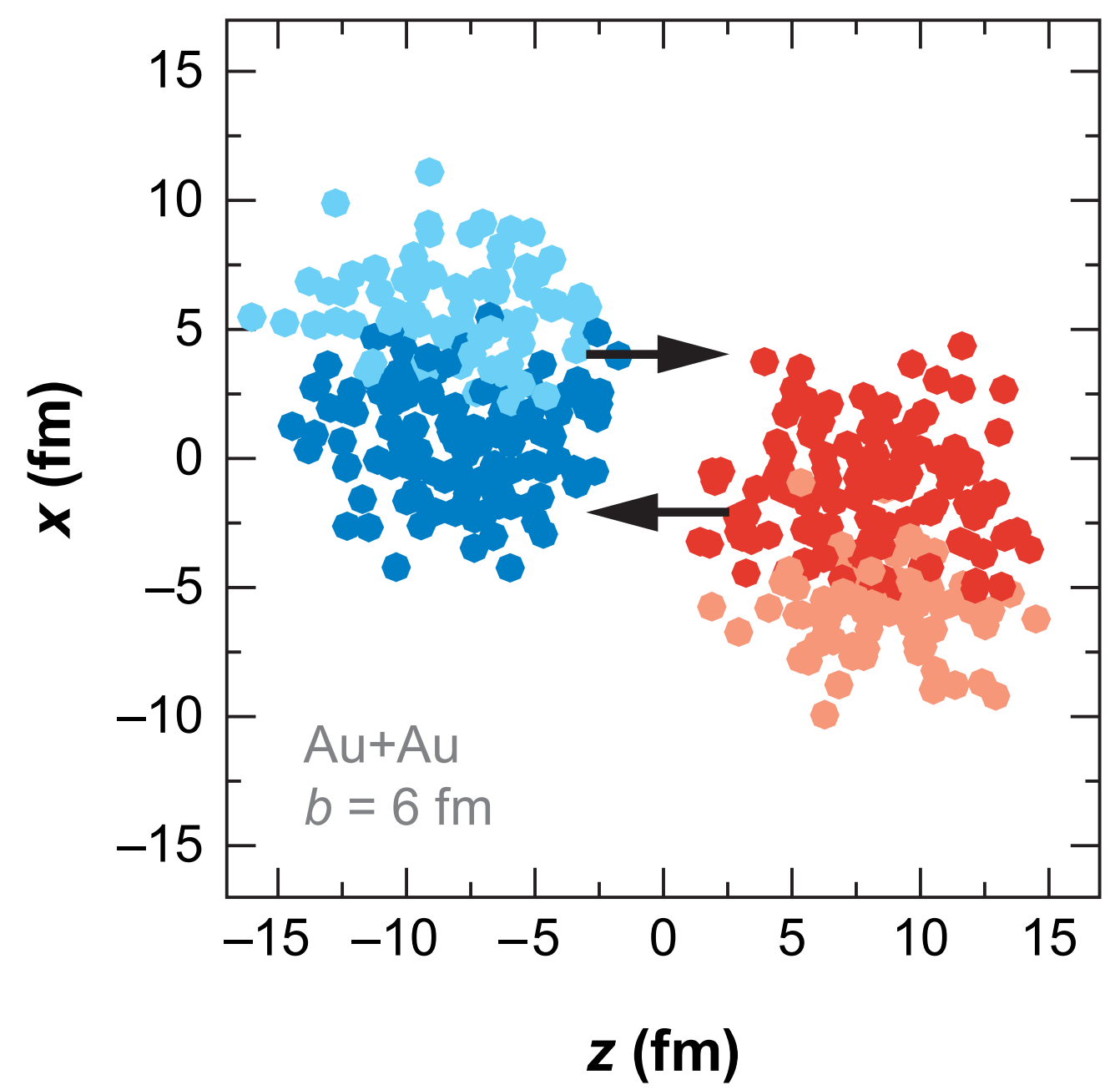

\section{PROJECT}

\section{MONTE CARLO GLALIBER PHOBOS (G ALVER \& ROLAND)}




\section{INITIAL S}

STATE

MATTERS

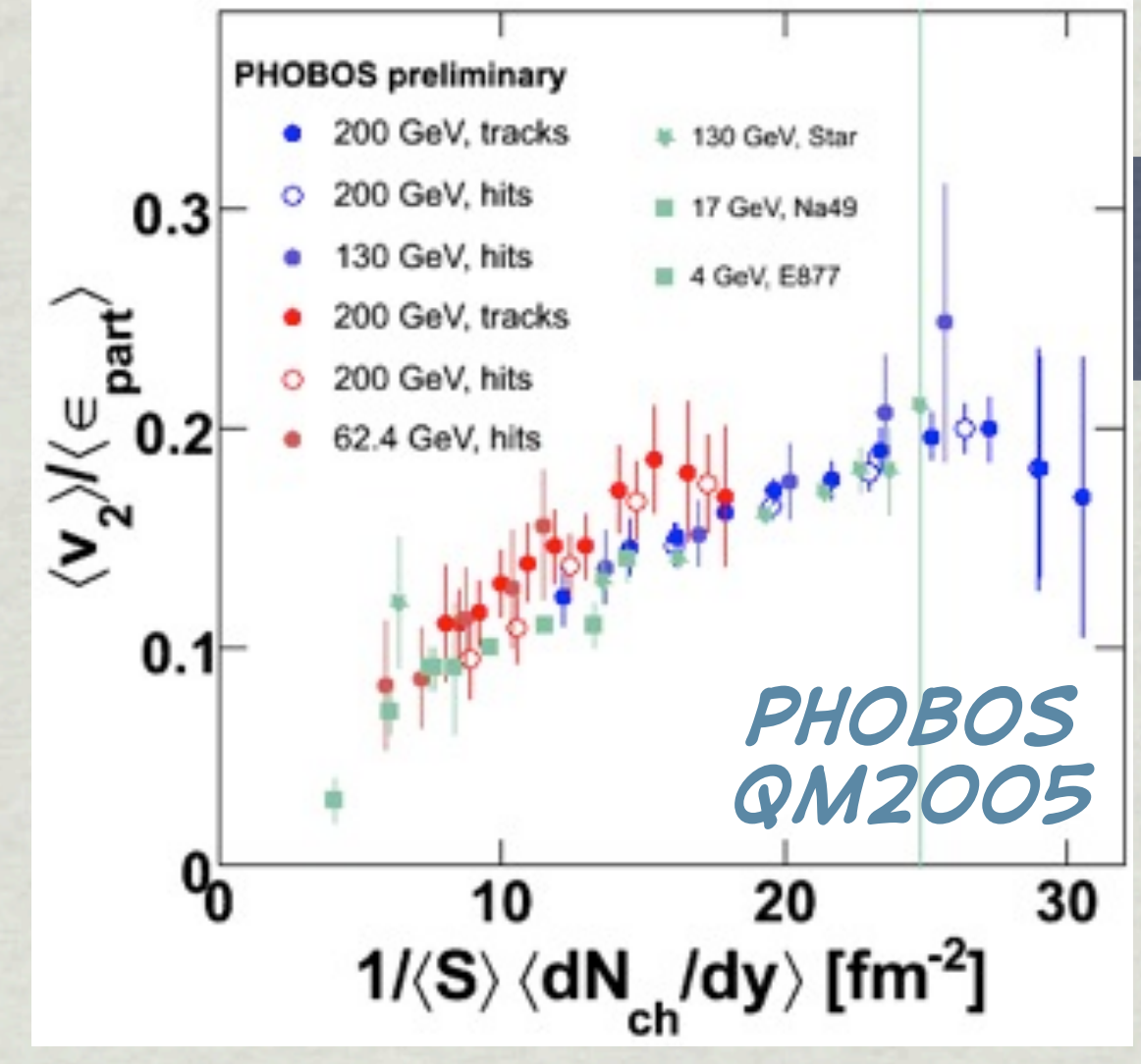

STAR, PRC 81054905 (2010)

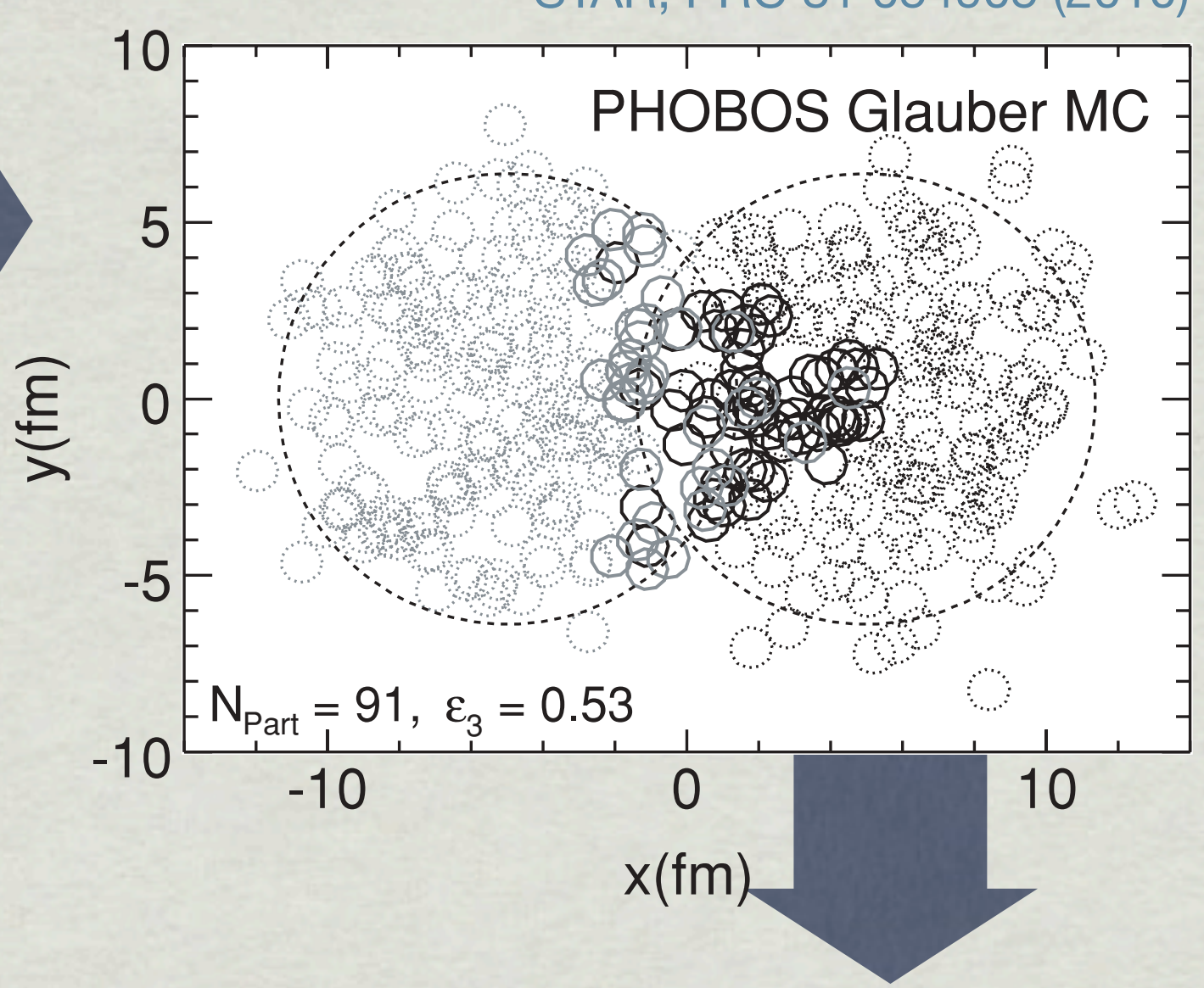

\section{PARTICIPANT ECCENTRICITY BROUGHT AL+AU \& CL+CU TOGETHER! (PHOBOS 2005)}

ALVER \& ROLAND WERE FIRST TO MAKE IT CLEAR THAT V 3 SHOLLD EXIST AND, MORE IMPORTANTLY, RIDGE AND CONE ARE "LEFT BEHIND" IF V 2 SLBTRACTED

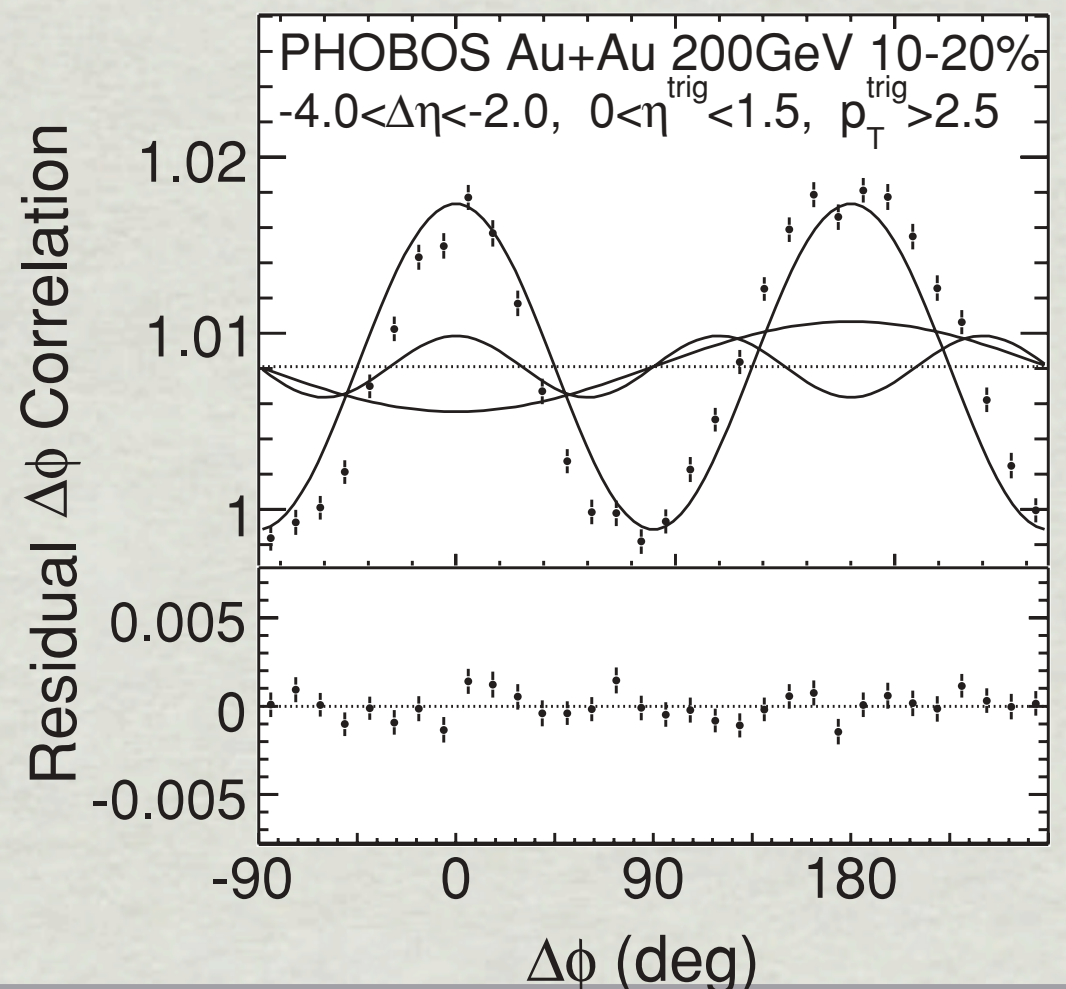




\section{THE RIDGE, POST $V_{3}$}

* ONCE SEEN, DIFFICLILT TO FORGET

* FLLICTLIATIONS IN THE INITIAL STATE PROVIDE SIMPLEST WAY TO HARMONIZE FLOW SYSTEMATICS

* THEY ARE ALSO THE SIMPLEST WAY TO LINDERSTAND THE RIDGE AND MACH CONE

...WAS THIS THE END OF THE RIDGE?? 
RECEIVED: September 22, 2010

ACCEPTED: September 23, 2010

Published: September 27, 2010

Observation of long-range, near-side angular correlations in proton-proton collisions at the LHC

The CMS collaboration

ABSTRACT: Results on two-particle angular correlations for charged particles emitted in proton-proton collisions at center-of-mass energies of $0.9,2.36$, and $7 \mathrm{TeV}$ are presented, using data collected with the CMS detector over a broad range of pseudorapidity $(\eta)$ and azimuthal angle $(\phi)$. Short-range correlations in $\Delta \eta$, which are studied in minimum bias

THE PP RIDGE CMS COLLABORATION 


\section{SEPTEMBER SUIRPRISE}
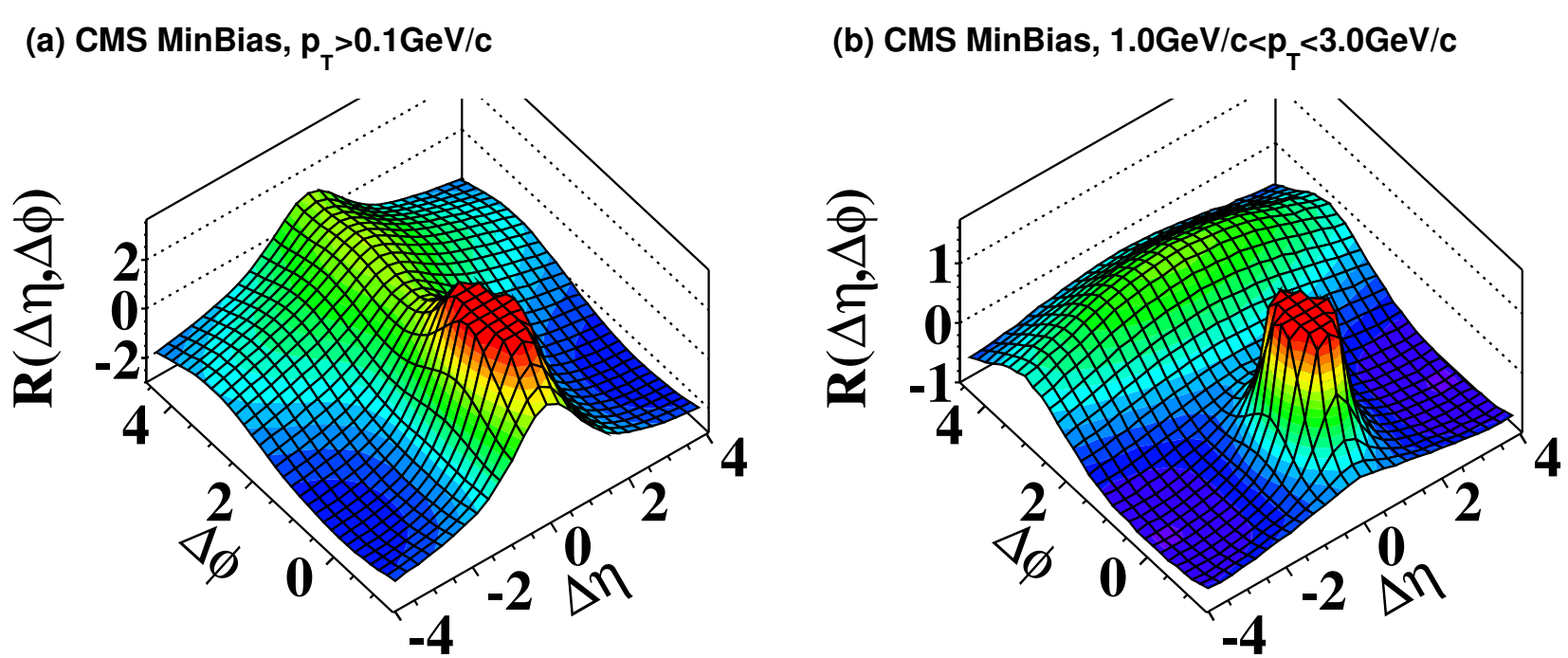

CMS, JHEP 1009091 (2010)

(c) $\mathrm{CMS} \mathrm{N} \geq 110, \mathrm{p}_{\mathrm{T}}>0.1 \mathrm{GeV} / \mathrm{c}$

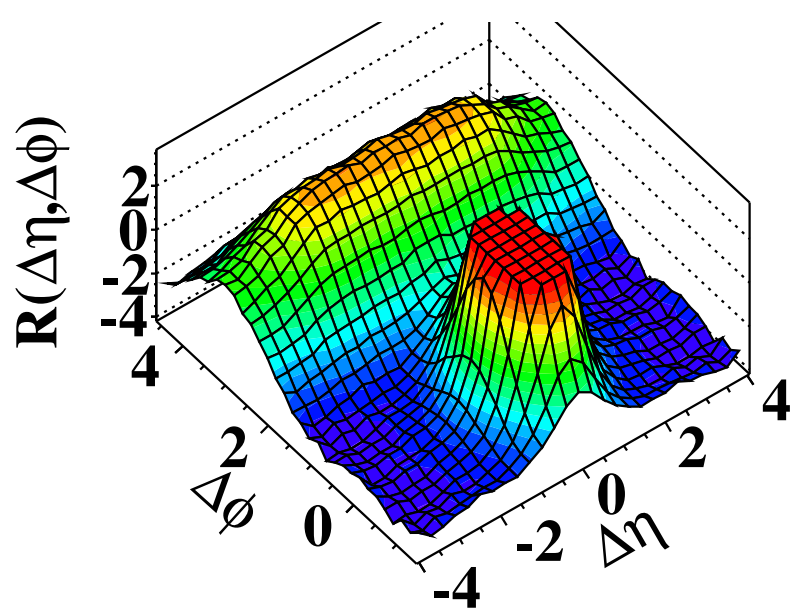

(d) $\mathrm{CMS} \mathrm{N} \geq 110,1.0 \mathrm{GeV} / \mathrm{c}<\mathrm{p}_{\mathrm{T}}<3.0 \mathrm{GeV} / \mathrm{c}$

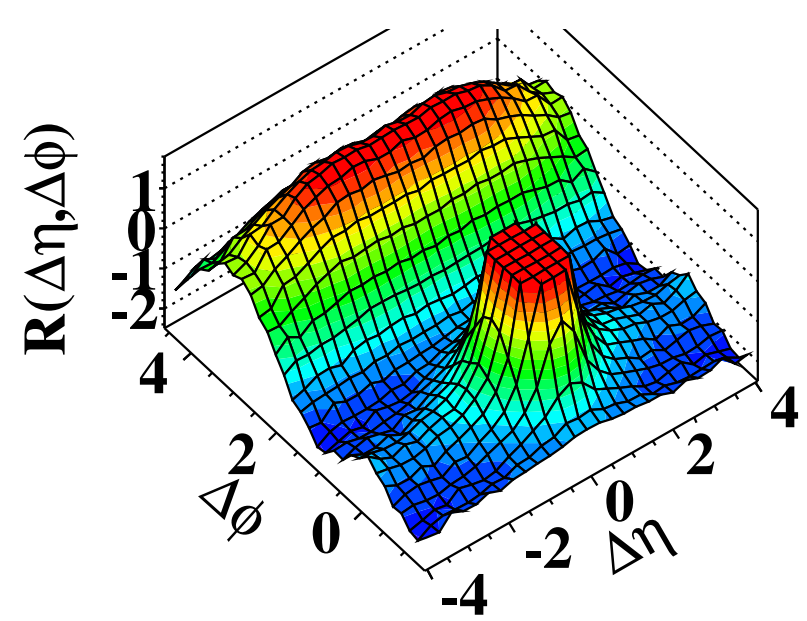

$$
\begin{gathered}
R(\Delta \eta, \Delta \phi)=\left\langle(\langle N\rangle-1)\left(\frac{S_{N}(\Delta \eta, \Delta \phi)}{B_{N}(\Delta \eta, \Delta \phi)}-1\right)\right\rangle_{\text {bins }} \\
S_{N}(\Delta \eta, \Delta \phi)=\frac{1}{N(N-1)} \frac{d^{2} N^{\text {signal }}}{d \Delta \eta d \Delta \phi} \\
B_{N}(\Delta \eta, \Delta \phi)=\frac{1}{N^{2}} \frac{d^{2} N^{\text {mixed }}}{d \Delta \eta d \Delta \phi}
\end{gathered}
$$

USING A SPECIAL HIGH MULTIPLICITY TRIGGER, A DATA SAMPLE LNAVAILABLE TO THE OTHER EXPERIMENTS REVEALED A RIDGE IN PP! 


\section{PHYSICS OF THE RIDGE}

* I DEFER TO MY THEORIST COLLEAGLIES, SPEAKING NEXT

* BLTT THE SAME DATA LED TO A WIDE VARIETY OF EXPLANATIONS

* PARTON SATLRATION (VENLGOPALAN ET AL)

* MLILTIPARTON INTERACTIONS (STRIKMAN)

* BREMSSTRAHLLNG IN STRONG FIELDS

* "JET-MEDILM" (HWA, WONG,...)

* HYDRODYNAMICS (WERNER, AVSAR, ETC.)

* AS WITH THE RHIC RIDGE, MANY

EXPLANATIONS BUT NO CLEAR WINNER

* ABSENCE OF CRISP PREDICTIONS CONFRONTING NEW PP RIDGE DATA 


\section{PP RIDGE VS. $P_{T}$}

CMS, PAS-HIN-2011-006 (Mar 2011)

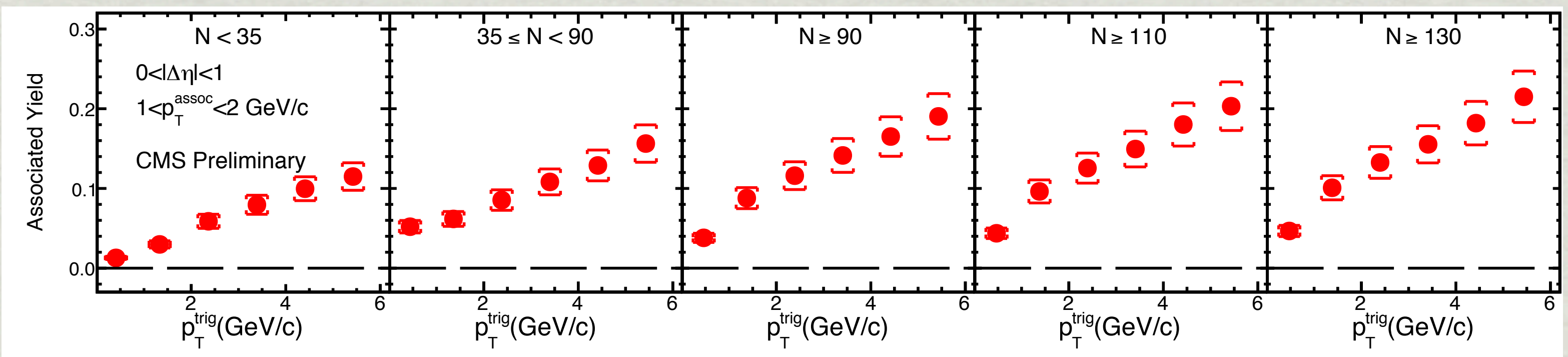

A SYSTEMATIC INCREASE IN JET REGION ( $\triangle E T A<1)$

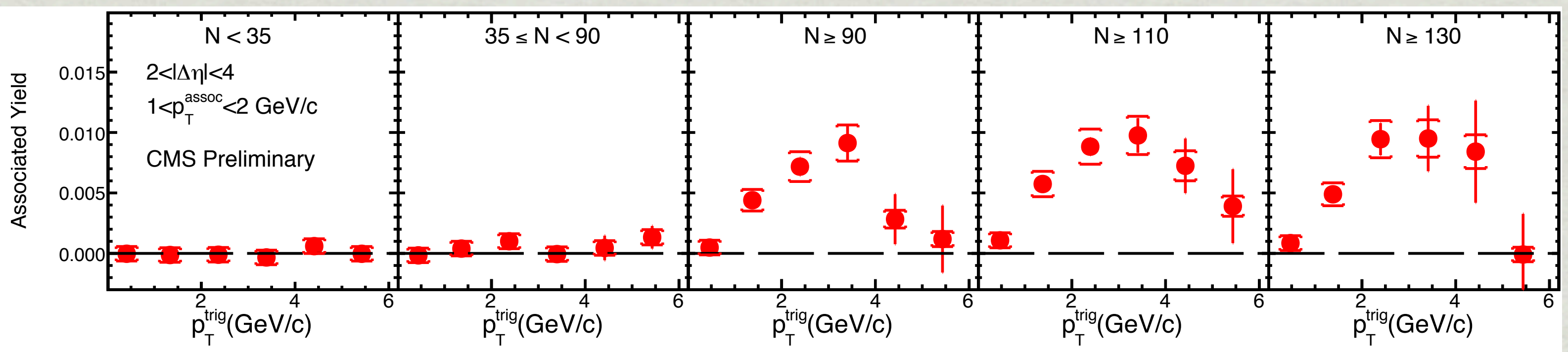

A CHARACTERISTIC PT DEPENDENCE IN "RIDGE" REGION: ONE WHICH LOOKED FAMILIAR FROM $A+A$ 

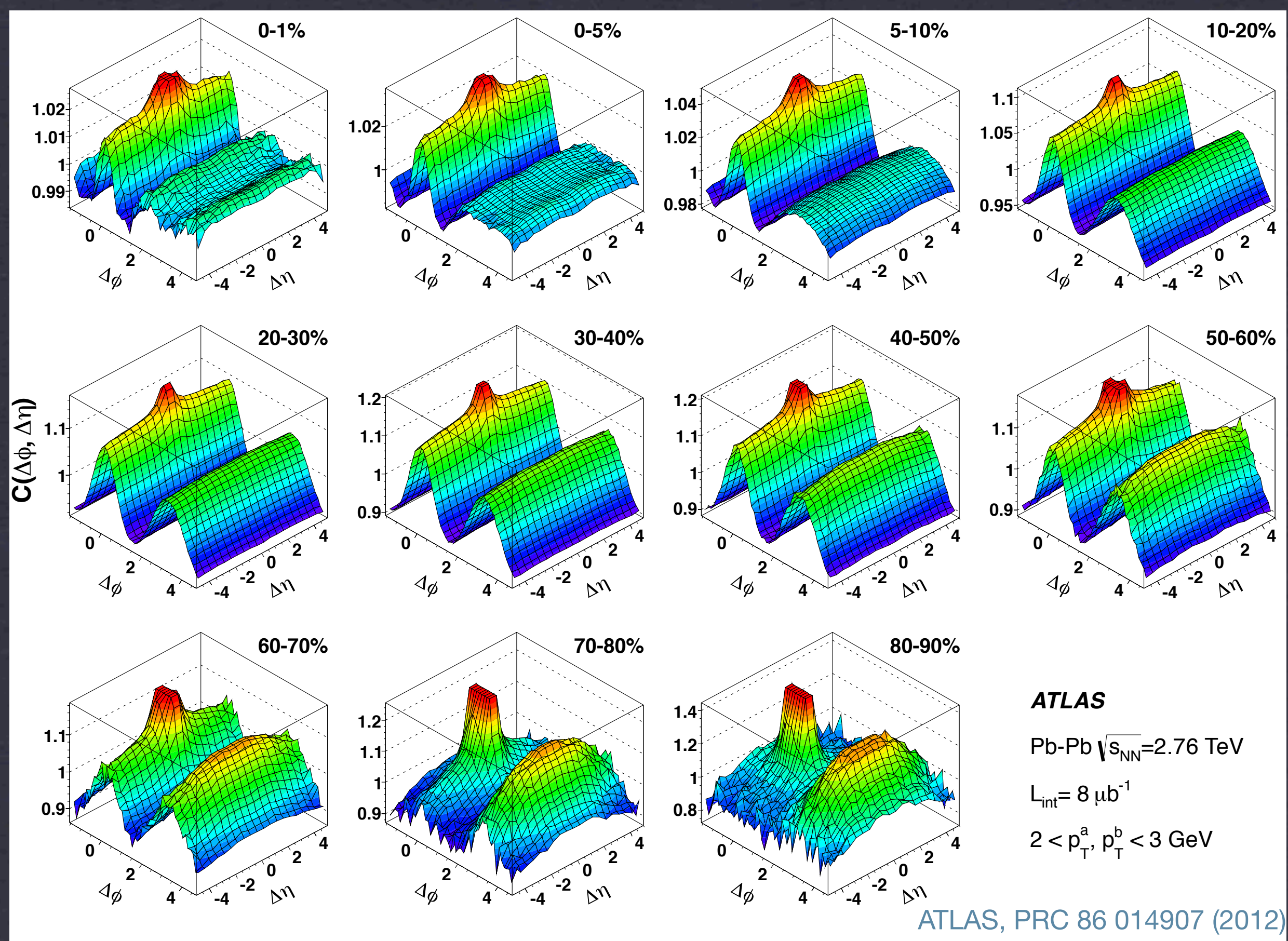

\section{PROJECT}

\section{THE PB+PB RIDGE ALICE, ATLAS, CMS}




\section{FOURIER DECOMPOSITION}

ATLAS, PRC 86014907 (2012)
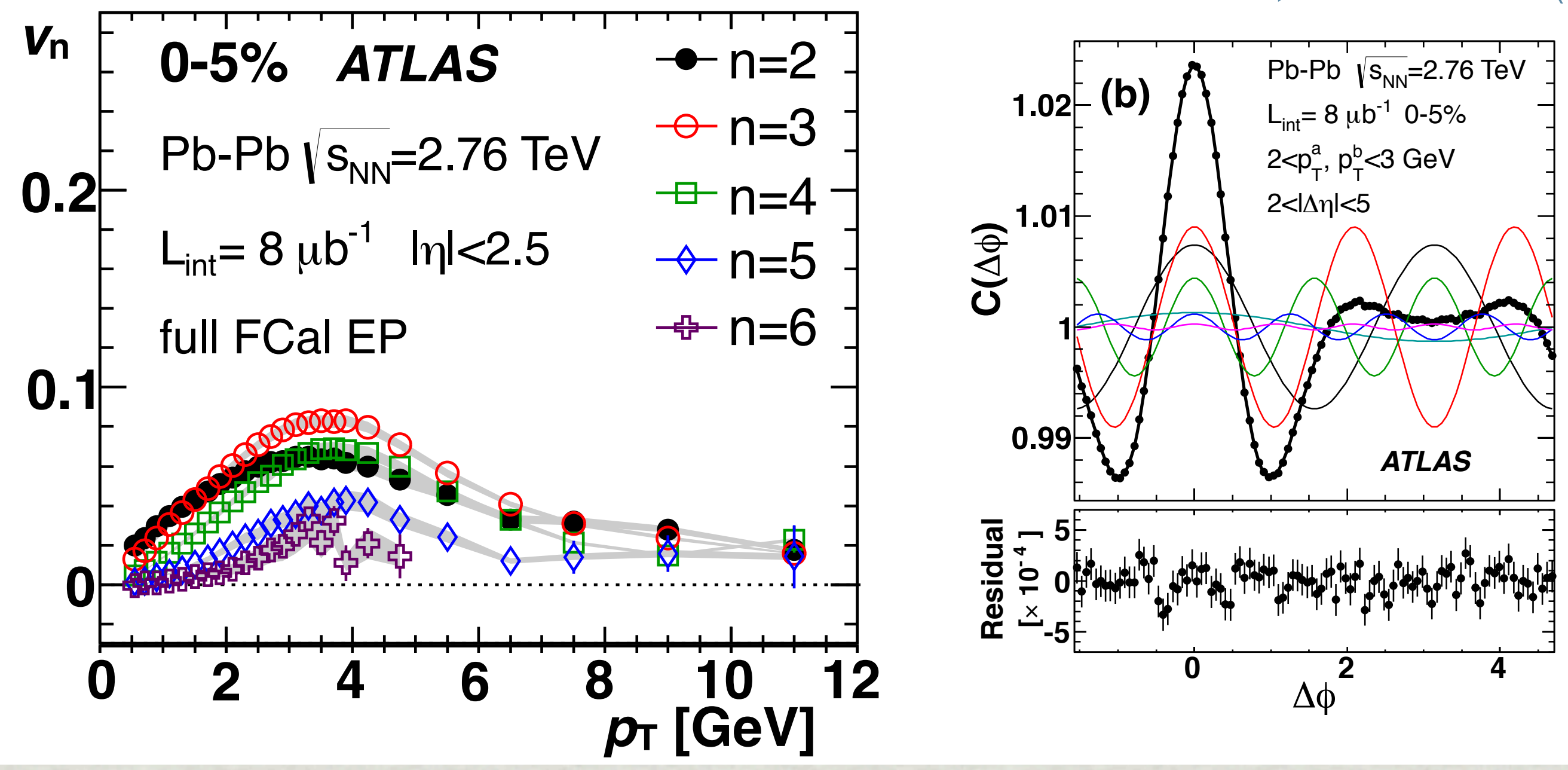

WHY STOP AT TRIANGULAR FLOW?

IN THIS VIEW, THE 2PC RIDGE IS SIMPLY ALL FOURIER COMPONENTS CONTRIBUTING AT $\triangle P H I=0$ !

AND NOTE VERY SIMILAR SHAPE OF PT DEPENDENCE TO PP RIDGE! 


\section{$V_{N}$ FROM FLOW}
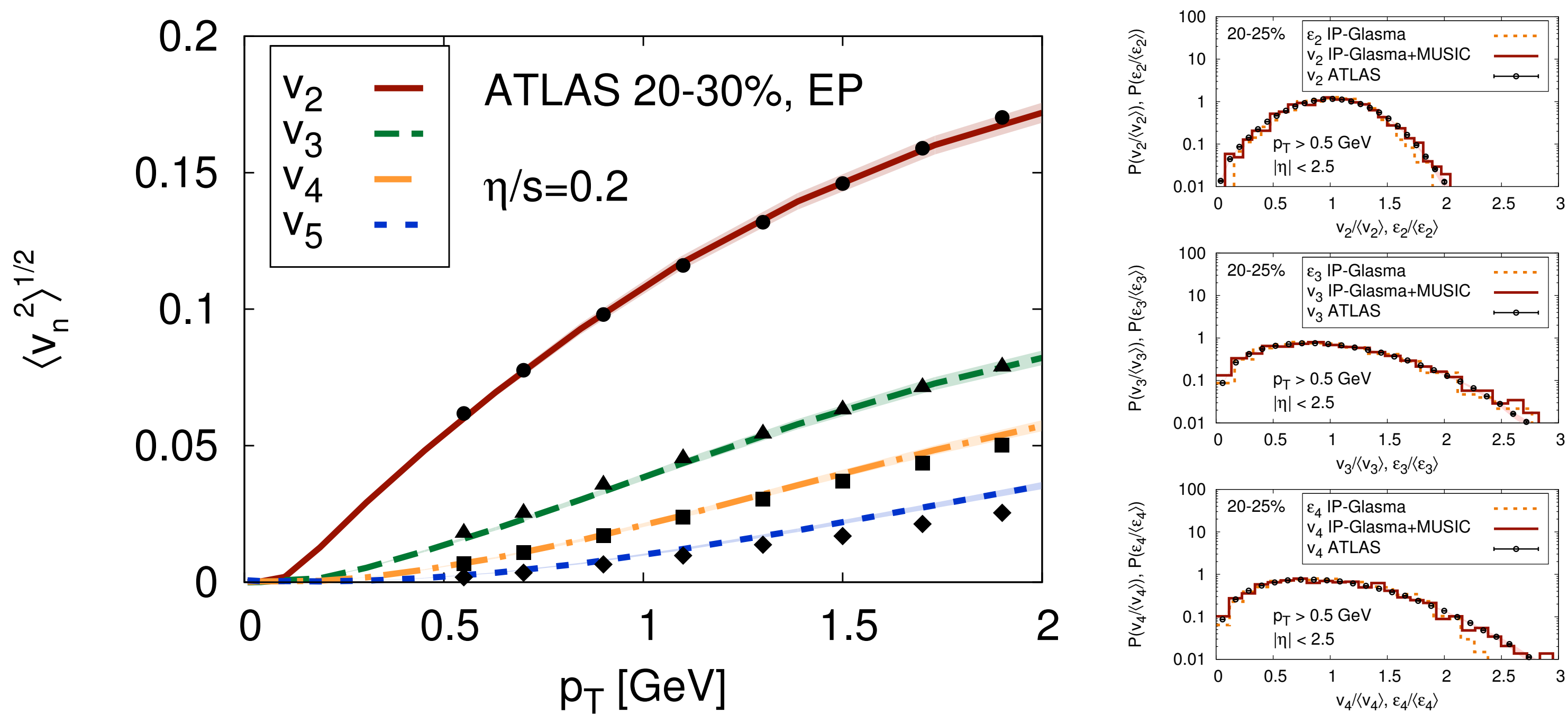

BOTH MEAN VALLES AND FLLCTUATIONS NICELY DESCRIBED BY EVENTWISE VISCOUS HYDRO WITH IP GLASMA IC. (E OTHERS)

(SCHENKE ET AL, HEINZ ET AL, LUZUM ET AL,...) 
WITH THE HIGHER-ORDER

HARMONICS...

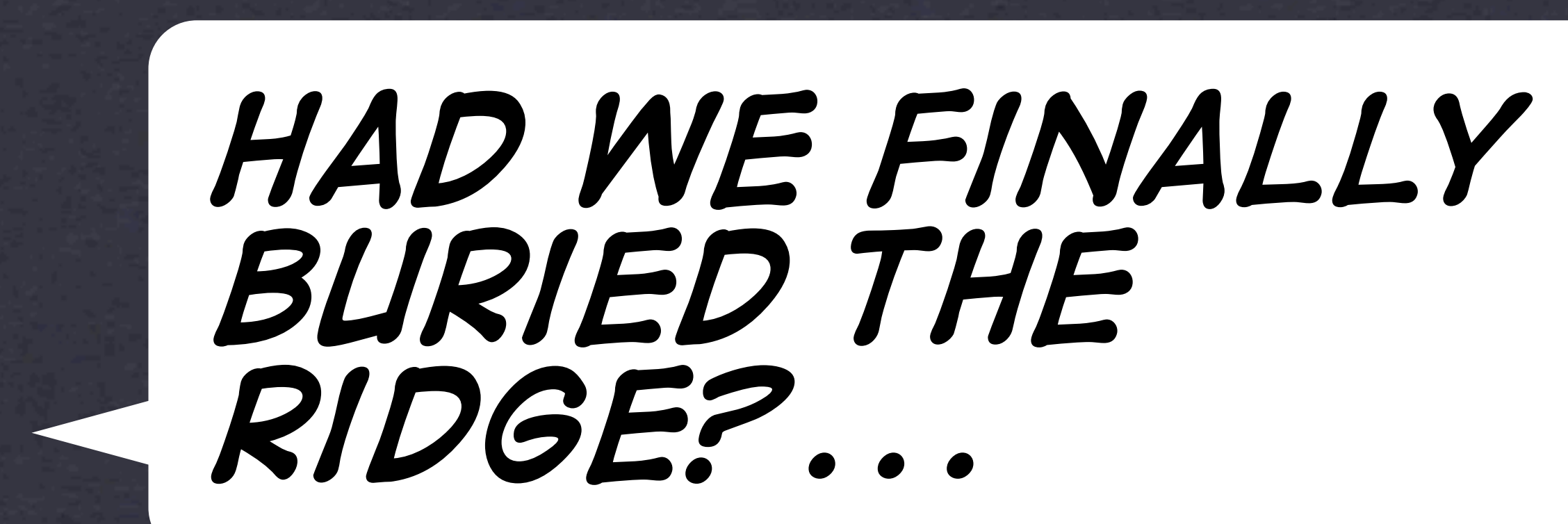

WHERE FLEAST IN A+A,
THE LEADING HYPOTHESIS 


\section{NOT SO FAST!...}

EUROPEAN ORGANIZATION FOR NUCLEAR RESEARCH (CERN)

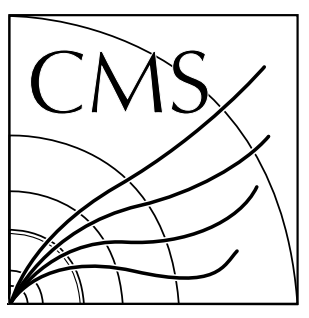

CMS-HIN-12-015

Observation of long-range, near-side angular correlations in $\mathrm{pPb}$ collisions at the LHC

The CMS Collaboration*

PROJECT

\section{DISCOVERY OF A P+PB RIDGE THE CMS COLLABORATION}




\section{EAT A MICROBARN OF LEAD, PROTONS!...}

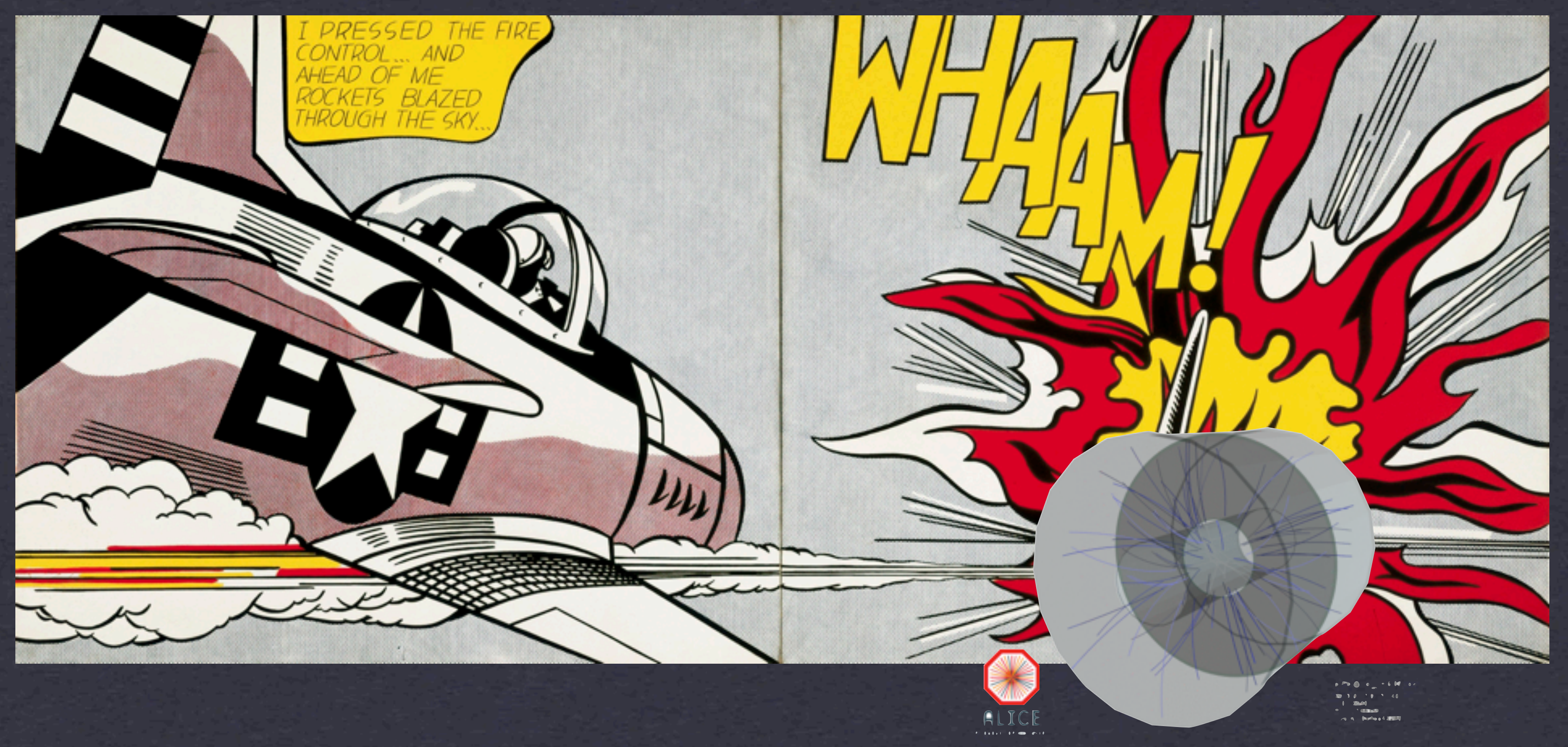

\section{PROJECT}

THE P+PB PILOT RLN

LARGE HADRON COLLIDER (J. JOWETT, ET AL) 


\section{RIDGE REDISCOVERED}

CMS, PLB 718795 (2013)
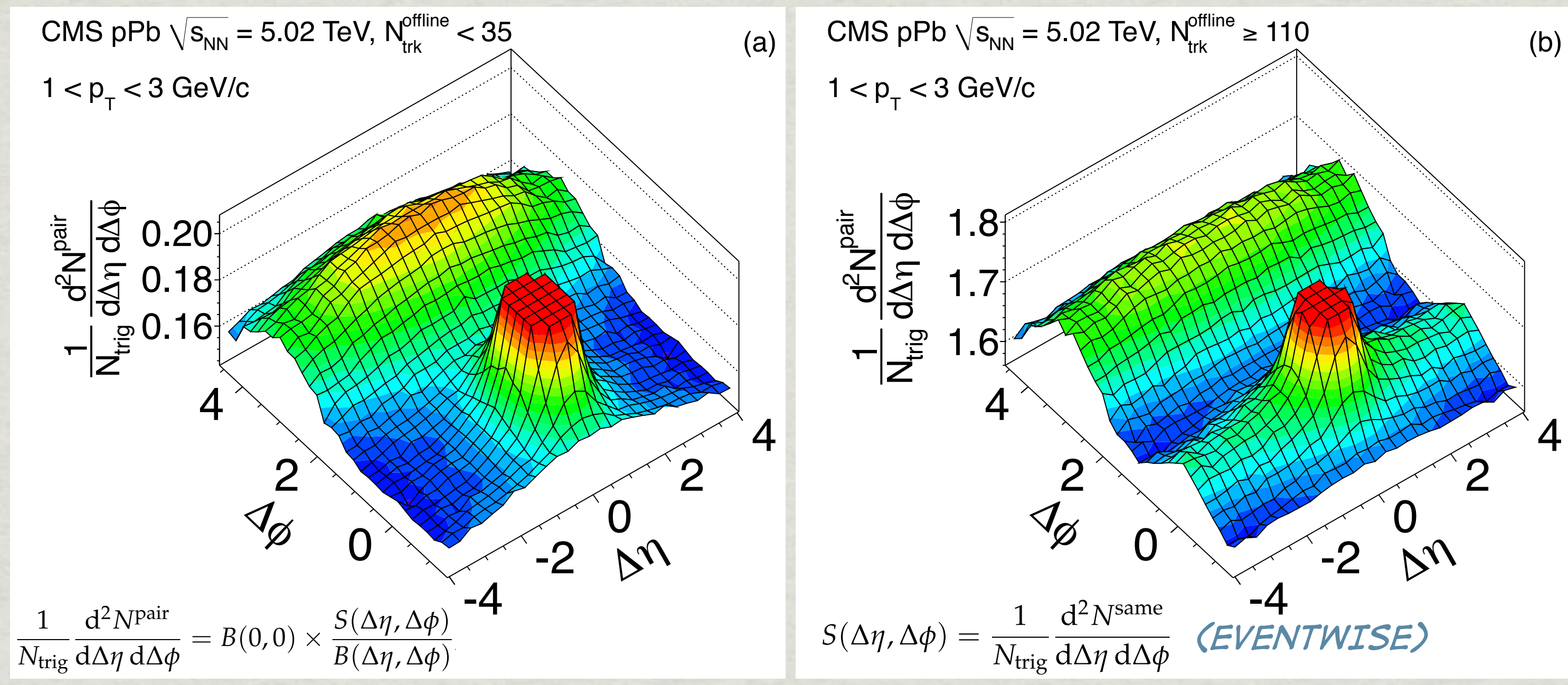

IN PP, RIDGE REQUIRED HIGHEST MULTIPLICITY COLLISIONS TO BE SEEN NEXT TO ENORMOUS NEAR-SIDE PEAK,

IN P+PB RIDGE WAS VISIBLE WITH RATHER MODEST MLILTIPLICITIES (DLE TO MLLTIPLE COLLISIONS) 


\section{CORRELATION FUNCTIONS}

\section{EQUAL MOMENTLM BINS}

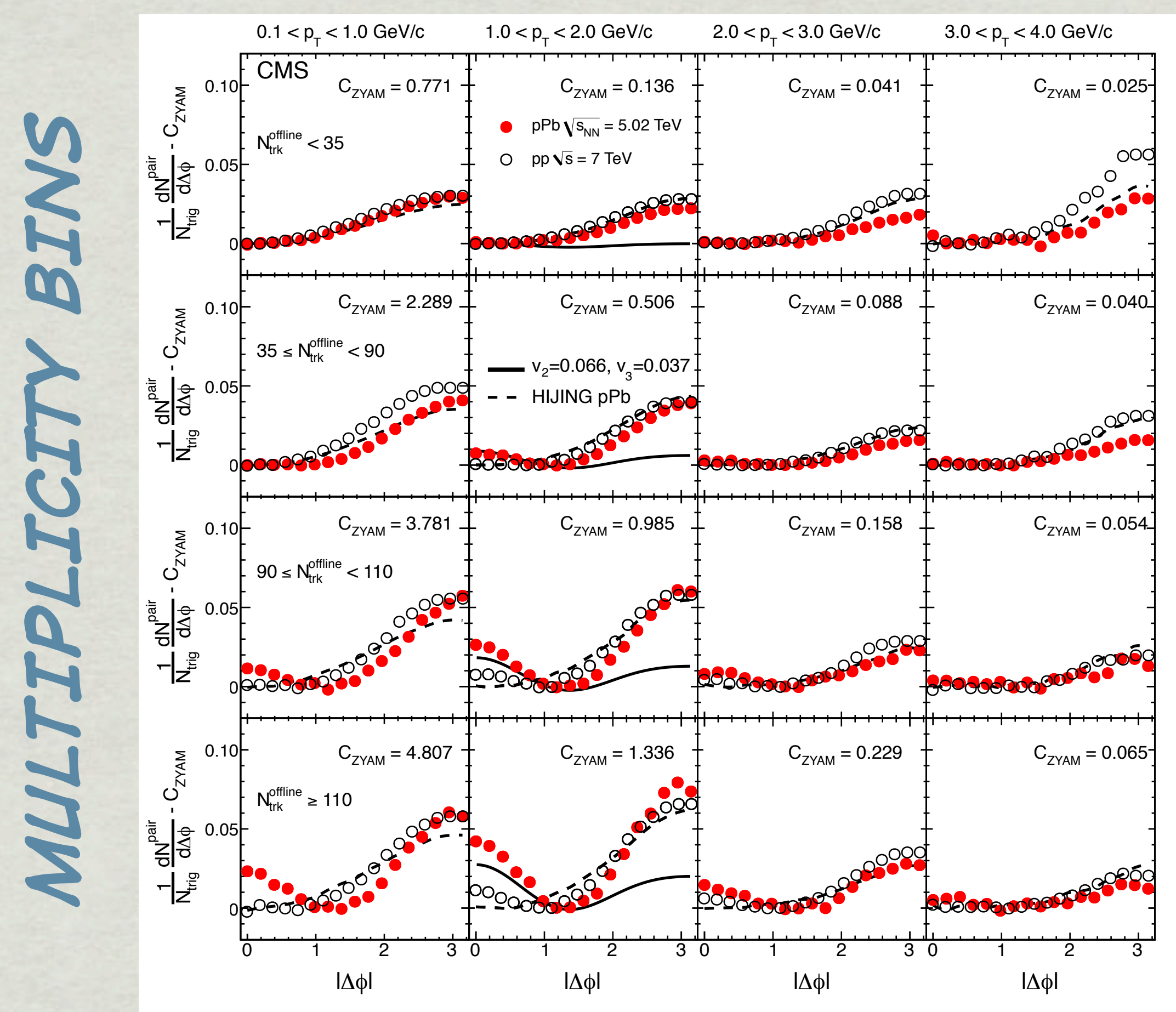

CMS, PLB 718795 (2013)

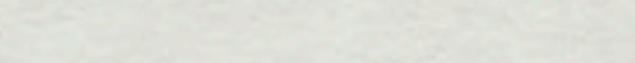




\section{CGC MODELING}

Dusling \& Venugopalan, prd 87054014 (2013)
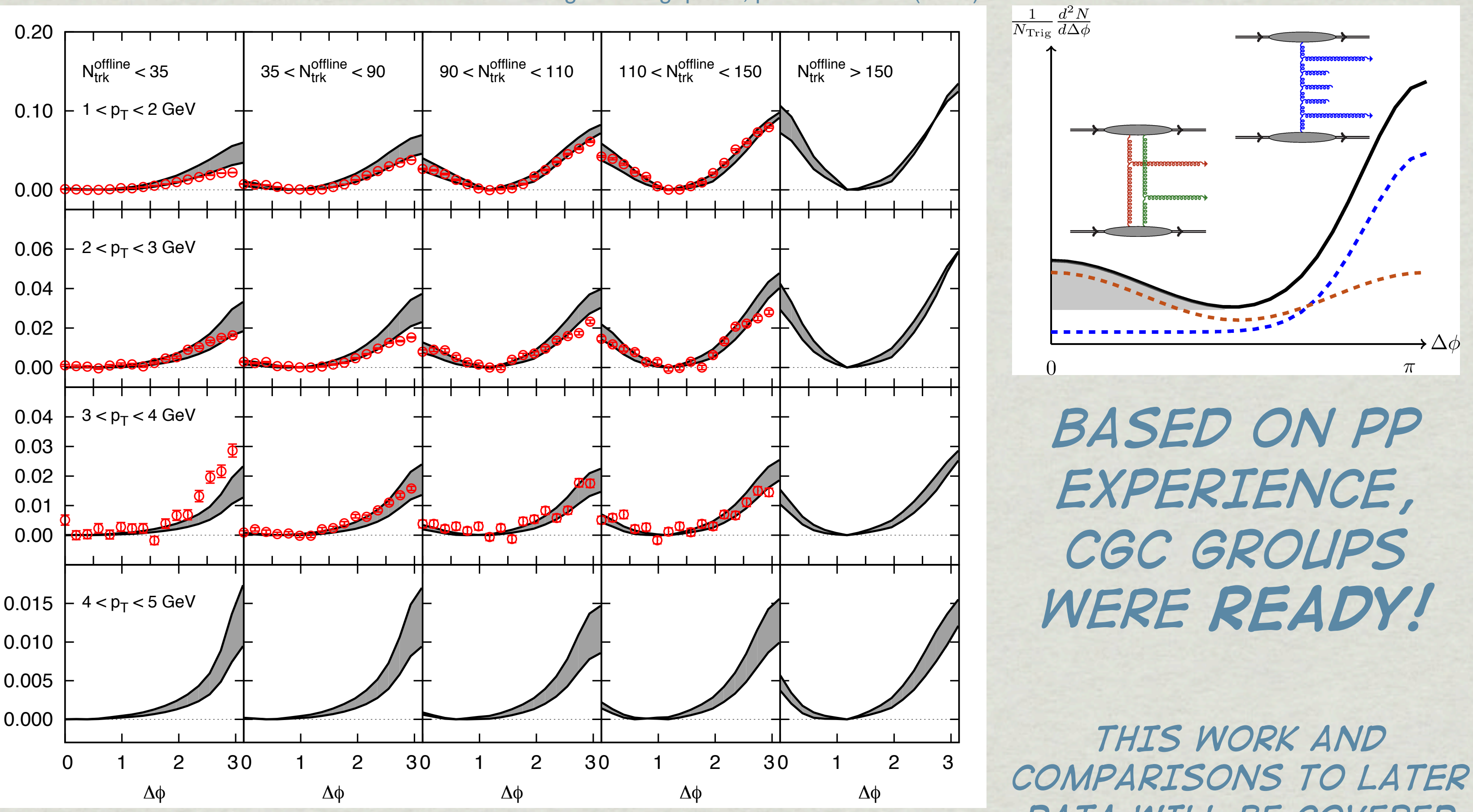

\section{BASED ON PP EXPERIENCE, CGC GROLIPS WERE READY!}

THIS WORK AND COMPARISONS TO LATER DATA WILL BE COVERED

ALSO WORK BY: KOVCHEGOV \& IN RAJUI'S TALK!... WERPERTNY 
MEANWHILE...

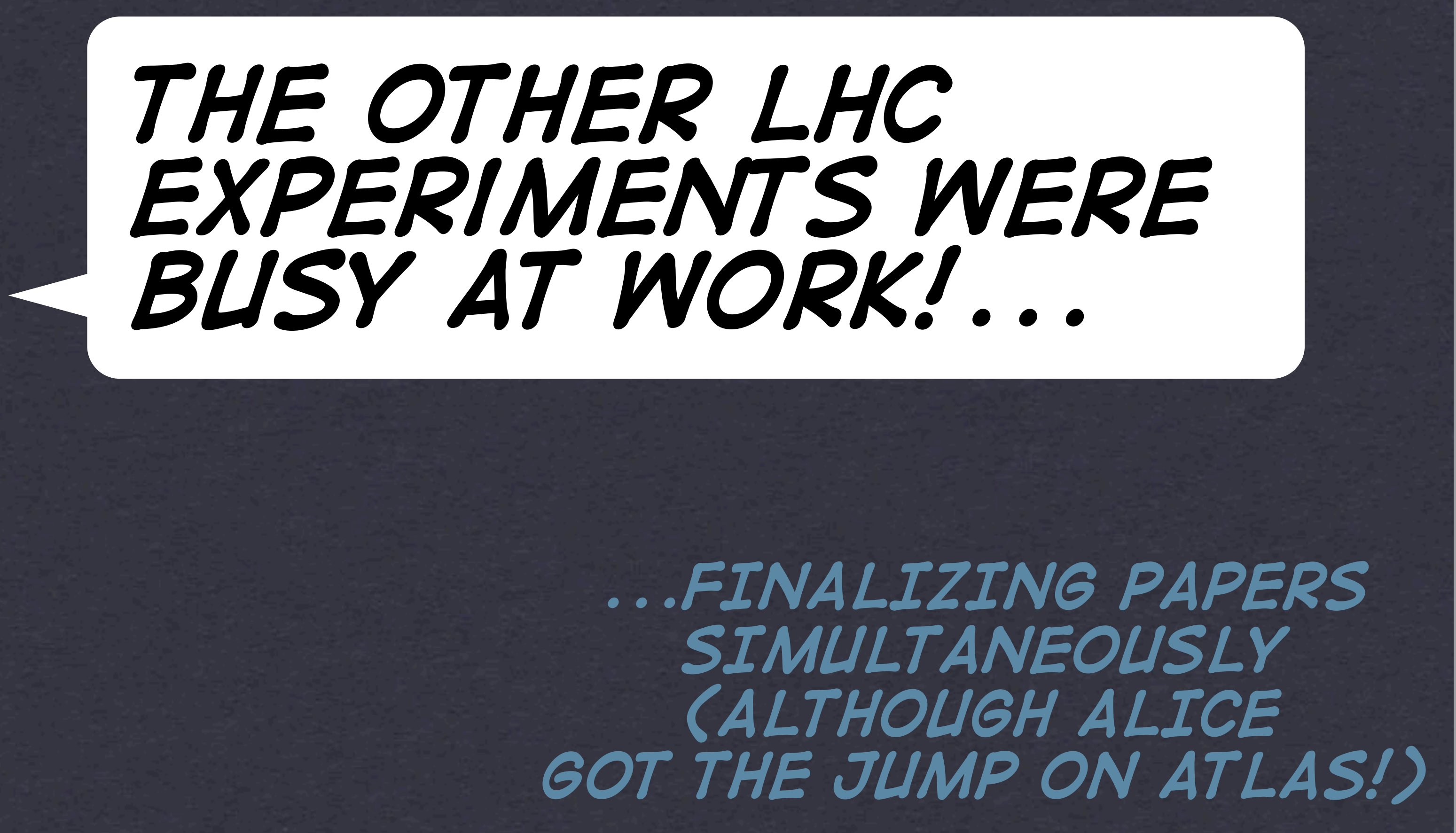

24 


\section{EUROPEAN ORGANIZATION FOR NUCLEAR RESEARCH}

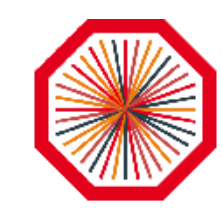

ALICE

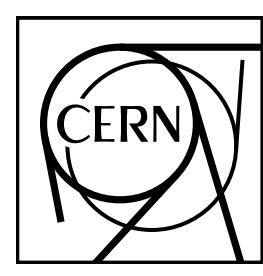

CERN-PH-EP-2012-359

03 Dec 2012

Long-range angular correlations on the near and away side in $\mathbf{p}-\mathrm{Pb}$ collisions at $\sqrt{\mathrm{s}_{\mathrm{NN}}}=\mathbf{5 . 0 2} \mathrm{TeV}$

\section{Abstract}

Angular correlations between charged trigger and associated particles are measured by the ALICE detector in $\mathrm{p}-\mathrm{Pb}$ collisions at a nucleon-nucleon centre-of-mass energy of $5.02 \mathrm{TeV}$ for transverse momentum ranges within $0.5<p_{\mathrm{T} \text {,assoc }}<p_{\mathrm{T} \text {,trig }}<4 \mathrm{GeV} / c$. The correlations

\section{THE P+PB RIDGE ALICE COLLABORATION}




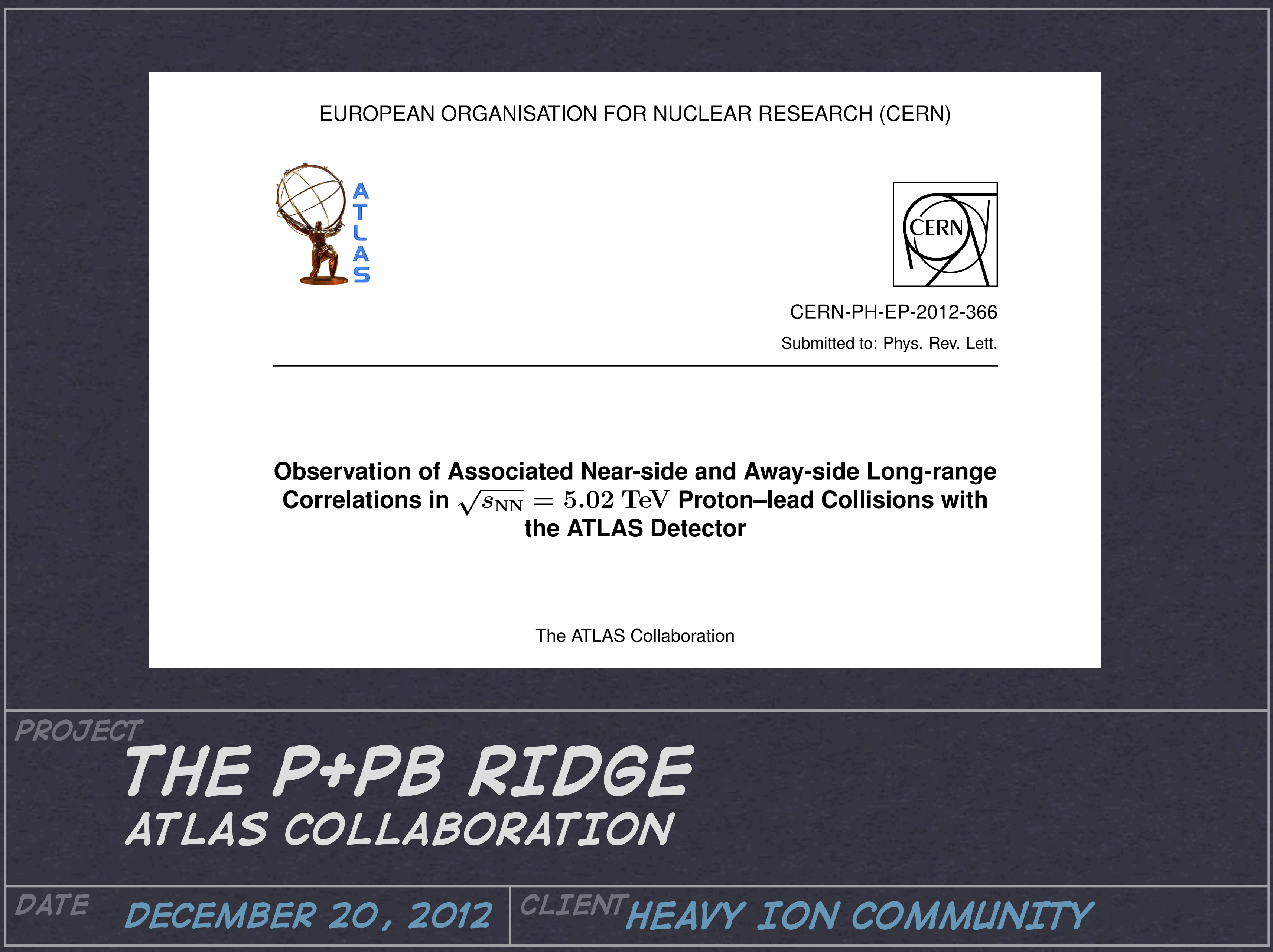




\section{"EVENT ACTIVITY"}

OVERALL ACTIVITY CHARACTERIZED BY ATLAS WITH ET SUM IN $3.2<n<4.9$,

AND ALICE WITH VOM, MLLTIPLICITY IN FORWARD DIRECTION
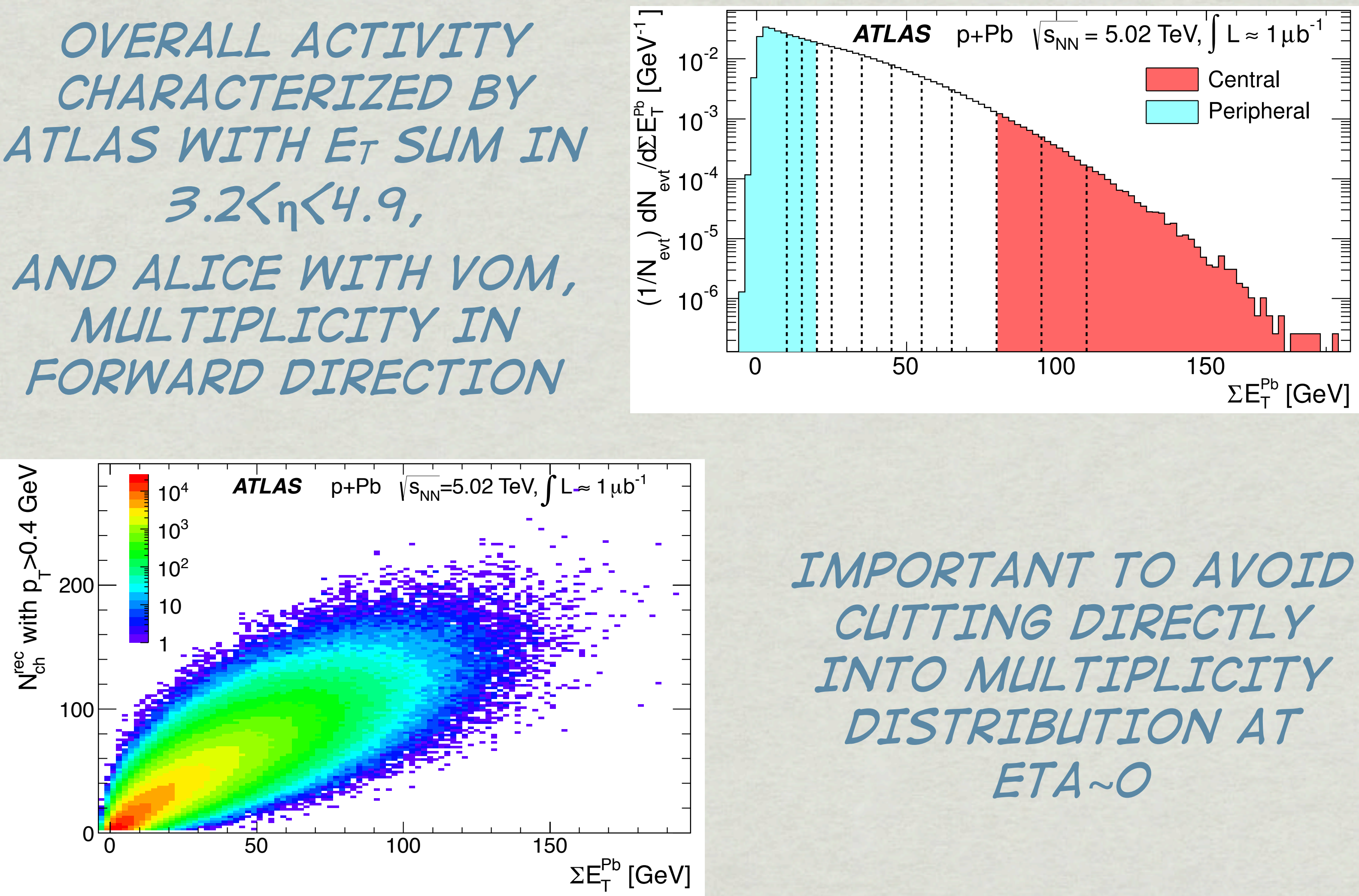

IMPORTANT TO AVOID CUTTING DIRECTLY INTO MULTIPLICITY DISTRIBLTION AT ETA O 


\section{SECOND OBSERVATION!}

ALICE, PLB 71929 (2013)
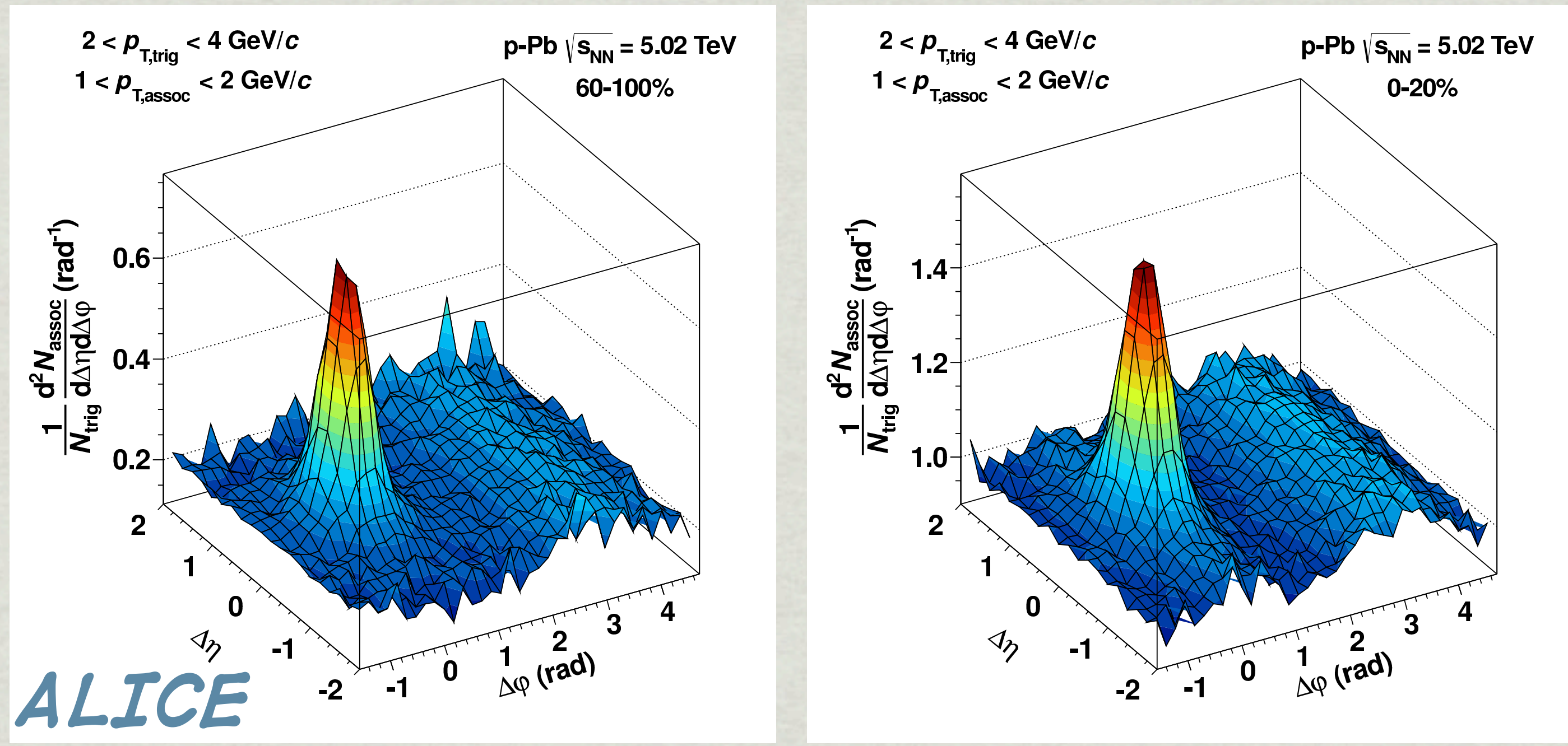

EVEN WITH A SMALLER $\triangle E T A ~ A C C E P T A N C E$, THE RIDGE WAS OBSERVED BY ALICE AT THE FOOT OF THE JET MOLINTAIN...

$\frac{1}{N_{\text {trig }}} \frac{\mathrm{d}^{2} N_{\text {assoc }}}{\mathrm{d} \Delta \eta \mathrm{d} \Delta \varphi}=\frac{S(\Delta \eta, \Delta \varphi)}{B(\Delta \eta, \Delta \varphi)} \quad S(\Delta \eta, \Delta \varphi)=1 / N_{\text {trig }} \mathrm{d}^{2} N_{\text {same }} / \mathrm{d} \Delta \eta \mathrm{d} \Delta \varphi \quad \begin{aligned} & \text { (SUMMED OVER } \\ & \text { MLILTIPLICITY CLASS) }\end{aligned}$ 


\section{ATLAS VS. CMS}

CMS

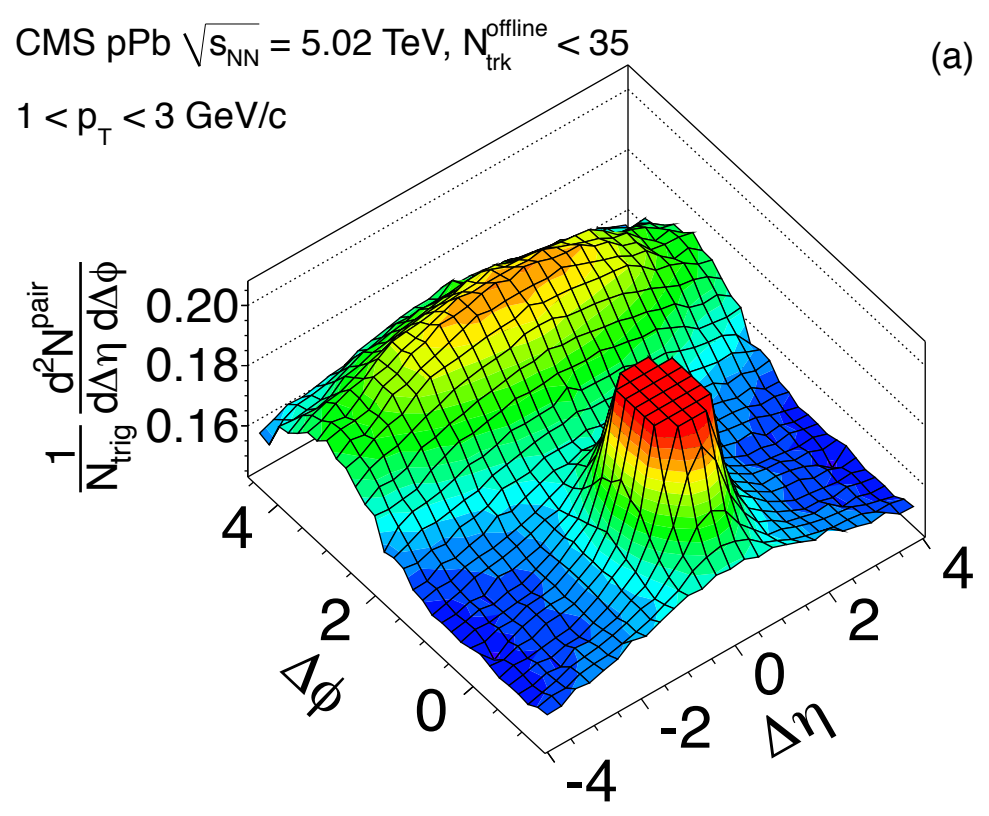

(a) $\mathrm{CMS} \mathrm{pPb} \sqrt{S_{\mathrm{NN}}}=5.02 \mathrm{TeV}, \mathrm{N}_{\mathrm{tth}}^{\text {pofline }} \geq 110$

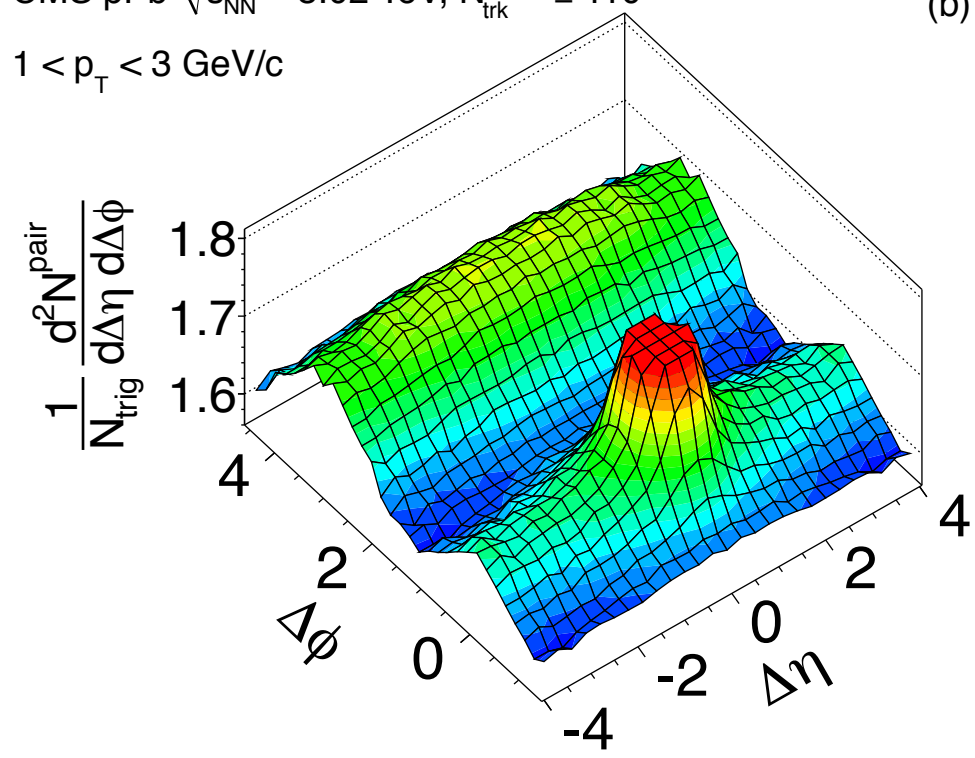

ATLAS 


\section{ATLAS VS. CMS}

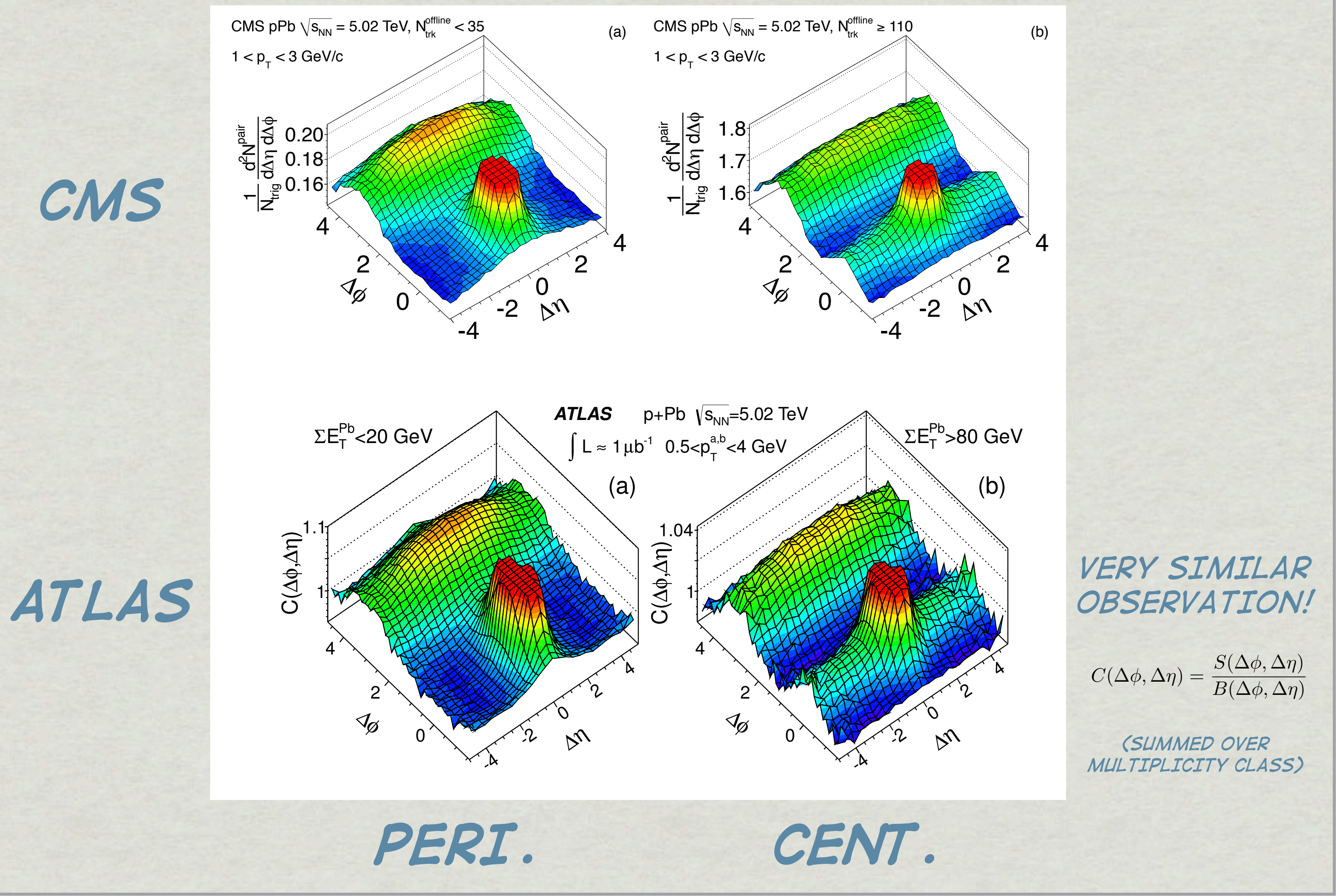


A TWIN?

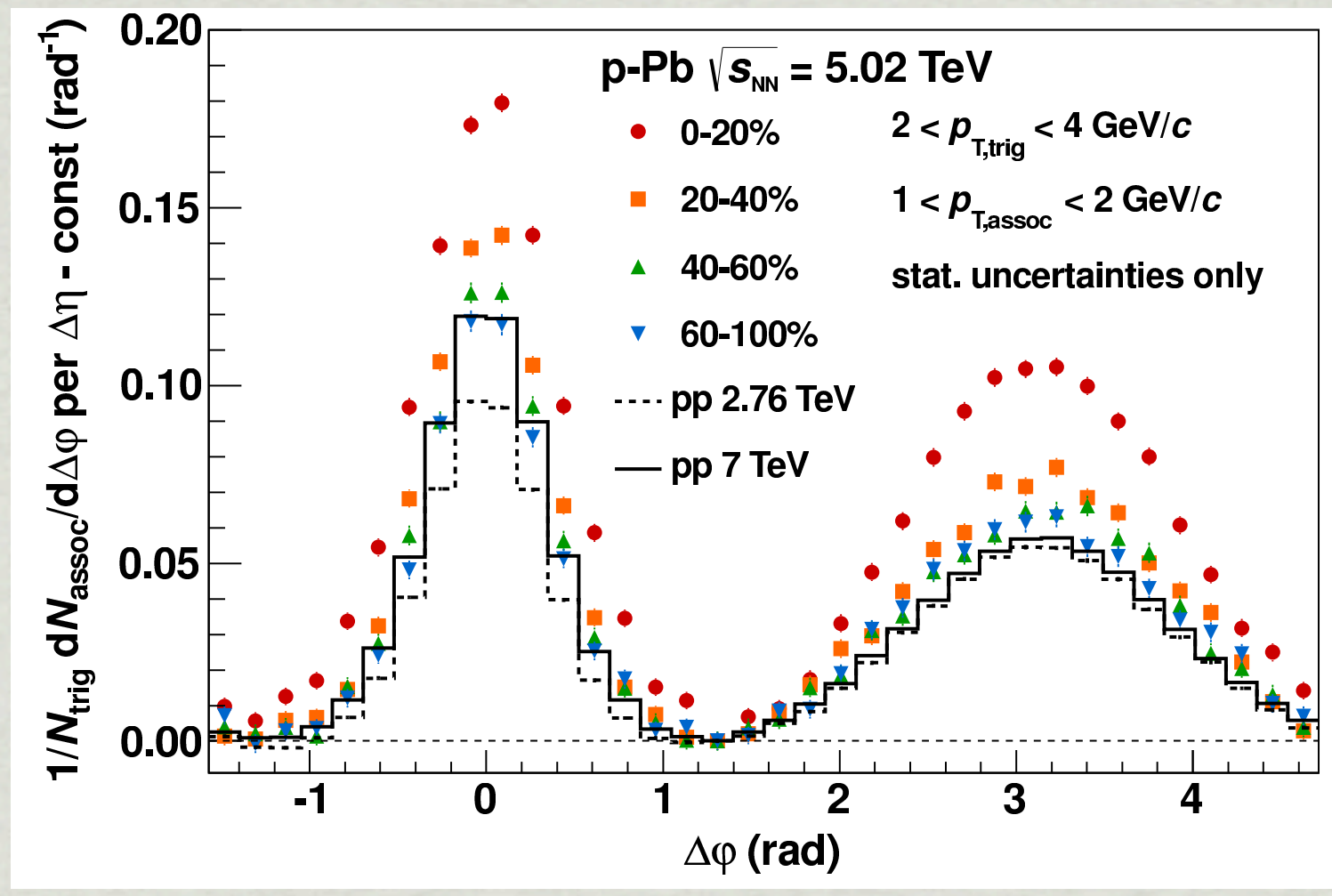

PERIPHERAL P+PB GAVE A SIMILAR YIELD AS SEEN IN PP AT SIMILAR ENERGIES...

\section{USE PERIPHERAL AS A PROXY FOR PP}

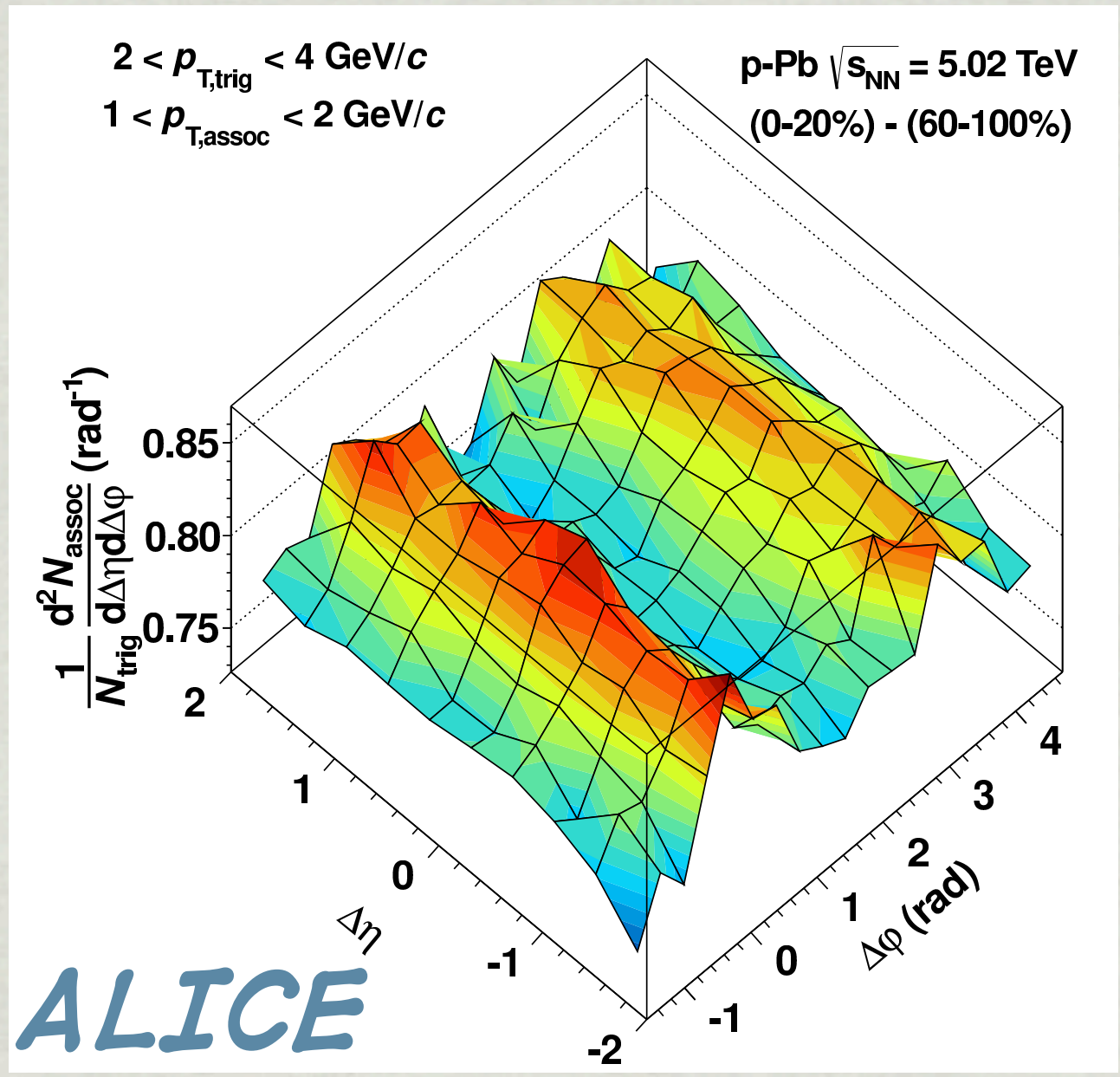

PERIPHERAL TURNED OUT TO SLBTRACT CLEANLY: LEAVING BEHIND TWO RIDGES! 


\section{ATLAS RECOIL REMOVAL}

ATLAS, arXiv:1212.5198 (2013)

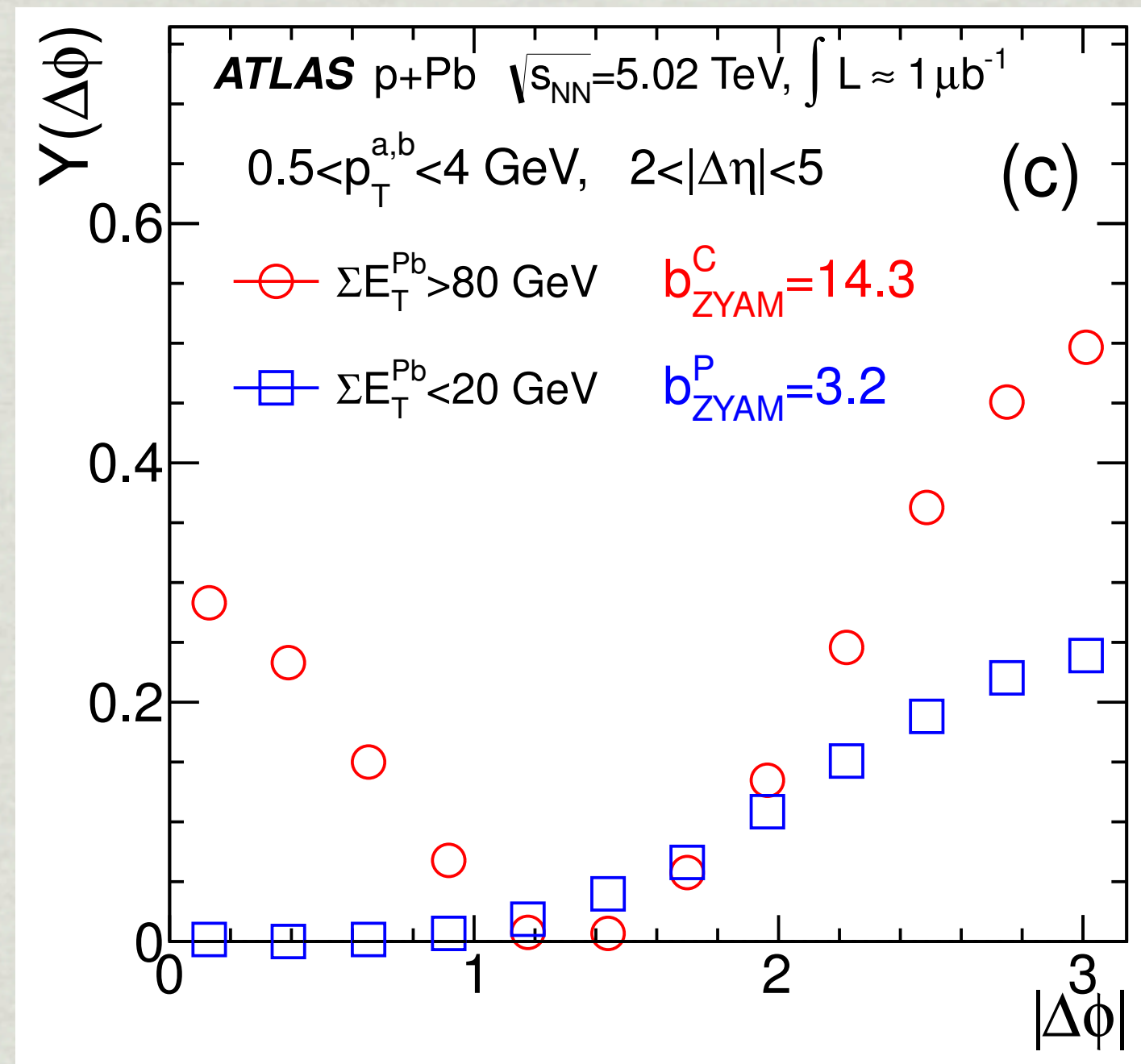

$Y(\Delta \phi)=\left(\frac{\int B(\Delta \phi) d \Delta \phi}{\pi N_{a}}\right) C(\Delta \phi)-b_{\mathrm{ZYAM}}$

(PER-TRIGGER YIELD, I.E. PAIRS/PARTICLE, AFTER ZYAM)

COMPARING $Y(\triangle \Phi)$ IN CENTRAL TO PERIPHERAL:

OBSERVED THE DIFFERENCE IN YIELDS

TO BE CONSTANT VS. CENTRALITY!

STRAIGHTFORWARD INTERPRETATION AS "RECOIL" 


\section{ATLAS RECOIL REMOVAL}

ATLAS, arXiv:1212.5198 (2013)
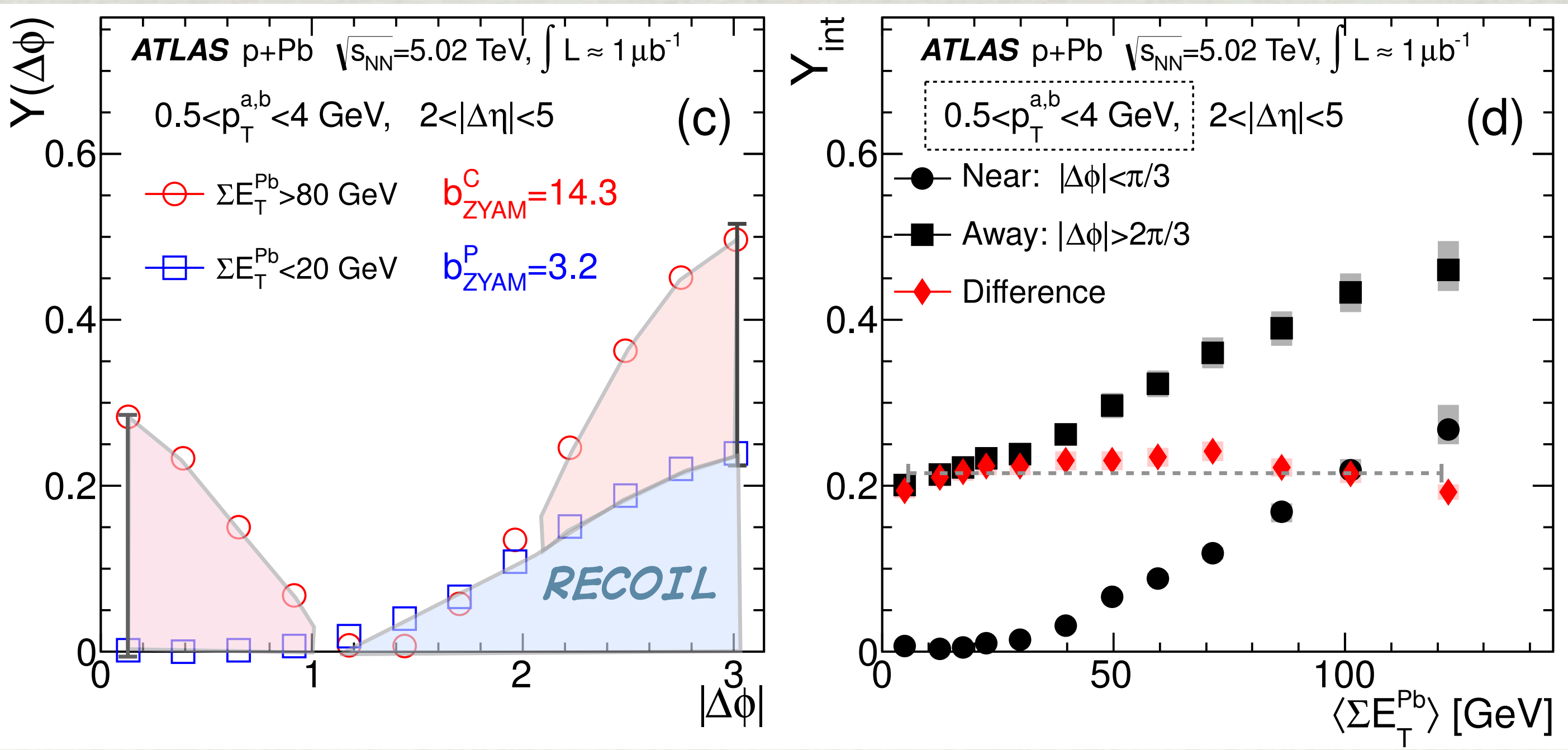

COMPARING $Y(\triangle \Phi)$ IN CENTRAL TO PERIPHERAL:

OBSERVED THE DIFFERENCE IN YIELDS TO BE CONSTANT VS. CENTRALITY!

STRAIGHTFORWARD INTERPRETATION AS "RECOIL" 


\section{AN IDENTICAL TWIN? (ALICE)}
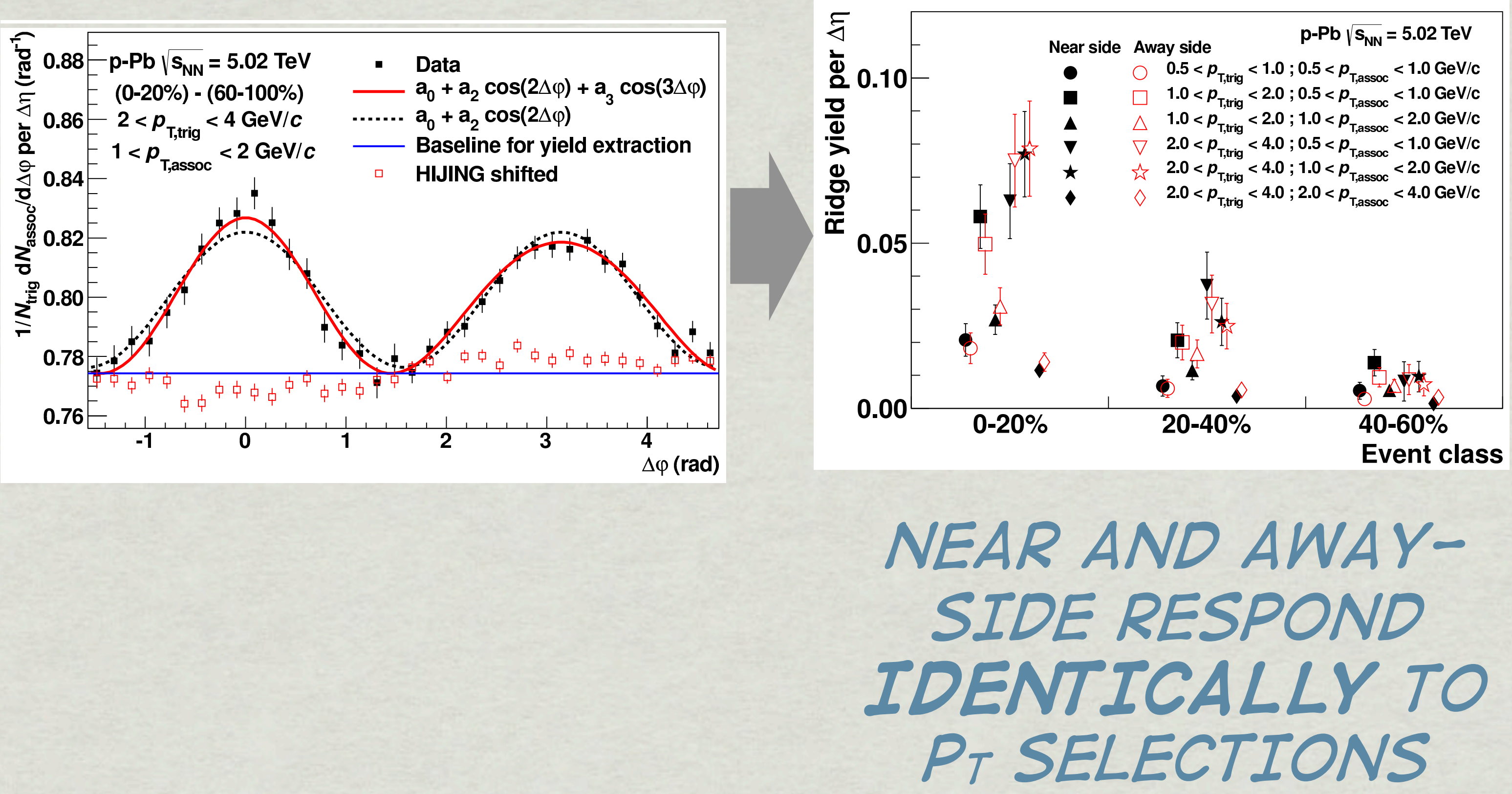


\section{AN IDENTICAL TWIN? (ATLAS)}

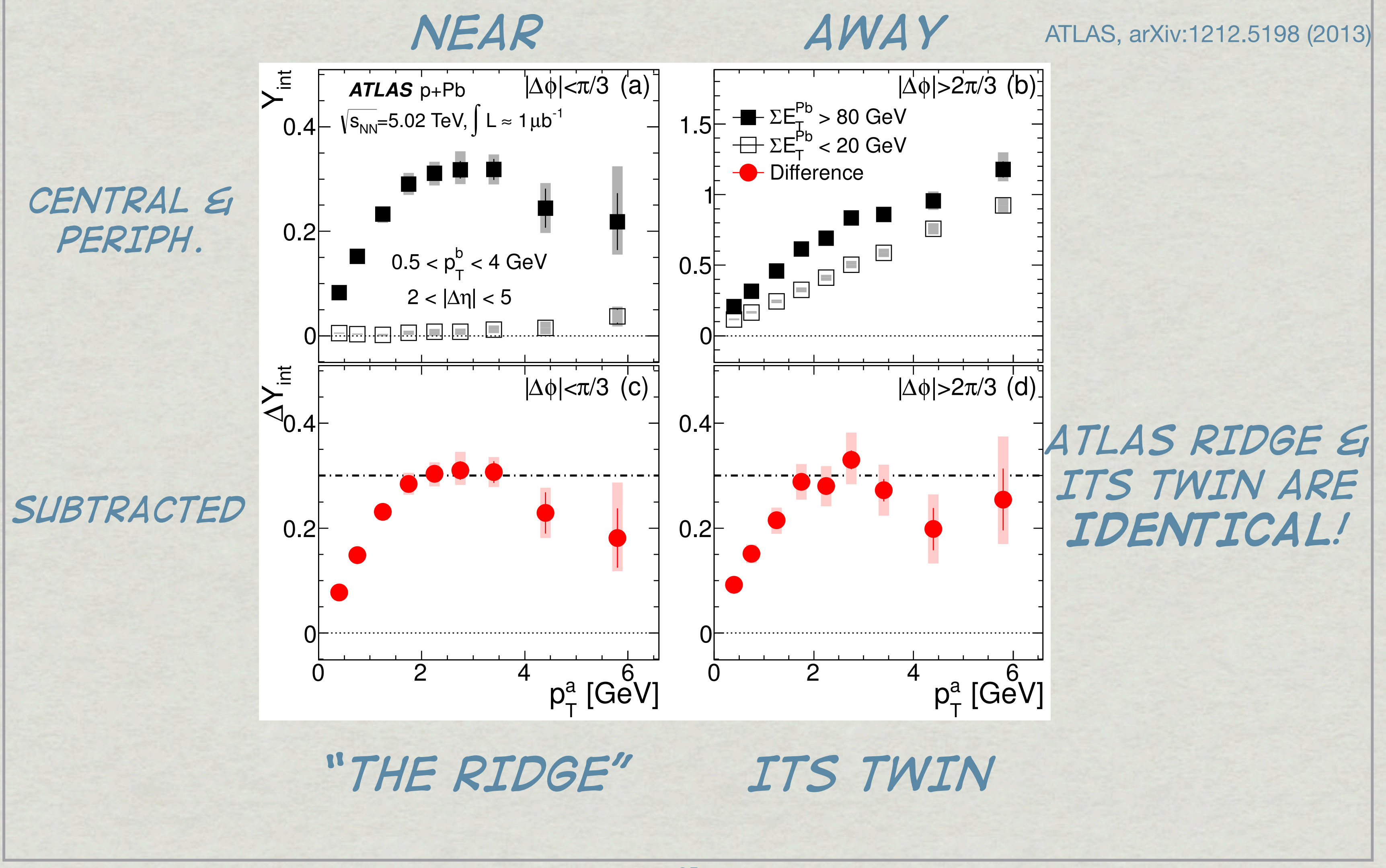




\section{FLOW IN P+PB? (ALICE)}
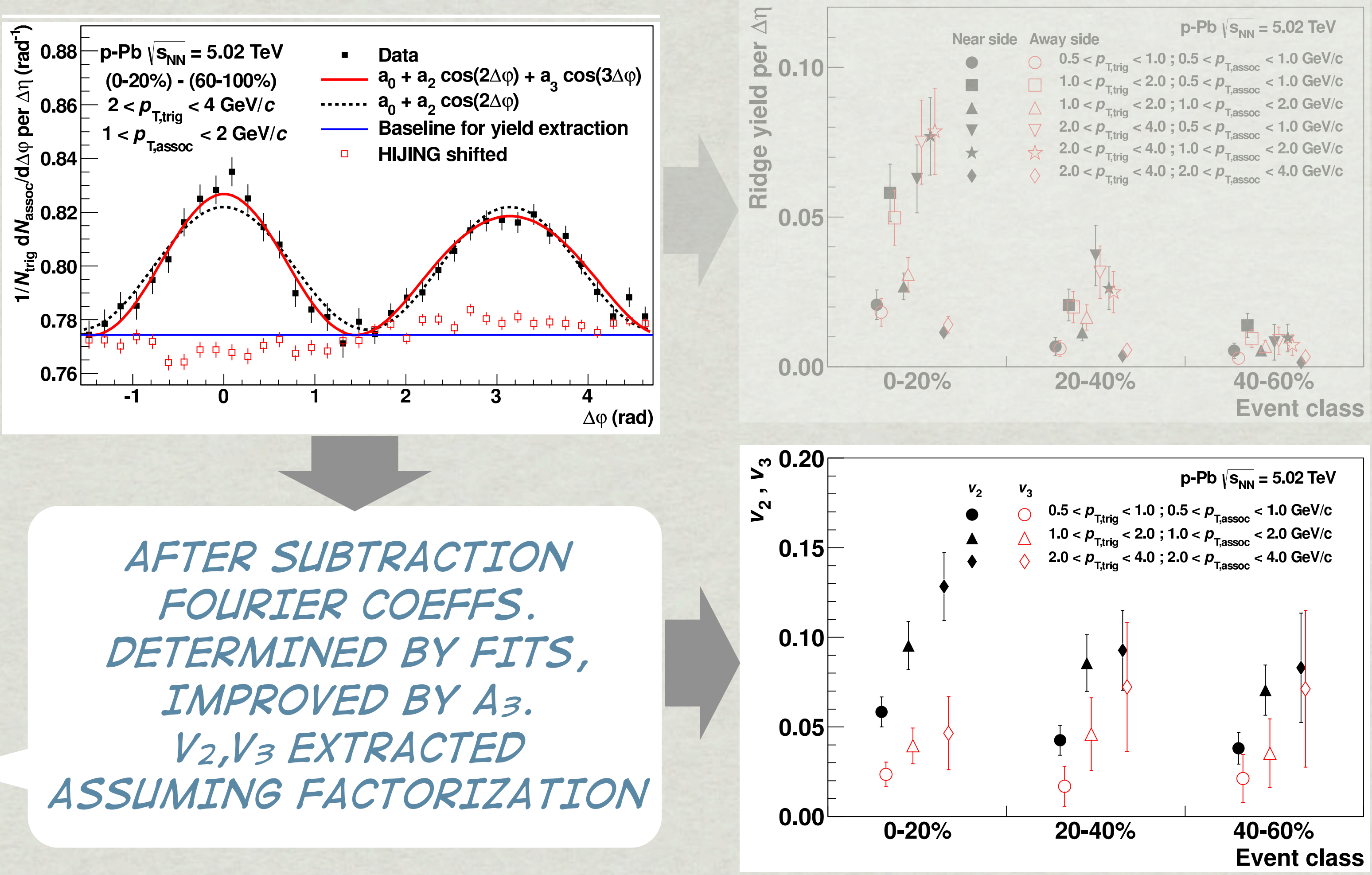

SIGNIFICANT $V_{2}$ AND $V_{3}$ CONTRIBUTIONS! 


\section{HOLY SNEAKY SINUSOIDS!}

FOR NEAR-INCLUSIVE ASSOCIATED PARTICLES (0.5-4 GEV), SINUSOID DOMINATES AFTER RECOIL SUBTRACTION FOR ALL PT INTERVALS

\section{SHOWN ARE:}

$a_{0}+2 a_{2} \cos 2 \Delta \phi$ (SOLID)

$a_{0}+2 a_{2} \cos 2 \Delta \phi+2 a_{3} \cos 3 \Delta \phi$ (DOTTED)

FOURIER COEFFS. CALCLILATED USING DFT:

$$
a_{n}=\langle\Delta Y(\Delta \phi) \cos n \Delta \phi\rangle
$$

\section{$P_{T}($ ASSOC $)=0.5-4$ GEV}

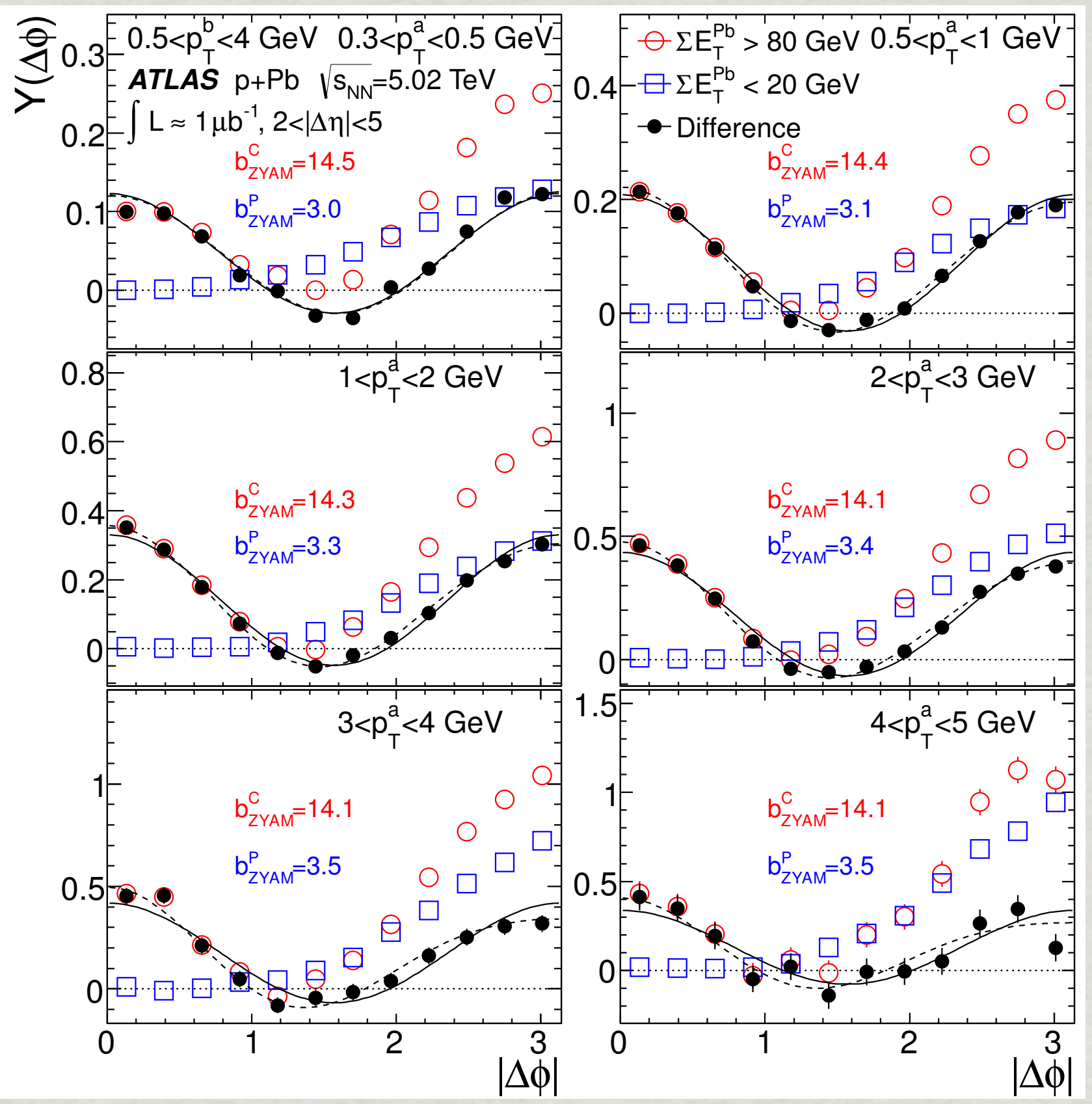

ATLAS, arXiv:1212.5198 (2013) 


\section{FLOW IN P+PB?}

(ATLAS

ATLAS, arXiv:1212.5198 (2013), accepted to PRL with new data!
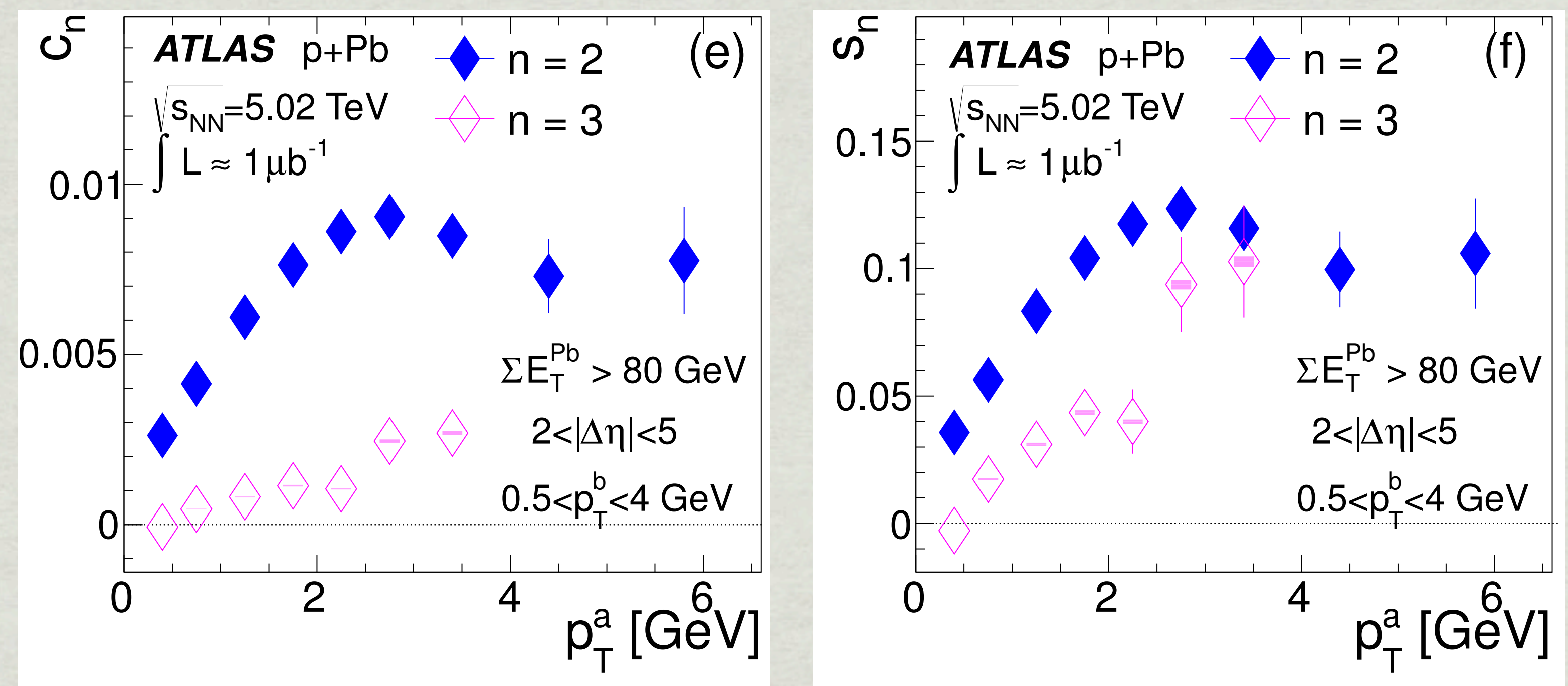

IF MODULATION OF 2PC FACTORIZES INTO CONVOLUTION OF SINGLE-PARTICLE SINUSOIDS, CAN EXPRESS MODULATION IN TERMS OF "SN" (SAME PROCEDURE USED IN HI TO EXTRACT $V N$ ): ...ATLAS EXTRACTS SIGNIFICANT $S_{2}$ AND $S_{3 !}$ 


\section{AT THE LHC, THE RIDGE IS NOT ALONE!}
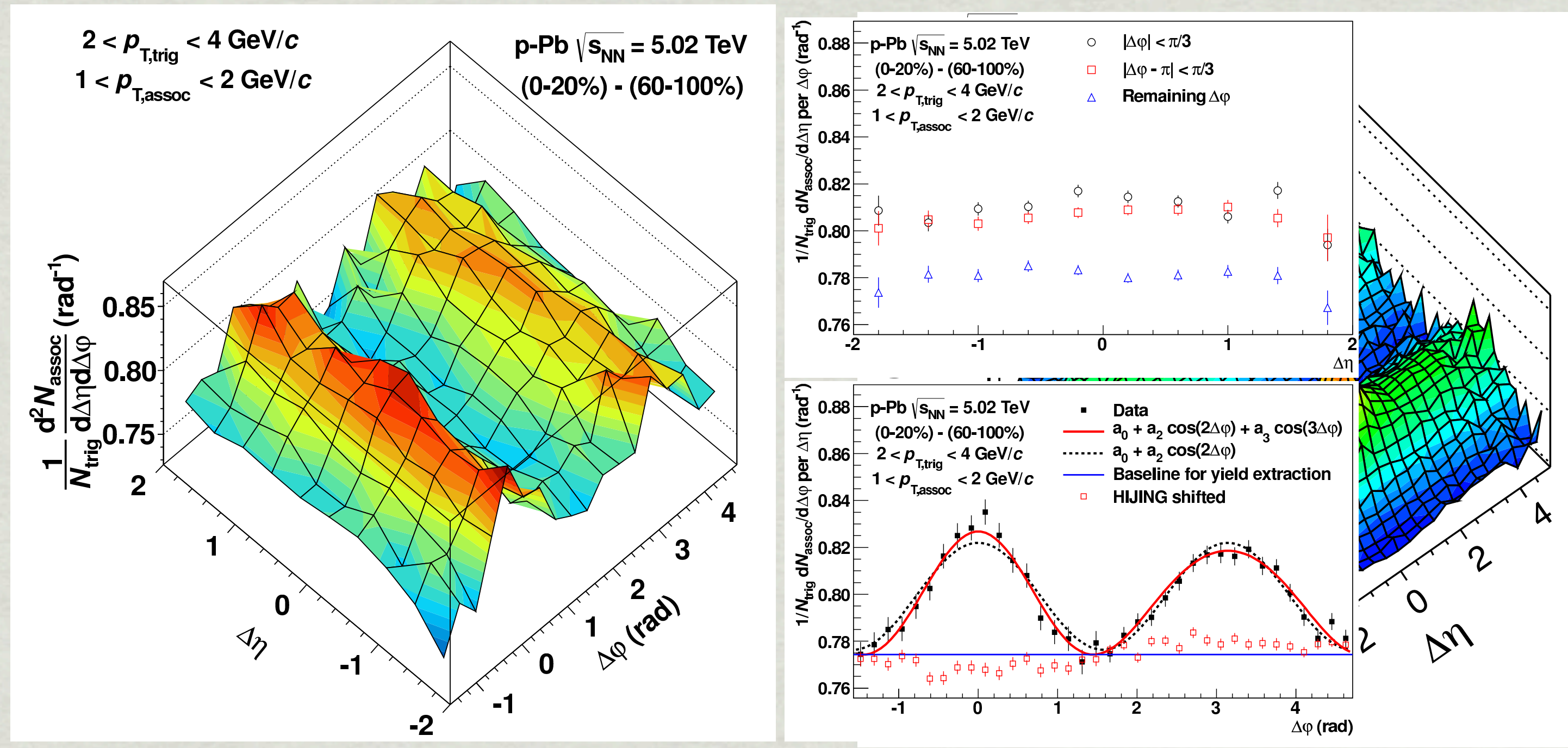
AND SOON AFTER, ACROSS THE ATLANTIC (E NEXT TO MY BNL OFFICE)... 
AND SOON AFTER, ACROSS THE ATLANTIC (E NEXT TO MY BNL OFFICE)...

HMM, IS

THIS IN $D+A L ?$ 
Quadrupole anisotropy in dihadron azimuthal correlations in central $d+\mathrm{Au}$ collisions at $\sqrt{s_{N N}}=200 \mathrm{GeV}$

A. Adare, ${ }^{13}$ C. Aidala, ${ }^{41,42}$ N.N. Ajitanand, ${ }^{58}$ Y. Akiba, ${ }^{54,}{ }^{55}$ H. Al-Bataineh, ${ }^{48}$ J. Alexander, ${ }^{58}$ A. Angerami, ${ }^{14}$ K. Aoki ${ }^{33,}{ }^{54}$ N. Apadula, ${ }^{59}$ Y. Aramaki, ${ }^{12,54}$ E.T. Atomssa,${ }^{34}$ R. Averbeck, ${ }^{59}$ T.C. Awes, ${ }^{50}$ B. Azmoun, ${ }^{7}$

V. Babintsev ${ }^{23}$ M. Bai ${ }^{6}$ G. Baksay, ${ }^{19}$ L. Baksay,${ }^{19}$ K.N. Barish ${ }^{8}$ B. Bassalleck, ${ }^{47}$ A.T. Basye, ${ }^{1}$ S. Bathe,${ }^{5,8,55}$ V. Baublis ${ }^{53}$ C. Baumann, ${ }^{43}$ A. Bazilevsky, ${ }^{7}$ S. Belikov,${ }^{7}$, R. Belmont,${ }^{63}$ R. Bennett,${ }^{59}$ J.H. Bhom, ${ }^{67}$ D.S. Blau, ${ }^{32}$ J.S. Bok, ${ }^{67}$ K. Boyle ${ }^{59}$ M.L. Brooks, ${ }^{37}$ H. Buesching, ${ }^{7}$ V. Bumazhnov, ${ }^{23}$ G. Bunce, ${ }^{7,}{ }^{55}$ S. Butsyk, ${ }^{37}$ S. Campbell, ${ }^{59}$ A. Caringi, ${ }^{44}$ C.-H. Chen, ${ }^{59}$ C.Y. Chi,${ }^{14}$ M. Chiu, ${ }^{7}$ I.J. Choi, ${ }^{67}$ J.B. Choi, ${ }^{10}$ R.K. Choudhury, ${ }^{4}$ P. Christiansen,${ }^{39}$ T. Chujo, ${ }^{62}$ P. Chung, ${ }^{58}$ O. Chvala,${ }^{8}$ V. Cianciolo, ${ }^{50}$ Z. Citron, ${ }^{59}$ B.A. Cole,${ }^{14}$ Z. Conesa del Valle, ${ }^{34}$ M. Connors,${ }^{59}$ M. Csanád, ${ }^{17}$ T. Csörgő, ${ }^{66}$ T. Dahms, ${ }^{59}$ S. Dairaku, ${ }^{33,}{ }^{54}$ I. Danchev, ${ }^{63}$ K. Das,${ }^{20}$ A. Datta, ${ }^{41}$ G. David, ${ }^{7}$ M.K. Dayananda, ${ }^{21}$ A. Denisov, ${ }^{23}$ A. Deshpande, ${ }^{55,}{ }^{59}$ E.J. Desmond,${ }^{7}$ K.V. Dharmawardane, ${ }^{48}$ O. Dietzsch, ${ }^{57}$ A. Dion, ${ }^{27}$ M. Donadelli, ${ }^{57}$ O. Drapier ${ }^{34}$ A. Drees, ${ }^{59}$ K.A. Drees,${ }^{6}$ J.M. Durham, ${ }^{59}$ A. Durum, ${ }^{23}$ D. Dutta, ${ }^{4}$ L. D'Orazio, ${ }^{40}$ S. Edwards ${ }^{20}$ Y.V. Efremenko, ${ }^{50}$ F. Ellinghaus, ${ }^{13}$ T. Engelmore, ${ }^{14}$ A. Enokizono, ${ }^{50}$ H. En'yo, ${ }^{54,55}$

S. Esumi ${ }^{62}$ B. Fadem, ${ }^{44}$ D.E. Fields,${ }^{47}$ M. Finger, ${ }^{9}$ M. Finger, Jr. ${ }^{9}$ F. Fleuret, ${ }^{34}$ S.L. Fokin, ${ }^{32}$ Z. Fraenkel, ${ }^{65,}$ * J.E. Frantz, ${ }^{49}, 59$ A. Franz,${ }^{7}$ A.D. Frawley ${ }^{20}$ K. Fujiwara, ${ }^{54}$ Y. Fukao, ${ }^{54}$ T. Fusayasu, ${ }^{46}$ I. Garishvili, ${ }^{60}$ A. Glenn, ${ }^{36}$ H. Gong, ${ }^{59}$ M. Gonin, ${ }^{34}$ Y. Goto, ${ }^{54,55}$ R. Granier de Cassagnac, ${ }^{34}$ N. Grau $,{ }^{2}, 14$ S.V. Greene, ${ }^{63}$ G. Grim, ${ }^{37}$ M. Grosse Perdekamp, ${ }^{24}$ T. Gunji, ${ }^{12}$ H.-A. Gustafsson, ${ }^{39,}{ }^{*}$ J.S. Haggerty ${ }^{7}$ K.I. Hahn, ${ }^{18}$ H. Hamagaki, ${ }^{12}$ J. Hamblen, ${ }^{60}$ R. Han, ${ }^{52}$ J. Hanks, ${ }^{14}$ E. Haslum, ${ }^{39}$ R. Hayano, ${ }^{12}$ X. He, ${ }^{21}$ M. Heffner,${ }^{36}$ T.K. Hemmick, ${ }^{59}$ T. Hester ${ }^{8}$ J.C. Hill, ${ }^{27}$ M. Hohlmann, ${ }^{19}$ W. Holzmann, ${ }^{14}$ K. Homma, ${ }^{22}$ B. Hong, ${ }^{31}$ T. Horaguchi, ${ }^{22}$ D. Hornback,${ }^{60}$ $\bullet \bullet$

\section{PROJECT}

\section{QUADRUPOLES IN D+AL PHENIX COLLABORATION}




\section{PHENIX D+AU}

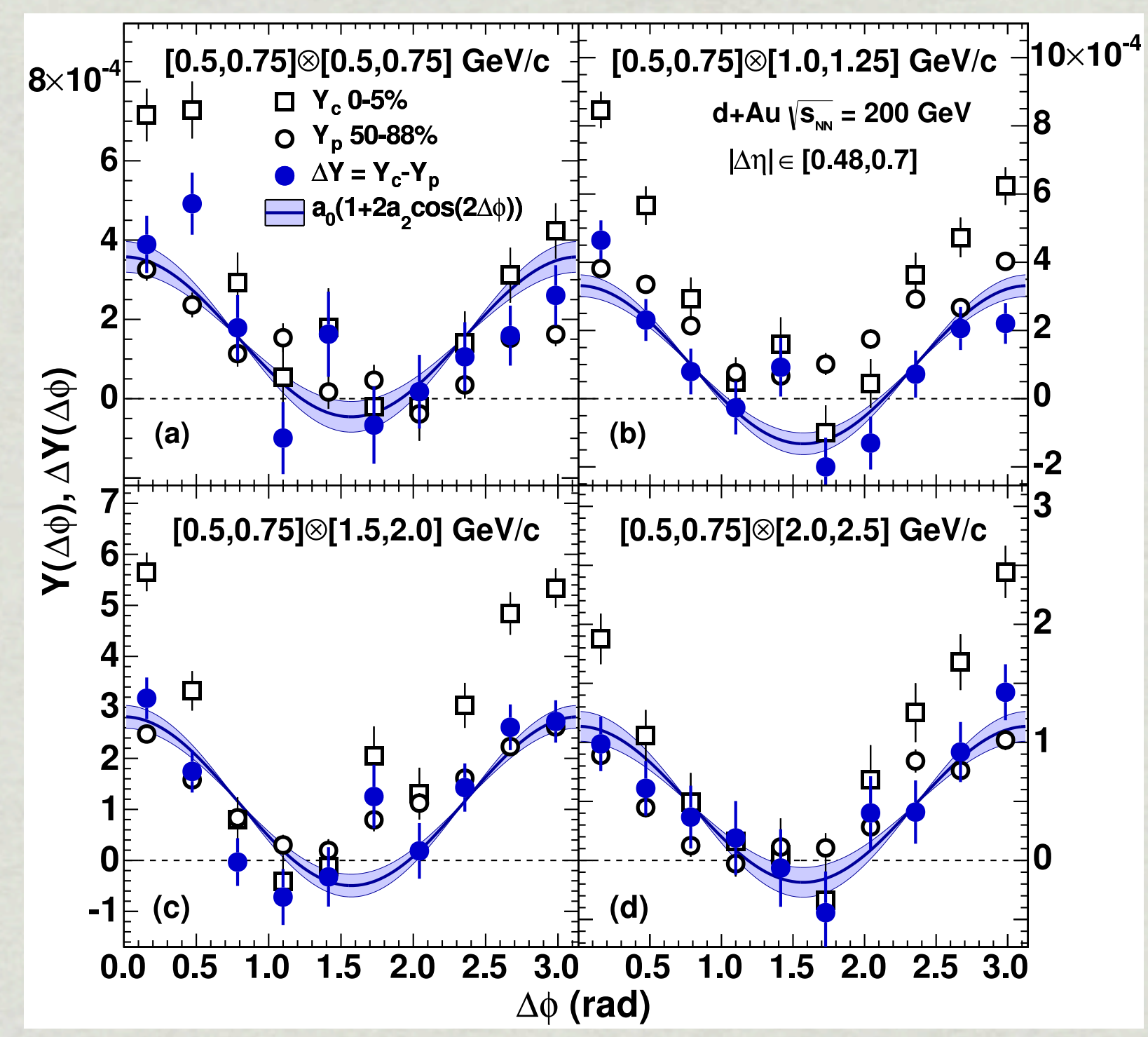

DESPITE SMALL ETA COVERAGE OF PHENIX, MADE GOOD LSE OF THEIR 1.6 BILLION EVENTS: CORRELATIONS WITH SOFT PARTICLES (0.5-0.75 GEV) ALSO EVINCE QUADRUPOLE MODULATION 


\section{MODLLATIONS@RHIC}

PHENIX, arXiv:1303.1794 (2013)

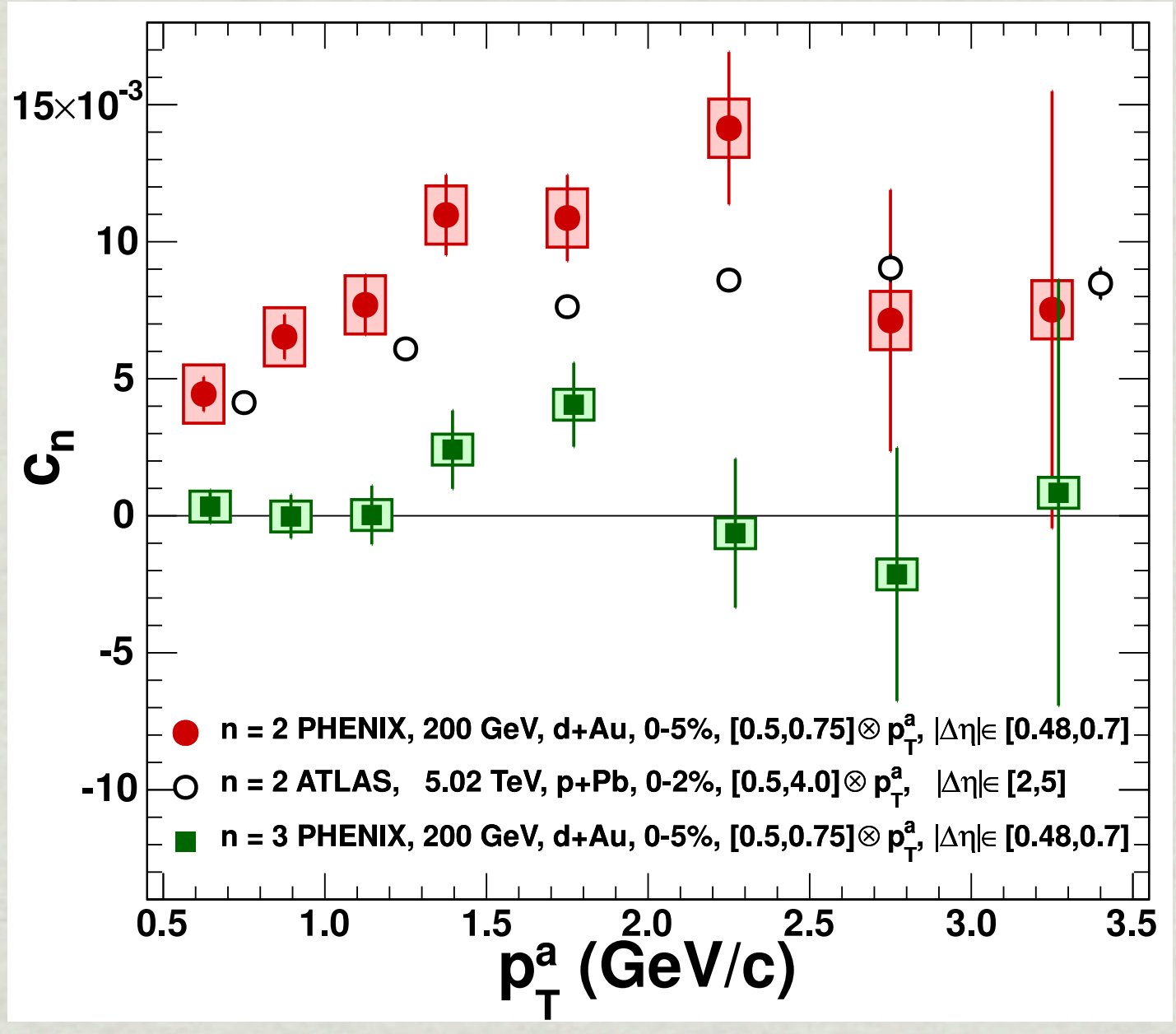

2PC MODULATIONS ARE OF SIMILAR ORDER TO THOSE SEEN QLHC

(QUANTITATIVE COMPARISON IS NOT POSSIBLE GIVEN DIFFERENT PT AND $\triangle E T A$ CLTSS)

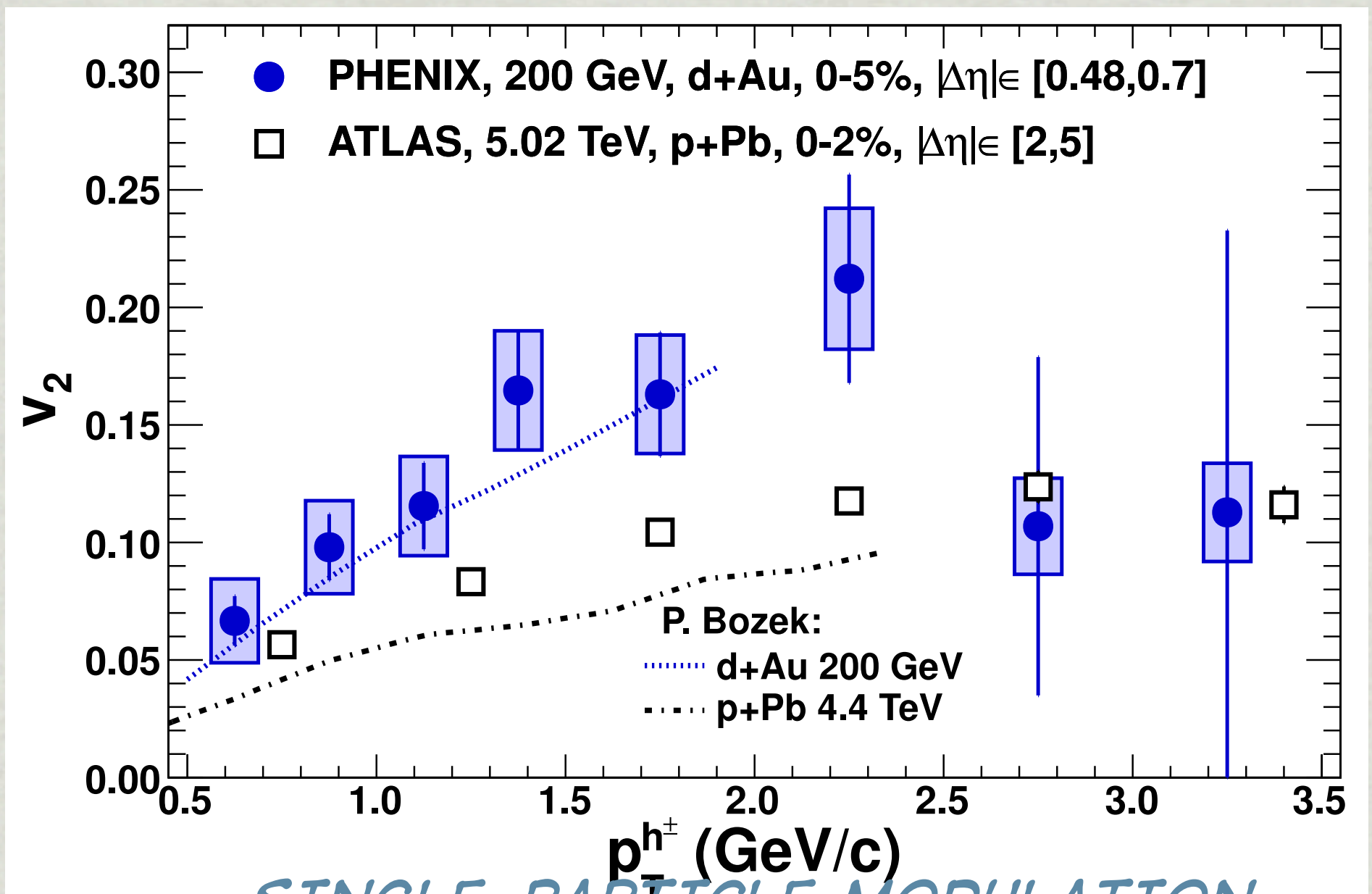

SINGLE-PART ICLE MODLLATION CAN BE DIRECTLY COMPARED, AND D+AL@RHIC IS FOUND TO HAVE LARGER V 2 THAN P+PB@LHC!...

REASONABLE AGREEMENT W/ HYDRO PREDICTIONS (N.B. LHC PREDICTIONS AT WRONG ENERGY!) 


\section{HYDRO SCALING OF P/D+A?}

PHENIX, arXiv:1303.1794 (2013)

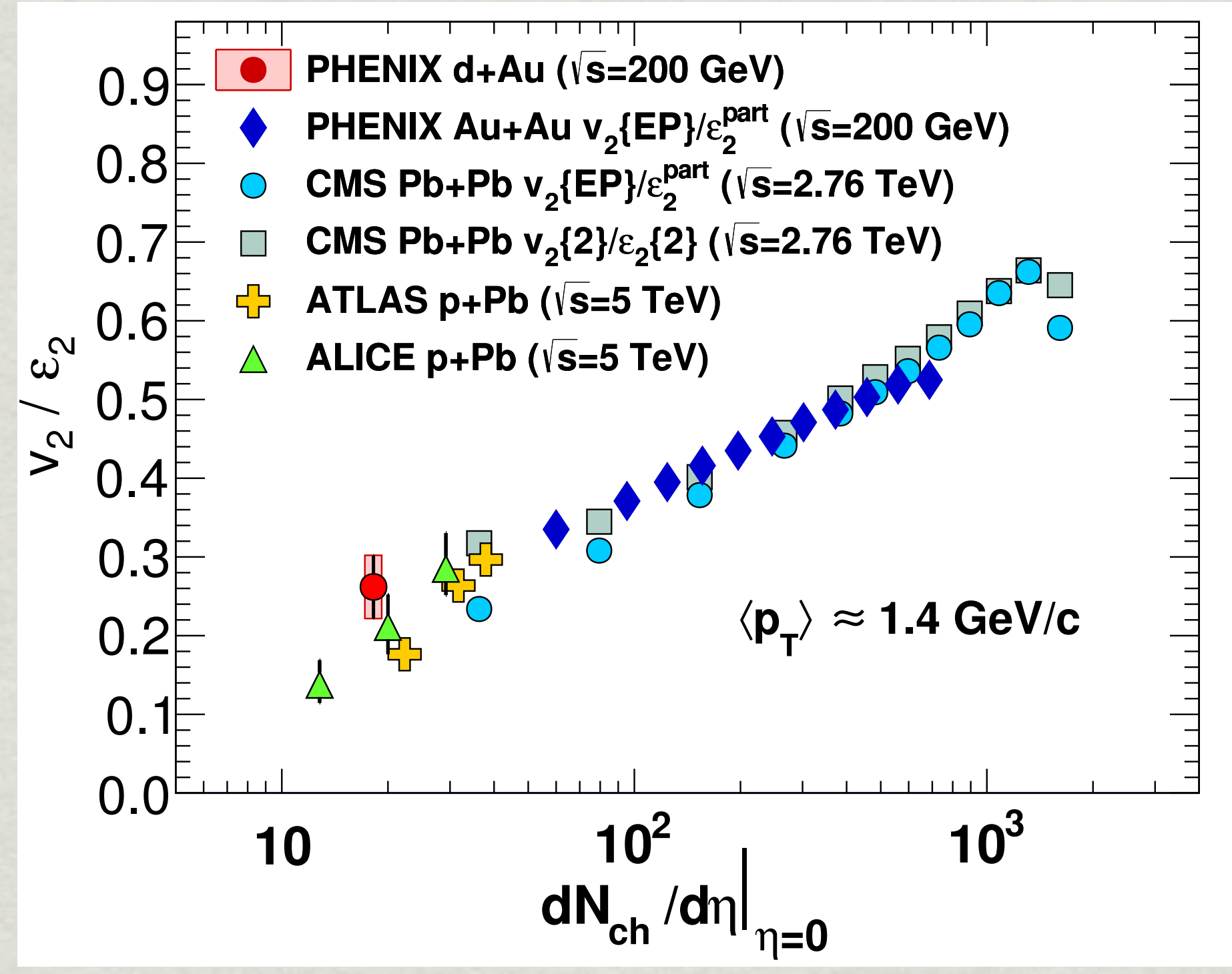

SIMILAR, BUT NOT IDENT ICAL TO OLDER VERSION (LEAVES OUT TRANSVERSE AREA).

DESPITE USE OF HIJING MULTIPLICITIES AND ECCENTRICITIES NOT PROVIDED BY THE EXPERIMENTS, DO P/D+A DATA SCALE TO THE O(50\%) LEVEL? 


\section{BLTT WAIT THERE'S MORE!}

FOR NO ADDITIONAL RLNNING, WE CAN ADD W TWO MORE PARTICLES! 


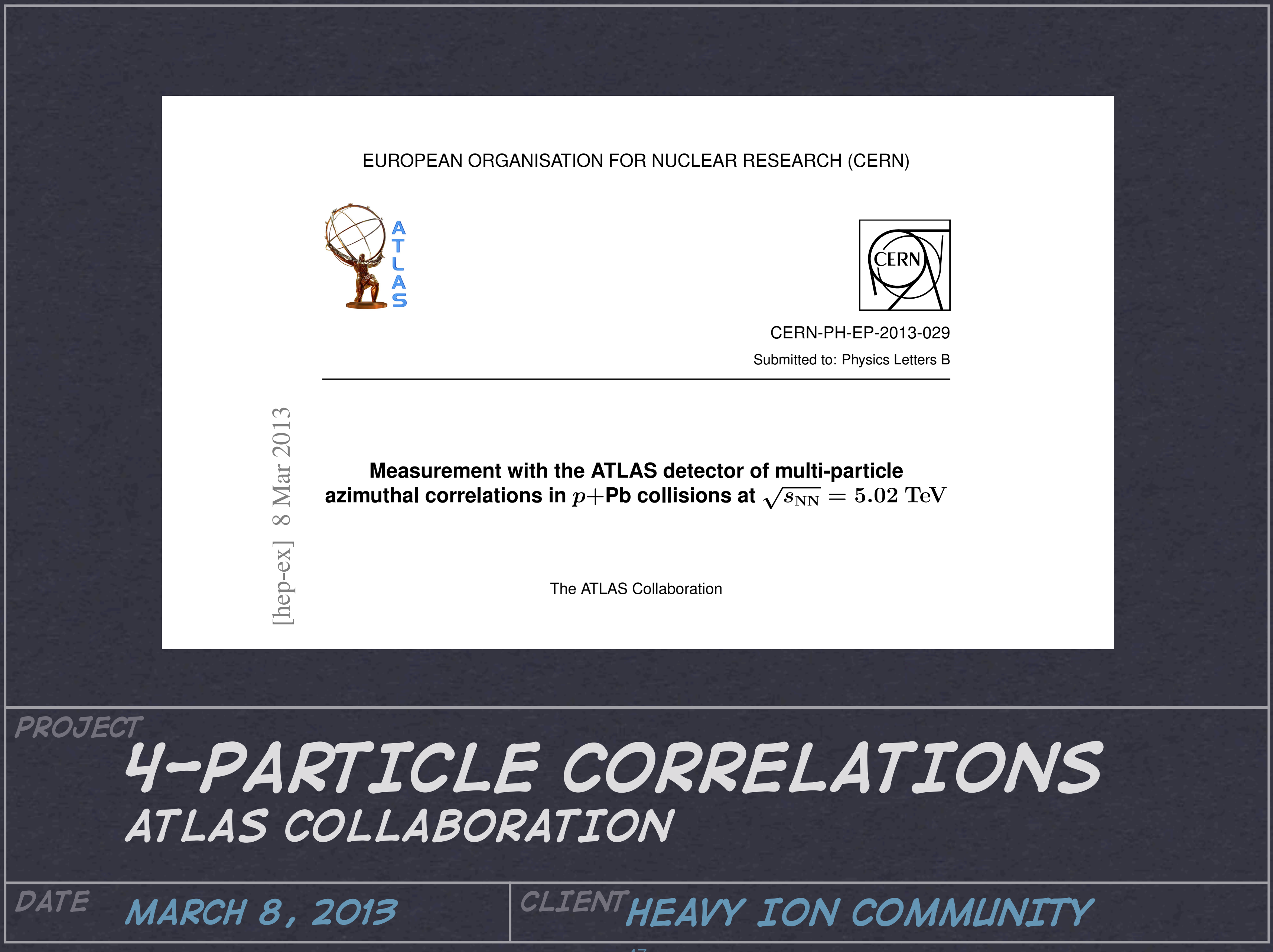




\section{CLMLILANTS IN P+PB}

IF THE EFFECT IS DUE TO FINAL-STATE DYNAMICS, SHOLLD OBSERVE TRLE MULTIPART ICLE CORRELATIONS: CUMULANTS ARE THE NATURAL WAY TO CHECK
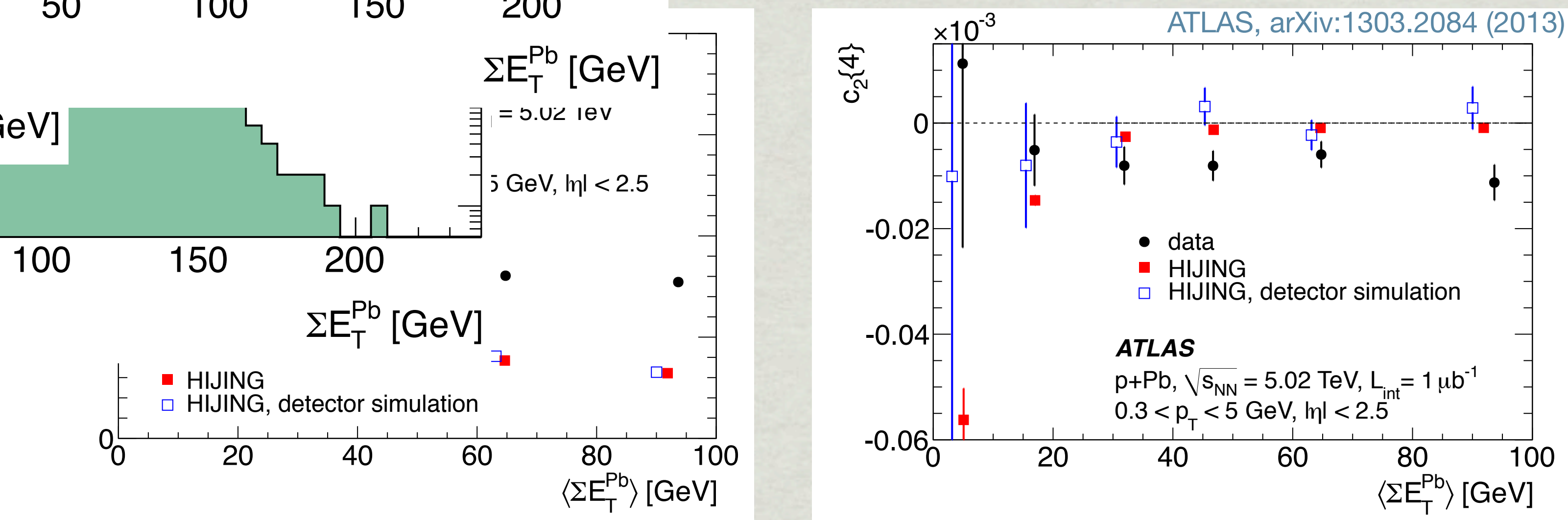

$$
\begin{aligned}
& \operatorname{corr}_{n}\{2\}=\left\langle\mathrm{e}^{\mathrm{i} n\left(\phi_{1}-\phi_{2}\right)}\right\rangle \\
& c_{n}\{2\}=\left\langle\operatorname{corr}_{n}\{2\}\right\rangle \\
& \operatorname{corr}_{n}\{4\}=\left\langle\mathrm{e}^{\mathrm{i} n\left(\phi_{1}+\phi_{2}-\phi_{3}-\phi_{4}\right)}\right\rangle \\
& c_{n}\{4\}=\left\langle\operatorname{corr}_{n}\{4\}\right\rangle-2 \cdot\left\langle\operatorname{corr}_{n}\{2\}\right\rangle^{2} \\
& v_{2}^{\text {ref }}\{2\}=\sqrt{c_{2}\{2\}} \\
& v_{2}^{\mathrm{ref}}\{4\}=\sqrt[4]{-c_{2}\{4\}} \\
& 4 \text { PARTICLE CLMULANT IS NEGATIVE }
\end{aligned}
$$

(BUT SO IS PERIPHERAL HIJING, UNLESS WE USE $|E T A|<1)$ 


\section{$P_{T}$ DEPENDENCE}

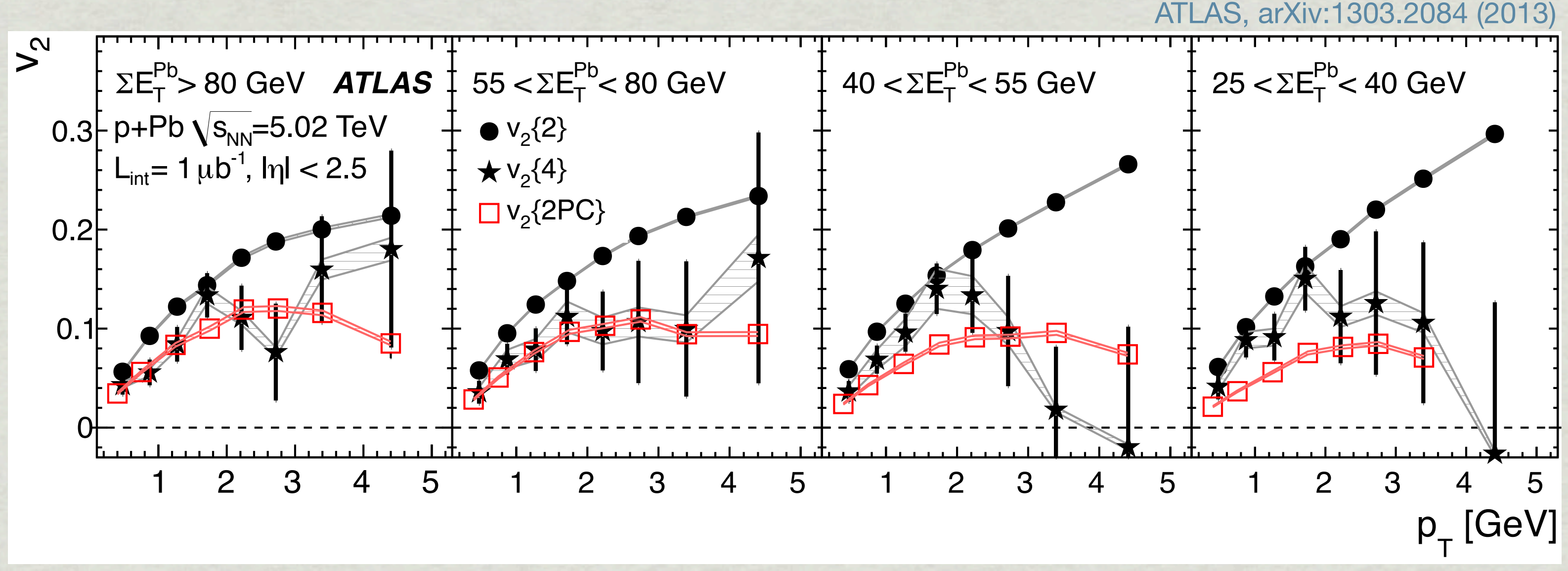

PT DEPENDENCE FROM CLMLILANTS SHOWS:

1) CLEAR DIJET CONTAMINATION IN 2-PARTICLE

2) SIGNIFICANT $4-P, A N D G O O D$ AGREEMENT OF 4-P WITH 2PC IN CENTRAL EVENTS

3) SIMILAR PT SHAPE FOR 4-P AS FOLND IN PB+PB (EP METHOD)

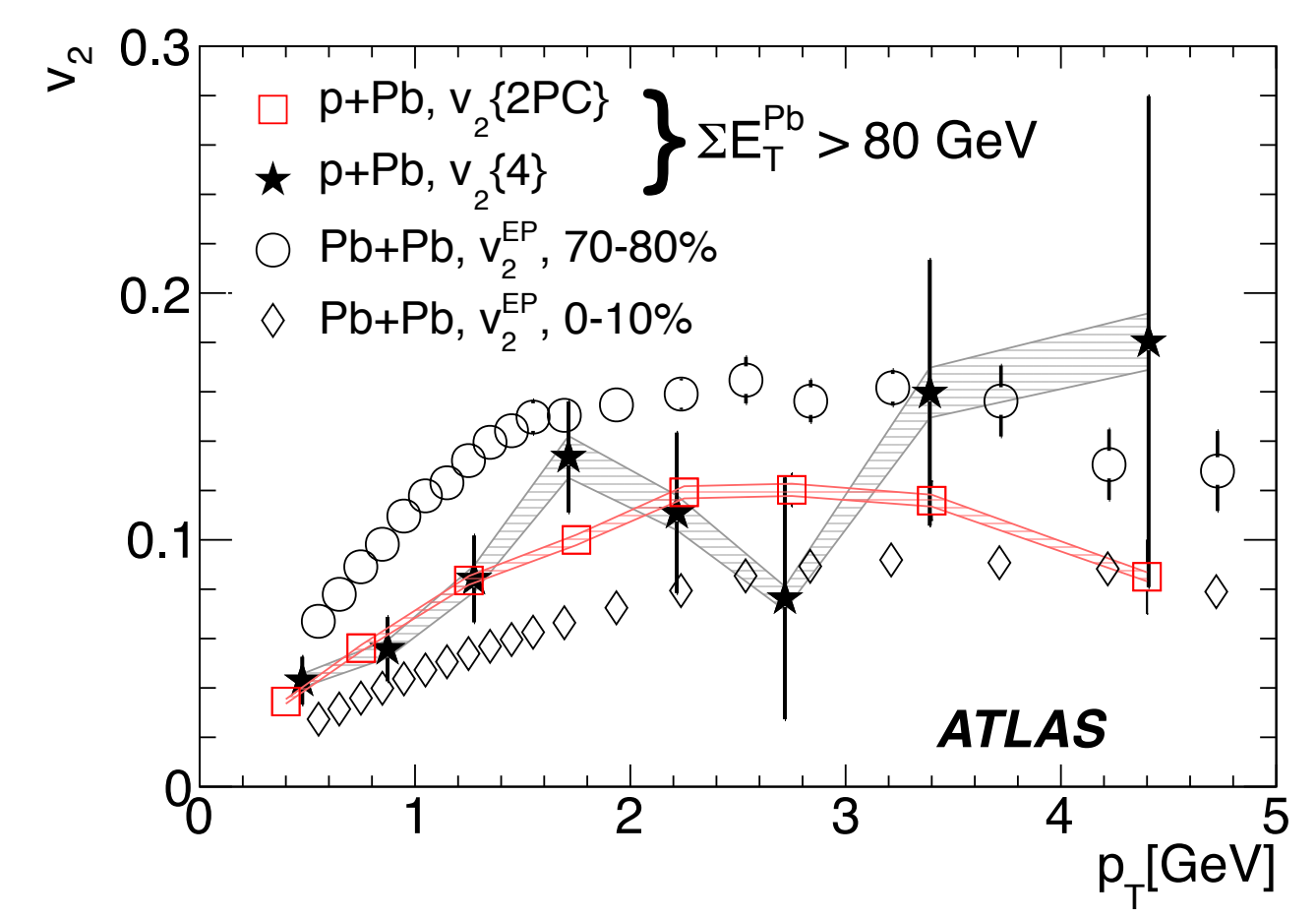




\section{INTEGRATED V2}

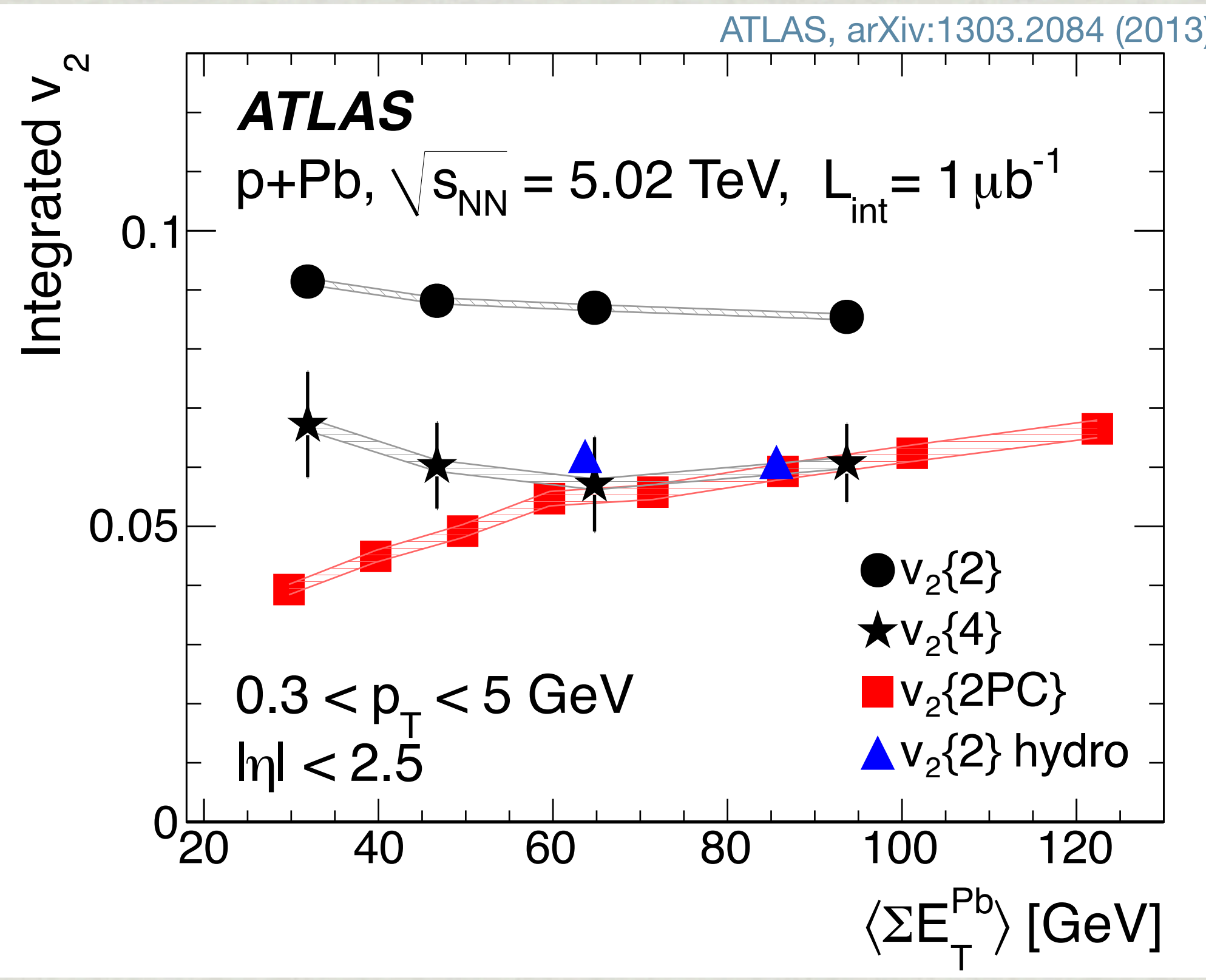

BOZEK ET AL, 5.02 TEV PRIVATE COMM.

AGREEMENT WITH HYDRO IN MORE CENTRAL EVENTS:

SLPPORT FOR FINAL STATE INTERACTIONS?

SOME ISSUES: 1) HYDRO V2 DECREASES WITH CENTRALITY

(DATA INCREASES) 2) SENSITIVITY OF CLMLLANTS TO FLUCTUATIONS IN SMALL SYSTEMS 


\section{THE STORY SO FAR}

* RIDGE DISCOVERED IN AL+AL @ RHIC

* "EXPLAINED" BY TRIANGLILAR FLOW, I.E. FLLICTLIATIONS IN THE INITIAL STATE

* STRONG SUPPORT FROM LHC PB+PB

* RIDGE REDISCOVERED IN PP

* LET A MILLION EXPLANATIONS BLOOM, FROM CGC TO HYDRO

* NEAR-SIDE RIDGE DISCOVERED IN P+PB

* IDENTICAL AWAY-SIDE RIDGE - > ONE PHENOMENON!

* CGC INTERFERENCE GRAPHS?

* HYDRODYNAMIC RESPONSE TO FLLCTLIATIONS?

* D+AU DATA SHOW THE "DOUBLE RIDGE", I.E. QLIADRLIPOLE MODLLATIONS

* HYDRO PREDICTIONS, FLOW SCALING

* 4-PARTICLE CLIMLILANTS TILT TOWARDS HYDRO INTERPRETATION OF THE DATA...FOR NOW 


\section{STAY TUNED, FOR THE NEXT EPISODE!}




\section{IMPORTANT QLIESTIONS!}

* P+PB WAS SLIPPOSED TO BE ABOUT INITIAL STATE (I.E. CGC) BUT WE HAVE A SLRPRISING HINT OF FINAL STATE DYNAMICS (I.E. FLOW)

* CGC EI FLOW BOTH CLAIM TO HAVE DESCRIPTIVE AND PREDICTIVE POWER

* LIMITS OF HYDRODYNAMICS

* CAN THERMALIZATION BE ACHIEVED FOR SUCH SMALL SPACE/TIME SCALES?

* aRE VISCOLIS CORRECTIONS TOO LARGE?

* WHAT ABOLT CLMLLANTS?

* SCOPEIPREDICTIVE POWER OF CGC APPROACH

* IF MODEL IS COMPLETE, THEN PREDICTIONS ARE ESSENTIAL

* WHAT ABOLIT MLILTIPARTICLE EFFECTS? V3?

* WHAT ABOUT PP?

* IN LIGHT OF P+PB, P+P RIDGE SLIGGESTS THAT WE SHOLILD BE THINKING MORE CAREFLLLY ABOLIT PP INITIAL STATE

* HOW TO HANDLE GEOMETRY /FLLCTLATIONS IN PP?

* EXPLANATION FOR LONG RANGE CORRELATIONS

* CGC (FLUXX TLBES) VS. HYDRO (BLILT IN, BLT 3+1D??) 


\section{AVENLIES FOR PROGRESS}

* EVEN MORE MLILTIPARTICLE OBSERVABLES $V_{2}(G)$, AND HIGHER ORDER MODLLATION

* EVEN LONGER RANGE CORRELATIONS

* PREDICTIONS FOR ETA DEPENDENCE FROM CGC OR HYDRO?

* PARTICLE SPECIES DEPENDENCE (CQ SCALING?)

* A COMPREHENSIVE DESCRIPTION, FROM SMALL TO LARGE SYSTEMS, ESPECIALLY WHERE THEY OVERLAP IN SIZE/DENSITY

* PREDICTIONS ARE CRLCIAL, SINCE VERY DIFFERENT APPROACHES ARE ABLE TO DESCRIBE THE SAME DATA! 
A PARTING (PERSONAL) QLESTION: WOULD WE HAVE IMAGINED DISCUSSING 4-PART ICLE CLMULANTS IN P+PB $Q$ LHC?

I DIDNT, BLT IT'S A VERY EXCITING MOMENT!

$$
\begin{aligned}
& \text { "THE BEST LAID SCHEMES } \\
& \text { OF MICE AND MEN GANG } \\
& \text { AFT AGLEY..." }
\end{aligned}
$$

("THE BEST LAID PLANS OF MICE AND MEN OFTEN GO ASTRAY")

IT SEEMS THE SAME IS TRLE FOR MICE (PROTONS), MAMMOTHS (IONS), AND THE RIDGE! 


\section{THANKS!!}

(SPECIAL THANKS TO J. JIA FOR DISCLSSIONS) 


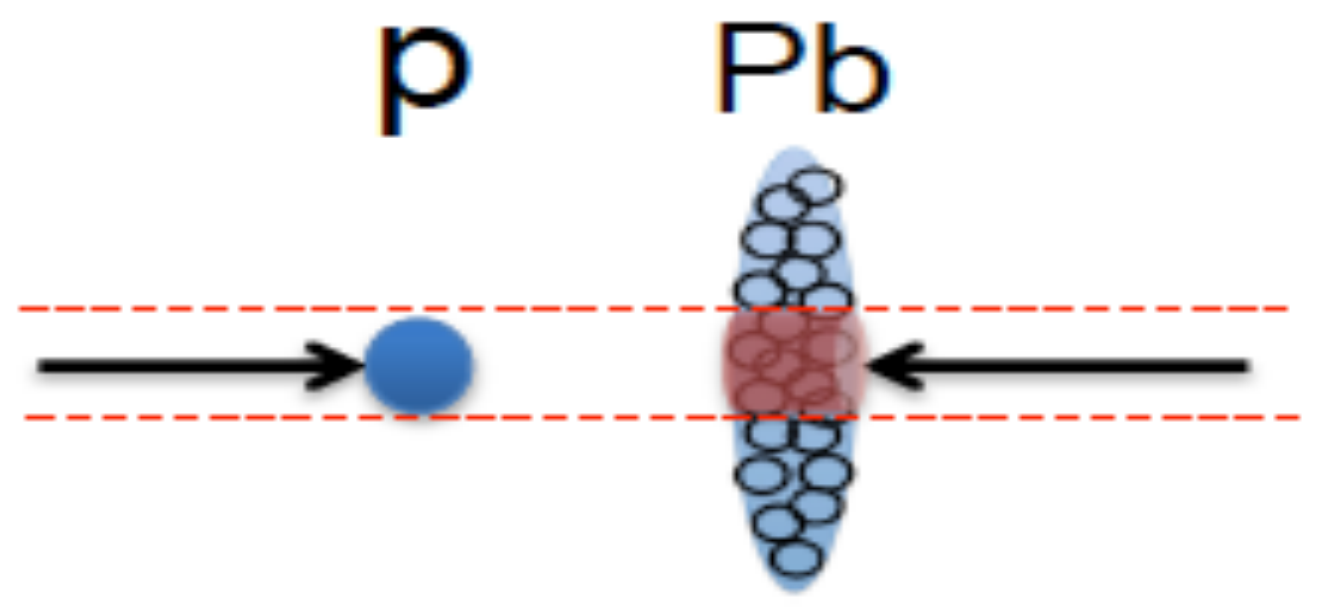

\title{
Large ridges in small systems: gluon entanglement in the Glasma
}

\author{
Raju Venugopalan \\ Brookhaven National Laboratory
}




\section{Talk outline}

$\diamond$ Multi-particle dynamics in dense-dense systems: an ab initio CGC EFT approach

$\diamond$ The ridge in $p+p$ collisions: Glasma + BFKL - QCD contributions

$\diamond$ The ridge in $A+A$ collisions: the dominance of flow generated from the Glasma pedestal (IP-Glasma model)

$\diamond$ The ridge in $p / d+A$ : like $p+p$ or $A+A$ ?

Relative role of Glasma + flow contributions 


\section{High multiplicity events:}

\section{"dense-dense" hadron-hadron collisions}

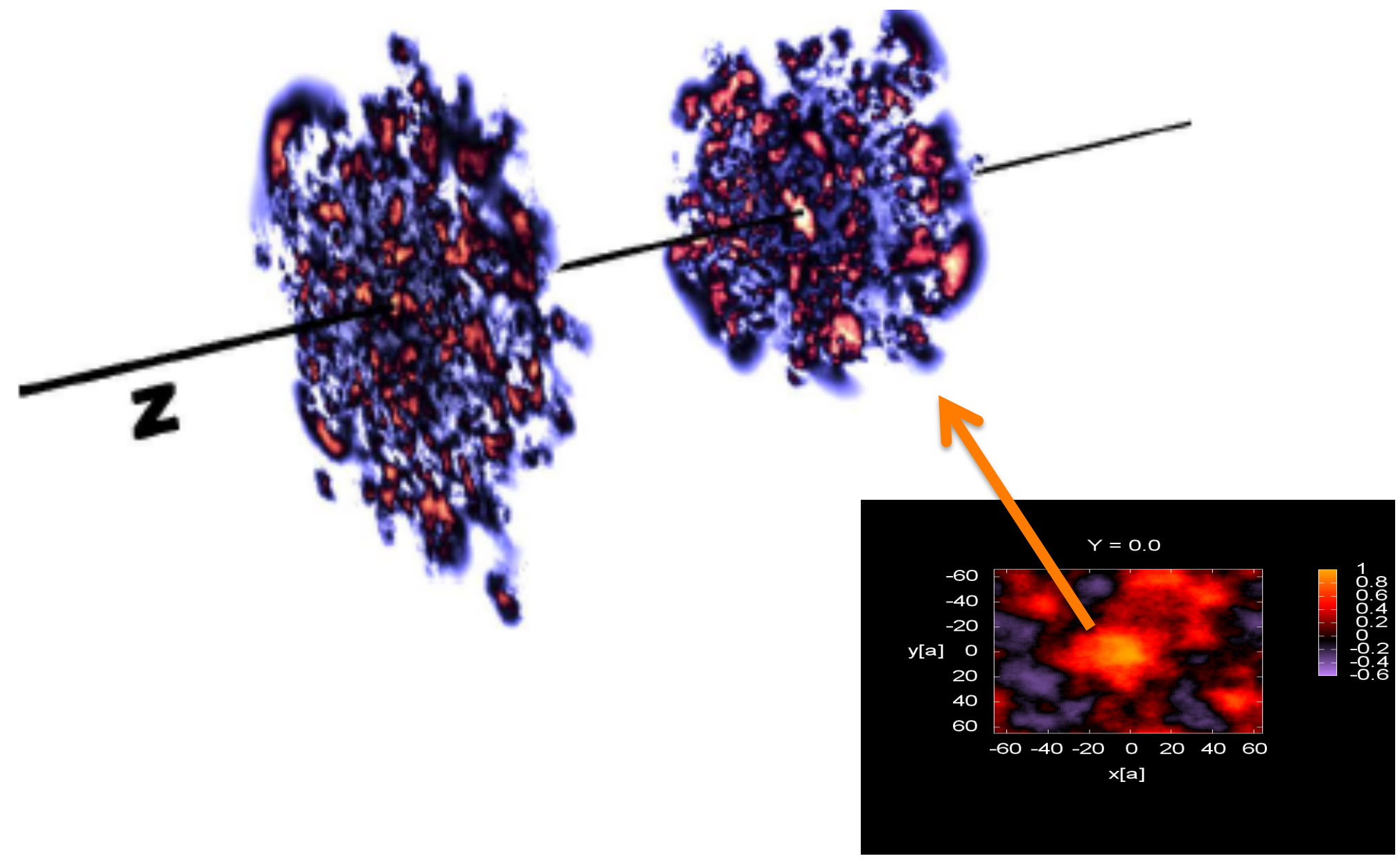

Incoming nuclei are Color Glass Condensates: Highly occupied gluon states with maximal occupancy allowed in QCD 


\section{High parton densities: multi-particle production}

Counting powers of "effective" color charge density $\boldsymbol{\rho}=\mathbf{g} \mathbf{n}_{\text {occ }}$

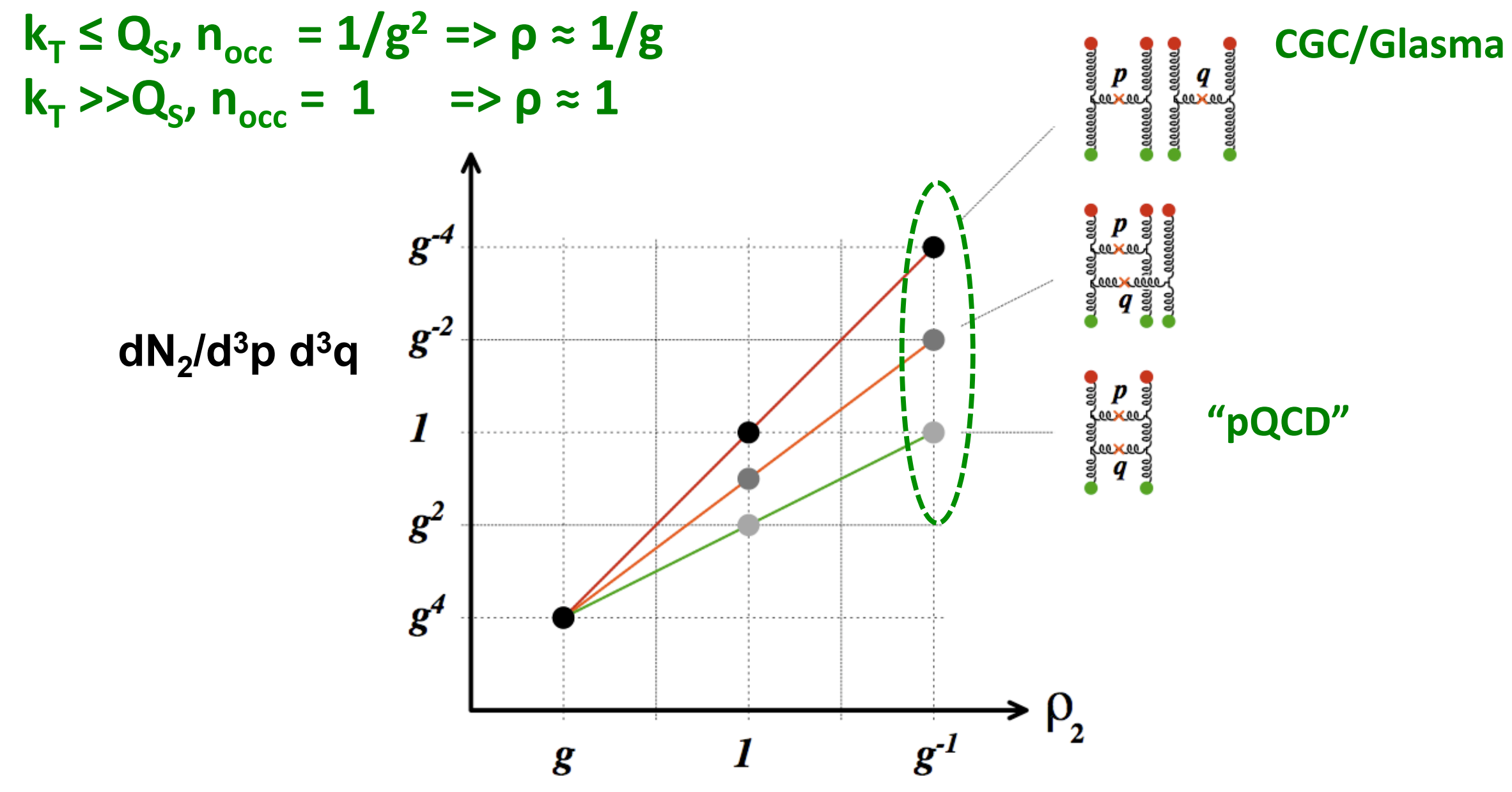

"Dense-dense" limit relevant for high multiplicity events: $\rho_{1}, \rho_{2} \sim 1 / g$ 


\section{High multiplicity events: single inclusive production}

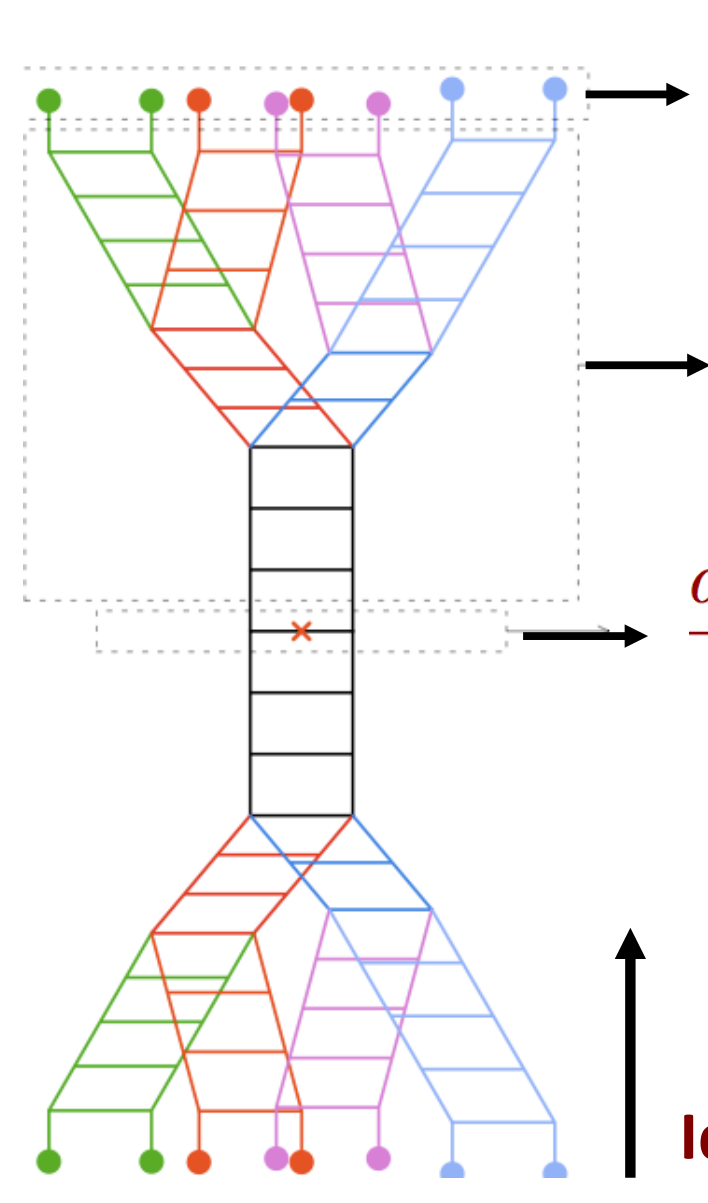

Full JIMWLK+YM evolution feasible Lappi, PLB 703 (2011)209

$\checkmark$ In practice: approximations of varying rigor

Identical evol. for nucleus 2

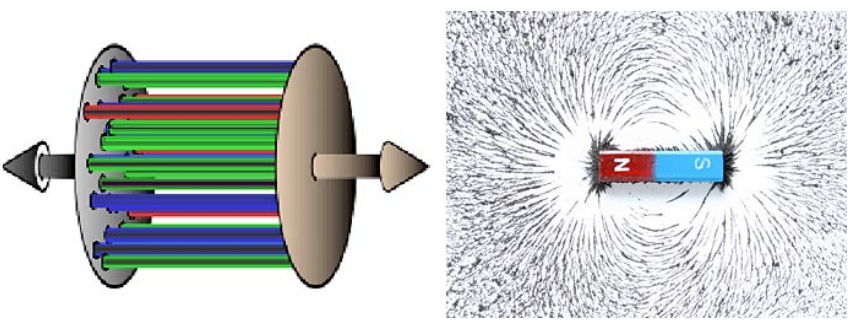

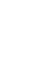

Gelis,Lappi,RV

arXiv:0804.2630 [hep-ph]; arXiv:0807.1306 [hep-ph] arXiv:0810.4829 [hep-ph]
Computed from Yang-Mills Glasma gluon fields

Krasnitz, Nara, RV; Lappi 


\section{High multiplicity events: two particle correlations}
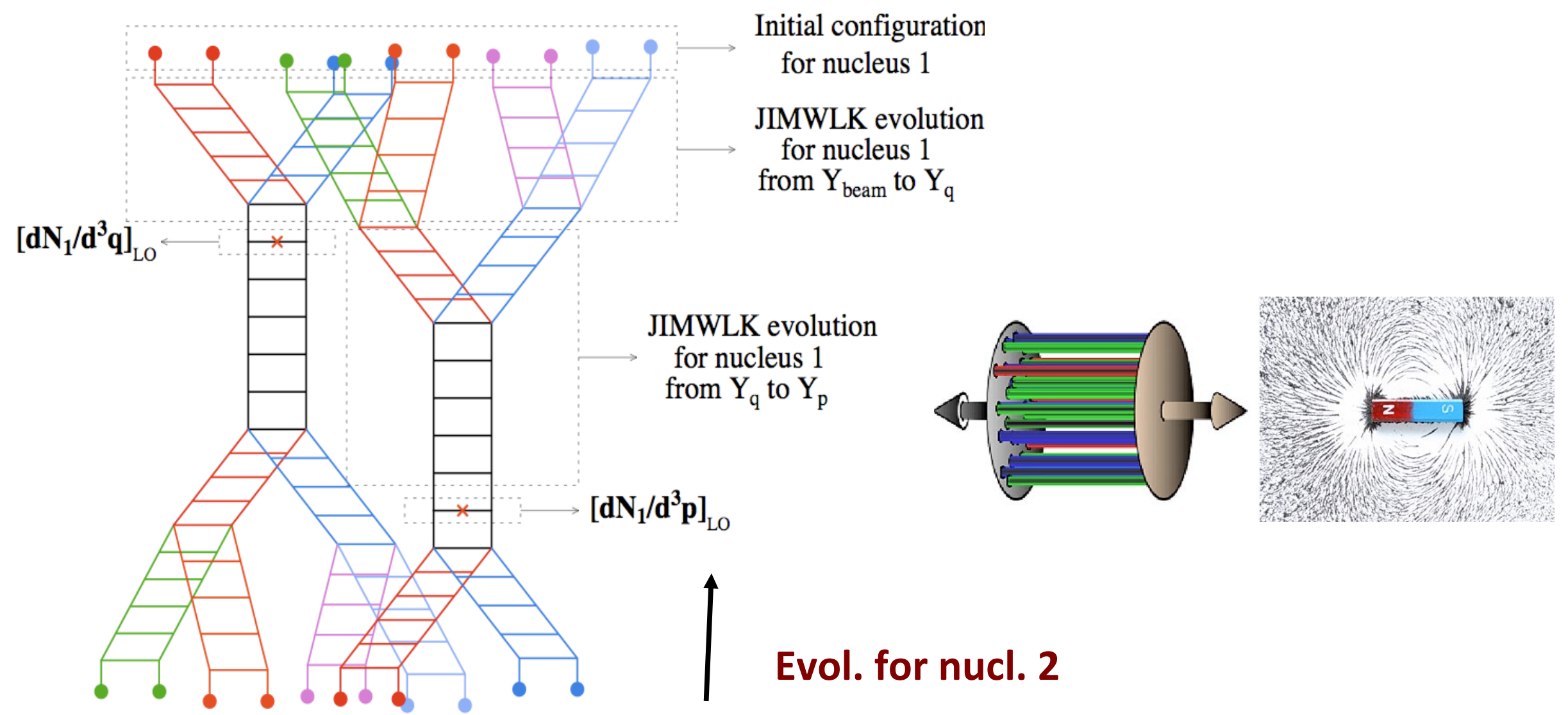

Full YM+JIMWLK evolution - not available yet Lappi,Schenke,RV in progress

Approximations: BK Gaussian truncation approximation for $k_{T} \geq Q_{S} ; Y M$ results for MV model available for all $k_{T}$

Dusling,Gelis,Lappi,RV:0911.2720; Lappi,Srednyak,RV:0911.2068; Kovchegov,Wertepny: 1212.1195 


\section{The saturated hadron: Glasma graphs -I}

RG evolution:

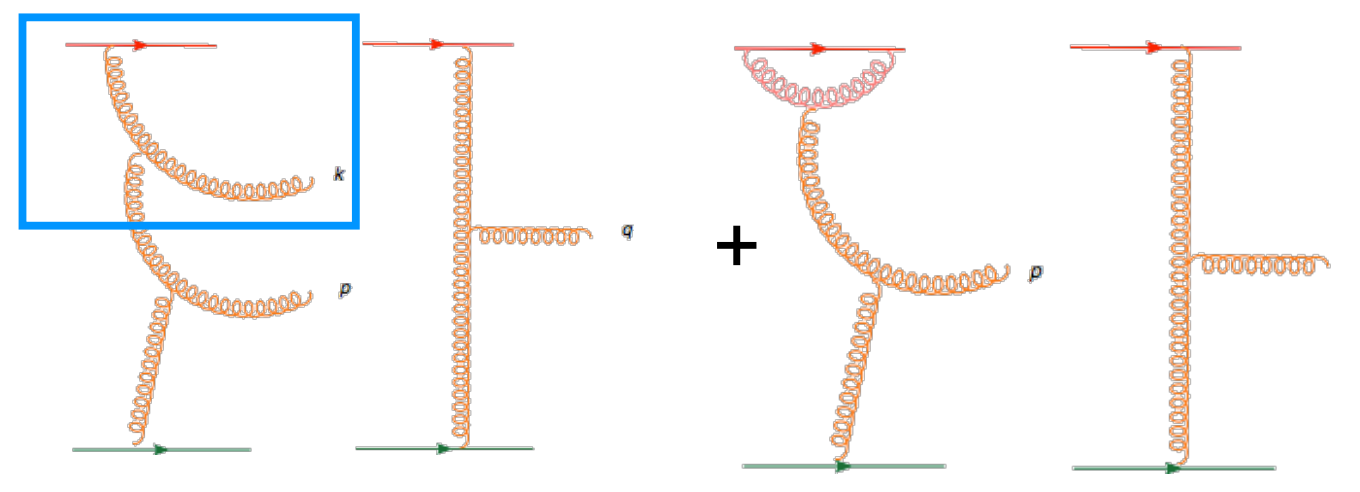

$+\ldots$ Keeping leading logs to all orders (NLO+NNLO+...)

$=$ LO graph with evolved sources

avg. over sources in each event and over all events gives correlation

$$
\left\langle\frac{d N_{2}}{d^{3} p d^{3} q}\right\rangle_{\mathrm{LLogs}}=\left.\left.\int\left[d \rho_{1}\right]\left[d \rho_{2}\right] W_{Y_{1}}\left[\rho_{1}\right] W_{Y_{2}}\left[\rho_{2}\right] \frac{d N}{d^{3} p}\right|_{\mathrm{LO}} \frac{d N}{d^{3} q}\right|_{\mathrm{LO}}
$$

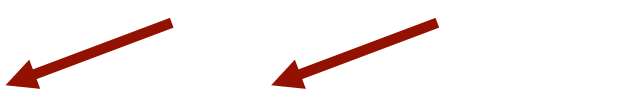

From solns. of Yang-Mills eqns. with two light cone sources Includes all mult. scat. contributions $\left(g \rho_{1}\right)^{n}$ and $\left(g \rho_{2}\right)^{n}$

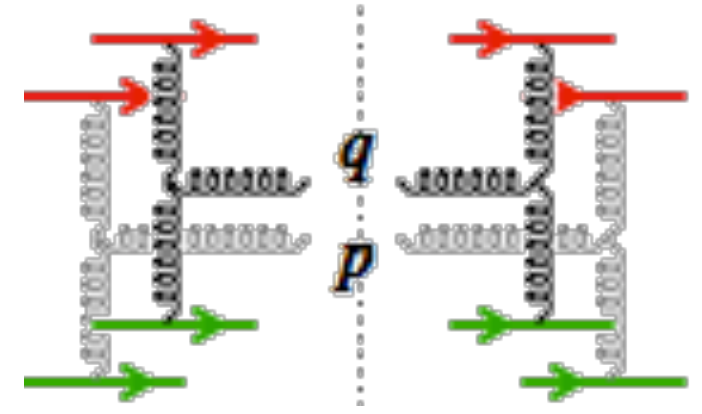




\section{The saturated hadron: Glasma graphs -II}
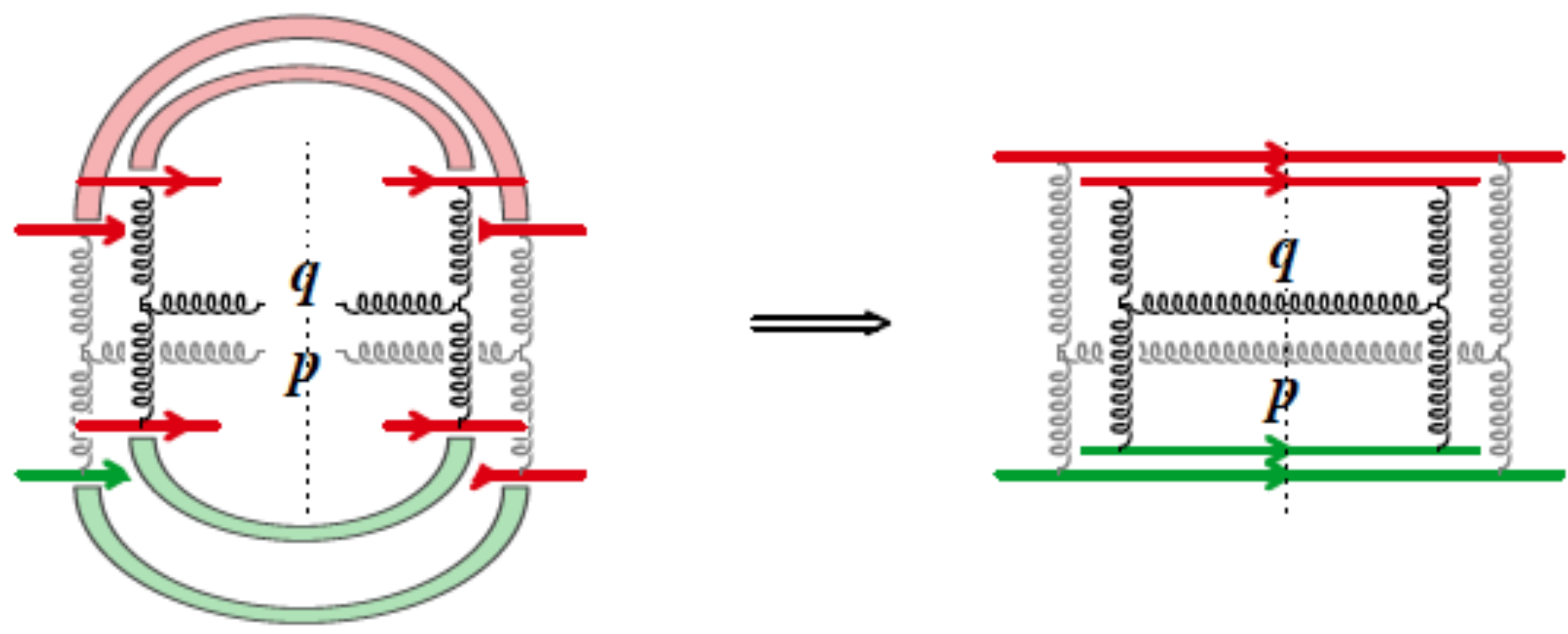

Disconnected graphs: $\quad d^{2} N_{\text {pedestal }} \approx N_{\text {incl. }} \times N_{\text {incl. }}$

Dominant contribution to the "uncollimated" pedestal

Other sources in CGC EFT - 4 particle correlations

Kovner, Lublinsky, 1211:1298 


\section{The saturated hadron: Glasma graphs-III}

Correlations are induced by color fluctuations that vary event to event for Gaussian weight functionals in $\rho$, have color screening radius $\sim 1 / Q_{S}$
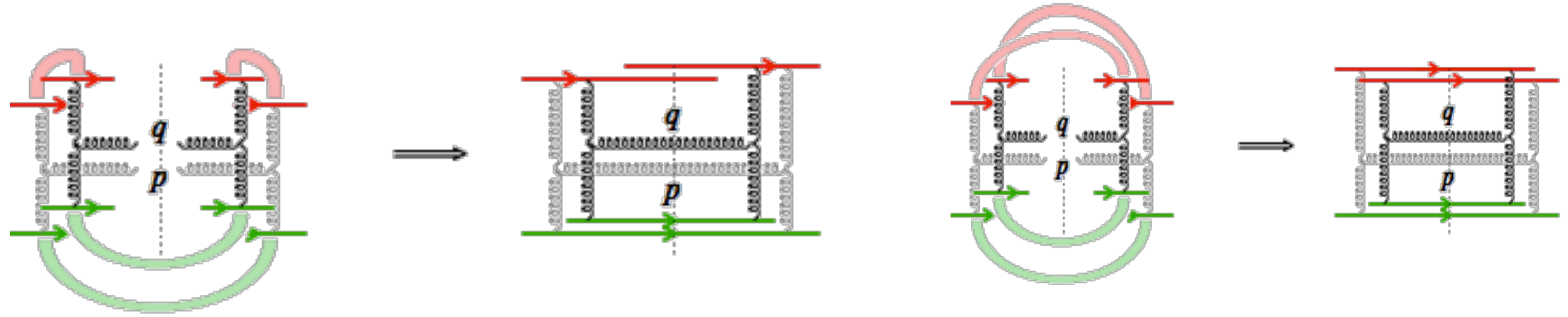

Glasma graphs generate long range rapidity correlations, are suppressed for $Q_{S} \ll p_{T}$ by powers of $\alpha_{S}$ AND $N_{C}$ (At high $p_{T}$, large $x$ or large impact parameters)

However recall: coupling of sources to fields with $k_{T} \leq Q_{S}=1 / g$ not $g$ for high occupancy fields (central impact parameters, small $x$, low $p_{T}$, large nuclei) 


\section{The saturated hadron: Glasma graphs-III}

Correlations are induced by color fluctuations that vary event to event for Gaussian weight functionals in $\rho$, have color screening radius $\sim 1 / Q_{S}$
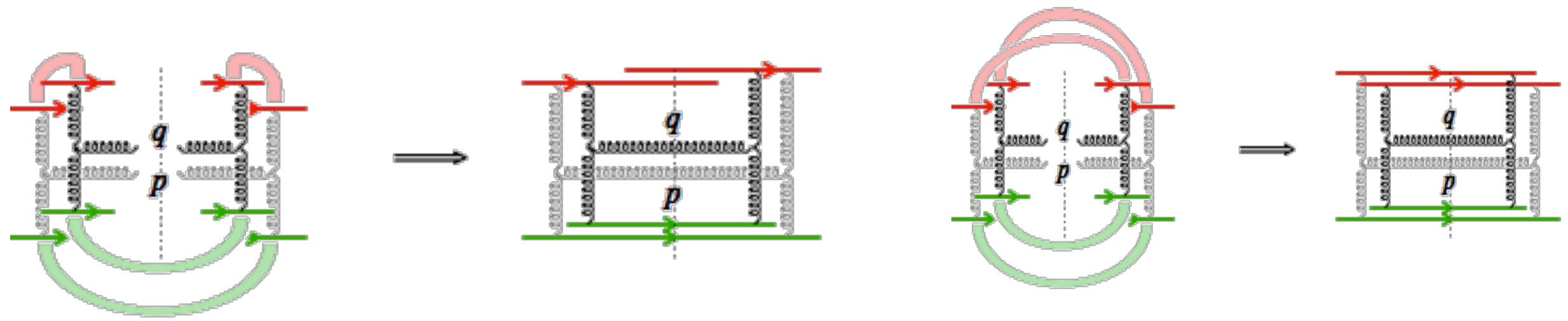

$\checkmark$ Glasma graphs enhanced for high multiplicity events by $\alpha_{s}^{-8}$ -- a factor of $10^{5}$ !

Collimated contributions competitive with pQCD back-to-back graphs for high multiplicity events 


\section{2-particle $\Rightarrow$ n-particle correlations}

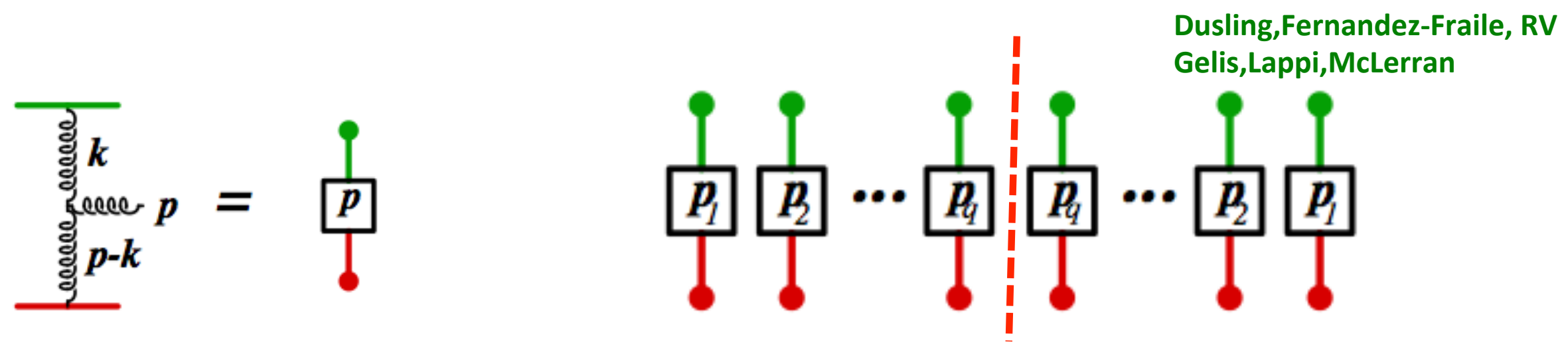

Multiplicity distribution: Leading combinatorics -> negative binomial dist.

$$
\begin{gathered}
P_{n}^{\mathrm{N} . \mathrm{B}}(\bar{n}, k)=\frac{\Gamma(k+n)}{\Gamma(k) \Gamma(n+1)} \frac{\bar{n}^{n} k^{k}}{(\bar{n}+k)^{n+k}} \\
k=\zeta \frac{\left(N_{c}^{2}-1\right) Q_{S}^{2} S_{\perp}}{2 \pi} \quad \begin{array}{l}
\mathbf{k}=\mathbf{1}: \text { Bose-Einstein } \\
\mathbf{k}=\infty
\end{array}
\end{gathered}
$$

From Yang-Mills: $\zeta \sim 1 / 6$ 


\section{High Multiplicity pp collisions}

미

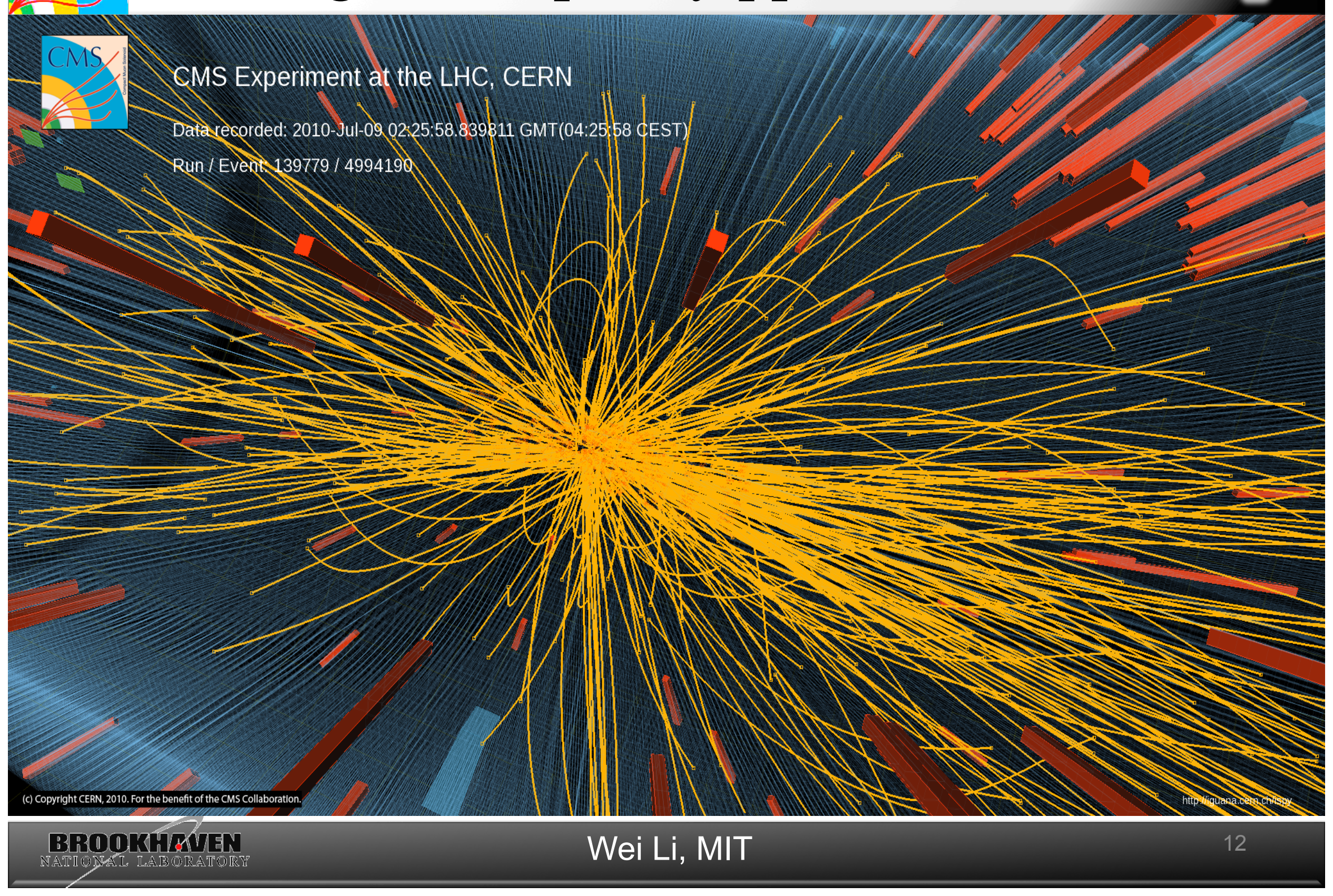




\section{n-particle Glasma correlations describe $p+p$ multiplicity dist.}

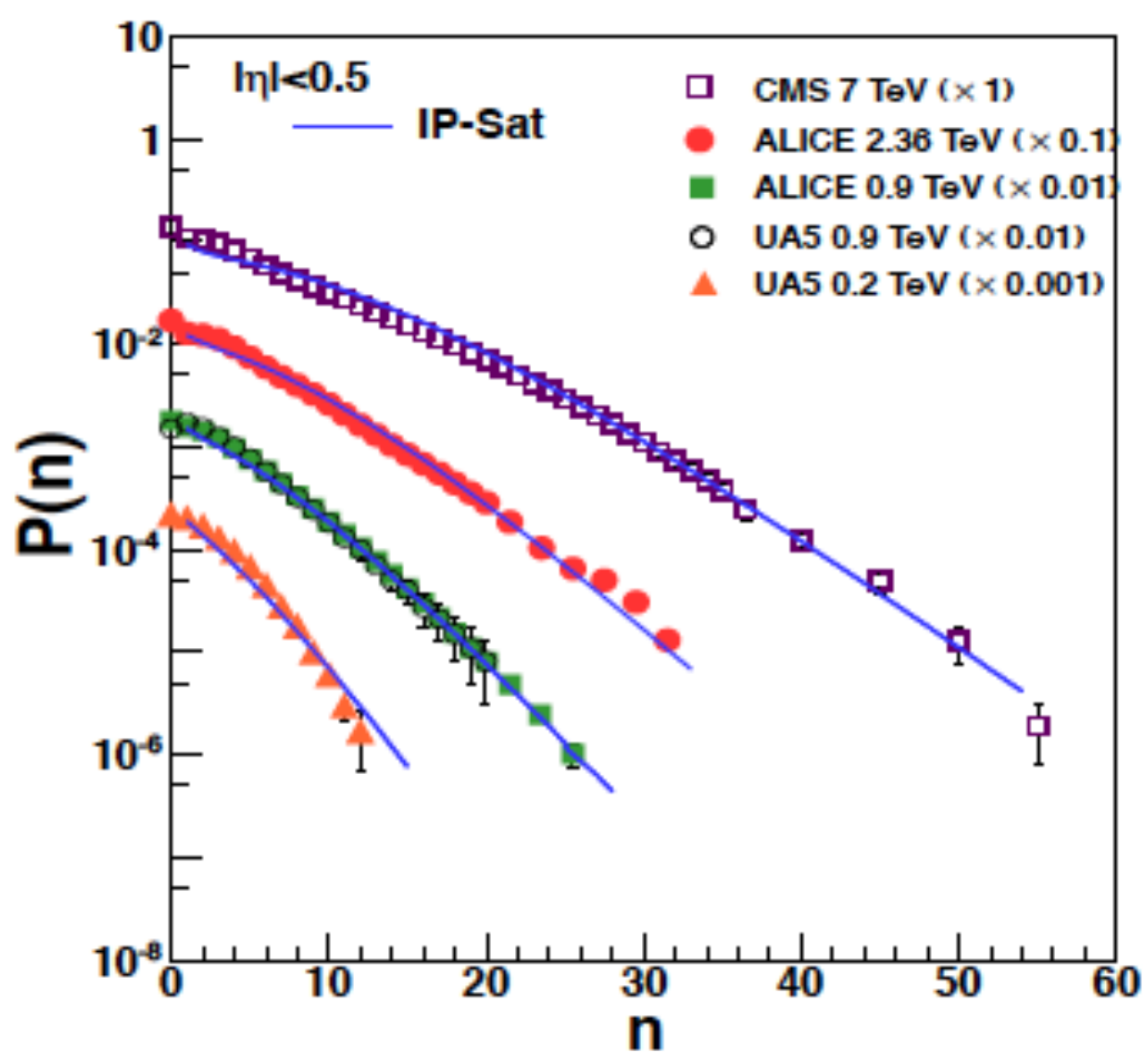

Tribedy, RV

1112.2445

Approx: $\mathrm{k}_{\mathrm{T}}$ factorization; more detailed $\mathrm{YM}$ treatment in progress 


\section{High multiplicity events in $p+p$}
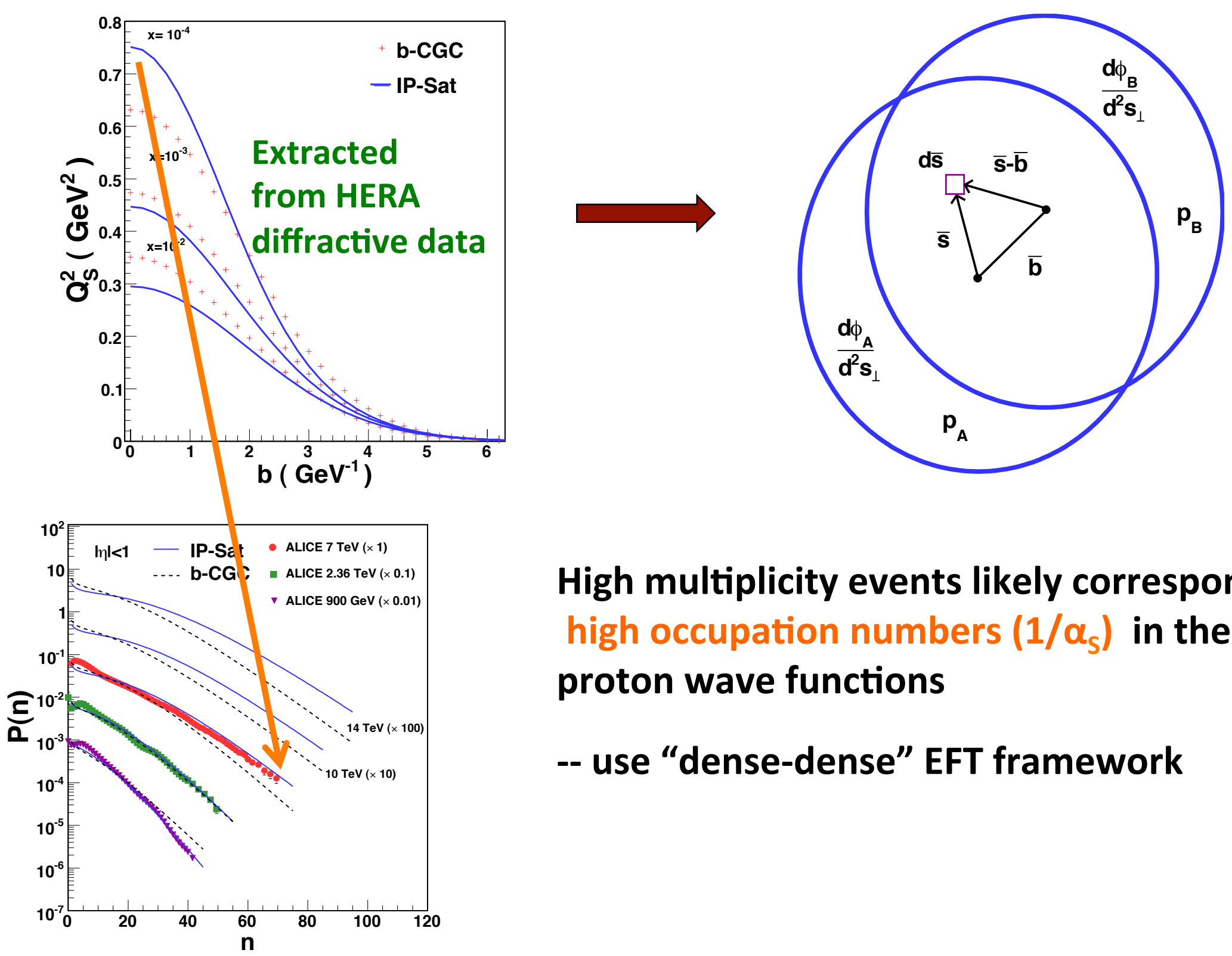

High multiplicity events likely correspond to high occupation numbers $\left(1 / \alpha_{s}\right)$ in the proton wave functions

-- use "dense-dense" EFT framework 


\section{The ridge in high mult. $p+p$ collisions}

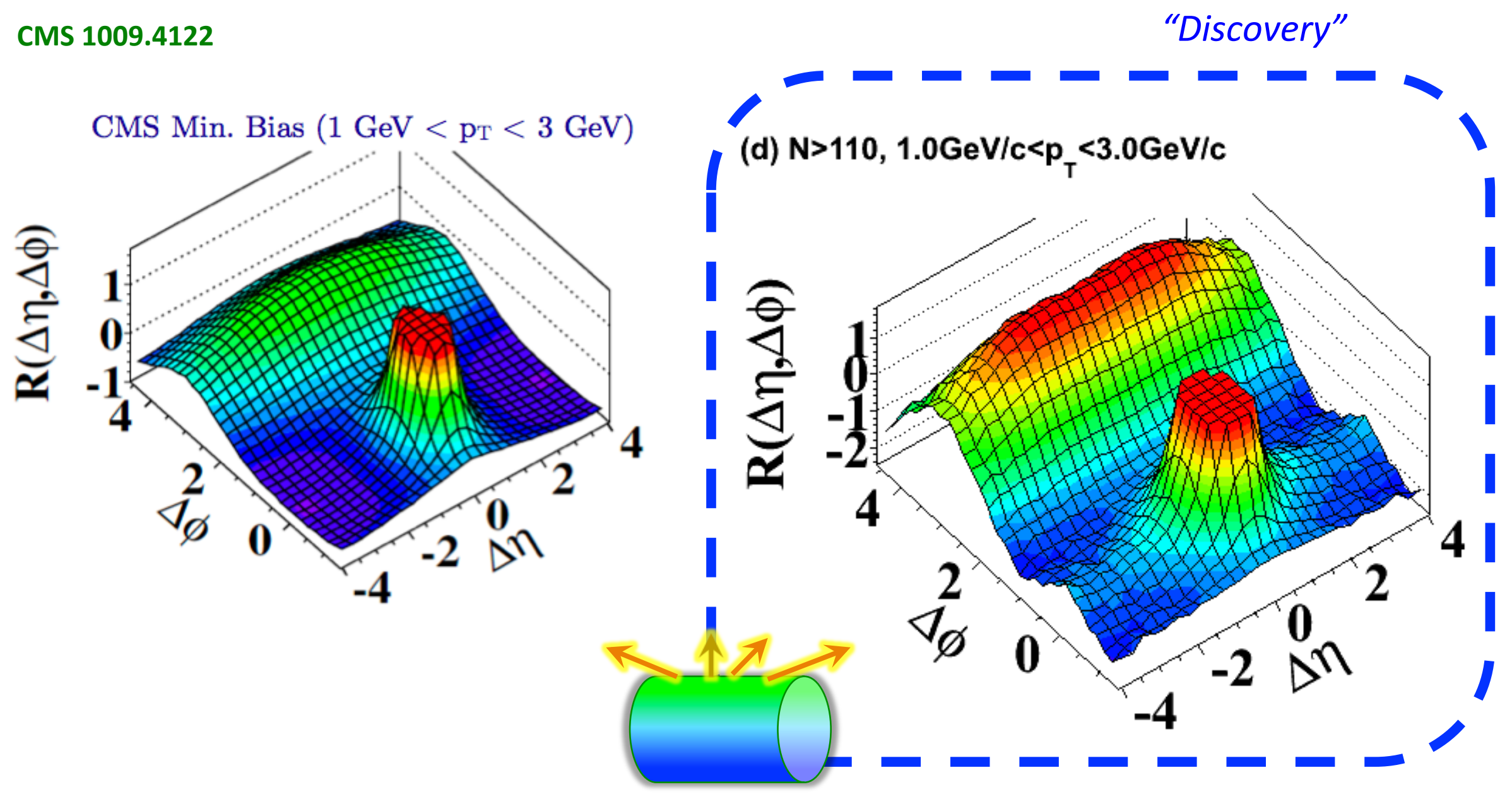




\section{Anatomy of long range di-hadron collimation}

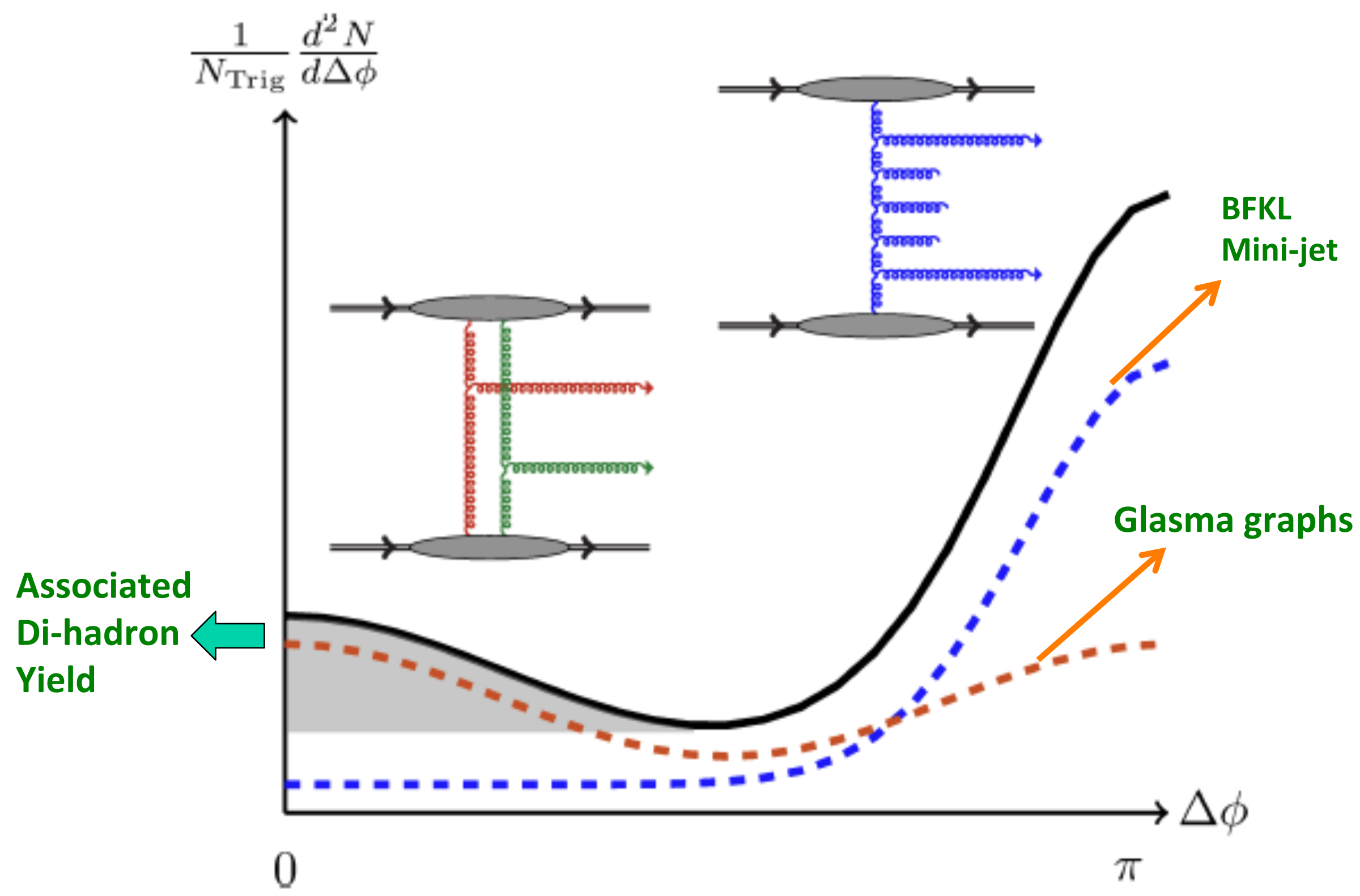




\section{Long range di-hadron Glasma correlations}

Dumitru,Dusling,Gelis,Jalilian-Marian,Lappi,RV, arXiv:1009.5295

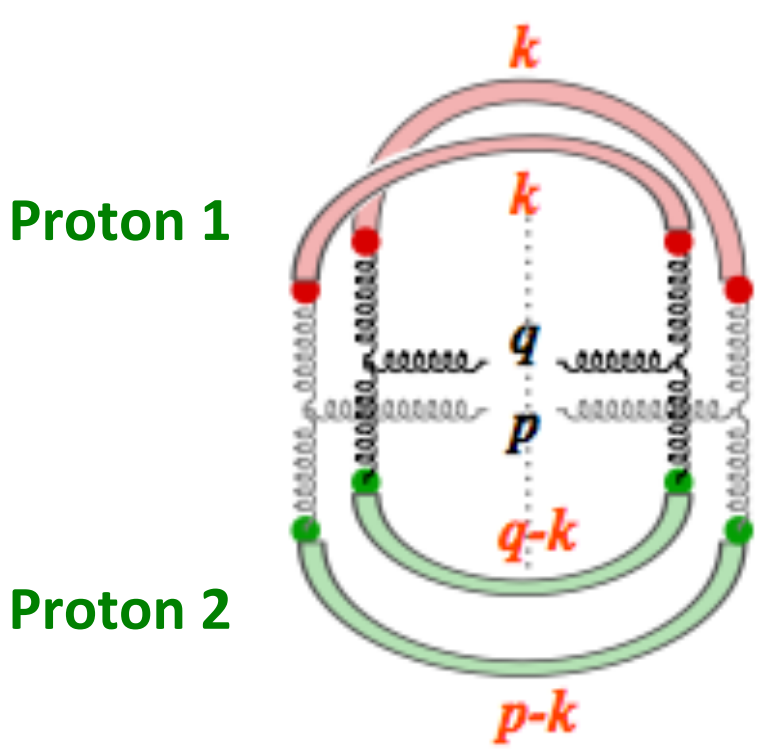

$\begin{aligned} C(\mathbf{p}, \mathbf{q}) & \propto \frac{g^{4}}{\mathbf{p}_{\perp}^{2} \mathbf{q}_{\perp}^{2}} \int \mathrm{d}^{2} \mathbf{k}_{1 \perp} \Phi_{A_{1}}^{2}\left(y_{p}, \mathbf{k}_{1 \perp}\right) \Phi_{A_{2}}\left(y_{p}, \mathbf{p}_{\perp}-\mathbf{k}_{1 \perp}\right) \Phi_{A_{2}}\left(y_{q}, \mathbf{q}_{\perp}-\mathbf{k}_{1 \perp}\right) \\ & + \text { permutations }\end{aligned}$

RG evolution of two particle correlations $C(p, q)$ expressed in terms of "unintegrated gluon distributions" in the proton 


\section{Collimated Glasma yield}

$C(\mathbf{p}, \mathbf{q}) \propto \frac{g^{4}}{\mathbf{p}_{\perp}^{2} \mathbf{q}_{\perp}^{2}} \int \mathrm{d}^{2} \mathbf{k}_{1 \perp} \Phi_{A_{1}}^{2}\left(y_{p}, \mathbf{k}_{1 \perp}\right) \Phi_{A_{2}}\left(y_{p}, \mathbf{p}_{\perp}-\mathbf{k}_{1 \perp}\right) \Phi_{A_{2}}\left(y_{q}, \mathbf{q}_{\perp}-\mathbf{k}_{1 \perp}\right)$

+ permutations
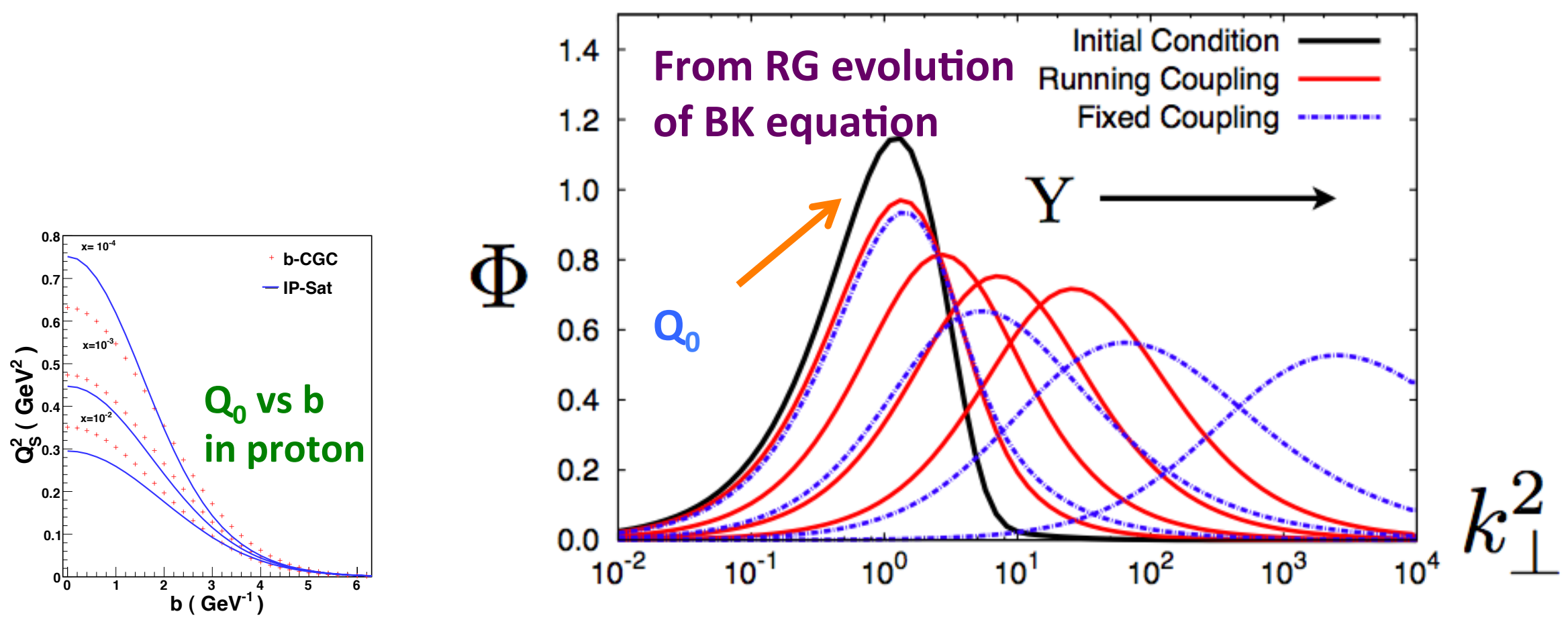

Dominant contribution from $\quad\left|\mathrm{p}_{\mathrm{T}}-\mathrm{k}_{\mathrm{T}}\right| \sim\left|\mathrm{q}_{\mathrm{T}}-\mathrm{k}_{\mathrm{T}}\right| \sim\left|\mathrm{k}_{\mathrm{T}}\right| \sim \mathrm{Q}_{\mathrm{S}}$

This gives a collimation for $\Delta \Phi \approx 0$ and $\pi$ 


\section{Angular structure from (mini-) jet radiation}

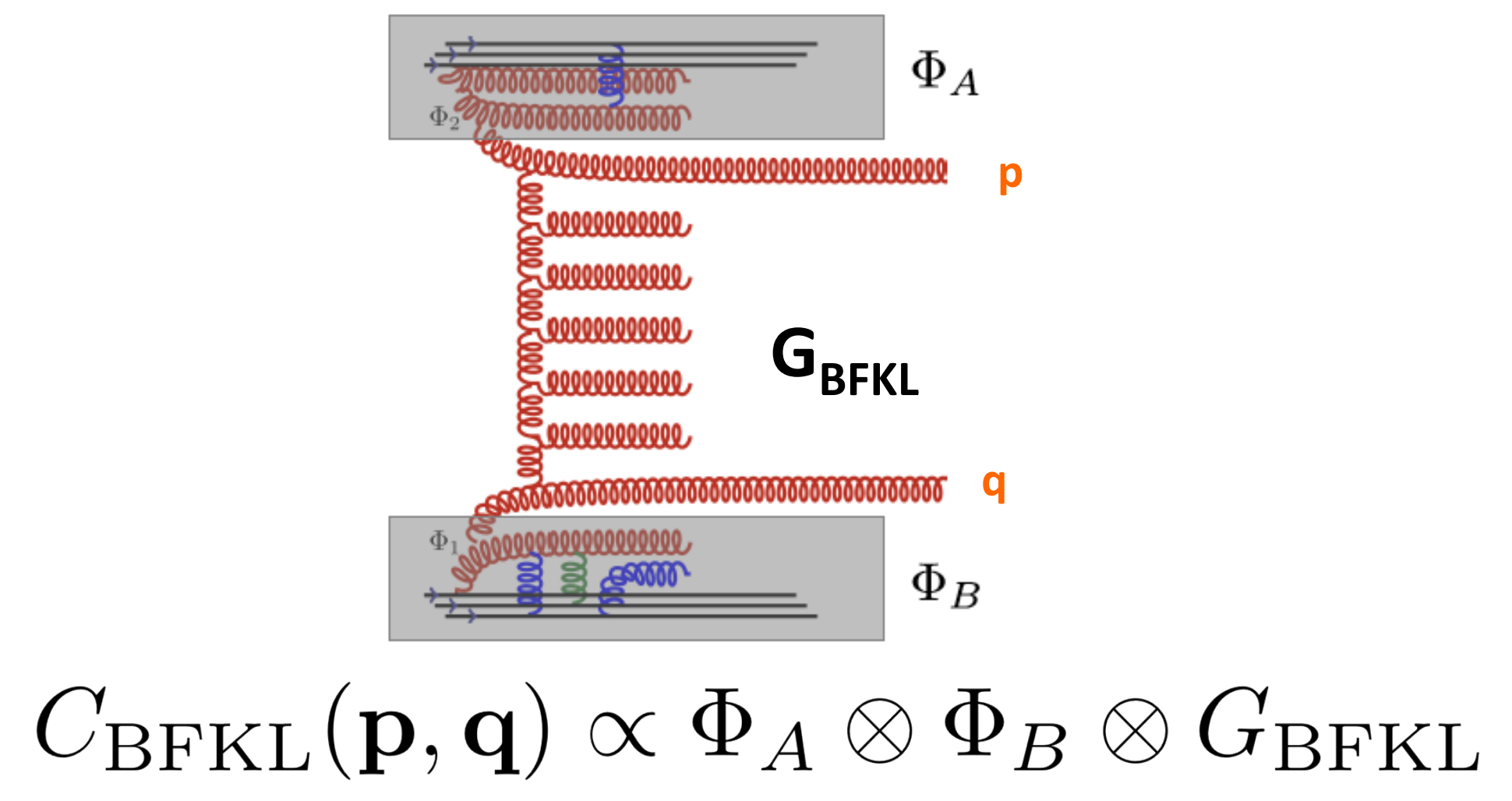

Mini-jets: $O(1)$ in high multiplicity events

- give an angular collimation at $\Delta \Phi \cong \pi$

LHC results also test the structure of bremsstrahlung radiation between jets 


\section{Quantitative description of ridge}

$$
\begin{aligned}
& \frac{d^{2} N}{d \Delta \phi}=K \int_{-2.4}^{+2.4} d \eta_{p} d \eta_{q} \mathcal{A}\left(\eta_{p}, \eta_{q}\right) \\
& \times \int_{p_{T}^{\min }}^{p_{T}^{\max }} \frac{d p_{T}^{2}}{2} \int_{q_{T}^{\min }}^{q_{T}^{\max }} \frac{d q_{T}^{2}}{2} \int d \phi_{p} \int d \phi_{q} \delta\left(\phi_{p}-\phi_{q}-\Delta \phi\right) \\
& \times \int_{0}^{1} d z_{1} d z_{2} \frac{D\left(z_{1}\right)}{z_{1}^{2}} \frac{D\left(z_{2}\right)}{z_{2}^{2}} \frac{d^{2} N_{\mathrm{Glasma}}^{\text {corr. }}}{d^{2} \mathbf{p}_{T} d^{2} \mathbf{q}_{T} d \eta_{p} d \eta_{q}}\left(\frac{p_{\mathrm{T}}}{z_{1}}, \frac{q_{\mathrm{T}}}{z_{2}}, \Delta \phi\right) \quad N_{\mathrm{trig}}=\int_{-2.4}^{+2.4} d \eta \int_{p_{T}^{\min }}^{p_{T}^{\max }} d^{2} \mathbf{p}_{T} \int_{0}^{1} d z \frac{D(z)}{z^{2}} \frac{d N}{d \eta d^{2} \mathbf{p}_{T}}\left(\frac{p_{\mathrm{T}}}{z}\right)
\end{aligned}
$$

$$
\text { Assoc. Yield }=\frac{1}{N_{\text {trig }}} \int_{0}^{\Delta \phi_{\min } .} d \Delta \phi \frac{d^{2} N}{d \Delta \phi}-\left.\frac{d^{2} N}{d \Delta \phi}\right|_{\Delta \phi_{\min }}
$$

Dependence on transverse area cancels in ratio...
Rarer and rarer gluon configurations probed In the proton

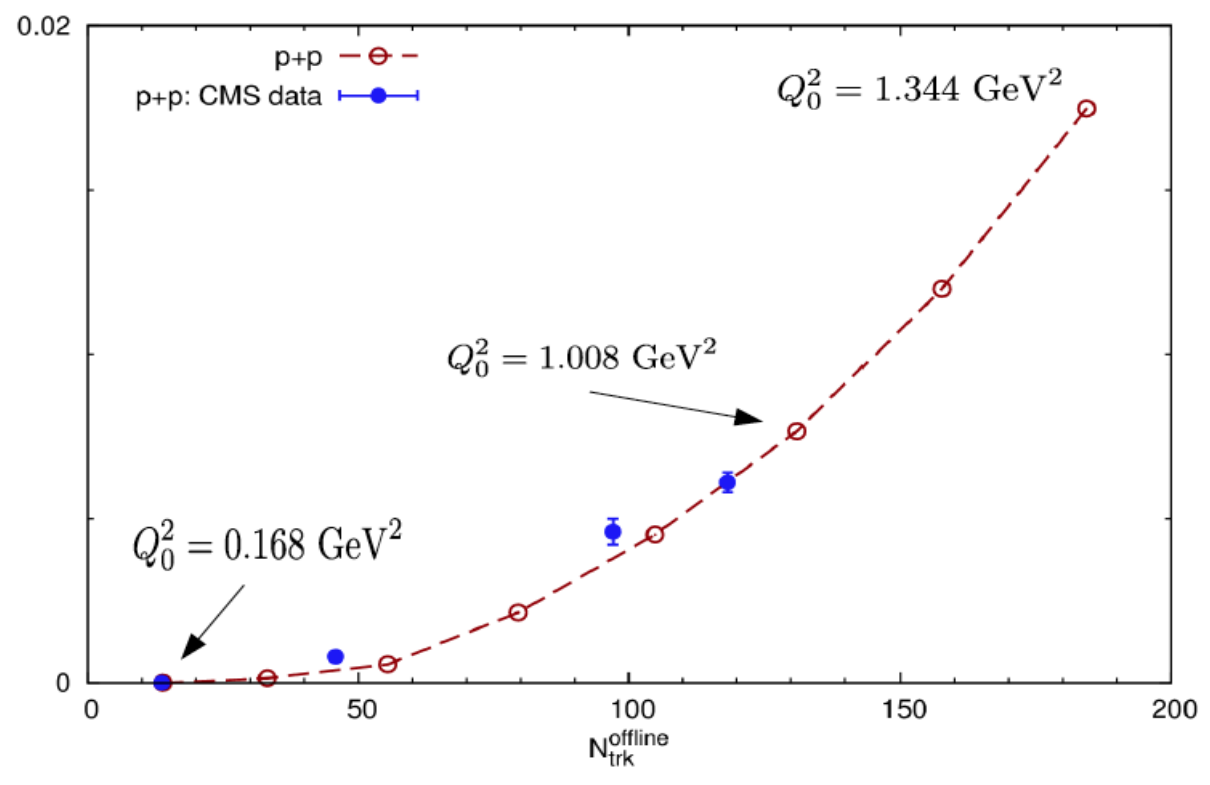


CMS data: JHEP 1009, 091 (2010); PLB 718, 795 (2013)

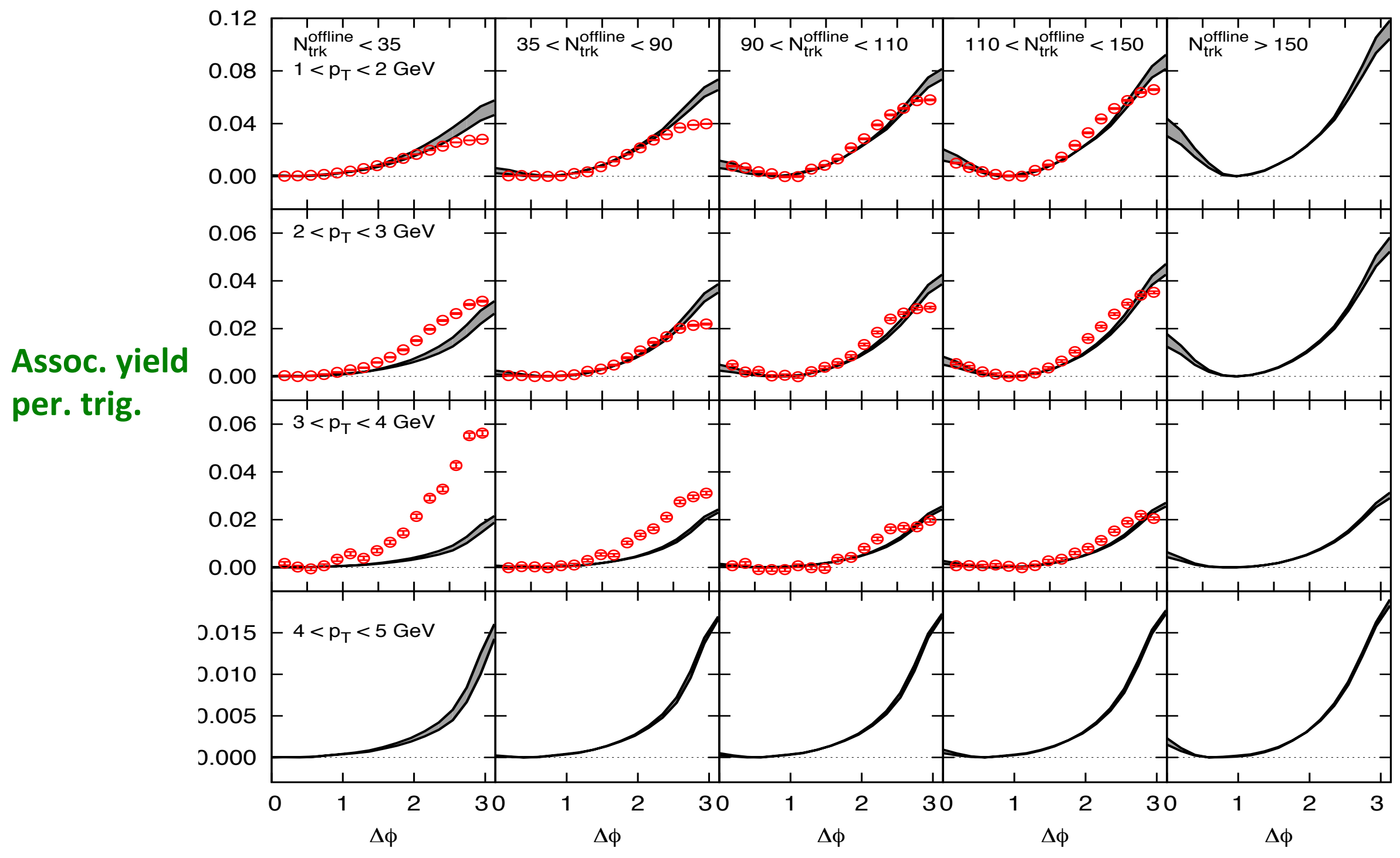

$\mathrm{K}_{\mathrm{BFKL}}=\mathrm{K}_{\text {Glasma }}=1$ KKP fragmentation

Dusling,RV, PRD 87, 051502 (R) (2013); arXiv:1302.7018 


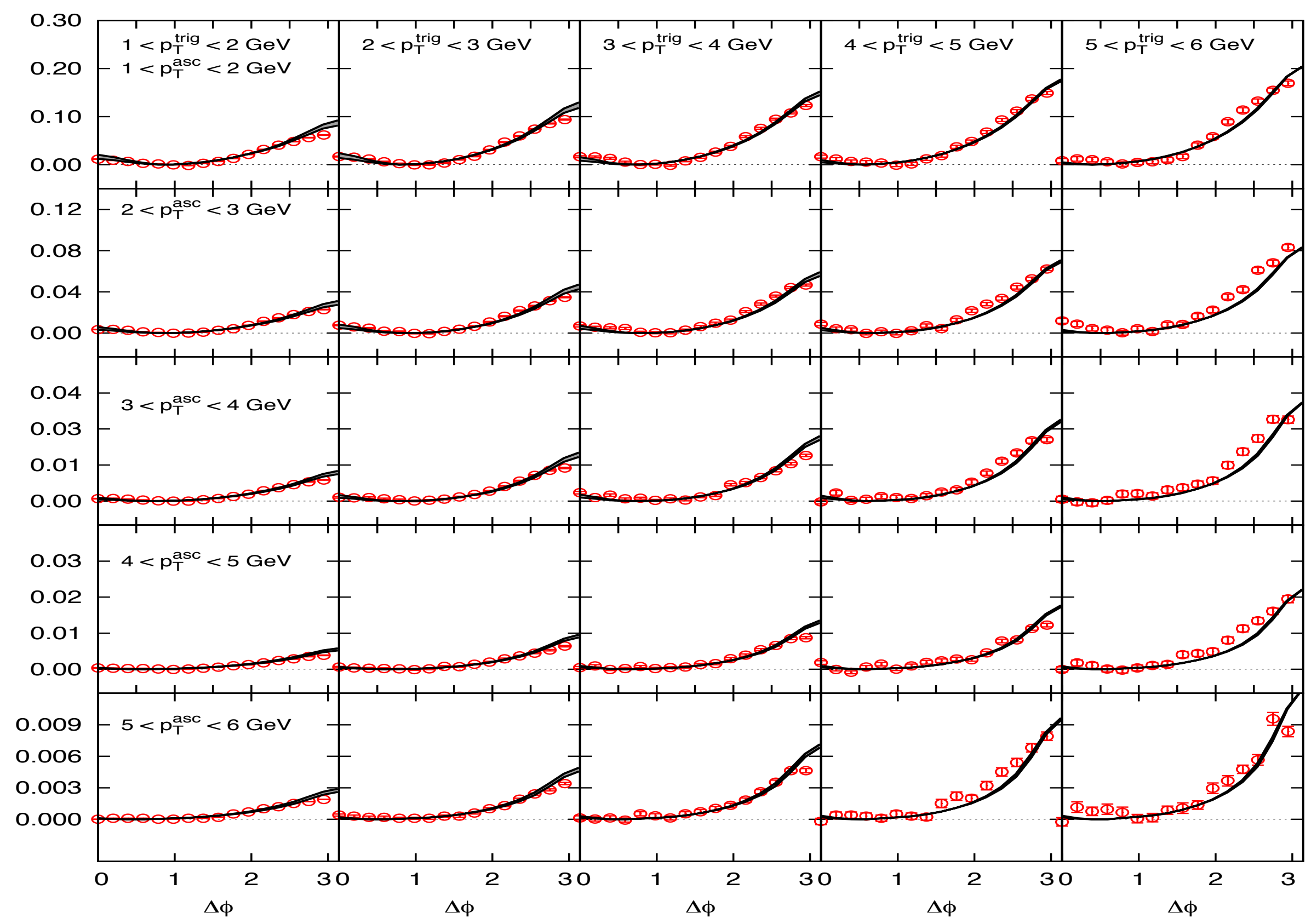




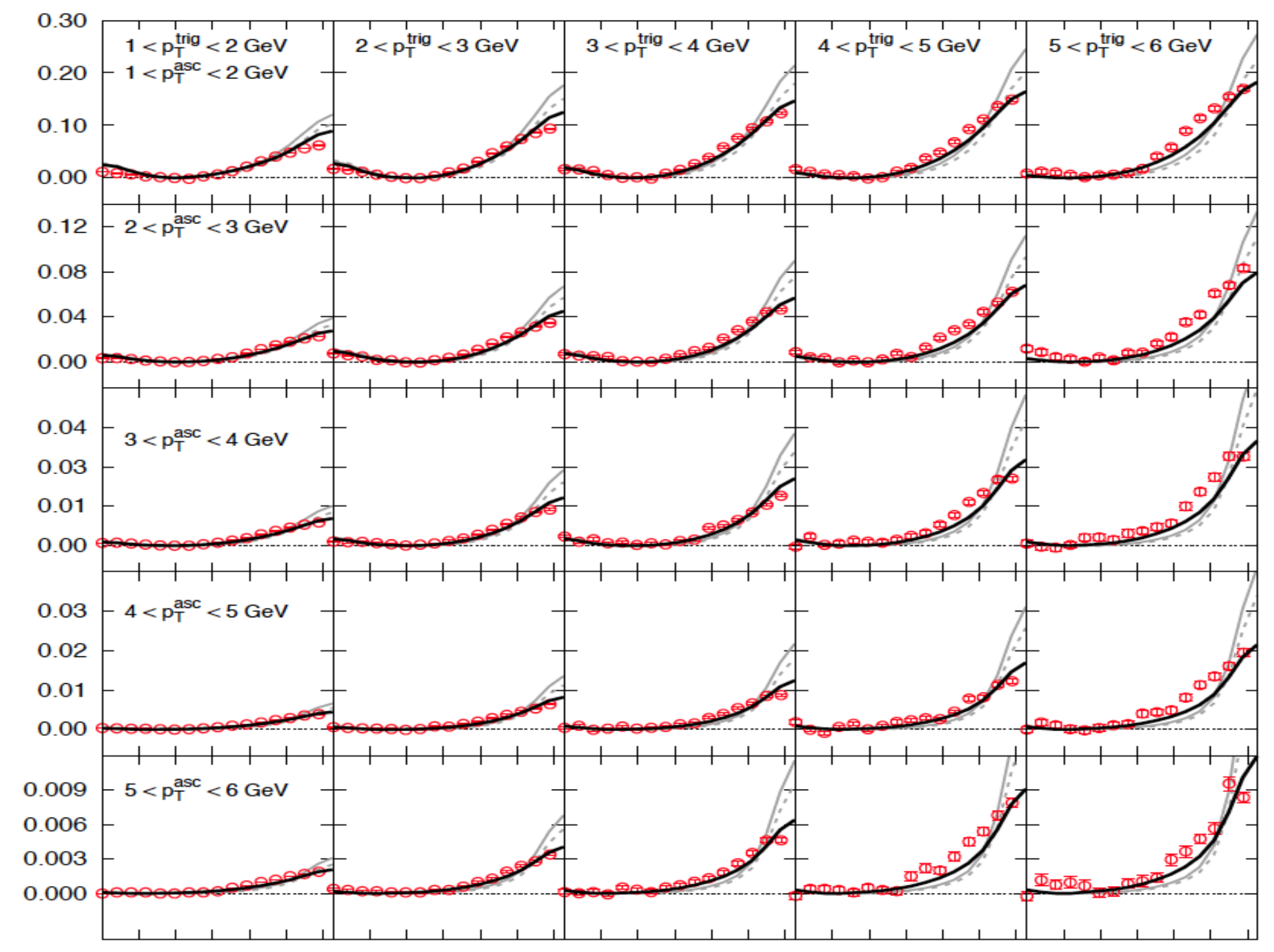

Grey curves: 2 -> 4 QCD (Multi-Regge) QCD results

- no emission in $\Delta \eta$ between jets 


\section{$p+p$}

In $p+p$ we are seeing the intrinsic collimation from a single flux tube

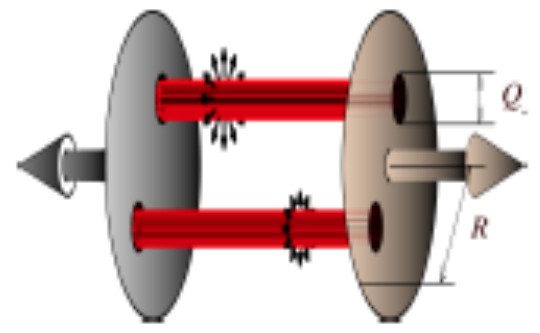

Assoc. Yield $\left[\times 10^{-2}\right]$

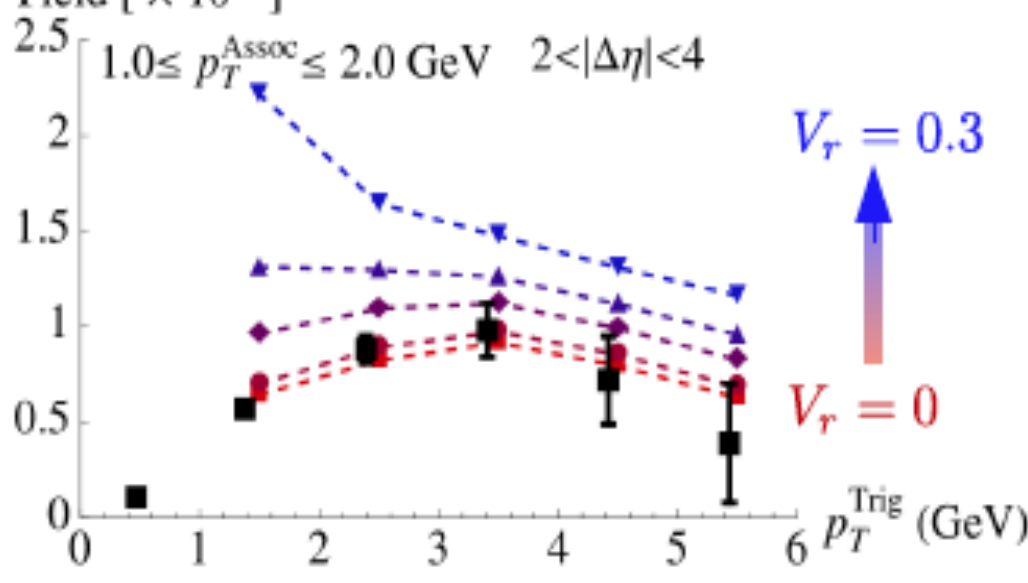

Increasing transverse flow in $p+p$ creates a discrepancy with data.
VS

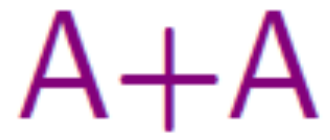

In $A+A$ there are many such tubes each with an intrinsic correlation enhanced by flow

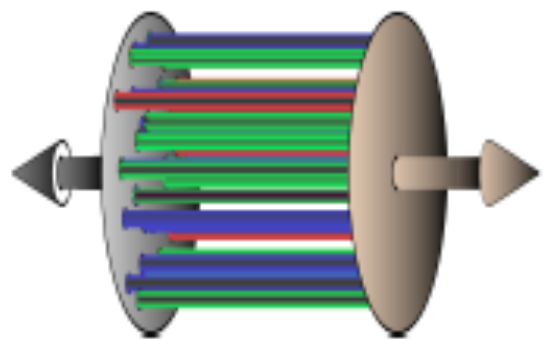

Assoc. Yield

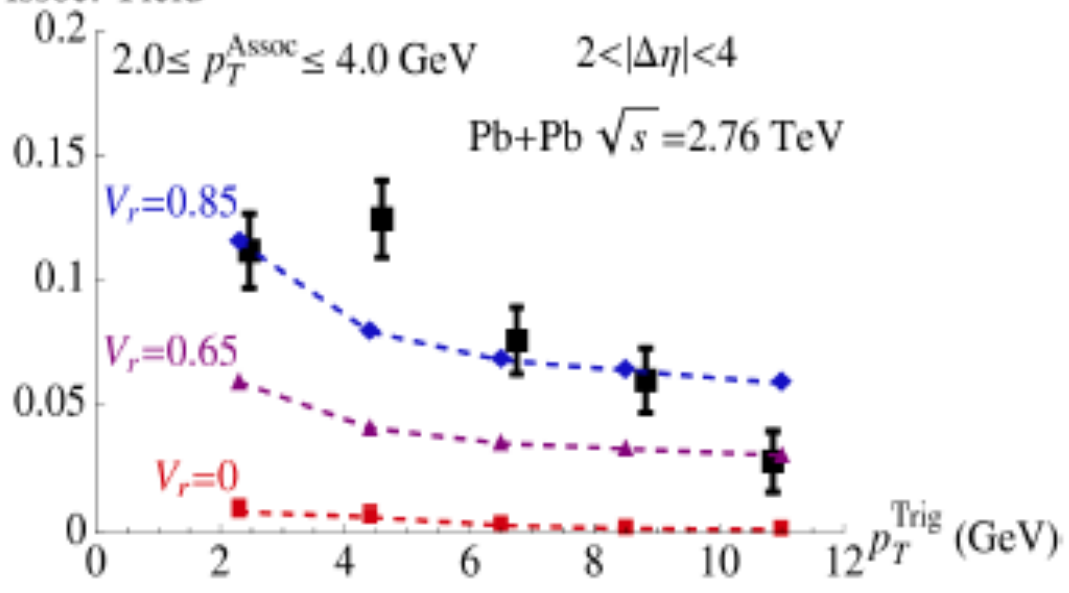

Yet, transverse flow is needed to explain identical measurements in $\mathrm{Pb}+\mathrm{Pb}$ 


\section{Analysis of "pilot run" on proton lead collisions}

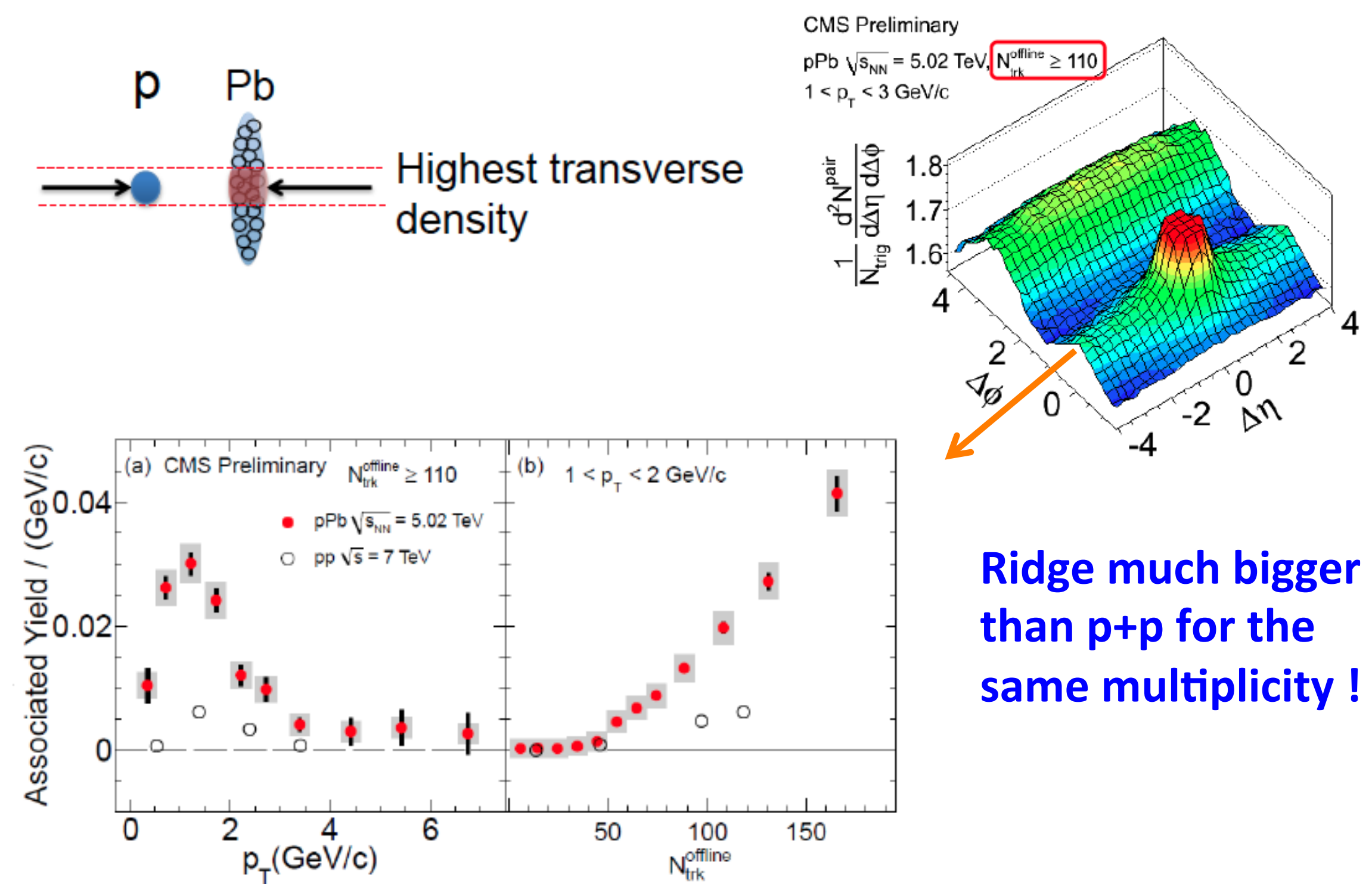




\section{CMS $\mathrm{p}+\mathrm{Pb}$ nearside per trigger yield-I}

Dusling, RV: PRD87, 054014 (2013); arXiv:1302.7018

$\mathrm{Q}_{0}{ }^{2}$ (lead) $=\mathrm{N}_{\text {Part }}{ }^{\mathrm{Pb}} * \mathrm{Q}_{0}{ }^{2}$ (proton)

\# of "wounded" nucleons in

\section{Lead nucleus}

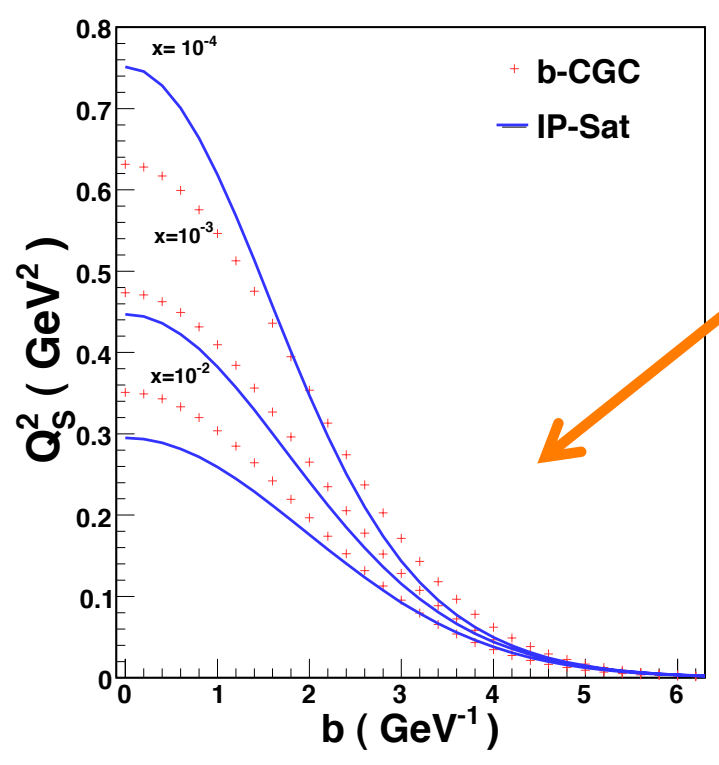

Associated Yield $\left(1.0 \leq \mathrm{p}_{\mathrm{T}}[\mathrm{GeV}] \leq 2.0\right)$

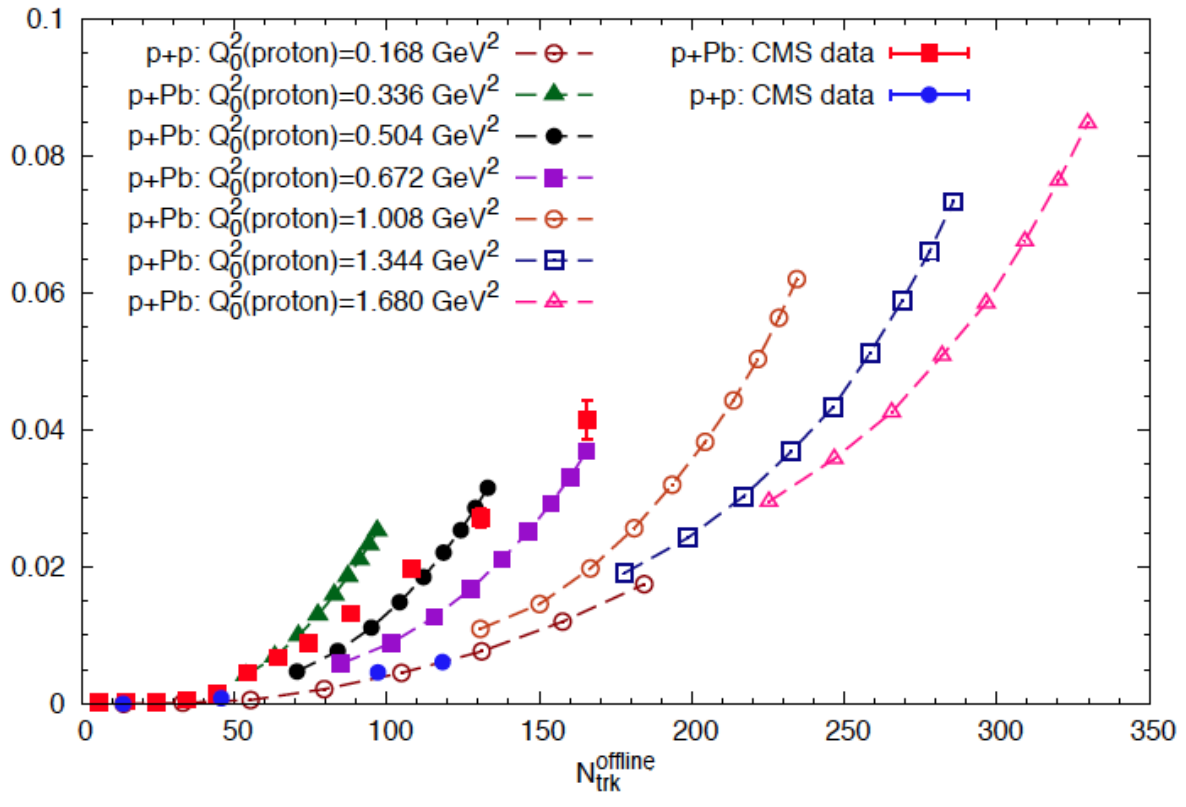




\section{CMS $\mathrm{p}+\mathrm{Pb}$ nearside yield per trigger-II}

Dusling, RV: 1302.7018

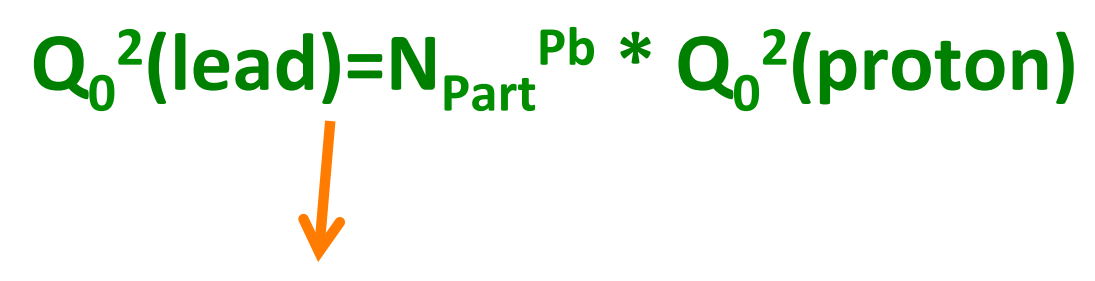

\# of "wounded" nucleons in Lead nucleus
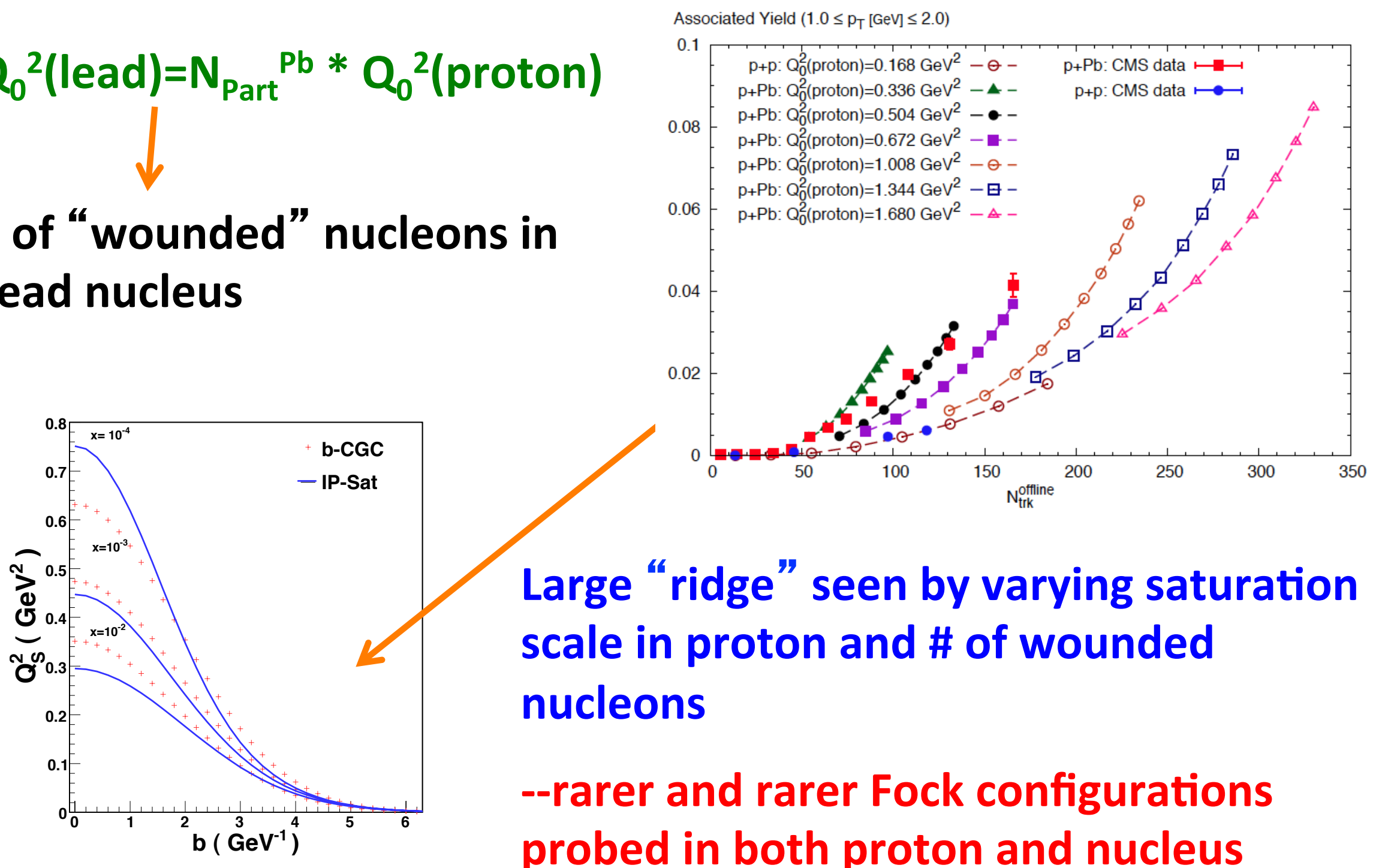

Large "ridge" seen by varying saturation scale in proton and \# of wounded nucleons

--rarer and rarer Fock configurations probed in both proton and nucleus 


\section{$\mathrm{CMS} \mathrm{p}+\mathrm{Pb}$ nearside yield per trigger-III}

Associated Yield

Dusling, RV: 1211.3701

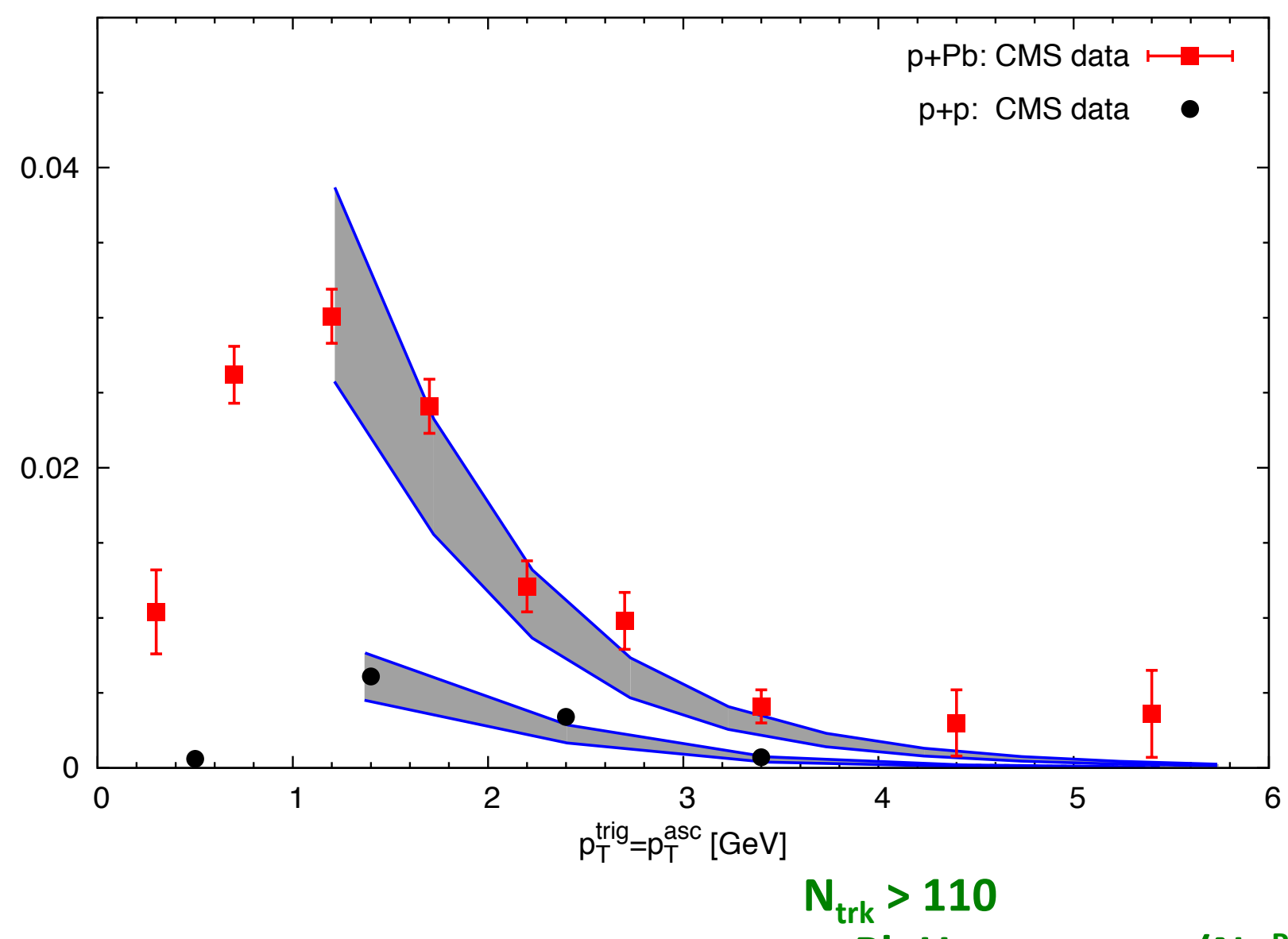

1302.7018

p+Pb Upper curve: $\left(\mathrm{Np}^{\text {Part }}, \mathrm{N}_{\mathrm{A}}^{\text {Part }}\right)=(3,22)$

p+Pb Lower curve: $\left(\mathrm{Np}^{\text {Part }}, \mathrm{N}_{\mathrm{A}}{ }^{\text {Part }}\right)=(4,14)$

$p+p N_{\text {part }}=5,6$ 


\section{$\mathrm{CMS} \mathrm{p}+\mathrm{Pb}$ yield compared to Glasma + BFKL}

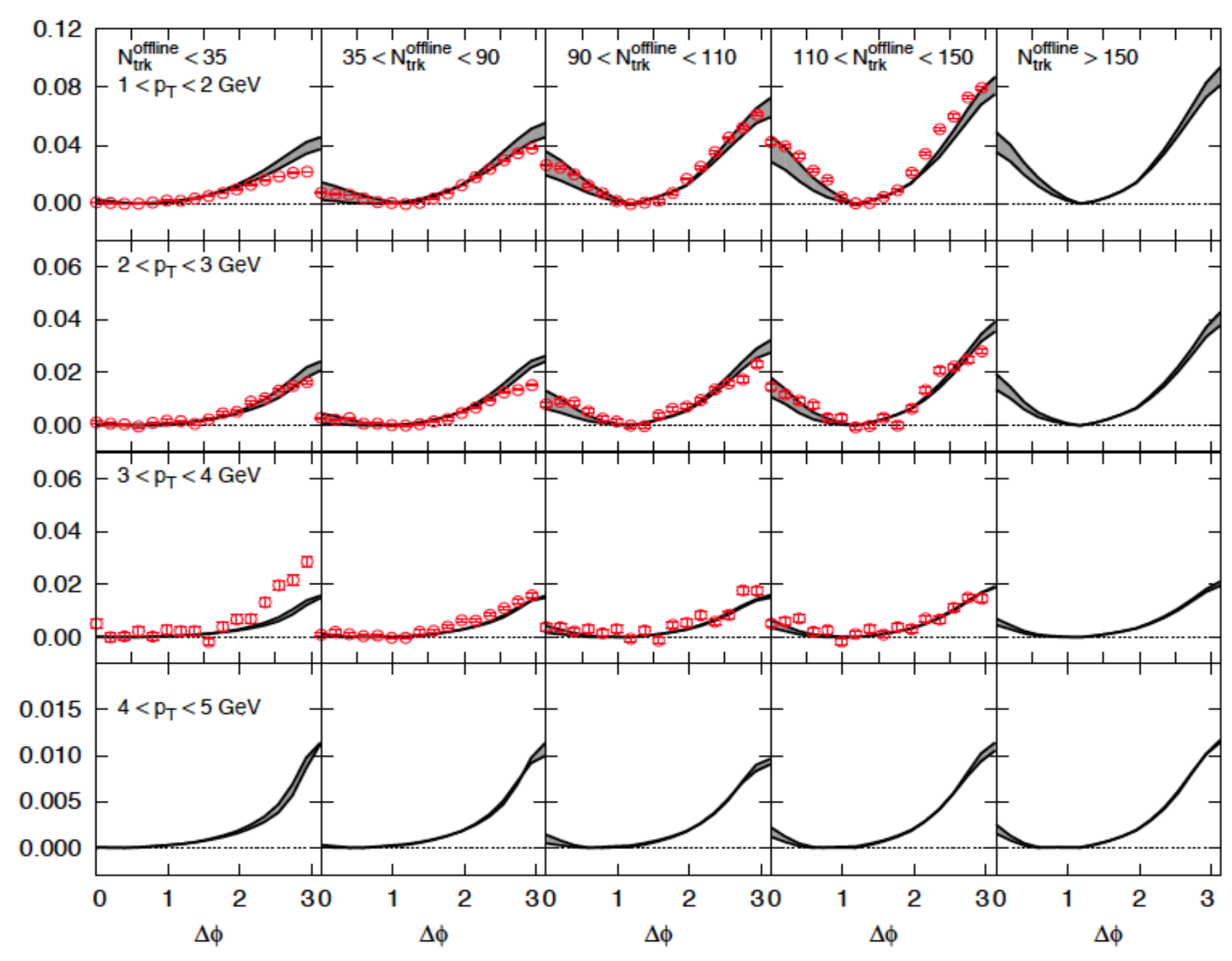

Dusling, RV: 1211.3701

1302.7018

Smoking gun for gluon saturation and BFKL dynamics ? 


\section{ALICE data on the $\mathrm{p}+\mathrm{Pb}$ ridge}

ALICE coll. PLB 719, 29 (2013)

Different acceptance $(|\Delta \eta|<1.8)$ than CMS $(2<|\eta|<4)$ and ATLAS $(2<|\eta|<5)$.

ALICE subtracts away-side "jet" contribution at $40-60 \%$ centrality from most central events -can interpret as $v_{2}$

-this gives symmetric "dipole" shape of correlation - expected for Glasma contribution

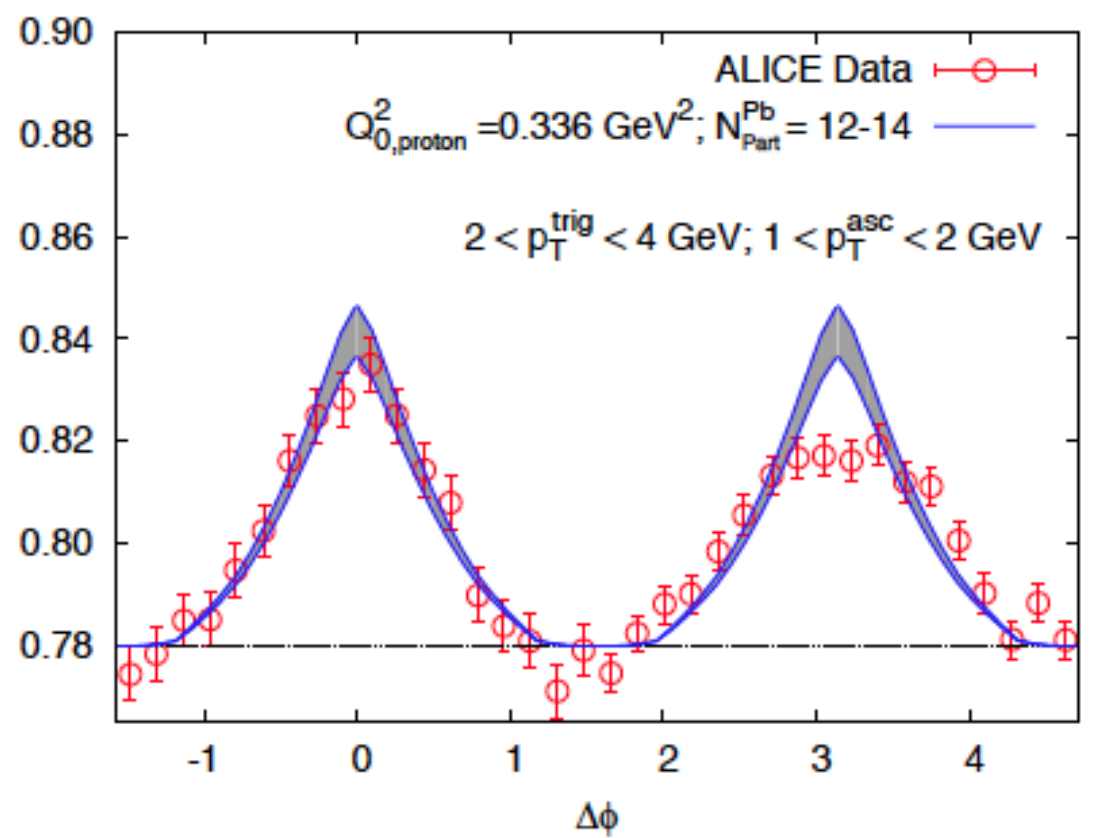

Different analysis technique from CMS/ATLAS

-- same normalization as for CMS/ATLAS

Curves for $\mathrm{Q}_{0 \text {, proton }}{ }^{2}=0.336 \mathrm{GeV}^{2}$ $\& N_{\text {part }}^{\mathrm{Pb}}=12-14$ 


\section{Comparison to ATLAS $\mathrm{p}+\mathrm{Pb}$ ridge}

ATLAS coll. arXiv: 1212.5198. PRL in press
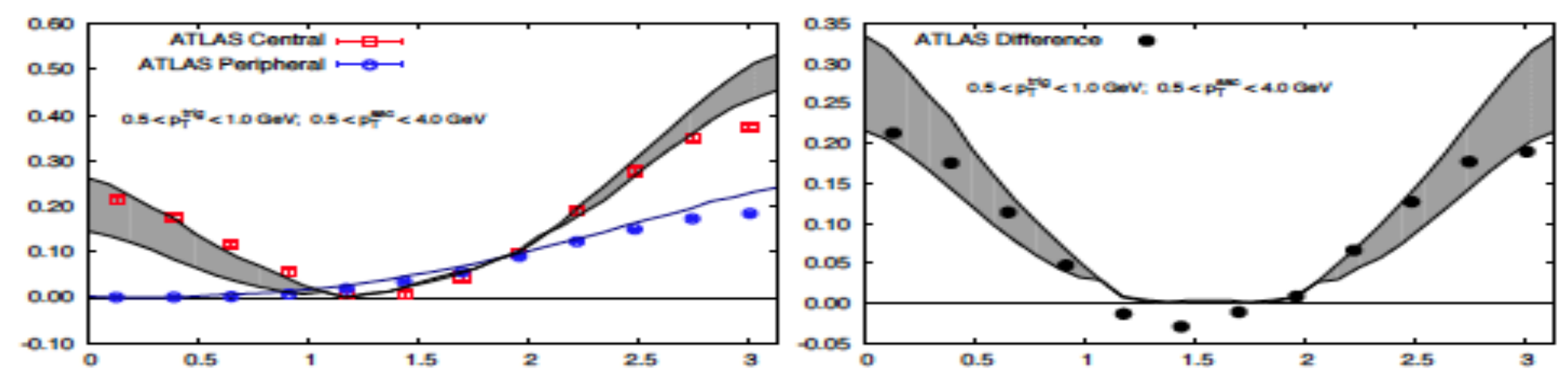

$\mathrm{p}+\mathrm{A}$ centralities
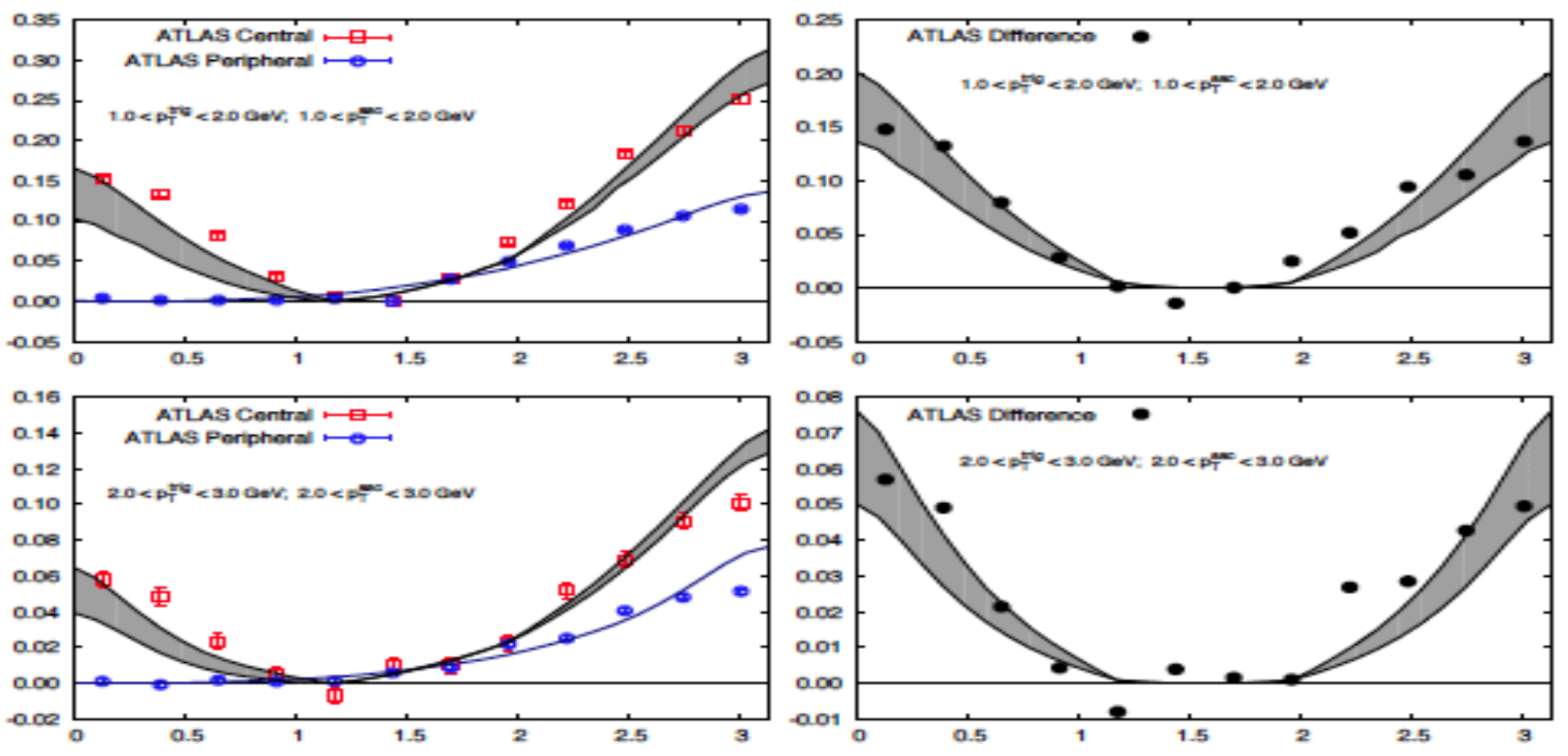


\section{Comparison to ATLAS $\mathrm{p}+\mathrm{Pb}$ ridge}

ATLAS coll. arXiv: 1212.5198
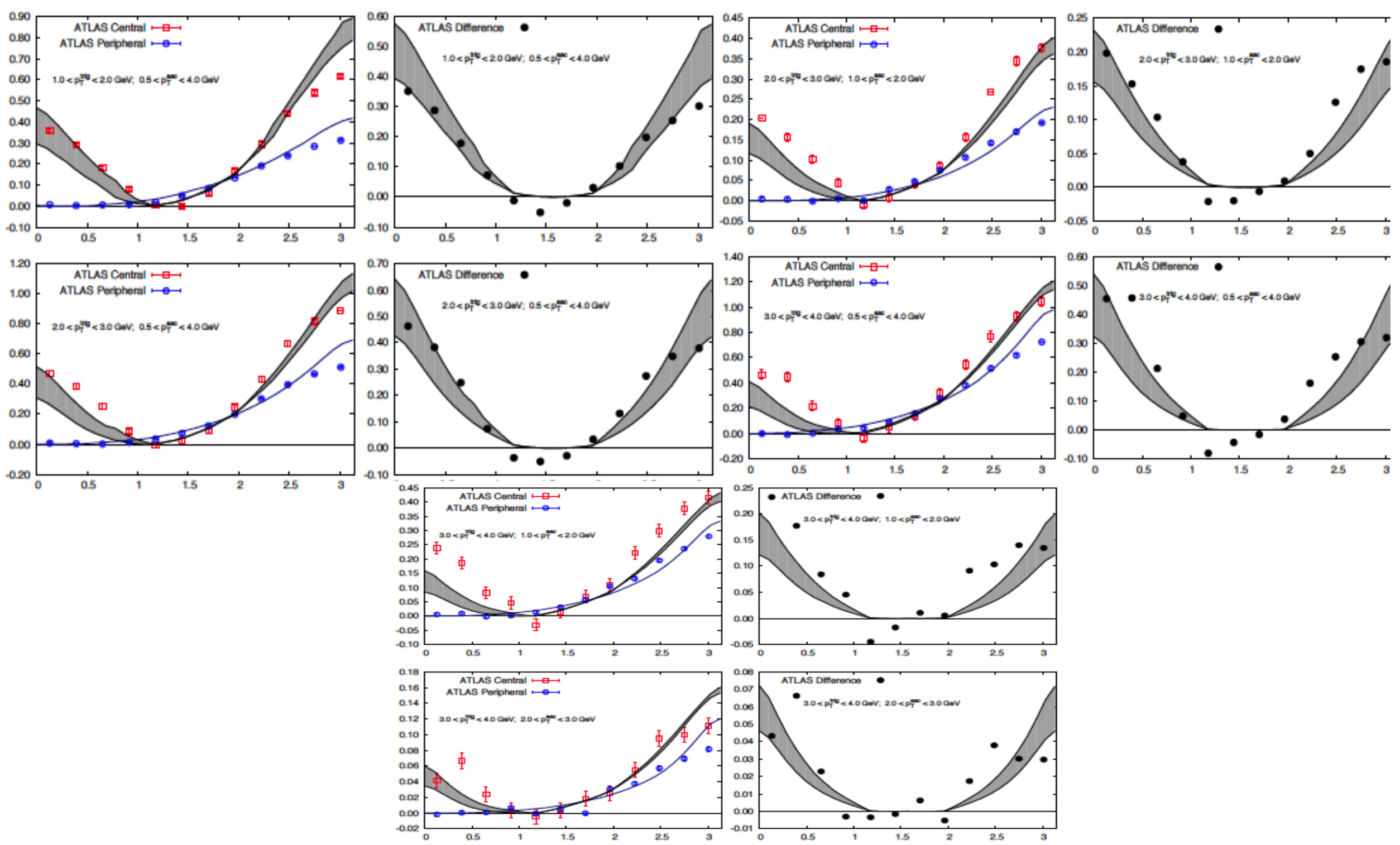


\section{Physics underlying the ridge}

Look at ratio of yield at $\Delta \Phi_{p q}=0$ to $\Delta \phi_{p q}=\pi$ for $\left|p_{T}\right|=\left|q_{T}\right|$

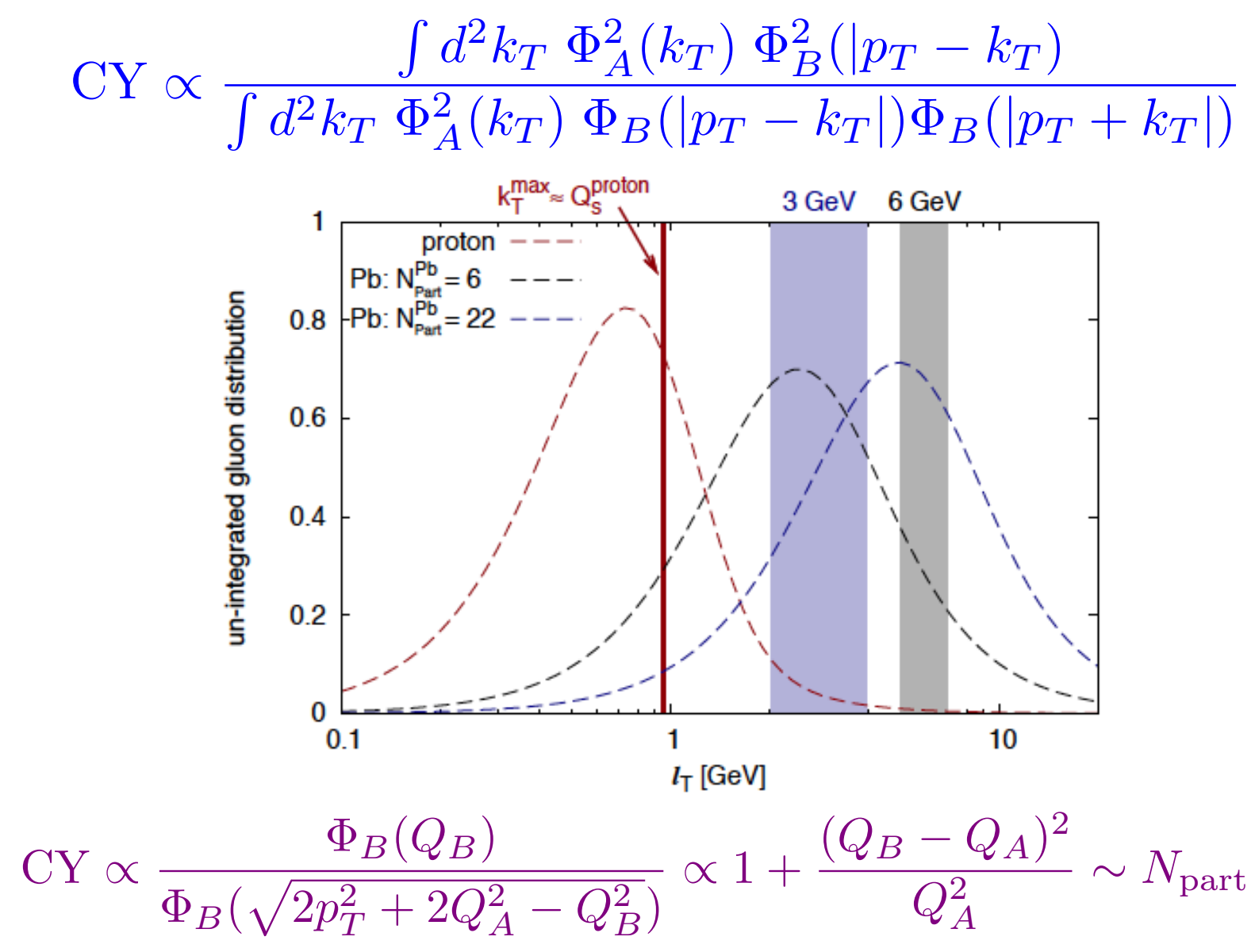

As seen in the LHC $\mathrm{p}+\mathrm{Pb}$ data... 


\section{RHIC d+Au data}
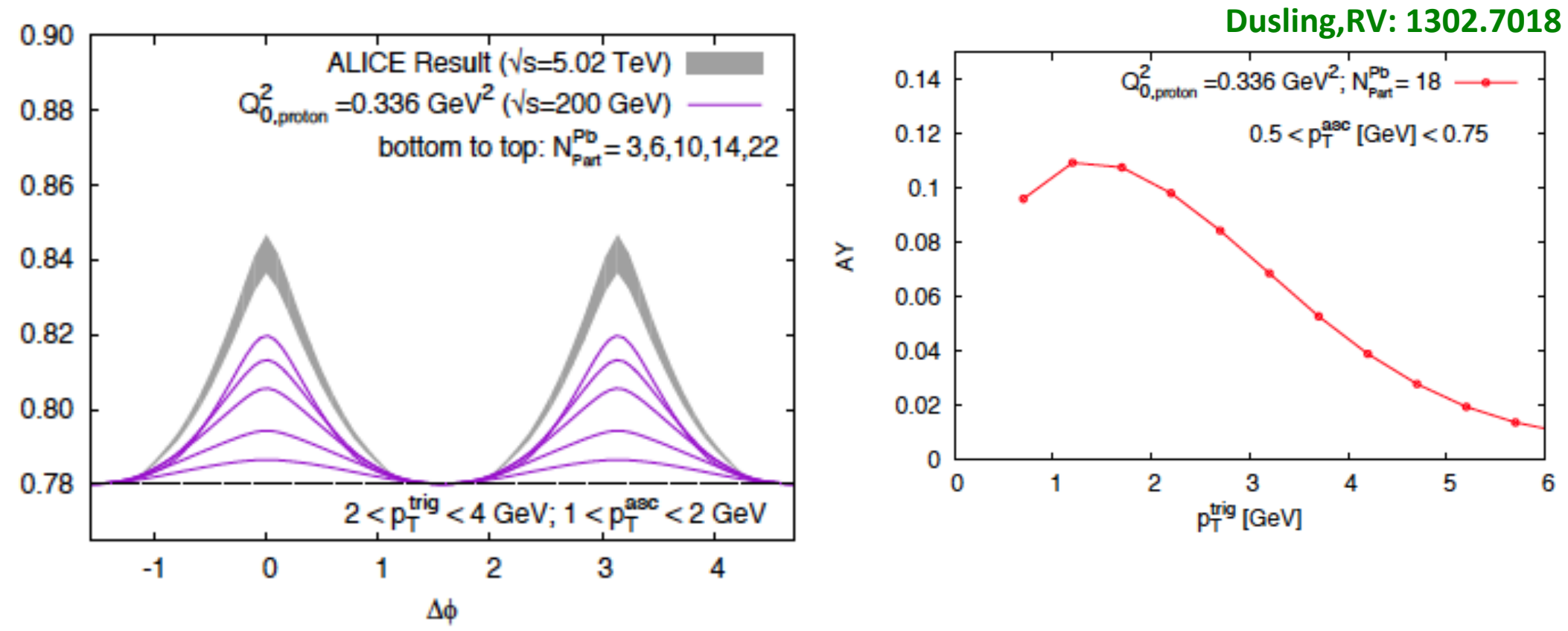

PHENIX has measured large v2 in 0-5\% d+Au collisions: arXiv:1303.1794

We compute correlated yield

$$
\begin{array}{r}
d^{2} N_{\text {corr }} \approx d^{2} N_{\text {pedestal }} \mathbf{v}_{2}{ }^{2} \\
\longrightarrow \text { Strong function of } p_{T}{ }^{a}, p_{T}{ }^{b}-\text { larger at LHC }
\end{array}
$$

Extraction of $d^{2} N_{\text {corr }}$ would allow quantitative study in our framework 


\section{Are there signficant final state effects ?}

Why is jet unmodified while Glasma graph (which generates the $v_{2}$ ) is ?
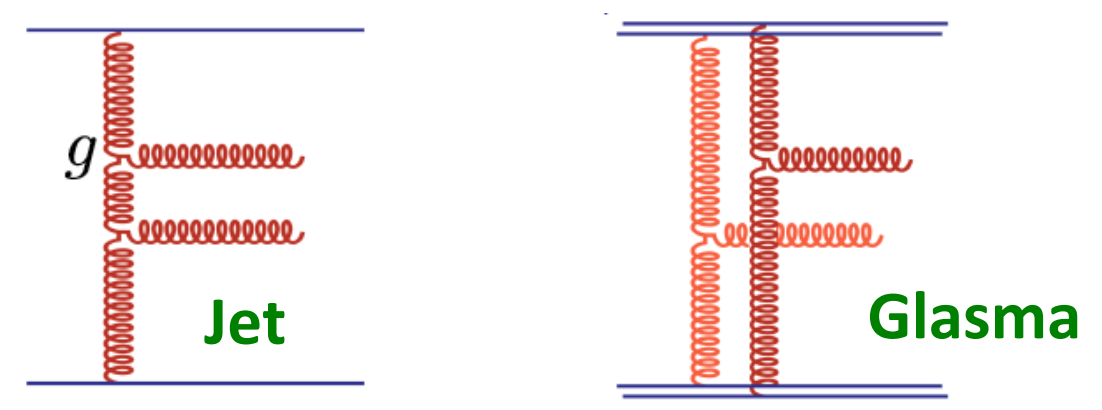

Appears quite different in $p+p$ and $p / A$ versus $A+A$
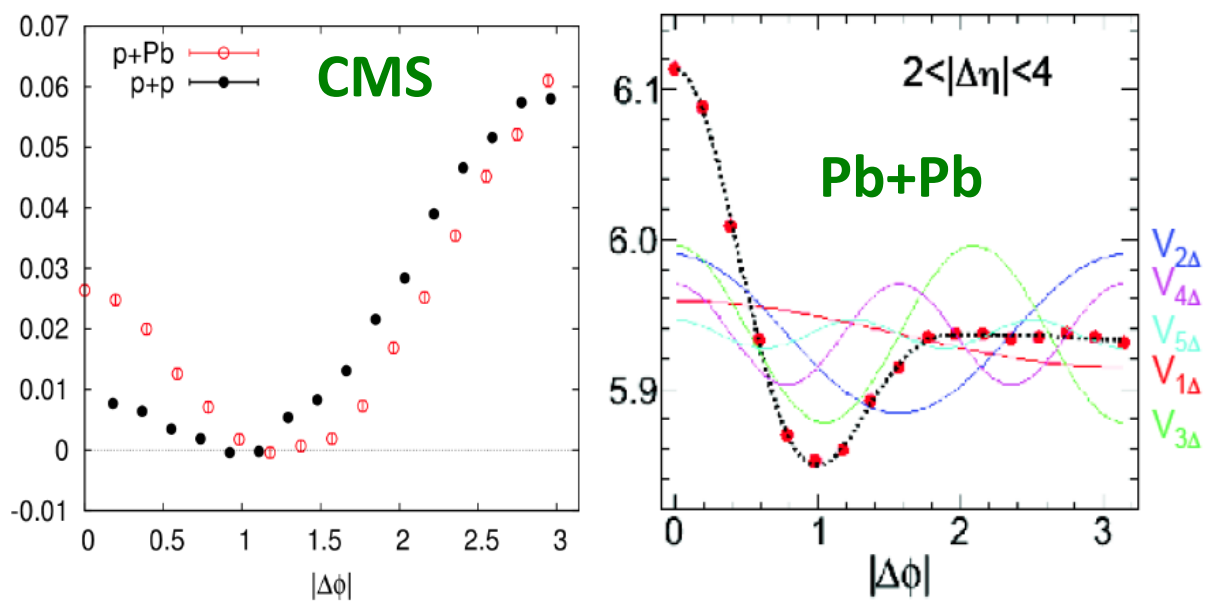


\section{Are there signficant final state effects ?}

Why is jet unmodified while Glasma graph (which generates the $v_{2}$ ) is ?

Appears quite different in $p+p$ and $p / A$ versus $A+A$

Sizes are the same in $p+p$ and $p+\mathrm{Pb}$ whose $" v_{2}$ " is 4 times larger

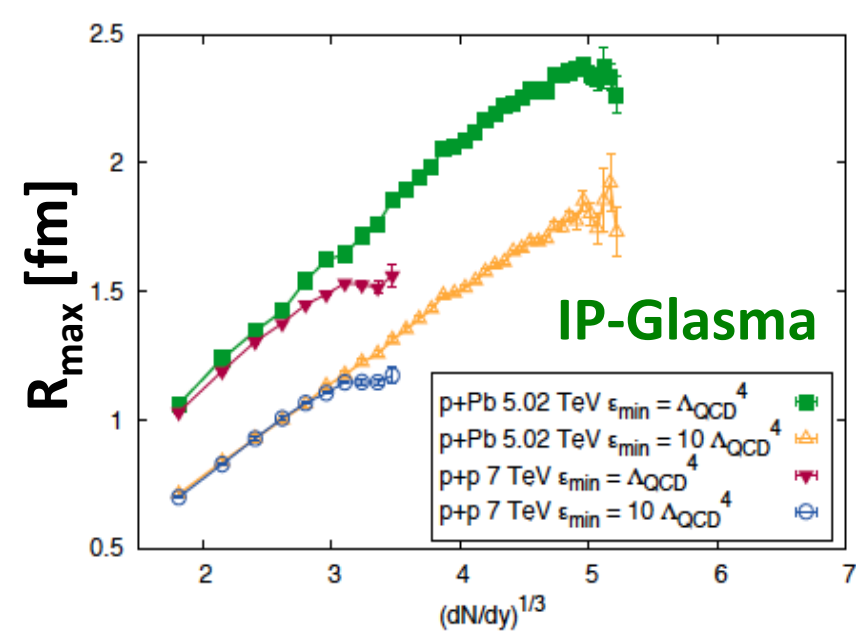

ALICE Data p-p 900GeV p-p 7TeV

$\triangle \mathrm{Pb}-\mathrm{Pb} 2.76 \mathrm{TeV}$ STAR Data

$\checkmark \mathrm{Cu}-\mathrm{Cu}$ 62.4GeV $\mathrm{Cu}-\mathrm{Cu} 200 \mathrm{GeV}$

$\checkmark$ Au-Au 62.4GeV

- Au-Au $200 \mathrm{GeV}$
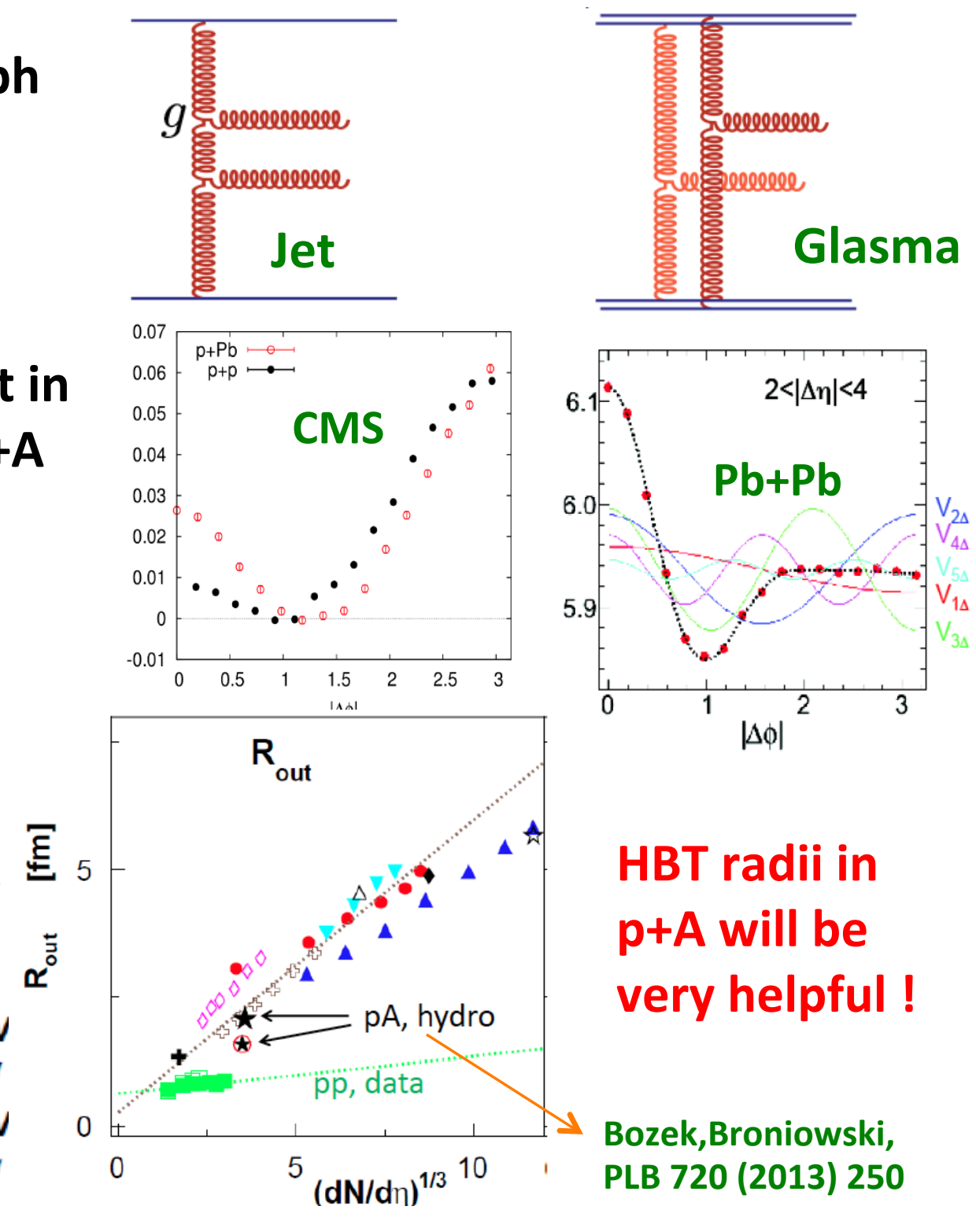

$\mathrm{HBT}$ radii in $p+A$ will be very helpful ! PLB 720 (2013) 250 


\section{Role of flow in $p+p, p / d+A$}

$\diamond$ Is there room for flow in these systems ? See next talk by Piotr Bozek

$\diamond$ Our view: hydro results very sensitive to initial conditions

-- significant differences seen between IP-Glasma and MC-Glauber models

-- flow in $p+p$ is small and at most $50 \%$ less than $p+A$ even for

"nearly ideal" flow simulations

-- large viscous corrections in small size systems

-- talk on Wednesday by Bjoern Schenke Bzdak,Schenke,Tribedy,RV, arXiv:1304.3403 


\section{Role of flow in $p+p, p / d+A$}

$\diamond$ Is there room for flow in these systems ? See next talk by Piotr Bozek

$\diamond$ Our view: hydro results very sensitive to initial conditions

-- significant differences seen between IP-Glasma and MC-Glauber models

-- flow in $p+p$ is small and at most $50 \%$ less than $p+A$ even for

"nearly ideal" flow simulations

-- large viscous corrections in small size systems

-- talk on Wednesday by Bjoern Schenke Bzdak,Schenke,Tribedy,RV, arXiv:1304.3403

$\diamond$ Trend/magnitude of flow different from $\mathrm{p}+\mathrm{Pb}$ data - note Glasma $\eta / \mathrm{s}=0.08$ !
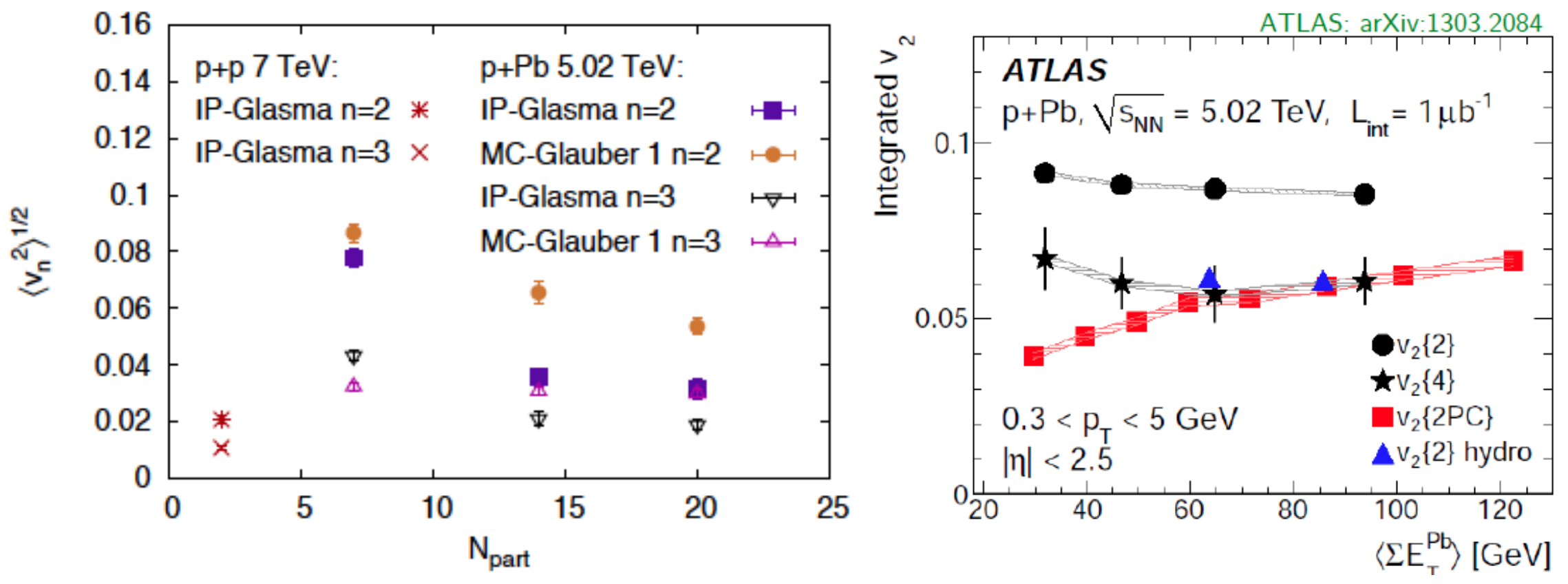


\section{Conclusions}

The Glasma framework provides a systematic description of multi-particle production in $p+p, p+A$ and $A+A$ collisions

The $p+p$ ridge can be quantitatively understood from gluon saturation enhanced quantum interference Glasma graphs + BFKL graphs

- The A+A ridge and $v_{n}$ moments are quantitatively described by IP-Glasma initial conditions + flow

The $\mathrm{p} / \mathrm{d}+\mathrm{A}$ ridge situation is not completely clear yet - but will be clarified soon. 2-part corr. data presented thus far are quantitatively described by Glasma+BFKL dynamics 


\section{EXTRA SLIDES}




\section{The big picture: many-body universal gluodynamics}

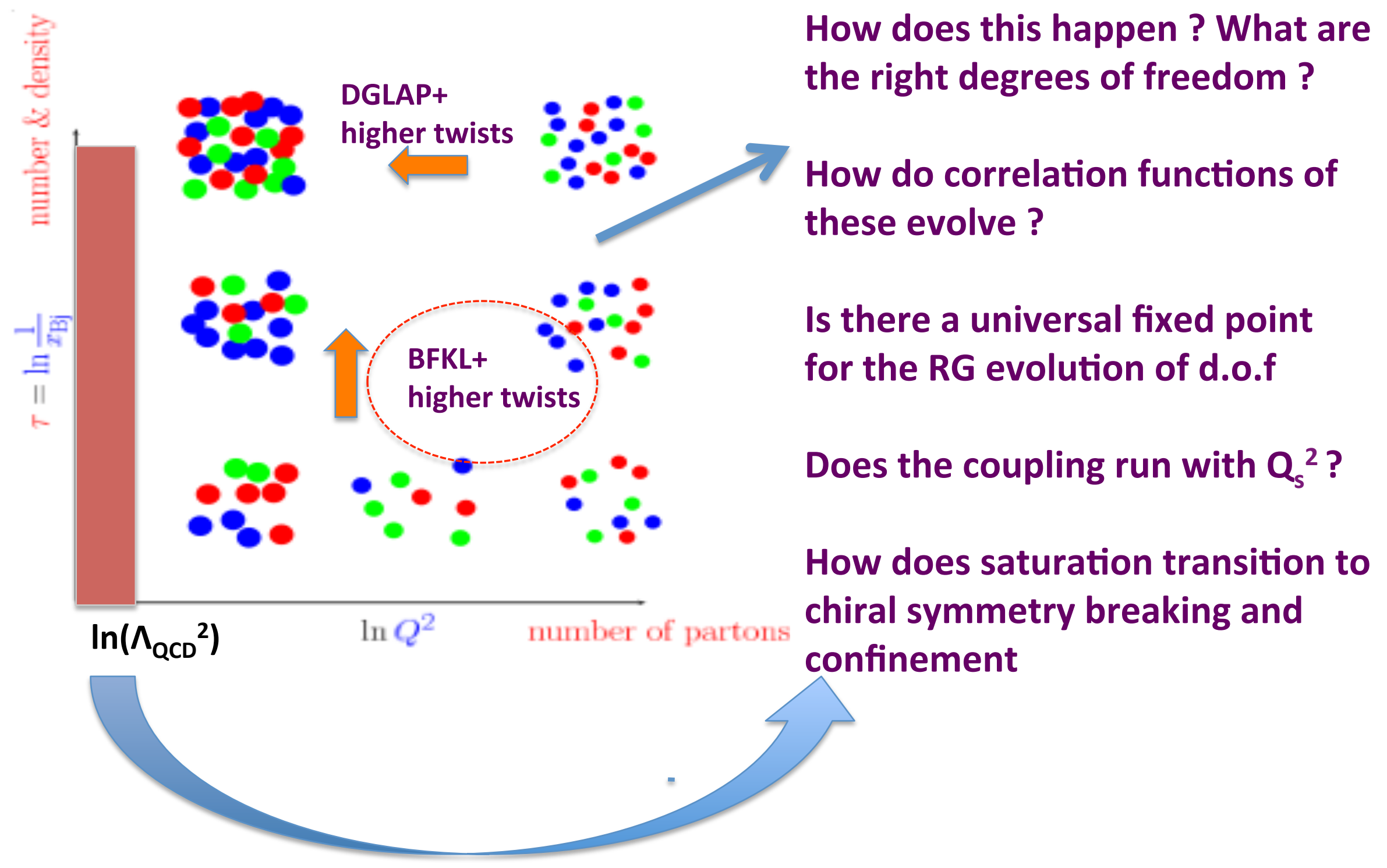




\section{Physics underlying systematics of the ridge}

For Glasma graphs

$$
d^{2} N \propto \int d^{2} k_{T} \Phi_{A}^{2}\left(k_{T}\right) \Phi_{B}\left(\left|p_{T}-k_{T}\right|\right) \Phi_{B}\left(\left|q_{T}-k_{T}\right|\right)
$$

For $\left|p_{T}\right|=\left|q_{T}\right|$, from the Cauchy-Schwarz inequality:

$$
\int d^{2} k_{T} \Phi_{A}^{2}\left(k_{T}\right) \Phi_{B}\left(\left|p_{T}-k_{T}\right|\right) \Phi_{B}\left(\left|q_{T}-k_{T}\right|\right) \leq \int d^{2} k_{T} \Phi_{A}^{2}\left(k_{T}\right) \Phi_{B}^{2}\left(\left|p_{T}-k_{T}\right|\right)
$$

Equality implies no collimation; satisfied only iff $\Phi_{B}\left(\left|p_{T}-k_{T}\right|\right) \propto \Phi_{B}\left(\left|q_{T}-k_{T}\right|\right)$

True only if $\Phi$ is flat in $k_{T}$ - for above fns. Else, there must be a collimation 


\section{Physics underlying the ridge}
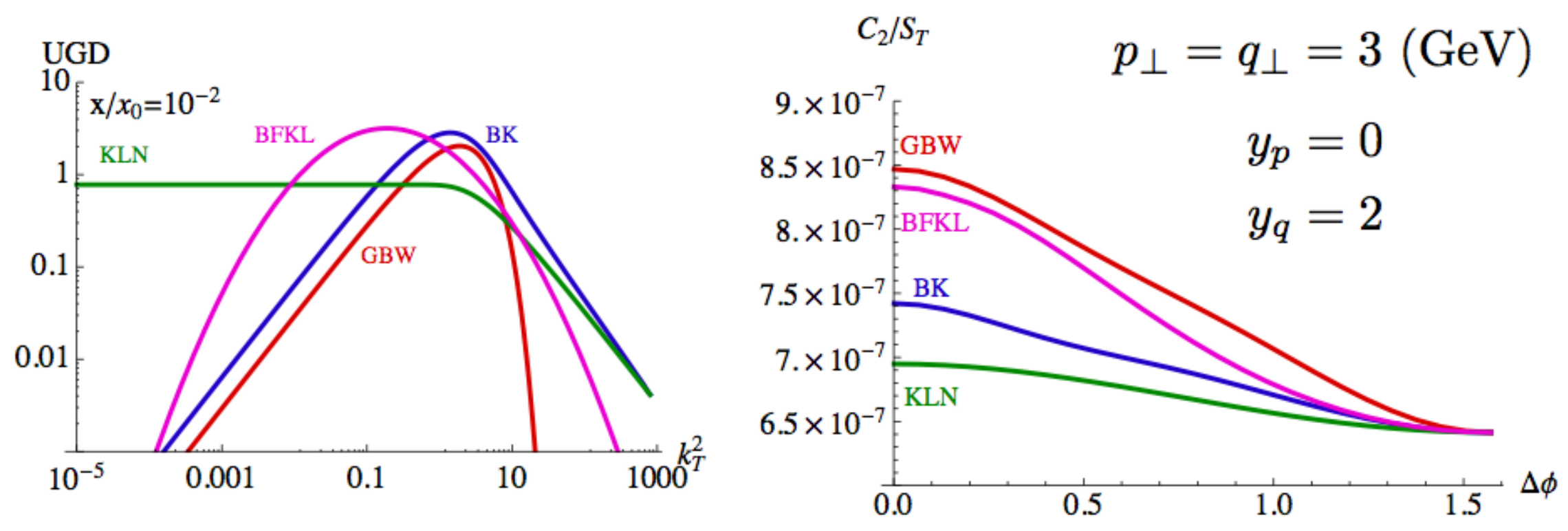


\section{Physics underlying the ridge}

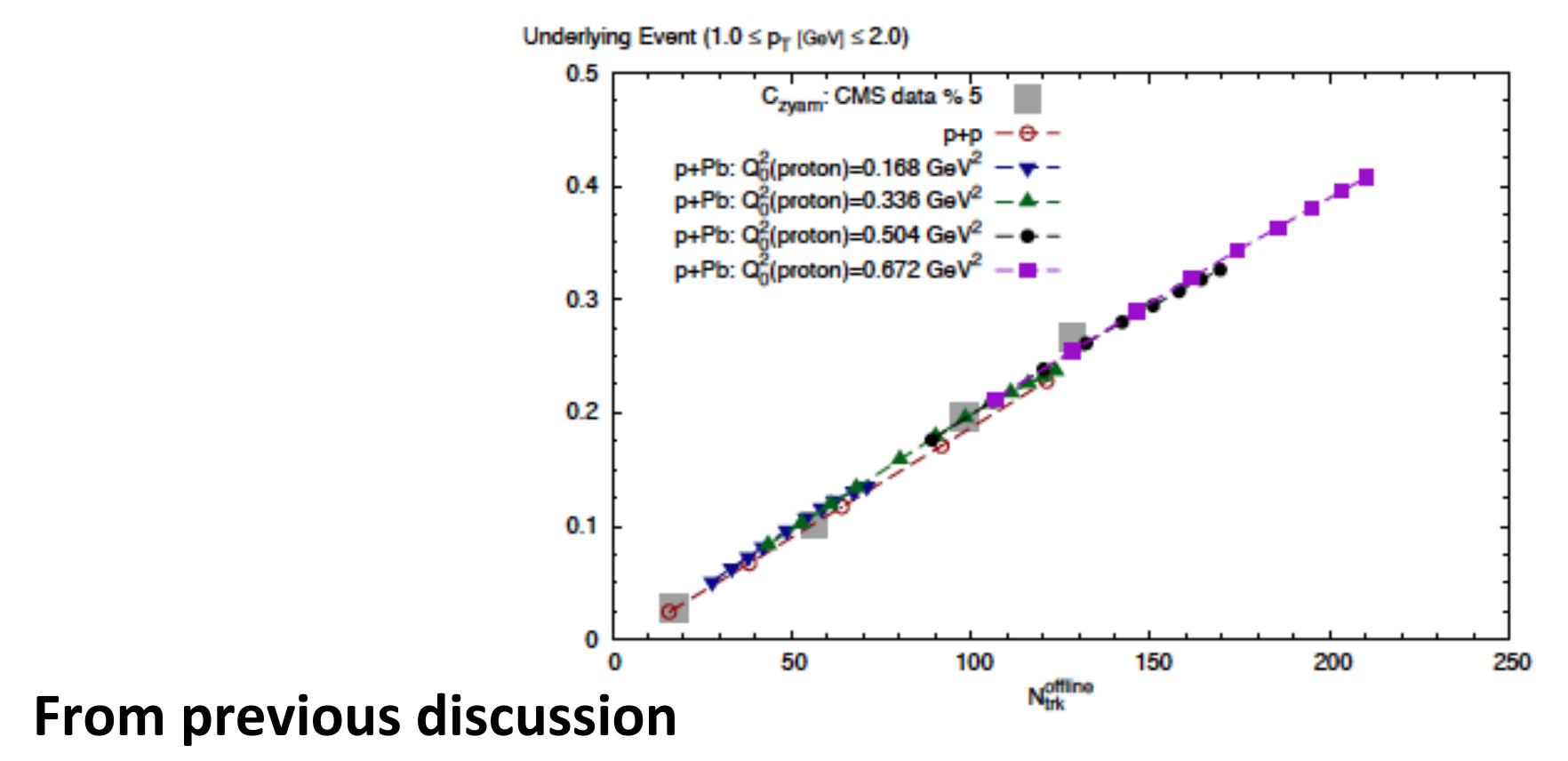

$$
\mathrm{UE} \propto \frac{\int d^{2} k_{T} \Phi_{A}^{2}\left(k_{T}\right) \Phi_{B}^{2}\left(\mid p_{T}-k_{T}\right)}{\int d^{2} k_{T} \Phi_{A}\left(k_{T}\right) \Phi_{B}\left(\left|p_{T}-k_{T}\right|\right)} \propto N_{\text {track }}
$$




\section{rcBK vs IP-Sat evolution}

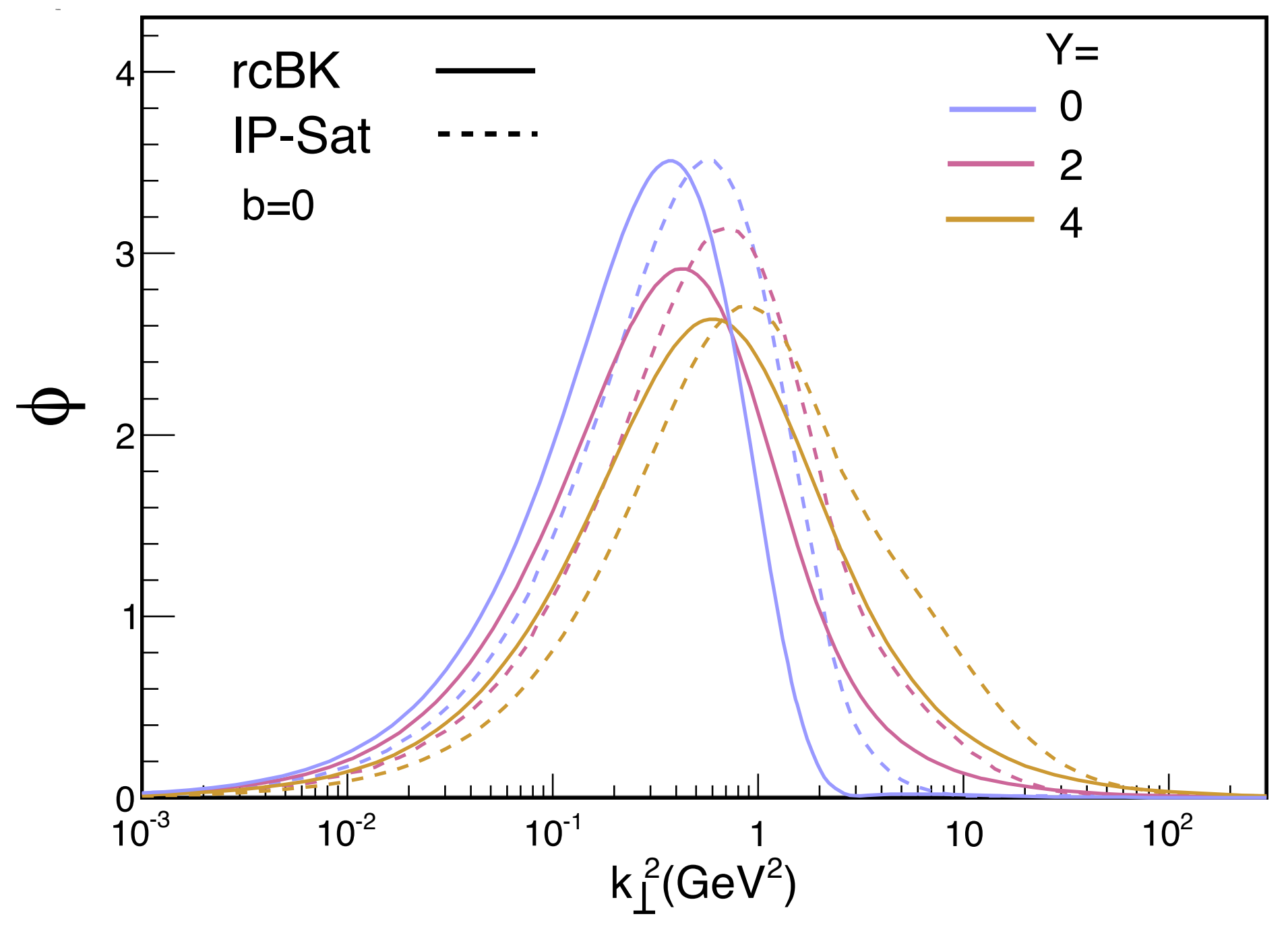


Jet Quenching at RHIC vs LHC in Light of Recent dAu vs pPb Controls

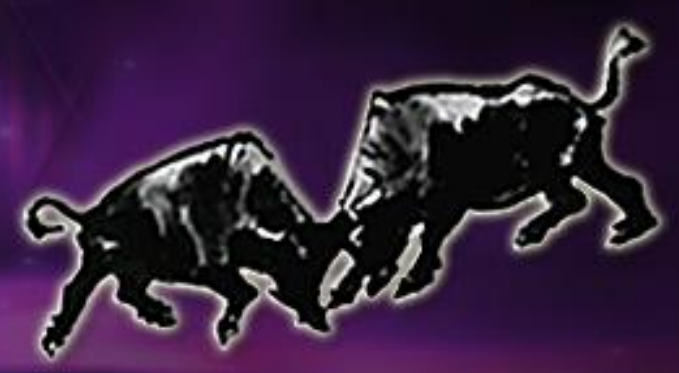

\title{
Particle Correlations at RHIC: Present \& Future
}

\author{
Fuqiang Wang \\ Purdue University
}




\section{Two types of correlations}

\section{FLOW}

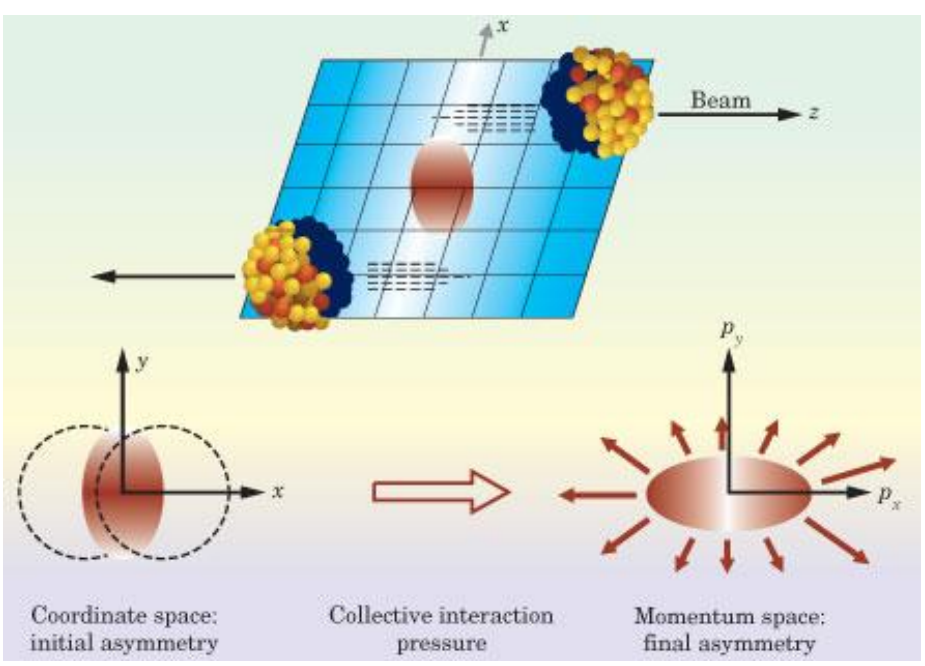

Initial-state anisotropy + fluctuations Hydrodynamic evolution

Final-state event-wise correlations Bulk medium properties

\section{JET QUENCHING}

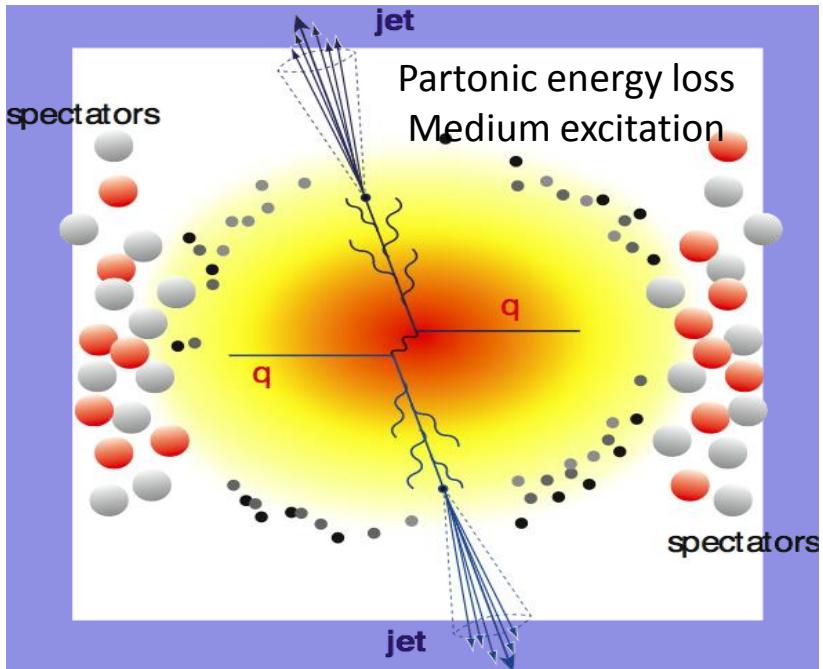

Initial-state hard processes Jet-medium interactions Final-state modified particle correlations Probe $\Delta \mathrm{E}$ mechanisms, medium properties 


\section{Flow and nonflow}

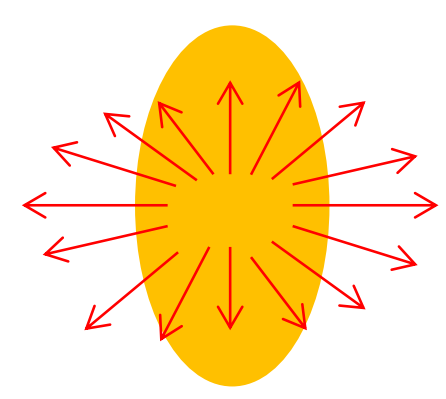

Flow due to hydrodynamic pressure

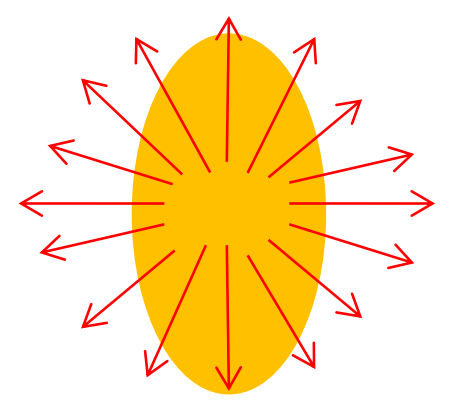

Anisotropy due to pathlength-dep. energy loss

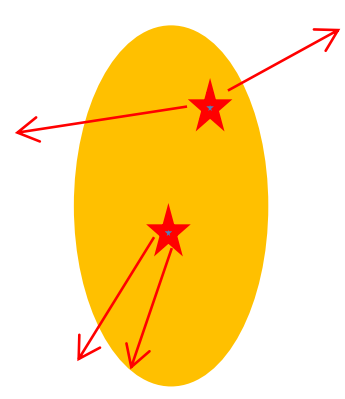

Nonflow correlations 


\section{High $\mathrm{p}_{\mathrm{T}}$ suppression \& re-emergence}
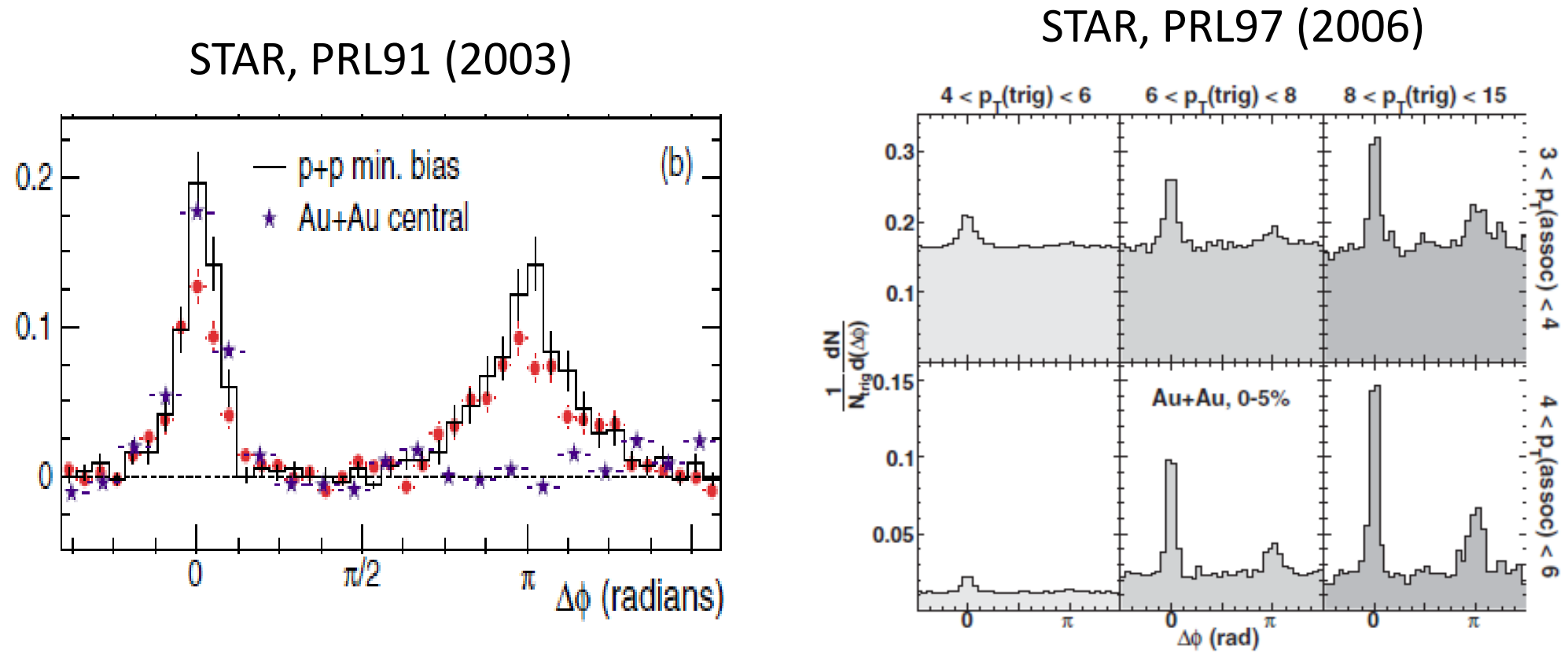

- Clear evidence of jet-quenching, and it's a final-state effect

- Finite probability of non-interacting jets at high $\mathrm{p}_{\mathrm{T}}$. 


\section{Low-intermediate $\mathrm{p}_{\mathrm{T}}$ broadening}

STAR, PRL 95 (2005); PRC82 (2010)

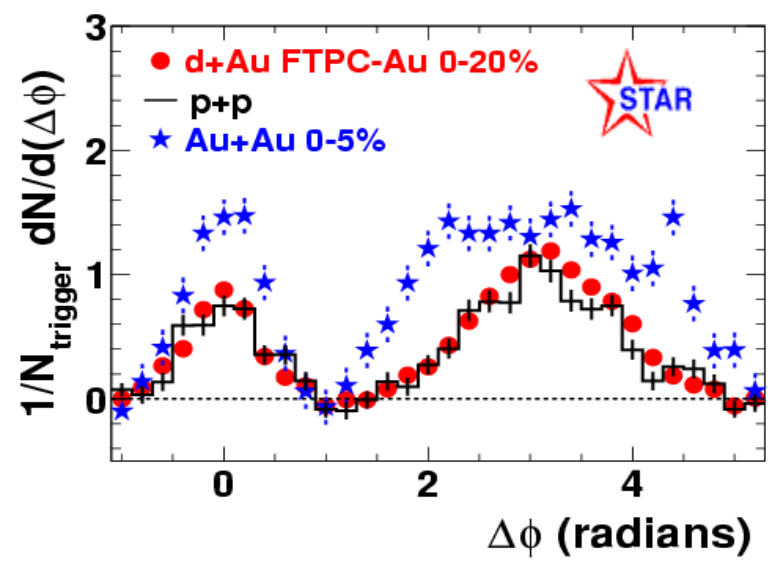

- Low $\mathrm{p}_{\mathrm{T}}$ enhancement and broadening

- Large- $\Delta \eta$ small- $\Delta \phi$ ridge correlation

- Away-side double peak

- $v_{2}$ subtracted but not higher harmonics

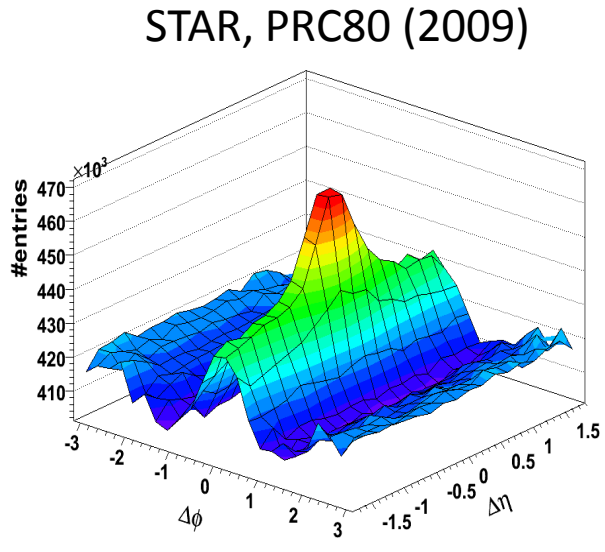

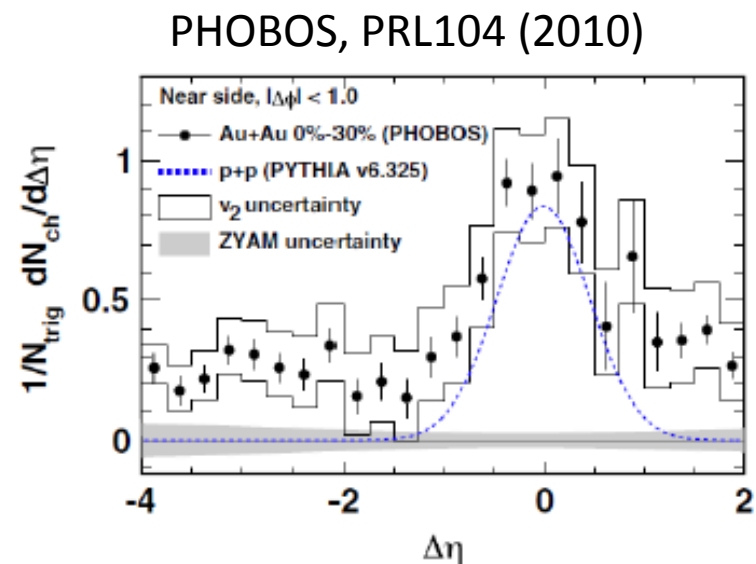

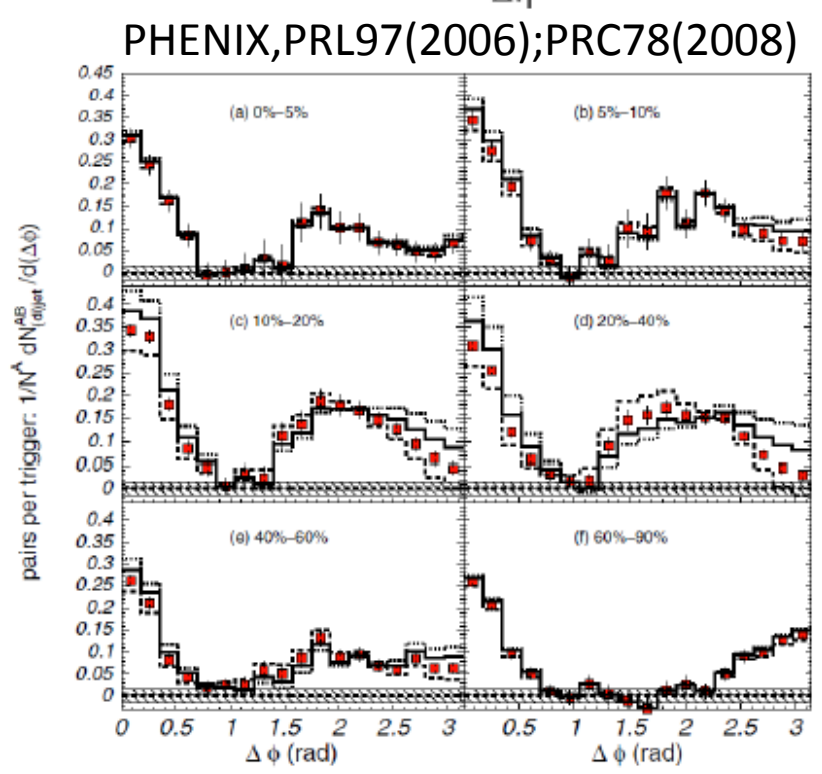




\section{All $v_{n}^{\prime}$ s are possible}

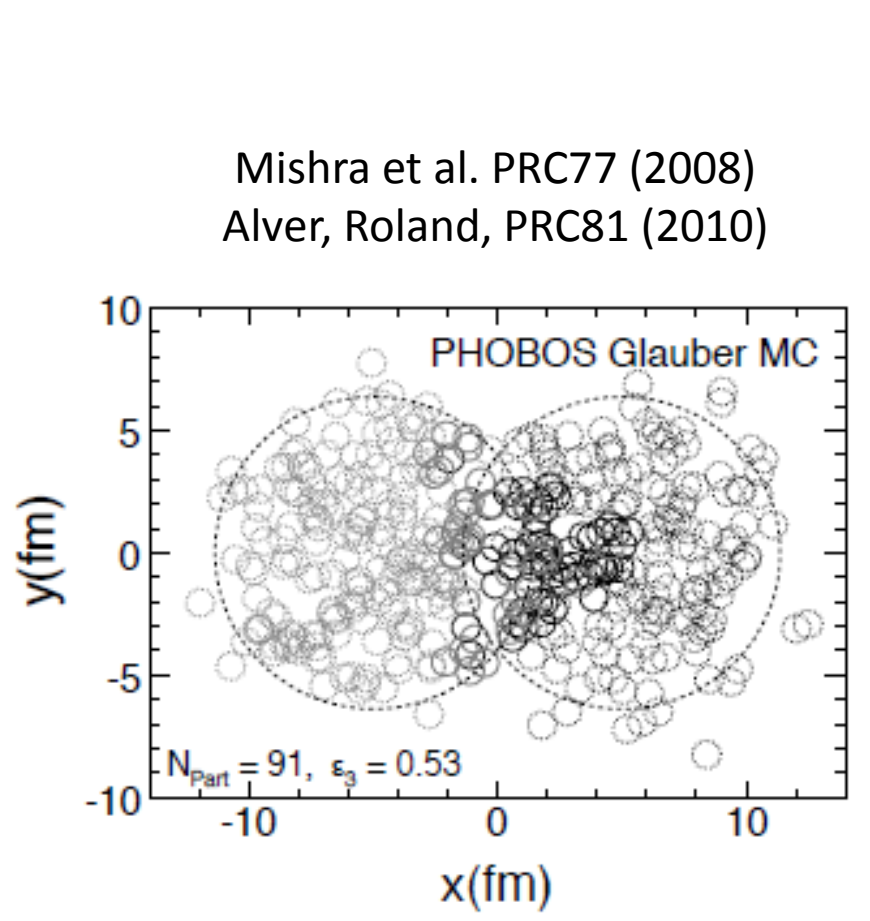

Schenke et al. PRL106 (2011)

Qiu, Heinz, PRC84 (2011)

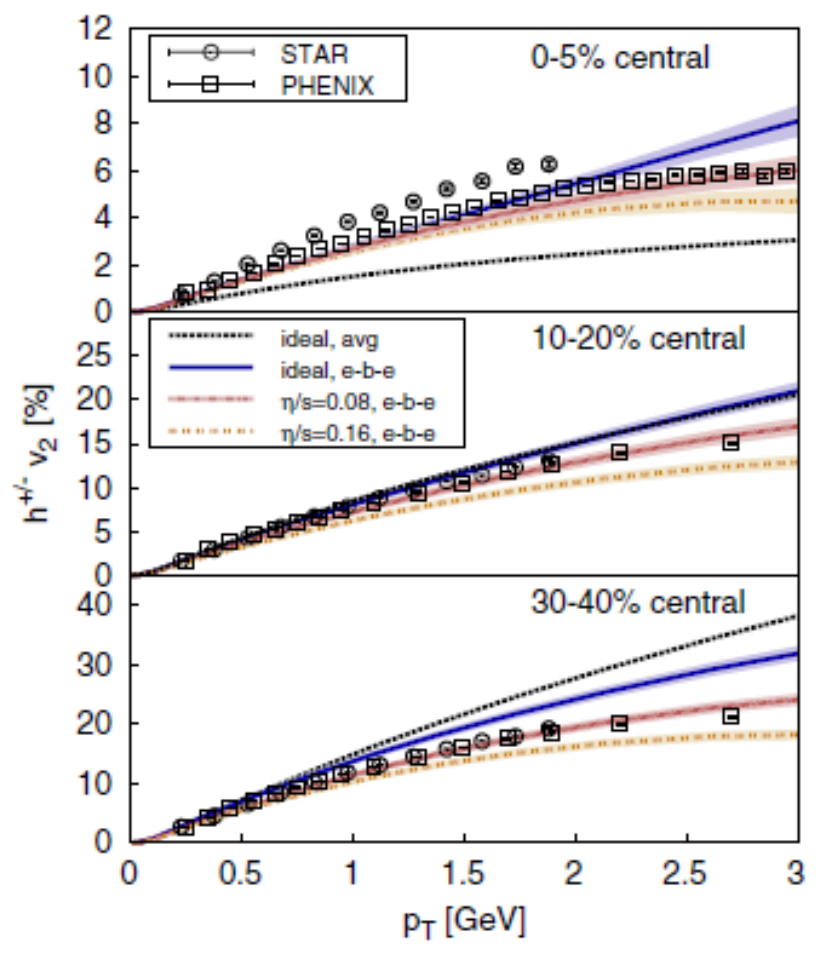

- Event-by-event fluctuations can generate initial-state triangular anisotropy

- Observable consequence in the final-state due to hydro evolution 


\section{$\mathrm{v}_{3}$ measurements at RHIC}

PHENIX, PRL107 (2011)

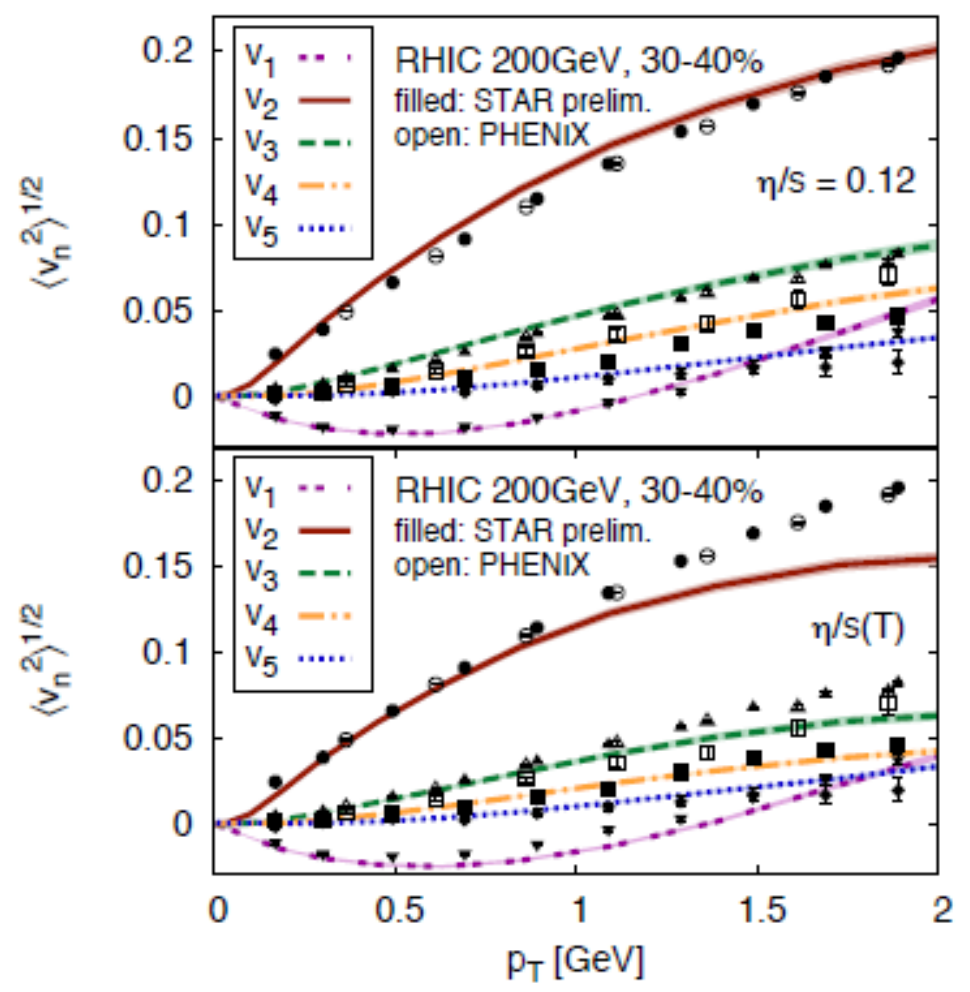

STAR, arXiv:1301.2187

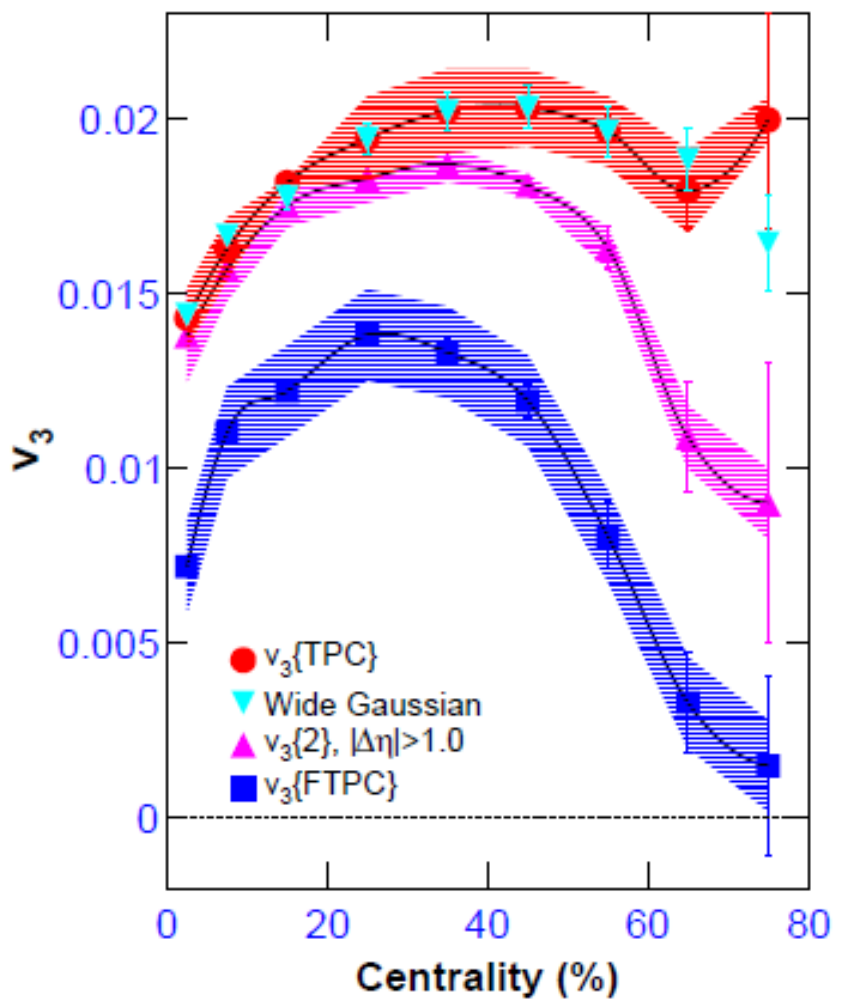

- There might be tension between STAR/PHENIX 


\section{$v_{3}$ decreases with $\Delta \eta$}

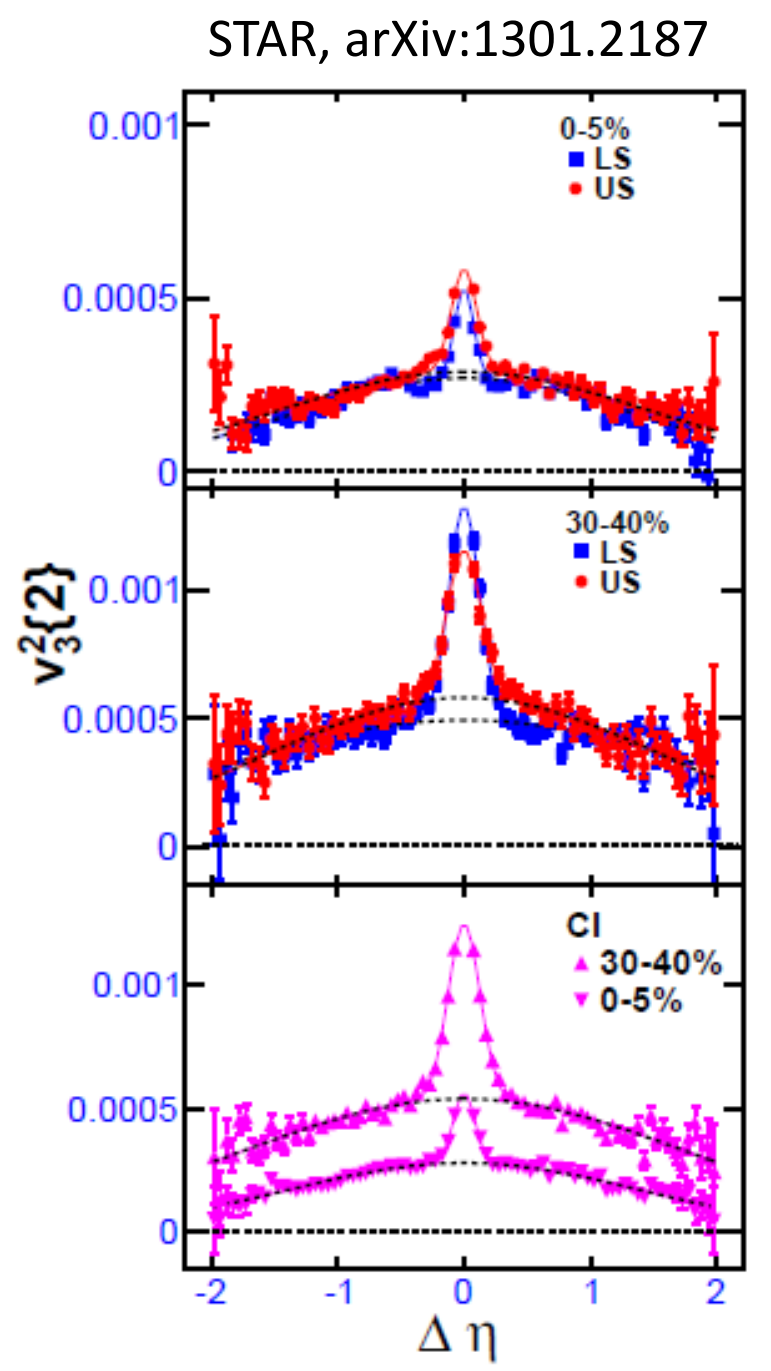

- $v_{3}$ decreases with $\Delta \eta$

- Consistent with untriggered correlations

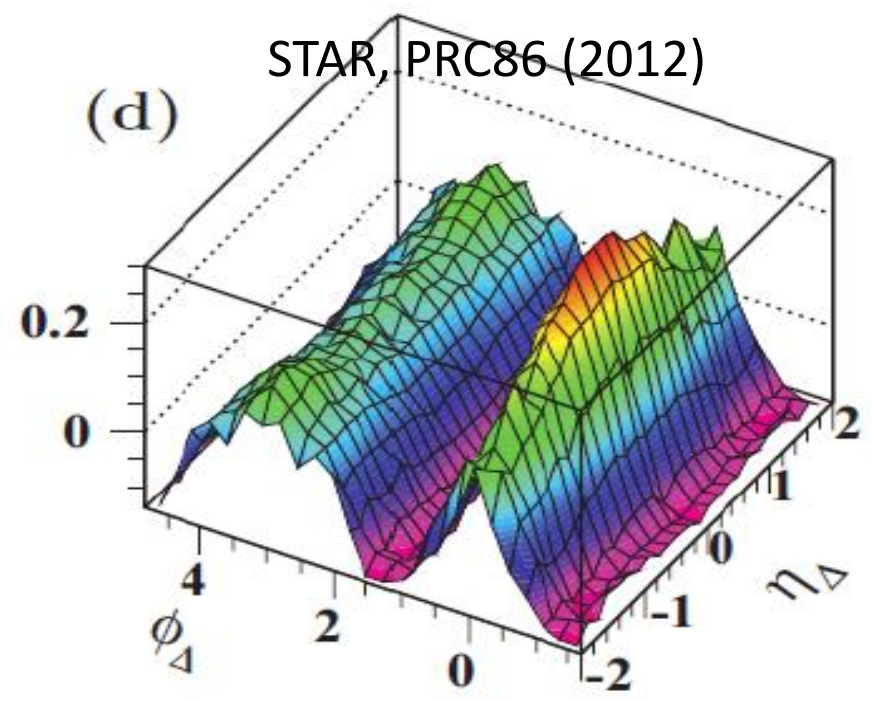




\section{$\mathrm{v}_{3}$ depends $\Delta \eta$}

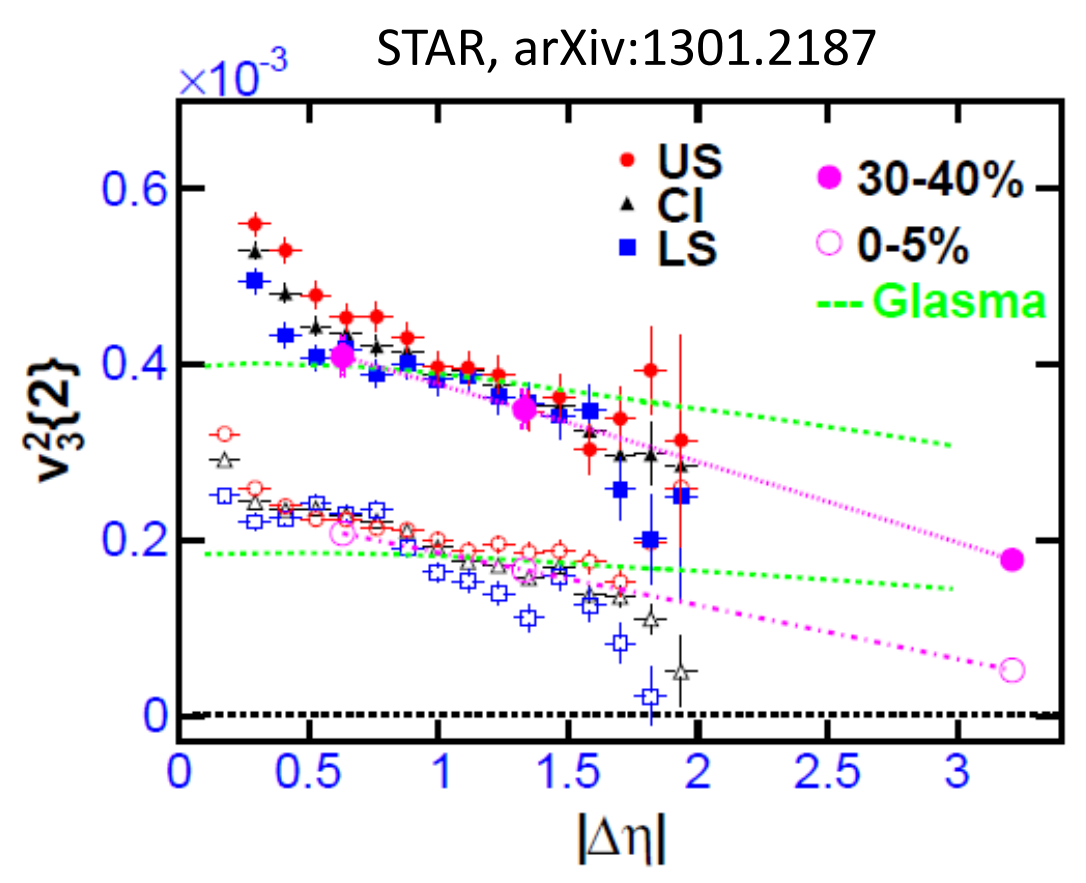

- Nonflow?
- $\Delta \eta$-dependent flow fluctuations? Harmonic planes may decorrelate over $\Delta \eta$

Bozek et al. PRC83 (2011)

Petersen et al. PRC84 (2011)

Xiao et al. PRC87 (2013)

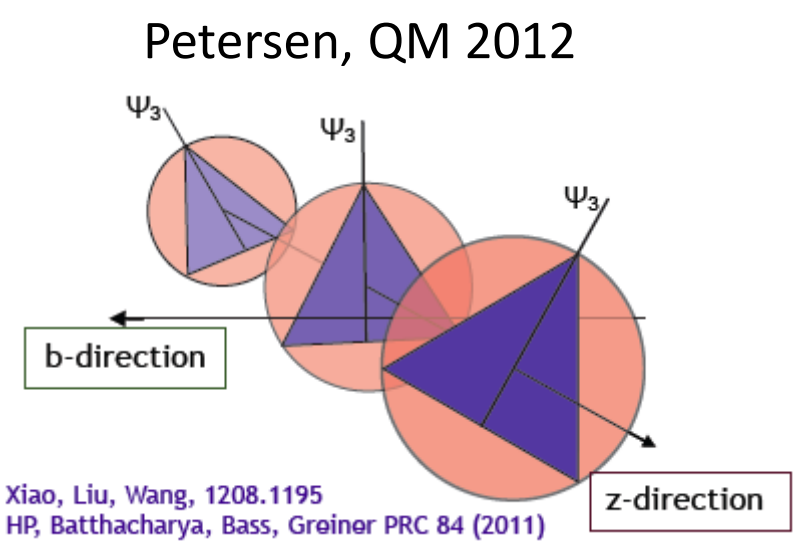

- $\Delta \eta$-gap method may not be suitable 


\section{Flow/nonflow vs. $\eta$}

x 10-4 Li Yi (STAR), QM12, arXiv:1210.6640

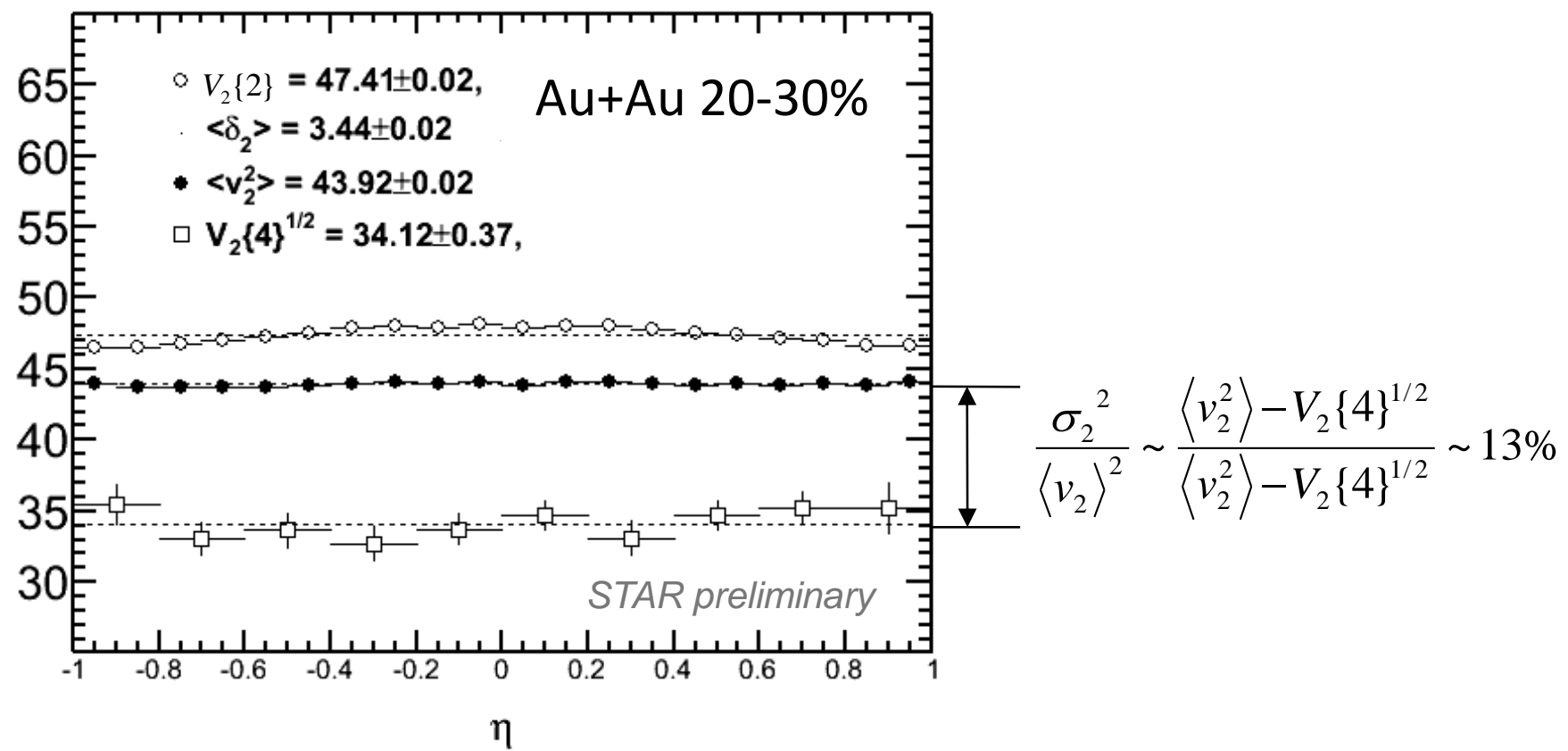

- Method does not assume flow shape vs. $\eta$

- Flow seems independent of $\eta$.

- $(\text { Nonflow/flow) })^{2}$ 4\%, (Nonflow/flow) 20\%,

- $\quad(\text { Fluctuation/flow) })^{2}$ 13\%, Fluctuation/flow 36\% 


\section{Why is 'flow' factorization so good? Because it is bootstrapped!}

$$
V_{n}\left(p_{T}^{a}, p_{T}^{b}\right) \times V_{n}\left(p_{T}^{r e f}, p_{T}^{r e f}\right) \approx V_{n}\left(p_{T}^{a}, p_{T}^{r e f}\right) \times V_{n}\left(p_{T}^{b}, p_{T}^{r e f}\right)
$$

Kikola et al. PRC86 (2012) 014901

Independent jet fragmentation $\rightarrow$ Jet correlation may approximately factorize!

$$
\left\langle\cos n\left(\phi_{i}-\phi_{j}\right)\right\rangle=\left\langle\cos n\left[\left(\phi_{i}-\psi_{j e t}\right)-\left(\phi_{j}-\psi_{j e t}\right)\right]\right\rangle=\left\langle\cos n\left(\phi_{i}-\psi_{j e t}\right)\right\rangle\left\langle\cos n\left(\phi_{j}-\psi_{j e t}\right)\right\rangle
$$

Anisotropic flow + non-flow: $\quad V_{2 \Delta}\left(p_{T}^{a}, p_{T}^{b}\right)=v_{2}\left(p_{T}^{a}\right) v_{2}\left(p_{T}^{b}\right)+\delta_{2}\left(p_{T}^{a}\right) \delta_{2}\left(p_{T}^{b}\right)$

$$
\begin{aligned}
& \frac{V_{n \Delta}\left(p_{T}^{b}, p_{T}^{a}\right)}{v_{n}^{\prime}\left(p_{T}^{a}\right) v_{n}^{\prime}\left(p_{T}^{b}\right)}-1 \\
& \quad=\frac{v_{n}\left(p_{T}^{b}\right) v_{n}\left(p_{T}^{c}\right)+\delta_{n}\left(p_{T}^{b}\right) \delta_{n}\left(p_{T}^{c}\right)}{\frac{v_{n}\left(p_{T}^{a}\right) v_{n}\left(p_{T}^{c}\right)+\delta_{n}\left(p_{T}^{a}\right) \delta_{n}\left(p_{T}^{c}\right)}{\sqrt{v_{n}^{2}\left(p_{T}^{c}\right)+\delta_{n}^{2}\left(p_{T}^{c}\right)}} \frac{\left.v_{T}^{b}\right) v_{n}\left(p_{T}^{c}\right)+\delta_{n}\left(p_{T}^{b}\right) \delta_{n}\left(p_{T}^{c}\right)}{\sqrt{v_{n}^{2}\left(p_{T}^{c}\right)+\delta_{n}^{2}\left(p_{T}^{c}\right)}}-1} \\
& \quad \approx\left(\frac{\delta_{n}\left(p_{T}^{a}\right)}{v_{n}\left(p_{T}^{a}\right)}-\frac{\delta_{n}\left(p_{T}^{c}\right)}{v_{n}\left(p_{T}^{c}\right)}\right)\left(\frac{\delta_{n}\left(p_{T}^{b}\right)}{v_{n}\left(p_{T}^{b}\right)}-\frac{\delta_{n}\left(p_{T}^{c}\right)}{v_{n}\left(p_{T}^{c}\right)}\right)
\end{aligned}
$$

- $\delta_{\mathrm{n}}\left(\mathrm{p}_{\mathrm{T}}\right) / \mathrm{v}_{\mathrm{n}}\left(\mathrm{p}_{\mathrm{T}}\right) \sim 20 \% \rightarrow$ deviation $\sim 10^{-2}-10^{-3}$

- $\delta_{n}\left(p_{T}\right) \propto v_{n}\left(p_{T}\right) \rightarrow$ precise factorization 


\section{Is it important to remove nonflow?}

Important because it affects extracted QGP medium property, such as $\eta / s$.

Hydro-data comparison (e.g. Uli Heinz): v/ $\varepsilon \rightarrow \eta / s \sim(1-2) * 1 / 4 \pi$ $20 \%$ error on $\varepsilon$ (Glauber vs CGC) $\rightarrow 100 \%$ uncertainty on $\eta / s$.

The question is how to reduce uncertainty in $\mathrm{v}_{2} / \varepsilon$ :

1. $\varepsilon$ from theoretical part

2. $v_{2}$ from experimental part

Song, Bass, Heinz, etal. PRL 106, 192301 (2011)

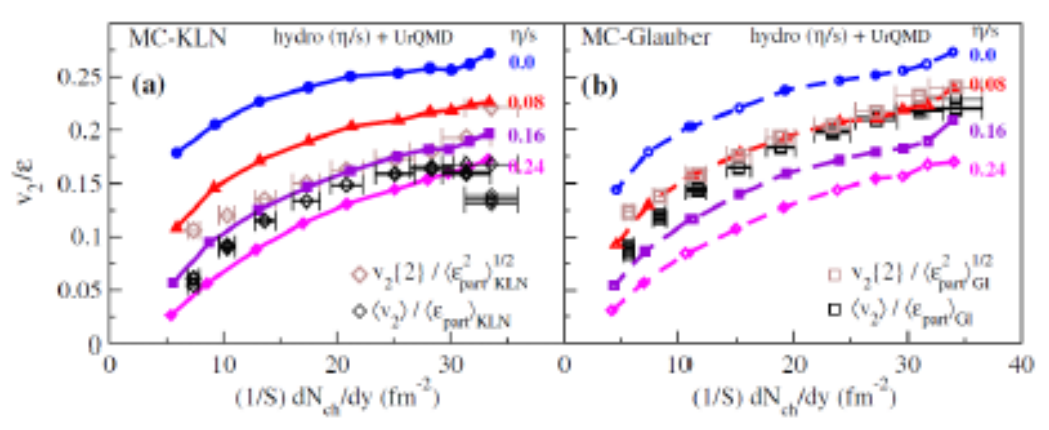

"The extraction of $\eta / s$ from a comparison with hydrodynamics thus requires careful treatment of both fluctuation and nonflow effects" 


\section{$\mathrm{v}_{\mathrm{n}}$ background subtraction}

- It's absolutely important to remove all sizeable $\mathrm{v}_{\mathrm{n}}$ 's in jet-correlations

- What $v_{n}$ to subtract? $v_{n}$ with minimal jet (nonflow) contributions

- But $v_{n}$ have to be measured by final-state particle correlations
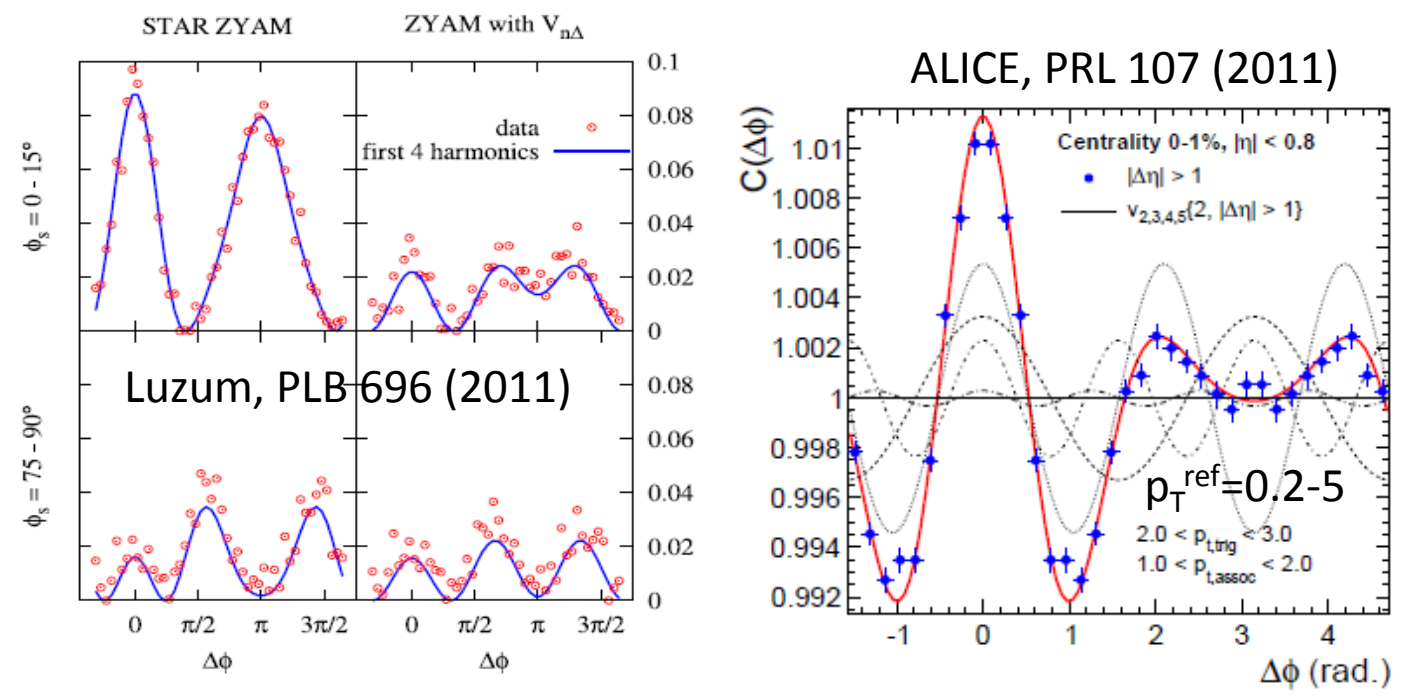

Word of caution: With nonvanishing odd harmonics, nothing really prevents people from fitting everything to $v_{n}$. Fine in itself, but dangerous if people subsequently take it as entirely hydro flow.

The real question is what's in $v_{n}$ ?

- Apply $\Delta \eta$-gap: may not work because of potential $\Delta \eta$-dep. flow fluctuations

- Viable way is to use as low $p_{T}$ reference particles as possible.

Disadvantage is flow is small at low $p_{T}$. 


\section{Dihadron correl. with vn subtractions}

Todoroki (PHENIX), QM12, arXiv:1304.2852

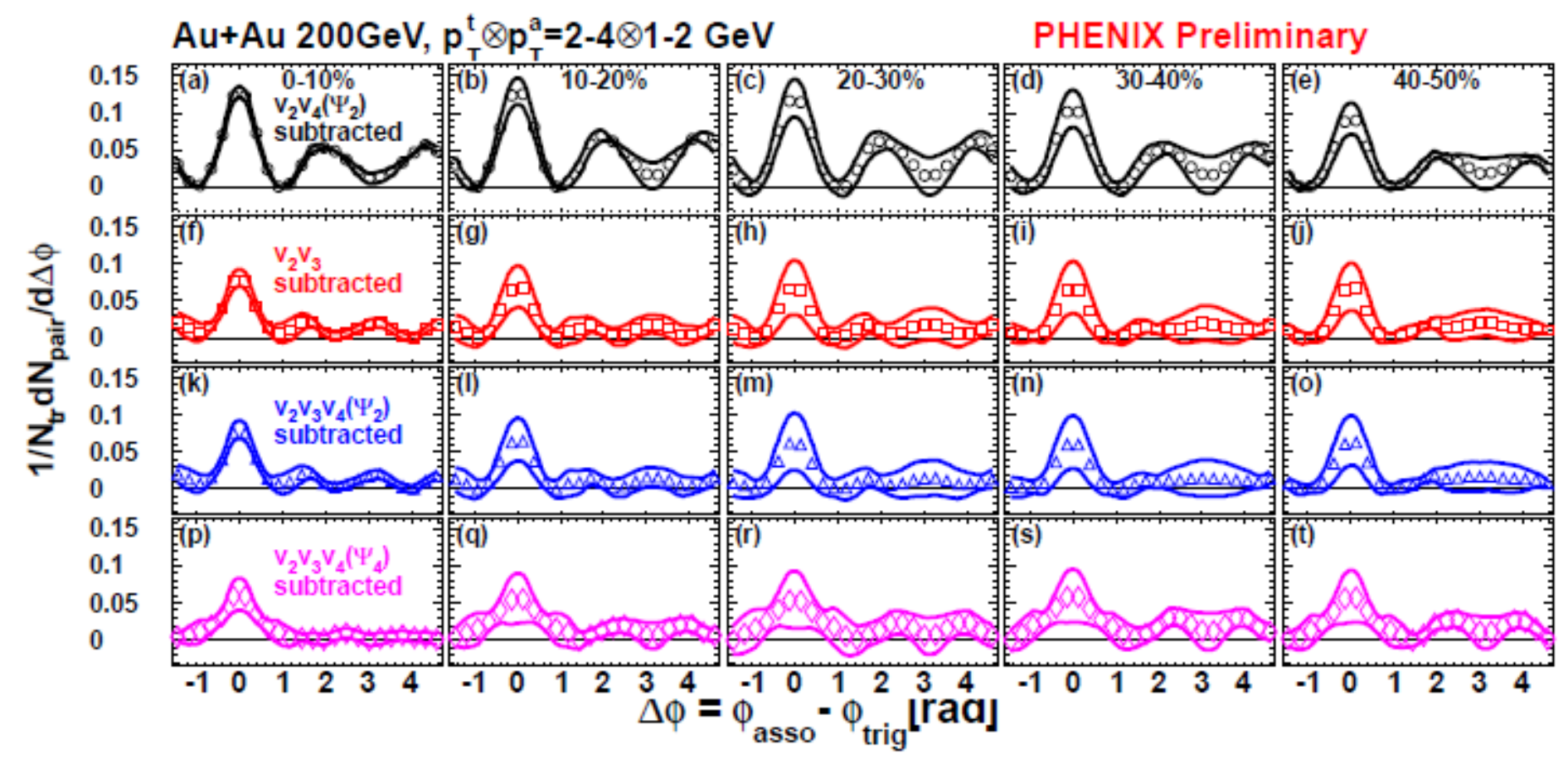




\section{Relative to the Event Plane}
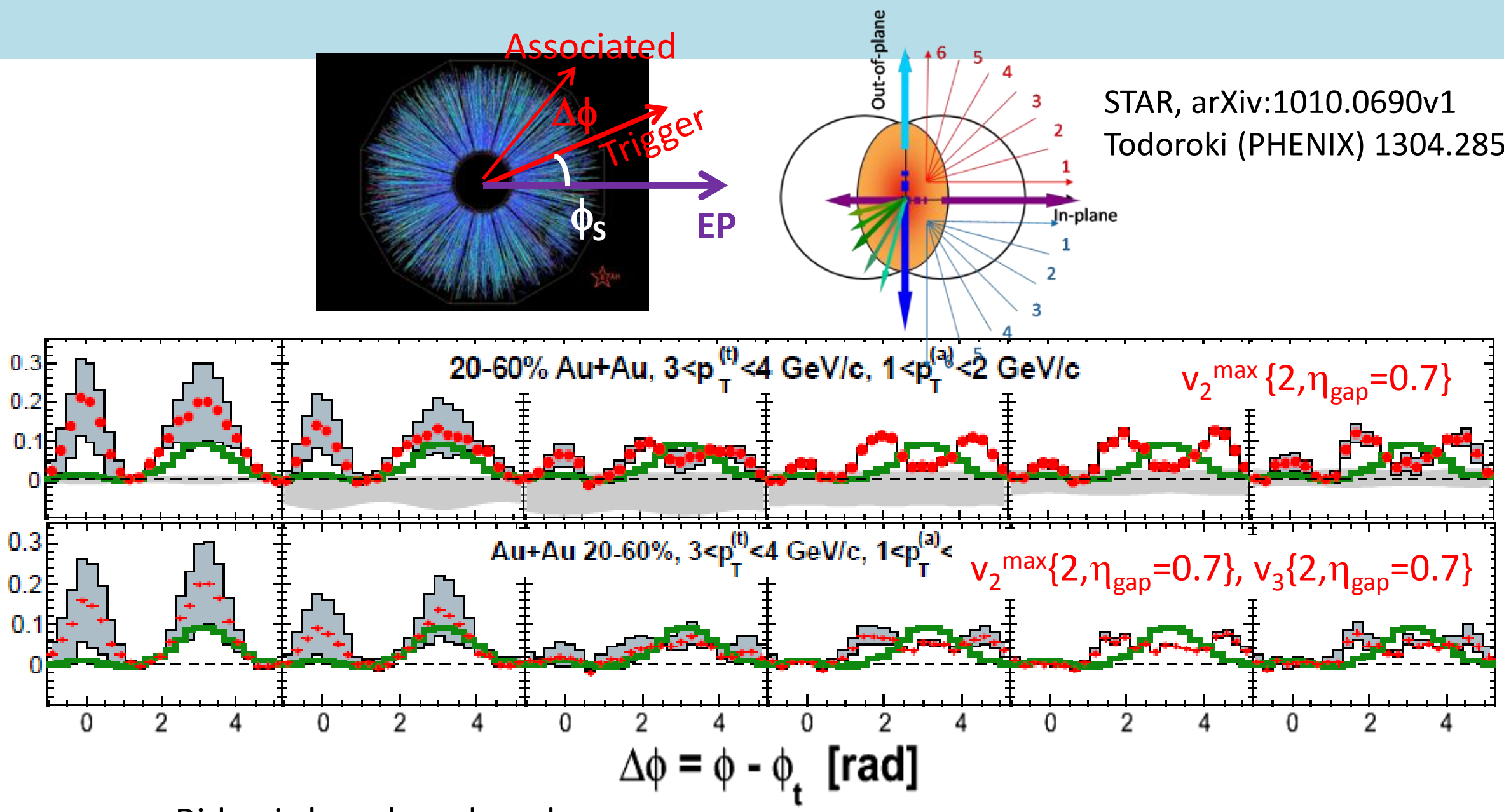

- Ridge is largely reduced.

- Evolution of structure seems to remain from in-plan to out-of-plane.

- Possible biases in EP reconstruction due to jet-correlations? 


\section{$v_{3}$ effect on 3-particle correl.}

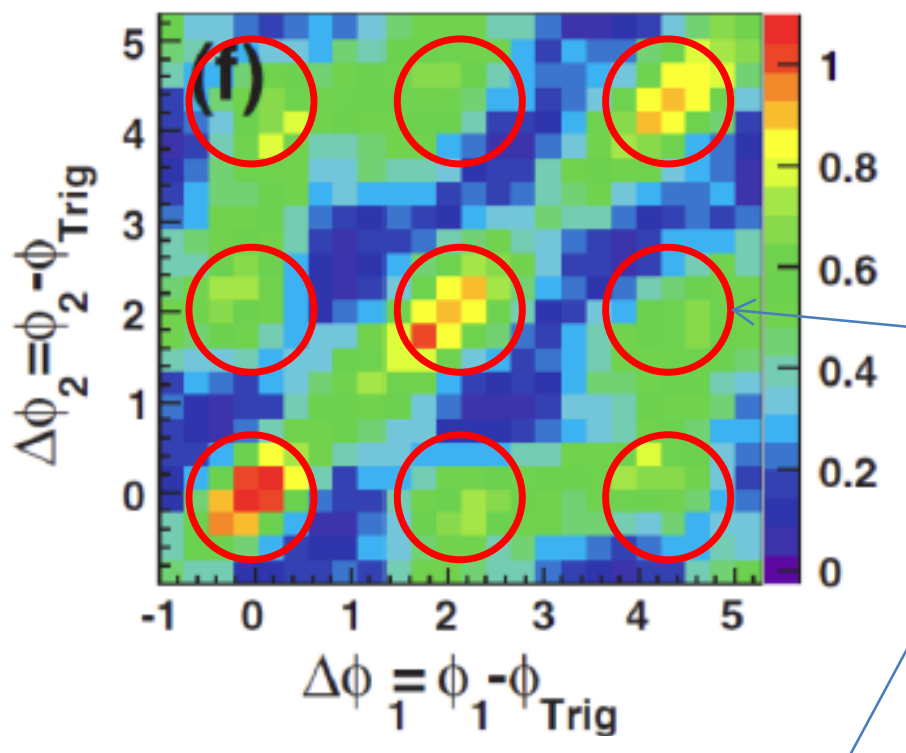

- v2 and v4 subtracted.

- Does v3 remove all of the off-diag. peak strength? Need further study.

- Deflected jets contributions (to diag. peaks) must be present.

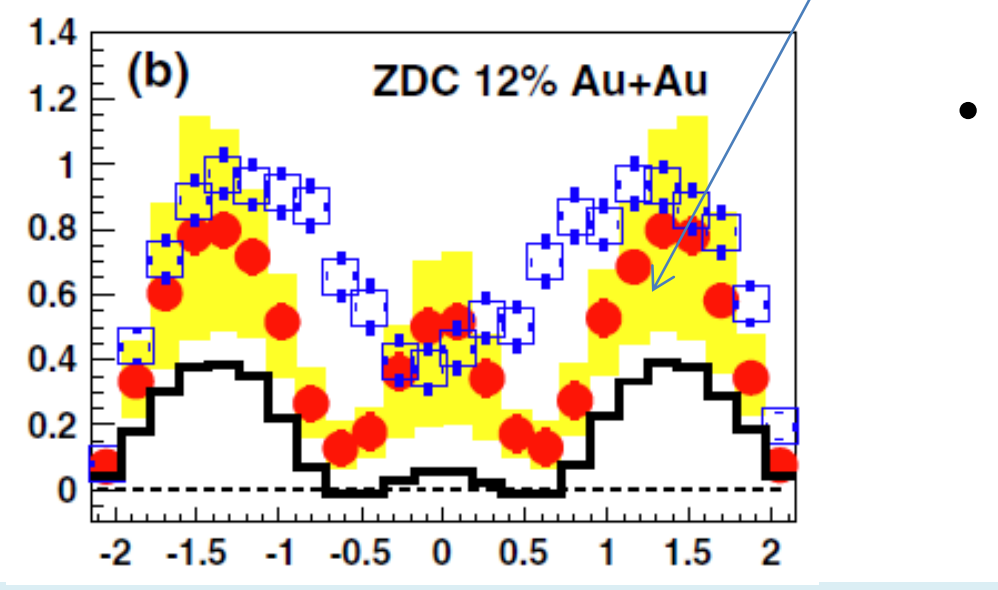

- Need to be followed up. 


\section{$\mathrm{pA}$ and $\mathrm{dA}$ ridge}

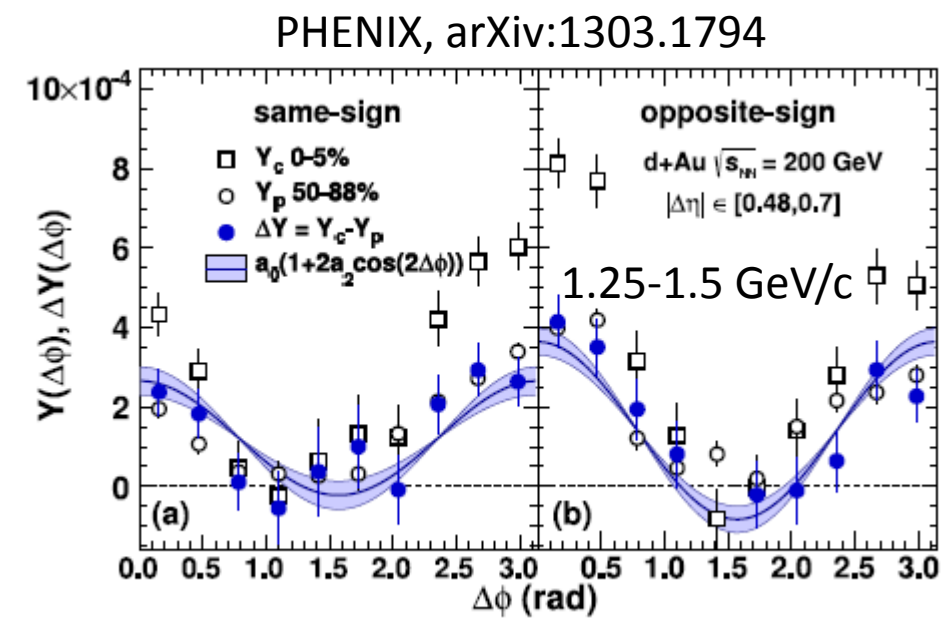

Bozek, PRC85 (2012)

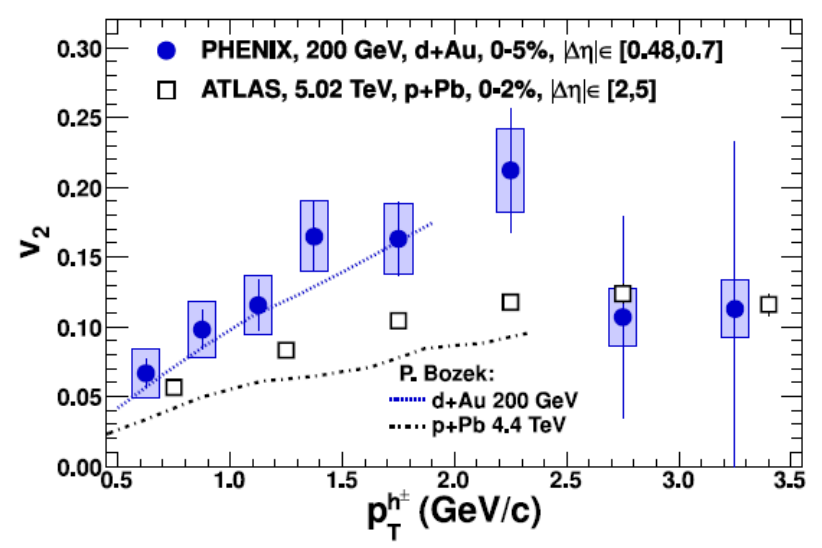

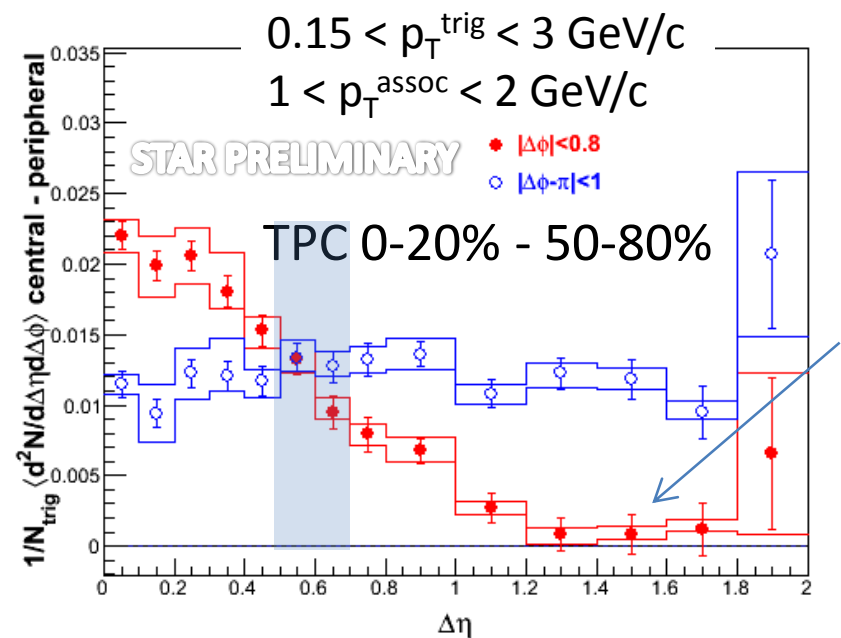

Minimal ridge
Dusling and Venugopalan, arXiv:1302.7018

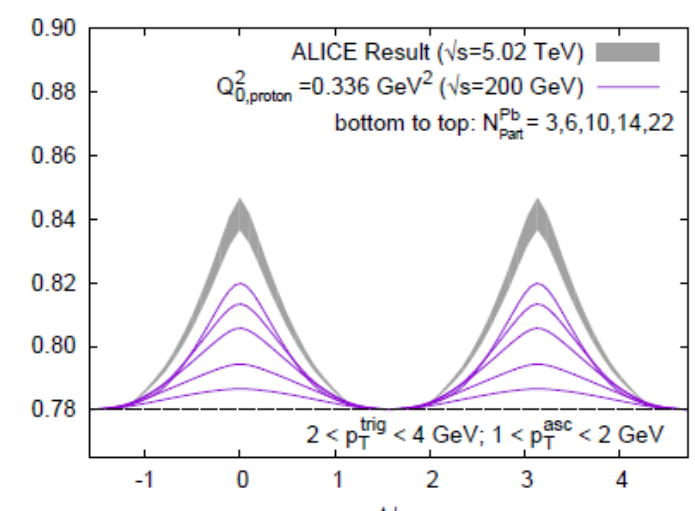

$\Delta \phi$

- $\mathrm{LHC}+\mathrm{RHIC}$ more stringent test on theoretical models. 


\section{Where is hydro bkgd to triggers?}

C. Shen et al. PRC82 (2010) 054904

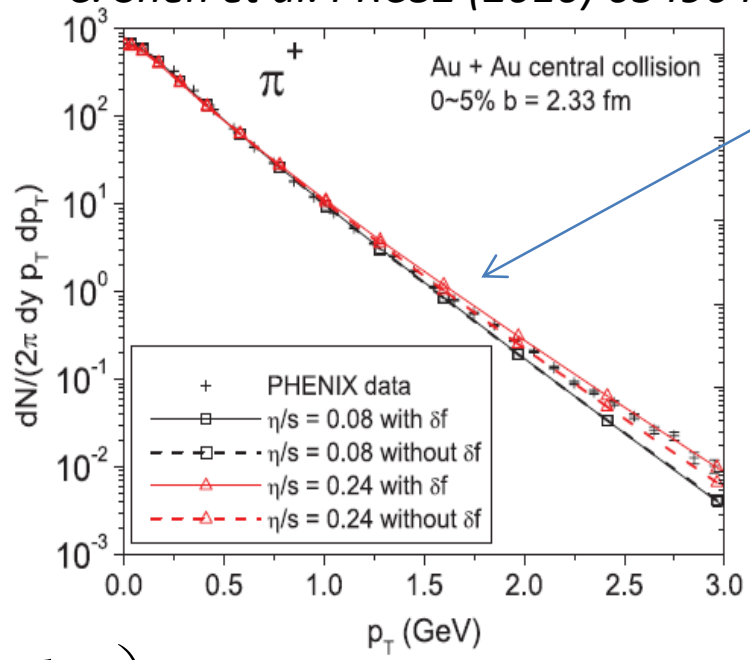

- Going to lower trigger $\mathrm{p}_{\mathrm{T}}$, expect large hydro contribution to particle production $\rightarrow$ Jet-like correlations should be reduced.

- Surprisingly similar $A u+A u$ and $d+A u$ near-side jet-like signal strength

- No evidence of trigger dilution from hydro background triggers

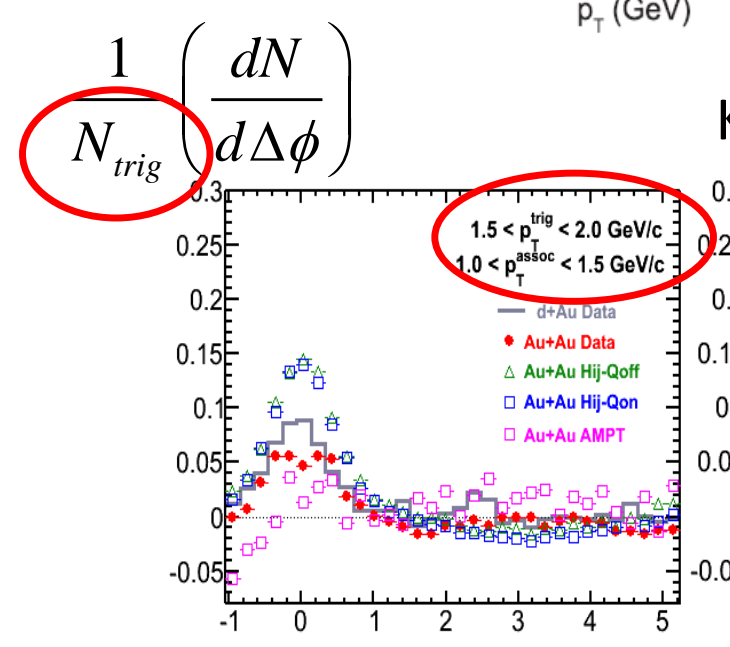

Konzer (STAR) QM 2012, STAR preliminary

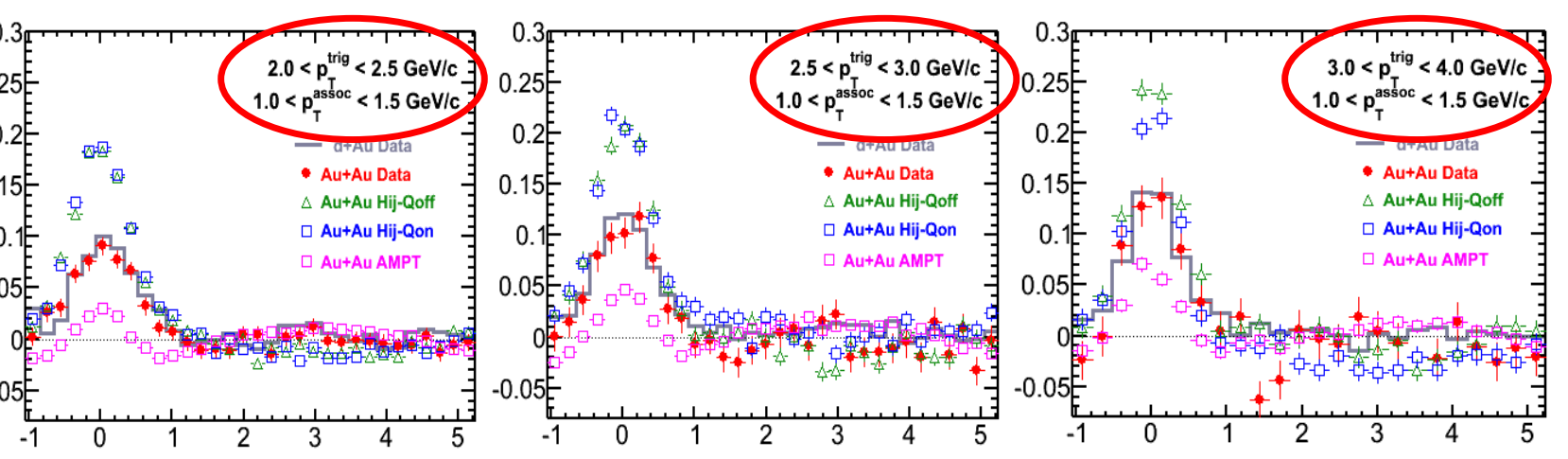




\section{Prospective future measurements}

- Hydro $v_{n}$ explains majority of ridge and double-peak correlations in heavy-ions. Does hydro $v_{n}$ explain all of the correlation signals?

- $v_{n}$ measurements with minimal nonflow: go to low $p_{T}$ reference particles, $\Delta \eta$-gap?

- Precision measurements of jet-correlations with $v_{n}$ subtraction

- Dihadron correlations w.r.t. EP with EP far removed in $\eta$.

- More clever way to separate flow/nonflow?

- $\quad \gamma$-jet with no flow background

- Heavy flavor induced correlations

- Can we learn something fundamental from small systems?

- How high dA multiplicity can we reach at RHIC? 


\title{
Harmonic jet tomography at RHIC+LHC
}

\author{
Xilin Zhang (Ohio U)
}

Jet Quenching Workshop, RIKEN BNL, April 2013

\author{
$X . Z$. and Jinfeng Liao, \\ PLB 713, 35 (2012) \\ arXiv:1208.6361, 1210.1245.
}




\section{Outline}

- Jet tomography: geometry and fluctuations; different jet energy loss models

- High-pt $\mathrm{Vn}$ at different centralities from RHIC to LHC

- Hard-soft correlation

- P-Pb case

- Conclusion and outlook 


\section{Jet tomography: geometry}
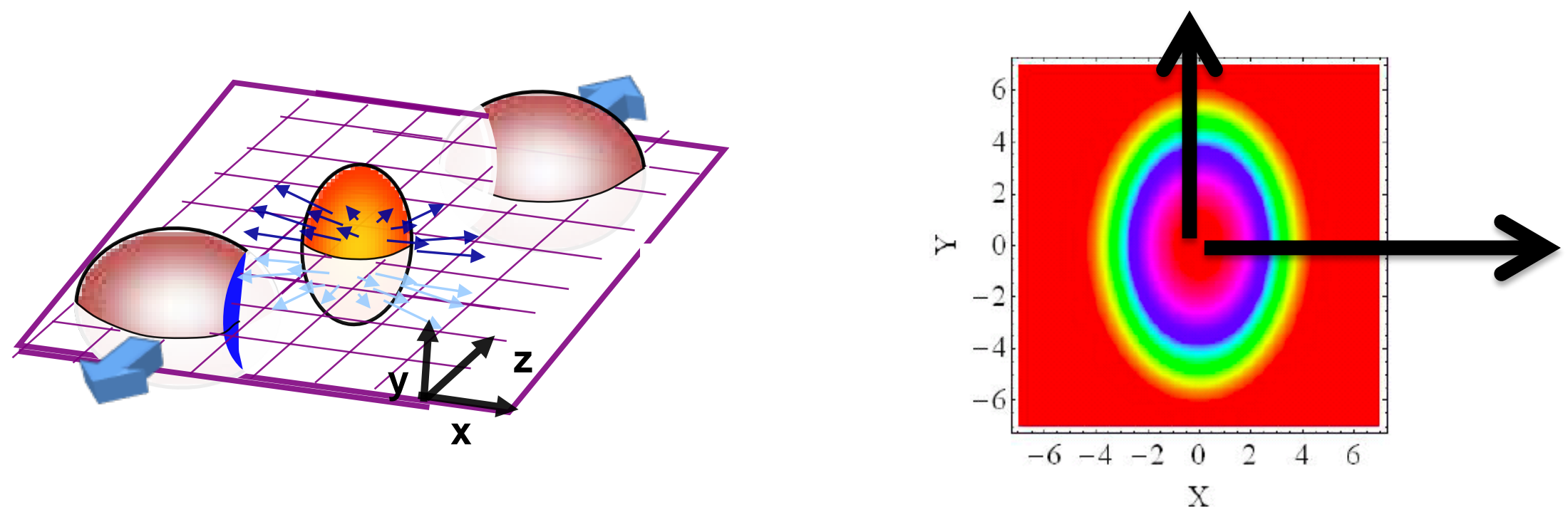

$d N^{h}$

$d \phi$

$\sim R_{A A}(\phi) \sim 1+2 \vee_{2} \cos \left[2\left(\phi-\Psi_{R}\right)\right]$

Can V2 be understood in a consistent picture from RHIC to LHC? 


\section{Jet tomography: High pt Vn!}

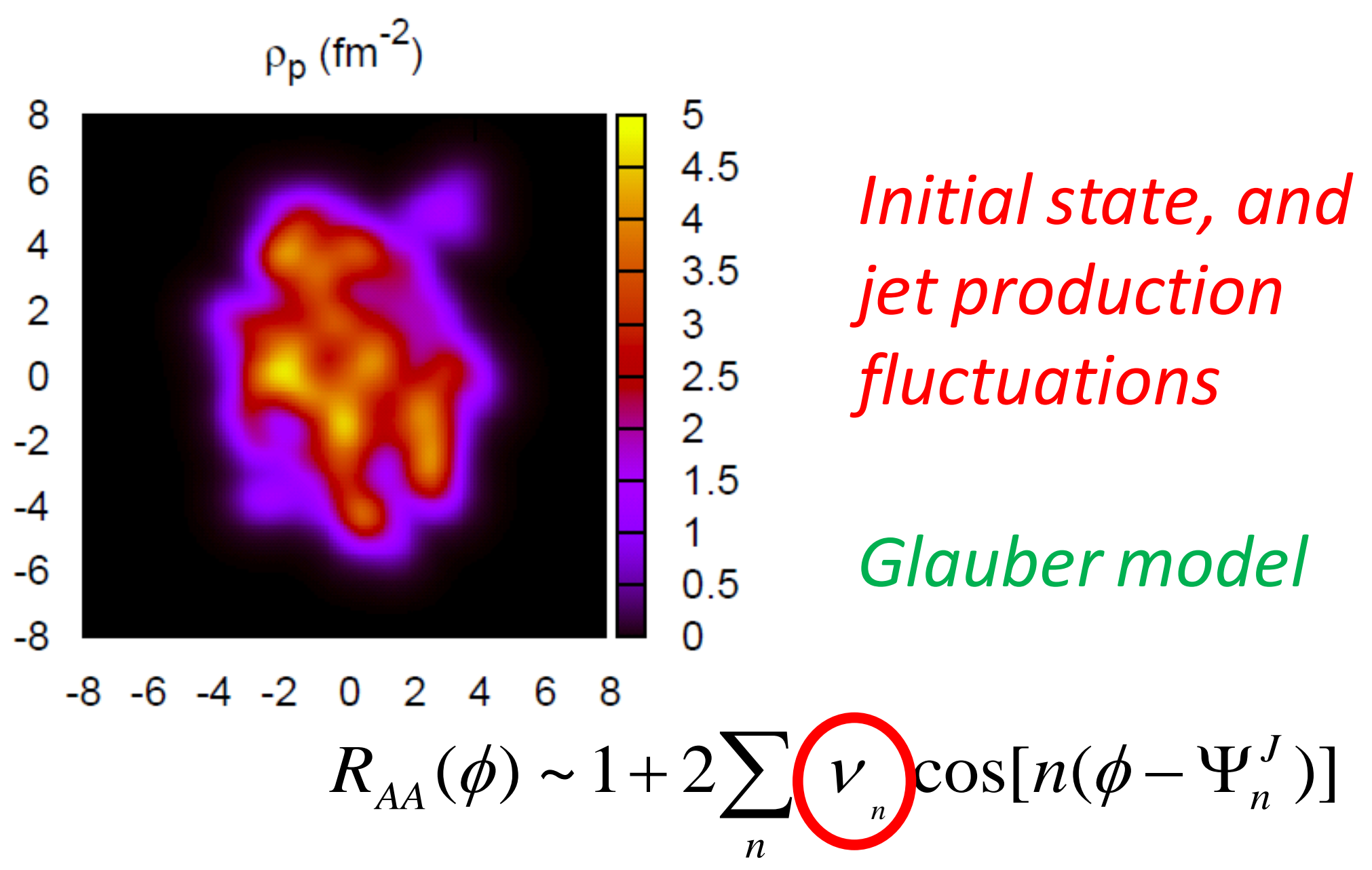

- Consistent picture and more information! -Hard-soft correlation. 


\section{Jet energy loss}

- Length dependence?

- Density dependence?

J. Liao and E. Shuryak, PRL 102, 202302 (2009)

$$
\Delta E=-E \kappa[s(l)] \times s(l) \times l^{m} \times \Delta l
$$

- NTcE model: $\mathrm{m}=1$. (Nontrivial matter near Tc)

- $\mathrm{L}^{\wedge} 2$ model: $\mathrm{m}=1, \kappa$ const. (pQCD)

- $L^{\wedge} 3$ model: $m=2, K$ const.

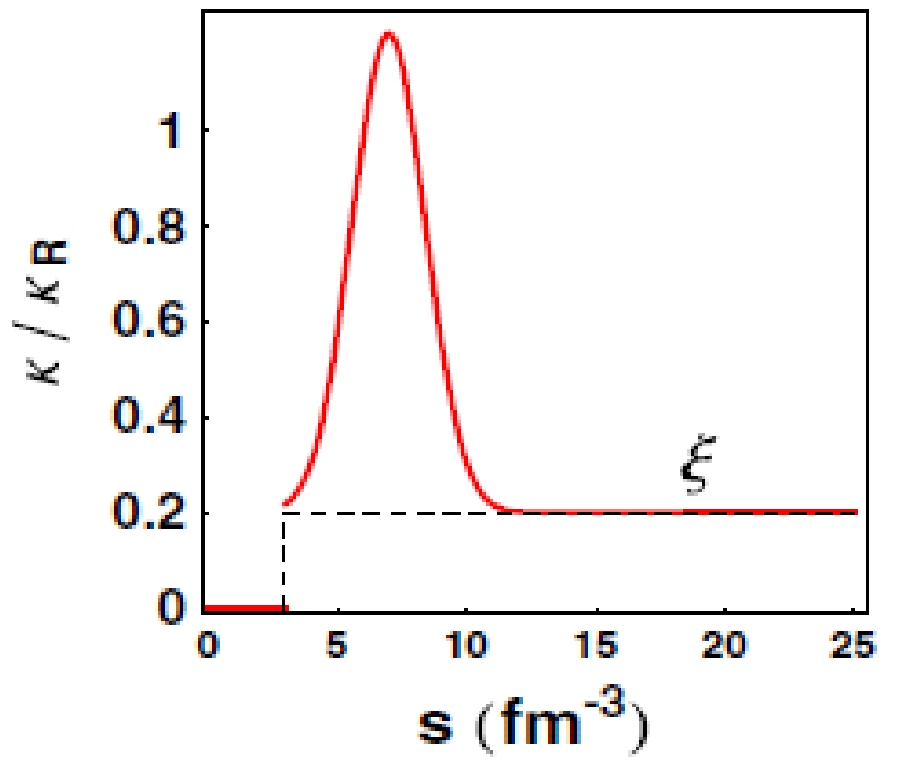
(AdS/QCD) Barbara Betz et.al., PRC 84, 024913 (2011) 


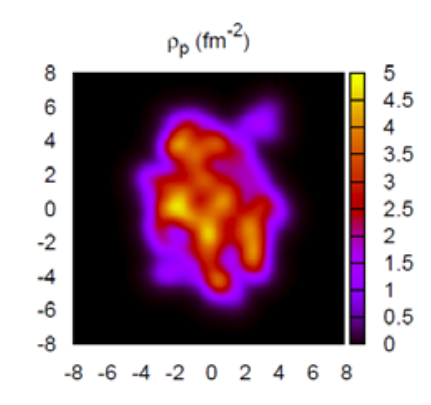

\section{MC: one event}

$$
E_{f}=E_{i} \times f_{\vec{P}}
$$

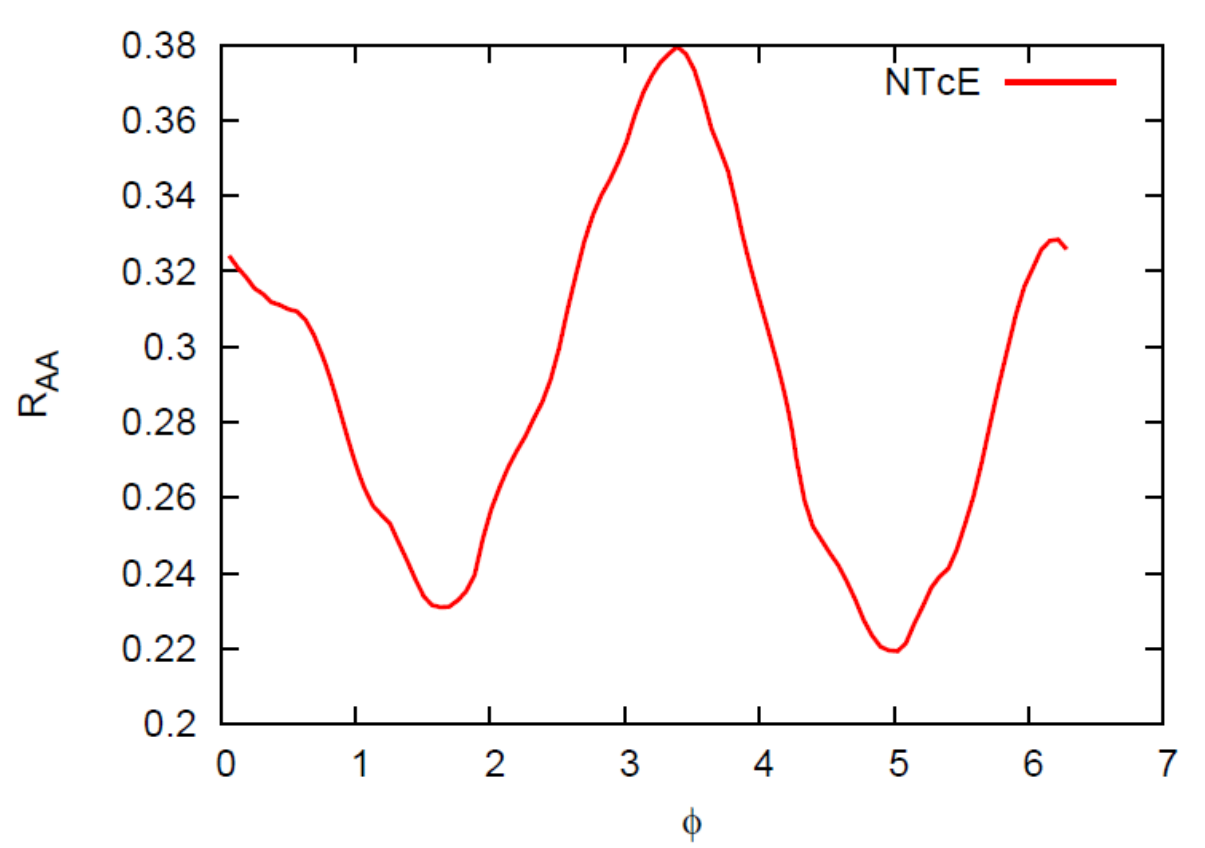

$R_{A A}=<\left(f_{\vec{P}}\right) \overbrace{}^{-2}>_{\vec{P}}$

Sum over $n=8.1$

all the jet (200 GeV),

spots and $n=6.0$

jetpaths (2.76 TeV)

$$
R_{A A}(\phi) \sim 1+2 \sum_{n} v_{n} \cos \left[n\left(\phi-\Psi_{n}^{J}\right)\right]
$$




\section{Results: V2, RHIC $200 \mathrm{GeV}$}

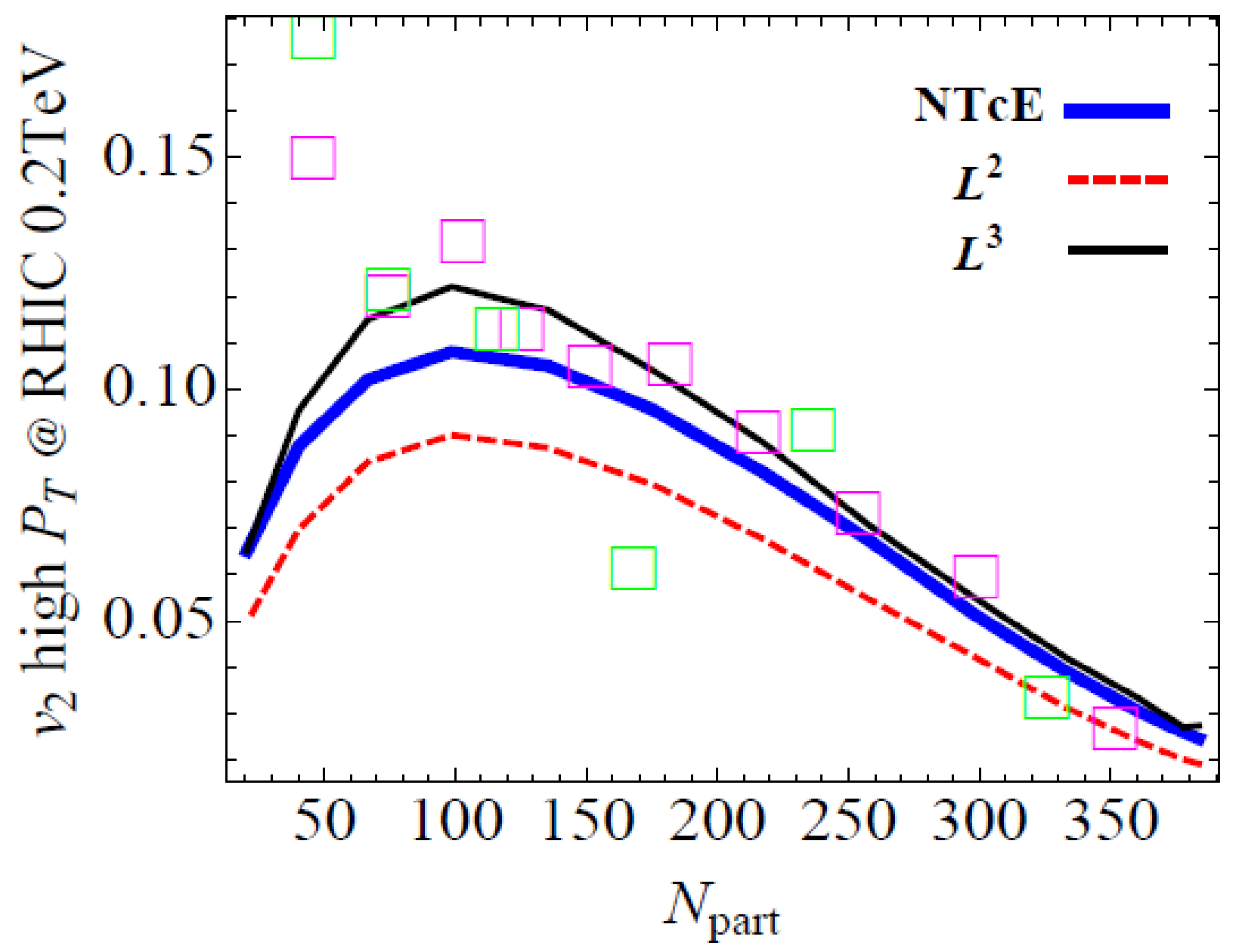

PHENIX, Pt: 6--9 GeV and > 9 GeV, PRL 105, 142301 (2010). 


\section{Results: V2, LHC 2.76 TeV}

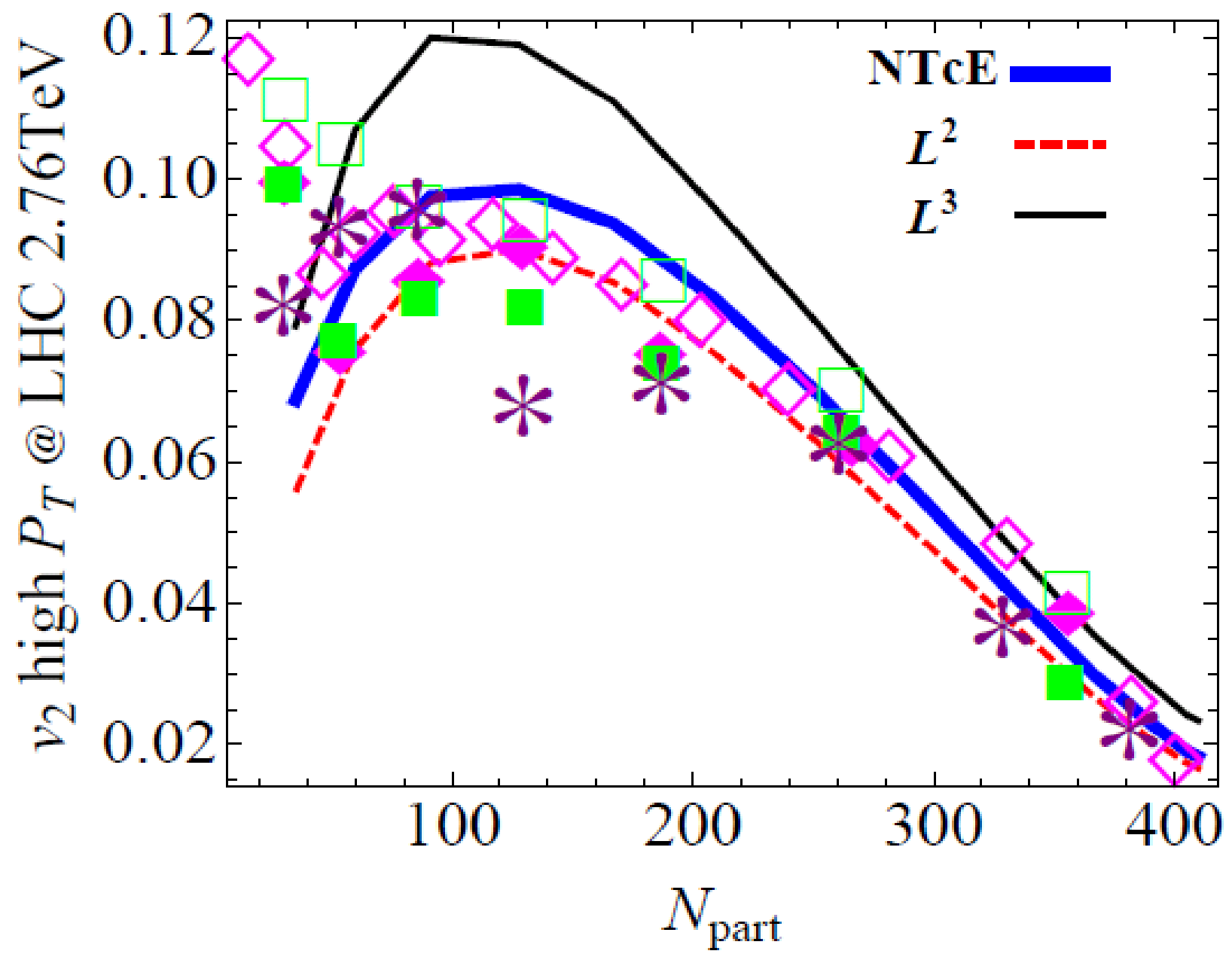

ATLAS, CMS, and ALICE, Pt: 10--20 GeV,

arXiv:1205.5761, 1204.1850, PLB 707, 330 (2012) 


\section{From RHIC to $\mathrm{LHC}$}
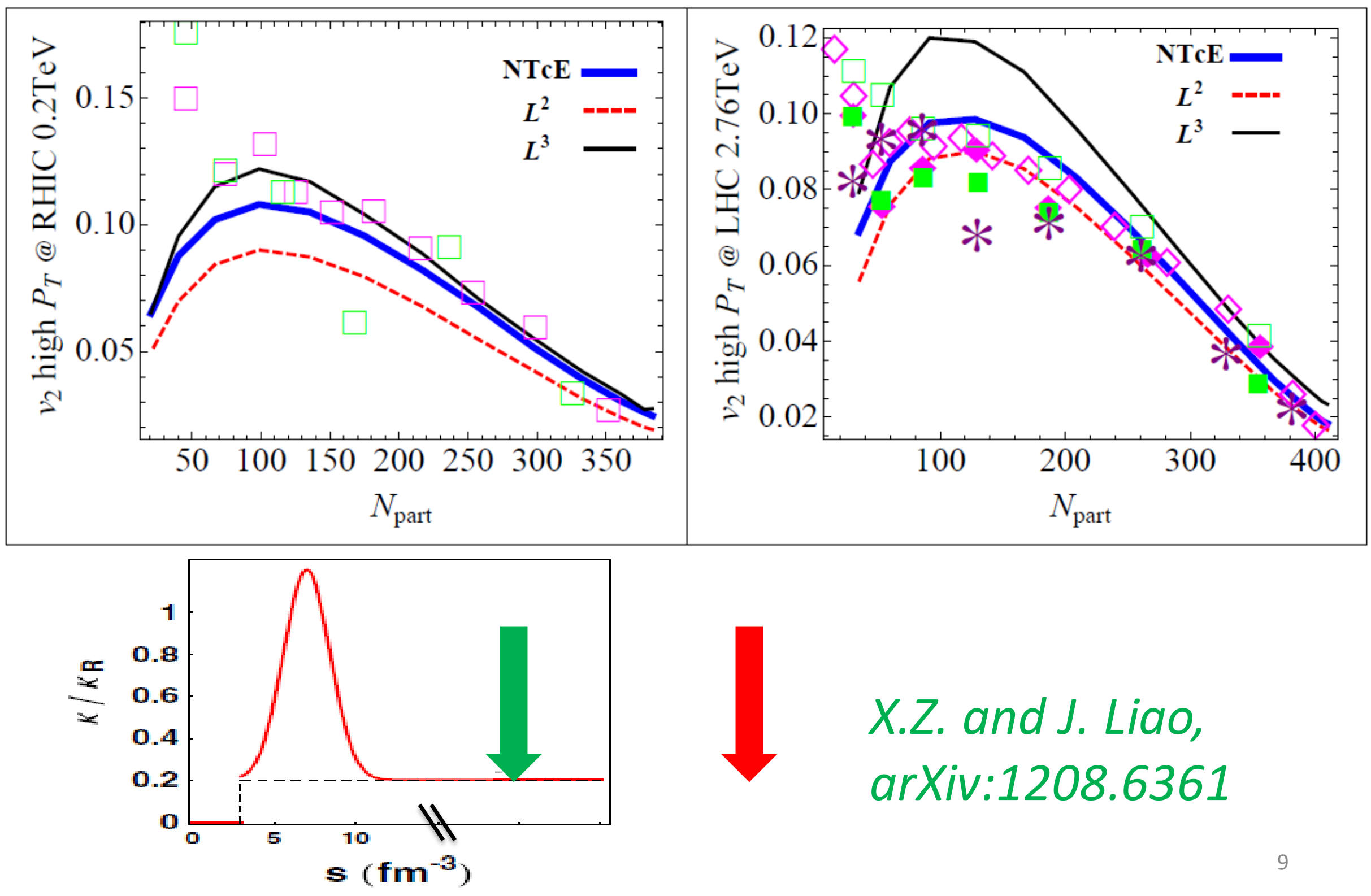

X.Z. and J. Liao, arXiv:1208.6361 
$69 \mathrm{GeV}, \mathrm{b}=6 \mathrm{fm}, \mathrm{t}=6 \mathrm{tau}$

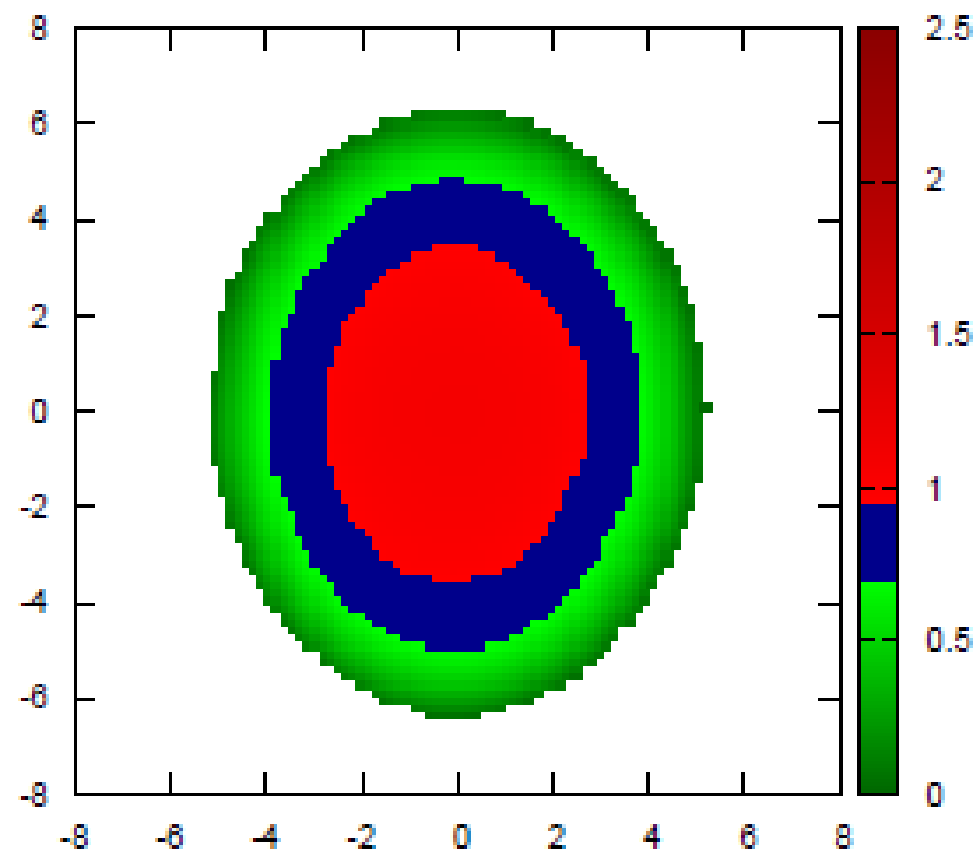

$200 \mathrm{GeV}, \mathrm{b}=6 \mathrm{fm}, \mathrm{t}=6 \mathrm{tau}$

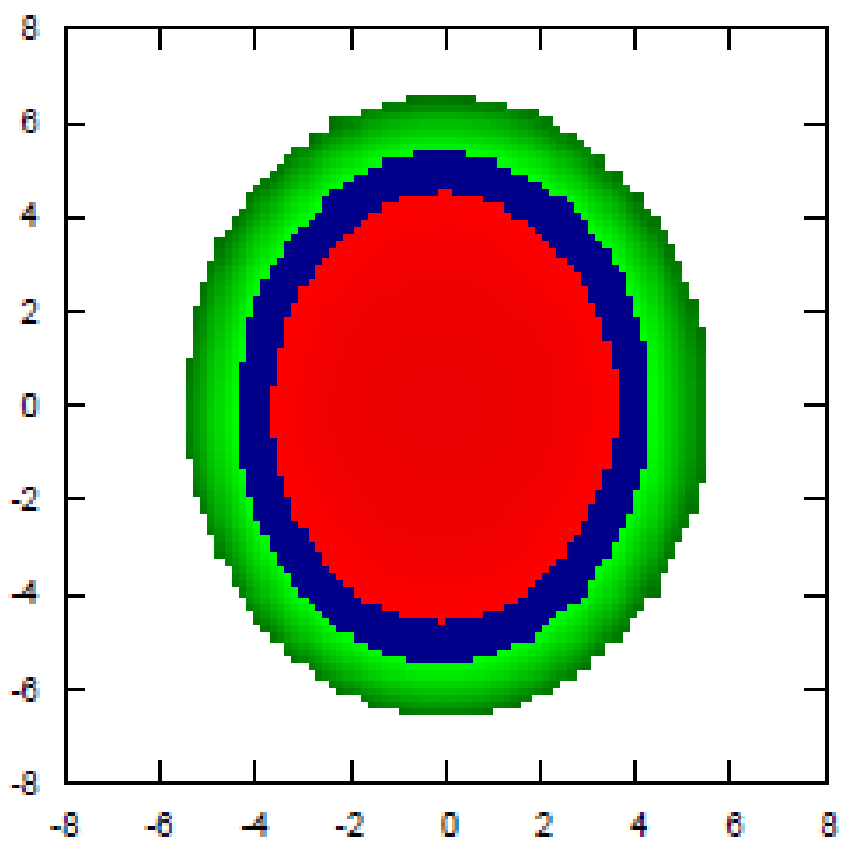

Entropy density

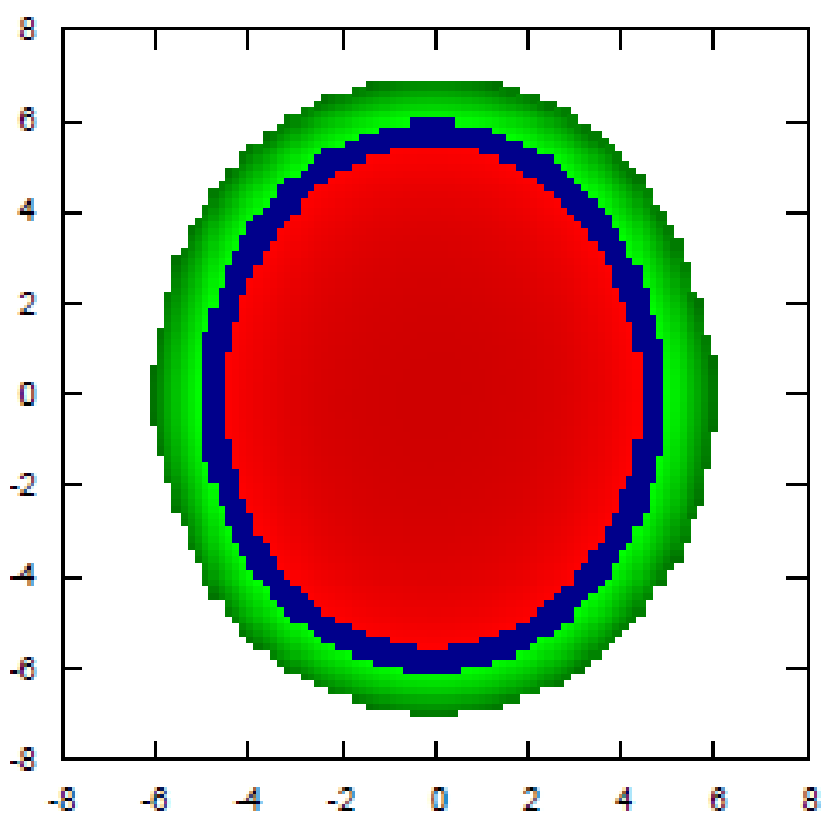




\section{Results: Vn, LHC 2.76 TeV}

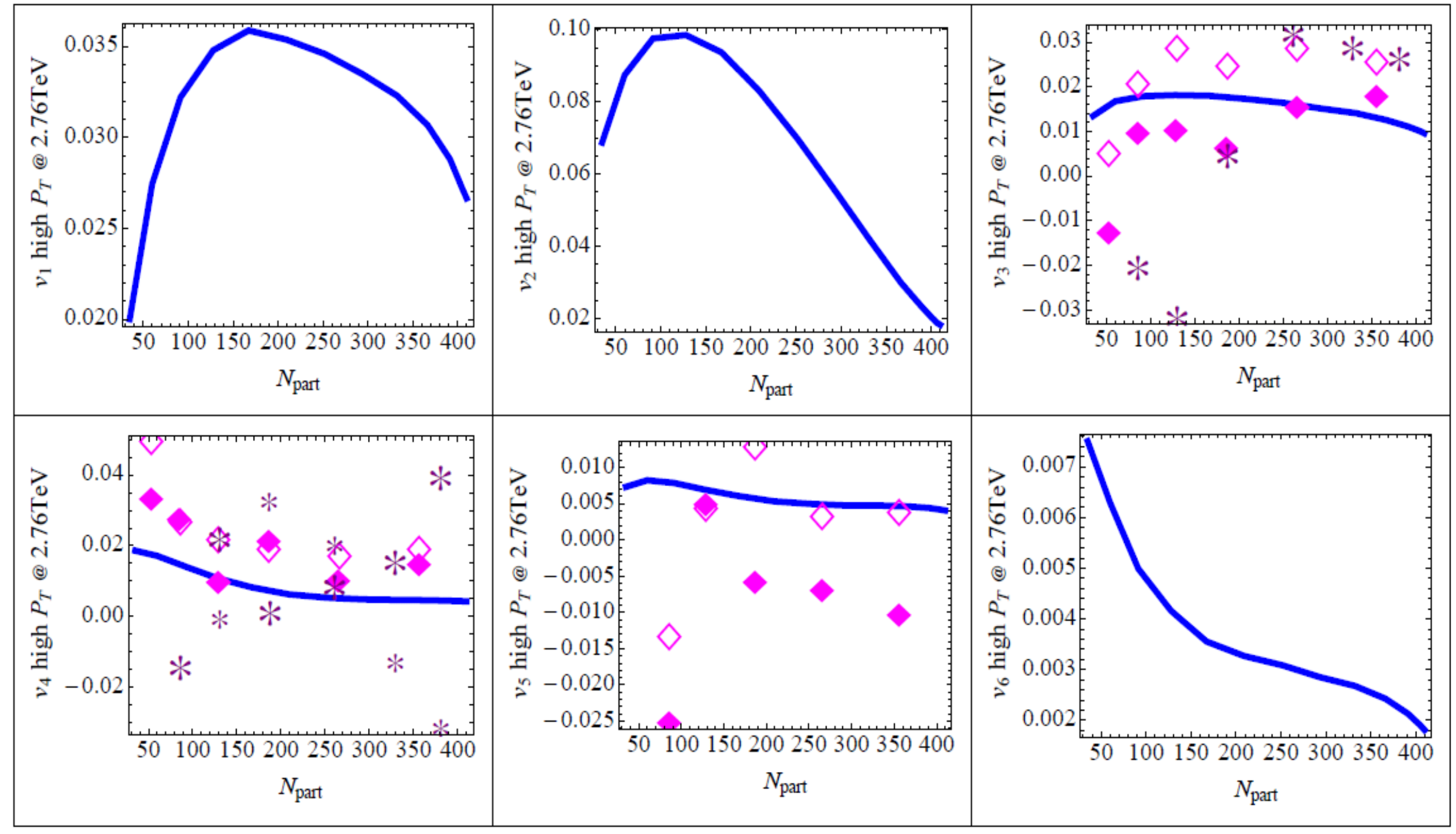




\section{Some details: Vn spectrum}
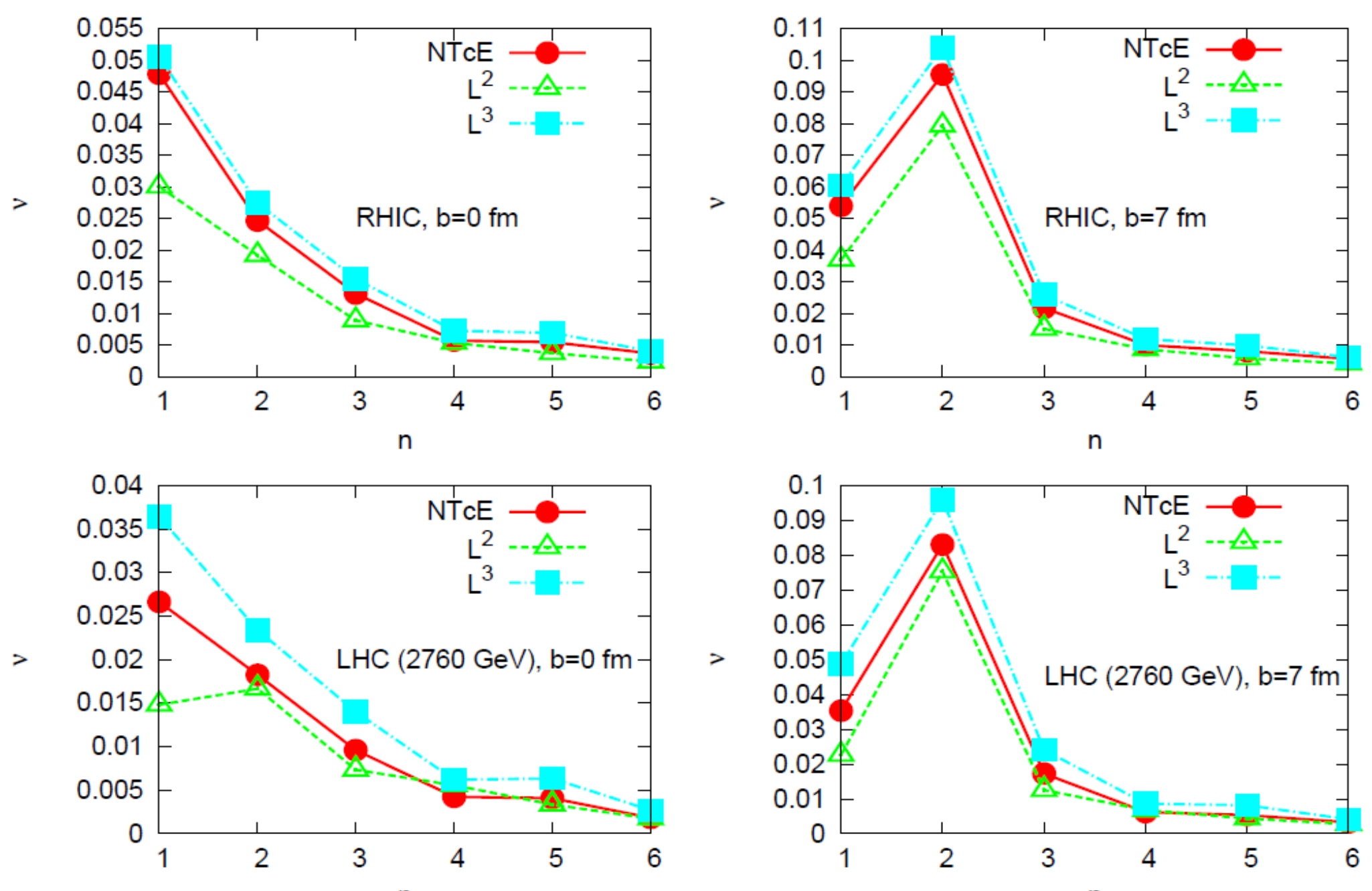

$X . Z^{n}$. and J. Liao, arXiv:1210.1245 


\section{Some details: angle dispersion}

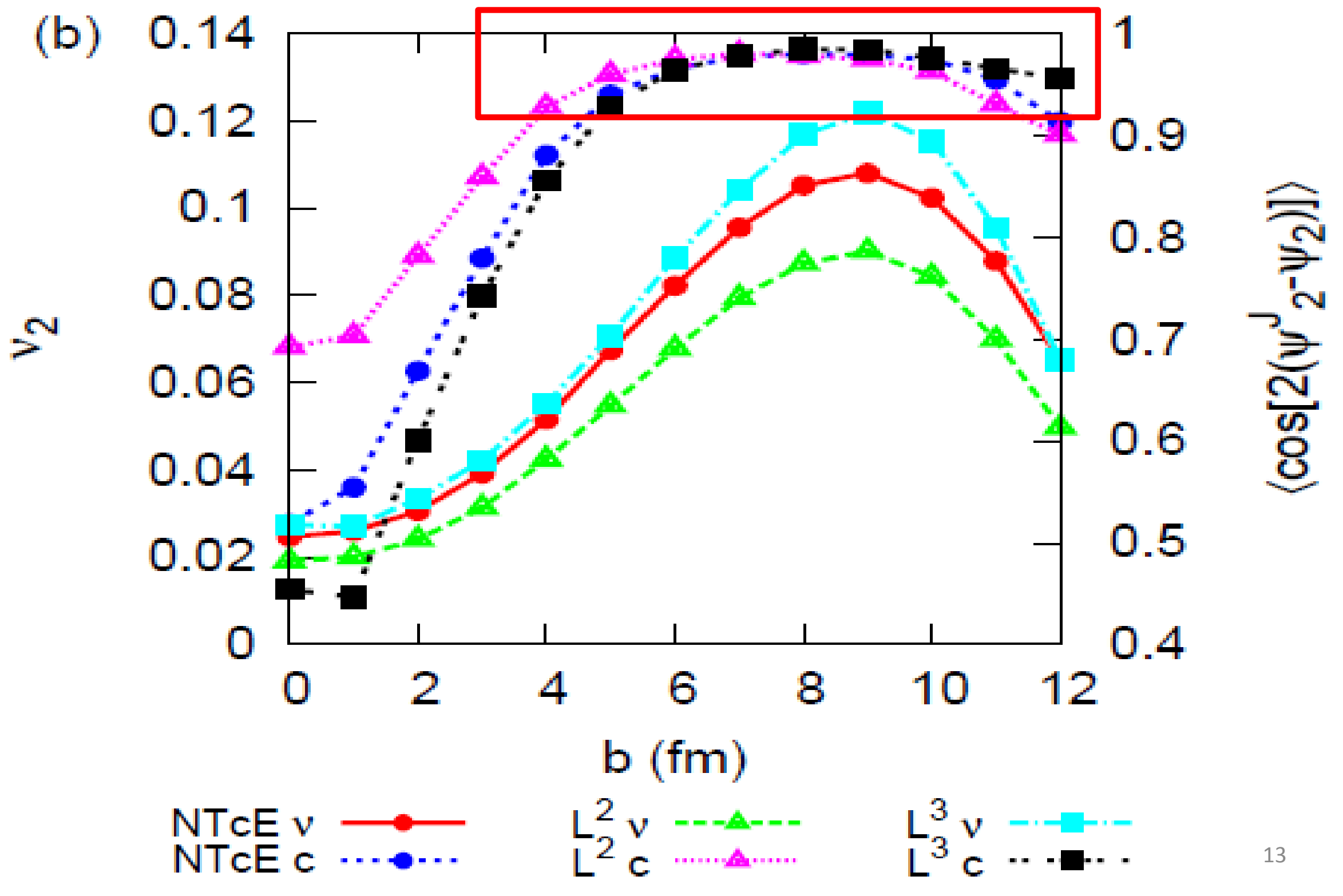




\section{Some details: angle dispersion}

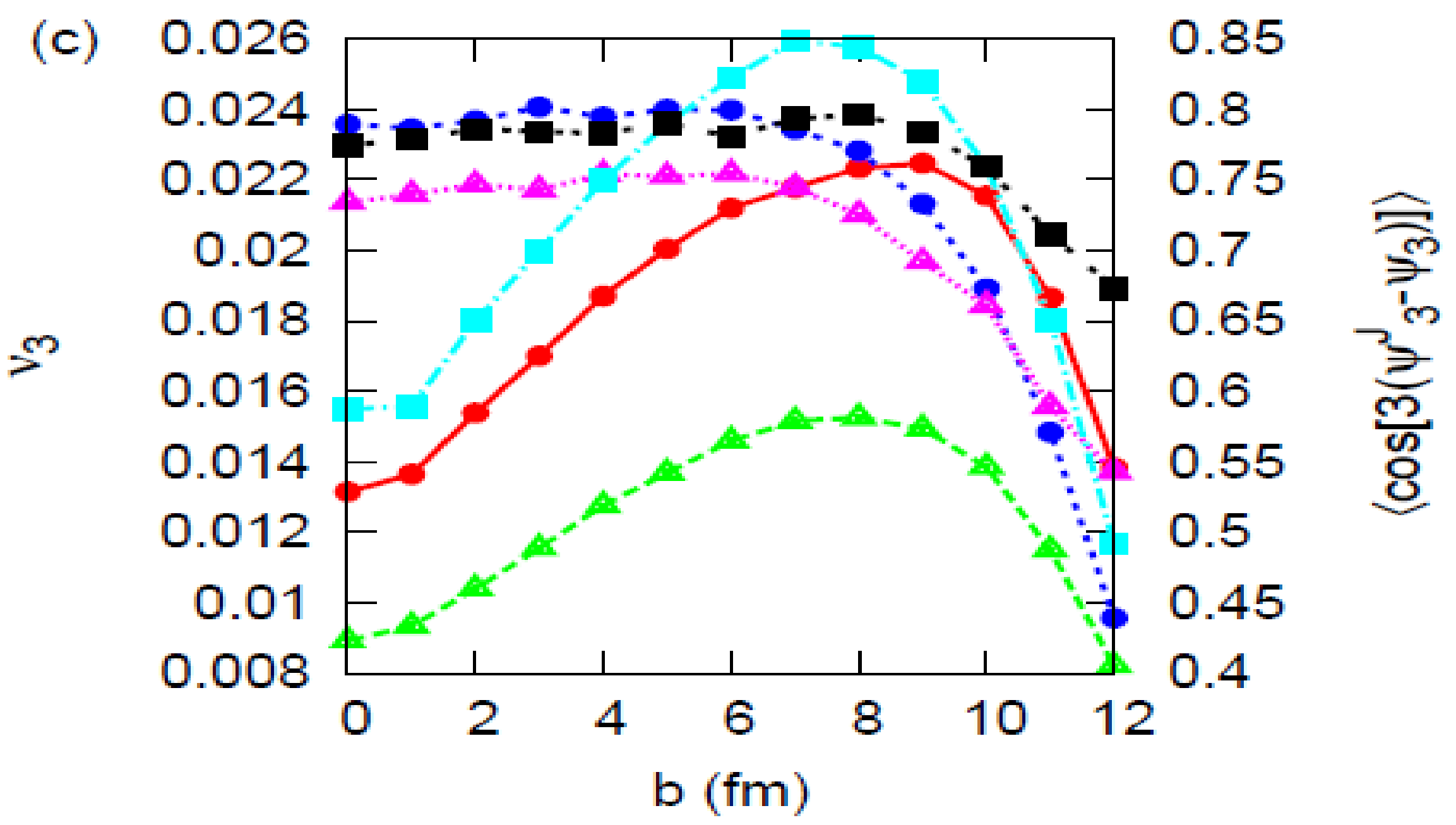

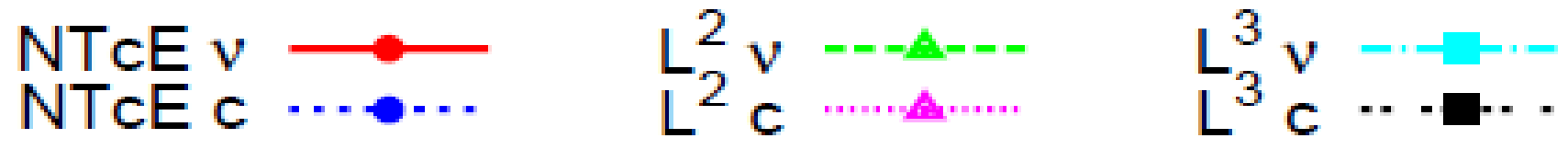




\section{Hard-soft Correlation}

$p_{T}^{t} 4-6, p_{T}^{a} 2-4,0-5 \%$ centrality,

$\mathrm{Pb}-\mathrm{Pb}$ (2.76 TeV)

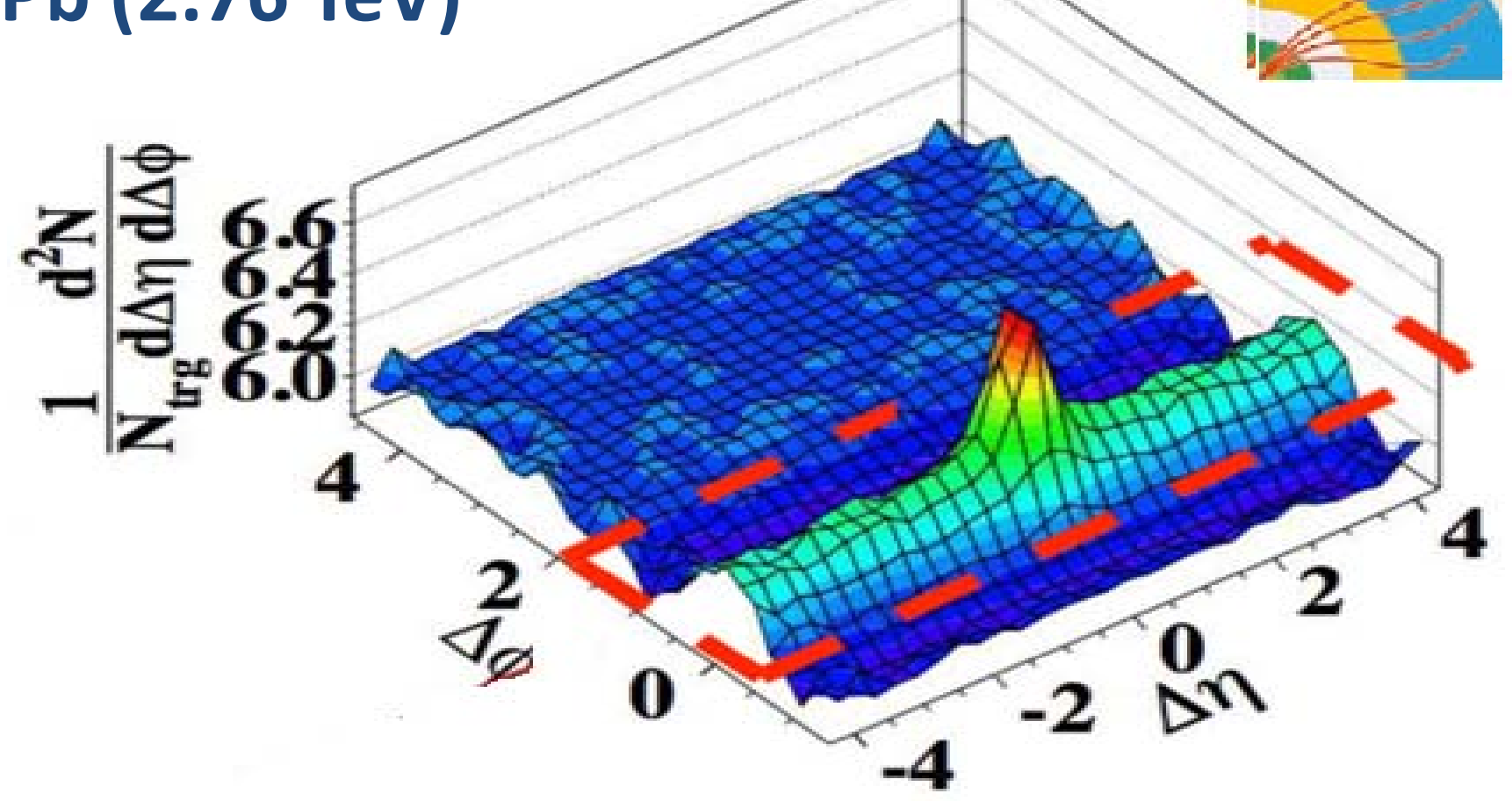

Hard-ridge phenomena

Flliptic flow not subtracted 


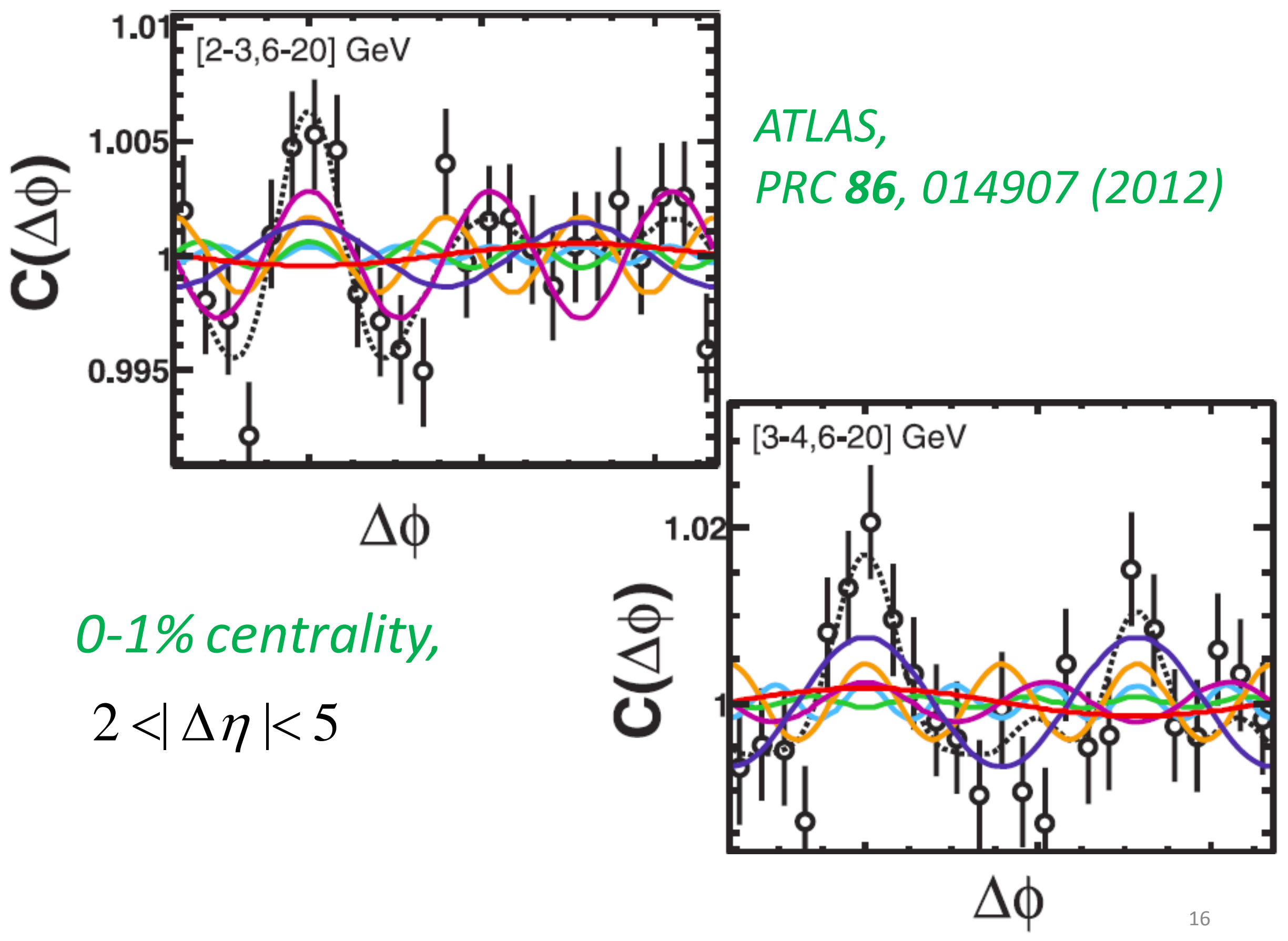




\section{Hard-soft Correlation}

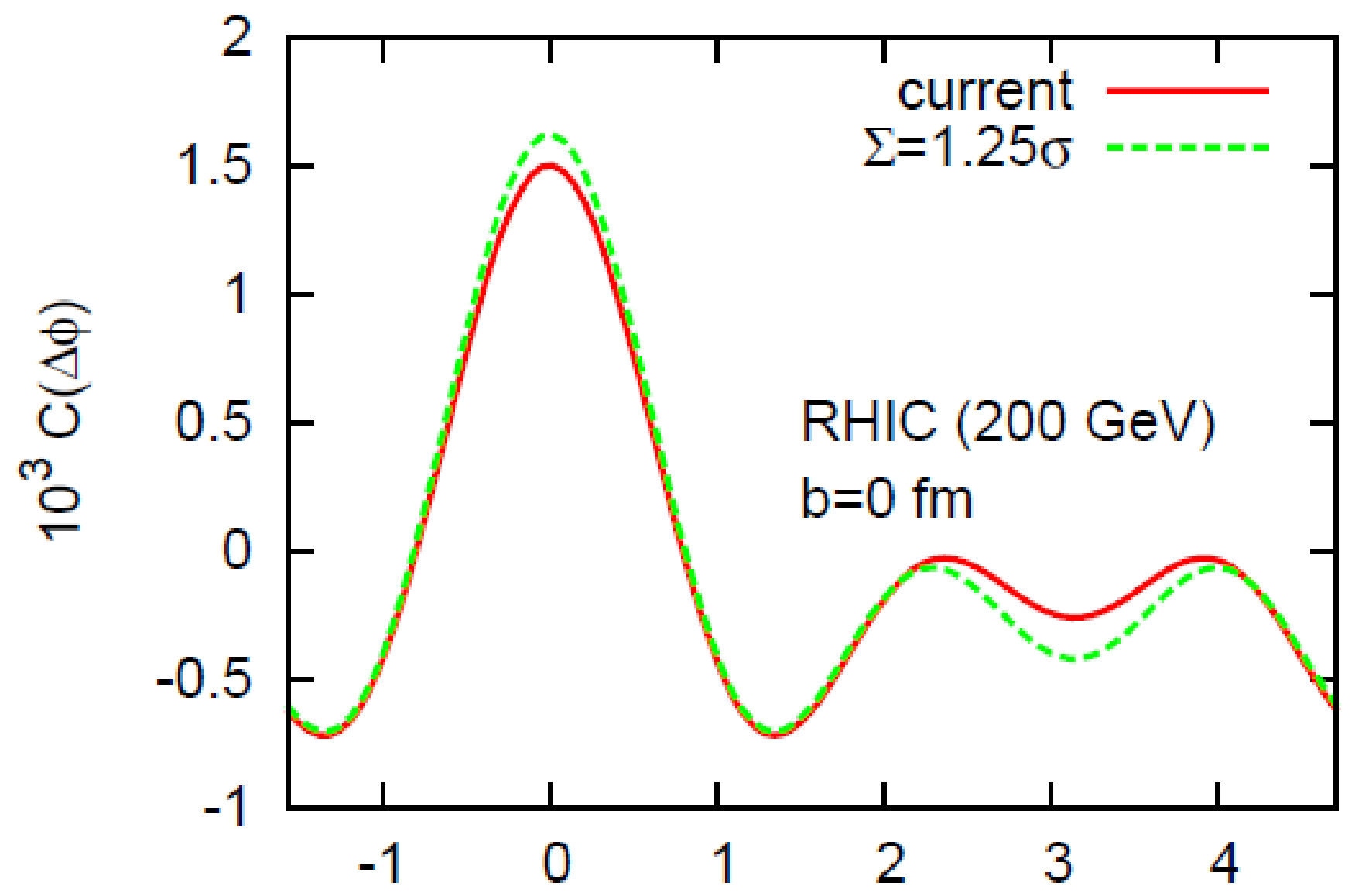

$$
\begin{array}{cl}
\frac{d N^{s}}{d \phi^{s}} \sim 1+2 \sum_{n} \nu_{n}^{s} \cos \left[n\left(\phi^{s}-\psi_{n}^{S}\right)\right], & <\frac{d N^{h \text { ard }}}{d y d \phi_{1}} \frac{d N^{\text {soft }}}{d y d \phi_{2}}> \\
\frac{d N^{h}}{d \phi^{h}} \sim 1+2 \sum_{m} \nu_{m}^{h} \cos \left[m\left(\phi^{h}-\psi_{m}^{J}\right)\right] & \sim 1+\sum_{n=1,2,3, \ldots}^{2<v_{n}^{h} v_{n}^{s}>\cos (n \Delta \phi)} \\
\text { X.Z. and J. Liao, PLB 713, } 35 \text { (2012). }
\end{array}
$$




\section{Hard-soft Correlation}

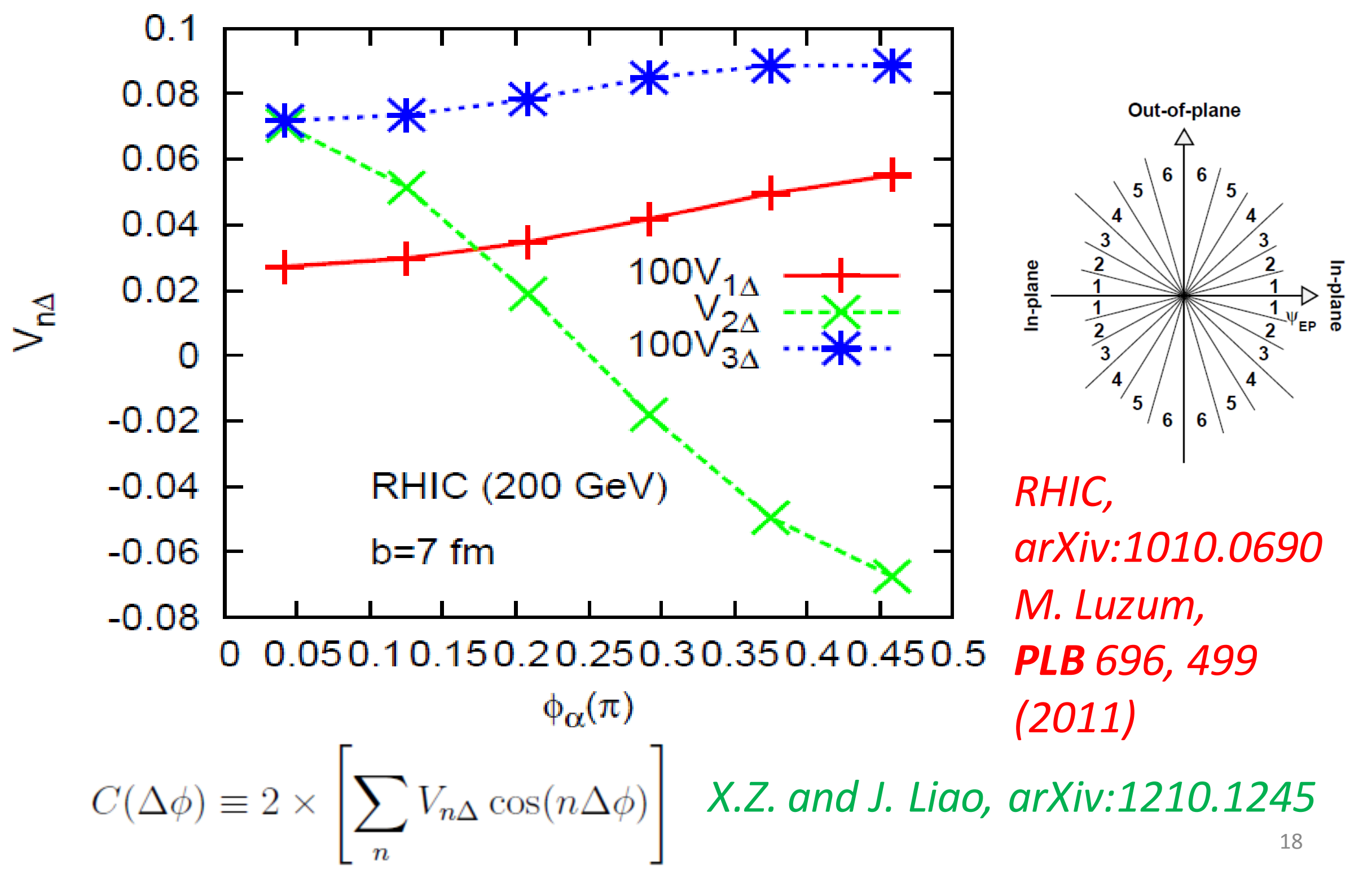




\section{P-Pb case (5.02 TeV): a try}

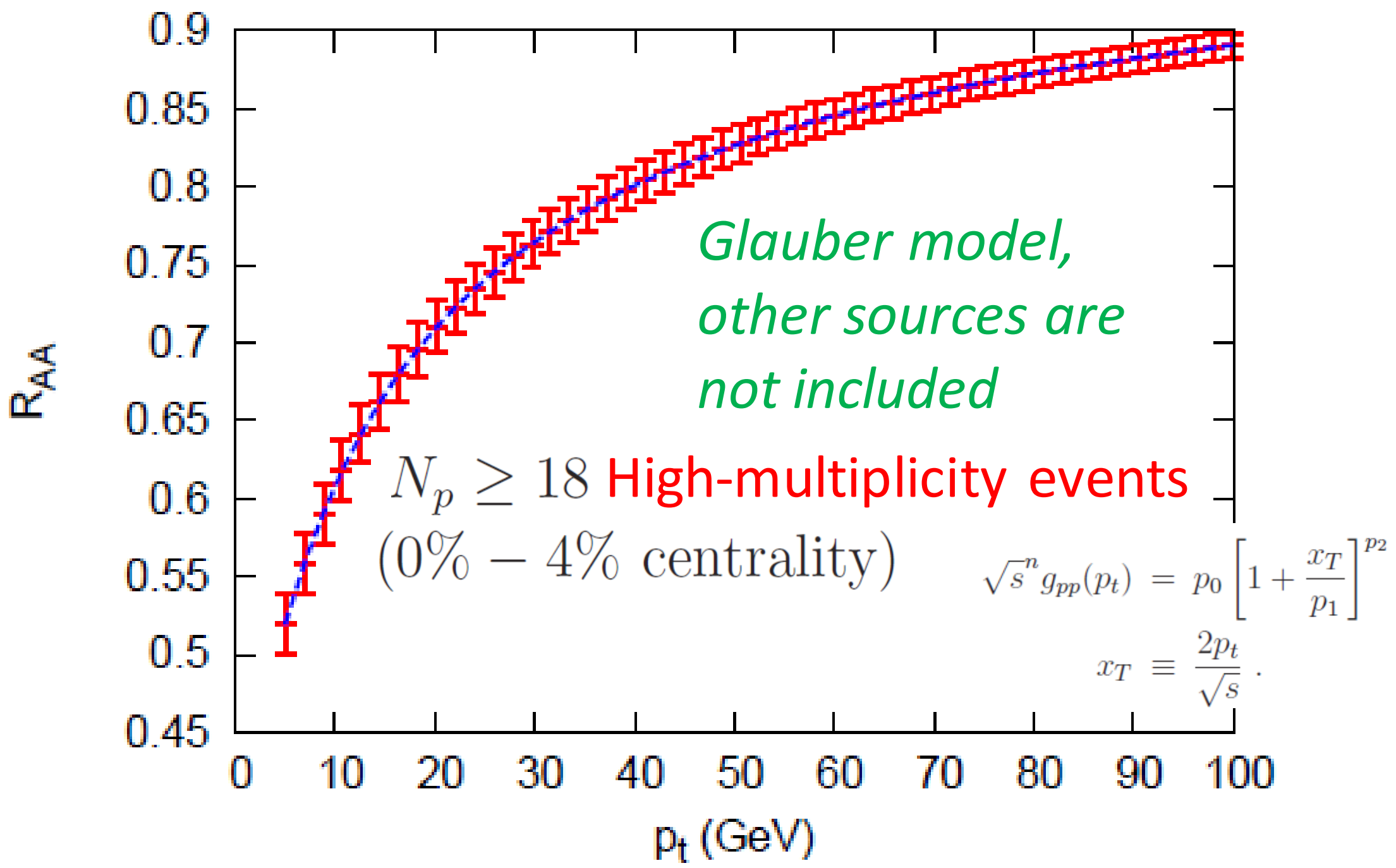

Piotr Bozek, PRC 85, 014911 (2012). 


\section{P-Pb case (5.02 TeV): a try}

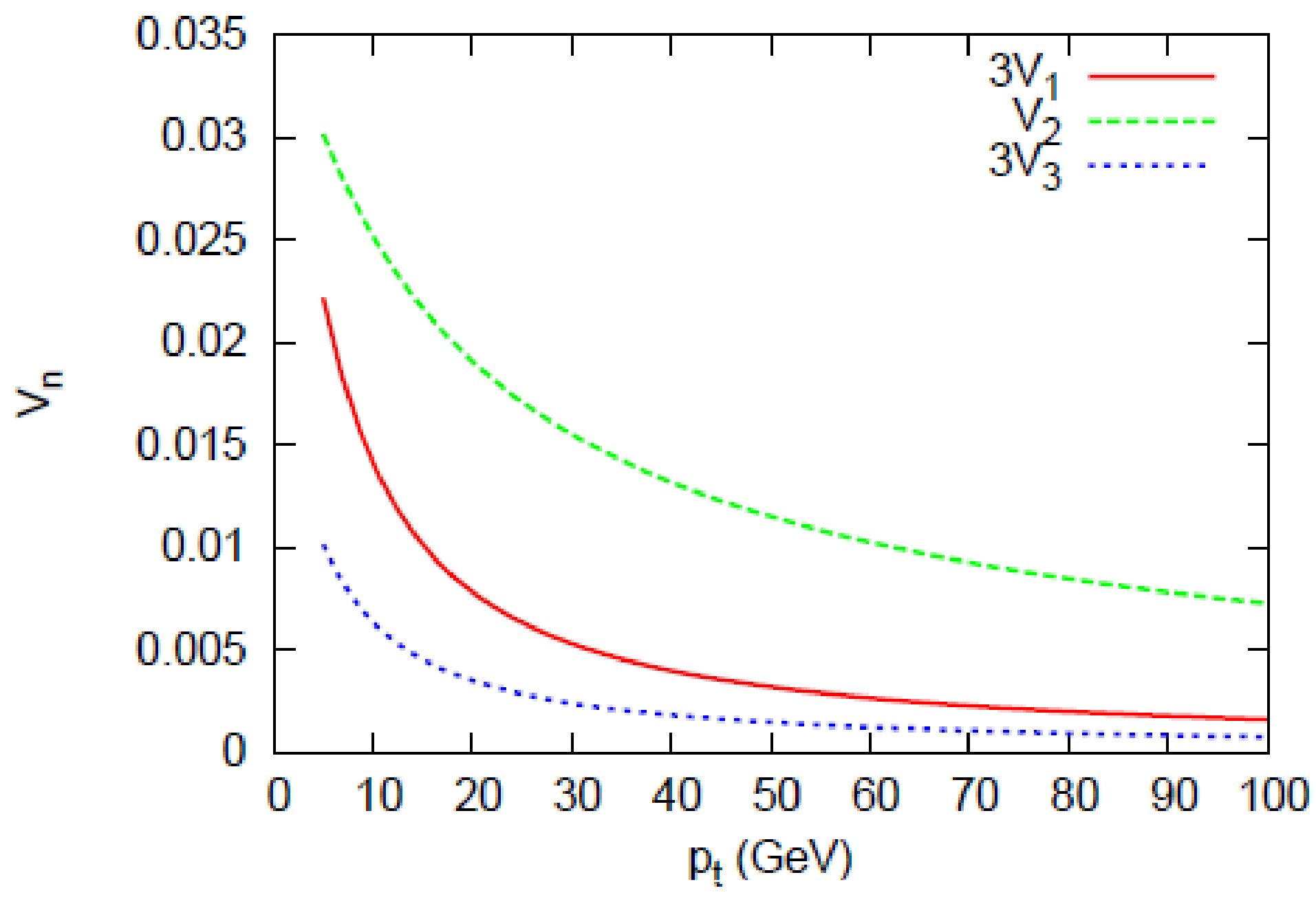

Final state interaction? 


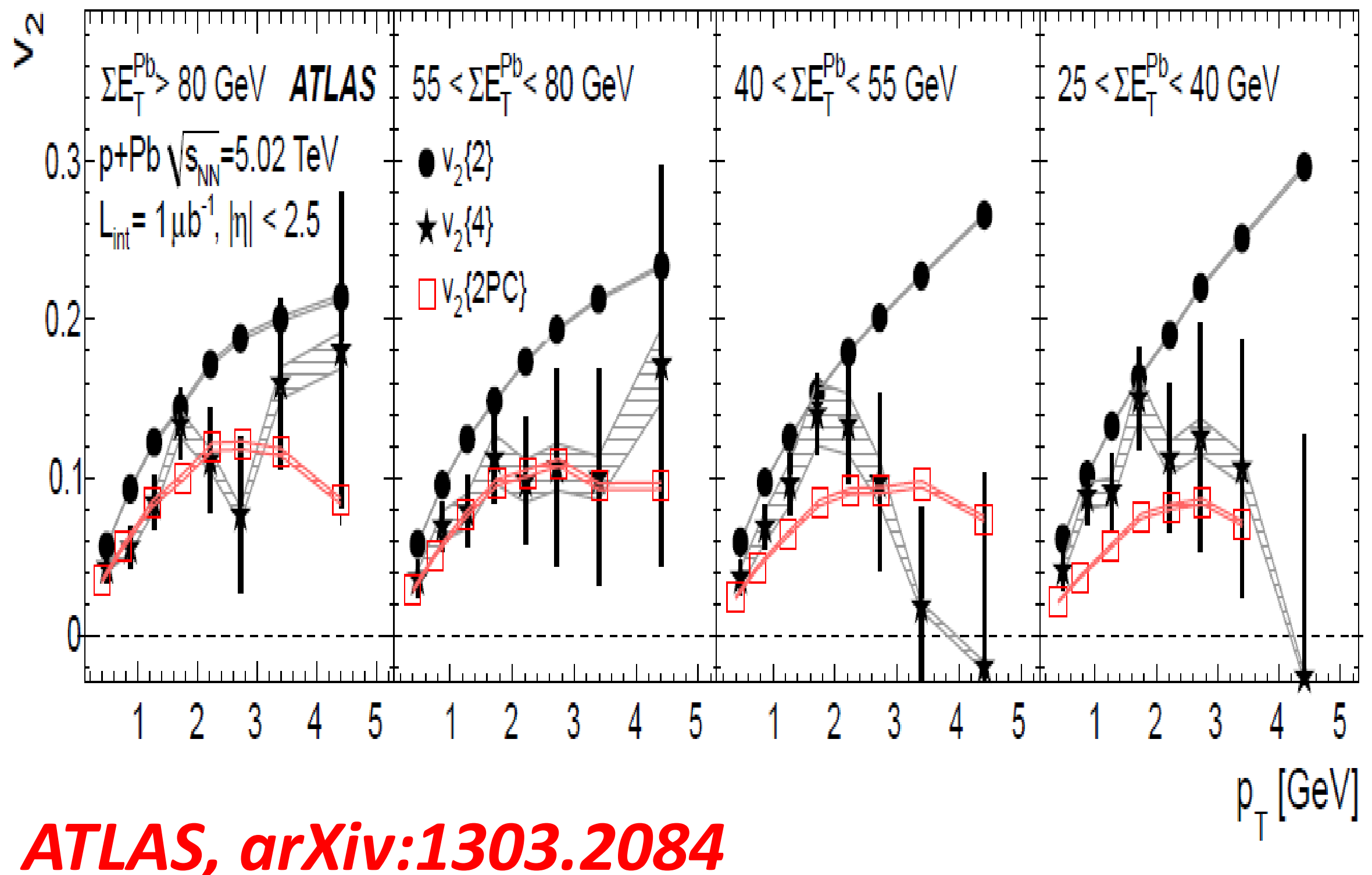




\section{P-Pb case (5.02 TeV): a try}

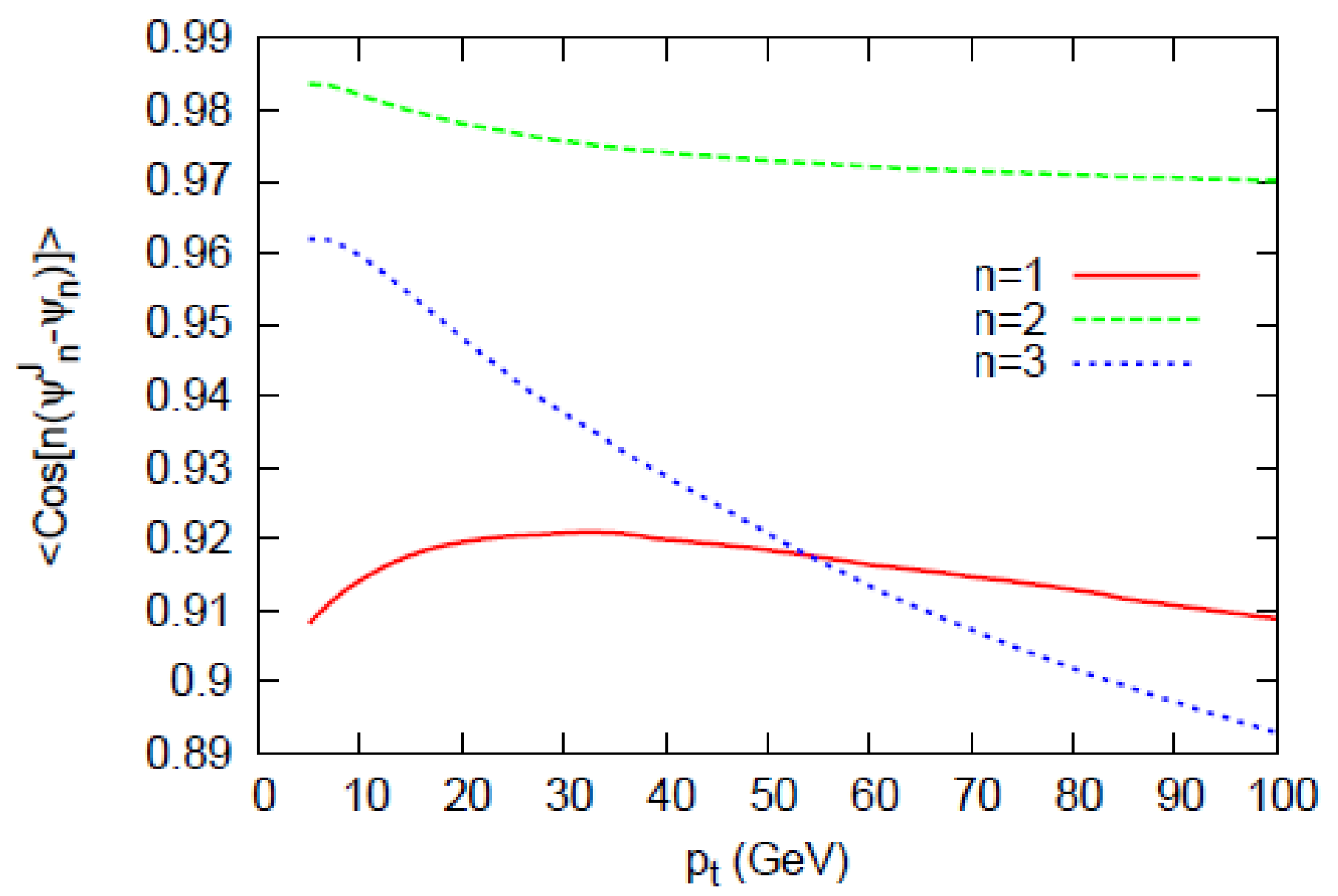

Angle dispersion 


\section{Conclusion and outlook}

- NTcE explains well the centrality-dependence of high-pt V2 from RHIC $200 \mathrm{GeV}$ to LHC 2.76 TeV (Vn?)

- Need more and better data on high-pt Vn

- Vn contribute to hard-soft correlation

- High-pt Raa and anisotropy are explored for high multiplicity $\mathrm{P}-\mathrm{Pb}$ events, data are needed

- Realistic matter evolution is needed (in progress)

- Connection between NTcE and QCD needs to be explored 


\section{Back up}




\section{Jet response at $b=0 \mathrm{fm}$}

Simple estimate

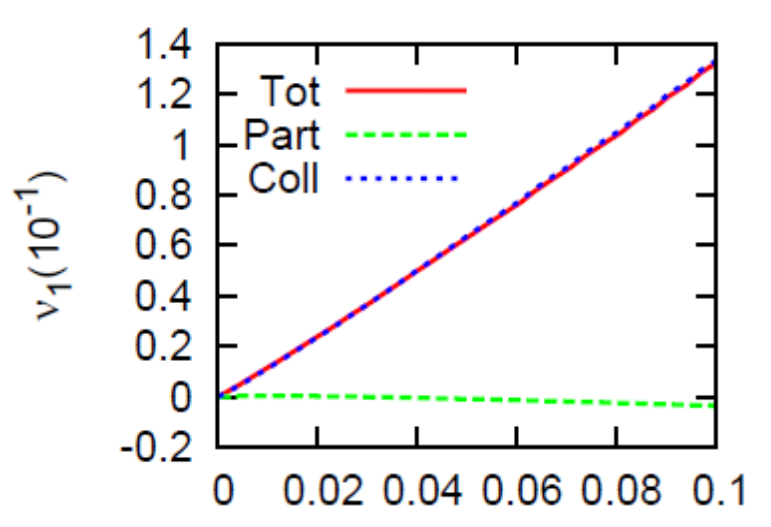

$\varepsilon_{1}$

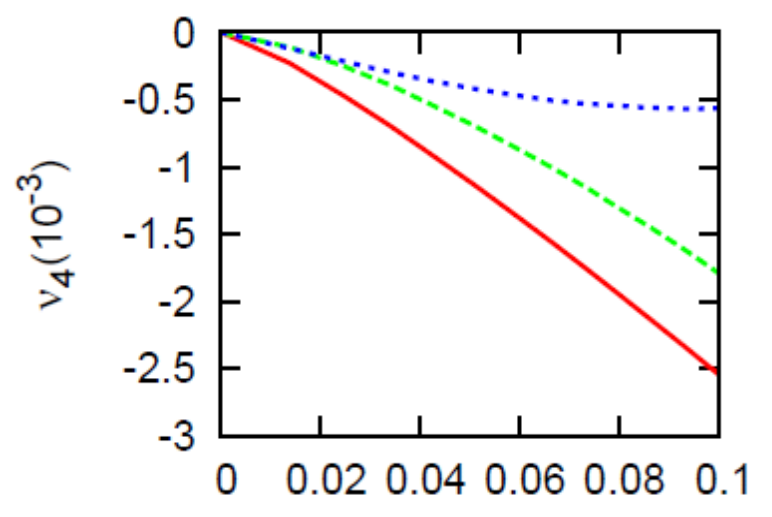

$\varepsilon_{4}$

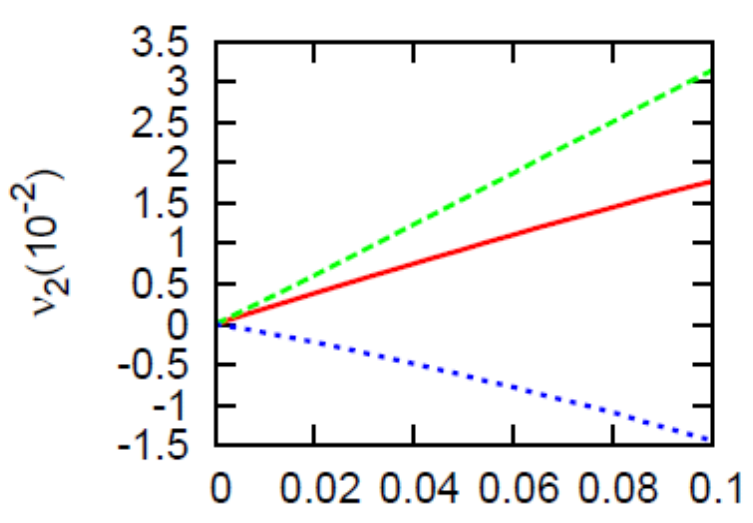

$\varepsilon_{2}$
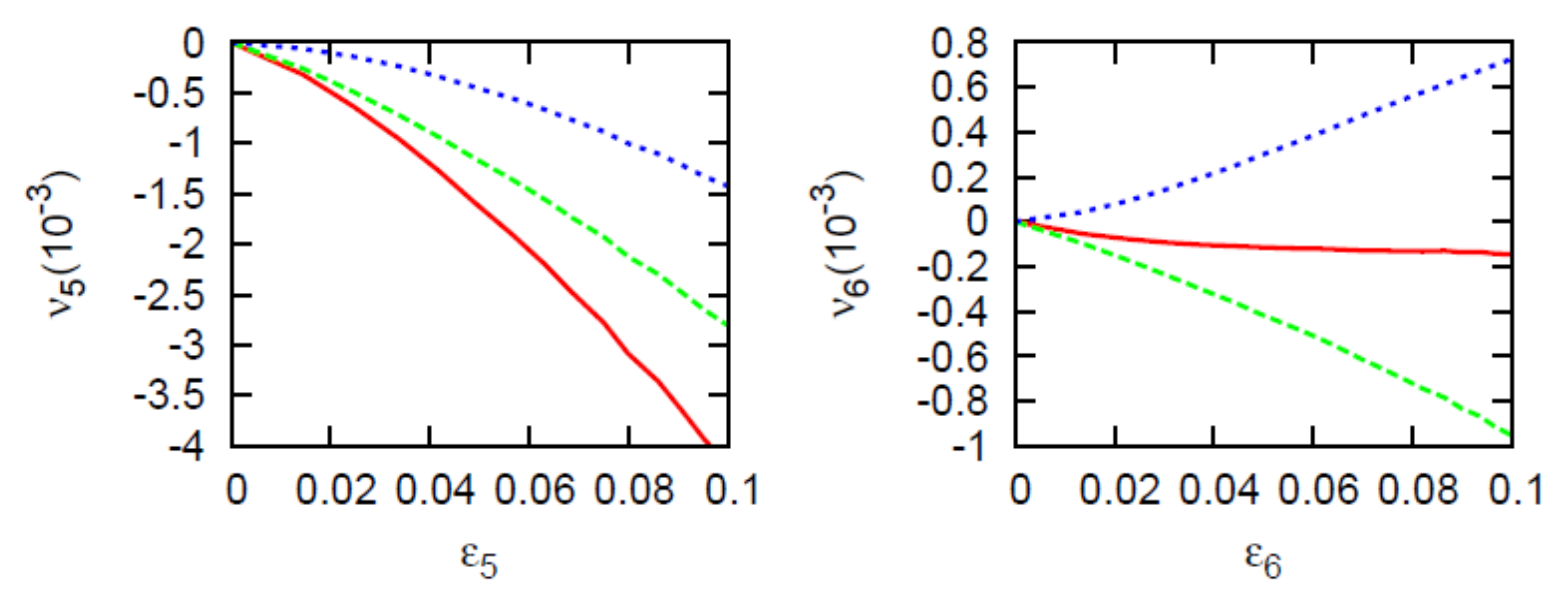

X.Z. and J. Liao, 2012

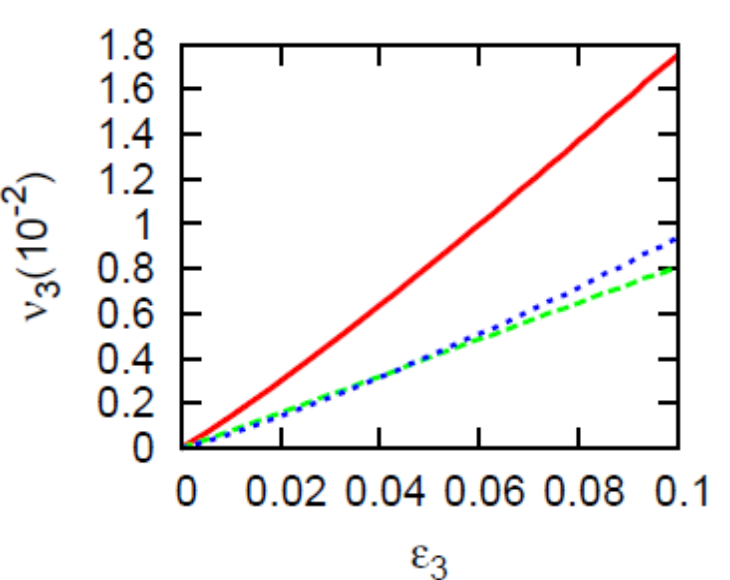




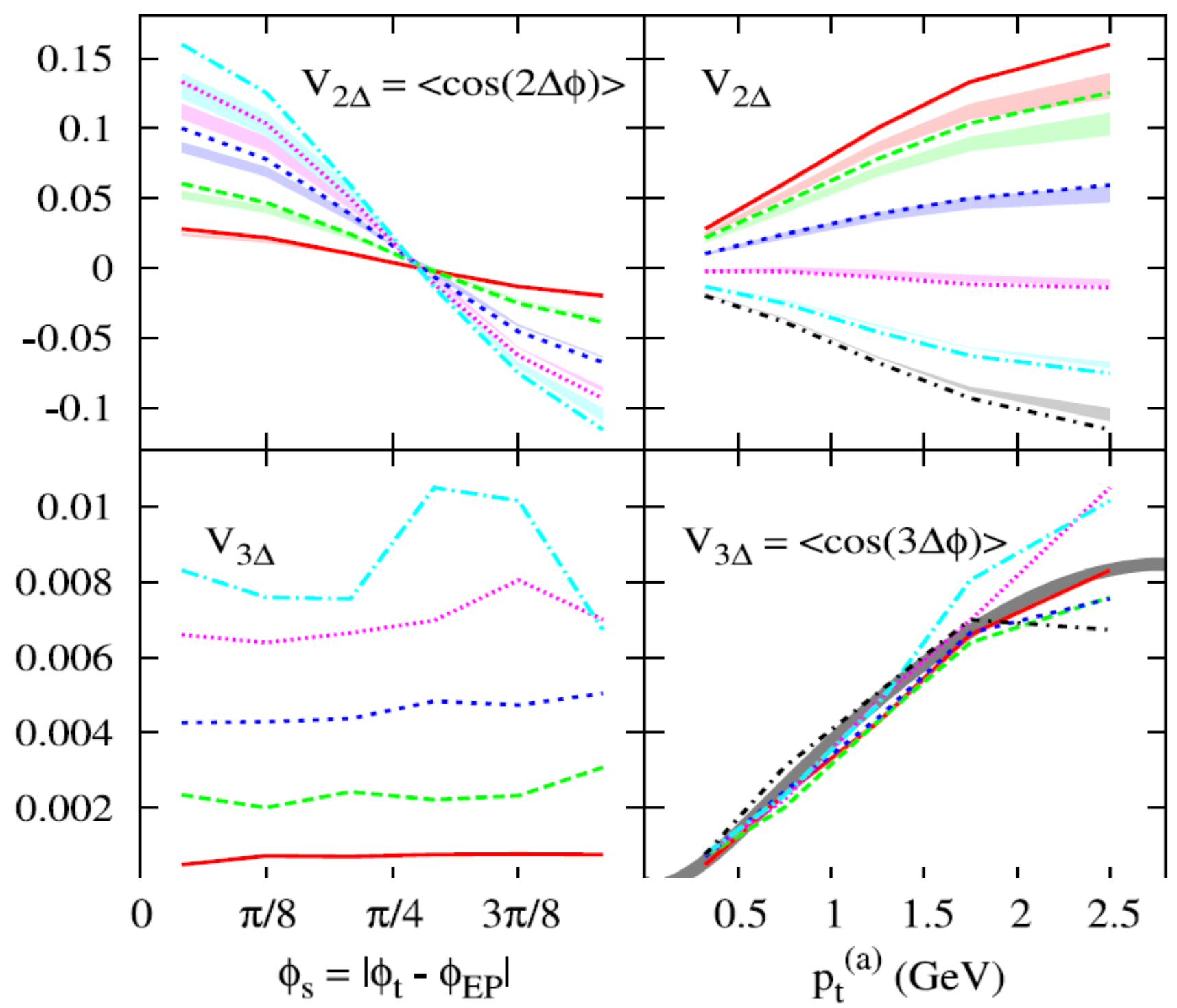

M. Luzum, PLB 696, 499 (2011) 

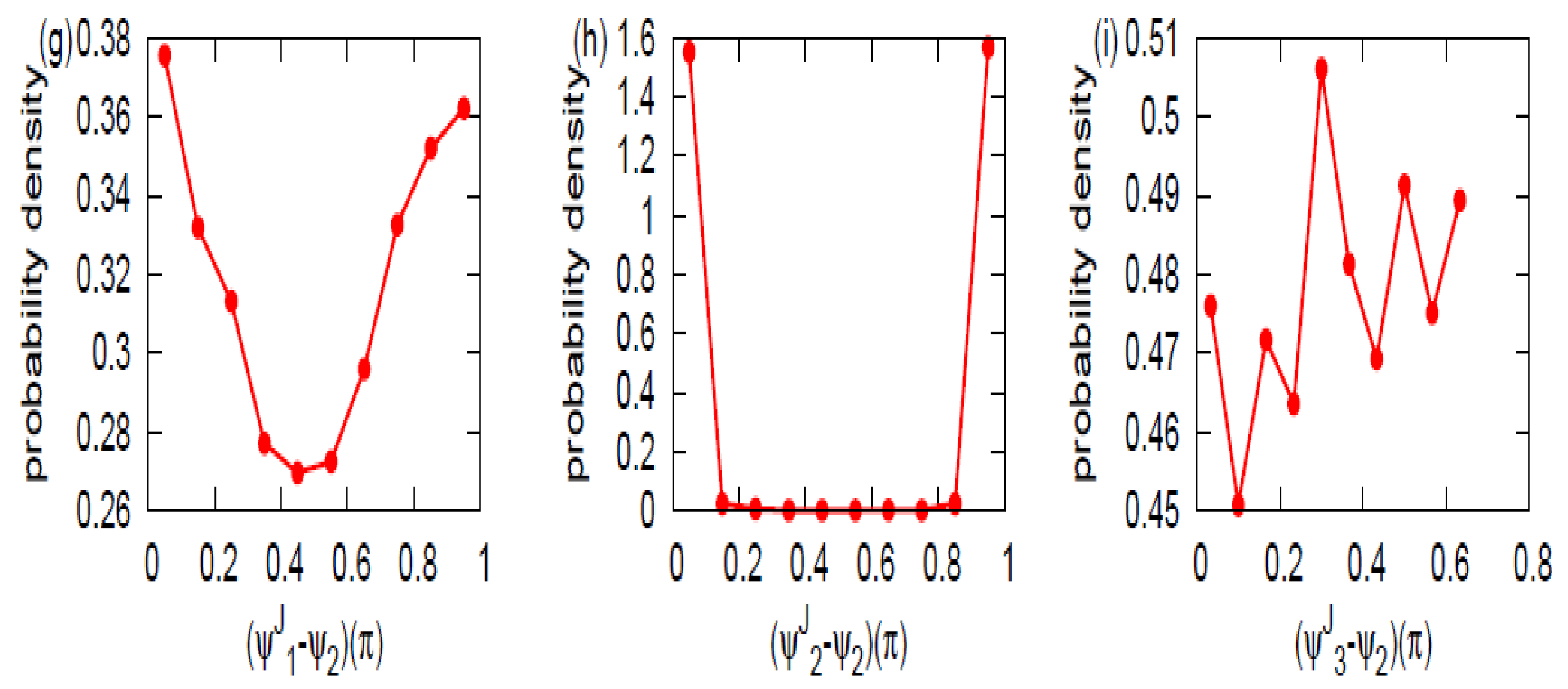

RHIC (200 GeV), b=7 fm 
Weak-coupling pQCD (Baier et al.):

$$
\hat{q}_{p Q C D}=\frac{8 \varsigma(3)}{\pi} \alpha_{S}^{2} N_{\text {color }}^{2} T^{3} \sim 0.94 \frac{G e V^{2}}{f m} \text { at } T=300 \mathrm{MeV}
$$

Strong-coupling N=4 SYM (Liu, Rajagopal and Wiedemann):

$$
\hat{q}_{\text {AdS /CFT }}=\frac{\pi^{\frac{3}{2}} \Gamma\left(\frac{3}{4}\right)}{\Gamma\left(\frac{5}{4}\right)} \sqrt{\alpha_{\text {SYM }} N_{\text {color }}} T^{3} \sim 4.5 \frac{G e V^{2}}{f m} \text { at } T=300 \mathrm{MeV}
$$

From P. Jacob's NNPSS2011 lecture 


\section{Jet tomography: $p t$ dependence}

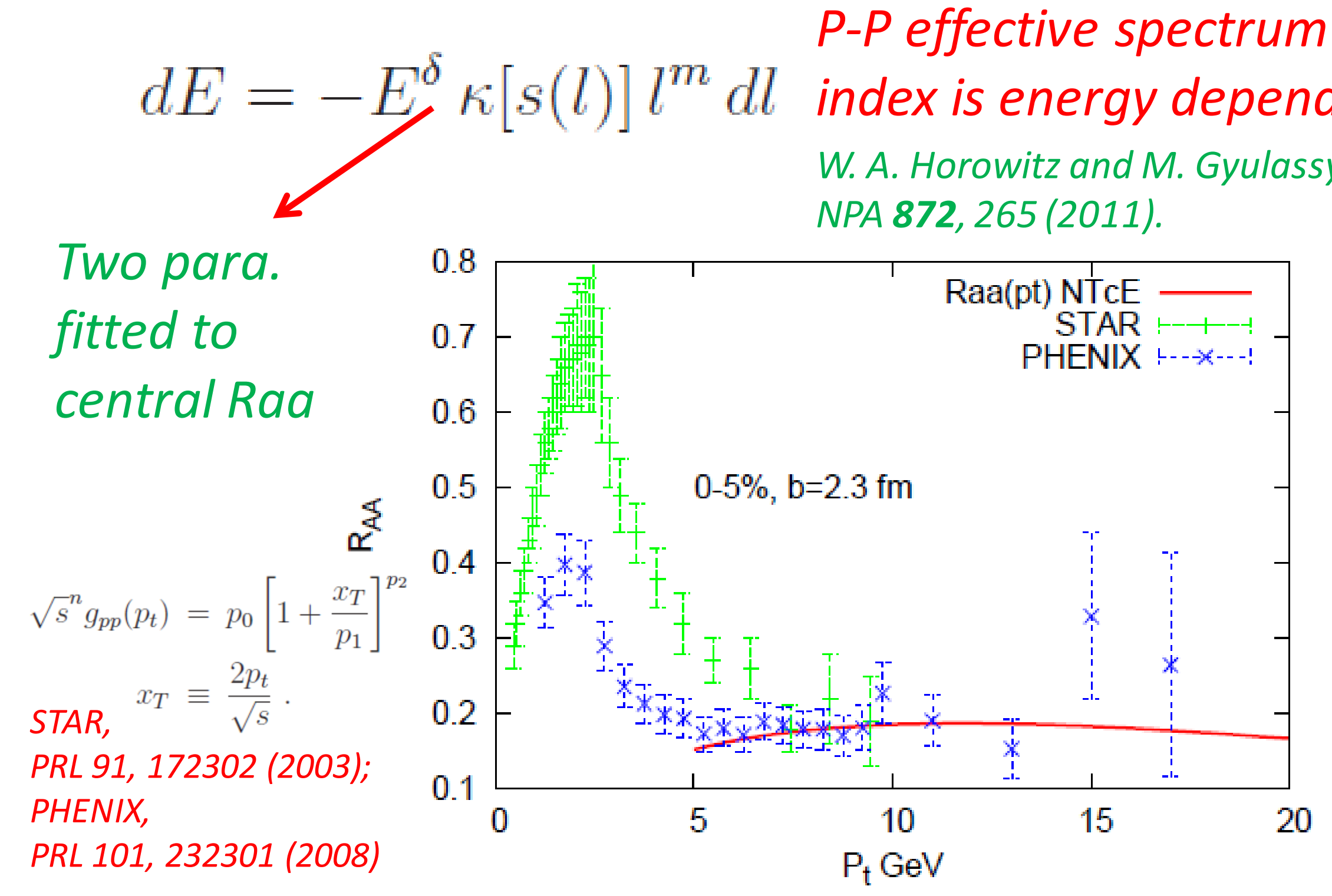




\section{Jet tomography: $\mathrm{pt}$ dependence}

P-P spectrum: QCD scaling formula.

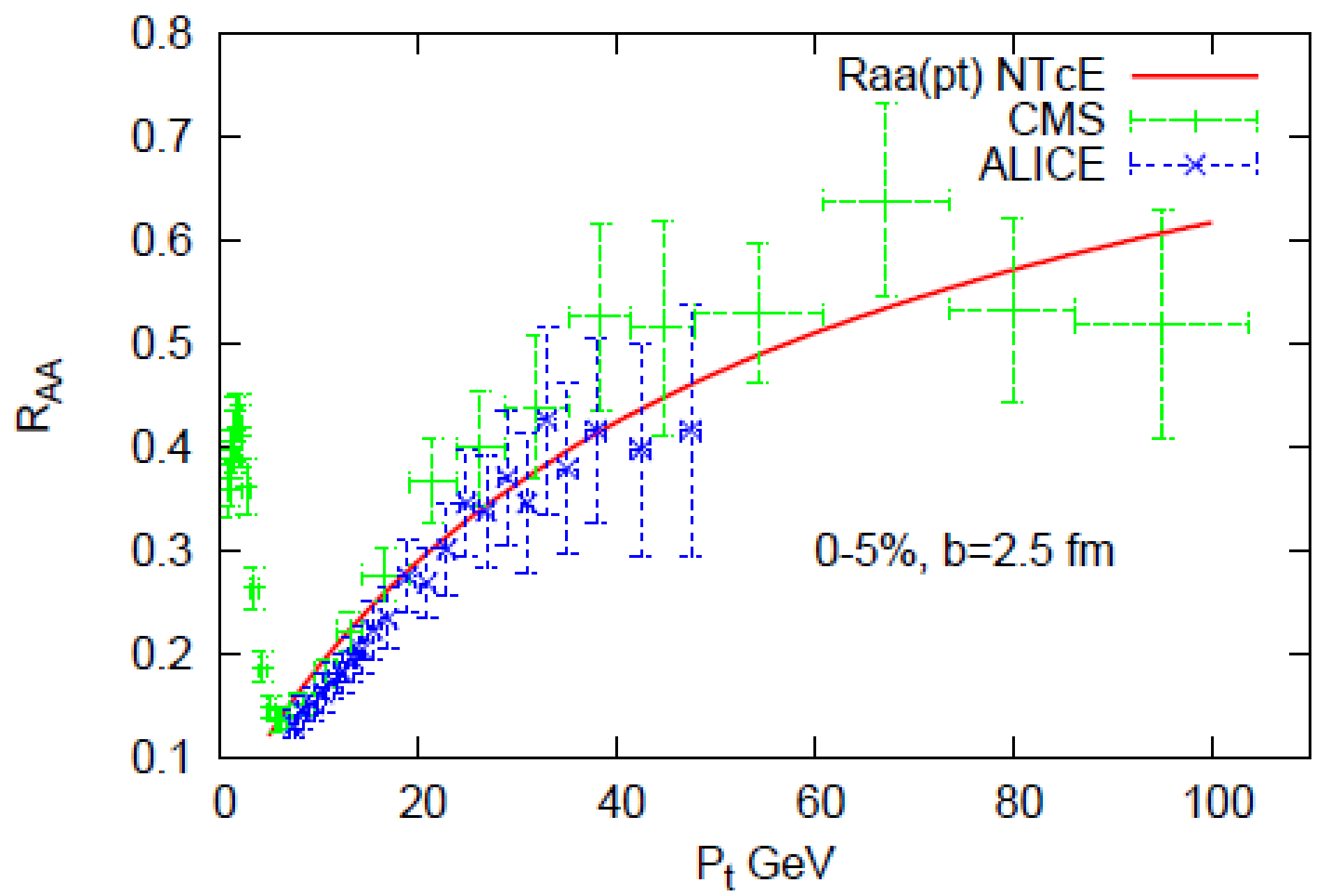

CMS, Eur. Phys. J. C 72, 1945 (2012); ALICE, arXiv:1208.2711 

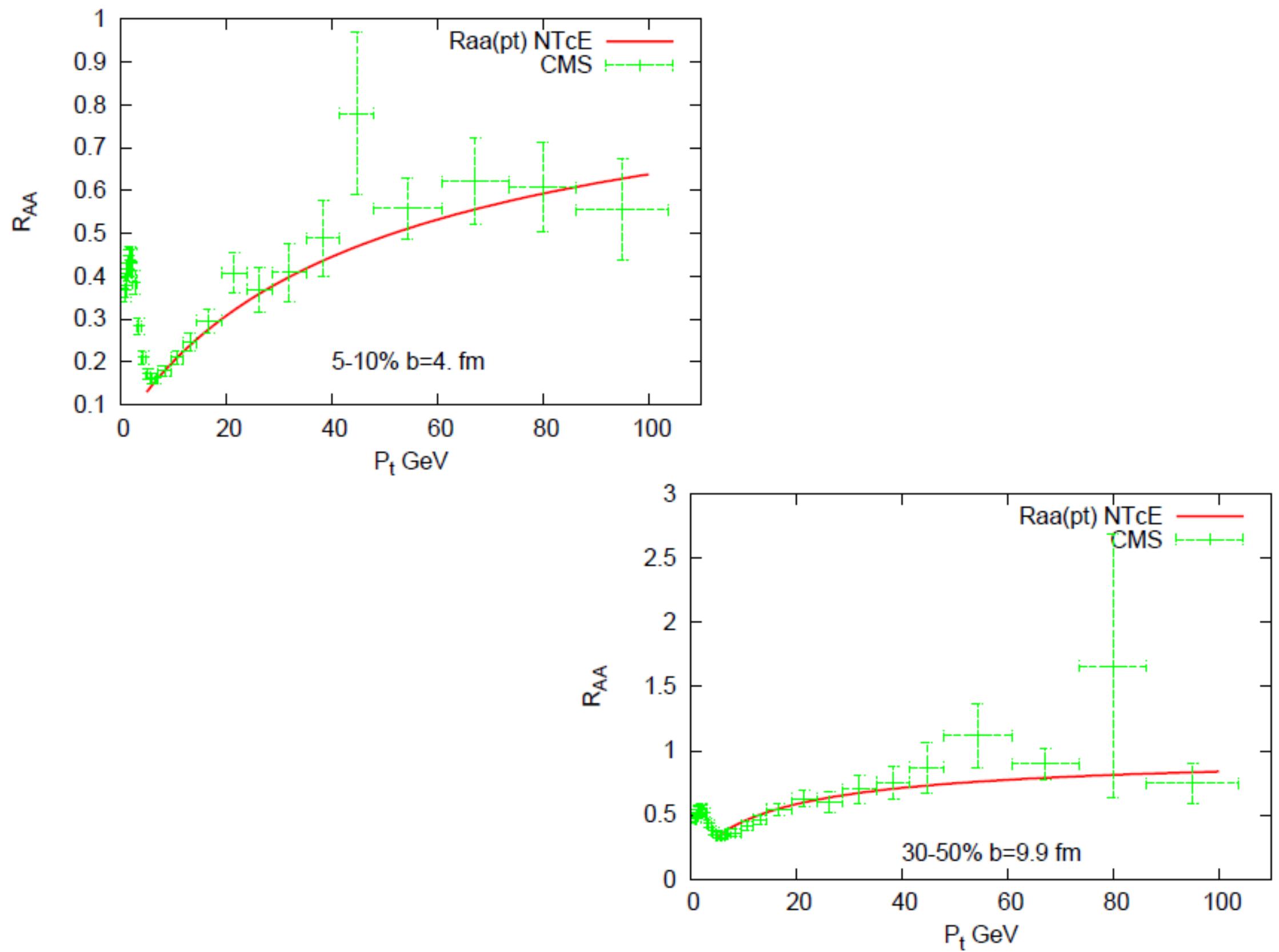


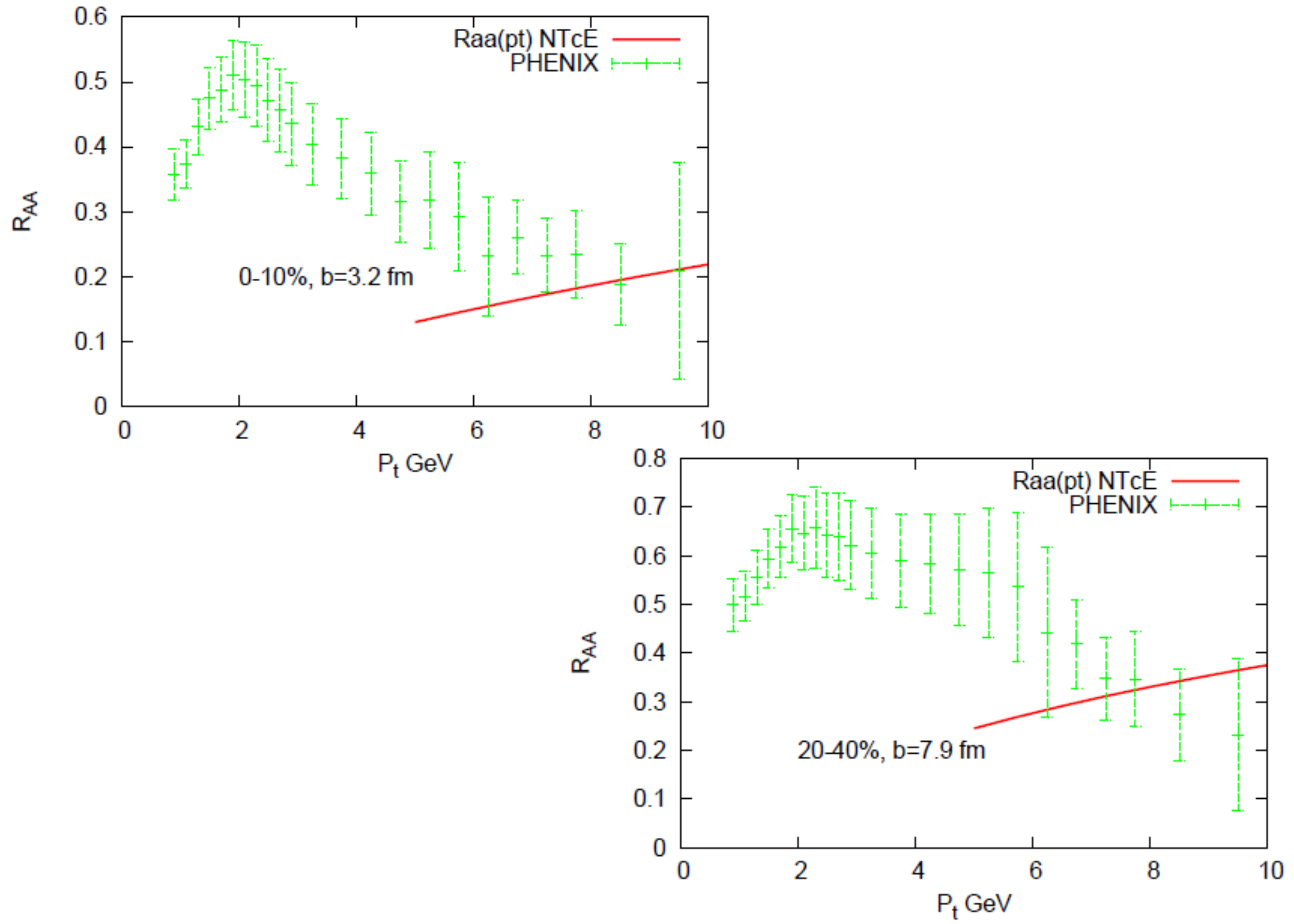




\section{Jet tomography: pt dependence}

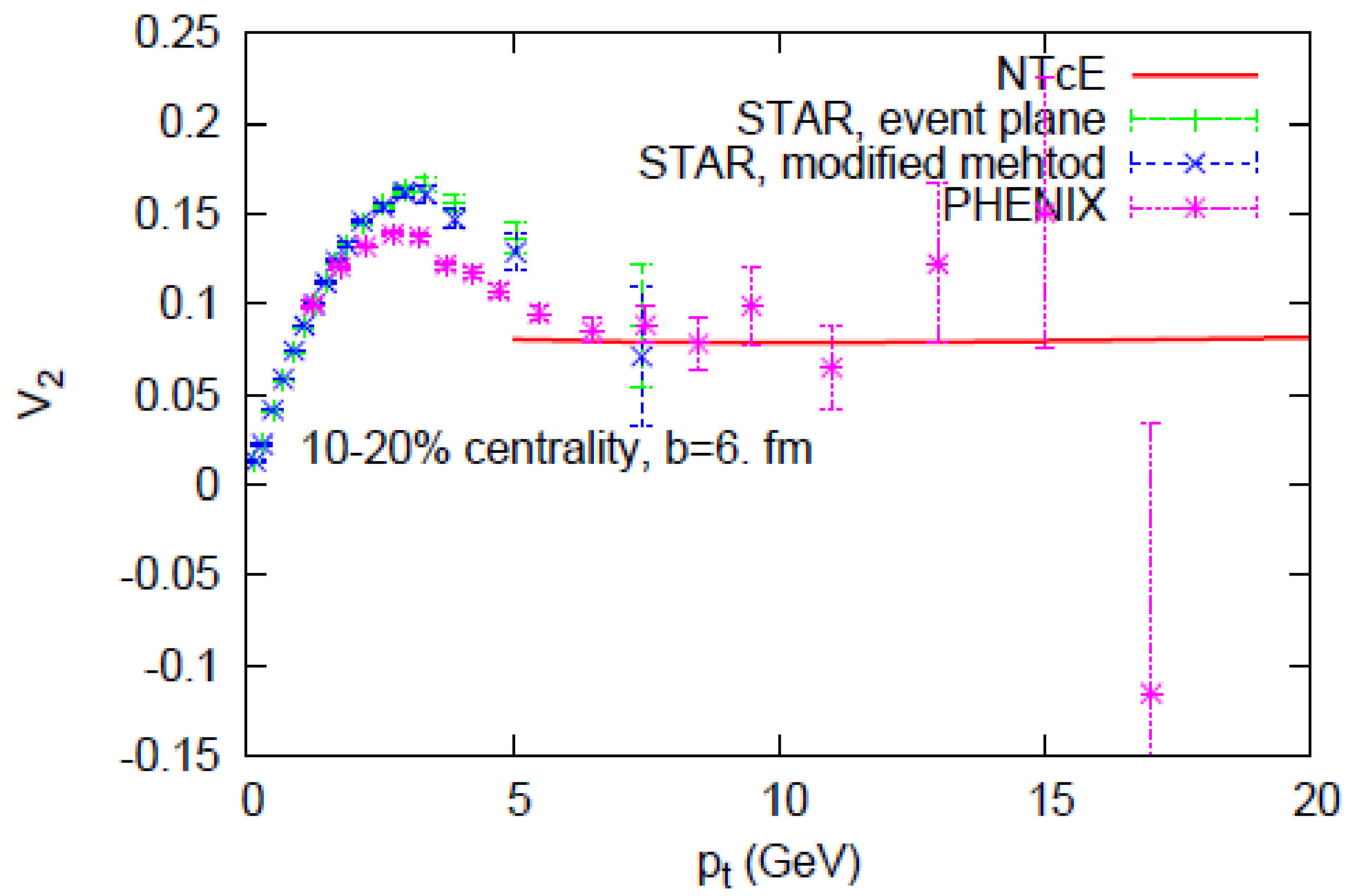

STAR, PRC 72, 014904 (2005); PHENIX, PRL 105, 142301 (2010). 


\section{Jet tomography: pt dependence}

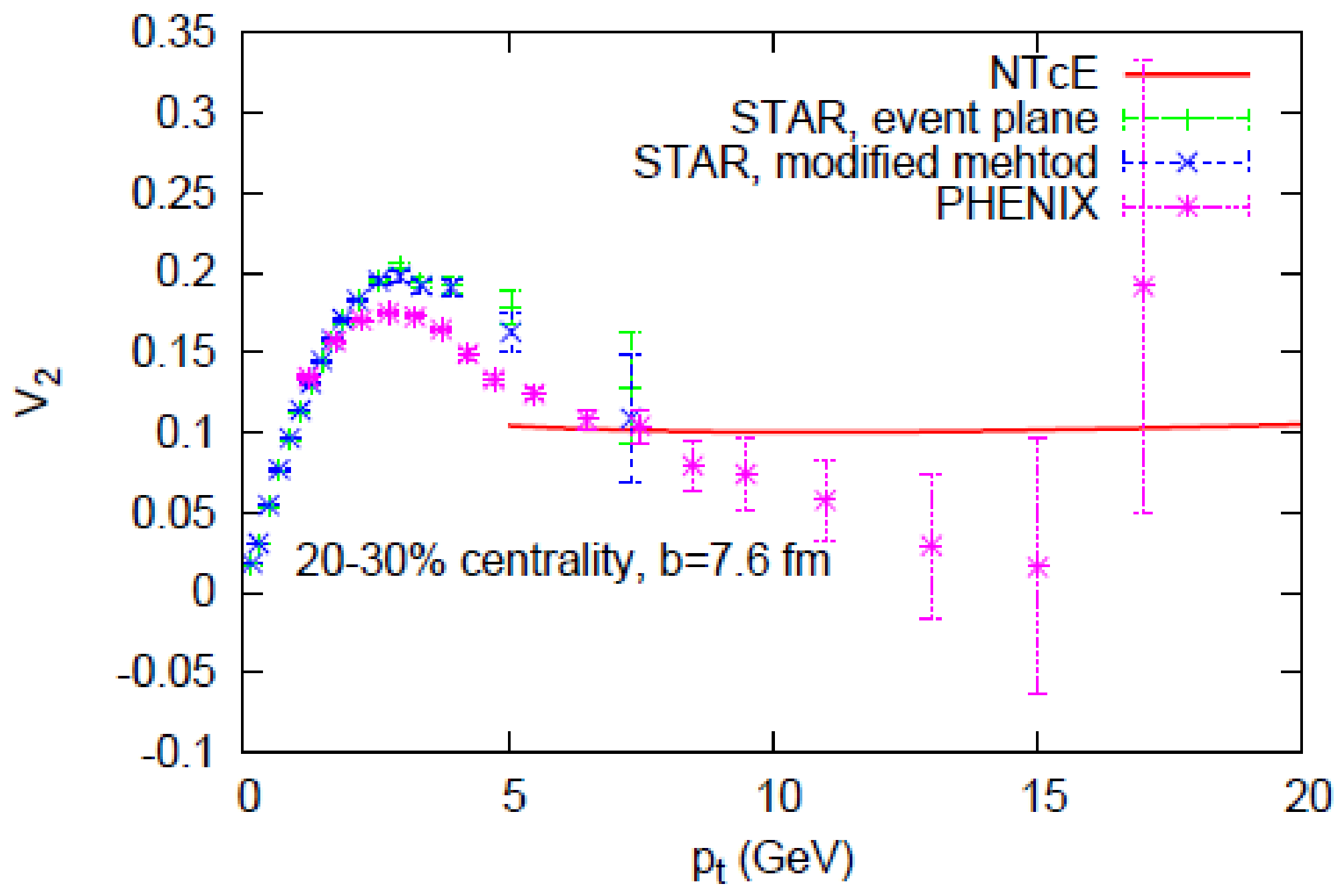




\section{Jet tomography: pt dependence}

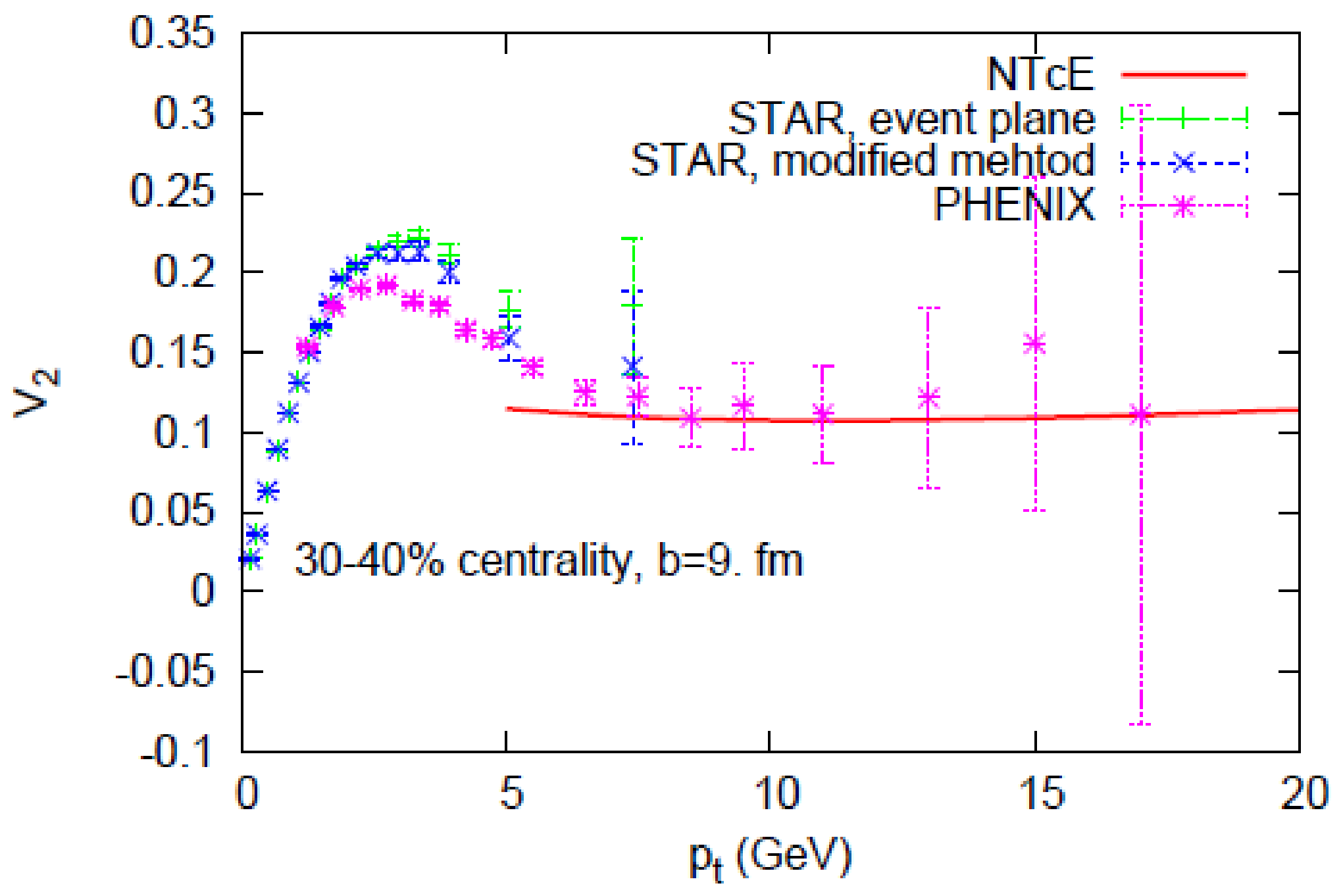




\section{Jet tomography: pt dependence}

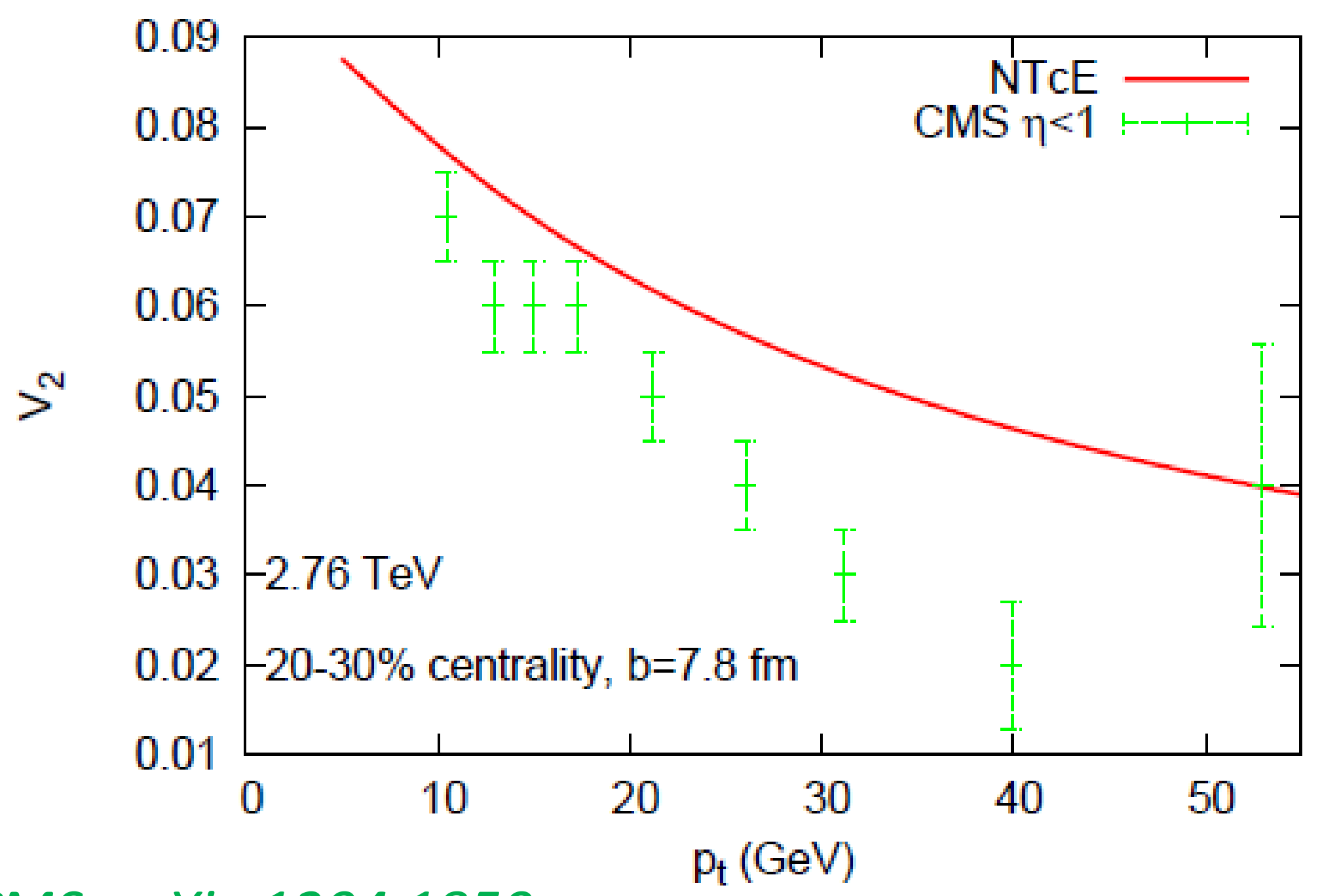

CMS, arXiv:1204.1850 


\section{Jet tomography: pt dependence}

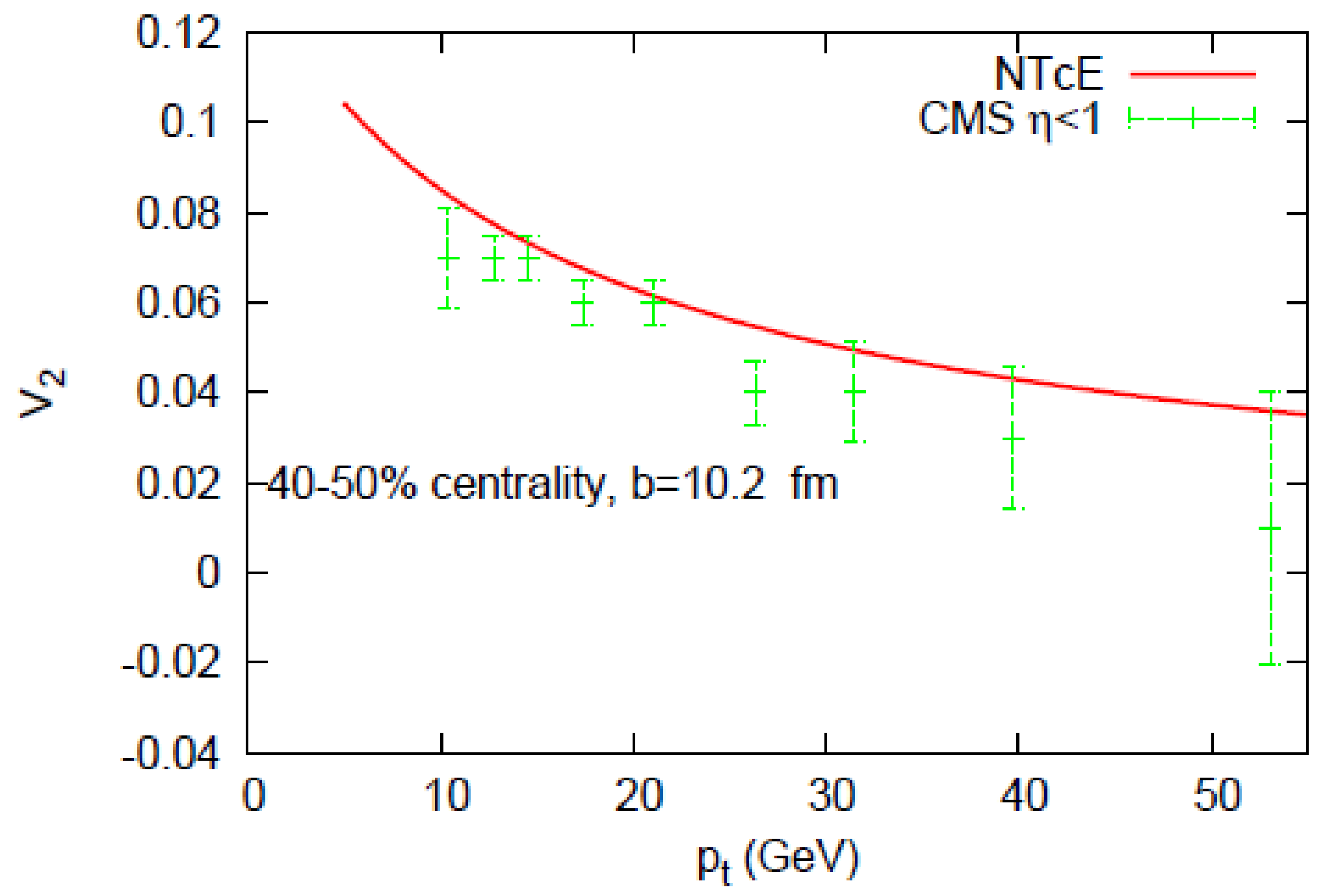




\section{Jet tomography: pt dependence}

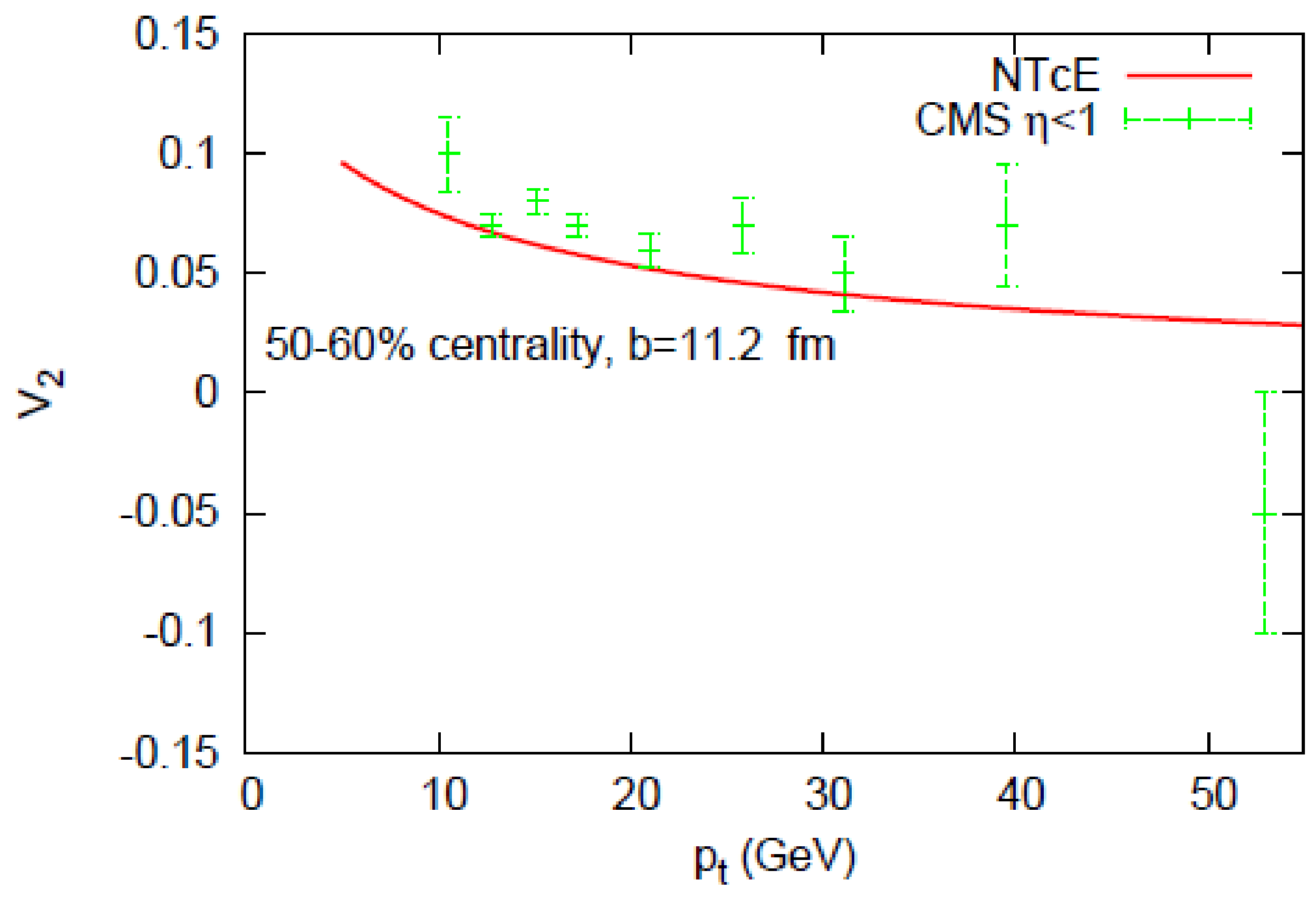




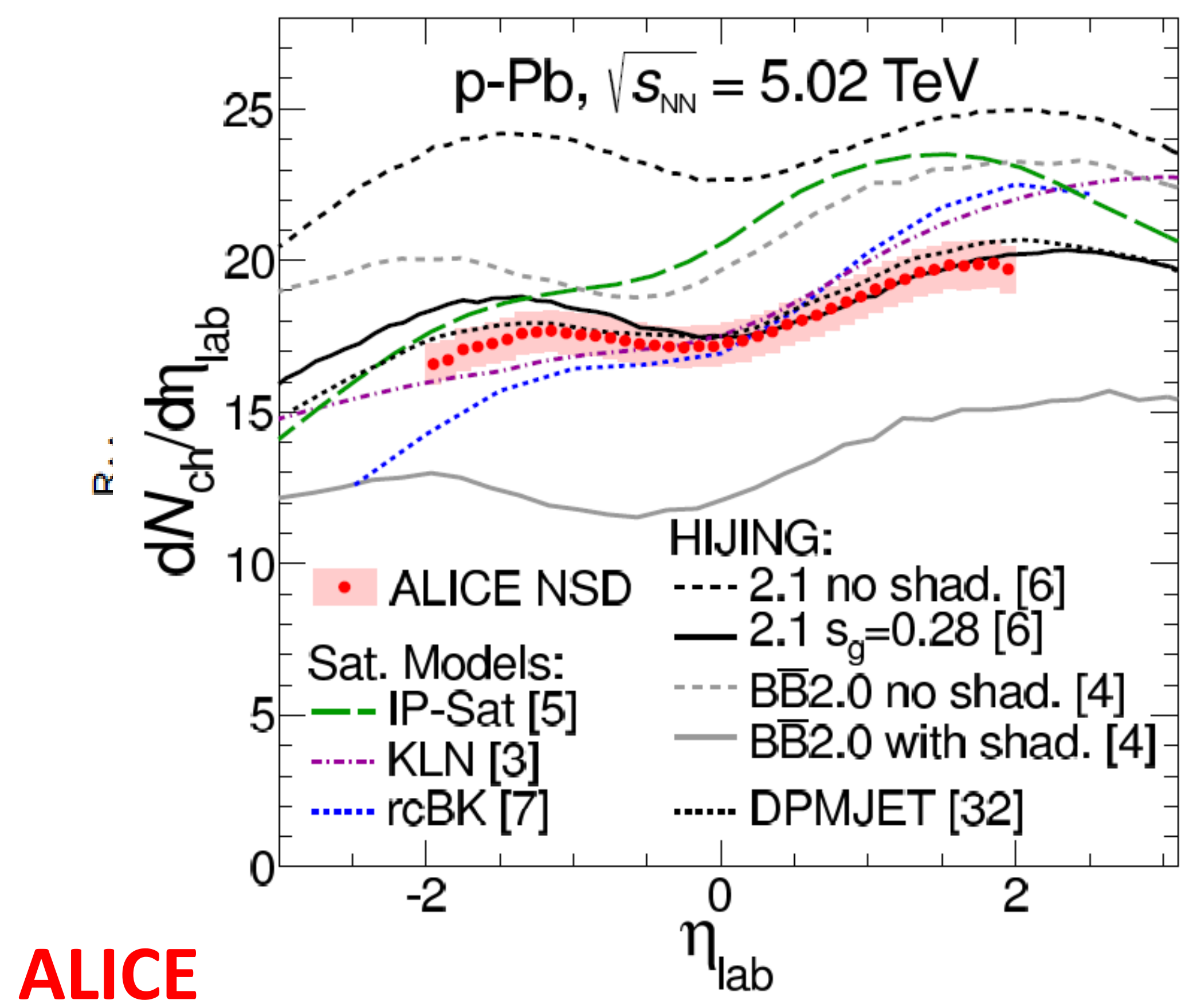



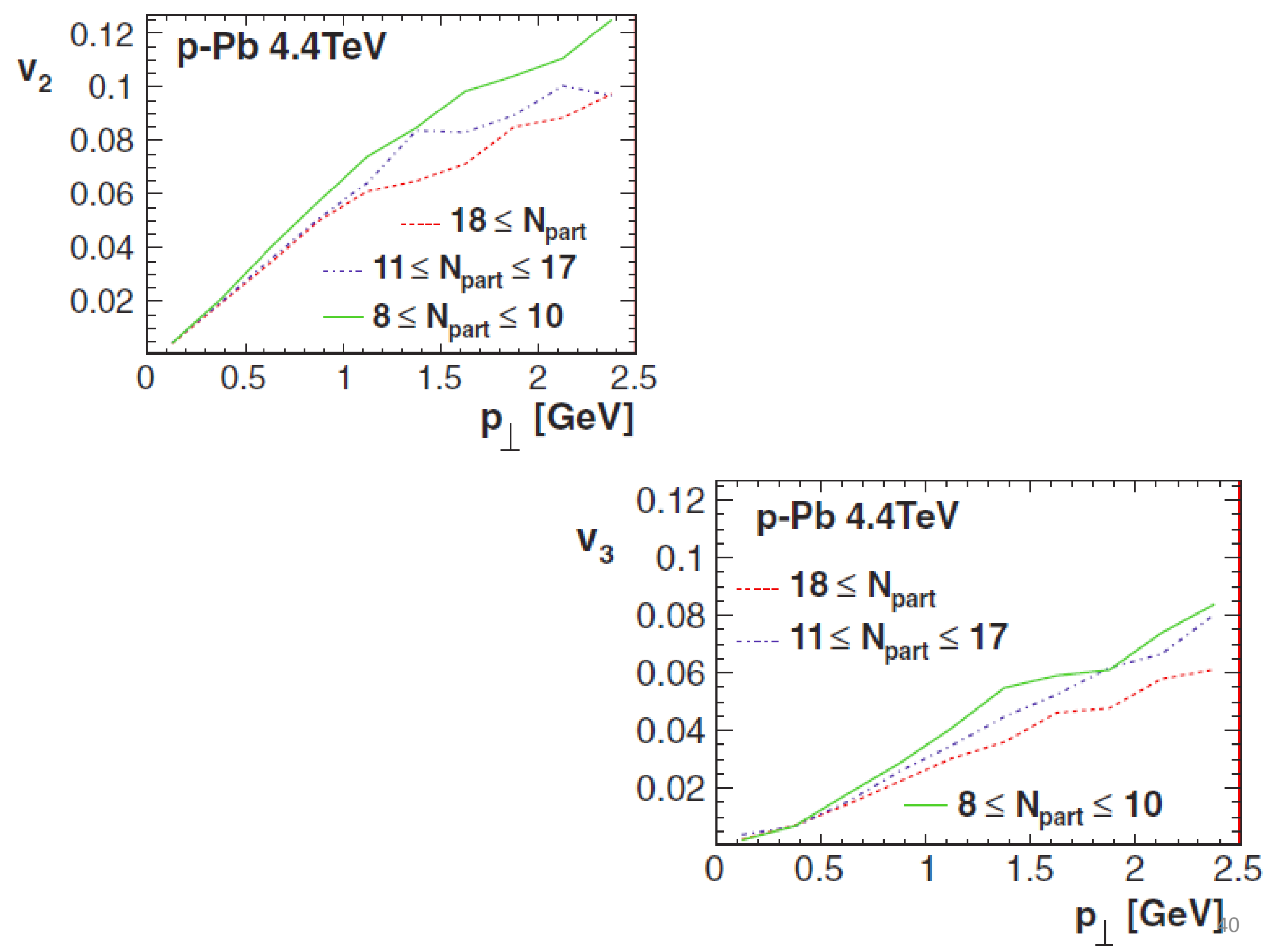


\section{Smaller profile}

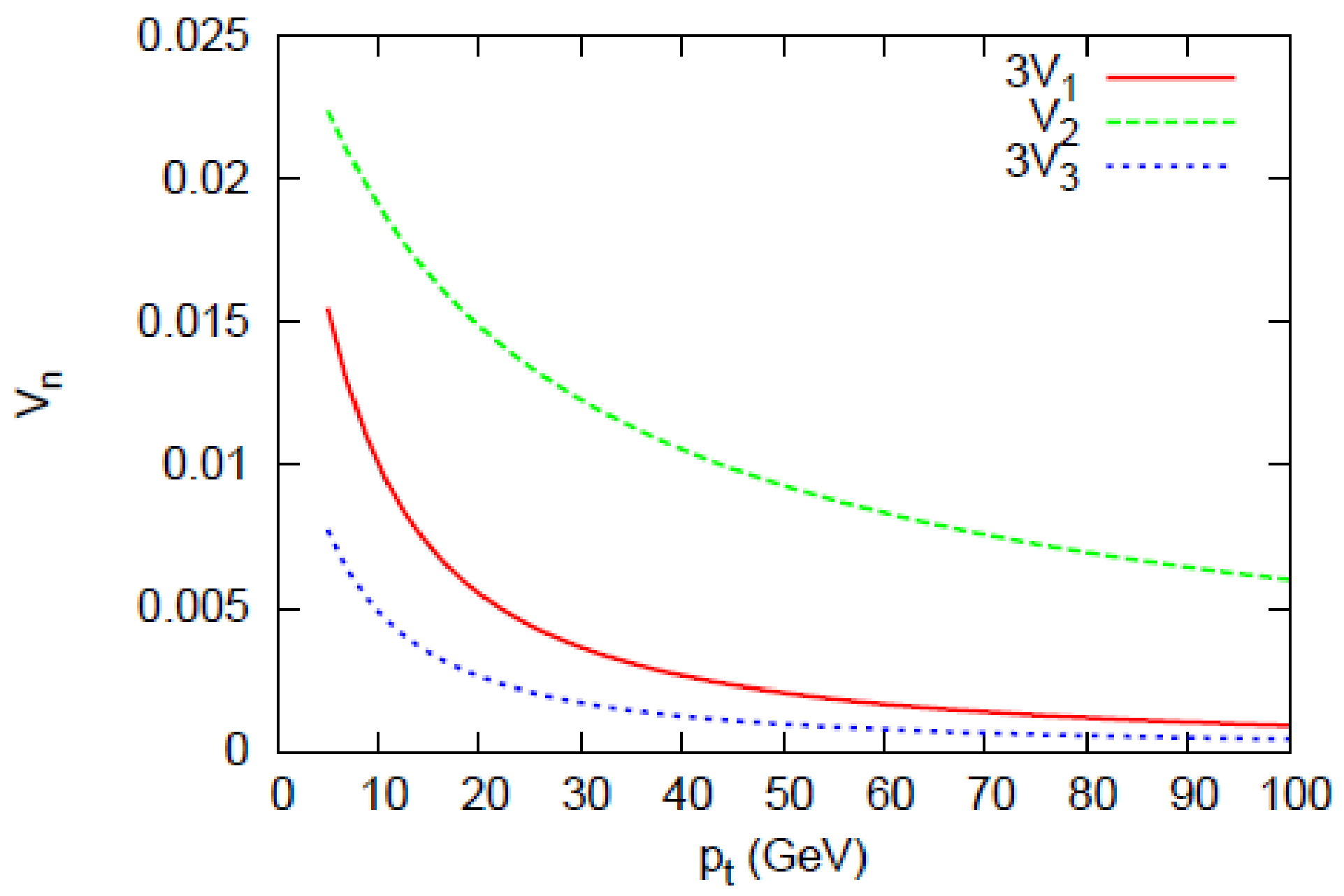




\section{Smaller profile}

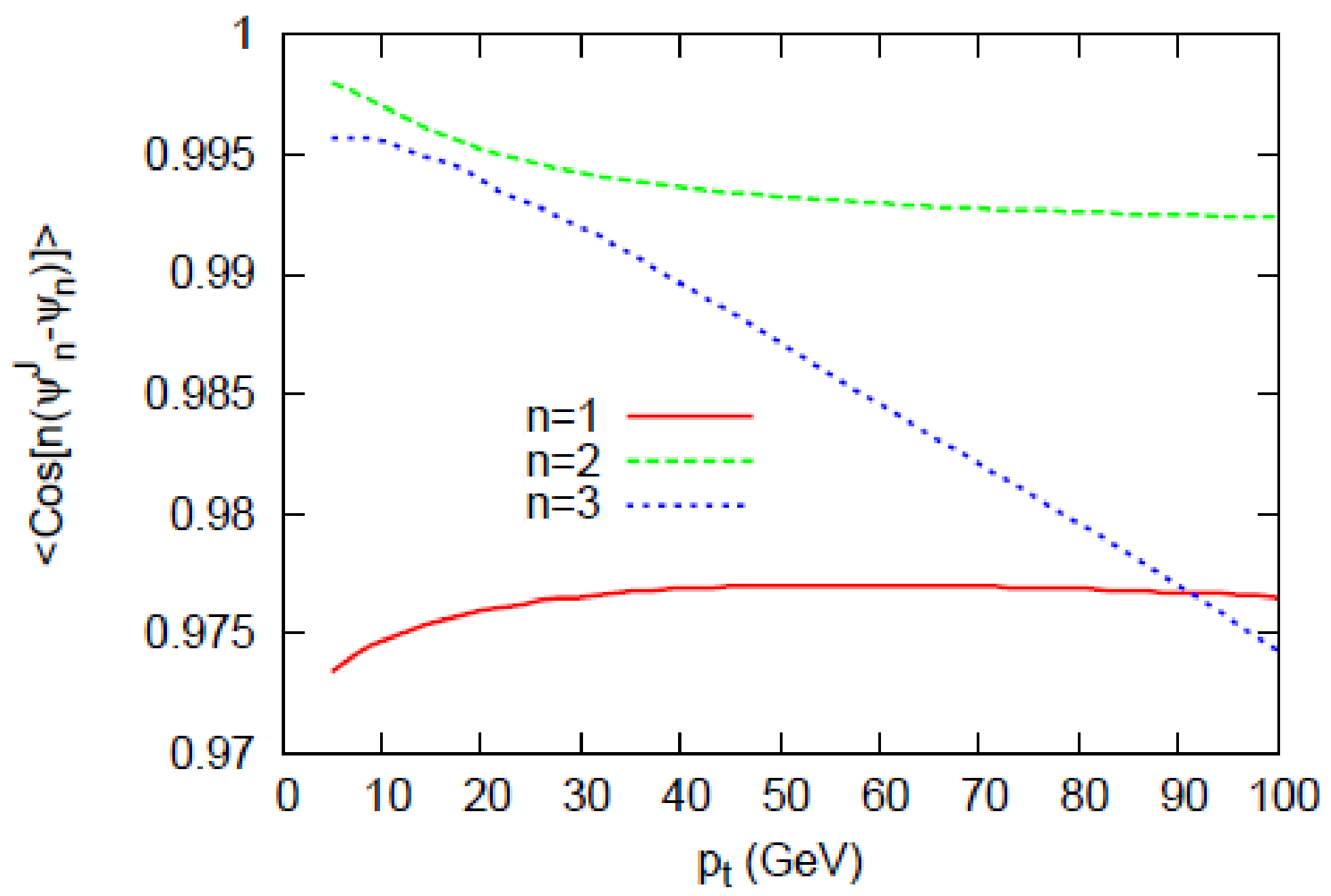




\section{Event activity}
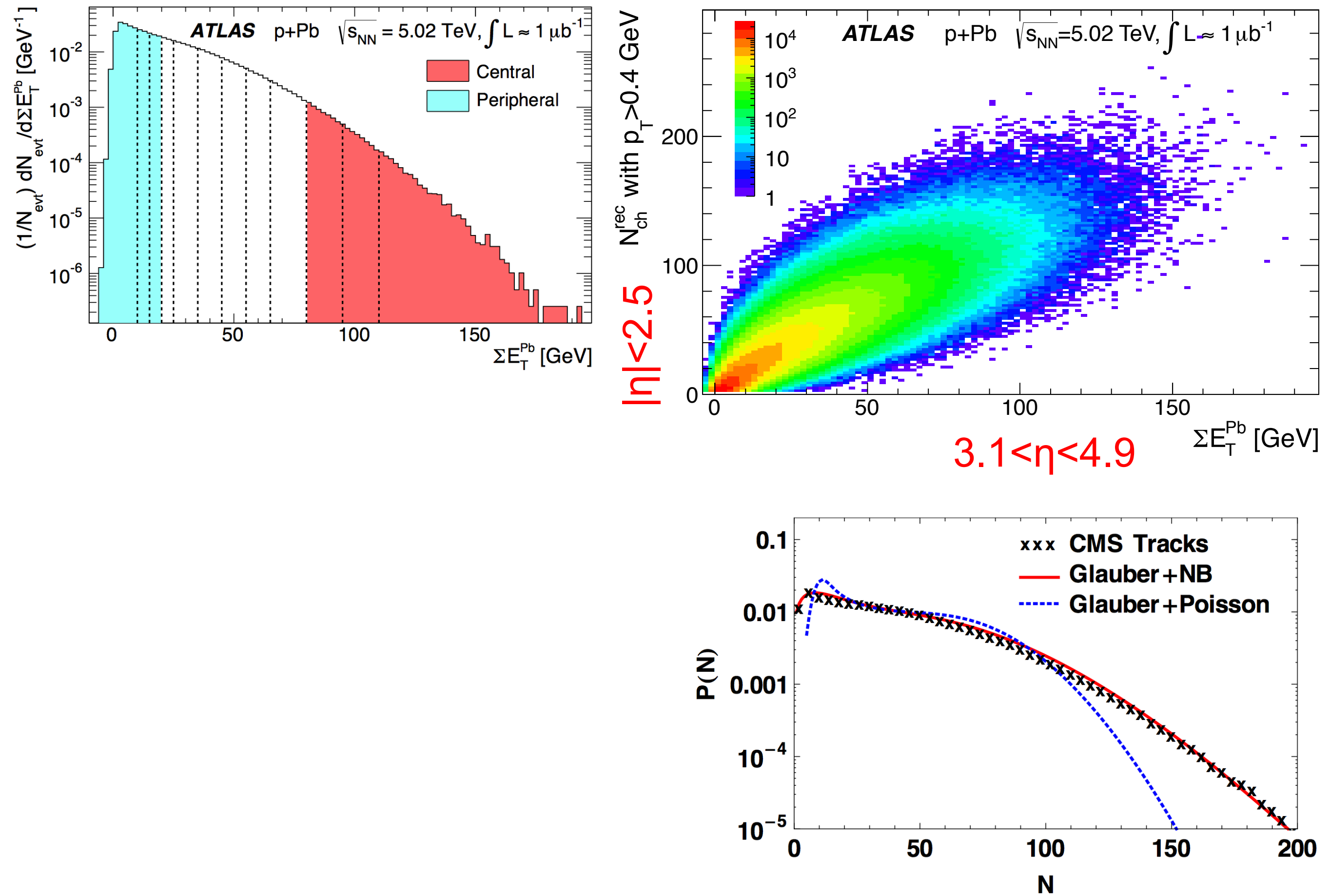


\section{Auto-correlation bias}
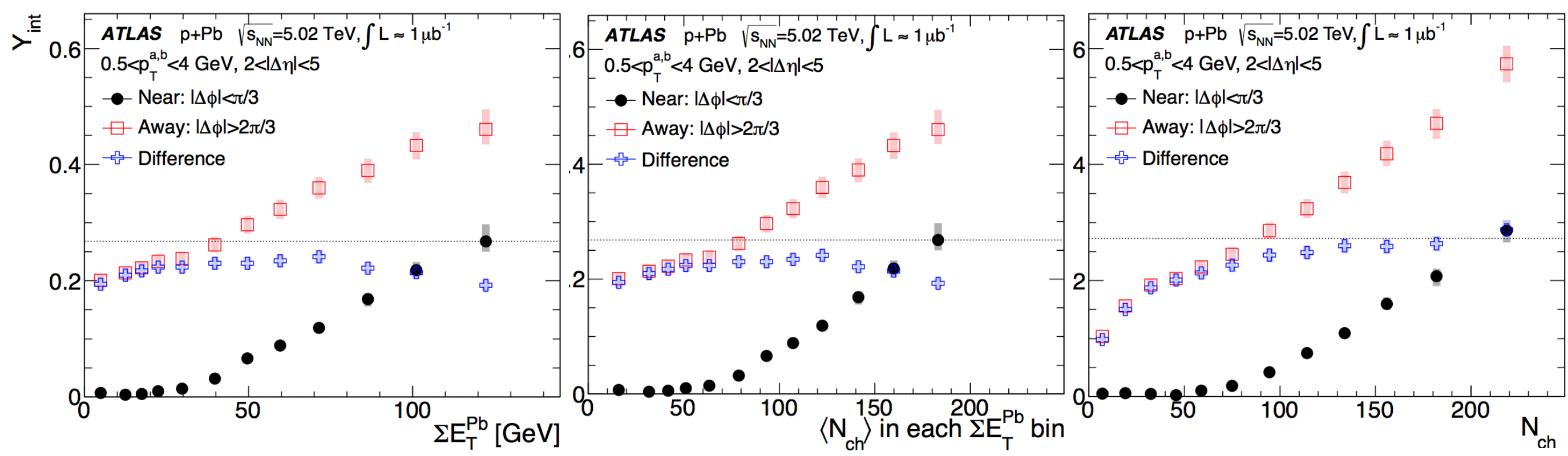

- No bias for long-range global correlations: i.e. the near-side ridge yield.

- Strong auto-correlations between Nch and short range correlation signal. 


\section{Illustrate bias with HIJING simulation}

- Hijing has no ridge, mostly short range correlations.

Clear bias effects in $\mathrm{N}_{\mathrm{ch}}$ based selections

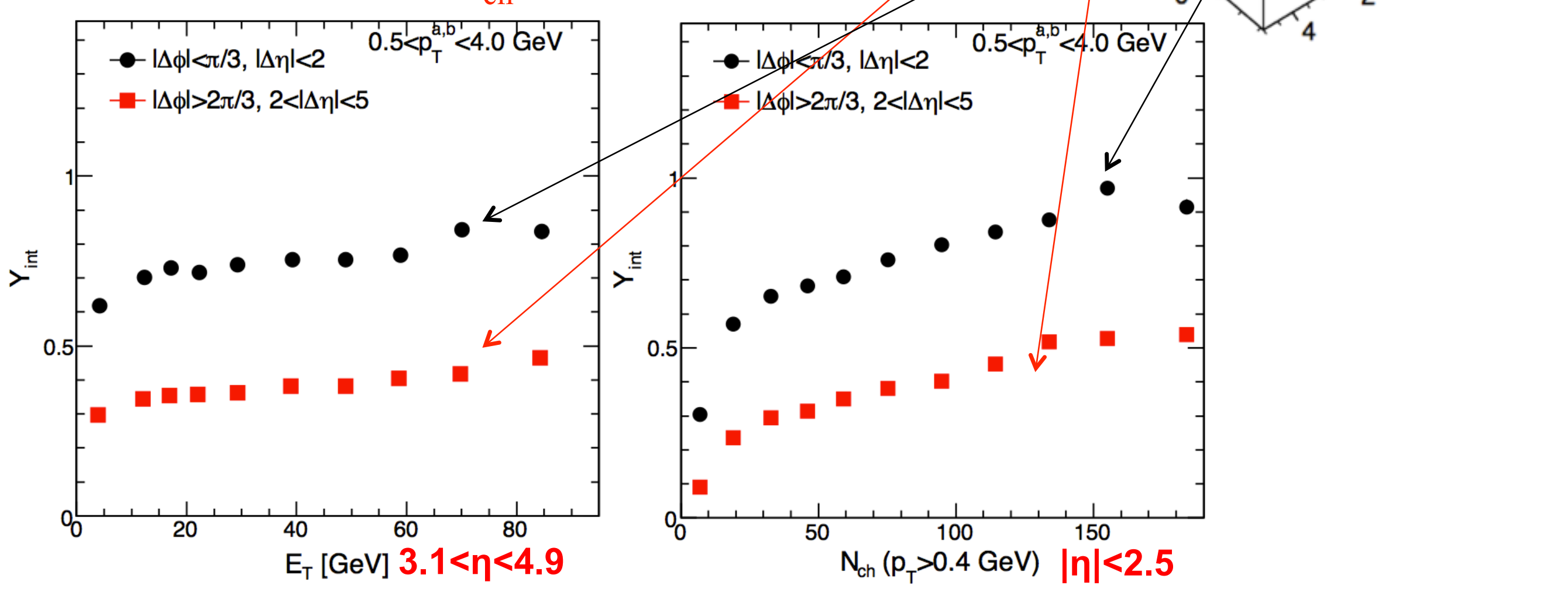




\section{$2 \mathrm{PC}$ in $\Delta \phi:$ peripheral}

- Define 1-D correlation function over the region $2<|\Delta \eta|<5$

- normalized to have mean value of 1

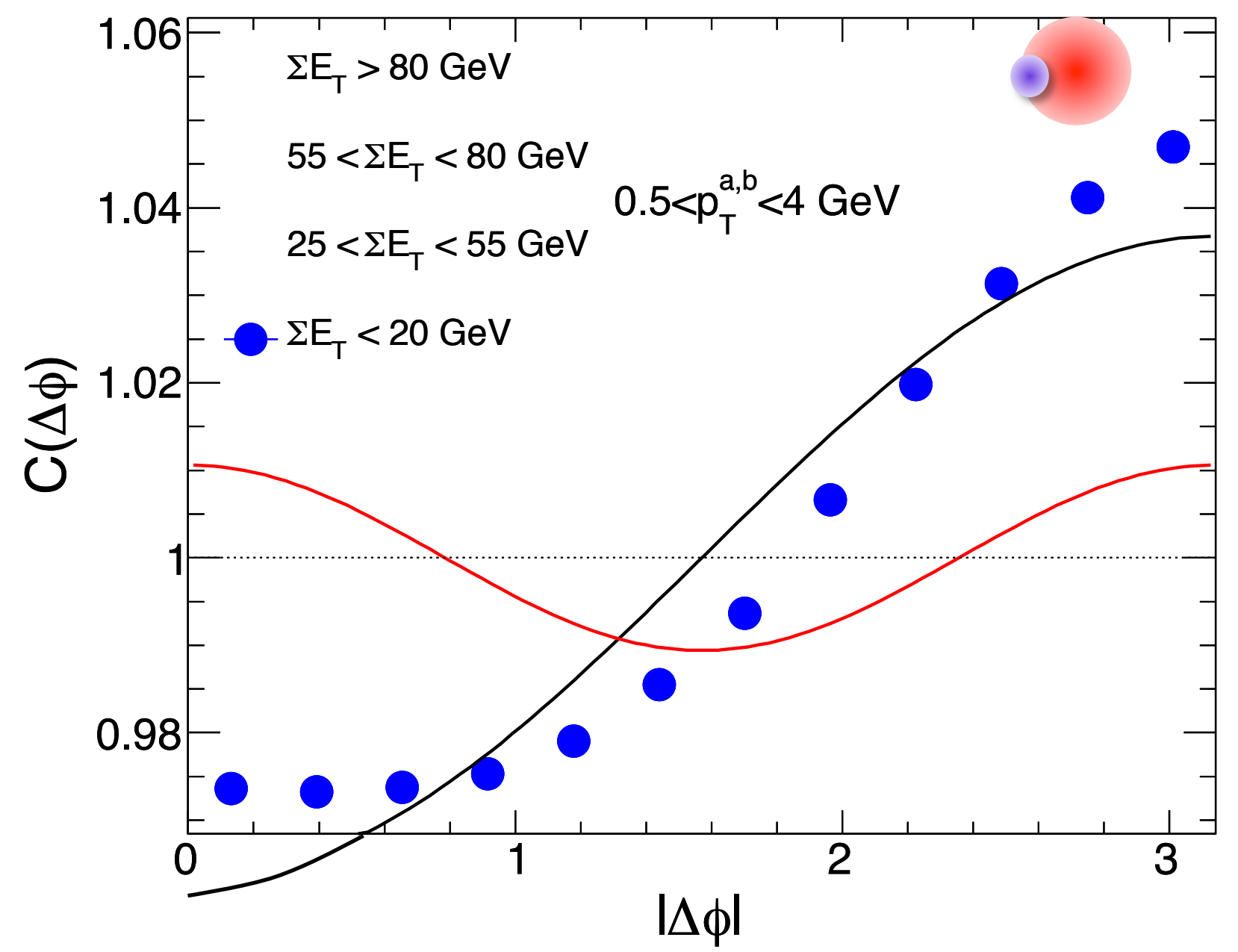

Dominated by a recoil contribution: $\propto-\frac{p_{T}^{a} p_{T}^{b} \cos \Delta \phi}{N_{b}} \quad$ N. Borghini 2000 Recoil can also has a small fake $v_{2}$ component (about $20 \%$ of the yield) 


\section{PC in $\Delta \phi:$ central}

- Define 1-D correlation function over the region $2<|\Delta \eta|<5$

- normalized to have mean value of 1

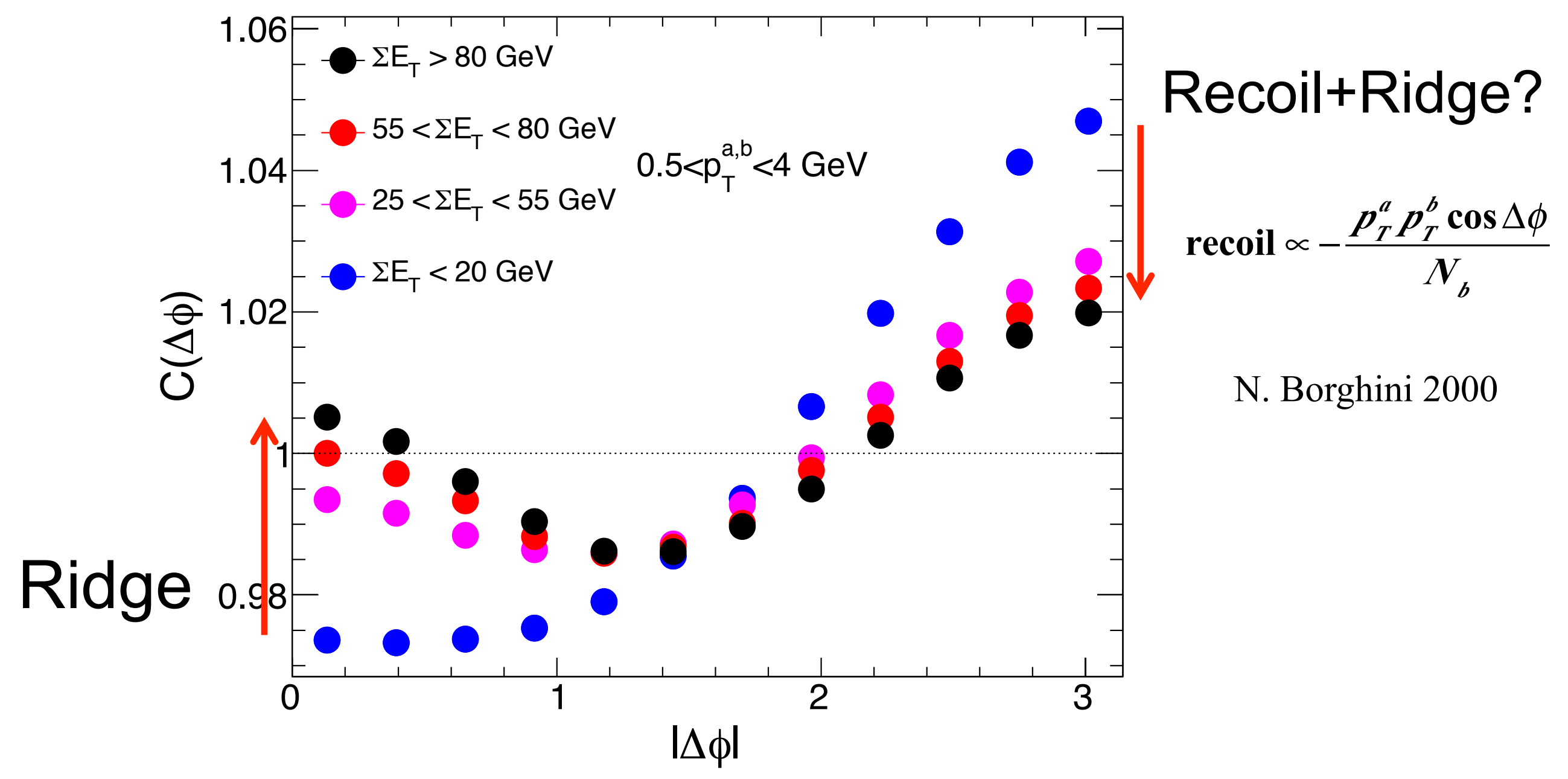

Need to quantify the recoil to the trigger $\rightarrow$ per-trigger yield

$$
\text { Corr } \times \mathbf{N}_{\mathbf{b}}=\frac{\mathbf{N}_{\text {pairs }}}{\mathbf{N}_{\mathbf{a}} \mathbf{N}_{\mathbf{b}}} \times \mathbf{N}_{\mathbf{b}}=\frac{\mathbf{N}_{\text {pairs }}}{\mathbf{N}_{\mathbf{a}}} \rightarrow \text { recoil is } \sim \text { constant }
$$




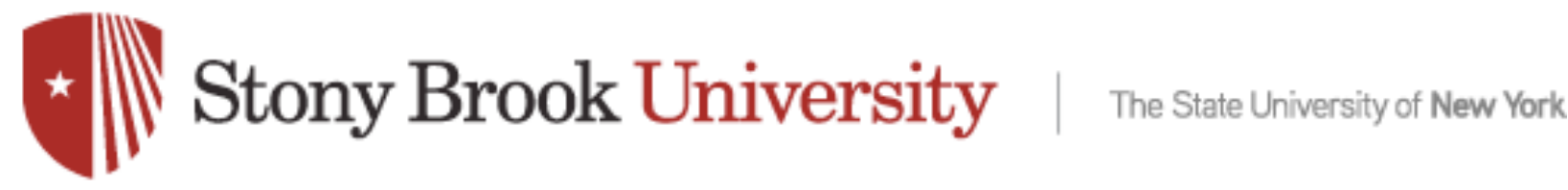

\section{Some personal opinions}

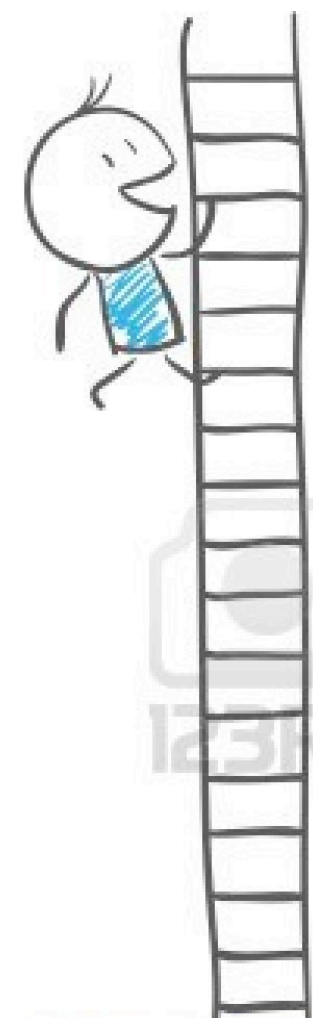

Jiangyong Jia

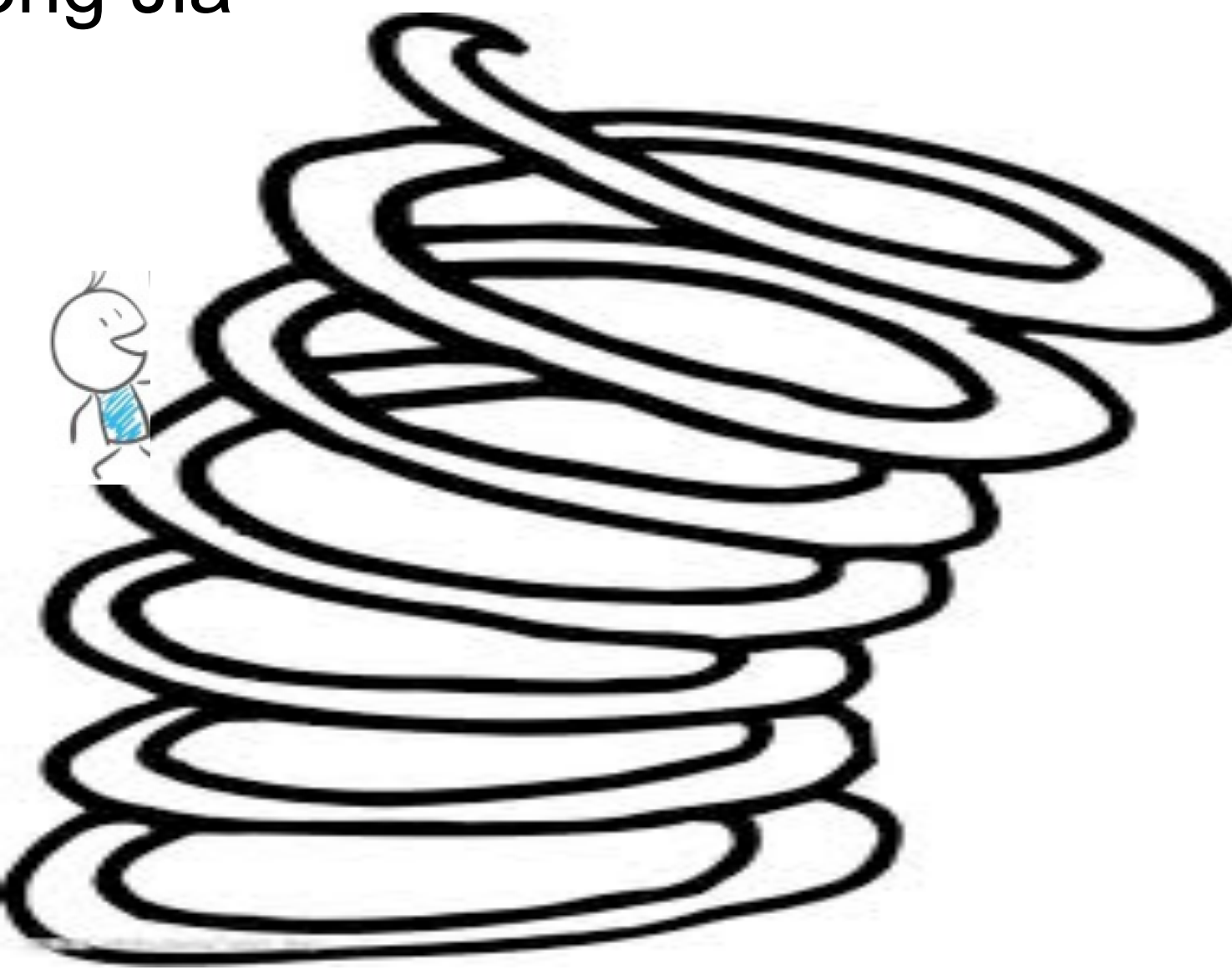




\section{State of affair 2003}

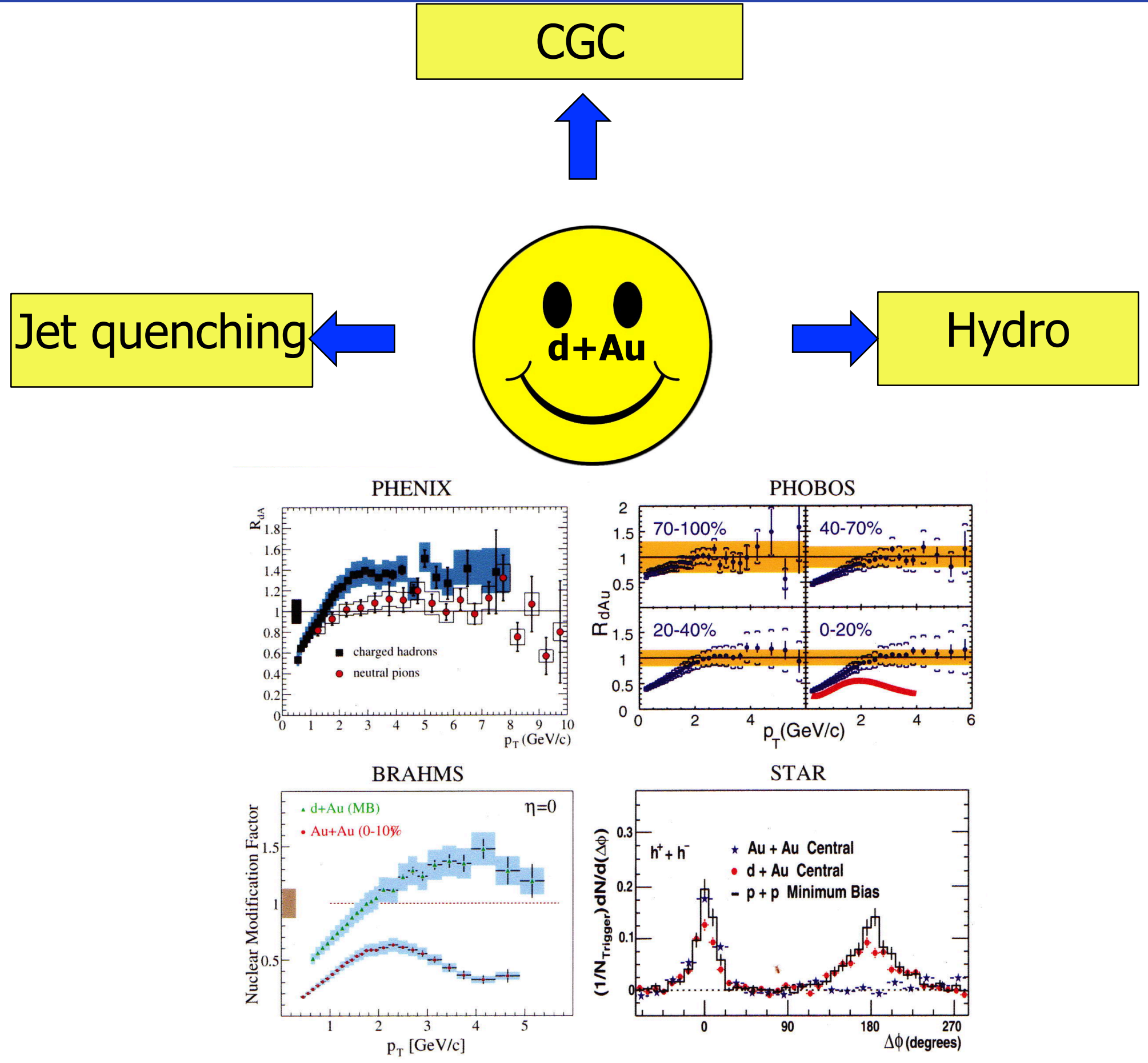




\section{State of affair 2013}

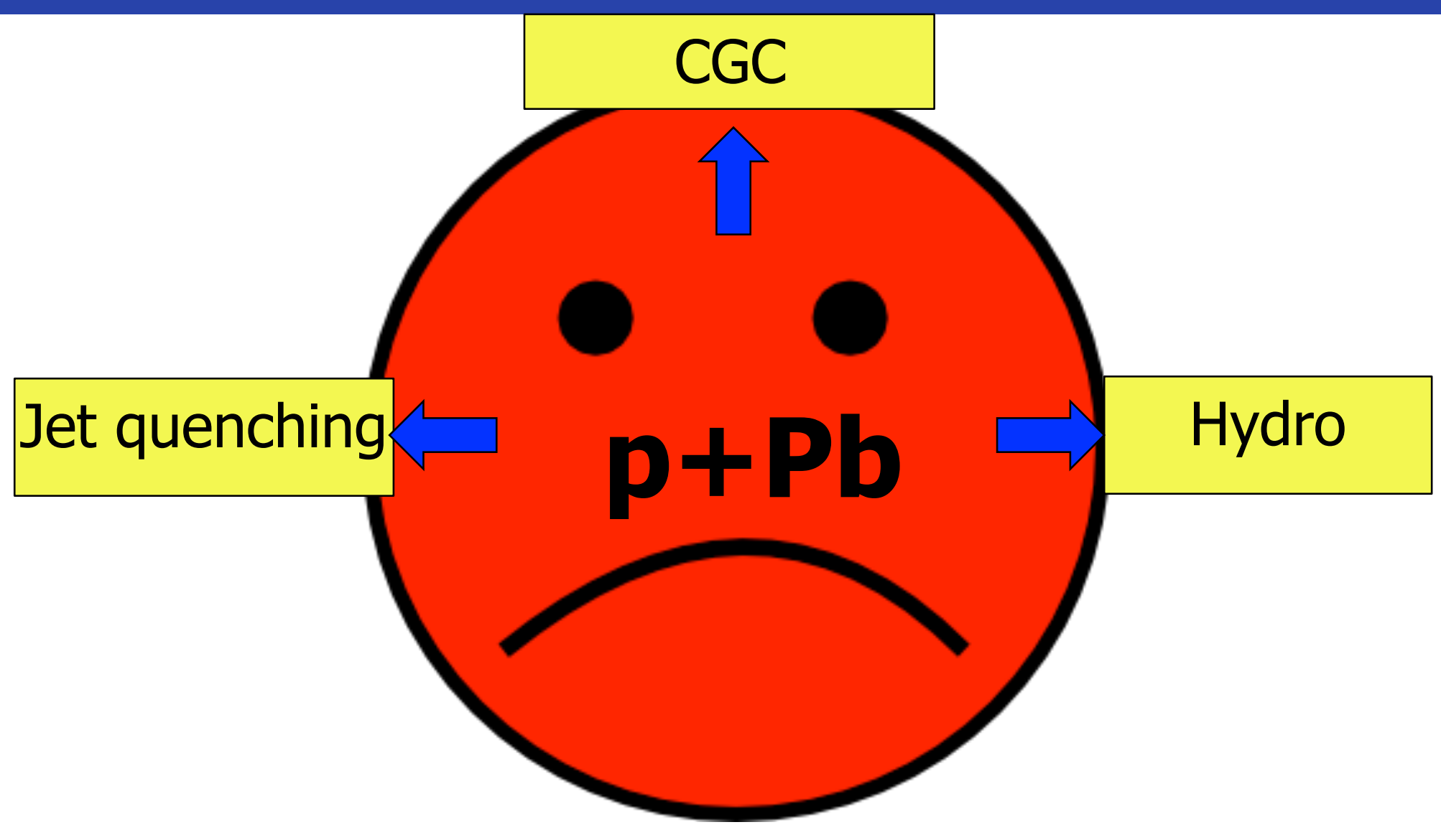




\section{State of affair 2013}

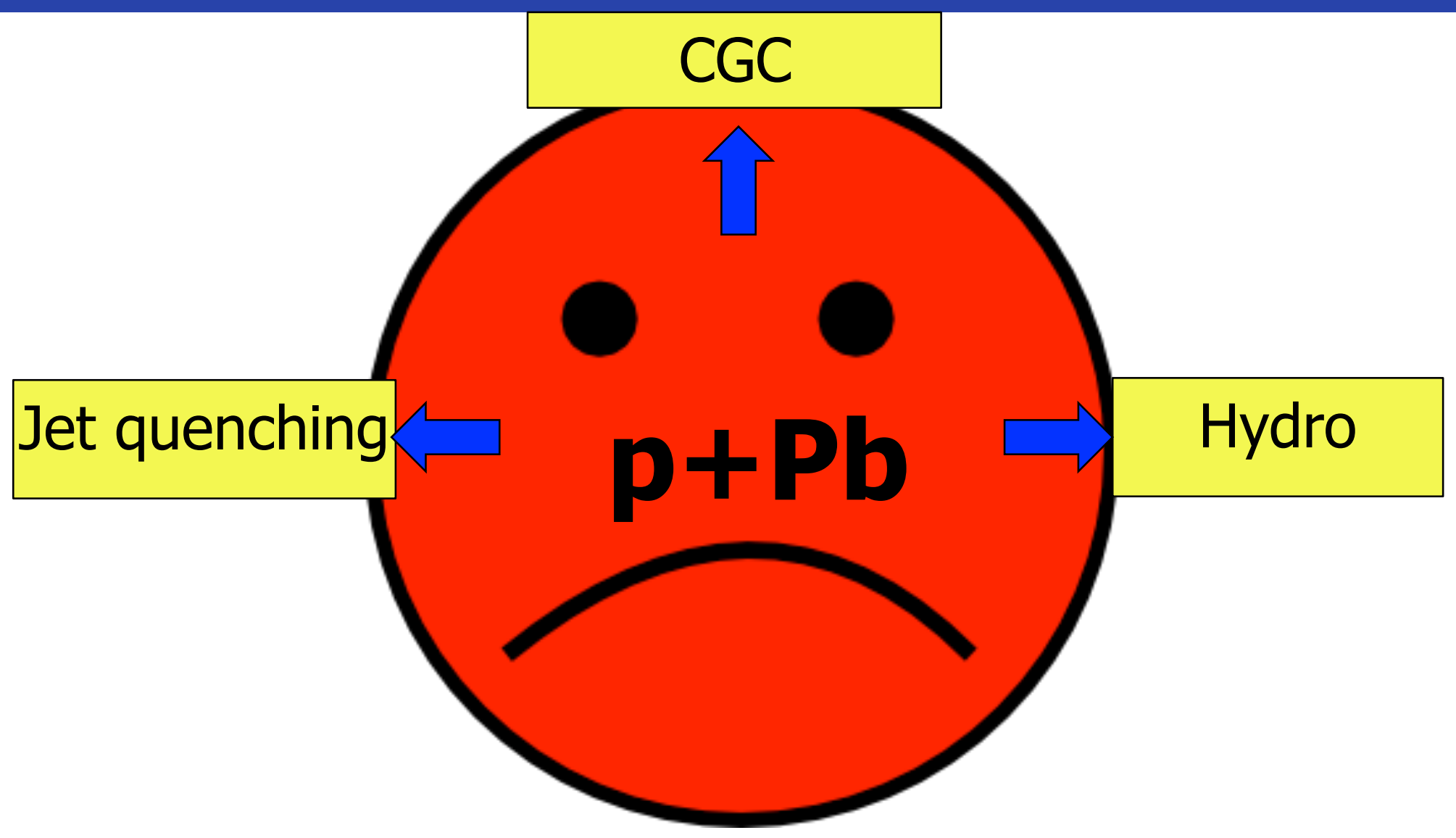

Miklos: My personal perspective is that instead of converging on a solid basis of null controls, we reached a maximal entropy state both theoretically and experimentally

- Theory : Indeed...

- Experiment : undigested information $\neq$ more entropy

What is the effective picture/theory of QCD in high-density system?? 


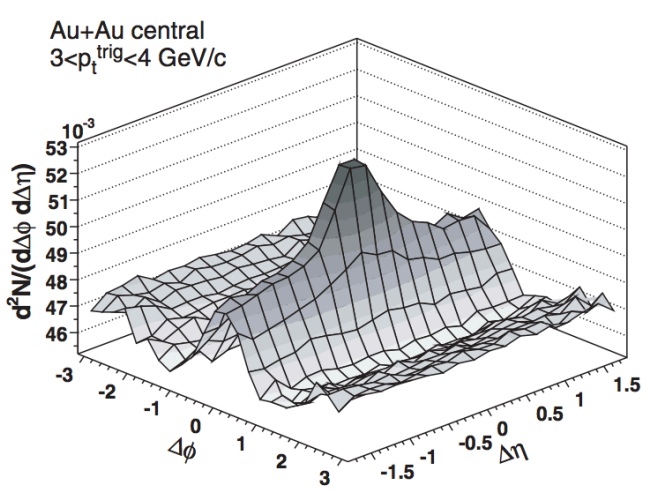

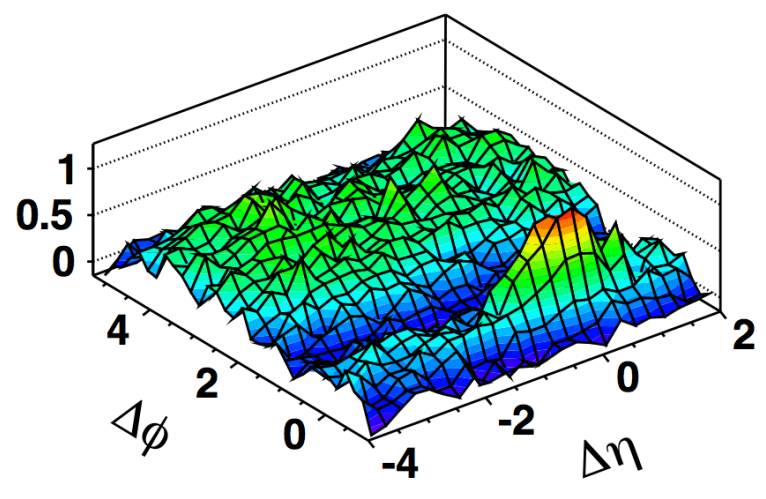

(b) $\mathrm{Au}+\mathrm{Au}$ 0\%-30\% (PHOBOS) (d) $\mathrm{CMS} \mathrm{N} \geq 110,1.0 \mathrm{GeV} / \mathrm{c}<\mathrm{p}_{\mathrm{T}}<3.0 \mathrm{GeV} / \mathrm{c}$

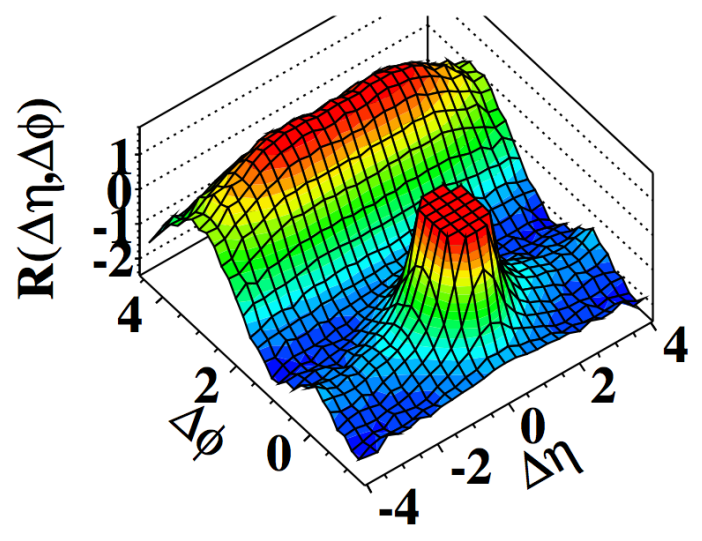

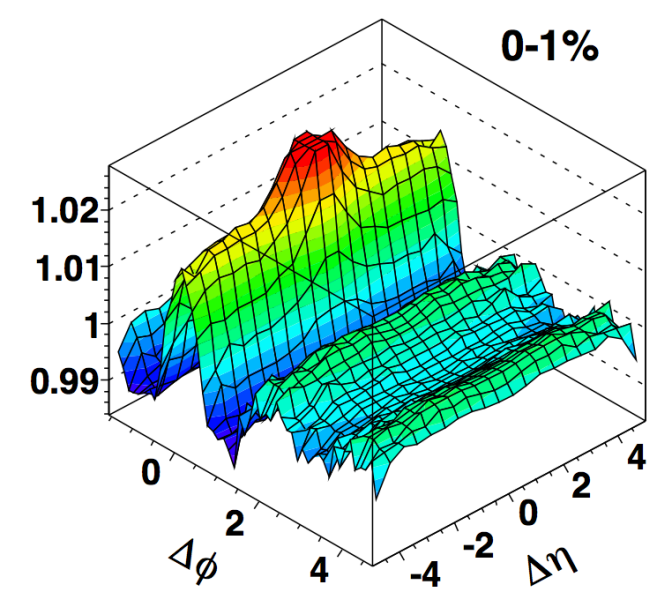
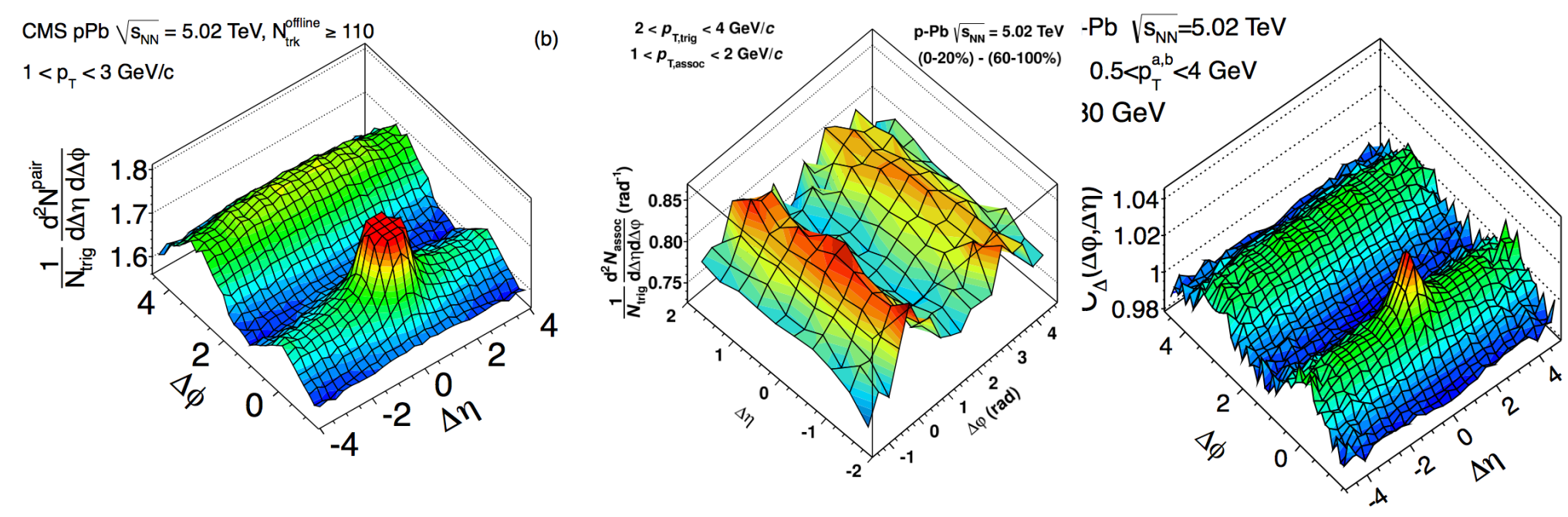


\section{leveraging RHIC/LHC differences}

1303.1794

initial entropy densitiy $0.30=$
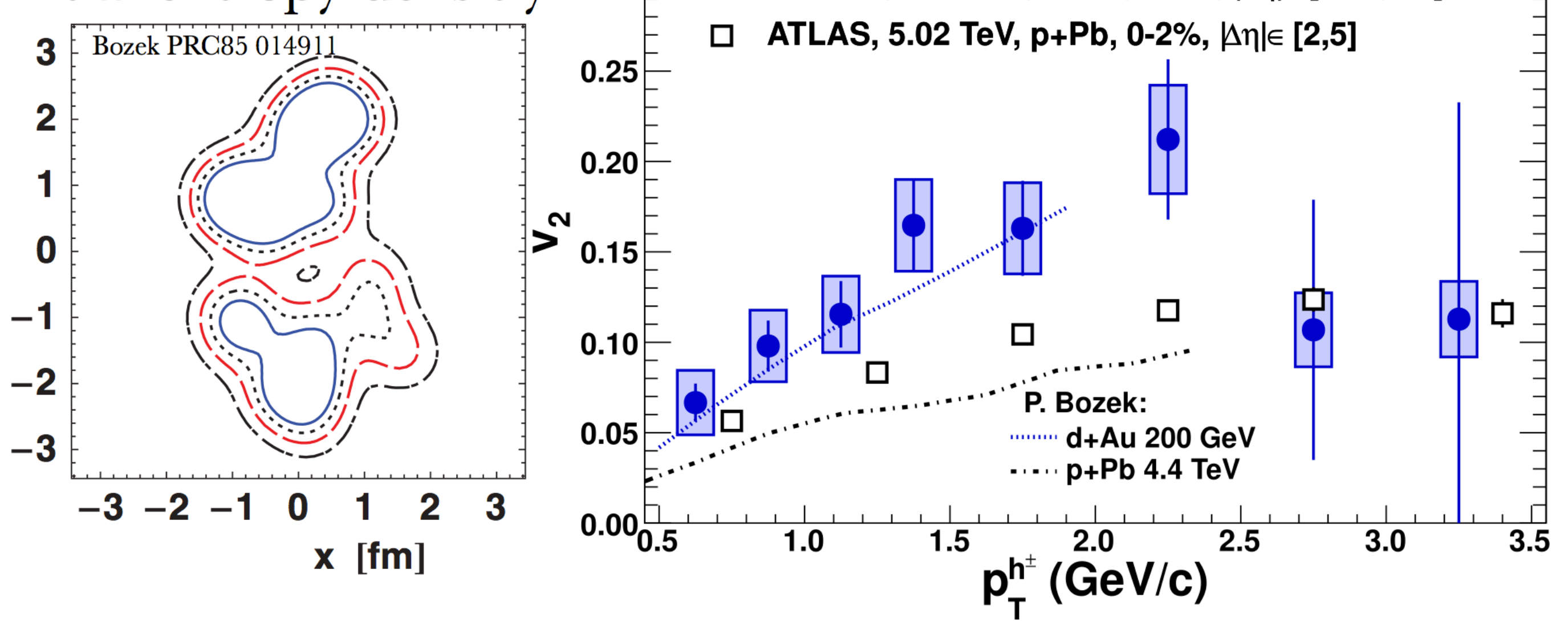

- PHENIX, $200 \mathrm{GeV}, \mathrm{d}+\mathrm{Au}, 0-5 \%, \Delta \eta \mid \in[0.48,0.7]$

ㄱ ATLAS, $5.02 \mathrm{TeV}, \mathrm{p}+\mathrm{Pb}, 0-2 \%, \Delta \eta \mid \in[2,5]$

- changing both the collision energy and the shape of the initial system as compared to the ridge seen in $\mathrm{pPb}$ 


\section{leveraging RHIC/LHC differences}

initial entropy densitiy 0.30

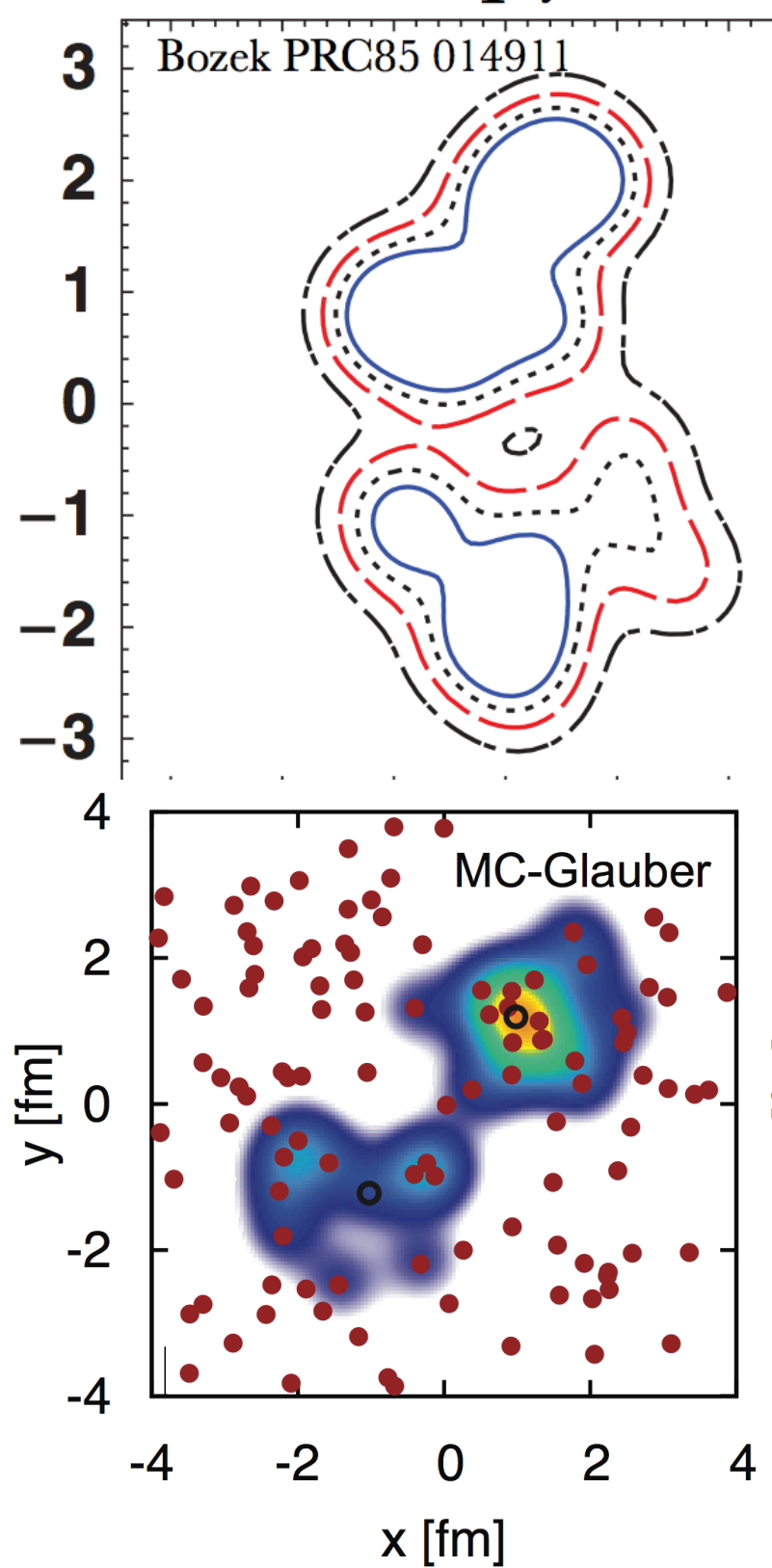

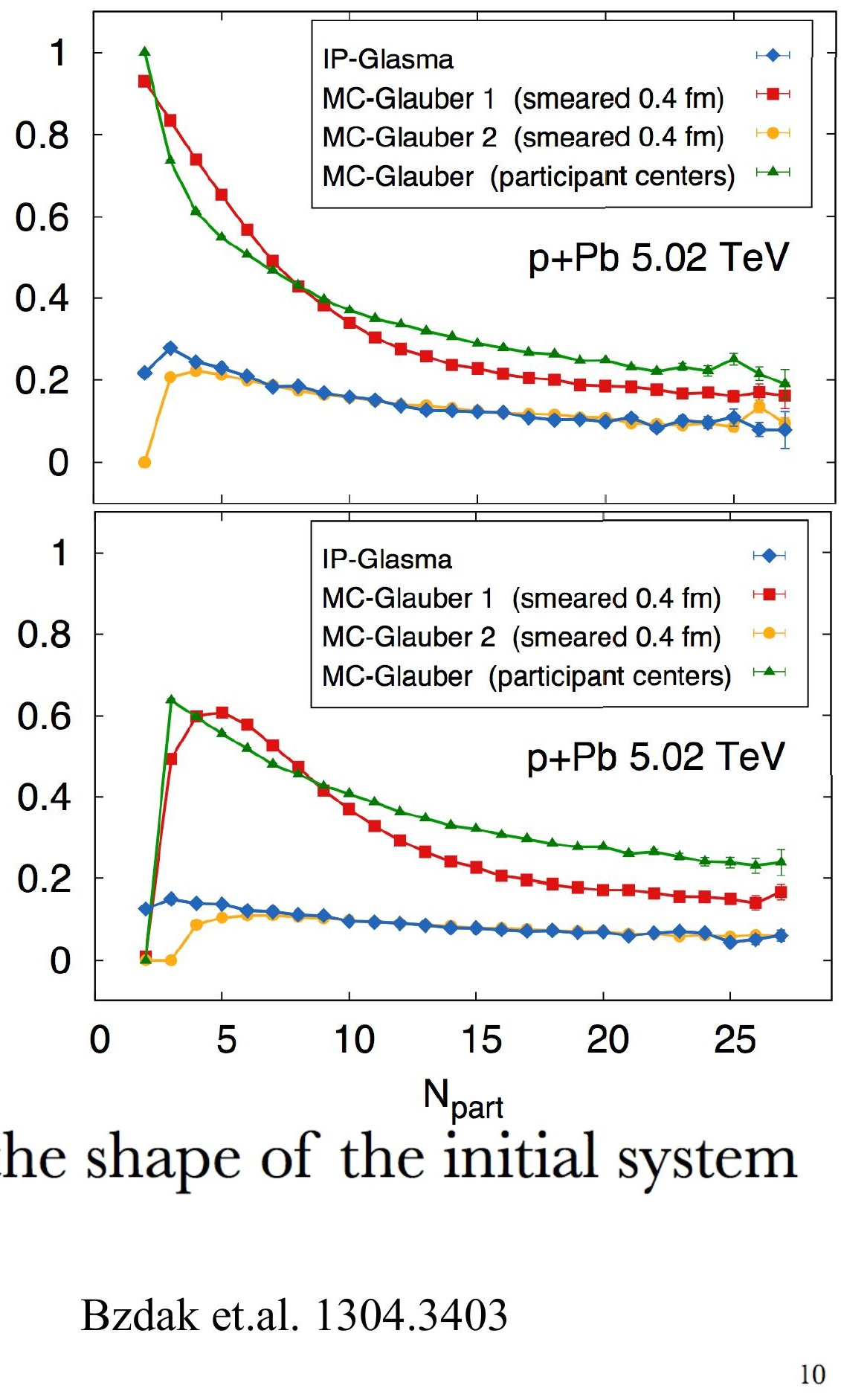




\section{Hydrodynamics in small system?}

- Flow depends on shape and size/gradient

$$
\stackrel{\rightleftarrows}{\rightleftarrows} \times \frac{F_{x}}{A}=-\eta \frac{\partial v_{x}}{\partial y}
$$

- HM pp and pA have larger density $(\eta / \mathrm{s})$ but much larger gradient!

$$
\rho(\phi, r)
$$

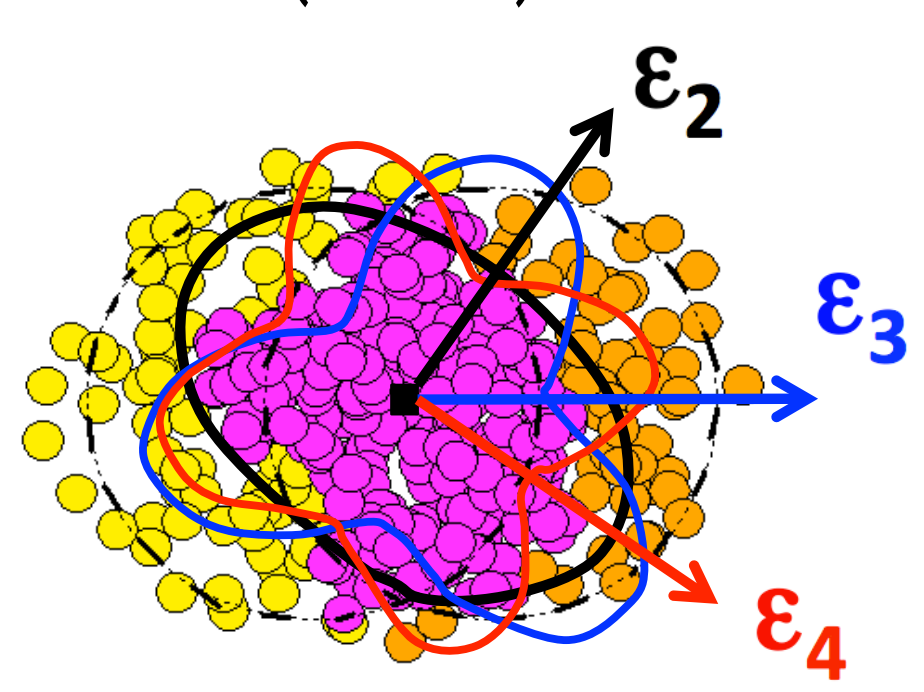

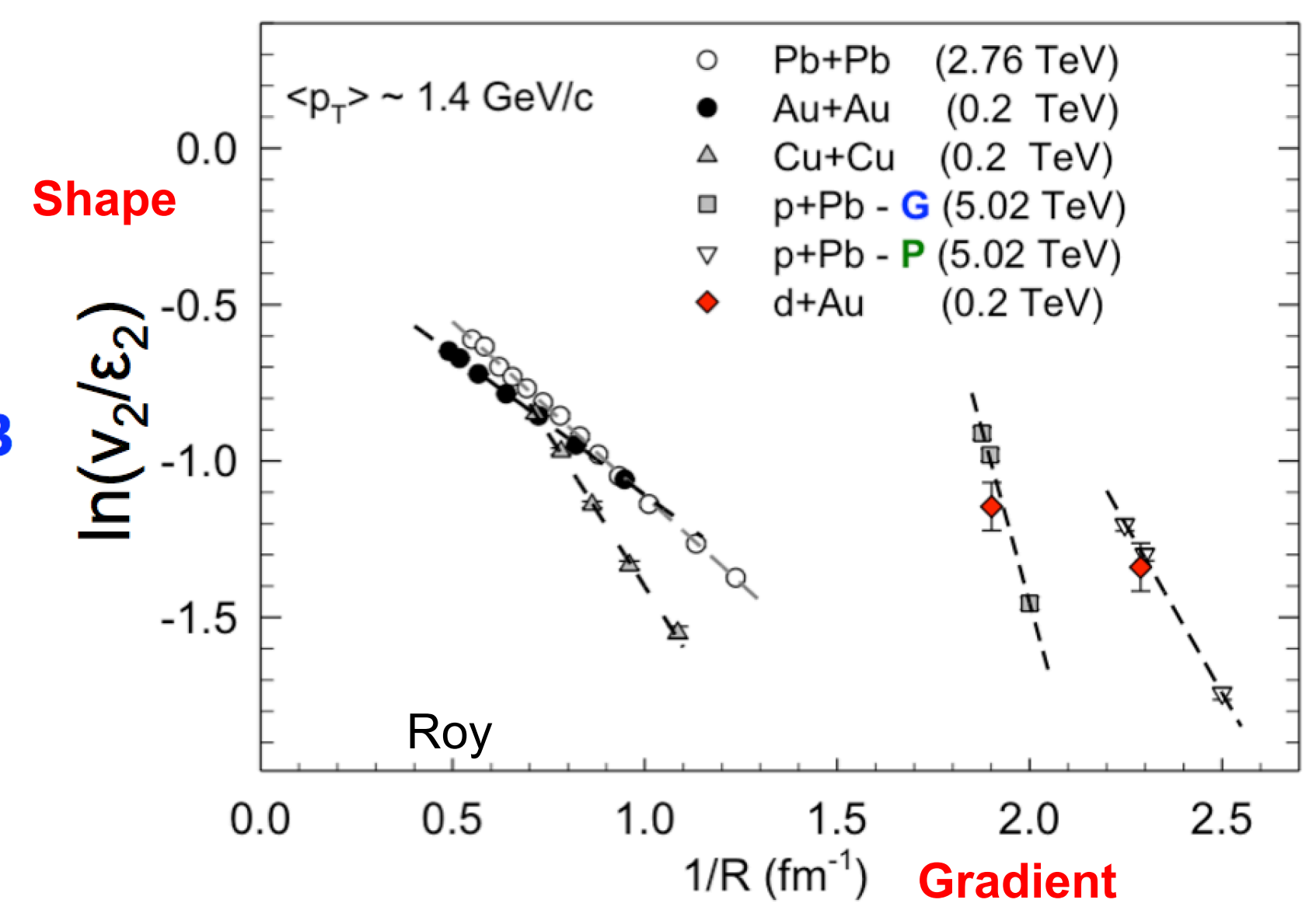




\section{$\mathrm{V}_{3}$ in $\mathrm{pPb}$ and $\mathrm{PbPb}$}

$\mathrm{PbPb}$

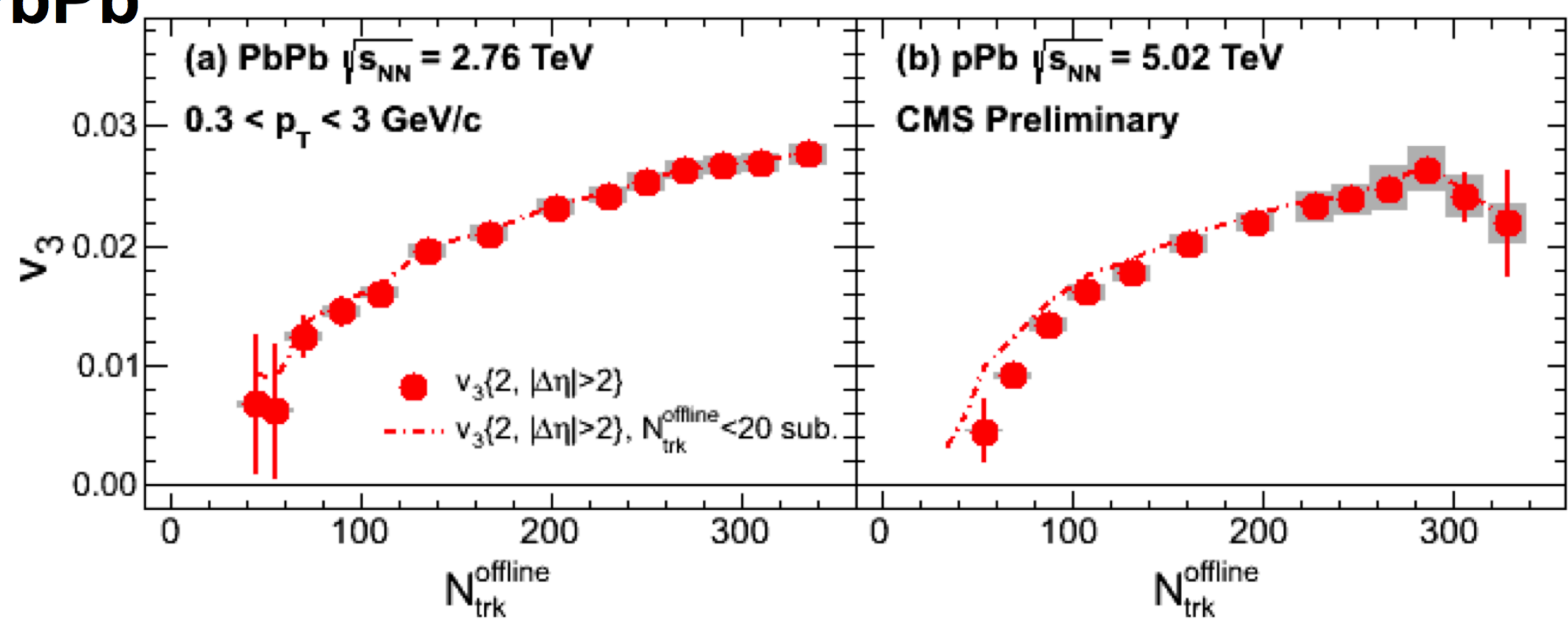

$\mathrm{pPb}$

$\mathrm{v}_{3}$ shows similar shape in $\mathrm{pPb}$ and $\mathrm{PbPb}$; magnitude comparable

"Peripheral subtraction" makes essentially no difference 


\section{$\mathrm{v}_{2}$ in $\mathrm{pPb}$ and $\mathrm{PbPb}$}

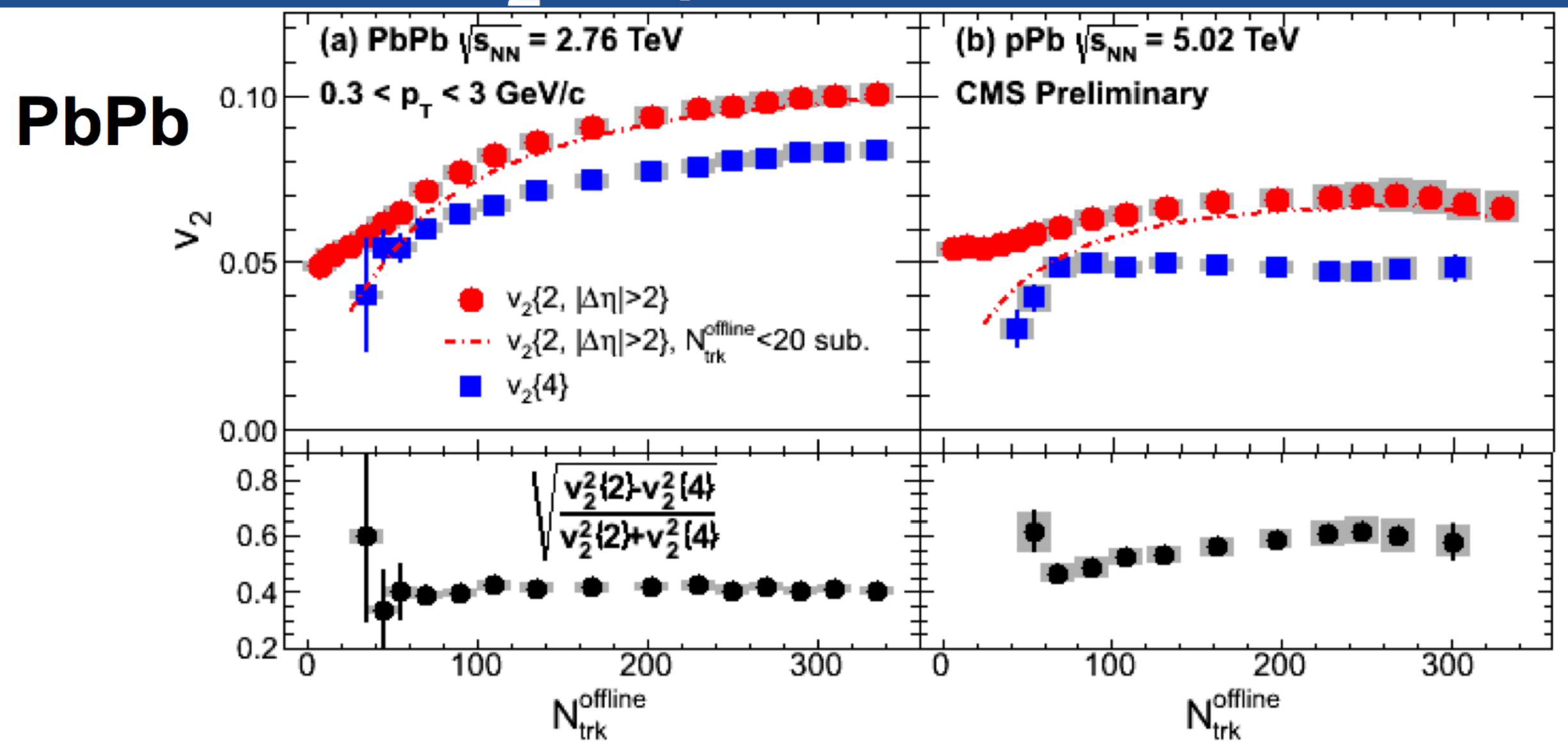

$\mathrm{pPb}$

"Fluctuations" larger in pPb,

$\begin{aligned} & v_{2}\{2\}=\sqrt{\left\langle v_{2}>^{2}+\sigma_{v_{2}}^{2}\right.} \\ & v_{2}\{4\}=\sqrt{<v_{2}>^{2}-\sigma_{v_{2}}^{2}}\end{aligned} \quad \frac{\sigma_{v_{2}}}{v_{2}}=\sqrt{\frac{v_{2}^{2}\{2\}-v_{2}^{2}\{4\}}{v_{2}^{2}\{2\}+v_{2}^{2}\{4\}}}$. with moderate multiplicity dependence 


\section{Flow fluctuation \& $v_{n}\{4\}$}

- Bessel-Gaussian

$$
\begin{aligned}
& p\left(\vec{v}_{n}\right) \propto \exp \left(\frac{-\left(\vec{v}_{n}-\overrightarrow{\bar{v}}_{n}\right)^{2}}{2 \delta_{n}^{2}}\right) \\
& p\left(v_{n}\right) \propto v_{n} \exp \left(\frac{-\left(v_{n}^{2}+\left(\bar{v}_{n}\right)^{2}\right.}{2 \delta_{n}^{2}}\right) I_{0}\left(\frac{v_{n} \bar{v}_{n}}{\delta_{n}^{2}}\right) \quad I_{0}(x) \approx e^{\frac{x^{2}}{4}}\left[1-\frac{x^{4}}{64}\right] \\
& \text { For pure fluctuations } \quad \bar{v}_{n}=0 \Rightarrow p\left(v_{n}\right) \propto v_{n} \exp \left(\frac{-\left(v_{n}^{2}\right)}{2 \delta_{n}^{2}}\right)
\end{aligned}
$$

- When $\alpha=\frac{\bar{v}_{n}}{\delta_{n}}<\approx 1$ distribution is very close to pure Gaussian with a redefinition of $\delta$

$$
\left(\begin{array}{c}
\delta_{n}^{2} \\
v_{n}^{R P}
\end{array}\right) \rightarrow\left(\begin{array}{c}
\delta_{n}^{2^{\prime}}=\delta_{n}^{2}+\left(\bar{v}_{n}\right)^{2} / 2 \\
\bar{v}_{n}{ }^{\prime}=0
\end{array}\right)
$$

Valid when $\quad \frac{v_{n} \bar{v}_{n}}{\delta_{n}^{2}}<<64^{1 / 4}=2.8$ or $v_{n} \ll 2.8 \delta_{n} \alpha$ 


\section{Flow fluctuation \& $v_{n}\{4\}$}

- Bessel-Gaussian

$$
\begin{aligned}
& p\left(\vec{v}_{n}\right) \propto \exp \left(\frac{-\left(\vec{v}_{n}-\overrightarrow{\bar{v}}_{n}\right)^{2}}{2 \delta_{n}^{2}}\right) \\
& p\left(v_{n}\right) \propto v_{n} \exp \left(\frac{-\left(v_{n}^{2}+\left(\bar{v}_{n}\right)^{2}\right.}{2 \delta_{n}^{2}}\right) I_{0}\left(\frac{v_{n} \bar{v}_{n}}{\delta_{n}^{2}}\right) I_{0}(x) \approx e^{\frac{x^{2}}{4}}\left[1-\frac{x^{4}}{64}\right] \\
& \text { For pure fluctuations } \bar{v}_{n}=0 \Rightarrow p\left(v_{n}\right) \propto v_{n} \exp \left(\frac{-\left(v_{n}^{2}\right)}{2 \delta_{n}^{2}}\right)
\end{aligned}
$$

- When $\alpha=\frac{\bar{v}_{n}}{\delta_{n}}<\approx 1$ distribution is very close to pure Gaussian with a redefinition of $\delta$

$$
\left(\begin{array}{c}
\delta_{n}^{2} \\
v_{n}^{R P}
\end{array}\right) \rightarrow\left(\begin{array}{c}
\delta_{n}^{2 \prime}=\delta_{n}^{2}+\left(\bar{v}_{n}\right)^{2} / 2 \\
\bar{v}_{n}{ }^{\prime}=0
\end{array}\right)
$$

$$
\text { Valid when } \quad \frac{v_{n} \bar{v}_{n}}{\delta_{n}^{2}} \ll<64^{1 / 4}=2.8 \text { or } v_{n} \ll 2.8 \delta_{n} \alpha
$$
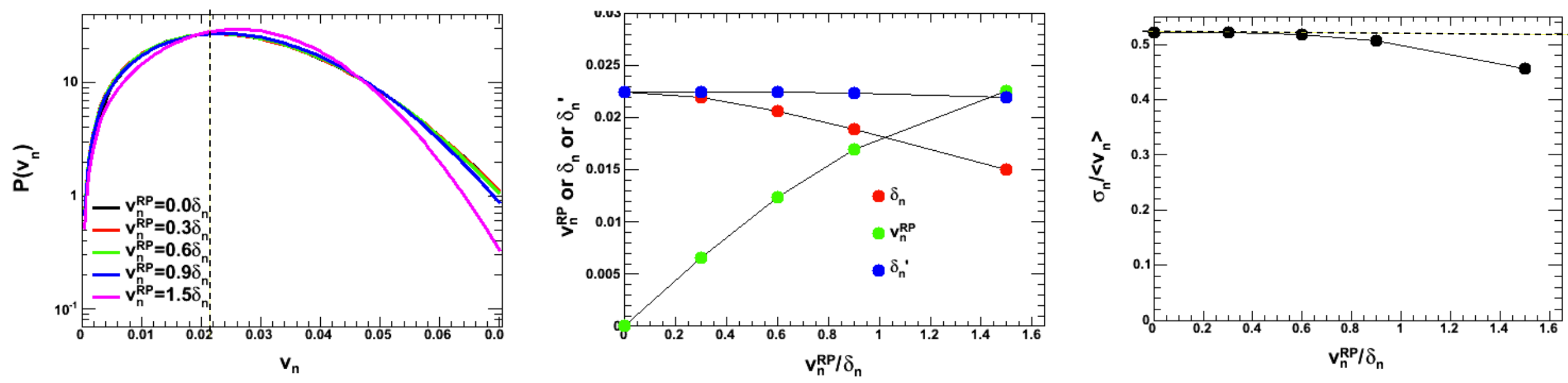


\section{Flow fluctuation \& $v_{\mathrm{n}}\{4\}$}

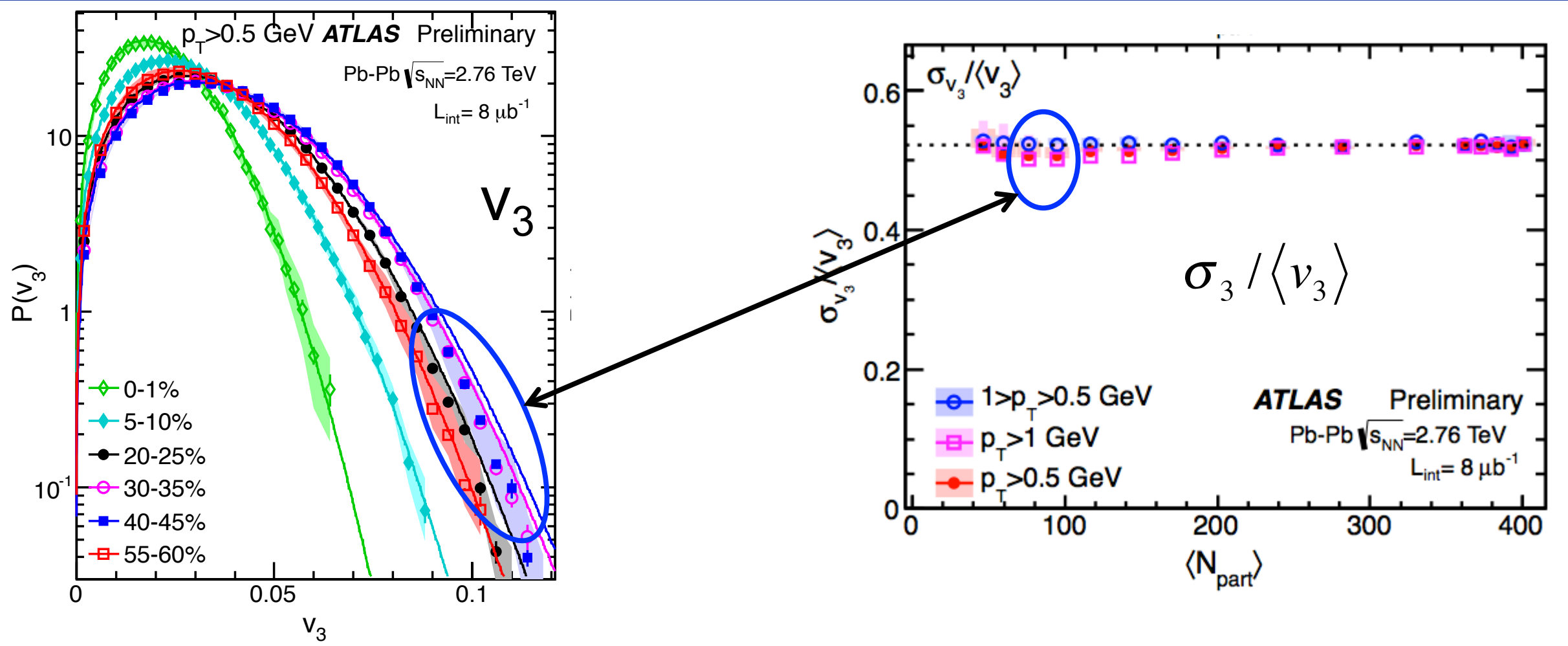

- Even a small deviation will give a large $\mathrm{v}_{\mathrm{n}}{ }^{\mathrm{RP}}$ or large $\mathrm{v}_{\mathrm{n}}\{4\}$

$$
v_{n}\{4\}=\left[2\left\langle v_{n}^{2}\right\rangle_{\uparrow}^{2}-\left\langle v_{n}^{4}\right\rangle\right]^{1 / 4}
$$

a $4 \%$ difference gives a $v_{n}\{4\}$ value of about $45 \%$ of $v_{n}\{2\}$ 


\section{Flow fluctuation \& $v_{\mathrm{n}}\{4\}$}

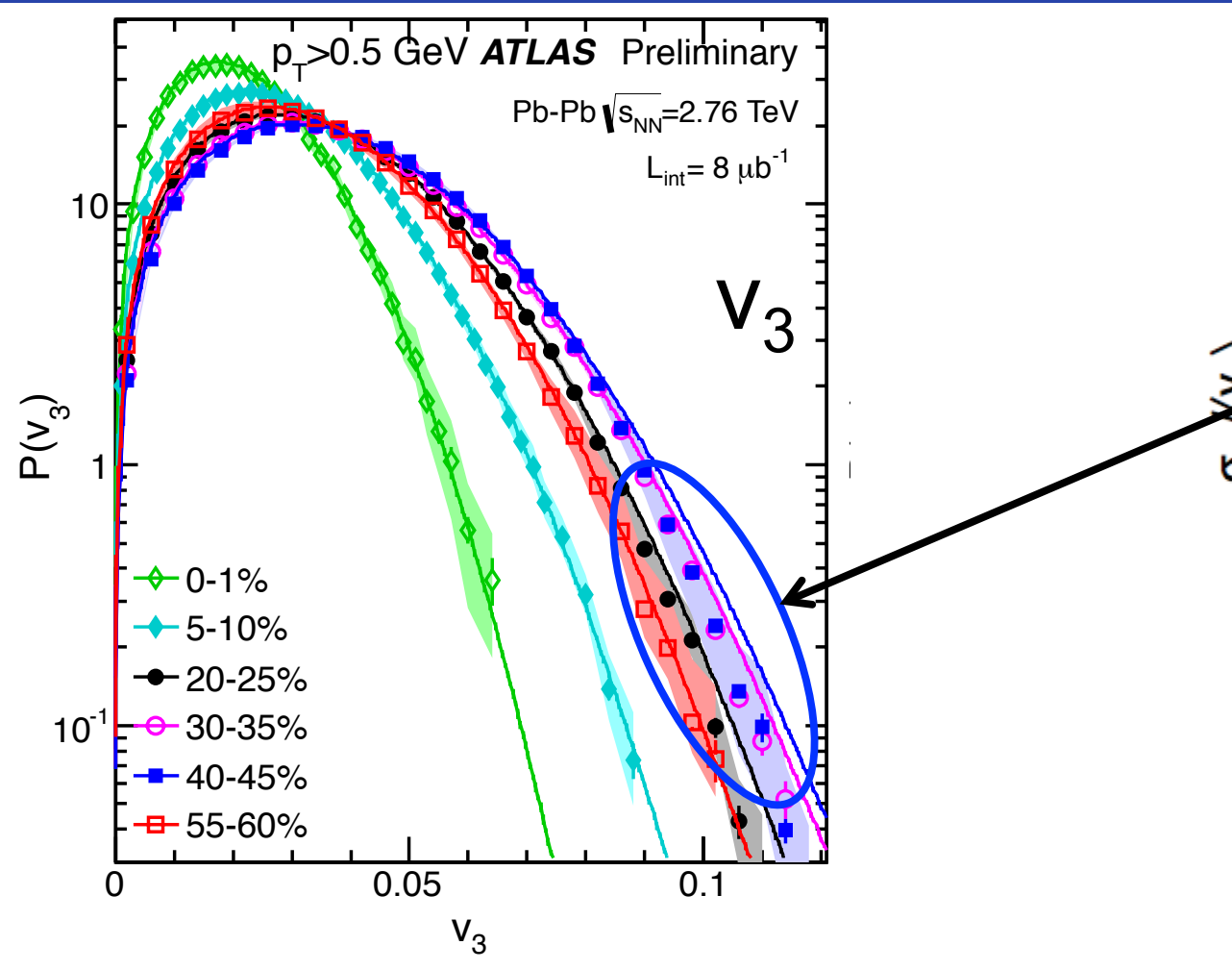

- Even a small deviation will give a large $\mathrm{v}_{\mathrm{n}}^{\mathrm{RP}}$ or large $\backslash\{4\}$

$$
v_{n}\{4\}=\left[2\left\langle v_{n}^{2}\right\rangle_{\uparrow}^{2}-\left\langle v_{n}^{4}\right\rangle\right]^{1 / 4}
$$

a $4 \%$ difference gives a $v_{n}\{4\}$ value of about $45 \%$ of $v_{n}\{2\}$

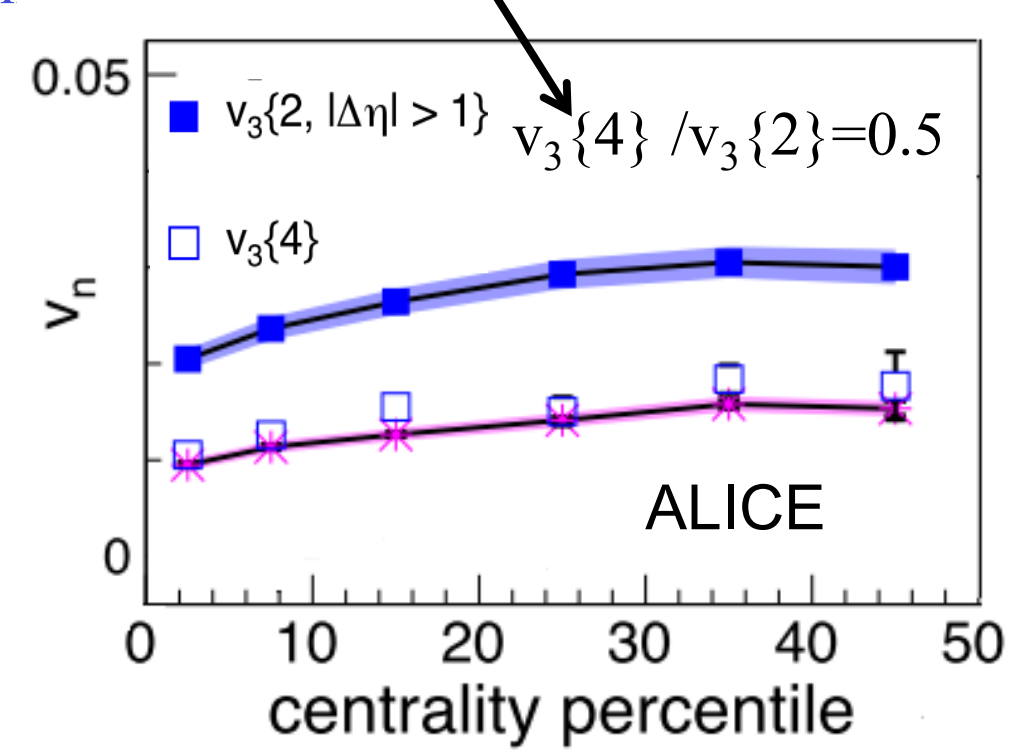




\section{$v_{2}\{4\}$ in $p+P b$}

- Just need a small deviation from Gaussian

- Small $\mathrm{N}_{\text {part }}$ or Negative binomial fluctuation (Bozek)

- Fluctuation of $\mathrm{M}$ and $\mathrm{v}_{\mathrm{n}}$ aren't de-coupled

$$
\frac{d N}{d \phi}=M\left[1+\sum_{n} 2 \mathrm{v}_{\mathrm{n}} \cos n\left(\phi-\Phi_{n}\right)\right]
$$
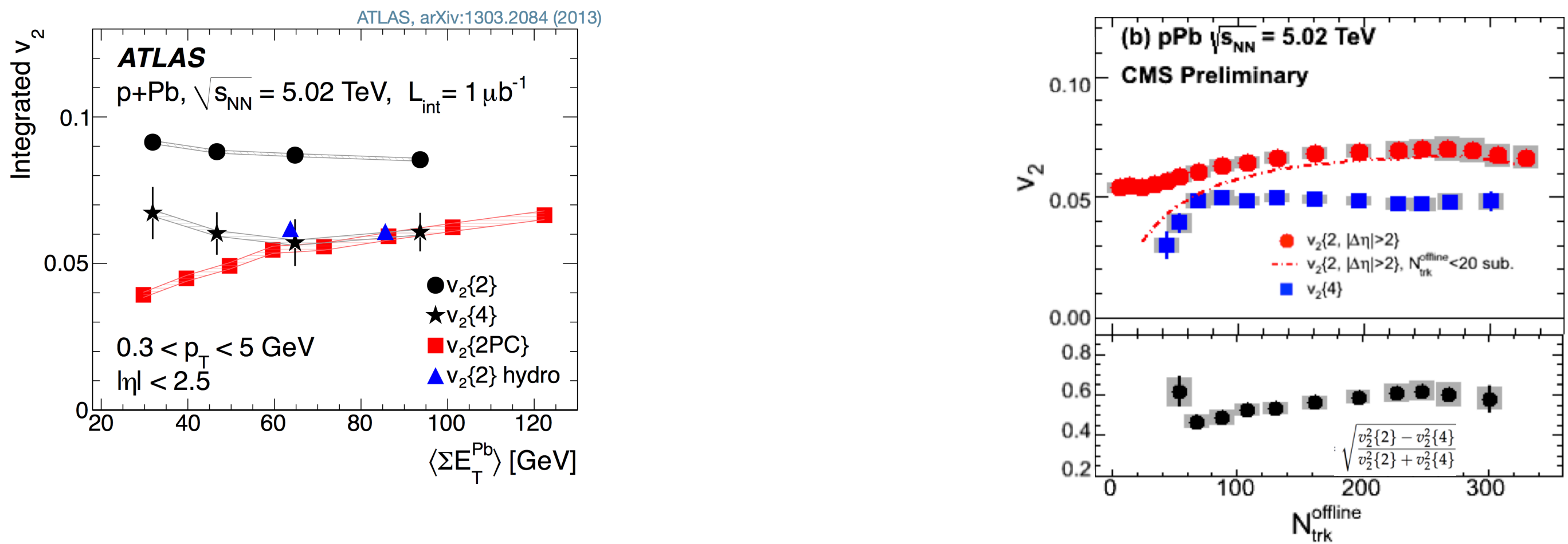


\section{Power spectrum}
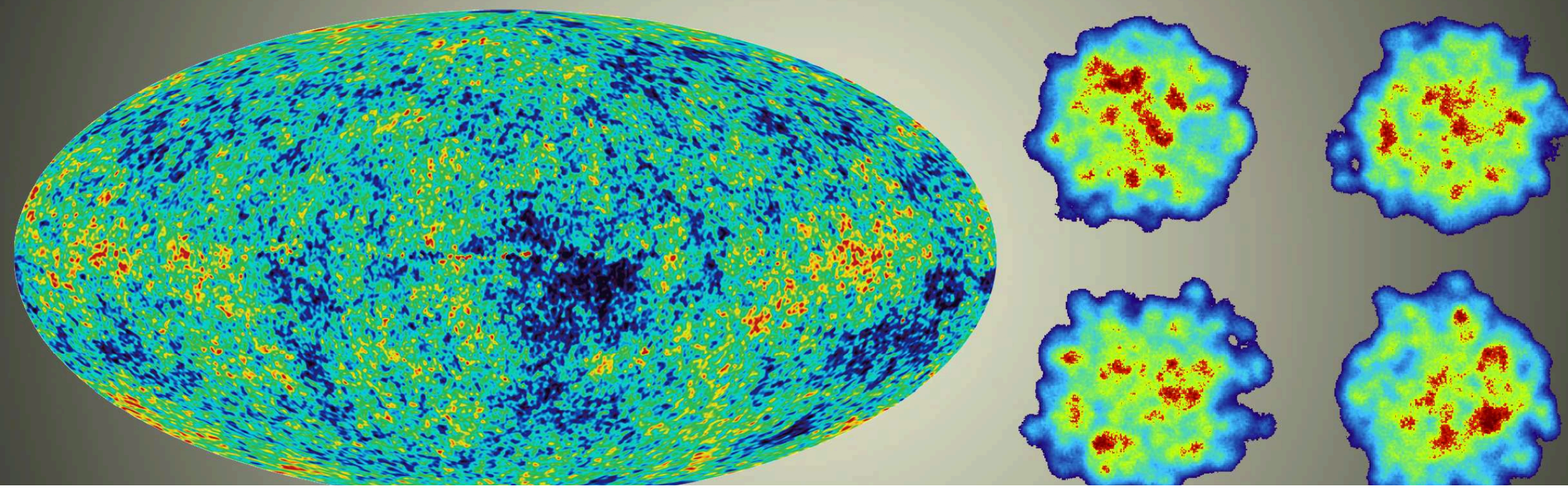

Planck 2013 CMB temperature power spectrum

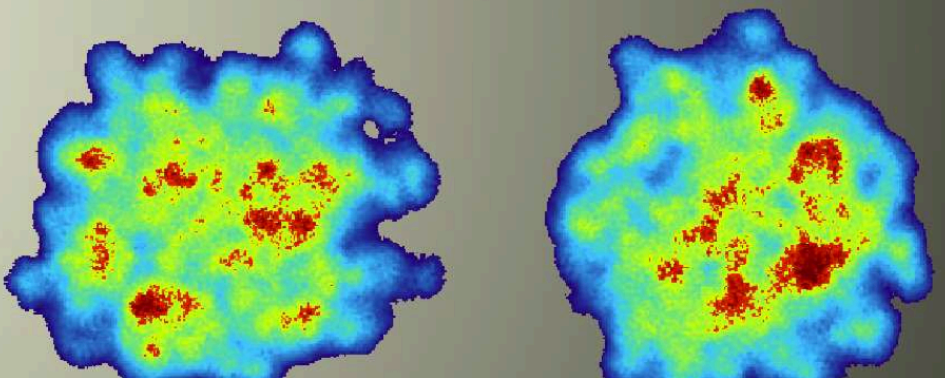
Angular scale
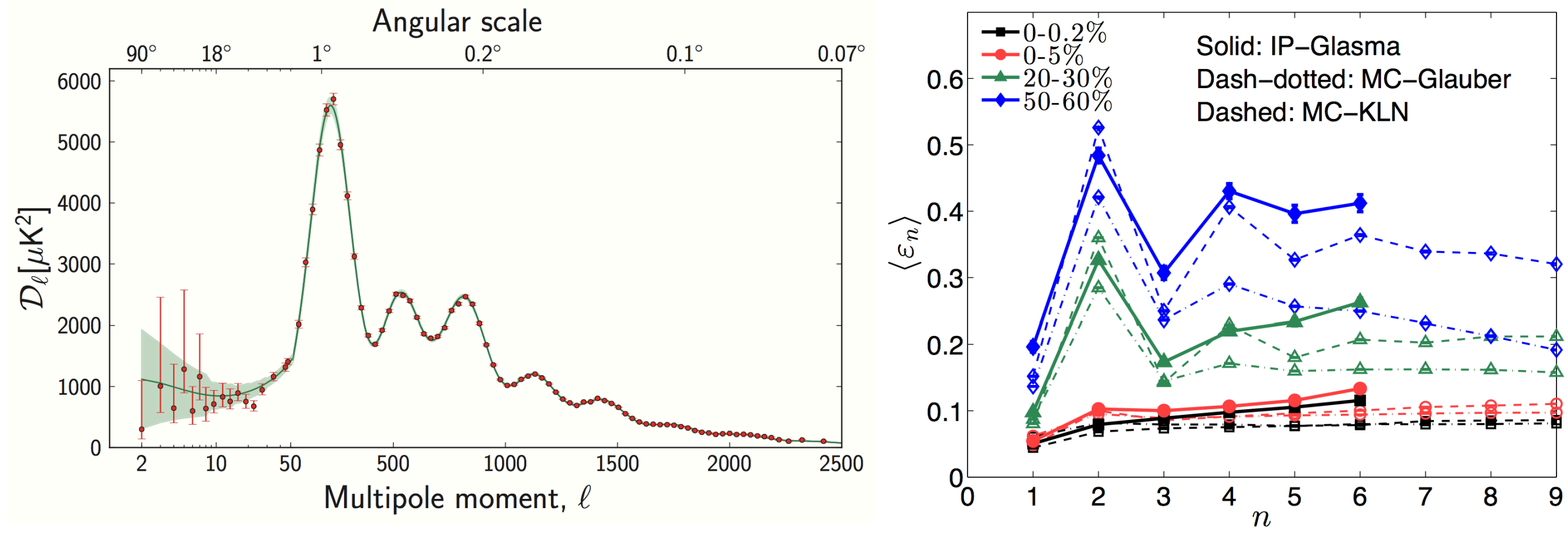


\section{Ripples of "little-bangs"}
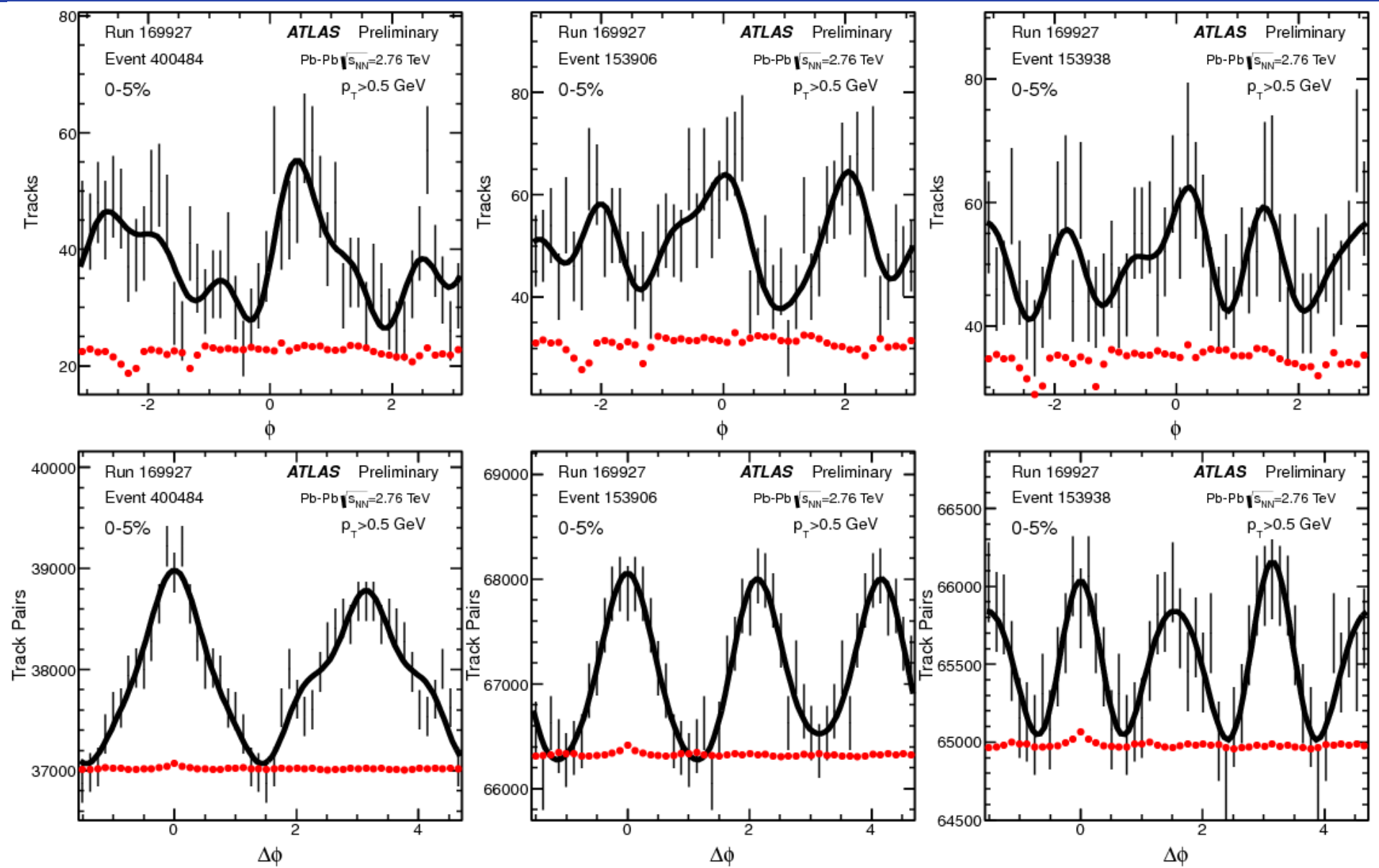

- Data-driven unfolding the key:

Sub-event "A" - Sub-event "B" $= \begin{cases}\text { Signal } & : \text { cancelled } \\ \text { Fluctuations+short-range corr. : } \sqrt{ } 2 \text { larger }\end{cases}$ $\left(\vec{v}_{n}^{\text {obs }}\right)^{\mathrm{a}}-\left(\vec{v}_{n}^{\text {obs }}\right)^{\mathrm{b}}=$ nonflow + noise $\left(\vec{v}_{n}^{\text {obs }}\right)^{\mathrm{a}}+\left(\vec{v}_{n}^{\mathrm{obs}}\right)^{\mathrm{b}}=2 \vec{v}_{n}$ J.Jia, S. Mohapatra: arxiv:1304.1471 


\section{Azimuthal power spectrum in hijing: (non-flow)}

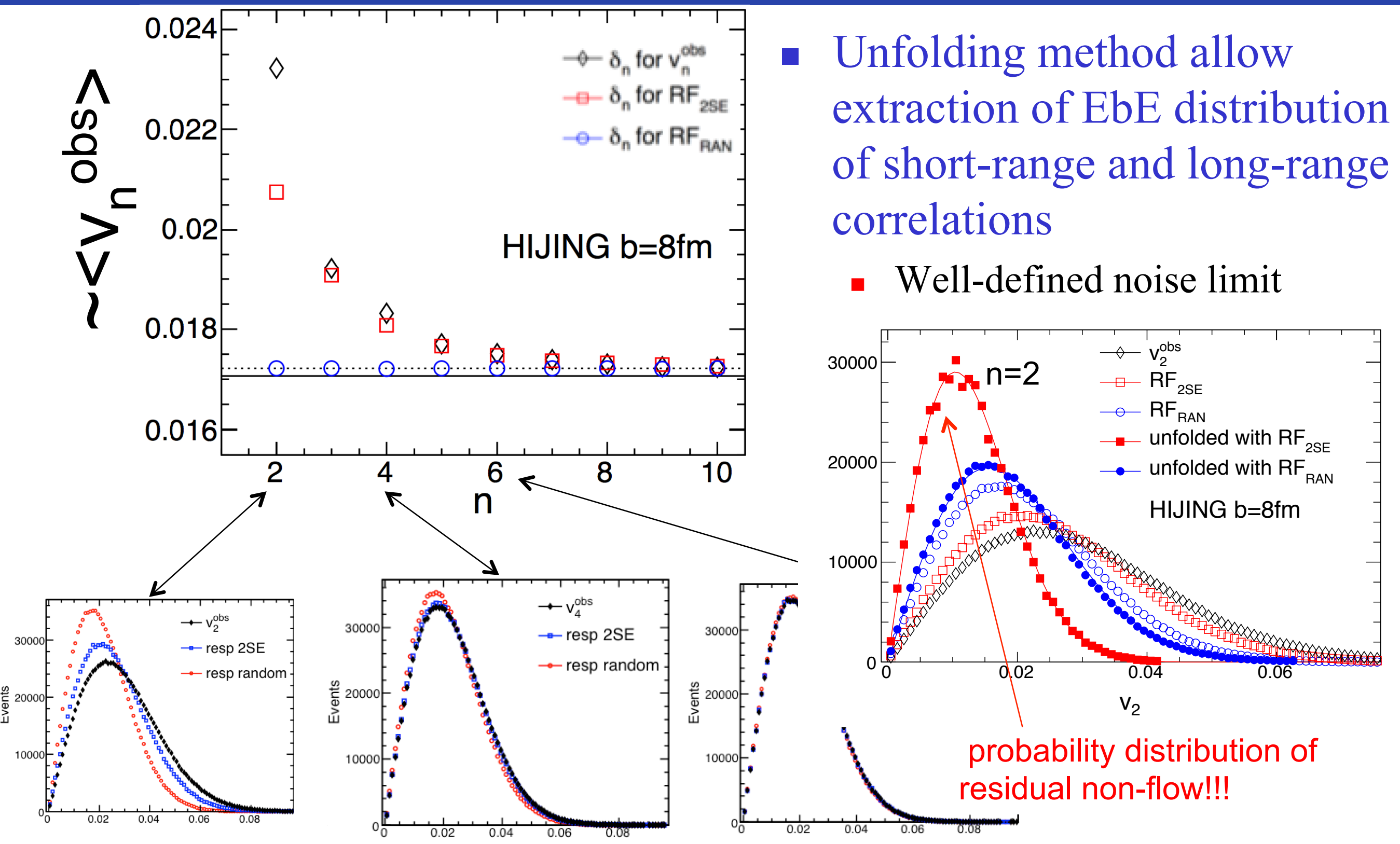




\section{Azimuthal power spectrum in AMPT: (flow+non-flow)}

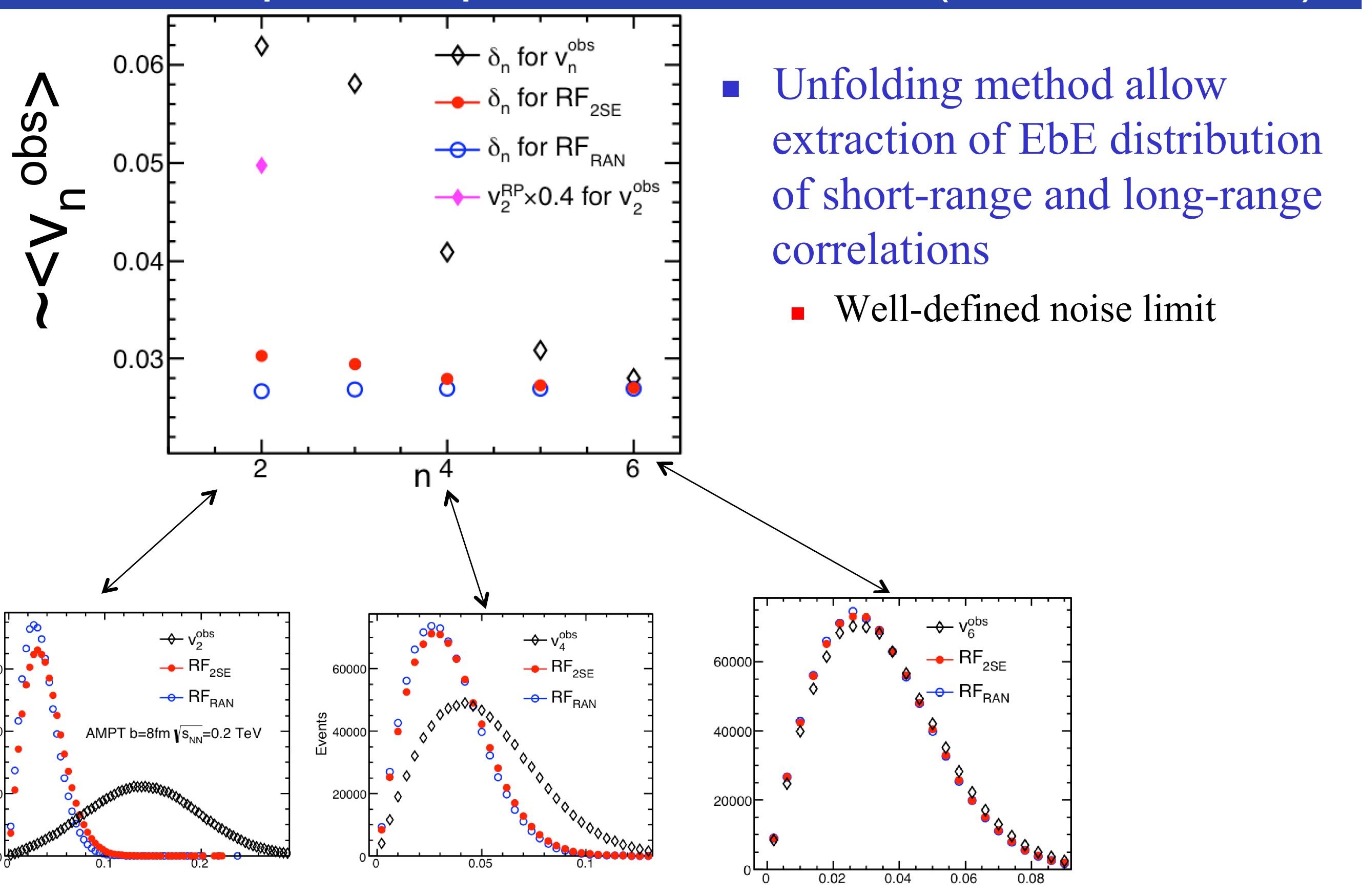




\section{AMPT unfolded distributions}

- Unfolded distribution scales well.
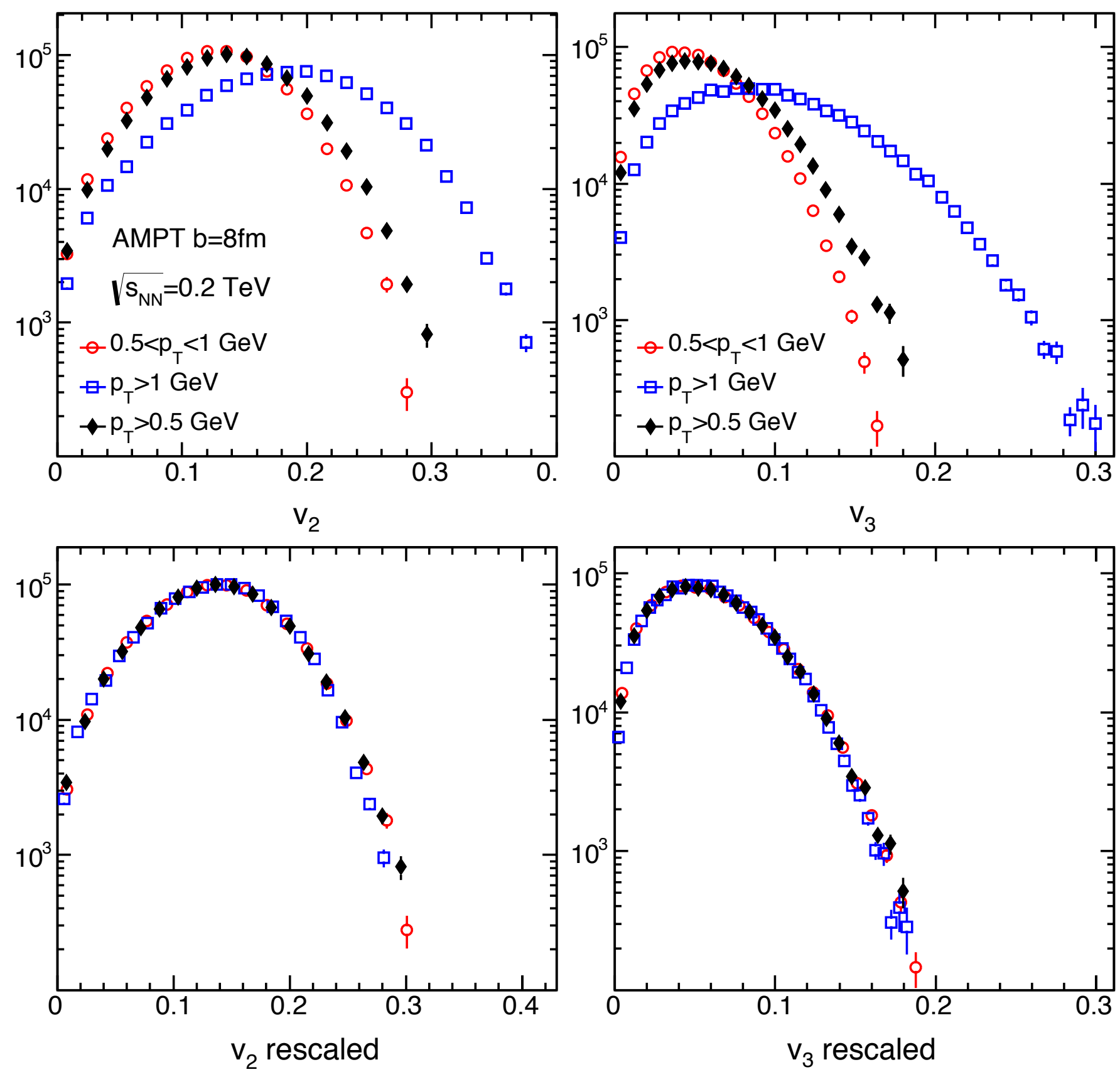


\section{It was a memorable workshop}

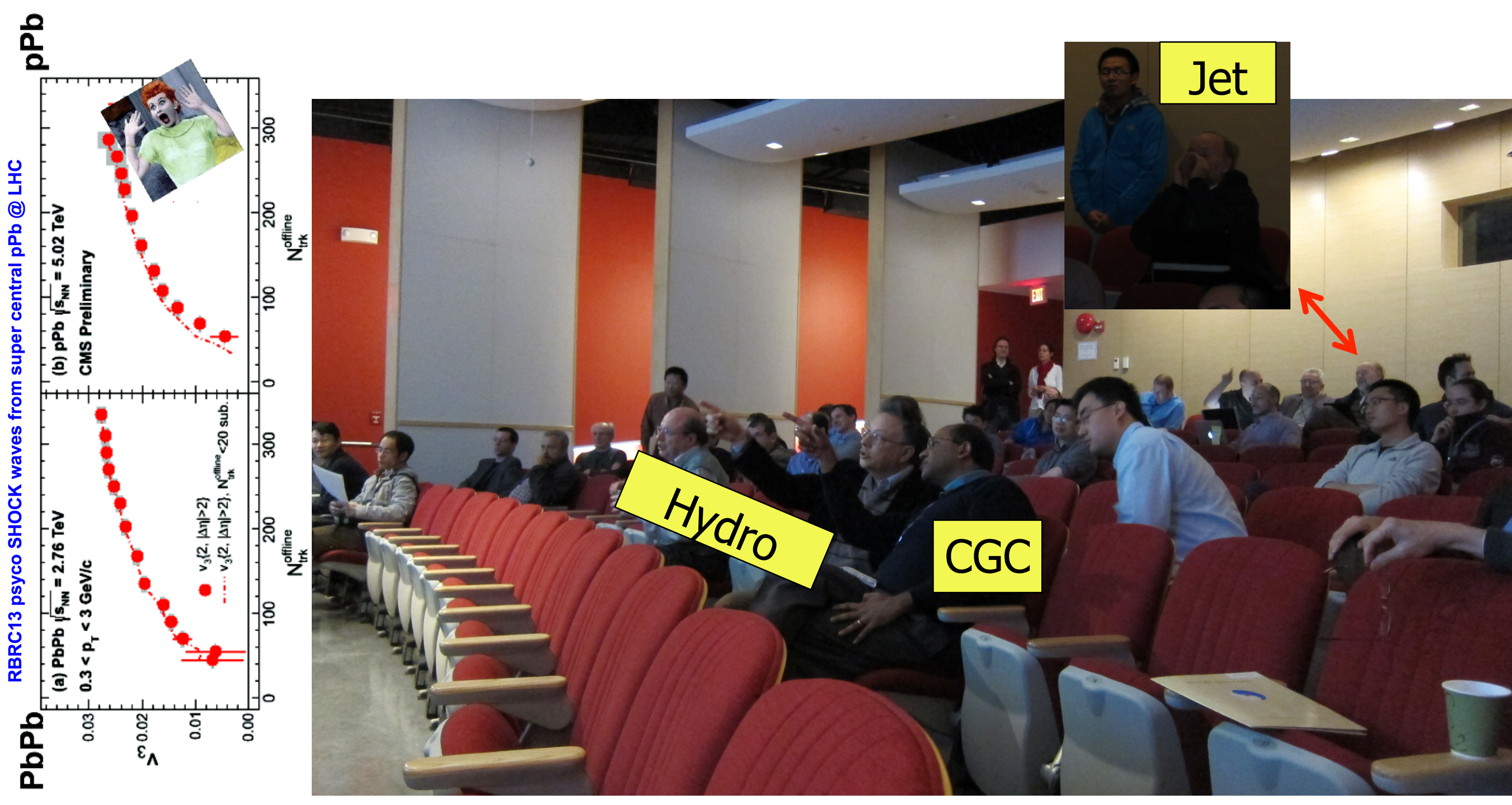


Decrease $\sqrt{s}:$ Saturation turns off $\left(Q_{s}(x) \sim \Lambda_{Q C D}\right)$, hydro phase shorter $\left(T_{\text {initial }}\right.$ decreases, $\tau_{0}$ increases $)$, maybe jumps with $\left(c_{s}, \eta / s\right.$ at deconfinement), Knudsen number $\sim(T R)^{-1}$ higher, chemical composition different $\left(\tau, K n \sim \mu_{B}\right.$ ? )

Increase rapidity: Saturation effects larger, hydro phase shorter (as above)

Decrease system size Slightly less saturation, $T_{0}\left(\sim N_{\text {part }}^{1 / 3}\right)$, bigger $R_{0}^{-1}$ Knudsen number

( $\mathrm{pA}$ : Higher y and smaller size!)

Increase $p_{T}$ first role of flow is increased, then tomographic regime 
Low $p_{T}$ harmonics the experimental situation
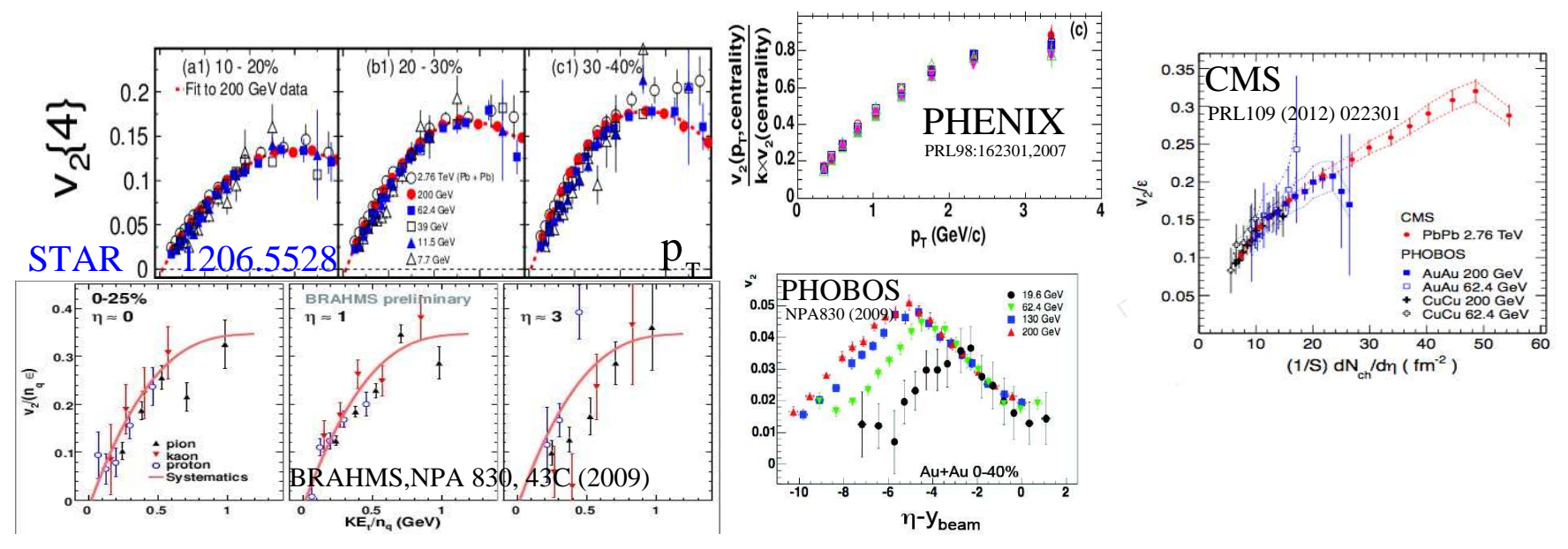

Here is what we know experimentally

$$
v_{2} \simeq \epsilon(b, A) F\left(p_{T}\right) \quad, \quad\langle v 2\rangle \simeq \int d p_{T} F\left(p_{T}\right) f\left(p_{T},\left\langle p_{T}\right\rangle_{y, A, b, \sqrt{s}}\right)
$$

$F\left(p_{T}\right)$ universal for all energies, $f\left(p_{T}\right)$ tracks mean momentum, $\sim \frac{1}{S} d N$ This is an experimental statement, as good as the error bars 


$$
\frac{v_{n}}{\epsilon_{n}} \sim c_{s} f \underbrace{\left(\frac{1}{T_{f}^{3} \tau_{0} R^{2}} \frac{d N}{d y}\right)}_{\sim \tanh (\ldots)}(1-\mathcal{O}(1) \frac{\eta}{s} \underbrace{\frac{1}{T R}}_{\text {no }})
$$

No change in any of these termsobserved, from SPS to RHIC!
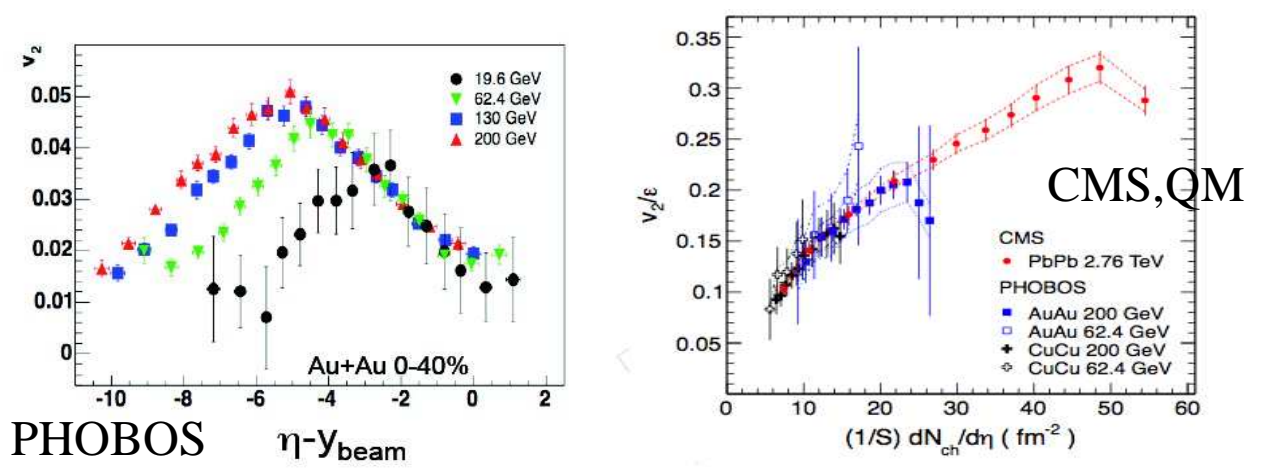

No sign of Knudsen number. $v_{3}$ of $\mathrm{pA}, \mathrm{AA}$ amazing, as $\epsilon_{3}^{p A} \simeq \epsilon_{3}^{A A}$, but $\mathrm{Kn} \mathcal{O}(10)$ bigger. Also no $\tau_{0}(\sqrt{s})$ seen. 
Low energy scan, STAR 1206.5528
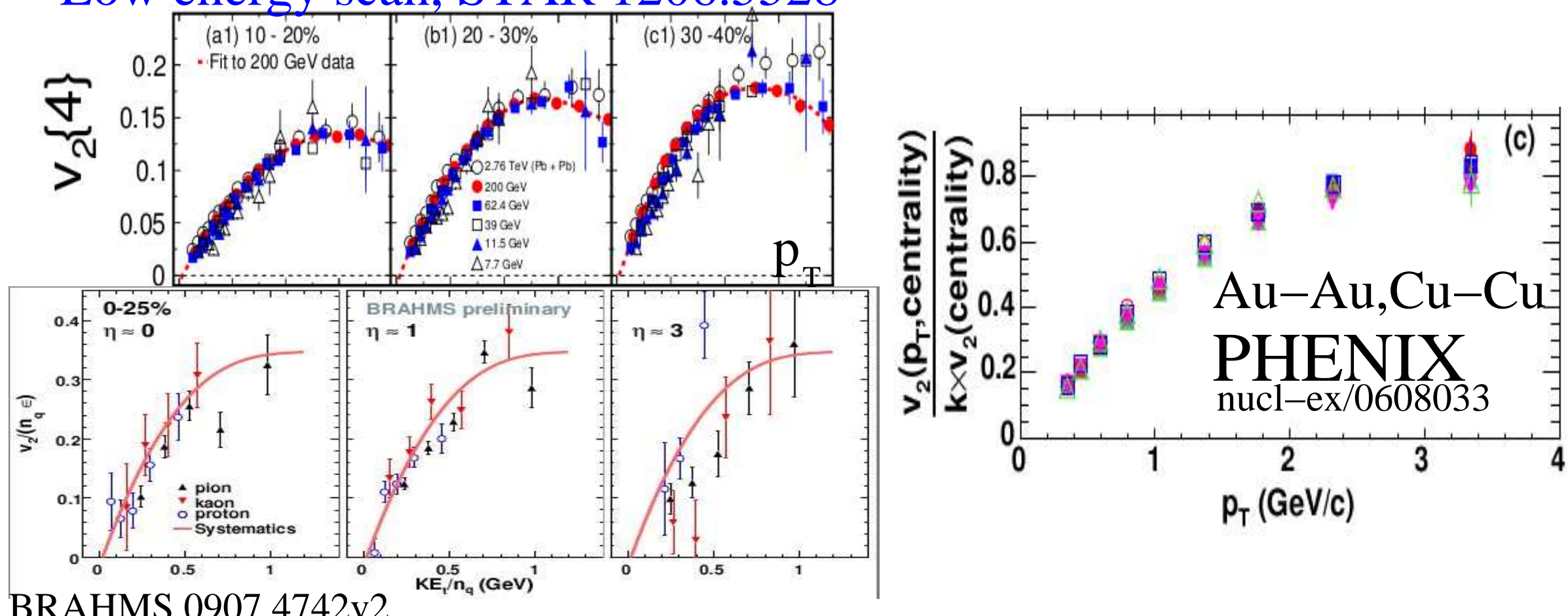

BRAHMS,0907.4742v2

Puzzle2: Why is $v_{2}\left(p_{T}\right)$ constant (at least at high $p_{T}$ )?

NB: this means $v_{2}\left(p_{T}\right) /\left\langle v_{2}\right\rangle$ independent of $N_{\text {part }}, y, \sqrt{s}$ 
Cooper-Frye

$$
\begin{aligned}
& v_{2}\left(p_{T}\right)=\int d \phi \cos (2 \phi)\left(E-p_{T}\left(\frac{d t}{d r}+\Delta \frac{d t}{d r}(\phi)\right)\right) e^{\left(-\frac{\gamma\left(E-p_{T}\left(u_{T}+\delta u_{T}(\phi)\right)\right)}{T}\right)} \\
& \simeq \int d \phi \cos ^{2}(2 \phi)[\underbrace{e^{-\frac{\gamma\left(E-p_{T} u_{T}\right)}{T}}}_{=0}-\underbrace{p_{T} \Delta \frac{d t}{d r}}_{\epsilon p_{T}}+\underbrace{\frac{\gamma \delta u_{T}(\phi) p_{T}}{T}}_{\sim}+\mathcal{O}\left(\epsilon^{2}\right)+\mathcal{O}(K n)]
\end{aligned}
$$

As long as $\frac{\delta v_{T}}{T} \sim \epsilon s^{0}$ (close to saturation of $\epsilon_{p}$ ), $v_{2}\left(p_{T}\right)$ independent of $\sqrt{s}$ In ideal and long-lived limit $\frac{\eta}{s T} \ll R$ in $v_{2}\left(p_{T}\right) \sim \epsilon \tanh \left(\epsilon \frac{p_{T}}{T_{f}}\right) \underset{p_{T} \gg T}{\rightarrow} p_{T}^{0}$

NB: deviations $\sim p_{T}$, more prominent at @high $p_{T}$ 


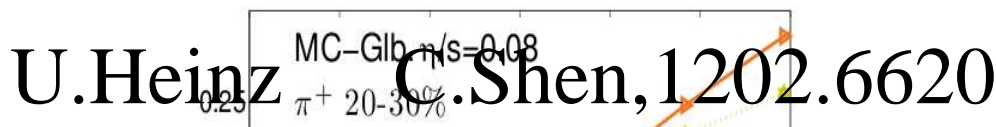
viscous hydro

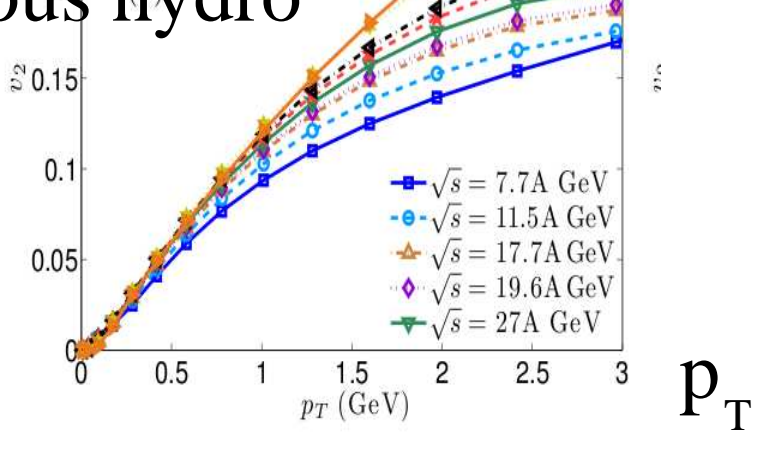

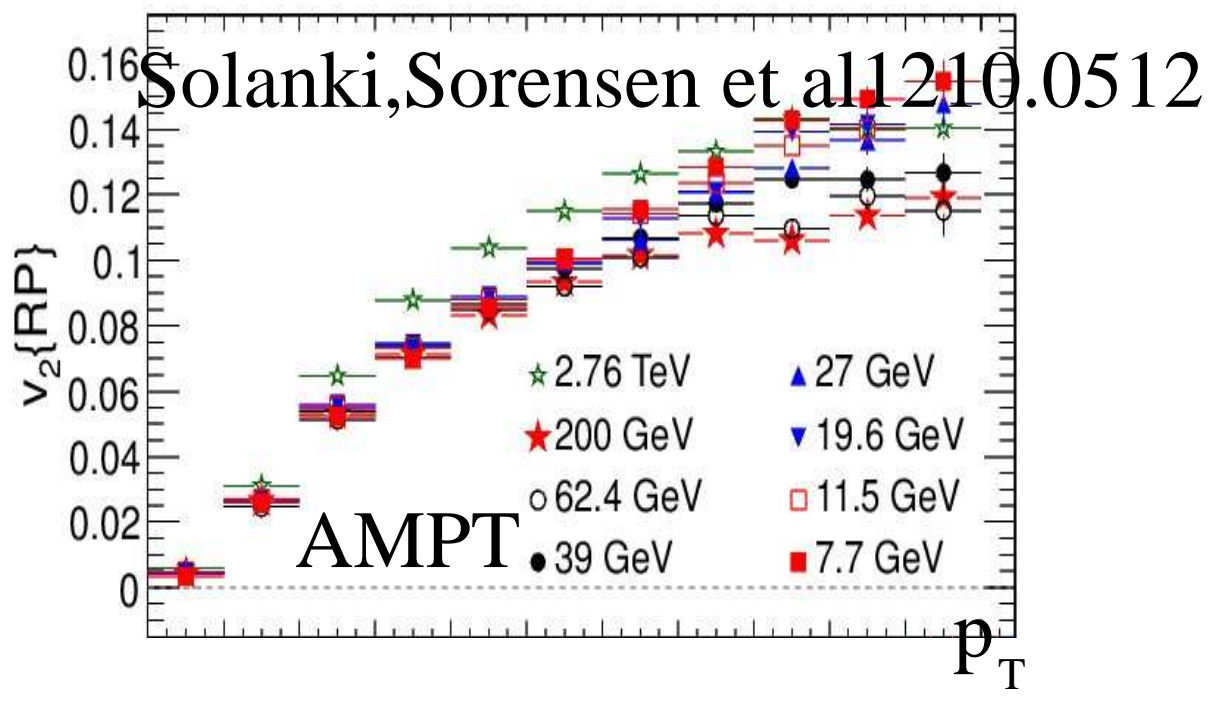

This is why all hydro and transport models generally give a systematic shift of $v_{2}\left(p_{T}\right)$ going up with $p_{T}$, as long as $p_{T} \sim \mathcal{O}(1)\left\langle p_{T}\right\rangle$. Thus this data-model disagreement not likely to go away! 
Go to high $p_{T}$ : At $K n(p T-T) \geq 1$ tomographic regime

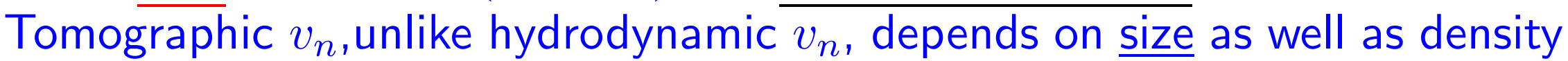

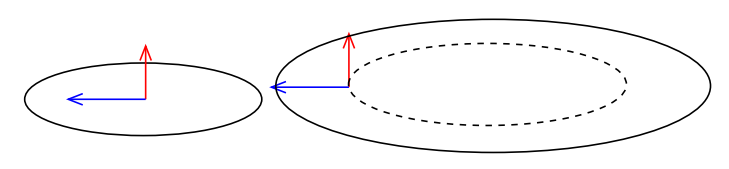

Take, as an initial condition, an elliptical distribution of matter at a given $\epsilon_{n}$, run jets through it and calculate $v_{n}$. Now increase $R$ with constant $\epsilon_{n}$

$$
\left.\frac{v_{n}}{\epsilon_{n}}\right|_{\text {tomo }} \rightarrow \frac{\text { Surface }}{\text { Volume }} \rightarrow 0 \quad,\left.\quad \frac{v_{n}}{\epsilon_{n}}\right|_{\text {hydro }} \rightarrow \text { constant }
$$

Role of "size" different in tomo vs hydro regime (M.Gyulassy, B.Betz,GT). $\frac{d E}{d \tau} \sim \kappa E^{a} T^{b} \tau^{c} \Rightarrow \Delta E \sim\left\langle E^{x}\right\rangle\left\langle T^{y}\right\rangle\langle R\rangle^{z} \neq f\left(\frac{1}{S} \frac{d N}{d y}\right)$

Volcanoes and waterfalls can only make this worse, as $\epsilon_{n}\left(T \sim T_{c}\right) \neq \epsilon_{n}^{\text {total }}$ 

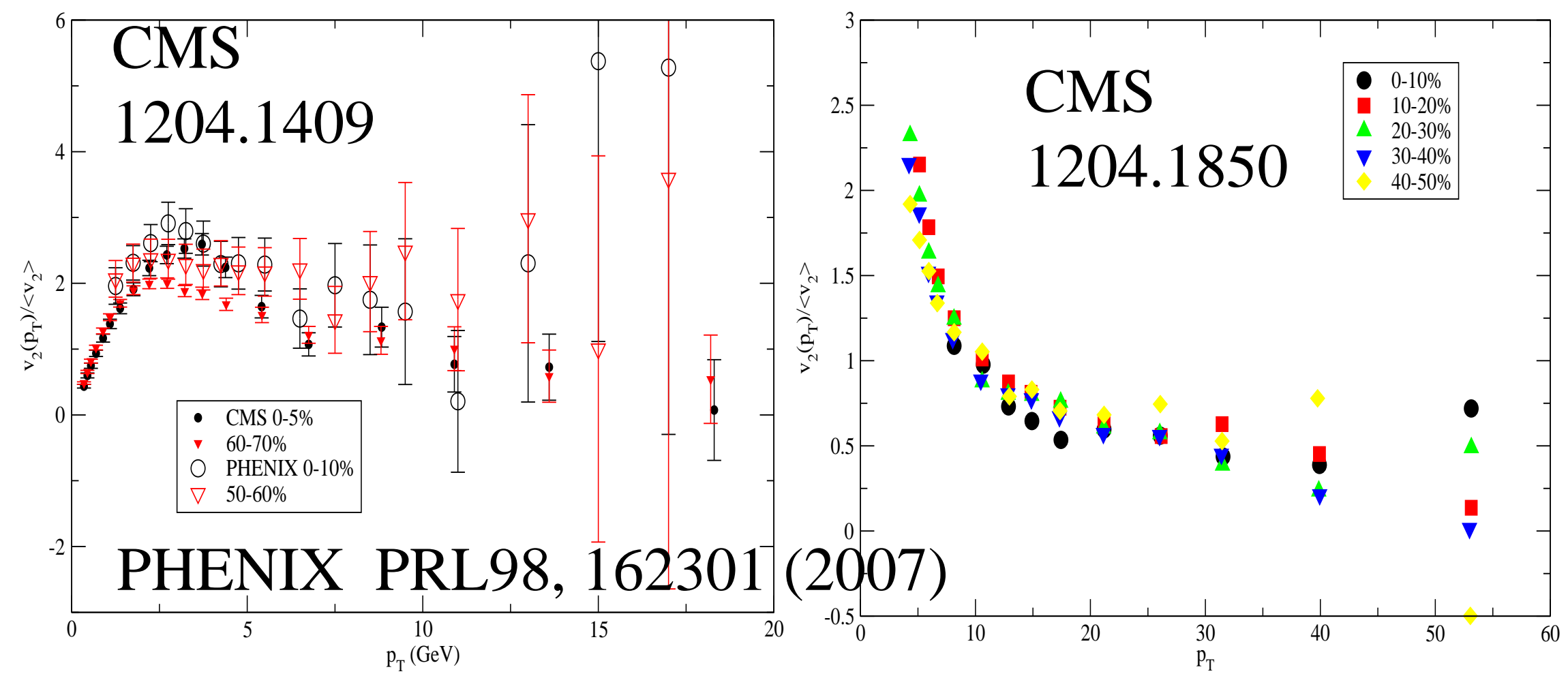

But, it seems no scaling break up to $p_{T} \sim 3.5 \mathrm{GeV}$, maybe til $20 \mathrm{GeV}$. Split@intermediate $p_{T}$, reunification (?) higher (but big errors!). If no scaling violation for all momenta, puzzle for tomography 

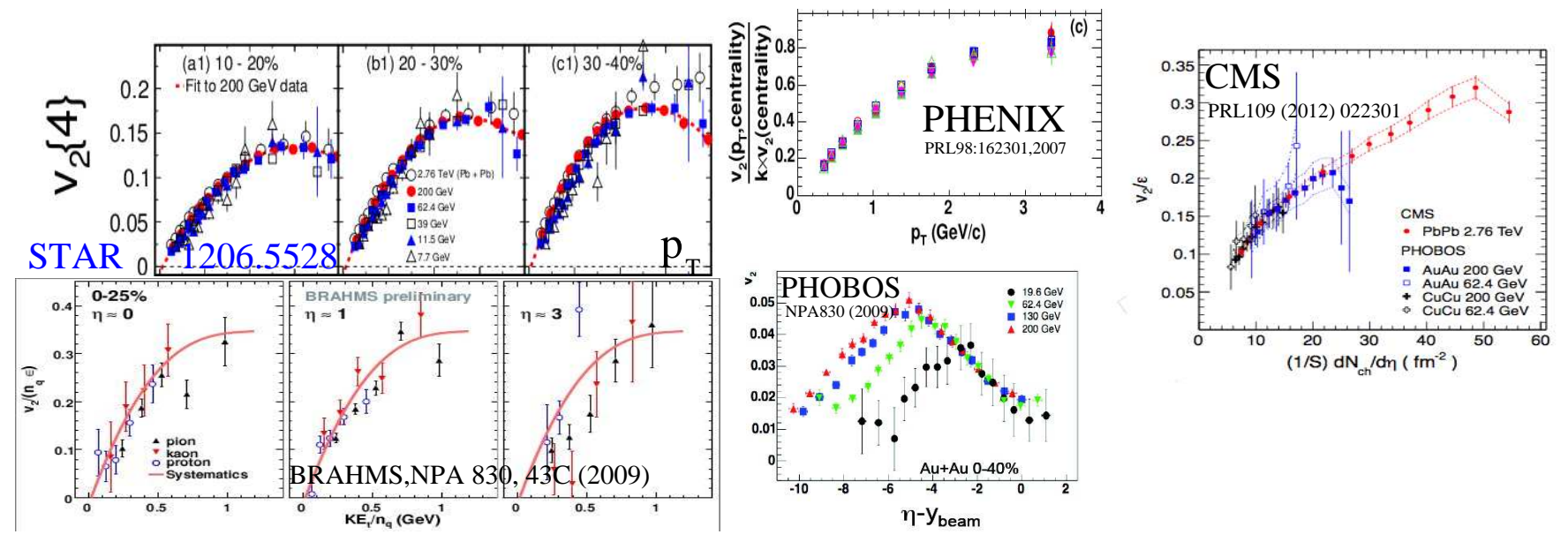

Anti-conclusion: Scalings puzzling for all popular models Unless scalings break (Experimentalists? ) model constrains on scalings more useful than "my model with 10 parameters fits this data at this $\sqrt{s}$ ". Remember Bjorken scaling/partons. QCD a descendant of a scaling non-trivial with bootstrap/Smatrix/...

More info: arXiv:1208.5996, arXiv:0911.4775 (PRC), nucl-th/0702013 (PRC) , extensive calculation with $A B C$ model using TITAN supercomputer in progress! 


\title{
$\mathrm{d}+\mathrm{Au}$ ridge at $\mathrm{RHIC}$ ?
}

\author{
Fuqiang Wang \\ For the STAR Collaboration
}




\section{D correlation functions \\ (mixed-event vertex-matched acceptance corrected)}
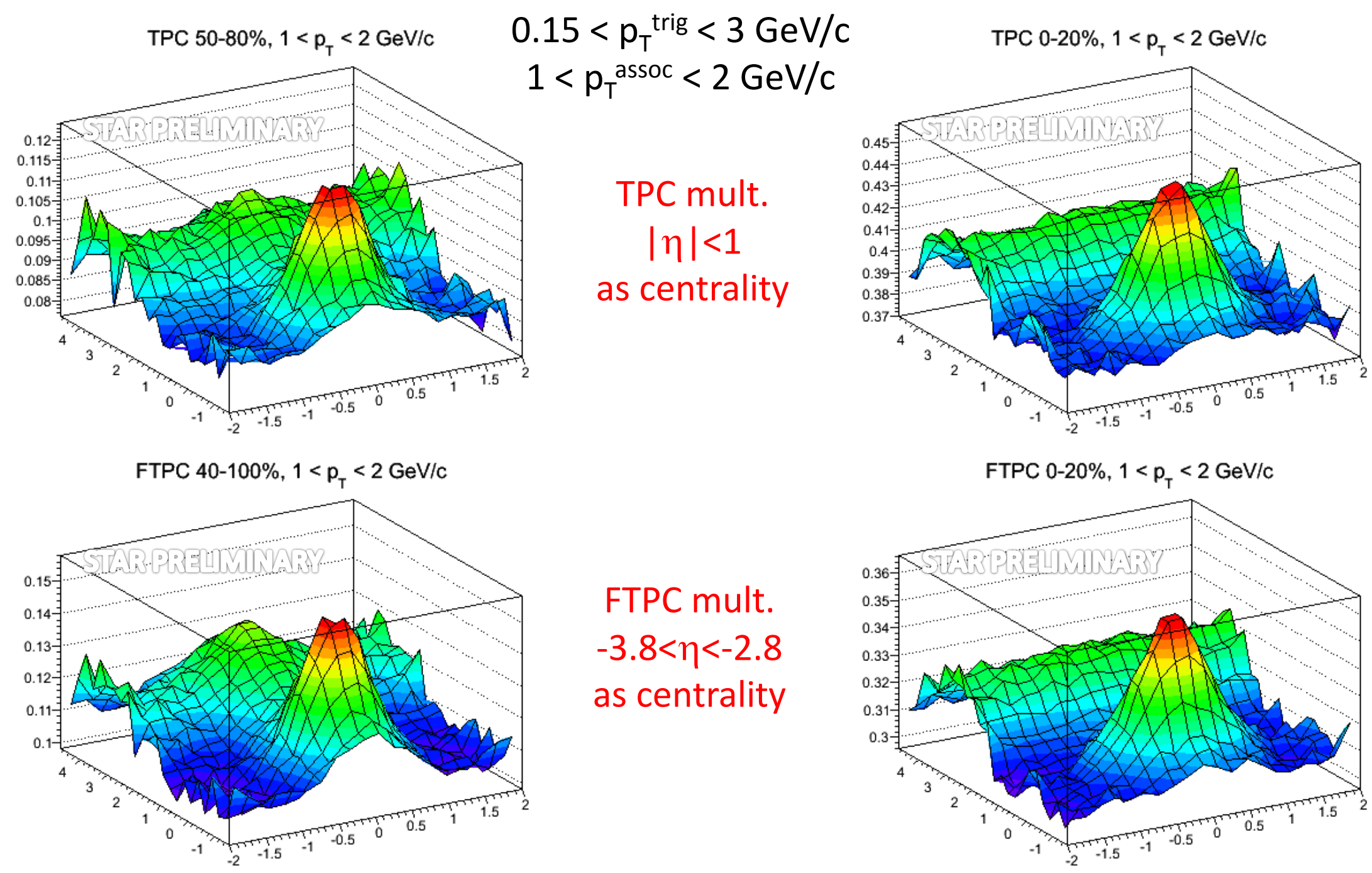

FTPC mult. $-3.8<\eta<-2.8$ as centrality

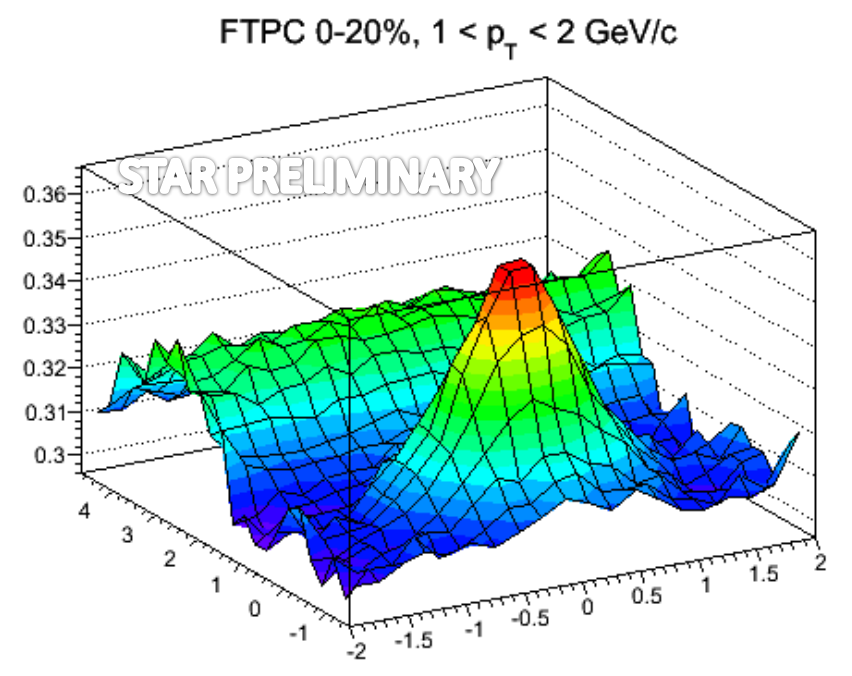




\section{$\Delta \phi$ projections in different $\Delta \eta$ (FTPC mult. $-3.8<\eta<-2.8$ as centrality)}

$0.15<\mathrm{p}_{\mathrm{T}}^{\text {trig }}<3 \mathrm{GeV} / \mathrm{c}, 1<\mathrm{p}_{\mathrm{T}}^{\text {assoc }}<2 \mathrm{GeV} / \mathrm{c}$

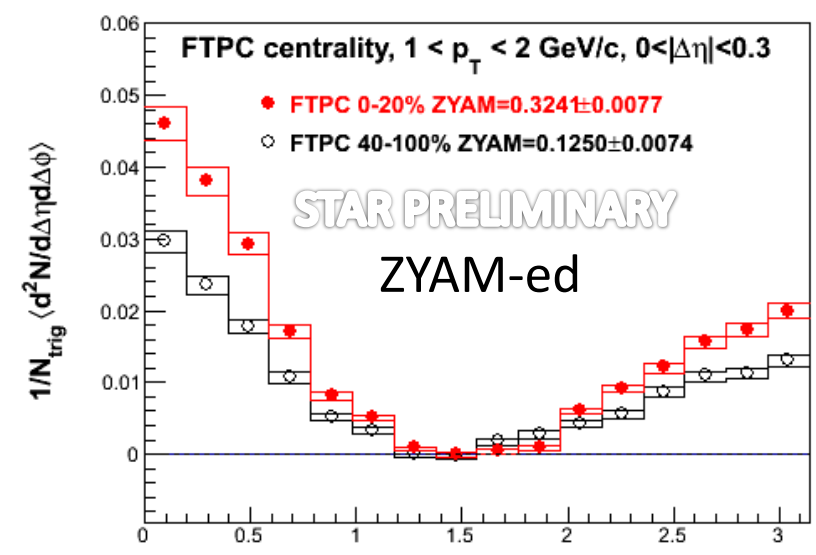

$\Delta \phi$

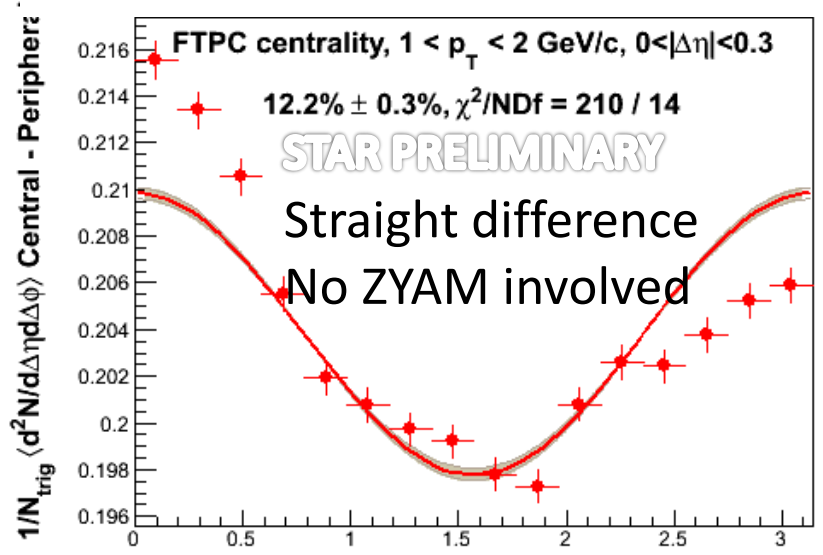

$\Delta \phi$

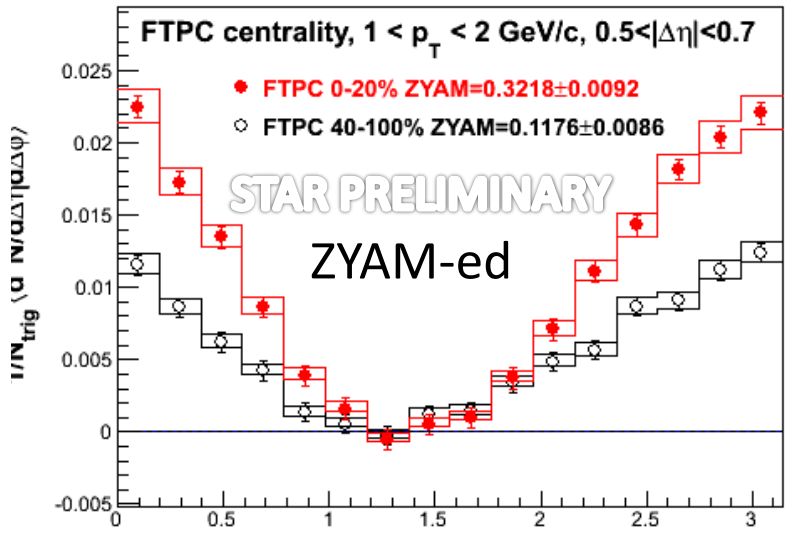

$\Delta \phi$

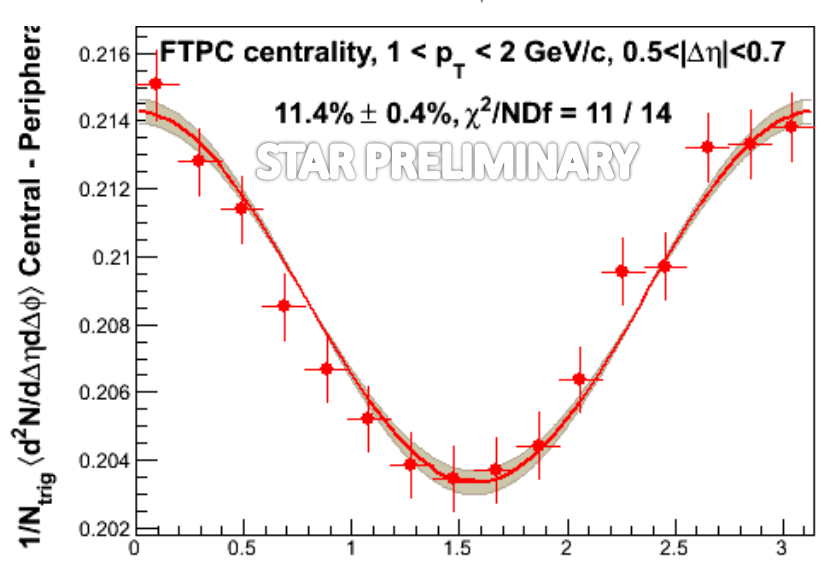

$\Delta \phi$

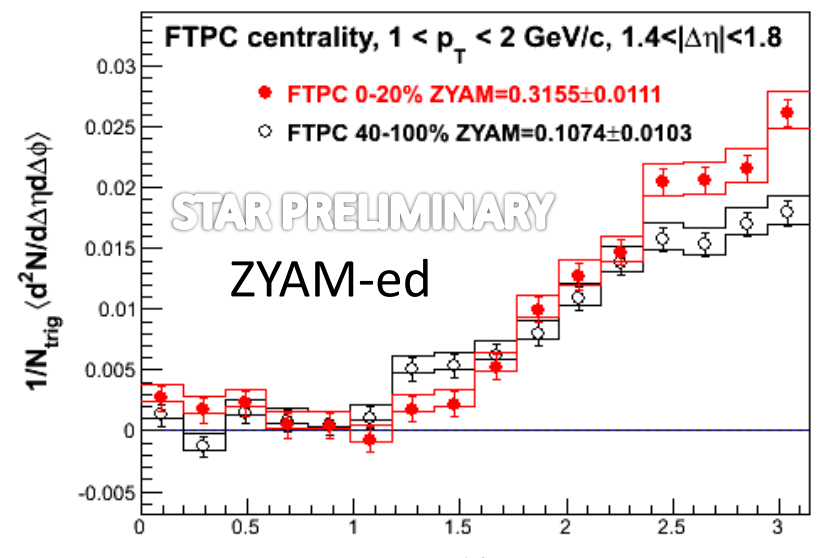

$\Delta \phi$

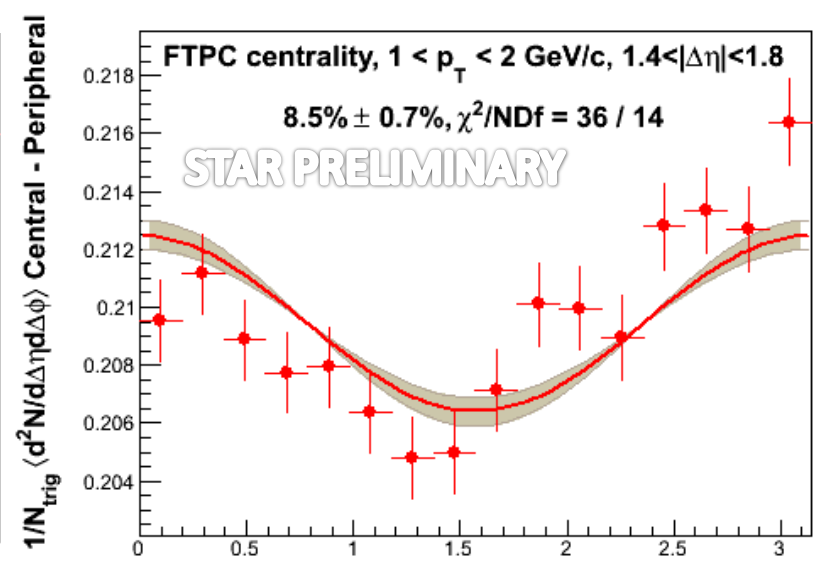

$\Delta \phi$

- ZYAM syst. error from different sizes of $\Delta \phi$ region for ZYAM.

- Efficiency corrected: $85 \pm 5 \%$. 


\section{$\Delta \eta$ projections in different $\Delta \phi$ (TPC mult. $|\eta|<1$ as centrality)}
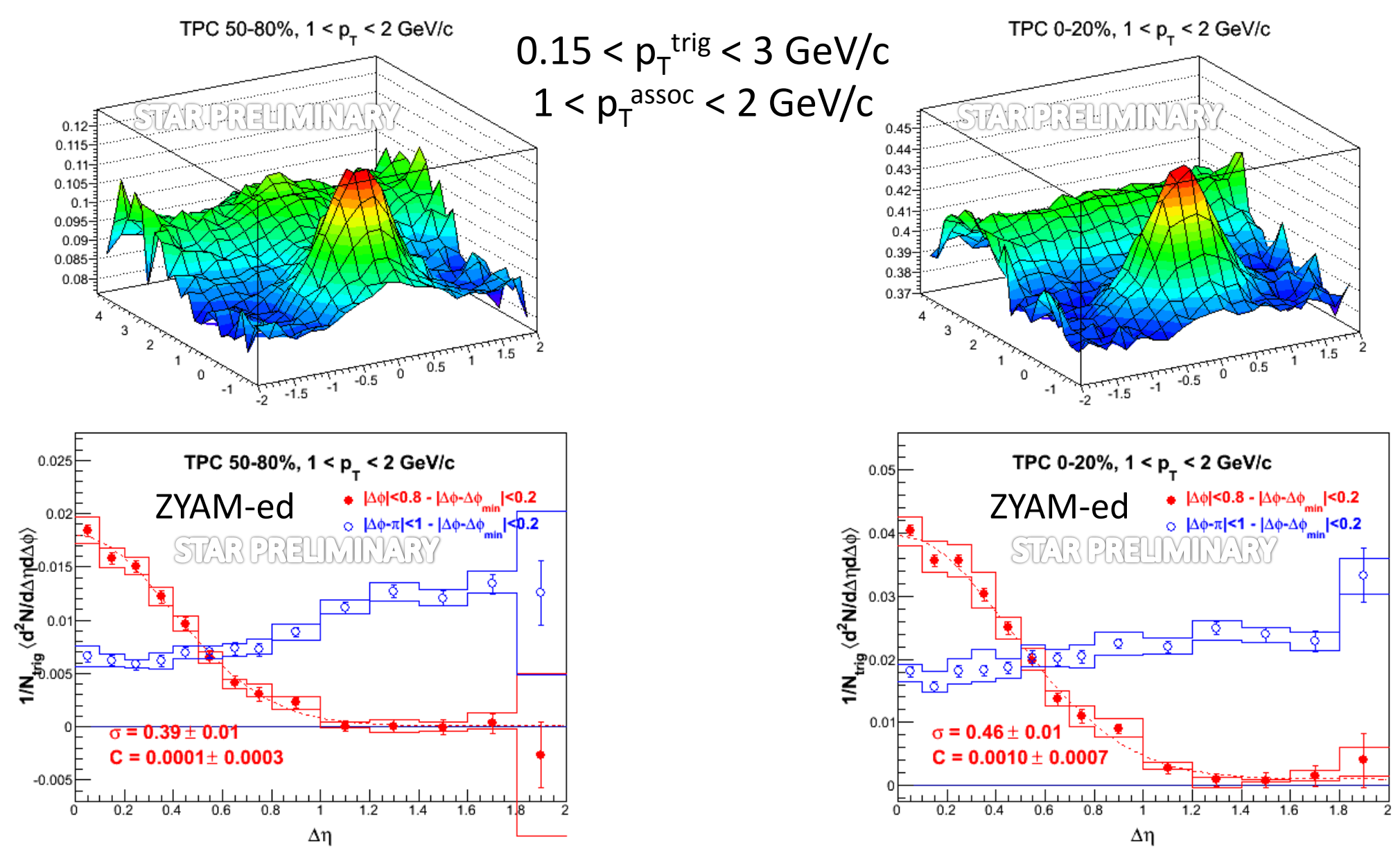

- ZYAM syst. error from different sizes of $\Delta \phi$ region for ZYAM.

- Efficiency corrected: $85 \pm 5 \%$. 


\section{$\Delta \eta$ projections in different $\Delta \phi$ (FTPC mult. $-3.8<\eta<-2.8$ as centrality)}
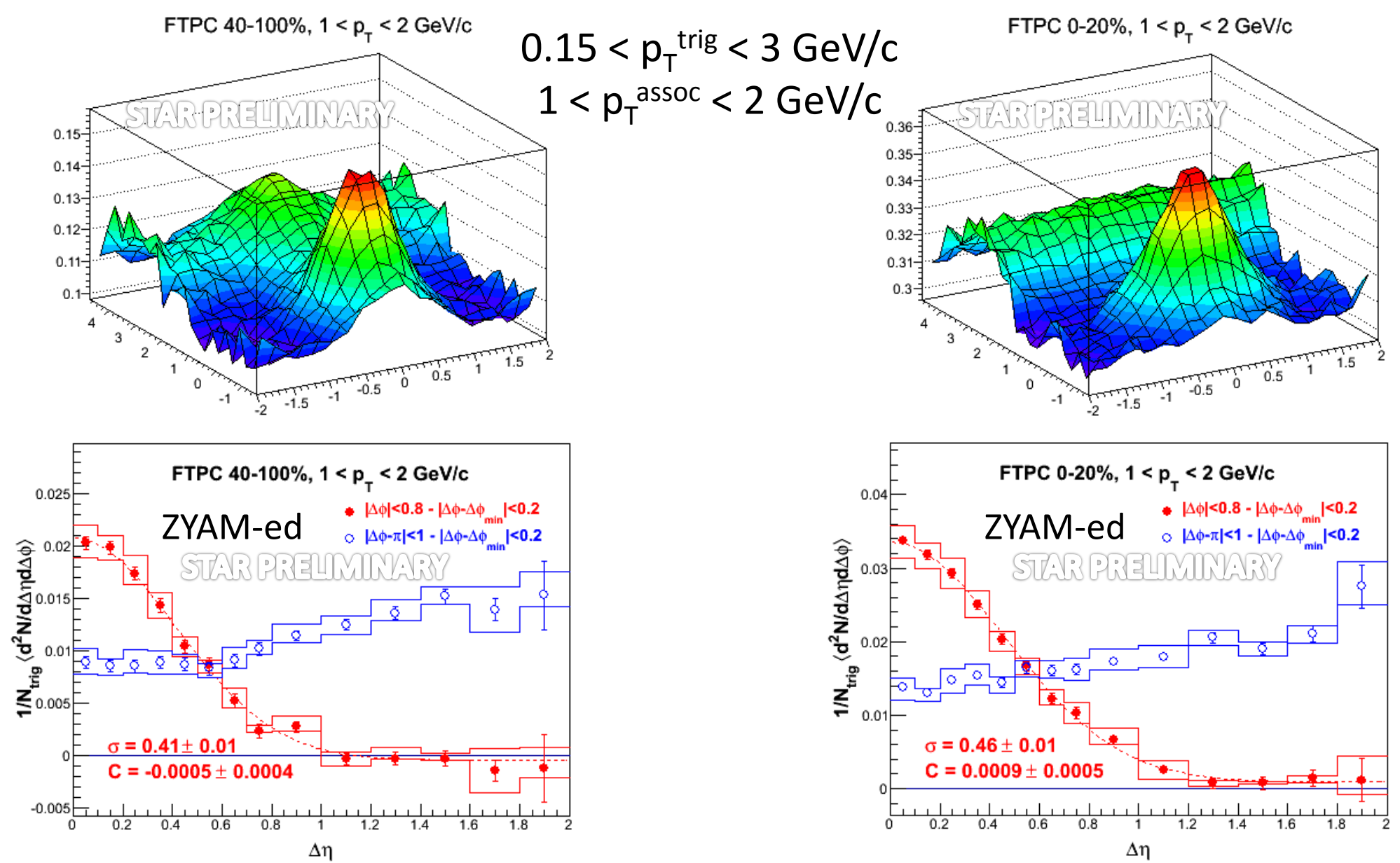

- ZYAM syst. error from different sizes of $\Delta \phi$ region for ZYAM.

- Efficiency corrected: $85 \pm 5 \%$. 


\section{Central - Peripheral}

$0.15<\mathrm{p}_{\mathrm{T}}^{\text {trig }}<3 \mathrm{GeV} / \mathrm{c}, 1<\mathrm{p}_{\mathrm{T}}^{\text {assoc }}<2 \mathrm{GeV} / \mathrm{c}$
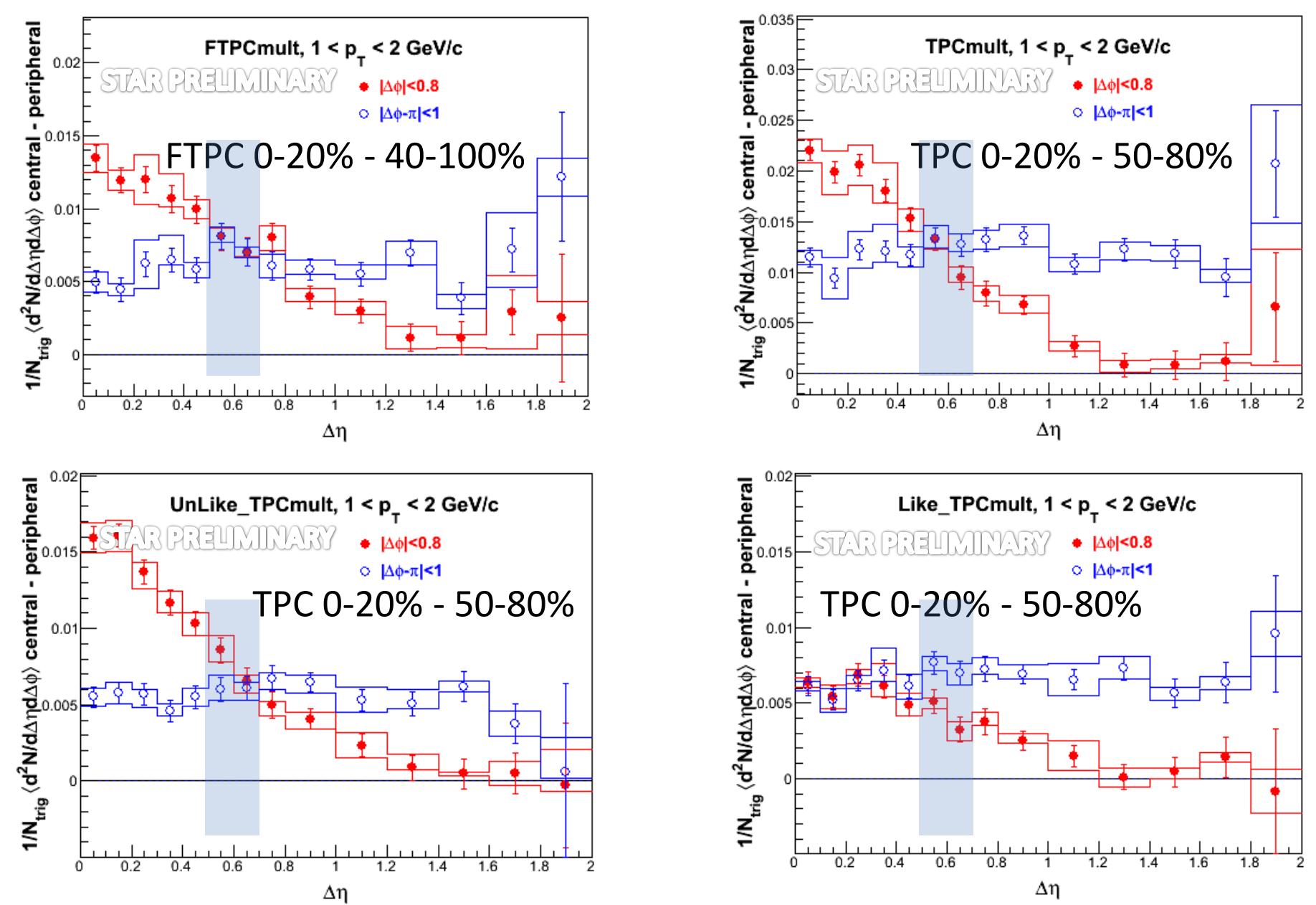

- ZYAM syst. error from different sizes of $\Delta \phi$ region for ZYAM.

- Efficiency corrected: $85 \pm 5 \%$. 


\section{Conditional yield vs multiplicity}

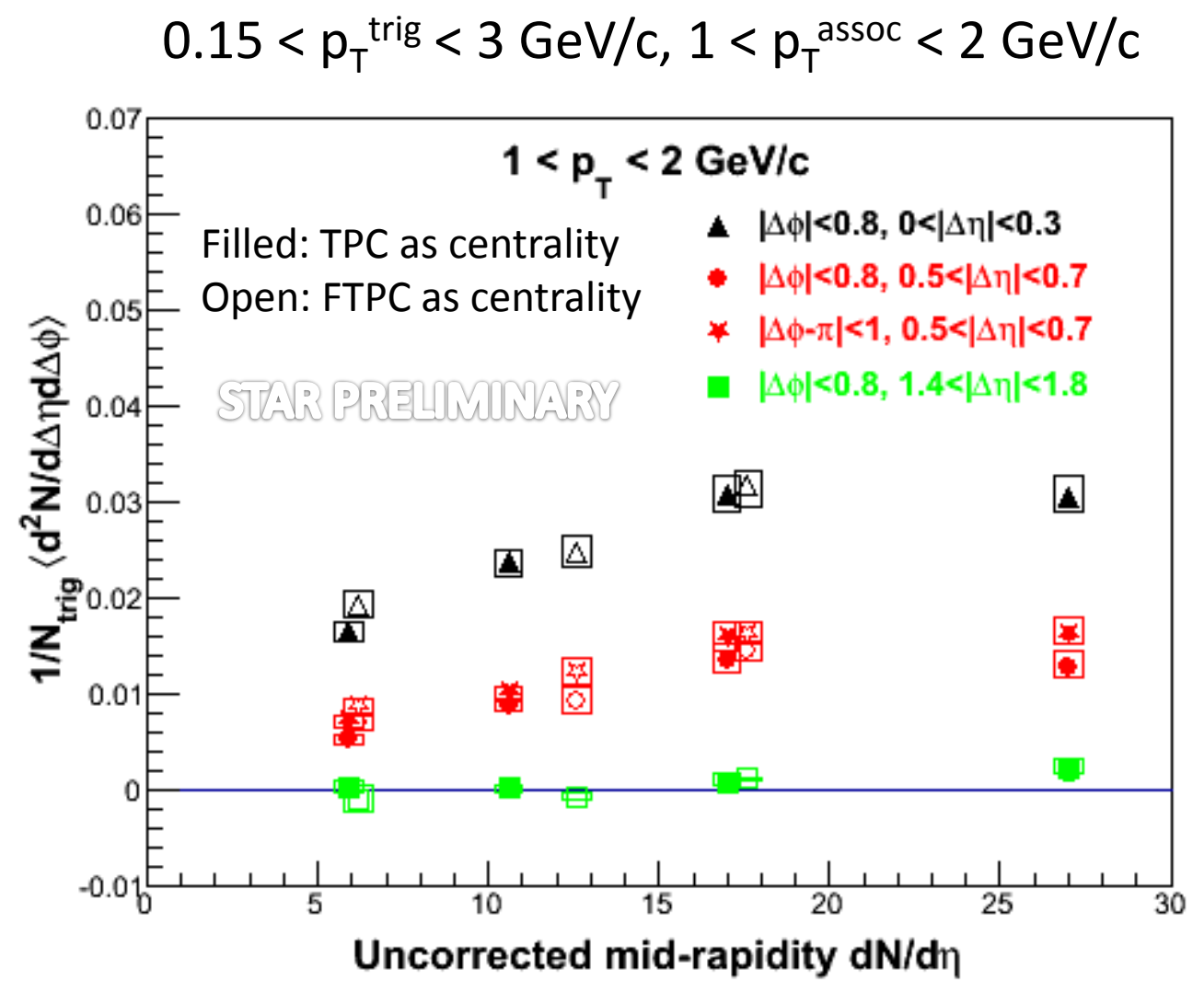




\section{Summary}

- Near-side Gaussian peak in $\Delta \eta$. Away-side approximately flat.

- Near-side large- $\Delta \eta$ yield consistent with zero.

- Central - peripheral excess resembles nearand away-side shapes and charge ordering.

- $d+A u$ data consistent with jet phenomenology. Large multiplicity events $\rightarrow$ larger-energy jets? 


\section{Jet Quenching at RHIC vs LHC in Light of Recent $\mathrm{dAu}$ vs $\mathrm{pPb}$ Controls}

April 15-17, 2013

Large Seminar Room, Physics, Bldg. 510

\section{MONDAY, April 15}

08:30-12:30 Session I. dAu/pPb Results, Ridge \& Interpretations

Chairperson: Jiangyong Jia

\begin{tabular}{|l|l|l|}
\hline 08:30-09:00 & & Registration \\
\hline 09:00-09:10 & Nicholas Samios & Welcome \\
\hline 09:10-09:40 & Wit Busza & History of pA from 1970 to 2013 \\
\hline 09:40-10:20 & Constantin Loizides & Recent LHC pPb results \\
\hline 10:20-11:00 & Peter Steinberg & The Ridge in pp/pA/AA at LHC \\
\hline 11:00-11:30 & \multicolumn{1}{|c|}{$\boldsymbol{C O F F E E}$} & $\boldsymbol{B R E A K}$ \\
\hline 11:30-12:00 & Raju Venugopalan & Ridge correlations: CGC perspective \\
\hline 12:00-12:30 & Piotr Bozek & Ridge correlations: hydro perspective \\
\hline
\end{tabular}

14:00-18:20 Session II. dAu/pPb Results, Ridge \& Interpretations Chairperson: Dmitri Kharzeev

\begin{tabular}{|c|c|c|}
\hline $14: 00-14: 30$ & Gabor David & Updates/puzzles of dAu at RHIC \\
\hline $14: 30-15: 00$ & Wolfram Fischer & Future pA: RHIC machine perspective \\
\hline $15: 00-15: 30$ & Gergely Barnafoldi & Cronin \& shadowing in $\mathrm{pA}$ \\
\hline $15: 30-16: 00$ & Boris Kopeliovich & Nuclear suppression in $\mathrm{pA}$ \\
\hline $16: 00-16: 30$ & \multicolumn{2}{|c|}{ COFFEE BREAK } \\
\hline $16: 30-17: 00$ & Adrian Dumitru & CGC predictions for $\mathrm{pp}, \mathrm{pA}$, and $\mathrm{AA}$ \\
\hline $17: 00-17: 30$ & Alex Kovner & Critical assessment of CGC \\
\hline $17: 30-18: 20$ & Dmitri Kharzeev & Brief introduction and moderated discussions \\
\hline
\end{tabular}




\section{TUESDAY, April 16}

09:00-12:00 Session III. Jet Quenching at LHC \& Opaqueness Evolution Chairperson: Andrea Dainese

\begin{tabular}{|l|l|l|}
\hline 09:00-09:30 & Gunther Roland & LHC jet quenching I \\
\hline 09:30-10:00 & Brian Cole & LHC jet quenching II \\
\hline 10:00-10:30 & Abhijit Majumder & Higher-twist predictions for pA and AA at LHC \\
\hline 10:30-11:00 & \multicolumn{2}{|c|}{ COFFEE } \\
BREAK \\
\hline 11:00-11:30 & Jinfeng Liao & Opaqueness evolution from color liberation \\
\hline 11:30-12:00 & Vladimir Skokov & Jet energy loss at Tc \\
\hline 12:00-12:30 & Denes Molnar & Hard and soft responses from parton transport \\
\hline
\end{tabular}

14:00-17:40 Session IV. RHIC/LHC Complementarity \& RHIC Future Chairperson: Peter Jacobs

\begin{tabular}{|l|l|l|}
\hline 14:00-14:30 & Anne Sickles & RHIC jet quenching: present \& future \\
\hline 14:30-15:00 & Joern Putschke & RHIC/LHC complementarity \\
\hline 15:00-15:30 & Fuqiang Wang & Particle correlations at RHIC: present \& future \\
\hline 15:30-15:50 & \multicolumn{2}{|c|}{ COFFEE } \\
\hline BREAK \\
15:50-16:20 & Roy Lacey & $\begin{array}{l}\text { Combined analysis of RHIC to LHC suppression } \\
\text { \& geometry }\end{array}$ \\
\hline 16:20-16:50 & Soumya Mohapatra & Event-by-event flow \& initial geometry from LHC \\
\hline 16:50-17:20 & Frasher Loshaj & Jet quenching \& fragmentation from RHIC to LHC \\
\hline 17:20-18:10 & Peter Jacobs & Brief introduction and moderated discussions \\
\hline
\end{tabular}

\section{Workshop Dinner: 18:30-20:00 @ Brookhaven Center, South Room}




\section{WEDNESDAY, April 17}

\section{9:00-12:30 Session V. Initial Conditions for Hard/Soft Observables Chairperson: Rob Pisarski}

\begin{tabular}{|l|l|l|}
\hline 09:00-09:30 & Ulrich Heinz & $\begin{array}{l}\text { Hydro with fluctuating initial condition \& } \\
\text { temperature dependent viscosity }\end{array}$ \\
\hline 09:30-10:00 & Bjorn Schenke & Initial conditions: IP-glasma vs KLN vs Glauber \\
\hline 10:00-10:30 & Barbara Betz & Raa and high p_t azimuthal anisotropy \\
\hline 10:30-11:00 & Xilin Zhang & Harmonic jet tomography at RHIC+LHC \\
\hline 11:00-11:30 & \multicolumn{1}{|c|}{ COFFEE } & BREAK \\
\hline 11:30-12:00 & Edward Shuryak & $\begin{array}{l}\text { Possible collective behavior \& hadron correlations } \\
\text { from pp to AA }\end{array}$ \\
\hline 12:00-12:30 & Yuri Kovchegov & $\begin{array}{l}\text { Long range rapidity correlations in heavy ion } \\
\text { collisions }\end{array}$ \\
\hline
\end{tabular}

\section{4:00-17:30 Session VI. Flavor \& BES \& Closing Remarks \\ Chairperson: Raju Venugopalan}

\begin{tabular}{|c|c|c|}
\hline 14:00-14:30 & Alessandro Buzzatti & Flavor tomography \\
\hline $14: 30-15: 00$ & Andrea Dainesee & Experimental status of flavor tomography \\
\hline $15: 00-15: 30$ & Helen Caines & Jet quenching \& initial geometry from RHIC BES \\
\hline $15: 30-16: 00$ & Elke Aschenauer & eRHIC as the future \\
\hline $16: 00-16: 30$ & \multicolumn{2}{|c|}{ COFFEE BREAK } \\
\hline $16: 30-17: 00$ & Jean Paul Blaizot & $\begin{array}{l}\text { In-medium QCD cascade: democratic branching } \\
\text { and wave turbulence }\end{array}$ \\
\hline $17: 00-17: 30$ & Miklos Gyulassy & Closing remarks \\
\hline
\end{tabular}




\begin{tabular}{|c|c|}
\hline \multicolumn{2}{|c|}{$\begin{array}{c}\text { Jet Quenching at RHIC vs LHC in Light of Recent dAu vs p } \\
\text { April 15-17, } 2013 \\
\text { List of Registered Participants }\end{array}$} \\
\hline Name & Affiliation \\
\hline Salvatore Aiola & Lawrence Berkeley National Laboratory \\
\hline Elke Aschenauer & Brookhaven National Laboratory \\
\hline Gergely Barnafoldi & Wigner RCP of the Hungarian Academy of Sciences \\
\hline Gokce Basar & Stony Brook University \\
\hline Jean-Paul Blaizot & CNRS \\
\hline Piotr Bozek & IFJ PAN / AGH Krakow Poland \\
\hline Wit Busza & Massachusetts Institute of Technology \\
\hline Alessandro Buzzatti & Lawrence Berkeley National Laboratory \\
\hline Adam Bzdak & Brookhaven National Laboratory \\
\hline Helen Caines & Yale University \\
\hline Chin-Hao Chen & Brookhaven National Laboratory \\
\hline William Christie & Brookhaven National Laboratory \\
\hline Brian Cole & Columbia University \\
\hline Megan Connors & Yale University \\
\hline Andrea Dainese & INFN - Padova \\
\hline Gabor David & Brookhaven National Laboratory \\
\hline L. Chanaka De Silva & University of Houston \\
\hline Abhay Deshpande & Stony Brook University \\
\hline Adrian Dumitru & Baruch College (CUNY) \& RIKEN BNL Research Center \\
\hline James Dunlop & Brookhaven National Laboratory \\
\hline Andrej Ficnar & Columbia University \\
\hline Wolfram Fischer & Brookhaven National Laboratory \\
\hline Miklos Gyulassy & Columbia University \\
\hline Ulrich Heinz & The Ohio State University \\
\hline Martin Hentschinski & Brookhaven National Laboratory \\
\hline Xu-Guang Huang & Indiana University \\
\hline Peter Jacobs & Lawrence Berkeley National Laboratory \\
\hline Jiangyong Jia & Brookhaven National Laboratory \\
\hline Kurt Jung & Purdue University \\
\hline Dmitri Kharzeev & Brookhaven National Laboratory \& Stony Brook University \\
\hline Boris Kopeliovich & UTFSM, Valparaiso, Chile \\
\hline Yuri Kovchegov & The Ohio State University \\
\hline Alex Kovner & University of Connecticut \\
\hline Roy Lacey & Stony Brook University \\
\hline Xuan Li & Temple University \\
\hline
\end{tabular}




\begin{tabular}{|c|c|}
\hline Jinfeng Liao & Indiana University \& RIKEN BNL Research Center \\
\hline Shu Lin & RIKEN BNL Research Center @ Brookhaven National Lab \\
\hline Tseh Liou & Columbia University \\
\hline Constantin Loizides & Lawrence Berkeley National Laboratory \\
\hline Ronald Longacre & Retiree, Brookhaven National Laboratory \\
\hline Frasher Loshaj & Stony Brook University \\
\hline Rongrong Ma & Yale University \\
\hline Abhijit Majumder & Wayne State University \\
\hline Mohammed Mia & Columbia University \\
\hline Alice Mignerey & University of Maryland \\
\hline Soumya Mohapatra & Stony Brook University \\
\hline Denes Molnar & Purdue University \\
\hline Mriganka Mouli Mondal & Texas A\&M University \\
\hline Berndt Mueller & Brookhaven National Laboratory \\
\hline Alice Ohlson & Yale University \\
\hline Robert Pak & Brookhaven National Laboratory \\
\hline Annalia Palumbo & Brookhaven National Laboratory \\
\hline Dennis Perepelitsa & Columbia University \\
\hline Elena Petreska & Baruch College \\
\hline Robert Pisarski & Brookhaven National Laboratory \\
\hline Joern Putschke & Wayne State University \\
\hline Rosi Reed & Yale University \\
\hline Gunther Roland & Massachusetts Institute of Technology \\
\hline Takao Sakaguchi & Brookhaven National Laboratory \\
\hline Bjoern Schenke & Brookhaven National Laboratory \\
\hline Edward Shuryak & Stony Brook University \\
\hline Anne Sickles & Brookhaven National Laboratory \\
\hline Vladimir Skokov & Brookhaven National Laboratory \\
\hline Peter Steinberg & Brookhaven National Laboratory \\
\hline Marco Stratmann & Brookhaven National Laboratory \\
\hline Deke Sun & Purdue University \\
\hline Michael Tannenbaum & Brookhaven National Laboratory \\
\hline Giorgio Torrieri & Columbia University \\
\hline Raju Venugopalan & Brookhaven National Laboratory \\
\hline Fuqiang Wang & Purdue University \\
\hline $\mathrm{Bin} \mathrm{Wu}$ & IPhT Saclay \\
\hline Jiechen Xu & Columbia University \\
\hline Lingshan Xu & CMS \\
\hline Zhenyu Ye & University of Illinois \\
\hline Xilin Zhang & Ohio University \\
\hline
\end{tabular}




\section{RIKEN BNL Research Center Proceedings}

Volume 114 - The The Physics of p $\uparrow+$ A Collisions at RHIC, January 7-9, 2013 - BNL-99107-2013

Volume 113 - Thermal Radiation Workshop, December 5-7, 2012 - BNL-99088-2013

Volume 112 - RBRC Scientific Review Committee Meeting, November 6-8, 2012 - BNL-99621-2013

Volume 111 - Forward Physics at RHIC, July 30-Aug 1, 2012 - BNL-98400-2012

Volume 110 - P- and CP-odd Effects in Hot and Dense Matter, June 25-27, 2012 - BNL-98398-2012

Volume 109 - New Horizons for Lattice Computations with Chiral Fermions, May 14-18, 2012 - BNL-98392-2012

Volume 108 - Hyperon-Hyperon Interactions and Searches for Exotic Di-Hyperons in Nuclear Collisions, February 29 - March 2, 2012 - BNL-97035-2012

Volume 107 - Future Directions in High Energy QCD, October 20-22, 2011 - BNL-98119-2011

Volume 106 - Fluctuations, Correlations and RHIC Low Energy Runs, October 3-5, 2011 - BNL-96514-2011

Volume 105 - Opportunities for Polarized He-3 in RHIC and EIC, September 28-30, 2011 - BNL-96418-2011

Volume 104 - Brookhaven Summer Program on Quarkonium Production in Elementary and Heavy Ion Collisions, June 6-18, 2011 - BNL-96171-2011

Volume 103 - Opportunities for Drell-Yan Physics at RHIC, May 11-13, 2011 - BNL-95236-2011-2011

Volume 102 - Initial State Fluctuations and Final-State Particle Correlations, February 2-4, 2011 - BNL-94704-2011

Volume 101 - RBRC Scientific Review Committee Meeting, October 27-29, 2010 - BNL-94589-2011

Volume 100 - Summer Program on Nucleon Spin Physics, July 14-27, 2010 - BNL-96163-2011

Volume 99 - The Physics of W and Z Bosons, June 24-25, 2010 - BNL-94287-2010

Volume 98 - Saturation, the Color Glass Condensate and the Glasma: What Have we Learned from RHIC? - May 10-12, 2010 BNL-94271-2010

Volume 97 - RBRC Scientific Review Committee Meeting, October 21-22, 2009 - BNL-90674-2009

Volume 96 - P- and CP-Odd Effects in Hot and Dense Matter, April 26-30, 2010 - BNL-94237-2010

Volume 95 - Progress in High-pT Physics at RHIC, March 17-19, 2010 - BNL-94214-2010

Volume 94 - Summer Program on Nucleon Spin Physics at LBL, June 1-12, 2009

Volume 93 - PHENIX Spinfest School 2009 at BNL - July 1-31, 2009. BNL-90343-2009 Link: PHENIXSpinfestSchool2009@B,BNL

Volume 92 - PKU-RBRC Workshop on Transverse Spin Physics, June 30 - July 4, 2008, Beijing, China - BNL-81685-2008

Volume 91 - RBRC Scientific Review Committee Meeting, November 17-18, 2008 - BNL-81556-2008

Volume 90 - PHENIX Spinfest School 2008 at BNL, August 4-8, 2008 - BNL-81478-2008

Volume 89 - Understanding QGP through Spectral Functions and Euclidean Correlators, April 23-25, 2008 - BNL-81318-2008

Volume 88 - Hydrodynamics in Heavy Ion Collisions and QCD Equation of State, April 21-22, 2008 - BNL-81307-2008

Volume 87 - RBRC Scientific Review Committee Meeting, November 5-6, 2007 - BNL-79570-2007

Volume 86 - Global Analysis of Polarized Parton Distributions in the RHIC Era, October 8, 2007 - BNL-79457-2007

Volume 85 - Parity-Violating Spin Asymmetries at RHIC-BNL, April 26-27, 2007 - BNL-79146-2007

Volume 84 - Domain Wall Fermions at Ten Years, March 15-17, 2007 - BNL 77857-2007

Volume 83 - QCD in Extreme Conditions, July 31 - August 2, 2006 - BNL-76933-2006

Volume 82 - RHIC Physics in the Context of the Standard Model, June 18-23, 2006 - BNL-76863-2006

Volume 81 - Parton Orbital Angular Momentum (Joint RBRC/University of New Mexico Workshop) February 24-26, 2006 BNL-75937-2006

Volume 80 - Can We Discover the QCD Critical Point at RHIC?, March 9-10, 2006 - BNL-75692-2006 
Volume 79 - Strangeness in Collisions, February 16-17, 2006 - BNL-79763-2008

Volume 78 - Heavy Flavor Productions and Hot/Dense Quark Matter, December 12-14, 2005 - BNL-76915-2006

Volume 77 - RBRC Scientific Review Committee Meeting, October 10-12, 2005 - BNL-52649-2005

Volume 76 - Odderon Searches at RHIC, September 27-29, 2005 - BNL-75092-2005

Volume 75 - Single Spin Asymmetries, June 1-3, 2005 - BNL-74717-2005

Volume 74 - RBRC QCDOC Computer Dedication and Symposium on RBRC QCDOC, May 26, 2005 - BNL-74813-2005

Volume 73 - Jet Correlations at RHIC, March 10-11, 2005 - BNL-73910-2005

Volume 72 - RHIC Spin Collaboration Meetings XXXI (January 14, 2005), XXXII (February 10, 2005), XXXIII (March 11, 2005) - BNL-73866-2005

Volume 71 - Classical and Quantum Aspects of the Color Glass Condensate - BNL-73793-2005

Volume 70 - Strongly Coupled Plasmas: Electromagnetic, Nuclear \& Atomic - BNL-73867-2005

Volume 69 - RBRC Scientific Review Committee - BNL-73546-2004

Volume 68 - Workshop on the Physics Programme of the RBRC and UKQCD QCDOC Machines - BNL-73604-2004

Volume 67 - High Performance Computing with BlueGene/L and QCDOC Architectures

Volume 66 - RHIC Spin Collaboration Meeting XXIX, October 8-9, 2004, Torino Italy - BNL-73534-2004

Volume 65 - RHIC Spin Collaboration Meetings XXVII (July 22, 2004), XXVIII (September 2, 2004), XXX (December 6, 2004) - BNL-73506-2004

Volume 64 - Theory Summer Program on RHIC Physics - BNL-73263-2004

Volume 63 - RHIC Spin Collaboration Meetings XXIV (May 21, 2004), XXV (May 27, 2004), XXVI (June 1, 2004) - BNL72397-2004

Volume 62 - New Discoveries at RHIC, May 14-15, 2004 - BNL- 72391-2004

Volume 61 - RIKEN-TODAI Mini Workshop on “Topics in Hadron Physics at RHIC”, March 23-24, 2004 - BNL-72336-2004

Volume 60 - Lattice QCD at Finite Temperature and Density - BNL-72083-2004

Volume 59 - RHIC Spin Collaboration Meeting XXI (January 22, 2004), XXII (February 27, 2004), XXIII (March 19, 2004)BNL-72382-2004

Volume 58 - RHIC Spin Collaboration Meeting XX - BNL-71900-2004

Volume 57 - High pt Physics at RHIC, December 2-6, 2003 - BNL-72069-2004

Volume 56 - RBRC Scientific Review Committee Meeting - BNL-71899-2003

Volume 55 - Collective Flow and QGP Properties - BNL-71898-2003

Volume 54 - RHIC Spin Collaboration Meetings XVII, XVIII, XIX - BNL-71751-2003

Volume 53 - Theory Studies for Polarized pp Scattering - BNL-71747-2003

Volume 52 - RIKEN School on QCD "Topics on the Proton" - BNL-71694-2003

Volume 51 - RHIC Spin Collaboration Meetings XV, XVI - BNL-71539-2003

Volume 50 - High Performance Computing with QCDOC and BlueGene - BNL-71147-2003

Volume 49 - RBRC Scientific Review Committee Meeting - BNL-52679

Volume 48 - RHIC Spin Collaboration Meeting XIV - BNL-71300-2003

Volume 47 - RHIC Spin Collaboration Meetings XII, XIII - BNL-71118-2003

Volume 46 - Large-Scale Computations in Nuclear Physics using the QCDOC - BNL-52678

Volume 45 - Summer Program: Current and Future Directions at RHIC - BNL-71035

Volume 44 - RHIC Spin Collaboration Meetings VIII, IX, X, XI - BNL-71117-2003

Volume 43 - RIKEN Winter School - Quark-Gluon Structure of the Nucleon and QCD - BNL-52672

Volume 42 - Baryon Dynamics at RHIC - BNL-52669

Volume 41 - Hadron Structure from Lattice QCD - BNL-52674

Volume 40 - Theory Studies for RHIC-Spin - BNL-52662 
Volume 39 - RHIC Spin Collaboration Meeting VII - BNL-52659

Volume 38 - RBRC Scientific Review Committee Meeting - BNL-52649

Volume 37 - RHIC Spin Collaboration Meeting VI (Part 2) - BNL-52660

Volume 36 - RHIC Spin Collaboration Meeting VI - BNL-52642

Volume 35 - RIKEN Winter School - Quarks, Hadrons and Nuclei - QCD Hard Processes and the Nucleon Spin - BNL-52643

Volume 34 - High Energy QCD: Beyond the Pomeron - BNL-52641

Volume 33 - Spin Physics at RHIC in Year-1 and Beyond - BNL-52635

Volume 32 - RHIC Spin Physics V - BNL-52628

Volume 31 - RHIC Spin Physics III \& IV Polarized Partons at High Q^2 Region - BNL-52617

Volume 30 - RBRC Scientific Review Committee Meeting - BNL-52603

Volume 29 - Future Transversity Measurements - BNL-52612

Volume 28 - Equilibrium \& Non-Equilibrium Aspects of Hot, Dense QCD - BNL-52613

Volume 27 - Predictions and Uncertainties for RHIC Spin Physics \& Event Generator for RHIC Spin Physics III - Towards Precision Spin Physics at RHIC - BNL-52596

Volume 26 - Circum-Pan-Pacific RIKEN Symposium on High Energy Spin Physics - BNL-52588

Volume 25 - RHIC Spin - BNL-52581

Volume 24 - Physics Society of Japan Biannual Meeting Symposium on QCD Physics at RIKEN BNL Research Center - BNL52578

Volume 23 - Coulomb and Pion-Asymmetry Polarimetry and Hadronic Spin Dependence at RHIC Energies - BNL-52589

Volume 22 - OSCAR II: Predictions for RHIC - BNL-52591

Volume 21 - RBRC Scientific Review Committee Meeting - BNL-52568

Volume 20 - Gauge-Invariant Variables in Gauge Theories - BNL-52590

Volume 19 - Numerical Algorithms at Non-Zero Chemical Potential - BNL-52573

Volume 18 - Event Generator for RHIC Spin Physics - BNL-52571

Volume 17 - Hard Parton Physics in High-Energy Nuclear Collisions - BNL-52574

Volume 16 - RIKEN Winter School - Structure of Hadrons - Introduction to QCD Hard Processes - BNL-52569

Volume 15 - QCD Phase Transitions - BNL-52561

Volume 14 - Quantum Fields In and Out of Equilibrium - BNL-52560

Volume 13 - Physics of the 1 Teraflop RIKEN-BNL-Columbia QCD Project First Anniversary Celebration - BNL-66299

Volume 12 - Quarkonium Production in Relativistic Nuclear Collisions - BNL-52559

Volume 11 - Event Generator for RHIC Spin Physics - BNL-66116

Volume 10 - Physics of Polarimetry at RHIC - BNL-65926

Volume 9 - High Density Matter in AGS, SPS and RHIC Collisions - BNL-65762

Volume 8 - Fermion Frontiers in Vector Lattice Gauge Theories - BNL-65634

Volume 7 - RHIC Spin Physics - BNL-65615

Volume 6 - Quarks and Gluons in the Nucleon - BNL-65234

Volume 5 - Color Superconductivity, Instantons and Parity (Non?)-Conservation at High Baryon Density - BNL-65105

Volume 4 - Inauguration Ceremony and Non-Equilibrium Many Body Dynamics - BNL-64912

Volume 3 - Hadron Spin-Flip at RHIC Energies - BNL-64724

Volume 2 - Perturbative QCD as a Probe of Hadron Structure - BNL-64723

Volume 1 - Open Standards for Cascade Models for RHIC - BNL-64722 


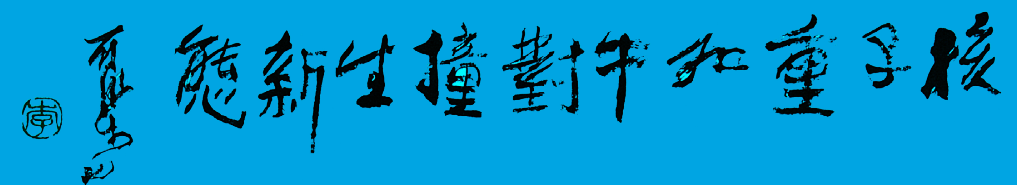 \\ il \\ ifi}

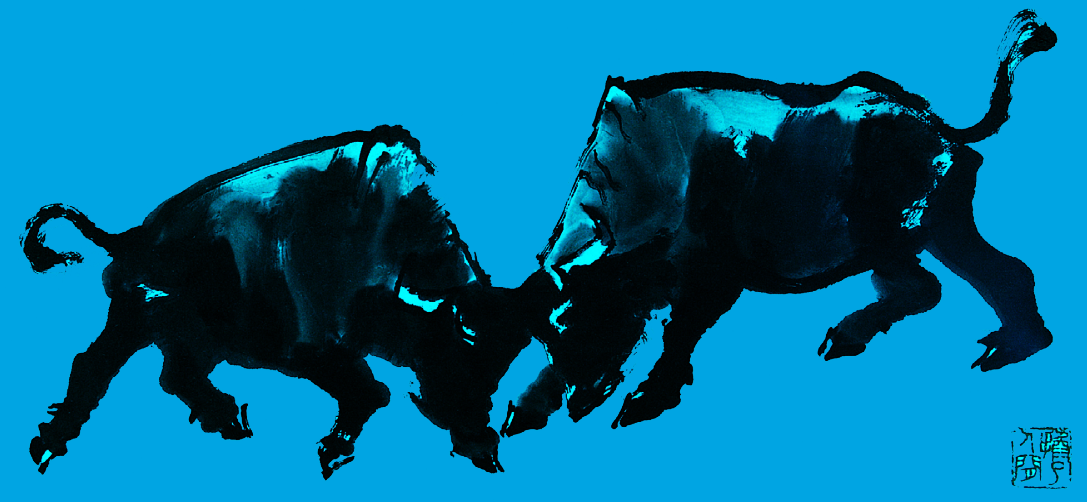

Li Keran

Nuclei as heavy as bulls

Through collision

Generate new states of matter.

T.D. Lee

\section{Organizers}

Miklos Gyulassy (Columbia University)

Jiangyong Jia (BNL)

Jinfeng Liao (IU / RBRC)

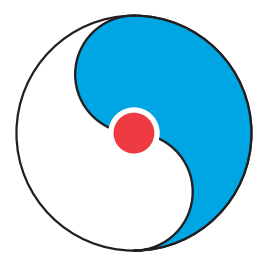

RIKEN BNL Research Center

Bldg. 510A, Brookhaven National Laboratory Upton, NY 11973-5000, USA 